\title{
BROOKHSWEN
}

NATIONAL LABORATORY

BNL-77286-2006-IR

\section{Recent References: \\ January 1, 2005 to December 31, 2005}

\author{
David F. Winchell \\ National Nuclear Data Center, Brookhaven National Laboratory
}

November 2006

\author{
Energy Sciences \& Technology Department \\ National Nuclear Data Center \\ Brookhaven National Laboratory \\ P.O. Box 5000 \\ Upton, NY 11973-5000 \\ www.bnl.gov
}

Notice: This manuscript has been authored by employees of Brookhaven Science Associates, LLC under Contract No. DE-AC02-98CH10886 with the U.S. Department of Energy. The publisher by accepting the manuscript for publication acknowledges that the United States Government retains a non-exclusive, paid-up, irrevocable, world-wide license to publish or reproduce the published form of this manuscript, or allow others to do so, for United States Government purposes. 


\section{DISCLAIMER}

This report was prepared as an account of work sponsored by an agency of the United States Government. Neither the United States Government nor any agency thereof, nor any of their employees, nor any of their contractors, subcontractors, or their employees, makes any warranty, express or implied, or assumes any legal liability or responsibility for the accuracy, completeness, or any third party's use or the results of such use of any information, apparatus, product, or process disclosed, or represents that its use would not infringe privately owned rights. Reference herein to any specific commercial product, process, or service by trade name, trademark, manufacturer, or otherwise, does not necessarily constitute or imply its endorsement, recommendation, or favoring by the United States Government or any agency thereof or its contractors or subcontractors. The views and opinions of authors expressed herein do not necessarily state or reflect those of the United States Government or any agency thereof. 


\title{
Recent References: January 1, 2005 to December 31, 2005
}

\author{
David F. Winchell \\ National Nuclear Data Center, Brookhaven National Laboratory
}

This document lists experimental references added to Nuclear Science References (NSR) during the period January 1, 2005 to December 31, 2005. The first section lists keynumbers and keywords sorted by mass and nuclide. The second section lists all references, ordered by keynumber.

For more information, and access to the most recent NSR updates, please visit the NSR web site at http://www.nndc.bnl.gov/nsr/.

\section{Contents}

Keynumbers and Keywords 2

$\begin{array}{ll}\text { References } & 339\end{array}$ 


\title{
Keynumbers and Keywords
}

\author{
$\mathrm{A}=1$ \\ ${ }^{1} \mathrm{n} \quad 2004 \mathrm{FI} 12$ \\ $2004 \mathrm{G056}$ \\ 2004ME23 \\ 2004NI18 \\ 2004SA64 \\ 2004WE17 \\ $2004 \mathrm{ZH} 42$ \\ NUCLEAR REACTIONS ${ }^{2} \mathrm{H}$ (polarized e, e'n), E=high; measured \\ asymmetry, polarization transfer. ${ }^{1} \mathrm{n}$ deduced electric form factor. \\ Comparison with previous work. JOUR FIZBE 13545 \\ NUCLEAR REACTIONS ${ }^{3} \mathrm{H}(\alpha, \mathrm{d} \alpha), \mathrm{E}=67.2 \mathrm{MeV}$; measured Ed, $\mathrm{E} \alpha$, \\ $\mathrm{d} \alpha$-coin, $\sigma(\theta) .{ }^{6} \mathrm{Li}$ deduced levels, widths. JOUR UKPJA 4916 \\ NUCLEAR REACTIONS ${ }^{1} \mathrm{H}\left(\pi^{-}, \pi^{0}\right)$, E at $105-177 \mathrm{MeV} / \mathrm{c}$; \\ measured $\sigma(\theta)$. JOUR FIZBE 13501 \\ NUCLEAR REACTIONS ${ }^{3} \mathrm{He}(\gamma, 2 \mathrm{p}), \mathrm{E}=0.35-1.55 \mathrm{GeV}$; measured Ep, \\ pp-coin, $\sigma, \sigma(\mathrm{E}, \theta)$; deduced reaction mechanism features. Tagged \\ photons. JOUR PRVCA 70064003 \\ NUCLEAR REACTIONS ${ }^{1} \mathrm{H}\left(\pi^{-}, \pi^{0}\right)$, E at $148-323 \mathrm{MeV} / \mathrm{c}$; measured \\ $\sigma, \sigma(\theta)$. Comparison with previous results. JOUR FIZBE 13405 \\ NUCLEAR REACTIONS ${ }^{2} \mathrm{H}$ (polarized e, e'n), $\mathrm{E}=2.3,3.5 \mathrm{GeV}$; \\ measured electron and neutron spectra, asymmetries. ${ }^{1} \mathrm{n}$ deduced \\ electric form factor. Comparison with previous results. Polarized \\ target. JOUR FIZBE 13531 \\ NUCLEAR REACTIONS ${ }^{3} \mathrm{He}($ polarized e, e), E=1.2 GeV; \\ ${ }^{3} \mathrm{He}$ (polarized e, e'X), $\mathrm{E}=5.7 \mathrm{GeV}$; measured asymmetries. ${ }^{1} \mathrm{n}$ deduced \\ spin asymmetries, polarized structure functions. Polarized target. \\ JOUR PRVCA 70065207 \\ 2005AB01 NUCLEAR REACTIONS ${ }^{1} \mathrm{H}\left(\mathrm{p}, \mathrm{p} \pi^{+}\right),\left(\mathrm{p}, \pi^{+}\right), \mathrm{E}=951 \mathrm{MeV}$; measured \\ missing mass spectra, $\sigma$; deduced D-state effects. JOUR PYLBB 61031 \\ 2005AH01 NUCLEAR REACTIONS ${ }^{1} \mathrm{H}\left(\gamma, \pi^{+} \gamma\right), \mathrm{E}=537-817 \mathrm{MeV}$; measured $\mathrm{E} \gamma$, \\ En, (pion) $\mathrm{n} \gamma$-coin, $\sigma(\theta)$; deduced pion polarizabilities. Tagged photon \\ beam, comparison with model predictions. JOUR ZAANE 23113 \\ 2005AH07 NUCLEAR REACTIONS ${ }^{1} \mathrm{H}\left(\right.$ polarized $\left.\gamma, \pi^{0}\right)$, (polarized $\left.\gamma, \pi^{+}\right), \mathrm{e} \approx$ \\ $340 \mathrm{MeV}$; measured $\sigma(\theta), \mathrm{G}$ asymmetries, related polarization \\ observables. Polarized target. JOUR ZAANE 26135 \\ 2005B035 RADIOACTIVITY ${ }^{1} \mathrm{n}\left(\beta^{-}\right)$; measured $\mathrm{E} \beta$. Plans for measurement of \\ time-reversal violating effects discussed. JOUR JRNBA 110461 \\ 2005BY03 RADIOACTIVITY ${ }^{1} \mathrm{n}\left(\beta^{-}\right)$; measured recoil proton spectra; deduced \\ electron-antineutrino angular correlation coefficient. JOUR JRNBA \\ 110395 \\ 2005BY04 RADIOACTIVITY ${ }^{1} \mathrm{n}\left(\beta^{-}\right)$; measured Ep, $\mathrm{E} \beta, \mathrm{E} \gamma, \mathrm{p} \gamma-, \beta \gamma-, \mathrm{p} \beta$-coin; \\ deduced upper limit for radiative decay branching ratio. JOUR \\ JRNBA 110415 \\ 2005DI03 NUCLEAR REACTIONS ${ }^{1,2} \mathrm{H}$ (polarized $\mathrm{e}^{+}$, $\mathrm{e}^{+}$'X), E=27.6 GeV; \\ ${ }^{1} \mathrm{H}\left(\right.$ polarized $\left.\mathrm{e}^{+}, \mathrm{e}^{+} \pi^{+}\right), \mathrm{E}=27.6 \mathrm{GeV}$; measured $\sigma$, polarization \\ observables. ${ }^{1} \mathrm{n},{ }^{1} \mathrm{H}$ deduced spin structure features. Polarized targets. \\ JOUR ZAANE 24 s01 23 \\ 2005DU14 NUCLEAR REACTIONS ${ }^{2} \mathrm{H}(\mathrm{p}, 2 \mathrm{p}), \mathrm{E}=16 \mathrm{MeV}$; measured $\sigma(\mathrm{E}, \theta)$ for \\ three kinematical configurations. Comparison with model predictions. \\ JOUR PRVCA 71054003 \\ 2005DZ03 RADIOACTIVITY ${ }^{1} \mathrm{n}\left(\beta^{-}\right)$; measured $\mathrm{T}_{1 / 2}$. Comparison with previous \\ results. JOUR JRNBA 110339
}




\section{$\mathrm{A}=1$ (continued)}

2005GL03

2005GR15

2005GR28

2005HA39

2005JA17

2005J021

2005KHZX

2005KI19

2005KR14

2005MI13

2005NI13

2005PEZZ

2005PR16

2005R002

2005R021
NUCLEAR REACTIONS ${ }^{2} \mathrm{H}$ (polarized e, e'n), E=660, 855, $883 \mathrm{MeV}$; measured asymmetry, polarization transfer. ${ }^{1} \mathrm{n}$ deduced electric form factor. JOUR ZAANE 24101 NUCLEAR REACTIONS ${ }^{1,2} \mathrm{H}$ (polarized $\gamma, \mathrm{X}$ ), $\mathrm{E} \approx 200-2900 \mathrm{MeV}$; measured helicity dependent photoabsorption $\sigma .{ }^{1} \mathrm{n},{ }^{1} \mathrm{H}$ deduced sum rule features. JOUR PPNPD 55375

NUCLEAR REACTIONS ${ }^{1} \mathrm{H}\left(\pi^{-}, \pi^{+} \pi^{-}\right),\left(\pi^{+}, 2 \pi^{+}\right), \mathrm{E}=243,264,284$, $305 \mathrm{MeV} ;{ }^{2} \mathrm{H},{ }^{12} \mathrm{C},{ }^{40} \mathrm{Ca},{ }^{208} \mathrm{~Pb}\left(\pi^{+}, 2 \pi^{+}\right),\left(\pi^{+}, \pi^{+} \pi^{-}\right), \mathrm{E}=283 \mathrm{MeV}$; $\mathrm{Sc}\left(\pi^{+}, 2 \pi^{+} \mathrm{X}\right),\left(\pi^{+}, \pi^{+} \pi^{-} \mathrm{X}\right), \mathrm{E}=243,264,284,305 \mathrm{MeV}$; measured invariant mass distributions, $\sigma(\theta)$, correlations; deduced partial chiral symmetry restoration. JOUR NUPAB 76380

NUCLEAR REACTIONS ${ }^{1} \mathrm{H}\left(\mathrm{e}^{+}, \mathrm{e}^{+} \pi^{+}\right), \mathrm{E}=27.6 \mathrm{GeV}$; measured $\sigma\left(\mathrm{Q}^{2}\right.$, $\mathrm{x})$. Comparison with model predictions. JOUR NUPAB $755557 \mathrm{c}$ NUCLEAR REACTIONS ${ }^{1} \mathrm{H}(\mathrm{n}, \mathrm{p}), \mathrm{E}=11 \mathrm{MeV}$; measured recoil proton spectra in scintillator. JOUR NIMAE 551245

NUCLEAR REACTIONS ${ }^{1} \mathrm{H}\left(\right.$ polarized e, e' $\left.\pi^{+}\right)$, (polarized e, e' $\pi^{0}$ ), $\mathrm{E}=1.515 \mathrm{GeV}$; measured $\sigma(\mathrm{E}, \theta)$, polarized longitudinal-transverse structure function; deduced sensitivity to Roper resonance. JOUR PRVCA 72058202

RADIOACTIVITY ${ }^{1} \mathrm{n}\left(\beta^{-}\right)$; measured $\beta \mathrm{p}-, \beta \mathrm{p} \gamma$-coin; deduced branching ratio for radiative decay. Comparison with model predictions. PREPRINT nucl-ex/0512001,12/1/2005

NUCLEAR REACTIONS ${ }^{2} \mathrm{H}(\mathrm{p}, 2 \mathrm{p}), \mathrm{E}=130 \mathrm{MeV}$; measured Ep, pp-coin, $\sigma\left(\theta_{1}, \theta_{2}\right)$; deduced three-nucleon force effects. JOUR PRVCA 72044006

NUCLEAR REACTIONS ${ }^{3} \mathrm{He}($ polarized e, e'), E=3.465-5.727 GeV; measured parallel and perpendicular cross section differences. ${ }^{1} \mathrm{n},{ }^{3} \mathrm{He}$ deduced momentum transfer dependence of spin structure function. JOUR PRLTA 95142002

NUCLEAR REACTIONS ${ }^{6,7} \mathrm{Li}\left({ }^{6} \mathrm{He}, \alpha^{6} \mathrm{He}\right),{ }^{6} \mathrm{Li}\left({ }^{6} \mathrm{He}, \mathrm{t} 2 \alpha\right), \mathrm{E}=18 \mathrm{MeV}$; measured excitation energy spectra. ${ }^{6,7} \mathrm{Li},{ }^{8,9,10} \mathrm{Be}$ deduced cluster states. JOUR NUPAB 753263

RADIOACTIVITY ${ }^{1} \mathrm{n}\left(\beta^{-}\right)$; measured $\mathrm{T}_{1 / 2}$. Cold neutrons, in-beam technique. JOUR PRVCA 71055502

NUCLEAR REACTIONS ${ }^{2} \mathrm{H}(\mathrm{p}, 2 \mathrm{p}), \mathrm{E}=6 \mathrm{MeV}$; measured Ep, pp-coin. ${ }^{1} \mathrm{H}(\mathrm{p}, \mathrm{p}), \mathrm{E}=0.3-0.8 \mathrm{MeV}$; deduced $\sigma$, Coulomb interaction effects. Trojan horse method. CONF Riken(Origin of Matter)

Proc,P513,Pellegriti

NUCLEAR REACTIONS ${ }^{1} \mathrm{H}\left(\pi^{-}, \pi^{0}\right)$, E at $716 \mathrm{MeV} / \mathrm{c}$; measured $\eta$-meson production associated $\mathrm{E} \gamma, \gamma \gamma$-coin, related data; deduced $\eta$-decay branching ratio. JOUR PRVCA 72025201

NUCLEAR REACTIONS ${ }^{2} \mathrm{H}(\gamma, \mathrm{p}), \mathrm{E}=0.5-3 \mathrm{GeV}$; measured Ep, $\sigma(\theta)$; deduced scaling features. JOUR PRLTA 94012301

NUCLEAR REACTIONS ${ }^{1} \mathrm{H}\left(\gamma, \pi^{+}\right)$, (polarized $\left.\gamma, \pi^{+}\right), \mathrm{E} \approx 400-800$

$\mathrm{MeV}$; measured unpolarized and helicity-dependent $\sigma, \sigma(\theta)$. JOUR

NUPAB $755451 \mathrm{c}$ 


\section{$\mathrm{A}=1$ (continued)}

2005R037 NUCLEAR REACTIONS ${ }^{1} \mathrm{H}\left({ }^{8} \mathrm{He},{ }^{8} \mathrm{He}\right)$, E not given; measured recoil proton spectrum; deduced excitation function. ${ }^{1} \mathrm{H}\left({ }^{6} \mathrm{He},{ }^{6} \mathrm{Li}\right)$, E not given; measured neutron spectrum, $\mathrm{n} \gamma$-coin; deduced excitation function. ${ }^{7,9} \mathrm{Li}$ deduced resonance parameters. ${ }^{7,9} \mathrm{He}$ deduced analog states features. JOUR NIMBE 241977

2005SAZS NUCLEAR REACTIONS ${ }^{1} \mathrm{H}(\mathrm{d}, 2 \mathrm{p}), \mathrm{E}=270 \mathrm{MeV}$; measured proton spin correlations; deduced violation of Bell's inequality. REPT CNS-REP-66,P32,Saito

2005SE01 RADIOACTIVITY ${ }^{1} \mathrm{n}\left(\beta^{-}\right)$; measured $\mathrm{T}_{1 / 2}$. Ultracold neutrons, comparison with previous results, model predictions. JOUR PYLBB 60572

2005SE16 NUCLEAR REACTIONS ${ }^{2} \mathrm{H},{ }^{3} \mathrm{He}$ (polarized e, e'n), E=high; measured asymmetries, polarization transfer. ${ }^{1} \mathrm{n}$ deduced electromagnetic form factor. Polarized target. JOUR NUPAB $755253 \mathrm{c}$

2005SE17 RADIOACTIVITY ${ }^{1} \mathrm{n}\left(\beta^{-}\right)$; measured $\mathrm{T}_{1 / 2}$. Comparison with previous results. JOUR JRNBA 110333

2005ST23 NUCLEAR REACTIONS ${ }^{1} \mathrm{H}\left(\pi^{-}, \pi^{0}\right)$, E at $649-752 \mathrm{MeV} / \mathrm{c}$; measured $\sigma, \sigma(\mathrm{E}, \theta)$; deduced $\eta$-meson contribution, other reaction mechanism features. Comparison with model predictions. JOUR PRVCA 72015205

2005TUZZ NUCLEAR REACTIONS ${ }^{2} \mathrm{H}\left({ }^{7} \mathrm{Li}, 2 \alpha\right),\left({ }^{6} \mathrm{Li},{ }^{3} \mathrm{He} \alpha\right),{ }^{6} \mathrm{Li}\left({ }^{6} \mathrm{Li}, 2 \alpha\right)$, E not given; measured particle spectra. ${ }^{7} \operatorname{Li}(\mathrm{p}, \alpha), \mathrm{E}(\mathrm{cm}) \approx 0.01-0.5 \mathrm{MeV}$; ${ }^{6} \mathrm{Li}(\mathrm{d}, \alpha),(\mathrm{p}, \alpha), \mathrm{E}(\mathrm{cm}) \approx 0.01-1 \mathrm{MeV}$; deduced astrophysical S-factors. CONF Riken(Origin of Matter) Proc,P553,Tumino 2005WI17 RADIOACTIVITY ${ }^{1} \mathrm{n}\left(\beta^{-}\right)$; measured $\mathrm{T}_{1 / 2}$. Trapped proton counting method. JOUR JRNBA 110327

2005YA05 NUCLEAR REACTIONS ${ }^{2} \mathrm{H}$ (polarized p, 2p), E=0.5, 0.8 GeV; measured $\operatorname{Ay}(\theta)$. Comparison with model predictions. JOUR PRLTA 94072304

$2005 Z$ Z03 NUCLEAR REACTIONS ${ }^{2} \mathrm{H}\left({ }^{8} \mathrm{Li},{ }^{9} \mathrm{Be}\right), \mathrm{E}=40.38 \mathrm{MeV}$; measured particle spectra, $\sigma(\theta)$; deduced astrophysical S-factors. JOUR CPLEE 222219

2005ZH14 NUCLEAR REACTIONS ${ }^{1} \mathrm{H}\left(\gamma, \pi^{+}\right),{ }^{2} \mathrm{H}\left(\gamma, \mathrm{p} \pi^{-}\right), \mathrm{E}=1.1-5.5 \mathrm{GeV}$; measured $\sigma(\mathrm{E}, \theta)$; deduced scaling behavior. ${ }^{1} \mathrm{n}\left(\gamma, \pi^{-}\right), \mathrm{E}=1.1-5.5$ $\mathrm{GeV}$; deduced $\sigma(\mathrm{E}, \theta)$, scaling behavior. JOUR PRVCA 71044603

${ }^{1} \mathrm{H} \quad$ 2004BEZP NUCLEAR REACTIONS ${ }^{1} \mathrm{H}\left({ }^{22} \mathrm{O}, \mathrm{p}\right),\left({ }^{22} \mathrm{O},{ }^{22} \mathrm{O}\right), \mathrm{E} \approx 47 \mathrm{MeV} /$ nucleon; measured particle spectra, $\sigma(\mathrm{E}, \theta) .{ }^{22} \mathrm{O}$ level deduced deformation parameter. MUST detector array. REPT IPNO-T-04-17,Becheva

2004FI12 NUCLEAR REACTIONS ${ }^{2} \mathrm{H}$ (polarized e, e'n), E=high; measured asymmetry, polarization transfer. ${ }^{1} \mathrm{n}$ deduced electric form factor. Comparison with previous work. JOUR FIZBE 13545

2004G058 NUCLEAR REACTIONS ${ }^{1} \mathrm{H}$ (polarized $\gamma, \pi^{+} \pi^{-}$), (polarized $\gamma$, $\left.\mathrm{K}^{+} \pi^{-}\right), \mathrm{E}=1.8-2.2 \mathrm{GeV}$; measured vector meson production associated particle spectra, angular distributions, asymmetries. Tagged photons. JOUR FIZBE 13553

2004KE18 NUCLEAR REACTIONS ${ }^{1} \mathrm{H}\left(\right.$ polarized e, e' $\left.\pi^{0}\right), \mathrm{E}=4.531 \mathrm{GeV}$; measured recoil polarization, response functions. JOUR FIZBE 1381 


\section{$\mathrm{A}=1$ (continued)}

2004SHZZ NUCLEAR REACTIONS ${ }^{1} \mathrm{H}$ (polarized n, n), $\mathrm{E}=1.39,1.69,1.89,1.99$ $\mathrm{GeV}$; measured $\sigma$, polarization, longitudinal cross-section difference. REPT JINR-E1-2004-87.Sharov

2004ST32 NUCLEAR REACTIONS ${ }^{1} \mathrm{H}$ (polarized $\gamma, \pi^{+} \pi^{-}$), E $=0.6-2.3 \mathrm{GeV}$; measured $\sigma(\theta)$, cross-section asymmetries. Tagged photons. JOUR FIZBE 13179

2004WE17 NUCLEAR REACTIONS ${ }^{2} \mathrm{H}$ (polarized e, e'n), E=2.3, $3.5 \mathrm{GeV}$; measured electron and neutron spectra, asymmetries. ${ }^{1} \mathrm{n}$ deduced electric form factor. Comparison with previous results. Polarized target. JOUR FIZBE 13531

2005AC22 NUCLEAR REACTIONS ${ }^{3} \mathrm{He}$ (polarized e, e'p), (polarized e, e'np), $\mathrm{E}=735 \mathrm{MeV}$; measured polarization observables; deduced final state interaction effects. Polarized target. JOUR ZAANE 25177

2005AH05 NUCLEAR REACTIONS ${ }^{1} \mathrm{H}\left(\right.$ polarized $\left.\gamma, 2 \pi^{0}\right), \mathrm{E}=400-800 \mathrm{MeV}$; measured unpolarized and helicity-dependent $\sigma$. Polarized target. JOUR PYLBB 624173

2005AH07 NUCLEAR REACTIONS ${ }^{1} \mathrm{H}\left(\right.$ polarized $\left.\gamma, \pi^{0}\right)$, (polarized $\left.\gamma, \pi^{+}\right)$, e $\approx$ $340 \mathrm{MeV}$; measured $\sigma(\theta), \mathrm{G}$ asymmetries, related polarization observables. Polarized target. JOUR ZAANE 26135

2005AK09 NUCLEAR REACTIONS ${ }^{1} \mathrm{H}\left(\mathrm{e}^{+}, \mathrm{e}^{+} \gamma\right), \mathrm{E}=$ high; measured $\sigma\left(\mathrm{Q}^{2}\right)$, $\sigma(\mathrm{W})$ for deeply virtual Compton scattering. Comparison with model predictions. JOUR ZCCNE 441

2005AL01 NUCLEAR REACTIONS ${ }^{1} \mathrm{H}(\mathrm{p}, \mathrm{p}), \mathrm{E}=0.45-2.5 \mathrm{GeV}$; measured analyzing powers vs energy, angle. Polarized target, comparisons with previous results. JOUR ZAANE 23351

2005AN30 NUCLEAR REACTIONS ${ }^{2} \mathrm{H}\left({ }^{7} \mathrm{Be}, 2 \alpha\right), \mathrm{E}=1.71,5.55 \mathrm{MeV}$; measured particle spectra, $\sigma .{ }^{7} \mathrm{Be}(\mathrm{d}, \mathrm{p}), \mathrm{E}(\mathrm{cm}) \approx 0.38,1.2 \mathrm{MeV}$; deduced astrophysical S-factors. Implications for primordial ${ }^{7} \mathrm{Li}$ abundance discussed. JOUR ASJOA 630 L105

2005AR21 NUCLEAR REACTIONS ${ }^{1} \mathrm{H}(\mathrm{e}, \mathrm{e}), \mathrm{E}=3.03 \mathrm{GeV}$; measured forward angle parity-violating asymmetries, strange-quark contributions. JOUR PRLTA 95092001

2005BA11 NUCLEAR REACTIONS ${ }^{1} \mathrm{H}\left({ }^{16} \mathrm{O}, \alpha^{12} \mathrm{C}\right)$, E at $3.25 \mathrm{GeV} / \mathrm{c} /$ nucleon; measured recoil proton spectra, angular distributions, charged fragment spectra; deduced reaction mechanism features. JOUR UKPJA 5016

2005BA25 NUCLEAR REACTIONS ${ }^{1} \mathrm{H}$ (polarized e, e), $\mathrm{E}=569.31,855.15 \mathrm{MeV}$; measured transverse spin asymmetry; deduced resonance contributions. JOUR ZAANE 24 s02 35

2005BA40 NUCLEAR REACTIONS ${ }^{1} \mathrm{H}\left({ }^{16} \mathrm{O}, \mathrm{X}\right){ }^{1} \mathrm{H} /{ }^{2} \mathrm{H} /{ }^{3} \mathrm{H} /{ }^{3} \mathrm{He} /{ }^{4} \mathrm{He} /{ }^{5} \mathrm{He}$ $/{ }^{6} \mathrm{He} /{ }^{5} \mathrm{Li} /{ }^{6} \mathrm{Li} /{ }^{7} \mathrm{Li} /{ }^{8} \mathrm{Li} /{ }^{7} \mathrm{Be} /{ }^{8} \mathrm{Be} /{ }^{9} \mathrm{Be} /{ }^{10} \mathrm{Be} /{ }^{9} \mathrm{~B} /{ }^{10} \mathrm{~B} /$ ${ }^{11} \mathrm{~B} /{ }^{12} \mathrm{~B} /{ }^{10} \mathrm{C} /{ }^{11} \mathrm{C} /{ }^{12} \mathrm{C} /{ }^{13} \mathrm{C} /{ }^{14} \mathrm{C} /{ }^{13} \mathrm{~N} /{ }^{14} \mathrm{~N} /{ }^{15} \mathrm{~N} /{ }^{14} \mathrm{O} /$ ${ }^{15} \mathrm{O} /{ }^{16} \mathrm{O}$, E at $3.25 \mathrm{GeV} / \mathrm{c} /$ nucleon; measured production $\sigma$. JOUR PZETA 81174

2005BA50 NUCLEAR REACTIONS ${ }^{1} \mathrm{H}$ (polarized p, p), $\mathrm{E}=0.45-2.5 \mathrm{GeV}$; measured spin correlation coefficients vs energy, angle; deduced scattering phase shifts, scattering amplitudes. Polarized target. JOUR PRVCA 71054002 


\section{$\mathrm{A}=1$ (continued)}

2005BA58 NUCLEAR REACTIONS ${ }^{1} \mathrm{H}$ (polarized e, e), E=854.3 MeV; measured single spin asymmetries; deduced form factor limits. JOUR NUPAB $755249 \mathrm{c}$

2005BA93 NUCLEAR REACTIONS ${ }^{1} \mathrm{H}\left(\gamma, \pi^{0}\right), \mathrm{E}=0.3-3.0 \mathrm{GeV}$; measured pion production $\sigma(\theta), \sigma$. Tagged photons. JOUR PRLTA 94012003

2005BAZV NUCLEAR REACTIONS ${ }^{1} \mathrm{H}(\mathrm{n}, \mathrm{n}), \mathrm{E}=15 \mathrm{MeV}$; measured recoil protons angular distributions. CONF Santa Fe (Nucl Data for Sci and Technol) Proc,Vol1,P834

2005BE12 NUCLEAR REACTIONS ${ }^{3} \mathrm{He}(\mathrm{e}$, e'np), E=high; measured proton spectra, missing energy, $\sigma(\mathrm{E}, \theta) .{ }^{3} \mathrm{He}$ deduced proton effective momentum density. JOUR PRLTA 94082305

2005BL09 NUCLEAR REACTIONS ${ }^{1} \mathrm{H}\left({ }^{6} \mathrm{He},{ }^{6} \mathrm{He}\right),\left({ }^{6} \mathrm{He},{ }^{6} \mathrm{He}\right), \mathrm{E}=15 \mathrm{MeV} /$ nucleon; measured $\sigma(\mathrm{q})$; deduced halo effect. ${ }^{1} \mathrm{H}\left({ }^{6} \mathrm{He}, \alpha\right), \mathrm{E}=25 \mathrm{MeV} /$ nucleon; measured $\sigma(\theta) .{ }^{2} \mathrm{H}\left({ }^{8} \mathrm{He},{ }^{6} \mathrm{Li}\right), \mathrm{E}=15 \mathrm{MeV} /$ nucleon; measured excitation energy spectrum; deduced possible resonance structure. ${ }^{1} \mathrm{H}\left({ }^{22} \mathrm{O},{ }^{22} \mathrm{O}^{\prime}\right), \mathrm{E}=46.6 \mathrm{MeV} /$ nucleon; measured $\sigma(\mathrm{E}, \theta)$. JOUR NUPAB $752279 \mathrm{c}$

2005B035 RADIOACTIVITY ${ }^{1} \mathrm{n}\left(\beta^{-}\right)$; measured $\mathrm{E} \beta$. Plans for measurement of time-reversal violating effects discussed. JOUR JRNBA 110461

2005BY03 RADIOACTIVITY ${ }^{1} \mathrm{n}\left(\beta^{-}\right)$; measured recoil proton spectra; deduced electron-antineutrino angular correlation coefficient. JOUR JRNBA 110395

2005BY04 RADIOACTIVITY ${ }^{1} \mathrm{n}\left(\beta^{-}\right)$; measured Ep, $\mathrm{E} \beta, \mathrm{E} \gamma, \mathrm{p} \gamma-, \beta \gamma_{-}, \mathrm{p} \beta$-coin; deduced upper limit for radiative decay branching ratio. JOUR JRNBA 110415

2005DA29 NUCLEAR REACTIONS ${ }^{1} \mathrm{H}\left(\gamma, \gamma^{\prime}\right)$, E=high; measured $\sigma$, polarization transfer asymmetry. JOUR NUPAB $755281 \mathrm{c}$

2005DEZT NUCLEAR REACTIONS ${ }^{1} \mathrm{H}\left(\pi^{+}, \pi^{+}\right),\left(\pi^{-}, \pi^{-}\right), \mathrm{E}=19,26,32,37,43$ $\mathrm{MeV}$; measured $\sigma(\theta)$; deduced real part of isospin forward scattering amplitude. PREPRINT nucl-ex/0512006,12/3/2005

2005DI03 NUCLEAR REACTIONS ${ }^{1,2} \mathrm{H}$ (polarized $\left.\mathrm{e}^{+}, \mathrm{e}^{+} \mathrm{X}\right), \mathrm{E}=27.6 \mathrm{GeV}$; ${ }^{1} \mathrm{H}\left(\right.$ polarized $\left.\mathrm{e}^{+}, \mathrm{e}^{+} \pi^{+}\right), \mathrm{E}=27.6 \mathrm{GeV}$; measured $\sigma$, polarization observables. ${ }^{1} \mathrm{n},{ }^{1} \mathrm{H}$ deduced spin structure features. Polarized targets. JOUR ZAANE 24 s01 23

2005D016 NUCLEAR REACTIONS ${ }^{1} \mathrm{H}\left({ }^{17} \mathrm{~B},{ }^{17} \mathrm{~B}^{\prime}\right), \mathrm{E}=43.8 \mathrm{MeV}$; measured $\mathrm{E} \gamma$, $\mathrm{I} \gamma$, (particle) $\gamma$-coin, $\sigma .{ }^{17} \mathrm{~B}$ deduced deformation parameters, decoupling of valence neutrons from core. JOUR PYLBB 62181

2005DZ03 RADIOACTIVITY ${ }^{1} \mathrm{n}\left(\beta^{-}\right)$; measured $\mathrm{T}_{1 / 2}$. Comparison with previous results. JOUR JRNBA 110339

2005EL07 NUCLEAR REACTIONS ${ }^{1} \mathrm{H}\left({ }^{19} \mathrm{C},{ }^{19} \mathrm{C}\right),\left({ }^{19} \mathrm{C},{ }^{18} \mathrm{CX}\right),\left({ }^{19} \mathrm{C},{ }^{17} \mathrm{CX}\right), \mathrm{E}$ $\approx 49.4 \mathrm{MeV} /$ nucleon; ${ }^{1} \mathrm{H}\left({ }^{17} \mathrm{C},{ }^{17} \mathrm{C}^{\prime}\right),\left({ }^{17} \mathrm{C},{ }^{16} \mathrm{CX}\right), \mathrm{E} \approx 43.3 \mathrm{MeV} /$ nucleon; measured $\mathrm{E} \gamma, \mathrm{I} \gamma, \gamma \gamma$-, (particle) $\gamma$-coin, $\sigma .{ }^{17,19} \mathrm{C}$ deduced levels, J, $\pi$. Comparison with shell model predictions. JOUR PYLBB 614174

2005ER01 NUCLEAR REACTIONS ${ }^{2} \mathrm{H}\left(\pi^{-}, 2 \pi^{-}\right), \mathrm{E}=430 \mathrm{MeV}$; measured pion and proton spectra, $\sigma, \sigma(\theta) .{ }^{1} \mathrm{n}\left(\pi^{-}, 2 \pi^{-}\right), \mathrm{E}=430 \mathrm{MeV}$; deduced $\sigma$. Comparisons with model predictions. JOUR ZAANE 23345 


\section{$\mathrm{A}=1$ (continued)}

2005GA45 NUCLEAR REACTIONS ${ }^{2} \mathrm{H}\left({ }^{44} \mathrm{Ar},{ }^{45} \mathrm{Ar}\right),\left({ }^{40} \mathrm{Ar},{ }^{41} \mathrm{Ar}\right), \mathrm{E}=10 \mathrm{MeV} /$ nucleon; measured particle spectra, $\sigma(\mathrm{E}, \theta) .{ }^{45} \mathrm{Ar}$ deduced levels, spectroscopic factors. JOUR JPGPE 31 S1623

2005GIZZ NUCLEAR REACTIONS ${ }^{1} \mathrm{H}\left({ }^{6} \mathrm{He}, \mathrm{t}\right),\left({ }^{6} \mathrm{He}, \alpha\right),\left({ }^{6} \mathrm{He},{ }^{6} \mathrm{He}\right), \mathrm{E}=150$ $\mathrm{MeV}$; measured particle spectra, $\sigma(\theta)$. ${ }^{6} \mathrm{He}$ deduced spectroscopic factors for cluster configurations. PREPRINT nucl-ex/0505007,5/04/2005

2005GL03 NUCLEAR REACTIONS ${ }^{2} \mathrm{H}$ (polarized e, e'n), E=660, 855, $883 \mathrm{MeV}$; measured asymmetry, polarization transfer. ${ }^{1} \mathrm{n}$ deduced electric form factor. JOUR ZAANE 24101

2005GR15 NUCLEAR REACTIONS ${ }^{1,2} \mathrm{H}$ (polarized $\gamma, \mathrm{X}$ ), E $\approx 200-2900 \mathrm{MeV}$; measured helicity dependent photoabsorption $\sigma .{ }^{1} \mathrm{n},{ }^{1} \mathrm{H}$ deduced sum rule features. JOUR PPNPD 55375

2005GR26 NUCLEAR REACTIONS ${ }^{1} \mathrm{H}(\mathrm{e}, \mathrm{e} \gamma), \mathrm{E}=$ high; measured $\sigma\left(\mathrm{Q}^{2}, \mathrm{~W}\right)$ for deeply virtual Compton scattering. JOUR ZCCNE $44 \mathrm{~S} 1$

2005GU18 NUCLEAR REACTIONS ${ }^{1} \mathrm{H}$ (polarized p, p), E (cm) $=200 \mathrm{GeV}$; measured analyzing power. Comparison with model predictions. JOUR NPBSE 14682

2005GU29 NUCLEAR REACTIONS ${ }^{2} \mathrm{H}\left({ }^{8} \mathrm{Li},{ }^{9} \mathrm{Li}\right), \mathrm{E}(\mathrm{cm})=7.8 \mathrm{MeV}$; measured $\sigma(\theta)$; deduced asymptotic normalization coefficient. ${ }^{8} \mathrm{~B}(\mathrm{p}, \gamma), \mathrm{E}=$ low; calculated astrophysical S-factor. DWBA analysis, inverse kinematics, comparison with data. JOUR NUPAB 761162

2005HA32 NUCLEAR REACTIONS ${ }^{1} \mathrm{H}$ (polarized $\gamma, \gamma$ ), $\mathrm{E} \approx 3.2 \mathrm{GeV}$; measured recoil proton polarization. JOUR PRLTA 94242001

2005HA37 NUCLEAR REACTIONS ${ }^{1,2} \mathrm{H}$ (polarized e, e'), E=high; measured analyzing powers; deduced form factors. Polarized targets. JOUR NUPAB $755257 \mathrm{c}$

2005HA64 NUCLEAR REACTIONS ${ }^{1} \mathrm{H}\left({ }^{6} \mathrm{He},{ }^{6} \mathrm{He}\right), \mathrm{E}=71 \mathrm{MeV} /$ nucleon; measured $\sigma(\theta)$, analyzing powers; deduced optical model parameters. ${ }^{6} \mathrm{He}$ deduced rms radius. Polarized target. Comparison with model predictions. JOUR ZAANE 25 s01 255

2005HAZU NUCLEAR REACTIONS ${ }^{1} \mathrm{H}\left({ }^{6} \mathrm{He},{ }^{6} \mathrm{He}\right), \mathrm{E}=71 \mathrm{MeV} /$ nucleon; measured $\sigma(\theta), \operatorname{Ay}(\theta)$. polarized target. CONF Argonne(Nuclei at the Limits),P360,Hatano

2005J002 NUCLEAR REACTIONS ${ }^{1} \mathrm{H}(\mathrm{n}, \mathrm{n}), \mathrm{E}=96 \mathrm{MeV}$; measured $\sigma(\theta)$. Comparison with model predictions. JOUR PRVCA 71024002

2005J012 NUCLEAR REACTIONS ${ }^{1} \mathrm{H}\left({ }^{10} \mathrm{C},{ }^{10} \mathrm{C}\right),\left({ }^{10} \mathrm{C},{ }^{10} \mathrm{C}\right), \mathrm{E}=45.3 \mathrm{MeV} /$ nucleon; ${ }^{1} \mathrm{H}\left({ }^{11} \mathrm{C},{ }^{11} \mathrm{C}\right),\left({ }^{11} \mathrm{C},{ }^{11} \mathrm{C}^{\prime}\right), \mathrm{E}=40.6 \mathrm{MeV} /$ nucleon; ${ }^{1} \mathrm{H}\left({ }^{12} \mathrm{C}\right.$, $\left.{ }^{12} \mathrm{C}\right),\left({ }^{12} \mathrm{C},{ }^{12} \mathrm{C}\right), \mathrm{E}=36.3 \mathrm{MeV} /$ nucleon; measured elastic and inelastic $\sigma(\theta) .{ }^{10,11} \mathrm{C}$ deduced radii, transition matrix elements. JOUR PRVCA 72014308

2005J021 NUCLEAR REACTIONS ${ }^{1} \mathrm{H}\left(\right.$ polarized e, e' $\left.\pi^{+}\right)$, (polarized e, e' $\pi^{0}$ ), $\mathrm{E}=1.515 \mathrm{GeV}$; measured $\sigma(\mathrm{E}, \theta)$, polarized longitudinal-transverse structure function; deduced sensitivity to Roper resonance. JOUR PRVCA 72058202

2005JOZX NUCLEAR REACTIONS ${ }^{1} \mathrm{H}(\mathrm{n}, \mathrm{n}), \mathrm{E}=96 \mathrm{MeV}$; measured $\sigma(\theta)$. Comparison with previous results and model predictions. CONF Santa Fe (Nucl Data for Sci and Technol) Proc,Vol1,P804 


\section{$\mathrm{A}=1$ (continued)}

2005KA25 NUCLEAR MOMENTS ${ }^{1,2} \mathrm{H}$; measured NMR spectra; deduced $\mu$ ratio. JOUR CJPHA 83405

2005KA26 NUCLEAR REACTIONS ${ }^{1} \mathrm{H}\left({ }^{19} \mathrm{C},{ }^{19} \mathrm{C}^{\prime}\right),\left({ }^{17} \mathrm{C},{ }^{17} \mathrm{C}^{\prime}\right),\left({ }^{17} \mathrm{~B},{ }^{17} \mathrm{~B}^{\prime}\right), \mathrm{E} \approx$ $53 \mathrm{MeV}$ / nucleon; measured prompt and delayed $\mathrm{E} \gamma, \mathrm{I} \gamma \cdot{ }^{17,19} \mathrm{C},{ }^{17} \mathrm{~B}$ deduced transitions. ${ }^{19} \mathrm{C}$ deduced no isomeric state. JOUR NUPAB 757315

2005KHZX RADIOACTIVITY ${ }^{1} \mathrm{n}\left(\beta^{-}\right)$; measured $\beta \mathrm{p}-, \beta \mathrm{p} \gamma$-coin; deduced branching ratio for radiative decay. Comparison with model predictions. PREPRINT nucl-ex/0512001,12/1/2005

$2005 K I 03$ NUCLEAR REACTIONS ${ }^{1} \mathrm{H}$ (polarized e, e), $\mathrm{E}=3 \mathrm{GeV}$; measured spin asymmetries. JOUR ZAANE 24 s02 39

2005LI19 NUCLEAR REACTIONS ${ }^{2} \mathrm{H}\left({ }^{8} \mathrm{Li},{ }^{9} \mathrm{Li}\right), \mathrm{E}=39 \mathrm{MeV}$; measured particle spectra, $\sigma(\theta) .{ }^{8} \operatorname{Li}(\mathrm{n}, \gamma), \mathrm{E}=$ low; deduced astrophysical reaction rates. JOUR PRVCA 71052801

2005MA13 NUCLEAR REACTIONS ${ }^{1} \mathrm{H}$ (polarized e, e), E=569.31, 855.15 MeV; measured transverse spin asymmetry; deduced intermediate states contributions. Comparison with model predictions. JOUR PRLTA 94 082001

2005MA19 NUCLEAR REACTIONS ${ }^{1} \mathrm{H}$ (polarized e, e), E=570.4, 854.3 MeV; measured parity violating asymmetry. JOUR ZAANE 24 s02 47

2005MA25 NUCLEAR REACTIONS ${ }^{1} \mathrm{H}$ (polarized e, e), E $=570.4 \mathrm{MeV}$; measured parity-violating asymmetry; deduced strangeness contribution. JOUR PRLTA 94152001

2005MA44 NUCLEAR REACTIONS ${ }^{1} \mathrm{H}$ (polarized e, e), E=570.4, 854.3 MeV; measured parity-violating single spin asymmetry. Comparison with model predictions. JOUR PPNPD 55320

2005MA48 NUCLEAR REACTIONS ${ }^{1} \mathrm{H}$ (polarized e, e), E=3 GeV; measured parity-violating asymmetries. JOUR NUPAB $755245 \mathrm{c}$

2005MAZM NUCLEAR REACTIONS ${ }^{2} \mathrm{H}\left({ }^{48} \mathrm{Ca},{ }^{49} \mathrm{Ca}\right), \mathrm{E}=105 \mathrm{MeV}$; measured $\mathrm{E} \gamma$, $\mathrm{I} \gamma$, (particle) $\gamma$-coin. ${ }^{48} \mathrm{Ca}$ (polarized d, p), $\mathrm{E}=14 \mathrm{MeV}$; measured proton spectra, $\sigma(\theta) .{ }^{49} \mathrm{Ca}$ deduced levels, J, $\pi$. REPT MLL 2004

Annual,P8,Maierbeck

2005MEZY NUCLEAR REACTIONS ${ }^{1,2} \mathrm{H}(\mathrm{n}, \mathrm{n}), \mathrm{E}=95 \mathrm{MeV}$; measured $\sigma(\theta)$; deduced three-nucleon force effects. CONF Santa Fe (Nucl Data for Sci and Technol) Proc,Vol1,P688

2005NA36 NUCLEAR REACTIONS ${ }^{2} \mathrm{H}, \mathrm{C}\left({ }^{7} \mathrm{Li},{ }^{7} \mathrm{Be}\right), \mathrm{E}=65 \mathrm{MeV} /$ nucleon; measured spin-flip and spin-nonflip particle spectra; deduced charge-exchange spin-flip $\sigma .{ }^{2} \mathrm{H}(\gamma, \mathrm{n}), \mathrm{E} \approx 1.5-10 \mathrm{MeV}$; deduced magnetic dipole $\sigma$. Comparison with previous results, model predictions. JOUR PRVCA 72041001

2005NI13 RADIOACTIVITY ${ }^{1} \mathrm{n}\left(\beta^{-}\right)$; measured $\mathrm{T}_{1 / 2}$. Cold neutrons, in-beam technique. JOUR PRVCA 71055502

20050KZZ NUCLEAR REACTIONS ${ }^{1} \mathrm{H}(\mathrm{p}, \mathrm{p}), \mathrm{E}=100 \mathrm{GeV}$; measured recoil proton spectra, analyzing power. Polarized target. PREPRINT nucl-ex/0502022,2/25/2005

20050N04 NUCLEAR REACTIONS ${ }^{1} \mathrm{H}\left({ }^{16} \mathrm{C},{ }^{16} \mathrm{C}^{\prime}\right), \mathrm{E}=33 \mathrm{MeV} /$ nucleon; measured $\mathrm{E} \gamma, \mathrm{I} \gamma$, (particle) $\gamma$-coin; deduced $\sigma \cdot{ }^{16} \mathrm{C}$ deduced deformation parameter. JOUR ZAANE 25 s01 347 


\section{$\mathrm{A}=1$ (continued)}

2005PEZZ NUCLEAR REACTIONS ${ }^{2} \mathrm{H}(\mathrm{p}, 2 \mathrm{p}), \mathrm{E}=6 \mathrm{MeV}$; measured Ep, pp-coin. ${ }^{1} \mathrm{H}(\mathrm{p}, \mathrm{p}), \mathrm{E}=0.3-0.8 \mathrm{MeV}$; deduced $\sigma$, Coulomb interaction effects. Trojan horse method. CONF Riken(Origin of Matter) Proc,P513,Pellegriti

2005PU02 NUCLEAR REACTIONS ${ }^{1} \mathrm{H}$ (polarized e, e), E=0.934-4.091 GeV; measured recoil proton spectra, polarization transfer, $\operatorname{Ay}(\theta) .{ }^{1} \mathrm{H}$ deduced elastic form factor ratio. Comparison with model predictions. JOUR PRVCA 71055202

2005QA01 NUCLEAR REACTIONS ${ }^{1} \mathrm{H}(\mathrm{e}, \mathrm{e}), \mathrm{E}=1.9-4.7 \mathrm{GeV}$; measured recoil proton spectra, $\sigma(\theta), \sigma .{ }^{1} \mathrm{H}$ deduced electromagnetic form factors. JOUR PRLTA 94142301

2005R037 NUCLEAR REACTIONS ${ }^{1} \mathrm{H}\left({ }^{8} \mathrm{He},{ }^{8} \mathrm{He}\right)$, E not given; measured recoil proton spectrum; deduced excitation function. ${ }^{1} \mathrm{H}\left({ }^{6} \mathrm{He},{ }^{6} \mathrm{Li}\right), \mathrm{E}$ not given; measured neutron spectrum, $\mathrm{n} \gamma$-coin; deduced excitation function. ${ }^{7,9} \mathrm{Li}$ deduced resonance parameters. ${ }^{7,9} \mathrm{He}$ deduced analog states features. JOUR NIMBE 241977

2005SA06 NUCLEAR REACTIONS ${ }^{1} \mathrm{H}(\mathrm{n}, \mathrm{n}), \mathrm{E}=194 \mathrm{MeV}$; measured $\sigma(\theta)$. Tagged beam, comparisons with previous results and model predictions. JOUR PRLTA 94082303

2005SAZT NUCLEAR REACTIONS ${ }^{1} \mathrm{H}(\alpha, \alpha), \mathrm{E}=80 \mathrm{MeV} /$ nucleon; measured p $\alpha$-coin, $\sigma(\theta)$; deduced target polarization. REPT RIKEN 2004 Annual,P36,Sakaguchi

2005SE01 RADIOACTIVITY ${ }^{1} \mathrm{n}\left(\beta^{-}\right)$; measured $\mathrm{T}_{1 / 2}$. Ultracold neutrons, comparison with previous results, model predictions. JOUR PYLBB 60572

2005SE04 NUCLEAR REACTIONS ${ }^{1} \mathrm{H}(\mathrm{e}, \mathrm{e}), \mathrm{E}=1.9-4.7 \mathrm{GeV}$; measured recoil proton spectra; deduced electromagnetic form factors. Comparison with spin-transfer measurements. JOUR ZAANE 24 s01 55

2005SE05 NUCLEAR REACTIONS ${ }^{2} \mathrm{H}(\mathrm{n}, \mathrm{n}),(\mathrm{n}, 2 \mathrm{n}), \mathrm{E}=13 \mathrm{MeV}$; measured En, nn-coin, $\sigma\left(\theta_{1}, \theta_{2}\right)$ for seven exit-channel configurations. Comparison with model predictions. JOUR PRVCA 71034006

2005SE17 RADIOACTIVITY ${ }^{1} \mathrm{n}\left(\beta^{-}\right)$; measured $\mathrm{T}_{1 / 2}$. Comparison with previous results. JOUR JRNBA 110333

2005SE22 NUCLEAR REACTIONS ${ }^{2} \mathrm{H}(\mathrm{p}, \mathrm{p}), \mathrm{E}=135 \mathrm{MeV} ;{ }^{1} \mathrm{H}(\mathrm{d}, \mathrm{d}), \mathrm{E}=135$ $\mathrm{MeV}$ / nucleon; measured $\sigma(\theta)$; deduced relativistic effects, three-nucleon force effects. Comparison with previous results. JOUR PRLTA 95162301

2005SEZV NUCLEAR REACTIONS ${ }^{2} \mathrm{H}(\mathrm{p}, \mathrm{p}), \mathrm{E}=135 \mathrm{MeV} ;{ }^{1} \mathrm{H}(\mathrm{d}, \mathrm{d}), \mathrm{E}=270$ $\mathrm{MeV}$; measured $\sigma(\theta)$. Comparison with model predictions and previous data. PREPRINT nucl-ex/0510005,10/3/2005

2005SP01 NUCLEAR REACTIONS ${ }^{1} \mathrm{H}\left(\mathrm{e}, \mathrm{e}^{\prime} \pi^{0}\right), \mathrm{E}=950 \mathrm{MeV}$; measured Ep, $\sigma(\theta) .{ }^{1} \mathrm{H}$ deduced quadrupole to dipole amplitude ratios. JOUR PRLTA 94022003

2005SP02 NUCLEAR REACTIONS ${ }^{1} \mathrm{H}$ (polarized e, e), E=200 MeV; ${ }^{2} \mathrm{H}$ (polarized e, e), $\mathrm{E}=125,200 \mathrm{MeV}$; measured asymmetries; deduced form factors. JOUR ZAANE $24 \mathrm{~s} 0251$

2005SPZZ NUCLEAR REACTIONS ${ }^{1} \mathrm{H}\left(\mathrm{p}, \mathrm{p} \pi^{+} \pi^{-}\right), \mathrm{E}=2.2 \mathrm{GeV}$; measured $\eta$-meson production associated missing mass spectra. CONF Bormio (XLIII Winter Meeting) Proc,P305 


\section{$\mathrm{A}=1$ (continued)}

2005SUZV NUCLEAR REACTIONS ${ }^{12} \mathrm{C}$ (polarized d, $\alpha$ ), E=130, $180 \mathrm{MeV}$; measured $\mathrm{E} \alpha$, asymmetry; deduced beam polarization. ${ }^{1} \mathrm{H}$ (polarized $\mathrm{d}$, d), $\mathrm{E}=130,180 \mathrm{MeV}$; measured analyzing powers. REPT CNS-REP-66,P34,Suda

2005TR09 NUCLEAR REACTIONS ${ }^{1} \mathrm{H}\left(\mathrm{n}, \mathrm{nK}^{+} \mathrm{K}^{-}\right)$, E at $5.2 \mathrm{GeV} / \mathrm{c}$; measured strangeness production associated invariant mass spectra; deduced resonance features. JOUR FECLA 12436

2005TU09 NUCLEAR REACTIONS ${ }^{2} \mathrm{H}\left({ }^{6} \mathrm{Li}, \mathrm{t} \alpha\right), \mathrm{E}=14 \mathrm{MeV}$; measured triton and $\alpha$ spectra. ${ }^{6} \mathrm{Li}(\mathrm{n}, \alpha), \mathrm{E} \approx 0-1 \mathrm{MeV}$; deduced $\sigma(\theta)$. JOUR ZAANE 25 s01 649

2005VIZY NUCLEAR REACTIONS ${ }^{1} \mathrm{H}(\mathrm{n}, \mathrm{n}), \mathrm{E}=194 \mathrm{MeV}$; measured $\sigma(\theta)$. Tagged neutron beam, comparison with previous results and model predictions. CONF Santa Fe (Nucl Data for Sci and Technol) Proc,Vol1,P820

2005WI17 RADIOACTIVITY ${ }^{1} \mathrm{n}\left(\beta^{-}\right)$; measured $\mathrm{T}_{1 / 2}$. Trapped proton counting method. JOUR JRNBA 110327

2005WU02 NUCLEAR REACTIONS ${ }^{1} \mathrm{H}\left(\gamma, \pi^{+} \pi^{-}\right), \mathrm{E}=0.5-2.6 \mathrm{GeV}$; measured total $\sigma, \sigma(\mathrm{E}, \theta)$; deduced $\rho^{0}$-meson and $\Delta$-baryon contributions. Tagged photons. JOUR ZAANE 23317

2005ZH14 NUCLEAR REACTIONS ${ }^{1} \mathrm{H}\left(\gamma, \pi^{+}\right),{ }^{2} \mathrm{H}\left(\gamma, \mathrm{p} \pi^{-}\right), \mathrm{E}=1.1-5.5 \mathrm{GeV}$; measured $\sigma(\mathrm{E}, \theta)$; deduced scaling behavior. ${ }^{1} \mathrm{n}\left(\gamma, \pi^{-}\right), \mathrm{E}=1.1-5.5$ $\mathrm{GeV}$; deduced $\sigma(\mathrm{E}, \theta)$, scaling behavior. JOUR PRVCA 71044603

$$
\mathrm{A}=\mathbf{2}
$$

$2 \mathrm{n}$

2005AM05

2005BA43

2005 CH50

${ }^{2} \mathrm{H}$

2004AZZW

2004AZZX

2004BUZY

2004FOZZ
NUCLEAR REACTIONS ${ }^{1} \mathrm{H}\left(\mathrm{p}-\right.$ bar, $\left.\mathrm{ee}^{+}\right), \mathrm{E}(\mathrm{cm}) \approx 3600 \mathrm{MeV}$; measured $\psi(2 \mathrm{~S})$ production associated invariant mass spectra, $\sigma(\theta)$; deduced helicity amplitude ratio. JOUR PYLBB 610177

NUCLEAR REACTIONS ${ }^{2} \mathrm{H}(\mathrm{d}, 2 \mathrm{p}), \mathrm{E}=171 \mathrm{MeV}$; measured Ep, pp-coin, $\sigma(\theta)$; deduced neutron-neutron scattering length. JOUR PRVCA 71044003

NUCLEAR REACTIONS ${ }^{1} \mathrm{H}\left({ }^{6} \mathrm{He}, \mathrm{p}\right),\left({ }^{6} \mathrm{He}, \mathrm{np}\right),\left({ }^{6} \mathrm{He}, \mathrm{p} \alpha\right), \mathrm{E}=717$ $\mathrm{MeV} /$ nucleon; ${ }^{1} \mathrm{H}\left({ }^{8} \mathrm{He}, \mathrm{p}\right),\left({ }^{8} \mathrm{He}, \mathrm{np}\right),\left({ }^{8} \mathrm{He}, \mathrm{p} \alpha\right), \mathrm{E}=671 \mathrm{MeV} /$ nucleon; measured recoil proton spectra, $\sigma(\mathrm{E}, \theta) .{ }^{6,8} \mathrm{He}$ deduced cluster configurations, spectroscopic factors. JOUR NUPAB 75943

NUCLEAR REACTIONS ${ }^{2} \mathrm{H}$ (polarized d, d'), E at $5.0 \mathrm{GeV} / \mathrm{c}$; measured vector and tensor analyzing powers. REPT

JINR-E1-2004-117,Azhgirey

NUCLEAR REACTIONS ${ }^{9} \mathrm{Be}(\mathrm{d}, \mathrm{pX}), \mathrm{E}$ at $5 \mathrm{GeV} / \mathrm{c}$; measured tensor analyzing power. ${ }^{2} \mathrm{H}$ deduced wave function features. REPT JINR-P1-2004-118,Azhgirey

NUCLEAR REACTIONS ${ }^{2} \mathrm{H}$ (polarized n, n), E=19.0 MeV; measured $\operatorname{Ay}(\theta)$. Comparison with model predictions. REPT TUNL-XLIII,P20,Buck NUCLEAR REACTIONS ${ }^{2} \mathrm{H}$ (polarized n, n), $\mathrm{E}=1.18,5.0,6.88,19$ $\mathrm{MeV}$; measured polarization, longitudinal cross-section difference. Polarized target. REPT TUNL-XLIII,P18,Foster 


\section{$\mathrm{A}=2$ (continued)}

2004S035 NUCLEAR REACTIONS ${ }^{7} \mathrm{Li}\left({ }^{7} \mathrm{Li}, 2 \alpha\right), \mathrm{E}=8,30 \mathrm{MeV} ;{ }^{9} \mathrm{Be}\left({ }^{7} \mathrm{Li},{ }^{7} \mathrm{Li}\right)$, $\left({ }^{7} \mathrm{Li}, \alpha^{6} \mathrm{Li}\right),\left({ }^{7} \mathrm{Li}, \alpha^{7} \mathrm{Li}\right), \mathrm{E}=52 \mathrm{MeV} ;{ }^{7} \mathrm{Li}\left({ }^{9} \mathrm{Be}, \alpha^{9} \mathrm{Be}\right),\left({ }^{9} \mathrm{Be}, \alpha^{10} \mathrm{Be}\right)$, $\mathrm{E}=70 \mathrm{MeV}$; measured excitation energy spectra. ${ }^{9,10} \mathrm{Be},{ }^{13,14} \mathrm{C}$ deduced excited states, cluster structures. JOUR FIZBE 13433

2005AB01 NUCLEAR REACTIONS ${ }^{1} \mathrm{H}\left(\mathrm{p}, \mathrm{p} \pi^{+}\right),\left(\mathrm{p}, \pi^{+}\right), \mathrm{E}=951 \mathrm{MeV}$; measured missing mass spectra, $\sigma$; deduced D-state effects. JOUR PYLBB 61031

2005AC22 NUCLEAR REACTIONS ${ }^{3} \mathrm{He}$ (polarized e, e'p), (polarized e, e'np), $\mathrm{E}=735 \mathrm{MeV}$; measured polarization observables; deduced final state interaction effects. Polarized target. JOUR ZAANE 25177

2005AG03 NUCLEAR REACTIONS ${ }^{2} \mathrm{H},{ }^{6} \mathrm{Li}\left(\right.$ polarized $\left.\mu^{+}, \mu^{+} \mathrm{X}\right), \mathrm{E}=160 \mathrm{GeV}$; measured longitudinal spin asymmetry. ${ }^{2} \mathrm{H}$ deduced spin structure function. Comparison with previous results. JOUR PYLBB 612154

2005AI06 NUCLEAR REACTIONS ${ }^{1} \mathrm{H}\left(\mathrm{e}^{+}, \mathrm{e}^{+} \mathrm{X}\right)$, E at $27.7 \mathrm{GeV} / \mathrm{c}$; measured tensor asymmetry. ${ }^{2} \mathrm{H}$ deduced tensor structure function. Polarized target. JOUR PRLTA 95242001

2005AT04 NUCLEAR REACTIONS ${ }^{2} \mathrm{H}(\mathrm{n}, \mathrm{n}$ '), E=low; measured production rate of ultracold neutrons with solid, liquid, and gaseous deuterium targets. JOUR PRVCA 71054601

2005BA40 NUCLEAR REACTIONS ${ }^{1} \mathrm{H}\left({ }^{16} \mathrm{O}, \mathrm{X}\right){ }^{1} \mathrm{H} /{ }^{2} \mathrm{H} /{ }^{3} \mathrm{H} /{ }^{3} \mathrm{He} /{ }^{4} \mathrm{He} /{ }^{5} \mathrm{He}$ $/{ }^{6} \mathrm{He} /{ }^{5} \mathrm{Li} /{ }^{6} \mathrm{Li} /{ }^{7} \mathrm{Li} /{ }^{8} \mathrm{Li} /{ }^{7} \mathrm{Be} /{ }^{8} \mathrm{Be} /{ }^{9} \mathrm{Be} /{ }^{10} \mathrm{Be} /{ }^{9} \mathrm{~B} /{ }^{10} \mathrm{~B} /$ ${ }^{11} \mathrm{~B} /{ }^{12} \mathrm{~B} /{ }^{10} \mathrm{C} /{ }^{11} \mathrm{C} /{ }^{12} \mathrm{C} /{ }^{13} \mathrm{C} /{ }^{14} \mathrm{C} /{ }^{13} \mathrm{~N} /{ }^{14} \mathrm{~N} /{ }^{15} \mathrm{~N} /{ }^{14} \mathrm{O} /$ ${ }^{15} \mathrm{O} /{ }^{16} \mathrm{O}$, E at $3.25 \mathrm{GeV} / \mathrm{c} /$ nucleon; measured production $\sigma$. JOUR PZETA 81174

2005CA42 NUCLEAR MOMENTS ${ }^{2} \mathrm{H},{ }^{15} \mathrm{~N}$; measured hfs; deduced parameters. JOUR APJSA 159181

2005CU06 NUCLEAR REACTIONS ${ }^{7} \mathrm{Li}\left({ }^{7} \mathrm{Li},{ }^{11} \mathrm{~B}\right),\left({ }^{7} \mathrm{Li},{ }^{12} \mathrm{~B}\right), \mathrm{E}=58 \mathrm{MeV} ;{ }^{12} \mathrm{C}$, ${ }^{16} \mathrm{O}\left({ }^{7} \mathrm{Li},{ }^{10} \mathrm{~B}\right), \mathrm{E}=58 \mathrm{MeV}$; measured particle spectra. ${ }^{10,11,12} \mathrm{~B}$ deduced relative yields for $\alpha+\mathrm{Li}$ and $\mathrm{H}+\mathrm{Be}$ decay channels from excited states. JOUR PRVCA 72044320

2005ER03 NUCLEAR REACTIONS ${ }^{2} \mathrm{H}$ (polarized p, p), $\mathrm{E}=108,120,135,150$, $170,190 \mathrm{MeV}$; measured $\sigma(\theta)$ and vector analyzing power; deduced three-nucleon forces contribution and necessity of inclusion. Comparisons with model predictions. JOUR PRVCA 71064004

2005G014 NUCLEAR REACTIONS ${ }^{3} \mathrm{He}(\alpha, \mathrm{p} \alpha), \mathrm{E}=27.2 \mathrm{MeV}$; measured Ep, E $\alpha$, $\mathrm{p} \alpha$-coin, $\sigma(\mathrm{E}, \theta) .{ }^{6} \mathrm{Li}$ deduced excited states energies, widths. JOUR UKPJA 50327

2005HA37 NUCLEAR REACTIONS ${ }^{1,2} \mathrm{H}$ (polarized e, e'), E=high; measured analyzing powers; deduced form factors. Polarized targets. JOUR NUPAB $755257 \mathrm{c}$

2005JE01 NUCLEAR REACTIONS ${ }^{2} \mathrm{H}\left({ }^{9} \mathrm{Li},{ }^{9} \mathrm{Li}\right),\left({ }^{9} \mathrm{Li}, \mathrm{np}\right),\left({ }^{9} \mathrm{Li}, \mathrm{nX}\right),\left({ }^{9} \mathrm{Li}, \mathrm{pX}\right)$, $\mathrm{E}=2.36 \mathrm{MeV} /$ nucleon; measured particle spectra, $\sigma(\theta) .{ }^{2} \mathrm{H}\left({ }^{9} \mathrm{Li}, \alpha \mathrm{X}\right)$, $\left({ }^{9} \mathrm{Li},{ }^{6} \mathrm{HeX}\right), \mathrm{E}=2.36 \mathrm{MeV} /$ nucleon; measured particle spectra. Post-accelerated radioactive beam. JOUR NUPAB 748374

2005KA25 NUCLEAR MOMENTS ${ }^{1,2} \mathrm{H}$; measured NMR spectra; deduced $\mu$ ratio. JOUR CJPHA 83405

2005LA30 NUCLEAR REACTIONS H, C(polarized d, pX), E at $9 \mathrm{GeV} \mathrm{/} \mathrm{c;}$ measured tensor analyzing power vs proton transverse momentum. ${ }^{2} \mathrm{H}$ deduced wave function features. JOUR PYLBB 62960 


\section{$\mathrm{A}=2$ (continued)}

2005MAZN NUCLEAR REACTIONS ${ }^{2} \mathrm{H}$ (polarized n, n), $\mathrm{E}=250 \mathrm{MeV}$; measured $\sigma(\theta), \operatorname{Ay}(\theta)$; deduced three-nucleon force effects. REPT CNS-REP-66,P38,Maeda

2005MEZY NUCLEAR REACTIONS ${ }^{1,2} \mathrm{H}(\mathrm{n}, \mathrm{n}), \mathrm{E}=95 \mathrm{MeV}$; measured $\sigma(\theta)$; deduced three-nucleon force effects. CONF Santa Fe (Nucl Data for Sci and Technol) Proc,Vol1,P688

$2005 \mathrm{MI} 13$ NUCLEAR REACTIONS ${ }^{6,7} \mathrm{Li}\left({ }^{6} \mathrm{He}, \alpha^{6} \mathrm{He}\right),{ }^{6} \mathrm{Li}\left({ }^{6} \mathrm{He}, \mathrm{t} 2 \alpha\right), \mathrm{E}=18 \mathrm{MeV}$; measured excitation energy spectra. ${ }^{6,7} \mathrm{Li},{ }^{8,9,10} \mathrm{Be}$ deduced cluster states. JOUR NUPAB 753263

2005RV01 NUCLEAR REACTIONS ${ }^{3} \mathrm{He}(\mathrm{e}, \mathrm{e}$ 'p), E=4806 MeV; measured $\sigma(\mathrm{E}$, $\theta)$, asymmetry; deduced final-state interaction effects, other reaction mechanism features. Comparison with model predictions. JOUR PRLTA 94192302

2005SA12 NUCLEAR REACTIONS ${ }^{3} \mathrm{He}(\mathrm{e}, \mathrm{e}$ 'p), E=4.8 GeV; measured $\sigma(\mathrm{E}, \theta)$, asymmetries. ${ }^{3} \mathrm{He}$ deduced bound state momentum distributions. Comparisons with model predictions. JOUR ZAANE 24 s01 81

2005SE05 NUCLEAR REACTIONS ${ }^{2} \mathrm{H}(\mathrm{n}, \mathrm{n}),(\mathrm{n}, 2 \mathrm{n}), \mathrm{E}=13 \mathrm{MeV}$; measured En, nn-coin, $\sigma\left(\theta_{1}, \theta_{2}\right)$ for seven exit-channel configurations. Comparison with model predictions. JOUR PRVCA 71034006

2005SE22 NUCLEAR REACTIONS ${ }^{2} \mathrm{H}(\mathrm{p}, \mathrm{p}), \mathrm{E}=135 \mathrm{MeV} ;{ }^{1} \mathrm{H}(\mathrm{d}, \mathrm{d}), \mathrm{E}=135$ $\mathrm{MeV} /$ nucleon; measured $\sigma(\theta)$; deduced relativistic effects, three-nucleon force effects. Comparison with previous results. JOUR PRLTA 95162301

2005SEZV NUCLEAR REACTIONS ${ }^{2} \mathrm{H}(\mathrm{p}, \mathrm{p}), \mathrm{E}=135 \mathrm{MeV} ;{ }^{1} \mathrm{H}(\mathrm{d}, \mathrm{d}), \mathrm{E}=270$ $\mathrm{MeV}$; measured $\sigma(\theta)$. Comparison with model predictions and previous data. PREPRINT nucl-ex/0510005,10/3/2005

2005SH51 NUCLEAR REACTIONS ${ }^{4} \mathrm{He}(\gamma, \mathrm{p}),(\gamma, \mathrm{n}),(\gamma, \mathrm{np}), \mathrm{E}=21.8-29.8 \mathrm{MeV}$; ${ }^{12} \mathrm{C}(\gamma, \mathrm{p}),(\gamma, \mathrm{n}), \mathrm{E}=22.3-32 \mathrm{MeV}$; measured charged particle spectra, photodisintegration $\sigma, \sigma(\theta)$. Monoenergetic pulsed photons, comparison with previous results and model predictions. JOUR PRVCA 72044004

2005SP02 NUCLEAR REACTIONS ${ }^{1} \mathrm{H}$ (polarized e, e), E=200 MeV; ${ }^{2} \mathrm{H}$ (polarized e, e), $\mathrm{E}=125,200 \mathrm{MeV}$; measured asymmetries; deduced form factors. JOUR ZAANE 24 s02 51

${ }^{3} \mathrm{n}$

2005AL15

${ }^{3} \mathrm{H}$

2004MIZR

20045035

$$
\mathrm{A}=3
$$

\section{$\mathrm{A}=3$}

NUCLEAR REACTIONS ${ }^{7} \mathrm{Li}\left({ }^{7} \mathrm{Li},{ }^{11} \mathrm{C}\right),\left({ }^{7} \mathrm{Li},{ }^{10} \mathrm{C}\right), \mathrm{E}=82 \mathrm{MeV}$; measured particle spectra; deduced resonance formation $\sigma$ upper limits. JOUR PZETA 8149 NUCLEAR REACTIONS ${ }^{4} \mathrm{He}\left({ }^{22} \mathrm{O},{ }^{23} \mathrm{~F}\right), \mathrm{E} \approx 35 \mathrm{MeV} /$ nucleon; measured $\mathrm{E} \gamma, \mathrm{I} \gamma$, (particle) $\gamma$-coin. ${ }^{23} \mathrm{~F}$ deduced levels, transitions. REPT CNS-REP-64,P269,Michimasa NUCLEAR REACTIONS ${ }^{7} \mathrm{Li}\left({ }^{7} \mathrm{Li}, 2 \alpha\right), \mathrm{E}=8,30 \mathrm{MeV} ;{ }^{9} \mathrm{Be}\left({ }^{7} \mathrm{Li},{ }^{7} \mathrm{Li}\right)$, $\left({ }^{7} \mathrm{Li}, \alpha^{6} \mathrm{Li}\right),\left({ }^{7} \mathrm{Li}, \alpha^{7} \mathrm{Li}\right), \mathrm{E}=52 \mathrm{MeV} ;{ }^{7} \mathrm{Li}\left({ }^{9} \mathrm{Be}, \alpha^{9} \mathrm{Be}\right),\left({ }^{9} \mathrm{Be}, \alpha^{10} \mathrm{Be}\right)$, $\mathrm{E}=70 \mathrm{MeV}$; measured excitation energy spectra. ${ }^{9,10} \mathrm{Be},{ }^{13,14} \mathrm{C}$ deduced excited states, cluster structures. JOUR FIZBE 13433 


\section{$\mathrm{A}=3$ (continued)}

2005BA40 NUCLEAR REACTIONS ${ }^{1} \mathrm{H}\left({ }^{16} \mathrm{O}, \mathrm{X}\right){ }^{1} \mathrm{H} /{ }^{2} \mathrm{H} /{ }^{3} \mathrm{H} /{ }^{3} \mathrm{He} /{ }^{4} \mathrm{He} /{ }^{5} \mathrm{He}$ $/{ }^{6} \mathrm{He} /{ }^{5} \mathrm{Li} /{ }^{6} \mathrm{Li} /{ }^{7} \mathrm{Li} /{ }^{8} \mathrm{Li} /{ }^{7} \mathrm{Be} /{ }^{8} \mathrm{Be} /{ }^{9} \mathrm{Be} /{ }^{10} \mathrm{Be} /{ }^{9} \mathrm{~B} /{ }^{10} \mathrm{~B} /$ ${ }^{11} \mathrm{~B} /{ }^{12} \mathrm{~B} /{ }^{10} \mathrm{C} /{ }^{11} \mathrm{C} /{ }^{12} \mathrm{C} /{ }^{13} \mathrm{C} /{ }^{14} \mathrm{C} /{ }^{13} \mathrm{~N} /{ }^{14} \mathrm{~N} /{ }^{15} \mathrm{~N} /{ }^{14} \mathrm{O} /$ ${ }^{15} \mathrm{O} /{ }^{16} \mathrm{O}$, E at $3.25 \mathrm{GeV} / \mathrm{c} /$ nucleon; measured production $\sigma$. JOUR PZETA 81174

2005BL09 NUCLEAR REACTIONS ${ }^{1} \mathrm{H}\left({ }^{6} \mathrm{He},{ }^{6} \mathrm{He}\right),\left({ }^{6} \mathrm{He},{ }^{6} \mathrm{He}\right), \mathrm{E}=15 \mathrm{MeV} /$ nucleon; measured $\sigma(\mathrm{q})$; deduced halo effect. ${ }^{1} \mathrm{H}\left({ }^{6} \mathrm{He}, \alpha\right), \mathrm{E}=25 \mathrm{MeV} /$ nucleon; measured $\sigma(\theta) .{ }^{2} \mathrm{H}\left({ }^{8} \mathrm{He},{ }^{6} \mathrm{Li}\right), \mathrm{E}=15 \mathrm{MeV} /$ nucleon; measured excitation energy spectrum; deduced possible resonance structure. ${ }^{1} \mathrm{H}\left({ }^{22} \mathrm{O},{ }^{22} \mathrm{O}^{\prime}\right), \mathrm{E}=46.6 \mathrm{MeV} /$ nucleon; measured $\sigma(\mathrm{E}, \theta)$. JOUR NUPAB $752279 \mathrm{c}$

2005 CU06 NUCLEAR REACTIONS ${ }^{7} \mathrm{Li}\left({ }^{7} \mathrm{Li},{ }^{11} \mathrm{~B}\right),\left({ }^{7} \mathrm{Li},{ }^{12} \mathrm{~B}\right), \mathrm{E}=58 \mathrm{MeV} ;{ }^{12} \mathrm{C}$, ${ }^{16} \mathrm{O}\left({ }^{7} \mathrm{Li},{ }^{10} \mathrm{~B}\right), \mathrm{E}=58 \mathrm{MeV}$; measured particle spectra. ${ }^{10,11,12} \mathrm{~B}$ deduced relative yields for $\alpha+\mathrm{Li}$ and $\mathrm{H}+\mathrm{Be}$ decay channels from excited states. JOUR PRVCA 72044320

2005GI07 NUCLEAR REACTIONS ${ }^{1} \mathrm{H}\left({ }^{6} \mathrm{He}, \alpha\right), \mathrm{E}=25 \mathrm{MeV} /$ nucleon; measured $\sigma(\theta)$; deduced particle transfer contributions, entrance potential dependence. ${ }^{6} \mathrm{He}$ deduced spectroscopic factors for $\mathrm{t}+\mathrm{t}$ and $\alpha+2 \mathrm{n}$ cluster configurations. ${ }^{1} \mathrm{H}\left({ }^{6} \mathrm{He}, \mathrm{p}\right), \mathrm{E}=25 \mathrm{MeV} /$ nucleon; measured $\sigma(\theta) .{ }^{3} \mathrm{He}(\alpha, \alpha), \mathrm{E}(\mathrm{cm})=28.7 \mathrm{MeV}$; calculated $\sigma(\theta)$. SPEG spectrometer and MUST array at GANIL. DWBA and coupled-channels calculations. JOUR PRVCA 71064311

2005GI18 NUCLEAR REACTIONS ${ }^{1} \mathrm{H}\left({ }^{6} \mathrm{He}, \alpha\right), \mathrm{E}=25 \mathrm{MeV} /$ nucleon; measured $\sigma(\theta)$; deduced particle transfer contributions, entrance potential dependence. ${ }^{6} \mathrm{He}$ deduced spectroscopic factors for $\mathrm{t}+\mathrm{t}$ and $\mathrm{a}+2 \mathrm{n}$ cluster configurations. SPEG spectrometer and MUST array at GANIL. DWBA and coupled-channels calculations. JOUR ZAANE 25 s01 267

2005GIZZ NUCLEAR REACTIONS ${ }^{1} \mathrm{H}\left({ }^{6} \mathrm{He}, \mathrm{t}\right),\left({ }^{6} \mathrm{He}, \alpha\right),\left({ }^{6} \mathrm{He},{ }^{6} \mathrm{He}\right), \mathrm{E}=150$ $\mathrm{MeV}$; measured particle spectra, $\sigma(\theta)$. ${ }^{6} \mathrm{He}$ deduced spectroscopic factors for cluster configurations. PREPRINT nucl-ex/0505007,5/04/2005

2005KI17 NUCLEAR REACTIONS ${ }^{4} \mathrm{He}(\gamma, \mathrm{p}),(\gamma, \mathrm{n}), \mathrm{E} \approx 27.6 \mathrm{MeV}$; measured particle spectra, tp-, $\left({ }^{3} \mathrm{He}\right) \mathrm{n}$-coin. Time projection chamber. JOUR NIMAE 552329

2005KR03 RADIOACTIVITY ${ }^{3} \mathrm{H}\left(\beta^{-}\right)$; measured $\mathrm{E} \beta$; deduced neutrino mass limit. JOUR ZCCNE 40447

2005LI29 NUCLEAR REACTIONS ${ }^{2} \mathrm{H}(\mathrm{d}, \mathrm{p}), \mathrm{E}=0.8-2.45 \mathrm{keV}$; measured charge particle yields; deduced reaction rate enhancement in titanium cathode. JOUR ZETFA 1271334

$2005 \mathrm{MI} 13 \quad$ NUCLEAR REACTIONS ${ }^{6,7} \mathrm{Li}\left({ }^{6} \mathrm{He}, \alpha^{6} \mathrm{He}\right),{ }^{6} \mathrm{Li}\left({ }^{6} \mathrm{He}, \mathrm{t} 2 \alpha\right), \mathrm{E}=18 \mathrm{MeV}$; measured excitation energy spectra. ${ }^{6,7} \mathrm{Li},{ }^{8,9,10} \mathrm{Be}$ deduced cluster states. JOUR NUPAB 753263

2005MI32 NUCLEAR REACTIONS ${ }^{4} \mathrm{He}\left({ }^{22} \mathrm{O},{ }^{23} \mathrm{~F}\right), \mathrm{E}=35 \mathrm{MeV} /$ nucleon; ${ }^{4} \mathrm{He}\left({ }^{23} \mathrm{~F},{ }^{23} \mathrm{~F}\right.$ '), $\mathrm{E}=41.5 \mathrm{MeV} /$ nucleon; ${ }^{4} \mathrm{He}\left({ }^{24} \mathrm{~F},{ }^{23} \mathrm{~F}\right), \mathrm{E}=36 \mathrm{MeV} /$ nucleon; measured $\mathrm{E} \gamma, \mathrm{I} \gamma$, (particle) $\gamma-, \gamma \gamma$-coin; deduced $\sigma(\mathrm{E}) \cdot{ }^{23} \mathrm{~F}$ deduced levels, J, $\pi$. DWBA analysis. JOUR ZAANE 25 s01 367 


\section{$\mathrm{A}=3$ (continued)}

2005MIZT NUCLEAR REACTIONS ${ }^{4} \mathrm{He}\left({ }^{22} \mathrm{O},{ }^{23} \mathrm{~F}\right),\left({ }^{23} \mathrm{~F},{ }^{23} \mathrm{~F}\right),\left({ }^{24} \mathrm{~F},{ }^{23} \mathrm{~F}\right)$, $\left({ }^{25} \mathrm{Ne},{ }^{23} \mathrm{~F}\right), \mathrm{E} \approx 35-43 \mathrm{MeV} /$ nucleon; measured $\mathrm{E} \gamma, \mathrm{I} \gamma$, (particle) $\gamma-$, $\gamma \gamma$-coin. ${ }^{4} \mathrm{He}\left({ }^{22} \mathrm{O},{ }^{23} \mathrm{~F}\right), \mathrm{E}=35 \mathrm{MeV} /$ nucleon; measured $\sigma(\theta) \cdot{ }^{23} \mathrm{~F}$ deduced levels, J, $\pi$, configurations. REPT CNS-REP-67,Michimasa

2005MIZU NUCLEAR REACTIONS ${ }^{4} \mathrm{He}\left({ }^{22} \mathrm{O},{ }^{23} \mathrm{~F}\right),\left({ }^{23} \mathrm{~F},{ }^{23} \mathrm{~F}\right),\left({ }^{24} \mathrm{~F},{ }^{23} \mathrm{~F}\right)$, E not given; measured $\mathrm{E} \gamma, \mathrm{I} \gamma, \gamma \gamma$-coin, $\sigma(\theta) .{ }^{23} \mathrm{~F}$ deduced levels, J, $\pi$. REPT CNS-REP-66,P26,Michimasa

2005MIZV NUCLEAR REACTIONS ${ }^{4} \mathrm{He}\left({ }^{22} \mathrm{O},{ }^{23} \mathrm{~F}\right), \mathrm{E} \approx 35 \mathrm{MeV} /$ nucleon; ${ }^{4} \mathrm{He}\left({ }^{23} \mathrm{~F},{ }^{23} \mathrm{~F}\right.$ '), $\mathrm{E} \approx 41.5 \mathrm{MeV} /$ nucleon; ${ }^{4} \mathrm{He}\left({ }^{24} \mathrm{~F},{ }^{23} \mathrm{~F}\right), \mathrm{E} \approx 36 \mathrm{MeV} /$ nucleon; measured $\mathrm{E} \gamma, \mathrm{I} \gamma, \gamma \gamma$-, (particle) $\gamma$-coin, angular distributions. ${ }^{23} \mathrm{~F}$ deduced levels, J, $\pi$. REPT RIKEN 2004 Annual,P51,Michimasa

2005RA27 NUCLEAR REACTIONS ${ }^{2} \mathrm{H}(\mathrm{d}, \mathrm{p}), \mathrm{E} \approx 4-23 \mathrm{keV}$; measured S-factors, electron screening effects for reactions in deuterated metals, temperature dependence. JOUR JPGPE 311141

2005RI13 NUCLEAR REACTIONS ${ }^{6} \mathrm{Li}(\mathrm{d}, \mathrm{pt}), \mathrm{E}=14 \mathrm{MeV}$; measured particle spectra, angular correlations. ${ }^{2} \mathrm{H}(\mathrm{d}, \mathrm{p}), \mathrm{E} \approx 50-2000 \mathrm{keV}$; deduced S-factors. Trojan horse method, comparison with previous results. JOUR NUPAB $758146 \mathrm{c}$

2005SH46 NUCLEAR REACTIONS ${ }^{4} \mathrm{He}\left({ }^{22} \mathrm{O},{ }^{23} \mathrm{~F}\right), \mathrm{E}=35 \mathrm{MeV} /$ nucleon; measured $\mathrm{E} \gamma, \mathrm{I} \gamma$, (particle) $\gamma$-coin, $\sigma(\theta) .{ }^{23} \mathrm{~F}$ deduced levels, $\mathrm{J}, \pi$. JOUR JPGPE 31 S1759

2005SH51 NUCLEAR REACTIONS ${ }^{4} \mathrm{He}(\gamma, \mathrm{p}),(\gamma, \mathrm{n}),(\gamma, \mathrm{np}), \mathrm{E}=21.8-29.8 \mathrm{MeV}$; ${ }^{12} \mathrm{C}(\gamma, \mathrm{p}),(\gamma, \mathrm{n}), \mathrm{E}=22.3-32 \mathrm{MeV}$; measured charged particle spectra, photodisintegration $\sigma, \sigma(\theta)$. Monoenergetic pulsed photons, comparison with previous results and model predictions. JOUR PRVCA 72044004

2005ST30 NUCLEAR REACTIONS ${ }^{4} \mathrm{He}\left(\mathrm{e}, \mathrm{e} \mathrm{p} \pi^{-}\right),\left(\mathrm{e}, \mathrm{e} \mathrm{p} \pi^{0}\right), \mathrm{E}=672 \mathrm{MeV}$; measured Ep, recoil spectra, $\sigma(\theta)$. Comparison with model predictions. JOUR PRLTA 95172501

2005TU09 NUCLEAR REACTIONS ${ }^{2} \mathrm{H}\left({ }^{6} \mathrm{Li}, \mathrm{t} \alpha\right), \mathrm{E}=14 \mathrm{MeV}$; measured triton and $\alpha$ spectra. ${ }^{6} \mathrm{Li}(\mathrm{n}, \alpha), \mathrm{E} \approx 0-1 \mathrm{MeV}$; deduced $\sigma(\theta)$. JOUR ZAANE 25 s01 649

2005VE08 NUCLEAR REACTIONS ${ }^{6} \operatorname{Li}(\mathrm{n}, \alpha), \mathrm{E}=$ reactor; measured triton spectra, angular distribution; deduced P-odd asymmetry coefficient. JOUR PZETA 82519

2005YA12 NUCLEAR REACTIONS ${ }^{6} \mathrm{Li}\left({ }^{3} \mathrm{He}, \mathrm{t}^{3} \mathrm{He}\right), \mathrm{E}=450 \mathrm{MeV} ;{ }^{6} \mathrm{Li}\left({ }^{7} \mathrm{Li}, \mathrm{t}^{7} \mathrm{Be}\right)$, $\mathrm{E}=455 \mathrm{MeV}$; measured particle spectra, angular correlations. ${ }^{6} \mathrm{He},{ }^{6} \mathrm{Li}$, ${ }^{6}$ Be deduced resonances. JOUR PRVCA 71064316

${ }^{3} \mathrm{He} \quad$ 2004CRZZ NUCLEAR REACTIONS ${ }^{3} \mathrm{He}($ polarized n, n), E=4.02, $5.54 \mathrm{MeV}$; measured $\operatorname{Ay}(\theta)$. REPT TUNL-XLIII,P23, Crowe

2004ZH42 NUCLEAR REACTIONS ${ }^{3} \mathrm{He}$ (polarized e, e), E=1.2 GeV;

${ }^{3} \mathrm{He}\left(\right.$ polarized e, e'X), $\mathrm{E}=5.7 \mathrm{GeV}$; measured asymmetries. ${ }^{1} \mathrm{n}$ deduced spin asymmetries, polarized structure functions. Polarized target. JOUR PRVCA 70065207

2005BA34 NUCLEAR REACTIONS ${ }^{136} \mathrm{Xe}\left(\mathrm{d},{ }^{3} \mathrm{HeX}\right){ }^{135} \mathrm{Xe}, \mathrm{E}=500 \mathrm{MeV} ;{ }^{1} \mathrm{H}(\mathrm{d}$, $\left.\pi^{0}\right), \mathrm{E}=500 \mathrm{MeV}$; measured helium spectra. ${ }^{135} \mathrm{Xe}$ deduced pionic state binding energy. JOUR YAFIA 68517 


\section{$\mathrm{A}=3$ (continued)}

2005Ba40 NUCLEAR REACTIONS ${ }^{1} \mathrm{H}\left({ }^{16} \mathrm{O}, \mathrm{X}\right){ }^{1} \mathrm{H} /{ }^{2} \mathrm{H} /{ }^{3} \mathrm{H} /{ }^{3} \mathrm{He} /{ }^{4} \mathrm{He} /{ }^{5} \mathrm{He}$ $/{ }^{6} \mathrm{He} /{ }^{5} \mathrm{Li} /{ }^{6} \mathrm{Li} /{ }^{7} \mathrm{Li} /{ }^{8} \mathrm{Li} /{ }^{7} \mathrm{Be} /{ }^{8} \mathrm{Be} /{ }^{9} \mathrm{Be} /{ }^{10} \mathrm{Be} /{ }^{9} \mathrm{~B} /{ }^{10} \mathrm{~B} /$ ${ }^{11} \mathrm{~B} /{ }^{12} \mathrm{~B} /{ }^{10} \mathrm{C} /{ }^{11} \mathrm{C} /{ }^{12} \mathrm{C} /{ }^{13} \mathrm{C} /{ }^{14} \mathrm{C} /{ }^{13} \mathrm{~N} /{ }^{14} \mathrm{~N} /{ }^{15} \mathrm{~N} /{ }^{14} \mathrm{O} /$ ${ }^{15} \mathrm{O} /{ }^{16} \mathrm{O}$, E at $3.25 \mathrm{GeV} / \mathrm{c} /$ nucleon; measured production $\sigma$. JOUR PZETA 81174

2005BE12 NUCLEAR REACTIONS ${ }^{3} \mathrm{He}$ (e, e'np), E=high; measured proton spectra, missing energy, $\sigma(\mathrm{E}, \theta) .{ }^{3} \mathrm{He}$ deduced proton effective momentum density. JOUR PRLTA 94082305

2005CA29 NUCLEAR REACTIONS ${ }^{12} \mathrm{C}(\mathrm{p}, \mathrm{X}), \mathrm{E}=180 \mathrm{MeV} ;{ }^{12} \mathrm{C}(\alpha, \mathrm{X}), \mathrm{E}=192.4$ $\mathrm{MeV}$; measured reaction $\sigma .{ }^{3,4} \mathrm{He}(\mathrm{p}, \mathrm{p}), \mathrm{E} \approx 40 \mathrm{MeV}$; measured $\sigma(\theta)$.

${ }^{40} \mathrm{Ca}\left({ }^{3} \mathrm{He},{ }^{3} \mathrm{He}\right.$ '), $\mathrm{E}=167 \mathrm{MeV}$; measured particle spectra. Modified attenuation technique for reaction cross section measurement. JOUR NIMAE 547541

2005CE02 NUCLEAR REACTIONS ${ }^{3} \mathrm{H}(\mathrm{p}, \mathrm{n}), \mathrm{E}=1.2-2.3 \mathrm{MeV}$; measured neutron spectra. JOUR NIMAE 540430

2005GI07 NUCLEAR REACTIONS ${ }^{1} \mathrm{H}\left({ }^{6} \mathrm{He}, \alpha\right), \mathrm{E}=25 \mathrm{MeV} /$ nucleon; measured $\sigma(\theta)$; deduced particle transfer contributions, entrance potential dependence. ${ }^{6} \mathrm{He}$ deduced spectroscopic factors for $\mathrm{t}+\mathrm{t}$ and $\alpha+2 \mathrm{n}$ cluster configurations. ${ }^{1} \mathrm{H}\left({ }^{6} \mathrm{He}, \mathrm{p}\right), \mathrm{E}=25 \mathrm{MeV} /$ nucleon; measured $\sigma(\theta) .{ }^{3} \mathrm{He}(\alpha, \alpha), \mathrm{E}(\mathrm{cm})=28.7 \mathrm{MeV}$; calculated $\sigma(\theta)$. SPEG spectrometer and MUST array at GANIL. DWBA and coupled-channels calculations. JOUR PRVCA 71064311

2005HA07 NUCLEAR REACTIONS ${ }^{2} \mathrm{H}(\mathrm{d}, \mathrm{n})$, E not given; measured En. Laser-generated plasma neutron source. JOUR NIMAE 540464

2005KE05 NUCLEAR REACTIONS ${ }^{3} \mathrm{He}(\mathrm{n}, \mathrm{n}), \mathrm{E}=$ low; measured coherent scattering length. JOUR JRNBA 110241

2005KI17 NUCLEAR REACTIONS ${ }^{4} \mathrm{He}(\gamma, \mathrm{p}),(\gamma, \mathrm{n}), \mathrm{E} \approx 27.6 \mathrm{MeV}$; measured particle spectra, tp-, $\left({ }^{3} \mathrm{He}\right)$ n-coin. Time projection chamber. JOUR NIMAE 552329

2005KLZZ NUCLEAR REACTIONS ${ }^{1} \mathrm{H}$ (polarized d, $\gamma$ ), $\mathrm{E}=29,45 \mathrm{MeV}$; measured vector and tensor analyzing powers. Comparison with model predictions. PREPRINT nucl-ex/0509008,9/05/2005

2005KR03 RADIOACTIVITY ${ }^{3} \mathrm{H}\left(\beta^{-}\right)$; measured $\mathrm{E} \beta$; deduced neutrino mass limit. JOUR ZCCNE 40447

2005KR14 NUCLEAR REACTIONS ${ }^{3} \mathrm{He}$ (polarized e, e'), E=3.465-5.727 GeV; measured parallel and perpendicular cross section differences. ${ }^{1} \mathrm{n},{ }^{3} \mathrm{He}$ deduced momentum transfer dependence of spin structure function. JOUR PRLTA 95142002

2005LE04 NUCLEAR REACTIONS Pb(p, X) ${ }^{3} \mathrm{He} /{ }^{4} \mathrm{He} /{ }^{21} \mathrm{Ne} /{ }^{22} \mathrm{Ne} /{ }^{36} \mathrm{Ar} /$ ${ }^{38} \mathrm{Ar} /{ }^{78} \mathrm{Kr} /{ }^{80} \mathrm{Kr} /{ }^{81} \mathrm{Kr} /{ }^{82} \mathrm{Kr} /{ }^{83} \mathrm{Kr} /{ }^{84} \mathrm{Kr} /{ }^{85} \mathrm{Kr} /{ }^{86} \mathrm{Kr} /{ }^{124} \mathrm{Xe}$ $/{ }^{126} \mathrm{Xe} /{ }^{128} \mathrm{Xe} /{ }^{129} \mathrm{Xe} /{ }^{130} \mathrm{Xe} /{ }^{131} \mathrm{Xe} /{ }^{132} \mathrm{Xe} /{ }^{134} \mathrm{Xe}, \mathrm{E}=44-2595$

$\mathrm{MeV}$; measured production $\sigma$; deduced reaction mechanism features. Mini-stack approach, comparisons with model predictions. JOUR NIMBE 2291

2005MAZQ NUCLEAR REACTIONS ${ }^{15} \mathrm{~N}(\mathrm{p}, \mathrm{n}), \mathrm{E}=5.1 \mathrm{MeV} ;{ }^{2} \mathrm{H}(\mathrm{d}, \mathrm{n}), \mathrm{E}=3.0$ $\mathrm{MeV}$; measured neutron spectra, transmission through iron spheres. CONF Santa Fe (Nucl Data for Sci and Technol) Proc,Vol1,P480 


\section{$\mathrm{A}=3$ (continued)}

2005ME03 NUCLEAR REACTIONS ${ }^{3} \mathrm{He}$ (polarized e, e'X), E=0.862-5.058 GeV; measured polarized $\sigma$; deduced sum rule features. ${ }^{3} \mathrm{He}$ deduced spin structure functions. Polarized target. JOUR ZAANE 24 s01 153

2005ME09 NUCLEAR REACTIONS ${ }^{1} \mathrm{H}$ (polarized d, $\gamma$ ), E=55, 66.5, $90 \mathrm{MeV} /$ nucleon; measured $\mathrm{E} \gamma$, (particle) $\gamma$-coin, vector and tensor analyzing powers. Comparison with model predictions. JOUR PYLBB 61718

2005MEZZ NUCLEAR REACTIONS ${ }^{1} \mathrm{H}$ (polarized d, $\gamma$ ), E $=55,66.5,90 \mathrm{MeV} /$ nucleon; measured $\mathrm{E} \gamma$, (particle) $\gamma$-coin, vector and tensor analyzing powers. Comparison with model predictions. PREPRINT nucl-ex/0501012,1/17/2005

2005NA14 NUCLEAR REACTIONS ${ }^{2} \mathrm{H}(\mathrm{d}, \mathrm{n}), \mathrm{E}>80 \mathrm{keV}$; measured neutron spectra, yields. Deuteron beam from electrostatic field of pyroelectric crystal in a deuterated atmosphere. JOUR NATUA 4341115

2005NI20 NUCLEAR REACTIONS ${ }^{4} \mathrm{He}(\gamma, \mathrm{n}), \mathrm{E}=23-42 \mathrm{MeV}$; measured $\sigma(\theta)$; deduced angle-integrated $\sigma$. Comparison with previous data and various model calculations. Liquid target, tagged photons. JOUR PYLBB 62665

2005NIZX NUCLEAR REACTIONS ${ }^{4} \mathrm{He}(\gamma, \mathrm{n}), \mathrm{E}=23-42 \mathrm{MeV}$; measured neutron spectra, $\sigma(\mathrm{E}, \theta)$; deduced parameters. Tagged photons, comparison with recoil-corrected continuum shell model and resonating group method predictions. PREPRINT nucl-ex/0506001,6/01/2005

2005SA12 NUCLEAR REACTIONS ${ }^{3} \mathrm{He}(\mathrm{e}, \mathrm{e}$ 'p), E=4.8 GeV; measured $\sigma(\mathrm{E}, \theta)$, asymmetries. ${ }^{3} \mathrm{He}$ deduced bound state momentum distributions. Comparisons with model predictions. JOUR ZAANE 24 s01 81

2005SH51 NUCLEAR REACTIONS ${ }^{4} \mathrm{He}(\gamma, \mathrm{p}),(\gamma, \mathrm{n}),(\gamma, \mathrm{np}), \mathrm{E}=21.8-29.8 \mathrm{MeV}$; ${ }^{12} \mathrm{C}(\gamma, \mathrm{p}),(\gamma, \mathrm{n}), \mathrm{E}=22.3-32 \mathrm{MeV}$; measured charged particle spectra, photodisintegration $\sigma, \sigma(\theta)$. Monoenergetic pulsed photons, comparison with previous results and model predictions. JOUR PRVCA 72044004

2005ST30 NUCLEAR REACTIONS ${ }^{4} \mathrm{He}\left(\mathrm{e}, \mathrm{e} \mathrm{p} \pi^{-}\right),\left(\mathrm{e}, \mathrm{e} \mathrm{p} \pi^{0}\right), \mathrm{E}=672 \mathrm{MeV}$; measured Ep, recoil spectra, $\sigma(\theta)$. Comparison with model predictions. JOUR PRLTA 95172501

2005TUZZ NUCLEAR REACTIONS ${ }^{2} \mathrm{H}\left({ }^{7} \mathrm{Li}, 2 \alpha\right),\left({ }^{6} \mathrm{Li},{ }^{3} \mathrm{He} \alpha\right),{ }^{6} \mathrm{Li}\left({ }^{6} \mathrm{Li}, 2 \alpha\right)$, E not given; measured particle spectra. ${ }^{7} \operatorname{Li}(\mathrm{p}, \alpha), \mathrm{E}(\mathrm{cm}) \approx 0.01-0.5 \mathrm{MeV}$; ${ }^{6} \mathrm{Li}(\mathrm{d}, \alpha),(\mathrm{p}, \alpha), \mathrm{E}(\mathrm{cm}) \approx 0.01-1 \mathrm{MeV}$; deduced astrophysical S-factors. CONF Riken(Origin of Matter) Proc,P553,Tumino 2005 YA12 NUCLEAR REACTIONS ${ }^{6} \mathrm{Li}\left({ }^{3} \mathrm{He}, \mathrm{t}^{3} \mathrm{He}\right), \mathrm{E}=450 \mathrm{MeV} ;{ }^{6} \mathrm{Li}\left({ }^{7} \mathrm{Li}, \mathrm{t}^{7} \mathrm{Be}\right)$, $\mathrm{E}=455 \mathrm{MeV}$; measured particle spectra, angular correlations. ${ }^{6} \mathrm{He},{ }^{6} \mathrm{Li}$, ${ }^{6}$ Be deduced resonances. JOUR PRVCA 71064316

$$
\mathrm{A}=4
$$

${ }^{4} \mathrm{n} \quad 2005$ AL15

NUCLEAR REACTIONS ${ }^{7} \mathrm{Li}\left({ }^{7} \mathrm{Li},{ }^{11} \mathrm{C}\right),\left({ }^{7} \mathrm{Li},{ }^{10} \mathrm{C}\right), \mathrm{E}=82 \mathrm{MeV}$; measured particle spectra; deduced resonance formation $\sigma$ upper limits. JOUR PZETA 8149 


\section{$\mathrm{A}=4$ (continued)}

2005BL09 NUCLEAR REACTIONS ${ }^{1} \mathrm{H}\left({ }^{6} \mathrm{He},{ }^{6} \mathrm{He}\right),\left({ }^{6} \mathrm{He},{ }^{6} \mathrm{He}\right), \mathrm{E}=15 \mathrm{MeV} /$ nucleon; measured $\sigma(\mathrm{q})$; deduced halo effect. ${ }^{1} \mathrm{H}\left({ }^{6} \mathrm{He}, \alpha\right), \mathrm{E}=25 \mathrm{MeV} /$ nucleon; measured $\sigma(\theta) .{ }^{2} \mathrm{H}\left({ }^{8} \mathrm{He},{ }^{6} \mathrm{Li}\right), \mathrm{E}=15 \mathrm{MeV} /$ nucleon; measured excitation energy spectrum; deduced possible resonance structure. ${ }^{1} \mathrm{H}\left({ }^{22} \mathrm{O},{ }^{22} \mathrm{O}^{\prime}\right), \mathrm{E}=46.6 \mathrm{MeV} /$ nucleon; measured $\sigma(\mathrm{E}, \theta)$. JOUR NUPAB $752279 \mathrm{c}$

2005CH50 NUCLEAR REACTIONS ${ }^{1} \mathrm{H}\left({ }^{6} \mathrm{He}, \mathrm{p}\right),\left({ }^{6} \mathrm{He}, \mathrm{np}\right),\left({ }^{6} \mathrm{He}, \mathrm{p} \alpha\right), \mathrm{E}=717$ $\mathrm{MeV} /$ nucleon; ${ }^{1} \mathrm{H}\left({ }^{8} \mathrm{He}, \mathrm{p}\right),\left({ }^{8} \mathrm{He}, \mathrm{np}\right),\left({ }^{8} \mathrm{He}, \mathrm{p} \alpha\right), \mathrm{E}=671 \mathrm{MeV} /$ nucleon; measured recoil proton spectra, $\sigma(\mathrm{E}, \theta) .{ }^{6,8} \mathrm{He}$ deduced cluster configurations, spectroscopic factors. JOUR NUPAB 75943

2005KI20 NUCLEAR REACTIONS ${ }^{4} \mathrm{He}\left(\pi^{+}, \pi^{-}\right), \mathrm{E}=120,150,180,240,270$ $\mathrm{MeV} ;{ }^{4} \mathrm{He}\left(\pi^{-}, \pi^{+}\right), \mathrm{E}=180,240 \mathrm{MeV}$; measured $\sigma(\mathrm{E}, \theta)$; deduced multiple scattering effects, total $\sigma$. JOUR PRVCA 72044608

${ }^{4} \mathrm{H} \quad 2005 \mathrm{GU} 17$ NUCLEAR REACTIONS ${ }^{9} \mathrm{Be}\left(\pi^{-}, \mathrm{ptX}\right),\left(\pi^{-}, \mathrm{dtX}\right),\left(\pi^{-}, 2 \mathrm{tX}\right), \mathrm{E}$ at rest; ${ }^{12} \mathrm{C}\left(\pi^{-}, \mathrm{ptX}\right),\left(\pi^{-}, \mathrm{dtX}\right),\left(\pi^{-}, 2 \mathrm{dX}\right), \mathrm{E}$ at rest; measured missing-mass spectra. ${ }^{4,5} \mathrm{H}$ deduced excited states energies, widths. JOUR ZAANE 24231

${ }^{4} \mathrm{He} \quad$ 2004BOZX NUCLEAR REACTIONS ${ }^{2} \mathrm{H}(\mathrm{t}, \mathrm{n}), \mathrm{E}=$ low; measured muon-catalyzed fusion rates for various temperatures and densities. REPT JINR-E15-2004-132,Bom

2004SA61 NUCLEAR REACTIONS ${ }^{2} \mathrm{H}$ (polarized d, $\gamma$ ), $\mathrm{E}<115 \mathrm{keV}$; measured $\mathrm{E} \gamma, \mathrm{I} \gamma, \sigma(\theta), \operatorname{Ay}(\theta), \mathrm{T}_{20}(\theta)$; deduced transition matrix elements. Comparison with resonating group model predictions. JOUR PRVCA 70064601

2005AL27 NUCLEAR REACTIONS ${ }^{2} \mathrm{H}\left({ }^{3} \mathrm{He}, \mathrm{p}\right), \mathrm{E}=0.5-6 \mathrm{MeV}$; measured Ep, $\sigma\left(\mathrm{E}, \theta=135^{\circ}\right)$. Application to depth profiling discussed. JOUR NIMBE 234169

2005ANZZ NUCLEAR REACTIONS ${ }^{4} \mathrm{He}$ (polarized e, e), E=3.03 GeV; measured parity-violating asymmetry. PREPRINT nucl-ex/0506010,6/07/2005

2005BA40 NUCLEAR REACTIONS ${ }^{1} \mathrm{H}\left({ }^{16} \mathrm{O}, \mathrm{X}\right){ }^{1} \mathrm{H} /{ }^{2} \mathrm{H} /{ }^{3} \mathrm{H} /{ }^{3} \mathrm{He} /{ }^{4} \mathrm{He} /{ }^{5} \mathrm{He}$ $/{ }^{6} \mathrm{He} /{ }^{5} \mathrm{Li} /{ }^{6} \mathrm{Li} /{ }^{7} \mathrm{Li} /{ }^{8} \mathrm{Li} /{ }^{7} \mathrm{Be} /{ }^{8} \mathrm{Be} /{ }^{9} \mathrm{Be} /{ }^{10} \mathrm{Be} /{ }^{9} \mathrm{~B} /{ }^{10} \mathrm{~B} /$ ${ }^{11} \mathrm{~B} /{ }^{12} \mathrm{~B} /{ }^{10} \mathrm{C} /{ }^{11} \mathrm{C} /{ }^{12} \mathrm{C} /{ }^{13} \mathrm{C} /{ }^{14} \mathrm{C} /{ }^{13} \mathrm{~N} /{ }^{14} \mathrm{~N} /{ }^{15} \mathrm{~N} /{ }^{14} \mathrm{O} /$ ${ }^{15} \mathrm{O} /{ }^{16} \mathrm{O}$, E at $3.25 \mathrm{GeV} / \mathrm{c} /$ nucleon; measured production $\sigma$. JOUR PZETA 81174

2005B015 NUCLEAR REACTIONS ${ }^{3} \mathrm{H}(\mathrm{d}, \mathrm{n}), \mathrm{E}=$ low; measured muon-catalyzed fusion rates, related quantities under a variety of $\mathrm{D} / \mathrm{T}$ mixture conditions. JOUR ZETFA 127752

2005BR04 NUCLEAR REACTIONS ${ }^{3} \mathrm{He}\left({ }^{3} \mathrm{He}, 2 \mathrm{p}\right), \mathrm{E}(\mathrm{cm}) \approx 10-1000 \mathrm{keV} ;{ }^{14} \mathrm{~N}(\mathrm{p}$, $\gamma), \mathrm{E} \approx 0.1-2.5 \mathrm{MeV}$; measured astrophysical S-factors. JOUR NPBSE 14360

2005BR15 NUCLEAR REACTIONS ${ }^{3} \mathrm{He}\left({ }^{3} \mathrm{He}, 2 \mathrm{p}\right), \mathrm{E}(\mathrm{cm}) \approx 16-100 \mathrm{keV}$; measured Ep, pp-coin, astrophysical S-factor. ${ }^{14} \mathrm{~N}(\mathrm{p}, \gamma), \mathrm{E}=130-240$ $\mathrm{keV}$; measured $\mathrm{E} \gamma$, astrophysical S-factor. JOUR NPBSE 14533

2005CA29 NUCLEAR REACTIONS ${ }^{12} \mathrm{C}(\mathrm{p}, \mathrm{X}), \mathrm{E}=180 \mathrm{MeV} ;{ }^{12} \mathrm{C}(\alpha, \mathrm{X}), \mathrm{E}=192.4$ $\mathrm{MeV}$; measured reaction $\sigma .{ }^{3,4} \mathrm{He}(\mathrm{p}, \mathrm{p}), \mathrm{E} \approx 40 \mathrm{MeV}$; measured $\sigma(\theta)$. ${ }^{40} \mathrm{Ca}\left({ }^{3} \mathrm{He},{ }^{3} \mathrm{He}\right), \mathrm{E}=167 \mathrm{MeV}$; measured particle spectra. Modified attenuation technique for reaction cross section measurement. JOUR NIMAE 547541 


\section{$\mathrm{A}=4$ (continued)}

2005CR05 NUCLEAR REACTIONS ${ }^{7} \operatorname{Li}(\mathrm{p}, \alpha), \mathrm{E}=30-100 \mathrm{keV}$; measured yields in various compounds; deduced electron screening effect, astrophysical S-factors. JOUR PYLBB 624181

2005DA12 NUCLEAR REACTIONS ${ }^{4} \mathrm{He}\left(\alpha, \alpha^{\prime}\right), \mathrm{E}=22.4,26.5 \mathrm{MeV}$; measured $\mathrm{E} \gamma, \mathrm{E} \alpha, \alpha \alpha-, \gamma \alpha$-coin; deduced resonance $\sigma .{ }^{8} \mathrm{Be}$ deduced transition B(E2), cluster structure. JOUR PRLTA 94122502

2005FR14 NUCLEAR REACTIONS ${ }^{12} \mathrm{C}\left({ }^{12} \mathrm{C},{ }^{8} \mathrm{Be}^{12} \mathrm{C}\right), \mathrm{E}=82-120 \mathrm{MeV}$; measured particle spectra, angular distributions. ${ }^{20} \mathrm{Ne}$ deduced possible resonance states energies, J, $\pi$. JOUR PRVCA 71047305

2005GIZZ NUCLEAR REACTIONS ${ }^{1} \mathrm{H}\left({ }^{6} \mathrm{He}, \mathrm{t}\right),\left({ }^{6} \mathrm{He}, \alpha\right),\left({ }^{6} \mathrm{He},{ }^{6} \mathrm{He}\right), \mathrm{E}=150$ $\mathrm{MeV}$; measured particle spectra, $\sigma(\theta) .{ }^{6} \mathrm{He}$ deduced spectroscopic factors for cluster configurations. PREPRINT nucl-ex/0505007,5/04/2005

2005LA25 NUCLEAR REACTIONS ${ }^{6} \mathrm{Li}\left({ }^{3} \mathrm{He}, \mathrm{p} \alpha\right), \mathrm{E}=5,6 \mathrm{MeV}$; measured Ep, $\mathrm{E} \alpha$, angular correlations. ${ }^{3} \mathrm{He}(\mathrm{d}, \mathrm{p}), \mathrm{E}=$ low; deduced astrophysical S-factor. JOUR NUPAB $75898 \mathrm{c}$

2005LE04 NUCLEAR REACTIONS Pb(p, X) ${ }^{3} \mathrm{He} /{ }^{4} \mathrm{He} /{ }^{21} \mathrm{Ne} /{ }^{22} \mathrm{Ne} /{ }^{36} \mathrm{Ar} /$ ${ }^{38} \mathrm{Ar} /{ }^{78} \mathrm{Kr} /{ }^{80} \mathrm{Kr} /{ }^{81} \mathrm{Kr} /{ }^{82} \mathrm{Kr} /{ }^{83} \mathrm{Kr} /{ }^{84} \mathrm{Kr} /{ }^{85} \mathrm{Kr} /{ }^{86} \mathrm{Kr} /{ }^{124} \mathrm{Xe}$ $/{ }^{126} \mathrm{Xe} /{ }^{128} \mathrm{Xe} /{ }^{129} \mathrm{Xe} /{ }^{130} \mathrm{Xe} /{ }^{131} \mathrm{Xe} /{ }^{132} \mathrm{Xe} /{ }^{134} \mathrm{Xe}, \mathrm{E}=44-2595$ $\mathrm{MeV}$; measured production $\sigma$; deduced reaction mechanism features. Mini-stack approach, comparisons with model predictions. JOUR NIMBE 2291

2005MI32 NUCLEAR REACTIONS ${ }^{4} \mathrm{He}\left({ }^{22} \mathrm{O},{ }^{23} \mathrm{~F}\right), \mathrm{E}=35 \mathrm{MeV} /$ nucleon; ${ }^{4} \mathrm{He}\left({ }^{23} \mathrm{~F},{ }^{23} \mathrm{~F}\right), \mathrm{E}=41.5 \mathrm{MeV} /$ nucleon; ${ }^{4} \mathrm{He}\left({ }^{24} \mathrm{~F},{ }^{23} \mathrm{~F}\right), \mathrm{E}=36 \mathrm{MeV} /$ nucleon; measured $\mathrm{E} \gamma, \mathrm{I} \gamma$, (particle) $\gamma$-, $\gamma \gamma$-coin; deduced $\sigma(\mathrm{E}) .{ }^{23} \mathrm{~F}$ deduced levels, J, $\pi$. DWBA analysis. JOUR ZAANE 25 s01 367 2005MIZT NUCLEAR REACTIONS ${ }^{4} \mathrm{He}\left({ }^{22} \mathrm{O},{ }^{23} \mathrm{~F}\right),\left({ }^{23} \mathrm{~F},{ }^{23} \mathrm{~F}\right),\left({ }^{24} \mathrm{~F},{ }^{23} \mathrm{~F}\right)$, $\left({ }^{25} \mathrm{Ne},{ }^{23} \mathrm{~F}\right), \mathrm{E} \approx 35-43 \mathrm{MeV} /$ nucleon; measured $\mathrm{E} \gamma, \mathrm{I} \gamma$, (particle) $\gamma-$, $\gamma \gamma$-coin. ${ }^{4} \mathrm{He}\left({ }^{22} \mathrm{O},{ }^{23} \mathrm{~F}\right), \mathrm{E}=35 \mathrm{MeV} /$ nucleon; measured $\sigma(\theta) \cdot{ }^{23} \mathrm{~F}$ deduced levels, J, $\pi$, configurations. REPT CNS-REP-67,Michimasa

2005MIZU NUCLEAR REACTIONS ${ }^{4} \mathrm{He}\left({ }^{22} \mathrm{O},{ }^{23} \mathrm{~F}\right),\left({ }^{23} \mathrm{~F},{ }^{23} \mathrm{~F}\right),\left({ }^{24} \mathrm{~F},{ }^{23} \mathrm{~F}\right)$, E not given; measured $\mathrm{E} \gamma, \mathrm{I} \gamma, \gamma \gamma$-coin, $\sigma(\theta) .{ }^{23} \mathrm{~F}$ deduced levels, J, $\pi$. REPT CNS-REP-66,P26,Michimasa

2005MIZV NUCLEAR REACTIONS ${ }^{4} \mathrm{He}\left({ }^{22} \mathrm{O},{ }^{23} \mathrm{~F}\right), \mathrm{E} \approx 35 \mathrm{MeV} /$ nucleon; ${ }^{4} \mathrm{He}\left({ }^{23} \mathrm{~F},{ }^{23} \mathrm{~F}\right.$ '), $\mathrm{E} \approx 41.5 \mathrm{MeV} /$ nucleon; ${ }^{4} \mathrm{He}\left({ }^{24} \mathrm{~F},{ }^{23} \mathrm{~F}\right), \mathrm{E} \approx 36 \mathrm{MeV} /$ nucleon; measured $\mathrm{E} \gamma, \mathrm{I} \gamma, \gamma \gamma$-, (particle) $\gamma$-coin, angular distributions. ${ }^{23} \mathrm{~F}$ deduced levels, J, $\pi$. REPT RIKEN 2004 Annual,P51,Michimasa

2005PA39 NUCLEAR REACTIONS ${ }^{1} \mathrm{H},{ }^{4} \mathrm{He}$ (polarized e, e), E=3 GeV; measured parity-violating asymmetries; deduced strange form factor limits. JOUR NUPAB $755241 \mathrm{c}$

2005RI13 NUCLEAR REACTIONS ${ }^{6} \mathrm{Li}(\mathrm{d}, \mathrm{pt}), \mathrm{E}=14 \mathrm{MeV}$; measured particle spectra, angular correlations. ${ }^{2} \mathrm{H}(\mathrm{d}, \mathrm{p}), \mathrm{E} \approx 50-2000 \mathrm{keV}$; deduced S-factors. Trojan horse method, comparison with previous results. JOUR NUPAB $758146 \mathrm{c}$

2005SIZY NUCLEAR REACTIONS ${ }^{238} \mathrm{U}(\mathrm{n}, \mathrm{nX}), \mathrm{E}=14 \mathrm{MeV}$; measured En, $\sigma(\mathrm{E}$, $\theta) .{ }^{3} \mathrm{H}(\mathrm{d}, \mathrm{n})$, E not given; measured neutron leakage spectrum from uranium sphere. Comparison with evaluated data. CONF Santa Fe (Nucl Data for Sci and Technol) Proc,Vol1,P67 


\section{$\mathrm{A}=4$ (continued)}

2005SU25 RADIOACTIVITY ${ }^{8} \mathrm{~B}(\mathrm{EC} \alpha)\left[\right.$ from $\left.{ }^{6} \mathrm{Li}\left({ }^{3} \mathrm{He}, \mathrm{n}\right)\right] ;{ }^{8} \mathrm{Li}\left(\beta^{-} \alpha\right)\left[\right.$ from ${ }^{7} \mathrm{Li}(\mathrm{d}$, p)]; measured $\beta$-NMR spectra; angular correlations; deduced limit on G-parity term. JOUR ZAANE 25 s01 709

2005TUZZ NUCLEAR REACTIONS ${ }^{2} \mathrm{H}\left({ }^{7} \mathrm{Li}, 2 \alpha\right),\left({ }^{6} \mathrm{Li},{ }^{3} \mathrm{He} \alpha\right),{ }^{6} \mathrm{Li}\left({ }^{6} \mathrm{Li}, 2 \alpha\right)$, E not given; measured particle spectra. ${ }^{7} \mathrm{Li}(\mathrm{p}, \alpha), \mathrm{E}(\mathrm{cm}) \approx 0.01-0.5 \mathrm{MeV}$; ${ }^{6} \mathrm{Li}(\mathrm{d}, \alpha),(\mathrm{p}, \alpha), \mathrm{E}(\mathrm{cm}) \approx 0.01-1 \mathrm{MeV}$; deduced astrophysical S-factors. CONF Riken(Origin of Matter) Proc,P553,Tumino 2005UEZZ NUCLEAR REACTIONS ${ }^{4} \mathrm{He}$ (polarized d, d), E=140, $270 \mathrm{MeV}$; measured $\sigma(\theta)$, tensor analyzing powers. REPT RIKEN 2004 Annual,P35,Uesaka

2005WRZZ NUCLEAR REACTIONS ${ }^{2} \mathrm{H}(\mathrm{d}, \mathrm{X})^{4} \mathrm{He}, \mathrm{E} \approx$ threshold; measured $\eta$ production $\sigma, \sigma(\theta)$. PREPRINT nucl-ex/0510056,10/20/2005

${ }^{4} \mathrm{Be} \quad 2005 \mathrm{KI} 20$ NUCLEAR REACTIONS ${ }^{4} \mathrm{He}\left(\pi^{+}, \pi^{-}\right), \mathrm{E}=120,150,180,240,270$ $\mathrm{MeV} ;{ }^{4} \mathrm{He}\left(\pi^{-}, \pi^{+}\right), \mathrm{E}=180,240 \mathrm{MeV}$; measured $\sigma(\mathrm{E}, \theta)$; deduced multiple scattering effects, total $\sigma$. JOUR PRVCA 72044608

\section{$\mathrm{A}=5$}

${ }^{5} \mathrm{H} \quad 2004 \mathrm{G054}$

NUCLEAR REACTIONS ${ }^{3} \mathrm{H}(\mathrm{t}, \mathrm{p}), \mathrm{E}=58 \mathrm{MeV}$; measured En, Ep, missing mass spectrum following residual nucleus decay. ${ }^{5} \mathrm{H}$ deduced levels, J, $\pi$. JOUR PRLTA 93262501

2005GOZY NUCLEAR REACTIONS ${ }^{3} \mathrm{H}(\mathrm{t}, \mathrm{p}), \mathrm{E}=57.7 \mathrm{MeV}$; measured particle spectra, angular correlations following residual nucleus decay. ${ }^{5} \mathrm{H}$ deduced ground-state energy, width, configuration. Cyclotron, mass-separator. CONF St Petersburg,P124,Golovkov 2005GU07 NUCLEAR REACTIONS ${ }^{9} \operatorname{Be}\left(\pi^{-}, \mathrm{dtX}\right),\left(\pi^{-}, \mathrm{ptX}\right),\left(\pi^{-}, \mathrm{pdX}\right),\left(\pi^{-}\right.$, $2 \mathrm{dX}), \mathrm{E}$ at rest; ${ }^{11} \mathrm{~B}\left(\pi^{-}, \mathrm{p} \alpha \mathrm{X}\right), \mathrm{E}$ at rest; measured missing mass spectra. ${ }^{5,6} \mathrm{H}$ deduced resonance parameters. JOUR YAFIA 68520 2005GU17 NUCLEAR REACTIONS ${ }^{9} \mathrm{Be}\left(\pi^{-}, \mathrm{ptX}\right),\left(\pi^{-}, \mathrm{dtX}\right),\left(\pi^{-}, 2 \mathrm{tX}\right), \mathrm{E}$ at rest; ${ }^{12} \mathrm{C}\left(\pi^{-}, \mathrm{ptX}\right),\left(\pi^{-}, \mathrm{dtX}\right),\left(\pi^{-}, 2 \mathrm{dX}\right), \mathrm{E}$ at rest; measured missing-mass spectra. ${ }^{4,5} \mathrm{H}$ deduced excited states energies, widths. JOUR ZAANE 24231

2005TE05 NUCLEAR REACTIONS ${ }^{3} \mathrm{H}(\mathrm{t}, \mathrm{p}), \mathrm{E}=58 \mathrm{MeV} ;{ }^{2} \mathrm{H}\left({ }^{6} \mathrm{He}, \mathrm{t}\right),\left({ }^{6} \mathrm{He},{ }^{3} \mathrm{He}\right)$, $\mathrm{E}=132 \mathrm{MeV}$; measured particle spectra, angular correlations following residual nucleus decay. ${ }^{5} \mathrm{He}$ deduced resonances J, $\pi$, IAS features. ${ }^{5} \mathrm{H}$ deduced ground-state resonance energy. JOUR ZAANE 25 s01 315

${ }^{5} \mathrm{He} \quad 2004 \mathrm{S035}$ NUCLEAR REACTIONS ${ }^{7} \mathrm{Li}\left({ }^{7} \mathrm{Li}, 2 \alpha\right), \mathrm{E}=8,30 \mathrm{MeV} ;{ }^{9} \mathrm{Be}\left({ }^{7} \mathrm{Li},{ }^{7} \mathrm{Li}\right)$, $\left({ }^{7} \mathrm{Li}, \alpha^{6} \mathrm{Li}\right),\left({ }^{7} \mathrm{Li}, \alpha^{7} \mathrm{Li}\right), \mathrm{E}=52 \mathrm{MeV} ;{ }^{7} \mathrm{Li}\left({ }^{9} \mathrm{Be}, \alpha^{9} \mathrm{Be}\right),\left({ }^{9} \mathrm{Be}, \alpha^{10} \mathrm{Be}\right)$, $\mathrm{E}=70 \mathrm{MeV}$; measured excitation energy spectra. ${ }^{9,10} \mathrm{Be},{ }^{13,14} \mathrm{C}$ deduced excited states, cluster structures. JOUR FIZBE 13433

2005BA40 NUCLEAR REACTIONS ${ }^{1} \mathrm{H}\left({ }^{16} \mathrm{O}, \mathrm{X}\right){ }^{1} \mathrm{H} /{ }^{2} \mathrm{H} /{ }^{3} \mathrm{H} /{ }^{3} \mathrm{He} /{ }^{4} \mathrm{He} /{ }^{5} \mathrm{He}$ $/{ }^{6} \mathrm{He} /{ }^{5} \mathrm{Li} /{ }^{6} \mathrm{Li} /{ }^{7} \mathrm{Li} /{ }^{8} \mathrm{Li} /{ }^{7} \mathrm{Be} /{ }^{8} \mathrm{Be} /{ }^{9} \mathrm{Be} /{ }^{10} \mathrm{Be} /{ }^{9} \mathrm{~B} /{ }^{10} \mathrm{~B} /$ ${ }^{11} \mathrm{~B} /{ }^{12} \mathrm{~B} /{ }^{10} \mathrm{C} /{ }^{11} \mathrm{C} /{ }^{12} \mathrm{C} /{ }^{13} \mathrm{C} /{ }^{14} \mathrm{C} /{ }^{13} \mathrm{~N} /{ }^{14} \mathrm{~N} /{ }^{15} \mathrm{~N} /{ }^{14} \mathrm{O} /$ ${ }^{15} \mathrm{O} /{ }^{16} \mathrm{O}$, E at $3.25 \mathrm{GeV} / \mathrm{c} /$ nucleon; measured production $\sigma$. JOUR PZETA 81174 


\section{$\mathrm{A}=5$ (continued)}

2005CH50 NUCLEAR REACTIONS ${ }^{1} \mathrm{H}\left({ }^{6} \mathrm{He}, \mathrm{p}\right),\left({ }^{6} \mathrm{He}, \mathrm{np}\right),\left({ }^{6} \mathrm{He}, \mathrm{p} \alpha\right), \mathrm{E}=717$ $\mathrm{MeV} /$ nucleon; ${ }^{1} \mathrm{H}\left({ }^{8} \mathrm{He}, \mathrm{p}\right),\left({ }^{8} \mathrm{He}, \mathrm{np}\right),\left({ }^{8} \mathrm{He}, \mathrm{p} \alpha\right), \mathrm{E}=671 \mathrm{MeV} /$ nucleon; measured recoil proton spectra, $\sigma(\mathrm{E}, \theta) .{ }^{6,8} \mathrm{He}$ deduced cluster configurations, spectroscopic factors. JOUR NUPAB 75943

2005KA23 NUCLEAR REACTIONS ${ }^{6} \mathrm{Li},{ }^{12} \mathrm{C}\left(\pi^{+}, \mathrm{K}^{+}\right),\left(\pi^{+}, \mathrm{pX}\right)$, E at $1.05 \mathrm{GeV}$ / c; measured excitation energy spectra, proton spectra following hypernucleus decay. ${ }^{5} \mathrm{He}$ deduced hypernucleus decay width. JOUR NUPAB $754173 \mathrm{c}$

2005MA45 RADIOACTIVITY ${ }^{5} \mathrm{He},{ }^{11} \mathrm{~B},{ }^{12} \mathrm{C}$; measured proton decay asymmetry parameters from polarized hypernuclei. JOUR NUPAB 754 168c

2005MI32 NUCLEAR REACTIONS ${ }^{4} \mathrm{He}\left({ }^{22} \mathrm{O},{ }^{23} \mathrm{~F}\right), \mathrm{E}=35 \mathrm{MeV} /$ nucleon; ${ }^{4} \mathrm{He}\left({ }^{23} \mathrm{~F},{ }^{23} \mathrm{~F}\right.$ '), $\mathrm{E}=41.5 \mathrm{MeV} /$ nucleon; ${ }^{4} \mathrm{He}\left({ }^{24} \mathrm{~F},{ }^{23} \mathrm{~F}\right), \mathrm{E}=36 \mathrm{MeV} /$ nucleon; measured $\mathrm{E} \gamma, \mathrm{I} \gamma$, (particle) $\gamma$-, $\gamma \gamma$-coin; deduced $\sigma(\mathrm{E}) .{ }^{23} \mathrm{~F}$ deduced levels, J, $\pi$. DWBA analysis. JOUR ZAANE 25 s01 367 2005MIZT NUCLEAR REACTIONS ${ }^{4} \mathrm{He}\left({ }^{22} \mathrm{O},{ }^{23} \mathrm{~F}\right),\left({ }^{23} \mathrm{~F},{ }^{23} \mathrm{~F}\right),\left({ }^{24} \mathrm{~F},{ }^{23} \mathrm{~F}\right)$, $\left({ }^{25} \mathrm{Ne},{ }^{23} \mathrm{~F}\right), \mathrm{E} \approx 35-43 \mathrm{MeV} /$ nucleon; measured $\mathrm{E} \gamma, \mathrm{I} \gamma$, (particle) $\gamma-$, $\gamma \gamma$-coin. ${ }^{4} \mathrm{He}\left({ }^{22} \mathrm{O},{ }^{23} \mathrm{~F}\right), \mathrm{E}=35 \mathrm{MeV} /$ nucleon; measured $\sigma(\theta) .{ }^{23} \mathrm{~F}$ deduced levels, J, $\pi$, configurations. REPT CNS-REP-67,Michimasa 2005MIZU NUCLEAR REACTIONS ${ }^{4} \mathrm{He}\left({ }^{22} \mathrm{O},{ }^{23} \mathrm{~F}\right),\left({ }^{23} \mathrm{~F},{ }^{23} \mathrm{~F}\right),\left({ }^{24} \mathrm{~F},{ }^{23} \mathrm{~F}\right)$, E not given; measured $\mathrm{E} \gamma, \mathrm{I} \gamma, \gamma \gamma$-coin, $\sigma(\theta) .{ }^{23} \mathrm{~F}$ deduced levels, J, $\pi$. REPT CNS-REP-66,P26,Michimasa

2005MIZV NUCLEAR REACTIONS ${ }^{4} \mathrm{He}\left({ }^{22} \mathrm{O},{ }^{23} \mathrm{~F}\right), \mathrm{E} \approx 35 \mathrm{MeV} /$ nucleon; ${ }^{4} \mathrm{He}\left({ }^{23} \mathrm{~F},{ }^{23} \mathrm{~F}\right.$ '), $\mathrm{E} \approx 41.5 \mathrm{MeV} /$ nucleon; ${ }^{4} \mathrm{He}\left({ }^{24} \mathrm{~F},{ }^{23} \mathrm{~F}\right), \mathrm{E} \approx 36 \mathrm{MeV} /$ nucleon; measured $\mathrm{E} \gamma, \mathrm{I} \gamma, \gamma \gamma$-, (particle) $\gamma$-coin, angular distributions. ${ }^{23} \mathrm{~F}$ deduced levels, J, $\pi$. REPT RIKEN 2004 Annual,P51,Michimasa $20050 K 02$ NUCLEAR REACTIONS ${ }^{6} \mathrm{Li}\left(\pi^{+}, \mathrm{K}^{+} \mathrm{p}\right),{ }^{12} \mathrm{C}\left(\pi^{+}, \mathrm{K}^{+}\right)$, E at $1.05 \mathrm{GeV}$ / c; measured nucleon-nucleon pair spectra, yields following hypernucleus decay; deduced hyperon decay widths. JOUR NUPAB $752196 \mathrm{c}$

20050K04 NUCLEAR REACTIONS ${ }^{6} \mathrm{Li},{ }^{12} \mathrm{C}\left(\pi^{+}, \mathrm{K}^{+}\right)$, E at $1.05 \mathrm{GeV} / \mathrm{c}$; measured excitation energy spectra, $\gamma$-spectra from neutral pion decay. ${ }^{5} \mathrm{He},{ }^{12} \mathrm{C}$ deduced hypernucleus decay branching ratios. JOUR NUPAB $754178 \mathrm{c}$

$20050 U 02$ NUCLEAR REACTIONS ${ }^{6} \mathrm{Li},{ }^{12} \mathrm{C}\left(\pi^{+}, \mathrm{K}^{+}\right)$, E not given; measured hypernucleus excitation energy spectra, nn-, np-coin following hypernucleus decay. ${ }^{5} \mathrm{He},{ }^{12} \mathrm{C}$ deduced hypernucleus decay widths, branching ratios. JOUR NUPAB $754157 \mathrm{c}$

2005PAZY NUCLEAR REACTIONS ${ }^{6} \mathrm{Li}\left(\mathrm{d},{ }^{3} \mathrm{He}\right),{ }^{7} \mathrm{Li}(\mathrm{d}, \alpha), \mathrm{E}=14.5 \mathrm{MeV}$; measured particle spectra, angular distributions. ${ }^{5} \mathrm{He}$ deduced excited state energy, width. CONF St Petersburg,P179,Pavlenko

2005S013 NUCLEAR REACTIONS ${ }^{16} \mathrm{O}\left({ }^{9} \mathrm{Be}, \alpha^{7} \mathrm{Be}\right),{ }^{7} \mathrm{Li}\left({ }^{9} \mathrm{Be}, \alpha^{7} \mathrm{Li}\right),\left({ }^{9} \mathrm{Be}, \mathrm{t} 2 \alpha\right)$, $\mathrm{E}=55,70 \mathrm{MeV}$; measured excitation energy spectra. ${ }^{11} \mathrm{~B},{ }^{11} \mathrm{C}$ deduced excited states energies, configurations. JOUR JPGPE 31 S1701

2005SOZZ NUCLEAR REACTIONS ${ }^{16} \mathrm{O}\left({ }^{9} \mathrm{Be}, \alpha^{7} \mathrm{Be}\right),{ }^{7} \mathrm{Li}\left({ }^{9} \mathrm{Be}, \alpha^{7} \mathrm{Li}\right),\left({ }^{9} \mathrm{Be}, \mathrm{t} 2 \alpha\right)$, $\mathrm{E}=55,70 \mathrm{MeV}$; measured particle spectra. ${ }^{11} \mathrm{C},{ }^{11} \mathrm{~B}$ deduced excited states energies, cluster structure, decay features. PREPRINT nucl-ex/0504026,4/25/2005 


\section{$\mathrm{A}=5$ (continued)}

2005TE05 NUCLEAR REACTIONS ${ }^{3} \mathrm{H}(\mathrm{t}, \mathrm{p}), \mathrm{E}=58 \mathrm{MeV} ;{ }^{2} \mathrm{H}\left({ }^{6} \mathrm{He}, \mathrm{t}\right),\left({ }^{6} \mathrm{He},{ }^{3} \mathrm{He}\right)$, $\mathrm{E}=132 \mathrm{MeV}$; measured particle spectra, angular correlations following residual nucleus decay. ${ }^{5} \mathrm{He}$ deduced resonances J, $\pi$, IAS features. ${ }^{5} \mathrm{H}$ deduced ground-state resonance energy. JOUR ZAANE 25 s01 315

${ }^{5} \mathrm{Li} \quad 2003 \mathrm{GO} 41$ NUCLEAR REACTIONS ${ }^{3} \mathrm{He}(\alpha, \mathrm{dt}), \mathrm{E}=67.2 \mathrm{MeV}$; measured particle spectra, dt-coin. ${ }^{5} \mathrm{Li}$ deduced excited states energies, widths. JOUR UKPJA 481035

2005BA40 NUCLEAR REACTIONS ${ }^{1} \mathrm{H}\left({ }^{16} \mathrm{O}, \mathrm{X}\right){ }^{1} \mathrm{H} /{ }^{2} \mathrm{H} /{ }^{3} \mathrm{H} /{ }^{3} \mathrm{He} /{ }^{4} \mathrm{He} /{ }^{5} \mathrm{He}$ $/{ }^{6} \mathrm{He} /{ }^{5} \mathrm{Li} /{ }^{6} \mathrm{Li} /{ }^{7} \mathrm{Li} /{ }^{8} \mathrm{Li} /{ }^{7} \mathrm{Be} /{ }^{8} \mathrm{Be} /{ }^{9} \mathrm{Be} /{ }^{10} \mathrm{Be} /{ }^{9} \mathrm{~B} /{ }^{10} \mathrm{~B} /$ ${ }^{11} \mathrm{~B} /{ }^{12} \mathrm{~B} /{ }^{10} \mathrm{C} /{ }^{11} \mathrm{C} /{ }^{12} \mathrm{C} /{ }^{13} \mathrm{C} /{ }^{14} \mathrm{C} /{ }^{13} \mathrm{~N} /{ }^{14} \mathrm{~N} /{ }^{15} \mathrm{~N} /{ }^{14} \mathrm{O} /$ ${ }^{15} \mathrm{O} /{ }^{16} \mathrm{O}$, E at $3.25 \mathrm{GeV} / \mathrm{c} /$ nucleon; measured production $\sigma$. JOUR PZETA 81174

\section{$\mathrm{A}=6$}

${ }^{6} \mathrm{H} \quad 2005 \mathrm{GU} 07$

NUCLEAR REACTIONS ${ }^{9} \mathrm{Be}\left(\pi^{-}, \mathrm{dtX}\right),\left(\pi^{-}, \mathrm{ptX}\right),\left(\pi^{-}, \operatorname{pdX}\right),\left(\pi^{-}\right.$, $2 \mathrm{dX}), \mathrm{E}$ at rest; ${ }^{11} \mathrm{~B}\left(\pi^{-}, \mathrm{p} \alpha \mathrm{X}\right), \mathrm{E}$ at rest; measured missing mass spectra. ${ }^{5,6} \mathrm{H}$ deduced resonance parameters. JOUR YAFIA 68520

${ }^{6} \mathrm{He} \quad 2004 \mathrm{SO} 035$ NUCLEAR REACTIONS ${ }^{7} \mathrm{Li}\left({ }^{7} \mathrm{Li}, 2 \alpha\right), \mathrm{E}=8,30 \mathrm{MeV} ;{ }^{9} \mathrm{Be}\left({ }^{7} \mathrm{Li},{ }^{7} \mathrm{Li}\right)$, $\left({ }^{7} \mathrm{Li}, \alpha^{6} \mathrm{Li}\right),\left({ }^{7} \mathrm{Li}, \alpha^{7} \mathrm{Li}\right), \mathrm{E}=52 \mathrm{MeV} ;{ }^{7} \mathrm{Li}\left({ }^{9} \mathrm{Be}, \alpha^{9} \mathrm{Be}\right),\left({ }^{9} \mathrm{Be}, \alpha^{10} \mathrm{Be}\right)$, $\mathrm{E}=70 \mathrm{MeV}$; measured excitation energy spectra. ${ }^{9,10} \mathrm{Be},{ }^{13,14} \mathrm{C}$ deduced excited states, cluster structures. JOUR FIZBE 13433

2005BA40 NUCLEAR REACTIONS ${ }^{1} \mathrm{H}\left({ }^{16} \mathrm{O}, \mathrm{X}\right){ }^{1} \mathrm{H} /{ }^{2} \mathrm{H} /{ }^{3} \mathrm{H} /{ }^{3} \mathrm{He} /{ }^{4} \mathrm{He} /{ }^{5} \mathrm{He}$ $/{ }^{6} \mathrm{He} /{ }^{5} \mathrm{Li} /{ }^{6} \mathrm{Li} /{ }^{7} \mathrm{Li} /{ }^{8} \mathrm{Li} /{ }^{7} \mathrm{Be} /{ }^{8} \mathrm{Be} /{ }^{9} \mathrm{Be} /{ }^{10} \mathrm{Be} /{ }^{9} \mathrm{~B} /{ }^{10} \mathrm{~B} /$ ${ }^{11} \mathrm{~B} /{ }^{12} \mathrm{~B} /{ }^{10} \mathrm{C} /{ }^{11} \mathrm{C} /{ }^{12} \mathrm{C} /{ }^{13} \mathrm{C} /{ }^{14} \mathrm{C} /{ }^{13} \mathrm{~N} /{ }^{14} \mathrm{~N} /{ }^{15} \mathrm{~N} /{ }^{14} \mathrm{O} /$ ${ }^{15} \mathrm{O} /{ }^{16} \mathrm{O}$, E at $3.25 \mathrm{GeV} / \mathrm{c} /$ nucleon; measured production $\sigma$. JOUR PZETA 81174

2005CH49 NUCLEAR REACTIONS $\mathrm{Pb}\left({ }^{6} \mathrm{He}, 2 \mathrm{n} \alpha\right), \mathrm{E}=240 \mathrm{MeV} /$ nucleon; measured $\mathrm{E} \alpha$, En, three-body energy and angular correlations; deduced role of final state interactions, other reaction mechanism features. ${ }^{6} \mathrm{He}$ deduced possible resonance. JOUR NUPAB 75923

2005 CH50 NUCLEAR REACTIONS ${ }^{1} \mathrm{H}\left({ }^{6} \mathrm{He}, \mathrm{p}\right),\left({ }^{6} \mathrm{He}, \mathrm{np}\right),\left({ }^{6} \mathrm{He}, \mathrm{p} \alpha\right), \mathrm{E}=717$ $\mathrm{MeV} /$ nucleon; ${ }^{1} \mathrm{H}\left({ }^{8} \mathrm{He}, \mathrm{p}\right),\left({ }^{8} \mathrm{He}, \mathrm{np}\right),\left({ }^{8} \mathrm{He}, \mathrm{p} \alpha\right), \mathrm{E}=671 \mathrm{MeV} /$ nucleon; measured recoil proton spectra, $\sigma(\mathrm{E}, \theta) .{ }^{6,8} \mathrm{He}$ deduced cluster configurations, spectroscopic factors. JOUR NUPAB 75943

2005GI07 NUCLEAR REACTIONS ${ }^{1} \mathrm{H}\left({ }^{6} \mathrm{He}, \alpha\right), \mathrm{E}=25 \mathrm{MeV} /$ nucleon; measured $\sigma(\theta)$; deduced particle transfer contributions, entrance potential dependence. ${ }^{6} \mathrm{He}$ deduced spectroscopic factors for $\mathrm{t}+\mathrm{t}$ and $\alpha+2 \mathrm{n}$ cluster configurations. ${ }^{1} \mathrm{H}\left({ }^{6} \mathrm{He}, \mathrm{p}\right), \mathrm{E}=25 \mathrm{MeV} /$ nucleon; measured $\sigma(\theta) .{ }^{3} \mathrm{He}(\alpha, \alpha), \mathrm{E}(\mathrm{cm})=28.7 \mathrm{MeV}$; calculated $\sigma(\theta)$. SPEG spectrometer and MUST array at GANIL. DWBA and coupled-channels calculations. JOUR PRVCA 71064311

2005GI18 NUCLEAR REACTIONS ${ }^{1} \mathrm{H}\left({ }^{6} \mathrm{He}, \alpha\right), \mathrm{E}=25 \mathrm{MeV} /$ nucleon; measured $\sigma(\theta)$; deduced particle transfer contributions, entrance potential dependence. ${ }^{6} \mathrm{He}$ deduced spectroscopic factors for $\mathrm{t}+\mathrm{t}$ and $\mathrm{a}+2 \mathrm{n}$ cluster configurations. SPEG spectrometer and MUST array at GANIL. DWBA and coupled-channels calculations. JOUR ZAANE 25 s01 267 


\section{$\mathrm{A}=6$ (continued)}

2005GIZZ NUCLEAR REACTIONS ${ }^{1} \mathrm{H}\left({ }^{6} \mathrm{He}, \mathrm{t}\right),\left({ }^{6} \mathrm{He}, \alpha\right),\left({ }^{6} \mathrm{He},{ }^{6} \mathrm{He}\right), \mathrm{E}=150$ $\mathrm{MeV}$; measured particle spectra, $\sigma(\theta)$. ${ }^{6} \mathrm{He}$ deduced spectroscopic factors for cluster configurations. PREPRINT nucl-ex/0505007,5/04/2005

2005HA64 NUCLEAR REACTIONS ${ }^{1} \mathrm{H}\left({ }^{6} \mathrm{He},{ }^{6} \mathrm{He}\right), \mathrm{E}=71 \mathrm{MeV} /$ nucleon; measured $\sigma(\theta)$, analyzing powers; deduced optical model parameters. ${ }^{6} \mathrm{He}$ deduced rms radius. Polarized target. Comparison with model predictions. JOUR ZAANE 25 s01 255

2005KI21 NUCLEAR REACTIONS ${ }^{1} \mathrm{H}\left({ }^{6} \mathrm{He}, \mathrm{p}\right),\left({ }^{8} \mathrm{He}, \mathrm{p}\right), \mathrm{E} \approx 700 \mathrm{MeV} /$ nucleon; measured recoil proton spectra, $\sigma(\mathrm{E}, \theta) .{ }^{6,8} \mathrm{He}$ deduced nuclear matter density distributions, charge radii, cluster configurations, spectroscopic factors. JOUR ZAANE 25 s01 215 2005SM04 RADIOACTIVITY ${ }^{6} \mathrm{He}\left(\beta^{-}\right)$[from ${ }^{7} \mathrm{Li}(\mathrm{p}, 2 \mathrm{p})$ ]; measured $\beta$-delayed deuteron and $\alpha$ spectra; deduced branching ratio. JOUR NIMAE 547 480

2005YA12 NUCLEAR REACTIONS ${ }^{6} \mathrm{Li}\left({ }^{3} \mathrm{He}, \mathrm{t}^{3} \mathrm{He}\right), \mathrm{E}=450 \mathrm{MeV} ;{ }^{6} \mathrm{Li}\left({ }^{7} \mathrm{Li}, \mathrm{t}^{7} \mathrm{Be}\right)$, $\mathrm{E}=455 \mathrm{MeV}$; measured particle spectra, angular correlations. ${ }^{6} \mathrm{He},{ }^{6} \mathrm{Li}$, ${ }^{6}$ Be deduced resonances. JOUR PRVCA 71064316

2005YE05 NUCLEAR REACTIONS ${ }^{9} \mathrm{Be}\left({ }^{6} \mathrm{He},{ }^{6} \mathrm{He}\right),\left({ }^{6} \mathrm{He},{ }^{5} \mathrm{He}\right),\left({ }^{6} \mathrm{He}, \alpha\right),\left({ }^{6} \mathrm{He}\right.$, $\alpha \mathrm{X}),\left({ }^{6} \mathrm{He}, \mathrm{tX}\right), \mathrm{E}=25 \mathrm{MeV} /$ nucleon; measured quasielastic, breakup, and transfer $\sigma(\theta)$. ${ }^{6} \mathrm{He}$ deduced two-triton configuration. JOUR JPGPE 31 S1647

${ }^{6} \mathrm{Li}$ 2004G056 NUCLEAR REACTIONS ${ }^{3} \mathrm{H}(\alpha, \mathrm{d} \alpha), \mathrm{E}=67.2 \mathrm{MeV}$; measured Ed, E $\alpha$, d $\alpha$-coin, $\sigma(\theta) .{ }^{6}$ Li deduced levels, widths. JOUR UKPJA 4916

2004 KU36 NUCLEAR REACTIONS ${ }^{3} \mathrm{He}\left({ }^{7} \mathrm{Li}, \alpha\right), \mathrm{E}=31.2 \mathrm{MeV}$; measured $\mathrm{E} \alpha$. ${ }^{6} \mathrm{Li}$ deduced resonance energies, widths. JOUR BJPHE 34933

$2005 \mathrm{AB0} 4 \quad$ NUCLEAR REACTIONS ${ }^{6,7} \mathrm{Li}\left(\pi^{-}, \mathrm{pX}\right),\left(\pi^{-}, \mathrm{dX}\right),\left(\pi^{-}, \mathrm{tX}\right), \mathrm{E}$ at $0.72,0.88 \mathrm{GeV} / \mathrm{c}$; measured particle spectra, $\sigma(\theta)$, missing energy.

${ }^{6,7} \mathrm{Li}$ deduced effective quasideuteron numbers. JOUR YAFIA 68503

2005BA40 NUCLEAR REACTIONS ${ }^{1} \mathrm{H}\left({ }^{16} \mathrm{O}, \mathrm{X}\right){ }^{1} \mathrm{H} /{ }^{2} \mathrm{H} /{ }^{3} \mathrm{H} /{ }^{3} \mathrm{He} /{ }^{4} \mathrm{He} /{ }^{5} \mathrm{He}$ $/{ }^{6} \mathrm{He} /{ }^{5} \mathrm{Li} /{ }^{6} \mathrm{Li} /{ }^{7} \mathrm{Li} /{ }^{8} \mathrm{Li} /{ }^{7} \mathrm{Be} /{ }^{8} \mathrm{Be} /{ }^{9} \mathrm{Be} /{ }^{10} \mathrm{Be} /{ }^{9} \mathrm{~B} /{ }^{10} \mathrm{~B} /$ ${ }^{11} \mathrm{~B} /{ }^{12} \mathrm{~B} /{ }^{10} \mathrm{C} /{ }^{11} \mathrm{C} /{ }^{12} \mathrm{C} /{ }^{13} \mathrm{C} /{ }^{14} \mathrm{C} /{ }^{13} \mathrm{~N} /{ }^{14} \mathrm{~N} /{ }^{15} \mathrm{~N} /{ }^{14} \mathrm{O} /$ ${ }^{15} \mathrm{O} /{ }^{16} \mathrm{O}$, E at $3.25 \mathrm{GeV} / \mathrm{c} /$ nucleon; measured production $\sigma$. JOUR PZETA 81174

2005B049 NUCLEAR REACTIONS ${ }^{1} \mathrm{H}\left({ }^{6} \mathrm{He}, \mathrm{n}\right)$, E not given; measured Doppler-shifted $\mathrm{E} \gamma, \mathrm{I} \gamma .{ }^{7} \mathrm{Li}$ deduced resonance features, IAS. JOUR ZAANE 25 s01 259

2005GEZZ NUCLEAR REACTIONS ${ }^{9} \mathrm{Be}(\mathrm{p}, \alpha), \mathrm{E}=3.1-5.24 \mathrm{MeV}$; measured $\sigma$. CONF St Petersburg,P171, Generalov

2005G014 NUCLEAR REACTIONS ${ }^{3} \mathrm{He}(\alpha, \mathrm{p} \alpha), \mathrm{E}=27.2 \mathrm{MeV}$; measured Ep, E $\alpha$, $\mathrm{p} \alpha$-coin, $\sigma(\mathrm{E}, \theta) .{ }^{6} \mathrm{Li}$ deduced excited states energies, widths. JOUR UKPJA 50327

2005MI13 NUCLEAR REACTIONS ${ }^{6,7} \mathrm{Li}\left({ }^{6} \mathrm{He}, \alpha^{6} \mathrm{He}\right),{ }^{6} \mathrm{Li}\left({ }^{6} \mathrm{He}, \mathrm{t} 2 \alpha\right), \mathrm{E}=18 \mathrm{MeV}$; measured excitation energy spectra. ${ }^{6,7} \mathrm{Li},{ }^{8,9,10} \mathrm{Be}$ deduced cluster states. JOUR NUPAB 753263

2005MIZT NUCLEAR REACTIONS ${ }^{4} \mathrm{He}\left({ }^{22} \mathrm{O},{ }^{23} \mathrm{~F}\right),\left({ }^{23} \mathrm{~F},{ }^{23} \mathrm{~F}^{\prime}\right),\left({ }^{24} \mathrm{~F},{ }^{23} \mathrm{~F}\right)$, $\left({ }^{25} \mathrm{Ne},{ }^{23} \mathrm{~F}\right), \mathrm{E} \approx 35-43 \mathrm{MeV} /$ nucleon; measured $\mathrm{E} \gamma, \mathrm{I} \gamma$, (particle) $\gamma-$, $\gamma \gamma$-coin. ${ }^{4} \mathrm{He}\left({ }^{22} \mathrm{O},{ }^{23} \mathrm{~F}\right), \mathrm{E}=35 \mathrm{MeV} /$ nucleon; measured $\sigma(\theta) .{ }^{23} \mathrm{~F}$ deduced levels, J, $\pi$, configurations. REPT CNS-REP-67,Michimasa 


\section{$\mathrm{A}=6$ (continued)}

2005N015 NUCLEAR MOMENTS ${ }^{6,7,8,9} \mathrm{Li}$; measured hfs, isotope shifts; deduced charge radii. Resonance ionization mass spectroscopy, comparison with model predictions. JOUR ZAANE 25 s01 199

2005SM04 RADIOACTIVITY ${ }^{6} \mathrm{He}\left(\beta^{-}\right)$[from $\left.{ }^{7} \mathrm{Li}(\mathrm{p}, 2 \mathrm{p})\right]$; measured $\beta$-delayed deuteron and $\alpha$ spectra; deduced branching ratio. JOUR NIMAE 547 480

2005VA27 NUCLEAR MOMENTS ${ }^{6,7} \mathrm{Li}$; measured hfs. JOUR CJPHA 83327 2005WAZW NUCLEAR REACTIONS Si $\left({ }^{6} \mathrm{Li}, \mathrm{X}\right),\left({ }^{7} \mathrm{Be}, \mathrm{X}\right),\left({ }^{10} \mathrm{~B}, \mathrm{X}\right),\left({ }^{9} \mathrm{C}, \mathrm{X}\right),\left({ }^{10} \mathrm{C}\right.$, $\mathrm{X}),\left({ }^{11} \mathrm{C}, \mathrm{X}\right),\left({ }^{12} \mathrm{~N}, \mathrm{X}\right),\left({ }^{13} \mathrm{O}, \mathrm{X}\right),\left({ }^{15} \mathrm{O}, \mathrm{X}\right),\left({ }^{17} \mathrm{Ne}, \mathrm{X}\right), \mathrm{E}=15-53 \mathrm{MeV} /$ nucleon; measured reaction and proton-removal $\sigma .{ }^{6} \mathrm{Li},{ }^{7} \mathrm{Be},{ }^{10} \mathrm{~B}$, ${ }^{9,10,11} \mathrm{C},{ }^{12} \mathrm{~N},{ }^{13,15} \mathrm{O},{ }^{17} \mathrm{Ne}$ deduced radii. Comparisons with model predictions. PREPRINT nucl-ex/0507025,7/18/2005

$2005 Y$ N12 NUCLEAR REACTIONS ${ }^{6} \mathrm{Li}\left({ }^{3} \mathrm{He}, \mathrm{t}{ }^{3} \mathrm{He}\right), \mathrm{E}=450 \mathrm{MeV} ;{ }^{6} \mathrm{Li}\left({ }^{7} \mathrm{Li}, \mathrm{t}^{7} \mathrm{Be}\right)$, $\mathrm{E}=455 \mathrm{MeV}$; measured particle spectra, angular correlations. ${ }^{6} \mathrm{He},{ }^{6} \mathrm{Li}$, ${ }^{6}$ Be deduced resonances. JOUR PRVCA 71064316

${ }^{6} \mathrm{Be} \quad 2004 \mathrm{GU} 21$ NUCLEAR REACTIONS ${ }^{9} \mathrm{Be}\left({ }^{14} \mathrm{~B},{ }^{13} \mathrm{BX}\right), \mathrm{E}=60 \mathrm{MeV} /$ nucleon; measured $\mathrm{E} \gamma, \mathrm{I} \gamma$, particle momentum distribution, $\sigma(\mathrm{E}) .{ }^{13} \mathrm{~B}$ deduced levels, J, $\pi$, asymptotic normalization coefficients. ${ }^{2} \mathrm{H}\left({ }^{8} \mathrm{~B}, \alpha\right), \mathrm{E}=28.5$ $\mathrm{MeV}$; measured E $\alpha$. JOUR BJPHE 341012

2005YA12 NUCLEAR REACTIONS ${ }^{6} \mathrm{Li}\left({ }^{3} \mathrm{He}, \mathrm{t}^{3} \mathrm{He}\right), \mathrm{E}=450 \mathrm{MeV} ;{ }^{6} \mathrm{Li}\left({ }^{7} \mathrm{Li}, \mathrm{t}^{7} \mathrm{Be}\right)$, $\mathrm{E}=455 \mathrm{MeV}$; measured particle spectra, angular correlations. ${ }^{6} \mathrm{He},{ }^{6} \mathrm{Li}$, ${ }^{6}$ Be deduced resonances. JOUR PRVCA 71064316

$$
\mathrm{A}=7
$$

${ }^{7} \mathrm{H} \quad 2005 \mathrm{GUZZ}$

NUCLEAR REACTIONS ${ }^{9} \mathrm{Be}\left(\pi^{-}, 2 \mathrm{pX}\right)$, E not given; measured charged particle spectra. ${ }^{7} \mathrm{H}$ deduced level energies, widths. Multilayer semiconductor spectrometer, LEP channel of LAMPF. CONF St Petersburg,P139,Gurov

${ }^{7} \mathrm{He} \quad 2005 \mathrm{CH} 50$ NUCLEAR REACTIONS ${ }^{1} \mathrm{H}\left({ }^{6} \mathrm{He}, \mathrm{p}\right),\left({ }^{6} \mathrm{He}, \mathrm{np}\right),\left({ }^{6} \mathrm{He}, \mathrm{p} \alpha\right), \mathrm{E}=717$ $\mathrm{MeV} /$ nucleon; ${ }^{1} \mathrm{H}\left({ }^{8} \mathrm{He}, \mathrm{p}\right),\left({ }^{8} \mathrm{He}, \mathrm{np}\right),\left({ }^{8} \mathrm{He}, \mathrm{p} \alpha\right), \mathrm{E}=671 \mathrm{MeV} /$ nucleon; measured recoil proton spectra, $\sigma(\mathrm{E}, \theta) .{ }^{6,8} \mathrm{He}$ deduced cluster configurations, spectroscopic factors. JOUR NUPAB 75943

2005R037 NUCLEAR REACTIONS ${ }^{1} \mathrm{H}\left({ }^{8} \mathrm{He},{ }^{8} \mathrm{He}\right)$, E not given; measured recoil proton spectrum; deduced excitation function. ${ }^{1} \mathrm{H}\left({ }^{6} \mathrm{He},{ }^{6} \mathrm{Li}\right)$, E not given; measured neutron spectrum, $\mathrm{n} \gamma$-coin; deduced excitation function. ${ }^{7,9} \mathrm{Li}$ deduced resonance parameters. ${ }^{7,9} \mathrm{He}$ deduced analog states features. JOUR NIMBE 241977

2005SK03 NUCLEAR REACTIONS ${ }^{1} \mathrm{H}\left({ }^{8} \mathrm{He}, \mathrm{p}\right),\left({ }^{8} \mathrm{He}, \mathrm{d}\right), \mathrm{E}=15.7 \mathrm{MeV} /$ nucleon; measured deuteron and proton spectra, $\sigma(\theta) .{ }^{8} \mathrm{He}(\mathrm{p}, \mathrm{p})$, $\mathrm{E}=15.7 \mathrm{MeV} /$ nucleon; deduced effect of coupling to pickup reaction. Coupled-channels framework, dynamic polarization potential. JOUR PYLBB 61982

2005WUZZ NUCLEAR REACTIONS ${ }^{2} \mathrm{H}\left({ }^{6} \mathrm{He}, \mathrm{p}\right), \mathrm{E}=69 \mathrm{MeV}$; measured particle spectra, angular distributions. ${ }^{7} \mathrm{He}$ deduced excited states. CONF Argonne(Nuclei at the Limits),P393, Wuosmaa 


\section{$\mathrm{A}=7$ (continued)}

${ }^{7} \mathrm{Li} \quad 2005 \mathrm{AB} 04$

2005BA40

2005BA96

2005B036

2005B049

2005FU13

2005GI03

2005GIZY

2005MI13

2005MI20

2005N015

2005RAZZ

2005R037

2005RU18
NUCLEAR REACTIONS ${ }^{6,7} \operatorname{Li}\left(\pi^{-}, \mathrm{pX}\right),\left(\pi^{-}, \mathrm{dX}\right),\left(\pi^{-}, \mathrm{tX}\right), \mathrm{E}$ at $0.72,0.88 \mathrm{GeV} / \mathrm{c}$; measured particle spectra, $\sigma(\theta)$, missing energy. ${ }^{6,7} \mathrm{Li}$ deduced effective quasideuteron numbers. JOUR YAFIA 68503 NUCLEAR REACTIONS ${ }^{1} \mathrm{H}\left({ }^{16} \mathrm{O}, \mathrm{X}\right){ }^{1} \mathrm{H} /{ }^{2} \mathrm{H} /{ }^{3} \mathrm{H} /{ }^{3} \mathrm{He} /{ }^{4} \mathrm{He} /{ }^{5} \mathrm{He}$ $/{ }^{6} \mathrm{He} /{ }^{5} \mathrm{Li} /{ }^{6} \mathrm{Li} /{ }^{7} \mathrm{Li} /{ }^{8} \mathrm{Li} /{ }^{7} \mathrm{Be} /{ }^{8} \mathrm{Be} /{ }^{9} \mathrm{Be} /{ }^{10} \mathrm{Be} /{ }^{9} \mathrm{~B} /{ }^{10} \mathrm{~B} /$ ${ }^{11} \mathrm{~B} /{ }^{12} \mathrm{~B} /{ }^{10} \mathrm{C} /{ }^{11} \mathrm{C} /{ }^{12} \mathrm{C} /{ }^{13} \mathrm{C} /{ }^{14} \mathrm{C} /{ }^{13} \mathrm{~N} /{ }^{14} \mathrm{~N} /{ }^{15} \mathrm{~N} /{ }^{14} \mathrm{O} /$ ${ }^{15} \mathrm{O} /{ }^{16} \mathrm{O}$, E at $3.25 \mathrm{GeV} / \mathrm{c} /$ nucleon; measured production $\sigma$. JOUR PZETA 81174 NUCLEAR REACTIONS ${ }^{7} \operatorname{Li}\left({ }^{7} \mathrm{Be},{ }^{7} \mathrm{Be}\right), \mathrm{E}(\mathrm{cm})=8.87,9.87 \mathrm{MeV}$; measured $\sigma(\theta)$; deduced optical model parameters. JOUR PRVCA 72 044602

NUCLEAR REACTIONS ${ }^{1} \mathrm{H}\left({ }^{6} \mathrm{He}, \gamma\right), \mathrm{E}<24 \mathrm{MeV}$; measured E $\gamma, \mathrm{I} \gamma$; deduced IAS formation $\sigma, \sigma(\theta)$. ${ }^{7} \mathrm{Li}$ deduced resonance parameters. Doppler-shift analysis technique. JOUR PRLTA 95132502

NUCLEAR REACTIONS ${ }^{1} \mathrm{H}\left({ }^{6} \mathrm{He}, \mathrm{n}\right)$, E not given; measured Doppler-shifted $\mathrm{E} \gamma, \mathrm{I} \gamma \cdot{ }^{7} \mathrm{Li}$ deduced resonance features, IAS. JOUR ZAANE 25 s01 259

RADIOACTIVITY ${ }^{7} \operatorname{Be}(\mathrm{EC})$ [from $\left.{ }^{7} \operatorname{Li}(\mathrm{p}, \mathrm{n})\right]$; measured $\mathrm{T}_{1 / 2}$ for source implanted in metals; deduced no environmental effect. JOUR NUPAB $758697 \mathrm{c}$

NUCLEAR REACTIONS ${ }^{10} \mathrm{~B}(\mathrm{n}, \alpha), \mathrm{E}=1.5-3.8 \mathrm{MeV}$; measured $\sigma$, $\sigma(\theta)$. Effects of particle leaking discussed. JOUR NIMAE 538550

NUCLEAR REACTIONS ${ }^{10} \mathrm{~B}(\mathrm{n}, \alpha), \mathrm{E}=1.5-5.6 \mathrm{MeV}$; measured $\mathrm{E} \alpha, \sigma$ ratio, excitation function. Comparison with previous results. CONF Santa Fe (Nucl Data for Sci and Technol) Proc,Vol1,P816

NUCLEAR REACTIONS ${ }^{6,7} \mathrm{Li}\left({ }^{6} \mathrm{He}, \alpha^{6} \mathrm{He}\right),{ }^{6} \mathrm{Li}\left({ }^{6} \mathrm{He}, \mathrm{t} 2 \alpha\right), \mathrm{E}=18 \mathrm{MeV}$; measured excitation energy spectra. ${ }^{6,7} \mathrm{Li},{ }^{8,9,10} \mathrm{Be}$ deduced cluster states. JOUR NUPAB 753263 NUCLEAR REACTIONS ${ }^{7} \mathrm{Li},{ }^{9} \mathrm{Be},{ }^{10,11} \mathrm{~B},{ }^{12} \mathrm{C}\left(\mathrm{K}^{-}, \mathrm{X}\right)$, E at rest; measured $\mathrm{E} \gamma, \mathrm{I} \gamma .{ }^{7} \mathrm{Li}$ deduced hypernucleus transition. Hyperball array. JOUR NUPAB 754 80c

NUCLEAR MOMENTS ${ }^{6,7,8,9} \mathrm{Li}$; measured hfs, isotope shifts; deduced charge radii. Resonance ionization mass spectroscopy, comparison with model predictions. JOUR ZAANE 25 s01 199

RADIOACTIVITY ${ }^{7} \mathrm{Be}(\mathrm{EC})$; measured $\mathrm{E} \gamma, \mathrm{I} \gamma, \mathrm{T}_{1 / 2}$ for source implanted in $\mathrm{C}_{60}$ and gold foil; deduced environmental effect. PREPRINT nucl-ex/0509021,9/15/2005 NUCLEAR REACTIONS ${ }^{1} \mathrm{H}\left({ }^{8} \mathrm{He},{ }^{8} \mathrm{He}\right)$, E not given; measured recoil proton spectrum; deduced excitation function. ${ }^{1} \mathrm{H}\left({ }^{6} \mathrm{He},{ }^{6} \mathrm{Li}\right)$, E not given; measured neutron spectrum, $\mathrm{n} \gamma$-coin; deduced excitation function. ${ }^{7,9} \mathrm{Li}$ deduced resonance parameters. ${ }^{7,9} \mathrm{He}$ deduced analog states features. JOUR NIMBE 241977

NUCLEAR REACTIONS ${ }^{7} \mathrm{Li}\left({ }^{11} \mathrm{~B}, \mathrm{X}\right), \mathrm{E}=44 \mathrm{MeV}$; measured particle spectra, charge distributions. ${ }^{7} \mathrm{Li}\left({ }^{11} \mathrm{~B},{ }^{11} \mathrm{~B}\right),\left({ }^{11} \mathrm{~B},{ }^{11} \mathrm{~B} '\right), \mathrm{E}=44 \mathrm{MeV}$; measured $\sigma(\mathrm{E}, \theta) ;{ }^{11} \mathrm{~B}\left({ }^{7} \mathrm{Li},{ }^{7} \mathrm{Li}\right),\left({ }^{7} \mathrm{Li},{ }^{7} \mathrm{Li} '\right), \mathrm{E}=34 \mathrm{MeV}$; analyzed $\sigma(\mathrm{E}$, $\theta)$; deduced optical model parameters, transfer channel contributions, reorientation effects. ${ }^{7} \mathrm{Li},{ }^{11} \mathrm{~B}$ deduced deformation parameters. Optical model and coupled-reaction-channels analysis. JOUR PRVCA 72034608 


\section{$\mathrm{A}=7$ (continued)}

2005SI02 NUCLEAR REACTIONS ${ }^{1} \mathrm{H}\left({ }^{7} \mathrm{Li}, \mathrm{p}\right), \mathrm{E}=2.28-5.7 \mathrm{MeV}$; measured recoil proton spectra, $\sigma\left(\theta=30,45^{\circ}\right)$. Al-backed melamine target. JOUR NIMBE 229180

2005TA19 NUCLEAR REACTIONS ${ }^{10} \mathrm{~B},{ }^{16} \mathrm{O}\left(\mathrm{K}^{-}, \pi^{-}\right)$, E at $0.93 \mathrm{GeV} / \mathrm{c}$; ${ }^{11} \mathrm{~B}\left(\pi^{+}, \mathrm{K}^{+}\right)$, E at $1.05 \mathrm{GeV} / \mathrm{c} ;{ }^{7} \mathrm{Li},{ }^{10} \mathrm{~B}\left(\mathrm{~K}^{-}, \gamma\right)$, E at rest; measured $\mathrm{E} \gamma, \mathrm{I} \gamma \cdot{ }^{7} \mathrm{Li},{ }^{9} \mathrm{Be},{ }^{10,11} \mathrm{~B},{ }^{16} \mathrm{O}$ deduced hypernucleus levels, J, $\pi$. Hyperball array. JOUR NUPAB $75458 \mathrm{c}$

2005VA27

2005ZH09

${ }^{7} \mathrm{Be} \quad$ 2004MAZP

2004NA42

2005BA40

2005BAZU

2005BU05

2005DA41

2005FU13

2005NA32

2005RAZZ

2005SE23

2005 SI 14
NUCLEAR MOMENTS ${ }^{6,7} \mathrm{Li}$; measured hfs. JOUR CJPHA 83327

RADIOACTIVITY ${ }^{7} \mathrm{Be}(\mathrm{EC})$; measured decay rates for source implanted in Pd and Au. JOUR CPLEE 22565 NUCLEAR REACTIONS C, ${ }^{27} \mathrm{Al}, \mathrm{Cu}, \mathrm{Ag},{ }^{197} \mathrm{Au}(\alpha, \mathrm{X}){ }^{7} \mathrm{Be}, \mathrm{E}=400$ $\mathrm{MeV}$; C, ${ }^{27} \mathrm{Al}, \mathrm{Cu}, \mathrm{Ag},{ }^{197} \mathrm{Au}(\mathrm{n}, \mathrm{X}){ }^{7} \mathrm{Be}, \mathrm{E}<500 \mathrm{MeV} ; \mathrm{Cu}, \mathrm{Ag}$, ${ }^{197} \mathrm{Au}(\alpha, \mathrm{X}){ }^{10} \mathrm{Be}, \mathrm{E}=400 \mathrm{MeV} ; \mathrm{Cu}, \mathrm{Ag},{ }^{197} \mathrm{Au}(\mathrm{n}, \mathrm{X}){ }^{10} \mathrm{Be}, \mathrm{E}<500$ $\mathrm{MeV}$; measured yields. REPT KEK Preprint 2004-90,Matsumura NUCLEAR REACTIONS ${ }^{4} \mathrm{He}\left({ }^{3} \mathrm{He}, \gamma\right), \mathrm{E}=1000-2300 \mathrm{keV}$; measured capture $\sigma$; deduced S-factors. Activation technique, astrophysical implications discussed. JOUR PRLTA 93262503

NUCLEAR REACTIONS ${ }^{1} \mathrm{H}\left({ }^{16} \mathrm{O}, \mathrm{X}\right){ }^{1} \mathrm{H} /{ }^{2} \mathrm{H} /{ }^{3} \mathrm{H} /{ }^{3} \mathrm{He} /{ }^{4} \mathrm{He} /{ }^{5} \mathrm{He}$ $/{ }^{6} \mathrm{He} /{ }^{5} \mathrm{Li} /{ }^{6} \mathrm{Li} /{ }^{7} \mathrm{Li} /{ }^{8} \mathrm{Li} /{ }^{7} \mathrm{Be} /{ }^{8} \mathrm{Be} /{ }^{9} \mathrm{Be} /{ }^{10} \mathrm{Be} /{ }^{9} \mathrm{~B} /{ }^{10} \mathrm{~B} /$ ${ }^{11} \mathrm{~B} /{ }^{12} \mathrm{~B} /{ }^{10} \mathrm{C} /{ }^{11} \mathrm{C} /{ }^{12} \mathrm{C} /{ }^{13} \mathrm{C} /{ }^{14} \mathrm{C} /{ }^{13} \mathrm{~N} /{ }^{14} \mathrm{~N} /{ }^{15} \mathrm{~N} /{ }^{14} \mathrm{O} /$ ${ }^{15} \mathrm{O} /{ }^{16} \mathrm{O}$, E at $3.25 \mathrm{GeV} / \mathrm{c} /$ nucleon; measured production $\sigma$. JOUR PZETA 81174 NUCLEAR REACTIONS C, W(p, nX), (d, nX), E=50, $70 \mathrm{MeV}$; Li(d, $\mathrm{nX}), \mathrm{E}=40 \mathrm{MeV}$; measured neutron spectra, $\sigma(\mathrm{E}, \theta)$, thick target yields. $\mathrm{Li}(\mathrm{d}, \mathrm{X})^{7} \mathrm{Be}, \mathrm{E} \approx 10-40 \mathrm{MeV}$; measured production $\sigma$. CONF Santa Fe (Nucl Data for Sci and Technol) Proc,Vol1,P884 NUCLEAR REACTIONS ${ }^{7} \operatorname{Li}(\mathrm{p}, \mathrm{n}), \mathrm{E}=1.88-2.0 \mathrm{MeV}$; measured neutron spectra, yields. JOUR NIMBE 229144

NUCLEAR REACTIONS H, C $\left({ }^{7} \mathrm{Li}, \mathrm{X}\right)^{7} \mathrm{Be}, \mathrm{E} \approx 25-30 \mathrm{MeV}$; measured yields. JOUR NIMBE 241953

RADIOACTIVITY ${ }^{7} \mathrm{Be}(\mathrm{EC})$ [from $\left.{ }^{7} \mathrm{Li}(\mathrm{p}, \mathrm{n})\right]$; measured $\mathrm{T}_{1 / 2}$ for source implanted in metals; deduced no environmental effect. JOUR NUPAB $758697 \mathrm{c}$

NUCLEAR REACTIONS ${ }^{4} \mathrm{He}\left({ }^{3} \mathrm{He}, \gamma\right), \mathrm{E}=1000-2300 \mathrm{keV}$; measured $\sigma$; deduced astrophysical S-factors. JOUR NUPAB $758689 \mathrm{c}$

RADIOACTIVITY ${ }^{7} \mathrm{Be}(\mathrm{EC})$; measured $\mathrm{E} \gamma, \mathrm{I} \gamma, \mathrm{T}_{1 / 2}$ for source implanted in $\mathrm{C}_{60}$ and gold foil; deduced environmental effect. PREPRINT nucl-ex/0509021,9/15/2005 NUCLEAR REACTIONS ${ }^{197} \mathrm{Au}(\mathrm{n}, \gamma), \mathrm{E}=$ spectrum; measured $\mathrm{E} \gamma, \mathrm{I} \gamma$; deduced neutron flux. ${ }^{7} \mathrm{Li}(\mathrm{p}, \mathrm{n}), \mathrm{E}$ not given; deduced neutron spectrum. ${ }^{62} \mathrm{Ni}(\mathrm{n}, \gamma), \mathrm{E} \approx 5.5-20 \mathrm{keV}$; measured $\sigma$; deduced Maxwellian-averaged $\sigma$. JOUR JUPSA 742981

NUCLEAR REACTIONS C, O, Si, Mg, $\mathrm{Al}(\mathrm{n}, \mathrm{X})^{7} \mathrm{Be}, \mathrm{E} \approx 0.1-750$ $\mathrm{MeV} ; \mathrm{O}, \mathrm{Si}, \mathrm{Mg}, \mathrm{Al}(\mathrm{n}, \mathrm{X}){ }^{22} \mathrm{Na} /{ }^{23} \mathrm{Na}, \mathrm{E} \approx 0.1-750 \mathrm{MeV} ;{ }^{197} \mathrm{Au}(\mathrm{n}$ $\mathrm{X}){ }^{194} \mathrm{Au} /{ }^{196} \mathrm{Au} /{ }^{198} \mathrm{Au}, \mathrm{E} \approx 0.1-750 \mathrm{MeV} ; \mathrm{Ti}, \mathrm{Fe}, \mathrm{Ni}, \mathrm{Cu}(\mathrm{n}, \mathrm{X}){ }^{46} \mathrm{Sc}$ $/{ }^{48} \mathrm{Sc}, \mathrm{E} \approx 0.1-750 \mathrm{MeV} ; \mathrm{Fe}, \mathrm{Ni}, \mathrm{Cu}(\mathrm{n}, \mathrm{X}){ }^{48} \mathrm{~V} /{ }^{51} \mathrm{Cr} /{ }^{52} \mathrm{Mn} /{ }^{54} \mathrm{Mn}$, $\mathrm{E} \approx 0.1-750 \mathrm{MeV} ; \mathrm{Ni}, \mathrm{Cu}(\mathrm{n}, \mathrm{X}){ }^{56} \mathrm{Ni} /{ }^{57} \mathrm{Ni} /{ }^{56} \mathrm{Co} /{ }^{57} \mathrm{Co} /{ }^{58} \mathrm{Co} /$ ${ }^{60} \mathrm{Co} /{ }^{59} \mathrm{Fe}, \mathrm{E} \approx 0.1-750 \mathrm{MeV}$; measured energy-integrated production $\sigma$. JOUR NIMBE 234419 


\section{$\mathrm{A}=7$ (continued)}

2005TIZX NUCLEAR REACTIONS Pb, ${ }^{208} \mathrm{~Pb}(\mathrm{p}, \mathrm{X}){ }^{203} \mathrm{~Pb} /{ }^{200} \mathrm{Tl} /{ }^{199} \mathrm{Tl} /$ ${ }^{196} \mathrm{Au} /{ }^{192} \mathrm{Ir} /{ }^{190} \mathrm{Ir} /{ }^{173} \mathrm{Lu} /{ }^{101 m} \mathrm{Rh} /{ }^{86} \mathrm{Rb} /{ }^{59} \mathrm{Fe} /{ }^{24} \mathrm{Na} /{ }^{7} \mathrm{Be}$, $\mathrm{E}=40-2600 \mathrm{MeV}$; measured excitation functions. Comparison with previous work and model predictions. Other reactions discussed. CONF Santa Fe (Nucl Data for Sci and Technol) Proc,Vol1,P1070 2005TIZY NUCLEAR REACTIONS Pb, ${ }^{208} \mathrm{~Pb},{ }^{209} \mathrm{Bi}(\mathrm{p}, \mathrm{X}){ }^{203} \mathrm{~Pb} /{ }^{200} \mathrm{Tl} /{ }^{199} \mathrm{Tl}$ $/{ }^{196} \mathrm{Au} /{ }^{192} \mathrm{Ir} /{ }^{190} \mathrm{Ir} /{ }^{173} \mathrm{Lu} /{ }^{101 m} \mathrm{Rh} /{ }^{86} \mathrm{Rb} /{ }^{59} \mathrm{Fe} /{ }^{24} \mathrm{Na} /{ }^{7} \mathrm{Be}$, $\mathrm{E}=40-2600 \mathrm{MeV}$; measured production $\sigma$. Comparison with model predictions. PREPRINT nucl-ex/0507009,7/05/2005

2005WAZW NUCLEAR REACTIONS Si $\left({ }^{6} \mathrm{Li}, \mathrm{X}\right),\left({ }^{7} \mathrm{Be}, \mathrm{X}\right),\left({ }^{10} \mathrm{~B}, \mathrm{X}\right),\left({ }^{9} \mathrm{C}, \mathrm{X}\right),\left({ }^{10} \mathrm{C}\right.$, $\mathrm{X}),\left({ }^{11} \mathrm{C}, \mathrm{X}\right),\left({ }^{12} \mathrm{~N}, \mathrm{X}\right),\left({ }^{13} \mathrm{O}, \mathrm{X}\right),\left({ }^{15} \mathrm{O}, \mathrm{X}\right),\left({ }^{17} \mathrm{Ne}, \mathrm{X}\right), \mathrm{E}=15-53 \mathrm{MeV} /$ nucleon; measured reaction and proton-removal $\sigma .{ }^{6} \mathrm{Li},{ }^{7} \mathrm{Be},{ }^{10} \mathrm{~B}$, ${ }^{9,10,11} \mathrm{C},{ }^{12} \mathrm{~N},{ }^{13,15} \mathrm{O},{ }^{17} \mathrm{Ne}$ deduced radii. Comparisons with model predictions. PREPRINT nucl-ex/0507025,7/18/2005

2005ZH09 RADIOACTIVITY ${ }^{7} \mathrm{Be}(\mathrm{EC})$; measured decay rates for source implanted in Pd and Au. JOUR CPLEE 22565

\section{$\mathrm{A}=8$}

$\quad{ }^{8} \mathrm{He} \quad 2005 \mathrm{CH} 50$

NUCLEAR REACTIONS ${ }^{1} \mathrm{H}\left({ }^{6} \mathrm{He}, \mathrm{p}\right),\left({ }^{6} \mathrm{He}, \mathrm{np}\right),\left({ }^{6} \mathrm{He}, \mathrm{p} \alpha\right), \mathrm{E}=717$ $\mathrm{MeV} /$ nucleon; ${ }^{1} \mathrm{H}\left({ }^{8} \mathrm{He}, \mathrm{p}\right),\left({ }^{8} \mathrm{He}, \mathrm{np}\right),\left({ }^{8} \mathrm{He}, \mathrm{p} \alpha\right), \mathrm{E}=671 \mathrm{MeV} /$ nucleon; measured recoil proton spectra, $\sigma(\mathrm{E}, \theta) .{ }^{6,8} \mathrm{He}$ deduced cluster configurations, spectroscopic factors. JOUR NUPAB 75943

2005KI21 NUCLEAR REACTIONS ${ }^{1} \mathrm{H}\left({ }^{6} \mathrm{He}, \mathrm{p}\right),\left({ }^{8} \mathrm{He}, \mathrm{p}\right), \mathrm{E} \approx 700 \mathrm{MeV} /$ nucleon; measured recoil proton spectra, $\sigma(\mathrm{E}, \theta) .{ }^{6,8} \mathrm{He}$ deduced nuclear matter density distributions, charge radii, cluster configurations, spectroscopic factors. JOUR ZAANE 25 s01 215 2005SK03 NUCLEAR REACTIONS ${ }^{1} \mathrm{H}\left({ }^{8} \mathrm{He}, \mathrm{p}\right),\left({ }^{8} \mathrm{He}, \mathrm{d}\right), \mathrm{E}=15.7 \mathrm{MeV} /$ nucleon; measured deuteron and proton spectra, $\sigma(\theta) .{ }^{8} \mathrm{He}(\mathrm{p}, \mathrm{p})$, $\mathrm{E}=15.7 \mathrm{MeV} /$ nucleon; deduced effect of coupling to pickup reaction. Coupled-channels framework, dynamic polarization potential. JOUR PYLBB 61982

${ }^{8} \mathrm{Li}$ 2005Ba40 NUClEAR REACTIONS ${ }^{1} \mathrm{H}\left({ }^{16} \mathrm{O}, \mathrm{X}\right){ }^{1} \mathrm{H} /{ }^{2} \mathrm{H} /{ }^{3} \mathrm{H} /{ }^{3} \mathrm{He} /{ }^{4} \mathrm{He} /{ }^{5} \mathrm{He}$ $/{ }^{6} \mathrm{He} /{ }^{5} \mathrm{Li} /{ }^{6} \mathrm{Li} /{ }^{7} \mathrm{Li} /{ }^{8} \mathrm{Li} /{ }^{7} \mathrm{Be} /{ }^{8} \mathrm{Be} /{ }^{9} \mathrm{Be} /{ }^{10} \mathrm{Be} /{ }^{9} \mathrm{~B} /{ }^{10} \mathrm{~B} /$ ${ }^{11} \mathrm{~B} /{ }^{12} \mathrm{~B} /{ }^{10} \mathrm{C} /{ }^{11} \mathrm{C} /{ }^{12} \mathrm{C} /{ }^{13} \mathrm{C} /{ }^{14} \mathrm{C} /{ }^{13} \mathrm{~N} /{ }^{14} \mathrm{~N} /{ }^{15} \mathrm{~N} /{ }^{14} \mathrm{O} /$ ${ }^{15} \mathrm{O} /{ }^{16} \mathrm{O}$, E at $3.25 \mathrm{GeV} / \mathrm{c} /$ nucleon; measured production $\sigma$. JOUR PZETA 81174

2005B045 RADIOACTIVITY ${ }^{8,9} \operatorname{Li}\left(\beta^{-}\right)$[from Ta(p, X)]; measured $\beta$-asymmetries, $\beta$-NMR spectra from polarized sources. ${ }^{8,9} \mathrm{Li}$ deduced quadrupole moments. ${ }^{9} \mathrm{Li}$ deduced $\mu$. Comparisons with previous results and model predictions. JOUR PRVCA 72044309

2005MU26 RADIOACTIVITY ${ }^{8,9} \operatorname{Li}\left(\beta^{-}\right)$[from $\left.\mathrm{C}\left({ }^{12} \mathrm{C}, \mathrm{X}\right)\right]$; measured $\mathrm{E} \gamma, \beta \gamma$-coin. ${ }^{9}$ Be levels deduced decay widths. Application to triple radiative capture discussed. JOUR NUPAB $758647 \mathrm{c}$

2005NA15 NUCLEAR REACTIONS ${ }^{7} \operatorname{Li}(\mathrm{n}, \gamma), \mathrm{E} \approx 10-80 \mathrm{keV}$; measured $\mathrm{E} \gamma, \mathrm{I} \gamma$, $\sigma$; deduced interaction potential features. ${ }^{7} \mathrm{Be}(\mathrm{p}, \gamma), \mathrm{E} \approx 0.1-3 \mathrm{MeV}$; calculated astrophysical S-factor. JOUR PRVCA 71055803 


\section{$\mathrm{A}=8$ (continued)}

2005N015 NUCLEAR MOMENTS ${ }^{6,7,8,9} \mathrm{Li}$; measured hfs, isotope shifts; deduced charge radii. Resonance ionization mass spectroscopy, comparison with model predictions. JOUR ZAANE 25 s01 199

2005SU25 RADIOACTIVITY ${ }^{8} \mathrm{~B}(\mathrm{EC} \alpha)\left[\right.$ from $\left.{ }^{6} \mathrm{Li}\left({ }^{3} \mathrm{He}, \mathrm{n}\right)\right] ;{ }^{8} \mathrm{Li}\left(\beta^{-} \alpha\right)\left[\right.$ from ${ }^{7} \mathrm{Li}(\mathrm{d}$, p)]; measured $\beta$-NMR spectra; angular correlations; deduced limit on G-parity term. JOUR ZAANE 25 s01 709

${ }^{8} \mathrm{Be} \quad 2004 \mathrm{AHZW}$ NUCLEAR REACTIONS ${ }^{7} \operatorname{Li}($ polarized d, n), E= $=160 \mathrm{keV}$; measured $\sigma(\theta)$, vector and tensor analyzing powers; deduced transition matrix elements. REPT TUNL-XLIII,P28,Ahmed

2004FR34 NUCLEAR REACTIONS ${ }^{12} \mathrm{C}\left({ }^{12} \mathrm{C}, 2^{8} \mathrm{Be}\right), \mathrm{E}=82-120 \mathrm{MeV}$; measured excitation energy spectra, angular correlations. ${ }^{16} \mathrm{O}$ deduced levels, $\mathrm{J}$, $\pi$. Comparison with model predictions. JOUR PRVCA 70064311

2005AN23 NUCLEAR REACTIONS ${ }^{2} \mathrm{H}\left({ }^{7} \mathrm{Be}, \mathrm{p}\right), \mathrm{E}=1.71,5.545 \mathrm{MeV}$; measured proton spectra. JOUR NUPAB $758775 \mathrm{c}$

2005AN30 NUCLEAR REACTIONS ${ }^{2} \mathrm{H}\left({ }^{7} \mathrm{Be}, 2 \alpha\right), \mathrm{E}=1.71,5.55 \mathrm{MeV}$; measured particle spectra, $\sigma .{ }^{7} \mathrm{Be}(\mathrm{d}, \mathrm{p}), \mathrm{E}(\mathrm{cm}) \approx 0.38,1.2 \mathrm{MeV}$; deduced astrophysical S-factors. Implications for primordial ${ }^{7} \mathrm{Li}$ abundance discussed. JOUR ASJOA 630 L105

2005AS04 NUCLEAR REACTIONS ${ }^{12} \mathrm{C}\left({ }^{10} \mathrm{Be}, 2 \alpha\right),\left({ }^{10} \mathrm{Be}, \mathrm{n} 2 \alpha\right), \mathrm{E}=30 \mathrm{MeV} /$ nucleon; measured $\mathrm{En}, \mathrm{E} \alpha$, relative energy spectra, $\sigma(\mathrm{E}) .{ }^{8,9} \mathrm{Be}$ deduced levels, J, $\pi$. Kinematically complete measurement. JOUR PRVCA 72024314

2005BA40 NUCLEAR REACTIONS ${ }^{1} \mathrm{H}\left({ }^{16} \mathrm{O}, \mathrm{X}\right){ }^{1} \mathrm{H} /{ }^{2} \mathrm{H} /{ }^{3} \mathrm{H} /{ }^{3} \mathrm{He} /{ }^{4} \mathrm{He} /{ }^{5} \mathrm{He}$ $/{ }^{6} \mathrm{He} /{ }^{5} \mathrm{Li} /{ }^{6} \mathrm{Li} /{ }^{7} \mathrm{Li} /{ }^{8} \mathrm{Li} /{ }^{7} \mathrm{Be} /{ }^{8} \mathrm{Be} /{ }^{9} \mathrm{Be} /{ }^{10} \mathrm{Be} /{ }^{9} \mathrm{~B} /{ }^{10} \mathrm{~B} /$ ${ }^{11} \mathrm{~B} /{ }^{12} \mathrm{~B} /{ }^{10} \mathrm{C} /{ }^{11} \mathrm{C} /{ }^{12} \mathrm{C} /{ }^{13} \mathrm{C} /{ }^{14} \mathrm{C} /{ }^{13} \mathrm{~N} /{ }^{14} \mathrm{~N} /{ }^{15} \mathrm{~N} /{ }^{14} \mathrm{O} /$ ${ }^{15} \mathrm{O} /{ }^{16} \mathrm{O}$, E at $3.25 \mathrm{GeV} / \mathrm{c} /$ nucleon; measured production $\sigma$. JOUR PZETA 81174

$2005 B 045 \quad$ RADIOACTIVITY ${ }^{8,9} \operatorname{Li}\left(\beta^{-}\right)$[from Ta(p, X)]; measured $\beta$-asymmetries, $\beta$-NMR spectra from polarized sources. ${ }^{8,9} \mathrm{Li}$ deduced quadrupole moments. ${ }^{9} \mathrm{Li}$ deduced $\mu$. Comparisons with previous results and model predictions. JOUR PRVCA 72044309

2005DA12 NUCLEAR REACTIONS ${ }^{4} \mathrm{He}\left(\alpha, \alpha^{\prime}\right), \mathrm{E}=22.4,26.5 \mathrm{MeV}$; measured $\mathrm{E} \gamma, \mathrm{E} \alpha, \alpha \alpha-, \gamma \alpha$-coin; deduced resonance $\sigma .{ }^{8} \mathrm{Be}$ deduced transition B(E2), cluster structure. JOUR PRLTA 94122502

$2005 \mathrm{MI} 13 \quad$ NUCLEAR REACTIONS ${ }^{6,7} \mathrm{Li}\left({ }^{6} \mathrm{He}, \alpha^{6} \mathrm{He}\right),{ }^{6} \mathrm{Li}\left({ }^{6} \mathrm{He}, \mathrm{t} 2 \alpha\right), \mathrm{E}=18 \mathrm{MeV}$; measured excitation energy spectra. ${ }^{6,7} \mathrm{Li},{ }^{8,9,10} \mathrm{Be}$ deduced cluster states. JOUR NUPAB 753263

2005MU26 RADIOACTIVITY ${ }^{8,9} \operatorname{Li}\left(\beta^{-}\right)$[from $\left.\mathrm{C}\left({ }^{12} \mathrm{C}, \mathrm{X}\right)\right]$; measured $\mathrm{E} \gamma, \beta \gamma$-coin. ${ }^{9}$ Be levels deduced decay widths. Application to triple radiative capture discussed. JOUR NUPAB $758647 \mathrm{c}$

2005RU03 NUCLEAR REACTIONS ${ }^{12} \mathrm{C}\left({ }^{11} \mathrm{~B},{ }^{15} \mathrm{~N}\right), \mathrm{E}=49 \mathrm{MeV}$; measured $\sigma(\mathrm{E}$, $\theta) ;{ }^{12} \mathrm{C}\left({ }^{11} \mathrm{~B},{ }^{8} \mathrm{Be}\right), \mathrm{E}(\mathrm{cm})=10-17 \mathrm{MeV}$; analyzed $\sigma(\mathrm{E}, \theta)$; deduced reaction mechanism features, optical model parameters. Coupled channels analysis. JOUR ZAANE 23445

2005SCZV NUCLEAR REACTIONS ${ }^{9} \mathrm{Be}\left({ }^{26} \mathrm{Mg},{ }^{27} \mathrm{Mg}\right), \mathrm{E}=57 \mathrm{MeV}$; measured $\mathrm{E} \gamma$, $\mathrm{I} \gamma, \alpha \alpha$-coin, $\sigma(\theta) .{ }^{27} \mathrm{Mg}$ deduced transitions. REPT MLL 2004 Annual,P4,Schwerdtfeger 


\section{$\mathrm{A}=8$ (continued)}

2005SPZY NUCLEAR REACTIONS ${ }^{12} \mathrm{C}\left({ }^{32} \mathrm{~S},{ }^{36} \mathrm{Ar}\right), \mathrm{E}=65 \mathrm{MeV} ;{ }^{12} \mathrm{C}\left({ }^{34} \mathrm{~S},{ }^{38} \mathrm{Ar}\right)$, $\mathrm{E}=67 \mathrm{MeV}$; measured $\mathrm{E} \gamma, \mathrm{I} \gamma(\theta, \mathrm{H}, \mathrm{t}), \alpha \gamma$-coin. ${ }^{36,38} \mathrm{Ar}$ levels deduced $\mathrm{g}$ factors. Transient field technique. Comparison with shell model predictions. REPT MLL 2004 Annual,P5,Speidel

${ }^{8} \mathrm{~B} \quad$ 2004REZY NUCLEAR REACTIONS ${ }^{1} \mathrm{H}\left({ }^{11} \mathrm{C}, \mathrm{p}\right),\left({ }^{11} \mathrm{C}, \alpha\right), \mathrm{E}^{*}=8.7-9.9 \mathrm{MeV}$; measured particle spectra, angular distributions, $\sigma .{ }^{8} \operatorname{Be}(\alpha, \mathrm{p})$, $\mathrm{E}^{*}=8.7-9.9 \mathrm{MeV}$; deduced excitation function, astrophysical reaction rates. REPT ANL-04/22,P3,Rehm

2005JU03 NUCLEAR REACTIONS ${ }^{7} \mathrm{Be}(\mathrm{p}, \gamma), \mathrm{E}(\mathrm{cm})=116-2460 \mathrm{MeV}$; measured $\sigma$; deduced astrophysical S-factors. Comparison with previous results. JOUR NPBSE 138112

$2005 \mathrm{LI} 40 \quad$ NUCLEAR REACTIONS ${ }^{2} \mathrm{H}\left({ }^{7} \mathrm{Be}, \mathrm{n}\right),\left({ }^{11} \mathrm{C}, \mathrm{n}\right),\left({ }^{8} \mathrm{Li}, \mathrm{p}\right), \mathrm{E} \approx 5.8-9.8$ $\mathrm{MeV}$; measured $\sigma(\theta)$, total $\sigma$; deduced astrophysical S-factors. JOUR NUPAB 758 110c

2005NA15 NUCLEAR REACTIONS ${ }^{7} \operatorname{Li}(\mathrm{n}, \gamma), \mathrm{E} \approx 10-80 \mathrm{keV}$; measured $\mathrm{E} \gamma, \mathrm{I} \gamma$, $\sigma$; deduced interaction potential features. ${ }^{7} \mathrm{Be}(\mathrm{p}, \gamma), \mathrm{E} \approx 0.1-3 \mathrm{MeV}$; calculated astrophysical S-factor. JOUR PRVCA 71055803

2005SCZX NUCLEAR REACTIONS ${ }^{208} \mathrm{~Pb}\left({ }^{8} \mathrm{~B}, \mathrm{p}^{7} \mathrm{Be}\right), \mathrm{E}=254 \mathrm{MeV} /$ nucleon; measured fragment spectra, angular correlations. ${ }^{7} \mathrm{Be}(\mathrm{p}, \gamma), \mathrm{E}=\mathrm{low}$; deduced astrophysical S-factor. PREPRINT nucl-ex/0508014,08/11/2005

2005SU25 RADIOACTIVITY ${ }^{8} \mathrm{~B}(\mathrm{EC} \alpha)\left[\right.$ from $\left.{ }^{6} \mathrm{Li}\left({ }^{3} \mathrm{He}, \mathrm{n}\right)\right] ;{ }^{8} \mathrm{Li}\left(\beta^{-} \alpha\right)\left[\right.$ from ${ }^{7} \mathrm{Li}(\mathrm{d}$, p)]; measured $\beta$-NMR spectra; angular correlations; deduced limit on G-parity term. JOUR ZAANE 25 s01 709

2005TA32 NUCLEAR REACTIONS Be, C, Al $\left({ }^{12} \mathrm{C}, \mathrm{X}\right), \mathrm{E}=30-200 \mathrm{MeV} /$ nucleon; $\mathrm{Be}\left({ }^{9} \mathrm{Be}, \mathrm{X}\right), \mathrm{E}=70-100 \mathrm{MeV} /$ nucleon; measured reaction $\sigma(\mathrm{E})$; deduced nucleon-nucleon interaction range. ${ }^{8} \mathrm{~B}$ deduced nuclear matter density distribution. Comparison with Glauber calculations. JOUR ZAANE 25 s01 217

$$
\mathrm{A}=\mathbf{9}
$$

${ }^{9} \mathrm{He} \quad$ 2005R037

NUCLEAR REACTIONS ${ }^{1} \mathrm{H}\left({ }^{8} \mathrm{He},{ }^{8} \mathrm{He}\right)$, E not given; measured recoil proton spectrum; deduced excitation function. ${ }^{1} \mathrm{H}\left({ }^{6} \mathrm{He},{ }^{6} \mathrm{Li}\right)$, E not given; measured neutron spectrum, $\mathrm{n} \gamma$-coin; deduced excitation function. ${ }^{7,9} \mathrm{Li}$ deduced resonance parameters. ${ }^{7,9} \mathrm{He}$ deduced analog states features. JOUR NIMBE 241977

${ }^{9} \mathrm{Li} \quad$ 2005B045 RADIOACTIVITY ${ }^{8,9} \mathrm{Li}\left(\beta^{-}\right)[$from $\mathrm{Ta}(\mathrm{p}, \mathrm{X})]$; measured $\beta$-asymmetries, $\beta$-NMR spectra from polarized sources. ${ }^{8,9} \mathrm{Li}$ deduced quadrupole moments. ${ }^{9} \mathrm{Li}$ deduced $\mu$. Comparisons with previous results and model predictions. JOUR PRVCA 72044309

2005JE01 NUCLEAR REACTIONS ${ }^{2} \mathrm{H}\left({ }^{9} \mathrm{Li},{ }^{9} \mathrm{Li}\right),\left({ }^{9} \mathrm{Li}, \mathrm{np}\right),\left({ }^{9} \mathrm{Li}, \mathrm{nX}\right),\left({ }^{9} \mathrm{Li}, \mathrm{pX}\right)$, $\mathrm{E}=2.36 \mathrm{MeV} /$ nucleon; measured particle spectra, $\sigma(\theta) .{ }^{2} \mathrm{H}\left({ }^{9} \mathrm{Li}, \alpha \mathrm{X}\right)$, $\left({ }^{9} \mathrm{Li},{ }^{6} \mathrm{HeX}\right), \mathrm{E}=2.36 \mathrm{MeV} /$ nucleon; measured particle spectra.

Post-accelerated radioactive beam. JOUR NUPAB 748374

$2005 \mathrm{LI} 19$ NUCLEAR REACTIONS ${ }^{2} \mathrm{H}\left({ }^{8} \mathrm{Li},{ }^{9} \mathrm{Li}\right), \mathrm{E}=39 \mathrm{MeV}$; measured particle spectra, $\sigma(\theta) .{ }^{8} \mathrm{Li}(\mathrm{n}, \gamma), \mathrm{E}=$ low; deduced astrophysical reaction rates. JOUR PRVCA 71052801 


\section{$\mathrm{A}=9$ (continued)}

2005LI35 NUCLEAR REACTIONS ${ }^{2} \mathrm{H}\left({ }^{8} \mathrm{Li}, \mathrm{p}\right), \mathrm{E}(\mathrm{cm})=7.8 \mathrm{MeV}$; measured $\sigma(\theta)$; deduced asymptotic normalization coefficients. ${ }^{9} \mathrm{C}$ deduced radius, density distributions, halo structure. JOUR CPLEE 221870

2005LI40 NUCLEAR REACTIONS ${ }^{2} \mathrm{H}\left({ }^{7} \mathrm{Be}, \mathrm{n}\right),\left({ }^{11} \mathrm{C}, \mathrm{n}\right),\left({ }^{8} \mathrm{Li}, \mathrm{p}\right), \mathrm{E} \approx 5.8-9.8$ $\mathrm{MeV}$; measured $\sigma(\theta)$, total $\sigma$; deduced astrophysical S-factors. JOUR NUPAB 758 110c

2005MU26 RADIOACTIVITY ${ }^{8,9} \operatorname{Li}\left(\beta^{-}\right)$[from $\left.\mathrm{C}\left({ }^{12} \mathrm{C}, \mathrm{X}\right)\right]$; measured $\mathrm{E} \gamma, \beta \gamma$-coin. ${ }^{9} \mathrm{Be}$ levels deduced decay widths. Application to triple radiative capture discussed. JOUR NUPAB $758647 \mathrm{c}$

2005N015 NUCLEAR MOMENTS ${ }^{6,7,8,9} \mathrm{Li}$; measured hfs, isotope shifts; deduced charge radii. Resonance ionization mass spectroscopy, comparison with model predictions. JOUR ZAANE 25 s01 199

2005PR11 RADIOACTIVITY ${ }^{9} \operatorname{Li}\left(\beta^{-}\right)$[from Ta(p, X)]; measured $\beta$-delayed $\mathrm{E} \alpha$, $\alpha \alpha$-coin; deduced $\beta$-decay branching ratios. ${ }^{9} \mathrm{Be}$ deduced levels, $\mathrm{J}, \pi$, resonance states. JOUR PYLBB 61843

2005R037 NUCLEAR REACTIONS ${ }^{1} \mathrm{H}\left({ }^{8} \mathrm{He},{ }^{8} \mathrm{He}\right)$, E not given; measured recoil proton spectrum; deduced excitation function. ${ }^{1} \mathrm{H}\left({ }^{6} \mathrm{He},{ }^{6} \mathrm{Li}\right)$, E not given; measured neutron spectrum, $\mathrm{n} \gamma$-coin; deduced excitation function. ${ }^{7,9} \mathrm{Li}$ deduced resonance parameters. ${ }^{7,9} \mathrm{He}$ deduced analog states features. JOUR NIMBE 241977

2005 WU03 NUCLEAR REACTIONS ${ }^{2} \mathrm{H}\left({ }^{8} \mathrm{Li}, \mathrm{p}\right), \mathrm{E} \approx 76 \mathrm{MeV}$; measured Ep, excitation energy spectra, $\sigma(\theta) .{ }^{9} \mathrm{Li}$ deduced levels, $\mathrm{J}, \pi$, spectroscopic factors. Comparison with model predictions. JOUR PRLTA 94082502

${ }^{9} \mathrm{Be} \quad 2004 \mathrm{~S} 035$ NUCLEAR REACTIONS ${ }^{7} \mathrm{Li}\left({ }^{7} \mathrm{Li}, 2 \alpha\right), \mathrm{E}=8,30 \mathrm{MeV} ;{ }^{9} \mathrm{Be}\left({ }^{7} \mathrm{Li},{ }^{7} \mathrm{Li}\right)$, $\left({ }^{7} \mathrm{Li}, \alpha^{6} \mathrm{Li}\right),\left({ }^{7} \mathrm{Li}, \alpha^{7} \mathrm{Li}\right), \mathrm{E}=52 \mathrm{MeV} ;{ }^{7} \mathrm{Li}\left({ }^{9} \mathrm{Be}, \alpha^{9} \mathrm{Be}\right),\left({ }^{9} \mathrm{Be}, \alpha^{10} \mathrm{Be}\right)$, $\mathrm{E}=70 \mathrm{MeV}$; measured excitation energy spectra. ${ }^{9,10} \mathrm{Be},{ }^{13,14} \mathrm{C}$ deduced excited states, cluster structures. JOUR FIZBE 13433

2005 AD35 NUCLEAR REACTIONS ${ }^{6} \mathrm{Li}\left({ }^{6} \mathrm{Li}, \alpha \mathrm{X}\right),\left({ }^{7} \mathrm{Li}, \alpha \mathrm{X}\right), \mathrm{E}=14-20 \mathrm{MeV}$; measured $\alpha$-spectra. ${ }^{12} \mathrm{C}(\mathrm{n}, \alpha), \mathrm{E}=72.8 \mathrm{MeV} ;{ }^{28} \mathrm{Si}\left({ }^{6} \mathrm{Li}, \alpha\right), \mathrm{E}=36 \mathrm{MeV}$; analyzed $\alpha$-spectra. Statistical model calculations. Target-projectile symmetry discussed. JOUR ZAANE 25 s01 299

2005AS04 NUCLEAR REACTIONS ${ }^{12} \mathrm{C}\left({ }^{10} \mathrm{Be}, 2 \alpha\right),\left({ }^{10} \mathrm{Be}, \mathrm{n} 2 \alpha\right), \mathrm{E}=30 \mathrm{MeV} /$ nucleon; measured $\mathrm{En}, \mathrm{E} \alpha$, relative energy spectra, $\sigma(\mathrm{E}) .{ }^{8,9} \mathrm{Be}$ deduced levels, J, $\pi$. Kinematically complete measurement. JOUR PRVCA 72024314

2005BA40 NUCLEAR REACTIONS ${ }^{1} \mathrm{H}\left({ }^{16} \mathrm{O}, \mathrm{X}\right){ }^{1} \mathrm{H} /{ }^{2} \mathrm{H} /{ }^{3} \mathrm{H} /{ }^{3} \mathrm{He} /{ }^{4} \mathrm{He} /{ }^{5} \mathrm{He}$ $/{ }^{6} \mathrm{He} /{ }^{5} \mathrm{Li} /{ }^{6} \mathrm{Li} /{ }^{7} \mathrm{Li} /{ }^{8} \mathrm{Li} /{ }^{7} \mathrm{Be} /{ }^{8} \mathrm{Be} /{ }^{9} \mathrm{Be} /{ }^{10} \mathrm{Be} /{ }^{9} \mathrm{~B} /{ }^{10} \mathrm{~B} /$ ${ }^{11} \mathrm{~B} /{ }^{12} \mathrm{~B} /{ }^{10} \mathrm{C} /{ }^{11} \mathrm{C} /{ }^{12} \mathrm{C} /{ }^{13} \mathrm{C} /{ }^{14} \mathrm{C} /{ }^{13} \mathrm{~N} /{ }^{14} \mathrm{~N} /{ }^{15} \mathrm{~N} /{ }^{14} \mathrm{O} /$ ${ }^{15} \mathrm{O} /{ }^{16} \mathrm{O}$, E at $3.25 \mathrm{GeV} / \mathrm{c} /$ nucleon; measured production $\sigma$. JOUR PZETA 81174

2005B045 RADIOACTIVITY ${ }^{8,9} \operatorname{Li}\left(\beta^{-}\right)$[from Ta(p, X)]; measured $\beta$-asymmetries, $\beta$-NMR spectra from polarized sources. ${ }^{8,9} \mathrm{Li}$ deduced quadrupole moments. ${ }^{9} \mathrm{Li}$ deduced $\mu$. Comparisons with previous results and model predictions. JOUR PRVCA 72044309

$2005 \mathrm{MI} 13$ NUCLEAR REACTIONS ${ }^{6,7} \mathrm{Li}\left({ }^{6} \mathrm{He}, \alpha^{6} \mathrm{He}\right),{ }^{6} \mathrm{Li}\left({ }^{6} \mathrm{He}, \mathrm{t} 2 \alpha\right), \mathrm{E}=18 \mathrm{MeV}$; measured excitation energy spectra. ${ }^{6,7} \mathrm{Li},{ }^{8,9,10} \mathrm{Be}$ deduced cluster states. JOUR NUPAB 753263 


\section{$\mathrm{A}=9$ (continued)}

2005MU26 RADIOACTIVITY ${ }^{8,9} \operatorname{Li}\left(\beta^{-}\right)$[from $\left.\mathrm{C}\left({ }^{12} \mathrm{C}, \mathrm{X}\right)\right]$; measured $\mathrm{E} \gamma, \beta \gamma$-coin. ${ }^{9}$ Be levels deduced decay widths. Application to triple radiative capture discussed. JOUR NUPAB $758647 \mathrm{c}$

2005PR11 RADIOACTIVITY ${ }^{9} \operatorname{Li}\left(\beta^{-}\right)$[from Ta(p, X)]; measured $\beta$-delayed $\mathrm{E} \alpha$, $\alpha \alpha$-coin; deduced $\beta$-decay branching ratios. ${ }^{9} \mathrm{Be}$ deduced levels, $\mathrm{J}, \pi$, resonance states. JOUR PYLBB 61843

2005TA19 NUCLEAR REACTIONS ${ }^{10} \mathrm{~B},{ }^{16} \mathrm{O}\left(\mathrm{K}^{-}, \pi^{-}\right)$, E at $0.93 \mathrm{GeV} / \mathrm{c}$;

${ }^{11} \mathrm{~B}\left(\pi^{+}, \mathrm{K}^{+}\right)$, E at $1.05 \mathrm{GeV} / \mathrm{c} ;{ }^{7} \mathrm{Li},{ }^{10} \mathrm{~B}\left(\mathrm{~K}^{-}, \gamma\right)$, E at rest; measured $\mathrm{E} \gamma, \mathrm{I} \gamma .{ }^{7} \mathrm{Li},{ }^{9} \mathrm{Be},{ }^{10,11} \mathrm{~B},{ }^{16} \mathrm{O}$ deduced hypernucleus levels, J, $\pi$.

Hyperball array. JOUR NUPAB $75458 \mathrm{c}$

2005WAZX NUCLEAR REACTIONS ${ }^{12} \mathrm{C}$ (polarized $\gamma$, pd), $\mathrm{E}=170-350 \mathrm{MeV}$; measured deuteron and proton spectra, polarization asymmetry; deduced reaction mechanism features. Tagged photons. PREPRINT nucl-ex/0506018,6/14/2005

2005YE01 NUCLEAR REACTIONS ${ }^{9} \mathrm{Be}\left({ }^{6} \mathrm{He},{ }^{6} \mathrm{He}\right), \mathrm{E}=25 \mathrm{MeV} /$ nucleon; measured quasielastic $\sigma(\theta)$; deduced optical model parameters, inelastic channels contribution. JOUR PRVCA 71014604

2005YE05 NUCLEAR REACTIONS ${ }^{9} \mathrm{Be}\left({ }^{6} \mathrm{He},{ }^{6} \mathrm{He}\right),\left({ }^{6} \mathrm{He},{ }^{5} \mathrm{He}\right),\left({ }^{6} \mathrm{He}, \alpha\right),\left({ }^{6} \mathrm{He}\right.$, $\alpha \mathrm{X}),\left({ }^{6} \mathrm{He}, \mathrm{tX}\right), \mathrm{E}=25 \mathrm{MeV} /$ nucleon; measured quasielastic, breakup, and transfer $\sigma(\theta) .{ }^{6} \mathrm{He}$ deduced two-triton configuration. JOUR JPGPE 31 S1647

${ }^{9} \mathrm{~B} \quad 2005 \mathrm{BA} 40$ NUCLEAR REACTIONS ${ }^{1} \mathrm{H}\left({ }^{16} \mathrm{O}, \mathrm{X}\right){ }^{1} \mathrm{H} /{ }^{2} \mathrm{H} /{ }^{3} \mathrm{H} /{ }^{3} \mathrm{He} /{ }^{4} \mathrm{He} /{ }^{5} \mathrm{He}$ $/{ }^{6} \mathrm{He} /{ }^{5} \mathrm{Li} /{ }^{6} \mathrm{Li} /{ }^{7} \mathrm{Li} /{ }^{8} \mathrm{Li} /{ }^{7} \mathrm{Be} /{ }^{8} \mathrm{Be} /{ }^{9} \mathrm{Be} /{ }^{10} \mathrm{Be} /{ }^{9} \mathrm{~B} /{ }^{10} \mathrm{~B} /$ ${ }^{11} \mathrm{~B} /{ }^{12} \mathrm{~B} /{ }^{10} \mathrm{C} /{ }^{11} \mathrm{C} /{ }^{12} \mathrm{C} /{ }^{13} \mathrm{C} /{ }^{14} \mathrm{C} /{ }^{13} \mathrm{~N} /{ }^{14} \mathrm{~N} /{ }^{15} \mathrm{~N} /{ }^{14} \mathrm{O} /$ ${ }^{15} \mathrm{O} /{ }^{16} \mathrm{O}$, E at $3.25 \mathrm{GeV} / \mathrm{c} /$ nucleon; measured production $\sigma$. JOUR PZETA 81174

${ }^{9} \mathrm{C} \quad 2005$ GU29 NUCLEAR REACTIONS ${ }^{2} \mathrm{H}\left({ }^{8} \mathrm{Li},{ }^{9} \mathrm{Li}\right), \mathrm{E}(\mathrm{cm})=7.8 \mathrm{MeV}$; measured $\sigma(\theta)$; deduced asymptotic normalization coefficient. ${ }^{8} \mathrm{~B}(\mathrm{p}, \gamma), \mathrm{E}=$ low; calculated astrophysical S-factor. DWBA analysis, inverse kinematics, comparison with data. JOUR NUPAB 761162

2005LI35 NUCLEAR REACTIONS ${ }^{2} \mathrm{H}\left({ }^{8} \mathrm{Li}, \mathrm{p}\right), \mathrm{E}(\mathrm{cm})=7.8 \mathrm{MeV}$; measured $\sigma(\theta)$; deduced asymptotic normalization coefficients. ${ }^{9} \mathrm{C}$ deduced radius, density distributions, halo structure. JOUR CPLEE 221870

2005WAZW NUCLEAR REACTIONS Si $\left({ }^{6} \mathrm{Li}, \mathrm{X}\right),\left({ }^{7} \mathrm{Be}, \mathrm{X}\right),\left({ }^{10} \mathrm{~B}, \mathrm{X}\right),\left({ }^{9} \mathrm{C}, \mathrm{X}\right),\left({ }^{10} \mathrm{C}\right.$, $\mathrm{X}),\left({ }^{11} \mathrm{C}, \mathrm{X}\right),\left({ }^{12} \mathrm{~N}, \mathrm{X}\right),\left({ }^{13} \mathrm{O}, \mathrm{X}\right),\left({ }^{15} \mathrm{O}, \mathrm{X}\right),\left({ }^{17} \mathrm{Ne}, \mathrm{X}\right), \mathrm{E}=15-53 \mathrm{MeV} /$ nucleon; measured reaction and proton-removal $\sigma .{ }^{6} \mathrm{Li},{ }^{7} \mathrm{Be},{ }^{10} \mathrm{~B}$, ${ }^{9,10,11} \mathrm{C},{ }^{12} \mathrm{~N},{ }^{13,15} \mathrm{O},{ }^{17} \mathrm{Ne}$ deduced radii. Comparisons with model predictions. PREPRINT nucl-ex/0507025,7/18/2005

$$
\mathrm{A}=\mathbf{1 0}
$$

${ }^{10} \mathrm{Li} \quad$ 2005SA03

NUCLEAR REACTIONS ${ }^{10} \mathrm{~B}\left(\pi^{-}, \mathrm{K}^{+}\right),{ }^{10} \mathrm{~B},{ }^{12} \mathrm{C}\left(\pi^{+}, \mathrm{K}^{+}\right)$, E at 1.05, $1.2 \mathrm{GeV} / \mathrm{c}$; measured missing mass spectra, hypernucleus production $\sigma$. JOUR PRLTA 94052502

${ }^{10} \mathrm{Be}$ 2004MAZP NUCLEAR REACTIONS C, ${ }^{27} \mathrm{Al}, \mathrm{Cu}, \mathrm{Ag},{ }^{197} \mathrm{Au}(\alpha, \mathrm{X}){ }^{7} \mathrm{Be}, \mathrm{E}=400$ $\mathrm{MeV}$; C, ${ }^{27} \mathrm{Al}, \mathrm{Cu}, \mathrm{Ag},{ }^{197} \mathrm{Au}(\mathrm{n}, \mathrm{X}){ }^{7} \mathrm{Be}, \mathrm{E}<500 \mathrm{MeV}$; Cu, Ag, ${ }^{197} \mathrm{Au}(\alpha, \mathrm{X}){ }^{10} \mathrm{Be}, \mathrm{E}=400 \mathrm{MeV} ; \mathrm{Cu}, \mathrm{Ag},{ }^{197} \mathrm{Au}(\mathrm{n}, \mathrm{X}){ }^{10} \mathrm{Be}, \mathrm{E}<500$ $\mathrm{MeV}$; measured yields. REPT KEK Preprint 2004-90,Matsumura 


\section{$\mathrm{A}=10$ (continued)}

2004MIZS NUCLEAR REACTIONS Fe(p, X) ${ }^{52} \mathrm{Mn}, \mathrm{E}<2.6 \mathrm{GeV} ; \mathrm{Pb}(\mathrm{p}, \mathrm{X}){ }^{10} \mathrm{Be}$, $\mathrm{E}<2.6 \mathrm{GeV} ;{ }^{209} \mathrm{Bi}(\mathrm{p}, 4 \mathrm{np}), \mathrm{E}<2.6 \mathrm{GeV} ; \mathrm{Pb}(\mathrm{n}, \mathrm{X}){ }^{196} \mathrm{Au} /{ }^{95} \mathrm{Zr}, \mathrm{E} \approx$ 70-180 MeV; measured excitation functions. Comparison with model predictions. REPT NEA/NSC/DOC(2004)14,P28,Michel

2004S035 NUCLEAR REACTIONS ${ }^{7} \mathrm{Li}\left({ }^{7} \mathrm{Li}, 2 \alpha\right), \mathrm{E}=8,30 \mathrm{MeV} ;{ }^{9} \mathrm{Be}\left({ }^{7} \mathrm{Li},{ }^{7} \mathrm{Li}\right)$, $\left({ }^{7} \mathrm{Li}, \alpha^{6} \mathrm{Li}\right),\left({ }^{7} \mathrm{Li}, \alpha^{7} \mathrm{Li}\right), \mathrm{E}=52 \mathrm{MeV} ;{ }^{7} \mathrm{Li}\left({ }^{9} \mathrm{Be}, \alpha^{9} \mathrm{Be}\right),\left({ }^{9} \mathrm{Be}, \alpha^{10} \mathrm{Be}\right)$, $\mathrm{E}=70 \mathrm{MeV}$; measured excitation energy spectra. ${ }^{9,10} \mathrm{Be},{ }^{13,14} \mathrm{C}$ deduced excited states, cluster structures. JOUR FIZBE 13433

2005BA40 NUCLEAR REACTIONS ${ }^{1} \mathrm{H}\left({ }^{16} \mathrm{O}, \mathrm{X}\right){ }^{1} \mathrm{H} /{ }^{2} \mathrm{H} /{ }^{3} \mathrm{H} /{ }^{3} \mathrm{He} /{ }^{4} \mathrm{He} /{ }^{5} \mathrm{He}$ $/{ }^{6} \mathrm{He} /{ }^{5} \mathrm{Li} /{ }^{6} \mathrm{Li} /{ }^{7} \mathrm{Li} /{ }^{8} \mathrm{Li} /{ }^{7} \mathrm{Be} /{ }^{8} \mathrm{Be} /{ }^{9} \mathrm{Be} /{ }^{10} \mathrm{Be} /{ }^{9} \mathrm{~B} /{ }^{10} \mathrm{~B} /$ ${ }^{11} \mathrm{~B} /{ }^{12} \mathrm{~B} /{ }^{10} \mathrm{C} /{ }^{11} \mathrm{C} /{ }^{12} \mathrm{C} /{ }^{13} \mathrm{C} /{ }^{14} \mathrm{C} /{ }^{13} \mathrm{~N} /{ }^{14} \mathrm{~N} /{ }^{15} \mathrm{~N} /{ }^{14} \mathrm{O} /$ ${ }^{15} \mathrm{O} /{ }^{16} \mathrm{O}$, E at $3.25 \mathrm{GeV} / \mathrm{c} /$ nucleon; measured production $\sigma$. JOUR PZETA 81174

2005HI03 RADIOACTIVITY ${ }^{11} \operatorname{Li}\left(\beta^{-}\right),\left(\beta^{-} \mathrm{n}\right)$; measured $\beta$-delayed $\mathrm{E} \gamma, \mathrm{En}$, asymmetry following decay of spin-polarized source. ${ }^{10,11} \mathrm{Be}$ deduced levels, J, $\pi$, S-factors. Comparison with antisymmetrized molecular dynamics model predictions. JOUR PYLBB 611239

$2005 \mathrm{MI} 13 \quad$ NUCLEAR REACTIONS ${ }^{6,7} \mathrm{Li}\left({ }^{6} \mathrm{He}, \alpha^{6} \mathrm{He}\right),{ }^{6} \mathrm{Li}\left({ }^{6} \mathrm{He}, \mathrm{t} 2 \alpha\right), \mathrm{E}=18 \mathrm{MeV}$; measured excitation energy spectra. ${ }^{6,7} \mathrm{Li},{ }^{8,9,10} \mathrm{Be}$ deduced cluster states. JOUR NUPAB 753263

2005SCZW NUCLEAR REACTIONS $\mathrm{Pb}(\mathrm{p}, \mathrm{X}){ }^{10} \mathrm{Be} /{ }^{26} \mathrm{Al} /{ }^{129} \mathrm{I} /{ }^{36} \mathrm{Cl}$, $\mathrm{E}=200-2600 \mathrm{MeV}$; measured excitation functions. Stacked foil activation, chemical separation. Comparison with model predictions. CONF Santa Fe (Nucl Data for Sci and Technol) Proc,Vol2,P1517 2005TA19 NUCLEAR REACTIONS ${ }^{10} \mathrm{~B},{ }^{16} \mathrm{O}\left(\mathrm{K}^{-}, \pi^{-}\right)$, E at $0.93 \mathrm{GeV} / \mathrm{c}$; ${ }^{11} \mathrm{~B}\left(\pi^{+}, \mathrm{K}^{+}\right)$, E at $1.05 \mathrm{GeV} / \mathrm{c} ;{ }^{7} \mathrm{Li},{ }^{10} \mathrm{~B}\left(\mathrm{~K}^{-}, \gamma\right)$, E at rest; measured $\mathrm{E} \gamma, \mathrm{I} \gamma \cdot{ }^{7} \mathrm{Li},{ }^{9} \mathrm{Be},{ }^{10,11} \mathrm{~B},{ }^{16} \mathrm{O}$ deduced hypernucleus levels, $\mathrm{J}, \pi$. Hyperball array. JOUR NUPAB $75458 \mathrm{c}$

2005YE05 NUCLEAR REACTIONS ${ }^{9} \mathrm{Be}\left({ }^{6} \mathrm{He},{ }^{6} \mathrm{He}\right),\left({ }^{6} \mathrm{He},{ }^{5} \mathrm{He}\right),\left({ }^{6} \mathrm{He}, \alpha\right),\left({ }^{6} \mathrm{He}\right.$, $\alpha \mathrm{X}),\left({ }^{6} \mathrm{He}, \mathrm{tX}\right), \mathrm{E}=25 \mathrm{MeV} /$ nucleon; measured quasielastic, breakup, and transfer $\sigma(\theta)$. ${ }^{6} \mathrm{He}$ deduced two-triton configuration. JOUR JPGPE 31 S1647

${ }^{10} \mathrm{~B}$ 2005Ba40 NUCLEAR REACTIONS ${ }^{1} \mathrm{H}\left({ }^{16} \mathrm{O}, \mathrm{X}\right){ }^{1} \mathrm{H} /{ }^{2} \mathrm{H} /{ }^{3} \mathrm{H} /{ }^{3} \mathrm{He} /{ }^{4} \mathrm{He} /{ }^{5} \mathrm{He}$ $/{ }^{6} \mathrm{He} /{ }^{5} \mathrm{Li} /{ }^{6} \mathrm{Li} /{ }^{7} \mathrm{Li} /{ }^{8} \mathrm{Li} /{ }^{7} \mathrm{Be} /{ }^{8} \mathrm{Be} /{ }^{9} \mathrm{Be} /{ }^{10} \mathrm{Be} /{ }^{9} \mathrm{~B} /{ }^{10} \mathrm{~B} /$ ${ }^{11} \mathrm{~B} /{ }^{12} \mathrm{~B} /{ }^{10} \mathrm{C} /{ }^{11} \mathrm{C} /{ }^{12} \mathrm{C} /{ }^{13} \mathrm{C} /{ }^{14} \mathrm{C} /{ }^{13} \mathrm{~N} /{ }^{14} \mathrm{~N} /{ }^{15} \mathrm{~N} /{ }^{14} \mathrm{O} /$ ${ }^{15} \mathrm{O} /{ }^{16} \mathrm{O}$, E at $3.25 \mathrm{GeV} / \mathrm{c} /$ nucleon; measured production $\sigma$. JOUR PZETA 81174

2005BE43 NUCLEAR REACTIONS ${ }^{10} \mathrm{~B}$ (polarized p, p), (polarized p, p'), E=197 $\mathrm{MeV}$; measured $\sigma(\mathrm{E}, \theta)$, analyzing power, polarization transfer coefficients. Comparison with model predictions. JOUR PRVCA 71 064607

2005 CU06 NUCLEAR REACTIONS ${ }^{7} \mathrm{Li}\left({ }^{7} \mathrm{Li},{ }^{11} \mathrm{~B}\right),\left({ }^{7} \mathrm{Li},{ }^{12} \mathrm{~B}\right), \mathrm{E}=58 \mathrm{MeV} ;{ }^{12} \mathrm{C}$, ${ }^{16} \mathrm{O}\left({ }^{7} \mathrm{Li},{ }^{10} \mathrm{~B}\right), \mathrm{E}=58 \mathrm{MeV}$; measured particle spectra. ${ }^{10,11,12} \mathrm{~B}$ deduced relative yields for $\alpha+\mathrm{Li}$ and $\mathrm{H}+\mathrm{Be}$ decay channels from excited states. JOUR PRVCA 72044320

2005GL05 NUCLEAR REACTIONS ${ }^{12} \mathrm{C}\left(\gamma, \pi^{-} \mathrm{p}\right),\left(\gamma, \pi^{-} 2 \mathrm{p}\right), \mathrm{E}=500 \mathrm{MeV}$ bremsstrahlung; measured Ep, pion spectra, $\sigma(\mathrm{E}, \theta)$; deduced reaction mechanism features. JOUR PZETA 81546 


\section{$\mathrm{A}=10$ (continued)}

2005SUZV NUCLEAR REACTIONS ${ }^{12} \mathrm{C}$ (polarized d, $\alpha$ ), $\mathrm{E}=130,180 \mathrm{MeV}$; measured $\mathrm{E} \alpha$, asymmetry; deduced beam polarization. ${ }^{1} \mathrm{H}$ (polarized $\mathrm{d}$, d), $\mathrm{E}=130,180 \mathrm{MeV}$; measured analyzing powers. REPT CNS-REP-66,P34,Suda

2005TA19 NUCLEAR REACTIONS ${ }^{10} \mathrm{~B},{ }^{16} \mathrm{O}\left(\mathrm{K}^{-}, \pi^{-}\right)$, E at $0.93 \mathrm{GeV} / \mathrm{c}$; ${ }^{11} \mathrm{~B}\left(\pi^{+}, \mathrm{K}^{+}\right)$, E at $1.05 \mathrm{GeV} / \mathrm{c} ;{ }^{7} \mathrm{Li},{ }^{10} \mathrm{~B}\left(\mathrm{~K}^{-}, \gamma\right)$, E at rest; measured $\mathrm{E} \gamma, \mathrm{I} \gamma .{ }^{7} \mathrm{Li},{ }^{9} \mathrm{Be},{ }^{10,11} \mathrm{~B},{ }^{16} \mathrm{O}$ deduced hypernucleus levels, J, $\pi$. Hyperball array. JOUR NUPAB $75458 \mathrm{c}$

2005WAZW NUCLEAR REACTIONS $\mathrm{Si}\left({ }^{6} \mathrm{Li}, \mathrm{X}\right),\left({ }^{7} \mathrm{Be}, \mathrm{X}\right),\left({ }^{10} \mathrm{~B}, \mathrm{X}\right),\left({ }^{9} \mathrm{C}, \mathrm{X}\right),\left({ }^{10} \mathrm{C}\right.$, $\mathrm{X}),\left({ }^{11} \mathrm{C}, \mathrm{X}\right),\left({ }^{12} \mathrm{~N}, \mathrm{X}\right),\left({ }^{13} \mathrm{O}, \mathrm{X}\right),\left({ }^{15} \mathrm{O}, \mathrm{X}\right),\left({ }^{17} \mathrm{Ne}, \mathrm{X}\right), \mathrm{E}=15-53 \mathrm{MeV} /$ nucleon; measured reaction and proton-removal $\sigma .{ }^{6} \mathrm{Li},{ }^{7} \mathrm{Be},{ }^{10} \mathrm{~B}$, ${ }^{9,10,11} \mathrm{C},{ }^{12} \mathrm{~N},{ }^{13,15} \mathrm{O},{ }^{17} \mathrm{Ne}$ deduced radii. Comparisons with model predictions. PREPRINT nucl-ex/0507025,7/18/2005

${ }^{10} \mathrm{C} \quad 2005 \mathrm{BA} 40$ NUCLEAR REACTIONS ${ }^{1} \mathrm{H}\left({ }^{16} \mathrm{O}, \mathrm{X}\right){ }^{1} \mathrm{H} /{ }^{2} \mathrm{H} /{ }^{3} \mathrm{H} /{ }^{3} \mathrm{He} /{ }^{4} \mathrm{He} /{ }^{5} \mathrm{He}$ $/{ }^{6} \mathrm{He} /{ }^{5} \mathrm{Li} /{ }^{6} \mathrm{Li} /{ }^{7} \mathrm{Li} /{ }^{8} \mathrm{Li} /{ }^{7} \mathrm{Be} /{ }^{8} \mathrm{Be} /{ }^{9} \mathrm{Be} /{ }^{10} \mathrm{Be} /{ }^{9} \mathrm{~B} /{ }^{10} \mathrm{~B} /$ ${ }^{11} \mathrm{~B} /{ }^{12} \mathrm{~B} /{ }^{10} \mathrm{C} /{ }^{11} \mathrm{C} /{ }^{12} \mathrm{C} /{ }^{13} \mathrm{C} /{ }^{14} \mathrm{C} /{ }^{13} \mathrm{~N} /{ }^{14} \mathrm{~N} /{ }^{15} \mathrm{~N} /{ }^{14} \mathrm{O} /$ ${ }^{15} \mathrm{O} /{ }^{16} \mathrm{O}$, E at $3.25 \mathrm{GeV} / \mathrm{c} /$ nucleon; measured production $\sigma$. JOUR PZETA 81174

2005J012 NUCLEAR REACTIONS ${ }^{1} \mathrm{H}\left({ }^{10} \mathrm{C},{ }^{10} \mathrm{C}\right),\left({ }^{10} \mathrm{C},{ }^{10} \mathrm{C}^{\prime}\right), \mathrm{E}=45.3 \mathrm{MeV} /$ nucleon; ${ }^{1} \mathrm{H}\left({ }^{11} \mathrm{C},{ }^{11} \mathrm{C}\right),\left({ }^{11} \mathrm{C},{ }^{11} \mathrm{C}\right), \mathrm{E}=40.6 \mathrm{MeV} /$ nucleon; ${ }^{1} \mathrm{H}\left({ }^{12} \mathrm{C}\right.$, $\left.{ }^{12} \mathrm{C}\right),\left({ }^{12} \mathrm{C},{ }^{12} \mathrm{C}^{\prime}\right), \mathrm{E}=36.3 \mathrm{MeV} /$ nucleon; measured elastic and inelastic $\sigma(\theta) .{ }^{10,11} \mathrm{C}$ deduced radii, transition matrix elements. JOUR PRVCA 72014308

2005SA44 RADIOACTIVITY ${ }^{46} \mathrm{~V}(\mathrm{EC})$; analyzed masses; deduced Q(EC), log ft. ${ }^{10} \mathrm{C},{ }^{14} \mathrm{O},{ }^{22} \mathrm{Mg},{ }^{26 m} \mathrm{Al},{ }^{34} \mathrm{Cl},{ }^{34} \mathrm{Ar},{ }^{38 m} \mathrm{~K},{ }^{42} \mathrm{Sc},{ }^{46} \mathrm{~V},{ }^{50} \mathrm{Mn},{ }^{54} \mathrm{Co},{ }^{74} \mathrm{Rb}$; compiled, analyzed log ft; deduced quark-mixing matrix element. JOUR PRLTA 95102501

2005WAZW NUCLEAR REACTIONS $\mathrm{Si}\left({ }^{6} \mathrm{Li}, \mathrm{X}\right),\left({ }^{7} \mathrm{Be}, \mathrm{X}\right),\left({ }^{10} \mathrm{~B}, \mathrm{X}\right),\left({ }^{9} \mathrm{C}, \mathrm{X}\right),\left({ }^{10} \mathrm{C}\right.$, $\mathrm{X}),\left({ }^{11} \mathrm{C}, \mathrm{X}\right),\left({ }^{12} \mathrm{~N}, \mathrm{X}\right),\left({ }^{13} \mathrm{O}, \mathrm{X}\right),\left({ }^{15} \mathrm{O}, \mathrm{X}\right),\left({ }^{17} \mathrm{Ne}, \mathrm{X}\right), \mathrm{E}=15-53 \mathrm{MeV} /$ nucleon; measured reaction and proton-removal $\sigma .{ }^{6} \mathrm{Li},{ }^{7} \mathrm{Be},{ }^{10} \mathrm{~B}$, ${ }^{9,10,11} \mathrm{C},{ }^{12} \mathrm{~N},{ }^{13,15} \mathrm{O},{ }^{17} \mathrm{Ne}$ deduced radii. Comparisons with model predictions. PREPRINT nucl-ex/0507025,7/18/2005

$$
\mathrm{A}=11
$$

${ }^{11} \mathrm{Li} \quad 2005 \mathrm{BB} 01$

ATOMIC MASSES ${ }^{11} \mathrm{Li}$; measured mass; deduced two-neutron separation energy. ${ }^{11} \mathrm{Be}$; measured mass. Transmission mass spectrometer. JOUR ZAANE 25 s01 31

2005 HI03 RADIOACTIVITY ${ }^{11} \operatorname{Li}\left(\beta^{-}\right),\left(\beta^{-} \mathrm{n}\right)$; measured $\beta$-delayed $\mathrm{E} \gamma$, En, asymmetry following decay of spin-polarized source. ${ }^{10,11}$ Be deduced levels, J, $\pi$, S-factors. Comparison with antisymmetrized molecular dynamics model predictions. JOUR PYLBB 611239

${ }^{11} \mathrm{Be} \quad 2005 \mathrm{BB} 01$ ATOMIC MASSES ${ }^{11} \mathrm{Li}$; measured mass; deduced two-neutron separation energy. ${ }^{11} \mathrm{Be}$; measured mass. Transmission mass spectrometer. JOUR ZAANE 25 s01 31 


\section{$\mathrm{A}=11$ (continued)}

2005HI03 RADIOACTIVITY ${ }^{11} \operatorname{Li}\left(\beta^{-}\right),\left(\beta^{-}\right.$n); measured $\beta$-delayed $\mathrm{E} \gamma$, En, asymmetry following decay of spin-polarized source. ${ }^{10,11}$ Be deduced levels, J, $\pi$, S-factors. Comparison with antisymmetrized molecular dynamics model predictions. JOUR PYLBB 611239

2005PA68 NUCLEAR REACTIONS C $\left({ }^{12} \mathrm{Be}, \mathrm{n}^{11} \mathrm{Be}\right), \mathrm{E}=39.3 \mathrm{MeV} /$ nucleon; measured En, E $\gamma$, projectile-like fragments spectra, relative energy spectra; deduced $\sigma(\mathrm{E}) .{ }^{11} \mathrm{Be}$ deduced excited states. ${ }^{12} \mathrm{Be}$ deduced ground state configuration. Kinematically complete measurement. JOUR ZAANE 25 s01 349

2005PAZV NUCLEAR REACTIONS C $\left({ }^{12} \mathrm{Be},{ }^{11} \mathrm{BeX}\right), \mathrm{E}(\mathrm{cm}) \approx 39.3 \mathrm{MeV}$; measured $\mathrm{E} \gamma, \mathrm{En}$, (particle) $\gamma$-, (particle)n-coin; deduced one-neutron removal $\sigma(\mathrm{E}) .{ }^{11}$ Be levels deduced spectroscopic factors. ${ }^{12} \mathrm{Be}$ deduced ground-state configuration. PREPRINT nucl-ex/0510048,10/16/2005

2005PAZZ NUCLEAR REACTIONS ${ }^{12} \mathrm{C}\left({ }^{12} \mathrm{Be}, \mathrm{n}^{11} \mathrm{Be}\right), \mathrm{E}=41 \mathrm{MeV} /$ nucleon; measured $\mathrm{E} \gamma$, I $\gamma$, particle spectra, $\sigma(\mathrm{E}) .{ }^{11} \mathrm{Be}$ deduced levels. ${ }^{12} \mathrm{Be}$ deduced ground state configuration. CONF Argonne(Nuclei at the Limits),P373,Pain

2005YE05 NUCLEAR REACTIONS ${ }^{9} \mathrm{Be}\left({ }^{6} \mathrm{He},{ }^{6} \mathrm{He}\right),\left({ }^{6} \mathrm{He},{ }^{5} \mathrm{He}\right),\left({ }^{6} \mathrm{He}, \alpha\right),\left({ }^{6} \mathrm{He}\right.$, $\alpha \mathrm{X}),\left({ }^{6} \mathrm{He}, \mathrm{tX}\right), \mathrm{E}=25 \mathrm{MeV} /$ nucleon; measured quasielastic, breakup, and transfer $\sigma(\theta)$. ${ }^{6} \mathrm{He}$ deduced two-triton configuration. JOUR JPGPE 31 S1647

${ }^{11} \mathrm{~B}$ 2004REZY NUCLEAR REACTIONS ${ }^{1} \mathrm{H}\left({ }^{11} \mathrm{C}, \mathrm{p}\right),\left({ }^{11} \mathrm{C}, \alpha\right), \mathrm{E}^{*}=8.7-9.9 \mathrm{MeV}$; measured particle spectra, angular distributions, $\sigma .{ }^{8} \mathrm{Be}(\alpha, \mathrm{p})$, $\mathrm{E}^{*}=8.7-9.9 \mathrm{MeV}$; deduced excitation function, astrophysical reaction rates. REPT ANL-04/22,P3,Rehm

2005BA40 NUCLEAR REACTIONS ${ }^{1} \mathrm{H}\left({ }^{16} \mathrm{O}, \mathrm{X}\right){ }^{1} \mathrm{H} /{ }^{2} \mathrm{H} /{ }^{3} \mathrm{H} /{ }^{3} \mathrm{He} /{ }^{4} \mathrm{He} /{ }^{5} \mathrm{He}$ $/{ }^{6} \mathrm{He} /{ }^{5} \mathrm{Li} /{ }^{6} \mathrm{Li} /{ }^{7} \mathrm{Li} /{ }^{8} \mathrm{Li} /{ }^{7} \mathrm{Be} /{ }^{8} \mathrm{Be} /{ }^{9} \mathrm{Be} /{ }^{10} \mathrm{Be} /{ }^{9} \mathrm{~B} /{ }^{10} \mathrm{~B} /$ ${ }^{11} \mathrm{~B} /{ }^{12} \mathrm{~B} /{ }^{10} \mathrm{C} /{ }^{11} \mathrm{C} /{ }^{12} \mathrm{C} /{ }^{13} \mathrm{C} /{ }^{14} \mathrm{C} /{ }^{13} \mathrm{~N} /{ }^{14} \mathrm{~N} /{ }^{15} \mathrm{~N} /{ }^{14} \mathrm{O} /$ ${ }^{15} \mathrm{O} /{ }^{16} \mathrm{O}$, E at $3.25 \mathrm{GeV} / \mathrm{c} /$ nucleon; measured production $\sigma$. JOUR PZETA 81174

2005 BU33 NUCLEAR REACTIONS ${ }^{11} \mathrm{~B}(\alpha, \alpha),\left(\alpha, \alpha^{\prime}\right), \mathrm{E}=40,50 \mathrm{MeV}$; measured $\mathrm{E} \alpha$, elastic and inelastic $\sigma(\theta)$; deduced optical model parameters. ${ }^{11} \mathrm{~B}$ deduced spectroscopic factors, deformation parameters. JOUR YAFIA 681356

2005 CU06 NUCLEAR REACTIONS ${ }^{7} \mathrm{Li}\left({ }^{7} \mathrm{Li},{ }^{11} \mathrm{~B}\right),\left({ }^{7} \mathrm{Li},{ }^{12} \mathrm{~B}\right), \mathrm{E}=58 \mathrm{MeV} ;{ }^{12} \mathrm{C}$, ${ }^{16} \mathrm{O}\left({ }^{7} \mathrm{Li},{ }^{10} \mathrm{~B}\right), \mathrm{E}=58 \mathrm{MeV}$; measured particle spectra. ${ }^{10,11,12} \mathrm{~B}$ deduced relative yields for $\alpha+\mathrm{Li}$ and $\mathrm{H}+\mathrm{Be}$ decay channels from excited states. JOUR PRVCA 72044320

2005ISZZ NUCLEAR REACTIONS ${ }^{4} \mathrm{He}\left({ }^{8} \mathrm{Li}, \mathrm{n}\right), \mathrm{E}=14.6 \mathrm{MeV} ;{ }^{4} \mathrm{He}\left({ }^{16} \mathrm{~N}, \mathrm{n}\right)$, $\mathrm{E}=32 \mathrm{MeV}$; measured En, excitation energy spectra. ${ }^{16} \mathrm{~N}(\alpha, \mathrm{n}), \mathrm{E}(\mathrm{cm})$ $\approx 1-4.5 \mathrm{MeV} ;{ }^{8} \mathrm{Li}(\alpha, \mathrm{n}), \mathrm{E} \approx 0.5-3 \mathrm{MeV}$; deduced excitation functions. CONF Riken(Origin of Matter) Proc,P316, Ishiyama

2005KAZU NUCLEAR REACTIONS ${ }^{11} \mathrm{~B}(\mathrm{~d}, \mathrm{~d}$ '), $\mathrm{E}=200 \mathrm{MeV}$; measured $\sigma(\mathrm{E}, \theta)$. ${ }^{11} \mathrm{~B}$ levels deduced isoscalar monopole and quadrupole strengths, cluster structure. Comparison with antisymmetrized molecular dynamics model predictions. PREPRINT nucl-ex/0512040,12/25/2005

2005KAZV NUCLEAR REACTIONS ${ }^{11} \mathrm{~B}$ (polarized d, d), (polarized d, d'), E=200 $\mathrm{MeV}$; measured $\sigma(\mathrm{E}, \theta) .{ }^{11} \mathrm{~B}$ deduced levels, $\mathrm{B}(\mathrm{E} 2)$. Comparison with model predictions. REPT CNS-REP-66,P40,Kawabata 


\section{$\mathrm{A}=11$ (continued)}

2005MA45 RADIOACTIVITY ${ }^{5} \mathrm{He},{ }^{11} \mathrm{~B},{ }^{12} \mathrm{C}$; measured proton decay asymmetry parameters from polarized hypernuclei. JOUR NUPAB $754168 \mathrm{c}$

2005ME05 NUCLEAR REACTIONS ${ }^{14} \mathrm{C}\left({ }^{11} \mathrm{~B},{ }^{11} \mathrm{~B}\right),\left({ }^{11} \mathrm{~B},{ }^{14} \mathrm{C}\right), \mathrm{E}=45 \mathrm{MeV}$; measured $\sigma(\mathrm{E}, \theta)$; deduced optical model parameters. ${ }^{14} \mathrm{C}$ levels deduced deformation parameters, single-particle structure. Coupled-channels analysis. JOUR NUPAB 75313

2005MI19 NUCLEAR REACTIONS ${ }^{11} \mathrm{~B}\left(\pi^{+}, \mathrm{K}^{+}\right)$, E not given; measured $\mathrm{E} \gamma, \mathrm{I} \gamma$, DSA. ${ }^{11} \mathrm{~B}$ deduced hypernucleus transitions. Hyperball array, comparison with model predictions. JOUR NUPAB $75475 \mathrm{c}$

2005M004 NUCLEAR REACTIONS ${ }^{12} \mathrm{C}$ (e, e'p), E=379-585 MeV; measured excitation energy spectra, momentum distributions; deduced longitudinal and transverse response functions. ${ }^{12} \mathrm{C}(\mathrm{e}, \mathrm{e} \mathrm{p}), \mathrm{E}=379-585$ $\mathrm{MeV} ;{ }^{12} \mathrm{C}(\gamma, \mathrm{p}), \mathrm{E}=61.8,71.6 \mathrm{MeV}$; analyzed transverse reduced $\sigma$, role of two-body currents. JOUR PRVCA 71014607

2005NIZU NUCLEAR REACTIONS ${ }^{4} \mathrm{He}\left({ }^{8} \mathrm{Li}, \mathrm{n}\right), \mathrm{E}(\mathrm{cm}) \approx 0.5 \mathrm{MeV}$; measured particle spectra. REPT CNS-REP-66,P9,Nishimura

2005PR02 NUCLEAR REACTIONS ${ }^{4} \mathrm{He},{ }^{12} \mathrm{C}$ (polarized e, e'p), $\mathrm{E}=2.261,4.461$

$\mathrm{GeV}$; measured single spin azimuthal asymmetries vs missing momentum, missing energy; deduced final state interaction effects. Comparisons with model predictions. JOUR NUPAB 748357

2005ROZX NUCLEAR REACTIONS ${ }^{12} \mathrm{C}(\mathrm{e}, \mathrm{e}$ 'p), $\mathrm{E}=3.123,3.298 \mathrm{GeV}$; measured electron and proton spectra; deduced nuclear transparency.

PREPRINT nucl-ex/0506007,6/05/2005

2005RU18 NUCLEAR REACTIONS ${ }^{7} \operatorname{Li}\left({ }^{11} \mathrm{~B}, \mathrm{X}\right), \mathrm{E}=44 \mathrm{MeV}$; measured particle spectra, charge distributions. ${ }^{7} \mathrm{Li}\left({ }^{11} \mathrm{~B},{ }^{11} \mathrm{~B}\right),\left({ }^{11} \mathrm{~B},{ }^{11} \mathrm{~B}\right), \mathrm{E}=44 \mathrm{MeV}$; measured $\sigma(\mathrm{E}, \theta) ;{ }^{11} \mathrm{~B}\left({ }^{7} \mathrm{Li},{ }^{7} \mathrm{Li}\right),\left({ }^{7} \mathrm{Li},{ }^{7} \mathrm{Li}\right), \mathrm{E}=34 \mathrm{MeV}$; analyzed $\sigma(\mathrm{E}$, $\theta)$; deduced optical model parameters, transfer channel contributions, reorientation effects. ${ }^{7} \mathrm{Li},{ }^{11} \mathrm{~B}$ deduced deformation parameters. Optical model and coupled-reaction-channels analysis. JOUR PRVCA 72034608

2005SH51 NUCLEAR REACTIONS ${ }^{4} \mathrm{He}(\gamma, \mathrm{p}),(\gamma, \mathrm{n}),(\gamma, \mathrm{np}), \mathrm{E}=21.8-29.8 \mathrm{MeV}$; ${ }^{12} \mathrm{C}(\gamma, \mathrm{p}),(\gamma, \mathrm{n}), \mathrm{E}=22.3-32 \mathrm{MeV}$; measured charged particle spectra, photodisintegration $\sigma, \sigma(\theta)$. Monoenergetic pulsed photons, comparison with previous results and model predictions. JOUR PRVCA 72044004

2005S013 NUCLEAR REACTIONS ${ }^{16} \mathrm{O}\left({ }^{9} \mathrm{Be}, \alpha^{7} \mathrm{Be}\right),{ }^{7} \mathrm{Li}\left({ }^{9} \mathrm{Be}, \alpha^{7} \mathrm{Li}\right),\left({ }^{9} \mathrm{Be}, \mathrm{t} 2 \alpha\right)$, $\mathrm{E}=55,70 \mathrm{MeV}$; measured excitation energy spectra. ${ }^{11} \mathrm{~B},{ }^{11} \mathrm{C}$ deduced excited states energies, configurations. JOUR JPGPE 31 S1701

2005SOZZ NUCLEAR REACTIONS ${ }^{16} \mathrm{O}\left({ }^{9} \mathrm{Be}, \alpha^{7} \mathrm{Be}\right),{ }^{7} \mathrm{Li}\left({ }^{9} \mathrm{Be}, \alpha^{7} \mathrm{Li}\right),\left({ }^{9} \mathrm{Be}, \mathrm{t} 2 \alpha\right)$, $\mathrm{E}=55,70 \mathrm{MeV}$; measured particle spectra. ${ }^{11} \mathrm{C},{ }^{11} \mathrm{~B}$ deduced excited states energies, cluster structure, decay features. PREPRINT nucl-ex/0504026,4/25/2005

2005TA19 NUCLEAR REACTIONS ${ }^{10} \mathrm{~B},{ }^{16} \mathrm{O}\left(\mathrm{K}^{-}, \pi^{-}\right)$, E at $0.93 \mathrm{GeV} / \mathrm{c}$; ${ }^{11} \mathrm{~B}\left(\pi^{+}, \mathrm{K}^{+}\right)$, E at $1.05 \mathrm{GeV} / \mathrm{c} ;{ }^{7} \mathrm{Li},{ }^{10} \mathrm{~B}\left(\mathrm{~K}^{-}, \gamma\right)$, E at rest; measured $\mathrm{E} \gamma, \mathrm{I} \gamma \cdot{ }^{7} \mathrm{Li},{ }^{9} \mathrm{Be},{ }^{10,11} \mathrm{~B},{ }^{16} \mathrm{O}$ deduced hypernucleus levels, $\mathrm{J}, \pi$. Hyperball array. JOUR NUPAB $75458 \mathrm{c}$

${ }^{11} \mathrm{C}$ 2004B047 NUCLEAR REACTIONS ${ }^{12} \mathrm{C}\left(\mathrm{e}, \mathrm{e}^{\prime} \pi^{-} \mathrm{p}\right), \mathrm{E}=855 \mathrm{MeV}$; measured $\Delta$-particle production associated carbon, pion, and proton spectra; deduced medium effects. JOUR FIZBE 13507 


\section{$\mathrm{A}=11$ (continued)}

2004REZY

2005BA40

2005J012

$2005 \mathrm{KI09}$

2005SH51

2005S013

2005SOZZ

${ }^{12} \mathrm{Be} \quad 2005 \mathrm{IMZZ}$
NUCLEAR REACTIONS ${ }^{1} \mathrm{H}\left({ }^{11} \mathrm{C}, \mathrm{p}\right),\left({ }^{11} \mathrm{C}, \alpha\right), \mathrm{E}^{*}=8.7-9.9 \mathrm{MeV}$; measured particle spectra, angular distributions, $\sigma .{ }^{8} \operatorname{Be}(\alpha, \mathrm{p})$, $\mathrm{E}^{*}=8.7-9.9 \mathrm{MeV}$; deduced excitation function, astrophysical reaction rates. REPT ANL-04/22,P3,Rehm NUCLEAR REACTIONS ${ }^{1} \mathrm{H}\left({ }^{16} \mathrm{O}, \mathrm{X}\right){ }^{1} \mathrm{H} /{ }^{2} \mathrm{H} /{ }^{3} \mathrm{H} /{ }^{3} \mathrm{He} /{ }^{4} \mathrm{He} /{ }^{5} \mathrm{He}$ $/{ }^{6} \mathrm{He} /{ }^{5} \mathrm{Li} /{ }^{6} \mathrm{Li} /{ }^{7} \mathrm{Li} /{ }^{8} \mathrm{Li} /{ }^{7} \mathrm{Be} /{ }^{8} \mathrm{Be} /{ }^{9} \mathrm{Be} /{ }^{10} \mathrm{Be} /{ }^{9} \mathrm{~B} /{ }^{10} \mathrm{~B} /$ ${ }^{11} \mathrm{~B} /{ }^{12} \mathrm{~B} /{ }^{10} \mathrm{C} /{ }^{11} \mathrm{C} /{ }^{12} \mathrm{C} /{ }^{13} \mathrm{C} /{ }^{14} \mathrm{C} /{ }^{13} \mathrm{~N} /{ }^{14} \mathrm{~N} /{ }^{15} \mathrm{~N} /{ }^{14} \mathrm{O} /$ ${ }^{15} \mathrm{O} /{ }^{16} \mathrm{O}$, E at $3.25 \mathrm{GeV} / \mathrm{c} /$ nucleon; measured production $\sigma$. JOUR PZETA 81174 NUCLEAR REACTIONS ${ }^{12} \mathrm{C}\left(\gamma, \pi^{-} \mathrm{p}\right),\left(\gamma, \pi^{-} 2 \mathrm{p}\right), \mathrm{E}=500 \mathrm{MeV}$ bremsstrahlung; measured Ep, pion spectra, $\sigma(\mathrm{E}, \theta)$; deduced reaction mechanism features. JOUR PZETA 81546 NUCLEAR REACTIONS ${ }^{1} \mathrm{H}\left({ }^{10} \mathrm{C},{ }^{10} \mathrm{C}\right),\left({ }^{10} \mathrm{C},{ }^{10} \mathrm{C}^{\prime}\right), \mathrm{E}=45.3 \mathrm{MeV} /$ nucleon; ${ }^{1} \mathrm{H}\left({ }^{11} \mathrm{C},{ }^{11} \mathrm{C}\right),\left({ }^{11} \mathrm{C},{ }^{11} \mathrm{C}\right), \mathrm{E}=40.6 \mathrm{MeV} /$ nucleon; ${ }^{1} \mathrm{H}\left({ }^{12} \mathrm{C}\right.$, $\left.{ }^{12} \mathrm{C}\right),\left({ }^{12} \mathrm{C},{ }^{12} \mathrm{C}^{\prime}\right), \mathrm{E}=36.3 \mathrm{MeV} /$ nucleon; measured elastic and inelastic $\sigma(\theta) .{ }^{10,11} \mathrm{C}$ deduced radii, transition matrix elements. JOUR PRVCA 72014308

NUCLEAR REACTIONS ${ }^{12} \mathrm{C}(\mathrm{p}, \mathrm{d}), \mathrm{E}=45 \mathrm{MeV}$; measured deuteron spectra, $\sigma(\mathrm{E}, \theta)$. JOUR KPSJA 461318

NUCLEAR REACTIONS ${ }^{4} \mathrm{He}(\gamma, \mathrm{p}),(\gamma, \mathrm{n}),(\gamma, \mathrm{np}), \mathrm{E}=21.8-29.8 \mathrm{MeV}$; ${ }^{12} \mathrm{C}(\gamma, \mathrm{p}),(\gamma, \mathrm{n}), \mathrm{E}=22.3-32 \mathrm{MeV}$; measured charged particle spectra, photodisintegration $\sigma, \sigma(\theta)$. Monoenergetic pulsed photons, comparison with previous results and model predictions. JOUR PRVCA 72044004 NUCLEAR REACTIONS ${ }^{16} \mathrm{O}\left({ }^{9} \mathrm{Be}, \alpha^{7} \mathrm{Be}\right),{ }^{7} \mathrm{Li}\left({ }^{9} \mathrm{Be}, \alpha^{7} \mathrm{Li}\right),\left({ }^{9} \mathrm{Be}, \mathrm{t} 2 \alpha\right)$, $\mathrm{E}=55,70 \mathrm{MeV}$; measured excitation energy spectra. ${ }^{11} \mathrm{~B},{ }^{11} \mathrm{C}$ deduced excited states energies, configurations. JOUR JPGPE 31 S1701

NUCLEAR REACTIONS ${ }^{16} \mathrm{O}\left({ }^{9} \mathrm{Be}, \alpha^{7} \mathrm{Be}\right),{ }^{7} \mathrm{Li}\left({ }^{9} \mathrm{Be}, \alpha^{7} \mathrm{Li}\right),\left({ }^{9} \mathrm{Be}, \mathrm{t} 2 \alpha\right)$, $\mathrm{E}=55,70 \mathrm{MeV}$; measured particle spectra. ${ }^{11} \mathrm{C},{ }^{11} \mathrm{~B}$ deduced excited states energies, cluster structure, decay features. PREPRINT nucl-ex/0504026,4/25/2005

NUCLEAR REACTIONS Si $\left({ }^{6} \mathrm{Li}, \mathrm{X}\right),\left({ }^{7} \mathrm{Be}, \mathrm{X}\right),\left({ }^{10} \mathrm{~B}, \mathrm{X}\right),\left({ }^{9} \mathrm{C}, \mathrm{X}\right),\left({ }^{10} \mathrm{C}\right.$, $\mathrm{X}),\left({ }^{11} \mathrm{C}, \mathrm{X}\right),\left({ }^{12} \mathrm{~N}, \mathrm{X}\right),\left({ }^{13} \mathrm{O}, \mathrm{X}\right),\left({ }^{15} \mathrm{O}, \mathrm{X}\right),\left({ }^{17} \mathrm{Ne}, \mathrm{X}\right), \mathrm{E}=15-53 \mathrm{MeV} /$ nucleon; measured reaction and proton-removal $\sigma \cdot{ }^{6} \mathrm{Li},{ }^{7} \mathrm{Be},{ }^{10} \mathrm{~B}$, ${ }^{9,10,11} \mathrm{C},{ }^{12} \mathrm{~N},{ }^{13,15} \mathrm{O},{ }^{17} \mathrm{Ne}$ deduced radii. Comparisons with model predictions. PREPRINT nucl-ex/0507025,7/18/2005

$$
\mathrm{A}=12
$$

NUCLEAR REACTIONS ${ }^{197} \mathrm{Au}\left({ }^{12} \mathrm{Be},{ }^{12} \mathrm{Be}\right), \mathrm{E}=40.3 \mathrm{MeV} /$ nucleon; measured $\mathrm{E} \gamma, \mathrm{I} \gamma$, (particle) $\gamma$-coin, DSA following projectile Coulomb excitation. ${ }^{12}$ Be deduced transition. REPT RIKEN 2004

Annual,P41,Imai

NUCLEAR REACTIONS C( $\left.{ }^{12} \mathrm{Be}, \mathrm{n}^{11} \mathrm{Be}\right), \mathrm{E}=39.3 \mathrm{MeV} /$ nucleon; measured En, E $\gamma$, projectile-like fragments spectra, relative energy spectra; deduced $\sigma(\mathrm{E}) .{ }^{11}$ Be deduced excited states. ${ }^{12}$ Be deduced ground state configuration. Kinematically complete measurement. JOUR ZAANE 25 s01 349 


\section{$\mathrm{A}=12$ (continued)}

2005PAZV NUCLEAR REACTIONS $\mathrm{C}\left({ }^{12} \mathrm{Be},{ }^{11} \mathrm{BeX}\right), \mathrm{E}(\mathrm{cm}) \approx 39.3 \mathrm{MeV}$; measured $\mathrm{E} \gamma$, En, (particle) $\gamma$-, (particle)n-coin; deduced one-neutron removal $\sigma(\mathrm{E}) .{ }^{11}$ Be levels deduced spectroscopic factors. ${ }^{12}$ Be deduced ground-state configuration. PREPRINT nucl-ex/0510048,10/16/2005

2005PAZZ NUCLEAR REACTIONS ${ }^{12} \mathrm{C}\left({ }^{12} \mathrm{Be}, \mathrm{n}^{11} \mathrm{Be}\right), \mathrm{E}=41 \mathrm{MeV} /$ nucleon; measured $\mathrm{E} \gamma, \mathrm{I} \gamma$, particle spectra, $\sigma(\mathrm{E}) .{ }^{11} \mathrm{Be}$ deduced levels. ${ }^{12} \mathrm{Be}$ deduced ground state configuration. CONF Argonne(Nuclei at the Limits),P373,Pain

${ }^{12} \mathrm{~B} \quad 2004 \mathrm{FU} 34$ NUCLEAR REACTIONS C(e, e' $\left.{ }^{+}\right), \mathrm{E}=1.8 \mathrm{GeV}$; measured missing mass spectrum. ${ }^{12} \mathrm{~B}$ deduced hypernucleus excited states. JOUR FIZBE 13645

2005BA40 NUCLEAR REACTIONS ${ }^{1} \mathrm{H}\left({ }^{16} \mathrm{O}, \mathrm{X}\right){ }^{1} \mathrm{H} /{ }^{2} \mathrm{H} /{ }^{3} \mathrm{H} /{ }^{3} \mathrm{He} /{ }^{4} \mathrm{He} /{ }^{5} \mathrm{He}$ $/{ }^{6} \mathrm{He} /{ }^{5} \mathrm{Li} /{ }^{6} \mathrm{Li} /{ }^{7} \mathrm{Li} /{ }^{8} \mathrm{Li} /{ }^{7} \mathrm{Be} /{ }^{8} \mathrm{Be} /{ }^{9} \mathrm{Be} /{ }^{10} \mathrm{Be} /{ }^{9} \mathrm{~B} /{ }^{10} \mathrm{~B} /$ ${ }^{11} \mathrm{~B} /{ }^{12} \mathrm{~B} /{ }^{10} \mathrm{C} /{ }^{11} \mathrm{C} /{ }^{12} \mathrm{C} /{ }^{13} \mathrm{C} /{ }^{14} \mathrm{C} /{ }^{13} \mathrm{~N} /{ }^{14} \mathrm{~N} /{ }^{15} \mathrm{~N} /{ }^{14} \mathrm{O} /$ ${ }^{15} \mathrm{O} /{ }^{16} \mathrm{O}$, E at $3.25 \mathrm{GeV} / \mathrm{c} /$ nucleon; measured production $\sigma$. JOUR PZETA 81174

2005 CU06 NUCLEAR REACTIONS ${ }^{7} \mathrm{Li}\left({ }^{7} \mathrm{Li},{ }^{11} \mathrm{~B}\right),\left({ }^{7} \mathrm{Li},{ }^{12} \mathrm{~B}\right), \mathrm{E}=58 \mathrm{MeV} ;{ }^{12} \mathrm{C}$, ${ }^{16} \mathrm{O}\left({ }^{7} \mathrm{Li},{ }^{10} \mathrm{~B}\right), \mathrm{E}=58 \mathrm{MeV}$; measured particle spectra. ${ }^{10,11,12} \mathrm{~B}$ deduced relative yields for $\alpha+\mathrm{Li}$ and $\mathrm{H}+\mathrm{Be}$ decay channels from excited states. JOUR PRVCA 72044320

2005DI16 RADIOACTIVITY ${ }^{12} \mathrm{~B}\left(\beta^{-}\right),\left(\beta^{-} 3 \alpha\right)$ [from Ta(p, X)]; measured $\beta$-delayed $\mathrm{E} \alpha, \alpha \alpha$-coin. ${ }^{12} \mathrm{C}$ deduced excited states, J, $\pi$. R-matrix analysis. JOUR NUPAB 7603

2005GA09 NUCLEAR REACTIONS ${ }^{9} \mathrm{Be},{ }^{12} \mathrm{C}\left(\mathrm{e}, \mathrm{e} \mathrm{K}^{+}\right), \mathrm{E}=4 \mathrm{GeV}$; measured hypernucleus production associated missing energy spectra. JOUR ZAANE 24 s01 91

2005KA06 NUCLEAR REACTIONS ${ }^{1} \mathrm{H}\left({ }^{17} \mathrm{~B}, \mathrm{X}\right){ }^{17} \mathrm{~B} /{ }^{15} \mathrm{~B} /{ }^{14} \mathrm{~B} /{ }^{13} \mathrm{~B} /{ }^{12} \mathrm{~B}, \mathrm{E} \approx$ $43 \mathrm{MeV} /$ nucleon; measured $\mathrm{E} \gamma, \mathrm{I} \gamma$, (particle) $\gamma$-coin, relative yields.

${ }^{15,17} \mathrm{~B}$ deduced levels, $\mathrm{J}, \pi$. Comparison with model predictions. JOUR PYLBB 608206

2005K013 NUCLEAR REACTIONS ${ }^{12} \mathrm{C}\left({ }^{17} \mathrm{~B},{ }^{17} \mathrm{~B}^{\prime}\right),\left({ }^{17} \mathrm{~B},{ }^{15} \mathrm{BX}\right),\left({ }^{15} \mathrm{~B},{ }^{15} \mathrm{~B}^{\prime}\right)$, $\left({ }^{17} \mathrm{~B},{ }^{14} \mathrm{BX}\right),\left({ }^{17} \mathrm{~B},{ }^{12} \mathrm{BX}\right),\left({ }^{15} \mathrm{~B},{ }^{14} \mathrm{BX}\right),\left({ }^{15} \mathrm{~B},{ }^{12} \mathrm{BX}\right), \mathrm{E} \approx 70 \mathrm{MeV} /$ nucleon; measured $\mathrm{E} \gamma$, I $\gamma$, (particle) $\gamma$-coin. ${ }^{12} \mathrm{C}\left({ }^{17} \mathrm{~B},{ }^{17} \mathrm{~B}^{\prime}\right),\left({ }^{15} \mathrm{~B},{ }^{15} \mathrm{~B}^{\prime}\right)$, $\mathrm{E} \approx 70 \mathrm{MeV}$; measured $\sigma(\mathrm{E}, \theta) .{ }^{15,17} \mathrm{~B}$ deduced levels, transitions, quadrupole deformation lengths. ${ }^{12,14} \mathrm{~B}$ deduced transitions. JOUR PRVCA 71044611

${ }^{12} \mathrm{C}$ 2005AG04 NUCLEAR REACTIONS ${ }^{6,7} \mathrm{Li},{ }^{12} \mathrm{C},{ }^{27} \mathrm{Al},{ }^{51} \mathrm{~V}\left(\mathrm{~K}^{-}, \pi^{-} \mathrm{X}\right), \mathrm{E}$ at rest; measured hypernucleus production associated mass spectra; deduced hypernucleus decay features. ${ }^{12} \mathrm{C}$ deduced hypernucleus binding energies. JOUR NUPAB 752 139c

2005 AG09 NUCLEAR REACTIONS ${ }^{6,7} \mathrm{Li},{ }^{12} \mathrm{C},{ }^{27} \mathrm{Al},{ }^{51} \mathrm{~V}\left(\mathrm{~K}^{-}, \pi^{-} \mathrm{X}\right)$, E at rest; measured hypernucleus production associated mass spectra; deduced hypernucleus decay features. ${ }^{12} \mathrm{C}$ deduced hypernucleus ground and excited state energies. JOUR NUPAB $754399 \mathrm{c}$

2005AG11 NUCLEAR REACTIONS ${ }^{12} \mathrm{C}\left(\mathrm{K}^{-}, \pi^{-}\right)$, E at rest; measured hypernucleus production associated excitation energy spectra. ${ }^{12} \mathrm{C}$ deduced hyperon binding energies. JOUR PYLBB 62235 


\section{$\mathrm{A}=12$ (continued)}

2005AL37 NUCLEAR REACTIONS ${ }^{12} \mathrm{C}\left({ }^{3} \mathrm{He}, \mathrm{t} \pi^{+}\right), \mathrm{E}=2 \mathrm{GeV}$; measured excitation energy spectra. ${ }^{1} \mathrm{H}\left(\mathrm{d}, \mathrm{d}^{\prime} \mathrm{X}\right),\left(\alpha, \alpha^{\prime} \mathrm{X}\right), \mathrm{E} \approx 1 \mathrm{GeV} /$ nucleon; measured missing mass spectra. JOUR NIMAE 551290

2005BA40 NUCLEAR REACTIONS ${ }^{1} \mathrm{H}\left({ }^{16} \mathrm{O}, \mathrm{X}\right){ }^{1} \mathrm{H} /{ }^{2} \mathrm{H} /{ }^{3} \mathrm{H} /{ }^{3} \mathrm{He} /{ }^{4} \mathrm{He} /{ }^{5} \mathrm{He}$ $/{ }^{6} \mathrm{He} /{ }^{5} \mathrm{Li} /{ }^{6} \mathrm{Li} /{ }^{7} \mathrm{Li} /{ }^{8} \mathrm{Li} /{ }^{7} \mathrm{Be} /{ }^{8} \mathrm{Be} /{ }^{9} \mathrm{Be} /{ }^{10} \mathrm{Be} /{ }^{9} \mathrm{~B} /{ }^{10} \mathrm{~B} /$ ${ }^{11} \mathrm{~B} /{ }^{12} \mathrm{~B} /{ }^{10} \mathrm{C} /{ }^{11} \mathrm{C} /{ }^{12} \mathrm{C} /{ }^{13} \mathrm{C} /{ }^{14} \mathrm{C} /{ }^{13} \mathrm{~N} /{ }^{14} \mathrm{~N} /{ }^{15} \mathrm{~N} /{ }^{14} \mathrm{O} /$ ${ }^{15} \mathrm{O} /{ }^{16} \mathrm{O}$, E at $3.25 \mathrm{GeV} / \mathrm{c} /$ nucleon; measured production $\sigma$. JOUR PZETA 81174

2005B022 NUCLEAR REACTIONS ${ }^{12} \mathrm{C}\left({ }^{3} \mathrm{He}, \mathrm{t} \pi \pi^{+}\right), \mathrm{E}=2 \mathrm{GeV} ;{ }^{12} \mathrm{C}\left({ }^{12} \mathrm{C},{ }^{12} \mathrm{~N} \pi^{-}\right)$, $\mathrm{E}=1.1 \mathrm{GeV} /$ nucleon; measured $\sigma(\mathrm{E}, \theta)$. JOUR NUPAB $755507 \mathrm{c}$

2005DA42 NUCLEAR REACTIONS ${ }^{12} \mathrm{C}\left({ }^{132} \mathrm{Te},{ }^{132} \mathrm{Te}\right),\left({ }^{130} \mathrm{Te},{ }^{130} \mathrm{Te}\right),\left({ }^{126} \mathrm{Te}\right.$, ${ }^{126} \mathrm{Te}$ '), $\left({ }^{122} \mathrm{Te},{ }^{122} \mathrm{Te}\right.$ '), $\mathrm{E}=3 \mathrm{MeV} /$ nucleon; measured $\mathrm{E} \gamma, \mathrm{I} \gamma(\theta)$, (particle) $\gamma$-coin following projectile Coulomb excitation. ${ }^{132} \mathrm{Te}$ level deduced g-factor. Recoil-in-vacuum technique. JOUR NIMBE 241971

2005DI16 RADIOACTIVITY ${ }^{12} \mathrm{~B}\left(\beta^{-}\right),\left(\beta^{-} 3 \alpha\right)$ [from Ta(p, X)]; measured $\beta$-delayed $\mathrm{E} \alpha, \alpha \alpha$-coin. ${ }^{12} \mathrm{C}$ deduced excited states, J, $\pi$. R-matrix analysis. JOUR NUPAB 7603

2005 G036 ATOMIC MASSES ${ }^{12} \mathrm{C},{ }^{16} \mathrm{O},{ }^{20} \mathrm{Ne},{ }^{32} \mathrm{~S},{ }^{36,40} \mathrm{Ar}$; measured masses. Cyclotron-based mass spectrometry. JOUR JPGPE 31 S1869

2005GR25 NUCLEAR REACTIONS ${ }^{64} \mathrm{Ni}\left({ }^{132} \mathrm{Sn}, \mathrm{X}\right),\left({ }^{134} \mathrm{Sn}, \mathrm{X}\right), \mathrm{E}=450-620 \mathrm{MeV}$; measured fusion $\sigma$. C $\left({ }^{130} \mathrm{Te},{ }^{130} \mathrm{Te}\right),\left({ }^{132} \mathrm{Te},{ }^{132} \mathrm{Te}\right), \mathrm{E}=3 \mathrm{MeV} /$ nucleon; measured $\mathrm{E} \gamma, \mathrm{I} \gamma$, (particle) $\gamma$-coin following projectile Coulomb excitation. ${ }^{132} \mathrm{Te}$ level deduced g factor. ${ }^{13} \mathrm{C}\left({ }^{134} \mathrm{Te},{ }^{135} \mathrm{Te}\right), \mathrm{E}=550$ $\mathrm{MeV}$; measured $\mathrm{E} \gamma, \mathrm{I} \gamma .{ }^{135} \mathrm{Te}$ level deduced J, $\pi$. JOUR JPGPE 31 S1639

2005KA23 NUCLEAR REACTIONS ${ }^{6} \mathrm{Li},{ }^{12} \mathrm{C}\left(\pi^{+}, \mathrm{K}^{+}\right),\left(\pi^{+}, \mathrm{pX}\right)$, E at $1.05 \mathrm{GeV}$ / c; measured excitation energy spectra, proton spectra following hypernucleus decay. ${ }^{5} \mathrm{He}$ deduced hypernucleus decay width. JOUR NUPAB $754173 \mathrm{c}$

2005KN02 RADIOACTIVITY ${ }^{13} \mathrm{O}\left(\beta^{+} \mathrm{p}\right)$ [from ${ }^{14} \mathrm{~N}(\mathrm{p}, 2 \mathrm{n})$ ]; measured $\beta$-delayed $\mathrm{Ep}$, Ip; deduced log $\mathrm{ft} .{ }^{13} \mathrm{~N}$ deduced branching ratios for proton decay from excited states. JOUR PRVCA 72044312

2005K013 NUCLEAR REACTIONS ${ }^{12} \mathrm{C}\left({ }^{17} \mathrm{~B},{ }^{17} \mathrm{~B}^{\prime}\right),\left({ }^{17} \mathrm{~B},{ }^{15} \mathrm{BX}\right),\left({ }^{15} \mathrm{~B},{ }^{15} \mathrm{~B}^{\prime}\right)$, $\left({ }^{17} \mathrm{~B},{ }^{14} \mathrm{BX}\right),\left({ }^{17} \mathrm{~B},{ }^{12} \mathrm{BX}\right),\left({ }^{15} \mathrm{~B},{ }^{14} \mathrm{BX}\right),\left({ }^{15} \mathrm{~B},{ }^{12} \mathrm{BX}\right), \mathrm{E} \approx 70 \mathrm{MeV} /$ nucleon; measured $\mathrm{E} \gamma, \mathrm{I} \gamma$, (particle) $\gamma$-coin. ${ }^{12} \mathrm{C}\left({ }^{17} \mathrm{~B},{ }^{17} \mathrm{~B}^{\prime}\right),\left({ }^{15} \mathrm{~B},{ }^{15} \mathrm{~B}^{\prime}\right)$, $\mathrm{E} \approx 70 \mathrm{MeV}$; measured $\sigma(\mathrm{E}, \theta) .{ }^{15,17} \mathrm{~B}$ deduced levels, transitions, quadrupole deformation lengths. ${ }^{12,14} \mathrm{~B}$ deduced transitions. JOUR PRVCA 71044611

$2005 \mathrm{KU} 36$ NUCLEAR REACTIONS ${ }^{15} \mathrm{~N}(\mathrm{p}, \alpha \gamma), \mathrm{E} \approx 429,897 \mathrm{keV}$; measured $\gamma$-ray yields for nitrogen in various materials; deduced depth profiles. JOUR NIMBE 240704

2005MA45 RADIOACTIVITY ${ }^{5} \mathrm{He},{ }^{11} \mathrm{~B},{ }^{12} \mathrm{C}$; measured proton decay asymmetry parameters from polarized hypernuclei. JOUR NUPAB $754168 \mathrm{c}$

$20050 K 02$ NUCLEAR REACTIONS ${ }^{6} \mathrm{Li}\left(\pi^{+}, \mathrm{K}^{+} \mathrm{p}\right),{ }^{12} \mathrm{C}\left(\pi^{+}, \mathrm{K}^{+}\right)$, E at $1.05 \mathrm{GeV}$ / c; measured nucleon-nucleon pair spectra, yields following hypernucleus decay; deduced hyperon decay widths. JOUR NUPAB $752196 \mathrm{c}$ 


\section{$\mathrm{A}=12$ (continued)}

$20050 K 04 \quad$ NUCLEAR REACTIONS ${ }^{6} \mathrm{Li},{ }^{12} \mathrm{C}\left(\pi^{+}, \mathrm{K}^{+}\right)$, E at $1.05 \mathrm{GeV} / \mathrm{c}$; measured excitation energy spectra, $\gamma$-spectra from neutral pion decay. ${ }^{5} \mathrm{He},{ }^{12} \mathrm{C}$ deduced hypernucleus decay branching ratios. JOUR NUPAB $754178 \mathrm{c}$

$20050 U 02$ NUCLEAR REACTIONS ${ }^{6} \mathrm{Li},{ }^{12} \mathrm{C}\left(\pi^{+}, \mathrm{K}^{+}\right)$, E not given; measured hypernucleus excitation energy spectra, nn-, np-coin following hypernucleus decay. ${ }^{5} \mathrm{He},{ }^{12} \mathrm{C}$ deduced hypernucleus decay widths, branching ratios. JOUR NUPAB $754157 \mathrm{c}$

2005PAZZ NUCLEAR REACTIONS ${ }^{12} \mathrm{C}\left({ }^{12} \mathrm{Be}, \mathrm{n}^{11} \mathrm{Be}\right), \mathrm{E}=41 \mathrm{MeV} /$ nucleon; measured $\mathrm{E} \gamma, \mathrm{I} \gamma$, particle spectra, $\sigma(\mathrm{E}) .{ }^{11} \mathrm{Be}$ deduced levels. ${ }^{12} \mathrm{Be}$ deduced ground state configuration. CONF Argonne(Nuclei at the Limits),P373,Pain

2005R029 NUCLEAR REACTIONS ${ }^{12} \mathrm{C}$ (polarized n, n), E=2.2-8.5 MeV; measured $\operatorname{Ay}(\theta)$. Comparison with previous data and model predictions. JOUR PRVCA 72024605

2005SA03 NUCLEAR REACTIONS ${ }^{10} \mathrm{~B}\left(\pi^{-}, \mathrm{K}^{+}\right),{ }^{10} \mathrm{~B},{ }^{12} \mathrm{C}\left(\pi^{+}, \mathrm{K}^{+}\right)$, E at 1.05, $1.2 \mathrm{GeV} / \mathrm{c}$; measured missing mass spectra, hypernucleus production $\sigma$. JOUR PRLTA 94052502

2005SA04 NUCLEAR REACTIONS ${ }^{12} \mathrm{C},{ }^{28} \mathrm{Si},{ }^{27} \mathrm{Al}, \mathrm{Fe}\left(\pi^{+}, \mathrm{K}^{+}\right)$, E at $1.06 \mathrm{GeV}$ / c; measured hypernucleus mass spectra, pion and proton spectra following hypernucleus decay. ${ }^{12} \mathrm{C},{ }^{28} \mathrm{Si},{ }^{27} \mathrm{Al}$, Fe deduced mesonic and nonmesonic hypernucleus decay widths. Comparison with model predictions. JOUR PRVCA 71025203

2005SAZX NUCLEAR REACTIONS ${ }^{12} \mathrm{C}\left({ }^{24} \mathrm{Mg},{ }^{20} \mathrm{Ne}\right),\left({ }^{24} \mathrm{Mg}, 2{ }^{12} \mathrm{C}\right), \mathrm{E}=130$ $\mathrm{MeV}$; measured $\mathrm{E} \gamma, \mathrm{I} \gamma$, (particle) $\gamma-$, (particle)(particle)-coin. ${ }^{16} \mathrm{O}$ deduced transitions, branching ratio. Euroball IV array. CONF Bormio (XLIII Winter Meeting) Proc,P224

$2005 \mathrm{~S} 014$ NUCLEAR REACTIONS ${ }^{12} \mathrm{C}\left({ }^{6} \mathrm{Li}, \mathrm{d} \alpha\right), \mathrm{E}=26 \mathrm{MeV} ;{ }^{59} \mathrm{Co}\left({ }^{6} \mathrm{Li}, \mathrm{d} \alpha\right)$, $\mathrm{E}=30 \mathrm{MeV}$; measured particle spectra, $\sigma(\theta(\alpha), \theta(\mathrm{d}))$, three-body final state correlations; deduced reaction mechanism features. JOUR BJPHE 35888

${ }^{12} \mathrm{~N} \quad 2005 \mathrm{LI} 40$ NUCLEAR REACTIONS ${ }^{2} \mathrm{H}\left({ }^{7} \mathrm{Be}, \mathrm{n}\right),\left({ }^{11} \mathrm{C}, \mathrm{n}\right),\left({ }^{8} \mathrm{Li}, \mathrm{p}\right), \mathrm{E} \approx 5.8-9.8$ $\mathrm{MeV}$; measured $\sigma(\theta)$, total $\sigma$; deduced astrophysical S-factors. JOUR NUPAB $758110 \mathrm{c}$

2005WAZW NUCLEAR REACTIONS Si $\left({ }^{6} \mathrm{Li}, \mathrm{X}\right),\left({ }^{7} \mathrm{Be}, \mathrm{X}\right),\left({ }^{10} \mathrm{~B}, \mathrm{X}\right),\left({ }^{9} \mathrm{C}, \mathrm{X}\right),\left({ }^{10} \mathrm{C}\right.$, $\mathrm{X}),\left({ }^{11} \mathrm{C}, \mathrm{X}\right),\left({ }^{12} \mathrm{~N}, \mathrm{X}\right),\left({ }^{13} \mathrm{O}, \mathrm{X}\right),\left({ }^{15} \mathrm{O}, \mathrm{X}\right),\left({ }^{17} \mathrm{Ne}, \mathrm{X}\right), \mathrm{E}=15-53 \mathrm{MeV} /$ nucleon; measured reaction and proton-removal $\sigma .{ }^{6} \mathrm{Li},{ }^{7} \mathrm{Be},{ }^{10} \mathrm{~B}$, ${ }^{9,10,11} \mathrm{C},{ }^{12} \mathrm{~N},{ }^{13,15} \mathrm{O},{ }^{17} \mathrm{Ne}$ deduced radii. Comparisons with model predictions. PREPRINT nucl-ex/0507025,7/18/2005

$$
\mathrm{A}=13
$$

${ }^{13} \mathrm{~B} \quad 2004 \mathrm{GU} 21$

NUCLEAR REACTIONS ${ }^{9} \mathrm{Be}\left({ }^{14} \mathrm{~B},{ }^{13} \mathrm{BX}\right), \mathrm{E}=60 \mathrm{MeV} /$ nucleon; measured $\mathrm{E} \gamma, \mathrm{I} \gamma$, particle momentum distribution, $\sigma(\mathrm{E}) .{ }^{13} \mathrm{~B}$ deduced levels, $\mathrm{J}, \pi$, asymptotic normalization coefficients. ${ }^{2} \mathrm{H}\left({ }^{8} \mathrm{~B}, \alpha\right), \mathrm{E}=28.5$ $\mathrm{MeV}$; measured E $\alpha$. JOUR BJPHE 341012

2005GEZY NUCLEAR REACTIONS ${ }^{11} \mathrm{~B}(\mathrm{t}, \mathrm{p}), \mathrm{E}=2.6-7 \mathrm{MeV}$; measured $\sigma$. CONF St Petersburg,P172,Generalov 


\section{$\mathrm{A}=13$ (continued)}

2005GEZY RADIOACTIVITY ${ }^{13} \mathrm{~B}\left(\beta^{-}\right)$[from ${ }^{11} \mathrm{~B}(\mathrm{t}, \mathrm{p})$ ]; measured $\mathrm{T}_{1 / 2}$. CONF St Petersburg,P172,Generalov

2005KA06 NUCLEAR REACTIONS ${ }^{1} \mathrm{H}\left({ }^{17} \mathrm{~B}, \mathrm{X}\right){ }^{17} \mathrm{~B} /{ }^{15} \mathrm{~B} /{ }^{14} \mathrm{~B} /{ }^{13} \mathrm{~B} /{ }^{12} \mathrm{~B}, \mathrm{E} \approx$ $43 \mathrm{MeV} /$ nucleon; measured $\mathrm{E} \gamma, \mathrm{I} \gamma$, (particle) $\gamma$-coin, relative yields.

${ }^{15,17} \mathrm{~B}$ deduced levels, $\mathrm{J}, \pi$. Comparison with model predictions. JOUR PYLBB 608206

${ }^{13} \mathrm{C} \quad 2004 \mathrm{~S} 035$

NUCLEAR REACTIONS ${ }^{7} \mathrm{Li}\left({ }^{7} \mathrm{Li}, 2 \alpha\right), \mathrm{E}=8,30 \mathrm{MeV} ;{ }^{9} \mathrm{Be}\left({ }^{7} \mathrm{Li},{ }^{7} \mathrm{Li}\right)$, $\left({ }^{7} \mathrm{Li}, \alpha^{6} \mathrm{Li}\right),\left({ }^{7} \mathrm{Li}, \alpha^{7} \mathrm{Li}\right), \mathrm{E}=52 \mathrm{MeV} ;{ }^{7} \mathrm{Li}\left({ }^{9} \mathrm{Be}, \alpha^{9} \mathrm{Be}\right),\left({ }^{9} \mathrm{Be}, \alpha^{10} \mathrm{Be}\right)$, $\mathrm{E}=70 \mathrm{MeV}$; measured excitation energy spectra. ${ }^{9,10} \mathrm{Be},{ }^{13,14} \mathrm{C}$ deduced excited states, cluster structures. JOUR FIZBE 13433

2005AN15 NUCLEAR MOMENTS ${ }^{13} \mathrm{C},{ }^{14,15} \mathrm{~N},{ }^{17} \mathrm{O},{ }^{19} \mathrm{~F},{ }^{31} \mathrm{P},{ }^{33} \mathrm{~S}$; measured NMR spectra; deduced $\mu$. JOUR CHPLB 411111

2005AS04 NUCLEAR REACTIONS ${ }^{12} \mathrm{C}\left({ }^{10} \mathrm{Be}, 2 \alpha\right),\left({ }^{10} \mathrm{Be}, \mathrm{n} 2 \alpha\right), \mathrm{E}=30 \mathrm{MeV} /$ nucleon; measured $\mathrm{En}, \mathrm{E} \alpha$, relative energy spectra, $\sigma(\mathrm{E}) .{ }^{8,9} \mathrm{Be}$ deduced levels, $\mathrm{J}, \pi$. Kinematically complete measurement. JOUR PRVCA 72024314

2005BA40 NUCLEAR REACTIONS ${ }^{1} \mathrm{H}\left({ }^{16} \mathrm{O}, \mathrm{X}\right){ }^{1} \mathrm{H} /{ }^{2} \mathrm{H} /{ }^{3} \mathrm{H} /{ }^{3} \mathrm{He} /{ }^{4} \mathrm{He} /{ }^{5} \mathrm{He}$ $/{ }^{6} \mathrm{He} /{ }^{5} \mathrm{Li} /{ }^{6} \mathrm{Li} /{ }^{7} \mathrm{Li} /{ }^{8} \mathrm{Li} /{ }^{7} \mathrm{Be} /{ }^{8} \mathrm{Be} /{ }^{9} \mathrm{Be} /{ }^{10} \mathrm{Be} /{ }^{9} \mathrm{~B} /{ }^{10} \mathrm{~B} /$ ${ }^{11} \mathrm{~B} /{ }^{12} \mathrm{~B} /{ }^{10} \mathrm{C} /{ }^{11} \mathrm{C} /{ }^{12} \mathrm{C} /{ }^{13} \mathrm{C} /{ }^{14} \mathrm{C} /{ }^{13} \mathrm{~N} /{ }^{14} \mathrm{~N} /{ }^{15} \mathrm{~N} /{ }^{14} \mathrm{O} /$ ${ }^{15} \mathrm{O} /{ }^{16} \mathrm{O}$, E at $3.25 \mathrm{GeV} / \mathrm{c} /$ nucleon; measured production $\sigma$. JOUR PZETA 81174

2005 CU06 NUCLEAR REACTIONS ${ }^{7} \mathrm{Li}\left({ }^{7} \mathrm{Li},{ }^{11} \mathrm{~B}\right),\left({ }^{7} \mathrm{Li},{ }^{12} \mathrm{~B}\right), \mathrm{E}=58 \mathrm{MeV} ;{ }^{12} \mathrm{C}$, ${ }^{16} \mathrm{O}\left({ }^{7} \mathrm{Li},{ }^{10} \mathrm{~B}\right), \mathrm{E}=58 \mathrm{MeV}$; measured particle spectra. ${ }^{10,11,12} \mathrm{~B}$ deduced relative yields for $\alpha+\mathrm{Li}$ and $\mathrm{H}+\mathrm{Be}$ decay channels from excited states. JOUR PRVCA 72044320

2005GEZY RADIOACTIVITY ${ }^{13} \mathrm{~B}\left(\beta^{-}\right)$[from $\left.{ }^{11} \mathrm{~B}(\mathrm{t}, \mathrm{p})\right]$; measured $\mathrm{T}_{1 / 2}$. CONF St Petersburg,P172,Generalov

2005TAZY NUCLEAR REACTIONS ${ }^{14} \mathrm{~N}\left({ }^{13} \mathrm{~N},{ }^{14} \mathrm{O}\right), \mathrm{E}=11.8 \mathrm{MeV} /$ nucleon; measured particle spectra; deduced asymptotic normalization coefficient. ${ }^{13} \mathrm{~N}(\mathrm{p}, \gamma), \mathrm{E}(\mathrm{cm}) \approx 0-600 \mathrm{keV}$; deduced astrophysical $\mathrm{S}$-factor, reaction rate. Implications for novae nucleosynthesis discussed. CONF Argonne(Nuclei at the Limits),P329,Tang

${ }^{13} \mathrm{~N} \quad 2005 \mathrm{BA} 40$ NUCLEAR REACTIONS ${ }^{1} \mathrm{H}\left({ }^{16} \mathrm{O}, \mathrm{X}\right){ }^{1} \mathrm{H} /{ }^{2} \mathrm{H} /{ }^{3} \mathrm{H} /{ }^{3} \mathrm{He} /{ }^{4} \mathrm{He} /{ }^{5} \mathrm{He}$ $/{ }^{6} \mathrm{He} /{ }^{5} \mathrm{Li} /{ }^{6} \mathrm{Li} /{ }^{7} \mathrm{Li} /{ }^{8} \mathrm{Li} /{ }^{7} \mathrm{Be} /{ }^{8} \mathrm{Be} /{ }^{9} \mathrm{Be} /{ }^{10} \mathrm{Be} /{ }^{9} \mathrm{~B} /{ }^{10} \mathrm{~B} /$ ${ }^{11} \mathrm{~B} /{ }^{12} \mathrm{~B} /{ }^{10} \mathrm{C} /{ }^{11} \mathrm{C} /{ }^{12} \mathrm{C} /{ }^{13} \mathrm{C} /{ }^{14} \mathrm{C} /{ }^{13} \mathrm{~N} /{ }^{14} \mathrm{~N} /{ }^{15} \mathrm{~N} /{ }^{14} \mathrm{O} /$ ${ }^{15} \mathrm{O} /{ }^{16} \mathrm{O}$, E at $3.25 \mathrm{GeV} / \mathrm{c} /$ nucleon; measured production $\sigma$. JOUR PZETA 81174

2005FE11 NUCLEAR REACTIONS ${ }^{1} \mathrm{H}\left({ }^{12} \mathrm{C}, \gamma\right), \mathrm{E}(\mathrm{cm})=206.8,229.5 \mathrm{keV}$; measured yields. Accelerator mass spectrometry. JOUR NIMBE 240 495

2005KN02 RADIOACTIVITY ${ }^{13} \mathrm{O}\left(\beta^{+} \mathrm{p}\right)$ [from ${ }^{14} \mathrm{~N}(\mathrm{p}, 2 \mathrm{n})$ ]; measured $\beta$-delayed $\mathrm{Ep}$, Ip; deduced log ft. ${ }^{13} \mathrm{~N}$ deduced branching ratios for proton decay from excited states. JOUR PRVCA 72044312

2005TEZX NUCLEAR REACTIONS ${ }^{1} \mathrm{H}\left({ }^{13} \mathrm{~N}, \mathrm{p}\right), \mathrm{E}=3.7 \mathrm{MeV} /$ nucleon; measured recoil proton spectra. ${ }^{14} \mathrm{O}$ deduced resonance energies. REPT CNS-REP-66,P5,Teranishi

2006LE01 NUCLEAR REACTIONS ${ }^{13} \mathrm{C}(\mathrm{p}, \mathrm{n}), \mathrm{E}=5-30 \mathrm{MeV}$; measured neutron yield. Comparison with previous results. JOUR NIMAE 556397 


\section{$\mathrm{A}=13$ (continued)}

${ }^{13} \mathrm{O} \quad$ 2005KN02 RADIOACTIVITY ${ }^{13} \mathrm{O}\left(\beta^{+} \mathrm{p}\right)\left[\right.$ from $\left.{ }^{14} \mathrm{~N}(\mathrm{p}, 2 \mathrm{n})\right]$; measured $\beta$-delayed $\mathrm{Ep}$, Ip; deduced log ft. ${ }^{13} \mathrm{~N}$ deduced branching ratios for proton decay from excited states. JOUR PRVCA 72044312

2005WAZW NUCLEAR REACTIONS $\mathrm{Si}\left({ }^{6} \mathrm{Li}, \mathrm{X}\right),\left({ }^{7} \mathrm{Be}, \mathrm{X}\right),\left({ }^{10} \mathrm{~B}, \mathrm{X}\right),\left({ }^{9} \mathrm{C}, \mathrm{X}\right),\left({ }^{10} \mathrm{C}\right.$, $\mathrm{X}),\left({ }^{11} \mathrm{C}, \mathrm{X}\right),\left({ }^{12} \mathrm{~N}, \mathrm{X}\right),\left({ }^{13} \mathrm{O}, \mathrm{X}\right),\left({ }^{15} \mathrm{O}, \mathrm{X}\right),\left({ }^{17} \mathrm{Ne}, \mathrm{X}\right), \mathrm{E}=15-53 \mathrm{MeV} /$ nucleon; measured reaction and proton-removal $\sigma .{ }^{6} \mathrm{Li},{ }^{7} \mathrm{Be},{ }^{10} \mathrm{~B}$, ${ }^{9,10,11} \mathrm{C},{ }^{12} \mathrm{~N},{ }^{13,15} \mathrm{O},{ }^{17} \mathrm{Ne}$ deduced radii. Comparisons with model predictions. PREPRINT nucl-ex/0507025,7/18/2005

\section{$\mathrm{A}=14$}

${ }^{14} \mathrm{~B} \quad 2005 \mathrm{KA06}$

$2005 K 013$

${ }^{14} \mathrm{C}$

2004S035

2005AS04

2005BA40

2005G010

2005 G030

$2005 \mathrm{MC} 12$

2005ME05
NUCLEAR REACTIONS ${ }^{1} \mathrm{H}\left({ }^{17} \mathrm{~B}, \mathrm{X}\right){ }^{17} \mathrm{~B} /{ }^{15} \mathrm{~B} /{ }^{14} \mathrm{~B} /{ }^{13} \mathrm{~B} /{ }^{12} \mathrm{~B}, \mathrm{E} \approx$ $43 \mathrm{MeV} /$ nucleon; measured $\mathrm{E} \gamma, \mathrm{I} \gamma$, (particle) $\gamma$-coin, relative yields. ${ }^{15,17} \mathrm{~B}$ deduced levels, $\mathrm{J}, \pi$. Comparison with model predictions. JOUR PYLBB 608206

NUCLEAR REACTIONS ${ }^{12} \mathrm{C}\left({ }^{17} \mathrm{~B},{ }^{17} \mathrm{~B}^{\prime}\right),\left({ }^{17} \mathrm{~B},{ }^{15} \mathrm{BX}\right),\left({ }^{15} \mathrm{~B},{ }^{15} \mathrm{~B}^{\prime}\right)$, $\left({ }^{17} \mathrm{~B},{ }^{14} \mathrm{BX}\right),\left({ }^{17} \mathrm{~B},{ }^{12} \mathrm{BX}\right),\left({ }^{15} \mathrm{~B},{ }^{14} \mathrm{BX}\right),\left({ }^{15} \mathrm{~B},{ }^{12} \mathrm{BX}\right), \mathrm{E} \approx 70 \mathrm{MeV} /$ nucleon; measured $\mathrm{E} \gamma, \mathrm{I} \gamma$, (particle) $\gamma$-coin. ${ }^{12} \mathrm{C}\left({ }^{17} \mathrm{~B},{ }^{17} \mathrm{~B}^{\prime}\right),\left({ }^{15} \mathrm{~B},{ }^{15} \mathrm{~B}^{\prime}\right)$, $\mathrm{E} \approx 70 \mathrm{MeV}$; measured $\sigma(\mathrm{E}, \theta) .{ }^{15,17} \mathrm{~B}$ deduced levels, transitions, quadrupole deformation lengths. ${ }^{12,14} \mathrm{~B}$ deduced transitions. JOUR PRVCA 71044611

NUCLEAR REACTIONS ${ }^{7} \mathrm{Li}\left({ }^{7} \mathrm{Li}, 2 \alpha\right), \mathrm{E}=8,30 \mathrm{MeV} ;{ }^{9} \mathrm{Be}\left({ }^{7} \mathrm{Li},{ }^{7} \mathrm{Li}\right)$, $\left({ }^{7} \mathrm{Li}, \alpha^{6} \mathrm{Li}\right),\left({ }^{7} \mathrm{Li}, \alpha^{7} \mathrm{Li}\right), \mathrm{E}=52 \mathrm{MeV} ;{ }^{7} \mathrm{Li}\left({ }^{9} \mathrm{Be}, \alpha^{9} \mathrm{Be}\right),\left({ }^{9} \mathrm{Be}, \alpha^{10} \mathrm{Be}\right)$, $\mathrm{E}=70 \mathrm{MeV}$; measured excitation energy spectra. ${ }^{9,10} \mathrm{Be},{ }^{13,14} \mathrm{C}$ deduced excited states, cluster structures. JOUR FIZBE 13433 NUCLEAR REACTIONS ${ }^{12} \mathrm{C}\left({ }^{10} \mathrm{Be}, 2 \alpha\right),\left({ }^{10} \mathrm{Be}, \mathrm{n} 2 \alpha\right), \mathrm{E}=30 \mathrm{MeV} /$ nucleon; measured $\mathrm{En}, \mathrm{E} \alpha$, relative energy spectra, $\sigma(\mathrm{E}) \cdot{ }^{8,9} \mathrm{Be}$ deduced levels, J, $\pi$. Kinematically complete measurement. JOUR PRVCA 72024314

NUCLEAR REACTIONS ${ }^{1} \mathrm{H}\left({ }^{16} \mathrm{O}, \mathrm{X}\right){ }^{1} \mathrm{H} /{ }^{2} \mathrm{H} /{ }^{3} \mathrm{H} /{ }^{3} \mathrm{He} /{ }^{4} \mathrm{He} /{ }^{5} \mathrm{He}$ $/{ }^{6} \mathrm{He} /{ }^{5} \mathrm{Li} /{ }^{6} \mathrm{Li} /{ }^{7} \mathrm{Li} /{ }^{8} \mathrm{Li} /{ }^{7} \mathrm{Be} /{ }^{8} \mathrm{Be} /{ }^{9} \mathrm{Be} /{ }^{10} \mathrm{Be} /{ }^{9} \mathrm{~B} /{ }^{10} \mathrm{~B} /$ ${ }^{11} \mathrm{~B} /{ }^{12} \mathrm{~B} /{ }^{10} \mathrm{C} /{ }^{11} \mathrm{C} /{ }^{12} \mathrm{C} /{ }^{13} \mathrm{C} /{ }^{14} \mathrm{C} /{ }^{13} \mathrm{~N} /{ }^{14} \mathrm{~N} /{ }^{15} \mathrm{~N} /{ }^{14} \mathrm{O} /$ ${ }^{15} \mathrm{O} /{ }^{16} \mathrm{O}$, E at $3.25 \mathrm{GeV} / \mathrm{c} /$ nucleon; measured production $\sigma$. JOUR PZETA 81174 NUCLEAR REACTIONS ${ }^{14} \mathrm{~N}\left(\mu^{-}, \nu\right)$, E at $65 \mathrm{MeV} / \mathrm{c}$; measured Doppler-shifted $\mathrm{E} \gamma, \mathrm{I} \gamma$; deduced recoil nucleus alignment. Comparison with model predictions. JOUR PRVCA 71035503

NUCLEAR REACTIONS ${ }^{14} \mathrm{C}(\alpha, \alpha), \mathrm{E}=16.3-19.2 \mathrm{MeV}$; measured $\sigma(\theta)$, excitation function. ${ }^{18} \mathrm{O}$ deduced levels, J, $\pi, \alpha$-cluster states. JOUR YAFIA 681123

NUCLEAR REACTIONS ${ }^{12} \mathrm{C}\left({ }^{16} \mathrm{O},{ }^{14} \mathrm{O}\right)$, E not given; measured excitation energy spectra. ${ }^{14} \mathrm{C}$ deduced decay branch widths. JOUR JPGPE 31 S1921

NUCLEAR REACTIONS ${ }^{14} \mathrm{C}\left({ }^{11} \mathrm{~B},{ }^{11} \mathrm{~B}\right),\left({ }^{11} \mathrm{~B},{ }^{14} \mathrm{C}\right), \mathrm{E}=45 \mathrm{MeV}$; measured $\sigma(\mathrm{E}, \theta)$; deduced optical model parameters. ${ }^{14} \mathrm{C}$ levels deduced deformation parameters, single-particle structure. Coupled-channels analysis. JOUR NUPAB 75313 


\section{$\mathrm{A}=14$ (continued)}

2005NE14 NUCLEAR REACTIONS ${ }^{14} \mathrm{~N}(\mathrm{~d}, 2 \mathrm{p}), \mathrm{E}=170 \mathrm{MeV} ;{ }^{14} \mathrm{~N}\left({ }^{3} \mathrm{He}, \mathrm{t}\right)$, $\mathrm{E}=420 \mathrm{MeV}$; measured excitation energy spectra; deduced isospin symmetry features. JOUR JPGPE 31 S1931

2005PA41 NUCLEAR REACTIONS ${ }^{16} \mathrm{O}\left(\mathrm{n},{ }^{3} \mathrm{He}\right),(\mathrm{n}, \mathrm{t}), \mathrm{E}=15.4-18.1 \mathrm{MeV}$; measured activation $\sigma$. Accelerator mass spectrometry. JOUR KPSJA 4723

$2005 \mathrm{~S} 013 \quad$ NUCLEAR REACTIONS ${ }^{16} \mathrm{O}\left({ }^{9} \mathrm{Be}, \alpha^{7} \mathrm{Be}\right),{ }^{7} \mathrm{Li}\left({ }^{9} \mathrm{Be}, \alpha^{7} \mathrm{Li}\right),\left({ }^{9} \mathrm{Be}, \mathrm{t} 2 \alpha\right)$, $\mathrm{E}=55,70 \mathrm{MeV}$; measured excitation energy spectra. ${ }^{11} \mathrm{~B},{ }^{11} \mathrm{C}$ deduced excited states energies, configurations. JOUR JPGPE 31 S1701

2005SOZZ NUCLEAR REACTIONS ${ }^{16} \mathrm{O}\left({ }^{9} \mathrm{Be}, \alpha{ }^{7} \mathrm{Be}\right),{ }^{7} \mathrm{Li}\left({ }^{9} \mathrm{Be}, \alpha^{7} \mathrm{Li}\right),\left({ }^{9} \mathrm{Be}, \mathrm{t} 2 \alpha\right)$, $\mathrm{E}=55,70 \mathrm{MeV}$; measured particle spectra. ${ }^{11} \mathrm{C},{ }^{11} \mathrm{~B}$ deduced excited states energies, cluster structure, decay features. PREPRINT nucl-ex/0504026,4/25/2005

${ }^{14} \mathrm{~N} \quad 2005$ AN15 NUCLEAR MOMENTS ${ }^{13} \mathrm{C},{ }^{14,15} \mathrm{~N},{ }^{17} \mathrm{O},{ }^{19} \mathrm{~F},{ }^{31} \mathrm{P},{ }^{33} \mathrm{~S}$; measured NMR spectra; deduced $\mu$. JOUR CHPLB 411111

2005BA40 NUCLEAR REACTIONS ${ }^{1} \mathrm{H}\left({ }^{16} \mathrm{O}, \mathrm{X}\right){ }^{1} \mathrm{H} /{ }^{2} \mathrm{H} /{ }^{3} \mathrm{H} /{ }^{3} \mathrm{He} /{ }^{4} \mathrm{He} /{ }^{5} \mathrm{He}$ $/{ }^{6} \mathrm{He} /{ }^{5} \mathrm{Li} /{ }^{6} \mathrm{Li} /{ }^{7} \mathrm{Li} /{ }^{8} \mathrm{Li} /{ }^{7} \mathrm{Be} /{ }^{8} \mathrm{Be} /{ }^{9} \mathrm{Be} /{ }^{10} \mathrm{Be} /{ }^{9} \mathrm{~B} /{ }^{10} \mathrm{~B} /$ ${ }^{11} \mathrm{~B} /{ }^{12} \mathrm{~B} /{ }^{10} \mathrm{C} /{ }^{11} \mathrm{C} /{ }^{12} \mathrm{C} /{ }^{13} \mathrm{C} /{ }^{14} \mathrm{C} /{ }^{13} \mathrm{~N} /{ }^{14} \mathrm{~N} /{ }^{15} \mathrm{~N} /{ }^{14} \mathrm{O} /$ ${ }^{15} \mathrm{O} /{ }^{16} \mathrm{O}$, E at $3.25 \mathrm{GeV} / \mathrm{c} /$ nucleon; measured production $\sigma$. JOUR PZETA 81174

2005BL23 NUCLEAR REACTIONS ${ }^{12} \mathrm{C},{ }^{14} \mathrm{~N}\left({ }^{17} \mathrm{~F},{ }^{17} \mathrm{~F}\right), \mathrm{E}=10 \mathrm{MeV} /$ nucleon; measured $\sigma(\theta)$; deduced parameters, reaction mechanism features. Double-folding procedure. JOUR PRVCA 72034606

2005CH44 NUCLEAR REACTIONS ${ }^{14} \mathrm{~N}(\alpha, \gamma), \mathrm{E}=1775 \mathrm{keV}$; measured $\mathrm{E} \gamma$, I $\gamma$. ${ }^{17,18} \mathrm{O}(\mathrm{p}, \alpha), \mathrm{E} \approx 190-205 \mathrm{keV}$; measured $\mathrm{E} \alpha, \sigma, \sigma(\theta)$; deduced resonance parameters. Astrophysical implications discussed. JOUR PRLTA 95031101

2005MA92 NUCLEAR REACTIONS ${ }^{13} \mathrm{C}(\mathrm{p}, \gamma), \mathrm{E} \approx 450-680 \mathrm{MeV}$; measured $\mathrm{E} \gamma$, $\mathrm{I} \gamma .{ }^{14} \mathrm{~N}$ deduced resonance width. Monolayer target. JOUR NIMAE 55531

2005PA41 NUCLEAR REACTIONS ${ }^{16} \mathrm{O}\left(\mathrm{n},{ }^{3} \mathrm{He}\right),(\mathrm{n}, \mathrm{t}), \mathrm{E}=15.4-18.1 \mathrm{MeV}$; measured activation $\sigma$. Accelerator mass spectrometry. JOUR KPSJA 4723

2005RA26 NUCLEAR MOMENTS ${ }^{14} \mathrm{~N}$; measured hfs; deduced parameters. JOUR CHPLB 415161

${ }^{14} \mathrm{O}$ 2005BA40 NUCLEAR REACTIONS ${ }^{1} \mathrm{H}\left({ }^{16} \mathrm{O}, \mathrm{X}\right){ }^{1} \mathrm{H} /{ }^{2} \mathrm{H} /{ }^{3} \mathrm{H} /{ }^{3} \mathrm{He} /{ }^{4} \mathrm{He} /{ }^{5} \mathrm{He}$ $/{ }^{6} \mathrm{He} /{ }^{5} \mathrm{Li} /{ }^{6} \mathrm{Li} /{ }^{7} \mathrm{Li} /{ }^{8} \mathrm{Li} /{ }^{7} \mathrm{Be} /{ }^{8} \mathrm{Be} /{ }^{9} \mathrm{Be} /{ }^{10} \mathrm{Be} /{ }^{9} \mathrm{~B} /{ }^{10} \mathrm{~B} /$ ${ }^{11} \mathrm{~B} /{ }^{12} \mathrm{~B} /{ }^{10} \mathrm{C} /{ }^{11} \mathrm{C} /{ }^{12} \mathrm{C} /{ }^{13} \mathrm{C} /{ }^{14} \mathrm{C} /{ }^{13} \mathrm{~N} /{ }^{14} \mathrm{~N} /{ }^{15} \mathrm{~N} /{ }^{14} \mathrm{O} /$ ${ }^{15} \mathrm{O} /{ }^{16} \mathrm{O}$, E at $3.25 \mathrm{GeV} / \mathrm{c} /$ nucleon; measured production $\sigma$. JOUR PZETA 81174

2005BAZP NUCLEAR REACTIONS ${ }^{4} \mathrm{He}\left({ }^{14} \mathrm{O}, \mathrm{X}\right), \mathrm{E}=60 \mathrm{MeV} /$ nucleon; measured particle spectra, $\sigma(\mathrm{E}, \theta) .{ }^{14} \mathrm{O}$ deduced $\mathrm{E} 0$ and $\mathrm{E} 1$ strength distributions. REPT CNS-REP-66,P28,Baba

2005BAZQ NUCLEAR REACTIONS He $\left({ }^{14} \mathrm{O}, \mathrm{X}\right), \mathrm{E}=60 \mathrm{MeV} /$ nucleon; measured particle spectra; deduced excitation energy spectrum. ${ }^{14} \mathrm{O}$ deduced electric multipole strength distributions. REPT RIKEN 2004 Annual,P48,Baba 


\section{$\mathrm{A}=14$ (continued)}

2005GU25 NUCLEAR REACTIONS ${ }^{1} \mathrm{H}\left({ }^{14} \mathrm{O}, \mathrm{p}\right), \mathrm{E}=120 \mathrm{MeV}$; measured recoil proton spectra, $\sigma(\theta)$. ${ }^{15} \mathrm{~F}$ deduced resonance energies, $\mathrm{J}, \pi$. JOUR PRVCA 72034312

2005NE05 NUCLEAR REACTIONS ${ }^{14} \mathrm{~N}\left({ }^{3} \mathrm{He}, \mathrm{t}\right), \mathrm{E}=140 \mathrm{MeV} /$ nucleon; measured triton spectra. ${ }^{14} \mathrm{O}$ deduced level energies, widths. JOUR PRVCA 71047303

2005NE14 NUCLEAR REACTIONS ${ }^{14} \mathrm{~N}(\mathrm{~d}, 2 \mathrm{p}), \mathrm{E}=170 \mathrm{MeV} ;{ }^{14} \mathrm{~N}\left({ }^{3} \mathrm{He}, \mathrm{t}\right)$, $\mathrm{E}=420 \mathrm{MeV}$; measured excitation energy spectra; deduced isospin symmetry features. JOUR JPGPE 31 S1931

2005SA44 RADIOACTIVITY ${ }^{46} \mathrm{~V}(\mathrm{EC})$; analyzed masses; deduced Q(EC), log ft. ${ }^{10} \mathrm{C},{ }^{14} \mathrm{O},{ }^{22} \mathrm{Mg},{ }^{26 m} \mathrm{Al},{ }^{34} \mathrm{Cl},{ }^{34} \mathrm{Ar},{ }^{38 m} \mathrm{~K},{ }^{42} \mathrm{Sc},{ }^{46} \mathrm{~V},{ }^{50} \mathrm{Mn},{ }^{54} \mathrm{Co},{ }^{74} \mathrm{Rb}$; compiled, analyzed log ft; deduced quark-mixing matrix element. JOUR PRLTA 95102501

2005TAZY NUCLEAR REACTIONS ${ }^{14} \mathrm{~N}\left({ }^{13} \mathrm{~N},{ }^{14} \mathrm{O}\right), \mathrm{E}=11.8 \mathrm{MeV} /$ nucleon; measured particle spectra; deduced asymptotic normalization coefficient. ${ }^{13} \mathrm{~N}(\mathrm{p}, \gamma), \mathrm{E}(\mathrm{cm}) \approx 0-600 \mathrm{keV}$; deduced astrophysical $\mathrm{S}$-factor, reaction rate. Implications for novae nucleosynthesis discussed. CONF Argonne(Nuclei at the Limits),P329,Tang 2005TEZX NUCLEAR REACTIONS ${ }^{1} \mathrm{H}\left({ }^{13} \mathrm{~N}, \mathrm{p}\right), \mathrm{E}=3.7 \mathrm{MeV} /$ nucleon; measured recoil proton spectra. ${ }^{14} \mathrm{O}$ deduced resonance energies. REPT CNS-REP-66,P5,Teranishi

$$
\mathrm{A}=15
$$

${ }^{15} \mathrm{~B} \quad$ 2005KA06

NUCLEAR REACTIONS ${ }^{1} \mathrm{H}\left({ }^{17} \mathrm{~B}, \mathrm{X}\right){ }^{17} \mathrm{~B} /{ }^{15} \mathrm{~B} /{ }^{14} \mathrm{~B} /{ }^{13} \mathrm{~B} /{ }^{12} \mathrm{~B}, \mathrm{E} \approx$ $43 \mathrm{MeV} /$ nucleon; measured $\mathrm{E} \gamma, \mathrm{I} \gamma$, (particle) $\gamma$-coin, relative yields.

${ }^{15,17} \mathrm{~B}$ deduced levels, $\mathrm{J}, \pi$. Comparison with model predictions. JOUR PYLBB 608206

2005K013 NUCLEAR REACTIONS ${ }^{12} \mathrm{C}\left({ }^{17} \mathrm{~B},{ }^{17} \mathrm{~B}^{\prime}\right),\left({ }^{17} \mathrm{~B},{ }^{15} \mathrm{BX}\right),\left({ }^{15} \mathrm{~B},{ }^{15} \mathrm{~B}^{\prime}\right)$, $\left({ }^{17} \mathrm{~B},{ }^{14} \mathrm{BX}\right),\left({ }^{17} \mathrm{~B},{ }^{12} \mathrm{BX}\right),\left({ }^{15} \mathrm{~B},{ }^{14} \mathrm{BX}\right),\left({ }^{15} \mathrm{~B},{ }^{12} \mathrm{BX}\right), \mathrm{E} \approx 70 \mathrm{MeV} /$ nucleon; measured $\mathrm{E} \gamma, \mathrm{I} \gamma$, (particle) $\gamma$-coin. ${ }^{12} \mathrm{C}\left({ }^{17} \mathrm{~B},{ }^{17} \mathrm{~B}^{\prime}\right),\left({ }^{15} \mathrm{~B},{ }^{15} \mathrm{~B}^{\prime}\right)$, $\mathrm{E} \approx 70 \mathrm{MeV}$; measured $\sigma(\mathrm{E}, \theta) .{ }^{15,17} \mathrm{~B}$ deduced levels, transitions, quadrupole deformation lengths. ${ }^{12,14} \mathrm{~B}$ deduced transitions. JOUR PRVCA 71044611

${ }^{15} \mathrm{C}$ 2005DA38 NUCLEAR REACTIONS $\mathrm{Pb}\left({ }^{17} \mathrm{C}, \mathrm{n}{ }^{16} \mathrm{C}\right),\left({ }^{23} \mathrm{O}, \mathrm{n}^{22} \mathrm{O}\right), \mathrm{E} \approx 400-600$ $\mathrm{MeV} /$ nucleon; measured $\mathrm{E} \gamma, \mathrm{I} \gamma$, Coulomb dissociation $\sigma \cdot{ }^{14} \mathrm{C}(\mathrm{n}, \gamma)$, $\mathrm{E}(\mathrm{cm})=23 \mathrm{keV}$; deduced capture $\sigma$. JOUR JPGPE $31 \mathrm{~S} 1583$

2005DA43 NUCLEAR REACTIONS $\mathrm{Pb}\left({ }^{17} \mathrm{C}, \mathrm{n}{ }^{16} \mathrm{C}\right),\left({ }^{23} \mathrm{O}, \mathrm{n}^{22} \mathrm{O}\right), \mathrm{E} \approx 400-600$ $\mathrm{MeV} /$ nucleon; measured $\mathrm{E} \gamma, \mathrm{I} \gamma$, Coulomb dissociation $\sigma .{ }^{14} \mathrm{C}(\mathrm{n}, \gamma)$, $\mathrm{E}(\mathrm{cm})=23 \mathrm{keV}$; deduced capture $\sigma$. JOUR ZAANE $25 \mathrm{~s} 01339$

2005NAZZ NUCLEAR REACTIONS $\mathrm{Pb}\left({ }^{15} \mathrm{C}, \mathrm{n}^{14} \mathrm{C}\right), \mathrm{E}=68 \mathrm{MeV} /$ nucleon; measured dissociation $\sigma$, relative energy spectra. ${ }^{14} \mathrm{C}(\mathrm{n}, \gamma)$, $\mathrm{E}(\mathrm{cm})=0-2.7 \mathrm{MeV}$; deduced $\sigma$. CONF Riken(Origin of Matter) Proc,P155,Nakamura

2005RE22 NUCLEAR REACTIONS ${ }^{14} \mathrm{C}(\mathrm{n}, \gamma), \mathrm{E}=30,150,500 \mathrm{keV}$; measured $\sigma$. Fast cyclic activation technique. JOUR NUPAB $758787 \mathrm{c}$

${ }^{15} \mathrm{~N}$ 2004HAZR NUCLEAR REACTIONS ${ }^{16} \mathrm{O}$ (e, e'p), E=199.53 MeV; measured $\sigma(\mathrm{E}$, $\theta)$, missing momentum spectra. JOUR KKYHB 371 


\section{$\mathrm{A}=15$ (continued)}

2005AN15 NUCLEAR MOMENTS ${ }^{13} \mathrm{C},{ }^{14,15} \mathrm{~N},{ }^{17} \mathrm{O},{ }^{19} \mathrm{~F},{ }^{31} \mathrm{P},{ }^{33} \mathrm{~S}$; measured NMR spectra; deduced $\mu$. JOUR CHPLB 411111

2005Ba40 NUCLEAR REACTIONS ${ }^{1} \mathrm{H}\left({ }^{16} \mathrm{O}, \mathrm{X}\right){ }^{1} \mathrm{H} /{ }^{2} \mathrm{H} /{ }^{3} \mathrm{H} /{ }^{3} \mathrm{He} /{ }^{4} \mathrm{He} /{ }^{5} \mathrm{He}$ $/{ }^{6} \mathrm{He} /{ }^{5} \mathrm{Li} /{ }^{6} \mathrm{Li} /{ }^{7} \mathrm{Li} /{ }^{8} \mathrm{Li} /{ }^{7} \mathrm{Be} /{ }^{8} \mathrm{Be} /{ }^{9} \mathrm{Be} /{ }^{10} \mathrm{Be} /{ }^{9} \mathrm{~B} /{ }^{10} \mathrm{~B} /$ ${ }^{11} \mathrm{~B} /{ }^{12} \mathrm{~B} /{ }^{10} \mathrm{C} /{ }^{11} \mathrm{C} /{ }^{12} \mathrm{C} /{ }^{13} \mathrm{C} /{ }^{14} \mathrm{C} /{ }^{13} \mathrm{~N} /{ }^{14} \mathrm{~N} /{ }^{15} \mathrm{~N} /{ }^{14} \mathrm{O} /$ ${ }^{15} \mathrm{O} /{ }^{16} \mathrm{O}$, E at $3.25 \mathrm{GeV} / \mathrm{c} /$ nucleon; measured production $\sigma$. JOUR PZETA 81174

2005CA42 NUCLEAR MOMENTS ${ }^{2} \mathrm{H},{ }^{15} \mathrm{~N}$; measured hfs; deduced parameters. JOUR APJSA 159181

2005CH44 NUCLEAR REACTIONS ${ }^{14} \mathrm{~N}(\alpha, \gamma), \mathrm{E}=1775 \mathrm{keV}$; measured $\mathrm{E} \gamma$, I $\gamma$. ${ }^{17,18} \mathrm{O}(\mathrm{p}, \alpha), \mathrm{E} \approx 190-205 \mathrm{keV} ;$ measured $\mathrm{E} \alpha, \sigma, \sigma(\theta)$; deduced resonance parameters. Astrophysical implications discussed. JOUR PRLTA 95031101

2005DE45 NUCLEAR REACTIONS ${ }^{2} \mathrm{H}\left({ }^{18} \mathrm{~F}, \mathrm{p} \alpha\right)$, E not given; measured excitation energy spectrum. ${ }^{19} \mathrm{~F}$ level deduced spectroscopic factor. ${ }^{18} \mathrm{~F}(\mathrm{p}, \alpha), \mathrm{E}(\mathrm{cm})=0-1 \mathrm{MeV}$; calculated astrophysical S-factor. JOUR NUPAB $758745 \mathrm{c}$

2005KI11 NUCLEAR REACTIONS ${ }^{16} \mathrm{O}\left(\mathrm{K}^{-}, \mathrm{n}\right),\left(\mathrm{K}^{-}, \mathrm{nX}\right), \mathrm{E}$ at $0.93 \mathrm{GeV} / \mathrm{c}$; measured neutron spectra; deduced kaonic nuclei. JOUR NUPAB 754 $383 \mathrm{c}$

2005K002 NUCLEAR REACTIONS ${ }^{16} \mathrm{O}$ (p, 2p), E=392 MeV; measured Ep, E $\gamma$, pp-, p $\gamma$-coin. ${ }^{15} \mathrm{~N}$ levels deduced $\gamma$-emission probabilities. JOUR NPBSE 13972

2005LA28 NUCLEAR REACTIONS ${ }^{2} \mathrm{H}\left({ }^{14} \mathrm{~N}, \mathrm{p}\right), \mathrm{E}=10.6 \mathrm{MeV} /$ nucleon; measured Ep, E $\gamma, \sigma(\theta)$. Comparison with previous results. JOUR JPGPE 31 S1691

2005RU03 NUCLEAR REACTIONS ${ }^{12} \mathrm{C}\left({ }^{11} \mathrm{~B},{ }^{15} \mathrm{~N}\right), \mathrm{E}=49 \mathrm{MeV}$; measured $\sigma(\mathrm{E}$, $\theta) ;{ }^{12} \mathrm{C}\left({ }^{11} \mathrm{~B},{ }^{8} \mathrm{Be}\right), \mathrm{E}(\mathrm{cm})=10-17 \mathrm{MeV}$; analyzed $\sigma(\mathrm{E}, \theta)$; deduced reaction mechanism features, optical model parameters. Coupled channels analysis. JOUR ZAANE 23445

2005SAZU NUCLEAR REACTIONS ${ }^{14} \mathrm{~N}(\mathrm{n}, \gamma)$, E=thermal; measured prompt $\mathrm{E} \gamma, \mathrm{I} \gamma$; deduced capture $\sigma$. Pair spectrometer, spectrum unfolding procedure. CONF Santa Fe (Nucl Data for Sci and Technol) Proc, Vol1,P1000

${ }^{15} \mathrm{O} 2004 \mathrm{CO27}$ NUCLEAR REACTIONS ${ }^{14} \mathrm{~N}(\mathrm{p}, \gamma), \mathrm{E}=$ low; measured astrophysical S-factors. Solid and gas targets. JOUR NIFCA 27423

2005BA40 NUCLEAR REACTIONS ${ }^{1} \mathrm{H}\left({ }^{16} \mathrm{O}, \mathrm{X}\right){ }^{1} \mathrm{H} /{ }^{2} \mathrm{H} /{ }^{3} \mathrm{H} /{ }^{3} \mathrm{He} /{ }^{4} \mathrm{He} /{ }^{5} \mathrm{He}$ $/{ }^{6} \mathrm{He} /{ }^{5} \mathrm{Li} /{ }^{6} \mathrm{Li} /{ }^{7} \mathrm{Li} /{ }^{8} \mathrm{Li} /{ }^{7} \mathrm{Be} /{ }^{8} \mathrm{Be} /{ }^{9} \mathrm{Be} /{ }^{10} \mathrm{Be} /{ }^{9} \mathrm{~B} /{ }^{10} \mathrm{~B} /$ ${ }^{11} \mathrm{~B} /{ }^{12} \mathrm{~B} /{ }^{10} \mathrm{C} /{ }^{11} \mathrm{C} /{ }^{12} \mathrm{C} /{ }^{13} \mathrm{C} /{ }^{14} \mathrm{C} /{ }^{13} \mathrm{~N} /{ }^{14} \mathrm{~N} /{ }^{15} \mathrm{~N} /{ }^{14} \mathrm{O} /$ ${ }^{15} \mathrm{O} /{ }^{16} \mathrm{O}$, E at $3.25 \mathrm{GeV} / \mathrm{c} /$ nucleon; measured production $\sigma$. JOUR PZETA 81174

2005BA82 NUCLEAR REACTIONS ${ }^{1} \mathrm{H}\left({ }^{18} \mathrm{~F}, \mathrm{p}\right), \mathrm{E}(\mathrm{cm}) \approx 0.3-1.3 \mathrm{MeV}$; measured proton spectra, $\sigma(\theta)$, excitation functions. ${ }^{19} \mathrm{Ne}$ deduced resonance parameters, excited state energy, J, $\pi \cdot{ }^{18} \mathrm{~F}(\mathrm{p}, \alpha),(\mathrm{p}, \gamma), \mathrm{E}=\mathrm{low}$; calculated astrophysical reaction rates. JOUR NUPAB $758737 \mathrm{c}$

2005BB05 NUCLEAR REACTIONS ${ }^{1} \mathrm{H}\left({ }^{18} \mathrm{~F}, \mathrm{p}\right), \mathrm{E}(\mathrm{cm}) \approx 0.3-1.3 \mathrm{MeV}$; measured Ep, $\sigma(\theta)$; deduced excitation functions. ${ }^{19} \mathrm{Ne}$ deduced resonance energies, $\mathrm{J}, \pi$, analog states. ${ }^{18} \mathrm{~F}(\mathrm{p}, \alpha),(\mathrm{p}, \gamma), \mathrm{E}=$ low; calculated astrophysical reaction rates. JOUR ZAANE 25 s01 643 


\section{$\mathrm{A}=15$ (continued)}

2005BR04 NUCLEAR REACTIONS ${ }^{3} \mathrm{He}\left({ }^{3} \mathrm{He}, 2 \mathrm{p}\right), \mathrm{E}(\mathrm{cm}) \approx 10-1000 \mathrm{keV} ;{ }^{14} \mathrm{~N}(\mathrm{p}$, $\gamma), \mathrm{E} \approx 0.1-2.5 \mathrm{MeV}$; measured astrophysical S-factors. JOUR NPBSE 14360

2005BR15 NUCLEAR REACTIONS ${ }^{3} \mathrm{He}\left({ }^{3} \mathrm{He}, 2 \mathrm{p}\right), \mathrm{E}(\mathrm{cm}) \approx 16-100 \mathrm{keV}$; measured Ep, pp-coin, astrophysical S-factor. ${ }^{14} \mathrm{~N}(\mathrm{p}, \gamma), \mathrm{E}=130-240$ $\mathrm{keV}$; measured $\mathrm{E} \gamma$, astrophysical S-factor. JOUR NPBSE 14533

$2005 \mathrm{CO} 16 \quad$ NUCLEAR REACTIONS ${ }^{14} \mathrm{~N}(\mathrm{p}, \gamma), \mathrm{E}=$ low; measured $\mathrm{E} \gamma$, I $\gamma$; deduced S-factors. JOUR NUPAB $758383 \mathrm{c}$

2005DE45 NUCLEAR REACTIONS ${ }^{2} \mathrm{H}\left({ }^{18} \mathrm{~F}, \mathrm{p} \alpha\right)$, E not given; measured excitation energy spectrum. ${ }^{19} \mathrm{~F}$ level deduced spectroscopic factor. ${ }^{18} \mathrm{~F}(\mathrm{p}, \alpha), \mathrm{E}(\mathrm{cm})=0-1 \mathrm{MeV}$; calculated astrophysical S-factor. JOUR NUPAB $758745 \mathrm{c}$

2005 IM02 NUCLEAR REACTIONS ${ }^{14} \mathrm{~N}(\mathrm{p}, \gamma), \mathrm{E}(\mathrm{cm})=119-367 \mathrm{keV}$; measured $\mathrm{E} \gamma, \mathrm{I} \gamma$, excitation functions; deduced astrophysical S-factors. R-matrix analysis. JOUR ZAANE 25455

2005IMZY NUCLEAR REACTIONS ${ }^{14} \mathrm{~N}(\mathrm{p}, \gamma)$, E $(\mathrm{cm})=119-367 \mathrm{keV}$; measured $\mathrm{E} \gamma, \mathrm{I} \gamma$, excitation functions; deduced astrophysical S-factors, reaction rates. PREPRINT nucl-ex/0509005,9/01/2005

2005K009 NUCLEAR REACTIONS ${ }^{2} \mathrm{H}\left({ }^{18} \mathrm{~F}, \mathrm{p}\right), \mathrm{E}=108.5 \mathrm{MeV}$; measured Ep, $\sigma(\theta) .{ }^{19} \mathrm{~F}$ levels deduced spectroscopic factors. ${ }^{19} \mathrm{Ne}$ calculated proton resonance widths. ${ }^{18} \mathrm{~F}(\mathrm{p}, \gamma),(\mathrm{p}, \alpha), \mathrm{E}=$ low; deduced astrophysical reaction rates. JOUR PRVCA 71032801

2005K031 NUCLEAR REACTIONS ${ }^{2} \mathrm{H}\left({ }^{18} \mathrm{~F}, \mathrm{p}\right), \mathrm{E}=108.49 \mathrm{MeV}$; measured particle spectra, $\sigma(\theta) .{ }^{19} \mathrm{~F}$ levels deduced spectroscopic factors. ${ }^{18} \mathrm{~F}(\mathrm{p}$, $\alpha), \mathrm{E}=$ low; calculated astrophysical reaction rates. JOUR NUPAB 758 $753 \mathrm{c}$

2005MAZQ NUCLEAR REACTIONS ${ }^{15} \mathrm{~N}(\mathrm{p}, \mathrm{n}), \mathrm{E}=5.1 \mathrm{MeV} ;{ }^{2} \mathrm{H}(\mathrm{d}, \mathrm{n}), \mathrm{E}=3.0$ $\mathrm{MeV}$; measured neutron spectra, transmission through iron spheres. CONF Santa Fe (Nucl Data for Sci and Technol) Proc,Vol1,P480

2005PR20 NUCLEAR REACTIONS ${ }^{14} \mathrm{~N}(\mathrm{p}, \gamma), \mathrm{E}=\mathrm{low}$; measured $\mathrm{E} \gamma$, I $\gamma$; deduced astrophysical S-factor. Solid and gas targets. JOUR JPGPE 31 S1537

2005RU04 NUCLEAR REACTIONS ${ }^{14} \mathrm{~N}(\mathrm{p}, \gamma), \mathrm{E}=155-524 \mathrm{keV}$; measured E $\gamma$, excitation function; deduced S-factor. R-matrix analysis, astrophysical implications discussed. JOUR PRLTA 94082503

2005WAZW NUCLEAR REACTIONS $\mathrm{Si}\left({ }^{6} \mathrm{Li}, \mathrm{X}\right),\left({ }^{7} \mathrm{Be}, \mathrm{X}\right),\left({ }^{10} \mathrm{~B}, \mathrm{X}\right),\left({ }^{9} \mathrm{C}, \mathrm{X}\right),\left({ }^{10} \mathrm{C}\right.$, $\mathrm{X}),\left({ }^{11} \mathrm{C}, \mathrm{X}\right),\left({ }^{12} \mathrm{~N}, \mathrm{X}\right),\left({ }^{13} \mathrm{O}, \mathrm{X}\right),\left({ }^{15} \mathrm{O}, \mathrm{X}\right),\left({ }^{17} \mathrm{Ne}, \mathrm{X}\right), \mathrm{E}=15-53 \mathrm{MeV} /$ nucleon; measured reaction and proton-removal $\sigma .{ }^{6} \mathrm{Li},{ }^{7} \mathrm{Be},{ }^{10} \mathrm{~B}$, ${ }^{9,10,11} \mathrm{C},{ }^{12} \mathrm{~N},{ }^{13,15} \mathrm{O},{ }^{17} \mathrm{Ne}$ deduced radii. Comparisons with model predictions. PREPRINT nucl-ex/0507025,7/18/2005

${ }^{15} \mathrm{~F} \quad 2005 \mathrm{GU} 25$ NUCLEAR REACTIONS ${ }^{1} \mathrm{H}\left({ }^{14} \mathrm{O}, \mathrm{p}\right), \mathrm{E}=120 \mathrm{MeV}$; measured recoil proton spectra, $\sigma(\theta) .{ }^{15} \mathrm{~F}$ deduced resonance energies, J, $\pi$. JOUR PRVCA 72034312 


\section{$\mathrm{A}=16$}

\begin{tabular}{|c|c|c|}
\hline${ }^{16} \mathrm{Be}$ & $2004 \mathrm{TH} 15$ & $\begin{array}{l}\text { NUCLEAR REACTIONS } \mathrm{Be}\left({ }^{40} \mathrm{Ar}, \mathrm{X}\right), \mathrm{E}=140 \mathrm{MeV} / \text { nucleon; } \\
\text { measured fragment isotopic yields; deduced no evidence for }{ }^{16} \mathrm{Be} \text {. } \\
{ }^{12} \mathrm{C}\left({ }^{24} \mathrm{~F}, \mathrm{X}\right),\left({ }^{25} \mathrm{~F}, \mathrm{X}\right),\left({ }^{26} \mathrm{~F}, \mathrm{X}\right){ }^{20} \mathrm{O} /{ }^{21} \mathrm{O} /{ }^{22} \mathrm{O} /{ }^{23} \mathrm{O} /{ }^{24} \mathrm{O}, \mathrm{E} \approx 50 \\
\mathrm{MeV} / \text { nucleon; measured yields; deduced no evidence for }{ }^{25} \mathrm{O} \text {. JOUR } \\
\text { APHPF } 21379\end{array}$ \\
\hline \multirow[t]{3}{*}{${ }^{16} \mathrm{C}$} & $2004 \mathrm{AS} 13$ & $\begin{array}{l}\text { NUCLEAR REACTIONS C }\left({ }^{16} \mathrm{C}, \mathrm{X}\right), \mathrm{E}=46 \mathrm{MeV} \text { / nucleon; measured } \\
\text { particle spectra, breakup and neutron removal } \sigma \text {; deduced reaction } \\
\text { mechanism features. }{ }^{16} \mathrm{C} \text { deduced no ground-state cluster structure. } \\
\text { Comparison with model predictions. JOUR PRVCA } 70064607\end{array}$ \\
\hline & 2005В039 & $\begin{array}{l}\text { NUCLEAR REACTIONS }{ }^{13,14} \mathrm{C}\left({ }^{12} \mathrm{C},{ }^{9} \mathrm{C}\right), \mathrm{E}=231 \mathrm{MeV} \text {; measured } \\
\text { excitation energy spectra. }{ }^{16,17} \mathrm{C} \text { deduced levels, J, } \pi \text {, configurations. } \\
\text { JOUR JPGPE } 31 \text { S1461 }\end{array}$ \\
\hline & 20050N04 & $\begin{array}{l}\text { NUCLEAR REACTIONS }{ }^{1} \mathrm{H}\left({ }^{16} \mathrm{C},{ }^{16} \mathrm{C}^{\prime}\right), \mathrm{E}=33 \mathrm{MeV} / \text { nucleon; } \\
\text { measured } \mathrm{E} \gamma \text {, I } \gamma \text {, (particle) } \gamma \text {-coin; deduced } \sigma .{ }^{16} \mathrm{C} \text { deduced } \\
\text { deformation parameter. JOUR ZAANE } 25 \mathrm{~s} 01347\end{array}$ \\
\hline${ }^{16} \mathrm{~N}$ & 2004TAZW & $\begin{array}{l}\left.\text { RADIOACTIVITY }{ }^{16} \mathrm{~N}\left(\beta^{-}\right) \text {from }{ }^{2} \mathrm{H}\left({ }^{15} \mathrm{~N}, \mathrm{n}\right)\right] ; \text { measured } \beta \text {-delayed } \\
\text { E } \alpha \text {. Gas-filled ionization chambers. REPT ANL- } 04 / 22, \mathrm{P} 5 \text {, Tang }\end{array}$ \\
\hline \multirow[t]{4}{*}{${ }^{16} \mathrm{O}$} & 2004FR34 & $\begin{array}{l}\text { NUCLEAR REACTIONS }{ }^{12} \mathrm{C}\left({ }^{12} \mathrm{C}, 2^{8} \mathrm{Be}\right), \mathrm{E}=82-120 \mathrm{MeV} \text {; measured } \\
\text { excitation energy spectra, angular correlations. }{ }^{16} \mathrm{O} \text { deduced levels, J, } \\
\pi \text {. Comparison with model predictions. JOUR PRVCA } 70064311\end{array}$ \\
\hline & 2004PE24 & $\begin{array}{l}\text { NUCLEAR REACTIONS }{ }^{16} \mathrm{O}\left(\text { polarized } \gamma, \gamma^{\prime}\right), \mathrm{E}=25-40 \mathrm{MeV} \text {; } \\
\text { measured polarization asymmetries; deduced resonance features. } \\
\text { JOUR PRVCA } 70064305\end{array}$ \\
\hline & 2004TAZW & $\begin{array}{l}\left.\text { RADIOACTIVITY }{ }^{16} \mathrm{~N}\left(\beta^{-}\right) \text {from }{ }^{2} \mathrm{H}\left({ }^{15} \mathrm{~N}, \mathrm{n}\right)\right] ; \text { measured } \beta \text {-delayed } \\
\text { E } \alpha \text {. Gas-filled ionization chambers. REPT ANL- } 04 / 22, \mathrm{P} 5 \text {, Tang }\end{array}$ \\
\hline & 2005BA40 & $\begin{array}{l}\text { NUCLEAR REACTIONS }{ }^{1} \mathrm{H}\left({ }^{16} \mathrm{O}, \mathrm{X}\right){ }^{1} \mathrm{H} /{ }^{2} \mathrm{H} /{ }^{3} \mathrm{H} /{ }^{3} \mathrm{He} /{ }^{4} \mathrm{He} /{ }^{5} \mathrm{He} \\
/{ }^{6} \mathrm{He} /{ }^{5} \mathrm{Li} /{ }^{6} \mathrm{Li} /{ }^{7} \mathrm{Li} /{ }^{8} \mathrm{Li} /{ }^{7} \mathrm{Be} /{ }^{8} \mathrm{Be} /{ }^{9} \mathrm{Be} /{ }^{10} \mathrm{Be} /{ }^{9} \mathrm{~B} /{ }^{10} \mathrm{~B} / \\
{ }^{11} \mathrm{~B} /{ }^{12} \mathrm{~B} /{ }^{10} \mathrm{C} /{ }^{11} \mathrm{C} /{ }^{12} \mathrm{C} /{ }^{13} \mathrm{C} /{ }^{14} \mathrm{C} /{ }^{13} \mathrm{~N} /{ }^{14} \mathrm{~N} /{ }^{15} \mathrm{~N} /{ }^{14} \mathrm{O} / \\
{ }^{15} \mathrm{O} /{ }^{16} \mathrm{O} \text {, E at } 3.25 \mathrm{GeV} / \mathrm{c} / \text { nucleon: measured production } \sigma .\end{array}$ \\
\hline
\end{tabular}
JOUR PZETA 81174

2005FUZW NUCLEAR REACTIONS ${ }^{4} \mathrm{He}\left({ }^{16} \mathrm{O}, \alpha\right), \mathrm{E}<32.5 \mathrm{MeV}$; measured recoil $\alpha$ spectrum. REPT CNS-REP-66,P13,Fujikawa

2005 G036 ATOMIC MASSES ${ }^{12} \mathrm{C},{ }^{16} \mathrm{O},{ }^{20} \mathrm{Ne},{ }^{32} \mathrm{~S},{ }^{36,40} \mathrm{Ar}$; measured masses. Cyclotron-based mass spectrometry. JOUR JPGPE 31 S1869

2005HA16 NUCLEAR REACTIONS ${ }^{12} \mathrm{C}(\alpha, \gamma), \mathrm{E}(\mathrm{cm})=0.89-2.8 \mathrm{MeV}$; measured $\sigma(\theta)$, S-factors; deduced astrophysical reaction rate. JOUR NUPAB $752514 \mathrm{c}$

2005HA48 NUCLEAR REACTIONS ${ }^{12} \mathrm{C}(\alpha, \gamma)$, E $(\mathrm{cm})=0.89-2.8 \mathrm{MeV}$; measured $\mathrm{E} \gamma, \mathrm{I} \gamma$, angular distributions; deduced S-factors for E1 and E2 capture. Eurogam and Gandi arrays, astrophysical implications discussed. JOUR NUPAB 758 363c

2005HAZN NUCLEAR REACTIONS ${ }^{13} \mathrm{C}(\alpha, \mathrm{n}), \mathrm{E}=0.8-8.0 \mathrm{MeV}$; measured $\sigma$. PREPRINT nucl-ex/0509014,9/09/2005

2005KH13 NUCLEAR REACTIONS ${ }^{16} \mathrm{O}\left({ }^{16} \mathrm{O},{ }^{16} \mathrm{O}\right.$ '), $\mathrm{E}=250,350,480,704,1120$ $\mathrm{MeV}$; measured $\sigma(\mathrm{E}, \theta)$; deduced refractive features. DWBA and folding-model analysis, nuclear rainbow. JOUR NUPAB 7593 


\section{$\mathrm{A}=16$ (continued)}

2005KHZZ NUCLEAR REACTIONS ${ }^{16} \mathrm{O}\left({ }^{16} \mathrm{O},{ }^{16} \mathrm{O}\right.$ '), $\mathrm{E}=250,350,480,704,1120$ $\mathrm{MeV}$; measured $\sigma(\mathrm{E}, \theta)$; deduced refractive features. DWBA and folding-model analyses, nuclear rainbow. PREPRINT nucl-ex/0504020,4/22/2005

2005KRZY NUCLEAR REACTIONS ${ }^{14} \mathrm{~N}\left({ }^{3} \mathrm{He}, \mathrm{p}\right), \mathrm{E}=2.4 \mathrm{MeV}$; measured E $\gamma$, Ep, $\mathrm{p} \gamma$-coin, electron-positron pair spectrum; deduced possible neutral boson production. REPT ATOMKI 2004 Annual,P3,Krasznahorkay 2005MA52 NUCLEAR REACTIONS ${ }^{12} \mathrm{C}(\alpha, \gamma), \mathrm{E}=2.27 \mathrm{MeV} ;{ }^{27} \mathrm{Al}(\mathrm{p}, \gamma), \mathrm{E}=2.05$ $\mathrm{MeV}$; measured $\mathrm{E} \gamma, \mathrm{I} \gamma(\theta)$. JOUR NIMAE 547411

2005MA69 NUCLEAR REACTIONS ${ }^{12} \mathrm{C}(\alpha, \gamma), \mathrm{E}(\mathrm{cm})=1.39,1.58 \mathrm{MeV}$; measured $\mathrm{E} \gamma, \mathrm{I} \gamma, \sigma(\mathrm{E} 2) / \sigma(\mathrm{E} 1)$. Pulsed beam. JOUR NUPAB 758 371c

2005MA71 NUCLEAR REACTIONS ${ }^{19} \mathrm{~F}(\mathrm{p}, \alpha), \mathrm{E}=1.95-2.10 \mathrm{MeV}$; measured $\mathrm{E} \gamma$, $\mathrm{I} \gamma, \gamma \gamma$-coin. ${ }^{16} \mathrm{O}$ level deduced decay branching ratio. Astrophysical implications discussed. JOUR NUPAB $758403 \mathrm{c}$

2005PL04 NUCLEAR REACTIONS ${ }^{12} \mathrm{C}(\alpha, \gamma), \mathrm{E}(\mathrm{cm})=1.0-1.5 \mathrm{MeV}$; measured $\mathrm{E} \gamma, \mathrm{I} \gamma$, angular distributions; deduced S-factors. JOUR NUPAB 758 $415 \mathrm{c}$

2005SAZX NUCLEAR REACTIONS ${ }^{12} \mathrm{C}\left({ }^{24} \mathrm{Mg},{ }^{20} \mathrm{Ne}\right),\left({ }^{24} \mathrm{Mg}, 2{ }^{12} \mathrm{C}\right), \mathrm{E}=130$ $\mathrm{MeV}$; measured $\mathrm{E} \gamma, \mathrm{I} \gamma$, (particle) $\gamma$-, (particle)(particle)-coin. ${ }^{16} \mathrm{O}$ deduced transitions, branching ratio. Euroball IV array. CONF Bormio (XLIII Winter Meeting) Proc,P224

2005SC17 NUCLEAR REACTIONS ${ }^{12} \mathrm{C}(\alpha, \gamma), \mathrm{E}(\mathrm{cm})=1.3-5.0 \mathrm{MeV}$; measured $\mathrm{E} \gamma, \mathrm{I} \gamma$, (recoil) $\gamma$-coin; deduced astrophysical S-factors. Recoil mass separator. JOUR NUPAB 758 367c

2005SC29 NUCLEAR REACTIONS ${ }^{4} \mathrm{He}\left({ }^{12} \mathrm{C}, \gamma\right), \mathrm{E}=0.7-5.0 \mathrm{MeV}$; measured total recoil spectra, $\sigma .{ }^{12} \mathrm{C}(\alpha, \gamma), \mathrm{E}=1.9-4.9 \mathrm{MeV}$; deduced astrophysical S-factors. JOUR ZAANE 26301

2005SCZT NUCLEAR REACTIONS ${ }^{4} \mathrm{He}\left({ }^{12} \mathrm{C}, \gamma\right)$, E not given; measured recoil particle spectra. ${ }^{12} \mathrm{C}(\alpha, \gamma), \mathrm{E}=1.9-4.9 \mathrm{MeV}$; deduced astrophysical S-factors, resonance features. PREPRINT nucl-ex/0511050,11/29/2005

2005SHZZ NUCLEAR REACTIONS ${ }^{12} \mathrm{C}(\alpha, \gamma), \mathrm{E}(\mathrm{cm})=1.3,1.5 \mathrm{MeV}$; measured $\mathrm{E} 1$ and $\mathrm{E} 2 \sigma, \sigma(\theta) .{ }^{12} \mathrm{C}(\alpha, \gamma), \mathrm{E}(\mathrm{cm}) \approx 1000-3000 \mathrm{keV}$; analyzed data; deduced astrophysical S-factors. CONF Riken(Origin of Matter) Proc,P217,Shima

2005TA19 NUCLEAR REACTIONS ${ }^{10} \mathrm{~B},{ }^{16} \mathrm{O}\left(\mathrm{K}^{-}, \pi^{-}\right)$, E at $0.93 \mathrm{GeV} / \mathrm{c}$; ${ }^{11} \mathrm{~B}\left(\pi^{+}, \mathrm{K}^{+}\right)$, E at $1.05 \mathrm{GeV} / \mathrm{c} ;{ }^{7} \mathrm{Li},{ }^{10} \mathrm{~B}\left(\mathrm{~K}^{-}, \gamma\right)$, E at rest; measured $\mathrm{E} \gamma, \mathrm{I} \gamma \cdot{ }^{7} \mathrm{Li},{ }^{9} \mathrm{Be},{ }^{10,11} \mathrm{~B},{ }^{16} \mathrm{O}$ deduced hypernucleus levels, $\mathrm{J}, \pi$. Hyperball array. JOUR NUPAB $75458 \mathrm{c}$

2005 UK01 NUCLEAR REACTIONS ${ }^{16} \mathrm{O}\left(\mathrm{K}^{-}, \pi^{-}\right)$, E at $0.93 \mathrm{GeV} / \mathrm{c}$; measured $\mathrm{E} \gamma, \mathrm{I} \gamma .{ }^{16} \mathrm{O}$ deduced hypernucleus levels, J, $\pi$. Hyperball array. JOUR NUPAB $75470 \mathrm{c}$

$$
\mathrm{A}=\mathbf{1 7}
$$

${ }^{17} \mathrm{~B}$ 2005D016 NUCLEAR REACTIONS ${ }^{1} \mathrm{H}\left({ }^{17} \mathrm{~B},{ }^{17} \mathrm{~B}^{\prime}\right), \mathrm{E}=43.8 \mathrm{MeV}$; measured $\mathrm{E} \gamma$, $\mathrm{I} \gamma$, (particle) $\gamma$-coin, $\sigma .{ }^{17} \mathrm{~B}$ deduced deformation parameters, decoupling of valence neutrons from core. JOUR PYLBB 62181 


\section{$\mathrm{A}=17$ (continued)}

2005KA06 NUCLEAR REACTIONS ${ }^{1} \mathrm{H}\left({ }^{17} \mathrm{~B}, \mathrm{X}\right){ }^{17} \mathrm{~B} /{ }^{15} \mathrm{~B} /{ }^{14} \mathrm{~B} /{ }^{13} \mathrm{~B} /{ }^{12} \mathrm{~B}, \mathrm{E} \approx$ $43 \mathrm{MeV} /$ nucleon; measured $\mathrm{E} \gamma, \mathrm{I} \gamma$, (particle) $\gamma$-coin, relative yields. ${ }^{15,17} \mathrm{~B}$ deduced levels, $\mathrm{J}, \pi$. Comparison with model predictions. JOUR PYLBB 608206

2005KA26 NUCLEAR REACTIONS ${ }^{1} \mathrm{H}\left({ }^{19} \mathrm{C},{ }^{19} \mathrm{C}^{\prime}\right),\left({ }^{17} \mathrm{C},{ }^{17} \mathrm{C}^{\prime}\right),\left({ }^{17} \mathrm{~B},{ }^{17} \mathrm{~B}^{\prime}\right), \mathrm{E} \approx$ $53 \mathrm{MeV}$ / nucleon; measured prompt and delayed $\mathrm{E} \gamma, \mathrm{I} \gamma \cdot{ }^{17,19} \mathrm{C},{ }^{17} \mathrm{~B}$ deduced transitions. ${ }^{19} \mathrm{C}$ deduced no isomeric state. JOUR NUPAB 757315

2005K013 NUCLEAR REACTIONS ${ }^{12} \mathrm{C}\left({ }^{17} \mathrm{~B},{ }^{17} \mathrm{~B}^{\prime}\right),\left({ }^{17} \mathrm{~B},{ }^{15} \mathrm{BX}\right),\left({ }^{15} \mathrm{~B},{ }^{15} \mathrm{~B}^{\prime}\right)$, $\left({ }^{17} \mathrm{~B},{ }^{14} \mathrm{BX}\right),\left({ }^{17} \mathrm{~B},{ }^{12} \mathrm{BX}\right),\left({ }^{15} \mathrm{~B},{ }^{14} \mathrm{BX}\right),\left({ }^{15} \mathrm{~B},{ }^{12} \mathrm{BX}\right), \mathrm{E} \approx 70 \mathrm{MeV} /$ nucleon; measured $\mathrm{E} \gamma, \mathrm{I} \gamma$, (particle) $\gamma$-coin. ${ }^{12} \mathrm{C}\left({ }^{17} \mathrm{~B},{ }^{17} \mathrm{~B}^{\prime}\right),\left({ }^{15} \mathrm{~B},{ }^{15} \mathrm{~B}^{\prime}\right)$, $\mathrm{E} \approx 70 \mathrm{MeV}$; measured $\sigma(\mathrm{E}, \theta) .{ }^{15,17} \mathrm{~B}$ deduced levels, transitions, quadrupole deformation lengths. ${ }^{12,14} \mathrm{~B}$ deduced transitions. JOUR PRVCA 71044611

${ }^{17} \mathrm{C}$ 2005B039 NUCLEAR REACTIONS ${ }^{13,14} \mathrm{C}\left({ }^{12} \mathrm{C},{ }^{9} \mathrm{C}\right), \mathrm{E}=231 \mathrm{MeV}$; measured excitation energy spectra. ${ }^{16,17} \mathrm{C}$ deduced levels, $\mathrm{J}, \pi$, configurations. JOUR JPGPE 31 S1461

2005EL07 NUCLEAR REACTIONS ${ }^{1} \mathrm{H}\left({ }^{19} \mathrm{C},{ }^{19} \mathrm{C}\right),\left({ }^{19} \mathrm{C},{ }^{18} \mathrm{CX}\right),\left({ }^{19} \mathrm{C},{ }^{17} \mathrm{CX}\right), \mathrm{E}$ $\approx 49.4 \mathrm{MeV} /$ nucleon; ${ }^{1} \mathrm{H}\left({ }^{17} \mathrm{C},{ }^{17} \mathrm{C}^{\prime}\right),\left({ }^{17} \mathrm{C},{ }^{16} \mathrm{CX}\right), \mathrm{E} \approx 43.3 \mathrm{MeV} /$ nucleon; measured $\mathrm{E} \gamma, \mathrm{I} \gamma, \gamma \gamma-$, (particle) $\gamma$-coin, $\sigma \cdot{ }^{17,19} \mathrm{C}$ deduced levels, J, $\pi$. Comparison with shell model predictions. JOUR PYLBB 614174

2005KA26 NUCLEAR REACTIONS ${ }^{1} \mathrm{H}\left({ }^{19} \mathrm{C},{ }^{19} \mathrm{C}^{\prime}\right),\left({ }^{17} \mathrm{C},{ }^{17} \mathrm{C}^{\prime}\right),\left({ }^{17} \mathrm{~B},{ }^{17} \mathrm{~B} '\right), \mathrm{E} \approx$ $53 \mathrm{MeV} /$ nucleon; measured prompt and delayed $\mathrm{E} \gamma, \mathrm{I} \gamma \cdot{ }^{17,19} \mathrm{C},{ }^{17} \mathrm{~B}$ deduced transitions. ${ }^{19} \mathrm{C}$ deduced no isomeric state. JOUR NUPAB 757315

${ }^{17} \mathrm{O}$ 2005AN15 NUCLEAR MOMENTS ${ }^{13} \mathrm{C},{ }^{14,15} \mathrm{~N},{ }^{17} \mathrm{O},{ }^{19} \mathrm{~F},{ }^{31} \mathrm{P},{ }^{33} \mathrm{~S}$; measured NMR spectra; deduced $\mu$. JOUR CHPLB 411111

2005DE54 NUCLEAR REACTIONS ${ }^{14} \mathrm{~N}(\alpha, \mathrm{p}), \mathrm{E}=4893-6047 \mathrm{keV}$; measured $\sigma\left(\theta=172^{\circ}\right)$. Application to nitrogen depth profiling discussed. JOUR NIMBE 240803

${ }^{17} \mathrm{~F} \quad 2005 \mathrm{AN} 24$ NUCLEAR REACTIONS ${ }^{14} \mathrm{~N},{ }^{12} \mathrm{C},{ }^{16} \mathrm{O}(\mathrm{d}, \mathrm{n})$, E not given; measured activation yields in plasma focus device. JOUR ARISE 63545

2005KU27 NUCLEAR REACTIONS ${ }^{4} \mathrm{He}\left({ }^{14} \mathrm{O}, \mathrm{p}\right), \mathrm{E}(\mathrm{cm}) \approx 1-3.6 \mathrm{MeV}$; measured proton spectrum. ${ }^{1} \mathrm{H}\left({ }^{23} \mathrm{Mg}, \mathrm{p}\right), \mathrm{E}(\mathrm{cm}) \approx 0.6-3.5 \mathrm{MeV}$; measured elastic $\sigma(\theta) .{ }^{24} \mathrm{Al}$ deduced excited states energies. JOUR NUPAB $758733 \mathrm{c}$

${ }^{17} \mathrm{Ne} \quad 2005 \mathrm{GEO} 6$ NUCLEAR MOMENTS ${ }^{17,19,21,23,25} \mathrm{Ne}$; measured hfs; deduced $\mu$, quadrupole moments. Collinear fast-beam laser spectroscopy, comparison with shell model predictions. JOUR PRVCA 71064319 2005KA51 NUCLEAR REACTIONS Be $\left({ }^{17} \mathrm{Ne},{ }^{15} \mathrm{OX}\right), \mathrm{E}=64 \mathrm{MeV} /$ nucleon; measured fragments longitudinal momentum distributions, interaction $\sigma .{ }^{17} \mathrm{Ne}$ deduced two-proton halo features. Few-body Glauber model analysis. JOUR ZAANE 25 s01 327

2005TA33 NUCLEAR REACTIONS ${ }^{9} \mathrm{Be},{ }^{12} \mathrm{C},{ }^{27} \mathrm{Al}\left({ }^{17} \mathrm{Ne}, \mathrm{X}\right), \mathrm{E}=42,62 \mathrm{MeV} /$ nucleon; measured interaction and reaction $\sigma .{ }^{17} \mathrm{Ne}$ deduced matter density distribution. JOUR ZAANE 25 s01 221 


\section{$\mathrm{A}=17$ (continued)}

2005WAZW NUCLEAR REACTIONS $\mathrm{Si}\left({ }^{6} \mathrm{Li}, \mathrm{X}\right),\left({ }^{7} \mathrm{Be}, \mathrm{X}\right),\left({ }^{10} \mathrm{~B}, \mathrm{X}\right),\left({ }^{9} \mathrm{C}, \mathrm{X}\right),\left({ }^{10} \mathrm{C}\right.$, $\mathrm{X}),\left({ }^{11} \mathrm{C}, \mathrm{X}\right),\left({ }^{12} \mathrm{~N}, \mathrm{X}\right),\left({ }^{13} \mathrm{O}, \mathrm{X}\right),\left({ }^{15} \mathrm{O}, \mathrm{X}\right),\left({ }^{17} \mathrm{Ne}, \mathrm{X}\right), \mathrm{E}=15-53 \mathrm{MeV} /$ nucleon; measured reaction and proton-removal $\sigma .{ }^{6} \mathrm{Li},{ }^{7} \mathrm{Be},{ }^{10} \mathrm{~B}$, ${ }^{9,10,11} \mathrm{C},{ }^{12} \mathrm{~N},{ }^{13,15} \mathrm{O},{ }^{17} \mathrm{Ne}$ deduced radii. Comparisons with model predictions. PREPRINT nucl-ex/0507025,7/18/2005

\section{$\mathrm{A}=18$}

${ }^{18} \mathrm{O} \quad$ 2005DE15

NUCLEAR REACTIONS ${ }^{1} \mathrm{H}\left({ }^{18} \mathrm{O}, \mathrm{p}\right),\left({ }^{18} \mathrm{Ne}, \mathrm{p}\right), \mathrm{E}(\mathrm{cm}) \approx 900-6000$ $\mathrm{keV}$; measured Ep, excitation functions, $\sigma\left(\theta=180^{\circ}\right)$. ${ }^{19} \mathrm{Na}$ deduced level energies, J, $\pi$, widths, two-proton emission features. JOUR ZAANE 24237

2005G030 NUCLEAR REACTIONS ${ }^{14} \mathrm{C}(\alpha, \alpha), \mathrm{E}=16.3-19.2 \mathrm{MeV}$; measured $\sigma(\theta)$, excitation function. ${ }^{18} \mathrm{O}$ deduced levels, $\mathrm{J}, \pi, \alpha$-cluster states. JOUR YAFIA 681123

2005N013 NUCLEAR REACTIONS ${ }^{2} \mathrm{H},{ }^{3,4} \mathrm{He},{ }^{6,7} \mathrm{Li},{ }^{9} \mathrm{Be},{ }^{10,11} \mathrm{~B},{ }^{16} \mathrm{O}$, ${ }^{19} \mathrm{~F}$ (polarized p, 2p), $\mathrm{E}=392 \mathrm{MeV}$; measured analyzing powers. Comparison with model predictions. JOUR PRVCA 72041602

${ }^{18} \mathrm{~F} \quad 2004 \mathrm{FOZY}$ NUCLEAR REACTIONS ${ }^{17} \mathrm{O}(\mathrm{p}, \gamma), \mathrm{E}=185-215 \mathrm{keV}$; measured $\mathrm{E} \gamma, \mathrm{I} \gamma$; deduced excitation function. ${ }^{18} \mathrm{~F}$ deduced resonance strengths. Astrophysical implications discussed. REPT TUNL-XLIII,P32,Fox

2005BA82 NUCLEAR REACTIONS ${ }^{1} \mathrm{H}\left({ }^{18} \mathrm{~F}, \mathrm{p}\right), \mathrm{E}(\mathrm{cm}) \approx 0.3-1.3 \mathrm{MeV}$; measured proton spectra, $\sigma(\theta)$, excitation functions. ${ }^{19} \mathrm{Ne}$ deduced resonance parameters, excited state energy, J, $\pi \cdot{ }^{18} \mathrm{~F}(\mathrm{p}, \alpha),(\mathrm{p}, \gamma), \mathrm{E}=$ low; calculated astrophysical reaction rates. JOUR NUPAB $758737 \mathrm{c}$

2005BB05 NUCLEAR REACTIONS ${ }^{1} \mathrm{H}\left({ }^{18} \mathrm{~F}, \mathrm{p}\right), \mathrm{E}(\mathrm{cm}) \approx 0.3-1.3 \mathrm{MeV}$; measured Ep, $\sigma(\theta)$; deduced excitation functions. ${ }^{19} \mathrm{Ne}$ deduced resonance energies, $\mathrm{J}, \pi$, analog states. ${ }^{18} \mathrm{~F}(\mathrm{p}, \alpha),(\mathrm{p}, \gamma), \mathrm{E}=$ low; calculated astrophysical reaction rates. JOUR ZAANE 25 s01 643

2005CH44 NUCLEAR REACTIONS ${ }^{14} \mathrm{~N}(\alpha, \gamma), \mathrm{E}=1775 \mathrm{keV}$; measured $\mathrm{E} \gamma$, I $\gamma$. ${ }^{17,18} \mathrm{O}(\mathrm{p}, \alpha), \mathrm{E} \approx 190-205 \mathrm{keV} ;$ measured $\mathrm{E} \alpha, \sigma, \sigma(\theta)$; deduced resonance parameters. Astrophysical implications discussed. JOUR PRLTA 95031101

2005FI01 NUCLEAR REACTIONS ${ }^{1} \mathrm{H}\left({ }^{17} \mathrm{O}, \gamma\right), \mathrm{E}=12.5 \mathrm{MeV}$; measured particle spectra; deduced resonance strength. Recoil separator, other reactions discussed. JOUR NUPAB 748351

$2005 \mathrm{~F} 003$ NUCLEAR REACTIONS ${ }^{17} \mathrm{O}(\mathrm{p}, \gamma)$, E=140-540 keV; measured E $\gamma$, I $\gamma$; deduced resonance parameters, excitation functions, thermonuclear reaction rates. JOUR PRVCA 71055801

2005HE04 NUCLEAR REACTIONS ${ }^{18} \mathrm{O}(\mathrm{p}, \mathrm{n}), \mathrm{E}=2582 \mathrm{keV}$; measured neutron spectrum. ${ }^{138} \mathrm{Ba}(\mathrm{n}, \gamma), \mathrm{E}=$ spectrum; measured Maxwellian-averaged $\sigma$. JOUR PRVCA 71025803

2005HE19 NUCLEAR REACTIONS ${ }^{18} \mathrm{O}(\mathrm{p}, \mathrm{n}), \mathrm{E}=2582 \mathrm{keV}$; measured neutron spectra. ${ }^{138} \mathrm{Ba},{ }^{139} \mathrm{La},{ }^{175} \mathrm{Lu}(\mathrm{n}, \gamma), \mathrm{E}=$ spectrum; measured $\sigma$. JOUR NUPAB $758529 \mathrm{c}$ 


\section{$\mathrm{A}=18$ (continued)}

2005IL02 NUCLEAR REACTIONS ${ }^{17} \mathrm{O}(\mathrm{p}, \gamma), \mathrm{E} \approx 190,519 \mathrm{keV}$; measured $\mathrm{E} \gamma$, $\mathrm{I} \gamma$; deduced resonance excitation functions. ${ }^{23} \mathrm{Na}(\mathrm{p}, \gamma), \mathrm{E} \approx 150 \mathrm{keV}$; measured $\mathrm{E} \gamma, \mathrm{I} \gamma, \gamma \gamma$-coin; deduced resonance strength upper limit. Astrophysical implications discussed. JOUR NUPAB $75873 \mathrm{c}$

${ }^{18} \mathrm{Ne} \quad 2004 \mathrm{SIZX}$

2005DE15

${ }^{19} \mathrm{C} \quad$ 2005EL07

${ }^{19} \mathrm{~N} \quad 2005 \mathrm{DOZX}$

${ }^{19} \mathrm{O} \quad 2005 \mathrm{KO} 43$

${ }^{19} \mathrm{~F} \quad 2005$ AN15

2005DE45

2005ISZZ
NUCLEAR REACTIONS ${ }^{1} \mathrm{H}\left({ }^{21} \mathrm{Na}, \alpha\right), \mathrm{E} \approx 113 \mathrm{MeV}$; measured $\mathrm{E} \alpha$, $\sigma(\mathrm{E}, \theta),\left({ }^{18} \mathrm{Ne}\right) \alpha$-coin. ${ }^{18} \mathrm{Ne}(\alpha, \mathrm{p}), \mathrm{E}(\mathrm{cm}) \approx 2.5 \mathrm{MeV}$; deduced angle-integrated $\sigma$. REPT ANL-04/22,P8,Sinha NUCLEAR REACTIONS ${ }^{1} \mathrm{H}\left({ }^{18} \mathrm{O}, \mathrm{p}\right),\left({ }^{18} \mathrm{Ne}, \mathrm{p}\right), \mathrm{E}(\mathrm{cm}) \approx 900-6000$ $\mathrm{keV}$; measured Ep, excitation functions, $\sigma\left(\theta=180^{\circ}\right)$. ${ }^{19} \mathrm{Na}$ deduced level energies, $\mathrm{J}, \pi$, widths, two-proton emission features. JOUR ZAANE 24237

NUCLEAR REACTIONS ${ }^{16} \mathrm{O}\left({ }^{3} \mathrm{He}, \mathrm{n}\right), \mathrm{E}=9.9-10.4 \mathrm{MeV}$; measured neutron spectra, $\sigma(\theta) .{ }^{18} \mathrm{Ne}$ deduced resonance energy, width. Comparison with previous results. JOUR PRVCA 72025802

$$
\mathrm{A}=19
$$

NUCLEAR REACTIONS ${ }^{1} \mathrm{H}\left({ }^{19} \mathrm{C},{ }^{19} \mathrm{C}\right),\left({ }^{19} \mathrm{C},{ }^{18} \mathrm{CX}\right),\left({ }^{19} \mathrm{C},{ }^{17} \mathrm{CX}\right), \mathrm{E}$ $\approx 49.4 \mathrm{MeV} /$ nucleon; ${ }^{1} \mathrm{H}\left({ }^{17} \mathrm{C},{ }^{17} \mathrm{C}\right),\left({ }^{17} \mathrm{C},{ }^{16} \mathrm{CX}\right), \mathrm{E} \approx 43.3 \mathrm{MeV} /$ nucleon; measured $\mathrm{E} \gamma, \mathrm{I} \gamma, \gamma \gamma$-, (particle) $\gamma$-coin, $\sigma \cdot{ }^{17,19} \mathrm{C}$ deduced levels, J, $\pi$. Comparison with shell model predictions. JOUR PYLBB 614174

NUCLEAR REACTIONS ${ }^{1} \mathrm{H}\left({ }^{19} \mathrm{C},{ }^{19} \mathrm{C}^{\prime}\right),\left({ }^{17} \mathrm{C},{ }^{17} \mathrm{C}^{\prime}\right),\left({ }^{17} \mathrm{~B},{ }^{17} \mathrm{~B} '\right), \mathrm{E} \approx$ $53 \mathrm{MeV}$ / nucleon; measured prompt and delayed $\mathrm{E} \gamma, \mathrm{I} \gamma \cdot{ }^{17,19} \mathrm{C},{ }^{17} \mathrm{~B}$ deduced transitions. ${ }^{19} \mathrm{C}$ deduced no isomeric state. JOUR NUPAB 757315

NUCLEAR REACTIONS ${ }^{9} \mathrm{Be}\left({ }^{36} \mathrm{~S}, \mathrm{X}\right){ }^{19} \mathrm{~N}$, E not given; measured $\mathrm{E} \gamma$, I $\gamma, \gamma \gamma$-, (recoil) $\gamma$-coin. ${ }^{19} \mathrm{~N}$ deduced levels. REPT ATOMKI 2004 Annual,P8,Dombradi NUCLEAR REACTIONS U(p, X) ${ }^{19} \mathrm{O} /{ }^{20} \mathrm{O} /{ }^{21} \mathrm{O} /{ }^{22} \mathrm{O}, \mathrm{E}=1.4 \mathrm{GeV}$; measured yields. JOUR ZAANE 25 s01 729

NUCLEAR MOMENTS ${ }^{13} \mathrm{C},{ }^{14,15} \mathrm{~N},{ }^{17} \mathrm{O},{ }^{19} \mathrm{~F},{ }^{31} \mathrm{P},{ }^{33} \mathrm{~S}$; measured NMR spectra; deduced $\mu$. JOUR CHPLB 411111

NUCLEAR REACTIONS ${ }^{2} \mathrm{H}\left({ }^{18} \mathrm{~F}, \mathrm{p} \alpha\right)$, E not given; measured excitation energy spectrum. ${ }^{19} \mathrm{~F}$ level deduced spectroscopic factor. ${ }^{18} \mathrm{~F}(\mathrm{p}, \alpha), \mathrm{E}(\mathrm{cm})=0-1 \mathrm{MeV}$; calculated astrophysical S-factor. JOUR NUPAB $758745 \mathrm{c}$

NUCLEAR REACTIONS ${ }^{4} \mathrm{He}\left({ }^{8} \mathrm{Li}, \mathrm{n}\right), \mathrm{E}=14.6 \mathrm{MeV} ;{ }^{4} \mathrm{He}\left({ }^{16} \mathrm{~N}, \mathrm{n}\right)$, $\mathrm{E}=32 \mathrm{MeV}$; measured En, excitation energy spectra. ${ }^{16} \mathrm{~N}(\alpha, \mathrm{n}), \mathrm{E}(\mathrm{cm})$ $\approx 1-4.5 \mathrm{MeV} ;{ }^{8} \mathrm{Li}(\alpha, \mathrm{n}), \mathrm{E} \approx 0.5-3 \mathrm{MeV}$; deduced excitation functions. CONF Riken(Origin of Matter) Proc,P316,Ishiyama NUCLEAR REACTIONS ${ }^{2} \mathrm{H}\left({ }^{18} \mathrm{~F}, \mathrm{p}\right), \mathrm{E}=108.5 \mathrm{MeV}$; measured Ep, $\sigma(\theta) .{ }^{19} \mathrm{~F}$ levels deduced spectroscopic factors. ${ }^{19} \mathrm{Ne}$ calculated proton resonance widths. ${ }^{18} \mathrm{~F}(\mathrm{p}, \gamma),(\mathrm{p}, \alpha), \mathrm{E}=$ low; deduced astrophysical reaction rates. JOUR PRVCA 71032801 


\section{$\mathrm{A}=19$ (continued)}

2005K031 NUCLEAR REACTIONS ${ }^{2} \mathrm{H}\left({ }^{18} \mathrm{~F}, \mathrm{p}\right), \mathrm{E}=108.49 \mathrm{MeV}$; measured particle spectra, $\sigma(\theta) .{ }^{19} \mathrm{~F}$ levels deduced spectroscopic factors. ${ }^{18} \mathrm{~F}(\mathrm{p}$, $\alpha), \mathrm{E}=$ low; calculated astrophysical reaction rates. JOUR NUPAB 758 $753 \mathrm{c}$

${ }^{19} \mathrm{Ne} \quad$ 2005BA82 NUCLEAR REACTIONS ${ }^{1} \mathrm{H}\left({ }^{18} \mathrm{~F}, \mathrm{p}\right), \mathrm{E}(\mathrm{cm}) \approx 0.3-1.3 \mathrm{MeV}$; measured proton spectra, $\sigma(\theta)$, excitation functions. ${ }^{19} \mathrm{Ne}$ deduced resonance parameters, excited state energy, $\mathrm{J}, \pi \cdot{ }^{18} \mathrm{~F}(\mathrm{p}, \alpha),(\mathrm{p}, \gamma), \mathrm{E}=\mathrm{low}$; calculated astrophysical reaction rates. JOUR NUPAB $758737 \mathrm{c}$

2005BB05 NUCLEAR REACTIONS ${ }^{1} \mathrm{H}\left({ }^{18} \mathrm{~F}, \mathrm{p}\right), \mathrm{E}(\mathrm{cm}) \approx 0.3-1.3 \mathrm{MeV}$; measured Ep, $\sigma(\theta)$; deduced excitation functions. ${ }^{19} \mathrm{Ne}$ deduced resonance energies, J, $\pi$, analog states. ${ }^{18} \mathrm{~F}(\mathrm{p}, \alpha),(\mathrm{p}, \gamma), \mathrm{E}=$ low; calculated astrophysical reaction rates. JOUR ZAANE 25 s01 643

2005GE06 NUCLEAR MOMENTS ${ }^{17,19,21,23,25} \mathrm{Ne}$; measured hfs; deduced $\mu$, quadrupole moments. Collinear fast-beam laser spectroscopy, comparison with shell model predictions. JOUR PRVCA 71064319 2005K009 NUCLEAR REACTIONS ${ }^{2} \mathrm{H}\left({ }^{18} \mathrm{~F}, \mathrm{p}\right), \mathrm{E}=108.5 \mathrm{MeV}$; measured Ep, $\sigma(\theta) .{ }^{19} \mathrm{~F}$ levels deduced spectroscopic factors. ${ }^{19} \mathrm{Ne}$ calculated proton resonance widths. ${ }^{18} \mathrm{~F}(\mathrm{p}, \gamma),(\mathrm{p}, \alpha), \mathrm{E}=$ low; deduced astrophysical reaction rates. JOUR PRVCA 71032801

2005 TA28 NUCLEAR REACTIONS ${ }^{17} \mathrm{O}\left({ }^{3} \mathrm{He}, \mathrm{n}\right), \mathrm{E}=3.0 \mathrm{MeV}$; measured $\mathrm{E} \gamma, \mathrm{I} \gamma$, $\mathrm{n} \gamma$-coin, DSA. ${ }^{19} \mathrm{Ne}$ levels deduced energies, $\mathrm{T}_{1 / 2}$. Astrophysical implications discussed. Comparison with model predictions. JOUR PRVCA 72041302

${ }^{19} \mathrm{Na}$ 2005DE15 NUCLEAR REACTIONS ${ }^{1} \mathrm{H}\left({ }^{18} \mathrm{O}, \mathrm{p}\right),\left({ }^{18} \mathrm{Ne}, \mathrm{p}\right), \mathrm{E}(\mathrm{cm}) \approx 900-6000$ $\mathrm{keV}$; measured Ep, excitation functions, $\sigma\left(\theta=180^{\circ}\right)$. ${ }^{19} \mathrm{Na}$ deduced level energies, $\mathrm{J}, \pi$, widths, two-proton emission features. JOUR ZAANE 24237

\section{$\mathrm{A}=\mathbf{2 0}$}

${ }^{20} \mathrm{O} \quad 2004 \mathrm{TH} 15$

NUCLEAR REACTIONS Be $\left({ }^{40} \mathrm{Ar}, \mathrm{X}\right), \mathrm{E}=140 \mathrm{MeV} /$ nucleon; measured fragment isotopic yields; deduced no evidence for ${ }^{16} \mathrm{Be}$. ${ }^{12} \mathrm{C}\left({ }^{24} \mathrm{~F}, \mathrm{X}\right),\left({ }^{25} \mathrm{~F}, \mathrm{X}\right),\left({ }^{26} \mathrm{~F}, \mathrm{X}\right){ }^{20} \mathrm{O} /{ }^{21} \mathrm{O} /{ }^{22} \mathrm{O} /{ }^{23} \mathrm{O} /{ }^{24} \mathrm{O}, \mathrm{E} \approx 50$ $\mathrm{MeV} /$ nucleon; measured yields; deduced no evidence for ${ }^{25} \mathrm{O}$. JOUR APHPF 21379

2005KO43 NUCLEAR REACTIONS U(p, X) ${ }^{19} \mathrm{O} /{ }^{20} \mathrm{O} /{ }^{21} \mathrm{O} /{ }^{22} \mathrm{O}, \mathrm{E}=1.4 \mathrm{GeV}$; measured yields. JOUR ZAANE 25 s01 729

2005WI05 NUCLEAR REACTIONS ${ }^{10} \mathrm{Be}\left({ }^{14} \mathrm{C}, \alpha\right), \mathrm{E}=21.4 \mathrm{MeV}$; measured $\mathrm{E} \gamma$, $\mathrm{E} \alpha, \alpha \gamma-, \gamma \gamma$-coin. ${ }^{20} \mathrm{O}$ deduced levels, J, $\pi$, core excitation. Comparison with shell model predictions. JOUR PRLTA 94132501 ${ }^{20} \mathrm{~F}$ 2005EG01 NUCLEAR REACTIONS ${ }^{14} \mathrm{~N},{ }^{19} \mathrm{~F}(\mathrm{n}, \gamma), \mathrm{E}=$ thermal; measured $\mathrm{E} \gamma$, I $\gamma$, capture $\sigma$. JOUR NIMAE 545296

${ }^{20} \mathrm{Ne}$ 2005BB06 NUCLEAR REACTIONS ${ }^{12} \mathrm{C}\left({ }^{12} \mathrm{C}, \mathrm{X}\right), \mathrm{E}=5.3-7 \mathrm{MeV}$; measured $\mathrm{E} \gamma$, $\mathrm{I} \gamma$, thick-target yields. ${ }^{12} \mathrm{C}\left({ }^{12} \mathrm{C}, \mathrm{p}\right),\left({ }^{12} \mathrm{C}, \alpha\right), \mathrm{E}=5.3-7 \mathrm{MeV}$; deduced $\sigma$. Astrophysical implications discussed. JOUR ZAANE 25 s01 645

2005FR14 NUCLEAR REACTIONS ${ }^{12} \mathrm{C}\left({ }^{12} \mathrm{C},{ }^{8} \mathrm{Be}^{12} \mathrm{C}\right), \mathrm{E}=82-120 \mathrm{MeV}$; measured particle spectra, angular distributions. ${ }^{20} \mathrm{Ne}$ deduced possible resonance states energies, J, $\pi$. JOUR PRVCA 71047305 


\section{$\mathrm{A}=20$ (continued)}

2005 G036 ATOMIC MASSES ${ }^{12} \mathrm{C},{ }^{16} \mathrm{O},{ }^{20} \mathrm{Ne},{ }^{32} \mathrm{~S},{ }^{36,40} \mathrm{Ar}$; measured masses. Cyclotron-based mass spectrometry. JOUR JPGPE 31 S1869

2005 IL03 NUCLEAR REACTIONS ${ }^{23} \mathrm{Na}(\mathrm{p}, \gamma),(\mathrm{p}, \alpha), \mathrm{E}=130-155 \mathrm{keV}$; measured $\mathrm{E} \gamma, \mathrm{I} \gamma$; deduced resonance strength upper limits, astrophysical reaction rates. JOUR JPGPE 31 S1785

2005ST09 NUCLEAR REACTIONS ${ }^{12} \mathrm{C}\left({ }^{12} \mathrm{C}, \alpha\right), \mathrm{E}=34.7 \mathrm{MeV} ;{ }^{12} \mathrm{C}\left({ }^{16} \mathrm{O}, \alpha\right)$, $\mathrm{E}=38.5 \mathrm{MeV} ;$ measured $\mathrm{E} \alpha, \mathrm{E} \gamma, \mathrm{I} \gamma(\theta, \mathrm{t}), \alpha \gamma$-coin; $\mathrm{Gd}\left({ }^{24} \mathrm{Mg},{ }^{24} \mathrm{Mg}\right)$, $\mathrm{E}=165 \mathrm{MeV}$; measured $\mathrm{E} \gamma, \mathrm{I} \gamma(\theta, \mathrm{t})$, (particle) $\gamma$-coin; deduced transient field strengths. JOUR PYLBB 61181

\begin{tabular}{|c|c|c|}
\hline${ }^{20} \mathrm{Na}$ & $2005 \mathrm{CO} 017$ & $\begin{array}{l}\text { NUCLEAR REACTIONS }{ }^{1} \mathrm{H}\left({ }^{19} \mathrm{Ne}, \gamma\right), \mathrm{E}=10 \mathrm{MeV} \text {; measured particle } \\
\text { spectra. }{ }^{20} \mathrm{Na} \text { deduced resonance strength. JOUR NUPAB } 758 \text { 741c }\end{array}$ \\
\hline & 2005RU15 & $\begin{array}{l}\text { NUCLEAR REACTIONS }{ }^{1} \mathrm{H}\left({ }^{20} \mathrm{Na}, \mathrm{p}\right),\left({ }^{21} \mathrm{Na}, \mathrm{p}\right), \mathrm{E}(\mathrm{cm}) \approx 500-1600 \\
\mathrm{keV} \text {; measured recoil proton spectra; deduced excitation functions. } \\
{ }^{22} \mathrm{Ne} \text { deduced resonance energies, widths. JOUR NUPAB } 758166 \mathrm{c}\end{array}$ \\
\hline${ }^{20} \mathrm{Mg}$ & 2005IWZX & $\begin{array}{l}\text { NUCLEAR REACTIONS } \mathrm{Pb}\left({ }^{20} \mathrm{Mg},{ }^{20} \mathrm{Mg} \text { ') }, \mathrm{E}=58 \mathrm{MeV} / \text { nucleon; }\right. \\
\text { measured } \mathrm{E} \gamma, \mathrm{I} \gamma,(\text { particle}) \gamma \text {-coin following projectile Coulomb } \\
\text { excitation. }{ }^{20} \mathrm{Mg} \text { deduced transition. REPT RIKEN } 2004 \\
\text { Annual,P58,Iwasa }\end{array}$ \\
\hline
\end{tabular}

$$
\mathrm{A}=\mathbf{2 1}
$$

${ }^{21} \mathrm{O} \quad 2004 \mathrm{TH} 15$

${ }^{21} \mathrm{Ne} \quad$ 2005GE06

2005LE04

${ }^{21} \mathrm{Na} \quad$ 2004SIZX

2005HEZT

2005RU01
NUCLEAR REACTIONS Be $\left({ }^{40} \mathrm{Ar}, \mathrm{X}\right), \mathrm{E}=140 \mathrm{MeV} /$ nucleon; measured fragment isotopic yields; deduced no evidence for ${ }^{16} \mathrm{Be}$. ${ }^{12} \mathrm{C}\left({ }^{24} \mathrm{~F}, \mathrm{X}\right),\left({ }^{25} \mathrm{~F}, \mathrm{X}\right),\left({ }^{26} \mathrm{~F}, \mathrm{X}\right){ }^{20} \mathrm{O} /{ }^{21} \mathrm{O} /{ }^{22} \mathrm{O} /{ }^{23} \mathrm{O} /{ }^{24} \mathrm{O}, \mathrm{E} \approx 50$ $\mathrm{MeV} /$ nucleon; measured yields; deduced no evidence for ${ }^{25} \mathrm{O}$. JOUR APHPF 21379

NUCLEAR REACTIONS U(p, X $){ }^{19} \mathrm{O} /{ }^{20} \mathrm{O} /{ }^{21} \mathrm{O} /{ }^{22} \mathrm{O}, \mathrm{E}=1.4 \mathrm{GeV}$; measured yields. JOUR ZAANE 25 s01 729

NUCLEAR MOMENTS ${ }^{17,19,21,23,25} \mathrm{Ne}$; measured hfs; deduced $\mu$, quadrupole moments. Collinear fast-beam laser spectroscopy, comparison with shell model predictions. JOUR PRVCA 71064319 NUCLEAR REACTIONS Pb(p, X) ${ }^{3} \mathrm{He} /{ }^{4} \mathrm{He} /{ }^{21} \mathrm{Ne} /{ }^{22} \mathrm{Ne} /{ }^{36} \mathrm{Ar} /$ ${ }^{38} \mathrm{Ar} /{ }^{78} \mathrm{Kr} /{ }^{80} \mathrm{Kr} /{ }^{81} \mathrm{Kr} /{ }^{82} \mathrm{Kr} /{ }^{83} \mathrm{Kr} /{ }^{84} \mathrm{Kr} /{ }^{85} \mathrm{Kr} /{ }^{86} \mathrm{Kr} /{ }^{124} \mathrm{Xe}$ $/{ }^{126} \mathrm{Xe} /{ }^{128} \mathrm{Xe} /{ }^{129} \mathrm{Xe} /{ }^{130} \mathrm{Xe} /{ }^{131} \mathrm{Xe} /{ }^{132} \mathrm{Xe} /{ }^{134} \mathrm{Xe}, \mathrm{E}=44-2595$ $\mathrm{MeV}$; measured production $\sigma$; deduced reaction mechanism features. Mini-stack approach, comparisons with model predictions. JOUR NIMBE 2291 NUCLEAR REACTIONS ${ }^{1} \mathrm{H}\left({ }^{21} \mathrm{Na}, \alpha\right), \mathrm{E} \approx 113 \mathrm{MeV}$; measured $\mathrm{E} \alpha$, $\sigma(\mathrm{E}, \theta),\left({ }^{18} \mathrm{Ne}\right) \alpha$-coin. ${ }^{18} \mathrm{Ne}(\alpha, \mathrm{p}), \mathrm{E}(\mathrm{cm}) \approx 2.5 \mathrm{MeV}$; deduced angle-integrated $\sigma$. REPT ANL-04/22,P8,Sinha NUCLEAR REACTIONS ${ }^{1} \mathrm{H}\left({ }^{21} \mathrm{Na}, \mathrm{p}\right), \mathrm{E} \approx 4 \mathrm{MeV} /$ nucleon; measured recoil proton spectra, $\sigma(\theta)$. ${ }^{22} \mathrm{Mg}$ deduced level energies, resonance features. REPT CNS-REP-66,P1,He NUCLEAR REACTIONS ${ }^{1} \mathrm{H}\left({ }^{21} \mathrm{Na}, \mathrm{p}\right), \mathrm{E}=580-1560 \mathrm{keV} /$ nucleon; measured elastic and inelastic recoil proton spectra, $\sigma(\theta) .{ }^{22} \mathrm{Mg}$ deduced resonance energies, widths, $\mathrm{J}, \pi$, analog states. ${ }^{21} \mathrm{Na}(\mathrm{p}, \gamma)$, $\mathrm{E}=$ low; calculated reaction rate. R-matrix analysis. JOUR PRVCA 71 025802 


\section{$\mathrm{A}=\mathbf{2 1}$ (continued)}

2005RU15 NUCLEAR REACTIONS ${ }^{1} \mathrm{H}\left({ }^{20} \mathrm{Na}, \mathrm{p}\right),\left({ }^{21} \mathrm{Na}, \mathrm{p}\right), \mathrm{E}(\mathrm{cm}) \approx 500-1600$ $\mathrm{keV}$; measured recoil proton spectra; deduced excitation functions. ${ }^{22} \mathrm{Ne}$ deduced resonance energies, widths. JOUR NUPAB 758 166c

\section{$\mathrm{A}=\mathbf{2 2}$}

${ }^{22} \mathrm{O} \quad$ 2004BEZP

${ }^{22} \mathrm{~F}$

${ }^{22} \mathrm{Ne}$

2005KE08

2005KE11

2005LE04

2005RU15

2005UG04

2005WE06
NUCLEAR REACTIONS ${ }^{1} \mathrm{H}\left({ }^{22} \mathrm{O}, \mathrm{p}\right),\left({ }^{22} \mathrm{O},{ }^{22} \mathrm{O}\right), \mathrm{E} \approx 47 \mathrm{MeV} /$ nucleon; measured particle spectra, $\sigma(\mathrm{E}, \theta) .{ }^{22} \mathrm{O}$ level deduced deformation parameter. MUST detector array. REPT IPNO-T-04-17,Becheva NUCLEAR REACTIONS Be $\left({ }^{40} \mathrm{Ar}, \mathrm{X}\right), \mathrm{E}=140 \mathrm{MeV} /$ nucleon; measured fragment isotopic yields; deduced no evidence for ${ }^{16} \mathrm{Be}$. ${ }^{12} \mathrm{C}\left({ }^{24} \mathrm{~F}, \mathrm{X}\right),\left({ }^{25} \mathrm{~F}, \mathrm{X}\right),\left({ }^{26} \mathrm{~F}, \mathrm{X}\right){ }^{20} \mathrm{O} /{ }^{21} \mathrm{O} /{ }^{22} \mathrm{O} /{ }^{23} \mathrm{O} /{ }^{24} \mathrm{O}, \mathrm{E} \approx 50$ $\mathrm{MeV} /$ nucleon; measured yields; deduced no evidence for ${ }^{25} \mathrm{O}$. JOUR APHPF 21379

2005K043 NUCLEAR REACTIONS U(p, X) ${ }^{19} \mathrm{O} /{ }^{20} \mathrm{O} /{ }^{21} \mathrm{O} /{ }^{22} \mathrm{O}, \mathrm{E}=1.4 \mathrm{GeV}$; measured yields. JOUR ZAANE 25 s01 729

RADIOACTIVITY ${ }^{22} \mathrm{O},{ }^{22} \mathrm{~F}\left(\beta^{-}\right)$[from $\mathrm{U}(\mathrm{p}, \mathrm{X})$ and subsequent decay]; measured $\mathrm{E} \gamma, \mathrm{I} \gamma, \gamma \gamma-, \beta \gamma$-coin, $\mathrm{T}_{1 / 2} \cdot{ }^{22} \mathrm{~F}$ deduced levels, $\mathrm{J}, \pi$, $\beta$-feeding intensities. ${ }^{22} \mathrm{Ne}$ deduced transitions. Mass separator, comparison with model predictions. JOUR JPGPE 31553

RADIOACTIVITY ${ }^{22} \mathrm{O},{ }^{22} \mathrm{~F}\left(\beta^{-}\right)$[from $\mathrm{U}(\mathrm{p}, \mathrm{X})$ and subsequent decay]; measured $\mathrm{E} \gamma, \mathrm{I} \gamma, \gamma \gamma-, \beta \gamma$-coin, $\mathrm{T}_{1 / 2} \cdot{ }^{22} \mathrm{~F}$ deduced levels, $\mathrm{J}, \pi$, $\beta$-feeding intensities. ${ }^{22} \mathrm{Ne}$ deduced transitions. Mass separator, comparison with model predictions. JOUR JPGPE 31553 NUCLEAR REACTIONS ${ }^{150} \mathrm{Nd}\left({ }^{26} \mathrm{Ne}, \mathrm{X}\right){ }^{22} \mathrm{Ne} /{ }^{23} \mathrm{Na} /{ }^{28} \mathrm{Mg}, \mathrm{E}=160$ $\mathrm{MeV}$; measured $\mathrm{E} \gamma, \mathrm{I} \gamma, \gamma \gamma-$, (particle) $\gamma$-coin. ${ }^{22} \mathrm{Ne},{ }^{23} \mathrm{Na},{ }^{28} \mathrm{Mg}$ deduced levels, J, $\pi$. Euroball IV array, fragment separator. JOUR JPGPE 31 S1903

NUCLEAR REACTIONS ${ }^{150} \mathrm{Nd}\left({ }^{26} \mathrm{Mg}, \mathrm{X}\right), \mathrm{E}=160 \mathrm{MeV}$; measured $\mathrm{E} \gamma$, $\mathrm{I} \gamma, \gamma \gamma$-, (particle) $\gamma$-coin. ${ }^{22} \mathrm{Ne},{ }^{23} \mathrm{Na}$ deduced levels, J, $\pi$. Euroball IV array, binary reaction spectrometer. JOUR ZAANE 25 s01 431 NUCLEAR REACTIONS $\mathrm{Pb}(\mathrm{p}, \mathrm{X}){ }^{3} \mathrm{He} /{ }^{4} \mathrm{He} /{ }^{21} \mathrm{Ne} /{ }^{22} \mathrm{Ne} /{ }^{36} \mathrm{Ar} /$ ${ }^{38} \mathrm{Ar} /{ }^{78} \mathrm{Kr} /{ }^{80} \mathrm{Kr} /{ }^{81} \mathrm{Kr} /{ }^{82} \mathrm{Kr} /{ }^{83} \mathrm{Kr} /{ }^{84} \mathrm{Kr} /{ }^{85} \mathrm{Kr} /{ }^{86} \mathrm{Kr} /{ }^{124} \mathrm{Xe}$ / ${ }^{126} \mathrm{Xe} /{ }^{128} \mathrm{Xe} /{ }^{129} \mathrm{Xe} /{ }^{130} \mathrm{Xe} /{ }^{131} \mathrm{Xe} /{ }^{132} \mathrm{Xe} /{ }^{134} \mathrm{Xe}, \mathrm{E}=44-2595$ $\mathrm{MeV}$; measured production $\sigma$; deduced reaction mechanism features. Mini-stack approach, comparisons with model predictions. JOUR NIMBE 2291

NUCLEAR REACTIONS ${ }^{1} \mathrm{H}\left({ }^{20} \mathrm{Na}, \mathrm{p}\right),\left({ }^{21} \mathrm{Na}, \mathrm{p}\right), \mathrm{E}(\mathrm{cm}) \approx 500-1600$ $\mathrm{keV}$; measured recoil proton spectra; deduced excitation functions. ${ }^{22} \mathrm{Ne}$ deduced resonance energies, widths. JOUR NUPAB 758 166c NUCLEAR REACTIONS ${ }^{19} \mathrm{~F}(\alpha, \mathrm{p}), \mathrm{E}=1238-2009 \mathrm{keV}$; measured yields; deduced astrophysical reaction rates. JOUR NUPAB $758577 \mathrm{c}$ RADIOACTIVITY ${ }^{22} \mathrm{O},{ }^{22} \mathrm{~F}\left(\beta^{-}\right)$[from $\mathrm{U}(\mathrm{p}, \mathrm{X})$ and subsequent decay]; measured $\mathrm{E} \gamma, \mathrm{I} \gamma, \gamma \gamma-, \beta \gamma$-coin, $\mathrm{T}_{1 / 2} \cdot{ }^{22} \mathrm{~F}$ deduced levels, $\mathrm{J}, \pi$, $\beta$-feeding intensities. ${ }^{22} \mathrm{Ne}$ deduced transitions. Mass separator, comparison with model predictions. JOUR JPGPE 31553 


\section{$\mathrm{A}=22$ (continued)}

${ }^{22} \mathrm{Na} \quad$ 2005SI14

NUCLEAR REACTIONS C, O, Si, Mg, Al(n, X) ${ }^{7} \mathrm{Be}, \mathrm{E} \approx 0.1-750$ $\mathrm{MeV} ; \mathrm{O}, \mathrm{Si}, \mathrm{Mg}, \mathrm{Al}(\mathrm{n}, \mathrm{X}){ }^{22} \mathrm{Na} /{ }^{23} \mathrm{Na}, \mathrm{E} \approx 0.1-750 \mathrm{MeV} ;{ }^{197} \mathrm{Au}(\mathrm{n}$, $\mathrm{X}){ }^{194} \mathrm{Au} /{ }^{196} \mathrm{Au} /{ }^{198} \mathrm{Au}, \mathrm{E} \approx 0.1-750 \mathrm{MeV} ; \mathrm{Ti}, \mathrm{Fe}, \mathrm{Ni}, \mathrm{Cu}(\mathrm{n}, \mathrm{X})^{46} \mathrm{Sc}$ $/{ }^{48} \mathrm{Sc}, \mathrm{E} \approx 0.1-750 \mathrm{MeV} ; \mathrm{Fe}, \mathrm{Ni}, \mathrm{Cu}(\mathrm{n}, \mathrm{X}){ }^{48} \mathrm{~V} /{ }^{51} \mathrm{Cr} /{ }^{52} \mathrm{Mn} /{ }^{54} \mathrm{Mn}$, $\mathrm{E} \approx 0.1-750 \mathrm{MeV} ; \mathrm{Ni}, \mathrm{Cu}(\mathrm{n}, \mathrm{X}){ }^{56} \mathrm{Ni} /{ }^{57} \mathrm{Ni} /{ }^{56} \mathrm{Co} /{ }^{57} \mathrm{Co} /{ }^{58} \mathrm{Co} /$ ${ }^{60} \mathrm{Co} /{ }^{59} \mathrm{Fe}, \mathrm{E} \approx 0.1-750 \mathrm{MeV}$; measured energy-integrated production $\sigma$. JOUR NIMBE 234419

NUCLEAR REACTIONS ${ }^{12} \mathrm{C}\left({ }^{12} \mathrm{C}, 2 \mathrm{n}\right)$, E not given; measured $\mathrm{E} \gamma, \mathrm{I} \gamma$, (recoil) $\gamma$-coin. ${ }^{22} \mathrm{Mg}$ deduced levels, $\mathrm{J}, \pi$, mass excess. Gammasphere array, mass separator. REPT ANL-04/22,P12,Seweryniak

$2005 \mathrm{CH} 30 \quad$ NUCLEAR REACTIONS ${ }^{1} \mathrm{H}\left({ }^{21} \mathrm{Na}, \gamma\right), \mathrm{E}(\mathrm{cm}) \approx 200-1100 \mathrm{keV}$; measured thick-target yield. ${ }^{21} \mathrm{Na}(\mathrm{p}, \gamma), \mathrm{E}=$ low; deduced resonance parameters, astrophysical reaction rate. JOUR NUPAB 752 510c

2005DAZW NUCLEAR REACTIONS ${ }^{1} \mathrm{H}\left({ }^{21} \mathrm{Na}, \gamma\right), \mathrm{E}(\mathrm{cm})=206-1101 \mathrm{keV}$; measured $\mathrm{E} \gamma, \mathrm{I} \gamma$, (particle) $\gamma$-coin; deduced thick-target yields, resonance strengths. CONF Santa Fe (Nucl Data for Sci and Technol) Proc, Vol2,P1345

2005HEZS NUCLEAR REACTIONS ${ }^{1} \mathrm{H}\left({ }^{22} \mathrm{Mg}, \mathrm{p}\right), \mathrm{E} \approx 4 \mathrm{MeV} /$ nucleon; measured recoil proton spectra, $\sigma(\theta) .{ }^{23} \mathrm{Al}$ deduced level energies, possible $\mathrm{J}, \pi$, resonance features. REPT CNS-REP-66,P3,He

2005HEZT NUCLEAR REACTIONS ${ }^{1} \mathrm{H}\left({ }^{21} \mathrm{Na}, \mathrm{p}\right), \mathrm{E} \approx 4 \mathrm{MeV} /$ nucleon; measured recoil proton spectra, $\sigma(\theta)$. ${ }^{22} \mathrm{Mg}$ deduced level energies, resonance features. REPT CNS-REP-66,P1,He

2005HEZZ NUCLEAR REACTIONS ${ }^{1} \mathrm{H}\left({ }^{22} \mathrm{Mg}, \mathrm{p}\right), \mathrm{E}(\mathrm{cm}) \approx 0.5-3.5 \mathrm{MeV}$; measured proton spectrum. ${ }^{23} \mathrm{Al}$ deduced levels, $\mathrm{J}, \pi$. CONF Riken(Origin of Matter) Proc,P481,He

2005PA31 NUCLEAR REACTIONS ${ }^{24} \mathrm{Mg},{ }^{28} \mathrm{Si}(\mathrm{p}, \mathrm{t}), \mathrm{E}=33 \mathrm{MeV}$; measured triton spectra; deduced reaction Q-values. ${ }^{22} \mathrm{Mg},{ }^{26} \mathrm{Si}$ deduced mass excesses. JOUR PRVCA 71055804

2005RU01 NUCLEAR REACTIONS ${ }^{1} \mathrm{H}\left({ }^{21} \mathrm{Na}, \mathrm{p}\right), \mathrm{E}=580-1560 \mathrm{keV} /$ nucleon; measured elastic and inelastic recoil proton spectra, $\sigma(\theta) .{ }^{22} \mathrm{Mg}$ deduced resonance energies, widths, $\mathrm{J}, \pi$, analog states. ${ }^{21} \mathrm{Na}(\mathrm{p}, \gamma)$, $\mathrm{E}=$ low; calculated reaction rate. R-matrix analysis. JOUR PRVCA 71 025802

2005SA44 RADIOACTIVITY ${ }^{46} \mathrm{~V}(\mathrm{EC})$; analyzed masses; deduced Q(EC), log ft. ${ }^{10} \mathrm{C},{ }^{14} \mathrm{O},{ }^{22} \mathrm{Mg},{ }^{26 m} \mathrm{Al},{ }^{34} \mathrm{Cl},{ }^{34} \mathrm{Ar},{ }^{38 m} \mathrm{~K},{ }^{42} \mathrm{Sc},{ }^{46} \mathrm{~V},{ }^{50} \mathrm{Mn},{ }^{54} \mathrm{Co},{ }^{74} \mathrm{Rb}$; compiled, analyzed log ft; deduced quark-mixing matrix element. JOUR PRLTA 95102501

2005SE02 NUCLEAR REACTIONS ${ }^{12} \mathrm{C}\left({ }^{12} \mathrm{C}, 2 \mathrm{n}\right), \mathrm{E}=52 \mathrm{MeV}$; measured $\mathrm{E} \gamma, \mathrm{I} \gamma$, (recoil) $\gamma$-coin. ${ }^{22} \mathrm{Mg}$ deduced levels, $\mathrm{J}, \pi$, mass excess. Implications for astrophysical reaction rate discussed. Gammasphere array. JOUR PRLTA 94032501

2005SHZY NUCLEAR REACTIONS ${ }^{24} \mathrm{Mg},{ }^{28} \mathrm{Si}\left(\alpha,{ }^{6} \mathrm{He}\right), \mathrm{E}=205 \mathrm{MeV}$; measured excitation energy spectra. ${ }^{22} \mathrm{Mg},{ }^{26} \mathrm{Si}$ deduced resonance energies.

Astrophysical implications discussed. CONF Riken(Origin of Matter)

Proc,P367,Shimizu

2005TR11 NUCLEAR REACTIONS ${ }^{1} \mathrm{H}\left({ }^{21} \mathrm{Na}, \gamma\right), \mathrm{E}(\mathrm{cm})=200-1135 \mathrm{keV}$; measured $\mathrm{E} \gamma, \mathrm{I} \gamma, \gamma \gamma$-coin. ${ }^{22} \mathrm{Mg}$ deduced levels, $\mathrm{J}, \pi$, resonance strengths. JOUR NUPAB $758729 \mathrm{c}$ 
${ }^{23} \mathrm{O}$ 2004TH15 NUCLEAR REACTIONS Be $\left({ }^{40} \mathrm{Ar}, \mathrm{X}\right), \mathrm{E}=140 \mathrm{MeV} /$ nucleon; measured fragment isotopic yields; deduced no evidence for ${ }^{16} \mathrm{Be}$. ${ }^{12} \mathrm{C}\left({ }^{24} \mathrm{~F}, \mathrm{X}\right),\left({ }^{25} \mathrm{~F}, \mathrm{X}\right),\left({ }^{26} \mathrm{~F}, \mathrm{X}\right){ }^{20} \mathrm{O} /{ }^{21} \mathrm{O} /{ }^{22} \mathrm{O} /{ }^{23} \mathrm{O} /{ }^{24} \mathrm{O}, \mathrm{E} \approx 50$ $\mathrm{MeV} /$ nucleon; measured yields; deduced no evidence for ${ }^{25} \mathrm{O}$. JOUR APHPF 21379

2005 C024 NUCLEAR REACTIONS C $\left({ }^{23} \mathrm{O},{ }^{22} \mathrm{OX}\right), \mathrm{E}=938 \mathrm{MeV} /$ nucleon; measured longitudinal momentum distributions, one-neutron removal $\sigma .{ }^{23} \mathrm{O}$ deduced ground-state $\mathrm{J}, \pi$, configuration. JOUR ZAANE 25 s01 343

2005N001 NUCLEAR REACTIONS $\mathrm{Pb}\left({ }^{23} \mathrm{O}, \mathrm{n}^{22} \mathrm{O}\right), \mathrm{E}=422 \mathrm{MeV} /$ nucleon; measured En, E $\gamma, \mathrm{I} \gamma$, (fragment) $\gamma-$, n $\gamma$-coin, $\sigma(\mathrm{E})$; deduced final-state interaction effects. ${ }^{23} \mathrm{O}$ deduced ground state $\mathrm{J}, \pi$, configuration, spectroscopic factor. JOUR PYLBB 60579

${ }^{23} \mathrm{~F}$ NUCLEAR REACTIONS ${ }^{4} \mathrm{He}\left({ }^{22} \mathrm{O},{ }^{23} \mathrm{~F}\right), \mathrm{E} \approx 35 \mathrm{MeV} /$ nucleon; measured $\mathrm{E} \gamma, \mathrm{I} \gamma$, (particle) $\gamma$-coin. ${ }^{23} \mathrm{~F}$ deduced levels, transitions. REPT CNS-REP-64,P269,Michimasa

2005 MI32 NUCLEAR REACTIONS ${ }^{4} \mathrm{He}\left({ }^{22} \mathrm{O},{ }^{23} \mathrm{~F}\right), \mathrm{E}=35 \mathrm{MeV} /$ nucleon; ${ }^{4} \mathrm{He}\left({ }^{23} \mathrm{~F},{ }^{23} \mathrm{~F}\right.$ ') $\mathrm{E}=41.5 \mathrm{MeV} /$ nucleon; ${ }^{4} \mathrm{He}\left({ }^{24} \mathrm{~F},{ }^{23} \mathrm{~F}\right), \mathrm{E}=36 \mathrm{MeV} /$ nucleon; measured $\mathrm{E} \gamma, \mathrm{I} \gamma$, (particle) $\gamma$-, $\gamma \gamma$-coin; deduced $\sigma(\mathrm{E}) .{ }^{23} \mathrm{~F}$ deduced levels, J, $\pi$. DWBA analysis. JOUR ZAANE 25 s01 367

2005MIZT NUCLEAR REACTIONS ${ }^{4} \mathrm{He}\left({ }^{22} \mathrm{O},{ }^{23} \mathrm{~F}\right),\left({ }^{23} \mathrm{~F},{ }^{23} \mathrm{~F}^{\prime}\right),\left({ }^{24} \mathrm{~F},{ }^{23} \mathrm{~F}\right)$, $\left({ }^{25} \mathrm{Ne},{ }^{23} \mathrm{~F}\right), \mathrm{E} \approx 35-43 \mathrm{MeV} /$ nucleon; measured $\mathrm{E} \gamma, \mathrm{I} \gamma$, (particle) $\gamma-$, $\gamma \gamma$-coin. ${ }^{4} \mathrm{He}\left({ }^{22} \mathrm{O},{ }^{23} \mathrm{~F}\right), \mathrm{E}=35 \mathrm{MeV} /$ nucleon; measured $\sigma(\theta) .{ }^{23} \mathrm{~F}$ deduced levels, J, $\pi$, configurations. REPT CNS-REP-67,Michimasa

2005MIZU NUCLEAR REACTIONS ${ }^{4} \mathrm{He}\left({ }^{22} \mathrm{O},{ }^{23} \mathrm{~F}\right),\left({ }^{23} \mathrm{~F},{ }^{23} \mathrm{~F}\right),\left({ }^{24} \mathrm{~F},{ }^{23} \mathrm{~F}\right)$, E not given; measured $\mathrm{E} \gamma, \mathrm{I} \gamma, \gamma \gamma$-coin, $\sigma(\theta) .{ }^{23} \mathrm{~F}$ deduced levels, J, $\pi$. REPT CNS-REP-66,P26,Michimasa

2005MIZV NUCLEAR REACTIONS ${ }^{4} \mathrm{He}\left({ }^{22} \mathrm{O},{ }^{23} \mathrm{~F}\right), \mathrm{E} \approx 35 \mathrm{MeV} /$ nucleon; ${ }^{4} \mathrm{He}\left({ }^{23} \mathrm{~F},{ }^{23} \mathrm{~F}\right.$ ' $), \mathrm{E} \approx 41.5 \mathrm{MeV} /$ nucleon; ${ }^{4} \mathrm{He}\left({ }^{24} \mathrm{~F},{ }^{23} \mathrm{~F}\right), \mathrm{E} \approx 36 \mathrm{MeV} /$ nucleon; measured $\mathrm{E} \gamma, \mathrm{I} \gamma, \gamma \gamma$-, (particle) $\gamma$-coin, angular distributions. ${ }^{23} \mathrm{~F}$ deduced levels, J, $\pi$. REPT RIKEN 2004 Annual,P51,Michimasa 2005SH46 NUCLEAR REACTIONS ${ }^{4} \mathrm{He}\left({ }^{22} \mathrm{O},{ }^{23} \mathrm{~F}\right), \mathrm{E}=35 \mathrm{MeV} /$ nucleon; measured $\mathrm{E} \gamma, \mathrm{I} \gamma$, (particle) $\gamma$-coin, $\sigma(\theta) .{ }^{23} \mathrm{~F}$ deduced levels, J, $\pi$. JOUR JPGPE 31 S1759

${ }^{23} \mathrm{Ne} \quad 2005 \mathrm{GE} 06$ NUCLEAR MOMENTS 17,19,21,23,25 Ne; measured hfs; deduced $\mu$, quadrupole moments. Collinear fast-beam laser spectroscopy, comparison with shell model predictions. JOUR PRVCA 71064319 2005K001 NUCLEAR REACTIONS ${ }^{13} \mathrm{C}\left({ }^{18} \mathrm{O},{ }^{8} \mathrm{Be}\right),\left({ }^{18} \mathrm{O}, 2 \alpha\right), \mathrm{E}=100 \mathrm{MeV}$; ${ }^{24} \mathrm{Mg}\left({ }^{28} \mathrm{Si},{ }^{12} \mathrm{C}\right),\left({ }^{28} \mathrm{Si}, 3 \alpha\right), \mathrm{E}=130 \mathrm{MeV}$; measured particle spectra, $\mathrm{E} \gamma, \mathrm{I} \gamma$, (particle) $\gamma$-coin; deduced cluster emission features. GASP, ISIS arrays. JOUR ZAANE 2319

${ }^{23} \mathrm{Na} \quad 2004 \mathrm{VO} 21$ NUCLEAR REACTIONS ${ }^{22} \mathrm{Ne}(\mathrm{p}, \gamma), \mathrm{E}=840-2220 \mathrm{keV}$; measured $\mathrm{E} \gamma$, I $\gamma$, excitation function. ${ }^{23} \mathrm{Na}$ deduced levels, J, $\pi$, IAS features. Comparison with model predictions. JOUR BRSPE 68210

2005BB06 NUCLEAR REACTIONS ${ }^{12} \mathrm{C}\left({ }^{12} \mathrm{C}, \mathrm{X}\right), \mathrm{E}=5.3-7 \mathrm{MeV}$; measured $\mathrm{E} \gamma$, I $\gamma$, thick-target yields. ${ }^{12} \mathrm{C}\left({ }^{12} \mathrm{C}, \mathrm{p}\right),\left({ }^{12} \mathrm{C}, \alpha\right), \mathrm{E}=5.3-7 \mathrm{MeV}$; deduced $\sigma$. Astrophysical implications discussed. JOUR ZAANE 25 s01 645 


\section{$\mathrm{A}=\mathbf{2 3}$ (continued)}

2005BE03 RADIOACTIVITY ${ }^{23} \mathrm{Na},{ }^{127} \mathrm{I}$; measured $\mathrm{T}_{1 / 2}$ lower limits for spontaneous decay to superdense state; deduced potential barrier features. NaI detectors. JOUR ZAANE 237

2005DE42 NUCLEAR REACTIONS ${ }^{26} \mathrm{Al}(\mathrm{n}, \alpha),(\mathrm{n}, \mathrm{p}), \mathrm{E}<140 \mathrm{keV}$; measured $\sigma$; deduced resonance features. ${ }^{36} \mathrm{Cl}(\mathrm{n}, \mathrm{p}),{ }^{26} \mathrm{Al}(\mathrm{n}, \alpha), \mathrm{E}=$ stellar; analyzed astrophysical reaction rates. JOUR NUPAB 758 80c

2005JE06 NUCLEAR REACTIONS ${ }^{12} \mathrm{C}\left({ }^{12} \mathrm{C}, \mathrm{p}\right),\left({ }^{12} \mathrm{C}, \mathrm{n}\right), \mathrm{E}=22 \mathrm{MeV}$; measured $\mathrm{E} \gamma, \mathrm{I} \gamma, \gamma \gamma$-coin. ${ }^{23} \mathrm{Mg}$ levels deduced $\mathrm{J}, \pi .{ }^{22} \mathrm{Na}(\mathrm{p}, \gamma), \mathrm{E}=\mathrm{low}$; calculated astrophysical reaction rate, resonance contributions. Gammasphere array. JOUR NUPAB $758749 \mathrm{c}$

2005KE08 NUCLEAR REACTIONS ${ }^{150} \mathrm{Nd}\left({ }^{26} \mathrm{Ne}, \mathrm{X}\right){ }^{22} \mathrm{Ne} /{ }^{23} \mathrm{Na} /{ }^{28} \mathrm{Mg}, \mathrm{E}=160$ $\mathrm{MeV}$; measured $\mathrm{E} \gamma, \mathrm{I} \gamma, \gamma \gamma$-, (particle) $\gamma$-coin. ${ }^{22} \mathrm{Ne},{ }^{23} \mathrm{Na},{ }^{28} \mathrm{Mg}$ deduced levels, J, $\pi$. Euroball IV array, fragment separator. JOUR JPGPE 31 S1903

2005KE11 NUCLEAR REACTIONS ${ }^{150} \mathrm{Nd}\left({ }^{26} \mathrm{Mg}, \mathrm{X}\right), \mathrm{E}=160 \mathrm{MeV}$; measured E $\gamma$, $\mathrm{I} \gamma, \gamma \gamma$-, (particle) $\gamma$-coin. ${ }^{22} \mathrm{Ne},{ }^{23} \mathrm{Na}$ deduced levels, J, $\pi$. Euroball IV array, binary reaction spectrometer. JOUR ZAANE 25 s01 431

2005SI14 NUCLEAR REACTIONS C, O, Si, Mg, $\mathrm{Al}(\mathrm{n}, \mathrm{X})^{7} \mathrm{Be}, \mathrm{E} \approx 0.1-750$ $\mathrm{MeV}$; O, Si, Mg, $\mathrm{Al}(\mathrm{n}, \mathrm{X}){ }^{22} \mathrm{Na} /{ }^{23} \mathrm{Na}, \mathrm{E} \approx 0.1-750 \mathrm{MeV} ;{ }^{197} \mathrm{Au}(\mathrm{n}$, $\mathrm{X}){ }^{194} \mathrm{Au} /{ }^{196} \mathrm{Au} /{ }^{198} \mathrm{Au}, \mathrm{E} \approx 0.1-750 \mathrm{MeV} ; \mathrm{Ti}, \mathrm{Fe}, \mathrm{Ni}, \mathrm{Cu}(\mathrm{n}, \mathrm{X}){ }^{46} \mathrm{Sc}$ $/{ }^{48} \mathrm{Sc}, \mathrm{E} \approx 0.1-750 \mathrm{MeV} ; \mathrm{Fe}, \mathrm{Ni}, \mathrm{Cu}(\mathrm{n}, \mathrm{X}){ }^{48} \mathrm{~V} /{ }^{51} \mathrm{Cr} /{ }^{52} \mathrm{Mn} /{ }^{54} \mathrm{Mn}$, $\mathrm{E} \approx 0.1-750 \mathrm{MeV} ; \mathrm{Ni}, \mathrm{Cu}(\mathrm{n}, \mathrm{X}){ }^{56} \mathrm{Ni} /{ }^{57} \mathrm{Ni} /{ }^{56} \mathrm{Co} /{ }^{57} \mathrm{Co} /{ }^{58} \mathrm{Co} /$ ${ }^{60} \mathrm{Co} /{ }^{59} \mathrm{Fe}, \mathrm{E} \approx 0.1-750 \mathrm{MeV}$; measured energy-integrated production $\sigma$. JOUR NIMBE 234419

${ }^{23} \mathrm{Mg}$ 2005JE06 NUCLEAR REACTIONS ${ }^{12} \mathrm{C}\left({ }^{12} \mathrm{C}, \mathrm{p}\right),\left({ }^{12} \mathrm{C}, \mathrm{n}\right), \mathrm{E}=22 \mathrm{MeV}$; measured $\mathrm{E} \gamma, \mathrm{I} \gamma, \gamma \gamma$-coin. ${ }^{23} \mathrm{Mg}$ levels deduced $\mathrm{J}, \pi .{ }^{22} \mathrm{Na}(\mathrm{p}, \gamma), \mathrm{E}=$ low; calculated astrophysical reaction rate, resonance contributions. Gammasphere array. JOUR NUPAB $758749 \mathrm{c}$

2005KU27 NUCLEAR REACTIONS ${ }^{4} \mathrm{He}\left({ }^{14} \mathrm{O}, \mathrm{p}\right), \mathrm{E}(\mathrm{cm}) \approx 1-3.6 \mathrm{MeV}$; measured proton spectrum. ${ }^{1} \mathrm{H}\left({ }^{23} \mathrm{Mg}, \mathrm{p}\right), \mathrm{E}(\mathrm{cm}) \approx 0.6-3.5 \mathrm{MeV}$; measured elastic $\sigma(\theta) .{ }^{24} \mathrm{Al}$ deduced excited states energies. JOUR NUPAB $758733 \mathrm{c}$

2005TEZY NUCLEAR REACTIONS ${ }^{1} \mathrm{H}\left({ }^{23} \mathrm{Mg}, \mathrm{p}\right), \mathrm{E}(\mathrm{cm}) \approx 0.5-3.5 \mathrm{MeV}$; measured recoil proton spectra, $\sigma(\theta)$. REPT RIKEN 2004 Annual,P59,Teranishi

2005TEZZ NUCLEAR REACTIONS ${ }^{1} \mathrm{H}\left({ }^{23} \mathrm{Mg}, \mathrm{p}\right),\left({ }^{24} \mathrm{Mg}, \mathrm{p}\right), \mathrm{E}(\mathrm{cm}) \approx 0.5-3.5$ $\mathrm{MeV}$; measured excitation functions, $\sigma(\theta)$; deduced resonance features. CONF Riken(Origin of Matter) Proc,P361,Teranishi

${ }^{23} \mathrm{Al} 2005 \mathrm{GO} 3 \quad$ NUCLEAR REACTIONS $\mathrm{Pb}\left({ }^{23} \mathrm{Al}, \mathrm{p}^{22} \mathrm{Mg}\right), \mathrm{E}=50 \mathrm{MeV} /$ nucleon; measured particles relative energy spectrum, $\mathrm{E} \gamma, \mathrm{I} \gamma$; deduced Coulomb dissociation $\sigma(\theta) .{ }^{23} \mathrm{Al}$ level deduced radiative width. JOUR NUPAB $758761 \mathrm{c}$

$2005 \mathrm{GO34}$ NUCLEAR REACTIONS ${ }^{208} \mathrm{~Pb}\left({ }^{23} \mathrm{Al}, \mathrm{p}{ }^{22} \mathrm{Mg}\right), \mathrm{E}=50 \mathrm{MeV} /$ nucleon; measured relative energy spectrum, $\sigma(\theta)$. ${ }^{23} \mathrm{Al}$ deduced excited state radiative width. Astrophysical implications discussed. JOUR JPGPE $31 \mathrm{~S} 1517$

2005HEZS NUCLEAR REACTIONS ${ }^{1} \mathrm{H}\left({ }^{22} \mathrm{Mg}, \mathrm{p}\right), \mathrm{E} \approx 4 \mathrm{MeV} /$ nucleon; measured recoil proton spectra, $\sigma(\theta) .{ }^{23} \mathrm{Al}$ deduced level energies, possible $\mathrm{J}, \pi$, resonance features. REPT CNS-REP-66,P3,He 


\section{$\mathrm{A}=23$ (continued)}

2005HEZZ

NUCLEAR REACTIONS ${ }^{1} \mathrm{H}\left({ }^{22} \mathrm{Mg}, \mathrm{p}\right), \mathrm{E}(\mathrm{cm}) \approx 0.5-3.5 \mathrm{MeV}$; measured proton spectrum. ${ }^{23} \mathrm{Al}$ deduced levels, J, $\pi$. CONF Riken(Origin of Matter) Proc,P481,He

\section{$\mathrm{A}=\mathbf{2 4}$}

${ }^{24} \mathrm{O} \quad 2004 \mathrm{TH} 15$

${ }^{24} \mathrm{Ne} \quad$ 2004KRZX

${ }^{24} \mathrm{Na} \quad$ 2005MU21

2005TIZY

2005 IL02

2005IL03

2005JE03

2005JEZZ
NUCLEAR REACTIONS Be $\left({ }^{40} \mathrm{Ar}, \mathrm{X}\right), \mathrm{E}=140 \mathrm{MeV} /$ nucleon; measured fragment isotopic yields; deduced no evidence for ${ }^{16} \mathrm{Be}$. ${ }^{12} \mathrm{C}\left({ }^{24} \mathrm{~F}, \mathrm{X}\right),\left({ }^{25} \mathrm{~F}, \mathrm{X}\right),\left({ }^{26} \mathrm{~F}, \mathrm{X}\right){ }^{20} \mathrm{O} /{ }^{21} \mathrm{O} /{ }^{22} \mathrm{O} /{ }^{23} \mathrm{O} /{ }^{24} \mathrm{O}, \mathrm{E} \approx 50$ $\mathrm{MeV}$ / nucleon; measured yields; deduced no evidence for ${ }^{25} \mathrm{O}$. JOUR APHPF 21379 NUCLEAR REACTIONS ${ }^{27} \mathrm{Al}(\mathrm{n}, \mathrm{n} 3 \mathrm{p}),{ }^{59} \mathrm{Co},{ }^{139} \mathrm{La},{ }^{129} \mathrm{I},{ }^{197} \mathrm{Au}$, ${ }^{237} \mathrm{~Np}(\mathrm{n}, \gamma),{ }^{59} \mathrm{Co},{ }^{127,129} \mathrm{I},{ }^{197} \mathrm{Au},{ }^{209} \mathrm{Bi}(\mathrm{n}, \mathrm{xn}),{ }^{235,238} \mathrm{U}(\mathrm{n}, \mathrm{F})$, $\mathrm{E}=$ spectrum; measured yields; deduced reaction rates. $\mathrm{Pb}(\mathrm{p}, \mathrm{nX})$, $\mathrm{E}=1.5 \mathrm{GeV}$; deduced neutron spectrum. REPT

JINR-E1-2004-79,Krivopustov NUCLEAR REACTIONS ${ }^{115} \mathrm{In}(\mathrm{n}, \mathrm{n}),{ }^{27} \mathrm{Al}(\mathrm{n}, \alpha),{ }^{93} \mathrm{Nb}(\mathrm{n}, 2 \mathrm{n}),(\mathrm{n}, 4 \mathrm{n})$, ${ }^{209} \operatorname{Bi}(\mathrm{n}, 4 \mathrm{n}),(\mathrm{n}, 5 \mathrm{n}),(\mathrm{n}, 6 \mathrm{n}),(\mathrm{n}, 7 \mathrm{n}), \mathrm{E} \approx 10-1000 \mathrm{MeV}$; measured reaction rates. Comparison with model predictions. JOUR NIMAE 547 555

NUCLEAR REACTIONS ${ }^{27} \mathrm{Al}(\mathrm{d}, \mathrm{X}){ }^{27} \mathrm{Mg} /{ }^{24} \mathrm{Na}, \mathrm{E}=22-40 \mathrm{MeV}$; $\mathrm{Cu}(\mathrm{d}, \mathrm{X}){ }^{62} \mathrm{Zn} /{ }^{63} \mathrm{Zn} /{ }^{61} \mathrm{Cu} /{ }^{64} \mathrm{Cu}, \mathrm{E}=22-40 \mathrm{MeV} ; \mathrm{W}(\mathrm{d}, \mathrm{X}){ }^{181} \mathrm{Re} /$ ${ }^{182} \operatorname{Re} /{ }^{183} \operatorname{Re} /{ }^{184} \operatorname{Re} /{ }^{186} \operatorname{Re} /{ }^{187} \mathrm{~W}, \mathrm{E}=22-40 \mathrm{MeV}$; measured activation $\sigma$. Comparison with previous results and model predictions. CONF Santa Fe (Nucl Data for Sci and Technol) Proc,Vol2,P1489 NUCLEAR REACTIONS Pb, ${ }^{208} \mathrm{~Pb}(\mathrm{p}, \mathrm{X}){ }^{203} \mathrm{~Pb} /{ }^{200} \mathrm{Tl} /{ }^{199} \mathrm{Tl} /$ ${ }^{196} \mathrm{Au} /{ }^{192} \mathrm{Ir} /{ }^{190} \mathrm{Ir} /{ }^{173} \mathrm{Lu} /{ }^{101 m} \mathrm{Rh} /{ }^{86} \mathrm{Rb} /{ }^{59} \mathrm{Fe} /{ }^{24} \mathrm{Na} /{ }^{7} \mathrm{Be}$, $\mathrm{E}=40-2600 \mathrm{MeV}$; measured excitation functions. Comparison with previous work and model predictions. Other reactions discussed. CONF Santa Fe (Nucl Data for Sci and Technol) Proc,Vol1,P1070 NUCLEAR REACTIONS Pb, ${ }^{208} \mathrm{~Pb},{ }^{209} \mathrm{Bi}(\mathrm{p}, \mathrm{X}){ }^{203} \mathrm{~Pb} /{ }^{200} \mathrm{Tl} /{ }^{199} \mathrm{Tl}$ $/{ }^{196} \mathrm{Au} /{ }^{192} \mathrm{Ir} /{ }^{190} \mathrm{Ir} /{ }^{173} \mathrm{Lu} /{ }^{101 m} \mathrm{Rh} /{ }^{86} \mathrm{Rb} /{ }^{59} \mathrm{Fe} /{ }^{24} \mathrm{Na} /{ }^{7} \mathrm{Be}$, $\mathrm{E}=40-2600 \mathrm{MeV}$; measured production $\sigma$. Comparison with model predictions. PREPRINT nucl-ex/0507009,7/05/2005

NUCLEAR REACTIONS ${ }^{17} \mathrm{O}(\mathrm{p}, \gamma), \mathrm{E} \approx 190,519 \mathrm{keV}$; measured $\mathrm{E} \gamma$, $\mathrm{I} \gamma$; deduced resonance excitation functions. ${ }^{23} \mathrm{Na}(\mathrm{p}, \gamma), \mathrm{E} \approx 150 \mathrm{keV}$; measured $\mathrm{E} \gamma, \mathrm{I} \gamma, \gamma \gamma$-coin; deduced resonance strength upper limit. Astrophysical implications discussed. JOUR NUPAB 758 73c NUCLEAR REACTIONS ${ }^{23} \mathrm{Na}(\mathrm{p}, \gamma),(\mathrm{p}, \alpha), \mathrm{E}=130-155 \mathrm{keV}$; measured $\mathrm{E} \gamma, \mathrm{I} \gamma$; deduced resonance strength upper limits, astrophysical reaction rates. JOUR JPGPE 31 S1785 NUCLEAR REACTIONS ${ }^{12} \mathrm{C}\left({ }^{12} \mathrm{C}, \gamma\right), \mathrm{E}(\mathrm{cm}) \approx 8 \mathrm{MeV}$; measured $\mathrm{E} \gamma$, $\mathrm{I} \gamma, \sigma$; deduced role of doorway states. Gammasphere array. JOUR PRVCA 71041301

NUCLEAR REACTIONS ${ }^{12} \mathrm{C}\left({ }^{12} \mathrm{C}, \gamma\right), \mathrm{E} \approx 16 \mathrm{MeV}$; measured $\mathrm{E} \gamma, \mathrm{I} \gamma$, $\sigma$; deduced role of doorway states. Gammasphere array, mass separator. CONF Argonne(Nuclei at the Limits),P367,Jenkins 


\section{$\mathrm{A}=\mathbf{2 4}$ (continued)}

2005ST09 NUCLEAR REACTIONS ${ }^{12} \mathrm{C}\left({ }^{12} \mathrm{C}, \alpha\right), \mathrm{E}=34.7 \mathrm{MeV} ;{ }^{12} \mathrm{C}\left({ }^{16} \mathrm{O}, \alpha\right)$, $\mathrm{E}=38.5 \mathrm{MeV}$; measured $\mathrm{E} \alpha, \mathrm{E} \gamma, \mathrm{I} \gamma(\theta, \mathrm{t}), \alpha \gamma$-coin; $\mathrm{Gd}\left({ }^{24} \mathrm{Mg},{ }^{24} \mathrm{Mg}{ }^{\prime}\right)$, $\mathrm{E}=165 \mathrm{MeV}$; measured $\mathrm{E} \gamma, \mathrm{I} \gamma(\theta, \mathrm{t})$, (particle) $\gamma$-coin; deduced transient field strengths. JOUR PYLBB 61181

2005TEZZ NUCLEAR REACTIONS ${ }^{1} \mathrm{H}\left({ }^{23} \mathrm{Mg}, \mathrm{p}\right),\left({ }^{24} \mathrm{Mg}, \mathrm{p}\right), \mathrm{E}(\mathrm{cm}) \approx 0.5-3.5$ $\mathrm{MeV}$; measured excitation functions, $\sigma(\theta)$; deduced resonance features. CONF Riken(Origin of Matter) Proc,P361,Teranishi

${ }^{24} \mathrm{Al} \quad 2005 \mathrm{KU} 27$ NUCLEAR REACTIONS ${ }^{4} \mathrm{He}\left({ }^{14} \mathrm{O}, \mathrm{p}\right), \mathrm{E}(\mathrm{cm}) \approx 1-3.6 \mathrm{MeV}$; measured proton spectrum. ${ }^{1} \mathrm{H}\left({ }^{23} \mathrm{Mg}, \mathrm{p}\right), \mathrm{E}(\mathrm{cm}) \approx 0.6-3.5 \mathrm{MeV}$; measured elastic $\sigma(\theta) .{ }^{24} \mathrm{Al}$ deduced excited states energies. JOUR NUPAB $758733 \mathrm{c}$

\section{$\mathrm{A}=\mathbf{2 5}$}

${ }^{25} \mathrm{O} \quad 2004 \mathrm{TH} 15$

NUCLEAR REACTIONS Be $\left({ }^{40} \mathrm{Ar}, \mathrm{X}\right), \mathrm{E}=140 \mathrm{MeV} /$ nucleon; measured fragment isotopic yields; deduced no evidence for ${ }^{16} \mathrm{Be}$. ${ }^{12} \mathrm{C}\left({ }^{24} \mathrm{~F}, \mathrm{X}\right),\left({ }^{25} \mathrm{~F}, \mathrm{X}\right),\left({ }^{26} \mathrm{~F}, \mathrm{X}\right){ }^{20} \mathrm{O} /{ }^{21} \mathrm{O} /{ }^{22} \mathrm{O} /{ }^{23} \mathrm{O} /{ }^{24} \mathrm{O}, \mathrm{E} \approx 50$ $\mathrm{MeV} /$ nucleon; measured yields; deduced no evidence for ${ }^{25} \mathrm{O}$. JOUR APHPF 21379

${ }^{25} \mathrm{Ne} \quad 2005 \mathrm{BE} 60$ NUCLEAR REACTIONS ${ }^{9} \mathrm{Be}, \mathrm{C}\left({ }^{36} \mathrm{~S}, \mathrm{X}\right){ }^{25} \mathrm{Ne} /{ }^{26} \mathrm{Ne} /{ }^{27} \mathrm{Ne} /{ }^{28} \mathrm{Ne}$, $\mathrm{E}=77.5 \mathrm{MeV} /$ nucleon; measured $\mathrm{E} \gamma, \mathrm{I} \gamma, \gamma \gamma$-coin. ${ }^{26,28} \mathrm{Ne}$ deduced levels, J, $\pi .{ }^{27,29} \mathrm{Ne}$ deduced excited states. Comparison with shell model predictions. JOUR PRVCA 72054316

2005CA44 NUCLEAR REACTIONS ${ }^{2} \mathrm{H}\left({ }^{24} \mathrm{Ne}, \mathrm{p}\right), \mathrm{E}=10 \mathrm{MeV} /$ nucleon; measured Ep, E $\gamma$, p $\gamma$-coin, $\sigma(\theta) .{ }^{25}$ Ne deduced levels, J, $\pi$. JOUR JPGPE 31 S1655

2005CA50 NUCLEAR REACTIONS ${ }^{2} \mathrm{H}\left({ }^{24} \mathrm{Ne}, \mathrm{p}\right), \mathrm{E}=10 \mathrm{MeV} /$ nucleon; measured Ep, E $\gamma$, p $\gamma$-coin, $\sigma(\theta) .{ }^{25} \mathrm{Ne}$ deduced levels, J, $\pi$. JOUR ZAANE 25 s01 245

2005GE06 NUCLEAR MOMENTS ${ }^{17,19,21,23,25}$ Ne; measured hfs; deduced $\mu$, quadrupole moments. Collinear fast-beam laser spectroscopy, comparison with shell model predictions. JOUR PRVCA 71064319

2005GIZX NUCLEAR REACTIONS $\mathrm{Pb}\left({ }^{26} \mathrm{Ne},{ }^{25} \mathrm{NeX}\right), \mathrm{E}=58 \mathrm{MeV} /$ nucleon; measured $\mathrm{E} \gamma, \mathrm{I} \gamma$, (particle) $\gamma$-coin. ${ }^{25} \mathrm{Ne}$ deduced levels, transitions. REPT RIKEN 2004 Annual,P53, Gibelin

${ }^{25} \mathrm{Al}$ 2005MO28 NUCLEAR REACTIONS ${ }^{1} \mathrm{H}\left({ }^{25} \mathrm{Al}, \mathrm{p}\right),\left({ }^{26} \mathrm{Si}, \mathrm{p}\right), \mathrm{E}(\mathrm{cm})=0.5-3 \mathrm{MeV}$; measured recoil proton spectra; deduced excitation functions. ${ }^{26} \mathrm{Si},{ }^{27} \mathrm{P}$ deduced levels, proton resonance states. Comparison with shell model predictions. JOUR NUPAB $758158 \mathrm{c}$

2005MOZU NUCLEAR REACTIONS ${ }^{1} \mathrm{H}\left({ }^{25} \mathrm{Al}, \mathrm{p}\right),\left({ }^{26} \mathrm{Si}, \mathrm{p}\right), \mathrm{E}(\mathrm{cm}) \approx 0.5-3 \mathrm{MeV}$; measured elastic $\sigma\left(\theta=180^{\circ}\right) .{ }^{27} \mathrm{P}$ deduced resonance energies, $\mathrm{J}, \pi$. REPT RIKEN 2004 Annual,P63,Moon

2005MOZZ NUCLEAR REACTIONS ${ }^{1} \mathrm{H}\left({ }^{25} \mathrm{Al}, \mathrm{p}\right), \mathrm{E}(\mathrm{cm}) \approx 0.5-3.44 \mathrm{MeV} /$ nucleon; ${ }^{1} \mathrm{H}\left({ }^{26} \mathrm{Si}, \mathrm{p}\right), \mathrm{E}(\mathrm{cm}) \approx 0.5-3.95 \mathrm{MeV} /$ nucleon; measured excitation functions; deduced resonance features. Thick target. CONF Riken(Origin of Matter) Proc,P505,Moon 


\section{$\mathrm{A}=\mathbf{2 6}$}

\begin{tabular}{|c|c|c|}
\hline \multirow[t]{2}{*}{${ }^{26} \mathrm{O}$} & $2005 \mathrm{SC} 20$ & $\begin{array}{l}\text { NUCLEAR REACTIONS } \mathrm{C}\left({ }^{27} \mathrm{~F}, \mathrm{X}\right),\left({ }^{29} \mathrm{Ne}, \mathrm{X}\right), \mathrm{E} \approx 90 \mathrm{MeV} / \\
\text { nucleon; measured fragment yields, production } \sigma \text { upper limits; deduced } \\
\text { no evidence for }{ }^{26} \mathrm{O},{ }^{28} \mathrm{~F} \text {. JOUR PRVCA } 72037601\end{array}$ \\
\hline & $2005 \mathrm{SCZY}$ & $\begin{array}{l}\text { NUCLEAR REACTIONS } \mathrm{C}\left({ }^{27} \mathrm{~F}, \mathrm{X}\right),\left({ }^{29} \mathrm{Ne}, \mathrm{X}\right), \mathrm{E} \approx 90 \mathrm{MeV} / \\
\text { nucleon; measured isotopic yields following proton-stripping reactions; } \\
\text { deduced no evidence for }{ }^{26} \mathrm{O},{ }^{28} \mathrm{~F} . \mathrm{PREPRINT} \\
\text { nucl-ex } / 0504007,4 / 5 / 2005\end{array}$ \\
\hline \multirow[t]{3}{*}{${ }^{26} \mathrm{Ne}$} & 2005BE60 & $\begin{array}{l}\text { NUCLEAR REACTIONS }{ }^{9} \mathrm{Be}, \mathrm{C}\left({ }^{36} \mathrm{~S}, \mathrm{X}\right){ }^{25} \mathrm{Ne} /{ }^{26} \mathrm{Ne} /{ }^{27} \mathrm{Ne} /{ }^{28} \mathrm{Ne} \text {, } \\
\mathrm{E}=77.5 \mathrm{MeV} / \text { nucleon; measured } \mathrm{E} \gamma, \mathrm{I} \gamma, \gamma \gamma \text {-coin. }{ }^{26,28} \mathrm{Ne} \text { deduced } \\
\text { levels, J, } \pi .{ }^{27,29} \mathrm{Ne} \text { deduced excited states. Comparison with shell } \\
\text { model predictions. JOUR PRVCA } 72054316\end{array}$ \\
\hline & 2005DOZW & $\begin{array}{l}\text { NUCLEAR REACTIONS }{ }^{9} \mathrm{Be}\left({ }^{36} \mathrm{~S}, \mathrm{X}\right){ }^{26} \mathrm{Ne} /{ }^{28} \mathrm{Ne}, \mathrm{E} \text { not given; } \\
\text { measured E } \gamma, \mathrm{I} \gamma, \gamma \gamma-\text {, (recoil) } \gamma \text {-coin. }{ }^{26,28} \mathrm{Ne} \text { deduced transitions. } \\
\text { REPT ATOMKI } 2004 \text { Annual,P10,Dombradi }\end{array}$ \\
\hline & 2005GAZT & $\begin{array}{l}\text { ATOMIC MASSES }{ }^{26} \mathrm{Ne},{ }^{26,27,28,29,30} \mathrm{Na},{ }^{29,30,31,32,33} \mathrm{Mg} \text {; measured } \\
\text { masses. Reanalysis of data using new calibration. PREPRINT } \\
\text { nucl-ex } / 0511007,11 / 2 / 2005\end{array}$ \\
\hline \multirow[t]{4}{*}{${ }^{26} \mathrm{Na}$} & 2005GAZT & $\begin{array}{l}\text { ATOMIC MASSES }{ }^{26} \mathrm{Ne},{ }^{26,27,28,29,30} \mathrm{Na},{ }^{29,30,31,32,33} \mathrm{Mg} \text {; measured } \\
\text { masses. Reanalysis of data using new calibration. PREPRINT } \\
\text { nucl-ex } / 0511007,11 / 2 / 2005\end{array}$ \\
\hline & 2005GR07 & $\begin{array}{l}\text { RADIOACTIVITY }{ }^{26} \mathrm{Na}\left(\beta^{-}\right)[\text {from } \mathrm{Si}, \mathrm{Ta}(\mathrm{p}, \mathrm{X})] ; \text { measured } \mathrm{E} \gamma, \mathrm{I} \gamma \\
\mathrm{T}_{1 / 2} ; \text { deduced log ft. }{ }^{26} \mathrm{Mg} \text { deduced levels, J, } \pi, \beta \text {-feeding intensities. } \\
\text { JOUR PRVCA } 71044309\end{array}$ \\
\hline & 2005WI20 & $\begin{array}{l}\text { RADIOACTIVITY }{ }^{26} \mathrm{Na}\left(\beta^{-}\right) ;{ }^{152} \mathrm{Eu}\left(\beta^{-}\right),(\mathrm{EC}) ; \text { measured } \mathrm{E} \gamma, \mathrm{I} \gamma, \gamma \gamma-\text {, } \\
\beta \gamma \text {-coin. }{ }^{152} \mathrm{Sm} \text { level deduced } \mathrm{T}_{1 / 2} \text {. JOUR JPGPE } 31 \mathrm{~S} 1979\end{array}$ \\
\hline & 2005ZEZZ & $\begin{array}{l}\text { NUCLEAR REACTIONS }{ }^{26} \mathrm{Mg}\left({ }^{3} \mathrm{He}, \mathrm{t}\right), \mathrm{E}=140 \mathrm{MeV} / \text { nucleon; } \\
{ }^{26} \mathrm{Mg}\left(\mathrm{t},{ }^{3} \mathrm{He}\right), \mathrm{E}=115 \mathrm{MeV} / \text { nucleon; measured excitation energy } \\
\text { spectra, } \sigma(\theta) ; \text { deduced Gamow-Teller strengths. PREPRINT } \\
\text { nucl-ex } / 0512025,12 / 20 / 2005\end{array}$ \\
\hline \multirow[t]{5}{*}{${ }^{26} \mathrm{Mg}$} & 2005BE61 & $\begin{array}{l}\text { NUCLEAR REACTIONS }{ }^{26} \mathrm{Mg}\left({ }^{76} \mathrm{Kr},{ }^{76} \mathrm{Kr}{ }^{9}\right), \mathrm{E}=230 \mathrm{MeV} \text {; measured } \\
\mathrm{E} \gamma, \mathrm{I} \gamma(\theta, \mathrm{H}, \mathrm{t}),(\text { particle}) \gamma \text {-coin following projectile Coulomb } \\
\text { excitation. }{ }^{76} \mathrm{Kr} \text { level deduced g factor. Transient field technique. } \\
\text { JOUR ZAANE } 25 \text { s01 } 203\end{array}$ \\
\hline & $2005 \mathrm{CH} 66$ & 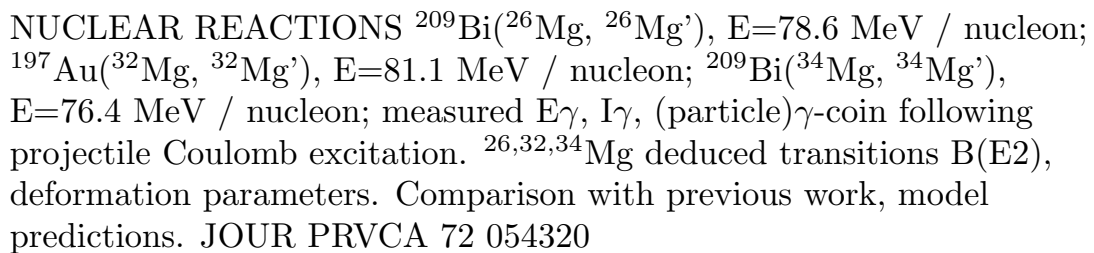 \\
\hline & 2005DE42 & $\begin{array}{l}\text { NUCLEAR REACTIONS }{ }^{26} \mathrm{Al}(\mathrm{n}, \alpha),(\mathrm{n}, \mathrm{p}), \mathrm{E}<140 \mathrm{keV} \text {; measured } \\
\sigma \text {; deduced resonance features. }{ }^{36} \mathrm{Cl}(\mathrm{n}, \mathrm{p}),{ }^{26} \mathrm{Al}(\mathrm{n}, \alpha), \mathrm{E}=\text { stellar; } \\
\text { analyzed astrophysical reaction rates. JOUR NUPAB } 75880 \mathrm{c}\end{array}$ \\
\hline & 2005GR07 & $\begin{array}{l}\text { RADIOACTIVITY }{ }^{26} \mathrm{Na}\left(\beta^{-}\right)[\text {from } \mathrm{Si}, \mathrm{Ta}(\mathrm{p}, \mathrm{X})] ; \text { measured } \mathrm{E} \gamma, \mathrm{I} \gamma \\
\mathrm{T}_{1 / 2} ; \text { deduced } \log \mathrm{ft} .{ }^{26} \mathrm{Mg} \text { deduced levels, } \mathrm{J}, \pi, \beta \text {-feeding intensities. } \\
\text { JOUR PRVCA } 71044309\end{array}$ \\
\hline & 2005SC01 & $\begin{array}{l}\text { RADIOACTIVITY }{ }^{26 m} \mathrm{Al}\left(\beta^{+}\right)\left[\text {from }{ }^{27} \mathrm{Al}(\gamma, \mathrm{n})\right] ; \text { measured } \mathrm{T}_{1 / 2} \text {. } \\
\text { JOUR NIMAE } 539191\end{array}$ \\
\hline
\end{tabular}




\section{$\mathrm{A}=\mathbf{2 6}$ (continued)}

2005WI20 RADIOACTIVITY ${ }^{26} \mathrm{Na}\left(\beta^{-}\right) ;{ }^{152} \mathrm{Eu}\left(\beta^{-}\right)$, (EC); measured $\mathrm{E} \gamma, \mathrm{I} \gamma, \gamma \gamma-$, $\beta \gamma$-coin. ${ }^{152} \mathrm{Sm}$ level deduced $\mathrm{T}_{1 / 2}$. JOUR JPGPE $31 \mathrm{~S} 1979$

${ }^{26} \mathrm{Al} \quad 2005 \mathrm{HE} 24 \quad$ NUCLEAR REACTIONS ${ }^{14} \mathrm{~N}\left({ }^{16} \mathrm{O}, \alpha\right), \mathrm{E}(\mathrm{cm})=6.6,7.9,9.5 \mathrm{MeV}$; measured $\sigma$. Accelerator mass spectrometry. JOUR NIMBE 240612

2005SA44 RADIOACTIVITY ${ }^{46} \mathrm{~V}(\mathrm{EC})$; analyzed masses; deduced $\mathrm{Q}(\mathrm{EC}), \log \mathrm{ft}$. ${ }^{10} \mathrm{C},{ }^{14} \mathrm{O},{ }^{22} \mathrm{Mg},{ }^{26 m} \mathrm{Al},{ }^{34} \mathrm{Cl},{ }^{34} \mathrm{Ar},{ }^{38 m} \mathrm{~K},{ }^{42} \mathrm{Sc},{ }^{46} \mathrm{~V},{ }^{50} \mathrm{Mn},{ }^{54} \mathrm{Co},{ }^{74} \mathrm{Rb}$; compiled, analyzed log ft; deduced quark-mixing matrix element. JOUR PRLTA 95102501

2005SC01 RADIOACTIVITY ${ }^{26 m} \mathrm{Al}\left(\beta^{+}\right)$[from ${ }^{27} \mathrm{Al}(\gamma, \mathrm{n})$ ]; measured $\mathrm{T}_{1 / 2}$. JOUR NIMAE 539191

2005SCZW NUCLEAR REACTIONS $\mathrm{Pb}(\mathrm{p}, \mathrm{X}){ }^{10} \mathrm{Be} /{ }^{26} \mathrm{Al} /{ }^{129} \mathrm{I} /{ }^{36} \mathrm{Cl}$, $\mathrm{E}=200-2600 \mathrm{MeV}$; measured excitation functions. Stacked foil activation, chemical separation. Comparison with model predictions. CONF Santa Fe (Nucl Data for Sci and Technol) Proc,Vol2,P1517

2005WAZU NUCLEAR REACTIONS ${ }^{27} \mathrm{Al}(\mathrm{n}, 2 \mathrm{n}), \mathrm{E}=13.6-14.9 \mathrm{MeV}$; measured $\sigma$. Accelerator mass spectrometry, other potential measurements discussed. CONF Santa Fe (Nucl Data for Sci and Technol) Proc,Vol1,P621

2005ZEZZ NUCLEAR REACTIONS ${ }^{26} \mathrm{Mg}\left({ }^{3} \mathrm{He}, \mathrm{t}\right), \mathrm{E}=140 \mathrm{MeV} /$ nucleon; ${ }^{26} \mathrm{Mg}\left(\mathrm{t},{ }^{3} \mathrm{He}\right), \mathrm{E}=115 \mathrm{MeV} /$ nucleon; measured excitation energy spectra, $\sigma(\theta)$; deduced Gamow-Teller strengths. PREPRINT nucl-ex/0512025,12/20/2005

${ }^{26} \mathrm{Si} \quad$ 2004PA42 NUCLEAR REACTIONS ${ }^{24} \mathrm{Mg}\left({ }^{3} \mathrm{He}, \mathrm{n}\right), \mathrm{E}=7.9,8.11,10.0 \mathrm{MeV}$; measured En, $\sigma(\theta) .{ }^{26} \mathrm{Si}$ deduced levels, J, $\pi .{ }^{25} \mathrm{Al}(\mathrm{p}, \gamma), \mathrm{E}=\mathrm{low}$; calculated astrophysical reaction rates. JOUR PRVCA 70065805 2005M028 NUCLEAR REACTIONS ${ }^{1} \mathrm{H}\left({ }^{25} \mathrm{Al}, \mathrm{p}\right),\left({ }^{26} \mathrm{Si}, \mathrm{p}\right), \mathrm{E}(\mathrm{cm})=0.5-3 \mathrm{MeV}$; measured recoil proton spectra; deduced excitation functions. ${ }^{26} \mathrm{Si},{ }^{27} \mathrm{P}$ deduced levels, proton resonance states. Comparison with shell model predictions. JOUR NUPAB 758 158c

2005MOZR NUCLEAR REACTIONS ${ }^{1} \mathrm{H}\left({ }^{26} \mathrm{Si}, \mathrm{p}\right), \mathrm{E} \approx 4 \mathrm{MeV} /$ nucleon; measured recoil proton spectra, $\sigma(\theta)$. ${ }^{27} \mathrm{P}$ deduced level energies, $\mathrm{J}, \pi$, resonance features. REPT CNS-REP-66,P6,Moon

2005MOZU NUCLEAR REACTIONS ${ }^{1} \mathrm{H}\left({ }^{25} \mathrm{Al}, \mathrm{p}\right),\left({ }^{26} \mathrm{Si}, \mathrm{p}\right), \mathrm{E}(\mathrm{cm}) \approx 0.5-3 \mathrm{MeV}$; measured elastic $\sigma\left(\theta=180^{\circ}\right)$. ${ }^{27} \mathrm{P}$ deduced resonance energies, $\mathrm{J}, \pi$. REPT RIKEN 2004 Annual,P63,Moon

2005MOZZ NUCLEAR REACTIONS ${ }^{1} \mathrm{H}\left({ }^{25} \mathrm{Al}, \mathrm{p}\right), \mathrm{E}(\mathrm{cm}) \approx 0.5-3.44 \mathrm{MeV} /$ nucleon; ${ }^{1} \mathrm{H}\left({ }^{26} \mathrm{Si}, \mathrm{p}\right), \mathrm{E}(\mathrm{cm}) \approx 0.5-3.95 \mathrm{MeV} /$ nucleon; measured excitation functions; deduced resonance features. Thick target. CONF Riken(Origin of Matter) Proc,P505,Moon

2005PA31 NUCLEAR REACTIONS ${ }^{24} \mathrm{Mg},{ }^{28} \mathrm{Si}(\mathrm{p}, \mathrm{t}), \mathrm{E}=33 \mathrm{MeV}$; measured triton spectra; deduced reaction Q-values. ${ }^{22} \mathrm{Mg},{ }^{26} \mathrm{Si}$ deduced mass excesses. JOUR PRVCA 71055804

2005SHZY NUCLEAR REACTIONS ${ }^{24} \mathrm{Mg},{ }^{28} \mathrm{Si}\left(\alpha,{ }^{6} \mathrm{He}\right), \mathrm{E}=205 \mathrm{MeV}$; measured excitation energy spectra. ${ }^{22} \mathrm{Mg},{ }^{26} \mathrm{Si}$ deduced resonance energies. Astrophysical implications discussed. CONF Riken(Origin of Matter) Proc,P367,Shimizu 


\section{$\mathrm{A}=\mathbf{2 7}$}

\begin{tabular}{|c|c|c|}
\hline${ }^{27} \mathrm{Ne}$ & 2005BE60 & $\begin{array}{l}\text { NUCLEAR REACTIONS }{ }^{9} \mathrm{Be}, \mathrm{C}\left({ }^{36} \mathrm{~S}, \mathrm{X}\right){ }^{25} \mathrm{Ne} /{ }^{26} \mathrm{Ne} /{ }^{27} \mathrm{Ne} /{ }^{28} \mathrm{Ne} \text {, } \\
\mathrm{E}=77.5 \mathrm{MeV} / \text { nucleon; measured } \mathrm{E} \gamma, \mathrm{I} \gamma, \gamma \gamma \text {-coin. }{ }^{26,28} \mathrm{Ne} \text { deduced } \\
\text { levels, J, } \pi .{ }^{27,29} \mathrm{Ne} \text { deduced excited states. Comparison with shell } \\
\text { model predictions. JOUR PRVCA } 72054316\end{array}$ \\
\hline${ }^{27} \mathrm{Na}$ & $2005 \mathrm{GAZT}$ & $\begin{array}{l}\text { ATOMIC MASSES }{ }^{26} \mathrm{Ne},{ }^{26,27,28,29,30} \mathrm{Na},{ }^{29,30,31,32,33} \mathrm{Mg} \text {; measured } \\
\text { masses. Reanalysis of data using new calibration. PREPRINT } \\
\text { nucl-ex } / 0511007,11 / 2 / 2005\end{array}$ \\
\hline${ }^{27} \mathrm{Mg}$ & 2005NAZY & $\begin{array}{l}\text { NUCLEAR REACTIONS }{ }^{27} \mathrm{Al}(\mathrm{d}, \mathrm{X}){ }^{27} \mathrm{Mg} /{ }^{24} \mathrm{Na}, \mathrm{E}=22-40 \mathrm{MeV} \\
\mathrm{Cu}(\mathrm{d}, \mathrm{X})^{62} \mathrm{Zn} /{ }^{63} \mathrm{Zn} /{ }^{61} \mathrm{Cu} /{ }^{64} \mathrm{Cu}, \mathrm{E}=22-40 \mathrm{MeV} ; \mathrm{W}(\mathrm{d}, \mathrm{X}){ }^{181} \mathrm{Re} / \\
{ }^{182} \mathrm{Re} /{ }^{183} \mathrm{Re} /{ }^{184} \mathrm{Re} /{ }^{186} \mathrm{Re} /{ }^{187} \mathrm{~W}, \mathrm{E}=22-40 \mathrm{MeV} ; \text { measured } \\
\text { activation } \sigma \text {. Comparison with previous results and model predictions. } \\
\text { CONF Santa Fe (Nucl Data for Sci and Technol) Proc,Vol2,P1489 }\end{array}$ \\
\hline & $2005 \mathrm{SCZV}$ & $\begin{array}{l}\text { NUCLEAR REACTIONS }{ }^{9} \mathrm{Be}\left({ }^{26} \mathrm{Mg},{ }^{27} \mathrm{Mg}\right), \mathrm{E}=57 \mathrm{MeV} ; \text { measured } \mathrm{E} \gamma \text {, } \\
\mathrm{I} \gamma, \alpha \alpha \text {-coin, } \sigma(\theta) .{ }^{27} \mathrm{Mg} \text { deduced transitions. REPT MLL } 2004 \\
\text { Annual,P4,Schwerdtfeger }\end{array}$ \\
\hline${ }^{27} \mathrm{Al}$ & $2005 \mathrm{SA} 04$ & $\begin{array}{l}\text { NUCLEAR REACTIONS }{ }^{12} \mathrm{C},{ }^{28} \mathrm{Si},{ }^{27} \mathrm{Al}, \mathrm{Fe}\left(\pi^{+}, \mathrm{K}^{+}\right), \mathrm{E} \text { at } 1.06 \mathrm{GeV} \\
\text { / c; measured hypernucleus mass spectra, pion and proton spectra } \\
\text { following hypernucleus decay. }{ }^{12} \mathrm{C},{ }^{28} \mathrm{Si},{ }^{27} \mathrm{Al} \text {, Fe deduced mesonic and } \\
\text { nonmesonic hypernucleus decay widths. Comparison with model } \\
\text { predictions. JOUR PRVCA } 71025203\end{array}$ \\
\hline${ }^{27} \mathrm{Si}$ & 2005LE30 & $\begin{array}{l}\text { NUCLEAR REACTIONS }{ }^{27} \mathrm{Al}\left({ }^{3} \mathrm{He}, \mathrm{t}\right), \mathrm{E}=25 \mathrm{MeV} \text {; measured Ep } \\
\text { following residual nucleus decay to ground and metastable states. }{ }^{27} \mathrm{Si} \\
\text { deduced resonance energy. Astrophysical implications discussed. JOUR } \\
\text { NUPAB } 75884 \mathrm{c}\end{array}$ \\
\hline & $2005 \mathrm{SA} 37$ & $\begin{array}{l}\text { NUCLEAR REACTIONS }{ }^{40} \mathrm{Ca}(\mathrm{e}, \mathrm{e} \cdot \mathrm{n}), \mathrm{E}=129 \mathrm{MeV} ; \text { measured En, } \\
\text { missing energy spectra, angular correlations, } \sigma(\mathrm{E}, \theta) ;{ }^{12} \mathrm{C},{ }^{28} \mathrm{Si}(\mathrm{e}, \mathrm{e} n) \text {, } \\
\text { E not given; analyzed data; deduced core excitation in giant resonance. } \\
\text { JOUR PRVCA } 71064313\end{array}$ \\
\hline \multirow[t]{5}{*}{${ }^{27} \mathrm{P}$} & $2005 \mathrm{MO} 28$ & $\begin{array}{l}\text { NUCLEAR REACTIONS }{ }^{1} \mathrm{H}\left({ }^{25} \mathrm{Al}, \mathrm{p}\right),\left({ }^{26} \mathrm{Si}, \mathrm{p}\right), \mathrm{E}(\mathrm{cm})=0.5-3 \mathrm{MeV} ; \\
\text { measured recoil proton spectra; deduced excitation functions. }{ }^{26} \mathrm{Si},{ }^{27} \mathrm{P} \\
\text { deduced levels, proton resonance states. Comparison with shell model } \\
\text { predictions. JOUR NUPAB } 758158 \mathrm{c}\end{array}$ \\
\hline & 2005MOZR & $\begin{array}{l}\text { NUCLEAR REACTIONS }{ }^{1} \mathrm{H}\left({ }^{26} \mathrm{Si}, \mathrm{p}\right), \mathrm{E} \approx 4 \mathrm{MeV} / \text { nucleon; measured } \\
\text { recoil proton spectra, } \sigma(\theta) .{ }^{27} \mathrm{P} \text { deduced level energies, } \mathrm{J}, \pi \text {, resonance } \\
\text { features. REPT CNS-REP-66,P6,Moon }\end{array}$ \\
\hline & 2005MOZU & $\begin{array}{l}\text { NUCLEAR REACTIONS }{ }^{1} \mathrm{H}\left({ }^{25} \mathrm{Al}, \mathrm{p}\right),\left({ }^{26} \mathrm{Si}, \mathrm{p}\right), \mathrm{E}(\mathrm{cm}) \approx 0.5-3 \mathrm{MeV} \\
\text { measured elastic } \sigma\left(\theta=180^{\circ}\right) .{ }^{27} \mathrm{P} \text { deduced resonance energies, J, } \pi \text {. } \\
\text { REPT RIKEN } 2004 \text { Annual,P63,Moon }\end{array}$ \\
\hline & 2005T011 & $\begin{array}{l}\text { NUCLEAR REACTIONS } \mathrm{Pb}\left({ }^{27} \mathrm{P}, \mathrm{p}^{26} \mathrm{Si}\right), \mathrm{E}=57 \mathrm{MeV} / \text { nucleon; } \\
\text { measured relative energy spectrum. }{ }^{27} \mathrm{P} \text { deduced excited state } \gamma \text {-decay } \\
\text { width. Comparison with previous results. JOUR NUPAB } 758 \text { 182c }\end{array}$ \\
\hline & $2005 \mathrm{TOZZ}$ & $\begin{array}{l}\text { NUCLEAR REACTIONS }{ }^{208} \mathrm{~Pb}\left({ }^{27} \mathrm{P}, \mathrm{p}^{26} \mathrm{Si}\right), \mathrm{E}=57 \mathrm{MeV} / \text { nucleon; } \\
\text { measured relative energy spectrum, } \sigma(\mathrm{E}) .{ }^{27} \mathrm{P} \text { deduced gamma decay } \\
\text { width of first excited state. CONF Riken(Origin of Matter) } \\
\text { Proc,P549,Togano }\end{array}$ \\
\hline
\end{tabular}




\section{$\mathrm{A}=\mathbf{2 8}$}

\begin{tabular}{|c|c|c|}
\hline \multirow[t]{2}{*}{${ }^{28} \mathrm{~F}$} & $2005 \mathrm{SC} 20$ & $\begin{array}{l}\text { NUCLEAR REACTIONS } \mathrm{C}\left({ }^{27} \mathrm{~F}, \mathrm{X}\right),\left({ }^{29} \mathrm{Ne}, \mathrm{X}\right), \mathrm{E} \approx 90 \mathrm{MeV} / \\
\text { nucleon; measured fragment yields, production } \sigma \text { upper limits; deduced } \\
\text { no evidence for }{ }^{26} \mathrm{O},{ }^{28} \mathrm{~F} \text {. JOUR PRVCA } 72037601\end{array}$ \\
\hline & $2005 \mathrm{SCZY}$ & $\begin{array}{l}\text { NUCLEAR REACTIONS } \mathrm{C}\left({ }^{27} \mathrm{~F}, \mathrm{X}\right),\left({ }^{29} \mathrm{Ne}, \mathrm{X}\right), \mathrm{E} \approx 90 \mathrm{MeV} / \\
\text { nucleon; measured isotopic yields following proton-stripping reactions; } \\
\text { deduced no evidence for }{ }^{26} \mathrm{O},{ }^{28} \mathrm{~F} . \mathrm{PREPRINT} \\
\text { nucl-ex } / 0504007,4 / 5 / 2005\end{array}$ \\
\hline \multirow[t]{4}{*}{${ }^{28} \mathrm{Ne}$} & 2005BE60 & $\begin{array}{l}\text { NUCLEAR REACTIONS }{ }^{9} \mathrm{Be}, \mathrm{C}\left({ }^{36} \mathrm{~S}, \mathrm{X}\right){ }^{25} \mathrm{Ne} /{ }^{26} \mathrm{Ne} /{ }^{27} \mathrm{Ne} /{ }^{28} \mathrm{Ne} \text {, } \\
\mathrm{E}=77.5 \mathrm{MeV} / \text { nucleon; measured } \mathrm{E} \gamma, \mathrm{I} \gamma, \gamma \gamma \text {-coin. }{ }^{26,28} \mathrm{Ne} \text { deduced } \\
\text { levels, J, } \pi .{ }^{27,29} \mathrm{Ne} \text { deduced excited states. Comparison with shell } \\
\text { model predictions. JOUR PRVCA } 72054316\end{array}$ \\
\hline & 2005DOZW & $\begin{array}{l}\text { NUCLEAR REACTIONS }{ }^{9} \mathrm{Be}\left({ }^{36} \mathrm{~S}, \mathrm{X}\right){ }^{26} \mathrm{Ne} /{ }^{28} \mathrm{Ne}, \mathrm{E} \text { not given; } \\
\text { measured E } \gamma, \mathrm{I} \gamma, \gamma \gamma-\text {, (recoil) } \gamma \text {-coin. }{ }^{26,28} \mathrm{Ne} \text { deduced transitions. } \\
\text { REPT ATOMKI } 2004 \text { Annual,P10,Dombradi }\end{array}$ \\
\hline & 2005IW02 & $\begin{array}{l}\text { NUCLEAR REACTIONS C, } \mathrm{Pb}\left({ }^{28} \mathrm{Ne},{ }^{28} \mathrm{Ne}\right), \mathrm{E}=46 \mathrm{MeV} / \text { nucleon; } \\
\text { measured } \mathrm{E} \gamma, \mathrm{I} \gamma, \text { (particle) } \gamma \text {-coin. }{ }^{28} \mathrm{Ne} \text { deduced transition } \mathrm{B}(\mathrm{E} 2) \text {, } \\
\text { suppressed collectivity. Comparison with neighboring nuclides, model } \\
\text { predictions. JOUR PYLBB } 620118\end{array}$ \\
\hline & 2005TR05 & $\begin{array}{l}\text { RADIOACTIVITY }{ }^{28,29} \mathrm{Ne}\left(\beta^{-}\right)\left[\text {from } \mathrm{Be}\left({ }^{48} \mathrm{Ca}, \mathrm{X}\right)\right] \text {; measured } \mathrm{E} \gamma, \mathrm{I} \gamma \\
\gamma \gamma-, \beta \gamma \text {-coin; deduced } \log \mathrm{ft} .{ }^{28,29} \mathrm{Na} \text { deduced levels, J, } \pi, \beta \text {-feeding } \\
\text { intensities, configurations, inverted shell structure. JOUR PRLTA } 94 \\
162501\end{array}$ \\
\hline \multirow[t]{2}{*}{${ }^{28} \mathrm{Na}$} & 2005GAZT & $\begin{array}{l}\text { ATOMIC MASSES }{ }^{26} \mathrm{Ne},{ }^{26,27,28,29,30} \mathrm{Na},{ }^{29,30,31,32,33} \mathrm{Mg} \text {; measured } \\
\text { masses. Reanalysis of data using new calibration. PREPRINT } \\
\text { nucl-ex/0511007,11/2/2005 }\end{array}$ \\
\hline & 2005TR05 & $\begin{array}{l}\text { RADIOACTIVITY }{ }^{28,29} \mathrm{Ne}\left(\beta^{-}\right)\left[\text {from } \mathrm{Be}\left({ }^{48} \mathrm{Ca}, \mathrm{X}\right)\right] \text {; measured } \mathrm{E} \gamma, \mathrm{I} \gamma \\
\gamma \gamma-, \beta \gamma \text {-coin; deduced } \log \mathrm{ft} .{ }^{28,29} \mathrm{Na} \text { deduced levels, J, } \pi, \beta \text {-feeding } \\
\text { intensities, configurations, inverted shell structure. JOUR PRLTA } 94 \\
162501\end{array}$ \\
\hline${ }^{28} \mathrm{Mg}$ & 2005KE08 & $\begin{array}{l}\text { NUCLEAR REACTIONS }{ }^{150} \mathrm{Nd}\left({ }^{26} \mathrm{Ne}, \mathrm{X}\right){ }^{22} \mathrm{Ne} /{ }^{23} \mathrm{Na} /{ }^{28} \mathrm{Mg}, \mathrm{E}=160 \\
\mathrm{MeV} \text {; measured } \mathrm{E} \gamma, \mathrm{I} \gamma, \gamma \gamma-,(\text { particle }) \gamma \text {-coin. }{ }^{22} \mathrm{Ne},{ }^{23} \mathrm{Na},{ }^{28} \mathrm{Mg} \\
\text { deduced levels, J, } \pi \text {. Euroball IV array, fragment separator. JOUR } \\
\text { JPGPE } 31 \mathrm{~S} 1903\end{array}$ \\
\hline${ }^{28} \mathrm{Al}$ & 2005GE07 & $\begin{array}{l}\text { NUCLEAR REACTIONS }{ }^{10} \mathrm{~B},{ }^{27} \mathrm{Al}(\text { polarized } \mathrm{n}, \gamma), \mathrm{E}=\mathrm{low} \text {; measured } \\
\text { parity-violating } \gamma \text {-ray asymmetry. JOUR JRNBA } 110215\end{array}$ \\
\hline \multirow[t]{3}{*}{${ }^{28} \mathrm{Si}$} & 2004MB08 & $\begin{array}{l}\text { NUCLEAR REACTIONS }{ }^{28} \mathrm{Si}\left({ }^{16} \mathrm{O},{ }^{16} \mathrm{O}^{\prime}\right), \mathrm{E}=40-46,71,73,75 \mathrm{MeV} \\
\text { measured E } \gamma, \mathrm{I} \gamma,(\text { particle }) \gamma \text {-coin, } \sigma(\theta) \text {. Gammasphere, Chico arrays. } \\
\text { JOUR BJPHE } 34885\end{array}$ \\
\hline & 2005MA52 & $\begin{array}{l}\text { NUCLEAR REACTIONS }{ }^{12} \mathrm{C}(\alpha, \gamma), \mathrm{E}=2.27 \mathrm{MeV} ;{ }^{27} \mathrm{Al}(\mathrm{p}, \gamma), \mathrm{E}=2.05 \\
\mathrm{MeV} ; \text { measured } \mathrm{E} \gamma, \mathrm{I} \gamma(\theta) . \text { JOUR NIMAE } 547411\end{array}$ \\
\hline & 2005SA04 & $\begin{array}{l}\text { NUCLEAR REACTIONS }{ }^{12} \mathrm{C},{ }^{28} \mathrm{Si},{ }^{27} \mathrm{Al}, \mathrm{Fe}\left(\pi^{+}, \mathrm{K}^{+}\right), \mathrm{E} \text { at } 1.06 \mathrm{GeV} \\
\text { / c; measured hypernucleus mass spectra, pion and proton spectra } \\
\text { following hypernucleus decay. }{ }^{12} \mathrm{C},{ }^{28} \mathrm{Si},{ }^{27} \mathrm{Al}, \mathrm{Fe} \text { deduced mesonic and } \\
\text { nonmesonic hypernucleus decay widths. Comparison with model } \\
\text { predictions. JOUR PRVCA } 71025203\end{array}$ \\
\hline
\end{tabular}

predictions. JOUR PRVCA 71025203 


\section{$\mathrm{A}=28$ (continued)}

2005WE01 NUCLEAR REACTIONS ${ }^{12} \mathrm{C}\left({ }^{29} \mathrm{P},{ }^{28} \mathrm{SiX}\right), \mathrm{E}=30.7 \mathrm{MeV} /$ nucleon; measured fragments parallel momentum distribution. ${ }^{28} \mathrm{Si},{ }^{29} \mathrm{P}$ deduced particle density distributions, related features. Glauber model and Skyrme-Hartree-Fock calculations. JOUR CPLEE 2261

\section{$\mathrm{A}=29$}

${ }^{29} \mathrm{Ne} \quad 2005 \mathrm{BE} 60$

NUCLEAR REACTIONS ${ }^{9} \mathrm{Be}, \mathrm{C}\left({ }^{36} \mathrm{~S}, \mathrm{X}\right){ }^{25} \mathrm{Ne} /{ }^{26} \mathrm{Ne} /{ }^{27} \mathrm{Ne} /{ }^{28} \mathrm{Ne}$, $\mathrm{E}=77.5 \mathrm{MeV} /$ nucleon; measured $\mathrm{E} \gamma$, I $\gamma$, $\gamma \gamma$-coin. ${ }^{26,28} \mathrm{Ne}$ deduced levels, J, $\pi .{ }^{27,29} \mathrm{Ne}$ deduced excited states. Comparison with shell model predictions. JOUR PRVCA 72054316

2005TR05 RADIOACTIVITY ${ }^{28,29} \mathrm{Ne}\left(\beta^{-}\right)$[from $\mathrm{Be}\left({ }^{48} \mathrm{Ca}, \mathrm{X}\right)$ ]; measured $\mathrm{E} \gamma, \mathrm{I} \gamma$, $\gamma \gamma$-, $\beta \gamma$-coin; deduced log ft. ${ }^{28,29} \mathrm{Na}$ deduced levels, J, $\pi, \beta$-feeding intensities, configurations, inverted shell structure. JOUR PRLTA 94 162501

2005TR13 RADIOACTIVITY ${ }^{29} \mathrm{Ne}\left(\beta^{-}\right)$[from $\left.\mathrm{Be}\left({ }^{48} \mathrm{Ca}, \mathrm{X}\right)\right]$; measured $\mathrm{E} \gamma, \mathrm{E} \beta$, $\gamma \gamma$-, $\beta \gamma$-coin; deduced log ft. ${ }^{29} \mathrm{Na}$ deduced levels, $\beta$-feeding intensities. Comparison with shell model calculations. JOUR ZAANE 25 s01 101 ATOMIC MASSES ${ }^{26} \mathrm{Ne},{ }^{26,27,28,29,30} \mathrm{Na},{ }^{29,30,31,32,33} \mathrm{Mg}$; measured masses. Reanalysis of data using new calibration. PREPRINT nucl-ex/0511007,11/2/2005

2005 TR05 RADIOACTIVITY ${ }^{28,29} \mathrm{Ne}\left(\beta^{-}\right)$[from $\left.\mathrm{Be}\left({ }^{48} \mathrm{Ca}, \mathrm{X}\right)\right]$; measured $\mathrm{E} \gamma, \mathrm{I} \gamma$, $\gamma \gamma$-, $\beta \gamma$-coin; deduced log ft. ${ }^{28,29} \mathrm{Na}$ deduced levels, J, $\pi, \beta$-feeding intensities, configurations, inverted shell structure. JOUR PRLTA 94 162501

2005TR13 RADIOACTIVITY ${ }^{29} \mathrm{Ne}\left(\beta^{-}\right)$[from $\left.\mathrm{Be}\left({ }^{48} \mathrm{Ca}, \mathrm{X}\right)\right]$; measured $\mathrm{E} \gamma, \mathrm{E} \beta$, $\gamma \gamma-, \beta \gamma$-coin; deduced log ft. ${ }^{29} \mathrm{Na}$ deduced levels, $\beta$-feeding intensities. Comparison with shell model calculations. JOUR ZAANE 25 s01 101

${ }^{29} \mathrm{Mg} \quad$ 2005GAZT ATOMIC MASSES ${ }^{26} \mathrm{Ne},{ }^{26,27,28,29,30} \mathrm{Na},{ }^{29,30,31,32,33} \mathrm{Mg}$; measured masses. Reanalysis of data using new calibration. PREPRINT nucl-ex/0511007,11/2/2005

${ }^{29} \mathrm{Si} \quad$ 2005DEZW NUCLEAR REACTIONS ${ }^{28} \mathrm{Si},{ }^{32} \mathrm{~S},{ }^{35} \mathrm{Cl}(\mathrm{n}, \gamma), \mathrm{E}=$ reactor; measured $\mathrm{E} \gamma, \mathrm{I} \gamma .{ }^{29} \mathrm{Si},{ }^{33} \mathrm{~S},{ }^{36} \mathrm{Cl}$ deduced binding energies. Flat-crystal spectrometer. PREPRINT nucl-ex/0507011,7/06/2005

2005JEZY NUCLEAR REACTIONS ${ }^{28} \mathrm{Si},{ }^{32} \mathrm{~S},{ }^{35} \mathrm{Cl}(\mathrm{n}, \gamma), \mathrm{E}=$ thermal; measured $\mathrm{E} \gamma, \mathrm{I} \gamma \cdot{ }^{29} \mathrm{Si},{ }^{33} \mathrm{~S},{ }^{36} \mathrm{Cl}$ deduced level energies, neutron binding energies. Double crystal spectrometers. CONF Santa Fe (Nucl Data for Sci and Technol) Proc,Vol1,P617

${ }^{29} \mathrm{P} \quad 2005 \mathrm{WE} 01$ NUCLEAR REACTIONS ${ }^{12} \mathrm{C}\left({ }^{29} \mathrm{P},{ }^{28} \mathrm{SiX}\right), \mathrm{E}=30.7 \mathrm{MeV} /$ nucleon; measured fragments parallel momentum distribution. ${ }^{28} \mathrm{Si},{ }^{29} \mathrm{P}$ deduced particle density distributions, related features. Glauber model and Skyrme-Hartree-Fock calculations. JOUR CPLEE 2261 
${ }^{30} \mathrm{Na} \quad$ 2005GAZT ATOMIC MASSES ${ }^{26} \mathrm{Ne},{ }^{26,27,28,29,30} \mathrm{Na},{ }^{29,30,31,32,33 \mathrm{Mg} \text {; measured }}$ masses. Reanalysis of data using new calibration. PREPRINT nucl-ex/0511007,11/2/2005

2005MA96 RADIOACTIVITY ${ }^{30,31,32} \mathrm{Na}\left(\beta^{-}\right) ;{ }^{31,32} \mathrm{Na}\left(\beta^{-} \mathrm{n}\right)$; measured $\mathrm{E} \gamma, \mathrm{I} \gamma$, $\gamma \gamma-, \beta \gamma$-coin. ${ }^{30,31,32} \mathrm{Mg}$ deduced levels $\mathrm{T}_{1 / 2}$. Ultra-fast timing techniques. JOUR ZAANE 25 s01 105

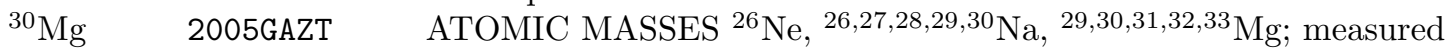
masses. Reanalysis of data using new calibration. PREPRINT nucl-ex/0511007,11/2/2005

2005MA96 RADIOACTIVITY ${ }^{30,31,32} \mathrm{Na}\left(\beta^{-}\right) ;{ }^{31,32} \mathrm{Na}\left(\beta^{-} \mathrm{n}\right)$; measured $\mathrm{E} \gamma, \mathrm{I} \gamma$, $\gamma \gamma-, \beta \gamma$-coin. ${ }^{30,31,32} \mathrm{Mg}$ deduced levels $\mathrm{T}_{1 / 2}$. Ultra-fast timing techniques. JOUR ZAANE 25 s01 105

2005NI09 NUCLEAR REACTIONS Ni( ${ }^{30} \mathrm{Mg},{ }^{30} \mathrm{Mg}$ '), $\mathrm{E}=2.25 \mathrm{MeV} /$ nucleon; measured $\mathrm{E} \gamma, \mathrm{I} \gamma$, (particle) $\gamma$-coin following projectile Coulomb excitation. ${ }^{30} \mathrm{Mg}$ deduced transition, B(E2). JOUR NUPAB 752 273c 2005NI11 NUCLEAR REACTIONS Ni( $\left({ }^{30} \mathrm{Mg},{ }^{30} \mathrm{Mg}\right.$ '), $\mathrm{E}=2.25 \mathrm{MeV} /$ nucleon; measured $\mathrm{E} \gamma, \mathrm{I} \gamma$, (particle) $\gamma$-coin following projectile Coulomb excitation. ${ }^{30} \mathrm{Mg}$ transition deduced B(E2). JOUR PRLTA 94172501 2005SC27 NUCLEAR REACTIONS Ni $\left({ }^{30} \mathrm{Mg},{ }^{30} \mathrm{Mg}\right.$ '), $\mathrm{E}=2.2 \mathrm{MeV} /$ nucleon; measured $\mathrm{E} \gamma, \mathrm{I} \gamma$, (particle) $\gamma$-coin following projectile Coulomb excitation. ${ }^{2}$ Hmeasured $\mathrm{E} \gamma, \mathrm{I} \gamma, \gamma \gamma-$, (particle) $\gamma$-coin. ${ }^{30} \mathrm{Mg}$ deduced transitions $\mathrm{B}(\mathrm{E} 2) .{ }^{31} \mathrm{Mg}$ deduced transitions. Miniball array. JOUR ZAANE 25 s01 397

${ }^{30} \mathrm{Al}$ 2005UE01 RADIOACTIVITY ${ }^{30,32} \mathrm{Al}\left(\beta^{-}\right)$[from ${ }^{40} \mathrm{Ar}$ fragmentation]; measured $\beta$-NMR spectra, $\mathrm{T}_{1 / 2}$; deduced $\mu$. JOUR PYLBB 615186

2005UE01 NUCLEAR MOMENTS ${ }^{30,32} \mathrm{Al}$; measured $\beta$-NMR spectra; deduced $\mu$. JOUR PYLBB 615186

${ }^{30} \mathrm{Si} \quad$ 2005UE01 RADIOACTIVITY ${ }^{30,32} \mathrm{Al}\left(\beta^{-}\right)$[from ${ }^{40} \mathrm{Ar}$ fragmentation]; measured $\beta$-NMR spectra, $\mathrm{T}_{1 / 2}$; deduced $\mu$. JOUR PYLBB 615186

${ }^{30} \mathrm{P} \quad 2005 \mathrm{AD} 35$ NUCLEAR REACTIONS ${ }^{6} \mathrm{Li}\left({ }^{6} \mathrm{Li}, \alpha \mathrm{X}\right),\left({ }^{7} \mathrm{Li}, \alpha \mathrm{X}\right), \mathrm{E}=14-20 \mathrm{MeV}$; measured $\alpha$-spectra. ${ }^{12} \mathrm{C}(\mathrm{n}, \alpha), \mathrm{E}=72.8 \mathrm{MeV} ;{ }^{28} \mathrm{Si}\left({ }^{6} \mathrm{Li}, \alpha\right), \mathrm{E}=36 \mathrm{MeV}$; analyzed $\alpha$-spectra. Statistical model calculations. Target-projectile symmetry discussed. JOUR ZAANE 25 s01 299

2005KA46 RADIOACTIVITY ${ }^{31} \mathrm{Cl}\left(\beta^{+} \mathrm{p}\right)$ [from $\mathrm{S}(\mathrm{p}, \mathrm{X}), \mathrm{E}=40 \mathrm{MeV}$; m measured $\beta$-delayed $\mathrm{E} \gamma, \mathrm{Ep} .{ }^{58} \mathrm{Zn}\left(\beta^{+}\right)$[from $\mathrm{Nb}(\mathrm{p}, \mathrm{X}), \mathrm{E}=1.4 \mathrm{GeV}$ ]; measured $\mathrm{E} \gamma, \mathrm{I} \gamma, \beta \gamma$-coin, $\mathrm{T}_{1 / 2} \cdot{ }^{58} \mathrm{Cu}$ deduced levels, $\beta$-feeding intensities. ${ }^{81 m} \mathrm{Kr}(\mathrm{EC}),(\mathrm{IT}) ;{ }^{81} \mathrm{Y},{ }^{81} \mathrm{Sr},{ }^{85} \mathrm{Nb},{ }^{85} \mathrm{Zr},{ }^{86} \mathrm{Mo},{ }^{86} \mathrm{Nb}(\mathrm{EC})$ [from $\mathrm{Ni}$, $\left.{ }^{54} \mathrm{Fe}\left({ }^{32} \mathrm{~S}, \mathrm{X}\right)\right]$; measured $\mathrm{E} \gamma, \mathrm{I} \gamma, \mathrm{E}($ ce $), \mathrm{I}(\mathrm{ce}), \mathrm{T}_{1 / 2} \cdot{ }^{81} \mathrm{Kr},{ }^{85} \mathrm{Zr},{ }^{85} \mathrm{Nb}$ deduced isomeric transitions $\mathrm{T}_{1 / 2}$, ICC. ${ }^{85} \mathrm{Zr},{ }^{86} \mathrm{Nb}$ deduced levels, J, $\pi$, ICC. ${ }^{81} \mathrm{Br}$ deduced neutrino capture rate. Mass-separated sources. JOUR ZAANE 25 s01 129

\section{$\mathrm{A}=31$}

${ }^{31} \mathrm{Na} \quad 2005 \mathrm{MA} 96$

RADIOACTIVITY ${ }^{30,31,32} \mathrm{Na}\left(\beta^{-}\right) ;{ }^{31,32} \mathrm{Na}\left(\beta^{-} \mathrm{n}\right)$; measured $\mathrm{E} \gamma, \mathrm{I} \gamma$, $\gamma \gamma$-, $\beta \gamma$-coin. ${ }^{30,31,32} \mathrm{Mg}$ deduced levels $\mathrm{T}_{1 / 2}$. Ultra-fast timing techniques. JOUR ZAANE 25 s01 105 


\section{$\mathrm{A}=31$ (continued)}

${ }^{31} \mathrm{Mg} \quad$ 2005GAZT

2005K041

2005MA86

2005MA96

2005NE01

2005SC27

${ }^{31} \mathrm{Al}$

2005K041

2005MA86

2005NE01

${ }^{31} \mathrm{P}$

2004V022

2005AN15

2005DEZZ

2005JE07
ATOMIC MASSES ${ }^{26} \mathrm{Ne},{ }^{26,27,28,29,30} \mathrm{Na},{ }^{29,30,31,32,33} \mathrm{Mg}$; measured masses. Reanalysis of data using new calibration. PREPRINT nucl-ex/0511007,11/2/2005

RADIOACTIVITY ${ }^{31} \mathrm{Mg}\left(\beta^{-}\right)$[from $\mathrm{U}(\mathrm{p}, \mathrm{X})$ ]; measured $\beta$-asymmetry and hfs, $\beta$-NMR spectra from polarized source. ${ }^{31} \mathrm{Mg}$ deduced ground-state $\mathrm{J}, \pi, \mu$. JOUR ZAANE 25 s01 193

RADIOACTIVITY ${ }^{31} \mathrm{Mg}\left(\beta^{-}\right)$[from $\left.\mathrm{Be}^{36} \mathrm{~S}, \mathrm{X}\right)$ ]; measured $\mathrm{E} \gamma, \mathrm{I} \gamma$, $\beta \gamma$-coin, $\mathrm{T}_{1 / 2}$; deduced log ft. ${ }^{31} \mathrm{Al}$ deduced levels, feeding intensities.

${ }^{31} \mathrm{Mg}$ deduced ground-state intruder configuration. JOUR PRVCA 72 044314

RADIOACTIVITY ${ }^{30,31,32} \mathrm{Na}\left(\beta^{-}\right) ;{ }^{31,32} \mathrm{Na}\left(\beta^{-} \mathrm{n}\right)$; measured $\mathrm{E} \gamma, \mathrm{I} \gamma$, $\gamma \gamma$-, $\beta \gamma$-coin. ${ }^{30,31,32} \mathrm{Mg}$ deduced levels $\mathrm{T}_{1 / 2}$. Ultra-fast timing techniques. JOUR ZAANE $25 \mathrm{~s} 01105$

RADIOACTIVITY ${ }^{31} \mathrm{Mg}\left(\beta^{-}\right)$[from $\mathrm{U}(\mathrm{p}, \mathrm{X})$ ]; measured $\beta$-asymmetry, $\beta$-NMR spectra from polarized source. ${ }^{31} \mathrm{Mg}$ deduced ground-state $\mathrm{J}$, $\pi, \mu$. JOUR PRLTA 94022501

NUCLEAR REACTIONS Ni( $\left({ }^{30} \mathrm{Mg},{ }^{30} \mathrm{Mg}\right.$ '), $\mathrm{E}=2.2 \mathrm{MeV} /$ nucleon; measured $\mathrm{E} \gamma, \mathrm{I} \gamma$, (particle) $\gamma$-coin following projectile Coulomb excitation. ${ }^{2} \mathrm{Hmeasured} \mathrm{E} \gamma, \mathrm{I} \gamma, \gamma \gamma$-, (particle) $\gamma$-coin. ${ }^{30} \mathrm{Mg}$ deduced transitions $\mathrm{B}(\mathrm{E} 2) .{ }^{31} \mathrm{Mg}$ deduced transitions. Miniball array. JOUR ZAANE 25 s01 397

RADIOACTIVITY ${ }^{31} \mathrm{Mg}\left(\beta^{-}\right)$[from $\mathrm{U}(\mathrm{p}, \mathrm{X})$ ]; measured $\beta$-asymmetry and hfs, $\beta$-NMR spectra from polarized source. ${ }^{31} \mathrm{Mg}$ deduced ground-state $\mathrm{J}, \pi, \mu$. JOUR ZAANE 25 s01 193 RADIOACTIVITY ${ }^{31} \mathrm{Mg}\left(\beta^{-}\right)$[from $\mathrm{Be}\left({ }^{36} \mathrm{~S}, \mathrm{X}\right)$ ]; measured $\mathrm{E} \gamma, \mathrm{I} \gamma$, $\beta \gamma$-coin, $\mathrm{T}_{1 / 2}$; deduced $\log \mathrm{ft} .{ }^{31} \mathrm{Al}$ deduced levels, feeding intensities. ${ }^{31} \mathrm{Mg}$ deduced ground-state intruder configuration. JOUR PRVCA 72 044314

RADIOACTIVITY ${ }^{31} \mathrm{Mg}\left(\beta^{-}\right)$[from $\mathrm{U}(\mathrm{p}, \mathrm{X})$ ]; measured $\beta$-asymmetry, $\beta$-NMR spectra from polarized source. ${ }^{31} \mathrm{Mg}$ deduced ground-state $\mathrm{J}$, $\pi, \mu$. JOUR PRLTA 94022501

NUCLEAR REACTIONS ${ }^{30} \mathrm{Si}(\mathrm{p}, \gamma), \mathrm{E}=1750-1905 \mathrm{keV}$; measured $\mathrm{E} \gamma$, $\mathrm{I} \gamma$, excitation function. ${ }^{31} \mathrm{P}$ deduced levels, $\mathrm{J}, \pi, \mathrm{B}(\mathrm{M} 1)$, IAR features. JOUR BRSPE 68218

NUCLEAR MOMENTS ${ }^{13} \mathrm{C},{ }^{14,15} \mathrm{~N},{ }^{17} \mathrm{O},{ }^{19} \mathrm{~F},{ }^{31} \mathrm{P},{ }^{33} \mathrm{~S}$; measured NMR spectra; deduced $\mu$. JOUR CHPLB 411111

NUCLEAR REACTIONS ${ }^{24} \mathrm{Mg}\left({ }^{16} \mathrm{O}, \mathrm{n} 2 \alpha\right),\left({ }^{16} \mathrm{O}, \mathrm{p} 2 \alpha\right),\left({ }^{16} \mathrm{O}, \mathrm{n} \alpha\right)$, $\left({ }^{16} \mathrm{O}, \mathrm{p} \alpha\right), \mathrm{E}=70 \mathrm{MeV}$; measured $\mathrm{E} \gamma, \mathrm{I} \gamma, \gamma \gamma-$, (charged particle) $\gamma_{-}$, (neutron) $\gamma$-coin. ${ }^{31} \mathrm{~S},{ }^{31} \mathrm{P},{ }^{35} \mathrm{Ar},{ }^{35} \mathrm{Cl}$ deduced levels, J, $\pi$, mirror energy differences. GASP, ISIS arrays. CONF Argonne(Nuclei at the Limits),P205,Della Vedova NUCLEAR REACTIONS ${ }^{12} \mathrm{C}\left({ }^{20} \mathrm{Ne}, \mathrm{p}\right),\left({ }^{20} \mathrm{Ne}, \mathrm{n}\right), \mathrm{E}=32 \mathrm{MeV}$; measured $\mathrm{E} \gamma, \mathrm{I} \gamma, \gamma \gamma$-, (recoil) $\gamma$-coin. ${ }^{31} \mathrm{~S},{ }^{31} \mathrm{P}$ deduced high-spin levels, $\mathrm{J}, \pi$. Gammasphere array, fragment mass analyzer. JOUR PRVCA 72 031303 


\section{$\mathrm{A}=31$ (continued)}

${ }^{31} \mathrm{~S}$ 2005DEZZ NUCLEAR REACTIONS ${ }^{24} \mathrm{Mg}\left({ }^{16} \mathrm{O}, \mathrm{n} 2 \alpha\right),\left({ }^{16} \mathrm{O}, \mathrm{p} 2 \alpha\right),\left({ }^{16} \mathrm{O}, \mathrm{n} \alpha\right)$, $\left({ }^{16} \mathrm{O}, \mathrm{p} \alpha\right), \mathrm{E}=70 \mathrm{MeV}$; measured $\mathrm{E} \gamma, \mathrm{I} \gamma, \gamma \gamma-$, (charged particle) $\gamma-$, (neutron) $\gamma$-coin. ${ }^{31} \mathrm{~S},{ }^{31} \mathrm{P},{ }^{35} \mathrm{Ar},{ }^{35} \mathrm{Cl}$ deduced levels, $\mathrm{J}, \pi$, mirror energy differences. GASP, ISIS arrays. CONF Argonne(Nuclei at the Limits),P205,Della Vedova 2005GA54 NUCLEAR REACTIONS ${ }^{9} \mathrm{Be}\left({ }^{32} \mathrm{~S},{ }^{31} \mathrm{SX}\right),\left({ }^{33} \mathrm{Cl},{ }^{32} \mathrm{ClX}\right),\left({ }^{32} \mathrm{Ar}\right.$, $\left.{ }^{31} \mathrm{ArX}\right),\left({ }^{34} \mathrm{Ar},{ }^{33} \mathrm{ArX}\right), \mathrm{E} \approx 65 \mathrm{MeV} /$ nucleon; measured $\mathrm{E} \gamma, \mathrm{I} \gamma$, (particle) $\gamma$-coin, particle momentum distributions; deduced one-neutron removal $\sigma \cdot{ }^{31} \mathrm{~S},{ }^{32} \mathrm{Cl},{ }^{31,33} \mathrm{Ar}$ levels deduced spectroscopic factors. Comparison with shell model predictions. JOUR ZAANE 25 s01 251 2005JE07 NUCLEAR REACTIONS ${ }^{12} \mathrm{C}\left({ }^{20} \mathrm{Ne}, \mathrm{p}\right),\left({ }^{20} \mathrm{Ne}, \mathrm{n}\right), \mathrm{E}=32 \mathrm{MeV}$; measured $\mathrm{E} \gamma, \mathrm{I} \gamma, \gamma \gamma$-, (recoil) $\gamma$-coin. ${ }^{31} \mathrm{~S},{ }^{31} \mathrm{P}$ deduced high-spin levels, $\mathrm{J}, \pi$. Gammasphere array, fragment mass analyzer. JOUR PRVCA 72 031303

${ }^{31} \mathrm{Cl}$ 2005KA46 RADIOACTIVITY ${ }^{31} \mathrm{Cl}\left(\beta^{+} \mathrm{p}\right)$ [from $\mathrm{S}(\mathrm{p}, \mathrm{X}), \mathrm{E}=40 \mathrm{MeV}$ ]; measured $\beta$-delayed $\mathrm{E} \gamma, \mathrm{Ep} .{ }^{58} \mathrm{Zn}\left(\beta^{+}\right)$[from $\mathrm{Nb}(\mathrm{p}, \mathrm{X}), \mathrm{E}=1.4 \mathrm{GeV}$ ]; measured $\mathrm{E} \gamma, \mathrm{I} \gamma, \beta \gamma$-coin, $\mathrm{T}_{1 / 2} .{ }^{58} \mathrm{Cu}$ deduced levels, $\beta$-feeding intensities. ${ }^{81 m} \mathrm{Kr}$ (EC), (IT); ${ }^{81} \mathrm{Y},{ }^{81} \mathrm{Sr},{ }^{85} \mathrm{Nb},{ }^{85} \mathrm{Zr},{ }^{86} \mathrm{Mo},{ }^{86} \mathrm{Nb}$ (EC) [from $\mathrm{Ni}$, $\left.{ }^{54} \mathrm{Fe}\left({ }^{32} \mathrm{~S}, \mathrm{X}\right)\right]$; measured $\mathrm{E} \gamma, \mathrm{I} \gamma, \mathrm{E}(\mathrm{ce}), \mathrm{I}(\mathrm{ce}), \mathrm{T}_{1 / 2} \cdot{ }^{81} \mathrm{Kr},{ }^{85} \mathrm{Zr},{ }^{85} \mathrm{Nb}$ deduced isomeric transitions $\mathrm{T}_{1 / 2}$, ICC. ${ }^{85} \mathrm{Zr},{ }^{86} \mathrm{Nb}$ deduced levels, $\mathrm{J}$, $\pi$, ICC. ${ }^{81} \mathrm{Br}$ deduced neutrino capture rate. Mass-separated sources. JOUR ZAANE 25 s01 129

${ }^{31} \mathrm{Ar}$ 2005GA54 NUCLEAR REACTIONS ${ }^{9} \mathrm{Be}\left({ }^{32} \mathrm{~S},{ }^{31} \mathrm{SX}\right),\left({ }^{33} \mathrm{Cl},{ }^{32} \mathrm{ClX}\right),\left({ }^{32} \mathrm{Ar}\right.$, $\left.{ }^{31} \mathrm{ArX}\right),\left({ }^{34} \mathrm{Ar},{ }^{33} \mathrm{ArX}\right), \mathrm{E} \approx 65 \mathrm{MeV} /$ nucleon; measured $\mathrm{E} \gamma, \mathrm{I} \gamma$, (particle) $\gamma$-coin, particle momentum distributions; deduced one-neutron removal $\sigma \cdot{ }^{31} \mathrm{~S},{ }^{32} \mathrm{Cl},{ }^{31,33} \mathrm{Ar}$ levels deduced spectroscopic factors. Comparison with shell model predictions. JOUR ZAANE 25 s01 251

\section{$\mathrm{A}=32$}

${ }^{32} \mathrm{Na} \quad$ 2005MA81

RADIOACTIVITY ${ }^{32} \mathrm{Na},{ }^{80} \mathrm{Ga}\left(\beta^{-}\right)$; measured $\mathrm{E} \gamma, \mathrm{I} \gamma, \gamma \gamma-, \beta \gamma$-coin. ${ }^{32} \mathrm{Mg},{ }^{80} \mathrm{Ge}$ levels deduced $\mathrm{T}_{1 / 2}$. Ultra-fast timing techniques. JOUR JPGPE 31 S1421

2005MA96 RADIOACTIVITY ${ }^{30,31,32} \mathrm{Na}\left(\beta^{-}\right) ;{ }^{31,32} \mathrm{Na}\left(\beta^{-} \mathrm{n}\right)$; measured $\mathrm{E} \gamma, \mathrm{I} \gamma$, $\gamma \gamma-, \beta \gamma$-coin. ${ }^{30,31,32} \mathrm{Mg}$ deduced levels $\mathrm{T}_{1 / 2}$. Ultra-fast timing techniques. JOUR ZAANE 25 s01 105

${ }^{32} \mathrm{Mg} \quad$ 2004C029 RADIOACTIVITY ${ }^{74} \operatorname{Kr}(\mathrm{EC}),\left(\beta^{+}\right)[$from $\mathrm{Nb}(\mathrm{p}, \mathrm{X})]$; measured $\beta \gamma$-coin; deduced Gamow-Teller strength distribution. ${ }^{33} \mathrm{Na}\left(\beta^{-}\right)$, $\left(\beta^{-} \mathrm{n}\right)$ [from $\left.\mathrm{U}(\mathrm{p}, \mathrm{X})\right]$; measured $\beta \gamma-, \mathrm{n} \beta-, \gamma \gamma$-coin, $\mathrm{T}_{1 / 2} .{ }^{33} \mathrm{Mg}$ deduced ground-state $\mathrm{J}, \pi$. Total absorption spectrometer. JOUR BJPHE 34 850

2005 CH66 NUCLEAR REACTIONS ${ }^{209} \mathrm{Bi}\left({ }^{26} \mathrm{Mg},{ }^{26} \mathrm{Mg}\right.$ '), E=78.6 MeV / nucleon; ${ }^{197} \mathrm{Au}\left({ }^{32} \mathrm{Mg},{ }^{32} \mathrm{Mg}\right.$ '), E=81.1 MeV / nucleon; ${ }^{209} \mathrm{Bi}\left({ }^{34} \mathrm{Mg},{ }^{34} \mathrm{Mg}\right.$ ') $\mathrm{E}=76.4 \mathrm{MeV} /$ nucleon; measured $\mathrm{E} \gamma, \mathrm{I} \gamma$, (particle) $\gamma$-coin following projectile Coulomb excitation. ${ }^{26,32,34} \mathrm{Mg}$ deduced transitions $\mathrm{B}(\mathrm{E} 2)$, deformation parameters. Comparison with previous work, model predictions. JOUR PRVCA 72054320 


\section{$\mathrm{A}=32$ (continued)}

2005GAZT ATOMIC MASSES ${ }^{26} \mathrm{Ne},{ }^{26,27,28,29,30} \mathrm{Na},{ }^{29,30,31,32,33} \mathrm{Mg}$; measured masses. Reanalysis of data using new calibration. PREPRINT nucl-ex/0511007,11/2/2005

2005MA81 RADIOACTIVITY ${ }^{32} \mathrm{Na},{ }^{80} \mathrm{Ga}\left(\beta^{-}\right)$; measured $\mathrm{E} \gamma, \mathrm{I} \gamma, \gamma \gamma-, \beta \gamma$-coin. ${ }^{32} \mathrm{Mg},{ }^{80} \mathrm{Ge}$ levels deduced $\mathrm{T}_{1 / 2}$. Ultra-fast timing techniques. JOUR JPGPE 31 S1421

2005MA96 RADIOACTIVITY ${ }^{30,31,32} \mathrm{Na}\left(\beta^{-}\right) ;{ }^{31,32} \mathrm{Na}\left(\beta^{-} \mathrm{n}\right)$; measured $\mathrm{E} \gamma$, I $\gamma$, $\gamma \gamma-, \beta \gamma$-coin. ${ }^{30,31,32} \mathrm{Mg}$ deduced levels $\mathrm{T}_{1 / 2}$. Ultra-fast timing techniques. JOUR ZAANE 25 s01 105

${ }^{32} \mathrm{Al}$ 2005UE01 RADIOACTIVITY ${ }^{30,32} \mathrm{Al}\left(\beta^{-}\right)$[from ${ }^{40} \mathrm{Ar}$ fragmentation]; measured $\beta$-NMR spectra, $\mathrm{T}_{1 / 2} ;$ deduced $\mu$. JOUR PYLBB 615186

2005UE01 NUCLEAR MOMENTS ${ }^{30,32} \mathrm{Al}$; measured $\beta$-NMR spectra; deduced $\mu$. JOUR PYLBB 615186

${ }^{32} \mathrm{Si}$ 2005UE01 RADIOACTIVITY ${ }^{30,32} \mathrm{Al}\left(\beta^{-}\right)$[from ${ }^{40} \mathrm{Ar}$ fragmentation]; measured $\beta$-NMR spectra, $\mathrm{T}_{1 / 2}$; deduced $\mu$. JOUR PYLBB 615186

${ }^{32} \mathrm{~S} \quad 2005 \mathrm{ADZW}$

2005 G036

2005SH38

NUCLEAR REACTIONS ${ }^{31} \mathrm{P}(\mathrm{p}, \gamma)$, E not given; measured $\mathrm{E} \gamma, \mathrm{I} \gamma \cdot{ }^{32} \mathrm{~S}$ deduced excited state energy. REPT Univ Washington Annual 2005,P58,Adelberger ATOMIC MASSES ${ }^{12} \mathrm{C},{ }^{16} \mathrm{O},{ }^{20} \mathrm{Ne},{ }^{32} \mathrm{~S},{ }^{36,40} \mathrm{Ar}$; measured masses. Cyclotron-based mass spectrometry. JOUR JPGPE 31 S1869 ATOMIC MASSES ${ }^{32,33} \mathrm{~S},{ }^{84,86} \mathrm{Kr},{ }^{129,132} \mathrm{Xe}$; measured masses. Penning trap. JOUR PLRAA 72022510

${ }^{32} \mathrm{Cl}$ 2005GA54 NUCLEAR REACTIONS ${ }^{9} \mathrm{Be}\left({ }^{32} \mathrm{~S},{ }^{31} \mathrm{SX}\right),\left({ }^{33} \mathrm{Cl},{ }^{32} \mathrm{ClX}\right),\left({ }^{32} \mathrm{Ar}\right.$, $\left.{ }^{31} \mathrm{ArX}\right),\left({ }^{34} \mathrm{Ar},{ }^{33} \mathrm{ArX}\right), \mathrm{E} \approx 65 \mathrm{MeV} /$ nucleon; measured $\mathrm{E} \gamma, \mathrm{I} \gamma$, (particle) $\gamma$-coin, particle momentum distributions; deduced one-neutron removal $\sigma \cdot{ }^{31} \mathrm{~S},{ }^{32} \mathrm{Cl},{ }^{31,33} \mathrm{Ar}$ levels deduced spectroscopic factors. Comparison with shell model predictions. JOUR ZAANE 25 s01 251

\section{$\mathrm{A}=33$}

${ }^{33} \mathrm{Na} \quad 2004 \mathrm{CO} 29$

${ }^{33} \mathrm{Mg} \quad 2004 \mathrm{CO} 29$

2005GAZT

${ }^{33} \mathrm{~S} \quad 2005$ AN15

2005DEZW
RADIOACTIVITY ${ }^{74} \mathrm{Kr}(\mathrm{EC}),\left(\beta^{+}\right)$[from $\mathrm{Nb}(\mathrm{p}, \mathrm{X})$ ]; measured $\beta \gamma$-coin; deduced Gamow-Teller strength distribution. ${ }^{33} \mathrm{Na}\left(\beta^{-}\right)$, $\left(\beta^{-} \mathrm{n}\right)$ [from $\left.\mathrm{U}(\mathrm{p}, \mathrm{X})\right]$; measured $\beta \gamma-, \mathrm{n} \beta-, \gamma \gamma$-coin, $\mathrm{T}_{1 / 2} .{ }^{33} \mathrm{Mg}$ deduced ground-state $\mathrm{J}, \pi$. Total absorption spectrometer. JOUR BJPHE 34 850 RADIOACTIVITY ${ }^{74} \mathrm{Kr}(\mathrm{EC}),\left(\beta^{+}\right)$[from $\mathrm{Nb}(\mathrm{p}, \mathrm{X})$ ]; measured $\beta \gamma$-coin; deduced Gamow-Teller strength distribution. ${ }^{33} \mathrm{Na}\left(\beta^{-}\right)$, $\left(\beta^{-} \mathrm{n}\right)$ [from $\mathrm{U}(\mathrm{p}, \mathrm{X})$ ]; measured $\beta \gamma-, \mathrm{n} \beta-, \gamma \gamma$-coin, $\mathrm{T}_{1 / 2} .{ }^{33} \mathrm{Mg}$ deduced ground-state $\mathrm{J}, \pi$. Total absorption spectrometer. JOUR BJPHE 34 850 ATOMIC MASSES ${ }^{26} \mathrm{Ne},{ }^{26,27,28,29,30} \mathrm{Na},{ }^{29,30,31,32,33} \mathrm{Mg}$; measured masses. Reanalysis of data using new calibration. PREPRINT nucl-ex/0511007,11/2/2005

NUCLEAR MOMENTS ${ }^{13} \mathrm{C},{ }^{14,15} \mathrm{~N},{ }^{17} \mathrm{O},{ }^{19} \mathrm{~F},{ }^{31} \mathrm{P},{ }^{33} \mathrm{~S}$; measured NMR spectra; deduced $\mu$. JOUR CHPLB 411111 NUCLEAR REACTIONS ${ }^{28} \mathrm{Si},{ }^{32} \mathrm{~S},{ }^{35} \mathrm{Cl}(\mathrm{n}, \gamma), \mathrm{E}=$ reactor; measured $\mathrm{E} \gamma, \mathrm{I} \gamma \cdot{ }^{29} \mathrm{Si},{ }^{33} \mathrm{~S},{ }^{36} \mathrm{Cl}$ deduced binding energies. Flat-crystal spectrometer. PREPRINT nucl-ex/0507011,7/06/2005 


\section{$\mathrm{A}=33$ (continued)}

2005JEZY NUCLEAR REACTIONS ${ }^{28} \mathrm{Si},{ }^{32} \mathrm{~S},{ }^{35} \mathrm{Cl}(\mathrm{n}, \gamma), \mathrm{E}=$ thermal; measured $\mathrm{E} \gamma, \mathrm{I} \gamma \cdot{ }^{29} \mathrm{Si},{ }^{33} \mathrm{~S},{ }^{36} \mathrm{Cl}$ deduced level energies, neutron binding energies. Double crystal spectrometers. CONF Santa Fe (Nucl Data for Sci and Technol) Proc,Vol1,P617

2005SH38 ATOMIC MASSES ${ }^{32,33} \mathrm{~S},{ }^{84,86} \mathrm{Kr},{ }^{129,132} \mathrm{Xe}$; measured masses. Penning trap. JOUR PLRAA 72022510

${ }^{33} \mathrm{Cl} \quad 2005 \mathrm{ADZV}$ NUCLEAR REACTIONS ${ }^{32} \mathrm{~S}(\mathrm{p}, \gamma), \mathrm{E} \approx 3.4 \mathrm{MeV}$; measured $\mathrm{E} \gamma, \mathrm{I} \gamma$. ${ }^{33} \mathrm{Cl}$ deduced transitions. REPT Univ Washington Annual 2005,P59,Adelberger

${ }^{33} \mathrm{Ar} \quad$ 2005GA54 NUCLEAR REACTIONS ${ }^{9} \mathrm{Be}\left({ }^{32} \mathrm{~S},{ }^{31} \mathrm{SX}\right),\left({ }^{33} \mathrm{Cl},{ }^{32} \mathrm{ClX}\right),\left({ }^{32} \mathrm{Ar}\right.$, $\left.{ }^{31} \mathrm{ArX}\right),\left({ }^{34} \mathrm{Ar},{ }^{33} \mathrm{ArX}\right), \mathrm{E} \approx 65 \mathrm{MeV} /$ nucleon; measured $\mathrm{E} \gamma, \mathrm{I} \gamma$, (particle) $\gamma$-coin, particle momentum distributions; deduced one-neutron removal $\sigma \cdot{ }^{31} \mathrm{~S},{ }^{32} \mathrm{Cl},{ }^{31,33} \mathrm{Ar}$ levels deduced spectroscopic factors. Comparison with shell model predictions. JOUR ZAANE 25 s01 251

\section{$\mathrm{A}=34$}

${ }^{34} \mathrm{Mg} \quad 2005 \mathrm{CH} 66$

NUCLEAR REACTIONS ${ }^{209} \mathrm{Bi}\left({ }^{26} \mathrm{Mg},{ }^{26} \mathrm{Mg}\right.$ '), $\mathrm{E}=78.6 \mathrm{MeV} /$ nucleon; ${ }^{197} \mathrm{Au}\left({ }^{32} \mathrm{Mg},{ }^{32} \mathrm{Mg}\right.$ '), E=81.1 MeV / nucleon; ${ }^{209} \mathrm{Bi}\left({ }^{34} \mathrm{Mg},{ }^{34} \mathrm{Mg}\right.$ ') $\mathrm{E}=76.4 \mathrm{MeV} /$ nucleon; measured $\mathrm{E} \gamma, \mathrm{I} \gamma$, (particle) $\gamma$-coin following projectile Coulomb excitation. ${ }^{26,32,34} \mathrm{Mg}$ deduced transitions $\mathrm{B}(\mathrm{E} 2)$, deformation parameters. Comparison with previous work, model predictions. JOUR PRVCA 72054320

${ }^{34} \mathrm{Al}$ 20050B04 NUCLEAR REACTIONS ${ }^{251} \mathrm{Cf}(\mathrm{n}, \mathrm{F}), \mathrm{E}=$ thermal; measured light charged particle yields, energy distributions following ternary fission; deduced evidence for ${ }^{34} \mathrm{Al},{ }^{36} \mathrm{Si}$. JOUR NUPAB 761173

${ }^{34} \mathrm{Si} \quad 2005 \mathrm{TI} 11$ RADIOACTIVITY ${ }^{35} \mathrm{Al}\left(\beta^{-}\right),\left(\beta^{-} \mathrm{n}\right)$ [from ${ }^{36} \mathrm{~S}$ fragmentation]; measured $\beta$-delayed $\mathrm{E} \gamma, \mathrm{En}, \mathrm{T}_{1 / 2}$, neutron emission probability; deduced log ft. ${ }^{34,35} \mathrm{Si}$ deduced levels, J, $\pi$, feeding intensities. JOUR JPGPE 31 S1965

${ }^{34} \mathrm{P} \quad$ 20050L02 NUCLEAR REACTIONS ${ }^{176} \mathrm{Yb}\left({ }^{36} \mathrm{~S}, \mathrm{X}\right){ }^{34} \mathrm{P}, \mathrm{E}=230 \mathrm{MeV}$; measured $\mathrm{E} \gamma, \mathrm{I} \gamma, \gamma \gamma$-coin. ${ }^{34} \mathrm{P}$ deduced levels, J, $\pi$, configurations. GASP array, level systematics in neighboring isotopes discussed. JOUR PRVCA 71 034316

$20050 \mathrm{~L} 04$ NUCLEAR REACTIONS ${ }^{176} \mathrm{Yb}\left({ }^{36} \mathrm{~S}, \mathrm{X}\right){ }^{34} \mathrm{P}, \mathrm{E}=230 \mathrm{MeV} ;{ }^{208} \mathrm{~Pb}\left({ }^{36} \mathrm{~S}\right.$, $\mathrm{X})^{36} \mathrm{~S} /{ }^{38} \mathrm{~S} /{ }^{34} \mathrm{P} /{ }^{36} \mathrm{P}, \mathrm{E}=215 \mathrm{MeV}$; measured $\mathrm{E} \gamma, \mathrm{I} \gamma, \gamma \gamma-$ (particle) $\gamma$-coin. ${ }^{34} \mathrm{P}$ deduced levels, J, $\pi$, configurations. JOUR JPGPE 31 S1935

${ }^{34} \mathrm{~S} \quad 2005 \mathrm{FU03} \quad$ NUCLEAR REACTIONS ${ }^{34} \mathrm{~S}\left({ }^{7} \mathrm{Li}, \mathrm{t} \alpha\right), \mathrm{E}=26 \mathrm{MeV}$; measured particle spectra, angular correlations. ${ }^{38} \mathrm{Ar}$ deduced $\alpha$-cluster states energies, J, $\pi$. JOUR PRVCA 71067602

2005MA03 NUCLEAR REACTIONS ${ }^{24} \mathrm{Mg}\left({ }^{16} \mathrm{O}, 2 \mathrm{p} \alpha\right), \mathrm{E}=70 \mathrm{MeV}$; measured $\mathrm{E} \gamma$, $\mathrm{I} \gamma, \gamma \gamma-$, (charged particle) $\gamma$-coin, DSA. ${ }^{34} \mathrm{~S}$ deduced high-spin levels, $\mathrm{J}$, $\pi, \mathrm{T}_{1 / 2}, \mathrm{~B}(\mathrm{M} 1), \mathrm{B}(\mathrm{E} 2)$. Comparison with shell model calculations. JOUR PRVCA 71014316 


\section{$\mathrm{A}=34$ (continued)}

\begin{tabular}{|c|c|c|}
\hline${ }^{34} \mathrm{Cl}$ & $2005 \mathrm{SA} 44$ & $\begin{array}{l}\text { RADIOACTIVITY }{ }^{46} \mathrm{~V}(\mathrm{EC}) \text {; analyzed masses; deduced } \mathrm{Q}(\mathrm{EC}), \log \mathrm{ft} . \\
{ }^{10} \mathrm{C},{ }^{14} \mathrm{O},{ }^{22} \mathrm{Mg},{ }^{26 m} \mathrm{Al},{ }^{34} \mathrm{Cl},{ }^{34} \mathrm{Ar},{ }^{38 m} \mathrm{~K},{ }^{42} \mathrm{Sc},{ }^{46} \mathrm{~V},{ }^{50} \mathrm{Mn},{ }^{54} \mathrm{Co},{ }^{74} \mathrm{Rb} \text {; } \\
\text { compiled, analyzed log ft; deduced quark-mixing matrix element. } \\
\text { JOUR PRLTA } 95102501\end{array}$ \\
\hline & $2005 \mathrm{SA} 44$ & $\begin{array}{l}\text { RADIOACTIVITY }{ }^{46} \mathrm{~V} \text { (EC); analyzed masses; deduced Q(EC), log ft. } \\
{ }^{10} \mathrm{C},{ }^{14} \mathrm{O},{ }^{22} \mathrm{Mg},{ }^{26 m} \mathrm{Al},{ }^{34} \mathrm{Cl},{ }^{34} \mathrm{Ar},{ }^{38 m} \mathrm{~K},{ }^{42} \mathrm{Sc},{ }^{46} \mathrm{~V},{ }^{50} \mathrm{Mn},{ }^{54} \mathrm{Co},{ }^{74} \mathrm{Rb} ;\end{array}$ \\
\hline
\end{tabular}
JOUR PRLTA 95102501

\section{$\mathrm{A}=35$}

${ }^{35} \mathrm{Al}$ 2005TI11 RADIOACTIVITY ${ }^{35} \mathrm{Al}\left(\beta^{-}\right),\left(\beta^{-} \mathrm{n}\right)$ [from ${ }^{36} \mathrm{~S}$ fragmentation]; measured $\beta$-delayed $\mathrm{E} \gamma$, En, $\mathrm{T}_{1 / 2}$, neutron emission probability; deduced $\log \mathrm{ft} .{ }^{34,35} \mathrm{Si}$ deduced levels, J, $\pi$, feeding intensities. JOUR JPGPE 31 S1965

${ }^{35} \mathrm{Si} \quad$ 2005TI11 RADIOACTIVITY ${ }^{35} \mathrm{Al}\left(\beta^{-}\right),\left(\beta^{-} \mathrm{n}\right)$ [from ${ }^{36} \mathrm{~S}$ fragmentation]; measured $\beta$-delayed $\mathrm{E} \gamma, \mathrm{En}, \mathrm{T}_{1 / 2}$, neutron emission probability; deduced $\log \mathrm{ft} .{ }^{34,35} \mathrm{Si}$ deduced levels, J, $\pi$, feeding intensities. JOUR JPGPE 31 S1965

${ }^{35} \mathrm{Cl}$ 2005DEZZ NUCLEAR REACTIONS ${ }^{24} \mathrm{Mg}\left({ }^{16} \mathrm{O}, \mathrm{n} 2 \alpha\right),\left({ }^{16} \mathrm{O}, \mathrm{p} 2 \alpha\right),\left({ }^{16} \mathrm{O}, \mathrm{n} \alpha\right)$, $\left({ }^{16} \mathrm{O}, \mathrm{p} \alpha\right), \mathrm{E}=70 \mathrm{MeV}$; measured $\mathrm{E} \gamma, \mathrm{I} \gamma, \gamma \gamma-$, (charged particle) $\gamma-$, (neutron) $\gamma$-coin. ${ }^{31} \mathrm{~S},{ }^{31} \mathrm{P},{ }^{35} \mathrm{Ar},{ }^{35} \mathrm{Cl}$ deduced levels, J, $\pi$, mirror energy differences. GASP, ISIS arrays. CONF Argonne(Nuclei at the Limits),P205,Della Vedova

2005EK01 NUCLEAR REACTIONS ${ }^{16} \mathrm{O}\left({ }^{24} \mathrm{Mg}, \mathrm{n} \alpha\right),\left({ }^{24} \mathrm{Mg}, \mathrm{p} \alpha\right), \mathrm{E}=60 \mathrm{MeV}$; ${ }^{28} \mathrm{Si}\left({ }^{32} \mathrm{~S}, \mathrm{n} 2 \alpha\right),\left({ }^{32} \mathrm{~S}, \mathrm{p} 2 \alpha\right), \mathrm{E}=130 \mathrm{MeV} ;{ }^{24} \mathrm{Mg}\left({ }^{40} \mathrm{Ca}, 2 \mathrm{np}\right),\left({ }^{40} \mathrm{Ca}, \mathrm{n} 2 \mathrm{p}\right)$, $\mathrm{E}=104 \mathrm{MeV}$; measured $\mathrm{E} \gamma, \mathrm{I} \gamma, \gamma \gamma-$, (charged particle) $\gamma_{-}$, (neutron) $\gamma$-coin. ${ }^{35} \mathrm{Ar},{ }^{35} \mathrm{Cl},{ }^{51} \mathrm{Fe},{ }^{51} \mathrm{Mn},{ }^{61} \mathrm{Ga},{ }^{61} \mathrm{Zn}$ deduced levels, J, $\pi$, mirror energy difference. Discussed electromagnetic spin-orbit effect. Large-scale shell model calculations. JOUR ZAANE 25 s01 363

2005KSZZ NUCLEAR REACTIONS ${ }^{12} \mathrm{C}\left({ }^{28} \mathrm{Si}, \mathrm{p} \alpha\right), \mathrm{E}=70,88 \mathrm{MeV}$; measured $\mathrm{E} \gamma$, $\mathrm{I} \gamma, \gamma \gamma$-coin, DSA. ${ }^{35} \mathrm{Cl}$ level deduced $\mathrm{T}_{1 / 2}$, isospin-mixing effects. PREPRINT nucl-ex/0507019,7/13/2005

${ }^{35} \mathrm{Ar}$ 2005DEZZ NUCLEAR REACTIONS ${ }^{24} \mathrm{Mg}\left({ }^{16} \mathrm{O}, \mathrm{n} 2 \alpha\right),\left({ }^{16} \mathrm{O}, \mathrm{p} 2 \alpha\right),\left({ }^{16} \mathrm{O}, \mathrm{n} \alpha\right)$, $\left({ }^{16} \mathrm{O}, \mathrm{p} \alpha\right), \mathrm{E}=70 \mathrm{MeV}$; measured $\mathrm{E} \gamma, \mathrm{I} \gamma, \gamma \gamma-$, (charged particle) $\gamma^{-}$, (neutron) $\gamma$-coin. ${ }^{31} \mathrm{~S},{ }^{31} \mathrm{P},{ }^{35} \mathrm{Ar},{ }^{35} \mathrm{Cl}$ deduced levels, $\mathrm{J}, \pi$, mirror energy differences. GASP, ISIS arrays. CONF Argonne(Nuclei at the Limits),P205,Della Vedova

2005EK01 NUCLEAR REACTIONS ${ }^{16} \mathrm{O}\left({ }^{24} \mathrm{Mg}, \mathrm{n} \alpha\right),\left({ }^{24} \mathrm{Mg}, \mathrm{p} \alpha\right), \mathrm{E}=60 \mathrm{MeV}$; ${ }^{28} \mathrm{Si}\left({ }^{32} \mathrm{~S}, \mathrm{n} 2 \alpha\right),\left({ }^{32} \mathrm{~S}, \mathrm{p} 2 \alpha\right), \mathrm{E}=130 \mathrm{MeV} ;{ }^{24} \mathrm{Mg}\left({ }^{40} \mathrm{Ca}, 2 \mathrm{np}\right),\left({ }^{40} \mathrm{Ca}, \mathrm{n} 2 \mathrm{p}\right)$, $\mathrm{E}=104 \mathrm{MeV}$; measured $\mathrm{E} \gamma, \mathrm{I} \gamma, \gamma \gamma-$, (charged particle) $\gamma_{-}$, (neutron) $\gamma$-coin. ${ }^{35} \mathrm{Ar},{ }^{35} \mathrm{Cl},{ }^{51} \mathrm{Fe},{ }^{51} \mathrm{Mn},{ }^{61} \mathrm{Ga},{ }^{61} \mathrm{Zn}$ deduced levels, J, $\pi$, mirror energy difference. Discussed electromagnetic spin-orbit effect. Large-scale shell model calculations. JOUR ZAANE 25 s01 363 


\section{$\mathrm{A}=36$}

${ }^{36} \mathrm{Si} \quad$ 2005CAZZ NUCLEAR REACTIONS ${ }^{1} \mathrm{H}\left({ }^{36} \mathrm{Si}, \mathrm{p}\right),\left({ }^{38} \mathrm{Si}, \mathrm{p}\right)$, E not given; measured particle spectra, (particle) $\gamma$-coin. ${ }^{36,38} \mathrm{Si}$ deduced excited states energies. CONF Argonne(Nuclei at the Limits),P127,Campbell 20050B04 NUCLEAR REACTIONS ${ }^{251} \mathrm{Cf}(\mathrm{n}, \mathrm{F}), \mathrm{E}=$ thermal; measured light charged particle yields, energy distributions following ternary fission; deduced evidence for ${ }^{34} \mathrm{Al},{ }^{36} \mathrm{Si}$. JOUR NUPAB 761173

${ }^{36} \mathrm{P} \quad 20050 \mathrm{~L} 04$
NUCLEAR REACTIONS ${ }^{176} \mathrm{Yb}\left({ }^{36} \mathrm{~S}, \mathrm{X}\right){ }^{34} \mathrm{P}, \mathrm{E}=230 \mathrm{MeV} ;{ }^{208} \mathrm{~Pb}\left({ }^{36} \mathrm{~S}\right.$, $\mathrm{X}){ }^{36} \mathrm{~S} /{ }^{38} \mathrm{~S} /{ }^{34} \mathrm{P} /{ }^{36} \mathrm{P}, \mathrm{E}=215 \mathrm{MeV}$; measured $\mathrm{E} \gamma, \mathrm{I} \gamma, \gamma \gamma-$, (particle) $\gamma$-coin. ${ }^{34} \mathrm{P}$ deduced levels, J, $\pi$, configurations. JOUR JPGPE 31 S1935

${ }^{36} \mathrm{~S} \quad$ 2005DE42 NUCLEAR REACTIONS ${ }^{26} \mathrm{Al}(\mathrm{n}, \alpha),(\mathrm{n}, \mathrm{p}), \mathrm{E}<140 \mathrm{keV}$; measured $\sigma$; deduced resonance features. ${ }^{36} \mathrm{Cl}(\mathrm{n}, \mathrm{p}),{ }^{26} \mathrm{Al}(\mathrm{n}, \alpha), \mathrm{E}=$ stellar; analyzed astrophysical reaction rates. JOUR NUPAB 758 80c 20050L04 NUCLEAR REACTIONS ${ }^{176} \mathrm{Yb}\left({ }^{36} \mathrm{~S}, \mathrm{X}\right){ }^{34} \mathrm{P}, \mathrm{E}=230 \mathrm{MeV} ;{ }^{208} \mathrm{~Pb}\left({ }^{36} \mathrm{~S}\right.$, $\mathrm{X}){ }^{36} \mathrm{~S} /{ }^{38} \mathrm{~S} /{ }^{34} \mathrm{P} /{ }^{36} \mathrm{P}, \mathrm{E}=215 \mathrm{MeV}$; measured $\mathrm{E} \gamma, \mathrm{I} \gamma, \gamma \gamma-$ (particle) $\gamma$-coin. ${ }^{34} \mathrm{P}$ deduced levels, $\mathrm{J}, \pi$, configurations. JOUR JPGPE 31 S1935

${ }^{36} \mathrm{Cl} \quad$ 2005BEZT NUCLEAR REACTIONS ${ }^{35} \mathrm{Cl}(\mathrm{n}, \gamma)$, E not given; measured $\mathrm{E} \gamma, \mathrm{I} \gamma$. ${ }^{36} \mathrm{Cl}$ deduced transitions, level energies, binding energy. ${ }^{52,54} \mathrm{Cr},{ }^{56} \mathrm{Fe}$, ${ }^{206} \mathrm{~Pb}(\mathrm{n}, \gamma)$, E not given; analyzed $\mathrm{E} \gamma \cdot{ }^{53,55} \mathrm{Cr},{ }^{57} \mathrm{Fe},{ }^{207} \mathrm{~Pb}$ deduced binding energies. GAMS4 spectrometer. CONF Santa Fe (Nucl Data for Sci and Technol) Proc,Vol1,P1074

2005DEZW NUCLEAR REACTIONS ${ }^{28} \mathrm{Si},{ }^{32} \mathrm{~S},{ }^{35} \mathrm{Cl}(\mathrm{n}, \gamma), \mathrm{E}=$ reactor; measured $\mathrm{E} \gamma, \mathrm{I} \gamma \cdot{ }^{29} \mathrm{Si},{ }^{33} \mathrm{~S},{ }^{36} \mathrm{Cl}$ deduced binding energies. Flat-crystal spectrometer. PREPRINT nucl-ex/0507011,7/06/2005

2005JEZY NUCLEAR REACTIONS ${ }^{28} \mathrm{Si},{ }^{32} \mathrm{~S},{ }^{35} \mathrm{Cl}(\mathrm{n}, \gamma)$, E=thermal; measured $\mathrm{E} \gamma, \mathrm{I} \gamma .{ }^{29} \mathrm{Si},{ }^{33} \mathrm{~S},{ }^{36} \mathrm{Cl}$ deduced level energies, neutron binding energies. Double crystal spectrometers. CONF Santa Fe (Nucl Data for Sci and Technol) Proc,Vol1,P617

2005SCZW NUCLEAR REACTIONS $\mathrm{Pb}(\mathrm{p}, \mathrm{X}){ }^{10} \mathrm{Be} /{ }^{26} \mathrm{Al} /{ }^{129} \mathrm{I} /{ }^{36} \mathrm{Cl}$, $\mathrm{E}=200-2600 \mathrm{MeV}$; measured excitation functions. Stacked foil activation, chemical separation. Comparison with model predictions. CONF Santa Fe (Nucl Data for Sci and Technol) Proc,Vol2,P1517

${ }^{36} \mathrm{Ar} \quad 2005 \mathrm{G036}$ ATOMIC MASSES ${ }^{12} \mathrm{C},{ }^{16} \mathrm{O},{ }^{20} \mathrm{Ne},{ }^{32} \mathrm{~S},{ }^{36,40} \mathrm{Ar}$; measured masses. Cyclotron-based mass spectrometry. JOUR JPGPE 31 S1869 2005LE04 NUCLEAR REACTIONS Pb(p, X) ${ }^{3} \mathrm{He} /{ }^{4} \mathrm{He} /{ }^{21} \mathrm{Ne} /{ }^{22} \mathrm{Ne} /{ }^{36} \mathrm{Ar} /$ ${ }^{38} \mathrm{Ar} /{ }^{78} \mathrm{Kr} /{ }^{80} \mathrm{Kr} /{ }^{81} \mathrm{Kr} /{ }^{82} \mathrm{Kr} /{ }^{83} \mathrm{Kr} /{ }^{84} \mathrm{Kr} /{ }^{85} \mathrm{Kr} /{ }^{86} \mathrm{Kr} /{ }^{124} \mathrm{Xe}$ $/{ }^{126} \mathrm{Xe} /{ }^{128} \mathrm{Xe} /{ }^{129} \mathrm{Xe} /{ }^{130} \mathrm{Xe} /{ }^{131} \mathrm{Xe} /{ }^{132} \mathrm{Xe} /{ }^{134} \mathrm{Xe}, \mathrm{E}=44-2595$ $\mathrm{MeV}$; measured production $\sigma$; deduced reaction mechanism features. Mini-stack approach, comparisons with model predictions. JOUR NIMBE 2291

2005SPZY NUCLEAR REACTIONS ${ }^{12} \mathrm{C}\left({ }^{32} \mathrm{~S},{ }^{36} \mathrm{Ar}\right), \mathrm{E}=65 \mathrm{MeV} ;{ }^{12} \mathrm{C}\left({ }^{34} \mathrm{~S},{ }^{38} \mathrm{Ar}\right)$, $\mathrm{E}=67 \mathrm{MeV}$; measured $\mathrm{E} \gamma, \mathrm{I} \gamma(\theta, \mathrm{H}, \mathrm{t}), \alpha \gamma$-coin. ${ }^{36,38} \mathrm{Ar}$ levels deduced $\mathrm{g}$ factors. Transient field technique. Comparison with shell model predictions. REPT MLL 2004 Annual,P5,Speidel 


\begin{tabular}{|c|c|c|}
\hline \multirow[b]{2}{*}{${ }^{37} \mathrm{Cl}$} & \multicolumn{2}{|r|}{$A=37$} \\
\hline & 2005HE03 & $\begin{array}{l}\text { RADIOACTIVITY }{ }^{37} \mathrm{~K}\left(\beta^{+}\right) ;{ }^{37} \operatorname{Ar}(\mathrm{EC}) ; \text { measured } \mathrm{T}_{1 / 2} \text {. JOUR } \\
\text { NJOPF } 744\end{array}$ \\
\hline${ }^{37} \mathrm{Ar}$ & 2005HE03 & $\begin{array}{l}\text { RADIOACTIVITY }{ }^{37} \mathrm{~K}\left(\beta^{+}\right) ;{ }^{37} \operatorname{Ar}(\mathrm{EC}) ; \text { measured } \mathrm{T}_{1 / 2} \text {. JOUR } \\
\text { NJOPF } 744\end{array}$ \\
\hline & 2005HE03 & $\begin{array}{l}\text { ATOMIC MASSES }{ }^{37} \mathrm{~K},{ }^{37} \mathrm{Ar} \text {; measured masses. Penning trap } \\
\text { spectrometer. JOUR NJOPF } 744\end{array}$ \\
\hline${ }^{37} \mathrm{~K}$ & 2005HE03 & $\begin{array}{l}\text { RADIOACTIVITY }{ }^{37} \mathrm{~K}\left(\beta^{+}\right) ;{ }^{37} \operatorname{Ar}(\mathrm{EC}) ; \text { measured } \mathrm{T}_{1 / 2} \text {. JOUR } \\
\text { NJOPF } 744\end{array}$ \\
\hline & $2005 \mathrm{HE} 03$ & $\begin{array}{l}\text { ATOMIC MASSES }{ }^{37} \mathrm{~K},{ }^{37} \mathrm{Ar} \text {; measured masses. Penning trap } \\
\text { spectrometer. JOUR NJOPF } 744\end{array}$ \\
\hline
\end{tabular}

\section{$\mathrm{A}=38$}

${ }^{38} \mathrm{Si} \quad$ 2005CAZZ

NUCLEAR REACTIONS ${ }^{1} \mathrm{H}\left({ }^{36} \mathrm{Si}, \mathrm{p}\right),\left({ }^{38} \mathrm{Si}, \mathrm{p}\right)$, E not given; measured particle spectra, (particle) $\gamma$-coin. ${ }^{36,38} \mathrm{Si}$ deduced excited states energies. CONF Argonne(Nuclei at the Limits),P127, Campbell ${ }^{38} \mathrm{~S} 20050 \mathrm{~L} 04 \quad \mathrm{NUCLEAR}$ REACTIONS ${ }^{176} \mathrm{Yb}\left({ }^{36} \mathrm{~S}, \mathrm{X}\right){ }^{34} \mathrm{P}, \mathrm{E}=230 \mathrm{MeV} ;{ }^{208} \mathrm{~Pb}\left({ }^{36} \mathrm{~S}\right.$,
$\mathrm{X})^{36} \mathrm{~S} /{ }^{38} \mathrm{~S} /{ }^{34} \mathrm{P} /{ }^{36} \mathrm{P}, \mathrm{E}=215 \mathrm{MeV} ;$ measured $\mathrm{E} \gamma, \mathrm{I} \gamma, \gamma \gamma-$, (particle) $\gamma$-coin. ${ }^{34} \mathrm{P}$ deduced levels, $\mathrm{J}, \pi$, configurations. JOUR JPGPE 31 S1935

${ }^{38} \mathrm{Ar} \quad 2005 \mathrm{FU} 03 \quad$ NUCLEAR REACTIONS ${ }^{34} \mathrm{~S}\left({ }^{7} \mathrm{Li}, \mathrm{t} \alpha\right), \mathrm{E}=26 \mathrm{MeV}$; measured particle spectra, angular correlations. ${ }^{38} \mathrm{Ar}$ deduced $\alpha$-cluster states energies, J, $\pi$. JOUR PRVCA 71067602

2005G011 RADIOACTIVITY ${ }^{38 m} \mathrm{~K}\left(\beta^{+}\right)$; measured $\mathrm{E} \beta$, recoil spectrum, (recoil) $\beta$-coin; deduced $\beta$ - $\nu$ correlation parameter. Magneto-optical trap. JOUR PRLTA 94142501

2005LE04 NUCLEAR REACTIONS Pb(p, X) ${ }^{3} \mathrm{He} /{ }^{4} \mathrm{He} /{ }^{21} \mathrm{Ne} /{ }^{22} \mathrm{Ne} /{ }^{36} \mathrm{Ar} /$ ${ }^{38} \mathrm{Ar} /{ }^{78} \mathrm{Kr} /{ }^{80} \mathrm{Kr} /{ }^{81} \mathrm{Kr} /{ }^{82} \mathrm{Kr} /{ }^{83} \mathrm{Kr} /{ }^{84} \mathrm{Kr} /{ }^{85} \mathrm{Kr} /{ }^{86} \mathrm{Kr} /{ }^{124} \mathrm{Xe}$ $/{ }^{126} \mathrm{Xe} /{ }^{128} \mathrm{Xe} /{ }^{129} \mathrm{Xe} /{ }^{130} \mathrm{Xe} /{ }^{131} \mathrm{Xe} /{ }^{132} \mathrm{Xe} /{ }^{134} \mathrm{Xe}, \mathrm{E}=44-2595$ $\mathrm{MeV}$; measured production $\sigma$; deduced reaction mechanism features. Mini-stack approach, comparisons with model predictions. JOUR NIMBE 2291

2005SPZY NUCLEAR REACTIONS ${ }^{12} \mathrm{C}\left({ }^{32} \mathrm{~S},{ }^{36} \mathrm{Ar}\right), \mathrm{E}=65 \mathrm{MeV} ;{ }^{12} \mathrm{C}\left({ }^{34} \mathrm{~S},{ }^{38} \mathrm{Ar}\right)$, $\mathrm{E}=67 \mathrm{MeV}$; measured $\mathrm{E} \gamma, \mathrm{I} \gamma(\theta, \mathrm{H}, \mathrm{t}), \alpha \gamma$-coin. ${ }^{36,38}$ Ar levels deduced $\mathrm{g}$ factors. Transient field technique. Comparison with shell model predictions. REPT MLL 2004 Annual,P5,Speidel

${ }^{38} \mathrm{~K} \quad 2005 \mathrm{GO} 11 \quad$ RADIOACTIVITY ${ }^{38 m} \mathrm{~K}\left(\beta^{+}\right)$; measured $\mathrm{E} \beta$, recoil spectrum, (recoil) $\beta$-coin; deduced $\beta$ - $\nu$ correlation parameter. Magneto-optical trap. JOUR PRLTA 94142501

2005SA44 RADIOACTIVITY ${ }^{46} \mathrm{~V}(\mathrm{EC})$; analyzed masses; deduced Q(EC), log ft. ${ }^{10} \mathrm{C},{ }^{14} \mathrm{O},{ }^{22} \mathrm{Mg},{ }^{26 m} \mathrm{Al},{ }^{34} \mathrm{Cl},{ }^{34} \mathrm{Ar},{ }^{38 m} \mathrm{~K},{ }^{42} \mathrm{Sc},{ }^{46} \mathrm{~V},{ }^{50} \mathrm{Mn},{ }^{54} \mathrm{Co},{ }^{74} \mathrm{Rb}$; compiled, analyzed log ft; deduced quark-mixing matrix element.

JOUR PRLTA 95102501 


\section{$\mathrm{A}=39$}

\begin{tabular}{|c|c|c|}
\hline${ }^{39} \mathrm{Ar}$ & 2005FUZX & $\begin{array}{l}\text { NUCLEAR REACTIONS }{ }^{3} \mathrm{He}\left({ }^{40} \mathrm{Ar}, \alpha\right), \mathrm{E}=4.5 \mathrm{MeV} / \text { nucleon; } \\
\text { measured yield. Application to half-life determination discussed. } \\
\text { REPT CNS-REP-66,P8,Fulop }\end{array}$ \\
\hline${ }^{39} \mathrm{Ca}$ & $2005 \mathrm{SA} 37$ & $\begin{array}{l}\text { NUCLEAR REACTIONS }{ }^{40} \mathrm{Ca}(\mathrm{e}, \mathrm{e} \mathrm{n}), \mathrm{E}=129 \mathrm{MeV} ; \text { measured En, } \\
\text { missing energy spectra, angular correlations, } \sigma(\mathrm{E}, \theta) ;{ }^{12} \mathrm{C},{ }^{28} \mathrm{Si}(\mathrm{e}, \mathrm{e} n) \text {, } \\
\text { E not given; analyzed data; deduced core excitation in giant resonance. } \\
\text { JOUR PRVCA } 71064313\end{array}$ \\
\hline
\end{tabular}

$$
\mathrm{A}=40
$$

${ }^{40} \mathrm{Ar} \quad 2005 \mathrm{G036}$

2005ST22

${ }^{40} \mathrm{Ca} \quad 2005 \mathrm{CA} 29$

${ }^{41} \mathrm{~K}$

2005GUZX

${ }^{42} \mathrm{Si}$

2005FR19

${ }^{42} \mathrm{~K}$

2005IDZZ
ATOMIC MASSES ${ }^{12} \mathrm{C},{ }^{16} \mathrm{O},{ }^{20} \mathrm{Ne},{ }^{32} \mathrm{~S},{ }^{36,40} \mathrm{Ar}$; measured masses. Cyclotron-based mass spectrometry. JOUR JPGPE 31 S1869 NUCLEAR REACTIONS C $\left({ }^{40} \mathrm{Ar},{ }^{40} \mathrm{Ar}\right), \mathrm{E}=80 \mathrm{MeV} ; \mathrm{C}\left({ }^{46} \mathrm{Ti},{ }^{46} \mathrm{Ti}\right)$, $\mathrm{E}=100 \mathrm{MeV}$; measured $\mathrm{E} \gamma, \mathrm{I} \gamma(\theta, \mathrm{H}, \mathrm{t})$, (particle) $\gamma$-coin following projectile Coulomb excitation. ${ }^{40} \mathrm{Ar}$ level deduced $\mathrm{g}$ factor, configuration. Transient field technique, comparison with shell model calculations. JOUR PRVCA 72014309

NUCLEAR REACTIONS ${ }^{12} \mathrm{C}(\mathrm{p}, \mathrm{X}), \mathrm{E}=180 \mathrm{MeV} ;{ }^{12} \mathrm{C}(\alpha, \mathrm{X}), \mathrm{E}=192.4$ $\mathrm{MeV}$; measured reaction $\sigma \cdot{ }^{3,4} \mathrm{He}(\mathrm{p}, \mathrm{p}), \mathrm{E} \approx 40 \mathrm{MeV}$; measured $\sigma(\theta)$. ${ }^{40} \mathrm{Ca}\left({ }^{3} \mathrm{He},{ }^{3} \mathrm{He}\right), \mathrm{E}=167 \mathrm{MeV}$; measured particle spectra. Modified attenuation technique for reaction cross section measurement. JOUR NIMAE 547541 NUCLEAR REACTIONS ${ }^{13} \mathrm{C}\left({ }^{18} \mathrm{O},{ }^{8} \mathrm{Be}\right),\left({ }^{18} \mathrm{O}, 2 \alpha\right), \mathrm{E}=100 \mathrm{MeV}$; ${ }^{24} \mathrm{Mg}\left({ }^{28} \mathrm{Si},{ }^{12} \mathrm{C}\right),\left({ }^{28} \mathrm{Si}, 3 \alpha\right), \mathrm{E}=130 \mathrm{MeV}$; measured particle spectra, $\mathrm{E} \gamma, \mathrm{I} \gamma$, (particle) $\gamma$-coin; deduced cluster emission features. GASP, ISIS arrays. JOUR ZAANE 2319

\section{$\mathrm{A}=41$}

NUCLEAR REACTIONS ${ }^{44} \mathrm{Ca}$ (polarized $\mathrm{p}, \alpha$ ), $\mathrm{E}=24.6 \mathrm{MeV}$; measured $\sigma(\theta), \operatorname{Ay}(\theta)$. DWBA analysis. REPT MLL 2004 Annual,P6,Guazzoni

\section{$\mathrm{A}=42$}

NUCLEAR REACTIONS Be $\left({ }^{44} \mathrm{~S}, \mathrm{X}\right){ }^{43} \mathrm{P} /{ }^{42} \mathrm{Si}, \mathrm{E}=98.6 \mathrm{MeV} /$ nucleon; $\mathrm{Be}\left({ }^{46} \mathrm{Ar}, \mathrm{X}\right)^{44} \mathrm{~S}, \mathrm{E}=98.1 \mathrm{MeV} /$ nucleon; measured particle spectra, $\mathrm{E} \gamma, \mathrm{I} \gamma$, (particle) $\gamma$-coin; deduced $\sigma .{ }^{43} \mathrm{P}$ deduced transition. ${ }^{42} \mathrm{Si},{ }^{43} \mathrm{P},{ }^{44} \mathrm{~S}$ deduced ground-state configurations, shell closure features. JOUR NATUA 435922

NUCLEAR REACTIONS ${ }^{9} \mathrm{Be}\left({ }^{37} \mathrm{P}, \mathrm{X}\right){ }^{42} \mathrm{~K}, \mathrm{E} \approx 5 \mathrm{MeV} /$ nucleon; ${ }^{9} \mathrm{Be}\left({ }^{46} \mathrm{Ar}, \mathrm{X}\right){ }^{49} \mathrm{Ti} /{ }^{50} \mathrm{Ti} /{ }^{51} \mathrm{Ti} /{ }^{46} \mathrm{Ca}, \mathrm{E} \approx 5 \mathrm{MeV} /$ nucleon; measured $\mathrm{E} \gamma, \mathrm{I} \gamma .{ }^{42} \mathrm{~K},{ }^{49,50,51} \mathrm{Ti},{ }^{46} \mathrm{Ca}$ deduced levels, J, $\pi .{ }^{9} \mathrm{Be}\left({ }^{46} \mathrm{Ar}\right.$, $\mathrm{xn}), \mathrm{E}=2-7 \mathrm{MeV} /$ nucleon; measured excitation functions. CONF Argonne(Nuclei at the Limits),P136,Ideguchi 


\section{$\mathrm{A}=42$ (continued)}

${ }^{42} \mathrm{Ca}$ 2004KMZZ NUCLEAR REACTIONS ${ }^{28} \mathrm{Si}\left({ }^{18} \mathrm{O}, \alpha\right), \mathrm{E}=105 \mathrm{MeV}$; measured $\mathrm{E} \gamma, \mathrm{I} \gamma$, $\gamma \gamma$-coin. ${ }^{46} \mathrm{Ti}$ deduced GDR strength distribution. ${ }^{42} \mathrm{Ca}$ deduced rotational band feeding intensities. Euroball IV, Hector arrays. PREPRINT nucl-ex/0412046,12/21/2004

$2005 \mathrm{CO} 25$ NUCLEAR REACTIONS ${ }^{208} \mathrm{~Pb}\left({ }^{40} \mathrm{Ca},{ }^{42} \mathrm{Ca}\right), \mathrm{E}=225 \mathrm{MeV}$; measured $\sigma(\mathrm{E}, \theta) .{ }^{42} \mathrm{Ca}$ deduced excited states configurations. ${ }^{208} \mathrm{~Pb}\left({ }^{90} \mathrm{Zr}, \mathrm{X}\right)$, $\mathrm{E}=560 \mathrm{MeV}$; measured $\mathrm{E} \gamma, \mathrm{I} \gamma$, (fragment) $\gamma$-coin, isotopic yields for projectile-like fragments. ${ }^{90} \mathrm{Zr}$ deduced transitions. JOUR ZAANE 25 s01 427

2005GUZW NUCLEAR REACTIONS ${ }^{45} \mathrm{Sc}$ (polarized p, $\alpha$ ), E=24.6 MeV; measured $\sigma(\theta), \operatorname{Ay}(\theta) .{ }^{42} \mathrm{Ca}$ levels deduced configurations. REPT MLL 2004 Annual,P7,Guazzoni

2005KM01 NUCLEAR REACTIONS ${ }^{28} \mathrm{Si}\left({ }^{18} \mathrm{O}, \mathrm{X}\right), \mathrm{E}=105 \mathrm{MeV}$; measured $\mathrm{E} \gamma, \mathrm{I} \gamma$. ${ }^{46} \mathrm{Ti}$ deduced GDR strength function. ${ }^{42} \mathrm{Ca}$ deduced feeding of highly-deformed rotational band from GDR decay. Euroball IV and Hector arrays. JOUR APOBB 361169

${ }^{42} \mathrm{Sc} \quad 2005 \mathrm{SA} 44$ RADIOACTIVITY ${ }^{46} \mathrm{~V}(\mathrm{EC})$; analyzed masses; deduced $\mathrm{Q}(\mathrm{EC}), \log \mathrm{ft}$. ${ }^{10} \mathrm{C},{ }^{14} \mathrm{O},{ }^{22} \mathrm{Mg},{ }^{26 m} \mathrm{Al},{ }^{34} \mathrm{Cl},{ }^{34} \mathrm{Ar},{ }^{38 m} \mathrm{~K},{ }^{42} \mathrm{Sc},{ }^{46} \mathrm{~V},{ }^{50} \mathrm{Mn},{ }^{54} \mathrm{Co},{ }^{74} \mathrm{Rb}$; compiled, analyzed log ft; deduced quark-mixing matrix element. JOUR PRLTA 95102501

$$
\mathrm{A}=43
$$

${ }^{43} \mathrm{P}$ 2005FR19 NUCLEAR REACTIONS Be $\left({ }^{44} \mathrm{~S}, \mathrm{X}\right){ }^{43} \mathrm{P} /{ }^{42} \mathrm{Si}, \mathrm{E}=98.6 \mathrm{MeV} /$ nucleon; $\mathrm{Be}\left({ }^{46} \mathrm{Ar}, \mathrm{X}\right){ }^{44} \mathrm{~S}, \mathrm{E}=98.1 \mathrm{MeV} /$ nucleon; measured particle spectra, $\mathrm{E} \gamma, \mathrm{I} \gamma$, (particle) $\gamma$-coin; deduced $\sigma .{ }^{43} \mathrm{P}$ deduced transition. ${ }^{42} \mathrm{Si},{ }^{43} \mathrm{P},{ }^{44} \mathrm{~S}$ deduced ground-state configurations, shell closure features. JOUR NATUA 435922

${ }^{43} \mathrm{Cr} \quad 2005 \mathrm{BL} 31$ RADIOACTIVITY ${ }^{45} \mathrm{Fe},{ }^{48} \mathrm{Ni},{ }^{54} \mathrm{Zn}(2 \mathrm{p})$ [from Ni $\left.\left({ }^{58} \mathrm{Ni}, \mathrm{X}\right)\right]$; measured proton spectra, $\mathrm{T}_{1 / 2}$. Comparison with theory. JOUR ZAANE $25 \mathrm{~s} 01$ 169

2005D020 RADIOACTIVITY ${ }^{45} \mathrm{Fe}(2 \mathrm{p})$ [from Ni( $\left.\left.{ }^{58} \mathrm{Ni}, \mathrm{X}\right)\right]$; measured $\mathrm{Ep}, \mathrm{T}_{1 / 2}$, branching ratio. ${ }^{48} \mathrm{Ni}$; measured decay energy, $\mathrm{T}_{1 / 2}$; deduced probable two-proton decay. Comparisons with model predictions. JOUR PRVCA 72054315

2005GI15 RADIOACTIVITY ${ }^{45} \mathrm{Fe},{ }^{54} \mathrm{Zn}(\mathrm{p}),(2 \mathrm{p})$ [from Ni $\left.\left({ }^{58} \mathrm{Ni}, \mathrm{X}\right)\right]$; measured proton spectra, $\mathrm{T}_{1 / 2}$. JOUR JPGPE $31 \mathrm{~S} 1509$

\section{$\mathrm{A}=44$}

${ }^{44} \mathrm{~S} \quad 2005 \mathrm{FR} 19$

NUCLEAR REACTIONS Be $\left({ }^{44} \mathrm{~S}, \mathrm{X}\right){ }^{43} \mathrm{P} /{ }^{42} \mathrm{Si}, \mathrm{E}=98.6 \mathrm{MeV} /$ nucleon; $\mathrm{Be}\left({ }^{46} \mathrm{Ar}, \mathrm{X}\right){ }^{44} \mathrm{~S}, \mathrm{E}=98.1 \mathrm{MeV} /$ nucleon; measured particle spectra, $\mathrm{E} \gamma, \mathrm{I} \gamma$, (particle) $\gamma$-coin; deduced $\sigma \cdot{ }^{43} \mathrm{P}$ deduced transition. ${ }^{42} \mathrm{Si},{ }^{43} \mathrm{P},{ }^{44} \mathrm{~S}$ deduced ground-state configurations, shell closure features. JOUR NATUA 435922 


\section{$\mathrm{A}=44$ (continued)}

2005GR30 RADIOACTIVITY ${ }^{44} \mathrm{~S}(\mathrm{IT})$ [from $\left.\mathrm{Be}\left({ }^{48} \mathrm{Ca}, \mathrm{X}\right)\right]$; measured $\mathrm{E}(\mathrm{ce}), \mathrm{T}_{1 / 2}$. ${ }^{44} \mathrm{~S}$ deduced levels, $\mathrm{J}, \pi$. Comparison with shell model calculations. JOUR ZAANE 25 s01 111

${ }^{44} \mathrm{Ca}$ 2005L006 NUCLEAR REACTIONS ${ }^{44} \mathrm{Ca}(\mathrm{p}, \mathrm{p}),(\mathrm{p}, \mathrm{p}$ '), $\mathrm{E}=2.50-3.53 \mathrm{MeV}$; measured Ep, $\sigma(\mathrm{E}, \theta) .{ }^{45} \mathrm{Sc}$ deduced resonance parameters, level densities. JOUR PRVCA 71064315

${ }^{44} \mathrm{Sc} \quad$ 2005LA19 NUCLEAR REACTIONS ${ }^{30} \mathrm{Si}\left({ }^{18} \mathrm{O}, 3 \mathrm{np}\right), \mathrm{E}=68 \mathrm{MeV}$; measured $\mathrm{E} \gamma$, $\mathrm{I} \gamma, \gamma \gamma$-, (recoil) $\gamma$-coin, $\gamma$-ray polarization. ${ }^{44} \mathrm{Sc}$ deduced high-spin levels, $\mathrm{J}, \pi, \mathrm{T}_{1 / 2}, \mathrm{~B}(\mathrm{E} 2)$, configurations. Euroball IV array. JOUR ZAANE 251

${ }^{44} \mathrm{Ti}$ 2005BrZU NUCLEAR REACTIONS Ti(p, X) ${ }^{44} \mathrm{Ti}, \mathrm{E}=21-29 \mathrm{MeV} ; \mathrm{Ni}(\mathrm{p}, \mathrm{X}){ }^{56} \mathrm{Ni}$, $\mathrm{E}=18-28 \mathrm{MeV} ; \mathrm{Zr}(\mathrm{p}, \mathrm{X})^{88} \mathrm{Zr}, \mathrm{E}=19-28 \mathrm{MeV}$; measured production $\sigma$. Activation technique, comparison with previous results. CONF Santa Fe (Nucl Data for Sci and Technol) Proc,Vol2,P1374

2005NA30 NUCLEAR REACTIONS ${ }^{4} \mathrm{He}\left({ }^{40} \mathrm{Ca}, \gamma\right), \mathrm{E}=72 \mathrm{MeV}$; measured yields. Radiochemical separation, accelerator mass spectrometry. Astrophysical implications discussed. JOUR NUPAB 758 411c 2005NAZW NUCLEAR REACTIONS ${ }^{4} \mathrm{He}\left({ }^{40} \mathrm{Ca}, \gamma\right), \mathrm{E}(\mathrm{cm})=0.6-1.2 \mathrm{MeV} /$ nucleon; measured yields. Radiochemical separation, accelerator mass spectrometry. Astrophysical implications discussed. PREPRINT nucl-ex/0509006,9/03/2005

${ }^{44} \mathrm{Mn} \quad$ 2005GI15 RADIOACTIVITY ${ }^{45} \mathrm{Fe},{ }^{54} \mathrm{Zn}(\mathrm{p}),(2 \mathrm{p})\left[\right.$ from Ni $\left.\left({ }^{58} \mathrm{Ni}, \mathrm{X}\right)\right]$; measured proton spectra, $\mathrm{T}_{1 / 2}$. JOUR JPGPE $31 \mathrm{~S} 1509$

\section{$\mathrm{A}=45$}

${ }^{45} \mathrm{Ar} \quad$ 2005GA18

NUCLEAR REACTIONS ${ }^{9} \mathrm{Be}\left({ }^{46} \mathrm{Ar},{ }^{45} \mathrm{ArX}\right), \mathrm{E}=70 \mathrm{MeV} /$ nucleon; measured $\mathrm{E} \gamma, \mathrm{I} \gamma$, fragments parallel momentum distributions following one-neutron removal; deduced dissipative effects. ${ }^{45} \mathrm{Ar}$ levels deduced branching ratios, spectroscopic factors. Comparison with eikonal theory. JOUR PRVCA 71051301

2005GA45 NUCLEAR REACTIONS ${ }^{2} \mathrm{H}\left({ }^{44} \mathrm{Ar},{ }^{45} \mathrm{Ar}\right),\left({ }^{40} \mathrm{Ar},{ }^{41} \mathrm{Ar}\right), \mathrm{E}=10 \mathrm{MeV} /$ nucleon; measured particle spectra, $\sigma(\mathrm{E}, \theta) .{ }^{45} \mathrm{Ar}$ deduced levels, spectroscopic factors. JOUR JPGPE 31 S1623

${ }^{45} \mathrm{Ca}$ 2005DAZX NUCLEAR REACTIONS ${ }^{48} \mathrm{Ti}(\mathrm{n}, \mathrm{n}$ '), (n, 2n), (n, p), (n, $\alpha), \mathrm{E}=1-250$ $\mathrm{MeV}$; measured $\mathrm{E} \gamma, \mathrm{I} \gamma$; deduced partial $\gamma$-ray transition $\sigma$. Other exit channels discussed. Comparison with model predictions. CONF Santa Fe (Nucl Data for Sci and Technol) Proc,Vol1,P1035

2005KEZZ NUCLEAR REACTIONS Ti(p, X) ${ }^{45} \mathrm{Ca}, \mathrm{E}=30-200 \mathrm{MeV} ;{ }^{85} \mathrm{Rb}(\mathrm{p}, 4 \mathrm{n})$, $\mathrm{E}=35-70 \mathrm{MeV}$; measured excitation functions. ${ }^{89} \mathrm{Y}(\mathrm{n}, \mathrm{p}), \mathrm{E}=$ fast; measured spectrum-averaged $\sigma$. Activation technique, radiochemical separation, x-ray spectrometry. CONF Santa Fe (Nucl Data for Sci and Technol) Proc,Vol1,P758

${ }^{45} \mathrm{Sc} \quad 2005 \mathrm{~L} 006 \quad$ NUCLEAR REACTIONS ${ }^{44} \mathrm{Ca}(\mathrm{p}, \mathrm{p}),(\mathrm{p}, \mathrm{p}), \mathrm{E}=2.50-3.53 \mathrm{MeV}$; measured Ep, $\sigma(\mathrm{E}, \theta) .{ }^{45} \mathrm{Sc}$ deduced resonance parameters, level densities. JOUR PRVCA 71064315 


\section{$\mathrm{A}=45$ (continued)}

${ }^{45} \mathrm{Fe} \quad 2005 \mathrm{BL} 31$

2005D020

2005D020

2005GI15

${ }^{46} \mathrm{Ca}$

2005IDZZ

${ }^{46} \mathrm{Sc}$

2005SI14

${ }^{46} \mathrm{Ti}$

2004KMZZ

2005KM01

2005SA44

2005SA44
RADIOACTIVITY ${ }^{45} \mathrm{Fe},{ }^{48} \mathrm{Ni},{ }^{54} \mathrm{Zn}(2 \mathrm{p})$ [from Ni( $\left.\left({ }^{58} \mathrm{Ni}, \mathrm{X}\right)\right]$; measured proton spectra, $\mathrm{T}_{1 / 2}$. Comparison with theory. JOUR ZAANE $25 \mathrm{~s} 01$ 169

NUCLEAR REACTIONS Ni( $\left.{ }^{58} \mathrm{Ni}, \mathrm{X}\right), \mathrm{E}=74.5 \mathrm{MeV} /$ nucleon; measured fragments isotopic yields; deduced evidence for ${ }^{48} \mathrm{Ni},{ }^{45} \mathrm{Fe}$. JOUR PRVCA 72054315 RADIOACTIVITY ${ }^{45} \mathrm{Fe}(2 \mathrm{p})$ [from $\mathrm{Ni}\left({ }^{58} \mathrm{Ni}, \mathrm{X}\right)$ ]; measured Ep, $\mathrm{T}_{1 / 2}$, branching ratio. ${ }^{48} \mathrm{Ni}$; measured decay energy, $\mathrm{T}_{1 / 2}$; deduced probable two-proton decay. Comparisons with model predictions. JOUR PRVCA 72054315

RADIOACTIVITY ${ }^{45} \mathrm{Fe},{ }^{54} \mathrm{Zn}(\mathrm{p}),(2 \mathrm{p})$ [from $\left.\mathrm{Ni}\left({ }^{58} \mathrm{Ni}, \mathrm{X}\right)\right]$; measured proton spectra, $\mathrm{T}_{1 / 2}$. JOUR JPGPE $31 \mathrm{~S} 1509$

\section{$\mathrm{A}=46$}

NUCLEAR REACTIONS ${ }^{1} \mathrm{H}, \mathrm{C}\left({ }^{46} \mathrm{Ar},{ }^{46} \mathrm{Ar}{ }^{\prime}\right), \mathrm{E} \approx 68 \mathrm{MeV} /$ nucleon; measured $\mathrm{E} \gamma, \mathrm{I} \gamma$, (particle) $\gamma$-coin. ${ }^{46} \mathrm{Ar}$ deduced levels, J, $\pi, \mathrm{B}(\mathrm{E} 2)$, deformation lengths. Optical model analysis. JOUR PRVCA 72024311 NUCLEAR REACTIONS ${ }^{9} \mathrm{Be}\left({ }^{37} \mathrm{P}, \mathrm{X}\right){ }^{42} \mathrm{~K}, \mathrm{E} \approx 5 \mathrm{MeV} /$ nucleon; ${ }^{9} \mathrm{Be}\left({ }^{46} \mathrm{Ar}, \mathrm{X}\right){ }^{49} \mathrm{Ti} /{ }^{50} \mathrm{Ti} /{ }^{51} \mathrm{Ti} /{ }^{46} \mathrm{Ca}, \mathrm{E} \approx 5 \mathrm{MeV} /$ nucleon; measured $\mathrm{E} \gamma, \mathrm{I} \gamma .{ }^{42} \mathrm{~K},{ }^{49,50,51} \mathrm{Ti},{ }^{46} \mathrm{Ca}$ deduced levels, J, $\pi .{ }^{9} \mathrm{Be}\left({ }^{46} \mathrm{Ar}\right.$, $\mathrm{xn}), \mathrm{E}=2-7 \mathrm{MeV} /$ nucleon; measured excitation functions. CONF Argonne(Nuclei at the Limits),P136,Ideguchi NUCLEAR REACTIONS $\mathrm{C}\left({ }^{46} \mathrm{Ca},{ }^{46} \mathrm{Ca}\right), \mathrm{E}=95 \mathrm{MeV}$; measured $\mathrm{E} \gamma$, $\mathrm{I} \gamma(\theta, \mathrm{H}, \mathrm{t})$, (particle) $\gamma$-coin following projectile Coulomb excitation. ${ }^{46} \mathrm{Ca}$ level deduced g-factor, configuration. Transient field technique, comparisons with neighboring isotopes. JOUR PYLBB 605265 NUCLEAR REACTIONS C, O, Si, Mg, $\mathrm{Al}(\mathrm{n}, \mathrm{X})^{7} \mathrm{Be}, \mathrm{E} \approx 0.1-750$ $\mathrm{MeV} ; \mathrm{O}, \mathrm{Si}, \mathrm{Mg}, \mathrm{Al}(\mathrm{n}, \mathrm{X}){ }^{22} \mathrm{Na} /{ }^{23} \mathrm{Na}, \mathrm{E} \approx 0.1-750 \mathrm{MeV} ;{ }^{197} \mathrm{Au}(\mathrm{n}$, $\mathrm{X}){ }^{194} \mathrm{Au} /{ }^{196} \mathrm{Au} /{ }^{198} \mathrm{Au}, \mathrm{E} \approx 0.1-750 \mathrm{MeV} ; \mathrm{Ti}, \mathrm{Fe}, \mathrm{Ni}, \mathrm{Cu}(\mathrm{n}, \mathrm{X}){ }^{46} \mathrm{Sc}$ $/{ }^{48} \mathrm{Sc}, \mathrm{E} \approx 0.1-750 \mathrm{MeV} ; \mathrm{Fe}, \mathrm{Ni}, \mathrm{Cu}(\mathrm{n}, \mathrm{X}){ }^{48} \mathrm{~V} /{ }^{51} \mathrm{Cr} /{ }^{52} \mathrm{Mn} /{ }^{54} \mathrm{Mn}$, $\mathrm{E} \approx 0.1-750 \mathrm{MeV} ; \mathrm{Ni}, \mathrm{Cu}(\mathrm{n}, \mathrm{X}){ }^{56} \mathrm{Ni} /{ }^{57} \mathrm{Ni} /{ }^{56} \mathrm{Co} /{ }^{57} \mathrm{Co} /{ }^{58} \mathrm{Co} /$ ${ }^{60} \mathrm{Co} /{ }^{59} \mathrm{Fe}, \mathrm{E} \approx 0.1-750 \mathrm{MeV}$; measured energy-integrated production $\sigma$. JOUR NIMBE 234419

NUCLEAR REACTIONS ${ }^{28} \mathrm{Si}\left({ }^{18} \mathrm{O}, \alpha\right), \mathrm{E}=105 \mathrm{MeV}$; measured $\mathrm{E} \gamma, \mathrm{I} \gamma$, $\gamma \gamma$-coin. ${ }^{46} \mathrm{Ti}$ deduced GDR strength distribution. ${ }^{42} \mathrm{Ca}$ deduced rotational band feeding intensities. Euroball IV, Hector arrays. PREPRINT nucl-ex/0412046,12/21/2004

NUCLEAR REACTIONS ${ }^{28} \mathrm{Si}\left({ }^{18} \mathrm{O}, \mathrm{X}\right), \mathrm{E}=105 \mathrm{MeV}$; measured $\mathrm{E} \gamma, \mathrm{I} \gamma$. ${ }^{46} \mathrm{Ti}$ deduced GDR strength function. ${ }^{42} \mathrm{Ca}$ deduced feeding of highly-deformed rotational band from GDR decay. Euroball IV and Hector arrays. JOUR APOBB 361169

ATOMIC MASSES ${ }^{46} \mathrm{Ti},{ }^{46} \mathrm{~V}$; measured masses; deduced Q(EC). Penning trap mass spectrometer. JOUR PRLTA 95102501 RADIOACTIVITY ${ }^{46} \mathrm{~V}(\mathrm{EC})$; analyzed masses; deduced Q(EC), log ft. ${ }^{10} \mathrm{C},{ }^{14} \mathrm{O},{ }^{22} \mathrm{Mg},{ }^{26 m} \mathrm{Al},{ }^{34} \mathrm{Cl},{ }^{34} \mathrm{Ar},{ }^{38 m} \mathrm{~K},{ }^{42} \mathrm{Sc},{ }^{46} \mathrm{~V},{ }^{50} \mathrm{Mn},{ }^{54} \mathrm{Co},{ }^{74} \mathrm{Rb} ;$ compiled, analyzed log ft; deduced quark-mixing matrix element. JOUR PRLTA 95102501 


\section{$\mathrm{A}=46$ (continued)}

${ }^{46} \mathrm{~V}$ 20040NZZ RADIOACTIVITY ${ }^{46} \mathrm{Cr}(\mathrm{EC})\left[\right.$ from $\left.\mathrm{Be}\left({ }^{50} \mathrm{Cr}, \mathrm{X}\right)\right]$; measured $\mathrm{E} \gamma, \mathrm{I} \gamma$, $\beta \gamma$-coin, $\mathrm{T}_{1 / 2}$; deduced Gamow-Teller transition, branching ratio, log ft. ${ }^{46} \mathrm{~V}$ deduced $\beta$-feeding intensity. Comparison with model predictions. REPT CNS-REP-64,P235,Onishi

20050N03 RADIOACTIVITY ${ }^{46} \mathrm{Cr}\left(\beta^{+}\right)$from $\left.{ }^{9} \mathrm{Be}\left({ }^{50} \mathrm{Cr}, \mathrm{X}\right)\right]$; measured $\mathrm{E} \gamma$, I $\gamma$, $\gamma \gamma-, \beta \gamma$-coin, $\mathrm{T}_{1 / 2}$; deduced Gamow-Teller decay branching ratio, $\mathrm{B}(\mathrm{GT}) .{ }^{46} \mathrm{~V}$ deduced transitions. Comparison with model predictions. JOUR PRVCA 72024308

2005SA44 ATOMIC MASSES ${ }^{46} \mathrm{Ti},{ }^{46} \mathrm{~V}$; measured masses; deduced Q(EC). Penning trap mass spectrometer. JOUR PRLTA 95102501

2005SA44 RADIOACTIVITY ${ }^{46} \mathrm{~V}(\mathrm{EC})$; analyzed masses; deduced Q(EC), log ft. ${ }^{10} \mathrm{C},{ }^{14} \mathrm{O},{ }^{22} \mathrm{Mg},{ }^{26 m} \mathrm{Al},{ }^{34} \mathrm{Cl},{ }^{34} \mathrm{Ar},{ }^{38 m} \mathrm{~K},{ }^{42} \mathrm{Sc},{ }^{46} \mathrm{~V},{ }^{50} \mathrm{Mn},{ }^{54} \mathrm{Co},{ }^{74} \mathrm{Rb}$; compiled, analyzed log ft; deduced quark-mixing matrix element. JOUR PRLTA 95102501

${ }^{46} \mathrm{Cr}$ 20040NZZ RADIOACTIVITY ${ }^{46} \mathrm{Cr}(\mathrm{EC})\left[\right.$ from $\left.\mathrm{Be}\left({ }^{50} \mathrm{Cr}, \mathrm{X}\right)\right]$; measured $\mathrm{E} \gamma, \mathrm{I} \gamma$, $\beta \gamma$-coin, $\mathrm{T}_{1 / 2} ;$ deduced Gamow-Teller transition, branching ratio, log ft. ${ }^{46} \mathrm{~V}$ deduced $\beta$-feeding intensity. Comparison with model predictions. REPT CNS-REP-64,P235,Onishi

20050N03 RADIOACTIVITY ${ }^{46} \mathrm{Cr}\left(\beta^{+}\right)$[from $\left.{ }^{9} \mathrm{Be}\left({ }^{50} \mathrm{Cr}, \mathrm{X}\right)\right]$; measured $\mathrm{E} \gamma$, I $\gamma$, $\gamma \gamma-, \beta \gamma$-coin, $\mathrm{T}_{1 / 2}$; deduced Gamow-Teller decay branching ratio, $\mathrm{B}(\mathrm{GT}) .{ }^{46} \mathrm{~V}$ deduced transitions. Comparison with model predictions. JOUR PRVCA 72024308

2005 YA26 NUCLEAR REACTIONS Pb $\left({ }^{46} \mathrm{Cr},{ }^{46} \mathrm{Cr}\right),\left({ }^{50} \mathrm{Fe},{ }^{50} \mathrm{Fe}\right),\left({ }^{54} \mathrm{Ni},{ }^{54} \mathrm{Ni} '\right)$, $\mathrm{E}=41-44 \mathrm{MeV} /$ nucleon; measured $\sigma(\theta), \mathrm{E} \gamma, \mathrm{I} \gamma$, (particle) $\gamma$-coin following projectile Coulomb excitation. ${ }^{46} \mathrm{Cr},{ }^{50} \mathrm{Fe},{ }^{54} \mathrm{Ni}$ deduced excitation B(E2). DWBA analysis. JOUR ZAANE 25 s01 409

${ }^{46} \mathrm{Fe} \quad 2005 \mathrm{BL} 31$ RADIOACTIVITY ${ }^{45} \mathrm{Fe},{ }^{48} \mathrm{Ni},{ }^{54} \mathrm{Zn}(2 \mathrm{p})\left[\right.$ [rom Ni($\left.\left.{ }^{58} \mathrm{Ni}, \mathrm{X}\right)\right]$; measured proton spectra, $\mathrm{T}_{1 / 2}$. Comparison with theory. JOUR ZAANE 25 s01 169

\section{$\mathrm{A}=47$}

${ }^{47} \mathrm{~K} \quad$ 2004ISZX NUCLEAR REACTIONS ${ }^{198} \mathrm{Pt}\left({ }^{48} \mathrm{Ca}, \mathrm{X}\right){ }^{47} \mathrm{~K} /{ }^{48} \mathrm{~K}, \mathrm{E}=8.5 \mathrm{MeV} /$ nucleon; measured prompt and delayed $\mathrm{E} \gamma, \mathrm{I} \gamma, \gamma \gamma$-, (particle) $\gamma$-coin. ${ }^{47,48} \mathrm{~K}$ deduced levels, $\mathrm{J}, \pi$, isomeric states $\mathrm{T}_{1 / 2}$. REPT CNS-REP-64,P27,Ishii ${ }^{47} \mathrm{Sc} \quad$ 2005DIZY NUCLEAR REACTIONS Fe(p, X) ${ }^{57} \mathrm{Co} /{ }^{56} \mathrm{Co} /{ }^{55} \mathrm{Co} /{ }^{54} \mathrm{Mn} /{ }^{52} \mathrm{Mn}$ $/{ }^{48} \mathrm{~V} /{ }^{51} \mathrm{Cr} /{ }^{48} \mathrm{Cr} /{ }^{47} \mathrm{Sc}, \mathrm{E} \approx 20-70 \mathrm{MeV}$; measured activation $\sigma$. Comparison with previous results. CONF Santa Fe (Nucl Data for Sci and Technol) Proc,Vol1,P1011

${ }^{47} \mathrm{Ti}$ 2005DAZX NUCLEAR REACTIONS ${ }^{48} \mathrm{Ti}(\mathrm{n}, \mathrm{n}),(\mathrm{n}, 2 \mathrm{n}),(\mathrm{n}, \mathrm{p}),(\mathrm{n}, \alpha), \mathrm{E}=1-250$ $\mathrm{MeV}$; measured $\mathrm{E} \gamma, \mathrm{I} \gamma$; deduced partial $\gamma$-ray transition $\sigma$. Other exit channels discussed. Comparison with model predictions. CONF Santa Fe (Nucl Data for Sci and Technol) Proc,Vol1,P1035 


\section{$\mathrm{A}=48$}

$\begin{array}{ll}{ }^{48} \mathrm{~K} & \\ & \text { 2004ISZX } \\ { }^{48} \mathrm{Ca} & 2005 Z D 02 \\ { }^{48} \mathrm{Sc} & \text { 2005DAZX }\end{array}$

2005SI14

${ }^{48} \mathrm{Ti} \quad$ 2005DAZX

2005PA23

2005TRZZ

2005VA31

2005ZD02

${ }^{48} \mathrm{~V}$

2005B010

2005CHZY

2005DIZY
NUCLEAR REACTIONS ${ }^{198} \mathrm{Pt}\left({ }^{48} \mathrm{Ca}, \mathrm{X}\right){ }^{47} \mathrm{~K} /{ }^{48} \mathrm{~K}, \mathrm{E}=8.5 \mathrm{MeV} /$ nucleon; measured prompt and delayed $\mathrm{E} \gamma, \mathrm{I} \gamma, \gamma \gamma$-, (particle) $\gamma$-coin. ${ }^{47,48} \mathrm{~K}$ deduced levels, $\mathrm{J}, \pi$, isomeric states $\mathrm{T}_{1 / 2}$. REPT CNS-REP-64,P27,Ishii RADIOACTIVITY ${ }^{48} \mathrm{Ca}\left(2 \beta^{-}\right)$; measured $0 \nu \beta \beta$-decay $\mathrm{T}_{1 / 2}$ lower limit. $\mathrm{CaWO}_{4}$ crystal scintillators. JOUR APHYE 23249

NUCLEAR REACTIONS ${ }^{48} \operatorname{Ti}\left(\mathrm{n}, \mathrm{n}^{\prime}\right),(\mathrm{n}, 2 \mathrm{n}),(\mathrm{n}, \mathrm{p}),(\mathrm{n}, \alpha), \mathrm{E}=1-250$ $\mathrm{MeV}$; measured $\mathrm{E} \gamma, \mathrm{I} \gamma$; deduced partial $\gamma$-ray transition $\sigma$. Other exit channels discussed. Comparison with model predictions. CONF Santa Fe (Nucl Data for Sci and Technol) Proc,Vol1,P1035

NUCLEAR REACTIONS C, O, Si, Mg, $\mathrm{Al}(\mathrm{n}, \mathrm{X})^{7} \mathrm{Be}, \mathrm{E} \approx 0.1-750$ $\mathrm{MeV} ; \mathrm{O}, \mathrm{Si}, \mathrm{Mg}, \mathrm{Al}(\mathrm{n}, \mathrm{X}){ }^{22} \mathrm{Na} /{ }^{23} \mathrm{Na}, \mathrm{E} \approx 0.1-750 \mathrm{MeV} ;{ }^{197} \mathrm{Au}(\mathrm{n}$, $\mathrm{X}){ }^{194} \mathrm{Au} /{ }^{196} \mathrm{Au} /{ }^{198} \mathrm{Au}, \mathrm{E} \approx 0.1-750 \mathrm{MeV} ; \mathrm{Ti}, \mathrm{Fe}, \mathrm{Ni}, \mathrm{Cu}(\mathrm{n}, \mathrm{X})^{46} \mathrm{Sc}$ $/{ }^{48} \mathrm{Sc}, \mathrm{E} \approx 0.1-750 \mathrm{MeV} ; \mathrm{Fe}, \mathrm{Ni}, \mathrm{Cu}(\mathrm{n}, \mathrm{X}){ }^{48} \mathrm{~V} /{ }^{51} \mathrm{Cr} /{ }^{52} \mathrm{Mn} /{ }^{54} \mathrm{Mn}$, $\mathrm{E} \approx 0.1-750 \mathrm{MeV} ; \mathrm{Ni}, \mathrm{Cu}(\mathrm{n}, \mathrm{X}){ }^{56} \mathrm{Ni} /{ }^{57} \mathrm{Ni} /{ }^{56} \mathrm{Co} /{ }^{57} \mathrm{Co} /{ }^{58} \mathrm{Co} /$ ${ }^{60} \mathrm{Co} /{ }^{59} \mathrm{Fe}, \mathrm{E} \approx 0.1-750 \mathrm{MeV}$; measured energy-integrated production $\sigma$. JOUR NIMBE 234419

NUCLEAR REACTIONS ${ }^{48} \operatorname{Ti}\left(\mathrm{n}, \mathrm{n}^{\prime}\right),(\mathrm{n}, 2 \mathrm{n}),(\mathrm{n}, \mathrm{p}),(\mathrm{n}, \alpha), \mathrm{E}=1-250$ $\mathrm{MeV}$; measured $\mathrm{E} \gamma, \mathrm{I} \gamma$; deduced partial $\gamma$-ray transition $\sigma$. Other exit channels discussed. Comparison with model predictions. CONF Santa Fe (Nucl Data for Sci and Technol) Proc,Vol1,P1035

NUCLEAR REACTIONS C $\left({ }^{78} \mathrm{Ge},{ }^{78} \mathrm{Ge}\right),\left({ }^{80} \mathrm{Ge},{ }^{80} \mathrm{Ge}\right), \mathrm{E}=2.24 \mathrm{MeV}$ / nucleon; ${ }^{48} \mathrm{Ti}\left({ }^{82} \mathrm{Ge},{ }^{82} \mathrm{Ge}\right), \mathrm{E}=220 \mathrm{MeV}$; measured $\mathrm{E} \gamma, \mathrm{I} \gamma$, (particle) $\gamma$-coin following projectile Coulomb excitation. ${ }^{78,80,82} \mathrm{Ge}$ deduced excitation $\mathrm{B}(\mathrm{E} 2)$. Systematic trends in $\mathrm{B}(\mathrm{E} 2)$ values discussed. JOUR PRLTA 94122501 RADIOACTIVITY ${ }^{48} \mathrm{~V}(\mathrm{EC}),\left(\beta^{+}\right)$[from Ti(p, X)]; measured $\mathrm{E} \gamma, \mathrm{I} \gamma$; deduced log ft. ${ }^{48} \mathrm{Ti}$ deduced transition intensities. CONF Santa Fe (Nucl Data for Sci and Technol) Proc,Vol1,P261 NUCLEAR REACTIONS ${ }^{48} \mathrm{Ti}\left({ }^{132} \mathrm{Sn},{ }^{132} \mathrm{Sn}\right.$ '), E=470-495 MeV; ${ }^{90} \mathrm{Zr}\left({ }^{134} \mathrm{Sn},{ }^{134} \mathrm{Sn}\right.$ '), $\mathrm{E}=400 \mathrm{MeV}$; measured $\mathrm{E} \gamma, \mathrm{I} \gamma$, (particle) $\gamma$-coin following projectile Coulomb excitation. ${ }^{132,134} \mathrm{Sn}$ deduced transitions B(E2). JOUR ZAANE 25 s01 391

RADIOACTIVITY ${ }^{48} \mathrm{Ca}\left(2 \beta^{-}\right)$; measured $0 \nu \beta \beta$-decay $\mathrm{T}_{1 / 2}$ lower limit. $\mathrm{CaWO}_{4}$ crystal scintillators. JOUR APHYE 23249

NUCLEAR REACTIONS Zn(p, X) ${ }^{64} \mathrm{Cu} /{ }^{57} \mathrm{Ni} /{ }^{56} \mathrm{Ni} /{ }^{52} \mathrm{Mn} /{ }^{54} \mathrm{Mn}$ $/{ }^{62} \mathrm{Zn} /{ }^{65} \mathrm{Zn} /{ }^{51} \mathrm{Cr} /{ }^{48} \mathrm{~V} /{ }^{55} \mathrm{Co} /{ }^{56} \mathrm{Co} /{ }^{57} \mathrm{Co} /{ }^{58} \mathrm{Co} /{ }^{60} \mathrm{Co} /{ }^{66} \mathrm{Ga}$ $/{ }^{67} \mathrm{Ga} /{ }^{52} \mathrm{Fe} /{ }^{59} \mathrm{Fe}, \mathrm{E} \approx 31-141 \mathrm{MeV}$; measured production $\sigma$. Stacked-foil activation. JOUR JRNCD 264101 NUCLEAR REACTIONS ${ }^{10} \mathrm{~B}\left({ }^{40} \mathrm{Ca}, \mathrm{X}\right){ }^{48} \mathrm{Mn} /{ }^{48} \mathrm{~V}, \mathrm{E}=110 \mathrm{MeV}$; measured $\mathrm{E} \gamma, \mathrm{I} \gamma, \gamma \gamma$-, (recoil) $\gamma$-coin. ${ }^{48} \mathrm{Mn},{ }^{48} \mathrm{~V}$ deduced levels, J, $\pi$, Coulomb energy differences. Gammasphere array, mass separator. CONF Argonne(Nuclei at the Limits),P199, Chandler NUCLEAR REACTIONS Fe(p, X) ${ }^{57} \mathrm{Co} /{ }^{56} \mathrm{Co} /{ }^{55} \mathrm{Co} /{ }^{54} \mathrm{Mn} /{ }^{52} \mathrm{Mn}$ $/{ }^{48} \mathrm{~V} /{ }^{51} \mathrm{Cr} /{ }^{48} \mathrm{Cr} /{ }^{47} \mathrm{Sc}, \mathrm{E} \approx 20-70 \mathrm{MeV}$; measured activation $\sigma$. Comparison with previous results. CONF Santa Fe (Nucl Data for Sci and Technol) Proc,Vol1,P1011 


\section{$\mathrm{A}=48$ (continued)}

2005MIZZ NUCLEAR REACTIONS Cu(n, X) ${ }^{56} \mathrm{Co}, \mathrm{E}=40-180 \mathrm{MeV} ; \mathrm{Fe}(\mathrm{n}$, $\mathrm{X})^{54} \mathrm{Mn} /{ }^{52} \mathrm{Mn} /{ }^{51} \mathrm{Cr} /{ }^{48} \mathrm{~V}, \mathrm{E} \approx 0-180 \mathrm{MeV} ; \mathrm{Pb}(\mathrm{n}, \mathrm{X}){ }^{196} \mathrm{Au} /{ }^{200} \mathrm{~Pb}$ $/{ }^{103} \mathrm{Ru}, \mathrm{E} \approx 40-180 \mathrm{MeV} ; \mathrm{U}(\mathrm{n}, \mathrm{X}){ }^{99} \mathrm{Mo}, \mathrm{E} \approx 0-180 \mathrm{MeV}$; measured excitation functions. Comparison with proton-induced reactions. CONF Santa Fe (Nucl Data for Sci and Technol) Proc,Vol1,P861 2005SI14 NUCLEAR REACTIONS C, O, Si, Mg, $\mathrm{Al}(\mathrm{n}, \mathrm{X})^{7} \mathrm{Be}, \mathrm{E} \approx 0.1-750$ $\mathrm{MeV} ; \mathrm{O}, \mathrm{Si}, \mathrm{Mg}, \mathrm{Al}(\mathrm{n}, \mathrm{X}){ }^{22} \mathrm{Na} /{ }^{23} \mathrm{Na}, \mathrm{E} \approx 0.1-750 \mathrm{MeV} ;{ }^{197} \mathrm{Au}(\mathrm{n}$, $\mathrm{X}){ }^{194} \mathrm{Au} /{ }^{196} \mathrm{Au} /{ }^{198} \mathrm{Au}, \mathrm{E} \approx 0.1-750 \mathrm{MeV} ; \mathrm{Ti}, \mathrm{Fe}, \mathrm{Ni}, \mathrm{Cu}(\mathrm{n}, \mathrm{X}){ }^{46} \mathrm{Sc}$ $/{ }^{48} \mathrm{Sc}, \mathrm{E} \approx 0.1-750 \mathrm{MeV} ; \mathrm{Fe}, \mathrm{Ni}, \mathrm{Cu}(\mathrm{n}, \mathrm{X}){ }^{48} \mathrm{~V} /{ }^{51} \mathrm{Cr} /{ }^{52} \mathrm{Mn} /{ }^{54} \mathrm{Mn}$, $\mathrm{E} \approx 0.1-750 \mathrm{MeV} ; \mathrm{Ni}, \mathrm{Cu}(\mathrm{n}, \mathrm{X}){ }^{56} \mathrm{Ni} /{ }^{57} \mathrm{Ni} /{ }^{56} \mathrm{Co} /{ }^{57} \mathrm{Co} /{ }^{58} \mathrm{Co} /$ ${ }^{60} \mathrm{Co} /{ }^{59} \mathrm{Fe}, \mathrm{E} \approx 0.1-750 \mathrm{MeV}$; measured energy-integrated production $\sigma$. JOUR NIMBE 234419

2005TRZZ RADIOACTIVITY ${ }^{48} \mathrm{~V}(\mathrm{EC}),\left(\beta^{+}\right)$[from Ti(p, X)]; measured $\mathrm{E} \gamma$, I $\gamma$; deduced log ft. ${ }^{48} \mathrm{Ti}$ deduced transition intensities. CONF Santa Fe (Nucl Data for Sci and Technol) Proc,Vol1,P261

${ }^{48} \mathrm{Cr} \quad 2005$ DIZY NUCLEAR REACTIONS Fe(p, X) ${ }^{57} \mathrm{Co} /{ }^{56} \mathrm{Co} /{ }^{55} \mathrm{Co} /{ }^{54} \mathrm{Mn} /{ }^{52} \mathrm{Mn}$ $/{ }^{48} \mathrm{~V} /{ }^{51} \mathrm{Cr} /{ }^{48} \mathrm{Cr} /{ }^{47} \mathrm{Sc}, \mathrm{E} \approx 20-70 \mathrm{MeV}$; measured activation $\sigma$. Comparison with previous results. CONF Santa Fe (Nucl Data for Sci and Technol) Proc,Vol1,P1011

${ }^{48} \mathrm{Mn} \quad 2005 \mathrm{CHZY}$ NUCLEAR REACTIONS ${ }^{10} \mathrm{~B}\left({ }^{40} \mathrm{Ca}, \mathrm{X}\right){ }^{48} \mathrm{Mn} /{ }^{48} \mathrm{~V}, \mathrm{E}=110 \mathrm{MeV}$; measured $\mathrm{E} \gamma$, I $\gamma, \gamma \gamma$-, (recoil) $\gamma$-coin. ${ }^{48} \mathrm{Mn},{ }^{48} \mathrm{~V}$ deduced levels, J, $\pi$, Coulomb energy differences. Gammasphere array, mass separator. CONF Argonne(Nuclei at the Limits),P199, Chandler RADIOACTIVITY ${ }^{45} \mathrm{Fe},{ }^{48} \mathrm{Ni},{ }^{54} \mathrm{Zn}(2 \mathrm{p})$ [from $\mathrm{Ni}\left({ }^{58} \mathrm{Ni}, \mathrm{X}\right)$ ]; measured proton spectra, $\mathrm{T}_{1 / 2}$. Comparison with theory. JOUR ZAANE $25 \mathrm{~s} 01$ 169

2005D020 NUCLEAR REACTIONS Ni( $\left.{ }^{58} \mathrm{Ni}, \mathrm{X}\right), \mathrm{E}=74.5 \mathrm{MeV} /$ nucleon; measured fragments isotopic yields; deduced evidence for ${ }^{48} \mathrm{Ni},{ }^{45} \mathrm{Fe}$. JOUR PRVCA 72054315

2005D020 RADIOACTIVITY ${ }^{45} \mathrm{Fe}(2 \mathrm{p})$ [from $\left.\mathrm{Ni}\left({ }^{58} \mathrm{Ni}, \mathrm{X}\right)\right]$; measured $\mathrm{Ep}, \mathrm{T}_{1 / 2}$, branching ratio. ${ }^{48} \mathrm{Ni}$; measured decay energy, $\mathrm{T}_{1 / 2}$; deduced probable two-proton decay. Comparisons with model predictions. JOUR PRVCA 72054315

2005GI15 NUCLEAR REACTIONS Ni $\left({ }^{58} \mathrm{Ni}, \mathrm{X}\right), \mathrm{E}=75 \mathrm{MeV} /$ nucleon; measured fragments isotopic yields; deduced evidence for ${ }^{48} \mathrm{Ni}$. JOUR JPGPE 31 S1509

\section{$\mathrm{A}=49$}

${ }^{49} \mathrm{Ca} \quad$ 2005MAZM

NUCLEAR REACTIONS ${ }^{2} \mathrm{H}\left({ }^{48} \mathrm{Ca},{ }^{49} \mathrm{Ca}\right), \mathrm{E}=105 \mathrm{MeV}$; measured $\mathrm{E} \gamma$, $\mathrm{I} \gamma$, (particle) $\gamma$-coin. ${ }^{48} \mathrm{Ca}$ (polarized d, p), $\mathrm{E}=14 \mathrm{MeV}$; measured proton spectra, $\sigma(\theta) .{ }^{49} \mathrm{Ca}$ deduced levels, J, $\pi$. REPT MLL 2004

Annual,P8,Maierbeck

${ }^{49} \mathrm{Ti}$ 2005ID03 NUCLEAR REACTIONS ${ }^{9} \mathrm{Be}\left({ }^{46} \mathrm{Ar}, 3 \mathrm{n}\right),\left({ }^{46} \mathrm{Ar}, 4 \mathrm{n}\right),\left({ }^{46} \mathrm{Ar}, 5 \mathrm{n}\right),\left({ }^{46} \mathrm{Ar}\right.$, $6 \mathrm{n}$ ), $\mathrm{E} \approx 2-6 \mathrm{MeV} /$ nucleon; measured $\mathrm{E} \gamma, \mathrm{I} \gamma$, (particle) $\gamma$-coin; deduced excitation functions. ${ }^{49,50,51} \mathrm{Ti}$ deduced high-spin levels, $\mathrm{J}, \pi$. JOUR ZAANE 25 s01 429 


\section{$\mathrm{A}=49$ (continued)}

2005IDZZ NUCLEAR REACTIONS ${ }^{9} \mathrm{Be}\left({ }^{37} \mathrm{P}, \mathrm{X}\right){ }^{42} \mathrm{~K}, \mathrm{E} \approx 5 \mathrm{MeV} /$ nucleon; ${ }^{9} \mathrm{Be}\left({ }^{46} \mathrm{Ar}, \mathrm{X}\right){ }^{49} \mathrm{Ti} /{ }^{50} \mathrm{Ti} /{ }^{51} \mathrm{Ti} /{ }^{46} \mathrm{Ca}, \mathrm{E} \approx 5 \mathrm{MeV} /$ nucleon; measured $\mathrm{E} \gamma, \mathrm{I} \gamma .{ }^{42} \mathrm{~K},{ }^{49,50,51} \mathrm{Ti},{ }^{46} \mathrm{Ca}$ deduced levels, $\mathrm{J}, \pi .{ }^{9} \mathrm{Be}\left({ }^{46} \mathrm{Ar}\right.$, $\mathrm{xn}), \mathrm{E}=2-7 \mathrm{MeV} /$ nucleon; measured excitation functions. CONF Argonne(Nuclei at the Limits),P136,Ideguchi

2005NIZT NUCLEAR REACTIONS ${ }^{9} \mathrm{Be}\left({ }^{46} \mathrm{Ar}, \mathrm{xn}\right), \mathrm{E} \approx 2.5-4.5 \mathrm{MeV} /$ nucleon; measured $\mathrm{E} \gamma, \mathrm{I} \gamma, \gamma \gamma$-coin; deduced excitation functions. ${ }^{49,50} \mathrm{Ti}$ deduced transitions. ${ }^{51} \mathrm{Ti}$ deduced levels, J, $\pi$. REPT CNS-REP-66,P22,Niikura

2005NIZV NUCLEAR REACTIONS ${ }^{9} \mathrm{Be}\left({ }^{46} \mathrm{Ar}, 3 \mathrm{n}\right),\left({ }^{46} \mathrm{Ar}, 4 \mathrm{n}\right),\left({ }^{46} \mathrm{Ar}, 5 \mathrm{n}\right),\left({ }^{46} \mathrm{Ar}\right.$, $6 \mathrm{n}), \mathrm{E}=2-7 \mathrm{MeV} /$ nucleon; measured excitation functions. Comparison with statistical model predictions. REPT RIKEN 2004 Annual,P67,Niikura

$$
\mathrm{A}=50
$$

${ }^{50} \mathrm{Ca} \quad$ 2005BR18

${ }^{50} \mathrm{Ti}$

2005BA14

2005ID03

2005IDZZ

2005NIZT

2005NIZV

2005YU07

${ }^{50} \mathrm{~V}$

2005BA14
NUCLEAR REACTIONS ${ }^{48} \mathrm{Ca}\left({ }^{48} \mathrm{Ca}, \mathrm{X}\right){ }^{50} \mathrm{Ca} /{ }^{51} \mathrm{Sc}, \mathrm{E}=210 \mathrm{MeV}$; ${ }^{208} \mathrm{~Pb}\left({ }^{48} \mathrm{Ca}, \mathrm{X}\right){ }^{50} \mathrm{Ca} /{ }^{51} \mathrm{Sc}, \mathrm{E}=280 \mathrm{MeV} ;{ }^{238} \mathrm{U}\left({ }^{48} \mathrm{Ca}, \mathrm{X}\right){ }^{50} \mathrm{Ca} /{ }^{51} \mathrm{Sc}$, $\mathrm{E}=330 \mathrm{MeV}$; measured $\mathrm{E} \gamma, \mathrm{I} \gamma, \gamma \gamma$-coin. ${ }^{50} \mathrm{Ca},{ }^{51} \mathrm{Sc}$ deduced levels, $\mathrm{J}, \pi$, configurations. GASP, Gammasphere arrays. JOUR APOBB 361343 NUCLEAR REACTIONS ${ }^{50} \mathrm{~V}(\mathrm{~d}, 2 \mathrm{p}), \mathrm{E}=171 \mathrm{MeV}$; measured Ep, pp-coin, $\sigma(\mathrm{E}, \theta) .{ }^{50} \mathrm{~V}$ deduced Gamow-Teller strength distribution. Comparison with model predictions. JOUR PRVCA 71024603 NUCLEAR REACTIONS ${ }^{9} \mathrm{Be}\left({ }^{46} \mathrm{Ar}, 3 \mathrm{n}\right),\left({ }^{46} \mathrm{Ar}, 4 \mathrm{n}\right),\left({ }^{46} \mathrm{Ar}, 5 \mathrm{n}\right),\left({ }^{46} \mathrm{Ar}\right.$, $6 \mathrm{n}), \mathrm{E} \approx 2-6 \mathrm{MeV} /$ nucleon; measured $\mathrm{E} \gamma, \mathrm{I} \gamma$, (particle) $\gamma$-coin; deduced excitation functions. ${ }^{49,50,51} \mathrm{Ti}$ deduced high-spin levels, $\mathrm{J}, \pi$. JOUR ZAANE 25 s01 429

NUCLEAR REACTIONS ${ }^{9} \mathrm{Be}\left({ }^{37} \mathrm{P}, \mathrm{X}\right){ }^{42} \mathrm{~K}, \mathrm{E} \approx 5 \mathrm{MeV} /$ nucleon; ${ }^{9} \mathrm{Be}\left({ }^{46} \mathrm{Ar}, \mathrm{X}\right){ }^{49} \mathrm{Ti} /{ }^{50} \mathrm{Ti} /{ }^{51} \mathrm{Ti} /{ }^{46} \mathrm{Ca}, \mathrm{E} \approx 5 \mathrm{MeV} /$ nucleon; measured $\mathrm{E} \gamma, \mathrm{I} \gamma .{ }^{42} \mathrm{~K},{ }^{49,50,51} \mathrm{Ti},{ }^{46} \mathrm{Ca}$ deduced levels, J, $\pi .{ }^{9} \mathrm{Be}\left({ }^{46} \mathrm{Ar}\right.$, $\mathrm{xn}), \mathrm{E}=2-7 \mathrm{MeV} /$ nucleon; measured excitation functions. CONF Argonne(Nuclei at the Limits),P136,Ideguchi

NUCLEAR REACTIONS ${ }^{9} \mathrm{Be}\left({ }^{46} \mathrm{Ar}, \mathrm{xn}\right), \mathrm{E} \approx 2.5-4.5 \mathrm{MeV} /$ nucleon; measured $\mathrm{E} \gamma, \mathrm{I} \gamma, \gamma \gamma$-coin; deduced excitation functions. ${ }^{49,50} \mathrm{Ti}$

deduced transitions. ${ }^{51} \mathrm{Ti}$ deduced levels, J, $\pi$. REPT

CNS-REP-66,P22,Niikura

NUCLEAR REACTIONS ${ }^{9} \mathrm{Be}\left({ }^{46} \mathrm{Ar}, 3 \mathrm{n}\right),\left({ }^{46} \mathrm{Ar}, 4 \mathrm{n}\right),\left({ }^{46} \mathrm{Ar}, 5 \mathrm{n}\right),\left({ }^{46} \mathrm{Ar}\right.$, $6 \mathrm{n}), \mathrm{E}=2-7 \mathrm{MeV} /$ nucleon; measured excitation functions. Comparison with statistical model predictions. REPT RIKEN 2004

Annual,P67,Niikura NUCLEAR REACTIONS ${ }^{50} \mathrm{Ti}\left({ }^{129} \mathrm{Sb},{ }^{129} \mathrm{Sb}\right),\left({ }^{129} \mathrm{Te},{ }^{129} \mathrm{Te}\right), \mathrm{E}=400$ $\mathrm{MeV}$; measured $\mathrm{E} \gamma, \mathrm{I} \gamma$, (particle) $\gamma$-coin following projectile Coulomb excitation. ${ }^{129} \mathrm{Te},{ }^{129} \mathrm{Sb}$ deduced transitions B(E2). Clarion, Hyball arrays. JOUR ZAANE 25 s01 395

NUCLEAR REACTIONS ${ }^{50} \mathrm{~V}(\mathrm{~d}, 2 \mathrm{p}), \mathrm{E}=171 \mathrm{MeV}$; measured Ep, pp-coin, $\sigma(\mathrm{E}, \theta) .{ }^{50} \mathrm{~V}$ deduced Gamow-Teller strength distribution. Comparison with model predictions. JOUR PRVCA 71024603 


\section{$\mathrm{A}=50$ (continued)}

2005SU07 NUCLEAR REACTIONS ${ }^{51} \mathrm{~V}\left({ }^{3} \mathrm{He},{ }^{3} \mathrm{He}\right),\left({ }^{3} \mathrm{He}, \alpha\right)$, E not given; measured $\mathrm{E} \gamma, \mathrm{I} \gamma \cdot{ }^{50,51} \mathrm{~V}$ deduced radiative strength functions, thermodynamic properties. JOUR APOBB 361197

2005SUZU NUCLEAR REACTIONS ${ }^{51} \mathrm{~V}\left({ }^{3} \mathrm{He},{ }^{3} \mathrm{He}\right),\left({ }^{3} \mathrm{He}, \alpha\right), \mathrm{E}=30 \mathrm{MeV}$; measured $\mathrm{E} \gamma, \mathrm{I} \gamma$, (particle) $\gamma$-coin. ${ }^{50,51} \mathrm{~V}$ deduced level densities, radiative strength functions, microcanonical entropies. PREPRINT nucl-ex/0511054,11/30/2005

${ }^{50} \mathrm{Cr} \quad$ 2005SAZY NUCLEAR REACTIONS ${ }^{197} \mathrm{Au}\left({ }^{54} \mathrm{Cr},{ }^{54} \mathrm{Cr}\right),\left({ }^{56} \mathrm{Cr},{ }^{56} \mathrm{Cr}\right),\left({ }^{58} \mathrm{Cr}\right.$, ${ }^{58} \mathrm{Cr}$ '), $\mathrm{E}=100 \mathrm{MeV} /$ nucleon; measured $\mathrm{E} \gamma, \mathrm{I} \gamma$, (particle) $\gamma$-coin following projectile Coulomb excitation. ${ }^{54,56,58} \mathrm{Cr}$ deduced transitions. $\mathrm{Be}\left({ }^{55} \mathrm{Ni}, \mathrm{X}\right){ }^{50} \mathrm{Cr}, \mathrm{E}=171 \mathrm{MeV} /$ nucleon; measured $\mathrm{E} \gamma, \mathrm{I} \gamma$, (particle) $\gamma$-coin. ${ }^{50} \mathrm{Cr}$ deduced transitions. $\mathrm{Be}\left({ }^{55} \mathrm{Ni}, \mathrm{X}\right),{ }^{197} \mathrm{Au}\left({ }^{108} \mathrm{Sn}\right.$, $\mathrm{X})$, E not given; measured fragment yields. CONF Argonne(Nuclei at the Limits),P151,Saito

2005W001 NUCLEAR REACTIONS ${ }^{197} \mathrm{Au}\left({ }^{84} \mathrm{Kr},{ }^{84} \mathrm{Kr}{ }^{\prime}\right),\left({ }^{56} \mathrm{Cr},{ }^{56} \mathrm{Cr}^{\prime}\right),\left({ }^{108} \mathrm{Sn}\right.$, ${ }^{108} \mathrm{Sn}$ '), $\mathrm{E}=113-142 \mathrm{MeV} /$ nucleon; measured $\mathrm{E} \gamma, \mathrm{I} \gamma$ following projectile Coulomb excitation. ${ }^{84} \mathrm{Kr},{ }^{56} \mathrm{Cr},{ }^{108} \mathrm{Sn}$ deduced transitions. ${ }^{9} \mathrm{Be}\left({ }^{55} \mathrm{Ni}, \mathrm{X}\right){ }^{54} \mathrm{Co} /{ }^{52} \mathrm{Fe} /{ }^{50} \mathrm{Cr}, \mathrm{E}=171 \mathrm{MeV} /$ nucleon; measured $\mathrm{E} \gamma$, I $\gamma$, (particle) $\gamma$-coin. JOUR NIMAE 537637

${ }^{50} \mathrm{Mn} \quad 2005 \mathrm{FU} 16$ NUCLEAR REACTIONS ${ }^{50} \mathrm{Cr}\left({ }^{3} \mathrm{He}, \mathrm{t}\right), \mathrm{E}=140 \mathrm{MeV} /$ nucleon; measured triton spectra; deduced Gamow-Teller transition strengths. ${ }^{50} \mathrm{Mn}$ deduced level energies. ${ }^{50} \mathrm{Fe}$ deduced $\beta$-decay intensities. Astrophysical implications discussed. JOUR PRLTA 95212501

2005SA44 RADIOACTIVITY ${ }^{46} \mathrm{~V}(\mathrm{EC})$; analyzed masses; deduced Q(EC), log ft. ${ }^{10} \mathrm{C},{ }^{14} \mathrm{O},{ }^{22} \mathrm{Mg},{ }^{26 m} \mathrm{Al},{ }^{34} \mathrm{Cl},{ }^{34} \mathrm{Ar},{ }^{38 m} \mathrm{~K},{ }^{42} \mathrm{Sc},{ }^{46} \mathrm{~V},{ }^{50} \mathrm{Mn},{ }^{54} \mathrm{Co},{ }^{74} \mathrm{Rb}$; compiled, analyzed log ft; deduced quark-mixing matrix element. JOUR PRLTA 95102501

${ }^{50} \mathrm{Fe} \quad 2005 \mathrm{FU} 16$ NUCLEAR REACTIONS ${ }^{50} \mathrm{Cr}\left({ }^{3} \mathrm{He}, \mathrm{t}\right), \mathrm{E}=140 \mathrm{MeV} /$ nucleon; measured triton spectra; deduced Gamow-Teller transition strengths. ${ }^{50} \mathrm{Mn}$ deduced level energies. ${ }^{50} \mathrm{Fe}$ deduced $\beta$-decay intensities. Astrophysical implications discussed. JOUR PRLTA 95212501 2005 YA26 NUCLEAR REACTIONS Pb $\left({ }^{46} \mathrm{Cr},{ }^{46} \mathrm{Cr}\right.$ '), $\left({ }^{50} \mathrm{Fe},{ }^{50} \mathrm{Fe}\right.$ '), $\left({ }^{54} \mathrm{Ni},{ }^{54} \mathrm{Ni}^{\prime}\right)$, $\mathrm{E}=41-44 \mathrm{MeV} /$ nucleon; measured $\sigma(\theta), \mathrm{E} \gamma, \mathrm{I} \gamma$, (particle) $\gamma$-coin following projectile Coulomb excitation. ${ }^{46} \mathrm{Cr},{ }^{50} \mathrm{Fe},{ }^{54} \mathrm{Ni}$ deduced excitation B(E2). DWBA analysis. JOUR ZAANE 25 s01 409

\section{$\mathrm{A}=51$}

${ }^{51} \mathrm{Sc} \quad 2005 \mathrm{BR} 18$

NUCLEAR REACTIONS ${ }^{48} \mathrm{Ca}\left({ }^{48} \mathrm{Ca}, \mathrm{X}\right){ }^{50} \mathrm{Ca} /{ }^{51} \mathrm{Sc}, \mathrm{E}=210 \mathrm{MeV}$; ${ }^{208} \mathrm{~Pb}\left({ }^{48} \mathrm{Ca}, \mathrm{X}\right){ }^{50} \mathrm{Ca} /{ }^{51} \mathrm{Sc}, \mathrm{E}=280 \mathrm{MeV} ;{ }^{238} \mathrm{U}\left({ }^{48} \mathrm{Ca}, \mathrm{X}\right){ }^{50} \mathrm{Ca} /{ }^{51} \mathrm{Sc}$, $\mathrm{E}=330 \mathrm{MeV}$; measured $\mathrm{E} \gamma, \mathrm{I} \gamma, \gamma \gamma$-coin. ${ }^{50} \mathrm{Ca},{ }^{51} \mathrm{Sc}$ deduced levels, $\mathrm{J}, \pi$, configurations. GASP, Gammasphere arrays. JOUR APOBB 361343

${ }^{51} \mathrm{Ti} 2005 \mathrm{ID} 03 \quad$ NUCLEAR REACTIONS ${ }^{9} \mathrm{Be}\left({ }^{46} \mathrm{Ar}, 3 \mathrm{n}\right),\left({ }^{46} \mathrm{Ar}, 4 \mathrm{n}\right),\left({ }^{46} \mathrm{Ar}, 5 \mathrm{n}\right),\left({ }^{46} \mathrm{Ar}\right.$, $6 \mathrm{n}$ ), $\mathrm{E} \approx 2-6 \mathrm{MeV} /$ nucleon; measured $\mathrm{E} \gamma, \mathrm{I} \gamma$, (particle) $\gamma$-coin; deduced excitation functions. ${ }^{49,50,51} \mathrm{Ti}$ deduced high-spin levels, $\mathrm{J}, \pi$. JOUR ZAANE 25 s01 429 


\section{$\mathrm{A}=51$ (continued)}

2005IDZZ NUCLEAR REACTIONS ${ }^{9} \mathrm{Be}\left({ }^{37} \mathrm{P}, \mathrm{X}\right){ }^{42} \mathrm{~K}, \mathrm{E} \approx 5 \mathrm{MeV} /$ nucleon; ${ }^{9} \mathrm{Be}\left({ }^{46} \mathrm{Ar}, \mathrm{X}\right){ }^{49} \mathrm{Ti} /{ }^{50} \mathrm{Ti} /{ }^{51} \mathrm{Ti} /{ }^{46} \mathrm{Ca}, \mathrm{E} \approx 5 \mathrm{MeV} /$ nucleon; measured $\mathrm{E} \gamma, \mathrm{I} \gamma .{ }^{42} \mathrm{~K},{ }^{49,50,51} \mathrm{Ti},{ }^{46} \mathrm{Ca}$ deduced levels, $\mathrm{J}, \pi .{ }^{9} \mathrm{Be}\left({ }^{46} \mathrm{Ar}\right.$, $\mathrm{xn}), \mathrm{E}=2-7 \mathrm{MeV} /$ nucleon; measured excitation functions. CONF Argonne(Nuclei at the Limits),P136,Ideguchi

2005NIZT NUCLEAR REACTIONS ${ }^{9} \mathrm{Be}\left({ }^{46} \mathrm{Ar}, \mathrm{xn}\right), \mathrm{E} \approx 2.5-4.5 \mathrm{MeV} /$ nucleon; measured $\mathrm{E} \gamma, \mathrm{I} \gamma, \gamma \gamma$-coin; deduced excitation functions. ${ }^{49,50} \mathrm{Ti}$ deduced transitions. ${ }^{51} \mathrm{Ti}$ deduced levels, $\mathrm{J}, \pi$. REPT CNS-REP-66,P22,Niikura

2005NIZV NUCLEAR REACTIONS ${ }^{9} \mathrm{Be}\left({ }^{46} \mathrm{Ar}, 3 \mathrm{n}\right),\left({ }^{46} \mathrm{Ar}, 4 \mathrm{n}\right),\left({ }^{46} \mathrm{Ar}, 5 \mathrm{n}\right),\left({ }^{46} \mathrm{Ar}\right.$, $6 \mathrm{n}), \mathrm{E}=2-7 \mathrm{MeV} /$ nucleon; measured excitation functions. Comparison with statistical model predictions. REPT RIKEN 2004 Annual,P67,Niikura

${ }^{51} \mathrm{~V} \quad 2005 \mathrm{Su} 07 \quad$ NUCLEAR REACTIONS ${ }^{51} \mathrm{~V}\left({ }^{3} \mathrm{He},{ }^{3} \mathrm{He}\right),\left({ }^{3} \mathrm{He}, \alpha\right)$, E not given; measured $\mathrm{E} \gamma, \mathrm{I} \gamma .{ }^{50,51} \mathrm{~V}$ deduced radiative strength functions, thermodynamic properties. JOUR APOBB 361197

2005SUZU NUCLEAR REACTIONS ${ }^{51} \mathrm{~V}\left({ }^{3} \mathrm{He},{ }^{3} \mathrm{He}\right),\left({ }^{3} \mathrm{He}, \alpha\right), \mathrm{E}=30 \mathrm{MeV}$; measured $\mathrm{E} \gamma, \mathrm{I} \gamma$, (particle) $\gamma$-coin. ${ }^{50,51} \mathrm{~V}$ deduced level densities, radiative strength functions, microcanonical entropies. PREPRINT nucl-ex/0511054,11/30/2005

${ }^{51} \mathrm{Cr} \quad$ 2005BAZR NUCLEAR REACTIONS ${ }^{107} \mathrm{Ag}(\alpha, \gamma), \mathrm{E}=7.8-11.9 \mathrm{MeV} ;{ }^{48} \mathrm{Ti}(\alpha, \mathrm{n}), \mathrm{E}$ $\approx 6.5-11.5 \mathrm{MeV}$; measured $\sigma$. Stacked-foil activation technique, comparison with model predictions. CONF Santa Fe (Nucl Data for Sci and Technol) Proc,Vol2,P1370

2005B010 NUCLEAR REACTIONS Zn(p, X) ${ }^{64} \mathrm{Cu} /{ }^{57} \mathrm{Ni} /{ }^{56} \mathrm{Ni} /{ }^{52} \mathrm{Mn} /{ }^{54} \mathrm{Mn}$ $/{ }^{62} \mathrm{Zn} /{ }^{65} \mathrm{Zn} /{ }^{51} \mathrm{Cr} /{ }^{48} \mathrm{~V} /{ }^{55} \mathrm{Co} /{ }^{56} \mathrm{Co} /{ }^{57} \mathrm{Co} /{ }^{58} \mathrm{Co} /{ }^{60} \mathrm{Co} /{ }^{66} \mathrm{Ga}$ $/{ }^{67} \mathrm{Ga} /{ }^{52} \mathrm{Fe} /{ }^{59} \mathrm{Fe}, \mathrm{E} \approx 31-141 \mathrm{MeV}$; measured production $\sigma$.

Stacked-foil activation. JOUR JRNCD 264101

2005DIZY NUCLEAR REACTIONS Fe(p, X) ${ }^{57} \mathrm{Co} /{ }^{56} \mathrm{Co} /{ }^{55} \mathrm{Co} /{ }^{54} \mathrm{Mn} /{ }^{52} \mathrm{Mn}$ $/{ }^{48} \mathrm{~V} /{ }^{51} \mathrm{Cr} /{ }^{48} \mathrm{Cr} /{ }^{47} \mathrm{Sc}, \mathrm{E} \approx 20-70 \mathrm{MeV}$; measured activation $\sigma$. Comparison with previous results. CONF Santa Fe (Nucl Data for Sci and Technol) Proc,Vol1,P1011

2005MIZZ NUCLEAR REACTIONS Cu(n, X) ${ }^{56} \mathrm{Co}, \mathrm{E}=40-180 \mathrm{MeV} ; \mathrm{Fe}(\mathrm{n}$, $\mathrm{X}){ }^{54} \mathrm{Mn} /{ }^{52} \mathrm{Mn} /{ }^{51} \mathrm{Cr} /{ }^{48} \mathrm{~V}, \mathrm{E} \approx 0-180 \mathrm{MeV} ; \mathrm{Pb}(\mathrm{n}, \mathrm{X}){ }^{196} \mathrm{Au} /{ }^{200} \mathrm{~Pb}$ $/{ }^{103} \mathrm{Ru}, \mathrm{E} \approx 40-180 \mathrm{MeV}$; U(n, X) ${ }^{99} \mathrm{Mo}, \mathrm{E} \approx 0-180 \mathrm{MeV}$; measured excitation functions. Comparison with proton-induced reactions. CONF Santa Fe (Nucl Data for Sci and Technol) Proc,Vol1,P861

2005SI14 NUCLEAR REACTIONS C, O, Si, Mg, Al(n, X) ${ }^{7} \mathrm{Be}, \mathrm{E} \approx 0.1-750$ $\mathrm{MeV} ; \mathrm{O}, \mathrm{Si}, \mathrm{Mg}, \mathrm{Al}(\mathrm{n}, \mathrm{X}){ }^{22} \mathrm{Na} /{ }^{23} \mathrm{Na}, \mathrm{E} \approx 0.1-750 \mathrm{MeV} ;{ }^{197} \mathrm{Au}(\mathrm{n}$, $\mathrm{X}){ }^{194} \mathrm{Au} /{ }^{196} \mathrm{Au} /{ }^{198} \mathrm{Au}, \mathrm{E} \approx 0.1-750 \mathrm{MeV} ; \mathrm{Ti}, \mathrm{Fe}, \mathrm{Ni}, \mathrm{Cu}(\mathrm{n}, \mathrm{X})^{46} \mathrm{Sc}$ $/{ }^{48} \mathrm{Sc}, \mathrm{E} \approx 0.1-750 \mathrm{MeV} ; \mathrm{Fe}, \mathrm{Ni}, \mathrm{Cu}(\mathrm{n}, \mathrm{X}){ }^{48} \mathrm{~V} /{ }^{51} \mathrm{Cr} /{ }^{52} \mathrm{Mn} /{ }^{54} \mathrm{Mn}$, $\mathrm{E} \approx 0.1-750 \mathrm{MeV} ; \mathrm{Ni}, \mathrm{Cu}(\mathrm{n}, \mathrm{X}){ }^{56} \mathrm{Ni} /{ }^{57} \mathrm{Ni} /{ }^{56} \mathrm{Co} /{ }^{57} \mathrm{Co} /{ }^{58} \mathrm{Co} /$ ${ }^{60} \mathrm{Co} /{ }^{59} \mathrm{Fe}, \mathrm{E} \approx 0.1-750 \mathrm{MeV}$; measured energy-integrated production $\sigma$. JOUR NIMBE 234419 


\section{$\mathrm{A}=51$ (continued)}

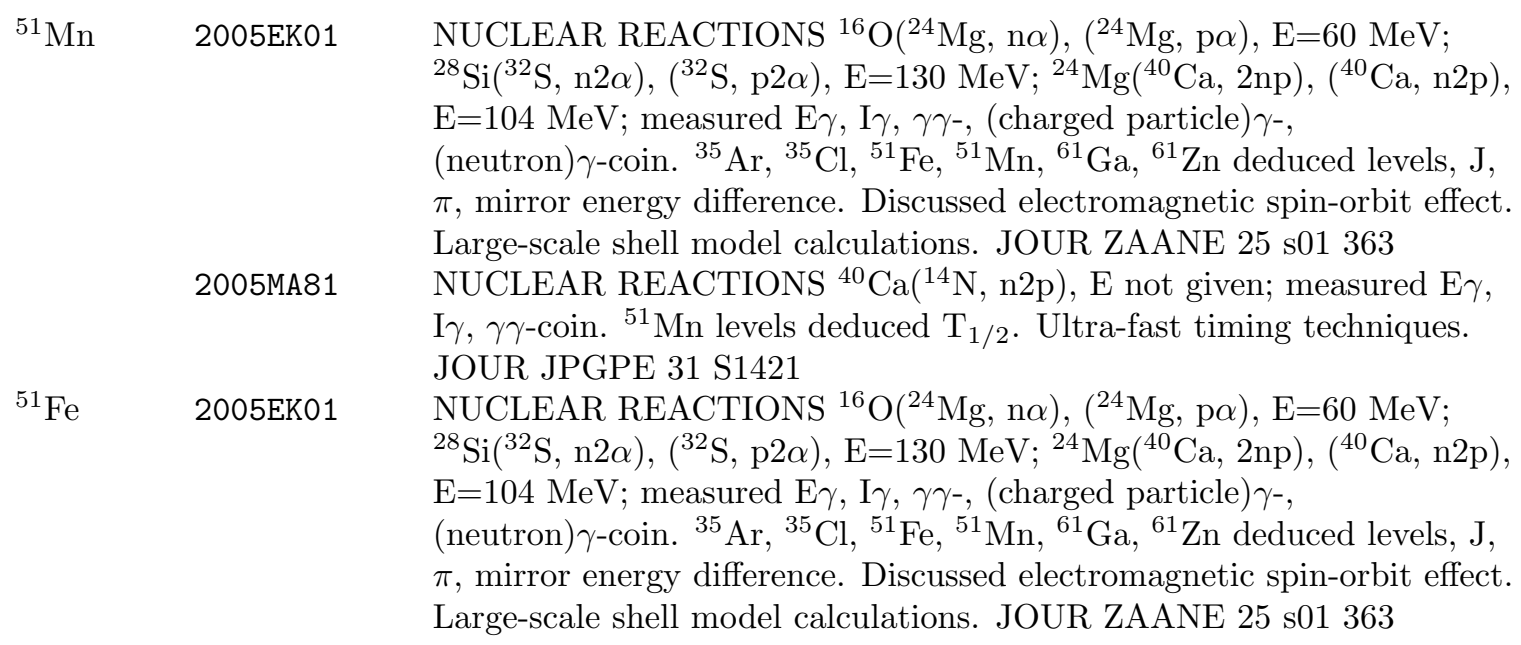

\section{$\mathrm{A}=52$}

${ }^{52} \mathrm{Ti}$ 2005DI05 NUCLEAR REACTIONS ${ }^{197} \mathrm{Au}\left({ }^{76} \mathrm{Ge},{ }^{76} \mathrm{Ge}\right),\left({ }^{52} \mathrm{Ti},{ }^{52} \mathrm{Ti}\right),\left({ }^{54} \mathrm{Ti}\right.$, $\left.{ }^{54} \mathrm{Ti}^{\prime}\right),\left({ }^{56} \mathrm{Ti},{ }^{56} \mathrm{Ti}^{\prime}\right), \mathrm{E} \approx 80-90 \mathrm{MeV}$; measured $\mathrm{E} \gamma, \mathrm{I} \gamma$, (particle) $\gamma$-coin following projectile Coulomb excitation. ${ }^{52,54,56} \mathrm{Ti}$ deduced transitions $\mathrm{B}(\mathrm{E} 2)$, subshell closures. Comparison with large-scale shell model calculations. JOUR PRVCA 71041302

2005DIZZ NUCLEAR REACTIONS ${ }^{238} \mathrm{U}\left({ }^{48} \mathrm{Ca}, \mathrm{X}\right){ }^{56} \mathrm{Ti}, \mathrm{E}=330 \mathrm{MeV}$; measured $\mathrm{E} \gamma, \mathrm{I} \gamma, \gamma \gamma$-coin. ${ }^{56} \mathrm{Ti}$ deduced levels, $\mathrm{J}, \pi .{ }^{197} \mathrm{Au}\left({ }^{76} \mathrm{Ge},{ }^{76} \mathrm{Ge}\right),\left({ }^{52} \mathrm{Ti}\right.$, $\left.{ }^{52} \mathrm{Ti}^{\prime}\right),\left({ }^{54} \mathrm{Ti},{ }^{54} \mathrm{Ti}^{\prime}\right),\left({ }^{56} \mathrm{Ti},{ }^{56} \mathrm{Ti}{ }^{\prime}\right), \mathrm{E} \approx 80-90 \mathrm{MeV}$; measured $\mathrm{E} \gamma, \mathrm{I} \gamma$, (particle) $\gamma$-coin following projectile Coulomb excitation. ${ }^{52,54,56} \mathrm{Ti}$, ${ }^{76} \mathrm{Ge},{ }^{197} \mathrm{Au}$ deduced transitions B(E2). CONF Argonne(Nuclei at the Limits),P131,Dinca

2005 ID03 NUCLEAR REACTIONS ${ }^{9} \mathrm{Be}\left({ }^{46} \mathrm{Ar}, 3 \mathrm{n}\right),\left({ }^{46} \mathrm{Ar}, 4 \mathrm{n}\right),\left({ }^{46} \mathrm{Ar}, 5 \mathrm{n}\right),\left({ }^{46} \mathrm{Ar}\right.$, $6 \mathrm{n}), \mathrm{E} \approx 2-6 \mathrm{MeV} /$ nucleon; measured $\mathrm{E} \gamma, \mathrm{I} \gamma$, (particle) $\gamma$-coin; deduced excitation functions. ${ }^{49,50,51} \mathrm{Ti}$ deduced high-spin levels, J, $\pi$. JOUR ZAANE 25 s01 429

2005NIZV NUCLEAR REACTIONS ${ }^{9} \mathrm{Be}\left({ }^{46} \mathrm{Ar}, 3 \mathrm{n}\right),\left({ }^{46} \mathrm{Ar}, 4 \mathrm{n}\right),\left({ }^{46} \mathrm{Ar}, 5 \mathrm{n}\right),\left({ }^{46} \mathrm{Ar}\right.$, $6 \mathrm{n}), \mathrm{E}=2-7 \mathrm{MeV} /$ nucleon; measured excitation functions. Comparison with statistical model predictions. REPT RIKEN 2004 Annual,P67,Niikura

${ }^{52} \mathrm{Mn} \quad$ 2004ADZW NUCLEAR REACTIONS ${ }^{209} \mathrm{Bi}(\mathrm{n}, 4 \mathrm{n}),(\mathrm{n}, 5 \mathrm{n}),(\mathrm{n}, 6 \mathrm{n}),(\mathrm{n}, 7 \mathrm{n}),(\mathrm{n}$, $9 \mathrm{n}),{ }^{232} \mathrm{Th}(\mathrm{n}, \gamma),{ }^{197} \mathrm{Au}(\mathrm{n}, 2 \mathrm{n}),(\mathrm{n}, 4 \mathrm{n}),(\mathrm{n}, 6 \mathrm{n}),(\mathrm{n}, 7 \mathrm{n}),(\mathrm{n}, \gamma),{ }^{115} \operatorname{In}(\mathrm{n}$, $5 \mathrm{n}),(\mathrm{n}, 6 \mathrm{n}),(\mathrm{n}, 7 \mathrm{n}),{ }^{59} \mathrm{Co}(\mathrm{n}, 2 \mathrm{n}),(\mathrm{n}, 3 \mathrm{n}),(\mathrm{n}, 4 \mathrm{n}),(\mathrm{n}, 5 \mathrm{n}),(\mathrm{n}, \gamma),(\mathrm{n}$, $\mathrm{p}),(\mathrm{n}, 6 \mathrm{n} 2 \mathrm{p}), \mathrm{E}=$ spectrum; measured $\mathrm{E} \gamma, \mathrm{I} \gamma$; deduced reaction rates. $\mathrm{Pb}(\mathrm{p}, \mathrm{nX}), \mathrm{E}=1 \mathrm{GeV}$; deduced spallation neutron spectrum. REPT JINR-E1-2004-16,Adam

2004MIZS NUCLEAR REACTIONS Fe(p, X) ${ }^{52} \mathrm{Mn}, \mathrm{E}<2.6 \mathrm{GeV} ; \mathrm{Pb}(\mathrm{p}, \mathrm{X})^{10} \mathrm{Be}$, $\mathrm{E}<2.6 \mathrm{GeV} ;{ }^{209} \mathrm{Bi}(\mathrm{p}, 4 \mathrm{np}), \mathrm{E}<2.6 \mathrm{GeV} ; \mathrm{Pb}(\mathrm{n}, \mathrm{X}){ }^{196} \mathrm{Au} /{ }^{95} \mathrm{Zr}, \mathrm{E} \approx$ 70-180 MeV; measured excitation functions. Comparison with model predictions. REPT NEA/NSC/DOC(2004)14,P28,Michel 


\section{$\mathrm{A}=52$ (continued)}

2004QAZZ NUCLEAR REACTIONS ${ }^{52} \mathrm{Cr}(\mathrm{p}, \mathrm{n}),\left({ }^{3} \mathrm{He}, \mathrm{t}\right),{ }^{54} \mathrm{Fe}(\mathrm{d}, \alpha),\left({ }^{3} \mathrm{He}, \mathrm{p} \alpha\right), \mathrm{E}$ $\approx 5-35 \mathrm{MeV}$; measured isomer production ratios. REPT NEA/NSC/DOC(2004)14,P11,Qaim

2005AD01 NUCLEAR REACTIONS ${ }^{209} \operatorname{Bi}(\mathrm{n}, 4 \mathrm{n}),(\mathrm{n}, 5 \mathrm{n}),(\mathrm{n}, 6 \mathrm{n}),(\mathrm{n}, 7 \mathrm{n}),(\mathrm{n}$, 9n), ${ }^{232} \mathrm{Th}(\mathrm{n}, \gamma),{ }^{197} \mathrm{Au}(\mathrm{n}, 2 \mathrm{n}),(\mathrm{n}, 4 \mathrm{n}),(\mathrm{n}, 6 \mathrm{n}),(\mathrm{n}, 7 \mathrm{n}),(\mathrm{n}, \gamma),{ }^{59} \mathrm{Co}(\mathrm{n}$, $2 \mathrm{n}),(\mathrm{n}, 3 \mathrm{n}),(\mathrm{n}, 4 \mathrm{n}),(\mathrm{n}, 5 \mathrm{n}),(\mathrm{n}, \mathrm{p}),(\mathrm{n}, 6 \mathrm{n} 2 \mathrm{p}),{ }^{115} \operatorname{In}(\mathrm{n}, 5 \mathrm{n}),(\mathrm{n}, 6 \mathrm{n}),(\mathrm{n}$, $7 \mathrm{n}), \mathrm{E}=$ spectrum; measured $\mathrm{E} \gamma, \mathrm{I} \gamma$; deduced reaction rates. Activation technique, spallation neutrons from $1 \mathrm{GeV}$ proton beam, comparison with model predictions. JOUR ZAANE 2361

2005B010 NUCLEAR REACTIONS Zn(p, X) ${ }^{64} \mathrm{Cu} /{ }^{57} \mathrm{Ni} /{ }^{56} \mathrm{Ni} /{ }^{52} \mathrm{Mn} /{ }^{54} \mathrm{Mn}$ $/{ }^{62} \mathrm{Zn} /{ }^{65} \mathrm{Zn} /{ }^{51} \mathrm{Cr} /{ }^{48} \mathrm{~V} /{ }^{55} \mathrm{Co} /{ }^{56} \mathrm{Co} /{ }^{57} \mathrm{Co} /{ }^{58} \mathrm{Co} /{ }^{60} \mathrm{Co} /{ }^{66} \mathrm{Ga}$ $/{ }^{67} \mathrm{Ga} /{ }^{52} \mathrm{Fe} /{ }^{59} \mathrm{Fe}, \mathrm{E} \approx 31-141 \mathrm{MeV}$; measured production $\sigma$. Stacked-foil activation. JOUR JRNCD 264101

2005DIZY NUCLEAR REACTIONS Fe(p, X) ${ }^{57} \mathrm{Co} /{ }^{56} \mathrm{Co} /{ }^{55} \mathrm{Co} /{ }^{54} \mathrm{Mn} /{ }^{52} \mathrm{Mn}$ $/{ }^{48} \mathrm{~V} /{ }^{51} \mathrm{Cr} /{ }^{48} \mathrm{Cr} /{ }^{47} \mathrm{Sc}, \mathrm{E} \approx 20-70 \mathrm{MeV}$; measured activation $\sigma$.

Comparison with previous results. CONF Santa Fe (Nucl Data for Sci and Technol) Proc,Vol1,P1011

2005MIZZ NUCLEAR REACTIONS Cu(n, X) ${ }^{56} \mathrm{Co}, \mathrm{E}=40-180 \mathrm{MeV} ; \mathrm{Fe}(\mathrm{n}$, $\mathrm{X}){ }^{54} \mathrm{Mn} /{ }^{52} \mathrm{Mn} /{ }^{51} \mathrm{Cr} /{ }^{48} \mathrm{~V}, \mathrm{E} \approx 0-180 \mathrm{MeV} ; \mathrm{Pb}(\mathrm{n}, \mathrm{X}){ }^{196} \mathrm{Au} /{ }^{200} \mathrm{~Pb}$ $/{ }^{103} \mathrm{Ru}, \mathrm{E} \approx 40-180 \mathrm{MeV} ; \mathrm{U}(\mathrm{n}, \mathrm{X}){ }^{99} \mathrm{Mo}, \mathrm{E} \approx 0-180 \mathrm{MeV}$; measured excitation functions. Comparison with proton-induced reactions. CONF Santa Fe (Nucl Data for Sci and Technol) Proc,Vol1,P861 2005SI14 NUCLEAR REACTIONS C, O, Si, Mg, Al(n, X) ${ }^{7} \mathrm{Be}, \mathrm{E} \approx 0.1-750$ $\mathrm{MeV} ; \mathrm{O}, \mathrm{Si}, \mathrm{Mg}, \mathrm{Al}(\mathrm{n}, \mathrm{X}){ }^{22} \mathrm{Na} /{ }^{23} \mathrm{Na}, \mathrm{E} \approx 0.1-750 \mathrm{MeV} ;{ }^{197} \mathrm{Au}(\mathrm{n}$, $\mathrm{X}){ }^{194} \mathrm{Au} /{ }^{196} \mathrm{Au} /{ }^{198} \mathrm{Au}, \mathrm{E} \approx 0.1-750 \mathrm{MeV}$; Ti, Fe, Ni, Cu(n, X ${ }^{46} \mathrm{Sc}$ $/{ }^{48} \mathrm{Sc}, \mathrm{E} \approx 0.1-750 \mathrm{MeV} ; \mathrm{Fe}, \mathrm{Ni}, \mathrm{Cu}(\mathrm{n}, \mathrm{X}){ }^{48} \mathrm{~V} /{ }^{51} \mathrm{Cr} /{ }^{52} \mathrm{Mn} /{ }^{54} \mathrm{Mn}$, $\mathrm{E} \approx 0.1-750 \mathrm{MeV} ; \mathrm{Ni}, \mathrm{Cu}(\mathrm{n}, \mathrm{X}){ }^{56} \mathrm{Ni} /{ }^{57} \mathrm{Ni} /{ }^{56} \mathrm{Co} /{ }^{57} \mathrm{Co} /{ }^{58} \mathrm{Co} /$ ${ }^{60} \mathrm{Co} /{ }^{59} \mathrm{Fe}, \mathrm{E} \approx 0.1-750 \mathrm{MeV}$; measured energy-integrated production $\sigma$. JOUR NIMBE 234419

${ }^{52} \mathrm{Fe} \quad 2005 \mathrm{~B} 010$ NUCLEAR REACTIONS Zn(p, X) ${ }^{64} \mathrm{Cu} /{ }^{57} \mathrm{Ni} /{ }^{56} \mathrm{Ni} /{ }^{52} \mathrm{Mn} /{ }^{54} \mathrm{Mn}$ $/{ }^{62} \mathrm{Zn} /{ }^{65} \mathrm{Zn} /{ }^{51} \mathrm{Cr} /{ }^{48} \mathrm{~V} /{ }^{55} \mathrm{Co} /{ }^{56} \mathrm{Co} /{ }^{57} \mathrm{Co} /{ }^{58} \mathrm{Co} /{ }^{60} \mathrm{Co} /{ }^{66} \mathrm{Ga}$ $/{ }^{67} \mathrm{Ga} /{ }^{52} \mathrm{Fe} /{ }^{59} \mathrm{Fe}, \mathrm{E} \approx 31-141 \mathrm{MeV}$; measured production $\sigma$. Stacked-foil activation. JOUR JRNCD 264101

2005GA15 NUCLEAR REACTIONS ${ }^{197} \mathrm{Au}\left({ }^{52} \mathrm{Fe},{ }^{52} \mathrm{Fe}\right),\left({ }^{54} \mathrm{Ni},{ }^{54} \mathrm{Ni}\right),\left({ }^{56} \mathrm{Ni}\right.$, $\left.{ }^{56} \mathrm{Ni}^{\prime}\right),\left({ }^{58} \mathrm{Ni},{ }^{58} \mathrm{Ni}\right.$ '), E not given; measured $\mathrm{E} \gamma, \mathrm{I} \gamma$, (particle) $\gamma$-coin following projectile Coulomb excitation. ${ }^{52} \mathrm{Fe},{ }^{54,56,58} \mathrm{Ni}$ transitions deduced $\mathrm{B}(\mathrm{E} 2) .{ }^{9} \mathrm{Be}\left({ }^{32} \mathrm{~S},{ }^{31} \mathrm{SX}\right),\left({ }^{33} \mathrm{Cl},{ }^{32} \mathrm{ClX}\right),\left({ }^{34} \mathrm{Ar},{ }^{33} \mathrm{ArX}\right)$, E not given; measured one-neutron removal $\sigma$. JOUR APOBB 361227

2005GA20 RADIOACTIVITY ${ }^{52} \mathrm{Fe}(\mathrm{IT})$ [from $\left.\mathrm{Si}\left({ }^{36} \mathrm{Ar}, \mathrm{X}\right)\right]$; measured $\mathrm{E} \gamma, \mathrm{I} \gamma$, $\gamma \gamma$-coin. ${ }^{52} \mathrm{Fe}$ deduced levels, $\mathrm{J}, \pi, \mathrm{T}_{1 / 2}, \mathrm{~B}(\mathrm{E} 4)$, yrast trap. Comparison with shell model predictions. JOUR PYLBB 61988 2005W001 NUCLEAR REACTIONS ${ }^{197} \mathrm{Au}\left({ }^{84} \mathrm{Kr},{ }^{84} \mathrm{Kr}\right),\left({ }^{56} \mathrm{Cr},{ }^{56} \mathrm{Cr}\right),\left({ }^{108} \mathrm{Sn}\right.$, ${ }^{108} \mathrm{Sn}$ '), $\mathrm{E}=113-142 \mathrm{MeV} /$ nucleon; measured $\mathrm{E} \gamma, \mathrm{I} \gamma$ following projectile Coulomb excitation. ${ }^{84} \mathrm{Kr},{ }^{56} \mathrm{Cr},{ }^{108} \mathrm{Sn}$ deduced transitions. ${ }^{9} \mathrm{Be}\left({ }^{55} \mathrm{Ni}, \mathrm{X}\right){ }^{54} \mathrm{Co} /{ }^{52} \mathrm{Fe} /{ }^{50} \mathrm{Cr}, \mathrm{E}=171 \mathrm{MeV} /$ nucleon; measured $\mathrm{E} \gamma$, I $\gamma$, (particle) $\gamma$-coin. JOUR NIMAE 537637

${ }^{52} \mathrm{Ni}$ 2005BL15 RADIOACTIVITY ${ }^{54} \mathrm{Zn}(2 \mathrm{p})$ [from $\mathrm{Ni}\left({ }^{58} \mathrm{Ni}, \mathrm{X}\right)$ ]; measured $\mathrm{Ep}, \mathrm{T}_{1 / 2}$, two-proton decay branching ratio. Comparison with model predictions. JOUR PRLTA 94232501 


\section{$\mathrm{A}=52$ (continued)}

2005BL31 RADIOACTIVITY ${ }^{45} \mathrm{Fe},{ }^{48} \mathrm{Ni},{ }^{54} \mathrm{Zn}(2 \mathrm{p})$ [from $\mathrm{Ni}\left({ }^{58} \mathrm{Ni}, \mathrm{X}\right)$ ]; measured proton spectra, $\mathrm{T}_{1 / 2}$. Comparison with theory. JOUR ZAANE 25 s01 169

2005BlzZ RADIOACTIVITY ${ }^{54} \mathrm{Zn}(2 \mathrm{p})$ [from Ni( $\left.{ }^{58} \mathrm{Ni}, \mathrm{X}\right)$ ]; measured $\mathrm{Ep}, \mathrm{T}_{1 / 2}$, two-proton decay branching ratio. Comparison with model predictions. PREPRINT nucl-ex/0505016,5/13/2005

2005GI15 RADIOACTIVITY ${ }^{45} \mathrm{Fe},{ }^{54} \mathrm{Zn}(\mathrm{p}),(2 \mathrm{p})$ [from Ni $\left.\left({ }^{58} \mathrm{Ni}, \mathrm{X}\right)\right]$; measured proton spectra, $\mathrm{T}_{1 / 2}$. JOUR JPGPE $31 \mathrm{~S} 1509$

\section{$\mathrm{A}=53$}

${ }^{53} \mathrm{Ti}$ 2005F014 NUCLEAR REACTIONS ${ }^{208} \mathrm{~Pb}\left({ }^{48} \mathrm{Ca}, \mathrm{X}\right), \mathrm{E}=305 \mathrm{MeV} ;{ }^{238} \mathrm{U}\left({ }^{48} \mathrm{Ca}\right.$, $\mathrm{X}), \mathrm{E}=330 \mathrm{MeV}$; measured $\mathrm{E} \gamma, \mathrm{I} \gamma, \gamma \gamma$-coin. ${ }^{53} \mathrm{Ti}$ deduced levels, $\mathrm{J}, \pi$. Gammasphere array, cross-coincidence with reaction partners.

Comparison with model predictions. JOUR PRVCA 72044315

${ }^{53} \mathrm{Cr} \quad$ 2005BEZT NUCLEAR REACTIONS ${ }^{35} \mathrm{Cl}(\mathrm{n}, \gamma)$, E not given; measured $\mathrm{E} \gamma, \mathrm{I} \gamma$. ${ }^{36} \mathrm{Cl}$ deduced transitions, level energies, binding energy. ${ }^{52,54} \mathrm{Cr},{ }^{56} \mathrm{Fe}$, ${ }^{206} \mathrm{~Pb}(\mathrm{n}, \gamma)$, E not given; analyzed $\mathrm{E} \gamma \cdot{ }^{53,55} \mathrm{Cr},{ }^{57} \mathrm{Fe},{ }^{207} \mathrm{~Pb}$ deduced binding energies. GAMS4 spectrometer. CONF Santa Fe (Nucl Data for Sci and Technol) Proc,Vol1,P1074

${ }^{53} \mathrm{Fe} \quad 2005 \mathrm{DU} 19$ NUCLEAR REACTIONS $\left.{ }^{28} \mathrm{Si}^{32} \mathrm{~S}, \mathrm{n} 2 \mathrm{p} \alpha\right), \mathrm{E}=125 \mathrm{MeV}$; measured $\mathrm{E} \gamma$, $\mathrm{I} \gamma, \gamma \gamma_{-}$, (charged particle) $\gamma-$, (neutron) $\gamma$-coin. ${ }^{24} \mathrm{Mg}\left({ }^{32} \mathrm{~S}, \mathrm{n} 2 \mathrm{p}\right), \mathrm{E}=95$ $\mathrm{MeV}$; measured Doppler-shifted $\mathrm{E} \gamma, \mathrm{I} \gamma, \gamma \gamma$-coin. ${ }^{53} \mathrm{Fe}$ deduced high-spin levels, J, $\pi, \mathrm{T}_{1 / 2}$, configurations. Gammasphere, Microball, GASP arrays, recoil-distance technique. Comparison with shell-model predictions. JOUR PRVCA 72014307

${ }^{53} \mathrm{Cu}$ 2005GI15 RADIOACTIVITY ${ }^{45} \mathrm{Fe},{ }^{54} \mathrm{Zn}(\mathrm{p}),(2 \mathrm{p})$ [from Ni $\left.\left({ }^{58} \mathrm{Ni}, \mathrm{X}\right)\right]$; measured proton spectra, $\mathrm{T}_{1 / 2}$. JOUR JPGPE $31 \mathrm{~S} 1509$

\section{$\mathrm{A}=54$}

$\begin{array}{ll}{ }^{54} \mathrm{Sc} & 2004 \mathrm{LI} 75 \\ { }^{54} \mathrm{Ti} & 2004 \mathrm{~F} 009\end{array}$

2004LI75 RADIOACTIVITY ${ }^{54,55,56} \mathrm{Sc}\left(\beta^{-}\right)$[from $\mathrm{Be}\left({ }^{86} \mathrm{Kr}, \mathrm{X}\right)$ ]; measured $\mathrm{E} \beta$,

RADIOACTIVITY ${ }^{54,55,56} \mathrm{Sc}\left(\beta^{-}\right)$[from $\mathrm{Be}\left({ }^{86} \mathrm{Kr}, \mathrm{X}\right)$ ]; measured $\mathrm{E} \beta$, $\mathrm{E} \gamma, \beta \gamma$-coin, $\mathrm{T}_{1 / 2} .{ }^{54,55,56} \mathrm{Ti}$ deduced levels, $\mathrm{J}, \pi$, configurations. Comparison with model predictions. JOUR PRVCA 70064303 NUCLEAR REACTIONS ${ }^{238} \mathrm{U}\left({ }^{48} \mathrm{Ca}, \mathrm{X}\right){ }^{54} \mathrm{Ti} /{ }^{56} \mathrm{Ti}, \mathrm{E}=330 \mathrm{MeV}$; measured $\mathrm{E} \gamma, \mathrm{I} \gamma, \gamma \gamma$-coin. ${ }^{54,56} \mathrm{Ti}$ deduced levels, $\mathrm{J}, \pi$, configurations. Gammasphere array. JOUR PRVCA 70064304 $\mathrm{E} \gamma, \beta \gamma$-coin, $\mathrm{T}_{1 / 2} .{ }^{54,55,56} \mathrm{Ti}$ deduced levels, $\mathrm{J}, \pi$, configurations. Comparison with model predictions. JOUR PRVCA 70064303 2005 DI05 NUCLEAR REACTIONS ${ }^{197} \mathrm{Au}\left({ }^{76} \mathrm{Ge},{ }^{76} \mathrm{Ge}\right),\left({ }^{52} \mathrm{Ti},{ }^{52} \mathrm{Ti}\right),\left({ }^{54} \mathrm{Ti}\right.$, ${ }^{54}{ }^{\mathrm{Ti}}$ '), $\left({ }^{56} \mathrm{Ti},{ }^{56} \mathrm{Ti}^{\prime}\right), \mathrm{E} \approx 80-90 \mathrm{MeV}$; measured $\mathrm{E} \gamma, \mathrm{I} \gamma$, (particle) $\gamma$-coin following projectile Coulomb excitation. ${ }^{52,54,56} \mathrm{Ti}$ deduced transitions $\mathrm{B}(\mathrm{E} 2)$, subshell closures. Comparison with large-scale shell model calculations. JOUR PRVCA 71041302 


\section{$\mathrm{A}=54$ (continued)}

2005DIZZ NUCLEAR REACTIONS ${ }^{238} \mathrm{U}\left({ }^{48} \mathrm{Ca}, \mathrm{X}\right){ }^{56} \mathrm{Ti}, \mathrm{E}=330 \mathrm{MeV}$; measured $\mathrm{E} \gamma, \mathrm{I} \gamma, \gamma \gamma$-coin. ${ }^{56} \mathrm{Ti}$ deduced levels, $\mathrm{J}, \pi .{ }^{197} \mathrm{Au}\left({ }^{76} \mathrm{Ge},{ }^{76} \mathrm{Ge}\right),\left({ }^{52} \mathrm{Ti}\right.$, $\left.{ }^{52} \mathrm{Ti}^{\prime}\right),\left({ }^{54} \mathrm{Ti},{ }^{54} \mathrm{Ti}^{\prime}\right),\left({ }^{56} \mathrm{Ti},{ }^{56} \mathrm{Ti}^{\prime}\right), \mathrm{E} \approx 80-90 \mathrm{MeV}$; measured $\mathrm{E} \gamma, \mathrm{I} \gamma$, (particle) $\gamma$-coin following projectile Coulomb excitation. ${ }^{52,54,56} \mathrm{Ti}$, ${ }^{76} \mathrm{Ge},{ }^{197} \mathrm{Au}$ deduced transitions B(E2). CONF Argonne(Nuclei at the Limits),P131,Dinca

${ }^{54} \mathrm{Cr} \quad$ 2005BE33

2005BU14

2006B001

${ }^{54} \mathrm{Mn} \quad$ 2005B010

2005DIZY

2005MIZZ
NUCLEAR REACTIONS ${ }^{197} \mathrm{Au}\left({ }^{54} \mathrm{Cr},{ }^{54} \mathrm{Cr}\right.$ '), E=136 MeV / nucleon; measured $\mathrm{E} \gamma, \mathrm{I} \gamma$, (particle) $\gamma$-coin following projectile Coulomb excitation. ${ }^{54} \mathrm{Cr}$ deduced transitions. JOUR APOBB 361235 NUCLEAR REACTIONS ${ }^{197} \mathrm{Au}\left({ }^{54} \mathrm{Cr},{ }^{54} \mathrm{Cr}\right),\left({ }^{56} \mathrm{Cr},{ }^{56} \mathrm{Cr}\right),\left({ }^{58} \mathrm{Cr}\right.$, ${ }^{58} \mathrm{Cr}$ '), $\mathrm{E} \approx 135 \mathrm{MeV} /$ nucleon; measured measured $\mathrm{E} \gamma, \mathrm{I} \gamma$, (particle) $\gamma$-coin following projectile Coulomb excitation. ${ }^{54,56,58} \mathrm{Cr}$ deduced transitions. JOUR APOBB 361249 NUCLEAR REACTIONS ${ }^{197} \mathrm{Au}\left({ }^{54} \mathrm{Cr},{ }^{54} \mathrm{Cr}\right),\left({ }^{56} \mathrm{Cr},{ }^{56} \mathrm{Cr}\right),\left({ }^{58} \mathrm{Cr}\right.$, ${ }^{58} \mathrm{Cr}$ '), $\mathrm{E} \approx 100 \mathrm{MeV} /$ nucleon; measured $\mathrm{E} \gamma, \mathrm{I} \gamma$, (particle) $\gamma$-coin following projectile Coulomb excitation. ${ }^{54,56,58} \mathrm{Cr}$ deduced transitions B(E2). Comparison with shell model predictions. JOUR PYLBB 622 29

2005HUZZ NUCLEAR REACTIONS ${ }^{197} \mathrm{Au}\left({ }^{54} \mathrm{Cr},{ }^{54} \mathrm{Cr}\right),\left({ }^{56} \mathrm{Cr},{ }^{56} \mathrm{Cr}\right),\left({ }^{5} \mathrm{Cr}\right.$, $\left.{ }^{58} \mathrm{Cr}^{\prime}\right), \mathrm{E} \approx 136 \mathrm{MeV} /$ nucleon; measured $\mathrm{E} \gamma, \mathrm{I} \gamma$, (particle) $\gamma$-coin following projectile Coulomb excitation. ${ }^{54,56,58} \mathrm{Cr}$ deduced levels, B(E2). CONF Bormio (XLIII Winter Meeting) Proc,P232 NUCLEAR REACTIONS ${ }^{197} \mathrm{Au}\left({ }^{54} \mathrm{Cr},{ }^{54} \mathrm{Cr}\right),\left({ }^{56} \mathrm{Cr},{ }^{56} \mathrm{Cr}\right),\left({ }^{58} \mathrm{Cr}\right.$, ${ }^{58} \mathrm{Cr}$ '), E $=100 \mathrm{MeV} /$ nucleon; measured $\mathrm{E} \gamma, \mathrm{I} \gamma$, (particle) $\gamma$-coin following projectile Coulomb excitation. ${ }^{54,56,58} \mathrm{Cr}$ deduced transitions. $\mathrm{Be}\left({ }^{55} \mathrm{Ni}, \mathrm{X}\right){ }^{50} \mathrm{Cr}, \mathrm{E}=171 \mathrm{MeV} /$ nucleon; measured $\mathrm{E} \gamma, \mathrm{I} \gamma$, (particle) $\gamma$-coin. ${ }^{50} \mathrm{Cr}$ deduced transitions. $\mathrm{Be}\left({ }^{55} \mathrm{Ni}, \mathrm{X}\right),{ }^{197} \mathrm{Au}\left({ }^{108} \mathrm{Sn}\right.$, $\mathrm{X})$, E not given; measured fragment yields. CONF Argonne(Nuclei at the Limits),P151,Saito

RADIOACTIVITY ${ }^{54} \mathrm{Mn},{ }^{65} \mathrm{Zn}(\mathrm{EC})$; measured $\beta \gamma$-coin. Triple to double coincidence ratio method. JOUR ARISE 64124

NUCLEAR REACTIONS Zn(p, X) ${ }^{64} \mathrm{Cu} /{ }^{57} \mathrm{Ni} /{ }^{56} \mathrm{Ni} /{ }^{52} \mathrm{Mn} /{ }^{54} \mathrm{Mn}$ $/{ }^{62} \mathrm{Zn} /{ }^{65} \mathrm{Zn} /{ }^{51} \mathrm{Cr} /{ }^{48} \mathrm{~V} /{ }^{55} \mathrm{Co} /{ }^{56} \mathrm{Co} /{ }^{57} \mathrm{Co} /{ }^{58} \mathrm{Co} /{ }^{60} \mathrm{Co} /{ }^{66} \mathrm{Ga}$ $/{ }^{67} \mathrm{Ga} /{ }^{52} \mathrm{Fe} /{ }^{59} \mathrm{Fe}, \mathrm{E} \approx 31-141 \mathrm{MeV}$; measured production $\sigma$.

Stacked-foil activation. JOUR JRNCD 264101 NUCLEAR REACTIONS Fe(p, X) ${ }^{57} \mathrm{Co} /{ }^{56} \mathrm{Co} /{ }^{55} \mathrm{Co} /{ }^{54} \mathrm{Mn} /{ }^{52} \mathrm{Mn}$ $/{ }^{48} \mathrm{~V} /{ }^{51} \mathrm{Cr} /{ }^{48} \mathrm{Cr} /{ }^{47} \mathrm{Sc}, \mathrm{E} \approx 20-70 \mathrm{MeV}$; measured activation $\sigma$. Comparison with previous results. CONF Santa Fe (Nucl Data for Sci and Technol) Proc,Vol1,P1011

NUCLEAR REACTIONS Cu(n, X) ${ }^{56} \mathrm{Co}, \mathrm{E}=40-180 \mathrm{MeV}$; $\mathrm{Fe}(\mathrm{n}$, $\mathrm{X}){ }^{54} \mathrm{Mn} /{ }^{52} \mathrm{Mn} /{ }^{51} \mathrm{Cr} /{ }^{48} \mathrm{~V}, \mathrm{E} \approx 0-180 \mathrm{MeV} ; \mathrm{Pb}(\mathrm{n}, \mathrm{X}){ }^{196} \mathrm{Au} /{ }^{200} \mathrm{~Pb}$ $/{ }^{103} \mathrm{Ru}, \mathrm{E} \approx 40-180 \mathrm{MeV} ; \mathrm{U}(\mathrm{n}, \mathrm{X}){ }^{99} \mathrm{Mo}, \mathrm{E} \approx 0-180 \mathrm{MeV}$; measured excitation functions. Comparison with proton-induced reactions. CONF Santa Fe (Nucl Data for Sci and Technol) Proc,Vol1,P861 


\section{$\mathrm{A}=54$ (continued)}

2005SI14 NUCLEAR REACTIONS C, O, Si, Mg, Al(n, X $)^{7} \mathrm{Be}, \mathrm{E} \approx 0.1-750$ $\mathrm{MeV} ; \mathrm{O}, \mathrm{Si}, \mathrm{Mg}, \mathrm{Al}(\mathrm{n}, \mathrm{X}){ }^{22} \mathrm{Na} /{ }^{23} \mathrm{Na}, \mathrm{E} \approx 0.1-750 \mathrm{MeV} ;{ }^{197} \mathrm{Au}(\mathrm{n}$, $\mathrm{X}){ }^{194} \mathrm{Au} /{ }^{196} \mathrm{Au} /{ }^{198} \mathrm{Au}, \mathrm{E} \approx 0.1-750 \mathrm{MeV} ; \mathrm{Ti}, \mathrm{Fe}, \mathrm{Ni}, \mathrm{Cu}(\mathrm{n}, \mathrm{X})^{46} \mathrm{Sc}$ $/{ }^{48} \mathrm{Sc}, \mathrm{E} \approx 0.1-750 \mathrm{MeV} ; \mathrm{Fe}, \mathrm{Ni}, \mathrm{Cu}(\mathrm{n}, \mathrm{X}){ }^{48} \mathrm{~V} /{ }^{51} \mathrm{Cr} /{ }^{52} \mathrm{Mn} /{ }^{54} \mathrm{Mn}$, $\mathrm{E} \approx 0.1-750 \mathrm{MeV} ; \mathrm{Ni}, \mathrm{Cu}(\mathrm{n}, \mathrm{X}){ }^{56} \mathrm{Ni} /{ }^{57} \mathrm{Ni} /{ }^{56} \mathrm{Co} /{ }^{57} \mathrm{Co} /{ }^{58} \mathrm{Co} /$ ${ }^{60} \mathrm{Co} /{ }^{59} \mathrm{Fe}, \mathrm{E} \approx 0.1-750 \mathrm{MeV}$; measured energy-integrated production $\sigma$. JOUR NIMBE 234419

2005SI32 NUCLEAR REACTIONS Cu(n, X $)^{54} \mathrm{Mn} /{ }^{59} \mathrm{Fe} /{ }^{56} \mathrm{Co} /{ }^{57} \mathrm{Co} /{ }^{58} \mathrm{Co}$ $/{ }^{60} \mathrm{Co}, \mathrm{E} \approx 70.7,110.8 \mathrm{MeV}$; measured $\sigma$. Comparison with previous results, model predictions. JOUR NIMBE 240617

2006B001 RADIOACTIVITY ${ }^{54} \mathrm{Mn},{ }^{65} \mathrm{Zn}(\mathrm{EC})$; measured $\beta \gamma$-coin. Triple to double coincidence ratio method. JOUR ARISE 64124

${ }^{54} \mathrm{Fe} \quad 2005 \mathrm{HA} 25$ NUCLEAR REACTIONS ${ }^{9} \mathrm{Be}\left({ }^{55} \mathrm{Ni}, \mathrm{X}\right){ }^{54} \mathrm{Ni}$, E not given; ${ }^{9} \mathrm{Be}\left({ }^{55} \mathrm{Co}\right.$, $\mathrm{X})^{54} \mathrm{Fe}, \mathrm{E}$ not given; measured $\mathrm{E} \gamma, \mathrm{I} \gamma$, (particle) $\gamma$-coin. Two-step fragmentation of ${ }^{58} \mathrm{Ni}$ primary beam. JOUR APOBB 361253

2005 TA27 NUCLEAR REACTIONS ${ }^{9} \mathrm{Be}\left({ }^{55} \mathrm{Ni}, \mathrm{X}\right),\left({ }^{55} \mathrm{Co}, \mathrm{X}\right), \mathrm{E} \approx 170 \mathrm{MeV} /$ nucleon; measured $\mathrm{E} \gamma, \mathrm{I} \gamma$, (fragment) $\gamma$-coin. ${ }^{54} \mathrm{Ni},{ }^{54} \mathrm{Fe}$ deduced transitions. JOUR JPGPE 31 S1527

${ }^{54} \mathrm{Co} \quad$ 2005SA44 RADIOACTIVITY ${ }^{46} \mathrm{~V}(\mathrm{EC})$; analyzed masses; deduced $\mathrm{Q}(\mathrm{EC})$, log ft. ${ }^{10} \mathrm{C},{ }^{14} \mathrm{O},{ }^{22} \mathrm{Mg},{ }^{26 m} \mathrm{Al},{ }^{34} \mathrm{Cl},{ }^{34} \mathrm{Ar},{ }^{38 m} \mathrm{~K},{ }^{42} \mathrm{Sc},{ }^{46} \mathrm{~V},{ }^{50} \mathrm{Mn},{ }^{54} \mathrm{Co},{ }^{74} \mathrm{Rb}$; compiled, analyzed log ft; deduced quark-mixing matrix element. JOUR PRLTA 95102501

2005W001 NUCLEAR REACTIONS ${ }^{197} \mathrm{Au}\left({ }^{84} \mathrm{Kr},{ }^{84} \mathrm{Kr}\right),\left({ }^{56} \mathrm{Cr},{ }^{56} \mathrm{Cr}{ }^{\prime}\right),\left({ }^{108} \mathrm{Sn}\right.$, ${ }^{108} \mathrm{Sn}$ '), $\mathrm{E}=113-142 \mathrm{MeV} /$ nucleon; measured $\mathrm{E} \gamma$, I $\gamma$ following projectile Coulomb excitation. ${ }^{84} \mathrm{Kr},{ }^{56} \mathrm{Cr},{ }^{108} \mathrm{Sn}$ deduced transitions. ${ }^{9} \mathrm{Be}\left({ }^{55} \mathrm{Ni}, \mathrm{X}\right){ }^{54} \mathrm{Co} /{ }^{52} \mathrm{Fe} /{ }^{50} \mathrm{Cr}, \mathrm{E}=171 \mathrm{MeV} /$ nucleon; measured $\mathrm{E} \gamma$, I $\gamma$, (particle) $\gamma$-coin. JOUR NIMAE 537637

${ }^{54} \mathrm{Ni} \quad 2005 \mathrm{GA} 15$ NUCLEAR REACTIONS ${ }^{197} \mathrm{Au}\left({ }^{52} \mathrm{Fe},{ }^{52} \mathrm{Fe}\right),\left({ }^{54} \mathrm{Ni},{ }^{54} \mathrm{Ni}\right),\left({ }^{56} \mathrm{Ni}\right.$, $\left.{ }^{56} \mathrm{Ni}^{\prime}\right),\left({ }^{58} \mathrm{Ni},{ }^{58} \mathrm{Ni}\right.$ '), E not given; measured $\mathrm{E} \gamma, \mathrm{I} \gamma$, (particle) $\gamma$-coin following projectile Coulomb excitation. ${ }^{52} \mathrm{Fe},{ }^{54,56,58} \mathrm{Ni}$ transitions deduced $\mathrm{B}(\mathrm{E} 2) .{ }^{9} \mathrm{Be}\left({ }^{32} \mathrm{~S},{ }^{31} \mathrm{SX}\right),\left({ }^{33} \mathrm{Cl},{ }^{32} \mathrm{ClX}\right),\left({ }^{34} \mathrm{Ar},{ }^{33} \mathrm{ArX}\right)$, E not given; measured one-neutron removal $\sigma$. JOUR APOBB 361227

2005HA25 NUCLEAR REACTIONS ${ }^{9} \mathrm{Be}\left({ }^{55} \mathrm{Ni}, \mathrm{X}\right){ }^{54} \mathrm{Ni}$, E not given; ${ }^{9} \mathrm{Be}\left({ }^{55} \mathrm{Co}\right.$, $\mathrm{X})^{54} \mathrm{Fe}, \mathrm{E}$ not given; measured $\mathrm{E} \gamma, \mathrm{I} \gamma$, (particle) $\gamma$-coin. Two-step fragmentation of ${ }^{58} \mathrm{Ni}$ primary beam. JOUR APOBB 361253

2005 TA27 NUCLEAR REACTIONS ${ }^{9} \mathrm{Be}\left({ }^{55} \mathrm{Ni}, \mathrm{X}\right),\left({ }^{55} \mathrm{Co}, \mathrm{X}\right), \mathrm{E} \approx 170 \mathrm{MeV} /$ nucleon; measured $\mathrm{E} \gamma, \mathrm{I} \gamma$, (fragment) $\gamma$-coin. ${ }^{54} \mathrm{Ni},{ }^{54} \mathrm{Fe}$ deduced transitions. JOUR JPGPE 31 S1527

2005 YA26 NUCLEAR REACTIONS Pb $\left({ }^{46} \mathrm{Cr},{ }^{46} \mathrm{Cr}\right),\left({ }^{50} \mathrm{Fe},{ }^{50} \mathrm{Fe} '\right),\left({ }^{54} \mathrm{Ni},{ }^{54} \mathrm{Ni}^{\prime}\right)$, $\mathrm{E}=41-44 \mathrm{MeV} /$ nucleon; measured $\sigma(\theta), \mathrm{E} \gamma, \mathrm{I} \gamma$, (particle) $\gamma$-coin following projectile Coulomb excitation. ${ }^{46} \mathrm{Cr},{ }^{50} \mathrm{Fe},{ }^{54} \mathrm{Ni}$ deduced excitation B(E2). DWBA analysis. JOUR ZAANE 25 s01 409

${ }^{54} \mathrm{Zn} \quad 2005 B L 15$ NUCLEAR REACTIONS Ni( $\left.{ }^{58} \mathrm{Ni}, \mathrm{X}\right), \mathrm{E}=74.5 \mathrm{MeV} /$ nucleon; measured fragment yields; deduced evidence for ${ }^{54} \mathrm{Zn}$. JOUR PRLTA 94232501

2005BL15 RADIOACTIVITY ${ }^{54} \mathrm{Zn}(2 \mathrm{p})$ [from $\mathrm{Ni}\left({ }^{58} \mathrm{Ni}, \mathrm{X}\right)$ ]; measured $\mathrm{Ep}, \mathrm{T}_{1 / 2}$, two-proton decay branching ratio. Comparison with model predictions. JOUR PRLTA 94232501 


\section{$\mathrm{A}=54$ (continued)}

2005BL31 RADIOACTIVITY ${ }^{45} \mathrm{Fe},{ }^{48} \mathrm{Ni},{ }^{54} \mathrm{Zn}(2 \mathrm{p})$ [from Ni( $\left.{ }^{58} \mathrm{Ni}, \mathrm{X}\right)$ ]; measured proton spectra, $\mathrm{T}_{1 / 2}$. Comparison with theory. JOUR ZAANE $25 \mathrm{~s} 01$ 169

2005BLZZ NUCLEAR REACTIONS Ni( $\left.{ }^{58} \mathrm{Ni}, \mathrm{X}\right), \mathrm{E}=74.5 \mathrm{MeV} /$ nucleon; measured fragment yields; deduced evidence for ${ }^{54} \mathrm{Zn}$. PREPRINT nucl-ex/0505016,5/13/2005

2005BlzZ RADIOACTIVITY ${ }^{54} \mathrm{Zn}(2 \mathrm{p})$ [from $\mathrm{Ni}\left({ }^{58} \mathrm{Ni}, \mathrm{X}\right)$ ]; measured $\mathrm{Ep}, \mathrm{T}_{1 / 2}$, two-proton decay branching ratio. Comparison with model predictions. PREPRINT nucl-ex/0505016,5/13/2005

2005GI15 RADIOACTIVITY ${ }^{45} \mathrm{Fe},{ }^{54} \mathrm{Zn}(\mathrm{p}),(2 \mathrm{p})$ [from Ni $\left.\left({ }^{58} \mathrm{Ni}, \mathrm{X}\right)\right]$; measured proton spectra, $\mathrm{T}_{1 / 2}$. JOUR JPGPE $31 \mathrm{~S} 1509$

\section{$\mathrm{A}=55$}

\begin{tabular}{|c|c|}
\hline${ }^{55} \mathrm{Sc}$ & $2004 \mathrm{LI} 75$ \\
\hline${ }^{55} \mathrm{Ti}$ & 2004LI75 \\
\hline${ }^{55} \mathrm{Cr}$ & 2005BEZT \\
\hline${ }^{55} \mathrm{Fe}$ & 2005MAZL \\
\hline${ }^{55} \mathrm{Co}$ & 2004ADZ \\
\hline
\end{tabular}

RADIOACTIVITY ${ }^{54,55,56} \mathrm{Sc}\left(\beta^{-}\right)$[from $\mathrm{Be}\left({ }^{86} \mathrm{Kr}, \mathrm{X}\right)$ ]; measured $\mathrm{E} \beta$, $\mathrm{E} \gamma, \beta \gamma$-coin, $\mathrm{T}_{1 / 2} .{ }^{54,55,56} \mathrm{Ti}$ deduced levels, $\mathrm{J}, \pi$, configurations. Comparison with model predictions. JOUR PRVCA 70064303 RADIOACTIVITY ${ }^{54,55,56} \mathrm{Sc}\left(\beta^{-}\right)$[from $\mathrm{Be}\left({ }^{86} \mathrm{Kr}, \mathrm{X}\right)$ ]; measured $\mathrm{E} \beta$, $\mathrm{E} \gamma, \beta \gamma$-coin, $\mathrm{T}_{1 / 2} .{ }^{54,55,56} \mathrm{Ti}$ deduced levels, $\mathrm{J}, \pi$, configurations. Comparison with model predictions. JOUR PRVCA 70064303 NUCLEAR REACTIONS ${ }^{35} \mathrm{Cl}(\mathrm{n}, \gamma)$, E not given; measured $\mathrm{E} \gamma, \mathrm{I} \gamma$. ${ }^{36} \mathrm{Cl}$ deduced transitions, level energies, binding energy. ${ }^{52,54} \mathrm{Cr},{ }^{56} \mathrm{Fe}$, ${ }^{206} \mathrm{~Pb}(\mathrm{n}, \gamma)$, E not given; analyzed $\mathrm{E} \gamma \cdot{ }^{53,55} \mathrm{Cr},{ }^{57} \mathrm{Fe},{ }^{207} \mathrm{~Pb}$ deduced binding energies. GAMS4 spectrometer. CONF Santa Fe (Nucl Data for Sci and Technol) Proc,Vol1,P1074

NUCLEAR REACTIONS ${ }^{58} \mathrm{Ni}$ (polarized p, d), E=24.6 MeV; measured $\sigma(\theta), \operatorname{Ay}(\theta) .{ }^{2} \mathrm{H}\left({ }^{54} \mathrm{Fe}, \mathrm{p}\right), \mathrm{E}=4.8 \mathrm{MeV} /$ nucleon; measured $\sigma(\theta)$. Other reactions discussed. REPT MLL 2004 Annual,P9,Mahgoub

NUCLEAR REACTIONS ${ }^{209} \operatorname{Bi}(\mathrm{n}, 4 \mathrm{n}),(\mathrm{n}, 5 \mathrm{n}),(\mathrm{n}, 6 \mathrm{n}),(\mathrm{n}, 7 \mathrm{n}),(\mathrm{n}$, 9n), ${ }^{232} \mathrm{Th}(\mathrm{n}, \gamma),{ }^{197} \mathrm{Au}(\mathrm{n}, 2 \mathrm{n}),(\mathrm{n}, 4 \mathrm{n}),(\mathrm{n}, 6 \mathrm{n}),(\mathrm{n}, 7 \mathrm{n}),(\mathrm{n}, \gamma),{ }^{115} \operatorname{In}(\mathrm{n}$, $5 \mathrm{n}),(\mathrm{n}, 6 \mathrm{n}),(\mathrm{n}, 7 \mathrm{n}),{ }^{59} \mathrm{Co}(\mathrm{n}, 2 \mathrm{n}),(\mathrm{n}, 3 \mathrm{n}),(\mathrm{n}, 4 \mathrm{n}),(\mathrm{n}, 5 \mathrm{n}),(\mathrm{n}, \gamma),(\mathrm{n}$, $\mathrm{p}),(\mathrm{n}, 6 \mathrm{n} 2 \mathrm{p}), \mathrm{E}=$ spectrum; measured $\mathrm{E} \gamma, \mathrm{I} \gamma$; deduced reaction rates. $\mathrm{Pb}(\mathrm{p}, \mathrm{nX}), \mathrm{E}=1 \mathrm{GeV}$; deduced spallation neutron spectrum. REPT JINR-E1-2004-16,Adam

2005AD01 NUCLEAR REACTIONS ${ }^{209} \operatorname{Bi}(\mathrm{n}, 4 \mathrm{n}),(\mathrm{n}, 5 \mathrm{n}),(\mathrm{n}, 6 \mathrm{n}),(\mathrm{n}, 7 \mathrm{n}),(\mathrm{n}$, 9n), ${ }^{232} \mathrm{Th}(\mathrm{n}, \gamma),{ }^{197} \mathrm{Au}(\mathrm{n}, 2 \mathrm{n}),(\mathrm{n}, 4 \mathrm{n}),(\mathrm{n}, 6 \mathrm{n}),(\mathrm{n}, 7 \mathrm{n}),(\mathrm{n}, \gamma),{ }^{59} \mathrm{Co}(\mathrm{n}$, 2n), (n, 3n), (n, 4n), (n, 5n), (n, p), (n, 6n2p), ${ }^{115} \operatorname{In}(\mathrm{n}, 5 \mathrm{n}),(\mathrm{n}, 6 \mathrm{n}),(\mathrm{n}$, $7 \mathrm{n}), \mathrm{E}=$ spectrum; measured $\mathrm{E} \gamma, \mathrm{I} \gamma$; deduced reaction rates. Activation technique, spallation neutrons from $1 \mathrm{GeV}$ proton beam, comparison with model predictions. JOUR ZAANE 2361

2005B010 NUCLEAR REACTIONS Zn(p, X) ${ }^{64} \mathrm{Cu} /{ }^{57} \mathrm{Ni} /{ }^{56} \mathrm{Ni} /{ }^{52} \mathrm{Mn} /{ }^{54} \mathrm{Mn}$ $/{ }^{62} \mathrm{Zn} /{ }^{65} \mathrm{Zn} /{ }^{51} \mathrm{Cr} /{ }^{48} \mathrm{~V} /{ }^{55} \mathrm{Co} /{ }^{56} \mathrm{Co} /{ }^{57} \mathrm{Co} /{ }^{58} \mathrm{Co} /{ }^{60} \mathrm{Co} /{ }^{66} \mathrm{Ga}$ $/{ }^{67} \mathrm{Ga} /{ }^{52} \mathrm{Fe} /{ }^{59} \mathrm{Fe}, \mathrm{E} \approx 31-141 \mathrm{MeV}$; measured production $\sigma$. Stacked-foil activation. JOUR JRNCD 264101

2005Dizy NUCLEAR REACTIONS Fe(p, X $){ }^{57} \mathrm{Co} /{ }^{56} \mathrm{Co} /{ }^{55} \mathrm{Co} /{ }^{54} \mathrm{Mn} /{ }^{52} \mathrm{Mn}$ $/{ }^{48} \mathrm{~V} /{ }^{51} \mathrm{Cr} /{ }^{48} \mathrm{Cr} /{ }^{47} \mathrm{Sc}, \mathrm{E} \approx 20-70 \mathrm{MeV}$; measured activation $\sigma$. Comparison with previous results. CONF Santa Fe (Nucl Data for Sci and Technol) Proc,Vol1,P1011 


\section{$\mathrm{A}=55$ (continued)}

${ }^{55} \mathrm{Ni} 2004 \mathrm{YU} 11 \quad$ NUCLEAR REACTIONS ${ }^{197} \mathrm{Au}\left({ }^{55} \mathrm{Ni},{ }^{55} \mathrm{Ni}\right), \mathrm{E}=84.8 \mathrm{MeV}$; measured $\mathrm{E} \gamma, \mathrm{I} \gamma$, (particle) $\gamma$-coin following projectile Coulomb excitation. ${ }^{55} \mathrm{Ni}$ deduced level, transition $\mathrm{B}(\mathrm{E} 2)$. Comparison with model predictions. JOUR PRVCA 70064321

\section{$\mathrm{A}=56$}

${ }^{56} \mathrm{Sc} \quad 2004 \mathrm{LI} 75$

2005MA93

${ }^{56} \mathrm{Ti}$

2004F009

2004 LI75

2005DI05

2005DIZZ

2005MA93

${ }^{56} \mathrm{Cr}$

2005BU14

2005BU29

2005GU27

2005HUZZ
RADIOACTIVITY ${ }^{54,55,56} \mathrm{Sc}\left(\beta^{-}\right)$[from $\mathrm{Be}\left({ }^{86} \mathrm{Kr}, \mathrm{X}\right)$ ]; measured $\mathrm{E} \beta$, $\mathrm{E} \gamma, \beta \gamma$-coin, $\mathrm{T}_{1 / 2} .{ }^{54,55,56} \mathrm{Ti}$ deduced levels, $\mathrm{J}, \pi$, configurations. Comparison with model predictions. JOUR PRVCA 70064303 RADIOACTIVITY ${ }^{56} \mathrm{Sc}\left(\beta^{-}\right)$[from $\left.\mathrm{Be}\left({ }^{78} \mathrm{Kr}, \mathrm{X}\right)\right]$; measured $\mathrm{E} \gamma, \mathrm{I} \gamma$, $\beta \gamma$-coin. ${ }^{56} \mathrm{Ti}$ deduced levels. Mass-separated source. JOUR NIMBE 241195

NUCLEAR REACTIONS ${ }^{238} \mathrm{U}\left({ }^{48} \mathrm{Ca}, \mathrm{X}\right){ }^{54} \mathrm{Ti} /{ }^{56} \mathrm{Ti}, \mathrm{E}=330 \mathrm{MeV}$; measured $\mathrm{E} \gamma, \mathrm{I} \gamma, \gamma \gamma$-coin. ${ }^{54,56} \mathrm{Ti}$ deduced levels, $\mathrm{J}, \pi$, configurations. Gammasphere array. JOUR PRVCA 70064304

RADIOACTIVITY ${ }^{54,55,56} \mathrm{Sc}\left(\beta^{-}\right)$[from $\mathrm{Be}\left({ }^{86} \mathrm{Kr}, \mathrm{X}\right)$ ]; measured $\mathrm{E} \beta$, $\mathrm{E} \gamma, \beta \gamma$-coin, $\mathrm{T}_{1 / 2} .{ }^{54,55,56} \mathrm{Ti}$ deduced levels, $\mathrm{J}, \pi$, configurations. Comparison with model predictions. JOUR PRVCA 70064303 NUCLEAR REACTIONS ${ }^{197} \mathrm{Au}\left({ }^{76} \mathrm{Ge},{ }^{76} \mathrm{Ge}\right.$ '), $\left({ }^{52} \mathrm{Ti},{ }^{52} \mathrm{Ti}\right),\left({ }^{54} \mathrm{Ti}\right.$, $\left.{ }^{54} \mathrm{Ti}^{\prime}\right),\left({ }^{56} \mathrm{Ti},{ }^{56} \mathrm{Ti}^{\prime}\right), \mathrm{E} \approx 80-90 \mathrm{MeV}$; measured $\mathrm{E} \gamma, \mathrm{I} \gamma$, (particle) $\gamma$-coin following projectile Coulomb excitation. ${ }^{52,54,56} \mathrm{Ti}$ deduced transitions $\mathrm{B}(\mathrm{E} 2)$, subshell closures. Comparison with large-scale shell model calculations. JOUR PRVCA 71041302

NUCLEAR REACTIONS ${ }^{238} \mathrm{U}\left({ }^{48} \mathrm{Ca}, \mathrm{X}\right){ }^{56} \mathrm{Ti}, \mathrm{E}=330 \mathrm{MeV}$; measured $\mathrm{E} \gamma, \mathrm{I} \gamma, \gamma \gamma$-coin. ${ }^{56} \mathrm{Ti}$ deduced levels, J, $\pi .{ }^{197} \mathrm{Au}\left({ }^{76} \mathrm{Ge},{ }^{76} \mathrm{Ge}\right),\left({ }^{52} \mathrm{Ti}\right.$, $\left.{ }^{52} \mathrm{Ti}^{\prime}\right),\left({ }^{54} \mathrm{Ti},{ }^{54} \mathrm{Ti}^{\prime}\right),\left({ }^{56} \mathrm{Ti},{ }^{56} \mathrm{Ti}^{\prime}\right), \mathrm{E} \approx 80-90 \mathrm{MeV}$; measured $\mathrm{E} \gamma, \mathrm{I} \gamma$, (particle) $\gamma$-coin following projectile Coulomb excitation. ${ }^{52,54,56} \mathrm{Ti}$, ${ }^{76} \mathrm{Ge},{ }^{197} \mathrm{Au}$ deduced transitions B(E2). CONF Argonne(Nuclei at the Limits),P131,Dinca

RADIOACTIVITY ${ }^{56} \mathrm{Sc}\left(\beta^{-}\right)$[from $\left.\mathrm{Be}\left({ }^{78} \mathrm{Kr}, \mathrm{X}\right)\right]$; measured $\mathrm{E} \gamma, \mathrm{I} \gamma$, $\beta \gamma$-coin. ${ }^{56} \mathrm{Ti}$ deduced levels. Mass-separated source. JOUR NIMBE 241195

NUCLEAR REACTIONS ${ }^{197} \mathrm{Au}\left({ }^{54} \mathrm{Cr},{ }^{54} \mathrm{Cr}\right),\left({ }^{56} \mathrm{Cr},{ }^{56} \mathrm{Cr}\right),\left({ }^{5} \mathrm{Cr}\right.$, ${ }^{58} \mathrm{Cr}$ '), $\mathrm{E} \approx 135 \mathrm{MeV} /$ nucleon; measured measured $\mathrm{E} \gamma, \mathrm{I} \gamma$, (particle) $\gamma$-coin following projectile Coulomb excitation. ${ }^{54,56,58} \mathrm{Cr}$ deduced transitions. JOUR APOBB 361249

NUCLEAR REACTIONS ${ }^{197} \mathrm{Au}\left({ }^{54} \mathrm{Cr},{ }^{54} \mathrm{Cr}\right),\left({ }^{56} \mathrm{Cr},{ }^{56} \mathrm{Cr}\right),\left({ }^{58} \mathrm{Cr}\right.$, ${ }^{58} \mathrm{Cr}$ '), $\mathrm{E} \approx 100 \mathrm{MeV} /$ nucleon; measured $\mathrm{E} \gamma, \mathrm{I} \gamma$, (particle) $\gamma$-coin following projectile Coulomb excitation. ${ }^{54,56,58} \mathrm{Cr}$ deduced transitions B(E2). Comparison with shell model predictions. JOUR PYLBB 622 29 ATOMIC MASSES ${ }^{56,57} \mathrm{Cr}$; measured masses. Penning trap mass spectrometer. JOUR JPGPE 31 S1765 NUCLEAR REACTIONS ${ }^{197} \mathrm{Au}\left({ }^{54} \mathrm{Cr},{ }^{54} \mathrm{Cr}\right),\left({ }^{56} \mathrm{Cr},{ }^{56} \mathrm{Cr}\right),\left({ }^{58} \mathrm{Cr}\right.$, ${ }^{58} \mathrm{Cr}^{\prime}$ ), $\mathrm{E} \approx 136 \mathrm{MeV} /$ nucleon; measured $\mathrm{E} \gamma, \mathrm{I} \gamma$, (particle) $\gamma$-coin following projectile Coulomb excitation. ${ }^{54,56,58} \mathrm{Cr}$ deduced levels, B(E2). CONF Bormio (XLIII Winter Meeting) Proc,P232 


\section{$\mathrm{A}=56$ (continued)}

2005SAZY NUCLEAR REACTIONS ${ }^{197} \mathrm{Au}\left({ }^{54} \mathrm{Cr},{ }^{54} \mathrm{Cr}\right.$ ' $),\left({ }^{56} \mathrm{Cr},{ }^{56} \mathrm{Cr}\right),\left({ }^{58} \mathrm{Cr}\right.$, ${ }^{58} \mathrm{Cr}$ '), $\mathrm{E}=100 \mathrm{MeV} /$ nucleon; measured $\mathrm{E} \gamma, \mathrm{I} \gamma$, (particle) $\gamma$-coin following projectile Coulomb excitation. ${ }^{54,56,58} \mathrm{Cr}$ deduced transitions. $\mathrm{Be}\left({ }^{55} \mathrm{Ni}, \mathrm{X}\right){ }^{50} \mathrm{Cr}, \mathrm{E}=171 \mathrm{MeV} /$ nucleon; measured $\mathrm{E} \gamma, \mathrm{I} \gamma$, (particle) $\gamma$-coin. ${ }^{50} \mathrm{Cr}$ deduced transitions. $\mathrm{Be}\left({ }^{55} \mathrm{Ni}, \mathrm{X}\right),{ }^{197} \mathrm{Au}\left({ }^{108} \mathrm{Sn}\right.$, $\mathrm{X})$, E not given; measured fragment yields. CONF Argonne(Nuclei at the Limits),P151,Saito 2005W001 NUCLEAR REACTIONS ${ }^{197} \mathrm{Au}\left({ }^{84} \mathrm{Kr},{ }^{84} \mathrm{Kr}\right),\left({ }^{56} \mathrm{Cr},{ }^{56} \mathrm{Cr}\right),\left({ }^{108} \mathrm{Sn}\right.$, ${ }^{108} \mathrm{Sn}$ '), E $=113-142 \mathrm{MeV} /$ nucleon; measured $\mathrm{E} \gamma, \mathrm{I} \gamma$ following projectile Coulomb excitation. ${ }^{84} \mathrm{Kr},{ }^{56} \mathrm{Cr},{ }^{108} \mathrm{Sn}$ deduced transitions. ${ }^{9} \mathrm{Be}\left({ }^{55} \mathrm{Ni}, \mathrm{X}\right){ }^{54} \mathrm{Co} /{ }^{52} \mathrm{Fe} /{ }^{50} \mathrm{Cr}, \mathrm{E}=171 \mathrm{MeV} /$ nucleon; measured $\mathrm{E} \gamma$, I $\gamma$, (particle) $\gamma$-coin. JOUR NIMAE 537637

${ }^{56} \mathrm{Mn} \quad 2004 \mathrm{Ag} 09$ NUCLEAR REACTIONS ${ }^{103} \mathrm{Rh}\left(\mathrm{n}, \mathrm{n}^{\prime}\right){ }^{103 m} \mathrm{Rh}, \mathrm{E} \approx 4.8 \mathrm{MeV} ;{ }^{115} \mathrm{In}(\mathrm{n}$, $\left.\mathrm{n}^{\prime}\right){ }^{115 m} \mathrm{In}, \mathrm{E} \approx 5 \mathrm{MeV} ;{ }^{232} \mathrm{Th},{ }^{238} \mathrm{U}(\mathrm{n}, \mathrm{F}), \mathrm{E} \approx 5 \mathrm{MeV} ;{ }^{24} \mathrm{Mg},{ }^{27} \mathrm{Al}$, ${ }^{46,47,48} \mathrm{Ti},{ }^{54,56} \mathrm{Fe},{ }^{58} \mathrm{Ni},{ }^{64} \mathrm{Zn}(\mathrm{n}, \mathrm{p}), \mathrm{E} \approx 2-8 \mathrm{MeV} ;{ }^{27} \mathrm{Al},{ }^{59} \mathrm{Co}(\mathrm{n}, \alpha), \mathrm{E}$ $\approx 8.3 \mathrm{MeV}$; measured activation $\sigma$. Spectrum average technique, comparison with previous results. JOUR RAACA 9263

2005GU37 ATOMIC MASSES ${ }^{56,57} \mathrm{Mn},{ }^{82 m} \mathrm{Rb},{ }^{92} \mathrm{Sr},{ }^{124,127} \mathrm{Cs},{ }^{130} \mathrm{Ba}$; measured masses. Penning trap mass spectrometer. JOUR ZAANE 25 s01 35 NUCLEAR REACTIONS ${ }^{3,4} \mathrm{He},{ }^{12} \mathrm{C},{ }^{56} \mathrm{Fe}(\mathrm{e}, \mathrm{e}), \mathrm{E} \approx 4.4-4.7 \mathrm{GeV}$; measured relative $\sigma\left(\mathrm{Q}^{2}, \mathrm{x}\right)$; deduced 2- and 3-nucleon short range correlation probabilities. PREPRINT nucl-ex/0508026,8/24/2005 2005NEZY NUCLEAR REACTIONS Fe, ${ }^{56} \mathrm{Fe}(\mathrm{n}, \mathrm{n} \gamma), \mathrm{E} \approx 14 \mathrm{MeV}$; measured absolute $\sigma$ for production of 847-keV $\gamma$-ray. $\mathrm{Cr}(\mathrm{n}, \mathrm{n} \gamma), \mathrm{E} \approx 14 \mathrm{MeV}$; measured relative $\sigma$ for production of 1434 -keV $\gamma$-ray. Comparison with previous results. CONF Santa Fe (Nucl Data for Sci and Technol) Proc, Vol1,P838

${ }^{56} \mathrm{Co} \quad$ 2004ADZW NUCLEAR REACTIONS ${ }^{209} \mathrm{Bi}(\mathrm{n}, 4 \mathrm{n}),(\mathrm{n}, 5 \mathrm{n}),(\mathrm{n}, 6 \mathrm{n}),(\mathrm{n}, 7 \mathrm{n}),(\mathrm{n}$, $9 \mathrm{n}),{ }^{232} \mathrm{Th}(\mathrm{n}, \gamma),{ }^{197} \mathrm{Au}(\mathrm{n}, 2 \mathrm{n}),(\mathrm{n}, 4 \mathrm{n}),(\mathrm{n}, 6 \mathrm{n}),(\mathrm{n}, 7 \mathrm{n}),(\mathrm{n}, \gamma),{ }^{115} \operatorname{In}(\mathrm{n}$, $5 \mathrm{n}),(\mathrm{n}, 6 \mathrm{n}),(\mathrm{n}, 7 \mathrm{n}),{ }^{59} \mathrm{Co}(\mathrm{n}, 2 \mathrm{n}),(\mathrm{n}, 3 \mathrm{n}),(\mathrm{n}, 4 \mathrm{n}),(\mathrm{n}, 5 \mathrm{n}),(\mathrm{n}, \gamma),(\mathrm{n}$, $\mathrm{p}),(\mathrm{n}, 6 \mathrm{n} 2 \mathrm{p}), \mathrm{E}=$ spectrum; measured $\mathrm{E} \gamma, \mathrm{I} \gamma$; deduced reaction rates. $\mathrm{Pb}(\mathrm{p}, \mathrm{nX}), \mathrm{E}=1 \mathrm{GeV}$; deduced spallation neutron spectrum. REPT JINR-E1-2004-16,Adam

2005AD01 NUCLEAR REACTIONS ${ }^{209} \operatorname{Bi}(\mathrm{n}, 4 \mathrm{n}),(\mathrm{n}, 5 \mathrm{n}),(\mathrm{n}, 6 \mathrm{n}),(\mathrm{n}, 7 \mathrm{n}),(\mathrm{n}$, 9n), ${ }^{232} \mathrm{Th}(\mathrm{n}, \gamma),{ }^{197} \mathrm{Au}(\mathrm{n}, 2 \mathrm{n}),(\mathrm{n}, 4 \mathrm{n}),(\mathrm{n}, 6 \mathrm{n}),(\mathrm{n}, 7 \mathrm{n}),(\mathrm{n}, \gamma),{ }^{59} \mathrm{Co}(\mathrm{n}$, 2n), (n, 3n), (n, 4n), (n, 5n), (n, p), (n, 6n2p), ${ }^{115} \operatorname{In}(\mathrm{n}, 5 \mathrm{n}),(\mathrm{n}, 6 \mathrm{n}),(\mathrm{n}$, $7 \mathrm{n}), \mathrm{E}=$ spectrum; measured $\mathrm{E} \gamma, \mathrm{I} \gamma$; deduced reaction rates. Activation technique, spallation neutrons from $1 \mathrm{GeV}$ proton beam, comparison with model predictions. JOUR ZAANE 2361

2005B010 NUCLEAR REACTIONS Zn(p, X) ${ }^{64} \mathrm{Cu} /{ }^{57} \mathrm{Ni} /{ }^{56} \mathrm{Ni} /{ }^{52} \mathrm{Mn} /{ }^{54} \mathrm{Mn}$ $/{ }^{62} \mathrm{Zn} /{ }^{65} \mathrm{Zn} /{ }^{51} \mathrm{Cr} /{ }^{48} \mathrm{~V} /{ }^{55} \mathrm{Co} /{ }^{56} \mathrm{Co} /{ }^{57} \mathrm{Co} /{ }^{58} \mathrm{Co} /{ }^{60} \mathrm{Co} /{ }^{66} \mathrm{Ga}$ $/{ }^{67} \mathrm{Ga} /{ }^{52} \mathrm{Fe} /{ }^{59} \mathrm{Fe}, \mathrm{E} \approx 31-141 \mathrm{MeV}$; measured production $\sigma$. Stacked-foil activation. JOUR JRNCD 264101

2005Dizy NUCLEAR REACTIONS Fe $(\mathrm{p}, \mathrm{X}){ }^{57} \mathrm{Co} /{ }^{56} \mathrm{Co} /{ }^{55} \mathrm{Co} /{ }^{54} \mathrm{Mn} /{ }^{52} \mathrm{Mn}$ $/{ }^{48} \mathrm{~V} /{ }^{51} \mathrm{Cr} /{ }^{48} \mathrm{Cr} /{ }^{47} \mathrm{Sc}, \mathrm{E} \approx 20-70 \mathrm{MeV}$; measured activation $\sigma$. Comparison with previous results. CONF Santa Fe (Nucl Data for Sci and Technol) Proc,Vol1,P1011 


\section{$\mathrm{A}=56$ (continued)}

2005MIZZ NUCLEAR REACTIONS Cu(n, X) ${ }^{56} \mathrm{Co}, \mathrm{E}=40-180 \mathrm{MeV} ; \mathrm{Fe}(\mathrm{n}$, $\mathrm{X}){ }^{54} \mathrm{Mn} /{ }^{52} \mathrm{Mn} /{ }^{51} \mathrm{Cr} /{ }^{48} \mathrm{~V}, \mathrm{E} \approx 0-180 \mathrm{MeV} ; \mathrm{Pb}(\mathrm{n}, \mathrm{X}){ }^{196} \mathrm{Au} /{ }^{200} \mathrm{~Pb}$ $/{ }^{103} \mathrm{Ru}, \mathrm{E} \approx 40-180 \mathrm{MeV} ; \mathrm{U}(\mathrm{n}, \mathrm{X}){ }^{99} \mathrm{Mo}, \mathrm{E} \approx 0-180 \mathrm{MeV}$; measured excitation functions. Comparison with proton-induced reactions. CONF Santa Fe (Nucl Data for Sci and Technol) Proc,Vol1,P861 2005SEZW NUCLEAR REACTIONS ${ }^{58} \mathrm{Ni}(\mathrm{n}, \mathrm{t}),{ }^{59} \mathrm{Co}(\mathrm{n}, \mathrm{p}),{ }^{63} \mathrm{Cu}(\mathrm{n}, \alpha), \mathrm{E}=14-20$ $\mathrm{MeV}$; measured activation $\sigma$. Comparison with previous results. CONF Santa Fe (Nucl Data for Sci and Technol) Proc,Vol1,P1019 2005SI14 NUCLEAR REACTIONS C, O, Si, Mg, $\mathrm{Al}(\mathrm{n}, \mathrm{X})^{7} \mathrm{Be}, \mathrm{E} \approx 0.1-750$ $\mathrm{MeV} ; \mathrm{O}, \mathrm{Si}, \mathrm{Mg}, \mathrm{Al}(\mathrm{n}, \mathrm{X}){ }^{22} \mathrm{Na} /{ }^{23} \mathrm{Na}, \mathrm{E} \approx 0.1-750 \mathrm{MeV} ;{ }^{197} \mathrm{Au}(\mathrm{n}$, $\mathrm{X}){ }^{194} \mathrm{Au} /{ }^{196} \mathrm{Au} /{ }^{198} \mathrm{Au}, \mathrm{E} \approx 0.1-750 \mathrm{MeV} ; \mathrm{Ti}, \mathrm{Fe}, \mathrm{Ni}, \mathrm{Cu}(\mathrm{n}, \mathrm{X}){ }^{46} \mathrm{Sc}$ $/{ }^{48} \mathrm{Sc}, \mathrm{E} \approx 0.1-750 \mathrm{MeV} ; \mathrm{Fe}, \mathrm{Ni}, \mathrm{Cu}(\mathrm{n}, \mathrm{X}){ }^{48} \mathrm{~V} /{ }^{51} \mathrm{Cr} /{ }^{52} \mathrm{Mn} /{ }^{54} \mathrm{Mn}$, $\mathrm{E} \approx 0.1-750 \mathrm{MeV} ; \mathrm{Ni}, \mathrm{Cu}(\mathrm{n}, \mathrm{X}){ }^{56} \mathrm{Ni} /{ }^{57} \mathrm{Ni} /{ }^{56} \mathrm{Co} /{ }^{57} \mathrm{Co} /{ }^{58} \mathrm{Co} /$ ${ }^{60} \mathrm{Co} /{ }^{59} \mathrm{Fe}, \mathrm{E} \approx 0.1-750 \mathrm{MeV}$; measured energy-integrated production $\sigma$. JOUR NIMBE 234419

2005SI21 NUCLEAR REACTIONS Ni $(\alpha, \mathrm{X}){ }^{62} \mathrm{Zn} /{ }^{61} \mathrm{Cu} /{ }^{56} \mathrm{Ni} /{ }^{57} \mathrm{Ni} /{ }^{56} \mathrm{Co} /$ ${ }^{58} \mathrm{Co}, \mathrm{E}=21-50 \mathrm{MeV}$; measured excitation functions. Stacked-foil activation, comparison with model predictions. JOUR IMPEE 14611 2005SI32 NUCLEAR REACTIONS Cu(n, X $)^{54} \mathrm{Mn} /{ }^{59} \mathrm{Fe} /{ }^{56} \mathrm{Co} /{ }^{57} \mathrm{Co} /{ }^{58} \mathrm{Co}$ $/{ }^{60} \mathrm{Co}, \mathrm{E} \approx 70.7,110.8 \mathrm{MeV}$; measured $\sigma$. Comparison with previous results, model predictions. JOUR NIMBE 240617

2005ZHZZ NUCLEAR REACTIONS ${ }^{56,57} \mathrm{Fe},{ }^{90,94} \mathrm{Zr}(\mathrm{p}, \mathrm{n}), \mathrm{E}=7-11 \mathrm{MeV}$; measured En, $\sigma(\mathrm{E}) .{ }^{56,57} \mathrm{Co},{ }^{90,94} \mathrm{Nb}$ deduced level densities. Statistical equilibrium and pre-equilibrium model analysis. CONF Santa Fe (Nucl Data for Sci and Technol) Proc,Vol1,P931

${ }^{56} \mathrm{Ni}$ 2005B010 NUCLEAR REACTIONS Zn(p, X) ${ }^{64} \mathrm{Cu} /{ }^{57} \mathrm{Ni} /{ }^{56} \mathrm{Ni} /{ }^{52} \mathrm{Mn} /{ }^{54} \mathrm{Mn}$ $/{ }^{62} \mathrm{Zn} /{ }^{65} \mathrm{Zn} /{ }^{51} \mathrm{Cr} /{ }^{48} \mathrm{~V} /{ }^{55} \mathrm{Co} /{ }^{56} \mathrm{Co} /{ }^{57} \mathrm{Co} /{ }^{58} \mathrm{Co} /{ }^{60} \mathrm{Co} /{ }^{66} \mathrm{Ga}$ $/{ }^{67} \mathrm{Ga} /{ }^{52} \mathrm{Fe} /{ }^{59} \mathrm{Fe}, \mathrm{E} \approx 31-141 \mathrm{MeV}$; measured production $\sigma$.

Stacked-foil activation. JOUR JRNCD 264101

2005BRZU NUCLEAR REACTIONS Ti(p, X) ${ }^{44} \mathrm{Ti}, \mathrm{E}=21-29 \mathrm{MeV} ; \mathrm{Ni}(\mathrm{p}, \mathrm{X})^{56} \mathrm{Ni}$, $\mathrm{E}=18-28 \mathrm{MeV} ; \mathrm{Zr}(\mathrm{p}, \mathrm{X})^{88} \mathrm{Zr}, \mathrm{E}=19-28 \mathrm{MeV}$; measured production $\sigma$. Activation technique, comparison with previous results. CONF Santa Fe (Nucl Data for Sci and Technol) Proc,Vol2,P1374

$2005 \mathrm{GA} 15$ NUCLEAR REACTIONS ${ }^{197} \mathrm{Au}\left({ }^{52} \mathrm{Fe},{ }^{52} \mathrm{Fe}\right),\left({ }^{54} \mathrm{Ni},{ }^{54} \mathrm{Ni}\right),\left({ }^{56} \mathrm{Ni}\right.$, $\left.{ }^{56} \mathrm{Ni}^{\prime}\right),\left({ }^{58} \mathrm{Ni},{ }^{58} \mathrm{Ni}\right.$ '), E not given; measured $\mathrm{E} \gamma, \mathrm{I} \gamma$, (particle) $\gamma$-coin following projectile Coulomb excitation. ${ }^{52} \mathrm{Fe},{ }^{54,56,58} \mathrm{Ni}$ transitions deduced $\mathrm{B}(\mathrm{E} 2) .{ }^{9} \mathrm{Be}\left({ }^{32} \mathrm{~S},{ }^{31} \mathrm{SX}\right),\left({ }^{33} \mathrm{Cl},{ }^{32} \mathrm{ClX}\right),\left({ }^{34} \mathrm{Ar},{ }^{33} \mathrm{ArX}\right)$, E not given; measured one-neutron removal $\sigma$. JOUR APOBB 361227

2005SI14 NUCLEAR REACTIONS C, O, Si, Mg, $\mathrm{Al}(\mathrm{n}, \mathrm{X})^{7} \mathrm{Be}, \mathrm{E} \approx 0.1-750$ $\mathrm{MeV} ; \mathrm{O}, \mathrm{Si}, \mathrm{Mg}, \mathrm{Al}(\mathrm{n}, \mathrm{X}){ }^{22} \mathrm{Na} /{ }^{23} \mathrm{Na}, \mathrm{E} \approx 0.1-750 \mathrm{MeV} ;{ }^{197} \mathrm{Au}(\mathrm{n}$ $\mathrm{X}){ }^{194} \mathrm{Au} /{ }^{196} \mathrm{Au} /{ }^{198} \mathrm{Au}, \mathrm{E} \approx 0.1-750 \mathrm{MeV} ; \mathrm{Ti}, \mathrm{Fe}, \mathrm{Ni}, \mathrm{Cu}(\mathrm{n}, \mathrm{X})^{46} \mathrm{Sc}$ $/{ }^{48} \mathrm{Sc}, \mathrm{E} \approx 0.1-750 \mathrm{MeV} ; \mathrm{Fe}, \mathrm{Ni}, \mathrm{Cu}(\mathrm{n}, \mathrm{X}){ }^{48} \mathrm{~V} /{ }^{51} \mathrm{Cr} /{ }^{52} \mathrm{Mn} /{ }^{54} \mathrm{Mn}$, $\mathrm{E} \approx 0.1-750 \mathrm{MeV} ; \mathrm{Ni}, \mathrm{Cu}(\mathrm{n}, \mathrm{X}){ }^{56} \mathrm{Ni} /{ }^{57} \mathrm{Ni} /{ }^{56} \mathrm{Co} /{ }^{57} \mathrm{Co} /{ }^{58} \mathrm{Co} /$ ${ }^{60} \mathrm{Co} /{ }^{59} \mathrm{Fe}, \mathrm{E} \approx 0.1-750 \mathrm{MeV}$; measured energy-integrated production $\sigma$. JOUR NIMBE 234419

2005SI21 NUCLEAR REACTIONS Ni $(\alpha, \mathrm{X}){ }^{62} \mathrm{Zn} /{ }^{61} \mathrm{Cu} /{ }^{56} \mathrm{Ni} /{ }^{57} \mathrm{Ni} /{ }^{56} \mathrm{Co} /$ ${ }^{58} \mathrm{Co}, \mathrm{E}=21-50 \mathrm{MeV}$; measured excitation functions. Stacked-foil activation, comparison with model predictions. JOUR IMPEE 14611 


\section{$\mathrm{A}=56$ (continued)}

2005TAZZ NUCLEAR REACTIONS ${ }^{13} \mathrm{C},{ }^{56} \mathrm{Fe}\left({ }^{11} \mathrm{~B},{ }^{11} \mathrm{Li}\right), \mathrm{E}=758 \mathrm{MeV}$; measured $\sigma(\mathrm{E})$; deduced Gamow-Teller resonance, IAS features. CONF Riken(Origin of Matter) Proc,P533,Takahisa

\section{$\mathrm{A}=57$}

${ }^{57} \mathrm{Sc}$

2005GA01

${ }^{57} \mathrm{Ti}$

2005GA01

${ }^{57} \mathrm{~V}$

${ }^{57} \mathrm{Cr}$

2005DE34

2005LI53

${ }^{57} \mathrm{Mn} \quad 2005 \mathrm{GU} 37$

${ }^{57} \mathrm{Fe} \quad$ 2005BEZT

${ }^{57} \mathrm{Co} \quad 2004 \mathrm{ADZW}$
RADIOACTIVITY ${ }^{57,58} \mathrm{Sc},{ }^{58,59,60} \mathrm{Ti},{ }^{61} \mathrm{~V},{ }^{62,63,64,65,66} \mathrm{Cr}\left(\beta^{-}\right)$[from $\left.{ }^{58} \mathrm{Ni}\left({ }^{76} \mathrm{Ge}, \mathrm{X}\right)\right]$; measured $\mathrm{E} \gamma, \mathrm{E} \beta, \beta \gamma$-coin, $\mathrm{T}_{1 / 2} \cdot{ }^{58} \mathrm{~V},{ }^{61} \mathrm{Cr},{ }^{62} \mathrm{Mn}$ deduced levels, J, $\pi .{ }^{58} \mathrm{~V},{ }^{61} \mathrm{Cr},{ }^{62,63,64,65} \mathrm{Mn}$ deduced transitions. ${ }^{60} \mathrm{~V}$, ${ }^{62} \mathrm{Mn}$ deduced isomeric states. Comparison with model predictions. JOUR ZAANE 2341 NUCLEAR REACTIONS ${ }^{58} \mathrm{Ni}\left({ }^{76} \mathrm{Ge}, \mathrm{X}\right){ }^{57} \mathrm{Sc} /{ }^{58} \mathrm{Sc} /{ }^{58} \mathrm{Ti} /{ }^{59} \mathrm{Ti} /$ ${ }^{60} \mathrm{Ti} /{ }^{60} \mathrm{~V} /{ }^{61} \mathrm{~V} /{ }^{62} \mathrm{~V} /{ }^{63} \mathrm{~V} /{ }^{62} \mathrm{Cr} /{ }^{63} \mathrm{Cr} /{ }^{64} \mathrm{Cr} /{ }^{65} \mathrm{Cr} /{ }^{66} \mathrm{Cr} /{ }^{65} \mathrm{Mn}$ / ${ }^{66} \mathrm{Mn}, \mathrm{E}=61.8 \mathrm{MeV}$ / nucleon; measured yields. JOUR ZAANE 2341 RADIOACTIVITY ${ }^{57,58} \mathrm{Sc},{ }^{58,59,60} \mathrm{Ti},{ }^{61} \mathrm{~V},{ }^{62,63,64,65,66} \mathrm{Cr}\left(\beta^{-}\right)$[from $\left.{ }^{58} \mathrm{Ni}\left({ }^{76} \mathrm{Ge}, \mathrm{X}\right)\right]$; measured $\mathrm{E} \gamma, \mathrm{E} \beta, \beta \gamma$-coin, $\mathrm{T}_{1 / 2} .{ }^{58} \mathrm{~V},{ }^{61} \mathrm{Cr},{ }^{62} \mathrm{Mn}$ deduced levels, J, $\pi .{ }^{58} \mathrm{~V},{ }^{61} \mathrm{Cr},{ }^{62,63,64,65} \mathrm{Mn}$ deduced transitions. ${ }^{60} \mathrm{~V}$, ${ }^{62} \mathrm{Mn}$ deduced isomeric states. Comparison with model predictions. JOUR ZAANE 2341 RADIOACTIVITY ${ }^{57} \mathrm{Ti},{ }^{59} \mathrm{~V},{ }^{59} \mathrm{Cr}\left(\beta^{-}\right)$[from $\left.\mathrm{Be}\left({ }^{86} \mathrm{Kr}, \mathrm{X}\right)\right]$; measured $\beta$-delayed $\mathrm{E} \gamma, \mathrm{I} \gamma, \gamma \gamma$-coin, $\mathrm{T}_{1 / 2}$; deduced log ft. ${ }^{57} \mathrm{~V},{ }^{59} \mathrm{Cr},{ }^{59} \mathrm{Mn}$ deduced levels, $\beta$-feeding intensities, deformation. Comparison with shell-model predictions. JOUR PRVCA 72054321

RADIOACTIVITY ${ }^{57} \mathrm{Ti},{ }^{59} \mathrm{~V},{ }^{59} \mathrm{Cr}\left(\beta^{-}\right)$[from $\mathrm{Be}\left({ }^{86} \mathrm{Kr}, \mathrm{X}\right)$ ]; measured $\beta$-delayed $\mathrm{E} \gamma, \mathrm{I} \gamma, \gamma \gamma$-coin, $\mathrm{T}_{1 / 2}$; deduced $\log \mathrm{ft} .{ }^{57} \mathrm{~V},{ }^{59} \mathrm{Cr},{ }^{59} \mathrm{Mn}$ deduced levels, $\beta$-feeding intensities, deformation. Comparison with shell-model predictions. JOUR PRVCA 72054321

NUCLEAR REACTIONS ${ }^{14} \mathrm{C}\left({ }^{48} \mathrm{Ca}, \mathrm{n} \alpha\right), \mathrm{E}=130 \mathrm{MeV}$; measured $\mathrm{E} \gamma$, $\mathrm{I} \gamma, \gamma \gamma$-, (recoil) $\gamma$-coin. ${ }^{57} \mathrm{Cr}$ deduced high-spin levels, J, $\pi$, configurations. Gammasphere array, mass separator. JOUR PYLBB 622151 ATOMIC MASSES ${ }^{56,57} \mathrm{Cr}$; measured masses. Penning trap mass spectrometer. JOUR JPGPE 31 S1765 ATOMIC MASSES ${ }^{56,57} \mathrm{Mn},{ }^{82 m} \mathrm{Rb},{ }^{92} \mathrm{Sr},{ }^{124,127} \mathrm{Cs},{ }^{130} \mathrm{Ba}$; measured masses. Penning trap mass spectrometer. JOUR ZAANE 25 s01 35 NUCLEAR REACTIONS ${ }^{35} \mathrm{Cl}(\mathrm{n}, \gamma)$, E not given; measured $\mathrm{E} \gamma$, I $\gamma$. ${ }^{36} \mathrm{Cl}$ deduced transitions, level energies, binding energy. ${ }^{52,54} \mathrm{Cr},{ }^{56} \mathrm{Fe}$, ${ }^{206} \mathrm{~Pb}(\mathrm{n}, \gamma)$, E not given; analyzed $\mathrm{E} \gamma \cdot{ }^{53,55} \mathrm{Cr},{ }^{57} \mathrm{Fe},{ }^{207} \mathrm{~Pb}$ deduced binding energies. GAMS4 spectrometer. CONF Santa Fe (Nucl Data for Sci and Technol) Proc,Vol1,P1074

NUCLEAR REACTIONS ${ }^{209} \operatorname{Bi}(\mathrm{n}, 4 \mathrm{n}),(\mathrm{n}, 5 \mathrm{n}),(\mathrm{n}, 6 \mathrm{n}),(\mathrm{n}, 7 \mathrm{n}),(\mathrm{n}$, 9n), ${ }^{232} \mathrm{Th}(\mathrm{n}, \gamma),{ }^{197} \mathrm{Au}(\mathrm{n}, 2 \mathrm{n}),(\mathrm{n}, 4 \mathrm{n}),(\mathrm{n}, 6 \mathrm{n}),(\mathrm{n}, 7 \mathrm{n}),(\mathrm{n}, \gamma),{ }^{115} \mathrm{In}(\mathrm{n}$, 5n), (n, 6n), (n, 7n), ${ }^{59} \operatorname{Co}(\mathrm{n}, 2 \mathrm{n}),(\mathrm{n}, 3 \mathrm{n}),(\mathrm{n}, 4 \mathrm{n}),(\mathrm{n}, 5 \mathrm{n}),(\mathrm{n}, \gamma),(\mathrm{n}$, $\mathrm{p}),(\mathrm{n}, 6 \mathrm{n} 2 \mathrm{p}), \mathrm{E}=$ spectrum; measured $\mathrm{E} \gamma, \mathrm{I} \gamma$; deduced reaction rates. $\mathrm{Pb}(\mathrm{p}, \mathrm{nX}), \mathrm{E}=1 \mathrm{GeV}$; deduced spallation neutron spectrum. REPT JINR-E1-2004-16,Adam 


\section{$\mathrm{A}=57$ (continued)}

2005AD01 NUCLEAR REACTIONS ${ }^{209} \operatorname{Bi}(\mathrm{n}, 4 \mathrm{n}),(\mathrm{n}, 5 \mathrm{n}),(\mathrm{n}, 6 \mathrm{n}),(\mathrm{n}, 7 \mathrm{n}),(\mathrm{n}$, 9n), ${ }^{232} \mathrm{Th}(\mathrm{n}, \gamma),{ }^{197} \mathrm{Au}(\mathrm{n}, 2 \mathrm{n}),(\mathrm{n}, 4 \mathrm{n}),(\mathrm{n}, 6 \mathrm{n}),(\mathrm{n}, 7 \mathrm{n}),(\mathrm{n}, \gamma),{ }^{59} \mathrm{Co}(\mathrm{n}$, 2n), (n, 3n), (n, 4n), (n, 5n), (n, p), (n, 6n2p), ${ }^{115} \operatorname{In}(\mathrm{n}, 5 \mathrm{n}),(\mathrm{n}, 6 \mathrm{n}),(\mathrm{n}$, $7 \mathrm{n}), \mathrm{E}=$ spectrum; measured $\mathrm{E} \gamma, \mathrm{I} \gamma$; deduced reaction rates. Activation technique, spallation neutrons from $1 \mathrm{GeV}$ proton beam, comparison with model predictions. JOUR ZAANE 2361

2005B010 NUCLEAR REACTIONS Zn(p, X) ${ }^{64} \mathrm{Cu} /{ }^{57} \mathrm{Ni} /{ }^{56} \mathrm{Ni} /{ }^{52} \mathrm{Mn} /{ }^{54} \mathrm{Mn}$ $/{ }^{62} \mathrm{Zn} /{ }^{65} \mathrm{Zn} /{ }^{51} \mathrm{Cr} /{ }^{48} \mathrm{~V} /{ }^{55} \mathrm{Co} /{ }^{56} \mathrm{Co} /{ }^{57} \mathrm{Co} /{ }^{58} \mathrm{Co} /{ }^{60} \mathrm{Co} /{ }^{66} \mathrm{Ga}$ $/{ }^{67} \mathrm{Ga} /{ }^{52} \mathrm{Fe} /{ }^{59} \mathrm{Fe}, \mathrm{E} \approx 31-141 \mathrm{MeV}$; measured production $\sigma$.

Stacked-foil activation. JOUR JRNCD 264101

2005DIZY NUCLEAR REACTIONS Fe(p, X) ${ }^{57} \mathrm{Co} /{ }^{56} \mathrm{Co} /{ }^{55} \mathrm{Co} /{ }^{54} \mathrm{Mn} /{ }^{52} \mathrm{Mn}$ $/{ }^{48} \mathrm{~V} /{ }^{51} \mathrm{Cr} /{ }^{48} \mathrm{Cr} /{ }^{47} \mathrm{Sc}, \mathrm{E} \approx 20-70 \mathrm{MeV}$; measured activation $\sigma$. Comparison with previous results. CONF Santa Fe (Nucl Data for Sci and Technol) Proc,Vol1,P1011

2005SI14 NUCLEAR REACTIONS C, O, Si, Mg, $\mathrm{Al}(\mathrm{n}, \mathrm{X})^{7} \mathrm{Be}, \mathrm{E} \approx 0.1-750$ $\mathrm{MeV} ; \mathrm{O}, \mathrm{Si}, \mathrm{Mg}, \mathrm{Al}(\mathrm{n}, \mathrm{X}){ }^{22} \mathrm{Na} /{ }^{23} \mathrm{Na}, \mathrm{E} \approx 0.1-750 \mathrm{MeV} ;{ }^{197} \mathrm{Au}(\mathrm{n}$, $\mathrm{X})^{194} \mathrm{Au} /{ }^{196} \mathrm{Au} /{ }^{198} \mathrm{Au}, \mathrm{E} \approx 0.1-750 \mathrm{MeV} ; \mathrm{Ti}, \mathrm{Fe}, \mathrm{Ni}, \mathrm{Cu}(\mathrm{n}, \mathrm{X})^{46} \mathrm{Sc}$ $/{ }^{48} \mathrm{Sc}, \mathrm{E} \approx 0.1-750 \mathrm{MeV} ; \mathrm{Fe}, \mathrm{Ni}, \mathrm{Cu}(\mathrm{n}, \mathrm{X}){ }^{48} \mathrm{~V} /{ }^{51} \mathrm{Cr} /{ }^{52} \mathrm{Mn} /{ }^{54} \mathrm{Mn}$, $\mathrm{E} \approx 0.1-750 \mathrm{MeV} ; \mathrm{Ni}, \mathrm{Cu}(\mathrm{n}, \mathrm{X}){ }^{56} \mathrm{Ni} /{ }^{57} \mathrm{Ni} /{ }^{56} \mathrm{Co} /{ }^{57} \mathrm{Co} /{ }^{58} \mathrm{Co} /$ ${ }^{60} \mathrm{Co} /{ }^{59} \mathrm{Fe}, \mathrm{E} \approx 0.1-750 \mathrm{MeV}$; measured energy-integrated production $\sigma$. JOUR NIMBE 234419

2005SI32 NUCLEAR REACTIONS Cu(n, X ${ }^{54} \mathrm{Mn} /{ }^{59} \mathrm{Fe} /{ }^{56} \mathrm{Co} /{ }^{57} \mathrm{Co} /{ }^{58} \mathrm{Co}$ $/{ }^{60} \mathrm{Co}, \mathrm{E} \approx 70.7,110.8 \mathrm{MeV}$; measured $\sigma$. Comparison with previous results, model predictions. JOUR NIMBE 240617

2005ZHZZ NUCLEAR REACTIONS ${ }^{56,57} \mathrm{Fe},{ }^{90,94} \mathrm{Zr}(\mathrm{p}, \mathrm{n}), \mathrm{E}=7-11 \mathrm{MeV}$; measured En, $\sigma(\mathrm{E}) .{ }^{56,57} \mathrm{Co},{ }^{90,94} \mathrm{Nb}$ deduced level densities. Statistical equilibrium and pre-equilibrium model analysis. CONF Santa Fe (Nucl Data for Sci and Technol) Proc,Vol1,P931

${ }^{57} \mathrm{Ni}$ 2005B010 NUCLEAR REACTIONS Zn(p, X) ${ }^{64} \mathrm{Cu} /{ }^{57} \mathrm{Ni} /{ }^{56} \mathrm{Ni} /{ }^{52} \mathrm{Mn} /{ }^{54} \mathrm{Mn}$ $/{ }^{62} \mathrm{Zn} /{ }^{65} \mathrm{Zn} /{ }^{51} \mathrm{Cr} /{ }^{48} \mathrm{~V} /{ }^{55} \mathrm{Co} /{ }^{56} \mathrm{Co} /{ }^{57} \mathrm{Co} /{ }^{58} \mathrm{Co} /{ }^{60} \mathrm{Co} /{ }^{66} \mathrm{Ga}$ $/{ }^{67} \mathrm{Ga} /{ }^{52} \mathrm{Fe} /{ }^{59} \mathrm{Fe}, \mathrm{E} \approx 31-141 \mathrm{MeV}$; measured production $\sigma$. Stacked-foil activation. JOUR JRNCD 264101

2005MAZL NUCLEAR REACTIONS ${ }^{58} \mathrm{Ni}$ (polarized p, d), E $=24.6 \mathrm{MeV}$; measured $\sigma(\theta), \operatorname{Ay}(\theta) .{ }^{2} \mathrm{H}\left({ }^{54} \mathrm{Fe}, \mathrm{p}\right), \mathrm{E}=4.8 \mathrm{MeV} /$ nucleon; measured $\sigma(\theta)$. Other reactions discussed. REPT MLL 2004 Annual,P9,Mahgoub

2005SI14 NUCLEAR REACTIONS C, O, Si, Mg, Al(n, X) ${ }^{7} \mathrm{Be}, \mathrm{E} \approx 0.1-750$ $\mathrm{MeV} ; \mathrm{O}, \mathrm{Si}, \mathrm{Mg}, \mathrm{Al}(\mathrm{n}, \mathrm{X}){ }^{22} \mathrm{Na} /{ }^{23} \mathrm{Na}, \mathrm{E} \approx 0.1-750 \mathrm{MeV} ;{ }^{197} \mathrm{Au}(\mathrm{n}$, $\mathrm{X}){ }^{194} \mathrm{Au} /{ }^{196} \mathrm{Au} /{ }^{198} \mathrm{Au}, \mathrm{E} \approx 0.1-750 \mathrm{MeV} ; \mathrm{Ti}, \mathrm{Fe}, \mathrm{Ni}, \mathrm{Cu}(\mathrm{n}, \mathrm{X})^{46} \mathrm{Sc}$ $/{ }^{48} \mathrm{Sc}, \mathrm{E} \approx 0.1-750 \mathrm{MeV} ; \mathrm{Fe}, \mathrm{Ni}, \mathrm{Cu}(\mathrm{n}, \mathrm{X}){ }^{48} \mathrm{~V} /{ }^{51} \mathrm{Cr} /{ }^{52} \mathrm{Mn} /{ }^{54} \mathrm{Mn}$, $\mathrm{E} \approx 0.1-750 \mathrm{MeV} ; \mathrm{Ni}, \mathrm{Cu}(\mathrm{n}, \mathrm{X}){ }^{56} \mathrm{Ni} /{ }^{57} \mathrm{Ni} /{ }^{56} \mathrm{Co} /{ }^{57} \mathrm{Co} /{ }^{58} \mathrm{Co} /$ ${ }^{60} \mathrm{Co} /{ }^{59} \mathrm{Fe}, \mathrm{E} \approx 0.1-750 \mathrm{MeV}$; measured energy-integrated production $\sigma$. JOUR NIMBE 234419

2005SI21 NUCLEAR REACTIONS Ni $(\alpha, \mathrm{X}){ }^{62} \mathrm{Zn} /{ }^{61} \mathrm{Cu} /{ }^{56} \mathrm{Ni} /{ }^{57} \mathrm{Ni} /{ }^{56} \mathrm{Co} /$ ${ }^{58} \mathrm{Co}, \mathrm{E}=21-50 \mathrm{MeV}$; measured excitation functions. Stacked-foil activation, comparison with model predictions. JOUR IMPEE 14611 
$\mathrm{A}=58$

${ }^{58} \mathrm{Sc} \quad$ 2005GA01 RADIOACTIVITY ${ }^{57,58} \mathrm{Sc},{ }^{58,59,60} \mathrm{Ti},{ }^{61} \mathrm{~V},{ }^{62,63,64,65,66} \mathrm{Cr}\left(\beta^{-}\right)[\mathrm{from}$ $\left.{ }^{58} \mathrm{Ni}\left({ }^{76} \mathrm{Ge}, \mathrm{X}\right)\right]$; measured $\mathrm{E} \gamma, \mathrm{E} \beta, \beta \gamma$-coin, $\mathrm{T}_{1 / 2} \cdot{ }^{58} \mathrm{~V},{ }^{61} \mathrm{Cr},{ }^{62} \mathrm{Mn}$ deduced levels, J, $\pi .{ }^{58} \mathrm{~V},{ }^{61} \mathrm{Cr},{ }^{62,63,64,65} \mathrm{Mn}$ deduced transitions. ${ }^{60} \mathrm{~V}$, ${ }^{62} \mathrm{Mn}$ deduced isomeric states. Comparison with model predictions. JOUR ZAANE 2341

2005GA01 NUCLEAR REACTIONS ${ }^{58} \mathrm{Ni}\left({ }^{76} \mathrm{Ge}, \mathrm{X}\right){ }^{57} \mathrm{Sc} /{ }^{58} \mathrm{Sc} /{ }^{58} \mathrm{Ti} /{ }^{59} \mathrm{Ti} /$ ${ }^{60} \mathrm{Ti} /{ }^{60} \mathrm{~V} /{ }^{61} \mathrm{~V} /{ }^{62} \mathrm{~V} /{ }^{63} \mathrm{~V} /{ }^{62} \mathrm{Cr} /{ }^{63} \mathrm{Cr} /{ }^{64} \mathrm{Cr} /{ }^{65} \mathrm{Cr} /{ }^{66} \mathrm{Cr} /{ }^{65} \mathrm{Mn}$ / ${ }^{66} \mathrm{Mn}, \mathrm{E}=61.8 \mathrm{MeV}$ / nucleon; measured yields. JOUR ZAANE 2341 ${ }^{58} \mathrm{Ti}$ 2005GA01 RADIOACTIVITY ${ }^{57,58} \mathrm{Sc},{ }^{58,59,60} \mathrm{Ti},{ }^{61} \mathrm{~V},{ }^{62,63,64,65,66} \mathrm{Cr}\left(\beta^{-}\right)[$from $\left.{ }^{58} \mathrm{Ni}\left({ }^{76} \mathrm{Ge}, \mathrm{X}\right)\right]$; measured $\mathrm{E} \gamma, \mathrm{E} \beta, \beta \gamma$-coin, $\mathrm{T}_{1 / 2} \cdot{ }^{58} \mathrm{~V},{ }^{61} \mathrm{Cr},{ }^{62} \mathrm{Mn}$ deduced levels, J, $\pi .{ }^{58} \mathrm{~V},{ }^{61} \mathrm{Cr},{ }^{62,63,64,65} \mathrm{Mn}$ deduced transitions. ${ }^{60} \mathrm{~V}$, ${ }^{62} \mathrm{Mn}$ deduced isomeric states. Comparison with model predictions. JOUR ZAANE 2341

2005GA01 NUCLEAR REACTIONS ${ }^{58} \mathrm{Ni}\left({ }^{76} \mathrm{Ge}, \mathrm{X}\right){ }^{57} \mathrm{Sc} /{ }^{58} \mathrm{Sc} /{ }^{58} \mathrm{Ti} /{ }^{59} \mathrm{Ti} /$ ${ }^{60} \mathrm{Ti} /{ }^{60} \mathrm{~V} /{ }^{61} \mathrm{~V} /{ }^{62} \mathrm{~V} /{ }^{63} \mathrm{~V} /{ }^{62} \mathrm{Cr} /{ }^{63} \mathrm{Cr} /{ }^{64} \mathrm{Cr} /{ }^{65} \mathrm{Cr} /{ }^{66} \mathrm{Cr} /{ }^{65} \mathrm{Mn}$ / ${ }^{66} \mathrm{Mn}, \mathrm{E}=61.8 \mathrm{MeV}$ / nucleon; measured yields. JOUR ZAANE 2341

${ }^{58} \mathrm{~V}$ RADIOACTIVITY ${ }^{57,58} \mathrm{Sc},{ }^{58,59,60} \mathrm{Ti},{ }^{61} \mathrm{~V},{ }^{62,63,64,65,66} \mathrm{Cr}\left(\beta^{-}\right)$[from $\left.{ }^{58} \mathrm{Ni}\left({ }^{76} \mathrm{Ge}, \mathrm{X}\right)\right]$; measured $\mathrm{E} \gamma, \mathrm{E} \beta, \beta \gamma$-coin, $\mathrm{T}_{1 / 2} \cdot{ }^{58} \mathrm{~V},{ }^{61} \mathrm{Cr},{ }^{62} \mathrm{Mn}$ deduced levels, J, $\pi .{ }^{58} \mathrm{~V},{ }^{61} \mathrm{Cr},{ }^{62,63,64,65} \mathrm{Mn}$ deduced transitions. ${ }^{60} \mathrm{~V}$, ${ }^{62} \mathrm{Mn}$ deduced isomeric states. Comparison with model predictions. JOUR ZAANE 2341

${ }^{58} \mathrm{Cr} \quad 2005 B U 14$ NUCLEAR REACTIONS ${ }^{197} \mathrm{Au}\left({ }^{54} \mathrm{Cr},{ }^{54} \mathrm{Cr}\right),\left({ }^{56} \mathrm{Cr},{ }^{56} \mathrm{Cr}\right),\left({ }^{58} \mathrm{Cr}\right.$, $\left.{ }^{58} \mathrm{Cr}^{\prime}\right), \mathrm{E} \approx 135 \mathrm{MeV} /$ nucleon; measured measured $\mathrm{E} \gamma, \mathrm{I} \gamma$, (particle) $\gamma$-coin following projectile Coulomb excitation. ${ }^{54,56,58} \mathrm{Cr}$ deduced transitions. JOUR APOBB 361249

2005BU29 NUCLEAR REACTIONS ${ }^{197} \mathrm{Au}\left({ }^{54} \mathrm{Cr},{ }^{54} \mathrm{Cr}\right),\left({ }^{56} \mathrm{Cr},{ }^{56} \mathrm{Cr}\right),\left({ }^{58} \mathrm{Cr}\right.$, $\left.{ }^{58} \mathrm{Cr}^{\prime}\right), \mathrm{E} \approx 100 \mathrm{MeV} /$ nucleon; measured $\mathrm{E} \gamma, \mathrm{I} \gamma$, (particle) $\gamma$-coin following projectile Coulomb excitation. ${ }^{54,56,58} \mathrm{Cr}$ deduced transitions B(E2). Comparison with shell model predictions. JOUR PYLBB 622 29

2005GA44 NUCLEAR REACTIONS ${ }^{208} \mathrm{~Pb}\left({ }^{90} \mathrm{Zr}, \mathrm{X}\right){ }^{90} \mathrm{Zr} /{ }^{92} \mathrm{Zr} /{ }^{88} \mathrm{Sr}, \mathrm{E}=560$ $\mathrm{MeV} ;{ }^{238} \mathrm{U}\left({ }^{64} \mathrm{Ni}, \mathrm{X}\right){ }^{58} \mathrm{Cr}, \mathrm{E}=400 \mathrm{MeV}$; measured $\mathrm{E} \gamma, \mathrm{I} \gamma, \gamma \gamma-$, (recoil) $\gamma$-coin. ${ }^{58} \mathrm{Cr},{ }^{90,92} \mathrm{Zr},{ }^{88} \mathrm{Sr}$ deduced transitions. Clara array, mass separator. JOUR JPGPE 31 S1443

2005GA56 NUCLEAR REACTIONS ${ }^{238} \mathrm{U}\left({ }^{82} \mathrm{Se}, \mathrm{X}\right), \mathrm{E}=505 \mathrm{MeV} ;{ }^{238} \mathrm{U}\left({ }^{64} \mathrm{Ni}, \mathrm{X}\right)$, $\mathrm{E}=400 \mathrm{MeV}$; measured $\mathrm{E} \gamma, \mathrm{I} \gamma, \gamma \gamma$-, (fragment) $\gamma$-coin, projectile-like fragments isotopic yields. ${ }^{58} \mathrm{Cr},{ }^{80} \mathrm{As},{ }^{82} \mathrm{Ge},{ }^{84} \mathrm{Se}$ deduced levels, J, $\pi$. Clara array, Prisma spectrometer. JOUR ZAANE 25 s01 421

2005HUZZ NUCLEAR REACTIONS ${ }^{197} \mathrm{Au}\left({ }^{54} \mathrm{Cr},{ }^{54} \mathrm{Cr}\right),\left({ }^{56} \mathrm{Cr},{ }^{56} \mathrm{Cr}\right),\left({ }^{58} \mathrm{Cr}\right.$, $\left.{ }^{58} \mathrm{Cr}^{\prime}\right), \mathrm{E} \approx 136 \mathrm{MeV} /$ nucleon; measured $\mathrm{E} \gamma, \mathrm{I} \gamma$, (particle) $\gamma$-coin following projectile Coulomb excitation. ${ }^{54,56,58} \mathrm{Cr}$ deduced levels, B(E2). CONF Bormio (XLIII Winter Meeting) Proc,P232 


\section{$\mathrm{A}=58$ (continued)}

2005SAZY NUCLEAR REACTIONS ${ }^{197} \mathrm{Au}\left({ }^{54} \mathrm{Cr},{ }^{54} \mathrm{Cr}\right.$ ' $),\left({ }^{56} \mathrm{Cr},{ }^{56} \mathrm{Cr}\right),\left({ }^{58} \mathrm{Cr}\right.$, ${ }^{58} \mathrm{Cr}$ '), $\mathrm{E}=100 \mathrm{MeV} /$ nucleon; measured $\mathrm{E} \gamma, \mathrm{I} \gamma$, (particle) $\gamma$-coin following projectile Coulomb excitation. ${ }^{54,56,58} \mathrm{Cr}$ deduced transitions. $\mathrm{Be}\left({ }^{55} \mathrm{Ni}, \mathrm{X}\right){ }^{50} \mathrm{Cr}, \mathrm{E}=171 \mathrm{MeV} /$ nucleon; measured $\mathrm{E} \gamma, \mathrm{I} \gamma$, (particle) $\gamma$-coin. ${ }^{50} \mathrm{Cr}$ deduced transitions. $\mathrm{Be}\left({ }^{55} \mathrm{Ni}, \mathrm{X}\right),{ }^{197} \mathrm{Au}\left({ }^{108} \mathrm{Sn}\right.$, $\mathrm{X}), \mathrm{E}$ not given; measured fragment yields. CONF Argonne(Nuclei at the Limits),P151,Saito

${ }^{58} \mathrm{Co} \quad$ 2004ADZW NUCLEAR REACTIONS ${ }^{209} \operatorname{Bi}(\mathrm{n}, 4 \mathrm{n}),(\mathrm{n}, 5 \mathrm{n}),(\mathrm{n}, 6 \mathrm{n}),(\mathrm{n}, 7 \mathrm{n}),(\mathrm{n}$, 9n), ${ }^{232} \mathrm{Th}(\mathrm{n}, \gamma),{ }^{197} \mathrm{Au}(\mathrm{n}, 2 \mathrm{n}),(\mathrm{n}, 4 \mathrm{n}),(\mathrm{n}, 6 \mathrm{n}),(\mathrm{n}, 7 \mathrm{n}),(\mathrm{n}, \gamma),{ }^{115} \mathrm{In}(\mathrm{n}$, 5n), (n, 6n), (n, 7n), ${ }^{59} \mathrm{Co}(\mathrm{n}, 2 \mathrm{n}),(\mathrm{n}, 3 \mathrm{n}),(\mathrm{n}, 4 \mathrm{n}),(\mathrm{n}, 5 \mathrm{n}),(\mathrm{n}, \gamma),(\mathrm{n}$, $\mathrm{p}),(\mathrm{n}, 6 \mathrm{n} 2 \mathrm{p}), \mathrm{E}=$ spectrum; measured $\mathrm{E} \gamma, \mathrm{I} \gamma$; deduced reaction rates. $\mathrm{Pb}(\mathrm{p}, \mathrm{nX}), \mathrm{E}=1 \mathrm{GeV}$; deduced spallation neutron spectrum. REPT JINR-E1-2004-16,Adam

2005AD01 NUCLEAR REACTIONS ${ }^{209} \operatorname{Bi}(\mathrm{n}, 4 \mathrm{n}),(\mathrm{n}, 5 \mathrm{n}),(\mathrm{n}, 6 \mathrm{n}),(\mathrm{n}, 7 \mathrm{n}),(\mathrm{n}$, 9n), ${ }^{232} \mathrm{Th}(\mathrm{n}, \gamma),{ }^{197} \mathrm{Au}(\mathrm{n}, 2 \mathrm{n}),(\mathrm{n}, 4 \mathrm{n}),(\mathrm{n}, 6 \mathrm{n}),(\mathrm{n}, 7 \mathrm{n}),(\mathrm{n}, \gamma),{ }^{59} \mathrm{Co}(\mathrm{n}$, $2 \mathrm{n}),(\mathrm{n}, 3 \mathrm{n}),(\mathrm{n}, 4 \mathrm{n}),(\mathrm{n}, 5 \mathrm{n}),(\mathrm{n}, \mathrm{p}),(\mathrm{n}, 6 \mathrm{n} 2 \mathrm{p}),{ }^{115} \operatorname{In}(\mathrm{n}, 5 \mathrm{n}),(\mathrm{n}, 6 \mathrm{n}),(\mathrm{n}$, $7 \mathrm{n}), \mathrm{E}=$ spectrum; measured $\mathrm{E} \gamma, \mathrm{I} \gamma$; deduced reaction rates. Activation technique, spallation neutrons from $1 \mathrm{GeV}$ proton beam, comparison with model predictions. JOUR ZAANE 2361

2005B010 NUCLEAR REACTIONS Zn(p, X) ${ }^{64} \mathrm{Cu} /{ }^{57} \mathrm{Ni} /{ }^{56} \mathrm{Ni} /{ }^{52} \mathrm{Mn} /{ }^{54} \mathrm{Mn}$ $/{ }^{62} \mathrm{Zn} /{ }^{65} \mathrm{Zn} /{ }^{51} \mathrm{Cr} /{ }^{48} \mathrm{~V} /{ }^{55} \mathrm{Co} /{ }^{56} \mathrm{Co} /{ }^{57} \mathrm{Co} /{ }^{58} \mathrm{Co} /{ }^{60} \mathrm{Co} /{ }^{66} \mathrm{Ga}$ $/{ }^{67} \mathrm{Ga} /{ }^{52} \mathrm{Fe} /{ }^{59} \mathrm{Fe}, \mathrm{E} \approx 31-141 \mathrm{MeV}$; measured production $\sigma$.

Stacked-foil activation. JOUR JRNCD 264101

2005HA03 NUCLEAR REACTIONS ${ }^{58} \mathrm{Ni}(\mathrm{d}, 2 \mathrm{p}), \mathrm{E}=170 \mathrm{MeV}$; measured Ep, pp-coin, $\sigma(\mathrm{E}, \theta) .{ }^{58} \mathrm{Co}$ deduced levels, Gamow-Teller strengths, related features. ${ }^{58} \mathrm{Ni},{ }^{58} \mathrm{Co}$ deduced analog states Coulomb displacement energy. Comparison with large-scale shell model calculations. JOUR PRVCA 71014606

2005SI14 NUCLEAR REACTIONS C, O, Si, Mg, Al(n, X) ${ }^{7} \mathrm{Be}, \mathrm{E} \approx 0.1-750$ $\mathrm{MeV} ; \mathrm{O}, \mathrm{Si}, \mathrm{Mg}, \mathrm{Al}(\mathrm{n}, \mathrm{X}){ }^{22} \mathrm{Na} /{ }^{23} \mathrm{Na}, \mathrm{E} \approx 0.1-750 \mathrm{MeV} ;{ }^{197} \mathrm{Au}(\mathrm{n}$, $\mathrm{X}){ }^{194} \mathrm{Au} /{ }^{196} \mathrm{Au} /{ }^{198} \mathrm{Au}, \mathrm{E} \approx 0.1-750 \mathrm{MeV} ; \mathrm{Ti}, \mathrm{Fe}, \mathrm{Ni}, \mathrm{Cu}(\mathrm{n}, \mathrm{X}){ }^{46} \mathrm{Sc}$ $/{ }^{48} \mathrm{Sc}, \mathrm{E} \approx 0.1-750 \mathrm{MeV} ; \mathrm{Fe}, \mathrm{Ni}, \mathrm{Cu}(\mathrm{n}, \mathrm{X}){ }^{48} \mathrm{~V} /{ }^{51} \mathrm{Cr} /{ }^{52} \mathrm{Mn} /{ }^{54} \mathrm{Mn}$, $\mathrm{E} \approx 0.1-750 \mathrm{MeV} ; \mathrm{Ni}, \mathrm{Cu}(\mathrm{n}, \mathrm{X}){ }^{56} \mathrm{Ni} /{ }^{57} \mathrm{Ni} /{ }^{56} \mathrm{Co} /{ }^{57} \mathrm{Co} /{ }^{58} \mathrm{Co} /$ ${ }^{60} \mathrm{Co} /{ }^{59} \mathrm{Fe}, \mathrm{E} \approx 0.1-750 \mathrm{MeV}$; measured energy-integrated production $\sigma$. JOUR NIMBE 234419

2005Si21 NUCLEAR REACTIONS Ni $(\alpha, \mathrm{X}){ }^{62} \mathrm{Zn} /{ }^{61} \mathrm{Cu} /{ }^{56} \mathrm{Ni} /{ }^{57} \mathrm{Ni} /{ }^{56} \mathrm{Co} /$ ${ }^{58} \mathrm{Co}, \mathrm{E}=21-50 \mathrm{MeV}$; measured excitation functions. Stacked-foil activation, comparison with model predictions. JOUR IMPEE 14611 2005SI28 NUCLEAR REACTIONS ${ }^{51} \mathrm{~V}\left({ }^{10} \mathrm{~B}, 2 \mathrm{np}\right), \mathrm{E}=33,36 \mathrm{MeV}$; measured $\mathrm{E} \gamma, \mathrm{I} \gamma, \gamma \gamma$-coin, DSA. ${ }^{58}$ Co deduced levels, $\mathrm{J}, \pi, \mathrm{T}_{1 / 2}, \mathrm{~B}(\mathrm{M} 1)$. Comparison with shell model predictions. JOUR JPGPE 31 S1577 2005SI32 NUCLEAR REACTIONS Cu(n, X) ${ }^{54} \mathrm{Mn} /{ }^{59} \mathrm{Fe} /{ }^{56} \mathrm{Co} /{ }^{57} \mathrm{Co} /{ }^{58} \mathrm{Co}$ $/{ }^{60} \mathrm{Co}, \mathrm{E} \approx 70.7,110.8 \mathrm{MeV}$; measured $\sigma$. Comparison with previous results, model predictions. JOUR NIMBE 240617

2005SI37 NUCLEAR REACTIONS ${ }^{51} \mathrm{~V}\left({ }^{10} \mathrm{~B}, 2 \mathrm{np}\right), \mathrm{E}=33 \mathrm{MeV}$; measured $\mathrm{E} \gamma, \mathrm{I} \gamma$, $\gamma \gamma-$, (charged particle) $\gamma$-coin. ${ }^{58} \mathrm{Co}$ deduced levels, $\mathrm{J}, \pi$, configurations. Comparison with shell model predictions. JOUR BJPHE 35821 


\section{$\mathrm{A}=58$ (continued)}

2005ZE04 NUCLEAR REACTIONS ${ }^{58} \mathrm{Ni}\left(\mathrm{t},{ }^{3} \mathrm{He}\right), \mathrm{E}=112 \mathrm{MeV} /$ nucleon; measured $\sigma(\mathrm{E}, \theta)$; deduced Gamow-Teller strength distribution. JOUR NUPAB $75867 \mathrm{c}$

${ }^{58} \mathrm{Ni} \quad 2005 \mathrm{ALO3}$

NUCLEAR REACTIONS ${ }^{58} \mathrm{Ni}\left({ }^{16} \mathrm{O},{ }^{16} \mathrm{O}\right),\left({ }^{16} \mathrm{O},{ }^{16} \mathrm{O}\right),\left({ }^{16} \mathrm{O},{ }^{12} \mathrm{C}\right)$, $\mathrm{E}=46 \mathrm{MeV} ;{ }^{58} \mathrm{Ni}\left({ }^{18} \mathrm{O},{ }^{18} \mathrm{O}\right),\left({ }^{18} \mathrm{O},{ }^{18} \mathrm{O}\right),\left({ }^{18} \mathrm{O},{ }^{17} \mathrm{O}\right),\left({ }^{18} \mathrm{O},{ }^{16} \mathrm{O}\right), \mathrm{E}=46$ $\mathrm{MeV}$; measured elastic, inelastic, and transfer $\sigma(\mathrm{E}, \theta)$.

Coupled-channels analysis, comparison with previous results. JOUR NUPAB 74859

2005AL45 NUCLEAR REACTIONS ${ }^{58} \mathrm{Ni}\left({ }^{16} \mathrm{O},{ }^{16} \mathrm{O}\right),\left({ }^{16} \mathrm{O},{ }^{16} \mathrm{O}\right),\left({ }^{16} \mathrm{O}, \mathrm{X}\right),\left({ }^{18} \mathrm{O}\right.$, $\left.{ }^{18} \mathrm{O}\right),\left({ }^{18} \mathrm{O},{ }^{18} \mathrm{O}\right),\left({ }^{18} \mathrm{O}, \mathrm{X}\right), \mathrm{E}=46 \mathrm{MeV}$; measured elastic, inelastic, and transfer $\sigma(\theta)$. Comparison with model predictions. JOUR BJPHE 35909

$2005 \mathrm{CO} 22$ NUCLEAR REACTIONS ${ }^{58} \mathrm{Ni}(\mathrm{p}, \mathrm{p} \gamma),(\mathrm{p}, \mathrm{n} \gamma), \mathrm{E}=14 \mathrm{MeV}$; measured prompt and delayed $\mathrm{E} \gamma, \mathrm{I} \gamma .{ }^{58} \mathrm{Cu}$ level deduced $\mathrm{T}_{1 / 2}, \mathrm{~B}(\mathrm{E} 2)$, collective features. Comparison with model predictions. JOUR PRVCA 72 054305

2005GA15 NUCLEAR REACTIONS ${ }^{197} \mathrm{Au}\left({ }^{52} \mathrm{Fe},{ }^{52} \mathrm{Fe}\right),\left({ }^{54} \mathrm{Ni},{ }^{54} \mathrm{Ni}\right),\left({ }^{56} \mathrm{Ni}\right.$, $\left.{ }^{56} \mathrm{Ni}^{\prime}\right),\left({ }^{58} \mathrm{Ni},{ }^{58} \mathrm{Ni}\right.$ '), E not given; measured $\mathrm{E} \gamma, \mathrm{I} \gamma$, (particle) $\gamma$-coin following projectile Coulomb excitation. ${ }^{52} \mathrm{Fe},{ }^{54,56,58} \mathrm{Ni}$ transitions deduced $\mathrm{B}(\mathrm{E} 2) .{ }^{9} \mathrm{Be}\left({ }^{32} \mathrm{~S},{ }^{31} \mathrm{SX}\right),\left({ }^{33} \mathrm{Cl},{ }^{32} \mathrm{ClX}\right),\left({ }^{34} \mathrm{Ar},{ }^{33} \mathrm{ArX}\right)$, E not given; measured one-neutron removal $\sigma$. JOUR APOBB 361227

2005HA03 NUCLEAR REACTIONS ${ }^{58} \mathrm{Ni}(\mathrm{d}, 2 \mathrm{p}), \mathrm{E}=170 \mathrm{MeV}$; measured Ep, pp-coin, $\sigma(\mathrm{E}, \theta) .{ }^{58} \mathrm{Co}$ deduced levels, Gamow-Teller strengths, related features. ${ }^{58} \mathrm{Ni},{ }^{58} \mathrm{Co}$ deduced analog states Coulomb displacement energy. Comparison with large-scale shell model calculations. JOUR PRVCA 71014606

2005H010 NUCLEAR REACTIONS ${ }^{58} \mathrm{Ni}$ (polarized p, p'), (polarized p, p), $\mathrm{E}=172 \mathrm{MeV}$; measured elastic and inelastic $\sigma(\mathrm{E}, \theta)$, analyzing powers. Comparison with model predictions. JOUR PYLBB 612165

${ }^{58} \mathrm{Cu} \quad 2005 \mathrm{CO} 22$ NUCLEAR REACTIONS ${ }^{58} \mathrm{Ni}(\mathrm{p}, \mathrm{p} \gamma),(\mathrm{p}, \mathrm{n} \gamma), \mathrm{E}=14 \mathrm{MeV}$; measured prompt and delayed $\mathrm{E} \gamma, \mathrm{I} \gamma .{ }^{58} \mathrm{Cu}$ level deduced $\mathrm{T}_{1 / 2}, \mathrm{~B}(\mathrm{E} 2)$, collective features. Comparison with model predictions. JOUR PRVCA 72 054305

2005KA46 RADIOACTIVITY ${ }^{31} \mathrm{Cl}\left(\beta^{+} \mathrm{p}\right)$ [from $\mathrm{S}(\mathrm{p}, \mathrm{X}), \mathrm{E}=40 \mathrm{MeV}$ ]; measured $\beta$-delayed $\mathrm{E} \gamma, \mathrm{Ep} .{ }^{58} \mathrm{Zn}\left(\beta^{+}\right)$[from $\mathrm{Nb}(\mathrm{p}, \mathrm{X}), \mathrm{E}=1.4 \mathrm{GeV}$ ]; measured $\mathrm{E} \gamma, \mathrm{I} \gamma, \beta \gamma$-coin, $\mathrm{T}_{1 / 2} .{ }^{58} \mathrm{Cu}$ deduced levels, $\beta$-feeding intensities. ${ }^{81 m} \mathrm{Kr}(\mathrm{EC}),(\mathrm{IT}) ;{ }^{81} \mathrm{Y},{ }^{81} \mathrm{Sr},{ }^{85} \mathrm{Nb},{ }^{85} \mathrm{Zr},{ }^{86} \mathrm{Mo},{ }^{86} \mathrm{Nb}(\mathrm{EC})$ [from $\mathrm{Ni}$, $\left.{ }^{54} \mathrm{Fe}\left({ }^{32} \mathrm{~S}, \mathrm{X}\right)\right]$; measured $\mathrm{E} \gamma, \mathrm{I} \gamma, \mathrm{E}(\mathrm{ce}), \mathrm{I}(\mathrm{ce}), \mathrm{T}_{1 / 2} \cdot{ }^{81} \mathrm{Kr},{ }^{85} \mathrm{Zr},{ }^{85} \mathrm{Nb}$ deduced isomeric transitions $\mathrm{T}_{1 / 2}$, ICC. ${ }^{85} \mathrm{Zr},{ }^{86} \mathrm{Nb}$ deduced levels, $\mathrm{J}$, $\pi$, ICC. ${ }^{81} \mathrm{Br}$ deduced neutrino capture rate. Mass-separated sources. JOUR ZAANE 25 s01 129

${ }^{58} \mathrm{Zn} \quad$ 2005KA46 RADIOACTIVITY ${ }^{31} \mathrm{Cl}\left(\beta^{+} \mathrm{p}\right)$ [from $\mathrm{S}(\mathrm{p}, \mathrm{X}), \mathrm{E}=40 \mathrm{MeV}$ ]; measured $\beta$-delayed $\mathrm{E} \gamma, \mathrm{Ep} .{ }^{58} \mathrm{Zn}\left(\beta^{+}\right)$[from $\mathrm{Nb}(\mathrm{p}, \mathrm{X}), \mathrm{E}=1.4 \mathrm{GeV}$ ]; measured $\mathrm{E} \gamma, \mathrm{I} \gamma, \beta \gamma$-coin, $\mathrm{T}_{1 / 2} .{ }^{58} \mathrm{Cu}$ deduced levels, $\beta$-feeding intensities. ${ }^{81 m} \mathrm{Kr}(\mathrm{EC}),(\mathrm{IT}) ;{ }^{81} \mathrm{Y},{ }^{81} \mathrm{Sr},{ }^{85} \mathrm{Nb},{ }^{85} \mathrm{Zr},{ }^{86} \mathrm{Mo},{ }^{86} \mathrm{Nb}$ (EC) [from $\mathrm{Ni}$, $\left.{ }^{54} \mathrm{Fe}\left({ }^{32} \mathrm{~S}, \mathrm{X}\right)\right]$; measured $\mathrm{E} \gamma, \mathrm{I} \gamma, \mathrm{E}(\mathrm{ce}), \mathrm{I}(\mathrm{ce}), \mathrm{T}_{1 / 2} \cdot{ }^{81} \mathrm{Kr},{ }^{85} \mathrm{Zr},{ }^{85} \mathrm{Nb}$ deduced isomeric transitions $\mathrm{T}_{1 / 2}$, ICC. ${ }^{85} \mathrm{Zr},{ }^{86} \mathrm{Nb}$ deduced levels, $\mathrm{J}$, $\pi$, ICC. ${ }^{81} \mathrm{Br}$ deduced neutrino capture rate. Mass-separated sources. JOUR ZAANE 25 s01 129 


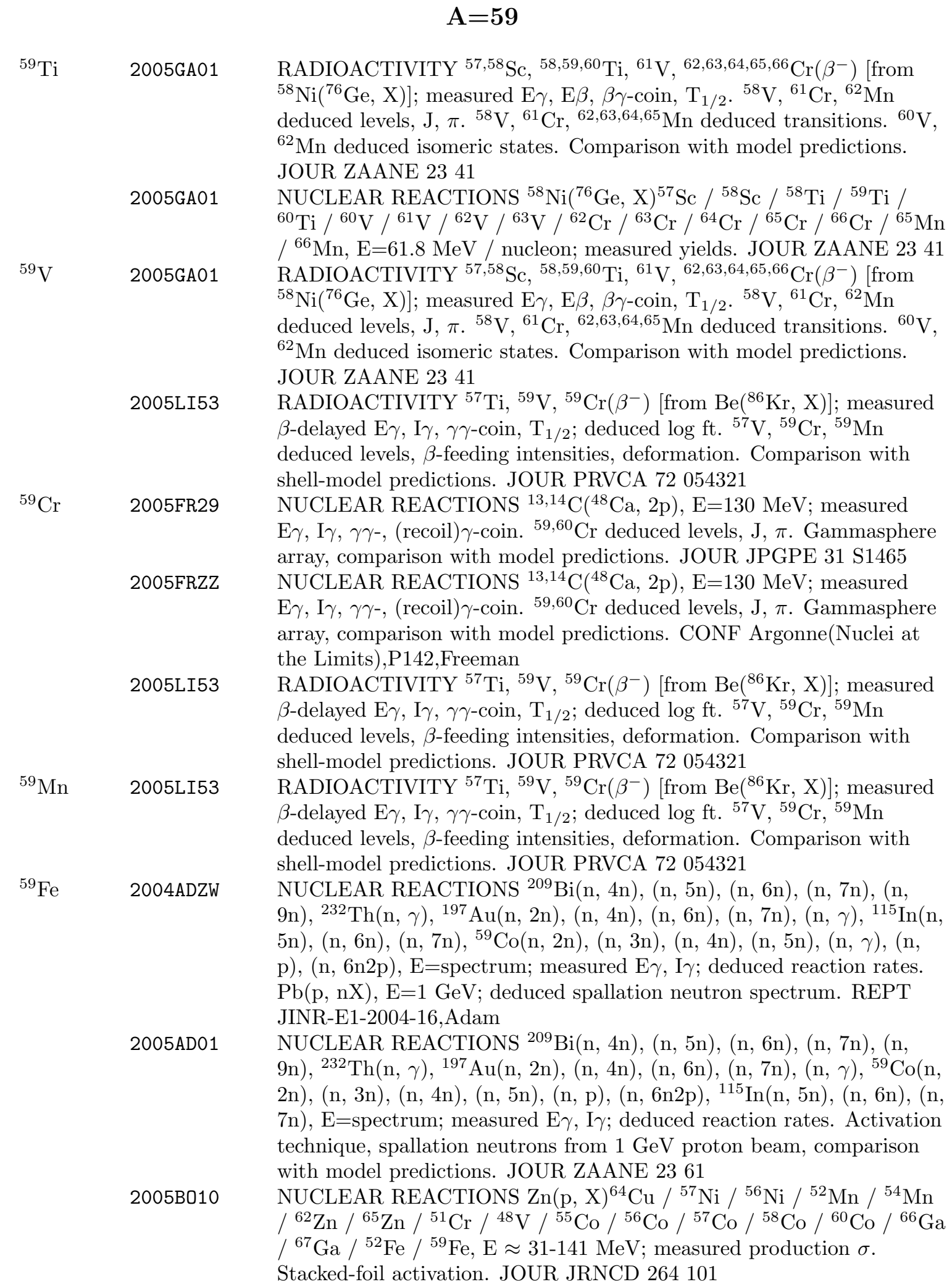




\section{$\mathrm{A}=59$ (continued)}

2005SEZW NUCLEAR REACTIONS ${ }^{58} \mathrm{Ni}(\mathrm{n}, \mathrm{t}),{ }^{59} \mathrm{Co}(\mathrm{n}, \mathrm{p}),{ }^{63} \mathrm{Cu}(\mathrm{n}, \alpha), \mathrm{E}=14-20$ $\mathrm{MeV}$; measured activation $\sigma$. Comparison with previous results. CONF Santa Fe (Nucl Data for Sci and Technol) Proc,Vol1,P1019

2005SI14 NUCLEAR REACTIONS C, O, Si, Mg, $\mathrm{Al}(\mathrm{n}, \mathrm{X})^{7} \mathrm{Be}, \mathrm{E} \approx 0.1-750$ $\mathrm{MeV} ; \mathrm{O}, \mathrm{Si}, \mathrm{Mg}, \mathrm{Al}(\mathrm{n}, \mathrm{X}){ }^{22} \mathrm{Na} /{ }^{23} \mathrm{Na}, \mathrm{E} \approx 0.1-750 \mathrm{MeV} ;{ }^{197} \mathrm{Au}(\mathrm{n}$, $\mathrm{X}){ }^{194} \mathrm{Au} /{ }^{196} \mathrm{Au} /{ }^{198} \mathrm{Au}, \mathrm{E} \approx 0.1-750 \mathrm{MeV} ; \mathrm{Ti}, \mathrm{Fe}, \mathrm{Ni}, \mathrm{Cu}(\mathrm{n}, \mathrm{X})^{46} \mathrm{Sc}$ $/{ }^{48} \mathrm{Sc}, \mathrm{E} \approx 0.1-750 \mathrm{MeV} ; \mathrm{Fe}, \mathrm{Ni}, \mathrm{Cu}(\mathrm{n}, \mathrm{X}){ }^{48} \mathrm{~V} /{ }^{51} \mathrm{Cr} /{ }^{52} \mathrm{Mn} /{ }^{54} \mathrm{Mn}$, $\mathrm{E} \approx 0.1-750 \mathrm{MeV} ; \mathrm{Ni}, \mathrm{Cu}(\mathrm{n}, \mathrm{X}){ }^{56} \mathrm{Ni} /{ }^{57} \mathrm{Ni} /{ }^{56} \mathrm{Co} /{ }^{57} \mathrm{Co} /{ }^{58} \mathrm{Co} /$ ${ }^{60} \mathrm{Co} /{ }^{59} \mathrm{Fe}, \mathrm{E} \approx 0.1-750 \mathrm{MeV}$; measured energy-integrated production $\sigma$. JOUR NIMBE 234419

2005SI32 NUCLEAR REACTIONS Cu(n, X $)^{54} \mathrm{Mn} /{ }^{59} \mathrm{Fe} /{ }^{56} \mathrm{Co} /{ }^{57} \mathrm{Co} /{ }^{58} \mathrm{Co}$ $/{ }^{60} \mathrm{Co}, \mathrm{E} \approx 70.7,110.8 \mathrm{MeV}$; measured $\sigma$. Comparison with previous results, model predictions. JOUR NIMBE 240617

2005TIZX NUCLEAR REACTIONS Pb, ${ }^{208} \mathrm{~Pb}(\mathrm{p}, \mathrm{X}){ }^{203} \mathrm{~Pb} /{ }^{200} \mathrm{Tl} /{ }^{199} \mathrm{Tl} /$ ${ }^{196} \mathrm{Au} /{ }^{192} \mathrm{Ir} /{ }^{190} \mathrm{Ir} /{ }^{173} \mathrm{Lu} /{ }^{101 m} \mathrm{Rh} /{ }^{86} \mathrm{Rb} /{ }^{59} \mathrm{Fe} /{ }^{24} \mathrm{Na} /{ }^{7} \mathrm{Be}$, $\mathrm{E}=40-2600 \mathrm{MeV}$; measured excitation functions. Comparison with previous work and model predictions. Other reactions discussed. CONF Santa Fe (Nucl Data for Sci and Technol) Proc,Vol1,P1070 2005TIZY NUCLEAR REACTIONS Pb, ${ }^{208} \mathrm{~Pb},{ }^{209} \mathrm{Bi}(\mathrm{p}, \mathrm{X}){ }^{203} \mathrm{~Pb} /{ }^{200} \mathrm{Tl} /{ }^{199} \mathrm{Tl}$ $/{ }^{196} \mathrm{Au} /{ }^{192} \mathrm{Ir} /{ }^{190} \mathrm{Ir} /{ }^{173} \mathrm{Lu} /{ }^{101 m} \mathrm{Rh} /{ }^{86} \mathrm{Rb} /{ }^{59} \mathrm{Fe} /{ }^{24} \mathrm{Na} /{ }^{7} \mathrm{Be}$, $\mathrm{E}=40-2600 \mathrm{MeV}$; measured production $\sigma$. Comparison with model predictions. PREPRINT nucl-ex/0507009,7/05/2005

${ }^{59} \mathrm{Co} \quad 2004 \mathrm{~S} 036 \quad$ NUCLEAR REACTIONS ${ }^{59} \mathrm{Co}\left({ }^{6} \mathrm{Li}, \mathrm{X}\right),\left({ }^{7} \mathrm{Li}, \mathrm{X}\right), \mathrm{E}=12-26 \mathrm{MeV}$; measured fusion $\sigma$; deduced breakup effects. ${ }^{59} \mathrm{Co}\left({ }^{6} \mathrm{Li}, \mathrm{d} \alpha\right), \mathrm{E}=26$ $\mathrm{MeV}$; measured $\mathrm{E} \alpha, \mathrm{Ed}, \mathrm{d} \alpha$-coin. JOUR BJPHE 34907

2005S014 NUCLEAR REACTIONS ${ }^{12} \mathrm{C}\left({ }^{6} \mathrm{Li}, \mathrm{d} \alpha\right), \mathrm{E}=26 \mathrm{MeV} ;{ }^{59} \mathrm{Co}\left({ }^{6} \mathrm{Li}, \mathrm{d} \alpha\right)$, $\mathrm{E}=30 \mathrm{MeV}$; measured particle spectra, $\sigma(\theta(\alpha), \theta(\mathrm{d}))$, three-body final state correlations; deduced reaction mechanism features. JOUR BJPHE 35888

${ }^{59} \mathrm{Ni} \quad 2005 \mathrm{ALO3}$ NUCLEAR REACTIONS ${ }^{58} \mathrm{Ni}\left({ }^{16} \mathrm{O},{ }^{16} \mathrm{O}\right),\left({ }^{16} \mathrm{O},{ }^{16} \mathrm{O}\right),\left({ }^{16} \mathrm{O},{ }^{12} \mathrm{C}\right)$, $\mathrm{E}=46 \mathrm{MeV} ;{ }^{58} \mathrm{Ni}\left({ }^{18} \mathrm{O},{ }^{18} \mathrm{O}\right),\left({ }^{18} \mathrm{O},{ }^{18} \mathrm{O}\right),\left({ }^{18} \mathrm{O},{ }^{17} \mathrm{O}\right),\left({ }^{18} \mathrm{O},{ }^{16} \mathrm{O}\right), \mathrm{E}=46$ $\mathrm{MeV}$; measured elastic, inelastic, and transfer $\sigma(\mathrm{E}, \theta)$.

Coupled-channels analysis, comparison with previous results. JOUR NUPAB 74859

${ }^{59} \mathrm{Ga}$ 2005ST29 NUCLEAR REACTIONS ${ }^{9} \mathrm{Be}\left({ }^{78} \mathrm{Kr}, \mathrm{X}\right){ }^{60} \mathrm{Ge} /{ }^{61} \mathrm{Ge} /{ }^{62} \mathrm{Ge} /{ }^{63} \mathrm{Ge} /$ ${ }^{64} \mathrm{Ge} /{ }^{64} \mathrm{Se} /{ }^{65} \mathrm{Se} /{ }^{66} \mathrm{Se} /{ }^{67} \mathrm{Se} /{ }^{68} \mathrm{Se}, \mathrm{E}=140 \mathrm{MeV} /$ nucleon; measured production $\sigma$, isotopic yields; deduced no evidence for ${ }^{59} \mathrm{Ga}$, ${ }^{63}$ As. ${ }^{60} \mathrm{Ge},{ }^{64}$ Se deduced $\mathrm{T}_{1 / 2}$ lower limits. ${ }^{59} \mathrm{Ga},{ }^{63}$ As deduced $\mathrm{T}_{1 / 2}$ upper limits. JOUR PYLBB 62732

2005St34 NUCLEAR REACTIONS ${ }^{9} \mathrm{Be}\left({ }^{78} \mathrm{Kr}, \mathrm{X}\right){ }^{60} \mathrm{Ge} /{ }^{61} \mathrm{Ge} /{ }^{62} \mathrm{Ge} /{ }^{63} \mathrm{Ge} /$ ${ }^{64} \mathrm{Ge} /{ }^{64} \mathrm{Se} /{ }^{65} \mathrm{Se} /{ }^{66} \mathrm{Se} /{ }^{67} \mathrm{Se} /{ }^{68} \mathrm{Se}, \mathrm{E}=140 \mathrm{MeV} /$ nucleon; measured production $\sigma$, isotopic yields; deduced no evidence for ${ }^{59} \mathrm{Ga}$, ${ }^{63}$ As. JOUR ZAANE 25 s01 335 


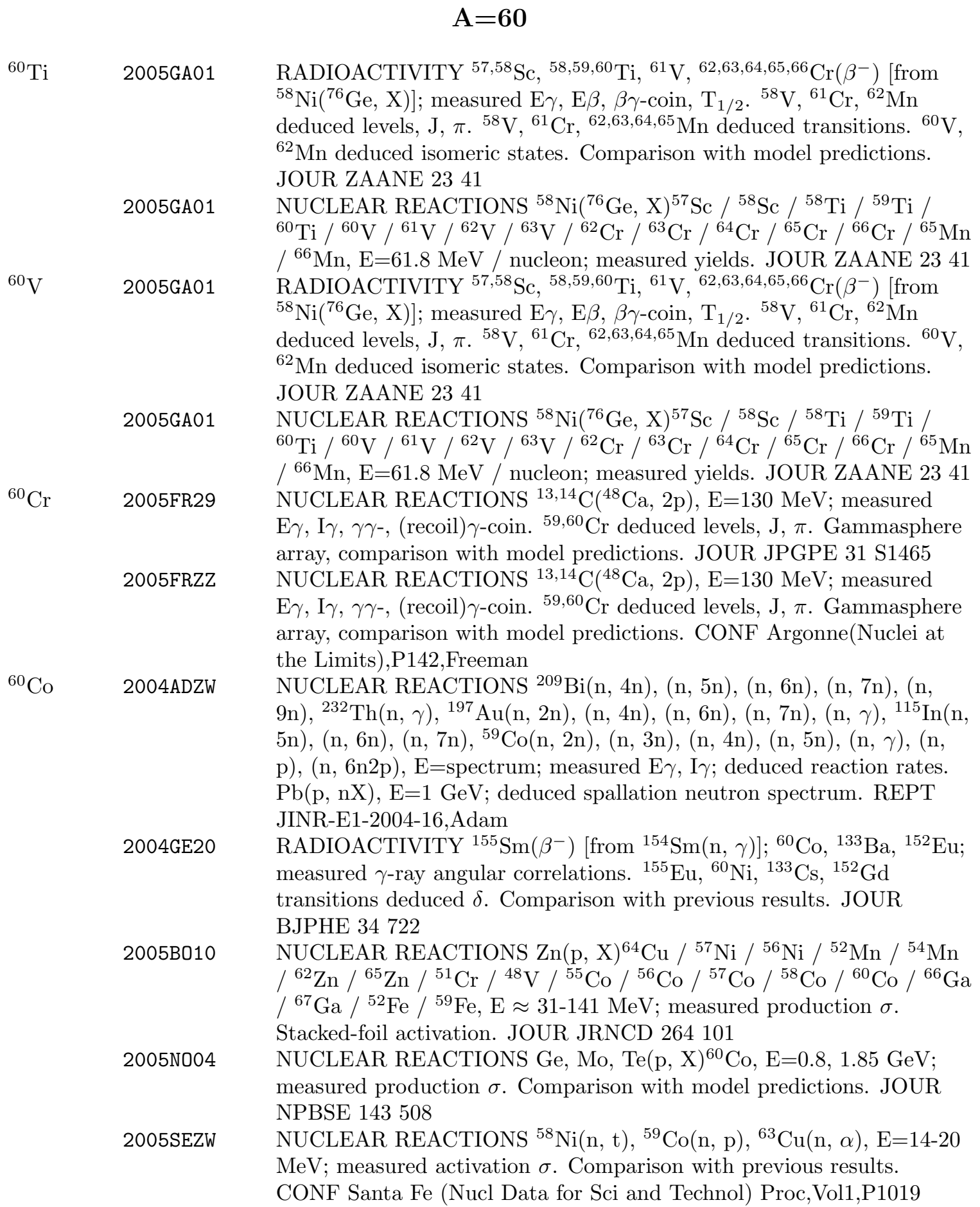




\section{$\mathrm{A}=60$ (continued)}

2005SI14 NUCLEAR REACTIONS C, O, Si, Mg, Al(n, X $)^{7} \mathrm{Be}, \mathrm{E} \approx 0.1-750$ $\mathrm{MeV} ; \mathrm{O}, \mathrm{Si}, \mathrm{Mg}, \mathrm{Al}(\mathrm{n}, \mathrm{X}){ }^{22} \mathrm{Na} /{ }^{23} \mathrm{Na}, \mathrm{E} \approx 0.1-750 \mathrm{MeV} ;{ }^{197} \mathrm{Au}(\mathrm{n}$, $\mathrm{X}){ }^{194} \mathrm{Au} /{ }^{196} \mathrm{Au} /{ }^{198} \mathrm{Au}, \mathrm{E} \approx 0.1-750 \mathrm{MeV} ; \mathrm{Ti}, \mathrm{Fe}, \mathrm{Ni}, \mathrm{Cu}(\mathrm{n}, \mathrm{X})^{46} \mathrm{Sc}$ $/{ }^{48} \mathrm{Sc}, \mathrm{E} \approx 0.1-750 \mathrm{MeV} ; \mathrm{Fe}, \mathrm{Ni}, \mathrm{Cu}(\mathrm{n}, \mathrm{X}){ }^{48} \mathrm{~V} /{ }^{51} \mathrm{Cr} /{ }^{52} \mathrm{Mn} /{ }^{54} \mathrm{Mn}$, $\mathrm{E} \approx 0.1-750 \mathrm{MeV} ; \mathrm{Ni}, \mathrm{Cu}(\mathrm{n}, \mathrm{X}){ }^{56} \mathrm{Ni} /{ }^{57} \mathrm{Ni} /{ }^{56} \mathrm{Co} /{ }^{57} \mathrm{Co} /{ }^{58} \mathrm{Co} /$ ${ }^{60} \mathrm{Co} /{ }^{59} \mathrm{Fe}, \mathrm{E} \approx 0.1-750 \mathrm{MeV}$; measured energy-integrated production $\sigma$. JOUR NIMBE 234419

2005SI32 NUCLEAR REACTIONS Cu(n, X $)^{54} \mathrm{Mn} /{ }^{59} \mathrm{Fe} /{ }^{56} \mathrm{Co} /{ }^{57} \mathrm{Co} /{ }^{58} \mathrm{Co}$ $/{ }^{60} \mathrm{Co}, \mathrm{E} \approx 70.7,110.8 \mathrm{MeV}$; measured $\sigma$. Comparison with previous results, model predictions. JOUR NIMBE 240617

${ }^{60} \mathrm{Ni}$ 2004GE20 RADIOACTIVITY ${ }^{155} \mathrm{Sm}\left(\beta^{-}\right)\left[\right.$from $\left.{ }^{154} \mathrm{Sm}(\mathrm{n}, \gamma)\right] ;{ }^{60} \mathrm{Co},{ }^{133} \mathrm{Ba},{ }^{152} \mathrm{Eu}$; measured $\gamma$-ray angular correlations. ${ }^{155} \mathrm{Eu},{ }^{60} \mathrm{Ni},{ }^{133} \mathrm{Cs},{ }^{152} \mathrm{Gd}$ transitions deduced $\delta$. Comparison with previous results. JOUR BJPHE 34722

2005AL03 NUCLEAR REACTIONS ${ }^{58} \mathrm{Ni}\left({ }^{16} \mathrm{O},{ }^{16} \mathrm{O}\right),\left({ }^{16} \mathrm{O},{ }^{16} \mathrm{O}\right),\left(\left({ }^{16} \mathrm{O},{ }^{12} \mathrm{C}\right)\right.$, $\mathrm{E}=46 \mathrm{MeV} ;{ }^{58} \mathrm{Ni}\left({ }^{18} \mathrm{O},{ }^{18} \mathrm{O}\right),\left({ }^{18} \mathrm{O},{ }^{18} \mathrm{O}\right),\left({ }^{18} \mathrm{O},{ }^{17} \mathrm{O}\right),\left({ }^{18} \mathrm{O},{ }^{16} \mathrm{O}\right), \mathrm{E}=46$ $\mathrm{MeV}$; measured elastic, inelastic, and transfer $\sigma(\mathrm{E}, \theta)$.

Coupled-channels analysis, comparison with previous results. JOUR NUPAB 74859

2005WI23 NUCLEAR REACTIONS ${ }^{100} \mathrm{Mo}\left({ }^{11} \mathrm{~B}\right.$, xnypz $\left.\alpha\right){ }^{104} \mathrm{Rh} /{ }^{105} \mathrm{Rh} /{ }^{107} \mathrm{Pd} /$ ${ }^{108} \mathrm{Pd}, \mathrm{E}=43 \mathrm{MeV} ;{ }^{51} \mathrm{~V}\left({ }^{16} \mathrm{O}\right.$, xnypz $\left.\alpha\right){ }^{60} \mathrm{Ni} /{ }^{61} \mathrm{Ni} /{ }^{61} \mathrm{Cu} /{ }^{62} \mathrm{Cu}, \mathrm{E}=70$ $\mathrm{MeV}$; measured $\mathrm{E} \gamma, \mathrm{I} \gamma, \gamma \gamma$-, (charged particle) $\gamma$-coin; deduced $\gamma$-ray yield ratios. Application to exit channel determination discussed. JOUR BJPHE 35898

${ }^{60} \mathrm{Ge} \quad$ 2005St29 NUCLEAR REACTIONS ${ }^{9} \mathrm{Be}\left({ }^{78} \mathrm{Kr}, \mathrm{X}\right){ }^{60} \mathrm{Ge} /{ }^{61} \mathrm{Ge} /{ }^{62} \mathrm{Ge} /{ }^{63} \mathrm{Ge} /$ ${ }^{64} \mathrm{Ge} /{ }^{64} \mathrm{Se} /{ }^{65} \mathrm{Se} /{ }^{66} \mathrm{Se} /{ }^{67} \mathrm{Se} /{ }^{68} \mathrm{Se}, \mathrm{E}=140 \mathrm{MeV} /$ nucleon; measured production $\sigma$, isotopic yields; deduced no evidence for ${ }^{59} \mathrm{Ga}$, ${ }^{63}$ As. ${ }^{60} \mathrm{Ge},{ }^{64}$ Se deduced $\mathrm{T}_{1 / 2}$ lower limits. ${ }^{59} \mathrm{Ga},{ }^{63}$ As deduced $\mathrm{T}_{1 / 2}$ upper limits. JOUR PYLBB 62732

2005ST34 NUCLEAR REACTIONS ${ }^{9} \mathrm{Be}\left({ }^{78} \mathrm{Kr}, \mathrm{X}\right){ }^{60} \mathrm{Ge} /{ }^{61} \mathrm{Ge} /{ }^{62} \mathrm{Ge} /{ }^{63} \mathrm{Ge} /$ ${ }^{64} \mathrm{Ge} /{ }^{64} \mathrm{Se} /{ }^{65} \mathrm{Se} /{ }^{66} \mathrm{Se} /{ }^{67} \mathrm{Se} /{ }^{68} \mathrm{Se}, \mathrm{E}=140 \mathrm{MeV} /$ nucleon; measured production $\sigma$, isotopic yields; deduced no evidence for ${ }^{59} \mathrm{Ga}$, ${ }^{63}$ As. JOUR ZAANE 25 s01 335

\section{$\mathrm{A}=61$}

${ }^{61} \mathrm{~V}$

2005GA01

RADIOACTIVITY ${ }^{57,58} \mathrm{Sc},{ }^{58,59,60} \mathrm{Ti},{ }^{61} \mathrm{~V},{ }^{62,63,64,65,66} \mathrm{Cr}\left(\beta^{-}\right)$[from $\left.{ }^{58} \mathrm{Ni}\left({ }^{76} \mathrm{Ge}, \mathrm{X}\right)\right]$; measured $\mathrm{E} \gamma, \mathrm{E} \beta, \beta \gamma$-coin, $\mathrm{T}_{1 / 2} \cdot{ }^{58} \mathrm{~V},{ }^{61} \mathrm{Cr},{ }^{62} \mathrm{Mn}$ deduced levels, J, $\pi .{ }^{58} \mathrm{~V},{ }^{61} \mathrm{Cr},{ }^{62,63,64,65} \mathrm{Mn}$ deduced transitions. ${ }^{60} \mathrm{~V}$, ${ }^{62} \mathrm{Mn}$ deduced isomeric states. Comparison with model predictions. JOUR ZAANE 2341

2005GA01 NUCLEAR REACTIONS ${ }^{58} \mathrm{Ni}\left({ }^{76} \mathrm{Ge}, \mathrm{X}\right){ }^{57} \mathrm{Sc} /{ }^{58} \mathrm{Sc} /{ }^{58} \mathrm{Ti} /{ }^{59} \mathrm{Ti} /$ ${ }^{60} \mathrm{Ti} /{ }^{60} \mathrm{~V} /{ }^{61} \mathrm{~V} /{ }^{62} \mathrm{~V} /{ }^{63} \mathrm{~V} /{ }^{62} \mathrm{Cr} /{ }^{63} \mathrm{Cr} /{ }^{64} \mathrm{Cr} /{ }^{65} \mathrm{Cr} /{ }^{66} \mathrm{Cr} /{ }^{65} \mathrm{Mn}$ / ${ }^{66} \mathrm{Mn}, \mathrm{E}=61.8 \mathrm{MeV} /$ nucleon; measured yields. JOUR ZAANE 2341 


\section{$\mathrm{A}=61$ (continued)}

${ }^{61} \mathrm{Cr} \quad 2005 \mathrm{GA} 01$
${ }^{61} \mathrm{Ni} \quad 2005 \mathrm{R} 011$

2005WI23

${ }^{61} \mathrm{Cu}$

2005NAZY

2005SI21

2005WI23

${ }^{61} \mathrm{Zn}$

2005EK01

${ }^{61} \mathrm{Ga}$

2005AN03

2005EK01

2005RU06

${ }^{61} \mathrm{Ge} \quad 2005 \mathrm{ST} 29$
RADIOACTIVITY ${ }^{57,58} \mathrm{Sc},{ }^{58,59,60} \mathrm{Ti},{ }^{61} \mathrm{~V},{ }^{62,63,64,65,66} \mathrm{Cr}\left(\beta^{-}\right)$[from $\left.{ }^{58} \mathrm{Ni}\left({ }^{76} \mathrm{Ge}, \mathrm{X}\right)\right]$; measured $\mathrm{E} \gamma, \mathrm{E} \beta, \beta \gamma$-coin, $\mathrm{T}_{1 / 2} .{ }^{58} \mathrm{~V},{ }^{61} \mathrm{Cr},{ }^{62} \mathrm{Mn}$ deduced levels, J, $\pi .{ }^{58} \mathrm{~V},{ }^{61} \mathrm{Cr},{ }^{62,63,64,65} \mathrm{Mn}$ deduced transitions. ${ }^{60} \mathrm{~V}$, ${ }^{62} \mathrm{Mn}$ deduced isomeric states. Comparison with model predictions. JOUR ZAANE 2341 NUCLEAR REACTIONS ${ }^{61} \mathrm{Ni}\left(\gamma, \gamma^{\prime}\right), \mathrm{E} \approx 67.41 \mathrm{keV}$; measured $\mathrm{E} \gamma$, $\mathrm{I} \gamma(\mathrm{t}) .{ }^{61} \mathrm{Ni}$ level deduced $\mathrm{T}_{1 / 2}$. Synchrotron radiation, nuclear lighthouse effect. JOUR PRBMD 71140401 NUCLEAR REACTIONS ${ }^{100} \mathrm{Mo}\left({ }^{11} \mathrm{~B}\right.$, xnypz $\left.\alpha\right){ }^{104} \mathrm{Rh} /{ }^{105} \mathrm{Rh} /{ }^{107} \mathrm{Pd} /$ ${ }^{108} \mathrm{Pd}, \mathrm{E}=43 \mathrm{MeV} ;{ }^{51} \mathrm{~V}\left({ }^{16} \mathrm{O}\right.$, xnypz $\left.\alpha\right){ }^{60} \mathrm{Ni} /{ }^{61} \mathrm{Ni} /{ }^{61} \mathrm{Cu} /{ }^{62} \mathrm{Cu}, \mathrm{E}=70$ $\mathrm{MeV}$; measured $\mathrm{E} \gamma, \mathrm{I} \gamma, \gamma \gamma$-, (charged particle) $\gamma$-coin; deduced $\gamma$-ray yield ratios. Application to exit channel determination discussed. JOUR BJPHE 35898 NUCLEAR REACTIONS ${ }^{27} \mathrm{Al}(\mathrm{d}, \mathrm{X}){ }^{27} \mathrm{Mg} /{ }^{24} \mathrm{Na}, \mathrm{E}=22-40 \mathrm{MeV}$; $\mathrm{Cu}(\mathrm{d}, \mathrm{X}){ }^{62} \mathrm{Zn} /{ }^{63} \mathrm{Zn} /{ }^{61} \mathrm{Cu} /{ }^{64} \mathrm{Cu}, \mathrm{E}=22-40 \mathrm{MeV} ; \mathrm{W}(\mathrm{d}, \mathrm{X}){ }^{181} \mathrm{Re} /$ ${ }^{182} \operatorname{Re} /{ }^{183} \operatorname{Re} /{ }^{184} \operatorname{Re} /{ }^{186} \operatorname{Re} /{ }^{187} \mathrm{~W}, \mathrm{E}=22-40 \mathrm{MeV}$; measured activation $\sigma$. Comparison with previous results and model predictions. CONF Santa Fe (Nucl Data for Sci and Technol) Proc,Vol2,P1489 NUCLEAR REACTIONS Ni $(\alpha, \mathrm{X}){ }^{62} \mathrm{Zn} /{ }^{61} \mathrm{Cu} /{ }^{56} \mathrm{Ni} /{ }^{57} \mathrm{Ni} /{ }^{56} \mathrm{Co} /$ ${ }^{58} \mathrm{Co}, \mathrm{E}=21-50 \mathrm{MeV}$; measured excitation functions. Stacked-foil activation, comparison with model predictions. JOUR IMPEE 14611 NUCLEAR REACTIONS ${ }^{100} \mathrm{Mo}\left({ }^{11} \mathrm{~B}\right.$, xnypz $\left.\alpha\right){ }^{104} \mathrm{Rh} /{ }^{105} \mathrm{Rh} /{ }^{107} \mathrm{Pd} /$ ${ }^{108} \mathrm{Pd}, \mathrm{E}=43 \mathrm{MeV} ;{ }^{51} \mathrm{~V}\left({ }^{16} \mathrm{O}\right.$, xnypz $\left.\alpha\right){ }^{60} \mathrm{Ni} /{ }^{61} \mathrm{Ni} /{ }^{61} \mathrm{Cu} /{ }^{62} \mathrm{Cu}, \mathrm{E}=70$ $\mathrm{MeV}$; measured $\mathrm{E} \gamma, \mathrm{I} \gamma, \gamma \gamma$-, (charged particle) $\gamma$-coin; deduced $\gamma$-ray yield ratios. Application to exit channel determination discussed. JOUR BJPHE 35898 NUCLEAR REACTIONS ${ }^{16} \mathrm{O}\left({ }^{24} \mathrm{Mg}, \mathrm{n} \alpha\right),\left({ }^{24} \mathrm{Mg}, \mathrm{p} \alpha\right), \mathrm{E}=60 \mathrm{MeV}$; ${ }^{28} \mathrm{Si}\left({ }^{32} \mathrm{~S}, \mathrm{n} 2 \alpha\right),\left({ }^{32} \mathrm{~S}, \mathrm{p} 2 \alpha\right), \mathrm{E}=130 \mathrm{MeV} ;{ }^{24} \mathrm{Mg}\left({ }^{40} \mathrm{Ca}, 2 \mathrm{np}\right),\left({ }^{40} \mathrm{Ca}, \mathrm{n} 2 \mathrm{p}\right)$, $\mathrm{E}=104 \mathrm{MeV}$; measured $\mathrm{E} \gamma, \mathrm{I} \gamma, \gamma \gamma-$, (charged particle) $\gamma-$, (neutron) $\gamma$-coin. ${ }^{35} \mathrm{Ar},{ }^{35} \mathrm{Cl},{ }^{51} \mathrm{Fe},{ }^{51} \mathrm{Mn},{ }^{61} \mathrm{Ga},{ }^{61} \mathrm{Zn}$ deduced levels, J, $\pi$, mirror energy difference. Discussed electromagnetic spin-orbit effect. Large-scale shell model calculations. JOUR ZAANE 25 s01 363 NUCLEAR REACTIONS ${ }^{24} \mathrm{Mg}\left({ }^{40} \mathrm{Ca}, 2 \mathrm{np}\right), \mathrm{E}=104 \mathrm{MeV}$; measured $\mathrm{E} \gamma, \mathrm{I} \gamma, \gamma \gamma$-, (recoil) $\gamma$-coin. ${ }^{61} \mathrm{Ga}$ deduced levels, $\mathrm{J}, \pi$, analog states features. Clarion array, mass separator. JOUR PRVCA 71011303 NUCLEAR REACTIONS ${ }^{16} \mathrm{O}\left({ }^{24} \mathrm{Mg}, \mathrm{n} \alpha\right),\left({ }^{24} \mathrm{Mg}, \mathrm{p} \alpha\right), \mathrm{E}=60 \mathrm{MeV}$; ${ }^{28} \mathrm{Si}\left({ }^{32} \mathrm{~S}, \mathrm{n} 2 \alpha\right),\left({ }^{32} \mathrm{~S}, \mathrm{p} 2 \alpha\right), \mathrm{E}=130 \mathrm{MeV} ;{ }^{24} \mathrm{Mg}\left({ }^{40} \mathrm{Ca}, 2 \mathrm{np}\right),\left({ }^{40} \mathrm{Ca}, \mathrm{n} 2 \mathrm{p}\right)$, $\mathrm{E}=104 \mathrm{MeV}$; measured $\mathrm{E} \gamma, \mathrm{I} \gamma, \gamma \gamma-$, (charged particle) $\gamma_{-}$, (neutron) $\gamma$-coin. ${ }^{35} \mathrm{Ar},{ }^{35} \mathrm{Cl},{ }^{51} \mathrm{Fe},{ }^{51} \mathrm{Mn},{ }^{61} \mathrm{Ga},{ }^{61} \mathrm{Zn}$ deduced levels, J, $\pi$, mirror energy difference. Discussed electromagnetic spin-orbit effect. Large-scale shell model calculations. JOUR ZAANE 25 s01 363 NUCLEAR REACTIONS ${ }^{24} \mathrm{Mg}\left({ }^{40} \mathrm{Ca}, 2 \mathrm{np}\right),\left({ }^{40} \mathrm{Ca}, 2 \mathrm{n}\right), \mathrm{E}=104 \mathrm{MeV}$; measured $\mathrm{E} \gamma, \mathrm{I} \gamma, \gamma \gamma$-, (recoil) $\gamma$-coin. ${ }^{61} \mathrm{Ga},{ }^{62} \mathrm{Ge}$ deduced levels, transitions. JOUR NUPAB 752 241c NUCLEAR REACTIONS ${ }^{9} \mathrm{Be}\left({ }^{78} \mathrm{Kr}, \mathrm{X}\right){ }^{60} \mathrm{Ge} /{ }^{61} \mathrm{Ge} /{ }^{62} \mathrm{Ge} /{ }^{63} \mathrm{Ge} /$ ${ }^{64} \mathrm{Ge} /{ }^{64} \mathrm{Se} /{ }^{65} \mathrm{Se} /{ }^{66} \mathrm{Se} /{ }^{67} \mathrm{Se} /{ }^{68} \mathrm{Se}, \mathrm{E}=140 \mathrm{MeV} /$ nucleon; measured production $\sigma$, isotopic yields; deduced no evidence for ${ }^{59} \mathrm{Ga}$, ${ }^{63}$ As. ${ }^{60} \mathrm{Ge},{ }^{64}$ Se deduced $\mathrm{T}_{1 / 2}$ lower limits. ${ }^{59} \mathrm{Ga},{ }^{63}$ As deduced $\mathrm{T}_{1 / 2}$ upper limits. JOUR PYLBB 62732 


\section{$\mathrm{A}=61$ (continued)}

2005ST34 NUCLEAR REACTIONS ${ }^{9} \mathrm{Be}\left({ }^{78} \mathrm{Kr}, \mathrm{X}\right){ }^{60} \mathrm{Ge} /{ }^{61} \mathrm{Ge} /{ }^{62} \mathrm{Ge} /{ }^{63} \mathrm{Ge} /$ ${ }^{64} \mathrm{Ge} /{ }^{64} \mathrm{Se} /{ }^{65} \mathrm{Se} /{ }^{66} \mathrm{Se} /{ }^{67} \mathrm{Se} /{ }^{68} \mathrm{Se}, \mathrm{E}=140 \mathrm{MeV} /$ nucleon; measured production $\sigma$, isotopic yields; deduced no evidence for ${ }^{59} \mathrm{Ga}$, ${ }^{63}$ As. JOUR ZAANE 25 s01 335

\section{$\mathrm{A}=62$}

$\begin{array}{ll}{ }^{62} \mathrm{~V} & 2005 \mathrm{GA} 01 \\ { }^{62} \mathrm{Cr} & 2005 \mathrm{GA} 01\end{array}$

NUCLEAR REACTIONS ${ }^{58} \mathrm{Ni}\left({ }^{76} \mathrm{Ge}, \mathrm{X}\right){ }^{57} \mathrm{Sc} /{ }^{58} \mathrm{Sc} /{ }^{58} \mathrm{Ti} /{ }^{59} \mathrm{Ti} /$ ${ }^{60} \mathrm{Ti} /{ }^{60} \mathrm{~V} /{ }^{61} \mathrm{~V} /{ }^{62} \mathrm{~V} /{ }^{63} \mathrm{~V} /{ }^{62} \mathrm{Cr} /{ }^{63} \mathrm{Cr} /{ }^{64} \mathrm{Cr} /{ }^{65} \mathrm{Cr} /{ }^{66} \mathrm{Cr} /{ }^{65} \mathrm{Mn}$ $/{ }^{66} \mathrm{Mn}, \mathrm{E}=61.8 \mathrm{MeV} /$ nucleon; measured yields. JOUR ZAANE 2341 RADIOACTIVITY ${ }^{57,58} \mathrm{Sc},{ }^{58,59,60} \mathrm{Ti},{ }^{61} \mathrm{~V},{ }^{62,63,64,65,66} \mathrm{Cr}\left(\beta^{-}\right)$[from $\left.{ }^{58} \mathrm{Ni}\left({ }^{76} \mathrm{Ge}, \mathrm{X}\right)\right]$; measured $\mathrm{E} \gamma, \mathrm{E} \beta, \beta \gamma$-coin, $\mathrm{T}_{1 / 2} \cdot{ }^{58} \mathrm{~V},{ }^{61} \mathrm{Cr},{ }^{62} \mathrm{Mn}$ deduced levels, J, $\pi .{ }^{58} \mathrm{~V},{ }^{61} \mathrm{Cr},{ }^{62,63,64,65} \mathrm{Mn}$ deduced transitions. ${ }^{60} \mathrm{~V}$, ${ }^{62} \mathrm{Mn}$ deduced isomeric states. Comparison with model predictions. JOUR ZAANE 2341

2005GA01 NUCLEAR REACTIONS ${ }^{58} \mathrm{Ni}\left({ }^{76} \mathrm{Ge}, \mathrm{X}\right){ }^{57} \mathrm{Sc} /{ }^{58} \mathrm{Sc} /{ }^{58} \mathrm{Ti} /{ }^{59} \mathrm{Ti} /$ ${ }^{60} \mathrm{Ti} /{ }^{60} \mathrm{~V} /{ }^{61} \mathrm{~V} /{ }^{62} \mathrm{~V} /{ }^{63} \mathrm{~V} /{ }^{62} \mathrm{Cr} /{ }^{63} \mathrm{Cr} /{ }^{64} \mathrm{Cr} /{ }^{65} \mathrm{Cr} /{ }^{66} \mathrm{Cr} /{ }^{65} \mathrm{Mn}$ / ${ }^{66} \mathrm{Mn}, \mathrm{E}=61.8 \mathrm{MeV} /$ nucleon; measured yields. JOUR ZAANE 2341 ${ }^{62} \mathrm{Mn} \quad 2005 \mathrm{GA} 01$ RADIOACTIVITY ${ }^{57,58} \mathrm{Sc},{ }^{58,59,60} \mathrm{Ti},{ }^{61} \mathrm{~V},{ }^{62,63,64,65,66} \mathrm{Cr}\left(\beta^{-}\right)$[from $\left.{ }^{58} \mathrm{Ni}\left({ }^{76} \mathrm{Ge}, \mathrm{X}\right)\right]$; measured $\mathrm{E} \gamma, \mathrm{E} \beta, \beta \gamma$-coin, $\mathrm{T}_{1 / 2} \cdot{ }^{58} \mathrm{~V},{ }^{61} \mathrm{Cr},{ }^{62} \mathrm{Mn}$ deduced levels, J, $\pi .{ }^{58} \mathrm{~V},{ }^{61} \mathrm{Cr},{ }^{62,63,64,65} \mathrm{Mn}$ deduced transitions. ${ }^{60} \mathrm{~V}$, ${ }^{62} \mathrm{Mn}$ deduced isomeric states. Comparison with model predictions. JOUR ZAANE 2341

${ }^{62} \mathrm{Co}$ 2005PE12 NUCLEAR REACTIONS ${ }^{197} \mathrm{Au}\left({ }^{65} \mathrm{Cu}, \mathrm{X}\right){ }^{62} \mathrm{Co} /{ }^{63} \mathrm{Co}, \mathrm{E} \approx 400-460$ $\mathrm{MeV}$; measured yields. Ion-guide isotope separator. JOUR NIMAE 546 418

2005T014
NUCLEAR REACTIONS ${ }^{62} \mathrm{Ni}(\nu, \gamma), \mathrm{E}=5.5-90 \mathrm{keV}$; measured $\mathrm{E} \gamma$, capture $\sigma$; deduced Maxwellian-averaged $\sigma$. JOUR ASJOA 623 L153 ATOMIC MASSES ${ }^{62} \mathrm{Ga},{ }^{62} \mathrm{Zn},{ }^{62} \mathrm{Cu}$; measured masses. ${ }^{62} \mathrm{Ga}$ deduced $\mathrm{Q}$ (EC) for superallowed $\beta$-decay. Penning trap. PREPRINT nucl-ex/0512010,12/12/2005

2005MAZP NUCLEAR REACTIONS ${ }^{64} \mathrm{Zn}(\mathrm{n}, \mathrm{p}),{ }^{64} \mathrm{Zn},{ }^{63,65} \mathrm{Cu}(\mathrm{n}, 2 \mathrm{n}), \mathrm{E} \approx 10-15$ $\mathrm{MeV}$; measured $\sigma$. Comparison with previous results. CONF Santa Fe (Nucl Data for Sci and Technol) Proc,Vol1,P609

2005PE23 NUCLEAR REACTIONS ${ }^{197} \mathrm{Au}\left({ }^{65} \mathrm{Cu}, \mathrm{X}\right){ }^{62} \mathrm{Cu} /{ }^{63} \mathrm{Cu}, \mathrm{E}=443 \mathrm{MeV}$; measured yields. JOUR ZAANE 25 s01 749

2005WI23 NUCLEAR REACTIONS ${ }^{100} \mathrm{Mo}\left({ }^{11} \mathrm{~B}\right.$, xnypz $\left.\alpha\right){ }^{104} \mathrm{Rh} /{ }^{105} \mathrm{Rh} /{ }^{107} \mathrm{Pd} /$ ${ }^{108} \mathrm{Pd}, \mathrm{E}=43 \mathrm{MeV} ;{ }^{51} \mathrm{~V}\left({ }^{16} \mathrm{O}\right.$, xnypz $\left.\alpha\right){ }^{60} \mathrm{Ni} /{ }^{61} \mathrm{Ni} /{ }^{61} \mathrm{Cu} /{ }^{62} \mathrm{Cu}, \mathrm{E}=70$ $\mathrm{MeV}$; measured $\mathrm{E} \gamma, \mathrm{I} \gamma, \gamma \gamma$-, (charged particle) $\gamma$-coin; deduced $\gamma$-ray yield ratios. Application to exit channel determination discussed. JOUR BJPHE 35898

2005AL03 NUCLEAR REACTIONS ${ }^{58} \mathrm{Ni}\left({ }^{16} \mathrm{O},{ }^{16} \mathrm{O}\right),\left({ }^{16} \mathrm{O},{ }^{16} \mathrm{O}\right),\left({ }^{16} \mathrm{O},{ }^{12} \mathrm{C}\right)$, $\mathrm{E}=46 \mathrm{MeV} ;{ }^{58} \mathrm{Ni}\left({ }^{18} \mathrm{O},{ }^{18} \mathrm{O}\right),\left({ }^{18} \mathrm{O},{ }^{18} \mathrm{O}\right),\left({ }^{18} \mathrm{O},{ }^{17} \mathrm{O}\right),\left({ }^{18} \mathrm{O},{ }^{16} \mathrm{O}\right), \mathrm{E}=46$ $\mathrm{MeV}$; measured elastic, inelastic, and transfer $\sigma(\mathrm{E}, \theta)$.

Coupled-channels analysis, comparison with previous results. JOUR NUPAB 74859 


\section{$\mathrm{A}=62$ (continued)}

2005B010 NUCLEAR REACTIONS Zn(p, X) ${ }^{64} \mathrm{Cu} /{ }^{57} \mathrm{Ni} /{ }^{56} \mathrm{Ni} /{ }^{52} \mathrm{Mn} /{ }^{54} \mathrm{Mn}$ $/{ }^{62} \mathrm{Zn} /{ }^{65} \mathrm{Zn} /{ }^{51} \mathrm{Cr} /{ }^{48} \mathrm{~V} /{ }^{55} \mathrm{Co} /{ }^{56} \mathrm{Co} /{ }^{57} \mathrm{Co} /{ }^{58} \mathrm{Co} /{ }^{60} \mathrm{Co} /{ }^{66} \mathrm{Ga}$ $/{ }^{67} \mathrm{Ga} /{ }^{52} \mathrm{Fe} /{ }^{59} \mathrm{Fe}, \mathrm{E} \approx 31-141 \mathrm{MeV}$; measured production $\sigma$. Stacked-foil activation. JOUR JRNCD 264101

2005CA06 RADIOACTIVITY ${ }^{62} \mathrm{Ga}(\mathrm{EC})$ [from $\left.{ }^{64} \mathrm{Zn}(\mathrm{p}, 3 \mathrm{n})\right]$; measured $\mathrm{E} \gamma, \mathrm{I} \gamma$, $\beta \gamma$-coin, $\mathrm{T}_{1 / 2}$; deduced branching ratios. ${ }^{62} \mathrm{Zn}$ deduced levels, $\beta$-feeding intensities. JOUR ZAANE 23409

2005ERZZ ATOMIC MASSES ${ }^{62} \mathrm{Ga},{ }^{62} \mathrm{Zn},{ }^{62} \mathrm{Cu}$; measured masses. ${ }^{62}$ Ga deduced $\mathrm{Q}$ (EC) for superallowed $\beta$-decay. Penning trap. PREPRINT nucl-ex/0512010,12/12/2005

2005HY04 RADIOACTIVITY ${ }^{62} \mathrm{Ga}\left(\beta^{+}\right)$; measured $\mathrm{T}_{1 / 2}$. Comparison with previous results. JOUR JPGPE $31 \mathrm{~S} 1885$

2005NAZY NUCLEAR REACTIONS ${ }^{27} \mathrm{Al}(\mathrm{d}, \mathrm{X})^{27} \mathrm{Mg} /{ }^{24} \mathrm{Na}, \mathrm{E}=22-40 \mathrm{MeV}$; $\mathrm{Cu}(\mathrm{d}, \mathrm{X}){ }^{62} \mathrm{Zn} /{ }^{63} \mathrm{Zn} /{ }^{61} \mathrm{Cu} /{ }^{64} \mathrm{Cu}, \mathrm{E}=22-40 \mathrm{MeV} ; \mathrm{W}(\mathrm{d}, \mathrm{X}){ }^{181} \mathrm{Re} /$ ${ }^{182} \operatorname{Re} /{ }^{183} \operatorname{Re} /{ }^{184} \operatorname{Re} /{ }^{186} \operatorname{Re} /{ }^{187} \mathrm{~W}, \mathrm{E}=22-40 \mathrm{MeV}$; measured activation $\sigma$. Comparison with previous results and model predictions. CONF Santa Fe (Nucl Data for Sci and Technol) Proc,Vol2,P1489

2005SI21 NUCLEAR REACTIONS Ni $(\alpha, \mathrm{X}){ }^{62} \mathrm{Zn} /{ }^{61} \mathrm{Cu} /{ }^{56} \mathrm{Ni} /{ }^{57} \mathrm{Ni} /{ }^{56} \mathrm{Co} /$ ${ }^{58} \mathrm{Co}, \mathrm{E}=21-50 \mathrm{MeV}$; measured excitation functions. Stacked-foil activation, comparison with model predictions. JOUR IMPEE 14611

${ }^{62} \mathrm{Ga} \quad$ 2005CA06 RADIOACTIVITY ${ }^{62} \mathrm{Ga}(\mathrm{EC})$ [from ${ }^{64} \mathrm{Zn}(\mathrm{p}, 3 \mathrm{n})$ ]; measured $\mathrm{E} \gamma, \mathrm{I} \gamma$, $\beta \gamma$-coin, $\mathrm{T}_{1 / 2}$; deduced branching ratios. ${ }^{62} \mathrm{Zn}$ deduced levels, $\beta$-feeding intensities. JOUR ZAANE 23409

2005ERZZ ATOMIC MASSES ${ }^{62} \mathrm{Ga},{ }^{62} \mathrm{Zn},{ }^{62} \mathrm{Cu}$; measured masses. ${ }^{62} \mathrm{Ga}$ deduced $\mathrm{Q}(\mathrm{EC})$ for superallowed $\beta$-decay. Penning trap. PREPRINT nucl-ex/0512010,12/12/2005

2005HY04 RADIOACTIVITY ${ }^{62} \mathrm{Ga}\left(\beta^{+}\right)$; measured $\mathrm{T}_{1 / 2}$. Comparison with previous results. JOUR JPGPE $31 \mathrm{~S} 1885$

${ }^{62} \mathrm{Ge} \quad$ 2005RU06 NUCLEAR REACTIONS ${ }^{24} \mathrm{Mg}\left({ }^{40} \mathrm{Ca}, 2 \mathrm{np}\right),\left({ }^{40} \mathrm{Ca}, 2 \mathrm{n}\right), \mathrm{E}=104 \mathrm{MeV}$; measured $\mathrm{E} \gamma$, I $\gamma, \gamma \gamma$-, (recoil) $\gamma$-coin. ${ }^{61} \mathrm{Ga},{ }^{62} \mathrm{Ge}$ deduced levels, transitions. JOUR NUPAB $752241 \mathrm{c}$

2005ST29 NUCLEAR REACTIONS ${ }^{9} \mathrm{Be}\left({ }^{78} \mathrm{Kr}, \mathrm{X}\right){ }^{60} \mathrm{Ge} /{ }^{61} \mathrm{Ge} /{ }^{62} \mathrm{Ge} /{ }^{63} \mathrm{Ge} /$ ${ }^{64} \mathrm{Ge} /{ }^{64} \mathrm{Se} /{ }^{65} \mathrm{Se} /{ }^{66} \mathrm{Se} /{ }^{67} \mathrm{Se} /{ }^{68} \mathrm{Se}, \mathrm{E}=140 \mathrm{MeV} /$ nucleon; measured production $\sigma$, isotopic yields; deduced no evidence for ${ }^{59} \mathrm{Ga}$, ${ }^{63}$ As. ${ }^{60} \mathrm{Ge},{ }^{64}$ Se deduced $\mathrm{T}_{1 / 2}$ lower limits. ${ }^{59} \mathrm{Ga},{ }^{63}$ As deduced $\mathrm{T}_{1 / 2}$ upper limits. JOUR PYLBB 62732

2005ST34 NUCLEAR REACTIONS ${ }^{9} \mathrm{Be}\left({ }^{78} \mathrm{Kr}, \mathrm{X}\right){ }^{60} \mathrm{Ge} /{ }^{61} \mathrm{Ge} /{ }^{62} \mathrm{Ge} /{ }^{63} \mathrm{Ge} /$ ${ }^{64} \mathrm{Ge} /{ }^{64} \mathrm{Se} /{ }^{65} \mathrm{Se} /{ }^{66} \mathrm{Se} /{ }^{67} \mathrm{Se} /{ }^{68} \mathrm{Se}, \mathrm{E}=140 \mathrm{MeV} /$ nucleon; measured production $\sigma$, isotopic yields; deduced no evidence for ${ }^{59} \mathrm{Ga}$, ${ }^{63}$ As. JOUR ZAANE 25 s01 335

\section{$\mathrm{A}=63$}

${ }^{63} \mathrm{~V} \quad 2005 \mathrm{GA01}$

NUCLEAR REACTIONS ${ }^{58} \mathrm{Ni}\left({ }^{76} \mathrm{Ge}, \mathrm{X}\right){ }^{57} \mathrm{Sc} /{ }^{58} \mathrm{Sc} /{ }^{58} \mathrm{Ti} /{ }^{59} \mathrm{Ti} /$ ${ }^{60} \mathrm{Ti} /{ }^{60} \mathrm{~V} /{ }^{61} \mathrm{~V} /{ }^{62} \mathrm{~V} /{ }^{63} \mathrm{~V} /{ }^{62} \mathrm{Cr} /{ }^{63} \mathrm{Cr} /{ }^{64} \mathrm{Cr} /{ }^{65} \mathrm{Cr} /{ }^{66} \mathrm{Cr} /{ }^{65} \mathrm{Mn}$ / ${ }^{66} \mathrm{Mn}, \mathrm{E}=61.8 \mathrm{MeV} /$ nucleon; measured yields. JOUR ZAANE 2341 


\section{$\mathrm{A}=63$ (continued)}

${ }^{63} \mathrm{Cr} \quad$ 2005GA01

2005GA01

2005NIZZ

${ }^{63} \mathrm{Mn}$

2005GA01

2005NIZZ

${ }^{63} \mathrm{Co}$

2005PE12

2005PE12

${ }^{63} \mathrm{Ni}$

2004AHZZ

2005GE09

2005NA08

2005NA31

2005PE12

2005PE23

2005SE23

${ }^{63} \mathrm{Cu}$

2005PE23
RADIOACTIVITY ${ }^{57,58} \mathrm{Sc},{ }^{58,59,60} \mathrm{Ti},{ }^{61} \mathrm{~V},{ }^{62,63,64,65,66} \mathrm{Cr}\left(\beta^{-}\right)$[from $\left.{ }^{58} \mathrm{Ni}\left({ }^{76} \mathrm{Ge}, \mathrm{X}\right)\right]$; measured $\mathrm{E} \gamma, \mathrm{E} \beta, \beta \gamma$-coin, $\mathrm{T}_{1 / 2} .{ }^{58} \mathrm{~V},{ }^{61} \mathrm{Cr},{ }^{62} \mathrm{Mn}$ deduced levels, J, $\pi .{ }^{58} \mathrm{~V},{ }^{61} \mathrm{Cr},{ }^{62,63,64,65} \mathrm{Mn}$ deduced transitions. ${ }^{60} \mathrm{~V}$, ${ }^{62} \mathrm{Mn}$ deduced isomeric states. Comparison with model predictions. JOUR ZAANE 2341 NUCLEAR REACTIONS ${ }^{58} \mathrm{Ni}\left({ }^{76} \mathrm{Ge}, \mathrm{X}\right){ }^{57} \mathrm{Sc} /{ }^{58} \mathrm{Sc} /{ }^{58} \mathrm{Ti} /{ }^{59} \mathrm{Ti} /$ ${ }^{60} \mathrm{Ti} /{ }^{60} \mathrm{~V} /{ }^{61} \mathrm{~V} /{ }^{62} \mathrm{~V} /{ }^{63} \mathrm{~V} /{ }^{62} \mathrm{Cr} /{ }^{63} \mathrm{Cr} /{ }^{64} \mathrm{Cr} /{ }^{65} \mathrm{Cr} /{ }^{66} \mathrm{Cr} /{ }^{65} \mathrm{Mn}$ / ${ }^{66} \mathrm{Mn}, \mathrm{E}=61.8 \mathrm{MeV}$ / nucleon; measured yields. JOUR ZAANE 2341 RADIOACTIVITY ${ }^{63,64} \mathrm{Cr},{ }^{65,66} \mathrm{Mn},{ }^{67,68} \mathrm{Fe},{ }^{69,70} \mathrm{Co}\left(\beta^{-}\right)$[from $\left.\mathrm{Be}\left({ }^{86} \mathrm{Kr}, \mathrm{X}\right)\right]$; measured $\mathrm{E} \beta, \mathrm{T}_{1 / 2}$. Fragment separator, comparisons with previous results and model predictions. CONF Riken(Origin of Matter) Proc,P304,Nishimura RADIOACTIVITY ${ }^{57,58} \mathrm{Sc},{ }^{58,59,60} \mathrm{Ti},{ }^{61} \mathrm{~V},{ }^{62,63,64,65,66} \mathrm{Cr}\left(\beta^{-}\right)$[from $\left.{ }^{58} \mathrm{Ni}\left({ }^{76} \mathrm{Ge}, \mathrm{X}\right)\right]$; measured $\mathrm{E} \gamma, \mathrm{E} \beta, \beta \gamma$-coin, $\mathrm{T}_{1 / 2} .{ }^{58} \mathrm{~V},{ }^{61} \mathrm{Cr},{ }^{62} \mathrm{Mn}$ deduced levels, J, $\pi .{ }^{58} \mathrm{~V},{ }^{61} \mathrm{Cr},{ }^{62,63,64,65} \mathrm{Mn}$ deduced transitions. ${ }^{60} \mathrm{~V}$, ${ }^{62} \mathrm{Mn}$ deduced isomeric states. Comparison with model predictions. JOUR ZAANE 2341 RADIOACTIVITY ${ }^{63,64} \mathrm{Cr},{ }^{65,66} \mathrm{Mn},{ }^{67,68} \mathrm{Fe},{ }^{69,70} \mathrm{Co}\left(\beta^{-}\right)$[from $\left.\mathrm{Be}\left({ }^{86} \mathrm{Kr}, \mathrm{X}\right)\right]$; measured $\mathrm{E} \beta, \mathrm{T}_{1 / 2}$. Fragment separator, comparisons with previous results and model predictions. CONF Riken(Origin of Matter) Proc,P304,Nishimura NUCLEAR REACTIONS ${ }^{197} \mathrm{Au}\left({ }^{65} \mathrm{Cu}, \mathrm{X}\right){ }^{62} \mathrm{Co} /{ }^{63} \mathrm{Co}, \mathrm{E} \approx 400-460$ $\mathrm{MeV}$; measured yields. Ion-guide isotope separator. JOUR NIMAE 546 418

RADIOACTIVITY ${ }^{63} \mathrm{Co}\left(\beta^{-}\right)$[from ${ }^{197} \mathrm{Au}\left({ }^{65} \mathrm{Cu}, \mathrm{X}\right)$ ]; measured $\beta$-delayed $\mathrm{E} \gamma, \mathrm{I} \gamma$. Ion-guide isotope separator. JOUR NIMAE 546418 NUCLEAR REACTIONS ${ }^{62} \mathrm{Ni}(\mathrm{n}, \gamma), \mathrm{E}=$ low; measured capture $\sigma$ for neutron spectrum with $\mathrm{kT}=25 \mathrm{keV}$. Accelerator mass spectrometry. REPT ANL-04/22,P15,Ahmad NUCLEAR REACTIONS ${ }^{62,64} \mathrm{Ni}(\mathrm{d}, \mathrm{p})$, E not given; measured $\mathrm{E} \gamma$, $\mathrm{I} \gamma(\theta, \mathrm{H}, \mathrm{t}) .{ }^{65} \mathrm{Ni}$ deduced isomeric state $\mathrm{g}$ factor. Time dependent perturbed angular correlation technique, comparison with model predictions. JOUR JPGPE 31 S1439

NUCLEAR REACTIONS ${ }^{62} \mathrm{Ni}(\mathrm{n}, \gamma), \mathrm{E}=$ spectrum; measured total $\sigma$. Fast-neutron activation, accelerator mass spectrometry. Astrophysical implications discussed. JOUR PRLTA 94092504 NUCLEAR REACTIONS ${ }^{62} \mathrm{Ni}(\mathrm{n}, \gamma), \mathrm{E}=5,5-90 \mathrm{MeV}$; measured $\mathrm{E} \gamma$, I $\gamma$, capture $\sigma$; deduced Maxwellian averaged $\sigma$. JOUR NUPAB 758 $537 \mathrm{c}$

RADIOACTIVITY ${ }^{63} \mathrm{Co}\left(\beta^{-}\right)$[from ${ }^{197} \mathrm{Au}\left({ }^{65} \mathrm{Cu}, \mathrm{X}\right)$ ]; measured $\beta$-delayed $\mathrm{E} \gamma, \mathrm{I} \gamma$. Ion-guide isotope separator. JOUR NIMAE 546418 RADIOACTIVITY ${ }^{63} \mathrm{Cu}\left(\right.$ EC) [from ${ }^{197} \mathrm{Au}\left({ }^{65} \mathrm{Cu}, \mathrm{X}\right)$ ]; measured $\beta$-delayed E $\gamma$, I $\gamma$. JOUR ZAANE 25 s01 749 NUCLEAR REACTIONS ${ }^{197} \mathrm{Au}(\mathrm{n}, \gamma), \mathrm{E}=$ spectrum; measured $\mathrm{E} \gamma, \mathrm{I} \gamma$; deduced neutron flux. ${ }^{7} \operatorname{Li}(\mathrm{p}, \mathrm{n})$, E not given; deduced neutron spectrum. ${ }^{62} \mathrm{Ni}(\mathrm{n}, \gamma), \mathrm{E} \approx 5.5-20 \mathrm{keV}$; measured $\sigma$; deduced Maxwellian-averaged $\sigma$. JOUR JUPSA 742981 RADIOACTIVITY ${ }^{63} \mathrm{Cu}(\mathrm{EC})$ [from ${ }^{197} \mathrm{Au}\left({ }^{65} \mathrm{Cu}, \mathrm{X}\right)$ ]; measured $\beta$-delayed $\mathrm{E} \gamma, \mathrm{I} \gamma$. JOUR ZAANE 25 s01 749 


\section{$\mathrm{A}=63$ (continued)}

2005PE23 NUCLEAR REACTIONS ${ }^{197} \mathrm{Au}\left({ }^{65} \mathrm{Cu}, \mathrm{X}\right){ }^{62} \mathrm{Cu} /{ }^{63} \mathrm{Cu}, \mathrm{E}=443 \mathrm{MeV}$; measured yields. JOUR ZAANE 25 s01 749

\begin{tabular}{|c|c|c|}
\hline \multirow[b]{2}{*}{${ }^{63} \mathrm{Zn}$} & \multirow[b]{2}{*}{ 2005NAZY } & \\
\hline & & $\begin{array}{l}\text { NUCLEAR REACTIONS }{ }^{27} \mathrm{Al}(\mathrm{d}, \mathrm{X}){ }^{27} \mathrm{Mg} /{ }^{24} \mathrm{Na}, \mathrm{E}=22-40 \mathrm{MeV} \\
\mathrm{Cu}(\mathrm{d}, \mathrm{X}){ }^{62} \mathrm{Zn} /{ }^{63} \mathrm{Zn} /{ }^{61} \mathrm{Cu} /{ }^{64} \mathrm{Cu}, \mathrm{E}=22-40 \mathrm{MeV} ; \mathrm{W}(\mathrm{d}, \mathrm{X}){ }^{181} \mathrm{Re} / \\
{ }^{182} \mathrm{Re} /{ }^{183} \mathrm{Re} /{ }^{184} \mathrm{Re} /{ }^{186} \mathrm{Re} /{ }^{187} \mathrm{~W}, \mathrm{E}=22-40 \mathrm{MeV} ; \text { measured } \\
\text { activation } \sigma . \text { Comparison with previous results and model predictions. } \\
\text { CONF Santa Fe (Nucl Data for Sci and Technol) Proc,Vol2,P1489 }\end{array}$ \\
\hline \multirow[t]{2}{*}{${ }^{63} \mathrm{Ge}$} & 2005ST29 & $\begin{array}{l}\text { NUCLEAR REACTIONS }{ }^{9} \mathrm{Be}\left({ }^{78} \mathrm{Kr}, \mathrm{X}\right){ }^{60} \mathrm{Ge} /{ }^{61} \mathrm{Ge} /{ }^{62} \mathrm{Ge} /{ }^{63} \mathrm{Ge} / \\
{ }^{64} \mathrm{Ge} /{ }^{64} \mathrm{Se} /{ }^{65} \mathrm{Se} /{ }^{66} \mathrm{Se} /{ }^{67} \mathrm{Se} /{ }^{68} \mathrm{Se}, \mathrm{E}=140 \mathrm{MeV} / \text { nucleon; } \\
\text { measured production } \sigma \text {, isotopic yields; deduced no evidence for }{ }^{59} \mathrm{Ga} \text {, } \\
{ }^{63} \text { As. }{ }^{60} \mathrm{Ge},{ }^{64} \mathrm{Se} \text { deduced } \mathrm{T}_{1 / 2} \text { lower limits. }{ }^{59} \mathrm{Ga},{ }^{63} \text { As deduced } \mathrm{T}_{1 / 2} \\
\text { upper limits. JOUR PYLBB } 62732\end{array}$ \\
\hline & 2005ST34 & $\begin{array}{l}\text { NUCLEAR REACTIONS }{ }^{9} \mathrm{Be}\left({ }^{78} \mathrm{Kr}, \mathrm{X}\right){ }^{60} \mathrm{Ge} /{ }^{61} \mathrm{Ge} /{ }^{62} \mathrm{Ge} /{ }^{63} \mathrm{Ge} / \\
{ }^{64} \mathrm{Ge} /{ }^{64} \mathrm{Se} /{ }^{65} \mathrm{Se} /{ }^{66} \mathrm{Se} /{ }^{67} \mathrm{Se} /{ }^{68} \mathrm{Se}, \mathrm{E}=140 \mathrm{MeV} / \text { nucleon; } \\
\text { measured production } \sigma \text {, isotopic yields; deduced no evidence for }{ }^{59} \mathrm{Ga} \text {, } \\
{ }^{63} \mathrm{As} \text {. JOUR ZAANE } 25 \text { s01 } 335\end{array}$ \\
\hline \multirow[t]{2}{*}{${ }^{63} \mathrm{As}$} & 2005ST29 & $\begin{array}{l}\text { NUCLEAR REACTIONS }{ }^{9} \mathrm{Be}\left({ }^{78} \mathrm{Kr}, \mathrm{X}\right){ }^{60} \mathrm{Ge} /{ }^{61} \mathrm{Ge} /{ }^{62} \mathrm{Ge} /{ }^{63} \mathrm{Ge} / \\
{ }^{64} \mathrm{Ge} /{ }^{64} \mathrm{Se} /{ }^{65} \mathrm{Se} /{ }^{66} \mathrm{Se} /{ }^{67} \mathrm{Se} /{ }^{68} \mathrm{Se}, \mathrm{E}=140 \mathrm{MeV} / \text { nucleon; } \\
\text { measured production } \sigma \text {, isotopic yields; deduced no evidence for }{ }^{59} \mathrm{Ga} \text {, } \\
{ }^{63} \text { As. }{ }^{60} \mathrm{Ge},{ }^{64} \mathrm{Se} \text { deduced } \mathrm{T}_{1 / 2} \text { lower limits. }{ }^{59} \mathrm{Ga},{ }^{63} \text { As deduced } \mathrm{T}_{1 / 2} \\
\text { upper limits. JOUR PYLBB } 62732\end{array}$ \\
\hline & 2005ST34 & $\begin{array}{l}\text { NUCLEAR REACTIONS }{ }^{9} \mathrm{Be}\left({ }^{78} \mathrm{Kr}, \mathrm{X}\right){ }^{60} \mathrm{Ge} /{ }^{61} \mathrm{Ge} /{ }^{62} \mathrm{Ge} /{ }^{63} \mathrm{Ge} / \\
{ }^{64} \mathrm{Ge} /{ }^{64} \mathrm{Se} /{ }^{65} \mathrm{Se} /{ }^{66} \mathrm{Se} /{ }^{67} \mathrm{Se} /{ }^{68} \mathrm{Se}, \mathrm{E}=140 \mathrm{MeV} / \text { nucleon; } \\
\text { measured production } \sigma \text {, isotopic yields; deduced no evidence for }{ }^{59} \mathrm{Ga} \text {, } \\
{ }^{63} \text { As. JOUR ZAANE } 25 \text { s01 } 335\end{array}$ \\
\hline
\end{tabular}

\section{$\mathrm{A}=64$}

${ }^{64} \mathrm{Cr} \quad 2005 \mathrm{GA0} 01$

RADIOACTIVITY ${ }^{57,58} \mathrm{Sc},{ }^{58,59,60} \mathrm{Ti},{ }^{61} \mathrm{~V},{ }^{62,63,64,65,66} \mathrm{Cr}\left(\beta^{-}\right)$[from $\left.{ }^{58} \mathrm{Ni}\left({ }^{76} \mathrm{Ge}, \mathrm{X}\right)\right]$; measured $\mathrm{E} \gamma, \mathrm{E} \beta, \beta \gamma$-coin, $\mathrm{T}_{1 / 2} \cdot{ }^{58} \mathrm{~V},{ }^{61} \mathrm{Cr},{ }^{62} \mathrm{Mn}$ deduced levels, J, $\pi .{ }^{58} \mathrm{~V},{ }^{61} \mathrm{Cr},{ }^{62,63,64,65} \mathrm{Mn}$ deduced transitions. ${ }^{60} \mathrm{~V}$, ${ }^{62} \mathrm{Mn}$ deduced isomeric states. Comparison with model predictions. JOUR ZAANE 2341

2005GA01 NUCLEAR REACTIONS ${ }^{58} \mathrm{Ni}\left({ }^{76} \mathrm{Ge}, \mathrm{X}\right){ }^{57} \mathrm{Sc} /{ }^{58} \mathrm{Sc} /{ }^{58} \mathrm{Ti} /{ }^{59} \mathrm{Ti} /$ ${ }^{60} \mathrm{Ti} /{ }^{60} \mathrm{~V} /{ }^{61} \mathrm{~V} /{ }^{62} \mathrm{~V} /{ }^{63} \mathrm{~V} /{ }^{62} \mathrm{Cr} /{ }^{63} \mathrm{Cr} /{ }^{64} \mathrm{Cr} /{ }^{65} \mathrm{Cr} /{ }^{66} \mathrm{Cr} /{ }^{65} \mathrm{Mn}$ / ${ }^{66} \mathrm{Mn}, \mathrm{E}=61.8 \mathrm{MeV} /$ nucleon; measured yields. JOUR ZAANE 2341

2005NIZZ RADIOACTIVITY ${ }^{63,64} \mathrm{Cr},{ }^{65,66} \mathrm{Mn},{ }^{67,68} \mathrm{Fe},{ }^{69,70} \mathrm{Co}\left(\beta^{-}\right)$[from $\left.\mathrm{Be}\left({ }^{86} \mathrm{Kr}, \mathrm{X}\right)\right]$; measured $\mathrm{E} \beta, \mathrm{T}_{1 / 2}$. Fragment separator, comparisons with previous results and model predictions. CONF Riken(Origin of Matter) Proc,P304,Nishimura

${ }^{64} \mathrm{Mn}$ 2005GA01 RADIOACTIVITY ${ }^{57,58} \mathrm{Sc},{ }^{58,59,60} \mathrm{Ti},{ }^{61} \mathrm{~V},{ }^{62,63,64,65,66} \mathrm{Cr}\left(\beta^{-}\right)$[from $\left.{ }^{58} \mathrm{Ni}\left({ }^{76} \mathrm{Ge}, \mathrm{X}\right)\right]$; measured $\mathrm{E} \gamma, \mathrm{E} \beta, \beta \gamma$-coin, $\mathrm{T}_{1 / 2} \cdot{ }^{58} \mathrm{~V},{ }^{61} \mathrm{Cr},{ }^{62} \mathrm{Mn}$ deduced levels, J, $\pi .{ }^{58} \mathrm{~V},{ }^{61} \mathrm{Cr},{ }^{62,63,64,65} \mathrm{Mn}$ deduced transitions. ${ }^{60} \mathrm{~V}$, ${ }^{62} \mathrm{Mn}$ deduced isomeric states. Comparison with model predictions. JOUR ZAANE 2341 


\section{$\mathrm{A}=64$ (continued)}

2005NIZZ RADIOACTIVITY ${ }^{63,64} \mathrm{Cr},{ }^{65,66} \mathrm{Mn},{ }^{67,68} \mathrm{Fe},{ }^{69,70} \mathrm{Co}\left(\beta^{-}\right)$[from $\left.\mathrm{Be}\left({ }^{86} \mathrm{Kr}, \mathrm{X}\right)\right]$; measured $\mathrm{E} \beta, \mathrm{T}_{1 / 2}$. Fragment separator, comparisons with previous results and model predictions. CONF Riken(Origin of Matter) Proc,P304,Nishimura

${ }^{64} \mathrm{Ni} \quad 2005 \mathrm{ZU} 01$ RADIOACTIVITY ${ }^{120} \mathrm{Te}\left(\beta^{+} \mathrm{EC}\right) ;{ }^{64} \mathrm{Zn},{ }^{106,108} \mathrm{Cd},{ }^{120} \mathrm{Te}(2 \mathrm{EC})$; measured $\mathrm{T}_{1 / 2}$ lower limits. JOUR NPBSE 138236

${ }^{64} \mathrm{Cu} \quad$ 2004AG09 NUCLEAR REACTIONS ${ }^{103} \mathrm{Rh}\left(\mathrm{n}, \mathrm{n}\right.$ ') ${ }^{103 m} \mathrm{Rh}, \mathrm{E} \approx 4.8 \mathrm{MeV} ;{ }^{115} \mathrm{In}(\mathrm{n}$, n') ${ }^{115 m} \mathrm{In}, \mathrm{E} \approx 5 \mathrm{MeV} ;{ }^{232} \mathrm{Th},{ }^{238} \mathrm{U}(\mathrm{n}, \mathrm{F}), \mathrm{E} \approx 5 \mathrm{MeV} ;{ }^{24} \mathrm{Mg},{ }^{27} \mathrm{Al}$, ${ }^{46,47,48} \mathrm{Ti},{ }^{54,56} \mathrm{Fe},{ }^{58} \mathrm{Ni},{ }^{64} \mathrm{Zn}(\mathrm{n}, \mathrm{p}), \mathrm{E} \approx 2-8 \mathrm{MeV} ;{ }^{27} \mathrm{Al},{ }^{59} \mathrm{Co}(\mathrm{n}, \alpha), \mathrm{E}$ $\approx 8.3 \mathrm{MeV}$; measured activation $\sigma$. Spectrum average technique, comparison with previous results. JOUR RAACA 9263

2005B010 NUCLEAR REACTIONS Zn(p, X) ${ }^{64} \mathrm{Cu} /{ }^{57} \mathrm{Ni} /{ }^{56} \mathrm{Ni} /{ }^{52} \mathrm{Mn} /{ }^{54} \mathrm{Mn}$ $/{ }^{62} \mathrm{Zn} /{ }^{65} \mathrm{Zn} /{ }^{51} \mathrm{Cr} /{ }^{48} \mathrm{~V} /{ }^{55} \mathrm{Co} /{ }^{56} \mathrm{Co} /{ }^{57} \mathrm{Co} /{ }^{58} \mathrm{Co} /{ }^{60} \mathrm{Co} /{ }^{66} \mathrm{Ga}$ $/{ }^{67} \mathrm{Ga} /{ }^{52} \mathrm{Fe} /{ }^{59} \mathrm{Fe}, \mathrm{E} \approx 31-141 \mathrm{MeV}$; measured production $\sigma$. Stacked-foil activation. JOUR JRNCD 264101

2005HIZX NUCLEAR REACTIONS ${ }^{66} \mathrm{Zn}(\mathrm{d}, \alpha), \mathrm{E}=5-14 \mathrm{MeV} ; \mathrm{Ce}\left({ }^{3} \mathrm{He}, \mathrm{xn}\right){ }^{140} \mathrm{Nd}$, $\mathrm{E}=16-35 \mathrm{MeV} ;{ }^{141} \mathrm{Ce}(\mathrm{p}, 2 \mathrm{n}), \mathrm{E}=10-45 \mathrm{MeV} ;{ }^{192} \mathrm{Os}(\mathrm{p}, \mathrm{n}), \mathrm{E}=6-19 \mathrm{MeV}$; measured excitation functions; deduced thick-target yields. Stacked-foil activation technique. CONF Santa Fe (Nucl Data for Sci and Technol) Proc, Vol2,P1631

2005MAZP NUCLEAR REACTIONS ${ }^{64} \mathrm{Zn}(\mathrm{n}, \mathrm{p}),{ }^{64} \mathrm{Zn},{ }^{63,65} \mathrm{Cu}(\mathrm{n}, 2 \mathrm{n}), \mathrm{E} \approx 10-15$ $\mathrm{MeV}$; measured $\sigma$. Comparison with previous results. CONF Santa Fe (Nucl Data for Sci and Technol) Proc,Vol1,P609

2005NAZY NUCLEAR REACTIONS ${ }^{27} \mathrm{Al}(\mathrm{d}, \mathrm{X})^{27} \mathrm{Mg} /{ }^{24} \mathrm{Na}, \mathrm{E}=22-40 \mathrm{MeV}$; $\mathrm{Cu}(\mathrm{d}, \mathrm{X}){ }^{62} \mathrm{Zn} /{ }^{63} \mathrm{Zn} /{ }^{61} \mathrm{Cu} /{ }^{64} \mathrm{Cu}, \mathrm{E}=22-40 \mathrm{MeV} ; \mathrm{W}(\mathrm{d}, \mathrm{X}){ }^{181} \mathrm{Re} /$ ${ }^{182} \operatorname{Re} /{ }^{183} \operatorname{Re} /{ }^{184} \operatorname{Re} /{ }^{186} \operatorname{Re} /{ }^{187} \mathrm{~W}, \mathrm{E}=22-40 \mathrm{MeV}$; measured activation $\sigma$. Comparison with previous results and model predictions. CONF Santa Fe (Nucl Data for Sci and Technol) Proc,Vol2,P1489

2005 P017 NUCLEAR REACTIONS ${ }^{64} \mathrm{Ni}\left({ }^{3} \mathrm{He}, \mathrm{t}\right), \mathrm{E}=140 \mathrm{MeV}$ / nucleon; measured triton spectra, $\sigma(\theta) .{ }^{64} \mathrm{Cu}$ deduced levels, J, $\pi$, Gamow-Teller strength distribution. JOUR JPGPE 31 S1945

2005SHZS NUCLEAR REACTIONS ${ }^{65} \mathrm{Cu}\left({ }^{6} \mathrm{Li}, \mathrm{d} \alpha\right),\left({ }^{6} \mathrm{Li},{ }^{7} \mathrm{Li}\right),\left({ }^{6} \mathrm{Li},{ }^{3} \mathrm{He}\right),\left({ }^{6} \mathrm{Li}\right.$, $\alpha),\left({ }^{6} \mathrm{Li}, \alpha \mathrm{X}\right),\left({ }^{7} \mathrm{Li}, \mathrm{t} \alpha\right),\left({ }^{7} \mathrm{Li}, \mathrm{d} \alpha\right),\left({ }^{7} \mathrm{Li},{ }^{6} \mathrm{Li}\right),\left({ }^{7} \mathrm{Li},{ }^{6} \mathrm{He}\right),\left({ }^{7} \mathrm{Li}, \alpha\right),\left({ }^{7} \mathrm{Li}\right.$, $\alpha \mathrm{X}), \mathrm{E}=25 \mathrm{MeV}$; measured particle spectra, $\sigma, \sigma(\theta)$; deduced reaction mechanism features. Comparison with coupled channels predictions. PREPRINT nucl-ex/0512032,12/21/2005

2005SZ04 NUCLEAR REACTIONS Zn, ${ }^{68} \mathrm{Zn}(\mathrm{p}, \mathrm{X}){ }^{64} \mathrm{Cu}, \mathrm{E} \approx 18-100 \mathrm{MeV}$; ${ }^{66} \mathrm{Zn}(\mathrm{p}, \mathrm{n} 2 \mathrm{p}), \mathrm{E} \approx 35-100 \mathrm{MeV}$; measured production $\sigma$. Stacked-foil activation technique. JOUR NIMBE 240625

${ }^{64} \mathrm{Zn} \quad 2005 \mathrm{CH} 60$ ATOMIC MASSES ${ }^{64} \mathrm{Zn},{ }^{64} \mathrm{Ga},{ }^{68} \mathrm{Ge},{ }^{68} \mathrm{As},{ }^{68,72} \mathrm{Se},{ }^{76} \mathrm{Kr},{ }^{76} \mathrm{Rb},{ }^{80} \mathrm{Sr}$, ${ }^{80} \mathrm{Y}$; measured masses. Direct time-of-flight technique, comparison with previous results. JOUR JPGPE 31 S1771

2005 G009 NUCLEAR REACTIONS ${ }^{64} \mathrm{Zn}\left({ }^{6} \mathrm{Li}, \mathrm{X}\right),\left({ }^{7} \mathrm{Li}, \mathrm{X}\right),\left({ }^{9} \mathrm{Be}, \mathrm{X}\right),\left({ }^{16} \mathrm{O}, \mathrm{X}\right), \mathrm{E}$ $\approx 16-69 \mathrm{MeV}$; measured fusion and reaction $\sigma$; deduced reaction mechanism features. ${ }^{64} \mathrm{Zn}\left({ }^{9} \mathrm{Be},{ }^{9} \mathrm{Be}\right), \mathrm{E}=17-28 \mathrm{MeV} ;{ }^{64} \mathrm{Zn}\left({ }^{16} \mathrm{O},{ }^{16} \mathrm{O}\right)$, $\mathrm{E}=40-64 \mathrm{MeV}$; measured elastic $\sigma(\theta)$. Coupled channels analysis. JOUR PRVCA 71034608 


\section{$\mathrm{A}=64$ (continued)}

2005LE12 NUCLEAR REACTIONS C $\left({ }^{64} \mathrm{Zn},{ }^{64} \mathrm{Zn}\right),\left({ }^{68} \mathrm{Zn},{ }^{68} \mathrm{Zn}\right), \mathrm{E}=180 \mathrm{MeV}$; measured $\mathrm{E} \gamma, \mathrm{I} \gamma(\theta, \mathrm{H}, \mathrm{t}), \gamma \gamma-$, (particle) $\gamma$-coin, DSA following projectile Coulomb excitation. ${ }^{64,68} \mathrm{Zn}$ levels deduced $\mathrm{g}$ factors, $\mathrm{T}_{1 / 2}$, $\mathrm{B}(\mathrm{E} 2)$. Transient-field technique, large-scale shell model calculations. JOUR PRVCA 71034303

2005LE38 NUCLEAR REACTIONS C( $\left.{ }^{68} \mathrm{Zn},{ }^{68} \mathrm{Zn}\right), \mathrm{E}=180 \mathrm{MeV}$; measured E $\gamma$, $\mathrm{I} \gamma(\theta, \mathrm{H}, \mathrm{t})$ (particle) $\gamma$-coin following projectile Coulomb excitation. ${ }^{68} \mathrm{Zn}$ levels deduced $\mathrm{g}$ factors, $\mathrm{T}_{1 / 2}, \mathrm{~B}(\mathrm{E} 2)$, configurations. ${ }^{64} \mathrm{Zn}$ levels analyzed $\mathrm{g}$ factors, B(E2). Large-scale shell-model calculations. JOUR PRVCA 72044301

2005ZU01 RADIOACTIVITY ${ }^{120} \mathrm{Te}\left(\beta^{+} \mathrm{EC}\right) ;{ }^{64} \mathrm{Zn},{ }^{106,108} \mathrm{Cd},{ }^{120} \mathrm{Te}(2 \mathrm{EC})$; measured $\mathrm{T}_{1 / 2}$ lower limits. JOUR NPBSE 138236

\section{${ }^{64} \mathrm{Ga} \quad 2005 \mathrm{CH} 60$}

${ }^{64} \mathrm{Ge} \quad$ 2005CL08 ATOMIC MASSES ${ }^{64} \mathrm{Zn},{ }^{64} \mathrm{Ga},{ }^{68} \mathrm{Ge},{ }^{68} \mathrm{As},{ }^{68,72} \mathrm{Se},{ }^{76} \mathrm{Kr},{ }^{76} \mathrm{Rb},{ }^{80} \mathrm{Sr}$, ${ }^{80} \mathrm{Y}$; measured masses. Direct time-of-flight technique, comparison with previous results. JOUR JPGPE 31 S1771

ATOMIC MASSES ${ }^{64} \mathrm{Ge},{ }^{68} \mathrm{Se}$; analyzed masses; deduced effective $\mathrm{T}_{1 / 2} \cdot{ }^{90,91} \mathrm{Mo},{ }^{90,91,92,93} \mathrm{Tc},{ }^{93,94} \mathrm{Ru},{ }^{94,95} \mathrm{Rh},{ }^{104,105,106,107} \mathrm{In}$, $104,105,107,108$ Sn, ${ }^{107,108}$ Sb; measured masses. Penning trap, astrophysical implications discussed. JOUR ZAANE 25 s01 629 2005ST29 NUCLEAR REACTIONS ${ }^{9} \mathrm{Be}\left({ }^{78} \mathrm{Kr}, \mathrm{X}\right){ }^{60} \mathrm{Ge} /{ }^{61} \mathrm{Ge} /{ }^{62} \mathrm{Ge} /{ }^{63} \mathrm{Ge} /$ ${ }^{64} \mathrm{Ge} /{ }^{64} \mathrm{Se} /{ }^{65} \mathrm{Se} /{ }^{66} \mathrm{Se} /{ }^{67} \mathrm{Se} /{ }^{68} \mathrm{Se}, \mathrm{E}=140 \mathrm{MeV} /$ nucleon; measured production $\sigma$, isotopic yields; deduced no evidence for ${ }^{59} \mathrm{Ga}$, ${ }^{63}$ As. ${ }^{60} \mathrm{Ge},{ }^{64}$ Se deduced $\mathrm{T}_{1 / 2}$ lower limits. ${ }^{59} \mathrm{Ga},{ }^{63}$ As deduced $\mathrm{T}_{1 / 2}$ upper limits. JOUR PYLBB 62732

2005ST34 NUCLEAR REACTIONS ${ }^{9} \mathrm{Be}\left({ }^{78} \mathrm{Kr}, \mathrm{X}\right){ }^{60} \mathrm{Ge} /{ }^{61} \mathrm{Ge} /{ }^{62} \mathrm{Ge} /{ }^{63} \mathrm{Ge} /$ ${ }^{64} \mathrm{Ge} /{ }^{64} \mathrm{Se} /{ }^{65} \mathrm{Se} /{ }^{66} \mathrm{Se} /{ }^{67} \mathrm{Se} /{ }^{68} \mathrm{Se}, \mathrm{E}=140 \mathrm{MeV} /$ nucleon; measured production $\sigma$, isotopic yields; deduced no evidence for ${ }^{59} \mathrm{Ga}$, ${ }^{63}$ As. JOUR ZAANE 25 s01 335

${ }^{64} \mathrm{Se} \quad 2005 \mathrm{ST} 29$ NUCLEAR REACTIONS ${ }^{9} \mathrm{Be}\left({ }^{78} \mathrm{Kr}, \mathrm{X}\right){ }^{60} \mathrm{Ge} /{ }^{61} \mathrm{Ge} /{ }^{62} \mathrm{Ge} /{ }^{63} \mathrm{Ge} /$ ${ }^{64} \mathrm{Ge} /{ }^{64} \mathrm{Se} /{ }^{65} \mathrm{Se} /{ }^{66} \mathrm{Se} /{ }^{67} \mathrm{Se} /{ }^{68} \mathrm{Se}, \mathrm{E}=140 \mathrm{MeV} /$ nucleon; measured production $\sigma$, isotopic yields; deduced no evidence for ${ }^{59} \mathrm{Ga}$, ${ }^{63}$ As. ${ }^{60} \mathrm{Ge},{ }^{64}$ Se deduced $\mathrm{T}_{1 / 2}$ lower limits. ${ }^{59} \mathrm{Ga},{ }^{63}$ As deduced $\mathrm{T}_{1 / 2}$ upper limits. JOUR PYLBB 62732

2005ST34 NUCLEAR REACTIONS ${ }^{9} \mathrm{Be}\left({ }^{78} \mathrm{Kr}, \mathrm{X}\right){ }^{60} \mathrm{Ge} /{ }^{61} \mathrm{Ge} /{ }^{62} \mathrm{Ge} /{ }^{63} \mathrm{Ge} /$ ${ }^{64} \mathrm{Ge} /{ }^{64} \mathrm{Se} /{ }^{65} \mathrm{Se} /{ }^{66} \mathrm{Se} /{ }^{67} \mathrm{Se} /{ }^{68} \mathrm{Se}, \mathrm{E}=140 \mathrm{MeV} /$ nucleon; measured production $\sigma$, isotopic yields; deduced no evidence for ${ }^{59} \mathrm{Ga}$, ${ }^{63}$ As. JOUR ZAANE 25 s01 335

\section{$\mathrm{A}=65$}

${ }^{65} \mathrm{Cr} \quad$ 2005GA01

RADIOACTIVITY ${ }^{57,58} \mathrm{Sc},{ }^{58,59,60} \mathrm{Ti},{ }^{61} \mathrm{~V},{ }^{62,63,64,65,66} \mathrm{Cr}\left(\beta^{-}\right)$[from $\left.{ }^{58} \mathrm{Ni}\left({ }^{76} \mathrm{Ge}, \mathrm{X}\right)\right]$; measured $\mathrm{E} \gamma, \mathrm{E} \beta, \beta \gamma$-coin, $\mathrm{T}_{1 / 2} .{ }^{58} \mathrm{~V},{ }^{61} \mathrm{Cr},{ }^{62} \mathrm{Mn}$ deduced levels, J, $\pi .{ }^{58} \mathrm{~V},{ }^{61} \mathrm{Cr},{ }^{62,63,64,65} \mathrm{Mn}$ deduced transitions. ${ }^{60} \mathrm{~V}$, ${ }^{62} \mathrm{Mn}$ deduced isomeric states. Comparison with model predictions. JOUR ZAANE 2341

2005GA01 NUCLEAR REACTIONS ${ }^{58} \mathrm{Ni}\left({ }^{76} \mathrm{Ge}, \mathrm{X}\right)^{57} \mathrm{Sc} /{ }^{58} \mathrm{Sc} /{ }^{58} \mathrm{Ti} /{ }^{59} \mathrm{Ti} /$ ${ }^{60} \mathrm{Ti} /{ }^{60} \mathrm{~V} /{ }^{61} \mathrm{~V} /{ }^{62} \mathrm{~V} /{ }^{63} \mathrm{~V} /{ }^{62} \mathrm{Cr} /{ }^{63} \mathrm{Cr} /{ }^{64} \mathrm{Cr} /{ }^{65} \mathrm{Cr} /{ }^{66} \mathrm{Cr} /{ }^{65} \mathrm{Mn}$ / ${ }^{66} \mathrm{Mn}, \mathrm{E}=61.8 \mathrm{MeV} /$ nucleon; measured yields. JOUR ZAANE 2341 


\section{$\mathrm{A}=65$ (continued)}

\begin{tabular}{|c|c|c|}
\hline \multirow[t]{3}{*}{${ }^{65} \mathrm{Mn}$} & 2005GA01 & $\begin{array}{l}\text { RADIOACTIVITY }{ }^{57,58} \mathrm{Sc},{ }^{58,59,60} \mathrm{Ti},{ }^{61} \mathrm{~V},{ }^{62,63,64,65,66} \mathrm{Cr}\left(\beta^{-}\right)[\text {from } \\
\left.{ }^{58} \mathrm{Ni}\left({ }^{76} \mathrm{Ge}, \mathrm{X}\right)\right] ; \text { measured } \mathrm{E} \gamma, \mathrm{E} \beta, \beta \gamma \text {-coin, } \mathrm{T}_{1 / 2} \cdot{ }^{58} \mathrm{~V},{ }^{61} \mathrm{Cr},{ }^{62} \mathrm{Mn} \\
\text { deduced levels, J, } \pi .{ }^{58} \mathrm{~V},{ }^{61} \mathrm{Cr},{ }^{62,63,64,65} \mathrm{Mn} \text { deduced transitions. }{ }^{60} \mathrm{~V} \text {, } \\
\text { 62 Mn deduced isomeric states. Comparison with model predictions. } \\
\text { JOUR ZAANE } 2341\end{array}$ \\
\hline & 2005 GA01 & $\begin{array}{l}\text { NUCLEAR REACTIONS }{ }^{58} \mathrm{Ni}\left({ }^{76} \mathrm{Ge}, \mathrm{X}\right){ }^{57} \mathrm{Sc} /{ }^{58} \mathrm{Sc} /{ }^{58} \mathrm{Ti} /{ }^{59} \mathrm{Ti} / \\
{ }^{60} \mathrm{Ti} /{ }^{60} \mathrm{~V} /{ }^{61} \mathrm{~V} /{ }^{62} \mathrm{~V} /{ }^{63} \mathrm{~V} /{ }^{62} \mathrm{Cr} /{ }^{63} \mathrm{Cr} /{ }^{64} \mathrm{Cr} /{ }^{65} \mathrm{Cr} /{ }^{66} \mathrm{Cr} /{ }^{65} \mathrm{Mn} \\
/{ }^{66} \mathrm{Mn}, \mathrm{E}=61.8 \mathrm{MeV} / \text { nucleon; measured yields. JOUR ZAANE } 2341\end{array}$ \\
\hline & 2005 NIZZ & $\begin{array}{l}\text { RADIOACTIVITY }{ }^{63,64} \mathrm{Cr},{ }^{65,66} \mathrm{Mn},{ }^{67,68} \mathrm{Fe},{ }^{69,70} \mathrm{Co}\left(\beta^{-}\right)[\text {from } \\
\left.\mathrm{Be}\left({ }^{86} \mathrm{Kr}, \mathrm{X}\right)\right] \text {; measured } \mathrm{E} \beta, \mathrm{T}_{1 / 2} \text {. Fragment separator, comparisons } \\
\text { with previous results and model predictions. CONF Riken(Origin of } \\
\text { Matter) Proc,P304,Nishimura }\end{array}$ \\
\hline${ }^{65} \mathrm{Fe}$ & 2005 NIZZ & $\begin{array}{l}\text { RADIOACTIVITY }{ }^{63,64} \mathrm{Cr},{ }^{65,66} \mathrm{Mn},{ }^{67,68} \mathrm{Fe},{ }^{69,70} \mathrm{Co}\left(\beta^{-}\right) \text {[from } \\
\left.\mathrm{Be}\left({ }^{86} \mathrm{Kr}, \mathrm{X}\right)\right] \text {; measured } \mathrm{E} \beta, \mathrm{T}_{1 / 2} \text {. Fragment separator, comparisons } \\
\text { with previous results and model predictions. CONF Riken(Origin of } \\
\text { Matter) Proc,P304,Nishimura }\end{array}$ \\
\hline${ }^{65} \mathrm{Ni}$ & 2005GE09 & $\begin{array}{l}\text { NUCLEAR REACTIONS }{ }^{62,64} \mathrm{Ni}(\mathrm{d}, \mathrm{p}) \text {, E not given; measured } \mathrm{E} \gamma \text {, } \\
\mathrm{I} \gamma(\theta, \mathrm{H}, \mathrm{t}) .{ }^{65} \mathrm{Ni} \text { deduced isomeric state g factor. Time dependent } \\
\text { perturbed angular correlation technique, comparison with model } \\
\text { predictions. JOUR JPGPE } 31 \mathrm{~S} 1439\end{array}$ \\
\hline \multirow[t]{4}{*}{${ }^{65} \mathrm{Cu}$} & 2005BEZX & $\begin{array}{l}\text { RADIOACTIVITY }{ }^{65} \mathrm{Zn}\left(\beta^{+}\right),(\mathrm{EC}) \text {; measured } \mathrm{E} \gamma, \mathrm{I} \gamma, \beta \gamma \text {-coin; } \\
\text { deduced } \gamma \text {-emission intensities. }{ }^{65} \mathrm{Cu} \text { levels deduced } \beta \text {-feeding } \\
\text { intensities. EUROMET project } 721 \text {. REPT CEA-R- } 6081 \text {,Be }\end{array}$ \\
\hline & 2005IW01 & $\begin{array}{l}\text { RADIOACTIVITY }{ }^{65} \mathrm{Zn}\left(\beta^{+}\right),(\mathrm{EC}) ; \text { measured } \mathrm{E} \gamma, \mathrm{I} \gamma,(\mathrm{X} \text {-ray }) \gamma \text {-coin; } \\
\text { deduced } \gamma \text {-ray emission probability. }{ }^{241} \mathrm{Am}(\alpha) ; \text { measured } \mathrm{E} \gamma, \mathrm{I} \gamma \\
\alpha \gamma \text {-coin; deduced } \gamma \text {-ray emission probabilities. }{ }^{65} \mathrm{Cu},{ }^{237} \mathrm{~Np} \text { deduced } \\
\text { transitions. JOUR ARISE } 63107\end{array}$ \\
\hline & 2005SHZS & $\begin{array}{l}\text { NUCLEAR REACTIONS }{ }^{65} \mathrm{Cu}\left({ }^{6} \mathrm{Li}, \mathrm{d} \alpha\right),\left({ }^{6} \mathrm{Li},{ }^{7} \mathrm{Li}\right),\left({ }^{6} \mathrm{Li},{ }^{3} \mathrm{He}\right),\left({ }^{6} \mathrm{Li},\right. \\
\alpha),\left({ }^{6} \mathrm{Li}, \alpha \mathrm{X}\right),\left({ }^{7} \mathrm{Li}, \mathrm{t} \alpha\right),\left({ }^{7} \mathrm{Li}, \mathrm{d} \alpha\right),\left({ }^{7} \mathrm{Li},{ }^{6} \mathrm{Li}\right),\left({ }^{7} \mathrm{Li},{ }^{6} \mathrm{He}\right),\left({ }^{7} \mathrm{Li}, \alpha\right),\left({ }^{7} \mathrm{Li} \text {, }\right. \\
\alpha \mathrm{X}), \mathrm{E}=25 \mathrm{MeV} \text { measured particle spectra, } \sigma, \sigma(\theta) ; \text { deduced reaction } \\
\text { mechanism features. Comparison with coupled channels predictions. } \\
\text { PREPRINT nucl-ex/0512032,12/21/2005 }\end{array}$ \\
\hline & 2006B001 & $\begin{array}{l}\text { RADIOACTIVITY }{ }^{54} \mathrm{Mn},{ }^{65} \mathrm{Zn}(\mathrm{EC}) ; \text { measured } \beta \gamma \text {-coin. Triple to } \\
\text { double coincidence ratio method. JOUR ARISE } 64124\end{array}$ \\
\hline \multirow[t]{4}{*}{${ }^{65} \mathrm{Zn}$} & 2005BEZX & $\begin{array}{l}\text { RADIOACTIVITY }{ }^{65} \mathrm{Zn}\left(\beta^{+}\right),(\mathrm{EC}) \text {; measured } \mathrm{E} \gamma, \mathrm{I} \gamma, \beta \gamma \text {-coin; } \\
\text { deduced } \gamma \text {-emission intensities. }{ }^{65} \mathrm{Cu} \text { levels deduced } \beta \text {-feeding } \\
\text { intensities. EUROMET project } 721 \text {. REPT CEA-R-6081,Be }\end{array}$ \\
\hline & 2005B010 & $\begin{array}{l}\text { NUCLEAR REACTIONS Zn }(\mathrm{p}, \mathrm{X}){ }^{64} \mathrm{Cu} /{ }^{57} \mathrm{Ni} /{ }^{56} \mathrm{Ni} /{ }^{52} \mathrm{Mn} /{ }^{54} \mathrm{Mn} \\
/{ }^{62} \mathrm{Zn} /{ }^{65} \mathrm{Zn} /{ }^{51} \mathrm{Cr} /{ }^{48} \mathrm{~V} /{ }^{55} \mathrm{Co} /{ }^{56} \mathrm{Co} /{ }^{57} \mathrm{Co} /{ }^{58} \mathrm{Co} /{ }^{60} \mathrm{Co} /{ }^{66} \mathrm{Ga} \\
/{ }^{67} \mathrm{Ga} /{ }^{52} \mathrm{Fe} /{ }^{59} \mathrm{Fe}, \mathrm{E} \approx 31-141 \mathrm{MeV} ; \text { measured production } \sigma . \\
\text { Stacked-foil activation. JOUR JRNCD } 264101\end{array}$ \\
\hline & 2005IW01 & $\begin{array}{l}\text { RADIOACTIVITY }{ }^{65} \mathrm{Zn}\left(\beta^{+}\right),(\mathrm{EC}) ; \text { measured } \mathrm{E} \gamma, \mathrm{I} \gamma,(\mathrm{X} \text {-ray }) \gamma \text {-coin; } \\
\text { deduced } \gamma \text {-ray emission probability. }{ }^{241} \mathrm{Am}(\alpha) ; \text { measured } \mathrm{E} \gamma, \mathrm{I} \gamma \\
\alpha \gamma \text {-coin; deduced } \gamma \text {-ray emission probabilities. }{ }^{65} \mathrm{Cu},{ }^{237} \mathrm{~Np} \text { deduced } \\
\text { transitions. JOUR ARISE } 63107\end{array}$ \\
\hline & 2006B001 & $\begin{array}{l}\text { RADIOACTIVITY }{ }^{54} \mathrm{Mn},{ }^{65} \mathrm{Zn}(\mathrm{EC}) ; \text { measured } \beta \gamma \text {-coin. Triple to } \\
\text { double coincidence ratio method. JOUR ARISE } 64124\end{array}$ \\
\hline
\end{tabular}




\section{$\mathrm{A}=65$ (continued)}

${ }^{65} \mathrm{Se}$

2005ST29

2005ST34

${ }^{66} \mathrm{Cr}$

2005GA01

2005GA01

${ }^{66} \mathrm{Mn}$

2005GA01

2005 NIZZ

${ }^{66} \mathrm{Fe}$

2005NIZZ

${ }^{66} \mathrm{Cu}$

2005SHZS

${ }^{66} \mathrm{Zn}$

2005SHZS
NUCLEAR REACTIONS ${ }^{9} \mathrm{Be}\left({ }^{78} \mathrm{Kr}, \mathrm{X}\right){ }^{60} \mathrm{Ge} /{ }^{61} \mathrm{Ge} /{ }^{62} \mathrm{Ge} /{ }^{63} \mathrm{Ge} /$ ${ }^{64} \mathrm{Ge} /{ }^{64} \mathrm{Se} /{ }^{65} \mathrm{Se} /{ }^{66} \mathrm{Se} /{ }^{67} \mathrm{Se} /{ }^{68} \mathrm{Se}, \mathrm{E}=140 \mathrm{MeV} /$ nucleon; measured production $\sigma$, isotopic yields; deduced no evidence for ${ }^{59} \mathrm{Ga}$, ${ }^{63}$ As. ${ }^{60} \mathrm{Ge},{ }^{64}$ Se deduced $\mathrm{T}_{1 / 2}$ lower limits. ${ }^{59} \mathrm{Ga},{ }^{63} \mathrm{As}$ deduced $\mathrm{T}_{1 / 2}$ upper limits. JOUR PYLBB 62732

NUCLEAR REACTIONS ${ }^{9} \mathrm{Be}\left({ }^{78} \mathrm{Kr}, \mathrm{X}\right){ }^{60} \mathrm{Ge} /{ }^{61} \mathrm{Ge} /{ }^{62} \mathrm{Ge} /{ }^{63} \mathrm{Ge} /$ ${ }^{64} \mathrm{Ge} /{ }^{64} \mathrm{Se} /{ }^{65} \mathrm{Se} /{ }^{66} \mathrm{Se} /{ }^{67} \mathrm{Se} /{ }^{68} \mathrm{Se}, \mathrm{E}=140 \mathrm{MeV} /$ nucleon; measured production $\sigma$, isotopic yields; deduced no evidence for ${ }^{59} \mathrm{Ga}$, ${ }^{63}$ As. JOUR ZAANE 25 s01 335

\section{$\mathrm{A}=66$}

RADIOACTIVITY ${ }^{57,58} \mathrm{Sc},{ }^{58,59,60} \mathrm{Ti},{ }^{61} \mathrm{~V},{ }^{62,63,64,65,66} \mathrm{Cr}\left(\beta^{-}\right)$[from $\left.{ }^{58} \mathrm{Ni}\left({ }^{76} \mathrm{Ge}, \mathrm{X}\right)\right]$; measured $\mathrm{E} \gamma, \mathrm{E} \beta, \beta \gamma$-coin, $\mathrm{T}_{1 / 2} .{ }^{58} \mathrm{~V},{ }^{61} \mathrm{Cr},{ }^{62} \mathrm{Mn}$ deduced levels, J, $\pi \cdot{ }^{58} \mathrm{~V},{ }^{61} \mathrm{Cr},{ }^{62,63,64,65} \mathrm{Mn}$ deduced transitions. ${ }^{60} \mathrm{~V}$, ${ }^{62} \mathrm{Mn}$ deduced isomeric states. Comparison with model predictions. JOUR ZAANE 2341 NUCLEAR REACTIONS ${ }^{58} \mathrm{Ni}\left({ }^{76} \mathrm{Ge}, \mathrm{X}\right){ }^{57} \mathrm{Sc} /{ }^{58} \mathrm{Sc} /{ }^{58} \mathrm{Ti} /{ }^{59} \mathrm{Ti} /$ ${ }^{60} \mathrm{Ti} /{ }^{60} \mathrm{~V} /{ }^{61} \mathrm{~V} /{ }^{62} \mathrm{~V} /{ }^{63} \mathrm{~V} /{ }^{62} \mathrm{Cr} /{ }^{63} \mathrm{Cr} /{ }^{64} \mathrm{Cr} /{ }^{65} \mathrm{Cr} /{ }^{66} \mathrm{Cr} /{ }^{65} \mathrm{Mn}$ $/{ }^{66} \mathrm{Mn}, \mathrm{E}=61.8 \mathrm{MeV} /$ nucleon; measured yields. JOUR ZAANE 2341 RADIOACTIVITY ${ }^{57,58} \mathrm{Sc},{ }^{58,59,60} \mathrm{Ti},{ }^{61} \mathrm{~V},{ }^{62,63,64,65,66} \mathrm{Cr}\left(\beta^{-}\right)$[from $\left.{ }^{58} \mathrm{Ni}\left({ }^{76} \mathrm{Ge}, \mathrm{X}\right)\right]$; measured $\mathrm{E} \gamma, \mathrm{E} \beta, \beta \gamma$-coin, $\mathrm{T}_{1 / 2} \cdot{ }^{58} \mathrm{~V},{ }^{61} \mathrm{Cr},{ }^{62} \mathrm{Mn}$ deduced levels, J, $\pi .{ }^{58} \mathrm{~V},{ }^{61} \mathrm{Cr},{ }^{62,63,64,65} \mathrm{Mn}$ deduced transitions. ${ }^{60} \mathrm{~V}$, ${ }^{62} \mathrm{Mn}$ deduced isomeric states. Comparison with model predictions. JOUR ZAANE 2341

NUCLEAR REACTIONS ${ }^{58} \mathrm{Ni}\left({ }^{76} \mathrm{Ge}, \mathrm{X}\right){ }^{57} \mathrm{Sc} /{ }^{58} \mathrm{Sc} /{ }^{58} \mathrm{Ti} /{ }^{59} \mathrm{Ti} /$ ${ }^{60} \mathrm{Ti} /{ }^{60} \mathrm{~V} /{ }^{61} \mathrm{~V} /{ }^{62} \mathrm{~V} /{ }^{63} \mathrm{~V} /{ }^{62} \mathrm{Cr} /{ }^{63} \mathrm{Cr} /{ }^{64} \mathrm{Cr} /{ }^{65} \mathrm{Cr} /{ }^{66} \mathrm{Cr} /{ }^{65} \mathrm{Mn}$ / ${ }^{66} \mathrm{Mn}, \mathrm{E}=61.8 \mathrm{MeV}$ / nucleon; measured yields. JOUR ZAANE 2341 RADIOACTIVITY ${ }^{63,64} \mathrm{Cr},{ }^{65,66} \mathrm{Mn},{ }^{67,68} \mathrm{Fe},{ }^{69,70} \mathrm{Co}\left(\beta^{-}\right)$[from $\left.\mathrm{Be}\left({ }^{86} \mathrm{Kr}, \mathrm{X}\right)\right]$; measured $\mathrm{E} \beta, \mathrm{T}_{1 / 2}$. Fragment separator, comparisons with previous results and model predictions. CONF Riken(Origin of Matter) Proc,P304,Nishimura

RADIOACTIVITY ${ }^{63,64} \mathrm{Cr},{ }^{65,66} \mathrm{Mn},{ }^{67,68} \mathrm{Fe},{ }^{69,70} \mathrm{Co}\left(\beta^{-}\right)$[from $\left.\mathrm{Be}\left({ }^{86} \mathrm{Kr}, \mathrm{X}\right)\right]$; measured $\mathrm{E} \beta, \mathrm{T}_{1 / 2}$. Fragment separator, comparisons with previous results and model predictions. CONF Riken(Origin of Matter) Proc,P304,Nishimura NUCLEAR REACTIONS ${ }^{65} \mathrm{Cu}\left({ }^{6} \mathrm{Li}, \mathrm{d} \alpha\right),\left({ }^{6} \mathrm{Li},{ }^{7} \mathrm{Li}\right),\left({ }^{6} \mathrm{Li},{ }^{3} \mathrm{He}\right),\left({ }^{6} \mathrm{Li}\right.$, $\alpha),\left({ }^{6} \mathrm{Li}, \alpha \mathrm{X}\right),\left({ }^{7} \mathrm{Li}, \mathrm{t} \alpha\right),\left({ }^{7} \mathrm{Li}, \mathrm{d} \alpha\right),\left({ }^{7} \mathrm{Li},{ }^{6} \mathrm{Li}\right),\left({ }^{7} \mathrm{Li},{ }^{6} \mathrm{He}\right),\left({ }^{7} \mathrm{Li}, \alpha\right),\left({ }^{7} \mathrm{Li}\right.$, $\alpha \mathrm{X}), \mathrm{E}=25 \mathrm{MeV}$; measured particle spectra, $\sigma, \sigma(\theta)$; deduced reaction mechanism features. Comparison with coupled channels predictions. PREPRINT nucl-ex/0512032,12/21/2005

NUCLEAR REACTIONS ${ }^{65} \mathrm{Cu}\left({ }^{6} \mathrm{Li}, \mathrm{d} \alpha\right),\left({ }^{6} \mathrm{Li},{ }^{7} \mathrm{Li}\right),\left({ }^{6} \mathrm{Li},{ }^{3} \mathrm{He}\right),\left({ }^{6} \mathrm{Li}\right.$, $\alpha),\left({ }^{6} \mathrm{Li}, \alpha \mathrm{X}\right),\left({ }^{7} \mathrm{Li}, \mathrm{t} \alpha\right),\left({ }^{7} \mathrm{Li}, \mathrm{d} \alpha\right),\left({ }^{7} \mathrm{Li},{ }^{6} \mathrm{Li}\right),\left({ }^{7} \mathrm{Li},{ }^{6} \mathrm{He}\right),\left({ }^{7} \mathrm{Li}, \alpha\right),\left({ }^{7} \mathrm{Li}\right.$, $\alpha \mathrm{X}), \mathrm{E}=25 \mathrm{MeV}$; measured particle spectra, $\sigma, \sigma(\theta)$; deduced reaction mechanism features. Comparison with coupled channels predictions. PREPRINT nucl-ex/0512032,12/21/2005 


\section{$\mathrm{A}=66$ (continued)}

\begin{tabular}{|c|c|c|}
\hline \multirow[t]{2}{*}{${ }^{66} \mathrm{Ga}$} & 2005B010 & $\begin{array}{l}\text { NUCLEAR REACTIONS Zn }(\mathrm{p}, \mathrm{X}){ }^{64} \mathrm{Cu} /{ }^{57} \mathrm{Ni} /{ }^{56} \mathrm{Ni} /{ }^{52} \mathrm{Mn} /{ }^{54} \mathrm{Mn} \\
/{ }^{62} \mathrm{Zn} /{ }^{65} \mathrm{Zn} /{ }^{51} \mathrm{Cr} /{ }^{48} \mathrm{~V} /{ }^{55} \mathrm{Co} /{ }^{56} \mathrm{Co} /{ }^{57} \mathrm{Co} /{ }^{58} \mathrm{Co} /{ }^{60} \mathrm{Co} /{ }^{66} \mathrm{Ga} \\
/{ }^{67} \mathrm{Ga} /{ }^{52} \mathrm{Fe} /{ }^{59} \mathrm{Fe}, \mathrm{E} \approx 31-141 \mathrm{MeV} \text {; measured production } \sigma .\end{array}$ \\
\hline & 2005SZ02 & $\begin{array}{l}\text { NUCLEAR REACTIONS }{ }^{66} \mathrm{Zn}(\mathrm{p}, \mathrm{n}),{ }^{68} \mathrm{Zn}(\mathrm{p}, 2 \mathrm{n}),(\mathrm{p}, 3 \mathrm{n}), \mathrm{E} \approx 5-100 \\
\mathrm{MeV} ; \mathrm{Zn}(\mathrm{p}, \mathrm{X}){ }^{66} \mathrm{Ga} /{ }^{67} \mathrm{Ga}, \mathrm{E} \approx 5-100 \mathrm{MeV} ; \text { measured production } \sigma . \\
\text { Stacked-foil activation, comparison with previous results. JOUR } \\
\text { NIMBE } 234375\end{array}$ \\
\hline \multirow[t]{2}{*}{${ }^{66} \mathrm{Se}$} & 2005ST29 & $\begin{array}{l}\text { NUCLEAR REACTIONS }{ }^{9} \mathrm{Be}\left({ }^{78} \mathrm{Kr}, \mathrm{X}\right){ }^{60} \mathrm{Ge} /{ }^{61} \mathrm{Ge} /{ }^{62} \mathrm{Ge} /{ }^{63} \mathrm{Ge} / \\
{ }^{64} \mathrm{Ge} /{ }^{64} \mathrm{Se} /{ }^{65} \mathrm{Se} /{ }^{66} \mathrm{Se} /{ }^{67} \mathrm{Se} /{ }^{68} \mathrm{Se}, \mathrm{E}=140 \mathrm{MeV} / \text { nucleon; } \\
\text { measured production } \sigma \text {, isotopic yields; deduced no evidence for } \\
{ }^{59} \mathrm{Ga} \text {, } \\
{ }^{63} \text { As. }{ }^{60} \mathrm{Ge},{ }^{64} \mathrm{Se} \text { deduced } \mathrm{T}_{1 / 2} \text { lower limits. }{ }^{59} \mathrm{Ga},{ }^{63} \mathrm{As} \text { deduced } \mathrm{T}_{1 / 2} \\
\text { upper limits. JOUR PYLBB } 62732\end{array}$ \\
\hline & 2005ST34 & 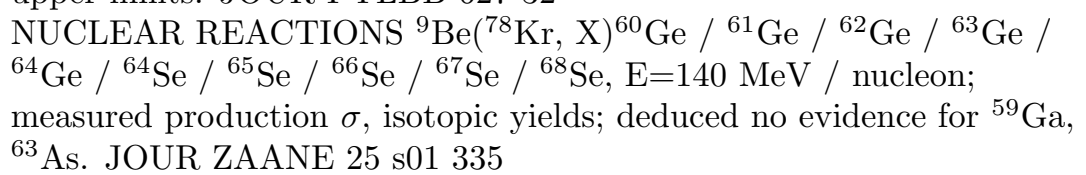 \\
\hline
\end{tabular}

\section{$\mathrm{A}=67$}

\begin{tabular}{|c|c|c|}
\hline${ }^{67} \mathrm{Fe}$ & 2005 NIZZ & RADIOACTIVITY ${ }^{63,64} \mathrm{Cr},{ }^{65,66} \mathrm{Mn},{ }^{67,68} \mathrm{Fe},{ }^{69,70} \mathrm{Co}\left(\beta^{-}\right)[$from \\
\hline & & $\begin{array}{l}\left.\mathrm{Be}\left({ }^{86} \mathrm{Kr}, \mathrm{X}\right)\right] \text {; measured } \mathrm{E} \beta, \mathrm{T}_{1 / 2} \text {. Fragment separator, comparisons } \\
\text { with previous results and model predictions. CONF Riken(Origin of } \\
\text { Matter) Proc,P } 304, \text { Nishimura }\end{array}$ \\
\hline${ }^{67} \mathrm{Co}$ & 2005 NIZZ & $\begin{array}{l}\text { RADIOACTIVITY }{ }^{63,64} \mathrm{Cr},{ }^{65,66} \mathrm{Mn},{ }^{67,68} \mathrm{Fe},{ }^{69,70} \mathrm{Co}\left(\beta^{-}\right) \text {[from } \\
\left.\mathrm{Be}\left({ }^{86} \mathrm{Kr}, \mathrm{X}\right)\right] \text {; measured } \mathrm{E} \beta, \mathrm{T}_{1 / 2} \text {. Fragment separator, comparisons } \\
\text { with previous results and model predictions. CONF Riken(Origin of } \\
\text { Matter) Proc,P304,Nishimura }\end{array}$ \\
\hline${ }^{67} \mathrm{Zn}$ & 2005SHZS & $\begin{array}{l}\text { NUCLEAR REACTIONS }{ }^{65} \mathrm{Cu}\left({ }^{6} \mathrm{Li}, \mathrm{d} \alpha\right),\left({ }^{6} \mathrm{Li},{ }^{7} \mathrm{Li}\right),\left({ }^{6} \mathrm{Li},{ }^{3} \mathrm{He}\right),\left({ }^{6} \mathrm{Li},\right. \\
\alpha),\left({ }^{6} \mathrm{Li}, \alpha \mathrm{X}\right),\left({ }^{7} \mathrm{Li}, \mathrm{t} \alpha\right),\left({ }^{7} \mathrm{Li}, \mathrm{d} \alpha\right),\left({ }^{7} \mathrm{Li},{ }^{6} \mathrm{Li}\right),\left({ }^{7} \mathrm{Li},{ }^{6} \mathrm{He}\right),\left({ }^{7} \mathrm{Li}, \alpha\right),\left({ }^{7} \mathrm{Li},\right. \\
\alpha \mathrm{X}), \mathrm{E}=25 \mathrm{MeV} \text {; measured particle spectra, } \sigma, \sigma(\theta) ; \text { deduced reaction } \\
\text { mechanism features. Comparison with coupled channels predictions. } \\
\text { PREPRINT nucl-ex } / 0512032,12 / 21 / 2005\end{array}$ \\
\hline${ }^{67} \mathrm{Ga}$ & 2005ВА30 & $\begin{array}{l}\text { NUCLEAR REACTIONS }{ }^{63} \mathrm{Cu}(\alpha, \gamma), \mathrm{E}=5.9-8.7 \mathrm{MeV} \text {; measured } \sigma \text {. } \\
\text { Stacked-foil activation technique, comparison with model predictions. } \\
\text { Astrophysical implications discussed. JOUR PRVCA } 71035801\end{array}$ \\
\hline & 2005BAZS & $\begin{array}{l}\text { NUCLEAR REACTIONS }{ }^{63} \mathrm{Cu}(\alpha, \gamma), \mathrm{E}=5.9-8.7 \mathrm{MeV} \text {; measured } \sigma \text {. } \\
\text { Activation technique, comparison with model predictions. CONF } \\
\text { Santa Fe (Nucl Data for Sci and Technol) Proc,Vol2,P1366 }\end{array}$ \\
\hline & 2005B010 & $\begin{array}{l}\text { NUCLEAR REACTIONS Zn }(\mathrm{p}, \mathrm{X}){ }^{64} \mathrm{Cu} /{ }^{57} \mathrm{Ni} /{ }^{56} \mathrm{Ni} /{ }^{52} \mathrm{Mn} /{ }^{54} \mathrm{Mn} \\
/{ }^{62} \mathrm{Zn} /{ }^{65} \mathrm{Zn} /{ }^{51} \mathrm{Cr} /{ }^{48} \mathrm{~V} /{ }^{55} \mathrm{Co} /{ }^{56} \mathrm{Co} /{ }^{57} \mathrm{Co} /{ }^{58} \mathrm{Co} /{ }^{60} \mathrm{Co} /{ }^{66} \mathrm{Ga} \\
/{ }^{67} \mathrm{Ga} /{ }^{52} \mathrm{Fe} /{ }^{59} \mathrm{Fe}, \mathrm{E} \approx 31-141 \mathrm{MeV} ; \text { measured production } \sigma . \\
\text { Stacked-foil activation. JOUR JRNCD } 264101\end{array}$ \\
\hline & 2005SZ02 & $\begin{array}{l}\text { NUCLEAR REACTIONS }{ }^{66} \mathrm{Zn}(\mathrm{p}, \mathrm{n}),{ }^{68} \mathrm{Zn}(\mathrm{p}, 2 \mathrm{n}),(\mathrm{p}, 3 \mathrm{n}), \mathrm{E} \approx 5-100 \\
\mathrm{MeV} ; \mathrm{Zn}(\mathrm{p}, \mathrm{X}){ }^{66} \mathrm{Ga} /{ }^{67} \mathrm{Ga}, \mathrm{E} \approx 5-100 \mathrm{MeV} ; \text { measured production } \sigma . \\
\text { Stacked-foil activation, comparison with previous results. JOUR } \\
\text { NIMBE } 234375\end{array}$ \\
\hline
\end{tabular}




\section{$\mathrm{A}=67$ (continued)}

${ }^{67} \mathrm{Se}$

2005ST29

2005ST34

${ }^{68} \mathrm{Co}$

${ }^{68} \mathrm{Zn}$

${ }^{68} \mathrm{Ge}$
2005NIZZ

2005LE12

2005LE38

2005LEZX

2005SHZS

$2005 N I Z Z$

$2005 \mathrm{CH} 60$

2005LE19
NUCLEAR REACTIONS ${ }^{9} \mathrm{Be}\left({ }^{78} \mathrm{Kr}, \mathrm{X}\right){ }^{60} \mathrm{Ge} /{ }^{61} \mathrm{Ge} /{ }^{62} \mathrm{Ge} /{ }^{63} \mathrm{Ge} /$ ${ }^{64} \mathrm{Ge} /{ }^{64} \mathrm{Se} /{ }^{65} \mathrm{Se} /{ }^{66} \mathrm{Se} /{ }^{67} \mathrm{Se} /{ }^{68} \mathrm{Se}, \mathrm{E}=140 \mathrm{MeV} /$ nucleon; measured production $\sigma$, isotopic yields; deduced no evidence for ${ }^{59} \mathrm{Ga}$, ${ }^{63}$ As. ${ }^{60} \mathrm{Ge},{ }^{64}$ Se deduced $\mathrm{T}_{1 / 2}$ lower limits. ${ }^{59} \mathrm{Ga},{ }^{63} \mathrm{As}$ deduced $\mathrm{T}_{1 / 2}$ upper limits. JOUR PYLBB 62732

NUCLEAR REACTIONS ${ }^{9} \mathrm{Be}\left({ }^{78} \mathrm{Kr}, \mathrm{X}\right){ }^{60} \mathrm{Ge} /{ }^{61} \mathrm{Ge} /{ }^{62} \mathrm{Ge} /{ }^{63} \mathrm{Ge} /$ ${ }^{64} \mathrm{Ge} /{ }^{64} \mathrm{Se} /{ }^{65} \mathrm{Se} /{ }^{66} \mathrm{Se} /{ }^{67} \mathrm{Se} /{ }^{68} \mathrm{Se}, \mathrm{E}=140 \mathrm{MeV} /$ nucleon; measured production $\sigma$, isotopic yields; deduced no evidence for ${ }^{59} \mathrm{Ga}$, ${ }^{63}$ As. JOUR ZAANE 25 s01 335

\section{$\mathrm{A}=68$}

RADIOACTIVITY ${ }^{63,64} \mathrm{Cr},{ }^{65,66} \mathrm{Mn},{ }^{67,68} \mathrm{Fe},{ }^{69,70} \mathrm{Co}\left(\beta^{-}\right)$[from $\left.\mathrm{Be}\left({ }^{86} \mathrm{Kr}, \mathrm{X}\right)\right]$; measured $\mathrm{E} \beta, \mathrm{T}_{1 / 2}$. Fragment separator, comparisons with previous results and model predictions. CONF Riken(Origin of Matter) Proc,P304,Nishimura

RADIOACTIVITY ${ }^{63,64} \mathrm{Cr},{ }^{65,66} \mathrm{Mn},{ }^{67,68} \mathrm{Fe},{ }^{69,70} \mathrm{Co}\left(\beta^{-}\right)$[from $\left.\mathrm{Be}\left({ }^{86} \mathrm{Kr}, \mathrm{X}\right)\right]$; measured $\mathrm{E} \beta, \mathrm{T}_{1 / 2}$. Fragment separator, comparisons with previous results and model predictions. CONF Riken(Origin of Matter) Proc,P304,Nishimura

NUCLEAR REACTIONS C( ${ }^{64} \mathrm{Zn},{ }^{64} \mathrm{Zn}$ '), $\left({ }^{68} \mathrm{Zn},{ }^{68} \mathrm{Zn}\right.$ '), E=180 MeV; measured $\mathrm{E} \gamma, \mathrm{I} \gamma(\theta, \mathrm{H}, \mathrm{t}), \gamma \gamma-$, (particle) $\gamma$-coin, DSA following projectile Coulomb excitation. ${ }^{64,68} \mathrm{Zn}$ levels deduced $\mathrm{g}$ factors, $\mathrm{T}_{1 / 2}$, B(E2). Transient-field technique, large-scale shell model calculations. JOUR PRVCA 71034303

NUCLEAR REACTIONS C( ${ }^{68} \mathrm{Zn},{ }^{68} \mathrm{Zn}$ '), E $=180 \mathrm{MeV}$; measured $\mathrm{E} \gamma$, $\mathrm{I} \gamma(\theta, \mathrm{H}, \mathrm{t})$ (particle) $\gamma$-coin following projectile Coulomb excitation.

${ }^{68} \mathrm{Zn}$ levels deduced $\mathrm{g}$ factors, $\mathrm{T}_{1 / 2}, \mathrm{~B}(\mathrm{E} 2)$, configurations. ${ }^{64} \mathrm{Zn}$ levels analyzed $\mathrm{g}$ factors, B(E2). Large-scale shell-model calculations. JOUR PRVCA 72044301

NUCLEAR REACTIONS C $\left({ }^{68} \mathrm{Zn},{ }^{68} \mathrm{Zn}\right), \mathrm{E}=180 \mathrm{MeV}$; measured $\mathrm{E} \gamma$, $\mathrm{I} \gamma(\theta, \mathrm{H}, \mathrm{t})$, (particle) $\gamma$-coin, DSA following projectile Coulomb excitation. ${ }^{68} \mathrm{Zn}$ levels deduced $\mathrm{T}_{1 / 2}$, g factors. Transient field technique, comparison with shell model predictions. PREPRINT nucl-ex/0506006,6/05/2005 NUCLEAR REACTIONS ${ }^{65} \mathrm{Cu}\left({ }^{6} \mathrm{Li}, \mathrm{d} \alpha\right),\left({ }^{6} \mathrm{Li},{ }^{7} \mathrm{Li}\right),\left({ }^{6} \mathrm{Li},{ }^{3} \mathrm{He}\right),\left({ }^{6} \mathrm{Li}\right.$, $\alpha),\left({ }^{6} \mathrm{Li}, \alpha \mathrm{X}\right),\left({ }^{7} \mathrm{Li}, \mathrm{t} \alpha\right),\left({ }^{7} \mathrm{Li}, \mathrm{d} \alpha\right),\left({ }^{7} \mathrm{Li},{ }^{6} \mathrm{Li}\right),\left({ }^{7} \mathrm{Li},{ }^{6} \mathrm{He}\right),\left({ }^{7} \mathrm{Li}, \alpha\right),\left({ }^{7} \mathrm{Li}\right.$, $\alpha \mathrm{X}), \mathrm{E}=25 \mathrm{MeV}$; measured particle spectra, $\sigma, \sigma(\theta)$; deduced reaction mechanism features. Comparison with coupled channels predictions. PREPRINT nucl-ex/0512032,12/21/2005 ATOMIC MASSES ${ }^{64} \mathrm{Zn},{ }^{64} \mathrm{Ga},{ }^{68} \mathrm{Ge},{ }^{68} \mathrm{As},{ }^{68,72} \mathrm{Se},{ }^{76} \mathrm{Kr},{ }^{76} \mathrm{Rb},{ }^{80} \mathrm{Sr}$, ${ }^{80} \mathrm{Y}$; measured masses. Direct time-of-flight technique, comparison with previous results. JOUR JPGPE 31 S1771

NUCLEAR REACTIONS ${ }^{12} \mathrm{C}\left({ }^{64} \mathrm{Zn}, 2 \alpha\right), \mathrm{E}=180 \mathrm{MeV}$; measured $\mathrm{E} \gamma$, $\mathrm{I} \gamma(\theta, \mathrm{H}, \mathrm{t}), \alpha \alpha-, \alpha \gamma$-coin. ${ }^{68} \mathrm{Ge}$ deduced level energies, B(E2), g factor. Transient field technique. JOUR PRVCA 71044316 


\section{$\mathrm{A}=68$ (continued)}

\begin{tabular}{|c|c|c|}
\hline \multirow[t]{2}{*}{${ }^{68} \mathrm{As}$} & $2005 \mathrm{CH} 60$ & $\begin{array}{l}\text { ATOMIC MASSES }{ }^{64} \mathrm{Zn},{ }^{64} \mathrm{Ga},{ }^{68} \mathrm{Ge},{ }^{68} \mathrm{As},{ }^{68}, 72 \mathrm{Se},{ }^{76} \mathrm{Kr},{ }^{76} \mathrm{Rb},{ }^{80} \mathrm{Sr} \text {, } \\
{ }^{80} \mathrm{Y} \text {; measured masses. Direct time-of-flight technique, comparison with } \\
\text { previous results. JOUR JPGPE } 31 \mathrm{~S} 1771\end{array}$ \\
\hline & 2005ST08 & $\begin{array}{l}\text { NUCLEAR REACTIONS }{ }^{40} \mathrm{Ca}\left({ }^{32} \mathrm{~S}, \mathrm{n} 3 \mathrm{p}\right), \mathrm{E}=95,105 \mathrm{MeV} \text {; measured } \\
\mathrm{E} \gamma, \mathrm{I} \gamma, \gamma \gamma-, \text { (charged particle) } \gamma-\text {-, (neutron }) \gamma \text {-coin. }{ }^{68} \mathrm{As} \text { deduced } \\
\text { high-spin levels, J, } \pi \text {, configurations. Euroball and Euclides arrays, } \\
\text { total Routhian surface calculations. JOUR ZAANE } 241\end{array}$ \\
\hline \multirow[t]{4}{*}{${ }^{68} \mathrm{Se}$} & $2005 \mathrm{CH} 60$ & $\begin{array}{l}\text { ATOMIC MASSES }{ }^{64} \mathrm{Zn},{ }^{64} \mathrm{Ga},{ }^{68} \mathrm{Ge},{ }^{68} \mathrm{As},{ }^{68,72} \mathrm{Se},{ }^{76} \mathrm{Kr},{ }^{76} \mathrm{Rb},{ }^{80} \mathrm{Sr} \text {, } \\
{ }^{80} \mathrm{Y} \text {; measured masses. Direct time-of-flight technique, comparison with } \\
\text { previous results. JOUR JPGPE } 31 \mathrm{~S} 1771\end{array}$ \\
\hline & 2005CL08 & 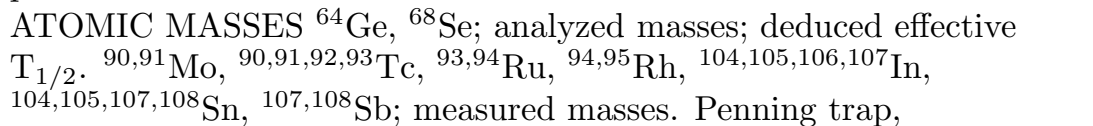 \\
\hline & 2005ST29 & $\begin{array}{l}\text { astrophysical implications discussed. JOUR ZAANE } 25 \text { s01 } 629 \\
\text { NUCLEAR REACTIONS }{ }^{9} \mathrm{Be}\left({ }^{78} \mathrm{Kr}, \mathrm{X}\right){ }^{60} \mathrm{Ge} /{ }^{61} \mathrm{Ge} /{ }^{62} \mathrm{Ge} /{ }^{63} \mathrm{Ge} / \\
{ }^{64} \mathrm{Ge} /{ }^{64} \mathrm{Se} /{ }^{65} \mathrm{Se} /{ }^{66} \mathrm{Se} /{ }^{67} \mathrm{Se} /{ }^{68} \mathrm{Se}, \mathrm{E}=140 \mathrm{MeV} / \text { nucleon; } \\
\text { measured production } \sigma \text {, isotopic yields; deduced no evidence for }{ }^{59} \mathrm{Ga} \text {, } \\
{ }^{63} \text { As. }{ }^{60} \mathrm{Ge},{ }^{64} \mathrm{Se} \text { deduced T } 1 / 2 \text { lower limits. }{ }^{59} \mathrm{Ga},{ }^{63} \text { As deduced } \mathrm{T}_{1 / 2} \\
\text { upper limits. JOUR PYLBB } 62732\end{array}$ \\
\hline & 2005ST34 & $\begin{array}{l}\text { NUCLEAR REACTIONS }{ }^{9} \mathrm{Be}\left({ }^{78} \mathrm{Kr}, \mathrm{X}\right){ }^{60} \mathrm{Ge} /{ }^{61} \mathrm{Ge} /{ }^{62} \mathrm{Ge} /{ }^{63} \mathrm{Ge} / \\
{ }^{64} \mathrm{Ge} /{ }^{64} \mathrm{Se} /{ }^{65} \mathrm{Se} /{ }^{66} \mathrm{Se} /{ }^{67} \mathrm{Se} /{ }^{68} \mathrm{Se}, \mathrm{E}=140 \mathrm{MeV} / \text { nucleon; } \\
\text { measured production } \sigma \text {, isotopic yields; deduced no evidence for }{ }^{59} \mathrm{Ga} \text {, } \\
{ }^{63} \text { As. JOUR ZAANE } 25 \mathrm{~s} 01335\end{array}$ \\
\hline
\end{tabular}

\section{$\mathrm{A}=69$}

${ }^{69} \mathrm{Co} \quad 2005$ NIZZ

RADIOACTIVITY ${ }^{63,64} \mathrm{Cr},{ }^{65,66} \mathrm{Mn},{ }^{67,68} \mathrm{Fe},{ }^{69,70} \mathrm{Co}\left(\beta^{-}\right)$[from $\left.\operatorname{Be}\left({ }^{86} \mathrm{Kr}, \mathrm{X}\right)\right]$; measured $\mathrm{E} \beta, \mathrm{T}_{1 / 2}$. Fragment separator, comparisons with previous results and model predictions. CONF Riken(Origin of Matter) Proc,P304,Nishimura

${ }^{69} \mathrm{Ni}$ 2005NIZZ RADIOACTIVITY ${ }^{63,64} \mathrm{Cr},{ }^{65,66} \mathrm{Mn},{ }^{67,68} \mathrm{Fe},{ }^{69,70} \mathrm{Co}\left(\beta^{-}\right)$[from $\left.\mathrm{Be}\left({ }^{86} \mathrm{Kr}, \mathrm{X}\right)\right]$; measured $\mathrm{E} \beta, \mathrm{T}_{1 / 2}$. Fragment separator, comparisons with previous results and model predictions. CONF Riken(Origin of Matter) Proc,P304,Nishimura

\section{$\mathrm{A}=70$}

${ }^{70} \mathrm{Co} \quad 2005 \mathrm{NIZZ}$

RADIOACTIVITY ${ }^{63,64} \mathrm{Cr},{ }^{65,66} \mathrm{Mn},{ }^{67,68} \mathrm{Fe},{ }^{69,70} \mathrm{Co}\left(\beta^{-}\right)$[from $\left.\mathrm{Be}\left({ }^{86} \mathrm{Kr}, \mathrm{X}\right)\right]$; measured $\mathrm{E} \beta, \mathrm{T}_{1 / 2}$. Fragment separator, comparisons with previous results and model predictions. CONF Riken(Origin of Matter) Proc,P304,Nishimura

${ }^{70} \mathrm{Ni}$ 2004PEZW NUCLEAR REACTIONS ${ }^{208} \mathrm{~Pb}\left({ }^{70} \mathrm{Ni},{ }^{70} \mathrm{Ni}\right),\left({ }^{74} \mathrm{Zn},{ }^{74} \mathrm{Zn}\right),\left({ }^{76} \mathrm{Ge}\right.$, ${ }^{76} \mathrm{Ge}$ '), $\mathrm{E} \approx 40 \mathrm{MeV} /$ nucleon; measured $\mathrm{E} \gamma, \mathrm{I} \gamma$, (particle) $\gamma$-coin following projectile Coulomb excitation. ${ }^{70} \mathrm{Ni},{ }^{74} \mathrm{Zn}$ deduced transitions B(E2). REPT IPNO-T-05-02,Perru 


\section{$\mathrm{A}=70$ (continued)}

2005MA95 RADIOACTIVITY ${ }^{71,72,73,74} \mathrm{Co}\left(\beta^{-}\right),\left(\beta^{-} \mathrm{n}\right)$ [from ${ }^{9} \mathrm{Be}\left({ }^{86} \mathrm{Kr}, \mathrm{X}\right)$ ]; measured $\mathrm{E} \gamma, \mathrm{E} \beta, \beta \gamma$-coin; deduced $\beta \mathrm{n}$ branching fraction. ${ }_{70,71,72,73,74} \mathrm{Ni}$ deduced levels, J, $\pi$. JOUR ZAANE 25 s01 93

2005MAZX RADIOACTIVITY ${ }^{71,72,73,74} \mathrm{Co}\left(\beta^{-}\right),\left(\beta^{-} \mathrm{n}\right)$ [from ${ }^{9} \mathrm{Be}\left({ }^{86} \mathrm{Kr}, \mathrm{X}\right)$ ]; measured $\beta$-delayed $\mathrm{E} \gamma, \mathrm{I} \gamma$; deduced branching ratios. ${ }^{70,71} \mathrm{Ni}$ deduced transitions. ${ }^{76} \mathrm{Ni}(\mathrm{IT})\left[\right.$ from $\left.{ }^{9} \mathrm{Be}\left({ }^{86} \mathrm{Kr}, \mathrm{X}\right)\right]$; measured $\mathrm{E} \gamma, \mathrm{I} \gamma, \mathrm{T}_{1 / 2} .{ }^{76} \mathrm{Ni}$ deduced levels. CONF Argonne(Nuclei at the Limits),P164,Mazzocchi 2005NIZZ RADIOACTIVITY ${ }^{63,64} \mathrm{Cr},{ }^{65,66} \mathrm{Mn},{ }^{67,68} \mathrm{Fe},{ }^{69,70} \mathrm{Co}\left(\beta^{-}\right)$[from $\left.\mathrm{Be}\left({ }^{86} \mathrm{Kr}, \mathrm{X}\right)\right]$; measured $\mathrm{E} \beta, \mathrm{T}_{1 / 2}$. Fragment separator, comparisons with previous results and model predictions. CONF Riken(Origin of Matter) Proc,P304,Nishimura

${ }^{70} \mathrm{Ga} \quad$ 2005WA29 NUCLEAR REACTIONS ${ }^{71} \mathrm{Ga}(\mathrm{n}, 2 \mathrm{n}), \mathrm{E}=13.5,14.1,14.7 \mathrm{MeV}$; measured $\sigma$. Activation technique, comparison with previous results. JOUR PRVCA 72037604

${ }^{70} \mathrm{Ge} \quad 2004 \mathrm{KO} 64$ NUCLEAR REACTIONS ${ }^{6} \mathrm{Li},{ }^{16} \mathrm{O},{ }^{32} \mathrm{~S},{ }^{50,51} \mathrm{~V},{ }^{70,72} \mathrm{Ge}(\mathrm{d}, \mathrm{d})$, (d, d'), $\mathrm{E}=171 \mathrm{MeV} ;{ }^{90} \mathrm{Zr},{ }^{116} \mathrm{Sn}(\mathrm{d}, \mathrm{d}),(\mathrm{d}, \mathrm{d}$ '), $\mathrm{E}=183 \mathrm{MeV}$; measured $\sigma(\theta)$; deduced optical model parameters. JOUR PRVCA 70067601

2005BA13 NUCLEAR REACTIONS ${ }^{70,72,74} \mathrm{Ge}\left({ }^{6} \mathrm{Li},{ }^{6} \mathrm{Li}\right),\left({ }^{6} \mathrm{Li},{ }^{6} \mathrm{Li}\right), \mathrm{E}=28 \mathrm{MeV}$; measured $\sigma(\mathrm{E}, \theta)$, elastic $\sigma(\theta)$; deduced Coulomb-nuclear interference effects. ${ }^{70,72,74}$ Ge levels deduced $\mathrm{B}(\mathrm{E} 2)$ / $\mathrm{B}(\mathrm{IS} 2)$ ratio, mixed-symmetry effects. JOUR PRVCA 71024303

\section{$\mathrm{A}=71$}

\section{${ }^{71} \mathrm{Co} \quad$ 2005MA95}

2005MAZX

${ }^{71} \mathrm{Ni}$

2005MA95

RADIOACTIVITY ${ }^{71,72,73,74} \mathrm{Co}\left(\beta^{-}\right),\left(\beta^{-} \mathrm{n}\right)$ [from $\left.{ }^{9} \mathrm{Be}\left({ }^{86} \mathrm{Kr}, \mathrm{X}\right)\right]$; measured $\mathrm{E} \gamma, \mathrm{E} \beta, \beta \gamma$-coin; deduced $\beta \mathrm{n}$ branching fraction.

70,71,72,73,74 Ni deduced levels, J, $\pi$. JOUR ZAANE 25 s01 93 RADIOACTIVITY ${ }^{71,72,73,74} \mathrm{Co}\left(\beta^{-}\right),\left(\beta^{-} \mathrm{n}\right)$ [from $\left.{ }^{9} \mathrm{Be}\left({ }^{86} \mathrm{Kr}, \mathrm{X}\right)\right]$; measured $\beta$-delayed $\mathrm{E} \gamma, \mathrm{I} \gamma$; deduced branching ratios. ${ }^{70,71} \mathrm{Ni}$ deduced transitions. ${ }^{76} \mathrm{Ni}(\mathrm{IT})\left[\right.$ from $\left.{ }^{9} \mathrm{Be}\left({ }^{86} \mathrm{Kr}, \mathrm{X}\right)\right]$; measured $\mathrm{E} \gamma, \mathrm{I} \gamma, \mathrm{T}_{1 / 2} .{ }^{76} \mathrm{Ni}$ deduced levels. CONF Argonne(Nuclei at the Limits),P164,Mazzocchi RADIOACTIVITY ${ }^{71,72,73,74} \mathrm{Co}\left(\beta^{-}\right),\left(\beta^{-} \mathrm{n}\right)$ [from $\left.{ }^{9} \mathrm{Be}\left({ }^{86} \mathrm{Kr}, \mathrm{X}\right)\right]$; measured $\mathrm{E} \gamma, \mathrm{E} \beta, \beta \gamma$-coin; deduced $\beta \mathrm{n}$ branching fraction. 70,71,72,73,74 Ni deduced levels, J, $\pi$. JOUR ZAANE 25 s01 93

2005MAZX RADIOACTIVITY ${ }^{71,72,73,74} \mathrm{Co}\left(\beta^{-}\right),\left(\beta^{-} \mathrm{n}\right)$ [from ${ }^{9} \mathrm{Be}\left({ }^{86} \mathrm{Kr}, \mathrm{X}\right)$ ]; measured $\beta$-delayed $\mathrm{E} \gamma$, I $\gamma$; deduced branching ratios. ${ }^{70,71} \mathrm{Ni}$ deduced transitions. ${ }^{76} \mathrm{Ni}(\mathrm{IT})\left[\right.$ from $\left.{ }^{9} \mathrm{Be}\left({ }^{86} \mathrm{Kr}, \mathrm{X}\right)\right]$; measured $\mathrm{E} \gamma, \mathrm{I} \gamma, \mathrm{T}_{1 / 2} .{ }^{76} \mathrm{Ni}$ deduced levels. CONF Argonne(Nuclei at the Limits),P164,Mazzocchi

${ }^{71} \mathrm{Ge} \quad 2004 \mathrm{HO} 25$ NUCLEAR REACTIONS ${ }^{70,73} \mathrm{Ge}(\mathrm{n}, \gamma), \mathrm{E}=$ thermal; measured $\mathrm{E} \gamma, \mathrm{I} \gamma$, $\gamma \gamma$-coin. ${ }^{71,74} \mathrm{Ge}$ deduced transitions, two-quantum cascade intensities. JOUR BRSPE 681324

2005SE14 RADIOACTIVITY ${ }^{71} \operatorname{As}\left(\beta^{+}\right)$; measured $\mathrm{E} \gamma, \mathrm{E} \beta, \beta$-decay angular distributions from oriented nuclei; deduced $\beta$-asymmetry parameter. ${ }^{71}$ As deduced ground-state admixture. Low-temperature nuclear orientation technique. JOUR PRVCA 71064310 


\section{$\mathrm{A}=71$ (continued)}

${ }^{71} \mathrm{As} \quad$ 2005SE14 NUCLEAR MOMENTS ${ }^{71}$ As; measured E $\gamma, \mathrm{E} \beta, \beta$-decay angular distributions from oriented nuclei; deduced $\beta$-asymmetry parameter. ${ }^{71}$ As deduced ground-state admixture. Low-temperature nuclear orientation technique. JOUR PRVCA 71064310

2005SE14 RADIOACTIVITY ${ }^{71} \mathrm{As}\left(\beta^{+}\right)$; measured $\mathrm{E} \gamma, \mathrm{E} \beta, \beta$-decay angular distributions from oriented nuclei; deduced $\beta$-asymmetry parameter. ${ }^{71}$ As deduced ground-state admixture. Low-temperature nuclear orientation technique. JOUR PRVCA 71064310

${ }^{71} \mathrm{Br} \quad 2005 \mathrm{FI} 10$ NUCLEAR REACTIONS ${ }^{40} \mathrm{Ca}\left({ }^{40} \mathrm{Ca}, \mathrm{p} 2 \alpha\right), \mathrm{E}=160 \mathrm{MeV}$; measured $\mathrm{E} \gamma, \mathrm{I} \gamma, \gamma \gamma$-, (charged particle) $\gamma$-coin. ${ }^{71} \mathrm{Br}$ deduced levels, J, $\pi, \delta$, rotational bands, shape coexistence features. ${ }^{71} \mathrm{Kr}$; analyzed data; deduced ground-state J, $\pi$. Gammasphere, Microball arrays. JOUR PRVCA 72024321

${ }^{71} \mathrm{Kr} \quad 2005 \mathrm{FI} 10$ NUCLEAR REACTIONS ${ }^{40} \mathrm{Ca}\left({ }^{40} \mathrm{Ca}, \mathrm{p} 2 \alpha\right), \mathrm{E}=160 \mathrm{MeV}$; measured $\mathrm{E} \gamma, \mathrm{I} \gamma, \gamma \gamma-$, (charged particle) $\gamma$-coin. ${ }^{71} \mathrm{Br}$ deduced levels, $\mathrm{J}, \pi, \delta$, rotational bands, shape coexistence features. ${ }^{71} \mathrm{Kr}$; analyzed data; deduced ground-state J, $\pi$. Gammasphere, Microball arrays. JOUR PRVCA 72024321

\section{$\mathrm{A}=72$}

\section{${ }^{72} \mathrm{Co}$ \\ 2005MA59}

2005MA95

2005MAZX

${ }^{72} \mathrm{Ni}$

2005MA59

2005MAZX

${ }^{72} \mathrm{Zn}$

2005DE12

RADIOACTIVITY ${ }^{72,74} \mathrm{Co}\left(\beta^{-}\right)$[from $\left.\mathrm{Be}\left({ }^{86} \mathrm{Kr}, \mathrm{X}\right)\right]$; measured $\mathrm{E} \gamma, \mathrm{I} \gamma$, $\beta \gamma$-coin. ${ }^{76} \mathrm{Ni}(\mathrm{IT})$ [from $\mathrm{Be}\left({ }^{86} \mathrm{Kr}, \mathrm{X}\right)$ ]; measured $\mathrm{E} \gamma, \mathrm{I} \gamma .{ }^{72,74,76} \mathrm{Ni}$ deduced levels, $\mathrm{J}, \pi$. Level systematics in neighboring isotopes discussed. JOUR PYLBB 62245 RADIOACTIVITY ${ }^{71,72,73,74} \mathrm{Co}\left(\beta^{-}\right),\left(\beta^{-} \mathrm{n}\right)$ [from $\left.{ }^{9} \mathrm{Be}\left({ }^{86} \mathrm{Kr}, \mathrm{X}\right)\right]$; measured $\mathrm{E} \gamma, \mathrm{E} \beta, \beta \gamma$-coin; deduced $\beta \mathrm{n}$ branching fraction. 70,71,72,73,74 Ni deduced levels, J, $\pi$. JOUR ZAANE 25 s01 93 RADIOACTIVITY ${ }^{71,72,73,74} \mathrm{Co}\left(\beta^{-}\right),\left(\beta^{-} \mathrm{n}\right)$ [from $\left.{ }^{9} \mathrm{Be}\left({ }^{86} \mathrm{Kr}, \mathrm{X}\right)\right]$; measured $\beta$-delayed $\mathrm{E} \gamma, \mathrm{I} \gamma$; deduced branching ratios. ${ }^{70,71} \mathrm{Ni}$ deduced transitions. ${ }^{76} \mathrm{Ni}(\mathrm{IT})\left[\right.$ from $\left.{ }^{9} \mathrm{Be}\left({ }^{86} \mathrm{Kr}, \mathrm{X}\right)\right]$; measured $\mathrm{E} \gamma, \mathrm{I} \gamma, \mathrm{T}_{1 / 2} .{ }^{76} \mathrm{Ni}$ deduced levels. CONF Argonne(Nuclei at the Limits),P164,Mazzocchi RADIOACTIVITY ${ }^{72,74} \mathrm{Co}\left(\beta^{-}\right)$[from $\left.\mathrm{Be}\left({ }^{86} \mathrm{Kr}, \mathrm{X}\right)\right]$; measured $\mathrm{E} \gamma, \mathrm{I} \gamma$, $\beta \gamma$-coin. ${ }^{76} \mathrm{Ni}(\mathrm{IT})$ [from $\mathrm{Be}\left({ }^{86} \mathrm{Kr}, \mathrm{X}\right)$ ]; measured $\mathrm{E} \gamma, \mathrm{I} \gamma .{ }^{72,74,76} \mathrm{Ni}$ deduced levels, $\mathrm{J}, \pi$. Level systematics in neighboring isotopes discussed. JOUR PYLBB 62245

2005MA95 RADIOACTIVITY ${ }^{71,72,73,74} \mathrm{Co}\left(\beta^{-}\right),\left(\beta^{-} \mathrm{n}\right)$ [from $\left.{ }^{9} \mathrm{Be}\left({ }^{86} \mathrm{Kr}, \mathrm{X}\right)\right]$; measured $\mathrm{E} \gamma, \mathrm{E} \beta, \beta \gamma$-coin; deduced $\beta \mathrm{n}$ branching fraction. 70,71,72,73,74 Ni deduced levels, J, $\pi$. JOUR ZAANE 25 s01 93 RADIOACTIVITY ${ }^{71,72,73,74} \mathrm{Co}\left(\beta^{-}\right),\left(\beta^{-} \mathrm{n}\right)$ [from $\left.{ }^{9} \mathrm{Be}\left({ }^{86} \mathrm{Kr}, \mathrm{X}\right)\right]$; measured $\beta$-delayed $\mathrm{E} \gamma, \mathrm{I} \gamma$; deduced branching ratios. ${ }^{70,71} \mathrm{Ni}$ deduced transitions. ${ }^{76} \mathrm{Ni}(\mathrm{IT})$ [from ${ }^{9} \mathrm{Be}\left({ }^{86} \mathrm{Kr}, \mathrm{X}\right)$ ]; measured $\mathrm{E} \gamma, \mathrm{I} \gamma, \mathrm{T}_{1 / 2} \cdot{ }^{76} \mathrm{Ni}$ deduced levels. CONF Argonne(Nuclei at the Limits),P164,Mazzocchi NUCLEAR REACTIONS ${ }^{238} \mathrm{U}\left({ }^{82} \mathrm{Se}, \mathrm{X}\right), \mathrm{E}=505 \mathrm{MeV}$; measured fragments isotopic yields. ${ }^{238} \mathrm{U}\left({ }^{82} \mathrm{Se}, \mathrm{X}\right){ }^{72} \mathrm{Zn} /{ }^{84} \mathrm{Se} /{ }^{85} \mathrm{Br}, \mathrm{E}=505$ $\mathrm{MeV}$; measured $\mathrm{E} \gamma$, I $\gamma$, (particle) $\gamma$-coin. ${ }^{72} \mathrm{Zn},{ }^{84} \mathrm{Se},{ }^{85} \mathrm{Br}$ deduced levels, J, $\pi$. JOUR NUPAB 751 533c 


\section{$\mathrm{A}=72$ (continued)}

${ }^{72} \mathrm{Ga}$

2005LI02

2005SH03

${ }^{72} \mathrm{Ge}$

2004K064

2005BA13

2005LI02

2005SH03

${ }^{72} \mathrm{As}$

${ }^{72} \mathrm{Se}$

${ }^{72} \mathrm{Kr}$

2005LI09

$2005 \mathrm{CH} 60$

2005CLZZ

2005GA22

RADIOACTIVITY ${ }^{72} \mathrm{Ga}\left(\beta^{-}\right)$[from ${ }^{71} \mathrm{Ga}(\mathrm{n}, \gamma)$ ]; measured $\beta$-delayed $\mathrm{E} \gamma, \mathrm{I} \gamma, \gamma \gamma$-coin; deduced log ft. ${ }^{72} \mathrm{Ge}$ deduced levels, J, $\pi, \beta$-feeding intensities. JOUR CHPHD 1495

RADIOACTIVITY ${ }^{72} \mathrm{Ga}\left(\beta^{-}\right)$[from ${ }^{71} \mathrm{Ga}(\mathrm{n}, \gamma)$ ]; measured $\beta$-delayed $\mathrm{E} \gamma, \mathrm{I} \gamma, \gamma \gamma$-coin; deduced log ft. ${ }^{72} \mathrm{Ge}$ deduced levels, J, $\pi, \beta$-feeding intensities. JOUR JUPSA 74299

NUCLEAR REACTIONS ${ }^{6} \mathrm{Li},{ }^{16} \mathrm{O},{ }^{32} \mathrm{~S},{ }^{50,51} \mathrm{~V},{ }^{70,72} \mathrm{Ge}(\mathrm{d}, \mathrm{d})$, (d, d'), $\mathrm{E}=171 \mathrm{MeV} ;{ }^{90} \mathrm{Zr},{ }^{116} \mathrm{Sn}(\mathrm{d}, \mathrm{d}),(\mathrm{d}, \mathrm{d}$ '), $\mathrm{E}=183 \mathrm{MeV}$; measured $\sigma(\theta)$; deduced optical model parameters. JOUR PRVCA 70067601 NUCLEAR REACTIONS ${ }^{70,72,74} \mathrm{Ge}\left({ }^{6} \mathrm{Li},{ }^{6} \mathrm{Li}\right),\left({ }^{6} \mathrm{Li},{ }^{6} \mathrm{Li}\right), \mathrm{E}=28 \mathrm{MeV}$; measured $\sigma(\mathrm{E}, \theta)$, elastic $\sigma(\theta)$; deduced Coulomb-nuclear interference effects. ${ }^{70,72,74}$ Ge levels deduced B(E2) / B(IS2) ratio,

mixed-symmetry effects. JOUR PRVCA 71024303

NUCLEAR REACTIONS ${ }^{208} \mathrm{~Pb}\left({ }^{74} \mathrm{Kr},{ }^{74} \mathrm{Kr}\right.$ '), $\left({ }^{76} \mathrm{Kr},{ }^{76} \mathrm{Kr}{ }^{\prime}\right), \mathrm{E}=4.5$ $\mathrm{MeV} /$ nucleon; measured $\mathrm{E} \gamma$, I $\gamma$, (particle) $\gamma$-coin following projectile Coulomb excitation. ${ }^{74,76} \mathrm{Kr}$ deduced levels, $\mathrm{J}, \pi$, quadrupole moments. ${ }^{208} \mathrm{~Pb}\left({ }^{72} \mathrm{Ge},{ }^{72} \mathrm{Ge}\right)$, E not given; measured $\mathrm{E} \gamma, \mathrm{I} \gamma, \mathrm{E}(\mathrm{ce}), \mathrm{I}(\mathrm{ce})$, (particle) $\gamma$-coin following projectile Coulomb excitation. ${ }^{72} \mathrm{Ge}$ deduced transitions. Exogam array. JOUR APOBB 361281

RADIOACTIVITY ${ }^{72} \mathrm{Ga}\left(\beta^{-}\right)$[from ${ }^{71} \mathrm{Ga}(\mathrm{n}, \gamma)$ ]; measured $\beta$-delayed $\mathrm{E} \gamma, \mathrm{I} \gamma, \gamma \gamma$-coin; deduced $\log \mathrm{ft} .{ }^{72} \mathrm{Ge}$ deduced levels, J, $\pi, \beta$-feeding intensities. JOUR CHPHD 1495

RADIOACTIVITY ${ }^{72} \mathrm{As}(\mathrm{EC})$ [from ${ }^{72} \mathrm{Ge}(\mathrm{p}, \mathrm{n})$ ]; measured $\mathrm{E} \gamma, \mathrm{I} \gamma$, $\gamma \gamma$-coin; deduced log ft. ${ }^{72}$ Ge deduced levels, J, $\pi$. JOUR CHPHD 14 487

RADIOACTIVITY ${ }^{72} \mathrm{Ga}\left(\beta^{-}\right)$[from ${ }^{71} \mathrm{Ga}(\mathrm{n}, \gamma)$ ]; measured $\beta$-delayed $\mathrm{E} \gamma, \mathrm{I} \gamma, \gamma \gamma$-coin; deduced log ft. ${ }^{72} \mathrm{Ge}$ deduced levels, $\mathrm{J}, \pi, \beta$-feeding intensities. JOUR JUPSA 74299 RADIOACTIVITY ${ }^{72} \mathrm{As}(\mathrm{EC})$ [from ${ }^{72} \mathrm{Ge}(\mathrm{p}, \mathrm{n})$ ]; measured $\mathrm{E} \gamma, \mathrm{I} \gamma$, $\gamma \gamma$-coin; deduced log ft. ${ }^{72} \mathrm{Ge}$ deduced levels, J, $\pi$. JOUR CHPHD 14 487 ATOMIC MASSES ${ }^{64} \mathrm{Zn},{ }^{64} \mathrm{Ga},{ }^{68} \mathrm{Ge},{ }^{68} \mathrm{As},{ }^{68,72} \mathrm{Se},{ }^{76} \mathrm{Kr},{ }^{76} \mathrm{Rb},{ }^{80} \mathrm{Sr}$, ${ }^{80} \mathrm{Y}$; measured masses. Direct time-of-flight technique, comparison with previous results. JOUR JPGPE 31 S1771

NUCLEAR REACTIONS Be $\left({ }^{78} \mathrm{Kr}, \mathrm{X}\right){ }^{72} \mathrm{Kr} /{ }^{74} \mathrm{Kr}, \mathrm{E}=73 \mathrm{MeV}$; measured delayed $\mathrm{E} \gamma, \mathrm{I} \gamma, \mathrm{E}(\mathrm{ce}), \mathrm{I}(\mathrm{ce})$, (recoil) $\gamma-$, (recoil)(ce)-coin. ${ }^{72,74} \mathrm{Kr}$ deduced isomeric levels, $\mathrm{J}, \pi, \mathrm{T}_{1 / 2}$, E0 strength. ${ }^{72} \mathrm{Kr}$ deduced shape isomer. ${ }^{208} \mathrm{~Pb}\left({ }^{76} \mathrm{Kr},{ }^{76} \mathrm{Kr}\right),\left({ }^{74} \mathrm{Kr},{ }^{74} \mathrm{Kr}\right), \mathrm{E} \approx 4.5 \mathrm{MeV} /$ nucleon; measured $\mathrm{E} \gamma, \mathrm{I} \gamma$, (particle) $\gamma$-coin following projectile Coulomb excitation. ${ }^{74,76} \mathrm{Kr}$ deduced levels, J, $\pi$. CONF Argonne(Nuclei at the Limits),P55, Clement NUCLEAR REACTIONS ${ }^{197} \mathrm{Au}\left({ }^{72} \mathrm{Kr},{ }^{72} \mathrm{Kr}\right.$ '), E=69.3 MeV / nucleon; ${ }^{197} \mathrm{Au}\left({ }^{78} \mathrm{Kr},{ }^{78} \mathrm{Kr}\right.$ '), E $=57.4 \mathrm{MeV} /$ nucleon; measured $\mathrm{E} \gamma, \mathrm{I} \gamma$, (particle) $\gamma$-coin following projectile Coulomb excitation. ${ }^{72,78} \mathrm{Kr}$ deduced excitation $\mathrm{B}(\mathrm{E} 2)$, quadrupole moments, deformation. Comparison with shell-model Monte Carlo predictions. JOUR PRLTA 95022502 ATOMIC MASSES ${ }^{72,73,74} \mathrm{Kr},{ }^{73} \mathrm{Rb},{ }^{74} \mathrm{Sr}$; measured masses. Penning trap mass spectrometer. JOUR ZAANE 25 s01 41 


\section{$\mathrm{A}=73$}

\begin{tabular}{|c|c|c|}
\hline \multirow[t]{2}{*}{${ }^{73} \mathrm{Co}$} & 2005MA95 & $\begin{array}{l}\text { RADIOACTIVITY }{ }^{71,72,73,74} \mathrm{Co}\left(\beta^{-}\right),\left(\beta^{-} \mathrm{n}\right)\left[\text { from }{ }^{9} \mathrm{Be}\left({ }^{86} \mathrm{Kr}, \mathrm{X}\right)\right] \\
\text { measured } \mathrm{E} \gamma, \mathrm{E} \beta, \beta \gamma \text {-coin; deduced } \beta \mathrm{n} \text { branching fraction. } \\
70,71,72,73,74 \mathrm{Ni} \text { deduced levels, J, } \pi \text {. JOUR ZAANE } 25 \text { s01 } 93\end{array}$ \\
\hline & 2005MAZX & $\begin{array}{l}\text { RADIOACTIVITY }{ }^{71,72,73,74} \mathrm{Co}\left(\beta^{-}\right),\left(\beta^{-} \mathrm{n}\right)\left[\text { from }{ }^{9} \mathrm{Be}\left({ }^{86} \mathrm{Kr}, \mathrm{X}\right)\right] \text {; } \\
\text { measured } \beta \text {-delayed } \mathrm{E} \gamma, \mathrm{I} \gamma ; \text { deduced branching ratios. }{ }^{70,71} \mathrm{Ni} \text { deduced } \\
\text { transitions. }{ }^{76} \mathrm{Ni}(\mathrm{IT})\left[\text { from }{ }^{9} \mathrm{Be}\left({ }^{86} \mathrm{Kr}, \mathrm{X}\right)\right] \text {; measured } \mathrm{E} \gamma, \mathrm{I} \gamma, \mathrm{T}_{1 / 2} \cdot{ }^{76} \mathrm{Ni} \\
\text { deduced levels. CONF Argonne(Nuclei at the Limits),P164,Mazzocchi }\end{array}$ \\
\hline \multirow[t]{2}{*}{${ }^{73} \mathrm{Ni}$} & 2005MA95 & $\begin{array}{l}\text { RADIOACTIVITY }{ }^{71,72,73,74} \mathrm{Co}\left(\beta^{-}\right),\left(\beta^{-} \mathrm{n}\right)\left[\text { from }{ }^{9} \mathrm{Be}\left({ }^{86} \mathrm{Kr}, \mathrm{X}\right)\right] \\
\text { measured } \mathrm{E} \gamma, \mathrm{E} \beta, \beta \gamma \text {-coin; deduced } \beta \text { n branching fraction. } \\
70,71,72,73,74 \mathrm{Ni} \text { deduced levels, J, } \pi \text {. JOUR ZAANE } 25 \text { s01 } 93\end{array}$ \\
\hline & 2005MAZX & $\begin{array}{l}\text { RADIOACTIVITY }{ }^{71,72,73,74} \mathrm{Co}\left(\beta^{-}\right),\left(\beta^{-} \mathrm{n}\right)\left[\text { from }{ }^{9} \mathrm{Be}\left({ }^{86} \mathrm{Kr}, \mathrm{X}\right)\right] \\
\text { measured } \beta \text {-delayed } \mathrm{E} \gamma, \mathrm{I} \gamma \text {; deduced branching ratios. } \\
\text { transitions. }{ }^{76} \mathrm{Ni}(\mathrm{IT})[\text { from deduced } \\
\left.{ }^{9} \mathrm{Be}\left({ }^{86} \mathrm{Kr}, \mathrm{X}\right)\right] \text {; measured } \mathrm{E} \gamma, \mathrm{I} \gamma, \mathrm{T}_{1 / 2} \cdot{ }^{76} \mathrm{Ni} \\
\text { deduced levels. CONF Argonne(Nuclei at the Limits),P164,Mazzocchi }\end{array}$ \\
\hline${ }^{73} \mathrm{Ge}$ & 2004VA37 & $\begin{array}{l}\text { RADIOACTIVITY }{ }^{73} \mathrm{Ge}\left(\beta^{-}\right) \text {; measured } \mathrm{T}_{1 / 2} \text { lower limit for } \\
\text { charge-nonconserving } \beta \text {-decay. JOUR BRSPE } 681255\end{array}$ \\
\hline $73 \Lambda_{0}$ & 2004VA37 & $\begin{array}{l}\text { RADIOACTIVITY }{ }^{73} \mathrm{Ge}\left(\beta^{-}\right) \text {; measured } \mathrm{T}_{1 / 2} \text { lower limit for } \\
\text { charge-nonconserving } \beta \text {-decay. JOUR BRSPE } 681255\end{array}$ \\
\hline${ }^{73} \mathrm{Kr}$ & 2005R039 & $\begin{array}{l}\text { ATOMIC MASSES }{ }^{72,73,74} \mathrm{Kr},{ }^{73} \mathrm{Rb},{ }^{74} \mathrm{Sr} \text {; measured masses. Penning } \\
\text { trap mass spectrometer. JOUR ZAANE } 25 \mathrm{~s} 0141\end{array}$ \\
\hline $\mathrm{Rb}$ & 2005R039 & $\begin{array}{l}\text { ATOMIC MASSES }{ }^{72,73,74} \mathrm{Kr},{ }^{73} \mathrm{Rb},{ }^{74} \mathrm{Sr} \text {; measured masses. Penning } \\
\text { trap mass spectrometer. JOUR ZAANE } 25 \mathrm{~s} 0141\end{array}$ \\
\hline
\end{tabular}

\section{$\mathrm{A}=74$}

${ }^{74} \mathrm{Co} \quad$ 2005MA59

RADIOACTIVITY ${ }^{72,74} \mathrm{Co}\left(\beta^{-}\right)$[from $\left.\mathrm{Be}\left({ }^{86} \mathrm{Kr}, \mathrm{X}\right)\right]$; measured $\mathrm{E} \gamma, \mathrm{I} \gamma$, $\beta \gamma$-coin. ${ }^{76} \mathrm{Ni}(\mathrm{IT})\left[\right.$ from $\left.\mathrm{Be}\left({ }^{86} \mathrm{Kr}, \mathrm{X}\right)\right]$; measured $\mathrm{E} \gamma, \mathrm{I} \gamma .{ }^{72,74,76} \mathrm{Ni}$ deduced levels, $\mathrm{J}, \pi$. Level systematics in neighboring isotopes discussed. JOUR PYLBB 62245

2005MA95 RADIOACTIVITY ${ }^{71,72,73,74} \mathrm{Co}\left(\beta^{-}\right),\left(\beta^{-} \mathrm{n}\right)$ [from $\left.{ }^{9} \mathrm{Be}\left({ }^{86} \mathrm{Kr}, \mathrm{X}\right)\right]$; measured $\mathrm{E} \gamma, \mathrm{E} \beta, \beta \gamma$-coin; deduced $\beta \mathrm{n}$ branching fraction. 70,71,72,73,74 Ni deduced levels, J, $\pi$. JOUR ZAANE 25 s01 93

2005MAZX NUCLEAR REACTIONS ${ }^{9} \mathrm{Be}\left({ }^{86} \mathrm{Kr}, \mathrm{X}\right), \mathrm{E}=140 \mathrm{MeV} /$ nucleon; measured fragment yields; deduced evidence for ${ }^{74} \mathrm{Co},{ }^{76} \mathrm{Ni}$. CONF Argonne(Nuclei at the Limits),P164,Mazzocchi 2005MAZX RADIOACTIVITY ${ }^{71,72,73,74} \mathrm{Co}\left(\beta^{-}\right),\left(\beta^{-} \mathrm{n}\right)$ [from $\left.{ }^{9} \mathrm{Be}\left({ }^{86} \mathrm{Kr}, \mathrm{X}\right)\right]$; measured $\beta$-delayed $\mathrm{E} \gamma, \mathrm{I} \gamma$; deduced branching ratios. ${ }^{70,71} \mathrm{Ni}$ deduced transitions. ${ }^{76} \mathrm{Ni}(\mathrm{IT})\left[\right.$ from $\left.{ }^{9} \mathrm{Be}\left({ }^{86} \mathrm{Kr}, \mathrm{X}\right)\right]$; measured $\mathrm{E} \gamma, \mathrm{I} \gamma, \mathrm{T}_{1 / 2} \cdot{ }^{76} \mathrm{Ni}$ deduced levels. CONF Argonne(Nuclei at the Limits),P164,Mazzocchi

${ }^{74} \mathrm{Ni} \quad 2005 \mathrm{MA59}$ RADIOACTIVITY ${ }^{72,74} \mathrm{Co}\left(\beta^{-}\right)$[from $\left.\mathrm{Be}\left({ }^{86} \mathrm{Kr}, \mathrm{X}\right)\right]$; measured $\mathrm{E} \gamma, \mathrm{I} \gamma$, $\beta \gamma$-coin. ${ }^{76} \mathrm{Ni}(\mathrm{IT})\left[\right.$ from $\left.\mathrm{Be}\left({ }^{86} \mathrm{Kr}, \mathrm{X}\right)\right]$; measured $\mathrm{E} \gamma, \mathrm{I} \gamma .{ }^{72,74,76} \mathrm{Ni}$ deduced levels, $\mathrm{J}, \pi$. Level systematics in neighboring isotopes discussed. JOUR PYLBB 62245

2005MA95 RADIOACTIVITY ${ }^{71,72,73,74} \mathrm{Co}\left(\beta^{-}\right),\left(\beta^{-} \mathrm{n}\right)$ [from ${ }^{9} \mathrm{Be}\left({ }^{86} \mathrm{Kr}, \mathrm{X}\right)$ ]; measured $\mathrm{E} \gamma, \mathrm{E} \beta, \beta \gamma$-coin; deduced $\beta \mathrm{n}$ branching fraction. 70,71,72,73,74 Ni deduced levels, J, $\pi$. JOUR ZAANE 25 s01 93 


\section{$\mathrm{A}=74$ (continued)}

2005MAZX RADIOACTIVITY ${ }^{71,72,73,74} \mathrm{Co}\left(\beta^{-}\right),\left(\beta^{-} \mathrm{n}\right)$ [from ${ }^{9} \mathrm{Be}\left({ }^{86} \mathrm{Kr}, \mathrm{X}\right)$ ]; measured $\beta$-delayed $\mathrm{E} \gamma, \mathrm{I} \gamma$; deduced branching ratios. ${ }^{70,71} \mathrm{Ni}$ deduced transitions. ${ }^{76} \mathrm{Ni}(\mathrm{IT})\left[\right.$ from $\left.{ }^{9} \mathrm{Be}\left({ }^{86} \mathrm{Kr}, \mathrm{X}\right)\right]$; measured $\mathrm{E} \gamma, \mathrm{I} \gamma, \mathrm{T}_{1 / 2} .{ }^{76} \mathrm{Ni}$ deduced levels. CONF Argonne(Nuclei at the Limits),P164,Mazzocchi

${ }^{74} \mathrm{Cu} \quad$ 2005VA19

${ }^{74} \mathrm{Zn}$

2004PEZW

2005VA19

${ }^{74} \mathrm{Ge}$

$2004 \mathrm{HO} 25$

2005BA13

${ }^{74} \mathrm{Br}$

$2004 \mathrm{CO} 29$

${ }^{74} \mathrm{Kr}$

$2004 \mathrm{CO} 29$

2005CLZZ

2005G015

$2005 \mathrm{GO} 43$
RADIOACTIVITY ${ }^{74,76,78} \mathrm{Cu}\left(\beta^{-}\right) ;{ }^{78} \mathrm{Cu}\left(\beta^{-} \mathrm{n}\right)$ [from ${ }^{238} \mathrm{U}(\mathrm{n}, \mathrm{F}),(\mathrm{p}$,

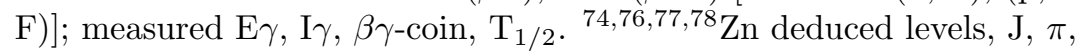
configurations. Mass separator, comparisons with model predictions. JOUR PRVCA 71054307 NUCLEAR REACTIONS ${ }^{208} \mathrm{~Pb}\left({ }^{70} \mathrm{Ni},{ }^{70} \mathrm{Ni}^{\prime}\right),\left({ }^{74} \mathrm{Zn},{ }^{74} \mathrm{Zn}\right),\left({ }^{76} \mathrm{Ge}\right.$, ${ }^{76} \mathrm{Ge}$ '), $\mathrm{E} \approx 40 \mathrm{MeV} /$ nucleon; measured $\mathrm{E} \gamma, \mathrm{I} \gamma$, (particle) $\gamma$-coin following projectile Coulomb excitation. ${ }^{70} \mathrm{Ni},{ }^{74} \mathrm{Zn}$ deduced transitions B(E2). REPT IPNO-T-05-02,Perru

RADIOACTIVITY ${ }^{74,76,78} \mathrm{Cu}\left(\beta^{-}\right) ;{ }^{78} \mathrm{Cu}\left(\beta^{-} \mathrm{n}\right)$ [from ${ }^{238} \mathrm{U}(\mathrm{n}, \mathrm{F})$, (p, $\mathrm{F})]$; measured $\mathrm{E} \gamma, \mathrm{I} \gamma, \beta \gamma$-coin, $\mathrm{T}_{1 / 2} \cdot{ }^{74,76,77,78} \mathrm{Zn}$ deduced levels, $\mathrm{J}, \pi$, configurations. Mass separator, comparisons with model predictions. JOUR PRVCA 71054307

NUCLEAR REACTIONS ${ }^{70,73} \mathrm{Ge}(\mathrm{n}, \gamma), \mathrm{E}=$ thermal; measured $\mathrm{E} \gamma, \mathrm{I} \gamma$, $\gamma \gamma$-coin. ${ }^{71,74} \mathrm{Ge}$ deduced transitions, two-quantum cascade intensities. JOUR BRSPE 681324

NUCLEAR REACTIONS ${ }^{70,72,74} \mathrm{Ge}\left({ }^{6} \mathrm{Li},{ }^{6} \mathrm{Li}\right),\left({ }^{6} \mathrm{Li},{ }^{6} \mathrm{Li}{ }^{\prime}\right), \mathrm{E}=28 \mathrm{MeV}$; measured $\sigma(\mathrm{E}, \theta)$, elastic $\sigma(\theta)$; deduced Coulomb-nuclear interference effects. ${ }^{70,72,74}$ Ge levels deduced $\mathrm{B}(\mathrm{E} 2)$ / B(IS2) ratio, mixed-symmetry effects. JOUR PRVCA 71024303

RADIOACTIVITY ${ }^{74} \mathrm{Kr}(\mathrm{EC}),\left(\beta^{+}\right)$[from $\mathrm{Nb}(\mathrm{p}, \mathrm{X})$ ]; measured $\beta \gamma$-coin; deduced Gamow-Teller strength distribution. ${ }^{33} \mathrm{Na}\left(\beta^{-}\right)$, $\left(\beta^{-} \mathrm{n}\right)$ [from $\mathrm{U}(\mathrm{p}, \mathrm{X})$ ]; measured $\beta \gamma-, \mathrm{n} \beta-, \gamma \gamma$-coin, $\mathrm{T}_{1 / 2} .{ }^{33} \mathrm{Mg}$ deduced ground-state $\mathrm{J}, \pi$. Total absorption spectrometer. JOUR BJPHE 34 850

RADIOACTIVITY ${ }^{74} \mathrm{Kr}(\mathrm{EC}),\left(\beta^{+}\right)$[from $\left.\mathrm{Nb}(\mathrm{p}, \mathrm{X})\right]$; measured $\beta \gamma$-coin; deduced Gamow-Teller strength distribution. ${ }^{33} \mathrm{Na}\left(\beta^{-}\right)$, $\left(\beta^{-} \mathrm{n}\right)$ [from $\mathrm{U}(\mathrm{p}, \mathrm{X})$ ]; measured $\beta \gamma-, \mathrm{n} \beta-, \gamma \gamma$-coin, $\mathrm{T}_{1 / 2} .{ }^{33} \mathrm{Mg}$ deduced ground-state $\mathrm{J}, \pi$. Total absorption spectrometer. JOUR BJPHE 34 850

NUCLEAR REACTIONS Be $\left({ }^{78} \mathrm{Kr}, \mathrm{X}\right){ }^{72} \mathrm{Kr} /{ }^{74} \mathrm{Kr}, \mathrm{E}=73 \mathrm{MeV}$; measured delayed $\mathrm{E} \gamma, \mathrm{I} \gamma, \mathrm{E}(\mathrm{ce}), \mathrm{I}(\mathrm{ce})$, (recoil) $\gamma$-, (recoil)(ce)-coin. ${ }^{72,74} \mathrm{Kr}$ deduced isomeric levels, $\mathrm{J}, \pi, \mathrm{T}_{1 / 2}$, E0 strength. ${ }^{72} \mathrm{Kr}$ deduced shape isomer. ${ }^{208} \mathrm{~Pb}\left({ }^{76} \mathrm{Kr},{ }^{76} \mathrm{Kr}\right),\left({ }^{74} \mathrm{Kr},{ }^{74} \mathrm{Kr}\right), \mathrm{E} \approx 4.5 \mathrm{MeV} /$ nucleon; measured $\mathrm{E} \gamma, \mathrm{I} \gamma$, (particle) $\gamma$-coin following projectile Coulomb excitation. ${ }^{74,76} \mathrm{Kr}$ deduced levels, J, $\pi$. CONF Argonne(Nuclei at the Limits),P55, Clement NUCLEAR REACTIONS ${ }^{208} \mathrm{~Pb}\left({ }^{74} \mathrm{Kr},{ }^{74} \mathrm{Kr}\right.$ '), $\left({ }^{76} \mathrm{Kr},{ }^{76} \mathrm{Kr}\right.$ '), E=4.5 $\mathrm{MeV} /$ nucleon; measured $\mathrm{E} \gamma, \mathrm{I} \gamma$, (particle) $\gamma$-coin following projectile Coulomb excitation. ${ }^{74,76} \mathrm{Kr}$ deduced levels, $\mathrm{J}, \pi$, quadrupole moments. ${ }^{208} \mathrm{~Pb}\left({ }^{72} \mathrm{Ge},{ }^{72} \mathrm{Ge}\right.$ '), E not given; measured $\mathrm{E} \gamma, \mathrm{I} \gamma, \mathrm{E}(\mathrm{ce}), \mathrm{I}(\mathrm{ce})$, (particle) $\gamma$-coin following projectile Coulomb excitation. ${ }^{72} \mathrm{Ge}$ deduced transitions. Exogam array. JOUR APOBB 361281 NUCLEAR REACTIONS ${ }^{40} \mathrm{Ca}\left({ }^{40} \mathrm{Ca}, 2 \mathrm{p} \alpha\right),\left({ }^{40} \mathrm{Ca}, 4 \mathrm{p}\right), \mathrm{E}=147 \mathrm{MeV}$; measured Doppler-shifted $\mathrm{E} \gamma, \mathrm{I} \gamma, \gamma \gamma$-coin. ${ }^{74,76} \mathrm{Kr}$ levels deduced $\mathrm{T}_{1 / 2}$, B(E2). GASP array, recoil-distance technique. JOUR ZAANE 26153 


\section{$\mathrm{A}=74$ (continued)}

2005K011 NUCLEAR REACTIONS ${ }^{208} \mathrm{~Pb}\left({ }^{74} \mathrm{Kr},{ }^{74} \mathrm{Kr}\right.$ '), $\left({ }^{76} \mathrm{Kr},{ }^{76} \mathrm{Kr}{ }^{\prime}\right), \mathrm{E} \approx 350$ $\mathrm{MeV}$; measured $\mathrm{E} \gamma, \mathrm{I} \gamma$, (particle) $\gamma$-coin following projectile Coulomb excitation. ${ }^{74,76} \mathrm{Kr}$ deduced levels, J, $\pi$, quadrupole moments. Exogam array. JOUR NUPAB $752255 \mathrm{c}$

2005R039 ATOMIC MASSES ${ }^{72,73,74} \mathrm{Kr},{ }^{73} \mathrm{Rb},{ }^{74} \mathrm{Sr}$; measured masses. Penning trap mass spectrometer. JOUR ZAANE 25 s01 41

2005VA30 NUCLEAR REACTIONS ${ }^{40} \mathrm{Ca}\left({ }^{40} \mathrm{Ca}, 2 \mathrm{p} \alpha\right), \mathrm{E}=165,185 \mathrm{MeV}$; measured $\mathrm{E} \gamma, \mathrm{I} \gamma, \gamma \gamma-$, (charged particle) $\gamma-$, (neutron) $\gamma$-coin, DSA. ${ }^{74} \mathrm{Kr}$ deduced high-spin levels, $\mathrm{J}, \pi, \mathrm{T}_{1 / 2}$, transition quadrupole moments, configurations, nontermination of rotational bands. Euroball III, ISIS, Gammasphere, and Microball arrays. JOUR PRLTA 95232501

${ }^{74} \mathrm{Rb} \quad$ 2005SA44 RADIOACTIVITY ${ }^{46} \mathrm{~V}(\mathrm{EC})$; analyzed masses; deduced Q(EC), log ft. ${ }^{10} \mathrm{C},{ }^{14} \mathrm{O},{ }^{22} \mathrm{Mg},{ }^{26 m} \mathrm{Al},{ }^{34} \mathrm{Cl},{ }^{34} \mathrm{Ar},{ }^{38 m} \mathrm{~K},{ }^{42} \mathrm{Sc},{ }^{46} \mathrm{~V},{ }^{50} \mathrm{Mn},{ }^{54} \mathrm{Co},{ }^{74} \mathrm{Rb}$; compiled, analyzed log ft; deduced quark-mixing matrix element. JOUR PRLTA 95102501

${ }^{74} \mathrm{Sr} \quad 2005 \mathrm{R} 039$ ATOMIC MASSES ${ }^{72,73,74} \mathrm{Kr},{ }^{73} \mathrm{Rb},{ }^{74} \mathrm{Sr}$; measured masses. Penning trap mass spectrometer. JOUR ZAANE 25 s01 41

$$
\mathrm{A}=75
$$

$\begin{array}{ll}{ }^{75} \mathrm{Ni} & 2005 \mathrm{HO08} \\ { }^{75} \mathrm{Cu} & 2005 \mathrm{HO08} \\ { }^{75} \mathrm{As} & 2005 \mathrm{RA} 29 \\ { }^{75} \mathrm{Se} & 2005 \mathrm{RA} 29 \\ & \\ { }^{75} \mathrm{Br} & 2004 \mathrm{SC} 48\end{array}$

RADIOACTIVITY ${ }^{75,76,77,78} \mathrm{Ni},{ }^{77,78} \mathrm{Cu}\left(\beta^{-}\right)$[from $\mathrm{Be}\left({ }^{86} \mathrm{Kr}, \mathrm{X}\right)$ ]; measured $\mathrm{T}_{1 / 2}$. Astrophysical implications discussed. JOUR PRLTA 94112501 RADIOACTIVITY ${ }^{75,76,77,78} \mathrm{Ni},{ }^{77,78} \mathrm{Cu}\left(\beta^{-}\right)$[from $\mathrm{Be}\left({ }^{86} \mathrm{Kr}, \mathrm{X}\right)$ ]; measured $\mathrm{T}_{1 / 2}$. Astrophysical implications discussed. JOUR PRLTA 94112501 RADIOACTIVITY ${ }^{75} \mathrm{Se}(\mathrm{EC})$; measured $\mathrm{E} \gamma, \mathrm{I} \gamma, \mathrm{E}(\mathrm{ce}), \mathrm{I}(\mathrm{ce})$; deduced $\log \mathrm{ft} .{ }^{75} \mathrm{As}$ deduced levels, J, $\pi, \mathrm{ICC}, \mathrm{B}(\mathrm{E} 2), \delta$. Mini-orange spectrometer. JOUR ZAANE 2641

RADIOACTIVITY ${ }^{75} \mathrm{Se}(\mathrm{EC})$; measured $\mathrm{E} \gamma, \mathrm{I} \gamma, \mathrm{E}(\mathrm{ce}), \mathrm{I}(\mathrm{ce})$; deduced $\log \mathrm{ft} .{ }^{75}$ As deduced levels, J, $\pi$, ICC, B(E2), $\delta$. Mini-orange spectrometer. JOUR ZAANE 2641 NUCLEAR REACTIONS ${ }^{78} \operatorname{Kr}(\mathrm{d}, \mathrm{n}),(\mathrm{d}, \mathrm{p}),(\mathrm{d}, \alpha),(\mathrm{d}, \mathrm{n} \alpha), \mathrm{E} \approx 4-13$ $\mathrm{MeV}$; measured excitation functions. Stacked gas cell activation technique. JOUR RAACA 92203$$
\mathrm{A}=76
$$

${ }^{76} \mathrm{Ni} \quad 2005 \mathrm{HOO}$

RADIOACTIVITY ${ }^{75,76,77,78} \mathrm{Ni},{ }^{77,78} \mathrm{Cu}\left(\beta^{-}\right)$[from $\left.\mathrm{Be}\left({ }^{86} \mathrm{Kr}, \mathrm{X}\right)\right]$; measured $\mathrm{T}_{1 / 2}$. Astrophysical implications discussed. JOUR PRLTA 94112501

2005MA59 RADIOACTIVITY ${ }^{72,74} \mathrm{Co}\left(\beta^{-}\right)$[from $\left.\mathrm{Be}\left({ }^{86} \mathrm{Kr}, \mathrm{X}\right)\right]$; measured $\mathrm{E} \gamma$, I $\gamma$, $\beta \gamma$-coin. ${ }^{76} \mathrm{Ni}(\mathrm{IT})$ [from $\mathrm{Be}\left({ }^{86} \mathrm{Kr}, \mathrm{X}\right)$ ]; measured $\mathrm{E} \gamma, \mathrm{I} \gamma$. ${ }^{72,74,76} \mathrm{Ni}$ deduced levels, $\mathrm{J}, \pi$. Level systematics in neighboring isotopes discussed. JOUR PYLBB 62245 


\section{$\mathrm{A}=76$ (continued)}

2005MAZX NUCLEAR REACTIONS ${ }^{9} \mathrm{Be}\left({ }^{86} \mathrm{Kr}, \mathrm{X}\right), \mathrm{E}=140 \mathrm{MeV} /$ nucleon; measured fragment yields; deduced evidence for ${ }^{74} \mathrm{Co},{ }^{76} \mathrm{Ni}$. CONF Argonne(Nuclei at the Limits),P164,Mazzocchi

2005MAZX RADIOACTIVITY ${ }^{71,72,73,74} \mathrm{Co}\left(\beta^{-}\right),\left(\beta^{-} \mathrm{n}\right)$ [from ${ }^{9} \mathrm{Be}\left({ }^{86} \mathrm{Kr}, \mathrm{X}\right)$ ]; measured $\beta$-delayed $\mathrm{E} \gamma, \mathrm{I} \gamma$; deduced branching ratios. ${ }^{70,71} \mathrm{Ni}$ deduced transitions. ${ }^{76} \mathrm{Ni}(\mathrm{IT})$ [from $\left.{ }^{9} \mathrm{Be}\left({ }^{86} \mathrm{Kr}, \mathrm{X}\right)\right]$; measured $\mathrm{E} \gamma, \mathrm{I} \gamma, \mathrm{T}_{1 / 2} \cdot{ }^{76} \mathrm{Ni}$ deduced levels. CONF Argonne(Nuclei at the Limits),P164,Mazzocchi ${ }^{76} \mathrm{Cu} \quad 2005 \mathrm{HOO}$ RADIOACTIVITY ${ }^{75,76,77,78} \mathrm{Ni},{ }^{77,78} \mathrm{Cu}\left(\beta^{-}\right)$[from $\mathrm{Be}\left({ }^{86} \mathrm{Kr}, \mathrm{X}\right)$ ]; measured $\mathrm{T}_{1 / 2}$. Astrophysical implications discussed. JOUR PRLTA 94112501

2005VA19 RADIOACTIVITY ${ }^{74,76,78} \mathrm{Cu}\left(\beta^{-}\right) ;{ }^{78} \mathrm{Cu}\left(\beta^{-} \mathrm{n}\right)$ [from ${ }^{238} \mathrm{U}(\mathrm{n}, \mathrm{F})$, (p, $\mathrm{F})]$; measured $\mathrm{E} \gamma, \mathrm{I} \gamma, \beta \gamma$-coin, $\mathrm{T}_{1 / 2} .{ }^{74,76,77,78} \mathrm{Zn}$ deduced levels, $\mathrm{J}, \pi$, configurations. Mass separator, comparisons with model predictions. JOUR PRVCA 71054307

${ }^{76} \mathrm{Zn} \quad$ 2005VA19 RADIOACTIVITY ${ }^{74,76,78} \mathrm{Cu}\left(\beta^{-}\right) ;{ }^{78} \mathrm{Cu}\left(\beta^{-} \mathrm{n}\right)$ [from ${ }^{238} \mathrm{U}(\mathrm{n}, \mathrm{F}),(\mathrm{p}$, $\mathrm{F})]$; measured $\mathrm{E} \gamma, \mathrm{I} \gamma, \beta \gamma$-coin, $\mathrm{T}_{1 / 2} \cdot{ }^{74,76,77,78} \mathrm{Zn}$ deduced levels, J, $\pi$, configurations. Mass separator, comparisons with model predictions.

JOUR PRVCA 71054307

${ }^{76} \mathrm{Ge} \quad 2005 \mathrm{BA} 60$ RADIOACTIVITY ${ }^{76} \mathrm{Ge}\left(2 \beta^{-}\right)$; measured $2 \nu \beta \beta$-decay $\mathrm{T}_{1 / 2}$, $0 \nu \beta \beta$-decay $\mathrm{T}_{1 / 2}$ lower limit. JOUR FECLA 12521

2005DIZZ NUCLEAR REACTIONS ${ }^{238} \mathrm{U}\left({ }^{48} \mathrm{Ca}, \mathrm{X}\right){ }^{56} \mathrm{Ti}, \mathrm{E}=330 \mathrm{MeV}$; measured $\mathrm{E} \gamma, \mathrm{I} \gamma, \gamma \gamma$-coin. ${ }^{56} \mathrm{Ti}$ deduced levels, $\mathrm{J}, \pi .{ }^{197} \mathrm{Au}\left({ }^{76} \mathrm{Ge},{ }^{76} \mathrm{Ge}\right),\left({ }^{52} \mathrm{Ti}\right.$, $\left.{ }^{52} \mathrm{Ti}^{\prime}\right),\left({ }^{54} \mathrm{Ti},{ }^{54} \mathrm{Ti}^{\prime}\right),\left({ }^{56} \mathrm{Ti},{ }^{56} \mathrm{Ti}^{\prime}\right), \mathrm{E} \approx 80-90 \mathrm{MeV}$; measured $\mathrm{E} \gamma, \mathrm{I} \gamma$, (particle) $\gamma$-coin following projectile Coulomb excitation. ${ }^{52,54,56} \mathrm{Ti}$, ${ }^{76} \mathrm{Ge},{ }^{197} \mathrm{Au}$ deduced transitions B(E2). CONF Argonne(Nuclei at the Limits),P131,Dinca

2005IW03 NUCLEAR REACTIONS Pb $\left({ }^{76} \mathrm{Ge},{ }^{76} \mathrm{Ge}\right),\left({ }^{78} \mathrm{Ge},{ }^{78} \mathrm{Ge}\right),\left({ }^{80} \mathrm{Ge}\right.$, $\left.{ }^{80} \mathrm{Ge}\right),\left({ }^{82} \mathrm{Ge},{ }^{82} \mathrm{Ge}\right), \mathrm{E} \approx 40 \mathrm{MeV} /$ nucleon; measured $\mathrm{E} \gamma, \mathrm{I} \gamma$, (particle) $\gamma$-coin following projectile Coulomb excitation. ${ }^{76,78,80,82} \mathrm{Ge}$ deduced transitions B(E2). JOUR ZAANE $25 \mathrm{~s} 01415$

2005KL02 RADIOACTIVITY ${ }^{76} \mathrm{Ge}\left(2 \beta^{-}\right)$; measured $0 \nu \beta \beta$-decay $\mathrm{T}_{1 / 2}$; deduced non-conservation of lepton number. JOUR NPBSE 143229

${ }^{76} \mathrm{Se} \quad$ 2005BA60 RADIOACTIVITY ${ }^{76} \mathrm{Ge}\left(2 \beta^{-}\right)$; measured $2 \nu \beta \beta$-decay $\mathrm{T}_{1 / 2}$, $0 \nu \beta \beta$-decay $\mathrm{T}_{1 / 2}$ lower limit. JOUR FECLA 12521

2005KL02 RADIOACTIVITY ${ }^{76} \mathrm{Ge}\left(2 \beta^{-}\right)$; measured $0 \nu \beta \beta$-decay $\mathrm{T}_{1 / 2}$; deduced non-conservation of lepton number. JOUR NPBSE 143229

${ }^{76} \mathrm{Br} \quad 2004 \mathrm{SC} 48$ NUCLEAR REACTIONS ${ }^{78} \operatorname{Kr}(\mathrm{d}, \mathrm{n}),(\mathrm{d}, \mathrm{p}),(\mathrm{d}, \alpha),(\mathrm{d}, \mathrm{n} \alpha), \mathrm{E} \approx 4-13$ $\mathrm{MeV}$; measured excitation functions. Stacked gas cell activation technique. JOUR RAACA 92203

2004SCZU NUCLEAR REACTIONS ${ }^{76} \mathrm{Se}(\mathrm{p}, \mathrm{n}), \mathrm{E} \approx 5-40 \mathrm{MeV} ;{ }^{78} \mathrm{Kr}(\mathrm{d}, \alpha), \mathrm{E} \approx$ 4-14 MeV; measured $\sigma .{ }^{126} \mathrm{Te}(\mathrm{p}, 3 \mathrm{n}), \mathrm{E}=8-70 \mathrm{MeV} ;{ }^{85} \mathrm{Rb}(\mathrm{p}, 4 \mathrm{n})$, $\mathrm{E}=44-66 \mathrm{MeV}$; measured yields. REPT NEA/NSC/DOC(2004)14,P13,Scholten

${ }^{76} \mathrm{Kr} \quad$ 2005BE61 NUCLEAR REACTIONS ${ }^{26} \mathrm{Mg}\left({ }^{76} \mathrm{Kr},{ }^{76} \mathrm{Kr}\right.$ '), E=230 MeV; measured $\mathrm{E} \gamma, \mathrm{I} \gamma(\theta, \mathrm{H}, \mathrm{t}),($ particle $) \gamma$-coin following projectile Coulomb excitation. ${ }^{76} \mathrm{Kr}$ level deduced $\mathrm{g}$ factor. Transient field technique. JOUR ZAANE 25 s01 203 


\section{$\mathrm{A}=76$ (continued)}

2005CH60 ATOMIC MASSES ${ }^{64} \mathrm{Zn},{ }^{64} \mathrm{Ga},{ }^{68} \mathrm{Ge},{ }^{68} \mathrm{As},{ }^{68,72} \mathrm{Se},{ }^{76} \mathrm{Kr},{ }^{76} \mathrm{Rb},{ }^{80} \mathrm{Sr}$, ${ }^{80} \mathrm{Y}$; measured masses. Direct time-of-flight technique, comparison with previous results. JOUR JPGPE 31 S1771

2005CLZZ NUCLEAR REACTIONS Be $\left({ }^{78} \mathrm{Kr}, \mathrm{X}\right){ }^{72} \mathrm{Kr} /{ }^{74} \mathrm{Kr}, \mathrm{E}=73 \mathrm{MeV}$; measured delayed $\mathrm{E} \gamma, \mathrm{I} \gamma, \mathrm{E}(\mathrm{ce}), \mathrm{I}(\mathrm{ce})$, (recoil) $\gamma$-, (recoil)(ce)-coin. ${ }^{72,74} \mathrm{Kr}$ deduced isomeric levels, $\mathrm{J}, \pi, \mathrm{T}_{1 / 2}$, E0 strength. ${ }^{72} \mathrm{Kr}$ deduced shape isomer. ${ }^{208} \mathrm{~Pb}\left({ }^{76} \mathrm{Kr},{ }^{76} \mathrm{Kr}\right),\left({ }^{74} \mathrm{Kr},{ }^{74} \mathrm{Kr}{ }^{\prime}\right), \mathrm{E} \approx 4.5 \mathrm{MeV} /$ nucleon; measured $\mathrm{E} \gamma, \mathrm{I} \gamma$, (particle) $\gamma$-coin following projectile Coulomb excitation. ${ }^{74,76} \mathrm{Kr}$ deduced levels, J, $\pi$. CONF Argonne(Nuclei at the Limits),P55,Clement

2005GI17 RADIOACTIVITY ${ }^{76} \mathrm{Rb}\left(\beta^{+}\right)$, (EC) [from $\mathrm{Nb}(\mathrm{p}, \mathrm{X})$ ]; measured $\mathrm{E} \gamma, \mathrm{I} \gamma$, $\mathrm{E}(\mathrm{ce}), \mathrm{I}(\mathrm{ce}), \gamma \gamma-, \beta \gamma$-coin; deduced log ft. ${ }^{76} \mathrm{Kr}$ deduced levels $\mathrm{J}, \pi$, $\mathrm{T}_{1 / 2}$, ICC. ${ }^{76} \mathrm{Rb}$ deduced ground state $\mathrm{J}, \pi$. JOUR PRVCA 72044308

2005 G015 NUCLEAR REACTIONS ${ }^{208} \mathrm{~Pb}\left({ }^{74} \mathrm{Kr},{ }^{74} \mathrm{Kr}{ }^{\prime}\right),\left({ }^{76} \mathrm{Kr},{ }^{76} \mathrm{Kr}\right.$ '), E=4.5 $\mathrm{MeV} /$ nucleon; measured $\mathrm{E} \gamma, \mathrm{I} \gamma$, (particle) $\gamma$-coin following projectile Coulomb excitation. ${ }^{74,76} \mathrm{Kr}$ deduced levels, $\mathrm{J}, \pi$, quadrupole moments. ${ }^{208} \mathrm{~Pb}\left({ }^{72} \mathrm{Ge},{ }^{72} \mathrm{Ge}\right.$ '), E not given; measured $\mathrm{E} \gamma, \mathrm{I} \gamma, \mathrm{E}(\mathrm{ce}), \mathrm{I}(\mathrm{ce})$, (particle) $\gamma$-coin following projectile Coulomb excitation. ${ }^{72} \mathrm{Ge}$ deduced transitions. Exogam array. JOUR APOBB 361281

2005 G043 NUCLEAR REACTIONS ${ }^{40} \mathrm{Ca}\left({ }^{40} \mathrm{Ca}, 2 \mathrm{p} \alpha\right),\left({ }^{40} \mathrm{Ca}, 4 \mathrm{p}\right), \mathrm{E}=147 \mathrm{MeV}$; measured Doppler-shifted $\mathrm{E} \gamma, \mathrm{I} \gamma, \gamma \gamma$-coin. ${ }^{74,76} \mathrm{Kr}$ levels deduced $\mathrm{T}_{1 / 2}$, B(E2). GASP array, recoil-distance technique. JOUR ZAANE 26153 $2005 \mathrm{KO} 11$ NUCLEAR REACTIONS ${ }^{208} \mathrm{~Pb}\left({ }^{74} \mathrm{Kr},{ }^{74} \mathrm{Kr}\right.$ '), $\left({ }^{76} \mathrm{Kr},{ }^{76} \mathrm{Kr}\right.$ '), $\mathrm{E} \approx 350$ $\mathrm{MeV}$; measured $\mathrm{E} \gamma, \mathrm{I} \gamma$, (particle) $\gamma$-coin following projectile Coulomb excitation. ${ }^{74,76} \mathrm{Kr}$ deduced levels, J, $\pi$, quadrupole moments. Exogam array. JOUR NUPAB $752255 \mathrm{c}$

2005VA09 NUCLEAR REACTIONS ${ }^{40} \mathrm{Ca}\left({ }^{40} \mathrm{Ca}, 4 \mathrm{p}\right), \mathrm{E}=165 \mathrm{MeV}$; measured $\mathrm{E} \gamma$, $\mathrm{I} \gamma, \gamma \gamma-$, (charged particle) $\gamma$-coin, DSA. ${ }^{76} \mathrm{Kr}$ deduced high-spin levels, $\mathrm{J}, \pi, \mathrm{T}_{1 / 2}$, transition quadrupole moments, configurations.

Gammasphere, Microball arrays, comparison with cranked mean-field model predictions. JOUR PRVCA 71034311

2005VA18 NUCLEAR REACTIONS ${ }^{40} \mathrm{Ca}\left({ }^{40} \mathrm{Ca}, 4 \mathrm{p}\right), \mathrm{E}=165 \mathrm{MeV}$; measured $\mathrm{E} \gamma$, $\mathrm{I} \gamma, \gamma \gamma$-, (charged particle) $\gamma$-coin, DSA. ${ }^{76} \mathrm{Kr}$ deduced high-spin levels, $\mathrm{J}, \pi, \mathrm{T}_{1 / 2}$, configurations. Gammasphere, Microball arrays. JOUR APOBB 361339

${ }^{76} \mathrm{Rb} \quad 2005 \mathrm{CH} 60 \quad$ ATOMIC MASSES ${ }^{64} \mathrm{Zn},{ }^{64} \mathrm{Ga},{ }^{68} \mathrm{Ge},{ }^{68} \mathrm{As},{ }^{68,72} \mathrm{Se},{ }^{76} \mathrm{Kr},{ }^{76} \mathrm{Rb},{ }^{80} \mathrm{Sr}$, ${ }^{80} \mathrm{Y}$; measured masses. Direct time-of-flight technique, comparison with previous results. JOUR JPGPE 31 S1771

2005GI17 RADIOACTIVITY ${ }^{76} \mathrm{Rb}\left(\beta^{+}\right)$, (EC) [from $\mathrm{Nb}(\mathrm{p}, \mathrm{X})$ ]; measured $\mathrm{E} \gamma, \mathrm{I} \gamma$, $\mathrm{E}(\mathrm{ce}), \mathrm{I}(\mathrm{ce}), \gamma \gamma-, \beta \gamma$-coin; deduced log ft. ${ }^{76} \mathrm{Kr}$ deduced levels $\mathrm{J}, \pi$, $\mathrm{T}_{1 / 2}$, ICC. ${ }^{76} \mathrm{Rb}$ deduced ground state $\mathrm{J}, \pi$. JOUR PRVCA 72044308 2005RU07 RADIOACTIVITY ${ }^{76} \mathrm{Sr}(\mathrm{EC}),\left(\beta^{+}\right)$[from $\left.\mathrm{Nb}(\mathrm{p}, \mathrm{X})\right]$; measured $\mathrm{E} \beta, \mathrm{I} \beta$, $\mathrm{E} \gamma$; deduced Gamow-Teller strength distribution. ${ }^{76} \mathrm{Sr}$ deduced ground-state deformation. Total absorption technique. JOUR NUPAB 752 251c

${ }^{76} \mathrm{Sr} \quad$ 2005RU07 RADIOACTIVITY ${ }^{76} \mathrm{Sr}(\mathrm{EC}),\left(\beta^{+}\right)[$from $\mathrm{Nb}(\mathrm{p}, \mathrm{X})] ;$ measured $\mathrm{E} \beta, \mathrm{I} \beta$, E $\gamma$; deduced Gamow-Teller strength distribution. ${ }^{76} \mathrm{Sr}$ deduced ground-state deformation. Total absorption technique. JOUR NUPAB $752251 \mathrm{c}$ 


\section{$\mathrm{A}=76$ (continued)}

2005SI34

ATOMIC MASSES ${ }^{76,77,80,81,86,88} \mathrm{Sr},{ }^{124,129,130,131,132} \mathrm{Sn}$; measured masses. Penning trap mass spectrometer, comparison with previous results. JOUR NUPAB 76345

\section{$\mathrm{A}=77$}

\begin{tabular}{|c|c|c|}
\hline${ }^{77} \mathrm{Ni}$ & $2005 \mathrm{HO0}$ & $\begin{array}{l}\left.\text { RADIOACTIVITY }{ }^{75,76,77,78} \mathrm{Ni},{ }^{77,78} \mathrm{Cu}\left(\beta^{-}\right) \text {[from } \mathrm{Be}\left({ }^{86} \mathrm{Kr}, \mathrm{X}\right)\right] \\
\text { measured } \mathrm{T}_{1 / 2} . \text { Astrophysical implications discussed. JOUR PRLTA } \\
94112501\end{array}$ \\
\hline${ }^{77} \mathrm{Cu}$ & $2005 \mathrm{HOO}$ & $\begin{array}{l}\left.\text { RADIOACTIVITY }{ }^{75,76,77,78} \mathrm{Ni},{ }^{77,78} \mathrm{Cu}\left(\beta^{-}\right) \text {[from } \mathrm{Be}\left({ }^{86} \mathrm{Kr}, \mathrm{X}\right)\right] \\
\text { measured } \mathrm{T}_{1 / 2} \text {. Astrophysical implications discussed. JOUR PRLTA } \\
94112501\end{array}$ \\
\hline \multirow[t]{2}{*}{${ }^{77} \mathrm{Zn}$} & $2005 \mathrm{HOO}$ & $\begin{array}{l}\text { RADIOACTIVITY }{ }^{75,76,77,78} \mathrm{Ni},{ }^{77,78} \mathrm{Cu}\left(\beta^{-}\right)\left[\text {from } \mathrm{Be}\left({ }^{86} \mathrm{Kr}, \mathrm{X}\right)\right] \\
\text { measured } \mathrm{T}_{1 / 2} \text {. Astrophysical implications discussed. JOUR PRLTA } \\
94112501\end{array}$ \\
\hline & 2005VA19 & $\begin{array}{l}\text { RADIOACTIVITY } 74,76,78 \mathrm{Cu}\left(\beta^{-}\right) ;{ }^{78} \mathrm{Cu}\left(\beta^{-} \mathrm{n}\right)\left[\text { from }{ }^{238} \mathrm{U}(\mathrm{n}, \mathrm{F}),(\mathrm{p},\right. \\
\mathrm{F})] \text {; measured } \mathrm{E} \gamma, \mathrm{I} \gamma, \beta \gamma \text {-coin, } \mathrm{T}_{1 / 2} \cdot{ }^{74,76,77,78} \mathrm{Zn} \text { deduced levels, J, } \pi \text {, } \\
\text { configurations. Mass separator, comparisons with model predictions. } \\
\text { JOUR PRVCA } 71054307\end{array}$ \\
\hline${ }^{77} \mathrm{As}$ & 2005LU07 & $\begin{array}{l}\text { NUCLEAR REACTIONS }{ }^{238} \mathrm{U}\left({ }^{82} \mathrm{Se}, \mathrm{X}\right), \mathrm{E}=505 \mathrm{MeV} \text {; measured } \mathrm{E} \gamma \text {, } \\
\mathrm{I} \gamma \text {, fragments isotopic yields. }{ }^{77,78,79,80,81,82,83} \mathrm{As} \text { deduced transitions. } \\
{ }^{192} \mathrm{Os}\left({ }^{82} \mathrm{Se}, \mathrm{X}\right), \mathrm{E}=460 \mathrm{MeV} \text {; measured } \mathrm{E} \gamma, \mathrm{I} \gamma, \gamma \gamma \text {-coin. }{ }^{80} \mathrm{As},{ }^{87} \mathrm{Rb} \text {, } \\
{ }^{84} \mathrm{Se} \text { deduced levels. Fragment separator. JOUR APOBB } 361301\end{array}$ \\
\hline${ }^{77} \mathrm{~S}$ & 2005SI34 & $\begin{array}{l}\text { ATOMIC MASSES } 76,77,80,81,86,88 \mathrm{Sr},{ }^{124,129,130,131,132} \mathrm{Sn} \text {; measured } \\
\text { masses. Penning trap mass spectrometer, comparison with previous } \\
\text { results. JOUR NUPAB } 76345\end{array}$ \\
\hline
\end{tabular}

\section{$\mathrm{A}=78$}

${ }^{78} \mathrm{Ni} \quad 2005 \mathrm{HOO}$

RADIOACTIVITY ${ }^{75,76,77,78} \mathrm{Ni},{ }^{77,78} \mathrm{Cu}\left(\beta^{-}\right)$[from $\mathrm{Be}\left({ }^{86} \mathrm{Kr}, \mathrm{X}\right)$ ]; measured $\mathrm{T}_{1 / 2}$. Astrophysical implications discussed. JOUR PRLTA 94112501

2005SC28 RADIOACTIVITY ${ }^{78} \mathrm{Ni}\left(\beta^{-}\right)$[from ${ }^{86} \mathrm{Kr}$ fragmentation]; measured $\mathrm{T}_{1 / 2}$. Comparison with model predictions, astrophysical implications discussed. JOUR ZAANE 25 s01 639

${ }^{78} \mathrm{Cu} \quad 2005 \mathrm{HO08}$ RADIOACTIVITY ${ }^{75,76,77,78} \mathrm{Ni},{ }^{77,78} \mathrm{Cu}\left(\beta^{-}\right)$[from $\left.\mathrm{Be}\left({ }^{86} \mathrm{Kr}, \mathrm{X}\right)\right]$; measured $\mathrm{T}_{1 / 2}$. Astrophysical implications discussed. JOUR PRLTA 94112501

2005SC28 RADIOACTIVITY ${ }^{78} \mathrm{Ni}\left(\beta^{-}\right)$[from ${ }^{86} \mathrm{Kr}$ fragmentation]; measured $\mathrm{T}_{1 / 2}$. Comparison with model predictions, astrophysical implications discussed. JOUR ZAANE 25 s01 639

2005VA19 RADIOACTIVITY ${ }^{74,76,78} \mathrm{Cu}\left(\beta^{-}\right) ;{ }^{78} \mathrm{Cu}\left(\beta^{-}\right.$n) [from ${ }^{238} \mathrm{U}(\mathrm{n}, \mathrm{F})$, (p, $\mathrm{F})]$; measured $\mathrm{E} \gamma, \mathrm{I} \gamma, \beta \gamma$-coin, $\mathrm{T}_{1 / 2} .{ }^{74,76,77,78} \mathrm{Zn}$ deduced levels, $\mathrm{J}, \pi$, configurations. Mass separator, comparisons with model predictions. JOUR PRVCA 71054307 


\section{$\mathrm{A}=78$ (continued)}

${ }^{78} \mathrm{Zn} \quad 2005 \mathrm{HOO8} \quad \mathrm{RADIOACTIVITY}{ }^{75,76,77,78} \mathrm{Ni},{ }^{77,78} \mathrm{Cu}\left(\beta^{-}\right)$[from $\left.\mathrm{Be}\left({ }^{86} \mathrm{Kr}, \mathrm{X}\right)\right]$; measured $\mathrm{T}_{1 / 2}$. Astrophysical implications discussed. JOUR PRLTA 94112501

2005VA19 RADIOACTIVITY ${ }^{74,76,78} \mathrm{Cu}\left(\beta^{-}\right) ;{ }^{78} \mathrm{Cu}\left(\beta^{-} \mathrm{n}\right)$ [from ${ }^{238} \mathrm{U}(\mathrm{n}, \mathrm{F}),(\mathrm{p}$, $\mathrm{F})]$; measured $\mathrm{E} \gamma, \mathrm{I} \gamma, \beta \gamma$-coin, $\mathrm{T}_{1 / 2} .{ }^{74,76,77,78} \mathrm{Zn}$ deduced levels, J, $\pi$, configurations. Mass separator, comparisons with model predictions. JOUR PRVCA 71054307

${ }^{78} \mathrm{Ge} \quad$ 2005BE17 RADIOACTIVITY ${ }^{127} \mathrm{I}\left({ }^{24} \mathrm{Ne}\right),\left({ }^{28} \mathrm{Mg}\right),\left({ }^{30} \mathrm{Mg}\right),\left({ }^{32} \mathrm{Si}\right),\left({ }^{34} \mathrm{Si}\right),\left({ }^{48} \mathrm{Ca}\right)$, $\left({ }^{49} \mathrm{Sc}\right)$; measured cluster decay $\mathrm{T}_{1 / 2}$ lower limits. JOUR ZAANE 2451 2005IW03 NUCLEAR REACTIONS Pb $\left({ }^{76} \mathrm{Ge},{ }^{76} \mathrm{Ge}\right),\left({ }^{78} \mathrm{Ge},{ }^{78} \mathrm{Ge}\right),\left({ }^{80} \mathrm{Ge}\right.$, $\left.{ }^{80} \mathrm{Ge}^{\prime}\right),\left({ }^{82} \mathrm{Ge},{ }^{82} \mathrm{Ge}{ }^{\prime}\right), \mathrm{E} \approx 40 \mathrm{MeV} /$ nucleon; measured $\mathrm{E} \gamma, \mathrm{I} \gamma$, (particle) $\gamma$-coin following projectile Coulomb excitation. ${ }^{76,78,80,82} \mathrm{Ge}$ deduced transitions B(E2). JOUR ZAANE 25 s01 415

2005PA23 NUCLEAR REACTIONS C $\left({ }^{78} \mathrm{Ge},{ }^{78} \mathrm{Ge}\right),\left({ }^{80} \mathrm{Ge},{ }^{80} \mathrm{Ge}\right), \mathrm{E}=2.24 \mathrm{MeV}$ / nucleon; ${ }^{48} \mathrm{Ti}\left({ }^{82} \mathrm{Ge},{ }^{82} \mathrm{Ge}\right), \mathrm{E}=220 \mathrm{MeV}$; measured $\mathrm{E} \gamma, \mathrm{I} \gamma$, (particle) $\gamma$-coin following projectile Coulomb excitation. ${ }^{78,80,82} \mathrm{Ge}$ deduced excitation B(E2). Systematic trends in B(E2) values discussed. JOUR PRLTA 94122501

${ }^{78} \mathrm{As} \quad 2005 \mathrm{LU} 07$ NUCLEAR REACTIONS ${ }^{238} \mathrm{U}\left({ }^{82} \mathrm{Se}, \mathrm{X}\right), \mathrm{E}=505 \mathrm{MeV}$; measured $\mathrm{E} \gamma$, I $\gamma$, fragments isotopic yields. ${ }^{77,78,79,80,81,82,83}$ As deduced transitions. ${ }^{192} \mathrm{Os}\left({ }^{82} \mathrm{Se}, \mathrm{X}\right), \mathrm{E}=460 \mathrm{MeV}$; measured $\mathrm{E} \gamma, \mathrm{I} \gamma, \gamma \gamma$-coin. ${ }^{80} \mathrm{As},{ }^{87} \mathrm{Rb}$, ${ }^{84}$ Se deduced levels. Fragment separator. JOUR APOBB 361301

${ }^{78} \mathrm{Se} \quad 2005 \mathrm{GAZV}$ RADIOACTIVITY ${ }^{78} \mathrm{Kr}(2 \mathrm{EC})$; measured $2 \mathrm{~K}(2 \nu)$-capture $\mathrm{T}_{1 / 2}$ lower limit. PREPRINT nucl-ex/0510070,10/26/2005

${ }^{78} \mathrm{Kr} \quad 2005 \mathrm{GA} 22$ NUCLEAR REACTIONS ${ }^{197} \mathrm{Au}\left({ }^{72} \mathrm{Kr},{ }^{72} \mathrm{Kr}\right.$ '), E=69.3 MeV / nucleon; ${ }^{197} \mathrm{Au}\left({ }^{78} \mathrm{Kr},{ }^{78} \mathrm{Kr}\right.$ '), E=57.4 MeV / nucleon; measured $\mathrm{E} \gamma, \mathrm{I} \gamma$, (particle) $\gamma$-coin following projectile Coulomb excitation. ${ }^{72,78} \mathrm{Kr}$ deduced excitation $\mathrm{B}(\mathrm{E} 2)$, quadrupole moments, deformation. Comparison with shell-model Monte Carlo predictions. JOUR PRLTA 95022502

2005GAZV RADIOACTIVITY ${ }^{78} \mathrm{Kr}(2 \mathrm{EC})$; measured $2 \mathrm{~K}(2 \nu)$-capture $\mathrm{T}_{1 / 2}$ lower limit. PREPRINT nucl-ex/0510070,10/26/2005

2005LE04 NUCLEAR REACTIONS Pb(p, X) ${ }^{3} \mathrm{He} /{ }^{4} \mathrm{He} /{ }^{21} \mathrm{Ne} /{ }^{22} \mathrm{Ne} /{ }^{36} \mathrm{Ar} /$ ${ }^{38} \mathrm{Ar} /{ }^{78} \mathrm{Kr} /{ }^{80} \mathrm{Kr} /{ }^{81} \mathrm{Kr} /{ }^{82} \mathrm{Kr} /{ }^{83} \mathrm{Kr} /{ }^{84} \mathrm{Kr} /{ }^{85} \mathrm{Kr} /{ }^{86} \mathrm{Kr} /{ }^{124} \mathrm{Xe}$ $/{ }^{126} \mathrm{Xe} /{ }^{128} \mathrm{Xe} /{ }^{129} \mathrm{Xe} /{ }^{130} \mathrm{Xe} /{ }^{131} \mathrm{Xe} /{ }^{132} \mathrm{Xe} /{ }^{134} \mathrm{Xe}, \mathrm{E}=44-2595$ $\mathrm{MeV}$; measured production $\sigma$; deduced reaction mechanism features. Mini-stack approach, comparisons with model predictions. JOUR NIMBE 2291

2005SC26 ATOMIC MASSES $78,80,82,83,84,86 \mathrm{Kr}$; measured masses. Penning trap mass spectrometer. JOUR ZAANE 25 s01 51

\section{$\mathrm{A}=79$}

${ }^{79} \mathrm{As}$ 2005BE17

RADIOACTIVITY ${ }^{127} \mathrm{I}\left({ }^{24} \mathrm{Ne}\right),\left({ }^{28} \mathrm{Mg}\right),\left({ }^{30} \mathrm{Mg}\right),\left({ }^{32} \mathrm{Si}\right),\left({ }^{34} \mathrm{Si}\right),\left({ }^{48} \mathrm{Ca}\right)$, $\left({ }^{49} \mathrm{Sc}\right)$; measured cluster decay $\mathrm{T}_{1 / 2}$ lower limits. JOUR ZAANE 2451 


\section{$\mathrm{A}=79$ (continued)}

2005LU07 NUCLEAR REACTIONS ${ }^{238} \mathrm{U}\left({ }^{82} \mathrm{Se}, \mathrm{X}\right), \mathrm{E}=505 \mathrm{MeV}$; measured E $\gamma$, I $\gamma$, fragments isotopic yields. $77,78,79,80,81,82,83$ As deduced transitions. ${ }^{192} \mathrm{Os}\left({ }^{82} \mathrm{Se}, \mathrm{X}\right), \mathrm{E}=460 \mathrm{MeV}$; measured $\mathrm{E} \gamma, \mathrm{I} \gamma, \gamma \gamma$-coin. ${ }^{80} \mathrm{As},{ }^{87} \mathrm{Rb}$, ${ }^{84}$ Se deduced levels. Fragment separator. JOUR APOBB 361301

${ }^{79} \mathrm{Kr} \quad$ 2004SC48 NUCLEAR REACTIONS ${ }^{78} \mathrm{Kr}(\mathrm{d}, \mathrm{n}),(\mathrm{d}, \mathrm{p}),(\mathrm{d}, \alpha),(\mathrm{d}, \mathrm{n} \alpha), \mathrm{E} \approx 4-13$ $\mathrm{MeV}$; measured excitation functions. Stacked gas cell activation technique. JOUR RAACA 92203

${ }^{79} \mathrm{Rb} \quad 2004 \mathrm{SC} 48 \quad$ NUCLEAR REACTIONS ${ }^{78} \mathrm{Kr}(\mathrm{d}, \mathrm{n}),(\mathrm{d}, \mathrm{p}),(\mathrm{d}, \alpha),(\mathrm{d}, \mathrm{n} \alpha), \mathrm{E} \approx 4-13$ $\mathrm{MeV}$; measured excitation functions. Stacked gas cell activation technique. JOUR RAACA 92203

\section{$\mathrm{A}=80$}

${ }^{80} \mathrm{Ga}$ 2005MA81 RADIOACTIVITY ${ }^{32} \mathrm{Na},{ }^{80} \mathrm{Ga}\left(\beta^{-}\right)$; measured $\mathrm{E} \gamma, \mathrm{I} \gamma, \gamma \gamma-, \beta \gamma$-coin. ${ }^{32} \mathrm{Mg},{ }^{80} \mathrm{Ge}$ levels deduced $\mathrm{T}_{1 / 2}$. Ultra-fast timing techniques. JOUR JPGPE 31 S1421

${ }^{80} \mathrm{Ge} \quad 2005$ IW03 NUCLEAR REACTIONS Pb $\left({ }^{76} \mathrm{Ge},{ }^{76} \mathrm{Ge}\right),\left({ }^{78} \mathrm{Ge},{ }^{78} \mathrm{Ge}\right),\left({ }^{80} \mathrm{Ge}\right.$, $\left.{ }^{80} \mathrm{Ge} '\right),\left({ }^{82} \mathrm{Ge},{ }^{82} \mathrm{Ge}^{\prime}\right), \mathrm{E} \approx 40 \mathrm{MeV} /$ nucleon; measured $\mathrm{E} \gamma, \mathrm{I} \gamma$, (particle) $\gamma$-coin following projectile Coulomb excitation. ${ }^{76,78,80,82} \mathrm{Ge}$ deduced transitions B(E2). JOUR ZAANE 25 s01 415

2005MA81 RADIOACTIVITY ${ }^{32} \mathrm{Na},{ }^{80} \mathrm{Ga}\left(\beta^{-}\right)$; measured $\mathrm{E} \gamma, \mathrm{I} \gamma, \gamma \gamma$-, $\beta \gamma$-coin. ${ }^{32} \mathrm{Mg},{ }^{80} \mathrm{Ge}$ levels deduced $\mathrm{T}_{1 / 2}$. Ultra-fast timing techniques. JOUR JPGPE 31 S1421

2005PA23 NUCLEAR REACTIONS C $\left({ }^{78} \mathrm{Ge},{ }^{78} \mathrm{Ge}\right),\left({ }^{80} \mathrm{Ge},{ }^{80} \mathrm{Ge}\right), \mathrm{E}=2.24 \mathrm{MeV}$ / nucleon; ${ }^{48} \mathrm{Ti}\left({ }^{82} \mathrm{Ge},{ }^{82} \mathrm{Ge}\right), \mathrm{E}=220 \mathrm{MeV}$; measured $\mathrm{E} \gamma, \mathrm{I} \gamma$, (particle) $\gamma$-coin following projectile Coulomb excitation. ${ }^{78,80,82} \mathrm{Ge}$ deduced excitation $\mathrm{B}(\mathrm{E} 2)$. Systematic trends in $\mathrm{B}(\mathrm{E} 2)$ values discussed. JOUR PRLTA 94122501

${ }^{80} \mathrm{As} \quad$ 2005GA56 NUCLEAR REACTIONS ${ }^{238} \mathrm{U}\left({ }^{82} \mathrm{Se}, \mathrm{X}\right), \mathrm{E}=505 \mathrm{MeV} ;{ }^{238} \mathrm{U}\left({ }^{64} \mathrm{Ni}, \mathrm{X}\right)$, $\mathrm{E}=400 \mathrm{MeV}$; measured $\mathrm{E} \gamma, \mathrm{I} \gamma, \gamma \gamma-$, (fragment) $\gamma$-coin, projectile-like fragments isotopic yields. ${ }^{58} \mathrm{Cr},{ }^{80} \mathrm{As},{ }^{82} \mathrm{Ge},{ }^{84} \mathrm{Se}$ deduced levels, $\mathrm{J}, \pi$. Clara array, Prisma spectrometer. JOUR ZAANE 25 s01 421

2005LU07 NUCLEAR REACTIONS ${ }^{238} \mathrm{U}\left({ }^{82} \mathrm{Se}, \mathrm{X}\right), \mathrm{E}=505 \mathrm{MeV}$; measured E $\gamma$, I $\gamma$, fragments isotopic yields. ${ }^{77,78,79,80,81,82,83}$ As deduced transitions. ${ }^{192} \mathrm{Os}\left({ }^{82} \mathrm{Se}, \mathrm{X}\right), \mathrm{E}=460 \mathrm{MeV}$; measured $\mathrm{E} \gamma, \mathrm{I} \gamma, \gamma \gamma$-coin. ${ }^{80} \mathrm{As},{ }^{87} \mathrm{Rb}$, ${ }^{84}$ Se deduced levels. Fragment separator. JOUR APOBB 361301 ${ }^{80} \mathrm{Kr}$ 2005LE04 NUCLEAR REACTIONS $\mathrm{Pb}(\mathrm{p}, \mathrm{X}){ }^{3} \mathrm{He} /{ }^{4} \mathrm{He} /{ }^{21} \mathrm{Ne} /{ }^{22} \mathrm{Ne} /{ }^{36} \mathrm{Ar} /$ ${ }^{38} \mathrm{Ar} /{ }^{78} \mathrm{Kr} /{ }^{80} \mathrm{Kr} /{ }^{81} \mathrm{Kr} /{ }^{82} \mathrm{Kr} /{ }^{83} \mathrm{Kr} /{ }^{84} \mathrm{Kr} /{ }^{85} \mathrm{Kr} /{ }^{86} \mathrm{Kr} /{ }^{124} \mathrm{Xe}$ $/{ }^{126} \mathrm{Xe} /{ }^{128} \mathrm{Xe} /{ }^{129} \mathrm{Xe} /{ }^{130} \mathrm{Xe} /{ }^{131} \mathrm{Xe} /{ }^{132} \mathrm{Xe} /{ }^{134} \mathrm{Xe}, \mathrm{E}=44-2595$ $\mathrm{MeV}$; measured production $\sigma$; deduced reaction mechanism features. Mini-stack approach, comparisons with model predictions. JOUR NIMBE 2291

2005SC26 ATOMIC MASSES $78,80,82,83,84,86 \mathrm{Kr}$; measured masses. Penning trap mass spectrometer. JOUR ZAANE 25 s01 51

${ }^{80} \mathrm{Sr} \quad 2005 \mathrm{CH} 60 \quad$ ATOMIC MASSES ${ }^{64} \mathrm{Zn},{ }^{64} \mathrm{Ga},{ }^{68} \mathrm{Ge},{ }^{68} \mathrm{As},{ }^{68,72} \mathrm{Se},{ }^{76} \mathrm{Kr},{ }^{76} \mathrm{Rb},{ }^{80} \mathrm{Sr}$, ${ }^{80} \mathrm{Y}$; measured masses. Direct time-of-flight technique, comparison with previous results. JOUR JPGPE 31 S1771 


\section{$\mathrm{A}=80$ (continued)}

2005SI34 ATOMIC MASSES ${ }^{76,77,80,81,86,88} \mathrm{Sr},{ }^{124,129,130,131,132} \mathrm{Sn}$; measured masses. Penning trap mass spectrometer, comparison with previous results. JOUR NUPAB 76345

2005 XU04 RADIOACTIVITY ${ }^{81} \mathrm{Zr},{ }^{85} \mathrm{Mo},{ }^{89} \mathrm{Ru},{ }^{92} \mathrm{Rh},{ }^{93} \mathrm{Pd},{ }^{121} \mathrm{Ce},{ }^{125} \mathrm{Nd}$, ${ }^{128} \mathrm{Pm},{ }^{129} \mathrm{Sm},{ }^{135,137} \mathrm{Gd},{ }^{139} \mathrm{Dy},{ }^{142} \mathrm{Ho},{ }^{149} \mathrm{Yb}\left(\beta^{+} \mathrm{p}\right)$; measured $\beta$-delayed $\mathrm{E} \gamma, \mathrm{I} \gamma$, proton spectra, $\mathrm{p} \gamma$-coin, $\mathrm{T}_{1 / 2}$. Comparison with model predictions. JOUR PRVCA 71054318

${ }^{80} \mathrm{Y}$ 2005CH60 ATOMIC MASSES ${ }^{64} \mathrm{Zn},{ }^{64} \mathrm{Ga},{ }^{68} \mathrm{Ge},{ }^{68} \mathrm{As},{ }^{68,72} \mathrm{Se},{ }^{76} \mathrm{Kr},{ }^{76} \mathrm{Rb},{ }^{80} \mathrm{Sr}$, ${ }^{80} \mathrm{Y}$; measured masses. Direct time-of-flight technique, comparison with previous results. JOUR JPGPE 31 S1771

\section{$\mathrm{A}=81$}

${ }^{81} \mathrm{Zn}$

2004VE14

${ }^{81} \mathrm{Ga}$

2004VE14

${ }^{81} \mathrm{As}$

2005LU07

${ }^{81} \mathrm{Br}$

2005KA39

2005KA46

${ }^{81} \mathrm{Kr}$

2005KA39

2005KA39
RADIOACTIVITY ${ }^{83} \mathrm{Ga},{ }^{81} \mathrm{Zn}\left(\beta^{-}\right)$[from $\left.\mathrm{U}(\mathrm{d}, \mathrm{X})\right]$; measured $\mathrm{E} \gamma, \mathrm{I} \gamma$, $\gamma \gamma-, \beta \gamma$-coin, $\mathrm{T}_{1 / 2} .{ }^{81} \mathrm{Ga},{ }^{83} \mathrm{As}$ deduced levels. JOUR BJPHE 34979 RADIOACTIVITY ${ }^{83} \mathrm{Ga},{ }^{81} \mathrm{Zn}\left(\beta^{-}\right)$[from $\left.\mathrm{U}(\mathrm{d}, \mathrm{X})\right]$; measured $\mathrm{E} \gamma, \mathrm{I} \gamma$, $\gamma \gamma-, \beta \gamma$-coin, $\mathrm{T}_{1 / 2} \cdot{ }^{81} \mathrm{Ga},{ }^{83} \mathrm{As}$ deduced levels. JOUR BJPHE 34979 NUCLEAR REACTIONS ${ }^{238} \mathrm{U}\left({ }^{82} \mathrm{Se}, \mathrm{X}\right), \mathrm{E}=505 \mathrm{MeV}$; measured E $\gamma$, $\mathrm{I} \gamma$, fragments isotopic yields. $77,78,79,80,81,82,83$ As deduced transitions. ${ }^{192} \mathrm{Os}\left({ }^{82} \mathrm{Se}, \mathrm{X}\right), \mathrm{E}=460 \mathrm{MeV}$; measured $\mathrm{E} \gamma, \mathrm{I} \gamma, \gamma \gamma$-coin. ${ }^{80} \mathrm{As},{ }^{87} \mathrm{Rb}$, ${ }^{84}$ Se deduced levels. Fragment separator. JOUR APOBB 361301 RADIOACTIVITY ${ }^{81 m} \mathrm{Kr}$ (EC), (IT); ${ }^{81} \mathrm{Y},{ }^{81} \mathrm{Sr},{ }^{85} \mathrm{Nb},{ }^{85} \mathrm{Zr},{ }^{86} \mathrm{Mo}$, ${ }^{86} \mathrm{Nb}(\mathrm{EC})\left[\right.$ from $\mathrm{Ni},{ }^{54} \mathrm{Fe}\left({ }^{32} \mathrm{~S}, \mathrm{X}\right)$ ]; measured $\mathrm{E} \gamma, \mathrm{I} \gamma, \mathrm{E}(\mathrm{ce}), \mathrm{I}(\mathrm{ce}), \mathrm{T}_{1 / 2}$. ${ }^{81} \mathrm{Kr},{ }^{85} \mathrm{Zr},{ }^{85} \mathrm{Nb}$ deduced isomeric transitions $\mathrm{T}_{1 / 2}$, ICC. ${ }^{85} \mathrm{Zr},{ }^{86} \mathrm{Nb}$ deduced levels, J, $\pi$, ICC. ${ }^{81} \mathrm{Br}$ deduced neutrino capture rate. Astrophysical implications discussed. JOUR ZAANE 25355 RADIOACTIVITY ${ }^{31} \mathrm{Cl}\left(\beta^{+} \mathrm{p}\right)$ [from $\mathrm{S}(\mathrm{p}, \mathrm{X}), \mathrm{E}=40 \mathrm{MeV}$ ]; measured $\beta$-delayed $\mathrm{E} \gamma, \mathrm{Ep} .{ }^{58} \mathrm{Zn}\left(\beta^{+}\right)$[from $\mathrm{Nb}(\mathrm{p}, \mathrm{X}), \mathrm{E}=1.4 \mathrm{GeV}$; measured $\mathrm{E} \gamma, \mathrm{I} \gamma, \beta \gamma$-coin, $\mathrm{T}_{1 / 2} \cdot{ }^{58} \mathrm{Cu}$ deduced levels, $\beta$-feeding intensities. ${ }^{81 m} \mathrm{Kr}(\mathrm{EC}),(\mathrm{IT}) ;{ }^{81} \mathrm{Y},{ }^{81} \mathrm{Sr},{ }^{85} \mathrm{Nb},{ }^{85} \mathrm{Zr},{ }^{86} \mathrm{Mo},{ }^{86} \mathrm{Nb}(\mathrm{EC})$ [from Ni, $\left.{ }^{54} \mathrm{Fe}\left({ }^{32} \mathrm{~S}, \mathrm{X}\right)\right]$; measured $\mathrm{E} \gamma, \mathrm{I} \gamma, \mathrm{E}(\mathrm{ce}), \mathrm{I}(\mathrm{ce}), \mathrm{T}_{1 / 2} \cdot{ }^{81} \mathrm{Kr},{ }^{85} \mathrm{Zr},{ }^{85} \mathrm{Nb}$ deduced isomeric transitions $\mathrm{T}_{1 / 2}$, ICC. ${ }^{85} \mathrm{Zr},{ }^{86} \mathrm{Nb}$ deduced levels, J, $\pi$, ICC. ${ }^{81} \mathrm{Br}$ deduced neutrino capture rate. Mass-separated sources. JOUR ZAANE 25 s01 129 RADIOACTIVITY ${ }^{81 m} \mathrm{Kr}$ (EC), (IT); ${ }^{81} \mathrm{Y},{ }^{81} \mathrm{Sr},{ }^{85} \mathrm{Nb},{ }^{85} \mathrm{Zr},{ }^{86} \mathrm{Mo}$, ${ }^{86} \mathrm{Nb}(\mathrm{EC})\left[\right.$ from $\mathrm{Ni},{ }^{54} \mathrm{Fe}\left({ }^{32} \mathrm{~S}, \mathrm{X}\right)$ ]; measured $\mathrm{E} \gamma, \mathrm{I} \gamma, \mathrm{E}($ ce $), \mathrm{I}($ ce $), \mathrm{T}_{1 / 2}$. ${ }^{81} \mathrm{Kr},{ }^{85} \mathrm{Zr},{ }^{85} \mathrm{Nb}$ deduced isomeric transitions $\mathrm{T}_{1 / 2}$, ICC. ${ }^{85} \mathrm{Zr},{ }^{86} \mathrm{Nb}$ deduced levels, J, $\pi$, ICC. ${ }^{81} \mathrm{Br}$ deduced neutrino capture rate. Astrophysical implications discussed. JOUR ZAANE 25355 NUCLEAR REACTIONS ${ }^{54} \mathrm{Fe}\left({ }^{32} \mathrm{~S}, \mathrm{X}\right){ }^{81} \mathrm{Zr} /{ }^{81} \mathrm{Y} /{ }^{81} \mathrm{Sr} /{ }^{81 m} \mathrm{Kr}$, $\mathrm{E}=150-170 \mathrm{MeV} ; \mathrm{Ni}\left({ }^{32} \mathrm{~S}, \mathrm{X}\right){ }^{85} \mathrm{Nb} /{ }^{85 m} \mathrm{Nb} /{ }^{85 Z r} /{ }^{85 m} \mathrm{Zr} /{ }^{86} \mathrm{Mo} /$ ${ }^{86} \mathrm{Nb}, \mathrm{E}=150-170 \mathrm{MeV}$; measured yields. JOUR ZAANE 25355 


\section{$\mathrm{A}=81$ (continued)}

2005KA46 RADIOACTIVITY ${ }^{31} \mathrm{Cl}\left(\beta^{+} \mathrm{p}\right)$ [from $\mathrm{S}(\mathrm{p}, \mathrm{X}), \mathrm{E}=40 \mathrm{MeV}$ ]; measured $\beta$-delayed $\mathrm{E} \gamma, \mathrm{Ep} .{ }^{58} \mathrm{Zn}\left(\beta^{+}\right)$[from $\mathrm{Nb}(\mathrm{p}, \mathrm{X}), \mathrm{E}=1.4 \mathrm{GeV}$ ]; measured $\mathrm{E} \gamma, \mathrm{I} \gamma, \beta \gamma$-coin, $\mathrm{T}_{1 / 2} .{ }^{58} \mathrm{Cu}$ deduced levels, $\beta$-feeding intensities. ${ }^{81 m} \mathrm{Kr}(\mathrm{EC}),(\mathrm{IT}) ;{ }^{81} \mathrm{Y},{ }^{81} \mathrm{Sr},{ }^{85} \mathrm{Nb},{ }^{85} \mathrm{Zr},{ }^{86} \mathrm{Mo},{ }^{86} \mathrm{Nb}(\mathrm{EC})$ [from Ni, $\left.{ }^{54} \mathrm{Fe}\left({ }^{32} \mathrm{~S}, \mathrm{X}\right)\right]$; measured $\mathrm{E} \gamma, \mathrm{I} \gamma, \mathrm{E}(\mathrm{ce}), \mathrm{I}(\mathrm{ce}), \mathrm{T}_{1 / 2} \cdot{ }^{81} \mathrm{Kr},{ }^{85} \mathrm{Zr},{ }^{85} \mathrm{Nb}$ deduced isomeric transitions $\mathrm{T}_{1 / 2}$, ICC. ${ }^{85} \mathrm{Zr},{ }^{86} \mathrm{Nb}$ deduced levels, J, $\pi$, ICC. ${ }^{81} \mathrm{Br}$ deduced neutrino capture rate. Mass-separated sources. JOUR ZAANE 25 s01 129

2005LE04 NUCLEAR REACTIONS Pb(p, X) ${ }^{3} \mathrm{He} /{ }^{4} \mathrm{He} /{ }^{21} \mathrm{Ne} /{ }^{22} \mathrm{Ne} /{ }^{36} \mathrm{Ar} /$ ${ }^{38} \mathrm{Ar} /{ }^{78} \mathrm{Kr} /{ }^{80} \mathrm{Kr} /{ }^{81} \mathrm{Kr} /{ }^{82} \mathrm{Kr} /{ }^{83} \mathrm{Kr} /{ }^{84} \mathrm{Kr} /{ }^{85} \mathrm{Kr} /{ }^{86} \mathrm{Kr} /{ }^{124} \mathrm{Xe}$ $/{ }^{126} \mathrm{Xe} /{ }^{128} \mathrm{Xe} /{ }^{129} \mathrm{Xe} /{ }^{130} \mathrm{Xe} /{ }^{131} \mathrm{Xe} /{ }^{132} \mathrm{Xe} /{ }^{134} \mathrm{Xe}, \mathrm{E}=44-2595$ $\mathrm{MeV}$; measured production $\sigma$; deduced reaction mechanism features. Mini-stack approach, comparisons with model predictions. JOUR NIMBE 2291

2005MUZY NUCLEAR REACTIONS ${ }^{84} \mathrm{Kr}(\mathrm{n}, \mathrm{X}), \mathrm{E}=0-400 \mathrm{keV}$; measured total $\sigma$. ${ }^{82,84,86} \mathrm{Kr}(\mathrm{n}, \gamma), \mathrm{E}=0-400 \mathrm{keV} ;{ }^{80,83} \mathrm{Kr}(\mathrm{n}, \gamma), \mathrm{E}=0-5 \mathrm{keV}$; measured capture $\sigma .{ }^{80,82,83,84,86} \mathrm{Kr}(\mathrm{n}, \gamma), \mathrm{E}=5-100 \mathrm{keV}$; deduced Maxwellian-averaged $\sigma$. Astrophysical implications discussed. CONF Santa Fe (Nucl Data for Sci and Technol) Proc,Vol2,P1327

${ }^{81} \mathrm{Rb} \quad$ 2004KA68 NUCLEAR REACTIONS ${ }^{85} \mathrm{Rb}(\mathrm{p}, \mathrm{np}),(\mathrm{p}, 2 \mathrm{np}),(\mathrm{p}, 3 \mathrm{np}),(\mathrm{p}, 4 \mathrm{np}), \mathrm{E}$ $\approx 17-100 \mathrm{MeV}$; measured excitation functions. Activation technique, comparison with model predictions. JOUR RAACA 92449

2005KA39 RADIOACTIVITY ${ }^{81 m} \mathrm{Kr}$ (EC), (IT); ${ }^{81} \mathrm{Y},{ }^{81} \mathrm{Sr},{ }^{85} \mathrm{Nb},{ }^{85} \mathrm{Zr},{ }^{86} \mathrm{Mo}$, ${ }^{86} \mathrm{Nb}(\mathrm{EC})\left[\right.$ from $\left.\mathrm{Ni},{ }^{54} \mathrm{Fe}\left({ }^{32} \mathrm{~S}, \mathrm{X}\right)\right]$; measured $\mathrm{E} \gamma, \mathrm{I} \gamma, \mathrm{E}(\mathrm{ce}), \mathrm{I}(\mathrm{ce}), \mathrm{T}_{1 / 2}$. ${ }^{81} \mathrm{Kr},{ }^{85} \mathrm{Zr},{ }^{85} \mathrm{Nb}$ deduced isomeric transitions $\mathrm{T}_{1 / 2}$, ICC. ${ }^{85} \mathrm{Zr},{ }^{86} \mathrm{Nb}$ deduced levels, J, $\pi$, ICC. ${ }^{81} \mathrm{Br}$ deduced neutrino capture rate. Astrophysical implications discussed. JOUR ZAANE 25355

2005KA46 RADIOACTIVITY ${ }^{31} \mathrm{Cl}\left(\beta^{+} \mathrm{p}\right)$ [from $\mathrm{S}(\mathrm{p}, \mathrm{X}), \mathrm{E}=40 \mathrm{MeV}$ ]; measured $\beta$-delayed $\mathrm{E} \gamma, \mathrm{Ep} .{ }^{58} \mathrm{Zn}\left(\beta^{+}\right)$[from $\mathrm{Nb}(\mathrm{p}, \mathrm{X}), \mathrm{E}=1.4 \mathrm{GeV}$ ]; measured $\mathrm{E} \gamma, \mathrm{I} \gamma, \beta \gamma$-coin, $\mathrm{T}_{1 / 2} .{ }^{58} \mathrm{Cu}$ deduced levels, $\beta$-feeding intensities. ${ }^{81 m} \mathrm{Kr}$ (EC), (IT); ${ }^{81} \mathrm{Y},{ }^{81} \mathrm{Sr},{ }^{85} \mathrm{Nb},{ }^{85} \mathrm{Zr},{ }^{86} \mathrm{Mo},{ }^{86} \mathrm{Nb}(\mathrm{EC})$ [from Ni, $\left.{ }^{54} \mathrm{Fe}\left({ }^{32} \mathrm{~S}, \mathrm{X}\right)\right]$; measured $\mathrm{E} \gamma, \mathrm{I} \gamma, \mathrm{E}(\mathrm{ce}), \mathrm{I}(\mathrm{ce}), \mathrm{T}_{1 / 2} \cdot{ }^{81} \mathrm{Kr},{ }^{85} \mathrm{Zr},{ }^{85} \mathrm{Nb}$ deduced isomeric transitions $\mathrm{T}_{1 / 2}$, ICC. ${ }^{85} \mathrm{Zr},{ }^{86} \mathrm{Nb}$ deduced levels, $\mathrm{J}$, $\pi$, ICC. ${ }^{81} \mathrm{Br}$ deduced neutrino capture rate. Mass-separated sources. JOUR ZAANE 25 s01 129

${ }^{81} \mathrm{Sr}$ 2005KA39 RADIOACTIVITY ${ }^{81 m} \mathrm{Kr}(\mathrm{EC}),(\mathrm{IT}) ;{ }^{81} \mathrm{Y},{ }^{81} \mathrm{Sr},{ }^{85} \mathrm{Nb},{ }^{85} \mathrm{Zr},{ }^{86} \mathrm{Mo}$, ${ }^{86} \mathrm{Nb}(\mathrm{EC})\left[\right.$ from $\left.\mathrm{Ni},{ }^{54} \mathrm{Fe}\left({ }^{32} \mathrm{~S}, \mathrm{X}\right)\right]$; measured $\mathrm{E} \gamma, \mathrm{I} \gamma, \mathrm{E}(\mathrm{ce}), \mathrm{I}(\mathrm{ce}), \mathrm{T}_{1 / 2}$. ${ }^{81} \mathrm{Kr},{ }^{85} \mathrm{Zr},{ }^{85} \mathrm{Nb}$ deduced isomeric transitions $\mathrm{T}_{1 / 2}$, ICC. ${ }^{85} \mathrm{Zr},{ }^{86} \mathrm{Nb}$ deduced levels, J, $\pi$, ICC. ${ }^{81} \mathrm{Br}$ deduced neutrino capture rate. Astrophysical implications discussed. JOUR ZAANE 25355

2005KA39 NUCLEAR REACTIONS ${ }^{54} \mathrm{Fe}\left({ }^{32} \mathrm{~S}, \mathrm{X}\right){ }^{81} \mathrm{Zr} /{ }^{81} \mathrm{Y} /{ }^{81} \mathrm{Sr} /{ }^{81 m} \mathrm{Kr}$, $\mathrm{E}=150-170 \mathrm{MeV} ; \mathrm{Ni}\left({ }^{32} \mathrm{~S}, \mathrm{X}\right){ }^{85} \mathrm{Nb} /{ }^{85 m} \mathrm{Nb} /{ }^{85 Z r} /{ }^{85 m} \mathrm{Zr} /{ }^{86} \mathrm{Mo} /$ ${ }^{86} \mathrm{Nb}, \mathrm{E}=150-170 \mathrm{MeV}$; measured yields. JOUR ZAANE 25355 


\section{$\mathrm{A}=81$ (continued)}

2005KA46 RADIOACTIVITY ${ }^{31} \mathrm{Cl}\left(\beta^{+} \mathrm{p}\right)$ [from $\mathrm{S}(\mathrm{p}, \mathrm{X}), \mathrm{E}=40 \mathrm{MeV}$ ]; measured $\beta$-delayed $\mathrm{E} \gamma, \mathrm{Ep} .{ }^{58} \mathrm{Zn}\left(\beta^{+}\right)$[from $\mathrm{Nb}(\mathrm{p}, \mathrm{X}), \mathrm{E}=1.4 \mathrm{GeV}$ ]; measured $\mathrm{E} \gamma, \mathrm{I} \gamma, \beta \gamma$-coin, $\mathrm{T}_{1 / 2} .{ }^{58} \mathrm{Cu}$ deduced levels, $\beta$-feeding intensities. ${ }^{81 m} \mathrm{Kr}(\mathrm{EC}),(\mathrm{IT}) ;{ }^{81} \mathrm{Y},{ }^{81} \mathrm{Sr},{ }^{85} \mathrm{Nb},{ }^{85} \mathrm{Zr},{ }^{86} \mathrm{Mo},{ }^{86} \mathrm{Nb}(\mathrm{EC})$ [from Ni, $\left.{ }^{54} \mathrm{Fe}\left({ }^{32} \mathrm{~S}, \mathrm{X}\right)\right]$; measured $\mathrm{E} \gamma, \mathrm{I} \gamma, \mathrm{E}(\mathrm{ce}), \mathrm{I}(\mathrm{ce}), \mathrm{T}_{1 / 2} \cdot{ }^{81} \mathrm{Kr},{ }^{85} \mathrm{Zr},{ }^{85} \mathrm{Nb}$ deduced isomeric transitions $\mathrm{T}_{1 / 2}$, ICC. ${ }^{85} \mathrm{Zr},{ }^{86} \mathrm{Nb}$ deduced levels, J, $\pi$, ICC. ${ }^{81} \mathrm{Br}$ deduced neutrino capture rate. Mass-separated sources. JOUR ZAANE 25 s01 129

2005SI34 ATOMIC MASSES ${ }^{76,77,80,81,86,88} \mathrm{Sr},{ }^{124,129,130,131,132} \mathrm{Sn}$; measured masses. Penning trap mass spectrometer, comparison with previous results. JOUR NUPAB 76345

${ }^{81} \mathrm{Y}$ 2005KA39 RADIOACTIVITY ${ }^{81 m} \mathrm{Kr}(\mathrm{EC}),(\mathrm{IT}) ;{ }^{81} \mathrm{Y},{ }^{81} \mathrm{Sr},{ }^{85} \mathrm{Nb},{ }^{85} \mathrm{Zr},{ }^{86} \mathrm{Mo}$, ${ }^{86} \mathrm{Nb}(\mathrm{EC})\left[\right.$ from $\left.\mathrm{Ni},{ }^{54} \mathrm{Fe}\left({ }^{32} \mathrm{~S}, \mathrm{X}\right)\right]$; measured $\mathrm{E} \gamma, \mathrm{I} \gamma, \mathrm{E}(\mathrm{ce}), \mathrm{I}(\mathrm{ce}), \mathrm{T}_{1 / 2}$.

${ }^{81} \mathrm{Kr},{ }^{85} \mathrm{Zr},{ }^{85} \mathrm{Nb}$ deduced isomeric transitions $\mathrm{T}_{1 / 2}$, ICC. ${ }^{85} \mathrm{Zr},{ }^{86} \mathrm{Nb}$ deduced levels, J, $\pi$, ICC. ${ }^{81} \mathrm{Br}$ deduced neutrino capture rate. Astrophysical implications discussed. JOUR ZAANE 25355

2005KA39 NUCLEAR REACTIONS ${ }^{54} \mathrm{Fe}\left({ }^{32} \mathrm{~S}, \mathrm{X}\right){ }^{81} \mathrm{Zr} /{ }^{81} \mathrm{Y} /{ }^{81} \mathrm{Sr} /{ }^{81 m} \mathrm{Kr}$, $\mathrm{E}=150-170 \mathrm{MeV} ; \mathrm{Ni}\left({ }^{32} \mathrm{~S}, \mathrm{X}\right){ }^{85} \mathrm{Nb} /{ }^{85 m} \mathrm{Nb} /{ }^{85 Z r} /{ }^{85 m} \mathrm{Zr} /{ }^{86} \mathrm{Mo} /$ ${ }^{86} \mathrm{Nb}, \mathrm{E}=150-170 \mathrm{MeV}$; measured yields. JOUR ZAANE 25355

2005KA46 RADIOACTIVITY ${ }^{31} \mathrm{Cl}\left(\beta^{+} \mathrm{p}\right)$ [from $\mathrm{S}(\mathrm{p}, \mathrm{X}), \mathrm{E}=40 \mathrm{MeV}$ ]; measured $\beta$-delayed $\mathrm{E} \gamma, \mathrm{Ep} .{ }^{58} \mathrm{Zn}\left(\beta^{+}\right)$[from $\mathrm{Nb}(\mathrm{p}, \mathrm{X}), \mathrm{E}=1.4 \mathrm{GeV}$ ]; measured $\mathrm{E} \gamma, \mathrm{I} \gamma, \beta \gamma$-coin, $\mathrm{T}_{1 / 2} .{ }^{58} \mathrm{Cu}$ deduced levels, $\beta$-feeding intensities. ${ }^{81 m} \mathrm{Kr}(\mathrm{EC}),(\mathrm{IT}) ;{ }^{81} \mathrm{Y},{ }^{81} \mathrm{Sr},{ }^{85} \mathrm{Nb},{ }^{85} \mathrm{Zr},{ }^{86} \mathrm{Mo},{ }^{86} \mathrm{Nb}(\mathrm{EC})$ [from Ni, $\left.{ }^{54} \mathrm{Fe}\left({ }^{32} \mathrm{~S}, \mathrm{X}\right)\right]$; measured $\mathrm{E} \gamma, \mathrm{I} \gamma, \mathrm{E}(\mathrm{ce}), \mathrm{I}(\mathrm{ce}), \mathrm{T}_{1 / 2} \cdot{ }^{81} \mathrm{Kr},{ }^{85} \mathrm{Zr},{ }^{85} \mathrm{Nb}$ deduced isomeric transitions $\mathrm{T}_{1 / 2}$, ICC. ${ }^{85} \mathrm{Zr},{ }^{86} \mathrm{Nb}$ deduced levels, $\mathrm{J}$, $\pi$, ICC. ${ }^{81} \mathrm{Br}$ deduced neutrino capture rate. Mass-separated sources. JOUR ZAANE 25 s01 129

${ }^{81} \mathrm{Zr} \quad 2005 \mathrm{KA} 39$ NUCLEAR REACTIONS ${ }^{54} \mathrm{Fe}\left({ }^{32} \mathrm{~S}, \mathrm{X}\right){ }^{81} \mathrm{Zr} /{ }^{81} \mathrm{Y} /{ }^{81} \mathrm{Sr} /{ }^{81 m} \mathrm{Kr}$, $\mathrm{E}=150-170 \mathrm{MeV} ; \mathrm{Ni}\left({ }^{32} \mathrm{~S}, \mathrm{X}\right){ }^{85} \mathrm{Nb} /{ }^{85 m} \mathrm{Nb} /{ }^{85 Z r} /{ }^{85 m} \mathrm{Zr} /{ }^{86} \mathrm{Mo} /$ ${ }^{86} \mathrm{Nb}, \mathrm{E}=150-170 \mathrm{MeV}$; measured yields. JOUR ZAANE 25355 2005XU04 RADIOACTIVITY ${ }^{81} \mathrm{Zr},{ }^{85} \mathrm{Mo},{ }^{89} \mathrm{Ru},{ }^{92} \mathrm{Rh},{ }^{93} \mathrm{Pd},{ }^{121} \mathrm{Ce},{ }^{125} \mathrm{Nd}$, ${ }^{128} \mathrm{Pm},{ }^{129} \mathrm{Sm},{ }^{135,137} \mathrm{Gd},{ }^{139} \mathrm{Dy},{ }^{142} \mathrm{Ho},{ }^{149} \mathrm{Yb}\left(\beta^{+} \mathrm{p}\right)$; measured $\beta$-delayed $\mathrm{E} \gamma, \mathrm{I} \gamma$, proton spectra, $\mathrm{p} \gamma$-coin, $\mathrm{T}_{1 / 2}$. Comparison with model predictions. JOUR PRVCA 71054318

\section{$\mathrm{A}=82$}

$\begin{array}{ll}{ }^{82} \mathrm{Ga} & \text { 2004PEZW } \\ { }^{82} \mathrm{Ge} & \text { 2004PEZW }\end{array}$

RADIOACTIVITY ${ }^{82,83} \mathrm{Ga}\left(\beta^{-}\right)$[from ${ }^{238} \mathrm{U}(\mathrm{n}, \mathrm{F})$ ]; measured $\mathrm{E} \gamma, \mathrm{I} \gamma$, $\gamma \gamma-, \beta \gamma$-coin, $\mathrm{T}_{1 / 2} .{ }^{82,83} \mathrm{Ge}$ deduced levels, configurations. REPT IPNO-T-05-02,Perru RADIOACTIVITY ${ }^{82,83} \mathrm{Ga}\left(\beta^{-}\right)$[from ${ }^{238} \mathrm{U}(\mathrm{n}, \mathrm{F})$ ]; measured $\mathrm{E} \gamma, \mathrm{I} \gamma$, $\gamma \gamma-, \beta \gamma$-coin, $\mathrm{T}_{1 / 2} \cdot{ }^{82,83} \mathrm{Ge}$ deduced levels, configurations. REPT IPNO-T-05-02,Perru

2005GA56 NUCLEAR REACTIONS ${ }^{238} \mathrm{U}\left({ }^{82} \mathrm{Se}, \mathrm{X}\right), \mathrm{E}=505 \mathrm{MeV} ;{ }^{238} \mathrm{U}\left({ }^{64} \mathrm{Ni}, \mathrm{X}\right)$, $\mathrm{E}=400 \mathrm{MeV}$; measured $\mathrm{E} \gamma, \mathrm{I} \gamma, \gamma \gamma$-, (fragment) $\gamma$-coin, projectile-like fragments isotopic yields. ${ }^{58} \mathrm{Cr},{ }^{80} \mathrm{As},{ }^{82} \mathrm{Ge},{ }^{84} \mathrm{Se}$ deduced levels, J, $\pi$. Clara array, Prisma spectrometer. JOUR ZAANE 25 s01 421 


\section{$\mathrm{A}=82$ (continued)}

2005IW03 NUCLEAR REACTIONS Pb $\left({ }^{76} \mathrm{Ge},{ }^{76} \mathrm{Ge}\right),\left({ }^{78} \mathrm{Ge},{ }^{78} \mathrm{Ge}\right),\left({ }^{80} \mathrm{Ge}\right.$, $\left.{ }^{80} \mathrm{Ge} '\right),\left({ }^{82} \mathrm{Ge},{ }^{82} \mathrm{Ge}^{\prime}\right), \mathrm{E} \approx 40 \mathrm{MeV} /$ nucleon; measured $\mathrm{E} \gamma, \mathrm{I} \gamma$, (particle) $\gamma$-coin following projectile Coulomb excitation. ${ }^{76,78,80,82} \mathrm{Ge}$ deduced transitions B(E2). JOUR ZAANE 25 s01 415

2005PA23 NUCLEAR REACTIONS C $\left({ }^{78} \mathrm{Ge},{ }^{78} \mathrm{Ge}\right),\left({ }^{80} \mathrm{Ge},{ }^{80} \mathrm{Ge}\right.$ '), $\mathrm{E}=2.24 \mathrm{MeV}$ / nucleon; ${ }^{48} \mathrm{Ti}\left({ }^{82} \mathrm{Ge},{ }^{82} \mathrm{Ge}\right), \mathrm{E}=220 \mathrm{MeV}$; measured $\mathrm{E} \gamma, \mathrm{I} \gamma$, (particle) $\gamma$-coin following projectile Coulomb excitation. ${ }^{78,80,82} \mathrm{Ge}$ deduced excitation $\mathrm{B}(\mathrm{E} 2)$. Systematic trends in $\mathrm{B}(\mathrm{E} 2)$ values discussed. JOUR PRLTA 94122501

${ }^{82} \mathrm{As}$ 2005LU07 NUCLEAR REACTIONS ${ }^{238} \mathrm{U}\left({ }^{82} \mathrm{Se}, \mathrm{X}\right), \mathrm{E}=505 \mathrm{MeV}$; measured E $\gamma$, I $\gamma$, fragments isotopic yields. ${ }^{77,78,79,80,81,82,83}$ As deduced transitions. ${ }^{192} \mathrm{Os}\left({ }^{82} \mathrm{Se}, \mathrm{X}\right), \mathrm{E}=460 \mathrm{MeV}$; measured $\mathrm{E} \gamma, \mathrm{I} \gamma, \gamma \gamma$-coin. ${ }^{80} \mathrm{As},{ }^{87} \mathrm{Rb}$, ${ }^{84}$ Se deduced levels. Fragment separator. JOUR APOBB 361301

${ }^{82} \mathrm{Se} \quad 2005 \mathrm{AR} 27$ RADIOACTIVITY ${ }^{82} \mathrm{Se},{ }^{100} \mathrm{Mo}\left(2 \beta^{-}\right)$; measured $2 \nu \beta \beta$-decay $\mathrm{T}_{1 / 2}$, $0 \nu \beta \beta$-decay $\mathrm{T}_{1 / 2}$ lower limits; deduced neutrino mass limits. JOUR PRLTA 95182302

2005BA33 RADIOACTIVITY ${ }^{82} \mathrm{Se},{ }^{100} \mathrm{Mo},{ }^{116} \mathrm{Cd},{ }^{150} \mathrm{Nd}\left(2 \beta^{-}\right)$; measured $2 \nu \beta \beta$-decay $\mathrm{T}_{1 / 2}, 0 \nu \beta \beta$-decay $\mathrm{T}_{1 / 2}$ lower limits. JOUR YAFIA 68443

2005SA07 RADIOACTIVITY ${ }^{82} \mathrm{Se},{ }^{96} \mathrm{Zr},{ }^{100} \mathrm{Mo},{ }^{116} \mathrm{Cd},{ }^{150} \mathrm{Nd}\left(2 \beta^{-}\right)$; measured $2 \nu \beta \beta$-decay $\mathrm{T}_{1 / 2} .{ }^{82} \mathrm{Se},{ }^{100} \mathrm{Mo}\left(2 \beta^{-}\right)$; measured $0 \nu \beta \beta$-decay $\mathrm{T}_{1 / 2}$ lower limits; deduced neutrino mass limits. JOUR NPBSE 143221

2005SHZW RADIOACTIVITY ${ }^{100} \mathrm{Mo},{ }^{82} \mathrm{Se}\left(2 \beta^{-}\right)$; measured $0 \nu \beta \beta$-decay $\mathrm{T}_{1 / 2}$ limits. NEMO-3 detector, underground laboratory in Modane. CONF St Petersburg,P42,Shitov

2005SI06 RADIOACTIVITY ${ }^{82} \mathrm{Se},{ }^{96} \mathrm{Zr},{ }^{100} \mathrm{Mo},{ }^{116} \mathrm{Cd},{ }^{150} \mathrm{Nd}\left(2 \beta^{-}\right)$; measured $2 \nu \beta \beta$-decay $\mathrm{T}_{1 / 2} .{ }^{82} \mathrm{Se},{ }^{100} \mathrm{Mo}\left(2 \beta^{-}\right)$; measured $0 \nu \beta \beta$-decay $\mathrm{T}_{1 / 2}$ lower limits; deduced neutrino mass limits. JOUR NPBSE 145272

${ }^{82} \mathrm{Kr} \quad 2005$ AR27 RADIOACTIVITY ${ }^{82} \mathrm{Se},{ }^{100} \mathrm{Mo}\left(2 \beta^{-}\right)$; measured $2 \nu \beta \beta$-decay $\mathrm{T}_{1 / 2}$, $0 \nu \beta \beta$-decay $\mathrm{T}_{1 / 2}$ lower limits; deduced neutrino mass limits. JOUR PRLTA 95182302

2005BA33 RADIOACTIVITY ${ }^{82} \mathrm{Se},{ }^{100} \mathrm{Mo},{ }^{116} \mathrm{Cd},{ }^{150} \mathrm{Nd}\left(2 \beta^{-}\right)$; measured $2 \nu \beta \beta$-decay $\mathrm{T}_{1 / 2}, 0 \nu \beta \beta$-decay $\mathrm{T}_{1 / 2}$ lower limits. JOUR YAFIA 68443 2005LE04 NUCLEAR REACTIONS Pb(p, X) ${ }^{3} \mathrm{He} /{ }^{4} \mathrm{He} /{ }^{21} \mathrm{Ne} /{ }^{22} \mathrm{Ne} /{ }^{36} \mathrm{Ar} /$ ${ }^{38} \mathrm{Ar} /{ }^{78} \mathrm{Kr} /{ }^{80} \mathrm{Kr} /{ }^{81} \mathrm{Kr} /{ }^{82} \mathrm{Kr} /{ }^{83} \mathrm{Kr} /{ }^{84} \mathrm{Kr} /{ }^{85} \mathrm{Kr} /{ }^{86} \mathrm{Kr} /{ }^{124} \mathrm{Xe}$ $/{ }^{126} \mathrm{Xe} /{ }^{128} \mathrm{Xe} /{ }^{129} \mathrm{Xe} /{ }^{130} \mathrm{Xe} /{ }^{131} \mathrm{Xe} /{ }^{132} \mathrm{Xe} /{ }^{134} \mathrm{Xe}, \mathrm{E}=44-2595$ $\mathrm{MeV}$; measured production $\sigma$; deduced reaction mechanism features. Mini-stack approach, comparisons with model predictions. JOUR NIMBE 2291

2005SA07 RADIOACTIVITY ${ }^{82} \mathrm{Se},{ }^{96} \mathrm{Zr},{ }^{100} \mathrm{Mo},{ }^{116} \mathrm{Cd},{ }^{150} \mathrm{Nd}\left(2 \beta^{-}\right)$; measured $2 \nu \beta \beta$-decay $\mathrm{T}_{1 / 2} .{ }^{82} \mathrm{Se},{ }^{100} \mathrm{Mo}\left(2 \beta^{-}\right)$; measured $0 \nu \beta \beta$-decay $\mathrm{T}_{1 / 2}$ lower limits; deduced neutrino mass limits. JOUR NPBSE 143221

2005 SC26 ATOMIC MASSES ${ }^{78,80,82,83,84,86} \mathrm{Kr}$; measured masses. Penning trap mass spectrometer. JOUR ZAANE $25 \mathrm{~s} 0151$

2005SHZW RADIOACTIVITY ${ }^{100} \mathrm{Mo},{ }^{82} \mathrm{Se}\left(2 \beta^{-}\right)$; measured $0 \nu \beta \beta$-decay $\mathrm{T}_{1 / 2}$ limits. NEMO-3 detector, underground laboratory in Modane. CONF St Petersburg,P42,Shitov

2005SI06 RADIOACTIVITY ${ }^{82} \mathrm{Se},{ }^{96} \mathrm{Zr},{ }^{100} \mathrm{Mo},{ }^{116} \mathrm{Cd},{ }^{150} \mathrm{Nd}\left(2 \beta^{-}\right)$; measured $2 \nu \beta \beta$-decay $\mathrm{T}_{1 / 2} .{ }^{82} \mathrm{Se},{ }^{100} \mathrm{Mo}\left(2 \beta^{-}\right)$; measured $0 \nu \beta \beta$-decay $\mathrm{T}_{1 / 2}$ lower limits; deduced neutrino mass limits. JOUR NPBSE 145272 


\section{$\mathrm{A}=82$ (continued)}

\begin{tabular}{|c|c|c|}
\hline \multirow[t]{2}{*}{${ }^{82} \mathrm{Rb}$} & 2004KA68 & $\begin{array}{l}\text { NUCLEAR REACTIONS }{ }^{85} \mathrm{Rb}(\mathrm{p}, \mathrm{np}),(\mathrm{p}, 2 \mathrm{np}),(\mathrm{p}, 3 \mathrm{np}),(\mathrm{p}, 4 \mathrm{np}), \mathrm{E} \\
\approx 17-100 \mathrm{MeV} \text {; measured excitation functions. Activation technique, } \\
\text { comparison with model predictions. JOUR RAACA } 92449\end{array}$ \\
\hline & 2005 GU37 & $\begin{array}{l}\text { ATOMIC MASSES }{ }^{56,57} \mathrm{Mn},{ }^{82 m} \mathrm{Rb},{ }^{92} \mathrm{Sr},{ }^{124,127} \mathrm{Cs},{ }^{130} \mathrm{Ba} \text {; measured } \\
\text { masses. Penning trap mass spectrometer. JOUR ZAANE } 25 \text { s01 } 35\end{array}$ \\
\hline \multirow[t]{3}{*}{${ }^{82} \mathrm{Sr}$} & $2004 \mathrm{SCZU}$ & $\begin{array}{l}\text { NUCLEAR REACTIONS }{ }^{76} \mathrm{Se}(\mathrm{p}, \mathrm{n}), \mathrm{E} \approx 5-40 \mathrm{MeV} ;{ }^{78} \mathrm{Kr}(\mathrm{d}, \alpha), \mathrm{E} \approx \\
4-14 \mathrm{MeV} ; \text { measured } \sigma .{ }^{126} \mathrm{Te}(\mathrm{p}, 3 \mathrm{n}), \mathrm{E}=8-70 \mathrm{MeV} ;{ }^{85} \mathrm{Rb}(\mathrm{p}, 4 \mathrm{n}), \\
\mathrm{E}=44-66 \mathrm{MeV} ; \text { measured yields. REPT } \\
\text { NEA/NSC/DOC(2004)14.P13.Scholten }\end{array}$ \\
\hline & 2005KEZZ & $\begin{array}{l}\text { NUCLEAR REACTIONS Ti }(\mathrm{p}, \mathrm{X}){ }^{45} \mathrm{Ca}, \mathrm{E}=30-200 \mathrm{MeV} ;{ }^{85} \mathrm{Rb}(\mathrm{p}, 4 \mathrm{n}) \text {, } \\
\mathrm{E}=35-70 \mathrm{MeV} \text {; measured excitation functions. }{ }^{89} \mathrm{Y}(\mathrm{n}, \mathrm{p}), \mathrm{E}=\mathrm{fast} ; \\
\text { measured spectrum-averaged } \sigma \text {. Activation technique, radiochemical } \\
\text { separation, x-ray spectrometry. CONF Santa Fe (Nucl Data for Sci and } \\
\text { Technol) Proc.Voll.P758 }\end{array}$ \\
\hline & 2005UD02 & $\begin{array}{l}\text { NUCLEAR REACTIONS }{ }^{89} \mathrm{Y}(\mathrm{p}, \mathrm{X}){ }^{89} \mathrm{Zr} /{ }^{88} \mathrm{Zr} /{ }^{86} \mathrm{Zr} /{ }^{88} \mathrm{Y} /{ }^{87} \mathrm{Y} / \\
{ }^{87 m} \mathrm{Y} /{ }^{86} \mathrm{Y} /{ }^{85} \mathrm{Sr} /{ }^{83} \mathrm{Sr} /{ }^{82} \mathrm{Sr} /{ }^{84} \mathrm{Rb} /{ }^{83} \mathrm{Rb}, \mathrm{E}=15-80 \mathrm{MeV} ; \\
\text { measured excitation functions; deduced integral yields. Stacked-foil } \\
\text { activation technique. JOUR ARISE } 63367\end{array}$ \\
\hline
\end{tabular}

\section{$\mathrm{A}=83$}

\begin{tabular}{|c|c|c|}
\hline${ }^{83} \mathrm{Ga}$ & 2004PEZW & $\begin{array}{l}\text { RADIOACTIVITY }{ }^{82,83} \mathrm{Ga}\left(\beta^{-}\right)\left[\text {from }{ }^{238} \mathrm{U}(\mathrm{n}, \mathrm{F})\right] \text {; measured } \mathrm{E} \gamma, \mathrm{I} \gamma \text {, } \\
\gamma \gamma-, \beta \gamma \text {-coin, } \mathrm{T}_{1 / 2} \cdot{ }^{82,83} \mathrm{Ge} \text { deduced levels, configurations. REPT }\end{array}$ \\
\hline & & IPNO-T-05-02,Perru \\
\hline & 2004VE14 & $\begin{array}{l}\text { RADIOACTIVITY }{ }^{83} \mathrm{Ga},{ }^{81} \mathrm{Zn}\left(\beta^{-}\right)[\text {from U(d, X)]; measured E } \gamma, \mathrm{I} \gamma \\
\gamma \gamma-, \beta \gamma \text {, coin, } \mathrm{T}_{1 / 2} .{ }^{81} \mathrm{Ga},{ }^{83} \mathrm{As} \text { deduced levels. JOUR BJPHE } 34979\end{array}$ \\
\hline${ }^{83} \mathrm{Ge}$ & 2004PEZW & $\begin{array}{l}\text { RADIOACTIVITY }{ }^{82,83} \mathrm{Ga}\left(\beta^{-}\right)\left[\text {from }{ }^{238} \mathrm{U}(\mathrm{n}, \mathrm{F})\right] ; \text { measured } \mathrm{E} \gamma, \mathrm{I} \gamma \\
\gamma \gamma-, \beta \gamma \text {-coin, } \mathrm{T}_{1 / 2} \cdot{ }^{82,83} \mathrm{Ge} \text { deduced levels, configurations. REPT } \\
\text { IPNO-T-05-02,Perru }\end{array}$ \\
\hline & 2004VE14 & $\begin{array}{l}\text { RADIOACTIVITY }{ }^{83} \mathrm{Ga},{ }^{81} \mathrm{Zn}\left(\beta^{-}\right)[\text {from U(d, X)]; measured E } \gamma, \mathrm{I} \gamma \\
\gamma \gamma-, \beta \gamma \text {-coin, } T_{1 / 2} .{ }^{81} \mathrm{Ga},{ }^{83} \mathrm{As} \text { deduced levels. JOUR BJPHE } 34979\end{array}$ \\
\hline & 2005CI07 & $\begin{array}{l}\text { NUCLEAR REACTIONS }{ }^{2} \mathrm{H}\left({ }^{82} \mathrm{Ge}, \mathrm{p}\right),\left({ }^{84} \mathrm{Se}, \mathrm{p}\right), \mathrm{E}=4 \mathrm{MeV} / \text { nucleon; } \\
\text { measured Ep, } \sigma(\theta) .{ }^{83} \mathrm{Ge},{ }^{85} \mathrm{Se} \text { deduced ground and excited states } \\
\text { energies, J, } \pi \text {. JOUR NIMBE } 241200\end{array}$ \\
\hline & 2005JOZZ & $\begin{array}{l}\text { NUCLEAR REACTIONS }{ }^{2} \mathrm{H}\left({ }^{82} \mathrm{Ge}, \mathrm{p}\right),\left({ }^{84} \mathrm{Se}, \mathrm{p}\right), \mathrm{E}=4 \mathrm{MeV} / \text { nucleon; } \\
\text { measured } \sigma(\mathrm{E}, \theta) \cdot{ }^{83} \mathrm{Ge},{ }^{85} \mathrm{Se} \text { deduced ground and excited states } \\
\text { energies, L. }{ }^{2} \mathrm{H}\left({ }^{124} \mathrm{Sn}, \mathrm{p}\right), \mathrm{E}=562 \mathrm{MeV} ; \text { measured } \sigma(\mathrm{E}, \theta) .{ }^{125} \mathrm{Sn} \text { levels } \\
\text { deduced spectroscopic factors. CONF Argonne(Nuclei at the } \\
\text { Limits),P176,Jones }\end{array}$ \\
\hline & 2005ТН03 & $\begin{array}{l}\text { NUCLEAR REACTIONS }{ }^{2} \mathrm{H}\left({ }^{82} \mathrm{Ge}, \mathrm{p}\right), \mathrm{E}=330 \mathrm{MeV} \text {; measured proton } \\
\text { spectra, } \sigma(\theta) \text {, Q value. }{ }^{83} \mathrm{Ge} \text { deduced levels, } \mathrm{J}, \pi \text {, spectroscopic factors, } \\
\text { mass excess. JOUR PRVCA } 71021302\end{array}$ \\
\hline & 2005 TH12 & $\begin{array}{l}\text { NUCLEAR REACTIONS }{ }^{2} \mathrm{H}\left({ }^{82} \mathrm{Ge}, \mathrm{p}\right), \mathrm{E}=4 \mathrm{MeV} / \text { nucleon; }{ }^{2} \mathrm{H}\left({ }^{84} \mathrm{Se} \text {, }\right. \\
\mathrm{p}), \mathrm{E}=4.5 \mathrm{MeV} / \text { nucleon; measured Ep, recoil particle spectrum, } \\
\text { proton angular distribution. }{ }^{83} \mathrm{Ge},{ }^{85} \mathrm{Se} \text { deduced levels J, } \pi \text {, } \\
\text { spectroscopic factors. DWBA analysis. JOUR ZAANE } 25 \text { s01 } 371\end{array}$ \\
\hline 83 & 2004VE14 & $\begin{array}{l}\text { RADIOACTIVITY }{ }^{83} \mathrm{Ga},{ }^{81} \mathrm{Zn}\left(\beta^{-}\right)[\text {from U(d, X)]; measured E } \gamma, \mathrm{I} \gamma \\
\gamma \gamma-, \beta \gamma \text {-coin, } \mathrm{T}_{1 / 2} \cdot{ }^{81} \mathrm{Ga},{ }^{83} \mathrm{As} \text { deduced levels. JOUR BJPHE } 34979\end{array}$ \\
\hline
\end{tabular}




\section{$\mathrm{A}=83$ (continued)}

2005LU07 NUCLEAR REACTIONS ${ }^{238} \mathrm{U}\left({ }^{82} \mathrm{Se}, \mathrm{X}\right), \mathrm{E}=505 \mathrm{MeV}$; measured E $\gamma$, I $\gamma$, fragments isotopic yields. $77,78,79,80,81,82,83$ As deduced transitions. ${ }^{192} \mathrm{Os}\left({ }^{82} \mathrm{Se}, \mathrm{X}\right), \mathrm{E}=460 \mathrm{MeV}$; measured $\mathrm{E} \gamma, \mathrm{I} \gamma, \gamma \gamma$-coin. ${ }^{80} \mathrm{As},{ }^{87} \mathrm{Rb}$, ${ }^{84}$ Se deduced levels. Fragment separator. JOUR APOBB 361301

${ }^{83} \mathrm{Kr} \quad$ 2005LE04 NUCLEAR REACTIONS $\mathrm{Pb}(\mathrm{p}, \mathrm{X}){ }^{3} \mathrm{He} /{ }^{4} \mathrm{He} /{ }^{21} \mathrm{Ne} /{ }^{22} \mathrm{Ne} /{ }^{36} \mathrm{Ar} /$ ${ }^{38} \mathrm{Ar} /{ }^{78} \mathrm{Kr} /{ }^{80} \mathrm{Kr} /{ }^{81} \mathrm{Kr} /{ }^{82} \mathrm{Kr} /{ }^{83} \mathrm{Kr} /{ }^{84} \mathrm{Kr} /{ }^{85} \mathrm{Kr} /{ }^{86} \mathrm{Kr} /{ }^{124} \mathrm{Xe}$ $/{ }^{126} \mathrm{Xe} /{ }^{128} \mathrm{Xe} /{ }^{129} \mathrm{Xe} /{ }^{130} \mathrm{Xe} /{ }^{131} \mathrm{Xe} /{ }^{132} \mathrm{Xe} /{ }^{134} \mathrm{Xe}, \mathrm{E}=44-2595$ $\mathrm{MeV}$; measured production $\sigma$; deduced reaction mechanism features. Mini-stack approach, comparisons with model predictions. JOUR NIMBE 2291

2005MUZY NUCLEAR REACTIONS ${ }^{84} \mathrm{Kr}(\mathrm{n}, \mathrm{X}), \mathrm{E}=0-400 \mathrm{keV}$; measured total $\sigma$. ${ }^{82,84,86} \mathrm{Kr}(\mathrm{n}, \gamma), \mathrm{E}=0-400 \mathrm{keV} ;{ }^{80,83} \mathrm{Kr}(\mathrm{n}, \gamma), \mathrm{E}=0-5 \mathrm{keV}$; measured capture $\sigma .{ }^{80,82,83,84,86} \mathrm{Kr}(\mathrm{n}, \gamma), \mathrm{E}=5-100 \mathrm{keV}$; deduced Maxwellian-averaged $\sigma$. Astrophysical implications discussed. CONF Santa Fe (Nucl Data for Sci and Technol) Proc,Vol2,P1327

2005SC26 ATOMIC MASSES ${ }^{78,80,82,83,84,86} \mathrm{Kr}$; measured masses. Penning trap mass spectrometer. JOUR ZAANE 25 s01 51

${ }^{83} \mathrm{Rb} \quad$ 2004KA68 NUCLEAR REACTIONS ${ }^{85} \mathrm{Rb}(\mathrm{p}, \mathrm{np}),(\mathrm{p}, 2 \mathrm{np}),(\mathrm{p}, 3 \mathrm{np}),(\mathrm{p}, 4 \mathrm{np}), \mathrm{E}$ $\approx 17-100 \mathrm{MeV}$; measured excitation functions. Activation technique, comparison with model predictions. JOUR RAACA 92449

2005UD02 NUCLEAR REACTIONS ${ }^{89} \mathrm{Y}(\mathrm{p}, \mathrm{X}){ }^{89} \mathrm{Zr} /{ }^{88} \mathrm{Zr} /{ }^{86} \mathrm{Zr} /{ }^{88} \mathrm{Y} /{ }^{87} \mathrm{Y} /$ ${ }^{87 m} \mathrm{Y} /{ }^{86} \mathrm{Y} /{ }^{85} \mathrm{Sr} /{ }^{83} \mathrm{Sr} /{ }^{82} \mathrm{Sr} /{ }^{84} \mathrm{Rb} /{ }^{83} \mathrm{Rb}, \mathrm{E}=15-80 \mathrm{MeV}$; measured excitation functions; deduced integral yields. Stacked-foil activation technique. JOUR ARISE 63367

${ }^{83} \mathrm{Sr} \quad 2005 \mathrm{DU} 23$ NUCLEAR REACTIONS Ge $\left({ }^{18} \mathrm{O}, \mathrm{X}\right)^{83 m} \mathrm{Sr} /{ }^{83} \mathrm{Y} /{ }^{84 m} \mathrm{Y} /{ }^{88 m} \mathrm{Y} /$ ${ }^{85} \mathrm{Zr} /{ }^{87} \mathrm{Zr}, \mathrm{E}=82.8 \mathrm{GeV} ;{ }^{84} \mathrm{Se}\left({ }^{18} \mathrm{O}, \mathrm{X}\right)^{86 m} \mathrm{Y} /{ }^{85} \mathrm{Zr} /{ }^{87} \mathrm{Nb} /{ }^{87 m} \mathrm{Nb} /$ ${ }^{88} \mathrm{Nb} /{ }^{88} \mathrm{Mo}, \mathrm{E}=82.7 \mathrm{MeV} ;{ }^{124} \mathrm{Sn}\left({ }^{50} \mathrm{Ti}, \mathrm{X}\right){ }^{168 m} \mathrm{Lu} /{ }^{167} \mathrm{Hf} /{ }^{168} \mathrm{Hf}$, $\mathrm{E}=223.7 \mathrm{MeV} ;{ }^{116} \mathrm{Sn}\left({ }^{50} \mathrm{Ti}, \mathrm{X}\right){ }^{162} \mathrm{Tm} /{ }^{161} \mathrm{Yb} /{ }^{162} \mathrm{Yb} /{ }^{163} \mathrm{Yb} /{ }^{162} \mathrm{Lu} /$ ${ }^{162} \mathrm{Hf}, \mathrm{E}=224.4 \mathrm{MeV}$; measured delayed $\mathrm{E} \gamma, \mathrm{I} \gamma$ following residual nucleus decay. Physical preseparation technique. JOUR NIMAE 551 528

2005UD02 NUCLEAR REACTIONS ${ }^{89} \mathrm{Y}(\mathrm{p}, \mathrm{X}){ }^{89} \mathrm{Zr} /{ }^{88} \mathrm{Zr} /{ }^{86} \mathrm{Zr} /{ }^{88} \mathrm{Y} /{ }^{87} \mathrm{Y} /$ ${ }^{87 m} \mathrm{Y} /{ }^{86} \mathrm{Y} /{ }^{85} \mathrm{Sr} /{ }^{83} \mathrm{Sr} /{ }^{82} \mathrm{Sr} /{ }^{84} \mathrm{Rb} /{ }^{83} \mathrm{Rb}, \mathrm{E}=15-80 \mathrm{MeV}$; measured excitation functions; deduced integral yields. Stacked-foil activation technique. JOUR ARISE 63367

${ }^{83} \mathrm{Y} \quad 2005 \mathrm{DU} 23$ NUCLEAR REACTIONS Ge $\left({ }^{18} \mathrm{O}, \mathrm{X}\right)^{83 m} \mathrm{Sr} /{ }^{83} \mathrm{Y} /{ }^{84 m} \mathrm{Y} /{ }^{88 m} \mathrm{Y} /$ ${ }^{85} \mathrm{Zr} /{ }^{87} \mathrm{Zr}, \mathrm{E}=82.8 \mathrm{GeV} ;{ }^{84} \mathrm{Se}\left({ }^{18} \mathrm{O}, \mathrm{X}\right){ }^{86 m} \mathrm{Y} /{ }^{85} \mathrm{Zr} /{ }^{87} \mathrm{Nb} /{ }^{87 m} \mathrm{Nb} /$ ${ }^{88} \mathrm{Nb} /{ }^{88} \mathrm{Mo}, \mathrm{E}=82.7 \mathrm{MeV} ;{ }^{124} \mathrm{Sn}\left({ }^{50} \mathrm{Ti}, \mathrm{X}\right){ }^{168 m} \mathrm{Lu} /{ }^{167} \mathrm{Hf} /{ }^{168} \mathrm{Hf}$, $\mathrm{E}=223.7 \mathrm{MeV} ;{ }^{116} \mathrm{Sn}\left({ }^{50} \mathrm{Ti}, \mathrm{X}\right){ }^{162} \mathrm{Tm} /{ }^{161} \mathrm{Yb} /{ }^{162} \mathrm{Yb} /{ }^{163} \mathrm{Yb} /{ }^{162} \mathrm{Lu} /$ ${ }^{162} \mathrm{Hf}, \mathrm{E}=224.4 \mathrm{MeV}$; measured delayed $\mathrm{E} \gamma, \mathrm{I} \gamma$ following residual nucleus decay. Physical preseparation technique. JOUR NIMAE 551 528

2005 YU04 NUCLEAR REACTIONS ${ }^{58} \mathrm{Ni}\left({ }^{28} \mathrm{Si}, 3 \mathrm{p}\right), \mathrm{E}=98 \mathrm{MeV}$; measured E $\gamma$, $\mathrm{I} \gamma(\theta, \mathrm{H}, \mathrm{t}), \gamma \gamma$-coin. ${ }^{83} \mathrm{Y}$ deduced g-factors for rotational band levels. Transient field technique, comparison with cranking model predictions. JOUR CPLEE 221628 


\section{$\mathrm{A}=84$}

${ }^{84} \mathrm{Se}$

2005DE12

2005LU07

${ }^{84} \mathrm{Br}$

2005BEZW

${ }^{84} \mathrm{Kr}$

2005LE04

2005MUZY

$2005 \mathrm{SC} 26$

2005SH38

2005W001

${ }^{84} \mathrm{Rb}$

2004KA68

2005PA33

2005UD02
NUCLEAR REACTIONS ${ }^{238} \mathrm{U}\left({ }^{82} \mathrm{Se}, \mathrm{X}\right), \mathrm{E}=505 \mathrm{MeV}$; measured fragments isotopic yields. ${ }^{238} \mathrm{U}\left({ }^{82} \mathrm{Se}, \mathrm{X}\right){ }^{72} \mathrm{Zn} /{ }^{84} \mathrm{Se} /{ }^{85} \mathrm{Br}, \mathrm{E}=505$ $\mathrm{MeV}$; measured $\mathrm{E} \gamma, \mathrm{I} \gamma$, (particle) $\gamma$-coin. ${ }^{72} \mathrm{Zn},{ }^{84} \mathrm{Se},{ }^{85} \mathrm{Br}$ deduced levels, J, $\pi$. JOUR NUPAB $751533 \mathrm{c}$ NUCLEAR REACTIONS ${ }^{238} \mathrm{U}\left({ }^{82} \mathrm{Se}, \mathrm{X}\right), \mathrm{E}=505 \mathrm{MeV} ;{ }^{238} \mathrm{U}\left({ }^{64} \mathrm{Ni}, \mathrm{X}\right)$, $\mathrm{E}=400 \mathrm{MeV}$; measured $\mathrm{E} \gamma, \mathrm{I} \gamma, \gamma \gamma-$, (fragment) $\gamma$-coin, projectile-like fragments isotopic yields. ${ }^{58} \mathrm{Cr},{ }^{80} \mathrm{As},{ }^{82} \mathrm{Ge},{ }^{84} \mathrm{Se}$ deduced levels, $\mathrm{J}, \pi$. Clara array, Prisma spectrometer. JOUR ZAANE 25 s01 421

NUCLEAR REACTIONS ${ }^{238} \mathrm{U}\left({ }^{82} \mathrm{Se}, \mathrm{X}\right), \mathrm{E}=505 \mathrm{MeV}$; measured $\mathrm{E} \gamma$, $\mathrm{I} \gamma$, fragments isotopic yields. ${ }^{77,78,79,80,81,82,83}$ As deduced transitions. ${ }^{192} \mathrm{Os}\left({ }^{82} \mathrm{Se}, \mathrm{X}\right), \mathrm{E}=460 \mathrm{MeV}$; measured $\mathrm{E} \gamma, \mathrm{I} \gamma, \gamma \gamma$-coin. ${ }^{80} \mathrm{As},{ }^{87} \mathrm{Rb}$, ${ }^{84}$ Se deduced levels. Fragment separator. JOUR APOBB 361301 NUCLEAR REACTIONS ${ }^{238} \mathrm{U}(\gamma, \mathrm{F}){ }^{84} \mathrm{Br} /{ }^{130} \mathrm{Sb} /{ }^{132} \mathrm{Sb} /{ }^{131} \mathrm{Te} /$ ${ }^{133} \mathrm{Te} /{ }^{134} \mathrm{I} /{ }^{135} \mathrm{Xe}, \mathrm{E}=16 \mathrm{MeV}$ bremsstrahlung; ${ }^{237} \mathrm{~Np}(\gamma, \mathrm{F}){ }^{134} \mathrm{I} /$ ${ }^{135} \mathrm{Xe}, \mathrm{E}=16 \mathrm{MeV}$ bremsstrahlung; measured $\mathrm{E} \gamma, \mathrm{I} \gamma$; deduced isomer yield ratios, fission fragments mean angular momenta. CONF Santa Fe (Nucl Data for Sci and Technol) Proc,Vol1,P641 NUCLEAR REACTIONS Pb(p, X) ${ }^{3} \mathrm{He} /{ }^{4} \mathrm{He} /{ }^{21} \mathrm{Ne} /{ }^{22} \mathrm{Ne} /{ }^{36} \mathrm{Ar} /$ ${ }^{38} \mathrm{Ar} /{ }^{78} \mathrm{Kr} /{ }^{80} \mathrm{Kr} /{ }^{81} \mathrm{Kr} /{ }^{82} \mathrm{Kr} /{ }^{83} \mathrm{Kr} /{ }^{84} \mathrm{Kr} /{ }^{85} \mathrm{Kr} /{ }^{86} \mathrm{Kr} /{ }^{124} \mathrm{Xe}$ $/{ }^{126} \mathrm{Xe} /{ }^{128} \mathrm{Xe} /{ }^{129} \mathrm{Xe} /{ }^{130} \mathrm{Xe} /{ }^{131} \mathrm{Xe} /{ }^{132} \mathrm{Xe} /{ }^{134} \mathrm{Xe}, \mathrm{E}=44-2595$ $\mathrm{MeV}$; measured production $\sigma$; deduced reaction mechanism features. Mini-stack approach, comparisons with model predictions. JOUR NIMBE 2291

NUCLEAR REACTIONS ${ }^{84} \mathrm{Kr}(\mathrm{n}, \mathrm{X}), \mathrm{E}=0-400 \mathrm{keV}$; measured total $\sigma$. ${ }^{82,84,86} \mathrm{Kr}(\mathrm{n}, \gamma), \mathrm{E}=0-400 \mathrm{keV} ;{ }^{80,83} \mathrm{Kr}(\mathrm{n}, \gamma), \mathrm{E}=0-5 \mathrm{keV}$; measured capture $\sigma .{ }^{80,82,83,84,86} \mathrm{Kr}(\mathrm{n}, \gamma), \mathrm{E}=5-100 \mathrm{keV}$; deduced Maxwellian-averaged $\sigma$. Astrophysical implications discussed. CONF Santa Fe (Nucl Data for Sci and Technol) Proc,Vol2,P1327 ATOMIC MASSES $78,80,82,83,84,86 \mathrm{Kr}$; measured masses. Penning trap mass spectrometer. JOUR ZAANE 25 s01 51

ATOMIC MASSES ${ }^{32,33} \mathrm{~S},{ }^{84,86} \mathrm{Kr},{ }^{129,132} \mathrm{Xe}$; measured masses. Penning trap. JOUR PLRAA 72022510 NUCLEAR REACTIONS ${ }^{197} \mathrm{Au}\left({ }^{84} \mathrm{Kr},{ }^{84} \mathrm{Kr}\right),\left({ }^{56} \mathrm{Cr},{ }^{56} \mathrm{Cr}\right),\left({ }^{108} \mathrm{Sn}\right.$, ${ }^{108} \mathrm{Sn}$ '), E=113-142 MeV / nucleon; measured $\mathrm{E} \gamma$, I $\gamma$ following projectile Coulomb excitation. ${ }^{84} \mathrm{Kr},{ }^{56} \mathrm{Cr},{ }^{108} \mathrm{Sn}$ deduced transitions. ${ }^{9} \mathrm{Be}\left({ }^{55} \mathrm{Ni}, \mathrm{X}\right){ }^{54} \mathrm{Co} /{ }^{52} \mathrm{Fe} /{ }^{50} \mathrm{Cr}, \mathrm{E}=171 \mathrm{MeV} /$ nucleon; measured $\mathrm{E} \gamma$, I $\gamma$, (particle) $\gamma$-coin. JOUR NIMAE 537637

NUCLEAR REACTIONS ${ }^{85} \mathrm{Rb}(\mathrm{p}, \mathrm{np}),(\mathrm{p}, 2 \mathrm{np}),(\mathrm{p}, 3 \mathrm{np}),(\mathrm{p}, 4 \mathrm{np}), \mathrm{E}$ $\approx 17-100 \mathrm{MeV}$; measured excitation functions. Activation technique, comparison with model predictions. JOUR RAACA 92449 NUCLEAR REACTIONS ${ }^{85,87} \operatorname{Rb}(\gamma, \mathrm{n}), \mathrm{E}=13-30 \mathrm{MeV}$ bremsstrahlung; measured isomeric yield ratios. Activation technique. JOUR AENGA 98238 NUCLEAR REACTIONS ${ }^{89} \mathrm{Y}(\mathrm{p}, \mathrm{X}){ }^{89} \mathrm{Zr} /{ }^{88} \mathrm{Zr} /{ }^{86} \mathrm{Zr} /{ }^{88} \mathrm{Y} /{ }^{87} \mathrm{Y} /$ ${ }^{87 m} \mathrm{Y} /{ }^{86} \mathrm{Y} /{ }^{85} \mathrm{Sr} /{ }^{83} \mathrm{Sr} /{ }^{82} \mathrm{Sr} /{ }^{84} \mathrm{Rb} /{ }^{83} \mathrm{Rb}, \mathrm{E}=15-80 \mathrm{MeV}$; measured excitation functions; deduced integral yields. Stacked-foil activation technique. JOUR ARISE 63367 


\section{$\mathrm{A}=84$ (continued)}

\begin{tabular}{|c|c|c|}
\hline & 2005DU23 & $\begin{array}{l}\text { NUCLEAR REACTIONS Ge }\left({ }^{18} \mathrm{O}, \mathrm{X}\right)^{83 m} \mathrm{Sr} /{ }^{83} \mathrm{Y} /{ }^{84 m} \mathrm{Y} /{ }^{88 m} \mathrm{Y} / \\
{ }^{85} \mathrm{Zr} /{ }^{87} \mathrm{Zr}, \mathrm{E}=82.8 \mathrm{GeV} ;{ }^{84} \mathrm{Se}\left({ }^{18} \mathrm{O}, \mathrm{X}\right){ }^{86 m} \mathrm{Y} /{ }^{85} \mathrm{Zr} /{ }^{87} \mathrm{Nb} /{ }^{87 m} \mathrm{Nb} / \\
{ }^{88} \mathrm{Nb} /{ }^{88} \mathrm{Mo}, \mathrm{E}=82.7 \mathrm{MeV} ;{ }^{124} \mathrm{Sn}\left({ }^{50} \mathrm{Ti}, \mathrm{X}\right){ }^{168 m} \mathrm{Lu} /{ }^{167} \mathrm{Hf} /{ }^{168} \mathrm{Hf} \\
\mathrm{E}=223.7 \mathrm{MeV} ;{ }^{116} \mathrm{Sn}\left({ }^{50} \mathrm{Ti}, \mathrm{X}\right)^{162} \mathrm{Tm} /{ }^{161} \mathrm{Yb} /{ }^{162} \mathrm{Yb} /{ }^{163} \mathrm{Yb} /{ }^{162} \mathrm{Lu} / \\
{ }^{162} \mathrm{Hf}, \mathrm{E}=224.4 \mathrm{MeV} \text {; measured delayed } \mathrm{E} \gamma, \mathrm{I} \gamma \text { following residual } \\
\text { nucleus decay. Physical preseparation technique. JOUR NIMAE } 551 \\
528\end{array}$ \\
\hline & 20051002 & $\begin{array}{l}\text { NUCLEAR REACTIONS }{ }^{84} \mathrm{Sr}(\mathrm{p}, \mathrm{n}), \mathrm{E}=13.5 \mathrm{MeV} \text {; measured } \mathrm{E} \gamma, \mathrm{I} \gamma(\theta \text {, } \\
\mathrm{H}, \mathrm{t}) .{ }^{84} \mathrm{Y} \text { deduced levels, J, } \pi \text {, configurations, } \mathrm{g} \text { factors, isomeric } \\
\text { states } \mathrm{T}_{1 / 2} \text {. Time-differential perturbed angular distribution method. } \\
\text { JOUR PRVCA } 72044313\end{array}$ \\
\hline & $2005 \mathrm{CHZZ}$ & $\begin{array}{l}\text { NUCLEAR REACTIONS }{ }^{58} \mathrm{Ni}\left({ }^{32} \mathrm{~S}, 2 \mathrm{p} \alpha\right), \mathrm{E}=140 \mathrm{MeV} \text {; measured } \mathrm{E} \gamma \text {, } \\
\mathrm{I} \gamma, \gamma \gamma-\text {, (charged particle) } \gamma \text {-coin. }{ }^{84} \mathrm{Zr} \text { deduced high-spin levels, } \mathrm{J}, \pi \text {, } \\
\text { superdeformed band, linking transitions, band mixing features. } \\
\text { Gammasphere, Microball arrays. CONF Argonne(Nuclei at the } \\
\text { Limits),P40,Chiara }\end{array}$ \\
\hline & 2005XU04 & $\begin{array}{l}\text { RADIOACTIVITY }{ }^{81} \mathrm{Zr},{ }^{85} \mathrm{Mo},{ }^{89} \mathrm{Ru},{ }^{92} \mathrm{Rh},{ }^{93} \mathrm{Pd},{ }^{121} \mathrm{Ce},{ }^{125} \mathrm{Nd} \text {, } \\
{ }^{128} \mathrm{Pm},{ }^{129} \mathrm{Sm},{ }^{135,137} \mathrm{Gd},{ }^{139} \mathrm{Dy},{ }^{142} \mathrm{Ho},{ }^{149} \mathrm{Yb}\left(\beta^{+} \mathrm{p}\right) ; \text { measured } \\
\beta \text {-delayed E } \gamma \text {, I } \gamma \text {, proton spectra, p } \gamma \text {-coin, } \mathrm{T}_{1 / 2} \text {. Comparison with } \\
\text { model predictions. JOUR PRVCA } 71054318\end{array}$ \\
\hline
\end{tabular}

$$
\mathrm{A}=85
$$

${ }^{85} \mathrm{Se} \quad 2005 \mathrm{CI07}$

NUCLEAR REACTIONS ${ }^{2} \mathrm{H}\left({ }^{82} \mathrm{Ge}, \mathrm{p}\right),\left({ }^{84} \mathrm{Se}, \mathrm{p}\right), \mathrm{E}=4 \mathrm{MeV} /$ nucleon; measured Ep, $\sigma(\theta) .{ }^{83} \mathrm{Ge},{ }^{85} \mathrm{Se}$ deduced ground and excited states energies, J, $\pi$. JOUR NIMBE 241200

2005JOZZ NUCLEAR REACTIONS ${ }^{2} \mathrm{H}\left({ }^{82} \mathrm{Ge}, \mathrm{p}\right),\left({ }^{84} \mathrm{Se}, \mathrm{p}\right), \mathrm{E}=4 \mathrm{MeV} /$ nucleon; measured $\sigma(\mathrm{E}, \theta) .{ }^{83} \mathrm{Ge},{ }^{85} \mathrm{Se}$ deduced ground and excited states energies, L. ${ }^{2} \mathrm{H}\left({ }^{124} \mathrm{Sn}, \mathrm{p}\right), \mathrm{E}=562 \mathrm{MeV}$; measured $\sigma(\mathrm{E}, \theta) .{ }^{125} \mathrm{Sn}$ levels deduced spectroscopic factors. CONF Argonne(Nuclei at the Limits),P176,Jones

2005TH09 NUCLEAR REACTIONS ${ }^{2} \mathrm{H}\left({ }^{84} \mathrm{Se}, \mathrm{p}\right),\left({ }^{124} \mathrm{Sn}, \mathrm{p}\right), \mathrm{E}=4.5 \mathrm{MeV} /$ nucleon; measured recoil proton spectra, $\sigma(\mathrm{E}, \theta) .{ }^{85} \mathrm{Se},{ }^{125} \mathrm{Sn}$ deduced levels, J, $\pi$. JOUR NUPAB 758 663c

2005TH12 NUCLEAR REACTIONS ${ }^{2} \mathrm{H}\left({ }^{82} \mathrm{Ge}, \mathrm{p}\right), \mathrm{E}=4 \mathrm{MeV} /$ nucleon; ${ }^{2} \mathrm{H}\left({ }^{84} \mathrm{Se}\right.$, p), $\mathrm{E}=4.5 \mathrm{MeV} /$ nucleon; measured Ep, recoil particle spectrum, proton angular distribution. ${ }^{83} \mathrm{Ge},{ }^{85} \mathrm{Se}$ deduced levels $\mathrm{J}, \pi$, spectroscopic factors. DWBA analysis. JOUR ZAANE 25 s01 371

${ }^{85} \mathrm{Br} \quad 2005 \mathrm{DE} 12$ NUCLEAR REACTIONS ${ }^{238} \mathrm{U}\left({ }^{82} \mathrm{Se}, \mathrm{X}\right), \mathrm{E}=505 \mathrm{MeV}$; measured fragments isotopic yields. ${ }^{238} \mathrm{U}\left({ }^{82} \mathrm{Se}, \mathrm{X}\right){ }^{72} \mathrm{Zn} /{ }^{84} \mathrm{Se} /{ }^{85} \mathrm{Br}, \mathrm{E}=505$ $\mathrm{MeV}$; measured $\mathrm{E} \gamma$, I $\gamma$, (particle) $\gamma$-coin. ${ }^{72} \mathrm{Zn},{ }^{84} \mathrm{Se},{ }^{85} \mathrm{Br}$ deduced levels, J, $\pi$. JOUR NUPAB $751533 \mathrm{c}$

2005F005 NUCLEAR REACTIONS ${ }^{173} \mathrm{Yb}\left({ }^{24} \mathrm{Mg}, \mathrm{X}\right), \mathrm{E}=134.5 \mathrm{MeV} ;{ }^{176} \mathrm{Yb}\left({ }^{23} \mathrm{Na}\right.$, $\mathrm{X}), \mathrm{E}=129 \mathrm{MeV} ;{ }^{208} \mathrm{~Pb}\left({ }^{18} \mathrm{O}, \mathrm{X}\right), \mathrm{E}=91 \mathrm{MeV}$; measured $\mathrm{E} \gamma, \mathrm{I} \gamma, \gamma \gamma$-coin following compound nucleus fission. ${ }^{85} \mathrm{Br},{ }^{87} \mathrm{Rb}$ deduced high-spin levels, J, $\pi$, configurations. Comparison with shell model predictions. JOUR PRVCA 71064312 


\section{$\mathrm{A}=85$ (continued)}

${ }^{85} \mathrm{Kr}$ 2005LE04 NUCLEAR REACTIONS $\mathrm{Pb}(\mathrm{p}, \mathrm{X}){ }^{3} \mathrm{He} /{ }^{4} \mathrm{He} /{ }^{21} \mathrm{Ne} /{ }^{22} \mathrm{Ne} /{ }^{36} \mathrm{Ar} /$ ${ }^{38} \mathrm{Ar} /{ }^{78} \mathrm{Kr} /{ }^{80} \mathrm{Kr} /{ }^{81} \mathrm{Kr} /{ }^{82} \mathrm{Kr} /{ }^{83} \mathrm{Kr} /{ }^{84} \mathrm{Kr} /{ }^{85} \mathrm{Kr} /{ }^{86} \mathrm{Kr} /{ }^{124} \mathrm{Xe}$ $/{ }^{126} \mathrm{Xe} /{ }^{128} \mathrm{Xe} /{ }^{129} \mathrm{Xe} /{ }^{130} \mathrm{Xe} /{ }^{131} \mathrm{Xe} /{ }^{132} \mathrm{Xe} /{ }^{134} \mathrm{Xe}, \mathrm{E}=44-2595$ $\mathrm{MeV}$; measured production $\sigma$; deduced reaction mechanism features. Mini-stack approach, comparisons with model predictions. JOUR NIMBE 2291

2005MUZY NUCLEAR REACTIONS ${ }^{84} \mathrm{Kr}(\mathrm{n}, \mathrm{X}), \mathrm{E}=0-400 \mathrm{keV}$; measured total $\sigma$. ${ }^{82,84,86} \mathrm{Kr}(\mathrm{n}, \gamma), \mathrm{E}=0-400 \mathrm{keV} ;{ }^{80,83} \mathrm{Kr}(\mathrm{n}, \gamma), \mathrm{E}=0-5 \mathrm{keV}$; measured capture $\sigma .{ }^{80,82,83,84,86} \mathrm{Kr}(\mathrm{n}, \gamma), \mathrm{E}=5-100 \mathrm{keV}$; deduced

Maxwellian-averaged $\sigma$. Astrophysical implications discussed. CONF Santa Fe (Nucl Data for Sci and Technol) Proc,Vol2,P1327

${ }^{85} \mathrm{Rb} \quad$ 2005KR15 NUCLEAR MOMENTS ${ }^{85,87} \mathrm{Rb}$; measured excited-state hfs. Electromagnetically induced transparency. JOUR EULEE 72221

${ }^{85} \mathrm{Sr} \quad 2005 \mathrm{DI} 15$

2005UD02

${ }^{85} \mathrm{Y} \quad 2005 K A 39$

NUCLEAR REACTIONS ${ }^{74} \mathrm{Se},{ }^{84} \mathrm{Sr}(\mathrm{n}, \gamma)$, E=spectrum; measured $\sigma$, isomer ratio. Activation technique. JOUR NUPAB 758 513c

NUCLEAR REACTIONS ${ }^{89} \mathrm{Y}(\mathrm{p}, \mathrm{X})^{89} \mathrm{Zr} /{ }^{88} \mathrm{Zr} /{ }^{86} \mathrm{Zr} /{ }^{88} \mathrm{Y} /{ }^{87} \mathrm{Y} /$ ${ }^{87 m} \mathrm{Y} /{ }^{86} \mathrm{Y} /{ }^{85} \mathrm{Sr} /{ }^{83} \mathrm{Sr} /{ }^{82} \mathrm{Sr} /{ }^{84} \mathrm{Rb} /{ }^{83} \mathrm{Rb}, \mathrm{E}=15-80 \mathrm{MeV}$; measured excitation functions; deduced integral yields. Stacked-foil activation technique. JOUR ARISE 63367

RADIOACTIVITY ${ }^{81 m} \mathrm{Kr}$ (EC), (IT); ${ }^{81} \mathrm{Y},{ }^{81} \mathrm{Sr},{ }^{85} \mathrm{Nb},{ }^{85} \mathrm{Zr},{ }^{86} \mathrm{Mo}$, ${ }^{86} \mathrm{Nb}(\mathrm{EC})$ [from $\mathrm{Ni},{ }^{54} \mathrm{Fe}\left({ }^{32} \mathrm{~S}, \mathrm{X}\right)$ ]; measured $\mathrm{E} \gamma, \mathrm{I} \gamma, \mathrm{E}(\mathrm{ce}), \mathrm{I}(\mathrm{ce}), \mathrm{T}_{1 / 2}$.

${ }^{81} \mathrm{Kr},{ }^{85} \mathrm{Zr},{ }^{85} \mathrm{Nb}$ deduced isomeric transitions $\mathrm{T}_{1 / 2}$, ICC. ${ }^{85} \mathrm{Zr},{ }^{86} \mathrm{Nb}$ deduced levels, J, $\pi$, ICC. ${ }^{81} \mathrm{Br}$ deduced neutrino capture rate.

Astrophysical implications discussed. JOUR ZAANE 25355

2005KA46 RADIOACTIVITY ${ }^{31} \mathrm{Cl}\left(\beta^{+} \mathrm{p}\right)$ [from $\mathrm{S}(\mathrm{p}, \mathrm{X}), \mathrm{E}=40 \mathrm{MeV}$ ]; measured $\beta$-delayed $\mathrm{E} \gamma, \mathrm{Ep} .{ }^{58} \mathrm{Zn}\left(\beta^{+}\right)$[from $\mathrm{Nb}(\mathrm{p}, \mathrm{X}), \mathrm{E}=1.4 \mathrm{GeV}$ ]; measured $\mathrm{E} \gamma, \mathrm{I} \gamma, \beta \gamma$-coin, $\mathrm{T}_{1 / 2} .{ }^{58} \mathrm{Cu}$ deduced levels, $\beta$-feeding intensities. ${ }^{81 m} \mathrm{Kr}(\mathrm{EC}),(\mathrm{IT}) ;{ }^{81} \mathrm{Y},{ }^{81} \mathrm{Sr},{ }^{85} \mathrm{Nb},{ }^{85} \mathrm{Zr},{ }^{86} \mathrm{Mo},{ }^{86} \mathrm{Nb}(\mathrm{EC})$ [from $\mathrm{Ni}$, $\left.{ }^{54} \mathrm{Fe}\left({ }^{32} \mathrm{~S}, \mathrm{X}\right)\right]$; measured $\mathrm{E} \gamma, \mathrm{I} \gamma, \mathrm{E}(\mathrm{ce}), \mathrm{I}(\mathrm{ce}), \mathrm{T}_{1 / 2} \cdot{ }^{81} \mathrm{Kr},{ }^{85} \mathrm{Zr},{ }^{85} \mathrm{Nb}$ deduced isomeric transitions $\mathrm{T}_{1 / 2}$, ICC. ${ }^{85} \mathrm{Zr},{ }^{86} \mathrm{Nb}$ deduced levels, $\mathrm{J}$, $\pi$, ICC. ${ }^{81} \mathrm{Br}$ deduced neutrino capture rate. Mass-separated sources. JOUR ZAANE 25 s01 129

${ }^{85} \mathrm{Zr} \quad 2005 \mathrm{DU} 23$ NUCLEAR REACTIONS Ge $\left({ }^{18} \mathrm{O}, \mathrm{X}\right)^{83 m} \mathrm{Sr} /{ }^{83} \mathrm{Y} /{ }^{84 m} \mathrm{Y} /{ }^{88 m} \mathrm{Y} /$ ${ }^{85} \mathrm{Zr} /{ }^{87} \mathrm{Zr}, \mathrm{E}=82.8 \mathrm{GeV} ;{ }^{84} \mathrm{Se}\left({ }^{18} \mathrm{O}, \mathrm{X}\right){ }^{86 m} \mathrm{Y} /{ }^{85} \mathrm{Zr} /{ }^{87} \mathrm{Nb} /{ }^{87 m} \mathrm{Nb} /$ ${ }^{88} \mathrm{Nb} /{ }^{88} \mathrm{Mo}, \mathrm{E}=82.7 \mathrm{MeV} ;{ }^{124} \mathrm{Sn}\left({ }^{50} \mathrm{Ti}, \mathrm{X}\right){ }^{168 m} \mathrm{Lu} /{ }^{167} \mathrm{Hf} /{ }^{168} \mathrm{Hf}$, $\mathrm{E}=223.7 \mathrm{MeV} ;{ }^{116} \mathrm{Sn}\left({ }^{50} \mathrm{Ti}, \mathrm{X}\right){ }^{162} \mathrm{Tm} /{ }^{161} \mathrm{Yb} /{ }^{162} \mathrm{Yb} /{ }^{163} \mathrm{Yb} /{ }^{162} \mathrm{Lu} /$ ${ }^{162} \mathrm{Hf}, \mathrm{E}=224.4 \mathrm{MeV}$; measured delayed $\mathrm{E} \gamma$, I $\gamma$ following residual nucleus decay. Physical preseparation technique. JOUR NIMAE 551 528

2005KA39 RADIOACTIVITY ${ }^{81 m} \mathrm{Kr}$ (EC), (IT); ${ }^{81} \mathrm{Y},{ }^{81} \mathrm{Sr},{ }^{85} \mathrm{Nb},{ }^{85} \mathrm{Zr},{ }^{86} \mathrm{Mo}$, ${ }^{86} \mathrm{Nb}(\mathrm{EC})$ [from $\left.\mathrm{Ni},{ }^{54} \mathrm{Fe}\left({ }^{32} \mathrm{~S}, \mathrm{X}\right)\right]$; measured $\mathrm{E} \gamma, \mathrm{I} \gamma, \mathrm{E}(\mathrm{ce}), \mathrm{I}\left(\right.$ ce), $\mathrm{T}_{1 / 2}$. ${ }^{81} \mathrm{Kr},{ }^{85} \mathrm{Zr},{ }^{85} \mathrm{Nb}$ deduced isomeric transitions $\mathrm{T}_{1 / 2}$, ICC. ${ }^{85} \mathrm{Zr},{ }^{86} \mathrm{Nb}$ deduced levels, J, $\pi$, ICC. ${ }^{81} \mathrm{Br}$ deduced neutrino capture rate. Astrophysical implications discussed. JOUR ZAANE 25355 


\section{$\mathrm{A}=85$ (continued)}

2005KA46 RADIOACTIVITY ${ }^{31} \mathrm{Cl}\left(\beta^{+} \mathrm{p}\right)$ [from $\mathrm{S}(\mathrm{p}, \mathrm{X}), \mathrm{E}=40 \mathrm{MeV}$ ]; measured $\beta$-delayed $\mathrm{E} \gamma, \mathrm{Ep} .{ }^{58} \mathrm{Zn}\left(\beta^{+}\right)$[from $\mathrm{Nb}(\mathrm{p}, \mathrm{X}), \mathrm{E}=1.4 \mathrm{GeV}$ ]; measured $\mathrm{E} \gamma, \mathrm{I} \gamma, \beta \gamma$-coin, $\mathrm{T}_{1 / 2} .{ }^{58} \mathrm{Cu}$ deduced levels, $\beta$-feeding intensities. ${ }^{81 m} \mathrm{Kr}(\mathrm{EC}),(\mathrm{IT}) ;{ }^{81} \mathrm{Y},{ }^{81} \mathrm{Sr},{ }^{85} \mathrm{Nb},{ }^{85} \mathrm{Zr},{ }^{86} \mathrm{Mo},{ }^{86} \mathrm{Nb}(\mathrm{EC})$ [from $\mathrm{Ni}$, $\left.{ }^{54} \mathrm{Fe}\left({ }^{32} \mathrm{~S}, \mathrm{X}\right)\right]$; measured $\mathrm{E} \gamma, \mathrm{I} \gamma, \mathrm{E}(\mathrm{ce}), \mathrm{I}(\mathrm{ce}), \mathrm{T}_{1 / 2} \cdot{ }^{81} \mathrm{Kr},{ }^{85} \mathrm{Zr},{ }^{85} \mathrm{Nb}$ deduced isomeric transitions $\mathrm{T}_{1 / 2}$, ICC. ${ }^{85} \mathrm{Zr},{ }^{86} \mathrm{Nb}$ deduced levels, J, $\pi$, ICC. ${ }^{81} \mathrm{Br}$ deduced neutrino capture rate. Mass-separated sources. JOUR ZAANE 25 s01 129

${ }^{85} \mathrm{Nb}$ 2005KA39 RADIOACTIVITY ${ }^{81 m} \mathrm{Kr}$ (EC), (IT); ${ }^{81} \mathrm{Y},{ }^{81} \mathrm{Sr},{ }^{85} \mathrm{Nb},{ }^{85} \mathrm{Zr},{ }^{86} \mathrm{Mo}$, ${ }^{86} \mathrm{Nb}(\mathrm{EC})\left[\right.$ from $\left.\mathrm{Ni},{ }^{54} \mathrm{Fe}\left({ }^{32} \mathrm{~S}, \mathrm{X}\right)\right]$; measured $\mathrm{E} \gamma, \mathrm{I} \gamma, \mathrm{E}(\mathrm{ce}), \mathrm{I}(\mathrm{ce}), \mathrm{T}_{1 / 2}$. ${ }^{81} \mathrm{Kr},{ }^{85} \mathrm{Zr},{ }^{85} \mathrm{Nb}$ deduced isomeric transitions $\mathrm{T}_{1 / 2}$, ICC. ${ }^{85} \mathrm{Zr},{ }^{86} \mathrm{Nb}$ deduced levels, J, $\pi$, ICC. ${ }^{81} \mathrm{Br}$ deduced neutrino capture rate. Astrophysical implications discussed. JOUR ZAANE 25355 2005KA39 NUCLEAR REACTIONS ${ }^{54} \mathrm{Fe}\left({ }^{32} \mathrm{~S}, \mathrm{X}\right){ }^{81} \mathrm{Zr} /{ }^{81} \mathrm{Y} /{ }^{81} \mathrm{Sr} /{ }^{81 m} \mathrm{Kr}$, $\mathrm{E}=150-170 \mathrm{MeV} ; \mathrm{Ni}\left({ }^{32} \mathrm{~S}, \mathrm{X}\right){ }^{85} \mathrm{Nb} /{ }^{85 m} \mathrm{Nb} /{ }^{85 Z r} /{ }^{85 m} \mathrm{Zr} /{ }^{86} \mathrm{Mo} /$ ${ }^{86} \mathrm{Nb}, \mathrm{E}=150-170 \mathrm{MeV}$; measured yields. JOUR ZAANE 25355

2005KA46 RADIOACTIVITY ${ }^{31} \mathrm{Cl}\left(\beta^{+} \mathrm{p}\right)$ [from $\mathrm{S}(\mathrm{p}, \mathrm{X}), \mathrm{E}=40 \mathrm{MeV}$ ]; measured $\beta$-delayed $\mathrm{E} \gamma, \mathrm{Ep} .{ }^{58} \mathrm{Zn}\left(\beta^{+}\right)$[from $\mathrm{Nb}(\mathrm{p}, \mathrm{X}), \mathrm{E}=1.4 \mathrm{GeV}$ ]; measured $\mathrm{E} \gamma, \mathrm{I} \gamma, \beta \gamma$-coin, $\mathrm{T}_{1 / 2} .{ }^{58} \mathrm{Cu}$ deduced levels, $\beta$-feeding intensities. ${ }^{81 m} \mathrm{Kr}(\mathrm{EC}),(\mathrm{IT}) ;{ }^{81} \mathrm{Y},{ }^{81} \mathrm{Sr},{ }^{85} \mathrm{Nb},{ }^{85} \mathrm{Zr},{ }^{86} \mathrm{Mo},{ }^{86} \mathrm{Nb}(\mathrm{EC})$ [from Ni, $\left.{ }^{54} \mathrm{Fe}\left({ }^{32} \mathrm{~S}, \mathrm{X}\right)\right]$; measured $\mathrm{E} \gamma, \mathrm{I} \gamma, \mathrm{E}(\mathrm{ce}), \mathrm{I}(\mathrm{ce}), \mathrm{T}_{1 / 2} \cdot{ }^{81} \mathrm{Kr},{ }^{85} \mathrm{Zr},{ }^{85} \mathrm{Nb}$ deduced isomeric transitions $\mathrm{T}_{1 / 2}$, ICC. ${ }^{85} \mathrm{Zr},{ }^{86} \mathrm{Nb}$ deduced levels, $\mathrm{J}$, $\pi$, ICC. ${ }^{81} \mathrm{Br}$ deduced neutrino capture rate. Mass-separated sources. JOUR ZAANE 25 s01 129

${ }^{85} \mathrm{Mo}$ 2005XU04 RADIOACTIVITY ${ }^{81} \mathrm{Zr},{ }^{85} \mathrm{Mo},{ }^{89} \mathrm{Ru},{ }^{92} \mathrm{Rh},{ }^{93} \mathrm{Pd},{ }^{121} \mathrm{Ce},{ }^{125} \mathrm{Nd}$, ${ }^{128} \mathrm{Pm},{ }^{129} \mathrm{Sm},{ }^{135,137} \mathrm{Gd},{ }^{139} \mathrm{Dy},{ }^{142} \mathrm{Ho},{ }^{149} \mathrm{Yb}\left(\beta^{+} \mathrm{p}\right)$; measured $\beta$-delayed $\mathrm{E} \gamma, \mathrm{I} \gamma$, proton spectra, $\mathrm{p} \gamma$-coin, $\mathrm{T}_{1 / 2}$. Comparison with model predictions. JOUR PRVCA 71054318

\section{$\mathrm{A}=86$}

${ }^{86} \mathrm{Kr} \quad$ 2005LE04

NUCLEAR REACTIONS $\mathrm{Pb}(\mathrm{p}, \mathrm{X}){ }^{3} \mathrm{He} /{ }^{4} \mathrm{He} /{ }^{21} \mathrm{Ne} /{ }^{22} \mathrm{Ne} /{ }^{36} \mathrm{Ar} /$ ${ }^{38} \mathrm{Ar} /{ }^{78} \mathrm{Kr} /{ }^{80} \mathrm{Kr} /{ }^{81} \mathrm{Kr} /{ }^{82} \mathrm{Kr} /{ }^{83} \mathrm{Kr} /{ }^{84} \mathrm{Kr} /{ }^{85} \mathrm{Kr} /{ }^{86} \mathrm{Kr} /{ }^{124} \mathrm{Xe}$ $/{ }^{126} \mathrm{Xe} /{ }^{128} \mathrm{Xe} /{ }^{129} \mathrm{Xe} /{ }^{130} \mathrm{Xe} /{ }^{131} \mathrm{Xe} /{ }^{132} \mathrm{Xe} /{ }^{134} \mathrm{Xe}, \mathrm{E}=44-2595$ $\mathrm{MeV}$; measured production $\sigma$; deduced reaction mechanism features. Mini-stack approach, comparisons with model predictions. JOUR NIMBE 2291

2005SC26 ATOMIC MASSES $78,80,82,83,84,86 \mathrm{Kr}$; measured masses. Penning trap mass spectrometer. JOUR ZAANE 25 s01 51

2005SH38 ATOMIC MASSES ${ }^{32,33} \mathrm{~S},{ }^{84,86} \mathrm{Kr},{ }^{129,132} \mathrm{Xe}$; measured masses. Penning trap. JOUR PLRAA 72022510

${ }^{86} \mathrm{Rb}$ 2005PA33 NUCLEAR REACTIONS ${ }^{85,87} \mathrm{Rb}(\gamma, \mathrm{n}), \mathrm{E}=13-30 \mathrm{MeV}$ bremsstrahlung; measured isomeric yield ratios. Activation technique. JOUR AENGA 98238 


\section{$\mathrm{A}=86$ (continued)}

2005TIZX NUCLEAR REACTIONS Pb, ${ }^{208} \mathrm{~Pb}(\mathrm{p}, \mathrm{X}){ }^{203} \mathrm{~Pb} /{ }^{200} \mathrm{Tl} /{ }^{199} \mathrm{Tl} /$ ${ }^{196} \mathrm{Au} /{ }^{192} \mathrm{Ir} /{ }^{190} \mathrm{Ir} /{ }^{173} \mathrm{Lu} /{ }^{101 m} \mathrm{Rh} /{ }^{86} \mathrm{Rb} /{ }^{59} \mathrm{Fe} /{ }^{24} \mathrm{Na} /{ }^{7} \mathrm{Be}$, $\mathrm{E}=40-2600 \mathrm{MeV}$; measured excitation functions. Comparison with previous work and model predictions. Other reactions discussed. CONF Santa Fe (Nucl Data for Sci and Technol) Proc,Vol1,P1070 2005TIZY NUCLEAR REACTIONS Pb, ${ }^{208} \mathrm{~Pb},{ }^{209} \mathrm{Bi}(\mathrm{p}, \mathrm{X}){ }^{203} \mathrm{~Pb} /{ }^{200} \mathrm{Tl} /{ }^{199} \mathrm{Tl}$ $/{ }^{196} \mathrm{Au} /{ }^{192} \mathrm{Ir} /{ }^{190} \mathrm{Ir} /{ }^{173} \mathrm{Lu} /{ }^{101 m} \mathrm{Rh} /{ }^{86} \mathrm{Rb} /{ }^{59} \mathrm{Fe} /{ }^{24} \mathrm{Na} /{ }^{7} \mathrm{Be}$, $\mathrm{E}=40-2600 \mathrm{MeV}$; measured production $\sigma$. Comparison with model predictions. PREPRINT nucl-ex/0507009,7/05/2005

\begin{tabular}{|c|c|c|}
\hline${ }^{86} \mathrm{Sr}$ & 2005SI34 & $\begin{array}{l}\text { ATOMIC MASSES }{ }^{76,77,80,81,86,88} \mathrm{Sr},{ }^{124,129,130,131,132} \mathrm{Sn} \text {; measured } \\
\text { masses. Penning trap mass spectrometer, comparison with previous } \\
\text { results. JOUR NUPAB } 76345\end{array}$ \\
\hline \multirow[t]{2}{*}{${ }^{86} \mathrm{Y}$} & 2005DU23 & $\begin{array}{l}\text { NUCLEAR REACTIONS Ge }\left({ }^{18} \mathrm{O}, \mathrm{X}\right){ }^{83 m} \mathrm{Sr} /{ }^{83} \mathrm{Y} /{ }^{84 m} \mathrm{Y} /{ }^{88 m} \mathrm{Y} / \\
{ }^{85} \mathrm{Zr} /{ }^{87} \mathrm{Zr}, \mathrm{E}=82.8 \mathrm{GeV} ;{ }^{84} \mathrm{Se}\left({ }^{18} \mathrm{O}, \mathrm{X}\right){ }^{86 m} \mathrm{Y} /{ }^{85} \mathrm{Zr} /{ }^{87} \mathrm{Nb} /{ }^{87 m} \mathrm{Nb} / \\
{ }^{88} \mathrm{Nb} /{ }^{88} \mathrm{Mo}, \mathrm{E}=82.7 \mathrm{MeV} ;{ }^{124} \mathrm{Sn}\left({ }^{50} \mathrm{Ti}, \mathrm{X}\right){ }^{168 m} \mathrm{Lu} /{ }^{167} \mathrm{Hf} /{ }^{168} \mathrm{Hf}, \\
\mathrm{E}=223.7 \mathrm{MeV} ;{ }^{116} \mathrm{Sn}\left({ }^{50} \mathrm{Ti}, \mathrm{X}\right){ }^{162} \mathrm{Tm} /{ }^{161} \mathrm{Yb} /{ }^{162} \mathrm{Yb} /{ }^{163} \mathrm{Yb} /{ }^{162} \mathrm{Lu} / \\
{ }^{162} \mathrm{Hf}, \mathrm{E}=224.4 \mathrm{MeV} ; \text { measured delayed } \mathrm{E} \gamma, \mathrm{I} \gamma \text { following residual } \\
\text { nucleus decay. Physical preseparation technique. JOUR NIMAE } 551 \\
528\end{array}$ \\
\hline & 2005UD02 & $\begin{array}{l}\text { NUCLEAR REACTIONS }{ }^{89} \mathrm{Y}\left(\mathrm{p}, \mathrm{X}{ }^{89} \mathrm{Zr} /{ }^{88} \mathrm{Zr} /{ }^{86} \mathrm{Zr} /{ }^{88} \mathrm{Y} /{ }^{87} \mathrm{Y} /\right. \\
{ }^{87 m} \mathrm{Y} /{ }^{86} \mathrm{Y} /{ }^{85} \mathrm{Sr} /{ }^{83} \mathrm{Sr} /{ }^{82} \mathrm{Sr} /{ }^{84} \mathrm{Rb} /{ }^{83} \mathrm{Rb}, \mathrm{E}=15-80 \mathrm{MeV} ; \\
\text { measured excitation functions; deduced integral yields. Stacked-foil } \\
\text { activation technique. JOUR ARISE } 63367\end{array}$ \\
\hline \multirow[t]{4}{*}{${ }^{86} \mathrm{Zr}$} & 2005BI25 & $\begin{array}{l}\text { NUCLEAR MOMENTS } 86,87,88,89,90,91,92,93,94,95,96,97,98,99,100,101,102 \mathrm{Zr} \text {; } \\
\text { measured charge radii. }{ }^{176} \mathrm{Yb} \text {; measured isomer shift. Ion-beam cooler, } \\
\text { laser spectroscopy. JOUR ZAANE } 25 \text { s01 } 187\end{array}$ \\
\hline & 2005KA39 & $\begin{array}{l}\text { RADIOACTIVITY }{ }^{81 m} \mathrm{Kr}(\mathrm{EC}),(\mathrm{IT}) ;{ }^{81} \mathrm{Y},{ }^{81} \mathrm{Sr},{ }^{85} \mathrm{Nb},{ }^{85} \mathrm{Zr},{ }^{86} \mathrm{Mo} \text {, } \\
{ }^{86} \mathrm{Nb}(\mathrm{EC})\left[\text { from Ni},{ }^{54} \mathrm{Fe}\left({ }^{32} \mathrm{~S}, \mathrm{X}\right)\right] ; \text { measured } \mathrm{E} \gamma, \mathrm{I} \gamma, \mathrm{E}(\mathrm{ce}), \mathrm{I}(\mathrm{ce}), \mathrm{T}_{1 / 2} \cdot \\
{ }^{81} \mathrm{Kr},{ }^{85} \mathrm{Zr},{ }^{85} \mathrm{Nb} \text { deduced isomeric transitions } \mathrm{T}_{1 / 2} \text {, ICC. }{ }^{85} \mathrm{Zr},{ }^{86} \mathrm{Nb} \\
\text { deduced levels, J, } \pi \text {, ICC. }{ }^{81} \mathrm{Br} \text { deduced neutrino capture rate. } \\
\text { Astrophysical implications discussed. JOUR ZAANE } 25355\end{array}$ \\
\hline & $2005 K A 46$ & $\begin{array}{l}\left.\text { RADIOACTIVITY }{ }^{31} \mathrm{Cl}\left(\beta^{+} \mathrm{p}\right) \text { [from } \mathrm{S}(\mathrm{p}, \mathrm{X}), \mathrm{E}=40 \mathrm{MeV}\right] ; \text { measured } \\
\left.\beta \text {-delayed E } \gamma, \mathrm{Ep} .{ }^{58} \mathrm{Zn}\left(\beta^{+}\right) \text {[from } \mathrm{Nb}(\mathrm{p}, \mathrm{X}), \mathrm{E}=1.4 \mathrm{GeV}\right] ; \text { measured } \\
\mathrm{E} \gamma, \mathrm{I} \gamma, \beta \gamma \text {-coin, } \mathrm{T}_{1 / 2} \cdot{ }^{58} \mathrm{Cu} \text { deduced levels, } \beta \text {-feeding intensities. } \\
{ }^{81 m} \mathrm{Kr}(\mathrm{EC}),(\mathrm{IT}) ;{ }^{81} \mathrm{Y},{ }^{81} \mathrm{Sr},{ }^{85} \mathrm{Nb},{ }^{85} \mathrm{Zr},{ }^{86} \mathrm{Mo},{ }^{86} \mathrm{Nb}(\mathrm{EC})[\text { from } \mathrm{Ni} \text {, } \\
\left.{ }^{54} \mathrm{Fe}\left({ }^{32} \mathrm{~S}, \mathrm{X}\right)\right] ; \text { measured } \mathrm{E} \gamma, \mathrm{I} \gamma, \mathrm{E}(\mathrm{ce}), \mathrm{I}(\mathrm{ce}), \mathrm{T}_{1 / 2} \cdot{ }^{81} \mathrm{Kr},{ }^{85} \mathrm{Zr},{ }^{85} \mathrm{Nb} \\
\text { deduced isomeric transitions } \mathrm{T}_{1 / 2}, \mathrm{ICC} .{ }^{85} \mathrm{Zr},{ }^{86} \mathrm{Nb} \text { deduced levels, J, } \\
\pi \text {, ICC. }{ }^{81} \mathrm{Br} \text { deduced neutrino capture rate. Mass-separated sources. } \\
\text { JOUR ZAANE } 25 \text { s01 } 129\end{array}$ \\
\hline & 2005UD02 & $\begin{array}{l}\text { NUCLEAR REACTIONS }{ }^{89} \mathrm{Y}(\mathrm{p}, \mathrm{X})^{89} \mathrm{Zr} /{ }^{88} \mathrm{Zr} /{ }^{86} \mathrm{Zr} /{ }^{88} \mathrm{Y} /{ }^{87} \mathrm{Y} / \\
{ }^{87 m} \mathrm{Y} /{ }^{86} \mathrm{Y} /{ }^{85} \mathrm{Sr} /{ }^{83} \mathrm{Sr} /{ }^{82} \mathrm{Sr} /{ }^{84} \mathrm{Rb} /{ }^{83} \mathrm{Rb}, \mathrm{E}=15-80 \mathrm{MeV} ; \\
\text { measured excitation functions; deduced integral yields. Stacked-foil } \\
\text { activation technique. JOUR ARISE } 63367\end{array}$ \\
\hline${ }^{86} \mathrm{~N}$ & 2005КА39 & $\begin{array}{l}\text { RADIOACTIVITY }{ }^{81 m} \mathrm{Kr}(\mathrm{EC}),(\mathrm{IT}) ;{ }^{81} \mathrm{Y},{ }^{81} \mathrm{Sr},{ }^{85} \mathrm{Nb},{ }^{85} \mathrm{Zr},{ }^{86} \mathrm{Mo} \text {, } \\
{ }^{86} \mathrm{Nb}(\mathrm{EC})\left[\mathrm{from} \mathrm{Ni},{ }^{54} \mathrm{Fe}\left({ }^{32} \mathrm{~S}, \mathrm{X}\right)\right] ; \text { measured } \mathrm{E} \gamma, \mathrm{I} \gamma, \mathrm{E}(\mathrm{ce}), \mathrm{I}(\mathrm{ce}), \mathrm{T}_{1 / 2} \cdot \\
{ }^{81} \mathrm{Kr},{ }^{85} \mathrm{Zr},{ }^{85} \mathrm{Nb} \text { deduced isomeric transitions } \mathrm{T}_{1 / 2}, \mathrm{ICC} .{ }^{85} \mathrm{Zr},{ }^{86} \mathrm{Nb} \\
\text { deduced levels, J, } \pi \text {, ICC. }{ }^{81} \mathrm{Br} \text { deduced neutrino capture rate. } \\
\text { Astrophysical implications discussed. JOUR ZAANE } 25355\end{array}$ \\
\hline
\end{tabular}
Astrophysical implications discussed. JOUR ZAANE 25355 


\section{$\mathrm{A}=86$ (continued)}

2005KA46 RADIOACTIVITY ${ }^{31} \mathrm{Cl}\left(\beta^{+} \mathrm{p}\right)$ [from $\mathrm{S}(\mathrm{p}, \mathrm{X}), \mathrm{E}=40 \mathrm{MeV}$ ]; measured $\beta$-delayed $\mathrm{E} \gamma, \mathrm{Ep} .{ }^{58} \mathrm{Zn}\left(\beta^{+}\right)$[from $\mathrm{Nb}(\mathrm{p}, \mathrm{X}), \mathrm{E}=1.4 \mathrm{GeV}$ ]; measured $\mathrm{E} \gamma, \mathrm{I} \gamma, \beta \gamma$-coin, $\mathrm{T}_{1 / 2} .{ }^{58} \mathrm{Cu}$ deduced levels, $\beta$-feeding intensities. ${ }^{81 m} \mathrm{Kr}(\mathrm{EC}),(\mathrm{IT}) ;{ }^{81} \mathrm{Y},{ }^{81} \mathrm{Sr},{ }^{85} \mathrm{Nb},{ }^{85} \mathrm{Zr},{ }^{86} \mathrm{Mo},{ }^{86} \mathrm{Nb}(\mathrm{EC})$ [from Ni, $\left.{ }^{54} \mathrm{Fe}\left({ }^{32} \mathrm{~S}, \mathrm{X}\right)\right]$; measured $\mathrm{E} \gamma, \mathrm{I} \gamma, \mathrm{E}(\mathrm{ce}), \mathrm{I}(\mathrm{ce}), \mathrm{T}_{1 / 2} \cdot{ }^{81} \mathrm{Kr},{ }^{85} \mathrm{Zr},{ }^{85} \mathrm{Nb}$ deduced isomeric transitions $\mathrm{T}_{1 / 2}$, ICC. ${ }^{85} \mathrm{Zr},{ }^{86} \mathrm{Nb}$ deduced levels, J, $\pi$, ICC. ${ }^{81} \mathrm{Br}$ deduced neutrino capture rate. Mass-separated sources. JOUR ZAANE 25 s01 129

${ }^{86} \mathrm{Mo} \quad 2005 \mathrm{KA} 39$ RADIOACTIVITY ${ }^{81 m} \mathrm{Kr}$ (EC), (IT); ${ }^{81} \mathrm{Y},{ }^{81} \mathrm{Sr},{ }^{85} \mathrm{Nb},{ }^{85} \mathrm{Zr},{ }^{86} \mathrm{Mo}$, ${ }^{86} \mathrm{Nb}(\mathrm{EC})$ [from $\mathrm{Ni},{ }^{54} \mathrm{Fe}\left({ }^{32} \mathrm{~S}, \mathrm{X}\right)$ ]; measured $\mathrm{E} \gamma, \mathrm{I} \gamma, \mathrm{E}\left(\right.$ ce) $, \mathrm{I}($ ce $), \mathrm{T}_{1 / 2}$. ${ }^{81} \mathrm{Kr},{ }^{85} \mathrm{Zr},{ }^{85} \mathrm{Nb}$ deduced isomeric transitions $\mathrm{T}_{1 / 2}$, ICC. ${ }^{85} \mathrm{Zr},{ }^{86} \mathrm{Nb}$ deduced levels, J, $\pi$, ICC. ${ }^{81} \mathrm{Br}$ deduced neutrino capture rate. Astrophysical implications discussed. JOUR ZAANE 25355

2005KA46 RADIOACTIVITY ${ }^{31} \mathrm{Cl}\left(\beta^{+} \mathrm{p}\right)$ [from $\mathrm{S}(\mathrm{p}, \mathrm{X}), \mathrm{E}=40 \mathrm{MeV}$; measured $\beta$-delayed $\mathrm{E} \gamma, \mathrm{Ep} .{ }^{58} \mathrm{Zn}\left(\beta^{+}\right)$[from $\mathrm{Nb}(\mathrm{p}, \mathrm{X}), \mathrm{E}=1.4 \mathrm{GeV}$ ]; measured $\mathrm{E} \gamma, \mathrm{I} \gamma, \beta \gamma$-coin, $\mathrm{T}_{1 / 2} .{ }^{58} \mathrm{Cu}$ deduced levels, $\beta$-feeding intensities. ${ }^{81 m} \mathrm{Kr}$ (EC), (IT); ${ }^{81} \mathrm{Y},{ }^{81} \mathrm{Sr},{ }^{85} \mathrm{Nb},{ }^{85} \mathrm{Zr},{ }^{86} \mathrm{Mo},{ }^{86} \mathrm{Nb}(\mathrm{EC})$ [from Ni, $\left.{ }^{54} \mathrm{Fe}\left({ }^{32} \mathrm{~S}, \mathrm{X}\right)\right]$; measured $\mathrm{E} \gamma, \mathrm{I} \gamma, \mathrm{E}(\mathrm{ce}), \mathrm{I}(\mathrm{ce}), \mathrm{T}_{1 / 2} \cdot{ }^{81} \mathrm{Kr},{ }^{85} \mathrm{Zr},{ }^{85} \mathrm{Nb}$ deduced isomeric transitions $\mathrm{T}_{1 / 2}$, ICC. ${ }^{85} \mathrm{Zr},{ }^{86} \mathrm{Nb}$ deduced levels, $\mathrm{J}$, $\pi$, ICC. ${ }^{81} \mathrm{Br}$ deduced neutrino capture rate. Mass-separated sources. JOUR ZAANE 25 s01 129

\section{$\mathrm{A}=87$}

$\begin{array}{ll}{ }^{87} \mathrm{Kr} & \\ & 2005 \mathrm{MUZY} \\ { }^{87} \mathrm{Rb} & 2005 \mathrm{~F} 005\end{array}$

NUCLEAR REACTIONS ${ }^{84} \mathrm{Kr}(\mathrm{n}, \mathrm{X}), \mathrm{E}=0-400 \mathrm{keV}$; measured total $\sigma$. ${ }^{82,84,86} \mathrm{Kr}(\mathrm{n}, \gamma), \mathrm{E}=0-400 \mathrm{keV} ;{ }^{80,83} \mathrm{Kr}(\mathrm{n}, \gamma), \mathrm{E}=0-5 \mathrm{keV}$; measured capture $\sigma .{ }^{80,82,83,84,86} \mathrm{Kr}(\mathrm{n}, \gamma), \mathrm{E}=5-100 \mathrm{keV}$; deduced Maxwellian-averaged $\sigma$. Astrophysical implications discussed. CONF Santa Fe (Nucl Data for Sci and Technol) Proc,Vol2,P1327 NUCLEAR REACTIONS ${ }^{173} \mathrm{Yb}\left({ }^{24} \mathrm{Mg}, \mathrm{X}\right), \mathrm{E}=134.5 \mathrm{MeV} ;{ }^{176} \mathrm{Yb}\left({ }^{23} \mathrm{Na}\right.$, $\mathrm{X}), \mathrm{E}=129 \mathrm{MeV} ;{ }^{208} \mathrm{~Pb}\left({ }^{18} \mathrm{O}, \mathrm{X}\right), \mathrm{E}=91 \mathrm{MeV}$; measured $\mathrm{E} \gamma, \mathrm{I} \gamma, \gamma \gamma$-coin following compound nucleus fission. ${ }^{85} \mathrm{Br},{ }^{87} \mathrm{Rb}$ deduced high-spin levels, $\mathrm{J}, \pi$, configurations. Comparison with shell model predictions. JOUR PRVCA 71064312

2005KR15 NUCLEAR MOMENTS ${ }^{85,87} \mathrm{Rb}$; measured excited-state hfs. Electromagnetically induced transparency. JOUR EULEE 72221 2005LU07 NUCLEAR REACTIONS ${ }^{238} \mathrm{U}\left({ }^{82} \mathrm{Se}, \mathrm{X}\right), \mathrm{E}=505 \mathrm{MeV}$; measured E $\gamma$, $\mathrm{I} \gamma$, fragments isotopic yields. $77,78,79,80,81,82,83 \mathrm{As}$ deduced transitions. ${ }^{192} \mathrm{Os}\left({ }^{82} \mathrm{Se}, \mathrm{X}\right), \mathrm{E}=460 \mathrm{MeV}$; measured $\mathrm{E} \gamma, \mathrm{I} \gamma, \gamma \gamma$-coin. ${ }^{80} \mathrm{As},{ }^{87} \mathrm{Rb}$, ${ }^{84}$ Se deduced levels. Fragment separator. JOUR APOBB 361301

${ }^{87} \mathrm{Sr} \quad$ 2005SEZX
NUCLEAR REACTIONS ${ }^{90,94} \operatorname{Zr}(\mathrm{n}, \alpha),{ }^{90,91,92,94} \mathrm{Zr}(\mathrm{n}, \mathrm{p}),{ }^{91,92} \mathrm{Zr}(\mathrm{n}$, $\mathrm{np}+\mathrm{d}), \mathrm{E}=14-20 \mathrm{MeV}$; measured activation $\sigma$. Comparison with model predictions. CONF Santa Fe (Nucl Data for Sci and Technol) Proc, Vol1,P981

${ }^{87} \mathrm{Y}$ 2005UD02 NUCLEAR REACTIONS ${ }^{89} \mathrm{Y}(\mathrm{p}, \mathrm{X}){ }^{89} \mathrm{Zr} /{ }^{88} \mathrm{Zr} /{ }^{86} \mathrm{Zr} /{ }^{88} \mathrm{Y} /{ }^{87} \mathrm{Y} /$ ${ }^{87 m} \mathrm{Y} /{ }^{86} \mathrm{Y} /{ }^{85} \mathrm{Sr} /{ }^{83} \mathrm{Sr} /{ }^{82} \mathrm{Sr} /{ }^{84} \mathrm{Rb} /{ }^{83} \mathrm{Rb}, \mathrm{E}=15-80 \mathrm{MeV}$; measured excitation functions; deduced integral yields. Stacked-foil activation technique. JOUR ARISE 63367 


\section{$\mathrm{A}=87$ (continued)}

${ }^{87} \mathrm{Zr} \quad 2005 \mathrm{BI} 25$

2005DU23

${ }^{87} \mathrm{Nb}$

2005DU23

${ }^{88} \mathrm{Kr}$

2005ADZZ

${ }^{88} \mathrm{Sr}$

2004KA62

$2005 \mathrm{GA} 44$

${ }^{88} \mathrm{Y}$

NUCLEAR MOMENTS 86,87,88,89,90,91,92,93,94,95,96,97,98,99,100,101,102 Zr; measured charge radii. ${ }^{176} \mathrm{Yb}$; measured isomer shift. Ion-beam cooler, laser spectroscopy. JOUR ZAANE 25 s01 187

NUCLEAR REACTIONS Ge $\left({ }^{18} \mathrm{O}, \mathrm{X}\right)^{83 m} \mathrm{Sr} /{ }^{83} \mathrm{Y} /{ }^{84 m} \mathrm{Y} /{ }^{88 m} \mathrm{Y} /$ ${ }^{85} \mathrm{Zr} /{ }^{87} \mathrm{Zr}, \mathrm{E}=82.8 \mathrm{GeV} ;{ }^{84} \mathrm{Se}\left({ }^{18} \mathrm{O}, \mathrm{X}\right)^{86 m} \mathrm{Y} /{ }^{85} \mathrm{Zr} /{ }^{87} \mathrm{Nb} /{ }^{87 m} \mathrm{Nb} /$ ${ }^{88} \mathrm{Nb} /{ }^{88} \mathrm{Mo}, \mathrm{E}=82.7 \mathrm{MeV} ;{ }^{124} \mathrm{Sn}\left({ }^{50} \mathrm{Ti}, \mathrm{X}\right){ }^{168 m} \mathrm{Lu} /{ }^{167} \mathrm{Hf} /{ }^{168} \mathrm{Hf}$, $\mathrm{E}=223.7 \mathrm{MeV} ;{ }^{116} \mathrm{Sn}\left({ }^{50} \mathrm{Ti}, \mathrm{X}\right){ }^{162} \mathrm{Tm} /{ }^{161} \mathrm{Yb} /{ }^{162} \mathrm{Yb} /{ }^{163} \mathrm{Yb} /{ }^{162} \mathrm{Lu} /$ ${ }^{162} \mathrm{Hf}, \mathrm{E}=224.4 \mathrm{MeV}$; measured delayed $\mathrm{E} \gamma$, I $\gamma$ following residual nucleus decay. Physical preseparation technique. JOUR NIMAE 551 528

NUCLEAR REACTIONS Ge $\left({ }^{18} \mathrm{O}, \mathrm{X}\right){ }^{83 m} \mathrm{Sr} /{ }^{83} \mathrm{Y} /{ }^{84 m} \mathrm{Y} /{ }^{88 m} \mathrm{Y} /$ ${ }^{85} \mathrm{Zr} /{ }^{87} \mathrm{Zr}, \mathrm{E}=82.8 \mathrm{GeV} ;{ }^{84} \mathrm{Se}\left({ }^{18} \mathrm{O}, \mathrm{X}\right){ }^{86 m} \mathrm{Y} /{ }^{85} \mathrm{Zr} /{ }^{87} \mathrm{Nb} /{ }^{87 m} \mathrm{Nb} /$ ${ }^{88} \mathrm{Nb} /{ }^{88} \mathrm{Mo}, \mathrm{E}=82.7 \mathrm{MeV} ;{ }^{124} \mathrm{Sn}\left({ }^{50} \mathrm{Ti}, \mathrm{X}\right){ }^{168 m} \mathrm{Lu} /{ }^{167} \mathrm{Hf} /{ }^{168} \mathrm{Hf}$, $\mathrm{E}=223.7 \mathrm{MeV} ;{ }^{116} \mathrm{Sn}\left({ }^{50} \mathrm{Ti}, \mathrm{X}\right){ }^{162} \mathrm{Tm} /{ }^{161} \mathrm{Yb} /{ }^{162} \mathrm{Yb} /{ }^{163} \mathrm{Yb} /{ }^{162} \mathrm{Lu} /$ ${ }^{162} \mathrm{Hf}, \mathrm{E}=224.4 \mathrm{MeV}$; measured delayed $\mathrm{E} \gamma, \mathrm{I} \gamma$ following residual nucleus decay. Physical preseparation technique. JOUR NIMAE 551 528

\section{$\mathrm{A}=88$}

NUCLEAR REACTIONS ${ }^{129} \mathrm{I}(\mathrm{n}, 7 \mathrm{n}),(\mathrm{n}, 6 \mathrm{n}),(\mathrm{n}, 4 \mathrm{n}),(\mathrm{n}, \gamma), \mathrm{E}=$ fast; ${ }^{237} \mathrm{~Np}(\mathrm{n}, \gamma), \mathrm{E}=$ fast; measured yields. ${ }^{237} \mathrm{~Np}(\mathrm{n}, \mathrm{F}){ }^{91} \mathrm{Sr} /{ }^{97} \mathrm{Zr} /{ }^{132} \mathrm{Te} /$ ${ }^{133} \mathrm{I} /{ }^{135} \mathrm{I}, \mathrm{E}=$ fast; ${ }^{238} \mathrm{Pu}(\mathrm{n}, \mathrm{F}){ }^{97} \mathrm{Zr} /{ }^{129} \mathrm{Sb} /{ }^{132} \mathrm{I} /{ }^{133} \mathrm{I} /{ }^{135} \mathrm{Xe} /$ ${ }^{105} \mathrm{Ru}, \mathrm{E}=$ fast; ${ }^{239} \mathrm{Pu}(\mathrm{n}, \mathrm{F}){ }^{88} \mathrm{Kr} /{ }^{91} \mathrm{Sr} /{ }^{92} \mathrm{Sr} /{ }^{92} \mathrm{Y} /{ }^{97} \mathrm{Zr} /{ }^{99} \mathrm{Mo} /$ ${ }^{103} \mathrm{Ru} /{ }^{105} \mathrm{Ru} /{ }^{128} \mathrm{Sb} /{ }^{129} \mathrm{Sb} /{ }^{132} \mathrm{Te} /{ }^{131} \mathrm{I} /{ }^{132} \mathrm{I} /{ }^{133} \mathrm{I} /{ }^{135} \mathrm{I} /{ }^{135} \mathrm{Xe}$ $/{ }^{143} \mathrm{Ce} /{ }^{140} \mathrm{Ba} /{ }^{140} \mathrm{La}, \mathrm{E}=$ fast; measured fission fragment yields. Secondary neutrons from proton irradiation. JINR nuclotron. CONF St Petersburg,P195,Adam NUCLEAR REACTIONS ${ }^{88} \mathrm{Sr}\left(\gamma, \gamma^{\prime}\right), \mathrm{E}=6.8 \mathrm{MeV}$ bremsstrahlung; measured $\mathrm{E} \gamma, \mathrm{I} \gamma \cdot{ }^{88} \mathrm{Sr}$ deduced levels, J, $\pi$, configurations, $\mathrm{B}(\mathrm{E} 1)$, $\mathrm{B}(\mathrm{M} 1), \mathrm{B}(\mathrm{E} 2)$. Comparison with model predictions. JOUR PRVCA 70 064307

NUCLEAR REACTIONS ${ }^{208} \mathrm{~Pb}\left({ }^{90} \mathrm{Zr}, \mathrm{X}\right){ }^{90} \mathrm{Zr} /{ }^{92} \mathrm{Zr} /{ }^{88} \mathrm{Sr}, \mathrm{E}=560$ $\mathrm{MeV} ;{ }^{238} \mathrm{U}\left({ }^{64} \mathrm{Ni}, \mathrm{X}\right){ }^{58} \mathrm{Cr}, \mathrm{E}=400 \mathrm{MeV}$; measured $\mathrm{E} \gamma, \mathrm{I} \gamma, \gamma \gamma-$, (recoil) $\gamma$-coin. ${ }^{58} \mathrm{Cr},{ }^{90,92} \mathrm{Zr},{ }^{88} \mathrm{Sr}$ deduced transitions. Clara array, mass separator. JOUR JPGPE 31 S1443

2005SI34 ATOMIC MASSES $76,77,80,81,86,88 \mathrm{Sr},{ }^{124,129,130,131,132} \mathrm{Sn}$; measured masses. Penning trap mass spectrometer, comparison with previous results. JOUR NUPAB 76345

2005DU23 NUCLEAR REACTIONS Ge $\left({ }^{18} \mathrm{O}, \mathrm{X}\right)^{83 m} \mathrm{Sr} /{ }^{83} \mathrm{Y} /{ }^{84 m} \mathrm{Y} /{ }^{88 m} \mathrm{Y} /$ ${ }^{85} \mathrm{Zr} /{ }^{87} \mathrm{Zr}, \mathrm{E}=82.8 \mathrm{GeV} ;{ }^{84} \mathrm{Se}\left({ }^{18} \mathrm{O}, \mathrm{X}\right){ }^{86 m} \mathrm{Y} /{ }^{85} \mathrm{Zr} /{ }^{87} \mathrm{Nb} /{ }^{87 m} \mathrm{Nb} /$ ${ }^{88} \mathrm{Nb} /{ }^{88} \mathrm{Mo}, \mathrm{E}=82.7 \mathrm{MeV} ;{ }^{124} \mathrm{Sn}\left({ }^{50} \mathrm{Ti}, \mathrm{X}\right){ }^{168 m} \mathrm{Lu} /{ }^{167} \mathrm{Hf} /{ }^{168} \mathrm{Hf}$, $\mathrm{E}=223.7 \mathrm{MeV} ;{ }^{116} \mathrm{Sn}\left({ }^{50} \mathrm{Ti}, \mathrm{X}\right){ }^{162} \mathrm{Tm} /{ }^{161} \mathrm{Yb} /{ }^{162} \mathrm{Yb} /{ }^{163} \mathrm{Yb} /{ }^{162} \mathrm{Lu} /$ ${ }^{162} \mathrm{Hf}, \mathrm{E}=224.4 \mathrm{MeV}$; measured delayed $\mathrm{E} \gamma$, I $\gamma$ following residual nucleus decay. Physical preseparation technique. JOUR NIMAE 551 528 


\section{$\mathrm{A}=88$ (continued)}

2005TAZT NUCLEAR REACTIONS Mo, Nb, Zr, Y $(\mathrm{p}, \mathrm{X})^{88} \mathrm{Zr} /{ }^{88} \mathrm{Y}, \mathrm{E} \approx 20-80$ $\mathrm{MeV} ; \mathrm{Mo}, \mathrm{Zr}, \mathrm{Y}(\mathrm{d}, \mathrm{X})^{88} \mathrm{Zr} /{ }^{88} \mathrm{Y}, \mathrm{E} \approx 5-50 \mathrm{MeV}$; measured excitation functions; deduced thick-target yields. Comparison with previous results. Stacked-foil activation technique. CONF Santa Fe (Nucl Data for Sci and Technol) Proc,Vol2,P1658

2005UD02 NUCLEAR REACTIONS ${ }^{89} \mathrm{Y}(\mathrm{p}, \mathrm{X}){ }^{89} \mathrm{Zr} /{ }^{88} \mathrm{Zr} /{ }^{86} \mathrm{Zr} /{ }^{88} \mathrm{Y} /{ }^{87} \mathrm{Y} /$ ${ }^{87 m} \mathrm{Y} /{ }^{86} \mathrm{Y} /{ }^{85} \mathrm{Sr} /{ }^{83} \mathrm{Sr} /{ }^{82} \mathrm{Sr} /{ }^{84} \mathrm{Rb} /{ }^{83} \mathrm{Rb}, \mathrm{E}=15-80 \mathrm{MeV}$;

measured excitation functions; deduced integral yields. Stacked-foil activation technique. JOUR ARISE 63367

${ }^{88} \mathrm{Zr} \quad 2005 \mathrm{BI} 25$ NUCLEAR MOMENTS 86,87,88,89,90,91,92,93,94,95,96,97,98,99,100,101,102 Zr; measured charge radii. ${ }^{176} \mathrm{Yb}$; measured isomer shift. Ion-beam cooler, laser spectroscopy. JOUR ZAANE 25 s01 187

2005BRZU NUCLEAR REACTIONS Ti(p, X $)^{44} \mathrm{Ti}, \mathrm{E}=21-29 \mathrm{MeV} ; \mathrm{Ni}(\mathrm{p}, \mathrm{X})^{56} \mathrm{Ni}$, $\mathrm{E}=18-28 \mathrm{MeV} ; \mathrm{Zr}(\mathrm{p}, \mathrm{X})^{88} \mathrm{Zr}, \mathrm{E}=19-28 \mathrm{MeV}$; measured production $\sigma$. Activation technique, comparison with previous results. CONF Santa Fe (Nucl Data for Sci and Technol) Proc,Vol2,P1374

2005TAZT NUCLEAR REACTIONS Mo, Nb, Zr, Y $(\mathrm{p}, \mathrm{X})^{88} \mathrm{Zr} /{ }^{88} \mathrm{Y}, \mathrm{E} \approx 20-80$ $\mathrm{MeV} ; \mathrm{Mo}, \mathrm{Zr}, \mathrm{Y}(\mathrm{d}, \mathrm{X})^{88} \mathrm{Zr} /{ }^{88} \mathrm{Y}, \mathrm{E} \approx 5-50 \mathrm{MeV}$; measured excitation functions; deduced thick-target yields. Comparison with previous results. Stacked-foil activation technique. CONF Santa Fe (Nucl Data for Sci and Technol) Proc,Vol2,P1658

2005UD02 NUCLEAR REACTIONS ${ }^{89} \mathrm{Y}(\mathrm{p}, \mathrm{X})^{89} \mathrm{Zr} /{ }^{88} \mathrm{Zr} /{ }^{86} \mathrm{Zr} /{ }^{88} \mathrm{Y} /{ }^{87} \mathrm{Y} /$ ${ }^{87 m} \mathrm{Y} /{ }^{86} \mathrm{Y} /{ }^{85} \mathrm{Sr} /{ }^{83} \mathrm{Sr} /{ }^{82} \mathrm{Sr} /{ }^{84} \mathrm{Rb} /{ }^{83} \mathrm{Rb}, \mathrm{E}=15-80 \mathrm{MeV}$; measured excitation functions; deduced integral yields. Stacked-foil activation technique. JOUR ARISE 63367

2005WA31 NUCLEAR REACTIONS ${ }^{92,98,100} \mathrm{Mo}\left(\gamma, \gamma^{\prime}\right), \mathrm{E}=13.2 \mathrm{MeV}$ bremsstrahlung; measured $\mathrm{E} \gamma, \mathrm{I} \gamma .{ }^{92,100} \mathrm{Mo},{ }^{197} \mathrm{Au}(\gamma, \mathrm{n}),{ }^{92} \mathrm{Mo}(\gamma, \mathrm{p})$, $(\gamma, \alpha), \mathrm{E} \approx 11.8-16.5 \mathrm{MeV}$ bremsstrahlung; measured integrated $\sigma$. JOUR JPGPE 31 S1969

${ }^{88} \mathrm{Nb} \quad 2005 \mathrm{DU} 23$ NUCLEAR REACTIONS Ge $\left({ }^{18} \mathrm{O}, \mathrm{X}\right)^{83 m} \mathrm{Sr} /{ }^{83} \mathrm{Y} /{ }^{84 m} \mathrm{Y} /{ }^{88 m} \mathrm{Y} /$ ${ }^{85} \mathrm{Zr} /{ }^{87} \mathrm{Zr}, \mathrm{E}=82.8 \mathrm{GeV} ;{ }^{84} \mathrm{Se}\left({ }^{18} \mathrm{O}, \mathrm{X}\right){ }^{86 m} \mathrm{Y} /{ }^{85} \mathrm{Zr} /{ }^{87} \mathrm{Nb} /{ }^{87 m} \mathrm{Nb} /$ ${ }^{88} \mathrm{Nb} /{ }^{88} \mathrm{Mo}, \mathrm{E}=82.7 \mathrm{MeV} ;{ }^{124} \mathrm{Sn}\left({ }^{50} \mathrm{Ti}, \mathrm{X}\right){ }^{168 m} \mathrm{Lu} /{ }^{167} \mathrm{Hf} /{ }^{168} \mathrm{Hf}$, $\mathrm{E}=223.7 \mathrm{MeV} ;{ }^{116} \mathrm{Sn}\left({ }^{50} \mathrm{Ti}, \mathrm{X}\right){ }^{162} \mathrm{Tm} /{ }^{161} \mathrm{Yb} /{ }^{162} \mathrm{Yb} /{ }^{163} \mathrm{Yb} /{ }^{162} \mathrm{Lu} /$ ${ }^{162} \mathrm{Hf}, \mathrm{E}=224.4 \mathrm{MeV}$; measured delayed $\mathrm{E} \gamma$, I $\gamma$ following residual nucleus decay. Physical preseparation technique. JOUR NIMAE 551 528

${ }^{88} \mathrm{Mo}$ 2005DU23 NUCLEAR REACTIONS Ge( $\left({ }^{18} \mathrm{O}, \mathrm{X}\right){ }^{83 m} \mathrm{Sr} /{ }^{83} \mathrm{Y} /{ }^{84 m} \mathrm{Y} /{ }^{88 m} \mathrm{Y} /$ ${ }^{85} \mathrm{Zr} /{ }^{87} \mathrm{Zr}, \mathrm{E}=82.8 \mathrm{GeV} ;{ }^{84} \mathrm{Se}\left({ }^{18} \mathrm{O}, \mathrm{X}\right){ }^{86 m} \mathrm{Y} /{ }^{85} \mathrm{Zr} /{ }^{87} \mathrm{Nb} /{ }^{87 m} \mathrm{Nb} /$ ${ }^{88} \mathrm{Nb} /{ }^{88} \mathrm{Mo}, \mathrm{E}=82.7 \mathrm{MeV} ;{ }^{124} \mathrm{Sn}\left({ }^{50} \mathrm{Ti}, \mathrm{X}\right){ }^{168 m} \mathrm{Lu} /{ }^{167} \mathrm{Hf} /{ }^{168} \mathrm{Hf}$, $\mathrm{E}=223.7 \mathrm{MeV} ;{ }^{116} \mathrm{Sn}\left({ }^{50} \mathrm{Ti}, \mathrm{X}\right){ }^{162} \mathrm{Tm} /{ }^{161} \mathrm{Yb} /{ }^{162} \mathrm{Yb} /{ }^{163} \mathrm{Yb} /{ }^{162} \mathrm{Lu} /$ ${ }^{162} \mathrm{Hf}, \mathrm{E}=224.4 \mathrm{MeV}$; measured delayed $\mathrm{E} \gamma$, I $\gamma$ following residual nucleus decay. Physical preseparation technique. JOUR NIMAE 551 528

2005XU04 RADIOACTIVITY ${ }^{81} \mathrm{Zr},{ }^{85} \mathrm{Mo},{ }^{89} \mathrm{Ru},{ }^{92} \mathrm{Rh},{ }^{93} \mathrm{Pd},{ }^{121} \mathrm{Ce},{ }^{125} \mathrm{Nd}$, ${ }^{128} \mathrm{Pm},{ }^{129} \mathrm{Sm},{ }^{135,137} \mathrm{Gd},{ }^{139} \mathrm{Dy},{ }^{142} \mathrm{Ho},{ }^{149} \mathrm{Yb}\left(\beta^{+} \mathrm{p}\right)$; measured $\beta$-delayed $\mathrm{E} \gamma, \mathrm{I} \gamma$, proton spectra, $\mathrm{p} \gamma$-coin, $\mathrm{T}_{1 / 2}$. Comparison with model predictions. JOUR PRVCA 71054318 


\section{$\mathrm{A}=89$}

${ }^{89} \mathrm{Kr}$

2004GA60

$2005 G A 25$

${ }^{89} \mathrm{Rb}$

${ }^{89} \mathrm{Sr}$

2004SP06

2005KEZZ

${ }^{89} \mathrm{Y}$

2005BI25

2005RE09
NUCLEAR REACTIONS ${ }^{237} \mathrm{~Np}(\gamma, \mathrm{F}){ }^{135} \mathrm{Xe} /{ }^{137} \mathrm{Xe} /{ }^{138} \mathrm{Xe} /{ }^{139} \mathrm{Xe} /$ ${ }^{140} \mathrm{Xe} /{ }^{141} \mathrm{Xe} /{ }^{142} \mathrm{Xe} /{ }^{89} \mathrm{Kr} /{ }^{91} \mathrm{Kr} /{ }^{92} \mathrm{Kr} /{ }^{93} \mathrm{Kr}, \mathrm{E}=25 \mathrm{MeV}$ bremsstrahlung; measured fission yields, isotopic distribution parameters. Comparison with results from other targets. JOUR BRSPE 681298

2004GAZV NUCLEAR REACTIONS ${ }^{237} \mathrm{~Np},{ }^{243} \mathrm{Am}(\gamma, \mathrm{F}){ }^{89} \mathrm{Kr} /{ }^{91} \mathrm{Kr} /{ }^{92} \mathrm{Kr} /$ ${ }^{93} \mathrm{Kr} /{ }^{135} \mathrm{Xe} /{ }^{137} \mathrm{Xe} /{ }^{138} \mathrm{Xe} /{ }^{139} \mathrm{Xe} /{ }^{140} \mathrm{Xe} /{ }^{141} \mathrm{Xe} /{ }^{142} \mathrm{Xe}$, $\mathrm{E}_{\max }=25 \mathrm{MeV}$; measured $\mathrm{E} \gamma$, I $\gamma$; deduced fission fragment yields. REPT JINR-P15-2004-119, Gangrsky NUCLEAR REACTIONS ${ }^{248} \mathrm{Cm}(\gamma, \mathrm{F}){ }^{89} \mathrm{Kr} /{ }^{91} \mathrm{Kr} /{ }^{92} \mathrm{Kr} /{ }^{93} \mathrm{Kr} /$ ${ }^{135} \mathrm{Xe} /{ }^{137} \mathrm{Xe} /{ }^{138} \mathrm{Xe} /{ }^{139} \mathrm{Xe} /{ }^{140} \mathrm{Xe} /{ }^{141} \mathrm{Xe} /{ }^{142} \mathrm{Xe}, \mathrm{E}=25 \mathrm{MeV}$ bremsstrahlung; measured $\mathrm{E} \gamma, \mathrm{I} \gamma$; deduced yields. JOUR FECLA 125 44 NUCLEAR REACTIONS ${ }^{237} \mathrm{~Np},{ }^{243} \mathrm{Am}(\gamma, \mathrm{F}){ }^{135} \mathrm{Xe} /{ }^{137} \mathrm{Xe} /{ }^{138} \mathrm{Xe} /$ ${ }^{139} \mathrm{Xe} /{ }^{140} \mathrm{Xe} /{ }^{141} \mathrm{Xe} /{ }^{142} \mathrm{Xe} /{ }^{89} \mathrm{Kr} /{ }^{91} \mathrm{Kr} /{ }^{92} \mathrm{Kr} /{ }^{93} \mathrm{Kr}, \mathrm{E}=25 \mathrm{MeV}$ bremsstrahlung; measured fission yields, isotopic distribution parameters. JOUR YAFIA 681475 NUCLEAR REACTIONS ${ }^{238} \mathrm{U}(\mathrm{p}, \mathrm{F}){ }^{89} \mathrm{Rb} /{ }^{90} \mathrm{Rb} /{ }^{91} \mathrm{Rb} /{ }^{93} \mathrm{Rb} /$ ${ }^{94} \mathrm{Rb} /{ }^{95} \mathrm{Rb} /{ }^{139} \mathrm{Cs} /{ }^{140} \mathrm{Cs} /{ }^{141} \mathrm{Cs} /{ }^{142} \mathrm{Cs} /{ }^{144} \mathrm{Cs} /{ }^{145} \mathrm{Cs}, \mathrm{E}=1$ $\mathrm{GeV}$; measured yields. JOUR ZAANE 23257

NUCLEAR REACTIONS ${ }^{64,67} \mathrm{Zn},{ }^{89} \mathrm{Y}(\mathrm{n}, \mathrm{p}), \mathrm{E}=14 \mathrm{MeV}$; measured $\sigma$. Comparison with results using fission neutrons. JOUR RAACA 92183 NUCLEAR REACTIONS Ti(p, X) ${ }^{45} \mathrm{Ca}, \mathrm{E}=30-200 \mathrm{MeV} ;{ }^{85} \mathrm{Rb}(\mathrm{p}, 4 \mathrm{n})$, $\mathrm{E}=35-70 \mathrm{MeV}$; measured excitation functions. ${ }^{89} \mathrm{Y}(\mathrm{n}, \mathrm{p}), \mathrm{E}=$ fast; measured spectrum-averaged $\sigma$. Activation technique, radiochemical separation, x-ray spectrometry. CONF Santa Fe (Nucl Data for Sci and Technol) Proc,Vol1,P758

2005KIZV NUCLEAR REACTIONS ${ }^{89} \mathrm{Y}(\alpha, \alpha), \mathrm{E}=16.165 \mathrm{MeV}$; measured $\sigma(\theta)$. REPT ATOMKI 2004 Annual,P14,Kiss

2005WAZS NUCLEAR REACTIONS ${ }^{82} \mathrm{Se}\left({ }^{17} \mathrm{~N}, \mathrm{X}\right), \mathrm{E}=104 \mathrm{MeV}$; measured prompt and delayed $\mathrm{E} \gamma, \mathrm{I} \gamma, \gamma \gamma$-coin. ${ }^{89,90} \mathrm{Y},{ }^{93} \mathrm{Nb}$ deduced transitions, possible high-spin isomers. REPT CNS-REP-66,P15,Wakabayashi NUCLEAR MOMENTS 86,87,88,89,90,91,92,93,94,95,96,97,98,99,100,101,102 Zr; measured charge radii. ${ }^{176} \mathrm{Yb}$; measured isomer shift. Ion-beam cooler, laser spectroscopy. JOUR ZAANE 25 s01 187

NUCLEAR REACTIONS ${ }^{92,94} \mathrm{Mo}(\mathrm{n}, 2 \mathrm{n}),{ }^{92,100} \mathrm{Mo}(\mathrm{n}, \alpha),{ }^{95,96,97} \mathrm{Mo}(\mathrm{n}$, p), ${ }^{96,97,98} \mathrm{Mo}(\mathrm{n}, \mathrm{np}+\mathrm{d}), \mathrm{E} \approx 13.5-21 \mathrm{MeV}$; measured activation $\sigma$; deduced reaction mechanism features. ${ }^{93} \mathrm{Nb}(\mathrm{p}, \mathrm{n}),(\mathrm{p}, \gamma), \mathrm{E} \approx 1-6$ $\mathrm{MeV} ;{ }^{92,93,94,95,96,97,98,100} \mathrm{Mo},{ }^{93} \mathrm{Nb}(\mathrm{n}, \gamma), \mathrm{E}<4 \mathrm{MeV} ;{ }^{92,94,100} \mathrm{Mo}(\mathrm{n}$, 2n), ${ }^{92,94,95,96,97,98} \mathrm{Mo}(\mathrm{n}, \mathrm{p}),{ }^{92,94,95,96,97,98,100} \mathrm{Mo}(\mathrm{n}, \mathrm{np}+\mathrm{d})$, ${ }_{92,98,100} \mathrm{Mo}(\mathrm{n}, \alpha), \mathrm{E}<21 \mathrm{MeV}$; compiled, analyzed $\sigma$. Analysis with local and global approaches compared. JOUR PRVCA 71044617 NUCLEAR REACTIONS ${ }^{89} \mathrm{Y}(\mathrm{p}, \mathrm{X})^{89} \mathrm{Zr} /{ }^{88} \mathrm{Zr} /{ }^{86} \mathrm{Zr} /{ }^{88} \mathrm{Y} /{ }^{87} \mathrm{Y} /$ ${ }^{87 m} \mathrm{Y} /{ }^{86} \mathrm{Y} /{ }^{85} \mathrm{Sr} /{ }^{83} \mathrm{Sr} /{ }^{82} \mathrm{Sr} /{ }^{84} \mathrm{Rb} /{ }^{83} \mathrm{Rb}, \mathrm{E}=15-80 \mathrm{MeV}$; measured excitation functions; deduced integral yields. Stacked-foil activation technique. JOUR ARISE 63367 


\section{$\mathrm{A}=89$ (continued)}

${ }^{89} \mathrm{Ru}$

2005XU04

${ }^{90} \mathrm{Rb}$

${ }^{90} \mathrm{Y}$

2005SEZX

2005WAZS

2005YA11

${ }^{90} \mathrm{Zr}$

2005BI25

2005CH53

$2005 \mathrm{CO} 25$

2005GA44

2005HU10

2005VA31

${ }^{90} \mathrm{Nb}$
RADIOACTIVITY ${ }^{81} \mathrm{Zr},{ }^{85} \mathrm{Mo},{ }^{89} \mathrm{Ru},{ }^{92} \mathrm{Rh},{ }^{93} \mathrm{Pd},{ }^{121} \mathrm{Ce},{ }^{125} \mathrm{Nd}$, ${ }^{128} \mathrm{Pm},{ }^{129} \mathrm{Sm},{ }^{135,137} \mathrm{Gd},{ }^{139} \mathrm{Dy},{ }^{142} \mathrm{Ho},{ }^{149} \mathrm{Yb}\left(\beta^{+} \mathrm{p}\right)$; measured $\beta$-delayed $\mathrm{E} \gamma, \mathrm{I} \gamma$, proton spectra, $\mathrm{p} \gamma$-coin, $\mathrm{T}_{1 / 2}$. Comparison with model predictions. JOUR PRVCA 71054318

\section{$\mathrm{A}=\mathbf{9 0}$}

NUCLEAR REACTIONS ${ }^{238} \mathrm{U}(\mathrm{p}, \mathrm{F}){ }^{89} \mathrm{Rb} /{ }^{90} \mathrm{Rb} /{ }^{91} \mathrm{Rb} /{ }^{93} \mathrm{Rb} /$ ${ }^{94} \mathrm{Rb} /{ }^{95} \mathrm{Rb} /{ }^{139} \mathrm{Cs} /{ }^{140} \mathrm{Cs} /{ }^{141} \mathrm{Cs} /{ }^{142} \mathrm{Cs} /{ }^{144} \mathrm{Cs} /{ }^{145} \mathrm{Cs}, \mathrm{E}=1$ $\mathrm{GeV}$; measured yields. JOUR ZAANE 23257

NUCLEAR REACTIONS ${ }^{90,94} \mathrm{Zr}(\mathrm{n}, \alpha),{ }^{90,91,92,94} \mathrm{Zr}(\mathrm{n}, \mathrm{p}),{ }^{91,92} \mathrm{Zr}(\mathrm{n}$, $\mathrm{np}+\mathrm{d}), \mathrm{E}=14-20 \mathrm{MeV}$; measured activation $\sigma$. Comparison with model predictions. CONF Santa Fe (Nucl Data for Sci and Technol) Proc,Vol1,P981 NUCLEAR REACTIONS ${ }^{82} \mathrm{Se}\left({ }^{17} \mathrm{~N}, \mathrm{X}\right), \mathrm{E}=104 \mathrm{MeV}$; measured prompt and delayed $\mathrm{E} \gamma, \mathrm{I} \gamma, \gamma \gamma$-coin. ${ }^{89,90} \mathrm{Y},{ }^{93} \mathrm{Nb}$ deduced transitions, possible high-spin isomers. REPT CNS-REP-66,P15,Wakabayashi NUCLEAR REACTIONS ${ }^{90} \mathrm{Zr}(\mathrm{n}, \mathrm{p}), \mathrm{E}=293 \mathrm{MeV}$; measured $\sigma(\mathrm{E}, \theta)$; ${ }^{90} \mathrm{Zr}(\mathrm{p}, \mathrm{n}), \mathrm{E}=295 \mathrm{MeV}$; analyzed $\sigma(\mathrm{E}, \theta)$; deduced Gamow-Teller strengths, quenching factor. JOUR PYLBB 615193 NUCLEAR MOMENTS 86,87,88,89,90,91,92,93,94,95,96,97,98,99,100,101,102 Zr; measured charge radii. ${ }^{176} \mathrm{Yb}$; measured isomer shift. Ion-beam cooler, laser spectroscopy. JOUR ZAANE 25 s01 187 NUCLEAR REACTIONS ${ }^{92} \operatorname{Zr}\left(\alpha, \alpha^{\prime}\right),\left(\alpha,{ }^{3} \mathrm{HeX}\right),(\alpha, \mathrm{tX}),(\alpha, \mathrm{dX}),(\alpha$, $\mathrm{pX}), \mathrm{E}=51 \mathrm{MeV}$; measured particle spectra. ${ }^{92} \operatorname{Zr}\left(\alpha, \alpha^{\prime}\right),(\alpha, \mathrm{xn} \alpha)$, $\mathrm{E}=51 \mathrm{MeV}$; measured $\mathrm{E} \gamma, \mathrm{I} \gamma, \alpha \gamma$-coin. ${ }^{90,91,92} \mathrm{Zr}$ deduced transitions. Surrogate reaction technique. JOUR NUPAB 758 126c

NUCLEAR REACTIONS ${ }^{208} \mathrm{~Pb}\left({ }^{40} \mathrm{Ca},{ }^{42} \mathrm{Ca}\right), \mathrm{E}=225 \mathrm{MeV}$; measured $\sigma(\mathrm{E}, \theta) .{ }^{42} \mathrm{Ca}$ deduced excited states configurations. ${ }^{208} \mathrm{~Pb}\left({ }^{90} \mathrm{Zr}, \mathrm{X}\right)$, $\mathrm{E}=560 \mathrm{MeV}$; measured $\mathrm{E} \gamma, \mathrm{I} \gamma$, (fragment) $\gamma$-coin, isotopic yields for projectile-like fragments. ${ }^{90} \mathrm{Zr}$ deduced transitions. JOUR ZAANE 25 s01 427

NUCLEAR REACTIONS ${ }^{208} \mathrm{~Pb}\left({ }^{90} \mathrm{Zr}, \mathrm{X}\right){ }^{90} \mathrm{Zr} /{ }^{92} \mathrm{Zr} /{ }^{88} \mathrm{Sr}, \mathrm{E}=560$ $\mathrm{MeV} ;{ }^{238} \mathrm{U}\left({ }^{64} \mathrm{Ni}, \mathrm{X}\right){ }^{58} \mathrm{Cr}, \mathrm{E}=400 \mathrm{MeV}$; measured $\mathrm{E} \gamma, \mathrm{I} \gamma, \gamma \gamma-$, (recoil) $\gamma$-coin. ${ }^{58} \mathrm{Cr},{ }^{90,92} \mathrm{Zr},{ }^{88} \mathrm{Sr}$ deduced transitions. Clara array, mass separator. JOUR JPGPE 31 S1443

NUCLEAR REACTIONS ${ }^{90} \mathrm{Zr},{ }^{116} \mathrm{Sn},{ }^{208} \mathrm{~Pb}(\alpha, \alpha$ 'n), E=200 MeV; ${ }^{208} \mathrm{~Pb}(\alpha, \alpha$ p $), \mathrm{E}=200 \mathrm{MeV}$; measured $\mathrm{E} \alpha, \sigma(\theta), \mathrm{p} \alpha-$, n $\alpha$-coin. ${ }^{90} \mathrm{Zr}$, ${ }^{116} \mathrm{Sn},{ }^{208} \mathrm{~Pb}$ deduced isoscalar GDR parameters, particle decay features. JOUR APOBB 361115

NUCLEAR REACTIONS ${ }^{48} \mathrm{Ti}\left({ }^{132} \mathrm{Sn},{ }^{132} \mathrm{Sn}\right), \mathrm{E}=470-495 \mathrm{MeV}$; ${ }^{90} \mathrm{Zr}\left({ }^{134} \mathrm{Sn},{ }^{134} \mathrm{Sn}\right.$ '), $\mathrm{E}=400 \mathrm{MeV}$; measured $\mathrm{E} \gamma, \mathrm{I} \gamma$, (particle) $\gamma$-coin following projectile Coulomb excitation. ${ }^{132,134} \mathrm{Sn}$ deduced transitions B(E2). JOUR ZAANE 25 s01 391

2004ZH45 NUCLEAR REACTIONS ${ }^{90,94} \mathrm{Zr}$ (p, n), E=7-11 MeV; measured En, $\sigma(\mathrm{E}, \theta)$, excitation functions. ${ }^{90,94} \mathrm{Nb}$ deduced level densities, shell effects. Optical-statistical analysis. JOUR BRSPE 681319 


\section{$\mathrm{A}=90$ (continued)}

2005ALZZ NUCLEAR REACTIONS ${ }^{93} \mathrm{Nb}(\gamma, \mathrm{n}),(\gamma, 3 \mathrm{n}),, \mathrm{E}=50 \mathrm{MeV}$ bremsstrahlung; measured $\mathrm{E} \gamma, \mathrm{I} \gamma$; deduced yield ratio. HPGe detectors, microtron. CONF St Petersburg,P56,Aliev

$2005 \mathrm{CH} 65$ NUCLEAR REACTIONS ${ }^{63} \mathrm{Cu}\left({ }^{31} \mathrm{P}, \mathrm{n} 3 \mathrm{p}\right), \mathrm{E}=120,125 \mathrm{MeV}$; measured prompt and delayed $\mathrm{E} \gamma, \mathrm{I} \gamma, \gamma \gamma-$, (recoil) $\gamma$-coin. ${ }^{90} \mathrm{Nb}$ deduced levels, $\mathrm{J}$, $\pi$, configurations, isomeric states $\mathrm{T}_{1 / 2}, \mathrm{~B}(\mathrm{E} 2)$. Large-basis shell model calculations. JOUR PRVCA 72054309

2005CU07 NUCLEAR REACTIONS ${ }^{76} \mathrm{Ge}\left({ }^{19} \mathrm{~F}, 5 \mathrm{n}\right), \mathrm{E}=80 \mathrm{MeV}$; measured $\mathrm{E} \gamma, \mathrm{I} \gamma$, $\gamma \gamma$-coin. ${ }^{90} \mathrm{Nb}$ deduced high-spin levels, $\mathrm{J}, \pi$, configurations.

Semi-empirical shell model calculations. JOUR PRVCA 72044322

2005MU21 NUCLEAR REACTIONS ${ }^{115} \operatorname{In}(\mathrm{n}, \mathrm{n}),{ }^{27} \mathrm{Al}(\mathrm{n}, \alpha),{ }^{93} \mathrm{Nb}(\mathrm{n}, 2 \mathrm{n}),(\mathrm{n}, 4 \mathrm{n})$, ${ }^{209} \operatorname{Bi}(\mathrm{n}, 4 \mathrm{n}),(\mathrm{n}, 5 \mathrm{n}),(\mathrm{n}, 6 \mathrm{n}),(\mathrm{n}, 7 \mathrm{n}), \mathrm{E} \approx 10-1000 \mathrm{MeV}$; measured reaction rates. Comparison with model predictions. JOUR NIMAE 547 555

2005YA11 NUCLEAR REACTIONS ${ }^{90} \mathrm{Zr}(\mathrm{n}, \mathrm{p}), \mathrm{E}=293 \mathrm{MeV}$; measured $\sigma(\mathrm{E}, \theta)$; ${ }^{90} \mathrm{Zr}(\mathrm{p}, \mathrm{n}), \mathrm{E}=295 \mathrm{MeV}$; analyzed $\sigma(\mathrm{E}, \theta)$; deduced Gamow-Teller strengths, quenching factor. JOUR PYLBB 615193

2005ZHZZ NUCLEAR REACTIONS ${ }^{56,57} \mathrm{Fe},{ }^{90,94} \mathrm{Zr}(\mathrm{p}, \mathrm{n}), \mathrm{E}=7-11 \mathrm{MeV}$; measured En, $\sigma(\mathrm{E}) .{ }^{56,57} \mathrm{Co},{ }^{90,94} \mathrm{Nb}$ deduced level densities. Statistical equilibrium and pre-equilibrium model analysis. CONF Santa Fe (Nucl Data for Sci and Technol) Proc,Vol1,P931

\begin{tabular}{|c|c|c|}
\hline${ }^{90} \mathrm{Mo}$ & 2005CL08 & $\begin{array}{l}\text { ATOMIC MASSES }{ }^{64} \mathrm{Ge},{ }^{68} \mathrm{Se} \text {; analyzed masses; deduced effective } \\
\mathrm{T}_{1 / 2} \cdot{ }^{90,91} \mathrm{Mo},{ }^{90,91,92,93} \mathrm{Tc},{ }^{93,94} \mathrm{Ru},{ }^{94,95} \mathrm{Rh},{ }^{104,105,106,107} \mathrm{In}, \\
104,105,107,108 \mathrm{Sn}\end{array}$ \\
\hline & & astrophysical implications discussed. JOUR ZAANE 25 s01 629 \\
\hline${ }^{90} \mathrm{Tc}$ & 2005CL08 & $\begin{array}{l}\text { ATOMIC MASSES }{ }^{64} \mathrm{Ge},{ }^{68} \mathrm{Se} \text {; analyzed masses; deduced effective } \\
\mathrm{T}_{1 / 2 .} \cdot{ }^{90,91} \mathrm{Mo},{ }^{90,91,92,93} \mathrm{Tc},{ }^{93,94} \mathrm{Ru},{ }^{94,95} \mathrm{Rh},{ }^{104,105,106,107} \mathrm{In}, \\
{ }^{104,105,107,108} \mathrm{Sn},{ }^{107,108} \mathrm{Sb} \text {; measured masses. Penning trap, }\end{array}$ \\
\hline
\end{tabular}

\section{$\mathrm{A}=91$}

${ }^{91} \mathrm{Kr} \quad 2004 \mathrm{GA} 60$

NUCLEAR REACTIONS ${ }^{237} \mathrm{~Np}(\gamma, \mathrm{F}){ }^{135} \mathrm{Xe} /{ }^{137} \mathrm{Xe} /{ }^{138} \mathrm{Xe} /{ }^{139} \mathrm{Xe} /$ ${ }^{140} \mathrm{Xe} /{ }^{141} \mathrm{Xe} /{ }^{142} \mathrm{Xe} /{ }^{89} \mathrm{Kr} /{ }^{91} \mathrm{Kr} /{ }^{92} \mathrm{Kr} /{ }^{93} \mathrm{Kr}, \mathrm{E}=25 \mathrm{MeV}$ bremsstrahlung; measured fission yields, isotopic distribution parameters. Comparison with results from other targets. JOUR BRSPE 681298

2004GAZV NUCLEAR REACTIONS ${ }^{237} \mathrm{~Np},{ }^{243} \mathrm{Am}(\gamma, \mathrm{F}){ }^{89} \mathrm{Kr} /{ }^{91} \mathrm{Kr} /{ }^{92} \mathrm{Kr} /$ ${ }^{93} \mathrm{Kr} /{ }^{135} \mathrm{Xe} /{ }^{137} \mathrm{Xe} /{ }^{138} \mathrm{Xe} /{ }^{139} \mathrm{Xe} /{ }^{140} \mathrm{Xe} /{ }^{141} \mathrm{Xe} /{ }^{142} \mathrm{Xe}$, $\mathrm{E}_{\max }=25 \mathrm{MeV}$; measured $\mathrm{E} \gamma$, I $\gamma$; deduced fission fragment yields. REPT JINR-P15-2004-119, Gangrsky 2005GA25 NUCLEAR REACTIONS ${ }^{248} \mathrm{Cm}(\gamma, \mathrm{F}){ }^{89} \mathrm{Kr} /{ }^{91} \mathrm{Kr} /{ }^{92} \mathrm{Kr} /{ }^{93} \mathrm{Kr} /$ ${ }^{135} \mathrm{Xe} /{ }^{137} \mathrm{Xe} /{ }^{138} \mathrm{Xe} /{ }^{139} \mathrm{Xe} /{ }^{140} \mathrm{Xe} /{ }^{141} \mathrm{Xe} /{ }^{142} \mathrm{Xe}, \mathrm{E}=25 \mathrm{MeV}$ bremsstrahlung; measured $\mathrm{E} \gamma, \mathrm{I} \gamma$; deduced yields. JOUR FECLA 125 44 


\section{$\mathrm{A}=91$ (continued)}

2005GA50 NUCLEAR REACTIONS ${ }^{237} \mathrm{~Np},{ }^{243} \mathrm{Am}(\gamma, \mathrm{F}){ }^{135} \mathrm{Xe} /{ }^{137} \mathrm{Xe} /{ }^{138} \mathrm{Xe} /$ ${ }^{139} \mathrm{Xe} /{ }^{140} \mathrm{Xe} /{ }^{141} \mathrm{Xe} /{ }^{142} \mathrm{Xe} /{ }^{89} \mathrm{Kr} /{ }^{91} \mathrm{Kr} /{ }^{92} \mathrm{Kr} /{ }^{93} \mathrm{Kr}, \mathrm{E}=25 \mathrm{MeV}$ bremsstrahlung; measured fission yields, isotopic distribution parameters. JOUR YAFIA 681475

$\begin{array}{ll}{ }^{91} \mathrm{Rb} & \text { 2005AN01 } \\ { }^{91} \mathrm{Sr} & \text { 2005ADZZ }\end{array}$
NUCLEAR REACTIONS ${ }^{238} \mathrm{U}(\mathrm{p}, \mathrm{F}){ }^{89} \mathrm{Rb} /{ }^{90} \mathrm{Rb} /{ }^{91} \mathrm{Rb} /{ }^{93} \mathrm{Rb} /$ ${ }^{94} \mathrm{Rb} /{ }^{95} \mathrm{Rb} /{ }^{139} \mathrm{Cs} /{ }^{140} \mathrm{Cs} /{ }^{141} \mathrm{Cs} /{ }^{142} \mathrm{Cs} /{ }^{144} \mathrm{Cs} /{ }^{145} \mathrm{Cs}, \mathrm{E}=1$ $\mathrm{GeV}$; measured yields. JOUR ZAANE 23257 NUCLEAR REACTIONS ${ }^{129} \mathrm{I}(\mathrm{n}, 7 \mathrm{n}),(\mathrm{n}, 6 \mathrm{n}),(\mathrm{n}, 4 \mathrm{n}),(\mathrm{n}, \gamma), \mathrm{E}=$ fast; ${ }^{237} \mathrm{~Np}(\mathrm{n}, \gamma), \mathrm{E}=$ fast; measured yields. ${ }^{237} \mathrm{~Np}(\mathrm{n}, \mathrm{F}){ }^{91} \mathrm{Sr} /{ }^{97} \mathrm{Zr} /{ }^{132} \mathrm{Te} /$ ${ }^{133} \mathrm{I} /{ }^{135} \mathrm{I}, \mathrm{E}=$ fast; ${ }^{238} \mathrm{Pu}(\mathrm{n}, \mathrm{F}){ }^{97} \mathrm{Zr} /{ }^{129} \mathrm{Sb} /{ }^{132} \mathrm{I} /{ }^{133} \mathrm{I} /{ }^{135} \mathrm{Xe} /$ ${ }^{105} \mathrm{Ru}, \mathrm{E}=$ fast; ${ }^{239} \mathrm{Pu}(\mathrm{n}, \mathrm{F}){ }^{88} \mathrm{Kr} /{ }^{91} \mathrm{Sr} /{ }^{92} \mathrm{Sr} /{ }^{92} \mathrm{Y} /{ }^{97} \mathrm{Zr} /{ }^{99} \mathrm{Mo} /$ ${ }^{103} \mathrm{Ru} /{ }^{105} \mathrm{Ru} /{ }^{128} \mathrm{Sb} /{ }^{129} \mathrm{Sb} /{ }^{132} \mathrm{Te} /{ }^{131} \mathrm{I} /{ }^{132} \mathrm{I} /{ }^{133} \mathrm{I} /{ }^{135} \mathrm{I} /{ }^{135} \mathrm{Xe}$ $/{ }^{143} \mathrm{Ce} /{ }^{140} \mathrm{Ba} /{ }^{140} \mathrm{La}, \mathrm{E}=$ fast; measured fission fragment yields. Secondary neutrons from proton irradiation. JINR nuclotron. CONF St Petersburg,P195,Adam

2005SEZX NUCLEAR REACTIONS ${ }^{90,94} \operatorname{Zr}(\mathrm{n}, \alpha),{ }^{90,91,92,94} \mathrm{Zr}(\mathrm{n}, \mathrm{p}),{ }^{91,92} \mathrm{Zr}(\mathrm{n}$, $\mathrm{np}+\mathrm{d}), \mathrm{E}=14-20 \mathrm{MeV}$; measured activation $\sigma$. Comparison with model predictions. CONF Santa Fe (Nucl Data for Sci and Technol)

Proc, Vol1,P981

${ }^{91} \mathrm{Y} \quad 2005 \mathrm{BU} 08$

NUCLEAR REACTIONS ${ }^{82} \mathrm{Se}\left({ }^{12} \mathrm{C}, 2 \mathrm{np}\right), \mathrm{E}=38 \mathrm{MeV} ;{ }^{82} \mathrm{Se}\left({ }^{16} \mathrm{O}, 2 \mathrm{np}\right)$, $\mathrm{E}=48 \mathrm{MeV}$; measured $\mathrm{E} \gamma, \mathrm{I} \gamma, \gamma \gamma-$, (charged particle) $\gamma-$,

(neutron) $\gamma$-coin. ${ }^{12} \mathrm{C},{ }^{16} \mathrm{O}\left({ }^{82} \mathrm{Se}, \mathrm{X}\right){ }^{91} \mathrm{Y} /{ }^{95} \mathrm{Nb}, \mathrm{E}=470 \mathrm{MeV}$; measured $\mathrm{E} \gamma, \mathrm{I} \gamma, \gamma \gamma$-coin. ${ }^{91} \mathrm{Y},{ }^{95} \mathrm{Nb}$ deduced high-spin levels, J, $\pi$,

configurations. GASP array, comparison with shell model predictions, level systematics in neighboring isotones discussed. JOUR PRVCA 71 034315

2005SEZX NUCLEAR REACTIONS ${ }^{90,94} \operatorname{Zr}(\mathrm{n}, \alpha),{ }^{90,91,92,94} \mathrm{Zr}(\mathrm{n}, \mathrm{p}),{ }^{91,92} \mathrm{Zr}(\mathrm{n}$, $\mathrm{np}+\mathrm{d}), \mathrm{E}=14-20 \mathrm{MeV}$; measured activation $\sigma$. Comparison with model predictions. CONF Santa Fe (Nucl Data for Sci and Technol)

Proc,Vol1,P981

${ }^{91} \mathrm{Zr} \quad 2005 \mathrm{BI} 25$ NUCLEAR MOMENTS 86,87,88,89,90,91,92,93,94,95,96,97,98,99,100,101,102Zr; measured charge radii. ${ }^{176} \mathrm{Yb}$; measured isomer shift. Ion-beam cooler, laser spectroscopy. JOUR ZAANE 25 s01 187

2005CH53 NUCLEAR REACTIONS ${ }^{92} \operatorname{Zr}(\alpha, \alpha),\left(\alpha,{ }^{3} \mathrm{HeX}\right),(\alpha, \mathrm{tX}),(\alpha, \mathrm{dX}),(\alpha$, $\mathrm{pX}), \mathrm{E}=51 \mathrm{MeV}$; measured particle spectra. ${ }^{92} \mathrm{Zr}\left(\alpha, \alpha^{\prime}\right),(\alpha, \mathrm{xn} \alpha)$, $\mathrm{E}=51 \mathrm{MeV} ;$ measured $\mathrm{E} \gamma, \mathrm{I} \gamma, \alpha \gamma$-coin. ${ }^{90,91,92} \mathrm{Zr}$ deduced transitions. Surrogate reaction technique. JOUR NUPAB 758 126c

2005FUZV NUCLEAR REACTIONS ${ }^{82} \mathrm{Se}\left({ }^{16} \mathrm{O}, 3 \mathrm{n} \alpha\right)$, E not given; measured prompt and delayed $\mathrm{E} \gamma, \mathrm{I} \gamma, \gamma \gamma$-coin. ${ }^{91} \mathrm{Zr}$ deduced high-spin levels. REPT CNS-REP-66,P17,Fukuchi

2005MOZW NUCLEAR REACTIONS ${ }^{90,91,92,94,96} \mathrm{Zr}(\mathrm{n}, \gamma), \mathrm{E}<100 \mathrm{keV}$; measured $\mathrm{E} \gamma, \mathrm{I} \gamma$, capture yields. CONF Santa Fe (Nucl Data for Sci and Technol) Proc,Vol1,P880

2005TA23 NUCLEAR REACTIONS ${ }^{90,91,92,94,96} \mathrm{Zr}(\mathrm{n}, \gamma), \mathrm{E}<1 \mathrm{MeV}$; measured $\mathrm{E} \gamma, \mathrm{I} \gamma$, capture yields. JOUR NUPAB $758573 \mathrm{c}$

${ }^{91} \mathrm{Nb} 2005 \mathrm{WA} 31$ NUCLEAR REACTIONS ${ }^{92,98,100} \mathrm{Mo}\left(\gamma, \gamma^{\prime}\right), \mathrm{E}=13.2 \mathrm{MeV}$ bremsstrahlung; measured $\mathrm{E} \gamma, \mathrm{I} \gamma .{ }^{92,100} \mathrm{Mo},{ }^{197} \mathrm{Au}(\gamma, \mathrm{n}),{ }^{92} \mathrm{Mo}(\gamma, \mathrm{p})$, $(\gamma, \alpha), \mathrm{E} \approx 11.8-16.5 \mathrm{MeV}$ bremsstrahlung; measured integrated $\sigma$. JOUR JPGPE 31 S1969 


\section{$\mathbf{A}=91$ (continued)}

\begin{tabular}{|c|c|c|}
\hline \multirow[t]{2}{*}{${ }^{91} \mathrm{Mo}$} & 2005CL08 & $\begin{array}{l}\text { ATOMIC MASSES }{ }^{64} \mathrm{Ge},{ }^{68} \mathrm{Se} \text {; analyzed masses; deduced effective } \\
\mathrm{T}_{1 / 2} \cdot{ }^{90,91} \mathrm{Mo},{ }^{90,91,92,93} \mathrm{Tc},{ }^{93,94} \mathrm{Ru},{ }^{94,95} \mathrm{Rh},{ }^{104,105,106,107} \mathrm{In}, \\
104,105,107,108 \mathrm{Sn},{ }^{107,108} \mathrm{Sb} \text { measured masses. Penning trap, }\end{array}$ \\
\hline & 2005RE09 & $\begin{array}{l}\text { astrophysical implications discussed. JOUR ZAANE } 25 \text { s01 } 629 \\
\text { NUCLEAR REACTIONS }{ }^{92,94} \mathrm{Mo}(\mathrm{n}, 2 \mathrm{n}),{ }^{92,100} \mathrm{Mo}(\mathrm{n}, \alpha),{ }^{95,96,97} \mathrm{Mo}(\mathrm{n}, \\
\mathrm{p}),{ }^{96,97,98} \mathrm{Mo}(\mathrm{n}, \mathrm{np}+\mathrm{d}), \mathrm{E} \approx 13.5-21 \mathrm{MeV} ; \text { measured activation } \sigma ; \\
\text { deduced reaction mechanism features. }{ }^{93} \mathrm{Nb}(\mathrm{p}, \mathrm{n}),(\mathrm{p}, \gamma), \mathrm{E} \approx 1-6 \\
\mathrm{MeV} ;{ }^{92,93,94,95,96,97,98,100} \mathrm{Mo},{ }^{93} \mathrm{Nb}(\mathrm{n}, \gamma), \mathrm{E}<4 \mathrm{MeV} ;{ }^{92,94,100} \mathrm{Mo}(\mathrm{n}, \\
2 \mathrm{n}),{ }^{92,94,95,96,97,98} \mathrm{Mo}(\mathrm{n}, \mathrm{p}),{ }^{92,94,95,96,97,98,100} \mathrm{Mo}(\mathrm{n}, \mathrm{np}+\mathrm{d}), \\
92,98,100 \mathrm{Mo}(\mathrm{n}, \alpha), \mathrm{E}<21 \mathrm{MeV} ; \text { compiled, analyzed } \sigma \text {. Analysis with }\end{array}$ \\
\hline${ }^{91} \mathrm{Tc}$ & 2005CL08 & $\begin{array}{l}\text { local and global approaches compared. JOUR PRVCA } 71044617 \\
\text { ATOMIC MASSES }{ }^{64} \mathrm{Ge},{ }^{68} \mathrm{Se} \text {; analyzed masses; deduced effective } \\
\mathrm{T}_{1 / 2} \cdot{ }^{90,91} \mathrm{Mo},{ }^{90,91,92,93} \mathrm{Tc},{ }^{93,94} \mathrm{Ru},{ }^{94,95} \mathrm{Rh},{ }^{104,105,106,107} \mathrm{In}, \\
104,105,107,108 \mathrm{Sn},{ }^{107,108} \mathrm{Sb} \text {; measured masses. Penning trap, } \\
\text { astrophvsical implications discussed. JOUR ZAANE } 25 \text { s01 } 629\end{array}$ \\
\hline & 2005XU04 & $\begin{array}{l}\text { RADIOACTIVITY }{ }^{81} \mathrm{Zr},{ }^{85} \mathrm{Mo},{ }^{89} \mathrm{Ru},{ }^{92} \mathrm{Rh},{ }^{93} \mathrm{Pd},{ }^{121} \mathrm{Ce},{ }^{125} \mathrm{Nd} \text {, } \\
{ }^{128} \mathrm{Pm},{ }^{129} \mathrm{Sm},{ }^{135,137} \mathrm{Gd},{ }^{139} \mathrm{Dy},{ }^{142} \mathrm{Ho},{ }^{149} \mathrm{Yb}\left(\beta^{+} \mathrm{p}\right) ; \text { measured } \\
\beta \text {-delayed E } \gamma, \mathrm{I} \gamma \text {, proton spectra, p } \gamma \text {-coin, } \mathrm{T}_{1 / 2} \text {. Comparison with } \\
\text { model predictions. JOUR PRVCA } 71054318\end{array}$ \\
\hline${ }^{91} \mathrm{Rh}$ & 2005MA55 & $\begin{array}{l}\text { NUCLEAR REACTIONS }{ }^{54} \mathrm{Fe}\left({ }^{40} \mathrm{Ca}, 2 \mathrm{np}\right), \mathrm{E}=130 \mathrm{MeV} \text {; measured } \mathrm{E} \gamma \text {, } \\
\mathrm{I} \gamma, \gamma \gamma-,\left(\text { charged particle) } \gamma-\text {, (neutron) } \gamma \text {-coin. }{ }^{91} \mathrm{Rh} \text { deduced high-spin }\right. \\
\text { levels, J, } \pi \text {, configurations, possible isomeric state. GASP, ISIS arrays, } \\
\text { comparison with shell model predictions. JOUR PRVCA } 72014302\end{array}$ \\
\hline
\end{tabular}

\section{$\mathrm{A}=92$}

${ }^{92} \mathrm{Kr} \quad 2004 \mathrm{GA} 60$

NUCLEAR REACTIONS ${ }^{237} \mathrm{~Np}(\gamma, \mathrm{F}){ }^{135} \mathrm{Xe} /{ }^{137} \mathrm{Xe} /{ }^{138} \mathrm{Xe} /{ }^{139} \mathrm{Xe} /$ ${ }^{140} \mathrm{Xe} /{ }^{141} \mathrm{Xe} /{ }^{142} \mathrm{Xe} /{ }^{89} \mathrm{Kr} /{ }^{91} \mathrm{Kr} /{ }^{92} \mathrm{Kr} /{ }^{93} \mathrm{Kr}, \mathrm{E}=25 \mathrm{MeV}$ bremsstrahlung; measured fission yields, isotopic distribution parameters. Comparison with results from other targets. JOUR BRSPE 681298

2004GAZV NUCLEAR REACTIONS ${ }^{237} \mathrm{~Np},{ }^{243} \mathrm{Am}(\gamma, \mathrm{F}){ }^{89} \mathrm{Kr} /{ }^{91} \mathrm{Kr} /{ }^{92} \mathrm{Kr} /$ ${ }^{93} \mathrm{Kr} /{ }^{135} \mathrm{Xe} /{ }^{137} \mathrm{Xe} /{ }^{138} \mathrm{Xe} /{ }^{139} \mathrm{Xe} /{ }^{140} \mathrm{Xe} /{ }^{141} \mathrm{Xe} /{ }^{142} \mathrm{Xe}$, $\mathrm{E}_{\max }=25 \mathrm{MeV}$; measured $\mathrm{E} \gamma, \mathrm{I} \gamma$; deduced fission fragment yields. REPT JINR-P15-2004-119, Gangrsky 2005GA25 NUCLEAR REACTIONS ${ }^{248} \mathrm{Cm}(\gamma, \mathrm{F}){ }^{89} \mathrm{Kr} /{ }^{91} \mathrm{Kr} /{ }^{92} \mathrm{Kr} /{ }^{93} \mathrm{Kr} /$ ${ }^{135} \mathrm{Xe} /{ }^{137} \mathrm{Xe} /{ }^{138} \mathrm{Xe} /{ }^{139} \mathrm{Xe} /{ }^{140} \mathrm{Xe} /{ }^{141} \mathrm{Xe} /{ }^{142} \mathrm{Xe}, \mathrm{E}=25 \mathrm{MeV}$ bremsstrahlung; measured $\mathrm{E} \gamma, \mathrm{I} \gamma$; deduced yields. JOUR FECLA 125 44

2005GA50 NUCLEAR REACTIONS ${ }^{237} \mathrm{~Np},{ }^{243} \mathrm{Am}(\gamma, \mathrm{F}){ }^{135} \mathrm{Xe} /{ }^{137} \mathrm{Xe} /{ }^{138} \mathrm{Xe} /$ ${ }^{139} \mathrm{Xe} /{ }^{140} \mathrm{Xe} /{ }^{141} \mathrm{Xe} /{ }^{142} \mathrm{Xe} /{ }^{89} \mathrm{Kr} /{ }^{91} \mathrm{Kr} /{ }^{92} \mathrm{Kr} /{ }^{93} \mathrm{Kr}, \mathrm{E}=25 \mathrm{MeV}$ bremsstrahlung; measured fission yields, isotopic distribution parameters. JOUR YAFIA 681475 


\section{$\mathrm{A}=92$ (continued)}

${ }^{92} \mathrm{Sr} \quad$ 2005ADZZ NUCLEAR REACTIONS ${ }^{129} \mathrm{I}(\mathrm{n}, 7 \mathrm{n}),(\mathrm{n}, 6 \mathrm{n}),(\mathrm{n}, 4 \mathrm{n}),(\mathrm{n}, \gamma), \mathrm{E}=$ fast; ${ }^{237} \mathrm{~Np}(\mathrm{n}, \gamma), \mathrm{E}=$ fast; measured yields. ${ }^{237} \mathrm{~Np}(\mathrm{n}, \mathrm{F}){ }^{91} \mathrm{Sr} /{ }^{97} \mathrm{Zr} /{ }^{132} \mathrm{Te} /$ ${ }^{133} \mathrm{I} /{ }^{135} \mathrm{I}, \mathrm{E}=$ fast; ${ }^{238} \mathrm{Pu}(\mathrm{n}, \mathrm{F}){ }^{97} \mathrm{Zr} /{ }^{129} \mathrm{Sb} /{ }^{132} \mathrm{I} /{ }^{133} \mathrm{I} /{ }^{135} \mathrm{Xe} /$ ${ }^{105} \mathrm{Ru}, \mathrm{E}=$ fast; ${ }^{239} \mathrm{Pu}(\mathrm{n}, \mathrm{F}){ }^{88} \mathrm{Kr} /{ }^{91} \mathrm{Sr} /{ }^{92} \mathrm{Sr} /{ }^{92} \mathrm{Y} /{ }^{97} \mathrm{Zr} /{ }^{99} \mathrm{Mo} /$ ${ }^{103} \mathrm{Ru} /{ }^{105} \mathrm{Ru} /{ }^{128} \mathrm{Sb} /{ }^{129} \mathrm{Sb} /{ }^{132} \mathrm{Te} /{ }^{131} \mathrm{I} /{ }^{132} \mathrm{I} /{ }^{133} \mathrm{I} /{ }^{135} \mathrm{I} /{ }^{135} \mathrm{Xe}$ $/{ }^{143} \mathrm{Ce} /{ }^{140} \mathrm{Ba} /{ }^{140} \mathrm{La}, \mathrm{E}=$ fast; measured fission fragment yields. Secondary neutrons from proton irradiation. JINR nuclotron. CONF St Petersburg,P195,Adam

2005 GU37 ATOMIC MASSES ${ }^{56,57} \mathrm{Mn},{ }^{82 m} \mathrm{Rb},{ }^{92} \mathrm{Sr},{ }^{124,127} \mathrm{Cs},{ }^{130} \mathrm{Ba}$; measured masses. Penning trap mass spectrometer. JOUR ZAANE 25 s01 35

${ }^{92} \mathrm{Y} \quad 2005 \mathrm{ADZZ}$
NUCLEAR REACTIONS ${ }^{129} \mathrm{I}(\mathrm{n}, 7 \mathrm{n}),(\mathrm{n}, 6 \mathrm{n}),(\mathrm{n}, 4 \mathrm{n}),(\mathrm{n}, \gamma), \mathrm{E}=$ fast; ${ }^{237} \mathrm{~Np}(\mathrm{n}, \gamma), \mathrm{E}=$ fast; measured yields. ${ }^{237} \mathrm{~Np}(\mathrm{n}, \mathrm{F}){ }^{91} \mathrm{Sr} /{ }^{97} \mathrm{Zr} /{ }^{132} \mathrm{Te} /$ ${ }^{133} \mathrm{I} /{ }^{135} \mathrm{I}, \mathrm{E}=$ fast; ${ }^{238} \mathrm{Pu}(\mathrm{n}, \mathrm{F}){ }^{97} \mathrm{Zr} /{ }^{129} \mathrm{Sb} /{ }^{132} \mathrm{I} /{ }^{133} \mathrm{I} /{ }^{135} \mathrm{Xe} /$ ${ }^{105} \mathrm{Ru}, \mathrm{E}=$ fast; ${ }^{239} \mathrm{Pu}(\mathrm{n}, \mathrm{F}){ }^{88} \mathrm{Kr} /{ }^{91} \mathrm{Sr} /{ }^{92} \mathrm{Sr} /{ }^{92} \mathrm{Y} /{ }^{97} \mathrm{Zr} /{ }^{99} \mathrm{Mo} /$ ${ }^{103} \mathrm{Ru} /{ }^{105} \mathrm{Ru} /{ }^{128} \mathrm{Sb} /{ }^{129} \mathrm{Sb} /{ }^{132} \mathrm{Te} /{ }^{131} \mathrm{I} /{ }^{132} \mathrm{I} /{ }^{133} \mathrm{I} /{ }^{135} \mathrm{I} /{ }^{135} \mathrm{Xe}$ $/{ }^{143} \mathrm{Ce} /{ }^{140} \mathrm{Ba} /{ }^{140} \mathrm{La}, \mathrm{E}=$ fast; measured fission fragment yields. Secondary neutrons from proton irradiation. JINR nuclotron. CONF St Petersburg,P195,Adam 2005SEZX NUCLEAR REACTIONS ${ }^{90,94} \mathrm{Zr}(\mathrm{n}, \alpha),{ }^{90,91,92,94} \mathrm{Zr}(\mathrm{n}, \mathrm{p}),{ }^{91,92} \mathrm{Zr}(\mathrm{n}$, $\mathrm{np}+\mathrm{d}), \mathrm{E}=14-20 \mathrm{MeV}$; measured activation $\sigma$. Comparison with model predictions. CONF Santa Fe (Nucl Data for Sci and Technol) Proc, Vol1,P981

2005BI25 NUCLEAR MOMENTS 86,87,88,89,90,91,92,93,94,95,96,97,98,99,100,101,102Zr; measured charge radii. ${ }^{176} \mathrm{Yb}$; measured isomer shift. Ion-beam cooler, laser spectroscopy. JOUR ZAANE 25 s01 187

2005CH53 NUCLEAR REACTIONS ${ }^{92} \operatorname{Zr}(\alpha, \alpha),\left(\alpha,{ }^{3} \mathrm{HeX}\right),(\alpha, \mathrm{tX}),(\alpha, \mathrm{dX}),(\alpha$, $\mathrm{pX}), \mathrm{E}=51 \mathrm{MeV}$; measured particle spectra. ${ }^{92} \operatorname{Zr}\left(\alpha, \alpha^{\prime}\right),(\alpha, \operatorname{xn} \alpha)$, $\mathrm{E}=51 \mathrm{MeV}$; measured $\mathrm{E} \gamma, \mathrm{I} \gamma, \alpha \gamma$-coin. ${ }^{90,91,92} \mathrm{Zr}$ deduced transitions. Surrogate reaction technique. JOUR NUPAB $758126 \mathrm{c}$

2005FR17 NUCLEAR REACTIONS ${ }^{92} \mathrm{Zr}(\mathrm{n}, \mathrm{n} \gamma), \mathrm{E}=2.2,3.9 \mathrm{MeV}$; measured $\mathrm{E} \gamma$, $\mathrm{I} \gamma$, angular distributions, DSA. ${ }^{92} \mathrm{Zr}(\mathrm{n}, \mathrm{n} \gamma), \mathrm{E}=2.6-3.9 \mathrm{MeV}$; measured excitation functions. ${ }^{92} \mathrm{Zr}$ deduced levels, $\mathrm{J}, \pi, \mathrm{T}_{1 / 2}, \delta$. Comparison with model predictions, neighboring nuclides. JOUR PRVCA 71054304 2005GA44 NUCLEAR REACTIONS ${ }^{208} \mathrm{~Pb}\left({ }^{90} \mathrm{Zr}, \mathrm{X}\right){ }^{90} \mathrm{Zr} /{ }^{92} \mathrm{Zr} /{ }^{88} \mathrm{Sr}, \mathrm{E}=560$ $\mathrm{MeV} ;{ }^{238} \mathrm{U}\left({ }^{64} \mathrm{Ni}, \mathrm{X}\right){ }^{58} \mathrm{Cr}, \mathrm{E}=400 \mathrm{MeV}$; measured $\mathrm{E} \gamma, \mathrm{I} \gamma, \gamma \gamma-$ (recoil) $\gamma$-coin. ${ }^{58} \mathrm{Cr},{ }^{90,92} \mathrm{Zr},{ }^{88} \mathrm{Sr}$ deduced transitions. Clara array, mass separator. JOUR JPGPE 31 S1443

2005LA13 NUCLEAR REACTIONS Zr, ${ }^{91} \mathrm{Zr}(\mathrm{n}, \gamma), \mathrm{E} \approx 0.1-5000 \mathrm{eV}$; measured $\mathrm{E} \gamma$, capture $\sigma$, baseline shift effect. JOUR NIMAE 543502

2005MOZW NUCLEAR REACTIONS ${ }^{90,91,92,94,96} \mathrm{Zr}(\mathrm{n}, \gamma), \mathrm{E}<100 \mathrm{keV}$; measured $\mathrm{E} \gamma$, I $\gamma$, capture yields. CONF Santa Fe (Nucl Data for Sci and Technol) Proc,Vol1,P880

$20050 \mathrm{H} 04$ NUCLEAR REACTIONS ${ }^{91,92} \mathrm{Zr}(\mathrm{n}, \gamma), \mathrm{E}=15-550 \mathrm{keV}$; measured $\mathrm{E} \gamma$, $\gamma$-ray multiplicity, capture $\sigma$. JOUR JNSTA 42333 


\section{$\mathrm{A}=92$ (continued)}

2005PA48 NUCLEAR REACTIONS ${ }^{176} \mathrm{Yb}\left({ }^{28} \mathrm{Si}, \mathrm{X}\right){ }^{92} \mathrm{Zr} /{ }^{93} \mathrm{Zr} /{ }^{94} \mathrm{Zr} /{ }^{95} \mathrm{Zr} /$ ${ }^{96} \mathrm{Zr}, \mathrm{E}=145 \mathrm{MeV} ;{ }^{176} \mathrm{Yb}\left({ }^{31} \mathrm{P}, \mathrm{X}\right){ }^{92} \mathrm{Zr} /{ }^{93} \mathrm{Zr} /{ }^{94} \mathrm{Zr} /{ }^{95} \mathrm{Zr} /{ }^{96} \mathrm{Zr}$, $\mathrm{E}=152 \mathrm{MeV}$; measured $\mathrm{E} \gamma, \mathrm{I} \gamma, \gamma \gamma$-coin. ${ }^{92,93,94,95,96} \mathrm{Zr}$ deduced high-spin levels, J, $\pi$, configurations. Eurogam II and Euroball IV arrays, comparisons with shell-model predictions. JOUR PRVCA 72 024304

2005TA23 NUCLEAR REACTIONS ${ }^{90,91,92,94,96} \mathrm{Zr}(\mathrm{n}, \gamma), \mathrm{E}<1 \mathrm{MeV}$; measured $\mathrm{E} \gamma, \mathrm{I} \gamma$, capture yields. JOUR NUPAB $758573 \mathrm{c}$

${ }^{92} \mathrm{Nb} \quad$ 20040DZZ

2005MU21

2005RE09

2005FU01

2005RUZZ

2005WA31

${ }^{92} \mathrm{Tc} \quad$ 2005CL08

${ }^{92} \mathrm{Ru} \quad 2005 \mathrm{XU} 04$

${ }^{92} \mathrm{Rh} \quad$ 2005MUZX
NUCLEAR REACTIONS ${ }^{82} \mathrm{Se}\left({ }^{17} \mathrm{~N}, 7 \mathrm{n}\right),\left({ }^{17} \mathrm{~N}, 6 \mathrm{n}\right), \mathrm{E} \approx 104 \mathrm{MeV}$; measured $\mathrm{E} \gamma, \mathrm{I} \gamma \cdot{ }^{92,93} \mathrm{Nb}$ deduced transitions. REPT CNS-REP-64,P289,Odahara

NUCLEAR REACTIONS ${ }^{93} \mathrm{Nb}(\gamma, \mathrm{n}),(\gamma, 3 \mathrm{n}),, \mathrm{E}=50 \mathrm{MeV}$ bremsstrahlung; measured $\mathrm{E} \gamma, \mathrm{I} \gamma$; deduced yield ratio. HPGe detectors, microtron. CONF St Petersburg,P56,Aliev NUCLEAR REACTIONS ${ }^{115} \mathrm{In}(\mathrm{n}, \mathrm{n}),{ }^{27} \mathrm{Al}(\mathrm{n}, \alpha),{ }^{93} \mathrm{Nb}(\mathrm{n}, 2 \mathrm{n}),(\mathrm{n}, 4 \mathrm{n})$, ${ }^{209} \operatorname{Bi}(\mathrm{n}, 4 \mathrm{n}),(\mathrm{n}, 5 \mathrm{n}),(\mathrm{n}, 6 \mathrm{n}),(\mathrm{n}, 7 \mathrm{n}), \mathrm{E} \approx 10-1000 \mathrm{MeV}$; measured reaction rates. Comparison with model predictions. JOUR NIMAE 547 555

NUCLEAR REACTIONS ${ }^{92,94} \mathrm{Mo}(\mathrm{n}, 2 \mathrm{n}),{ }^{92,100} \mathrm{Mo}(\mathrm{n}, \alpha),{ }^{95,96,97} \mathrm{Mo}(\mathrm{n}$, p), ${ }^{96,97,98} \mathrm{Mo}(\mathrm{n}, \mathrm{np}+\mathrm{d}), \mathrm{E} \approx 13.5-21 \mathrm{MeV}$; measured activation $\sigma$; deduced reaction mechanism features. ${ }^{93} \mathrm{Nb}(\mathrm{p}, \mathrm{n}),(\mathrm{p}, \gamma), \mathrm{E} \approx 1-6$ $\mathrm{MeV} ;{ }^{92,93,94,95,96,97,98,100} \mathrm{Mo},{ }^{93} \mathrm{Nb}(\mathrm{n}, \gamma), \mathrm{E}<4 \mathrm{MeV} ;{ }^{92,94,100} \mathrm{Mo}(\mathrm{n}$, 2n), ${ }^{92,94,95,96,97,98} \mathrm{Mo}(\mathrm{n}, \mathrm{p}),{ }^{92,94,95,96,97,98,100} \mathrm{Mo}(\mathrm{n}, \mathrm{np}+\mathrm{d})$, ${ }_{92,98,100} \mathrm{Mo}(\mathrm{n}, \alpha), \mathrm{E}<21 \mathrm{MeV}$; compiled, analyzed $\sigma$. Analysis with local and global approaches compared. JOUR PRVCA 71044617 NUCLEAR REACTIONS ${ }^{82} \mathrm{Se}\left({ }^{16} \mathrm{O}, 5 \mathrm{n}\right),\left({ }^{16} \mathrm{O}, 6 \mathrm{n}\right), \mathrm{E}=100 \mathrm{MeV}$; measured prompt and delayed $\mathrm{E} \gamma$, I $\gamma, \gamma \gamma$-coin, $\gamma$-ray linear polarization. ${ }^{93}$ Mo deduced high-spin levels, $\mathrm{J}, \pi$, configurations, isomeric states $\mathrm{T}_{1 / 2} .{ }^{92}$ Mo deduced levels, $\mathrm{J}, \pi$. JOUR ZAANE 24249 NUCLEAR REACTIONS ${ }^{92,98,100} \mathrm{Mo}\left(\gamma, \gamma^{\prime}\right), \mathrm{E}=14 \mathrm{MeV}$ bremsstrahlung; measured $\mathrm{E} \gamma, \mathrm{I} \gamma .{ }^{92,98,100}$ Mo deduced dipole strength functions, resonance features. PREPRINT nucl-ex/0512027,12/20/2005 NUCLEAR REACTIONS ${ }^{92,98,100} \mathrm{Mo}\left(\gamma, \gamma^{\prime}\right), \mathrm{E}=13.2 \mathrm{MeV}$ bremsstrahlung; measured $\mathrm{E} \gamma, \mathrm{I} \gamma .{ }^{92,100} \mathrm{Mo},{ }^{197} \mathrm{Au}(\gamma, \mathrm{n}),{ }^{92} \mathrm{Mo}(\gamma, \mathrm{p})$, $(\gamma, \alpha), \mathrm{E} \approx 11.8-16.5 \mathrm{MeV}$ bremsstrahlung; measured integrated $\sigma$.

JOUR JPGPE 31 S1969

ATOMIC MASSES ${ }^{64} \mathrm{Ge},{ }^{68} \mathrm{Se}$; analyzed masses; deduced effective $\mathrm{T}_{1 / 2} .{ }^{90,91} \mathrm{Mo},{ }^{90,91,92,93} \mathrm{Tc},{ }^{93,94} \mathrm{Ru},{ }^{94,95} \mathrm{Rh},{ }^{104,105,106,107} \mathrm{In}$, $104,105,107,108$ Sn, ${ }^{107,108} \mathrm{Sb}$; measured masses. Penning trap, astrophysical implications discussed. JOUR ZAANE 25 s01 629 RADIOACTIVITY ${ }^{81} \mathrm{Zr},{ }^{85} \mathrm{Mo},{ }^{89} \mathrm{Ru},{ }^{92} \mathrm{Rh},{ }^{93} \mathrm{Pd},{ }^{121} \mathrm{Ce},{ }^{125} \mathrm{Nd}$, ${ }^{128} \mathrm{Pm},{ }^{129} \mathrm{Sm},{ }^{135,137} \mathrm{Gd},{ }^{139} \mathrm{Dy},{ }^{142} \mathrm{Ho},{ }^{149} \mathrm{Yb}\left(\beta^{+} \mathrm{p}\right)$; measured $\beta$-delayed $\mathrm{E} \gamma, \mathrm{I} \gamma$, proton spectra, $\mathrm{p} \gamma$-coin, $\mathrm{T}_{1 / 2}$. Comparison with model predictions. JOUR PRVCA 71054318 RADIOACTIVITY ${ }^{94 m} \mathrm{Ag}(2 \mathrm{p})$ [from ${ }^{58} \mathrm{Ni}\left({ }^{40} \mathrm{Ca}, 3 \mathrm{np}\right)$ ]; measured $\mathrm{E} \gamma$, Ep, pp-, $\gamma \gamma$-, p $\gamma$-coin; deduced two-proton decay branching ratio. ${ }^{92} \mathrm{Rh}$ deduced levels, J, $\pi$. REPT GSI 2005-1,P87,Mukha 


\section{$\mathrm{A}=92$ (continued)}

2005XU04 RADIOACTIVITY ${ }^{81} \mathrm{Zr},{ }^{85} \mathrm{Mo},{ }^{89} \mathrm{Ru},{ }^{92} \mathrm{Rh},{ }^{93} \mathrm{Pd},{ }^{121} \mathrm{Ce},{ }^{125} \mathrm{Nd}$, ${ }^{128} \mathrm{Pm},{ }^{129} \mathrm{Sm},{ }^{135,137} \mathrm{Gd},{ }^{139} \mathrm{Dy},{ }^{142} \mathrm{Ho},{ }^{149} \mathrm{Yb}\left(\beta^{+} \mathrm{p}\right)$; measured $\beta$-delayed $\mathrm{E} \gamma, \mathrm{I} \gamma$, proton spectra, $\mathrm{p} \gamma$-coin, $\mathrm{T}_{1 / 2}$. Comparison with model predictions. JOUR PRVCA 71054318

\section{$\mathrm{A}=93$}

${ }^{93} \mathrm{Kr} \quad 2004 \mathrm{GA} 60$

NUCLEAR REACTIONS ${ }^{237} \mathrm{~Np}(\gamma, \mathrm{F}){ }^{135} \mathrm{Xe} /{ }^{137} \mathrm{Xe} /{ }^{138} \mathrm{Xe} /{ }^{139} \mathrm{Xe} /$ ${ }^{140} \mathrm{Xe} /{ }^{141} \mathrm{Xe} /{ }^{142} \mathrm{Xe} /{ }^{89} \mathrm{Kr} /{ }^{91} \mathrm{Kr} /{ }^{92} \mathrm{Kr} /{ }^{93} \mathrm{Kr}, \mathrm{E}=25 \mathrm{MeV}$ bremsstrahlung; measured fission yields, isotopic distribution parameters. Comparison with results from other targets. JOUR BRSPE 681298

2004GAZV NUCLEAR REACTIONS ${ }^{237} \mathrm{~Np},{ }^{243} \mathrm{Am}(\gamma, \mathrm{F}){ }^{89} \mathrm{Kr} /{ }^{91} \mathrm{Kr} /{ }^{92} \mathrm{Kr} /$ ${ }^{93} \mathrm{Kr} /{ }^{135} \mathrm{Xe} /{ }^{137} \mathrm{Xe} /{ }^{138} \mathrm{Xe} /{ }^{139} \mathrm{Xe} /{ }^{140} \mathrm{Xe} /{ }^{141} \mathrm{Xe} /{ }^{142} \mathrm{Xe}$, $\mathrm{E}_{\max }=25 \mathrm{MeV}$; measured $\mathrm{E} \gamma, \mathrm{I} \gamma$; deduced fission fragment yields. REPT JINR-P15-2004-119, Gangrsky

2005GA25 NUCLEAR REACTIONS ${ }^{248} \mathrm{Cm}(\gamma, \mathrm{F}){ }^{89} \mathrm{Kr} /{ }^{91} \mathrm{Kr} /{ }^{92} \mathrm{Kr} /{ }^{93} \mathrm{Kr} /$ ${ }^{135} \mathrm{Xe} /{ }^{137} \mathrm{Xe} /{ }^{138} \mathrm{Xe} /{ }^{139} \mathrm{Xe} /{ }^{140} \mathrm{Xe} /{ }^{141} \mathrm{Xe} /{ }^{142} \mathrm{Xe}, \mathrm{E}=25 \mathrm{MeV}$ bremsstrahlung; measured $\mathrm{E} \gamma, \mathrm{I} \gamma$; deduced yields. JOUR FECLA 125 44

2005GA50 NUCLEAR REACTIONS ${ }^{237} \mathrm{~Np},{ }^{243} \mathrm{Am}(\gamma, \mathrm{F}){ }^{135} \mathrm{Xe} /{ }^{137} \mathrm{Xe} /{ }^{138} \mathrm{Xe} /$ ${ }^{139} \mathrm{Xe} /{ }^{140} \mathrm{Xe} /{ }^{141} \mathrm{Xe} /{ }^{142} \mathrm{Xe} /{ }^{89} \mathrm{Kr} /{ }^{91} \mathrm{Kr} /{ }^{92} \mathrm{Kr} /{ }^{93} \mathrm{Kr}, \mathrm{E}=25 \mathrm{MeV}$ bremsstrahlung; measured fission yields, isotopic distribution parameters. JOUR YAFIA 681475

${ }^{93} \mathrm{Rb}$ 2005AN01 NUCLEAR REACTIONS ${ }^{238} \mathrm{U}(\mathrm{p}, \mathrm{F}){ }^{89} \mathrm{Rb} /{ }^{90} \mathrm{Rb} /{ }^{91} \mathrm{Rb} /{ }^{93} \mathrm{Rb} /$ ${ }^{94} \mathrm{Rb} /{ }^{95} \mathrm{Rb} /{ }^{139} \mathrm{Cs} /{ }^{140} \mathrm{Cs} /{ }^{141} \mathrm{Cs} /{ }^{142} \mathrm{Cs} /{ }^{144} \mathrm{Cs} /{ }^{145} \mathrm{Cs}, \mathrm{E}=1$

$\mathrm{GeV}$; measured yields. JOUR ZAANE 23257

${ }^{93} \mathrm{Y} \quad 2005 \mathrm{BE} 17$

${ }^{93} \mathrm{Zr} \quad 2005 B I 25$ RADIOACTIVITY ${ }^{127} \mathrm{I}\left({ }^{24} \mathrm{Ne}\right),\left({ }^{28} \mathrm{Mg}\right),\left({ }^{30} \mathrm{Mg}\right),\left({ }^{32} \mathrm{Si}\right),\left({ }^{34} \mathrm{Si}\right),\left({ }^{48} \mathrm{Ca}\right)$ $\left({ }^{49} \mathrm{Sc}\right)$; measured cluster decay $\mathrm{T}_{1 / 2}$ lower limits. JOUR ZAANE 2451 NUCLEAR MOMENTS 86,87,88,89,90,91,92,93,94,95,96,97,98,99,100,101,102 Zr; measured charge radii. ${ }^{176} \mathrm{Yb}$; measured isomer shift. Ion-beam cooler, laser spectroscopy. JOUR ZAANE 25 s01 187

2005MOZW NUCLEAR REACTIONS ${ }^{90,91,92,94,96} \mathrm{Zr}(\mathrm{n}, \gamma), \mathrm{E}<100 \mathrm{keV}$; measured $\mathrm{E} \gamma$, I $\gamma$, capture yields. CONF Santa Fe (Nucl Data for Sci and Technol) Proc, Vol1,P880

20050H04 NUCLEAR REACTIONS ${ }^{91,92} \mathrm{Zr}(\mathrm{n}, \gamma), \mathrm{E}=15-550 \mathrm{keV}$; measured $\mathrm{E} \gamma$, $\gamma$-ray multiplicity, capture $\sigma$. JOUR JNSTA 42333

20050HZX NUCLEAR REACTIONS ${ }^{92} \mathrm{Zr}(\mathrm{n}, \gamma), \mathrm{E}=15-90,550 \mathrm{keV}$; measured E $\gamma$, capture $\sigma$. CONF Santa Fe (Nucl Data for Sci and Technol)

Proc, Vol1,P945

2005PA48 NUCLEAR REACTIONS ${ }^{176} \mathrm{Yb}\left({ }^{28} \mathrm{Si}, \mathrm{X}\right){ }^{92} \mathrm{Zr} /{ }^{93} \mathrm{Zr} /{ }^{94} \mathrm{Zr} /{ }^{95} \mathrm{Zr} /$ ${ }^{96} \mathrm{Zr}, \mathrm{E}=145 \mathrm{MeV} ;{ }^{176} \mathrm{Yb}\left({ }^{31} \mathrm{P}, \mathrm{X}\right){ }^{92} \mathrm{Zr} /{ }^{93} \mathrm{Zr} /{ }^{94} \mathrm{Zr} /{ }^{95} \mathrm{Zr} /{ }^{96} \mathrm{Zr}$, $\mathrm{E}=152 \mathrm{MeV}$; measured $\mathrm{E} \gamma, \mathrm{I} \gamma, \gamma \gamma$-coin. ${ }^{92,93,94,95,96} \mathrm{Zr}$ deduced high-spin levels, J, $\pi$, configurations. Eurogam II and Euroball IV arrays, comparisons with shell-model predictions. JOUR PRVCA 72 024304

2005TA23 NUCLEAR REACTIONS ${ }^{90,91,92,94,96} \mathrm{Zr}(\mathrm{n}, \gamma), \mathrm{E}<1 \mathrm{MeV}$; measured $\mathrm{E} \gamma, \mathrm{I} \gamma$, capture yields. JOUR NUPAB $758573 \mathrm{c}$ 


\section{$\mathrm{A}=93$ (continued)}

\begin{tabular}{|c|c|c|}
\hline \multirow[t]{3}{*}{${ }^{93} \mathrm{Nb}$} & 20040DZZ & $\begin{array}{l}\text { NUCLEAR REACTIONS }{ }^{82} \mathrm{Se}\left({ }^{17} \mathrm{~N}, 7 \mathrm{n}\right),\left({ }^{17} \mathrm{~N}, 6 \mathrm{n}\right), \mathrm{E} \approx 104 \mathrm{MeV} ; \\
\text { measured } \mathrm{E} \gamma, \mathrm{I} \gamma .{ }^{92,93} \mathrm{Nb} \text { deduced transitions. REPT }\end{array}$ \\
\hline & $2005 \mathrm{MC} 13$ & $\begin{array}{l}\text { NUCLEAR REACTIONS }{ }^{93} \mathrm{Nb}\left(\mathrm{n}, \mathrm{n}^{\prime}\right), \mathrm{E}=1.5-2.6 \mathrm{MeV} ;{ }^{94} \mathrm{Zr}(\mathrm{p}, 2 \mathrm{n}) \text {, } \\
\mathrm{E}=11.5-19 \mathrm{MeV} \text {; measured } \mathrm{E} \gamma, \mathrm{I} \gamma, \mathrm{DSA} \text {, branching ratios, excitation } \\
\text { functions. }{ }^{93} \mathrm{Nb} \text { deduced levels } \mathrm{J}, \pi, \mathrm{T}_{1 / 2} \text {, mixed-symmetry states. } \\
\text { JOUR ZAANE } 25 \text { s01 } 377\end{array}$ \\
\hline & 2005WAZS & $\begin{array}{l}\text { NUCLEAR REACTIONS }{ }^{82} \mathrm{Se}\left({ }^{17} \mathrm{~N}, \mathrm{X}\right), \mathrm{E}=104 \mathrm{MeV} \text {; measured } \\
\text { prompt and delayed } \mathrm{E} \gamma, \mathrm{I} \gamma, \gamma \gamma \text {-coin. }{ }^{89,90} \mathrm{Y},{ }^{93} \mathrm{Nb} \text { deduced transitions, } \\
\text { possible high-spin isomers. REPT CNS-REP- } 66, \mathrm{P} 15 \text {, Wakabayashi }\end{array}$ \\
\hline \multirow[t]{6}{*}{${ }^{93} \mathrm{Mo}$} & 2004FUZX & $\begin{array}{l}\text { NUCLEAR REACTIONS }{ }^{82} \mathrm{Se}\left({ }^{16} \mathrm{O}, 5 \mathrm{n}\right), \mathrm{E}=100 \mathrm{MeV} \text {; measured } \mathrm{E} \gamma \text {, } \\
\mathrm{I} \gamma, \gamma \gamma \text {-coin. }{ }^{93} \mathrm{Mo} \text { deduced high-spin levels, J, } \pi \text {, configurations, } \\
\text { isomeric states } \mathrm{T}_{1 / 2} . \text { REPT CNS-REP- } 64, \mathrm{P} 109, \text { Fukuchi }\end{array}$ \\
\hline & $2005 \mathrm{CHZW}$ & $\begin{array}{l}\text { NUCLEAR REACTIONS }{ }^{94,96} \mathrm{Mo}\left({ }^{3} \mathrm{He},{ }^{3} \mathrm{He}\right),\left({ }^{3} \mathrm{He}, \alpha\right), \mathrm{E}=30 \mathrm{MeV} \\
97,98 \mathrm{Mo}\left({ }^{3} \mathrm{He},{ }^{3} \mathrm{He}\right),\left({ }^{3} \mathrm{He}, \alpha\right), \mathrm{E}=45 \mathrm{MeV} ; \text { measured particle spectra, } \\
\mathrm{E} \gamma, \mathrm{I} \gamma \text {, (particle) } \gamma \text {-coin. }{ }^{93,94,95,96,97,98} \mathrm{Mo} \text { deduced level density } \\
\text { parameters, thermodynamic quantities. PREPRINT } \\
\text { nucl-ex/0507007,7/04/2005 }\end{array}$ \\
\hline & 2005FU01 & $\begin{array}{l}\text { NUCLEAR REACTIONS }{ }^{82} \mathrm{Se}\left({ }^{16} \mathrm{O}, 5 \mathrm{n}\right),\left({ }^{16} \mathrm{O}, 6 \mathrm{n}\right), \mathrm{E}=100 \mathrm{MeV} \text {; } \\
\text { measured prompt and delayed } \mathrm{E} \gamma, \mathrm{I} \gamma, \gamma \gamma \text {-coin, } \gamma \text {-ray linear } \\
\text { polarization. }{ }^{93} \mathrm{Mo} \text { deduced high-spin levels, J, } \pi \text {, configurations, } \\
\text { isomeric states } \mathrm{T}_{1 / 2} .{ }^{92} \text { Mo deduced levels, J, } \pi \text {. JOUR ZAANE } 24249\end{array}$ \\
\hline & 2005GU16 & $\begin{array}{l}\text { NUCLEAR REACTIONS }{ }^{94,96} \mathrm{Mo}\left({ }^{3} \mathrm{He},{ }^{3} \mathrm{He}\right),\left({ }^{3} \mathrm{He}, \alpha\right), \mathrm{E}=30 \mathrm{MeV} \text {; } \\
97,98 \mathrm{Mo}\left({ }^{3} \mathrm{He},{ }^{3} \mathrm{He}\right),\left({ }^{3} \mathrm{He}, \alpha\right), \mathrm{E}=45 \mathrm{MeV} ; \text { measured particle spectra, } \\
\mathrm{E} \gamma, \mathrm{I} \gamma \text {, (particle) } \gamma \text {-coin. }{ }^{93,94,95,96,97,98} \mathrm{Mo} \text { deduced radiative strength } \\
\text { functions. JOUR PRVCA } 71044307\end{array}$ \\
\hline & 2005RE09 & $\begin{array}{l}\text { NUCLEAR REACTIONS }{ }^{92,94} \mathrm{Mo}(\mathrm{n}, 2 \mathrm{n}),{ }^{92,100} \mathrm{Mo}(\mathrm{n}, \alpha),{ }^{95,96,97} \mathrm{Mo}(\mathrm{n}, \\
\mathrm{p}),{ }^{96,97,98} \mathrm{Mo}(\mathrm{n}, \mathrm{np}+\mathrm{d}), \mathrm{E} \approx 13.5-21 \mathrm{MeV} \text {; measured activation } \sigma ; \\
\text { deduced reaction mechanism features. }{ }^{93} \mathrm{Nb}(\mathrm{p}, \mathrm{n}),(\mathrm{p}, \gamma), \mathrm{E} \approx 1-6 \\
\mathrm{MeV} ;{ }^{92,93,94,95,96,97,98,100} \mathrm{Mo},{ }^{93} \mathrm{Nb}(\mathrm{n}, \gamma), \mathrm{E}<4 \mathrm{MeV} ;{ }^{92,94,100} \mathrm{Mo}(\mathrm{n}, \\
2 \mathrm{n}),{ }^{92,94,95,96,97,98} \mathrm{Mo}(\mathrm{n}, \mathrm{p}),{ }^{92,94,95,96,97,98,100} \mathrm{Mo}(\mathrm{n}, \mathrm{np}+\mathrm{d}), \\
92,98,100 \mathrm{Mo}(\mathrm{n}, \alpha), \mathrm{E}<21 \mathrm{MeV} \text {; compiled, analyzed } \sigma \text {. Analysis with }\end{array}$ \\
\hline & & local and global approaches compared. JOUR PRVCA 71044617 \\
\hline \multirow[t]{3}{*}{${ }^{93} \mathrm{Tc}$} & 2005CL08 & $\begin{array}{l}\text { ATOMIC MASSES }{ }^{64} \mathrm{Ge},{ }^{68} \mathrm{Se} \text {; analyzed masses; deduced effective } \\
\mathrm{T}_{1 / 2 .} \cdot{ }^{90,91} \mathrm{Mo},{ }^{90,91,92,93} \mathrm{Tc},{ }^{93,94} \mathrm{Ru},{ }^{94,95} \mathrm{Rh},{ }^{104,105,106,107} \mathrm{In}, \\
{ }_{104,105,107,108} \mathrm{Sn},{ }^{107,108} \mathrm{Sb} \text {; measured masses. Penning trap, } \\
\text { astrophysical implications discussed. JOUR ZAANE } 25 \mathrm{~s} 01629\end{array}$ \\
\hline & 2005NA28 & $\begin{array}{l}\text { RADIOACTIVITY }{ }^{93 m} \mathrm{Tc}(\mathrm{IT}) \text { [ }\left[\text { rom }{ }^{45} \mathrm{Sc}\left({ }^{52} \mathrm{Cr}, 2 \mathrm{n} 2 \mathrm{p}\right)\right] \text {; measured } \mathrm{E} \gamma \\
\mathrm{I} \gamma, \gamma \text { asymmetry from polarized nucleus decay. }{ }^{93} \mathrm{Tc} \text { deduced parity } \\
\text { nonconservation in isomeric state decay. JOUR PRVCA } 72027303\end{array}$ \\
\hline & 2005NA43 & $\begin{array}{l}\text { RADIOACTIVITY }{ }^{93 m} \mathrm{Tc}(\mathrm{IT})\left[\text { from }{ }^{45} \mathrm{Sc}\left({ }^{52} \mathrm{Cr}, 2 \mathrm{n} 2 \mathrm{p}\right)\right] \text {; measured } \gamma \text {-ray } \\
\text { anisotropies from oriented source; deduced parity-nonconserving matrix } \\
\text { element. JOUR ZAANE } 25 \mathrm{~s} 01703\end{array}$ \\
\hline${ }^{93} \mathrm{Ru}$ & 2005CL08 & $\begin{array}{l}\text { ATOMIC MASSES }{ }^{64} \mathrm{Ge},{ }^{68} \mathrm{Se} \text {; analyzed masses; deduced effective } \\
\mathrm{T}_{1 / 2 .} \cdot{ }^{90,91} \mathrm{Mo},{ }^{90,91,92,93} \mathrm{Tc},{ }^{93,94} \mathrm{Ru},{ }^{94,95} \mathrm{Rh},{ }^{104,105,106,107} \mathrm{In}, \\
{ }_{104,105,107,108} \mathrm{Sn},{ }^{107,108} \mathrm{Sb} \text {; measured masses. Penning trap, }\end{array}$ \\
\hline
\end{tabular}




\section{$\mathrm{A}=93$ (continued)}

${ }^{93} \mathrm{Pd} \quad$ 2005MU15 RADIOACTIVITY ${ }^{94 m} \mathrm{Ag}$ (p) [from $\left.{ }^{58} \mathrm{Ni}\left({ }^{40} \mathrm{Ca}, 3 \mathrm{np}\right)\right]$; measured Ep, $\gamma \gamma$-, $\mathrm{p} \gamma$-coin, $\mathrm{T}_{1 / 2}$ following decay of high-spin isomer; deduced branching ratios, Q-value. ${ }^{94} \mathrm{Ag}$ deduced isomer configuration, deformation. ${ }^{93} \mathrm{Pd}$ deduced levels. JOUR PRLTA 95022501

2005XU04 RADIOACTIVITY ${ }^{81} \mathrm{Zr},{ }^{85} \mathrm{Mo},{ }^{89} \mathrm{Ru},{ }^{92} \mathrm{Rh},{ }^{93} \mathrm{Pd},{ }^{121} \mathrm{Ce},{ }^{125} \mathrm{Nd}$, ${ }^{128} \mathrm{Pm},{ }^{129} \mathrm{Sm},{ }^{135,137} \mathrm{Gd},{ }^{139} \mathrm{Dy},{ }^{142} \mathrm{Ho},{ }^{149} \mathrm{Yb}\left(\beta^{+} \mathrm{p}\right)$; measured $\beta$-delayed $\mathrm{E} \gamma, \mathrm{I} \gamma$, proton spectra, $\mathrm{p} \gamma$-coin, $\mathrm{T}_{1 / 2}$. Comparison with model predictions. JOUR PRVCA 71054318

\section{$\mathrm{A}=94$}

\begin{tabular}{|c|c|c|}
\hline${ }^{94} \mathrm{Rb}$ & 2005AN01 & $\begin{array}{l}\text { NUCLEAR REACTIONS }{ }^{238} \mathrm{U}(\mathrm{p}, \mathrm{F}){ }^{89} \mathrm{Rb} /{ }^{90} \mathrm{Rb} /{ }^{91} \mathrm{Rb} /{ }^{93} \mathrm{Rb} / \\
{ }^{94} \mathrm{Rb} /{ }^{95} \mathrm{Rb} /{ }^{139} \mathrm{Cs} /{ }^{140} \mathrm{Cs} /{ }^{141} \mathrm{Cs} /{ }^{142} \mathrm{Cs} /{ }^{144} \mathrm{Cs} /{ }^{145} \mathrm{Cs}, \mathrm{E}=1 \\
\mathrm{GeV} \text { : measured vields. JOUR ZAANE } 23257\end{array}$ \\
\hline${ }^{94} \mathrm{Y}$ & 2005SEZX & $\begin{array}{l}\text { NUCLEAR REACTIONS }{ }^{90,94} \mathrm{Zr}(\mathrm{n}, \alpha),{ }^{90,91,92,94} \mathrm{Zr}(\mathrm{n}, \mathrm{p}),{ }^{91,92} \mathrm{Zr}(\mathrm{n}, \\
\mathrm{np}+\mathrm{d}), \mathrm{E}=14-20 \mathrm{MeV} \text {; measured activation } \sigma . \text { Comparison with model } \\
\text { predictions. CONF Santa Fe (Nucl Data for Sci and Technol) } \\
\text { Proc,Vol1,P981 }\end{array}$ \\
\hline${ }^{94} \mathrm{Zr}$ & 2005BI25 & $\begin{array}{l}\text { NUCLEAR MOMENTS } 86,87,88,89,90,91,92,93,94,95,96,97,98,99,100,101,102 \mathrm{Zr} \text {; } \\
\text { measured charge radii. }{ }^{176} \mathrm{Yb} \text {; measured isomer shift. Ion-beam cooler, } \\
\text { laser spectroscopy. JOUR ZAANE } 25 \text { s01 } 187\end{array}$ \\
\hline & 2005PA48 & $\begin{array}{l}\text { NUCLEAR REACTIONS }{ }^{176} \mathrm{Yb}\left({ }^{28} \mathrm{Si}, \mathrm{X}\right){ }^{92} \mathrm{Zr} /{ }^{93} \mathrm{Zr} /{ }^{94} \mathrm{Zr} /{ }^{95} \mathrm{Zr} / \\
{ }^{96} \mathrm{Zr}, \mathrm{E}=145 \mathrm{MeV} ;{ }^{176} \mathrm{Yb}\left({ }^{31} \mathrm{P}, \mathrm{X}\right){ }^{92} \mathrm{Zr} /{ }^{93} \mathrm{Zr} /{ }^{94} \mathrm{Zr} /{ }^{95} \mathrm{Zr} /{ }^{96} \mathrm{Zr} \text {, } \\
\mathrm{E}=152 \mathrm{MeV} ; \text { measured } \mathrm{E} \gamma, \mathrm{I} \gamma, \gamma \gamma \text {-coin. }{ }^{92,93,94,95,96} \mathrm{Zr} \text { deduced } \\
\text { high-spin levels, J, } \pi \text {, configurations. Eurogam II and Euroball IV } \\
\text { arrays, comparisons with shell-model predictions. JOUR PRVCA } 72 \\
024304\end{array}$ \\
\hline \multirow[t]{3}{*}{${ }^{94} \mathrm{Nb}$} & $2004 \mathrm{ZH} 45$ & $\begin{array}{l}\text { NUCLEAR REACTIONS }{ }^{90,94} \mathrm{Zr}(\mathrm{p}, \mathrm{n}), \mathrm{E}=7-11 \mathrm{MeV} \text {; measured En, } \\
\sigma(\mathrm{E}, \theta) \text {, excitation functions. }{ }^{90,94} \mathrm{Nb} \text { deduced level densities, shell } \\
\text { effects. Optical-statistical analysis. JOUR BRSPE } 681319\end{array}$ \\
\hline & 2005RE09 & $\begin{array}{l}\text { NUCLEAR REACTIONS }{ }^{92,94} \mathrm{Mo}(\mathrm{n}, 2 \mathrm{n}),{ }^{92,100} \mathrm{Mo}(\mathrm{n}, \alpha),{ }^{95,96,97} \mathrm{Mo}(\mathrm{n}, \\
\mathrm{p}),{ }^{96,97,98} \mathrm{Mo}(\mathrm{n}, \mathrm{np}+\mathrm{d}), \mathrm{E} \approx 13.5-21 \mathrm{MeV} \text {; measured activation } \sigma ; \\
\text { deduced reaction mechanism features. }{ }^{93} \mathrm{Nb}(\mathrm{p}, \mathrm{n}),(\mathrm{p}, \gamma), \mathrm{E} \approx 1-6 \\
\mathrm{MeV} ;{ }^{92,93,94,95,96,97,98,100} \mathrm{Mo},{ }^{93} \mathrm{Nb}(\mathrm{n}, \gamma), \mathrm{E}<4 \mathrm{MeV} ;{ }^{92,94,100} \mathrm{Mo}(\mathrm{n}, \\
2 \mathrm{n}),{ }^{92,94,95,96,97,98} \mathrm{Mo}(\mathrm{n}, \mathrm{p}),{ }^{92,94,95,96,97,98,100} \mathrm{Mo}(\mathrm{n}, \mathrm{np}+\mathrm{d}), \\
92,98,100 \mathrm{Mo}(\mathrm{n}, \alpha), \mathrm{E}<21 \mathrm{MeV} \text {; compiled, analyzed } \sigma \text {. Analysis with }\end{array}$ \\
\hline & $2005 \mathrm{ZHZZ}$ & $\begin{array}{l}\text { local and global approaches compared. JOUR PRVCA } 71044617 \\
\text { NUCLEAR REACTIONS }{ }^{56,57} \mathrm{Fe},{ }^{90,94} \mathrm{Zr}(\mathrm{p}, \mathrm{n}), \mathrm{E}=7-11 \mathrm{MeV} ; \\
\text { measured En, } \sigma(\mathrm{E}) .{ }^{56,57} \mathrm{Co},{ }^{90,94} \mathrm{Nb} \text { deduced level densities. Statistical } \\
\text { equilibrium and pre-equilibrium model analysis. CONF Santa Fe (Nucl } \\
\text { Data for Sci and Technol) Proc,Vol1,P931 }\end{array}$ \\
\hline${ }^{94} \mathrm{Mo}$ & $2005 \mathrm{CHZW}$ & $\begin{array}{l}\text { NUCLEAR REACTIONS }{ }^{94,96} \mathrm{Mo}\left({ }^{3} \mathrm{He},{ }^{3} \mathrm{He}\right),\left({ }^{3} \mathrm{He}, \alpha\right), \mathrm{E}=30 \mathrm{MeV} \\
97,98 \mathrm{Mo}\left({ }^{3} \mathrm{He},{ }^{3} \mathrm{He}\right),\left({ }^{3} \mathrm{He}, \alpha\right), \mathrm{E}=45 \mathrm{MeV} ; \text { measured particle spectra, } \\
\mathrm{E} \gamma, \mathrm{I} \gamma,(\text { particle }) \gamma \text {-coin. }{ }^{93,94,95,96,97,98} \mathrm{Mo} \text { deduced level density } \\
\text { parameters, thermodynamic quantities. PREPRINT } \\
\text { nucl-ex/0507007,7/04/2005 }\end{array}$ \\
\hline
\end{tabular}
nucl-ex/0507007,7/04/2005 


\section{$\mathrm{A}=94$ (continued)}

2005 GU16 NUCLEAR REACTIONS ${ }^{94,96} \mathrm{Mo}\left({ }^{3} \mathrm{He},{ }^{3} \mathrm{He}\right),\left({ }^{3} \mathrm{He}, \alpha\right), \mathrm{E}=30 \mathrm{MeV}$; $\left.{ }^{97,98} \mathrm{Mo}\left({ }^{3} \mathrm{He},{ }^{3} \mathrm{He}\right)^{\prime}\right),\left({ }^{3} \mathrm{He}, \alpha\right), \mathrm{E}=45 \mathrm{MeV}$; measured particle spectra, $\mathrm{E} \gamma, \mathrm{I} \gamma$, (particle) $\gamma$-coin. ${ }^{93,94,95,96,97,98}$ Mo deduced radiative strength functions. JOUR PRVCA 71044307

2005RE09 NUCLEAR REACTIONS ${ }^{92,94} \mathrm{Mo}(\mathrm{n}, 2 \mathrm{n}),{ }^{92,100} \mathrm{Mo}(\mathrm{n}, \alpha),{ }^{95,96,97} \mathrm{Mo}(\mathrm{n}$, p), ${ }^{96,97,98} \mathrm{Mo}(\mathrm{n}, \mathrm{np}+\mathrm{d}), \mathrm{E} \approx 13.5-21 \mathrm{MeV}$; measured activation $\sigma$; deduced reaction mechanism features. ${ }^{93} \mathrm{Nb}(\mathrm{p}, \mathrm{n}),(\mathrm{p}, \gamma), \mathrm{E} \approx 1-6$ $\mathrm{MeV} ;{ }^{92,93,94,95,96,97,98,100} \mathrm{Mo},{ }^{93} \mathrm{Nb}(\mathrm{n}, \gamma), \mathrm{E}<4 \mathrm{MeV} ;{ }^{92,94,100} \mathrm{Mo}(\mathrm{n}$, 2n), ${ }^{92,94,95,96,97,98} \mathrm{Mo}(\mathrm{n}, \mathrm{p}),{ }^{92,94,95,96,97,98,100} \mathrm{Mo}(\mathrm{n}, \mathrm{np}+\mathrm{d})$, ${ }_{92,98,100} \mathrm{Mo}(\mathrm{n}, \alpha), \mathrm{E}<21 \mathrm{MeV}$; compiled, analyzed $\sigma$. Analysis with local and global approaches compared. JOUR PRVCA 71044617

\section{${ }^{94} \mathrm{Ru}$ \\ 2005CL08}

${ }^{94} \mathrm{Rh}$

2005CL08

${ }^{94} \mathrm{Ag}$

2005MU15

2005MUZX

${ }^{95} \mathrm{Sr}$

${ }^{95} \mathrm{Y}$

${ }^{95} \mathrm{Zr}$

2005AN01 ATOMIC MASSES ${ }^{64} \mathrm{Ge},{ }^{68} \mathrm{Se}$; analyzed masses; deduced effective $\mathrm{T}_{1 / 2} \cdot{ }^{90,91} \mathrm{Mo},{ }^{90,91,92,93} \mathrm{Tc},{ }^{93,94} \mathrm{Ru},{ }^{94,95} \mathrm{Rh},{ }^{104,105,106,107} \mathrm{In}$, ${ }_{104,105,107,108} \mathrm{Sn},{ }^{107,108} \mathrm{Sb}$; measured masses. Penning trap, astrophysical implications discussed. JOUR ZAANE 25 s01 629 ATOMIC MASSES ${ }^{64} \mathrm{Ge},{ }^{68} \mathrm{Se}$; analyzed masses; deduced effective $\mathrm{T}_{1 / 2} \cdot{ }^{90,91} \mathrm{Mo},{ }^{90,91,92,93} \mathrm{Tc},{ }^{93,94} \mathrm{Ru},{ }^{94,95} \mathrm{Rh},{ }^{104,105,106,107} \mathrm{In}$, ${ }_{104,105,107,108}$ Sn, ${ }^{107,108}$ Sb; measured masses. Penning trap, astrophysical implications discussed. JOUR ZAANE 25 s01 629 RADIOACTIVITY ${ }^{94 m} \mathrm{Ag}$ (p) [from ${ }^{58} \mathrm{Ni}\left({ }^{40} \mathrm{Ca}, 3 \mathrm{np}\right)$ ]; measured $\mathrm{Ep}$, $\gamma \gamma$-, $\mathrm{p} \gamma$-coin, $\mathrm{T}_{1 / 2}$ following decay of high-spin isomer; deduced branching ratios, Q-value. ${ }^{94} \mathrm{Ag}$ deduced isomer configuration, deformation. ${ }^{93} \mathrm{Pd}$ deduced levels. JOUR PRLTA 95022501 RADIOACTIVITY ${ }^{94 m} \mathrm{Ag}(2 \mathrm{p})$ [from $\left.{ }^{58} \mathrm{Ni}\left({ }^{40} \mathrm{Ca}, 3 \mathrm{np}\right)\right]$; measured $\mathrm{E} \gamma$, Ep, pp-, $\gamma \gamma-$, p $\gamma$-coin; deduced two-proton decay branching ratio. ${ }^{92} \mathrm{Rh}$ deduced levels, J, $\pi$. REPT GSI 2005-1,P87,Mukha

\section{$\mathrm{A}=95$}

NUCLEAR REACTIONS ${ }^{238} \mathrm{U}(\mathrm{p}, \mathrm{F}){ }^{89} \mathrm{Rb} /{ }^{90} \mathrm{Rb} /{ }^{91} \mathrm{Rb} /{ }^{93} \mathrm{Rb} /$ ${ }^{94} \mathrm{Rb} /{ }^{95} \mathrm{Rb} /{ }^{139} \mathrm{Cs} /{ }^{140} \mathrm{Cs} /{ }^{141} \mathrm{Cs} /{ }^{142} \mathrm{Cs} /{ }^{144} \mathrm{Cs} /{ }^{145} \mathrm{Cs}, \mathrm{E}=1$ $\mathrm{GeV}$; measured yields. JOUR ZAANE 23257

2005HW06 RADIOACTIVITY ${ }^{252} \mathrm{Cf}(\mathrm{SF})$; measured E $\gamma, \mathrm{I} \gamma, \gamma \gamma$-coin. ${ }^{95,97} \mathrm{Sr},{ }^{99} \mathrm{Zr}$, ${ }^{108} \mathrm{Tc},{ }^{133,134} \mathrm{Te},{ }^{137} \mathrm{Xe}$ levels deduced $\mathrm{T}_{1 / 2}$. Gammasphere array, time-gated triple-coincidence method. JOUR ZAANE 25 s01 463 2005BE17 RADIOACTIVITY ${ }^{127} \mathrm{I}\left({ }^{24} \mathrm{Ne}\right),\left({ }^{28} \mathrm{Mg}\right),\left({ }^{30} \mathrm{Mg}\right),\left({ }^{32} \mathrm{Si}\right),\left({ }^{34} \mathrm{Si}\right),\left({ }^{48} \mathrm{Ca}\right)$, $\left({ }^{49} \mathrm{Sc}\right)$; measured cluster decay $\mathrm{T}_{1 / 2}$ lower limits. JOUR ZAANE 2451 2004MIZS NUCLEAR REACTIONS Fe(p, X) ${ }^{52} \mathrm{Mn}, \mathrm{E}<2.6 \mathrm{GeV} ; \mathrm{Pb}(\mathrm{p}, \mathrm{X}){ }^{10} \mathrm{Be}$, $\mathrm{E}<2.6 \mathrm{GeV} ;{ }^{209} \mathrm{Bi}(\mathrm{p}, 4 \mathrm{np}), \mathrm{E}<2.6 \mathrm{GeV} ; \mathrm{Pb}(\mathrm{n}, \mathrm{X}){ }^{196} \mathrm{Au} /{ }^{95} \mathrm{Zr}, \mathrm{E} \approx$ 70-180 MeV; measured excitation functions. Comparison with model predictions. REPT NEA/NSC/DOC(2004)14,P28,Michel

2005BI25 NUCLEAR MOMENTS 86,87,88,89,90,91,92,93,94,95,96,97,98,99,100,101,102 Zr; measured charge radii. ${ }^{176} \mathrm{Yb}$; measured isomer shift. Ion-beam cooler, laser spectroscopy. JOUR ZAANE 25 s01 187

2005MOZW NUCLEAR REACTIONS ${ }^{90,91,92,94,96} \mathrm{Zr}(\mathrm{n}, \gamma), \mathrm{E}<100 \mathrm{keV}$; measured $\mathrm{E} \gamma$, I $\gamma$, capture yields. CONF Santa Fe (Nucl Data for Sci and Technol) Proc,Vol1,P880 


\section{$\mathrm{A}=95$ (continued)}

2005PA48 NUCLEAR REACTIONS ${ }^{176} \mathrm{Yb}\left({ }^{28} \mathrm{Si}, \mathrm{X}\right){ }^{92} \mathrm{Zr} /{ }^{93} \mathrm{Zr} /{ }^{94} \mathrm{Zr} /{ }^{95} \mathrm{Zr} /$ ${ }^{96} \mathrm{Zr}, \mathrm{E}=145 \mathrm{MeV} ;{ }^{176} \mathrm{Yb}\left({ }^{31} \mathrm{P}, \mathrm{X}\right){ }^{92} \mathrm{Zr} /{ }^{93} \mathrm{Zr} /{ }^{94} \mathrm{Zr} /{ }^{95} \mathrm{Zr} /{ }^{96} \mathrm{Zr}$, $\mathrm{E}=152 \mathrm{MeV}$; measured $\mathrm{E} \gamma, \mathrm{I} \gamma, \gamma \gamma$-coin. ${ }^{92,93,94,95,96} \mathrm{Zr}$ deduced high-spin levels, J, $\pi$, configurations. Eurogam II and Euroball IV arrays, comparisons with shell-model predictions. JOUR PRVCA 72 024304

2005RE09 NUCLEAR REACTIONS ${ }^{92,94} \mathrm{Mo}(\mathrm{n}, 2 \mathrm{n}),{ }^{92,100} \mathrm{Mo}(\mathrm{n}, \alpha),{ }^{95,96,97} \mathrm{Mo}(\mathrm{n}$, p), ${ }^{96,97,98} \mathrm{Mo}(\mathrm{n}, \mathrm{np}+\mathrm{d}), \mathrm{E} \approx 13.5-21 \mathrm{MeV}$; measured activation $\sigma$; deduced reaction mechanism features. ${ }^{93} \mathrm{Nb}(\mathrm{p}, \mathrm{n}),(\mathrm{p}, \gamma), \mathrm{E} \approx 1-6$ $\mathrm{MeV} ;{ }^{92,93,94,95,96,97,98,100} \mathrm{Mo},{ }^{93} \mathrm{Nb}(\mathrm{n}, \gamma), \mathrm{E}<4 \mathrm{MeV} ;{ }^{92,94,100} \mathrm{Mo}(\mathrm{n}$, 2n), ${ }^{92,94,95,96,97,98} \mathrm{Mo}(\mathrm{n}, \mathrm{p}),{ }^{92,94,95,96,97,98,100} \mathrm{Mo}(\mathrm{n}, \mathrm{np}+\mathrm{d})$, ${ }^{92,98,100} \mathrm{Mo}(\mathrm{n}, \alpha), \mathrm{E}<21 \mathrm{MeV}$; compiled, analyzed $\sigma$. Analysis with local and global approaches compared. JOUR PRVCA 71044617 2005TA23 NUCLEAR REACTIONS ${ }^{90,91,92,94,96} \mathrm{Zr}(\mathrm{n}, \gamma), \mathrm{E}<1 \mathrm{MeV}$; measured $\mathrm{E} \gamma, \mathrm{I} \gamma$, capture yields. JOUR NUPAB $758573 \mathrm{c}$

2005UOzZ NUCLEAR REACTIONS U(p, F $){ }^{95} \mathrm{Zr} /{ }^{115} \mathrm{Cd} /{ }^{134} \mathrm{Cs} /{ }^{136} \mathrm{Cs} /{ }^{137} \mathrm{Cs}$ $/{ }^{147} \mathrm{Nd}, \mathrm{E} \approx 20-70 \mathrm{MeV}$; measured production $\sigma$. Stacked-foil activation technique, comparison with model predictions. CONF Santa Fe (Nucl Data for Sci and Technol) Proc,Vol2,P1547

${ }^{95} \mathrm{Nb} \quad 2005 \mathrm{BU} 08$ NUCLEAR REACTIONS ${ }^{82} \mathrm{Se}\left({ }^{12} \mathrm{C}, 2 \mathrm{np}\right), \mathrm{E}=38 \mathrm{MeV} ;{ }^{82} \mathrm{Se}\left({ }^{16} \mathrm{O}, 2 \mathrm{np}\right)$, $\mathrm{E}=48 \mathrm{MeV}$; measured $\mathrm{E} \gamma, \mathrm{I} \gamma, \gamma \gamma-$, (charged particle) $\gamma-$,

(neutron) $\gamma$-coin. ${ }^{12} \mathrm{C},{ }^{16} \mathrm{O}\left({ }^{82} \mathrm{Se}, \mathrm{X}\right){ }^{91} \mathrm{Y} /{ }^{95} \mathrm{Nb}, \mathrm{E}=470 \mathrm{MeV}$; measured $\mathrm{E} \gamma, \mathrm{I} \gamma, \gamma \gamma$-coin. ${ }^{91} \mathrm{Y},{ }^{95} \mathrm{Nb}$ deduced high-spin levels, J, $\pi$,

configurations. GASP array, comparison with shell model predictions, level systematics in neighboring isotones discussed. JOUR PRVCA 71 034315

2005RA30 NUCLEAR REACTIONS ${ }^{93} \mathrm{Nb}(\mathrm{t}, \mathrm{p}), \mathrm{E}=12 \mathrm{MeV}$; measured Ep, $\sigma(\mathrm{E}$, $\theta) .{ }^{95} \mathrm{Nb}$ deduced levels, $\mathrm{J}, \pi$. Comparison with previous results and model predictions. JOUR PRVCA 72054303

2005RE09 NUCLEAR REACTIONS ${ }^{92,94} \mathrm{Mo}(\mathrm{n}, 2 \mathrm{n}),{ }^{92,100} \mathrm{Mo}(\mathrm{n}, \alpha),{ }^{95,96,97} \mathrm{Mo}(\mathrm{n}$, p), ${ }^{96,97,98} \mathrm{Mo}(\mathrm{n}, \mathrm{np}+\mathrm{d}), \mathrm{E} \approx 13.5-21 \mathrm{MeV}$; measured activation $\sigma$; deduced reaction mechanism features. ${ }^{93} \mathrm{Nb}(\mathrm{p}, \mathrm{n}),(\mathrm{p}, \gamma), \mathrm{E} \approx 1-6$ $\mathrm{MeV} ;{ }^{92,93,94,95,96,97,98,100} \mathrm{Mo},{ }^{93} \mathrm{Nb}(\mathrm{n}, \gamma), \mathrm{E}<4 \mathrm{MeV} ;{ }^{92,94,100} \mathrm{Mo}(\mathrm{n}$, 2n), ${ }^{92,94,95,96,97,98} \mathrm{Mo}(\mathrm{n}, \mathrm{p}),{ }^{92,94,95,96,97,98,100} \mathrm{Mo}(\mathrm{n}, \mathrm{np}+\mathrm{d})$,

${ }_{92,98,100} \mathrm{Mo}(\mathrm{n}, \alpha), \mathrm{E}<21 \mathrm{MeV}$; compiled, analyzed $\sigma$. Analysis with local and global approaches compared. JOUR PRVCA 71044617

${ }^{95} \mathrm{Mo} \quad$ 2005CHzw NUCLEAR REACTIONS ${ }^{94,96} \mathrm{Mo}\left({ }^{3} \mathrm{He},{ }^{3} \mathrm{He}\right),\left({ }^{3} \mathrm{He}, \alpha\right), \mathrm{E}=30 \mathrm{MeV}$; ${ }^{97,98} \mathrm{Mo}\left({ }^{3} \mathrm{He},{ }^{3} \mathrm{He}\right),\left({ }^{3} \mathrm{He}, \alpha\right), \mathrm{E}=45 \mathrm{MeV}$; measured particle spectra, $\mathrm{E} \gamma, \mathrm{I} \gamma$, (particle) $\gamma$-coin. ${ }^{93,94,95,96,97,98}$ Mo deduced level density parameters, thermodynamic quantities. PREPRINT nucl-ex/0507007,7/04/2005

2005 GU16 NUCLEAR REACTIONS ${ }^{94,96} \mathrm{Mo}\left({ }^{3} \mathrm{He},{ }^{3} \mathrm{He}\right),\left({ }^{3} \mathrm{He}, \alpha\right), \mathrm{E}=30 \mathrm{MeV}$; ${ }^{97,98} \mathrm{Mo}\left({ }^{3} \mathrm{He},{ }^{3} \mathrm{He}\right),\left({ }^{3} \mathrm{He}, \alpha\right), \mathrm{E}=45 \mathrm{MeV}$; measured particle spectra, $\mathrm{E} \gamma, \mathrm{I} \gamma$, (particle) $\gamma$-coin. ${ }^{93,94,95,96,97,98} \mathrm{Mo}$ deduced radiative strength functions. JOUR PRVCA 71044307

2005HA49 NUCLEAR REACTIONS ${ }^{92} \mathrm{Mo}(\alpha, \gamma), \mathrm{E}=9 \mathrm{MeV} ;{ }^{91} \mathrm{Zr}(\alpha, \gamma), \mathrm{E}=10.5$ $\mathrm{MeV} ;{ }^{118} \mathrm{Sn}(\alpha, \gamma), \mathrm{E}=11.5 \mathrm{MeV}$; measured $\mathrm{E} \gamma, \mathrm{I} \gamma \cdot{ }^{91} \mathrm{Zr},{ }^{118} \mathrm{Sn}(\alpha, \gamma)$, $\mathrm{E}(\mathrm{cm}) \approx 9-11 \mathrm{MeV}$; measured $\sigma$. Comparison with model predictions. JOUR NUPAB $758505 \mathrm{c}$ 


\section{$\mathrm{A}=95$ (continued)}

\begin{tabular}{|c|c|c|}
\hline${ }^{95} \mathrm{Tc}$ & 2005MU22 & $\begin{array}{l}\text { NUCLEAR REACTIONS }{ }^{93} \mathrm{Nb}(\alpha, 2 \mathrm{n}), \mathrm{E} \approx 20-120 \mathrm{MeV} ; \text { measured } \\
\text { excitation function, isomer yield ratio. Activation technique, } \\
\text { comparison with model predictions. JOUR PRVCA } 72014609\end{array}$ \\
\hline${ }^{95} \mathrm{Rh}$ & 2005CL08 & $\begin{array}{l}\text { ATOMIC MASSES }{ }^{64} \mathrm{Ge},{ }^{68} \mathrm{Se} \text {; analyzed masses; deduced effective } \\
\mathrm{T}_{1 / 2} \cdot{ }^{90,91} \mathrm{Mo},{ }^{90,91,92,93} \mathrm{Tc},{ }^{93,94} \mathrm{Ru},{ }^{94,95} \mathrm{Rh},{ }^{104,105,106,107} \mathrm{In}, \\
{ }_{104,105,107,108} \mathrm{Sn},{ }^{107,108} \mathrm{Sb} \text {; measured masses. Penning trap, }\end{array}$ \\
\hline${ }^{95} \mathrm{Pd}$ & 2005 HA 45 & $\begin{array}{l}\text { astrophysical implications discussed. JOUR ZAANE } 25 \text { s01 } 629 \\
\text { RADIOACTIVITY }{ }^{95} \mathrm{Ag}(\mathrm{EC})\left[\text { from }{ }^{58} \mathrm{Ni}\left({ }^{40} \mathrm{Ca}, 2 \mathrm{np}\right)\right] ; \text { measured } \\
\beta \text {-delayed } \mathrm{E} \gamma, \mathrm{I} \gamma, \gamma \gamma-, \beta \gamma \text {-coin. }{ }^{95} \mathrm{Pd} \text { deduced levels, J, } \pi \text {. Mass } \\
\text { separator. Comparison with shell-model predictions. JOUR PRVCA } 72 \\
024303\end{array}$ \\
\hline${ }^{95} \mathrm{Ag}$ & $2005 \mathrm{HA} 45$ & $\begin{array}{l}\text { RADIOACTIVITY }{ }^{95} \mathrm{Ag}(\mathrm{EC})\left[\text { from }{ }^{58} \mathrm{Ni}\left({ }^{40} \mathrm{Ca}, 2 \mathrm{np}\right)\right] ; \text { measured } \\
\beta \text {-delayed } \mathrm{E} \gamma, \mathrm{I} \gamma, \gamma \gamma-, \beta \gamma \text {-coin. }{ }^{95} \mathrm{Pd} \text { deduced levels, J, } \pi \text {. Mass } \\
\text { separator. Comparison with shell-model predictions. JOUR PRVCA } 72 \\
024303\end{array}$ \\
\hline
\end{tabular}

\section{$\mathrm{A}=96$}

\begin{tabular}{|c|c|c|}
\hline${ }^{96} \mathrm{Rb}$ & 2005PI13 & $\begin{array}{l}\text { NUCLEAR REACTIONS }{ }^{241} \mathrm{Pu}(\mathrm{n}, \mathrm{F}){ }^{96} \mathrm{Rb}, \mathrm{E}=\text { thermal; measured } \\
\text { delayed } \mathrm{E} \gamma, \mathrm{I} \gamma, \mathrm{E}(\mathrm{ce}), \mathrm{I}(\mathrm{ce}), \gamma \gamma-,(\mathrm{ce}) \gamma \text {-coin, X-ray spectra. }{ }^{96} \mathrm{Rb} \\
\text { deduced levels, J, } \pi \text {, configurations, deformation, isomer } \mathrm{T}_{1 / 2} \text {. Mass } \\
\text { separator, comparisons with neighboring nuclides. JOUR PRVCA } 71 \\
064327\end{array}$ \\
\hline${ }^{96} \mathrm{Sr}$ & 2004WU08 & $\begin{array}{l}\text { NUCLEAR REACTIONS }{ }^{238} \mathrm{U}(\alpha, \mathrm{F}){ }^{96} \mathrm{Sr} /{ }^{97} \mathrm{Sr} /{ }^{98} \mathrm{Zr} /{ }^{99} \mathrm{Zr}, \mathrm{E}=30 \\
\mathrm{MeV} \text {; measured } \mathrm{E} \gamma, \mathrm{I} \gamma, \gamma \gamma-\text {, (fragment }) \gamma \text {-coin. }{ }^{96,97} \mathrm{Sr},{ }^{98,99} \mathrm{Zr} \text { deduced } \\
\text { high-spin levels, J, } \pi \text {, configurations. Gammasphere, Chico arrays. } \\
\text { JOUR PRVCA } 70064312\end{array}$ \\
\hline \multirow[t]{5}{*}{${ }^{96} \mathrm{Zr}$} & 2005BI25 & $\begin{array}{l}\text { NUCLEAR MOMENTS } 86,87,88,89,90,91,92,93,94,95,96,97,98,99,100,101,102 \mathrm{Zr} \text {; } \\
\text { measured charge radii. }{ }^{176} \mathrm{Yb} \text {; measured isomer shift. Ion-beam cooler, } \\
\text { laser spectroscopy. JOUR ZAANE } 25 \text { s01 } 187\end{array}$ \\
\hline & 2005PA48 & $\begin{array}{l}\text { NUCLEAR REACTIONS }{ }^{176} \mathrm{Yb}\left({ }^{28} \mathrm{Si}, \mathrm{X}\right){ }^{92} \mathrm{Zr} /{ }^{93} \mathrm{Zr} /{ }^{94} \mathrm{Zr} /{ }^{95} \mathrm{Zr} / \\
{ }^{96} \mathrm{Zr}, \mathrm{E}=145 \mathrm{MeV} ;{ }^{176} \mathrm{Yb}\left({ }^{31} \mathrm{P}, \mathrm{X}\right){ }^{92} \mathrm{Zr} /{ }^{93} \mathrm{Zr} /{ }^{94} \mathrm{Zr} /{ }^{95} \mathrm{Zr} /{ }^{96} \mathrm{Zr} \text {, } \\
\mathrm{E}=152 \mathrm{MeV} \text {; measured } \mathrm{E} \gamma, \mathrm{I} \gamma, \gamma \gamma \text {-coin. }{ }^{92,93,94,95,96} \mathrm{Zr} \text { deduced } \\
\text { high-spin levels, J, } \pi \text {, configurations. Eurogam II and Euroball IV } \\
\text { arrays, comparisons with shell-model predictions. JOUR PRVCA } 72 \\
024304\end{array}$ \\
\hline & 2005SA07 & $\begin{array}{l}\text { RADIOACTIVITY }{ }^{82} \mathrm{Se},{ }^{96} \mathrm{Zr},{ }^{100} \mathrm{Mo},{ }^{116} \mathrm{Cd},{ }^{150} \mathrm{Nd}\left(2 \beta^{-}\right) \text {; measured } \\
2 \nu \beta \beta \text {-decay } \mathrm{T}_{1 / 2} \cdot{ }^{82} \mathrm{Se},{ }^{100} \mathrm{Mo}\left(2 \beta^{-}\right) \text {; measured } 0 \nu \beta \beta \text {-decay } \mathrm{T}_{1 / 2} \text { lower } \\
\text { limits; deduced neutrino mass limits. JOUR NPBSE } 143221\end{array}$ \\
\hline & 2005SI06 & $\begin{array}{l}\text { RADIOACTIVITY }{ }^{82} \mathrm{Se},{ }^{96} \mathrm{Zr},{ }^{100} \mathrm{Mo},{ }^{116} \mathrm{Cd},{ }^{150} \mathrm{Nd}\left(2 \beta^{-}\right) \text {; measured } \\
2 \nu \beta \beta \text {-decay } \mathrm{T}_{1 / 2} \cdot{ }^{82} \mathrm{Se},{ }^{100} \mathrm{Mo}\left(2 \beta^{-}\right) \text {; measured } 0 \nu \beta \beta \text {-decay } \mathrm{T}_{1 / 2} \text { lower } \\
\text { limits; deduced neutrino mass limits. JOUR NPBSE } 145272\end{array}$ \\
\hline & 2005SM08 & 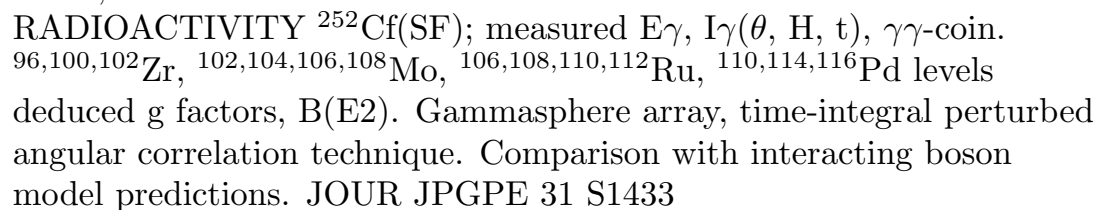 \\
\hline
\end{tabular}




\section{$\mathrm{A}=96$ (continued)}

\begin{tabular}{|c|c|c|}
\hline${ }^{96} \mathrm{Nb}$ & 2005RE09 & $\begin{array}{l}\text { NUCLEAR REACTIONS }{ }^{92,94} \mathrm{Mo}(\mathrm{n}, 2 \mathrm{n}),{ }^{92,100} \mathrm{Mo}(\mathrm{n}, \alpha),{ }^{95,96,97} \mathrm{Mo}(\mathrm{n}, \\
\mathrm{p}),{ }^{96,97,98} \mathrm{Mo}(\mathrm{n}, \mathrm{np}+\mathrm{d}), \mathrm{E} \approx 13.5-21 \mathrm{MeV} ; \text { measured activation } \sigma ; \\
\text { deduced reaction mechanism features. }{ }^{93} \mathrm{Nb}(\mathrm{p}, \mathrm{n}),(\mathrm{p}, \gamma), \mathrm{E} \approx 1-6 \\
\mathrm{MeV} ;{ }^{92,93,94,95,96,97,98,100} \mathrm{Mo},{ }^{93} \mathrm{Nb}(\mathrm{n}, \gamma), \mathrm{E}<4 \mathrm{MeV} ;{ }^{92,94,100} \mathrm{Mo}(\mathrm{n}, \\
2 \mathrm{n}),{ }^{92,94,95,96,97,98} \mathrm{Mo}(\mathrm{n}, \mathrm{p}),{ }^{92,94,95,96,97,98,100} \mathrm{Mo}(\mathrm{n}, \mathrm{np}+\mathrm{d}), \\
92,98,100 \mathrm{Mo}(\mathrm{n}, \alpha), \mathrm{E}<21 \mathrm{MeV} \text {; compiled, analyzed } \sigma . \text { Analysis with } \\
\text { local and olobal anproaches compared. JOUR PRVCA } 71044617\end{array}$ \\
\hline \multirow[t]{5}{*}{${ }^{96} \mathrm{Mo}$} & 2005CHZW & $\begin{array}{l}\text { local and global approaches compared. JOUR PRVCA } 71044617 \\
\text { NUCLEAR REACTIONS }{ }^{44,96} \mathrm{Mo}\left({ }^{3} \mathrm{He},{ }^{3} \mathrm{He}\right),\left({ }^{3} \mathrm{He}, \alpha\right), \mathrm{E}=30 \mathrm{MeV} \text {; } \\
97,98 \mathrm{Mo}\left({ }^{3} \mathrm{He},{ }^{3} \mathrm{He}\right),\left({ }^{3} \mathrm{He}, \alpha\right), \mathrm{E}=45 \mathrm{MeV} ; \text { measured particle spectra, } \\
\mathrm{E} \gamma, \mathrm{I} \gamma,(\text { particle }) \gamma \text {-coin. }{ }^{93,94,95,96,97,98} \mathrm{Mo} \text { deduced level density } \\
\text { parameters, thermodynamic quantities. PREPRINT } \\
\text { nucl-ex/0507007,7/04/2005 }\end{array}$ \\
\hline & 2005 GU16 & $\begin{array}{l}\text { NUCLEAR REACTIONS }{ }^{94,96} \mathrm{Mo}\left({ }^{3} \mathrm{He},{ }^{3} \mathrm{He}\right),\left({ }^{3} \mathrm{He}, \alpha\right), \mathrm{E}=30 \mathrm{MeV} \\
97,98 \mathrm{Mo}\left({ }^{3} \mathrm{He},{ }^{3} \mathrm{He}\right),\left({ }^{3} \mathrm{He}, \alpha\right), \mathrm{E}=45 \mathrm{MeV} ; \text { measured particle spectra, } \\
\mathrm{E} \gamma, \mathrm{I} \gamma \text {, (particle) } \gamma \text {-coin. }{ }^{93,94,95,96,97,98} \mathrm{Mo} \text { deduced radiative strength } \\
\text { functions. JOUR PRVCA } 71044307\end{array}$ \\
\hline & $2005 \mathrm{SA} 07$ & $\begin{array}{l}\text { RADIOACTIVITY }{ }^{82} \mathrm{Se},{ }^{96} \mathrm{Zr},{ }^{100} \mathrm{Mo},{ }^{116} \mathrm{Cd},{ }^{150} \mathrm{Nd}\left(2 \beta^{-}\right) \text {; measured } \\
2 \nu \beta \beta \text {-decay } \mathrm{T}_{1 / 2} \cdot{ }^{82} \mathrm{Se},{ }^{100} \mathrm{Mo}\left(2 \beta^{-}\right) \text {; measured } 0 \nu \beta \beta \text {-decay } \mathrm{T}_{1 / 2} \text { lower } \\
\text { limits; deduced neutrino mass limits. JOUR NPBSE } 143221\end{array}$ \\
\hline & 2005SI06 & $\begin{array}{l}\text { RADIOACTIVITY }{ }^{82} \mathrm{Se},{ }^{96} \mathrm{Zr},{ }^{100} \mathrm{Mo},{ }^{116} \mathrm{Cd},{ }^{150} \mathrm{Nd}\left(2 \beta^{-}\right) \text {; measured } \\
2 \nu \beta \beta \text {-decay } \mathrm{T}_{1 / 2} \cdot{ }^{82} \mathrm{Se},{ }^{100} \mathrm{Mo}\left(2 \beta^{-}\right) \text {; measured } 0 \nu \beta \beta \text {-decay } \mathrm{T}_{1 / 2} \text { lower } \\
\text { limits; deduced neutrino mass limits. JOUR NPBSE } 145272\end{array}$ \\
\hline & 2005ZI02 & $\begin{array}{l}\text { NUCLEAR REACTIONS }{ }^{96} \mathrm{Mo}\left({ }^{20} \mathrm{Ne},{ }^{20} \mathrm{Ne}\right) \\
\mathrm{MeV} /\left({ }^{40} \mathrm{Ar},{ }^{40} \mathrm{Ar}{ }^{\prime}\right), \mathrm{E}=2.5 \\
\text { (particleon; } \gamma \text {-coin following Coulomb excitation. }{ }^{96} \mathrm{M} \text { Mo deduced } \\
\text { transitions. JOUR APOBB } 361289\end{array}$ \\
\hline${ }^{96} \mathrm{Ru}$ & $2005 \mathrm{HA} 49$ & $\begin{array}{l}\text { NUCLEAR REACTIONS }{ }^{92} \mathrm{Mo}(\alpha, \gamma), \mathrm{E}=9 \mathrm{MeV} ;{ }^{91} \mathrm{Zr}(\alpha, \gamma), \mathrm{E}=10.5 \\
\mathrm{MeV} ;{ }^{118} \mathrm{Sn}(\alpha, \gamma), \mathrm{E}=11.5 \mathrm{MeV} ; \text { measured } \mathrm{E} \gamma, \mathrm{I} \gamma .{ }^{91} \mathrm{Zr},{ }^{118} \mathrm{Sn}(\alpha, \gamma), \\
\mathrm{E}(\mathrm{cm}) \approx 9-11 \mathrm{MeV} ; \text { measured } \sigma . \text { Comparison with model predictions. } \\
\text { JOUR NUPAB } 758505 \mathrm{c}\end{array}$ \\
\hline
\end{tabular}

\section{$\mathrm{A}=\mathbf{9 7}$}

${ }^{97} \mathrm{Sr} \quad 2004 \mathrm{WU} 08$

2005HW06

${ }^{97} \mathrm{Zr} \quad 2005 \mathrm{ADZZ}$
NUCLEAR REACTIONS ${ }^{238} \mathrm{U}(\alpha, \mathrm{F}){ }^{96} \mathrm{Sr} /{ }^{97} \mathrm{Sr} /{ }^{98} \mathrm{Zr} /{ }^{99} \mathrm{Zr}, \mathrm{E}=30$ $\mathrm{MeV}$; measured $\mathrm{E} \gamma, \mathrm{I} \gamma, \gamma \gamma$-, (fragment) $\gamma$-coin. ${ }^{96,97} \mathrm{Sr},{ }^{98,99} \mathrm{Zr}$ deduced high-spin levels, $J, \pi$, configurations. Gammasphere, Chico arrays. JOUR PRVCA 70064312 RADIOACTIVITY ${ }^{252} \mathrm{Cf}(\mathrm{SF})$; measured $\mathrm{E} \gamma, \mathrm{I} \gamma, \gamma \gamma$-coin. ${ }^{95,97} \mathrm{Sr},{ }^{99} \mathrm{Zr}$, ${ }^{108} \mathrm{Tc},{ }^{133,134} \mathrm{Te},{ }^{137} \mathrm{Xe}$ levels deduced $\mathrm{T}_{1 / 2}$. Gammasphere array, time-gated triple-coincidence method. JOUR ZAANE 25 s01 463 NUCLEAR REACTIONS ${ }^{129} \mathrm{I}(\mathrm{n}, 7 \mathrm{n}),(\mathrm{n}, 6 \mathrm{n}),(\mathrm{n}, 4 \mathrm{n}),(\mathrm{n}, \gamma), \mathrm{E}=$ fast; ${ }^{237} \mathrm{~Np}(\mathrm{n}, \gamma), \mathrm{E}=$ fast; measured yields. ${ }^{237} \mathrm{~Np}(\mathrm{n}, \mathrm{F}){ }^{91} \mathrm{Sr} /{ }^{97} \mathrm{Zr} /{ }^{132} \mathrm{Te} /$ ${ }^{133} \mathrm{I} /{ }^{135} \mathrm{I}, \mathrm{E}=$ fast; ${ }^{238} \mathrm{Pu}(\mathrm{n}, \mathrm{F}){ }^{97} \mathrm{Zr} /{ }^{129} \mathrm{Sb} /{ }^{132} \mathrm{I} /{ }^{133} \mathrm{I} /{ }^{135} \mathrm{Xe} /$ ${ }^{105} \mathrm{Ru}, \mathrm{E}=$ fast; ${ }^{239} \mathrm{Pu}(\mathrm{n}, \mathrm{F}){ }^{88} \mathrm{Kr} /{ }^{91} \mathrm{Sr} /{ }^{92} \mathrm{Sr} /{ }^{92} \mathrm{Y} /{ }^{97} \mathrm{Zr} /{ }^{99} \mathrm{Mo} /$ ${ }^{103} \mathrm{Ru} /{ }^{105} \mathrm{Ru} /{ }^{128} \mathrm{Sb} /{ }^{129} \mathrm{Sb} /{ }^{132} \mathrm{Te} /{ }^{131} \mathrm{I} /{ }^{132} \mathrm{I} /{ }^{133} \mathrm{I} /{ }^{135} \mathrm{I} /{ }^{135} \mathrm{Xe}$ $/{ }^{143} \mathrm{Ce} /{ }^{140} \mathrm{Ba} /{ }^{140} \mathrm{La}, \mathrm{E}=$ fast; measured fission fragment yields. Secondary neutrons from proton irradiation. JINR nuclotron. CONF St Petersburg,P195,Adam 


\section{$\mathrm{A}=97$ (continued)}

2005BI25 NUCLEAR MOMENTS 86,87,88,89,90,91,92,93,94,95,96,97,98,99,100,101,102Zr; measured charge radii. ${ }^{176} \mathrm{Yb}$; measured isomer shift. Ion-beam cooler, laser spectroscopy. JOUR ZAANE 25 s01 187

2005HU14 RADIOACTIVITY ${ }^{97} \operatorname{Zr}\left(\beta^{-}\right)$[from $\left.\operatorname{Zr}(\mathrm{n}, \mathrm{X})\right]$; measured $\mathrm{T}_{1 / 2}$. JOUR JRNCD 265499

2005MOZW NUCLEAR REACTIONS ${ }^{90,91,92,94,96} \mathrm{Zr}(\mathrm{n}, \gamma), \mathrm{E}<100 \mathrm{keV}$; measured $\mathrm{E} \gamma, \mathrm{I} \gamma$, capture yields. CONF Santa Fe (Nucl Data for Sci and Technol) Proc, Vol1,P880

2005RE09 NUCLEAR REACTIONS ${ }^{92,94} \mathrm{Mo}(\mathrm{n}, 2 \mathrm{n}),{ }^{92,100} \mathrm{Mo}(\mathrm{n}, \alpha),{ }^{95,96,97} \mathrm{Mo}(\mathrm{n}$, p), ${ }^{96,97,98} \mathrm{Mo}(\mathrm{n}, \mathrm{np}+\mathrm{d}), \mathrm{E} \approx 13.5-21 \mathrm{MeV}$; measured activation $\sigma$; deduced reaction mechanism features. ${ }^{93} \mathrm{Nb}(\mathrm{p}, \mathrm{n}),(\mathrm{p}, \gamma), \mathrm{E} \approx 1-6$ $\mathrm{MeV} ;{ }^{92,93,94,95,96,97,98,100} \mathrm{Mo},{ }^{93} \mathrm{Nb}(\mathrm{n}, \gamma), \mathrm{E}<4 \mathrm{MeV} ;{ }^{92,94,100} \mathrm{Mo}(\mathrm{n}$, 2n), ${ }^{92,94,95,96,97,98} \mathrm{Mo}(\mathrm{n}, \mathrm{p}),{ }^{92,94,95,96,97,98,100} \mathrm{Mo}(\mathrm{n}, \mathrm{np}+\mathrm{d})$, 92,98,100 $\mathrm{Mo}(\mathrm{n}, \alpha), \mathrm{E}<21 \mathrm{MeV}$; compiled, analyzed $\sigma$. Analysis with local and global approaches compared. JOUR PRVCA 71044617

2005TA23 NUCLEAR REACTIONS ${ }^{90,91,92,94,96} \mathrm{Zr}(\mathrm{n}, \gamma), \mathrm{E}<1 \mathrm{MeV}$; measured $\mathrm{E} \gamma, \mathrm{I} \gamma$, capture yields. JOUR NUPAB 758 573c

${ }^{97} \mathrm{Nb} \quad 2005 \mathrm{BE} 17$

2005HU14

2005RE09

${ }^{97}$ Mo $\quad 2005 \mathrm{CHZW}$

2005GU16 RADIOACTIVITY ${ }^{127} \mathrm{I}\left({ }^{24} \mathrm{Ne}\right),\left({ }^{28} \mathrm{Mg}\right),\left({ }^{30} \mathrm{Mg}\right),\left({ }^{32} \mathrm{Si}\right),\left({ }^{34} \mathrm{Si}\right),\left({ }^{48} \mathrm{Ca}\right)$, $\left({ }^{49} \mathrm{Sc}\right)$; measured cluster decay $\mathrm{T}_{1 / 2}$ lower limits. JOUR ZAANE 2451 RADIOACTIVITY ${ }^{97} \operatorname{Zr}\left(\beta^{-}\right)$[from $\mathrm{Zr}(\mathrm{n}, \mathrm{X})$ ]; measured $\mathrm{T}_{1 / 2}$. JOUR JRNCD 265499 NUCLEAR REACTIONS ${ }^{92,94} \mathrm{Mo}(\mathrm{n}, 2 \mathrm{n}),{ }^{92,100} \mathrm{Mo}(\mathrm{n}, \alpha),{ }^{95,96,97} \mathrm{Mo}(\mathrm{n}$, p), ${ }^{96,97,98} \mathrm{Mo}(\mathrm{n}, \mathrm{np}+\mathrm{d}), \mathrm{E} \approx 13.5-21 \mathrm{MeV}$; measured activation $\sigma$; deduced reaction mechanism features. ${ }^{93} \mathrm{Nb}(\mathrm{p}, \mathrm{n}),(\mathrm{p}, \gamma), \mathrm{E} \approx 1-6$

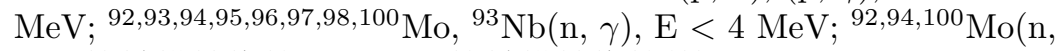
2n), ${ }^{92,94,95,96,97,98} \mathrm{Mo}(\mathrm{n}, \mathrm{p}),{ }^{92,94,95,96,97,98,100} \mathrm{Mo}(\mathrm{n}, \mathrm{np}+\mathrm{d})$, 92,98,100 $\mathrm{Mo}(\mathrm{n}, \alpha), \mathrm{E}<21 \mathrm{MeV}$; compiled, analyzed $\sigma$. Analysis with local and global approaches compared. JOUR PRVCA 71044617 NUCLEAR REACTIONS ${ }^{94,96} \mathrm{Mo}\left({ }^{3} \mathrm{He},{ }^{3} \mathrm{He}\right),\left({ }^{3} \mathrm{He}, \alpha\right), \mathrm{E}=30 \mathrm{MeV}$; ${ }^{97,98} \mathrm{Mo}\left({ }^{3} \mathrm{He},{ }^{3} \mathrm{He}\right),\left({ }^{3} \mathrm{He}, \alpha\right), \mathrm{E}=45 \mathrm{MeV}$; measured particle spectra, $\mathrm{E} \gamma, \mathrm{I} \gamma$, (particle) $\gamma$-coin. ${ }^{93,94,95,96,97,98}$ Mo deduced level density parameters, thermodynamic quantities. PREPRINT nucl-ex/0507007,7/04/2005 NUCLEAR REACTIONS ${ }^{94,96} \mathrm{Mo}\left({ }^{3} \mathrm{He},{ }^{3} \mathrm{He}\right),\left({ }^{3} \mathrm{He}, \alpha\right), \mathrm{E}=30 \mathrm{MeV}$; ${ }_{97,98} \mathrm{Mo}\left({ }^{3} \mathrm{He},{ }^{3} \mathrm{He}\right),\left({ }^{3} \mathrm{He}, \alpha\right), \mathrm{E}=45 \mathrm{MeV}$; measured particle spectra, $\mathrm{E} \gamma, \mathrm{I} \gamma$, (particle) $\gamma$-coin. ${ }^{93,94,95,96,97,98} \mathrm{Mo}$ deduced radiative strength functions. JOUR PRVCA 71044307

${ }^{97} \mathrm{Ru} \quad 2005 \mathrm{UD} 01$ NUCLEAR REACTIONS Ag(p, X) ${ }^{106 m} \mathrm{Ag} /{ }^{105} \mathrm{Ag} /{ }^{103} \mathrm{Pd} /{ }^{101} \mathrm{Pd} /$ ${ }^{100} \mathrm{Pd} /{ }^{105} \mathrm{Rh} /{ }^{102} \mathrm{Rh} /{ }^{101 m} \mathrm{Rh} /{ }^{100} \mathrm{Rh} /{ }^{99} \mathrm{Rh} /{ }^{97} \mathrm{Ru}, \mathrm{E}=11-80$ $\mathrm{MeV}$; measured excitation functions. Stacked-foil activation. JOUR ARISE 62533

${ }^{97} \mathrm{Rh} \quad 2005 \mathrm{T015}$ NUCLEAR REACTIONS ${ }^{93} \mathrm{Nb}\left({ }^{12} \mathrm{C}, \mathrm{X}\right){ }^{97} \mathrm{Rh} /{ }^{99} \mathrm{Rh}, \mathrm{E}=55.7-77.5$ $\mathrm{MeV} ;{ }^{89} \mathrm{Y}\left({ }^{16} \mathrm{O}, \mathrm{X}\right){ }^{99} \mathrm{Rh}, \mathrm{E}=68-81 \mathrm{MeV}$; measured isomeric $\sigma$ ratios following complete and incomplete fusion; deduced angular momentum transfer. Recoil catcher technique. JOUR PRAMC 641 


\section{$\mathrm{A}=98$}

\begin{tabular}{|c|c|c|}
\hline${ }^{98} \mathrm{Sr}$ & $2005 F 017$ & $\begin{array}{l}\text { RADIOACTIVITY }{ }^{252} \mathrm{Cf}(\mathrm{SF}) ; \text { measured } \mathrm{E} \gamma, \mathrm{I} \gamma, \gamma \gamma \text {-coin. }{ }^{98} \mathrm{Sr} \\
{ }^{102,104} \mathrm{Zr},{ }^{137} \mathrm{Xe},{ }^{143} \mathrm{Ba},{ }^{152} \mathrm{Ce} \text { levels deduced } \mathrm{T}_{1 / 2} \text {. Gammasphere }\end{array}$ \\
\hline & & array, time-gated triple-coincidence method. JOUR ZAANE 25 s01 465 \\
\hline${ }^{98} \mathrm{Zr}$ & 2004WU08 & $\begin{array}{l}\text { NUCLEAR REACTIONS }{ }^{238} \mathrm{U}(\alpha, \mathrm{F}){ }^{96} \mathrm{Sr} /{ }^{97} \mathrm{Sr} /{ }^{98} \mathrm{Zr} /{ }^{99} \mathrm{Zr}, \mathrm{E}=30 \\
\mathrm{MeV} \text {; measured } \mathrm{E} \gamma, \mathrm{I} \gamma, \gamma \gamma-\text {, (fragment) } \gamma \text {-coin. }{ }^{96,97} \mathrm{Sr},{ }^{98,99} \mathrm{Zr} \text { deduced } \\
\text { high-spin levels, J, } \pi \text {, configurations. Gammasphere, Chico arrays. } \\
\text { JOUR PRVCA } 70064312\end{array}$ \\
\hline & 2005BI25 & $\begin{array}{l}\text { NUCLEAR MOMENTS } 86,87,88,89,90,91,92,93,94,95,96,97,98,99,100,101,102 \mathrm{Zr} \text {; } \\
\text { measured charge radii. }{ }^{176} \mathrm{Yb} \text {; measured isomer shift. Ion-beam cooler, } \\
\text { laser spectroscopy. JOUR ZAANE } 25 \text { s01 } 187\end{array}$ \\
\hline & 2005J022 & ATOMIC MASSES ${ }^{98,99,100,101,102,103,104,105} \mathrm{Zr}$; measured masses. \\
\hline & & Penning trap mass spectrometer. JOUR ZAANE 25 s01 27 \\
\hline${ }^{98} \mathrm{Nb}$ & 2005RE09 & $\begin{array}{l}\text { NUCLEAR REACTIONS }{ }^{92,94} \mathrm{Mo}(\mathrm{n}, 2 \mathrm{n}),{ }^{92,100} \mathrm{Mo}(\mathrm{n}, \alpha),{ }^{95,96,97} \mathrm{Mo}(\mathrm{n}, \\
\mathrm{p}),{ }^{96,97,98} \mathrm{Mo}(\mathrm{n}, \mathrm{np}+\mathrm{d}), \mathrm{E} \approx 13.5-21 \mathrm{MeV} \text { measured activation } \sigma ; \\
\text { deduced reaction mechanism features. }{ }^{93} \mathrm{Nb}(\mathrm{p}, \mathrm{n}),(\mathrm{p}, \gamma), \mathrm{E} \approx 1-6 \\
\mathrm{MeV} ;{ }^{92,93,94,95,96,97,98,100} \mathrm{Mo},{ }^{93} \mathrm{Nb}(\mathrm{n}, \gamma), \mathrm{E}<4 \mathrm{MeV} ;{ }^{92,94,100} \mathrm{Mo}(\mathrm{n}, \\
2 \mathrm{n}),{ }^{92,94,95,96,97,98} \mathrm{Mo}(\mathrm{n}, \mathrm{p}),{ }^{92,94,95,96,97,98,100} \mathrm{Mo}(\mathrm{n}, \mathrm{np}+\mathrm{d}), \\
92,98,100 \mathrm{Mo}(\mathrm{n}, \alpha), \mathrm{E}<21 \mathrm{MeV} \text {; compiled, analyzed } \sigma \text {. Analysis with }\end{array}$ \\
\hline & & local and global approaches compared. JOUR PRVCA 71044617 \\
\hline${ }^{98} \mathrm{Mo}$ & $2005 \mathrm{CHZW}$ & $\begin{array}{l}\text { NUCLEAR REACTIONS }{ }^{94,96} \mathrm{Mo}\left({ }^{3} \mathrm{He},{ }^{3} \mathrm{He}\right),\left({ }^{3} \mathrm{He}, \alpha\right), \mathrm{E}=30 \mathrm{MeV} \text {; } \\
97,98 \mathrm{Mo}\left({ }^{3} \mathrm{He},{ }^{3} \mathrm{He}\right),\left({ }^{3} \mathrm{He}, \alpha\right), \mathrm{E}=45 \mathrm{MeV} \text {; measured particle spectra, } \\
\mathrm{E} \gamma, \mathrm{I} \gamma \text {, (particle) } \gamma \text {-coin. }{ }^{93,94,95,96,97,98} \mathrm{Mo} \text { deduced level density } \\
\text { parameters, thermodynamic quantities. PREPRINT } \\
\text { nucl-ex } / 0507007,7 / 04 / 2005\end{array}$ \\
\hline & $2005 G U 16$ & $\begin{array}{l}\text { NUCLEAR REACTIONS }{ }^{94,96} \mathrm{Mo}\left({ }^{3} \mathrm{He},{ }^{3} \mathrm{He}\right),\left({ }^{3} \mathrm{He}, \alpha\right), \mathrm{E}=30 \mathrm{MeV} \text {; } \\
97,98 \mathrm{Mo}\left({ }^{3} \mathrm{He},{ }^{3} \mathrm{He}\right),\left({ }^{3} \mathrm{He}, \alpha\right), \mathrm{E}=45 \mathrm{MeV} ; \text { measured particle spectra, } \\
\mathrm{E} \gamma, \mathrm{I} \gamma \text {, (particle) } \gamma \text {-coin. }{ }^{93,94,95,96,97,98} \mathrm{Mo} \text { deduced radiative strength } \\
\text { functions. JOUR PRVCA } 71044307\end{array}$ \\
\hline & 2005RU14 & $\begin{array}{l}\text { NUCLEAR REACTIONS }{ }^{98,100} \mathrm{Mo}(\gamma, \gamma), \mathrm{E}=3.2-3.8 \mathrm{MeV} \\
\text { bremsstrahlung; measured } \mathrm{E} \gamma, \mathrm{I} \gamma .{ }^{98,100} \text { Mo deduced levels, J, } \pi \text {, } \\
\text { branching ratios, transition probabilities, shape isomer configuration } \\
\text { mixing features. JOUR PRLTA } 95062501\end{array}$ \\
\hline & 2005RUZZ & $\begin{array}{l}\text { NUCLEAR REACTIONS }{ }^{92,98,100} \mathrm{Mo}\left(\gamma, \gamma^{\prime}\right), \mathrm{E}=14 \mathrm{MeV} \\
\text { bremsstrahlung; measured } \mathrm{E} \gamma, \mathrm{I} \gamma \cdot{ }^{92,98,100} \mathrm{Mo} \text { deduced dipole strength } \\
\text { functions, resonance features. PREPRINT nucl-ex/0512027,12/20/2005 }\end{array}$ \\
\hline & 2005WA31 & $\begin{array}{l}\text { NUCLEAR REACTIONS }{ }^{92,98,100} \mathrm{Mo}\left(\gamma, \gamma^{\prime}\right), \mathrm{E}=13.2 \mathrm{MeV} \\
\text { bremsstrahlung; measured } \mathrm{E} \gamma, \mathrm{I} \gamma .{ }^{92,100} \mathrm{Mo},{ }^{197} \mathrm{Au}(\gamma, \mathrm{n}),{ }^{92} \mathrm{Mo}(\gamma, \mathrm{p}), \\
(\gamma, \alpha), \mathrm{E} \approx 11.8-16.5 \mathrm{MeV} \text { bremsstrahlung; measured integrated } \sigma . \\
\text { JOUR JPGPE } 31 \mathrm{~S} 1969\end{array}$ \\
\hline
\end{tabular}

\section{$\mathrm{A}=99$}

${ }^{99} \mathrm{Y} \quad$ 2005LH01

RADIOACTIVITY ${ }^{99} \mathrm{Y}\left(\beta^{-}\right)$; measured $\beta$-delayed $\mathrm{E} \gamma, \mathrm{I} \gamma, \gamma \gamma$-coin; deduced logft. ${ }^{99} \mathrm{Zr}$ deduced levels, $\mathrm{J}, \pi$, configurations. Interacting boson-fermion model calculations. JOUR PRVCA 72034308 


\section{$\mathrm{A}=99$ (continued)}

2005LU21 RADIOACTIVITY ${ }^{252} \mathrm{Cf}(\mathrm{SF})$; measured E $\gamma$, I $\gamma, \gamma \gamma$-coin. ${ }^{99,101} \mathrm{Y}$, ${ }^{101,105} \mathrm{Nb}$ deduced levels, J, $\pi$, configurations, rotational bands, shape transition features. Gammasphere array, triaxial-rotor-plus-quasiparticle calculations. JOUR JPGPE 311303

2005LU24 RADIOACTIVITY ${ }^{252} \mathrm{Cf}(\mathrm{SF})$; measured $\mathrm{E} \gamma, \mathrm{I} \gamma, \gamma \gamma$-coin. ${ }^{99,101} \mathrm{Y}$, ${ }^{101,105} \mathrm{Nb}$ deduced levels, J, $\pi$, configurations, deformation.

Gammasphere array, triaxial-rotor-plus-particle calculations. JOUR ZAANE 25 s01 469

${ }^{99} \mathrm{Zr} \quad$ 2004WU08 NUCLEAR REACTIONS ${ }^{238} \mathrm{U}(\alpha, \mathrm{F}){ }^{96} \mathrm{Sr} /{ }^{97} \mathrm{Sr} /{ }^{98} \mathrm{Zr} /{ }^{99} \mathrm{Zr}, \mathrm{E}=30$ $\mathrm{MeV}$; measured $\mathrm{E} \gamma, \mathrm{I} \gamma, \gamma \gamma$-, (fragment) $\gamma$-coin. ${ }^{96,97} \mathrm{Sr},{ }^{98,99} \mathrm{Zr}$ deduced high-spin levels, J, $\pi$, configurations. Gammasphere, Chico arrays.

JOUR PRVCA 70064312

2005 BI25 NUCLEAR MOMENTS 86,87,88,89,90,91,92,93,94,95,96,97,98,99,100,101,102 Zr; measured charge radii. ${ }^{176} \mathrm{Yb}$; measured isomer shift. Ion-beam cooler, laser spectroscopy. JOUR ZAANE 25 s01 187

2005HW06 RADIOACTIVITY ${ }^{252} \mathrm{Cf}(\mathrm{SF})$; measured $\mathrm{E} \gamma, \mathrm{I} \gamma, \gamma \gamma$-coin. ${ }^{95,97} \mathrm{Sr},{ }^{99} \mathrm{Zr}$, ${ }^{108} \mathrm{Tc},{ }^{133,134} \mathrm{Te},{ }^{137} \mathrm{Xe}$ levels deduced $\mathrm{T}_{1 / 2}$. Gammasphere array, time-gated triple-coincidence method. JOUR ZAANE 25 s01 463 2005J022 ATOMIC MASSES ${ }^{98,99,100,101,102,103,104,105} \mathrm{Zr}$; measured masses. Penning trap mass spectrometer. JOUR ZAANE 25 s01 27

2005LH01 RADIOACTIVITY ${ }^{99} \mathrm{Y}\left(\beta^{-}\right)$; measured $\beta$-delayed E $\gamma, \mathrm{I} \gamma, \gamma \gamma$-coin; deduced logft. ${ }^{99} \mathrm{Zr}$ deduced levels, $\mathrm{J}, \pi$, configurations. Interacting boson-fermion model calculations. JOUR PRVCA 72034308

$\begin{array}{ll}{ }^{99} \mathrm{Nb} & \text { 2005BE17 } \\ { }^{99} \mathrm{Mo} & \text { 2005ADZZ }\end{array}$

2005MIZZ RADIOACTIVITY ${ }^{127} \mathrm{I}\left({ }^{24} \mathrm{Ne}\right),\left({ }^{28} \mathrm{Mg}\right),\left({ }^{30} \mathrm{Mg}\right),\left({ }^{32} \mathrm{Si}\right),\left({ }^{34} \mathrm{Si}\right),\left({ }^{48} \mathrm{Ca}\right)$, $\left({ }^{49} \mathrm{Sc}\right)$; measured cluster decay $\mathrm{T}_{1 / 2}$ lower limits. JOUR ZAANE 2451 NUCLEAR REACTIONS ${ }^{129} \mathrm{I}(\mathrm{n}, 7 \mathrm{n}),(\mathrm{n}, 6 \mathrm{n}),(\mathrm{n}, 4 \mathrm{n}),(\mathrm{n}, \gamma), \mathrm{E}=$ fast; ${ }^{237} \mathrm{~Np}(\mathrm{n}, \gamma), \mathrm{E}=$ fast; measured yields. ${ }^{237} \mathrm{~Np}(\mathrm{n}, \mathrm{F}){ }^{91} \mathrm{Sr} /{ }^{97} \mathrm{Zr} /{ }^{132} \mathrm{Te} /$ ${ }^{133} \mathrm{I} /{ }^{135} \mathrm{I}, \mathrm{E}=$ fast; ${ }^{238} \mathrm{Pu}(\mathrm{n}, \mathrm{F}){ }^{97} \mathrm{Zr} /{ }^{129} \mathrm{Sb} /{ }^{132} \mathrm{I} /{ }^{133} \mathrm{I} /{ }^{135} \mathrm{Xe} /$ ${ }^{105} \mathrm{Ru}, \mathrm{E}=$ fast; ${ }^{239} \mathrm{Pu}(\mathrm{n}, \mathrm{F}){ }^{88} \mathrm{Kr} /{ }^{91} \mathrm{Sr} /{ }^{92} \mathrm{Sr} /{ }^{92} \mathrm{Y} /{ }^{97} \mathrm{Zr} /{ }^{99} \mathrm{Mo} /$ ${ }^{103} \mathrm{Ru} /{ }^{105} \mathrm{Ru} /{ }^{128} \mathrm{Sb} /{ }^{129} \mathrm{Sb} /{ }^{132} \mathrm{Te} /{ }^{131} \mathrm{I} /{ }^{132} \mathrm{I} /{ }^{133} \mathrm{I} /{ }^{135} \mathrm{I} /{ }^{135} \mathrm{Xe}$ $/{ }^{143} \mathrm{Ce} /{ }^{140} \mathrm{Ba} /{ }^{140} \mathrm{La}, \mathrm{E}=$ fast; measured fission fragment yields. Secondary neutrons from proton irradiation. JINR nuclotron. CONF St Petersburg,P195,Adam NUCLEAR REACTIONS Cu(n, X) ${ }^{56} \mathrm{Co}, \mathrm{E}=40-180 \mathrm{MeV} ; \mathrm{Fe}(\mathrm{n}$, $\mathrm{X}){ }^{54} \mathrm{Mn} /{ }^{52} \mathrm{Mn} /{ }^{51} \mathrm{Cr} /{ }^{48} \mathrm{~V}, \mathrm{E} \approx 0-180 \mathrm{MeV} ; \mathrm{Pb}(\mathrm{n}, \mathrm{X}){ }^{196} \mathrm{Au} /{ }^{200} \mathrm{~Pb}$ / ${ }^{103} \mathrm{Ru}, \mathrm{E} \approx 40-180 \mathrm{MeV} ; \mathrm{U}(\mathrm{n}, \mathrm{X}){ }^{99} \mathrm{Mo}, \mathrm{E} \approx 0-180 \mathrm{MeV}$; measured excitation functions. Comparison with proton-induced reactions. CONF Santa Fe (Nucl Data for Sci and Technol) Proc,Vol1,P861 2005RE09 NUCLEAR REACTIONS ${ }^{92,94} \mathrm{Mo}(\mathrm{n}, 2 \mathrm{n}),{ }^{92,100} \mathrm{Mo}(\mathrm{n}, \alpha),{ }^{95,96,97} \mathrm{Mo}(\mathrm{n}$, p), ${ }^{96,97,98} \mathrm{Mo}(\mathrm{n}, \mathrm{np}+\mathrm{d}), \mathrm{E} \approx 13.5-21 \mathrm{MeV}$; measured activation $\sigma$; deduced reaction mechanism features. ${ }^{93} \mathrm{Nb}(\mathrm{p}, \mathrm{n}),(\mathrm{p}, \gamma), \mathrm{E} \approx 1-6$ $\mathrm{MeV} ;{ }^{92,93,94,95,96,97,98,100} \mathrm{Mo},{ }^{93} \mathrm{Nb}(\mathrm{n}, \gamma), \mathrm{E}<4 \mathrm{MeV} ;{ }^{92,94,100} \mathrm{Mo}(\mathrm{n}$, 2n), ${ }^{92,94,95,96,97,98} \mathrm{Mo}(\mathrm{n}, \mathrm{p}),{ }^{92,94,95,96,97,98,100} \mathrm{Mo}(\mathrm{n}, \mathrm{np}+\mathrm{d})$, ${ }_{92,98,100} \mathrm{Mo}(\mathrm{n}, \alpha), \mathrm{E}<21 \mathrm{MeV}$; compiled, analyzed $\sigma$. Analysis with local and global approaches compared. JOUR PRVCA 71044617 ${ }^{99} \mathrm{Ru}$ 2004R047 NUCLEAR REACTIONS ${ }^{99,101} \mathrm{Ru}\left(\mathrm{d}, \mathrm{d}^{\prime}\right), \mathrm{E}=13 \mathrm{MeV}$; measured $\sigma(\mathrm{E}$, $\theta$ ). ${ }^{99,101} \mathrm{Ru}$ levels deduced deformation lengths, Coulomb-nuclear interference parameters. DWBA-deformed optical model analysis. JOUR BJPHE 34760 


\section{$\mathrm{A}=99$ (continued)}

${ }^{99} \mathrm{Rh} \quad 2005 \mathrm{TO} 15 \quad$ NUCLEAR REACTIONS ${ }^{93} \mathrm{Nb}\left({ }^{12} \mathrm{C}, \mathrm{X}\right){ }^{97} \mathrm{Rh} /{ }^{99} \mathrm{Rh}, \mathrm{E}=55.7-77.5$ $\mathrm{MeV} ;{ }^{89} \mathrm{Y}\left({ }^{16} \mathrm{O}, \mathrm{X}\right){ }^{99} \mathrm{Rh}, \mathrm{E}=68-81 \mathrm{MeV}$; measured isomeric $\sigma$ ratios following complete and incomplete fusion; deduced angular momentum transfer. Recoil catcher technique. JOUR PRAMC 641

2005UD01 NUCLEAR REACTIONS Ag(p, X) ${ }^{106 m} \mathrm{Ag} /{ }^{105} \mathrm{Ag} /{ }^{103} \mathrm{Pd} /{ }^{101} \mathrm{Pd} /$ ${ }^{100} \mathrm{Pd} /{ }^{105} \mathrm{Rh} /{ }^{102} \mathrm{Rh} /{ }^{101 m} \mathrm{Rh} /{ }^{100} \mathrm{Rh} /{ }^{99} \mathrm{Rh} /{ }^{97} \mathrm{Ru}, \mathrm{E}=11-80$ $\mathrm{MeV}$; measured excitation functions. Stacked-foil activation. JOUR ARISE 62533

\section{$\mathrm{A}=\mathbf{1 0 0}$}

\section{${ }^{100} \mathrm{Zr} \quad 2005 \mathrm{BI} 25$}

2005JA12

2005J022

2005SM08

${ }^{100} \mathrm{Mo} \quad$ 2005AR27

2005BA01

2005BA33

2005HOZW

2005RU14

2005RUZZ

2005SA07

2005SHZW
NUCLEAR MOMENTS 86,87,88,89,90,91,92,93,94,95,96,97,98,99,100,101,102 Zr; measured charge radii. ${ }^{176} \mathrm{Yb}$; measured isomer shift. Ion-beam cooler, laser spectroscopy. JOUR ZAANE 25 s01 187

RADIOACTIVITY ${ }^{252} \mathrm{Cf}(\mathrm{SF})$; measured $\mathrm{E} \gamma, \mathrm{I} \gamma, \alpha \gamma-, \gamma \gamma$-coin for $\alpha$-accompanied ternary fission; deduced fission fragments average angular momentum. ${ }^{100,102} \mathrm{Zr},{ }^{106} \mathrm{Mo},{ }^{144,146} \mathrm{Ba},{ }^{138,140,142} \mathrm{Xe}$; deduced transition intensities. Gammasphere array. JOUR ZAANE 24373 ATOMIC MASSES ${ }^{98,99,100,101,102,103,104,105} \mathrm{Zr}$; measured masses. Penning trap mass spectrometer. JOUR ZAANE 25 s01 27 RADIOACTIVITY ${ }^{252} \mathrm{Cf}(\mathrm{SF})$; measured $\mathrm{E} \gamma, \mathrm{I} \gamma(\theta, \mathrm{H}, \mathrm{t}), \gamma \gamma$-coin. 96,100,102 Zr, ${ }^{102,104,106,108} \mathrm{Mo},{ }^{106,108,110,112} \mathrm{Ru},{ }^{110,114,116} \mathrm{Pd}$ levels deduced $\mathrm{g}$ factors, $\mathrm{B}(\mathrm{E} 2)$. Gammasphere array, time-integral perturbed angular correlation technique. Comparison with interacting boson model predictions. JOUR JPGPE 31 S1433

RADIOACTIVITY ${ }^{82} \mathrm{Se},{ }^{100} \mathrm{Mo}\left(2 \beta^{-}\right)$; measured $2 \nu \beta \beta$-decay $\mathrm{T}_{1 / 2}$, $0 \nu \beta \beta$-decay $\mathrm{T}_{1 / 2}$ lower limits; deduced neutrino mass limits. JOUR PRLTA 95182302

RADIOACTIVITY ${ }^{100} \mathrm{Mo}\left(2 \beta^{-}\right)$; measured $2 \nu 2 \beta$-decay $\mathrm{E} \beta, \mathrm{T}_{1 / 2}$. JOUR NPBSE 138207 RADIOACTIVITY ${ }^{82} \mathrm{Se},{ }^{100} \mathrm{Mo},{ }^{116} \mathrm{Cd},{ }^{150} \mathrm{Nd}\left(2 \beta^{-}\right)$; measured $2 \nu \beta \beta$-decay $\mathrm{T}_{1 / 2}, 0 \nu \beta \beta$-decay $\mathrm{T}_{1 / 2}$ lower limits. JOUR YAFIA 68443 RADIOACTIVITY ${ }^{100} \mathrm{Mo}\left(2 \beta^{-}\right)$; measured $\mathrm{E} \gamma, \mathrm{I} \gamma, \gamma \gamma$-coin, $\mathrm{T}_{1 / 2}$ for decay to excited states. ${ }^{100} \mathrm{Ru}$ levels deduced feeding intensities. PREPRINT nucl-ex/0512030,12/20/2005

NUCLEAR REACTIONS ${ }^{98,100} \mathrm{Mo}\left(\gamma, \gamma^{\prime}\right), \mathrm{E}=3.2-3.8 \mathrm{MeV}$ bremsstrahlung; measured $\mathrm{E} \gamma, \mathrm{I} \gamma .{ }^{98,100}$ Mo deduced levels, J, $\pi$, branching ratios, transition probabilities, shape isomer configuration mixing features. JOUR PRLTA 95062501

NUCLEAR REACTIONS ${ }^{92,98,100} \mathrm{Mo}\left(\gamma, \gamma^{\prime}\right), \mathrm{E}=14 \mathrm{MeV}$ bremsstrahlung; measured $\mathrm{E} \gamma, \mathrm{I} \gamma .{ }^{92,98,100}$ Mo deduced dipole strength functions, resonance features. PREPRINT nucl-ex/0512027,12/20/2005 RADIOACTIVITY ${ }^{82} \mathrm{Se},{ }^{96} \mathrm{Zr},{ }^{100} \mathrm{Mo},{ }^{116} \mathrm{Cd},{ }^{150} \mathrm{Nd}\left(2 \beta^{-}\right)$; measured $2 \nu \beta \beta$-decay $\mathrm{T}_{1 / 2} .{ }^{82} \mathrm{Se},{ }^{100} \mathrm{Mo}\left(2 \beta^{-}\right)$; measured $0 \nu \beta \beta$-decay $\mathrm{T}_{1 / 2}$ lower limits; deduced neutrino mass limits. JOUR NPBSE 143221 RADIOACTIVITY ${ }^{100} \mathrm{Mo},{ }^{82} \mathrm{Se}\left(2 \beta^{-}\right)$; measured $0 \nu \beta \beta$-decay $\mathrm{T}_{1 / 2}$ limits. NEMO-3 detector, underground laboratory in Modane. CONF St Petersburg,P42,Shitov 


\section{$\mathrm{A}=100$ (continued)}

2005SI06 RADIOACTIVITY ${ }^{82} \mathrm{Se},{ }^{96} \mathrm{Zr},{ }^{100} \mathrm{Mo},{ }^{116} \mathrm{Cd},{ }^{150} \mathrm{Nd}\left(2 \beta^{-}\right)$; measured $2 \nu \beta \beta$-decay $\mathrm{T}_{1 / 2} .{ }^{82} \mathrm{Se},{ }^{100} \mathrm{Mo}\left(2 \beta^{-}\right)$; measured $0 \nu \beta \beta$-decay $\mathrm{T}_{1 / 2}$ lower limits; deduced neutrino mass limits. JOUR NPBSE 145272

2005WA31 NUCLEAR REACTIONS ${ }^{92,98,100} \mathrm{Mo}\left(\gamma, \gamma^{\prime}\right), \mathrm{E}=13.2 \mathrm{MeV}$ bremsstrahlung; measured $\mathrm{E} \gamma, \mathrm{I} \gamma .{ }^{92,100} \mathrm{Mo},{ }^{197} \mathrm{Au}(\gamma, \mathrm{n}),{ }^{92} \mathrm{Mo}(\gamma, \mathrm{p})$, $(\gamma, \alpha), \mathrm{E} \approx 11.8-16.5 \mathrm{MeV}$ bremsstrahlung; measured integrated $\sigma$. JOUR JPGPE 31 S1969

2005WR01 NUCLEAR REACTIONS ${ }^{100} \mathrm{Mo}\left({ }^{40} \mathrm{Ar},{ }^{40} \mathrm{Ar}\right.$ '), E $=90 \mathrm{MeV}$; measured $\mathrm{E} \gamma, \mathrm{I} \gamma$, (particle) $\gamma$-coin following Coulomb excitation. ${ }^{100}$ Mo deduced levels, J, $\pi$. JOUR IMPEE 14359

${ }^{100} \mathrm{Tc} \quad 2004 \mathrm{FU} 30 \quad$ NUCLEAR REACTIONS ${ }^{99} \mathrm{Tc}(\mathrm{n}, \gamma), \mathrm{E}=$ thermal; measured $\mathrm{E} \gamma, \mathrm{I} \gamma$, capture $\sigma .{ }^{100} \mathrm{Tc}$ deduced levels, J, $\pi$. JOUR JNSTA 411033

2005FUZY NUCLEAR REACTIONS ${ }^{99} \mathrm{Tc}(\mathrm{n}, \gamma), \mathrm{E}=$ thermal; measured prompt and delayed $\mathrm{E} \gamma, \mathrm{I} \gamma$; deduced capture $\sigma$, reaction $\sigma$ lower limit. CONF Santa Fe (Nucl Data for Sci and Technol) Proc,Vol2,P1454

2005FUZY RADIOACTIVITY ${ }^{100} \mathrm{Tc}\left(\beta^{-}\right)$[from $\left.{ }^{99} \mathrm{Tc}(\mathrm{n}, \gamma)\right]$; measured $\mathrm{E} \gamma$, I $\gamma$. ${ }^{100} \mathrm{Ru}$ deduced transitions. CONF Santa Fe (Nucl Data for Sci and Technol) Proc,Vol2,P1454

2005J004 NUCLEAR REACTIONS ${ }^{96} \mathrm{Zr}\left({ }^{7} \mathrm{Li}, 3 \mathrm{n}\right), \mathrm{E}=27 \mathrm{MeV}$; measured $\mathrm{E} \gamma$, I $\gamma$, $\gamma \gamma$-coin. ${ }^{100}$ Tc deduced levels, J, $\pi, \mathrm{B}(\mathrm{M} 1) / \mathrm{B}(\mathrm{E} 2)$, chiral partner bands. Total Routhian surface and core quasi-particle coupling model calculations. JOUR ZAANE 2423

${ }^{100} \mathrm{Ru}$ 2005AR27 RADIOACTIVITY ${ }^{82} \mathrm{Se},{ }^{100} \mathrm{Mo}\left(2 \beta^{-}\right)$; measured $2 \nu \beta \beta$-decay $\mathrm{T}_{1 / 2}$, $0 \nu \beta \beta$-decay $\mathrm{T}_{1 / 2}$ lower limits; deduced neutrino mass limits. JOUR PRLTA 95182302

2005BA01 RADIOACTIVITY ${ }^{100} \mathrm{Mo}\left(2 \beta^{-}\right)$; measured $2 \nu 2 \beta$-decay $\mathrm{E} \beta, \mathrm{T}_{1 / 2}$. JOUR NPBSE 138207

2005BA33 RADIOACTIVITY ${ }^{82} \mathrm{Se},{ }^{100} \mathrm{Mo},{ }^{116} \mathrm{Cd},{ }^{150} \mathrm{Nd}\left(2 \beta^{-}\right)$; measured $2 \nu \beta \beta$-decay $\mathrm{T}_{1 / 2}, 0 \nu \beta \beta$-decay $\mathrm{T}_{1 / 2}$ lower limits. JOUR YAFIA 68443

2005FUZY RADIOACTIVITY ${ }^{100} \mathrm{Tc}\left(\beta^{-}\right)$[from $\left.{ }^{99} \mathrm{Tc}(\mathrm{n}, \gamma)\right]$; measured $\mathrm{E} \gamma$, I $\gamma$. ${ }^{100} \mathrm{Ru}$ deduced transitions. CONF Santa Fe (Nucl Data for Sci and Technol) Proc,Vol2,P1454

2005HOZW RADIOACTIVITY ${ }^{100} \mathrm{Mo}\left(2 \beta^{-}\right)$; measured $\mathrm{E} \gamma, \mathrm{I} \gamma, \gamma \gamma$-coin, $\mathrm{T}_{1 / 2}$ for decay to excited states. ${ }^{100} \mathrm{Ru}$ levels deduced feeding intensities. PREPRINT nucl-ex/0512030,12/20/2005

2005SA07 RADIOACTIVITY ${ }^{82} \mathrm{Se},{ }^{96} \mathrm{Zr},{ }^{100} \mathrm{Mo},{ }^{116} \mathrm{Cd},{ }^{150} \mathrm{Nd}\left(2 \beta^{-}\right)$; measured $2 \nu \beta \beta$-decay $\mathrm{T}_{1 / 2} .{ }^{82} \mathrm{Se},{ }^{100} \mathrm{Mo}\left(2 \beta^{-}\right)$; measured $0 \nu \beta \beta$-decay $\mathrm{T}_{1 / 2}$ lower limits; deduced neutrino mass limits. JOUR NPBSE 143221

2005SHZW RADIOACTIVITY ${ }^{100} \mathrm{Mo},{ }^{82} \mathrm{Se}\left(2 \beta^{-}\right)$; measured $0 \nu \beta \beta$-decay $\mathrm{T}_{1 / 2}$ limits. NEMO-3 detector, underground laboratory in Modane. CONF St Petersburg,P42,Shitov

2005SI06 RADIOACTIVITY ${ }^{82} \mathrm{Se},{ }^{96} \mathrm{Zr},{ }^{100} \mathrm{Mo},{ }^{116} \mathrm{Cd},{ }^{150} \mathrm{Nd}\left(2 \beta^{-}\right)$; measured $2 \nu \beta \beta$-decay $\mathrm{T}_{1 / 2} .{ }^{82} \mathrm{Se},{ }^{100} \mathrm{Mo}\left(2 \beta^{-}\right)$; measured $0 \nu \beta \beta$-decay $\mathrm{T}_{1 / 2}$ lower limits; deduced neutrino mass limits. JOUR NPBSE 145272

${ }^{100} \mathrm{Rh}$ 2005UD01 NUCLEAR REACTIONS Ag(p, X) ${ }^{106 m} \mathrm{Ag} /{ }^{105} \mathrm{Ag} /{ }^{103} \mathrm{Pd} /{ }^{101} \mathrm{Pd} /$ ${ }^{100} \mathrm{Pd} /{ }^{105} \mathrm{Rh} /{ }^{102} \mathrm{Rh} /{ }^{101 m} \mathrm{Rh} /{ }^{100} \mathrm{Rh} /{ }^{99} \mathrm{Rh} /{ }^{97} \mathrm{Ru}, \mathrm{E}=11-80$ $\mathrm{MeV}$; measured excitation functions. Stacked-foil activation. JOUR ARISE 62533 


\section{$\mathrm{A}=100$ (continued)}

\begin{tabular}{|c|c|c|}
\hline \multirow[t]{2}{*}{${ }^{100} \mathrm{Pd}$} & \multirow[t]{2}{*}{ 2005UD01 } & $\begin{array}{l}\text { NUCLEAR REACTIONS } \mathrm{Ag}(\mathrm{p}, \mathrm{X}){ }^{106 m} \mathrm{Ag} /{ }^{105} \mathrm{Ag} /{ }^{103} \mathrm{Pd} /{ }^{101} \mathrm{Pd} / \\
{ }^{100} \mathrm{Pd} /{ }^{105} \mathrm{Rh} /{ }^{102} \mathrm{Rh} /{ }^{101 m} \mathrm{Rh} /{ }^{100} \mathrm{Rh} /{ }^{99} \mathrm{Rh} /{ }^{97} \mathrm{Ru}, \mathrm{E}=11-80\end{array}$ \\
\hline & & $\begin{array}{l}\text { MeV; measured excitation functions. Stacked-foil activation. JOUR } \\
\text { ARISE } 62533\end{array}$ \\
\hline${ }^{100} \mathrm{In}$ & $2005 \mathrm{KA} 47$ & $\begin{array}{l}\left.\text { RADIOACTIVITY }{ }^{102} \mathrm{Sn}\left(\beta^{+}\right) \text {from }{ }^{58} \mathrm{Ni}\left({ }^{50} \mathrm{Cr}, \mathrm{X}\right)\right] \text {; measured } \mathrm{E} \gamma, \mathrm{I} \gamma \\
\gamma \gamma \text {-coin, } \mathrm{E} \beta, \mathrm{B}(\mathrm{GT}) \cdot{ }^{102} \mathrm{In} \text { levels deduced } \beta \text {-feeding intensities, log ft, } \\
\text { hindrance factor. }{ }^{100} \mathrm{Sn}\left(\beta^{+}\right) \text {; analyzed data; deduced } \mathrm{B}(\mathrm{GT}) \text {, hindrance } \\
\text { factor. JOUR ZAANE } 25 \text { s01 } 135\end{array}$ \\
\hline \multirow[t]{2}{*}{${ }^{100} \mathrm{Sn}$} & $2005 \mathrm{KA} 47$ & $\begin{array}{l}\left.\text { RADIOACTIVITY }{ }^{102} \mathrm{Sn}\left(\beta^{+}\right) \text {[from }{ }^{58} \mathrm{Ni}\left({ }^{50} \mathrm{Cr}, \mathrm{X}\right)\right] \text {; measured } \mathrm{E} \gamma, \mathrm{I} \gamma \text {, } \\
\gamma \gamma \text {-coin, } \mathrm{E} \beta, \mathrm{B}(\mathrm{GT}) \cdot{ }^{102} \mathrm{In} \text { levels deduced } \beta \text {-feeding intensities, log ft, } \\
\text { hindrance factor. }{ }^{100} \mathrm{Sn}\left(\beta^{+}\right) \text {; analyzed data; deduced } \mathrm{B}(\mathrm{GT}) \text {, hindrance } \\
\text { factor. JOUR ZAANE } 25 \mathrm{~s} 01135\end{array}$ \\
\hline & $2005 \mathrm{KA} 47$ & $\begin{array}{l}\text { NUCLEAR REACTIONS }{ }^{58} \mathrm{Ni}\left({ }^{50} \mathrm{Cr}, \mathrm{X}\right){ }^{101} \mathrm{Sn} /{ }^{102} \mathrm{Sn} /{ }^{103} \mathrm{Sn} /{ }^{104} \mathrm{Sn} / \\
{ }^{105} \mathrm{Sn}, \mathrm{E} \approx 5 \mathrm{MeV} / \text { nucleon; measured production } \sigma .{ }^{58} \mathrm{Ni}\left({ }^{50} \mathrm{Cr} \text {, }\right. \\
\mathrm{X})^{100} \mathrm{Sn}, \mathrm{E}=5.8 \mathrm{MeV} / \text { nucleon; deduced approximate production } \sigma . \\
\text { JOUR ZAANE } 25 \mathrm{~s} 01135\end{array}$ \\
\hline
\end{tabular}

\section{$\mathrm{A}=\mathbf{1 0 1}$}

${ }^{101} \mathrm{Y} \quad 2005 \mathrm{LU} 21$

RADIOACTIVITY ${ }^{252} \mathrm{Cf}(\mathrm{SF}) ;$ measured $\mathrm{E} \gamma, \mathrm{I} \gamma, \gamma \gamma$-coin. ${ }^{99,101} \mathrm{Y}$, ${ }^{101,105} \mathrm{Nb}$ deduced levels, J, $\pi$, configurations, rotational bands, shape transition features. Gammasphere array, triaxial-rotor-plus-quasiparticle calculations. JOUR JPGPE 311303

2005LU24 RADIOACTIVITY ${ }^{252} \mathrm{Cf}(\mathrm{SF})$; measured E $\gamma$, I $\gamma, \gamma \gamma$-coin. ${ }^{99,101} \mathrm{Y}$, ${ }^{101,105} \mathrm{Nb}$ deduced levels, $\mathrm{J}, \pi$, configurations, deformation.

Gammasphere array, triaxial-rotor-plus-particle calculations. JOUR ZAANE 25 s01 469

${ }^{101} \mathrm{Zr} \quad$ 2005BI25 NUCLEAR MOMENTS 86,87,88,89,90,91,92,93,94,95,96,97,98,99,100,101,102 Zr; measured charge radii. ${ }^{176} \mathrm{Yb}$; measured isomer shift. Ion-beam cooler, laser spectroscopy. JOUR ZAANE 25 s01 187

2005J022 ATOMIC MASSES ${ }^{98,99,100,101,102,103,104,105} \mathrm{Zr}$; measured masses. Penning trap mass spectrometer. JOUR ZAANE 25 s01 27

${ }^{101} \mathrm{Nb}$ 2005LU21 RADIOACTIVITY ${ }^{252} \mathrm{Cf}(\mathrm{SF}) ;$ measured $\mathrm{E} \gamma, \mathrm{I} \gamma, \gamma \gamma$-coin. ${ }^{99,101} \mathrm{Y}$, ${ }^{101,105} \mathrm{Nb}$ deduced levels, J, $\pi$, configurations, rotational bands, shape transition features. Gammasphere array, triaxial-rotor-plus-quasiparticle calculations. JOUR JPGPE 311303 2005LU24 RADIOACTIVITY ${ }^{252} \mathrm{Cf}(\mathrm{SF}) ;$ measured $\mathrm{E} \gamma, \mathrm{I} \gamma, \gamma \gamma$-coin. ${ }^{99,101} \mathrm{Y}$, ${ }^{101,105} \mathrm{Nb}$ deduced levels, J, $\pi$, configurations, deformation.

Gammasphere array, triaxial-rotor-plus-particle calculations. JOUR ZAANE 25 s01 469

${ }^{101} \mathrm{Mo}$ 2005RE11 NUCLEAR REACTIONS ${ }^{100} \mathrm{Mo}\left({ }^{136} \mathrm{Xe}, \mathrm{X}\right){ }^{101} \mathrm{Mo} /{ }^{103} \mathrm{Ru} /{ }^{104} \mathrm{Ru}$, $\mathrm{E}=700 \mathrm{MeV}$; measured $\mathrm{E} \gamma, \mathrm{I} \gamma, \gamma \gamma-$, (particle) $\gamma$-coin. ${ }^{101} \mathrm{Mo},{ }^{103,104} \mathrm{Ru}$ deduced high-spin levels, J, $\pi$, configurations. Gammasphere, Chico arrays. JOUR APOBB 361313

${ }^{101} \mathrm{Ru}$ 2004R047 NUCLEAR REACTIONS ${ }^{99,101} \mathrm{Ru}(\mathrm{d}, \mathrm{d}$ '), $\mathrm{E}=13 \mathrm{MeV}$; measured $\sigma(\mathrm{E}$, $\theta) .{ }^{99,101} \mathrm{Ru}$ levels deduced deformation lengths, Coulomb-nuclear interference parameters. DWBA-deformed optical model analysis. JOUR BJPHE 34760 


\section{$\mathrm{A}=101$ (continued)}

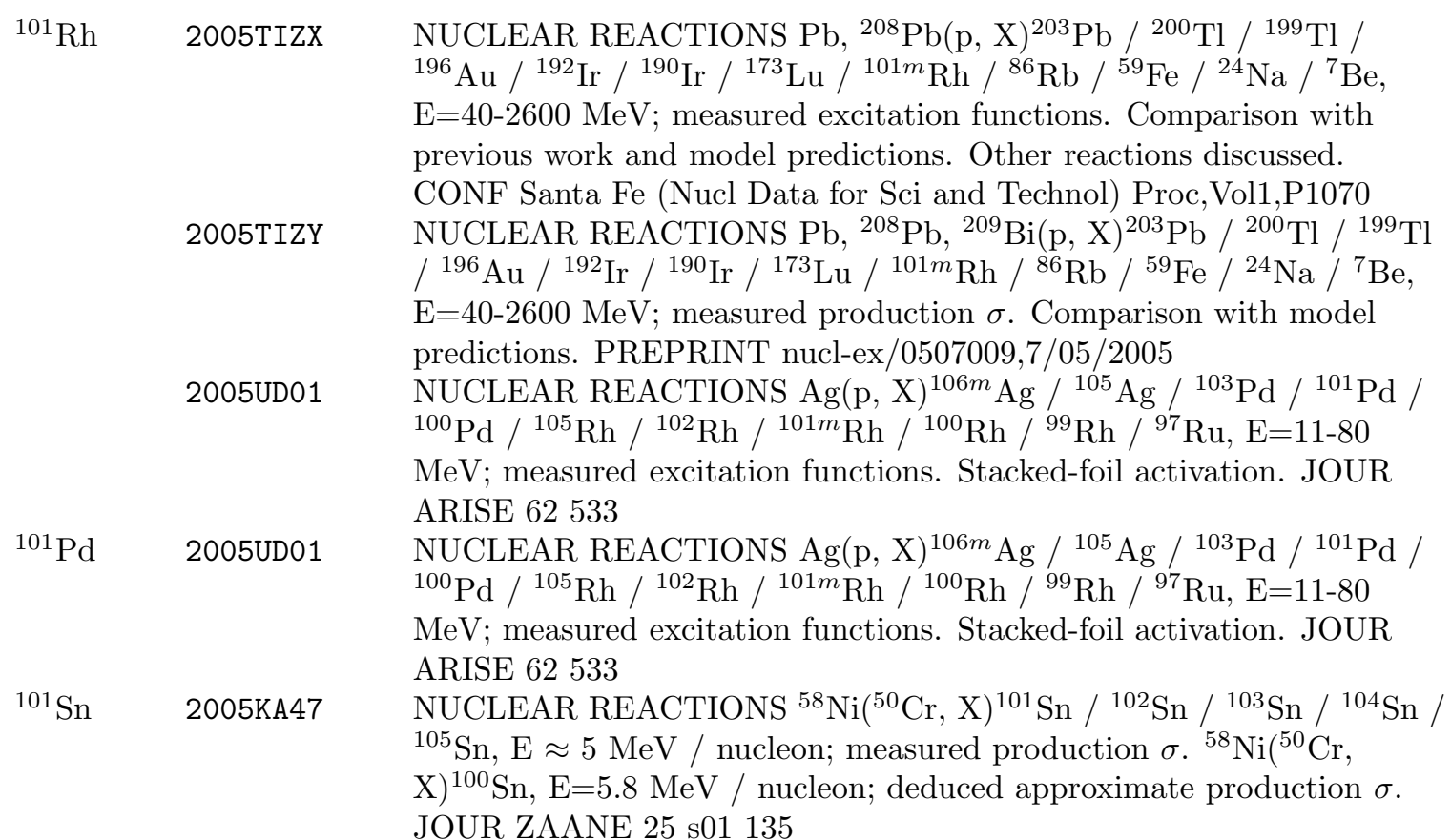

\section{$\mathrm{A}=102$}

${ }^{102} \mathrm{Zr} \quad 2005 \mathrm{BI} 25$

NUCLEAR MOMENTS 86,87,88,89,90,91,92,93,94,95,96,97,98,99,100,101,102Zr; measured charge radii. ${ }^{176} \mathrm{Yb}$; measured isomer shift. Ion-beam cooler, laser spectroscopy. JOUR ZAANE 25 s01 187

$2005 \mathrm{~F} 017$ RADIOACTIVITY ${ }^{252} \mathrm{Cf}(\mathrm{SF})$; measured $\mathrm{E} \gamma, \mathrm{I} \gamma, \gamma \gamma$-coin. ${ }^{98} \mathrm{Sr}$, ${ }^{102,104} \mathrm{Zr},{ }^{137} \mathrm{Xe},{ }^{143} \mathrm{Ba},{ }^{152} \mathrm{Ce}$ levels deduced $\mathrm{T}_{1 / 2}$. Gammasphere array, time-gated triple-coincidence method. JOUR ZAANE 25 s01 465

2005JA12 RADIOACTIVITY ${ }^{252} \mathrm{Cf}(\mathrm{SF})$; measured $\mathrm{E} \gamma, \mathrm{I} \gamma, \alpha \gamma-, \gamma \gamma$-coin for $\alpha$-accompanied ternary fission; deduced fission fragments average angular momentum. ${ }^{100,102} \mathrm{Zr},{ }^{106} \mathrm{Mo},{ }^{144,146} \mathrm{Ba},{ }^{138,140,142} \mathrm{Xe}$; deduced transition intensities. Gammasphere array. JOUR ZAANE 24373

2005J022 ATOMIC MASSES ${ }^{98,99,100,101,102,103,104,105} \mathrm{Zr}$; measured masses. Penning trap mass spectrometer. JOUR ZAANE 25 s01 27

2005SM08 RADIOACTIVITY ${ }^{252} \mathrm{Cf}(\mathrm{SF})$; measured $\mathrm{E} \gamma, \mathrm{I} \gamma(\theta, \mathrm{H}, \mathrm{t}), \gamma \gamma$-coin. 96,100,102 Zr, ${ }^{102,104,106,108} \mathrm{Mo},{ }^{106,108,110,112} \mathrm{Ru},{ }^{110,114,116} \mathrm{Pd}$ levels deduced $\mathrm{g}$ factors, $\mathrm{B}(\mathrm{E} 2)$. Gammasphere array, time-integral perturbed angular correlation technique. Comparison with interacting boson model predictions. JOUR JPGPE 31 S1433

${ }^{102} \mathrm{Mo}$ 2005SM08 RADIOACTIVITY ${ }^{252} \mathrm{Cf}(\mathrm{SF}) ;$ measured $\mathrm{E} \gamma, \mathrm{I} \gamma(\theta, \mathrm{H}, \mathrm{t}), \gamma \gamma$-coin. ${ }^{96,100,102} \mathrm{Zr},{ }^{102,104,106,108} \mathrm{Mo},{ }^{106,108,110,112} \mathrm{Ru},{ }^{110,114,116} \mathrm{Pd}$ levels deduced $\mathrm{g}$ factors, $\mathrm{B}(\mathrm{E} 2)$. Gammasphere array, time-integral perturbed angular correlation technique. Comparison with interacting boson model predictions. JOUR JPGPE 31 S1433 


\section{$\mathrm{A}=102$ (continued)}

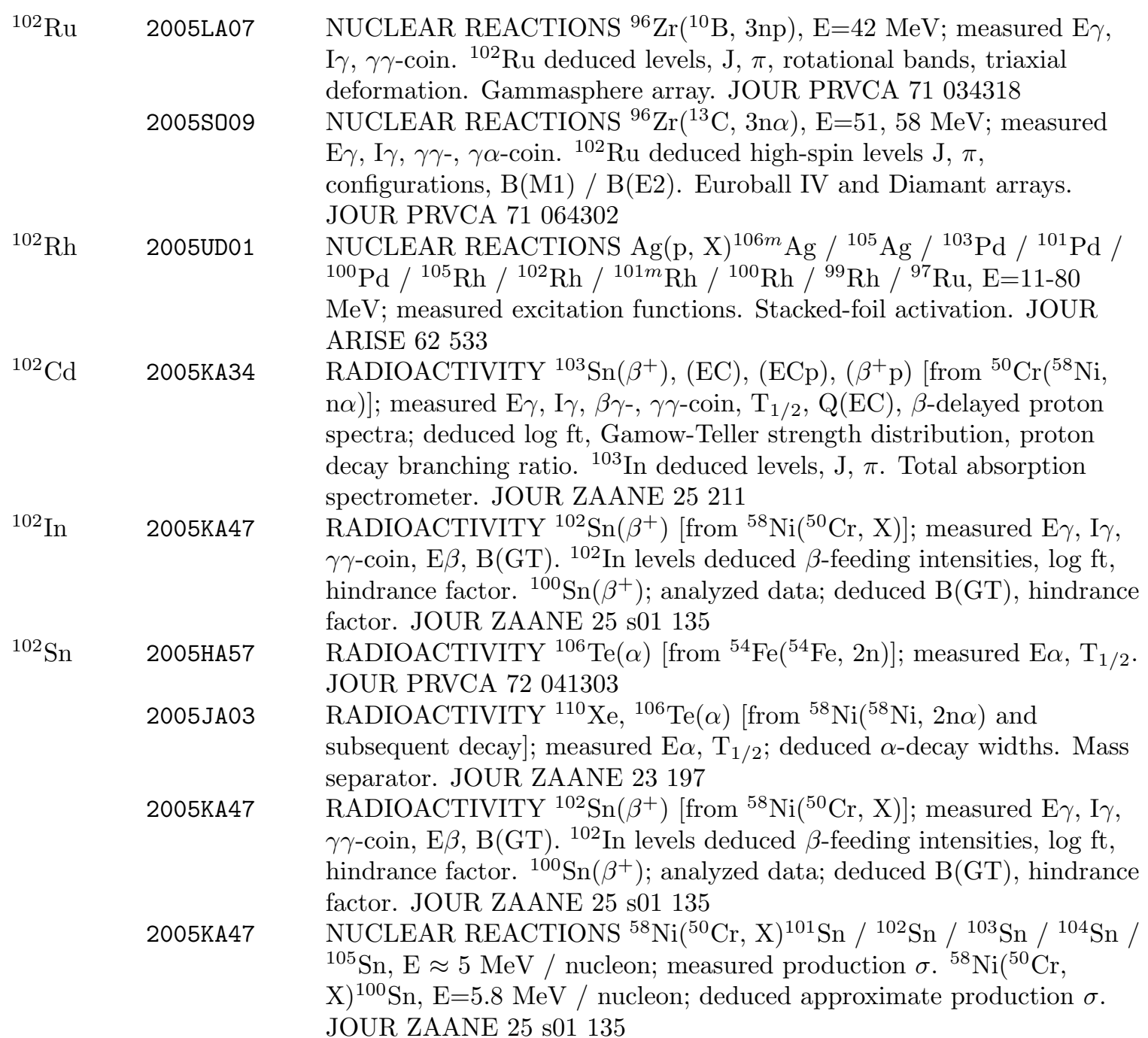

$$
\mathrm{A}=\mathbf{1 0 3}
$$

$\begin{array}{ll}{ }^{103} \mathrm{Zr} & \text { 2005J022 } \\ { }^{103} \mathrm{Tc} & 2005 \mathrm{BE} 17 \\ { }^{103} \mathrm{Ru} & 2005 \mathrm{ADZZ}\end{array}$

ATOMIC MASSES 98,99,100,101,102,103,104,105 Zr; measured masses. Penning trap mass spectrometer. JOUR ZAANE 25 s01 27 RADIOACTIVITY ${ }^{127} \mathrm{I}\left({ }^{24} \mathrm{Ne}\right),\left({ }^{28} \mathrm{Mg}\right),\left({ }^{30} \mathrm{Mg}\right),\left({ }^{32} \mathrm{Si}\right),\left({ }^{34} \mathrm{Si}\right),\left({ }^{48} \mathrm{Ca}\right)$, $\left({ }^{49} \mathrm{Sc}\right)$; measured cluster decay $\mathrm{T}_{1 / 2}$ lower limits. JOUR ZAANE 2451 NUCLEAR REACTIONS ${ }^{129} \mathrm{I}(\mathrm{n}, 7 \mathrm{n}),(\mathrm{n}, 6 \mathrm{n}),(\mathrm{n}, 4 \mathrm{n}),(\mathrm{n}, \gamma), \mathrm{E}=$ fast; ${ }^{237} \mathrm{~Np}(\mathrm{n}, \gamma), \mathrm{E}=$ fast; measured yields. ${ }^{237} \mathrm{~Np}(\mathrm{n}, \mathrm{F}){ }^{91} \mathrm{Sr} /{ }^{97} \mathrm{Zr} /{ }^{132} \mathrm{Te} /$ ${ }^{133} \mathrm{I} /{ }^{135} \mathrm{I}, \mathrm{E}=$ fast; ${ }^{238} \mathrm{Pu}(\mathrm{n}, \mathrm{F}){ }^{97} \mathrm{Zr} /{ }^{129} \mathrm{Sb} /{ }^{132} \mathrm{I} /{ }^{133} \mathrm{I} /{ }^{135} \mathrm{Xe} /$ ${ }^{105} \mathrm{Ru}, \mathrm{E}=$ fast; ${ }^{239} \mathrm{Pu}(\mathrm{n}, \mathrm{F}){ }^{88} \mathrm{Kr} /{ }^{91} \mathrm{Sr} /{ }^{92} \mathrm{Sr} /{ }^{92} \mathrm{Y} /{ }^{97} \mathrm{Zr} /{ }^{99} \mathrm{Mo} /$ ${ }^{103} \mathrm{Ru} /{ }^{105} \mathrm{Ru} /{ }^{128} \mathrm{Sb} /{ }^{129} \mathrm{Sb} /{ }^{132} \mathrm{Te} /{ }^{131} \mathrm{I} /{ }^{132} \mathrm{I} /{ }^{133} \mathrm{I} /{ }^{135} \mathrm{I} /{ }^{135} \mathrm{Xe}$ $/{ }^{143} \mathrm{Ce} /{ }^{140} \mathrm{Ba} /{ }^{140} \mathrm{La}, \mathrm{E}=$ fast; measured fission fragment yields. Secondary neutrons from proton irradiation. JINR nuclotron. CONF St Petersburg,P195,Adam 


\section{$\mathrm{A}=103$ (continued)}

2005MIZZ NUCLEAR REACTIONS Cu(n, X) ${ }^{56} \mathrm{Co}, \mathrm{E}=40-180 \mathrm{MeV} ; \mathrm{Fe}(\mathrm{n}$, $\mathrm{X}){ }^{54} \mathrm{Mn} /{ }^{52} \mathrm{Mn} /{ }^{51} \mathrm{Cr} /{ }^{48} \mathrm{~V}, \mathrm{E} \approx 0-180 \mathrm{MeV} ; \mathrm{Pb}(\mathrm{n}, \mathrm{X}){ }^{196} \mathrm{Au} /{ }^{200} \mathrm{~Pb}$ $/{ }^{103} \mathrm{Ru}, \mathrm{E} \approx 40-180 \mathrm{MeV} ; \mathrm{U}(\mathrm{n}, \mathrm{X}){ }^{99} \mathrm{Mo}, \mathrm{E} \approx 0-180 \mathrm{MeV}$; measured excitation functions. Comparison with proton-induced reactions. CONF Santa Fe (Nucl Data for Sci and Technol) Proc,Vol1,P861 2005RE11 NUCLEAR REACTIONS ${ }^{100} \mathrm{Mo}\left({ }^{136} \mathrm{Xe}, \mathrm{X}\right){ }^{101} \mathrm{Mo} /{ }^{103} \mathrm{Ru} /{ }^{104} \mathrm{Ru}$, $\mathrm{E}=700 \mathrm{MeV}$; measured $\mathrm{E} \gamma$, I $\gamma, \gamma \gamma-$, (particle) $\gamma$-coin. ${ }^{101} \mathrm{Mo},{ }^{103,104} \mathrm{Ru}$ deduced high-spin levels, J, $\pi$, configurations. Gammasphere, Chico arrays. JOUR APOBB 361313

${ }^{103} \mathrm{Rh}$ 2004AG09 NUCLEAR REACTIONS ${ }^{103} \mathrm{Rh}\left(\mathrm{n}, \mathrm{n}^{\prime}\right)^{103 m} \mathrm{Rh}, \mathrm{E} \approx 4.8 \mathrm{MeV} ;{ }^{115} \mathrm{In}(\mathrm{n}$, $\left.\mathrm{n}^{\prime}\right){ }^{115 m} \mathrm{In}, \mathrm{E} \approx 5 \mathrm{MeV} ;{ }^{232} \mathrm{Th},{ }^{238} \mathrm{U}(\mathrm{n}, \mathrm{F}), \mathrm{E} \approx 5 \mathrm{MeV} ;{ }^{24} \mathrm{Mg},{ }^{27} \mathrm{Al}$, ${ }^{46,47,48} \mathrm{Ti},{ }^{54,56} \mathrm{Fe},{ }^{58} \mathrm{Ni},{ }^{64} \mathrm{Zn}(\mathrm{n}, \mathrm{p}), \mathrm{E} \approx 2-8 \mathrm{MeV} ;{ }^{27} \mathrm{Al},{ }^{59} \mathrm{Co}(\mathrm{n}, \alpha), \mathrm{E}$ $\approx 8.3 \mathrm{MeV}$; measured activation $\sigma$. Spectrum average technique, comparison with previous results. JOUR RAACA 9263

2005BRZV NUCLEAR REACTIONS ${ }^{103} \mathrm{Rh}(\mathrm{n}, \mathrm{n}),(\mathrm{n}, \gamma), \mathrm{E}=0.01-1000 \mathrm{eV}$; measured capture and transmission $\sigma .{ }^{103} \mathrm{Rh}$ deduced resonance parameters. CONF Santa Fe (Nucl Data for Sci and Technol) Proc,Vol1,P953 2005 CH62 NUCLEAR REACTIONS ${ }^{103} \mathrm{Rh}\left(\gamma, \gamma^{\prime}\right), \mathrm{E} \approx 40 \mathrm{keV}$; measured $\mathrm{E} \gamma$, $\mathrm{X}$-ray spectra; deduced ICC. Isomer production via bremsstrahlung spectra. JOUR CPLEE 222530

2005DU15 RADIOACTIVITY ${ }^{103} \mathrm{Pd}(\mathrm{EC})$ [from ${ }^{102} \mathrm{Pd}(\mathrm{n}, \gamma)$ ]; measured $\mathrm{E} \gamma$, I $\gamma$. ${ }^{103} \mathrm{Rh}$ deduced levels, $\beta$-feeding intensities. JOUR PRVCA 71054322

${ }^{103} \mathrm{Pd} \quad$ 2004hizz NUCLEAR REACTIONS ${ }^{102} \mathrm{Ru}\left({ }^{3} \mathrm{He}, 2 \mathrm{n}\right),{ }^{100} \mathrm{Ru}(\alpha, \mathrm{n}),{ }^{103} \mathrm{Rh}(\mathrm{d}, 2 \mathrm{n})$, $(\mathrm{p}, \mathrm{n}), \mathrm{E} \approx 5-35 \mathrm{MeV}$; analyzed excitation functions, yields. $\mathrm{Ce}\left({ }^{3} \mathrm{He}\right.$, $\mathrm{xn}){ }^{140} \mathrm{Nd}, \mathrm{E}<27 \mathrm{MeV} ;{ }^{141} \operatorname{Pr}(\mathrm{p}, 2 \mathrm{n}), \mathrm{E}<23 \mathrm{MeV}$; measured yields. ${ }^{192} \mathrm{Os}(\mathrm{p}, \mathrm{n}), \mathrm{E} \approx 6-20$; measured $\sigma$. REPT NEA/NSC/DOC(2004)14,P15,Hilgers

2005DU15 NUCLEAR REACTIONS ${ }^{102,108} \mathrm{Pd}(\mathrm{n}, \gamma), \mathrm{E}=$ reactor; measured thermal and resonance capture $\sigma$; deduced resonance integrals. Activation technique. JOUR PRVCA 71054322

2005DU15 RADIOACTIVITY ${ }^{103} \mathrm{Pd}(\mathrm{EC})$ [from ${ }^{102} \mathrm{Pd}(\mathrm{n}, \gamma)$ ]; measured $\mathrm{E} \gamma$, I $\gamma$. ${ }^{103} \mathrm{Rh}$ deduced levels, $\beta$-feeding intensities. JOUR PRVCA 71054322 2005SKZZ NUCLEAR REACTIONS ${ }^{100} \mathrm{Ru}(\alpha, \mathrm{n}), \mathrm{E}=12-25 \mathrm{MeV} ;{ }^{101} \mathrm{Ru}(\alpha, 2 \mathrm{n})$, $\mathrm{E}=15-25 \mathrm{MeV} ;{ }^{101} \mathrm{Ru}\left({ }^{3} \mathrm{He}, \mathrm{n}\right), \mathrm{E}=15-34 \mathrm{MeV} ;{ }^{102} \mathrm{Ru}\left({ }^{3} \mathrm{He}, 2 \mathrm{n}\right)$, $\mathrm{E}=15-34 \mathrm{MeV}$; measured excitation functions; deduced thick-target yields. Stacked-foil activation technique. CONF Santa Fe (Nucl Data for Sci and Technol) Proc,Vol2,P1634

2005UD01 NUCLEAR REACTIONS Ag(p, X) ${ }^{106 m} \mathrm{Ag} /{ }^{105} \mathrm{Ag} /{ }^{103} \mathrm{Pd} /{ }^{101} \mathrm{Pd} /$ ${ }^{100} \mathrm{Pd} /{ }^{105} \mathrm{Rh} /{ }^{102} \mathrm{Rh} /{ }^{101 m} \mathrm{Rh} /{ }^{100} \mathrm{Rh} /{ }^{99} \mathrm{Rh} /{ }^{97} \mathrm{Ru}, \mathrm{E}=11-80$ $\mathrm{MeV}$; measured excitation functions. Stacked-foil activation. JOUR ARISE 62533

${ }^{103} \mathrm{Ag}$ 2004HE35 NUCLEAR REACTIONS Pd(p, xn $)^{103} \mathrm{Ag}, \mathrm{E} \approx 15-37 \mathrm{MeV}$; Pd(d, $\mathrm{xn}){ }^{103} \mathrm{Ag}, \mathrm{E} \approx 5-20 \mathrm{MeV}$; measured excitation functions. Stacked-foil activation technique. JOUR RAACA 92215

2005HE05 NUCLEAR REACTIONS Pd( $\alpha$, xnyp $)^{103} \mathrm{Ag} /{ }^{104} \mathrm{Ag} /{ }^{105} \mathrm{Ag} /{ }^{106 m} \mathrm{Ag}$ / ${ }^{110 m} \mathrm{Ag} /{ }^{111} \mathrm{Ag} /{ }^{112} \mathrm{Ag} /{ }^{104} \mathrm{Cd} /{ }^{105} \mathrm{Cd} /{ }^{111 m} \mathrm{Cd}, \mathrm{E}=10-37 \mathrm{MeV}$; measured $\sigma$. Stacked-foil activation, comparison with model predictions. JOUR NIMBE 229321 


\section{$\mathrm{A}=103$ (continued)}

2005HEZW NUCLEAR REACTIONS Pd $(\alpha, \mathrm{X}){ }^{103} \mathrm{Ag} /{ }^{105} \mathrm{Ag} /{ }^{106 m} \mathrm{Ag} /{ }^{110 m} \mathrm{Ag} /$ ${ }^{111} \mathrm{Ag} /{ }^{112} \mathrm{Ag} /{ }^{104} \mathrm{Cd} /{ }^{105} \mathrm{Cd} /{ }^{111 m} \mathrm{Cd}, \mathrm{E} \approx 20-37 \mathrm{MeV}$; measured production $\sigma$. Activation technique, comparison with model predictions. CONF Santa Fe (Nucl Data for Sci and Technol) Proc,Vol1,P961

${ }^{103} \mathrm{In} \quad$ 2005KA34

2005KA48

${ }^{103} \mathrm{Sn}$

2004HA59

2005KA34

2005KA47

2005KA48

${ }^{104} \mathrm{Mo}$

2005SM08

${ }^{104} \mathrm{Ru}$

2005RE11
RADIOACTIVITY ${ }^{103} \mathrm{Sn}\left(\beta^{+}\right),(\mathrm{EC}),(\mathrm{ECp}),\left(\beta^{+} \mathrm{p}\right)\left[\right.$ from ${ }^{50} \mathrm{Cr}\left({ }^{58} \mathrm{Ni}\right.$, $\mathrm{n} \alpha)]$; measured $\mathrm{E} \gamma, \mathrm{I} \gamma, \beta \gamma$-, $\gamma \gamma$-coin, $\mathrm{T}_{1 / 2}, \mathrm{Q}(\mathrm{EC}), \beta$-delayed proton spectra; deduced log ft, Gamow-Teller strength distribution, proton decay branching ratio. ${ }^{103} \mathrm{In}$ deduced levels, $\mathrm{J}, \pi$. Total absorption spectrometer. JOUR ZAANE 25211

RADIOACTIVITY ${ }^{103} \mathrm{Sn}\left(\beta^{+}\right)$, (EC) [from ${ }^{58} \mathrm{Ni}\left({ }^{50} \mathrm{Cr}, \mathrm{X}\right), \mathrm{E}=5 \mathrm{MeV} /$ nucleon]; measured $\mathrm{E} \gamma, \mathrm{I} \gamma, \gamma \gamma-, \beta \gamma$-coin, $\mathrm{E} \beta, \mathrm{B}(\mathrm{GT}), \mathrm{T}_{1 / 2} .{ }^{103} \mathrm{In}$ deduced levels, $\mathrm{J}, \pi$, hindrance factor. ${ }^{105} \mathrm{Sn}\left(\beta^{+}\right)$, (EC) [from ${ }^{58} \mathrm{Ni}\left({ }^{50} \mathrm{Cr}, \mathrm{X}\right), \mathrm{E}=5 \mathrm{MeV} /$ nucleon]; analyzed data; deduced $\mathrm{B}(\mathrm{GT})$, hindrance factor. JOUR ZAANE 25 s01 139

RADIOACTIVITY ${ }^{107} \mathrm{Te}(\alpha)$ [from $\left.{ }^{58} \mathrm{Ni}\left({ }^{52} \mathrm{Cr}, 3 \mathrm{n}\right)\right]$; measured $\mathrm{E} \alpha, \mathrm{I} \alpha$. JOUR PRVCA 70064314 RADIOACTIVITY ${ }^{103} \mathrm{Sn}\left(\beta^{+}\right),(\mathrm{EC}),(\mathrm{ECp}),\left(\beta^{+} \mathrm{p}\right)\left[\right.$ from ${ }^{50} \mathrm{Cr}\left({ }^{58} \mathrm{Ni}\right.$, $\mathrm{n} \alpha)]$; measured $\mathrm{E} \gamma, \mathrm{I} \gamma, \beta \gamma$-, $\gamma \gamma$-coin, $\mathrm{T}_{1 / 2}, \mathrm{Q}(\mathrm{EC}), \beta$-delayed proton spectra; deduced log ft, Gamow-Teller strength distribution, proton decay branching ratio. ${ }^{103} \mathrm{In}$ deduced levels, $\mathrm{J}, \pi$. Total absorption spectrometer. JOUR ZAANE 25211

NUCLEAR REACTIONS ${ }^{58} \mathrm{Ni}\left({ }^{50} \mathrm{Cr}, \mathrm{X}\right){ }^{101} \mathrm{Sn} /{ }^{102} \mathrm{Sn} /{ }^{103} \mathrm{Sn} /{ }^{104} \mathrm{Sn} /$ ${ }^{105} \mathrm{Sn}, \mathrm{E} \approx 5 \mathrm{MeV} /$ nucleon; measured production $\sigma .{ }^{58} \mathrm{Ni}\left({ }^{50} \mathrm{Cr}\right.$, $\mathrm{X})^{100} \mathrm{Sn}, \mathrm{E}=5.8 \mathrm{MeV} /$ nucleon; deduced approximate production $\sigma$. JOUR ZAANE 25 s01 135

RADIOACTIVITY ${ }^{103} \mathrm{Sn}\left(\beta^{+}\right)$, (EC) [from ${ }^{58} \mathrm{Ni}\left({ }^{50} \mathrm{Cr}, \mathrm{X}\right), \mathrm{E}=5 \mathrm{MeV} /$ nucleon]; measured $\mathrm{E} \gamma, \mathrm{I} \gamma, \gamma \gamma-, \beta \gamma$-coin, $\mathrm{E} \beta, \mathrm{B}(\mathrm{GT}), \mathrm{T}_{1 / 2} \cdot{ }^{103} \mathrm{In}$ deduced levels, $\mathrm{J}, \pi$, hindrance factor. ${ }^{105} \mathrm{Sn}\left(\beta^{+}\right)$, (EC) [from ${ }^{58} \mathrm{Ni}\left({ }^{50} \mathrm{Cr}, \mathrm{X}\right), \mathrm{E}=5 \mathrm{MeV} /$ nucleon]; analyzed data; deduced $\mathrm{B}(\mathrm{GT})$, hindrance factor. JOUR ZAANE 25 s01 139

\section{$\mathrm{A}=104$}

RADIOACTIVITY ${ }^{252} \mathrm{Cf}(\mathrm{SF})$; measured $\mathrm{E} \gamma, \mathrm{I} \gamma, \gamma \gamma$-coin. ${ }^{98} \mathrm{Sr}$, ${ }^{102,104} \mathrm{Zr},{ }^{137} \mathrm{Xe},{ }^{143} \mathrm{Ba},{ }^{152} \mathrm{Ce}$ levels deduced $\mathrm{T}_{1 / 2}$. Gammasphere array, time-gated triple-coincidence method. JOUR ZAANE 25 s01 465 ATOMIC MASSES 98,99,100,101,102,103,104,105 Zr; measured masses. Penning trap mass spectrometer. JOUR ZAANE 25 s01 27

RADIOACTIVITY ${ }^{252} \mathrm{Cf}(\mathrm{SF})$; measured $\mathrm{E} \gamma, \mathrm{I} \gamma(\theta, \mathrm{H}, \mathrm{t}), \gamma \gamma$-coin. 96,100,102 Zr, ${ }^{102,104,106,108} \mathrm{Mo},{ }^{106,108,110,112} \mathrm{Ru},{ }^{110,114,116} \mathrm{Pd}$ levels deduced $\mathrm{g}$ factors, $\mathrm{B}(\mathrm{E} 2)$. Gammasphere array, time-integral perturbed angular correlation technique. Comparison with interacting boson model predictions. JOUR JPGPE 31 S1433 NUCLEAR REACTIONS ${ }^{100} \mathrm{Mo}\left({ }^{136} \mathrm{Xe}, \mathrm{X}\right){ }^{101} \mathrm{Mo} /{ }^{103} \mathrm{Ru} /{ }^{104} \mathrm{Ru}$, $\mathrm{E}=700 \mathrm{MeV}$; measured $\mathrm{E} \gamma, \mathrm{I} \gamma, \gamma \gamma-$, (particle) $\gamma$-coin. ${ }^{101} \mathrm{Mo},{ }^{103,104} \mathrm{Ru}$ deduced high-spin levels, J, $\pi$, configurations. Gammasphere, Chico arrays. JOUR APOBB 361313 


\section{$\mathrm{A}=104$ (continued)}

${ }^{104} \mathrm{Rh}$ 2005BRzV NUCLEAR REACTIONS ${ }^{103} \mathrm{Rh}(\mathrm{n}, \mathrm{n}),(\mathrm{n}, \gamma), \mathrm{E}=0.01-1000 \mathrm{eV}$; measured capture and transmission $\sigma .{ }^{103} \mathrm{Rh}$ deduced resonance parameters. CONF Santa Fe (Nucl Data for Sci and Technol) Proc,Vol1,P953

2005WI23 NUCLEAR REACTIONS ${ }^{100} \mathrm{Mo}\left({ }^{11} \mathrm{~B}\right.$, xnypz $\left.\alpha\right){ }^{104} \mathrm{Rh} /{ }^{105} \mathrm{Rh} /{ }^{107} \mathrm{Pd} /$ ${ }^{108} \mathrm{Pd}, \mathrm{E}=43 \mathrm{MeV} ;{ }^{51} \mathrm{~V}\left({ }^{16} \mathrm{O}\right.$, xnypz $\left.\alpha\right){ }^{60} \mathrm{Ni} /{ }^{61} \mathrm{Ni} /{ }^{61} \mathrm{Cu} /{ }^{62} \mathrm{Cu}, \mathrm{E}=70$ $\mathrm{MeV}$; measured $\mathrm{E} \gamma, \mathrm{I} \gamma, \gamma \gamma-$, (charged particle) $\gamma$-coin; deduced $\gamma$-ray yield ratios. Application to exit channel determination discussed. JOUR BJPHE 35898

${ }^{104} \mathrm{Pd} \quad 2004 \mathrm{RO} 48 \quad$ NUCLEAR REACTIONS ${ }^{104,106,108,110} \mathrm{Pd}(\mathrm{d}, \mathrm{d}$ '), E=13 MeV; measured $\sigma(\mathrm{E}, \theta) .{ }^{104,106,108,110} \mathrm{Pd}$ levels deduced $\mathrm{B}(\mathrm{E} 2)$, deformation lengths, Coulomb-nuclear interference parameters. DWBA-deformed optical model analysis. JOUR BJPHE 34777

2005BEZS NUCLEAR REACTIONS ${ }^{108} \mathrm{Pd}\left({ }^{122} \mathrm{Cd},{ }^{122} \mathrm{Cd}\right),{ }^{104} \mathrm{Pd}\left({ }^{124} \mathrm{Cd},{ }^{124} \mathrm{Cd}\right.$ '), $\left({ }^{126} \mathrm{Cd},{ }^{126} \mathrm{Cd}\right.$ '), E not given; measured $\mathrm{E} \gamma, \mathrm{I} \gamma$, (particle) $\gamma$-coin following projectile Coulomb excitation. ${ }^{122,124} \mathrm{Cd}$ levels deduced excitation B(E2). REPT MLL 2004 Annual,P14,Behrens

${ }^{104} \mathrm{Ag} \quad 2005 \mathrm{HE} 05 \quad$ NUCLEAR REACTIONS Pd $\left.(\alpha, \mathrm{xnyp})\right)^{103} \mathrm{Ag} /{ }^{104} \mathrm{Ag} /{ }^{105} \mathrm{Ag} /{ }^{106 m} \mathrm{Ag}$
$/{ }^{110 m} \mathrm{Ag} /{ }^{111} \mathrm{Ag} /{ }^{112} \mathrm{Ag} /{ }^{104} \mathrm{Cd} /{ }^{105} \mathrm{Cd} /{ }^{111 m} \mathrm{Cd}, \mathrm{E}=10-37 \mathrm{MeV}$; measured $\sigma$. Stacked-foil activation, comparison with model predictions. JOUR NIMBE 229321

${ }^{104} \mathrm{Cd}$ 2005HE05 NUCLEAR REACTIONS Pd( $\alpha$, xnyp $)^{103} \mathrm{Ag} /{ }^{104} \mathrm{Ag} /{ }^{105} \mathrm{Ag} /{ }^{106 m} \mathrm{Ag}$ $/{ }^{110 m} \mathrm{Ag} /{ }^{111} \mathrm{Ag} /{ }^{112} \mathrm{Ag} /{ }^{104} \mathrm{Cd} /{ }^{105} \mathrm{Cd} /{ }^{111 m} \mathrm{Cd}, \mathrm{E}=10-37 \mathrm{MeV}$; measured $\sigma$. Stacked-foil activation, comparison with model predictions. JOUR NIMBE 229321

2005HEZW NUCLEAR REACTIONS Pd $(\alpha, \mathrm{X}){ }^{103} \mathrm{Ag} /{ }^{105} \mathrm{Ag} /{ }^{106 m} \mathrm{Ag} /{ }^{110 m} \mathrm{Ag} /$ ${ }^{111} \mathrm{Ag} /{ }^{112} \mathrm{Ag} /{ }^{104} \mathrm{Cd} /{ }^{105} \mathrm{Cd} /{ }^{111 m} \mathrm{Cd}, \mathrm{E} \approx 20-37 \mathrm{MeV}$; measured production $\sigma$. Activation technique, comparison with model predictions. CONF Santa Fe (Nucl Data for Sci and Technol) Proc, Vol1,P961

${ }^{104}$ In $\quad$ 2005CL08 ATOMIC MASSES ${ }^{64} \mathrm{Ge},{ }^{68} \mathrm{Se}$; analyzed masses; deduced effective $\mathrm{T}_{1 / 2} \cdot{ }^{90,91} \mathrm{Mo},{ }^{90,91,92,93} \mathrm{Tc},{ }^{93,94} \mathrm{Ru},{ }^{94,95} \mathrm{Rh},{ }^{104,105,106,107} \mathrm{In}$, $104,105,107,108 \mathrm{Sn},{ }^{107,108} \mathrm{Sb}$; measured masses. Penning trap, astrophysical implications discussed. JOUR ZAANE 25 s01 629

${ }^{104} \mathrm{Sn} \quad$ 2005CL08 ATOMIC MASSES ${ }^{64} \mathrm{Ge},{ }^{68} \mathrm{Se}$; analyzed masses; deduced effective $\mathrm{T}_{1 / 2} \cdot{ }^{90,91} \mathrm{Mo},{ }^{90,91,92,93} \mathrm{Tc},{ }^{93,94} \mathrm{Ru},{ }^{94,95} \mathrm{Rh},{ }^{104,105,106,107} \mathrm{In}$, ${ }_{104,105,107,108}$ Sn, ${ }^{107,108}$ Sb; measured masses. Penning trap, astrophysical implications discussed. JOUR ZAANE 25 s01 629

2005KA47 NUCLEAR REACTIONS ${ }^{58} \mathrm{Ni}\left({ }^{50} \mathrm{Cr}, \mathrm{X}\right){ }^{101} \mathrm{Sn} /{ }^{102} \mathrm{Sn} /{ }^{103} \mathrm{Sn} /{ }^{104} \mathrm{Sn} /$ ${ }^{105} \mathrm{Sn}, \mathrm{E} \approx 5 \mathrm{MeV} /$ nucleon; measured production $\sigma \cdot{ }^{58} \mathrm{Ni}\left({ }^{50} \mathrm{Cr}\right.$, $\mathrm{X})^{100} \mathrm{Sn}, \mathrm{E}=5.8 \mathrm{MeV} /$ nucleon; deduced approximate production $\sigma$. JOUR ZAANE 25 s01 135

2005LI47 RADIOACTIVITY ${ }^{105} \mathrm{Sb}(\mathrm{p})$ [from $\left.{ }^{50} \mathrm{Cr}\left({ }^{58} \mathrm{Ni}, 2 \mathrm{np}\right)\right]$; measured $\mathrm{Ep}$; deduced upper limit for proton decay branching ratio. JOUR PRVCA 72047301

2005LIZY RADIOACTIVITY ${ }^{105} \mathrm{Sb}(\mathrm{p})$ [from $\left.{ }^{50} \mathrm{Cr}\left({ }^{58} \mathrm{Ni}, 2 \mathrm{np}\right)\right]$; measured Ep; deduced upper limit for proton decay branching ratio. REPT GSI 2005-1,P85,Liu 


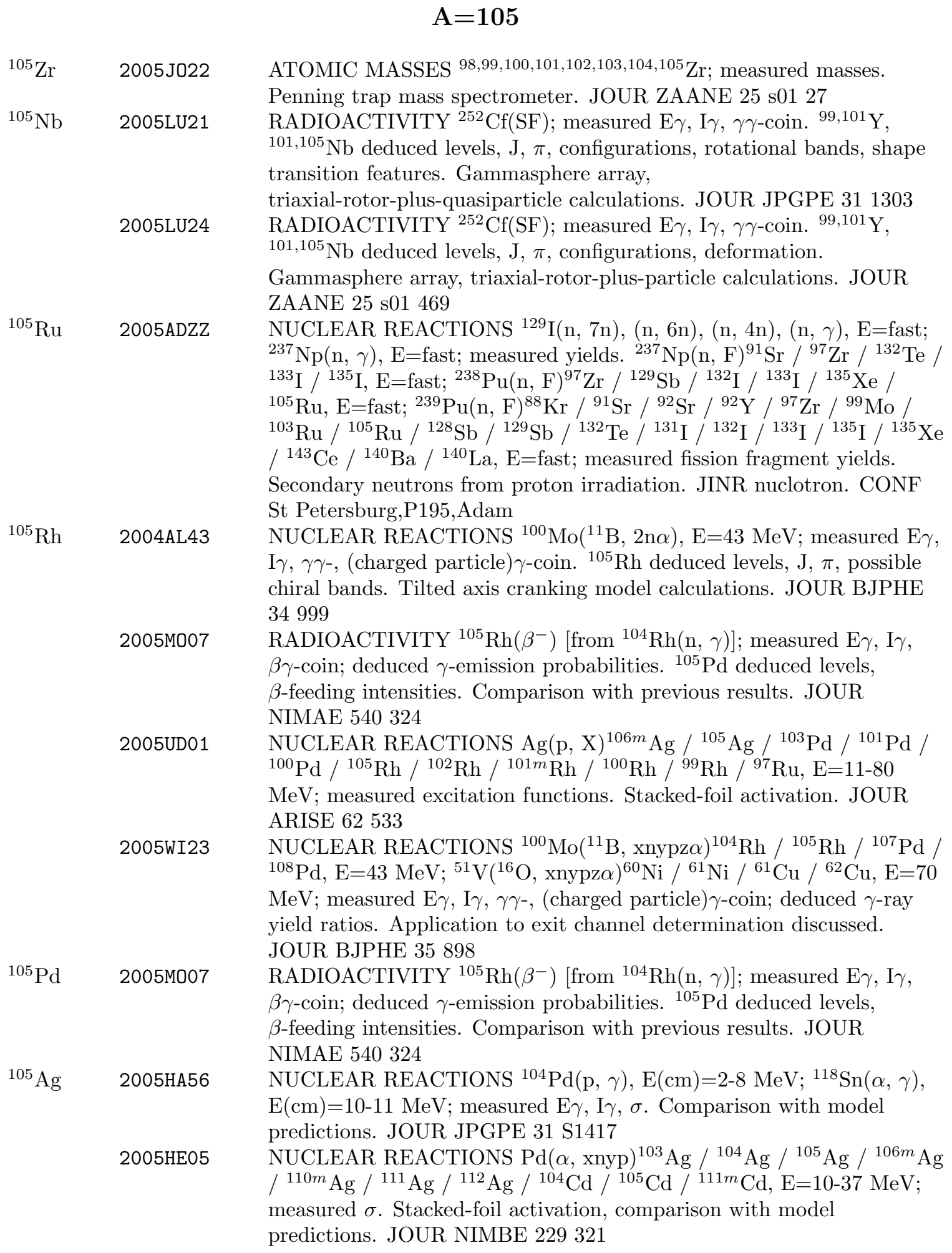




\section{$\mathrm{A}=105$ (continued)}

2005HEZW NUCLEAR REACTIONS Pd $(\alpha, \mathrm{X}){ }^{103} \mathrm{Ag} /{ }^{105} \mathrm{Ag} /{ }^{106 m} \mathrm{Ag} /{ }^{110 m} \mathrm{Ag} /$ ${ }^{111} \mathrm{Ag} /{ }^{112} \mathrm{Ag} /{ }^{104} \mathrm{Cd} /{ }^{105} \mathrm{Cd} /{ }^{111 m} \mathrm{Cd}, \mathrm{E} \approx 20-37 \mathrm{MeV}$; measured production $\sigma$. Activation technique, comparison with model predictions. CONF Santa Fe (Nucl Data for Sci and Technol) Proc,Vol1,P961

2005UD01 NUCLEAR REACTIONS Ag(p, X) ${ }^{106 m} \mathrm{Ag} /{ }^{105} \mathrm{Ag} /{ }^{103} \mathrm{Pd} /{ }^{101} \mathrm{Pd} /$ ${ }^{100} \mathrm{Pd} /{ }^{105} \mathrm{Rh} /{ }^{102} \mathrm{Rh} /{ }^{101 m} \mathrm{Rh} /{ }^{100} \mathrm{Rh} /{ }^{99} \mathrm{Rh} /{ }^{97} \mathrm{Ru}, \mathrm{E}=11-80$

$\mathrm{MeV}$; measured excitation functions. Stacked-foil activation. JOUR ARISE 62533

${ }^{105} \mathrm{Cd}$ 2005HE05 NUCLEAR REACTIONS Pd( $\alpha$, xnyp $)^{103} \mathrm{Ag} /{ }^{104} \mathrm{Ag} /{ }^{105} \mathrm{Ag} /{ }^{106 m} \mathrm{Ag}$ $/{ }^{110 m} \mathrm{Ag} /{ }^{111} \mathrm{Ag} /{ }^{112} \mathrm{Ag} /{ }^{104} \mathrm{Cd} /{ }^{105} \mathrm{Cd} /{ }^{111 m} \mathrm{Cd}, \mathrm{E}=10-37 \mathrm{MeV}$; measured $\sigma$. Stacked-foil activation, comparison with model predictions. JOUR NIMBE 229321

2005HEZW NUCLEAR REACTIONS $\operatorname{Pd}(\alpha, \mathrm{X}){ }^{103} \mathrm{Ag} /{ }^{105} \mathrm{Ag} /{ }^{106 m} \mathrm{Ag} /{ }^{110 m} \mathrm{Ag} /$ ${ }^{111} \mathrm{Ag} /{ }^{112} \mathrm{Ag} /{ }^{104} \mathrm{Cd} /{ }^{105} \mathrm{Cd} /{ }^{111 m} \mathrm{Cd}, \mathrm{E} \approx 20-37 \mathrm{MeV}$; measured production $\sigma$. Activation technique, comparison with model predictions. CONF Santa Fe (Nucl Data for Sci and Technol) Proc,Vol1,P961

${ }^{105} \mathrm{In} \quad$ 2005CL08 ATOMIC MASSES ${ }^{64} \mathrm{Ge},{ }^{68} \mathrm{Se}$; analyzed masses; deduced effective $\mathrm{T}_{1 / 2} \cdot{ }^{90,91} \mathrm{Mo},{ }^{90,91,92,93} \mathrm{Tc},{ }^{93,94} \mathrm{Ru},{ }^{94,95} \mathrm{Rh},{ }^{104,105,106,107} \mathrm{In}$,

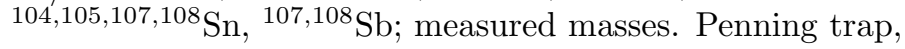
astrophysical implications discussed. JOUR ZAANE 25 s01 629 2005KA48 RADIOACTIVITY ${ }^{103} \mathrm{Sn}\left(\beta^{+}\right)$, (EC) [from ${ }^{58} \mathrm{Ni}\left({ }^{50} \mathrm{Cr}, \mathrm{X}\right), \mathrm{E}=5 \mathrm{MeV} /$ nucleon]; measured $\mathrm{E} \gamma, \mathrm{I} \gamma, \gamma \gamma-, \beta \gamma$-coin, $\mathrm{E} \beta, \mathrm{B}(\mathrm{GT}), \mathrm{T}_{1 / 2} .{ }^{103} \mathrm{In}$ deduced levels, $\mathrm{J}, \pi$, hindrance factor. ${ }^{105} \mathrm{Sn}\left(\beta^{+}\right)$, (EC) [from ${ }^{58} \mathrm{Ni}\left({ }^{50} \mathrm{Cr}, \mathrm{X}\right), \mathrm{E}=5 \mathrm{MeV} /$ nucleon]; analyzed data; deduced $\mathrm{B}(\mathrm{GT})$, hindrance factor. JOUR ZAANE 25 s01 139

${ }^{105} \mathrm{Sn} \quad$ 2005CL08 ATOMIC MASSES ${ }^{64} \mathrm{Ge},{ }^{68} \mathrm{Se}$; analyzed masses; deduced effective $\mathrm{T}_{1 / 2} \cdot{ }^{90,91} \mathrm{Mo},{ }^{90,91,92,93} \mathrm{Tc},{ }^{93,94} \mathrm{Ru},{ }^{94,95} \mathrm{Rh},{ }^{104,105,106,107} \mathrm{In}$, $104,105,107,108 \mathrm{Sn},{ }^{107,108} \mathrm{Sb}$; measured masses. Penning trap, astrophysical implications discussed. JOUR ZAANE 25 s01 629

2005KA47 NUCLEAR REACTIONS ${ }^{58} \mathrm{Ni}\left({ }^{50} \mathrm{Cr}, \mathrm{X}\right){ }^{101} \mathrm{Sn} /{ }^{102} \mathrm{Sn} /{ }^{103} \mathrm{Sn} /{ }^{104} \mathrm{Sn} /$ ${ }^{105} \mathrm{Sn}, \mathrm{E} \approx 5 \mathrm{MeV} /$ nucleon; measured production $\sigma \cdot{ }^{58} \mathrm{Ni}\left({ }^{50} \mathrm{Cr}\right.$, $\mathrm{X})^{100} \mathrm{Sn}, \mathrm{E}=5.8 \mathrm{MeV} /$ nucleon; deduced approximate production $\sigma$. JOUR ZAANE 25 s01 135

2005KA48 RADIOACTIVITY ${ }^{103} \mathrm{Sn}\left(\beta^{+}\right),(\mathrm{EC})\left[\right.$ from ${ }^{58} \mathrm{Ni}\left({ }^{50} \mathrm{Cr}, \mathrm{X}\right), \mathrm{E}=5 \mathrm{MeV} /$ nucleon]; measured $\mathrm{E} \gamma, \mathrm{I} \gamma, \gamma \gamma-, \beta \gamma$-coin, $\mathrm{E} \beta, \mathrm{B}(\mathrm{GT}), \mathrm{T}_{1 / 2} .{ }^{103} \mathrm{In}$ deduced levels, $\mathrm{J}, \pi$, hindrance factor. ${ }^{105} \mathrm{Sn}\left(\beta^{+}\right)$, (EC) [from ${ }^{58} \mathrm{Ni}\left({ }^{50} \mathrm{Cr}, \mathrm{X}\right), \mathrm{E}=5 \mathrm{MeV} /$ nucleon]; analyzed data; deduced $\mathrm{B}(\mathrm{GT})$, hindrance factor. JOUR ZAANE 25 s01 139

${ }^{105} \mathrm{Sb}$ 2005LI47 NUCLEAR REACTIONS ${ }^{50} \mathrm{Cr}\left({ }^{58} \mathrm{Ni}, 2 \mathrm{np}\right), \mathrm{E}=222,255 \mathrm{MeV}$; measured delayed Ep. ${ }^{105} \mathrm{Sb}$ deduced upper limit for proton decay branching ratio. JOUR PRVCA 72047301

2005LI47 RADIOACTIVITY ${ }^{105} \mathrm{Sb}(\mathrm{p})$ [from $\left.{ }^{50} \mathrm{Cr}\left({ }^{58} \mathrm{Ni}, 2 \mathrm{np}\right)\right]$; measured Ep; deduced upper limit for proton decay branching ratio. JOUR PRVCA 72047301

2005LIZY NUCLEAR REACTIONS ${ }^{50} \mathrm{Cr}\left({ }^{58} \mathrm{Ni}, 2 \mathrm{np}\right), \mathrm{E}=222,255 \mathrm{MeV}$; measured delayed Ep. ${ }^{105} \mathrm{Sb}$ deduced upper limit for proton decay branching ratio. REPT GSI 2005-1,P85,Liu 


\section{$\mathrm{A}=105$ (continued)}

2005LIZY

RADIOACTIVITY ${ }^{105} \mathrm{Sb}(\mathrm{p})$ [from $\left.{ }^{50} \mathrm{Cr}\left({ }^{58} \mathrm{Ni}, 2 \mathrm{np}\right)\right]$; measured Ep; deduced upper limit for proton decay branching ratio. REPT GSI 2005-1,P85,Liu

\section{$\mathrm{A}=106$}

${ }^{106} \mathrm{Mo} \quad$ 2005JA12

2005SM08

2005ZH36

${ }^{106} \mathrm{Ru} \quad$ 2005SM08

${ }^{106} \mathrm{Pd} \quad$ 2004BRZV

2004R048

2005BRZX

2005ZU01

${ }^{106} \mathrm{Ag} \quad 2005 \mathrm{HE} 05$

2005HEZW

2005J020
RADIOACTIVITY ${ }^{252} \mathrm{Cf}(\mathrm{SF})$; measured $\mathrm{E} \gamma, \mathrm{I} \gamma, \alpha \gamma-, \gamma \gamma$-coin for $\alpha$-accompanied ternary fission; deduced fission fragments average angular momentum. ${ }^{100,102} \mathrm{Zr},{ }^{106} \mathrm{Mo},{ }^{144,146} \mathrm{Ba},{ }^{138,140,142} \mathrm{Xe}$; deduced transition intensities. Gammasphere array. JOUR ZAANE 24373 RADIOACTIVITY ${ }^{252} \mathrm{Cf}(\mathrm{SF})$; measured $\mathrm{E} \gamma, \mathrm{I} \gamma(\theta, \mathrm{H}, \mathrm{t}), \gamma \gamma$-coin. 96,100,102 Zr, ${ }^{102,104,106,108} \mathrm{Mo},{ }^{106,108,110,112} \mathrm{Ru},{ }^{110,114,116} \mathrm{Pd}$ levels deduced $\mathrm{g}$ factors, $\mathrm{B}(\mathrm{E} 2)$. Gammasphere array, time-integral perturbed angular correlation technique. Comparison with interacting boson model predictions. JOUR JPGPE 31 S1433

RADIOACTIVITY ${ }^{252} \mathrm{Cf}(\mathrm{SF})$; measured E $\gamma$, I $\gamma, \gamma \gamma$-coin. ${ }^{106} \mathrm{Mo}$ deduced high-spin levels, J, $\pi$, chiral vibrational bands. Gammasphere array, tilted-axis cranking model analysis. JOUR ZAANE 25 s01 459

RADIOACTIVITY ${ }^{252} \mathrm{Cf}(\mathrm{SF})$; measured $\mathrm{E} \gamma, \mathrm{I} \gamma(\theta, \mathrm{H}, \mathrm{t}), \gamma \gamma$-coin. ${ }^{96,100,102} \mathrm{Zr},{ }^{102,104,106,108} \mathrm{Mo},{ }^{106,108,110,112} \mathrm{Ru},{ }^{110,114,116} \mathrm{Pd}$ levels

deduced $\mathrm{g}$ factors, B(E2). Gammasphere array, time-integral perturbed angular correlation technique. Comparison with interacting boson model predictions. JOUR JPGPE 31 S1433

RADIOACTIVITY ${ }^{106} \mathrm{Cd}(2 \mathrm{EC})$; measured $\mathrm{T}_{1 / 2}$ lower limit. REPT JINR-P6-2004-219,Brudanin NUCLEAR REACTIONS ${ }^{104,106,108,110} \mathrm{Pd}(\mathrm{d}$, d'), $\mathrm{E}=13 \mathrm{MeV}$; measured $\sigma(\mathrm{E}, \theta) .{ }^{104,106,108,110} \mathrm{Pd}$ levels deduced $\mathrm{B}(\mathrm{E} 2)$, deformation lengths, Coulomb-nuclear interference parameters. DWBA-deformed optical model analysis. JOUR BJPHE 34777 RADIOACTIVITY ${ }^{106} \mathrm{Cd}(2 \mathrm{EC})$; measured $\mathrm{T}_{1 / 2}$ limit. Modane underground laboratory. CONF St Petersburg,P299,Brudanin RADIOACTIVITY ${ }^{120} \mathrm{Te}\left(\beta^{+} \mathrm{EC}\right) ;{ }^{64} \mathrm{Zn},{ }^{106,108} \mathrm{Cd},{ }^{120} \mathrm{Te}(2 \mathrm{EC})$; measured $\mathrm{T}_{1 / 2}$ lower limits. JOUR NPBSE 138236 NUCLEAR REACTIONS Pd( $\alpha$, xnyp $){ }^{103} \mathrm{Ag} /{ }^{104} \mathrm{Ag} /{ }^{105} \mathrm{Ag} /{ }^{106 m} \mathrm{Ag}$ $/{ }^{110 m} \mathrm{Ag} /{ }^{111} \mathrm{Ag} /{ }^{112} \mathrm{Ag} /{ }^{104} \mathrm{Cd} /{ }^{105} \mathrm{Cd} /{ }^{111 m} \mathrm{Cd}, \mathrm{E}=10-37 \mathrm{MeV}$; measured $\sigma$. Stacked-foil activation, comparison with model predictions. JOUR NIMBE 229321

NUCLEAR REACTIONS $\operatorname{Pd}(\alpha, \mathrm{X}){ }^{103} \mathrm{Ag} /{ }^{105} \mathrm{Ag} /{ }^{106 m} \mathrm{Ag} /{ }^{110 m} \mathrm{Ag} /$ ${ }^{111} \mathrm{Ag} /{ }^{112} \mathrm{Ag} /{ }^{104} \mathrm{Cd} /{ }^{105} \mathrm{Cd} /{ }^{111 m} \mathrm{Cd}, \mathrm{E} \approx 20-37 \mathrm{MeV}$; measured production $\sigma$. Activation technique, comparison with model predictions. CONF Santa Fe (Nucl Data for Sci and Technol) Proc, Vol1,P961 NUCLEAR REACTIONS ${ }^{100} \mathrm{Mo}\left({ }^{10} \mathrm{~B}, 4 \mathrm{n}\right), \mathrm{E}=42 \mathrm{MeV}$; measured $\mathrm{E} \gamma$, $\mathrm{I} \gamma, \gamma \gamma$-coin. ${ }^{106} \mathrm{Ag}$ deduced high-spin levels, $\mathrm{J}, \pi$, configurations, possible triaxial rotation. Gammasphere array. JOUR JPGPE 31 S1895 


\section{$\mathrm{A}=106$ (continued)}

2005UD01 NUCLEAR REACTIONS Ag(p, X) ${ }^{106 m} \mathrm{Ag} /{ }^{105} \mathrm{Ag} /{ }^{103} \mathrm{Pd} /{ }^{101} \mathrm{Pd} /$ ${ }^{100} \mathrm{Pd} /{ }^{105} \mathrm{Rh} /{ }^{102} \mathrm{Rh} /{ }^{101 m} \mathrm{Rh} /{ }^{100} \mathrm{Rh} /{ }^{99} \mathrm{Rh} /{ }^{97} \mathrm{Ru}, \mathrm{E}=11-80$ $\mathrm{MeV}$; measured excitation functions. Stacked-foil activation. JOUR ARISE 62533

${ }^{106} \mathrm{Cd}$ 2004BRZV RADIOACTIVITY ${ }^{106} \mathrm{Cd}(2 \mathrm{EC})$; measured $\mathrm{T}_{1 / 2}$ lower limit. REPT JINR-P6-2004-219,Brudanin

2005BRZX RADIOACTIVITY ${ }^{106} \mathrm{Cd}(2 \mathrm{EC})$; measured $\mathrm{T}_{1 / 2}$ limit. Modane underground laboratory. CONF St Petersburg,P299,Brudanin $2005 \mathrm{GYO} 3$ NUCLEAR REACTIONS ${ }^{106} \mathrm{Cd}(\alpha, \gamma), \mathrm{E}=8-12.5 \mathrm{MeV}$; measured $\mathrm{E} \gamma$, $\mathrm{I} \gamma, \sigma \cdot{ }^{106} \mathrm{Cd}(\alpha, \alpha), \mathrm{E} \approx 15,17,19 \mathrm{MeV} ;$ measured $\sigma(\theta)$. Astrophysical implications discussed, comparison with model predictions. JOUR NUPAB $758517 \mathrm{c}$

2005SI23 NUCLEAR REACTIONS ${ }^{62} \mathrm{Ni}\left({ }^{48} \mathrm{Ca}, 4 \mathrm{n}\right), \mathrm{E}=183,207 \mathrm{MeV} ;{ }^{96} \mathrm{Zr}\left({ }^{16} \mathrm{O}\right.$, $4 \mathrm{n}), \mathrm{E}=72 \mathrm{MeV}$; measured $\mathrm{E} \gamma, \mathrm{I} \gamma, \gamma \gamma$-coin, DSA. ${ }^{106,108} \mathrm{Cd}$ deduced high-spin levels, $\mathrm{J}, \pi, \mathrm{T}_{1 / 2}, \mathrm{~B}(\mathrm{E} 2)$, configurations. Gammasphere array. JOUR PRVCA 72024318

2005ZU01 RADIOACTIVITY ${ }^{120} \mathrm{Te}\left(\beta^{+} \mathrm{EC}\right) ;{ }^{64} \mathrm{Zn},{ }^{106,108} \mathrm{Cd},{ }^{120} \mathrm{Te}(2 \mathrm{EC})$; measured $\mathrm{T}_{1 / 2}$ lower limits. JOUR NPBSE 138236

${ }^{106} \mathrm{In} \quad$ 2005CL08 ATOMIC MASSES ${ }^{64} \mathrm{Ge},{ }^{68} \mathrm{Se}$; analyzed masses; deduced effective $\mathrm{T}_{1 / 2} .{ }^{90,91} \mathrm{Mo},{ }^{90,91,92,93} \mathrm{Tc},{ }^{93,94} \mathrm{Ru},{ }^{94,95} \mathrm{Rh},{ }^{104,105,106,107} \mathrm{In}$, $104,105,107,108 \mathrm{Sn},{ }^{107,108} \mathrm{Sb}$; measured masses. Penning trap, astrophysical implications discussed. JOUR ZAANE 25 s01 629

${ }^{106} \mathrm{Sn} \quad$ 2005MIZW RADIOACTIVITY ${ }^{106,107} \mathrm{Sb}(\mathrm{EC}),\left(\beta^{+}\right)$[from ${ }^{58} \mathrm{Ni}\left({ }^{58} \mathrm{Ni}, \mathrm{X}\right)$ ]; measured $\mathrm{E} \gamma, \mathrm{I} \gamma, \beta \gamma-, \gamma \gamma$-coin. ${ }^{106,107} \mathrm{Sn}$ deduced levels, configurations. Total absorption spectrometer. REPT GSI 2005-1,P84,Miernik

${ }^{106} \mathrm{Sb}$ 2005MIZW RADIOACTIVITY ${ }^{106,107} \mathrm{Sb}(\mathrm{EC}),\left(\beta^{+}\right)$[from $\left.{ }^{58} \mathrm{Ni}\left({ }^{58} \mathrm{Ni}, \mathrm{X}\right)\right]$; measured $\mathrm{E} \gamma, \mathrm{I} \gamma, \beta \gamma-, \gamma \gamma$-coin. ${ }^{106,107}$ Sn deduced levels, configurations. Total absorption spectrometer. REPT GSI 2005-1,P84,Miernik

$2005 \mathrm{~S} 006$ NUCLEAR REACTIONS ${ }^{54} \mathrm{Fe}\left({ }^{58} \mathrm{Ni}, \mathrm{np} \alpha\right), \mathrm{E}=240 \mathrm{MeV}$; measured $\mathrm{E} \gamma$, $\mathrm{I} \gamma, \gamma \gamma-$, (charged particle) $\gamma-$-, (neutron) $\gamma$-coin, $\gamma$-ray polarization. ${ }^{106} \mathrm{Sb}$ deduced high-spin levels, J, $\pi$, configurations. Euroball, ISIS arrays. JOUR NUPAB 753251

${ }^{106} \mathrm{Te}$ 2005HA57 NUCLEAR REACTIONS ${ }^{54} \mathrm{Fe}\left({ }^{54} \mathrm{Fe}, 2 \mathrm{n}\right), \mathrm{E}=182 \mathrm{MeV}$; measured $\mathrm{E} \gamma$, I $\gamma$, (recoil) $\gamma$-coin; deduced $\sigma .{ }^{106} \mathrm{Te}$ deduced levels, possible vibrational excitation. Recoil-decay tagging, level systematics in Te isotopes discussed. JOUR PRVCA 72041303

2005HA57 RADIOACTIVITY ${ }^{106} \mathrm{Te}(\alpha)\left[\right.$ from $\left.{ }^{54} \mathrm{Fe}\left({ }^{54} \mathrm{Fe}, 2 \mathrm{n}\right)\right]$; measured $\mathrm{E} \alpha, \mathrm{T}_{1 / 2}$. JOUR PRVCA 72041303

2005JA03 RADIOACTIVITY ${ }^{110} \mathrm{Xe},{ }^{106} \mathrm{Te}(\alpha)\left[\right.$ from ${ }^{58} \mathrm{Ni}\left({ }^{58} \mathrm{Ni}, 2 \mathrm{n} \alpha\right)$ and subsequent decay]; measured $\mathrm{E} \alpha, \mathrm{T}_{1 / 2}$; deduced $\alpha$-decay widths. Mass separator. JOUR ZAANE 23197

\section{$\mathrm{A}=\mathbf{1 0 7}$}

${ }^{107} \mathrm{Mo} \quad$ 2005UR02 RADIOACTIVITY ${ }^{248} \mathrm{Cm}(\mathrm{SF}) ;$ measured $\mathrm{E} \gamma, \mathrm{I} \gamma, \gamma \gamma$-coin, angular correlations. ${ }^{107}$ Mo deduced high-spin levels, J, $\pi$, configurations. Eurogam2 array. JOUR PRVCA 72027302 


\section{$\mathrm{A}=107$ (continued)}

\begin{tabular}{|c|c|c|}
\hline${ }^{107} \mathrm{Pd}$ & 2005WI23 & $\begin{array}{l}\text { NUCLEAR REACTIONS }{ }^{100} \mathrm{Mo}\left({ }^{11} \mathrm{~B} \text {, xnypz } \alpha\right){ }^{104} \mathrm{Rh} /{ }^{105} \mathrm{Rh} /{ }^{107} \mathrm{Pd} / \\
{ }^{108} \mathrm{Pd}, \mathrm{E}=43 \mathrm{MeV} ;{ }^{51} \mathrm{~V}\left({ }^{16} \mathrm{O} \text {, xnypz } \alpha\right)^{60} \mathrm{Ni} /{ }^{61} \mathrm{Ni} /{ }^{61} \mathrm{Cu} /{ }^{62} \mathrm{Cu}, \mathrm{E}=70 \\
\mathrm{MeV} \text {; measured } \mathrm{E} \gamma, \mathrm{I} \gamma, \gamma \gamma-\text {, (charged particle }) \gamma \text {-coin; deduced } \gamma \text {-ray } \\
\text { yield ratios. Application to exit channel determination discussed. } \\
\text { JOUR BJPHE } 35898\end{array}$ \\
\hline${ }^{107} \mathrm{Cd}$ & 2005AN26 & $\begin{array}{l}\text { NUCLEAR REACTIONS }{ }^{98} \mathrm{Mo}\left({ }^{12} \mathrm{C}, 3 \mathrm{n}\right), \mathrm{E}=60 \mathrm{MeV} \text {; measured } \\
\text { Doppler-shifted } \mathrm{E} \gamma \text {, I } \gamma, \gamma \gamma \text {-coin. }{ }^{107} \mathrm{Cd} \text { levels deduced } \mathrm{T}_{1 / 2}, \mathrm{~B}(\mathrm{E} 2) \text {. } \\
\text { Recoil-distance method. JOUR JPGPE } 31 \mathrm{~S} 1563\end{array}$ \\
\hline \multirow[t]{2}{*}{${ }^{107} \mathrm{In}$} & 2005CL08 & $\begin{array}{l}\text { ATOMIC MASSES }{ }^{64} \mathrm{Ge},{ }^{68} \mathrm{Se} ; \text { analyzed masses; deduced effective } \\
\mathrm{T}_{1 / 2} \cdot{ }^{90,91} \mathrm{Mo},{ }^{90,91,92,93} \mathrm{Tc},{ }^{93,94} \mathrm{Ru},{ }^{94,95} \mathrm{Rh},{ }^{104,105,106,107} \mathrm{In}, \\
104,105,107,108 \mathrm{Sn},{ }^{107,108} \mathrm{Sb} \text {; measured masses. Penning trap, } \\
\text { astrophysical implications discussed. JOUR ZAANE } 25 \text { s01 } 629\end{array}$ \\
\hline & 2005IDZY & $\begin{array}{l}\text { NUCLEAR REACTIONS }{ }^{58} \mathrm{Ni}\left({ }^{52} \mathrm{Cr}, 3 \mathrm{p}\right), \mathrm{E}=187 \mathrm{MeV} \text {; measured } \mathrm{E} \gamma \text {, } \\
\text { I } \gamma, \gamma \gamma-,(\text { recoil }) \gamma \text {-coin. }{ }^{107} \mathrm{In} \text { deduced rotational band, configurations. } \\
\text { Jurosphere array, recoil separator, total Routhian surface calculations. } \\
\text { REPT CNS-REP-66,P19,Ideguchi }\end{array}$ \\
\hline \multirow[t]{2}{*}{${ }^{107} \mathrm{Sn}$} & 2005CL08 & 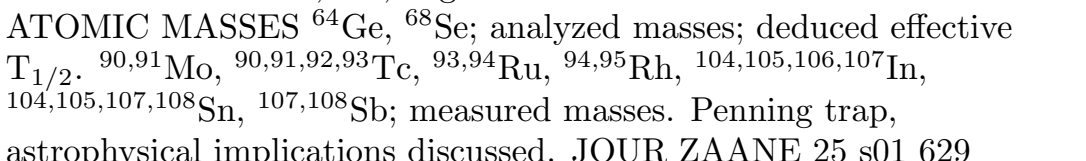 \\
\hline & 2005MIZW & $\begin{array}{l}\left.\text { RADIOACTIVITY }{ }^{106,107} \mathrm{Sb}(\mathrm{EC}),\left(\beta^{+}\right) \text {[from }{ }^{58} \mathrm{Ni}\left({ }^{58} \mathrm{Ni}, \mathrm{X}\right)\right] \text {; measured } \\
\mathrm{E} \gamma, \mathrm{I} \gamma, \beta \gamma-, \gamma \gamma \text {-coin. }{ }^{106,107} \mathrm{Sn} \text { deduced levels, configurations. Total } \\
\text { absorption spectrometer. REPT GSI 2005-1,P84,Miernik }\end{array}$ \\
\hline \multirow[t]{2}{*}{${ }^{107} \mathrm{Sb}$} & 2005CL08 & $\begin{array}{l}\text { ATOMIC MASSES }{ }^{64} \mathrm{Ge},{ }^{68} \mathrm{Se} \text {; analyzed masses; deduced effective } \\
\mathrm{T}_{1 / 2} \cdot{ }^{90,91} \mathrm{Mo},{ }^{90,91,92,93} \mathrm{Tc},{ }^{93,94} \mathrm{Ru},{ }^{94,95} \mathrm{Rh},{ }^{104,105,106,107} \mathrm{In}, \\
{ }^{104,105,107,108} \mathrm{Sn},{ }^{107,108} \mathrm{Sb} \text {; measured masses. Penning trap, } \\
\text { astrophysical implications discussed. JOUR ZAANE } 25 \mathrm{~s} 01629\end{array}$ \\
\hline & 2005MIZW & $\begin{array}{l}\text { RADIOACTIVITY }{ }^{106,107} \mathrm{Sb}(\mathrm{EC}),\left(\beta^{+}\right)\left[\text {from }{ }^{58} \mathrm{Ni}\left({ }^{58} \mathrm{Ni}, \mathrm{X}\right)\right] \text {; measured } \\
\mathrm{E} \gamma, \mathrm{I} \gamma, \beta \gamma-, \gamma \gamma \text {-coin. }{ }^{106,107} \mathrm{Sn} \text { deduced levels, configurations. Total } \\
\text { absorption spectrometer. REPT GSI 2005-1,P84,Miernik }\end{array}$ \\
\hline \multirow[t]{2}{*}{${ }^{107} \mathrm{Te}$} & 2004HA59 & $\begin{array}{l}\text { NUCLEAR REACTIONS }{ }^{58} \mathrm{Ni}\left({ }^{52} \mathrm{Cr}, 3 \mathrm{n}\right), \mathrm{E}=187 \mathrm{MeV} \text {; measured } \mathrm{E} \gamma \text {, } \\
\mathrm{I} \gamma, \gamma \gamma-,(\text { recoil }) \gamma \text {-coin. }{ }^{107} \mathrm{Te} \text { deduced transitions, excited state. } \\
\text { Jurogam array, recoil-decay tagging. JOUR PRVCA } 70064314\end{array}$ \\
\hline & 2004HA59 & $\begin{array}{l}\text { RADIOACTIVITY }{ }^{107} \mathrm{Te}(\alpha)\left[\text { from }{ }^{58} \mathrm{Ni}\left({ }^{52} \mathrm{Cr}, 3 \mathrm{n}\right)\right] \text {; measured } \mathrm{E} \alpha, \mathrm{I} \alpha . \\
\text { JOUR PRVCA } 70064314\end{array}$ \\
\hline
\end{tabular}

\section{$\mathrm{A}=\mathbf{1 0 8}$}

${ }^{108} \mathrm{Mo}$ 2005SM08 RADIOACTIVITY ${ }^{252} \mathrm{Cf}(\mathrm{SF}) ;$ measured $\mathrm{E} \gamma, \mathrm{I} \gamma(\theta, \mathrm{H}, \mathrm{t}), \gamma \gamma$-coin. ${ }^{96,100,102} \mathrm{Zr},{ }^{102,104,106,108} \mathrm{Mo},{ }^{106,108,110,112} \mathrm{Ru},{ }^{110,114,116} \mathrm{Pd}$ levels deduced $\mathrm{g}$ factors, B(E2). Gammasphere array, time-integral perturbed angular correlation technique. Comparison with interacting boson model predictions. JOUR JPGPE 31 S1433

${ }^{108} \mathrm{Tc} \quad 2005 \mathrm{HW} 06$ RADIOACTIVITY ${ }^{252} \mathrm{Cf}(\mathrm{SF}) ;$ measured $\mathrm{E} \gamma, \mathrm{I} \gamma, \gamma \gamma$-coin. ${ }^{95,97} \mathrm{Sr},{ }^{99} \mathrm{Zr}$, ${ }^{108} \mathrm{Tc},{ }^{133,134} \mathrm{Te},{ }^{137} \mathrm{Xe}$ levels deduced $\mathrm{T}_{1 / 2}$. Gammasphere array, time-gated triple-coincidence method. JOUR ZAANE 25 s01 463 


\section{$\mathrm{A}=108$ (continued)}

${ }^{108} \mathrm{Ru}$ 2005SM08 RADIOACTIVITY ${ }^{252} \mathrm{Cf}(\mathrm{SF}) ;$ measured $\mathrm{E} \gamma, \mathrm{I} \gamma(\theta, \mathrm{H}, \mathrm{t}), \gamma \gamma$-coin. ${ }^{96,100,102} \mathrm{Zr},{ }^{102,104,106,108} \mathrm{Mo},{ }^{106,108,110,112} \mathrm{Ru},{ }^{110,114,116} \mathrm{Pd}$ levels deduced $\mathrm{g}$ factors, $\mathrm{B}(\mathrm{E} 2)$. Gammasphere array, time-integral perturbed angular correlation technique. Comparison with interacting boson model predictions. JOUR JPGPE 31 S1433

${ }^{108} \mathrm{Pd}$ 2004AL44 NUCLEAR REACTIONS ${ }^{100} \mathrm{Mo}\left({ }^{11} \mathrm{~B}, 2 \mathrm{np}\right), \mathrm{E}=43 \mathrm{MeV}$; measured E $\gamma$, $\mathrm{I} \gamma, \gamma \gamma$-, (charged particle) $\gamma$-coin. ${ }^{108} \mathrm{Pd}$ deduced levels, $\mathrm{J}, \pi$, configurations. Cranking model analysis. JOUR BJPHE 341005

2004R048 NUCLEAR REACTIONS ${ }^{104,106,108,110} \mathrm{Pd}$ (d, d'), E=13 MeV; measured $\sigma(\mathrm{E}, \theta) .{ }^{104,106,108,110} \mathrm{Pd}$ levels deduced $\mathrm{B}(\mathrm{E} 2)$, deformation lengths, Coulomb-nuclear interference parameters. DWBA-deformed optical model analysis. JOUR BJPHE 34777

2005AL25 NUCLEAR REACTIONS ${ }^{100} \mathrm{Mo}\left({ }^{11} \mathrm{~B}, 2 \mathrm{np}\right), \mathrm{E}=43 \mathrm{MeV}$; measured E $\gamma$, $\mathrm{I} \gamma, \gamma \gamma$-, (charged particle) $\gamma$-coin. ${ }^{108} \mathrm{Pd}$ deduced high-spin levels, $\mathrm{J}, \pi$, configurations. Total Routhian surface calculations. JOUR PRVCA 71 054315

2005BEZS NUCLEAR REACTIONS ${ }^{108} \mathrm{Pd}\left({ }^{122} \mathrm{Cd},{ }^{122} \mathrm{Cd}\right),{ }^{104} \mathrm{Pd}\left({ }^{124} \mathrm{Cd},{ }^{124} \mathrm{Cd}\right.$ '), $\left({ }^{126} \mathrm{Cd},{ }^{126} \mathrm{Cd}\right.$ '), E not given; measured $\mathrm{E} \gamma, \mathrm{I} \gamma$, (particle) $\gamma$-coin following projectile Coulomb excitation. ${ }^{122,124} \mathrm{Cd}$ levels deduced excitation B(E2). REPT MLL 2004 Annual,P14,Behrens

2005WI23 NUCLEAR REACTIONS ${ }^{100} \mathrm{Mo}\left({ }^{11} \mathrm{~B}\right.$, xnypz $\left.\alpha\right){ }^{104} \mathrm{Rh} /{ }^{105} \mathrm{Rh} /{ }^{107} \mathrm{Pd} /$ ${ }^{108} \mathrm{Pd}, \mathrm{E}=43 \mathrm{MeV} ;{ }^{51} \mathrm{~V}\left({ }^{16} \mathrm{O}\right.$, xnypz $\left.\alpha\right){ }^{60} \mathrm{Ni} /{ }^{61} \mathrm{Ni} /{ }^{61} \mathrm{Cu} /{ }^{62} \mathrm{Cu}, \mathrm{E}=70$ $\mathrm{MeV}$; measured $\mathrm{E} \gamma, \mathrm{I} \gamma, \gamma \gamma$-, (charged particle) $\gamma$-coin; deduced $\gamma$-ray yield ratios. Application to exit channel determination discussed. JOUR BJPHE 35898

2005ZU01 RADIOACTIVITY ${ }^{120} \mathrm{Te}\left(\beta^{+} \mathrm{EC}\right) ;{ }^{64} \mathrm{Zn},{ }^{106,108} \mathrm{Cd},{ }^{120} \mathrm{Te}(2 \mathrm{EC})$; measured $\mathrm{T}_{1 / 2}$ lower limits. JOUR NPBSE 138236

${ }^{108} \mathrm{Cd}$ 2005DA16 NUCLEAR REACTIONS ${ }^{100} \mathrm{Mo}\left({ }^{13} \mathrm{C}, 5 \mathrm{n}\right), \mathrm{E}=65 \mathrm{MeV}$; measured $\mathrm{E} \gamma$, $\mathrm{I} \gamma, \gamma \gamma$-coin, DSA. ${ }^{108} \mathrm{Cd}$ deduced high-spin levels, J, $\pi$, B(E2), antimagnetic rotation. Total Routhian surface calculations. JOUR PRVCA 71041305

2005FA06 NUCLEAR REACTIONS ${ }^{64} \mathrm{Ni}\left({ }^{48} \mathrm{Ca}, 4 \mathrm{n}\right), \mathrm{E}=207 \mathrm{MeV}$; measured $\mathrm{E} \gamma$, $\mathrm{I} \gamma, \gamma \gamma$-coin. ${ }^{108} \mathrm{Cd}$ deduced rotational bands transitions, quadrupole moments. JOUR NUPAB $752231 \mathrm{c}$

2005SI23 NUCLEAR REACTIONS ${ }^{62} \mathrm{Ni}\left({ }^{48} \mathrm{Ca}, 4 \mathrm{n}\right), \mathrm{E}=183,207 \mathrm{MeV} ;{ }^{96} \mathrm{Zr}\left({ }^{16} \mathrm{O}\right.$, $4 \mathrm{n}), \mathrm{E}=72 \mathrm{MeV}$; measured $\mathrm{E} \gamma, \mathrm{I} \gamma, \gamma \gamma$-coin, DSA. ${ }^{106,108} \mathrm{Cd}$ deduced high-spin levels, $\mathrm{J}, \pi, \mathrm{T}_{1 / 2}, \mathrm{~B}(\mathrm{E} 2)$, configurations. Gammasphere array. JOUR PRVCA 72024318

2005ZU01 RADIOACTIVITY ${ }^{120} \mathrm{Te}\left(\beta^{+} \mathrm{EC}\right) ;{ }^{64} \mathrm{Zn},{ }^{106,108} \mathrm{Cd},{ }^{120} \mathrm{Te}(2 \mathrm{EC})$; measured $\mathrm{T}_{1 / 2}$ lower limits. JOUR NPBSE 138236

${ }^{108} \mathrm{Sn} \quad 2005 \mathrm{CL} 08$ ATOMIC MASSES ${ }^{64} \mathrm{Ge},{ }^{68} \mathrm{Se}$; analyzed masses; deduced effective $\mathrm{T}_{1 / 2} \cdot{ }^{90,91} \mathrm{Mo},{ }^{90,91,92,93} \mathrm{Tc},{ }^{93,94} \mathrm{Ru},{ }^{94,95} \mathrm{Rh},{ }^{104,105,106,107} \mathrm{In}$, ${ }_{104,105,107,108} \mathrm{Sn},{ }^{107,108} \mathrm{Sb}$; measured masses. Penning trap, astrophysical implications discussed. JOUR ZAANE 25 s01 629 2005W001 NUCLEAR REACTIONS ${ }^{197} \mathrm{Au}\left({ }^{84} \mathrm{Kr},{ }^{84} \mathrm{Kr}\right),\left({ }^{56} \mathrm{Cr},{ }^{56} \mathrm{Cr}\right),\left({ }^{108} \mathrm{Sn}\right.$, ${ }^{108}{ }^{\mathrm{Sn}}$ '), $\mathrm{E}=113-142 \mathrm{MeV} /$ nucleon; measured $\mathrm{E} \gamma$, I $\gamma$ following projectile Coulomb excitation. ${ }^{84} \mathrm{Kr},{ }^{56} \mathrm{Cr},{ }^{108} \mathrm{Sn}$ deduced transitions. ${ }^{9} \mathrm{Be}\left({ }^{55} \mathrm{Ni}, \mathrm{X}\right){ }^{54} \mathrm{Co} /{ }^{52} \mathrm{Fe} /{ }^{50} \mathrm{Cr}, \mathrm{E}=171 \mathrm{MeV} /$ nucleon; measured $\mathrm{E} \gamma$, I $\gamma$, (particle) $\gamma$-coin. JOUR NIMAE 537637 


\section{$\mathrm{A}=108$ (continued)}

$\begin{array}{cl}{ }^{108} \mathrm{Sb} \text { 2005CL08 } & \text { ATOMIC MASSES }{ }^{64} \mathrm{Ge},{ }^{68} \mathrm{Se} \text {; analyzed masses; deduced effective } \\ & \mathrm{T}_{1 / 2} \cdot{ }^{90,91} \mathrm{Mo},{ }^{90,91,92,93} \mathrm{Tc},{ }^{33,94} \mathrm{Ru},{ }^{94,95} \mathrm{Rh},{ }^{104,105,106,107} \mathrm{In}, \\ & 104,105,107,108 \mathrm{Sn},{ }^{107,108} \mathrm{Sb} \text {; measured masses. Penning trap, } \\ & \text { astrophysical implications discussed. JOUR ZAANE } 25 \text { s01 } 629\end{array}$

\section{$\mathrm{A}=109$}

${ }^{109} \mathrm{Tc}$ 2005UR01 RADIOACTIVITY ${ }^{248} \mathrm{Cm}(\mathrm{SF}) ;$ measured $\mathrm{E} \gamma, \mathrm{I} \gamma, \gamma \gamma$-coin. ${ }^{109,110,111} \mathrm{Tc},{ }^{135} \mathrm{I}$ deduced transitions. ${ }^{111} \mathrm{Tc}$ deduced levels, J, $\pi$, configurations. Eurogam2 array. Level systematics in neighboring nuclides discussed. JOUR ZAANE 24161

${ }^{109} \mathrm{Pd}$ 2005DU15 NUCLEAR REACTIONS ${ }^{102,108} \mathrm{Pd}(\mathrm{n}, \gamma), \mathrm{E}=$ reactor; measured thermal and resonance capture $\sigma$; deduced resonance integrals. Activation technique. JOUR PRVCA 71054322

${ }^{109} \mathrm{Cd} \quad$ 2005GY02 RADIOACTIVITY ${ }^{109} \mathrm{In},{ }^{110} \mathrm{Sn}(\mathrm{EC})\left[\right.$ from $\left.{ }^{106} \mathrm{Cd}(\alpha, \gamma),(\alpha, \mathrm{p})\right]$; measured $\mathrm{E} \gamma, \mathrm{I} \gamma, \mathrm{T}_{1 / 2}$. JOUR PRVCA 71057302

2005GYZZ RADIOACTIVITY ${ }^{109} \mathrm{In},{ }^{110} \mathrm{Sn}(\mathrm{EC})\left[\right.$ from $\left.{ }^{106} \mathrm{Cd}(\alpha, \mathrm{X})\right]$; measured $\mathrm{E} \gamma$, $\mathrm{I} \gamma, \mathrm{T}_{1 / 2}$. PREPRINT nucl-ex/0503012,3/18/2005

${ }^{109}$ In $\quad$ 2004ADZW NUCLEAR REACTIONS ${ }^{209} \operatorname{Bi}(\mathrm{n}, 4 \mathrm{n}),(\mathrm{n}, 5 \mathrm{n}),(\mathrm{n}, 6 \mathrm{n}),(\mathrm{n}, 7 \mathrm{n}),(\mathrm{n}$, 9n), ${ }^{232} \mathrm{Th}(\mathrm{n}, \gamma),{ }^{197} \mathrm{Au}(\mathrm{n}, 2 \mathrm{n}),(\mathrm{n}, 4 \mathrm{n}),(\mathrm{n}, 6 \mathrm{n}),(\mathrm{n}, 7 \mathrm{n}),(\mathrm{n}, \gamma),{ }^{115} \operatorname{In}(\mathrm{n}$, 5n), (n, 6n), (n, 7n), ${ }^{59} \mathrm{Co}(\mathrm{n}, 2 \mathrm{n}),(\mathrm{n}, 3 \mathrm{n}),(\mathrm{n}, 4 \mathrm{n}),(\mathrm{n}, 5 \mathrm{n}),(\mathrm{n}, \gamma),(\mathrm{n}$, $\mathrm{p}),(\mathrm{n}, 6 \mathrm{n} 2 \mathrm{p}), \mathrm{E}=$ spectrum; measured $\mathrm{E} \gamma, \mathrm{I} \gamma$; deduced reaction rates. $\mathrm{Pb}(\mathrm{p}, \mathrm{nX}), \mathrm{E}=1 \mathrm{GeV}$; deduced spallation neutron spectrum. REPT JINR-E1-2004-16,Adam

2005AD01 NUCLEAR REACTIONS ${ }^{209} \operatorname{Bi}(\mathrm{n}, 4 \mathrm{n}),(\mathrm{n}, 5 \mathrm{n}),(\mathrm{n}, 6 \mathrm{n}),(\mathrm{n}, 7 \mathrm{n}),(\mathrm{n}$, $9 \mathrm{n}),{ }^{232} \mathrm{Th}(\mathrm{n}, \gamma),{ }^{197} \mathrm{Au}(\mathrm{n}, 2 \mathrm{n}),(\mathrm{n}, 4 \mathrm{n}),(\mathrm{n}, 6 \mathrm{n}),(\mathrm{n}, 7 \mathrm{n}),(\mathrm{n}, \gamma),{ }^{59} \mathrm{Co}(\mathrm{n}$, 2n), (n, 3n), (n, 4n), (n, 5n), (n, p), (n, 6n2p), ${ }^{115} \operatorname{In}(\mathrm{n}, 5 \mathrm{n}),(\mathrm{n}, 6 \mathrm{n}),(\mathrm{n}$, $7 \mathrm{n}), \mathrm{E}=$ spectrum; measured $\mathrm{E} \gamma, \mathrm{I} \gamma$; deduced reaction rates. Activation technique, spallation neutrons from $1 \mathrm{GeV}$ proton beam, comparison with model predictions. JOUR ZAANE 2361

2005GY02 RADIOACTIVITY ${ }^{109} \mathrm{In},{ }^{110} \mathrm{Sn}(\mathrm{EC})\left[\right.$ from $\left.{ }^{106} \mathrm{Cd}(\alpha, \gamma),(\alpha, \mathrm{p})\right]$; measured $\mathrm{E} \gamma, \mathrm{I} \gamma, \mathrm{T}_{1 / 2}$. JOUR PRVCA 71057302

2005GYZZ RADIOACTIVITY ${ }^{109} \mathrm{In},{ }^{110} \mathrm{Sn}(\mathrm{EC})\left[\right.$ from $\left.{ }^{106} \mathrm{Cd}(\alpha, \mathrm{X})\right]$; measured $\mathrm{E} \gamma$, $\mathrm{I} \gamma, \mathrm{T}_{1 / 2}$. PREPRINT nucl-ex/0503012,3/18/2005

\section{$\mathrm{A}=\mathbf{1 1 0}$}

${ }^{110} \mathrm{Tc} \quad 2005 \mathrm{UR} 01$

RADIOACTIVITY ${ }^{248} \mathrm{Cm}(\mathrm{SF}) ;$ measured $\mathrm{E} \gamma, \mathrm{I} \gamma, \gamma \gamma$-coin. ${ }^{109,110,111} \mathrm{Tc},{ }^{135} \mathrm{I}$ deduced transitions. ${ }^{111} \mathrm{Tc}$ deduced levels, J, $\pi$, configurations. Eurogam2 array. Level systematics in neighboring nuclides discussed. JOUR ZAANE 24161

${ }^{110} \mathrm{Ru}$ 2005SM08 RADIOACTIVITY ${ }^{252} \mathrm{Cf}(\mathrm{SF}) ;$ measured $\mathrm{E} \gamma, \mathrm{I} \gamma(\theta, \mathrm{H}, \mathrm{t}), \gamma \gamma$-coin. ${ }^{96,100,102} \mathrm{Zr},{ }^{102,104,106,108} \mathrm{Mo},{ }^{106,108,110,112} \mathrm{Ru},{ }^{110,114,116} \mathrm{Pd}$ levels deduced g factors, B(E2). Gammasphere array, time-integral perturbed angular correlation technique. Comparison with interacting boson model predictions. JOUR JPGPE 31 S1433 


\section{$\mathrm{A}=110$ (continued)}

${ }^{110} \mathrm{Pd}$ 2004R048 NUCLEAR REACTIONS ${ }^{104,106,108,110} \mathrm{Pd}(\mathrm{d}, \mathrm{d}$ '), E=13 MeV; measured $\sigma(\mathrm{E}, \theta)$. ${ }^{104,106,108,110} \mathrm{Pd}$ levels deduced $\mathrm{B}(\mathrm{E} 2)$, deformation lengths, Coulomb-nuclear interference parameters. DWBA-deformed optical model analysis. JOUR BJPHE 34777

2005SM08 RADIOACTIVITY ${ }^{252} \mathrm{Cf}(\mathrm{SF})$; measured $\mathrm{E} \gamma, \mathrm{I} \gamma(\theta, \mathrm{H}, \mathrm{t}), \gamma \gamma$-coin. ${ }_{96,100,102} \mathrm{Zr},{ }^{102,104,106,108} \mathrm{Mo},{ }^{106,108,110,112} \mathrm{Ru},{ }^{110,114,116} \mathrm{Pd}$ levels deduced $\mathrm{g}$ factors, $\mathrm{B}(\mathrm{E} 2)$. Gammasphere array, time-integral perturbed angular correlation technique. Comparison with interacting boson model predictions. JOUR JPGPE 31 S1433

${ }^{110} \mathrm{Ag}$ 2005HE05 NUCLEAR REACTIONS Pd $(\alpha, \text { xnyp })^{103} \mathrm{Ag} /{ }^{104} \mathrm{Ag} /{ }^{105} \mathrm{Ag} /{ }^{106 m} \mathrm{Ag}$ $/{ }^{110 m} \mathrm{Ag} /{ }^{111} \mathrm{Ag} /{ }^{112} \mathrm{Ag} /{ }^{104} \mathrm{Cd} /{ }^{105} \mathrm{Cd} /{ }^{111 m} \mathrm{Cd}, \mathrm{E}=10-37 \mathrm{MeV}$; measured $\sigma$. Stacked-foil activation, comparison with model predictions. JOUR NIMBE 229321

2005HEZW NUCLEAR REACTIONS $\operatorname{Pd}(\alpha, \mathrm{X}){ }^{103} \mathrm{Ag} /{ }^{105} \mathrm{Ag} /{ }^{106 m} \mathrm{Ag} /{ }^{110 m} \mathrm{Ag} /$ ${ }^{111} \mathrm{Ag} /{ }^{112} \mathrm{Ag} /{ }^{104} \mathrm{Cd} /{ }^{105} \mathrm{Cd} /{ }^{111 m} \mathrm{Cd}, \mathrm{E} \approx 20-37 \mathrm{MeV}$; measured production $\sigma$. Activation technique, comparison with model predictions. CONF Santa Fe (Nucl Data for Sci and Technol) Proc,Vol1,P961

${ }^{110} \mathrm{Cd} \quad 2005 \mathrm{~K} 032$
NUCLEAR REACTIONS ${ }^{110,111,112,114,116} \mathrm{Cd}\left(\gamma, \gamma^{\prime}\right), \mathrm{E} \approx 2.7-4.1 \mathrm{MeV}$ bremsstrahlung; measured $\mathrm{E} \gamma, \mathrm{I} \gamma, \gamma$-ray polarization. $110,111,112,114,116 \mathrm{Cd}$ deduced levels, $\mathrm{J}, \pi$, excitation B(M1), B(E1). JOUR PRVCA 72034302

2005LU06 NUCLEAR REACTIONS ${ }^{110,116} \mathrm{Cd},{ }^{112,124} \mathrm{Sn}\left(\alpha, \alpha^{\prime}\right), \mathrm{E}=240 \mathrm{MeV}$; measured $\mathrm{E} \alpha, \sigma(\theta) .{ }^{110,116} \mathrm{Cd},{ }^{112,124} \mathrm{Sn}$ deduced electric monopole strength distributions, resonance parameters. Comparison with model predictions. JOUR APOBB 361107

${ }^{110} \mathrm{In} \quad$ 2004ADZW NUCLEAR REACTIONS ${ }^{209} \operatorname{Bi}(\mathrm{n}, 4 \mathrm{n}),(\mathrm{n}, 5 \mathrm{n}),(\mathrm{n}, 6 \mathrm{n}),(\mathrm{n}, 7 \mathrm{n}),(\mathrm{n}$, 9n), ${ }^{232} \mathrm{Th}(\mathrm{n}, \gamma),{ }^{197} \mathrm{Au}(\mathrm{n}, 2 \mathrm{n}),(\mathrm{n}, 4 \mathrm{n}),(\mathrm{n}, 6 \mathrm{n}),(\mathrm{n}, 7 \mathrm{n}),(\mathrm{n}, \gamma),{ }^{115} \operatorname{In}(\mathrm{n}$, 5n), (n, 6n), (n, 7n), ${ }^{59} \mathrm{Co}(\mathrm{n}, 2 \mathrm{n}),(\mathrm{n}, 3 \mathrm{n}),(\mathrm{n}, 4 \mathrm{n}),(\mathrm{n}, 5 \mathrm{n}),(\mathrm{n}, \gamma),(\mathrm{n}$, $\mathrm{p}),(\mathrm{n}, 6 \mathrm{n} 2 \mathrm{p}), \mathrm{E}=$ spectrum; measured $\mathrm{E} \gamma, \mathrm{I} \gamma$; deduced reaction rates. $\mathrm{Pb}(\mathrm{p}, \mathrm{nX}), \mathrm{E}=1 \mathrm{GeV}$; deduced spallation neutron spectrum. REPT JINR-E1-2004-16,Adam

2005AD01 NUCLEAR REACTIONS ${ }^{209} \mathrm{Bi}(\mathrm{n}, 4 \mathrm{n}),(\mathrm{n}, 5 \mathrm{n}),(\mathrm{n}, 6 \mathrm{n}),(\mathrm{n}, 7 \mathrm{n}),(\mathrm{n}$, 9n), ${ }^{232} \mathrm{Th}(\mathrm{n}, \gamma),{ }^{197} \mathrm{Au}(\mathrm{n}, 2 \mathrm{n}),(\mathrm{n}, 4 \mathrm{n}),(\mathrm{n}, 6 \mathrm{n}),(\mathrm{n}, 7 \mathrm{n}),(\mathrm{n}, \gamma),{ }^{59} \mathrm{Co}(\mathrm{n}$, 2n), (n, 3n), (n, 4n), (n, 5n), (n, p), (n, 6n2p), ${ }^{115} \operatorname{In}(\mathrm{n}, 5 \mathrm{n}),(\mathrm{n}, 6 \mathrm{n}),(\mathrm{n}$, $7 \mathrm{n}), \mathrm{E}=$ spectrum; measured $\mathrm{E} \gamma, \mathrm{I} \gamma$; deduced reaction rates. Activation technique, spallation neutrons from $1 \mathrm{GeV}$ proton beam, comparison with model predictions. JOUR ZAANE 2361

2005GY02 RADIOACTIVITY ${ }^{109} \mathrm{In},{ }^{110} \mathrm{Sn}(\mathrm{EC})$ [from ${ }^{106} \mathrm{Cd}(\alpha, \gamma),(\alpha, \mathrm{p})$ ]; measured $\mathrm{E} \gamma, \mathrm{I} \gamma, \mathrm{T}_{1 / 2}$. JOUR PRVCA 71057302

2005GYZZ RADIOACTIVITY ${ }^{109} \mathrm{In},{ }^{110} \mathrm{Sn}(\mathrm{EC})\left[\right.$ from $\left.{ }^{106} \mathrm{Cd}(\alpha, \mathrm{X})\right]$; measured $\mathrm{E} \gamma$, $\mathrm{I} \gamma, \mathrm{T}_{1 / 2}$. PREPRINT nucl-ex/0503012,3/18/2005 


\section{$\mathrm{A}=110$ (continued)}

${ }^{110} \mathrm{Sn}$ 2005BA18 NUCLEAR REACTIONS ${ }^{112} \mathrm{Sn}(\mathrm{d}, \mathrm{p}),(\mathrm{d}, 3 \mathrm{np}),(\mathrm{p}, 2 \mathrm{np}),{ }^{118} \mathrm{Sn}(\mathrm{d}, 2 \mathrm{n})$, (d, 3n), (d, 5n), (d, 2np), (d, 6np), (d, 9np), (p, n), (p, 3n), (p, 4n), (p, np), (p, 5np), (p, 8np), ${ }^{120} \mathrm{Sn}(\mathrm{d}, 2 \mathrm{n}),(\mathrm{d}, 4 \mathrm{n}),(\mathrm{d}, 6 \mathrm{n}),(\mathrm{d}, 7 \mathrm{n}),(\mathrm{d}, 4 \mathrm{np})$, (d, 8np), (d, 11np), (p, n), (p, 3n), (p, 5n), (p, 6n), (p, 3np), (p, 7np), (p, 10np), ${ }^{124} \operatorname{Sn}(\mathrm{d}, 2 \mathrm{n}),(\mathrm{d}, 4 \mathrm{n}),(\mathrm{d}, 6 \mathrm{n}),(\mathrm{d}, 8 \mathrm{n}),(\mathrm{d}, 10 \mathrm{n}),(\mathrm{d}, 11 \mathrm{n}),(\mathrm{d}$, $2 \mathrm{np}),(\mathrm{d}, 8 \mathrm{np}),(\mathrm{d}, 12 \mathrm{np}),(\mathrm{d}, 15 \mathrm{np}),(\mathrm{p}, \mathrm{n}),(\mathrm{p}, 3 \mathrm{n}),(\mathrm{p}, 5 \mathrm{n}),(\mathrm{p}, 7 \mathrm{n}),(\mathrm{p}$, 9n), (p, 10n), (p, np), (p, 7np), (p, 11np), (p, 14np), E=3.65 GeV / nucleon; measured $\sigma .{ }^{120} \mathrm{Sn}(\mathrm{p}, \mathrm{X}), \mathrm{E}=0.66 \mathrm{GeV}$; measured spallation fragments mass distribution. Activation technique, comparison with model predictions. JOUR YAFIA 68195

2005GY02 RADIOACTIVITY ${ }^{109} \mathrm{In},{ }^{110} \mathrm{Sn}(\mathrm{EC})\left[\right.$ from $\left.{ }^{106} \mathrm{Cd}(\alpha, \gamma),(\alpha, \mathrm{p})\right]$; measured $\mathrm{E} \gamma, \mathrm{I} \gamma, \mathrm{T}_{1 / 2}$. JOUR PRVCA 71057302

2005GY03 NUCLEAR REACTIONS ${ }^{106} \mathrm{Cd}(\alpha, \gamma), \mathrm{E}=8-12.5 \mathrm{MeV}$; measured $\mathrm{E} \gamma$, $\mathrm{I} \gamma, \sigma .{ }^{106} \mathrm{Cd}(\alpha, \alpha), \mathrm{E} \approx 15,17,19 \mathrm{MeV} ;$ measured $\sigma(\theta)$. Astrophysical implications discussed, comparison with model predictions. JOUR NUPAB $758517 \mathrm{c}$

2005GYZY NUCLEAR REACTIONS ${ }^{106} \mathrm{Cd}(\alpha, \gamma), \mathrm{E}(\mathrm{cm}) \approx 8-12 \mathrm{MeV}$; measured capture $\sigma$. Activation technique, comparison with model predictions. REPT ATOMKI 2004 Annual,P19,Gyurky

2005GYZZ RADIOACTIVITY ${ }^{109} \mathrm{In},{ }^{110} \mathrm{Sn}(\mathrm{EC})$ [from $\left.{ }^{106} \mathrm{Cd}(\alpha, \mathrm{X})\right]$; measured $\mathrm{E} \gamma$, $\mathrm{I} \gamma, \mathrm{T}_{1 / 2}$. PREPRINT nucl-ex/0503012,3/18/2005

2005W003 NUCLEAR REACTIONS ${ }^{98} \mathrm{Mo}\left({ }^{16} \mathrm{O}, 3 \mathrm{n}\right),\left({ }^{16} \mathrm{O}, 4 \mathrm{n}\right), \mathrm{E}=60,70,75,80$

$\mathrm{MeV}$; measured prompt and delayed $\mathrm{E} \gamma, \mathrm{I} \gamma, \gamma \gamma$-coin, excitation functions. ${ }^{110,111}$ Sn deduced high-spin levels, J, $\pi$, configurations, isomeric states. Osiris-II array, total Routhian surface calculations. JOUR ZAANE 24259

${ }^{110} \mathrm{Xe} \quad$ 2005JA03 RADIOACTIVITY ${ }^{110} \mathrm{Xe},{ }^{106} \mathrm{Te}(\alpha)\left[\right.$ from ${ }^{58} \mathrm{Ni}\left({ }^{58} \mathrm{Ni}, 2 \mathrm{n} \alpha\right)$ and subsequent decay]; measured $\mathrm{E} \alpha, \mathrm{T}_{1 / 2}$; deduced $\alpha$-decay widths. Mass separator. JOUR ZAANE 23197

\section{$\mathrm{A}=\mathbf{1 1 1}$}

${ }^{111} \mathrm{Tc} \quad$ 2005UR01 RADIOACTIVITY ${ }^{248} \mathrm{Cm}(\mathrm{SF}) ;$ measured $\mathrm{E} \gamma, \mathrm{I} \gamma, \gamma \gamma$-coin. ${ }^{109,110,111} \mathrm{Tc},{ }^{135} \mathrm{I}$ deduced transitions. ${ }^{111} \mathrm{Tc}$ deduced levels, J, $\pi$, configurations. Eurogam2 array. Level systematics in neighboring nuclides discussed. JOUR ZAANE 24161

${ }^{111} \mathrm{Ag} \quad 2005 \mathrm{HE} 05 \quad$ NUCLEAR REACTIONS Pd $(\alpha, \text { xnyp })^{103} \mathrm{Ag} /{ }^{104} \mathrm{Ag} /{ }^{105} \mathrm{Ag} /{ }^{106 m} \mathrm{Ag}$ / ${ }^{110 m} \mathrm{Ag} /{ }^{111} \mathrm{Ag} /{ }^{112} \mathrm{Ag} /{ }^{104} \mathrm{Cd} /{ }^{105} \mathrm{Cd} /{ }^{111 m} \mathrm{Cd}, \mathrm{E}=10-37 \mathrm{MeV}$; measured $\sigma$. Stacked-foil activation, comparison with model predictions. JOUR NIMBE 229321

2005HEZW NUCLEAR REACTIONS $\operatorname{Pd}(\alpha, \mathrm{X}){ }^{103} \mathrm{Ag} /{ }^{105} \mathrm{Ag} /{ }^{106 m} \mathrm{Ag} /{ }^{110 m} \mathrm{Ag} /$ ${ }^{111} \mathrm{Ag} /{ }^{112} \mathrm{Ag} /{ }^{104} \mathrm{Cd} /{ }^{105} \mathrm{Cd} /{ }^{111 m} \mathrm{Cd}, \mathrm{E} \approx 20-37 \mathrm{MeV}$; measured production $\sigma$. Activation technique, comparison with model predictions. CONF Santa Fe (Nucl Data for Sci and Technol) Proc,Vol1,P961 


\section{$\mathrm{A}=111$ (continued)}

${ }^{111} \mathrm{Cd}$ 2005HE05 NUCLEAR REACTIONS Pd( $\alpha$, xnyp $)^{103} \mathrm{Ag} /{ }^{104} \mathrm{Ag} /{ }^{105} \mathrm{Ag} /{ }^{106 m} \mathrm{Ag}$ $/{ }^{110 m} \mathrm{Ag} /{ }^{111} \mathrm{Ag} /{ }^{112} \mathrm{Ag} /{ }^{104} \mathrm{Cd} /{ }^{105} \mathrm{Cd} /{ }^{111 m} \mathrm{Cd}, \mathrm{E}=10-37 \mathrm{MeV}$; measured $\sigma$. Stacked-foil activation, comparison with model predictions. JOUR NIMBE 229321

2005HEZW NUCLEAR REACTIONS $\mathrm{Pd}(\alpha, \mathrm{X}){ }^{103} \mathrm{Ag} /{ }^{105} \mathrm{Ag} /{ }^{106 m} \mathrm{Ag} /{ }^{110 m} \mathrm{Ag} /$ ${ }^{111} \mathrm{Ag} /{ }^{112} \mathrm{Ag} /{ }^{104} \mathrm{Cd} /{ }^{105} \mathrm{Cd} /{ }^{111 m} \mathrm{Cd}, \mathrm{E} \approx 20-37 \mathrm{MeV}$; measured production $\sigma$. Activation technique, comparison with model predictions. CONF Santa Fe (Nucl Data for Sci and Technol) Proc,Vol1,P961

2005K032 NUCLEAR REACTIONS ${ }^{110,111,112,114,116} \mathrm{Cd}\left(\gamma, \gamma^{\prime}\right), \mathrm{E} \approx 2.7-4.1 \mathrm{MeV}$ bremsstrahlung; measured $\mathrm{E} \gamma, \mathrm{I} \gamma, \gamma$-ray polarization.

${ }_{110,111,112,114,116} \mathrm{Cd}$ deduced levels, J, $\pi$, excitation B(M1), B(E1).

JOUR PRVCA 72034302

${ }^{111} \mathrm{In} \quad$ 2004ADZW NUCLEAR REACTIONS ${ }^{209} \operatorname{Bi}(\mathrm{n}, 4 \mathrm{n}),(\mathrm{n}, 5 \mathrm{n}),(\mathrm{n}, 6 \mathrm{n}),(\mathrm{n}, 7 \mathrm{n}),(\mathrm{n}$, 9n), ${ }^{232} \mathrm{Th}(\mathrm{n}, \gamma),{ }^{197} \mathrm{Au}(\mathrm{n}, 2 \mathrm{n}),(\mathrm{n}, 4 \mathrm{n}),(\mathrm{n}, 6 \mathrm{n}),(\mathrm{n}, 7 \mathrm{n}),(\mathrm{n}, \gamma),{ }^{115} \operatorname{In}(\mathrm{n}$, $5 \mathrm{n}),(\mathrm{n}, 6 \mathrm{n}),(\mathrm{n}, 7 \mathrm{n}),{ }^{59} \mathrm{Co}(\mathrm{n}, 2 \mathrm{n}),(\mathrm{n}, 3 \mathrm{n}),(\mathrm{n}, 4 \mathrm{n}),(\mathrm{n}, 5 \mathrm{n}),(\mathrm{n}, \gamma),(\mathrm{n}$, $\mathrm{p}),(\mathrm{n}, 6 \mathrm{n} 2 \mathrm{p}), \mathrm{E}=$ spectrum; measured $\mathrm{E} \gamma, \mathrm{I} \gamma$; deduced reaction rates. $\mathrm{Pb}(\mathrm{p}, \mathrm{nX}), \mathrm{E}=1 \mathrm{GeV}$; deduced spallation neutron spectrum. REPT JINR-E1-2004-16,Adam

2005AD01 NUCLEAR REACTIONS ${ }^{209} \operatorname{Bi}(\mathrm{n}, 4 \mathrm{n}),(\mathrm{n}, 5 \mathrm{n}),(\mathrm{n}, 6 \mathrm{n}),(\mathrm{n}, 7 \mathrm{n}),(\mathrm{n}$, 9n), ${ }^{232} \mathrm{Th}(\mathrm{n}, \gamma),{ }^{197} \mathrm{Au}(\mathrm{n}, 2 \mathrm{n}),(\mathrm{n}, 4 \mathrm{n}),(\mathrm{n}, 6 \mathrm{n}),(\mathrm{n}, 7 \mathrm{n}),(\mathrm{n}, \gamma),{ }^{59} \mathrm{Co}(\mathrm{n}$, 2n), (n, 3n), (n, 4n), (n, 5n), (n, p), (n, 6n2p), ${ }^{115} \operatorname{In}(\mathrm{n}, 5 \mathrm{n}),(\mathrm{n}, 6 \mathrm{n}),(\mathrm{n}$, $7 \mathrm{n}), \mathrm{E}=$ spectrum; measured $\mathrm{E} \gamma, \mathrm{I} \gamma$; deduced reaction rates. Activation technique, spallation neutrons from $1 \mathrm{GeV}$ proton beam, comparison with model predictions. JOUR ZAANE 2361

2005BAZR NUCLEAR REACTIONS ${ }^{107} \operatorname{Ag}(\alpha, \gamma), \mathrm{E}=7.8-11.9 \mathrm{MeV} ;{ }^{48} \mathrm{Ti}(\alpha, \mathrm{n}), \mathrm{E}$ $\approx 6.5-11.5 \mathrm{MeV}$; measured $\sigma$. Stacked-foil activation technique, comparison with model predictions. CONF Santa Fe (Nucl Data for Sci and Technol) Proc,Vol2,P1370

2005TAZS NUCLEAR REACTIONS Sn, Cd(p, X) ${ }^{111} \mathrm{In} /{ }^{114 m} \mathrm{In}, \mathrm{E}=10-80 \mathrm{MeV}$; measured excitation functions; deduced integral yields. Comparison with model predictions and previous work. Stacked-foil activation technique. CONF Santa Fe (Nucl Data for Sci and Technol) Proc,Vol2,P1662

${ }^{111} \mathrm{Sn} \quad 2005 \mathrm{~W} 003 \quad$ NUCLEAR REACTIONS ${ }^{98} \mathrm{Mo}\left({ }^{16} \mathrm{O}, 3 \mathrm{n}\right),\left({ }^{16} \mathrm{O}, 4 \mathrm{n}\right), \mathrm{E}=60,70,75,80$ $\mathrm{MeV}$; measured prompt and delayed $\mathrm{E} \gamma, \mathrm{I} \gamma, \gamma \gamma$-coin, excitation functions. ${ }^{110,111}$ Sn deduced high-spin levels, J, $\pi$, configurations, isomeric states. Osiris-II array, total Routhian surface calculations. JOUR ZAANE 24259

${ }^{111} \mathrm{Sb} \quad 2005 \mathrm{SH} 24 \quad$ RADIOACTIVITY ${ }^{111} \mathrm{Te}\left(\beta^{+}\right),(\mathrm{EC})\left[\right.$ from $\left.{ }^{58} \mathrm{Ni}\left({ }^{56} \mathrm{Fe}, \mathrm{n} 2 \mathrm{p}\right)\right]$; measured $\mathrm{E} \gamma, \mathrm{I} \gamma, \gamma \gamma$-coin, $\mathrm{T}_{1 / 2} .{ }^{111} \mathrm{Sb}$ deduced levels, $\mathrm{J}, \pi$. Mass separator, comparisons with shell-model predictions and level systematics in neighboring isotopes. JOUR PRVCA 71064323

2005SH53 RADIOACTIVITY ${ }^{111} \mathrm{Te}\left(\beta^{+}\right)\left[\right.$from $\left.{ }^{58} \mathrm{Ni}\left({ }^{56} \mathrm{Fe}, 2 \mathrm{pn}\right)\right] ;{ }^{135} \mathrm{Sn}\left(\beta^{-}\right)$, $\left(\beta^{-} \mathrm{n}\right)$ [from $\left.\mathrm{U}(\mathrm{p}, \mathrm{F}), \mathrm{E}=1.4 \mathrm{GeV}\right]$; measured $\mathrm{E} \gamma, \mathrm{I} \gamma, \gamma \gamma$-coin following decay of mass-separated sources. ${ }^{111,134,135} \mathrm{Sb}$ deduced levels, J, $\pi$. Comparison with model calculations. JOUR ZAANE 25 s01 121 


\section{$\mathrm{A}=111$ (continued)}

${ }^{111} \mathrm{Te}$ 2005Sh24 RADIOACTIVITY ${ }^{111} \mathrm{Te}\left(\beta^{+}\right),(\mathrm{EC})$ [from $\left.{ }^{58} \mathrm{Ni}\left({ }^{56} \mathrm{Fe}, \mathrm{n} 2 \mathrm{p}\right)\right]$; measured $\mathrm{E} \gamma, \mathrm{I} \gamma, \gamma \gamma$-coin, $\mathrm{T}_{1 / 2} .{ }^{111} \mathrm{Sb}$ deduced levels, J, $\pi$. Mass separator, comparisons with shell-model predictions and level systematics in neighboring isotopes. JOUR PRVCA 71064323

2005SH53 RADIOACTIVITY ${ }^{111} \mathrm{Te}\left(\beta^{+}\right)\left[\right.$from $\left.{ }^{58} \mathrm{Ni}\left({ }^{56} \mathrm{Fe}, 2 \mathrm{pn}\right)\right] ;{ }^{135} \mathrm{Sn}\left(\beta^{-}\right)$, $\left(\beta^{-} \mathrm{n}\right)$ [from $\mathrm{U}(\mathrm{p}, \mathrm{F}), \mathrm{E}=1.4 \mathrm{GeV}$ ]; measured $\mathrm{E} \gamma, \mathrm{I} \gamma, \gamma \gamma$-coin following decay of mass-separated sources. ${ }^{111,134,135} \mathrm{Sb}$ deduced levels, J, $\pi$. Comparison with model calculations. JOUR ZAANE 25 s01 121

\section{$\mathrm{A}=\mathbf{1 1 2}$}

${ }^{112} \mathrm{Ru} \quad$ 2005SM08

${ }^{112} \mathrm{Ag} \quad$ 2005HE05

2005HEZW

${ }^{112} \mathrm{Cd} \quad$ 2005K032

${ }^{112} \mathrm{Sn} \quad 2004 \mathrm{KU} 30$

2005KU28

2005KU37

2005LU06
RADIOACTIVITY ${ }^{252} \mathrm{Cf}(\mathrm{SF})$; measured $\mathrm{E} \gamma, \mathrm{I} \gamma(\theta, \mathrm{H}, \mathrm{t}), \gamma \gamma$-coin. ${ }_{96,100,102} \mathrm{Zr},{ }^{102,104,106,108} \mathrm{Mo},{ }^{106,108,110,112} \mathrm{Ru},{ }^{110,114,116} \mathrm{Pd}$ levels deduced $\mathrm{g}$ factors, $\mathrm{B}(\mathrm{E} 2)$. Gammasphere array, time-integral perturbed angular correlation technique. Comparison with interacting boson model predictions. JOUR JPGPE 31 S1433

NUCLEAR REACTIONS Pd $(\alpha$, xnyp $){ }^{103} \mathrm{Ag} /{ }^{104} \mathrm{Ag} /{ }^{105} \mathrm{Ag} /{ }^{106 m} \mathrm{Ag}$ $/{ }^{110 m} \mathrm{Ag} /{ }^{111} \mathrm{Ag} /{ }^{112} \mathrm{Ag} /{ }^{104} \mathrm{Cd} /{ }^{105} \mathrm{Cd} /{ }^{111 m} \mathrm{Cd}, \mathrm{E}=10-37 \mathrm{MeV}$; measured $\sigma$. Stacked-foil activation, comparison with model predictions. JOUR NIMBE 229321

NUCLEAR REACTIONS Pd $(\alpha, \mathrm{X}){ }^{103} \mathrm{Ag} /{ }^{105} \mathrm{Ag} /{ }^{106 m} \mathrm{Ag} /{ }^{110 m} \mathrm{Ag} /$ ${ }^{111} \mathrm{Ag} /{ }^{112} \mathrm{Ag} /{ }^{104} \mathrm{Cd} /{ }^{105} \mathrm{Cd} /{ }^{111 m} \mathrm{Cd}, \mathrm{E} \approx 20-37 \mathrm{MeV}$; measured production $\sigma$. Activation technique, comparison with model predictions. CONF Santa Fe (Nucl Data for Sci and Technol) Proc,Vol1,P961 NUCLEAR REACTIONS ${ }^{110,111,112,114,116} \mathrm{Cd}\left(\gamma, \gamma^{\prime}\right), \mathrm{E} \approx 2.7-4.1 \mathrm{MeV}$ bremsstrahlung; measured $\mathrm{E} \gamma, \mathrm{I} \gamma, \gamma$-ray polarization.

${ }_{110,111,112,114,116} \mathrm{Cd}$ deduced levels, J, $\pi$, excitation B(M1), B(E1).

JOUR PRVCA 72034302

NUCLEAR REACTIONS ${ }^{112,114,120,124} \operatorname{Sn}(\alpha, \alpha),(\alpha, \alpha), \mathrm{E} \approx 50 \mathrm{MeV}$; measured $\sigma(\mathrm{E}, \theta)$; deduced optical model parameters. ${ }^{112,114,120,124} \mathrm{Sn}$ deduced transition strengths, deformation parameters, related features. JOUR UKPJA 49841

NUCLEAR REACTIONS ${ }^{112} \mathrm{Sn}(\alpha, \alpha), \mathrm{E}=14.4,19.5 \mathrm{MeV} ;{ }^{124} \mathrm{Sn}(\alpha, \alpha)$, $\mathrm{E}=19.5 \mathrm{MeV}$; measured elastic $\sigma(\theta)$; deduced optical potential parameters. ${ }^{112} \mathrm{Sn}(\alpha, \gamma), \mathrm{E}(\mathrm{cm})=7-11 \mathrm{MeV}$; calculated astrophysical S-factors, reaction rates. JOUR PRVCA 71065802

NUCLEAR REACTIONS ${ }^{112} \mathrm{Sn}(\mathrm{n}, \mathrm{n} \gamma), \mathrm{E}=2.5-4.0 \mathrm{MeV}$; measured $\mathrm{E} \gamma$, $\mathrm{I} \gamma, \gamma \gamma$-coin, angular distributions, excitation functions, DSA. ${ }^{112} \mathrm{Sn}$ deduced levels, J, $\pi, \delta, \mathrm{T}_{1 / 2}$, B(M1), B(E2). JOUR PRVCA 72034313 NUCLEAR REACTIONS ${ }^{112} \mathrm{Sn}(\mathrm{n}, \mathrm{n} \gamma), \mathrm{E}=2.5-4.0 \mathrm{MeV}$; measured $\mathrm{E} \gamma$, $\mathrm{I} \gamma, \gamma \gamma$-coin, DSA, excitation functions, angular distributions. ${ }^{112} \mathrm{Sn}$ deduced levels, $\mathrm{J}, \pi, \mathrm{T}_{1 / 2}$. JOUR ZAANE $25 \mathrm{~s} 01443$ NUCLEAR REACTIONS ${ }^{110,116} \mathrm{Cd},{ }^{112,124} \mathrm{Sn}\left(\alpha, \alpha^{\prime}\right), \mathrm{E}=240 \mathrm{MeV}$; measured $\mathrm{E} \alpha, \sigma(\theta) .{ }^{110,116} \mathrm{Cd},{ }^{112,124} \mathrm{Sn}$ deduced electric monopole strength distributions, resonance parameters. Comparison with model predictions. JOUR APOBB 361107 


\section{$\mathrm{A}=112$ (continued)}

2005PYZZ NUCLEAR REACTIONS ${ }^{112} \mathrm{Sn}\left(\gamma, \gamma^{\prime}\right), \mathrm{E}=3.8 \mathrm{MeV}$ bremsstrahlung; measured $\mathrm{E} \gamma, \mathrm{I} \gamma .{ }^{112} \mathrm{Sn}$ level deduced $\mathrm{B}(\mathrm{E} 1)$, decay width, two-phonon configuration. PREPRINT nucl-ex/0512013,12/8/2005

${ }^{112} \mathrm{Sb}$ 2005DE02 NUCLEAR REACTIONS ${ }^{89} \mathrm{Y}\left({ }^{30} \mathrm{Si}, 3 \mathrm{n} \alpha\right), \mathrm{E}=120 \mathrm{MeV}$; measured $\mathrm{E} \gamma$, $\mathrm{I} \gamma, \gamma \gamma$-coin, DSA. ${ }^{112} \mathrm{Sb}$ levels deduced $\mathrm{T}_{1 / 2}, \mathrm{~B}(\mathrm{M} 1), \mathrm{B}(\mathrm{E} 2)$. Comparisons with tilted axis cranking model predictions. JOUR PRVCA 71017303

${ }^{112} \mathrm{Te}$ 2005JA10 RADIOACTIVITY ${ }^{113} \mathrm{Xe}\left(\beta^{+} \mathrm{p}\right),(\mathrm{ECp})\left[\right.$ from $\left.{ }^{58} \mathrm{Ni}\left({ }^{58} \mathrm{Ni}, \mathrm{n} 2 \mathrm{p}\right)\right]$; measured $\beta$-delayed $\mathrm{E} \gamma, \mathrm{Ep}, \mathrm{X}$-ray spectra, $\mathrm{Q}$ values. ${ }^{113} \mathrm{I}$ deduced level widths, $\mathrm{T}_{1 / 2}$, branching ratios for proton decay. ${ }^{112} \mathrm{Te}$ levels deduced feeding intensities. Comparison with statistical model predictions. JOUR ZAANE 24205

\section{$\mathrm{A}=\mathbf{1 1 3}$}

${ }^{113} \mathrm{Pd} \quad 2005 \mathrm{~F} 009$

${ }^{113} \mathrm{Cd} \quad$ 2005BU20

${ }^{113} \mathrm{In} \quad$ 2004MB03

2005GOZX

2005NA37

${ }^{113} \mathrm{Sn} \quad$ 2005BA18

2005PA22
RADIOACTIVITY ${ }^{252} \mathrm{Cf}(\mathrm{SF})$; measured $\mathrm{E} \gamma, \mathrm{I} \gamma, \gamma \gamma$-coin. ${ }^{113,115,117} \mathrm{Pd}$ deduced levels, J, $\pi$. Gammasphere array. JOUR PRVCA 72014315 NUCLEAR REACTIONS ${ }^{112} \mathrm{Cd}$ (polarized d, p), $\mathrm{E}=22 \mathrm{MeV}$; ${ }^{114} \mathrm{Cd}$ (polarized d, t), $\mathrm{E}=25 \mathrm{MeV}$; measured particle spectra, $\sigma(\theta)$, $\operatorname{Ay}(\theta) .{ }^{113} \mathrm{Cd}$ deduced levels, $\mathrm{J}, \pi$, spectroscopic factors, configurations. Interacting Boson Fermion model and Quadrupole Phonon model calculations. JOUR NUPAB 75654

RADIOACTIVITY ${ }^{113} \mathrm{Cd}\left(\beta^{-}\right)$; measured $\mathrm{E} \beta, \mathrm{T}_{1 / 2}$. CdZnTe detectors. PREPRINT nucl-ex/0508016,08/12/2005 NUCLEAR MOMENTS ${ }^{113,115} \mathrm{In},{ }^{153,155} \mathrm{Eu},{ }^{185,187} \mathrm{Re},{ }^{203,205} \mathrm{Tl}$, ${ }^{209,211} \mathrm{Fr}$; measured hfs; deduced hyperfine magnetic anomaly, relative radii. Laser resonance fluorescence. JOUR BRSPE 68157

RADIOACTIVITY ${ }^{113} \mathrm{Cd}\left(\beta^{-}\right)$; measured $\mathrm{E} \beta, \mathrm{T}_{1 / 2}$. CdZnTe detectors. PREPRINT nucl-ex/0508016,08/12/2005 NUCLEAR REACTIONS ${ }^{100} \mathrm{Mo}\left({ }^{18} \mathrm{O}, 4 \mathrm{np}\right), \mathrm{E}=95 \mathrm{MeV} ;{ }^{110} \mathrm{Pd}\left({ }^{7} \mathrm{Li}\right.$, $4 \mathrm{n}), \mathrm{E}=36 \mathrm{MeV}$; measured $\mathrm{E} \gamma, \mathrm{I} \gamma, \gamma \gamma$-coin. ${ }^{113} \mathrm{In}$ deduced high-spin levels, J, $\pi$, configurations, shape coexistence. Cranked mean-field calculations. JOUR PRVCA 72044304

NUCLEAR REACTIONS ${ }^{112} \mathrm{Sn}(\mathrm{d}, \mathrm{p}),(\mathrm{d}, 3 \mathrm{np}),(\mathrm{p}, 2 \mathrm{np}),{ }^{118} \mathrm{Sn}(\mathrm{d}, 2 \mathrm{n})$, (d, 3n), (d, 5n), (d, 2np), (d, 6np), (d, 9np), (p, n), (p, 3n), (p, 4n), (p, np), (p, 5np), (p, 8np), ${ }^{120} \mathrm{Sn}(\mathrm{d}, 2 \mathrm{n}),(\mathrm{d}, 4 \mathrm{n}),(\mathrm{d}, 6 \mathrm{n}),(\mathrm{d}, 7 \mathrm{n}),(\mathrm{d}, 4 \mathrm{np})$, (d, 8np), (d, 11np), (p, n), (p, 3n), (p, 5n), (p, 6n), (p, 3np), (p, 7np), (p, 10np), ${ }^{124} \mathrm{Sn}(\mathrm{d}, 2 \mathrm{n}),(\mathrm{d}, 4 \mathrm{n}),(\mathrm{d}, 6 \mathrm{n}),(\mathrm{d}, 8 \mathrm{n}),(\mathrm{d}, 10 \mathrm{n}),(\mathrm{d}, 11 \mathrm{n}),(\mathrm{d}$, 2np), (d, 8np), (d, 12np), (d, 15np), (p, n), (p, 3n), (p, 5n), (p, 7n), (p, 9n), (p, 10n), (p, np), (p, 7np), (p, 11np), (p, 14np), E=3.65 GeV / nucleon; measured $\sigma .{ }^{120} \mathrm{Sn}(\mathrm{p}, \mathrm{X}), \mathrm{E}=0.66 \mathrm{GeV}$; measured spallation fragments mass distribution. Activation technique, comparison with model predictions. JOUR YAFIA 68195

NUCLEAR REACTIONS ${ }^{114} \mathrm{Cd}(\alpha, 2 \mathrm{n}),(\alpha, 3 \mathrm{n}),(\alpha, 4 \mathrm{n}),(\alpha, 5 \mathrm{n})$, $\mathrm{E}=35,40,45,50,55$; measured $\mathrm{E} \gamma$, En, $\sigma, \sigma(\theta)$; deduced equilibrium and pre-equilibrium contributions, related reaction mechanism features. JOUR PRVCA 71034605 


\section{$\mathrm{A}=113$ (continued)}

\begin{tabular}{|c|c|c|}
\hline${ }^{113} \mathrm{I}$ & $2005 \mathrm{JA} 10$ & $\begin{array}{l}\text { RADIOACTIVITY }{ }^{113} \mathrm{Xe}\left(\beta^{+} \mathrm{p}\right),(\mathrm{ECp})\left[\text { from }{ }^{58} \mathrm{Ni}\left({ }^{58} \mathrm{Ni}, \mathrm{n} 2 \mathrm{p}\right)\right] \\
\text { measured } \beta \text {-delayed } \mathrm{E} \gamma, \mathrm{Ep}, \mathrm{X} \text {-ray spectra, } \mathrm{Q} \text { values. }{ }^{113} \mathrm{I} \text { deduced } \\
\text { level widths, } \mathrm{T}_{1 / 2} \text {, branching ratios for proton decay. }{ }^{112} \mathrm{Te} \text { levels } \\
\text { deduced feeding intensities. Comparison with statistical model } \\
\text { predictions. JOUR ZAANE } 24205\end{array}$ \\
\hline${ }^{113} \mathrm{Xe}$ & $2005 \mathrm{JA} 10$ & $\begin{array}{l}\text { RADIOACTIVITY }{ }^{113} \mathrm{Xe}\left(\beta^{+} \mathrm{p}\right),(\mathrm{ECp})\left[\text { from }{ }^{58} \mathrm{Ni}\left({ }^{58} \mathrm{Ni}, \mathrm{n} 2 \mathrm{p}\right)\right] \\
\text { measured } \beta \text {-delayed } \mathrm{E} \gamma, \mathrm{Ep}, \mathrm{X} \text {-ray spectra, } \mathrm{Q} \text { values. }{ }^{113} \mathrm{I} \text { deduced } \\
\text { level widths, } \mathrm{T}_{1 / 2} \text {, branching ratios for proton decay. }{ }^{112} \mathrm{Te} \text { levels } \\
\text { deduced feeding intensities. Comparison with statistical model } \\
\text { predictions. JOUR ZAANE } 24205\end{array}$ \\
\hline
\end{tabular}

\section{$\mathrm{A}=114$}

${ }^{114} \mathrm{Pd} \quad$ 2005SM08 RADIOACTIVITY ${ }^{252} \mathrm{Cf}(\mathrm{SF}) ;$ measured $\mathrm{E} \gamma, \mathrm{I} \gamma(\theta, \mathrm{H}, \mathrm{t}), \gamma \gamma$-coin. ${ }^{96,100,102} \mathrm{Zr},{ }^{102,104,106,108} \mathrm{Mo},{ }^{106,108,110,112} \mathrm{Ru},{ }^{110,114,116} \mathrm{Pd}$ levels deduced $\mathrm{g}$ factors, B(E2). Gammasphere array, time-integral perturbed angular correlation technique. Comparison with interacting boson model predictions. JOUR JPGPE 31 S1433

${ }^{114} \mathrm{Cd} \quad$ 2005K032 NUCLEAR REACTIONS ${ }^{110,111,112,114,116} \mathrm{Cd}\left(\gamma, \gamma^{\prime}\right), \mathrm{E} \approx 2.7-4.1 \mathrm{MeV}$ bremsstrahlung; measured $\mathrm{E} \gamma, \mathrm{I} \gamma, \gamma$-ray polarization.

${ }_{110,111,112,114,116} \mathrm{Cd}$ deduced levels, J, $\pi$, excitation B(M1), B(E1).

JOUR PRVCA 72034302

${ }^{114}$ In $\quad$ 2005TAZS

NUCLEAR REACTIONS Sn, Cd(p, X) ${ }^{111} \mathrm{In} /{ }^{114 m} \mathrm{In}, \mathrm{E}=10-80 \mathrm{MeV}$; measured excitation functions; deduced integral yields. Comparison with model predictions and previous work. Stacked-foil activation technique. CONF Santa Fe (Nucl Data for Sci and Technol)

Proc,Vol2,P1662

${ }^{114} \mathrm{Sn} \quad 2004 \mathrm{KU} 30 \quad$ NUCLEAR REACTIONS ${ }^{112,114,120,124} \mathrm{Sn}(\alpha, \alpha),\left(\alpha, \alpha^{\prime}\right), \mathrm{E} \approx 50 \mathrm{MeV}$; measured $\sigma(\mathrm{E}, \theta)$; deduced optical model parameters. ${ }^{112,114,120,124} \mathrm{Sn}$ deduced transition strengths, deformation parameters, related features. JOUR UKPJA 49841

2005PA22 NUCLEAR REACTIONS ${ }^{114} \mathrm{Cd}(\alpha, 2 \mathrm{n}),(\alpha, 3 \mathrm{n}),(\alpha, 4 \mathrm{n}),(\alpha, 5 \mathrm{n})$, $\mathrm{E}=35,40,45,50,55$; measured $\mathrm{E} \gamma$, En, $\sigma, \sigma(\theta)$; deduced equilibrium and pre-equilibrium contributions, related reaction mechanism features. JOUR PRVCA 71034605

${ }^{114} \mathrm{Te}$ 2005MO20 NUCLEAR REACTIONS ${ }^{93} \mathrm{Nb}\left({ }^{24} \mathrm{Mg}, 2 \mathrm{np}\right), \mathrm{E}=90 \mathrm{MeV}$; measured Doppler-shifted E $\gamma$, I $\gamma, \gamma \gamma$-coin. ${ }^{114}$ Te deduced levels, J, $\pi, \mathrm{T}_{1 / 2}$, $\mathrm{B}(\mathrm{E} 2)$. Recoil-distance technique. Comparison with model predictions and level systematics in neighboring nuclides. JOUR PRVCA 71 064324

\section{$\mathrm{A}=115$}

${ }^{115} \mathrm{Pd} \quad 2005 \mathrm{~F} 009 \quad$ RADIOACTIVITY ${ }^{252} \mathrm{Cf}(\mathrm{SF}) ;$ measured $\mathrm{E} \gamma, \mathrm{I} \gamma, \gamma \gamma$-coin. ${ }^{113,115,117} \mathrm{Pd}$ deduced levels, J, $\pi$. Gammasphere array. JOUR PRVCA 72014315 


\section{$\mathrm{A}=115$ (continued)}

${ }^{115} \mathrm{Cd}$ 2005UOzZ NUCLEAR REACTIONS U(p, F $){ }^{95} \mathrm{Zr} /{ }^{115} \mathrm{Cd} /{ }^{134} \mathrm{Cs} /{ }^{136} \mathrm{Cs} /{ }^{137} \mathrm{Cs}$ $/{ }^{147} \mathrm{Nd}, \mathrm{E} \approx 20-70 \mathrm{MeV}$; measured production $\sigma$. Stacked-foil activation technique, comparison with model predictions. CONF Santa Fe (Nucl Data for Sci and Technol) Proc,Vol2,P1547

2005VIZZ RADIOACTIVITY ${ }^{115} \mathrm{Cd}\left(\beta^{-}\right) ;{ }^{117 m} \mathrm{Sn},{ }^{125 m} \mathrm{Te}(\mathrm{IT})$; measured $\mathrm{I} \gamma$, (X-ray) $\gamma$-coin. ${ }^{115} \mathrm{In},{ }^{117} \mathrm{Sn},{ }^{125} \mathrm{Te}$ transitions deduced ICC. CONF St Petersburg,P65, Vishnevsky

${ }^{115} \mathrm{In} \quad$ 2004AG09 NUCLEAR REACTIONS ${ }^{103} \mathrm{Rh}\left(\mathrm{n}, \mathrm{n}^{\prime}\right){ }^{103 m} \mathrm{Rh}, \mathrm{E} \approx 4.8 \mathrm{MeV} ;{ }^{115} \mathrm{In}(\mathrm{n}$, $\left.\mathrm{n}^{\prime}\right){ }^{115 m} \mathrm{In}, \mathrm{E} \approx 5 \mathrm{MeV} ;{ }^{232} \mathrm{Th},{ }^{238} \mathrm{U}(\mathrm{n}, \mathrm{F}), \mathrm{E} \approx 5 \mathrm{MeV} ;{ }^{24} \mathrm{Mg},{ }^{27} \mathrm{Al}$, ${ }^{46,47,48} \mathrm{Ti},{ }^{54,56} \mathrm{Fe},{ }^{58} \mathrm{Ni},{ }^{64} \mathrm{Zn}(\mathrm{n}, \mathrm{p}), \mathrm{E} \approx 2-8 \mathrm{MeV} ;{ }^{27} \mathrm{Al},{ }^{59} \mathrm{Co}(\mathrm{n}, \alpha), \mathrm{E}$ $\approx 8.3 \mathrm{MeV}$; measured activation $\sigma$. Spectrum average technique, comparison with previous results. JOUR RAACA 9263

2004MB03 NUCLEAR MOMENTS ${ }^{113,115} \mathrm{In},{ }^{153,155} \mathrm{Eu},{ }^{185,187} \mathrm{Re},{ }^{203,205} \mathrm{Tl}$, ${ }^{209,211} \mathrm{Fr}$; measured hfs; deduced hyperfine magnetic anomaly, relative radii. Laser resonance fluorescence. JOUR BRSPE 68157

2005CA03 RADIOACTIVITY ${ }^{115} \operatorname{In}\left(\beta^{-}\right)$; measured $\beta$-delayed $\mathrm{E} \gamma$, I $\gamma$; deduced branching ratio and $\mathrm{Q} \beta$ for decay to excited level, limit on charge-nonconserving decay. ${ }^{115} \mathrm{Sn}$ level deduced energy, $\beta$-feeding intensity. JOUR NUPAB 748333

2005CAZW RADIOACTIVITY ${ }^{115} \operatorname{In}\left(\beta^{-}\right)$; measured $\beta$-delayed $\mathrm{E} \gamma, \mathrm{I} \gamma$; deduced branching ratio, partial $\mathrm{T}_{1 / 2}$, and $\mathrm{Q}$-value for decay to first excited state. ${ }^{115} \mathrm{Sn}$ deduced level energy. Implications for neutrino mass limits discussed. PREPRINT nucl-ex/0509020,9/15/2005

2005MU21 NUCLEAR REACTIONS ${ }^{115} \mathrm{In}(\mathrm{n}, \mathrm{n}),{ }^{27} \mathrm{Al}(\mathrm{n}, \alpha),{ }^{93} \mathrm{Nb}(\mathrm{n}, 2 \mathrm{n}),(\mathrm{n}, 4 \mathrm{n})$, ${ }^{209} \operatorname{Bi}(\mathrm{n}, 4 \mathrm{n}),(\mathrm{n}, 5 \mathrm{n}),(\mathrm{n}, 6 \mathrm{n}),(\mathrm{n}, 7 \mathrm{n}), \mathrm{E} \approx 10-1000 \mathrm{MeV}$; measured reaction rates. Comparison with model predictions. JOUR NIMAE 547 555

2005VIZZ RADIOACTIVITY ${ }^{115} \mathrm{Cd}\left(\beta^{-}\right) ;{ }^{117 m} \mathrm{Sn},{ }^{125 m} \mathrm{Te}(\mathrm{IT}) ;$ measured I $\gamma$, (X-ray) $\gamma$-coin. ${ }^{115} \mathrm{In},{ }^{117} \mathrm{Sn},{ }^{125} \mathrm{Te}$ transitions deduced ICC. CONF St Petersburg,P65,Vishnevsky

${ }^{115} \mathrm{Sn} \quad 2005 \mathrm{CA03} \quad \mathrm{RADIOACTIVITY}{ }^{115} \operatorname{In}\left(\beta^{-}\right)$; measured $\beta$-delayed $\mathrm{E} \gamma$, I $\gamma$; deduced branching ratio and $\mathrm{Q} \beta$ for decay to excited level, limit on charge-nonconserving decay. ${ }^{115} \mathrm{Sn}$ level deduced energy, $\beta$-feeding intensity. JOUR NUPAB 748333

2005CAZW RADIOACTIVITY ${ }^{115} \operatorname{In}\left(\beta^{-}\right)$; measured $\beta$-delayed $\mathrm{E} \gamma$, I $\gamma$; deduced branching ratio, partial $\mathrm{T}_{1 / 2}$, and $\mathrm{Q}$-value for decay to first excited state. ${ }^{115} \mathrm{Sn}$ deduced level energy. Implications for neutrino mass limits discussed. PREPRINT nucl-ex/0509020,9/15/2005

2005PA22 NUCLEAR REACTIONS ${ }^{114} \operatorname{Cd}(\alpha, 2 \mathrm{n}),(\alpha, 3 \mathrm{n}),(\alpha, 4 \mathrm{n}),(\alpha, 5 \mathrm{n})$, $\mathrm{E}=35,40,45,50,55$; measured $\mathrm{E} \gamma$, En, $\sigma, \sigma(\theta)$; deduced equilibrium and pre-equilibrium contributions, related reaction mechanism features. JOUR PRVCA 71034605 


\section{$\mathrm{A}=115$ (continued)}

${ }^{115} \mathrm{Sb}$ 2005BA18 NUCLEAR REACTIONS ${ }^{112} \mathrm{Sn}(\mathrm{d}, \mathrm{p}),(\mathrm{d}, 3 \mathrm{np}),(\mathrm{p}, 2 \mathrm{np}),{ }^{118} \mathrm{Sn}(\mathrm{d}, 2 \mathrm{n})$, (d, 3n), (d, 5n), (d, 2np), (d, 6np), (d, 9np), (p, n), (p, 3n), (p, 4n), (p, np), (p, 5np), (p, 8np), ${ }^{120} \mathrm{Sn}(\mathrm{d}, 2 \mathrm{n}),(\mathrm{d}, 4 \mathrm{n}),(\mathrm{d}, 6 \mathrm{n}),(\mathrm{d}, 7 \mathrm{n}),(\mathrm{d}, 4 \mathrm{np})$, (d, 8np), (d, 11np), (p, n), (p, 3n), (p, 5n), (p, 6n), (p, 3np), (p, 7np), (p, 10np), ${ }^{124} \operatorname{Sn}(\mathrm{d}, 2 \mathrm{n}),(\mathrm{d}, 4 \mathrm{n}),(\mathrm{d}, 6 \mathrm{n}),(\mathrm{d}, 8 \mathrm{n}),(\mathrm{d}, 10 \mathrm{n}),(\mathrm{d}, 11 \mathrm{n}),(\mathrm{d}$, 2np), (d, 8np), (d, 12np), (d, 15np), (p, n), (p, 3n), (p, 5n), (p, 7n), (p, 9n), (p, 10n), (p, np), (p, 7np), (p, 11np), (p, 14np), E=3.65 GeV / nucleon; measured $\sigma .{ }^{120} \mathrm{Sn}(\mathrm{p}, \mathrm{X}), \mathrm{E}=0.66 \mathrm{GeV}$; measured spallation fragments mass distribution. Activation technique, comparison with model predictions. JOUR YAFIA 68195

\section{$\mathrm{A}=116$}

${ }^{116} \mathrm{Pd} \quad$ 2005SM08 RADIOACTIVITY ${ }^{252} \mathrm{Cf}(\mathrm{SF}) ;$ measured $\mathrm{E} \gamma, \mathrm{I} \gamma(\theta, \mathrm{H}, \mathrm{t}), \gamma \gamma$-coin. ${ }^{96,100,102} \mathrm{Zr},{ }^{102,104,106,108} \mathrm{Mo},{ }^{106,108,110,112} \mathrm{Ru},{ }^{110,114,116} \mathrm{Pd}$ levels

deduced $\mathrm{g}$ factors, $\mathrm{B}(\mathrm{E} 2)$. Gammasphere array, time-integral perturbed angular correlation technique. Comparison with interacting boson model predictions. JOUR JPGPE 31 S1433

${ }^{116} \mathrm{Ag} \quad$ 2005BA94 RADIOACTIVITY ${ }^{116 m} \mathrm{Ag}(\mathrm{IT}),{ }^{116} \mathrm{Ag}\left(\beta^{-}\right)$[from $\mathrm{U}(\mathrm{p}, \mathrm{F})$ ]; measured $\mathrm{E}(\mathrm{ce}), \mathrm{I}(\mathrm{ce}), \mathrm{E} \gamma, \mathrm{I} \gamma .{ }^{116} \mathrm{Ag}$ deduced levels, $\mathrm{J}, \pi, \mathrm{ICC}$, isomer $\mathrm{T}_{1 / 2}$. ${ }^{116} \mathrm{Cd}$ deduced transitions. JOUR PRVCA 72044306

2005RI19 RADIOACTIVITY ${ }^{116,118,120} \mathrm{Ag}\left(\beta^{-}\right)$; measured $\mathrm{E} \gamma, \mathrm{I} \gamma, \beta \gamma$-coin. ${ }^{116,118,120} \mathrm{Cd}$ deduced levels, J, $\pi$. Three-phonon states discussed. JOUR ZAANE 25 s01 119

${ }^{116} \mathrm{Cd}$ 2005BA33 RADIOACTIVITY ${ }^{82} \mathrm{Se},{ }^{100} \mathrm{Mo},{ }^{116} \mathrm{Cd},{ }^{150} \mathrm{Nd}\left(2 \beta^{-}\right)$; measured $2 \nu \beta \beta$-decay $\mathrm{T}_{1 / 2}, 0 \nu \beta \beta$-decay $\mathrm{T}_{1 / 2}$ lower limits. JOUR YAFIA 68443 2005BA94 RADIOACTIVITY ${ }^{116 m} \mathrm{Ag}(\mathrm{IT}),{ }^{116} \mathrm{Ag}\left(\beta^{-}\right)$[from $\mathrm{U}(\mathrm{p}, \mathrm{F})$ ]; measured $\mathrm{E}(\mathrm{ce}), \mathrm{I}(\mathrm{ce}), \mathrm{E} \gamma, \mathrm{I} \gamma .{ }^{116} \mathrm{Ag}$ deduced levels, $\mathrm{J}, \pi, \mathrm{ICC}$, isomer $\mathrm{T}_{1 / 2}$. ${ }^{116} \mathrm{Cd}$ deduced transitions. JOUR PRVCA 72044306

2005DA03 RADIOACTIVITY ${ }^{116} \mathrm{Cd}\left(2 \beta^{-}\right)$; measured $2 \nu 2 \beta$-decay $\mathrm{T}_{1 / 2}$, $0 \nu 2 \beta$-decay $\mathrm{T}_{1 / 2}$ lower limit. JOUR NPBSE 138230

2005K032 NUCLEAR REACTIONS ${ }^{110,111,112,114,116} \mathrm{Cd}\left(\gamma, \gamma^{\prime}\right), \mathrm{E} \approx 2.7-4.1 \mathrm{MeV}$ bremsstrahlung; measured $\mathrm{E} \gamma, \mathrm{I} \gamma, \gamma$-ray polarization.

${ }_{110,111,112,114,116} \mathrm{Cd}$ deduced levels, J, $\pi$, excitation B(M1), B(E1).

JOUR PRVCA 72034302

2005LU06 NUCLEAR REACTIONS ${ }^{110,116} \mathrm{Cd},{ }^{112,124} \mathrm{Sn}\left(\alpha, \alpha^{\prime}\right), \mathrm{E}=240 \mathrm{MeV}$; measured $\mathrm{E} \alpha, \sigma(\theta) .{ }^{110,116} \mathrm{Cd},{ }^{112,124} \mathrm{Sn}$ deduced electric monopole strength distributions, resonance parameters. Comparison with model predictions. JOUR APOBB 361107

2005RA13 NUCLEAR REACTIONS ${ }^{116} \mathrm{Sn}(\mathrm{d}, 2 \mathrm{p}), \mathrm{E}=183 \mathrm{MeV}$; measured Ep, $\sigma(\mathrm{E}, \theta) .{ }^{116} \mathrm{In}$ levels deduced Gamow-Teller strength distribution. ${ }^{116} \mathrm{Cd}$ deduced $2 \beta$-decay matrix elements. JOUR PRVCA 71054313

2005RI19 RADIOACTIVITY ${ }^{116,118,120} \mathrm{Ag}\left(\beta^{-}\right)$; measured $\mathrm{E} \gamma, \mathrm{I} \gamma, \beta \gamma$-coin. ${ }^{116,118,120} \mathrm{Cd}$ deduced levels, J, $\pi$. Three-phonon states discussed. JOUR ZAANE 25 s01 119

2005SA07 RADIOACTIVITY ${ }^{82} \mathrm{Se},{ }^{96} \mathrm{Zr},{ }^{100} \mathrm{Mo},{ }^{116} \mathrm{Cd},{ }^{150} \mathrm{Nd}\left(2 \beta^{-}\right)$; measured $2 \nu \beta \beta$-decay $\mathrm{T}_{1 / 2} .{ }^{82} \mathrm{Se},{ }^{100} \mathrm{Mo}\left(2 \beta^{-}\right)$; measured $0 \nu \beta \beta$-decay $\mathrm{T}_{1 / 2}$ lower limits; deduced neutrino mass limits. JOUR NPBSE 143221 


\section{$\mathrm{A}=116$ (continued)}

2005SI06 RADIOACTIVITY ${ }^{82} \mathrm{Se},{ }^{96} \mathrm{Zr},{ }^{100} \mathrm{Mo},{ }^{116} \mathrm{Cd},{ }^{150} \mathrm{Nd}\left(2 \beta^{-}\right)$; measured $2 \nu \beta \beta$-decay $\mathrm{T}_{1 / 2} .{ }^{82} \mathrm{Se},{ }^{100} \mathrm{Mo}\left(2 \beta^{-}\right)$; measured $0 \nu \beta \beta$-decay $\mathrm{T}_{1 / 2}$ lower limits; deduced neutrino mass limits. JOUR NPBSE 145272

${ }^{116} \mathrm{In}$ 2005RA13 NUCLEAR REACTIONS ${ }^{116} \mathrm{Sn}(\mathrm{d}, 2 \mathrm{p}), \mathrm{E}=183 \mathrm{MeV}$; measured Ep, $\sigma(\mathrm{E}, \theta) .{ }^{116} \mathrm{In}$ levels deduced Gamow-Teller strength distribution. ${ }^{116} \mathrm{Cd}$ deduced $2 \beta$-decay matrix elements. JOUR PRVCA 71054313

${ }^{116} \mathrm{Sn} \quad 2004 \mathrm{KO64}$ NUCLEAR REACTIONS ${ }^{6} \mathrm{Li},{ }^{16} \mathrm{O},{ }^{32} \mathrm{~S},{ }^{50,51} \mathrm{~V},{ }^{70,72} \mathrm{Ge}(\mathrm{d}, \mathrm{d})$, (d, d'), $\mathrm{E}=171 \mathrm{MeV} ;{ }^{90} \mathrm{Zr},{ }^{116} \mathrm{Sn}(\mathrm{d}, \mathrm{d}),(\mathrm{d}, \mathrm{d}$ '), $\mathrm{E}=183 \mathrm{MeV}$; measured $\sigma(\theta)$; deduced optical model parameters. JOUR PRVCA 70067601

2005BA33 RADIOACTIVITY ${ }^{82} \mathrm{Se},{ }^{100} \mathrm{Mo},{ }^{116} \mathrm{Cd},{ }^{150} \mathrm{Nd}\left(2 \beta^{-}\right)$; measured $2 \nu \beta \beta$-decay $\mathrm{T}_{1 / 2}, 0 \nu \beta \beta$-decay $\mathrm{T}_{1 / 2}$ lower limits. JOUR YAFIA 68443

2005DA03 RADIOACTIVITY ${ }^{116} \mathrm{Cd}\left(2 \beta^{-}\right)$; measured $2 \nu 2 \beta$-decay $\mathrm{T}_{1 / 2}$, $0 \nu 2 \beta$-decay $\mathrm{T}_{1 / 2}$ lower limit. JOUR NPBSE 138230

2005HU10 NUCLEAR REACTIONS ${ }^{90} \mathrm{Zr},{ }^{116} \mathrm{Sn},{ }^{208} \mathrm{~Pb}(\alpha, \alpha$ 'n), E=200 MeV; ${ }^{208} \mathrm{~Pb}(\alpha, \alpha$ p $), \mathrm{E}=200 \mathrm{MeV}$; measured $\mathrm{E} \alpha, \sigma(\theta), \mathrm{p} \alpha-$, n $\alpha$-coin. ${ }^{90} \mathrm{Zr}$, ${ }^{116} \mathrm{Sn},{ }^{208} \mathrm{~Pb}$ deduced isoscalar GDR parameters, particle decay features. JOUR APOBB 361115

2005PA22 NUCLEAR REACTIONS ${ }^{114} \mathrm{Cd}(\alpha, 2 \mathrm{n}),(\alpha, 3 \mathrm{n}),(\alpha, 4 \mathrm{n}),(\alpha, 5 \mathrm{n})$, $\mathrm{E}=35,40,45,50,55$; measured $\mathrm{E} \gamma$, En, $\sigma, \sigma(\theta)$; deduced equilibrium and pre-equilibrium contributions, related reaction mechanism features. JOUR PRVCA 71034605

2005SA07 RADIOACTIVITY ${ }^{82} \mathrm{Se},{ }^{96} \mathrm{Zr},{ }^{100} \mathrm{Mo},{ }^{116} \mathrm{Cd},{ }^{150} \mathrm{Nd}\left(2 \beta^{-}\right)$; measured $2 \nu \beta \beta$-decay $\mathrm{T}_{1 / 2} .{ }^{82} \mathrm{Se},{ }^{100} \mathrm{Mo}\left(2 \beta^{-}\right)$; measured $0 \nu \beta \beta$-decay $\mathrm{T}_{1 / 2}$ lower limits; deduced neutrino mass limits. JOUR NPBSE 143221

2005SI06 RADIOACTIVITY ${ }^{82} \mathrm{Se},{ }^{96} \mathrm{Zr},{ }^{100} \mathrm{Mo},{ }^{116} \mathrm{Cd},{ }^{150} \mathrm{Nd}\left(2 \beta^{-}\right)$; measured $2 \nu \beta \beta$-decay $\mathrm{T}_{1 / 2} .{ }^{82} \mathrm{Se},{ }^{100} \mathrm{Mo}\left(2 \beta^{-}\right)$; measured $0 \nu \beta \beta$-decay $\mathrm{T}_{1 / 2}$ lower limits; deduced neutrino mass limits. JOUR NPBSE 145272

${ }^{116} \mathrm{Sb}$ 2005BA18 NUCLEAR REACTIONS ${ }^{112} \mathrm{Sn}(\mathrm{d}, \mathrm{p}),(\mathrm{d}, 3 \mathrm{np}),(\mathrm{p}, 2 \mathrm{np}),{ }^{118} \mathrm{Sn}(\mathrm{d}, 2 \mathrm{n})$, (d, 3n), (d, 5n), (d, 2np), (d, 6np), (d, 9np), (p, n), (p, 3n), (p, 4n), (p, $\mathrm{np}),(\mathrm{p}, 5 \mathrm{np}),(\mathrm{p}, 8 \mathrm{np}),{ }^{120} \operatorname{Sn}(\mathrm{d}, 2 \mathrm{n}),(\mathrm{d}, 4 \mathrm{n}),(\mathrm{d}, 6 \mathrm{n}),(\mathrm{d}, 7 \mathrm{n}),(\mathrm{d}, 4 \mathrm{np})$, (d, 8np), (d, 11np), (p, n), (p, 3n), (p, 5n), (p, 6n), (p, 3np), (p, 7np), (p, 10np), ${ }^{124} \operatorname{Sn}(\mathrm{d}, 2 \mathrm{n}),(\mathrm{d}, 4 \mathrm{n}),(\mathrm{d}, 6 \mathrm{n}),(\mathrm{d}, 8 \mathrm{n}),(\mathrm{d}, 10 \mathrm{n}),(\mathrm{d}, 11 \mathrm{n}),(\mathrm{d}$, 2np), (d, 8np), (d, 12np), (d, 15np), (p, n), (p, 3n), (p, 5n), (p, 7n), (p, 9n), (p, 10n), (p, np), (p, 7np), (p, 11np), (p, 14np), E=3.65 GeV / nucleon; measured $\sigma \cdot{ }^{120} \mathrm{Sn}(\mathrm{p}, \mathrm{X}), \mathrm{E}=0.66 \mathrm{GeV}$; measured spallation fragments mass distribution. Activation technique, comparison with model predictions. JOUR YAFIA 68195

${ }^{116} \mathrm{Te}$ 2005GA21 NUCLEAR REACTIONS ${ }^{112} \mathrm{Sn}(\alpha, \alpha), \mathrm{E}=14.4,19.5 \mathrm{MeV} ;{ }^{124} \mathrm{Sn}(\alpha, \alpha)$, $\mathrm{E}=19.5 \mathrm{MeV}$; measured elastic $\sigma(\theta)$; deduced optical potential parameters. ${ }^{112} \mathrm{Sn}(\alpha, \gamma), \mathrm{E}(\mathrm{cm})=7-11 \mathrm{MeV}$; calculated astrophysical S-factors, reaction rates. JOUR PRVCA 71065802

${ }^{116} \mathrm{Xe}$ 2005JA06 RADIOACTIVITY ${ }^{117} \mathrm{Ba}\left(\beta^{+} \mathrm{p}\right)\left[\right.$ from $\left.{ }^{63} \mathrm{Cu}\left({ }^{58} \mathrm{Ni}, 3 \mathrm{np}\right)\right]$; measured $\mathrm{E} \gamma$, $\mathrm{E} \beta, \beta \gamma$-coin, $\beta$-delayed proton spectra; deduced Q. ${ }^{117} \mathrm{Cs}$ deduced $\beta$-feeding intensities, proton decay branching ratios, resonance structure. Total absorption spectrometer, comparison with model predictions. JOUR ZAANE 23401 


\section{$\mathrm{A}=117$}

${ }^{117} \mathrm{Pd} \quad 2005 \mathrm{~F} 009 \quad$ RADIOACTIVITY ${ }^{252} \mathrm{Cf}(\mathrm{SF}) ;$ measured $\mathrm{E} \gamma, \mathrm{I} \gamma, \gamma \gamma$-coin. ${ }^{113,115,117} \mathrm{Pd}$ deduced levels, J, $\pi$. Gammasphere array. JOUR PRVCA 72014315

${ }^{117} \mathrm{Sn} \quad 2005 \mathrm{BA} 18$ NUCLEAR REACTIONS ${ }^{112} \mathrm{Sn}(\mathrm{d}, \mathrm{p}),(\mathrm{d}, 3 \mathrm{np}),(\mathrm{p}, 2 \mathrm{np}),{ }^{118} \mathrm{Sn}(\mathrm{d}, 2 \mathrm{n})$, (d, 3n), (d, 5n), (d, 2np), (d, 6np), (d, 9np), (p, n), (p, 3n), (p, 4n), (p, np), (p, 5np), (p, 8np), ${ }^{120} \mathrm{Sn}(\mathrm{d}, 2 \mathrm{n}),(\mathrm{d}, 4 \mathrm{n}),(\mathrm{d}, 6 \mathrm{n}),(\mathrm{d}, 7 \mathrm{n}),(\mathrm{d}, 4 \mathrm{np})$, (d, 8np), (d, 11np), (p, n), (p, 3n), (p, 5n), (p, 6n), (p, 3np), (p, 7np), (p, 10np), ${ }^{124} \operatorname{Sn}(\mathrm{d}, 2 \mathrm{n}),(\mathrm{d}, 4 \mathrm{n}),(\mathrm{d}, 6 \mathrm{n}),(\mathrm{d}, 8 \mathrm{n}),(\mathrm{d}, 10 \mathrm{n}),(\mathrm{d}, 11 \mathrm{n}),(\mathrm{d}$, 2np), (d, 8np), (d, 12np), (d, 15np), (p, n), (p, 3n), (p, 5n), (p, 7n), (p, 9n), (p, 10n), (p, np), (p, 7np), (p, 11np), (p, 14np), E=3.65 GeV / nucleon; measured $\sigma .{ }^{120} \mathrm{Sn}(\mathrm{p}, \mathrm{X}), \mathrm{E}=0.66 \mathrm{GeV}$; measured spallation fragments mass distribution. Activation technique, comparison with model predictions. JOUR YAFIA 68195

2005HE08 NUCLEAR REACTIONS ${ }^{100} \mathrm{Mo}\left({ }^{17} \mathrm{O}\right.$, xnyp), $\mathrm{E}=78.8 \mathrm{MeV} ;{ }^{100} \mathrm{Mo}\left({ }^{18} \mathrm{O}\right.$, xnyp), $\mathrm{E}=95.0 \mathrm{MeV}$; measured $\mathrm{E} \gamma, \mathrm{I} \gamma$, (evaporation residue) $\gamma$-coin. ${ }^{117,118}$ Sn deduced GDR widths, temperature and spin dependence features. Comparison with model predictions. JOUR NUPAB 750175 2005VIZZ RADIOACTIVITY ${ }^{115} \mathrm{Cd}\left(\beta^{-}\right) ;{ }^{117 m} \mathrm{Sn},{ }^{125 m} \mathrm{Te}(\mathrm{IT})$; measured $\mathrm{I} \gamma$, (X-ray) $\gamma$-coin. ${ }^{115} \mathrm{In},{ }^{117} \mathrm{Sn},{ }^{125} \mathrm{Te}$ transitions deduced ICC. CONF St Petersburg,P65,Vishnevsky

${ }^{117} \mathrm{Sb} \quad$ 2005BA18 NUCLEAR REACTIONS ${ }^{112} \mathrm{Sn}(\mathrm{d}, \mathrm{p}),(\mathrm{d}, 3 \mathrm{np}),(\mathrm{p}, 2 \mathrm{np}),{ }^{118} \mathrm{Sn}(\mathrm{d}, 2 \mathrm{n})$, (d, 3n), (d, 5n), (d, 2np), (d, 6np), (d, 9np), (p, n), (p, 3n), (p, 4n), (p, np), (p, 5np), (p, 8np), ${ }^{120} \mathrm{Sn}(\mathrm{d}, 2 \mathrm{n}),(\mathrm{d}, 4 \mathrm{n}),(\mathrm{d}, 6 \mathrm{n}),(\mathrm{d}, 7 \mathrm{n}),(\mathrm{d}, 4 \mathrm{np})$, (d, 8np), (d, 11np), (p, n), (p, 3n), (p, 5n), (p, 6n), (p, 3np), (p, 7np), (p, 10np), ${ }^{124} \operatorname{Sn}(\mathrm{d}, 2 \mathrm{n}),(\mathrm{d}, 4 \mathrm{n}),(\mathrm{d}, 6 \mathrm{n}),(\mathrm{d}, 8 \mathrm{n}),(\mathrm{d}, 10 \mathrm{n}),(\mathrm{d}, 11 \mathrm{n}),(\mathrm{d}$, 2np), (d, 8np), (d, 12np), (d, 15np), (p, n), (p, 3n), (p, 5n), (p, 7n), (p, 9n), (p, 10n), (p, np), (p, 7np), (p, 11np), (p, 14np), E=3.65 GeV / nucleon; measured $\sigma .{ }^{120} \mathrm{Sn}(\mathrm{p}, \mathrm{X}), \mathrm{E}=0.66 \mathrm{GeV}$; measured spallation fragments mass distribution. Activation technique, comparison with model predictions. JOUR YAFIA 68195

${ }^{117} \mathrm{Cs} \quad$ 2005JA06

RADIOACTIVITY ${ }^{117} \mathrm{Ba}\left(\beta^{+} \mathrm{p}\right)$ [from $\left.{ }^{63} \mathrm{Cu}\left({ }^{58} \mathrm{Ni}, 3 \mathrm{np}\right)\right]$; measured $\mathrm{E} \gamma$, $\mathrm{E} \beta, \beta \gamma$-coin, $\beta$-delayed proton spectra; deduced Q. ${ }^{117} \mathrm{Cs}$ deduced $\beta$-feeding intensities, proton decay branching ratios, resonance structure. Total absorption spectrometer, comparison with model predictions. JOUR ZAANE 23401

${ }^{117} \mathrm{Ba}$ 2005JA06 RADIOACTIVITY ${ }^{117} \mathrm{Ba}\left(\beta^{+} \mathrm{p}\right)\left[\right.$ from $\left.{ }^{63} \mathrm{Cu}\left({ }^{58} \mathrm{Ni}, 3 \mathrm{np}\right)\right] ;$ measured $\mathrm{E} \gamma$, $\mathrm{E} \beta, \beta \gamma$-coin, $\beta$-delayed proton spectra; deduced Q. ${ }^{117} \mathrm{Cs}$ deduced $\beta$-feeding intensities, proton decay branching ratios, resonance structure. Total absorption spectrometer, comparison with model predictions. JOUR ZAANE 23401

\section{$\mathrm{A}=\mathbf{1 1 8}$}

\begin{tabular}{|c|c|c|}
\hline${ }^{118} \mathrm{Ag}$ & 2005RI19 & $\begin{array}{l}\text { RADIOACTIVITY }{ }^{116,118,120} \mathrm{Ag}\left(\beta^{-}\right) \text {; measured } \mathrm{E} \gamma, \mathrm{I} \gamma, \beta \gamma \text {-coin. } \\
{ }_{116,118,120} \mathrm{Cd} \text { deduced levels, } \mathrm{J}, \pi \text {. Three-phonon states discussed. }\end{array}$ \\
\hline${ }^{118} \mathrm{Cd}$ & 2005RI19 & $\begin{array}{l}\text { JOUR ZAANE } 25 \text { s01 } 119 \\
\text { RADIOACTIVITY }{ }^{116,118,120} \mathrm{Ag}\left(\beta^{-}\right) \text {; measured E } \gamma, \mathrm{I} \gamma, \beta \gamma \text {-coin. } \\
116,118,120\end{array}$ \\
\hline & & JOUR ZAANE 25 s01 119 \\
\hline
\end{tabular}




\section{$\mathrm{A}=118$ (continued)}

\begin{abstract}
${ }^{118} \mathrm{Sn} \quad$ 2005HE08
${ }^{118} \mathrm{Sb} \quad$ 2005BA18

NUCLEAR REACTIONS ${ }^{100} \mathrm{Mo}\left({ }^{17} \mathrm{O}\right.$, xnyp $), \mathrm{E}=78.8 \mathrm{MeV} ;{ }^{100} \mathrm{Mo}\left({ }^{18} \mathrm{O}\right.$, xnyp), $\mathrm{E}=95.0 \mathrm{MeV}$; measured $\mathrm{E} \gamma, \mathrm{I} \gamma$, (evaporation residue) $\gamma$-coin. ${ }^{117,118} \mathrm{Sn}$ deduced GDR widths, temperature and spin dependence features. Comparison with model predictions. JOUR NUPAB 750175 NUCLEAR REACTIONS ${ }^{112} \mathrm{Sn}(\mathrm{d}, \mathrm{p}),(\mathrm{d}, 3 \mathrm{np}),(\mathrm{p}, 2 \mathrm{np}),{ }^{118} \mathrm{Sn}(\mathrm{d}, 2 \mathrm{n})$, (d, 3n), (d, 5n), (d, 2np), (d, 6np), (d, 9np), (p, n), (p, 3n), (p, 4n), (p, $\mathrm{np}),(\mathrm{p}, 5 \mathrm{np}),(\mathrm{p}, 8 \mathrm{np}),{ }^{120} \mathrm{Sn}(\mathrm{d}, 2 \mathrm{n}),(\mathrm{d}, 4 \mathrm{n}),(\mathrm{d}, 6 \mathrm{n}),(\mathrm{d}, 7 \mathrm{n}),(\mathrm{d}, 4 \mathrm{np})$, (d, 8np), (d, 11np), (p, n), (p, 3n), (p, 5n), (p, 6n), (p, 3np), (p, 7np), (p, 10np), ${ }^{124} \operatorname{Sn}(\mathrm{d}, 2 \mathrm{n}),(\mathrm{d}, 4 \mathrm{n}),(\mathrm{d}, 6 \mathrm{n}),(\mathrm{d}, 8 \mathrm{n}),(\mathrm{d}, 10 \mathrm{n}),(\mathrm{d}, 11 \mathrm{n}),(\mathrm{d}$, 2np), (d, 8np), (d, 12np), (d, 15np), (p, n), (p, 3n), (p, 5n), (p, 7n), (p, 9n), (p, 10n), (p, np), (p, 7np), (p, 11np), (p, 14np), E=3.65 GeV / nucleon; measured $\sigma .{ }^{120} \mathrm{Sn}(\mathrm{p}, \mathrm{X}), \mathrm{E}=0.66 \mathrm{GeV}$; measured spallation fragments mass distribution. Activation technique, comparison with model predictions. JOUR YAFIA 68195
\end{abstract}

\section{$\mathrm{A}=119$}

$\begin{array}{ll}{ }^{119} \mathrm{Rh} & 2005 \mathrm{MO} 0 \\ { }^{119} \mathrm{Pd} & 2005 \mathrm{MO} 0 \\ { }^{119} \mathrm{In} & 2005 \mathrm{GU} 32\end{array}$

RADIOACTIVITY ${ }^{119} \mathrm{Rh}\left(\beta^{-}\right)$[from Be $\left({ }^{136} \mathrm{Xe}, \mathrm{X}\right)$ ]; measured $\mathrm{T}_{1 / 2}$. JOUR NUPAB $758643 \mathrm{c}$

RADIOACTIVITY ${ }^{119} \mathrm{Rh}\left(\beta^{-}\right)$[from $\mathrm{Be}\left({ }^{136} \mathrm{Xe}, \mathrm{X}\right)$ ]; measured $\mathrm{T}_{1 / 2}$. JOUR NUPAB $758643 \mathrm{c}$

NUCLEAR REACTIONS ${ }^{122} \mathrm{Sn},{ }^{123} \mathrm{Sb}$ (polarized p, $\alpha$ ), E=24 MeV; measured $\mathrm{E} \alpha, \sigma(\theta), \operatorname{Ay}(\theta) .{ }^{119} \mathrm{In},{ }^{120} \mathrm{Sn}$ deduced homologous states features. JOUR PRVCA 72044604

\section{$\mathrm{A}=120$}

$\begin{array}{ll}{ }^{120} \mathrm{Ag} & 2005 \mathrm{RI} 19 \\ { }^{120} \mathrm{Cd} & \\ & 2005 \mathrm{RI} 19 \\ { }^{120} \mathrm{Sn} & 2004 \mathrm{KU} 30\end{array}$

RADIOACTIVITY ${ }^{116,118,120} \mathrm{Ag}\left(\beta^{-}\right)$; measured $\mathrm{E} \gamma, \mathrm{I} \gamma, \beta \gamma$-coin. ${ }^{116,118,120} \mathrm{Cd}$ deduced levels, J, $\pi$. Three-phonon states discussed. JOUR ZAANE 25 s01 119

RADIOACTIVITY ${ }^{116,118,120} \mathrm{Ag}\left(\beta^{-}\right)$; measured $\mathrm{E} \gamma, \mathrm{I} \gamma, \beta \gamma$-coin. ${ }^{116,118,120} \mathrm{Cd}$ deduced levels, $\mathrm{J}, \pi$. Three-phonon states discussed. JOUR ZAANE 25 s01 119 NUCLEAR REACTIONS ${ }^{112,114,120,124} \operatorname{Sn}(\alpha, \alpha),(\alpha, \alpha), \mathrm{E} \approx 50 \mathrm{MeV}$; measured $\sigma(\mathrm{E}, \theta)$; deduced optical model parameters. ${ }^{112,114,120,124} \mathrm{Sn}$ deduced transition strengths, deformation parameters, related features. JOUR UKPJA 49841

2005GU32 NUCLEAR REACTIONS ${ }^{122} \mathrm{Sn},{ }^{123} \mathrm{Sb}$ (polarized $\mathrm{p}, \alpha$ ), E=24 MeV; measured $\mathrm{E} \alpha, \sigma(\theta), \operatorname{Ay}(\theta) .{ }^{119} \mathrm{In},{ }^{120} \mathrm{Sn}$ deduced homologous states features. JOUR PRVCA 72044604

2005ZU01 RADIOACTIVITY ${ }^{120} \mathrm{Te}\left(\beta^{+} \mathrm{EC}\right) ;{ }^{64} \mathrm{Zn},{ }^{106,108} \mathrm{Cd},{ }^{120} \mathrm{Te}(2 \mathrm{EC})$; measured $\mathrm{T}_{1 / 2}$ lower limits. JOUR NPBSE 138236 


\section{$\mathrm{A}=120$ (continued)}

${ }^{120} \mathrm{Sb}$ 2005BA18 NUCLEAR REACTIONS ${ }^{112} \mathrm{Sn}(\mathrm{d}, \mathrm{p}),(\mathrm{d}, 3 \mathrm{np}),(\mathrm{p}, 2 \mathrm{np}),{ }^{118} \mathrm{Sn}(\mathrm{d}, 2 \mathrm{n})$, (d, 3n), (d, 5n), (d, 2np), (d, 6np), (d, 9np), (p, n), (p, 3n), (p, 4n), (p, np), (p, 5np), (p, 8np), ${ }^{120} \mathrm{Sn}(\mathrm{d}, 2 \mathrm{n}),(\mathrm{d}, 4 \mathrm{n}),(\mathrm{d}, 6 \mathrm{n}),(\mathrm{d}, 7 \mathrm{n}),(\mathrm{d}, 4 \mathrm{np})$, (d, 8np), (d, 11np), (p, n), (p, 3n), (p, 5n), (p, 6n), (p, 3np), (p, 7np), (p, 10np), ${ }^{124} \operatorname{Sn}(\mathrm{d}, 2 \mathrm{n}),(\mathrm{d}, 4 \mathrm{n}),(\mathrm{d}, 6 \mathrm{n}),(\mathrm{d}, 8 \mathrm{n}),(\mathrm{d}, 10 \mathrm{n}),(\mathrm{d}, 11 \mathrm{n}),(\mathrm{d}$, 2np), (d, 8np), (d, 12np), (d, 15np), (p, n), (p, 3n), (p, 5n), (p, 7n), (p, 9n), (p, 10n), (p, np), (p, 7np), (p, 11np), (p, 14np), E=3.65 GeV / nucleon; measured $\sigma .{ }^{120} \mathrm{Sn}(\mathrm{p}, \mathrm{X}), \mathrm{E}=0.66 \mathrm{GeV}$; measured spallation fragments mass distribution. Activation technique, comparison with model predictions. JOUR YAFIA 68195

2005BIZZ NUCLEAR REACTIONS ${ }^{81} \mathrm{Br},{ }^{121} \mathrm{Sb}(\gamma, \mathrm{n}), \mathrm{E}=9-18 \mathrm{MeV}$; measured isomer production $\sigma$. Microtron. CONF St Petersburg,P214,Bigan

${ }^{120} \mathrm{Te} \quad 2005 \mathrm{ZU} 01 \quad$ RADIOACTIVITY ${ }^{120} \mathrm{Te}\left(\beta^{+} \mathrm{EC}\right) ;{ }^{44} \mathrm{Zn},{ }^{106,108} \mathrm{Cd},{ }^{120} \mathrm{Te}(2 \mathrm{EC}) ;$ measured $\mathrm{T}_{1 / 2}$ lower limits. JOUR NPBSE 138236

${ }^{120} \mathrm{I}$ 2003MOZS NUCLEAR REACTIONS ${ }^{118} \mathrm{Sn}\left({ }^{6} \mathrm{Li}, 4 \mathrm{n}\right), \mathrm{E}=48 \mathrm{MeV}$; measured $\mathrm{E} \gamma$, $\mathrm{I} \gamma, \gamma \gamma$-coin. ${ }^{120} \mathrm{I}$ deduced high-spin levels, J, $\pi$, configurations, B(M1) / B(E2). REPT ANU-P/1564 2002 Annual,P11,Moon

${ }^{120} \mathrm{Ba}$ 2005XU04 RADIOACTIVITY ${ }^{81} \mathrm{Zr},{ }^{85} \mathrm{Mo},{ }^{89} \mathrm{Ru},{ }^{92} \mathrm{Rh},{ }^{93} \mathrm{Pd},{ }^{121} \mathrm{Ce},{ }^{125} \mathrm{Nd}$, ${ }^{128} \mathrm{Pm},{ }^{129} \mathrm{Sm},{ }^{135,137} \mathrm{Gd},{ }^{139} \mathrm{Dy},{ }^{142} \mathrm{Ho},{ }^{149} \mathrm{Yb}\left(\beta^{+} \mathrm{p}\right)$; measured $\beta$-delayed $\mathrm{E} \gamma, \mathrm{I} \gamma$, proton spectra, $\mathrm{p} \gamma$-coin, $\mathrm{T}_{1 / 2}$. Comparison with model predictions. JOUR PRVCA 71054318

${ }^{120} \mathrm{Ce} \quad$ 2005R019 RADIOACTIVITY ${ }^{121} \mathrm{Pr}(\mathrm{p})$ [from $\left.{ }^{92} \mathrm{Mo}\left({ }^{36} \mathrm{Ar}, 6 \mathrm{np}\right)\right]$; measured Ep, $\mathrm{T}_{1 / 2} .{ }^{121} \mathrm{Pr}$ deduced ground-state $\mathrm{J}$, deformation. Comparison with previous results. JOUR PRLTA 95032502

\section{$\mathrm{A}=121$}

${ }^{121} \mathrm{Sb} \quad 2005 \mathrm{P} 003$

NUCLEAR REACTIONS ${ }^{238} \mathrm{U}\left({ }^{12} \mathrm{C}, \mathrm{X}\right), \mathrm{E}=90 \mathrm{MeV} ;{ }^{208} \mathrm{~Pb}\left({ }^{18} \mathrm{O}, \mathrm{X}\right)$, $\mathrm{E}=85 \mathrm{MeV} ;{ }^{176} \mathrm{Yb}\left({ }^{31} \mathrm{P}, \mathrm{X}\right), \mathrm{E}=152 \mathrm{MeV}$; measured prompt and delayed $\mathrm{E} \gamma, \mathrm{I} \gamma, \gamma \gamma$-coin. ${ }^{121,123,125,127} \mathrm{Sb}$ deduced high-spin levels, J, $\pi$, configurations. ${ }^{123,125,127} \mathrm{Sb}$ deduced isomeric states energies, $\mathrm{T}_{1 / 2}$. Euroball III and IV arrays. JOUR ZAANE 2439

${ }^{121} \mathrm{Ce}$ 2005XU04 RADIOACTIVITY ${ }^{81} \mathrm{Zr},{ }^{85} \mathrm{Mo},{ }^{89} \mathrm{Ru},{ }^{92} \mathrm{Rh},{ }^{93} \mathrm{Pd},{ }^{121} \mathrm{Ce},{ }^{125} \mathrm{Nd}$, ${ }^{128} \mathrm{Pm},{ }^{129} \mathrm{Sm},{ }^{135,137} \mathrm{Gd},{ }^{139} \mathrm{Dy},{ }^{142} \mathrm{Ho},{ }^{149} \mathrm{Yb}\left(\beta^{+} \mathrm{p}\right)$; measured $\beta$-delayed $\mathrm{E} \gamma, \mathrm{I} \gamma$, proton spectra, $\mathrm{p} \gamma$-coin, $\mathrm{T}_{1 / 2}$. Comparison with model predictions. JOUR PRVCA 71054318

${ }^{121} \mathrm{Pr}$ 2005R019 RADIOACTIVITY ${ }^{121} \mathrm{Pr}(\mathrm{p})$ [from $\left.{ }^{92} \mathrm{Mo}\left({ }^{36} \mathrm{Ar}, 6 \mathrm{np}\right)\right]$; measured Ep, $\mathrm{T}_{1 / 2} \cdot{ }^{121} \mathrm{Pr}$ deduced ground-state $\mathrm{J}$, deformation. Comparison with previous results. JOUR PRLTA 95032502

\section{$\mathrm{A}=122$}

${ }^{122} \mathrm{Cd}$ 2005BEZS NUCLEAR REACTIONS ${ }^{108} \mathrm{Pd}\left({ }^{122} \mathrm{Cd},{ }^{122} \mathrm{Cd}\right),{ }^{104} \mathrm{Pd}\left({ }^{124} \mathrm{Cd},{ }^{124} \mathrm{Cd}\right.$ '), $\left({ }^{126} \mathrm{Cd},{ }^{126} \mathrm{Cd}\right.$ '), E not given; measured $\mathrm{E} \gamma, \mathrm{I} \gamma$, (particle) $\gamma$-coin following projectile Coulomb excitation. ${ }^{122,124} \mathrm{Cd}$ levels deduced excitation B(E2). REPT MLL 2004 Annual,P14,Behrens 


\section{$\mathrm{A}=122$ (continued)}

${ }^{122} \mathrm{Sb}$ 2005BA18 NUCLEAR REACTIONS ${ }^{112} \mathrm{Sn}(\mathrm{d}, \mathrm{p}),(\mathrm{d}, 3 \mathrm{np}),(\mathrm{p}, 2 \mathrm{np}),{ }^{118} \mathrm{Sn}(\mathrm{d}, 2 \mathrm{n})$, (d, 3n), (d, 5n), (d, 2np), (d, 6np), (d, 9np), (p, n), (p, 3n), (p, 4n), (p, np), (p, 5np), (p, 8np), ${ }^{120} \mathrm{Sn}(\mathrm{d}, 2 \mathrm{n}),(\mathrm{d}, 4 \mathrm{n}),(\mathrm{d}, 6 \mathrm{n}),(\mathrm{d}, 7 \mathrm{n}),(\mathrm{d}, 4 \mathrm{np})$, (d, 8np), (d, 11np), (p, n), (p, 3n), (p, 5n), (p, 6n), (p, 3np), (p, 7np), (p, 10np), ${ }^{124} \mathrm{Sn}(\mathrm{d}, 2 \mathrm{n}),(\mathrm{d}, 4 \mathrm{n}),(\mathrm{d}, 6 \mathrm{n}),(\mathrm{d}, 8 \mathrm{n}),(\mathrm{d}, 10 \mathrm{n}),(\mathrm{d}, 11 \mathrm{n}),(\mathrm{d}$, 2np), (d, 8np), (d, 12np), (d, 15np), (p, n), (p, 3n), (p, 5n), (p, 7n), (p, 9n), (p, 10n), (p, np), (p, 7np), (p, 11np), (p, 14np), E=3.65 GeV / nucleon; measured $\sigma .{ }^{120} \mathrm{Sn}(\mathrm{p}, \mathrm{X}), \mathrm{E}=0.66 \mathrm{GeV}$; measured spallation fragments mass distribution. Activation technique, comparison with model predictions. JOUR YAFIA 68195

${ }^{122} \mathrm{Te}$ 2005HA49 NUCLEAR REACTIONS ${ }^{92} \mathrm{Mo}(\alpha, \gamma), \mathrm{E}=9 \mathrm{MeV} ;{ }^{91} \mathrm{Zr}(\alpha, \gamma), \mathrm{E}=10.5$ $\mathrm{MeV} ;{ }^{118} \mathrm{Sn}(\alpha, \gamma), \mathrm{E}=11.5 \mathrm{MeV}$; measured $\mathrm{E} \gamma, \mathrm{I} \gamma .{ }^{91} \mathrm{Zr},{ }^{118} \mathrm{Sn}(\alpha, \gamma)$, $\mathrm{E}(\mathrm{cm}) \approx 9-11 \mathrm{MeV}$; measured $\sigma$. Comparison with model predictions. JOUR NUPAB $758505 \mathrm{c}$

2005HA56 NUCLEAR REACTIONS ${ }^{104} \mathrm{Pd}(\mathrm{p}, \gamma), \mathrm{E}(\mathrm{cm})=2-8 \mathrm{MeV} ;{ }^{118} \mathrm{Sn}(\alpha, \gamma)$, $\mathrm{E}(\mathrm{cm})=10-11 \mathrm{MeV}$; measured $\mathrm{E} \gamma, \mathrm{I} \gamma, \sigma$. Comparison with model predictions. JOUR JPGPE 31 S1417

2005HI04 NUCLEAR REACTIONS ${ }^{122} \mathrm{Te}(\mathrm{n}, \mathrm{n}$ '), $\mathrm{E}=1.72,2.80,3.35 \mathrm{MeV}$; measured $\mathrm{E} \gamma, \mathrm{I} \gamma$, DSA; deduced excitation functions. ${ }^{122} \mathrm{Te}$ deduced levels, $\mathrm{J}, \pi, \mathrm{T}_{1 / 2}, \mathrm{~B}(\mathrm{M} 1)$, B(E2). Comparison with interacting boson model predictions. JOUR PRVCA 71034307

2005HIZZ NUCLEAR REACTIONS ${ }^{122} \mathrm{Te}(\mathrm{n}, \mathrm{n}$ '), E=1.72, 2.80, $3.35 \mathrm{MeV}$; measured Doppler-shifted $\mathrm{E} \gamma, \mathrm{I} \gamma$, DSA. ${ }^{122}$ Te deduced levels, J, $\pi$, $\mathrm{T}_{1 / 2}, \mathrm{~B}(\mathrm{E} 1), \mathrm{B}(\mathrm{M} 1), \mathrm{B}(\mathrm{E} 2)$. PC S F Hicks, $1 / 3 / 2005$

${ }^{122} \mathrm{I}$ 2003MOZR NUCLEAR REACTIONS ${ }^{120} \mathrm{Sn}\left({ }^{7} \mathrm{Li}, 5 \mathrm{n}\right), \mathrm{E}=58 \mathrm{MeV}$; measured $\mathrm{E} \gamma$, $\mathrm{I} \gamma, \gamma \gamma$-coin. ${ }^{122} \mathrm{I}$ deduced high-spin levels, $\mathrm{J}, \pi$. Level systematics in neighboring isotopes discussed. REPT ANU-P/1564 2002

Annual,P13,Moon

2004MOZT NUCLEAR REACTIONS ${ }^{120} \mathrm{Sn}\left({ }^{7} \mathrm{Li}, 5 \mathrm{n}\right), \mathrm{E}=58 \mathrm{MeV}$; measured not given. ${ }^{122} \mathrm{I}$ deduced levels, J, $\pi$. PC C B Moon, $2 / 24 / 2004$

NUCLEAR REACTIONS ${ }^{64} \mathrm{Ni}\left({ }^{64} \mathrm{Ni}, 2 \mathrm{n}\right),\left({ }^{64} \mathrm{Ni}, 2 \mathrm{n} \alpha\right), \mathrm{E}=255,261$

$\mathrm{MeV}$; measured $\mathrm{E} \gamma, \mathrm{I} \gamma, \gamma \gamma$-, (particle) $\gamma$-coin. ${ }^{122} \mathrm{Xe}$ deduced possible hyperdeformed structure. Euroball IV and Diamant arrays. JOUR APOBB 361033

${ }^{122} \mathrm{Cs} 2005 \mathrm{KU} 34$ NUCLEAR REACTIONS ${ }^{107} \mathrm{Ag}\left({ }^{19} \mathrm{~F}, 3 \mathrm{np}\right), \mathrm{E}=93 \mathrm{MeV}$; measured $\mathrm{E} \gamma$, $\mathrm{I} \gamma, \gamma \gamma$-coin. ${ }^{122} \mathrm{Cs}$ deduced levels, $\mathrm{J}, \pi$, configurations. Comparison with model predictions. JOUR PRVCA 72044319

2005 UU01 NUCLEAR REACTIONS ${ }^{107} \mathrm{Ag}\left({ }^{19} \mathrm{~F}, 3 \mathrm{np}\right), \mathrm{E}=85 \mathrm{MeV}$; measured $\mathrm{E} \gamma$, $\mathrm{I} \gamma, \gamma \gamma$-coin. ${ }^{122} \mathrm{Cs}$ deduced high-spin levels, J, $\pi$, configurations, B(M1) / B(E2), chiral doublet bands. Level systematics in neighboring isotopes compared. JOUR JPGPE 31 B1

${ }^{122} \mathrm{Ce}$ 2005SM07 NUCLEAR REACTIONS ${ }^{64} \mathrm{Zn}\left({ }^{64} \mathrm{Zn}, 2 \mathrm{n} \alpha\right), \mathrm{E}=260 \mathrm{MeV}$; measured $\mathrm{E} \gamma, \mathrm{I} \gamma, \gamma \gamma_{-}, \mathrm{n} \gamma-$, (charged particle) $\gamma-$, (recoil) $\gamma$-coin. ${ }^{122} \mathrm{Ce}$ deduced levels, J, $\pi$, rotational band, angular distribution ratios, transition multipolarities, quadrupole deformation parameter. Microball and Gammasphere arrays, comparison with Woods-Saxon cranking predictions. JOUR PYLBB 625203 


\section{$\mathrm{A}=123$}

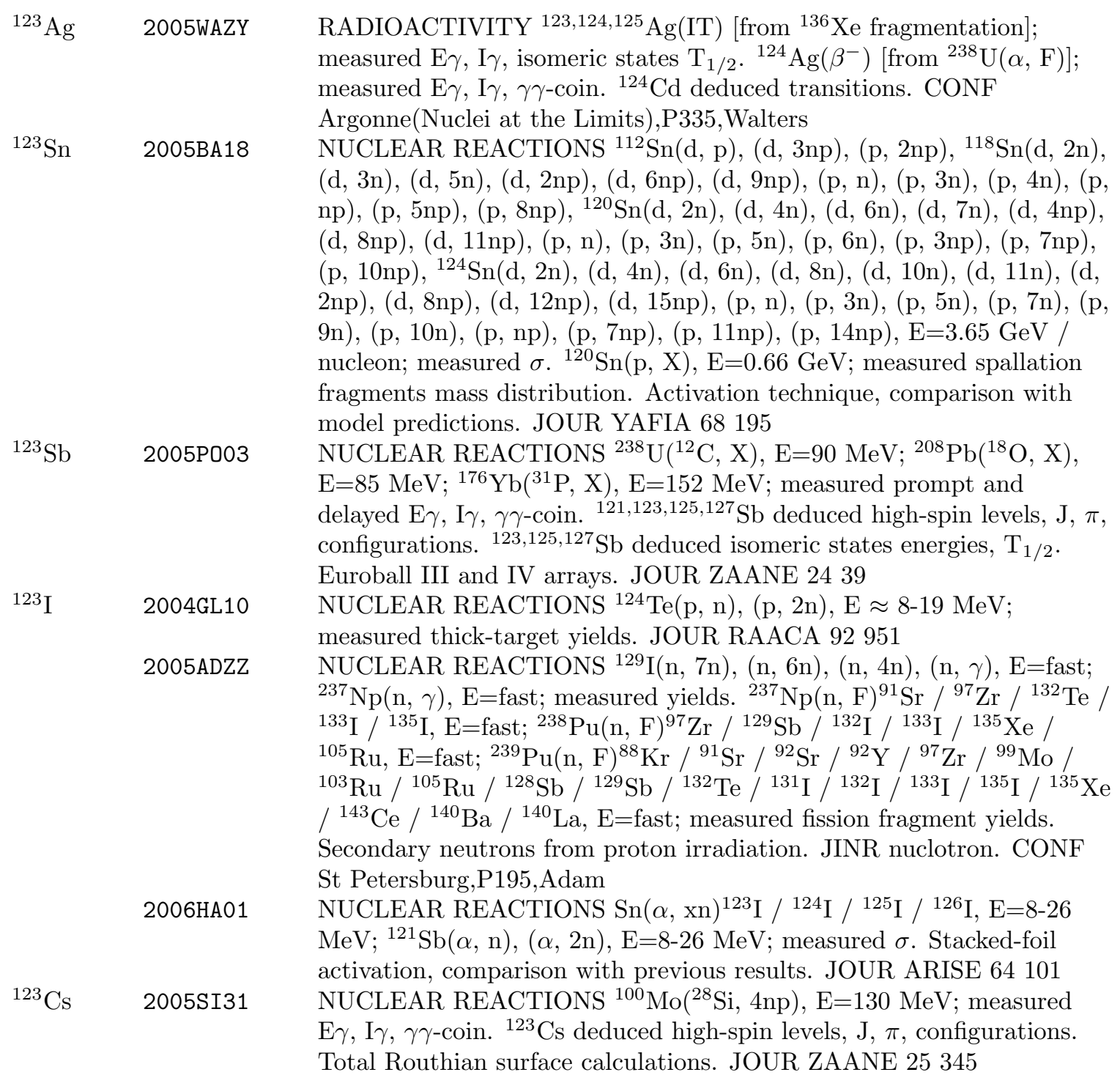

\section{$\mathrm{A}=\mathbf{1 2 4}$}

${ }^{124} \mathrm{Ag} \quad$ 2005KA45

RADIOACTIVITY ${ }^{124,126} \mathrm{Ag}\left(\beta^{-}\right)$; measured $\mathrm{E} \gamma, \mathrm{I} \gamma, \gamma \gamma$-coin following decay of mass-separated sources. ${ }^{124,126} \mathrm{Cd}$ deduced levels, J, $\pi$. Comparison with shell-model predictions. JOUR ZAANE 25 s01 117 2005WAZY RADIOACTIVITY ${ }^{123,124,125} \mathrm{Ag}$ (IT) [from ${ }^{136}$ Xe fragmentation]; measured $\mathrm{E} \gamma, \mathrm{I} \gamma$, isomeric states $\mathrm{T}_{1 / 2} \cdot{ }^{124} \mathrm{Ag}\left(\beta^{-}\right)\left[\right.$from $\left.{ }^{238} \mathrm{U}(\alpha, \mathrm{F})\right]$; measured $\mathrm{E} \gamma, \mathrm{I} \gamma, \gamma \gamma$-coin. ${ }^{124} \mathrm{Cd}$ deduced transitions. CONF Argonne(Nuclei at the Limits),P335, Walters 


\section{$\mathrm{A}=124$ (continued)}

${ }^{124} \mathrm{Cd}$ 2005BEzS NUCLEAR REACTIONS ${ }^{108} \mathrm{Pd}\left({ }^{122} \mathrm{Cd},{ }^{122} \mathrm{Cd}\right.$ ' $),{ }^{104} \mathrm{Pd}\left({ }^{124} \mathrm{Cd},{ }^{124} \mathrm{Cd}\right.$ ' $)$, $\left({ }^{126} \mathrm{Cd},{ }^{126} \mathrm{Cd}^{\prime}\right)$, E not given; measured $\mathrm{E} \gamma, \mathrm{I} \gamma$, (particle) $\gamma$-coin following projectile Coulomb excitation. ${ }^{122,124} \mathrm{Cd}$ levels deduced excitation B(E2). REPT MLL 2004 Annual,P14,Behrens

2005KA45 RADIOACTIVITY ${ }^{124,126} \mathrm{Ag}\left(\beta^{-}\right)$; measured $\mathrm{E} \gamma, \mathrm{I} \gamma, \gamma \gamma$-coin following decay of mass-separated sources. ${ }^{124,126} \mathrm{Cd}$ deduced levels, $\mathrm{J}, \pi$.

Comparison with shell-model predictions. JOUR ZAANE 25 s01 117

2005WAZY RADIOACTIVITY ${ }^{123,124,125} \mathrm{Ag}$ (IT) [from ${ }^{136}$ Xe fragmentation]; measured $\mathrm{E} \gamma, \mathrm{I} \gamma$, isomeric states $\mathrm{T}_{1 / 2} \cdot{ }^{124} \mathrm{Ag}\left(\beta^{-}\right)$[from $\left.{ }^{238} \mathrm{U}(\alpha, \mathrm{F})\right]$; measured $\mathrm{E} \gamma, \mathrm{I} \gamma, \gamma \gamma$-coin. ${ }^{124} \mathrm{Cd}$ deduced transitions. $\mathrm{CONF}$ Argonne(Nuclei at the Limits),P335, Walters

${ }^{124} \mathrm{Sn} \quad 2004 \mathrm{KU} 30 \quad$ NUCLEAR REACTIONS ${ }^{112,114,120,124} \mathrm{Sn}(\alpha, \alpha),\left(\alpha, \alpha^{\prime}\right), \mathrm{E} \approx 50 \mathrm{MeV}$ measured $\sigma(\mathrm{E}, \theta)$; deduced optical model parameters. ${ }^{112,114,120,124} \mathrm{Sn}$ deduced transition strengths, deformation parameters, related features. JOUR UKPJA 49841

2005BA02 NUCLEAR REACTIONS ${ }^{124} \mathrm{Sn}(\mathrm{n}, \mathrm{n} \gamma), \mathrm{E}=2.2-4.5 \mathrm{MeV}$; measured E $\gamma$, $\mathrm{I} \gamma, \gamma \gamma$-coin, excitation functions, angular distributions, DSA. ${ }^{124} \mathrm{Sn}$ deduced levels, $\mathrm{J}, \pi, \mathrm{T}_{1 / 2}$, two- and three-phonon excitations. JOUR NUPAB 747206

2005GA21 NUCLEAR REACTIONS ${ }^{112} \operatorname{Sn}(\alpha, \alpha), \mathrm{E}=14.4,19.5 \mathrm{MeV} ;{ }^{124} \operatorname{Sn}(\alpha, \alpha)$, $\mathrm{E}=19.5 \mathrm{MeV}$; measured elastic $\sigma(\theta)$; deduced optical potential parameters. ${ }^{112} \mathrm{Sn}(\alpha, \gamma), \mathrm{E}(\mathrm{cm})=7-11 \mathrm{MeV}$; calculated astrophysical S-factors, reaction rates. JOUR PRVCA 71065802

2005LU06 NUCLEAR REACTIONS ${ }^{110,116} \mathrm{Cd},{ }^{112,124} \mathrm{Sn}\left(\alpha, \alpha^{\prime}\right), \mathrm{E}=240 \mathrm{MeV}$; measured $\mathrm{E} \alpha, \sigma(\theta) .{ }^{110,116} \mathrm{Cd},{ }^{112,124} \mathrm{Sn}$ deduced electric monopole strength distributions, resonance parameters. Comparison with model predictions. JOUR APOBB 361107

2005 SI34 ATOMIC MASSES ${ }^{76,77,80,81,86,88} \mathrm{Sr},{ }^{124,129,130,131,132} \mathrm{Sn}$; measured masses. Penning trap mass spectrometer, comparison with previous results. JOUR NUPAB 76345

${ }^{124} \mathrm{Sb}$ 2005BA18 NUCLEAR REACTIONS ${ }^{112} \mathrm{Sn}(\mathrm{d}, \mathrm{p}),(\mathrm{d}, 3 \mathrm{np}),(\mathrm{p}, 2 \mathrm{np}),{ }^{118} \mathrm{Sn}(\mathrm{d}, 2 \mathrm{n})$, (d, 3n), (d, 5n), (d, 2np), (d, 6np), (d, 9np), (p, n), (p, 3n), (p, 4n), (p, np), (p, 5np), (p, 8np), ${ }^{120} \mathrm{Sn}(\mathrm{d}, 2 \mathrm{n}),(\mathrm{d}, 4 \mathrm{n}),(\mathrm{d}, 6 \mathrm{n}),(\mathrm{d}, 7 \mathrm{n}),(\mathrm{d}, 4 \mathrm{np})$, (d, 8np), (d, 11np), (p, n), (p, 3n), (p, 5n), (p, 6n), (p, 3np), (p, 7np), (p, 10np), ${ }^{124} \operatorname{Sn}(\mathrm{d}, 2 \mathrm{n}),(\mathrm{d}, 4 \mathrm{n}),(\mathrm{d}, 6 \mathrm{n}),(\mathrm{d}, 8 \mathrm{n}),(\mathrm{d}, 10 \mathrm{n}),(\mathrm{d}, 11 \mathrm{n}),(\mathrm{d}$, 2np), (d, 8np), (d, 12np), (d, 15np), (p, n), (p, 3n), (p, 5n), (p, 7n), (p, 9n), (p, 10n), (p, np), (p, 7np), (p, 11np), (p, 14np), E=3.65 GeV / nucleon; measured $\sigma .{ }^{120} \mathrm{Sn}(\mathrm{p}, \mathrm{X}), \mathrm{E}=0.66 \mathrm{GeV}$; measured spallation fragments mass distribution. Activation technique, comparison with model predictions. JOUR YAFIA 68195

${ }^{124} \mathrm{I}$ 2003MOZQ NUCLEAR REACTIONS ${ }^{122} \mathrm{Sn}\left({ }^{7} \mathrm{Li}, 5 \mathrm{n}\right)$, E not given; measured $\mathrm{E} \gamma$, I $\gamma, \gamma \gamma$-coin. ${ }^{124}$ I deduced high-spin levels, $\mathrm{J}, \pi$, configurations. REPT ANU-P/1564 2002 Annual,P15,Moon

2004GL10 NUCLEAR REACTIONS ${ }^{124} \mathrm{Te}(\mathrm{p}, \mathrm{n}),(\mathrm{p}, 2 \mathrm{n}), \mathrm{E} \approx 8-19 \mathrm{MeV}$; measured thick-target yields. JOUR RAACA 92951

2004SCZU NUCLEAR REACTIONS ${ }^{76} \mathrm{Se}(\mathrm{p}, \mathrm{n}), \mathrm{E} \approx 5-40 \mathrm{MeV} ;{ }^{78} \mathrm{Kr}(\mathrm{d}, \alpha), \mathrm{E} \approx$ 4-14 MeV; measured $\sigma .{ }^{126} \mathrm{Te}(\mathrm{p}, 3 \mathrm{n}), \mathrm{E}=8-70 \mathrm{MeV} ;{ }^{85} \mathrm{Rb}(\mathrm{p}, 4 \mathrm{n})$, $\mathrm{E}=44-66 \mathrm{MeV}$; measured yields. REPT NEA/NSC/DOC(2004)14,P13,Scholten 


\section{$\mathrm{A}=124$ (continued)}

2005ADZZ NUCLEAR REACTIONS ${ }^{129} \mathrm{I}(\mathrm{n}, 7 \mathrm{n}),(\mathrm{n}, 6 \mathrm{n}),(\mathrm{n}, 4 \mathrm{n}),(\mathrm{n}, \gamma), \mathrm{E}=$ fast; ${ }^{237} \mathrm{~Np}(\mathrm{n}, \gamma), \mathrm{E}=$ fast; measured yields. ${ }^{237} \mathrm{~Np}(\mathrm{n}, \mathrm{F}){ }^{91} \mathrm{Sr} /{ }^{97} \mathrm{Zr} /{ }^{132} \mathrm{Te} /$ ${ }^{133} \mathrm{I} /{ }^{135} \mathrm{I}, \mathrm{E}=$ fast; ${ }^{238} \mathrm{Pu}(\mathrm{n}, \mathrm{F}){ }^{97} \mathrm{Zr} /{ }^{129} \mathrm{Sb} /{ }^{132} \mathrm{I} /{ }^{133} \mathrm{I} /{ }^{135} \mathrm{Xe} /$ ${ }^{105} \mathrm{Ru}, \mathrm{E}=$ fast; ${ }^{239} \mathrm{Pu}(\mathrm{n}, \mathrm{F}){ }^{88} \mathrm{Kr} /{ }^{91} \mathrm{Sr} /{ }^{92} \mathrm{Sr} /{ }^{92} \mathrm{Y} /{ }^{97} \mathrm{Zr} /{ }^{99} \mathrm{Mo} /$ ${ }^{103} \mathrm{Ru} /{ }^{105} \mathrm{Ru} /{ }^{128} \mathrm{Sb} /{ }^{129} \mathrm{Sb} /{ }^{132} \mathrm{Te} /{ }^{131} \mathrm{I} /{ }^{132} \mathrm{I} /{ }^{133} \mathrm{I} /{ }^{135} \mathrm{I} /{ }^{135} \mathrm{Xe}$ $/{ }^{143} \mathrm{Ce} /{ }^{140} \mathrm{Ba} /{ }^{140} \mathrm{La}, \mathrm{E}=$ fast; measured fission fragment yields. Secondary neutrons from proton irradiation. JINR nuclotron. CONF St Petersburg,P195,Adam

2006HA01 NUCLEAR REACTIONS $\operatorname{Sn}(\alpha, \mathrm{xn}){ }^{123} \mathrm{I} /{ }^{124} \mathrm{I} /{ }^{125} \mathrm{I} /{ }^{126} \mathrm{I}, \mathrm{E}=8-26$ $\mathrm{MeV} ;{ }^{121} \mathrm{Sb}(\alpha, \mathrm{n}),(\alpha, 2 \mathrm{n}), \mathrm{E}=8-26 \mathrm{MeV}$; measured $\sigma$. Stacked-foil activation, comparison with previous results. JOUR ARISE 64101

${ }^{124} \mathrm{Xe} \quad$ 2005LE04

${ }^{124} \mathrm{Cs} \quad$ 2005GU37

${ }^{124} \mathrm{Ba} \quad$ 2005AL20

2005MA84

${ }^{124} \mathrm{Ce} \quad$ 2005XU04

2005WAZY

${ }^{125} \mathrm{Sn}$

2004J019

2005J023

2005JOZZ
NUCLEAR REACTIONS $\mathrm{Pb}(\mathrm{p}, \mathrm{X}){ }^{3} \mathrm{He} /{ }^{4} \mathrm{He} /{ }^{21} \mathrm{Ne} /{ }^{22} \mathrm{Ne} /{ }^{36} \mathrm{Ar} /$ ${ }^{38} \mathrm{Ar} /{ }^{78} \mathrm{Kr} /{ }^{80} \mathrm{Kr} /{ }^{81} \mathrm{Kr} /{ }^{82} \mathrm{Kr} /{ }^{83} \mathrm{Kr} /{ }^{84} \mathrm{Kr} /{ }^{85} \mathrm{Kr} /{ }^{86} \mathrm{Kr} /{ }^{124} \mathrm{Xe}$ $/{ }^{126} \mathrm{Xe} /{ }^{128} \mathrm{Xe} /{ }^{129} \mathrm{Xe} /{ }^{130} \mathrm{Xe} /{ }^{131} \mathrm{Xe} /{ }^{132} \mathrm{Xe} /{ }^{134} \mathrm{Xe}, \mathrm{E}=44-2595$ $\mathrm{MeV}$; measured production $\sigma$; deduced reaction mechanism features. Mini-stack approach, comparisons with model predictions. JOUR NIMBE 2291 ATOMIC MASSES ${ }^{56,57} \mathrm{Mn},{ }^{82 m} \mathrm{Rb},{ }^{92} \mathrm{Sr},{ }^{124,127} \mathrm{Cs},{ }^{130} \mathrm{Ba}$; measured masses. Penning trap mass spectrometer. JOUR ZAANE 25 s01 35 NUCLEAR REACTIONS ${ }^{64} \mathrm{Ni}\left({ }^{64} \mathrm{Ni}, 4 \mathrm{n}\right), \mathrm{E}=255,261 \mathrm{MeV}$; measured $\mathrm{E} \gamma, \mathrm{I} \gamma, \gamma \gamma$-coin. ${ }^{124} \mathrm{Ba}$ deduced high-spin levels, J, $\pi$. Euroball IV and Diamant arrays. JOUR APOBB 361029

NUCLEAR REACTIONS ${ }^{64} \mathrm{Ni}\left({ }^{64} \mathrm{Ni}, 3 \mathrm{n}\right),\left({ }^{64} \mathrm{Ni}, 4 \mathrm{n}\right), \mathrm{E}=255-261 \mathrm{MeV}$; measured $\mathrm{E} \gamma, \mathrm{I} \gamma, \gamma \gamma$-coin. ${ }^{124,125} \mathrm{Ba}$ deduced levels, J, $\pi$, octupole correlations. Euroball and Diamant arrays. JOUR JPGPE 31 S1729 RADIOACTIVITY ${ }^{81} \mathrm{Zr},{ }^{85} \mathrm{Mo},{ }^{89} \mathrm{Ru},{ }^{92} \mathrm{Rh},{ }^{93} \mathrm{Pd},{ }^{121} \mathrm{Ce},{ }^{125} \mathrm{Nd}$, ${ }^{128} \mathrm{Pm},{ }^{129} \mathrm{Sm},{ }^{135,137} \mathrm{Gd},{ }^{139} \mathrm{Dy},{ }^{142} \mathrm{Ho},{ }^{149} \mathrm{Yb}\left(\beta^{+} \mathrm{p}\right)$; measured $\beta$-delayed $\mathrm{E} \gamma, \mathrm{I} \gamma$, proton spectra, $\mathrm{p} \gamma$-coin, $\mathrm{T}_{1 / 2}$. Comparison with model predictions. JOUR PRVCA 71054318

\section{$\mathrm{A}=125$}

RADIOACTIVITY ${ }^{123,124,125} \mathrm{Ag}$ (IT) [from ${ }^{136}$ Xe fragmentation]; measured $\mathrm{E} \gamma, \mathrm{I} \gamma$, isomeric states $\mathrm{T}_{1 / 2} \cdot{ }^{124} \mathrm{Ag}\left(\beta^{-}\right)$[from $\left.{ }^{238} \mathrm{U}(\alpha, \mathrm{F})\right]$; measured $\mathrm{E} \gamma, \mathrm{I} \gamma, \gamma \gamma$-coin. ${ }^{124} \mathrm{Cd}$ deduced transitions. CONF Argonne(Nuclei at the Limits),P335, Walters NUCLEAR REACTIONS ${ }^{2} \mathrm{H}\left({ }^{124} \mathrm{Sn}, \mathrm{p}\right), \mathrm{E}=562 \mathrm{MeV}$; measured $\mathrm{Ep}$, $\sigma(\theta) .{ }^{125} \mathrm{Sn}$ deduced levels, spectroscopic factors. DWBA analysis. Comparison with previous results. JOUR PRVCA 70067602 NUCLEAR REACTIONS ${ }^{2} \mathrm{H}\left({ }^{124} \mathrm{Sn}, \mathrm{p}\right), \mathrm{E}=4.5 \mathrm{MeV} /$ nucleon; measured $\sigma(\theta) .{ }^{125} \mathrm{Sn}$ levels deduced spectroscopic factors. DWBA analysis. JOUR ZAANE 25 s01 283

NUCLEAR REACTIONS ${ }^{2} \mathrm{H}\left({ }^{82} \mathrm{Ge}, \mathrm{p}\right),\left({ }^{84} \mathrm{Se}, \mathrm{p}\right), \mathrm{E}=4 \mathrm{MeV} /$ nucleon; measured $\sigma(\mathrm{E}, \theta) .{ }^{83} \mathrm{Ge},{ }^{85} \mathrm{Se}$ deduced ground and excited states energies, L. ${ }^{2} \mathrm{H}\left({ }^{124} \mathrm{Sn}, \mathrm{p}\right), \mathrm{E}=562 \mathrm{MeV}$; measured $\sigma(\mathrm{E}, \theta) .{ }^{125} \mathrm{Sn}$ levels deduced spectroscopic factors. CONF Argonne(Nuclei at the Limits),P176,Jones 


\section{$\mathrm{A}=125$ (continued)}

2005LE34 NUCLEAR MOMENTS

$125,125 m, 126,127,127 m, 128,129,129 m, 130,130 m, 131,131 m, 132 \mathrm{Sn}$; measured isotope shifts; deduced charge radii, dynamical effects.

$125,125 m, 127,127 m, 129,129 m, 130 m, 131,131 m \mathrm{Sn}$; measured $\mu$, quadrupole moments. Laser spectroscopy, mean-field calculations. JOUR PRVCA 72034305

2005 TH09 NUCLEAR REACTIONS ${ }^{2} \mathrm{H}\left({ }^{84} \mathrm{Se}, \mathrm{p}\right),\left({ }^{124} \mathrm{Sn}, \mathrm{p}\right), \mathrm{E}=4.5 \mathrm{MeV} /$ nucleon; measured recoil proton spectra, $\sigma(\mathrm{E}, \theta) .{ }^{85} \mathrm{Se},{ }^{125} \mathrm{Sn}$ deduced levels, J, $\pi$. JOUR NUPAB 758 663c

${ }^{125} \mathrm{Sb} \quad 2005 \mathrm{JU} 12$ NUCLEAR REACTIONS ${ }^{124} \mathrm{Sn}\left({ }^{7} \mathrm{Li}, 2 \mathrm{n} \alpha\right), \mathrm{E}=37 \mathrm{MeV}$; measured delayed $\mathrm{E} \gamma, \mathrm{I} \gamma, \mathrm{E}(\mathrm{ce}), \mathrm{I}(\mathrm{ce}) .{ }^{125} \mathrm{Sb}$ deduced levels, J, $\pi$, configurations, isomeric states $\mathrm{T}_{1 / 2}$, ICC. Level systematics in neighboring isotopes compared. JOUR JPGPE 31 S1899

$2005 \mathrm{P003}$ NUCLEAR REACTIONS ${ }^{238} \mathrm{U}\left({ }^{12} \mathrm{C}, \mathrm{X}\right), \mathrm{E}=90 \mathrm{MeV} ;{ }^{208} \mathrm{~Pb}\left({ }^{18} \mathrm{O}, \mathrm{X}\right)$, $\mathrm{E}=85 \mathrm{MeV} ;{ }^{176} \mathrm{Yb}\left({ }^{31} \mathrm{P}, \mathrm{X}\right), \mathrm{E}=152 \mathrm{MeV}$; measured prompt and delayed $\mathrm{E} \gamma, \mathrm{I} \gamma, \gamma \gamma$-coin. ${ }^{121,123,125,127} \mathrm{Sb}$ deduced high-spin levels, J, $\pi$, configurations. ${ }^{123,125,127} \mathrm{Sb}$ deduced isomeric states energies, $\mathrm{T}_{1 / 2}$. Euroball III and IV arrays. JOUR ZAANE 2439

${ }^{125} \mathrm{Te} \quad 2004 \mathrm{G059}$

$2005 P 009$

2005VIZZ

${ }^{125} \mathrm{I}$

2006HA01

${ }^{125} \mathrm{Xe} \quad 2005 \mathrm{HAZW}$

${ }^{125} \mathrm{Ba} \quad 2005 \mathrm{MA} 84$

${ }^{125} \mathrm{Nd} \quad 2005 \mathrm{XU} 04$

2005XU04
RADIOACTIVITY ${ }^{125 m} \mathrm{Te}(\mathrm{IT})$; measured $\mathrm{T}_{1 / 2}$, non-exponential decay features. JOUR BRSPE 681335

RADIOACTIVITY ${ }^{125} \mathrm{I}(\mathrm{EC})$; measured E $\gamma$, electron and X-ray spectra, sum energy spectra. JOUR NIMAE 544584

RADIOACTIVITY ${ }^{115} \mathrm{Cd}\left(\beta^{-}\right) ;{ }^{117 m} \mathrm{Sn},{ }^{125 m} \mathrm{Te}(\mathrm{IT})$; measured $\mathrm{I} \gamma$, (X-ray) $\gamma$-coin. ${ }^{115} \mathrm{In},{ }^{117} \mathrm{Sn},{ }^{125} \mathrm{Te}$ transitions deduced ICC. CONF St Petersburg,P65,Vishnevsky

RADIOACTIVITY ${ }^{125} \mathrm{I}(\mathrm{EC})$; measured $\mathrm{E} \gamma$, electron and X-ray spectra, sum energy spectra. JOUR NIMAE 544584

NUCLEAR REACTIONS Sn $(\alpha, \mathrm{xn}){ }^{123} \mathrm{I} /{ }^{124} \mathrm{I} /{ }^{125} \mathrm{I} /{ }^{126} \mathrm{I}, \mathrm{E}=8-26$ $\mathrm{MeV} ;{ }^{121} \mathrm{Sb}(\alpha, \mathrm{n}),(\alpha, 2 \mathrm{n}), \mathrm{E}=8-26 \mathrm{MeV}$; measured $\sigma$. Stacked-foil activation, comparison with previous results. JOUR ARISE 64101 NUCLEAR REACTIONS ${ }^{82} \mathrm{Se}\left({ }^{48} \mathrm{Ca}, 4 \mathrm{n}\right),\left({ }^{48} \mathrm{Ca}, 5 \mathrm{n}\right), \mathrm{E}=185,195,205$ $\mathrm{MeV}$; measured $\mathrm{E} \gamma, \mathrm{I} \gamma, \gamma \gamma$-coin. ${ }^{126} \mathrm{Xe}$ deduced high-spin levels, J, $\pi$, deformation. Euroball, Gammasphere arrays, potential energy surface calculations. CONF Argonne(Nuclei at the Limits),P46,Hansen NUCLEAR REACTIONS ${ }^{64} \mathrm{Ni}\left({ }^{64} \mathrm{Ni}, 3 \mathrm{n}\right),\left({ }^{64} \mathrm{Ni}, 4 \mathrm{n}\right), \mathrm{E}=255-261 \mathrm{MeV}$; measured $\mathrm{E} \gamma, \mathrm{I} \gamma, \gamma \gamma$-coin. ${ }^{124,125} \mathrm{Ba}$ deduced levels, J, $\pi$, octupole correlations. Euroball and Diamant arrays. JOUR JPGPE 31 S1729 RADIOACTIVITY ${ }^{81} \mathrm{Zr},{ }^{85} \mathrm{Mo},{ }^{89} \mathrm{Ru},{ }^{92} \mathrm{Rh},{ }^{93} \mathrm{Pd},{ }^{121} \mathrm{Ce},{ }^{125} \mathrm{Nd}$, ${ }^{128} \mathrm{Pm},{ }^{129} \mathrm{Sm},{ }^{135,137} \mathrm{Gd},{ }^{139} \mathrm{Dy},{ }^{142} \mathrm{Ho},{ }^{149} \mathrm{Yb}\left(\beta^{+} \mathrm{p}\right)$; measured $\beta$-delayed $\mathrm{E} \gamma, \mathrm{I} \gamma$, proton spectra, $\mathrm{p} \gamma$-coin, $\mathrm{T}_{1 / 2}$. Comparison with model predictions. JOUR PRVCA 71054318 NUCLEAR REACTIONS ${ }^{92} \mathrm{Mo},{ }^{106} \mathrm{Cd}\left({ }^{32} \mathrm{~S}, 3 \mathrm{n}\right), \mathrm{E}=151 \mathrm{MeV}$; ${ }^{92} \mathrm{Mo}\left({ }^{36} \mathrm{Ar}, 3 \mathrm{n}\right), \mathrm{E}=169 \mathrm{MeV} ;{ }^{96} \mathrm{Ru}\left({ }^{36} \mathrm{Ar}, 3 \mathrm{n}\right)$, ( $\left.{ }^{36} \mathrm{Ar}, 3 \mathrm{np}\right), \mathrm{E}=165,174$ $\mathrm{MeV} ;{ }^{106} \mathrm{Cd}\left({ }^{36} \mathrm{Ar}, 3 \mathrm{n}\right),\left({ }^{36} \mathrm{Ar}, \mathrm{n} \alpha\right), \mathrm{E}=176 \mathrm{MeV} ;{ }^{106} \mathrm{Cd}\left({ }^{40} \mathrm{Ca}, 4 \mathrm{n}\right)$, $\mathrm{E}=202 \mathrm{MeV} ;{ }^{112} \mathrm{Sn}\left({ }^{40} \mathrm{Ca}, 3 \mathrm{n}\right), \mathrm{E}=185 \mathrm{MeV}$; measured $\sigma$. JOUR PRVCA 71054318 


\section{$\mathrm{A}=126$}

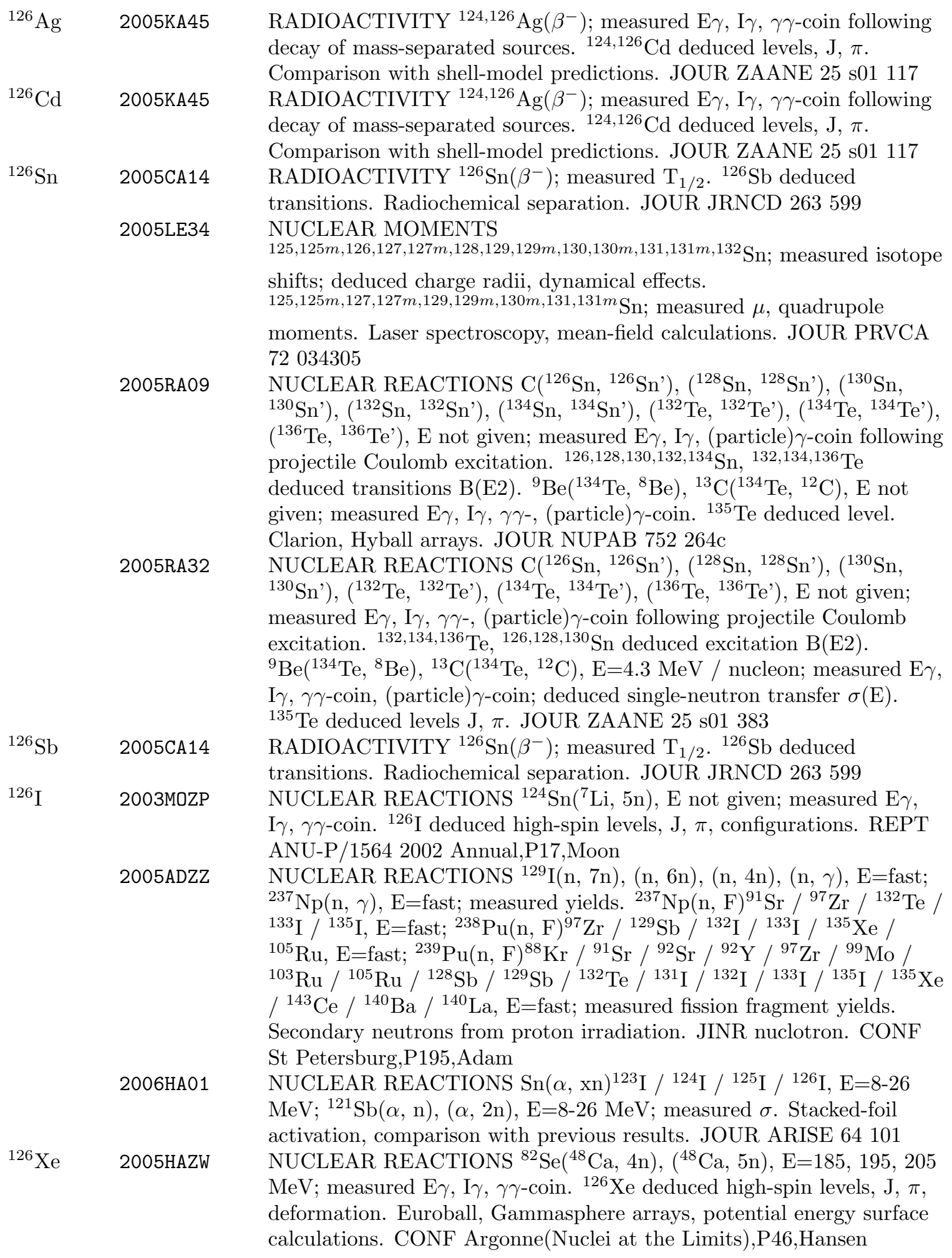




\section{$\mathrm{A}=126$ (continued)}

2005LE04 NUCLEAR REACTIONS Pb(p, X) ${ }^{3} \mathrm{He} /{ }^{4} \mathrm{He} /{ }^{21} \mathrm{Ne} /{ }^{22} \mathrm{Ne} /{ }^{36} \mathrm{Ar} /$ ${ }^{38} \mathrm{Ar} /{ }^{78} \mathrm{Kr} /{ }^{80} \mathrm{Kr} /{ }^{81} \mathrm{Kr} /{ }^{82} \mathrm{Kr} /{ }^{83} \mathrm{Kr} /{ }^{84} \mathrm{Kr} /{ }^{85} \mathrm{Kr} /{ }^{86} \mathrm{Kr} /{ }^{124} \mathrm{Xe}$ $/{ }^{126} \mathrm{Xe} /{ }^{128} \mathrm{Xe} /{ }^{129} \mathrm{Xe} /{ }^{130} \mathrm{Xe} /{ }^{131} \mathrm{Xe} /{ }^{132} \mathrm{Xe} /{ }^{134} \mathrm{Xe}, \mathrm{E}=44-2595$ $\mathrm{MeV}$; measured production $\sigma$; deduced reaction mechanism features. Mini-stack approach, comparisons with model predictions. JOUR NIMBE 2291

$\begin{array}{ccl}{ }^{126} \mathrm{Cs} & \text { 2005PI08 } & \text { NUCLEAR MOMENTS }{ }^{126} \mathrm{Cs} ; \text { measured hfs; deduced } \mu . \\ & & \text { Bohr-Weisskopf effect. Atomic beam magnetic resonance. JOUR } \\ & \text { NUPAB } 7533 \\ & \text { NUCLEAR REACTIONS }{ }^{64} \mathrm{Ni}\left({ }^{64} \mathrm{Ni}, 2 \mathrm{n}\right),\left({ }^{64} \mathrm{Ni}, 2 \mathrm{n} \alpha\right), \mathrm{E}=255,261 \\ { }^{126} \mathrm{Ba} & \text { 2005NY02 } & \text { MeV; measured } \mathrm{E} \gamma, \mathrm{I} \gamma, \gamma \gamma-,(\text { particle }) \gamma \text {-coin. }{ }^{122} \mathrm{Xe} \text { deduced possible } \\ & & \text { hyperdeformed structure. Euroball IV and Diamant arrays. JOUR } \\ & \text { APOBB } 361033\end{array}$

\section{$\mathrm{A}=127$}

${ }^{127} \mathrm{Sn}$

2005LE34

${ }^{127} \mathrm{Sb}$

2005P003

${ }^{127} \mathrm{Te}$

$2005 \mathrm{HO} 15$

${ }^{127} \mathrm{I}$

2005BE03

2005BE17

${ }^{127} \mathrm{Cs} \quad$ 2005GU37

${ }^{127} \mathrm{La}$

2005 II01

${ }^{127} \mathrm{Ce} \quad$ 2003WIZU

\section{NUCLEAR MOMENTS}

$125,125 m, 126,127,127 m, 128,129,129 m, 130,130 m, 131,131 m, 132 \mathrm{Sn}$; measured isotope

shifts; deduced charge radii, dynamical effects.

$125,125 m, 127,127 m, 129,129 m, 130 m, 131,131 m \mathrm{Sn}$; measured $\mu$, quadrupole moments. Laser spectroscopy, mean-field calculations. JOUR PRVCA 72034305

NUCLEAR REACTIONS ${ }^{238} \mathrm{U}\left({ }^{12} \mathrm{C}, \mathrm{X}\right), \mathrm{E}=90 \mathrm{MeV} ;{ }^{208} \mathrm{~Pb}\left({ }^{18} \mathrm{O}, \mathrm{X}\right)$, $\mathrm{E}=85 \mathrm{MeV} ;{ }^{176} \mathrm{Yb}\left({ }^{31} \mathrm{P}, \mathrm{X}\right), \mathrm{E}=152 \mathrm{MeV}$; measured prompt and delayed $\mathrm{E} \gamma, \mathrm{I} \gamma, \gamma \gamma$-coin. ${ }^{121,123,125,127} \mathrm{Sb}$ deduced high-spin levels, J, $\pi$, configurations. ${ }^{123,125,127} \mathrm{Sb}$ deduced isomeric states energies, $\mathrm{T}_{1 / 2}$. Euroball III and IV arrays. JOUR ZAANE 2439

NUCLEAR REACTIONS ${ }^{126} \mathrm{Te}(\mathrm{n}, \gamma), \mathrm{E}=$ thermal; measured $\mathrm{E} \gamma, \mathrm{I} \gamma$, $\gamma \gamma$-coin. ${ }^{126} \mathrm{Te}$ (polarized d, p), $\mathrm{E}=20 \mathrm{MeV}$; measured proton spectra, $\sigma(\theta), \operatorname{Ay}(\theta) .{ }^{127}$ Te deduced levels, J, $\pi, \gamma$-branching ratios, binding energy, spectroscopic factors. DWBA and coupled-channels analysis, interacting boson-fermion and quasiparticle phonon model calculations. JOUR NUPAB 756249

RADIOACTIVITY ${ }^{23} \mathrm{Na},{ }^{127} \mathrm{I}$; measured $\mathrm{T}_{1 / 2}$ lower limits for spontaneous decay to superdense state; deduced potential barrier features. NaI detectors. JOUR ZAANE 237

RADIOACTIVITY ${ }^{127} \mathrm{I}\left({ }^{24} \mathrm{Ne}\right),\left({ }^{28} \mathrm{Mg}\right),\left({ }^{30} \mathrm{Mg}\right),\left({ }^{32} \mathrm{Si}\right),\left({ }^{34} \mathrm{Si}\right),\left({ }^{48} \mathrm{Ca}\right)$, $\left({ }^{49} \mathrm{Sc}\right)$; measured cluster decay $\mathrm{T}_{1 / 2}$ lower limits. JOUR ZAANE 2451 ATOMIC MASSES ${ }^{56,57} \mathrm{Mn},{ }^{82 m} \mathrm{Rb},{ }^{92} \mathrm{Sr},{ }^{124,127} \mathrm{Cs},{ }^{130} \mathrm{Ba}$; measured masses. Penning trap mass spectrometer. JOUR ZAANE 25 s01 35 RADIOACTIVITY ${ }^{127} \mathrm{Ce}\left(\beta^{+}\right)$, (EC) [from $\left.\mathrm{Mo}\left({ }^{35} \mathrm{Cl}, \mathrm{xnp}\right)\right]$; measured $\mathrm{E} \gamma, \mathrm{I} \gamma, \mathrm{E}(\mathrm{ce}), \mathrm{I}(\mathrm{ce}), \beta \gamma-, \gamma \gamma$-coin, $\mathrm{T}_{1 / 2} .{ }^{127} \mathrm{La}$ deduced levels, J, $\pi$, $\mathrm{T}_{1 / 2}$, configurations. Comparison with Nilsson model predictions. JOUR ZAANE 2333

NUCLEAR REACTIONS ${ }^{106} \mathrm{Cd}\left({ }^{24} \mathrm{Mg}\right.$, n2p), E not given; measured $\mathrm{E} \gamma, \mathrm{I} \gamma, \gamma \gamma-$, (charged particle) $\gamma$-coin. ${ }^{127} \mathrm{Ce}$ deduced high-spin levels, J, $\pi$. REPT ANU-P/1564 2002 Annual,P18,Wilson 


\section{$\mathrm{A}=127$ (continued)}

2005 II01 RADIOACTIVITY ${ }^{127} \mathrm{Ce}\left(\beta^{+}\right)$, (EC) [from $\mathrm{Mo}\left({ }^{35} \mathrm{Cl}\right.$, xnp)]; measured $\mathrm{E} \gamma, \mathrm{I} \gamma, \mathrm{E}(\mathrm{ce}), \mathrm{I}(\mathrm{ce}), \beta \gamma-, \gamma \gamma$-coin, $\mathrm{T}_{1 / 2} .{ }^{127} \mathrm{La}$ deduced levels, J, $\pi$, $\mathrm{T}_{1 / 2}$, configurations. Comparison with Nilsson model predictions. JOUR ZAANE 2333

${ }^{127} \mathrm{Pr}$ 2005XU04 RADIOACTIVITY ${ }^{81} \mathrm{Zr},{ }^{85} \mathrm{Mo},{ }^{89} \mathrm{Ru},{ }^{92} \mathrm{Rh},{ }^{93} \mathrm{Pd},{ }^{121} \mathrm{Ce},{ }^{125} \mathrm{Nd}$, ${ }^{128} \mathrm{Pm},{ }^{129} \mathrm{Sm},{ }^{135,137} \mathrm{Gd},{ }^{139} \mathrm{Dy},{ }^{142} \mathrm{Ho},{ }^{149} \mathrm{Yb}\left(\beta^{+} \mathrm{p}\right)$; measured $\beta$-delayed $\mathrm{E} \gamma, \mathrm{I} \gamma$, proton spectra, $\mathrm{p} \gamma$-coin, $\mathrm{T}_{1 / 2}$. Comparison with model predictions. JOUR PRVCA 71054318

\section{$\mathrm{A}=128$}

\section{${ }^{128} \mathrm{Sn} \quad$ 2005LE34 NUCLEAR MOMENTS}

$125,125 m, 126,127,127 m, 128,129,129 m, 130,130 m, 131,131 m, 132 \mathrm{Sn}$; measured isotope shifts; deduced charge radii, dynamical effects.

$125,125 m, 127,127 m, 129,129 m, 130 m, 131,131 m \mathrm{Sn}$; measured $\mu$, quadrupole moments. Laser spectroscopy, mean-field calculations. JOUR PRVCA 72034305

2005RA09 NUCLEAR REACTIONS C( $\left.{ }^{126} \mathrm{Sn},{ }^{126} \mathrm{Sn}\right),\left({ }^{128} \mathrm{Sn},{ }^{128} \mathrm{Sn}\right),\left({ }^{130} \mathrm{Sn}\right.$, ${ }^{130} \mathrm{Sn}$ '), ( ${ }^{132} \mathrm{Sn},{ }^{132} \mathrm{Sn}$ '), ( ${ }^{134} \mathrm{Sn},{ }^{134} \mathrm{Sn}$ '), ( $\left.{ }^{132} \mathrm{Te},{ }^{132} \mathrm{Te}\right),\left({ }^{134} \mathrm{Te},{ }^{134} \mathrm{Te}\right.$ '), $\left({ }^{136} \mathrm{Te},{ }^{136} \mathrm{Te}\right)$, E not given; measured $\mathrm{E} \gamma, \mathrm{I} \gamma$, (particle) $\gamma$-coin following projectile Coulomb excitation. ${ }^{126,128,130,132,134} \mathrm{Sn},{ }^{132,134,136} \mathrm{Te}$ deduced transitions $\mathrm{B}(\mathrm{E} 2) .{ }^{9} \mathrm{Be}\left({ }^{134} \mathrm{Te},{ }^{8} \mathrm{Be}\right),{ }^{13} \mathrm{C}\left({ }^{134} \mathrm{Te},{ }^{12} \mathrm{C}\right)$, E not given; measured $\mathrm{E} \gamma, \mathrm{I} \gamma, \gamma \gamma$-, (particle) $\gamma$-coin. ${ }^{135} \mathrm{Te}$ deduced level. Clarion, Hyball arrays. JOUR NUPAB 752 264c

2005RA32 NUCLEAR REACTIONS C $\left({ }^{126} \mathrm{Sn},{ }^{126} \mathrm{Sn}\right),\left({ }^{128} \mathrm{Sn},{ }^{128} \mathrm{Sn}\right),\left({ }^{130} \mathrm{Sn}\right.$, ${ }^{130} \mathrm{Sn}$ '), ( ${ }^{132} \mathrm{Te},{ }^{132} \mathrm{Te}$ '), ( ${ }^{134} \mathrm{Te},{ }^{134} \mathrm{Te}$ '), ( ${ }^{136} \mathrm{Te},{ }^{136} \mathrm{Te}$ '), E not given; measured $\mathrm{E} \gamma, \mathrm{I} \gamma, \gamma \gamma$-, (particle) $\gamma$-coin following projectile Coulomb excitation. ${ }^{132,134,136} \mathrm{Te},{ }^{126,128,130} \mathrm{Sn}$ deduced excitation $\mathrm{B}(\mathrm{E} 2)$.

${ }^{9} \mathrm{Be}\left({ }^{134} \mathrm{Te},{ }^{8} \mathrm{Be}\right),{ }^{13} \mathrm{C}\left({ }^{134} \mathrm{Te},{ }^{12} \mathrm{C}\right), \mathrm{E}=4.3 \mathrm{MeV} /$ nucleon; measured $\mathrm{E} \gamma$, $\mathrm{I} \gamma, \gamma \gamma$-coin, (particle) $\gamma$-coin; deduced single-neutron transfer $\sigma(\mathrm{E})$.

${ }^{135}$ Te deduced levels J, $\pi$. JOUR ZAANE 25 s01 383

${ }^{128} \mathrm{Sb} \quad 2005 \mathrm{ADZZ}$ NUCLEAR REACTIONS ${ }^{129} \mathrm{I}(\mathrm{n}, 7 \mathrm{n})$, (n, 6n), (n, 4n), (n, $\left.\gamma\right), \mathrm{E}=$ fast; ${ }^{237} \mathrm{~Np}(\mathrm{n}, \gamma), \mathrm{E}=$ fast; measured yields. ${ }^{237} \mathrm{~Np}(\mathrm{n}, \mathrm{F}){ }^{91} \mathrm{Sr} /{ }^{97} \mathrm{Zr} /{ }^{132} \mathrm{Te} /$ ${ }^{133} \mathrm{I} /{ }^{135} \mathrm{I}, \mathrm{E}=$ fast; ${ }^{238} \mathrm{Pu}(\mathrm{n}, \mathrm{F}){ }^{97} \mathrm{Zr} /{ }^{129} \mathrm{Sb} /{ }^{132} \mathrm{I} /{ }^{133} \mathrm{I} /{ }^{135} \mathrm{Xe} /$ ${ }^{105} \mathrm{Ru}, \mathrm{E}=$ fast; ${ }^{239} \mathrm{Pu}(\mathrm{n}, \mathrm{F}){ }^{88} \mathrm{Kr} /{ }^{91} \mathrm{Sr} /{ }^{92} \mathrm{Sr} /{ }^{92} \mathrm{Y} /{ }^{97} \mathrm{Zr} /{ }^{99} \mathrm{Mo} /$ ${ }^{103} \mathrm{Ru} /{ }^{105} \mathrm{Ru} /{ }^{128} \mathrm{Sb} /{ }^{129} \mathrm{Sb} /{ }^{132} \mathrm{Te} /{ }^{131} \mathrm{I} /{ }^{132} \mathrm{I} /{ }^{133} \mathrm{I} /{ }^{135} \mathrm{I} /{ }^{135} \mathrm{Xe}$ $/{ }^{143} \mathrm{Ce} /{ }^{140} \mathrm{Ba} /{ }^{140} \mathrm{La}, \mathrm{E}=$ fast; measured fission fragment yields. Secondary neutrons from proton irradiation. JINR nuclotron. CONF St Petersburg,P195,Adam

2005NA05 NUCLEAR REACTIONS ${ }^{232} \mathrm{Th},{ }^{232,238} \mathrm{U},{ }^{238,240} \mathrm{Pu},{ }^{244} \mathrm{Cm}(\mathrm{n}, \mathrm{F}){ }^{128} \mathrm{Sb}$ / ${ }^{130} \mathrm{Sb} /{ }^{132} \mathrm{Sb} /{ }^{131} \mathrm{Te} /{ }^{133} \mathrm{Te} /{ }^{132} \mathrm{I} /{ }^{134} \mathrm{I} /{ }^{136} \mathrm{I} /{ }^{135} \mathrm{Xe} /{ }^{138} \mathrm{Cs}$, $\mathrm{E}=$ thermal, fast; measured isomer yield ratios; deduced fission fragment angular momenta. Spin-dependent statistical model analysis. JOUR PRVCA 71014304 


\section{$\mathrm{A}=128$ (continued)}

${ }^{128} \mathrm{Xe}$ 2005LE04 NUCLEAR REACTIONS $\mathrm{Pb}(\mathrm{p}, \mathrm{X}){ }^{3} \mathrm{He} /{ }^{4} \mathrm{He} /{ }^{21} \mathrm{Ne} /{ }^{22} \mathrm{Ne} /{ }^{36} \mathrm{Ar} /$ ${ }^{38} \mathrm{Ar} /{ }^{78} \mathrm{Kr} /{ }^{80} \mathrm{Kr} /{ }^{81} \mathrm{Kr} /{ }^{82} \mathrm{Kr} /{ }^{83} \mathrm{Kr} /{ }^{84} \mathrm{Kr} /{ }^{85} \mathrm{Kr} /{ }^{86} \mathrm{Kr} /{ }^{124} \mathrm{Xe}$ $/{ }^{126} \mathrm{Xe} /{ }^{128} \mathrm{Xe} /{ }^{129} \mathrm{Xe} /{ }^{130} \mathrm{Xe} /{ }^{131} \mathrm{Xe} /{ }^{132} \mathrm{Xe} /{ }^{134} \mathrm{Xe}, \mathrm{E}=44-2595$ $\mathrm{MeV}$; measured production $\sigma$; deduced reaction mechanism features. Mini-stack approach, comparisons with model predictions. JOUR NIMBE 2291

${ }^{128} \mathrm{Cs}$ 2005GR10 NUCLEAR REACTIONS ${ }^{122} \mathrm{Sn}\left({ }^{14} \mathrm{~N}, 4 \mathrm{n}\right), \mathrm{E}=70 \mathrm{MeV} ;{ }^{122} \mathrm{Sn}\left({ }^{10} \mathrm{~B}, 4 \mathrm{n}\right)$, $\mathrm{E}=55 \mathrm{MeV}$; measured $\mathrm{E} \gamma, \mathrm{I} \gamma, \gamma \gamma$-coin, DSA. ${ }^{128} \mathrm{Cs},{ }^{132} \mathrm{La}$ levels deduced $\mathrm{T}_{1 / 2}, \mathrm{~B}(\mathrm{E} 2), \mathrm{B}(\mathrm{M} 1)$, chirality. Osiris II array. JOUR IMPEE 14347

2005SR02 NUCLEAR REACTIONS ${ }^{122} \mathrm{Sn}\left({ }^{14} \mathrm{~N}, 4 \mathrm{n}\right), \mathrm{E}=70 \mathrm{MeV} ;{ }^{122} \mathrm{Sn}\left({ }^{10} \mathrm{~B}, 4 \mathrm{n}\right)$, $\mathrm{E}=55 \mathrm{MeV}$; measured $\mathrm{E} \gamma, \mathrm{I} \gamma, \gamma \gamma$-coin, DSA. ${ }^{132} \mathrm{La},{ }^{128} \mathrm{Cs}$ deduced levels, $\mathrm{J}, \pi, \mathrm{T}_{1 / 2}$, rotational bands, intraband $\mathrm{B}(\mathrm{M} 1), \mathrm{B}(\mathrm{E} 2) .{ }^{128} \mathrm{Cs}$ deduced possible chiral bands. Osiris II array. JOUR APOBB 361063 ${ }^{128} \mathrm{Nd}$ 2005XU04 RADIOACTIVITY ${ }^{81} \mathrm{Zr},{ }^{85} \mathrm{Mo},{ }^{89} \mathrm{Ru},{ }^{92} \mathrm{Rh},{ }^{93} \mathrm{Pd},{ }^{121} \mathrm{Ce},{ }^{125} \mathrm{Nd}$, ${ }^{128} \mathrm{Pm},{ }^{129} \mathrm{Sm},{ }^{135,137} \mathrm{Gd},{ }^{139} \mathrm{Dy},{ }^{142} \mathrm{Ho},{ }^{149} \mathrm{Yb}\left(\beta^{+} \mathrm{p}\right)$; measured $\beta$-delayed $\mathrm{E} \gamma, \mathrm{I} \gamma$, proton spectra, $\mathrm{p} \gamma$-coin, $\mathrm{T}_{1 / 2}$. Comparison with model predictions. JOUR PRVCA 71054318

${ }^{128} \mathrm{Pm}$ 2005XU04 RADIOACTIVITY ${ }^{81} \mathrm{Zr},{ }^{85} \mathrm{Mo},{ }^{89} \mathrm{Ru},{ }^{92} \mathrm{Rh},{ }^{93} \mathrm{Pd},{ }^{121} \mathrm{Ce},{ }^{125} \mathrm{Nd}$, ${ }^{128} \mathrm{Pm},{ }^{129} \mathrm{Sm},{ }^{135,137} \mathrm{Gd},{ }^{139} \mathrm{Dy},{ }^{142} \mathrm{Ho},{ }^{149} \mathrm{Yb}\left(\beta^{+} \mathrm{p}\right)$; measured $\beta$-delayed $\mathrm{E} \gamma, \mathrm{I} \gamma$, proton spectra, $\mathrm{p} \gamma$-coin, $\mathrm{T}_{1 / 2}$. Comparison with model predictions. JOUR PRVCA 71054318

2005XU04 NUCLEAR REACTIONS ${ }^{92} \mathrm{Mo},{ }^{106} \mathrm{Cd}\left({ }^{32} \mathrm{~S}, 3 \mathrm{n}\right), \mathrm{E}=151 \mathrm{MeV}$; ${ }^{92} \mathrm{Mo}\left({ }^{36} \mathrm{Ar}, 3 \mathrm{n}\right), \mathrm{E}=169 \mathrm{MeV} ;{ }^{96} \mathrm{Ru}\left({ }^{36} \mathrm{Ar}, 3 \mathrm{n}\right),\left({ }^{36} \mathrm{Ar}, 3 \mathrm{np}\right), \mathrm{E}=165,174$ $\mathrm{MeV} ;{ }^{106} \mathrm{Cd}\left({ }^{36} \mathrm{Ar}, 3 \mathrm{n}\right),\left({ }^{36} \mathrm{Ar}, \mathrm{n} \alpha\right), \mathrm{E}=176 \mathrm{MeV} ;{ }^{106} \mathrm{Cd}\left({ }^{40} \mathrm{Ca}, 4 \mathrm{n}\right)$, $\mathrm{E}=202 \mathrm{MeV} ;{ }^{112} \mathrm{Sn}\left({ }^{40} \mathrm{Ca}, 3 \mathrm{n}\right), \mathrm{E}=185 \mathrm{MeV}$; measured $\sigma$. JOUR PRVCA 71054318

\section{$\mathrm{A}=129$}

${ }^{129} \mathrm{Sn} \quad$ 2005LE34 NUCLEAR MOMENTS

$125,125 m, 126,127,127 m, 128,129,129 m, 130,130 m, 131,131 m, 132 \mathrm{Sn}$; measured isotope shifts; deduced charge radii, dynamical effects.

$125,125 m, 127,127 m, 129,129 m, 130 m, 131,131 m \mathrm{Sn}$; measured $\mu$, quadrupole moments. Laser spectroscopy, mean-field calculations. JOUR PRVCA 72034305

2005SI34 ATOMIC MASSES $76,77,80,81,86,88 \mathrm{Sr},{ }^{124,129,130,131,132} \mathrm{Sn}$; measured masses. Penning trap mass spectrometer, comparison with previous results. JOUR NUPAB 76345

${ }^{129} \mathrm{Sb}$ 2005ADZZ NUCLEAR REACTIONS ${ }^{129} \mathrm{I}(\mathrm{n}, 7 \mathrm{n}),(\mathrm{n}, 6 \mathrm{n}),(\mathrm{n}, 4 \mathrm{n}),(\mathrm{n}, \gamma), \mathrm{E}=$ fast; ${ }^{237} \mathrm{~Np}(\mathrm{n}, \gamma), \mathrm{E}=$ fast; measured yields. ${ }^{237} \mathrm{~Np}(\mathrm{n}, \mathrm{F}){ }^{91} \mathrm{Sr} /{ }^{97} \mathrm{Zr} /{ }^{132} \mathrm{Te} /$ ${ }^{133} \mathrm{I} /{ }^{135} \mathrm{I}, \mathrm{E}=$ fast; ${ }^{238} \mathrm{Pu}(\mathrm{n}, \mathrm{F}){ }^{97} \mathrm{Zr} /{ }^{129} \mathrm{Sb} /{ }^{132} \mathrm{I} /{ }^{133} \mathrm{I} /{ }^{135} \mathrm{Xe} /$ ${ }^{105} \mathrm{Ru}, \mathrm{E}=$ fast; ${ }^{239} \mathrm{Pu}(\mathrm{n}, \mathrm{F}){ }^{88} \mathrm{Kr} /{ }^{91} \mathrm{Sr} /{ }^{92} \mathrm{Sr} /{ }^{92} \mathrm{Y} /{ }^{97} \mathrm{Zr} /{ }^{99} \mathrm{Mo} /$ ${ }^{103} \mathrm{Ru} /{ }^{105} \mathrm{Ru} /{ }^{128} \mathrm{Sb} /{ }^{129} \mathrm{Sb} /{ }^{132} \mathrm{Te} /{ }^{131} \mathrm{I} /{ }^{132} \mathrm{I} /{ }^{133} \mathrm{I} /{ }^{135} \mathrm{I} /{ }^{135} \mathrm{Xe}$ $/{ }^{143} \mathrm{Ce} /{ }^{140} \mathrm{Ba} /{ }^{140} \mathrm{La}, \mathrm{E}=$ fast; measured fission fragment yields. Secondary neutrons from proton irradiation. JINR nuclotron. CONF St Petersburg,P195,Adam 


\section{$\mathrm{A}=129$ (continued)}

2005YU07 NUCLEAR REACTIONS ${ }^{50} \mathrm{Ti}\left({ }^{129} \mathrm{Sb},{ }^{129} \mathrm{Sb}\right),\left({ }^{129} \mathrm{Te},{ }^{129} \mathrm{Te}\right), \mathrm{E}=400$ $\mathrm{MeV}$; measured $\mathrm{E} \gamma, \mathrm{I} \gamma$, (particle) $\gamma$-coin following projectile Coulomb excitation. ${ }^{129} \mathrm{Te},{ }^{129} \mathrm{Sb}$ deduced transitions B(E2). Clarion, Hyball arrays. JOUR ZAANE 25 s01 395

${ }^{129} \mathrm{Te} \quad 2005$ YUO7 NUCLEAR REACTIONS ${ }^{50} \mathrm{Ti}\left({ }^{129} \mathrm{Sb},{ }^{129} \mathrm{Sb}^{\prime}\right),\left({ }^{129} \mathrm{Te},{ }^{129} \mathrm{Te}\right), \mathrm{E}=400$ $\mathrm{MeV}$; measured $\mathrm{E} \gamma, \mathrm{I} \gamma$, (particle) $\gamma$-coin following projectile Coulomb excitation. ${ }^{129} \mathrm{Te},{ }^{129} \mathrm{Sb}$ deduced transitions B(E2). Clarion, Hyball arrays. JOUR ZAANE 25 s01 395

${ }^{129} \mathrm{I} \quad$ 2005SCZW NUCLEAR REACTIONS $\mathrm{Pb}(\mathrm{p}, \mathrm{X}){ }^{10} \mathrm{Be} /{ }^{26} \mathrm{Al} /{ }^{129} \mathrm{I} /{ }^{36} \mathrm{Cl}$, $\mathrm{E}=200-2600 \mathrm{MeV}$; measured excitation functions. Stacked foil activation, chemical separation. Comparison with model predictions. CONF Santa Fe (Nucl Data for Sci and Technol) Proc,Vol2,P1517 ${ }^{129} \mathrm{Xe}$ 2005LE04 NUCLEAR REACTIONS $\mathrm{Pb}(\mathrm{p}, \mathrm{X}){ }^{3} \mathrm{He} /{ }^{4} \mathrm{He} /{ }^{21} \mathrm{Ne} /{ }^{22} \mathrm{Ne} /{ }^{36} \mathrm{Ar} /$ ${ }^{38} \mathrm{Ar} /{ }^{78} \mathrm{Kr} /{ }^{80} \mathrm{Kr} /{ }^{81} \mathrm{Kr} /{ }^{82} \mathrm{Kr} /{ }^{83} \mathrm{Kr} /{ }^{84} \mathrm{Kr} /{ }^{85} \mathrm{Kr} /{ }^{86} \mathrm{Kr} /{ }^{124} \mathrm{Xe}$ $/{ }^{126} \mathrm{Xe} /{ }^{128} \mathrm{Xe} /{ }^{129} \mathrm{Xe} /{ }^{130} \mathrm{Xe} /{ }^{131} \mathrm{Xe} /{ }^{132} \mathrm{Xe} /{ }^{134} \mathrm{Xe}, \mathrm{E}=44-2595$ $\mathrm{MeV}$; measured production $\sigma$; deduced reaction mechanism features. Mini-stack approach, comparisons with model predictions. JOUR NIMBE 2291

2005SH38 ATOMIC MASSES ${ }^{32,33} \mathrm{~S},{ }^{84,86} \mathrm{Kr},{ }^{129,132}$ Xe; measured masses. Penning trap. JOUR PLRAA 72022510

2005W004 NUCLEAR MOMENTS ${ }^{129,131}$ Xe; measured hfs; deduced role of nuclear spin in photoionization. JOUR PLRAA 71052504

${ }^{129} \mathrm{Sm} 2005 \mathrm{XU} 04 \quad$ RADIOACTIVITY ${ }^{81} \mathrm{Zr},{ }^{85} \mathrm{Mo},{ }^{89} \mathrm{Ru},{ }^{92} \mathrm{Rh},{ }^{93} \mathrm{Pd},{ }^{121} \mathrm{Ce},{ }^{125} \mathrm{Nd}$, ${ }^{128} \mathrm{Pm},{ }^{129} \mathrm{Sm},{ }^{135,137} \mathrm{Gd},{ }^{139} \mathrm{Dy},{ }^{142} \mathrm{Ho},{ }^{149} \mathrm{Yb}\left(\beta^{+} \mathrm{p}\right)$; measured $\beta$-delayed $\mathrm{E} \gamma, \mathrm{I} \gamma$, proton spectra, $\mathrm{p} \gamma$-coin, $\mathrm{T}_{1 / 2}$. Comparison with model predictions. JOUR PRVCA 71054318

2005XU04 NUCLEAR REACTIONS ${ }^{92} \mathrm{Mo},{ }^{106} \mathrm{Cd}\left({ }^{32} \mathrm{~S}, 3 \mathrm{n}\right), \mathrm{E}=151 \mathrm{MeV}$; ${ }^{92} \mathrm{Mo}\left({ }^{36} \mathrm{Ar}, 3 \mathrm{n}\right), \mathrm{E}=169 \mathrm{MeV} ;{ }^{96} \mathrm{Ru}\left({ }^{36} \mathrm{Ar}, 3 \mathrm{n}\right),\left({ }^{36} \mathrm{Ar}, 3 \mathrm{np}\right), \mathrm{E}=165,174$ $\mathrm{MeV} ;{ }^{106} \mathrm{Cd}\left({ }^{36} \mathrm{Ar}, 3 \mathrm{n}\right),\left({ }^{36} \mathrm{Ar}, \mathrm{n} \alpha\right), \mathrm{E}=176 \mathrm{MeV} ;{ }^{106} \mathrm{Cd}\left({ }^{40} \mathrm{Ca}, 4 \mathrm{n}\right)$, $\mathrm{E}=202 \mathrm{MeV} ;{ }^{112} \mathrm{Sn}\left({ }^{40} \mathrm{Ca}, 3 \mathrm{n}\right), \mathrm{E}=185 \mathrm{MeV}$; measured $\sigma$. JOUR PRVCA 71054318

\section{$\mathrm{A}=130$}

${ }^{130} \mathrm{Sn} \quad 2005 \mathrm{AD} 29 \quad$ NUCLEAR REACTIONS Be $\left({ }^{238} \mathrm{U}, \mathrm{X}\right), \mathrm{E}$ not given; measured fragment yields. ${ }^{12} \mathrm{C},{ }^{208} \mathrm{~Pb}\left({ }^{130} \mathrm{Sn}, \mathrm{nX}\right),\left({ }^{132} \mathrm{Sn}, \mathrm{nX}\right), \mathrm{E} \approx 500 \mathrm{MeV} /$ nucleon; measured En, $\mathrm{E} \gamma, \mathrm{n} \gamma$-coin; deduced Coulomb dissociation $\sigma(\mathrm{E}) .{ }^{130,132} \mathrm{Sn}$ deduced dipole strength distributions, pygmy and giant dipole resonance parameters. JOUR PRLTA 95132501

2005ADZX NUCLEAR REACTIONS Pb( $\left.{ }^{130} \mathrm{Sn},{ }^{130} \mathrm{Sn}\right),\left({ }^{132} \mathrm{Sn},{ }^{132} \mathrm{Sn}^{\prime}\right), \mathrm{E}^{*} \approx 5-30$ $\mathrm{MeV}$; measured $\Sigma(\mathrm{E})$ following projectile Coulomb excitation. ${ }^{130,132} \mathrm{Sn}(\gamma, \mathrm{nX}), \mathrm{E}^{*} \approx 5-30 \mathrm{MeV}$; deduced photo-neutron $\sigma .{ }^{130,132} \mathrm{Sn}$ deduced pygmy and GDR energies. REPT GSI 2005-1,P94,Adrich 


\section{$\mathrm{A}=130$ (continued)}

2005LE34 NUCLEAR MOMENTS

$125,125 m, 126,127,127 m, 128,129,129 m, 130,130 m, 131,131 m, 132 \mathrm{Sn}$; measured isotope shifts; deduced charge radii, dynamical effects.

$125,125 m, 127,127 m, 129,129 m, 130 m, 131,131 m \mathrm{Sn}$; measured $\mu$, quadrupole moments. Laser spectroscopy, mean-field calculations. JOUR PRVCA 72034305

2005RA09 NUCLEAR REACTIONS C( $\left.{ }^{126} \mathrm{Sn},{ }^{126} \mathrm{Sn}\right),\left({ }^{128} \mathrm{Sn},{ }^{128} \mathrm{Sn}\right),\left({ }^{130} \mathrm{Sn}\right.$, ${ }^{130} \mathrm{Sn}$ '), ( $\left.{ }^{132} \mathrm{Sn},{ }^{132} \mathrm{Sn}\right)$, ( ${ }^{134} \mathrm{Sn},{ }^{134} \mathrm{Sn}$ '), ( ${ }^{132} \mathrm{Te},{ }^{132} \mathrm{Te}$ '), ( ${ }^{134} \mathrm{Te},{ }^{134} \mathrm{Te}$ '), $\left({ }^{136} \mathrm{Te},{ }^{136} \mathrm{Te}\right), \mathrm{E}$ not given; measured $\mathrm{E} \gamma, \mathrm{I} \gamma$, (particle) $\gamma$-coin following projectile Coulomb excitation. ${ }^{126,128,130,132,134} \mathrm{Sn},{ }^{132,134,136} \mathrm{Te}$ deduced transitions $\mathrm{B}(\mathrm{E} 2) .{ }^{9} \mathrm{Be}\left({ }^{134} \mathrm{Te},{ }^{8} \mathrm{Be}\right),{ }^{13} \mathrm{C}\left({ }^{134} \mathrm{Te},{ }^{12} \mathrm{C}\right)$, E not given; measured $\mathrm{E} \gamma, \mathrm{I} \gamma, \gamma \gamma$-, (particle) $\gamma$-coin. ${ }^{135} \mathrm{Te}$ deduced level. Clarion, Hyball arrays. JOUR NUPAB 752 264c

2005RA32 NUCLEAR REACTIONS C $\left({ }^{126} \mathrm{Sn},{ }^{126} \mathrm{Sn}\right),\left({ }^{128} \mathrm{Sn},{ }^{128} \mathrm{Sn}\right),\left({ }^{130} \mathrm{Sn}\right.$, ${ }^{130} \mathrm{Sn}$ '), ( $\left.{ }^{132} \mathrm{Te},{ }^{132} \mathrm{Te}\right),\left({ }^{134} \mathrm{Te},{ }^{134} \mathrm{Te}\right),\left({ }^{136} \mathrm{Te},{ }^{136} \mathrm{Te}\right)$, E not given; measured $\mathrm{E} \gamma, \mathrm{I} \gamma, \gamma \gamma$-, (particle) $\gamma$-coin following projectile Coulomb excitation. ${ }^{132,134,136} \mathrm{Te},{ }^{126,128,130} \mathrm{Sn}$ deduced excitation $\mathrm{B}(\mathrm{E} 2)$.

${ }^{9} \mathrm{Be}\left({ }^{134} \mathrm{Te},{ }^{8} \mathrm{Be}\right),{ }^{13} \mathrm{C}\left({ }^{134} \mathrm{Te},{ }^{12} \mathrm{C}\right), \mathrm{E}=4.3 \mathrm{MeV} /$ nucleon; measured $\mathrm{E} \gamma$, $\mathrm{I} \gamma, \gamma \gamma$-coin, (particle) $\gamma$-coin; deduced single-neutron transfer $\sigma(\mathrm{E})$.

${ }^{135}$ Te deduced levels J, $\pi$. JOUR ZAANE 25 s01 383

2005SI34 ATOMIC MASSES ${ }^{76,77,80,81,86,88} \mathrm{Sr},{ }^{124,129,130,131,132} \mathrm{Sn}$; measured masses. Penning trap mass spectrometer, comparison with previous results. JOUR NUPAB 76345

${ }^{130} \mathrm{Sb} \quad 2005 B E Z W$ NUCLEAR REACTIONS ${ }^{238} \mathrm{U}(\gamma, \mathrm{F}){ }^{84} \mathrm{Br} /{ }^{130} \mathrm{Sb} /{ }^{132} \mathrm{Sb} /{ }^{131} \mathrm{Te} /$ ${ }^{133} \mathrm{Te} /{ }^{134} \mathrm{I} /{ }^{135} \mathrm{Xe}, \mathrm{E}=16 \mathrm{MeV}$ bremsstrahlung; ${ }^{237} \mathrm{~Np}(\gamma, \mathrm{F}){ }^{134} \mathrm{I} /$ ${ }^{135} \mathrm{Xe}, \mathrm{E}=16 \mathrm{MeV}$ bremsstrahlung; measured $\mathrm{E} \gamma, \mathrm{I} \gamma$; deduced isomer yield ratios, fission fragments mean angular momenta. CONF Santa Fe (Nucl Data for Sci and Technol) Proc,Vol1,P641

2005NA05 NUCLEAR REACTIONS ${ }^{232} \mathrm{Th},{ }^{232,238} \mathrm{U},{ }^{238,240} \mathrm{Pu},{ }^{244} \mathrm{Cm}(\mathrm{n}, \mathrm{F}){ }^{128} \mathrm{Sb}$ $/{ }^{130} \mathrm{Sb} /{ }^{132} \mathrm{Sb} /{ }^{131} \mathrm{Te} /{ }^{133} \mathrm{Te} /{ }^{132} \mathrm{I} /{ }^{134} \mathrm{I} /{ }^{136} \mathrm{I} /{ }^{135} \mathrm{Xe} /{ }^{138} \mathrm{Cs}$, $\mathrm{E}=$ thermal, fast; measured isomer yield ratios; deduced fission fragment angular momenta. Spin-dependent statistical model analysis. JOUR PRVCA 71014304

${ }^{130} \mathrm{Te} \quad$ 2005AR25 RADIOACTIVITY ${ }^{130} \mathrm{Te}\left(2 \beta^{-}\right)$; measured $0 \nu \beta \beta$-decay $\mathrm{T}_{1 / 2}$ lower limit. JOUR PRLTA 95142501

2005PI02 RADIOACTIVITY ${ }^{130} \mathrm{Te}\left(2 \beta^{-}\right)$; measured $0 \nu 2 \beta$-decay $\mathrm{T}_{1 / 2}$ lower limit. JOUR NPBSE 138210

${ }^{130} \mathrm{I} \quad$ 2005ADZZ NUCLEAR REACTIONS ${ }^{129} \mathrm{I}(\mathrm{n}, 7 \mathrm{n}),(\mathrm{n}, 6 \mathrm{n}),(\mathrm{n}, 4 \mathrm{n}),(\mathrm{n}, \gamma), \mathrm{E}=\mathrm{fast}$; ${ }^{237} \mathrm{~Np}(\mathrm{n}, \gamma), \mathrm{E}=$ fast; measured yields. ${ }^{237} \mathrm{~Np}(\mathrm{n}, \mathrm{F}){ }^{91} \mathrm{Sr} /{ }^{97} \mathrm{Zr} /{ }^{132} \mathrm{Te} /$ ${ }^{133} \mathrm{I} /{ }^{135} \mathrm{I}, \mathrm{E}=$ fast; ${ }^{238} \mathrm{Pu}(\mathrm{n}, \mathrm{F}){ }^{97} \mathrm{Zr} /{ }^{129} \mathrm{Sb} /{ }^{132} \mathrm{I} /{ }^{133} \mathrm{I} /{ }^{135} \mathrm{Xe} /$ ${ }^{105} \mathrm{Ru}, \mathrm{E}=$ fast; ${ }^{239} \mathrm{Pu}(\mathrm{n}, \mathrm{F}){ }^{88} \mathrm{Kr} /{ }^{91} \mathrm{Sr} /{ }^{92} \mathrm{Sr} /{ }^{92} \mathrm{Y} /{ }^{97} \mathrm{Zr} /{ }^{99} \mathrm{Mo} /$ ${ }^{103} \mathrm{Ru} /{ }^{105} \mathrm{Ru} /{ }^{128} \mathrm{Sb} /{ }^{129} \mathrm{Sb} /{ }^{132} \mathrm{Te} /{ }^{131} \mathrm{I} /{ }^{132} \mathrm{I} /{ }^{133} \mathrm{I} /{ }^{135} \mathrm{I} /{ }^{135} \mathrm{Xe}$ $/{ }^{143} \mathrm{Ce} /{ }^{140} \mathrm{Ba} /{ }^{140} \mathrm{La}, \mathrm{E}=$ fast; measured fission fragment yields. Secondary neutrons from proton irradiation. JINR nuclotron. CONF St Petersburg,P195,Adam

2005BEZV NUCLEAR REACTIONS ${ }^{99} \mathrm{Tc},{ }^{129} \mathrm{I}(\mathrm{n}, \gamma), \mathrm{E}=\mathrm{cold}$; measured $\mathrm{E} \gamma, \mathrm{I} \gamma$; deduced thermal capture $\sigma$. CONF Santa Fe (Nucl Data for Sci and Technol) Proc, Vol1,P744 


\section{$\mathrm{A}=130$ (continued)}

2005BEZV RADIOACTIVITY ${ }^{130,130 m} \mathrm{I}\left(\beta^{-}\right)$[from ${ }^{129} \mathrm{I}(\mathrm{n}, \gamma)$ ]; measured $\mathrm{E} \gamma, \mathrm{I} \gamma$, $\mathrm{T}_{1 / 2}$. CONF Santa Fe (Nucl Data for Sci and Technol) Proc,Vol1,P744 2005UN01 NUCLEAR REACTIONS ${ }^{128} \mathrm{Te}\left({ }^{14} \mathrm{~N}, 4 \mathrm{n}\right),\left({ }^{14} \mathrm{~N}, 5 \mathrm{n}\right),\left({ }^{14} \mathrm{~N}, 4 \mathrm{np}\right),\left({ }^{14} \mathrm{~N}\right.$, $5 \mathrm{n} \alpha),\left({ }^{14} \mathrm{~N}, 6 \mathrm{n} \alpha\right),\left({ }^{14} \mathrm{~N}, \mathrm{n} 2 \mathrm{p} \alpha\right),\left({ }^{14} \mathrm{~N}, \mathrm{n} 2 \mathrm{p} 2 \alpha\right),\left({ }^{14} \mathrm{~N}, 3 \alpha\right), \mathrm{E} \approx 64-90 ;$ measured excitation functions; deduced reaction mechanism features. Activation technique, comparison with model predictions. JOUR IMPEE 14775

${ }^{130} \mathrm{Xe}$ 2005AR25 RADIOACTIVITY ${ }^{130} \mathrm{Te}\left(2 \beta^{-}\right)$; measured $0 \nu \beta \beta$-decay $\mathrm{T}_{1 / 2}$ lower limit. JOUR PRLTA 95142501

2005BEZV RADIOACTIVITY ${ }^{130,130 m} \mathrm{I}\left(\beta^{-}\right)$[from $\left.{ }^{129} \mathrm{I}(\mathrm{n}, \gamma)\right]$; measured $\mathrm{E} \gamma, \mathrm{I} \gamma$, $\mathrm{T}_{1 / 2}$. CONF Santa Fe (Nucl Data for Sci and Technol) Proc,Vol1,P744

2005LE04 NUCLEAR REACTIONS Pb(p, X) ${ }^{3} \mathrm{He} /{ }^{4} \mathrm{He} /{ }^{21} \mathrm{Ne} /{ }^{22} \mathrm{Ne} /{ }^{36} \mathrm{Ar} /$ ${ }^{38} \mathrm{Ar} /{ }^{78} \mathrm{Kr} /{ }^{80} \mathrm{Kr} /{ }^{81} \mathrm{Kr} /{ }^{82} \mathrm{Kr} /{ }^{83} \mathrm{Kr} /{ }^{84} \mathrm{Kr} /{ }^{85} \mathrm{Kr} /{ }^{86} \mathrm{Kr} /{ }^{124} \mathrm{Xe}$ $/{ }^{126} \mathrm{Xe} /{ }^{128} \mathrm{Xe} /{ }^{129} \mathrm{Xe} /{ }^{130} \mathrm{Xe} /{ }^{131} \mathrm{Xe} /{ }^{132} \mathrm{Xe} /{ }^{134} \mathrm{Xe}, \mathrm{E}=44-2595$ $\mathrm{MeV}$; measured production $\sigma$; deduced reaction mechanism features. Mini-stack approach, comparisons with model predictions. JOUR NIMBE 2291

2005PI02 RADIOACTIVITY ${ }^{130} \mathrm{Te}\left(2 \beta^{-}\right)$; measured $0 \nu 2 \beta$-decay $\mathrm{T}_{1 / 2}$ lower limit. JOUR NPBSE 138210

${ }^{130} \mathrm{Cs} \quad 2005 \mathrm{SI} 13 \quad$ NUCLEAR REACTIONS ${ }^{124} \mathrm{Sn}\left({ }^{11} \mathrm{~B}, 5 \mathrm{n}\right), \mathrm{E}=60 \mathrm{MeV}$; measured $\mathrm{E} \gamma$, $\mathrm{I} \gamma, \gamma \gamma$-coin. ${ }^{130} \mathrm{Cs}$ deduced high-spin levels, J, $\pi$, B(M1) / B(E2), chiral structure. Euroball IV array. JOUR JPGPE 31541

${ }^{130} \mathrm{Ba} \quad 2005 \mathrm{GU} 37$ ATOMIC MASSES ${ }^{56,57} \mathrm{Mn},{ }^{82 m} \mathrm{Rb},{ }^{92} \mathrm{Sr},{ }^{124,127} \mathrm{Cs},{ }^{130} \mathrm{Ba}$; measured masses. Penning trap mass spectrometer. JOUR ZAANE 25 s01 35

\section{$\mathrm{A}=131$}

${ }^{131} \mathrm{Sn} \quad$ 2005LE34 NUCLEAR MOMENTS

$125,125 m, 126,127,127 m, 128,129,129 m, 130,130 m, 131,131 m, 132 \mathrm{Sn}$; measured isotope shifts; deduced charge radii, dynamical effects.

$125,125 m, 127,127 m, 129,129 m, 130 m, 131,131 m \mathrm{Sn}$; measured $\mu$, quadrupole moments. Laser spectroscopy, mean-field calculations. JOUR PRVCA 72034305

2005SI34 ATOMIC MASSES $76,77,80,81,86,88 \mathrm{Sr},{ }^{124,129,130,131,132} \mathrm{Sn}$; measured masses. Penning trap mass spectrometer, comparison with previous results. JOUR NUPAB 76345

${ }^{131} \mathrm{Te}$ 2005BEZW NUCLEAR REACTIONS ${ }^{238} \mathrm{U}(\gamma, \mathrm{F}){ }^{84} \mathrm{Br} /{ }^{130} \mathrm{Sb} /{ }^{132} \mathrm{Sb} /{ }^{131} \mathrm{Te} /$ ${ }^{133} \mathrm{Te} /{ }^{134} \mathrm{I} /{ }^{135} \mathrm{Xe}, \mathrm{E}=16 \mathrm{MeV}$ bremsstrahlung; ${ }^{237} \mathrm{~Np}(\gamma, \mathrm{F}){ }^{134} \mathrm{I} /$

${ }^{135} \mathrm{Xe}, \mathrm{E}=16 \mathrm{MeV}$ bremsstrahlung; measured $\mathrm{E} \gamma, \mathrm{I} \gamma$; deduced isomer yield ratios, fission fragments mean angular momenta. CONF Santa Fe (Nucl Data for Sci and Technol) Proc,Vol1,P641

2005NA05 NUCLEAR REACTIONS ${ }^{232} \mathrm{Th},{ }^{232,238} \mathrm{U},{ }^{238,240} \mathrm{Pu},{ }^{244} \mathrm{Cm}(\mathrm{n}, \mathrm{F}){ }^{128} \mathrm{Sb}$ $/{ }^{130} \mathrm{Sb} /{ }^{132} \mathrm{Sb} /{ }^{131} \mathrm{Te} /{ }^{133} \mathrm{Te} /{ }^{132} \mathrm{I} /{ }^{134} \mathrm{I} /{ }^{136} \mathrm{I} /{ }^{135} \mathrm{Xe} /{ }^{138} \mathrm{Cs}$, $\mathrm{E}=$ thermal, fast; measured isomer yield ratios; deduced fission fragment angular momenta. Spin-dependent statistical model analysis. JOUR PRVCA 71014304 


\section{$\mathrm{A}=131$ (continued)}

\begin{tabular}{|c|c|c|}
\hline \multirow[t]{2}{*}{${ }^{131} \mathrm{I}$} & 2005ADZZ & $\begin{array}{l}\text { NUCLEAR REACTIONS }{ }^{129} \mathrm{I}(\mathrm{n}, 7 \mathrm{n}),(\mathrm{n}, 6 \mathrm{n}),(\mathrm{n}, 4 \mathrm{n}),(\mathrm{n}, \gamma), \mathrm{E}=\text { fast; } \\
{ }^{237} \mathrm{~Np}(\mathrm{n}, \gamma), \mathrm{E}=\text { fast; measured yields. }{ }^{237} \mathrm{~Np}(\mathrm{n}, \mathrm{F}){ }^{91} \mathrm{Sr} /{ }^{97} \mathrm{Zr} /{ }^{132} \mathrm{Te} / \\
\\
{ }^{133} \mathrm{I} /{ }^{135} \mathrm{I}, \mathrm{E}=\text { fast; }{ }^{238} \mathrm{Pu}(\mathrm{n}, \mathrm{F}){ }^{97} \mathrm{Zr} /{ }^{129} \mathrm{Sb} /{ }^{132} \mathrm{I} /{ }^{133} \mathrm{I} /{ }^{135} \mathrm{Xe} / \\
{ }^{105} \mathrm{Ru}, \mathrm{E}=\mathrm{fast} ;{ }^{239} \mathrm{Pu}(\mathrm{n}, \mathrm{F}){ }^{88} \mathrm{Kr} /{ }^{91} \mathrm{Sr} /{ }^{92} \mathrm{Sr} /{ }^{92} \mathrm{Y} /{ }^{97} \mathrm{Zr} /{ }^{99} \mathrm{Mo} / \\
\\
{ }^{103} \mathrm{Ru} /{ }^{105} \mathrm{Ru} /{ }^{128} \mathrm{Sb} /{ }^{129} \mathrm{Sb} /{ }^{132} \mathrm{Te} /{ }^{131} \mathrm{I} /{ }^{132} \mathrm{I} /{ }^{133} \mathrm{I} /{ }^{135} \mathrm{I} /{ }^{135} \mathrm{Xe} \\
/{ }^{143} \mathrm{Ce} /{ }^{140} \mathrm{Ba} /{ }^{140} \mathrm{La}, \mathrm{E}=\text { fast; measured fission fragment yields. } \\
\text { Secondary neutrons from proton irradiation. JINR nuclotron. CONF } \\
\text { St Petersburg,P195,Adam }\end{array}$ \\
\hline & 2005UN01 & $\begin{array}{l}\text { NUCLEAR REACTIONS }{ }^{128} \mathrm{Te}\left({ }^{14} \mathrm{~N}, 4 \mathrm{n}\right),\left({ }^{14} \mathrm{~N}, 5 \mathrm{n}\right),\left({ }^{14} \mathrm{~N}, 4 \mathrm{np}\right),\left({ }^{14} \mathrm{~N},\right. \\
5 \mathrm{n} \alpha),\left({ }^{14} \mathrm{~N}, 6 \mathrm{n} \alpha\right),\left({ }^{14} \mathrm{~N}, \mathrm{n} 2 \mathrm{p} \alpha\right),\left({ }^{14} \mathrm{~N}, \mathrm{n} 2 \mathrm{p} 2 \alpha\right),\left({ }^{14} \mathrm{~N}, 3 \alpha\right), \mathrm{E} \approx 64-90 ; \\
\text { measured excitation functions; deduced reaction mechanism features. } \\
\text { Activation technique, comparison with model predictions. JOUR } \\
\text { IMPEE } 14775\end{array}$ \\
\hline \multirow[t]{2}{*}{${ }^{131} \mathrm{Xe}$} & 2005LE04 & $\begin{array}{l}\text { NUCLEAR REACTIONS } \mathrm{Pb}(\mathrm{p}, \mathrm{X}){ }^{3} \mathrm{He} /{ }^{4} \mathrm{He} /{ }^{21} \mathrm{Ne} /{ }^{22} \mathrm{Ne} /{ }^{36} \mathrm{Ar} / \\
{ }^{88} \mathrm{Ar} /{ }^{78} \mathrm{Kr} /{ }^{80} \mathrm{Kr} /{ }^{81} \mathrm{Kr} /{ }^{82} \mathrm{Kr} /{ }^{83} \mathrm{Kr} /{ }^{84} \mathrm{Kr} /{ }^{85} \mathrm{Kr} /{ }^{86} \mathrm{Kr} /{ }^{124} \mathrm{Xe} \\
/{ }^{126} \mathrm{Xe} /{ }^{128} \mathrm{Xe} /{ }^{129} \mathrm{Xe} /{ }^{130} \mathrm{Xe} /{ }^{131} \mathrm{Xe} /{ }^{132} \mathrm{Xe} /{ }^{134} \mathrm{Xe}, \mathrm{E}=44-2595 \\
\mathrm{MeV} \text {; measured production } \sigma \text {; deduced reaction mechanism features. } \\
\text { Mini-stack approach, comparisons with model predictions. JOUR } \\
\text { NIMBE } 2291\end{array}$ \\
\hline & 2005W004 & $\begin{array}{l}\text { NUCLEAR MOMENTS }{ }^{129,131} \text { Xe; measured hfs; deduced role of } \\
\text { nuclear spin in photoionization. JOUR PLRAA } 71052504\end{array}$ \\
\hline $1 C_{1}$ & $2005 \mathrm{KU} 10$ & $\begin{array}{l}\text { NUCLEAR REACTIONS }{ }^{124} \mathrm{Sn}\left({ }^{11} \mathrm{~B}, 4 \mathrm{n}\right), \mathrm{E}=57 \mathrm{MeV} \text {; measured } \mathrm{E} \gamma \text {, } \\
\mathrm{I} \gamma, \gamma \gamma \text {-coin. }{ }^{131} \mathrm{Cs} \text { deduced high-spin levels, } \mathrm{J}, \pi \text {, configurations } \mathrm{B}(\mathrm{M} 1) \\
\text { / B(E2). Total Routhian surface and tilted axis cranking model } \\
\text { calculations. JOUR ZAANE } 2413\end{array}$ \\
\hline${ }^{11} \mathrm{Ce}$ & 2005РA30 & $\begin{array}{l}\text { NUCLEAR REACTIONS }{ }^{100} \mathrm{Mo}\left({ }^{36} \mathrm{~S}, 4 \mathrm{n}\right),\left({ }^{36} \mathrm{~S}, 5 \mathrm{n}\right), \mathrm{E}=160,165 \mathrm{MeV} \text {; } \\
\text { measured } \mathrm{E} \gamma, \mathrm{I} \gamma, \gamma \gamma \text {-coin. }{ }^{131,132} \mathrm{Ce} \text { deduced high-spin levels, J, } \pi \text {, } \\
\text { superdeformed bands, configurations, band termination features. } \\
\text { Euroball IV array, cranked mean-field calculations. JOUR PRVCA } 71 \\
054309\end{array}$ \\
\hline
\end{tabular}

\section{$\mathrm{A}=132$}

${ }^{132} \mathrm{Sn} \quad 2005 \mathrm{AD} 29$

NUCLEAR REACTIONS Be $\left({ }^{238} \mathrm{U}, \mathrm{X}\right)$, E not given; measured fragment yields. ${ }^{12} \mathrm{C},{ }^{208} \mathrm{~Pb}\left({ }^{130} \mathrm{Sn}, \mathrm{nX}\right),\left({ }^{132} \mathrm{Sn}, \mathrm{nX}\right), \mathrm{E} \approx 500 \mathrm{MeV} /$ nucleon; measured En, E $\gamma$, n $\gamma$-coin; deduced Coulomb dissociation $\sigma(\mathrm{E}) .{ }^{130,132} \mathrm{Sn}$ deduced dipole strength distributions, pygmy and giant dipole resonance parameters. JOUR PRLTA 95132501

2005ADZX NUCLEAR REACTIONS Pb( $\left.{ }^{130} \mathrm{Sn},{ }^{130} \mathrm{Sn}\right),\left({ }^{132} \mathrm{Sn},{ }^{132} \mathrm{Sn}^{\prime}\right), \mathrm{E}^{*} \approx 5-30$ $\mathrm{MeV}$; measured $\Sigma(\mathrm{E})$ following projectile Coulomb excitation. ${ }^{130,132} \mathrm{Sn}(\gamma, \mathrm{nX}), \mathrm{E}^{*} \approx 5-30 \mathrm{MeV}$; deduced photo-neutron $\sigma \cdot{ }^{130,132} \mathrm{Sn}$ deduced pygmy and GDR energies. REPT GSI 2005-1,P94,Adrich 2005LE34 NUCLEAR MOMENTS $125,125 m, 126,127,127 m, 128,129,129 m, 130,130 m, 131,131 m, 132 \mathrm{Sn}$; measured isotope shifts; deduced charge radii, dynamical effects. $125,125 m, 127,127 m, 129,129 m, 130 m, 131,131 m \mathrm{Sn}$; measured $\mu$, quadrupole moments. Laser spectroscopy, mean-field calculations. JOUR PRVCA 72034305 


\section{$\mathrm{A}=132$ (continued)}

2005RA09 NUCLEAR REACTIONS C $\left({ }^{126} \mathrm{Sn},{ }^{126} \mathrm{Sn}^{\prime}\right),\left({ }^{128} \mathrm{Sn},{ }^{128} \mathrm{Sn}^{\prime}\right),\left({ }^{130} \mathrm{Sn}\right.$, ${ }^{130} \mathrm{Sn}$ '), ( ${ }^{132} \mathrm{Sn},{ }^{132} \mathrm{Sn}$ '), ( ${ }^{134} \mathrm{Sn},{ }^{134} \mathrm{Sn}$ '), $\left({ }^{132} \mathrm{Te},{ }^{132} \mathrm{Te}\right),\left({ }^{134} \mathrm{Te},{ }^{134} \mathrm{Te}\right)$, $\left({ }^{136} \mathrm{Te},{ }^{136} \mathrm{Te}\right), \mathrm{E}$ not given; measured $\mathrm{E} \gamma$, I $\gamma$, (particle) $\gamma$-coin following projectile Coulomb excitation. ${ }^{126,128,130,132,134} \mathrm{Sn},{ }^{132,134,136} \mathrm{Te}$ deduced transitions $\mathrm{B}(\mathrm{E} 2) .{ }^{9} \mathrm{Be}\left({ }^{134} \mathrm{Te},{ }^{8} \mathrm{Be}\right),{ }^{13} \mathrm{C}\left({ }^{134} \mathrm{Te},{ }^{12} \mathrm{C}\right)$, E not given; measured $\mathrm{E} \gamma, \mathrm{I} \gamma, \gamma \gamma-$, (particle) $\gamma$-coin. ${ }^{135} \mathrm{Te}$ deduced level. Clarion, Hyball arrays. JOUR NUPAB $752264 \mathrm{c}$

2005SI34 ATOMIC MASSES ${ }^{76,77,80,81,86,88} \mathrm{Sr}$, ${ }^{124,129,130,131,132} \mathrm{Sn}$; measured masses. Penning trap mass spectrometer, comparison with previous results. JOUR NUPAB 76345

2005VA31 NUCLEAR REACTIONS ${ }^{48} \mathrm{Ti}\left({ }^{132} \mathrm{Sn},{ }^{132} \mathrm{Sn}\right), \mathrm{E}=470-495 \mathrm{MeV}$; ${ }^{90} \mathrm{Zr}\left({ }^{134} \mathrm{Sn},{ }^{134} \mathrm{Sn}\right.$ '), E=400 MeV; measured $\mathrm{E} \gamma, \mathrm{I} \gamma$, (particle) $\gamma$-coin following projectile Coulomb excitation. ${ }^{132,134} \mathrm{Sn}$ deduced transitions B(E2). JOUR ZAANE 25 s01 391

${ }^{132} \mathrm{Sb}$ 2005BEZW NUCLEAR REACTIONS ${ }^{238} \mathrm{U}(\gamma, \mathrm{F}){ }^{84} \mathrm{Br} /{ }^{130} \mathrm{Sb} /{ }^{132} \mathrm{Sb} /{ }^{131} \mathrm{Te} /$ ${ }^{133} \mathrm{Te} /{ }^{134} \mathrm{I} /{ }^{135} \mathrm{Xe}, \mathrm{E}=16 \mathrm{MeV}$ bremsstrahlung; ${ }^{237} \mathrm{~Np}(\gamma, \mathrm{F}){ }^{134} \mathrm{I} /$ ${ }^{135} \mathrm{Xe}, \mathrm{E}=16 \mathrm{MeV}$ bremsstrahlung; measured $\mathrm{E} \gamma, \mathrm{I} \gamma$; deduced isomer yield ratios, fission fragments mean angular momenta. CONF Santa Fe (Nucl Data for Sci and Technol) Proc,Vol1,P641

2005HU08 RADIOACTIVITY ${ }^{132} \mathrm{Sb}\left(\beta^{-}\right)$[from $\mathrm{U}(\mathrm{p}, \mathrm{F})$ ]; measured $\mathrm{E} \gamma, \mathrm{I} \gamma$, $\gamma \gamma$-coin. ${ }^{132}$ Te deduced levels, J, $\pi$. Clarion array, comparison with model predictions. JOUR PRVCA 71044311

2005NA05 NUCLEAR REACTIONS ${ }^{232} \mathrm{Th},{ }^{232,238} \mathrm{U},{ }^{238,240} \mathrm{Pu},{ }^{244} \mathrm{Cm}(\mathrm{n}, \mathrm{F}){ }^{128} \mathrm{Sb}$ $/{ }^{130} \mathrm{Sb} /{ }^{132} \mathrm{Sb} /{ }^{131} \mathrm{Te} /{ }^{133} \mathrm{Te} /{ }^{132} \mathrm{I} /{ }^{134} \mathrm{I} /{ }^{136} \mathrm{I} /{ }^{135} \mathrm{Xe} /{ }^{138} \mathrm{Cs}$, $\mathrm{E}=$ thermal, fast; measured isomer yield ratios; deduced fission fragment angular momenta. Spin-dependent statistical model analysis. JOUR PRVCA 71014304

2005ZA14 RADIOACTIVITY ${ }^{132} \mathrm{Sb}\left(\beta^{-}\right)$; measured $\mathrm{E} \gamma, \mathrm{I} \gamma, \gamma \gamma$-coin. ${ }^{132} \mathrm{Te}$ deduced levels, J, $\pi$. Comparisons with quasiparticle RPA calculations with density-dependent pairing. Clarion array. JOUR ZAANE 25 s01 389

${ }^{132} \mathrm{Te} \quad 2005 \mathrm{ADZZ} \quad$ NUCLEAR REACTIONS ${ }^{129} \mathrm{I}(\mathrm{n}, 7 \mathrm{n}),(\mathrm{n}, 6 \mathrm{n}),(\mathrm{n}, 4 \mathrm{n}),(\mathrm{n}, \gamma), \mathrm{E}=$ fast; ${ }^{237} \mathrm{~Np}(\mathrm{n}, \gamma), \mathrm{E}=$ fast; measured yields. ${ }^{237} \mathrm{~Np}(\mathrm{n}, \mathrm{F}){ }^{91} \mathrm{Sr} /{ }^{97} \mathrm{Zr} /{ }^{132} \mathrm{Te} /$ ${ }^{133} \mathrm{I} /{ }^{135} \mathrm{I}, \mathrm{E}=$ fast; ${ }^{238} \mathrm{Pu}(\mathrm{n}, \mathrm{F}){ }^{97} \mathrm{Zr} /{ }^{129} \mathrm{Sb} /{ }^{132} \mathrm{I} /{ }^{133} \mathrm{I} /{ }^{135} \mathrm{Xe} /$ ${ }^{105} \mathrm{Ru}, \mathrm{E}=$ fast; ${ }^{239} \mathrm{Pu}(\mathrm{n}, \mathrm{F})^{88} \mathrm{Kr} /{ }^{91} \mathrm{Sr} /{ }^{92} \mathrm{Sr} /{ }^{92} \mathrm{Y} /{ }^{97} \mathrm{Zr} /{ }^{99} \mathrm{Mo} /$ ${ }^{103} \mathrm{Ru} /{ }^{105} \mathrm{Ru} /{ }^{128} \mathrm{Sb} /{ }^{129} \mathrm{Sb} /{ }^{132} \mathrm{Te} /{ }^{131} \mathrm{I} /{ }^{132} \mathrm{I} /{ }^{133} \mathrm{I} /{ }^{135} \mathrm{I} /{ }^{135} \mathrm{Xe}$ $/{ }^{143} \mathrm{Ce} /{ }^{140} \mathrm{Ba} /{ }^{140} \mathrm{La}, \mathrm{E}=$ fast; measured fission fragment yields. Secondary neutrons from proton irradiation. JINR nuclotron. CONF St Petersburg,P195,Adam

2005DA42 NUCLEAR REACTIONS ${ }^{12} \mathrm{C}\left({ }^{132} \mathrm{Te},{ }^{132} \mathrm{Te}\right),\left({ }^{130} \mathrm{Te},{ }^{130} \mathrm{Te}\right),\left({ }^{126} \mathrm{Te}\right.$, $\left.{ }^{126} \mathrm{Te}^{\prime}\right),\left({ }^{122} \mathrm{Te},{ }^{122} \mathrm{Te}\right.$ '), $\mathrm{E}=3 \mathrm{MeV} /$ nucleon; measured $\mathrm{E} \gamma, \mathrm{I} \gamma(\theta)$, (particle) $\gamma$-coin following projectile Coulomb excitation. ${ }^{132} \mathrm{Te}$ level deduced g-factor. Recoil-in-vacuum technique. JOUR NIMBE 241971

2005GR25 NUCLEAR REACTIONS ${ }^{64} \mathrm{Ni}\left({ }^{132} \mathrm{Sn}, \mathrm{X}\right),\left({ }^{134} \mathrm{Sn}, \mathrm{X}\right), \mathrm{E}=450-620 \mathrm{MeV}$; measured fusion $\sigma . \mathrm{C}\left({ }^{130} \mathrm{Te},{ }^{130} \mathrm{Te}\right),\left({ }^{132} \mathrm{Te},{ }^{132} \mathrm{Te}\right), \mathrm{E}=3 \mathrm{MeV} /$ nucleon; measured $\mathrm{E} \gamma$, I $\gamma$, (particle) $\gamma$-coin following projectile Coulomb excitation. ${ }^{132} \mathrm{Te}$ level deduced g factor. ${ }^{13} \mathrm{C}\left({ }^{134} \mathrm{Te},{ }^{135} \mathrm{Te}\right), \mathrm{E}=550$ $\mathrm{MeV}$; measured $\mathrm{E} \gamma, \mathrm{I} \gamma .{ }^{135} \mathrm{Te}$ level deduced J, $\pi$. JOUR JPGPE 31 S1639 


\section{$\mathrm{A}=132$ (continued)}

2005HU08 RADIOACTIVITY ${ }^{132} \mathrm{Sb}\left(\beta^{-}\right)$[from U(p, F)]; measured $\mathrm{E} \gamma, \mathrm{I} \gamma$, $\gamma \gamma$-coin. ${ }^{132} \mathrm{Te}$ deduced levels, $\mathrm{J}, \pi$. Clarion array, comparison with model predictions. JOUR PRVCA 71044311

2005RA09 NUCLEAR REACTIONS C ( $\left.{ }^{126} \mathrm{Sn},{ }^{126} \mathrm{Sn}\right),\left({ }^{128} \mathrm{Sn},{ }^{128} \mathrm{Sn}\right),\left({ }^{130} \mathrm{Sn}\right.$, ${ }^{130} \mathrm{Sn}$ '), ( ${ }^{132} \mathrm{Sn},{ }^{132} \mathrm{Sn}$ '), $\left({ }^{134} \mathrm{Sn},{ }^{134} \mathrm{Sn}\right.$ '), $\left({ }^{132} \mathrm{Te},{ }^{132} \mathrm{Te}\right),\left({ }^{134} \mathrm{Te},{ }^{134} \mathrm{Te}\right)$, $\left({ }^{136} \mathrm{Te},{ }^{136} \mathrm{Te}\right.$ '), E not given; measured $\mathrm{E} \gamma$, I $\gamma$, (particle) $\gamma$-coin following projectile Coulomb excitation. ${ }^{126,128,130,132,134} \mathrm{Sn},{ }^{132,134,136} \mathrm{Te}$ deduced transitions $\mathrm{B}(\mathrm{E} 2) .{ }^{9} \mathrm{Be}\left({ }^{134} \mathrm{Te},{ }^{8} \mathrm{Be}\right),{ }^{13} \mathrm{C}\left({ }^{134} \mathrm{Te},{ }^{12} \mathrm{C}\right)$, E not given; measured $\mathrm{E} \gamma, \mathrm{I} \gamma, \gamma \gamma-$, (particle) $\gamma$-coin. ${ }^{135} \mathrm{Te}$ deduced level. Clarion, Hyball arrays. JOUR NUPAB 752 264c

2005RA32 NUCLEAR REACTIONS C ( $\left.{ }^{126} \mathrm{Sn},{ }^{126} \mathrm{Sn}\right),\left({ }^{128} \mathrm{Sn},{ }^{128} \mathrm{Sn}\right),\left({ }^{130} \mathrm{Sn}\right.$, ${ }^{130} \mathrm{Sn}$ '), $\left({ }^{132} \mathrm{Te},{ }^{132} \mathrm{Te}\right),\left({ }^{134} \mathrm{Te},{ }^{134} \mathrm{Te}\right),\left({ }^{136} \mathrm{Te},{ }^{136} \mathrm{Te}\right.$ '), E not given; measured $\mathrm{E} \gamma, \mathrm{I} \gamma, \gamma \gamma$-, (particle) $\gamma$-coin following projectile Coulomb excitation. ${ }^{132,134,136} \mathrm{Te},{ }^{126,128,130} \mathrm{Sn}$ deduced excitation $\mathrm{B}(\mathrm{E} 2)$. ${ }^{9} \mathrm{Be}\left({ }^{134} \mathrm{Te},{ }^{8} \mathrm{Be}\right),{ }^{13} \mathrm{C}\left({ }^{134} \mathrm{Te},{ }^{12} \mathrm{C}\right), \mathrm{E}=4.3 \mathrm{MeV} /$ nucleon; measured $\mathrm{E} \gamma$, $\mathrm{I} \gamma, \gamma \gamma$-coin, (particle) $\gamma$-coin; deduced single-neutron transfer $\sigma(\mathrm{E})$. ${ }^{135}$ Te deduced levels J, $\pi$. JOUR ZAANE 25 s01 383

2005ST18 NUCLEAR REACTIONS C( $\left.{ }^{132} \mathrm{Te},{ }^{132} \mathrm{Te}\right),\left({ }^{122} \mathrm{Te},{ }^{122} \mathrm{Te}\right),\left({ }^{126} \mathrm{Te}\right.$, ${ }^{126} \mathrm{Te}$ '), $\left({ }^{130} \mathrm{Te},{ }^{130} \mathrm{Te}\right.$ '), $\mathrm{E}=3 \mathrm{MeV} /$ nucleon; measured $\mathrm{E} \gamma, \mathrm{I} \gamma(\theta, \phi)$, (particle) $\gamma$-coin following projectile Coulomb excitation; deduced parameters. ${ }^{132} \mathrm{Te}$ level deduced g factor. Clarion, Hyball arrays, recoil-in-vacuum technique. JOUR PRLTA 94192501

2005ST33 NUCLEAR REACTIONS C $\left({ }^{132} \mathrm{Te},{ }^{132} \mathrm{Te}\right.$ '), $\left({ }^{122} \mathrm{Te},{ }^{122} \mathrm{Te}\right),\left({ }^{126} \mathrm{Te}\right.$, $\left.{ }^{126} \mathrm{Te}^{\prime}\right),\left({ }^{130} \mathrm{Te},{ }^{130} \mathrm{Te}\right.$ '), E $=3 \mathrm{MeV} /$ nucleon; measured $\mathrm{E} \gamma, \mathrm{I} \gamma(\theta, \phi)$, (particle) $\gamma$-coin following projectile Coulomb excitation; deduced parameters. ${ }^{132} \mathrm{Te}$ level deduced g factor. Clarion, Hyball arrays, recoil-in-vacuum technique. JOUR ZAANE 25 s01 205

2005ZA14 RADIOACTIVITY ${ }^{132} \mathrm{Sb}\left(\beta^{-}\right)$; measured $\mathrm{E} \gamma, \mathrm{I} \gamma, \gamma \gamma$-coin. ${ }^{132} \mathrm{Te}$ deduced levels, J, $\pi$. Comparisons with quasiparticle RPA calculations with density-dependent pairing. Clarion array. JOUR ZAANE 25 s01 389

${ }^{132} \mathrm{I} \quad$ 2005ADZZ NUCLEAR REACTIONS ${ }^{129} \mathrm{I}(\mathrm{n}, 7 \mathrm{n}),(\mathrm{n}, 6 \mathrm{n}),(\mathrm{n}, 4 \mathrm{n}),(\mathrm{n}, \gamma), \mathrm{E}=$ fast; ${ }^{237} \mathrm{~Np}(\mathrm{n}, \gamma), \mathrm{E}=$ fast; measured yields. ${ }^{237} \mathrm{~Np}(\mathrm{n}, \mathrm{F}){ }^{91} \mathrm{Sr} /{ }^{97} \mathrm{Zr} /{ }^{132} \mathrm{Te} /$ ${ }^{133} \mathrm{I} /{ }^{135} \mathrm{I}, \mathrm{E}=$ fast; ${ }^{238} \mathrm{Pu}(\mathrm{n}, \mathrm{F}){ }^{97} \mathrm{Zr} /{ }^{129} \mathrm{Sb} /{ }^{132} \mathrm{I} /{ }^{133} \mathrm{I} /{ }^{135} \mathrm{Xe} /$ ${ }^{105} \mathrm{Ru}, \mathrm{E}=$ fast; ${ }^{239} \mathrm{Pu}(\mathrm{n}, \mathrm{F}){ }^{88} \mathrm{Kr} /{ }^{91} \mathrm{Sr} /{ }^{92} \mathrm{Sr} /{ }^{92} \mathrm{Y} /{ }^{97} \mathrm{Zr} /{ }^{99} \mathrm{Mo} /$ ${ }^{103} \mathrm{Ru} /{ }^{105} \mathrm{Ru} /{ }^{128} \mathrm{Sb} /{ }^{129} \mathrm{Sb} /{ }^{132} \mathrm{Te} /{ }^{131} \mathrm{I} /{ }^{132} \mathrm{I} /{ }^{133} \mathrm{I} /{ }^{135} \mathrm{I} /{ }^{135} \mathrm{Xe}$ $/{ }^{143} \mathrm{Ce} /{ }^{140} \mathrm{Ba} /{ }^{140} \mathrm{La}, \mathrm{E}=$ fast; measured fission fragment yields. Secondary neutrons from proton irradiation. JINR nuclotron. CONF St Petersburg,P195,Adam

2005NA05 NUCLEAR REACTIONS ${ }^{232} \mathrm{Th},{ }^{232,238} \mathrm{U},{ }^{238,240} \mathrm{Pu},{ }^{244} \mathrm{Cm}(\mathrm{n}, \mathrm{F}){ }^{128} \mathrm{Sb}$ / ${ }^{130} \mathrm{Sb} /{ }^{132} \mathrm{Sb} /{ }^{131} \mathrm{Te} /{ }^{133} \mathrm{Te} /{ }^{132} \mathrm{I} /{ }^{134} \mathrm{I} /{ }^{136} \mathrm{I} /{ }^{135} \mathrm{Xe} /{ }^{138} \mathrm{Cs}$, $\mathrm{E}=$ thermal, fast; measured isomer yield ratios; deduced fission fragment angular momenta. Spin-dependent statistical model analysis. JOUR PRVCA 71014304 


\section{$\mathrm{A}=132$ (continued)}

${ }^{132} \mathrm{Xe}$ 2005LE04 NUCLEAR REACTIONS $\mathrm{Pb}(\mathrm{p}, \mathrm{X}){ }^{3} \mathrm{He} /{ }^{4} \mathrm{He} /{ }^{21} \mathrm{Ne} /{ }^{22} \mathrm{Ne} /{ }^{36} \mathrm{Ar} /$ ${ }^{38} \mathrm{Ar} /{ }^{78} \mathrm{Kr} /{ }^{80} \mathrm{Kr} /{ }^{81} \mathrm{Kr} /{ }^{82} \mathrm{Kr} /{ }^{83} \mathrm{Kr} /{ }^{84} \mathrm{Kr} /{ }^{85} \mathrm{Kr} /{ }^{86} \mathrm{Kr} /{ }^{124} \mathrm{Xe}$ $/{ }^{126} \mathrm{Xe} /{ }^{128} \mathrm{Xe} /{ }^{129} \mathrm{Xe} /{ }^{130} \mathrm{Xe} /{ }^{131} \mathrm{Xe} /{ }^{132} \mathrm{Xe} /{ }^{134} \mathrm{Xe}, \mathrm{E}=44-2595$ $\mathrm{MeV}$; measured production $\sigma$; deduced reaction mechanism features. Mini-stack approach, comparisons with model predictions. JOUR NIMBE 2291

2005SH38 ATOMIC MASSES ${ }^{32,33} \mathrm{~S},{ }^{84,86} \mathrm{Kr},{ }^{129,132}$ Xe; measured masses. Penning trap. JOUR PLRAA 72022510

${ }^{132} \mathrm{La}$ 2005GR10 NUCLEAR REACTIONS ${ }^{122} \mathrm{Sn}\left({ }^{14} \mathrm{~N}, 4 \mathrm{n}\right), \mathrm{E}=70 \mathrm{MeV} ;{ }^{122} \mathrm{Sn}\left({ }^{10} \mathrm{~B}, 4 \mathrm{n}\right)$, $\mathrm{E}=55 \mathrm{MeV}$; measured $\mathrm{E} \gamma, \mathrm{I} \gamma, \gamma \gamma$-coin, DSA. ${ }^{128} \mathrm{Cs},{ }^{132}$ La levels deduced $\mathrm{T}_{1 / 2}, \mathrm{~B}(\mathrm{E} 2), \mathrm{B}(\mathrm{M} 1)$, chirality. Osiris II array. JOUR IMPEE 14347

2005SR02 NUCLEAR REACTIONS ${ }^{122} \mathrm{Sn}\left({ }^{14} \mathrm{~N}, 4 \mathrm{n}\right), \mathrm{E}=70 \mathrm{MeV} ;{ }^{122} \mathrm{Sn}\left({ }^{10} \mathrm{~B}, 4 \mathrm{n}\right)$, $\mathrm{E}=55 \mathrm{MeV}$; measured $\mathrm{E} \gamma, \mathrm{I} \gamma, \gamma \gamma$-coin, DSA. ${ }^{132} \mathrm{La},{ }^{128} \mathrm{Cs}$ deduced levels, J, $\pi, \mathrm{T}_{1 / 2}$, rotational bands, intraband B(M1), B(E2). ${ }^{128} \mathrm{Cs}$ deduced possible chiral bands. Osiris II array. JOUR APOBB 361063 2005UN01 NUCLEAR REACTIONS ${ }^{128} \mathrm{Te}\left({ }^{14} \mathrm{~N}, 4 \mathrm{n}\right),\left({ }^{14} \mathrm{~N}, 5 \mathrm{n}\right),\left({ }^{14} \mathrm{~N}, 4 \mathrm{np}\right),\left({ }^{14} \mathrm{~N}\right.$, $5 \mathrm{n} \alpha),\left({ }^{14} \mathrm{~N}, 6 \mathrm{n} \alpha\right),\left({ }^{14} \mathrm{~N}, \mathrm{n} 2 \mathrm{p} \alpha\right),\left({ }^{14} \mathrm{~N}, \mathrm{n} 2 \mathrm{p} 2 \alpha\right),\left({ }^{14} \mathrm{~N}, 3 \alpha\right), \mathrm{E} \approx 64-90 ;$ measured excitation functions; deduced reaction mechanism features. Activation technique, comparison with model predictions. JOUR IMPEE 14775

${ }^{132} \mathrm{Ce} \quad$ 2005CA23 NUCLEAR REACTIONS ${ }^{198} \mathrm{Pt}\left({ }^{18} \mathrm{O}, \mathrm{xn}\right), \mathrm{E}=96 \mathrm{MeV}$; measured prompt and delayed $\mathrm{E} \gamma, \mathrm{I} \gamma .{ }^{216} \mathrm{Rn}$ deduced GDR parameters. ${ }^{68} \mathrm{Zn}\left({ }^{64} \mathrm{Ni}, \mathrm{X}\right), \mathrm{E}=300,400,500 \mathrm{MeV} ;{ }^{116} \mathrm{Sn}\left({ }^{16} \mathrm{O}, \mathrm{X}\right), \mathrm{E}=130,250 \mathrm{MeV}$; measured $\mathrm{E} \gamma, \mathrm{I} \gamma .{ }^{132} \mathrm{Ce}$ deduced GDR features, entrance channel effects. JOUR APOBB 361145

2005GR09 NUCLEAR REACTIONS ${ }^{68} \mathrm{Zn}\left({ }^{64} \mathrm{Ni}, \mathrm{X}\right), \mathrm{E}=300,400,500 \mathrm{MeV}$; ${ }^{116} \mathrm{Sn}\left({ }^{16} \mathrm{O}, \mathrm{X}\right), \mathrm{E}=130,250 \mathrm{MeV}$; measured $\mathrm{E} \gamma, \mathrm{E} \alpha$, light charged particle and evaporation residue spectra. ${ }^{132} \mathrm{Ce}$ deduced GDR features, possible pre-equilibrium effects. JOUR APOBB 361155

2005PA30 NUCLEAR REACTIONS ${ }^{100} \mathrm{Mo}\left({ }^{36} \mathrm{~S}, 4 \mathrm{n}\right),\left({ }^{36} \mathrm{~S}, 5 \mathrm{n}\right), \mathrm{E}=160,165 \mathrm{MeV}$; measured $\mathrm{E} \gamma, \mathrm{I} \gamma, \gamma \gamma$-coin. ${ }^{131,132} \mathrm{Ce}$ deduced high-spin levels, J, $\pi$, superdeformed bands, configurations, band termination features. Euroball IV array, cranked mean-field calculations. JOUR PRVCA 71 054309

2005WI19 NUCLEAR REACTIONS ${ }^{68} \mathrm{Zn}\left({ }^{64} \mathrm{Ni}, \mathrm{X}\right), \mathrm{E}=300,400,500 \mathrm{MeV}$; measured $\mathrm{E} \gamma, \mathrm{I} \gamma$, (particle) $\gamma$-coin. ${ }^{132} \mathrm{Ce}$ deduced GDR parameters. JOUR JPGPE 31 S1973

\section{$\mathrm{A}=133$}

${ }^{133} \mathrm{Te}$ 2005BEZW NUCLEAR REACTIONS ${ }^{238} \mathrm{U}(\gamma, \mathrm{F}){ }^{84} \mathrm{Br} /{ }^{130} \mathrm{Sb} /{ }^{132} \mathrm{Sb} /{ }^{131} \mathrm{Te} /$ ${ }^{133} \mathrm{Te} /{ }^{134} \mathrm{I} /{ }^{135} \mathrm{Xe}, \mathrm{E}=16 \mathrm{MeV}$ bremsstrahlung; ${ }^{237} \mathrm{~Np}(\gamma, \mathrm{F}){ }^{134} \mathrm{I} /$ ${ }^{135} \mathrm{Xe}, \mathrm{E}=16 \mathrm{MeV}$ bremsstrahlung; measured $\mathrm{E} \gamma, \mathrm{I} \gamma$; deduced isomer yield ratios, fission fragments mean angular momenta. CONF Santa Fe (Nucl Data for Sci and Technol) Proc,Vol1,P641 


\section{$\mathrm{A}=133$ (continued)}

2005HW06 RADIOACTIVITY ${ }^{252} \mathrm{Cf}(\mathrm{SF})$; measured E $\gamma, \mathrm{I} \gamma, \gamma \gamma$-coin. ${ }^{95,97} \mathrm{Sr},{ }^{99} \mathrm{Zr}$, ${ }^{108} \mathrm{Tc},{ }^{133,134} \mathrm{Te},{ }^{137} \mathrm{Xe}$ levels deduced $\mathrm{T}_{1 / 2}$. Gammasphere array, time-gated triple-coincidence method. JOUR ZAANE 25 s01 463

2005NA05 NUCLEAR REACTIONS ${ }^{232} \mathrm{Th},{ }^{232,238} \mathrm{U},{ }^{238,240} \mathrm{Pu},{ }^{244} \mathrm{Cm}(\mathrm{n}, \mathrm{F}){ }^{128} \mathrm{Sb}$ $/{ }^{130} \mathrm{Sb} /{ }^{132} \mathrm{Sb} /{ }^{131} \mathrm{Te} /{ }^{133} \mathrm{Te} /{ }^{132} \mathrm{I} /{ }^{134} \mathrm{I} /{ }^{136} \mathrm{I} /{ }^{135} \mathrm{Xe} /{ }^{138} \mathrm{Cs}$, $\mathrm{E}=$ thermal, fast; measured isomer yield ratios; deduced fission fragment angular momenta. Spin-dependent statistical model analysis. JOUR PRVCA 71014304

${ }^{133} \mathrm{I} \quad 2005$ ADZZ NUCLEAR REACTIONS ${ }^{129} \mathrm{I}(\mathrm{n}, 7 \mathrm{n}),(\mathrm{n}, 6 \mathrm{n}),(\mathrm{n}, 4 \mathrm{n}),(\mathrm{n}, \gamma), \mathrm{E}=$ fast; ${ }^{237} \mathrm{~Np}(\mathrm{n}, \gamma), \mathrm{E}=$ fast; measured yields. ${ }^{237} \mathrm{~Np}(\mathrm{n}, \mathrm{F}){ }^{91} \mathrm{Sr} /{ }^{97} \mathrm{Zr} /{ }^{132} \mathrm{Te} /$ ${ }^{133} \mathrm{I} /{ }^{135} \mathrm{I}, \mathrm{E}=$ fast; ${ }^{238} \mathrm{Pu}(\mathrm{n}, \mathrm{F}){ }^{97} \mathrm{Zr} /{ }^{129} \mathrm{Sb} /{ }^{132} \mathrm{I} /{ }^{133} \mathrm{I} /{ }^{135} \mathrm{Xe} /$ ${ }^{105} \mathrm{Ru}, \mathrm{E}=$ fast; ${ }^{239} \mathrm{Pu}(\mathrm{n}, \mathrm{F}){ }^{88} \mathrm{Kr} /{ }^{91} \mathrm{Sr} /{ }^{92} \mathrm{Sr} /{ }^{92} \mathrm{Y} /{ }^{97} \mathrm{Zr} /{ }^{99} \mathrm{Mo} /$ ${ }^{103} \mathrm{Ru} /{ }^{105} \mathrm{Ru} /{ }^{128} \mathrm{Sb} /{ }^{129} \mathrm{Sb} /{ }^{132} \mathrm{Te} /{ }^{131} \mathrm{I} /{ }^{132} \mathrm{I} /{ }^{133} \mathrm{I} /{ }^{135} \mathrm{I} /{ }^{135} \mathrm{Xe}$ $/{ }^{143} \mathrm{Ce} /{ }^{140} \mathrm{Ba} /{ }^{140} \mathrm{La}, \mathrm{E}=$ fast; measured fission fragment yields. Secondary neutrons from proton irradiation. JINR nuclotron. CONF St Petersburg,P195,Adam

${ }^{133} \mathrm{Cs} \quad 2004 \mathrm{GE} 20$ RADIOACTIVITY ${ }^{155} \mathrm{Sm}\left(\beta^{-}\right)$[from ${ }^{154} \mathrm{Sm}(\mathrm{n}, \gamma)$ ]; ${ }^{60} \mathrm{Co},{ }^{133} \mathrm{Ba},{ }^{152} \mathrm{Eu}$; measured $\gamma$-ray angular correlations. ${ }^{155} \mathrm{Eu},{ }^{60} \mathrm{Ni},{ }^{133} \mathrm{Cs},{ }^{152} \mathrm{Gd}$ transitions deduced $\delta$. Comparison with previous results. JOUR BJPHE 34722

2005DA40 NUCLEAR MOMENTS ${ }^{133} \mathrm{Cs}$; measured hfs; deduced constants. JOUR EULEE 72740

${ }^{133} \mathrm{Ba} \quad$ 2004GE20 RADIOACTIVITY ${ }^{155} \mathrm{Sm}\left(\beta^{-}\right)\left[\right.$from $\left.{ }^{154} \mathrm{Sm}(\mathrm{n}, \gamma)\right] ;{ }^{60} \mathrm{Co},{ }^{133} \mathrm{Ba},{ }^{152} \mathrm{Eu}$ measured $\gamma$-ray angular correlations. ${ }^{155} \mathrm{Eu},{ }^{60} \mathrm{Ni},{ }^{133} \mathrm{Cs},{ }^{152} \mathrm{Gd}$ transitions deduced $\delta$. Comparison with previous results. JOUR BJPHE 34722

${ }^{133} \mathrm{La} \quad$ 2005UN01 NUCLEAR REACTIONS ${ }^{128} \mathrm{Te}\left({ }^{14} \mathrm{~N}, 4 \mathrm{n}\right),\left({ }^{14} \mathrm{~N}, 5 \mathrm{n}\right),\left({ }^{14} \mathrm{~N}, 4 \mathrm{np}\right),\left({ }^{14} \mathrm{~N}\right.$, $5 \mathrm{n} \alpha),\left({ }^{14} \mathrm{~N}, 6 \mathrm{n} \alpha\right),\left({ }^{14} \mathrm{~N}, \mathrm{n} 2 \mathrm{p} \alpha\right),\left({ }^{14} \mathrm{~N}, \mathrm{n} 2 \mathrm{p} 2 \alpha\right),\left({ }^{14} \mathrm{~N}, 3 \alpha\right), \mathrm{E} \approx 64-90 ;$ measured excitation functions; deduced reaction mechanism features. Activation technique, comparison with model predictions. JOUR IMPEE 14775

${ }^{133} \mathrm{Nd}$ 2005PE18 NUCLEAR REACTIONS ${ }^{104} \mathrm{Pd}\left({ }^{32} \mathrm{~S}, \mathrm{n} 2 \mathrm{p}\right), \mathrm{E}=135 \mathrm{MeV}$; measured Doppler-shifted E $\gamma, \mathrm{I} \gamma, \gamma \gamma$-coin. ${ }^{133} \mathrm{Nd}$ levels deduced $\mathrm{T}_{1 / 2}, \mathrm{~B}(\mathrm{E} 2)$, decay-out mechanism for highly deformed rotational band. GASP array, recoil-distance method. JOUR PRVCA 72031304

\section{$\mathrm{A}=\mathbf{1 3 4}$}

${ }^{134} \mathrm{Sn} \quad$ 2005RA09

NUCLEAR REACTIONS C ( $\left.{ }^{126} \mathrm{Sn},{ }^{126} \mathrm{Sn}^{\prime}\right),\left({ }^{128} \mathrm{Sn},{ }^{128} \mathrm{Sn}\right),\left({ }^{130} \mathrm{Sn}\right.$, ${ }^{130} \mathrm{Sn}$ '), ( $\left.{ }^{132} \mathrm{Sn},{ }^{132} \mathrm{Sn}^{\prime}\right),\left({ }^{134} \mathrm{Sn},{ }^{134} \mathrm{Sn}\right),\left({ }^{132} \mathrm{Te},{ }^{132} \mathrm{Te}\right),\left({ }^{134} \mathrm{Te},{ }^{134} \mathrm{Te}\right)$, $\left({ }^{136} \mathrm{Te},{ }^{136} \mathrm{Te}\right.$ '), E not given; measured $\mathrm{E} \gamma$, I $\gamma$, (particle) $\gamma$-coin following projectile Coulomb excitation. ${ }^{126,128,130,132,134} \mathrm{Sn},{ }^{132,134,136} \mathrm{Te}$ deduced transitions $\mathrm{B}(\mathrm{E} 2) .{ }^{9} \mathrm{Be}\left({ }^{134} \mathrm{Te},{ }^{8} \mathrm{Be}\right),{ }^{13} \mathrm{C}\left({ }^{134} \mathrm{Te},{ }^{12} \mathrm{C}\right)$, E not given; measured $\mathrm{E} \gamma, \mathrm{I} \gamma, \gamma \gamma-$, (particle) $\gamma$-coin. ${ }^{135} \mathrm{Te}$ deduced level. Clarion, Hyball arrays. JOUR NUPAB $752264 \mathrm{c}$

2005SH23 RADIOACTIVITY ${ }^{134} \operatorname{Sn}\left(\beta^{-}\right) ;{ }^{135} \operatorname{Sn}\left(\beta^{-}\right),\left(\beta^{-} \mathrm{n}\right)$; measured $\mathrm{E} \gamma, \mathrm{I} \gamma$, $\beta \gamma-, \gamma \gamma$-coin. ${ }^{134} \mathrm{Sb}$ deduced levels, $\mathrm{J}, \pi, \beta$-decaying isomeric state. Mass separator, shell model calculations. JOUR PRVCA 71064321 


\section{$\mathrm{A}=134$ (continued)}

2005VA31 NUCLEAR REACTIONS ${ }^{48} \mathrm{Ti}\left({ }^{132} \mathrm{Sn},{ }^{132} \mathrm{Sn}\right), \mathrm{E}=470-495 \mathrm{MeV}$; ${ }^{90} \mathrm{Zr}\left({ }^{134} \mathrm{Sn},{ }^{134} \mathrm{Sn}\right.$ '), $\mathrm{E}=400 \mathrm{MeV}$; measured $\mathrm{E} \gamma, \mathrm{I} \gamma$, (particle) $\gamma$-coin following projectile Coulomb excitation. ${ }^{132,134} \mathrm{Sn}$ deduced transitions B(E2). JOUR ZAANE 25 s01 391

${ }^{134} \mathrm{Sb} \quad 2005 \mathrm{SH} 23 \quad$ RADIOACTIVITY ${ }^{134} \operatorname{Sn}\left(\beta^{-}\right) ;{ }^{135} \mathrm{Sn}\left(\beta^{-}\right),\left(\beta^{-} \mathrm{n}\right) ;$ measured $\mathrm{E} \gamma, \mathrm{I} \gamma$, $\beta \gamma$-, $\gamma \gamma$-coin. ${ }^{134} \mathrm{Sb}$ deduced levels, $\mathrm{J}, \pi, \beta$-decaying isomeric state. Mass separator, shell model calculations. JOUR PRVCA 71064321 2005SH53 RADIOACTIVITY ${ }^{111} \mathrm{Te}\left(\beta^{+}\right)\left[\right.$from $\left.{ }^{58} \mathrm{Ni}\left({ }^{56} \mathrm{Fe}, 2 \mathrm{pn}\right)\right] ;{ }^{135} \mathrm{Sn}\left(\beta^{-}\right)$, $\left(\beta^{-} \mathrm{n}\right)[$ from $\mathrm{U}(\mathrm{p}, \mathrm{F}), \mathrm{E}=1.4 \mathrm{GeV}]$; measured $\mathrm{E} \gamma, \mathrm{I} \gamma, \gamma \gamma$-coin following decay of mass-separated sources. ${ }^{111,134,135} \mathrm{Sb}$ deduced levels, J, $\pi$. Comparison with model calculations. JOUR ZAANE 25 s01 121

${ }^{134} \mathrm{Te} \quad 2005 \mathrm{HW} 06$

2005RA09 RADIOACTIVITY ${ }^{252} \mathrm{Cf}(\mathrm{SF})$; measured $\mathrm{E} \gamma, \mathrm{I} \gamma, \gamma \gamma$-coin. ${ }^{95,97} \mathrm{Sr},{ }^{99} \mathrm{Zr}$, ${ }^{108} \mathrm{Tc},{ }^{133,134} \mathrm{Te},{ }^{137} \mathrm{Xe}$ levels deduced $\mathrm{T}_{1 / 2}$. Gammasphere array, time-gated triple-coincidence method. JOUR ZAANE 25 s01 463 NUCLEAR REACTIONS C ( $\left.{ }^{126} \mathrm{Sn},{ }^{126} \mathrm{Sn}\right),\left({ }^{128} \mathrm{Sn},{ }^{128} \mathrm{Sn}\right),\left({ }^{130} \mathrm{Sn}\right.$, ${ }^{130} \mathrm{Sn}$ '), ( ${ }^{132} \mathrm{Sn},{ }^{132} \mathrm{Sn}$ '), ( $\left.{ }^{134} \mathrm{Sn},{ }^{134} \mathrm{Sn}\right),\left({ }^{132} \mathrm{Te},{ }^{132} \mathrm{Te}\right),\left({ }^{134} \mathrm{Te},{ }^{134} \mathrm{Te}\right)$, $\left({ }^{136} \mathrm{Te},{ }^{136} \mathrm{Te}\right.$ '), E not given; measured $\mathrm{E} \gamma$, I $\gamma$, (particle) $\gamma$-coin following projectile Coulomb excitation. ${ }^{126,128,130,132,134} \mathrm{Sn},{ }^{132,134,136} \mathrm{Te}$ deduced transitions $\mathrm{B}(\mathrm{E} 2) .{ }^{9} \mathrm{Be}\left({ }^{134} \mathrm{Te},{ }^{8} \mathrm{Be}\right),{ }^{13} \mathrm{C}\left({ }^{134} \mathrm{Te},{ }^{12} \mathrm{C}\right)$, E not given; measured $\mathrm{E} \gamma, \mathrm{I} \gamma, \gamma \gamma-$, (particle) $\gamma$-coin. ${ }^{135} \mathrm{Te}$ deduced level. Clarion, Hyball arrays. JOUR NUPAB 752 264c

2005RA32 NUCLEAR REACTIONS C ( $\left.{ }^{126} \mathrm{Sn},{ }^{126} \mathrm{Sn}\right),\left({ }^{128} \mathrm{Sn},{ }^{128} \mathrm{Sn}\right),\left({ }^{130} \mathrm{Sn}\right.$, ${ }^{130} \mathrm{Sn}$ '), $\left({ }^{132} \mathrm{Te},{ }^{132} \mathrm{Te}\right.$ '), $\left({ }^{134} \mathrm{Te},{ }^{134} \mathrm{Te}\right),\left({ }^{136} \mathrm{Te},{ }^{136} \mathrm{Te}\right), \mathrm{E}$ not given; measured $\mathrm{E} \gamma, \mathrm{I} \gamma, \gamma \gamma-$, (particle) $\gamma$-coin following projectile Coulomb excitation. ${ }^{132,134,136} \mathrm{Te},{ }^{126,128,130} \mathrm{Sn}$ deduced excitation $\mathrm{B}(\mathrm{E} 2)$. ${ }^{9} \mathrm{Be}\left({ }^{134} \mathrm{Te},{ }^{8} \mathrm{Be}\right),{ }^{13} \mathrm{C}\left({ }^{134} \mathrm{Te},{ }^{12} \mathrm{C}\right), \mathrm{E}=4.3 \mathrm{MeV} /$ nucleon; measured $\mathrm{E} \gamma$, $\mathrm{I} \gamma, \gamma \gamma$-coin, (particle) $\gamma$-coin; deduced single-neutron transfer $\sigma(\mathrm{E})$.

${ }^{135}$ Te deduced levels J, $\pi$. JOUR ZAANE 25 s01 383

${ }^{134} \mathrm{I} \quad 2005 B E Z W$ NUCLEAR REACTIONS ${ }^{238} \mathrm{U}(\gamma, \mathrm{F}){ }^{84} \mathrm{Br} /{ }^{130} \mathrm{Sb} /{ }^{132} \mathrm{Sb} /{ }^{131} \mathrm{Te} /$ ${ }^{133} \mathrm{Te} /{ }^{134} \mathrm{I} /{ }^{135} \mathrm{Xe}, \mathrm{E}=16 \mathrm{MeV}$ bremsstrahlung; ${ }^{237} \mathrm{~Np}(\gamma, \mathrm{F}){ }^{134} \mathrm{I} /$ ${ }^{135} \mathrm{Xe}, \mathrm{E}=16 \mathrm{MeV}$ bremsstrahlung; measured $\mathrm{E} \gamma, \mathrm{I} \gamma$; deduced isomer yield ratios, fission fragments mean angular momenta. CONF Santa Fe (Nucl Data for Sci and Technol) Proc,Vol1,P641

2005NA05 NUCLEAR REACTIONS ${ }^{232} \mathrm{Th},{ }^{232,238} \mathrm{U},{ }^{238,240} \mathrm{Pu},{ }^{244} \mathrm{Cm}(\mathrm{n}, \mathrm{F}){ }^{128} \mathrm{Sb}$ $/{ }^{130} \mathrm{Sb} /{ }^{132} \mathrm{Sb} /{ }^{131} \mathrm{Te} /{ }^{133} \mathrm{Te} /{ }^{132} \mathrm{I} /{ }^{134} \mathrm{I} /{ }^{136} \mathrm{I} /{ }^{135} \mathrm{Xe} /{ }^{138} \mathrm{Cs}$, $\mathrm{E}=$ thermal, fast; measured isomer yield ratios; deduced fission fragment angular momenta. Spin-dependent statistical model analysis. JOUR PRVCA 71014304

${ }^{134} \mathrm{Xe} \quad$ 2005LE04 NUCLEAR REACTIONS $\mathrm{Pb}(\mathrm{p}, \mathrm{X}){ }^{3} \mathrm{He} /{ }^{4} \mathrm{He} /{ }^{21} \mathrm{Ne} /{ }^{22} \mathrm{Ne} /{ }^{36} \mathrm{Ar} /$ ${ }^{38} \mathrm{Ar} /{ }^{78} \mathrm{Kr} /{ }^{80} \mathrm{Kr} /{ }^{81} \mathrm{Kr} /{ }^{82} \mathrm{Kr} /{ }^{83} \mathrm{Kr} /{ }^{84} \mathrm{Kr} /{ }^{85} \mathrm{Kr} /{ }^{86} \mathrm{Kr} /{ }^{124} \mathrm{Xe}$ / ${ }^{126} \mathrm{Xe} /{ }^{128} \mathrm{Xe} /{ }^{129} \mathrm{Xe} /{ }^{130} \mathrm{Xe} /{ }^{131} \mathrm{Xe} /{ }^{132} \mathrm{Xe} /{ }^{134} \mathrm{Xe}, \mathrm{E}=44-2595$

$\mathrm{MeV}$; measured production $\sigma$; deduced reaction mechanism features. Mini-stack approach, comparisons with model predictions. JOUR NIMBE 2291

${ }^{134} \mathrm{Cs} \quad 2005 U 0 Z Z$ NUCLEAR REACTIONS U(p, F) ${ }^{95} \mathrm{Zr} /{ }^{115} \mathrm{Cd} /{ }^{134} \mathrm{Cs} /{ }^{136} \mathrm{Cs} /{ }^{137} \mathrm{Cs}$ $/{ }^{147} \mathrm{Nd}, \mathrm{E} \approx 20-70 \mathrm{MeV}$; measured production $\sigma$. Stacked-foil activation technique, comparison with model predictions. CONF Santa Fe (Nucl Data for Sci and Technol) Proc,Vol2,P1547 


\section{$\mathrm{A}=134$ (continued)}

${ }^{134} \mathrm{Pr} \quad$ 2005T022 NUCLEAR REACTIONS ${ }^{119} \mathrm{Sn}\left({ }^{19} \mathrm{~F}, 4 \mathrm{n}\right), \mathrm{E}=83,87 \mathrm{MeV}$; measured $\mathrm{E} \gamma, \mathrm{I} \gamma, \gamma \gamma$-coin, DSA. ${ }^{134} \mathrm{Pr}$ levels deduced $\mathrm{T}_{1 / 2}, \mathrm{~B}(\mathrm{M} 1), \mathrm{B}(\mathrm{E} 2)$, mixing ratios. Euroball IV array, recoil-distance and Doppler-shift attenuation techniques. JOUR ZAANE 25 s01 447

2005TOZY NUCLEAR REACTIONS ${ }^{119} \mathrm{Sn}\left({ }^{19} \mathrm{~F}, 4 \mathrm{n}\right), \mathrm{E}=83,87 \mathrm{MeV}$; measured Doppler-shifted $\mathrm{E} \gamma, \mathrm{I} \gamma, \gamma \gamma$-coin. ${ }^{134} \mathrm{Pr}$ deduced high-spin levels $\mathrm{T}_{1 / 2}$, B(E2), B(M1), chiral symmetry features. Recoil-distance and Doppler-shift attenuation techniques. CONF Argonne(Nuclei at the Limits),P93,Tonev

${ }^{134} \mathrm{Sm} \quad 2005 \mathrm{XU} 04 \quad$ RADIOACTIVITY ${ }^{81} \mathrm{Zr},{ }^{85} \mathrm{Mo},{ }^{89} \mathrm{Ru},{ }^{92} \mathrm{Rh},{ }^{93} \mathrm{Pd},{ }^{121} \mathrm{Ce},{ }^{125} \mathrm{Nd}$, ${ }^{128} \mathrm{Pm},{ }^{129} \mathrm{Sm},{ }^{135,137} \mathrm{Gd},{ }^{139} \mathrm{Dy},{ }^{142} \mathrm{Ho},{ }^{149} \mathrm{Yb}\left(\beta^{+} \mathrm{p}\right)$; measured $\beta$-delayed $\mathrm{E} \gamma, \mathrm{I} \gamma$, proton spectra, $\mathrm{p} \gamma$-coin, $\mathrm{T}_{1 / 2}$. Comparison with model predictions. JOUR PRVCA 71054318

\section{$\mathrm{A}=135$}

${ }^{135} \mathrm{Sn} \quad 2005 \mathrm{~K} 040$

RADIOACTIVITY ${ }^{135} \operatorname{Sn}\left(\beta^{-}\right)$[from ${ }^{235} \mathrm{U}(\mathrm{n}, \mathrm{F}), \mathrm{E}=$ thermal]; measured $\mathrm{E} \gamma, \mathrm{I} \gamma, \beta \gamma$-coin following decay of mass-separated sources. ${ }^{135} \mathrm{Sb}$ deduced levels, $\mathrm{J}, \pi, \mathrm{T}_{1 / 2}, \mathrm{~B}(\mathrm{M} 1)$, configurations. Comparison with shell model calculations. JOUR ZAANE 25 s01 123

2005SH23 RADIOACTIVITY ${ }^{134} \operatorname{Sn}\left(\beta^{-}\right) ;{ }^{135} \operatorname{Sn}\left(\beta^{-}\right),\left(\beta^{-} \mathrm{n}\right)$; measured $\mathrm{E} \gamma, \mathrm{I} \gamma$, $\beta \gamma$-, $\gamma \gamma$-coin. ${ }^{134} \mathrm{Sb}$ deduced levels, $\mathrm{J}, \pi, \beta$-decaying isomeric state. Mass separator, shell model calculations. JOUR PRVCA 71064321

2005SH36 RADIOACTIVITY ${ }^{135} \mathrm{Sn}\left(\beta^{-}\right)$[from U(n, F)]; measured $\mathrm{E} \gamma, \mathrm{I} \gamma$, $\gamma \gamma$-coin. ${ }^{135} \mathrm{Sb}$ deduced levels, $\mathrm{J}, \pi$. Resonance ionization, mass separator. Comparisons with shell-model predictions. JOUR PRVCA 72024305

2005SH53 RADIOACTIVITY ${ }^{111} \mathrm{Te}\left(\beta^{+}\right)$[from $\left.{ }^{58} \mathrm{Ni}\left({ }^{56} \mathrm{Fe}, 2 \mathrm{pn}\right)\right] ;{ }^{135} \mathrm{Sn}\left(\beta^{-}\right)$, $\left(\beta^{-} \mathrm{n}\right)$ [from $\left.\mathrm{U}(\mathrm{p}, \mathrm{F}), \mathrm{E}=1.4 \mathrm{GeV}\right]$; measured $\mathrm{E} \gamma, \mathrm{I} \gamma, \gamma \gamma$-coin following decay of mass-separated sources. ${ }^{111,134,135} \mathrm{Sb}$ deduced levels, J, $\pi$. Comparison with model calculations. JOUR ZAANE 25 s01 121

$\begin{array}{ll}{ }^{135} \mathrm{Sb} & 2005 \mathrm{KO} 40 \\ & \mathrm{RADIOACTIVITY}{ }^{135} \mathrm{Sn}\left(\beta^{-}\right)\left[\text {from }{ }^{235} \mathrm{U}(\mathrm{n}, \mathrm{F}), \mathrm{E}=\text { thermal]; measured }\right. \\ & \mathrm{E} \gamma, \mathrm{I} \gamma, \beta \gamma \text {-coin following decay of mass-separated sources. }{ }^{135} \mathrm{Sb}\end{array}$ deduced levels, $\mathrm{J}, \pi, \mathrm{T}_{1 / 2}, \mathrm{~B}(\mathrm{M} 1)$, configurations. Comparison with shell model calculations. JOUR ZAANE 25 s01 123

2005SH23 RADIOACTIVITY ${ }^{134} \operatorname{Sn}\left(\beta^{-}\right) ;{ }^{135} \mathrm{Sn}\left(\beta^{-}\right),\left(\beta^{-} \mathrm{n}\right) ;$ measured $\mathrm{E} \gamma, \mathrm{I} \gamma$, $\beta \gamma-, \gamma \gamma$-coin. ${ }^{134} \mathrm{Sb}$ deduced levels, $\mathrm{J}, \pi, \beta$-decaying isomeric state. Mass separator, shell model calculations. JOUR PRVCA 71064321

2005SH36 RADIOACTIVITY ${ }^{135} \operatorname{Sn}\left(\beta^{-}\right)[$from $\mathrm{U}(\mathrm{n}, \mathrm{F})]$; measured $\mathrm{E} \gamma, \mathrm{I} \gamma$, $\gamma \gamma$-coin. ${ }^{135} \mathrm{Sb}$ deduced levels, $\mathrm{J}, \pi$. Resonance ionization, mass separator. Comparisons with shell-model predictions. JOUR PRVCA 72024305

2005SH53 RADIOACTIVITY ${ }^{111} \mathrm{Te}\left(\beta^{+}\right)\left[\right.$from $\left.{ }^{58} \mathrm{Ni}\left({ }^{56} \mathrm{Fe}, 2 \mathrm{pn}\right)\right] ;{ }^{135} \mathrm{Sn}\left(\beta^{-}\right)$, $\left(\beta^{-} \mathrm{n}\right)$ [from $\left.\mathrm{U}(\mathrm{p}, \mathrm{F}), \mathrm{E}=1.4 \mathrm{GeV}\right]$; measured $\mathrm{E} \gamma, \mathrm{I} \gamma, \gamma \gamma$-coin following decay of mass-separated sources. ${ }^{111,134,135} \mathrm{Sb}$ deduced levels, J, $\pi$. Comparison with model calculations. JOUR ZAANE 25 s01 121 


\section{$\mathrm{A}=135$ (continued)}

${ }^{135} \mathrm{Te} \quad$ 2005GR25 NUCLEAR REACTIONS ${ }^{64} \mathrm{Ni}\left({ }^{132} \mathrm{Sn}, \mathrm{X}\right),\left({ }^{134} \mathrm{Sn}, \mathrm{X}\right), \mathrm{E}=450-620 \mathrm{MeV}$; measured fusion $\sigma$. C $\left({ }^{130} \mathrm{Te},{ }^{130} \mathrm{Te}\right),\left({ }^{132} \mathrm{Te},{ }^{132} \mathrm{Te}\right), \mathrm{E}=3 \mathrm{MeV} /$ nucleon; measured $\mathrm{E} \gamma, \mathrm{I} \gamma$, (particle) $\gamma$-coin following projectile Coulomb excitation. ${ }^{132} \mathrm{Te}$ level deduced g factor. ${ }^{13} \mathrm{C}\left({ }^{134} \mathrm{Te},{ }^{135} \mathrm{Te}\right), \mathrm{E}=550$ $\mathrm{MeV} ;$ measured $\mathrm{E} \gamma, \mathrm{I} \gamma$. ${ }^{135} \mathrm{Te}$ level deduced J, $\pi$. JOUR JPGPE 31 S1639

2005RA09 NUCLEAR REACTIONS C ( $\left.{ }^{126} \mathrm{Sn},{ }^{126} \mathrm{Sn}\right),\left({ }^{128} \mathrm{Sn},{ }^{128} \mathrm{Sn}\right),\left({ }^{130} \mathrm{Sn}\right.$, $\left.{ }^{130} \mathrm{Sn}^{\prime}\right),\left({ }^{132} \mathrm{Sn},{ }^{132} \mathrm{Sn}\right),\left({ }^{134} \mathrm{Sn},{ }^{134} \mathrm{Sn}\right),\left({ }^{132} \mathrm{Te},{ }^{132} \mathrm{Te}\right),\left({ }^{134} \mathrm{Te},{ }^{134} \mathrm{Te}{ }^{\prime}\right)$, $\left({ }^{136} \mathrm{Te},{ }^{136} \mathrm{Te}\right), \mathrm{E}$ not given; measured $\mathrm{E} \gamma$, I $\gamma$, (particle) $\gamma$-coin following projectile Coulomb excitation. ${ }^{126,128,130,132,134} \mathrm{Sn},{ }^{132,134,136} \mathrm{Te}$ deduced transitions $\mathrm{B}(\mathrm{E} 2) .{ }^{9} \mathrm{Be}\left({ }^{134} \mathrm{Te},{ }^{8} \mathrm{Be}\right),{ }^{13} \mathrm{C}\left({ }^{134} \mathrm{Te},{ }^{12} \mathrm{C}\right)$, E not given; measured $\mathrm{E} \gamma, \mathrm{I} \gamma, \gamma \gamma-$, (particle) $\gamma$-coin. ${ }^{135} \mathrm{Te}$ deduced level. Clarion, Hyball arrays. JOUR NUPAB 752 264c

2005RA32 NUCLEAR REACTIONS C $\left({ }^{126} \mathrm{Sn},{ }^{126} \mathrm{Sn}^{\prime}\right),\left({ }^{128} \mathrm{Sn},{ }^{128} \mathrm{Sn}^{\prime}\right),\left({ }^{130} \mathrm{Sn}\right.$, ${ }^{130} \mathrm{Sn}$ '), $\left({ }^{132} \mathrm{Te},{ }^{132} \mathrm{Te}\right),\left({ }^{134} \mathrm{Te},{ }^{134} \mathrm{Te}\right),\left({ }^{136} \mathrm{Te},{ }^{136} \mathrm{Te}\right)$, E not given; measured $\mathrm{E} \gamma, \mathrm{I} \gamma, \gamma \gamma-$, (particle) $\gamma$-coin following projectile Coulomb excitation. ${ }^{132,134,136} \mathrm{Te},{ }^{126,128,130} \mathrm{Sn}$ deduced excitation $\mathrm{B}(\mathrm{E} 2)$.

${ }^{9} \mathrm{Be}\left({ }^{134} \mathrm{Te},{ }^{8} \mathrm{Be}\right),{ }^{13} \mathrm{C}\left({ }^{134} \mathrm{Te},{ }^{12} \mathrm{C}\right), \mathrm{E}=4.3 \mathrm{MeV} /$ nucleon; measured $\mathrm{E} \gamma$, $\mathrm{I} \gamma, \gamma \gamma$-coin, (particle) $\gamma$-coin; deduced single-neutron transfer $\sigma(\mathrm{E})$.

${ }^{135}$ Te deduced levels J, $\pi$. JOUR ZAANE 25 s01 383

${ }^{135} \mathrm{I} \quad 2005 \mathrm{ADZZ}$ NUCLEAR REACTIONS ${ }^{129} \mathrm{I}(\mathrm{n}, 7 \mathrm{n}),(\mathrm{n}, 6 \mathrm{n}),(\mathrm{n}, 4 \mathrm{n}),(\mathrm{n}, \gamma), \mathrm{E}=$ fast; ${ }^{237} \mathrm{~Np}(\mathrm{n}, \gamma), \mathrm{E}=$ fast; measured yields. ${ }^{237} \mathrm{~Np}(\mathrm{n}, \mathrm{F}){ }^{91} \mathrm{Sr} /{ }^{97} \mathrm{Zr} /{ }^{132} \mathrm{Te} /$ ${ }^{133} \mathrm{I} /{ }^{135} \mathrm{I}, \mathrm{E}=$ fast; ${ }^{238} \mathrm{Pu}(\mathrm{n}, \mathrm{F}){ }^{97} \mathrm{Zr} /{ }^{129} \mathrm{Sb} /{ }^{132} \mathrm{I} /{ }^{133} \mathrm{I} /{ }^{135} \mathrm{Xe} /$ ${ }^{105} \mathrm{Ru}, \mathrm{E}=$ fast; ${ }^{239} \mathrm{Pu}(\mathrm{n}, \mathrm{F}){ }^{88} \mathrm{Kr} /{ }^{91} \mathrm{Sr} /{ }^{92} \mathrm{Sr} /{ }^{92} \mathrm{Y} /{ }^{97} \mathrm{Zr} /{ }^{99} \mathrm{Mo} /$ ${ }^{103} \mathrm{Ru} /{ }^{105} \mathrm{Ru} /{ }^{128} \mathrm{Sb} /{ }^{129} \mathrm{Sb} /{ }^{132} \mathrm{Te} /{ }^{131} \mathrm{I} /{ }^{132} \mathrm{I} /{ }^{133} \mathrm{I} /{ }^{135} \mathrm{I} /{ }^{135} \mathrm{Xe}$ $/{ }^{143} \mathrm{Ce} /{ }^{140} \mathrm{Ba} /{ }^{140} \mathrm{La}, \mathrm{E}=$ fast; measured fission fragment yields. Secondary neutrons from proton irradiation. JINR nuclotron. CONF St Petersburg,P195,Adam

2005GAZW NUCLEAR REACTIONS ${ }^{232} \mathrm{Th},{ }^{238} \mathrm{U},{ }^{243} \mathrm{Am},{ }^{248} \mathrm{Cm}(\gamma, \mathrm{F}){ }^{135 m} \mathrm{Xe} /$ ${ }^{135} \mathrm{Xe} /{ }^{135} \mathrm{I}, \mathrm{E}=25 \mathrm{MeV}$ bremsstrahlung; measured yield ratios. Microtron, gas flow transport. CONF St Petersburg,P66,Gangrsky

2005UR01 RADIOACTIVITY ${ }^{248} \mathrm{Cm}(\mathrm{SF})$; measured E $\gamma, \mathrm{I} \gamma, \gamma \gamma$-coin. ${ }^{109,110,111} \mathrm{Tc},{ }^{135} \mathrm{I}$ deduced transitions. ${ }^{111} \mathrm{Tc}$ deduced levels, J, $\pi$, configurations. Eurogam2 array. Level systematics in neighboring nuclides discussed. JOUR ZAANE 24161

${ }^{135} \mathrm{Xe} \quad$ 2004GA60 NUCLEAR REACTIONS ${ }^{237} \mathrm{~Np}(\gamma, \mathrm{F}){ }^{135} \mathrm{Xe} /{ }^{137} \mathrm{Xe} /{ }^{138} \mathrm{Xe} /{ }^{139} \mathrm{Xe} /$ ${ }^{140} \mathrm{Xe} /{ }^{141} \mathrm{Xe} /{ }^{142} \mathrm{Xe} /{ }^{89} \mathrm{Kr} /{ }^{91} \mathrm{Kr} /{ }^{92} \mathrm{Kr} /{ }^{93} \mathrm{Kr}, \mathrm{E}=25 \mathrm{MeV}$ bremsstrahlung; measured fission yields, isotopic distribution parameters. Comparison with results from other targets. JOUR BRSPE 681298

2004GAZV NUCLEAR REACTIONS ${ }^{237} \mathrm{~Np},{ }^{243} \mathrm{Am}(\gamma, \mathrm{F}){ }^{89} \mathrm{Kr} /{ }^{91} \mathrm{Kr} /{ }^{92} \mathrm{Kr} /$ ${ }^{93} \mathrm{Kr} /{ }^{135} \mathrm{Xe} /{ }^{137} \mathrm{Xe} /{ }^{138} \mathrm{Xe} /{ }^{139} \mathrm{Xe} /{ }^{140} \mathrm{Xe} /{ }^{141} \mathrm{Xe} /{ }^{142} \mathrm{Xe}$, $\mathrm{E}_{\text {max }}=25 \mathrm{MeV}$; measured $\mathrm{E} \gamma$, I $\gamma$; deduced fission fragment yields. REPT JINR-P15-2004-119, Gangrsky 


\section{$\mathrm{A}=135$ (continued)}

2005ADZZ NUCLEAR REACTIONS ${ }^{129} \mathrm{I}(\mathrm{n}, 7 \mathrm{n}),(\mathrm{n}, 6 \mathrm{n}),(\mathrm{n}, 4 \mathrm{n}),(\mathrm{n}, \gamma), \mathrm{E}=$ fast; ${ }^{237} \mathrm{~Np}(\mathrm{n}, \gamma), \mathrm{E}=$ fast; measured yields. ${ }^{237} \mathrm{~Np}(\mathrm{n}, \mathrm{F}){ }^{91} \mathrm{Sr} /{ }^{97} \mathrm{Zr} /{ }^{132} \mathrm{Te} /$ ${ }^{133} \mathrm{I} /{ }^{135} \mathrm{I}, \mathrm{E}=$ fast; ${ }^{238} \mathrm{Pu}(\mathrm{n}, \mathrm{F}){ }^{97} \mathrm{Zr} /{ }^{129} \mathrm{Sb} /{ }^{132} \mathrm{I} /{ }^{133} \mathrm{I} /{ }^{135} \mathrm{Xe} /$ ${ }^{105} \mathrm{Ru}, \mathrm{E}=$ fast; ${ }^{239} \mathrm{Pu}(\mathrm{n}, \mathrm{F}){ }^{88} \mathrm{Kr} /{ }^{91} \mathrm{Sr} /{ }^{92} \mathrm{Sr} /{ }^{92} \mathrm{Y} /{ }^{97} \mathrm{Zr} /{ }^{99} \mathrm{Mo} /$ ${ }^{103} \mathrm{Ru} /{ }^{105} \mathrm{Ru} /{ }^{128} \mathrm{Sb} /{ }^{129} \mathrm{Sb} /{ }^{132} \mathrm{Te} /{ }^{131} \mathrm{I} /{ }^{132} \mathrm{I} /{ }^{133} \mathrm{I} /{ }^{135} \mathrm{I} /{ }^{135} \mathrm{Xe}$ $/{ }^{143} \mathrm{Ce} /{ }^{140} \mathrm{Ba} /{ }^{140} \mathrm{La}, \mathrm{E}=$ fast; measured fission fragment yields. Secondary neutrons from proton irradiation. JINR nuclotron. CONF St Petersburg,P195,Adam

2005BA34 NUCLEAR REACTIONS ${ }^{136} \mathrm{Xe}\left(\mathrm{d},{ }^{3} \mathrm{HeX}\right){ }^{135} \mathrm{Xe}, \mathrm{E}=500 \mathrm{MeV} ;{ }^{1} \mathrm{H}(\mathrm{d}$, $\left.\pi^{0}\right), \mathrm{E}=500 \mathrm{MeV}$; measured helium spectra. ${ }^{135} \mathrm{Xe}$ deduced pionic state binding energy. JOUR YAFIA 68517

2005BEZW NUCLEAR REACTIONS ${ }^{238} \mathrm{U}(\gamma, \mathrm{F}){ }^{84} \mathrm{Br} /{ }^{130} \mathrm{Sb} /{ }^{132} \mathrm{Sb} /{ }^{131} \mathrm{Te} /$ ${ }^{133} \mathrm{Te} /{ }^{134} \mathrm{I} /{ }^{135} \mathrm{Xe}, \mathrm{E}=16 \mathrm{MeV}$ bremsstrahlung; ${ }^{237} \mathrm{~Np}(\gamma, \mathrm{F}){ }^{134} \mathrm{I} /$ ${ }^{135} \mathrm{Xe}, \mathrm{E}=16 \mathrm{MeV}$ bremsstrahlung; measured $\mathrm{E} \gamma$, I $\gamma$; deduced isomer yield ratios, fission fragments mean angular momenta. CONF Santa Fe (Nucl Data for Sci and Technol) Proc,Vol1,P641

2005GA25 NUCLEAR REACTIONS ${ }^{248} \mathrm{Cm}(\gamma, \mathrm{F}){ }^{89} \mathrm{Kr} /{ }^{91} \mathrm{Kr} /{ }^{92} \mathrm{Kr} /{ }^{93} \mathrm{Kr} /$ ${ }^{135} \mathrm{Xe} /{ }^{137} \mathrm{Xe} /{ }^{138} \mathrm{Xe} /{ }^{139} \mathrm{Xe} /{ }^{140} \mathrm{Xe} /{ }^{141} \mathrm{Xe} /{ }^{142} \mathrm{Xe}, \mathrm{E}=25 \mathrm{MeV}$ bremsstrahlung; measured $\mathrm{E} \gamma, \mathrm{I} \gamma$; deduced yields. JOUR FECLA 125 44

2005GA50 NUCLEAR REACTIONS ${ }^{237} \mathrm{~Np},{ }^{243} \mathrm{Am}(\gamma, \mathrm{F}){ }^{135} \mathrm{Xe} /{ }^{137} \mathrm{Xe} /{ }^{138} \mathrm{Xe} /$ ${ }^{139} \mathrm{Xe} /{ }^{140} \mathrm{Xe} /{ }^{141} \mathrm{Xe} /{ }^{142} \mathrm{Xe} /{ }^{89} \mathrm{Kr} /{ }^{91} \mathrm{Kr} /{ }^{92} \mathrm{Kr} /{ }^{93} \mathrm{Kr}, \mathrm{E}=25 \mathrm{MeV}$ bremsstrahlung; measured fission yields, isotopic distribution parameters. JOUR YAFIA 681475 2005GAZW NUCLEAR REACTIONS ${ }^{232} \mathrm{Th},{ }^{238} \mathrm{U},{ }^{243} \mathrm{Am},{ }^{248} \mathrm{Cm}(\gamma, \mathrm{F}){ }^{135 m} \mathrm{Xe} /$ ${ }^{135} \mathrm{Xe} /{ }^{135} \mathrm{I}, \mathrm{E}=25 \mathrm{MeV}$ bremsstrahlung; measured yield ratios. Microtron, gas flow transport. CONF St Petersburg,P66,Gangrsky 2005NA05 NUCLEAR REACTIONS ${ }^{232} \mathrm{Th},{ }^{232,238} \mathrm{U},{ }^{238,240} \mathrm{Pu},{ }^{244} \mathrm{Cm}(\mathrm{n}, \mathrm{F}){ }^{128} \mathrm{Sb}$ / ${ }^{130} \mathrm{Sb} /{ }^{132} \mathrm{Sb} /{ }^{131} \mathrm{Te} /{ }^{133} \mathrm{Te} /{ }^{132} \mathrm{I} /{ }^{134} \mathrm{I} /{ }^{136} \mathrm{I} /{ }^{135} \mathrm{Xe} /{ }^{138} \mathrm{Cs}$, $\mathrm{E}=$ thermal, fast; measured isomer yield ratios; deduced fission fragment angular momenta. Spin-dependent statistical model analysis. JOUR PRVCA 71014304

${ }^{135} \mathrm{Cs}$ 2005UN01 NUCLEAR REACTIONS ${ }^{128} \mathrm{Te}\left({ }^{14} \mathrm{~N}, 4 \mathrm{n}\right),\left({ }^{14} \mathrm{~N}, 5 \mathrm{n}\right),\left({ }^{14} \mathrm{~N}, 4 \mathrm{np}\right),\left({ }^{14} \mathrm{~N}\right.$, $5 \mathrm{n} \alpha),\left({ }^{14} \mathrm{~N}, 6 \mathrm{n} \alpha\right),\left({ }^{14} \mathrm{~N}, \mathrm{n} 2 \mathrm{p} \alpha\right),\left({ }^{14} \mathrm{~N}, \mathrm{n} 2 \mathrm{p} 2 \alpha\right),\left({ }^{14} \mathrm{~N}, 3 \alpha\right), \mathrm{E} \approx 64-90 ;$ measured excitation functions; deduced reaction mechanism features. Activation technique, comparison with model predictions. JOUR IMPEE 14775

${ }^{135} \mathrm{Ce}$ 2005JAzZ NUCLEAR REACTIONS ${ }^{124} \mathrm{Sn}\left({ }^{16} \mathrm{O}, 5 \mathrm{n}\right), \mathrm{E}=80 \mathrm{MeV}$; measured $\mathrm{E} \gamma$, $\mathrm{I} \gamma, \gamma \gamma$-coin, DSA. ${ }^{135} \mathrm{Ce}$ deduced high-spin levels, $\mathrm{J}, \pi, \mathrm{T}_{1 / 2}, \mathrm{~B}(\mathrm{M} 1)$, $\mathrm{B}(\mathrm{E} 2)$, chiral doublet bands. CONF Argonne(Nuclei at the Limits),P99,Jain 2005VEZZ NUCLEAR REACTIONS $\operatorname{Pr}(\mathrm{p}, \mathrm{X}){ }^{139} \mathrm{Nd} /{ }^{138} \mathrm{Nd} /{ }^{139} \mathrm{Ce} /{ }^{135} \mathrm{Ce} /$ ${ }^{137} \operatorname{Pr} /{ }^{138 m} \mathrm{Pr}, \mathrm{E}=20-100 \mathrm{MeV}$; La(p, X) ${ }^{139} \mathrm{Ce}, \mathrm{E}=0-20 \mathrm{MeV}$; measured excitation functions. Comparison with model predictions. CONF Santa Fe (Nucl Data for Sci and Technol) Proc,Vol2,P1650

${ }^{135} \mathrm{Gd} \quad 2005 \mathrm{XU} 04 \quad$ RADIOACTIVITY ${ }^{81} \mathrm{Zr},{ }^{85} \mathrm{Mo},{ }^{89} \mathrm{Ru},{ }^{92} \mathrm{Rh},{ }^{93} \mathrm{Pd},{ }^{121} \mathrm{Ce},{ }^{125} \mathrm{Nd}$, ${ }^{128} \mathrm{Pm},{ }^{129} \mathrm{Sm},{ }^{135,137} \mathrm{Gd},{ }^{139} \mathrm{Dy},{ }^{142} \mathrm{Ho},{ }^{149} \mathrm{Yb}\left(\beta^{+} \mathrm{p}\right)$; measured $\beta$-delayed $\mathrm{E} \gamma, \mathrm{I} \gamma$, proton spectra, $\mathrm{p} \gamma$-coin, $\mathrm{T}_{1 / 2}$. Comparison with model predictions. JOUR PRVCA 71054318 


\section{$\mathrm{A}=135$ (continued)}

2005XU04 NUCLEAR REACTIONS ${ }^{92} \mathrm{Mo},{ }^{106} \mathrm{Cd}\left({ }^{32} \mathrm{~S}, 3 \mathrm{n}\right), \mathrm{E}=151 \mathrm{MeV}$; ${ }^{92} \mathrm{Mo}\left({ }^{36} \mathrm{Ar}, 3 \mathrm{n}\right), \mathrm{E}=169 \mathrm{MeV} ;{ }^{96} \mathrm{Ru}\left({ }^{36} \mathrm{Ar}, 3 \mathrm{n}\right),\left({ }^{36} \mathrm{Ar}, 3 \mathrm{np}\right), \mathrm{E}=165,174$ $\mathrm{MeV} ;{ }^{106} \mathrm{Cd}\left({ }^{36} \mathrm{Ar}, 3 \mathrm{n}\right),\left({ }^{36} \mathrm{Ar}, \mathrm{n} \alpha\right), \mathrm{E}=176 \mathrm{MeV} ;{ }^{106} \mathrm{Cd}\left({ }^{40} \mathrm{Ca}, 4 \mathrm{n}\right)$, $\mathrm{E}=202 \mathrm{MeV} ;{ }^{112} \mathrm{Sn}\left({ }^{40} \mathrm{Ca}, 3 \mathrm{n}\right), \mathrm{E}=185 \mathrm{MeV}$; measured $\sigma$. JOUR PRVCA 71054318

\section{$\mathrm{A}=136$}

${ }^{136} \mathrm{Te} \quad$ 2005RA09

NUCLEAR REACTIONS C ( $\left.{ }^{126} \mathrm{Sn},{ }^{126} \mathrm{Sn}^{\prime}\right),\left({ }^{128} \mathrm{Sn},{ }^{128} \mathrm{Sn}\right),\left({ }^{130} \mathrm{Sn}\right.$, $\left.{ }^{130} \mathrm{Sn}^{\prime}\right),\left({ }^{132} \mathrm{Sn},{ }^{132} \mathrm{Sn}\right)$ ') $\left({ }^{134} \mathrm{Sn},{ }^{134} \mathrm{Sn}\right),\left({ }^{132} \mathrm{Te},{ }^{132} \mathrm{Te}\right),\left({ }^{134} \mathrm{Te},{ }^{134} \mathrm{Te}\right)$, $\left({ }^{136} \mathrm{Te},{ }^{136} \mathrm{Te}\right.$ '), E not given; measured $\mathrm{E} \gamma, \mathrm{I} \gamma$, (particle) $\gamma$-coin following projectile Coulomb excitation. ${ }^{126,128,130,132,134} \mathrm{Sn},{ }^{132,134,136} \mathrm{Te}$ deduced transitions $\mathrm{B}(\mathrm{E} 2) .{ }^{9} \mathrm{Be}\left({ }^{134} \mathrm{Te},{ }^{8} \mathrm{Be}\right),{ }^{13} \mathrm{C}\left({ }^{134} \mathrm{Te},{ }^{12} \mathrm{C}\right)$, E not given; measured $\mathrm{E} \gamma, \mathrm{I} \gamma, \gamma \gamma-$, (particle) $\gamma$-coin. ${ }^{135} \mathrm{Te}$ deduced level. Clarion, Hyball arrays. JOUR NUPAB 752 264c

2005RA32 NUCLEAR REACTIONS C $\left({ }^{126} \mathrm{Sn},{ }^{126} \mathrm{Sn}\right),\left({ }^{128} \mathrm{Sn},{ }^{128} \mathrm{Sn}^{\prime}\right),\left({ }^{130} \mathrm{Sn}\right.$, $\left.{ }^{130} \mathrm{Sn}\right),\left({ }^{132} \mathrm{Te},{ }^{132} \mathrm{Te}\right),\left({ }^{134} \mathrm{Te},{ }^{134} \mathrm{Te}\right),\left({ }^{136} \mathrm{Te},{ }^{136} \mathrm{Te}\right.$ '), E not given; measured $\mathrm{E} \gamma, \mathrm{I} \gamma, \gamma \gamma$-, (particle) $\gamma$-coin following projectile Coulomb excitation. ${ }^{132,134,136} \mathrm{Te},{ }^{126,128,130} \mathrm{Sn}$ deduced excitation $\mathrm{B}(\mathrm{E} 2)$. ${ }^{9} \mathrm{Be}\left({ }^{134} \mathrm{Te},{ }^{8} \mathrm{Be}\right),{ }^{13} \mathrm{C}\left({ }^{134} \mathrm{Te},{ }^{12} \mathrm{C}\right), \mathrm{E}=4.3 \mathrm{MeV} /$ nucleon; measured $\mathrm{E} \gamma$, $\mathrm{I} \gamma, \gamma \gamma$-coin, (particle) $\gamma$-coin; deduced single-neutron transfer $\sigma(\mathrm{E})$.

${ }^{135}$ Te deduced levels J, $\pi$. JOUR ZAANE 25 s01 383

${ }^{136} \mathrm{I}$ 2005NA05 NUCLEAR REACTIONS ${ }^{232} \mathrm{Th},{ }^{232,238} \mathrm{U},{ }^{238,240} \mathrm{Pu},{ }^{244} \mathrm{Cm}(\mathrm{n}, \mathrm{F}){ }^{128} \mathrm{Sb}$ $/{ }^{130} \mathrm{Sb} /{ }^{132} \mathrm{Sb} /{ }^{131} \mathrm{Te} /{ }^{133} \mathrm{Te} /{ }^{132} \mathrm{I} /{ }^{134} \mathrm{I} /{ }^{136} \mathrm{I} /{ }^{135} \mathrm{Xe} /{ }^{138} \mathrm{Cs}$, $\mathrm{E}=$ thermal, fast; measured isomer yield ratios; deduced fission fragment angular momenta. Spin-dependent statistical model analysis. JOUR PRVCA 71014304

${ }^{136} \mathrm{Xe} \quad 2005 \mathrm{GAZU}$ RADIOACTIVITY ${ }^{136} \mathrm{Xe}\left(2 \beta^{-}\right)$; measured $0 \nu \beta \beta$-decay and $2 \nu \beta \beta$-decay $\mathrm{T}_{1 / 2}$ lower limits. PREPRINT nucl-ex/0510071,10/26/2005

${ }^{136} \mathrm{Cs} \quad$ 2005UOZZ NUCLEAR REACTIONS U(p, F $){ }^{95} \mathrm{Zr} /{ }^{115} \mathrm{Cd} /{ }^{134} \mathrm{Cs} /{ }^{136} \mathrm{Cs} /{ }^{137} \mathrm{Cs}$ $/{ }^{147} \mathrm{Nd}, \mathrm{E} \approx 20-70 \mathrm{MeV}$; measured production $\sigma$. Stacked-foil activation technique, comparison with model predictions. CONF Santa Fe (Nucl Data for Sci and Technol) Proc,Vol2,P1547

${ }^{136} \mathrm{Ba} \quad 2005 \mathrm{GAZU}$ RADIOACTIVITY ${ }^{136} \mathrm{Xe}\left(2 \beta^{-}\right)$; measured $0 \nu \beta \beta$-decay and $2 \nu \beta \beta$-decay $\mathrm{T}_{1 / 2}$ lower limits. PREPRINT nucl-ex/0510071,10/26/2005

${ }^{136} \mathrm{La} \quad 2005 \mathrm{BH} 06$ NUCLEAR REACTIONS ${ }^{130} \mathrm{Te}\left({ }^{11} \mathrm{~B}, 5 \mathrm{n}\right), \mathrm{E}=52 \mathrm{MeV}$; measured $\mathrm{E} \gamma$, $\mathrm{I} \gamma, \gamma \gamma$-coin. ${ }^{136} \mathrm{La}$ deduced high-spin levels, $\mathrm{J}, \pi$, configurations. Two-quasiparticle-rotor model calculation. JOUR NUPAB 750199 2005ZH16 NUCLEAR REACTIONS ${ }^{130} \mathrm{Te}\left({ }^{11} \mathrm{~B}, 5 \mathrm{n}\right), \mathrm{E}=60 \mathrm{MeV}$; measured $\mathrm{E} \gamma$, I $\gamma, \gamma \gamma$-coin. ${ }^{136} \mathrm{La}$ deduced high-spin levels, J, $\pi$, configurations. JOUR ZAANE 24199

${ }^{136} \mathrm{Ce}$ 2005LA29 NUCLEAR REACTIONS ${ }^{124} \mathrm{Sn}\left({ }^{16} \mathrm{O}, 4 \mathrm{n}\right), \mathrm{E}=80 \mathrm{MeV}$; measured $\mathrm{E} \gamma$, $\mathrm{I} \gamma, \gamma \gamma$-coin, $\gamma$-ray polarization, DSA. ${ }^{136} \mathrm{Ce}$ deduced high-spin levels, I, $\pi, \mathrm{T}_{1 / 2}, \mathrm{~B}(\mathrm{M} 1), \mathrm{B}(\mathrm{E} 2)$, transition quadrupole moments. ${ }^{124} \mathrm{Sn}\left({ }^{16} \mathrm{O}\right.$, $4 \mathrm{n}), \mathrm{E}=65-98 \mathrm{MeV}$; measured $\mathrm{E} \gamma$, excitation functions. Comparisons with cranking model predictions. JOUR NUPAB 7611 


\section{$\mathrm{A}=136$ (continued)}

${ }^{136} \mathrm{Sm}$ 2005XU04 RADIOACTIVITY ${ }^{81} \mathrm{Zr},{ }^{85} \mathrm{Mo},{ }^{89} \mathrm{Ru},{ }^{92} \mathrm{Rh},{ }^{93} \mathrm{Pd},{ }^{121} \mathrm{Ce},{ }^{125} \mathrm{Nd}$, ${ }^{128} \mathrm{Pm},{ }^{129} \mathrm{Sm},{ }^{135,137} \mathrm{Gd},{ }^{139} \mathrm{Dy},{ }^{142} \mathrm{Ho},{ }^{149} \mathrm{Yb}\left(\beta^{+} \mathrm{p}\right)$; measured $\beta$-delayed $\mathrm{E} \gamma, \mathrm{I} \gamma$, proton spectra, $\mathrm{p} \gamma$-coin, $\mathrm{T}_{1 / 2}$. Comparison with model predictions. JOUR PRVCA 71054318

\section{$\mathrm{A}=137$}

${ }^{137} \mathrm{Xe} \quad 2004 \mathrm{GA} 60$

NUCLEAR REACTIONS ${ }^{237} \mathrm{~Np}(\gamma, \mathrm{F}){ }^{135} \mathrm{Xe} /{ }^{137} \mathrm{Xe} /{ }^{138} \mathrm{Xe} /{ }^{139} \mathrm{Xe} /$ ${ }^{140} \mathrm{Xe} /{ }^{141} \mathrm{Xe} /{ }^{142} \mathrm{Xe} /{ }^{89} \mathrm{Kr} /{ }^{91} \mathrm{Kr} /{ }^{92} \mathrm{Kr} /{ }^{93} \mathrm{Kr}, \mathrm{E}=25 \mathrm{MeV}$ bremsstrahlung; measured fission yields, isotopic distribution parameters. Comparison with results from other targets. JOUR BRSPE 681298

2004GAZV NUCLEAR REACTIONS ${ }^{237} \mathrm{~Np},{ }^{243} \mathrm{Am}(\gamma, \mathrm{F}){ }^{89} \mathrm{Kr} /{ }^{91} \mathrm{Kr} /{ }^{92} \mathrm{Kr} /$ ${ }^{93} \mathrm{Kr} /{ }^{135} \mathrm{Xe} /{ }^{137} \mathrm{Xe} /{ }^{138} \mathrm{Xe} /{ }^{139} \mathrm{Xe} /{ }^{140} \mathrm{Xe} /{ }^{141} \mathrm{Xe} /{ }^{142} \mathrm{Xe}$, $\mathrm{E}_{\max }=25 \mathrm{MeV}$; measured $\mathrm{E} \gamma$, I $\gamma$; deduced fission fragment yields. REPT JINR-P15-2004-119, Gangrsky

2005 F017 RADIOACTIVITY ${ }^{252} \mathrm{Cf}(\mathrm{SF})$; measured $\mathrm{E} \gamma, \mathrm{I} \gamma, \gamma \gamma$-coin. ${ }^{98} \mathrm{Sr}$, ${ }^{102,104} \mathrm{Zr},{ }^{137} \mathrm{Xe},{ }^{143} \mathrm{Ba},{ }^{152} \mathrm{Ce}$ levels deduced $\mathrm{T}_{1 / 2}$. Gammasphere array, time-gated triple-coincidence method. JOUR ZAANE 25 s01 465 2005GA25 NUCLEAR REACTIONS ${ }^{248} \mathrm{Cm}(\gamma, \mathrm{F}){ }^{89} \mathrm{Kr} /{ }^{91} \mathrm{Kr} /{ }^{92} \mathrm{Kr} /{ }^{93} \mathrm{Kr} /$ ${ }^{135} \mathrm{Xe} /{ }^{137} \mathrm{Xe} /{ }^{138} \mathrm{Xe} /{ }^{139} \mathrm{Xe} /{ }^{140} \mathrm{Xe} /{ }^{141} \mathrm{Xe} /{ }^{142} \mathrm{Xe}, \mathrm{E}=25 \mathrm{MeV}$ bremsstrahlung; measured $\mathrm{E} \gamma, \mathrm{I} \gamma$; deduced yields. JOUR FECLA 125 44

2005GA50 NUCLEAR REACTIONS ${ }^{237} \mathrm{~Np},{ }^{243} \mathrm{Am}(\gamma, \mathrm{F}){ }^{135} \mathrm{Xe} /{ }^{137} \mathrm{Xe} /{ }^{138} \mathrm{Xe} /$ ${ }^{139} \mathrm{Xe} /{ }^{140} \mathrm{Xe} /{ }^{141} \mathrm{Xe} /{ }^{142} \mathrm{Xe} /{ }^{89} \mathrm{Kr} /{ }^{91} \mathrm{Kr} /{ }^{92} \mathrm{Kr} /{ }^{93} \mathrm{Kr}, \mathrm{E}=25 \mathrm{MeV}$ bremsstrahlung; measured fission yields, isotopic distribution parameters. JOUR YAFIA 681475

2005HW06 RADIOACTIVITY ${ }^{252} \mathrm{Cf}(\mathrm{SF}) ;$ measured $\mathrm{E} \gamma, \mathrm{I} \gamma, \gamma \gamma$-coin. ${ }^{95,97} \mathrm{Sr},{ }^{99} \mathrm{Zr}$, ${ }^{108} \mathrm{Tc},{ }^{133,134} \mathrm{Te},{ }^{137} \mathrm{Xe}$ levels deduced $\mathrm{T}_{1 / 2}$. Gammasphere array, time-gated triple-coincidence method. JOUR ZAANE 25 s01 463

${ }^{137} \mathrm{Cs} \quad 2005 \mathrm{UOZZ}$ NUCLEAR REACTIONS U(p, F $){ }^{95} \mathrm{Zr} /{ }^{115} \mathrm{Cd} /{ }^{134} \mathrm{Cs} /{ }^{136} \mathrm{Cs} /{ }^{137} \mathrm{Cs}$ $/{ }^{147} \mathrm{Nd}, \mathrm{E} \approx 20-70 \mathrm{MeV}$; measured production $\sigma$. Stacked-foil activation technique, comparison with model predictions. CONF Santa Fe (Nucl Data for Sci and Technol) Proc,Vol2,P1547

${ }^{137} \mathrm{Ce} \quad$ 2005UN01 NUCLEAR REACTIONS ${ }^{128} \mathrm{Te}\left({ }^{14} \mathrm{~N}, 4 \mathrm{n}\right),\left({ }^{14} \mathrm{~N}, 5 \mathrm{n}\right),\left({ }^{14} \mathrm{~N}, 4 \mathrm{np}\right),\left({ }^{14} \mathrm{~N}\right.$, $5 \mathrm{n} \alpha),\left({ }^{14} \mathrm{~N}, 6 \mathrm{n} \alpha\right),\left({ }^{14} \mathrm{~N}, \mathrm{n} 2 \mathrm{p} \alpha\right),\left({ }^{14} \mathrm{~N}, \mathrm{n} 2 \mathrm{p} 2 \alpha\right),\left({ }^{14} \mathrm{~N}, 3 \alpha\right), \mathrm{E} \approx 64-90$ measured excitation functions; deduced reaction mechanism features. Activation technique, comparison with model predictions. JOUR IMPEE 14775

${ }^{137} \mathrm{Pr}$ 2005UN01 NUCLEAR REACTIONS ${ }^{128} \mathrm{Te}\left({ }^{14} \mathrm{~N}, 4 \mathrm{n}\right),\left({ }^{14} \mathrm{~N}, 5 \mathrm{n}\right),\left({ }^{14} \mathrm{~N}, 4 \mathrm{np}\right),\left({ }^{14} \mathrm{~N}\right.$, $5 \mathrm{n} \alpha),\left({ }^{14} \mathrm{~N}, 6 \mathrm{n} \alpha\right),\left({ }^{14} \mathrm{~N}, \mathrm{n} 2 \mathrm{p} \alpha\right),\left({ }^{14} \mathrm{~N}, \mathrm{n} 2 \mathrm{p} 2 \alpha\right),\left({ }^{14} \mathrm{~N}, 3 \alpha\right), \mathrm{E} \approx 64-90$ measured excitation functions; deduced reaction mechanism features. Activation technique, comparison with model predictions. JOUR IMPEE 14775

2005VEZZ NUCLEAR REACTIONS $\operatorname{Pr}(\mathrm{p}, \mathrm{X}){ }^{139} \mathrm{Nd} /{ }^{138} \mathrm{Nd} /{ }^{139} \mathrm{Ce} /{ }^{135} \mathrm{Ce} /$ ${ }^{137} \operatorname{Pr} /{ }^{138 m} \operatorname{Pr}, \mathrm{E}=20-100 \mathrm{MeV} ; \mathrm{La}(\mathrm{p}, \mathrm{X}){ }^{139} \mathrm{Ce}, \mathrm{E}=0-20 \mathrm{MeV}$; measured excitation functions. Comparison with model predictions. CONF Santa Fe (Nucl Data for Sci and Technol) Proc,Vol2,P1650 


\section{$\mathrm{A}=137$ (continued)}

$\begin{array}{cl}{ }^{137} \mathrm{Gd} & \text { 2005XU04 } \\ & \text { RADIOACTIVITY }{ }^{81} \mathrm{Zr},{ }^{85} \mathrm{Mo},{ }^{89} \mathrm{Ru},{ }^{92} \mathrm{Rh},{ }^{93} \mathrm{Pd},{ }^{121} \mathrm{Ce},{ }^{125} \mathrm{Nd}, \\ & \beta \text {-delayed E } \gamma, \mathrm{I} \gamma, \text { proton spectra, } \mathrm{p} \gamma \text {-coin, } \mathrm{T}_{1 / 2} . \text { Comparison with } \\ & \text { model predictions. JOUR PRVCA } 71054318 \\ & \text { NUCLEAR REACTIONS }{ }^{92} \mathrm{Mo},{ }^{106} \mathrm{Cd}\left({ }^{32} \mathrm{~S}, 3 \mathrm{n}\right), \mathrm{E}=151 \mathrm{MeV} ; \\ & { }^{92} \mathrm{Mo}\left({ }^{36} \mathrm{Ar}, 3 \mathrm{n}\right), \mathrm{E}=169 \mathrm{MeV} ;{ }^{96} \mathrm{Ru}\left({ }^{36} \mathrm{Ar}, 3 \mathrm{n}\right),\left({ }^{36} \mathrm{Ar}, 3 \mathrm{np}\right), \mathrm{E}=165,174 \\ & \mathrm{MeV} ;{ }^{106} \mathrm{Cd}\left({ }^{36} \mathrm{Ar}, 3 \mathrm{n}\right),\left({ }^{36} \mathrm{Ar}, \mathrm{n} \alpha\right), \mathrm{E}=176 \mathrm{MeV} ;{ }^{106} \mathrm{Cd}\left({ }^{40} \mathrm{Ca}, 4 \mathrm{n}\right), \\ & \mathrm{E}=202 \mathrm{MeV} ;{ }^{112} \mathrm{Sn}\left({ }^{40} \mathrm{Ca}, 3 \mathrm{n}\right), \mathrm{E}=185 \mathrm{MeV} ; \text { measured } \sigma . \text { JOUR } \\ & \text { PRVCA } 71054318\end{array}$

$$
\mathrm{A}=\mathbf{1 3 8}
$$

${ }^{138} \mathrm{Xe} \quad 2004 \mathrm{GA} 60$

NUCLEAR REACTIONS ${ }^{237} \mathrm{~Np}(\gamma, \mathrm{F}){ }^{135} \mathrm{Xe} /{ }^{137} \mathrm{Xe} /{ }^{138} \mathrm{Xe} /{ }^{139} \mathrm{Xe} /$ ${ }^{140} \mathrm{Xe} /{ }^{141} \mathrm{Xe} /{ }^{142} \mathrm{Xe} /{ }^{89} \mathrm{Kr} /{ }^{91} \mathrm{Kr} /{ }^{92} \mathrm{Kr} /{ }^{93} \mathrm{Kr}, \mathrm{E}=25 \mathrm{MeV}$ bremsstrahlung; measured fission yields, isotopic distribution parameters. Comparison with results from other targets. JOUR BRSPE 681298

2004GAZV NUCLEAR REACTIONS ${ }^{237} \mathrm{~Np},{ }^{243} \mathrm{Am}(\gamma, \mathrm{F}){ }^{89} \mathrm{Kr} /{ }^{91} \mathrm{Kr} /{ }^{92} \mathrm{Kr} /$ ${ }^{93} \mathrm{Kr} /{ }^{135} \mathrm{Xe} /{ }^{137} \mathrm{Xe} /{ }^{138} \mathrm{Xe} /{ }^{139} \mathrm{Xe} /{ }^{140} \mathrm{Xe} /{ }^{141} \mathrm{Xe} /{ }^{142} \mathrm{Xe}$, $\mathrm{E}_{\max }=25 \mathrm{MeV}$; measured $\mathrm{E} \gamma$, I $\gamma$; deduced fission fragment yields. REPT JINR-P15-2004-119, Gangrsky 2005GA25 NUCLEAR REACTIONS ${ }^{248} \mathrm{Cm}(\gamma, \mathrm{F}){ }^{89} \mathrm{Kr} /{ }^{91} \mathrm{Kr} /{ }^{92} \mathrm{Kr} /{ }^{93} \mathrm{Kr} /$ ${ }^{135} \mathrm{Xe} /{ }^{137} \mathrm{Xe} /{ }^{138} \mathrm{Xe} /{ }^{139} \mathrm{Xe} /{ }^{140} \mathrm{Xe} /{ }^{141} \mathrm{Xe} /{ }^{142} \mathrm{Xe}, \mathrm{E}=25 \mathrm{MeV}$ bremsstrahlung; measured $\mathrm{E} \gamma, \mathrm{I} \gamma$; deduced yields. JOUR FECLA 125 44

2005GA50 NUCLEAR REACTIONS ${ }^{237} \mathrm{~Np},{ }^{243} \mathrm{Am}(\gamma, \mathrm{F}){ }^{135} \mathrm{Xe} /{ }^{137} \mathrm{Xe} /{ }^{138} \mathrm{Xe} /$ ${ }^{139} \mathrm{Xe} /{ }^{140} \mathrm{Xe} /{ }^{141} \mathrm{Xe} /{ }^{142} \mathrm{Xe} /{ }^{89} \mathrm{Kr} /{ }^{91} \mathrm{Kr} /{ }^{92} \mathrm{Kr} /{ }^{93} \mathrm{Kr}, \mathrm{E}=25 \mathrm{MeV}$ bremsstrahlung; measured fission yields, isotopic distribution parameters. JOUR YAFIA 681475

2005JA12 RADIOACTIVITY ${ }^{252} \mathrm{Cf}(\mathrm{SF})$; measured $\mathrm{E} \gamma, \mathrm{I} \gamma, \alpha \gamma-, \gamma \gamma$-coin for $\alpha$-accompanied ternary fission; deduced fission fragments average angular momentum. ${ }^{100,102} \mathrm{Zr},{ }^{106} \mathrm{Mo},{ }^{144,146} \mathrm{Ba},{ }^{138,140,142} \mathrm{Xe}$; deduced transition intensities. Gammasphere array. JOUR ZAANE 24373

${ }^{138} \mathrm{Cs}$ 2005NA05 NUCLEAR REACTIONS ${ }^{232} \mathrm{Th},{ }^{232,238} \mathrm{U},{ }^{238,240} \mathrm{Pu},{ }^{244} \mathrm{Cm}(\mathrm{n}, \mathrm{F}){ }^{128} \mathrm{Sb}$ $/{ }^{130} \mathrm{Sb} /{ }^{132} \mathrm{Sb} /{ }^{131} \mathrm{Te} /{ }^{133} \mathrm{Te} /{ }^{132} \mathrm{I} /{ }^{134} \mathrm{I} /{ }^{136} \mathrm{I} /{ }^{135} \mathrm{Xe} /{ }^{138} \mathrm{Cs}$, $\mathrm{E}=$ thermal, fast; measured isomer yield ratios; deduced fission fragment angular momenta. Spin-dependent statistical model analysis. JOUR PRVCA 71014304

${ }^{138} \operatorname{Pr} \quad$ 2005GA14 NUCLEAR REACTIONS ${ }^{128} \mathrm{Te}\left({ }^{14} \mathrm{~N}, 4 \mathrm{n}\right), \mathrm{E}=55-65 \mathrm{MeV}$; measured $\mathrm{E} \gamma, \mathrm{I} \gamma, \gamma \gamma$-coin. ${ }^{138} \operatorname{Pr}$ deduced high-spin levels, J, $\pi, \mathrm{B}(\mathrm{M} 1) / \mathrm{B}(\mathrm{E} 2)$, configurations. Comparison with particle-rotor model predictions. JOUR ZAANE 24173

2005UN01 NUCLEAR REACTIONS ${ }^{128} \mathrm{Te}\left({ }^{14} \mathrm{~N}, 4 \mathrm{n}\right),\left({ }^{14} \mathrm{~N}, 5 \mathrm{n}\right),\left({ }^{14} \mathrm{~N}, 4 \mathrm{np}\right),\left({ }^{14} \mathrm{~N}\right.$, $5 \mathrm{n} \alpha),\left({ }^{14} \mathrm{~N}, 6 \mathrm{n} \alpha\right),\left({ }^{14} \mathrm{~N}, \mathrm{n} 2 \mathrm{p} \alpha\right),\left({ }^{14} \mathrm{~N}, \mathrm{n} 2 \mathrm{p} 2 \alpha\right),\left({ }^{14} \mathrm{~N}, 3 \alpha\right), \mathrm{E} \approx 64-90 ;$ measured excitation functions; deduced reaction mechanism features. Activation technique, comparison with model predictions. JOUR IMPEE 14775 


\section{$\mathrm{A}=138$ (continued)}

2005VEZZ NUCLEAR REACTIONS $\operatorname{Pr}(\mathrm{p}, \mathrm{X}){ }^{139} \mathrm{Nd} /{ }^{138} \mathrm{Nd} /{ }^{139} \mathrm{Ce} /{ }^{135} \mathrm{Ce} /$ ${ }^{137} \operatorname{Pr} /{ }^{138 m} \operatorname{Pr}, \mathrm{E}=20-100 \mathrm{MeV} ; \mathrm{La}(\mathrm{p}, \mathrm{X}){ }^{139} \mathrm{Ce}, \mathrm{E}=0-20 \mathrm{MeV}$; measured excitation functions. Comparison with model predictions. CONF Santa Fe (Nucl Data for Sci and Technol) Proc,Vol2,P1650 ${ }^{138} \mathrm{Nd} \quad$ 2005VEZZ
$\quad{ }^{137} \mathrm{Pr} /{ }^{138 m} \mathrm{Pr}, \mathrm{E}=20-100 \mathrm{MeV} ; \mathrm{La}(\mathrm{p}, \mathrm{X}){ }^{139} \mathrm{Ce}, \mathrm{E}=0-20 \mathrm{MeV}$ measured excitation functions. Comparison with model predictions. CONF Santa Fe (Nucl Data for Sci and Technol) Proc,Vol2,P1650 ${ }^{138} \mathrm{Gd} 2005 \mathrm{XU} 04 \quad$ RADIOACTIVITY ${ }^{81} \mathrm{Zr},{ }^{85} \mathrm{Mo},{ }^{89} \mathrm{Ru},{ }^{92} \mathrm{Rh},{ }^{93} \mathrm{Pd},{ }^{121} \mathrm{Ce},{ }^{125} \mathrm{Nd}$, ${ }^{128} \mathrm{Pm},{ }^{129} \mathrm{Sm},{ }^{135,137} \mathrm{Gd},{ }^{139} \mathrm{Dy},{ }^{142} \mathrm{Ho},{ }^{149} \mathrm{Yb}\left(\beta^{+} \mathrm{p}\right)$; measured $\beta$-delayed $\mathrm{E} \gamma, \mathrm{I} \gamma$, proton spectra, $\mathrm{p} \gamma$-coin, $\mathrm{T}_{1 / 2}$. Comparison with model predictions. JOUR PRVCA 71054318

\section{$\mathrm{A}=139$}

${ }^{139} \mathrm{Xe} \quad 2004 \mathrm{GA} 60$

NUCLEAR REACTIONS ${ }^{237} \mathrm{~Np}(\gamma, \mathrm{F}){ }^{135} \mathrm{Xe} /{ }^{137} \mathrm{Xe} /{ }^{138} \mathrm{Xe} /{ }^{139} \mathrm{Xe} /$ ${ }^{140} \mathrm{Xe} /{ }^{141} \mathrm{Xe} /{ }^{142} \mathrm{Xe} /{ }^{89} \mathrm{Kr} /{ }^{91} \mathrm{Kr} /{ }^{92} \mathrm{Kr} /{ }^{93} \mathrm{Kr}, \mathrm{E}=25 \mathrm{MeV}$ bremsstrahlung; measured fission yields, isotopic distribution parameters. Comparison with results from other targets. JOUR BRSPE 681298

2004GAZV NUCLEAR REACTIONS ${ }^{237} \mathrm{~Np},{ }^{243} \mathrm{Am}(\gamma, \mathrm{F}){ }^{89} \mathrm{Kr} /{ }^{91} \mathrm{Kr} /{ }^{92} \mathrm{Kr} /$ ${ }^{93} \mathrm{Kr} /{ }^{135} \mathrm{Xe} /{ }^{137} \mathrm{Xe} /{ }^{138} \mathrm{Xe} /{ }^{139} \mathrm{Xe} /{ }^{140} \mathrm{Xe} /{ }^{141} \mathrm{Xe} /{ }^{142} \mathrm{Xe}$, $\mathrm{E}_{\max }=25 \mathrm{MeV}$; measured $\mathrm{E} \gamma$, I $\gamma$; deduced fission fragment yields. REPT JINR-P15-2004-119, Gangrsky 2005GA25 NUCLEAR REACTIONS ${ }^{248} \mathrm{Cm}(\gamma, \mathrm{F}){ }^{89} \mathrm{Kr} /{ }^{91} \mathrm{Kr} /{ }^{92} \mathrm{Kr} /{ }^{93} \mathrm{Kr} /$ ${ }^{135} \mathrm{Xe} /{ }^{137} \mathrm{Xe} /{ }^{138} \mathrm{Xe} /{ }^{139} \mathrm{Xe} /{ }^{140} \mathrm{Xe} /{ }^{141} \mathrm{Xe} /{ }^{142} \mathrm{Xe}, \mathrm{E}=25 \mathrm{MeV}$ bremsstrahlung; measured $\mathrm{E} \gamma, \mathrm{I} \gamma$; deduced yields. JOUR FECLA 125 44

2005GA50 NUCLEAR REACTIONS ${ }^{237} \mathrm{~Np},{ }^{243} \mathrm{Am}(\gamma, \mathrm{F}){ }^{135} \mathrm{Xe} /{ }^{137} \mathrm{Xe} /{ }^{138} \mathrm{Xe} /$ ${ }^{139} \mathrm{Xe} /{ }^{140} \mathrm{Xe} /{ }^{141} \mathrm{Xe} /{ }^{142} \mathrm{Xe} /{ }^{89} \mathrm{Kr} /{ }^{91} \mathrm{Kr} /{ }^{92} \mathrm{Kr} /{ }^{93} \mathrm{Kr}, \mathrm{E}=25 \mathrm{MeV}$ bremsstrahlung; measured fission yields, isotopic distribution parameters. JOUR YAFIA 681475

${ }^{139} \mathrm{Cs}$ 2005AN01 NUCLEAR REACTIONS ${ }^{238} \mathrm{U}(\mathrm{p}, \mathrm{F}){ }^{89} \mathrm{Rb} /{ }^{90} \mathrm{Rb} /{ }^{91} \mathrm{Rb} /{ }^{93} \mathrm{Rb} /$ ${ }^{94} \mathrm{Rb} /{ }^{95} \mathrm{Rb} /{ }^{139} \mathrm{Cs} /{ }^{140} \mathrm{Cs} /{ }^{141} \mathrm{Cs} /{ }^{142} \mathrm{Cs} /{ }^{144} \mathrm{Cs} /{ }^{145} \mathrm{Cs}, \mathrm{E}=1$ $\mathrm{GeV}$; measured yields. JOUR ZAANE 23257

${ }^{139} \mathrm{Ba}$ 2005HE04 NUCLEAR REACTIONS ${ }^{18} \mathrm{O}(\mathrm{p}, \mathrm{n}), \mathrm{E}=2582 \mathrm{keV}$; measured neutron spectrum. ${ }^{138} \mathrm{Ba}(\mathrm{n}, \gamma), \mathrm{E}=$ spectrum; measured Maxwellian-averaged $\sigma$. JOUR PRVCA 71025803

${ }^{139} \mathrm{La}$ 2005SK04 NUCLEAR REACTIONS ${ }^{131} \mathrm{Xe},{ }^{139} \mathrm{La}(\mathrm{n}, \mathrm{n}), \mathrm{E}=$ low; measured neutron transmission spectra through polarized targets. Plans for measurement of time-reversal violating effects discussed. JOUR JRNBA 110471

${ }^{139} \mathrm{Ce}$ 2005VEzZ NUCLEAR REACTIONS Pr(p, X) ${ }^{139} \mathrm{Nd} /{ }^{138} \mathrm{Nd} /{ }^{139} \mathrm{Ce} /{ }^{135} \mathrm{Ce} /$ ${ }^{137} \operatorname{Pr} /{ }^{138 m} \operatorname{Pr}, \mathrm{E}=20-100 \mathrm{MeV}$; $\mathrm{La}(\mathrm{p}, \mathrm{X}){ }^{139} \mathrm{Ce}, \mathrm{E}=0-20 \mathrm{MeV}$; measured excitation functions. Comparison with model predictions. CONF Santa Fe (Nucl Data for Sci and Technol) Proc,Vol2,P1650 


\section{$\mathrm{A}=139$ (continued)}

\begin{tabular}{|c|c|c|}
\hline${ }^{139} \mathrm{Pr}$ & 2005YU06 & $\begin{array}{l}\text { NUCLEAR REACTIONS }{ }^{130} \mathrm{Te}\left({ }^{14} \mathrm{~N}, 4 \mathrm{n}\right),\left({ }^{14} \mathrm{~N}, 5 \mathrm{n}\right), \mathrm{E}=55-65 \mathrm{MeV} \text {; } \\
\text { measured E } \gamma, \mathrm{I} \gamma, \gamma \gamma \text {-coin; deduced } \gamma \text {-ray excitation functions. }{ }^{140} \mathrm{Pr} \\
\text { deduced high-spin levels, J, } \pi \text {, configurations. Level systematics in } \\
\text { neighboring nuclides discussed. JOUR CPLEE } 221873\end{array}$ \\
\hline${ }^{139} \mathrm{Nd}$ & 2005VEZZ & $\begin{array}{l}\text { NUCLEAR REACTIONS } \operatorname{Pr}(\mathrm{p}, \mathrm{X}){ }^{139} \mathrm{Nd} /{ }^{138} \mathrm{Nd} /{ }^{139} \mathrm{Ce} /{ }^{135} \mathrm{Ce} / \\
{ }^{137} \mathrm{Pr} /{ }^{138 m} \mathrm{Pr}, \mathrm{E}=20-100 \mathrm{MeV} ; \mathrm{La}(\mathrm{p}, \mathrm{X}){ }^{139} \mathrm{Ce}, \mathrm{E}=0-20 \mathrm{MeV} ; \\
\text { measured excitation functions. Comparison with model predictions. } \\
\text { CONF Santa Fe (Nucl Data for Sci and Technol) Proc,Vol2,P1650 }\end{array}$ \\
\hline${ }^{139} \mathrm{Dy}$ & 2005XU04 & $\begin{array}{l}\text { RADIOACTIVITY }{ }^{81} \mathrm{Zr},{ }^{85} \mathrm{Mo},{ }^{89} \mathrm{Ru},{ }^{92} \mathrm{Rh},{ }^{93} \mathrm{Pd},{ }^{121} \mathrm{Ce},{ }^{125} \mathrm{Nd} \text {, } \\
{ }^{128} \mathrm{Pm},{ }^{129} \mathrm{Sm},{ }^{135,137} \mathrm{Gd},{ }^{139} \mathrm{Dy},{ }^{142} \mathrm{Ho},{ }^{149} \mathrm{Yb}\left(\beta^{+} \mathrm{p}\right) ; \text { measured } \\
\beta \text {-delayed E } \gamma \text {, I } \gamma \text {, proton spectra, p } \gamma \text {-coin, } \mathrm{T}_{1 / 2} \text {. Comparison with } \\
\text { model predictions. JOUR PRVCA } 71054318\end{array}$ \\
\hline & 2005XU04 & $\begin{array}{l}\text { NUCLEAR REACTIONS }{ }^{92} \mathrm{Mo},{ }^{106} \mathrm{Cd}\left({ }^{32} \mathrm{~S}, 3 \mathrm{n}\right), \mathrm{E}=151 \mathrm{MeV} ; \\
{ }^{92} \mathrm{Mo}\left({ }^{36} \mathrm{Ar}, 3 \mathrm{n}\right), \mathrm{E}=169 \mathrm{MeV} ;{ }^{96} \mathrm{Ru}\left({ }^{36} \mathrm{Ar}, 3 \mathrm{n}\right),\left({ }^{36} \mathrm{Ar}, 3 \mathrm{np}\right), \mathrm{E}=165,174 \\
\mathrm{MeV} ;{ }^{106} \mathrm{Cd}\left({ }^{36} \mathrm{Ar}, 3 \mathrm{n}\right),\left({ }^{36} \mathrm{Ar}, \mathrm{n} \alpha\right), \mathrm{E}=176 \mathrm{MeV} ;{ }^{106} \mathrm{Cd}\left({ }^{40} \mathrm{Ca}, 4 \mathrm{n}\right), \\
\mathrm{E}=202 \mathrm{MeV} ;{ }^{112} \mathrm{Sn}\left({ }^{40} \mathrm{Ca}, 3 \mathrm{n}\right), \mathrm{E}=185 \mathrm{MeV} ; \text { measured } \sigma . \text { JOUR } \\
\text { PRVCA } 71054318\end{array}$ \\
\hline
\end{tabular}

\section{$\mathrm{A}=140$}

${ }^{140} \mathrm{Xe} \quad 2004 \mathrm{GA} 60$

2004GAZV

2005 GA25

2005JA12

${ }^{140} \mathrm{Cs} \quad$ 2005AN01
NUCLEAR REACTIONS ${ }^{237} \mathrm{~Np}(\gamma, \mathrm{F}){ }^{135} \mathrm{Xe} /{ }^{137} \mathrm{Xe} /{ }^{138} \mathrm{Xe} /{ }^{139} \mathrm{Xe} /$ ${ }^{140} \mathrm{Xe} /{ }^{141} \mathrm{Xe} /{ }^{142} \mathrm{Xe} /{ }^{89} \mathrm{Kr} /{ }^{91} \mathrm{Kr} /{ }^{92} \mathrm{Kr} /{ }^{93} \mathrm{Kr}, \mathrm{E}=25 \mathrm{MeV}$ bremsstrahlung; measured fission yields, isotopic distribution parameters. Comparison with results from other targets. JOUR BRSPE 681298 NUCLEAR REACTIONS ${ }^{237} \mathrm{~Np},{ }^{243} \mathrm{Am}(\gamma, \mathrm{F}){ }^{89} \mathrm{Kr} /{ }^{91} \mathrm{Kr} /{ }^{92} \mathrm{Kr} /$ ${ }^{93} \mathrm{Kr} /{ }^{135} \mathrm{Xe} /{ }^{137} \mathrm{Xe} /{ }^{138} \mathrm{Xe} /{ }^{139} \mathrm{Xe} /{ }^{140} \mathrm{Xe} /{ }^{141} \mathrm{Xe} /{ }^{142} \mathrm{Xe}$, $\mathrm{E}_{\max }=25 \mathrm{MeV}$; measured $\mathrm{E} \gamma, \mathrm{I} \gamma$; deduced fission fragment yields. REPT JINR-P15-2004-119, Gangrsky NUCLEAR REACTIONS ${ }^{248} \mathrm{Cm}(\gamma, \mathrm{F}){ }^{89} \mathrm{Kr} /{ }^{91} \mathrm{Kr} /{ }^{92} \mathrm{Kr} /{ }^{93} \mathrm{Kr} /$ ${ }^{135} \mathrm{Xe} /{ }^{137} \mathrm{Xe} /{ }^{138} \mathrm{Xe} /{ }^{139} \mathrm{Xe} /{ }^{140} \mathrm{Xe} /{ }^{141} \mathrm{Xe} /{ }^{142} \mathrm{Xe}, \mathrm{E}=25 \mathrm{MeV}$ bremsstrahlung; measured $\mathrm{E} \gamma, \mathrm{I} \gamma$; deduced yields. JOUR FECLA 125 44 NUCLEAR REACTIONS ${ }^{237} \mathrm{~Np},{ }^{243} \mathrm{Am}(\gamma, \mathrm{F}){ }^{135} \mathrm{Xe} /{ }^{137} \mathrm{Xe} /{ }^{138} \mathrm{Xe} /$ ${ }^{139} \mathrm{Xe} /{ }^{140} \mathrm{Xe} /{ }^{141} \mathrm{Xe} /{ }^{142} \mathrm{Xe} /{ }^{89} \mathrm{Kr} /{ }^{91} \mathrm{Kr} /{ }^{92} \mathrm{Kr} /{ }^{93} \mathrm{Kr}, \mathrm{E}=25 \mathrm{MeV}$ bremsstrahlung; measured fission yields, isotopic distribution parameters. JOUR YAFIA 681475 RADIOACTIVITY ${ }^{252} \mathrm{Cf}(\mathrm{SF})$; measured $\mathrm{E} \gamma, \mathrm{I} \gamma, \alpha \gamma-, \gamma \gamma$-coin for $\alpha$-accompanied ternary fission; deduced fission fragments average angular momentum. ${ }^{100,102} \mathrm{Zr},{ }^{106} \mathrm{Mo},{ }^{144,146} \mathrm{Ba},{ }^{138,140,142} \mathrm{Xe}$; deduced transition intensities. Gammasphere array. JOUR ZAANE 24373 NUCLEAR REACTIONS ${ }^{238} \mathrm{U}(\mathrm{p}, \mathrm{F}){ }^{89} \mathrm{Rb} /{ }^{90} \mathrm{Rb} /{ }^{91} \mathrm{Rb} /{ }^{93} \mathrm{Rb} /$ ${ }^{94} \mathrm{Rb} /{ }^{95} \mathrm{Rb} /{ }^{139} \mathrm{Cs} /{ }^{140} \mathrm{Cs} /{ }^{141} \mathrm{Cs} /{ }^{142} \mathrm{Cs} /{ }^{144} \mathrm{Cs} /{ }^{145} \mathrm{Cs}, \mathrm{E}=1$ $\mathrm{GeV}$; measured yields. JOUR ZAANE 23257 


\section{$\mathrm{A}=140$ (continued)}

${ }^{140} \mathrm{Ba} \quad 2005 \mathrm{ADZZ}$

${ }^{140} \mathrm{La} \quad 2005 \mathrm{ADZZ}$

${ }^{140} \mathrm{Pr} \quad$ 2005HIZX

${ }^{140} \mathrm{Nd} \quad 2004 \mathrm{HIZZ}$

2004NE13

${ }^{140} \mathrm{Eu} \quad 2005 \mathrm{TA3} 1$

${ }^{140}$ Dy $\quad 2005$ BI24
NUCLEAR REACTIONS ${ }^{129} \mathrm{I}(\mathrm{n}, 7 \mathrm{n}),(\mathrm{n}, 6 \mathrm{n}),(\mathrm{n}, 4 \mathrm{n}),(\mathrm{n}, \gamma), \mathrm{E}=$ fast; ${ }^{237} \mathrm{~Np}(\mathrm{n}, \gamma), \mathrm{E}=$ fast; measured yields. ${ }^{237} \mathrm{~Np}(\mathrm{n}, \mathrm{F}){ }^{91} \mathrm{Sr} /{ }^{97} \mathrm{Zr} /{ }^{132} \mathrm{Te} /$ ${ }^{133} \mathrm{I} /{ }^{135} \mathrm{I}, \mathrm{E}=$ fast; ${ }^{238} \mathrm{Pu}(\mathrm{n}, \mathrm{F}){ }^{97} \mathrm{Zr} /{ }^{129} \mathrm{Sb} /{ }^{132} \mathrm{I} /{ }^{133} \mathrm{I} /{ }^{135} \mathrm{Xe} /$ ${ }^{105} \mathrm{Ru}, \mathrm{E}=$ fast; ${ }^{239} \mathrm{Pu}(\mathrm{n}, \mathrm{F}){ }^{88} \mathrm{Kr} /{ }^{91} \mathrm{Sr} /{ }^{92} \mathrm{Sr} /{ }^{92} \mathrm{Y} /{ }^{97} \mathrm{Zr} /{ }^{99} \mathrm{Mo} /$ ${ }^{103} \mathrm{Ru} /{ }^{105} \mathrm{Ru} /{ }^{128} \mathrm{Sb} /{ }^{129} \mathrm{Sb} /{ }^{132} \mathrm{Te} /{ }^{131} \mathrm{I} /{ }^{132} \mathrm{I} /{ }^{133} \mathrm{I} /{ }^{135} \mathrm{I} /{ }^{135} \mathrm{Xe}$ $/{ }^{143} \mathrm{Ce} /{ }^{140} \mathrm{Ba} /{ }^{140} \mathrm{La}, \mathrm{E}=$ fast; measured fission fragment yields. Secondary neutrons from proton irradiation. JINR nuclotron. CONF St Petersburg,P195,Adam

NUCLEAR REACTIONS ${ }^{129} \mathrm{I}(\mathrm{n}, 7 \mathrm{n}),(\mathrm{n}, 6 \mathrm{n}),(\mathrm{n}, 4 \mathrm{n}),(\mathrm{n}, \gamma), \mathrm{E}=$ fast; ${ }^{237} \mathrm{~Np}(\mathrm{n}, \gamma), \mathrm{E}=$ fast; measured yields. ${ }^{237} \mathrm{~Np}(\mathrm{n}, \mathrm{F}){ }^{91} \mathrm{Sr} /{ }^{97} \mathrm{Zr} /{ }^{132} \mathrm{Te} /$ ${ }^{133} \mathrm{I} /{ }^{135} \mathrm{I}, \mathrm{E}=$ fast; ${ }^{238} \mathrm{Pu}(\mathrm{n}, \mathrm{F}){ }^{97} \mathrm{Zr} /{ }^{129} \mathrm{Sb} /{ }^{132} \mathrm{I} /{ }^{133} \mathrm{I} /{ }^{135} \mathrm{Xe} /$ ${ }^{105} \mathrm{Ru}, \mathrm{E}=$ fast; ${ }^{239} \mathrm{Pu}(\mathrm{n}, \mathrm{F}){ }^{88} \mathrm{Kr} /{ }^{91} \mathrm{Sr} /{ }^{92} \mathrm{Sr} /{ }^{92} \mathrm{Y} /{ }^{97} \mathrm{Zr} /{ }^{99} \mathrm{Mo} /$ ${ }^{103} \mathrm{Ru} /{ }^{105} \mathrm{Ru} /{ }^{128} \mathrm{Sb} /{ }^{129} \mathrm{Sb} /{ }^{132} \mathrm{Te} /{ }^{131} \mathrm{I} /{ }^{132} \mathrm{I} /{ }^{133} \mathrm{I} /{ }^{135} \mathrm{I} /{ }^{135} \mathrm{Xe}$ $/{ }^{143} \mathrm{Ce} /{ }^{140} \mathrm{Ba} /{ }^{140} \mathrm{La}, \mathrm{E}=$ fast; measured fission fragment yields. Secondary neutrons from proton irradiation. JINR nuclotron. CONF St Petersburg,P195,Adam

NUCLEAR REACTIONS ${ }^{66} \mathrm{Zn}(\mathrm{d}, \alpha), \mathrm{E}=5-14 \mathrm{MeV}$; $\mathrm{Ce}\left({ }^{3} \mathrm{He}, \mathrm{xn}\right){ }^{140} \mathrm{Nd}$, $\mathrm{E}=16-35 \mathrm{MeV} ;{ }^{141} \mathrm{Ce}(\mathrm{p}, 2 \mathrm{n}), \mathrm{E}=10-45 \mathrm{MeV} ;{ }^{192} \mathrm{Os}(\mathrm{p}, \mathrm{n}), \mathrm{E}=6-19 \mathrm{MeV}$; measured excitation functions; deduced thick-target yields. Stacked-foil activation technique. CONF Santa Fe (Nucl Data for Sci and Technol) Proc, Vol2,P1631

NUCLEAR REACTIONS ${ }^{130} \mathrm{Te}\left({ }^{14} \mathrm{~N}, 4 \mathrm{n}\right),\left({ }^{14} \mathrm{~N}, 5 \mathrm{n}\right), \mathrm{E}=55-65 \mathrm{MeV}$; measured $\mathrm{E} \gamma, \mathrm{I} \gamma, \gamma \gamma$-coin; deduced $\gamma$-ray excitation functions. ${ }^{140} \mathrm{Pr}$ deduced high-spin levels, J, $\pi$, configurations. Level systematics in neighboring nuclides discussed. JOUR CPLEE 221873

NUCLEAR REACTIONS ${ }^{102} \mathrm{Ru}\left({ }^{3} \mathrm{He}, 2 \mathrm{n}\right),{ }^{100} \mathrm{Ru}(\alpha, \mathrm{n}),{ }^{103} \mathrm{Rh}(\mathrm{d}, 2 \mathrm{n})$, $(\mathrm{p}, \mathrm{n}), \mathrm{E} \approx 5-35 \mathrm{MeV}$; analyzed excitation functions, yields. $\mathrm{Ce}\left({ }^{3} \mathrm{He}\right.$, xn $)^{140} \mathrm{Nd}, \mathrm{E}<27 \mathrm{MeV} ;{ }^{141} \operatorname{Pr}(\mathrm{p}, 2 \mathrm{n}), \mathrm{E}<23 \mathrm{MeV}$; measured yields. ${ }^{192} \mathrm{Os}(\mathrm{p}, \mathrm{n}), \mathrm{E} \approx 6-20$; measured $\sigma$. REPT

NEA/NSC/DOC(2004)14,P15,Hilgers NUCLEAR REACTIONS ${ }^{96} \mathrm{Zr}\left({ }^{48} \mathrm{Ca}, 4 \mathrm{n}\right), \mathrm{E}=195 \mathrm{MeV}$; measured $\mathrm{E} \gamma$, $\mathrm{I} \gamma, \gamma \gamma$-coin, fractional Doppler shifts. ${ }^{140} \mathrm{Nd}$ deduced superdeformed band transitions, quadrupole moments, configurations. Euroball array. JOUR PRVCA 70064315

NUCLEAR REACTIONS ${ }^{66} \mathrm{Zn}(\mathrm{d}, \alpha), \mathrm{E}=5-14 \mathrm{MeV}$; $\mathrm{Ce}\left({ }^{3} \mathrm{He}, \mathrm{xn}\right){ }^{140} \mathrm{Nd}$, $\mathrm{E}=16-35 \mathrm{MeV} ;{ }^{141} \mathrm{Ce}(\mathrm{p}, 2 \mathrm{n}), \mathrm{E}=10-45 \mathrm{MeV} ;{ }^{192} \mathrm{Os}(\mathrm{p}, \mathrm{n}), \mathrm{E}=6-19 \mathrm{MeV}$; measured excitation functions; deduced thick-target yields. Stacked-foil activation technique. CONF Santa Fe (Nucl Data for Sci and Technol) Proc, Vol2,P1631

RADIOACTIVITY ${ }^{140 m} \mathrm{Eu},{ }^{142 m} \mathrm{~Tb},{ }^{144 m} \mathrm{Ho}(\mathrm{IT})$ [from ${ }^{54} \mathrm{Fe}\left({ }^{92} \mathrm{Mo}, \mathrm{X}\right)$ ]; measured X-ray spectra, E $\gamma, \gamma \gamma$-coin, $\mathrm{E}(\mathrm{ce}), \mathrm{T}_{1 / 2} \cdot{ }^{140} \mathrm{Eu},{ }^{142} \mathrm{~Tb},{ }^{144} \mathrm{Ho}$ dlevels, J, $\pi$, configurations. Mass-separated sources. JOUR ZAANE $25 \mathrm{~s} 01151$

RADIOACTIVITY ${ }^{141} \mathrm{Ho},{ }^{144,145,146} \mathrm{Tm}(\mathrm{p})\left[\right.$ from ${ }^{92} \mathrm{Mo}\left({ }^{54} \mathrm{Fe}\right.$, xnyp) and ${ }^{92} \mathrm{Mo}\left({ }^{58} \mathrm{Ni}\right.$, xnyp $\left.)\right]$; measured $\mathrm{Ep}, \mathrm{T}_{1 / 2}$; deduced branching ratios. ${ }^{141} \mathrm{Ho},{ }^{140} \mathrm{Dy},{ }^{145,146} \mathrm{Tm},{ }^{144,145} \mathrm{Er}$ deduced levels, configurations.

JOUR NIMBE 241185 


\section{$\mathrm{A}=141$}

${ }^{141} \mathrm{Xe} \quad 2004 \mathrm{GA} 60 \quad$ NUCLEAR REACTIONS ${ }^{237} \mathrm{~Np}(\gamma, \mathrm{F}){ }^{135} \mathrm{Xe} /{ }^{137} \mathrm{Xe} /{ }^{138} \mathrm{Xe} /{ }^{139} \mathrm{Xe} /$ ${ }^{140} \mathrm{Xe} /{ }^{141} \mathrm{Xe} /{ }^{142} \mathrm{Xe} /{ }^{89} \mathrm{Kr} /{ }^{91} \mathrm{Kr} /{ }^{92} \mathrm{Kr} /{ }^{93} \mathrm{Kr}, \mathrm{E}=25 \mathrm{MeV}$ bremsstrahlung; measured fission yields, isotopic distribution parameters. Comparison with results from other targets. JOUR BRSPE 681298

2004GAZV NUCLEAR REACTIONS ${ }^{237} \mathrm{~Np},{ }^{243} \mathrm{Am}(\gamma, \mathrm{F}){ }^{89} \mathrm{Kr} /{ }^{91} \mathrm{Kr} /{ }^{92} \mathrm{Kr} /$ ${ }^{93} \mathrm{Kr} /{ }^{135} \mathrm{Xe} /{ }^{137} \mathrm{Xe} /{ }^{138} \mathrm{Xe} /{ }^{139} \mathrm{Xe} /{ }^{140} \mathrm{Xe} /{ }^{141} \mathrm{Xe} /{ }^{142} \mathrm{Xe}$, $\mathrm{E}_{\max }=25 \mathrm{MeV}$; measured $\mathrm{E} \gamma, \mathrm{I} \gamma$; deduced fission fragment yields. REPT JINR-P15-2004-119, Gangrsky 2005GA25 NUCLEAR REACTIONS ${ }^{248} \mathrm{Cm}(\gamma, \mathrm{F}){ }^{89} \mathrm{Kr} /{ }^{91} \mathrm{Kr} /{ }^{92} \mathrm{Kr} /{ }^{93} \mathrm{Kr} /$ ${ }^{135} \mathrm{Xe} /{ }^{137} \mathrm{Xe} /{ }^{138} \mathrm{Xe} /{ }^{139} \mathrm{Xe} /{ }^{140} \mathrm{Xe} /{ }^{141} \mathrm{Xe} /{ }^{142} \mathrm{Xe}, \mathrm{E}=25 \mathrm{MeV}$ bremsstrahlung; measured $\mathrm{E} \gamma, \mathrm{I} \gamma$; deduced yields. JOUR FECLA 125 44

2005GA50 NUCLEAR REACTIONS ${ }^{237} \mathrm{~Np},{ }^{243} \mathrm{Am}(\gamma, \mathrm{F}){ }^{135} \mathrm{Xe} /{ }^{137} \mathrm{Xe} /{ }^{138} \mathrm{Xe} /$ ${ }^{139} \mathrm{Xe} /{ }^{140} \mathrm{Xe} /{ }^{141} \mathrm{Xe} /{ }^{142} \mathrm{Xe} /{ }^{89} \mathrm{Kr} /{ }^{91} \mathrm{Kr} /{ }^{92} \mathrm{Kr} /{ }^{93} \mathrm{Kr}, \mathrm{E}=25 \mathrm{MeV}$ bremsstrahlung; measured fission yields, isotopic distribution parameters. JOUR YAFIA 681475

${ }^{141} \mathrm{Cs} \quad$ 2005AN01 NUCLEAR REACTIONS ${ }^{238} \mathrm{U}(\mathrm{p}, \mathrm{F}){ }^{89} \mathrm{Rb} /{ }^{90} \mathrm{Rb} /{ }^{91} \mathrm{Rb} /{ }^{93} \mathrm{Rb} /$ ${ }^{94} \mathrm{Rb} /{ }^{95} \mathrm{Rb} /{ }^{139} \mathrm{Cs} /{ }^{140} \mathrm{Cs} /{ }^{141} \mathrm{Cs} /{ }^{142} \mathrm{Cs} /{ }^{144} \mathrm{Cs} /{ }^{145} \mathrm{Cs}, \mathrm{E}=1$ $\mathrm{GeV}$; measured yields. JOUR ZAANE 23257

${ }^{141} \mathrm{~Tb} \quad 2004 \mathrm{ME} 25$ NUCLEAR REACTIONS ${ }^{92} \mathrm{Mo}\left({ }^{54} \mathrm{Fe}, \mathrm{p} \alpha\right), \mathrm{E}=240 \mathrm{MeV}$; measured $\mathrm{E} \gamma$, $\mathrm{I} \gamma, \gamma \gamma-$, (charged particle) $\gamma$-coin. ${ }^{141} \mathrm{~Tb}$ deduced high-spin levels, J, $\pi$, configurations. GASP, ISIS arrays, cranking model analysis. JOUR BJPHE 341002

2005XU04 RADIOACTIVITY ${ }^{81} \mathrm{Zr},{ }^{85} \mathrm{Mo},{ }^{89} \mathrm{Ru},{ }^{92} \mathrm{Rh},{ }^{93} \mathrm{Pd},{ }^{121} \mathrm{Ce},{ }^{125} \mathrm{Nd}$, ${ }^{128} \mathrm{Pm},{ }^{129} \mathrm{Sm},{ }^{135,137} \mathrm{Gd},{ }^{139} \mathrm{Dy},{ }^{142} \mathrm{Ho},{ }^{149} \mathrm{Yb}\left(\beta^{+} \mathrm{p}\right)$; measured $\beta$-delayed $\mathrm{E} \gamma, \mathrm{I} \gamma$, proton spectra, $\mathrm{p} \gamma$-coin, $\mathrm{T}_{1 / 2}$. Comparison with model predictions. JOUR PRVCA 71054318

${ }^{141} \mathrm{Ho}$ 2005BI24 RADIOACTIVITY ${ }^{141} \mathrm{Ho},{ }^{144,145,146} \mathrm{Tm}(\mathrm{p})\left[\right.$ from ${ }^{92} \mathrm{Mo}\left({ }^{54} \mathrm{Fe}, \mathrm{xnyp}\right)$ and ${ }^{92} \mathrm{Mo}\left({ }^{58} \mathrm{Ni}\right.$, xnyp $\left.)\right]$; measured Ep, $\mathrm{T}_{1 / 2}$; deduced branching ratios. ${ }^{141} \mathrm{Ho},{ }^{140} \mathrm{Dy},{ }^{145,146} \mathrm{Tm},{ }^{144,145} \mathrm{Er}$ deduced levels, configurations. JOUR NIMBE 241185

\section{$\mathrm{A}=142$}

${ }^{142} \mathrm{Xe} \quad 2004 \mathrm{GA} 6$

NUCLEAR REACTIONS ${ }^{237} \mathrm{~Np}(\gamma, \mathrm{F}){ }^{135} \mathrm{Xe} /{ }^{137} \mathrm{Xe} /{ }^{138} \mathrm{Xe} /{ }^{139} \mathrm{Xe} /$ ${ }^{140} \mathrm{Xe} /{ }^{141} \mathrm{Xe} /{ }^{142} \mathrm{Xe} /{ }^{89} \mathrm{Kr} /{ }^{91} \mathrm{Kr} /{ }^{92} \mathrm{Kr} /{ }^{93} \mathrm{Kr}, \mathrm{E}=25 \mathrm{MeV}$ bremsstrahlung; measured fission yields, isotopic distribution parameters. Comparison with results from other targets. JOUR BRSPE 681298

2004GAZV NUCLEAR REACTIONS ${ }^{237} \mathrm{~Np},{ }^{243} \mathrm{Am}(\gamma, \mathrm{F}){ }^{89} \mathrm{Kr} /{ }^{91} \mathrm{Kr} /{ }^{92} \mathrm{Kr} /$ ${ }^{93} \mathrm{Kr} /{ }^{135} \mathrm{Xe} /{ }^{137} \mathrm{Xe} /{ }^{138} \mathrm{Xe} /{ }^{139} \mathrm{Xe} /{ }^{140} \mathrm{Xe} /{ }^{141} \mathrm{Xe} /{ }^{142} \mathrm{Xe}$, $\mathrm{E}_{\max }=25 \mathrm{MeV}$; measured $\mathrm{E} \gamma, \mathrm{I} \gamma$; deduced fission fragment yields. REPT JINR-P15-2004-119, Gangrsky 2005GA25 NUCLEAR REACTIONS ${ }^{248} \mathrm{Cm}(\gamma, \mathrm{F}){ }^{89} \mathrm{Kr} /{ }^{91} \mathrm{Kr} /{ }^{92} \mathrm{Kr} /{ }^{93} \mathrm{Kr} /$ ${ }^{135} \mathrm{Xe} /{ }^{137} \mathrm{Xe} /{ }^{138} \mathrm{Xe} /{ }^{139} \mathrm{Xe} /{ }^{140} \mathrm{Xe} /{ }^{141} \mathrm{Xe} /{ }^{142} \mathrm{Xe}, \mathrm{E}=25 \mathrm{MeV}$ bremsstrahlung; measured E $\gamma, \mathrm{I} \gamma$; deduced yields. JOUR FECLA 125 44 


\section{$\mathrm{A}=142$ (continued)}

2005GA50 NUCLEAR REACTIONS ${ }^{237} \mathrm{~Np},{ }^{243} \mathrm{Am}(\gamma, \mathrm{F}){ }^{135} \mathrm{Xe} /{ }^{137} \mathrm{Xe} /{ }^{138} \mathrm{Xe} /$ ${ }^{139} \mathrm{Xe} /{ }^{140} \mathrm{Xe} /{ }^{141} \mathrm{Xe} /{ }^{142} \mathrm{Xe} /{ }^{89} \mathrm{Kr} /{ }^{91} \mathrm{Kr} /{ }^{92} \mathrm{Kr} /{ }^{93} \mathrm{Kr}, \mathrm{E}=25 \mathrm{MeV}$ bremsstrahlung; measured fission yields, isotopic distribution parameters. JOUR YAFIA 681475

2005JA12 RADIOACTIVITY ${ }^{252} \mathrm{Cf}(\mathrm{SF})$; measured $\mathrm{E} \gamma, \mathrm{I} \gamma, \alpha \gamma$-, $\gamma \gamma$-coin for $\alpha$-accompanied ternary fission; deduced fission fragments average angular momentum. ${ }^{100,102} \mathrm{Zr},{ }^{106} \mathrm{Mo},{ }^{144,146} \mathrm{Ba},{ }^{138,140,142} \mathrm{Xe}$; deduced transition intensities. Gammasphere array. JOUR ZAANE 24373 ${ }^{142} \mathrm{Cs}$ 2005AN01 NUCLEAR REACTIONS ${ }^{238} \mathrm{U}(\mathrm{p}, \mathrm{F}){ }^{89} \mathrm{Rb} /{ }^{90} \mathrm{Rb} /{ }^{91} \mathrm{Rb} /{ }^{93} \mathrm{Rb} /$ ${ }^{94} \mathrm{Rb} /{ }^{95} \mathrm{Rb} /{ }^{139} \mathrm{Cs} /{ }^{140} \mathrm{Cs} /{ }^{141} \mathrm{Cs} /{ }^{142} \mathrm{Cs} /{ }^{144} \mathrm{Cs} /{ }^{145} \mathrm{Cs}, \mathrm{E}=1$ $\mathrm{GeV}$; measured yields. JOUR ZAANE 23257

${ }^{142} \mathrm{Ba} \quad 2005 \mathrm{BI} 02$ RADIOACTIVITY ${ }^{252} \mathrm{Cf}(\mathrm{SF})$; measured Doppler-shifted $\mathrm{E} \gamma, \mathrm{I} \gamma$, (particle) $\gamma$-, $\gamma \gamma$-coin. ${ }^{142,144} \mathrm{Ba}$ levels deduced $\mathrm{T}_{1 / 2}$, transition quadrupole moments. Euroball, Saphir arrays, differential Doppler shift method. JOUR PRVCA 71011301

${ }^{142} \mathrm{Nd}$ 2005MA10 NUCLEAR MOMENTS ${ }^{142,143,144,145,146,148,150} \mathrm{Nd}$; measured hfs, isotope shifts. JOUR CHPHD 14511

2005R035 NUCLEAR MOMENTS ${ }^{142,143,144,145,146,148,150} \mathrm{Nd}$; measured hfs, isotope shifts. JOUR CJPHA 83841

${ }^{142} \mathrm{Gd} \quad 2005 \mathrm{PA} 07$ NUCLEAR REACTIONS ${ }^{114} \mathrm{Sn}\left({ }^{32} \mathrm{~S}, 2 \mathrm{n} 2 \mathrm{p}\right), \mathrm{E}=160 \mathrm{MeV}$; measured $\mathrm{E} \gamma, \mathrm{I} \gamma, \gamma \gamma-$, (charged particle) $\gamma$-coin, DSA. ${ }^{142} \mathrm{Gd}$ deduced high-spin levels, J, $\pi$, configurations, $\mathrm{T}_{1 / 2}, \mathrm{~B}(\mathrm{M} 1), \mathrm{B}(\mathrm{E} 2)$. Euroball IV and Euclides arrays, comparisons with model predictions. JOUR ZAANE 23191

2006DR01 NUCLEAR REACTIONS ${ }^{99} \mathrm{Ru}\left({ }^{48} \mathrm{Ti}, 3 \mathrm{n} 2 \mathrm{p}\right), \mathrm{E}=240 \mathrm{MeV}$; measured $\mathrm{E} \gamma, \mathrm{I} \gamma, \gamma$-ray linear polarization. JOUR NIMAE 556182

${ }^{142} \mathrm{~Tb} 2005 \mathrm{RI} 17$ NUCLEAR REACTIONS ${ }^{92} \mathrm{Mo}\left({ }^{54} \mathrm{Fe}, \mathrm{xnypz} \alpha\right), \mathrm{E}=245 \mathrm{MeV}$; measured prompt and delayed $\mathrm{E} \gamma, \mathrm{I} \gamma, \gamma \gamma-$, (recoil) $\gamma$-coin. ${ }^{142} \mathrm{~Tb},{ }^{163} \mathrm{Dy}$ deduced transitions. ${ }^{143}$ Dy deduced isomeric state $\mathrm{T}_{1 / 2}$. Jurogam array. JOUR JPGPE 31 S1949

2005RYZZ RADIOACTIVITY ${ }^{146,146 m} \mathrm{Tm}(\mathrm{p})$ [from $\left.{ }^{92} \mathrm{Mo}\left({ }^{58} \mathrm{Ni}, \mathrm{xnp}\right)\right]$; measured proton spectra. ${ }^{146} \mathrm{Tm},{ }^{145} \mathrm{Er}$ deduced levels, configurations.

${ }^{142 m} \mathrm{~Tb}(\mathrm{IT})$; measured conversion electron spectra; deduced levels, $\mathrm{J}, \pi$. CONF Argonne(Nuclei at the Limits),P223,Rykaczewski

2005TA31 RADIOACTIVITY ${ }^{140 m} \mathrm{Eu},{ }^{142 m} \mathrm{~Tb},{ }^{144 m} \mathrm{Ho}(\mathrm{IT})$ [from ${ }^{54} \mathrm{Fe}\left({ }^{92} \mathrm{Mo}, \mathrm{X}\right)$ ]; measured X-ray spectra, $\mathrm{E} \gamma, \gamma \gamma$-coin, $\mathrm{E}(\mathrm{ce}), \mathrm{T}_{1 / 2} \cdot{ }^{140} \mathrm{Eu},{ }^{142} \mathrm{~Tb},{ }^{144} \mathrm{Ho}$ dlevels, J, $\pi$, configurations. Mass-separated sources. JOUR ZAANE $25 \mathrm{~s} 01151$

${ }^{142} \mathrm{Ho}$ 2005XU04 RADIOACTIVITY ${ }^{81} \mathrm{Zr},{ }^{85} \mathrm{Mo},{ }^{89} \mathrm{Ru},{ }^{92} \mathrm{Rh},{ }^{93} \mathrm{Pd},{ }^{121} \mathrm{Ce},{ }^{125} \mathrm{Nd}$, ${ }^{128} \mathrm{Pm},{ }^{129} \mathrm{Sm},{ }^{135,137} \mathrm{Gd},{ }^{139} \mathrm{Dy},{ }^{142} \mathrm{Ho},{ }^{149} \mathrm{Yb}\left(\beta^{+} \mathrm{p}\right)$; measured $\beta$-delayed $\mathrm{E} \gamma, \mathrm{I} \gamma$, proton spectra, $\mathrm{p} \gamma$-coin, $\mathrm{T}_{1 / 2}$. Comparison with model predictions. JOUR PRVCA 71054318

${ }^{142} \mathrm{Er} 2005 \mathrm{XU04} \quad$ NUCLEAR REACTIONS ${ }^{92} \mathrm{Mo},{ }^{106} \mathrm{Cd}\left({ }^{32} \mathrm{~S}, 3 \mathrm{n}\right), \mathrm{E}=151 \mathrm{MeV}$; ${ }^{92} \mathrm{Mo}\left({ }^{36} \mathrm{Ar}, 3 \mathrm{n}\right), \mathrm{E}=169 \mathrm{MeV} ;{ }^{96} \mathrm{Ru}\left({ }^{36} \mathrm{Ar}, 3 \mathrm{n}\right),\left({ }^{36} \mathrm{Ar}, 3 \mathrm{np}\right), \mathrm{E}=165,174$ $\mathrm{MeV} ;{ }^{106} \mathrm{Cd}\left({ }^{36} \mathrm{Ar}, 3 \mathrm{n}\right),\left({ }^{36} \mathrm{Ar}, \mathrm{n} \alpha\right), \mathrm{E}=176 \mathrm{MeV} ;{ }^{106} \mathrm{Cd}\left({ }^{40} \mathrm{Ca}, 4 \mathrm{n}\right)$, $\mathrm{E}=202 \mathrm{MeV} ;{ }^{112} \mathrm{Sn}\left({ }^{40} \mathrm{Ca}, 3 \mathrm{n}\right), \mathrm{E}=185 \mathrm{MeV}$; measured $\sigma$. JOUR PRVCA 71054318 


\section{$\mathrm{A}=143$}

\begin{tabular}{|c|c|c|}
\hline${ }^{143} \mathrm{Ba}$ & $2005 \mathrm{~F} 017$ & $\begin{array}{l}\text { RADIOACTIVITY }{ }^{252} \mathrm{Cf}(\mathrm{SF}) \text {; measured } \mathrm{E} \gamma, \mathrm{I} \gamma, \gamma \gamma \text {-coin. }{ }^{98} \mathrm{Sr} \\
{ }^{102,104} \mathrm{Zr},{ }^{137} \mathrm{Xe},{ }^{143} \mathrm{Ba},{ }^{152} \mathrm{Ce} \text { levels deduced } \mathrm{T}_{1 / 2 .} \text { Gammasphere }\end{array}$ \\
\hline & & array, time-gated triple-coincidence method. JOUR ZAANE 25 s01 465 \\
\hline${ }^{143} \mathrm{Ce}$ & 2005ADZZ & $\begin{array}{l}\text { NUCLEAR REACTIONS }{ }^{129} \mathrm{I}(\mathrm{n}, 7 \mathrm{n}),(\mathrm{n}, 6 \mathrm{n}),(\mathrm{n}, 4 \mathrm{n}),(\mathrm{n}, \gamma), \mathrm{E}=\text { fast; } \\
{ }^{237} \mathrm{~Np}(\mathrm{n}, \gamma), \mathrm{E}=\text { fast; measured yields. }{ }^{237} \mathrm{~Np}(\mathrm{n}, \mathrm{F}){ }^{91} \mathrm{Sr} /{ }^{97} \mathrm{Zr} /{ }^{132} \mathrm{Te} / \\
\\
{ }^{133} \mathrm{I} /{ }^{135} \mathrm{I}, \mathrm{E}=\text { fast; }{ }^{238} \mathrm{Pu}(\mathrm{n}, \mathrm{F}){ }^{97} \mathrm{Zr} /{ }^{129} \mathrm{Sb} /{ }^{132} \mathrm{I} /{ }^{133} \mathrm{I} /{ }^{135} \mathrm{Xe} / \\
{ }^{105} \mathrm{Ru}, \mathrm{E}=\text { fast; }{ }^{239} \mathrm{Pu}(\mathrm{n}, \mathrm{F}){ }^{88} \mathrm{Kr} /{ }^{91} \mathrm{Sr} /{ }^{92} \mathrm{Sr} /{ }^{92} \mathrm{Y} /{ }^{97} \mathrm{Zr} /{ }^{99} \mathrm{Mo} / \\
\\
{ }^{103} \mathrm{Ru} /{ }^{105} \mathrm{Ru} /{ }^{128} \mathrm{Sb} /{ }^{129} \mathrm{Sb} /{ }^{132} \mathrm{Te} /{ }^{131} \mathrm{I} /{ }^{132} \mathrm{I} /{ }^{133} \mathrm{I} /{ }^{135} \mathrm{I} /{ }^{135} \mathrm{Xe} \\
/{ }^{143} \mathrm{Ce} /{ }^{140} \mathrm{Ba} /{ }^{140} \mathrm{La}, \mathrm{E}=\text { fast; measured fission fragment yields. } \\
\text { Secondary neutrons from proton irradiation. JINR nuclotron. CONF } \\
\text { St Petersburg,P195,Adam }\end{array}$ \\
\hline \multirow[t]{4}{*}{${ }^{143} \mathrm{Nd}$} & $2003 \mathrm{KI} 26$ & $\begin{array}{l}\text { RADIOACTIVITY }{ }^{147} \mathrm{Sm}(\alpha) \text {; measured } \mathrm{E} \alpha, \mathrm{T}_{1 / 2} \text {. Comparison with } \\
\text { previous results. JOUR JNRSA } 4, \text { No } 1,5\end{array}$ \\
\hline & 2004WAZW & $\begin{array}{l}\text { NUCLEAR REACTIONS } \mathrm{Mg}\left({ }^{132} \mathrm{Xe}, \mathrm{xn}\right){ }^{149} \mathrm{Dy}, \mathrm{E}=7 \mathrm{MeV} / \text { nucleon; } \\
{ }^{12} \mathrm{C}\left({ }^{136} \mathrm{Xe}, 5 \mathrm{n}\right), \mathrm{E}=6.5 \mathrm{MeV} / \text { nucleon; measured } \mathrm{E} \gamma, \mathrm{I} \gamma(\theta, \mathrm{H}, \mathrm{t}) . \\
{ }^{149} \mathrm{Dy},{ }^{143} \mathrm{Nd} \text { deduced high-spin isomers g-factors. Time-differential } \\
\text { perturbed angular distribution method. REPT }\end{array}$ \\
\hline & 2005MA10 & $\begin{array}{l}\text { NUCLEAR MOMENTS }{ }^{142,143,144,145,146,148,150} \mathrm{Nd} \text {; measured hfs, } \\
\text { isotope shifts. JOUR CHPHD } 14511\end{array}$ \\
\hline & 2005R035 & $\begin{array}{l}\text { NUCLEAR MOMENTS }{ }^{142,143,144,145,146,148,150} \mathrm{Nd} \text {; measured hfs, } \\
\text { isotope shifts. JOUR CJPHA } 83841\end{array}$ \\
\hline${ }^{143} \mathrm{Pm}$ & $2005 \mathrm{AF} 02$ & $\begin{array}{l}\text { NUCLEAR REACTIONS }{ }^{141} \operatorname{Pr}(\alpha, \mathrm{n}),(\alpha, 2 \mathrm{n}), \mathrm{E}=15-45 \mathrm{MeV} \text {; } \\
\text { measured } \sigma . \text { Stacked-foil activation technique. Comparison with model } \\
\text { predictions. JOUR JUPSA } 741150\end{array}$ \\
\hline${ }^{143} \mathrm{Gd}$ & 2005BA64 & $\begin{array}{l}\text { NUCLEAR MOMENTS }{ }^{143 m, 145,145 m} \text { Gd; measured isotope shifts, hfs; } \\
\text { deduced radii, } \mu \text {. Laser spectroscopy. JOUR PRVCA } 72017301\end{array}$ \\
\hline${ }^{143} \mathrm{Dy}$ & 2005RI17 & $\begin{array}{l}\text { NUCLEAR REACTIONS }{ }^{92} \mathrm{Mo}\left({ }^{54} \mathrm{Fe} \text {, xnypz } \alpha\right), \mathrm{E}=245 \mathrm{MeV} \text {; measured } \\
\text { prompt and delayed } \mathrm{E} \gamma, \mathrm{I} \gamma, \gamma \gamma-,(\text { recoil }) \gamma-\text { coin. }{ }^{142} \mathrm{~Tb},{ }^{163} \mathrm{Dy} \text { deduced } \\
\text { transitions. }{ }^{143} \mathrm{Dy} \text { deduced isomeric state } \mathrm{T}_{1 / 2} . \text { Jurogam array. JOUR } \\
\text { JPGPE } 31 \mathrm{~S} 1949\end{array}$ \\
\hline \multirow[t]{2}{*}{${ }^{143} \operatorname{Er}$} & 2005BI24 & $\begin{array}{l}\text { RADIOACTIVITY }{ }^{141} \mathrm{Ho},{ }^{144,145,146} \mathrm{Tm}(\mathrm{p}) \text { [from }{ }^{92} \mathrm{Mo}\left({ }^{54} \mathrm{Fe}, \text { xnyp }\right) \\
\left.\text { and }{ }^{92} \mathrm{Mo}\left({ }^{58} \mathrm{Ni}, \text { xnyp }\right)\right] ; \text { measured Ep, } \mathrm{T}_{1 / 2} ; \text { deduced branching ratios. } \\
{ }^{141} \mathrm{Ho},{ }^{140} \mathrm{Dy},{ }^{145,146} \mathrm{Tm},{ }^{144,145} \mathrm{Er} \text { deduced levels, configurations. } \\
\text { JOUR NIMBE } 241185\end{array}$ \\
\hline & 2005GR32 & $\begin{array}{l}\text { RADIOACTIVITY }{ }^{144} \mathrm{Tm}(\mathrm{p})\left[\text { from }{ }^{58} \mathrm{Ni}\left({ }^{92} \mathrm{Mo}, \mathrm{p} 5 \mathrm{n}\right), \mathrm{E}=340 \mathrm{MeV}\right] \\
\text { measured proton spectra, } \mathrm{T}_{1 / 2} \text {; deduced fine structure. JOUR ZAANE } \\
25 \text { s01 } 145\end{array}$ \\
\hline
\end{tabular}

\section{$\mathrm{A}=144$}

${ }^{144} \mathrm{Cs} \quad$ 2005AN01

NUCLEAR REACTIONS ${ }^{238} \mathrm{U}(\mathrm{p}, \mathrm{F}){ }^{89} \mathrm{Rb} /{ }^{90} \mathrm{Rb} /{ }^{91} \mathrm{Rb} /{ }^{93} \mathrm{Rb} /$ ${ }^{94} \mathrm{Rb} /{ }^{95} \mathrm{Rb} /{ }^{139} \mathrm{Cs} /{ }^{140} \mathrm{Cs} /{ }^{141} \mathrm{Cs} /{ }^{142} \mathrm{Cs} /{ }^{144} \mathrm{Cs} /{ }^{145} \mathrm{Cs}, \mathrm{E}=1$ $\mathrm{GeV}$; measured yields. JOUR ZAANE 23257

${ }^{144} \mathrm{Ba}$ 2005BI02 RADIOACTIVITY ${ }^{252} \mathrm{Cf}(\mathrm{SF}) ;$ measured Doppler-shifted $\mathrm{E} \gamma, \mathrm{I} \gamma$, (particle) $\gamma-, \gamma \gamma$-coin. ${ }^{142,144} \mathrm{Ba}$ levels deduced $\mathrm{T}_{1 / 2}$, transition quadrupole moments. Euroball, Saphir arrays, differential Doppler shift method. JOUR PRVCA 71011301 


\section{$\mathrm{A}=144$ (continued)}

2005JA12 RADIOACTIVITY ${ }^{252} \mathrm{Cf}(\mathrm{SF})$; measured $\mathrm{E} \gamma, \mathrm{I} \gamma, \alpha \gamma-, \gamma \gamma$-coin for $\alpha$-accompanied ternary fission; deduced fission fragments average angular momentum. ${ }^{100,102} \mathrm{Zr},{ }^{106} \mathrm{Mo},{ }^{144,146} \mathrm{Ba},{ }^{138,140,142} \mathrm{Xe}$; deduced transition intensities. Gammasphere array. JOUR ZAANE 24373

2005SH49 RADIOACTIVITY ${ }^{252} \mathrm{Cf}(\mathrm{SF})$; measured Doppler-shifted $\mathrm{E} \gamma, \mathrm{I} \gamma, \gamma \gamma$-, (fragment) $\gamma$-coin. ${ }^{144} \mathrm{Ba}$ deduced transitions $\mathrm{T}_{1 / 2}, \mathrm{~B}(\mathrm{E} 2)$, transition dipole, quadrupole, and octupole moments for alternating-parity band. Gammasphere array, cluster-model analysis. JOUR ZAANE 25387 ${ }^{144} \mathrm{Nd}$ 2005MA10 NUCLEAR MOMENTS ${ }^{142,143,144,145,146,148,150} \mathrm{Nd}$; measured hfs, isotope shifts. JOUR CHPHD 14511

2005R035 NUCLEAR MOMENTS ${ }^{142,143,144,145,146,148,150} \mathrm{Nd}$; measured hfs, isotope shifts. JOUR CJPHA 83841

${ }^{144} \mathrm{Pm} \quad$ 2005AF02 NUCLEAR REACTIONS ${ }^{141} \operatorname{Pr}(\alpha, \mathrm{n}),(\alpha, 2 \mathrm{n}), \mathrm{E}=15-45 \mathrm{MeV}$; measured $\sigma$. Stacked-foil activation technique. Comparison with model predictions. JOUR JUPSA 741150

${ }^{144} \mathrm{Ho} \quad 2005 \mathrm{TA} 31$ RADIOACTIVITY ${ }^{140 m} \mathrm{Eu},{ }^{142 m} \mathrm{~Tb},{ }^{144 m} \mathrm{Ho}(\mathrm{IT})$ [from ${ }^{54} \mathrm{Fe}\left({ }^{92} \mathrm{Mo}, \mathrm{X}\right)$ ]; measured X-ray spectra, $\mathrm{E} \gamma, \gamma \gamma$-coin, $\mathrm{E}(\mathrm{ce}), \mathrm{T}_{1 / 2} \cdot{ }^{140} \mathrm{Eu},{ }^{142} \mathrm{~Tb},{ }^{144} \mathrm{Ho}$ dlevels, J, $\pi$, configurations. Mass-separated sources. JOUR ZAANE 25 s01 151

${ }^{144} \mathrm{Er} \quad$ 2004SEZW

RADIOACTIVITY ${ }^{145,147} \mathrm{Tm}(\mathrm{p})$; measured Ep. REPT ANL-04/22,P27,Seweryniak

2005BI24 RADIOACTIVITY ${ }^{141} \mathrm{Ho},{ }^{144,145,146} \mathrm{Tm}$ (p) [from ${ }^{92} \mathrm{Mo}\left({ }^{54} \mathrm{Fe}\right.$, xnyp) and ${ }^{92} \mathrm{Mo}\left({ }^{58} \mathrm{Ni}\right.$, xnyp)]; measured $\mathrm{Ep}, \mathrm{T}_{1 / 2}$; deduced branching ratios. ${ }^{141} \mathrm{Ho},{ }^{140} \mathrm{Dy},{ }^{145,146} \mathrm{Tm},{ }^{144,145} \mathrm{Er}$ deduced levels, configurations. JOUR NIMBE 241185

2005ROZY RADIOACTIVITY ${ }^{145,146} \mathrm{Tm}$ (p) [from ${ }^{58} \mathrm{Ni}\left({ }^{92} \mathrm{Mo}\right.$, xnp)]; measured Ep, $\mathrm{p} \gamma$-coin, $\mathrm{T}_{1 / 2} .{ }^{144,145} \mathrm{Er}$ deduced levels, feeding intensities. CONF Argonne(Nuclei at the Limits),P217,Robinson

2005SE26 RADIOACTIVITY ${ }^{145} \mathrm{Tm}(\mathrm{p})$ [from $\left.{ }^{58} \mathrm{Ni}\left({ }^{92} \mathrm{Mo}, 4 \mathrm{np}\right)\right]$; measured Ep, $\mathrm{E} \gamma, \mathrm{p} \gamma$-coin. JOUR ZAANE 25 s01 159

${ }^{144} \mathrm{Tm} \quad$ 2005BI24 RADIOACTIVITY ${ }^{141} \mathrm{Ho},{ }^{144,145,146} \mathrm{Tm}(\mathrm{p})$ [from ${ }^{92} \mathrm{Mo}\left({ }^{54} \mathrm{Fe}\right.$, xnyp) and ${ }^{92} \mathrm{Mo}\left({ }^{58} \mathrm{Ni}\right.$, xnyp)]; measured $\mathrm{Ep}, \mathrm{T}_{1 / 2}$; deduced branching ratios. ${ }^{141} \mathrm{Ho},{ }^{140} \mathrm{Dy},{ }^{145,146} \mathrm{Tm},{ }^{144,145} \mathrm{Er}$ deduced levels, configurations. JOUR NIMBE 241185

2005GR32 RADIOACTIVITY ${ }^{144} \mathrm{Tm}$ (p) [from ${ }^{58} \mathrm{Ni}\left({ }^{92} \mathrm{Mo}, \mathrm{p} 5 \mathrm{n}\right), \mathrm{E}=340 \mathrm{MeV}$ ]; measured proton spectra, $\mathrm{T}_{1 / 2}$; deduced fine structure. JOUR ZAANE $25 \mathrm{~s} 01145$

2005RYZZ NUCLEAR REACTIONS ${ }^{92} \mathrm{Mo}\left({ }^{58} \mathrm{Ni}, \mathrm{X}\right), \mathrm{E}=340 \mathrm{MeV}$; measured delayed Ep, (recoil)(proton)-coin. ${ }^{144} \mathrm{Tm}$ deduced possible proton decay. CONF Argonne(Nuclei at the Limits),P223,Rykaczewski

\section{$\mathrm{A}=145$}

${ }^{145} \mathrm{Cs} \quad$ 2005ANO1

NUCLEAR REACTIONS ${ }^{238} \mathrm{U}(\mathrm{p}, \mathrm{F}){ }^{89} \mathrm{Rb} /{ }^{90} \mathrm{Rb} /{ }^{91} \mathrm{Rb} /{ }^{93} \mathrm{Rb} /$ ${ }^{94} \mathrm{Rb} /{ }^{95} \mathrm{Rb} /{ }^{139} \mathrm{Cs} /{ }^{140} \mathrm{Cs} /{ }^{141} \mathrm{Cs} /{ }^{142} \mathrm{Cs} /{ }^{144} \mathrm{Cs} /{ }^{145} \mathrm{Cs}, \mathrm{E}=1$ $\mathrm{GeV}$; measured yields. JOUR ZAANE 23257

${ }^{145} \mathrm{Nd} \quad$ 2005MA10 NUCLEAR MOMENTS ${ }^{142,143,144,145,146,148,150} \mathrm{Nd}$; measured hfs, isotope shifts. JOUR CHPHD 14511 


\section{$\mathrm{A}=145$ (continued)}

2005R035 NUCLEAR MOMENTS ${ }^{142,143,144,145,146,148,150} \mathrm{Nd}$; measured hfs, isotope shifts. JOUR CJPHA 83841

$\begin{array}{ccl}{ }^{145} \mathrm{Gd} & \text { 2005BA64 } & \begin{array}{l}\text { NUCLEAR MOMENTS }{ }^{143 m, 145,145 m} \mathrm{Gd} \text {; measured isotope shifts, hfs; } \\ \text { deduced radii, } \mu \text {. Laser spectroscopy. JOUR PRVCA } 72 \text { 017301 }\end{array} \\ & \text { RADIOACTIVITY }{ }^{146} \mathrm{Tm}(\mathrm{p}) ; \text { measured Ep. REPT } \\ { }^{145} \mathrm{Er} & \text { 2004DAZX } & \begin{array}{l}\text { ANL-04/22,P29,Davids } \\ \text { RADIOACTIVITY }{ }^{146} \mathrm{Tm}(\mathrm{p})\left[\text { from }{ }^{58} \mathrm{Ni}\left({ }^{92} \mathrm{Mo}, \mathrm{p} 3 \mathrm{n}\right), \mathrm{E}=297 \mathrm{MeV}\right] ;\end{array} \\ & 2005 \mathrm{BB} 02 & \end{array}$
measured proton spectra, $\mathrm{T}_{1 / 2}$; deduced fine structure, decay branching ratios. ${ }^{145}$ Er deduced levels, configurations. JOUR ZAANE 25 s01 149 2005BI24 RADIOACTIVITY ${ }^{141} \mathrm{Ho},{ }^{144,145,146} \mathrm{Tm}(\mathrm{p})\left[\right.$ from ${ }^{92} \mathrm{Mo}\left({ }^{54} \mathrm{Fe}\right.$, xnyp) and ${ }^{92} \mathrm{Mo}\left({ }^{58} \mathrm{Ni}\right.$, xnyp)]; measured $\mathrm{Ep}, \mathrm{T}_{1 / 2}$; deduced branching ratios. ${ }^{141} \mathrm{Ho},{ }^{140} \mathrm{Dy},{ }^{145,146} \mathrm{Tm},{ }^{144,145} \mathrm{Er}$ deduced levels, configurations. JOUR NIMBE 241185

2005R040 RADIOACTIVITY ${ }^{146} \mathrm{Tm}(\mathrm{p})\left[\right.$ from $\left.{ }^{58} \mathrm{Ni}\left({ }^{92} \mathrm{Mo}, \mathrm{X}\right)\right]$; measured $\mathrm{E} \gamma, \mathrm{Ep}$, $\mathrm{T}_{1 / 2}$ following proton decay from ground and excited states. ${ }^{146} \mathrm{Tm}$, ${ }^{145}$ Er deduced levels, J, $\pi$, configurations. JOUR ZAANE 25 s01 155

2005ROZY RADIOACTIVITY ${ }^{145,146} \mathrm{Tm}(\mathrm{p})$ [from ${ }^{58} \mathrm{Ni}\left({ }^{92} \mathrm{Mo}\right.$, xnp)]; measured Ep, $\mathrm{p} \gamma$-coin, $\mathrm{T}_{1 / 2} .{ }^{144,145} \mathrm{Er}$ deduced levels, feeding intensities. CONF Argonne(Nuclei at the Limits),P217,Robinson

2005RYZZ RADIOACTIVITY ${ }^{146,146 m} \mathrm{Tm}$ (p) [from ${ }^{92} \mathrm{Mo}\left({ }^{58} \mathrm{Ni}\right.$, xnp)]; measured proton spectra. ${ }^{146} \mathrm{Tm},{ }^{145} \mathrm{Er}$ deduced levels, configurations.

${ }^{142 m} \mathrm{~Tb}(\mathrm{IT})$; measured conversion electron spectra; deduced levels, $\mathrm{J}, \pi$. CONF Argonne(Nuclei at the Limits),P223,Rykaczewski

${ }^{145} \mathrm{Tm}$ 2004SEZW RADIOACTIVITY ${ }^{145,147} \mathrm{Tm}(\mathrm{p})$; measured Ep. REPT ANL-04/22,P27,Seweryniak

2005BI24 RADIOACTIVITY ${ }^{141} \mathrm{Ho},{ }^{144,145,146} \mathrm{Tm}$ (p) [from ${ }^{92} \mathrm{Mo}\left({ }^{54} \mathrm{Fe}\right.$, xnyp) and ${ }^{92} \mathrm{Mo}\left({ }^{58} \mathrm{Ni}\right.$, xnyp)]; measured Ep, $\mathrm{T}_{1 / 2}$; deduced branching ratios. ${ }^{141} \mathrm{Ho},{ }^{140} \mathrm{Dy},{ }^{145,146} \mathrm{Tm},{ }^{144,145} \mathrm{Er}$ deduced levels, configurations. JOUR NIMBE 241185

2005ROZY NUCLEAR REACTIONS ${ }^{58} \mathrm{Ni}\left({ }^{92} \mathrm{Mo}, 2 \mathrm{np}\right),\left({ }^{92} \mathrm{Mo}, 3 \mathrm{np}\right),\left({ }^{92} \mathrm{Mo}, 4 \mathrm{np}\right)$, $\mathrm{E}$ not given; measured $\mathrm{E} \gamma, \mathrm{I} \gamma$, (recoil) $\gamma$-coin. ${ }^{145,147} \mathrm{Tm}$ deduced levels, $\mathrm{J}, \pi$, rotational bands. Recoil decay tagging, Gammasphere array. CONF Argonne(Nuclei at the Limits),P217,Robinson

2005ROZY RADIOACTIVITY ${ }^{145,146} \mathrm{Tm}$ (p) [from ${ }^{58} \mathrm{Ni}\left({ }^{92} \mathrm{Mo}\right.$, xnp)]; measured Ep, $\mathrm{p} \gamma$-coin, $\mathrm{T}_{1 / 2} \cdot{ }^{144,145} \mathrm{Er}$ deduced levels, feeding intensities. CONF Argonne(Nuclei at the Limits),P217,Robinson

2005SE26 NUCLEAR REACTIONS ${ }^{58} \mathrm{Ni}\left({ }^{92} \mathrm{Mo}, 2 \mathrm{np}\right), \mathrm{E}=512 \mathrm{MeV} ;{ }^{58} \mathrm{Ni}\left({ }^{92} \mathrm{Mo}\right.$, $3 \mathrm{np}), \mathrm{E}=460 \mathrm{MeV} ;{ }^{58} \mathrm{Ni}\left({ }^{92} \mathrm{Mo}, 4 \mathrm{np}\right), \mathrm{E}=417 \mathrm{MeV}$; measured $\mathrm{E} \gamma, \mathrm{I} \gamma$, $\gamma \gamma-$, (recoil) $\gamma$-coin. ${ }^{145,146,147} \mathrm{Tm}$ deduced levels, J, $\pi$, proton-decay features. Gammasphere array, recoil-decay tagging. Comparison with Particle Rotor model predictions. JOUR ZAANE 25 s01 159

2005SE26 RADIOACTIVITY ${ }^{145} \mathrm{Tm}(\mathrm{p})$ [from $\left.{ }^{58} \mathrm{Ni}\left({ }^{92} \mathrm{Mo}, 4 \mathrm{np}\right)\right]$; measured $\mathrm{Ep}$, $\mathrm{E} \gamma, \mathrm{p} \gamma$-coin. JOUR ZAANE 25 s01 159 


\section{$\mathrm{A}=146$}

\begin{tabular}{|c|c|c|}
\hline${ }^{146} \mathrm{Ba}$ & 2005JA12 & $\begin{array}{l}\text { RADIOACTIVITY }{ }^{252} \mathrm{Cf}(\mathrm{SF}) \text {; measured } \mathrm{E} \gamma, \mathrm{I} \gamma, \alpha \gamma-, \gamma \gamma \text {-coin for } \\
\alpha \text {-accompanied ternary fission; deduced fission fragments average } \\
\text { angular momentum. }{ }^{100,102} \mathrm{Zr},{ }^{106} \mathrm{Mo},{ }^{144,146} \mathrm{Ba},{ }^{138,140,142} \mathrm{Xe} \text {; deduced } \\
\text { transition intensities. Gammasphere array. JOUR ZAANE } 24373\end{array}$ \\
\hline \multirow[t]{2}{*}{${ }^{146} \mathrm{Nd}$} & 2005MA10 & $\begin{array}{l}\text { NUCLEAR MOMENTS }{ }^{142,143,144,145,146,148,150} \mathrm{Nd} \text {; measured hfs, } \\
\text { isotope shifts. JOUR CHPHD } 14511\end{array}$ \\
\hline & 2005R035 & $\begin{array}{l}\text { NUCLEAR MOMENTS } 142,143,144,145,146,148,150 \mathrm{Nd} \text {; measured hfs, } \\
\text { isotope shifts. JOUR CJPHA } 83841\end{array}$ \\
\hline${ }^{146} \mathrm{Er}$ & 2004SEZW & $\begin{array}{l}\text { RADIOACTIVITY }{ }^{145,147} \mathrm{Tm}(\mathrm{p}) ; \text { measured Ep. REPT } \\
\text { ANL-04/22,P27,Seweryniak }\end{array}$ \\
\hline \multirow[t]{2}{*}{${ }^{146} \mathrm{Tm}$} & 2004DAZX & $\begin{array}{l}\text { RADIOACTIVITY }{ }^{146} \mathrm{Tm}(\mathrm{p}) ; \text { measured Ep. REPT } \\
\text { ANL-04/22,P29,Davids }\end{array}$ \\
\hline & 2005BВ02 & RADIOACTIVITY ${ }^{146} \mathrm{Tm}(\mathrm{p})$ [from ${ }^{58} \mathrm{Ni}\left({ }^{92} \mathrm{Mo}, \mathrm{p} 3 \mathrm{n}\right), \mathrm{E}=297 \mathrm{MeV}$; \\
\hline
\end{tabular}
measured proton spectra, $\mathrm{T}_{1 / 2}$; deduced fine structure, decay branching ratios. ${ }^{145}$ Er deduced levels, configurations. JOUR ZAANE 25 s01 149

$2005 B 124$ RADIOACTIVITY ${ }^{141} \mathrm{Ho},{ }^{144,145,146} \mathrm{Tm}(\mathrm{p})\left[\right.$ from ${ }^{92} \mathrm{Mo}\left({ }^{54} \mathrm{Fe}\right.$, xnyp) and ${ }^{92} \mathrm{Mo}\left({ }^{58} \mathrm{Ni}\right.$, xnyp $\left.)\right]$; measured Ep, $\mathrm{T}_{1 / 2}$; deduced branching ratios. ${ }^{141} \mathrm{Ho},{ }^{140} \mathrm{Dy},{ }^{145,146} \mathrm{Tm},{ }^{144,145} \mathrm{Er}$ deduced levels, configurations. JOUR NIMBE 241185

2005R040 NUCLEAR REACTIONS ${ }^{58} \mathrm{Ni}\left({ }^{92} \mathrm{Mo}, 3 \mathrm{np}\right)$, E not given; measured $\mathrm{E} \gamma$, I $\gamma, \gamma \gamma$-, (recoil) $\gamma$-coin. ${ }^{146} \mathrm{Tm}$ deduced levels, J, $\pi$. Gammasphere array, recoil-decay tagging. JOUR ZAANE 25 s01 155

2005R040 RADIOACTIVITY ${ }^{146} \mathrm{Tm}(\mathrm{p})\left[\right.$ from $\left.{ }^{58} \mathrm{Ni}\left({ }^{92} \mathrm{Mo}, \mathrm{X}\right)\right]$; measured E $\gamma, \mathrm{Ep}$, $\mathrm{T}_{1 / 2}$ following proton decay from ground and excited states. ${ }^{146} \mathrm{Tm}$, ${ }^{145}$ Er deduced levels, J, $\pi$, configurations. JOUR ZAANE 25 s01 155

2005ROZY NUCLEAR REACTIONS ${ }^{58} \mathrm{Ni}\left({ }^{92} \mathrm{Mo}, 2 \mathrm{np}\right),\left({ }^{92} \mathrm{Mo}, 3 \mathrm{np}\right),\left({ }^{92} \mathrm{Mo}, 4 \mathrm{np}\right)$, E not given; measured $\mathrm{E} \gamma, \mathrm{I} \gamma$, (recoil) $\gamma$-coin. ${ }^{145,147} \mathrm{Tm}$ deduced levels, $\mathrm{J}, \pi$, rotational bands. Recoil decay tagging, Gammasphere array. CONF Argonne(Nuclei at the Limits),P217,Robinson

2005ROZY RADIOACTIVITY ${ }^{145,146} \mathrm{Tm}(\mathrm{p})$ [from $\left.{ }^{58} \mathrm{Ni}\left({ }^{92} \mathrm{Mo}, \mathrm{xnp}\right)\right]$; measured Ep, $\mathrm{p} \gamma$-coin, $\mathrm{T}_{1 / 2} \cdot{ }^{144,145} \mathrm{Er}$ deduced levels, feeding intensities. CONF Argonne(Nuclei at the Limits),P217,Robinson

2005RYZZ RADIOACTIVITY ${ }^{146,146 m} \mathrm{Tm}(\mathrm{p})$ [from $\left.{ }^{92} \mathrm{Mo}\left({ }^{58} \mathrm{Ni}, \mathrm{xnp}\right)\right]$; measured proton spectra. ${ }^{146} \mathrm{Tm},{ }^{145} \mathrm{Er}$ deduced levels, configurations.

${ }^{142 m} \mathrm{~Tb}(\mathrm{IT})$; measured conversion electron spectra; deduced levels, J, $\pi$. CONF Argonne(Nuclei at the Limits),P223,Rykaczewski

2005SE26 NUCLEAR REACTIONS ${ }^{58} \mathrm{Ni}\left({ }^{92} \mathrm{Mo}, 2 \mathrm{np}\right), \mathrm{E}=512 \mathrm{MeV} ;{ }^{58} \mathrm{Ni}\left({ }^{92} \mathrm{Mo}\right.$, $3 \mathrm{np}), \mathrm{E}=460 \mathrm{MeV} ;{ }^{58} \mathrm{Ni}\left({ }^{92} \mathrm{Mo}, 4 \mathrm{np}\right), \mathrm{E}=417 \mathrm{MeV}$; measured $\mathrm{E} \gamma$, I $\gamma$, $\gamma \gamma$-, (recoil) $\gamma$-coin. ${ }^{145,146,147} \mathrm{Tm}$ deduced levels, J, $\pi$, proton-decay features. Gammasphere array, recoil-decay tagging. Comparison with Particle Rotor model predictions. JOUR ZAANE 25 s01 159

\section{$\mathrm{A}=147$}

${ }^{147} \mathrm{Cs} \quad 2005 \mathrm{SY} 01$

RADIOACTIVITY ${ }^{147} \mathrm{Cs}\left(\beta^{-}\right)$[from ${ }^{235} \mathrm{U}(\mathrm{n}, \mathrm{F})$ ]; measured $\beta$-delayed $\mathrm{E} \gamma, \mathrm{I} \gamma, \gamma \gamma-$, (X-ray) $\gamma$-coin, $\mathrm{T}_{1 / 2} .{ }^{147} \mathrm{Ba}$ deduced levels, $\mathrm{J}, \pi, \mathrm{T}_{1 / 2}$, B(M1), B(E2). JOUR ZAANE 23481 


\section{$\mathrm{A}=147$ (continued)}

\begin{tabular}{|c|c|c|}
\hline${ }^{147} \mathrm{Ba}$ & 2005SY01 & $\begin{array}{l}\left.\text { RADIOACTIVITY }{ }^{147} \mathrm{Cs}\left(\beta^{-}\right) \text {from }{ }^{235} \mathrm{U}(\mathrm{n}, \mathrm{F})\right] ; \text { measured } \beta \text {-delayed } \\
\mathrm{E} \gamma, \mathrm{I} \gamma, \gamma \gamma-,\left(\mathrm{X} \text {-ray) } \gamma \text {-coin, } \mathrm{T}_{1 / 2} \cdot{ }^{147} \mathrm{Ba} \text { deduced levels, } \mathrm{J}, \pi, \mathrm{T}_{1 / 2},\right. \\
\mathrm{B}(\mathrm{M} 1), \mathrm{B}(\mathrm{E} 2) \text {. JOUR ZAANE } 23{ }^{481}\end{array}$ \\
\hline${ }^{147} \mathrm{Nd}$ & 2005UOZZ & $\begin{array}{l}\text { NUCLEAR REACTIONS U(p, F })^{95} \mathrm{Zr} /{ }^{115} \mathrm{Cd} /{ }^{134} \mathrm{Cs} /{ }^{136} \mathrm{Cs} /{ }^{137} \mathrm{Cs} \\
/{ }^{147} \mathrm{Nd}, \mathrm{E} \approx 20-70 \mathrm{MeV} \text {; measured production } \sigma . \text { Stacked-foil } \\
\text { activation technique, comparison with model predictions. CONF Santa } \\
\text { Fe (Nucl Data for Sci and Technol) Proc,Vol2,P1547 }\end{array}$ \\
\hline${ }^{147} \mathrm{Sm}$ & $2003 K I 26$ & $\begin{array}{l}\text { RADIOACTIVITY }{ }^{147} \mathrm{Sm}(\alpha) \text {; measured } \mathrm{E} \alpha, \mathrm{T}_{1 / 2} \text {. Comparison with } \\
\text { previous results. JOUR JNRSA } 4, \text { No } 1,5\end{array}$ \\
\hline${ }^{147} \mathrm{Dy}$ & 2005GE10 & $\begin{array}{l}\text { ATOMIC MASSES }{ }^{147,147 m} \text { Dy; measured masses for ground and } \\
\text { isomeric states. Schottky mass spectrometry. JOUR JPGPE } 31 \text { S1779 }\end{array}$ \\
\hline \multirow[t]{3}{*}{${ }^{147} \mathrm{Tm}$} & 2004SEZW & $\begin{array}{l}\text { RADIOACTIVITY }{ }^{145,147} \mathrm{Tm}(\mathrm{p}) ; \text { measured Ep. REPT } \\
\text { ANL-04/22,P27,Seweryniak }\end{array}$ \\
\hline & 2005ROZY & $\begin{array}{l}\text { NUCLEAR REACTIONS }{ }^{58} \mathrm{Ni}\left({ }^{92} \mathrm{Mo}, 2 \mathrm{np}\right),\left({ }^{92} \mathrm{Mo}, 3 \mathrm{np}\right),\left({ }^{92} \mathrm{Mo}, 4 \mathrm{np}\right) \text {, } \\
\mathrm{E} \text { not given; measured } \mathrm{E} \gamma, \mathrm{I} \gamma \text {, (recoil) } \gamma \text {-coin. }{ }^{145,147} \mathrm{Tm} \text { deduced levels, } \\
\mathrm{J}, \pi \text {, rotational bands. Recoil decay tagging, Gammasphere array. } \\
\text { CONF Argonne(Nuclei at the Limits),P217,Robinson }\end{array}$ \\
\hline & 2005SE26 & $\begin{array}{l}\text { NUCLEAR REACTIONS }{ }^{58} \mathrm{Ni}\left({ }^{92} \mathrm{Mo}, 2 \mathrm{np}\right), \mathrm{E}=512 \mathrm{MeV} ;{ }^{58} \mathrm{Ni}\left({ }^{92} \mathrm{Mo} \text {, }\right. \\
3 \mathrm{np}), \mathrm{E}=460 \mathrm{MeV} ;{ }^{58} \mathrm{Ni}\left({ }^{92} \mathrm{Mo}, 4 \mathrm{np}\right), \mathrm{E}=417 \mathrm{MeV} ; \text { measured } \mathrm{E} \gamma, \mathrm{I} \gamma, \\
\gamma \gamma-,(\text { recoil }) \gamma \text {-coin. }{ }^{145,146,147} \mathrm{Tm} \text { deduced levels, J, } \pi \text {, proton-decay } \\
\text { features. Gammasphere array, recoil-decay tagging. Comparison with } \\
\text { Particle Rotor model predictions. JOUR ZAANE } 25 \mathrm{~s} 01159\end{array}$ \\
\hline
\end{tabular}

$$
\mathrm{A}=\mathbf{1 4 8}
$$

${ }^{148} \mathrm{Nd}$ 2005MA10 NUCLEAR MOMENTS ${ }^{142,143,144,145,146,148,150} \mathrm{Nd}$; measured hfs, isotope shifts. JOUR CHPHD 14511

2005R035 NUCLEAR MOMENTS ${ }^{142,143,144,145,146,148,150} \mathrm{Nd}$; measured hfs, isotope shifts. JOUR CJPHA 83841

${ }^{148} \mathrm{Sm}$ 2005DA20 NUCLEAR REACTIONS ${ }^{147} \mathrm{Sm}(\mathrm{n}, \gamma), \mathrm{E} \approx$ resonance; measured capture $\sigma$. Minimization of statistical error discussed. JOUR NIMAE 544659

2005LI14 NUCLEAR REACTIONS ${ }^{148} \mathrm{Sm}\left(\gamma, \gamma^{\prime}\right), \mathrm{E}=3.2 \mathrm{MeV}$ bremsstrahlung; measured $\mathrm{E} \gamma, \mathrm{I} \gamma .{ }^{148} \mathrm{Sm}$ deduced levels, J, $\pi, \mathrm{B}(\mathrm{M} 1), \mathrm{B}(\mathrm{E} 1), \mathrm{B}(\mathrm{E} 2)$, mixed-symmetry state. Nuclear resonance fluorescence, interacting boson model. JOUR PRVCA 71044318

${ }^{148} \mathrm{Gd}$ 2005KE07 NUCLEAR REACTIONS W, Ta, Au(p, X) ${ }^{148} \mathrm{Gd}, \mathrm{E}=600,800 \mathrm{MeV}$; measured cumulative production $\sigma$. Comparison with previous results, model predictions. JOUR NUPAB 760225

${ }^{148} \mathrm{~Tb}$ 2004AL35 RADIOACTIVITY ${ }^{148} \mathrm{Dy}(\mathrm{EC}),\left(\beta^{+}\right)\left[\right.$from $\left.{ }^{93} \mathrm{Nb}\left({ }^{58} \mathrm{Ni}, 3 \mathrm{p}\right)\right]$; measured $\mathrm{E} \gamma, \mathrm{I} \gamma,\left(\mathrm{X}\right.$-ray) $\gamma-, \beta \gamma$-coin; deduced log ft. ${ }^{148} \mathrm{~Tb}$ levels deduced $\beta$-feeding intensities, Gamow-Teller strength distribution, resonant state features. Total absorption spectrometer, comparison with previous results. JOUR PRVCA 70064301 


\section{$\mathrm{A}=148$ (continued)}

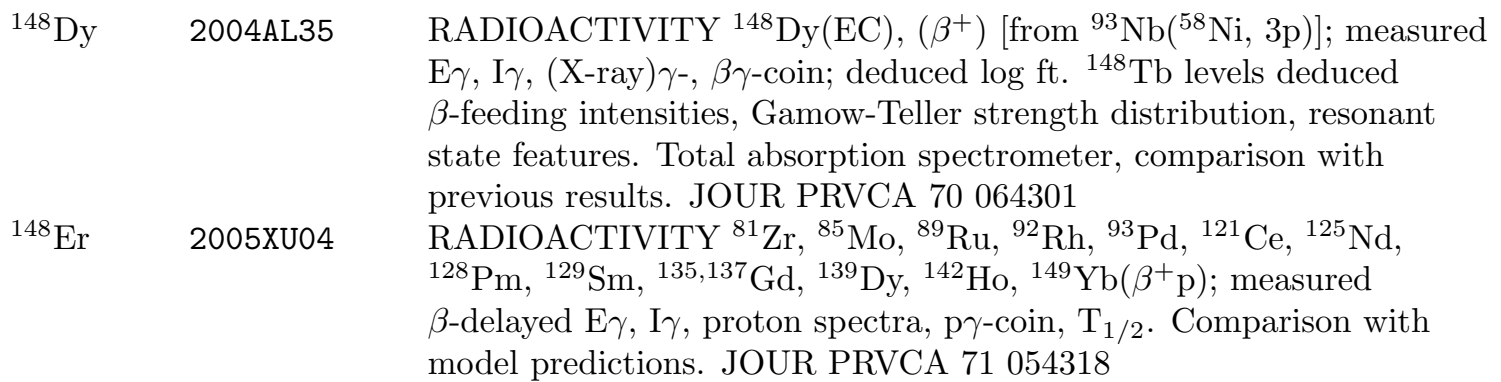

$$
\mathrm{A}=149
$$

${ }^{149}$ Dy 2004WAzw NUCLEAR REACTIONS $\mathrm{Mg}\left({ }^{132} \mathrm{Xe}, \mathrm{xn}\right){ }^{149} \mathrm{Dy}, \mathrm{E}=7 \mathrm{MeV} /$ nucleon; ${ }^{12} \mathrm{C}\left({ }^{136} \mathrm{Xe}, 5 \mathrm{n}\right), \mathrm{E}=6.5 \mathrm{MeV} /$ nucleon; measured $\mathrm{E} \gamma, \mathrm{I} \gamma(\theta, \mathrm{H}, \mathrm{t})$. ${ }^{149} \mathrm{Dy},{ }^{143} \mathrm{Nd}$ deduced high-spin isomers g-factors. Time-differential perturbed angular distribution method. REPT CNS-REP-64,P243,Watanabe

${ }^{149} \mathrm{Yb} \quad 2005 \mathrm{XU} 04$ RADIOACTIVITY ${ }^{81} \mathrm{Zr},{ }^{85} \mathrm{Mo},{ }^{89} \mathrm{Ru},{ }^{92} \mathrm{Rh},{ }^{93} \mathrm{Pd},{ }^{121} \mathrm{Ce},{ }^{125} \mathrm{Nd}$, ${ }^{128} \mathrm{Pm},{ }^{129} \mathrm{Sm},{ }^{135,137} \mathrm{Gd},{ }^{139} \mathrm{Dy},{ }^{142} \mathrm{Ho},{ }^{149} \mathrm{Yb}\left(\beta^{+} \mathrm{p}\right)$; measured $\beta$-delayed $\mathrm{E} \gamma, \mathrm{I} \gamma$, proton spectra, $\mathrm{p} \gamma$-coin, $\mathrm{T}_{1 / 2}$. Comparison with model predictions. JOUR PRVCA 71054318

2005XU04 NUCLEAR REACTIONS ${ }^{92} \mathrm{Mo},{ }^{106} \mathrm{Cd}\left({ }^{32} \mathrm{~S}, 3 \mathrm{n}\right), \mathrm{E}=151 \mathrm{MeV}$; ${ }^{92} \mathrm{Mo}\left({ }^{36} \mathrm{Ar}, 3 \mathrm{n}\right), \mathrm{E}=169 \mathrm{MeV} ;{ }^{96} \mathrm{Ru}\left({ }^{36} \mathrm{Ar}, 3 \mathrm{n}\right),\left({ }^{36} \mathrm{Ar}, 3 \mathrm{np}\right), \mathrm{E}=165,174$ $\mathrm{MeV} ;{ }^{106} \mathrm{Cd}\left({ }^{36} \mathrm{Ar}, 3 \mathrm{n}\right),\left({ }^{36} \mathrm{Ar}, \mathrm{n} \alpha\right), \mathrm{E}=176 \mathrm{MeV} ;{ }^{106} \mathrm{Cd}\left({ }^{40} \mathrm{Ca}, 4 \mathrm{n}\right)$, $\mathrm{E}=202 \mathrm{MeV} ;{ }^{112} \mathrm{Sn}\left({ }^{40} \mathrm{Ca}, 3 \mathrm{n}\right), \mathrm{E}=185 \mathrm{MeV}$; measured $\sigma$. JOUR PRVCA 71054318

\section{$\mathrm{A}=\mathbf{1 5 0}$}

${ }^{150} \mathrm{Nd} \quad 2005 \mathrm{BA33}$

2005MA10

2005R035

2005SA07

2005SI06

${ }^{150} \mathrm{Sm} \quad$ 2005BA33

2005SA07
RADIOACTIVITY ${ }^{82} \mathrm{Se},{ }^{100} \mathrm{Mo},{ }^{116} \mathrm{Cd},{ }^{150} \mathrm{Nd}\left(2 \beta^{-}\right)$; measured $2 \nu \beta \beta$-decay $\mathrm{T}_{1 / 2}, 0 \nu \beta \beta$-decay $\mathrm{T}_{1 / 2}$ lower limits. JOUR YAFIA 68443

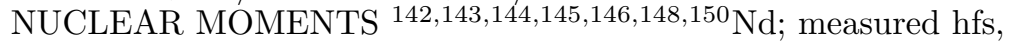
isotope shifts. JOUR CHPHD 14511

NUCLEAR MOMENTS ${ }^{142,143,144,145,146,148,150} \mathrm{Nd}$; measured hfs, isotope shifts. JOUR CJPHA 83841

RADIOACTIVITY ${ }^{82} \mathrm{Se},{ }^{96} \mathrm{Zr},{ }^{100} \mathrm{Mo},{ }^{116} \mathrm{Cd},{ }^{150} \mathrm{Nd}\left(2 \beta^{-}\right)$; measured $2 \nu \beta \beta$-decay $\mathrm{T}_{1 / 2} .{ }^{82} \mathrm{Se},{ }^{100} \mathrm{Mo}\left(2 \beta^{-}\right)$; measured $0 \nu \beta \beta$-decay $\mathrm{T}_{1 / 2}$ lower limits; deduced neutrino mass limits. JOUR NPBSE 143221

RADIOACTIVITY ${ }^{82} \mathrm{Se},{ }^{96} \mathrm{Zr},{ }^{100} \mathrm{Mo},{ }^{116} \mathrm{Cd},{ }^{150} \mathrm{Nd}\left(2 \beta^{-}\right)$; measured $2 \nu \beta \beta$-decay $\mathrm{T}_{1 / 2} .{ }^{82} \mathrm{Se},{ }^{100} \mathrm{Mo}\left(2 \beta^{-}\right)$; measured $0 \nu \beta \beta$-decay $\mathrm{T}_{1 / 2}$ lower limits; deduced neutrino mass limits. JOUR NPBSE 145272

RADIOACTIVITY ${ }^{82} \mathrm{Se},{ }^{100} \mathrm{Mo},{ }^{116} \mathrm{Cd},{ }^{150} \mathrm{Nd}\left(2 \beta^{-}\right)$; measured $2 \nu \beta \beta$-decay $\mathrm{T}_{1 / 2}, 0 \nu \beta \beta$-decay $\mathrm{T}_{1 / 2}$ lower limits. JOUR YAFIA 68443 RADIOACTIVITY ${ }^{82} \mathrm{Se},{ }^{96} \mathrm{Zr},{ }^{100} \mathrm{Mo},{ }^{116} \mathrm{Cd},{ }^{150} \mathrm{Nd}\left(2 \beta^{-}\right)$; measured $2 \nu \beta \beta$-decay $\mathrm{T}_{1 / 2} .{ }^{82} \mathrm{Se},{ }^{100} \mathrm{Mo}\left(2 \beta^{-}\right)$; measured $0 \nu \beta \beta$-decay $\mathrm{T}_{1 / 2}$ lower limits; deduced neutrino mass limits. JOUR NPBSE 143221 


\section{$\mathrm{A}=150$ (continued)}

2005SI06 RADIOACTIVITY ${ }^{82} \mathrm{Se},{ }^{96} \mathrm{Zr},{ }^{100} \mathrm{Mo},{ }^{116} \mathrm{Cd},{ }^{150} \mathrm{Nd}\left(2 \beta^{-}\right)$; measured $2 \nu \beta \beta$-decay $\mathrm{T}_{1 / 2} .{ }^{82} \mathrm{Se},{ }^{100} \mathrm{Mo}\left(2 \beta^{-}\right)$; measured $0 \nu \beta \beta$-decay $\mathrm{T}_{1 / 2}$ lower limits; deduced neutrino mass limits. JOUR NPBSE 145272

\section{$\mathrm{A}=151$}

${ }^{151} \mathrm{Sm} \quad$ 2005BU21

NUCLEAR REACTIONS ${ }^{149,151} \mathrm{Sm}(\mathrm{t}, \mathrm{p}), \mathrm{E}=15 \mathrm{MeV}$; measured proton spectra, $\sigma(\mathrm{E}, \theta)$; deduced $\mathrm{L}=0$ transition strengths. ${ }^{151,153} \mathrm{Sm}$ deduced levels, L, J, $\pi$, configurations. JOUR NUPAB 756308

\section{$\mathrm{A}=152$}

${ }^{152} \mathrm{Ce} \quad 2005 \mathrm{~F} 017 \quad$ RADIOACTIVITY ${ }^{252} \mathrm{Cf}(\mathrm{SF}) ;$ measured $\mathrm{E} \gamma, \mathrm{I} \gamma, \gamma \gamma$-coin. ${ }^{98} \mathrm{Sr}$, ${ }^{102,104} \mathrm{Zr},{ }^{137} \mathrm{Xe},{ }^{143} \mathrm{Ba},{ }^{152} \mathrm{Ce}$ levels deduced $\mathrm{T}_{1 / 2}$. Gammasphere array, time-gated triple-coincidence method. JOUR ZAANE 25 s01 465 ${ }^{152} \mathrm{Sm} \quad 2004 \mathrm{KU} 35$ RADIOACTIVITY ${ }^{238} \mathrm{Pu},{ }^{226} \mathrm{Ra}(\alpha) ;{ }^{152} \mathrm{Eu}(\mathrm{EC})$; measured low-energy electron spectra, angular distributions, (electron) $\alpha-$, (electron) $\gamma-$, (electron)(X-ray)-coin. JOUR BRSPE 681358

2005GA47 NUCLEAR REACTIONS ${ }^{150} \mathrm{Nd}(\alpha, 2 \mathrm{n}), \mathrm{E}=22.5 \mathrm{MeV} ;{ }^{152} \mathrm{Sm}(\mathrm{n}, \mathrm{n}), \mathrm{E}$ not given; measured $\mathrm{E} \gamma, \mathrm{I} \gamma, \gamma \gamma$-coin. ${ }^{152} \mathrm{Sm}$ deduced levels, $\mathrm{J}, \pi$, octupole and hexadecapole bands. JOUR JPGPE 31 S1855

2005KU17 RADIOACTIVITY ${ }^{152,152 m} \mathrm{Eu}(\mathrm{EC})$ [from $\left.{ }^{151} \mathrm{Eu}(\mathrm{n}, \gamma)\right]$; measured $\mathrm{E} \gamma$, $\mathrm{I} \gamma, \gamma \gamma$-coin. ${ }^{152} \mathrm{Sm}$ deduced levels, $\mathrm{J}, \pi$, rotational band, pairing isomer. JOUR PRVCA 71041303

2005KU17 NUCLEAR REACTIONS ${ }^{208} \mathrm{~Pb}\left({ }^{152} \mathrm{Sm},{ }^{152} \mathrm{Sm}\right.$ '), E=652 MeV; measured $\mathrm{E} \gamma, \mathrm{I} \gamma$, (particle) $\gamma-, \gamma \gamma$-coin following projectile Coulomb excitation. ${ }^{152} \mathrm{Sm}$ deduced levels, $\mathrm{J}, \pi, \mathrm{B}(\mathrm{E} 2)$, rotational band, pairing isomer. Gammasphere, Chico arrays, level systematics in neighboring nuclides discussed. JOUR PRVCA 71041303

2005MA73 NUCLEAR REACTIONS ${ }^{151} \mathrm{Sm}(\mathrm{n}, \gamma), \mathrm{E} \approx 0-1 \mathrm{MeV}$; measured capture $\sigma$. Astrophysical implication discussed. JOUR NUPAB 758 $533 \mathrm{c}$

2005WI20 RADIOACTIVITY ${ }^{26} \mathrm{Na}\left(\beta^{-}\right) ;{ }^{152} \mathrm{Eu}\left(\beta^{-}\right)$, (EC); measured $\mathrm{E} \gamma, \mathrm{I} \gamma, \gamma \gamma-$, $\beta \gamma$-coin. ${ }^{152} \mathrm{Sm}$ level deduced $\mathrm{T}_{1 / 2}$. JOUR JPGPE $31 \mathrm{~S} 1979$

${ }^{152} \mathrm{Eu}$ 2004GE20 RADIOACTIVITY ${ }^{155} \mathrm{Sm}\left(\beta^{-}\right)\left[\right.$from $\left.{ }^{154} \mathrm{Sm}(\mathrm{n}, \gamma)\right] ;{ }^{60} \mathrm{Co},{ }^{133} \mathrm{Ba},{ }^{152} \mathrm{Eu}$; measured $\gamma$-ray angular correlations. ${ }^{155} \mathrm{Eu},{ }^{60} \mathrm{Ni},{ }^{133} \mathrm{Cs},{ }^{152} \mathrm{Gd}$ transitions deduced $\delta$. Comparison with previous results. JOUR BJPHE 34722

2004 KU35 RADIOACTIVITY ${ }^{238} \mathrm{Pu},{ }^{226} \mathrm{Ra}(\alpha) ;{ }^{152} \mathrm{Eu}(\mathrm{EC})$; measured low-energy electron spectra, angular distributions, (electron) $\alpha-$, (electron) $\gamma-$, (electron)(X-ray)-coin. JOUR BRSPE 681358

2005KU17 RADIOACTIVITY ${ }^{152,152 m} \mathrm{Eu}(\mathrm{EC})$ [from ${ }^{151} \mathrm{Eu}(\mathrm{n}, \gamma)$ ]; measured $\mathrm{E} \gamma$, $\mathrm{I} \gamma, \gamma \gamma$-coin. ${ }^{152} \mathrm{Sm}$ deduced levels, J, $\pi$, rotational band, pairing isomer. JOUR PRVCA 71041303

2005WI20 RADIOACTIVITY ${ }^{26} \mathrm{Na}\left(\beta^{-}\right) ;{ }^{152} \mathrm{Eu}\left(\beta^{-}\right)$, (EC); measured $\mathrm{E} \gamma, \mathrm{I} \gamma, \gamma \gamma$, $\beta \gamma$-coin. ${ }^{152} \mathrm{Sm}$ level deduced $\mathrm{T}_{1 / 2}$. JOUR JPGPE $31 \mathrm{~S} 1979$ 


\section{$\mathrm{A}=152$ (continued)}

${ }^{152} \mathrm{Gd} \quad$ 2004GE20

${ }^{152}$ Dy $\quad$ 2004LAZW

2005LAZZ

${ }^{153} \mathrm{Eu}$

2004MB03

2005BU02

2005BU21

\section{$\mathrm{A}=154$}

${ }^{154} \mathrm{Sm} \quad$ 2005DEZV

${ }^{154} \mathrm{Eu} \quad$ 2005KUZX BJPHE 34722

ANL-04/22,P51,Lauritsen Limits),P34,Lauritsen

\section{$\mathrm{A}=153$} 747131 Petersburg,P53,Demidov
RADIOACTIVITY ${ }^{155} \mathrm{Sm}\left(\beta^{-}\right)$[from ${ }^{154} \mathrm{Sm}(\mathrm{n}, \gamma)$ ]; ${ }^{60} \mathrm{Co},{ }^{133} \mathrm{Ba},{ }^{152} \mathrm{Eu}$; measured $\gamma$-ray angular correlations. ${ }^{155} \mathrm{Eu},{ }^{60} \mathrm{Ni},{ }^{133} \mathrm{Cs},{ }^{152} \mathrm{Gd}$ transitions deduced $\delta$. Comparison with previous results. JOUR

NUCLEAR REACTIONS ${ }^{148} \mathrm{Nd}\left({ }^{9} \mathrm{Be}, 5 \mathrm{n}\right), \mathrm{E}=42,45,48,52,55 \mathrm{MeV}$; measured $\mathrm{E} \gamma, \mathrm{I} \gamma$; deduced excitation function. ${ }^{148} \mathrm{Nd}\left({ }^{9} \mathrm{Be}, 5 \mathrm{n}\right), \mathrm{E}=54$ $\mathrm{MeV}$; measured $\mathrm{E} \gamma, \mathrm{I} \gamma, \gamma \gamma$-coin. ${ }^{152} \mathrm{Gd}$ deduced high-spin levels, J, $\pi$, configuration, quadrupole deformation. Total Routhian surface calculations. JOUR PRVCA 72024317

RADIOACTIVITY ${ }^{26} \mathrm{Na}\left(\beta^{-}\right) ;{ }^{152} \mathrm{Eu}\left(\beta^{-}\right)$, (EC); measured $\mathrm{E} \gamma, \mathrm{I} \gamma, \gamma \gamma-$, $\beta \gamma$-coin. ${ }^{152} \mathrm{Sm}$ level deduced $\mathrm{T}_{1 / 2}$. JOUR JPGPE $31 \mathrm{~S} 1979$

NUCLEAR REACTIONS ${ }^{108} \mathrm{Pd}\left({ }^{48} \mathrm{Ca}, 4 \mathrm{n}\right), \mathrm{E}=194 \mathrm{MeV}$; measured $\mathrm{E} \gamma$, $\mathrm{I} \gamma, \gamma \gamma$-coin. ${ }^{152} \mathrm{Dy}$ deduced ridge widths, quadrupole moments, rotational damping features for deformed and superdeformed quasicontinuum spectra. Gammasphere array. REPT

NUCLEAR REACTIONS ${ }^{108} \mathrm{Pd}\left({ }^{48} \mathrm{Ca}, 4 \mathrm{n}\right), \mathrm{E}=194 \mathrm{MeV}$; measured $\mathrm{E} \gamma$, $\mathrm{I} \gamma, \gamma \gamma$-coin, quasi-continuum spectra. ${ }^{152}$ Dy deduced superdeformed band rotational damping width, decay-out features. Gammasphere array, Monte Carlo analysis. CONF Argonne(Nuclei at the

NUCLEAR REACTIONS ${ }^{149,151} \mathrm{Sm}(\mathrm{t}, \mathrm{p}), \mathrm{E}=15 \mathrm{MeV}$; measured proton spectra, $\sigma(\mathrm{E}, \theta)$; deduced $\mathrm{L}=0$ transition strengths. ${ }^{151,153} \mathrm{Sm}$ deduced levels, L, J, $\pi$, configurations. JOUR NUPAB 756308 NUCLEAR MOMENTS ${ }^{113,115} \mathrm{In},{ }^{153,155} \mathrm{Eu},{ }^{185,187} \mathrm{Re},{ }^{203,205} \mathrm{Tl}$, ${ }^{209,211} \mathrm{Fr}$; measured hfs; deduced hyperfine magnetic anomaly, relative radii. Laser resonance fluorescence. JOUR BRSPE 68157 NUCLEAR REACTIONS ${ }^{154} \mathrm{Gd}(\mathrm{t}, \alpha), \mathrm{E}=15 \mathrm{MeV} ;{ }^{152} \mathrm{Sm}\left({ }^{3} \mathrm{He}, \mathrm{d}\right)$, $\mathrm{E}=24 \mathrm{MeV} ;{ }^{152} \mathrm{Sm}(\alpha, \mathrm{t}), \mathrm{E}=25 \mathrm{MeV}$; measured particle spectra, $\sigma(\mathrm{E}$, $\theta) .{ }^{153} \mathrm{Eu}$ deduced levels, l-values, spectroscopic strengths, configurations. Nilsson model with Coriolis mixing. JOUR NUPAB

NUCLEAR REACTIONS ${ }^{154} \mathrm{Sm}(\mathrm{n}, \mathrm{n} \gamma) \mathrm{E}=$ reactor; measured $\mathrm{E} \gamma$, $\mathrm{I} \gamma(\theta) .{ }^{154} \mathrm{Sm}$ deduced levels, $\delta$, configurations. Reactor. CONF St

RADIOACTIVITY ${ }^{154} \mathrm{Eu}\left(\beta^{-}\right)$; measured $\mathrm{E} \gamma, \mathrm{I} \gamma, \gamma \gamma$-coin. ${ }^{154} \mathrm{Gd}$ deduced transition intensities. Application as relative efficiency calibration source discussed. CONF Santa Fe (Nucl Data for Sci and Technol) Proc,Vol1,P830 


\section{$\mathrm{A}=154$ (continued)}

${ }^{154} \mathrm{Gd}$ 2005BEZU NUCLEAR REACTIONS ${ }^{157} \mathrm{Gd}\left({ }^{3} \mathrm{He}, \alpha\right),\left({ }^{3} \mathrm{He}, 2 \mathrm{n} \alpha\right),\left({ }^{3} \mathrm{He}, \mathrm{n}^{3} \mathrm{He}\right)$, $\mathrm{E}=45 \mathrm{MeV}$; measured $\mathrm{E} \gamma, \mathrm{I} \gamma$, (particle) $\gamma$-coin. ${ }^{236,238} \mathrm{U}(\mathrm{d}, \mathrm{pF}),(\mathrm{d}$, d'F), $\mathrm{E}=24,32 \mathrm{MeV}$; measured $\mathrm{E} \gamma, \mathrm{I} \gamma$, (particle) $\gamma$-coin; deduced fission probability ratios. Surrogate reactions, Gammasphere and STARS arrays. CONF Santa Fe (Nucl Data for Sci and Technol) Proc,Vol1,P890

2005KUZX RADIOACTIVITY ${ }^{154} \mathrm{Eu}\left(\beta^{-}\right)$; measured $\mathrm{E} \gamma, \mathrm{I} \gamma, \gamma \gamma$-coin. ${ }^{154} \mathrm{Gd}$ deduced transition intensities. Application as relative efficiency calibration source discussed. CONF Santa Fe (Nucl Data for Sci and Technol) Proc,Vol1,P830

\section{$\mathrm{A}=155$}

${ }^{155} \mathrm{Sm} \quad$ 2004GE20

RADIOACTIVITY ${ }^{155} \mathrm{Sm}\left(\beta^{-}\right)$[from ${ }^{154} \mathrm{Sm}(\mathrm{n}, \gamma)$ ]; ${ }^{60} \mathrm{Co},{ }^{133} \mathrm{Ba},{ }^{152} \mathrm{Eu}$; measured $\gamma$-ray angular correlations. ${ }^{155} \mathrm{Eu},{ }^{60} \mathrm{Ni},{ }^{133} \mathrm{Cs},{ }^{152} \mathrm{Gd}$ transitions deduced $\delta$. Comparison with previous results. JOUR BJPHE 34722

2005RA33 RADIOACTIVITY ${ }^{155} \mathrm{Sm}\left(\beta^{-}\right)$[from $\left.{ }^{154} \mathrm{Sm}(\mathrm{n}, \gamma)\right]$; measured $\mathrm{E} \gamma$, I $\gamma$, $\gamma \gamma$-coin; deduced log ft. ${ }^{155} \mathrm{Eu}$ deduced levels, $\mathrm{J}, \pi, \beta$-feeding intensities. JOUR BJPHE 35839

${ }^{155} \mathrm{Eu} \quad 2004 \mathrm{GE} 20$ RADIOACTIVITY ${ }^{155} \mathrm{Sm}\left(\beta^{-}\right)$[from $\left.{ }^{154} \mathrm{Sm}(\mathrm{n}, \gamma)\right] ;{ }^{60} \mathrm{Co},{ }^{133} \mathrm{Ba},{ }^{152} \mathrm{Eu}$; measured $\gamma$-ray angular correlations. ${ }^{155} \mathrm{Eu},{ }^{60} \mathrm{Ni},{ }^{133} \mathrm{Cs},{ }^{152} \mathrm{Gd}$ transitions deduced $\delta$. Comparison with previous results. JOUR BJPHE 34722

2004 MB03 NUCLEAR MOMENTS ${ }^{113,115} \mathrm{In},{ }^{153,155} \mathrm{Eu},{ }^{185,187} \mathrm{Re},{ }^{203,205} \mathrm{Tl}$, ${ }^{209,211} \mathrm{Fr}$; measured hfs; deduced hyperfine magnetic anomaly, relative radii. Laser resonance fluorescence. JOUR BRSPE 68157

2005RA33 RADIOACTIVITY ${ }^{155} \mathrm{Sm}\left(\beta^{-}\right)$[from $\left.{ }^{154} \mathrm{Sm}(\mathrm{n}, \gamma)\right]$; measured $\mathrm{E} \gamma, \mathrm{I} \gamma$, $\gamma \gamma$-coin; deduced log ft. ${ }^{155} \mathrm{Eu}$ deduced levels, J, $\pi, \beta$-feeding intensities. JOUR BJPHE 35839

$$
\mathrm{A}=156
$$

${ }^{156} \mathrm{Gd}$ 2005BEZU NUCLEAR REACTIONS ${ }^{157} \mathrm{Gd}\left({ }^{3} \mathrm{He}, \alpha\right),\left({ }^{3} \mathrm{He}, 2 \mathrm{n} \alpha\right),\left({ }^{3} \mathrm{He}, \mathrm{n}^{3} \mathrm{He}\right)$, $\mathrm{E}=45 \mathrm{MeV}$; measured $\mathrm{E} \gamma, \mathrm{I} \gamma$, (particle) $\gamma$-coin. ${ }^{236,238} \mathrm{U}(\mathrm{d}, \mathrm{pF}),(\mathrm{d}$, d'F), $\mathrm{E}=24,32 \mathrm{MeV}$; measured $\mathrm{E} \gamma, \mathrm{I} \gamma$, (particle) $\gamma$-coin; deduced fission probability ratios. Surrogate reactions, Gammasphere and STARS arrays. CONF Santa Fe (Nucl Data for Sci and Technol) Proc,Vol1,P890

${ }^{156} \mathrm{Ho} \quad$ 2005KAZY RADIOACTIVITY ${ }^{156,158,160} \operatorname{Er}(\mathrm{EC})$; measured prompt and delayed $\mathrm{E} \gamma, \mathrm{I} \gamma, \gamma \gamma$-coin. ${ }^{156,158,160}$ Ho levels deduced $\mathrm{T}_{1 / 2}$. HPGe detectors, YaSNAPP-2 ISOL complex. CONF St Petersburg,P58,Kalinnikov ${ }^{156} \mathrm{Er} \quad$ 2005KAZY RADIOACTIVITY ${ }^{156,158,160} \operatorname{Er}(\mathrm{EC})$; measured prompt and delayed $\mathrm{E} \gamma, \mathrm{I} \gamma, \gamma \gamma$-coin. ${ }^{156,158,160}$ Ho levels deduced $\mathrm{T}_{1 / 2}$. HPGe detectors, YaSNAPP-2 ISOL complex. CONF St Petersburg,P58,Kalinnikov 


\section{$\mathrm{A}=156$ (continued)}

${ }^{156} \mathrm{Hf}$ 2005SE11 NUCLEAR REACTIONS ${ }^{102} \mathrm{Pd}\left({ }^{58} \mathrm{Ni}, 2 \mathrm{n}\right),\left({ }^{58} \mathrm{Ni}, 2 \mathrm{np}\right),\left({ }^{58} \mathrm{Ni}, 2 \mathrm{n} 2 \mathrm{p}\right)$ $\mathrm{E}=270 \mathrm{MeV}$; measured $\mathrm{E} \gamma, \mathrm{I} \gamma, \gamma \gamma-$, (recoil) $\gamma$-coin. ${ }^{156} \mathrm{Hf},{ }^{157} \mathrm{Ta},{ }^{158} \mathrm{~W}$ deduced levels, $\mathrm{J}, \pi$, isomeric states $\mathrm{T}_{1 / 2}$. Gammasphere array, recoil-decay tagging, shell model calculations. JOUR PRVCA 71 054319

\section{$\mathrm{A}=157$}

${ }^{157} \mathrm{Er}$

${ }^{157} \mathrm{Lu}$

2005SC22

${ }^{157} \mathrm{Ta}$

2005SE11

$\mathrm{A}=158$

${ }^{158} \mathrm{Gd} \quad$ 2005ME19

2005MI28

${ }^{158} \mathrm{Ho} \quad$ 2005KAZY

${ }^{158} \mathrm{Er} \quad$ 2005KAZY

${ }^{158} \mathrm{~W} \quad 2005 \mathrm{SE} 11$ 054319 JUPSA 743122
NUCLEAR REACTIONS ${ }^{114} \mathrm{Cd}\left({ }^{48} \mathrm{Ca}, 5 \mathrm{n}\right), \mathrm{E}=215 \mathrm{MeV}$; measured $\mathrm{E} \gamma$, $\mathrm{I} \gamma, \gamma \gamma$-coin. ${ }^{157} \mathrm{Er}$ deduced high-spin levels, $\mathrm{J}, \pi$, band termination features. Gammasphere array. JOUR JPGPE 31 S1735 RADIOACTIVITY ${ }^{167,167 m, 169,169 m} \operatorname{Ir},{ }^{165,165 m} \operatorname{Re},{ }^{161} \mathrm{Ta}(\alpha)$ [from ${ }^{92} \mathrm{Mo}\left({ }^{78} \mathrm{Kr}, 2 \mathrm{np}\right)$ and ${ }^{112} \mathrm{Sn}\left({ }^{58} \mathrm{Ni}, \mathrm{p}\right)$ and subsequent decay]; measured $\mathrm{E} \alpha, \mathrm{E} \gamma, \alpha \gamma$-coin, $\mathrm{T}_{1 / 2}$; deduced spectroscopic factors. ${ }^{167,167 m} \operatorname{Ir}(\mathrm{p})$ [from $\left.{ }^{112} \mathrm{Sn}\left({ }^{58} \mathrm{Ni}, 2 \mathrm{np}\right)\right]$; measured $\mathrm{Ep}, \mathrm{T}_{1 / 2}$; deduced spectroscopic factors. Jurogam array, mass separator. JOUR JPGPE 31 S1719 NUCLEAR REACTIONS ${ }^{102} \mathrm{Pd}\left({ }^{58} \mathrm{Ni}, 2 \mathrm{n}\right),\left({ }^{58} \mathrm{Ni}, 2 \mathrm{np}\right),\left({ }^{58} \mathrm{Ni}, 2 \mathrm{n} 2 \mathrm{p}\right)$, $\mathrm{E}=270 \mathrm{MeV}$; measured $\mathrm{E} \gamma, \mathrm{I} \gamma, \gamma \gamma-$, (recoil) $\gamma$-coin. ${ }^{156} \mathrm{Hf},{ }^{157} \mathrm{Ta},{ }^{158} \mathrm{~W}$ deduced levels, $\mathrm{J}, \pi$, isomeric states $\mathrm{T}_{1 / 2}$. Gammasphere array, recoil-decay tagging, shell model calculations. JOUR PRVCA 71

NUCLEAR REACTIONS ${ }^{160} \mathrm{Gd},{ }^{164} \mathrm{Dy},{ }^{170} \mathrm{Er},{ }^{178} \mathrm{Hf},{ }^{186} \mathrm{~W},{ }^{192} \mathrm{Os}(\mathrm{p}$, t), $\mathrm{E}=25 \mathrm{MeV}$; measured triton spectra, $\sigma(\theta) .{ }^{158} \mathrm{Gd},{ }^{162} \mathrm{Dy},{ }^{168} \mathrm{Er}$, ${ }^{176} \mathrm{Hf},{ }^{184} \mathrm{~W},{ }^{190} \mathrm{Os}$ deduced $0^{+}$level energies. JOUR JPGPE $31 \mathrm{~S} 1399$ NUCLEAR REACTIONS ${ }^{158} \mathrm{Gd}$ (X-ray, X-ray), $\mathrm{E} \approx 79.5 \mathrm{keV}$; measured delayed X-ray spectrum. ${ }^{158} \mathrm{Gd}$ deduced excited state energy, $\mathrm{T}_{1 / 2}$. Synchrotron radiation, comparison with previous results. JOUR

RADIOACTIVITY ${ }^{156,158,160} \operatorname{Er}(\mathrm{EC})$; measured prompt and delayed $\mathrm{E} \gamma, \mathrm{I} \gamma, \gamma \gamma$-coin. ${ }^{156,158,160}$ Ho levels deduced $\mathrm{T}_{1 / 2}$. HPGe detectors, YaSNAPP-2 ISOL complex. CONF St Petersburg,P58,Kalinnikov RADIOACTIVITY ${ }^{156,158,160} \operatorname{Er}(\mathrm{EC})$; measured prompt and delayed $\mathrm{E} \gamma, \mathrm{I} \gamma, \gamma \gamma$-coin. ${ }^{156,158,160}$ Ho levels deduced $\mathrm{T}_{1 / 2}$. HPGe detectors, YaSNAPP-2 ISOL complex. CONF St Petersburg,P58,Kalinnikov NUCLEAR REACTIONS ${ }^{102} \mathrm{Pd}\left({ }^{58} \mathrm{Ni}, 2 \mathrm{n}\right),\left({ }^{58} \mathrm{Ni}, 2 \mathrm{np}\right),\left({ }^{58} \mathrm{Ni}, 2 \mathrm{n} 2 \mathrm{p}\right)$, $\mathrm{E}=270 \mathrm{MeV}$; measured $\mathrm{E} \gamma, \mathrm{I} \gamma, \gamma \gamma-$, (recoil) $\gamma$-coin. ${ }^{156} \mathrm{Hf},{ }^{157} \mathrm{Ta},{ }^{158} \mathrm{~W}$ deduced levels, $\mathrm{J}, \pi$, isomeric states $\mathrm{T}_{1 / 2}$. Gammasphere array, recoil-decay tagging, shell model calculations. JOUR PRVCA 71 054319 


\begin{tabular}{|c|c|c|}
\hline \multicolumn{3}{|r|}{$A=159$} \\
\hline${ }^{159} \mathrm{Pm}$ & 2005 IC02 & $\begin{array}{l}\text { RADIOACTIVITY }{ }^{159} \mathrm{Pm},{ }^{162} \mathrm{Sm},{ }^{166} \mathrm{Gd},{ }^{166} \mathrm{~Tb}\left(\beta^{-}\right)\left[\text {from }{ }^{238} \mathrm{U}(\mathrm{p}, \mathrm{F})\right] \\
\text { measured } \mathrm{E} \gamma, \mathrm{I} \gamma, \beta \gamma-, \gamma \gamma \text {-coin, } \mathrm{T}_{1 / 2} \cdot{ }^{166} \mathrm{~Tb} \text { deduced levels, } \beta \text {-feeding } \\
\text { intensities. JOUR PRVCA } 71067302\end{array}$ \\
\hline${ }^{159} \mathrm{Sm}$ & 2005 IC02 & $\begin{array}{l}\text { RADIOACTIVITY }{ }^{159} \mathrm{Pm},{ }^{162} \mathrm{Sm},{ }^{166} \mathrm{Gd},{ }^{166} \mathrm{~Tb}\left(\beta^{-}\right)\left[\text {from }{ }^{238} \mathrm{U}(\mathrm{p}, \mathrm{F})\right] \\
\text { measured } \mathrm{E} \gamma, \mathrm{I} \gamma, \beta \gamma-, \gamma \gamma \text {-coin, } \mathrm{T}_{1 / 2} \cdot{ }^{166} \mathrm{~Tb} \text { deduced levels, } \beta \text {-feeding } \\
\text { intensities. JOUR PRVCA } 71067302\end{array}$ \\
\hline \multicolumn{3}{|r|}{$A=160$} \\
\hline \multirow[t]{2}{*}{${ }^{160} \mathrm{Ho}$} & 2005KAZX & $\begin{array}{l}\text { RADIOACTIVITY }{ }^{160 m} \mathrm{Ho}(\mathrm{IT}) \text {; measured } \gamma \gamma \text {-coin, } \mathrm{T}_{1 / 2} \text {. YASNAPP } \\
\text { setup. CONF St Petersburg,P72,Kalinnikov }\end{array}$ \\
\hline & 2005KAZY & $\begin{array}{l}\text { RADIOACTIVITY }{ }^{156,158,160} \mathrm{Er}(\mathrm{EC}) \text {; measured prompt and delayed } \\
\mathrm{E} \gamma, \mathrm{I} \gamma, \gamma \gamma \text {-coin. }{ }^{156,158,160} \mathrm{Ho} \text { levels deduced } \mathrm{T}_{1 / 2} . \text { HPGe detectors, } \\
\text { YaSNAPP-2 ISOL complex. CONF St Petersburg,P58,Kalinnikov }\end{array}$ \\
\hline \multirow[t]{2}{*}{${ }^{160} \mathrm{Er}$} & 2005KAZY & $\begin{array}{l}\text { RADIOACTIVITY }{ }^{156,158,160} \mathrm{Er}(\mathrm{EC}) \text {; measured prompt and delayed } \\
\mathrm{E} \gamma, \mathrm{I} \gamma, \gamma \gamma \text {-coin. }{ }^{156,158,160} \mathrm{Ho} \text { levels deduced } \mathrm{T}_{1 / 2} \text {. HPGe detectors, } \\
\text { YaSNAPP-2 ISOL complex. CONF St Petersburg,P } 58, \text { Kalinnikov }\end{array}$ \\
\hline & 2005W006 & $\begin{array}{l}\text { RADIOACTIVITY }{ }^{160} \mathrm{Yb},{ }^{160} \mathrm{Tm}(\mathrm{EC})\left[\text { from }{ }^{147} \mathrm{Sm}\left({ }^{18} \mathrm{O}, 5 \mathrm{n}\right) \text { and }\right. \\
\text { subsequent decay]; measured } \beta \text {-delayed } \mathrm{E} \gamma, \mathrm{I} \gamma(\theta, \mathrm{H}, \mathrm{t}), \gamma \gamma \text {-coin. }{ }^{160} \mathrm{Er} \\
\text { level deduced g factor. Perturbed angular correlation technique, } \\
\text { systematics in neighboring nuclides discussed. JOUR PRVCA } 72 \\
027301\end{array}$ \\
\hline \multirow[t]{2}{*}{${ }^{160} \mathrm{Tm}$} & 2005LA32 & $\begin{array}{l}\text { NUCLEAR REACTIONS }{ }^{130} \mathrm{Te}\left({ }^{35} \mathrm{Cl}, 5 \mathrm{n}\right), \mathrm{E}=170 \mathrm{MeV} \text {; measured } \mathrm{E} \gamma \text {, } \\
\text { I } \gamma, \gamma \gamma \text {-coin. }{ }^{160} \mathrm{Tm} \text { deduced high-spin levels, } \mathrm{J}, \pi \text {, configurations. } \\
\text { Euroball array. JOUR PRVCA } 72057303\end{array}$ \\
\hline & 2005W006 & $\begin{array}{l}\text { RADIOACTIVITY }{ }^{160} \mathrm{Yb},{ }^{160} \mathrm{Tm}(\mathrm{EC})\left[\operatorname{from}{ }^{147} \mathrm{Sm}\left({ }^{18} \mathrm{O}, 5 \mathrm{n}\right) \text { and }\right. \\
\text { subsequent decay]; measured } \beta \text {-delayed } \mathrm{E} \gamma, \mathrm{I} \gamma(\theta, \mathrm{H}, \mathrm{t}), \gamma \gamma \text {-coin. }{ }^{160} \mathrm{Er} \\
\text { level deduced g factor. Perturbed angular correlation technique, } \\
\text { systematics in neighboring nuclides discussed. JOUR PRVCA } 72 \\
027301\end{array}$ \\
\hline \multirow[t]{2}{*}{${ }^{160} \mathrm{Yb}$} & 2005BA88 & $\begin{array}{l}\text { NUCLEAR REACTIONS }{ }^{208} \mathrm{~Pb}(\mathrm{p}, \gamma), \mathrm{E}=11.9 \mathrm{MeV} ; \text { measured } \mathrm{E} \gamma, \mathrm{I} \gamma \\
{ }^{147} \mathrm{Sm}\left({ }^{16} \mathrm{O}, 3 \mathrm{n}\right), \mathrm{E}=73 \mathrm{MeV} \text {; measured } \mathrm{E} \gamma, \mathrm{I} \gamma, \gamma \gamma \text {-coin. }{ }^{160} \mathrm{Yb} \text { deduced } \\
\text { high-spin levels, J, } \pi \text {. Afrodite array. JOUR JPGPE } 31 \mathrm{~S} 1747\end{array}$ \\
\hline & 2005W006 & $\begin{array}{l}\text { RADIOACTIVITY }{ }^{160} \mathrm{Yb},{ }^{160} \mathrm{Tm}(\mathrm{EC})\left[\text { from }{ }^{147} \mathrm{Sm}\left({ }^{18} \mathrm{O}, 5 \mathrm{n}\right) \text { and }\right. \\
\text { subsequent decay]; measured } \beta \text {-delayed } \mathrm{E} \gamma, \mathrm{I} \gamma(\theta, \mathrm{H}, \mathrm{t}), \gamma \gamma \text {-coin. }{ }^{160} \mathrm{Er} \\
\text { level deduced g factor. Perturbed angular correlation technique, } \\
\text { systematics in neighboring nuclides discussed. JOUR PRVCA } 72 \\
027301\end{array}$ \\
\hline
\end{tabular}




\section{$\mathrm{A}=161$}

\begin{tabular}{|c|c|c|}
\hline${ }^{161} \mathrm{Yb}$ & 2005DU23 & $\begin{array}{l}\left.\text { NUCLEAR REACTIONS Ge( }{ }^{18} \mathrm{O}, \mathrm{X}\right)^{83 m} \mathrm{Sr} /{ }^{83} \mathrm{Y} /{ }^{84 m} \mathrm{Y} /{ }^{88 m} \mathrm{Y} / \\
{ }^{85} \mathrm{Zr} /{ }^{87} \mathrm{Zr}, \mathrm{E}=82.8 \mathrm{GeV} ;{ }^{84} \mathrm{Se}\left({ }^{18} \mathrm{O}, \mathrm{X}\right){ }^{86 m} \mathrm{Y} /{ }^{85} \mathrm{Zr} /{ }^{87} \mathrm{Nb} /{ }^{87 m} \mathrm{Nb} / \\
{ }^{88} \mathrm{Nb} /{ }^{88} \mathrm{Mo}, \mathrm{E}=82.7 \mathrm{MeV} ;{ }^{124} \mathrm{Sn}\left({ }^{50} \mathrm{Ti}, \mathrm{X}\right){ }^{168 m} \mathrm{Lu} /{ }^{167} \mathrm{Hf} /{ }^{168} \mathrm{Hf}, \\
\mathrm{E}=223.7 \mathrm{MeV} ;{ }^{116} \mathrm{Sn}\left({ }^{50} \mathrm{Ti}, \mathrm{X}\right)^{162} \mathrm{Tm} /{ }^{161} \mathrm{Yb} /{ }^{162} \mathrm{Yb} /{ }^{163} \mathrm{Yb} /{ }^{162} \mathrm{Lu} / \\
{ }^{162} \mathrm{Hf}, \mathrm{E}=224.4 \mathrm{MeV} \text {; measured delayed } \mathrm{E} \gamma, \mathrm{I} \gamma \text { following residual } \\
\text { nucleus decay. Physical preseparation technique. JOUR NIMAE } 551 \\
528\end{array}$ \\
\hline${ }^{161} \mathrm{Lu}$ & 2005BR14 & $\begin{array}{l}\text { NUCLEAR REACTIONS }{ }^{139} \mathrm{La}\left({ }^{28} \mathrm{Si}, 6 \mathrm{n}\right), \mathrm{E}=175 \mathrm{MeV} \text {; measured E } \gamma \text {, } \\
\mathrm{I} \gamma, \gamma \gamma \text {-coin. }{ }^{161} \mathrm{Lu} \text { deduced high-spin levels, } \mathrm{J}, \pi \text {, configurations, } \\
\text { triaxial superdeformed bands, possible wobbling excitation. Euroball } \\
\text { array, total Routhian surface calculation, level systematics in } \\
\text { neighboring isotopes discussed. JOUR ZAANE } 24167\end{array}$ \\
\hline${ }^{161} \mathrm{Ta}$ & $2005 \mathrm{SC} 22$ & $\begin{array}{l}\text { RADIOACTIVITY }{ }^{167,167 m, 169,169 m} \mathrm{Ir},{ }^{165,165 m} \mathrm{Re},{ }^{161} \mathrm{Ta}(\alpha) \text { [from } \\
{ }^{92} \mathrm{Mo}\left({ }^{78} \mathrm{Kr}, 2 \mathrm{np}\right) \text { and }{ }^{112} \mathrm{Sn}\left({ }^{58} \mathrm{Ni}, \mathrm{p}\right) \text { and subsequent decay]; measured } \\
\mathrm{E} \alpha, \mathrm{E} \gamma, \alpha \gamma \text {-coin, } \mathrm{T}_{1 / 2} ; \text { deduced spectroscopic factors. }{ }^{167,167 m} \mathrm{Ir}(\mathrm{p}) \\
{\left[\text { from }{ }^{112} \mathrm{Sn}\left({ }^{58} \mathrm{Ni}, 2 \mathrm{np}\right)\right] \text {; measured } \mathrm{Ep}, \mathrm{T}_{1 / 2} \text {; deduced spectroscopic }} \\
\text { factors. Jurogam array, mass separator. JOUR JPGPE } 31 \mathrm{~S} 1719\end{array}$ \\
\hline
\end{tabular}

\section{$\mathrm{A}=162$}

\begin{tabular}{|c|c|c|}
\hline${ }^{162} \mathrm{Sm}$ & 2005IC02 & $\begin{array}{l}\text { RADIOACTIVITY }{ }^{159} \mathrm{Pm},{ }^{162} \mathrm{Sm},{ }^{166} \mathrm{Gd},{ }^{166} \mathrm{~Tb}\left(\beta^{-}\right)\left[\text {from }{ }^{238} \mathrm{U}(\mathrm{p}, \mathrm{F})\right] \\
\text { measured E } \gamma, \mathrm{I} \gamma, \beta \gamma-, \gamma \gamma \text {-coin, } \mathrm{T}_{1 / 2} .{ }^{166} \mathrm{~Tb} \text { deduced levels, } \beta \text {-feeding } \\
\text { intensities. JOUR PRVCA } 71067302\end{array}$ \\
\hline${ }^{162} \mathrm{Eu}$ & 2005IC02 & $\begin{array}{l}\text { RADIOACTIVITY }{ }^{159} \mathrm{Pm},{ }^{162} \mathrm{Sm},{ }^{166} \mathrm{Gd},{ }^{166} \mathrm{~Tb}\left(\beta^{-}\right)\left[\text {from }{ }^{238} \mathrm{U}(\mathrm{p}, \mathrm{F})\right] \\
\text { measured E } \gamma, \mathrm{I} \gamma, \beta \gamma-, \gamma \gamma \text {-coin, } \mathrm{T}_{1 / 2} .{ }^{166} \mathrm{~Tb} \text { deduced levels, } \beta \text {-feeding } \\
\text { intensities. JOUR PRVCA } 71067302\end{array}$ \\
\hline${ }^{162} \mathrm{Gd}$ & 2005J024 & $\begin{array}{l}\text { RADIOACTIVITY }{ }^{252} \mathrm{Cf}(\mathrm{SF}) \text {; measured } \mathrm{E} \gamma, \mathrm{I} \gamma, \gamma \gamma \text {-coin. }{ }^{162,164} \mathrm{Gd} \\
\text { deduced levels, J, } \pi \text {. Gammasphere array, level systematics in } \\
\text { neighboring nuclides discussed. JOUR ZAANE } 25 \text { s01 } 467\end{array}$ \\
\hline \multirow[t]{2}{*}{${ }^{162} \mathrm{Dy}$} & 2004 KI23 & $\begin{array}{l}\text { NUCLEAR REACTIONS }{ }^{161,162,163,164} \mathrm{Dy}(\mathrm{n}, \gamma), \mathrm{E}=550 \mathrm{keV} \text {; measured } \\
\mathrm{E} \gamma, \mathrm{I} \gamma \text {, capture } \sigma . \text { JOUR KPSJA } 451474\end{array}$ \\
\hline & 2005ME19 & $\begin{array}{l}\text { NUCLEAR REACTIONS }{ }^{160} \mathrm{Gd},{ }^{164} \mathrm{Dy},{ }^{170} \mathrm{Er},{ }^{178} \mathrm{Hf},{ }^{186} \mathrm{~W},{ }^{192} \mathrm{Os}(\mathrm{p}, \\
\mathrm{t}), \mathrm{E}=25 \mathrm{MeV} \text {; measured triton spectra, } \sigma(\theta) .{ }^{158} \mathrm{Gd},{ }^{162} \mathrm{Dy},{ }^{168} \mathrm{Er}, \\
{ }^{176} \mathrm{Hf},{ }^{184} \mathrm{~W},{ }^{190} \text { Os deduced } 0^{+} \text {level energies. JOUR JPGPE } 31 \mathrm{~S} 1399\end{array}$ \\
\hline${ }^{162} \mathrm{Tm}$ & 2005DU23 & $\begin{array}{l}\text { NUCLEAR REACTIONS Ge }\left({ }^{18} \mathrm{O}, \mathrm{X}\right){ }^{83 m} \mathrm{Sr} /{ }^{83} \mathrm{Y} /{ }^{84 m} \mathrm{Y} /{ }^{88 m} \mathrm{Y} / \\
{ }^{85} \mathrm{Zr} /{ }^{87} \mathrm{Zr}, \mathrm{E}=82.8 \mathrm{GeV} ;{ }^{84} \mathrm{Se}\left({ }^{18} \mathrm{O}, \mathrm{X}\right){ }^{86 m} \mathrm{Y} /{ }^{85} \mathrm{Zr} /{ }^{87} \mathrm{Nb} /{ }^{87 m} \mathrm{Nb} / \\
{ }^{88} \mathrm{Nb} /{ }^{88} \mathrm{Mo}, \mathrm{E}=82.7 \mathrm{MeV} ;{ }^{124} \mathrm{Sn}\left({ }^{50} \mathrm{Ti}, \mathrm{X}\right){ }^{168 m} \mathrm{Lu} /{ }^{167} \mathrm{Hf} /{ }^{168} \mathrm{Hf}, \\
\mathrm{E}=223.7 \mathrm{MeV} ;{ }^{116} \mathrm{Sn}\left({ }^{50} \mathrm{Ti}, \mathrm{X}\right){ }^{162} \mathrm{Tm} /{ }^{161} \mathrm{Yb} /{ }^{162} \mathrm{Yb} /{ }^{163} \mathrm{Yb} /{ }^{162} \mathrm{Lu} / \\
{ }^{162} \mathrm{Hf}, \mathrm{E}=224.4 \mathrm{MeV} \text {; measured delayed } \mathrm{E} \gamma, \mathrm{I} \gamma \text { following residual } \\
\text { nucleus decay. Physical preseparation technique. JOUR NIMAE } 551 \\
528\end{array}$ \\
\hline${ }^{162} \mathrm{Yb}$ & 2005DU23 & $\begin{array}{l}\text { NUCLEAR REACTIONS Ge }\left({ }^{18} \mathrm{O}, \mathrm{X}\right){ }^{83}{ }^{m} \mathrm{Sr} /{ }^{83} \mathrm{Y} /{ }^{84 m} \mathrm{Y} /{ }^{88 m} \mathrm{Y} / \\
{ }^{85} \mathrm{Zr} /{ }^{87} \mathrm{Zr}, \mathrm{E}=82.8 \mathrm{GeV} ;{ }^{84} \mathrm{Se}\left({ }^{18} \mathrm{O}, \mathrm{X}\right){ }^{86 m} \mathrm{Y} /{ }^{85} \mathrm{Zr} /{ }^{87} \mathrm{Nb} /{ }^{87 m} \mathrm{Nb} / \\
{ }^{88} \mathrm{Nb} /{ }^{88} \mathrm{Mo}, \mathrm{E}=82.7 \mathrm{MeV} ;{ }^{124} \mathrm{Sn}\left({ }^{50} \mathrm{Ti}, \mathrm{X}\right){ }^{168 m} \mathrm{Lu} /{ }^{167} \mathrm{Hf} /{ }^{168} \mathrm{Hf}, \\
\mathrm{E}=223.7 \mathrm{MeV} ;{ }^{116} \mathrm{Sn}\left({ }^{50} \mathrm{Ti}, \mathrm{X}\right){ }^{162} \mathrm{Tm} /{ }^{161} \mathrm{Yb} /{ }^{162} \mathrm{Yb} /{ }^{163} \mathrm{Yb} /{ }^{162} \mathrm{Lu} / \\
{ }^{162} \mathrm{Hf}, \mathrm{E}=224.4 \mathrm{MeV} \text {; measured delayed } \mathrm{E} \gamma, \mathrm{I} \gamma \text { following residual } \\
\text { nucleus decay. Physical preseparation technique. JOUR NIMAE } 551 \\
528\end{array}$ \\
\hline
\end{tabular}




\section{$\mathrm{A}=162$ (continued)}

\begin{tabular}{|c|c|c|}
\hline${ }^{162} \mathrm{Lu}$ & 2005DU23 & $\begin{array}{l}\left.\text { NUCLEAR REACTIONS Ge( }{ }^{18} \mathrm{O}, \mathrm{X}\right){ }^{83 m} \mathrm{Sr} /{ }^{83} \mathrm{Y} /{ }^{84 m} \mathrm{Y} /{ }^{88 m} \mathrm{Y} / \\
\\
{ }^{85} \mathrm{Zr} /{ }^{87} \mathrm{Zr}, \mathrm{E}=82.8 \mathrm{GeV} ;{ }^{84} \mathrm{Se}\left({ }^{18} \mathrm{O}, \mathrm{X}\right){ }^{86 m} \mathrm{Y} /{ }^{85} \mathrm{Zr} /{ }^{87} \mathrm{Nb} /{ }^{87 m} \mathrm{Nb} / \\
{ }^{88} \mathrm{Nb} /{ }^{88} \mathrm{Mo}, \mathrm{E}=82.7 \mathrm{MeV} ;{ }^{124} \mathrm{Sn}\left({ }^{50} \mathrm{Ti}, \mathrm{X}\right)^{168 m} \mathrm{Lu} /{ }^{167} \mathrm{Hf} /{ }^{168} \mathrm{Hf} \\
\mathrm{E}=223.7 \mathrm{MeV} ;{ }^{116} \mathrm{Sn}\left({ }^{50} \mathrm{Ti}, \mathrm{X}\right){ }^{162} \mathrm{Tm} /{ }^{161} \mathrm{Yb} /{ }^{162} \mathrm{Yb} /{ }^{163} \mathrm{Yb} /{ }^{162} \mathrm{Lu} / \\
{ }^{162} \mathrm{Hf}, \mathrm{E}=224.4 \mathrm{MeV} \text {; measured delayed } \mathrm{E} \gamma, \mathrm{I} \gamma \text { following residual } \\
\text { nucleus decay. Physical preseparation technique. JOUR NIMAE } 551 \\
528\end{array}$ \\
\hline${ }^{162} \mathrm{Hf}$ & 2005DU23 & $\begin{array}{l}\text { NUCLEAR REACTIONS Ge }\left({ }^{18} \mathrm{O}, \mathrm{X}\right){ }^{83 m} \mathrm{Sr} /{ }^{83} \mathrm{Y} /{ }^{84 m} \mathrm{Y} /{ }^{88 m} \mathrm{Y} / \\
{ }^{85} \mathrm{Zr} /{ }^{87} \mathrm{Zr}, \mathrm{E}=82.8 \mathrm{GeV} ;{ }^{84} \mathrm{Se}\left({ }^{18} \mathrm{O}, \mathrm{X}\right){ }^{86 m} \mathrm{Y} /{ }^{85} \mathrm{Zr} /{ }^{87} \mathrm{Nb} /{ }^{87 m} \mathrm{Nb} / \\
{ }^{88} \mathrm{Nb} /{ }^{88} \mathrm{Mo}, \mathrm{E}=82.7 \mathrm{MeV} ;{ }^{124} \mathrm{Sn}\left({ }^{50} \mathrm{Ti}, \mathrm{X}\right){ }^{168 m} \mathrm{Lu} /{ }^{167} \mathrm{Hf} /{ }^{168} \mathrm{Hf} \\
\mathrm{E}=223.7 \mathrm{MeV} ;{ }^{116} \mathrm{Sn}\left({ }^{50} \mathrm{Ti}, \mathrm{X}\right){ }^{162} \mathrm{Tm} /{ }^{161} \mathrm{Yb} /{ }^{162} \mathrm{Yb} /{ }^{163} \mathrm{Yb} /{ }^{162} \mathrm{Lu} / \\
{ }^{162} \mathrm{Hf}, \mathrm{E}=224.4 \mathrm{MeV} \text {; measured delayed } \mathrm{E} \gamma, \mathrm{I} \gamma \text { following residual } \\
\text { nucleus decay. Physical preseparation technique. JOUR NIMAE } 551 \\
528\end{array}$ \\
\hline
\end{tabular}

\section{$\mathrm{A}=\mathbf{1 6 3}$}

${ }^{163}$ Dy $\quad 2004$ KI23

2005RI17

${ }^{163} \mathrm{Er}$

2005BE34

2005BR10

2005LE21

2005LE35

2005LEZZ
NUCLEAR REACTIONS ${ }^{161,162,163,164} \operatorname{Dy}(\mathrm{n}, \gamma), \mathrm{E}=550 \mathrm{keV}$; measured $\mathrm{E} \gamma, \mathrm{I} \gamma$, capture $\sigma$. JOUR KPSJA 451474

NUCLEAR REACTIONS ${ }^{92} \mathrm{Mo}\left({ }^{54} \mathrm{Fe}\right.$, xnypz $\left.\alpha\right), \mathrm{E}=245 \mathrm{MeV}$; measured prompt and delayed $\mathrm{E} \gamma, \mathrm{I} \gamma, \gamma \gamma$-, (recoil) $\gamma$-coin. ${ }^{142} \mathrm{~Tb},{ }^{163} \mathrm{Dy}$ deduced transitions. ${ }^{143}$ Dy deduced isomeric state $\mathrm{T}_{1 / 2}$. Jurogam array. JOUR JPGPE 31 S1949

NUCLEAR REACTIONS ${ }^{150} \mathrm{Nd}\left({ }^{18} \mathrm{O}, 5 \mathrm{n}\right), \mathrm{E}=87,93 \mathrm{MeV}$; measured $\mathrm{E} \gamma, \mathrm{I} \gamma, \gamma \gamma$-coin. ${ }^{163} \mathrm{Er}$ deduced K-mixing features vs temperature in quasi-continuum spectra. Euroball array, fluctuation analysis, band-mixing calculations. JOUR PYLBB 615160 NUCLEAR REACTIONS ${ }^{150} \mathrm{Nd}\left({ }^{18} \mathrm{O}, 5 \mathrm{n}\right), \mathrm{E}=87,93 \mathrm{MeV}$; measured $\mathrm{E} \gamma, \mathrm{I} \gamma, \gamma \gamma$-coin. ${ }^{163} \mathrm{Er}$ deduced K-mixing features vs temperature in quasi-continuum spectra. Euroball array. JOUR NUPAB $752227 \mathrm{c}$ NUCLEAR REACTIONS ${ }^{150} \mathrm{Nd}\left({ }^{18} \mathrm{O}, 5 \mathrm{n}\right), \mathrm{E}=87,93 \mathrm{MeV}$; measured $\mathrm{E} \gamma, \mathrm{I} \gamma, \gamma \gamma$-coin. ${ }^{163} \mathrm{Er}$ deduced compound and rotational damping widths, dependence on K-quantum number, order-to-chaos transition. Euroball array. JOUR APOBB 361121

NUCLEAR REACTIONS ${ }^{150} \mathrm{Nd}\left({ }^{18} \mathrm{O}, 5 \mathrm{n}\right), \mathrm{E}=87,93 \mathrm{MeV}$; measured $\mathrm{E} \gamma, \mathrm{I} \gamma, \gamma \gamma$-coin. ${ }^{163} \mathrm{Er}$ deduced quasi-continuum high-spin spectra, rotational bands excitation energy, compound and rotational damping widths vs K-quantum number, order-to-chaos transition features. Euroball array, comparison with model predictions. JOUR PRVCA 72 034307 NUCLEAR REACTIONS ${ }^{150} \mathrm{Nd}\left({ }^{18} \mathrm{O}, 5 \mathrm{n}\right), \mathrm{E}=87,93 \mathrm{MeV}$; measured $\mathrm{E} \gamma, \mathrm{I} \gamma, \gamma \gamma$-coin. ${ }^{163} \mathrm{Er}$ deduced compound and rotational damping widths, dependence on K-quantum number. Euroball array. CONF Argonne(Nuclei at the Limits),P309,Leoni 


\section{$\mathrm{A}=163$ (continued)}

\begin{tabular}{|c|c|c|}
\hline${ }^{163} \mathrm{Yb}$ & 2005DU23 & $\begin{array}{l}\text { NUCLEAR REACTIONS Ge }\left({ }^{18} \mathrm{O}, \mathrm{X}\right){ }^{83 m} \mathrm{Sr} /{ }^{83} \mathrm{Y} /{ }^{84 m} \mathrm{Y} /{ }^{88 m} \mathrm{Y} / \\
{ }^{85} \mathrm{Zr} /{ }^{87} \mathrm{Zr}, \mathrm{E}=82.8 \mathrm{GeV} ;{ }^{84} \mathrm{Se}\left({ }^{18} \mathrm{O}, \mathrm{X}\right){ }^{86 m} \mathrm{Y} /{ }^{85} \mathrm{Zr} /{ }^{87} \mathrm{Nb} /{ }^{87 m} \mathrm{Nb} / \\
{ }^{88} \mathrm{Nb} /{ }^{88} \mathrm{Mo}, \mathrm{E}=82.7 \mathrm{MeV} ;{ }^{124} \mathrm{Sn}\left({ }^{50} \mathrm{Ti}, \mathrm{X}\right){ }^{168 m} \mathrm{Lu} /{ }^{167} \mathrm{Hf} /{ }^{168} \mathrm{Hf}, \\
\mathrm{E}=223.7 \mathrm{MeV} ;{ }^{116} \mathrm{Sn}\left({ }^{50} \mathrm{Ti}, \mathrm{X}\right){ }^{162} \mathrm{Tm} /{ }^{161} \mathrm{Yb} /{ }^{162} \mathrm{Yb} /{ }^{163} \mathrm{Yb} /{ }^{162} \mathrm{Lu} / \\
{ }^{162} \mathrm{Hf}, \mathrm{E}=224.4 \mathrm{MeV} \text {; measured delayed } \mathrm{E} \gamma, \mathrm{I} \gamma \text { following residual } \\
\text { nucleus decay. Physical preseparation technique. JOUR NIMAE } 551 \\
528\end{array}$ \\
\hline${ }^{163} \mathrm{Lu}$ & $2005 \mathrm{GOZZ}$ & $\begin{array}{l}\text { NUCLEAR REACTIONS }{ }^{123} \mathrm{Sb}\left({ }^{44} \mathrm{Ca}, 4 \mathrm{n}\right), \mathrm{E}=190 \mathrm{MeV} \text {; measured } \mathrm{E} \gamma \text {, } \\
\mathrm{I} \gamma, \gamma \gamma \text {-coin, DSA. }{ }^{163} \mathrm{Lu} \text { deduced triaxial superdeformed bands } \\
\text { transitions } \mathrm{T}_{1 / 2}, \mathrm{~B}(\mathrm{E} 2), \mathrm{B}(\mathrm{M} 1) \text {, quadrupole moments. Gammasphere } \\
\text { array. Comparison with model predictions. CONF Argonne(Nuclei at } \\
\text { the Limits),P9, Gorgen }\end{array}$ \\
\hline${ }^{163} \mathrm{Re}$ & $2005 \mathrm{SC} 22$ & $\begin{array}{l}\text { RADIOACTIVITY }{ }^{167,167 m, 169,169 m} \mathrm{Ir},{ }^{165,165 m} \mathrm{Re},{ }^{161} \mathrm{Ta}(\alpha)[\text { from } \\
{ }^{92} \mathrm{Mo}\left({ }^{78} \mathrm{Kr}, 2 \mathrm{np}\right) \text { and }{ }^{112} \mathrm{Sn}\left({ }^{58} \mathrm{Ni}, \mathrm{p}\right) \text { and subsequent decay]; measured } \\
\mathrm{E} \alpha, \mathrm{E} \gamma, \alpha \gamma-\text { coin, } \mathrm{T}_{1 / 2} ; \text { deduced spectroscopic factors. }{ }^{167,167 m} \mathrm{Ir}(\mathrm{p}) \\
{\left[\text { from }{ }^{112} \mathrm{Sn}\left({ }^{58} \mathrm{Ni}, 2 \mathrm{np}\right)\right] ; \text { measured Ep, } \mathrm{T}_{1 / 2} \text {; deduced spectroscopic }} \\
\text { factors. Jurogam array, mass separator. JOUR JPGPE } 31 \mathrm{~S} 1719\end{array}$ \\
\hline
\end{tabular}

\section{$\mathrm{A}=164$}

${ }^{164} \mathrm{Gd} \quad$ 2005J024 RADIOACTIVITY ${ }^{252} \mathrm{Cf}(\mathrm{SF}) ;$ measured $\mathrm{E} \gamma, \mathrm{I} \gamma, \gamma \gamma$-coin. ${ }^{162,164} \mathrm{Gd}$ deduced levels, J, $\pi$. Gammasphere array, level systematics in neighboring nuclides discussed. JOUR ZAANE 25 s01 467
${ }^{164}$ Dy 2004KI23 NUCLEAR REACTIONS ${ }^{161,162,163,164} \mathrm{Dy}(\mathrm{n}, \gamma), \mathrm{E}=550 \mathrm{keV}$; measured $\mathrm{E} \gamma, \mathrm{I} \gamma$, capture $\sigma$. JOUR KPSJA 451474

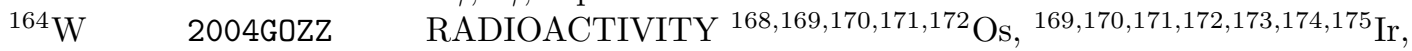
${ }_{170,171,172,173,174,175,176,177} \mathrm{Pt},{ }^{173,174,175,176,177} \mathrm{Au}$, ${ }_{174,175,176,177,178} \mathrm{Hg}(\alpha)$ [from ${ }^{92,94} \mathrm{Mo}\left({ }^{84} \mathrm{Sr}\right.$, xnyp) and subsequent decay]; measured $\mathrm{E} \alpha, \mathrm{E} \gamma, \alpha-\gamma$-coin, $\mathrm{T}_{1 / 2} .{ }^{165} \mathrm{Ta},{ }^{165,167} \mathrm{~W}$, ${ }^{165,166,167,168,171} \mathrm{Re},{ }^{169,170,171} \mathrm{Os},{ }^{169,170,171,172,175} \mathrm{Ir},{ }^{173,175} \mathrm{Pt},{ }^{174,176} \mathrm{Au}$ deduced levels, J, $\pi$. THESIS J Goon,University of Tennessee

\section{$\mathrm{A}=\mathbf{1 6 5}$}

${ }^{165}$ Dy 2004KI23 NUCLEAR REACTIONS $161,162,163,164 \mathrm{Dy}(\mathrm{n}, \gamma), \mathrm{E}=550 \mathrm{keV}$; measured $\mathrm{E} \gamma, \mathrm{I} \gamma$, capture $\sigma$. JOUR KPSJA 451474

2005BU07 NUCLEAR REACTIONS ${ }^{163} \mathrm{Dy},{ }^{177} \mathrm{Hf}(\mathrm{t}, \mathrm{p}), \mathrm{E}=17 \mathrm{MeV}$; measured $\sigma(\mathrm{Ep}, \theta) .{ }^{165} \mathrm{Dy},{ }^{179} \mathrm{Hf}$ deduced levels, L-values, $\mathrm{L}=0$ strengths. Enriched targets, magnetic spectrograph. Systematic trends in neighboring nuclides discussed. JOUR NUPAB 750185

2005KA33 NUCLEAR REACTIONS ${ }^{164} \operatorname{Dy}(\mathrm{n}, \gamma), \mathrm{E}=$ thermal; measured capture $\sigma$, resonance integral. Activation method. JOUR NIMAE 550626

${ }^{165} \mathrm{Er}$ 2004BE58 NUCLEAR REACTIONS ${ }^{165} \mathrm{Ho}(\mathrm{p}, \mathrm{n}), \mathrm{E} \approx 8-18 \mathrm{MeV}$; measured excitation function; deduced thick-target yield. Activation technique. JOUR RAACA 92219 


\section{$\mathrm{A}=165$ (continued)}

\begin{tabular}{|c|c|c|}
\hline${ }^{165} \mathrm{Lu}$ & 2005ANO4 & $\begin{array}{l}\text { NUCLEAR REACTIONS }{ }^{139} \mathrm{La}\left({ }^{30} \mathrm{Si}, 4 \mathrm{n}\right), \mathrm{E}=135 \mathrm{MeV} \text {; measured } \\
\text { Doppler-shifted } \mathrm{E} \gamma, \mathrm{I} \gamma, \gamma \gamma \text {-coin. }{ }^{165} \mathrm{Lu} \text { levels deduced } \mathrm{T}_{1 / 2} \text {, transition } \\
\text { quadrupole moments, B(E2). GASP array, total Routhian surface } \\
\text { calculations. JOUR PRVCA } 71014312\end{array}$ \\
\hline${ }^{165} \mathrm{Ta}$ & $2004 \mathrm{GOZZ}$ & $\begin{array}{l}\text { RADIOACTIVITY } 168,169,170,171,172 \mathrm{Os},{ }^{169,170,171,172,173,174,175} \mathrm{Ir}, \\
{ }^{170,171,172,173,174,175,176,177} \mathrm{Pt},{ }^{173,174,175,176,177} \mathrm{Au}, \\
{ }^{174,175,176,177,178} \mathrm{Hg}(\alpha)\left[\text { from }{ }^{92,94} \mathrm{Mo}\left({ }^{84} \mathrm{Sr}, \mathrm{xnyp}\right) \text { and subsequent }\right. \\
\text { decay]; measured } \mathrm{E} \alpha, \mathrm{E} \gamma, \alpha-\gamma \text {-coin, } \mathrm{T}_{1 / 2} \cdot{ }^{165} \mathrm{Ta},{ }^{165,167} \mathrm{~W}, \\
\\
{ }^{165,166,167,168,171} \mathrm{Re},{ }^{169,170,171} \mathrm{Os},{ }^{169,170,171,172,175} \mathrm{Ir},{ }^{173,175} \mathrm{Pt},{ }^{174,176} \mathrm{Au}\end{array}$ \\
\hline${ }^{165} \mathrm{~W}$ & $2004 \mathrm{GOZZ}$ & $\begin{array}{l}\text { deduced levels, J, } \pi \text {. THESIS J Goon,University of Tennessee } \\
\text { RADIOACTIVITY }{ }^{168,169,170,171,172} \mathrm{Os},{ }^{169,170,171,172,173,174,175} \mathrm{Ir}, \\
{ }^{170,171,172,173,174,175,176,177} \mathrm{Pt},{ }^{173,174,175,176,177} \mathrm{Au}, \\
{ }^{174,175,176,177,178} \mathrm{Hg}(\alpha)\left[\text { from }{ }^{92,94} \mathrm{Mo}\left({ }^{84} \mathrm{Sr}, \text { xnyp) and subsequent }\right.\right. \\
\text { decay]; measured } \mathrm{E} \alpha \text {, E } \gamma, \alpha \text { - } \gamma \text {-coin, } \mathrm{T}_{1 / 2} \cdot{ }^{165} \mathrm{Ta},{ }^{165,167} \mathrm{~W} \\
165,166,167,168,171 \mathrm{Re},{ }^{169,170,171} \mathrm{Os},{ }^{169,170,171,172,175} \mathrm{Ir},{ }^{173,175} \mathrm{Pt},{ }^{174,176} \mathrm{Au}\end{array}$ \\
\hline${ }^{165} \mathrm{Re}$ & $2004 \mathrm{GOZZ}$ & $\begin{array}{l}\text { deduced levels, J, } \pi \text {. THESIS J Goon,University of Tennessee } \\
\text { RADIOACTIVITY }{ }^{168,169,170,171,172} \mathrm{Os},{ }^{169,170,171,172,173,174,175} \mathrm{Ir}, \\
\text { 170,171,172,173,174,175,176,177} \mathrm{Pt},{ }^{173,174,175,176,177} \mathrm{Au}, \\
{ }^{174,175,176,177,178} \mathrm{Hg}(\alpha)\left[\text { from }{ }^{92,94} \mathrm{Mo}\left({ }^{84} \mathrm{Sr}, \mathrm{xnyp}\right) \text { and subsequent }\right. \\
\text { decay]; measured } \mathrm{E} \alpha, \mathrm{E} \gamma, \alpha \text { - } \gamma \text {-coin, } \mathrm{T}_{1 / 2} \cdot{ }^{165} \mathrm{Ta},{ }^{165,167} \mathrm{~W}, \\
{ }_{165,166,167,168,171} \mathrm{Re},{ }^{169,170,171} \mathrm{Os},{ }^{169,170,171,172,175} \mathrm{Ir},{ }^{173,175} \mathrm{Pt},{ }^{174,176} \mathrm{Au}\end{array}$ \\
\hline & $2005 \mathrm{SC} 22$ & $\begin{array}{l}\text { deduced levels, J, } \pi \text {. THESIS J Goon,University of Tennessee } \\
\text { RADIOACTIVITY }{ }^{167,167 m, 169,169 m} \mathrm{Ir},{ }^{165,165 m} \mathrm{Re},{ }^{161} \mathrm{Ta}(\alpha) \text { [from } \\
{ }^{92} \mathrm{Mo}\left({ }^{78} \mathrm{Kr}, 2 \mathrm{np}\right) \text { and }{ }^{112} \mathrm{Sn}\left({ }^{58} \mathrm{Ni}, \mathrm{p}\right) \text { and subsequent decay]; measured } \\
\mathrm{E} \alpha, \mathrm{E} \gamma, \alpha \gamma \text {-coin, } \mathrm{T}_{1 / 2} ; \text { deduced spectroscopic factors. }{ }^{167,167 m} \operatorname{Ir}(\mathrm{p}) \\
{\left[\text { from }{ }^{112} \mathrm{Sn}\left({ }^{58} \mathrm{Ni}, 2 \mathrm{np}\right)\right] ; \text { measured Ep, T }{ }_{1 / 2} \text {; deduced spectroscopic }} \\
\text { factors. Jurogam array, mass separator. JOUR JPGPE } 31 \mathrm{~S} 1719\end{array}$ \\
\hline
\end{tabular}

\section{$\mathrm{A}=\mathbf{1 6 6}$}

\begin{tabular}{|c|c|c|}
\hline${ }^{166} \mathrm{Gd}$ & 2005IC02 & $\begin{array}{l}\text { RADIOACTIVITY }{ }^{159} \mathrm{Pm},{ }^{162} \mathrm{Sm},{ }^{166} \mathrm{Gd},{ }^{166} \mathrm{~Tb}\left(\beta^{-}\right)\left[\text {from }{ }^{238} \mathrm{U}(\mathrm{p}, \mathrm{F})\right] \\
\text { measured } \mathrm{E} \gamma, \mathrm{I} \gamma, \beta \gamma-, \gamma \gamma \text {-coin, } \mathrm{T}_{1 / 2} \cdot{ }^{166} \mathrm{~Tb} \text { deduced levels, } \beta \text {-feeding } \\
\text { intensities. JOUR PRVCA } 71067302\end{array}$ \\
\hline${ }^{166} \mathrm{~Tb}$ & $2005 \mathrm{IC} 02$ & $\begin{array}{l}\text { RADIOACTIVITY }{ }^{159} \mathrm{Pm},{ }^{162} \mathrm{Sm},{ }^{166} \mathrm{Gd},{ }^{166} \mathrm{~Tb}\left(\beta^{-}\right)\left[\text {from }{ }^{238} \mathrm{U}(\mathrm{p}, \mathrm{F})\right] \text {; } \\
\text { measured } \mathrm{E} \gamma, \mathrm{I} \gamma, \beta \gamma-, \gamma \gamma \text {-coin, } \mathrm{T}_{1 / 2} \cdot{ }^{166} \mathrm{~Tb} \text { deduced levels, } \beta \text {-feeding } \\
\text { intensities. JOUR PRVCA } 71067302\end{array}$ \\
\hline${ }^{166} \mathrm{Dy}$ & 2005 IC02 & $\begin{array}{l}\text { RADIOACTIVITY }{ }^{159} \mathrm{Pm},{ }^{162} \mathrm{Sm},{ }^{166} \mathrm{Gd},{ }^{166} \mathrm{~Tb}\left(\beta^{-}\right)\left[\text {from }{ }^{238} \mathrm{U}(\mathrm{p}, \mathrm{F})\right] \\
\text { measured } \mathrm{E} \gamma, \mathrm{I} \gamma, \beta \gamma-, \gamma \gamma \text {-coin, } \mathrm{T}_{1 / 2} \cdot{ }^{166} \mathrm{~Tb} \text { deduced levels, } \beta \text {-feeding } \\
\text { intensities. JOUR PRVCA } 71067302\end{array}$ \\
\hline${ }^{166} \mathrm{Er}$ & 2005BU37 & $\begin{array}{l}\text { NUCLEAR REACTIONS }{ }^{164} \mathrm{Dy}\left({ }^{7} \mathrm{Li} \text {, xnyp), } \mathrm{E}=55 \mathrm{MeV} \text {; measured } \mathrm{E} \gamma \text {, }\right. \\
\mathrm{I} \gamma, \gamma \gamma-\text { (charged particle) } \gamma \text {-coin. }{ }^{167} \mathrm{Tm} \text { deduced high-spin levels, J, } \pi \text {, } \\
\text { configurations. }{ }^{166} \mathrm{Er} \text { deduced rotational band features. GASP, ISIS } \\
\text { arrays. JOUR JPGPE } 31 \mathrm{~S} 1827\end{array}$ \\
\hline${ }^{166} \mathrm{Yb}$ & 2005DEZX & $\begin{array}{l}\text { NUCLEAR REACTIONS }{ }^{124} \mathrm{Sn}\left({ }^{48} \mathrm{Ca}, 4 \mathrm{n}\right),\left({ }^{48} \mathrm{Ca}, 5 \mathrm{n}\right),\left({ }^{48} \mathrm{Ca}, 6 \mathrm{n}\right), \\
\mathrm{E}=215 \mathrm{MeV} ; \text { measured } \mathrm{E} \gamma, \mathrm{I} \gamma, \gamma \gamma \text {-coin. }{ }^{166,167,168} \mathrm{Yb} \text { deduced } \\
\text { transition energy correlations, level spacing and interaction potential } \\
\text { features, order-to-chaos transition. Gammasphere array. CONF } \\
\text { Argonne(Nuclei at the Limits).P303,Deleplanque }\end{array}$ \\
\hline
\end{tabular}




\section{$\mathrm{A}=166$ (continued)}

2005ST03 NUCLEAR REACTIONS ${ }^{124} \mathrm{Sn}\left({ }^{48} \mathrm{Ca}, 4 \mathrm{n}\right),\left({ }^{48} \mathrm{Ca}, 5 \mathrm{n}\right),\left({ }^{48} \mathrm{Ca}, 6 \mathrm{n}\right)$, $\mathrm{E}=215 \mathrm{MeV}$; measured $\mathrm{E} \gamma, \mathrm{I} \gamma, \gamma \gamma$-coin. ${ }^{166,167,168} \mathrm{Yb}$ deduced transition energy correlations, level spacing and interaction potential features, order-to-chaos transition. Gammasphere array. JOUR PRLTA 94042501 $\begin{array}{ll}{ }^{166} \mathrm{Lu} & \text { RADIOACTIVITY }{ }^{166} \mathrm{Hf}\left(\beta^{+}\right),(\mathrm{EC})\left[\text { from }{ }^{159} \mathrm{~Tb}\left({ }^{16} \mathrm{O}, 9 \mathrm{n}\right)\right] \text {; measured } \\ & \mathrm{E} \gamma, \mathrm{I} \gamma, \gamma \gamma \text {-coin. }{ }^{166} \mathrm{Hf} \text { deduced levels, J, } \pi, \mathrm{X}(5) \text { symmetry features. }\end{array}$ JOUR PRVCA 71024309

${ }^{166} \mathrm{Hf}$ 2005MC01 RADIOACTIVITY ${ }^{166} \mathrm{Hf}\left(\beta^{+}\right),(\mathrm{EC})\left[\right.$ from $\left.{ }^{159} \mathrm{~Tb}\left({ }^{16} \mathrm{O}, 9 \mathrm{n}\right)\right]$; measured $\mathrm{E} \gamma, \mathrm{I} \gamma, \gamma \gamma$-coin. ${ }^{166} \mathrm{Hf}$ deduced levels, J, $\pi, \mathrm{X}(5)$ symmetry features. JOUR PRVCA 71024309

${ }^{166} \mathrm{~W}$ 2004GOZZ RADIOACTIVITY $168,169,170,171,172 \mathrm{Os},{ }^{169,170,171,172,173,174,175} \mathrm{Ir}$, ${ }_{170,171,172,173,174,175,176,177} \mathrm{Pt},{ }^{173,174,175,176,177} \mathrm{Au}$, ${ }_{174,175,176,177,178} \mathrm{Hg}(\alpha)$ [from ${ }^{92,94} \mathrm{Mo}\left({ }^{84} \mathrm{Sr}\right.$, xnyp) and subsequent decay]; measured $\mathrm{E} \alpha, \mathrm{E} \gamma, \alpha$ - $\gamma$-coin, $\mathrm{T}_{1 / 2} .{ }^{165} \mathrm{Ta},{ }^{165,167} \mathrm{~W}$, ${ }^{165,166,167,168,171} \mathrm{Re},{ }^{169,170,171} \mathrm{Os},{ }^{169,170,171,172,175} \mathrm{Ir},{ }^{173,175} \mathrm{Pt},{ }^{174,176} \mathrm{Au}$ deduced levels, J, $\pi$. THESIS J Goon,University of Tennessee ${ }^{166} \mathrm{Re} \quad 2004 \mathrm{GOZZ}$ RADIOACTIVITY ${ }^{168,169,170,171,172}$ Os, ${ }^{169,170,171,172,173,174,175} \mathrm{Ir}$,

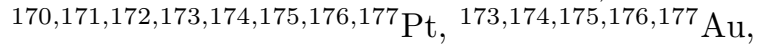
${ }^{174,175,176,177,178} \mathrm{Hg}(\alpha)$ [from ${ }^{92,94} \mathrm{Mo}\left({ }^{84} \mathrm{Sr}\right.$, xnyp) and subsequent decay]; measured $\mathrm{E} \alpha, \mathrm{E} \gamma, \alpha-\gamma$-coin, $\mathrm{T}_{1 / 2} .{ }^{165} \mathrm{Ta},{ }^{165,167} \mathrm{~W}$, ${ }^{165,166,167,168,171} \mathrm{Re},{ }^{169,170,171} \mathrm{Os},{ }^{169,170,171,172,175} \mathrm{Ir},{ }^{173,175} \mathrm{Pt},{ }^{174,176} \mathrm{Au}$ deduced levels, J, $\pi$. THESIS J Goon,University of Tennessee ${ }^{166} \mathrm{Os} \quad$ 2004GOZZ RADIOACTIVITY $168,169,170,171,172 \mathrm{Os}, 169,170,171,172,173,174,175 \mathrm{Ir}$, ${ }_{170,171,172,173,174,175,176,177} \mathrm{Pt},{ }^{173,174,175,176,177} \mathrm{Au}$, ${ }_{174,175,176,177,178} \mathrm{Hg}(\alpha)$ [from ${ }^{92,94} \mathrm{Mo}\left({ }^{84} \mathrm{Sr}\right.$, xnyp) and subsequent decay]; measured $\mathrm{E} \alpha, \mathrm{E} \gamma, \alpha-\gamma$-coin, $\mathrm{T}_{1 / 2} .{ }^{165} \mathrm{Ta},{ }^{165,167} \mathrm{~W}$, ${ }^{165,166,167,168,171} \mathrm{Re},{ }^{169,170,171} \mathrm{Os},{ }^{169,170,171,172,175} \mathrm{Ir},{ }^{173,175} \mathrm{Pt},{ }^{174,176} \mathrm{Au}$ deduced levels, J, $\pi$. THESIS J Goon,University of Tennessee 2005SC22 RADIOACTIVITY ${ }^{167,167 m, 169,169 m} \mathrm{Ir},{ }^{165,165 m} \mathrm{Re},{ }^{161} \mathrm{Ta}(\alpha)$ [from ${ }^{92} \mathrm{Mo}\left({ }^{78} \mathrm{Kr}, 2 \mathrm{np}\right)$ and ${ }^{112} \mathrm{Sn}\left({ }^{58} \mathrm{Ni}, \mathrm{p}\right)$ and subsequent decay]; measured $\mathrm{E} \alpha, \mathrm{E} \gamma, \alpha \gamma$-coin, $\mathrm{T}_{1 / 2}$; deduced spectroscopic factors. ${ }^{167,167 m} \operatorname{Ir}(\mathrm{p})$ [from $\left.{ }^{112} \mathrm{Sn}\left({ }^{58} \mathrm{Ni}, 2 \mathrm{np}\right)\right]$; measured Ep, $\mathrm{T}_{1 / 2}$; deduced spectroscopic factors. Jurogam array, mass separator. JOUR JPGPE 31 S1719

\section{$\mathrm{A}=167$}

${ }^{167} \mathrm{Tm}$ 2005BU37 NUCLEAR REACTIONS ${ }^{164} \mathrm{Dy}\left({ }^{7} \mathrm{Li}\right.$, xnyp), E $=55 \mathrm{MeV}$; measured $\mathrm{E} \gamma$, I $\gamma, \gamma \gamma$-, (charged particle) $\gamma$-coin. ${ }^{167} \mathrm{Tm}$ deduced high-spin levels, J, $\pi$, configurations. ${ }^{166} \mathrm{Er}$ deduced rotational band features. GASP, ISIS arrays. JOUR JPGPE 31 S1827

${ }^{167} \mathrm{Yb}$ 2005DEZX NUCLEAR REACTIONS ${ }^{124} \mathrm{Sn}\left({ }^{48} \mathrm{Ca}, 4 \mathrm{n}\right),\left({ }^{48} \mathrm{Ca}, 5 \mathrm{n}\right),\left({ }^{48} \mathrm{Ca}, 6 \mathrm{n}\right)$, $\mathrm{E}=215 \mathrm{MeV}$; measured $\mathrm{E} \gamma, \mathrm{I} \gamma, \gamma \gamma$-coin. ${ }^{166,167,168} \mathrm{Yb}$ deduced transition energy correlations, level spacing and interaction potential features, order-to-chaos transition. Gammasphere array. CONF Argonne(Nuclei at the Limits),P303,Deleplanque 


\section{$\mathrm{A}=167$ (continued)}

2005ST03 NUCLEAR REACTIONS ${ }^{124} \mathrm{Sn}\left({ }^{48} \mathrm{Ca}, 4 \mathrm{n}\right),\left({ }^{48} \mathrm{Ca}, 5 \mathrm{n}\right),\left({ }^{48} \mathrm{Ca}, 6 \mathrm{n}\right)$, $\mathrm{E}=215 \mathrm{MeV}$; measured $\mathrm{E} \gamma, \mathrm{I} \gamma, \gamma \gamma$-coin. ${ }^{166,167,168} \mathrm{Yb}$ deduced transition energy correlations, level spacing and interaction potential features, order-to-chaos transition. Gammasphere array. JOUR PRLTA 94042501

${ }^{167} \mathrm{Lu} \quad$ 2005AM02

${ }^{167} \mathrm{Hf}$

2005DU23

${ }^{167} \mathrm{~W}$

2004GOZZ

${ }^{167} \mathrm{Re}$

2004GOZZ

${ }^{167} \mathrm{Os}$

2004GOZZ

${ }^{167} \mathrm{Ir}$

$2005 \mathrm{SC} 22$

2005SC22
NUCLEAR REACTIONS ${ }^{123} \mathrm{Sb}\left({ }^{48} \mathrm{Ca}, 4 \mathrm{n}\right), \mathrm{E}=203 \mathrm{MeV}$; measured E $\gamma$, $\mathrm{I} \gamma, \gamma \gamma$-coin. ${ }^{167} \mathrm{Lu}$ deduced high-spin levels, J, $\pi$, triaxial superdeformed bands, configurations. Gammasphere array. JOUR PRVCA 71011302 NUCLEAR REACTIONS ${ }^{123} \mathrm{Sb}\left({ }^{48} \mathrm{Ca}, 4 \mathrm{n}\right), \mathrm{E}=203 \mathrm{MeV}$; measured $\mathrm{E} \gamma$, I $\gamma, \gamma \gamma$-coin, DSA. ${ }^{167} \mathrm{Lu}$ deduced triaxial superdeformed band transition quadrupole moment. Gammasphere array. JOUR JPGPE 31 S1873 NUCLEAR REACTIONS Ge( $\left.{ }^{18} \mathrm{O}, \mathrm{X}\right)^{83 m} \mathrm{Sr} /{ }^{83} \mathrm{Y} /{ }^{84 m} \mathrm{Y} /{ }^{88 m} \mathrm{Y} /$ ${ }^{85} \mathrm{Zr} /{ }^{87} \mathrm{Zr}, \mathrm{E}=82.8 \mathrm{GeV} ;{ }^{84} \mathrm{Se}\left({ }^{18} \mathrm{O}, \mathrm{X}\right){ }^{86 m} \mathrm{Y} /{ }^{85} \mathrm{Zr} /{ }^{87} \mathrm{Nb} /{ }^{87 m} \mathrm{Nb} /$ ${ }^{88} \mathrm{Nb} /{ }^{88} \mathrm{Mo}, \mathrm{E}=82.7 \mathrm{MeV} ;{ }^{124} \mathrm{Sn}\left({ }^{50} \mathrm{Ti}, \mathrm{X}\right){ }^{168 m} \mathrm{Lu} /{ }^{167} \mathrm{Hf} /{ }^{168} \mathrm{Hf}$, $\mathrm{E}=223.7 \mathrm{MeV} ;{ }^{116} \mathrm{Sn}\left({ }^{50} \mathrm{Ti}, \mathrm{X}\right){ }^{162} \mathrm{Tm} /{ }^{161} \mathrm{Yb} /{ }^{162} \mathrm{Yb} /{ }^{163} \mathrm{Yb} /{ }^{162} \mathrm{Lu} /$ ${ }^{162} \mathrm{Hf}, \mathrm{E}=224.4 \mathrm{MeV}$; measured delayed $\mathrm{E} \gamma, \mathrm{I} \gamma$ following residual nucleus decay. Physical preseparation technique. JOUR NIMAE 551 528

RADIOACTIVITY ${ }^{168,169,170,171,172}$ Os, ${ }^{169,170,171,172,173,174,175} \mathrm{Ir}$, $170,171,172,173,174,175,176,177 \mathrm{Pt},{ }^{173,174,175,176,177} \mathrm{Au}$,

${ }_{174,175,176,177,178} \mathrm{Hg}(\alpha)$ [from ${ }^{92,94} \mathrm{Mo}\left({ }^{84} \mathrm{Sr}\right.$, xnyp) and subsequent decay]; measured E $\alpha, \mathrm{E} \gamma, \alpha$ - $\gamma$-coin, $\mathrm{T}_{1 / 2} .{ }^{165} \mathrm{Ta},{ }^{165,167} \mathrm{~W}$, ${ }_{165,166,167,168,171} \operatorname{Re},{ }^{169,170,171} \mathrm{Os},{ }^{169,170,171,172,175} \mathrm{Ir},{ }^{173,175} \mathrm{Pt},{ }^{174,176} \mathrm{Au}$ deduced levels, J, $\pi$. THESIS J Goon,University of Tennessee

RADIOACTIVITY ${ }^{168,169,170,171,172}$ Os, ${ }^{169,170,171,172,173,174,175} \mathrm{Ir}$, $170,171,172,173,174,175,176,177 \mathrm{Pt},{ }^{173,174,175,176,177} \mathrm{Au}$, ${ }_{174,175,176,177,178} \mathrm{Hg}(\alpha)$ [from ${ }^{92,94} \mathrm{Mo}\left({ }^{84} \mathrm{Sr}\right.$, xnyp) and subsequent decay]; measured E $\alpha, \mathrm{E} \gamma, \alpha$ - $\gamma$-coin, $\mathrm{T}_{1 / 2} .{ }^{165} \mathrm{Ta},{ }^{165,167} \mathrm{~W}$, ${ }_{165,166,167,168,171} \mathrm{Re},{ }^{169,170,171} \mathrm{Os},{ }^{169,170,171,172,175} \mathrm{Ir},{ }^{173,175} \mathrm{Pt},{ }^{174,176} \mathrm{Au}$ deduced levels, J, $\pi$. THESIS J Goon,University of Tennessee RADIOACTIVITY $168,169,170,171,172$ Os, ${ }^{169,170,171,172,173,174,175} \mathrm{Ir}$, $170,171,172,173,174,175,176,177 \mathrm{Pt},{ }^{173,174,175,176,177} \mathrm{Au}$, $174,175,176,177,178 \mathrm{Hg}(\alpha)$ [from ${ }^{92,94} \mathrm{Mo}\left({ }^{84} \mathrm{Sr}\right.$, xnyp) and subsequent decay]; measured E $\alpha, \mathrm{E} \gamma, \alpha$ - $\gamma$-coin, $\mathrm{T}_{1 / 2} \cdot{ }^{165} \mathrm{Ta},{ }^{165,167} \mathrm{~W}$, ${ }_{165,166,167,168,171} \operatorname{Re},{ }^{169,170,171}$ Os, ${ }^{169,170,171,172,175} \mathrm{Ir},{ }^{173,175} \mathrm{Pt},{ }^{174,176} \mathrm{Au}$ deduced levels, J, $\pi$. THESIS J Goon,University of Tennessee NUCLEAR REACTIONS ${ }^{92} \mathrm{Mo}\left({ }^{78} \mathrm{Kr}, 2 \mathrm{np}\right), \mathrm{E}=360 \mathrm{MeV} ;{ }^{112} \mathrm{Sn}\left({ }^{58} \mathrm{Ni}\right.$, p), $\mathrm{E}=266 \mathrm{MeV}$; measured $\mathrm{E} \gamma, \mathrm{I} \gamma, \gamma \gamma$-, (recoil) $\gamma$-coin. ${ }^{167,169} \mathrm{Ir}$ deduced transitions. Recoil-decay tagging, Jurogam array. JOUR JPGPE 31 S1719 RADIOACTIVITY ${ }^{167,167 m, 169,169 m} \mathrm{Ir},{ }^{165,165 m} \mathrm{Re},{ }^{161} \mathrm{Ta}(\alpha)$ [from ${ }^{92} \mathrm{Mo}\left({ }^{78} \mathrm{Kr}, 2 \mathrm{np}\right)$ and ${ }^{112} \mathrm{Sn}\left({ }^{58} \mathrm{Ni}, \mathrm{p}\right)$ and subsequent decay]; measured $\mathrm{E} \alpha, \mathrm{E} \gamma, \alpha \gamma$-coin, $\mathrm{T}_{1 / 2}$; deduced spectroscopic factors. ${ }^{167,167 m} \operatorname{Ir}(\mathrm{p})$ [from $\left.{ }^{112} \mathrm{Sn}\left({ }^{58} \mathrm{Ni}, 2 \mathrm{np}\right)\right]$; measured Ep, $\mathrm{T}_{1 / 2}$; deduced spectroscopic factors. Jurogam array, mass separator. JOUR JPGPE 31 S1719 


\section{$\mathrm{A}=\mathbf{1 6 8}$}

${ }^{168} \mathrm{Er} \quad 2005 \mathrm{BUZZ} \quad$ NUCLEAR REACTIONS ${ }^{170} \mathrm{Er}(\mathrm{p}, \mathrm{t}), \mathrm{E}=25.0 \mathrm{MeV}$; measured $\mathrm{E} \gamma, \mathrm{I} \gamma$, $\sigma(\theta) .{ }^{168} \mathrm{Er}$ deduced $0^{+}$states energies. REPT MLL 2004

Annual,P16,Bucurescu

2005ME19 NUCLEAR REACTIONS ${ }^{160} \mathrm{Gd},{ }^{164} \mathrm{Dy},{ }^{170} \mathrm{Er},{ }^{178} \mathrm{Hf},{ }^{186} \mathrm{~W},{ }^{192} \mathrm{Os}(\mathrm{p}$, $\mathrm{t}), \mathrm{E}=25 \mathrm{MeV}$; measured triton spectra, $\sigma(\theta) .{ }^{158} \mathrm{Gd},{ }^{162} \mathrm{Dy},{ }^{168} \mathrm{Er}$, ${ }^{176} \mathrm{Hf},{ }^{184} \mathrm{~W},{ }^{190} \mathrm{Os}$ deduced $0^{+}$level energies. JOUR JPGPE $31 \mathrm{~S} 1399$ ${ }^{168} \mathrm{Yb}$ 2005DEZX NUCLEAR REACTIONS ${ }^{124} \mathrm{Sn}\left({ }^{48} \mathrm{Ca}, 4 \mathrm{n}\right),\left({ }^{48} \mathrm{Ca}, 5 \mathrm{n}\right),\left({ }^{48} \mathrm{Ca}, 6 \mathrm{n}\right)$, $\mathrm{E}=215 \mathrm{MeV}$; measured $\mathrm{E} \gamma, \mathrm{I} \gamma, \gamma \gamma$-coin. ${ }^{166,167,168} \mathrm{Yb}$ deduced transition energy correlations, level spacing and interaction potential features, order-to-chaos transition. Gammasphere array. CONF Argonne(Nuclei at the Limits),P303,Deleplanque

2005ST03 NUCLEAR REACTIONS ${ }^{124} \mathrm{Sn}\left({ }^{48} \mathrm{Ca}, 4 \mathrm{n}\right),\left({ }^{48} \mathrm{Ca}, 5 \mathrm{n}\right),\left({ }^{48} \mathrm{Ca}, 6 \mathrm{n}\right)$, $\mathrm{E}=215 \mathrm{MeV}$; measured $\mathrm{E} \gamma, \mathrm{I} \gamma, \gamma \gamma$-coin. ${ }^{166,167,168} \mathrm{Yb}$ deduced transition energy correlations, level spacing and interaction potential features, order-to-chaos transition. Gammasphere array. JOUR PRLTA 94042501

${ }^{168} \mathrm{Lu} \quad 2005 \mathrm{DU} 23$ NUCLEAR REACTIONS Ge $\left({ }^{18} \mathrm{O}, \mathrm{X}\right)^{83 m} \mathrm{Sr} /{ }^{83} \mathrm{Y} /{ }^{84 m} \mathrm{Y} /{ }^{88 m} \mathrm{Y} /$ ${ }^{85} \mathrm{Zr} /{ }^{87} \mathrm{Zr}, \mathrm{E}=82.8 \mathrm{GeV} ;{ }^{84} \mathrm{Se}\left({ }^{18} \mathrm{O}, \mathrm{X}\right)^{86 m} \mathrm{Y} /{ }^{85} \mathrm{Zr} /{ }^{87} \mathrm{Nb} /{ }^{87 m} \mathrm{Nb} /$ ${ }^{88} \mathrm{Nb} /{ }^{88} \mathrm{Mo}, \mathrm{E}=82.7 \mathrm{MeV} ;{ }^{124} \mathrm{Sn}\left({ }^{50} \mathrm{Ti}, \mathrm{X}\right){ }^{168 m} \mathrm{Lu} /{ }^{167} \mathrm{Hf} /{ }^{168} \mathrm{Hf}$, $\mathrm{E}=223.7 \mathrm{MeV} ;{ }^{116} \mathrm{Sn}\left({ }^{50} \mathrm{Ti}, \mathrm{X}\right){ }^{162} \mathrm{Tm} /{ }^{161} \mathrm{Yb} /{ }^{162} \mathrm{Yb} /{ }^{163} \mathrm{Yb} /{ }^{162} \mathrm{Lu} /$ ${ }^{162} \mathrm{Hf}, \mathrm{E}=224.4 \mathrm{MeV}$; measured delayed $\mathrm{E} \gamma$, I $\gamma$ following residual nucleus decay. Physical preseparation technique. JOUR NIMAE 551 528

${ }^{168} \mathrm{Hf}$ 2005DU23 NUCLEAR REACTIONS Ge $\left({ }^{18} \mathrm{O}, \mathrm{X}\right)^{83 m} \mathrm{Sr} /{ }^{83} \mathrm{Y} /{ }^{84 m} \mathrm{Y} /{ }^{88 m} \mathrm{Y} /$ ${ }^{85} \mathrm{Zr} /{ }^{87} \mathrm{Zr}, \mathrm{E}=82.8 \mathrm{GeV} ;{ }^{84} \mathrm{Se}\left({ }^{18} \mathrm{O}, \mathrm{X}\right){ }^{86 m} \mathrm{Y} /{ }^{85} \mathrm{Zr} /{ }^{87} \mathrm{Nb} /{ }^{87 m} \mathrm{Nb} /$ ${ }^{88} \mathrm{Nb} /{ }^{88} \mathrm{Mo}, \mathrm{E}=82.7 \mathrm{MeV} ;{ }^{124} \mathrm{Sn}\left({ }^{50} \mathrm{Ti}, \mathrm{X}\right){ }^{168 m} \mathrm{Lu} /{ }^{167} \mathrm{Hf} /{ }^{168} \mathrm{Hf}$, $\mathrm{E}=223.7 \mathrm{MeV} ;{ }^{116} \mathrm{Sn}\left({ }^{50} \mathrm{Ti}, \mathrm{X}\right){ }^{162} \mathrm{Tm} /{ }^{161} \mathrm{Yb} /{ }^{162} \mathrm{Yb} /{ }^{163} \mathrm{Yb} /{ }^{162} \mathrm{Lu} /$ ${ }^{162} \mathrm{Hf}, \mathrm{E}=224.4 \mathrm{MeV}$; measured delayed $\mathrm{E} \gamma, \mathrm{I} \gamma$ following residual nucleus decay. Physical preseparation technique. JOUR NIMAE 551 528

${ }^{168} \mathrm{~W} \quad 2002 \mathrm{DU} 22$ RADIOACTIVITY ${ }^{197,197 m, 198,199 m, 200,201 m} \mathrm{Po}(\alpha) ;{ }^{172,173} \mathrm{Os}(\alpha)$ [from $\left.{ }^{156} \mathrm{Dy}\left({ }^{22} \mathrm{Ne}, \mathrm{xn}\right)\right] ;{ }^{183,184,185} \mathrm{Hg}(\alpha)$ [from $\left.{ }^{168} \mathrm{Yb}\left({ }^{22} \mathrm{Ne}, \mathrm{xn}\right)\right]$; measured $\mathrm{E} \alpha, \mathrm{T}_{1 / 2}$. JOUR NIMAE 479631

2004GOZZ RADIOACTIVITY ${ }^{168,169,170,171,172} \mathrm{Os},{ }^{169,170,171,172,173,174,175} \mathrm{Ir}$, ${ }_{170,171,172,173,174,175,176,177} \mathrm{Pt},{ }^{173,174,175,176,177} \mathrm{Au}$, ${ }_{174,175,176,177,178} \mathrm{Hg}(\alpha)$ [from ${ }^{92,94} \mathrm{Mo}\left({ }^{84} \mathrm{Sr}\right.$, xnyp) and subsequent decay]; measured $\mathrm{E} \alpha, \mathrm{E} \gamma, \alpha-\gamma$-coin, $\mathrm{T}_{1 / 2} .{ }^{165} \mathrm{Ta},{ }^{165,167} \mathrm{~W}$, ${ }_{165,166,167,168,171} \mathrm{Re},{ }^{169,170,171} \mathrm{Os},{ }^{169,170,171,172,175} \mathrm{Ir},{ }^{173,175} \mathrm{Pt},{ }^{174,176} \mathrm{Au}$ deduced levels, J, $\pi$. THESIS J Goon,University of Tennessee

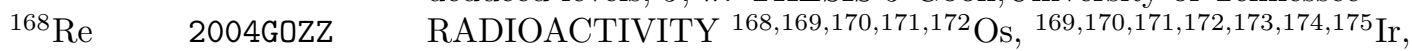
${ }_{170,171,172,173,174,175,176,177} \mathrm{Pt},{ }^{173,174,175,176,177} \mathrm{Au}$, ${ }_{174,175,176,177,178} \mathrm{Hg}(\alpha)$ [from ${ }^{92,94} \mathrm{Mo}\left({ }^{84} \mathrm{Sr}\right.$, xnyp) and subsequent decay]; measured $\mathrm{E} \alpha, \mathrm{E} \gamma, \alpha$ - $\gamma$-coin, $\mathrm{T}_{1 / 2} .{ }^{165} \mathrm{Ta},{ }^{165,167} \mathrm{~W}$, ${ }^{165,166,167,168,171} \mathrm{Re},{ }^{169,170,171} \mathrm{Os},{ }^{169,170,171,172,175} \mathrm{Ir},{ }^{173,175} \mathrm{Pt},{ }^{174,176} \mathrm{Au}$ deduced levels, J, $\pi$. THESIS J Goon,University of Tennessee 


\section{$\mathrm{A}=168$ (continued)}

$\begin{aligned}{ }^{168} \mathrm{Os} \quad \text { 2004GOZZ } & \text { RADIOACTIVITY } 168,169,170,171,172 \mathrm{Os},{ }^{169,170,171,172,173,174,175} \mathrm{Ir}, \\ & 170,171,172,173,174,175,176,177 \mathrm{Pt},{ }^{173,174,175,176,177} \mathrm{Au}, \\ & 174,175,176,177,178 \mathrm{Hg}(\alpha)\left[\mathrm{from}{ }^{92,94} \mathrm{Mo}\left({ }^{84} \mathrm{Sr}, \mathrm{xnyp}\right) \text { and subsequent }\right. \\ & \text { decay } ; \text { measured } \mathrm{E} \alpha, \mathrm{E} \gamma, \alpha-\gamma \text {-coin, } \mathrm{T}_{1} \cdot{ }^{165} \mathrm{Ta},{ }^{165,167} \mathrm{~W}, \\ & 165,166,167,168,171 \mathrm{Re},{ }^{169,170,171} \mathrm{Os},{ }^{169,170,171,172,175} \mathrm{Ir},{ }^{173,175} \mathrm{Pt},{ }^{174,176} \mathrm{Au} \\ & \text { deduced levels, J, } \pi . \text { THESIS J Goon,University of Tennessee }\end{aligned}$

\section{$\mathrm{A}=\mathbf{1 6 9}$}

${ }^{169} \mathrm{Tm} \quad$ 2005ALZX

2005BA10

${ }^{169} \mathrm{Yb} \quad$ 2005SP04

${ }^{169} \mathrm{~W}$

2002DU22

${ }^{169} \mathrm{Re}$

2004GOZZ

${ }^{169} \mathrm{Os}$

2004GOZZ

${ }^{169} \mathrm{Ir}$

2004GOZZ

2005SC22

$2005 \mathrm{SC} 22$
NUCLEAR REACTIONS ${ }^{169} \mathrm{Tm}(\mathrm{n}, \mathrm{n}),(\mathrm{n}, \mathrm{n}$ '), E=600 MeV; measured En, $\sigma(\theta)$. CONF Santa Fe (Nucl Data for Sci and Technol)

Proc,Vol1,P1054

NUCLEAR MOMENTS ${ }^{169} \mathrm{Tm}$; measured hfs. JOUR PHSTB 71159

NUCLEAR REACTIONS ${ }^{169} \mathrm{Tm}(\mathrm{p}, \mathrm{n}), \mathrm{E}=5-45 \mathrm{MeV}$; measured excitation function; deduced integral yield. Stacked-foil activation. JOUR ARISE 63235

RADIOACTIVITY ${ }^{197,197 m, 198,199 m, 200,201 m} \mathrm{Po}(\alpha) ;{ }^{172,173} \mathrm{Os}(\alpha)$ [from $\left.{ }^{156} \mathrm{Dy}\left({ }^{22} \mathrm{Ne}, \mathrm{xn}\right)\right] ;{ }^{183,184,185} \mathrm{Hg}(\alpha)$ [from ${ }^{168} \mathrm{Yb}\left({ }^{22} \mathrm{Ne}, \mathrm{xn}\right)$ ]; measured $\mathrm{E} \alpha, \mathrm{T}_{1 / 2}$. JOUR NIMAE 479631

RADIOACTIVITY ${ }^{168,169,170,171,172}$ Os, ${ }^{169,170,171,172,173,174,175} \mathrm{Ir}$, 170,171,172,173,174,175,176,177 Pt, ${ }^{173,174,175,176,177} \mathrm{Au}$,

${ }^{174,175,176,177,178} \mathrm{Hg}(\alpha)$ [from ${ }^{92,94} \mathrm{Mo}\left({ }^{84} \mathrm{Sr}\right.$, xnyp) and subsequent decay]; measured $\mathrm{E} \alpha, \mathrm{E} \gamma, \alpha$ - $\gamma$-coin, $\mathrm{T}_{1 / 2} .{ }^{165} \mathrm{Ta},{ }^{165,167} \mathrm{~W}$, ${ }_{165,166,167,168,171} \mathrm{Re},{ }^{169,170,171} \mathrm{Os},{ }^{169,170,171,172,175} \mathrm{Ir},{ }^{173,175} \mathrm{Pt},{ }^{174,176} \mathrm{Au}$ deduced levels, $J, \pi$. THESIS J Goon,University of Tennessee RADIOACTIVITY $168,169,170,171,172$ Os, ${ }^{169,170,171,172,173,174,175} \mathrm{Ir}$, ${ }_{170,171,172,173,174,175,176,177} \mathrm{Pt},{ }^{173,174,175,176,177} \mathrm{Au}$, 174,175,176,177,178 $\mathrm{Hg}(\alpha)$ [from ${ }^{92,94} \mathrm{Mo}\left({ }^{84} \mathrm{Sr}\right.$, xnyp) and subsequent decay]; measured $\mathrm{E} \alpha, \mathrm{E} \gamma, \alpha$ - $\gamma$-coin, $\mathrm{T}_{1 / 2} .{ }^{165} \mathrm{Ta},{ }^{165,167} \mathrm{~W}$, ${ }_{165,166,167,168,171} \mathrm{Re},{ }^{169,170,171} \mathrm{Os},{ }^{169,170,171,172,175} \mathrm{Ir},{ }^{173,175} \mathrm{Pt},{ }^{174,176} \mathrm{Au}$ deduced levels, J, $\pi$. THESIS J Goon,University of Tennessee RADIOACTIVITY ${ }^{168,169,170,171,172}$ Os, ${ }^{169,170,171,172,173,174,175} \mathrm{Ir}$, ${ }_{170,171,172,173,174,175,176,177} \mathrm{Pt},{ }^{173,174,175,176,177} \mathrm{Au}$, $174,175,176,177,178 \mathrm{Hg}(\alpha)$ [from ${ }^{92,94} \mathrm{Mo}\left({ }^{84} \mathrm{Sr}\right.$, xnyp) and subsequent decay]; measured $\mathrm{E} \alpha, \mathrm{E} \gamma, \alpha$ - $\gamma$-coin, $\mathrm{T}_{1 / 2} .{ }^{165} \mathrm{Ta},{ }^{165,167} \mathrm{~W}$, ${ }_{165,166,167,168,171} \mathrm{Re},{ }^{169,170,171} \mathrm{Os},{ }^{169,170,171,172,175} \mathrm{Ir},{ }^{173,175} \mathrm{Pt},{ }^{174,176} \mathrm{Au}$ deduced levels, J, $\pi$. THESIS J Goon,University of Tennessee NUCLEAR REACTIONS ${ }^{92} \mathrm{Mo}\left({ }^{78} \mathrm{Kr}, 2 \mathrm{np}\right), \mathrm{E}=360 \mathrm{MeV} ;{ }^{112} \mathrm{Sn}\left({ }^{58} \mathrm{Ni}\right.$, p), $\mathrm{E}=266 \mathrm{MeV}$; measured $\mathrm{E} \gamma, \mathrm{I} \gamma, \gamma \gamma$-, (recoil) $\gamma$-coin. ${ }^{167,169} \mathrm{Ir}$ deduced transitions. Recoil-decay tagging, Jurogam array. JOUR JPGPE 31 S1719 RADIOACTIVITY ${ }^{167,167 m, 169,169 m} \mathrm{Ir},{ }^{165,165 m} \mathrm{Re},{ }^{161} \mathrm{Ta}(\alpha)$ [from ${ }^{92} \mathrm{Mo}\left({ }^{78} \mathrm{Kr}, 2 \mathrm{np}\right)$ and ${ }^{112} \mathrm{Sn}\left({ }^{58} \mathrm{Ni}, \mathrm{p}\right)$ and subsequent decay]; measured $\mathrm{E} \alpha, \mathrm{E} \gamma, \alpha \gamma$-coin, $\mathrm{T}_{1 / 2}$; deduced spectroscopic factors. ${ }^{167,167 m} \operatorname{Ir}(\mathrm{p})$ [from ${ }^{112} \mathrm{Sn}\left({ }^{58} \mathrm{Ni}, 2 \mathrm{np}\right)$ ]; measured Ep, $\mathrm{T}_{1 / 2}$; deduced spectroscopic factors. Jurogam array, mass separator. JOUR JPGPE 31 S1719 


\section{$\mathrm{A}=169$ (continued)}

${ }^{169} \mathrm{Pt}$ 2005J018 NUCLEAR REACTIONS Sn $\left({ }^{58} \mathrm{Ni}, \mathrm{xn}\right){ }^{169} \mathrm{Pt} /{ }^{170} \mathrm{Pt} /{ }^{171} \mathrm{Pt} /{ }^{172} \mathrm{Pt} /$ ${ }^{173} \mathrm{Pt}, \mathrm{E}=266 \mathrm{MeV}$; measured $\mathrm{E} \gamma, \mathrm{I} \gamma, \gamma \gamma$-, (recoil) $\gamma$-coin.

${ }^{169,170,171,172,173} \mathrm{Pt}$ deduced levels, J, $\pi$. Recoil-decay tagging. JOUR

JPGPE 31 S1715

\section{$\mathrm{A}=170$}

${ }^{170} \mathrm{Re} \quad 2004 \mathrm{GOZZ}$

RADIOACTIVITY ${ }^{168,169,170,171,172}$ Os, ${ }^{169,170,171,172,173,174,175} \mathrm{Ir}$, $170,171,172,173,174,175,176,177 \mathrm{Pt},{ }^{173,174,175,176,177} \mathrm{Au}$,

$174,175,176,177,178 \mathrm{Hg}(\alpha)$ [from ${ }^{92,94} \mathrm{Mo}\left({ }^{84} \mathrm{Sr}\right.$, xnyp) and subsequent decay]; measured $\mathrm{E} \alpha, \mathrm{E} \gamma, \alpha$ - $\gamma$-coin, $\mathrm{T}_{1 / 2} .{ }^{165} \mathrm{Ta},{ }^{165,167} \mathrm{~W}$, ${ }_{165,166,167,168,171} \mathrm{Re},{ }^{169,170,171} \mathrm{Os},{ }^{169,170,171,172,175} \mathrm{Ir},{ }^{173,175} \mathrm{Pt},{ }^{174,176} \mathrm{Au}$ deduced levels, J, $\pi$. THESIS J Goon,University of Tennessee 2004 WA35 NUCLEAR REACTIONS ${ }^{142} \mathrm{Nd}\left({ }^{32} \mathrm{~S}, 3 \mathrm{np}\right), \mathrm{E}=155,166 \mathrm{MeV}$; measured $\mathrm{E} \gamma, \mathrm{I} \gamma, \gamma \gamma-$, (X-ray) $\gamma$-coin. ${ }^{170}$ Re deduced high-spin levels, $\mathrm{J}$, $\pi$, configurations. Level systematics in neighboring nuclides discussed.

JOUR PRVCA 70064306

${ }^{170} \mathrm{Os} \quad$ 2004GOZZ RADIOACTIVITY $168,169,170,171,172$ Os, ${ }^{169,170,171,172,173,174,175} \mathrm{Ir}$, $170,171,172,173,174,175,176,177 \mathrm{Pt},{ }^{173,174,175,176,177} \mathrm{Au}$, $174,175,176,177,178 \mathrm{Hg}(\alpha)$ [from ${ }^{92,94} \mathrm{Mo}\left({ }^{84} \mathrm{Sr}\right.$, xnyp) and subsequent decay]; measured $\mathrm{E} \alpha, \mathrm{E} \gamma, \alpha$ - $\gamma$-coin, $\mathrm{T}_{1 / 2} .{ }^{165} \mathrm{Ta},{ }^{165,167} \mathrm{~W}$, ${ }_{165,166,167,168,171} \mathrm{Re},{ }^{169,170,171} \mathrm{Os},{ }^{169,170,171,172,175} \mathrm{Ir},{ }^{173,175} \mathrm{Pt},{ }^{174,176} \mathrm{Au}$ deduced levels, J, $\pi$. THESIS J Goon,University of Tennessee ${ }^{170} \mathrm{Ir} \quad$ 2004GOZZ RADIOACTIVITY $168,169,170,171,172 \mathrm{Os},{ }^{169,170,171,172,173,174,175} \mathrm{Ir}$, ${ }_{170,171,172,173,174,175,176,177} \mathrm{Pt},{ }^{173,174,175,176,177} \mathrm{Au}$, ${ }_{174,175,176,177,178} \mathrm{Hg}(\alpha)$ [from ${ }^{92,94} \mathrm{Mo}\left({ }^{84} \mathrm{Sr}\right.$, xnyp) and subsequent decay]; measured $\mathrm{E} \alpha, \mathrm{E} \gamma, \alpha$ - $\gamma$-coin, $\mathrm{T}_{1 / 2} .{ }^{165} \mathrm{Ta},{ }^{165,167} \mathrm{~W}$, ${ }^{165,166,167,168,171} \mathrm{Re},{ }^{169,170,171} \mathrm{Os},{ }^{169,170,171,172,175} \mathrm{Ir},{ }^{173,175} \mathrm{Pt},{ }^{174,176} \mathrm{Au}$ deduced levels, J, $\pi$. THESIS J Goon,University of Tennessee

${ }^{170} \mathrm{Pt} \quad$ 2004GOZZ

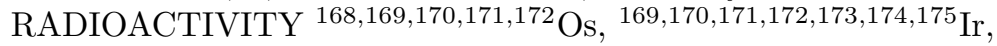
${ }_{170,171,172,173,174,175,176,177} \mathrm{Pt},{ }^{173,174,175,176,177} \mathrm{Au}$, ${ }_{174,175,176,177,178} \mathrm{Hg}(\alpha)$ [from ${ }^{92,94} \mathrm{Mo}\left({ }^{84} \mathrm{Sr}\right.$, xnyp) and subsequent decay]; measured $\mathrm{E} \alpha, \mathrm{E} \gamma, \alpha-\gamma$-coin, $\mathrm{T}_{1 / 2} .{ }^{165} \mathrm{Ta},{ }^{165,167} \mathrm{~W}$, ${ }_{165,166,167,168,171} \mathrm{Re},{ }^{169,170,171} \mathrm{Os},{ }^{169,170,171,172,175} \mathrm{Ir},{ }^{173,175} \mathrm{Pt},{ }^{174,176} \mathrm{Au}$ deduced levels, J, $\pi$. THESIS J Goon,University of Tennessee 2005J018 NUCLEAR REACTIONS Sn $\left({ }^{58} \mathrm{Ni}, \mathrm{xn}\right){ }^{169} \mathrm{Pt} /{ }^{170} \mathrm{Pt} /{ }^{171} \mathrm{Pt} /{ }^{172} \mathrm{Pt} /$ ${ }^{173} \mathrm{Pt}, \mathrm{E}=266 \mathrm{MeV}$; measured $\mathrm{E} \gamma, \mathrm{I} \gamma, \gamma \gamma-$, (recoil) $\gamma$-coin. ${ }_{169,170,171,172,173} \mathrm{Pt}$ deduced levels, J, $\pi$. Recoil-decay tagging. JOUR JPGPE 31 S1715

\section{$\mathrm{A}=171$}

${ }^{171} \mathrm{Yb} \quad$ 2005AG15 NUCLEAR REACTIONS ${ }^{57} \mathrm{Fe},{ }^{171} \mathrm{Yb}\left({ }^{3} \mathrm{He},{ }^{3} \mathrm{He}\right), \mathrm{E}=38-45 \mathrm{MeV}$; ${ }^{117} \mathrm{Sn},{ }^{172} \mathrm{Yb}\left({ }^{3} \mathrm{He}, \alpha\right), \mathrm{E}=38-45 \mathrm{MeV}$; measured $\mathrm{E} \gamma$, I $\gamma$, (particle) $\gamma$-coin; deduced radiative strength functions. JOUR NIMBE 241180 


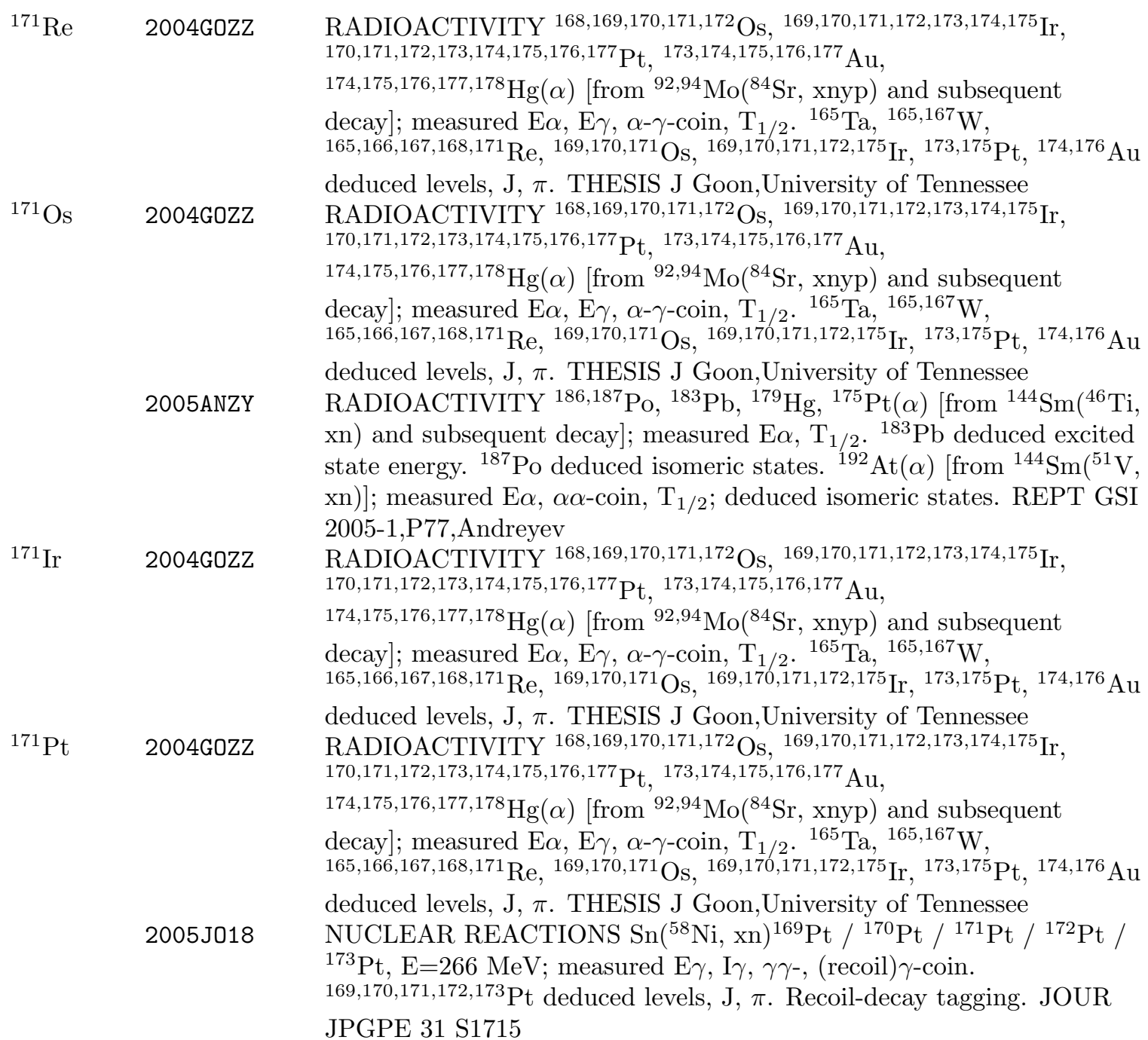

\section{$\mathrm{A}=172$}

${ }^{172} \mathrm{Yb}$ 2005SA15 NUCLEAR REACTIONS ${ }^{172,174} \mathrm{Yb}$ (polarized $\left.\gamma, \gamma^{\prime}\right), \mathrm{E}=2930,3005$, $3550 \mathrm{keV}$; measured $\mathrm{E} \gamma, \mathrm{I} \gamma$, asymmetries. ${ }^{172,174} \mathrm{Yb}$ levels deduced $\pi$. Parity and branching ratio systematics discussed. JOUR PRVCA 71 034304

2005SAZZ NUCLEAR REACTIONS ${ }^{172,174} \mathrm{Yb}$ (polarized $\left.\gamma, \gamma^{\prime}\right), \mathrm{E}=2930,3005$, $3550 \mathrm{keV}$; measured $\mathrm{E} \gamma, \mathrm{I} \gamma$, asymmetries. ${ }^{172,174} \mathrm{Yb}$ levels deduced $\pi$. Comparison with previous results. PREPRINT nucl-ex/0501006,1/11/2005

2005VE07 NUCLEAR REACTIONS ${ }^{170} \mathrm{Er}\left({ }^{7} \mathrm{Li}, 3 \mathrm{np}\right),\left({ }^{7} \mathrm{Li}, 4 \mathrm{np}\right),\left({ }^{7} \mathrm{Li}, 3 \mathrm{nd}\right),\left({ }^{7} \mathrm{Li}\right.$, 2nt), E $=51 \mathrm{MeV}$; measured $\mathrm{E} \gamma, \mathrm{I} \gamma$, (charged particle) $\gamma$-coin. ${ }^{172,173} \mathrm{Yb}$ deduced high-spin levels, J, $\pi$, configurations, absence of a static pair field. GASP, ISIS arrays. JOUR ZAANE 2619 


\section{$\mathrm{A}=172$ (continued)}

${ }^{172} \mathrm{Hf}$ 2005KA52 NUCLEAR REACTIONS ${ }^{177} \mathrm{Hf}(\mathrm{n}, \gamma), \mathrm{E}=$ thermal, resonance; ${ }^{178} \mathrm{Hf}(\mathrm{n}$, n' $\gamma)$, E $>3 \mathrm{MeV}$; measured isomer production $\sigma$. Ta, W, ${ }^{186} \mathrm{~W}, \operatorname{Re}(\mathrm{p}$, $\mathrm{X}){ }^{179 m} \mathrm{Hf} /{ }^{178 m} \mathrm{Hf} /{ }^{177 m} \mathrm{Lu} /{ }^{178} \mathrm{~W} /{ }^{175} \mathrm{Hf} /{ }^{172} \mathrm{Hf} /{ }^{173} \mathrm{Lu}, \mathrm{E}=650$ $\mathrm{MeV}$; analyzed yields, isomer ratios. ${ }^{176} \mathrm{Yb}(\alpha, 2 \mathrm{n}), \mathrm{E}<36 \mathrm{MeV}$; measured isomer yield. Other reactions discussed. JOUR YAFIA 68 1827

${ }^{172} \mathrm{Os} \quad$ 2002DU22

RADIOACTIVITY ${ }^{197,197 m, 198,199 m, 200,201 m} \mathrm{Po}(\alpha) ;{ }^{172,173} \mathrm{Os}(\alpha)$ [from $\left.{ }^{156} \mathrm{Dy}\left({ }^{22} \mathrm{Ne}, \mathrm{xn}\right)\right] ;{ }^{183,184,185} \mathrm{Hg}(\alpha)$ [from $\left.{ }^{168} \mathrm{Yb}\left({ }^{22} \mathrm{Ne}, \mathrm{xn}\right)\right]$; measured $\mathrm{E} \alpha, \mathrm{T}_{1 / 2}$. JOUR NIMAE 479631

2002DU22 NUCLEAR REACTIONS ${ }^{156} \mathrm{Dy}\left({ }^{22} \mathrm{Ne}, 5 \mathrm{n}\right),\left({ }^{22} \mathrm{Ne}, 6 \mathrm{n}\right), \mathrm{E}=127 \mathrm{MeV}$; ${ }^{162} \operatorname{Er}\left({ }^{18} \mathrm{O}, 6 \mathrm{n}\right),\left({ }^{18} \mathrm{O}, 7 \mathrm{n}\right), \mathrm{E}=116 \mathrm{MeV}$; measured radiochemical yields. JOUR NIMAE 479631

2004GOZZ RADIOACTIVITY ${ }^{168,169,170,171,172}$ Os, ${ }^{169,170,171,172,173,174,175} \mathrm{Ir}$, ${ }_{170,171,172,173,174,175,176,177} \mathrm{Pt},{ }^{173,174,175,176,177} \mathrm{Au}$, ${ }_{174,175,176,177,178} \mathrm{Hg}(\alpha)$ [from ${ }^{92,94} \mathrm{Mo}\left({ }^{84} \mathrm{Sr}\right.$, xnyp) and subsequent decay]; measured $\mathrm{E} \alpha, \mathrm{E} \gamma, \alpha$ - $\gamma$-coin, $\mathrm{T}_{1 / 2} .{ }^{165} \mathrm{Ta},{ }^{165,167} \mathrm{~W}$, ${ }^{165,166,167,168,171} \mathrm{Re},{ }^{169,170,171} \mathrm{Os},{ }^{169,170,171,172,175} \mathrm{Ir},{ }^{173,175} \mathrm{Pt},{ }^{174,176} \mathrm{Au}$ deduced levels, J, $\pi$. THESIS J Goon,University of Tennessee

${ }^{172} \mathrm{Ir} \quad$ 2004GOZZ RADIOACTIVITY ${ }^{168,169,170,171,172}$ Os, ${ }^{169,170,171,172,173,174,175} \mathrm{Ir}$, $170,171,172,173,174,175,176,177 \mathrm{Pt},{ }^{173,174,175,176,177} \mathrm{Au}$, 174,175,176,177,178 $\mathrm{Hg}(\alpha)$ [from ${ }^{92,94} \mathrm{Mo}\left({ }^{84} \mathrm{Sr}\right.$, xnyp) and subsequent decay]; measured $\mathrm{E} \alpha, \mathrm{E} \gamma, \alpha$ - $\gamma$-coin, $\mathrm{T}_{1 / 2} .{ }^{165} \mathrm{Ta},{ }^{165,167} \mathrm{~W}$, ${ }^{165,166,167,168,171} \mathrm{Re},{ }^{169,170,171} \mathrm{Os},{ }^{169,170,171,172,175} \mathrm{Ir},{ }^{173,175} \mathrm{Pt},{ }^{174,176} \mathrm{Au}$ deduced levels, J, $\pi$. THESIS J Goon,University of Tennessee ${ }^{172} \mathrm{Pt} \quad$ 2004GOZZ RADIOACTIVITY $168,169,170,171,172 \mathrm{Os}, 169,170,171,172,173,174,175 \mathrm{Ir}$, ${ }_{170,171,172,173,174,175,176,177} \mathrm{Pt},{ }^{173,174,175,176,177} \mathrm{Au}$, ${ }_{174,175,176,177,178} \mathrm{Hg}(\alpha)$ [from ${ }^{92,94} \mathrm{Mo}\left({ }^{84} \mathrm{Sr}\right.$, xnyp) and subsequent decay]; measured $\mathrm{E} \alpha, \mathrm{E} \gamma, \alpha-\gamma$-coin, $\mathrm{T}_{1 / 2} .{ }^{165} \mathrm{Ta},{ }^{165,167} \mathrm{~W}$, ${ }_{165,166,167,168,171} \mathrm{Re},{ }^{169,170,171} \mathrm{Os},{ }^{169,170,171,172,175} \mathrm{Ir},{ }^{173,175} \mathrm{Pt},{ }^{174,176} \mathrm{Au}$ deduced levels, J, $\pi$. THESIS J Goon,University of Tennessee 2005J018 NUCLEAR REACTIONS Sn $\left({ }^{58} \mathrm{Ni}, \mathrm{xn}\right){ }^{169} \mathrm{Pt} /{ }^{170} \mathrm{Pt} /{ }^{171} \mathrm{Pt} /{ }^{172} \mathrm{Pt} /$ ${ }^{173} \mathrm{Pt}, \mathrm{E}=266 \mathrm{MeV}$; measured $\mathrm{E} \gamma, \mathrm{I} \gamma, \gamma \gamma-$, (recoil) $\gamma$-coin. ${ }_{169,170,171,172,173} \mathrm{Pt}$ deduced levels, J, $\pi$. Recoil-decay tagging. JOUR JPGPE 31 S1715

\section{$\mathrm{A}=\mathbf{1 7 3}$}

${ }^{173} \mathrm{Yb}$ 2005TE04 NUCLEAR REACTIONS ${ }^{172,173} \mathrm{Yb}(\mathrm{n}, \gamma), \mathrm{E}=$ resonance; measured E $\gamma$, $\mathrm{I} \gamma$, capture yields. ${ }^{173} \mathrm{Yb}$ deduced resonance energies, J, $\pi .{ }^{173,174} \mathrm{Yb}$ deduced levels, J, $\pi$. JOUR NUPAB 76331

2005VE07 NUCLEAR REACTIONS ${ }^{170} \operatorname{Er}\left({ }^{7} \mathrm{Li}, 3 \mathrm{np}\right),\left({ }^{7} \mathrm{Li}, 4 \mathrm{np}\right),\left({ }^{7} \mathrm{Li}, 3 \mathrm{nd}\right),\left({ }^{7} \mathrm{Li}\right.$, 2nt), $\mathrm{E}=51 \mathrm{MeV}$; measured $\mathrm{E} \gamma, \mathrm{I} \gamma$, (charged particle) $\gamma$-coin. ${ }^{172,173} \mathrm{Yb}$ deduced high-spin levels, $\mathrm{J}, \pi$, configurations, absence of a static pair field. GASP, ISIS arrays. JOUR ZAANE 2619 


\section{$\mathrm{A}=173$ (continued)}

${ }^{173} \mathrm{Lu} \quad$ 2005KA52 NUCLEAR REACTIONS ${ }^{177} \mathrm{Hf}(\mathrm{n}, \gamma), \mathrm{E}=$ thermal, resonance; ${ }^{178} \mathrm{Hf}(\mathrm{n}$, n' $\gamma)$, E $>3 \mathrm{MeV}$; measured isomer production $\sigma$. Ta, W, ${ }^{186} \mathrm{~W}, \operatorname{Re}(\mathrm{p}$, $\mathrm{X}){ }^{179 m} \mathrm{Hf} /{ }^{178 m} \mathrm{Hf} /{ }^{177 m} \mathrm{Lu} /{ }^{178} \mathrm{~W} /{ }^{175} \mathrm{Hf} /{ }^{172} \mathrm{Hf} /{ }^{173} \mathrm{Lu}, \mathrm{E}=650$ $\mathrm{MeV}$; analyzed yields, isomer ratios. ${ }^{176} \mathrm{Yb}(\alpha, 2 \mathrm{n}), \mathrm{E}<36 \mathrm{MeV}$; measured isomer yield. Other reactions discussed. JOUR YAFIA 68 1827

2005TIZX NUCLEAR REACTIONS Pb, ${ }^{208} \mathrm{~Pb}(\mathrm{p}, \mathrm{X}){ }^{203} \mathrm{~Pb} /{ }^{200} \mathrm{Tl} /{ }^{199} \mathrm{Tl} /$ ${ }^{196} \mathrm{Au} /{ }^{192} \mathrm{Ir} /{ }^{190} \mathrm{Ir} /{ }^{173} \mathrm{Lu} /{ }^{101 m} \mathrm{Rh} /{ }^{86} \mathrm{Rb} /{ }^{59} \mathrm{Fe} /{ }^{24} \mathrm{Na} /{ }^{7} \mathrm{Be}$, $\mathrm{E}=40-2600 \mathrm{MeV}$; measured excitation functions. Comparison with previous work and model predictions. Other reactions discussed. CONF Santa Fe (Nucl Data for Sci and Technol) Proc,Vol1,P1070 2005TIZY NUCLEAR REACTIONS Pb, ${ }^{208} \mathrm{~Pb},{ }^{209} \mathrm{Bi}(\mathrm{p}, \mathrm{X}){ }^{203} \mathrm{~Pb} /{ }^{200} \mathrm{Tl} /{ }^{199} \mathrm{Tl}$ / ${ }^{196} \mathrm{Au} /{ }^{192} \mathrm{Ir} /{ }^{190} \mathrm{Ir} /{ }^{173} \mathrm{Lu} /{ }^{101 m} \mathrm{Rh} /{ }^{86} \mathrm{Rb} /{ }^{59} \mathrm{Fe} /{ }^{24} \mathrm{Na} /{ }^{7} \mathrm{Be}$, $\mathrm{E}=40-2600 \mathrm{MeV}$; measured production $\sigma$. Comparison with model predictions. PREPRINT nucl-ex/0507009,7/05/2005

${ }^{173} \mathrm{Hf}$ 2005HA05 NUCLEAR REACTIONS ${ }^{130} \mathrm{Te}\left({ }^{48} \mathrm{Ca}, 4 \mathrm{n}\right),\left({ }^{48} \mathrm{Ca}, 5 \mathrm{n}\right), \mathrm{E}=200,205$ $\mathrm{MeV}$; measured $\mathrm{E} \gamma, \mathrm{I} \gamma, \gamma \gamma$-coin, DSA. ${ }^{174} \mathrm{Hf}$ deduced superdeformed bands transitions, $\mathrm{T}_{1 / 2}$, quadrupole moments. ${ }^{173} \mathrm{Hf}$ deduced superdeformed band transitions. Gammasphere array, comparisons with model predictions. JOUR PYLBB 60831

2005HAZX NUCLEAR REACTIONS ${ }^{130} \mathrm{Te}\left({ }^{48} \mathrm{Ca}, 4 \mathrm{n}\right),\left({ }^{48} \mathrm{Ca}, 5 \mathrm{n}\right), \mathrm{E}=200,205$ $\mathrm{MeV}$; measured $\mathrm{E} \gamma, \mathrm{I} \gamma, \gamma \gamma$-coin, DSA. ${ }^{174} \mathrm{Hf}$ deduced superdeformed bands transitions, $\mathrm{T}_{1 / 2}$, quadrupole moments. ${ }^{173} \mathrm{Hf}$ deduced superdeformed band transitions. Gammasphere array, comparisons with model predictions. CONF Argonne(Nuclei at the Limits),P15,Hartley

${ }^{173} \mathrm{Os} \quad$ 2002DU22 RADIOACTIVITY $197,197 m, 198,199 m, 200,201 \mathrm{~m} \operatorname{Po}(\alpha) ;{ }^{172,173} \mathrm{Os}(\alpha)$ [from $\left.{ }^{156} \mathrm{Dy}\left({ }^{22} \mathrm{Ne}, \mathrm{xn}\right)\right] ;{ }^{183,184,185} \mathrm{Hg}(\alpha)$ [from $\left.{ }^{168} \mathrm{Yb}\left({ }^{22} \mathrm{Ne}, \mathrm{xn}\right)\right]$; measured $\mathrm{E} \alpha, \mathrm{T}_{1 / 2}$. JOUR NIMAE 479631

2002DU22 NUCLEAR REACTIONS ${ }^{156} \mathrm{Dy}\left({ }^{22} \mathrm{Ne}, 5 \mathrm{n}\right),\left({ }^{22} \mathrm{Ne}, 6 \mathrm{n}\right), \mathrm{E}=127 \mathrm{MeV}$; ${ }^{162} \operatorname{Er}\left({ }^{18} \mathrm{O}, 6 \mathrm{n}\right),\left({ }^{18} \mathrm{O}, 7 \mathrm{n}\right), \mathrm{E}=116 \mathrm{MeV}$; measured radiochemical yields. JOUR NIMAE 479631

2004GOZZ RADIOACTIVITY $168,169,170,171,172 \mathrm{Os},{ }^{169,170,171,172,173,174,175} \mathrm{Ir}$, $170,171,172,173,174,175,176,177 \mathrm{Pt},{ }^{173,174,175,176,177} \mathrm{Au}$, $174,175,176,177,178 \mathrm{Hg}(\alpha)$ [from ${ }^{92,94} \mathrm{Mo}\left({ }^{84} \mathrm{Sr}\right.$, xnyp) and subsequent decay]; measured $\mathrm{E} \alpha, \mathrm{E} \gamma, \alpha$ - $\gamma$-coin, $\mathrm{T}_{1 / 2} .{ }^{165} \mathrm{Ta},{ }^{165,167} \mathrm{~W}$, ${ }_{165,166,167,168,171} \mathrm{Re},{ }^{169,170,171} \mathrm{Os},{ }^{169,170,171,172,175} \mathrm{Ir},{ }^{173,175} \mathrm{Pt},{ }^{174,176} \mathrm{Au}$ deduced levels, J, $\pi$. THESIS J Goon,University of Tennessee

${ }^{173} \mathrm{Ir}$ 2004GOZZ RADIOACTIVITY $168,169,170,171,172$ Os, ${ }^{169,170,171,172,173,174,175} \mathrm{Ir}$, ${ }_{170,171,172,173,174,175,176,177} \mathrm{Pt},{ }^{173,174,175,176,177} \mathrm{Au}$, ${ }_{174,175,176,177,178} \mathrm{Hg}(\alpha)$ [from ${ }^{92,94} \mathrm{Mo}\left({ }^{84} \mathrm{Sr}\right.$, xnyp) and subsequent decay]; measured $\mathrm{E} \alpha, \mathrm{E} \gamma, \alpha$ - $\gamma$-coin, $\mathrm{T}_{1 / 2} .{ }^{165} \mathrm{Ta},{ }^{165,167} \mathrm{~W}$, ${ }_{165,166,167,168,171} \mathrm{Re},{ }^{169,170,171} \mathrm{Os},{ }^{169,170,171,172,175} \mathrm{Ir},{ }^{173,175} \mathrm{Pt},{ }^{174,176} \mathrm{Au}$ deduced levels, J, $\pi$. THESIS J Goon,University of Tennessee 2005CA43 NUCLEAR REACTIONS ${ }^{92} \mathrm{Mo}\left({ }^{84} \mathrm{Sr}, \mathrm{n} 2 \mathrm{p}\right),\left({ }^{84} \mathrm{Sr}, 3 \mathrm{p}\right),\left({ }^{84} \mathrm{Sr}, 2 \mathrm{np}\right)$, ${ }^{104} \mathrm{Ru}\left({ }^{84} \mathrm{Kr}, 2 \mathrm{np}\right),{ }^{90} \mathrm{Zr}\left({ }^{90} \mathrm{Zr}, \mathrm{n}\right),\left({ }^{90} \mathrm{Zr}, \mathrm{p}\right)$, E not given; ${ }^{92} \mathrm{Mo}\left({ }^{90} \mathrm{Zr}, \mathrm{n}\right)$, $\left({ }^{90} \mathrm{Zr}, \mathrm{p}\right), \mathrm{E}=385 \mathrm{MeV}$; measured $\mathrm{E} \gamma, \mathrm{I} \gamma, \gamma \gamma-$, (recoil) $\gamma$-coin. ${ }^{179} \mathrm{Hg}$ deduced high-spin levels, J, $\pi$. Gammasphere array, fragment separator. JOUR JPGPE 31 S1599 


\section{$\mathrm{A}=173$ (continued)}

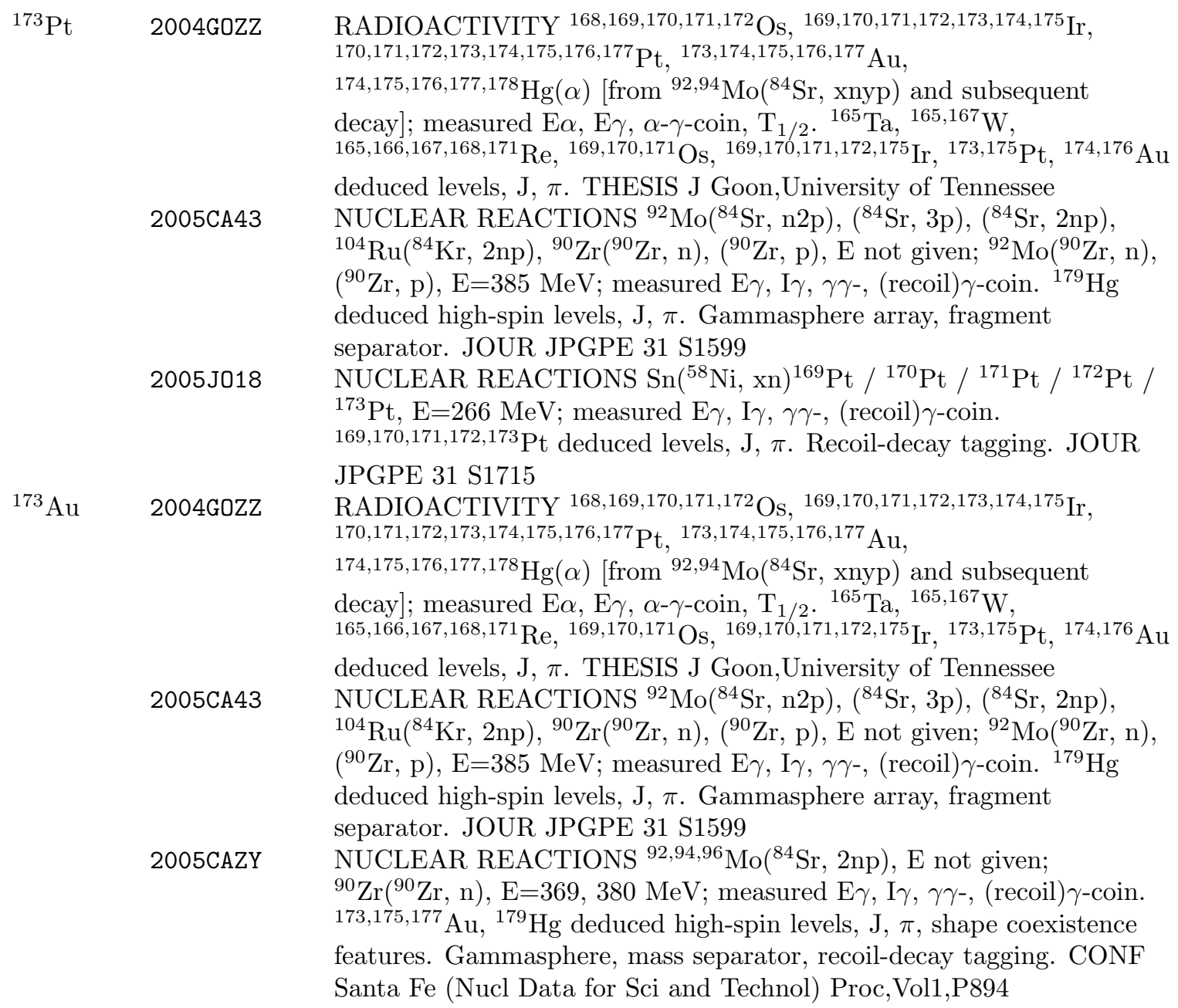

\section{$\mathrm{A}=174$}

${ }^{174} \mathrm{Er} \quad$ 2005CA02 RADIOACTIVITY ${ }^{188} \mathrm{Ta},{ }^{190} \mathrm{~W},{ }^{192,193} \mathrm{Re},{ }^{195} \mathrm{Os},{ }^{197,198} \mathrm{Ir}$, ${ }^{200,201,202} \mathrm{Pt},{ }^{203} \mathrm{Au}(\mathrm{IT})$ [from $\mathrm{Be}\left({ }^{208} \mathrm{~Pb}, \mathrm{X}\right)$ ]; measured $\mathrm{E} \gamma, \mathrm{I} \gamma, \gamma \gamma$-coin, $\mathrm{T}_{1 / 2} \cdot{ }^{188} \mathrm{Ta},{ }^{190} \mathrm{~W},{ }^{192,193} \mathrm{Re},{ }^{195} \mathrm{Os},{ }^{197,198} \mathrm{Ir},{ }^{200,201,202} \mathrm{Pt},{ }^{203} \mathrm{Au}$ deduced transitions. ${ }^{190} \mathrm{~W},{ }^{200,201,202} \mathrm{Pt}$ deduced levels, J, $\pi .{ }^{174,175} \mathrm{Er}$, ${ }^{185} \mathrm{Hf},{ }^{191,194} \mathrm{Re},{ }^{199} \operatorname{Ir}(\mathrm{IT})$ [from $\mathrm{Be}\left({ }^{208} \mathrm{~Pb}, \mathrm{X}\right)$ ]; measured $\mathrm{E} \gamma, \mathrm{I} \gamma$. JOUR ZAANE 23201

${ }^{174} \mathrm{Tm} \quad 2005 \mathrm{CH} 67 \quad$ RADIOACTIVITY ${ }^{174} \mathrm{Tm}(\mathrm{IT})$ [from Ta(p, X), E=500 MeV]; measured $\mathrm{E} \gamma, \mathrm{I} \gamma, \gamma \gamma$-coin following decay of mass-separated sources; deduced $\mathrm{T}_{1 / 2}$. Discussed K-hindrance and Nilsson configuration of new level. JOUR ZAANE 25 s01 125 


\section{$\mathrm{A}=174$ (continued)}

${ }^{174} \mathrm{Yb}$ 2005DR05 NUCLEAR REACTIONS ${ }^{175,176} \mathrm{Lu},{ }^{174} \mathrm{Yb}\left({ }^{136} \mathrm{Xe}, \mathrm{X}\right){ }^{174} \mathrm{Yb}, \mathrm{E}=6 \mathrm{MeV}$ / nucleon; ${ }^{173} \mathrm{Yb}\left({ }^{18} \mathrm{O},{ }^{17} \mathrm{O}\right)$, E not given; measured prompt and delayed $\mathrm{E} \gamma, \mathrm{I} \gamma, \gamma \gamma$-coin. ${ }^{174} \mathrm{Yb}$ deduced high-spin levels, $\mathrm{J}, \pi, \delta$, isomers $\mathrm{T}_{1 / 2}$, configurations. Gammasphere, Caesar arrays. JOUR PRVCA 71 044326

2005DRZY NUCLEAR REACTIONS ${ }^{175,176} \mathrm{Lu}\left({ }^{136} \mathrm{Xe}, \mathrm{X}\right){ }^{174} \mathrm{Yb}, \mathrm{E}=6 \mathrm{MeV} /$ nucleon; ${ }^{174} \mathrm{Yb}\left({ }^{136} \mathrm{Xe},{ }^{136} \mathrm{Xe}\right), \mathrm{E}=6 \mathrm{MeV} /$ nucleon; ${ }^{173} \mathrm{Yb}\left({ }^{18} \mathrm{O},{ }^{17} \mathrm{O}\right)$, $\mathrm{E}$ not given; measured prompt and delayed $\mathrm{E} \gamma, \mathrm{I} \gamma, \gamma \gamma$-coin. ${ }^{174} \mathrm{Yb}$ deduced high-spin levels, $\mathrm{J}, \pi$, configurations, isomeric states $\mathrm{T}_{1 / 2}$, transition strengths. Gammasphere array. PREPRINT ANU-P/1648,Dracoulis

2005GR22 NUCLEAR REACTIONS ${ }^{173} \mathrm{Yb}(\mathrm{n}, \gamma)$, E=resonance; measured E $\gamma$, I $\gamma$. ${ }^{174} \mathrm{Yb}$ deduced levels, $\mathrm{J}, \pi$, transition intensities and multipolarities, resonance features. Radiative capture, average resonance capture, neutron filtered beams, pair spectrometer. JOUR NUPAB 757287 2005SA15 NUCLEAR REACTIONS ${ }^{172,174} \mathrm{Yb}$ (polarized $\left.\gamma, \gamma^{\prime}\right), \mathrm{E}=2930,3005$, $3550 \mathrm{keV}$; measured $\mathrm{E} \gamma, \mathrm{I} \gamma$, asymmetries. ${ }^{172,174} \mathrm{Yb}$ levels deduced $\pi$. Parity and branching ratio systematics discussed. JOUR PRVCA 71 034304

2005SAZZ NUCLEAR REACTIONS ${ }^{172,174} \mathrm{Yb}$ (polarized $\gamma, \gamma^{\prime}$ ), $\mathrm{E}=2930,3005$, $3550 \mathrm{keV}$; measured $\mathrm{E} \gamma, \mathrm{I} \gamma$, asymmetries. ${ }^{172,174} \mathrm{Yb}$ levels deduced $\pi$. Comparison with previous results. PREPRINT nucl-ex/0501006,1/11/2005

$2005 T E 04 \quad$ NUCLEAR REACTIONS ${ }^{172,173} \mathrm{Yb}(\mathrm{n}, \gamma), \mathrm{E}=$ resonance; measured E $\gamma$, $\mathrm{I} \gamma$, capture yields. ${ }^{173} \mathrm{Yb}$ deduced resonance energies, $\mathrm{J}, \pi .{ }^{173,174} \mathrm{Yb}$ deduced levels, J, $\pi$. JOUR NUPAB 76331

${ }^{174} \mathrm{Hf}$ 2005HA05 NUCLEAR REACTIONS ${ }^{130} \mathrm{Te}\left({ }^{48} \mathrm{Ca}, 4 \mathrm{n}\right),\left({ }^{48} \mathrm{Ca}, 5 \mathrm{n}\right), \mathrm{E}=200,205$ $\mathrm{MeV}$; measured $\mathrm{E} \gamma$, I $\gamma, \gamma \gamma$-coin, DSA. ${ }^{174} \mathrm{Hf}$ deduced superdeformed bands transitions, $\mathrm{T}_{1 / 2}$, quadrupole moments. ${ }^{173} \mathrm{Hf}$ deduced superdeformed band transitions. Gammasphere array, comparisons with model predictions. JOUR PYLBB 60831

2005HAZX NUCLEAR REACTIONS ${ }^{130} \mathrm{Te}\left({ }^{48} \mathrm{Ca}, 4 \mathrm{n}\right),\left({ }^{48} \mathrm{Ca}, 5 \mathrm{n}\right), \mathrm{E}=200,205$ $\mathrm{MeV}$; measured $\mathrm{E} \gamma, \mathrm{I} \gamma, \gamma \gamma$-coin, DSA. ${ }^{174} \mathrm{Hf}$ deduced superdeformed bands transitions, $\mathrm{T}_{1 / 2}$, quadrupole moments. ${ }^{173} \mathrm{Hf}$ deduced superdeformed band transitions. Gammasphere array, comparisons with model predictions. CONF Argonne(Nuclei at the Limits),P15,Hartley

2005ME01 NUCLEAR REACTIONS Hf(n, X), E=0.1-100 eV; measured total neutron $\sigma .{ }^{174,176,177,178,179,180} \mathrm{Hf}$ deduced resonance parameters. Comparison with previous results. JOUR KPSJA 46401

2005TRZY NUCLEAR REACTIONS Hf(n, n), (n, $\gamma), \mathrm{E} \approx 0.005-200 \mathrm{eV}$; measured transmission and capture $\sigma .{ }^{174,176,177,178,179,180} \mathrm{Hf}$ deduced resonance parameters, capture resonance integrals. CONF Santa Fe (Nucl Data for Sci and Technol) Proc,Vol1,P949

${ }^{174} \mathrm{Re} \quad 2005 \mathrm{ZH} 32 \quad$ NUCLEAR REACTIONS ${ }^{152} \mathrm{Sm}\left({ }^{27} \mathrm{Al}, 5 \mathrm{n}\right), \mathrm{E}=125,132,140 \mathrm{MeV}$; measured $\mathrm{E} \gamma, \mathrm{I} \gamma, \gamma \gamma$-coin; deduced excitation functions. ${ }^{174} \mathrm{Re}$ deduced high-spin levels, J, $\pi$, configurations, signature inversion. Level systematics in neighboring nuclides discussed. JOUR CPLEE 222788 


\section{$\mathrm{A}=174$ (continued)}

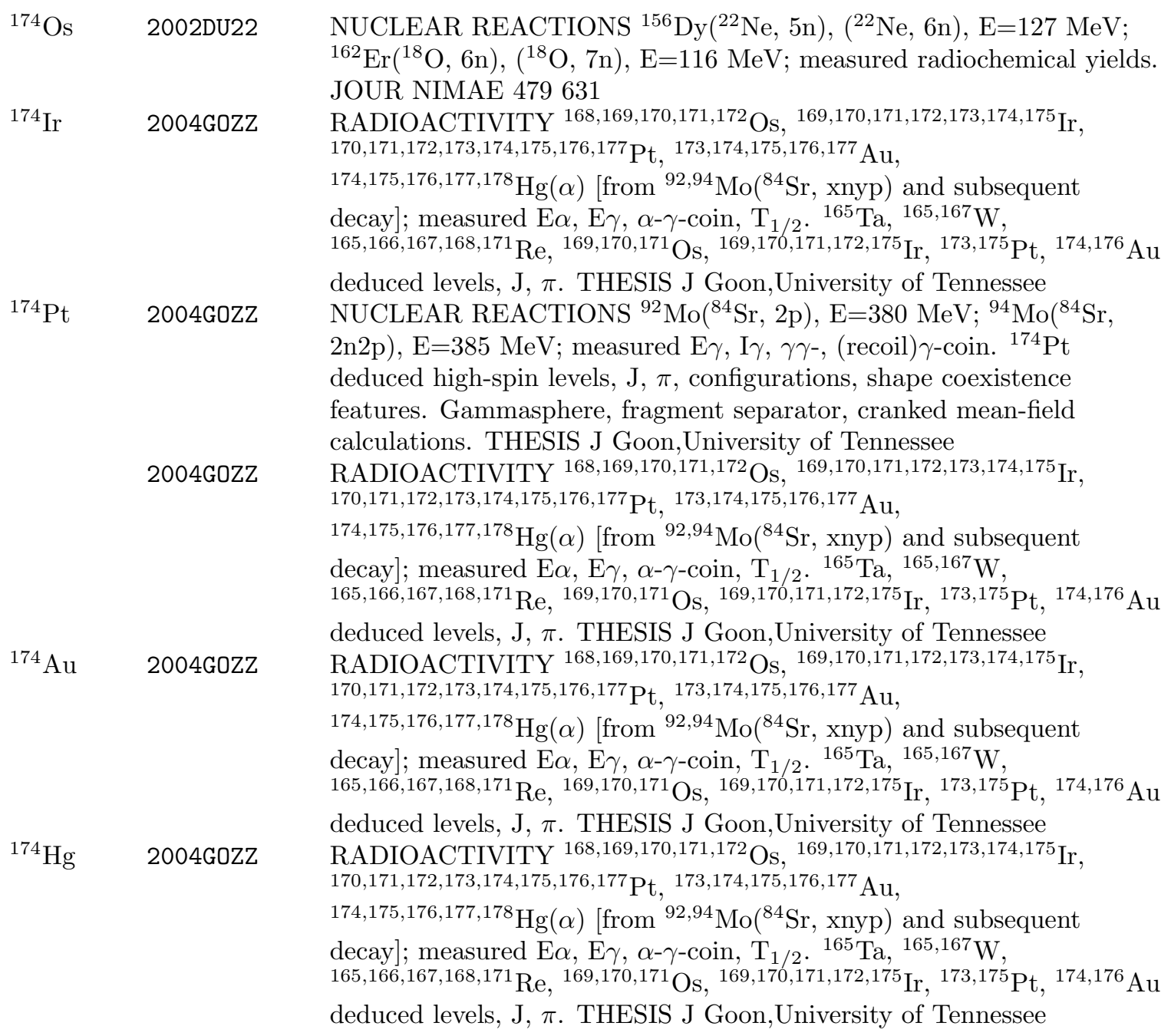

\section{$\mathrm{A}=\mathbf{1 7 5}$}

${ }^{175} \mathrm{Er} \quad$ 2005CA02 RADIOACTIVITY ${ }^{188} \mathrm{Ta},{ }^{190} \mathrm{~W},{ }^{192,193} \mathrm{Re},{ }^{195} \mathrm{Os},{ }^{197,198} \mathrm{Ir}$, ${ }^{200,201,202} \mathrm{Pt},{ }^{203} \mathrm{Au}(\mathrm{IT})$ [from $\mathrm{Be}\left({ }^{208} \mathrm{~Pb}, \mathrm{X}\right)$ ]; measured $\mathrm{E} \gamma, \mathrm{I} \gamma, \gamma \gamma$-coin, $\mathrm{T}_{1 / 2} \cdot{ }^{188} \mathrm{Ta},{ }^{190} \mathrm{~W},{ }^{192,193} \mathrm{Re},{ }^{195} \mathrm{Os},{ }^{197,198} \mathrm{Ir},{ }^{200,201,202} \mathrm{Pt},{ }^{203} \mathrm{Au}$ deduced transitions. ${ }^{190} \mathrm{~W},{ }^{200,201,202} \mathrm{Pt}$ deduced levels, J, $\pi .{ }^{174,175} \mathrm{Er}$, ${ }^{185} \mathrm{Hf},{ }^{191,194} \mathrm{Re},{ }^{199} \operatorname{Ir}(\mathrm{IT})$ [from $\left.\mathrm{Be}\left({ }^{208} \mathrm{~Pb}, \mathrm{X}\right)\right]$; measured $\mathrm{E} \gamma, \mathrm{I} \gamma$.

JOUR ZAANE 23201

${ }^{175} \mathrm{Yb}$ 2005NC01 NUCLEAR REACTIONS ${ }^{176} \mathrm{Yb}\left({ }^{136} \mathrm{Xe}, \mathrm{X}\right){ }^{175} \mathrm{Yb} /{ }^{176} \mathrm{Yb} /{ }^{177} \mathrm{Yb}$, $\mathrm{E}=750 \mathrm{MeV}$; measured $\mathrm{E} \gamma, \mathrm{I} \gamma, \gamma \gamma$-coin. ${ }^{175,176,177} \mathrm{Yb}$ deduced high-spin levels, J, $\pi$, configurations, gK-gR. Afrodite array. JOUR ZAANE 26265 


\section{$\mathrm{A}=175$ (continued)}

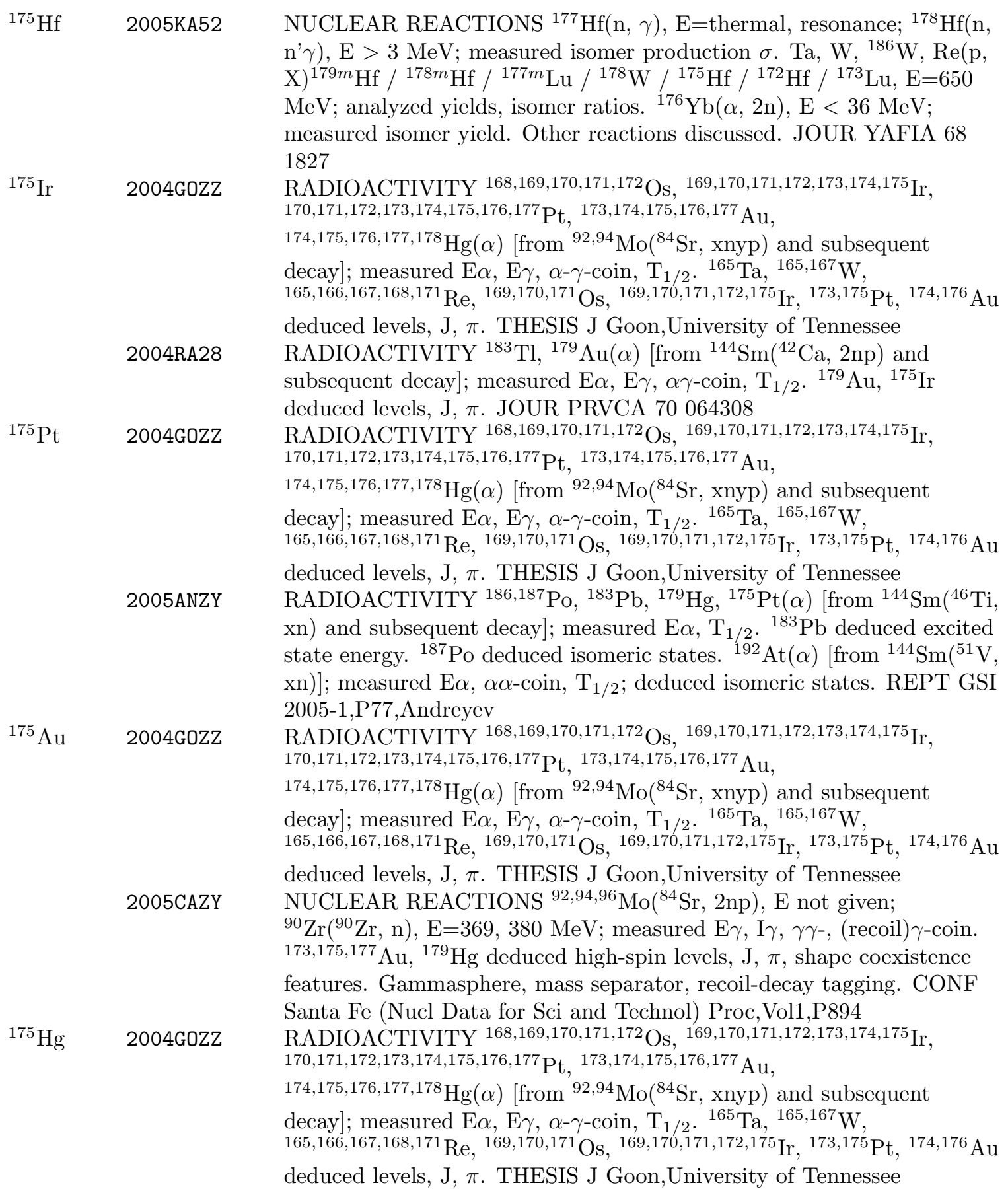

\section{$\mathrm{A}=176$}

${ }^{176} \mathrm{Yb}$ 2005AM04 RADIOACTIVITY ${ }^{176} \mathrm{Lu}\left(\beta^{-}\right),\left(\beta^{+}\right)$; measured isotope ratios; deduced decay branch upper limit. JOUR GCACA 69465 


\section{$\mathrm{A}=176$ (continued)}

2005BI25 NUCLEAR MOMENTS 86,87,88,89,90,91,92,93,94,95,96,97,98,99,100,101,102 Zr; measured charge radii. ${ }^{176} \mathrm{Yb}$; measured isomer shift. Ion-beam cooler, laser spectroscopy. JOUR ZAANE 25 s01 187

2005NC01 NUCLEAR REACTIONS ${ }^{176} \mathrm{Yb}\left({ }^{136} \mathrm{Xe}, \mathrm{X}\right){ }^{175} \mathrm{Yb} /{ }^{176} \mathrm{Yb} /{ }^{177} \mathrm{Yb}$, $\mathrm{E}=750 \mathrm{MeV}$; measured $\mathrm{E} \gamma, \mathrm{I} \gamma, \gamma \gamma$-coin. ${ }^{175,176,177} \mathrm{Yb}$ deduced high-spin levels, J, $\pi$, configurations, gK-gR. Afrodite array. JOUR ZAANE 26265

${ }^{176} \mathrm{Lu} \quad$ 2005AM04

2005HE19

${ }^{176} \mathrm{Hf} \quad 2004 \mathrm{CO} 26$

2005AM04

2005ME01

2005ME19

${ }^{176} \mathrm{Os} \quad 2005 \mathrm{DE} 48$

${ }^{176} \operatorname{Ir} \quad$ 2005WA25
RADIOACTIVITY ${ }^{176} \mathrm{Lu}\left(\beta^{-}\right),\left(\beta^{+}\right)$; measured isotope ratios; deduced decay branch upper limit. JOUR GCACA 69465

NUCLEAR REACTIONS ${ }^{18} \mathrm{O}(\mathrm{p}, \mathrm{n}), \mathrm{E}=2582 \mathrm{keV}$; measured neutron spectra. ${ }^{138} \mathrm{Ba},{ }^{139} \mathrm{La},{ }^{175} \mathrm{Lu}(\mathrm{n}, \gamma), \mathrm{E}=$ spectrum; measured $\sigma$. JOUR NUPAB $758529 \mathrm{c}$

RADIOACTIVITY ${ }^{180} \mathrm{~W}(\alpha)$; measured $\mathrm{E} \alpha, \mathrm{T}_{1 / 2}$, Q-value.

${ }_{182,183,184,186} \mathrm{~W}(\alpha)$; measured $\mathrm{T}_{1 / 2}$ lower limits. $\mathrm{CaWO}_{4}$ crystals. JOUR PRVCA 70064606

RADIOACTIVITY ${ }^{176} \mathrm{Lu}\left(\beta^{-}\right),\left(\beta^{+}\right)$; measured isotope ratios; deduced decay branch upper limit. JOUR GCACA 69465

NUCLEAR REACTIONS Hf(n, X), E=0.1-100 eV; measured total neutron $\sigma .{ }^{174,176,177,178,179,180} \mathrm{Hf}$ deduced resonance parameters.

Comparison with previous results. JOUR KPSJA 46401

NUCLEAR REACTIONS ${ }^{160} \mathrm{Gd},{ }^{164} \mathrm{Dy},{ }^{170} \mathrm{Er},{ }^{178} \mathrm{Hf},{ }^{186} \mathrm{~W},{ }^{192} \mathrm{Os}(\mathrm{p}$, $\mathrm{t}), \mathrm{E}=25 \mathrm{MeV}$; measured triton spectra, $\sigma(\theta) .{ }^{158} \mathrm{Gd},{ }^{162} \mathrm{Dy},{ }^{168} \mathrm{Er}$, ${ }^{176} \mathrm{Hf},{ }^{184} \mathrm{~W},{ }^{190} \mathrm{Os}$ deduced $0^{+}$level energies. JOUR JPGPE $31 \mathrm{~S} 1399$ NUCLEAR REACTIONS Hf(n, n), (n, $\gamma), \mathrm{E} \approx 0.005-200 \mathrm{eV}$; measured transmission and capture $\sigma .{ }^{174,176,177,178,179,180} \mathrm{Hf}$ deduced resonance parameters, capture resonance integrals. CONF Santa Fe (Nucl Data for Sci and Technol) Proc,Vol1,P949

NUCLEAR REACTIONS ${ }^{164,166,168} \operatorname{Er}\left({ }^{16} \mathrm{O}, 4 \mathrm{n}\right), \mathrm{E}=80 \mathrm{MeV}$; measured prompt and delayed $\mathrm{E} \gamma, \mathrm{I} \gamma .{ }^{154} \mathrm{Sm}\left({ }^{29} \mathrm{Si}, 5 \mathrm{n}\right), \mathrm{E}=158 \mathrm{MeV}$; measured Doppler-shifted E $\gamma$, I $\gamma, \gamma \gamma$-coin. ${ }^{176,178,180}$ Os deduced levels $\mathrm{T}_{1 / 2}$, transition quadrupole moments, symmetry features. Electronic timing and recoil distance techniques, GASP array, interacting boson model and general collective model predictions. JOUR JPGPE 31 S1427 NUCLEAR REACTIONS ${ }^{164,166,168} \operatorname{Er}\left({ }^{16} \mathrm{O}, 4 \mathrm{n}\right), \mathrm{E}=80 \mathrm{MeV}$; measured prompt and delayed $\mathrm{E} \gamma$, I $\gamma, \gamma \gamma$-coin. ${ }^{166} \operatorname{Er}\left({ }^{16} \mathrm{O}, 4 \mathrm{n}\right), \mathrm{E}=80 \mathrm{MeV}$; measured Doppler-shifted E $\gamma$, I $\gamma, \gamma \gamma$-coin. ${ }^{176,178,180}$ Os deduced levels, $\mathrm{J}, \pi, \mathrm{T}_{1 / 2}, \mathrm{~B}(\mathrm{E} 2)$. Pulsed-beam and recoil-distance techniques. JOUR PRVCA 72034306

2005WA25 RADIOACTIVITY ${ }^{176,176 m} \operatorname{Ir}\left(\beta^{+}\right)$, (EC) [from ${ }^{146} \mathrm{Nd}\left({ }^{35} \mathrm{Cl}, 5 \mathrm{n}\right)$ ]; measured $\mathrm{E} \gamma, \mathrm{I} \gamma, \gamma \gamma$-coin, $\mathrm{T}_{1 / 2} .{ }^{176}$ Os deduced levels, $\mathrm{J}, \pi .{ }^{176} \mathrm{Ir}$ deduced low-spin isomeric state. JOUR CPLEE 222211 RADIOACTIVITY ${ }^{176,176 m} \operatorname{Ir}\left(\beta^{+}\right)$, (EC) [from ${ }^{146} \mathrm{Nd}\left({ }^{35} \mathrm{Cl}, 5 \mathrm{n}\right)$ ]; measured $\mathrm{E} \gamma, \mathrm{I} \gamma, \gamma \gamma$-coin, $\mathrm{T}_{1 / 2} .{ }^{176}$ Os deduced levels, J, $\pi .{ }^{176} \mathrm{Ir}$ deduced low-spin isomeric state. JOUR CPLEE 222211 


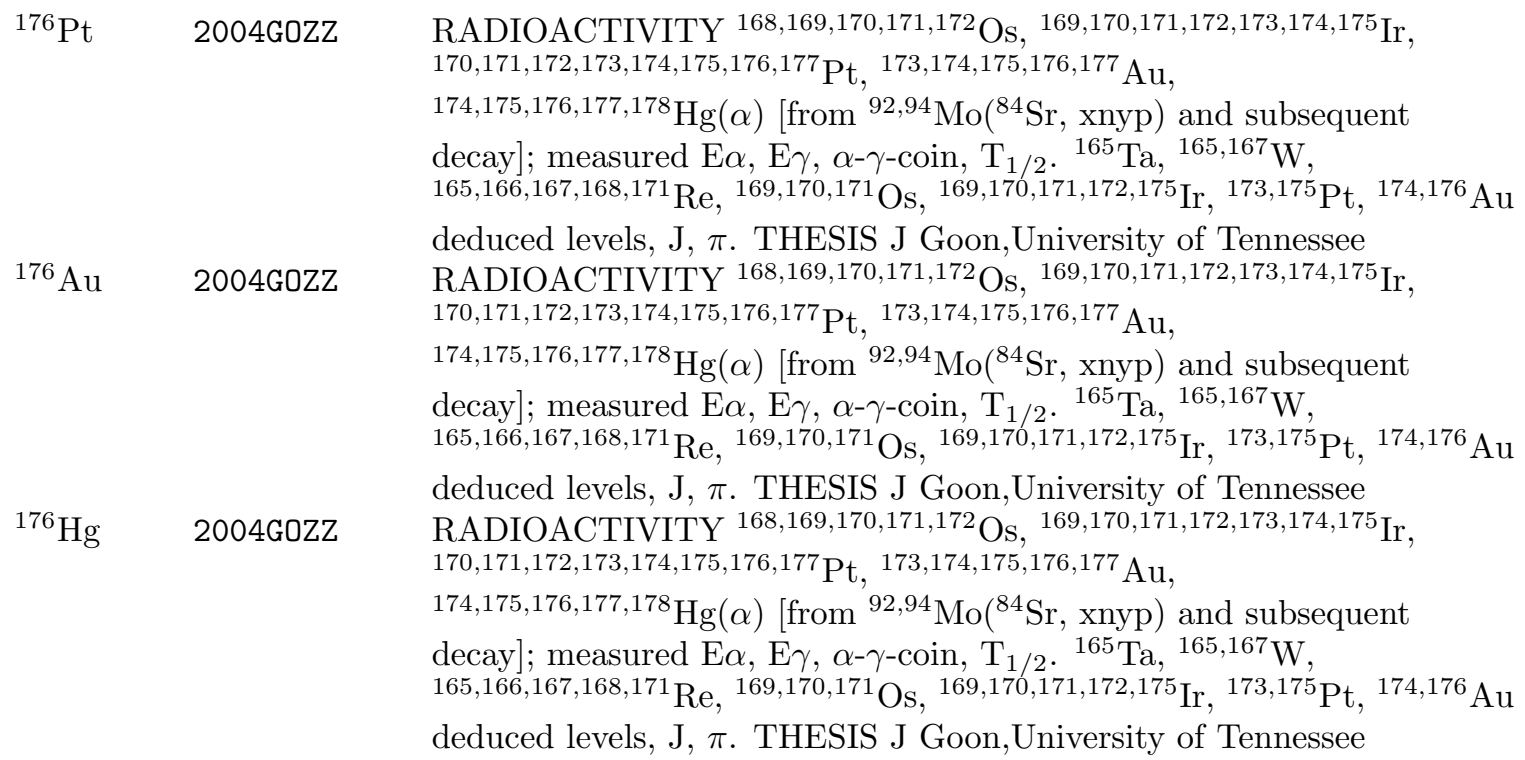

\section{$\mathrm{A}=\mathbf{1 7 7}$}

\section{${ }^{177} \mathrm{Yb} \quad 2005 \mathrm{NC0}$}

${ }^{177} \mathrm{Lu}$

2005KA52

${ }^{177} \mathrm{Hf} \quad 2005 \mathrm{ME01}$

2005TRZY

2005WIZZ

${ }^{177} \mathrm{Pt} \quad 2004 \mathrm{GOZZ}$

NUCLEAR REACTIONS ${ }^{176} \mathrm{Yb}\left({ }^{136} \mathrm{Xe}, \mathrm{X}\right){ }^{175} \mathrm{Yb} /{ }^{176} \mathrm{Yb} /{ }^{177} \mathrm{Yb}$, $\mathrm{E}=750 \mathrm{MeV}$; measured $\mathrm{E} \gamma, \mathrm{I} \gamma, \gamma \gamma$-coin. ${ }^{175,176,177} \mathrm{Yb}$ deduced high-spin levels, J, $\pi$, configurations, gK-gR. Afrodite array. JOUR ZAANE 26265 NUCLEAR REACTIONS ${ }^{177} \mathrm{Hf}(\mathrm{n}, \gamma), \mathrm{E}=$ thermal, resonance; ${ }^{178} \mathrm{Hf}(\mathrm{n}$, $\mathrm{n} \gamma), \mathrm{E}>3 \mathrm{MeV}$; measured isomer production $\sigma$. Ta, W, ${ }^{186} \mathrm{~W}, \operatorname{Re}(\mathrm{p}$, $\mathrm{X})^{179 m} \mathrm{Hf} /{ }^{178 m} \mathrm{Hf} /{ }^{177 m} \mathrm{Lu} /{ }^{178} \mathrm{~W} /{ }^{175} \mathrm{Hf} /{ }^{172} \mathrm{Hf} /{ }^{173} \mathrm{Lu}, \mathrm{E}=650$ $\mathrm{MeV}$; analyzed yields, isomer ratios. ${ }^{176} \mathrm{Yb}(\alpha, 2 \mathrm{n}), \mathrm{E}<36 \mathrm{MeV}$; measured isomer yield. Other reactions discussed. JOUR YAFIA 68 1827 NUCLEAR REACTIONS Hf(n, X), E=0.1-100 eV; measured total neutron $\sigma .{ }^{174,176,177,178,179,180} \mathrm{Hf}$ deduced resonance parameters. Comparison with previous results. JOUR KPSJA 46401

NUCLEAR REACTIONS Hf(n, n), $(\mathrm{n}, \gamma), \mathrm{E} \approx 0.005-200 \mathrm{eV}$; measured transmission and capture $\sigma .{ }^{174,176,177,178,179,180} \mathrm{Hf}$ deduced resonance parameters, capture resonance integrals. CONF Santa Fe (Nucl Data for Sci and Technol) Proc,Vol1,P949 NUCLEAR REACTIONS ${ }^{175,176} \mathrm{Lu},{ }^{176,177,178,179,180} \mathrm{Hf}(\mathrm{n}, \gamma), \mathrm{E}=3-225$ $\mathrm{keV}$; measured capture $\sigma$; deduced Maxwellian averaged $\sigma$.

Comparison with previous results, astrophysical implications discussed. CONF Santa Fe (Nucl Data for Sci and Technol) Proc,Vol2,P1315 RADIOACTIVITY ${ }^{168,169,170,171,172}$ Os, ${ }^{169,170,171,172,173,174,175} \mathrm{Ir}$, $170,171,172,173,174,175,176,177 \mathrm{Pt},{ }^{173,174,175,176,177} \mathrm{Au}$, $174,175,176,177,178 \mathrm{Hg}(\alpha)$ [from ${ }^{92,94} \mathrm{Mo}\left({ }^{84} \mathrm{Sr}\right.$, xnyp) and subsequent decay]; measured $\mathrm{E} \alpha, \mathrm{E} \gamma, \alpha-\gamma$-coin, $\mathrm{T}_{1 / 2} \cdot{ }^{165} \mathrm{Ta},{ }^{165,167} \mathrm{~W}$, ${ }_{165,166,167,168,171} \operatorname{Re},{ }^{169,170,171}$ Os, ${ }^{169,170,171,172,175} \mathrm{Ir},{ }^{173,175} \mathrm{Pt},{ }^{174,176} \mathrm{Au}$ deduced levels, J, $\pi$. THESIS J Goon,University of Tennessee 


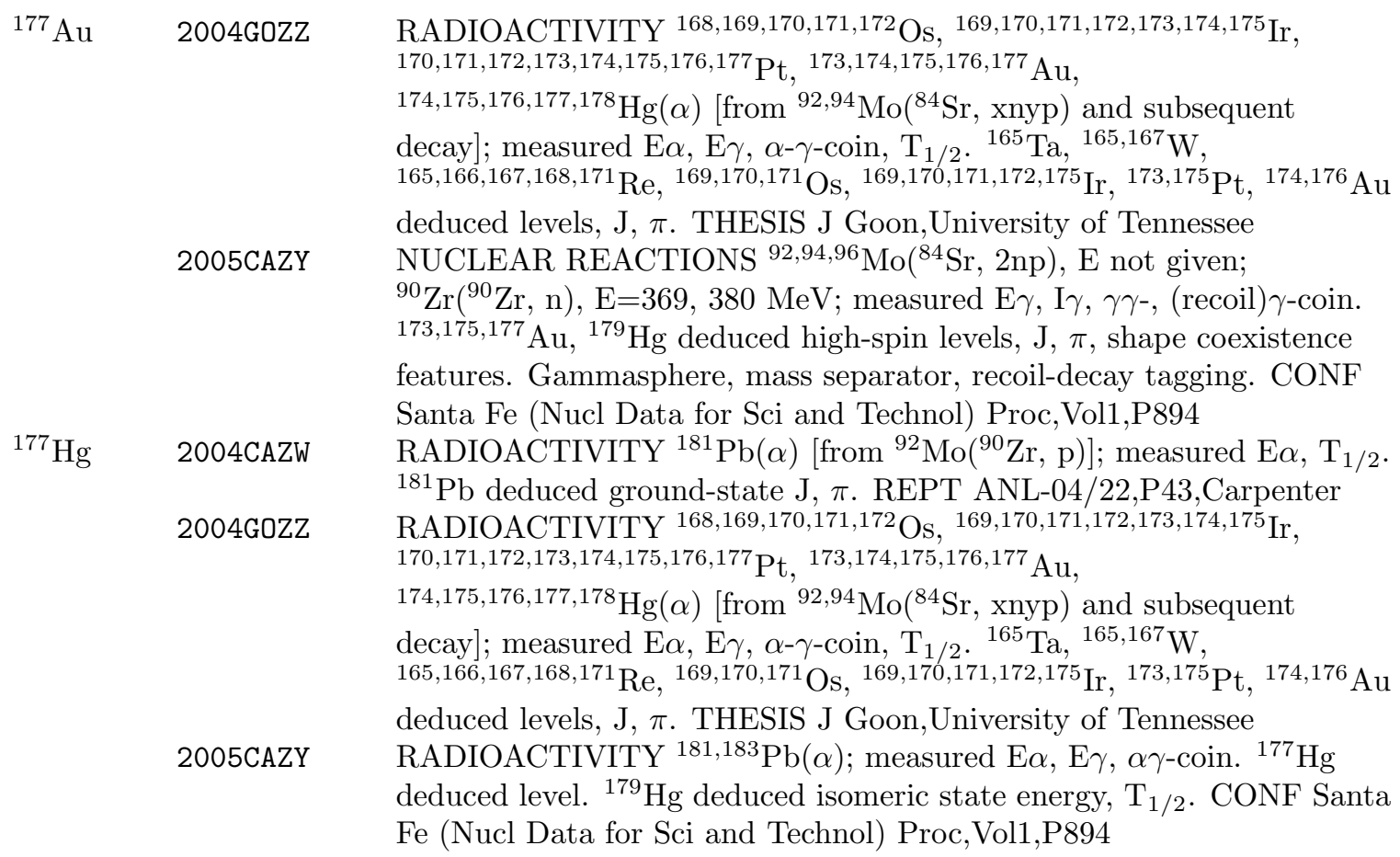

\section{$\mathrm{A}=\mathbf{1 7 8}$}

${ }^{178} \mathrm{Hf} \quad 2004 \mathrm{CO} 26$

2005KA52

2005ME01

2005TRZY

2005WIZZ
RADIOACTIVITY ${ }^{180} \mathrm{~W}(\alpha)$; measured $\mathrm{E} \alpha, \mathrm{T}_{1 / 2}$, Q-value. ${ }_{182,183,184,186} \mathrm{~W}(\alpha)$; measured $\mathrm{T}_{1 / 2}$ lower limits. $\mathrm{CaWO}_{4}$ crystals. JOUR PRVCA 70064606 NUCLEAR REACTIONS ${ }^{177} \mathrm{Hf}(\mathrm{n}, \gamma), \mathrm{E}=$ thermal, resonance; ${ }^{178} \mathrm{Hf}(\mathrm{n}$, n' $\gamma), \mathrm{E}>3 \mathrm{MeV}$; measured isomer production $\sigma$. Ta, W, ${ }^{186} \mathrm{~W}, \operatorname{Re}(\mathrm{p}$, $\mathrm{X}){ }^{179 m} \mathrm{Hf} /{ }^{178 m} \mathrm{Hf} /{ }^{177 m} \mathrm{Lu} /{ }^{178} \mathrm{~W} /{ }^{175} \mathrm{Hf} /{ }^{172} \mathrm{Hf} /{ }^{173} \mathrm{Lu}, \mathrm{E}=650$ $\mathrm{MeV}$; analyzed yields, isomer ratios. ${ }^{176} \mathrm{Yb}(\alpha, 2 \mathrm{n}), \mathrm{E}<36 \mathrm{MeV}$; measured isomer yield. Other reactions discussed. JOUR YAFIA 68 1827 NUCLEAR REACTIONS Hf(n, X), E=0.1-100 eV; measured total neutron $\sigma .{ }^{174,176,177,178,179,180} \mathrm{Hf}$ deduced resonance parameters. Comparison with previous results. JOUR KPSJA 46401 NUCLEAR REACTIONS Hf(n, n), (n, $\gamma), \mathrm{E} \approx 0.005-200 \mathrm{eV}$; measured transmission and capture $\sigma .{ }^{174,176,177,178,179,180} \mathrm{Hf}$ deduced resonance parameters, capture resonance integrals. CONF Santa Fe (Nucl Data for Sci and Technol) Proc,Vol1,P949 NUCLEAR REACTIONS ${ }^{175,176} \mathrm{Lu},{ }^{176,177,178,179,180} \mathrm{Hf}(\mathrm{n}, \gamma), \mathrm{E}=3-225$ $\mathrm{keV}$; measured capture $\sigma$; deduced Maxwellian averaged $\sigma$. Comparison with previous results, astrophysical implications discussed. CONF Santa Fe (Nucl Data for Sci and Technol) Proc,Vol2,P1315 


\section{$\mathrm{A}=178$ (continued)}

\begin{tabular}{|c|c|c|}
\hline${ }^{178} \mathrm{~W}$ & 2005KA52 & $\begin{array}{l}\text { NUCLEAR REACTIONS }{ }^{177} \mathrm{Hf}(\mathrm{n}, \gamma), \mathrm{E}=\text { thermal, resonance, }{ }^{178} \mathrm{Hf}(\mathrm{n} \text {, } \\
\mathrm{n} \gamma), \mathrm{E}>3 \mathrm{MeV} ; \text { measured isomer production } \sigma . \text { Ta, W, }{ }^{186} \mathrm{~W}, \mathrm{Re}(\mathrm{p} \text {, } \\
\mathrm{X}){ }^{179 m} \mathrm{Hf} /{ }^{178 m} \mathrm{Hf} /{ }^{177 m} \mathrm{Lu} /{ }^{178} \mathrm{~W} /{ }^{175} \mathrm{Hf} /{ }^{172} \mathrm{Hf} /{ }^{173} \mathrm{Lu}, \mathrm{E}=650 \\
\mathrm{MeV} \text {; analyzed yields, isomer ratios. }{ }^{176} \mathrm{Yb}(\alpha, 2 \mathrm{n}), \mathrm{E}<36 \mathrm{MeV} ; \\
\text { measured isomer yield. Other reactions discussed. JOUR YAFIA } 68 \\
1827\end{array}$ \\
\hline \multirow[t]{2}{*}{${ }^{178} \mathrm{Os}$} & 2005DE48 & $\begin{array}{l}\text { NUCLEAR REACTIONS }{ }^{164,166,168} \mathrm{Er}\left({ }^{16} \mathrm{O}, 4 \mathrm{n}\right), \mathrm{E}=80 \mathrm{MeV} \text {; measured } \\
\text { prompt and delayed } \mathrm{E} \gamma, \mathrm{I} \gamma \cdot{ }^{154} \mathrm{Sm}\left({ }^{29} \mathrm{Si}, 5 \mathrm{n}\right), \mathrm{E}=158 \mathrm{MeV} \text {; measured } \\
\text { Doppler-shifted } \mathrm{E} \gamma, \mathrm{I} \gamma, \gamma \gamma \text {-coin. }{ }^{176,178,180} \mathrm{Os} \text { deduced levels } \mathrm{T}_{1 / 2} \text {, } \\
\text { transition quadrupole moments, symmetry features. Electronic timing } \\
\text { and recoil distance techniques, GASP array, interacting boson model } \\
\text { and general collective model predictions. JOUR JPGPE } 31 \mathrm{~S} 1427\end{array}$ \\
\hline & 2005М033 & $\begin{array}{l}\text { NUCLEAR REACTIONS }{ }^{164,166,168} \operatorname{Er}\left({ }^{16} \mathrm{O}, 4 \mathrm{n}\right), \mathrm{E}=80 \mathrm{MeV} \text {; measured } \\
\text { prompt and delayed } \mathrm{E} \gamma, \mathrm{I} \gamma, \gamma \gamma \text {-coin. }{ }^{166} \mathrm{Er}\left({ }^{16} \mathrm{O}, 4 \mathrm{n}\right), \mathrm{E}=80 \mathrm{MeV} \text {; } \\
\text { measured Doppler-shifted } \mathrm{E} \gamma, \mathrm{I} \gamma, \gamma \gamma \text {-coin. }{ }^{176,178,180} \mathrm{Os} \text { deduced levels, } \\
\mathrm{J}, \pi, \mathrm{T}_{1 / 2} \text {, B(E2). Pulsed-beam and recoil-distance techniques. JOUR } \\
\text { PRVCA } 72034306\end{array}$ \\
\hline${ }^{178} \mathrm{Hg}$ & $2004 \mathrm{GOZZ}$ & $\begin{array}{l}\text { RADIOACTIVITY }{ }^{168,169,170,171,172} \mathrm{Os},{ }^{169,170,171,172,173,174,175} \mathrm{Ir} \\
170,171,172,173,174,175,176,177 \mathrm{Pt},{ }^{173,174,175,176,177} \mathrm{Au},\end{array}$ \\
\hline & & $\begin{array}{l}174,175,176,177,178 \mathrm{Hg}(\alpha)\left[\text { from }{ }^{92,94} \mathrm{Mo}\left({ }^{84} \mathrm{Sr}, \mathrm{xnyp}\right) \text { and subsequent }\right. \\
\text { decay]; measured } \mathrm{E} \alpha, \mathrm{E} \gamma, \alpha-\gamma \text {-coin, } \mathrm{T}_{1 / 2} \cdot{ }^{165} \mathrm{Ta},{ }^{165,167} \mathrm{~W} \\
\\
{ }^{165,166,167,168,171} \mathrm{Re},{ }^{169,170,171} \mathrm{Os},{ }^{169,170,171,172,175} \mathrm{Ir},{ }^{173,175} \mathrm{Pt},{ }^{174,176} \mathrm{Au}\end{array}$ \\
\hline
\end{tabular}

\section{$\mathrm{A}=\mathbf{1 7 9}$}

${ }^{179} \mathrm{Hf} \quad 2004 \mathrm{CO} 26$

RADIOACTIVITY ${ }^{180} \mathrm{~W}(\alpha)$; measured $\mathrm{E} \alpha, \mathrm{T}_{1 / 2}$, Q-value. $182,183,184,186 \mathrm{~W}(\alpha)$; measured $\mathrm{T}_{1 / 2}$ lower limits. $\mathrm{CaWO}_{4}$ crystals. JOUR PRVCA 70064606

2005BU07 NUCLEAR REACTIONS ${ }^{163} \mathrm{Dy},{ }^{177} \mathrm{Hf}(\mathrm{t}, \mathrm{p}), \mathrm{E}=17 \mathrm{MeV}$; measured $\sigma(\mathrm{Ep}, \theta) .{ }^{165} \mathrm{Dy},{ }^{179} \mathrm{Hf}$ deduced levels, L-values, $\mathrm{L}=0$ strengths. Enriched targets, magnetic spectrograph. Systematic trends in neighboring nuclides discussed. JOUR NUPAB 750185

2005KA52 NUCLEAR REACTIONS ${ }^{177} \mathrm{Hf}(\mathrm{n}, \gamma), \mathrm{E}=$ thermal, resonance; ${ }^{178} \mathrm{Hf}(\mathrm{n}$, n' $\gamma), \mathrm{E}>3 \mathrm{MeV}$; measured isomer production $\sigma$. Ta, W, ${ }^{186} \mathrm{~W}, \operatorname{Re}(\mathrm{p}$, $\mathrm{X}){ }^{179 m} \mathrm{Hf} /{ }^{178 m} \mathrm{Hf} /{ }^{177 m} \mathrm{Lu} /{ }^{178} \mathrm{~W} /{ }^{175} \mathrm{Hf} /{ }^{172} \mathrm{Hf} /{ }^{173} \mathrm{Lu}, \mathrm{E}=650$ $\mathrm{MeV}$; analyzed yields, isomer ratios. ${ }^{176} \mathrm{Yb}(\alpha, 2 \mathrm{n}), \mathrm{E}<36 \mathrm{MeV}$; measured isomer yield. Other reactions discussed. JOUR YAFIA 68 1827

2005ME01 NUCLEAR REACTIONS Hf(n, X), E=0.1-100 eV; measured total neutron $\sigma .{ }^{174,176,177,178,179,180} \mathrm{Hf}$ deduced resonance parameters. Comparison with previous results. JOUR KPSJA 46401

2005TRZY NUCLEAR REACTIONS Hf(n, n), (n, $\gamma), \mathrm{E} \approx 0.005-200 \mathrm{eV}$; measured transmission and capture $\sigma .{ }^{174,176,177,178,179,180} \mathrm{Hf}$ deduced resonance parameters, capture resonance integrals. CONF Santa Fe (Nucl Data for Sci and Technol) Proc,Vol1,P949 


\section{$\mathrm{A}=179$ (continued)}

2005WIZZ

$\begin{array}{ll}{ }^{179} \mathrm{Pt} & \quad 2002 \mathrm{DU} 22 \\ { }^{179} \mathrm{Au} & \text { 2004RA28 }\end{array}$

2005CA43

${ }^{179} \mathrm{Hg} \quad$ 2005ANZY

2005CAZY

2005CAZY

${ }^{180} \mathrm{Hf}$

\begin{abstract}
$2004 C 026$
\end{abstract}
2005ME01

2005TRZY
NUCLEAR REACTIONS ${ }^{175,176} \mathrm{Lu},{ }^{176,177,178,179,180} \mathrm{Hf}(\mathrm{n}, \gamma), \mathrm{E}=3-225$ $\mathrm{keV}$; measured capture $\sigma$; deduced Maxwellian averaged $\sigma$.

Comparison with previous results, astrophysical implications discussed. CONF Santa Fe (Nucl Data for Sci and Technol) Proc,Vol2,P1315 RADIOACTIVITY ${ }^{197,197 m, 198,199 m, 200,201 m} \operatorname{Po}(\alpha) ;{ }^{172,173} \operatorname{Os}(\alpha)$ [from $\left.{ }^{156} \mathrm{Dy}\left({ }^{22} \mathrm{Ne}, \mathrm{xn}\right)\right] ;{ }^{183,184,185} \mathrm{Hg}(\alpha)$ [from $\left.{ }^{168} \mathrm{Yb}\left({ }^{22} \mathrm{Ne}, \mathrm{xn}\right)\right]$; measured $\mathrm{E} \alpha, \mathrm{T}_{1 / 2}$. JOUR NIMAE 479631

RADIOACTIVITY ${ }^{183} \mathrm{Tl},{ }^{179} \mathrm{Au}(\alpha)$ [from ${ }^{144} \mathrm{Sm}\left({ }^{42} \mathrm{Ca}, 2 \mathrm{np}\right)$ and subsequent decay]; measured $\mathrm{E} \alpha, \mathrm{E} \gamma, \alpha \gamma$-coin, $\mathrm{T}_{1 / 2} .{ }^{179} \mathrm{Au},{ }^{175} \mathrm{Ir}$ deduced levels, J, $\pi$. JOUR PRVCA 70064308

NUCLEAR REACTIONS ${ }^{92} \mathrm{Mo}\left({ }^{84} \mathrm{Sr}, \mathrm{n} 2 \mathrm{p}\right),\left({ }^{84} \mathrm{Sr}, 3 \mathrm{p}\right),\left({ }^{84} \mathrm{Sr}, 2 \mathrm{np}\right)$, ${ }^{104} \mathrm{Ru}\left({ }^{84} \mathrm{Kr}, 2 \mathrm{np}\right),{ }^{90} \mathrm{Zr}\left({ }^{90} \mathrm{Zr}, \mathrm{n}\right),\left({ }^{90} \mathrm{Zr}, \mathrm{p}\right)$, E not given; ${ }^{92} \mathrm{Mo}\left({ }^{90} \mathrm{Zr}, \mathrm{n}\right)$, $\left({ }^{90} \mathrm{Zr}, \mathrm{p}\right), \mathrm{E}=385 \mathrm{MeV}$; measured $\mathrm{E} \gamma, \mathrm{I} \gamma, \gamma \gamma-$, (recoil) $\gamma$-coin. ${ }^{179} \mathrm{Hg}$ deduced high-spin levels, J, $\pi$. Gammasphere array, fragment separator. JOUR JPGPE 31 S1599 RADIOACTIVITY ${ }^{186,187} \mathrm{Po},{ }^{183} \mathrm{~Pb},{ }^{179} \mathrm{Hg},{ }^{175} \mathrm{Pt}(\alpha)$ from ${ }^{144} \mathrm{Sm}\left({ }^{46} \mathrm{Ti}\right.$, $\mathrm{xn})$ and subsequent decay]; measured $\mathrm{E} \alpha, \mathrm{T}_{1 / 2} .{ }^{183} \mathrm{~Pb}$ deduced excited state energy. ${ }^{187}$ Po deduced isomeric states. ${ }^{192} \mathrm{At}(\alpha)\left[\right.$ from ${ }^{144} \mathrm{Sm}\left({ }^{51} \mathrm{~V}\right.$, $\mathrm{xn})]$; measured $\mathrm{E} \alpha, \alpha \alpha$-coin, $\mathrm{T}_{1 / 2}$; deduced isomeric states. REPT GSI 2005-1,P77,Andreyev NUCLEAR REACTIONS ${ }^{92} \mathrm{Mo}\left({ }^{84} \mathrm{Sr}, \mathrm{n} 2 \mathrm{p}\right),\left({ }^{84} \mathrm{Sr}, 3 \mathrm{p}\right),\left({ }^{84} \mathrm{Sr}, 2 \mathrm{np}\right)$, ${ }^{104} \mathrm{Ru}\left({ }^{84} \mathrm{Kr}, 2 \mathrm{np}\right),{ }^{90} \mathrm{Zr}\left({ }^{90} \mathrm{Zr}, \mathrm{n}\right),\left({ }^{90} \mathrm{Zr}, \mathrm{p}\right)$, E not given; ${ }^{92} \mathrm{Mo}\left({ }^{90} \mathrm{Zr}, \mathrm{n}\right)$, $\left({ }^{90} \mathrm{Zr}, \mathrm{p}\right), \mathrm{E}=385 \mathrm{MeV}$; measured $\mathrm{E} \gamma, \mathrm{I} \gamma, \gamma \gamma-$, (recoil) $\gamma$-coin. ${ }^{179} \mathrm{Hg}$ deduced high-spin levels, J, $\pi$. Gammasphere array, fragment separator. JOUR JPGPE 31 S1599 NUCLEAR REACTIONS ${ }^{92,94,96} \mathrm{Mo}\left({ }^{84} \mathrm{Sr}, 2 \mathrm{np}\right)$, E not given; ${ }^{90} \mathrm{Zr}\left({ }^{90} \mathrm{Zr}, \mathrm{n}\right), \mathrm{E}=369,380 \mathrm{MeV}$; measured $\mathrm{E} \gamma, \mathrm{I} \gamma, \gamma \gamma-$, (recoil) $\gamma$-coin. ${ }^{173,175,177} \mathrm{Au},{ }^{179} \mathrm{Hg}$ deduced high-spin levels, J, $\pi$, shape coexistence features. Gammasphere, mass separator, recoil-decay tagging. CONF Santa Fe (Nucl Data for Sci and Technol) Proc,Vol1,P894 RADIOACTIVITY ${ }^{181,183} \mathrm{~Pb}(\alpha)$; measured $\mathrm{E} \alpha, \mathrm{E} \gamma, \alpha \gamma$-coin. ${ }^{177} \mathrm{Hg}$ deduced level. ${ }^{179} \mathrm{Hg}$ deduced isomeric state energy, $\mathrm{T}_{1 / 2}$. CONF Santa Fe (Nucl Data for Sci and Technol) Proc,Vol1,P894

$$
\mathrm{A}=\mathbf{1 8 0}
$$

RADIOACTIVITY ${ }^{180} \mathrm{~W}(\alpha)$; measured E $\alpha, \mathrm{T}_{1 / 2}, \mathrm{Q}$-value. $182,183,184,186 \mathrm{~W}(\alpha)$; measured $\mathrm{T}_{1 / 2}$ lower limits. CaWO $\mathrm{Ca}_{4}$ crystals. JOUR PRVCA 70064606 NUCLEAR REACTIONS Hf(n, X), E=0.1-100 eV; measured total neutron $\sigma .{ }^{174,176,177,178,179,180}$ Hf deduced resonance parameters. Comparison with previous results. JOUR KPSJA 46401 NUCLEAR REACTIONS Hf(n, n), $(\mathrm{n}, \gamma), \mathrm{E} \approx 0.005-200 \mathrm{eV}$; measured transmission and capture $\sigma .{ }^{174,176,177,178,179,180} \mathrm{Hf}$ deduced resonance parameters, capture resonance integrals. CONF Santa Fe (Nucl Data for Sci and Technol) Proc,Vol1,P949 


\section{$\mathrm{A}=180$ (continued)}

2005WIZZ

$\begin{array}{ll}{ }^{180} \mathrm{~W} & 2004 \mathrm{CO} 26 \\ { }^{180} \mathrm{Re} & 2005 E L 10\end{array}$

${ }^{180} \mathrm{Os} \quad 2005 \mathrm{DE} 48$

$2005 \mathrm{M033}$

${ }^{180} \mathrm{Pt} \quad 2002 \mathrm{DU} 22$

NUCLEAR REACTIONS ${ }^{175,176} \mathrm{Lu},{ }^{176,177,178,179,180} \mathrm{Hf}(\mathrm{n}, \gamma), \mathrm{E}=3-225$ $\mathrm{keV}$; measured capture $\sigma$; deduced Maxwellian averaged $\sigma$.

Comparison with previous results, astrophysical implications discussed. CONF Santa Fe (Nucl Data for Sci and Technol) Proc,Vol2,P1315 RADIOACTIVITY ${ }^{180} \mathrm{~W}(\alpha)$; measured E $\alpha, \mathrm{T}_{1 / 2}$, Q-value. $182,183,184,186 \mathrm{~W}(\alpha)$; measured $\mathrm{T}_{1 / 2}$ lower limits. CaWO 4 crystals. JOUR PRVCA 70064606 NUCLEAR REACTIONS ${ }^{174} \mathrm{Yb}\left({ }^{11} \mathrm{~B}, 5 \mathrm{n}\right), \mathrm{E}=71 \mathrm{MeV}$; measured $\mathrm{E} \gamma$, $\mathrm{I} \gamma, \mathrm{E}(\mathrm{ce}), \mathrm{I}(\mathrm{ce}), \gamma \gamma$-, (ce) $\gamma$-coin. ${ }^{180}$ Re deduced high-spin levels, J, $\pi$, ICC, configurations, K-forbidden transitions. Potential energy surface calculations. JOUR PRVCA 72054306

NUCLEAR REACTIONS ${ }^{164,166,168} \operatorname{Er}\left({ }^{16} \mathrm{O}, 4 \mathrm{n}\right), \mathrm{E}=80 \mathrm{MeV}$; measured prompt and delayed $\mathrm{E} \gamma, \mathrm{I} \gamma \cdot{ }^{154} \mathrm{Sm}\left({ }^{29} \mathrm{Si}, 5 \mathrm{n}\right), \mathrm{E}=158 \mathrm{MeV}$; measured Doppler-shifted $\mathrm{E} \gamma, \mathrm{I} \gamma, \gamma \gamma$-coin. ${ }^{176,178,180}$ Os deduced levels $\mathrm{T}_{1 / 2}$, transition quadrupole moments, symmetry features. Electronic timing and recoil distance techniques, GASP array, interacting boson model and general collective model predictions. JOUR JPGPE 31 S1427 NUCLEAR REACTIONS ${ }^{164,166,168} \operatorname{Er}\left({ }^{16} \mathrm{O}, 4 \mathrm{n}\right), \mathrm{E}=80 \mathrm{MeV}$; measured prompt and delayed $\mathrm{E} \gamma, \mathrm{I} \gamma, \gamma \gamma$-coin. ${ }^{166} \operatorname{Er}\left({ }^{16} \mathrm{O}, 4 \mathrm{n}\right), \mathrm{E}=80 \mathrm{MeV}$;

measured Doppler-shifted $\mathrm{E} \gamma, \mathrm{I} \gamma, \gamma \gamma$-coin. ${ }^{176,178,180}$ Os deduced levels, $\mathrm{J}, \pi, \mathrm{T}_{1 / 2}, \mathrm{~B}(\mathrm{E} 2)$. Pulsed-beam and recoil-distance techniques. JOUR PRVCA 72034306

RADIOACTIVITY ${ }^{197,197 m, 198,199 m, 200,201 m} \operatorname{Po}(\alpha) ;{ }^{172,173} \operatorname{Os}(\alpha)$ [from $\left.{ }^{156} \mathrm{Dy}\left({ }^{22} \mathrm{Ne}, \mathrm{xn}\right)\right] ;{ }^{183,184,185} \mathrm{Hg}(\alpha)$ [from ${ }^{168} \mathrm{Yb}\left({ }^{22} \mathrm{Ne}, \mathrm{xn}\right)$ ]; measured $\mathrm{E} \alpha, \mathrm{T}_{1 / 2}$. JOUR NIMAE 479631

\section{$A=181$}

${ }^{181} \mathrm{Hf} \quad 2005 W I Z Z$

NUCLEAR REACTIONS ${ }^{175,176} \mathrm{Lu},{ }^{176,177,178,179,180} \mathrm{Hf}(\mathrm{n}, \gamma), \mathrm{E}=3-225$ $\mathrm{keV}$; measured capture $\sigma$; deduced Maxwellian averaged $\sigma$.

Comparison with previous results, astrophysical implications discussed. CONF Santa Fe (Nucl Data for Sci and Technol) Proc,Vol2,P1315

${ }^{181} \operatorname{Re} \quad 2005 N A Z Y$

NUCLEAR REACTIONS ${ }^{27} \mathrm{Al}(\mathrm{d}, \mathrm{X}){ }^{27} \mathrm{Mg} /{ }^{24} \mathrm{Na}, \mathrm{E}=22-40 \mathrm{MeV}$; $\mathrm{Cu}(\mathrm{d}, \mathrm{X}){ }^{62} \mathrm{Zn} /{ }^{63} \mathrm{Zn} /{ }^{61} \mathrm{Cu} /{ }^{64} \mathrm{Cu}, \mathrm{E}=22-40 \mathrm{MeV} ; \mathrm{W}(\mathrm{d}, \mathrm{X}){ }^{181} \mathrm{Re} /$ ${ }^{182} \operatorname{Re} /{ }^{183} \operatorname{Re} /{ }^{184} \operatorname{Re} /{ }^{186} \operatorname{Re} /{ }^{187} \mathrm{~W}, \mathrm{E}=22-40 \mathrm{MeV}$; measured activation $\sigma$. Comparison with previous results and model predictions. CONF Santa Fe (Nucl Data for Sci and Technol) Proc,Vol2,P1489 NUCLEAR REACTIONS ${ }^{150} \mathrm{Nd}\left({ }^{36} \mathrm{~S}, 3 \mathrm{n}\right),\left({ }^{36} \mathrm{~S}, 5 \mathrm{n}\right)$, E not given; measured prompt and delayed $\mathrm{E} \gamma, \mathrm{I} \gamma, \gamma \gamma$-coin. ${ }^{181,183}$ Os deduced levels, J, $\pi$, configurations. Comparison with tilted axis cranking model predictions. JOUR JPGPE 31 S1709
${ }^{181} \mathrm{Pt}$ 2002DU22 RADIOACTIVITY ${ }^{197,197 m, 198,199 m, 200,201 m} \mathrm{Po}(\alpha) ;{ }^{172,173} \mathrm{Os}(\alpha)[\mathrm{from}$ $\left.{ }^{156} \mathrm{Dy}\left({ }^{22} \mathrm{Ne}, \mathrm{xn}\right)\right] ;{ }^{183,184,185} \mathrm{Hg}(\alpha)$ [from $\left.{ }^{168} \mathrm{Yb}\left({ }^{22} \mathrm{Ne}, \mathrm{xn}\right)\right]$; measured $\mathrm{E} \alpha, \mathrm{T}_{1 / 2}$. JOUR NIMAE 479631




\section{$\mathrm{A}=181$ (continued)}

\begin{tabular}{|c|c|c|}
\hline${ }^{181} \mathrm{Tl}$ & $2005 \mathrm{CA} 43$ & $\begin{array}{l}\text { NUCLEAR REACTIONS }{ }^{92} \mathrm{Mo}\left({ }^{84} \mathrm{Sr}, \mathrm{n} 2 \mathrm{p}\right),\left({ }^{84} \mathrm{Sr}, 3 \mathrm{p}\right),\left({ }^{84} \mathrm{Sr}, 2 \mathrm{np}\right) \text {, } \\
{ }^{104} \mathrm{Ru}\left({ }^{84} \mathrm{Kr}, 2 \mathrm{np}\right),{ }^{90} \mathrm{Zr}\left({ }^{90} \mathrm{Zr}, \mathrm{n}\right),\left({ }^{90} \mathrm{Zr}, \mathrm{p}\right), \mathrm{E} \text { not given; }{ }^{92} \mathrm{Mo}\left({ }^{90} \mathrm{Zr}, \mathrm{n}\right) \text {, } \\
\left.\left({ }^{90} \mathrm{Zr}, \mathrm{p}\right), \mathrm{E}=385 \mathrm{MeV} \text {; measured } \mathrm{E} \gamma, \mathrm{I} \gamma, \gamma \gamma-\text { (recoil }\right) \gamma \text {-coin. }{ }^{179} \mathrm{Hg} \\
\text { deduced high-spin levels, J, } \pi \text {. Gammasphere array, fragment } \\
\text { separator. JOUR JPGPE } 31 \text { S1599 }\end{array}$ \\
\hline \multirow[t]{3}{*}{${ }^{181} \mathrm{~Pb}$} & 2004CAZW & $\begin{array}{l}\mathrm{RADIOACTIVITY}{ }^{181} \mathrm{~Pb}(\alpha)\left[\text { from }{ }^{92} \mathrm{Mo}\left({ }^{90} \mathrm{Zr}, \mathrm{p}\right)\right] ; \text { measured } \mathrm{E} \alpha, \mathrm{T}_{1 / 2} \text {. } \\
\text { 181 } \mathrm{Pb} \text { deduced ground-state J, } \pi \text {. REPT ANL-04/22,P43,Carpenter }\end{array}$ \\
\hline & $2005 \mathrm{CA} 43$ & $\begin{array}{l}\text { NUCLEAR REACTIONS }{ }^{92} \mathrm{Mo}\left({ }^{84} \mathrm{Sr}, \mathrm{n} 2 \mathrm{p}\right),\left({ }^{84} \mathrm{Sr}, 3 \mathrm{p}\right),\left({ }^{84} \mathrm{Sr}, 2 \mathrm{np}\right) \text {, } \\
{ }^{104} \mathrm{Ru}\left({ }^{84} \mathrm{Kr}, 2 \mathrm{np}\right),{ }^{90} \mathrm{Zr}\left({ }^{90} \mathrm{Zr}, \mathrm{n}\right),\left({ }^{90} \mathrm{Zr}, \mathrm{p}\right), \mathrm{E} \text { not given; }{ }^{92} \mathrm{Mo}\left({ }^{90} \mathrm{Zr}, \mathrm{n}\right), \\
\left.\left({ }^{90} \mathrm{Zr}, \mathrm{p}\right), \mathrm{E}=385 \mathrm{MeV} \text { measured } \mathrm{E} \gamma, \mathrm{I} \gamma, \gamma \gamma-\text {, (recoil }\right) \gamma \text {-coin. }{ }^{179} \mathrm{Hg} \\
\text { deduced high-spin levels, J, } \pi \text {. Gammasphere array, fragment } \\
\text { separator. JOUR JPGPE } 31 \text { S1599 }\end{array}$ \\
\hline & $2005 \mathrm{CAZY}$ & $\begin{array}{l}\text { RADIOACTIVITY }{ }^{181,183} \mathrm{~Pb}(\alpha) \text {; measured } \mathrm{E} \alpha, \mathrm{E} \gamma, \alpha \gamma \text {-coin. }{ }^{177} \mathrm{Hg} \\
\text { deduced level. }{ }^{179} \mathrm{Hg} \text { deduced isomeric state energy, } \mathrm{T}_{1 / 2} \text {. CONF Santa } \\
\text { Fe (Nucl Data for Sci and Technol) Proc,Vol1,P894 }\end{array}$ \\
\hline
\end{tabular}

$$
\mathrm{A}=182
$$

${ }^{182} \mathrm{Hf} \quad 2004 \mathrm{CO} 26$

RADIOACTIVITY ${ }^{180} \mathrm{~W}(\alpha)$; measured $\mathrm{E} \alpha, \mathrm{T}_{1 / 2}$, Q-value.

${ }_{182,183,184,186} \mathrm{~W}(\alpha)$; measured $\mathrm{T}_{1 / 2}$ lower limits. $\mathrm{CaWO}_{4}$ crystals. JOUR PRVCA 70064606

2005V017 RADIOACTIVITY ${ }^{182} \mathrm{Hf}\left(\beta^{-}\right)$; measured $\mathrm{T}_{1 / 2}$. New accelerator mass spectrometry measurement discussed. JOUR NUPAB 758 340c

${ }^{182} \mathrm{Ta} 2005 \mathrm{~V} 017 \quad \mathrm{RADIOACTIVITY}{ }^{182} \mathrm{Hf}\left(\beta^{-}\right)$; measured $\mathrm{T}_{1 / 2}$. New accelerator mass spectrometry measurement discussed. JOUR NUPAB 758 340c

${ }^{182} \mathrm{~W} \quad 2004 \mathrm{CO} 26 \quad$ RADIOACTIVITY ${ }^{180} \mathrm{~W}(\alpha)$; measured $\mathrm{E} \alpha, \mathrm{T}_{1 / 2}$, Q-value. ${ }_{182,183,184,186} \mathrm{~W}(\alpha)$; measured $\mathrm{T}_{1 / 2}$ lower limits. $\mathrm{CaWO}_{4}$ crystals. JOUR PRVCA 70064606

${ }^{182} \mathrm{Re}$ 2005NAZY NUCLEAR REACTIONS ${ }^{27} \mathrm{Al}(\mathrm{d}, \mathrm{X}){ }^{27} \mathrm{Mg} /{ }^{24} \mathrm{Na}, \mathrm{E}=22-40 \mathrm{MeV}$; $\mathrm{Cu}(\mathrm{d}, \mathrm{X}){ }^{62} \mathrm{Zn} /{ }^{63} \mathrm{Zn} /{ }^{61} \mathrm{Cu} /{ }^{64} \mathrm{Cu}, \mathrm{E}=22-40 \mathrm{MeV} ; \mathrm{W}(\mathrm{d}, \mathrm{X}){ }^{181} \mathrm{Re} /$ ${ }^{182} \operatorname{Re} /{ }^{183} \operatorname{Re} /{ }^{184} \operatorname{Re} /{ }^{186} \operatorname{Re} /{ }^{187} \mathrm{~W}, \mathrm{E}=22-40 \mathrm{MeV}$; measured activation $\sigma$. Comparison with previous results and model predictions. CONF Santa Fe (Nucl Data for Sci and Technol) Proc,Vol2,P1489

${ }^{182} \mathrm{~Pb}$ 2005ANZY RADIOACTIVITY ${ }^{186,187} \mathrm{Po},{ }^{183} \mathrm{~Pb},{ }^{179} \mathrm{Hg},{ }^{175} \mathrm{Pt}(\alpha)$ [from ${ }^{144} \mathrm{Sm}\left({ }^{46} \mathrm{Ti}\right.$, $\mathrm{xn})$ and subsequent decay]; measured $\mathrm{E} \alpha, \mathrm{T}_{1 / 2} .{ }^{183} \mathrm{~Pb}$ deduced excited state energy. ${ }^{187}$ Po deduced isomeric states. ${ }^{192} \mathrm{At}(\alpha)\left[\mathrm{from}{ }^{144} \mathrm{Sm}\left({ }^{51} \mathrm{~V}\right.\right.$, $\mathrm{xn})]$; measured $\mathrm{E} \alpha, \alpha \alpha$-coin, $\mathrm{T}_{1 / 2}$; deduced isomeric states. REPT GSI 2005-1,P77,Andreyev

\section{$\mathrm{A}=183$}

${ }^{183} \mathrm{~W} \quad 2004 \mathrm{CO} 26 \quad$ RADIOACTIVITY ${ }^{180} \mathrm{~W}(\alpha)$; measured $\mathrm{E} \alpha, \mathrm{T}_{1 / 2}$, Q-value. $182,183,184,186 \mathrm{~W}(\alpha)$; measured $\mathrm{T}_{1 / 2}$ lower limits. $\mathrm{CaWO}_{4}$ crystals. JOUR PRVCA 70064606 


\section{$\mathrm{A}=183$ (continued)}

\begin{tabular}{|c|c|c|}
\hline \multirow[t]{2}{*}{${ }^{183} \mathrm{Re}$} & 2005CL07 & $\begin{array}{l}\text { NUCLEAR REACTIONS }{ }^{184} \mathrm{~W}\left({ }^{7} \mathrm{Li}, \mathrm{xn}\right),\left({ }^{7} \mathrm{Li}, \mathrm{xnp}\right),\left({ }^{7} \mathrm{Li}, \mathrm{xn} \alpha\right) \text {, } \\
\mathrm{E}=35-70 \mathrm{MeV} \text {; calculated } \sigma .{ }^{184} \mathrm{~W}\left({ }^{7} \mathrm{Li}, \mathrm{X}\right){ }^{184} \mathrm{Os} /{ }^{185} \mathrm{Os} /{ }^{186} \mathrm{Os} / \\
{ }^{188} \mathrm{Os} /{ }^{184} \mathrm{Ir} /{ }^{185} \mathrm{Ir} /{ }^{186} \mathrm{Ir} /{ }^{183} \mathrm{Re} /{ }^{185} \mathrm{Re}, \mathrm{E}=40-70 \mathrm{MeV} ; \text { measured } \\
\mathrm{E} \gamma, \mathrm{I} \gamma, \gamma \gamma-,(\text { charged particle }) \gamma \text {-coin, particle yield ratios. }{ }^{160} \mathrm{Gd}\left({ }^{7} \mathrm{Li} \text {, }\right. \\
\text { xnp), E }=35-65 \mathrm{MeV} \text {; analyzed } \sigma . \text { Liberace, Stars arrays. JOUR } \\
\text { PRVCA } 72054605\end{array}$ \\
\hline & 2005NAZY & $\begin{array}{l}\text { NUCLEAR REACTIONS }{ }^{27} \mathrm{Al}(\mathrm{d}, \mathrm{X}){ }^{27} \mathrm{Mg} /{ }^{24} \mathrm{Na}, \mathrm{E}=22-40 \mathrm{MeV} \\
\mathrm{Cu}(\mathrm{d}, \mathrm{X})^{62} \mathrm{Zn} /{ }^{63} \mathrm{Zn} /{ }^{61} \mathrm{Cu} /{ }^{64} \mathrm{Cu}, \mathrm{E}=22-40 \mathrm{MeV} ; \mathrm{W}(\mathrm{d}, \mathrm{X})^{181} \mathrm{Re} / \\
{ }^{182} \mathrm{Re} /{ }^{183} \mathrm{Re} /{ }^{184} \mathrm{Re} /{ }^{186} \mathrm{Re} /{ }^{187} \mathrm{~W}, \mathrm{E}=22-40 \mathrm{MeV} ; \text { measured } \\
\text { activation } \sigma . \text { Comparison with previous results and model predictions. } \\
\text { CONF Santa Fe (Nucl Data for Sci and Technol) Proc,Vol2,P1489 }\end{array}$ \\
\hline${ }^{183} \mathrm{Os}$ & 2005CU05 & $\begin{array}{l}\text { NUCLEAR REACTIONS }{ }^{150} \mathrm{Nd}\left({ }^{36} \mathrm{~S}, 3 \mathrm{n}\right),\left({ }^{36} \mathrm{~S}, 5 \mathrm{n}\right) \text {, E not given; } \\
\text { measured prompt and delayed } \mathrm{E} \gamma, \mathrm{I} \gamma, \gamma \gamma \text {-coin. }{ }^{181,183} \text { Os deduced } \\
\text { levels, J, } \pi \text {, configurations. Comparison with tilted axis cranking model } \\
\text { predictions. JOUR JPGPE } 31 \mathrm{~S} 1709\end{array}$ \\
\hline${ }^{183} \mathrm{Ir}$ & 2005FOZZ & $\begin{array}{l}\text { NUCLEAR REACTIONS }{ }^{191} \operatorname{Ir}\left(\mathrm{n}, \mathrm{n}^{\prime}\right),(\mathrm{n}, 2 \mathrm{n}),(\mathrm{n}, 3 \mathrm{n}),(\mathrm{n}, 4 \mathrm{n}),(\mathrm{n}, 5 \mathrm{n}) \text {, } \\
(\mathrm{n}, 6 \mathrm{n}),(\mathrm{n}, 7 \mathrm{n}),(\mathrm{n}, 8 \mathrm{n}),(\mathrm{n}, 9 \mathrm{n}), \mathrm{E}=1-300 \mathrm{MeV} ; \text { measured } \mathrm{E} \gamma, \mathrm{I} \gamma \\
\text { deduced } \gamma \text {-ray production } \sigma . \text { CONF Santa Fe (Nucl Data for Sci and } \\
\text { Technol) Proc,Vol1,P898 }\end{array}$ \\
\hline${ }^{183} \mathrm{Au}$ & $2005 \mathrm{~S} 001$ & $\begin{array}{l}\text { NUCLEAR REACTIONS }{ }^{159} \mathrm{~Tb}\left({ }^{29} \mathrm{Si}, 5 \mathrm{n}\right), \mathrm{E}=140 \mathrm{MeV} \text {; measured } \mathrm{E} \gamma \text {, } \\
\text { I } \gamma, \gamma \gamma \text {-coin. }{ }^{183} \mathrm{Au} \text { deduced high-spin levels, J, } \pi \text {, configurations. } \\
\text { GASP array. JOUR PRVCA } 71017302\end{array}$ \\
\hline${ }^{183} \mathrm{Hg}$ & 2002DU22 & $\begin{array}{l}\left.\text { RADIOACTIVITY }{ }^{197,197 m, 198,199 m, 200,201 m} \mathrm{Po}(\alpha) ;{ }^{172,173} \mathrm{Dy}\left({ }^{22} \mathrm{Ne}(\alpha) \text { xn }\right)\right] ;{ }^{183,184,185} \mathrm{Hg}(\alpha)[\text { from } \\
\left.{ }^{168} \mathrm{Yb}\left({ }^{22} \mathrm{Ne}, \mathrm{xn}\right)\right] ; \text { measured } \\
\mathrm{E} \alpha, \mathrm{T}_{1 / 2} \text {. JOUR NIMAE } 479631\end{array}$ \\
\hline \multirow[t]{2}{*}{${ }^{183} \mathrm{Tl}$} & 2004RA28 & $\begin{array}{l}\text { NUCLEAR REACTIONS }{ }^{144} \mathrm{Sm}\left({ }^{42} \mathrm{Ca}, 2 \mathrm{np}\right), \mathrm{E}=195,200 \mathrm{MeV} \\
\text { measured } \mathrm{E} \gamma, \mathrm{I} \gamma, \gamma \gamma-,(\text { recoil }) \gamma \text {-coin. }{ }^{183} \mathrm{Tl} \text { deduced high-spin levels, J, } \\
\pi \text {, configurations. Jurosphere array, recoil-decay tagging. JOUR } \\
\text { PRVCA } 70064308\end{array}$ \\
\hline & 2004RA28 & $\begin{array}{l}\text { RADIOACTIVITY }{ }^{183} \mathrm{Tl},{ }^{179} \mathrm{Au}(\alpha)\left[\text { from }{ }^{144} \mathrm{Sm}\left({ }^{42} \mathrm{Ca}, 2 \mathrm{np}\right) \text { and }\right. \\
\text { subsequent decay]; measured } \mathrm{E} \alpha, \mathrm{E} \gamma, \alpha \gamma \text {-coin, } \mathrm{T}_{1 / 2} \cdot{ }^{179} \mathrm{Au},{ }^{175} \mathrm{Ir} \\
\text { deduced levels, J, } \pi \text {. JOUR PRVCA } 70064308\end{array}$ \\
\hline \multirow[t]{2}{*}{${ }^{183} \mathrm{~Pb}$} & 2005AN17 & $\begin{array}{l}\text { NUCLEAR REACTIONS }{ }^{142} \mathrm{Nd}\left({ }^{52} \mathrm{Cr}, 3 \mathrm{n}\right),\left({ }^{52} \mathrm{Cr}, 4 \mathrm{n}\right),\left({ }^{52} \mathrm{Cr}, 5 \mathrm{n}\right), \\
\left({ }^{52} \mathrm{Cr}, 6 \mathrm{n}\right),\left({ }^{52} \mathrm{Cr}, \mathrm{np}\right),\left({ }^{52} \mathrm{Cr}, 2 \mathrm{np}\right),\left({ }^{52} \mathrm{Cr}, 3 \mathrm{np}\right),\left({ }^{52} \mathrm{Cr}, 4 \mathrm{np}\right),\left({ }^{52} \mathrm{Cr},\right. \\
\left.5^{5 \mathrm{np}}\right),\left({ }^{52} \mathrm{Cr}, 6 \mathrm{np}\right), \mathrm{E}=220-310 \mathrm{MeV} ;{ }^{142} \mathrm{Nd}\left({ }^{50} \mathrm{Cr}, 3 \mathrm{n}\right),\left({ }^{50} \mathrm{Cr}, 4 \mathrm{n}\right),\left({ }^{50} \mathrm{Cr},\right. \\
2 \mathrm{np}),\left({ }^{50} \mathrm{Cr}, 3 \mathrm{np}\right),\left({ }^{50} \mathrm{Cr}, 4 \mathrm{np}\right),\left({ }^{50} \mathrm{Cr}, 5 \mathrm{np}\right), \mathrm{E}=230-285 \mathrm{MeV} ; \\
{ }^{92} \mathrm{Mo}\left({ }^{98} \mathrm{Mo}, 2 \mathrm{np}\right),\left({ }^{98} \mathrm{Mo}, 3 \mathrm{np}\right), \mathrm{E}=427-460 \mathrm{MeV} ;{ }^{93} \mathrm{Nb}\left({ }^{95} \mathrm{Mo}, \mathrm{n}\right), \\
\left({ }^{95} \mathrm{Mo}, 2 \mathrm{n}\right),\left({ }^{95} \mathrm{Mo}, 3 \mathrm{n}\right),\left({ }^{95} \mathrm{Mo}, \mathrm{p}\right),\left({ }^{95} \mathrm{Mo}, \mathrm{np}\right),\left({ }^{95} \mathrm{Mo}, 2 \mathrm{np}\right),\left({ }^{95} \mathrm{Mo},\right. \\
3 \mathrm{np}),\left({ }^{95} \mathrm{Mo}, 4 \mathrm{np}\right), \mathrm{E}=375-456 \mathrm{MeV} ;{ }^{93} \mathrm{Nb}\left({ }^{94} \mathrm{Mo}, 2 \mathrm{n}\right),\left({ }^{94} \mathrm{Mo}, 3 \mathrm{n}\right), \\
\left({ }^{94} \mathrm{Mo}, \mathrm{np}\right),\left({ }^{94} \mathrm{Mo}, 2 \mathrm{np}\right),\left({ }^{94} \mathrm{Mo}, 3 \mathrm{np}\right), \mathrm{E}=405-450 \mathrm{MeV} ;{ }^{144} \mathrm{Sm}\left({ }^{46} \mathrm{Ti},\right. \\
3 \mathrm{n}),\left({ }^{46} \mathrm{Ti}, 4 \mathrm{n}\right), \mathrm{E}=202-242 \mathrm{MeV} ; \text { measured } \sigma . \text { Velocity filter, } \\
\text { comparison with statistical model predictions. JOUR PRVCA } 72 \\
014612\end{array}$ \\
\hline & 2005ANZY & $\begin{array}{l}\text { RADIOACTIVITY }{ }^{186,187} \mathrm{Po},{ }^{183} \mathrm{~Pb},{ }^{179} \mathrm{Hg},{ }^{175} \mathrm{Pt}(\alpha) \text { [from }{ }^{144} \mathrm{Sm}\left({ }^{46} \mathrm{Ti} \text {, }\right. \\
\text { xn) and subsequent decay]; measured } \mathrm{E} \alpha, \mathrm{T}_{1 / 2} \cdot{ }^{183} \mathrm{~Pb} \text { deduced excited } \\
\text { state energy. }{ }^{187} \mathrm{Po} \text { deduced isomeric states. }{ }^{192} \mathrm{At}(\alpha) \text { [from }{ }^{144} \mathrm{Sm}\left({ }^{51} \mathrm{~V} \text {, }\right. \\
\text { xn)]; measured } \mathrm{E} \alpha, \alpha \alpha \text {-coin, } \mathrm{T}_{1 / 2} \text {; deduced isomeric states. REPT GSI } \\
\text { 2005-1,P77, Andreyev }\end{array}$ \\
\hline
\end{tabular}




\section{$\mathrm{A}=183$ (continued)}

2005CAZY RADIOACTIVITY ${ }^{181,183} \mathrm{~Pb}(\alpha)$; measured $\mathrm{E} \alpha, \mathrm{E} \gamma, \alpha \gamma$-coin. ${ }^{177} \mathrm{Hg}$ deduced level. ${ }^{179} \mathrm{Hg}$ deduced isomeric state energy, $\mathrm{T}_{1 / 2}$. CONF Santa Fe (Nucl Data for Sci and Technol) Proc,Vol1,P894

\section{$\mathrm{A}=\mathbf{1 8 4}$}

${ }^{184} \mathrm{~W} \quad 2004 \mathrm{CO} 26$

2004L022

2005ME19

${ }^{184} \operatorname{Re}$

2004GA57

2005WH04

${ }^{184} \mathrm{Os} \quad$ 2005CL07

${ }^{184} \mathrm{Ir}$

2005CL07

2005FOZZ
RADIOACTIVITY ${ }^{180} \mathrm{~W}(\alpha)$; measured $\mathrm{E} \alpha, \mathrm{T}_{1 / 2}$, Q-value. ${ }_{182,183,184,186} \mathrm{~W}(\alpha)$; measured $\mathrm{T}_{1 / 2}$ lower limits. $\mathrm{CaWO}_{4}$ crystals. JOUR PRVCA 70064606 NUCLEAR REACTIONS ${ }^{183} \mathrm{~W}(\mathrm{n}, \gamma), \mathrm{E}=$ thermal; measured $\mathrm{E} \gamma, \mathrm{I} \gamma$. ${ }^{184} \mathrm{~W}$ deduced levels, $\mathrm{J}, \pi$, neutron binding energy. JOUR BRSPE 68 1292 NUCLEAR REACTIONS ${ }^{160} \mathrm{Gd},{ }^{164} \mathrm{Dy},{ }^{170} \mathrm{Er},{ }^{178} \mathrm{Hf},{ }^{186} \mathrm{~W},{ }^{192} \mathrm{Os}(\mathrm{p}$, t), $\mathrm{E}=25 \mathrm{MeV}$; measured triton spectra, $\sigma(\theta) .{ }^{158} \mathrm{Gd},{ }^{162} \mathrm{Dy},{ }^{168} \mathrm{Er}$, ${ }^{176} \mathrm{Hf},{ }^{184} \mathrm{~W},{ }^{190}$ Os deduced $0^{+}$level energies. JOUR JPGPE $31 \mathrm{~S} 1399$ NUCLEAR REACTIONS ${ }^{185} \mathrm{Re},{ }^{191} \mathrm{Ir},{ }^{197} \mathrm{Au}(\gamma, \mathrm{n}), \mathrm{E}=22 \mathrm{MeV}$ bremsstrahlung; ${ }^{185} \mathrm{Re},{ }^{191} \mathrm{Ir},{ }^{197} \mathrm{Au}(\mathrm{n}, 2 \mathrm{n}), \mathrm{E}=14.7 \mathrm{MeV} ;{ }^{181} \mathrm{Ta}(\alpha, \mathrm{n})$, $\mathrm{E}=18 \mathrm{MeV} ;{ }^{190} \mathrm{Os},{ }^{196} \mathrm{Pt}(\mathrm{d}, \mathrm{n}), \mathrm{E}=13,14 \mathrm{MeV} ;$ measured $\mathrm{E} \gamma, \mathrm{I} \gamma$; deduced isomer production ratios. Activation method. JOUR BRSPE 68187

NUCLEAR REACTIONS ${ }^{27} \mathrm{Al}(\mathrm{d}, \mathrm{X}){ }^{27} \mathrm{Mg} /{ }^{24} \mathrm{Na}, \mathrm{E}=22-40 \mathrm{MeV}$; $\mathrm{Cu}(\mathrm{d}, \mathrm{X}){ }^{62} \mathrm{Zn} /{ }^{63} \mathrm{Zn} /{ }^{61} \mathrm{Cu} /{ }^{64} \mathrm{Cu}, \mathrm{E}=22-40 \mathrm{MeV} ; \mathrm{W}(\mathrm{d}, \mathrm{X}){ }^{181} \mathrm{Re} /$ ${ }^{182} \operatorname{Re} /{ }^{183} \operatorname{Re} /{ }^{184} \operatorname{Re} /{ }^{186} \operatorname{Re} /{ }^{187} \mathrm{~W}, \mathrm{E}=22-40 \mathrm{MeV}$; measured activation $\sigma$. Comparison with previous results and model predictions. CONF Santa Fe (Nucl Data for Sci and Technol) Proc,Vol2,P1489 NUCLEAR REACTIONS ${ }^{180} \mathrm{Hf}\left({ }^{7} \mathrm{Li}, 3 \mathrm{n}\right), \mathrm{E}=30 \mathrm{MeV}$; measured prompt and delayed $\mathrm{E} \gamma, \mathrm{I} \gamma, \gamma \gamma$-coin, DCO ratios. ${ }^{184}$ Re deduced levels, $\mathrm{J}, \pi, \mathrm{T}_{1 / 2}$, gK - gR, configurations, rotational bands. Comparison with Nilsson-type blocked BCS calculations. JOUR NUPAB 7631

NUCLEAR REACTIONS ${ }^{184} \mathrm{~W}\left({ }^{7} \mathrm{Li}, \mathrm{xn}\right),\left({ }^{7} \mathrm{Li}, \mathrm{xnp}\right),\left({ }^{7} \mathrm{Li}, \mathrm{xn} \alpha\right)$, $\mathrm{E}=35-70 \mathrm{MeV}$; calculated $\sigma .{ }^{184} \mathrm{~W}\left({ }^{7} \mathrm{Li}, \mathrm{X}\right){ }^{184} \mathrm{Os} /{ }^{185} \mathrm{Os} /{ }^{186} \mathrm{Os} /$ ${ }^{188} \mathrm{Os} /{ }^{184} \mathrm{Ir} /{ }^{185} \mathrm{Ir} /{ }^{186} \mathrm{Ir} /{ }^{183} \mathrm{Re} /{ }^{185} \mathrm{Re}, \mathrm{E}=40-70 \mathrm{MeV}$; measured $\mathrm{E} \gamma, \mathrm{I} \gamma, \gamma \gamma-$, (charged particle) $\gamma$-coin, particle yield ratios. ${ }^{160} \mathrm{Gd}\left({ }^{7} \mathrm{Li}\right.$, xnp), $\mathrm{E}=35-65 \mathrm{MeV}$; analyzed $\sigma$. Liberace, Stars arrays. JOUR PRVCA 72054605 NUCLEAR REACTIONS ${ }^{184} \mathrm{~W}\left({ }^{7} \mathrm{Li}, \mathrm{xn}\right),\left({ }^{7} \mathrm{Li}, \mathrm{xnp}\right),\left({ }^{7} \mathrm{Li}, \mathrm{xn} \alpha\right)$, $\mathrm{E}=35-70 \mathrm{MeV}$; calculated $\sigma .{ }^{184} \mathrm{~W}\left({ }^{7} \mathrm{Li}, \mathrm{X}\right){ }^{184} \mathrm{Os} /{ }^{185} \mathrm{Os} /{ }^{186} \mathrm{Os} /$ ${ }^{188} \mathrm{Os} /{ }^{184} \mathrm{Ir} /{ }^{185} \mathrm{Ir} /{ }^{186} \mathrm{Ir} /{ }^{183} \mathrm{Re} /{ }^{185} \mathrm{Re}, \mathrm{E}=40-70 \mathrm{MeV}$; measured $\mathrm{E} \gamma, \mathrm{I} \gamma, \gamma \gamma-$, (charged particle) $\gamma$-coin, particle yield ratios. ${ }^{160} \mathrm{Gd}\left({ }^{7} \mathrm{Li}\right.$, $\mathrm{xnp}), \mathrm{E}=35-65 \mathrm{MeV}$; analyzed $\sigma$. Liberace, Stars arrays. JOUR PRVCA 72054605 NUCLEAR REACTIONS ${ }^{191} \operatorname{Ir}(\mathrm{n}, \mathrm{n}$ '), (n, 2n), (n, 3n), (n, 4n), (n, 5n), (n, 6n), (n, 7n), (n, 8n), (n, 9n), E=1-300 MeV; measured E $\gamma, \mathrm{I} \gamma$; deduced $\gamma$-ray production $\sigma$. CONF Santa Fe (Nucl Data for Sci and Technol) Proc,Vol1,P898 


\section{$\mathrm{A}=184$ (continued)}

${ }^{184} \mathrm{Au}$ 2005SA40 RADIOACTIVITY ${ }^{184} \mathrm{Hg}\left(\beta^{+}\right),(\mathrm{EC})$ [from $\left.\mathrm{Pb}(\mathrm{p}, \mathrm{X})\right]$; measured $\mathrm{E} \gamma$, $\mathrm{I} \gamma, \gamma \gamma$-coin, E(ce), I(ce); deduced log ft. ${ }^{184} \mathrm{Au}$ deduced levels, $\mathrm{J}, \pi$, configurations. Mass separator, comparisons with model predictions. JOUR ZAANE 255

$2005 \mathrm{ZH} 30$ NUCLEAR REACTIONS ${ }^{159} \mathrm{~Tb}\left({ }^{29} \mathrm{Si}, 4 \mathrm{n}\right), \mathrm{E}=140 \mathrm{MeV}$; measured $\mathrm{E} \gamma$, $\mathrm{I} \gamma, \gamma \gamma$-coin. ${ }^{184} \mathrm{Au}$ deduced high-spin levels, $\mathrm{J}, \pi$, configurations, signature inversion. GASP array. JOUR JPGPE 31 S1545

${ }^{184} \mathrm{Hg} \quad$ 2002DU22 RADIOACTIVITY ${ }^{197,197 m, 198,199 m, 200,201 m} \mathrm{Po}(\alpha) ;{ }^{172,173} \mathrm{Os}(\alpha)$ [from $\left.{ }^{156} \mathrm{Dy}\left({ }^{22} \mathrm{Ne}, \mathrm{xn}\right)\right] ;{ }^{183,184,185} \mathrm{Hg}(\alpha)$ [from $\left.{ }^{168} \mathrm{Yb}\left({ }^{22} \mathrm{Ne}, \mathrm{xn}\right)\right]$; measured $\mathrm{E} \alpha, \mathrm{T}_{1 / 2}$. JOUR NIMAE 479631

2005SA40 RADIOACTIVITY ${ }^{184} \mathrm{Hg}\left(\beta^{+}\right)$, (EC) [from $\left.\mathrm{Pb}(\mathrm{p}, \mathrm{X})\right]$; measured $\mathrm{E} \gamma$, $\mathrm{I} \gamma, \gamma \gamma$-coin, E(ce), I(ce); deduced log ft. ${ }^{184} \mathrm{Au}$ deduced levels, $\mathrm{J}, \pi$, configurations. Mass separator, comparisons with model predictions. JOUR ZAANE 255

${ }^{184} \mathrm{Tl}$ 2005VA04 RADIOACTIVITY ${ }^{189} \mathrm{Po}(\alpha)\left[\right.$ from $\left.{ }^{142} \mathrm{Nd}\left({ }^{52} \mathrm{Cr}, 5 \mathrm{n}\right),\left({ }^{50} \mathrm{Cr}, 3 \mathrm{n}\right)\right]$; measured $\mathrm{E} \alpha, \mathrm{E} \gamma, \mathrm{E}(\mathrm{ce}), \alpha \gamma$-coin. ${ }^{185} \mathrm{~Pb}$ deduced levels, J, $\pi$, ICC, $\mathrm{T}_{1 / 2}$, configurations. ${ }^{188,189,190,191} \mathrm{Bi},{ }^{189,190} \mathrm{Po}(\alpha)$ [from ${ }^{142} \mathrm{Nd}\left({ }^{52} \mathrm{Cr}\right.$, $\left.\mathrm{X}),\left({ }^{50} \mathrm{Cr}, \mathrm{X}\right)\right]$; measured $\mathrm{E} \alpha, \mathrm{I} \alpha$. Potential energy surface calculations, level systematics in neighboring isotopes discussed. JOUR ZAANE 24 57

${ }^{184} \mathrm{~Pb}$ 2005AN17 NUCLEAR REACTIONS ${ }^{142} \mathrm{Nd}\left({ }^{52} \mathrm{Cr}, 3 \mathrm{n}\right),\left({ }^{52} \mathrm{Cr}, 4 \mathrm{n}\right),\left({ }^{52} \mathrm{Cr}, 5 \mathrm{n}\right)$, $\left({ }^{52} \mathrm{Cr}, 6 \mathrm{n}\right),\left({ }^{52} \mathrm{Cr}, \mathrm{np}\right),\left({ }^{52} \mathrm{Cr}, 2 \mathrm{np}\right),\left({ }^{52} \mathrm{Cr}, 3 \mathrm{np}\right),\left({ }^{52} \mathrm{Cr}, 4 \mathrm{np}\right),\left({ }^{52} \mathrm{Cr}\right.$, $5 \mathrm{np}),\left({ }^{52} \mathrm{Cr}, 6 \mathrm{np}\right), \mathrm{E}=220-310 \mathrm{MeV} ;{ }^{142} \mathrm{Nd}\left({ }^{50} \mathrm{Cr}, 3 \mathrm{n}\right),\left({ }^{50} \mathrm{Cr}, 4 \mathrm{n}\right),\left({ }^{50} \mathrm{Cr}\right.$, $2 \mathrm{np}),\left({ }^{50} \mathrm{Cr}, 3 \mathrm{np}\right),\left({ }^{50} \mathrm{Cr}, 4 \mathrm{np}\right),\left({ }^{50} \mathrm{Cr}, 5 \mathrm{np}\right), \mathrm{E}=230-285 \mathrm{MeV}$; ${ }^{92} \mathrm{Mo}\left({ }^{98} \mathrm{Mo}, 2 \mathrm{np}\right),\left({ }^{98} \mathrm{Mo}, 3 \mathrm{np}\right), \mathrm{E}=427-460 \mathrm{MeV} ;{ }^{93} \mathrm{Nb}\left({ }^{95} \mathrm{Mo}, \mathrm{n}\right)$, $\left({ }^{95} \mathrm{Mo}, 2 \mathrm{n}\right),\left({ }^{95} \mathrm{Mo}, 3 \mathrm{n}\right),\left({ }^{95} \mathrm{Mo}, \mathrm{p}\right),\left({ }^{95} \mathrm{Mo}, \mathrm{np}\right),\left({ }^{95} \mathrm{Mo}, 2 \mathrm{np}\right),\left({ }^{95} \mathrm{Mo}\right.$, 3np), $\left({ }^{95} \mathrm{Mo}, 4 \mathrm{np}\right), \mathrm{E}=375-456 \mathrm{MeV} ;{ }^{93} \mathrm{Nb}\left({ }^{94} \mathrm{Mo}, 2 \mathrm{n}\right),\left({ }^{94} \mathrm{Mo}, 3 \mathrm{n}\right)$, $\left({ }^{94} \mathrm{Mo}, \mathrm{np}\right),\left({ }^{94} \mathrm{Mo}, 2 \mathrm{np}\right),\left({ }^{94} \mathrm{Mo}, 3 \mathrm{np}\right), \mathrm{E}=405-450 \mathrm{MeV} ;{ }^{144} \mathrm{Sm}\left({ }^{46} \mathrm{Ti}\right.$, $3 \mathrm{n}),\left({ }^{46} \mathrm{Ti}, 4 \mathrm{n}\right), \mathrm{E}=202-242 \mathrm{MeV}$; measured $\sigma$. Velocity filter, comparison with statistical model predictions. JOUR PRVCA 72 014612

2005UU03 RADIOACTIVITY ${ }^{188,190,192,194,196,198,200,202,204} \mathrm{Po}$, $191,193,195,197,199$ At, ${ }^{196,198,200,202,204,206} \mathrm{Rn},{ }^{199,201,203,205,207} \operatorname{Fr}(\alpha)$; measured reduced widths using gas filled recoil separator; deduced hindrance factors, proton intruder states and deformation effects. JOUR ZAANE 25 s01 179

${ }^{184} \mathrm{Bi}$ 2005AN17 NUCLEAR REACTIONS ${ }^{142} \mathrm{Nd}\left({ }^{52} \mathrm{Cr}, 3 \mathrm{n}\right),\left({ }^{52} \mathrm{Cr}, 4 \mathrm{n}\right),\left({ }^{52} \mathrm{Cr}, 5 \mathrm{n}\right)$, $\left({ }^{52} \mathrm{Cr}, 6 \mathrm{n}\right),\left({ }^{52} \mathrm{Cr}, \mathrm{np}\right),\left({ }^{52} \mathrm{Cr}, 2 \mathrm{np}\right),\left({ }^{52} \mathrm{Cr}, 3 \mathrm{np}\right),\left({ }^{52} \mathrm{Cr}, 4 \mathrm{np}\right),\left({ }^{52} \mathrm{Cr}\right.$, $5 \mathrm{np}),\left({ }^{52} \mathrm{Cr}, 6 \mathrm{np}\right), \mathrm{E}=220-310 \mathrm{MeV} ;{ }^{142} \mathrm{Nd}\left({ }^{50} \mathrm{Cr}, 3 \mathrm{n}\right),\left({ }^{50} \mathrm{Cr}, 4 \mathrm{n}\right),\left({ }^{50} \mathrm{Cr}\right.$, $2 \mathrm{np}),\left({ }^{50} \mathrm{Cr}, 3 \mathrm{np}\right),\left({ }^{50} \mathrm{Cr}, 4 \mathrm{np}\right),\left({ }^{50} \mathrm{Cr}, 5 \mathrm{np}\right), \mathrm{E}=230-285 \mathrm{MeV}$; ${ }^{92} \mathrm{Mo}\left({ }^{98} \mathrm{Mo}, 2 \mathrm{np}\right),\left({ }^{98} \mathrm{Mo}, 3 \mathrm{np}\right), \mathrm{E}=427-460 \mathrm{MeV} ;{ }^{93} \mathrm{Nb}\left({ }^{95} \mathrm{Mo}, \mathrm{n}\right)$, $\left({ }^{95} \mathrm{Mo}, 2 \mathrm{n}\right),\left({ }^{95} \mathrm{Mo}, 3 \mathrm{n}\right),\left({ }^{95} \mathrm{Mo}, \mathrm{p}\right),\left({ }^{95} \mathrm{Mo}, \mathrm{np}\right),\left({ }^{95} \mathrm{Mo}, 2 \mathrm{np}\right),\left({ }^{95} \mathrm{Mo}\right.$, 3np), ( $\left.{ }^{95} \mathrm{Mo}, 4 \mathrm{np}\right), \mathrm{E}=375-456 \mathrm{MeV} ;{ }^{93} \mathrm{Nb}\left({ }^{94} \mathrm{Mo}, 2 \mathrm{n}\right),\left({ }^{94} \mathrm{Mo}, 3 \mathrm{n}\right)$, $\left({ }^{94} \mathrm{Mo}, \mathrm{np}\right),\left({ }^{94} \mathrm{Mo}, 2 \mathrm{np}\right),\left({ }^{94} \mathrm{Mo}, 3 \mathrm{np}\right), \mathrm{E}=405-450 \mathrm{MeV} ;{ }^{144} \mathrm{Sm}\left({ }^{46} \mathrm{Ti}\right.$, $3 \mathrm{n}),\left({ }^{46} \mathrm{Ti}, 4 \mathrm{n}\right), \mathrm{E}=202-242 \mathrm{MeV}$; measured $\sigma$. Velocity filter, comparison with statistical model predictions. JOUR PRVCA 72 014612 


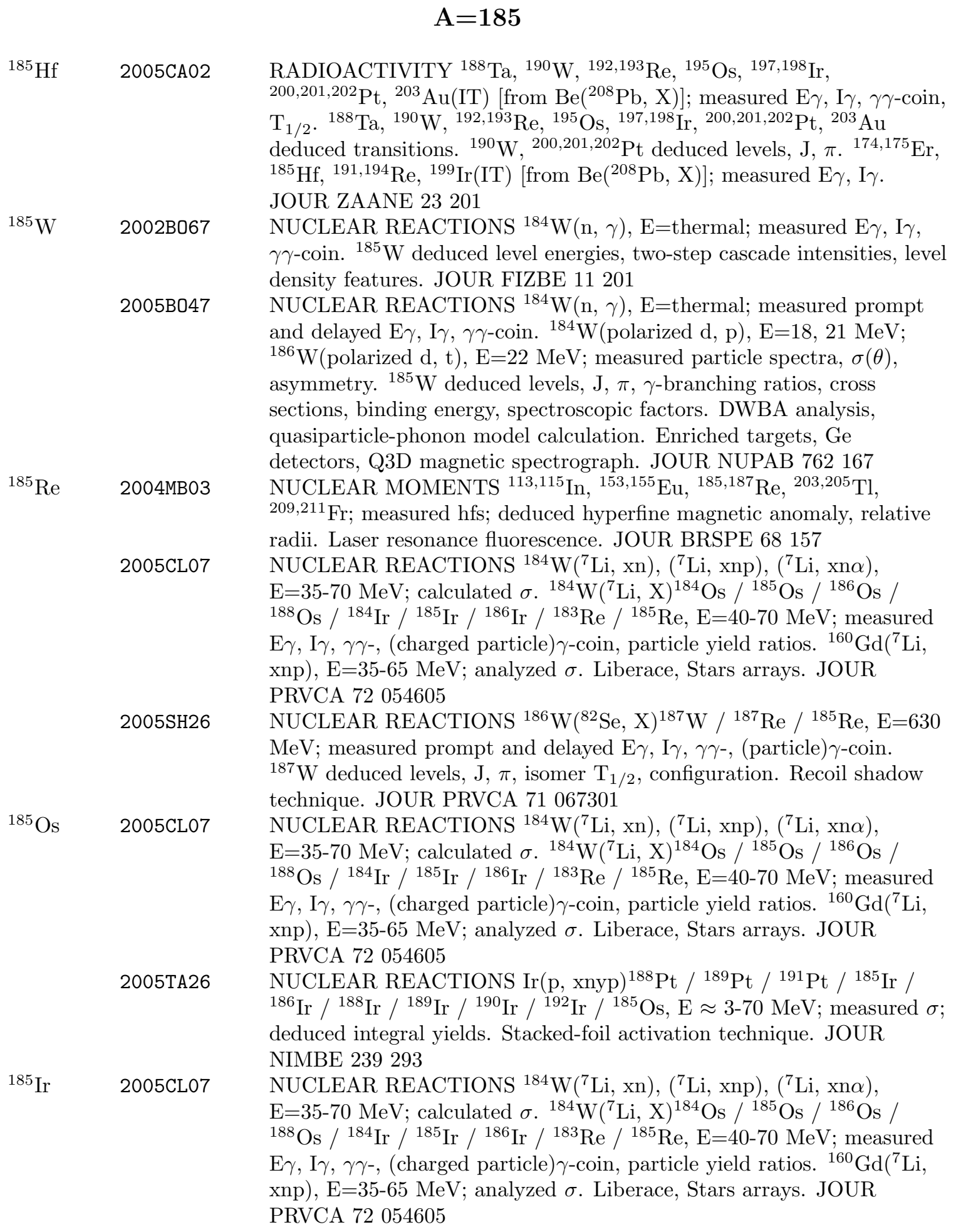




\section{$\mathrm{A}=185$ (continued)}

2005FOZZ NUCLEAR REACTIONS ${ }^{191} \operatorname{Ir}(\mathrm{n}, \mathrm{n}),(\mathrm{n}, 2 \mathrm{n}),(\mathrm{n}, 3 \mathrm{n}),(\mathrm{n}, 4 \mathrm{n}),(\mathrm{n}, 5 \mathrm{n})$, (n, 6n), (n, 7n), (n, 8n), (n, 9n), E=1-300 MeV; measured E $\gamma, \mathrm{I} \gamma$; deduced $\gamma$-ray production $\sigma$. CONF Santa Fe (Nucl Data for Sci and Technol) Proc,Vol1,P898

2005 TA26 NUCLEAR REACTIONS Ir $(\mathrm{p}, \mathrm{xnyp}){ }^{188} \mathrm{Pt} /{ }^{189} \mathrm{Pt} /{ }^{191} \mathrm{Pt} /{ }^{185} \mathrm{Ir} /$ ${ }^{186} \operatorname{Ir} /{ }^{188} \operatorname{Ir} /{ }^{189} \operatorname{Ir} /{ }^{190} \operatorname{Ir} /{ }^{192} \operatorname{Ir} /{ }^{185} \mathrm{Os}, \mathrm{E} \approx 3-70 \mathrm{MeV}$; measured $\sigma ;$ deduced integral yields. Stacked-foil activation technique. JOUR NIMBE 239293

${ }^{185} \mathrm{Au} \quad$ 2005CA43 NUCLEAR REACTIONS ${ }^{92} \mathrm{Mo}\left({ }^{84} \mathrm{Sr}, \mathrm{n} 2 \mathrm{p}\right),\left({ }^{84} \mathrm{Sr}, 3 \mathrm{p}\right),\left({ }^{84} \mathrm{Sr}, 2 \mathrm{np}\right)$, ${ }^{104} \mathrm{Ru}\left({ }^{84} \mathrm{Kr}, 2 \mathrm{np}\right),{ }^{90} \mathrm{Zr}\left({ }^{90} \mathrm{Zr}, \mathrm{n}\right),\left({ }^{90} \mathrm{Zr}, \mathrm{p}\right)$, E not given; ${ }^{92} \mathrm{Mo}\left({ }^{90} \mathrm{Zr}, \mathrm{n}\right)$, $\left({ }^{90} \mathrm{Zr}, \mathrm{p}\right), \mathrm{E}=385 \mathrm{MeV}$; measured $\mathrm{E} \gamma, \mathrm{I} \gamma, \gamma \gamma-$, (recoil $) \gamma$-coin. ${ }^{179} \mathrm{Hg}$ deduced high-spin levels, J, $\pi$. Gammasphere array, fragment separator. JOUR JPGPE 31 S1599

${ }^{185} \mathrm{Hg}$ 2002DU22 RADIOACTIVITY ${ }^{197,197 m, 198,199 m, 200,201 m} \mathrm{Po}(\alpha) ;{ }^{172,173} \mathrm{Os}(\alpha)$ [from $\left.{ }^{156} \mathrm{Dy}\left({ }^{22} \mathrm{Ne}, \mathrm{xn}\right)\right] ;{ }^{183,184,185} \mathrm{Hg}(\alpha)$ [from $\left.{ }^{168} \mathrm{Yb}\left({ }^{22} \mathrm{Ne}, \mathrm{xn}\right)\right]$; measured $\mathrm{E} \alpha, \mathrm{T}_{1 / 2}$. JOUR NIMAE 479631

${ }^{185} \mathrm{Tl}$ 2005VA04 RADIOACTIVITY ${ }^{189} \mathrm{Po}(\alpha)\left[\right.$ from $\left.{ }^{142} \mathrm{Nd}\left({ }^{52} \mathrm{Cr}, 5 \mathrm{n}\right),\left({ }^{50} \mathrm{Cr}, 3 \mathrm{n}\right)\right]$; measured $\mathrm{E} \alpha, \mathrm{E} \gamma, \mathrm{E}(\mathrm{ce}), \alpha \gamma$-coin. ${ }^{185} \mathrm{~Pb}$ deduced levels, J, $\pi$, ICC, $\mathrm{T}_{1 / 2}$, configurations. ${ }^{188,189,190,191} \mathrm{Bi},{ }^{189,190} \mathrm{Po}(\alpha)$ [from ${ }^{142} \mathrm{Nd}\left({ }^{52} \mathrm{Cr}\right.$, $\left.\mathrm{X}),\left({ }^{50} \mathrm{Cr}, \mathrm{X}\right)\right]$; measured $\mathrm{E} \alpha, \mathrm{I} \alpha$. Potential energy surface calculations, level systematics in neighboring isotopes discussed. JOUR ZAANE 24 57

${ }^{185} \mathrm{~Pb}$ 2005AN17 NUCLEAR REACTIONS ${ }^{142} \mathrm{Nd}\left({ }^{52} \mathrm{Cr}, 3 \mathrm{n}\right),\left({ }^{52} \mathrm{Cr}, 4 \mathrm{n}\right),\left({ }^{52} \mathrm{Cr}, 5 \mathrm{n}\right)$, $\left({ }^{52} \mathrm{Cr}, 6 \mathrm{n}\right),\left({ }^{52} \mathrm{Cr}, \mathrm{np}\right),\left({ }^{52} \mathrm{Cr}, 2 \mathrm{np}\right),\left({ }^{52} \mathrm{Cr}, 3 \mathrm{np}\right),\left({ }^{52} \mathrm{Cr}, 4 \mathrm{np}\right),\left({ }^{52} \mathrm{Cr}\right.$, $5 \mathrm{np}),\left({ }^{52} \mathrm{Cr}, 6 \mathrm{np}\right), \mathrm{E}=220-310 \mathrm{MeV} ;{ }^{142} \mathrm{Nd}\left({ }^{50} \mathrm{Cr}, 3 \mathrm{n}\right),\left({ }^{50} \mathrm{Cr}, 4 \mathrm{n}\right),\left({ }^{50} \mathrm{Cr}\right.$, $2 \mathrm{np}),\left({ }^{50} \mathrm{Cr}, 3 \mathrm{np}\right),\left({ }^{50} \mathrm{Cr}, 4 \mathrm{np}\right),\left({ }^{50} \mathrm{Cr}, 5 \mathrm{np}\right), \mathrm{E}=230-285 \mathrm{MeV}$; ${ }^{92} \mathrm{Mo}\left({ }^{98} \mathrm{Mo}, 2 \mathrm{np}\right),\left({ }^{98} \mathrm{Mo}, 3 \mathrm{np}\right), \mathrm{E}=427-460 \mathrm{MeV} ;{ }^{93} \mathrm{Nb}\left({ }^{95} \mathrm{Mo}, \mathrm{n}\right)$, $\left({ }^{95} \mathrm{Mo}, 2 \mathrm{n}\right),\left({ }^{95} \mathrm{Mo}, 3 \mathrm{n}\right),\left({ }^{95} \mathrm{Mo}, \mathrm{p}\right),\left({ }^{95} \mathrm{Mo}, \mathrm{np}\right),\left({ }^{95} \mathrm{Mo}, 2 \mathrm{np}\right),\left({ }^{95} \mathrm{Mo}\right.$, 3np), $\left({ }^{95} \mathrm{Mo}, 4 \mathrm{np}\right), \mathrm{E}=375-456 \mathrm{MeV} ;{ }^{93} \mathrm{Nb}\left({ }^{94} \mathrm{Mo}, 2 \mathrm{n}\right),\left({ }^{94} \mathrm{Mo}, 3 \mathrm{n}\right)$, $\left({ }^{94} \mathrm{Mo}, \mathrm{np}\right),\left({ }^{94} \mathrm{Mo}, 2 \mathrm{np}\right),\left({ }^{94} \mathrm{Mo}, 3 \mathrm{np}\right), \mathrm{E}=405-450 \mathrm{MeV} ;{ }^{144} \mathrm{Sm}\left({ }^{46} \mathrm{Ti}\right.$, $3 \mathrm{n}),\left({ }^{46} \mathrm{Ti}, 4 \mathrm{n}\right), \mathrm{E}=202-242 \mathrm{MeV}$; measured $\sigma$. Velocity filter, comparison with statistical model predictions. JOUR PRVCA 72 014612

2005VA04 RADIOACTIVITY ${ }^{189} \mathrm{Po}(\alpha)$ [from $\left.{ }^{142} \mathrm{Nd}\left({ }^{52} \mathrm{Cr}, 5 \mathrm{n}\right),\left({ }^{50} \mathrm{Cr}, 3 \mathrm{n}\right)\right]$; measured $\mathrm{E} \alpha, \mathrm{E} \gamma, \mathrm{E}(\mathrm{ce}), \alpha \gamma$-coin. ${ }^{185} \mathrm{~Pb}$ deduced levels, J, $\pi$, ICC, $\mathrm{T}_{1 / 2}$, configurations. ${ }^{188,189,190,191} \mathrm{Bi},{ }^{189,190} \mathrm{Po}(\alpha)$ [from ${ }^{142} \mathrm{Nd}\left({ }^{52} \mathrm{Cr}\right.$, $\left.\mathrm{X}),\left({ }^{50} \mathrm{Cr}, \mathrm{X}\right)\right]$; measured $\mathrm{E} \alpha, \mathrm{I} \alpha$. Potential energy surface calculations, level systematics in neighboring isotopes discussed. JOUR ZAANE 24 57 


\section{$\mathrm{A}=185$ (continued)}

${ }^{185} \mathrm{Bi}$ 2005AN17 NUCLEAR REACTIONS ${ }^{142} \mathrm{Nd}\left({ }^{52} \mathrm{Cr}, 3 \mathrm{n}\right),\left({ }^{52} \mathrm{Cr}, 4 \mathrm{n}\right),\left({ }^{52} \mathrm{Cr}, 5 \mathrm{n}\right)$, $\left({ }^{52} \mathrm{Cr}, 6 \mathrm{n}\right),\left({ }^{52} \mathrm{Cr}, \mathrm{np}\right),\left({ }^{52} \mathrm{Cr}, 2 \mathrm{np}\right),\left({ }^{52} \mathrm{Cr}, 3 \mathrm{np}\right),\left({ }^{52} \mathrm{Cr}, 4 \mathrm{np}\right),\left({ }^{52} \mathrm{Cr}\right.$, 5np), $\left({ }^{52} \mathrm{Cr}, 6 \mathrm{np}\right), \mathrm{E}=220-310 \mathrm{MeV} ;{ }^{142} \mathrm{Nd}\left({ }^{50} \mathrm{Cr}, 3 \mathrm{n}\right),\left({ }^{50} \mathrm{Cr}, 4 \mathrm{n}\right),\left({ }^{50} \mathrm{Cr}\right.$, $2 \mathrm{np}),\left({ }^{50} \mathrm{Cr}, 3 \mathrm{np}\right),\left({ }^{50} \mathrm{Cr}, 4 \mathrm{np}\right),\left({ }^{50} \mathrm{Cr}, 5 \mathrm{np}\right), \mathrm{E}=230-285 \mathrm{MeV}$; ${ }^{92} \mathrm{Mo}\left({ }^{98} \mathrm{Mo}, 2 \mathrm{np}\right),\left({ }^{98} \mathrm{Mo}, 3 \mathrm{np}\right), \mathrm{E}=427-460 \mathrm{MeV} ;{ }^{93} \mathrm{Nb}\left({ }^{95} \mathrm{Mo}, \mathrm{n}\right)$, $\left({ }^{95} \mathrm{Mo}, 2 \mathrm{n}\right),\left({ }^{95} \mathrm{Mo}, 3 \mathrm{n}\right),\left({ }^{95} \mathrm{Mo}, \mathrm{p}\right),\left({ }^{95} \mathrm{Mo}, \mathrm{np}\right),\left({ }^{95} \mathrm{Mo}, 2 \mathrm{np}\right),\left({ }^{95} \mathrm{Mo}\right.$, 3np), ( $\left.{ }^{95} \mathrm{Mo}, 4 \mathrm{np}\right), \mathrm{E}=375-456 \mathrm{MeV} ;{ }^{93} \mathrm{Nb}\left({ }^{94} \mathrm{Mo}, 2 \mathrm{n}\right),\left({ }^{94} \mathrm{Mo}, 3 \mathrm{n}\right)$, $\left({ }^{94} \mathrm{Mo}, \mathrm{np}\right),\left({ }^{94} \mathrm{Mo}, 2 \mathrm{np}\right),\left({ }^{94} \mathrm{Mo}, 3 \mathrm{np}\right), \mathrm{E}=405-450 \mathrm{MeV} ;{ }^{144} \mathrm{Sm}\left({ }^{46} \mathrm{Ti}\right.$, $3 \mathrm{n}),\left({ }^{46} \mathrm{Ti}, 4 \mathrm{n}\right), \mathrm{E}=202-242 \mathrm{MeV}$; measured $\sigma$. Velocity filter, comparison with statistical model predictions. JOUR PRVCA 72 014612

2005GEZW ATOMIC MASSES ${ }^{235}$ Ac; measured mass, $\mathrm{T}_{1 / 2}$. $185,186,187,188,189,190,191,192,193,194,195,196 \mathrm{Bi}$; measured masses, proton separation energies. ${ }^{207 m} \mathrm{Tl}$; measured $\mathrm{T}_{1 / 2}$. Stored beams, Schottky mass spectrometry. PREPRINT nucl-ex/0510009,10/4/2005

\section{$\mathrm{A}=\mathbf{1 8 6}$}

${ }^{186} \mathrm{~W} \quad 2004 \mathrm{C026}$

${ }^{186} \operatorname{Re} \quad 2005 A L Z Y$

2005HA60

2005HAZZ

2005NAZY

${ }^{186}$ Os $\quad 2005$ CL07

2005CL07

RADIOACTIVITY ${ }^{180} \mathrm{~W}(\alpha)$; measured E $\alpha, \mathrm{T}_{1 / 2}$, Q-value. ${ }_{182,183,184,186} \mathrm{~W}(\alpha)$; measured $\mathrm{T}_{1 / 2}$ lower limits. $\mathrm{CaWO}_{4}$ crystals. JOUR PRVCA 70064606

NUCLEAR REACTIONS ${ }^{186} \mathrm{~W}(\mathrm{~d}, 2 \mathrm{n}), \mathrm{E}=12.8-5.9 \mathrm{MeV}$; measured yields. Stacked foil activation. CONF St Petersburg,P181,Alekseev NUCLEAR REACTIONS ${ }^{185} \operatorname{Re}(\mathrm{n}, \gamma), \mathrm{E}=$ thermal; measured isomer yield ratio. Activation technique, astrophysical implications discussed. JOUR ASJOA 628533

NUCLEAR REACTIONS ${ }^{185} \operatorname{Re}(\mathrm{n}, \gamma), \mathrm{E}=$ thermal; measured capture $\sigma$ to ground and isomeric states. Astrophysical implications discussed. CONF Riken(Origin of Matter) Proc,P208,Hayakawa NUCLEAR REACTIONS ${ }^{27} \mathrm{Al}(\mathrm{d}, \mathrm{X}){ }^{27} \mathrm{Mg} /{ }^{24} \mathrm{Na}, \mathrm{E}=22-40 \mathrm{MeV}$; $\mathrm{Cu}(\mathrm{d}, \mathrm{X}){ }^{62} \mathrm{Zn} /{ }^{63} \mathrm{Zn} /{ }^{61} \mathrm{Cu} /{ }^{64} \mathrm{Cu}, \mathrm{E}=22-40 \mathrm{MeV} ; \mathrm{W}(\mathrm{d}, \mathrm{X}){ }^{181} \mathrm{Re} /$ ${ }^{182} \operatorname{Re} /{ }^{183} \operatorname{Re} /{ }^{184} \operatorname{Re} /{ }^{186} \operatorname{Re} /{ }^{187} \mathrm{~W}, \mathrm{E}=22-40 \mathrm{MeV}$; measured activation $\sigma$. Comparison with previous results and model predictions. CONF Santa Fe (Nucl Data for Sci and Technol) Proc,Vol2,P1489 NUCLEAR REACTIONS ${ }^{184} \mathrm{~W}\left({ }^{7} \mathrm{Li}, \mathrm{xn}\right),\left({ }^{7} \mathrm{Li}, \mathrm{xnp}\right),\left({ }^{7} \mathrm{Li}, \mathrm{xn} \alpha\right)$, $\mathrm{E}=35-70 \mathrm{MeV}$; calculated $\sigma .{ }^{184} \mathrm{~W}\left({ }^{7} \mathrm{Li}, \mathrm{X}\right){ }^{184} \mathrm{Os} /{ }^{185} \mathrm{Os} /{ }^{186} \mathrm{Os} /$ ${ }^{188} \mathrm{Os} /{ }^{184} \mathrm{Ir} /{ }^{185} \mathrm{Ir} /{ }^{186} \mathrm{Ir} /{ }^{183} \mathrm{Re} /{ }^{185} \mathrm{Re}, \mathrm{E}=40-70 \mathrm{MeV}$; measured $\mathrm{E} \gamma, \mathrm{I} \gamma, \gamma \gamma-$, (charged particle) $\gamma$-coin, particle yield ratios. ${ }^{160} \mathrm{Gd}\left({ }^{7} \mathrm{Li}\right.$, xnp), E=35-65 MeV; analyzed $\sigma$. Liberace, Stars arrays. JOUR PRVCA 72054605

NUCLEAR REACTIONS ${ }^{184} \mathrm{~W}\left({ }^{7} \mathrm{Li}, \mathrm{xn}\right),\left({ }^{7} \mathrm{Li}, \mathrm{xnp}\right),\left({ }^{7} \mathrm{Li}, \mathrm{xn} \alpha\right)$, $\mathrm{E}=35-70 \mathrm{MeV}$; calculated $\sigma .{ }^{184} \mathrm{~W}\left({ }^{7} \mathrm{Li}, \mathrm{X}\right){ }^{184} \mathrm{Os} /{ }^{185} \mathrm{Os} /{ }^{186} \mathrm{Os} /$ ${ }^{188} \mathrm{Os} /{ }^{184} \mathrm{Ir} /{ }^{185} \mathrm{Ir} /{ }^{186} \mathrm{Ir} /{ }^{183} \mathrm{Re} /{ }^{185} \mathrm{Re}, \mathrm{E}=40-70 \mathrm{MeV}$; measured $\mathrm{E} \gamma, \mathrm{I} \gamma, \gamma \gamma-$, (charged particle) $\gamma$-coin, particle yield ratios. ${ }^{160} \mathrm{Gd}\left({ }^{7} \mathrm{Li}\right.$, $\mathrm{xnp}), \mathrm{E}=35-65 \mathrm{MeV}$; analyzed $\sigma$. Liberace, Stars arrays. JOUR PRVCA 72054605 


\section{$\mathrm{A}=186$ (continued)}

2005FOZZ NUCLEAR REACTIONS ${ }^{191} \operatorname{Ir}(\mathrm{n}, \mathrm{n}),(\mathrm{n}, 2 \mathrm{n}),(\mathrm{n}, 3 \mathrm{n}),(\mathrm{n}, 4 \mathrm{n}),(\mathrm{n}, 5 \mathrm{n})$, (n, 6n), (n, 7n), (n, 8n), (n, 9n), E=1-300 MeV; measured E $\gamma, \mathrm{I} \gamma$; deduced $\gamma$-ray production $\sigma$. CONF Santa Fe (Nucl Data for Sci and Technol) Proc,Vol1,P898

2005 TA26 NUCLEAR REACTIONS Ir $(\mathrm{p}, \mathrm{xnyp}){ }^{188} \mathrm{Pt} /{ }^{189} \mathrm{Pt} /{ }^{191} \mathrm{Pt} /{ }^{185} \mathrm{Ir} /$ ${ }^{186} \operatorname{Ir} /{ }^{188} \operatorname{Ir} /{ }^{189} \operatorname{Ir} /{ }^{190} \operatorname{Ir} /{ }^{192} \operatorname{Ir} /{ }^{185} \mathrm{Os}, \mathrm{E} \approx 3-70 \mathrm{MeV}$; measured $\sigma$; deduced integral yields. Stacked-foil activation technique. JOUR NIMBE 239293

2005TAZV NUCLEAR REACTIONS Ir $(\mathrm{p}, \mathrm{X}){ }^{188} \mathrm{Pt} /{ }^{189} \mathrm{Pt} /{ }^{191} \mathrm{Pt} /{ }^{186} \mathrm{Ir} /{ }^{187} \mathrm{Ir}$ $/{ }^{188} \operatorname{Ir} /{ }^{189} \operatorname{Ir} /{ }^{190} \operatorname{Ir} /{ }^{192} \mathrm{Ir}, \mathrm{E} \approx 10-70 \mathrm{MeV}$; measured excitation functions; deduced integral yields. Stacked-foil activation technique. CONF Santa Fe (Nucl Data for Sci and Technol) Proc,Vol1,P1023 RADIOACTIVITY ${ }^{189} \mathrm{Po}(\alpha)$ [from ${ }^{142} \mathrm{Nd}\left({ }^{52} \mathrm{Cr}, 5 \mathrm{n}\right),\left({ }^{50} \mathrm{Cr}, 3 \mathrm{n}\right)$ ]; measured $\mathrm{E} \alpha, \mathrm{E} \gamma, \mathrm{E}(\mathrm{ce}), \alpha \gamma$-coin. ${ }^{185} \mathrm{~Pb}$ deduced levels, J, $\pi$, ICC, $\mathrm{T}_{1 / 2}$, configurations. ${ }^{188,189,190,191} \mathrm{Bi},{ }^{189,190} \mathrm{Po}(\alpha)$ [from ${ }^{142} \mathrm{Nd}\left({ }^{52} \mathrm{Cr}\right.$, $\left.\mathrm{X}),\left({ }^{50} \mathrm{Cr}, \mathrm{X}\right)\right]$; measured $\mathrm{E} \alpha, \mathrm{I} \alpha$. Potential energy surface calculations, level systematics in neighboring isotopes discussed. JOUR ZAANE 24 57

${ }^{186} \mathrm{~Pb}$ 2005AN17 NUCLEAR REACTIONS ${ }^{142} \mathrm{Nd}\left({ }^{52} \mathrm{Cr}, 3 \mathrm{n}\right),\left({ }^{52} \mathrm{Cr}, 4 \mathrm{n}\right),\left({ }^{52} \mathrm{Cr}, 5 \mathrm{n}\right)$, $\left({ }^{52} \mathrm{Cr}, 6 \mathrm{n}\right),\left({ }^{52} \mathrm{Cr}, \mathrm{np}\right),\left({ }^{52} \mathrm{Cr}, 2 \mathrm{np}\right),\left({ }^{52} \mathrm{Cr}, 3 \mathrm{np}\right),\left({ }^{52} \mathrm{Cr}, 4 \mathrm{np}\right),\left({ }^{52} \mathrm{Cr}\right.$, $5 \mathrm{np}),\left({ }^{52} \mathrm{Cr}, 6 \mathrm{np}\right), \mathrm{E}=220-310 \mathrm{MeV} ;{ }^{142} \mathrm{Nd}\left({ }^{50} \mathrm{Cr}, 3 \mathrm{n}\right),\left({ }^{50} \mathrm{Cr}, 4 \mathrm{n}\right),\left({ }^{50} \mathrm{Cr}\right.$, $2 \mathrm{np}),\left({ }^{50} \mathrm{Cr}, 3 \mathrm{np}\right),\left({ }^{50} \mathrm{Cr}, 4 \mathrm{np}\right),\left({ }^{50} \mathrm{Cr}, 5 \mathrm{np}\right), \mathrm{E}=230-285 \mathrm{MeV}$;

${ }^{92} \mathrm{Mo}\left({ }^{98} \mathrm{Mo}, 2 \mathrm{np}\right),\left({ }^{98} \mathrm{Mo}, 3 \mathrm{np}\right), \mathrm{E}=427-460 \mathrm{MeV} ;{ }^{93} \mathrm{Nb}\left({ }^{95} \mathrm{Mo}, \mathrm{n}\right)$, $\left({ }^{95} \mathrm{Mo}, 2 \mathrm{n}\right),\left({ }^{95} \mathrm{Mo}, 3 \mathrm{n}\right),\left({ }^{95} \mathrm{Mo}, \mathrm{p}\right),\left({ }^{95} \mathrm{Mo}, \mathrm{np}\right),\left({ }^{95} \mathrm{Mo}, 2 \mathrm{np}\right),\left({ }^{95} \mathrm{Mo}\right.$, 3np), ( $\left.{ }^{95} \mathrm{Mo}, 4 \mathrm{np}\right), \mathrm{E}=375-456 \mathrm{MeV} ;{ }^{93} \mathrm{Nb}\left({ }^{94} \mathrm{Mo}, 2 \mathrm{n}\right),\left({ }^{94} \mathrm{Mo}, 3 \mathrm{n}\right)$, $\left({ }^{94} \mathrm{Mo}, \mathrm{np}\right),\left({ }^{94} \mathrm{Mo}, 2 \mathrm{np}\right),\left({ }^{94} \mathrm{Mo}, 3 \mathrm{np}\right), \mathrm{E}=405-450 \mathrm{MeV} ;{ }^{144} \mathrm{Sm}\left({ }^{46} \mathrm{Ti}\right.$, $3 \mathrm{n}),\left({ }^{46} \mathrm{Ti}, 4 \mathrm{n}\right), \mathrm{E}=202-242 \mathrm{MeV}$; measured $\sigma$. Velocity filter, comparison with statistical model predictions. JOUR PRVCA 72 014612

2005PA42 NUCLEAR REACTIONS ${ }^{106} \mathrm{Pd}\left({ }^{83} \mathrm{Kr}, 3 \mathrm{n}\right), \mathrm{E}=355 \mathrm{MeV}$; measured E $\gamma$, $\mathrm{I} \gamma, \gamma \gamma$-, (recoil) $\gamma$-coin. ${ }^{186} \mathrm{~Pb}$ deduced levels, $\mathrm{J}, \pi$, oblate deformation. Jurogam array, recoil-decay tagging, interacting boson model calculations. JOUR PRVCA 72011304

2005PA69 NUCLEAR REACTIONS ${ }^{106} \mathrm{Pd}\left({ }^{83} \mathrm{Kr}, 3 \mathrm{n}\right), \mathrm{E}=355 \mathrm{MeV}$; measured E $\gamma$, $\mathrm{I} \gamma, \gamma \gamma$-, (recoil) $\gamma$-coin; deduced production $\sigma .{ }^{186} \mathrm{~Pb}$ deduced levels, J, $\pi$, deformation. Jurogam array, recoil-decay tagging. JOUR ZAANE $25 \mathrm{~s} 01449$

2005UU03 RADIOACTIVITY ${ }^{188,190,192,194,196,198,200,202,204} \mathrm{Po}$ 191,193,195,197,199 At, ${ }^{196,198,200,202,204,206} \mathrm{Rn},{ }^{199,201,203,205,207} \operatorname{Fr}(\alpha)$; measured reduced widths using gas filled recoil separator; deduced hindrance factors, proton intruder states and deformation effects. JOUR ZAANE 25 s01 179

2005VA04 RADIOACTIVITY ${ }^{189} \mathrm{Po}(\alpha)$ [from $\left.{ }^{142} \mathrm{Nd}\left({ }^{52} \mathrm{Cr}, 5 \mathrm{n}\right),\left({ }^{50} \mathrm{Cr}, 3 \mathrm{n}\right)\right]$; measured $\mathrm{E} \alpha, \mathrm{E} \gamma, \mathrm{E}(\mathrm{ce}), \alpha \gamma$-coin. ${ }^{185} \mathrm{~Pb}$ deduced levels, J, $\pi$, ICC, $\mathrm{T}_{1 / 2}$, configurations. ${ }^{188,189,190,191} \mathrm{Bi},{ }^{189,190} \mathrm{Po}(\alpha)$ [from ${ }^{142} \mathrm{Nd}\left({ }^{52} \mathrm{Cr}\right.$, $\left.\mathrm{X}),\left({ }^{50} \mathrm{Cr}, \mathrm{X}\right)\right]$; measured $\mathrm{E} \alpha, \mathrm{I} \alpha$. Potential energy surface calculations, level systematics in neighboring isotopes discussed. JOUR ZAANE 24 57 


\section{$\mathrm{A}=186$ (continued)}

${ }^{186} \mathrm{Bi}$ 2005AN17 NUCLEAR REACTIONS ${ }^{142} \mathrm{Nd}\left({ }^{52} \mathrm{Cr}, 3 \mathrm{n}\right),\left({ }^{52} \mathrm{Cr}, 4 \mathrm{n}\right),\left({ }^{52} \mathrm{Cr}, 5 \mathrm{n}\right)$, $\left({ }^{52} \mathrm{Cr}, 6 \mathrm{n}\right),\left({ }^{52} \mathrm{Cr}, \mathrm{np}\right),\left({ }^{52} \mathrm{Cr}, 2 \mathrm{np}\right),\left({ }^{52} \mathrm{Cr}, 3 \mathrm{np}\right),\left({ }^{52} \mathrm{Cr}, 4 \mathrm{np}\right),\left({ }^{52} \mathrm{Cr}\right.$, $5 \mathrm{np}),\left({ }^{52} \mathrm{Cr}, 6 \mathrm{np}\right), \mathrm{E}=220-310 \mathrm{MeV} ;{ }^{142} \mathrm{Nd}\left({ }^{50} \mathrm{Cr}, 3 \mathrm{n}\right),\left({ }^{50} \mathrm{Cr}, 4 \mathrm{n}\right),\left({ }^{50} \mathrm{Cr}\right.$, $2 \mathrm{np}),\left({ }^{50} \mathrm{Cr}, 3 \mathrm{np}\right),\left({ }^{50} \mathrm{Cr}, 4 \mathrm{np}\right),\left({ }^{50} \mathrm{Cr}, 5 \mathrm{np}\right), \mathrm{E}=230-285 \mathrm{MeV}$; ${ }^{92} \mathrm{Mo}\left({ }^{98} \mathrm{Mo}, 2 \mathrm{np}\right),\left({ }^{98} \mathrm{Mo}, 3 \mathrm{np}\right), \mathrm{E}=427-460 \mathrm{MeV} ;{ }^{93} \mathrm{Nb}\left({ }^{95} \mathrm{Mo}, \mathrm{n}\right)$, $\left({ }^{95} \mathrm{Mo}, 2 \mathrm{n}\right),\left({ }^{95} \mathrm{Mo}, 3 \mathrm{n}\right),\left({ }^{95} \mathrm{Mo}, \mathrm{p}\right),\left({ }^{95} \mathrm{Mo}, \mathrm{np}\right),\left({ }^{95} \mathrm{Mo}, 2 \mathrm{np}\right),\left({ }^{95} \mathrm{Mo}\right.$, 3np), ( $\left.{ }^{95} \mathrm{Mo}, 4 \mathrm{np}\right), \mathrm{E}=375-456 \mathrm{MeV} ;{ }^{93} \mathrm{Nb}\left({ }^{94} \mathrm{Mo}, 2 \mathrm{n}\right),\left({ }^{94} \mathrm{Mo}, 3 \mathrm{n}\right)$, $\left({ }^{94} \mathrm{Mo}, \mathrm{np}\right),\left({ }^{94} \mathrm{Mo}, 2 \mathrm{np}\right),\left({ }^{94} \mathrm{Mo}, 3 \mathrm{np}\right), \mathrm{E}=405-450 \mathrm{MeV} ;{ }^{144} \mathrm{Sm}\left({ }^{46} \mathrm{Ti}\right.$, $3 \mathrm{n}),\left({ }^{46} \mathrm{Ti}, 4 \mathrm{n}\right), \mathrm{E}=202-242 \mathrm{MeV}$; measured $\sigma$. Velocity filter, comparison with statistical model predictions. JOUR PRVCA 72 014612

2005GEZW ATOMIC MASSES ${ }^{235}$ Ac; measured mass, $T_{1 / 2}$. $185,186,187,188,189,190,191,192,193,194,195,196 \mathrm{Bi}$; measured masses, proton separation energies. ${ }^{207 m} \mathrm{Tl}$; measured $\mathrm{T}_{1 / 2}$. Stored beams, Schottky mass spectrometry. PREPRINT nucl-ex/0510009,10/4/2005

${ }^{186} \mathrm{Po}$ 2005AN17 NUCLEAR REACTIONS ${ }^{142} \mathrm{Nd}\left({ }^{52} \mathrm{Cr}, 3 \mathrm{n}\right),\left({ }^{52} \mathrm{Cr}, 4 \mathrm{n}\right),\left({ }^{52} \mathrm{Cr}, 5 \mathrm{n}\right)$, $\left({ }^{52} \mathrm{Cr}, 6 \mathrm{n}\right),\left({ }^{52} \mathrm{Cr}, \mathrm{np}\right),\left({ }^{52} \mathrm{Cr}, 2 \mathrm{np}\right),\left({ }^{52} \mathrm{Cr}, 3 \mathrm{np}\right),\left({ }^{52} \mathrm{Cr}, 4 \mathrm{np}\right),\left({ }^{52} \mathrm{Cr}\right.$, $5 \mathrm{np}),\left({ }^{52} \mathrm{Cr}, 6 \mathrm{np}\right), \mathrm{E}=220-310 \mathrm{MeV} ;{ }^{142} \mathrm{Nd}\left({ }^{50} \mathrm{Cr}, 3 \mathrm{n}\right),\left({ }^{50} \mathrm{Cr}, 4 \mathrm{n}\right),\left({ }^{50} \mathrm{Cr}\right.$, $2 \mathrm{np}),\left({ }^{50} \mathrm{Cr}, 3 \mathrm{np}\right),\left({ }^{50} \mathrm{Cr}, 4 \mathrm{np}\right),\left({ }^{50} \mathrm{Cr}, 5 \mathrm{np}\right), \mathrm{E}=230-285 \mathrm{MeV}$; ${ }^{92} \mathrm{Mo}\left({ }^{98} \mathrm{Mo}, 2 \mathrm{np}\right),\left({ }^{98} \mathrm{Mo}\right.$, 3np $), \mathrm{E}=427-460 \mathrm{MeV} ;{ }^{93} \mathrm{Nb}\left({ }^{95} \mathrm{Mo}, \mathrm{n}\right)$, $\left({ }^{95} \mathrm{Mo}, 2 \mathrm{n}\right),\left({ }^{95} \mathrm{Mo}, 3 \mathrm{n}\right),\left({ }^{95} \mathrm{Mo}, \mathrm{p}\right),\left({ }^{95} \mathrm{Mo}, \mathrm{np}\right),\left({ }^{95} \mathrm{Mo}, 2 \mathrm{np}\right),\left({ }^{95} \mathrm{Mo}\right.$, 3np), ( $\left.{ }^{95} \mathrm{Mo}, 4 \mathrm{np}\right), \mathrm{E}=375-456 \mathrm{MeV} ;{ }^{93} \mathrm{Nb}\left({ }^{94} \mathrm{Mo}, 2 \mathrm{n}\right),\left({ }^{94} \mathrm{Mo}, 3 \mathrm{n}\right)$, $\left({ }^{94} \mathrm{Mo}, \mathrm{np}\right),\left({ }^{94} \mathrm{Mo}, 2 \mathrm{np}\right),\left({ }^{94} \mathrm{Mo}, 3 \mathrm{np}\right), \mathrm{E}=405-450 \mathrm{MeV} ;{ }^{144} \mathrm{Sm}\left({ }^{46} \mathrm{Ti}\right.$, $3 \mathrm{n}),\left({ }^{46} \mathrm{Ti}, 4 \mathrm{n}\right), \mathrm{E}=202-242 \mathrm{MeV}$; measured $\sigma$. Velocity filter, comparison with statistical model predictions. JOUR PRVCA 72 014612

2005ANZY NUCLEAR REACTIONS ${ }^{144} \mathrm{Sm}\left({ }^{46} \mathrm{Ti}\right.$, xn), E not given; measured $\mathrm{E} \gamma$, $\mathrm{E} \alpha, \alpha \alpha-, \alpha \gamma$-coin following residual nucleus decay; deduced evidence for ${ }^{186,187}$ Po. ${ }^{144} \mathrm{Sm}\left({ }^{51} \mathrm{~V}, \mathrm{xn}\right)$, E not given; measured $\mathrm{E} \gamma, \mathrm{E} \alpha, \alpha \alpha-$, $\alpha \gamma$-coin following residual nucleus decay; deduced evidence for ${ }^{192} \mathrm{At}$. REPT GSI 2005-1,P77,Andreyev

2005ANZY RADIOACTIVITY ${ }^{186,187} \mathrm{Po},{ }^{183} \mathrm{~Pb},{ }^{179} \mathrm{Hg},{ }^{175} \mathrm{Pt}(\alpha)$ [from ${ }^{144} \mathrm{Sm}\left({ }^{46} \mathrm{Ti}\right.$, $\mathrm{xn})$ and subsequent decay]; measured $\mathrm{E} \alpha, \mathrm{T}_{1 / 2} .{ }^{183} \mathrm{~Pb}$ deduced excited state energy. ${ }^{187} \mathrm{Po}$ deduced isomeric states. ${ }^{192} \mathrm{At}(\alpha)\left[\right.$ from ${ }^{144} \mathrm{Sm}\left({ }^{51} \mathrm{~V}\right.$, $\mathrm{xn})]$; measured $\mathrm{E} \alpha, \alpha \alpha$-coin, $\mathrm{T}_{1 / 2}$; deduced isomeric states. REPT GSI 2005-1,P77,Andreyev

\section{$\mathrm{A}=187$}

${ }^{187} \mathrm{~W}$ 2005NAZY NUCLEAR REACTIONS ${ }^{27} \mathrm{Al}(\mathrm{d}, \mathrm{X})^{27} \mathrm{Mg} /{ }^{24} \mathrm{Na}, \mathrm{E}=22-40 \mathrm{MeV}$; $\mathrm{Cu}(\mathrm{d}, \mathrm{X}){ }^{62} \mathrm{Zn} /{ }^{63} \mathrm{Zn} /{ }^{61} \mathrm{Cu} /{ }^{64} \mathrm{Cu}, \mathrm{E}=22-40 \mathrm{MeV} ; \mathrm{W}(\mathrm{d}, \mathrm{X}){ }^{181} \mathrm{Re} /$ ${ }^{182} \operatorname{Re} /{ }^{183} \operatorname{Re} /{ }^{184} \operatorname{Re} /{ }^{186} \operatorname{Re} /{ }^{187} \mathrm{~W}, \mathrm{E}=22-40 \mathrm{MeV}$; measured activation $\sigma$. Comparison with previous results and model predictions. CONF Santa Fe (Nucl Data for Sci and Technol) Proc,Vol2,P1489

2005SH26 NUCLEAR REACTIONS ${ }^{186} \mathrm{~W}\left({ }^{82} \mathrm{Se}, \mathrm{X}\right){ }^{187} \mathrm{~W} /{ }^{187} \mathrm{Re} /{ }^{185} \mathrm{Re}, \mathrm{E}=630$ $\mathrm{MeV}$; measured prompt and delayed $\mathrm{E} \gamma, \mathrm{I} \gamma, \gamma \gamma-$, (particle) $\gamma$-coin. ${ }^{187} \mathrm{~W}$ deduced levels, $\mathrm{J}, \pi$, isomer $\mathrm{T}_{1 / 2}$, configuration. Recoil shadow technique. JOUR PRVCA 71067301 


\section{$\mathrm{A}=187$ (continued)}

\begin{tabular}{|c|c|c|}
\hline \multirow[t]{3}{*}{${ }^{187} \operatorname{Re}$} & 2004MB03 & $\begin{array}{l}\text { NUCLEAR MOMENTS }{ }^{113,115} \mathrm{In},{ }^{153,155} \mathrm{Eu},{ }^{185,187} \mathrm{Re},{ }^{203,205} \mathrm{Tl} \text {, } \\
{ }^{209,211} \mathrm{Fr} \text {; measured hfs; deduced hyperfine magnetic anomaly, relative }\end{array}$ \\
\hline & $2005 \mathrm{SH} 26$ & NUCLEAR REACTIONS ${ }^{186} \mathrm{~W}\left({ }^{82} \mathrm{Se}, \mathrm{X}\right)^{187} \mathrm{~W} /{ }^{187} \mathrm{Re} /{ }^{185} \mathrm{Re}, \mathrm{E}=630$ \\
\hline & & $\begin{array}{l}\mathrm{MeV} ; \text { measured prompt and delayed } \mathrm{E} \gamma, \mathrm{I} \gamma, \gamma \gamma-, \text { (particle) } \gamma \text {-coin. } \\
{ }^{187} \mathrm{~W} \text { deduced levels, J, } \pi \text {, isomer } \mathrm{T}_{1 / 2} \text {, configuration. Recoil shadow } \\
\text { technique. JOUR PRVCA } 71067301\end{array}$ \\
\hline \multirow[t]{6}{*}{${ }^{187} \mathrm{Os}$} & $2005 \mathrm{AB} 22$ & $\begin{array}{l}\text { NUCLEAR REACTIONS }{ }^{186,187} \mathrm{Os}(\mathrm{n}, \gamma), \mathrm{E} \approx 1-1000 \mathrm{keV} ; \text { measured } \\
\text { capture } \sigma . \text { Astrophysical implications discussed. JOUR NUPAB } 758 \\
501 \mathrm{c}\end{array}$ \\
\hline & 2005MOZV & $\begin{array}{l}\text { NUCLEAR REACTIONS }{ }^{186,187,188} \mathrm{Os}(\mathrm{n}, \gamma), \mathrm{E}<1 \mathrm{MeV} \text {; measured } \\
\text { capture } \sigma \text {. Comparison with previous results, astrophysical } \\
\text { implications discussed. CONF Santa Fe (Nucl Data for Sci and } \\
\text { Technol) Proc,Vol2,P1335 }\end{array}$ \\
\hline & 2005SE19 & $\begin{array}{l}\text { NUCLEAR REACTIONS }{ }^{186,187,188} \mathrm{Os}(\mathrm{n}, \gamma), \mathrm{E}=10-90 \mathrm{keV} \text {; measured } \\
\mathrm{E} \gamma, \mathrm{I} \gamma . \text { JOUR NUPAB } 758553 \mathrm{c}\end{array}$ \\
\hline & 2005SH37 & $\begin{array}{l}\text { NUCLEAR REACTIONS }{ }^{186} \mathrm{~W},{ }^{187} \mathrm{Re},{ }^{188} \mathrm{Os}(\gamma, \mathrm{n}), \mathrm{E}=7.3-10.9 \mathrm{MeV} \text {; } \\
\text { measured } \sigma .{ }^{185} \mathrm{~W},{ }^{186} \mathrm{Re},{ }^{187} \mathrm{Os}(\mathrm{n}, \gamma), \mathrm{E}<100 \mathrm{keV} \text { c calculated capture } \\
\sigma \text {. Astrophysical implications discussed. JOUR PRVCA } 72025808\end{array}$ \\
\hline & $2005 \mathrm{SH} 41$ & $\begin{array}{l}\text { NUCLEAR REACTIONS }{ }^{188} \mathrm{Os}(\gamma, \mathrm{n}), \mathrm{E}=8-11 \mathrm{MeV} \text {; measured } \sigma \\
\text { deduced parameters. }{ }^{187} \mathrm{Os}(\mathrm{n}, \gamma), \mathrm{E}=5-50 \mathrm{keV} \text {; calculated capture } \sigma \text {. } \\
\text { Astrophysical implications discussed. JOUR NUPAB } 758561 \mathrm{c}\end{array}$ \\
\hline & 2005SHZX & $\begin{array}{l}\text { NUCLEAR REACTIONS }{ }^{186} \mathrm{~W},{ }^{187} \mathrm{Re},{ }^{188} \mathrm{Os}(\gamma, \mathrm{n}), \mathrm{E} \approx 7.3-10.9 \mathrm{MeV} \text {; } \\
\text { measured } \sigma \text {; deduced parameters. Hauser-Feshbach model, implications } \\
\text { for cosmochronology discussed. PREPRINT } \\
\text { nucl-ex } / 0506027,6 / 30 / 2005\end{array}$ \\
\hline \multirow[t]{2}{*}{${ }^{187} \operatorname{Ir}$} & 2005FOZZ & $\begin{array}{l}\left.\text { NUCLEAR REACTIONS }{ }^{191} \operatorname{Ir}(\mathrm{n}, \mathrm{n})^{\prime}\right),(\mathrm{n}, 2 \mathrm{n}),(\mathrm{n}, 3 \mathrm{n}),(\mathrm{n}, 4 \mathrm{n}),(\mathrm{n}, 5 \mathrm{n}) \text {, } \\
(\mathrm{n}, 6 \mathrm{n}),(\mathrm{n}, 7 \mathrm{n}),(\mathrm{n}, 8 \mathrm{n}),(\mathrm{n}, 9 \mathrm{n}), \mathrm{E}=1-300 \mathrm{MeV} ; \text { measured } \mathrm{E} \gamma, \mathrm{I} \gamma ; \\
\text { deduced } \gamma \text {-ray production } \sigma . \text { CONF Santa Fe (Nucl Data for Sci and } \\
\text { Technol) Proc,Vol1,P898 }\end{array}$ \\
\hline & 2005TAZV & $\begin{array}{l}\text { NUCLEAR REACTIONS Ir }(\mathrm{p}, \mathrm{X}){ }^{188} \mathrm{Pt} /{ }^{189} \mathrm{Pt} /{ }^{191} \mathrm{Pt} /{ }^{186} \mathrm{Ir} /{ }^{187} \mathrm{Ir} \\
/{ }^{188} \mathrm{Ir} /{ }^{189} \mathrm{Ir} /{ }^{190} \mathrm{Ir} /{ }^{192} \mathrm{Ir}, \mathrm{E} \approx 10-70 \mathrm{MeV} \text {; measured excitation } \\
\text { functions; deduced integral yields. Stacked-foil activation technique. } \\
\text { CONF Santa Fe (Nucl Data for Sci and Technol) Proc,Vol1,P1023 }\end{array}$ \\
\hline \multirow[t]{2}{*}{${ }^{187} \mathrm{Tl}$} & $2005 \mathrm{CH} 38$ & $\begin{array}{l}\text { NUCLEAR REACTIONS }{ }^{159} \mathrm{~Tb}\left({ }^{32} \mathrm{~S}, 4 \mathrm{n}\right), \mathrm{E}=154 \mathrm{MeV} \text {; measured } \\
\text { Doppler-shifted } \mathrm{E} \gamma, \mathrm{I} \gamma .{ }^{187} \mathrm{Tl} \text { deduced high-spin levels, J, } \pi \text {, } \\
\text { configurations, } \mathrm{T}_{1 / 2}, \text { transition quadrupole moments, B(E2), shape } \\
\text { coexistence. Comparison with model predictions. JOUR PRVCA } 71 \\
054324\end{array}$ \\
\hline & 2005VA04 & $\begin{array}{l}\left.\text { RADIOACTIVITY }{ }^{189} \mathrm{Po}(\alpha) \text { from }{ }^{142} \mathrm{Nd}\left({ }^{52} \mathrm{Cr}, 5 \mathrm{n}\right),\left({ }^{50} \mathrm{Cr}, 3 \mathrm{n}\right)\right] \\
\text { measured } \mathrm{E} \alpha, \mathrm{E} \gamma, \mathrm{E}(\mathrm{ce}), \alpha \gamma-\mathrm{coin} .{ }^{185} \mathrm{~Pb} \text { deduced levels, } \mathrm{J}, \pi, \mathrm{ICC}, \\
\mathrm{T}_{1 / 2}, \text { configurations. }{ }^{188,189,190,191} \mathrm{Bi},{ }^{189,190} \mathrm{Po}(\alpha)\left[\text { from }{ }^{142} \mathrm{Nd}\left({ }^{52} \mathrm{Cr} \text {, }\right.\right. \\
\left.\mathrm{X}),\left({ }^{50} \mathrm{Cr}, \mathrm{X}\right)\right] \text {; measured } \mathrm{E} \alpha, \mathrm{I} \alpha . \text { Potential energy surface calculations, } \\
\text { level systematics in neighboring isotopes discussed. JOUR ZAANE } 24 \\
57\end{array}$ \\
\hline
\end{tabular}
57 


\section{$\mathrm{A}=187$ (continued)}

${ }^{187} \mathrm{~Pb}$ 2005AN17 NUCLEAR REACTIONS ${ }^{142} \mathrm{Nd}\left({ }^{52} \mathrm{Cr}, 3 \mathrm{n}\right),\left({ }^{52} \mathrm{Cr}, 4 \mathrm{n}\right),\left({ }^{52} \mathrm{Cr}, 5 \mathrm{n}\right)$, $\left({ }^{52} \mathrm{Cr}, 6 \mathrm{n}\right),\left({ }^{52} \mathrm{Cr}, \mathrm{np}\right),\left({ }^{52} \mathrm{Cr}, 2 \mathrm{np}\right),\left({ }^{52} \mathrm{Cr}, 3 \mathrm{np}\right),\left({ }^{52} \mathrm{Cr}, 4 \mathrm{np}\right),\left({ }^{52} \mathrm{Cr}\right.$, $5 \mathrm{np}),\left({ }^{52} \mathrm{Cr}, 6 \mathrm{np}\right), \mathrm{E}=220-310 \mathrm{MeV} ;{ }^{142} \mathrm{Nd}\left({ }^{50} \mathrm{Cr}, 3 \mathrm{n}\right),\left({ }^{50} \mathrm{Cr}, 4 \mathrm{n}\right),\left({ }^{50} \mathrm{Cr}\right.$, $2 \mathrm{np}),\left({ }^{50} \mathrm{Cr}, 3 \mathrm{np}\right),\left({ }^{50} \mathrm{Cr}, 4 \mathrm{np}\right),\left({ }^{50} \mathrm{Cr}, 5 \mathrm{np}\right), \mathrm{E}=230-285 \mathrm{MeV}$; ${ }^{92} \mathrm{Mo}\left({ }^{98} \mathrm{Mo}, 2 \mathrm{np}\right),\left({ }^{98} \mathrm{Mo}, 3 \mathrm{np}\right), \mathrm{E}=427-460 \mathrm{MeV} ;{ }^{93} \mathrm{Nb}\left({ }^{95} \mathrm{Mo}, \mathrm{n}\right)$, $\left({ }^{95} \mathrm{Mo}, 2 \mathrm{n}\right),\left({ }^{95} \mathrm{Mo}, 3 \mathrm{n}\right),\left({ }^{95} \mathrm{Mo}, \mathrm{p}\right),\left({ }^{95} \mathrm{Mo}, \mathrm{np}\right),\left({ }^{95} \mathrm{Mo}, 2 \mathrm{np}\right),\left({ }^{95} \mathrm{Mo}\right.$, 3np), $\left({ }^{95} \mathrm{Mo}, 4 \mathrm{np}\right), \mathrm{E}=375-456 \mathrm{MeV} ;{ }^{93} \mathrm{Nb}\left({ }^{94} \mathrm{Mo}, 2 \mathrm{n}\right),\left({ }^{94} \mathrm{Mo}, 3 \mathrm{n}\right)$, $\left({ }^{94} \mathrm{Mo}, \mathrm{np}\right),\left({ }^{94} \mathrm{Mo}, 2 \mathrm{np}\right),\left({ }^{94} \mathrm{Mo}, 3 \mathrm{np}\right), \mathrm{E}=405-450 \mathrm{MeV} ;{ }^{144} \mathrm{Sm}\left({ }^{46} \mathrm{Ti}\right.$, $3 \mathrm{n}),\left({ }^{46} \mathrm{Ti}, 4 \mathrm{n}\right), \mathrm{E}=202-242 \mathrm{MeV}$; measured $\sigma$. Velocity filter, comparison with statistical model predictions. JOUR PRVCA 72 014612

2005WE11 ATOMIC MASSES ${ }^{187,187 m} \mathrm{~Pb}$; measured masses. ${ }^{187} \mathrm{~Pb}$ deduced isomeric state energy. Penning trap mass spectrometer. JOUR PYLAA 34781

${ }^{187} \mathrm{Bi}$ 2005AN17 NUCLEAR REACTIONS ${ }^{142} \mathrm{Nd}\left({ }^{52} \mathrm{Cr}, 3 \mathrm{n}\right),\left({ }^{52} \mathrm{Cr}, 4 \mathrm{n}\right),\left({ }^{52} \mathrm{Cr}, 5 \mathrm{n}\right)$, $\left({ }^{52} \mathrm{Cr}, 6 \mathrm{n}\right),\left({ }^{52} \mathrm{Cr}, \mathrm{np}\right),\left({ }^{52} \mathrm{Cr}, 2 \mathrm{np}\right),\left({ }^{52} \mathrm{Cr}, 3 \mathrm{np}\right),\left({ }^{52} \mathrm{Cr}, 4 \mathrm{np}\right),\left({ }^{52} \mathrm{Cr}\right.$, $5 \mathrm{np}),\left({ }^{52} \mathrm{Cr}, 6 \mathrm{np}\right), \mathrm{E}=220-310 \mathrm{MeV} ;{ }^{142} \mathrm{Nd}\left({ }^{50} \mathrm{Cr}, 3 \mathrm{n}\right),\left({ }^{50} \mathrm{Cr}, 4 \mathrm{n}\right),\left({ }^{50} \mathrm{Cr}\right.$, $2 \mathrm{np}),\left({ }^{50} \mathrm{Cr}, 3 \mathrm{np}\right),\left({ }^{50} \mathrm{Cr}, 4 \mathrm{np}\right),\left({ }^{50} \mathrm{Cr}, 5 \mathrm{np}\right), \mathrm{E}=230-285 \mathrm{MeV}$; ${ }^{92} \mathrm{Mo}\left({ }^{98} \mathrm{Mo}, 2 \mathrm{np}\right),\left({ }^{98} \mathrm{Mo}, 3 \mathrm{np}\right), \mathrm{E}=427-460 \mathrm{MeV} ;{ }^{93} \mathrm{Nb}\left({ }^{95} \mathrm{Mo}, \mathrm{n}\right)$, $\left({ }^{95} \mathrm{Mo}, 2 \mathrm{n}\right),\left({ }^{95} \mathrm{Mo}, 3 \mathrm{n}\right),\left({ }^{95} \mathrm{Mo}, \mathrm{p}\right),\left({ }^{95} \mathrm{Mo}, \mathrm{np}\right),\left({ }^{95} \mathrm{Mo}, 2 \mathrm{np}\right),\left({ }^{95} \mathrm{Mo}\right.$, 3np), ( $\left.{ }^{95} \mathrm{Mo}, 4 \mathrm{np}\right), \mathrm{E}=375-456 \mathrm{MeV} ;{ }^{93} \mathrm{Nb}\left({ }^{94} \mathrm{Mo}, 2 \mathrm{n}\right),\left({ }^{94} \mathrm{Mo}, 3 \mathrm{n}\right)$, $\left({ }^{94} \mathrm{Mo}, \mathrm{np}\right),\left({ }^{94} \mathrm{Mo}, 2 \mathrm{np}\right),\left({ }^{94} \mathrm{Mo}, 3 \mathrm{np}\right), \mathrm{E}=405-450 \mathrm{MeV} ;{ }^{144} \mathrm{Sm}\left({ }^{46} \mathrm{Ti}\right.$, $3 \mathrm{n}),\left({ }^{46} \mathrm{Ti}, 4 \mathrm{n}\right), \mathrm{E}=202-242 \mathrm{MeV}$; measured $\sigma$. Velocity filter, comparison with statistical model predictions. JOUR PRVCA 72 014612

2005GEZW ATOMIC MASSES ${ }^{235}$ Ac; measured mass, $\mathrm{T}_{1 / 2}$. $185,186,187,188,189,190,191,192,193,194,195,196 \mathrm{Bi}$; measured masses, proton separation energies. ${ }^{207 m} \mathrm{Tl}$; measured $\mathrm{T}_{1 / 2}$. Stored beams, Schottky mass spectrometry. PREPRINT nucl-ex/0510009,10/4/2005

2005KE10 RADIOACTIVITY ${ }^{191,193,195} \operatorname{At}(\alpha)$; measured $\mathrm{E} \alpha, \mathrm{E} \gamma, \gamma \alpha$-coin. 191,193,195 At deduced levels, J, $\pi$, configurations, proton separation energies. ${ }^{187,189,191} \mathrm{Bi}$ deduced levels $\mathrm{J}, \pi$, configurations. Comparison with theory. JOUR ZAANE 25 s01 181

2005UU03 RADIOACTIVITY ${ }^{188,190,192,194,196,198,200,202,204} \mathrm{Po}$, 191,193,195,197,199 At, ${ }^{196,198,200,202,204,206} \mathrm{Rn},{ }^{199,201,203,205,207} \operatorname{Fr}(\alpha)$; measured reduced widths using gas filled recoil separator; deduced hindrance factors, proton intruder states and deformation effects. JOUR ZAANE 25 s01 179

${ }^{187} \mathrm{Po}$ 2005AN17 NUCLEAR REACTIONS ${ }^{142} \mathrm{Nd}\left({ }^{52} \mathrm{Cr}, 3 \mathrm{n}\right),\left({ }^{52} \mathrm{Cr}, 4 \mathrm{n}\right),\left({ }^{52} \mathrm{Cr}, 5 \mathrm{n}\right)$, $\left({ }^{52} \mathrm{Cr}, 6 \mathrm{n}\right),\left({ }^{52} \mathrm{Cr}, \mathrm{np}\right),\left({ }^{52} \mathrm{Cr}, 2 \mathrm{np}\right),\left({ }^{52} \mathrm{Cr}, 3 \mathrm{np}\right),\left({ }^{52} \mathrm{Cr}, 4 \mathrm{np}\right),\left({ }^{52} \mathrm{Cr}\right.$, $5 \mathrm{np}),\left({ }^{52} \mathrm{Cr}, 6 \mathrm{np}\right), \mathrm{E}=220-310 \mathrm{MeV} ;{ }^{142} \mathrm{Nd}\left({ }^{50} \mathrm{Cr}, 3 \mathrm{n}\right),\left({ }^{50} \mathrm{Cr}, 4 \mathrm{n}\right),\left({ }^{50} \mathrm{Cr}\right.$, $2 \mathrm{np}),\left({ }^{50} \mathrm{Cr}, 3 \mathrm{np}\right),\left({ }^{50} \mathrm{Cr}, 4 \mathrm{np}\right),\left({ }^{50} \mathrm{Cr}, 5 \mathrm{np}\right), \mathrm{E}=230-285 \mathrm{MeV}$; ${ }^{92} \mathrm{Mo}\left({ }^{98} \mathrm{Mo}, 2 \mathrm{np}\right),\left({ }^{98} \mathrm{Mo}, 3 \mathrm{np}\right), \mathrm{E}=427-460 \mathrm{MeV} ;{ }^{93} \mathrm{Nb}\left({ }^{95} \mathrm{Mo}, \mathrm{n}\right)$, $\left({ }^{95} \mathrm{Mo}, 2 \mathrm{n}\right),\left({ }^{95} \mathrm{Mo}, 3 \mathrm{n}\right),\left({ }^{95} \mathrm{Mo}, \mathrm{p}\right),\left({ }^{95} \mathrm{Mo}, \mathrm{np}\right),\left({ }^{95} \mathrm{Mo}, 2 \mathrm{np}\right),\left({ }^{95} \mathrm{Mo}\right.$, $3 \mathrm{np}),\left({ }^{95} \mathrm{Mo}, 4 \mathrm{np}\right), \mathrm{E}=375-456 \mathrm{MeV} ;{ }^{93} \mathrm{Nb}\left({ }^{94} \mathrm{Mo}, 2 \mathrm{n}\right),\left({ }^{94} \mathrm{Mo}, 3 \mathrm{n}\right)$, $\left({ }^{94} \mathrm{Mo}, \mathrm{np}\right),\left({ }^{94} \mathrm{Mo}, 2 \mathrm{np}\right),\left({ }^{94} \mathrm{Mo}, 3 \mathrm{np}\right), \mathrm{E}=405-450 \mathrm{MeV} ;{ }^{144} \mathrm{Sm}\left({ }^{46} \mathrm{Ti}\right.$, $3 \mathrm{n}),\left({ }^{46} \mathrm{Ti}, 4 \mathrm{n}\right), \mathrm{E}=202-242 \mathrm{MeV}$; measured $\sigma$. Velocity filter, comparison with statistical model predictions. JOUR PRVCA 72 014612 


\section{$\mathrm{A}=187$ (continued)}

2005ANZY NUCLEAR REACTIONS ${ }^{144} \mathrm{Sm}\left({ }^{46} \mathrm{Ti}\right.$, xn), E not given; measured $\mathrm{E} \gamma$, $\mathrm{E} \alpha, \alpha \alpha-, \alpha \gamma$-coin following residual nucleus decay; deduced evidence for ${ }^{186,187}$ Po. ${ }^{144} \mathrm{Sm}\left({ }^{51} \mathrm{~V}, \mathrm{xn}\right)$, E not given; measured $\mathrm{E} \gamma, \mathrm{E} \alpha, \alpha \alpha-$, $\alpha \gamma$-coin following residual nucleus decay; deduced evidence for ${ }^{192} \mathrm{At}$. REPT GSI 2005-1,P77,Andreyev

2005ANZY RADIOACTIVITY ${ }^{186,187} \mathrm{Po},{ }^{183} \mathrm{~Pb},{ }^{179} \mathrm{Hg},{ }^{175} \mathrm{Pt}(\alpha)$ from ${ }^{144} \mathrm{Sm}\left({ }^{46} \mathrm{Ti}\right.$, $\mathrm{xn})$ and subsequent decay]; measured $\mathrm{E} \alpha, \mathrm{T}_{1 / 2} .{ }^{183} \mathrm{~Pb}$ deduced excited state energy. ${ }^{187}$ Po deduced isomeric states. ${ }^{192} \mathrm{At}(\alpha)\left[\right.$ from ${ }^{144} \mathrm{Sm}\left({ }^{51} \mathrm{~V}\right.$, $\mathrm{xn})]$; measured $\mathrm{E} \alpha, \alpha \alpha$-coin, $\mathrm{T}_{1 / 2}$; deduced isomeric states. REPT GSI 2005-1,P77,Andreyev

\section{$\mathrm{A}=188$}

${ }^{188} \mathrm{Ta} \quad 2005 \mathrm{CA} 02$

Os 2004M054

2005 AB22

2005CL07

2005MOZV

2005SH37

2005SH41

${ }^{188} \mathrm{Ir} \quad 2005 \mathrm{FOZZ}$
RADIOACTIVITY ${ }^{188} \mathrm{Ta},{ }^{190} \mathrm{~W},{ }^{192,193} \mathrm{Re},{ }^{195} \mathrm{Os},{ }^{197,198} \mathrm{Ir}$, $200,201,202 \mathrm{Pt},{ }^{203} \mathrm{Au}(\mathrm{IT})$ [from $\mathrm{Be}\left({ }^{208} \mathrm{~Pb}, \mathrm{X}\right)$ ]; measured $\mathrm{E} \gamma, \mathrm{I} \gamma, \gamma \gamma$-coin, $\mathrm{T}_{1 / 2} \cdot{ }^{188} \mathrm{Ta},{ }^{190} \mathrm{~W},{ }^{192,193} \mathrm{Re},{ }^{195} \mathrm{Os},{ }^{197,198} \mathrm{Ir},{ }^{200,201,202} \mathrm{Pt},{ }^{203} \mathrm{Au}$ deduced transitions. ${ }^{190} \mathrm{~W},{ }^{200,201,202} \mathrm{Pt}$ deduced levels, J, $\pi .{ }^{174,175} \mathrm{Er}$, ${ }^{185} \mathrm{Hf},{ }^{191,194} \mathrm{Re},{ }^{199} \operatorname{Ir}(\mathrm{IT})$ [from $\mathrm{Be}\left({ }^{208} \mathrm{~Pb}, \mathrm{X}\right)$ ]; measured $\mathrm{E} \gamma, \mathrm{I} \gamma$.

JOUR ZAANE 23201

NUCLEAR REACTIONS ${ }^{192} \mathrm{Os}\left({ }^{82} \mathrm{Se}, \mathrm{X}\right){ }^{188} \mathrm{Os} /{ }^{190} \mathrm{Os}, \mathrm{E}=460 \mathrm{MeV}$; measured $\mathrm{E} \gamma, \mathrm{I} \gamma, \gamma \gamma$-coin. ${ }^{188,190}$ Os deduced high-spin levels, J, $\pi$. GASP array. JOUR BJPHE 34792

NUCLEAR REACTIONS ${ }^{186,187} \mathrm{Os}(\mathrm{n}, \gamma), \mathrm{E} \approx 1-1000 \mathrm{keV}$; measured capture $\sigma$. Astrophysical implications discussed. JOUR NUPAB 758 $501 \mathrm{c}$

NUCLEAR REACTIONS ${ }^{184} \mathrm{~W}\left({ }^{7} \mathrm{Li}, \mathrm{xn}\right),\left({ }^{7} \mathrm{Li}, \mathrm{xnp}\right),\left({ }^{7} \mathrm{Li}, \mathrm{xn} \alpha\right)$, $\mathrm{E}=35-70 \mathrm{MeV}$; calculated $\sigma .{ }^{184} \mathrm{~W}\left({ }^{7} \mathrm{Li}, \mathrm{X}\right){ }^{184} \mathrm{Os} /{ }^{185} \mathrm{Os} /{ }^{186} \mathrm{Os} /$ ${ }^{188} \mathrm{Os} /{ }^{184} \mathrm{Ir} /{ }^{185} \mathrm{Ir} /{ }^{186} \mathrm{Ir} /{ }^{183} \mathrm{Re} /{ }^{185} \mathrm{Re}, \mathrm{E}=40-70 \mathrm{MeV}$; measured $\mathrm{E} \gamma, \mathrm{I} \gamma, \gamma \gamma$-, (charged particle) $\gamma$-coin, particle yield ratios. ${ }^{160} \mathrm{Gd}\left({ }^{7} \mathrm{Li}\right.$, $\mathrm{xnp}), \mathrm{E}=35-65 \mathrm{MeV}$; analyzed $\sigma$. Liberace, Stars arrays. JOUR PRVCA 72054605

NUCLEAR REACTIONS ${ }^{186,187,188}$ Os (n, $\gamma$ ), E $<1 \mathrm{MeV}$; measured capture $\sigma$. Comparison with previous results, astrophysical implications discussed. CONF Santa Fe (Nucl Data for Sci and Technol) Proc, Vol2,P1335

NUCLEAR REACTIONS ${ }^{186,187,188} \mathrm{Os}(\mathrm{n}, \gamma)$, E=10-90 keV; measured $\mathrm{E} \gamma, \mathrm{I} \gamma . \mathrm{JOUR}$ NUPAB $758553 \mathrm{c}$

NUCLEAR REACTIONS ${ }^{186} \mathrm{~W},{ }^{187} \mathrm{Re},{ }^{188} \mathrm{Os}(\gamma, \mathrm{n}), \mathrm{E}=7.3-10.9 \mathrm{MeV}$; measured $\sigma .{ }^{185} \mathrm{~W},{ }^{186} \mathrm{Re},{ }^{187} \mathrm{Os}(\mathrm{n}, \gamma), \mathrm{E}<100 \mathrm{keV}$; calculated capture $\sigma$. Astrophysical implications discussed. JOUR PRVCA 72025808 NUCLEAR REACTIONS ${ }^{188} \mathrm{Os}(\gamma, \mathrm{n}), \mathrm{E}=8-11 \mathrm{MeV}$; measured $\sigma$; deduced parameters. ${ }^{187} \mathrm{Os}(\mathrm{n}, \gamma), \mathrm{E}=5-50 \mathrm{keV}$; calculated capture $\sigma$. Astrophysical implications discussed. JOUR NUPAB 758 561c NUCLEAR REACTIONS ${ }^{191} \operatorname{Ir}(\mathrm{n}, \mathrm{n}$ '), (n, 2n), (n, 3n), (n, 4n), (n, 5n), $(\mathrm{n}, 6 \mathrm{n}),(\mathrm{n}, 7 \mathrm{n}),(\mathrm{n}, 8 \mathrm{n}),(\mathrm{n}, 9 \mathrm{n}), \mathrm{E}=1-300 \mathrm{MeV}$; measured $\mathrm{E} \gamma, \mathrm{I} \gamma$; deduced $\gamma$-ray production $\sigma$. CONF Santa Fe (Nucl Data for Sci and Technol) Proc, Vol1,P898 


\section{$\mathrm{A}=188$ (continued)}

2005 TA26 NUCLEAR REACTIONS Ir(p, xnyp $)^{188} \mathrm{Pt} /{ }^{189} \mathrm{Pt} /{ }^{191} \mathrm{Pt} /{ }^{185} \mathrm{Ir} /$ ${ }^{186} \operatorname{Ir} /{ }^{188} \operatorname{Ir} /{ }^{189} \operatorname{Ir} /{ }^{190} \operatorname{Ir} /{ }^{192} \operatorname{Ir} /{ }^{185} \mathrm{Os}, \mathrm{E} \approx 3-70 \mathrm{MeV}$; measured $\sigma$; deduced integral yields. Stacked-foil activation technique. JOUR NIMBE 239293

2005TAZV NUCLEAR REACTIONS Ir $(\mathrm{p}, \mathrm{X}){ }^{188} \mathrm{Pt} /{ }^{189} \mathrm{Pt} /{ }^{191} \mathrm{Pt} /{ }^{186} \mathrm{Ir} /{ }^{187} \mathrm{Ir}$ $/{ }^{188} \operatorname{Ir} /{ }^{189} \operatorname{Ir} /{ }^{190} \operatorname{Ir} /{ }^{192} \mathrm{Ir}, \mathrm{E} \approx 10-70 \mathrm{MeV}$; measured excitation functions; deduced integral yields. Stacked-foil activation technique. CONF Santa Fe (Nucl Data for Sci and Technol) Proc,Vol1,P1023 ${ }^{188} \mathrm{Pt} \quad 2005 \mathrm{TA26}$ NUCLEAR REACTIONS $\operatorname{Ir}(\mathrm{p}, \mathrm{xnyp}){ }^{188} \mathrm{Pt} /{ }^{189} \mathrm{Pt} /{ }^{191} \mathrm{Pt} /{ }^{185} \mathrm{Ir} /$ ${ }^{186} \operatorname{Ir} /{ }^{188} \operatorname{Ir} /{ }^{189} \operatorname{Ir} /{ }^{190} \operatorname{Ir} /{ }^{192} \operatorname{Ir} /{ }^{185} \mathrm{Os}, \mathrm{E} \approx 3-70 \mathrm{MeV}$; measured $\sigma$; deduced integral yields. Stacked-foil activation technique. JOUR NIMBE 239293

2005TAZV NUCLEAR REACTIONS Ir $(\mathrm{p}, \mathrm{X}){ }^{188} \mathrm{Pt} /{ }^{189} \mathrm{Pt} /{ }^{191} \mathrm{Pt} /{ }^{186} \mathrm{Ir} /{ }^{187} \mathrm{Ir}$ $/{ }^{188} \operatorname{Ir} /{ }^{189} \operatorname{Ir} /{ }^{190} \operatorname{Ir} /{ }^{192} \mathrm{Ir}, \mathrm{E} \approx 10-70 \mathrm{MeV}$; measured excitation functions; deduced integral yields. Stacked-foil activation technique. CONF Santa Fe (Nucl Data for Sci and Technol) Proc,Vol1,P1023

${ }^{188} \mathrm{~Pb}$ 2005GR35 NUCLEAR REACTIONS ${ }^{108} \mathrm{Pd}\left({ }^{83} \mathrm{Kr}, 3 \mathrm{n}\right), \mathrm{E}=340 \mathrm{MeV}$; measured Doppler-shifted $\mathrm{E} \gamma, \mathrm{I} \gamma$, (recoil) $\gamma$-coin. ${ }^{188} \mathrm{~Pb}$ levels deduced $\mathrm{T}_{1 / 2}$, $\mathrm{B}(\mathrm{E} 2)$, deformation. Jurogam array, mass separator, recoil-distance technique. JOUR ZAANE 25 s01 441

2005UU03 RADIOACTIVITY 188,190,192,194,196,198,200,202,204Po, 191,193,195,197,199 At, ${ }^{196,198,200,202,204,206} \mathrm{Rn},{ }^{199,201,203,205,207} \operatorname{Fr}(\alpha)$; measured reduced widths using gas filled recoil separator; deduced hindrance factors, proton intruder states and deformation effects. JOUR ZAANE 25 s01 179

${ }^{188} \mathrm{Bi}$ 2005AN17 NUCLEAR REACTIONS ${ }^{142} \mathrm{Nd}\left({ }^{52} \mathrm{Cr}, 3 \mathrm{n}\right),\left({ }^{52} \mathrm{Cr}, 4 \mathrm{n}\right),\left({ }^{52} \mathrm{Cr}, 5 \mathrm{n}\right)$, $\left({ }^{52} \mathrm{Cr}, 6 \mathrm{n}\right),\left({ }^{52} \mathrm{Cr}, \mathrm{np}\right),\left({ }^{52} \mathrm{Cr}, 2 \mathrm{np}\right),\left({ }^{52} \mathrm{Cr}, 3 \mathrm{np}\right),\left({ }^{52} \mathrm{Cr}, 4 \mathrm{np}\right),\left({ }^{52} \mathrm{Cr}\right.$, $5 \mathrm{np}),\left({ }^{52} \mathrm{Cr}, 6 \mathrm{np}\right), \mathrm{E}=220-310 \mathrm{MeV} ;{ }^{142} \mathrm{Nd}\left({ }^{50} \mathrm{Cr}, 3 \mathrm{n}\right),\left({ }^{50} \mathrm{Cr}, 4 \mathrm{n}\right),\left({ }^{50} \mathrm{Cr}\right.$, $2 \mathrm{np}),\left({ }^{50} \mathrm{Cr}, 3 \mathrm{np}\right),\left({ }^{50} \mathrm{Cr}, 4 \mathrm{np}\right),\left({ }^{50} \mathrm{Cr}, 5 \mathrm{np}\right), \mathrm{E}=230-285 \mathrm{MeV}$;

${ }^{92} \mathrm{Mo}\left({ }^{98} \mathrm{Mo}, 2 \mathrm{np}\right),\left({ }^{98} \mathrm{Mo}, 3 \mathrm{np}\right), \mathrm{E}=427-460 \mathrm{MeV} ;{ }^{93} \mathrm{Nb}\left({ }^{95} \mathrm{Mo}, \mathrm{n}\right)$, $\left({ }^{95} \mathrm{Mo}, 2 \mathrm{n}\right),\left({ }^{95} \mathrm{Mo}, 3 \mathrm{n}\right),\left({ }^{95} \mathrm{Mo}, \mathrm{p}\right),\left({ }^{95} \mathrm{Mo}, \mathrm{np}\right),\left({ }^{95} \mathrm{Mo}, 2 \mathrm{np}\right),\left({ }^{95} \mathrm{Mo}\right.$, 3np), ( $\left.{ }^{95} \mathrm{Mo}, 4 \mathrm{np}\right), \mathrm{E}=375-456 \mathrm{MeV} ;{ }^{93} \mathrm{Nb}\left({ }^{94} \mathrm{Mo}, 2 \mathrm{n}\right),\left({ }^{94} \mathrm{Mo}, 3 \mathrm{n}\right)$, ( $\left.{ }^{94} \mathrm{Mo}, \mathrm{np}\right),\left({ }^{94} \mathrm{Mo}, 2 \mathrm{np}\right),\left({ }^{94} \mathrm{Mo}, 3 \mathrm{np}\right), \mathrm{E}=405-450 \mathrm{MeV} ;{ }^{144} \mathrm{Sm}\left({ }^{46} \mathrm{Ti}\right.$, $3 \mathrm{n}),\left({ }^{46} \mathrm{Ti}, 4 \mathrm{n}\right), \mathrm{E}=202-242 \mathrm{MeV}$; measured $\sigma$. Velocity filter, comparison with statistical model predictions. JOUR PRVCA 72 014612

2005ANZY RADIOACTIVITY ${ }^{186,187} \mathrm{Po},{ }^{183} \mathrm{~Pb},{ }^{179} \mathrm{Hg},{ }^{175} \mathrm{Pt}(\alpha)$ [from ${ }^{144} \mathrm{Sm}\left({ }^{46} \mathrm{Ti}\right.$, $\mathrm{xn}$ ) and subsequent decay]; measured $\mathrm{E} \alpha, \mathrm{T}_{1 / 2} .{ }^{183} \mathrm{~Pb}$ deduced excited state energy. ${ }^{187}$ Po deduced isomeric states. ${ }^{192} \mathrm{At}(\alpha)\left[\right.$ from ${ }^{144} \mathrm{Sm}\left({ }^{51} \mathrm{~V}\right.$, $\mathrm{xn})]$; measured $\mathrm{E} \alpha, \alpha \alpha$-coin, $\mathrm{T}_{1 / 2}$; deduced isomeric states. REPT GSI 2005-1,P77,Andreyev

2005GEZW ATOMIC MASSES ${ }^{235}$ Ac; measured mass, $\mathrm{T}_{1 / 2}$. $185,186,187,188,189,190,191,192,193,194,195,196 \mathrm{Bi}$; measured masses, proton separation energies. ${ }^{207 m} \mathrm{Tl}$; measured $\mathrm{T}_{1 / 2}$. Stored beams, Schottky mass spectrometry. PREPRINT nucl-ex/0510009,10/4/2005 


\section{$\mathrm{A}=188$ (continued)}

2005VA04 RADIOACTIVITY ${ }^{189} \mathrm{Po}(\alpha)$ [from $\left.{ }^{142} \mathrm{Nd}\left({ }^{52} \mathrm{Cr}, 5 \mathrm{n}\right),\left({ }^{50} \mathrm{Cr}, 3 \mathrm{n}\right)\right]$; measured $\mathrm{E} \alpha, \mathrm{E} \gamma, \mathrm{E}(\mathrm{ce}), \alpha \gamma$-coin. ${ }^{185} \mathrm{~Pb}$ deduced levels, J, $\pi$, ICC, $\mathrm{T}_{1 / 2}$, configurations. ${ }^{188,189,190,191} \mathrm{Bi},{ }^{189,190} \mathrm{Po}(\alpha)$ [from ${ }^{142} \mathrm{Nd}\left({ }^{52} \mathrm{Cr}\right.$, $\left.\mathrm{X}),\left({ }^{50} \mathrm{Cr}, \mathrm{X}\right)\right]$; measured $\mathrm{E} \alpha, \mathrm{I} \alpha$. Potential energy surface calculations, level systematics in neighboring isotopes discussed. JOUR ZAANE 24 57

${ }^{188} \mathrm{Po}$ 2005AN17 NUCLEAR REACTIONS ${ }^{142} \mathrm{Nd}\left({ }^{52} \mathrm{Cr}, 3 \mathrm{n}\right),\left({ }^{52} \mathrm{Cr}, 4 \mathrm{n}\right),\left({ }^{52} \mathrm{Cr}, 5 \mathrm{n}\right)$, $\left({ }^{52} \mathrm{Cr}, 6 \mathrm{n}\right),\left({ }^{52} \mathrm{Cr}, \mathrm{np}\right),\left({ }^{52} \mathrm{Cr}, 2 \mathrm{np}\right),\left({ }^{52} \mathrm{Cr}, 3 \mathrm{np}\right),\left({ }^{52} \mathrm{Cr}, 4 \mathrm{np}\right),\left({ }^{52} \mathrm{Cr}\right.$, 5np), $\left({ }^{52} \mathrm{Cr}, 6 \mathrm{np}\right), \mathrm{E}=220-310 \mathrm{MeV} ;{ }^{142} \mathrm{Nd}\left({ }^{50} \mathrm{Cr}, 3 \mathrm{n}\right),\left({ }^{50} \mathrm{Cr}, 4 \mathrm{n}\right),\left({ }^{50} \mathrm{Cr}\right.$, $2 \mathrm{np}),\left({ }^{50} \mathrm{Cr}, 3 \mathrm{np}\right),\left({ }^{50} \mathrm{Cr}, 4 \mathrm{np}\right),\left({ }^{50} \mathrm{Cr}, 5 \mathrm{np}\right), \mathrm{E}=230-285 \mathrm{MeV}$;

${ }^{92} \mathrm{Mo}\left({ }^{98} \mathrm{Mo}, 2 \mathrm{np}\right),\left({ }^{98} \mathrm{Mo}, 3 \mathrm{np}\right), \mathrm{E}=427-460 \mathrm{MeV} ;{ }^{93} \mathrm{Nb}\left({ }^{95} \mathrm{Mo}, \mathrm{n}\right)$, $\left({ }^{95} \mathrm{Mo}, 2 \mathrm{n}\right),\left({ }^{95} \mathrm{Mo}, 3 \mathrm{n}\right),\left({ }^{95} \mathrm{Mo}, \mathrm{p}\right),\left({ }^{95} \mathrm{Mo}, \mathrm{np}\right),\left({ }^{95} \mathrm{Mo}, 2 \mathrm{np}\right),\left({ }^{95} \mathrm{Mo}\right.$, $3 \mathrm{np}),\left({ }^{95} \mathrm{Mo}, 4 \mathrm{np}\right), \mathrm{E}=375-456 \mathrm{MeV} ;{ }^{93} \mathrm{Nb}\left({ }^{94} \mathrm{Mo}, 2 \mathrm{n}\right),\left({ }^{94} \mathrm{Mo}, 3 \mathrm{n}\right)$, $\left({ }^{94} \mathrm{Mo}, \mathrm{np}\right),\left({ }^{94} \mathrm{Mo}, 2 \mathrm{np}\right),\left({ }^{94} \mathrm{Mo}, 3 \mathrm{np}\right), \mathrm{E}=405-450 \mathrm{MeV} ;{ }^{144} \mathrm{Sm}\left({ }^{46} \mathrm{Ti}\right.$, $3 \mathrm{n}),\left({ }^{46} \mathrm{Ti}, 4 \mathrm{n}\right), \mathrm{E}=202-242 \mathrm{MeV}$; measured $\sigma$. Velocity filter, comparison with statistical model predictions. JOUR PRVCA 72 014612

2005UU03 RADIOACTIVITY $188,190,192,194,196,198,200,202,204$ Po, 191,193,195,197,199 At, ${ }^{196,198,200,202,204,206} \mathrm{Rn},{ }^{199,201,203,205,207} \operatorname{Fr}(\alpha)$; measured reduced widths using gas filled recoil separator; deduced hindrance factors, proton intruder states and deformation effects. JOUR ZAANE 25 s01 179

\section{$\mathrm{A}=\mathbf{1 8 9}$}

${ }^{189} \mathrm{Os} \quad$ 2005MOZV

NUCLEAR REACTIONS ${ }^{186,187,188} \mathrm{Os}(\mathrm{n}, \gamma), \mathrm{E}<1 \mathrm{MeV}$; measured capture $\sigma$. Comparison with previous results, astrophysical implications discussed. CONF Santa Fe (Nucl Data for Sci and Technol) Proc,Vol2,P1335

2005SE19 NUCLEAR REACTIONS ${ }^{186,187,188} \mathrm{Os}(\mathrm{n}, \gamma), \mathrm{E}=10-90 \mathrm{keV}$; measured $\mathrm{E} \gamma, \mathrm{I} \gamma$. JOUR NUPAB 758 553c

${ }^{189} \mathrm{Ir} \quad$ 2005FOZZ NUCLEAR REACTIONS ${ }^{191} \operatorname{Ir}(\mathrm{n}, \mathrm{n}$ '), (n, 2n), (n, 3n), (n, 4n), (n, 5n), (n, 6n), (n, 7n), (n, 8n), (n, 9n), E=1-300 MeV; measured E $\gamma, \mathrm{I} \gamma$; deduced $\gamma$-ray production $\sigma$. CONF Santa Fe (Nucl Data for Sci and Technol) Proc,Vol1,P898

2005 TA26 NUCLEAR REACTIONS Ir(p, xnyp) ${ }^{188} \mathrm{Pt} /{ }^{189} \mathrm{Pt} /{ }^{191} \mathrm{Pt} /{ }^{185} \mathrm{Ir} /$ ${ }^{186} \operatorname{Ir} /{ }^{188} \operatorname{Ir} /{ }^{189} \operatorname{Ir} /{ }^{190} \operatorname{Ir} /{ }^{192} \operatorname{Ir} /{ }^{185} \mathrm{Os}, \mathrm{E} \approx 3-70 \mathrm{MeV}$; measured $\sigma$; deduced integral yields. Stacked-foil activation technique. JOUR NIMBE 239293

2005TAZV NUCLEAR REACTIONS Ir $(\mathrm{p}, \mathrm{X}){ }^{188} \mathrm{Pt} /{ }^{189} \mathrm{Pt} /{ }^{191} \mathrm{Pt} /{ }^{186} \mathrm{Ir} /{ }^{187} \mathrm{Ir}$ $/{ }^{188} \operatorname{Ir} /{ }^{189} \operatorname{Ir} /{ }^{190} \operatorname{Ir} /{ }^{192} \operatorname{Ir}, \mathrm{E} \approx 10-70 \mathrm{MeV}$; measured excitation functions; deduced integral yields. Stacked-foil activation technique. CONF Santa Fe (Nucl Data for Sci and Technol) Proc,Vol1,P1023 2005TAZW NUCLEAR REACTIONS Pt(p, X) ${ }^{195} \mathrm{Au} /{ }^{196} \mathrm{Au} /{ }^{198} \mathrm{Au} /{ }^{189} \mathrm{Ir} /$ ${ }^{190} \mathrm{Ir} /{ }^{192} \mathrm{Ir} /{ }^{194} \mathrm{Ir}, \mathrm{E} \approx 0-70 \mathrm{MeV} ; \mathrm{Pt}(\mathrm{d}, \mathrm{X}){ }^{192} \mathrm{Au} /{ }^{193} \mathrm{Au} /{ }^{194} \mathrm{Au} /$ ${ }^{195} \mathrm{Au} /{ }^{196} \mathrm{Au} /{ }^{195 m} \mathrm{Pt} /{ }^{197} \mathrm{Pt} /{ }^{192} \mathrm{Ir}, \mathrm{E} \approx 0-21 \mathrm{MeV} ; \mathrm{Pt}(\alpha, \mathrm{X}){ }^{195} \mathrm{Au}$ $/{ }^{196} \mathrm{Au}, \mathrm{E} \approx 0-38 \mathrm{MeV}$; measured activation $\sigma$; deduced integral yields. CONF Santa Fe (Nucl Data for Sci and Technol) Proc,Vol1,P1015 


\section{$\mathrm{A}=189$ (continued)}

${ }^{189} \mathrm{Pt}$ 2005TA26 NUCLEAR REACTIONS $\operatorname{Ir}(\mathrm{p}, \mathrm{xnyp}){ }^{188} \mathrm{Pt} /{ }^{189} \mathrm{Pt} /{ }^{191} \mathrm{Pt} /{ }^{185} \mathrm{Ir} /$ ${ }^{186} \operatorname{Ir} /{ }^{188} \operatorname{Ir} /{ }^{189} \operatorname{Ir} /{ }^{190} \operatorname{Ir} /{ }^{192} \operatorname{Ir} /{ }^{185} \mathrm{Os}, \mathrm{E} \approx 3-70 \mathrm{MeV}$; measured $\sigma$; deduced integral yields. Stacked-foil activation technique. JOUR NIMBE 239293

2005TAZV NUCLEAR REACTIONS Ir $(\mathrm{p}, \mathrm{X}){ }^{188} \mathrm{Pt} /{ }^{189} \mathrm{Pt} /{ }^{191} \mathrm{Pt} /{ }^{186} \mathrm{Ir} /{ }^{187} \mathrm{Ir}$ $/{ }^{188} \operatorname{Ir} /{ }^{189} \operatorname{Ir} /{ }^{190} \operatorname{Ir} /{ }^{192} \mathrm{Ir}, \mathrm{E} \approx 10-70 \mathrm{MeV}$; measured excitation functions; deduced integral yields. Stacked-foil activation technique. CONF Santa Fe (Nucl Data for Sci and Technol) Proc,Vol1,P1023 ${ }^{189} \mathrm{Tl}$ 2005DE01 RADIOACTIVITY ${ }^{200,201,203,205} \mathrm{Fr},{ }^{196,197,199,201} \mathrm{At},{ }^{193} \operatorname{Bi}(\alpha)$ [from $\mathrm{Th}(\mathrm{p}, \mathrm{X})$ and subsequent decay]; measured $\mathrm{E} \alpha, \mathrm{T}_{1 / 2} \cdot{ }^{201} \mathrm{Fr},{ }^{197} \mathrm{At}$, ${ }^{193} \mathrm{Bi},{ }^{189} \mathrm{Tl}$ deduced levels, J, $\pi$. JOUR ZAANE 23243

${ }^{189} \mathrm{~Pb}$ 2005BA51 NUCLEAR REACTIONS ${ }^{158} \mathrm{Gd}\left({ }^{36} \mathrm{Ar}, 5 \mathrm{n}\right), \mathrm{E}=178 \mathrm{MeV}$; measured $\mathrm{E} \gamma$, $\mathrm{I} \gamma, \gamma \gamma-$, (recoil) $\gamma$-coin. ${ }^{164} \mathrm{Er}\left({ }^{29} \mathrm{Si}, 4 \mathrm{n}\right), \mathrm{E}=140 \mathrm{MeV}$; measured delayed $\mathrm{E} \gamma, \mathrm{I} \gamma, \gamma \gamma$-coin. ${ }^{189} \mathrm{~Pb}$ deduced levels, $\mathrm{J}, \pi$, configurations, deformation, isomer $\mathrm{T}_{1 / 2}$. Level systematics in neighboring isotopes discussed. Recoil mass spectrometer, pulsed beams. JOUR PRVCA 71 054302

2005BAZY NUCLEAR REACTIONS ${ }^{158} \mathrm{Gd}\left({ }^{36} \mathrm{Ar}, 5 \mathrm{n}\right), \mathrm{E}=178 \mathrm{MeV}$; measured E $\gamma$, $\mathrm{I} \gamma, \gamma \gamma-$, (recoil) $\gamma$-coin. ${ }^{164} \mathrm{Er}\left({ }^{29} \mathrm{Si}, 4 \mathrm{n}\right), \mathrm{E}=140 \mathrm{MeV}$; measured delayed $\mathrm{E} \gamma, \mathrm{I} \gamma, \gamma \gamma$-coin. ${ }^{189} \mathrm{~Pb}$ deduced levels, $\mathrm{J}, \pi$, configurations, isomer $\mathrm{T}_{1 / 2}$. CONF Argonne(Nuclei at the Limits),P62, Baxter

2005BAZZ NUCLEAR REACTIONS ${ }^{158} \mathrm{Gd}\left({ }^{36} \mathrm{Ar}, 5 \mathrm{n}\right), \mathrm{E}=178 \mathrm{MeV}$; measured $\mathrm{E} \gamma$, I $\gamma, \gamma \gamma$-, (recoil) $\gamma$-coin. ${ }^{164} \mathrm{Er}\left({ }^{29} \mathrm{Si}, 4 \mathrm{n}\right), \mathrm{E}=140 \mathrm{MeV}$; measured prompt and delayed $\mathrm{E} \gamma, \mathrm{I} \gamma, \gamma \gamma$-coin. ${ }^{189} \mathrm{~Pb}$ deduced levels, $\mathrm{J}, \pi$, isomeric state $\mathrm{T}_{1 / 2}$, configurations. PREPRINT ANU-P/1634,Baxter

2005UU02 RADIOACTIVITY ${ }^{201,202,203,204} \mathrm{Ra},{ }^{197,198,199,200} \mathrm{Rn},{ }^{193,194,195,196} \mathrm{Po}$, ${ }^{201,202,203,204} \mathrm{Fr},{ }^{197,198,199,200} \mathrm{At}(\alpha)$ [from ${ }^{141} \mathrm{Pr}\left({ }^{63,65} \mathrm{Cu}\right.$, xnypz $\left.\alpha\right)$, ${ }^{170} \mathrm{Yb}\left({ }^{36} \mathrm{Ar}\right.$, xnypz $\left.\alpha\right)$, and subsequent decay]; measured $\mathrm{E} \alpha, \mathrm{T}_{1 / 2}$, $\alpha \alpha$-coin for ground and metastable state decay. ${ }^{193,195} \mathrm{Bi},{ }^{197,199} \mathrm{At}$, ${ }^{201,203} \mathrm{Fr}$ deduced levels, $\mathrm{J}, \pi$. Comparisons with previous results. JOUR PRVCA 71024306

${ }^{189} \mathrm{Bi}$ 2005AN17 NUCLEAR REACTIONS ${ }^{142} \mathrm{Nd}\left({ }^{52} \mathrm{Cr}, 3 \mathrm{n}\right),\left({ }^{52} \mathrm{Cr}, 4 \mathrm{n}\right),\left({ }^{52} \mathrm{Cr}, 5 \mathrm{n}\right)$, $\left({ }^{52} \mathrm{Cr}, 6 \mathrm{n}\right),\left({ }^{52} \mathrm{Cr}, \mathrm{np}\right),\left({ }^{52} \mathrm{Cr}, 2 \mathrm{np}\right),\left({ }^{52} \mathrm{Cr}, 3 \mathrm{np}\right),\left({ }^{52} \mathrm{Cr}, 4 \mathrm{np}\right),\left({ }^{52} \mathrm{Cr}\right.$, $5 \mathrm{np}),\left({ }^{52} \mathrm{Cr}, 6 \mathrm{np}\right), \mathrm{E}=220-310 \mathrm{MeV} ;{ }^{142} \mathrm{Nd}\left({ }^{50} \mathrm{Cr}, 3 \mathrm{n}\right),\left({ }^{50} \mathrm{Cr}, 4 \mathrm{n}\right),\left({ }^{50} \mathrm{Cr}\right.$, $2 \mathrm{np}),\left({ }^{50} \mathrm{Cr}, 3 \mathrm{np}\right),\left({ }^{50} \mathrm{Cr}, 4 \mathrm{np}\right),\left({ }^{50} \mathrm{Cr}, 5 \mathrm{np}\right), \mathrm{E}=230-285 \mathrm{MeV}$; ${ }^{92} \mathrm{Mo}\left({ }^{98} \mathrm{Mo}, 2 \mathrm{np}\right),\left({ }^{98} \mathrm{Mo}, 3 \mathrm{np}\right), \mathrm{E}=427-460 \mathrm{MeV} ;{ }^{93} \mathrm{Nb}\left({ }^{95} \mathrm{Mo}, \mathrm{n}\right)$, $\left({ }^{95} \mathrm{Mo}, 2 \mathrm{n}\right),\left({ }^{95} \mathrm{Mo}, 3 \mathrm{n}\right),\left({ }^{95} \mathrm{Mo}, \mathrm{p}\right),\left({ }^{95} \mathrm{Mo}, \mathrm{np}\right),\left({ }^{95} \mathrm{Mo}, 2 \mathrm{np}\right),\left({ }^{95} \mathrm{Mo}\right.$, $3 \mathrm{np}),\left({ }^{95} \mathrm{Mo}, 4 \mathrm{np}\right), \mathrm{E}=375-456 \mathrm{MeV} ;{ }^{93} \mathrm{Nb}\left({ }^{94} \mathrm{Mo}, 2 \mathrm{n}\right),\left({ }^{94} \mathrm{Mo}, 3 \mathrm{n}\right)$, $\left({ }^{94} \mathrm{Mo}, \mathrm{np}\right),\left({ }^{94} \mathrm{Mo}, 2 \mathrm{np}\right),\left({ }^{94} \mathrm{Mo}, 3 \mathrm{np}\right), \mathrm{E}=405-450 \mathrm{MeV} ;{ }^{144} \mathrm{Sm}\left({ }^{46} \mathrm{Ti}\right.$, $3 \mathrm{n}),\left({ }^{46} \mathrm{Ti}, 4 \mathrm{n}\right), \mathrm{E}=202-242 \mathrm{MeV}$; measured $\sigma$. Velocity filter, comparison with statistical model predictions. JOUR PRVCA 72 014612

2005GEZW ATOMIC MASSES ${ }^{235}$ Ac; measured mass, $T_{1 / 2}$. $185,186,187,188,189,190,191,192,193,194,195,196 \mathrm{Bi}$; measured masses, proton separation energies. ${ }^{207 m} \mathrm{Tl}$; measured $\mathrm{T}_{1 / 2}$. Stored beams, Schottky mass spectrometry. PREPRINT nucl-ex/0510009,10/4/2005 


\section{$\mathrm{A}=189$ (continued)}

2005KE10 RADIOACTIVITY ${ }^{191,193,195} \operatorname{At}(\alpha)$; measured E $\alpha, \mathrm{E} \gamma, \gamma \alpha$-coin. 191,193,195 At deduced levels, J, $\pi$, configurations, proton separation energies. ${ }^{187,189,191} \mathrm{Bi}$ deduced levels $\mathrm{J}, \pi$, configurations. Comparison with theory. JOUR ZAANE 25 s01 181

2005 UU03 RADIOACTIVITY ${ }^{188,190,192,194,196,198,200,202,204} \mathrm{Po}$, $191,193,195,197,199$ At, ${ }^{196,198,200,202,204,206} \mathrm{Rn},{ }^{199,201,203,205,207} \operatorname{Fr}(\alpha)$; measured reduced widths using gas filled recoil separator; deduced hindrance factors, proton intruder states and deformation effects. JOUR ZAANE 25 s01 179

2005VA04 RADIOACTIVITY ${ }^{189} \mathrm{Po}(\alpha)$ [from $\left.{ }^{142} \mathrm{Nd}\left({ }^{52} \mathrm{Cr}, 5 \mathrm{n}\right),\left({ }^{50} \mathrm{Cr}, 3 \mathrm{n}\right)\right]$; measured $\mathrm{E} \alpha, \mathrm{E} \gamma, \mathrm{E}(\mathrm{ce}), \alpha \gamma$-coin. ${ }^{185} \mathrm{~Pb}$ deduced levels, J, $\pi$, ICC, $\mathrm{T}_{1 / 2}$, configurations. ${ }^{188,189,190,191} \mathrm{Bi},{ }^{189,190} \mathrm{Po}(\alpha)$ [from ${ }^{142} \mathrm{Nd}\left({ }^{52} \mathrm{Cr}\right.$, $\left.\mathrm{X}),\left({ }^{50} \mathrm{Cr}, \mathrm{X}\right)\right]$; measured $\mathrm{E} \alpha, \mathrm{I} \alpha$. Potential energy surface calculations, level systematics in neighboring isotopes discussed. JOUR ZAANE 24 57

${ }^{189} \mathrm{Po}$ 2005AN17 NUCLEAR REACTIONS ${ }^{142} \mathrm{Nd}\left({ }^{52} \mathrm{Cr}, 3 \mathrm{n}\right),\left({ }^{52} \mathrm{Cr}, 4 \mathrm{n}\right),\left({ }^{52} \mathrm{Cr}, 5 \mathrm{n}\right)$, $\left({ }^{52} \mathrm{Cr}, 6 \mathrm{n}\right),\left({ }^{52} \mathrm{Cr}, \mathrm{np}\right),\left({ }^{52} \mathrm{Cr}, 2 \mathrm{np}\right),\left({ }^{52} \mathrm{Cr}, 3 \mathrm{np}\right),\left({ }^{52} \mathrm{Cr}, 4 \mathrm{np}\right),\left({ }^{52} \mathrm{Cr}\right.$, $5 \mathrm{np}),\left({ }^{52} \mathrm{Cr}, 6 \mathrm{np}\right), \mathrm{E}=220-310 \mathrm{MeV} ;{ }^{142} \mathrm{Nd}\left({ }^{50} \mathrm{Cr}, 3 \mathrm{n}\right),\left({ }^{50} \mathrm{Cr}, 4 \mathrm{n}\right),\left({ }^{50} \mathrm{Cr}\right.$, $2 \mathrm{np}),\left({ }^{50} \mathrm{Cr}, 3 \mathrm{np}\right),\left({ }^{50} \mathrm{Cr}, 4 \mathrm{np}\right),\left({ }^{50} \mathrm{Cr}, 5 \mathrm{np}\right), \mathrm{E}=230-285 \mathrm{MeV}$; ${ }^{92} \mathrm{Mo}\left({ }^{98} \mathrm{Mo}, 2 \mathrm{np}\right),\left({ }^{98} \mathrm{Mo}, 3 \mathrm{np}\right), \mathrm{E}=427-460 \mathrm{MeV} ;{ }^{93} \mathrm{Nb}\left({ }^{95} \mathrm{Mo}, \mathrm{n}\right)$, $\left({ }^{95} \mathrm{Mo}, 2 \mathrm{n}\right),\left({ }^{95} \mathrm{Mo}, 3 \mathrm{n}\right),\left({ }^{95} \mathrm{Mo}, \mathrm{p}\right),\left({ }^{95} \mathrm{Mo}, \mathrm{np}\right),\left({ }^{95} \mathrm{Mo}, 2 \mathrm{np}\right),\left({ }^{95} \mathrm{Mo}\right.$, $3 \mathrm{np}),\left({ }^{95} \mathrm{Mo}, 4 \mathrm{np}\right), \mathrm{E}=375-456 \mathrm{MeV} ;{ }^{93} \mathrm{Nb}\left({ }^{94} \mathrm{Mo}, 2 \mathrm{n}\right),\left({ }^{94} \mathrm{Mo}, 3 \mathrm{n}\right)$, ( $\left.{ }^{94} \mathrm{Mo}, \mathrm{np}\right),\left({ }^{94} \mathrm{Mo}, 2 \mathrm{np}\right),\left({ }^{94} \mathrm{Mo}, 3 \mathrm{np}\right), \mathrm{E}=405-450 \mathrm{MeV} ;{ }^{144} \mathrm{Sm}\left({ }^{46} \mathrm{Ti}\right.$, $3 \mathrm{n}),\left({ }^{46} \mathrm{Ti}, 4 \mathrm{n}\right), \mathrm{E}=202-242 \mathrm{MeV}$; measured $\sigma$. Velocity filter, comparison with statistical model predictions. JOUR PRVCA 72 014612

2005VA04 RADIOACTIVITY ${ }^{189} \mathrm{Po}(\alpha)$ [from $\left.{ }^{142} \mathrm{Nd}\left({ }^{52} \mathrm{Cr}, 5 \mathrm{n}\right),\left({ }^{50} \mathrm{Cr}, 3 \mathrm{n}\right)\right]$; measured $\mathrm{E} \alpha, \mathrm{E} \gamma, \mathrm{E}(\mathrm{ce}), \alpha \gamma$-coin. ${ }^{185} \mathrm{~Pb}$ deduced levels, J, $\pi$, ICC, $\mathrm{T}_{1 / 2}$, configurations. ${ }^{188,189,190,191} \mathrm{Bi},{ }^{189,190} \mathrm{Po}(\alpha)$ [from ${ }^{142} \mathrm{Nd}\left({ }^{52} \mathrm{Cr}\right.$, $\left.\mathrm{X}),\left({ }^{50} \mathrm{Cr}, \mathrm{X}\right)\right]$; measured $\mathrm{E} \alpha, \mathrm{I} \alpha$. Potential energy surface calculations, level systematics in neighboring isotopes discussed. JOUR ZAANE 24 57

\section{$\mathrm{A}=\mathbf{1 9 0}$}

${ }^{190} \mathrm{~W} \quad 2005 \mathrm{CA0} 2$

RADIOACTIVITY ${ }^{188} \mathrm{Ta},{ }^{190} \mathrm{~W},{ }^{192,193} \mathrm{Re},{ }^{195} \mathrm{Os},{ }^{197,198} \mathrm{Ir}$, ${ }^{200,201,202} \mathrm{Pt},{ }^{203} \mathrm{Au}$ (IT) [from $\mathrm{Be}\left({ }^{208} \mathrm{~Pb}, \mathrm{X}\right)$ ]; measured $\mathrm{E} \gamma, \mathrm{I} \gamma, \gamma \gamma$-coin, $\mathrm{T}_{1 / 2} \cdot{ }^{188} \mathrm{Ta},{ }^{190} \mathrm{~W},{ }^{192,193} \mathrm{Re},{ }^{195} \mathrm{Os},{ }^{197,198} \mathrm{Ir},{ }^{200,201,202} \mathrm{Pt},{ }^{203} \mathrm{Au}$ deduced transitions. ${ }^{190} \mathrm{~W},{ }^{200,201,202} \mathrm{Pt}$ deduced levels, J, $\pi .{ }^{174,175} \mathrm{Er}$, ${ }^{185} \mathrm{Hf},{ }^{191,194} \mathrm{Re},{ }^{199} \mathrm{Ir}(\mathrm{IT})$ [from $\left.\mathrm{Be}\left({ }^{208} \mathrm{~Pb}, \mathrm{X}\right)\right]$; measured $\mathrm{E} \gamma, \mathrm{I} \gamma$.

JOUR ZAANE 23201

${ }^{190} \mathrm{Os} \quad$ 2004MO54 NUCLEAR REACTIONS ${ }^{192} \mathrm{Os}\left({ }^{82} \mathrm{Se}, \mathrm{X}\right){ }^{188} \mathrm{Os} /{ }^{190} \mathrm{Os}, \mathrm{E}=460 \mathrm{MeV}$; measured $\mathrm{E} \gamma, \mathrm{I} \gamma, \gamma \gamma$-coin. ${ }^{188,190} \mathrm{Os}$ deduced high-spin levels, J, $\pi$. GASP array. JOUR BJPHE 34792

2005ME19 NUCLEAR REACTIONS ${ }^{160} \mathrm{Gd},{ }^{164} \mathrm{Dy},{ }^{170} \mathrm{Er},{ }^{178} \mathrm{Hf},{ }^{186} \mathrm{~W},{ }^{192} \mathrm{Os}(\mathrm{p}$, t), $\mathrm{E}=25 \mathrm{MeV}$; measured triton spectra, $\sigma(\theta) .{ }^{158} \mathrm{Gd},{ }^{162} \mathrm{Dy},{ }^{168} \mathrm{Er}$, ${ }^{176} \mathrm{Hf},{ }^{184} \mathrm{~W},{ }^{190} \mathrm{Os}$ deduced $0^{+}$level energies. JOUR JPGPE $31 \mathrm{~S} 1399$ 


\section{$\mathrm{A}=190$ (continued)}

${ }^{190} \mathrm{Ir} \quad$ 2005FOZZ NUCLEAR REACTIONS ${ }^{191} \operatorname{Ir}(\mathrm{n}, \mathrm{n}),(\mathrm{n}, 2 \mathrm{n}),(\mathrm{n}, 3 \mathrm{n}),(\mathrm{n}, 4 \mathrm{n}),(\mathrm{n}, 5 \mathrm{n})$, (n, 6n), (n, 7n), (n, 8n), (n, 9n), E=1-300 MeV; measured E $\gamma, \mathrm{I} \gamma$; deduced $\gamma$-ray production $\sigma$. CONF Santa Fe (Nucl Data for Sci and Technol) Proc,Vol1,P898

2005 TA26 NUCLEAR REACTIONS Ir(p, xnyp) ${ }^{188} \mathrm{Pt} /{ }^{189} \mathrm{Pt} /{ }^{191} \mathrm{Pt} /{ }^{185} \mathrm{Ir} /$ ${ }^{186} \operatorname{Ir} /{ }^{188} \operatorname{Ir} /{ }^{189} \operatorname{Ir} /{ }^{190} \operatorname{Ir} /{ }^{192} \operatorname{Ir} /{ }^{185} \mathrm{Os}, \mathrm{E} \approx 3-70 \mathrm{MeV}$; measured $\sigma$; deduced integral yields. Stacked-foil activation technique. JOUR NIMBE 239293

2005TAZV NUCLEAR REACTIONS Ir $(\mathrm{p}, \mathrm{X}){ }^{188} \mathrm{Pt} /{ }^{189} \mathrm{Pt} /{ }^{191} \mathrm{Pt} /{ }^{186} \mathrm{Ir} /{ }^{187} \mathrm{Ir}$ $/{ }^{188} \operatorname{Ir} /{ }^{189} \operatorname{Ir} /{ }^{190} \operatorname{Ir} /{ }^{192} \mathrm{Ir}, \mathrm{E} \approx 10-70 \mathrm{MeV}$; measured excitation functions; deduced integral yields. Stacked-foil activation technique. CONF Santa Fe (Nucl Data for Sci and Technol) Proc,Vol1,P1023 2005TAZW NUCLEAR REACTIONS Pt $(\mathrm{p}, \mathrm{X}){ }^{195} \mathrm{Au} /{ }^{196} \mathrm{Au} /{ }^{198} \mathrm{Au} /{ }^{189} \mathrm{Ir} /$ ${ }^{190} \mathrm{Ir} /{ }^{192} \mathrm{Ir} /{ }^{194} \mathrm{Ir}, \mathrm{E} \approx 0-70 \mathrm{MeV} ; \mathrm{Pt}(\mathrm{d}, \mathrm{X}){ }^{192} \mathrm{Au} /{ }^{193} \mathrm{Au} /{ }^{194} \mathrm{Au} /$ ${ }^{195} \mathrm{Au} /{ }^{196} \mathrm{Au} /{ }^{195 m} \mathrm{Pt} /{ }^{197} \mathrm{Pt} /{ }^{192} \mathrm{Ir}, \mathrm{E} \approx 0-21 \mathrm{MeV} ; \mathrm{Pt}(\alpha, \mathrm{X}){ }^{195} \mathrm{Au}$ $/{ }^{196} \mathrm{Au}, \mathrm{E} \approx 0-38 \mathrm{MeV}$; measured activation $\sigma$; deduced integral yields. CONF Santa Fe (Nucl Data for Sci and Technol) Proc,Vol1,P1015 2005TIZX NUCLEAR REACTIONS Pb, ${ }^{208} \mathrm{~Pb}(\mathrm{p}, \mathrm{X}){ }^{203} \mathrm{~Pb} /{ }^{200} \mathrm{Tl} /{ }^{199} \mathrm{Tl} /$ ${ }^{196} \mathrm{Au} /{ }^{192} \mathrm{Ir} /{ }^{190} \mathrm{Ir} /{ }^{173} \mathrm{Lu} /{ }^{101 m} \mathrm{Rh} /{ }^{86} \mathrm{Rb} /{ }^{59} \mathrm{Fe} /{ }^{24} \mathrm{Na} /{ }^{7} \mathrm{Be}$, $\mathrm{E}=40-2600 \mathrm{MeV}$; measured excitation functions. Comparison with previous work and model predictions. Other reactions discussed. CONF Santa Fe (Nucl Data for Sci and Technol) Proc,Vol1,P1070 2005TIZY NUCLEAR REACTIONS Pb, ${ }^{208} \mathrm{~Pb},{ }^{209} \mathrm{Bi}(\mathrm{p}, \mathrm{X}){ }^{203} \mathrm{~Pb} /{ }^{200} \mathrm{Tl} /{ }^{199} \mathrm{Tl}$ / ${ }^{196} \mathrm{Au} /{ }^{192} \mathrm{Ir} /{ }^{190} \mathrm{Ir} /{ }^{173} \mathrm{Lu} /{ }^{101 m} \mathrm{Rh} /{ }^{86} \mathrm{Rb} /{ }^{59} \mathrm{Fe} /{ }^{24} \mathrm{Na} /{ }^{7} \mathrm{Be}$, $\mathrm{E}=40-2600 \mathrm{MeV}$; measured production $\sigma$. Comparison with model predictions. PREPRINT nucl-ex/0507009,7/05/2005

${ }^{190} \mathrm{Pt} \quad$ 2005LEZW NUCLEAR REACTIONS ${ }^{188,190,194} \mathrm{Os},{ }^{194,196} \mathrm{Pt}(\alpha, 2 \mathrm{n}), \mathrm{E}=27 \mathrm{MeV}$; measured $\mathrm{E} \gamma, \mathrm{I} \gamma(\theta, \mathrm{H}, \mathrm{t}) .{ }^{190,192,194} \mathrm{Pt},{ }^{196,198} \mathrm{Hg}$ deduced isomeric states g-factors, configurations. Integral perturbed angular distribution method, HPGe detectors. CONF St Petersburg,P81,Levon

${ }^{190} \mathrm{Tl} \quad 2005 X I 06$ NUCLEAR REACTIONS ${ }^{160} \mathrm{Gd}\left({ }^{35} \mathrm{Cl}, 5 \mathrm{n}\right), \mathrm{E}=167,175 \mathrm{MeV}$; measured $\mathrm{E} \gamma, \mathrm{I} \gamma, \gamma \gamma$-coin. ${ }^{190} \mathrm{Tl}$ deduced levels, J, $\pi$, configurations, rotational band, signature inversion. Total Routhian surface calculations. JOUR PRVCA 72044302

2005ZH31 NUCLEAR REACTIONS ${ }^{160} \mathrm{Gd}\left({ }^{35} \mathrm{Cl}, 5 \mathrm{n}\right), \mathrm{E}=167 \mathrm{MeV}$; measured E $\gamma$, $\mathrm{I} \gamma, \gamma \gamma$-coin. ${ }^{190} \mathrm{Tl}$ deduced levels, J, $\pi$, configurations, rotational band signature inversion. Level systematics in neighboring isotopes discussed. JOUR JPGPE 31 S1985

${ }^{190} \mathrm{~Pb}$ 2005UU02 RADIOACTIVITY ${ }^{201,202,203,204} \mathrm{Ra},{ }^{197,198,199,200} \mathrm{Rn},{ }^{193,194,195,196} \mathrm{Po}$, ${ }^{201,202,203,204} \mathrm{Fr},{ }^{197,198,199,200} \operatorname{At}(\alpha)$ [from ${ }^{141} \operatorname{Pr}\left({ }^{63,65} \mathrm{Cu}\right.$, xnypz $\left.\alpha\right)$, ${ }^{170} \mathrm{Yb}\left({ }^{36} \mathrm{Ar}, \mathrm{xnypz} \alpha\right)$, and subsequent decay]; measured $\mathrm{E} \alpha, \mathrm{T}_{1 / 2}$, $\alpha \alpha$-coin for ground and metastable state decay. ${ }^{193,195} \mathrm{Bi},{ }^{197,199} \mathrm{At}$, ${ }^{201,203} \mathrm{Fr}$ deduced levels, $\mathrm{J}, \pi$. Comparisons with previous results. JOUR PRVCA 71024306

2005UU03 RADIOACTIVITY ${ }^{188,190,192,194,196,198,200,202,204} \mathrm{Po}$, ${ }_{191,193,195,197,199}$ At, ${ }^{196,198,200,202,204,206} \mathrm{Rn},{ }^{199,201,203,205,207} \operatorname{Fr}(\alpha)$; measured reduced widths using gas filled recoil separator; deduced hindrance factors, proton intruder states and deformation effects. JOUR ZAANE 25 s01 179 


\section{$\mathrm{A}=190$ (continued)}

2005WI10 NUCLEAR REACTIONS ${ }^{166} \operatorname{Er}\left({ }^{28} \mathrm{Si}, 4 \mathrm{n}\right), \mathrm{E}=143 \mathrm{MeV}$; measured E $\gamma$, $\mathrm{I} \gamma, \gamma \gamma$-coin. ${ }^{190} \mathrm{~Pb}$ deduced high-spin levels, $\mathrm{J}, \pi$, superdeformed band. Gammasphere array. JOUR ZAANE 24179

${ }^{190} \mathrm{Bi}$ 2005AN17 NUCLEAR REACTIONS ${ }^{142} \mathrm{Nd}\left({ }^{52} \mathrm{Cr}, 3 \mathrm{n}\right),\left({ }^{52} \mathrm{Cr}, 4 \mathrm{n}\right),\left({ }^{52} \mathrm{Cr}, 5 \mathrm{n}\right)$, $\left({ }^{52} \mathrm{Cr}, 6 \mathrm{n}\right),\left({ }^{52} \mathrm{Cr}, \mathrm{np}\right),\left({ }^{52} \mathrm{Cr}, 2 \mathrm{np}\right),\left({ }^{52} \mathrm{Cr}, 3 \mathrm{np}\right),\left({ }^{52} \mathrm{Cr}, 4 \mathrm{np}\right),\left({ }^{52} \mathrm{Cr}\right.$, $5 \mathrm{np}),\left({ }^{52} \mathrm{Cr}, 6 \mathrm{np}\right), \mathrm{E}=220-310 \mathrm{MeV} ;{ }^{142} \mathrm{Nd}\left({ }^{50} \mathrm{Cr}, 3 \mathrm{n}\right),\left({ }^{50} \mathrm{Cr}, 4 \mathrm{n}\right),\left({ }^{50} \mathrm{Cr}\right.$, $2 \mathrm{np}),\left({ }^{50} \mathrm{Cr}, 3 \mathrm{np}\right),\left({ }^{50} \mathrm{Cr}, 4 \mathrm{np}\right),\left({ }^{50} \mathrm{Cr}, 5 \mathrm{np}\right), \mathrm{E}=230-285 \mathrm{MeV}$; ${ }^{92} \mathrm{Mo}\left({ }^{98} \mathrm{Mo}, 2 \mathrm{np}\right),\left({ }^{98} \mathrm{Mo}, 3 \mathrm{np}\right), \mathrm{E}=427-460 \mathrm{MeV} ;{ }^{93} \mathrm{Nb}\left({ }^{95} \mathrm{Mo}, \mathrm{n}\right)$, $\left({ }^{95} \mathrm{Mo}, 2 \mathrm{n}\right),\left({ }^{95} \mathrm{Mo}, 3 \mathrm{n}\right),\left({ }^{95} \mathrm{Mo}, \mathrm{p}\right),\left({ }^{95} \mathrm{Mo}, \mathrm{np}\right),\left({ }^{95} \mathrm{Mo}, 2 \mathrm{np}\right),\left({ }^{95} \mathrm{Mo}\right.$, 3np), $\left({ }^{95} \mathrm{Mo}, 4 \mathrm{np}\right), \mathrm{E}=375-456 \mathrm{MeV} ;{ }^{93} \mathrm{Nb}\left({ }^{94} \mathrm{Mo}, 2 \mathrm{n}\right),\left({ }^{94} \mathrm{Mo}, 3 \mathrm{n}\right)$, $\left({ }^{94} \mathrm{Mo}, \mathrm{np}\right),\left({ }^{94} \mathrm{Mo}, 2 \mathrm{np}\right),\left({ }^{94} \mathrm{Mo}, 3 \mathrm{np}\right), \mathrm{E}=405-450 \mathrm{MeV} ;{ }^{144} \mathrm{Sm}\left({ }^{46} \mathrm{Ti}\right.$, $3 \mathrm{n}),\left({ }^{46} \mathrm{Ti}, 4 \mathrm{n}\right), \mathrm{E}=202-242 \mathrm{MeV}$; measured $\sigma$. Velocity filter, comparison with statistical model predictions. JOUR PRVCA 72 014612

2005GEZW ATOMIC MASSES ${ }^{235}$ Ac; measured mass, $\mathrm{T}_{1 / 2}$. $185,186,187,188,189,190,191,192,193,194,195,196 \mathrm{Bi}$; measured masses, proton separation energies. ${ }^{207 m} \mathrm{Tl}$; measured $\mathrm{T}_{1 / 2}$. Stored beams, Schottky mass spectrometry. PREPRINT nucl-ex/0510009,10/4/2005

2005VA04 RADIOACTIVITY ${ }^{189} \mathrm{Po}(\alpha)$ [from $\left.{ }^{142} \mathrm{Nd}\left({ }^{52} \mathrm{Cr}, 5 \mathrm{n}\right),\left({ }^{50} \mathrm{Cr}, 3 \mathrm{n}\right)\right]$; measured $\mathrm{E} \alpha, \mathrm{E} \gamma, \mathrm{E}(\mathrm{ce}), \alpha \gamma$-coin. ${ }^{185} \mathrm{~Pb}$ deduced levels, J, $\pi$, ICC, $\mathrm{T}_{1 / 2}$, configurations. ${ }^{188,189,190,191} \mathrm{Bi},{ }^{189,190} \mathrm{Po}(\alpha)$ [from ${ }^{142} \mathrm{Nd}\left({ }^{52} \mathrm{Cr}\right.$, $\left.\mathrm{X}),\left({ }^{50} \mathrm{Cr}, \mathrm{X}\right)\right]$; measured $\mathrm{E} \alpha, \mathrm{I} \alpha$. Potential energy surface calculations, level systematics in neighboring isotopes discussed. JOUR ZAANE 24 57

${ }^{190} \mathrm{Po} \quad$ 2005AN17 NUCLEAR REACTIONS ${ }^{142} \mathrm{Nd}\left({ }^{52} \mathrm{Cr}, 3 \mathrm{n}\right),\left({ }^{52} \mathrm{Cr}, 4 \mathrm{n}\right),\left({ }^{52} \mathrm{Cr}, 5 \mathrm{n}\right)$, $\left({ }^{52} \mathrm{Cr}, 6 \mathrm{n}\right),\left({ }^{52} \mathrm{Cr}, \mathrm{np}\right),\left({ }^{52} \mathrm{Cr}, 2 \mathrm{np}\right),\left({ }^{52} \mathrm{Cr}, 3 \mathrm{np}\right),\left({ }^{52} \mathrm{Cr}, 4 \mathrm{np}\right),\left({ }^{52} \mathrm{Cr}\right.$, 5np $),\left({ }^{52} \mathrm{Cr}, 6 \mathrm{np}\right), \mathrm{E}=220-310 \mathrm{MeV} ;{ }^{142} \mathrm{Nd}\left({ }^{50} \mathrm{Cr}, 3 \mathrm{n}\right),\left({ }^{50} \mathrm{Cr}, 4 \mathrm{n}\right),\left({ }^{50} \mathrm{Cr}\right.$, $2 \mathrm{np}),\left({ }^{50} \mathrm{Cr}, 3 \mathrm{np}\right),\left({ }^{50} \mathrm{Cr}, 4 \mathrm{np}\right),\left({ }^{50} \mathrm{Cr}, 5 \mathrm{np}\right), \mathrm{E}=230-285 \mathrm{MeV}$; ${ }^{92} \mathrm{Mo}\left({ }^{98} \mathrm{Mo}, 2 \mathrm{np}\right),\left({ }^{98} \mathrm{Mo}, 3 \mathrm{np}\right), \mathrm{E}=427-460 \mathrm{MeV} ;{ }^{93} \mathrm{Nb}\left({ }^{95} \mathrm{Mo}, \mathrm{n}\right)$, $\left({ }^{95} \mathrm{Mo}, 2 \mathrm{n}\right),\left({ }^{95} \mathrm{Mo}, 3 \mathrm{n}\right),\left({ }^{95} \mathrm{Mo}, \mathrm{p}\right),\left({ }^{95} \mathrm{Mo}, \mathrm{np}\right),\left({ }^{95} \mathrm{Mo}, 2 \mathrm{np}\right),\left({ }^{95} \mathrm{Mo}\right.$, 3np), ( $\left.{ }^{95} \mathrm{Mo}, 4 \mathrm{np}\right), \mathrm{E}=375-456 \mathrm{MeV} ;{ }^{93} \mathrm{Nb}\left({ }^{94} \mathrm{Mo}, 2 \mathrm{n}\right),\left({ }^{94} \mathrm{Mo}, 3 \mathrm{n}\right)$, $\left({ }^{94} \mathrm{Mo}, \mathrm{np}\right),\left({ }^{94} \mathrm{Mo}, 2 \mathrm{np}\right),\left({ }^{94} \mathrm{Mo}, 3 \mathrm{np}\right), \mathrm{E}=405-450 \mathrm{MeV} ;{ }^{144} \mathrm{Sm}\left({ }^{46} \mathrm{Ti}\right.$, $3 \mathrm{n}),\left({ }^{46} \mathrm{Ti}, 4 \mathrm{n}\right), \mathrm{E}=202-242 \mathrm{MeV}$; measured $\sigma$. Velocity filter, comparison with statistical model predictions. JOUR PRVCA 72 014612

2005UU03 RADIOACTIVITY ${ }^{188,190,192,194,196,198,200,202,204}$ Po, $191,193,195,197,199$ At, ${ }^{196,198,200,202,204,206} \mathrm{Rn},{ }^{199,201,203,205,207} \operatorname{Fr}(\alpha)$; measured reduced widths using gas filled recoil separator; deduced hindrance factors, proton intruder states and deformation effects. JOUR ZAANE 25 s01 179

2005VA04 RADIOACTIVITY ${ }^{189} \mathrm{Po}(\alpha)$ [from $\left.{ }^{142} \mathrm{Nd}\left({ }^{52} \mathrm{Cr}, 5 \mathrm{n}\right),\left({ }^{50} \mathrm{Cr}, 3 \mathrm{n}\right)\right]$; measured $\mathrm{E} \alpha, \mathrm{E} \gamma, \mathrm{E}(\mathrm{ce}), \alpha \gamma$-coin. ${ }^{185} \mathrm{~Pb}$ deduced levels, J, $\pi$, ICC, $\mathrm{T}_{1 / 2}$, configurations. ${ }^{188,189,190,191} \mathrm{Bi},{ }^{189,190} \mathrm{Po}(\alpha)$ [from ${ }^{142} \mathrm{Nd}\left({ }^{52} \mathrm{Cr}\right.$, $\left.\mathrm{X}),\left({ }^{50} \mathrm{Cr}, \mathrm{X}\right)\right]$; measured $\mathrm{E} \alpha, \mathrm{I} \alpha$. Potential energy surface calculations, level systematics in neighboring isotopes discussed. JOUR ZAANE 24 57 


\begin{tabular}{|c|c|c|}
\hline & \multicolumn{2}{|r|}{$A=191$} \\
\hline${ }^{191} \mathrm{Re}$ & 2005CA02 & $\begin{array}{l}\text { RADIOACTIVITY }{ }^{188} \mathrm{Ta},{ }^{190} \mathrm{~W},{ }^{192,193} \mathrm{Re},{ }^{195} \mathrm{Os},{ }^{197,198} \mathrm{Ir} \text {, } \\
{ }^{200,201,202} \mathrm{Pt},{ }^{203} \mathrm{Au}(\mathrm{IT})\left[\text { from Be }\left({ }^{208} \mathrm{~Pb}, \mathrm{X}\right)\right] \text {; measured } \mathrm{E} \gamma, \mathrm{I} \gamma, \gamma \gamma \text {-coin, } \\
\mathrm{T}_{1 / 2} \cdot{ }^{188} \mathrm{Ta},{ }^{190} \mathrm{~W},{ }^{192,193} \mathrm{Re},{ }^{195} \mathrm{Os},{ }^{197,198} \mathrm{Ir},{ }^{200,201,202} \mathrm{Pt},{ }^{203} \mathrm{Au} \\
\text { deduced transitions. }{ }^{190} \mathrm{~W},{ }^{200,201,202} \mathrm{Pt} \text { deduced levels, J, } \pi .{ }^{174,175} \mathrm{Er} \text {, } \\
{ }^{185} \mathrm{Hf},{ }^{191,194} \mathrm{Re},{ }^{199} \mathrm{Ir}(\mathrm{IT})\left[\text { from Be }\left({ }^{208} \mathrm{~Pb}, \mathrm{X}\right)\right] \text {; measured } \mathrm{E} \gamma, \mathrm{I} \gamma \text {. } \\
\text { JOUR ZAANE } 23201\end{array}$ \\
\hline${ }^{191}$ Os & 2005J019 & $\begin{array}{l}\text { NUCLEAR REACTIONS }{ }^{192} \mathrm{Os}\left({ }^{82} \mathrm{Se}, \mathrm{X}\right){ }^{191} \mathrm{Os}, \mathrm{E}=460 \mathrm{MeV} \text {; measured } \\
\mathrm{E} \gamma \text {, I } \gamma, \gamma \gamma \text {-coin. }{ }^{191} \text { Os deduced levels, J, } \pi \text {, branching ratios, } \\
\text { configurations, isomeric state features. GASP array. JOUR JPGPE } 31 \\
\text { S1891 }\end{array}$ \\
\hline & 2005 NI12 & $\begin{array}{l}\left.\text { RADIOACTIVITY }{ }^{191} \mathrm{Os}\left(\beta^{-}\right) \text {from }{ }^{190} \mathrm{Os}(\mathrm{n}, \gamma)\right] ; \text { measured } \mathrm{E} \gamma, \mathrm{I} \gamma \text {, } \\
\text { X-ray spectra. }{ }^{191} \mathrm{Ir} \text { transition deduced ICC, fluorescence yield. } \\
\text { Comparison with model predictions, }{ }^{193 m} \mathrm{Ir} \text { decay data. Need for } \\
\text { K-shell hole to be included in calculations discussed. JOUR PRVCA } 71 \\
054320\end{array}$ \\
\hline${ }^{191} \mathrm{Ir}$ & 2005 FOZZ & $\begin{array}{l}\text { NUCLEAR REACTIONS }{ }^{191} \operatorname{Ir}(\mathrm{n}, \mathrm{n}),(\mathrm{n}, 2 \mathrm{n}),(\mathrm{n}, 3 \mathrm{n}),(\mathrm{n}, 4 \mathrm{n}),(\mathrm{n}, 5 \mathrm{n}) \text {, } \\
(\mathrm{n}, 6 \mathrm{n}),(\mathrm{n}, 7 \mathrm{n}),(\mathrm{n}, 8 \mathrm{n}),(\mathrm{n}, 9 \mathrm{n}), \mathrm{E}=1-300 \mathrm{MeV} ; \text { measured } \mathrm{E} \gamma, \mathrm{I} \gamma ; \\
\text { deduced } \gamma \text {-ray production } \sigma . \text { CONF Santa Fe (Nucl Data for Sci and } \\
\text { Technol) Proc,Vol1,P898 }\end{array}$ \\
\hline & 2005NI12 & $\begin{array}{l}\left.\text { RADIOACTIVITY }{ }^{191} \mathrm{Os}\left(\beta^{-}\right) \text {[from }{ }^{190} \mathrm{Os}(\mathrm{n}, \gamma)\right] \text {; measured } \mathrm{E} \gamma, \mathrm{I} \gamma \text {, } \\
\text { X-ray spectra. }{ }^{191} \mathrm{Ir} \text { transition deduced ICC, fluorescence yield. } \\
\text { Comparison with model predictions, }{ }^{193 m} \mathrm{Ir} \text { decay data. Need for } \\
\text { K-shell hole to be included in calculations discussed. JOUR PRVCA } 71 \\
054320\end{array}$ \\
\hline${ }^{191} \mathrm{Pt}$ & $2005 K U 01$ & $\begin{array}{l}\text { NUCLEAR REACTIONS }{ }^{186} \mathrm{~W}\left({ }^{11} \mathrm{~B}, 5 \mathrm{np}\right), \mathrm{E}=85 \mathrm{MeV} \text {; measured } \mathrm{E} \gamma \text {, } \\
\mathrm{I} \gamma, \gamma \gamma \text {-coin. }{ }^{191} \mathrm{Pt} \text { deduced high-spin levels, J, } \pi \text {, configurations, shape } \\
\text { coexistence. Eurogam-II array, cranked mean-field calculations. JOUR } \\
\text { ZAANE } 2369\end{array}$ \\
\hline & 2005 TA26 & $\begin{array}{l}\text { NUCLEAR REACTIONS } \operatorname{Ir}(\mathrm{p}, \mathrm{xnyp}){ }^{188} \mathrm{Pt} /{ }^{189} \mathrm{Pt} /{ }^{191} \mathrm{Pt} /{ }^{185} \mathrm{Ir} / \\
{ }^{186} \mathrm{Ir} /{ }^{188} \mathrm{Ir} /{ }^{189} \mathrm{Ir} /{ }^{190} \mathrm{Ir} /{ }^{192} \mathrm{Ir} /{ }^{185} \mathrm{Os}, \mathrm{E} \approx 3-70 \mathrm{MeV} ; \text { measured } \sigma ; \\
\text { deduced integral yields. Stacked-foil activation technique. JOUR } \\
\text { NIMBE } 239293\end{array}$ \\
\hline & 2005TAZV & $\begin{array}{l}\text { NUCLEAR REACTIONS Ir }(\mathrm{p}, \mathrm{X}){ }^{188} \mathrm{Pt} /{ }^{189} \mathrm{Pt} /{ }^{191} \mathrm{Pt} /{ }^{186} \mathrm{Ir} /{ }^{187} \mathrm{Ir} \\
/{ }^{188} \mathrm{Ir} /{ }^{189} \mathrm{Ir} /{ }^{190} \mathrm{Ir} /{ }^{192} \mathrm{Ir}, \mathrm{E} \approx 10-70 \mathrm{MeV} \text {; measured excitation } \\
\text { functions; deduced integral yields. Stacked-foil activation technique. } \\
\text { CONF Santa Fe (Nucl Data for Sci and Technol) Proc,Vol1,P1023 }\end{array}$ \\
\hline & 2006DI01 & $\begin{array}{l}\text { NUCLEAR REACTIONS Pt }(\mathrm{d}, \mathrm{X}){ }^{191} \mathrm{Au} /{ }^{192} \mathrm{Au} /{ }^{193} \mathrm{Au} /{ }^{194} \mathrm{Au} / \\
{ }^{195} \mathrm{Au} /{ }^{196} \mathrm{Au} /{ }^{196 m} \mathrm{Au} /{ }^{198} \mathrm{Au} /{ }^{199} \mathrm{Au} /{ }^{191} \mathrm{Pt} /{ }^{195 m} \mathrm{Pt} /{ }^{197} \mathrm{Pt} / \\
{ }^{192} \mathrm{Ir}, \mathrm{E} \approx 10-40 \mathrm{MeV} \text {; measured production } \sigma . \text { Stacked-foil activation } \\
\text { technique, comparison with model predictions. JOUR NIMBE } 24320\end{array}$ \\
\hline${ }^{191} \mathrm{Au}$ & 2004ADZW & $\begin{array}{l}\text { NUCLEAR REACTIONS }{ }^{209} \mathrm{Bi}(\mathrm{n}, 4 \mathrm{n}),(\mathrm{n}, 5 \mathrm{n}),(\mathrm{n}, 6 \mathrm{n}),(\mathrm{n}, 7 \mathrm{n}),(\mathrm{n}, \\
9 \mathrm{n}),{ }^{232} \mathrm{Th}(\mathrm{n}, \gamma),{ }^{197} \mathrm{Au}(\mathrm{n}, 2 \mathrm{n}),(\mathrm{n}, 4 \mathrm{n}),(\mathrm{n}, 6 \mathrm{n}),(\mathrm{n}, 7 \mathrm{n}),(\mathrm{n}, \gamma),{ }^{115} \operatorname{In}(\mathrm{n}, \\
5 \mathrm{n}),(\mathrm{n}, 6 \mathrm{n}),(\mathrm{n}, 7 \mathrm{n}),{ }^{59} \mathrm{Co}(\mathrm{n}, 2 \mathrm{n}),(\mathrm{n}, 3 \mathrm{n}),(\mathrm{n}, 4 \mathrm{n}),(\mathrm{n}, 5 \mathrm{n}),(\mathrm{n}, \gamma),(\mathrm{n}, \\
\mathrm{p}),(\mathrm{n}, 6 \mathrm{n} 2 \mathrm{p}), \mathrm{E}=\mathrm{spectrum} ; \text { measured } \mathrm{E} \gamma, \mathrm{I} \gamma ; \text { deduced reaction rates. } \\
\mathrm{Pb}(\mathrm{p}, \mathrm{nX}), \mathrm{E}=1 \mathrm{GeV} ; \text { deduced spallation neutron spectrum. REPT } \\
\text { JINR-E1-2004-16,Adam }\end{array}$ \\
\hline
\end{tabular}




\section{$\mathrm{A}=191$ (continued)}

2005AD01 NUCLEAR REACTIONS ${ }^{209} \operatorname{Bi}(\mathrm{n}, 4 \mathrm{n}),(\mathrm{n}, 5 \mathrm{n}),(\mathrm{n}, 6 \mathrm{n}),(\mathrm{n}, 7 \mathrm{n}),(\mathrm{n}$, 9n), ${ }^{232} \mathrm{Th}(\mathrm{n}, \gamma),{ }^{197} \mathrm{Au}(\mathrm{n}, 2 \mathrm{n}),(\mathrm{n}, 4 \mathrm{n}),(\mathrm{n}, 6 \mathrm{n}),(\mathrm{n}, 7 \mathrm{n}),(\mathrm{n}, \gamma),{ }^{59} \mathrm{Co}(\mathrm{n}$, 2n), (n, 3n), (n, 4n), (n, 5n), (n, p), (n, 6n2p), ${ }^{115} \operatorname{In}(\mathrm{n}, 5 \mathrm{n}),(\mathrm{n}, 6 \mathrm{n}),(\mathrm{n}$, $7 \mathrm{n}), \mathrm{E}=$ spectrum; measured $\mathrm{E} \gamma, \mathrm{I} \gamma$; deduced reaction rates. Activation technique, spallation neutrons from $1 \mathrm{GeV}$ proton beam, comparison with model predictions. JOUR ZAANE 2361

2006DI01 NUCLEAR REACTIONS Pt $(\mathrm{d}, \mathrm{X}){ }^{191} \mathrm{Au} /{ }^{192} \mathrm{Au} /{ }^{193} \mathrm{Au} /{ }^{194} \mathrm{Au} /$ ${ }^{195} \mathrm{Au} /{ }^{196} \mathrm{Au} /{ }^{196 m} \mathrm{Au} /{ }^{198} \mathrm{Au} /{ }^{199} \mathrm{Au} /{ }^{191} \mathrm{Pt} /{ }^{195 m} \mathrm{Pt} /{ }^{197} \mathrm{Pt} /$ ${ }^{192} \mathrm{Ir}, \mathrm{E} \approx 10-40 \mathrm{MeV}$; measured production $\sigma$. Stacked-foil activation technique, comparison with model predictions. JOUR NIMBE 24320

${ }^{191} \mathrm{~Pb}$ 2005UU02 RADIOACTIVITY ${ }^{201,202,203,204} \mathrm{Ra},{ }^{197,198,199,200} \mathrm{Rn},{ }^{193,194,195,196} \mathrm{Po}$, ${ }^{201,202,203,204} \mathrm{Fr},{ }^{197,198,199,200} \mathrm{At}(\alpha)$ [from ${ }^{141} \operatorname{Pr}\left({ }^{63,65} \mathrm{Cu}\right.$, xnypz $\left.\alpha\right)$, ${ }^{170} \mathrm{Yb}\left({ }^{36} \mathrm{Ar}\right.$, xnypz $\left.\alpha\right)$, and subsequent decay]; measured $\mathrm{E} \alpha, \mathrm{T}_{1 / 2}$, $\alpha \alpha$-coin for ground and metastable state decay. ${ }^{193,195} \mathrm{Bi},{ }^{197,199} \mathrm{At}$, ${ }^{201,203} \mathrm{Fr}$ deduced levels, J, $\pi$. Comparisons with previous results. JOUR PRVCA 71024306

${ }^{191} \mathrm{Bi}$ 2005AN17 NUCLEAR REACTIONS ${ }^{142} \mathrm{Nd}\left({ }^{52} \mathrm{Cr}, 3 \mathrm{n}\right),\left({ }^{52} \mathrm{Cr}, 4 \mathrm{n}\right),\left({ }^{52} \mathrm{Cr}, 5 \mathrm{n}\right)$, $\left({ }^{52} \mathrm{Cr}, 6 \mathrm{n}\right),\left({ }^{52} \mathrm{Cr}, \mathrm{np}\right),\left({ }^{52} \mathrm{Cr}, 2 \mathrm{np}\right),\left({ }^{52} \mathrm{Cr}, 3 \mathrm{np}\right),\left({ }^{52} \mathrm{Cr}, 4 \mathrm{np}\right),\left({ }^{52} \mathrm{Cr}\right.$, $5 \mathrm{np}),\left({ }^{52} \mathrm{Cr}, 6 \mathrm{np}\right), \mathrm{E}=220-310 \mathrm{MeV} ;{ }^{142} \mathrm{Nd}\left({ }^{50} \mathrm{Cr}, 3 \mathrm{n}\right),\left({ }^{50} \mathrm{Cr}, 4 \mathrm{n}\right),\left({ }^{50} \mathrm{Cr}\right.$, $2 \mathrm{np}),\left({ }^{50} \mathrm{Cr}, 3 \mathrm{np}\right),\left({ }^{50} \mathrm{Cr}, 4 \mathrm{np}\right),\left({ }^{50} \mathrm{Cr}, 5 \mathrm{np}\right), \mathrm{E}=230-285 \mathrm{MeV}$; ${ }^{92} \mathrm{Mo}\left({ }^{98} \mathrm{Mo}, 2 \mathrm{np}\right),\left({ }^{98} \mathrm{Mo}, 3 \mathrm{np}\right), \mathrm{E}=427-460 \mathrm{MeV} ;{ }^{93} \mathrm{Nb}\left({ }^{95} \mathrm{Mo}, \mathrm{n}\right)$, $\left({ }^{95} \mathrm{Mo}, 2 \mathrm{n}\right),\left({ }^{95} \mathrm{Mo}, 3 \mathrm{n}\right),\left({ }^{95} \mathrm{Mo}, \mathrm{p}\right),\left({ }^{95} \mathrm{Mo}, \mathrm{np}\right),\left({ }^{95} \mathrm{Mo}, 2 \mathrm{np}\right),\left({ }^{95} \mathrm{Mo}\right.$, $3 \mathrm{np}),\left({ }^{95} \mathrm{Mo}, 4 \mathrm{np}\right), \mathrm{E}=375-456 \mathrm{MeV} ;{ }^{93} \mathrm{Nb}\left({ }^{94} \mathrm{Mo}, 2 \mathrm{n}\right),\left({ }^{94} \mathrm{Mo}, 3 \mathrm{n}\right)$, $\left({ }^{94} \mathrm{Mo}, \mathrm{np}\right),\left({ }^{94} \mathrm{Mo}, 2 \mathrm{np}\right),\left({ }^{94} \mathrm{Mo}, 3 \mathrm{np}\right), \mathrm{E}=405-450 \mathrm{MeV} ;{ }^{144} \mathrm{Sm}\left({ }^{46} \mathrm{Ti}\right.$, 3n), $\left({ }^{46} \mathrm{Ti}, 4 \mathrm{n}\right), \mathrm{E}=202-242 \mathrm{MeV}$; measured $\sigma$. Velocity filter, comparison with statistical model predictions. JOUR PRVCA 72 014612

2005GEZW ATOMIC MASSES ${ }^{235}$ Ac; measured mass, $\mathrm{T}_{1 / 2}$. $185,186,187,188,189,190,191,192,193,194,195,196 \mathrm{Bi}$; measured masses, proton separation energies. ${ }^{207 m} \mathrm{Tl}$; measured $\mathrm{T}_{1 / 2}$. Stored beams, Schottky mass spectrometry. PREPRINT nucl-ex/0510009,10/4/2005

2005KE10 RADIOACTIVITY ${ }^{191,193,195} \operatorname{At}(\alpha)$; measured $\mathrm{E} \alpha, \mathrm{E} \gamma, \gamma \alpha$-coin. 191,193,195 At deduced levels, J, $\pi$, configurations, proton separation energies. ${ }^{187,189,191} \mathrm{Bi}$ deduced levels $\mathrm{J}, \pi$, configurations. Comparison with theory. JOUR ZAANE 25 s01 181

2005UU03 RADIOACTIVITY ${ }^{188,190,192,194,196,198,200,202,204}$ Po, $191,193,195,197,199$ At, ${ }^{196,198,200,202,204,206} \mathrm{Rn},{ }^{199,201,203,205,207} \operatorname{Fr}(\alpha)$; measured reduced widths using gas filled recoil separator; deduced hindrance factors, proton intruder states and deformation effects. JOUR ZAANE 25 s01 179

2005VA04 RADIOACTIVITY ${ }^{189} \mathrm{Po}(\alpha)$ [from $\left.{ }^{142} \mathrm{Nd}\left({ }^{52} \mathrm{Cr}, 5 \mathrm{n}\right),\left({ }^{50} \mathrm{Cr}, 3 \mathrm{n}\right)\right]$; measured $\mathrm{E} \alpha, \mathrm{E} \gamma, \mathrm{E}(\mathrm{ce}), \alpha \gamma$-coin. ${ }^{185} \mathrm{~Pb}$ deduced levels, J, $\pi$, ICC, $\mathrm{T}_{1 / 2}$, configurations. ${ }^{188,189,190,191} \mathrm{Bi},{ }^{189,190} \mathrm{Po}(\alpha)$ [from ${ }^{142} \mathrm{Nd}\left({ }^{52} \mathrm{Cr}\right.$, $\left.\mathrm{X}),\left({ }^{50} \mathrm{Cr}, \mathrm{X}\right)\right]$; measured $\mathrm{E} \alpha, \mathrm{I} \alpha$. Potential energy surface calculations, level systematics in neighboring isotopes discussed. JOUR ZAANE 24 57 


\section{$\mathrm{A}=191$ (continued)}

${ }^{191} \mathrm{Po} \quad$ 2005AN17 NUCLEAR REACTIONS ${ }^{142} \mathrm{Nd}\left({ }^{52} \mathrm{Cr}, 3 \mathrm{n}\right),\left({ }^{52} \mathrm{Cr}, 4 \mathrm{n}\right),\left({ }^{52} \mathrm{Cr}, 5 \mathrm{n}\right)$, $\left({ }^{52} \mathrm{Cr}, 6 \mathrm{n}\right),\left({ }^{52} \mathrm{Cr}, \mathrm{np}\right),\left({ }^{52} \mathrm{Cr}, 2 \mathrm{np}\right),\left({ }^{52} \mathrm{Cr}, 3 \mathrm{np}\right),\left({ }^{52} \mathrm{Cr}, 4 \mathrm{np}\right),\left({ }^{52} \mathrm{Cr}\right.$, 5np), $\left({ }^{52} \mathrm{Cr}, 6 \mathrm{np}\right), \mathrm{E}=220-310 \mathrm{MeV} ;{ }^{142} \mathrm{Nd}\left({ }^{50} \mathrm{Cr}, 3 \mathrm{n}\right),\left({ }^{50} \mathrm{Cr}, 4 \mathrm{n}\right),\left({ }^{50} \mathrm{Cr}\right.$, $2 \mathrm{np}),\left({ }^{50} \mathrm{Cr}, 3 \mathrm{np}\right),\left({ }^{50} \mathrm{Cr}, 4 \mathrm{np}\right),\left({ }^{50} \mathrm{Cr}, 5 \mathrm{np}\right), \mathrm{E}=230-285 \mathrm{MeV}$; ${ }^{92} \mathrm{Mo}\left({ }^{98} \mathrm{Mo}, 2 \mathrm{np}\right),\left({ }^{98} \mathrm{Mo}, 3 \mathrm{np}\right), \mathrm{E}=427-460 \mathrm{MeV} ;{ }^{93} \mathrm{Nb}\left({ }^{95} \mathrm{Mo}, \mathrm{n}\right)$, $\left({ }^{95} \mathrm{Mo}, 2 \mathrm{n}\right),\left({ }^{95} \mathrm{Mo}, 3 \mathrm{n}\right),\left({ }^{95} \mathrm{Mo}, \mathrm{p}\right),\left({ }^{95} \mathrm{Mo}, \mathrm{np}\right),\left({ }^{95} \mathrm{Mo}, 2 \mathrm{np}\right),\left({ }^{95} \mathrm{Mo}\right.$, 3np), ( $\left.{ }^{95} \mathrm{Mo}, 4 \mathrm{np}\right), \mathrm{E}=375-456 \mathrm{MeV} ;{ }^{93} \mathrm{Nb}\left({ }^{94} \mathrm{Mo}, 2 \mathrm{n}\right),\left({ }^{94} \mathrm{Mo}, 3 \mathrm{n}\right)$, $\left({ }^{94} \mathrm{Mo}, \mathrm{np}\right),\left({ }^{94} \mathrm{Mo}, 2 \mathrm{np}\right),\left({ }^{94} \mathrm{Mo}, 3 \mathrm{np}\right), \mathrm{E}=405-450 \mathrm{MeV} ;{ }^{144} \mathrm{Sm}\left({ }^{46} \mathrm{Ti}\right.$, $3 \mathrm{n}),\left({ }^{46} \mathrm{Ti}, 4 \mathrm{n}\right), \mathrm{E}=202-242 \mathrm{MeV}$; measured $\sigma$. Velocity filter, comparison with statistical model predictions. JOUR PRVCA 72 014612

${ }^{191}$ At 2005KE10 NUCLEAR REACTIONS ${ }^{142} \mathrm{Nd}\left({ }^{56} \mathrm{Fe}, 2 \mathrm{np}\right), \mathrm{E}=262 \mathrm{MeV} ;{ }^{141} \mathrm{Pr}\left({ }^{56} \mathrm{Fe}\right.$, $4 \mathrm{n}), \mathrm{E}=266 \mathrm{MeV} ;{ }^{141} \operatorname{Pr}\left({ }^{54} \mathrm{Fe}, 4 \mathrm{n}\right), \mathrm{E}=260 \mathrm{MeV}$; measured production $\sigma$. JOUR ZAANE $25 \mathrm{~s} 01181$

2005KE10 RADIOACTIVITY ${ }^{191,193,195} \operatorname{At}(\alpha)$; measured E $\alpha, \mathrm{E} \gamma, \gamma \alpha$-coin. $191,193,195$ At deduced levels, J, $\pi$, configurations, proton separation energies. ${ }^{187,189,191} \mathrm{Bi}$ deduced levels $\mathrm{J}, \pi$, configurations. Comparison with theory. JOUR ZAANE 25 s01 181

2005UU03 RADIOACTIVITY 188,190,192,194,196,198,200,202,204 Po, $191,193,195,197,199$ At, ${ }^{196,198,200,202,204,206} \mathrm{Rn},{ }^{199,201,203,205,207} \operatorname{Fr}(\alpha)$; measured reduced widths using gas filled recoil separator; deduced hindrance factors, proton intruder states and deformation effects. JOUR ZAANE 25 s01 179

\section{$\mathrm{A}=192$}

${ }^{192} \operatorname{Re} \quad 2005 \mathrm{CA02}$

RADIOACTIVITY ${ }^{188} \mathrm{Ta},{ }^{190} \mathrm{~W},{ }^{192,193} \mathrm{Re},{ }^{195} \mathrm{Os},{ }^{197,198} \mathrm{Ir}$, ${ }_{200,201,202} \mathrm{Pt},{ }^{203} \mathrm{Au}(\mathrm{IT})$ [from $\left.\mathrm{Be}\left({ }^{208} \mathrm{~Pb}, \mathrm{X}\right)\right]$; measured $\mathrm{E} \gamma, \mathrm{I} \gamma, \gamma \gamma$-coin, $\mathrm{T}_{1 / 2} \cdot{ }^{188} \mathrm{Ta},{ }^{190} \mathrm{~W},{ }^{192,193} \mathrm{Re},{ }^{195} \mathrm{Os},{ }^{197,198} \mathrm{Ir},{ }^{200,201,202} \mathrm{Pt},{ }^{203} \mathrm{Au}$ deduced transitions. ${ }^{190} \mathrm{~W},{ }^{200,201,202} \mathrm{Pt}$ deduced levels, J, $\pi .{ }^{174,175} \mathrm{Er}$, ${ }^{185} \mathrm{Hf},{ }^{191,194} \mathrm{Re},{ }^{199} \mathrm{Ir}(\mathrm{IT})$ [from $\mathrm{Be}\left({ }^{208} \mathrm{~Pb}, \mathrm{X}\right)$ ]; measured $\mathrm{E} \gamma, \mathrm{I} \gamma$.

JOUR ZAANE 23201

${ }^{192} \mathrm{Ir} \quad 2004 \mathrm{HIZZ} \quad$ NUCLEAR REACTIONS ${ }^{102} \mathrm{Ru}\left({ }^{3} \mathrm{He}, 2 \mathrm{n}\right),{ }^{100} \mathrm{Ru}(\alpha, \mathrm{n}),{ }^{103} \mathrm{Rh}(\mathrm{d}, 2 \mathrm{n})$, $(\mathrm{p}, \mathrm{n}), \mathrm{E} \approx 5-35 \mathrm{MeV}$; analyzed excitation functions, yields. $\mathrm{Ce}\left({ }^{3} \mathrm{He}\right.$, $\mathrm{xn}){ }^{140} \mathrm{Nd}, \mathrm{E}<27 \mathrm{MeV} ;{ }^{141} \operatorname{Pr}(\mathrm{p}, 2 \mathrm{n}), \mathrm{E}<23 \mathrm{MeV}$; measured yields. ${ }^{192} \mathrm{Os}(\mathrm{p}, \mathrm{n}), \mathrm{E} \approx 6-20$; measured $\sigma$. REPT NEA/NSC/DOC(2004)14,P15,Hilgers

$2005 \mathrm{HIO}$ NUCLEAR REACTIONS ${ }^{192} \mathrm{Os}(\mathrm{p}, \mathrm{n}), \mathrm{E} \approx 6-20 \mathrm{MeV}$; measured $\sigma$; deduced thick-target yield. Stacked-foil activation, comparison with model predictions. JOUR ARISE 6393

2005HIZX NUCLEAR REACTIONS ${ }^{66} \mathrm{Zn}(\mathrm{d}, \alpha), \mathrm{E}=5-14 \mathrm{MeV}$; $\mathrm{Ce}\left({ }^{3} \mathrm{He}, \mathrm{xn}\right){ }^{140} \mathrm{Nd}$, $\mathrm{E}=16-35 \mathrm{MeV} ;{ }^{141} \mathrm{Ce}(\mathrm{p}, 2 \mathrm{n}), \mathrm{E}=10-45 \mathrm{MeV} ;{ }^{192} \mathrm{Os}(\mathrm{p}, \mathrm{n}), \mathrm{E}=6-19 \mathrm{MeV}$; measured excitation functions; deduced thick-target yields. Stacked-foil activation technique. CONF Santa Fe (Nucl Data for Sci and Technol) Proc,Vol2,P1631 


\section{$\mathrm{A}=192$ (continued)}

2005TA26 NUCLEAR REACTIONS Ir(p, xnyp ${ }^{188} \mathrm{Pt} /{ }^{189} \mathrm{Pt} /{ }^{191} \mathrm{Pt} /{ }^{185} \mathrm{Ir} /$ ${ }^{186} \operatorname{Ir} /{ }^{188} \operatorname{Ir} /{ }^{189} \operatorname{Ir} /{ }^{190} \operatorname{Ir} /{ }^{192} \operatorname{Ir} /{ }^{185} \mathrm{Os}, \mathrm{E} \approx 3-70 \mathrm{MeV}$; measured $\sigma$; deduced integral yields. Stacked-foil activation technique. JOUR NIMBE 239293

2005TAZV NUCLEAR REACTIONS Ir $(\mathrm{p}, \mathrm{X}){ }^{188} \mathrm{Pt} /{ }^{189} \mathrm{Pt} /{ }^{191} \mathrm{Pt} /{ }^{186} \mathrm{Ir} /{ }^{187} \mathrm{Ir}$ $/{ }^{188} \operatorname{Ir} /{ }^{189} \operatorname{Ir} /{ }^{190} \operatorname{Ir} /{ }^{192} \mathrm{Ir}, \mathrm{E} \approx 10-70 \mathrm{MeV}$; measured excitation functions; deduced integral yields. Stacked-foil activation technique. CONF Santa Fe (Nucl Data for Sci and Technol) Proc,Vol1,P1023 2005TAZW NUCLEAR REACTIONS Pt(p, X) ${ }^{195} \mathrm{Au} /{ }^{196} \mathrm{Au} /{ }^{198} \mathrm{Au} /{ }^{189} \mathrm{Ir} /$ ${ }^{190} \mathrm{Ir} /{ }^{192} \mathrm{Ir} /{ }^{194} \mathrm{Ir}, \mathrm{E} \approx 0-70 \mathrm{MeV} ; \mathrm{Pt}(\mathrm{d}, \mathrm{X}){ }^{192} \mathrm{Au} /{ }^{193} \mathrm{Au} /{ }^{194} \mathrm{Au} /$ ${ }^{195} \mathrm{Au} /{ }^{196} \mathrm{Au} /{ }^{195 m} \mathrm{Pt} /{ }^{197} \mathrm{Pt} /{ }^{192} \mathrm{Ir}, \mathrm{E} \approx 0-21 \mathrm{MeV} ; \mathrm{Pt}(\alpha, \mathrm{X}){ }^{195} \mathrm{Au}$ $/{ }^{196} \mathrm{Au}, \mathrm{E} \approx 0-38 \mathrm{MeV}$; measured activation $\sigma$; deduced integral yields. CONF Santa Fe (Nucl Data for Sci and Technol) Proc,Vol1,P1015 2005TIZX NUCLEAR REACTIONS Pb, ${ }^{208} \mathrm{~Pb}(\mathrm{p}, \mathrm{X}){ }^{203} \mathrm{~Pb} /{ }^{200} \mathrm{Tl} /{ }^{199} \mathrm{Tl} /$ ${ }^{196} \mathrm{Au} /{ }^{192} \mathrm{Ir} /{ }^{190} \mathrm{Ir} /{ }^{173} \mathrm{Lu} /{ }^{101 m} \mathrm{Rh} /{ }^{86} \mathrm{Rb} /{ }^{59} \mathrm{Fe} /{ }^{24} \mathrm{Na} /{ }^{7} \mathrm{Be}$, $\mathrm{E}=40-2600 \mathrm{MeV}$; measured excitation functions. Comparison with previous work and model predictions. Other reactions discussed. CONF Santa Fe (Nucl Data for Sci and Technol) Proc,Vol1,P1070 2005TIZY NUCLEAR REACTIONS Pb, ${ }^{208} \mathrm{~Pb},{ }^{209} \mathrm{Bi}(\mathrm{p}, \mathrm{X}){ }^{203} \mathrm{~Pb} /{ }^{200} \mathrm{Tl} /{ }^{199} \mathrm{Tl}$ $/{ }^{196} \mathrm{Au} /{ }^{192} \mathrm{Ir} /{ }^{190} \mathrm{Ir} /{ }^{173} \mathrm{Lu} /{ }^{101 m} \mathrm{Rh} /{ }^{86} \mathrm{Rb} /{ }^{59} \mathrm{Fe} /{ }^{24} \mathrm{Na} /{ }^{7} \mathrm{Be}$, $\mathrm{E}=40-2600 \mathrm{MeV}$; measured production $\sigma$. Comparison with model predictions. PREPRINT nucl-ex/0507009,7/05/2005

2006DI01 NUCLEAR REACTIONS Pt(d, X) $)^{191} \mathrm{Au} /{ }^{192} \mathrm{Au} /{ }^{193} \mathrm{Au} /{ }^{194} \mathrm{Au} /$ ${ }^{195} \mathrm{Au} /{ }^{196} \mathrm{Au} /{ }^{196 m} \mathrm{Au} /{ }^{198} \mathrm{Au} /{ }^{199} \mathrm{Au} /{ }^{191} \mathrm{Pt} /{ }^{195 m} \mathrm{Pt} /{ }^{197} \mathrm{Pt} /$ ${ }^{192} \mathrm{Ir}, \mathrm{E} \approx 10-40 \mathrm{MeV}$; measured production $\sigma$. Stacked-foil activation technique, comparison with model predictions. JOUR NIMBE 24320 ${ }^{192} \mathrm{Pt}$ 2005LEZW NUCLEAR REACTIONS ${ }^{188,190,194} \mathrm{Os},{ }^{194,196} \mathrm{Pt}(\alpha, 2 \mathrm{n}), \mathrm{E}=27 \mathrm{MeV}$; measured $\mathrm{E} \gamma, \mathrm{I} \gamma(\theta, \mathrm{H}, \mathrm{t}) .{ }^{190,192,194} \mathrm{Pt},{ }^{196,198} \mathrm{Hg}$ deduced isomeric states g-factors, configurations. Integral perturbed angular distribution method, HPGe detectors. CONF St Petersburg,P81,Levon

${ }^{192} \mathrm{Au}$ 2004ADZW NUCLEAR REACTIONS ${ }^{209} \mathrm{Bi}(\mathrm{n}, 4 \mathrm{n}),(\mathrm{n}, 5 \mathrm{n}),(\mathrm{n}, 6 \mathrm{n}),(\mathrm{n}, 7 \mathrm{n}),(\mathrm{n}$, 9n), ${ }^{232} \mathrm{Th}(\mathrm{n}, \gamma),{ }^{197} \mathrm{Au}(\mathrm{n}, 2 \mathrm{n}),(\mathrm{n}, 4 \mathrm{n}),(\mathrm{n}, 6 \mathrm{n}),(\mathrm{n}, 7 \mathrm{n}),(\mathrm{n}, \gamma),{ }^{115} \mathrm{In}(\mathrm{n}$, 5n), (n, 6n), (n, 7n), ${ }^{59} \mathrm{Co}(\mathrm{n}, 2 \mathrm{n}),(\mathrm{n}, 3 \mathrm{n}),(\mathrm{n}, 4 \mathrm{n}),(\mathrm{n}, 5 \mathrm{n}),(\mathrm{n}, \gamma),(\mathrm{n}$, $\mathrm{p}),(\mathrm{n}, 6 \mathrm{n} 2 \mathrm{p}), \mathrm{E}=$ spectrum; measured $\mathrm{E} \gamma, \mathrm{I} \gamma$; deduced reaction rates. $\mathrm{Pb}(\mathrm{p}, \mathrm{nX}), \mathrm{E}=1 \mathrm{GeV}$; deduced spallation neutron spectrum. REPT JINR-E1-2004-16,Adam

2005AD01 NUCLEAR REACTIONS ${ }^{209} \operatorname{Bi}(\mathrm{n}, 4 \mathrm{n}),(\mathrm{n}, 5 \mathrm{n}),(\mathrm{n}, 6 \mathrm{n}),(\mathrm{n}, 7 \mathrm{n}),(\mathrm{n}$, 9n), ${ }^{232} \mathrm{Th}(\mathrm{n}, \gamma),{ }^{197} \mathrm{Au}(\mathrm{n}, 2 \mathrm{n}),(\mathrm{n}, 4 \mathrm{n}),(\mathrm{n}, 6 \mathrm{n}),(\mathrm{n}, 7 \mathrm{n}),(\mathrm{n}, \gamma),{ }^{59} \mathrm{Co}(\mathrm{n}$, 2n), (n, 3n), (n, 4n), (n, 5n), (n, p), (n, 6n2p), ${ }^{115} \operatorname{In}(\mathrm{n}, 5 \mathrm{n}),(\mathrm{n}, 6 \mathrm{n}),(\mathrm{n}$, $7 \mathrm{n}), \mathrm{E}=$ spectrum; measured $\mathrm{E} \gamma, \mathrm{I} \gamma$; deduced reaction rates. Activation technique, spallation neutrons from $1 \mathrm{GeV}$ proton beam, comparison with model predictions. JOUR ZAANE 2361

2005TAZW NUCLEAR REACTIONS Pt(p, X) ${ }^{195} \mathrm{Au} /{ }^{196} \mathrm{Au} /{ }^{198} \mathrm{Au} /{ }^{189} \mathrm{Ir} /$ ${ }^{190} \mathrm{Ir} /{ }^{192} \mathrm{Ir} /{ }^{194} \mathrm{Ir}, \mathrm{E} \approx 0-70 \mathrm{MeV} ; \mathrm{Pt}(\mathrm{d}, \mathrm{X}){ }^{192} \mathrm{Au} /{ }^{193} \mathrm{Au} /{ }^{194} \mathrm{Au} /$ ${ }^{195} \mathrm{Au} /{ }^{196} \mathrm{Au} /{ }^{195 m} \mathrm{Pt} /{ }^{197} \mathrm{Pt} /{ }^{192} \mathrm{Ir}, \mathrm{E} \approx 0-21 \mathrm{MeV} ; \mathrm{Pt}(\alpha, \mathrm{X}){ }^{195} \mathrm{Au}$ $/{ }^{196} \mathrm{Au}, \mathrm{E} \approx 0-38 \mathrm{MeV}$; measured activation $\sigma$; deduced integral yields. CONF Santa Fe (Nucl Data for Sci and Technol) Proc,Vol1,P1015 


\section{$\mathrm{A}=192$ (continued)}

2006DI01 NUCLEAR REACTIONS Pt(d, X) $)^{191} \mathrm{Au} /{ }^{192} \mathrm{Au} /{ }^{193} \mathrm{Au} /{ }^{194} \mathrm{Au} /$ ${ }^{195} \mathrm{Au} /{ }^{196} \mathrm{Au} /{ }^{196 m} \mathrm{Au} /{ }^{198} \mathrm{Au} /{ }^{199} \mathrm{Au} /{ }^{191} \mathrm{Pt} /{ }^{195 m} \mathrm{Pt} /{ }^{197} \mathrm{Pt} /$

${ }^{192} \mathrm{Ir}, \mathrm{E} \approx 10-40 \mathrm{MeV}$; measured production $\sigma$. Stacked-foil activation technique, comparison with model predictions. JOUR NIMBE 24320

${ }^{192} \mathrm{~Pb} \quad$ 2004WIZX

2005UU02

2005WI01

${ }^{192} \mathrm{Bi} \quad 2005 \mathrm{AN} 17$

2005GEZW

${ }^{192} \mathrm{Po}$
NUCLEAR REACTIONS ${ }^{168} \operatorname{Er}\left({ }^{29} \mathrm{Si}, 5 \mathrm{n}\right), \mathrm{E}=154 \mathrm{MeV}$; measured $\mathrm{E} \gamma$, $\mathrm{I} \gamma, \gamma \gamma$-coin, DSA. ${ }^{192} \mathrm{~Pb}$ deduced superdeformed band levels $\mathrm{T}_{1 / 2}$, quadrupole moment. Gammasphere array, total Routhian surface calculations. PREPRINT ANU-P/1610,Wilson

RADIOACTIVITY ${ }^{201,202,203,204} \mathrm{Ra},{ }^{197,198,199,200} \mathrm{Rn},{ }^{193,194,195,196} \mathrm{Po}$, ${ }^{201,202,203,204} \mathrm{Fr},{ }^{197,198,199,200} \operatorname{At}(\alpha)$ [from ${ }^{141} \operatorname{Pr}\left({ }^{63,65} \mathrm{Cu}\right.$, xnypz $\left.\alpha\right)$,

${ }^{170} \mathrm{Yb}\left({ }^{36} \mathrm{Ar}\right.$, xnypz $\left.\alpha\right)$, and subsequent decay]; measured $\mathrm{E} \alpha, \mathrm{T}_{1 / 2}$, $\alpha \alpha$-coin for ground and metastable state decay. ${ }^{193,195} \mathrm{Bi},{ }^{197,199} \mathrm{At}$, ${ }^{201,203} \mathrm{Fr}$ deduced levels, J, $\pi$. Comparisons with previous results.

JOUR PRVCA 71024306

RADIOACTIVITY 188,190,192,194,196,198,200,202,204 Po 191,193,195,197,199 At, ${ }^{196,198,200,202,204,206}$ Rn, ${ }^{199,201,203,205,207} \operatorname{Fr}(\alpha)$; measured reduced widths using gas filled recoil separator; deduced hindrance factors, proton intruder states and deformation effects. JOUR ZAANE 25 s01 179 NUCLEAR REACTIONS ${ }^{168} \operatorname{Er}\left({ }^{29} \mathrm{Si}, 5 \mathrm{n}\right), \mathrm{E}=154 \mathrm{MeV}$; measured $\mathrm{E} \gamma$, $\mathrm{I} \gamma, \gamma \gamma$-coin, DSA. ${ }^{192} \mathrm{~Pb}$ deduced superdeformed band levels $\mathrm{T}_{1 / 2}$, quadrupole moment. Gammasphere array, total Routhian surface calculations. JOUR NUPAB 74812

NUCLEAR REACTIONS ${ }^{142} \mathrm{Nd}\left({ }^{52} \mathrm{Cr}, 3 \mathrm{n}\right),\left({ }^{52} \mathrm{Cr}, 4 \mathrm{n}\right),\left({ }^{52} \mathrm{Cr}, 5 \mathrm{n}\right)$, $\left({ }^{52} \mathrm{Cr}, 6 \mathrm{n}\right),\left({ }^{52} \mathrm{Cr}, \mathrm{np}\right),\left({ }^{52} \mathrm{Cr}, 2 \mathrm{np}\right),\left({ }^{52} \mathrm{Cr}, 3 \mathrm{np}\right),\left({ }^{52} \mathrm{Cr}, 4 \mathrm{np}\right),\left({ }^{52} \mathrm{Cr}\right.$, 5np $),\left({ }^{52} \mathrm{Cr}, 6 \mathrm{np}\right), \mathrm{E}=220-310 \mathrm{MeV} ;{ }^{142} \mathrm{Nd}\left({ }^{50} \mathrm{Cr}, 3 \mathrm{n}\right),\left({ }^{50} \mathrm{Cr}, 4 \mathrm{n}\right),\left({ }^{50} \mathrm{Cr}\right.$, $2 \mathrm{np}),\left({ }^{50} \mathrm{Cr}, 3 \mathrm{np}\right),\left({ }^{50} \mathrm{Cr}, 4 \mathrm{np}\right),\left({ }^{50} \mathrm{Cr}, 5 \mathrm{np}\right), \mathrm{E}=230-285 \mathrm{MeV}$; ${ }^{92} \mathrm{Mo}\left({ }^{98} \mathrm{Mo}, 2 \mathrm{np}\right),\left({ }^{98} \mathrm{Mo}, 3 \mathrm{np}\right), \mathrm{E}=427-460 \mathrm{MeV} ;{ }^{93} \mathrm{Nb}\left({ }^{95} \mathrm{Mo}, \mathrm{n}\right)$, $\left({ }^{95} \mathrm{Mo}, 2 \mathrm{n}\right),\left({ }^{95} \mathrm{Mo}, 3 \mathrm{n}\right),\left({ }^{95} \mathrm{Mo}, \mathrm{p}\right),\left({ }^{95} \mathrm{Mo}, \mathrm{np}\right),\left({ }^{95} \mathrm{Mo}, 2 \mathrm{np}\right),\left({ }^{95} \mathrm{Mo}\right.$, 3np), $\left({ }^{95} \mathrm{Mo}, 4 \mathrm{np}\right), \mathrm{E}=375-456 \mathrm{MeV} ;{ }^{93} \mathrm{Nb}\left({ }^{94} \mathrm{Mo}, 2 \mathrm{n}\right),\left({ }^{94} \mathrm{Mo}, 3 \mathrm{n}\right)$, $\left({ }^{94} \mathrm{Mo}, \mathrm{np}\right),\left({ }^{94} \mathrm{Mo}, 2 \mathrm{np}\right),\left({ }^{94} \mathrm{Mo}, 3 \mathrm{np}\right), \mathrm{E}=405-450 \mathrm{MeV} ;{ }^{144} \mathrm{Sm}\left({ }^{46} \mathrm{Ti}\right.$, $3 \mathrm{n}),\left({ }^{46} \mathrm{Ti}, 4 \mathrm{n}\right), \mathrm{E}=202-242 \mathrm{MeV}$; measured $\sigma$. Velocity filter, comparison with statistical model predictions. JOUR PRVCA 72 014612

RADIOACTIVITY ${ }^{200,201,203,205} \mathrm{Fr},{ }^{196,197,199,201} \mathrm{At},{ }^{193} \mathrm{Bi}(\alpha)$ [from $\mathrm{Th}(\mathrm{p}, \mathrm{X})$ and subsequent decay]; measured $\mathrm{E} \alpha, \mathrm{T}_{1 / 2} \cdot{ }^{201} \mathrm{Fr},{ }^{197} \mathrm{At}$, ${ }^{193} \mathrm{Bi},{ }^{189} \mathrm{Tl}$ deduced levels, J, $\pi$. JOUR ZAANE 23243 ATOMIC MASSES ${ }^{235}$ Ac; measured mass, $\mathrm{T}_{1 / 2}$. 185,186,187,188,189,190,191,192,193,194,195,196 $\mathrm{Bi}$; measured masses, proton separation energies. ${ }^{207 m} \mathrm{Tl}$; measured $\mathrm{T}_{1 / 2}$. Stored beams, Schottky mass spectrometry. PREPRINT nucl-ex/0510009,10/4/2005

RADIOACTIVITY 188,190,192,194,196,198,200,202,204 Po, 191,193,195,197,199 At, ${ }^{196,198,200,202,204,206}$ Rn, ${ }^{199,201,203,205,207} \operatorname{Fr}(\alpha)$; measured reduced widths using gas filled recoil separator; deduced hindrance factors, proton intruder states and deformation effects. JOUR ZAANE 25 s01 179 


\section{$\mathrm{A}=192$ (continued)}

${ }^{192}$ At 2005ANZY

2005ANZY

${ }^{193} \mathrm{Re}$

2005CA02

${ }^{193} \mathrm{Os}$

2002B066

2004ZA15

2005ZA15

${ }^{193} \operatorname{Ir}$

2004ZA15

2005KI01

2005ZA15

${ }^{193} \mathrm{Au} \quad$ 2005TAZW

2006DI01

${ }^{193} \mathrm{~Pb}$
NUCLEAR REACTIONS ${ }^{144} \mathrm{Sm}\left({ }^{46} \mathrm{Ti}\right.$, xn), E not given; measured $\mathrm{E} \gamma$, $\mathrm{E} \alpha, \alpha \alpha-, \alpha \gamma$-coin following residual nucleus decay; deduced evidence for ${ }^{186,187}$ Po. ${ }^{144} \mathrm{Sm}\left({ }^{51} \mathrm{~V}, \mathrm{xn}\right)$, E not given; measured $\mathrm{E} \gamma, \mathrm{E} \alpha, \alpha \alpha^{-}$, $\alpha \gamma$-coin following residual nucleus decay; deduced evidence for ${ }^{192} \mathrm{At}$. REPT GSI 2005-1,P77,Andreyev RADIOACTIVITY ${ }^{186,187} \mathrm{Po},{ }^{183} \mathrm{~Pb},{ }^{179} \mathrm{Hg},{ }^{175} \mathrm{Pt}(\alpha)$ [from ${ }^{144} \mathrm{Sm}\left({ }^{46} \mathrm{Ti}\right.$, $\mathrm{xn})$ and subsequent decay]; measured $\mathrm{E} \alpha, \mathrm{T}_{1 / 2} .{ }^{183} \mathrm{~Pb}$ deduced excited state energy. ${ }^{187}$ Po deduced isomeric states. ${ }^{192} \mathrm{At}(\alpha)\left[\mathrm{from}{ }^{144} \mathrm{Sm}\left({ }^{51} \mathrm{~V}\right.\right.$, $\mathrm{xn})]$; measured $\mathrm{E} \alpha, \alpha \alpha$-coin, $\mathrm{T}_{1 / 2}$; deduced isomeric states. REPT GSI 2005-1,P77,Andreyev

\section{$\mathrm{A}=\mathbf{1 9 3}$}

RADIOACTIVITY ${ }^{188} \mathrm{Ta},{ }^{190} \mathrm{~W},{ }^{192,193} \mathrm{Re},{ }^{195} \mathrm{Os},{ }^{197,198} \mathrm{Ir}$, ${ }^{200,201,202} \mathrm{Pt},{ }^{203} \mathrm{Au}(\mathrm{IT})$ [from $\mathrm{Be}\left({ }^{208} \mathrm{~Pb}, \mathrm{X}\right)$ ]; measured $\mathrm{E} \gamma, \mathrm{I} \gamma, \gamma \gamma$-coin, $\mathrm{T}_{1 / 2} .{ }^{188} \mathrm{Ta},{ }^{190} \mathrm{~W},{ }^{192,193} \mathrm{Re},{ }^{195} \mathrm{Os},{ }^{197,198} \mathrm{Ir},{ }^{200,201,202} \mathrm{Pt},{ }^{203} \mathrm{Au}$ deduced transitions. ${ }^{190} \mathrm{~W},{ }^{200,201,202} \mathrm{Pt}$ deduced levels, J, $\pi .{ }^{174,175} \mathrm{Er}$, ${ }^{185} \mathrm{Hf},{ }^{191,194} \mathrm{Re},{ }^{199} \operatorname{Ir}(\mathrm{IT})$ [from $\mathrm{Be}\left({ }^{208} \mathrm{~Pb}, \mathrm{X}\right)$ ]; measured $\mathrm{E} \gamma, \mathrm{I} \gamma$.

JOUR ZAANE 23201

NUCLEAR REACTIONS ${ }^{192} \mathrm{Os}(\mathrm{n}, \gamma), \mathrm{E}=$ thermal; measured $\mathrm{E} \gamma, \mathrm{I} \gamma$, $\gamma \gamma$-coin. ${ }^{193}$ Os deduced level energies, two-step cascade intensities, level density features. JOUR FIZBE 1183

RADIOACTIVITY ${ }^{193} \mathrm{Os}\left(\beta^{-}\right)$[from ${ }^{192} \mathrm{Os}(\mathrm{n}, \gamma)$ ]; measured $\mathrm{E} \gamma, \mathrm{I} \gamma$, $\gamma \gamma$-coin. ${ }^{193}$ Ir deduced levels, transition intensities. JOUR BJPHE 34 719

RADIOACTIVITY ${ }^{193} \mathrm{Os}\left(\beta^{-}\right)$[from $\left.{ }^{192} \mathrm{Os}(\mathrm{n}, \gamma)\right]$; measured $\mathrm{E} \gamma, \mathrm{I} \gamma$, $\gamma \gamma$-coin. ${ }^{193}$ Ir deduced levels, J, $\pi$. JOUR BJPHE 35843

RADIOACTIVITY ${ }^{193} \mathrm{Os}\left(\beta^{-}\right)$[from ${ }^{192} \mathrm{Os}(\mathrm{n}, \gamma)$ ]; measured $\mathrm{E} \gamma, \mathrm{I} \gamma$, $\gamma \gamma$-coin. ${ }^{193} \mathrm{Ir}$ deduced levels, transition intensities. JOUR BJPHE 34 719

NUCLEAR REACTIONS ${ }^{193} \operatorname{Ir}$ (X-ray, X-ray), (X-ray, $\gamma$ ), E=low; measured $\gamma$-spectra, X-ray spectra. ${ }^{193} \mathrm{Ir}$ deduced probability for nuclear excitation by electron transition. Synchrotron radiation, silicon avalanche photodiode. JOUR NUPAB 7483

RADIOACTIVITY ${ }^{193} \mathrm{Os}\left(\beta^{-}\right)$[from $\left.{ }^{192} \mathrm{Os}(\mathrm{n}, \gamma)\right]$; measured $\mathrm{E} \gamma, \mathrm{I} \gamma$, $\gamma \gamma$-coin. ${ }^{193} \mathrm{Ir}$ deduced levels, J, $\pi$. JOUR BJPHE 35843

NUCLEAR REACTIONS Pt $(\mathrm{p}, \mathrm{X}){ }^{195} \mathrm{Au} /{ }^{196} \mathrm{Au} /{ }^{198} \mathrm{Au} /{ }^{189} \mathrm{Ir} /$ ${ }^{190} \mathrm{Ir} /{ }^{192} \mathrm{Ir} /{ }^{194} \mathrm{Ir}, \mathrm{E} \approx 0-70 \mathrm{MeV} ; \mathrm{Pt}(\mathrm{d}, \mathrm{X}){ }^{192} \mathrm{Au} /{ }^{193} \mathrm{Au} /{ }^{194} \mathrm{Au} /$ ${ }^{195} \mathrm{Au} /{ }^{196} \mathrm{Au} /{ }^{195 m} \mathrm{Pt} /{ }^{197} \mathrm{Pt} /{ }^{192} \mathrm{Ir}, \mathrm{E} \approx 0-21 \mathrm{MeV} ; \mathrm{Pt}(\alpha, \mathrm{X}){ }^{195} \mathrm{Au}$ $/{ }^{196} \mathrm{Au}, \mathrm{E} \approx 0-38 \mathrm{MeV}$; measured activation $\sigma$; deduced integral yields. CONF Santa Fe (Nucl Data for Sci and Technol) Proc,Vol1,P1015 NUCLEAR REACTIONS Pt $(\mathrm{d}, \mathrm{X}){ }^{191} \mathrm{Au} /{ }^{192} \mathrm{Au} /{ }^{193} \mathrm{Au} /{ }^{194} \mathrm{Au} /$ ${ }^{195} \mathrm{Au} /{ }^{196} \mathrm{Au} /{ }^{196 m} \mathrm{Au} /{ }^{198} \mathrm{Au} /{ }^{199} \mathrm{Au} /{ }^{191} \mathrm{Pt} /{ }^{195 m} \mathrm{Pt} /{ }^{197} \mathrm{Pt} /$ ${ }^{192} \mathrm{Ir}, \mathrm{E} \approx 10-40 \mathrm{MeV}$; measured production $\sigma$. Stacked-foil activation technique, comparison with model predictions. JOUR NIMBE 24320 RADIOACTIVITY ${ }^{197,197 m, 198,199 m, 200,201 m} \mathrm{Po}(\alpha) ;{ }^{172,173} \mathrm{Os}(\alpha)$ [from $\left.{ }^{156} \mathrm{Dy}\left({ }^{22} \mathrm{Ne}, \mathrm{xn}\right)\right] ;{ }^{183,184,185} \mathrm{Hg}(\alpha)$ [from $\left.{ }^{168} \mathrm{Yb}\left({ }^{22} \mathrm{Ne}, \mathrm{xn}\right)\right]$; measured $\mathrm{E} \alpha, \mathrm{T}_{1 / 2}$. JOUR NIMAE 479631 


\section{$\mathrm{A}=193$ (continued)}

2005GL09 NUCLEAR REACTIONS ${ }^{170} \operatorname{Er}\left({ }^{28} \mathrm{Si}, 5 \mathrm{n}\right), \mathrm{E}=149 \mathrm{MeV}$; measured $\mathrm{E} \gamma$, $\mathrm{I} \gamma, \gamma \gamma$-coin, DSA. ${ }^{193} \mathrm{~Pb}$ deduced magnetic rotational band levels $\mathrm{T}_{1 / 2}$. GASP array, recoil-distance and Doppler-shift attenuation methods used. JOUR JPGPE 31 S1559

${ }^{193} \mathrm{Bi}$ 2004KE15 NUCLEAR REACTIONS ${ }^{1,2} \mathrm{H}, \mathrm{Ti}\left({ }^{208} \mathrm{~Pb}, \mathrm{X}\right){ }^{193} \mathrm{Bi} /{ }^{194} \mathrm{Bi} /{ }^{195} \mathrm{Bi} /$ ${ }^{196} \mathrm{Bi} /{ }^{197} \mathrm{Bi} /{ }^{198} \mathrm{Bi} /{ }^{199} \mathrm{Bi} /{ }^{200} \mathrm{Bi} /{ }^{201} \mathrm{Bi} /{ }^{202} \mathrm{Bi} /{ }^{203} \mathrm{Bi} /{ }^{204} \mathrm{Bi} /$ ${ }^{205} \mathrm{Bi} /{ }^{206} \mathrm{Bi} /{ }^{207} \mathrm{Bi} /{ }^{208} \mathrm{Bi}, \mathrm{E}=1 \mathrm{GeV} /$ nucleon; measured charge-pickup $\sigma$, velocity distributions; deduced reaction mechanism features. Comparison with model predictions and previous results. JOUR PRVCA 70064608

2005DE01 RADIOACTIVITY ${ }^{200,201,203,205} \mathrm{Fr},{ }^{196,197,199,201} \mathrm{At},{ }^{193} \operatorname{Bi}(\alpha)$ [from $\mathrm{Th}(\mathrm{p}, \mathrm{X})$ and subsequent decay]; measured $\mathrm{E} \alpha, \mathrm{T}_{1 / 2} \cdot{ }^{201} \mathrm{Fr},{ }^{197} \mathrm{At}$, ${ }^{193} \mathrm{Bi},{ }^{189} \mathrm{Tl}$ deduced levels, J, $\pi$. JOUR ZAANE 23243

2005GEZW ATOMIC MASSES ${ }^{235}$ Ac; measured mass, $\mathrm{T}_{1 / 2}$. $185,186,187,188,189,190,191,192,193,194,195,196 \mathrm{Bi}$; measured masses, proton separation energies. ${ }^{207 m} \mathrm{Tl}$; measured $\mathrm{T}_{1 / 2}$. Stored beams, Schottky mass spectrometry. PREPRINT nucl-ex/0510009,10/4/2005

2005 UU02 RADIOACTIVITY ${ }^{201,202,203,204} \mathrm{Ra},{ }^{197,198,199,200} \mathrm{Rn},{ }^{193,194,195,196} \mathrm{Po}$, ${ }^{201,202,203,204} \mathrm{Fr},{ }^{197,198,199,200} \mathrm{At}(\alpha)$ [from ${ }^{141} \operatorname{Pr}\left({ }^{63,65} \mathrm{Cu}\right.$, xnypz $\left.\alpha\right)$, ${ }^{170} \mathrm{Yb}\left({ }^{36} \mathrm{Ar}\right.$, xnypz $\left.\alpha\right)$, and subsequent decay]; measured $\mathrm{E} \alpha, \mathrm{T}_{1 / 2}$, $\alpha \alpha$-coin for ground and metastable state decay. ${ }^{193,195} \mathrm{Bi},{ }^{197,199} \mathrm{At}$, ${ }^{201,203} \mathrm{Fr}$ deduced levels, J, $\pi$. Comparisons with previous results.

JOUR PRVCA 71024306

2005 UU03 RADIOACTIVITY ${ }^{188,190,192,194,196,198,200,202,204} \mathrm{Po}$, 191,193,195,197,199 At, ${ }^{196,198,200,202,204,206} \mathrm{Rn},{ }^{199,201,203,205,207} \operatorname{Fr}(\alpha)$; measured reduced widths using gas filled recoil separator; deduced hindrance factors, proton intruder states and deformation effects. JOUR ZAANE 25 s01 179

${ }^{193} \mathrm{Po}$ 2005UU02 RADIOACTIVITY ${ }^{201,202,203,204} \mathrm{Ra},{ }^{197,198,199,200} \mathrm{Rn},{ }^{193,194,195,196} \mathrm{Po}$, ${ }^{201,202,203,204} \mathrm{Fr},{ }^{197,198,199,200} \operatorname{At}(\alpha)$ [from ${ }^{141} \operatorname{Pr}\left({ }^{63,65} \mathrm{Cu}\right.$, xnypz $\left.\alpha\right)$, ${ }^{170} \mathrm{Yb}\left({ }^{36} \mathrm{Ar}, \mathrm{xnypz} \alpha\right)$, and subsequent decay]; measured $\mathrm{E} \alpha, \mathrm{T}_{1 / 2}$, $\alpha \alpha$-coin for ground and metastable state decay. ${ }^{193,195} \mathrm{Bi},{ }^{197,199} \mathrm{At}$, ${ }^{201,203} \mathrm{Fr}$ deduced levels, J, $\pi$. Comparisons with previous results.

JOUR PRVCA 71024306

${ }^{193} \mathrm{At}$ 2005KE10 NUCLEAR REACTIONS ${ }^{142} \mathrm{Nd}\left({ }^{56} \mathrm{Fe}, 2 \mathrm{np}\right), \mathrm{E}=262 \mathrm{MeV} ;{ }^{141} \mathrm{Pr}\left({ }^{56} \mathrm{Fe}\right.$, $4 \mathrm{n}), \mathrm{E}=266 \mathrm{MeV} ;{ }^{141} \operatorname{Pr}\left({ }^{54} \mathrm{Fe}, 4 \mathrm{n}\right), \mathrm{E}=260 \mathrm{MeV}$; measured production $\sigma$. JOUR ZAANE $25 \mathrm{~s} 01181$

2005KE10 RADIOACTIVITY ${ }^{191,193,195} \operatorname{At}(\alpha)$; measured E $\alpha, \mathrm{E} \gamma, \gamma \alpha$-coin. 191,193,195 At deduced levels, J, $\pi$, configurations, proton separation energies. ${ }^{187,189,191} \mathrm{Bi}$ deduced levels $\mathrm{J}, \pi$, configurations. Comparison with theory. JOUR ZAANE 25 s01 181

2005UU03 RADIOACTIVITY ${ }^{188,190,192,194,196,198,200,202,204} \mathrm{Po}$, $191,193,195,197,199$ At, ${ }^{196,198,200,202,204,206} \mathrm{Rn},{ }^{199,201,203,205,207} \operatorname{Fr}(\alpha)$; measured reduced widths using gas filled recoil separator; deduced hindrance factors, proton intruder states and deformation effects. JOUR ZAANE 25 s01 179 


\section{$\mathrm{A}=194$}

${ }^{194} \mathrm{Re} \quad$ 2005CA02 RADIOACTIVITY ${ }^{188} \mathrm{Ta},{ }^{190} \mathrm{~W},{ }^{192,193} \mathrm{Re},{ }^{195} \mathrm{Os},{ }^{197,198} \mathrm{Ir}$, 200,201,202 Pt, ${ }^{203} \mathrm{Au}(\mathrm{IT})$ [from $\left.\mathrm{Be}\left({ }^{208} \mathrm{~Pb}, \mathrm{X}\right)\right]$; measured $\mathrm{E} \gamma, \mathrm{I} \gamma, \gamma \gamma$-coin, $\mathrm{T}_{1 / 2} \cdot{ }^{188} \mathrm{Ta},{ }^{190} \mathrm{~W},{ }^{192,193} \mathrm{Re},{ }^{195} \mathrm{Os},{ }^{197,198} \mathrm{Ir},{ }^{200,201,202} \mathrm{Pt},{ }^{203} \mathrm{Au}$ deduced transitions. ${ }^{190} \mathrm{~W},{ }^{200,201,202} \mathrm{Pt}$ deduced levels, J, $\pi .{ }^{174,175} \mathrm{Er}$, ${ }^{185} \mathrm{Hf},{ }^{191,194} \mathrm{Re},{ }^{199} \operatorname{Ir}(\mathrm{IT})$ [from $\left.\mathrm{Be}\left({ }^{208} \mathrm{~Pb}, \mathrm{X}\right)\right]$; measured $\mathrm{E} \gamma, \mathrm{I} \gamma$.

JOUR ZAANE 23201

${ }^{194} \mathrm{Ir} \quad$ 2005TAZW NUCLEAR REACTIONS Pt $(\mathrm{p}, \mathrm{X}){ }^{195} \mathrm{Au} /{ }^{196} \mathrm{Au} /{ }^{198} \mathrm{Au} /{ }^{189} \mathrm{Ir} /$ ${ }^{190} \mathrm{Ir} /{ }^{192} \mathrm{Ir} /{ }^{194} \mathrm{Ir}, \mathrm{E} \approx 0-70 \mathrm{MeV} ; \mathrm{Pt}(\mathrm{d}, \mathrm{X}){ }^{192} \mathrm{Au} /{ }^{193} \mathrm{Au} /{ }^{194} \mathrm{Au} /$ ${ }^{195} \mathrm{Au} /{ }^{196} \mathrm{Au} /{ }^{195 m} \mathrm{Pt} /{ }^{197} \mathrm{Pt} /{ }^{192} \mathrm{Ir}, \mathrm{E} \approx 0-21 \mathrm{MeV} ; \operatorname{Pt}(\alpha, \mathrm{X}){ }^{195} \mathrm{Au}$ $/{ }^{196} \mathrm{Au}, \mathrm{E} \approx 0-38 \mathrm{MeV}$; measured activation $\sigma$; deduced integral yields. CONF Santa Fe (Nucl Data for Sci and Technol) Proc,Vol1,P1015

${ }^{194} \mathrm{Pt} \quad$ 2005J011 NUCLEAR REACTIONS ${ }^{192} \mathrm{Os}\left({ }^{82} \mathrm{Se}, \mathrm{X}\right){ }^{194} \mathrm{Pt}, \mathrm{E}=460 \mathrm{MeV}$; measured $\mathrm{E} \gamma, \mathrm{I} \gamma, \gamma \gamma$-coin. ${ }^{194} \mathrm{Pt}$ deduced levels, J, $\pi$, configurations, B(E2). GASP array. JOUR APOBB 361323

2005LEZW NUCLEAR REACTIONS ${ }^{188,190,194}$ Os, ${ }^{194,196} \mathrm{Pt}(\alpha, 2 \mathrm{n}), \mathrm{E}=27 \mathrm{MeV}$; measured $\mathrm{E} \gamma, \mathrm{I} \gamma(\theta, \mathrm{H}, \mathrm{t}) .{ }^{190,192,194} \mathrm{Pt},{ }^{196,198} \mathrm{Hg}$ deduced isomeric states g-factors, configurations. Integral perturbed angular distribution method, HPGe detectors. CONF St Petersburg,P81,Levon

2005SH52 ATOMIC MASSES ${ }^{194,195,196,198}$ Pt; measured masses. Penning trap mass spectrometer. JOUR ZAANE 25 s01 45

${ }^{194} \mathrm{Au} \quad$ 2004ADZW NUCLEAR REACTIONS ${ }^{209} \operatorname{Bi}(\mathrm{n}, 4 \mathrm{n}),(\mathrm{n}, 5 \mathrm{n}),(\mathrm{n}, 6 \mathrm{n}),(\mathrm{n}, 7 \mathrm{n}),(\mathrm{n}$, $9 \mathrm{n}),{ }^{232} \operatorname{Th}(\mathrm{n}, \gamma),{ }^{197} \mathrm{Au}(\mathrm{n}, 2 \mathrm{n}),(\mathrm{n}, 4 \mathrm{n}),(\mathrm{n}, 6 \mathrm{n}),(\mathrm{n}, 7 \mathrm{n}),(\mathrm{n}, \gamma),{ }^{115} \operatorname{In}(\mathrm{n}$, $5 \mathrm{n}),(\mathrm{n}, 6 \mathrm{n}),(\mathrm{n}, 7 \mathrm{n}),{ }^{59} \operatorname{Co}(\mathrm{n}, 2 \mathrm{n}),(\mathrm{n}, 3 \mathrm{n}),(\mathrm{n}, 4 \mathrm{n}),(\mathrm{n}, 5 \mathrm{n}),(\mathrm{n}, \gamma),(\mathrm{n}$, $\mathrm{p}),(\mathrm{n}, 6 \mathrm{n} 2 \mathrm{p}), \mathrm{E}=$ spectrum; measured $\mathrm{E} \gamma, \mathrm{I} \gamma$; deduced reaction rates. $\mathrm{Pb}(\mathrm{p}, \mathrm{nX}), \mathrm{E}=1 \mathrm{GeV}$; deduced spallation neutron spectrum. REPT JINR-E1-2004-16,Adam

2005AD01 NUCLEAR REACTIONS ${ }^{209} \operatorname{Bi}(\mathrm{n}, 4 \mathrm{n}),(\mathrm{n}, 5 \mathrm{n}),(\mathrm{n}, 6 \mathrm{n}),(\mathrm{n}, 7 \mathrm{n}),(\mathrm{n}$, $9 \mathrm{n}),{ }^{232} \mathrm{Th}(\mathrm{n}, \gamma),{ }^{197} \mathrm{Au}(\mathrm{n}, 2 \mathrm{n}),(\mathrm{n}, 4 \mathrm{n}),(\mathrm{n}, 6 \mathrm{n}),(\mathrm{n}, 7 \mathrm{n}),(\mathrm{n}, \gamma),{ }^{59} \mathrm{Co}(\mathrm{n}$, $2 \mathrm{n}),(\mathrm{n}, 3 \mathrm{n}),(\mathrm{n}, 4 \mathrm{n}),(\mathrm{n}, 5 \mathrm{n}),(\mathrm{n}, \mathrm{p}),(\mathrm{n}, 6 \mathrm{n} 2 \mathrm{p}),{ }^{115} \operatorname{In}(\mathrm{n}, 5 \mathrm{n}),(\mathrm{n}, 6 \mathrm{n}),(\mathrm{n}$, $7 \mathrm{n}), \mathrm{E}=$ spectrum; measured $\mathrm{E} \gamma, \mathrm{I} \gamma$; deduced reaction rates. Activation technique, spallation neutrons from $1 \mathrm{GeV}$ proton beam, comparison with model predictions. JOUR ZAANE 2361

2005SI14 NUCLEAR REACTIONS C, O, Si, Mg, Al(n, X)7 Be, E $\approx$ 0.1-750 $\mathrm{MeV} ; \mathrm{O}, \mathrm{Si}, \mathrm{Mg}, \mathrm{Al}(\mathrm{n}, \mathrm{X}){ }^{22} \mathrm{Na} /{ }^{23} \mathrm{Na}, \mathrm{E} \approx 0.1-750 \mathrm{MeV} ;{ }^{197} \mathrm{Au}(\mathrm{n}$, $\mathrm{X}){ }^{194} \mathrm{Au} /{ }^{196} \mathrm{Au} /{ }^{198} \mathrm{Au}, \mathrm{E} \approx 0.1-750 \mathrm{MeV} ; \mathrm{Ti}, \mathrm{Fe}, \mathrm{Ni}, \mathrm{Cu}(\mathrm{n}, \mathrm{X})^{46} \mathrm{Sc}$ $/{ }^{48} \mathrm{Sc}, \mathrm{E} \approx 0.1-750 \mathrm{MeV} ; \mathrm{Fe}, \mathrm{Ni}, \mathrm{Cu}(\mathrm{n}, \mathrm{X}){ }^{48} \mathrm{~V} /{ }^{51} \mathrm{Cr} /{ }^{52} \mathrm{Mn} /{ }^{54} \mathrm{Mn}$, $\mathrm{E} \approx 0.1-750 \mathrm{MeV} ; \mathrm{Ni}, \mathrm{Cu}(\mathrm{n}, \mathrm{X}){ }^{56} \mathrm{Ni} /{ }^{57} \mathrm{Ni} /{ }^{56} \mathrm{Co} /{ }^{57} \mathrm{Co} /{ }^{58} \mathrm{Co} /$ ${ }^{60} \mathrm{Co} /{ }^{59} \mathrm{Fe}, \mathrm{E} \approx 0.1-750 \mathrm{MeV}$; measured energy-integrated production $\sigma$. JOUR NIMBE 234419

2005TAZW NUCLEAR REACTIONS Pt(p, X) ${ }^{195} \mathrm{Au} /{ }^{196} \mathrm{Au} /{ }^{198} \mathrm{Au} /{ }^{189} \mathrm{Ir} /$ ${ }^{190} \mathrm{Ir} /{ }^{192} \mathrm{Ir} /{ }^{194} \mathrm{Ir}, \mathrm{E} \approx 0-70 \mathrm{MeV} ; \mathrm{Pt}(\mathrm{d}, \mathrm{X}){ }^{192} \mathrm{Au} /{ }^{193} \mathrm{Au} /{ }^{194} \mathrm{Au} /$ ${ }^{195} \mathrm{Au} /{ }^{196} \mathrm{Au} /{ }^{195 m} \mathrm{Pt} /{ }^{197} \mathrm{Pt} /{ }^{192} \mathrm{Ir}, \mathrm{E} \approx 0-21 \mathrm{MeV} ; \operatorname{Pt}(\alpha, \mathrm{X}){ }^{195} \mathrm{Au}$ $/{ }^{196} \mathrm{Au}, \mathrm{E} \approx 0-38 \mathrm{MeV}$; measured activation $\sigma$; deduced integral yields. CONF Santa Fe (Nucl Data for Sci and Technol) Proc,Vol1,P1015

2006Di01 NUCLEAR REACTIONS Pt(d, X) ${ }^{191} \mathrm{Au} /{ }^{192} \mathrm{Au} /{ }^{193} \mathrm{Au} /{ }^{194} \mathrm{Au} /$ ${ }^{195} \mathrm{Au} /{ }^{196} \mathrm{Au} /{ }^{196 m} \mathrm{Au} /{ }^{198} \mathrm{Au} /{ }^{199} \mathrm{Au} /{ }^{191} \mathrm{Pt} /{ }^{195 m} \mathrm{Pt} /{ }^{197} \mathrm{Pt} /$ ${ }^{192} \mathrm{Ir}, \mathrm{E} \approx 10-40 \mathrm{MeV}$; measured production $\sigma$. Stacked-foil activation technique, comparison with model predictions. JOUR NIMBE 24320 


\section{$\mathrm{A}=194$ (continued)}

${ }^{194} \mathrm{Hg} \quad$ 2004KHZX NUCLEAR REACTIONS ${ }^{150} \mathrm{Nd}\left({ }^{48} \mathrm{Ca}, 4 \mathrm{n}\right)$, E not given; measured $\mathrm{E} \gamma$ $\mathrm{I} \gamma, \gamma \gamma$-coin. ${ }^{194} \mathrm{Hg}$ deduced spreading widths for excited superdeformed quasicontinuum transitions. Gammasphere array. REPT

ANL-04/22,P61,Khoo

${ }^{194} \mathrm{~Pb} \quad$ 2002DU22

RADIOACTIVITY ${ }^{197,197 m, 198,199 m, 200,201 m} \mathrm{Po}(\alpha) ;{ }^{172,173} \mathrm{Os}(\alpha)$ [from

$\left.{ }^{156} \mathrm{Dy}\left({ }^{22} \mathrm{Ne}, \mathrm{xn}\right)\right] ;{ }^{183,184,185} \mathrm{Hg}(\alpha)$ [from $\left.{ }^{168} \mathrm{Yb}\left({ }^{22} \mathrm{Ne}, \mathrm{xn}\right)\right]$; measured $\mathrm{E} \alpha, \mathrm{T}_{1 / 2}$. JOUR NIMAE 479631

2005DRZW NUCLEAR REACTIONS ${ }^{170} \operatorname{Er}\left({ }^{29} \mathrm{Si}, 5 \mathrm{n}\right), \mathrm{E}=147 \mathrm{MeV} ;{ }^{170} \mathrm{Er}\left({ }^{30} \mathrm{Si}\right.$, $4 \mathrm{n}), \mathrm{E}=138 \mathrm{MeV}$; measured prompt and delayed $\mathrm{E} \gamma, \mathrm{I} \gamma, \gamma \gamma$-coin. ${ }^{194,196} \mathrm{~Pb}$ deduced levels, $\mathrm{J}, \pi$, configurations, isomers $\mathrm{T}_{1 / 2}$ and decay $\mathrm{B}(\mathrm{E} 1), \mathrm{B}(\mathrm{E} 2), \mathrm{B}(\mathrm{E} 3)$. Caesar array, potential energy surface calculations. PREPRINT ANU-P/1662,Dracoulis 2005UU03 RADIOACTIVITY $188,190,192,194,196,198,200,202,204$ Po, 191,193,195,197,199 At, ${ }^{196,198,200,202,204,206} \mathrm{Rn},{ }^{199,201,203,205,207} \operatorname{Fr}(\alpha)$; measured reduced widths using gas filled recoil separator; deduced hindrance factors, proton intruder states and deformation effects.

JOUR ZAANE 25 s01 179

${ }^{194} \mathrm{Bi} \quad$ 2004KE15 NUCLEAR REACTIONS ${ }^{1,2} \mathrm{H}, \mathrm{Ti}\left({ }^{208} \mathrm{~Pb}, \mathrm{X}\right){ }^{193} \mathrm{Bi} /{ }^{194} \mathrm{Bi} /{ }^{195} \mathrm{Bi} /$ ${ }^{196} \mathrm{Bi} /{ }^{197} \mathrm{Bi} /{ }^{198} \mathrm{Bi} /{ }^{199} \mathrm{Bi} /{ }^{200} \mathrm{Bi} /{ }^{201} \mathrm{Bi} /{ }^{202} \mathrm{Bi} /{ }^{203} \mathrm{Bi} /{ }^{204} \mathrm{Bi} /$ ${ }^{205} \mathrm{Bi} /{ }^{206} \mathrm{Bi} /{ }^{207} \mathrm{Bi} /{ }^{208} \mathrm{Bi}, \mathrm{E}=1 \mathrm{GeV} /$ nucleon; measured charge-pickup $\sigma$, velocity distributions; deduced reaction mechanism features. Comparison with model predictions and previous results. JOUR PRVCA 70064608

2005GEZW ATOMIC MASSES ${ }^{235}$ Ac; measured mass, $\mathrm{T}_{1 / 2}$. $185,186,187,188,189,190,191,192,193,194,195,196 \mathrm{Bi}$; measured masses, proton separation energies. ${ }^{207 m} \mathrm{Tl}$; measured $\mathrm{T}_{1 / 2}$. Stored beams, Schottky mass spectrometry. PREPRINT nucl-ex/0510009,10/4/2005

2005 UU02 RADIOACTIVITY ${ }^{201,202,203,204} \mathrm{Ra}$, ${ }^{197,198,199,200} \mathrm{Rn},{ }^{193,194,195,196} \mathrm{Po}$, ${ }^{201,202,203,204} \mathrm{Fr},{ }^{197,198,199,200} \operatorname{At}(\alpha)$ [from ${ }^{141} \operatorname{Pr}\left({ }^{63,65} \mathrm{Cu}\right.$, xnypz $\left.\alpha\right)$, ${ }^{170} \mathrm{Yb}\left({ }^{36} \mathrm{Ar}\right.$, xnypz $\left.\alpha\right)$, and subsequent decay]; measured $\mathrm{E} \alpha, \mathrm{T}_{1 / 2}$, $\alpha \alpha$-coin for ground and metastable state decay. ${ }^{193,195} \mathrm{Bi},{ }^{197,199} \mathrm{At}$, ${ }^{201,203} \mathrm{Fr}$ deduced levels, J, $\pi$. Comparisons with previous results. JOUR PRVCA 71024306

${ }^{194} \mathrm{Po} \quad 2005 \mathrm{UUU} 22$ RADIOACTIVITY ${ }^{201,202,203,204} \mathrm{Ra},{ }^{197,198,199,200} \mathrm{Rn},{ }^{193,194,195,196} \mathrm{Po}$, ${ }^{201,202,203,204} \mathrm{Fr},{ }^{197,198,199,200} \mathrm{At}(\alpha)$ [from ${ }^{141} \mathrm{Pr}\left({ }^{63,65} \mathrm{Cu}\right.$, xnypz $\left.\alpha\right)$, ${ }^{170} \mathrm{Yb}\left({ }^{36} \mathrm{Ar}\right.$, xnypz $\left.\alpha\right)$, and subsequent decay]; measured $\mathrm{E} \alpha, \mathrm{T}_{1 / 2}$, $\alpha \alpha$-coin for ground and metastable state decay. ${ }^{193,195} \mathrm{Bi},{ }^{197,199} \mathrm{At}$, ${ }^{201,203} \mathrm{Fr}$ deduced levels, J, $\pi$. Comparisons with previous results. JOUR PRVCA 71024306

2005UU03 RADIOACTIVITY ${ }^{188,190,192,194,196,198,200,202,204} \mathrm{Po}$, 191,193,195,197,199 At, ${ }^{196,198,200,202,204,206} \mathrm{Rn},{ }^{199,201,203,205,207} \operatorname{Fr}(\alpha)$; measured reduced widths using gas filled recoil separator; deduced hindrance factors, proton intruder states and deformation effects. JOUR ZAANE 25 s01 179 


\section{$\mathrm{A}=195$}

${ }^{195} \mathrm{Os}$ 2005CA02 RADIOACTIVITY ${ }^{188} \mathrm{Ta},{ }^{190} \mathrm{~W},{ }^{192,193} \mathrm{Re},{ }^{195} \mathrm{Os},{ }^{197,198} \mathrm{Ir}$, 200,201,202 Pt, ${ }^{203} \mathrm{Au}(\mathrm{IT})$ [from $\left.\mathrm{Be}\left({ }^{208} \mathrm{~Pb}, \mathrm{X}\right)\right]$; measured $\mathrm{E} \gamma, \mathrm{I} \gamma, \gamma \gamma$-coin, $\mathrm{T}_{1 / 2} \cdot{ }^{188} \mathrm{Ta},{ }^{190} \mathrm{~W},{ }^{192,193} \mathrm{Re},{ }^{195} \mathrm{Os},{ }^{197,198} \mathrm{Ir},{ }^{200,201,202} \mathrm{Pt},{ }^{203} \mathrm{Au}$ deduced transitions. ${ }^{190} \mathrm{~W},{ }^{200,201,202} \mathrm{Pt}$ deduced levels, J, $\pi .{ }^{174,175} \mathrm{Er}$, ${ }^{185} \mathrm{Hf},{ }^{191,194} \mathrm{Re},{ }^{199} \operatorname{Ir}(\mathrm{IT})$ [from $\left.\mathrm{Be}\left({ }^{208} \mathrm{~Pb}, \mathrm{X}\right)\right]$; measured $\mathrm{E} \gamma, \mathrm{I} \gamma$.

JOUR ZAANE 23201

${ }^{195} \mathrm{Pt} \quad 2005 \mathrm{SH} 52$

2005TAZW

${ }^{195} \mathrm{Au} \quad 2003 \mathrm{HI} 23$

2005TAZW

${ }^{195} \mathrm{~Pb} \quad$ 2002DU22

2005J010

${ }^{195} \mathrm{Bi} \quad 2004 \mathrm{KE} 15$

2005DE01
ATOMIC MASSES ${ }^{194,195,196,198}$ Pt; measured masses. Penning trap mass spectrometer. JOUR ZAANE 25 s01 45 NUCLEAR REACTIONS Pt(p, X) ${ }^{195} \mathrm{Au} /{ }^{196} \mathrm{Au} /{ }^{198} \mathrm{Au} /{ }^{189} \mathrm{Ir} /$ ${ }^{190} \mathrm{Ir} /{ }^{192} \mathrm{Ir} /{ }^{194} \mathrm{Ir}, \mathrm{E} \approx 0-70 \mathrm{MeV} ; \mathrm{Pt}(\mathrm{d}, \mathrm{X}){ }^{192} \mathrm{Au} /{ }^{193} \mathrm{Au} /{ }^{194} \mathrm{Au} /$ ${ }^{195} \mathrm{Au} /{ }^{196} \mathrm{Au} /{ }^{195 m} \mathrm{Pt} /{ }^{197} \mathrm{Pt} /{ }^{192} \mathrm{Ir}, \mathrm{E} \approx 0-21 \mathrm{MeV} ; \operatorname{Pt}(\alpha, \mathrm{X}){ }^{195} \mathrm{Au}$ $/{ }^{196} \mathrm{Au}, \mathrm{E} \approx 0-38 \mathrm{MeV}$; measured activation $\sigma$; deduced integral yields. CONF Santa Fe (Nucl Data for Sci and Technol) Proc,Vol1,P1015 NUCLEAR REACTIONS Pt(d, X) ${ }^{191} \mathrm{Au} /{ }^{192} \mathrm{Au} /{ }^{193} \mathrm{Au} /{ }^{194} \mathrm{Au} /$ ${ }^{195} \mathrm{Au} /{ }^{196} \mathrm{Au} /{ }^{196 m} \mathrm{Au} /{ }^{198} \mathrm{Au} /{ }^{199} \mathrm{Au} /{ }^{191} \mathrm{Pt} /{ }^{195 m} \mathrm{Pt} /{ }^{197} \mathrm{Pt} /$

${ }^{192} \mathrm{Ir}, \mathrm{E} \approx 10-40 \mathrm{MeV}$; measured production $\sigma$. Stacked-foil activation technique, comparison with model predictions. JOUR NIMBE 24320 NUCLEAR REACTIONS ${ }^{197} \mathrm{Au}\left({ }^{208} \mathrm{~Pb}, \mathrm{X}\right){ }^{195} \mathrm{Au} /{ }^{196} \mathrm{Au}, \mathrm{E}=40,158$ $\mathrm{GeV} /$ nucleon; measured electromagnetic dissociation $\sigma$ for one- and two-neutron removal. JOUR UKPJA 481165

NUCLEAR REACTIONS Pt $(\mathrm{p}, \mathrm{X}){ }^{195} \mathrm{Au} /{ }^{196} \mathrm{Au} /{ }^{198} \mathrm{Au} /{ }^{189} \mathrm{Ir} /$ ${ }^{190} \mathrm{Ir} /{ }^{192} \mathrm{Ir} /{ }^{194} \mathrm{Ir}, \mathrm{E} \approx 0-70 \mathrm{MeV} ; \mathrm{Pt}(\mathrm{d}, \mathrm{X}){ }^{192} \mathrm{Au} /{ }^{193} \mathrm{Au} /{ }^{194} \mathrm{Au} /$ ${ }^{195} \mathrm{Au} /{ }^{196} \mathrm{Au} /{ }^{195 m} \mathrm{Pt} /{ }^{197} \mathrm{Pt} /{ }^{192} \mathrm{Ir}, \mathrm{E} \approx 0-21 \mathrm{MeV} ; \operatorname{Pt}(\alpha, \mathrm{X}){ }^{195} \mathrm{Au}$ $/{ }^{196} \mathrm{Au}, \mathrm{E} \approx 0-38 \mathrm{MeV}$; measured activation $\sigma$; deduced integral yields. CONF Santa Fe (Nucl Data for Sci and Technol) Proc,Vol1,P1015 NUCLEAR REACTIONS Pt(d, X) ${ }^{191} \mathrm{Au} /{ }^{192} \mathrm{Au} /{ }^{193} \mathrm{Au} /{ }^{194} \mathrm{Au} /$ ${ }^{195} \mathrm{Au} /{ }^{196} \mathrm{Au} /{ }^{196 m} \mathrm{Au} /{ }^{198} \mathrm{Au} /{ }^{199} \mathrm{Au} /{ }^{191} \mathrm{Pt} /{ }^{195 m} \mathrm{Pt} /{ }^{197} \mathrm{Pt} /$ ${ }^{192} \mathrm{Ir}, \mathrm{E} \approx 10-40 \mathrm{MeV}$; measured production $\sigma$. Stacked-foil activation technique, comparison with model predictions. JOUR NIMBE 24320 RADIOACTIVITY ${ }^{197,197 m, 198,199 m, 200,201 m} \mathrm{Po}(\alpha) ;{ }^{172,173} \mathrm{Os}(\alpha)$ [from $\left.{ }^{156} \mathrm{Dy}\left({ }^{22} \mathrm{Ne}, \mathrm{xn}\right)\right] ;{ }^{183,184,185} \mathrm{Hg}(\alpha)$ [from ${ }^{168} \mathrm{Yb}\left({ }^{22} \mathrm{Ne}, \mathrm{xn}\right)$; measured E $\alpha, \mathrm{T}_{1 / 2}$. JOUR NIMAE 479631

NUCLEAR REACTIONS ${ }^{174} \mathrm{Yb}\left({ }^{26} \mathrm{Mg}, 5 \mathrm{n}\right), \mathrm{E}=132 \mathrm{MeV}$; measured $\mathrm{E} \gamma, \mathrm{I} \gamma, \gamma \gamma$-coin. ${ }^{195} \mathrm{~Pb}$ deduced high-spin levels, J, $\pi$, superdeformed bands, quasi-continuum decay-out spectra. Gammasphere array.

JOUR PRVCA 71044310

NUCLEAR REACTIONS ${ }^{1,2} \mathrm{H}, \mathrm{Ti}\left({ }^{208} \mathrm{~Pb}, \mathrm{X}\right){ }^{193} \mathrm{Bi} /{ }^{194} \mathrm{Bi} /{ }^{195} \mathrm{Bi} /$ ${ }^{196} \mathrm{Bi} /{ }^{197} \mathrm{Bi} /{ }^{198} \mathrm{Bi} /{ }^{199} \mathrm{Bi} /{ }^{200} \mathrm{Bi} /{ }^{201} \mathrm{Bi} /{ }^{202} \mathrm{Bi} /{ }^{203} \mathrm{Bi} /{ }^{204} \mathrm{Bi} /$ ${ }^{205} \mathrm{Bi} /{ }^{206} \mathrm{Bi} /{ }^{207} \mathrm{Bi} /{ }^{208} \mathrm{Bi}, \mathrm{E}=1 \mathrm{GeV} /$ nucleon; measured charge-pickup $\sigma$, velocity distributions; deduced reaction mechanism features. Comparison with model predictions and previous results. JOUR PRVCA 70064608

RADIOACTIVITY ${ }^{200,201,203,205} \mathrm{Fr},{ }^{196,197,199,201} \mathrm{At},{ }^{193} \mathrm{Bi}(\alpha)$ [from $\mathrm{Th}(\mathrm{p}, \mathrm{X})$ and subsequent decay]; measured $\mathrm{E} \alpha, \mathrm{T}_{1 / 2} \cdot{ }^{201} \mathrm{Fr},{ }^{197} \mathrm{At}$, ${ }^{193} \mathrm{Bi},{ }^{189} \mathrm{Tl}$ deduced levels, J, $\pi$. JOUR ZAANE 23243 


\section{$\mathrm{A}=195$ (continued)}

2005GEZW ATOMIC MASSES ${ }^{235}$ Ac; measured mass, $\mathrm{T}_{1 / 2}$.

$185,186,187,188,189,190,191,192,193,194,195,196 \mathrm{Bi}$; measured masses, proton separation energies. ${ }^{207 m} \mathrm{Tl}$; measured $\mathrm{T}_{1 / 2}$. Stored beams, Schottky mass spectrometry. PREPRINT nucl-ex/0510009,10/4/2005

2005 RUO2 RADIOACTIVITY ${ }^{201,202,203,204} \mathrm{Ra},{ }^{197,198,199,200} \mathrm{Rn},{ }^{193,194,195,196} \mathrm{Po}$, ${ }^{201,202,203,204} \mathrm{Fr},{ }^{197,198,199,200} \mathrm{At}(\alpha)$ [from ${ }^{141} \mathrm{Pr}\left({ }^{63,65} \mathrm{Cu}\right.$, xnypz $\left.\alpha\right)$, ${ }^{170} \mathrm{Yb}\left({ }^{36} \mathrm{Ar}\right.$, xnypz $\left.\alpha\right)$, and subsequent decay]; measured $\mathrm{E} \alpha, \mathrm{T}_{1 / 2}$, $\alpha \alpha$-coin for ground and metastable state decay. ${ }^{193,195} \mathrm{Bi},{ }^{197,199} \mathrm{At}$, ${ }^{201,203} \mathrm{Fr}$ deduced levels, J, $\pi$. Comparisons with previous results. JOUR PRVCA 71024306

2005UU03 RADIOACTIVITY ${ }^{188,190,192,194,196,198,200,202,204} \mathrm{Po}$, ${ }_{191,193,195,197,199}$ At, ${ }^{196,198,200,202,204,206} \mathrm{Rn},{ }^{199,201,203,205,207} \operatorname{Fr}(\alpha)$; measured reduced widths using gas filled recoil separator; deduced hindrance factors, proton intruder states and deformation effects. JOUR ZAANE 25 s01 179

${ }^{195} \mathrm{Po} \quad 2005 \mathrm{UU} 02$ RADIOACTIVITY ${ }^{201,202,203,204} \mathrm{Ra},{ }^{197,198,199,200} \mathrm{Rn},{ }^{193,194,195,196} \mathrm{Po}$, ${ }^{201,202,203,204} \mathrm{Fr},{ }^{197,198,199,200} \operatorname{At}(\alpha)$ [from ${ }^{141} \operatorname{Pr}\left({ }^{63,65} \mathrm{Cu}\right.$, xnypz $\left.\alpha\right)$, ${ }^{170} \mathrm{Yb}\left({ }^{36} \mathrm{Ar}, \mathrm{xnypz} \alpha\right)$, and subsequent decay]; measured $\mathrm{E} \alpha, \mathrm{T}_{1 / 2}$, $\alpha \alpha$-coin for ground and metastable state decay. ${ }^{193,195} \mathrm{Bi},{ }^{197,199} \mathrm{At}$, ${ }^{201,203} \mathrm{Fr}$ deduced levels, $\mathrm{J}, \pi$. Comparisons with previous results. JOUR PRVCA 71024306

${ }^{195} \mathrm{At}$ 2005KE10 NUCLEAR REACTIONS ${ }^{142} \mathrm{Nd}\left({ }^{56} \mathrm{Fe}, 2 \mathrm{np}\right), \mathrm{E}=262 \mathrm{MeV} ;{ }^{141} \mathrm{Pr}\left({ }^{56} \mathrm{Fe}\right.$, $4 \mathrm{n}), \mathrm{E}=266 \mathrm{MeV} ;{ }^{141} \operatorname{Pr}\left({ }^{54} \mathrm{Fe}, 4 \mathrm{n}\right), \mathrm{E}=260 \mathrm{MeV}$; measured production $\sigma$. JOUR ZAANE $25 \mathrm{~s} 01181$

2005KE10 RADIOACTIVITY ${ }^{191,193,195} \operatorname{At}(\alpha)$; measured $\mathrm{E} \alpha, \mathrm{E} \gamma, \gamma \alpha$-coin. ${ }^{191,193,195}$ At deduced levels, J, $\pi$, configurations, proton separation energies. ${ }^{187,189,191} \mathrm{Bi}$ deduced levels $\mathrm{J}, \pi$, configurations. Comparison with theory. JOUR ZAANE 25 s01 181

2005UU03 RADIOACTIVITY $188,190,192,194,196,198,200,202,204 \mathrm{Po}$, 191,193,195,197,199 At, ${ }^{196,198,200,202,204,206} \mathrm{Rn},{ }^{199,201,203,205,207} \operatorname{Fr}(\alpha)$; measured reduced widths using gas filled recoil separator; deduced hindrance factors, proton intruder states and deformation effects. JOUR ZAANE 25 s01 179

$$
\mathrm{A}=\mathbf{1 9 6}
$$

${ }^{196} \mathrm{Pt} \quad 2005 \mathrm{SH} 52$

ATOMIC MASSES ${ }^{194,195,196,198}$ Pt; measured masses. Penning trap mass spectrometer. JOUR ZAANE 25 s01 45

${ }^{196} \mathrm{Au} \quad 2003 \mathrm{HI} 23$ NUCLEAR REACTIONS ${ }^{197} \mathrm{Au}\left({ }^{208} \mathrm{~Pb}, \mathrm{X}\right){ }^{195} \mathrm{Au} /{ }^{196} \mathrm{Au}, \mathrm{E}=40,158$ $\mathrm{GeV} /$ nucleon; measured electromagnetic dissociation $\sigma$ for one- and two-neutron removal. JOUR UKPJA 481165

2004ADZW NUCLEAR REACTIONS ${ }^{209} \operatorname{Bi}(\mathrm{n}, 4 \mathrm{n}),(\mathrm{n}, 5 \mathrm{n}),(\mathrm{n}, 6 \mathrm{n}),(\mathrm{n}, 7 \mathrm{n}),(\mathrm{n}$, 9n), ${ }^{232} \operatorname{Th}(\mathrm{n}, \gamma),{ }^{197} \mathrm{Au}(\mathrm{n}, 2 \mathrm{n}),(\mathrm{n}, 4 \mathrm{n}),(\mathrm{n}, 6 \mathrm{n}),(\mathrm{n}, 7 \mathrm{n}),(\mathrm{n}, \gamma),{ }^{115} \operatorname{In}(\mathrm{n}$, 5n), (n, 6n), (n, 7n), ${ }^{59} \operatorname{Co}(\mathrm{n}, 2 \mathrm{n}),(\mathrm{n}, 3 \mathrm{n}),(\mathrm{n}, 4 \mathrm{n}),(\mathrm{n}, 5 \mathrm{n}),(\mathrm{n}, \gamma),(\mathrm{n}$, $\mathrm{p}),(\mathrm{n}, 6 \mathrm{n} 2 \mathrm{p}), \mathrm{E}=$ spectrum; measured $\mathrm{E} \gamma, \mathrm{I} \gamma$; deduced reaction rates. $\mathrm{Pb}(\mathrm{p}, \mathrm{nX}), \mathrm{E}=1 \mathrm{GeV}$; deduced spallation neutron spectrum. REPT JINR-E1-2004-16,Adam 


\section{$\mathrm{A}=196$ (continued)}

2004GA57

2005AD01

2005DA34

2005LI13

2005MIZZ

2005SI14

2005TAZW

2005TIZX

2005TIZY
NUCLEAR REACTIONS ${ }^{185} \mathrm{Re},{ }^{191} \mathrm{Ir},{ }^{197} \mathrm{Au}(\gamma, \mathrm{n}), \mathrm{E}=22 \mathrm{MeV}$ bremsstrahlung; ${ }^{185} \mathrm{Re},{ }^{191} \mathrm{Ir},{ }^{197} \mathrm{Au}(\mathrm{n}, 2 \mathrm{n}), \mathrm{E}=14.7 \mathrm{MeV} ;{ }^{181} \mathrm{Ta}(\alpha, \mathrm{n})$, $\mathrm{E}=18 \mathrm{MeV} ;{ }^{190} \mathrm{Os},{ }^{196} \mathrm{Pt}(\mathrm{d}, \mathrm{n}), \mathrm{E}=13,14 \mathrm{MeV}$; measured $\mathrm{E} \gamma, \mathrm{I} \gamma$; deduced isomer production ratios. Activation method. JOUR BRSPE 68187 NUCLEAR REACTIONS Fe $(\mathrm{p}, \mathrm{X})^{52} \mathrm{Mn}, \mathrm{E}<2.6 \mathrm{GeV} ; \mathrm{Pb}(\mathrm{p}, \mathrm{X}){ }^{10} \mathrm{Be}$, $\mathrm{E}<2.6 \mathrm{GeV} ;{ }^{209} \mathrm{Bi}(\mathrm{p}, 4 \mathrm{np}), \mathrm{E}<2.6 \mathrm{GeV} ; \mathrm{Pb}(\mathrm{n}, \mathrm{X}){ }^{196} \mathrm{Au} /{ }^{95} \mathrm{Zr}, \mathrm{E} \approx$ 70-180 MeV; measured excitation functions. Comparison with model predictions. REPT NEA/NSC/DOC(2004)14,P28,Michel NUCLEAR REACTIONS ${ }^{209} \operatorname{Bi}(\mathrm{n}, 4 \mathrm{n}),(\mathrm{n}, 5 \mathrm{n}),(\mathrm{n}, 6 \mathrm{n}),(\mathrm{n}, 7 \mathrm{n}),(\mathrm{n}$, 9n), ${ }^{232} \mathrm{Th}(\mathrm{n}, \gamma),{ }^{197} \mathrm{Au}(\mathrm{n}, 2 \mathrm{n}),(\mathrm{n}, 4 \mathrm{n}),(\mathrm{n}, 6 \mathrm{n}),(\mathrm{n}, 7 \mathrm{n}),(\mathrm{n}, \gamma),{ }^{59} \mathrm{Co}(\mathrm{n}$, 2n), (n, 3n), (n, 4n), (n, 5n), (n, p), (n, 6n2p), ${ }^{115} \operatorname{In}(\mathrm{n}, 5 \mathrm{n}),(\mathrm{n}, 6 \mathrm{n}),(\mathrm{n}$, $7 \mathrm{n}), \mathrm{E}=$ spectrum; measured $\mathrm{E} \gamma, \mathrm{I} \gamma$; deduced reaction rates. Activation technique, spallation neutrons from $1 \mathrm{GeV}$ proton beam, comparison with model predictions. JOUR ZAANE 2361

NUCLEAR REACTIONS ${ }^{197} \mathrm{Au}\left({ }^{12} \mathrm{C},{ }^{11} \mathrm{C}\right),\left({ }^{12} \mathrm{C},{ }^{13} \mathrm{C}\right),\left({ }^{16} \mathrm{O},{ }^{15} \mathrm{O}\right)$, $\left({ }^{16} \mathrm{O},{ }^{17} \mathrm{O}\right), \mathrm{E} \approx 6-7 \mathrm{MeV} /$ nucleon; measured yield ratios, high spin yield fraction; deduced reaction mechanism features. Radiochemical and off-line spectrometric techniques. JOUR JRNCD 26679

NUCLEAR REACTIONS ${ }^{197} \mathrm{Au}(\gamma, \mathrm{n}), \mathrm{E}=$ spectrum; measured activation yield. Incident gammas from laser Compton scattering. JOUR JNSTA 42259

NUCLEAR REACTIONS Cu(n, X) ${ }^{56} \mathrm{Co}, \mathrm{E}=40-180 \mathrm{MeV} ; \mathrm{Fe}(\mathrm{n}$, $\mathrm{X}){ }^{54} \mathrm{Mn} /{ }^{52} \mathrm{Mn} /{ }^{51} \mathrm{Cr} /{ }^{48} \mathrm{~V}, \mathrm{E} \approx 0-180 \mathrm{MeV} ; \mathrm{Pb}(\mathrm{n}, \mathrm{X}){ }^{196} \mathrm{Au} /{ }^{200} \mathrm{~Pb}$ $/{ }^{103} \mathrm{Ru}, \mathrm{E} \approx 40-180 \mathrm{MeV} ; \mathrm{U}(\mathrm{n}, \mathrm{X}){ }^{99} \mathrm{Mo}, \mathrm{E} \approx 0-180 \mathrm{MeV}$; measured excitation functions. Comparison with proton-induced reactions. CONF Santa Fe (Nucl Data for Sci and Technol) Proc,Vol1,P861 NUCLEAR REACTIONS C, O, Si, Mg, $\mathrm{Al}(\mathrm{n}, \mathrm{X})^{7} \mathrm{Be}, \mathrm{E} \approx 0.1-750$ $\mathrm{MeV} ; \mathrm{O}, \mathrm{Si}, \mathrm{Mg}, \mathrm{Al}(\mathrm{n}, \mathrm{X}){ }^{22} \mathrm{Na} /{ }^{23} \mathrm{Na}, \mathrm{E} \approx 0.1-750 \mathrm{MeV} ;{ }^{197} \mathrm{Au}(\mathrm{n}$, $\mathrm{X}){ }^{194} \mathrm{Au} /{ }^{196} \mathrm{Au} /{ }^{198} \mathrm{Au}, \mathrm{E} \approx 0.1-750 \mathrm{MeV} ; \mathrm{Ti}, \mathrm{Fe}, \mathrm{Ni}, \mathrm{Cu}(\mathrm{n}, \mathrm{X}){ }^{46} \mathrm{Sc}$ $/{ }^{48} \mathrm{Sc}, \mathrm{E} \approx 0.1-750 \mathrm{MeV} ; \mathrm{Fe}, \mathrm{Ni}, \mathrm{Cu}(\mathrm{n}, \mathrm{X}){ }^{48} \mathrm{~V} /{ }^{51} \mathrm{Cr} /{ }^{52} \mathrm{Mn} /{ }^{54} \mathrm{Mn}$, $\mathrm{E} \approx 0.1-750 \mathrm{MeV} ; \mathrm{Ni}, \mathrm{Cu}(\mathrm{n}, \mathrm{X}){ }^{56} \mathrm{Ni} /{ }^{57} \mathrm{Ni} /{ }^{56} \mathrm{Co} /{ }^{57} \mathrm{Co} /{ }^{58} \mathrm{Co} /$ ${ }^{60} \mathrm{Co} /{ }^{59} \mathrm{Fe}, \mathrm{E} \approx 0.1-750 \mathrm{MeV}$; measured energy-integrated production $\sigma$. JOUR NIMBE 234419

NUCLEAR REACTIONS Pt $(\mathrm{p}, \mathrm{X}){ }^{195} \mathrm{Au} /{ }^{196} \mathrm{Au} /{ }^{198} \mathrm{Au} /{ }^{189} \mathrm{Ir} /$ ${ }^{190} \mathrm{Ir} /{ }^{192} \mathrm{Ir} /{ }^{194} \mathrm{Ir}, \mathrm{E} \approx 0-70 \mathrm{MeV} ; \mathrm{Pt}(\mathrm{d}, \mathrm{X}){ }^{192} \mathrm{Au} /{ }^{193} \mathrm{Au} /{ }^{194} \mathrm{Au} /$ ${ }^{195} \mathrm{Au} /{ }^{196} \mathrm{Au} /{ }^{195 m} \mathrm{Pt} /{ }^{197} \mathrm{Pt} /{ }^{192} \mathrm{Ir}, \mathrm{E} \approx 0-21 \mathrm{MeV} ; \operatorname{Pt}(\alpha, \mathrm{X}){ }^{195} \mathrm{Au}$ $/{ }^{196} \mathrm{Au}, \mathrm{E} \approx 0-38 \mathrm{MeV}$; measured activation $\sigma$; deduced integral yields. CONF Santa Fe (Nucl Data for Sci and Technol) Proc,Vol1,P1015 NUCLEAR REACTIONS Pb, ${ }^{208} \mathrm{~Pb}(\mathrm{p}, \mathrm{X}){ }^{203} \mathrm{~Pb} /{ }^{200} \mathrm{Tl} /{ }^{199} \mathrm{Tl} /$ ${ }^{196} \mathrm{Au} /{ }^{192} \mathrm{Ir} /{ }^{190} \mathrm{Ir} /{ }^{173} \mathrm{Lu} /{ }^{101 m} \mathrm{Rh} /{ }^{86} \mathrm{Rb} /{ }^{59} \mathrm{Fe} /{ }^{24} \mathrm{Na} /{ }^{7} \mathrm{Be}$, $\mathrm{E}=40-2600 \mathrm{MeV}$; measured excitation functions. Comparison with previous work and model predictions. Other reactions discussed. CONF Santa Fe (Nucl Data for Sci and Technol) Proc,Vol1,P1070 NUCLEAR REACTIONS Pb, ${ }^{208} \mathrm{~Pb},{ }^{209} \mathrm{Bi}(\mathrm{p}, \mathrm{X}){ }^{203} \mathrm{~Pb} /{ }^{200} \mathrm{Tl} /{ }^{199} \mathrm{Tl}$ / ${ }^{196} \mathrm{Au} /{ }^{192} \mathrm{Ir} /{ }^{190} \mathrm{Ir} /{ }^{173} \mathrm{Lu} /{ }^{101 m} \mathrm{Rh} /{ }^{86} \mathrm{Rb} /{ }^{59} \mathrm{Fe} /{ }^{24} \mathrm{Na} /{ }^{7} \mathrm{Be}$, $\mathrm{E}=40-2600 \mathrm{MeV}$; measured production $\sigma$. Comparison with model predictions. PREPRINT nucl-ex/0507009,7/05/2005 


\section{$\mathrm{A}=196$ (continued)}

2005WA31 NUCLEAR REACTIONS ${ }^{92,98,100} \mathrm{Mo}\left(\gamma, \gamma^{\prime}\right), \mathrm{E}=13.2 \mathrm{MeV}$ bremsstrahlung; measured $\mathrm{E} \gamma, \mathrm{I} \gamma .{ }^{92,100} \mathrm{Mo},{ }^{197} \mathrm{Au}(\gamma, \mathrm{n}),{ }^{92} \mathrm{Mo}(\gamma, \mathrm{p})$, $(\gamma, \alpha), \mathrm{E} \approx 11.8-16.5 \mathrm{MeV}$ bremsstrahlung; measured integrated $\sigma$. JOUR JPGPE 31 S1969

2006Di01 NUCLEAR REACTIONS Pt(d, X) ${ }^{191} \mathrm{Au} /{ }^{192} \mathrm{Au} /{ }^{193} \mathrm{Au} /{ }^{194} \mathrm{Au} /$ ${ }^{195} \mathrm{Au} /{ }^{196} \mathrm{Au} /{ }^{196 m} \mathrm{Au} /{ }^{198} \mathrm{Au} /{ }^{199} \mathrm{Au} /{ }^{191} \mathrm{Pt} /{ }^{195 m} \mathrm{Pt} /{ }^{197} \mathrm{Pt} /$ ${ }^{192} \mathrm{Ir}, \mathrm{E} \approx 10-40 \mathrm{MeV}$; measured production $\sigma$. Stacked-foil activation technique, comparison with model predictions. JOUR NIMBE 24320 ${ }^{196} \mathrm{Hg}$ 2005LEZW NUCLEAR REACTIONS ${ }^{188,190,194} \mathrm{Os},{ }^{194,196} \mathrm{Pt}(\alpha, 2 \mathrm{n}), \mathrm{E}=27 \mathrm{MeV}$; measured $\mathrm{E} \gamma, \mathrm{I} \gamma(\theta, \mathrm{H}, \mathrm{t}) .{ }^{190,192,194} \mathrm{Pt},{ }^{196,198} \mathrm{Hg}$ deduced isomeric states g-factors, configurations. Integral perturbed angular distribution method, HPGe detectors. CONF St Petersburg,P81,Levon

${ }^{196} \mathrm{~Pb}$ 2002DU22 RADIOACTIVITY ${ }^{197,197 m, 198,199 m, 200,201 m} \operatorname{Po}(\alpha) ;{ }^{172,173} \mathrm{Os}(\alpha)$ [from $\left.{ }^{156} \mathrm{Dy}\left({ }^{22} \mathrm{Ne}, \mathrm{xn}\right)\right] ;{ }^{183,184,185} \mathrm{Hg}(\alpha)$ [from ${ }^{168} \mathrm{Yb}\left({ }^{22} \mathrm{Ne}, \mathrm{xn}\right)$ ]; measured $\mathrm{E} \alpha, \mathrm{T}_{1 / 2}$. JOUR NIMAE 479631

2005DRZW NUCLEAR REACTIONS ${ }^{170} \operatorname{Er}\left({ }^{29} \mathrm{Si}, 5 \mathrm{n}\right), \mathrm{E}=147 \mathrm{MeV} ;{ }^{170} \operatorname{Er}\left({ }^{30} \mathrm{Si}\right.$, $4 \mathrm{n}), \mathrm{E}=138 \mathrm{MeV}$; measured prompt and delayed $\mathrm{E} \gamma, \mathrm{I} \gamma, \gamma \gamma$-coin. ${ }^{194,196} \mathrm{~Pb}$ deduced levels, $\mathrm{J}, \pi$, configurations, isomers $\mathrm{T}_{1 / 2}$ and decay $\mathrm{B}(\mathrm{E} 1), \mathrm{B}(\mathrm{E} 2), \mathrm{B}(\mathrm{E} 3)$. Caesar array, potential energy surface calculations. PREPRINT ANU-P/1662,Dracoulis

2005UU03 RADIOACTIVITY 188,190,192,194,196,198,200,202,204Po, 191,193,195,197,199 At, ${ }^{196,198,200,202,204,206} \mathrm{Rn},{ }^{199,201,203,205,207} \operatorname{Fr}(\alpha)$; measured reduced widths using gas filled recoil separator; deduced hindrance factors, proton intruder states and deformation effects. JOUR ZAANE 25 s01 179

2005WI21 NUCLEAR REACTIONS ${ }^{170} \operatorname{Er}\left({ }^{30} \mathrm{Si}, 4 \mathrm{n}\right), \mathrm{E}=144 \mathrm{MeV}$; measured prompt and delayed $\mathrm{E} \gamma, \mathrm{I} \gamma, \gamma \gamma$-coin. ${ }^{196} \mathrm{~Pb}$ deduced superdeformed band excitation energy, J, $\pi$. Euroball IV array, time-correlated spectroscopy. JOUR PRLTA 95182501

2005WIZY NUCLEAR REACTIONS ${ }^{170} \operatorname{Er}\left({ }^{30} \mathrm{Si}, 4 \mathrm{n}\right), \mathrm{E}=144 \mathrm{MeV}$; measured prompt and delayed $\mathrm{E} \gamma, \mathrm{I} \gamma, \gamma \gamma$-coin. ${ }^{196} \mathrm{~Pb}$ deduced superdeformed band excitation energy. Euroball IV array, time-correlated spectroscopy. Level systematics in neighboring nuclides discussed. PREPRINT ANU-P/1667,Wilson

${ }^{196} \mathrm{Bi}$ 2004KE15 NUCLEAR REACTIONS ${ }^{1,2} \mathrm{H}, \mathrm{Ti}\left({ }^{208} \mathrm{~Pb}, \mathrm{X}\right){ }^{193} \mathrm{Bi} /{ }^{194} \mathrm{Bi} /{ }^{195} \mathrm{Bi} /$ ${ }^{196} \mathrm{Bi} /{ }^{197} \mathrm{Bi} /{ }^{198} \mathrm{Bi} /{ }^{199} \mathrm{Bi} /{ }^{200} \mathrm{Bi} /{ }^{201} \mathrm{Bi} /{ }^{202} \mathrm{Bi} /{ }^{203} \mathrm{Bi} /{ }^{204} \mathrm{Bi} /$ ${ }^{205} \mathrm{Bi} /{ }^{206} \mathrm{Bi} /{ }^{207} \mathrm{Bi} /{ }^{208} \mathrm{Bi}, \mathrm{E}=1 \mathrm{GeV} /$ nucleon; measured charge-pickup $\sigma$, velocity distributions; deduced reaction mechanism features. Comparison with model predictions and previous results. JOUR PRVCA 70064608

2005GEZW ATOMIC MASSES ${ }^{235}$ Ac; measured mass, $T_{1 / 2}$. $185,186,187,188,189,190,191,192,193,194,195,196 \mathrm{Bi}$; measured masses, proton separation energies. ${ }^{207 m} \mathrm{Tl}$; measured $\mathrm{T}_{1 / 2}$. Stored beams, Schottky mass spectrometry. PREPRINT nucl-ex/0510009,10/4/2005 


\section{$\mathrm{A}=196$ (continued)}

2005UU02

${ }^{196} \mathrm{Po}$

$2005 U U 02$

2005UU03

${ }^{196} \mathrm{At}$

${ }^{196} \mathrm{Rn}$

$2005 U U 03$

2005DE01

${ }^{197} \mathrm{Pt} \quad$ 2005TAZW

2006DI01

${ }^{197} \mathrm{Au} \quad$ 2004GA57
RADIOACTIVITY ${ }^{201,202,203,204} \mathrm{Ra},{ }^{197,198,199,200} \mathrm{Rn},{ }^{193,194,195,196} \mathrm{Po}$, ${ }^{201,202,203,204} \mathrm{Fr},{ }^{197,198,199,200} \operatorname{At}(\alpha)\left[\right.$ from ${ }^{141} \operatorname{Pr}\left({ }^{63,65} \mathrm{Cu}\right.$, xnypz $\left.\alpha\right)$, ${ }^{170} \mathrm{Yb}\left({ }^{36} \mathrm{Ar}, \mathrm{xnypz} \alpha\right)$, and subsequent decay]; measured $\mathrm{E} \alpha, \mathrm{T}_{1 / 2}$, $\alpha \alpha$-coin for ground and metastable state decay. ${ }^{193,195} \mathrm{Bi},{ }^{197,199} \mathrm{At}$, ${ }^{201,203}$ Fr deduced levels, J, $\pi$. Comparisons with previous results. JOUR PRVCA 71024306

RADIOACTIVITY ${ }^{201,202,203,204} \mathrm{Ra},{ }^{197,198,199,200} \mathrm{Rn},{ }^{193,194,195,196} \mathrm{Po}$, ${ }^{201,202,203,204} \mathrm{Fr},{ }^{197,198,199,200} \operatorname{At}(\alpha)$ [from ${ }^{141} \operatorname{Pr}\left({ }^{63,65} \mathrm{Cu}\right.$, xnypz $\left.\alpha\right)$,

${ }^{170} \mathrm{Yb}\left({ }^{36} \mathrm{Ar}, \mathrm{xnypz} \alpha\right)$, and subsequent decay]; measured $\mathrm{E} \alpha, \mathrm{T}_{1 / 2}$, $\alpha \alpha$-coin for ground and metastable state decay. ${ }^{193,195} \mathrm{Bi},{ }^{197,199} \mathrm{At}$, ${ }^{201,203} \mathrm{Fr}$ deduced levels, J, $\pi$. Comparisons with previous results.

JOUR PRVCA 71024306

RADIOACTIVITY 188,190,192,194,196,198,200,202,204 Po, 191,193,195,197,199 At, ${ }^{196,198,200,202,204,206} \mathrm{Rn},{ }^{199,201,203,205,207} \operatorname{Fr}(\alpha)$; measured reduced widths using gas filled recoil separator; deduced hindrance factors, proton intruder states and deformation effects.

JOUR ZAANE 25 s01 179

RADIOACTIVITY ${ }^{200,201,203,205} \mathrm{Fr},{ }^{196,197,199,201} \mathrm{At},{ }^{193} \mathrm{Bi}(\alpha)$ [from $\mathrm{Th}(\mathrm{p}, \mathrm{X})$ and subsequent decay]; measured $\mathrm{E} \alpha, \mathrm{T}_{1 / 2} \cdot{ }^{201} \mathrm{Fr},{ }^{197} \mathrm{At}$, ${ }^{193} \mathrm{Bi},{ }^{189} \mathrm{Tl}$ deduced levels, J, $\pi$. JOUR ZAANE 23243

RADIOACTIVITY 188,190,192,194,196,198,200,202,204 Po, 191,193,195,197,199 At, ${ }^{196,198,200,202,204,206} \mathrm{Rn},{ }^{199,201,203,205,207} \operatorname{Fr}(\alpha)$; measured reduced widths using gas filled recoil separator; deduced hindrance factors, proton intruder states and deformation effects. JOUR ZAANE 25 s01 179

\section{$\mathrm{A}=197$}

RADIOACTIVITY ${ }^{188} \mathrm{Ta},{ }^{190} \mathrm{~W},{ }^{192,193} \mathrm{Re},{ }^{195} \mathrm{Os},{ }^{197,198} \mathrm{Ir}$, 200,201,202 Pt, ${ }^{203} \mathrm{Au}(\mathrm{IT})$ [from $\mathrm{Be}\left({ }^{208} \mathrm{~Pb}, \mathrm{X}\right)$ ]; measured $\mathrm{E} \gamma, \mathrm{I} \gamma, \gamma \gamma$-coin, $\mathrm{T}_{1 / 2} .{ }^{188} \mathrm{Ta},{ }^{190} \mathrm{~W},{ }^{192,193} \mathrm{Re},{ }^{195} \mathrm{Os},{ }^{197,198} \mathrm{Ir},{ }^{200,201,202} \mathrm{Pt},{ }^{203} \mathrm{Au}$ deduced transitions. ${ }^{190} \mathrm{~W},{ }^{200,201,202} \mathrm{Pt}$ deduced levels, J, $\pi .{ }^{174,175} \mathrm{Er}$, ${ }^{185} \mathrm{Hf},{ }^{191,194} \mathrm{Re},{ }^{199} \operatorname{Ir}(\mathrm{IT})$ [from $\left.\mathrm{Be}\left({ }^{208} \mathrm{~Pb}, \mathrm{X}\right)\right]$; measured $\mathrm{E} \gamma, \mathrm{I} \gamma$.

JOUR ZAANE 23201

NUCLEAR REACTIONS Pt $(\mathrm{p}, \mathrm{X}){ }^{195} \mathrm{Au} /{ }^{196} \mathrm{Au} /{ }^{198} \mathrm{Au} /{ }^{189} \mathrm{Ir} /$ ${ }^{190} \mathrm{Ir} /{ }^{192} \mathrm{Ir} /{ }^{194} \mathrm{Ir}, \mathrm{E} \approx 0-70 \mathrm{MeV} ; \mathrm{Pt}(\mathrm{d}, \mathrm{X}){ }^{192} \mathrm{Au} /{ }^{193} \mathrm{Au} /{ }^{194} \mathrm{Au} /$ ${ }^{195} \mathrm{Au} /{ }^{196} \mathrm{Au} /{ }^{195 m} \mathrm{Pt} /{ }^{197} \mathrm{Pt} /{ }^{192} \mathrm{Ir}, \mathrm{E} \approx 0-21 \mathrm{MeV} ; \operatorname{Pt}(\alpha, \mathrm{X}){ }^{195} \mathrm{Au}$ / ${ }^{196} \mathrm{Au}, \mathrm{E} \approx 0-38 \mathrm{MeV}$; measured activation $\sigma$; deduced integral yields. CONF Santa Fe (Nucl Data for Sci and Technol) Proc,Vol1,P1015 NUCLEAR REACTIONS Pt(d, X) ${ }^{191} \mathrm{Au} /{ }^{192} \mathrm{Au} /{ }^{193} \mathrm{Au} /{ }^{194} \mathrm{Au} /$ ${ }^{195} \mathrm{Au} /{ }^{196} \mathrm{Au} /{ }^{196 m} \mathrm{Au} /{ }^{198} \mathrm{Au} /{ }^{199} \mathrm{Au} /{ }^{191} \mathrm{Pt} /{ }^{195 m} \mathrm{Pt} /{ }^{197} \mathrm{Pt} /$ ${ }^{192} \mathrm{Ir}, \mathrm{E} \approx 10-40 \mathrm{MeV}$; measured production $\sigma$. Stacked-foil activation technique, comparison with model predictions. JOUR NIMBE 24320 NUCLEAR REACTIONS ${ }^{185} \mathrm{Re},{ }^{191} \mathrm{Ir},{ }^{197} \mathrm{Au}(\gamma, \mathrm{n}), \mathrm{E}=22 \mathrm{MeV}$ bremsstrahlung; ${ }^{185} \mathrm{Re},{ }^{191} \mathrm{Ir},{ }^{197} \mathrm{Au}(\mathrm{n}, 2 \mathrm{n}), \mathrm{E}=14.7 \mathrm{MeV} ;{ }^{181} \mathrm{Ta}(\alpha, \mathrm{n})$, $\mathrm{E}=18 \mathrm{MeV} ;{ }^{190} \mathrm{Os},{ }^{196} \mathrm{Pt}(\mathrm{d}, \mathrm{n}), \mathrm{E}=13,14 \mathrm{MeV}$; measured $\mathrm{E} \gamma, \mathrm{I} \gamma$; deduced isomer production ratios. Activation method. JOUR BRSPE 68187 


\section{$\mathrm{A}=197$ (continued)}

2004 YU11 NUCLEAR REACTIONS ${ }^{197} \mathrm{Au}\left({ }^{55} \mathrm{Ni},{ }^{55} \mathrm{Ni}\right.$ '), E=84.8 MeV; measured $\mathrm{E} \gamma, \mathrm{I} \gamma$, (particle) $\gamma$-coin following projectile Coulomb excitation. ${ }^{55} \mathrm{Ni}$ deduced level, transition $\mathrm{B}(\mathrm{E} 2)$. Comparison with model predictions. JOUR PRVCA 70064321

2005BE33 NUCLEAR REACTIONS ${ }^{197} \mathrm{Au}\left({ }^{54} \mathrm{Cr},{ }^{54} \mathrm{Cr}\right.$ '), E $=136 \mathrm{MeV} /$ nucleon; measured $\mathrm{E} \gamma, \mathrm{I} \gamma$, (particle) $\gamma$-coin following projectile Coulomb excitation. ${ }^{54} \mathrm{Cr}$ deduced transitions. JOUR APOBB 361235 2005BU14 NUCLEAR REACTIONS ${ }^{197} \mathrm{Au}\left({ }^{54} \mathrm{Cr},{ }^{54} \mathrm{Cr}\right),\left({ }^{56} \mathrm{Cr},{ }^{56} \mathrm{Cr}{ }^{\prime}\right),\left({ }^{58} \mathrm{Cr}\right.$, ${ }^{58} \mathrm{Cr}$ '), $\mathrm{E} \approx 135 \mathrm{MeV} /$ nucleon; measured measured $\mathrm{E} \gamma, \mathrm{I} \gamma$, (particle) $\gamma$-coin following projectile Coulomb excitation. ${ }^{54,56,58} \mathrm{Cr}$ deduced transitions. JOUR APOBB 361249

2005BU29 NUCLEAR REACTIONS ${ }^{197} \mathrm{Au}\left({ }^{54} \mathrm{Cr},{ }^{54} \mathrm{Cr}\right),\left({ }^{56} \mathrm{Cr},{ }^{56} \mathrm{Cr}\right),\left({ }^{58} \mathrm{Cr}\right.$, $\left.{ }^{58} \mathrm{Cr}^{\prime}\right), \mathrm{E} \approx 100 \mathrm{MeV} /$ nucleon; measured $\mathrm{E} \gamma, \mathrm{I} \gamma$, (particle) $\gamma$-coin following projectile Coulomb excitation. ${ }^{54,56,58} \mathrm{Cr}$ deduced transitions B(E2). Comparison with shell model predictions. JOUR PYLBB 622 29

$2005 \mathrm{CH} 66$ NUCLEAR REACTIONS ${ }^{209} \mathrm{Bi}\left({ }^{26} \mathrm{Mg},{ }^{26} \mathrm{Mg}\right.$ '), E=78.6 MeV / nucleon; ${ }^{197} \mathrm{Au}\left({ }^{32} \mathrm{Mg},{ }^{32} \mathrm{Mg}\right.$ '), $\mathrm{E}=81.1 \mathrm{MeV} /$ nucleon; ${ }^{209} \mathrm{Bi}\left({ }^{34} \mathrm{Mg},{ }^{34} \mathrm{Mg}\right.$ '), $\mathrm{E}=76.4 \mathrm{MeV} /$ nucleon; measured $\mathrm{E} \gamma, \mathrm{I} \gamma$, (particle) $\gamma$-coin following projectile Coulomb excitation. ${ }^{26,32,34} \mathrm{Mg}$ deduced transitions $\mathrm{B}(\mathrm{E} 2)$, deformation parameters. Comparison with previous work, model predictions. JOUR PRVCA 72054320

2005 DI05 NUCLEAR REACTIONS ${ }^{197} \mathrm{Au}\left({ }^{76} \mathrm{Ge},{ }^{76} \mathrm{Ge}\right),\left({ }^{52} \mathrm{Ti},{ }^{52} \mathrm{Ti}\right),\left({ }^{54} \mathrm{Ti}\right.$, ${ }^{54}{ }^{\mathrm{Ti}}$ '), $\left({ }^{56} \mathrm{Ti},{ }^{56} \mathrm{Ti}^{\prime}\right), \mathrm{E} \approx 80-90 \mathrm{MeV}$; measured $\mathrm{E} \gamma, \mathrm{I} \gamma$, (particle) $\gamma$-coin following projectile Coulomb excitation. ${ }^{52,54,56} \mathrm{Ti}$ deduced transitions $\mathrm{B}(\mathrm{E} 2)$, subshell closures. Comparison with large-scale shell model calculations. JOUR PRVCA 71041302

2005DIZZ NUCLEAR REACTIONS ${ }^{238} \mathrm{U}\left({ }^{48} \mathrm{Ca}, \mathrm{X}\right){ }^{56} \mathrm{Ti}, \mathrm{E}=330 \mathrm{MeV}$; measured $\mathrm{E} \gamma, \mathrm{I} \gamma, \gamma \gamma$-coin. ${ }^{56} \mathrm{Ti}$ deduced levels, J, $\pi .{ }^{197} \mathrm{Au}\left({ }^{76} \mathrm{Ge},{ }^{76} \mathrm{Ge}\right),\left({ }^{52} \mathrm{Ti}\right.$, $\left.{ }^{52} \mathrm{Ti}^{\prime}\right),\left({ }^{54} \mathrm{Ti},{ }^{54} \mathrm{Ti}\right.$ '), $\left({ }^{56} \mathrm{Ti},{ }^{56} \mathrm{Ti}\right.$ '), $\mathrm{E} \approx 80-90 \mathrm{MeV}$; measured $\mathrm{E} \gamma, \mathrm{I} \gamma$, (particle) $\gamma$-coin following projectile Coulomb excitation. ${ }^{52,54,56} \mathrm{Ti}$, ${ }^{76} \mathrm{Ge},{ }^{197} \mathrm{Au}$ deduced transitions B(E2). CONF Argonne(Nuclei at the Limits),P131,Dinca

2005F006 NUCLEAR REACTIONS ${ }^{197} \mathrm{Au}(\mathrm{n}, \mathrm{n} \gamma), \mathrm{E} \approx 2-12 \mathrm{MeV}$; measured E $\gamma$, $\mathrm{I} \gamma, \gamma \gamma$-coin, excitation functions. ${ }^{197} \mathrm{Au}$ deduced levels, $\mathrm{J}, \pi$, configurations. JOUR PRVCA 71064314 2005GA15 NUCLEAR REACTIONS ${ }^{197} \mathrm{Au}\left({ }^{52} \mathrm{Fe},{ }^{52} \mathrm{Fe}\right),\left({ }^{54} \mathrm{Ni},{ }^{54} \mathrm{Ni}\right),\left({ }^{56} \mathrm{Ni}\right.$, $\left.{ }^{56} \mathrm{Ni}^{\prime}\right),\left({ }^{58} \mathrm{Ni},{ }^{58} \mathrm{Ni}\right.$ '), E not given; measured $\mathrm{E} \gamma, \mathrm{I} \gamma,($ particle $) \gamma$-coin following projectile Coulomb excitation. ${ }^{52} \mathrm{Fe},{ }^{54,56,58} \mathrm{Ni}$ transitions deduced $\mathrm{B}(\mathrm{E} 2) .{ }^{9} \mathrm{Be}\left({ }^{32} \mathrm{~S},{ }^{31} \mathrm{SX}\right),\left({ }^{33} \mathrm{Cl},{ }^{32} \mathrm{ClX}\right),\left({ }^{34} \mathrm{Ar},{ }^{33} \mathrm{ArX}\right)$, E not given; measured one-neutron removal $\sigma$. JOUR APOBB 361227

2005GA22 NUCLEAR REACTIONS ${ }^{197} \mathrm{Au}\left({ }^{72} \mathrm{Kr},{ }^{72} \mathrm{Kr}\right.$ '), E=69.3 MeV / nucleon; ${ }^{197} \mathrm{Au}\left({ }^{78} \mathrm{Kr},{ }^{78} \mathrm{Kr}\right.$ '), $\mathrm{E}=57.4 \mathrm{MeV} /$ nucleon; measured $\mathrm{E} \gamma, \mathrm{I} \gamma$, (particle) $\gamma$-coin following projectile Coulomb excitation. ${ }^{72,78} \mathrm{Kr}$ deduced excitation $\mathrm{B}(\mathrm{E} 2)$, quadrupole moments, deformation. Comparison with shell-model Monte Carlo predictions. JOUR PRLTA 95022502 


\section{$\mathrm{A}=197$ (continued)}

2005HUZZ NUCLEAR REACTIONS ${ }^{197} \mathrm{Au}\left({ }^{54} \mathrm{Cr},{ }^{54} \mathrm{Cr}\right),\left({ }^{56} \mathrm{Cr},{ }^{56} \mathrm{Cr}\right),\left({ }^{58} \mathrm{Cr}\right.$, ${ }^{58} \mathrm{Cr}$ '), $\mathrm{E} \approx 136 \mathrm{MeV} /$ nucleon; measured $\mathrm{E} \gamma, \mathrm{I} \gamma$, (particle) $\gamma$-coin following projectile Coulomb excitation. ${ }^{54,56,58} \mathrm{Cr}$ deduced levels, B(E2). CONF Bormio (XLIII Winter Meeting) Proc,P232

2005IMZZ NUCLEAR REACTIONS ${ }^{197} \mathrm{Au}\left({ }^{12} \mathrm{Be},{ }^{12} \mathrm{Be}\right), \mathrm{E}=40.3 \mathrm{MeV} /$ nucleon; measured $\mathrm{E} \gamma, \mathrm{I} \gamma$, (particle) $\gamma$-coin, DSA following projectile Coulomb excitation. ${ }^{12}$ Be deduced transition. REPT RIKEN 2004

Annual,P41,Imai

2005SAZY NUCLEAR REACTIONS ${ }^{197} \mathrm{Au}\left({ }^{54} \mathrm{Cr},{ }^{54} \mathrm{Cr}\right),\left({ }^{56} \mathrm{Cr},{ }^{56} \mathrm{Cr}\right),\left({ }^{5} \mathrm{Cr}\right.$, ${ }^{58} \mathrm{Cr}$ '), $\mathrm{E}=100 \mathrm{MeV} /$ nucleon; measured $\mathrm{E} \gamma, \mathrm{I} \gamma$, (particle) $\gamma$-coin following projectile Coulomb excitation. ${ }^{54,56,58} \mathrm{Cr}$ deduced transitions. $\mathrm{Be}\left({ }^{55} \mathrm{Ni}, \mathrm{X}\right){ }^{50} \mathrm{Cr}, \mathrm{E}=171 \mathrm{MeV} /$ nucleon; measured $\mathrm{E} \gamma, \mathrm{I} \gamma$, (particle) $\gamma$-coin. ${ }^{50} \mathrm{Cr}$ deduced transitions. $\mathrm{Be}\left({ }^{55} \mathrm{Ni}, \mathrm{X}\right),{ }^{197} \mathrm{Au}\left({ }^{108} \mathrm{Sn}\right.$, $\mathrm{X})$, E not given; measured fragment yields. CONF Argonne(Nuclei at the Limits),P151,Saito

2005SMZX NUCLEAR REACTIONS ${ }^{197} \mathrm{Au}(\mathrm{n}, \mathrm{n}), \mathrm{E}=0.3-10 \mathrm{MeV}$; measured $\sigma(\theta)$. ${ }^{197} \mathrm{Au}(\mathrm{n}, \mathrm{X}), \mathrm{E} \approx 0-15 \mathrm{MeV}$; analyzed total $\sigma$. Optical-statistical and coupled-channels model analysis. REPT ANL/NDM-161,Smith 2005W001 NUCLEAR REACTIONS ${ }^{197} \mathrm{Au}\left({ }^{84} \mathrm{Kr},{ }^{84} \mathrm{Kr}\right),\left({ }^{56} \mathrm{Cr},{ }^{56} \mathrm{Cr}\right),\left({ }^{108} \mathrm{Sn}\right.$, ${ }^{108} \mathrm{Sn}$ '), E $=113-142 \mathrm{MeV} /$ nucleon; measured $\mathrm{E} \gamma$, I $\gamma$ following projectile Coulomb excitation. ${ }^{84} \mathrm{Kr},{ }^{56} \mathrm{Cr},{ }^{108} \mathrm{Sn}$ deduced transitions. ${ }^{9} \mathrm{Be}\left({ }^{55} \mathrm{Ni}, \mathrm{X}\right){ }^{54} \mathrm{Co} /{ }^{52} \mathrm{Fe} /{ }^{50} \mathrm{Cr}, \mathrm{E}=171 \mathrm{MeV} /$ nucleon; measured $\mathrm{E} \gamma$, I $\gamma$, (particle) $\gamma$-coin. JOUR NIMAE 537637

\begin{tabular}{|c|c|c|}
\hline \multirow[b]{2}{*}{${ }^{7} \mathrm{Ho}$} & \multirow[b]{2}{*}{$2003 \mathrm{MBO} 3$} & \\
\hline & & $\begin{array}{l}\text { NUCLEAR REACTIONS }{ }^{198} \mathrm{Pt},{ }^{198} \mathrm{Hg}(\gamma, \mathrm{n}), \mathrm{E}=8-17 \mathrm{MeV} \text {; measured } \\
\mathrm{E} \gamma \text {, I } \gamma \text {, isomer yield ratios. Comparison with model predictions. JOUP } \\
\text { UKPJA } 48403\end{array}$ \\
\hline \multirow[t]{3}{*}{${ }^{197} \mathrm{~Pb}$} & 2002DU22 & RADIOACTIVITY ${ }^{197,197 m, 198,199 m, 200,201 m} \operatorname{Po}(\alpha) ;{ }^{172,173} \operatorname{Os}(\alpha)$ [from \\
\hline & & $\left.{ }^{156} \mathrm{Dy}\left({ }^{22} \mathrm{Ne}, \mathrm{xn}\right)\right] ;{ }^{183,184,185} \mathrm{Hg}(\alpha)\left[\right.$ from $\left.{ }^{168} \mathrm{Yb}\left({ }^{22} \mathrm{Ne}, \mathrm{xn}\right)\right] ;$ measured \\
\hline & & $\mathrm{E} \alpha, \mathrm{T}_{1 / 2}$. JOUR NIMAE 479631 \\
\hline \multirow[t]{7}{*}{${ }^{197} \mathrm{Bi}$} & 2004KE15 & $\begin{array}{l}\text { NUCLEAR REACTIONS }{ }^{1,2} \mathrm{H}, \mathrm{Ti}\left({ }^{208} \mathrm{~Pb}, \mathrm{X}\right){ }^{193} \mathrm{Bi} /{ }^{194} \mathrm{Bi} /{ }^{195} \mathrm{Bi} / \\
{ }^{196} \mathrm{Bi} /{ }^{197} \mathrm{Bi} /{ }^{198} \mathrm{Bi} /{ }^{199} \mathrm{Bi} /{ }^{200} \mathrm{Bi} /{ }^{201} \mathrm{Bi} /{ }^{202} \mathrm{Bi} /{ }^{203} \mathrm{Bi} /{ }^{204} \mathrm{Bi} /\end{array}$ \\
\hline & & $\begin{array}{l}{ }^{205} \mathrm{Bi} /{ }^{206} \mathrm{Bi} /{ }^{207} \mathrm{Bi} /{ }^{208} \mathrm{Bi}, \mathrm{E}=1 \mathrm{GeV} / \text { nucleon; measured } \\
\text { charge-pickup } \sigma \text {, velocity distributions; deduced reaction mechanism }\end{array}$ \\
\hline & & $\begin{array}{l}\text { features. Comparison with model predictions and previous results. } \\
\text { JOUR PRVCA } 70064608\end{array}$ \\
\hline & 2005DE01 & RADIOACTIVITY ${ }^{200,201,203,205} \mathrm{Fr},{ }^{196,197,199,201} \mathrm{At},{ }^{193} \mathrm{Bi}(\alpha)$ [from \\
\hline & & $\begin{array}{l}\mathrm{Th}(\mathrm{p}, \mathrm{X}) \text { and subsequent decay]; measured E } \alpha, \mathrm{T}_{1 / 2} \cdot{ }^{201} \mathrm{Fr},{ }^{197} \mathrm{At}, \\
{ }^{193} \mathrm{Bi},{ }^{189} \mathrm{Tl} \text { deduced levels, J, } \pi \text {. JOUR ZAANE } 23243\end{array}$ \\
\hline & 2005MA51 & NUCLEAR REACTIONS ${ }^{181} \mathrm{Ta}\left({ }^{22} \mathrm{Ne}, 6 \mathrm{n}\right), \mathrm{E}=125 \mathrm{MeV} ;$ measured $\mathrm{E} \gamma$ \\
\hline & & $\begin{array}{l}\text { I } \gamma, \gamma \gamma \text {-coin. }{ }^{197} \mathrm{Bi} \text { deduced high-spin levels, } \mathrm{J}, \pi \text {, configurations, shears } \\
\text { band. Afrodite array, total Routhian surface calculations. JOUR } \\
\text { ZAANE } 2549\end{array}$ \\
\hline \multirow[t]{3}{*}{${ }^{197} \mathrm{Po}$} & 2002DU22 & RADIOACTIVITY $197,197 m, 198,199 m, 200,201 m \operatorname{Po}(\alpha) ;{ }^{172,173} \operatorname{Os}(\alpha)$ [from \\
\hline & & $\left.{ }^{156} \mathrm{Dy}\left({ }^{22} \mathrm{Ne}, \mathrm{xn}\right)\right] ;{ }^{183,184,185} \mathrm{Hg}(\alpha)$ [from $\left.{ }^{168} \mathrm{Yb}\left({ }^{22} \mathrm{Ne}, \mathrm{xn}\right)\right] ;$ measured \\
\hline & & $\mathrm{E} \alpha, \mathrm{T}_{1 / 2}$. JOUR NIMAE 479631 \\
\hline \multirow[t]{2}{*}{${ }^{197} \mathrm{At}$} & 2005DE01 & RADIOACTIVITY ${ }^{200,201,203,205} \mathrm{Fr},{ }^{196,197,199,201} \mathrm{At},{ }^{193} \mathrm{Bi}(\alpha)$ [from \\
\hline & & $\mathrm{Th}(\mathrm{p}, \mathrm{X})$ and subsequent decay]; measured $\mathrm{E} \alpha, \mathrm{T}_{1 / 2} \cdot{ }^{201} \mathrm{Fr},{ }^{197} \mathrm{At}$, \\
\hline
\end{tabular}




\section{$\mathrm{A}=197$ (continued)}

2005UU02

2005UU03

${ }^{197} \mathrm{Rn}$

2005UU02

2005CA02

${ }^{198} \mathrm{Pt}$

2005SH52

${ }^{198} \mathrm{Au}$

2004ADZW

2004 TA46

2005AD01

2005DA34
RADIOACTIVITY ${ }^{201,202,203,204} \mathrm{Ra},{ }^{197,198,199,200} \mathrm{Rn},{ }^{193,194,195,196} \mathrm{Po}$, ${ }^{201,202,203,204} \mathrm{Fr},{ }^{197,198,199,200} \operatorname{At}(\alpha)$ [from ${ }^{141} \operatorname{Pr}\left({ }^{63,65} \mathrm{Cu}\right.$, xnypz $\left.\alpha\right)$, ${ }^{170} \mathrm{Yb}\left({ }^{36} \mathrm{Ar}, \mathrm{xnypz} \alpha\right)$, and subsequent decay]; measured $\mathrm{E} \alpha, \mathrm{T}_{1 / 2}$, $\alpha \alpha$-coin for ground and metastable state decay. ${ }^{193,195} \mathrm{Bi},{ }^{197,199} \mathrm{At}$, ${ }^{201,203} \mathrm{Fr}$ deduced levels, J, $\pi$. Comparisons with previous results. JOUR PRVCA 71024306 RADIOACTIVITY 188,190,192,194,196,198,200,202,204 Po, 191,193,195,197,199 At, ${ }^{196,198,200,202,204,206} \mathrm{Rn},{ }^{199,201,203,205,207} \operatorname{Fr}(\alpha)$; measured reduced widths using gas filled recoil separator; deduced hindrance factors, proton intruder states and deformation effects. JOUR ZAANE 25 s01 179 RADIOACTIVITY ${ }^{201,202,203,204} \mathrm{Ra},{ }^{197,198,199,200} \mathrm{Rn},{ }^{193,194,195,196} \mathrm{Po}$, ${ }^{201,202,203,204} \mathrm{Fr},{ }^{197,198,199,200} \operatorname{At}(\alpha)$ [from ${ }^{141} \operatorname{Pr}\left({ }^{63,65} \mathrm{Cu}\right.$, xnypz $\left.\alpha\right)$, ${ }^{170} \mathrm{Yb}\left({ }^{36} \mathrm{Ar}, \mathrm{xnypz} \alpha\right)$, and subsequent decay]; measured $\mathrm{E} \alpha, \mathrm{T}_{1 / 2}$, $\alpha \alpha$-coin for ground and metastable state decay. ${ }^{193,195} \mathrm{Bi},{ }^{197,199} \mathrm{At}$, ${ }^{201,203}$ Fr deduced levels, J, $\pi$. Comparisons with previous results. JOUR PRVCA 71024306

\section{$\mathrm{A}=198$}

RADIOACTIVITY ${ }^{188} \mathrm{Ta},{ }^{190} \mathrm{~W},{ }^{192,193} \mathrm{Re},{ }^{195} \mathrm{Os},{ }^{197,198} \mathrm{Ir}$, $200,201,202 \mathrm{Pt},{ }^{203} \mathrm{Au}(\mathrm{IT})$ [from $\mathrm{Be}\left({ }^{208} \mathrm{~Pb}, \mathrm{X}\right)$ ]; measured $\mathrm{E} \gamma, \mathrm{I} \gamma, \gamma \gamma$-coin, $\mathrm{T}_{1 / 2} \cdot{ }^{188} \mathrm{Ta},{ }^{190} \mathrm{~W},{ }^{192,193} \mathrm{Re},{ }^{195} \mathrm{Os},{ }^{197,198} \mathrm{Ir},{ }^{200,201,202} \mathrm{Pt},{ }^{203} \mathrm{Au}$ deduced transitions. ${ }^{190} \mathrm{~W},{ }^{200,201,202} \mathrm{Pt}$ deduced levels, J, $\pi .{ }^{174,175} \mathrm{Er}$, ${ }^{185} \mathrm{Hf},{ }^{191,194} \mathrm{Re},{ }^{199} \operatorname{Ir}(\mathrm{IT})$ [from $\left.\mathrm{Be}\left({ }^{208} \mathrm{~Pb}, \mathrm{X}\right)\right]$; measured $\mathrm{E} \gamma, \mathrm{I} \gamma$. JOUR ZAANE 23201

ATOMIC MASSES ${ }^{194,195,196,198}$ Pt; measured masses. Penning trap mass spectrometer. JOUR ZAANE 25 s01 45

NUCLEAR REACTIONS ${ }^{209} \operatorname{Bi}(\mathrm{n}, 4 \mathrm{n}),(\mathrm{n}, 5 \mathrm{n}),(\mathrm{n}, 6 \mathrm{n}),(\mathrm{n}, 7 \mathrm{n}),(\mathrm{n}$, 9n), ${ }^{232} \operatorname{Th}(\mathrm{n}, \gamma),{ }^{197} \mathrm{Au}(\mathrm{n}, 2 \mathrm{n}),(\mathrm{n}, 4 \mathrm{n}),(\mathrm{n}, 6 \mathrm{n}),(\mathrm{n}, 7 \mathrm{n}),(\mathrm{n}, \gamma),{ }^{115} \operatorname{In}(\mathrm{n}$, $5 \mathrm{n}),(\mathrm{n}, 6 \mathrm{n}),(\mathrm{n}, 7 \mathrm{n}),{ }^{59} \operatorname{Co}(\mathrm{n}, 2 \mathrm{n}),(\mathrm{n}, 3 \mathrm{n}),(\mathrm{n}, 4 \mathrm{n}),(\mathrm{n}, 5 \mathrm{n}),(\mathrm{n}, \gamma),(\mathrm{n}$, $\mathrm{p}),(\mathrm{n}, 6 \mathrm{n} 2 \mathrm{p}), \mathrm{E}=$ spectrum; measured $\mathrm{E} \gamma, \mathrm{I} \gamma$; deduced reaction rates. $\mathrm{Pb}(\mathrm{p}, \mathrm{nX}), \mathrm{E}=1 \mathrm{GeV}$; deduced spallation neutron spectrum. REPT JINR-E1-2004-16,Adam NUCLEAR REACTIONS ${ }^{198} \mathrm{Pt}(\mathrm{p}, \mathrm{n}), \mathrm{E} \approx 6-37 \mathrm{MeV} ;{ }^{198} \mathrm{Pt}(\mathrm{d}, \mathrm{n}),(\mathrm{d}$, $2 \mathrm{n}), \mathrm{E} \approx 5-20 \mathrm{MeV}$; measured excitation functions. Activation technique. JOUR RAACA 92223 NUCLEAR REACTIONS ${ }^{209} \operatorname{Bi}(\mathrm{n}, 4 \mathrm{n}),(\mathrm{n}, 5 \mathrm{n}),(\mathrm{n}, 6 \mathrm{n}),(\mathrm{n}, 7 \mathrm{n}),(\mathrm{n}$, $9 \mathrm{n}),{ }^{232} \mathrm{Th}(\mathrm{n}, \gamma),{ }^{197} \mathrm{Au}(\mathrm{n}, 2 \mathrm{n}),(\mathrm{n}, 4 \mathrm{n}),(\mathrm{n}, 6 \mathrm{n}),(\mathrm{n}, 7 \mathrm{n}),(\mathrm{n}, \gamma),{ }^{59} \mathrm{Co}(\mathrm{n}$, 2n), (n, 3n), (n, 4n), (n, 5n), (n, p), (n, 6n2p), ${ }^{115} \operatorname{In}(\mathrm{n}, 5 \mathrm{n}),(\mathrm{n}, 6 \mathrm{n}),(\mathrm{n}$, $7 \mathrm{n}), \mathrm{E}=$ spectrum; measured $\mathrm{E} \gamma, \mathrm{I} \gamma$; deduced reaction rates. Activation technique, spallation neutrons from $1 \mathrm{GeV}$ proton beam, comparison with model predictions. JOUR ZAANE 2361

NUCLEAR REACTIONS ${ }^{197} \mathrm{Au}\left({ }^{12} \mathrm{C},{ }^{11} \mathrm{C}\right),\left({ }^{12} \mathrm{C},{ }^{13} \mathrm{C}\right),\left({ }^{16} \mathrm{O},{ }^{15} \mathrm{O}\right)$, $\left({ }^{16} \mathrm{O},{ }^{17} \mathrm{O}\right), \mathrm{E} \approx 6-7 \mathrm{MeV} / \mathrm{nucleon}$; measured yield ratios, high spin yield fraction; deduced reaction mechanism features. Radiochemical and off-line spectrometric techniques. JOUR JRNCD 26679 


\section{$\mathrm{A}=198$ (continued)}

2005MIZX NUCLEAR REACTIONS ${ }^{23} \mathrm{Na},{ }^{27} \mathrm{Al},{ }^{51} \mathrm{~V},{ }^{55} \mathrm{Mn},{ }^{64} \mathrm{Ni},{ }^{65} \mathrm{Cu},{ }^{141} \mathrm{Pr}$, ${ }^{186} \mathrm{~W},{ }^{197} \mathrm{Au}(\mathrm{n}, \gamma), \mathrm{E}=$ thermal; measured prompt and delayed $\mathrm{E} \gamma, \mathrm{I} \gamma$; deduced capture $\sigma$. Reliability of prompt $\gamma$-ray method discussed. CONF Santa Fe (Nucl Data for Sci and Technol) Proc,Vol1,P996

2005SE23 NUCLEAR REACTIONS ${ }^{197} \mathrm{Au}(\mathrm{n}, \gamma), \mathrm{E}=$ spectrum; measured $\mathrm{E} \gamma, \mathrm{I} \gamma$; deduced neutron flux. ${ }^{7} \operatorname{Li}(\mathrm{p}, \mathrm{n})$, E not given; deduced neutron spectrum. ${ }^{62} \mathrm{Ni}(\mathrm{n}, \gamma), \mathrm{E} \approx 5.5-20 \mathrm{keV}$; measured $\sigma$; deduced Maxwellian-averaged $\sigma$. JOUR JUPSA 742981

2005SI14 NUCLEAR REACTIONS C, O, Si, Mg, $\mathrm{Al}(\mathrm{n}, \mathrm{X})^{7} \mathrm{Be}, \mathrm{E} \approx 0.1-750$ $\mathrm{MeV} ; \mathrm{O}, \mathrm{Si}, \mathrm{Mg}, \mathrm{Al}(\mathrm{n}, \mathrm{X}){ }^{22} \mathrm{Na} /{ }^{23} \mathrm{Na}, \mathrm{E} \approx 0.1-750 \mathrm{MeV} ;{ }^{197} \mathrm{Au}(\mathrm{n}$, $\mathrm{X}){ }^{194} \mathrm{Au} /{ }^{196} \mathrm{Au} /{ }^{198} \mathrm{Au}, \mathrm{E} \approx 0.1-750 \mathrm{MeV} ; \mathrm{Ti}, \mathrm{Fe}, \mathrm{Ni}, \mathrm{Cu}(\mathrm{n}, \mathrm{X}){ }^{46} \mathrm{Sc}$ $/{ }^{48} \mathrm{Sc}, \mathrm{E} \approx 0.1-750 \mathrm{MeV} ; \mathrm{Fe}, \mathrm{Ni}, \mathrm{Cu}(\mathrm{n}, \mathrm{X}){ }^{48} \mathrm{~V} /{ }^{51} \mathrm{Cr} /{ }^{52} \mathrm{Mn} /{ }^{54} \mathrm{Mn}$, $\mathrm{E} \approx 0.1-750 \mathrm{MeV} ; \mathrm{Ni}, \mathrm{Cu}(\mathrm{n}, \mathrm{X}){ }^{56} \mathrm{Ni} /{ }^{57} \mathrm{Ni} /{ }^{56} \mathrm{Co} /{ }^{57} \mathrm{Co} /{ }^{58} \mathrm{Co} /$ ${ }^{60} \mathrm{Co} /{ }^{59} \mathrm{Fe}, \mathrm{E} \approx 0.1-750 \mathrm{MeV}$; measured energy-integrated production $\sigma$. JOUR NIMBE 234419

2005TAZW NUCLEAR REACTIONS Pt(p, X) ${ }^{195} \mathrm{Au} /{ }^{196} \mathrm{Au} /{ }^{198} \mathrm{Au} /{ }^{189} \mathrm{Ir} /$ ${ }^{190} \mathrm{Ir} /{ }^{192} \mathrm{Ir} /{ }^{194} \mathrm{Ir}, \mathrm{E} \approx 0-70 \mathrm{MeV} ; \mathrm{Pt}(\mathrm{d}, \mathrm{X}){ }^{192} \mathrm{Au} /{ }^{193} \mathrm{Au} /{ }^{194} \mathrm{Au} /$ ${ }^{195} \mathrm{Au} /{ }^{196} \mathrm{Au} /{ }^{195 m} \mathrm{Pt} /{ }^{197} \mathrm{Pt} /{ }^{192} \mathrm{Ir}, \mathrm{E} \approx 0-21 \mathrm{MeV} ; \mathrm{Pt}(\alpha, \mathrm{X}){ }^{195} \mathrm{Au}$ $/{ }^{196} \mathrm{Au}, \mathrm{E} \approx 0-38 \mathrm{MeV}$; measured activation $\sigma$; deduced integral yields. CONF Santa Fe (Nucl Data for Sci and Technol) Proc,Vol1,P1015

2006Di01 NUCLEAR REACTIONS Pt (d, X) $)^{191} \mathrm{Au} /{ }^{192} \mathrm{Au} /{ }^{193} \mathrm{Au} /{ }^{194} \mathrm{Au} /$ ${ }^{195} \mathrm{Au} /{ }^{196} \mathrm{Au} /{ }^{196 m} \mathrm{Au} /{ }^{198} \mathrm{Au} /{ }^{199} \mathrm{Au} /{ }^{191} \mathrm{Pt} /{ }^{195 m} \mathrm{Pt} /{ }^{197} \mathrm{Pt} /$ ${ }^{192} \mathrm{Ir}, \mathrm{E} \approx 10-40 \mathrm{MeV}$; measured production $\sigma$. Stacked-foil activation technique, comparison with model predictions. JOUR NIMBE 24320

${ }^{198} \mathrm{Hg} \quad$ 2005LEZW NUCLEAR REACTIONS ${ }^{188,190,194} \mathrm{Os},{ }^{194,196} \mathrm{Pt}(\alpha, 2 \mathrm{n}), \mathrm{E}=27 \mathrm{MeV}$; measured $\mathrm{E} \gamma, \mathrm{I} \gamma(\theta, \mathrm{H}, \mathrm{t}) .{ }^{190,192,194} \mathrm{Pt},{ }^{196,198} \mathrm{Hg}$ deduced isomeric states g-factors, configurations. Integral perturbed angular distribution method, HPGe detectors. CONF St Petersburg,P81,Levon

${ }^{198} \mathrm{~Pb} \quad 2005 \mathrm{UU} 03$

RADIOACTIVITY $188,190,192,194,196,198,200,202,204 \mathrm{Po}$, $191,193,195,197,199$ At, ${ }^{196,198,200,202,204,206} \mathrm{Rn},{ }^{199,201,203,205,207} \operatorname{Fr}(\alpha)$; measured reduced widths using gas filled recoil separator; deduced hindrance factors, proton intruder states and deformation effects. JOUR ZAANE 25 s01 179

${ }^{198} \mathrm{Bi}$ 2004KE15 NUCLEAR REACTIONS ${ }^{1,2} \mathrm{H}, \mathrm{Ti}\left({ }^{208} \mathrm{~Pb}, \mathrm{X}\right){ }^{193} \mathrm{Bi} /{ }^{194} \mathrm{Bi} /{ }^{195} \mathrm{Bi} /$ ${ }^{196} \mathrm{Bi} /{ }^{197} \mathrm{Bi} /{ }^{198} \mathrm{Bi} /{ }^{199} \mathrm{Bi} /{ }^{200} \mathrm{Bi} /{ }^{201} \mathrm{Bi} /{ }^{202} \mathrm{Bi} /{ }^{203} \mathrm{Bi} /{ }^{204} \mathrm{Bi} /$ ${ }^{205} \mathrm{Bi} /{ }^{206} \mathrm{Bi} /{ }^{207} \mathrm{Bi} /{ }^{208} \mathrm{Bi}, \mathrm{E}=1 \mathrm{GeV} /$ nucleon; measured charge-pickup $\sigma$, velocity distributions; deduced reaction mechanism features. Comparison with model predictions and previous results. JOUR PRVCA 70064608

${ }^{198} \mathrm{Po}$ 2002DU22 RADIOACTIVITY ${ }^{197,197 m, 198,199 m, 200,201 m} \mathrm{Po}(\alpha) ;{ }^{172,173} \operatorname{Os}(\alpha)$ [from $\left.{ }^{156} \mathrm{Dy}\left({ }^{22} \mathrm{Ne}, \mathrm{xn}\right)\right] ;{ }^{183,184,185} \mathrm{Hg}(\alpha)$ [from $\left.{ }^{168} \mathrm{Yb}\left({ }^{22} \mathrm{Ne}, \mathrm{xn}\right)\right]$; measured $\mathrm{E} \alpha, \mathrm{T}_{1 / 2}$. JOUR NIMAE 479631

2005J003 NUCLEAR REACTIONS ${ }^{174} \mathrm{Yb}\left({ }^{29} \mathrm{Si}, 5 \mathrm{n}\right), \mathrm{E}=148 \mathrm{MeV}$; measured $\mathrm{E} \gamma$, $\mathrm{I} \gamma, \gamma \gamma$-coin. ${ }^{198}$ Po deduced spin-energy entry distributions for superdeformed and normal-deformed rotational bands. Gammasphere array. JOUR PRVCA 71024317 


\section{$\mathrm{A}=198$ (continued)}

2005UU03 RADIOACTIVITY 188,190,192,194,196,198,200,202,204Po, 191,193,195,197,199 At, ${ }^{196,198,200,202,204,206}$ Rn, ${ }^{199,201,203,205,207} \operatorname{Fr}(\alpha)$; measured reduced widths using gas filled recoil separator; deduced hindrance factors, proton intruder states and deformation effects.

JOUR ZAANE 25 s01 179

${ }^{198}$ At $\quad 2005$ UU02 RADIOACTIVITY ${ }^{201,202,203,204} \mathrm{Ra},{ }^{197,198,199,200} \mathrm{Rn},{ }^{193,194,195,196} \mathrm{Po}$, ${ }^{201,202,203,204} \mathrm{Fr},{ }^{197,198,199,200} \operatorname{At}(\alpha)$ [from ${ }^{141} \operatorname{Pr}\left({ }^{63,65} \mathrm{Cu}\right.$, xnypz $\left.\alpha\right)$, ${ }^{170} \mathrm{Yb}\left({ }^{36} \mathrm{Ar}, \mathrm{xnypz} \alpha\right)$, and subsequent decay]; measured $\mathrm{E} \alpha, \mathrm{T}_{1 / 2}$, $\alpha \alpha$-coin for ground and metastable state decay. ${ }^{193,195} \mathrm{Bi},{ }^{197,199} \mathrm{At}$, ${ }^{201,203} \mathrm{Fr}$ deduced levels, J, $\pi$. Comparisons with previous results. JOUR PRVCA 71024306

${ }^{198} \mathrm{Rn} \quad 2005 U U 02 \quad$ NUCLEAR REACTIONS ${ }^{141} \operatorname{Pr}\left({ }^{65} \mathrm{Cu}\right.$, xnypz $\left.\alpha\right), \mathrm{E}=283-293 \mathrm{MeV}$; measured delayed $\mathrm{E} \alpha, \mathrm{I} \alpha, \alpha \alpha-$, (recoil) $\alpha$-coin; deduced evidence for ${ }^{199,200,201} \mathrm{Rn},{ }^{202,203,204} \mathrm{Fr},{ }^{203,204} \mathrm{Ra} .{ }^{141} \operatorname{Pr}\left({ }^{63} \mathrm{Cu}\right.$, xnypz $\left.\alpha\right), \mathrm{E}=278-288$ $\mathrm{MeV}$; measured delayed $\mathrm{E} \alpha, \mathrm{I} \alpha, \alpha \alpha-$, (recoil) $\alpha$-coin; deduced evidence for ${ }^{198,199,200,201,202} \mathrm{Rn},{ }^{201,202} \mathrm{Fr},{ }^{201,202} \mathrm{Ra} .{ }^{170} \mathrm{Yb}\left({ }^{36} \mathrm{Ar}, \mathrm{xnypz} \alpha\right)$, $\mathrm{E}=180-185 \mathrm{MeV}$; measured delayed $\mathrm{E} \alpha, \mathrm{I} \alpha, \alpha \alpha-$, (recoil) $\alpha$-coin; deduced evidence for ${ }^{201} \mathrm{Fr},{ }^{203} \mathrm{Ra}$. Gas-filled recoil separator. JOUR PRVCA 71024306

2005UU02 RADIOACTIVITY ${ }^{201,202,203,204} \mathrm{Ra},{ }^{197,198,199,200} \mathrm{Rn},{ }^{193,194,195,196}$ Po, ${ }^{201,202,203,204} \mathrm{Fr},{ }^{197,198,199,200} \operatorname{At}(\alpha)$ [from ${ }^{141} \operatorname{Pr}\left({ }^{63,65} \mathrm{Cu}\right.$, xnypz $\left.\alpha\right)$,

${ }^{170} \mathrm{Yb}\left({ }^{36} \mathrm{Ar}, \mathrm{xnypz} \alpha\right)$, and subsequent decay]; measured $\mathrm{E} \alpha, \mathrm{T}_{1 / 2}$, $\alpha \alpha$-coin for ground and metastable state decay. ${ }^{193,195} \mathrm{Bi},{ }^{197,199} \mathrm{At}$, ${ }^{201,203}$ Fr deduced levels, J, $\pi$. Comparisons with previous results. JOUR PRVCA 71024306

2005UU03 RADIOACTIVITY ${ }^{188,190,192,194,196,198,200,202,204}$ Po, 191,193,195,197,199 At, ${ }^{196,198,200,202,204,206} \mathrm{Rn},{ }^{199,201,203,205,207} \operatorname{Fr}(\alpha)$; measured reduced widths using gas filled recoil separator; deduced hindrance factors, proton intruder states and deformation effects.

JOUR ZAANE 25 s01 179

\section{$\mathrm{A}=199$}

${ }^{199} \operatorname{Ir} \quad 2005 \mathrm{CA02}$

RADIOACTIVITY ${ }^{188} \mathrm{Ta},{ }^{190} \mathrm{~W},{ }^{192,193} \mathrm{Re},{ }^{195} \mathrm{Os},{ }^{197,198} \mathrm{Ir}$, $200,201,202 \mathrm{Pt},{ }^{203} \mathrm{Au}(\mathrm{IT})$ [from $\mathrm{Be}\left({ }^{208} \mathrm{~Pb}, \mathrm{X}\right)$ ]; measured $\mathrm{E} \gamma, \mathrm{I} \gamma, \gamma \gamma$-coin, $\mathrm{T}_{1 / 2} \cdot{ }^{188} \mathrm{Ta},{ }^{190} \mathrm{~W},{ }^{192,193} \mathrm{Re},{ }^{195} \mathrm{Os},{ }^{197,198} \mathrm{Ir},{ }^{200,201,202} \mathrm{Pt},{ }^{203} \mathrm{Au}$ deduced transitions. ${ }^{190} \mathrm{~W},{ }^{200,201,202} \mathrm{Pt}$ deduced levels, J, $\pi .{ }^{174,175} \mathrm{Er}$, ${ }^{185} \mathrm{Hf},{ }^{191,194} \mathrm{Re},{ }^{199} \operatorname{Ir}(\mathrm{IT})$ [from $\left.\mathrm{Be}\left({ }^{208} \mathrm{~Pb}, \mathrm{X}\right)\right]$; measured $\mathrm{E} \gamma, \mathrm{I} \gamma$.

JOUR ZAANE 23201

${ }^{199} \mathrm{Au} \quad$ 2004TA46 NUCLEAR REACTIONS ${ }^{198} \operatorname{Pt}(\mathrm{p}, \mathrm{n}), \mathrm{E} \approx 6-37 \mathrm{MeV} ;{ }^{198} \operatorname{Pt}(\mathrm{d}, \mathrm{n}),(\mathrm{d}$, $2 \mathrm{n}), \mathrm{E} \approx 5-20 \mathrm{MeV}$; measured excitation functions. Activation technique. JOUR RAACA 92223

2006DI01 NUCLEAR REACTIONS Pt(d, X) ${ }^{191} \mathrm{Au} /{ }^{192} \mathrm{Au} /{ }^{193} \mathrm{Au} /{ }^{194} \mathrm{Au} /$ ${ }^{195} \mathrm{Au} /{ }^{196} \mathrm{Au} /{ }^{196 m} \mathrm{Au} /{ }^{198} \mathrm{Au} /{ }^{199} \mathrm{Au} /{ }^{191} \mathrm{Pt} /{ }^{195 m} \mathrm{Pt} /{ }^{197} \mathrm{Pt} /$ ${ }^{192} \mathrm{Ir}, \mathrm{E} \approx 10-40 \mathrm{MeV}$; measured production $\sigma$. Stacked-foil activation technique, comparison with model predictions. JOUR NIMBE 24320 


\section{$\mathrm{A}=199$ (continued)}

\begin{tabular}{|c|c|c|}
\hline \multirow[t]{4}{*}{${ }^{199} \mathrm{Hg}$} & 2005BIZY & $\begin{array}{l}\text { NUCLEAR REACTIONS }{ }^{113} \mathrm{In},{ }^{195} \mathrm{Pt},{ }^{199} \mathrm{Hg}(\gamma, \gamma \\
\text { measured isomer production } \sigma \text {. Microtron. CONF St }\end{array}$ \\
\hline & & Petersburg,P215,Bigan \\
\hline & $2005 \mathrm{HO} 16$ & $\begin{array}{l}\text { NUCLEAR REACTIONS }{ }^{200} \mathrm{Hg}(\gamma, \mathrm{n}), \mathrm{E} \approx 10-17 \mathrm{MeV} ;{ }^{199} \mathrm{Hg}\left(\gamma, \gamma^{\prime}\right), \mathrm{E} \\
\approx 4 \text {-10 MeV; measured isomer excitation } \sigma \text {. Comparison with } \\
\text { cascade-evaporation model predictions. JOUR UKPJA } 50649\end{array}$ \\
\hline & 20050S02 & $\begin{array}{l}\text { NUCLEAR MOMENTS }{ }^{199} \mathrm{Hg} \text {; measured electric quadrupole moment. } \\
\text { Comparison with model predictions. JOUR PRLTA } 94163001\end{array}$ \\
\hline \multirow[t]{3}{*}{${ }^{199} \mathrm{Tl}$} & 2005TIZX & $\begin{array}{l}\text { NUCLEAR REACTIONS Pb, }{ }^{208} \mathrm{~Pb}(\mathrm{p}, \mathrm{X})^{203} \mathrm{~Pb} /{ }^{200} \mathrm{Tl} /{ }^{199} \mathrm{Tl} / \\
{ }^{196} \mathrm{Au} /{ }^{192} \mathrm{Tr} /{ }^{190} \mathrm{Tr} /{ }^{173} \mathrm{~L} /{ }^{101 m} \mathrm{Rh} /{ }^{86} \mathrm{Rb} /{ }^{59} \mathrm{Fe} /{ }^{24} \mathrm{Na} /{ }^{7} \mathrm{Be}\end{array}$ \\
\hline & & $\begin{array}{l}\mathrm{E}=40-2600 \mathrm{MeV} \text {; measured excitation functions. Comparison with } \\
\text { previous work and model predictions. Other reactions discussed. } \\
\text { CONF Santa Fe (Nucl Data for Sci and Technol) Proc,Vol1,P1070 }\end{array}$ \\
\hline & 2005 TIZY & $\begin{array}{l}\text { NUCLEAR REACTIONS Pb, }{ }^{208} \mathrm{~Pb},{ }^{209} \mathrm{Bi}(\mathrm{p}, \mathrm{X}){ }^{203} \mathrm{~Pb} /{ }^{200} \mathrm{Tl} /{ }^{199} \mathrm{Tl} \\
/{ }^{196} \mathrm{Au} /{ }^{192} \mathrm{Ir} /{ }^{190} \mathrm{Ir} /{ }^{173} \mathrm{Lu} /{ }^{101 m} \mathrm{Rh} /{ }^{86} \mathrm{Rb} /{ }^{59} \mathrm{Fe} /{ }^{24} \mathrm{Na} /{ }^{7} \mathrm{Be}, \\
\mathrm{E}=40-2600 \mathrm{MeV} \text {; measured production } \sigma . \text { Comparison with model } \\
\text { predictions. PREPRINT nucl-ex/0507009,7/05/2005 }\end{array}$ \\
\hline${ }^{199} \mathrm{Bi}$ & 2004KE15 & $\begin{array}{l}\text { NUCLEAR REACTIONS }{ }^{1,2} \mathrm{H}, \mathrm{Ti}\left({ }^{208} \mathrm{~Pb}, \mathrm{X}\right){ }^{193} \mathrm{Bi} /{ }^{194} \mathrm{Bi} /{ }^{195} \mathrm{Bi} / \\
{ }^{196} \mathrm{Bi} /{ }^{197} \mathrm{Bi} /{ }^{198} \mathrm{Bi} /{ }^{199} \mathrm{Bi} /{ }^{200} \mathrm{Bi} /{ }^{201} \mathrm{Bi} /{ }^{202} \mathrm{Bi} /{ }^{203} \mathrm{Bi} /{ }^{204} \mathrm{Bi} / \\
\\
{ }^{205} \mathrm{Bi} /{ }^{206} \mathrm{Bi} /{ }^{207} \mathrm{Bi} /{ }^{208} \mathrm{Bi}, \mathrm{E}=1 \mathrm{GeV} / \text { nucleon} \text {; measured }\end{array}$ \\
\hline & & $\begin{array}{l}\text { charge-pickup } \sigma \text {, velocity distributions; deduced reaction mechanism } \\
\text { features. Comparison with model predictions and previous results. } \\
\text { JOUR PRVCA } 70064608\end{array}$ \\
\hline \multirow[t]{3}{*}{${ }^{199} \mathrm{Po}$} & 2002DU22 & RADIOACTIVITY ${ }^{197,197 m, 198,199 m, 200,201 m} \mathrm{Po}(\alpha) ;{ }^{172,173} \mathrm{Os}(\alpha)$ [from \\
\hline & & $\left.{ }^{156} \mathrm{Dy}\left({ }^{22} \mathrm{Ne}, \mathrm{xn}\right)\right] ;{ }^{183,184,185} \mathrm{Hg}(\alpha)\left[\right.$ from $\left.{ }^{168} \mathrm{Yb}\left({ }^{22} \mathrm{Ne}, \mathrm{xn}\right)\right] ;$ measured \\
\hline & & $\mathrm{E} \alpha, \mathrm{T}_{1 / 2}$. JOUR NIMAE 479631 \\
\hline \multirow[t]{7}{*}{${ }^{199} \mathrm{At}$} & 2005DE01 & RADIOACTIVITY ${ }^{200,201,203,205} \mathrm{Fr},{ }^{196,197,199,201} \mathrm{At},{ }^{193} \mathrm{Bi}(\alpha)$ [from \\
\hline & & $\begin{array}{l}\mathrm{Th}(\mathrm{p}, \mathrm{X}) \text { and subsequent decay]; measured } \mathrm{E} \alpha, \mathrm{T}_{1 / 2} \cdot{ }^{201} \mathrm{Fr},{ }^{197} \mathrm{At} \\
{ }^{193} \mathrm{Bi},{ }^{189} \mathrm{Tl} \text { deduced levels, J, } \pi \text {. JOUR ZAANE } 23243\end{array}$ \\
\hline & 2005UU02 & $\begin{array}{l}\text { RADIOACTIVITY }{ }^{201,202,203,204} \mathrm{Ra},{ }^{197,198,199,200} \mathrm{Rn},{ }^{193,194,195,196} \mathrm{Po} \text {, } \\
\text { 201,202,203,204} \mathrm{Fr},{ }^{197,198,199,200} \mathrm{At}(\alpha) \text { [from }{ }^{141} \operatorname{Pr}\left({ }^{63,65} \mathrm{Cu}, \text { xnypz } \alpha\right),\end{array}$ \\
\hline & & $\begin{array}{l}{ }^{170} \mathrm{Yb}\left({ }^{36} \mathrm{Ar} \text {, xnypz } \alpha\right) \text {, and subsequent decay]; measured } \mathrm{E} \alpha, \mathrm{T}_{1 / 2}, \\
\alpha \alpha \text {-coin for ground and metastable state decay. }{ }^{193,195} \mathrm{Bi},{ }^{197,199} \mathrm{At}, \\
{ }^{201,203} \mathrm{Fr} \text { deduced levels, J, } \pi \text {. Comparisons with previous results. }\end{array}$ \\
\hline & & JOUR PRVCA 71024306 \\
\hline & 2005UU03 & $\begin{array}{l}\text { RADIOACTIVITY }{ }^{188,190,192,194,196,198,200,202,204} \mathrm{Po}, \\
191,193,195,197,199 \text { At, }{ }^{196,198,200,202,204,206} \mathrm{Rn},{ }^{199,201,203,205,207} \mathrm{Fr}(\alpha)\end{array}$ \\
\hline & & $\begin{array}{l}\text { measured reduced widths using gas filled recoil separator; deduced } \\
\text { hindrance factors, proton intruder states and deformation effects. } \\
\text { JOUR ZAANE } 25 \text { s01 } 179\end{array}$ \\
\hline \multirow[t]{3}{*}{${ }^{199} \mathrm{Rn}$} & 2005UU02 & $\begin{array}{l}\text { NUCLEAR REACTIONS }{ }^{141} \operatorname{Pr}\left({ }^{65} \mathrm{Cu} \text {, xnypz } \alpha\right), \mathrm{E}=283-293 \mathrm{MeV} \\
\text { measured delayed } \mathrm{E} \alpha, \mathrm{I} \alpha, \alpha \alpha-,(\text { recoil }) \alpha \text {-coin; deduced evidence for } \\
\\
{ }^{199,200,201} \mathrm{Rn},{ }^{202,203,204} \mathrm{Fr},{ }^{203,204} \mathrm{Ra} .{ }^{141} \operatorname{Pr}\left({ }^{63} \mathrm{Cu} \text {, xnypz } \alpha\right), \mathrm{E}=278-288\end{array}$ \\
\hline & & $\begin{array}{l}\mathrm{MeV} \text {; measured delayed } \mathrm{E} \alpha, \mathrm{I} \alpha, \alpha \alpha-,(\text { recoil }) \alpha \text {-coin; deduced evidence } \\
\text { for }{ }^{198,199,200,201,202} \mathrm{Rn},{ }^{201,202} \mathrm{Fr},{ }^{201,202} \mathrm{Ra} .{ }^{170} \mathrm{Yb}\left({ }^{36} \mathrm{Ar} \text {, xnypz } \alpha\right)\end{array}$ \\
\hline & & $\begin{array}{l}\mathrm{E}=180-185 \mathrm{MeV} \text {; measured delayed } \mathrm{E} \alpha, \mathrm{I} \alpha, \alpha \alpha-\text {, (recoil) } \alpha \text {-coin; } \\
\text { deduced evidence for }{ }^{201} \mathrm{Fr},{ }^{203} \mathrm{Ra} \text {. Gas-filled recoil separator. JOUR } \\
\text { PRVCA } 71024306\end{array}$ \\
\hline
\end{tabular}




\section{$\mathrm{A}=199$ (continued)}

2005UU02

2005UU03

2005CA02

${ }^{200} \mathrm{Tl}$

2005TIZX

2005TIZY

${ }^{200} \mathrm{~Pb}$

2005 MIZZ

2005 UU03

2004KE15

${ }^{200} \mathrm{Po}$
RADIOACTIVITY ${ }^{201,202,203,204} \mathrm{Ra},{ }^{197,198,199,200} \mathrm{Rn},{ }^{193,194,195,196} \mathrm{Po}$, ${ }^{201,202,203,204} \mathrm{Fr},{ }^{197,198,199,200} \operatorname{At}(\alpha)\left[\right.$ from ${ }^{141} \operatorname{Pr}\left({ }^{63,65} \mathrm{Cu}\right.$, xnypz $\left.\alpha\right)$, ${ }^{170} \mathrm{Yb}\left({ }^{36} \mathrm{Ar}, \mathrm{xnypz} \alpha\right)$, and subsequent decay]; measured $\mathrm{E} \alpha, \mathrm{T}_{1 / 2}$, $\alpha \alpha$-coin for ground and metastable state decay. ${ }^{193,195} \mathrm{Bi},{ }^{197,199} \mathrm{At}$, ${ }^{201,203}$ Fr deduced levels, J, $\pi$. Comparisons with previous results. JOUR PRVCA 71024306

RADIOACTIVITY 188,190,192,194,196,198,200,202,204 Po, 191,193,195,197,199 At, ${ }^{196,198,200,202,204,206} \mathrm{Rn},{ }^{199,201,203,205,207} \operatorname{Fr}(\alpha)$; measured reduced widths using gas filled recoil separator; deduced hindrance factors, proton intruder states and deformation effects. JOUR ZAANE 25 s01 179

\section{$\mathrm{A}=\mathbf{2 0 0}$}

RADIOACTIVITY ${ }^{188} \mathrm{Ta},{ }^{190} \mathrm{~W},{ }^{192,193} \mathrm{Re},{ }^{195} \mathrm{Os},{ }^{197,198} \mathrm{Ir}$, $200,201,202 \mathrm{Pt},{ }^{203} \mathrm{Au}(\mathrm{IT})$ [from $\left.\mathrm{Be}\left({ }^{208} \mathrm{~Pb}, \mathrm{X}\right)\right]$; measured $\mathrm{E} \gamma, \mathrm{I} \gamma, \gamma \gamma$-coin, $\mathrm{T}_{1 / 2} .{ }^{188} \mathrm{Ta},{ }^{190} \mathrm{~W},{ }^{192,193} \mathrm{Re},{ }^{195} \mathrm{Os},{ }^{197,198} \mathrm{Ir},{ }^{200,201,202} \mathrm{Pt},{ }^{203} \mathrm{Au}$ deduced transitions. ${ }^{190} \mathrm{~W},{ }^{200,201,202} \mathrm{Pt}$ deduced levels, J, $\pi .{ }^{174,175} \mathrm{Er}$, ${ }^{185} \mathrm{Hf},{ }^{191,194} \mathrm{Re},{ }^{199} \operatorname{Ir}(\mathrm{IT})$ [from $\mathrm{Be}\left({ }^{208} \mathrm{~Pb}, \mathrm{X}\right)$ ]; measured $\mathrm{E} \gamma, \mathrm{I} \gamma$. JOUR ZAANE 23201 NUCLEAR REACTIONS Pb, ${ }^{208} \mathrm{~Pb}(\mathrm{p}, \mathrm{X}){ }^{203} \mathrm{~Pb} /{ }^{200} \mathrm{Tl} /{ }^{199} \mathrm{Tl} /$ ${ }^{196} \mathrm{Au} /{ }^{192} \mathrm{Ir} /{ }^{190} \mathrm{Ir} /{ }^{173} \mathrm{Lu} /{ }^{101 m} \mathrm{Rh} /{ }^{86} \mathrm{Rb} /{ }^{59} \mathrm{Fe} /{ }^{24} \mathrm{Na} /{ }^{7} \mathrm{Be}$, $\mathrm{E}=40-2600 \mathrm{MeV}$; measured excitation functions. Comparison with previous work and model predictions. Other reactions discussed. CONF Santa Fe (Nucl Data for Sci and Technol) Proc,Vol1,P1070 NUCLEAR REACTIONS Pb, ${ }^{208} \mathrm{~Pb},{ }^{209} \mathrm{Bi}(\mathrm{p}, \mathrm{X}){ }^{203} \mathrm{~Pb} /{ }^{200} \mathrm{Tl} /{ }^{199} \mathrm{Tl}$ / ${ }^{196} \mathrm{Au} /{ }^{192} \mathrm{Ir} /{ }^{190} \mathrm{Ir} /{ }^{173} \mathrm{Lu} /{ }^{101 m} \mathrm{Rh} /{ }^{86} \mathrm{Rb} /{ }^{59} \mathrm{Fe} /{ }^{24} \mathrm{Na} /{ }^{7} \mathrm{Be}$, $\mathrm{E}=40-2600 \mathrm{MeV}$; measured production $\sigma$. Comparison with model predictions. PREPRINT nucl-ex/0507009,7/05/2005

NUCLEAR REACTIONS Cu(n, X) ${ }^{56} \mathrm{Co}, \mathrm{E}=40-180 \mathrm{MeV} ; \mathrm{Fe}(\mathrm{n}$, $\mathrm{X}){ }^{54} \mathrm{Mn} /{ }^{52} \mathrm{Mn} /{ }^{51} \mathrm{Cr} /{ }^{48} \mathrm{~V}, \mathrm{E} \approx 0-180 \mathrm{MeV} ; \mathrm{Pb}(\mathrm{n}, \mathrm{X}){ }^{196} \mathrm{Au} /{ }^{200} \mathrm{~Pb}$ $/{ }^{103} \mathrm{Ru}, \mathrm{E} \approx 40-180 \mathrm{MeV} ; \mathrm{U}(\mathrm{n}, \mathrm{X}){ }^{99} \mathrm{Mo}, \mathrm{E} \approx 0-180 \mathrm{MeV}$; measured excitation functions. Comparison with proton-induced reactions. CONF Santa Fe (Nucl Data for Sci and Technol) Proc,Vol1,P861 RADIOACTIVITY ${ }^{188,190,192,194,196,198,200,202,204}$ Po, 191,193,195,197,199 At, ${ }^{196,198,200,202,204,206}$ Rn, ${ }^{199,201,203,205,207} \operatorname{Fr}(\alpha)$; measured reduced widths using gas filled recoil separator; deduced hindrance factors, proton intruder states and deformation effects.

JOUR ZAANE 25 s01 179

NUCLEAR REACTIONS ${ }^{1,2} \mathrm{H}, \mathrm{Ti}\left({ }^{208} \mathrm{~Pb}, \mathrm{X}\right){ }^{193} \mathrm{Bi} /{ }^{194} \mathrm{Bi} /{ }^{195} \mathrm{Bi} /$ ${ }^{196} \mathrm{Bi} /{ }^{197} \mathrm{Bi} /{ }^{198} \mathrm{Bi} /{ }^{199} \mathrm{Bi} /{ }^{200} \mathrm{Bi} /{ }^{201} \mathrm{Bi} /{ }^{202} \mathrm{Bi} /{ }^{203} \mathrm{Bi} /{ }^{204} \mathrm{Bi} /$ ${ }^{205} \mathrm{Bi} /{ }^{206} \mathrm{Bi} /{ }^{207} \mathrm{Bi} /{ }^{208} \mathrm{Bi}, \mathrm{E}=1 \mathrm{GeV} /$ nucleon; measured charge-pickup $\sigma$, velocity distributions; deduced reaction mechanism features. Comparison with model predictions and previous results. JOUR PRVCA 70064608 RADIOACTIVITY ${ }^{197,197 m, 198,199 m, 200,201 m} \mathrm{Po}(\alpha) ;{ }^{172,173} \mathrm{Os}(\alpha)$ [from ${ }^{156} \mathrm{Dy}\left({ }^{22} \mathrm{Ne}, \mathrm{xn}\right)$ ]; ${ }^{183,184,185} \mathrm{Hg}(\alpha)$ [from ${ }^{168} \mathrm{Yb}\left({ }^{22} \mathrm{Ne}, \mathrm{xn}\right)$ ]; measured $\mathrm{E} \alpha, \mathrm{T}_{1 / 2}$. JOUR NIMAE 479631 


\section{$\mathrm{A}=200$ (continued)}

2005UU03 RADIOACTIVITY ${ }^{188,190,192,194,196,198,200,202,204} \mathrm{Po}$ 191,193,195,197,199 At, ${ }^{196,198,200,202,204,206}$ Rn, ${ }^{199,201,203,205,207} \operatorname{Fr}(\alpha)$; measured reduced widths using gas filled recoil separator; deduced hindrance factors, proton intruder states and deformation effects. JOUR ZAANE 25 s01 179

${ }^{200} \mathrm{At} \quad 2005 \mathrm{UU} 02$ RADIOACTIVITY ${ }^{201,202,203,204} \mathrm{Ra},{ }^{197,198,199,200} \mathrm{Rn},{ }^{193,194,195,196} \mathrm{Po}$, ${ }^{201,202,203,204} \mathrm{Fr},{ }^{197,198,199,200} \operatorname{At}(\alpha)$ [from ${ }^{141} \operatorname{Pr}\left({ }^{63,65} \mathrm{Cu}\right.$, xnypz $\left.\alpha\right)$, ${ }^{170} \mathrm{Yb}\left({ }^{36} \mathrm{Ar}, \mathrm{xnypz} \alpha\right)$, and subsequent decay]; measured $\mathrm{E} \alpha, \mathrm{T}_{1 / 2}$, $\alpha \alpha$-coin for ground and metastable state decay. ${ }^{193,195} \mathrm{Bi},{ }^{197,199} \mathrm{At}$, ${ }^{201,203}$ Fr deduced levels, J, $\pi$. Comparisons with previous results. JOUR PRVCA 71024306

${ }^{200} \mathrm{Rn} \quad 2005 \mathrm{NU} 02 \quad$ NUCLEAR REACTIONS ${ }^{141} \operatorname{Pr}\left({ }^{65} \mathrm{Cu}\right.$, xnypz $\left.\alpha\right), \mathrm{E}=283-293 \mathrm{MeV}$; measured delayed $\mathrm{E} \alpha, \mathrm{I} \alpha, \alpha \alpha-$, (recoil) $\alpha$-coin; deduced evidence for ${ }^{199,200,201} \mathrm{Rn},{ }^{202,203,204} \mathrm{Fr},{ }^{203,204} \mathrm{Ra} .{ }^{141} \operatorname{Pr}\left({ }^{63} \mathrm{Cu}\right.$, xnypz $\left.\alpha\right), \mathrm{E}=278-288$ $\mathrm{MeV}$; measured delayed $\mathrm{E} \alpha, \mathrm{I} \alpha, \alpha \alpha-$, (recoil) $\alpha$-coin; deduced evidence for ${ }^{198,199,200,201,202} \mathrm{Rn},{ }^{201,202} \mathrm{Fr},{ }^{201,202} \mathrm{Ra} .{ }^{170} \mathrm{Yb}\left({ }^{36} \mathrm{Ar}, \mathrm{xnypz} \alpha\right)$, $\mathrm{E}=180-185 \mathrm{MeV}$; measured delayed $\mathrm{E} \alpha, \mathrm{I} \alpha, \alpha \alpha$-, (recoil) $\alpha$-coin; deduced evidence for ${ }^{201} \mathrm{Fr},{ }^{203} \mathrm{Ra}$. Gas-filled recoil separator. JOUR PRVCA 71024306

2005UU02 RADIOACTIVITY ${ }^{201,202,203,204} \mathrm{Ra},{ }^{197,198,199,200} \mathrm{Rn},{ }^{193,194,195,196}$ Po, ${ }^{201,202,203,204} \mathrm{Fr},{ }^{197,198,199,200} \operatorname{At}(\alpha)$ [from ${ }^{141} \operatorname{Pr}\left({ }^{63,65} \mathrm{Cu}\right.$, xnypz $\left.\alpha\right)$,

${ }^{170} \mathrm{Yb}\left({ }^{36} \mathrm{Ar}, \mathrm{xnypz} \alpha\right)$, and subsequent decay]; measured $\mathrm{E} \alpha, \mathrm{T}_{1 / 2}$, $\alpha \alpha$-coin for ground and metastable state decay. ${ }^{193,195} \mathrm{Bi},{ }^{197,199} \mathrm{At}$, ${ }^{201,203}$ Fr deduced levels, J, $\pi$. Comparisons with previous results. JOUR PRVCA 71024306

2005UU03 RADIOACTIVITY ${ }^{188,190,192,194,196,198,200,202,204}$ Po, 191,193,195,197,199 At, ${ }^{196,198,200,202,204,206} \mathrm{Rn},{ }^{199,201,203,205,207} \operatorname{Fr}(\alpha)$; measured reduced widths using gas filled recoil separator; deduced hindrance factors, proton intruder states and deformation effects.

JOUR ZAANE 25 s01 179

${ }^{200} \mathrm{Fr}$ 2005DE01 RADIOACTIVITY ${ }^{200,201,203,205} \mathrm{Fr},{ }^{196,197,199,201} \mathrm{At},{ }^{193} \operatorname{Bi}(\alpha)[$ from $\mathrm{Th}(\mathrm{p}, \mathrm{X})$ and subsequent decay]; measured $\mathrm{E} \alpha, \mathrm{T}_{1 / 2} \cdot{ }^{201} \mathrm{Fr},{ }^{197} \mathrm{At}$, ${ }^{193} \mathrm{Bi},{ }^{189} \mathrm{Tl}$ deduced levels, J, $\pi$. JOUR ZAANE 23243

\section{$\mathrm{A}=\mathbf{2 0 1}$}

${ }^{201} \mathrm{Pt}$ 2005CA02 RADIOACTIVITY ${ }^{188} \mathrm{Ta},{ }^{190} \mathrm{~W},{ }^{192,193} \mathrm{Re},{ }^{195} \mathrm{Os},{ }^{197,198} \mathrm{Ir}$, $200,201,202 \mathrm{Pt},{ }^{203} \mathrm{Au}(\mathrm{IT})$ [from $\mathrm{Be}\left({ }^{208} \mathrm{~Pb}, \mathrm{X}\right)$ ]; measured $\mathrm{E} \gamma, \mathrm{I} \gamma, \gamma \gamma$-coin, $\mathrm{T}_{1 / 2} \cdot{ }^{188} \mathrm{Ta},{ }^{190} \mathrm{~W},{ }^{192,193} \mathrm{Re},{ }^{195} \mathrm{Os},{ }^{197,198} \mathrm{Ir},{ }^{200,201,202} \mathrm{Pt},{ }^{203} \mathrm{Au}$ deduced transitions. ${ }^{190} \mathrm{~W},{ }^{200,201,202} \mathrm{Pt}$ deduced levels, J, $\pi .{ }^{174,175} \mathrm{Er}$, ${ }^{185} \mathrm{Hf},{ }^{191,194} \mathrm{Re},{ }^{199} \operatorname{Ir}(\mathrm{IT})$ [from $\left.\mathrm{Be}\left({ }^{208} \mathrm{~Pb}, \mathrm{X}\right)\right]$; measured $\mathrm{E} \gamma, \mathrm{I} \gamma$. JOUR ZAANE 23201

${ }^{201} \mathrm{Bi}$ 2004ADZW NUCLEAR REACTIONS ${ }^{209} \operatorname{Bi}(\mathrm{n}, 4 \mathrm{n}),(\mathrm{n}, 5 \mathrm{n}),(\mathrm{n}, 6 \mathrm{n}),(\mathrm{n}, 7 \mathrm{n}),(\mathrm{n}$, 9n), ${ }^{232} \operatorname{Th}(\mathrm{n}, \gamma),{ }^{197} \mathrm{Au}(\mathrm{n}, 2 \mathrm{n}),(\mathrm{n}, 4 \mathrm{n}),(\mathrm{n}, 6 \mathrm{n}),(\mathrm{n}, 7 \mathrm{n}),(\mathrm{n}, \gamma),{ }^{115} \operatorname{In}(\mathrm{n}$, 5n), (n, 6n), (n, 7n), ${ }^{59} \operatorname{Co}(\mathrm{n}, 2 \mathrm{n}),(\mathrm{n}, 3 \mathrm{n}),(\mathrm{n}, 4 \mathrm{n}),(\mathrm{n}, 5 \mathrm{n}),(\mathrm{n}, \gamma),(\mathrm{n}$, $\mathrm{p}),(\mathrm{n}, 6 \mathrm{n} 2 \mathrm{p}), \mathrm{E}=$ spectrum; measured $\mathrm{E} \gamma, \mathrm{I} \gamma$; deduced reaction rates. $\mathrm{Pb}(\mathrm{p}, \mathrm{nX}), \mathrm{E}=1 \mathrm{GeV}$; deduced spallation neutron spectrum. REPT JINR-E1-2004-16,Adam 


\section{$\mathrm{A}=201$ (continued)}

2004KE15 NUCLEAR REACTIONS ${ }^{1,2} \mathrm{H}, \mathrm{Ti}\left({ }^{208} \mathrm{~Pb}, \mathrm{X}\right){ }^{193} \mathrm{Bi} /{ }^{194} \mathrm{Bi} /{ }^{195} \mathrm{Bi} /$ ${ }^{196} \mathrm{Bi} /{ }^{197} \mathrm{Bi} /{ }^{198} \mathrm{Bi} /{ }^{199} \mathrm{Bi} /{ }^{200} \mathrm{Bi} /{ }^{201} \mathrm{Bi} /{ }^{202} \mathrm{Bi} /{ }^{203} \mathrm{Bi} /{ }^{204} \mathrm{Bi} /$ ${ }^{205} \mathrm{Bi} /{ }^{206} \mathrm{Bi} /{ }^{207} \mathrm{Bi} /{ }^{208} \mathrm{Bi}, \mathrm{E}=1 \mathrm{GeV} /$ nucleon; measured charge-pickup $\sigma$, velocity distributions; deduced reaction mechanism features. Comparison with model predictions and previous results. JOUR PRVCA 70064608

2005AD01 NUCLEAR REACTIONS ${ }^{209} \operatorname{Bi}(\mathrm{n}, 4 \mathrm{n}),(\mathrm{n}, 5 \mathrm{n}),(\mathrm{n}, 6 \mathrm{n}),(\mathrm{n}, 7 \mathrm{n}),(\mathrm{n}$, 9n), ${ }^{232} \mathrm{Th}(\mathrm{n}, \gamma),{ }^{197} \mathrm{Au}(\mathrm{n}, 2 \mathrm{n}),(\mathrm{n}, 4 \mathrm{n}),(\mathrm{n}, 6 \mathrm{n}),(\mathrm{n}, 7 \mathrm{n}),(\mathrm{n}, \gamma),{ }^{59} \mathrm{Co}(\mathrm{n}$, 2n), (n, 3n), (n, 4n), (n, 5n), (n, p), (n, 6n2p), ${ }^{115} \operatorname{In}(\mathrm{n}, 5 \mathrm{n}),(\mathrm{n}, 6 \mathrm{n}),(\mathrm{n}$, $7 \mathrm{n}), \mathrm{E}=$ spectrum; measured $\mathrm{E} \gamma, \mathrm{I} \gamma$; deduced reaction rates. Activation technique, spallation neutrons from $1 \mathrm{GeV}$ proton beam, comparison with model predictions. JOUR ZAANE 2361

${ }^{201}$ Po 2002DU22 RADIOACTIVITY ${ }^{197,197 m, 198,199 m, 200,201 m} \operatorname{Po}(\alpha) ;{ }^{172,173} \operatorname{Os}(\alpha)[$ from $\left.{ }^{156} \mathrm{Dy}\left({ }^{22} \mathrm{Ne}, \mathrm{xn}\right)\right] ;{ }^{183,184,185} \mathrm{Hg}(\alpha)$ [from $\left.{ }^{168} \mathrm{Yb}\left({ }^{22} \mathrm{Ne}, \mathrm{xn}\right)\right]$; measured $\mathrm{E} \alpha, \mathrm{T}_{1 / 2}$. JOUR NIMAE 479631

${ }^{201}$ At 2005DE01 RADIOACTIVITY ${ }^{200,201,203,205} \mathrm{Fr},{ }^{196,197,199,201} \mathrm{At},{ }^{193} \operatorname{Bi}(\alpha)[$ from $\mathrm{Th}(\mathrm{p}, \mathrm{X})$ and subsequent decay]; measured $\mathrm{E} \alpha, \mathrm{T}_{1 / 2} \cdot{ }^{201} \mathrm{Fr},{ }^{197} \mathrm{At}$, ${ }^{193} \mathrm{Bi},{ }^{189} \mathrm{Tl}$ deduced levels, J, $\pi$. JOUR ZAANE 23243

2005UU03 RADIOACTIVITY $188,190,192,194,196,198,200,202,204$ Po, 191,193,195,197,199 At, ${ }^{196,198,200,202,204,206} \mathrm{Rn},{ }^{199,201,203,205,207} \operatorname{Fr}(\alpha)$; measured reduced widths using gas filled recoil separator; deduced hindrance factors, proton intruder states and deformation effects. JOUR ZAANE 25 s01 179

${ }^{201} \mathrm{Rn} \quad$ 2005UU02 NUCLEAR REACTIONS ${ }^{141} \operatorname{Pr}\left({ }^{65} \mathrm{Cu}\right.$, xnypz $\left.\alpha\right), \mathrm{E}=283-293 \mathrm{MeV}$; measured delayed $\mathrm{E} \alpha, \mathrm{I} \alpha, \alpha \alpha$-, (recoil) $\alpha$-coin; deduced evidence for ${ }^{199,200,201} \mathrm{Rn},{ }^{202,203,204} \mathrm{Fr},{ }^{203,204} \mathrm{Ra} .{ }^{141} \mathrm{Pr}\left({ }^{63} \mathrm{Cu}\right.$, xnypz $\left.\alpha\right), \mathrm{E}=278-288$ $\mathrm{MeV}$; measured delayed $\mathrm{E} \alpha, \mathrm{I} \alpha, \alpha \alpha$, (recoil) $\alpha$-coin; deduced evidence for ${ }^{198,199,200,201,202} \mathrm{Rn},{ }^{201,202} \mathrm{Fr},{ }^{201,202} \mathrm{Ra}$. ${ }^{170} \mathrm{Yb}\left({ }^{36} \mathrm{Ar}\right.$, xnypz $\left.\alpha\right)$, $\mathrm{E}=180-185 \mathrm{MeV}$; measured delayed $\mathrm{E} \alpha, \mathrm{I} \alpha, \alpha \alpha-$, (recoil) $\alpha$-coin; deduced evidence for ${ }^{201} \mathrm{Fr},{ }^{203} \mathrm{Ra}$. Gas-filled recoil separator. JOUR PRVCA 71024306

${ }^{201} \mathrm{Fr} \quad$ 2005DE01 RADIOACTIVITY ${ }^{200,201,203,205} \mathrm{Fr},{ }^{196,197,199,201} \mathrm{At},{ }^{193} \operatorname{Bi}(\alpha)$ [from $\mathrm{Th}(\mathrm{p}, \mathrm{X})$ and subsequent decay]; measured $\mathrm{E} \alpha, \mathrm{T}_{1 / 2} \cdot{ }^{201} \mathrm{Fr},{ }^{197} \mathrm{At}$, ${ }^{193} \mathrm{Bi},{ }^{189} \mathrm{Tl}$ deduced levels, J, $\pi$. JOUR ZAANE 23243

2005UU02 NUCLEAR REACTIONS ${ }^{141} \operatorname{Pr}\left({ }^{65} \mathrm{Cu}\right.$, xnypz $\left.\alpha\right), \mathrm{E}=283-293 \mathrm{MeV}$; measured delayed $\mathrm{E} \alpha, \mathrm{I} \alpha, \alpha \alpha-$, (recoil) $\alpha$-coin; deduced evidence for ${ }^{199,200,201} \mathrm{Rn},{ }^{202,203,204} \mathrm{Fr},{ }^{203,204} \mathrm{Ra} .{ }^{141} \operatorname{Pr}\left({ }^{63} \mathrm{Cu}\right.$, xnypz $\left.\alpha\right), \mathrm{E}=278-288$ $\mathrm{MeV}$; measured delayed $\mathrm{E} \alpha, \mathrm{I} \alpha, \alpha \alpha$-, (recoil) $\alpha$-coin; deduced evidence for ${ }^{198,199,200,201,202} \mathrm{Rn},{ }^{201,202} \mathrm{Fr},{ }^{201,202} \mathrm{Ra} .{ }^{170} \mathrm{Yb}\left({ }^{36} \mathrm{Ar}, \mathrm{xnypz} \alpha\right)$, $\mathrm{E}=180-185 \mathrm{MeV}$; measured delayed $\mathrm{E} \alpha, \mathrm{I} \alpha, \alpha \alpha-$, (recoil) $\alpha$-coin; deduced evidence for ${ }^{201} \mathrm{Fr},{ }^{203} \mathrm{Ra}$. Gas-filled recoil separator. JOUR PRVCA 71024306

2005 RU02 RADIOACTIVITY ${ }^{201,202,203,204} \mathrm{Ra},{ }^{197,198,199,200} \mathrm{Rn},{ }^{193,194,195,196}$ Po, ${ }^{201,202,203,204} \mathrm{Fr},{ }^{197,198,199,200} \operatorname{At}(\alpha)$ [from ${ }^{141} \operatorname{Pr}\left({ }^{63,65} \mathrm{Cu}\right.$, xnypz $\left.\alpha\right)$,

${ }^{170} \mathrm{Yb}\left({ }^{36} \mathrm{Ar}, \operatorname{xnypz} \alpha\right)$, and subsequent decay]; measured $\mathrm{E} \alpha, \mathrm{T}_{1 / 2}$, $\alpha \alpha$-coin for ground and metastable state decay. ${ }^{193,195} \mathrm{Bi},{ }^{197,199} \mathrm{At}$, ${ }^{201,203}$ Fr deduced levels, J, $\pi$. Comparisons with previous results. JOUR PRVCA 71024306 


\section{$\mathrm{A}=201$ (continued)}

2005UU03 RADIOACTIVITY ${ }^{188,190,192,194,196,198,200,202,204} \mathrm{Po}$ 191,193,195,197,199 At, ${ }^{196,198,200,202,204,206}$ Rn, ${ }^{199,201,203,205,207} \operatorname{Fr}(\alpha)$; measured reduced widths using gas filled recoil separator; deduced hindrance factors, proton intruder states and deformation effects. JOUR ZAANE 25 s01 179

${ }^{201} \mathrm{Ra} \quad 2005 \mathrm{UU} 02$ NUCLEAR REACTIONS ${ }^{141} \operatorname{Pr}\left({ }^{65} \mathrm{Cu}\right.$, xnypz $\left.\alpha\right), \mathrm{E}=283-293 \mathrm{MeV}$; measured delayed $\mathrm{E} \alpha, \mathrm{I} \alpha, \alpha \alpha$-, (recoil) $\alpha$-coin; deduced evidence for ${ }^{199,200,201} \mathrm{Rn},{ }^{202,203,204} \mathrm{Fr},{ }^{203,204} \mathrm{Ra} .{ }^{141} \mathrm{Pr}\left({ }^{63} \mathrm{Cu}\right.$, xnypz $\left.\alpha\right), \mathrm{E}=278-288$ $\mathrm{MeV}$; measured delayed $\mathrm{E} \alpha, \mathrm{I} \alpha, \alpha \alpha-$, (recoil) $\alpha$-coin; deduced evidence for ${ }^{198,199,200,201,202} \mathrm{Rn},{ }^{201,202} \mathrm{Fr},{ }^{201,202} \mathrm{Ra} .{ }^{170} \mathrm{Yb}\left({ }^{36} \mathrm{Ar}\right.$, xnypz $\left.\alpha\right)$, $\mathrm{E}=180-185 \mathrm{MeV}$; measured delayed $\mathrm{E} \alpha, \mathrm{I} \alpha, \alpha \alpha-$, (recoil) $\alpha$-coin; deduced evidence for ${ }^{201} \mathrm{Fr},{ }^{203} \mathrm{Ra}$. Gas-filled recoil separator. JOUR PRVCA 71024306

2005UU02 RADIOACTIVITY ${ }^{201,202,203,204} \mathrm{Ra},{ }^{197,198,199,200} \mathrm{Rn},{ }^{193,194,195,196}$ Po, ${ }^{201,202,203,204} \mathrm{Fr},{ }^{197,198,199,200} \operatorname{At}(\alpha)$ [from ${ }^{141} \operatorname{Pr}\left({ }^{63,65} \mathrm{Cu}\right.$, xnypz $\left.\alpha\right)$, ${ }^{170} \mathrm{Yb}\left({ }^{36} \mathrm{Ar}, \mathrm{xnypz} \alpha\right)$, and subsequent decay]; measured $\mathrm{E} \alpha, \mathrm{T}_{1 / 2}$, $\alpha \alpha$-coin for ground and metastable state decay. ${ }^{193,195} \mathrm{Bi},{ }^{197,199} \mathrm{At}$, ${ }^{201,203}$ Fr deduced levels, J, $\pi$. Comparisons with previous results. JOUR PRVCA 71024306

\section{$A=202$}

${ }^{202} \mathrm{Pt} \quad 2005 \mathrm{CA02}$

2004KE15

${ }^{202} \mathrm{Po}$

2005 UU03

${ }^{202} \mathrm{Rn} \quad 2005 \mathrm{UU} 02$
RADIOACTIVITY ${ }^{188} \mathrm{Ta},{ }^{190} \mathrm{~W},{ }^{192,193} \mathrm{Re},{ }^{195} \mathrm{Os},{ }^{197,198} \mathrm{Ir}$, $200,201,202 \mathrm{Pt},{ }^{203} \mathrm{Au}(\mathrm{IT})$ [from $\mathrm{Be}\left({ }^{208} \mathrm{~Pb}, \mathrm{X}\right)$ ]; measured $\mathrm{E} \gamma, \mathrm{I} \gamma, \gamma \gamma$-coin, $\mathrm{T}_{1 / 2} \cdot{ }^{188} \mathrm{Ta},{ }^{190} \mathrm{~W},{ }^{192,193} \mathrm{Re},{ }^{195} \mathrm{Os},{ }^{197,198} \mathrm{Ir},{ }^{200,201,202} \mathrm{Pt},{ }^{203} \mathrm{Au}$ deduced transitions. ${ }^{190} \mathrm{~W},{ }^{200,201,202} \mathrm{Pt}$ deduced levels, J, $\pi .{ }^{174,175} \mathrm{Er}$, ${ }^{185} \mathrm{Hf},{ }^{191,194} \mathrm{Re},{ }^{199} \operatorname{Ir}(\mathrm{IT})$ [from $\mathrm{Be}\left({ }^{208} \mathrm{~Pb}, \mathrm{X}\right)$ ]; measured $\mathrm{E} \gamma, \mathrm{I} \gamma$.

JOUR ZAANE 23201

NUCLEAR REACTIONS ${ }^{1,2} \mathrm{H}$, Ti $\left({ }^{208} \mathrm{~Pb}, \mathrm{X}\right){ }^{193} \mathrm{Bi} /{ }^{194} \mathrm{Bi} /{ }^{195} \mathrm{Bi} /$ ${ }^{196} \mathrm{Bi} /{ }^{197} \mathrm{Bi} /{ }^{198} \mathrm{Bi} /{ }^{199} \mathrm{Bi} /{ }^{200} \mathrm{Bi} /{ }^{201} \mathrm{Bi} /{ }^{202} \mathrm{Bi} /{ }^{203} \mathrm{Bi} /{ }^{204} \mathrm{Bi} /$ ${ }^{205} \mathrm{Bi} /{ }^{206} \mathrm{Bi} /{ }^{207} \mathrm{Bi} /{ }^{208} \mathrm{Bi}, \mathrm{E}=1 \mathrm{GeV} /$ nucleon; measured charge-pickup $\sigma$, velocity distributions; deduced reaction mechanism features. Comparison with model predictions and previous results. JOUR PRVCA 70064608 RADIOACTIVITY ${ }^{188,190,192,194,196,198,200,202,204} \mathrm{Po}$, 191,193,195,197,199 At, ${ }^{196,198,200,202,204,206} \mathrm{Rn},{ }^{199,201,203,205,207} \operatorname{Fr}(\alpha)$; measured reduced widths using gas filled recoil separator; deduced hindrance factors, proton intruder states and deformation effects. JOUR ZAANE 25 s01 179 NUCLEAR REACTIONS ${ }^{141} \operatorname{Pr}\left({ }^{65} \mathrm{Cu}\right.$, xnypz $\left.\alpha\right), \mathrm{E}=283-293 \mathrm{MeV}$; measured delayed $\mathrm{E} \alpha, \mathrm{I} \alpha, \alpha \alpha-$, (recoil) $\alpha$-coin; deduced evidence for ${ }^{199,200,201} \mathrm{Rn},{ }^{202,203,204} \mathrm{Fr},{ }^{203,204} \mathrm{Ra} .{ }^{141} \operatorname{Pr}\left({ }^{63} \mathrm{Cu}\right.$, xnypz $\left.\alpha\right), \mathrm{E}=278-288$ $\mathrm{MeV}$; measured delayed E $\alpha, \mathrm{I} \alpha, \alpha \alpha-$, (recoil) $\alpha$-coin; deduced evidence for ${ }^{198,199,200,201,202} \mathrm{Rn},{ }^{201,202} \mathrm{Fr},{ }^{201,202} \mathrm{Ra} .{ }^{170} \mathrm{Yb}\left({ }^{36} \mathrm{Ar}\right.$, xnypz $\left.\alpha\right)$, $\mathrm{E}=180-185 \mathrm{MeV}$; measured delayed $\mathrm{E} \alpha, \mathrm{I} \alpha, \alpha \alpha-$, (recoil) $\alpha$-coin; deduced evidence for ${ }^{201} \mathrm{Fr},{ }^{203} \mathrm{Ra}$. Gas-filled recoil separator. JOUR PRVCA 71024306 


\section{$\mathrm{A}=202$ (continued)}

2005UU03 RADIOACTIVITY ${ }^{188,190,192,194,196,198,200,202,204} \mathrm{Po}$ 191,193,195,197,199 At, ${ }^{196,198,200,202,204,206}$ Rn, ${ }^{199,201,203,205,207} \operatorname{Fr}(\alpha)$; measured reduced widths using gas filled recoil separator; deduced hindrance factors, proton intruder states and deformation effects. JOUR ZAANE 25 s01 179

${ }^{202} \mathrm{Fr} \quad 2005 \mathrm{UU} 02$

2005UU02

2005UU02

2005UU02 NUCLEAR REACTIONS ${ }^{141} \operatorname{Pr}\left({ }^{65} \mathrm{Cu}\right.$, xnypz $\left.\alpha\right), \mathrm{E}=283-293 \mathrm{MeV}$; measured delayed $\mathrm{E} \alpha$, I $\alpha, \alpha \alpha-$, (recoil) $\alpha$-coin; deduced evidence for ${ }^{199,200,201} \mathrm{Rn},{ }^{202,203,204} \mathrm{Fr},{ }^{203,204} \mathrm{Ra} .{ }^{141} \mathrm{Pr}\left({ }^{63} \mathrm{Cu}\right.$, xnypz $\left.\alpha\right), \mathrm{E}=278-288$ $\mathrm{MeV}$; measured delayed E $\alpha, \mathrm{I} \alpha, \alpha \alpha-$, (recoil) $\alpha$-coin; deduced evidence for ${ }^{198,199,200,201,202} \mathrm{Rn},{ }^{201,202} \mathrm{Fr},{ }^{201,202} \mathrm{Ra} .{ }^{170} \mathrm{Yb}\left({ }^{36} \mathrm{Ar}\right.$, xnypz $\left.\alpha\right)$, $\mathrm{E}=180-185 \mathrm{MeV}$; measured delayed $\mathrm{E} \alpha, \mathrm{I} \alpha, \alpha \alpha-$, (recoil) $\alpha$-coin; deduced evidence for ${ }^{201} \mathrm{Fr},{ }^{203} \mathrm{Ra}$. Gas-filled recoil separator. JOUR PRVCA 71024306 RADIOACTIVITY ${ }^{201,202,203,204} \mathrm{Ra},{ }^{197,198,199,200} \mathrm{Rn},{ }^{193,194,195,196} \mathrm{Po}$, ${ }^{201,202,203,204} \mathrm{Fr},{ }^{197,198,199,200} \operatorname{At}(\alpha)$ [from ${ }^{141} \operatorname{Pr}\left({ }^{63,65} \mathrm{Cu}\right.$, xnypz $\left.\alpha\right)$, ${ }^{170} \mathrm{Yb}\left({ }^{36} \mathrm{Ar}, \mathrm{xnypz} \alpha\right)$, and subsequent decay]; measured $\mathrm{E} \alpha, \mathrm{T}_{1 / 2}$, $\alpha \alpha$-coin for ground and metastable state decay. ${ }^{193,195} \mathrm{Bi},{ }^{197,199} \mathrm{At}$, ${ }^{201,203}$ Fr deduced levels, J, $\pi$. Comparisons with previous results. JOUR PRVCA 71024306 NUCLEAR REACTIONS ${ }^{141} \operatorname{Pr}\left({ }^{65} \mathrm{Cu}\right.$, xnypz $\left.\alpha\right), \mathrm{E}=283-293 \mathrm{MeV}$; measured delayed $\mathrm{E} \alpha, \mathrm{I} \alpha, \alpha \alpha-$, (recoil) $\alpha$-coin; deduced evidence for ${ }^{199,200,201} \mathrm{Rn},{ }^{202,203,204} \mathrm{Fr},{ }^{203,204}$ Ra. ${ }^{141} \operatorname{Pr}\left({ }^{63} \mathrm{Cu}\right.$, xnypz $\left.\alpha\right), \mathrm{E}=278-288$ $\mathrm{MeV}$; measured delayed E $\alpha, \mathrm{I} \alpha, \alpha \alpha-$, (recoil) $\alpha$-coin; deduced evidence for ${ }^{198,199,200,201,202} \mathrm{Rn},{ }^{201,202} \mathrm{Fr},{ }^{201,202} \mathrm{Ra} .{ }^{170} \mathrm{Yb}\left({ }^{36} \mathrm{Ar}, \mathrm{xnypz} \alpha\right)$, $\mathrm{E}=180-185 \mathrm{MeV}$; measured delayed $\mathrm{E} \alpha, \mathrm{I} \alpha, \alpha \alpha$-, (recoil) $\alpha$-coin; deduced evidence for ${ }^{201} \mathrm{Fr},{ }^{203} \mathrm{Ra}$. Gas-filled recoil separator. JOUR PRVCA 71024306 RADIOACTIVITY ${ }^{201,202,203,204} \mathrm{Ra},{ }^{197,198,199,200} \mathrm{Rn},{ }^{193,194,195,196} \mathrm{Po}$, ${ }^{201,202,203,204} \mathrm{Fr},{ }^{197,198,199,200} \operatorname{At}(\alpha)\left[\right.$ from ${ }^{141} \operatorname{Pr}\left({ }^{63,65} \mathrm{Cu}\right.$, xnypz $\left.\alpha\right)$, ${ }^{170} \mathrm{Yb}\left({ }^{36} \mathrm{Ar}\right.$, xnypz $\left.\alpha\right)$, and subsequent decay]; measured $\mathrm{E} \alpha, \mathrm{T}_{1 / 2}$, $\alpha \alpha$-coin for ground and metastable state decay. ${ }^{193,195} \mathrm{Bi},{ }^{197,199} \mathrm{At}$, ${ }^{201,203} \mathrm{Fr}$ deduced levels, J, $\pi$. Comparisons with previous results. JOUR PRVCA 71024306

\section{$\mathrm{A}=\mathbf{2 0 3}$}

${ }^{203} \mathrm{Au} \quad 2005 \mathrm{CA} 02$

RADIOACTIVITY ${ }^{188} \mathrm{Ta},{ }^{190} \mathrm{~W},{ }^{192,193} \mathrm{Re},{ }^{195} \mathrm{Os},{ }^{197,198} \mathrm{Ir}$, $200,201,202 \mathrm{Pt},{ }^{203} \mathrm{Au}(\mathrm{IT})$ [from $\mathrm{Be}\left({ }^{208} \mathrm{~Pb}, \mathrm{X}\right)$ ]; measured $\mathrm{E} \gamma, \mathrm{I} \gamma, \gamma \gamma$-coin, $\mathrm{T}_{1 / 2} \cdot{ }^{188} \mathrm{Ta},{ }^{190} \mathrm{~W},{ }^{192,193} \mathrm{Re},{ }^{195} \mathrm{Os},{ }^{197,198} \mathrm{Ir},{ }^{200,201,202} \mathrm{Pt},{ }^{203} \mathrm{Au}$ deduced transitions. ${ }^{190} \mathrm{~W},{ }^{200,201,202} \mathrm{Pt}$ deduced levels, J, $\pi .{ }^{174,175} \mathrm{Er}$, ${ }^{185} \mathrm{Hf},{ }^{191,194} \mathrm{Re},{ }^{199} \operatorname{Ir}(\mathrm{IT})$ [from $\mathrm{Be}\left({ }^{208} \mathrm{~Pb}, \mathrm{X}\right)$ ]; measured $\mathrm{E} \gamma, \mathrm{I} \gamma$. JOUR ZAANE 23201 NUCLEAR MOMENTS ${ }^{113,115} \mathrm{In},{ }^{153,155} \mathrm{Eu},{ }^{185,187} \mathrm{Re},{ }^{203,205} \mathrm{Tl}$, ${ }^{209,211} \mathrm{Fr}$; measured hfs; deduced hyperfine magnetic anomaly, relative radii. Laser resonance fluorescence. JOUR BRSPE 68157 NUCLEAR REACTIONS ${ }^{190,192,198} \mathrm{Pt},{ }^{196,198,204} \mathrm{Hg},{ }^{204} \mathrm{~Pb}(\gamma, \mathrm{n})$, $\mathrm{E}=$ spectrum; measured reaction rates. Astrophysical implications discussed, comparison with model predictions. JOUR NUPAB 758 521c 


\section{$\mathrm{A}=203$ (continued)}

2005TIZX NUCLEAR REACTIONS Pb, ${ }^{208} \mathrm{~Pb}(\mathrm{p}, \mathrm{X}){ }^{203} \mathrm{~Pb} /{ }^{200} \mathrm{Tl} /{ }^{199} \mathrm{Tl} /$ ${ }^{196} \mathrm{Au} /{ }^{192} \mathrm{Ir} /{ }^{190} \mathrm{Ir} /{ }^{173} \mathrm{Lu} /{ }^{101 m} \mathrm{Rh} /{ }^{86} \mathrm{Rb} /{ }^{59} \mathrm{Fe} /{ }^{24} \mathrm{Na} /{ }^{7} \mathrm{Be}$, $\mathrm{E}=40-2600 \mathrm{MeV}$; measured excitation functions. Comparison with previous work and model predictions. Other reactions discussed. CONF Santa Fe (Nucl Data for Sci and Technol) Proc,Vol1,P1070 2005TIZY NUCLEAR REACTIONS Pb, ${ }^{208} \mathrm{~Pb},{ }^{209} \mathrm{Bi}(\mathrm{p}, \mathrm{X}){ }^{203} \mathrm{~Pb} /{ }^{200} \mathrm{Tl} /{ }^{199} \mathrm{Tl}$ $/{ }^{196} \mathrm{Au} /{ }^{192} \mathrm{Ir} /{ }^{190} \mathrm{Ir} /{ }^{173} \mathrm{Lu} /{ }^{101 m} \mathrm{Rh} /{ }^{86} \mathrm{Rb} /{ }^{59} \mathrm{Fe} /{ }^{24} \mathrm{Na} /{ }^{7} \mathrm{Be}$, $\mathrm{E}=40-2600 \mathrm{MeV}$; measured production $\sigma$. Comparison with model predictions. PREPRINT nucl-ex/0507009,7/05/2005 ${ }^{203} \mathrm{Bi} \quad$ 2004ADZW
$\quad$ NUCLEAR REACTIONS ${ }^{209} \mathrm{Bi}(\mathrm{n}, 4 \mathrm{n}),(\mathrm{n}, 5 \mathrm{n}),(\mathrm{n}, 6 \mathrm{n}),(\mathrm{n}, 7 \mathrm{n}),(\mathrm{n}$, 5n), (n, 6n), (n, 7n), ${ }^{59} \operatorname{Co}(\mathrm{n}, 2 \mathrm{n}),(\mathrm{n}, 3 \mathrm{n}),(\mathrm{n}, 4 \mathrm{n}),(\mathrm{n}, 5 \mathrm{n}),(\mathrm{n}, \gamma),(\mathrm{n}$, $\mathrm{p}),(\mathrm{n}, 6 \mathrm{n} 2 \mathrm{p}), \mathrm{E}=$ spectrum; measured $\mathrm{E} \gamma, \mathrm{I} \gamma$; deduced reaction rates. $\mathrm{Pb}(\mathrm{p}, \mathrm{nX}), \mathrm{E}=1 \mathrm{GeV}$; deduced spallation neutron spectrum. REPT JINR-E1-2004-16,Adam

2004KE15 NUCLEAR REACTIONS ${ }^{1,2} \mathrm{H}, \mathrm{Ti}\left({ }^{208} \mathrm{~Pb}, \mathrm{X}\right){ }^{193} \mathrm{Bi} /{ }^{194} \mathrm{Bi} /{ }^{195} \mathrm{Bi} /$ ${ }^{196} \mathrm{Bi} /{ }^{197} \mathrm{Bi} /{ }^{198} \mathrm{Bi} /{ }^{199} \mathrm{Bi} /{ }^{200} \mathrm{Bi} /{ }^{201} \mathrm{Bi} /{ }^{202} \mathrm{Bi} /{ }^{203} \mathrm{Bi} /{ }^{204} \mathrm{Bi} /$ ${ }^{205} \mathrm{Bi} /{ }^{206} \mathrm{Bi} /{ }^{207} \mathrm{Bi} /{ }^{208} \mathrm{Bi}, \mathrm{E}=1 \mathrm{GeV} /$ nucleon; measured charge-pickup $\sigma$, velocity distributions; deduced reaction mechanism features. Comparison with model predictions and previous results. JOUR PRVCA 70064608

2005AD01 NUCLEAR REACTIONS ${ }^{209} \operatorname{Bi}(\mathrm{n}, 4 \mathrm{n}),(\mathrm{n}, 5 \mathrm{n}),(\mathrm{n}, 6 \mathrm{n}),(\mathrm{n}, 7 \mathrm{n}),(\mathrm{n}$, 9n), ${ }^{232} \mathrm{Th}(\mathrm{n}, \gamma),{ }^{197} \mathrm{Au}(\mathrm{n}, 2 \mathrm{n}),(\mathrm{n}, 4 \mathrm{n}),(\mathrm{n}, 6 \mathrm{n}),(\mathrm{n}, 7 \mathrm{n}),(\mathrm{n}, \gamma),{ }^{59} \mathrm{Co}(\mathrm{n}$, 2n), (n, 3n), (n, 4n), (n, 5n), (n, p), (n, 6n2p), ${ }^{115} \operatorname{In}(\mathrm{n}, 5 \mathrm{n}),(\mathrm{n}, 6 \mathrm{n}),(\mathrm{n}$, $7 \mathrm{n}), \mathrm{E}=$ spectrum; measured $\mathrm{E} \gamma, \mathrm{I} \gamma$; deduced reaction rates. Activation technique, spallation neutrons from $1 \mathrm{GeV}$ proton beam, comparison with model predictions. JOUR ZAANE 2361

2005MU21 NUCLEAR REACTIONS ${ }^{115} \mathrm{In}(\mathrm{n}, \mathrm{n}),{ }^{27} \mathrm{Al}(\mathrm{n}, \alpha),{ }^{93} \mathrm{Nb}(\mathrm{n}, 2 \mathrm{n}),(\mathrm{n}, 4 \mathrm{n})$, ${ }^{209} \operatorname{Bi}(\mathrm{n}, 4 \mathrm{n}),(\mathrm{n}, 5 \mathrm{n}),(\mathrm{n}, 6 \mathrm{n}),(\mathrm{n}, 7 \mathrm{n}), \mathrm{E} \approx 10-1000 \mathrm{MeV}$; measured reaction rates. Comparison with model predictions. JOUR NIMAE 547 555

${ }^{203}$ At 2005UU03 RADIOACTIVITY ${ }^{188,190,192,194,196,198,200,202,204} \mathrm{Po}$, $191,193,195,197,199$ At, ${ }^{196,198,200,202,204,206} \mathrm{Rn},{ }^{199,201,203,205,207} \operatorname{Fr}(\alpha)$; measured reduced widths using gas filled recoil separator; deduced hindrance factors, proton intruder states and deformation effects.

JOUR ZAANE 25 s01 179

${ }^{203} \mathrm{Fr}$ 2005DE01 RADIOACTIVITY ${ }^{200,201,203,205} \mathrm{Fr},{ }^{196,197,199,201} \mathrm{At},{ }^{193} \operatorname{Bi}(\alpha)[$ from $\mathrm{Th}(\mathrm{p}, \mathrm{X})$ and subsequent decay]; measured $\mathrm{E} \alpha, \mathrm{T}_{1 / 2} \cdot{ }^{201} \mathrm{Fr},{ }^{197} \mathrm{At}$, ${ }^{193} \mathrm{Bi},{ }^{189} \mathrm{Tl}$ deduced levels, J, $\pi$. JOUR ZAANE 23243

2005 UU02 NUCLEAR REACTIONS ${ }^{141} \operatorname{Pr}\left({ }^{65} \mathrm{Cu}\right.$, xnypz $\left.\alpha\right), \mathrm{E}=283-293 \mathrm{MeV}$; measured delayed $\mathrm{E} \alpha, \mathrm{I} \alpha, \alpha \alpha$-, (recoil) $\alpha$-coin; deduced evidence for ${ }^{199,200,201} \mathrm{Rn},{ }^{202,203,204} \mathrm{Fr},{ }^{203,204} \mathrm{Ra} .{ }^{141} \mathrm{Pr}\left({ }^{63} \mathrm{Cu}\right.$, xnypz $\left.\alpha\right), \mathrm{E}=278-288$ $\mathrm{MeV}$; measured delayed $\mathrm{E} \alpha, \mathrm{I} \alpha, \alpha \alpha-$, (recoil) $\alpha$-coin; deduced evidence for ${ }^{198,199,200,201,202} \mathrm{Rn},{ }^{201,202} \mathrm{Fr},{ }^{201,202} \mathrm{Ra} .{ }^{170} \mathrm{Yb}\left({ }^{36} \mathrm{Ar}\right.$, xnypz $\alpha$ ), $\mathrm{E}=180-185 \mathrm{MeV}$; measured delayed $\mathrm{E} \alpha, \mathrm{I} \alpha, \alpha \alpha-$, (recoil) $\alpha$-coin; deduced evidence for ${ }^{201} \mathrm{Fr},{ }^{203} \mathrm{Ra}$. Gas-filled recoil separator. JOUR PRVCA 71024306 


\section{$\mathrm{A}=203$ (continued)}

2005UU02

2005UU03

${ }^{203} \mathrm{Ra}$

2005UU02

2005UU02

\section{$\mathrm{A}=\mathbf{2 0 4}$}

$\begin{array}{ll}{ }^{204} \mathrm{~Pb} & 2005 W A 34 \\ { }^{204} \mathrm{Bi} & 2004 \text { ADZW }\end{array}$

2004KE15

2005AD01 JOUR PRVCA 71024306 JOUR ZAANE 25 s01 179 PRVCA 71024306 JOUR PRVCA 71024306 JOUR ZDDNE 36249 JINR-E1-2004-16,Adam JOUR PRVCA 70064608
RADIOACTIVITY ${ }^{201,202,203,204} \mathrm{Ra},{ }^{197,198,199,200} \mathrm{Rn},{ }^{193,194,195,196} \mathrm{Po}$, ${ }^{201,202,203,204} \mathrm{Fr},{ }^{197,198,199,200} \operatorname{At}(\alpha)$ [from ${ }^{141} \operatorname{Pr}\left({ }^{63,65} \mathrm{Cu}\right.$, xnypz $\left.\alpha\right)$, ${ }^{170} \mathrm{Yb}\left({ }^{36} \mathrm{Ar}, \mathrm{xnypz} \alpha\right)$, and subsequent decay]; measured $\mathrm{E} \alpha, \mathrm{T}_{1 / 2}$, $\alpha \alpha$-coin for ground and metastable state decay. ${ }^{193,195} \mathrm{Bi},{ }^{197,199} \mathrm{At}$, ${ }^{201,203} \mathrm{Fr}$ deduced levels, J, $\pi$. Comparisons with previous results. RADIOACTIVITY 188,190,192,194,196,198,200,202,204 Po, 191,193,195,197,199 At, ${ }^{196,198,200,202,204,206} \mathrm{Rn},{ }^{199,201,203,205,207} \operatorname{Fr}(\alpha)$; measured reduced widths using gas filled recoil separator; deduced hindrance factors, proton intruder states and deformation effects. NUCLEAR REACTIONS ${ }^{141} \operatorname{Pr}\left({ }^{65} \mathrm{Cu}\right.$, xnypz $\left.\alpha\right), \mathrm{E}=283-293 \mathrm{MeV}$; measured delayed $\mathrm{E} \alpha, \mathrm{I} \alpha, \alpha \alpha-$, (recoil) $\alpha$-coin; deduced evidence for ${ }^{199,200,201} \mathrm{Rn},{ }^{202,203,204} \mathrm{Fr},{ }^{203,204} \mathrm{Ra} .{ }^{141} \mathrm{Pr}\left({ }^{63} \mathrm{Cu}\right.$, xnypz $\left.\alpha\right), \mathrm{E}=278-288$ $\mathrm{MeV}$; measured delayed $\mathrm{E} \alpha, \mathrm{I} \alpha, \alpha \alpha-$, (recoil) $\alpha$-coin; deduced evidence for ${ }^{198,199,200,201,202} \mathrm{Rn},{ }^{201,202} \mathrm{Fr},{ }^{201,202} \mathrm{Ra} .{ }^{170} \mathrm{Yb}\left({ }^{36} \mathrm{Ar}, \mathrm{xnypz} \alpha\right)$, $\mathrm{E}=180-185 \mathrm{MeV}$; measured delayed $\mathrm{E} \alpha, \mathrm{I} \alpha, \alpha \alpha$-, (recoil) $\alpha$-coin; deduced evidence for ${ }^{201} \mathrm{Fr},{ }^{203} \mathrm{Ra}$. Gas-filled recoil separator. JOUR

RADIOACTIVITY ${ }^{201,202,203,204} \mathrm{Ra},{ }^{197,198,199,200} \mathrm{Rn},{ }^{193,194,195,196}$ Po, ${ }^{201,202,203,204} \mathrm{Fr},{ }^{197,198,199,200} \operatorname{At}(\alpha)$ [from ${ }^{141} \operatorname{Pr}\left({ }^{63,65} \mathrm{Cu}\right.$, xnypz $\left.\alpha\right)$,

${ }^{170} \mathrm{Yb}\left({ }^{36} \mathrm{Ar}, \mathrm{xnypz} \alpha\right)$, and subsequent decay]; measured $\mathrm{E} \alpha, \mathrm{T}_{1 / 2}$, $\alpha \alpha$-coin for ground and metastable state decay. ${ }^{193,195} \mathrm{Bi},{ }^{197,199} \mathrm{At}$, ${ }^{201,203}$ Fr deduced levels, J, $\pi$. Comparisons with previous results.

NUCLEAR MOMENTS ${ }^{204,206,207,208} \mathrm{~Pb}$; measured hfs, isotope shifts. NUCLEAR REACTIONS ${ }^{209} \operatorname{Bi}(\mathrm{n}, 4 \mathrm{n}),(\mathrm{n}, 5 \mathrm{n}),(\mathrm{n}, 6 \mathrm{n}),(\mathrm{n}, 7 \mathrm{n}),(\mathrm{n}$, $9 \mathrm{n}),{ }^{232} \operatorname{Th}(\mathrm{n}, \gamma),{ }^{197} \mathrm{Au}(\mathrm{n}, 2 \mathrm{n}),(\mathrm{n}, 4 \mathrm{n}),(\mathrm{n}, 6 \mathrm{n}),(\mathrm{n}, 7 \mathrm{n}),(\mathrm{n}, \gamma),{ }^{115} \operatorname{In}(\mathrm{n}$, 5n), (n, 6n), (n, 7n), ${ }^{59} \operatorname{Co}(\mathrm{n}, 2 \mathrm{n}),(\mathrm{n}, 3 \mathrm{n}),(\mathrm{n}, 4 \mathrm{n}),(\mathrm{n}, 5 \mathrm{n}),(\mathrm{n}, \gamma),(\mathrm{n}$, $\mathrm{p}),(\mathrm{n}, 6 \mathrm{n} 2 \mathrm{p}), \mathrm{E}=$ spectrum; measured $\mathrm{E} \gamma, \mathrm{I} \gamma$; deduced reaction rates. $\mathrm{Pb}(\mathrm{p}, \mathrm{nX}), \mathrm{E}=1 \mathrm{GeV}$; deduced spallation neutron spectrum. REPT NUCLEAR REACTIONS ${ }^{1,2} \mathrm{H}, \mathrm{Ti}\left({ }^{208} \mathrm{~Pb}, \mathrm{X}\right){ }^{193} \mathrm{Bi} /{ }^{194} \mathrm{Bi} /{ }^{195} \mathrm{Bi} /$ ${ }^{196} \mathrm{Bi} /{ }^{197} \mathrm{Bi} /{ }^{198} \mathrm{Bi} /{ }^{199} \mathrm{Bi} /{ }^{200} \mathrm{Bi} /{ }^{201} \mathrm{Bi} /{ }^{202} \mathrm{Bi} /{ }^{203} \mathrm{Bi} /{ }^{204} \mathrm{Bi} /$ ${ }^{205} \mathrm{Bi} /{ }^{206} \mathrm{Bi} /{ }^{207} \mathrm{Bi} /{ }^{208} \mathrm{Bi}, \mathrm{E}=1 \mathrm{GeV} /$ nucleon; measured charge-pickup $\sigma$, velocity distributions; deduced reaction mechanism features. Comparison with model predictions and previous results. NUCLEAR REACTIONS ${ }^{209} \operatorname{Bi}(\mathrm{n}, 4 \mathrm{n}),(\mathrm{n}, 5 \mathrm{n}),(\mathrm{n}, 6 \mathrm{n}),(\mathrm{n}, 7 \mathrm{n}),(\mathrm{n}$, 9n), ${ }^{232} \operatorname{Th}(\mathrm{n}, \gamma),{ }^{197} \mathrm{Au}(\mathrm{n}, 2 \mathrm{n}),(\mathrm{n}, 4 \mathrm{n}),(\mathrm{n}, 6 \mathrm{n}),(\mathrm{n}, 7 \mathrm{n}),(\mathrm{n}, \gamma),{ }^{59} \mathrm{Co}(\mathrm{n}$, 2n), (n, 3n), (n, 4n), (n, 5n), (n, p), (n, 6n2p), ${ }^{115} \operatorname{In}(\mathrm{n}, 5 \mathrm{n}),(\mathrm{n}, 6 \mathrm{n}),(\mathrm{n}$, $7 \mathrm{n}), \mathrm{E}=$ spectrum; measured $\mathrm{E} \gamma, \mathrm{I} \gamma$; deduced reaction rates. Activation technique, spallation neutrons from $1 \mathrm{GeV}$ proton beam, comparison with model predictions. JOUR ZAANE 2361 


\section{$\mathrm{A}=204$ (continued)}

2005MU21 NUCLEAR REACTIONS ${ }^{115} \mathrm{In}(\mathrm{n}, \mathrm{n}),{ }^{27} \mathrm{Al}(\mathrm{n}, \alpha),{ }^{93} \mathrm{Nb}(\mathrm{n}, 2 \mathrm{n}),(\mathrm{n}, 4 \mathrm{n})$, ${ }^{209} \operatorname{Bi}(\mathrm{n}, 4 \mathrm{n}),(\mathrm{n}, 5 \mathrm{n}),(\mathrm{n}, 6 \mathrm{n}),(\mathrm{n}, 7 \mathrm{n}), \mathrm{E} \approx 10-1000 \mathrm{MeV}$; measured reaction rates. Comparison with model predictions. JOUR NIMAE 547 555

${ }^{204} \mathrm{Po}$ 2005UU03 RADIOACTIVITY ${ }^{188,190,192,194,196,198,200,202,204}$ Po, 191,193,195,197,199 At, ${ }^{196,198,200,202,204,206}$ Rn, ${ }^{199,201,203,205,207} \operatorname{Fr}(\alpha)$; measured reduced widths using gas filled recoil separator; deduced hindrance factors, proton intruder states and deformation effects. JOUR ZAANE 25 s01 179

${ }^{204} \mathrm{Rn} \quad 2005 \mathrm{UU} 03$ RADIOACTIVITY ${ }^{188,190,192,194,196,198,200,202,204}$ Po, 191,193,195,197,199 At, ${ }^{196,198,200,202,204,206} \mathrm{Rn},{ }^{199,201,203,205,207} \operatorname{Fr}(\alpha)$; measured reduced widths using gas filled recoil separator; deduced hindrance factors, proton intruder states and deformation effects. JOUR ZAANE 25 s01 179

${ }^{204} \mathrm{Fr} \quad 2005 \mathrm{UU} 02$ NUCLEAR REACTIONS ${ }^{141} \operatorname{Pr}\left({ }^{65} \mathrm{Cu}\right.$, xnypz $\left.\alpha\right), \mathrm{E}=283-293 \mathrm{MeV}$; measured delayed $\mathrm{E} \alpha, \mathrm{I} \alpha, \alpha \alpha-$, (recoil) $\alpha$-coin; deduced evidence for ${ }^{199,200,201} \mathrm{Rn},{ }^{202,203,204} \mathrm{Fr},{ }^{203,204} \mathrm{Ra} .{ }^{141} \mathrm{Pr}\left({ }^{63} \mathrm{Cu}\right.$, xnypz $\left.\alpha\right), \mathrm{E}=278-288$

$\mathrm{MeV}$; measured delayed $\mathrm{E} \alpha, \mathrm{I} \alpha, \alpha \alpha-$, (recoil) $\alpha$-coin; deduced evidence for ${ }^{198,199,200,201,202} \mathrm{Rn},{ }^{201,202} \mathrm{Fr},{ }^{201,202} \mathrm{Ra} .{ }^{170} \mathrm{Yb}\left({ }^{36} \mathrm{Ar}, \mathrm{xnypz} \alpha\right)$, $\mathrm{E}=180-185 \mathrm{MeV}$; measured delayed $\mathrm{E} \alpha, \mathrm{I} \alpha, \alpha \alpha-$, (recoil) $\alpha$-coin; deduced evidence for ${ }^{201} \mathrm{Fr},{ }^{203} \mathrm{Ra}$. Gas-filled recoil separator. JOUR PRVCA 71024306

2005 RUO2 RIOACTIVITY ${ }^{201,202,203,204} \mathrm{Ra},{ }^{197,198,199,200} \mathrm{Rn},{ }^{193,194,195,196}$ Po, ${ }^{201,202,203,204} \mathrm{Fr},{ }^{197,198,199,200} \operatorname{At}(\alpha)$ [from ${ }^{141} \operatorname{Pr}\left({ }^{63,65} \mathrm{Cu}\right.$, xnypz $\left.\alpha\right)$, ${ }^{170} \mathrm{Yb}\left({ }^{36} \mathrm{Ar}, \mathrm{xnypz} \alpha\right)$, and subsequent decay]; measured $\mathrm{E} \alpha, \mathrm{T}_{1 / 2}$, $\alpha \alpha$-coin for ground and metastable state decay. ${ }^{193,195} \mathrm{Bi},{ }^{197,199} \mathrm{At}$, ${ }^{201,203} \mathrm{Fr}$ deduced levels, J, $\pi$. Comparisons with previous results. JOUR PRVCA 71024306

${ }^{204} \mathrm{Ra} \quad 2005 U U 02 \quad$ NUCLEAR REACTIONS ${ }^{141} \operatorname{Pr}\left({ }^{65} \mathrm{Cu}\right.$, xnypz $\left.\alpha\right), \mathrm{E}=283-293 \mathrm{MeV}$; measured delayed $\mathrm{E} \alpha, \mathrm{I} \alpha, \alpha \alpha-$, (recoil) $\alpha$-coin; deduced evidence for ${ }^{199,200,201} \mathrm{Rn},{ }^{202,203,204} \mathrm{Fr},{ }^{203,204} \mathrm{Ra} .{ }^{141} \operatorname{Pr}\left({ }^{63} \mathrm{Cu}\right.$, xnypz $\left.\alpha\right), \mathrm{E}=278-288$ $\mathrm{MeV}$; measured delayed $\mathrm{E} \alpha, \mathrm{I} \alpha, \alpha \alpha-$, (recoil) $\alpha$-coin; deduced evidence for ${ }^{198,199,200,201,202} \mathrm{Rn},{ }^{201,202} \mathrm{Fr},{ }^{201,202} \mathrm{Ra} .{ }^{170} \mathrm{Yb}\left({ }^{36} \mathrm{Ar}, \mathrm{xnypz} \alpha\right)$, $\mathrm{E}=180-185 \mathrm{MeV}$; measured delayed $\mathrm{E} \alpha, \mathrm{I} \alpha, \alpha \alpha-$, (recoil) $\alpha$-coin; deduced evidence for ${ }^{201} \mathrm{Fr},{ }^{203} \mathrm{Ra}$. Gas-filled recoil separator. JOUR PRVCA 71024306

2005UU02 RADIOACTIVITY ${ }^{201,202,203,204} \mathrm{Ra},{ }^{197,198,199,200} \mathrm{Rn},{ }^{193,194,195,196}$ Po, ${ }^{201,202,203,204} \mathrm{Fr},{ }^{197,198,199,200} \operatorname{At}(\alpha)\left[\right.$ from ${ }^{141} \operatorname{Pr}\left({ }^{63,65} \mathrm{Cu}\right.$, xnypz $\left.\alpha\right)$, ${ }^{170} \mathrm{Yb}\left({ }^{36} \mathrm{Ar}, \mathrm{xnypz} \alpha\right)$, and subsequent decay]; measured $\mathrm{E} \alpha, \mathrm{T}_{1 / 2}$, $\alpha \alpha$-coin for ground and metastable state decay. ${ }^{193,195} \mathrm{Bi},{ }^{197,199} \mathrm{At}$, ${ }^{201,203} \mathrm{Fr}$ deduced levels, J, $\pi$. Comparisons with previous results. JOUR PRVCA 71024306

\section{$A=205$}

${ }^{205} \mathrm{Tl}$ 2004MB03 NUCLEAR MOMENTS ${ }^{113,115} \mathrm{In},{ }^{153,155} \mathrm{Eu},{ }^{185,187} \mathrm{Re},{ }^{203,205} \mathrm{Tl}$, ${ }^{209,211} \mathrm{Fr}$; measured hfs; deduced hyperfine magnetic anomaly, relative radii. Laser resonance fluorescence. JOUR BRSPE 68157 


\section{$\mathrm{A}=205$ (continued)}

2005LIZZ ATOMIC MASSES ${ }^{205} \mathrm{Tl},{ }^{220,221,222} \mathrm{At},{ }^{220,221,222,223} \mathrm{Rn},{ }^{223,224,225} \mathrm{Fr}$, $223,224,225,226,227,229,230,231$ Ra, ${ }^{227,229,230,231} \mathrm{Ac},{ }^{230,231} \mathrm{Th},{ }^{232} \mathrm{U}$; measured masses. Schottky mass spectrometry, ${ }^{238} \mathrm{U}$ fragmentation. REPT GSI 2005-1,P79,Litvinov

${ }^{205} \mathrm{~Pb} \quad 2004 \mathrm{KU} 33$

2005SMZZ

${ }^{205} \mathrm{Bi}$

2004ADZW

$2004 \mathrm{KE} 15$

$2004 K U 33$

2004MIZS

2005AD01

2005 MU21

2005SMZZ

${ }^{205} \mathrm{Fr}$

2005DE01
RADIOACTIVITY ${ }^{205} \mathrm{Bi}(\mathrm{EC})$ [from $\mathrm{Pb}, \mathrm{Bi}(\mathrm{p}, \mathrm{X})$ ]; measured $\mathrm{T}_{1 / 2}$. Comparison with previous results. JOUR RAACA 92233 NUCLEAR REACTIONS ${ }^{204,206,207,208} \mathrm{~Pb},{ }^{205} \mathrm{Tl}(\mathrm{n}, \mathrm{F}),(\mathrm{p}, \mathrm{F})$, $\mathrm{E}=30-180 \mathrm{MeV}$; measured fission $\sigma .{ }^{206} \mathrm{Tl},{ }^{205,206,207,208,209} \mathrm{~Pb}$, $205,207,208,209$ Bi; deduced fissility. CONF Santa Fe (Nucl Data for Sci and Technol) Proc, Vol1,P637 NUCLEAR REACTIONS ${ }^{209} \operatorname{Bi}(\mathrm{n}, 4 \mathrm{n}),(\mathrm{n}, 5 \mathrm{n}),(\mathrm{n}, 6 \mathrm{n}),(\mathrm{n}, 7 \mathrm{n}),(\mathrm{n}$, 9n), ${ }^{232} \operatorname{Th}(\mathrm{n}, \gamma),{ }^{197} \mathrm{Au}(\mathrm{n}, 2 \mathrm{n}),(\mathrm{n}, 4 \mathrm{n}),(\mathrm{n}, 6 \mathrm{n}),(\mathrm{n}, 7 \mathrm{n}),(\mathrm{n}, \gamma),{ }^{115} \operatorname{In}(\mathrm{n}$, $5 \mathrm{n}),(\mathrm{n}, 6 \mathrm{n}),(\mathrm{n}, 7 \mathrm{n}),{ }^{59} \operatorname{Co}(\mathrm{n}, 2 \mathrm{n}),(\mathrm{n}, 3 \mathrm{n}),(\mathrm{n}, 4 \mathrm{n}),(\mathrm{n}, 5 \mathrm{n}),(\mathrm{n}, \gamma),(\mathrm{n}$, $\mathrm{p}),(\mathrm{n}, 6 \mathrm{n} 2 \mathrm{p}), \mathrm{E}=$ spectrum; measured $\mathrm{E} \gamma, \mathrm{I} \gamma$; deduced reaction rates. $\mathrm{Pb}(\mathrm{p}, \mathrm{nX}), \mathrm{E}=1 \mathrm{GeV}$; deduced spallation neutron spectrum. REPT JINR-E1-2004-16,Adam NUCLEAR REACTIONS ${ }^{1,2} \mathrm{H}$, Ti $\left({ }^{208} \mathrm{~Pb}, \mathrm{X}\right){ }^{193} \mathrm{Bi} /{ }^{194} \mathrm{Bi} /{ }^{195} \mathrm{Bi} /$ ${ }^{196} \mathrm{Bi} /{ }^{197} \mathrm{Bi} /{ }^{198} \mathrm{Bi} /{ }^{199} \mathrm{Bi} /{ }^{200} \mathrm{Bi} /{ }^{201} \mathrm{Bi} /{ }^{202} \mathrm{Bi} /{ }^{203} \mathrm{Bi} /{ }^{204} \mathrm{Bi} /$ ${ }^{205} \mathrm{Bi} /{ }^{206} \mathrm{Bi} /{ }^{207} \mathrm{Bi} /{ }^{208} \mathrm{Bi}, \mathrm{E}=1 \mathrm{GeV} /$ nucleon; measured charge-pickup $\sigma$, velocity distributions; deduced reaction mechanism features. Comparison with model predictions and previous results. JOUR PRVCA 70064608 RADIOACTIVITY ${ }^{205} \mathrm{Bi}(\mathrm{EC})$ [from $\mathrm{Pb}, \mathrm{Bi}(\mathrm{p}, \mathrm{X})$ ]; measured $\mathrm{T}_{1 / 2}$. Comparison with previous results. JOUR RAACA 92233 NUCLEAR REACTIONS Fe $(\mathrm{p}, \mathrm{X}){ }^{52} \mathrm{Mn}, \mathrm{E}<2.6 \mathrm{GeV} ; \mathrm{Pb}(\mathrm{p}, \mathrm{X}){ }^{10} \mathrm{Be}$, $\mathrm{E}<2.6 \mathrm{GeV} ;{ }^{209} \mathrm{Bi}(\mathrm{p}, 4 \mathrm{np}), \mathrm{E}<2.6 \mathrm{GeV} ; \mathrm{Pb}(\mathrm{n}, \mathrm{X}){ }^{196} \mathrm{Au} /{ }^{95} \mathrm{Zr}, \mathrm{E} \approx$ 70-180 MeV; measured excitation functions. Comparison with model predictions. REPT NEA/NSC/DOC(2004)14,P28,Michel NUCLEAR REACTIONS ${ }^{209} \operatorname{Bi}(\mathrm{n}, 4 \mathrm{n}),(\mathrm{n}, 5 \mathrm{n}),(\mathrm{n}, 6 \mathrm{n}),(\mathrm{n}, 7 \mathrm{n}),(\mathrm{n}$, $9 \mathrm{n}),{ }^{232} \mathrm{Th}(\mathrm{n}, \gamma),{ }^{197} \mathrm{Au}(\mathrm{n}, 2 \mathrm{n}),(\mathrm{n}, 4 \mathrm{n}),(\mathrm{n}, 6 \mathrm{n}),(\mathrm{n}, 7 \mathrm{n}),(\mathrm{n}, \gamma),{ }^{59} \mathrm{Co}(\mathrm{n}$, $2 \mathrm{n}),(\mathrm{n}, 3 \mathrm{n}),(\mathrm{n}, 4 \mathrm{n}),(\mathrm{n}, 5 \mathrm{n}),(\mathrm{n}, \mathrm{p}),(\mathrm{n}, 6 \mathrm{n} 2 \mathrm{p}),{ }^{115} \operatorname{In}(\mathrm{n}, 5 \mathrm{n}),(\mathrm{n}, 6 \mathrm{n}),(\mathrm{n}$, $7 \mathrm{n}), \mathrm{E}=$ spectrum; measured $\mathrm{E} \gamma, \mathrm{I} \gamma$; deduced reaction rates. Activation technique, spallation neutrons from $1 \mathrm{GeV}$ proton beam, comparison with model predictions. JOUR ZAANE 2361

NUCLEAR REACTIONS ${ }^{115} \operatorname{In}(\mathrm{n}, \mathrm{n}),{ }^{27} \mathrm{Al}(\mathrm{n}, \alpha),{ }^{93} \mathrm{Nb}(\mathrm{n}, 2 \mathrm{n}),(\mathrm{n}, 4 \mathrm{n})$, ${ }^{209} \operatorname{Bi}(\mathrm{n}, 4 \mathrm{n}),(\mathrm{n}, 5 \mathrm{n}),(\mathrm{n}, 6 \mathrm{n}),(\mathrm{n}, 7 \mathrm{n}), \mathrm{E} \approx 10-1000 \mathrm{MeV}$; measured reaction rates. Comparison with model predictions. JOUR NIMAE 547 555

NUCLEAR REACTIONS ${ }^{204,206,207,208} \mathrm{~Pb},{ }^{205} \mathrm{Tl}(\mathrm{n}, \mathrm{F}),(\mathrm{p}, \mathrm{F})$, $\mathrm{E}=30-180 \mathrm{MeV}$; measured fission $\sigma .{ }^{206} \mathrm{Tl},{ }^{205,206,207,208,209} \mathrm{~Pb}$, 205,207,208,209 Bi; deduced fissility. CONF Santa Fe (Nucl Data for Sci and Technol) Proc, Vol1,P637

RADIOACTIVITY ${ }^{200,201,203,205} \mathrm{Fr},{ }^{196,197,199,201} \mathrm{At},{ }^{193} \operatorname{Bi}(\alpha)$ [from $\mathrm{Th}(\mathrm{p}, \mathrm{X})$ and subsequent decay]; measured $\mathrm{E} \alpha, \mathrm{T}_{1 / 2} \cdot{ }^{201} \mathrm{Fr},{ }^{197} \mathrm{At}$, ${ }^{193} \mathrm{Bi},{ }^{189} \mathrm{Tl}$ deduced levels, J, $\pi$. JOUR ZAANE 23243 


\section{$\mathrm{A}=205$ (continued)}

2005UU03 RADIOACTIVITY ${ }^{188,190,192,194,196,198,200,202,204} \mathrm{Po}$ 191,193,195,197,199 At, ${ }^{196,198,200,202,204,206} \mathrm{Rn},{ }^{199,201,203,205,207} \operatorname{Fr}(\alpha)$; measured reduced widths using gas filled recoil separator; deduced hindrance factors, proton intruder states and deformation effects. JOUR ZAANE 25 s01 179

\section{$A=206$}

${ }^{206} \mathrm{Tl} \quad 2005 \mathrm{SMZZ}$

${ }^{206} \mathrm{~Pb}$

$2005 \mathrm{CO} 25$

2005WA34

${ }^{206} \mathrm{Bi} \quad 2004 \mathrm{ADZW}$

2005AD01
NUCLEAR REACTIONS ${ }^{204,206,207,208} \mathrm{~Pb},{ }^{205} \mathrm{Tl}(\mathrm{n}, \mathrm{F}),(\mathrm{p}, \mathrm{F})$, $\mathrm{E}=30-180 \mathrm{MeV}$; measured fission $\sigma .{ }^{206} \mathrm{Tl},{ }^{205,206,207,208,209} \mathrm{~Pb}$, $205,207,208,209 \mathrm{Bi}$; deduced fissility. CONF Santa Fe (Nucl Data for Sci and Technol) Proc,Vol1,P637

NUCLEAR REACTIONS ${ }^{208} \mathrm{~Pb}\left({ }^{40} \mathrm{Ca},{ }^{42} \mathrm{Ca}\right), \mathrm{E}=225 \mathrm{MeV}$; measured $\sigma(\mathrm{E}, \theta) .{ }^{42} \mathrm{Ca}$ deduced excited states configurations. ${ }^{208} \mathrm{~Pb}\left({ }^{90} \mathrm{Zr}, \mathrm{X}\right)$, $\mathrm{E}=560 \mathrm{MeV}$; measured $\mathrm{E} \gamma, \mathrm{I} \gamma$, (fragment) $\gamma$-coin, isotopic yields for projectile-like fragments. ${ }^{90} \mathrm{Zr}$ deduced transitions. JOUR ZAANE 25 s01 427

2005PAZW NUCLEAR REACTIONS ${ }^{207} \mathrm{~Pb}(\mathrm{n}, 2 \mathrm{n}), \mathrm{E}<20 \mathrm{MeV} ;{ }^{232} \mathrm{Th}(\mathrm{n}, 5 \mathrm{n})$, $\mathrm{E}=29-42 \mathrm{MeV}$; measured $\mathrm{E} \gamma, \mathrm{I} \gamma \cdot{ }^{207} \mathrm{~Pb}(\mathrm{n}, 2 \mathrm{n}), \mathrm{E}=8-24 \mathrm{MeV}$; calculated $\sigma$. CONF Santa Fe (Nucl Data for Sci and Technol) Proc, Vol1,P876 NUCLEAR REACTIONS ${ }^{204,206,207,208} \mathrm{~Pb},{ }^{205} \mathrm{Tl}(\mathrm{n}, \mathrm{F}),(\mathrm{p}, \mathrm{F})$, $\mathrm{E}=30-180 \mathrm{MeV}$; measured fission $\sigma .{ }^{206} \mathrm{Tl},{ }^{205,206,207,208,209} \mathrm{~Pb}$, 205,207,208,209 Bi; deduced fissility. CONF Santa Fe (Nucl Data for Sci and Technol) Proc,Vol1,P637

NUCLEAR MOMENTS ${ }^{204,206,207,208} \mathrm{~Pb}$; measured hfs, isotope shifts. JOUR ZDDNE 36249

NUCLEAR REACTIONS ${ }^{209} \operatorname{Bi}(\mathrm{n}, 4 \mathrm{n}),(\mathrm{n}, 5 \mathrm{n}),(\mathrm{n}, 6 \mathrm{n}),(\mathrm{n}, 7 \mathrm{n}),(\mathrm{n}$, 9n), ${ }^{232} \mathrm{Th}(\mathrm{n}, \gamma),{ }^{197} \mathrm{Au}(\mathrm{n}, 2 \mathrm{n}),(\mathrm{n}, 4 \mathrm{n}),(\mathrm{n}, 6 \mathrm{n}),(\mathrm{n}, 7 \mathrm{n}),(\mathrm{n}, \gamma),{ }^{115} \operatorname{In}(\mathrm{n}$, $5 \mathrm{n}),(\mathrm{n}, 6 \mathrm{n}),(\mathrm{n}, 7 \mathrm{n}),{ }^{59} \mathrm{Co}(\mathrm{n}, 2 \mathrm{n}),(\mathrm{n}, 3 \mathrm{n}),(\mathrm{n}, 4 \mathrm{n}),(\mathrm{n}, 5 \mathrm{n}),(\mathrm{n}, \gamma),(\mathrm{n}$, $\mathrm{p}),(\mathrm{n}, 6 \mathrm{n} 2 \mathrm{p}), \mathrm{E}=$ spectrum; measured $\mathrm{E} \gamma, \mathrm{I} \gamma$; deduced reaction rates. $\mathrm{Pb}(\mathrm{p}, \mathrm{nX}), \mathrm{E}=1 \mathrm{GeV}$; deduced spallation neutron spectrum. REPT JINR-E1-2004-16,Adam NUCLEAR REACTIONS ${ }^{1,2} \mathrm{H}, \mathrm{Ti}\left({ }^{208} \mathrm{~Pb}, \mathrm{X}\right){ }^{193} \mathrm{Bi} /{ }^{194} \mathrm{Bi} /{ }^{195} \mathrm{Bi} /$ ${ }^{196} \mathrm{Bi} /{ }^{197} \mathrm{Bi} /{ }^{198} \mathrm{Bi} /{ }^{199} \mathrm{Bi} /{ }^{200} \mathrm{Bi} /{ }^{201} \mathrm{Bi} /{ }^{202} \mathrm{Bi} /{ }^{203} \mathrm{Bi} /{ }^{204} \mathrm{Bi} /$ ${ }^{205} \mathrm{Bi} /{ }^{206} \mathrm{Bi} /{ }^{207} \mathrm{Bi} /{ }^{208} \mathrm{Bi}, \mathrm{E}=1 \mathrm{GeV} /$ nucleon; measured charge-pickup $\sigma$, velocity distributions; deduced reaction mechanism features. Comparison with model predictions and previous results. JOUR PRVCA 70064608 NUCLEAR REACTIONS ${ }^{209} \operatorname{Bi}(\mathrm{n}, 4 \mathrm{n}),(\mathrm{n}, 5 \mathrm{n}),(\mathrm{n}, 6 \mathrm{n}),(\mathrm{n}, 7 \mathrm{n}),(\mathrm{n}$, 9n), ${ }^{232} \mathrm{Th}(\mathrm{n}, \gamma),{ }^{197} \mathrm{Au}(\mathrm{n}, 2 \mathrm{n}),(\mathrm{n}, 4 \mathrm{n}),(\mathrm{n}, 6 \mathrm{n}),(\mathrm{n}, 7 \mathrm{n}),(\mathrm{n}, \gamma),{ }^{59} \mathrm{Co}(\mathrm{n}$, 2n), (n, 3n), (n, 4n), (n, 5n), (n, p), (n, 6n2p), ${ }^{115} \operatorname{In}(\mathrm{n}, 5 \mathrm{n}),(\mathrm{n}, 6 \mathrm{n}),(\mathrm{n}$, $7 \mathrm{n}), \mathrm{E}=$ spectrum; measured $\mathrm{E} \gamma, \mathrm{I} \gamma$; deduced reaction rates. Activation technique, spallation neutrons from $1 \mathrm{GeV}$ proton beam, comparison with model predictions. JOUR ZAANE 2361 


\section{$\mathrm{A}=206$ (continued)}

2005MU21 NUCLEAR REACTIONS ${ }^{115} \mathrm{In}(\mathrm{n}, \mathrm{n}),{ }^{27} \mathrm{Al}(\mathrm{n}, \alpha),{ }^{93} \mathrm{Nb}(\mathrm{n}, 2 \mathrm{n}),(\mathrm{n}, 4 \mathrm{n})$, ${ }^{209} \operatorname{Bi}(\mathrm{n}, 4 \mathrm{n}),(\mathrm{n}, 5 \mathrm{n}),(\mathrm{n}, 6 \mathrm{n}),(\mathrm{n}, 7 \mathrm{n}), \mathrm{E} \approx 10-1000 \mathrm{MeV}$; measured reaction rates. Comparison with model predictions. JOUR NIMAE 547 555

${ }^{206} \mathrm{At}$ 2005KU06 RADIOACTIVITY ${ }^{210,211,212,213,214} \operatorname{Fr}(\alpha)$ [from ${ }^{209} \mathrm{Bi}\left({ }^{12} \mathrm{C}, \mathrm{xn}\right)$ and subsequent decay]; measured $\mathrm{E} \alpha, \mathrm{I} \alpha, \mathrm{E} \gamma, \mathrm{I} \gamma, \alpha \gamma$-coin, $\mathrm{T}_{1 / 2}$; deduced hindrance factors. ${ }^{206,207,208,209,210}$ At deduced levels, J, $\pi$. Comparison with previous results. JOUR ZAANE 23417

${ }^{206} \mathrm{Rn} \quad 2005$ UUU03 RADIOACTIVITY ${ }^{188,190,192,194,196,198,200,202,204} \mathrm{Po}$, 191,193,195,197,199 At, ${ }^{196,198,200,202,204,206} \mathrm{Rn},{ }^{199,201,203,205,207} \operatorname{Fr}(\alpha)$; measured reduced widths using gas filled recoil separator; deduced hindrance factors, proton intruder states and deformation effects. JOUR ZAANE 25 s01 179

\section{$\mathrm{A}=\mathbf{2 0 7}$}

${ }^{207} \mathrm{Tl} \quad$ 2005BOZU

2005GEZW

$2005 \mathrm{HU} 10$

$20050 H 08$

${ }^{207} \mathrm{~Pb}$

2005BEZT

2005BOZT

2005BOZU

2005BOZV

2005HU10
RADIOACTIVITY ${ }^{207,207 m} \mathrm{Tl}\left(\beta^{-}\right)$; measured ground-state and isomer decay $\mathrm{T}_{1 / 2}$ of fully-stripped ion. Time-resolved Schottky mass spectrometry. REPT GSI 2005-1,P81,Boutin ATOMIC MASSES ${ }^{235} \mathrm{Ac}$; measured mass, $\mathrm{T}_{1 / 2}$. 185,186,187,188,189,190,191,192,193,194,195,196 $\mathrm{Bi}$; measured masses, proton separation energies. ${ }^{207 \mathrm{~m}} \mathrm{Tl}$; measured $\mathrm{T}_{1 / 2}$. Stored beams, Schottky mass spectrometry. PREPRINT nucl-ex/0510009,10/4/2005 NUCLEAR REACTIONS ${ }^{90} \mathrm{Zr},{ }^{116} \mathrm{Sn},{ }^{208} \mathrm{~Pb}(\alpha, \alpha$ 'n), E=200 MeV; ${ }^{208} \mathrm{~Pb}\left(\alpha, \alpha\right.$ 'p), $\mathrm{E}=200 \mathrm{MeV}$; measured $\mathrm{E} \alpha, \sigma(\theta), \mathrm{p} \alpha-$, n $\alpha$-coin. ${ }^{90} \mathrm{Zr}$, ${ }^{116} \mathrm{Sn},{ }^{208} \mathrm{~Pb}$ deduced isoscalar GDR parameters, particle decay features. JOUR APOBB 361115 RADIOACTIVITY ${ }^{207} \mathrm{Tl}\left(\beta^{-}\right)$[from $\mathrm{Be}\left({ }^{208} \mathrm{~Pb}, \mathrm{X}\right)$ ]; measured ratio of bound-state and continuum-state decay rates for $\beta$-decay of bare ions. Comparison with model predictions. JOUR PRLTA 95052501

NUCLEAR REACTIONS ${ }^{35} \mathrm{Cl}(\mathrm{n}, \gamma)$, E not given; measured $\mathrm{E} \gamma, \mathrm{I} \gamma$. ${ }^{36} \mathrm{Cl}$ deduced transitions, level energies, binding energy. ${ }^{52,54} \mathrm{Cr},{ }^{56} \mathrm{Fe}$, ${ }^{206} \mathrm{~Pb}(\mathrm{n}, \gamma)$, E not given; analyzed $\mathrm{E} \gamma \cdot{ }^{53,55} \mathrm{Cr},{ }^{57} \mathrm{Fe},{ }^{207} \mathrm{~Pb}$ deduced binding energies. GAMS4 spectrometer. CONF Santa Fe (Nucl Data for Sci and Technol) Proc,Vol1,P1074

NUCLEAR REACTIONS ${ }^{206} \mathrm{~Pb}(\mathrm{n}, \mathrm{X}),(\mathrm{n}, \gamma), \mathrm{E}=0-600 \mathrm{keV}$; measured total and capture $\sigma$; deduced resonance parameters. ${ }^{206} \mathrm{~Pb},{ }^{209} \mathrm{Bi}(\mathrm{n}, \gamma)$, $\mathrm{E}=$ thermal; measured $\sigma$. THESIS A Borella, Gent Univ

RADIOACTIVITY ${ }^{207,207 m} \mathrm{Tl}\left(\beta^{-}\right)$; measured ground-state and isomer decay $\mathrm{T}_{1 / 2}$ of fully-stripped ion. Time-resolved Schottky mass spectrometry. REPT GSI 2005-1,P81,Boutin

NUCLEAR REACTIONS ${ }^{206} \mathrm{~Pb}(\mathrm{n}, \mathrm{X}),(\mathrm{n}, \gamma), \mathrm{E}=0-600 \mathrm{keV}$; measured total and capture $\sigma$; deduced resonance parameters. CONF Santa Fe (Nucl Data for Sci and Technol) Proc,Vol2,P1539 NUCLEAR REACTIONS ${ }^{90} \mathrm{Zr},{ }^{116} \mathrm{Sn},{ }^{208} \mathrm{~Pb}(\alpha, \alpha$ 'n), E=200 MeV; ${ }^{208} \mathrm{~Pb}\left(\alpha, \alpha\right.$ 'p), $\mathrm{E}=200 \mathrm{MeV}$; measured $\mathrm{E} \alpha, \sigma(\theta), \mathrm{p} \alpha-$, n $\alpha$-coin. ${ }^{90} \mathrm{Zr}$, ${ }^{116} \mathrm{Sn},{ }^{208} \mathrm{~Pb}$ deduced isoscalar GDR parameters, particle decay features. JOUR APOBB 361115 


\section{$\mathrm{A}=207$ (continued)}

20050H08 RADIOACTIVITY ${ }^{207} \mathrm{Tl}\left(\beta^{-}\right)$[from Be $\left({ }^{208} \mathrm{~Pb}, \mathrm{X}\right)$ ]; measured ratio of bound-state and continuum-state decay rates for $\beta$-decay of bare ions. Comparison with model predictions. JOUR PRLTA 95052501

2005SH22 NUCLEAR REACTIONS ${ }^{79} \mathrm{Br},{ }^{90} \mathrm{Zr},{ }^{197} \mathrm{Au},{ }^{207} \mathrm{~Pb}(\mathrm{n}, \mathrm{n}$ ') $, \mathrm{E}=2.54,3.1$ $\mathrm{MeV}$; measured $\sigma$. Pulsed beam. JOUR ANEND 32949

2005SHZU NUCLEAR REACTIONS ${ }^{79} \mathrm{Br},{ }^{90} \mathrm{Zr},{ }^{197} \mathrm{Au},{ }^{207} \mathrm{~Pb}(\mathrm{n}, \mathrm{n}$ '), $\mathrm{E}=2.54,3.1$ $\mathrm{MeV}$; measured isomer activation $\sigma$. CONF Santa Fe (Nucl Data for Sci and Technol) Proc,Vol1,P992

2005SMZZ NUCLEAR REACTIONS ${ }^{204,206,207,208} \mathrm{~Pb},{ }^{205} \mathrm{Tl}(\mathrm{n}, \mathrm{F}),(\mathrm{p}, \mathrm{F})$, $\mathrm{E}=30-180 \mathrm{MeV}$; measured fission $\sigma .{ }^{206} \mathrm{Tl},{ }^{205,206,207,208,209} \mathrm{~Pb}$, $205,207,208,209 \mathrm{Bi}$; deduced fissility. CONF Santa Fe (Nucl Data for Sci and Technol) Proc,Vol1,P637

2005WA06 NUCLEAR MOMENTS ${ }^{207} \mathrm{~Pb}$; measured hfs. Comparison with previous results and model predictions. JOUR PHSTB 71274

2005WA22 NUCLEAR MOMENTS ${ }^{207} \mathrm{~Pb}$; measured hfs. Comparison with previous results and model predictions. JOUR PHSTB 72200

2005WA34 NUCLEAR MOMENTS ${ }^{204,206,207,208} \mathrm{~Pb}$; measured hfs, isotope shifts. JOUR ZDDNE 36249

${ }^{207} \mathrm{Bi}$ 2004KE15 NUCLEAR REACTIONS ${ }^{1,2} \mathrm{H}, \mathrm{Ti}\left({ }^{208} \mathrm{~Pb}, \mathrm{X}\right){ }^{193} \mathrm{Bi} /{ }^{194} \mathrm{Bi} /{ }^{195} \mathrm{Bi} /$ ${ }^{196} \mathrm{Bi} /{ }^{197} \mathrm{Bi} /{ }^{198} \mathrm{Bi} /{ }^{199} \mathrm{Bi} /{ }^{200} \mathrm{Bi} /{ }^{201} \mathrm{Bi} /{ }^{202} \mathrm{Bi} /{ }^{203} \mathrm{Bi} /{ }^{204} \mathrm{Bi} /$ ${ }^{205} \mathrm{Bi} /{ }^{206} \mathrm{Bi} /{ }^{207} \mathrm{Bi} /{ }^{208} \mathrm{Bi}, \mathrm{E}=1 \mathrm{GeV} /$ nucleon; measured charge-pickup $\sigma$, velocity distributions; deduced reaction mechanism features. Comparison with model predictions and previous results.

JOUR PRVCA 70064608

2005SMZZ NUCLEAR REACTIONS ${ }^{204,206,207,208} \mathrm{~Pb},{ }^{205} \mathrm{Tl}(\mathrm{n}, \mathrm{F}),(\mathrm{p}, \mathrm{F})$, $\mathrm{E}=30-180 \mathrm{MeV}$; measured fission $\sigma .{ }^{206} \mathrm{Tl},{ }^{205,206,207,208,209} \mathrm{~Pb}$, 205,207,208,209 Bi; deduced fissility. CONF Santa Fe (Nucl Data for Sci and Technol) Proc,Vol1,P637

${ }^{207} \mathrm{At}$ 2005KU06 RADIOACTIVITY ${ }^{210,211,212,213,214} \mathrm{Fr}(\alpha)$ [from ${ }^{209} \mathrm{Bi}\left({ }^{12} \mathrm{C}, \mathrm{xn}\right)$ and subsequent decay]; measured $\mathrm{E} \alpha, \mathrm{I} \alpha, \mathrm{E} \gamma, \mathrm{I} \gamma, \alpha \gamma$-coin, $\mathrm{T}_{1 / 2}$; deduced hindrance factors. ${ }^{206,207,208,209,210}$ At deduced levels, $\mathrm{J}, \pi$. Comparison with previous results. JOUR ZAANE 23417

${ }^{207} \mathrm{Fr} \quad 2005 \mathrm{UU} 03 \quad$ RADIOACTIVITY ${ }^{188,190,192,194,196,198,200,202,204} \mathrm{Po}$, $191,193,195,197,199$ At, ${ }^{196,198,200,202,204,206} \mathrm{Rn},{ }^{199,201,203,205,207} \operatorname{Fr}(\alpha)$; measured reduced widths using gas filled recoil separator; deduced hindrance factors, proton intruder states and deformation effects.

JOUR ZAANE 25 s01 179

${ }^{207} \mathrm{Ac} \quad 2005 \mathrm{LI} 17 \quad$ NUCLEAR REACTIONS Be $\left({ }^{238} \mathrm{U}, \mathrm{X}\right){ }^{207} \mathrm{Ac} /{ }^{208} \mathrm{Ac} /{ }^{209} \mathrm{Ac} /{ }^{210} \mathrm{Ac} /$ ${ }^{211} \mathrm{Ac} /{ }^{212} \mathrm{Ac} /{ }^{213} \mathrm{Ac} /{ }^{214} \mathrm{Ac} /{ }^{215} \mathrm{Ac} /{ }^{216} \mathrm{Ac} /{ }^{217} \mathrm{Ac} /{ }^{218} \mathrm{Ac} /{ }^{219} \mathrm{Ac}$ $/{ }^{220} \mathrm{Ac} /{ }^{221} \mathrm{Ac} /{ }^{211} \mathrm{Th} /{ }^{212} \mathrm{Th} /{ }^{213} \mathrm{Th} /{ }^{214} \mathrm{Th} /{ }^{215} \mathrm{Th} /{ }^{216} \mathrm{Th} /$ ${ }^{217} \mathrm{Th} /{ }^{218} \mathrm{Th} /{ }^{219} \mathrm{Th} /{ }^{220} \mathrm{Th} /{ }^{221} \mathrm{Th} /{ }^{222} \mathrm{Th} /{ }^{223} \mathrm{Th} /{ }^{216} \mathrm{~Pa} /$ ${ }^{217} \mathrm{~Pa} /{ }^{218} \mathrm{~Pa} /{ }^{219} \mathrm{~Pa} /{ }^{220} \mathrm{~Pa} /{ }^{221} \mathrm{~Pa} /{ }^{222} \mathrm{~Pa} /{ }^{223} \mathrm{~Pa} /{ }^{224} \mathrm{~Pa} /{ }^{225} \mathrm{~Pa} /$ ${ }^{226} \mathrm{~Pa} /{ }^{227} \mathrm{~Pa}, \mathrm{E}=1 \mathrm{GeV} /$ nucleon; measured (fragment)(decay)-coin, fragment yields. Fragment separator. JOUR NIMAE 543591 


\section{$\mathrm{A}=\mathbf{2 0 8}$}

${ }^{208} \mathrm{Tl}$ 2005GR28 NUCLEAR REACTIONS ${ }^{1} \mathrm{H}\left(\pi^{-}, \pi^{+} \pi^{-}\right),\left(\pi^{+}, 2 \pi^{+}\right), \mathrm{E}=243,264,284$, $305 \mathrm{MeV} ;{ }^{2} \mathrm{H},{ }^{12} \mathrm{C},{ }^{40} \mathrm{Ca},{ }^{208} \mathrm{~Pb}\left(\pi^{+}, 2 \pi^{+}\right),\left(\pi^{+}, \pi^{+} \pi^{-}\right), \mathrm{E}=283 \mathrm{MeV}$; $\mathrm{Sc}\left(\pi^{+}, 2 \pi^{+} \mathrm{X}\right),\left(\pi^{+}, \pi^{+} \pi^{-} \mathrm{X}\right), \mathrm{E}=243,264,284,305 \mathrm{MeV}$; measured invariant mass distributions, $\sigma(\theta)$, correlations; deduced partial chiral symmetry restoration. JOUR NUPAB 76380

2005SZ03 RADIOACTIVITY ${ }^{212} \mathrm{~Pb},{ }^{208} \mathrm{Tl}\left(\beta^{-}\right) ;{ }^{212} \mathrm{Bi}(\alpha),\left(\beta^{-}\right)$; measured $\mathrm{E} \gamma, \mathrm{I} \gamma$. Application to superheavy element identification discussed. JOUR JRNCD 265367

2005VAZZ RADIOACTIVITY ${ }^{208} \mathrm{Tl}\left(\beta^{-}\right)$; measured $\mathrm{E} \gamma, \mathrm{I} \gamma, \gamma \gamma$-coin. ${ }^{208} \mathrm{~Pb}$ deduced transition intensities. CONF St Petersburg,P320,Brudanin 2002 LI68 NUCLEAR REACTIONS ${ }^{208} \mathrm{~Pb}\left({ }^{19} \mathrm{~F},{ }^{19} \mathrm{~F}\right), \mathrm{E}=88-102 \mathrm{MeV}$; measured $\sigma(\theta)$; deduced parameters, role of deformation in fusion reactions, threshold anomaly. JOUR JNRSA 3,No 1,27

2004PEZW NUCLEAR REACTIONS ${ }^{208} \mathrm{~Pb}\left({ }^{70} \mathrm{Ni},{ }^{70} \mathrm{Ni}\right),\left({ }^{74} \mathrm{Zn},{ }^{74} \mathrm{Zn}\right),\left({ }^{76} \mathrm{Ge}\right.$, ${ }^{76} \mathrm{Ge}$ '), $\mathrm{E} \approx 40 \mathrm{MeV} /$ nucleon; measured $\mathrm{E} \gamma, \mathrm{I} \gamma$, (particle) $\gamma$-coin following projectile Coulomb excitation. ${ }^{70} \mathrm{Ni},{ }^{74} \mathrm{Zn}$ deduced transitions B(E2). REPT IPNO-T-05-02,Perru

2005CLZZ NUCLEAR REACTIONS Be $\left({ }^{78} \mathrm{Kr}, \mathrm{X}\right){ }^{72} \mathrm{Kr} /{ }^{74} \mathrm{Kr}, \mathrm{E}=73 \mathrm{MeV}$; measured delayed $\mathrm{E} \gamma, \mathrm{I} \gamma, \mathrm{E}(\mathrm{ce}), \mathrm{I}(\mathrm{ce})$, (recoil) $\gamma$-, (recoil)(ce)-coin. ${ }^{72,74} \mathrm{Kr}$ deduced isomeric levels, $\mathrm{J}, \pi, \mathrm{T}_{1 / 2}$, E0 strength. ${ }^{72} \mathrm{Kr}$ deduced shape isomer. ${ }^{208} \mathrm{~Pb}\left({ }^{76} \mathrm{Kr},{ }^{76} \mathrm{Kr}{ }^{\prime}\right),\left({ }^{74} \mathrm{Kr},{ }^{74} \mathrm{Kr}{ }^{\prime}\right), \mathrm{E} \approx 4.5 \mathrm{MeV} /$ nucleon; measured $\mathrm{E} \gamma, \mathrm{I} \gamma$, (particle) $\gamma$-coin following projectile Coulomb excitation. ${ }^{74,76} \mathrm{Kr}$ deduced levels, J, $\pi$. CONF Argonne(Nuclei at the Limits),P55, Clement

2005FL02 NUCLEAR REACTIONS ${ }^{208} \mathrm{~Pb}\left({ }^{16} \mathrm{O},{ }^{16} \mathrm{O}\right),\left({ }^{16} \mathrm{O}, \alpha{ }^{12} \mathrm{C}\right), \mathrm{E}=60,80$ $\mathrm{MeV}$ / nucleon; measured particle spectra, $\sigma(\mathrm{E}, \theta)$, angular correlations; deduced reaction mechanism features. DWBA and coupled-channels analyses. JOUR PYLBB 615167

2005G015 NUCLEAR REACTIONS ${ }^{208} \mathrm{~Pb}\left({ }^{74} \mathrm{Kr},{ }^{74} \mathrm{Kr}\right.$ '), $\left({ }^{76} \mathrm{Kr},{ }^{76} \mathrm{Kr}\right.$ '), E=4.5 $\mathrm{MeV} /$ nucleon; measured $\mathrm{E} \gamma, \mathrm{I} \gamma$, (particle) $\gamma$-coin following projectile Coulomb excitation. ${ }^{74,76} \mathrm{Kr}$ deduced levels, $\mathrm{J}, \pi$, quadrupole moments. ${ }^{208} \mathrm{~Pb}\left({ }^{72} \mathrm{Ge},{ }^{72} \mathrm{Ge}\right)$, E not given; measured $\mathrm{E} \gamma, \mathrm{I} \gamma, \mathrm{E}(\mathrm{ce}), \mathrm{I}(\mathrm{ce})$, (particle) $\gamma$-coin following projectile Coulomb excitation. ${ }^{72}$ Ge deduced transitions. Exogam array. JOUR APOBB 361281

$2005 \mathrm{GO34}$ NUCLEAR REACTIONS ${ }^{208} \mathrm{~Pb}\left({ }^{23} \mathrm{Al}, \mathrm{p}^{22} \mathrm{Mg}\right), \mathrm{E}=50 \mathrm{MeV} /$ nucleon; measured relative energy spectrum, $\sigma(\theta) .{ }^{23} \mathrm{Al}$ deduced excited state radiative width. Astrophysical implications discussed. JOUR JPGPE $31 \mathrm{~S} 1517$

2005HIZY NUCLEAR REACTIONS ${ }^{12} \mathrm{C},{ }^{89} \mathrm{Y},{ }^{208} \mathrm{~Pb}(\mathrm{n}, \mathrm{n}), \mathrm{E}=96 \mathrm{MeV}$; measured $\sigma, \sigma(\theta)$. Comparison with model predictions. CONF Santa Fe (Nucl Data for Sci and Technol) Proc,Vol1,P853

2005HU10 NUCLEAR REACTIONS ${ }^{90} \mathrm{Zr},{ }^{116} \mathrm{Sn},{ }^{208} \mathrm{~Pb}(\alpha, \alpha$ 'n), E=200 MeV; ${ }^{208} \mathrm{~Pb}\left(\alpha, \alpha\right.$ 'p), $\mathrm{E}=200 \mathrm{MeV}$; measured $\mathrm{E} \alpha, \sigma(\theta), \mathrm{p} \alpha-, \mathrm{n} \alpha$-coin. ${ }^{90} \mathrm{Zr}$, ${ }^{116} \mathrm{Sn},{ }^{208} \mathrm{~Pb}$ deduced isoscalar GDR parameters, particle decay features. JOUR APOBB 361115

2005KAZZ NUCLEAR REACTIONS ${ }^{197} \mathrm{Au},{ }^{208} \mathrm{~Pb}\left({ }^{6} \mathrm{He},{ }^{6} \mathrm{He}\right), \mathrm{E}=27 \mathrm{MeV}$; measured $\sigma(\theta)$; deduced diffuseness parameters, long-range absorption mechanisms. Optical model. PREPRINT nucl-ex/0507024,7/18/2005 


\section{$\mathrm{A}=208$ (continued)}

2005K011 NUCLEAR REACTIONS ${ }^{208} \mathrm{~Pb}\left({ }^{74} \mathrm{Kr},{ }^{74} \mathrm{Kr}\right.$ '), $\left({ }^{76} \mathrm{Kr},{ }^{76} \mathrm{Kr}\right.$ '), E $\approx 350$ $\mathrm{MeV}$; measured $\mathrm{E} \gamma, \mathrm{I} \gamma$, (particle) $\gamma$-coin following projectile Coulomb excitation. ${ }^{74,76} \mathrm{Kr}$ deduced levels, J, $\pi$, quadrupole moments. Exogam array. JOUR NUPAB $752255 \mathrm{c}$

2005KU17 NUCLEAR REACTIONS ${ }^{208} \mathrm{~Pb}\left({ }^{152} \mathrm{Sm},{ }^{152} \mathrm{Sm}\right), \mathrm{E}=652 \mathrm{MeV}$; measured $\mathrm{E} \gamma, \mathrm{I} \gamma$, (particle) $\gamma$-, $\gamma \gamma$-coin following projectile Coulomb excitation. ${ }^{152} \mathrm{Sm}$ deduced levels, $\mathrm{J}, \pi, \mathrm{B}(\mathrm{E} 2)$, rotational band, pairing isomer. Gammasphere, Chico arrays, level systematics in neighboring nuclides discussed. JOUR PRVCA 71041303

20050R02 NUCLEAR REACTIONS ${ }^{208} \mathrm{~Pb}(\mathrm{p}, \mathrm{p}$ '), $\mathrm{E}=17.3 \mathrm{MeV}$; measured Ep, $\mathrm{E}(\mathrm{ce}),(\mathrm{ce}) \mathrm{p}$-coin. ${ }^{208} \mathrm{~Pb}$ deduced levels, electric monopole transitions, E3 / E0 branching ratio. JOUR JPGPE 31 S1705

2005R042 NUCLEAR REACTIONS ${ }^{208} \mathrm{~Pb}\left({ }^{17} \mathrm{~F},{ }^{17} \mathrm{~F}\right),\left({ }^{17} \mathrm{~F},{ }^{16} \mathrm{OX}\right), \mathrm{E}=90.4 \mathrm{Mev}$; measured $\sigma(\theta)$. JOUR ZAANE $25 \mathrm{~s} 01289$

2005SA52 NUCLEAR REACTIONS ${ }^{208} \mathrm{~Pb}\left({ }^{6} \mathrm{He},{ }^{6} \mathrm{He}\right),\left({ }^{6} \mathrm{He}, \alpha\right), \mathrm{E}=14,16,17,18$, $22 \mathrm{MeV}$; measured $\sigma(\theta)$; deduced reaction mechanism features. JOUR JPGPE 31 S1953

2005SCZX NUCLEAR REACTIONS ${ }^{208} \mathrm{~Pb}\left({ }^{8} \mathrm{~B}, \mathrm{p}{ }^{7} \mathrm{Be}\right), \mathrm{E}=254 \mathrm{MeV} /$ nucleon; measured fragment spectra, angular correlations. ${ }^{7} \mathrm{Be}(\mathrm{p}, \gamma), \mathrm{E}=$ low; deduced astrophysical S-factor. PREPRINT nucl-ex/0508014,08/11/2005

2005SMZZ NUCLEAR REACTIONS ${ }^{204,206,207,208} \mathrm{~Pb},{ }^{205} \mathrm{Tl}(\mathrm{n}, \mathrm{F}),(\mathrm{p}, \mathrm{F})$, $\mathrm{E}=30-180 \mathrm{MeV}$; measured fission $\sigma .{ }^{206} \mathrm{Tl},{ }^{205,206,207,208,209} \mathrm{~Pb}$, 205,207,208,209 Bi; deduced fissility. CONF Santa Fe (Nucl Data for Sci and Technol) Proc,Vol1,P637

2005SZ03 RADIOACTIVITY ${ }^{212} \mathrm{~Pb},{ }^{208} \mathrm{Tl}\left(\beta^{-}\right) ;{ }^{212} \mathrm{Bi}(\alpha),\left(\beta^{-}\right)$; measured $\mathrm{E} \gamma$, I $\gamma$. Application to superheavy element identification discussed. JOUR JRNCD 265367

2005TOZZ NUCLEAR REACTIONS ${ }^{208} \mathrm{~Pb}\left({ }^{27} \mathrm{P}, \mathrm{p}{ }^{26} \mathrm{Si}\right), \mathrm{E}=57 \mathrm{MeV} /$ nucleon; measured relative energy spectrum, $\sigma(\mathrm{E}) .{ }^{27} \mathrm{P}$ deduced gamma decay width of first excited state. CONF Riken(Origin of Matter) Proc,P549,Togano

2005VAZZ RADIOACTIVITY ${ }^{208} \mathrm{Tl}\left(\beta^{-}\right)$; measured $\mathrm{E} \gamma, \mathrm{I} \gamma, \gamma \gamma$-coin. ${ }^{208} \mathrm{~Pb}$ deduced transition intensities. CONF St Petersburg,P320,Brudanin 2005WA34 NUCLEAR MOMENTS ${ }^{204,206,207,208} \mathrm{~Pb}$; measured hfs, isotope shifts. JOUR ZDDNE 36249

$2005 Y$ 17 NUCLEAR REACTIONS ${ }^{208} \mathrm{~Pb}\left({ }^{7} \mathrm{Li},{ }^{7} \mathrm{Li} '\right), \mathrm{E}=150 \mathrm{MeV}$; measured particle spectra, $\sigma(\mathrm{E}, \theta) .{ }^{208} \mathrm{~Pb}$ deduced giant resonance features. JOUR JUPSA 742640

2005YAZW NUCLEAR REACTIONS ${ }^{208} \mathrm{~Pb}(\mathrm{n}, \mathrm{n} \gamma), \mathrm{E}=6.5 \mathrm{MeV}$; measured E $\gamma$, $\mathrm{I} \gamma .{ }^{208} \mathrm{~Pb}$ deduced levels, $\mathrm{J}, \pi, \mathrm{T}_{1 / 2}, \delta, \mathrm{B}(\mathrm{Ee}) / \mathrm{B}(\mathrm{M} 1) . \mathrm{PC}$ Yates, $11 / 29 / 2005$

${ }^{208} \mathrm{Bi}$ 2004KE15 NUCLEAR REACTIONS ${ }^{1,2} \mathrm{H}, \mathrm{Ti}\left({ }^{208} \mathrm{~Pb}, \mathrm{X}\right){ }^{193} \mathrm{Bi} /{ }^{194} \mathrm{Bi} /{ }^{195} \mathrm{Bi} /$ ${ }^{196} \mathrm{Bi} /{ }^{197} \mathrm{Bi} /{ }^{198} \mathrm{Bi} /{ }^{199} \mathrm{Bi} /{ }^{200} \mathrm{Bi} /{ }^{201} \mathrm{Bi} /{ }^{202} \mathrm{Bi} /{ }^{203} \mathrm{Bi} /{ }^{204} \mathrm{Bi} /$ ${ }^{205} \mathrm{Bi} /{ }^{206} \mathrm{Bi} /{ }^{207} \mathrm{Bi} /{ }^{208} \mathrm{Bi}, \mathrm{E}=1 \mathrm{GeV} /$ nucleon; measured charge-pickup $\sigma$, velocity distributions; deduced reaction mechanism features. Comparison with model predictions and previous results. JOUR PRVCA 70064608 


\section{$\mathrm{A}=208$ (continued)}

2005GR28 NUCLEAR REACTIONS ${ }^{1} \mathrm{H}\left(\pi^{-}, \pi^{+} \pi^{-}\right),\left(\pi^{+}, 2 \pi^{+}\right), \mathrm{E}=243,264,284$, $305 \mathrm{MeV} ;{ }^{2} \mathrm{H},{ }^{12} \mathrm{C},{ }^{40} \mathrm{Ca},{ }^{208} \mathrm{~Pb}\left(\pi^{+}, 2 \pi^{+}\right),\left(\pi^{+}, \pi^{+} \pi^{-}\right), \mathrm{E}=283 \mathrm{MeV}$; $\mathrm{Sc}\left(\pi^{+}, 2 \pi^{+} \mathrm{X}\right),\left(\pi^{+}, \pi^{+} \pi^{-} \mathrm{X}\right), \mathrm{E}=243,264,284,305 \mathrm{MeV}$; measured invariant mass distributions, $\sigma(\theta)$, correlations; deduced partial chiral symmetry restoration. JOUR NUPAB 76380

2005SMZZ NUCLEAR REACTIONS ${ }^{204,206,207,208} \mathrm{~Pb},{ }^{205} \mathrm{Tl}(\mathrm{n}, \mathrm{F}),(\mathrm{p}, \mathrm{F})$, $\mathrm{E}=30-180 \mathrm{MeV}$; measured fission $\sigma .{ }^{206} \mathrm{Tl},{ }^{205,206,207,208,209} \mathrm{~Pb}$, 205,207,208,209 Bi; deduced fissility. CONF Santa Fe (Nucl Data for Sci and Technol) Proc,Vol1,P637

${ }^{208} \mathrm{At}$ 2005KU06 RADIOACTIVITY ${ }^{210,211,212,213,214} \mathrm{Fr}(\alpha)$ from ${ }^{209} \mathrm{Bi}\left({ }^{12} \mathrm{C}\right.$, xn $)$ and subsequent decay]; measured $\mathrm{E} \alpha, \mathrm{I} \alpha, \mathrm{E} \gamma, \mathrm{I} \gamma, \alpha \gamma$-coin, $\mathrm{T}_{1 / 2}$; deduced hindrance factors. ${ }^{206,207,208,209,210}$ At deduced levels, J, $\pi$. Comparison with previous results. JOUR ZAANE 23417

${ }^{208} \mathrm{Fr} \quad 2005 \mathrm{CO} 02$ NUCLEAR REACTIONS ${ }^{197} \mathrm{Au}\left({ }^{18} \mathrm{O}, 4 \mathrm{n}\right),\left({ }^{18} \mathrm{O}, 5 \mathrm{n}\right),\left({ }^{18} \mathrm{O}, 6 \mathrm{n}\right),\left({ }^{18} \mathrm{O}\right.$, $7 \mathrm{n}), \mathrm{E}=75-130 \mathrm{MeV}$; measured delayed $\mathrm{E} \alpha$, excitation functions. Comparison with model predictions. JOUR PRVCA 71014609

${ }^{208} \mathrm{Ra} \quad$ 2005RE02 NUCLEAR REACTIONS ${ }^{182} \mathrm{~W}\left({ }^{30} \mathrm{Si}, 4 \mathrm{n}\right), \mathrm{E}=151 \mathrm{MeV}$; measured delayed $\mathrm{E} \gamma, \mathrm{I} \gamma$, (recoil) $\gamma$-coin. ${ }^{208} \mathrm{Ra}$ deduced levels, $\mathrm{J}, \pi$, isomeric state $\mathrm{T}_{1 / 2} \cdot{ }^{182,183,184,186} \mathrm{~W}\left({ }^{30} \mathrm{Si}, \mathrm{X}\right){ }^{210} \mathrm{Ra} /{ }^{209} \mathrm{Ra} /{ }^{208} \mathrm{Ra} /{ }^{209} \mathrm{Fr}$, $\mathrm{E}=151 \mathrm{MeV}$; measured delayed $\mathrm{E} \gamma$, I $\gamma$, (recoil) $\gamma$-coin. ${ }^{209} \mathrm{Fr},{ }^{209,210} \mathrm{Ra}$ deduced transitions. Level systematics in neighboring nuclides discussed. JOUR PRVCA 71014302

2005RE23 NUCLEAR REACTIONS ${ }^{182,184} \mathrm{~W}\left({ }^{30} \mathrm{Si}, 4 \mathrm{n}\right), \mathrm{E}=148 \mathrm{MeV}$; measured delayed $\mathrm{E} \gamma$, I $\gamma, \gamma \gamma$-, (recoil) $\gamma$-coin. ${ }^{208,210} \mathrm{Ra}$ deduced levels, $\mathrm{J}, \pi$, isomers $\mathrm{T}_{1 / 2}, \mathrm{~B}(\mathrm{E} 2)$. Mass separator. JOUR JPGPE $31 \mathrm{~S} 1605$

${ }^{208} \mathrm{Ac} \quad 2005 \mathrm{LI} 17$ NUCLEAR REACTIONS Be $\left({ }^{238} \mathrm{U}, \mathrm{X}\right){ }^{207} \mathrm{Ac} /{ }^{208} \mathrm{Ac} /{ }^{209} \mathrm{Ac} /{ }^{210} \mathrm{Ac} /$ ${ }^{211} \mathrm{Ac} /{ }^{212} \mathrm{Ac} /{ }^{213} \mathrm{Ac} /{ }^{214} \mathrm{Ac} /{ }^{215} \mathrm{Ac} /{ }^{216} \mathrm{Ac} /{ }^{217} \mathrm{Ac} /{ }^{218} \mathrm{Ac} /{ }^{219} \mathrm{Ac}$ $/{ }^{220} \mathrm{Ac} /{ }^{221} \mathrm{Ac} /{ }^{211} \mathrm{Th} /{ }^{212} \mathrm{Th} /{ }^{213} \mathrm{Th} /{ }^{214} \mathrm{Th} /{ }^{215} \mathrm{Th} /{ }^{216} \mathrm{Th} /$ ${ }^{217} \mathrm{Th} /{ }^{218} \mathrm{Th} /{ }^{219} \mathrm{Th} /{ }^{220} \mathrm{Th} /{ }^{221} \mathrm{Th} /{ }^{222} \mathrm{Th} /{ }^{223} \mathrm{Th} /{ }^{216} \mathrm{~Pa} /$ ${ }^{217} \mathrm{~Pa} /{ }^{218} \mathrm{~Pa} /{ }^{219} \mathrm{~Pa} /{ }^{220} \mathrm{~Pa} /{ }^{221} \mathrm{~Pa} /{ }^{222} \mathrm{~Pa} /{ }^{223} \mathrm{~Pa} /{ }^{224} \mathrm{~Pa} /{ }^{225} \mathrm{~Pa} /$ ${ }^{226} \mathrm{~Pa} /{ }^{227} \mathrm{~Pa}, \mathrm{E}=1 \mathrm{GeV} /$ nucleon; measured (fragment)(decay)-coin, fragment yields. Fragment separator. JOUR NIMAE 543591

\section{$\mathrm{A}=209$}

${ }^{209} \mathrm{~Pb}$ 2005SMZZ NUCLEAR REACTIONS ${ }^{204,206,207,208} \mathrm{~Pb},{ }^{205} \mathrm{Tl}(\mathrm{n}, \mathrm{F}),(\mathrm{p}, \mathrm{F})$, $\mathrm{E}=30-180 \mathrm{MeV}$; measured fission $\sigma .{ }^{206} \mathrm{Tl},{ }^{205,206,207,208,209} \mathrm{~Pb}$, 205,207,208,209 Bi; deduced fissility. CONF Santa Fe (Nucl Data for Sci and Technol) Proc,Vol1,P637

${ }^{209} \mathrm{Bi}$ 2005BA88 NUCLEAR REACTIONS ${ }^{208} \mathrm{~Pb}(\mathrm{p}, \gamma), \mathrm{E}=11.9 \mathrm{MeV}$; measured $\mathrm{E} \gamma, \mathrm{I} \gamma$. ${ }^{147} \mathrm{Sm}\left({ }^{16} \mathrm{O}, 3 \mathrm{n}\right), \mathrm{E}=73 \mathrm{MeV}$; measured $\mathrm{E} \gamma, \mathrm{I} \gamma, \gamma \gamma$-coin. ${ }^{160} \mathrm{Yb}$ deduced high-spin levels, J, $\pi$. Afrodite array. JOUR JPGPE 31 S1747

2005CH66 NUCLEAR REACTIONS ${ }^{209} \mathrm{Bi}\left({ }^{26} \mathrm{Mg},{ }^{26} \mathrm{Mg}\right.$ '), E=78.6 MeV / nucleon; ${ }^{197} \mathrm{Au}\left({ }^{32} \mathrm{Mg},{ }^{32} \mathrm{Mg}\right.$ '), E=81.1 MeV / nucleon; ${ }^{209} \mathrm{Bi}\left({ }^{34} \mathrm{Mg},{ }^{34} \mathrm{Mg}\right.$ ') $\mathrm{E}=76.4 \mathrm{MeV} /$ nucleon; measured $\mathrm{E} \gamma, \mathrm{I} \gamma$, (particle) $\gamma$-coin following projectile Coulomb excitation. ${ }^{26,32,34} \mathrm{Mg}$ deduced transitions $\mathrm{B}(\mathrm{E} 2)$, deformation parameters. Comparison with previous work, model predictions. JOUR PRVCA 72054320 


\section{$\mathrm{A}=209$ (continued)}

2005MIZY NUCLEAR REACTIONS ${ }^{209} \mathrm{Bi}(\mathrm{n}, \mathrm{n} \gamma)$, E=threshold-18 MeV; measured $\gamma$-ray production $\sigma$. Comparison with previous results and model predictions. CONF Santa Fe (Nucl Data for Sci and Technol) Proc, Vol1,P973

2005SMZZ NUCLEAR REACTIONS ${ }^{204,206,207,208} \mathrm{~Pb},{ }^{205} \mathrm{Tl}(\mathrm{n}, \mathrm{F}),(\mathrm{p}, \mathrm{F})$, $\mathrm{E}=30-180 \mathrm{MeV}$; measured fission $\sigma .{ }^{206} \mathrm{Tl},{ }^{205,206,207,208,209} \mathrm{~Pb}$, $205,207,208,209 \mathrm{Bi}$; deduced fissility. CONF Santa Fe (Nucl Data for Sci and Technol) Proc,Vol1,P637

${ }^{209} \mathrm{Po} \quad 2005 \mathrm{LI} 17$ RADIOACTIVITY ${ }^{216,217,221,222} \mathrm{Th},{ }^{216} \mathrm{Ac},{ }^{215} \mathrm{Ra},{ }^{214} \mathrm{Fr},{ }^{213} \mathrm{Rn}(\alpha)$ [from $\mathrm{Be}\left({ }^{238} \mathrm{U}, \mathrm{X}\right)$ and subsequent decay]; measured $\mathrm{E} \alpha, \mathrm{T}_{1 / 2}$. Fragment separator. JOUR NIMAE 543591

${ }^{209} \mathrm{At}$ 2005KU06 RADIOACTIVITY ${ }^{210,211,212,213,214} \operatorname{Fr}(\alpha)\left[\right.$ from ${ }^{209} \mathrm{Bi}\left({ }^{12} \mathrm{C}, \mathrm{xn}\right)$ and subsequent decay]; measured $\mathrm{E} \alpha, \mathrm{I} \alpha, \mathrm{E} \gamma, \mathrm{I} \gamma, \alpha \gamma$-coin, $\mathrm{T}_{1 / 2}$; deduced hindrance factors. ${ }^{206,207,208,209,210}$ At deduced levels, $\mathrm{J}, \pi$. Comparison with previous results. JOUR ZAANE 23417

${ }^{209} \mathrm{Rn} \quad 2005 \mathrm{KUZV}$ RADIOACTIVITY ${ }^{213} \operatorname{Ra}(\alpha)$ [from ${ }^{170} \operatorname{Er}\left({ }^{50} \mathrm{Ti}, \mathrm{X}\right)$ ]; measured $\mathrm{E} \gamma, \mathrm{E} \alpha$, $\alpha \gamma$-coin. ${ }^{209} \mathrm{Rn}$ deduced levels, J, $\pi$, ICC. REPT GSI 2005-1,P76,Kuusiniemi NUCLEAR MOMENTS ${ }^{113,115} \mathrm{In},{ }^{153,155} \mathrm{Eu},{ }^{185,187} \mathrm{Re},{ }^{203,205} \mathrm{Tl}$, ${ }^{209,211} \mathrm{Fr}$; measured hfs; deduced hyperfine magnetic anomaly, relative radii. Laser resonance fluorescence. JOUR BRSPE 68157

$2005 \mathrm{C002}$ NUCLEAR REACTIONS ${ }^{197} \mathrm{Au}\left({ }^{18} \mathrm{O}, 4 \mathrm{n}\right),\left({ }^{18} \mathrm{O}, 5 \mathrm{n}\right),\left({ }^{18} \mathrm{O}, 6 \mathrm{n}\right),\left({ }^{18} \mathrm{O}\right.$, $7 \mathrm{n}), \mathrm{E}=75-130 \mathrm{MeV}$; measured delayed $\mathrm{E} \alpha$, excitation functions. Comparison with model predictions. JOUR PRVCA 71014609 2005RE02 NUCLEAR REACTIONS ${ }^{182} \mathrm{~W}\left({ }^{30} \mathrm{Si}, 4 \mathrm{n}\right), \mathrm{E}=151 \mathrm{MeV}$; measured delayed $\mathrm{E} \gamma$, I $\gamma$, (recoil) $\gamma$-coin. ${ }^{208} \mathrm{Ra}$ deduced levels, $\mathrm{J}, \pi$, isomeric state $\mathrm{T}_{1 / 2} \cdot{ }^{182,183,184,186} \mathrm{~W}\left({ }^{30} \mathrm{Si}, \mathrm{X}\right){ }^{210} \mathrm{Ra} /{ }^{209} \mathrm{Ra} /{ }^{208} \mathrm{Ra} /{ }^{209} \mathrm{Fr}$, $\mathrm{E}=151 \mathrm{MeV}$; measured delayed $\mathrm{E} \gamma$, I $\gamma$, (recoil) $\gamma$-coin. ${ }^{209} \mathrm{Fr},{ }^{209,210} \mathrm{Ra}$ deduced transitions. Level systematics in neighboring nuclides discussed. JOUR PRVCA 71014302

${ }^{209} \mathrm{Ra} \quad$ 2005RE02 NUCLEAR REACTIONS ${ }^{182} \mathrm{~W}\left({ }^{30} \mathrm{Si}, 4 \mathrm{n}\right), \mathrm{E}=151 \mathrm{MeV}$; measured delayed $\mathrm{E} \gamma, \mathrm{I} \gamma$, (recoil) $\gamma$-coin. ${ }^{208} \mathrm{Ra}$ deduced levels, $\mathrm{J}, \pi$, isomeric state $\mathrm{T}_{1 / 2} \cdot{ }^{182,183,184,186} \mathrm{~W}\left({ }^{30} \mathrm{Si}, \mathrm{X}\right){ }^{210} \mathrm{Ra} /{ }^{209} \mathrm{Ra} /{ }^{208} \mathrm{Ra} /{ }^{209} \mathrm{Fr}$, $\mathrm{E}=151 \mathrm{MeV}$; measured delayed $\mathrm{E} \gamma$, I $\gamma$, (recoil) $\gamma$-coin. ${ }^{209} \mathrm{Fr},{ }^{209,210} \mathrm{Ra}$ deduced transitions. Level systematics in neighboring nuclides discussed. JOUR PRVCA 71014302

${ }^{209} \mathrm{Ac}$ 2005LI17 NUCLEAR REACTIONS Be $\left({ }^{238} \mathrm{U}, \mathrm{X}\right){ }^{207} \mathrm{Ac} /{ }^{208} \mathrm{Ac} /{ }^{209} \mathrm{Ac} /{ }^{210} \mathrm{Ac} /$ ${ }^{211} \mathrm{Ac} /{ }^{212} \mathrm{Ac} /{ }^{213} \mathrm{Ac} /{ }^{214} \mathrm{Ac} /{ }^{215} \mathrm{Ac} /{ }^{216} \mathrm{Ac} /{ }^{217} \mathrm{Ac} /{ }^{218} \mathrm{Ac} /{ }^{219} \mathrm{Ac}$ / ${ }^{220} \mathrm{Ac} /{ }^{221} \mathrm{Ac} /{ }^{211} \mathrm{Th} /{ }^{212} \mathrm{Th} /{ }^{213} \mathrm{Th} /{ }^{214} \mathrm{Th} /{ }^{215} \mathrm{Th} /{ }^{216} \mathrm{Th} /$ ${ }^{217} \mathrm{Th} /{ }^{218} \mathrm{Th} /{ }^{219} \mathrm{Th} /{ }^{220} \mathrm{Th} /{ }^{221} \mathrm{Th} /{ }^{222} \mathrm{Th} /{ }^{223} \mathrm{Th} /{ }^{216} \mathrm{~Pa} /$ ${ }^{217} \mathrm{~Pa} /{ }^{218} \mathrm{~Pa} /{ }^{219} \mathrm{~Pa} /{ }^{220} \mathrm{~Pa} /{ }^{221} \mathrm{~Pa} /{ }^{222} \mathrm{~Pa} /{ }^{223} \mathrm{~Pa} /{ }^{224} \mathrm{~Pa} /{ }^{225} \mathrm{~Pa} /$ ${ }^{226} \mathrm{~Pa} /{ }^{227} \mathrm{~Pa}, \mathrm{E}=1 \mathrm{GeV} /$ nucleon; measured (fragment)(decay)-coin, fragment yields. Fragment separator. JOUR NIMAE 543591 


\section{$A=210$}

\begin{tabular}{|c|c|c|}
\hline${ }^{210} \mathrm{~Pb}$ & 2005SA52 & $\begin{array}{l}\text { NUCLEAR REACTIONS }{ }^{208} \mathrm{~Pb}\left({ }^{6} \mathrm{He},{ }^{6} \mathrm{He}\right),\left({ }^{6} \mathrm{He}, \alpha\right), \mathrm{E}=14,16,17,18 \text {, } \\
22 \mathrm{MeV} \text {; measured } \sigma(\theta) \text {; deduced reaction mechanism features. JOUR } \\
\text { JPGPE } 31 \text { S1953 }\end{array}$ \\
\hline \multirow[t]{6}{*}{${ }^{210} \mathrm{Bi}$} & 2004RA29 & $\begin{array}{l}\text { NUCLEAR REACTIONS }{ }^{208} \mathrm{~Pb},{ }^{209} \mathrm{Bi}(\mathrm{n}, \gamma), \mathrm{E}=\text { spectrum; measured } \\
\sigma \text {. Astrophysical implications discussed. Activation technique. JOUR } \\
\text { PRVCA } 70065803\end{array}$ \\
\hline & 2005BO27 & $\begin{array}{l}\text { NUCLEAR REACTIONS }{ }^{209} \mathrm{Bi}(\mathrm{n}, \gamma), \mathrm{E}=\text { cold; measured } \mathrm{E} \gamma, \mathrm{I} \gamma \text {, } \\
\text { capture } \sigma \text {. JOUR JRNCD } 265267\end{array}$ \\
\hline & 2005BOZT & $\begin{array}{l}\text { NUCLEAR REACTIONS }{ }^{206} \mathrm{~Pb}(\mathrm{n}, \mathrm{X}),(\mathrm{n}, \gamma), \mathrm{E}=0-600 \mathrm{keV} \text {; measured } \\
\text { total and capture } \sigma \text {; deduced resonance parameters. }{ }^{206} \mathrm{~Pb},{ }^{209} \mathrm{Bi}(\mathrm{n}, \gamma) \text {, } \\
\mathrm{E}=\text { thermal; measured } \sigma \text {. THESIS A Borella, Gent Univ }\end{array}$ \\
\hline & 2005BOZW & $\begin{array}{l}\text { NUCLEAR REACTIONS }{ }^{209} \mathrm{Bi}(\mathrm{n}, \gamma), \mathrm{E}=\text { thermal; measured total } \\
\text { capture } \sigma \text {, partial } \sigma \text { for capture to ground and isomeric states. CONF } \\
\text { Santa Fe (Nucl Data for Sci and Technol) Proc,Vol1,P648 }\end{array}$ \\
\hline & 2005DE16 & $\begin{array}{l}\text { NUCLEAR REACTIONS }{ }^{209} \mathrm{Bi}\left({ }^{6} \mathrm{He}, \alpha\right),\left({ }^{6} \mathrm{He}, \mathrm{n} \alpha\right), \mathrm{E}=23.1 \mathrm{MeV} ; \\
\text { measured } \mathrm{E} \alpha, \mathrm{E} \nu, \text { n } \alpha \text {-coin, angular distributions following residual } \\
\text { nucleus decay; deduced two-neutron transfer } \sigma . \text { JOUR PRVCA } 71 \\
051601\end{array}$ \\
\hline & 2005DOZY & $\begin{array}{l}\text { NUCLEAR REACTIONS }{ }^{207} \mathrm{~Pb},{ }^{209} \mathrm{Bi}(\mathrm{n}, \gamma), \mathrm{E}=0-1 \mathrm{MeV} \text {; measured } \\
\text { capture } \sigma \text {. Comparison with previous results. CONF Santa Fe (Nucl } \\
\text { Data for Sci and Technol) Proc,Vol2,P1521 }\end{array}$ \\
\hline \multirow[t]{2}{*}{${ }^{210} \mathrm{Po}$} & 2005HE13 & $\begin{array}{l}\text { NUCLEAR REACTIONS }{ }^{209} \operatorname{Bi}(\alpha, 2 \mathrm{n}),(\alpha, 3 \mathrm{n}),(\alpha, 2 \mathrm{np}), \mathrm{E} \approx 20-40 \\
\mathrm{MeV} \text {; measured } \sigma \text {; deduced thick-target yields. Stacked-foil activation, } \\
\text { comparison with model predictions. JOUR ARISE } 631\end{array}$ \\
\hline & 2005HEZX & $\begin{array}{l}\text { NUCLEAR REACTIONS }{ }^{209} \mathrm{Bi}(\alpha, 2 \mathrm{n}),(\alpha, 3 \mathrm{n}), \mathrm{E} \approx 20-39 \mathrm{MeV} ; \\
{ }^{209} \mathrm{Bi}(\alpha, \mathrm{X}){ }^{210} \mathrm{Po}, \mathrm{E} \approx 20-39 \mathrm{MeV} ; \text { measured production } \sigma ; \text { deduced } \\
\text { thick target yields. Activation technique. CONF Santa Fe (Nucl Data } \\
\text { for Sci and Technol) Proc,Vol1,P957 }\end{array}$ \\
\hline \multirow[t]{4}{*}{${ }^{210} \mathrm{At}$} & $2005 \mathrm{HE} 13$ & $\begin{array}{l}\text { NUCLEAR REACTIONS }{ }^{209} \operatorname{Bi}(\alpha, 2 \mathrm{n}),(\alpha, 3 \mathrm{n}),(\alpha, 2 \mathrm{np}), \mathrm{E} \approx 20-40 \\
\mathrm{MeV} \text {; measured } \sigma \text {; deduced thick-target yields. Stacked-foil activation, } \\
\text { comparison with model predictions. JOUR ARISE } 631\end{array}$ \\
\hline & 2005HEZX & $\begin{array}{l}\text { NUCLEAR REACTIONS }{ }^{209} \mathrm{Bi}(\alpha, 2 \mathrm{n}),(\alpha, 3 \mathrm{n}), \mathrm{E} \approx 20-39 \mathrm{MeV} ; \\
{ }^{209} \mathrm{Bi}(\alpha, \mathrm{X})^{210} \mathrm{Po}, \mathrm{E} \approx 20-39 \mathrm{MeV} ; \text { measured production } \sigma ; \text { deduced } \\
\text { thick target yields. Activation technique. CONF Santa Fe (Nucl Data } \\
\text { for Sci and Technol) Proc,Vol1,P957 }\end{array}$ \\
\hline & 2005KU06 & $\begin{array}{l}\text { RADIOACTIVITY }{ }^{210,211,212,213,214} \operatorname{Fr}(\alpha)\left[\text { from }{ }^{209} \mathrm{Bi}\left({ }^{12} \mathrm{C}, \text { xn }\right) \text { and }\right. \\
\text { subsequent decay]; measured } \mathrm{E} \alpha, \mathrm{I} \alpha, \mathrm{E} \gamma, \mathrm{I} \gamma, \alpha \gamma \text {-coin, } \mathrm{T}_{1 / 2} ; \text { deduced } \\
\text { hindrance factors. }{ }^{206,207,208,209,210} \mathrm{At} \text { deduced levels, J, } \pi \text {. Comparison } \\
\text { with previous results. JOUR ZAANE } 23417\end{array}$ \\
\hline & 2005LI17 & $\begin{array}{l}\text { RADIOACTIVITY }{ }^{216,217,221,222} \mathrm{Th},{ }^{216} \mathrm{Ac},{ }^{215} \mathrm{Ra},{ }^{214} \mathrm{Fr},{ }^{213} \mathrm{Rn}(\alpha) \\
\text { [from Be }\left({ }^{238} \mathrm{U}, \mathrm{X}\right) \text { and subsequent decay]; measured } \mathrm{E} \alpha, \mathrm{T}_{1 / 2} \text {. } \\
\text { Fragment separator. JOUR NIMAE } 543591\end{array}$ \\
\hline${ }^{210} \mathrm{Rn}$ & 2005P010 & $\begin{array}{l}\text { NUCLEAR REACTIONS }{ }^{198} \mathrm{Pt}\left({ }^{17} \mathrm{O}, 5 \mathrm{n}\right), \mathrm{E}=96 \mathrm{MeV} \text {; measured } \\
\text { prompt and delayed } \mathrm{E} \gamma, \mathrm{I} \gamma, \gamma \gamma \text {-coin, } \mathrm{E}(\mathrm{ce}), \mathrm{I}(\mathrm{ce}) .{ }^{210} \mathrm{Rn} \text { deduced } \\
\text { high-spin levels, J, } \pi \text {, ICC, configurations. Enriched target, pulsed } \\
\text { beam, superconducting electron spectrometer. JOUR NUPAB } 75683\end{array}$ \\
\hline
\end{tabular}




\section{$\mathrm{A}=210$ (continued)}

2005POZZ NUCLEAR REACTIONS ${ }^{198} \mathrm{Pt}\left({ }^{17} \mathrm{O}, 5 \mathrm{n}\right), \mathrm{E}=96 \mathrm{MeV}$; measured prompt and delayed $\mathrm{E} \gamma, \mathrm{I} \gamma, \gamma \gamma$-coin, $\mathrm{E}(\mathrm{ce}), \mathrm{I}(\mathrm{ce}) .{ }^{210} \mathrm{Rn}$ deduced high-spin levels, J, $\pi$, ICC, configurations. Pulsed beam. PREPRINT ANU-P/1649,Poletti

${ }^{210} \mathrm{Fr} \quad 2005 \mathrm{CO} 02 \quad$ NUCLEAR REACTIONS ${ }^{197} \mathrm{Au}\left({ }^{18} \mathrm{O}, 4 \mathrm{n}\right),\left({ }^{18} \mathrm{O}, 5 \mathrm{n}\right),\left({ }^{18} \mathrm{O}, 6 \mathrm{n}\right),\left({ }^{18} \mathrm{O}\right.$, $7 \mathrm{n}), \mathrm{E}=75-130 \mathrm{MeV}$; measured delayed $\mathrm{E} \alpha$, excitation functions. Comparison with model predictions. JOUR PRVCA 71014609 2005KU06 RADIOACTIVITY ${ }^{210,211,212,213,214} \mathrm{Fr}(\alpha)$ [from ${ }^{209} \mathrm{Bi}\left({ }^{12} \mathrm{C}\right.$, xn) and subsequent decay]; measured $\mathrm{E} \alpha, \mathrm{I} \alpha, \mathrm{E} \gamma, \mathrm{I} \gamma, \alpha \gamma$-coin, $\mathrm{T}_{1 / 2}$; deduced hindrance factors. ${ }^{206,207,208,209,210}$ At deduced levels, J, $\pi$. Comparison with previous results. JOUR ZAANE 23417

${ }^{210} \mathrm{Ra} \quad 2005 \mathrm{RE} 02$ NUCLEAR REACTIONS ${ }^{182} \mathrm{~W}\left({ }^{30} \mathrm{Si}, 4 \mathrm{n}\right), \mathrm{E}=151 \mathrm{MeV}$; measured delayed $\mathrm{E} \gamma$, I $\gamma$, (recoil) $\gamma$-coin. ${ }^{208} \mathrm{Ra}$ deduced levels, $\mathrm{J}, \pi$, isomeric state $\mathrm{T}_{1 / 2} .{ }^{182,183,184,186} \mathrm{~W}\left({ }^{30} \mathrm{Si}, \mathrm{X}\right){ }^{210} \mathrm{Ra} /{ }^{209} \mathrm{Ra} /{ }^{208} \mathrm{Ra} /{ }^{209} \mathrm{Fr}$, $\mathrm{E}=151 \mathrm{MeV}$; measured delayed $\mathrm{E} \gamma, \mathrm{I} \gamma$, (recoil) $\gamma$-coin. ${ }^{209} \mathrm{Fr},{ }^{209,210} \mathrm{Ra}$ deduced transitions. Level systematics in neighboring nuclides discussed. JOUR PRVCA 71014302

2005RE23 NUCLEAR REACTIONS ${ }^{182,184} \mathrm{~W}\left({ }^{30} \mathrm{Si}, 4 \mathrm{n}\right), \mathrm{E}=148 \mathrm{MeV}$; measured delayed $\mathrm{E} \gamma$, I $\gamma, \gamma \gamma$-, (recoil) $\gamma$-coin. ${ }^{208,210} \mathrm{Ra}$ deduced levels, J, $\pi$, isomers $\mathrm{T}_{1 / 2}, \mathrm{~B}(\mathrm{E} 2)$. Mass separator. JOUR JPGPE $31 \mathrm{~S} 1605$

${ }^{210} \mathrm{Ac} \quad 2005 \mathrm{LI} 17$ NUCLEAR REACTIONS Be $\left({ }^{238} \mathrm{U}, \mathrm{X}\right){ }^{207} \mathrm{Ac} /{ }^{208} \mathrm{Ac} /{ }^{209} \mathrm{Ac} /{ }^{210} \mathrm{Ac} /$ ${ }^{211} \mathrm{Ac} /{ }^{212} \mathrm{Ac} /{ }^{213} \mathrm{Ac} /{ }^{214} \mathrm{Ac} /{ }^{215} \mathrm{Ac} /{ }^{216} \mathrm{Ac} /{ }^{217} \mathrm{Ac} /{ }^{218} \mathrm{Ac} /{ }^{219} \mathrm{Ac}$ $/{ }^{220} \mathrm{Ac} /{ }^{221} \mathrm{Ac} /{ }^{211} \mathrm{Th} /{ }^{212} \mathrm{Th} /{ }^{213} \mathrm{Th} /{ }^{214} \mathrm{Th} /{ }^{215} \mathrm{Th} /{ }^{216} \mathrm{Th} /$ ${ }^{217} \mathrm{Th} /{ }^{218} \mathrm{Th} /{ }^{219} \mathrm{Th} /{ }^{220} \mathrm{Th} /{ }^{221} \mathrm{Th} /{ }^{222} \mathrm{Th} /{ }^{223} \mathrm{Th} /{ }^{216} \mathrm{~Pa} /$ ${ }^{217} \mathrm{~Pa} /{ }^{218} \mathrm{~Pa} /{ }^{219} \mathrm{~Pa} /{ }^{220} \mathrm{~Pa} /{ }^{221} \mathrm{~Pa} /{ }^{222} \mathrm{~Pa} /{ }^{223} \mathrm{~Pa} /{ }^{224} \mathrm{~Pa} /{ }^{225} \mathrm{~Pa} /$ ${ }^{226} \mathrm{~Pa} /{ }^{227} \mathrm{~Pa}, \mathrm{E}=1 \mathrm{GeV} /$ nucleon; measured (fragment)(decay)-coin, fragment yields. Fragment separator. JOUR NIMAE 543591

\section{$\mathrm{A}=\mathbf{2 1 1}$}

${ }^{211} \mathrm{~Pb} \quad$ 2004LAZV

2005LA01

${ }^{211} \mathrm{Bi}$

${ }^{211}$ At $2005 \mathrm{HE} 13$
NUCLEAR REACTIONS ${ }^{238} \mathrm{U}\left({ }^{208} \mathrm{~Pb}, \mathrm{X}\right){ }^{211} \mathrm{~Pb}, \mathrm{E}=1360 \mathrm{MeV}$; measured prompt and delayed $\mathrm{E} \gamma, \mathrm{I} \gamma, \gamma \gamma$-coin. ${ }^{211} \mathrm{~Pb}$ deduced high-spin levels, $\mathrm{J}, \pi$, configurations, isomeric states $\mathrm{T}_{1 / 2}$. Gammasphere array. PREPRINT ANU-P/1637,Lane NUCLEAR REACTIONS ${ }^{238} \mathrm{U}\left({ }^{208} \mathrm{~Pb}, \mathrm{X}\right){ }^{211} \mathrm{~Pb}, \mathrm{E}=1360 \mathrm{MeV}$; measured prompt and delayed $\mathrm{E} \gamma, \mathrm{I} \gamma, \gamma \gamma$-coin. ${ }^{211} \mathrm{~Pb}$ deduced high-spin levels, $\mathrm{J}, \pi$, configurations, isomeric states $\mathrm{T}_{1 / 2}$. Gammasphere array. JOUR PYLBB 60634 NUCLEAR REACTIONS ${ }^{209} \mathrm{Bi}\left({ }^{6} \mathrm{He}, \alpha\right),\left({ }^{6} \mathrm{He}, \mathrm{n} \alpha\right), \mathrm{E}=23.1 \mathrm{MeV}$; measured $\mathrm{E} \alpha, \mathrm{E} \nu, \mathrm{n} \alpha$-coin, angular distributions following residual nucleus decay; deduced two-neutron transfer $\sigma$. JOUR PRVCA 71 051601

NUCLEAR REACTIONS ${ }^{209} \operatorname{Bi}(\alpha, 2 \mathrm{n}),(\alpha, 3 \mathrm{n}),(\alpha, 2 \mathrm{np}), \mathrm{E} \approx 20-40$ $\mathrm{MeV}$; measured $\sigma$; deduced thick-target yields. Stacked-foil activation, comparison with model predictions. JOUR ARISE 631 


\section{$\mathrm{A}=211$ (continued)}

2005HEZX NUCLEAR REACTIONS ${ }^{209} \operatorname{Bi}(\alpha, 2 \mathrm{n}),(\alpha, 3 \mathrm{n}), \mathrm{E} \approx 20-39 \mathrm{MeV}$; ${ }^{209} \operatorname{Bi}(\alpha, \mathrm{X}){ }^{210} \mathrm{Po}, \mathrm{E} \approx 20-39 \mathrm{MeV}$; measured production $\sigma$; deduced thick target yields. Activation technique. CONF Santa Fe (Nucl Data for Sci and Technol) Proc,Vol1,P957

${ }^{211} \mathrm{Rn} \quad$ 2005LI17 RADIOACTIVITY ${ }^{216,217,221,222} \mathrm{Th},{ }^{216} \mathrm{Ac},{ }^{215} \mathrm{Ra},{ }^{214} \mathrm{Fr},{ }^{213} \mathrm{Rn}(\alpha)$ [from $\mathrm{Be}\left({ }^{238} \mathrm{U}, \mathrm{X}\right)$ and subsequent decay]; measured $\mathrm{E} \alpha, \mathrm{T}_{1 / 2}$. Fragment separator. JOUR NIMAE 543591

${ }^{211} \mathrm{Fr} \quad 2004 \mathrm{MB03}$ NUCLEAR MOMENTS ${ }^{113,115} \mathrm{In},{ }^{153,155} \mathrm{Eu},{ }^{185,187} \mathrm{Re},{ }^{203,205} \mathrm{Tl}$, ${ }^{209,211} \mathrm{Fr}$; measured hfs; deduced hyperfine magnetic anomaly, relative radii. Laser resonance fluorescence. JOUR BRSPE 68157

$2005 \mathrm{C} 002$ NUCLEAR REACTIONS ${ }^{197} \mathrm{Au}\left({ }^{18} \mathrm{O}, 4 \mathrm{n}\right),\left({ }^{18} \mathrm{O}, 5 \mathrm{n}\right),\left({ }^{18} \mathrm{O}, 6 \mathrm{n}\right),\left({ }^{18} \mathrm{O}\right.$, $7 \mathrm{n}), \mathrm{E}=75-130 \mathrm{MeV}$; measured delayed $\mathrm{E} \alpha$, excitation functions. Comparison with model predictions. JOUR PRVCA 71014609 2005KU06 RADIOACTIVITY ${ }^{210,211,212,213,214} \operatorname{Fr}(\alpha)$ [from ${ }^{209} \operatorname{Bi}\left({ }^{12} \mathrm{C}\right.$, xn) and subsequent decay]; measured $\mathrm{E} \alpha, \mathrm{I} \alpha, \mathrm{E} \gamma, \mathrm{I} \gamma, \alpha \gamma$-coin, $\mathrm{T}_{1 / 2}$; deduced hindrance factors. ${ }^{206,207,208,209,210}$ At deduced levels, J, $\pi$. Comparison with previous results. JOUR ZAANE 23417

${ }^{211} \mathrm{Ra} \quad 2005 \mathrm{KU} 31$ RADIOACTIVITY ${ }^{215,216,216 m, 217} \mathrm{Th}(\alpha)$ [from $\left.{ }^{170} \operatorname{Er}\left({ }^{50} \mathrm{Ti}, \mathrm{xn}\right)\right]$; measured $\mathrm{E} \alpha, \mathrm{I} \alpha, \mathrm{E} \gamma, \mathrm{I} \gamma, \alpha \gamma$-coin, $\mathrm{T}_{1 / 2} .{ }^{211,212,213} \mathrm{Ra}$ deduced levels, J, $\pi$, ICC. JOUR ZAANE 25397

${ }^{211} \mathrm{Ac} \quad 2005 \mathrm{LI} 17$ NUCLEAR REACTIONS Be $\left({ }^{238} \mathrm{U}, \mathrm{X}\right){ }^{207} \mathrm{Ac} /{ }^{208} \mathrm{Ac} /{ }^{209} \mathrm{Ac} /{ }^{210} \mathrm{Ac} /$ ${ }^{211} \mathrm{Ac} /{ }^{212} \mathrm{Ac} /{ }^{213} \mathrm{Ac} /{ }^{214} \mathrm{Ac} /{ }^{215} \mathrm{Ac} /{ }^{216} \mathrm{Ac} /{ }^{217} \mathrm{Ac} /{ }^{218} \mathrm{Ac} /{ }^{219} \mathrm{Ac}$ $/{ }^{220} \mathrm{Ac} /{ }^{221} \mathrm{Ac} /{ }^{211} \mathrm{Th} /{ }^{212} \mathrm{Th} /{ }^{213} \mathrm{Th} /{ }^{214} \mathrm{Th} /{ }^{215} \mathrm{Th} /{ }^{216} \mathrm{Th} /$ ${ }^{217} \mathrm{Th} /{ }^{218} \mathrm{Th} /{ }^{219} \mathrm{Th} /{ }^{220} \mathrm{Th} /{ }^{221} \mathrm{Th} /{ }^{222} \mathrm{Th} /{ }^{223} \mathrm{Th} /{ }^{216} \mathrm{~Pa} /$ ${ }^{217} \mathrm{~Pa} /{ }^{218} \mathrm{~Pa} /{ }^{219} \mathrm{~Pa} /{ }^{220} \mathrm{~Pa} /{ }^{221} \mathrm{~Pa} /{ }^{222} \mathrm{~Pa} /{ }^{223} \mathrm{~Pa} /{ }^{224} \mathrm{~Pa} /{ }^{225} \mathrm{~Pa} /$ ${ }^{226} \mathrm{~Pa} /{ }^{227} \mathrm{~Pa}, \mathrm{E}=1 \mathrm{GeV} /$ nucleon; measured (fragment)(decay)-coin, fragment yields. Fragment separator. JOUR NIMAE 543591

${ }^{211} \mathrm{Th}$ 2005LI17 NUCLEAR REACTIONS Be $\left({ }^{238} \mathrm{U}, \mathrm{X}\right){ }^{207} \mathrm{Ac} /{ }^{208} \mathrm{Ac} /{ }^{209} \mathrm{Ac} /{ }^{210} \mathrm{Ac} /$ ${ }^{211} \mathrm{Ac} /{ }^{212} \mathrm{Ac} /{ }^{213} \mathrm{Ac} /{ }^{214} \mathrm{Ac} /{ }^{215} \mathrm{Ac} /{ }^{216} \mathrm{Ac} /{ }^{217} \mathrm{Ac} /{ }^{218} \mathrm{Ac} /{ }^{219} \mathrm{Ac}$ $/{ }^{220} \mathrm{Ac} /{ }^{221} \mathrm{Ac} /{ }^{211} \mathrm{Th} /{ }^{212} \mathrm{Th} /{ }^{213} \mathrm{Th} /{ }^{214} \mathrm{Th} /{ }^{215} \mathrm{Th} /{ }^{216} \mathrm{Th} /$ ${ }^{217} \mathrm{Th} /{ }^{218} \mathrm{Th} /{ }^{219} \mathrm{Th} /{ }^{220} \mathrm{Th} /{ }^{221} \mathrm{Th} /{ }^{222} \mathrm{Th} /{ }^{223} \mathrm{Th} /{ }^{216} \mathrm{~Pa} /$ ${ }^{217} \mathrm{~Pa} /{ }^{218} \mathrm{~Pa} /{ }^{219} \mathrm{~Pa} /{ }^{220} \mathrm{~Pa} /{ }^{221} \mathrm{~Pa} /{ }^{222} \mathrm{~Pa} /{ }^{223} \mathrm{~Pa} /{ }^{224} \mathrm{~Pa} /{ }^{225} \mathrm{~Pa} /$ ${ }^{226} \mathrm{~Pa} /{ }^{227} \mathrm{~Pa}, \mathrm{E}=1 \mathrm{GeV} /$ nucleon; measured (fragment)(decay)-coin, fragment yields. Fragment separator. JOUR NIMAE 543591

\section{$\mathrm{A}=\mathbf{2 1 2}$}

${ }^{212} \mathrm{~Pb} \quad 2005 \mathrm{SZ} 03$

RADIOACTIVITY ${ }^{212} \mathrm{~Pb},{ }^{208} \mathrm{Tl}\left(\beta^{-}\right) ;{ }^{212} \mathrm{Bi}(\alpha),\left(\beta^{-}\right)$; measured $\mathrm{E} \gamma, \mathrm{I} \gamma$. Application to superheavy element identification discussed. JOUR JRNCD 265367

${ }^{212} \mathrm{Bi}$ 2005BE38 NUCLEAR REACTIONS C, ${ }^{27} \mathrm{Al},{ }^{208} \mathrm{~Pb}\left({ }^{8} \mathrm{Li}, \alpha\right), \mathrm{E}=27.7 \mathrm{MeV}$; measured $\mathrm{E} \alpha, \sigma(\theta)$; deduced reaction mechanism features. JOUR PRVCA 71054610

$2005 \mathrm{SZ03}$ RADIOACTIVITY ${ }^{212} \mathrm{~Pb},{ }^{208} \mathrm{Tl}\left(\beta^{-}\right) ;{ }^{212} \mathrm{Bi}(\alpha),\left(\beta^{-}\right)$; measured $\mathrm{E} \gamma, \mathrm{I} \gamma$. Application to superheavy element identification discussed. JOUR JRNCD 265367 


\section{$\mathrm{A}=212$ (continued)}

\begin{tabular}{|c|c|c|}
\hline \multirow[t]{2}{*}{${ }^{212} \mathrm{Po}$} & $2005 \mathrm{GA} 46$ & $\begin{array}{l}\text { NUCLEAR REACTIONS }{ }^{208} \mathrm{~Pb},{ }^{209} \mathrm{Bi}\left({ }^{8} \mathrm{He}, 4 \mathrm{n}\right), \mathrm{E}=28 \mathrm{MeV} \text {; measured } \\
\mathrm{E} \gamma, \mathrm{I} \gamma, \gamma \gamma \text {-coin. }{ }^{212} \mathrm{Po},{ }^{213} \mathrm{At} \text { deduced levels, } \mathrm{J}, \pi \text {. Exogam array. }\end{array}$ \\
\hline & $2005 \mathrm{SZ} 03$ & $\begin{array}{l}\text { RADIOACTIVITY }{ }^{212} \mathrm{~Pb},{ }^{208} \mathrm{Tl}\left(\beta^{-}\right) ;{ }^{212} \mathrm{Bi}(\alpha),\left(\beta^{-}\right) ; \text {measured } \mathrm{E} \gamma, \mathrm{I} \gamma \\
\text { Application to superheavy element identification discussed. JOUR } \\
\text { JRNCD } 265367\end{array}$ \\
\hline \multirow[t]{2}{*}{${ }^{212} \mathrm{Fr}$} & 2005KU06 & $\begin{array}{l}\text { RADIOACTIVITY }{ }^{210,211,212,213,214} \operatorname{Fr}(\alpha)\left[\text { from }{ }^{209} \mathrm{Bi}\left({ }^{12} \mathrm{C}, \text { xn }\right) \text { and }\right. \\
\text { subsequent decay]; measured } \mathrm{E} \alpha, \mathrm{I} \alpha, \mathrm{E} \gamma, \mathrm{I} \gamma, \alpha \gamma \text {-coin, } \mathrm{T}_{1 / 2} ; \text { deduced } \\
\text { hindrance factors. }{ }^{206,207,208,209,210} \mathrm{At} \text { deduced levels, J, } \pi \text {. Comparison } \\
\text { with previous results. JOUR ZAANE } 23417\end{array}$ \\
\hline & 2005LI17 & $\begin{array}{l}\text { RADIOACTIVITY }{ }^{216,217,221,222} \mathrm{Th},{ }^{216} \mathrm{Ac},{ }^{215} \mathrm{Ra},{ }^{214} \mathrm{Fr},{ }^{213} \mathrm{Rn}(\alpha) \\
\text { [from Be }\left({ }^{238} \mathrm{U} \text {, X) and subsequent decay]; measured } \mathrm{E} \alpha, \mathrm{T}_{1 / 2} .\right. \\
\text { Fragment separator. JOUR NIMAE } 543591\end{array}$ \\
\hline \multirow[t]{4}{*}{${ }^{212} \mathrm{Ra}$} & 2005KU31 & $\begin{array}{l}\text { RADIOACTIVITY }{ }^{215,216,216 m, 217} \mathrm{Th}(\alpha)\left[\mathrm{from}^{170} \mathrm{Er}\left({ }^{50} \mathrm{Ti}, \mathrm{xn}\right)\right] \\
\text { measured } \mathrm{E} \alpha, \mathrm{I} \alpha, \mathrm{E} \gamma, \mathrm{I} \gamma, \alpha \gamma \text {-coin, } \mathrm{T}_{1 / 2} \cdot{ }^{211,212,213} \mathrm{Ra} \text { deduced levels, J, } \\
\pi, \text { ICC. JOUR ZAANE } 25397\end{array}$ \\
\hline & $2005 \mathrm{KUZZ}$ & $\begin{array}{l}\text { RADIOACTIVITY }{ }^{216,216 m} \mathrm{Th}(\alpha),(\mathrm{IT})\left[\text { from }{ }^{170} \mathrm{Er}\left({ }^{50} \mathrm{Ti}, 4 \mathrm{n}\right)\right] \\
{ }^{251,251 m} \mathrm{No},{ }^{247,247 m} \mathrm{Fm}(\alpha)\left[\text { from }{ }^{206} \mathrm{~Pb}\left({ }^{48} \mathrm{Ca}, 3 \mathrm{n}\right) \text { and subsequent }\right.\end{array}$ \\
\hline & & $\begin{array}{l}\text { decay]; }{ }^{257,257 m} \mathrm{Db},{ }^{253,253 m} \mathrm{Lr},{ }^{249} \mathrm{Md}(\alpha)\left[\text { from }{ }^{209} \mathrm{Bi}\left({ }^{50} \mathrm{Ti}, 2 \mathrm{n}\right) \text { and }\right. \\
\text { subsequent decay]; measured } \mathrm{E} \alpha, \mathrm{E} \gamma, \alpha \gamma \text {-coin, } \mathrm{T}_{1 / 2} . \mathrm{CONF} \\
\text { Argonne(Nuclei at the Limits),P231,Kuusiniemi }\end{array}$ \\
\hline & 2005LI17 & $\begin{array}{l}\text { RADIOACTIVITY }{ }^{216,217,221,222} \mathrm{Th},{ }^{216} \mathrm{Ac},{ }^{215} \mathrm{Ra},{ }^{214} \mathrm{Fr},{ }^{213} \mathrm{Rn}(\alpha) \\
\text { [from } \mathrm{Be}\left({ }^{238} \mathrm{U}, \mathrm{X}\right) \text { and subsequent decay]; measured } \mathrm{E} \alpha, \mathrm{T}_{1 / 2} . \\
\text { Fragment separator. JOUR NIMAE } 543591\end{array}$ \\
\hline${ }^{212} \mathrm{Ac}$ & 2005 LI17 & $\begin{array}{l}\text { NUCLEAR REACTIONS Be }\left({ }^{238} \mathrm{U}, \mathrm{X}\right){ }^{207} \mathrm{Ac} /{ }^{208} \mathrm{Ac} /{ }^{209} \mathrm{Ac} /{ }^{210} \mathrm{Ac} / \\
\\
{ }^{211} \mathrm{Ac} /{ }^{212} \mathrm{Ac} /{ }^{213} \mathrm{Ac} /{ }^{214} \mathrm{Ac} /{ }^{215} \mathrm{Ac} /{ }^{216} \mathrm{Ac} /{ }^{217} \mathrm{Ac} /{ }^{218} \mathrm{Ac} /{ }^{219} \mathrm{Ac} \\
/{ }^{220} \mathrm{Ac} /{ }^{221} \mathrm{Ac} /{ }^{211} \mathrm{Th} /{ }^{212} \mathrm{Th} /{ }^{213} \mathrm{Th} /{ }^{214} \mathrm{Th} /{ }^{215} \mathrm{Th} /{ }^{216} \mathrm{Th} / \\
{ }^{217} \mathrm{Th} /{ }^{218} \mathrm{Th} /{ }^{219} \mathrm{Th} /{ }^{220} \mathrm{Th} /{ }^{221} \mathrm{Th} /{ }^{222} \mathrm{Th} /{ }^{223} \mathrm{Th} /{ }^{216} \mathrm{~Pa} / \\
\\
{ }^{217} \mathrm{~Pa} /{ }^{218} \mathrm{~Pa} /{ }^{219} \mathrm{~Pa} /{ }^{220} \mathrm{~Pa} /{ }^{221} \mathrm{~Pa} /{ }^{222} \mathrm{~Pa} /{ }^{223} \mathrm{~Pa} /{ }^{224} \mathrm{~Pa} /{ }^{225} \mathrm{~Pa} / \\
\text { fragment yields. Fragment separator. JOUR NIMAE } 543 \mathrm{GeV} \text { / } 591\end{array}$ \\
\hline $2 \mathrm{Tt}$ & $2005 \mathrm{LI} 17$ & $\begin{array}{l}\text { NUCLEAR REACTIONS Be }\left({ }^{238} \mathrm{U}, \mathrm{X}\right){ }^{207} \mathrm{Ac} /{ }^{208} \mathrm{Ac} /{ }^{209} \mathrm{Ac} /{ }^{210} \mathrm{Ac} / \\
\\
{ }^{211} \mathrm{Ac} /{ }^{212} \mathrm{Ac} /{ }^{213} \mathrm{Ac} /{ }^{214} \mathrm{Ac} /{ }^{215} \mathrm{Ac} /{ }^{216} \mathrm{Ac} /{ }^{217} \mathrm{Ac} /{ }^{218} \mathrm{Ac} /{ }^{219} \mathrm{Ac} \\
/{ }^{220} \mathrm{Ac} /{ }^{221} \mathrm{Ac} /{ }^{211} \mathrm{Th} /{ }^{212} \mathrm{Th} /{ }^{213} \mathrm{Th} /{ }^{214} \mathrm{Th} /{ }^{215} \mathrm{Th} /{ }^{216} \mathrm{Th} / \\
{ }^{217} \mathrm{Th} /{ }^{218} \mathrm{Th} /{ }^{219} \mathrm{Th} /{ }^{220} \mathrm{Th} /{ }^{221} \mathrm{Th} /{ }^{222} \mathrm{Th} /{ }^{223} \mathrm{Th} /{ }^{216} \mathrm{~Pa} / \\
{ }^{217} \mathrm{~Pa} /{ }^{218} \mathrm{~Pa} /{ }^{219} \mathrm{~Pa} /{ }^{220} \mathrm{~Pa} /{ }^{221} \mathrm{~Pa} /{ }^{222} \mathrm{~Pa} /{ }^{223} \mathrm{~Pa} /{ }^{224} \mathrm{~Pa} /{ }^{225} \mathrm{~Pa} / \\
{ }^{226} \mathrm{~Pa} /{ }^{227} \mathrm{~Pa}, \mathrm{E}=1 \mathrm{GeV} / \text { nucleon} \text { / measured (fragment)(decay)-coin, } \\
\text { fragment yields. Fragment separator. JOUR NIMAE } 543591\end{array}$ \\
\hline
\end{tabular}

$$
\mathrm{A}=\mathbf{2 1 3}
$$
$\begin{array}{ll}{ }^{213} \mathrm{At} & \text { NUCLEAR REACTIONS }{ }^{208} \mathrm{~Pb},{ }^{209} \mathrm{Bi}\left({ }^{8} \mathrm{He}, 4 \mathrm{n}\right), \mathrm{E}=28 \mathrm{MeV} \text {; measured } \\ & \mathrm{E} \gamma, \mathrm{I} \gamma, \gamma \gamma \text {-coin. }{ }^{212} \mathrm{Po},{ }^{213} \mathrm{At} \text { deduced levels, J, } \pi \text {. Exogam array. }\end{array}$ JOUR JPGPE 31 S1851

${ }^{213} \mathrm{Rn} \quad$ 2005LI17 RADIOACTIVITY ${ }^{216,217,221,222} \mathrm{Th},{ }^{216} \mathrm{Ac},{ }^{215} \mathrm{Ra},{ }^{214} \mathrm{Fr},{ }^{213} \mathrm{Rn}(\alpha)$ [from $\mathrm{Be}\left({ }^{238} \mathrm{U}, \mathrm{X}\right)$ and subsequent decay]; measured $\mathrm{E} \alpha, \mathrm{T}_{1 / 2}$. Fragment separator. JOUR NIMAE 543591 


\section{$\mathrm{A}=\mathbf{2 1 3}$ (continued)}

\begin{tabular}{|c|c|c|}
\hline${ }^{213} \mathrm{Fr}$ & $2005 \mathrm{KU} 06$ & $\begin{array}{l}\text { RADIOACTIVITY }{ }^{210,211,212,213,214} \mathrm{Fr}(\alpha)\left[\text { from }{ }^{209} \mathrm{Bi}\left({ }^{12} \mathrm{C}, \mathrm{xn}\right) \text { and }\right. \\
\text { subsequent decay]; measured } \mathrm{E} \alpha, \mathrm{I} \alpha, \mathrm{E} \gamma, \mathrm{I} \gamma, \alpha \gamma \text {-coin, } \mathrm{T}_{1 / 2} ; \text { deduced } \\
\text { hindrance factors. } \\
\text { with previous results. JOUR ZAANE } 23417\end{array}$ \\
\hline \multirow[t]{3}{*}{${ }^{213} \mathrm{Ra}$} & $2005 \mathrm{KU} 31$ & $\begin{array}{l}\text { RADIOACTIVITY }{ }^{215,216,216 m, 217} \mathrm{Th}(\alpha)\left[{ }_{211,212,213}{ }^{170} \mathrm{Er}\left({ }^{50} \mathrm{Ti}, \mathrm{xn}\right)\right] \\
\text { measured } \mathrm{E} \alpha, \mathrm{I} \alpha, \mathrm{E} \gamma, \mathrm{I} \gamma, \alpha \gamma \text {-coin, } \mathrm{T}_{1 / 2} .{ }^{211},{ }^{2} \text { levels, J, } \\
\pi, \text { ICC. JOUR ZAANE } 25397\end{array}$ \\
\hline & $2005 \mathrm{KUZV}$ & $\begin{array}{l}\text { RADIOACTIVITY }{ }^{213} \mathrm{Ra}(\alpha)\left[\text { from }{ }^{170} \mathrm{Er}\left({ }^{50} \mathrm{Ti}, \mathrm{X}\right)\right] ; \text { measured } \mathrm{E} \gamma, \mathrm{E} \alpha, \\
\alpha \gamma \text {-coin. }{ }^{209} \mathrm{Rn} \text { deduced levels, J, } \pi, \text { ICC. REPT GSI } \\
\text { 2005-1,P76,Kuusiniemi }\end{array}$ \\
\hline & $2005 \operatorname{LI} 17$ & $\begin{array}{l}\text { RADIOACTIVITY }{ }^{216,217,221,222} \mathrm{Th},{ }^{216} \mathrm{Ac},{ }^{215} \mathrm{Ra},{ }^{214} \mathrm{Fr},{ }^{213} \mathrm{Rn}(\alpha) \\
\text { [from Be }\left({ }^{238} \mathrm{U}, \mathrm{X}\right) \text { and subsequent decay]; measured } \mathrm{E} \alpha, \mathrm{T}_{1 / 2} \text {. } \\
\text { Fragment separator. JOUR NIMAE } 543591\end{array}$ \\
\hline${ }^{213} \mathrm{Ac}$ & $2005 \operatorname{LI} 17$ & $\begin{array}{l}\text { NUCLEAR REACTIONS Be }\left({ }^{238} \mathrm{U}, \mathrm{X}\right){ }^{207} \mathrm{Ac} /{ }^{208} \mathrm{Ac} /{ }^{209} \mathrm{Ac} /{ }^{210} \mathrm{Ac} / \\
{ }^{211} \mathrm{Ac} /{ }^{212} \mathrm{Ac} /{ }^{213} \mathrm{Ac} /{ }^{214} \mathrm{Ac} /{ }^{215} \mathrm{Ac} /{ }^{216} \mathrm{Ac} /{ }^{217} \mathrm{Ac} /{ }^{218} \mathrm{Ac} /{ }^{219} \mathrm{Ac} \\
/{ }^{220} \mathrm{Ac} /{ }^{221} \mathrm{Ac} /{ }^{211} \mathrm{Th} /{ }^{212} \mathrm{Th} /{ }^{213} \mathrm{Th} /{ }^{214} \mathrm{Th} /{ }^{215} \mathrm{Th} /{ }^{216} \mathrm{Th} / \\
{ }^{217} \mathrm{Th} /{ }^{218} \mathrm{Th} /{ }^{219} \mathrm{Th} /{ }^{220} \mathrm{Th} /{ }^{221} \mathrm{Th} /{ }^{222} \mathrm{Th} /{ }^{223} \mathrm{Th} /{ }^{216} \mathrm{~Pa} / \\
{ }^{217} \mathrm{~Pa} /{ }^{218} \mathrm{~Pa} /{ }^{219} \mathrm{~Pa} /{ }^{220} \mathrm{~Pa} /{ }^{221} \mathrm{~Pa} /{ }^{222} \mathrm{~Pa} /{ }^{223} \mathrm{~Pa} /{ }^{224} \mathrm{~Pa} /{ }^{225} \mathrm{~Pa} / \\
{ }^{226} \mathrm{~Pa} /{ }^{227} \mathrm{~Pa}, \mathrm{E}=1 \mathrm{GeV} / \text { nucleon} \text {; measured (fragment)(decay)-coin, } \\
\text { fragment yields. Fragment separator. JOUR NIMAE } 543591\end{array}$ \\
\hline \multirow[t]{3}{*}{${ }^{213} \mathrm{Th}$} & 2005LE42 & $\begin{array}{l}\text { RADIOACTIVITY }{ }^{217,218,218 m, 219} \mathrm{U}(\alpha) \text {; measured } \mathrm{E} \alpha, \mathrm{T}_{1 / 2} . \\
{ }^{217,218,219} \mathrm{U} \text { deduced ground state } \mathrm{J}, \pi .{ }^{218} \mathrm{U} \text { deduced isomer } \mathrm{J}, \pi \text {. }\end{array}$ \\
\hline & & $\begin{array}{l}\text { Implications for } Z=92 \text { shell closure discussed. JOUR ZAANE } 25 \text { s01 } \\
183\end{array}$ \\
\hline & $2005 \operatorname{LI} 17$ & $\begin{array}{l}\text { NUCLEAR REACTIONS Be }\left({ }^{238} \mathrm{U}, \mathrm{X}\right){ }^{207} \mathrm{Ac} /{ }^{208} \mathrm{Ac} /{ }^{209} \mathrm{Ac} /{ }^{210} \mathrm{Ac} / \\
{ }^{211} \mathrm{Ac} /{ }^{212} \mathrm{Ac} /{ }^{213} \mathrm{Ac} /{ }^{214} \mathrm{Ac} /{ }^{215} \mathrm{Ac} /{ }^{216} \mathrm{Ac} /{ }^{217} \mathrm{Ac} /{ }^{218} \mathrm{Ac} /{ }^{219} \mathrm{Ac} \\
/{ }^{220} \mathrm{Ac} /{ }^{221} \mathrm{Ac} /{ }^{211} \mathrm{Th} /{ }^{212} \mathrm{Th} /{ }^{213} \mathrm{Th} /{ }^{214} \mathrm{Th} /{ }^{215} \mathrm{Th} /{ }^{216} \mathrm{Th} / \\
{ }^{217} \mathrm{Th} /{ }^{218} \mathrm{Th} /{ }^{219} \mathrm{Th} /{ }^{220} \mathrm{Th} /{ }^{221} \mathrm{Th} /{ }^{222} \mathrm{Th} /{ }^{223} \mathrm{Th} /{ }^{216} \mathrm{~Pa} / \\
\\
{ }^{217} \mathrm{~Pa} /{ }^{218} \mathrm{~Pa} /{ }^{219} \mathrm{~Pa} /{ }^{220} \mathrm{~Pa} /{ }^{221} \mathrm{~Pa} /{ }^{222} \mathrm{~Pa} /{ }^{223} \mathrm{~Pa} /{ }^{224} \mathrm{~Pa} /{ }^{225} \mathrm{~Pa} / \\
{ }^{226} \mathrm{~Pa} /{ }^{227} \mathrm{~Pa}, \mathrm{E}=1 \mathrm{GeV} / \text { nucleon} \text { / measured (fragment)(decay)-coin, } \\
\text { fragment yields. Fragment separator. JOUR NIMAE } 543591\end{array}$ \\
\hline
\end{tabular}

\section{$\mathrm{A}=\mathbf{2 1 4}$}

${ }^{214} \mathrm{Fr}$ 2005KU06 RADIOACTIVITY ${ }^{210,211,212,213,214} \operatorname{Fr}(\alpha)\left[\right.$ from ${ }^{209} \operatorname{Bi}\left({ }^{12} \mathrm{C}, \mathrm{xn}\right)$ and subsequent decay]; measured $\mathrm{E} \alpha, \mathrm{I} \alpha, \mathrm{E} \gamma, \mathrm{I} \gamma, \alpha \gamma$-coin, $\mathrm{T}_{1 / 2}$; deduced hindrance factors. ${ }^{206,207,208,209,210}$ At deduced levels, J, $\pi$. Comparison with previous results. JOUR ZAANE 23417

2005LI17 RADIOACTIVITY ${ }^{216,217,221,222} \mathrm{Th},{ }^{216} \mathrm{Ac},{ }^{215} \mathrm{Ra},{ }^{214} \mathrm{Fr},{ }^{213} \mathrm{Rn}(\alpha)$ [from $\mathrm{Be}\left({ }^{238} \mathrm{U}, \mathrm{X}\right)$ and subsequent decay]; measured $\mathrm{E} \alpha, \mathrm{T}_{1 / 2}$. Fragment separator. JOUR NIMAE 543591

${ }^{214} \mathrm{Ac} \quad 2005 \mathrm{LI} 17 \quad$ NUCLEAR REACTIONS Be $\left({ }^{238} \mathrm{U}, \mathrm{X}\right){ }^{207} \mathrm{Ac} /{ }^{208} \mathrm{Ac} /{ }^{209} \mathrm{Ac} /{ }^{210} \mathrm{Ac} /$ ${ }^{211} \mathrm{Ac} /{ }^{212} \mathrm{Ac} /{ }^{213} \mathrm{Ac} /{ }^{214} \mathrm{Ac} /{ }^{215} \mathrm{Ac} /{ }^{216} \mathrm{Ac} /{ }^{217} \mathrm{Ac} /{ }^{218} \mathrm{Ac} /{ }^{219} \mathrm{Ac}$ $/{ }^{220} \mathrm{Ac} /{ }^{221} \mathrm{Ac} /{ }^{211} \mathrm{Th} /{ }^{212} \mathrm{Th} /{ }^{213} \mathrm{Th} /{ }^{214} \mathrm{Th} /{ }^{215} \mathrm{Th} /{ }^{216} \mathrm{Th} /$ ${ }^{217} \mathrm{Th} /{ }^{218} \mathrm{Th} /{ }^{219} \mathrm{Th} /{ }^{220} \mathrm{Th} /{ }^{221} \mathrm{Th} /{ }^{222} \mathrm{Th} /{ }^{223} \mathrm{Th} /{ }^{216} \mathrm{~Pa} /$ ${ }^{217} \mathrm{~Pa} /{ }^{218} \mathrm{~Pa} /{ }^{219} \mathrm{~Pa} /{ }^{220} \mathrm{~Pa} /{ }^{221} \mathrm{~Pa} /{ }^{222} \mathrm{~Pa} /{ }^{223} \mathrm{~Pa} /{ }^{224} \mathrm{~Pa} /{ }^{225} \mathrm{~Pa} /$ ${ }^{226} \mathrm{~Pa} /{ }^{227} \mathrm{~Pa}, \mathrm{E}=1 \mathrm{GeV} /$ nucleon; measured (fragment)(decay)-coin, fragment yields. Fragment separator. JOUR NIMAE 543591 


\section{$\mathrm{A}=214$ (continued)}

${ }^{214} \mathrm{Th}$ 2005LE42 RADIOACTIVITY ${ }^{217,218,218 m, 219} \mathrm{U}(\alpha)$; measured $\mathrm{E} \alpha, \mathrm{T}_{1 / 2}$.

${ }^{217,218,219} \mathrm{U}$ deduced ground state $\mathrm{J}, \pi .{ }^{218} \mathrm{U}$ deduced isomer $\mathrm{J}, \pi$.

Implications for $\mathrm{Z}=92$ shell closure discussed. JOUR ZAANE 25 s01 183

2005LI17 NUCLEAR REACTIONS Be $\left({ }^{238} \mathrm{U}, \mathrm{X}\right){ }^{207} \mathrm{Ac} /{ }^{208} \mathrm{Ac} /{ }^{209} \mathrm{Ac} /{ }^{210} \mathrm{Ac} /$ ${ }^{211} \mathrm{Ac} /{ }^{212} \mathrm{Ac} /{ }^{213} \mathrm{Ac} /{ }^{214} \mathrm{Ac} /{ }^{215} \mathrm{Ac} /{ }^{216} \mathrm{Ac} /{ }^{217} \mathrm{Ac} /{ }^{218} \mathrm{Ac} /{ }^{219} \mathrm{Ac}$ $/{ }^{220} \mathrm{Ac} /{ }^{221} \mathrm{Ac} /{ }^{211} \mathrm{Th} /{ }^{212} \mathrm{Th} /{ }^{213} \mathrm{Th} /{ }^{214} \mathrm{Th} /{ }^{215} \mathrm{Th} /{ }^{216} \mathrm{Th} /$ ${ }^{217} \mathrm{Th} /{ }^{218} \mathrm{Th} /{ }^{219} \mathrm{Th} /{ }^{220} \mathrm{Th} /{ }^{221} \mathrm{Th} /{ }^{222} \mathrm{Th} /{ }^{223} \mathrm{Th} /{ }^{216} \mathrm{~Pa} /$ ${ }^{217} \mathrm{~Pa} /{ }^{218} \mathrm{~Pa} /{ }^{219} \mathrm{~Pa} /{ }^{220} \mathrm{~Pa} /{ }^{221} \mathrm{~Pa} /{ }^{222} \mathrm{~Pa} /{ }^{223} \mathrm{~Pa} /{ }^{224} \mathrm{~Pa} /{ }^{225} \mathrm{~Pa} /$ ${ }^{226} \mathrm{~Pa} /{ }^{227} \mathrm{~Pa}, \mathrm{E}=1 \mathrm{GeV} /$ nucleon; measured (fragment)(decay)-coin, fragment yields. Fragment separator. JOUR NIMAE 543591

\section{$\mathrm{A}=\mathbf{2 1 5}$}

${ }^{215} \mathrm{Ra}$ 2005LI17 RADIOACTIVITY ${ }^{216,217,221,222} \mathrm{Th},{ }^{216} \mathrm{Ac},{ }^{215} \mathrm{Ra},{ }^{214} \mathrm{Fr},{ }^{213} \mathrm{Rn}(\alpha)$ [from $\mathrm{Be}\left({ }^{238} \mathrm{U}, \mathrm{X}\right)$ and subsequent decay]; measured $\mathrm{E} \alpha, \mathrm{T}_{1 / 2}$. Fragment separator. JOUR NIMAE 543591

${ }^{215} \mathrm{Ac} \quad 2005 \mathrm{LI} 17$ NUCLEAR REACTIONS Be $\left({ }^{238} \mathrm{U}, \mathrm{X}\right){ }^{207} \mathrm{Ac} /{ }^{208} \mathrm{Ac} /{ }^{209} \mathrm{Ac} /{ }^{210} \mathrm{Ac} /$ ${ }^{211} \mathrm{Ac} /{ }^{212} \mathrm{Ac} /{ }^{213} \mathrm{Ac} /{ }^{214} \mathrm{Ac} /{ }^{215} \mathrm{Ac} /{ }^{216} \mathrm{Ac} /{ }^{217} \mathrm{Ac} /{ }^{218} \mathrm{Ac} /{ }^{219} \mathrm{Ac}$ / ${ }^{220} \mathrm{Ac} /{ }^{221} \mathrm{Ac} /{ }^{211} \mathrm{Th} /{ }^{212} \mathrm{Th} /{ }^{213} \mathrm{Th} /{ }^{214} \mathrm{Th} /{ }^{215} \mathrm{Th} /{ }^{216} \mathrm{Th} /$ ${ }^{217} \mathrm{Th} /{ }^{218} \mathrm{Th} /{ }^{219} \mathrm{Th} /{ }^{220} \mathrm{Th} /{ }^{221} \mathrm{Th} /{ }^{222} \mathrm{Th} /{ }^{223} \mathrm{Th} /{ }^{216} \mathrm{~Pa} /$ ${ }^{217} \mathrm{~Pa} /{ }^{218} \mathrm{~Pa} /{ }^{219} \mathrm{~Pa} /{ }^{220} \mathrm{~Pa} /{ }^{221} \mathrm{~Pa} /{ }^{222} \mathrm{~Pa} /{ }^{223} \mathrm{~Pa} /{ }^{224} \mathrm{~Pa} /{ }^{225} \mathrm{~Pa} /$ ${ }^{226} \mathrm{~Pa} /{ }^{227} \mathrm{~Pa}, \mathrm{E}=1 \mathrm{GeV} /$ nucleon; measured (fragment)(decay)-coin, fragment yields. Fragment separator. JOUR NIMAE 543591

${ }^{215} \mathrm{Th}$ 2005KU31 RADIOACTIVITY ${ }^{215,216,216 m, 217} \mathrm{Th}(\alpha)$ [from $\left.{ }^{170} \operatorname{Er}\left({ }^{50} \mathrm{Ti}, \mathrm{xn}\right)\right]$; measured $\mathrm{E} \alpha, \mathrm{I} \alpha, \mathrm{E} \gamma, \mathrm{I} \gamma, \alpha \gamma$-coin, $\mathrm{T}_{1 / 2} .{ }^{211,212,213} \mathrm{Ra}$ deduced levels, J, $\pi$, ICC. JOUR ZAANE 25397

2005LE42 RADIOACTIVITY ${ }^{217,218,218 m, 219} \mathrm{U}(\alpha)$; measured $\mathrm{E} \alpha, \mathrm{T}_{1 / 2}$. ${ }^{217,218,219} \mathrm{U}$ deduced ground state $\mathrm{J}, \pi .{ }^{218} \mathrm{U}$ deduced isomer $\mathrm{J}, \pi$. Implications for $\mathrm{Z}=92$ shell closure discussed. JOUR ZAANE 25 s01 183

2005LI17 NUCLEAR REACTIONS Be $\left({ }^{238} \mathrm{U}, \mathrm{X}\right){ }^{207} \mathrm{Ac} /{ }^{208} \mathrm{Ac} /{ }^{209} \mathrm{Ac} /{ }^{210} \mathrm{Ac} /$ ${ }^{211} \mathrm{Ac} /{ }^{212} \mathrm{Ac} /{ }^{213} \mathrm{Ac} /{ }^{214} \mathrm{Ac} /{ }^{215} \mathrm{Ac} /{ }^{216} \mathrm{Ac} /{ }^{217} \mathrm{Ac} /{ }^{218} \mathrm{Ac} /{ }^{219} \mathrm{Ac}$ $/{ }^{220} \mathrm{Ac} /{ }^{221} \mathrm{Ac} /{ }^{211} \mathrm{Th} /{ }^{212} \mathrm{Th} /{ }^{213} \mathrm{Th} /{ }^{214} \mathrm{Th} /{ }^{215} \mathrm{Th} /{ }^{216} \mathrm{Th} /$ ${ }^{217} \mathrm{Th} /{ }^{218} \mathrm{Th} /{ }^{219} \mathrm{Th} /{ }^{220} \mathrm{Th} /{ }^{221} \mathrm{Th} /{ }^{222} \mathrm{Th} /{ }^{223} \mathrm{Th} /{ }^{216} \mathrm{~Pa} /$ ${ }^{217} \mathrm{~Pa} /{ }^{218} \mathrm{~Pa} /{ }^{219} \mathrm{~Pa} /{ }^{220} \mathrm{~Pa} /{ }^{221} \mathrm{~Pa} /{ }^{222} \mathrm{~Pa} /{ }^{223} \mathrm{~Pa} /{ }^{224} \mathrm{~Pa} /{ }^{225} \mathrm{~Pa} /$ ${ }^{226} \mathrm{~Pa} /{ }^{227} \mathrm{~Pa}, \mathrm{E}=1 \mathrm{GeV} /$ nucleon; measured (fragment)(decay)-coin, fragment yields. Fragment separator. JOUR NIMAE 543591

\section{$\mathrm{A}=216$}

${ }^{216} \mathrm{Rn} \quad 2004 \mathrm{KMO} 1$

NUCLEAR REACTIONS ${ }^{198} \mathrm{Pt}\left({ }^{18} \mathrm{O}, \mathrm{X}\right), \mathrm{E}=96 \mathrm{MeV}$; measured prompt and delayed $\mathrm{E} \gamma, \mathrm{I} \gamma, \gamma \gamma$-coin. ${ }^{216} \mathrm{Rn}$ deduced GDR energy, width, deformation features. Hector array, comparison with model predictions. JOUR PRVCA 70064317 


\section{$\mathrm{A}=216$ (continued)}

2005CA23 NUCLEAR REACTIONS ${ }^{198} \mathrm{Pt}\left({ }^{18} \mathrm{O}\right.$, xn), E=96 MeV; measured prompt and delayed $\mathrm{E} \gamma, \mathrm{I} \gamma .{ }^{216} \mathrm{Rn}$ deduced GDR parameters. ${ }^{68} \mathrm{Zn}\left({ }^{64} \mathrm{Ni}, \mathrm{X}\right), \mathrm{E}=300,400,500 \mathrm{MeV} ;{ }^{116} \mathrm{Sn}\left({ }^{16} \mathrm{O}, \mathrm{X}\right), \mathrm{E}=130,250 \mathrm{MeV}$; measured $\mathrm{E} \gamma, \mathrm{I} \gamma .{ }^{132} \mathrm{Ce}$ deduced GDR features, entrance channel effects. JOUR APOBB 361145

${ }^{216} \mathrm{Ac} \quad 2005 \mathrm{LI} 17 \quad$ NUCLEAR REACTIONS Be $\left({ }^{238} \mathrm{U}, \mathrm{X}\right){ }^{207} \mathrm{Ac} /{ }^{208} \mathrm{Ac} /{ }^{209} \mathrm{Ac} /{ }^{210} \mathrm{Ac} /$ ${ }^{211} \mathrm{Ac} /{ }^{212} \mathrm{Ac} /{ }^{213} \mathrm{Ac} /{ }^{214} \mathrm{Ac} /{ }^{215} \mathrm{Ac} /{ }^{216} \mathrm{Ac} /{ }^{217} \mathrm{Ac} /{ }^{218} \mathrm{Ac} /{ }^{219} \mathrm{Ac}$ $/{ }^{220} \mathrm{Ac} /{ }^{221} \mathrm{Ac} /{ }^{211} \mathrm{Th} /{ }^{212} \mathrm{Th} /{ }^{213} \mathrm{Th} /{ }^{214} \mathrm{Th} /{ }^{215} \mathrm{Th} /{ }^{216} \mathrm{Th} /$ ${ }^{217} \mathrm{Th} /{ }^{218} \mathrm{Th} /{ }^{219} \mathrm{Th} /{ }^{220} \mathrm{Th} /{ }^{221} \mathrm{Th} /{ }^{222} \mathrm{Th} /{ }^{223} \mathrm{Th} /{ }^{216} \mathrm{~Pa} /$ ${ }^{217} \mathrm{~Pa} /{ }^{218} \mathrm{~Pa} /{ }^{219} \mathrm{~Pa} /{ }^{220} \mathrm{~Pa} /{ }^{221} \mathrm{~Pa} /{ }^{222} \mathrm{~Pa} /{ }^{223} \mathrm{~Pa} /{ }^{224} \mathrm{~Pa} /{ }^{225} \mathrm{~Pa} /$ ${ }^{226} \mathrm{~Pa} /{ }^{227} \mathrm{~Pa}, \mathrm{E}=1 \mathrm{GeV} /$ nucleon; measured (fragment)(decay)-coin, fragment yields. Fragment separator. JOUR NIMAE 543591

2005LI17 RADIOACTIVITY ${ }^{216,217,221,222} \mathrm{Th},{ }^{216} \mathrm{Ac},{ }^{215} \mathrm{Ra},{ }^{214} \mathrm{Fr},{ }^{213} \mathrm{Rn}(\alpha)$ [from $\mathrm{Be}\left({ }^{238} \mathrm{U}, \mathrm{X}\right)$ and subsequent decay]; measured $\mathrm{E} \alpha, \mathrm{T}_{1 / 2}$. Fragment separator. JOUR NIMAE 543591

${ }^{216} \mathrm{Th}$ 2002SU35 NUCLEAR REACTIONS ${ }^{206} \mathrm{~Pb}\left({ }^{48} \mathrm{Ca}, 2 \mathrm{n}\right),{ }^{186} \mathrm{~W}\left({ }^{34} \mathrm{~S}, 4 \mathrm{n}\right), \mathrm{E}$ not given; measured yields, focal-plane position spectra in recoil separator. JOUR NIMAE 48171

2005KU31 RADIOACTIVITY ${ }^{215,216,216 m, 217} \operatorname{Th}(\alpha)$ [from ${ }^{170} \operatorname{Er}\left({ }^{50} \mathrm{Ti}, \mathrm{xn}\right)$ ]; measured $\mathrm{E} \alpha, \mathrm{I} \alpha, \mathrm{E} \gamma, \mathrm{I} \gamma, \alpha \gamma$-coin, $\mathrm{T}_{1 / 2} .{ }^{211,212,213} \mathrm{Ra}$ deduced levels, $\mathrm{J}$, $\pi$, ICC. JOUR ZAANE 25397

2005KUZZ RADIOACTIVITY ${ }^{216,216 m} \mathrm{Th}(\alpha)$, (IT) [from ${ }^{170} \mathrm{Er}\left({ }^{50} \mathrm{Ti}, 4 \mathrm{n}\right)$ ]; ${ }^{251,251 m} \mathrm{No},{ }^{247,247 m} \mathrm{Fm}(\alpha)$ [from ${ }^{206} \mathrm{~Pb}\left({ }^{48} \mathrm{Ca}, 3 \mathrm{n}\right)$ and subsequent decay]; ${ }^{257,257 m} \mathrm{Db},{ }^{253,253 m} \mathrm{Lr},{ }^{249} \mathrm{Md}(\alpha)$ [from ${ }^{209} \mathrm{Bi}\left({ }^{50} \mathrm{Ti}, 2 \mathrm{n}\right)$ and subsequent decay]; measured $\mathrm{E} \alpha, \mathrm{E} \gamma, \alpha \gamma$-coin, $\mathrm{T}_{1 / 2}$. CONF Argonne(Nuclei at the Limits),P231,Kuusiniemi

2005LI17 NUCLEAR REACTIONS Be $\left({ }^{238} \mathrm{U}, \mathrm{X}\right){ }^{207} \mathrm{Ac} /{ }^{208} \mathrm{Ac} /{ }^{209} \mathrm{Ac} /{ }^{210} \mathrm{Ac} /$ ${ }^{211} \mathrm{Ac} /{ }^{212} \mathrm{Ac} /{ }^{213} \mathrm{Ac} /{ }^{214} \mathrm{Ac} /{ }^{215} \mathrm{Ac} /{ }^{216} \mathrm{Ac} /{ }^{217} \mathrm{Ac} /{ }^{218} \mathrm{Ac} /{ }^{219} \mathrm{Ac}$ / ${ }^{220} \mathrm{Ac} /{ }^{221} \mathrm{Ac} /{ }^{211} \mathrm{Th} /{ }^{212} \mathrm{Th} /{ }^{213} \mathrm{Th} /{ }^{214} \mathrm{Th} /{ }^{215} \mathrm{Th} /{ }^{216} \mathrm{Th} /$ ${ }^{217} \mathrm{Th} /{ }^{218} \mathrm{Th} /{ }^{219} \mathrm{Th} /{ }^{220} \mathrm{Th} /{ }^{221} \mathrm{Th} /{ }^{222} \mathrm{Th} /{ }^{223} \mathrm{Th} /{ }^{216} \mathrm{~Pa} /$ ${ }^{217} \mathrm{~Pa} /{ }^{218} \mathrm{~Pa} /{ }^{219} \mathrm{~Pa} /{ }^{220} \mathrm{~Pa} /{ }^{221} \mathrm{~Pa} /{ }^{222} \mathrm{~Pa} /{ }^{223} \mathrm{~Pa} /{ }^{224} \mathrm{~Pa} /{ }^{225} \mathrm{~Pa} /$ ${ }^{226} \mathrm{~Pa} /{ }^{227} \mathrm{~Pa}, \mathrm{E}=1 \mathrm{GeV} /$ nucleon; measured (fragment)(decay)-coin, fragment yields. Fragment separator. JOUR NIMAE 543591

2005LI17 RADIOACTIVITY ${ }^{216,217,221,222} \mathrm{Th},{ }^{216} \mathrm{Ac},{ }^{215} \mathrm{Ra},{ }^{214} \mathrm{Fr},{ }^{213} \mathrm{Rn}(\alpha)$ [from $\mathrm{Be}\left({ }^{238} \mathrm{U}, \mathrm{X}\right)$ and subsequent decay]; measured $\mathrm{E} \alpha, \mathrm{T}_{1 / 2}$. Fragment separator. JOUR NIMAE 543591

${ }^{216} \mathrm{~Pa}$ 2005LI17 NUCLEAR REACTIONS Be $\left({ }^{238} \mathrm{U}, \mathrm{X}\right){ }^{207} \mathrm{Ac} /{ }^{208} \mathrm{Ac} /{ }^{209} \mathrm{Ac} /{ }^{210} \mathrm{Ac} /$ ${ }^{211} \mathrm{Ac} /{ }^{212} \mathrm{Ac} /{ }^{213} \mathrm{Ac} /{ }^{214} \mathrm{Ac} /{ }^{215} \mathrm{Ac} /{ }^{216} \mathrm{Ac} /{ }^{217} \mathrm{Ac} /{ }^{218} \mathrm{Ac} /{ }^{219} \mathrm{Ac}$ / ${ }^{220} \mathrm{Ac} /{ }^{221} \mathrm{Ac} /{ }^{211} \mathrm{Th} /{ }^{212} \mathrm{Th} /{ }^{213} \mathrm{Th} /{ }^{214} \mathrm{Th} /{ }^{215} \mathrm{Th} /{ }^{216} \mathrm{Th} /$ ${ }^{217} \mathrm{Th} /{ }^{218} \mathrm{Th} /{ }^{219} \mathrm{Th} /{ }^{220} \mathrm{Th} /{ }^{221} \mathrm{Th} /{ }^{222} \mathrm{Th} /{ }^{223} \mathrm{Th} /{ }^{216} \mathrm{~Pa} /$ ${ }^{217} \mathrm{~Pa} /{ }^{218} \mathrm{~Pa} /{ }^{219} \mathrm{~Pa} /{ }^{220} \mathrm{~Pa} /{ }^{221} \mathrm{~Pa} /{ }^{222} \mathrm{~Pa} /{ }^{223} \mathrm{~Pa} /{ }^{224} \mathrm{~Pa} /{ }^{225} \mathrm{~Pa} /$ ${ }^{226} \mathrm{~Pa} /{ }^{227} \mathrm{~Pa}, \mathrm{E}=1 \mathrm{GeV} /$ nucleon; measured (fragment)(decay)-coin, fragment yields. Fragment separator. JOUR NIMAE 543591 


\section{$\mathrm{A}=\mathbf{2 1 7}$}

${ }^{217} \mathrm{Ra}$

${ }^{217} \mathrm{Ac}$

${ }^{217} \mathrm{Th}$

$2005 K U 31$

2005LI17

2005LI17

${ }^{217} \mathrm{~Pa} \quad 2005 \mathrm{LI} 17$

${ }^{217} \mathrm{U}$

2005LE42

2005LE42

\section{$\mathrm{A}=218$}

${ }^{218} \mathrm{Ra} \quad 2005 \mathrm{LI} 17$ JOUR ZAANE 25 s01 183 183
RADIOACTIVITY ${ }^{216,217,221,222} \mathrm{Th},{ }^{216} \mathrm{Ac},{ }^{215} \mathrm{Ra},{ }^{214} \mathrm{Fr},{ }^{213} \mathrm{Rn}(\alpha)$ [from $\operatorname{Be}\left({ }^{238} \mathrm{U}, \mathrm{X}\right)$ and subsequent decay]; measured $\mathrm{E} \alpha, \mathrm{T}_{1 / 2}$. Fragment separator. JOUR NIMAE 543591 NUCLEAR REACTIONS Be $\left({ }^{238} \mathrm{U}, \mathrm{X}\right){ }^{207} \mathrm{Ac} /{ }^{208} \mathrm{Ac} /{ }^{209} \mathrm{Ac} /{ }^{210} \mathrm{Ac} /$ ${ }^{211} \mathrm{Ac} /{ }^{212} \mathrm{Ac} /{ }^{213} \mathrm{Ac} /{ }^{214} \mathrm{Ac} /{ }^{215} \mathrm{Ac} /{ }^{216} \mathrm{Ac} /{ }^{217} \mathrm{Ac} /{ }^{218} \mathrm{Ac} /{ }^{219} \mathrm{Ac}$ / ${ }^{220} \mathrm{Ac} /{ }^{221} \mathrm{Ac} /{ }^{211} \mathrm{Th} /{ }^{212} \mathrm{Th} /{ }^{213} \mathrm{Th} /{ }^{214} \mathrm{Th} /{ }^{215} \mathrm{Th} /{ }^{216} \mathrm{Th} /$ ${ }^{217} \mathrm{Th} /{ }^{218} \mathrm{Th} /{ }^{219} \mathrm{Th} /{ }^{220} \mathrm{Th} /{ }^{221} \mathrm{Th} /{ }^{222} \mathrm{Th} /{ }^{223} \mathrm{Th} /{ }^{216} \mathrm{~Pa} /$ ${ }^{217} \mathrm{~Pa} /{ }^{218} \mathrm{~Pa} /{ }^{219} \mathrm{~Pa} /{ }^{220} \mathrm{~Pa} /{ }^{221} \mathrm{~Pa} /{ }^{222} \mathrm{~Pa} /{ }^{223} \mathrm{~Pa} /{ }^{224} \mathrm{~Pa} /{ }^{225} \mathrm{~Pa} /$ ${ }^{226} \mathrm{~Pa} /{ }^{227} \mathrm{~Pa}, \mathrm{E}=1 \mathrm{GeV} /$ nucleon; measured (fragment)(decay)-coin, fragment yields. Fragment separator. JOUR NIMAE 543591 RADIOACTIVITY ${ }^{215,216,216 m, 217} \mathrm{Th}(\alpha)$ [from $\left.{ }^{170} \operatorname{Er}\left({ }^{50} \mathrm{Ti}, \mathrm{xn}\right)\right]$; measured $\mathrm{E} \alpha, \mathrm{I} \alpha, \mathrm{E} \gamma, \mathrm{I} \gamma, \alpha \gamma$-coin, $\mathrm{T}_{1 / 2} \cdot{ }^{211,212,213} \mathrm{Ra}$ deduced levels, J, $\pi$, ICC. JOUR ZAANE 25397

NUCLEAR REACTIONS Be $\left({ }^{238} \mathrm{U}, \mathrm{X}\right){ }^{207} \mathrm{Ac} /{ }^{208} \mathrm{Ac} /{ }^{209} \mathrm{Ac} /{ }^{210} \mathrm{Ac} /$ ${ }^{211} \mathrm{Ac} /{ }^{212} \mathrm{Ac} /{ }^{213} \mathrm{Ac} /{ }^{214} \mathrm{Ac} /{ }^{215} \mathrm{Ac} /{ }^{216} \mathrm{Ac} /{ }^{217} \mathrm{Ac} /{ }^{218} \mathrm{Ac} /{ }^{219} \mathrm{Ac}$ $/{ }^{220} \mathrm{Ac} /{ }^{221} \mathrm{Ac} /{ }^{211} \mathrm{Th} /{ }^{212} \mathrm{Th} /{ }^{213} \mathrm{Th} /{ }^{214} \mathrm{Th} /{ }^{215} \mathrm{Th} /{ }^{216} \mathrm{Th} /$ ${ }^{217} \mathrm{Th} /{ }^{218} \mathrm{Th} /{ }^{219} \mathrm{Th} /{ }^{220} \mathrm{Th} /{ }^{221} \mathrm{Th} /{ }^{222} \mathrm{Th} /{ }^{223} \mathrm{Th} /{ }^{216} \mathrm{~Pa} /$ ${ }^{217} \mathrm{~Pa} /{ }^{218} \mathrm{~Pa} /{ }^{219} \mathrm{~Pa} /{ }^{220} \mathrm{~Pa} /{ }^{221} \mathrm{~Pa} /{ }^{222} \mathrm{~Pa} /{ }^{223} \mathrm{~Pa} /{ }^{224} \mathrm{~Pa} /{ }^{225} \mathrm{~Pa} /$ ${ }^{226} \mathrm{~Pa} /{ }^{227} \mathrm{~Pa}, \mathrm{E}=1 \mathrm{GeV} /$ nucleon; measured (fragment)(decay)-coin, fragment yields. Fragment separator. JOUR NIMAE 543591 RADIOACTIVITY ${ }^{216,217,221,222} \mathrm{Th},{ }^{216} \mathrm{Ac},{ }^{215} \mathrm{Ra},{ }^{214} \mathrm{Fr},{ }^{213} \mathrm{Rn}(\alpha)$ [from $\mathrm{Be}\left({ }^{238} \mathrm{U}, \mathrm{X}\right)$ and subsequent decay]; measured $\mathrm{E} \alpha, \mathrm{T}_{1 / 2}$. Fragment separator. JOUR NIMAE 543591

NUCLEAR REACTIONS Be $\left({ }^{238} \mathrm{U}, \mathrm{X}\right){ }^{207} \mathrm{Ac} /{ }^{208} \mathrm{Ac} /{ }^{209} \mathrm{Ac} /{ }^{210} \mathrm{Ac} /$ ${ }^{211} \mathrm{Ac} /{ }^{212} \mathrm{Ac} /{ }^{213} \mathrm{Ac} /{ }^{214} \mathrm{Ac} /{ }^{215} \mathrm{Ac} /{ }^{216} \mathrm{Ac} /{ }^{217} \mathrm{Ac} /{ }^{218} \mathrm{Ac} /{ }^{219} \mathrm{Ac}$ / ${ }^{220} \mathrm{Ac} /{ }^{221} \mathrm{Ac} /{ }^{211} \mathrm{Th} /{ }^{212} \mathrm{Th} /{ }^{213} \mathrm{Th} /{ }^{214} \mathrm{Th} /{ }^{215} \mathrm{Th} /{ }^{216} \mathrm{Th} /$ ${ }^{217} \mathrm{Th} /{ }^{218} \mathrm{Th} /{ }^{219} \mathrm{Th} /{ }^{220} \mathrm{Th} /{ }^{221} \mathrm{Th} /{ }^{222} \mathrm{Th} /{ }^{223} \mathrm{Th} /{ }^{216} \mathrm{~Pa} /$ ${ }^{217} \mathrm{~Pa} /{ }^{218} \mathrm{~Pa} /{ }^{219} \mathrm{~Pa} /{ }^{220} \mathrm{~Pa} /{ }^{221} \mathrm{~Pa} /{ }^{222} \mathrm{~Pa} /{ }^{223} \mathrm{~Pa} /{ }^{224} \mathrm{~Pa} /{ }^{225} \mathrm{~Pa} /$ ${ }^{226} \mathrm{~Pa} /{ }^{227} \mathrm{~Pa}, \mathrm{E}=1 \mathrm{GeV} /$ nucleon; measured (fragment)(decay)-coin, fragment yields. Fragment separator. JOUR NIMAE 543591

NUCLEAR REACTIONS ${ }^{182} \mathrm{~W}\left({ }^{40} \mathrm{Ar}, \mathrm{xn}\right){ }^{217} \mathrm{U} /{ }^{218} \mathrm{U} /{ }^{218 m} \mathrm{U} /{ }^{219} \mathrm{U}$, $\mathrm{E}=186 \mathrm{MeV}$; measured $\mathrm{E} \alpha, \alpha \alpha^{-}$, (recoil) $\alpha$-coin; deduced production $\sigma$.

RADIOACTIVITY ${ }^{217,218,218 m, 219} \mathrm{U}(\alpha)$; measured E $\alpha, \mathrm{T}_{1 / 2}$. $217,218,219 \mathrm{U}$ deduced ground state $\mathrm{J}, \pi \cdot{ }^{218} \mathrm{U}$ deduced isomer $\mathrm{J}, \pi$. Implications for $Z=92$ shell closure discussed. JOUR ZAANE 25 s01

RADIOACTIVITY ${ }^{216,217,221,222} \mathrm{Th},{ }^{216} \mathrm{Ac},{ }^{215} \mathrm{Ra},{ }^{214} \mathrm{Fr},{ }^{213} \mathrm{Rn}(\alpha)$ [from $\mathrm{Be}\left({ }^{238} \mathrm{U}, \mathrm{X}\right)$ and subsequent decay]; measured $\mathrm{E} \alpha, \mathrm{T}_{1 / 2}$. Fragment separator. JOUR NIMAE 543591 


\section{$\mathrm{A}=\mathbf{2 1 8}$ (continued)}

${ }^{218} \mathrm{Ac}$ 2005LI17 NUCLEAR REACTIONS Be $\left({ }^{238} \mathrm{U}, \mathrm{X}\right){ }^{207} \mathrm{Ac} /{ }^{208} \mathrm{Ac} /{ }^{209} \mathrm{Ac} /{ }^{210} \mathrm{Ac} /$ ${ }^{211} \mathrm{Ac} /{ }^{212} \mathrm{Ac} /{ }^{213} \mathrm{Ac} /{ }^{214} \mathrm{Ac} /{ }^{215} \mathrm{Ac} /{ }^{216} \mathrm{Ac} /{ }^{217} \mathrm{Ac} /{ }^{218} \mathrm{Ac} /{ }^{219} \mathrm{Ac}$ $/{ }^{220} \mathrm{Ac} /{ }^{221} \mathrm{Ac} /{ }^{211} \mathrm{Th} /{ }^{212} \mathrm{Th} /{ }^{213} \mathrm{Th} /{ }^{214} \mathrm{Th} /{ }^{215} \mathrm{Th} /{ }^{216} \mathrm{Th} /$ ${ }^{217} \mathrm{Th} /{ }^{218} \mathrm{Th} /{ }^{219} \mathrm{Th} /{ }^{220} \mathrm{Th} /{ }^{221} \mathrm{Th} /{ }^{222} \mathrm{Th} /{ }^{223} \mathrm{Th} /{ }^{216} \mathrm{~Pa} /$ ${ }^{217} \mathrm{~Pa} /{ }^{218} \mathrm{~Pa} /{ }^{219} \mathrm{~Pa} /{ }^{220} \mathrm{~Pa} /{ }^{221} \mathrm{~Pa} /{ }^{222} \mathrm{~Pa} /{ }^{223} \mathrm{~Pa} /{ }^{224} \mathrm{~Pa} /{ }^{225} \mathrm{~Pa} /$ ${ }^{226} \mathrm{~Pa} /{ }^{227} \mathrm{~Pa}, \mathrm{E}=1 \mathrm{GeV} /$ nucleon; measured (fragment)(decay)-coin, fragment yields. Fragment separator. JOUR NIMAE 543591

${ }^{218} \mathrm{Th}$ 2005LI17 NUCLEAR REACTIONS Be $\left({ }^{238} \mathrm{U}, \mathrm{X}\right){ }^{207} \mathrm{Ac} /{ }^{208} \mathrm{Ac} /{ }^{209} \mathrm{Ac} /{ }^{210} \mathrm{Ac} /$ ${ }^{211} \mathrm{Ac} /{ }^{212} \mathrm{Ac} /{ }^{213} \mathrm{Ac} /{ }^{214} \mathrm{Ac} /{ }^{215} \mathrm{Ac} /{ }^{216} \mathrm{Ac} /{ }^{217} \mathrm{Ac} /{ }^{218} \mathrm{Ac} /{ }^{219} \mathrm{Ac}$ $/{ }^{220} \mathrm{Ac} /{ }^{221} \mathrm{Ac} /{ }^{211} \mathrm{Th} /{ }^{212} \mathrm{Th} /{ }^{213} \mathrm{Th} /{ }^{214} \mathrm{Th} /{ }^{215} \mathrm{Th} /{ }^{216} \mathrm{Th} /$ ${ }^{217} \mathrm{Th} /{ }^{218} \mathrm{Th} /{ }^{219} \mathrm{Th} /{ }^{220} \mathrm{Th} /{ }^{221} \mathrm{Th} /{ }^{222} \mathrm{Th} /{ }^{223} \mathrm{Th} /{ }^{216} \mathrm{~Pa} /$ ${ }^{217} \mathrm{~Pa} /{ }^{218} \mathrm{~Pa} /{ }^{219} \mathrm{~Pa} /{ }^{220} \mathrm{~Pa} /{ }^{221} \mathrm{~Pa} /{ }^{222} \mathrm{~Pa} /{ }^{223} \mathrm{~Pa} /{ }^{224} \mathrm{~Pa} /{ }^{225} \mathrm{~Pa} /$ ${ }^{226} \mathrm{~Pa} /{ }^{227} \mathrm{~Pa}, \mathrm{E}=1 \mathrm{GeV} /$ nucleon; measured (fragment)(decay)-coin, fragment yields. Fragment separator. JOUR NIMAE 543591

${ }^{218} \mathrm{~Pa}$ 2005LI17 NUCLEAR REACTIONS Be $\left({ }^{238} \mathrm{U}, \mathrm{X}\right){ }^{207} \mathrm{Ac} /{ }^{208} \mathrm{Ac} /{ }^{209} \mathrm{Ac} /{ }^{210} \mathrm{Ac} /$ ${ }^{211} \mathrm{Ac} /{ }^{212} \mathrm{Ac} /{ }^{213} \mathrm{Ac} /{ }^{214} \mathrm{Ac} /{ }^{215} \mathrm{Ac} /{ }^{216} \mathrm{Ac} /{ }^{217} \mathrm{Ac} /{ }^{218} \mathrm{Ac} /{ }^{219} \mathrm{Ac}$ $/{ }^{220} \mathrm{Ac} /{ }^{221} \mathrm{Ac} /{ }^{211} \mathrm{Th} /{ }^{212} \mathrm{Th} /{ }^{213} \mathrm{Th} /{ }^{214} \mathrm{Th} /{ }^{215} \mathrm{Th} /{ }^{216} \mathrm{Th} /$ ${ }^{217} \mathrm{Th} /{ }^{218} \mathrm{Th} /{ }^{219} \mathrm{Th} /{ }^{220} \mathrm{Th} /{ }^{221} \mathrm{Th} /{ }^{222} \mathrm{Th} /{ }^{223} \mathrm{Th} /{ }^{216} \mathrm{~Pa} /$ ${ }^{217} \mathrm{~Pa} /{ }^{218} \mathrm{~Pa} /{ }^{219} \mathrm{~Pa} /{ }^{220} \mathrm{~Pa} /{ }^{221} \mathrm{~Pa} /{ }^{222} \mathrm{~Pa} /{ }^{223} \mathrm{~Pa} /{ }^{224} \mathrm{~Pa} /{ }^{225} \mathrm{~Pa} /$ ${ }^{226} \mathrm{~Pa} /{ }^{227} \mathrm{~Pa}, \mathrm{E}=1 \mathrm{GeV} /$ nucleon; measured (fragment)(decay)-coin, fragment yields. Fragment separator. JOUR NIMAE 543591

${ }^{218} \mathrm{U}$ 2005LE42 NUCLEAR REACTIONS ${ }^{182} \mathrm{~W}\left({ }^{40} \mathrm{Ar}, \mathrm{xn}\right){ }^{217} \mathrm{U} /{ }^{218} \mathrm{U} /{ }^{218 m} \mathrm{U} /{ }^{219} \mathrm{U}$, $\mathrm{E}=186 \mathrm{MeV}$; measured $\mathrm{E} \alpha, \alpha \alpha-$, (recoil) $\alpha$-coin; deduced production $\sigma$. JOUR ZAANE 25 s01 183

2005LE42 RADIOACTIVITY ${ }^{217,218,218 m, 219} \mathrm{U}(\alpha)$; measured $\mathrm{E} \alpha, \mathrm{T}_{1 / 2}$. ${ }^{217,218,219} \mathrm{U}$ deduced ground state $\mathrm{J}, \pi .{ }^{218} \mathrm{U}$ deduced isomer $\mathrm{J}, \pi$. Implications for $\mathrm{Z}=92$ shell closure discussed. JOUR ZAANE $25 \mathrm{~s} 01$ 183

\section{$A=219$}

${ }^{219} \mathrm{Rn} \quad$ 2005JOZY

RADIOACTIVITY ${ }^{227,228} \mathrm{Th},{ }^{223,224} \mathrm{Ra}(\alpha)$; measured $\mathrm{E} \gamma, \alpha \gamma$-coin, $\gamma$-ray linear polarization. CONF Argonne(Nuclei at the Limits),P348,Jones

${ }^{219} \mathrm{Ac} \quad 2005 \mathrm{LI} 17$ NUCLEAR REACTIONS Be $\left({ }^{238} \mathrm{U}, \mathrm{X}\right){ }^{207} \mathrm{Ac} /{ }^{208} \mathrm{Ac} /{ }^{209} \mathrm{Ac} /{ }^{210} \mathrm{Ac} /$ ${ }^{211} \mathrm{Ac} /{ }^{212} \mathrm{Ac} /{ }^{213} \mathrm{Ac} /{ }^{214} \mathrm{Ac} /{ }^{215} \mathrm{Ac} /{ }^{216} \mathrm{Ac} /{ }^{217} \mathrm{Ac} /{ }^{218} \mathrm{Ac} /{ }^{219} \mathrm{Ac}$ $/{ }^{220} \mathrm{Ac} /{ }^{221} \mathrm{Ac} /{ }^{211} \mathrm{Th} /{ }^{212} \mathrm{Th} /{ }^{213} \mathrm{Th} /{ }^{214} \mathrm{Th} /{ }^{215} \mathrm{Th} /{ }^{216} \mathrm{Th} /$ ${ }^{217} \mathrm{Th} /{ }^{218} \mathrm{Th} /{ }^{219} \mathrm{Th} /{ }^{220} \mathrm{Th} /{ }^{221} \mathrm{Th} /{ }^{222} \mathrm{Th} /{ }^{223} \mathrm{Th} /{ }^{216} \mathrm{~Pa} /$ ${ }^{217} \mathrm{~Pa} /{ }^{218} \mathrm{~Pa} /{ }^{219} \mathrm{~Pa} /{ }^{220} \mathrm{~Pa} /{ }^{221} \mathrm{~Pa} /{ }^{222} \mathrm{~Pa} /{ }^{223} \mathrm{~Pa} /{ }^{224} \mathrm{~Pa} /{ }^{225} \mathrm{~Pa} /$ ${ }^{226} \mathrm{~Pa} /{ }^{227} \mathrm{~Pa}, \mathrm{E}=1 \mathrm{GeV} /$ nucleon; measured (fragment)(decay)-coin, fragment yields. Fragment separator. JOUR NIMAE 543591

${ }^{219} \mathrm{Th}$ 2005LI17 NUCLEAR REACTIONS Be $\left({ }^{238} \mathrm{U}, \mathrm{X}\right){ }^{207} \mathrm{Ac} /{ }^{208} \mathrm{Ac} /{ }^{209} \mathrm{Ac} /{ }^{210} \mathrm{Ac} /$ ${ }^{211} \mathrm{Ac} /{ }^{212} \mathrm{Ac} /{ }^{213} \mathrm{Ac} /{ }^{214} \mathrm{Ac} /{ }^{215} \mathrm{Ac} /{ }^{216} \mathrm{Ac} /{ }^{217} \mathrm{Ac} /{ }^{218} \mathrm{Ac} /{ }^{219} \mathrm{Ac}$ / ${ }^{220} \mathrm{Ac} /{ }^{221} \mathrm{Ac} /{ }^{211} \mathrm{Th} /{ }^{212} \mathrm{Th} /{ }^{213} \mathrm{Th} /{ }^{214} \mathrm{Th} /{ }^{215} \mathrm{Th} /{ }^{216} \mathrm{Th} /$ ${ }^{217} \mathrm{Th} /{ }^{218} \mathrm{Th} /{ }^{219} \mathrm{Th} /{ }^{220} \mathrm{Th} /{ }^{221} \mathrm{Th} /{ }^{222} \mathrm{Th} /{ }^{223} \mathrm{Th} /{ }^{216} \mathrm{~Pa} /$ ${ }^{217} \mathrm{~Pa} /{ }^{218} \mathrm{~Pa} /{ }^{219} \mathrm{~Pa} /{ }^{220} \mathrm{~Pa} /{ }^{221} \mathrm{~Pa} /{ }^{222} \mathrm{~Pa} /{ }^{223} \mathrm{~Pa} /{ }^{224} \mathrm{~Pa} /{ }^{225} \mathrm{~Pa} /$ ${ }^{226} \mathrm{~Pa} /{ }^{227} \mathrm{~Pa}, \mathrm{E}=1 \mathrm{GeV} /$ nucleon; measured (fragment)(decay)-coin, fragment yields. Fragment separator. JOUR NIMAE 543591 


\section{$\mathrm{A}=219$ (continued)}

\begin{tabular}{|c|c|c|}
\hline${ }^{219} \mathrm{~Pa}$ & 2005LI17 & $\begin{array}{l}\text { NUCLEAR REACTIONS Be }\left({ }^{238} \mathrm{U}, \mathrm{X}\right)^{207} \mathrm{Ac} /{ }^{208} \mathrm{Ac} /{ }^{209} \mathrm{Ac} /{ }^{210} \mathrm{Ac} / \\
\\
{ }^{211} \mathrm{Ac} /{ }^{212} \mathrm{Ac} /{ }^{213} \mathrm{Ac} /{ }^{214} \mathrm{Ac} /{ }^{215} \mathrm{Ac} /{ }^{216} \mathrm{Ac} /{ }^{217} \mathrm{Ac} /{ }^{218} \mathrm{Ac} /{ }^{219} \mathrm{Ac} \\
/{ }^{220} \mathrm{Ac} /{ }^{221} \mathrm{Ac} /{ }^{211} \mathrm{Th} /{ }^{212} \mathrm{Th} /{ }^{213} \mathrm{Th} /{ }^{214} \mathrm{Th} /{ }^{215} \mathrm{Th} /{ }^{216} \mathrm{Th} / \\
{ }^{217} \mathrm{Th} /{ }^{218} \mathrm{Th} /{ }^{219} \mathrm{Th} /{ }^{220} \mathrm{Th} / /{ }^{221} \mathrm{Th} /{ }^{222} \mathrm{Th} /{ }^{223} \mathrm{Th} /{ }^{216} \mathrm{~Pa} / \\
\\
{ }^{217} \mathrm{~Pa} /{ }^{218} \mathrm{~Pa} /{ }^{219} \mathrm{~Pa} /{ }^{220} \mathrm{~Pa} /{ }^{221} \mathrm{~Pa} /{ }^{222} \mathrm{~Pa} /{ }^{223} \mathrm{~Pa} /{ }^{224} \mathrm{~Pa} /{ }^{225} \mathrm{~Pa} / \\
\text { fragment yields. Fragment separator. JOUR NIMAE } 543591\end{array}$ \\
\hline \multirow[t]{3}{*}{${ }^{219} \mathrm{U}$} & 2005LE42 & $\begin{array}{l}\text { NUCLEAR REACTIONS }{ }^{182} \mathrm{~W}\left({ }^{40} \mathrm{Ar}, \mathrm{xn}\right){ }^{217} \mathrm{U} /{ }^{218} \mathrm{U} /{ }^{218 m} \mathrm{U} /{ }^{219} \mathrm{U} \text {, } \\
\mathrm{E}=186 \mathrm{MeV} \text {; measured } \mathrm{E} \alpha, \alpha \alpha-,(\text { recoil }) \alpha \text {-coin; deduced production } \sigma \text {. } \\
\text { JOUR ZAANE } 25 \text { s01 } 183\end{array}$ \\
\hline & 2005LE42 & $\begin{array}{l}\text { RADIOACTIVITY }{ }^{217,218,218 m, 219} \mathrm{U}(\alpha) ; \text { measured } \mathrm{E} \alpha, \mathrm{T}_{1 / 2} \cdot \\
{ }_{217,218,219} \mathrm{U} \text { deduced ground state } \mathrm{J}, \pi .{ }^{218} \mathrm{U} \text { deduced isomer } \mathrm{J}, \pi\end{array}$ \\
\hline & & $\begin{array}{l}\text { Implications for } Z=92 \text { shell closure discussed. JOUR ZAANE } 25 \text { s01 } \\
183\end{array}$ \\
\hline
\end{tabular}

\section{$\mathrm{A}=\mathbf{2 2 0}$}

${ }^{220} \mathrm{At} \quad 2005 \mathrm{LIZZ}$

ATOMIC MASSES ${ }^{205} \mathrm{Tl},{ }^{220,221,222} \mathrm{At},{ }^{220,221,222,223} \mathrm{Rn},{ }^{223,224,225} \mathrm{Fr}$, ${ }^{223,224,225,226,227,229,230,231} \mathrm{Ra},{ }^{227,229,230,231} \mathrm{Ac},{ }^{230,231} \mathrm{Th},{ }^{232} \mathrm{U}$;

measured masses. Schottky mass spectrometry, ${ }^{238} \mathrm{U}$ fragmentation. REPT GSI 2005-1,P79,Litvinov

${ }^{220} \mathrm{Rn} \quad$ 2005JOZY RADIOACTIVITY ${ }^{227,228} \mathrm{Th},{ }^{223,224} \mathrm{Ra}(\alpha) ;$ measured E $\gamma, \alpha \gamma$-coin, $\gamma$-ray linear polarization. CONF Argonne(Nuclei at the Limits),P348,Jones

2005LIZZ ATOMIC MASSES ${ }^{205} \mathrm{Tl},{ }^{220,221,222} \mathrm{At},{ }^{220,221,222,223} \mathrm{Rn},{ }^{223,224,225} \mathrm{Fr}$, ${ }^{223,224,225,226,227,229,230,231} \mathrm{Ra},{ }^{227,229,230,231} \mathrm{Ac},{ }^{230,231} \mathrm{Th},{ }^{232} \mathrm{U}$;

measured masses. Schottky mass spectrometry, ${ }^{238} \mathrm{U}$ fragmentation. REPT GSI 2005-1,P79,Litvinov

${ }^{220} \mathrm{Ac} \quad 2005 \mathrm{LI} 17 \quad$ NUCLEAR REACTIONS Be $\left({ }^{238} \mathrm{U}, \mathrm{X}\right){ }^{207} \mathrm{Ac} /{ }^{208} \mathrm{Ac} /{ }^{209} \mathrm{Ac} /{ }^{210} \mathrm{Ac} /$ ${ }^{211} \mathrm{Ac} /{ }^{212} \mathrm{Ac} /{ }^{213} \mathrm{Ac} /{ }^{214} \mathrm{Ac} /{ }^{215} \mathrm{Ac} /{ }^{216} \mathrm{Ac} /{ }^{217} \mathrm{Ac} /{ }^{218} \mathrm{Ac} /{ }^{219} \mathrm{Ac}$ $/{ }^{220} \mathrm{Ac} /{ }^{221} \mathrm{Ac} /{ }^{211} \mathrm{Th} /{ }^{212} \mathrm{Th} /{ }^{213} \mathrm{Th} /{ }^{214} \mathrm{Th} /{ }^{215} \mathrm{Th} /{ }^{216} \mathrm{Th} /$ ${ }^{217} \mathrm{Th} /{ }^{218} \mathrm{Th} /{ }^{219} \mathrm{Th} /{ }^{220} \mathrm{Th} /{ }^{221} \mathrm{Th} /{ }^{222} \mathrm{Th} /{ }^{223} \mathrm{Th} /{ }^{216} \mathrm{~Pa} /$ ${ }^{217} \mathrm{~Pa} /{ }^{218} \mathrm{~Pa} /{ }^{219} \mathrm{~Pa} /{ }^{220} \mathrm{~Pa} /{ }^{221} \mathrm{~Pa} /{ }^{222} \mathrm{~Pa} /{ }^{223} \mathrm{~Pa} /{ }^{224} \mathrm{~Pa} /{ }^{225} \mathrm{~Pa} /$ ${ }^{226} \mathrm{~Pa} /{ }^{227} \mathrm{~Pa}, \mathrm{E}=1 \mathrm{GeV} /$ nucleon; measured (fragment)(decay)-coin, fragment yields. Fragment separator. JOUR NIMAE 543591

${ }^{220} \mathrm{Th} 2005 \mathrm{LI} 17$ NUCLEAR REACTIONS Be $\left({ }^{238} \mathrm{U}, \mathrm{X}\right){ }^{207} \mathrm{Ac} /{ }^{208} \mathrm{Ac} /{ }^{209} \mathrm{Ac} /{ }^{210} \mathrm{Ac} /$ ${ }^{211} \mathrm{Ac} /{ }^{212} \mathrm{Ac} /{ }^{213} \mathrm{Ac} /{ }^{214} \mathrm{Ac} /{ }^{215} \mathrm{Ac} /{ }^{216} \mathrm{Ac} /{ }^{217} \mathrm{Ac} /{ }^{218} \mathrm{Ac} /{ }^{219} \mathrm{Ac}$ / ${ }^{220} \mathrm{Ac} /{ }^{221} \mathrm{Ac} /{ }^{211} \mathrm{Th} /{ }^{212} \mathrm{Th} /{ }^{213} \mathrm{Th} /{ }^{214} \mathrm{Th} /{ }^{215} \mathrm{Th} /{ }^{216} \mathrm{Th} /$ ${ }^{217} \mathrm{Th} /{ }^{218} \mathrm{Th} /{ }^{219} \mathrm{Th} /{ }^{220} \mathrm{Th} /{ }^{221} \mathrm{Th} /{ }^{222} \mathrm{Th} /{ }^{223} \mathrm{Th} /{ }^{216} \mathrm{~Pa} /$ ${ }^{217} \mathrm{~Pa} /{ }^{218} \mathrm{~Pa} /{ }^{219} \mathrm{~Pa} /{ }^{220} \mathrm{~Pa} /{ }^{221} \mathrm{~Pa} /{ }^{222} \mathrm{~Pa} /{ }^{223} \mathrm{~Pa} /{ }^{224} \mathrm{~Pa} /{ }^{225} \mathrm{~Pa} /$ ${ }^{226} \mathrm{~Pa} /{ }^{227} \mathrm{~Pa}, \mathrm{E}=1 \mathrm{GeV} /$ nucleon; measured (fragment)(decay)-coin, fragment yields. Fragment separator. JOUR NIMAE 543591 


\section{$\mathrm{A}=220$ (continued)}

${ }^{220} \mathrm{~Pa}$ 2005LI17 NUCLEAR REACTIONS Be $\left({ }^{238} \mathrm{U}, \mathrm{X}\right){ }^{207} \mathrm{Ac} /{ }^{208} \mathrm{Ac} /{ }^{209} \mathrm{Ac} /{ }^{210} \mathrm{Ac} /$ ${ }^{211} \mathrm{Ac} /{ }^{212} \mathrm{Ac} /{ }^{213} \mathrm{Ac} /{ }^{214} \mathrm{Ac} /{ }^{215} \mathrm{Ac} /{ }^{216} \mathrm{Ac} /{ }^{217} \mathrm{Ac} /{ }^{218} \mathrm{Ac} /{ }^{219} \mathrm{Ac}$ $/{ }^{220} \mathrm{Ac} /{ }^{221} \mathrm{Ac} /{ }^{211} \mathrm{Th} /{ }^{212} \mathrm{Th} /{ }^{213} \mathrm{Th} /{ }^{214} \mathrm{Th} /{ }^{215} \mathrm{Th} /{ }^{216} \mathrm{Th} /$ ${ }^{217} \mathrm{Th} /{ }^{218} \mathrm{Th} /{ }^{219} \mathrm{Th} /{ }^{220} \mathrm{Th} /{ }^{221} \mathrm{Th} /{ }^{222} \mathrm{Th} /{ }^{223} \mathrm{Th} /{ }^{216} \mathrm{~Pa} /$ ${ }^{217} \mathrm{~Pa} /{ }^{218} \mathrm{~Pa} /{ }^{219} \mathrm{~Pa} /{ }^{220} \mathrm{~Pa} /{ }^{221} \mathrm{~Pa} /{ }^{222} \mathrm{~Pa} /{ }^{223} \mathrm{~Pa} /{ }^{224} \mathrm{~Pa} /{ }^{225} \mathrm{~Pa} /$ ${ }^{226} \mathrm{~Pa} /{ }^{227} \mathrm{~Pa}, \mathrm{E}=1 \mathrm{GeV} /$ nucleon; measured (fragment)(decay)-coin, fragment yields. Fragment separator. JOUR NIMAE 543591

\section{$\mathrm{A}=221$}

${ }^{221}$ At 2005LIZZ ATOMIC MASSES ${ }^{205} \mathrm{Tl},{ }^{220,221,222} \mathrm{At},{ }^{220,221,222,223} \mathrm{Rn},{ }^{223,224,225} \mathrm{Fr}$, $223,224,225,226,227,229,230,231$ Ra, ${ }^{227,229,230,231} \mathrm{Ac},{ }^{230,231} \mathrm{Th},{ }^{232} \mathrm{U}$; measured masses. Schottky mass spectrometry, ${ }^{238} \mathrm{U}$ fragmentation. REPT GSI 2005-1,P79,Litvinov

${ }^{221} \mathrm{Rn}$ 2005LIZZ ATOMIC MASSES ${ }^{205} \mathrm{Tl},{ }^{220,221,222} \mathrm{At},{ }^{220,221,222,223} \mathrm{Rn},{ }^{223,224,225} \mathrm{Fr}$, $223,224,225,226,227,229,230,231$ Ra, ${ }^{227,229,230,231} \mathrm{Ac},{ }^{230,231} \mathrm{Th},{ }^{232} \mathrm{U}$;

measured masses. Schottky mass spectrometry, ${ }^{238} \mathrm{U}$ fragmentation. REPT GSI 2005-1,P79,Litvinov

${ }^{221} \mathrm{Ac} \quad$ 2005LI17 NUCLEAR REACTIONS Be $\left({ }^{238} \mathrm{U}, \mathrm{X}\right){ }^{207} \mathrm{Ac} /{ }^{208} \mathrm{Ac} /{ }^{209} \mathrm{Ac} /{ }^{210} \mathrm{Ac} /$ ${ }^{211} \mathrm{Ac} /{ }^{212} \mathrm{Ac} /{ }^{213} \mathrm{Ac} /{ }^{214} \mathrm{Ac} /{ }^{215} \mathrm{Ac} /{ }^{216} \mathrm{Ac} /{ }^{217} \mathrm{Ac} /{ }^{218} \mathrm{Ac} /{ }^{219} \mathrm{Ac}$ $/{ }^{220} \mathrm{Ac} /{ }^{221} \mathrm{Ac} /{ }^{211} \mathrm{Th} /{ }^{212} \mathrm{Th} /{ }^{213} \mathrm{Th} /{ }^{214} \mathrm{Th} /{ }^{215} \mathrm{Th} /{ }^{216} \mathrm{Th} /$ ${ }^{217} \mathrm{Th} /{ }^{218} \mathrm{Th} /{ }^{219} \mathrm{Th} /{ }^{220} \mathrm{Th} /{ }^{221} \mathrm{Th} /{ }^{222} \mathrm{Th} /{ }^{223} \mathrm{Th} /{ }^{216} \mathrm{~Pa} /$ ${ }^{217} \mathrm{~Pa} /{ }^{218} \mathrm{~Pa} /{ }^{219} \mathrm{~Pa} /{ }^{220} \mathrm{~Pa} /{ }^{221} \mathrm{~Pa} /{ }^{222} \mathrm{~Pa} /{ }^{223} \mathrm{~Pa} /{ }^{224} \mathrm{~Pa} /{ }^{225} \mathrm{~Pa} /$ ${ }^{226} \mathrm{~Pa} /{ }^{227} \mathrm{~Pa}, \mathrm{E}=1 \mathrm{GeV} /$ nucleon; measured (fragment)(decay)-coin, fragment yields. Fragment separator. JOUR NIMAE 543591

${ }^{221} \mathrm{Th}$ 2005LI17 NUCLEAR REACTIONS Be $\left({ }^{238} \mathrm{U}, \mathrm{X}\right){ }^{207} \mathrm{Ac} /{ }^{208} \mathrm{Ac} /{ }^{209} \mathrm{Ac} /{ }^{210} \mathrm{Ac} /$ ${ }^{211} \mathrm{Ac} /{ }^{212} \mathrm{Ac} /{ }^{213} \mathrm{Ac} /{ }^{214} \mathrm{Ac} /{ }^{215} \mathrm{Ac} /{ }^{216} \mathrm{Ac} /{ }^{217} \mathrm{Ac} /{ }^{218} \mathrm{Ac} /{ }^{219} \mathrm{Ac}$ $/{ }^{220} \mathrm{Ac} /{ }^{221} \mathrm{Ac} /{ }^{211} \mathrm{Th} /{ }^{212} \mathrm{Th} /{ }^{213} \mathrm{Th} /{ }^{214} \mathrm{Th} /{ }^{215} \mathrm{Th} /{ }^{216} \mathrm{Th} /$ ${ }^{217} \mathrm{Th} /{ }^{218} \mathrm{Th} /{ }^{219} \mathrm{Th} /{ }^{220} \mathrm{Th} /{ }^{221} \mathrm{Th} /{ }^{222} \mathrm{Th} /{ }^{223} \mathrm{Th} /{ }^{216} \mathrm{~Pa} /$ ${ }^{217} \mathrm{~Pa} /{ }^{218} \mathrm{~Pa} /{ }^{219} \mathrm{~Pa} /{ }^{220} \mathrm{~Pa} /{ }^{221} \mathrm{~Pa} /{ }^{222} \mathrm{~Pa} /{ }^{223} \mathrm{~Pa} /{ }^{224} \mathrm{~Pa} /{ }^{225} \mathrm{~Pa} /$ ${ }^{226} \mathrm{~Pa} /{ }^{227} \mathrm{~Pa}, \mathrm{E}=1 \mathrm{GeV} /$ nucleon; measured (fragment)(decay)-coin, fragment yields. Fragment separator. JOUR NIMAE 543591

2005LI17 RADIOACTIVITY ${ }^{216,217,221,222} \mathrm{Th},{ }^{216} \mathrm{Ac},{ }^{215} \mathrm{Ra},{ }^{214} \mathrm{Fr},{ }^{213} \mathrm{Rn}(\alpha)$ [from $\mathrm{Be}\left({ }^{238} \mathrm{U}, \mathrm{X}\right)$ and subsequent decay]; measured $\mathrm{E} \alpha, \mathrm{T}_{1 / 2}$. Fragment separator. JOUR NIMAE 543591

${ }^{221} \mathrm{~Pa}$ 2005LI17 NUCLEAR REACTIONS Be $\left({ }^{238} \mathrm{U}, \mathrm{X}\right){ }^{207} \mathrm{Ac} /{ }^{208} \mathrm{Ac} /{ }^{209} \mathrm{Ac} /{ }^{210} \mathrm{Ac} /$ ${ }^{211} \mathrm{Ac} /{ }^{212} \mathrm{Ac} /{ }^{213} \mathrm{Ac} /{ }^{214} \mathrm{Ac} /{ }^{215} \mathrm{Ac} /{ }^{216} \mathrm{Ac} /{ }^{217} \mathrm{Ac} /{ }^{218} \mathrm{Ac} /{ }^{219} \mathrm{Ac}$ $/{ }^{220} \mathrm{Ac} /{ }^{221} \mathrm{Ac} /{ }^{211} \mathrm{Th} /{ }^{212} \mathrm{Th} /{ }^{213} \mathrm{Th} /{ }^{214} \mathrm{Th} /{ }^{215} \mathrm{Th} /{ }^{216} \mathrm{Th} /$ ${ }^{217} \mathrm{Th} /{ }^{218} \mathrm{Th} /{ }^{219} \mathrm{Th} /{ }^{220} \mathrm{Th} /{ }^{221} \mathrm{Th} /{ }^{222} \mathrm{Th} /{ }^{223} \mathrm{Th} /{ }^{216} \mathrm{~Pa} /$ ${ }^{217} \mathrm{~Pa} /{ }^{218} \mathrm{~Pa} /{ }^{219} \mathrm{~Pa} /{ }^{220} \mathrm{~Pa} /{ }^{221} \mathrm{~Pa} /{ }^{222} \mathrm{~Pa} /{ }^{223} \mathrm{~Pa} /{ }^{224} \mathrm{~Pa} /{ }^{225} \mathrm{~Pa} /$ ${ }^{226} \mathrm{~Pa} /{ }^{227} \mathrm{~Pa}, \mathrm{E}=1 \mathrm{GeV} /$ nucleon; measured (fragment)(decay)-coin, fragment yields. Fragment separator. JOUR NIMAE 543591 


\section{$\mathrm{A}=222$}

\begin{tabular}{|c|c|c|}
\hline \multirow[t]{2}{*}{${ }^{222} \mathrm{At}$} & 2005LIZZ & $\begin{array}{l}\text { ATOMIC MASSES }{ }^{205} \mathrm{Tl},{ }^{220,221,222} \mathrm{At},{ }^{220,221,222,223} \mathrm{Rn},{ }^{223,224,225} \mathrm{Fr} \text {, } \\
223,224,225,226,227,229,230,231 \mathrm{Ra},{ }^{227,229,230,231} \mathrm{Ac},{ }^{230,231} \mathrm{Th},{ }^{232} \mathrm{U}\end{array}$ \\
\hline & & $\begin{array}{l}\text { measured masses. Schottky mass spectrometry, }{ }^{238} \mathrm{U} \text { fragmentation. } \\
\text { REPT GSI 2005-1,P79,Litvinov }\end{array}$ \\
\hline \multirow[t]{3}{*}{${ }^{222} \mathrm{Rn}$} & 2004KU35 & $\begin{array}{l}\text { RADIOACTIVITY }{ }^{238} \mathrm{Pu},{ }^{226} \mathrm{Ra}(\alpha) ;{ }^{152} \mathrm{Eu}(\mathrm{EC}) ; \text { measured low-energy } \\
\text { electron spectra, angular distributions, (electron) } \alpha-\text {, (electron) } \gamma-\text {, } \\
\text { (electron)(X-ray)-coin. JOUR BRSPE } 681358\end{array}$ \\
\hline & 2005LIZZ & 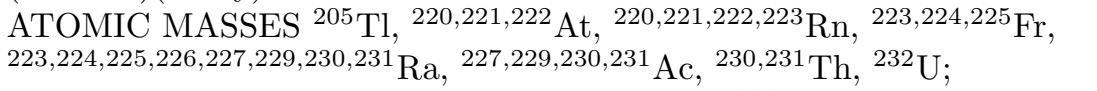 \\
\hline & & $\begin{array}{l}\text { measured masses. Schottky mass spectrometry, }{ }^{238} \mathrm{U} \text { fragmentation. } \\
\text { REPT GSI 2005-1,P79,Litvinov }\end{array}$ \\
\hline \multirow[t]{2}{*}{${ }^{222} \mathrm{Th}$} & 2005LI17 & $\begin{array}{l}\text { NUCLEAR REACTIONS Be }\left({ }^{238} \mathrm{U}, \mathrm{X}\right){ }^{207} \mathrm{Ac} /{ }^{208} \mathrm{Ac} /{ }^{209} \mathrm{Ac} /{ }^{210} \mathrm{Ac} / \\
{ }^{211} \mathrm{Ac} /{ }^{212} \mathrm{Ac} /{ }^{213} \mathrm{Ac} /{ }^{214} \mathrm{Ac} /{ }^{215} \mathrm{Ac} /{ }^{216} \mathrm{Ac} /{ }^{217} \mathrm{Ac} /{ }^{218} \mathrm{Ac} /{ }^{219} \mathrm{Ac} \\
/{ }^{220} \mathrm{Ac} /{ }^{221} \mathrm{Ac} /{ }^{211} \mathrm{Th} /{ }^{212} \mathrm{Th} /{ }^{213} \mathrm{Th} /{ }^{214} \mathrm{Th} /{ }^{215} \mathrm{Th} /{ }^{216} \mathrm{Th} / \\
{ }^{217} \mathrm{Th} /{ }^{218} \mathrm{Th} /{ }^{219} \mathrm{Th} /{ }^{220} \mathrm{Th} /{ }^{221} \mathrm{Th} /{ }^{222} \mathrm{Th} /{ }^{223} \mathrm{Th} /{ }^{216} \mathrm{~Pa} / \\
{ }^{217} \mathrm{~Pa} /{ }^{218} \mathrm{~Pa} /{ }^{219} \mathrm{~Pa} /{ }^{220} \mathrm{~Pa} /{ }^{221} \mathrm{~Pa} /{ }^{222} \mathrm{~Pa} /{ }^{223} \mathrm{~Pa} /{ }^{224} \mathrm{~Pa} /{ }^{225} \mathrm{~Pa} / \\
{ }^{226} \mathrm{~Pa} /{ }^{227} \mathrm{~Pa}, \mathrm{E}=1 \mathrm{GeV} / \text { nucleon} \text {; measured (fragment)(decay)-coin, } \\
\text { fragment yields. Fragment separator. JOUR NIMAE } 543591\end{array}$ \\
\hline & 2005LI17 & $\begin{array}{l}\text { RADIOACTIVITY }{ }^{216,217,221,222} \mathrm{Th},{ }^{216} \mathrm{Ac},{ }^{215} \mathrm{Ra},{ }^{214} \mathrm{Fr},{ }^{213} \mathrm{Rn}(\alpha) \\
\text { [from Be }\left({ }^{238} \mathrm{U}, \mathrm{X}\right) \text { and subsequent decay]; measured } \mathrm{E} \alpha, \mathrm{T}_{1 / 2} . \\
\text { Fragment separator. JOUR NIMAE } 543591\end{array}$ \\
\hline${ }^{222} \mathrm{~Pa}$ & 2005LI17 & $\begin{array}{l}\text { NUCLEAR REACTIONS Be }\left({ }^{238} \mathrm{U}, \mathrm{X}\right){ }^{207} \mathrm{Ac} /{ }^{208} \mathrm{Ac} /{ }^{209} \mathrm{Ac} /{ }^{210} \mathrm{Ac} / \\
{ }^{211} \mathrm{Ac} /{ }^{212} \mathrm{Ac} /{ }^{213} \mathrm{Ac} /{ }^{214} \mathrm{Ac} /{ }^{215} \mathrm{Ac} /{ }^{216} \mathrm{Ac} /{ }^{217} \mathrm{Ac} /{ }^{218} \mathrm{Ac} /{ }^{219} \mathrm{Ac} \\
/{ }^{220} \mathrm{Ac} /{ }^{221} \mathrm{Ac} /{ }^{211} \mathrm{Th} /{ }^{212} \mathrm{Th} /{ }^{213} \mathrm{Th} /{ }^{214} \mathrm{Th} /{ }^{215} \mathrm{Th} /{ }^{216} \mathrm{Th} / \\
{ }^{217} \mathrm{Th} /{ }^{218} \mathrm{Th} /{ }^{219} \mathrm{Th} /{ }^{220} \mathrm{Th} /{ }^{221} \mathrm{Th} /{ }^{222} \mathrm{Th} /{ }^{223} \mathrm{Th} /{ }^{216} \mathrm{~Pa} / \\
{ }^{217} \mathrm{~Pa} /{ }^{218} \mathrm{~Pa} /{ }^{219} \mathrm{~Pa} /{ }^{220} \mathrm{~Pa} /{ }^{221} \mathrm{~Pa} /{ }^{222} \mathrm{~Pa} /{ }^{223} \mathrm{~Pa} /{ }^{224} \mathrm{~Pa} /{ }^{225} \mathrm{~Pa} / \\
{ }^{226} \mathrm{~Pa} /{ }^{227} \mathrm{~Pa}, \mathrm{E}=1 \mathrm{GeV} / \text { nucleon} \text {; measured (fragment)(decay)-coin, } \\
\text { fragment yields. Fragment separator. JOUR NIMAE } 543591\end{array}$ \\
\hline
\end{tabular}

\section{$\mathrm{A}=223$}

${ }^{223} \mathrm{Rn}$ 2005LIZZ ATOMIC MASSES ${ }^{205} \mathrm{Tl},{ }^{220,221,222} \mathrm{At},{ }^{220,221,222,223} \mathrm{Rn},{ }^{223,224,225} \mathrm{Fr}$, ${ }^{223,224,225,226,227,229,230,231} \mathrm{Ra},{ }^{227,229,230,231} \mathrm{Ac},{ }^{230,231} \mathrm{Th},{ }^{232} \mathrm{U}$; measured masses. Schottky mass spectrometry, ${ }^{238} \mathrm{U}$ fragmentation. REPT GSI 2005-1,P79,Litvinov

${ }^{223} \mathrm{Fr}$ 2005LIZZ ATOMIC MASSES ${ }^{205} \mathrm{Tl},{ }^{220,221,222} \mathrm{At},{ }^{220,221,222,223} \mathrm{Rn},{ }^{223,224,225} \mathrm{Fr}$, $223,224,225,226,227,229,230,231 \mathrm{Ra},{ }^{227,229,230,231} \mathrm{Ac},{ }^{230,231} \mathrm{Th},{ }^{232} \mathrm{U}$; measured masses. Schottky mass spectrometry, ${ }^{238} \mathrm{U}$ fragmentation. REPT GSI 2005-1,P79,Litvinov

${ }^{223} \mathrm{Ra} \quad$ 2005JOZY RADIOACTIVITY ${ }^{227,228} \mathrm{Th},{ }^{223,224} \mathrm{Ra}(\alpha)$; measured E $\gamma, \alpha \gamma$-coin, $\gamma$-ray linear polarization. CONF Argonne(Nuclei at the Limits),P348,Jones

2005LIZZ ATOMIC MASSES ${ }^{205} \mathrm{Tl},{ }^{220,221,222} \mathrm{At},{ }^{220,221,222,223} \mathrm{Rn},{ }^{223,224,225} \mathrm{Fr}$, 223,224,225,226,227,229,230,231 Ra, ${ }^{227,229,230,231} \mathrm{Ac},{ }^{230,231} \mathrm{Th},{ }^{232} \mathrm{U}$; measured masses. Schottky mass spectrometry, ${ }^{238} \mathrm{U}$ fragmentation. REPT GSI 2005-1,P79,Litvinov 


\section{$\mathrm{A}=223$ (continued)}

\begin{tabular}{|c|c|c|}
\hline${ }^{223} \mathrm{Th}$ & 2005LI17 & $\begin{array}{l}\text { NUCLEAR REACTIONS Be }\left({ }^{238} \mathrm{U}, \mathrm{X}\right)^{207} \mathrm{Ac} /{ }^{208} \mathrm{Ac} /{ }^{209} \mathrm{Ac} /{ }^{210} \mathrm{Ac} / \\
\\
{ }^{211} \mathrm{Ac} /{ }^{212} \mathrm{Ac} /{ }^{213} \mathrm{Ac} /{ }^{214} \mathrm{Ac} /{ }^{215} \mathrm{Ac} /{ }^{216} \mathrm{Ac} /{ }^{217} \mathrm{Ac} /{ }^{218} \mathrm{Ac} /{ }^{219} \mathrm{Ac} \\
/{ }^{220} \mathrm{Ac} /{ }^{221} \mathrm{Ac} /{ }^{211} \mathrm{Th} /{ }^{212} \mathrm{Th} /{ }^{213} \mathrm{Th} /{ }^{214} \mathrm{Th} /{ }^{215} \mathrm{Th} /{ }^{216} \mathrm{Th} / \\
{ }^{217} \mathrm{Th} /{ }^{218} \mathrm{Th} /{ }^{219} \mathrm{Th} /{ }^{220} \mathrm{Th} / /{ }^{221} \mathrm{Th} /{ }^{222} \mathrm{Th} /{ }^{223} \mathrm{Th} /{ }^{216} \mathrm{~Pa} / \\
\\
{ }^{217} \mathrm{~Pa} /{ }^{218} \mathrm{~Pa} /{ }^{219} \mathrm{~Pa} /{ }^{220} \mathrm{~Pa} /{ }^{221} \mathrm{~Pa} /{ }^{222} \mathrm{~Pa} /{ }^{223} \mathrm{~Pa} /{ }^{224} \mathrm{~Pa} /{ }^{225} \mathrm{~Pa} / \\
\text { fragment yields. Fragment separator. JOUR NIMAE } 543591\end{array}$ \\
\hline${ }^{223} \mathrm{~Pa}$ & $2005 \operatorname{LI} 17$ & $\begin{array}{l}\text { NUCLEAR REACTIONS Be }\left({ }^{238} \mathrm{U}, \mathrm{X}\right){ }^{207} \mathrm{Ac} /{ }^{208} \mathrm{Ac} /{ }^{209} \mathrm{Ac} /{ }^{210} \mathrm{Ac} / \\
{ }^{211} \mathrm{Ac} /{ }^{212} \mathrm{Ac} /{ }^{213} \mathrm{Ac} /{ }^{214} \mathrm{Ac} /{ }^{215} \mathrm{Ac} /{ }^{216} \mathrm{Ac} /{ }^{217} \mathrm{Ac} /{ }^{218} \mathrm{Ac} /{ }^{219} \mathrm{Ac} \\
/{ }^{220} \mathrm{Ac} /{ }^{221} \mathrm{Ac} /{ }^{211} \mathrm{Th} /{ }^{212} \mathrm{Th} /{ }^{213} \mathrm{Th} /{ }^{214} \mathrm{Th} /{ }^{215} \mathrm{Th} /{ }^{216} \mathrm{Th} / \\
{ }^{217} \mathrm{Th} /{ }^{218} \mathrm{Th} /{ }^{219} \mathrm{Th} /{ }^{220} \mathrm{Th} /{ }^{221} \mathrm{Th} /{ }^{222} \mathrm{Th} /{ }^{223} \mathrm{Th} /{ }^{216} \mathrm{~Pa} / \\
{ }^{217} \mathrm{~Pa} /{ }^{218} \mathrm{~Pa} /{ }^{219} \mathrm{~Pa} /{ }^{220} \mathrm{~Pa} /{ }^{221} \mathrm{~Pa} /{ }^{222} \mathrm{~Pa} /{ }^{223} \mathrm{~Pa} /{ }^{224} \mathrm{~Pa} /{ }^{225} \mathrm{~Pa} / \\
\\
{ }^{226} \mathrm{~Pa} /{ }^{227} \mathrm{~Pa}, \mathrm{E}=1 \mathrm{GeV} / \text { nucleon} \text {; measured (fragment) (decay)-coin, } \\
\text { fragment yields. Fragment separator. JOUR NIMAE } 543591\end{array}$ \\
\hline
\end{tabular}

\section{$\mathrm{A}=224$}

${ }^{224} \mathrm{Fr} \quad$ 2005LIZZ

ATOMIC MASSES ${ }^{205} \mathrm{Tl},{ }^{220,221,222} \mathrm{At},{ }^{220,221,222,223} \mathrm{Rn},{ }^{223,224,225} \mathrm{Fr}$, ${ }^{223,224,225,226,227,229,230,231} \mathrm{Ra},{ }^{227,229,230,231} \mathrm{Ac},{ }^{230,231} \mathrm{Th},{ }^{232} \mathrm{U}$;

measured masses. Schottky mass spectrometry, ${ }^{238} \mathrm{U}$ fragmentation. REPT GSI 2005-1,P79,Litvinov

${ }^{224} \mathrm{Ra}$ 2005JOZY RADIOACTIVITY ${ }^{227,228} \mathrm{Th},{ }^{223,224} \mathrm{Ra}(\alpha) ;$ measured $\mathrm{E} \gamma, \alpha \gamma$-coin, $\gamma$-ray linear polarization. CONF Argonne(Nuclei at the Limits),P348,Jones

2005LIZZ ATOMIC MASSES ${ }^{205} \mathrm{Tl},{ }^{220,221,222} \mathrm{At},{ }^{220,221,222,223} \mathrm{Rn},{ }^{223,224,225} \mathrm{Fr}$, ${ }^{223,224,225,226,227,229,230,231} \mathrm{Ra},{ }^{227,229,230,231} \mathrm{Ac},{ }^{230,231} \mathrm{Th},{ }^{232} \mathrm{U}$;

measured masses. Schottky mass spectrometry, ${ }^{238} \mathrm{U}$ fragmentation. REPT GSI 2005-1,P79,Litvinov

${ }^{224} \mathrm{Th}$ 2005SE03 NUCLEAR REACTIONS ${ }^{176} \mathrm{Yb}\left({ }^{48} \mathrm{Ca}, \mathrm{X}\right), \mathrm{E}=206,219,256,259 \mathrm{MeV}$; measured $\mathrm{E} \gamma, \mathrm{I} \gamma$, (evaporation residue) $\gamma$-coin, $\gamma$-ray multiplicity and sum energy, fusion and evaporation residue $\sigma .{ }^{224} \mathrm{Th}$ deduced GDR parameters. Comparison with model predictions. JOUR NUPAB 750 245

${ }^{224} \mathrm{~Pa}$ 2005LI17 NUCLEAR REACTIONS Be $\left({ }^{238} \mathrm{U}, \mathrm{X}\right){ }^{207} \mathrm{Ac} /{ }^{208} \mathrm{Ac} /{ }^{209} \mathrm{Ac} /{ }^{210} \mathrm{Ac} /$ ${ }^{211} \mathrm{Ac} /{ }^{212} \mathrm{Ac} /{ }^{213} \mathrm{Ac} /{ }^{214} \mathrm{Ac} /{ }^{215} \mathrm{Ac} /{ }^{216} \mathrm{Ac} /{ }^{217} \mathrm{Ac} /{ }^{218} \mathrm{Ac} /{ }^{219} \mathrm{Ac}$ $/{ }^{220} \mathrm{Ac} /{ }^{221} \mathrm{Ac} /{ }^{211} \mathrm{Th} /{ }^{212} \mathrm{Th} /{ }^{213} \mathrm{Th} /{ }^{214} \mathrm{Th} /{ }^{215} \mathrm{Th} /{ }^{216} \mathrm{Th} /$ ${ }^{217} \mathrm{Th} /{ }^{218} \mathrm{Th} /{ }^{219} \mathrm{Th} /{ }^{220} \mathrm{Th} /{ }^{221} \mathrm{Th} /{ }^{222} \mathrm{Th} /{ }^{223} \mathrm{Th} /{ }^{216} \mathrm{~Pa} /$ ${ }^{217} \mathrm{~Pa} /{ }^{218} \mathrm{~Pa} /{ }^{219} \mathrm{~Pa} /{ }^{220} \mathrm{~Pa} /{ }^{221} \mathrm{~Pa} /{ }^{222} \mathrm{~Pa} /{ }^{223} \mathrm{~Pa} /{ }^{224} \mathrm{~Pa} /{ }^{225} \mathrm{~Pa} /$ ${ }^{226} \mathrm{~Pa} /{ }^{227} \mathrm{~Pa}, \mathrm{E}=1 \mathrm{GeV} /$ nucleon; measured (fragment)(decay)-coin, fragment yields. Fragment separator. JOUR NIMAE 543591

\section{$\mathrm{A}=225$}

${ }^{225} \mathrm{Fr}$ 2005LIZZ ATOMIC MASSES ${ }^{205} \mathrm{Tl},{ }^{220,221,222} \mathrm{At},{ }^{220,221,222,223} \mathrm{Rn},{ }^{223,224,225} \mathrm{Fr}$, ${ }^{223,224,225,226,227,229,230,231} \mathrm{Ra},{ }^{227,229,230,231} \mathrm{Ac},{ }^{230,231} \mathrm{Th},{ }^{232} \mathrm{U}$; measured masses. Schottky mass spectrometry, ${ }^{238} \mathrm{U}$ fragmentation. REPT GSI 2005-1,P79,Litvinov 


\section{$\mathrm{A}=225$ (continued)}

\begin{tabular}{|c|c|c|}
\hline${ }^{225} \mathrm{Ra}$ & 2005LIZZ & $\begin{array}{l}\text { ATOMIC MASSES }{ }^{205} \mathrm{Tl},{ }^{220,221,222} \mathrm{At},{ }^{220,221,222,223} \mathrm{Rn},{ }^{223,224,225} \mathrm{Fr} \\
{ }^{223,224,225,226,227,229,230,231} \mathrm{Ra},{ }^{227,229,230,231} \mathrm{Ac},{ }^{230,231} \mathrm{Th},{ }^{232} \mathrm{U}\end{array}$ \\
\hline & & $\begin{array}{l}\text { measured masses. Schottky mass spectrometry, }{ }^{238} \mathrm{U} \text { fragmentation. } \\
\text { REPT GSI 2005-1,P79,Litvinov }\end{array}$ \\
\hline${ }^{225} \mathrm{~Pa}$ & $2005 \operatorname{LI} 17$ & $\begin{array}{l}\text { NUCLEAR REACTIONS Be }\left({ }^{238} \mathrm{U}, \mathrm{X}\right){ }^{207} \mathrm{Ac} /{ }^{208} \mathrm{Ac} /{ }^{209} \mathrm{Ac} /{ }^{210} \mathrm{Ac} / \\
{ }^{211} \mathrm{Ac} /{ }^{212} \mathrm{Ac} /{ }^{213} \mathrm{Ac} /{ }^{214} \mathrm{Ac} /{ }^{215} \mathrm{Ac} /{ }^{216} \mathrm{Ac} /{ }^{217} \mathrm{Ac} /{ }^{218} \mathrm{Ac} /{ }^{219} \mathrm{Ac} \\
/{ }^{220} \mathrm{Ac} /{ }^{221} \mathrm{Ac} /{ }^{211} \mathrm{Th} /{ }^{212} \mathrm{Th} /{ }^{213} \mathrm{Th} /{ }^{214} \mathrm{Th} /{ }^{215} \mathrm{Th} /{ }^{216} \mathrm{Th} / \\
{ }^{217} \mathrm{Th} /{ }^{218} \mathrm{Th} /{ }^{219} \mathrm{Th} /{ }^{220} \mathrm{Th} /{ }^{221} \mathrm{Th} /{ }^{222} \mathrm{Th} /{ }^{223} \mathrm{Th} /{ }^{216} \mathrm{~Pa} / \\
\\
{ }^{217} \mathrm{~Pa} /{ }^{218} \mathrm{~Pa} /{ }^{219} \mathrm{~Pa} /{ }^{220} \mathrm{~Pa} /{ }^{221} \mathrm{~Pa} /{ }^{222} \mathrm{~Pa} /{ }^{223} \mathrm{~Pa} /{ }^{224} \mathrm{~Pa} /{ }^{225} \mathrm{~Pa} / \\
{ }^{226} \mathrm{~Pa} /{ }^{227} \mathrm{~Pa}, \mathrm{E}=1 \mathrm{GeV} / \text { nucleon} \text {; measured (fragment)(decay)-coin, } \\
\text { fragment yields. Fragment separator. JOUR NIMAE } 543591\end{array}$ \\
\hline
\end{tabular}

\section{$\mathrm{A}=226$}

${ }^{226} \mathrm{Ra} \quad 2004 \mathrm{KU} 35$

RADIOACTIVITY ${ }^{238} \mathrm{Pu},{ }^{226} \mathrm{Ra}(\alpha) ;{ }^{152} \mathrm{Eu}(\mathrm{EC})$; measured low-energy electron spectra, angular distributions, (electron) $\alpha-$, (electron) $\gamma-$, (electron)(X-ray)-coin. JOUR BRSPE 681358

2005LIZZ ATOMIC MASSES ${ }^{205} \mathrm{Tl},{ }^{220,221,222} \mathrm{At},{ }^{220,221,222,223} \mathrm{Rn},{ }^{223,224,225} \mathrm{Fr}$, $223,224,225,226,227,229,230,231$ Ra, ${ }^{227,229,230,231} \mathrm{Ac},{ }^{230,231} \mathrm{Th},{ }^{232} \mathrm{U}$; measured masses. Schottky mass spectrometry, ${ }^{238} \mathrm{U}$ fragmentation. REPT GSI 2005-1,P79,Litvinov

${ }^{226} \mathrm{~Pa}$ 2005LI17 NUCLEAR REACTIONS Be $\left({ }^{238} \mathrm{U}, \mathrm{X}\right){ }^{207} \mathrm{Ac} /{ }^{208} \mathrm{Ac} /{ }^{209} \mathrm{Ac} /{ }^{210} \mathrm{Ac} /$ ${ }^{211} \mathrm{Ac} /{ }^{212} \mathrm{Ac} /{ }^{213} \mathrm{Ac} /{ }^{214} \mathrm{Ac} /{ }^{215} \mathrm{Ac} /{ }^{216} \mathrm{Ac} /{ }^{217} \mathrm{Ac} /{ }^{218} \mathrm{Ac} /{ }^{219} \mathrm{Ac}$ / ${ }^{220} \mathrm{Ac} /{ }^{221} \mathrm{Ac} /{ }^{211} \mathrm{Th} /{ }^{212} \mathrm{Th} /{ }^{213} \mathrm{Th} /{ }^{214} \mathrm{Th} /{ }^{215} \mathrm{Th} /{ }^{216} \mathrm{Th} /$ ${ }^{217} \mathrm{Th} /{ }^{218} \mathrm{Th} /{ }^{219} \mathrm{Th} /{ }^{220} \mathrm{Th} /{ }^{221} \mathrm{Th} /{ }^{222} \mathrm{Th} /{ }^{223} \mathrm{Th} /{ }^{216} \mathrm{~Pa} /$ ${ }^{217} \mathrm{~Pa} /{ }^{218} \mathrm{~Pa} /{ }^{219} \mathrm{~Pa} /{ }^{220} \mathrm{~Pa} /{ }^{221} \mathrm{~Pa} /{ }^{222} \mathrm{~Pa} /{ }^{223} \mathrm{~Pa} /{ }^{224} \mathrm{~Pa} /{ }^{225} \mathrm{~Pa} /$ ${ }^{226} \mathrm{~Pa} /{ }^{227} \mathrm{~Pa}, \mathrm{E}=1 \mathrm{GeV} /$ nucleon; measured (fragment)(decay)-coin, fragment yields. Fragment separator. JOUR NIMAE 543591

\section{$\mathrm{A}=227$}

${ }^{227} \mathrm{Ra}$ 2005LIZZ ATOMIC MASSES ${ }^{205} \mathrm{Tl},{ }^{220,221,222} \mathrm{At},{ }^{220,221,222,223} \mathrm{Rn},{ }^{223,224,225} \mathrm{Fr}$, ${ }^{223,224,225,226,227,229,230,231} \mathrm{Ra},{ }^{227,229,230,231} \mathrm{Ac},{ }^{230,231} \mathrm{Th},{ }^{232} \mathrm{U}$; measured masses. Schottky mass spectrometry, ${ }^{238} \mathrm{U}$ fragmentation. REPT GSI 2005-1,P79,Litvinov

${ }^{227} \mathrm{Ac}$ 2005LIZZ ATOMIC MASSES ${ }^{205} \mathrm{Tl},{ }^{220,221,222} \mathrm{At},{ }^{220,221,222,223} \mathrm{Rn},{ }^{223,224,225} \mathrm{Fr}$, ${ }_{223,224,225,226,227,229,230,231} \mathrm{Ra},{ }^{227,229,230,231} \mathrm{Ac},{ }^{230,231} \mathrm{Th},{ }^{232} \mathrm{U}$; measured masses. Schottky mass spectrometry, ${ }^{238} \mathrm{U}$ fragmentation. REPT GSI 2005-1,P79,Litvinov

${ }^{227} \mathrm{Th}$ 2005JOZY RADIOACTIVITY ${ }^{227,228} \mathrm{Th},{ }^{223,224} \mathrm{Ra}(\alpha)$; measured $\mathrm{E} \gamma, \alpha \gamma$-coin, $\gamma$-ray linear polarization. CONF Argonne(Nuclei at the Limits),P348,Jones 


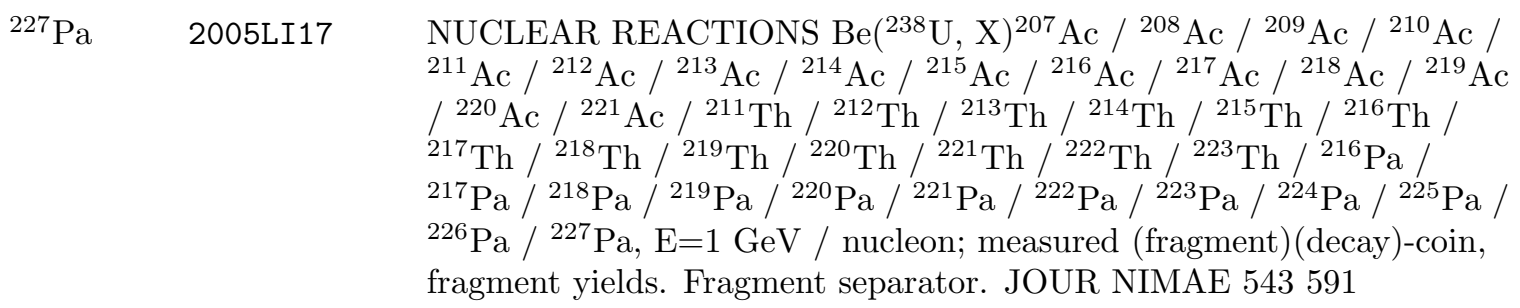

$$
\mathrm{A}=228
$$

${ }^{228} \mathrm{Th} \quad$ 2005JOZY

2005PAZW NUCLEAR REACTIONS ${ }^{207} \mathrm{~Pb}(\mathrm{n}, 2 \mathrm{n}), \mathrm{E}<20 \mathrm{MeV} ;{ }^{232} \mathrm{Th}(\mathrm{n}, 5 \mathrm{n})$, $\mathrm{E}=29-42 \mathrm{MeV}$; measured $\mathrm{E} \gamma, \mathrm{I} \gamma .{ }^{207} \mathrm{~Pb}(\mathrm{n}, 2 \mathrm{n}), \mathrm{E}=8-24 \mathrm{MeV}$; calculated $\sigma$. CONF Santa Fe (Nucl Data for Sci and Technol) Proc, Vol1,P876

$$
\mathrm{A}=229
$$

\section{${ }^{229} \mathrm{Ra} \quad 2005 \mathrm{HE} 26$}

2005LIZZ

${ }^{229} \mathrm{Ac}$

2005LIZZ

${ }^{229} \mathrm{~Np} \quad$ 2002AS08

$\begin{array}{ll}{ }^{230} \mathrm{Fr} & 2005 \mathrm{HE} 26 \\ { }^{230} \mathrm{Ra} & 2005 \mathrm{HE} 26 \\ & 2005 \mathrm{LIZZ}\end{array}$

\section{$\mathrm{A}=\mathbf{2 3 0}$}

ATOMIC MASSES ${ }^{229,230,231,232}$ Ra, ${ }^{230} \mathrm{Fr}$; measured masses. Penning trap mass spectrometer. JOUR ZAANE 25 s01 17

ATOMIC MASSES ${ }^{205} \mathrm{Tl},{ }^{220,221,222} \mathrm{At},{ }^{220,221,222,223} \mathrm{Rn},{ }^{223,224,225} \mathrm{Fr}$, ${ }_{223,224,225,226,227,229,230,231} \mathrm{Ra},{ }^{227,229,230,231} \mathrm{Ac},{ }^{230,231} \mathrm{Th},{ }^{232} \mathrm{U}$;

measured masses. Schottky mass spectrometry, ${ }^{238} \mathrm{U}$ fragmentation.

REPT GSI 2005-1,P79,Litvinov

ATOMIC MASSES ${ }^{205} \mathrm{Tl},{ }^{220,221,222} \mathrm{At},{ }^{220,221,222,223} \mathrm{Rn},{ }^{223,224,225} \mathrm{Fr}$, ${ }^{223,224,225,226,227,229,230,231} \mathrm{Ra},{ }^{227,229,230,231} \mathrm{Ac},{ }^{230,231} \mathrm{Th},{ }^{232} \mathrm{U}$;

measured masses. Schottky mass spectrometry, ${ }^{238} \mathrm{U}$ fragmentation.

REPT GSI 2005-1,P79,Litvinov

RADIOACTIVITY ${ }^{235,236} \mathrm{Am}$ (EC) [from ${ }^{233,235} \mathrm{U}\left({ }^{6} \mathrm{Li}, \mathrm{xn}\right)$ ]; measured prompt and delayed $\mathrm{E} \gamma, \mathrm{I} \gamma, \gamma \gamma$-coin; deduced $\log \mathrm{ft} .{ }^{235,236} \mathrm{Pu}$ deduced levels, J, $\pi$, configurations. ${ }^{233,234,235,236} \mathrm{Am},{ }^{237,238} \mathrm{Cm}(\alpha)$ [from $\left.{ }^{233,235} \mathrm{U},{ }^{237} \mathrm{~Np}\left({ }^{6} \mathrm{Li}, \mathrm{xn}\right)\right]$; measured $\mathrm{E} \alpha, \mathrm{T}_{1 / 2}$. Isotope separator. JOUR JNRSA 3,No 1,187

ATOMIC MASSES $229,230,231,232 \mathrm{Ra},{ }^{230} \mathrm{Fr}$; measured masses. Penning trap mass spectrometer. JOUR ZAANE 25 s01 17

ATOMIC MASSES ${ }^{229,230,231,232} \mathrm{Ra},{ }^{230} \mathrm{Fr}$; measured masses. Penning trap mass spectrometer. JOUR ZAANE 25 s01 17

ATOMIC MASSES ${ }^{205} \mathrm{Tl},{ }^{220,221,222} \mathrm{At},{ }^{220,221,222,223} \mathrm{Rn},{ }^{223,224,225} \mathrm{Fr}$, ${ }^{223,224,225,226,227,229,230,231} \mathrm{Ra},{ }^{227,229,230,231} \mathrm{Ac},{ }^{230,231} \mathrm{Th},{ }^{232} \mathrm{U}$;

measured masses. Schottky mass spectrometry, ${ }^{238} \mathrm{U}$ fragmentation. REPT GSI 2005-1,P79,Litvinov 


\section{$\mathrm{A}=230$ (continued)}

\begin{tabular}{|c|c|c|}
\hline \multirow[t]{2}{*}{$230 \mathrm{~A}$} & $2005 \mathrm{LIZZ}$ & $\begin{array}{l}\text { ATOMIC MASSES }{ }^{205} \mathrm{Tl},{ }^{220,221,222} \mathrm{At},{ }^{220,221,222,223} \mathrm{Rn},{ }^{223,224,225} \mathrm{Fr}, \\
\end{array}$ \\
\hline & & $\begin{array}{l}\text { measured masses. Schottky mass spectrometry, }{ }^{238} \mathrm{U} \text { fragmentation. } \\
\text { REPT GSI 2005-1,P79,Litvinov }\end{array}$ \\
\hline \multirow[t]{3}{*}{${ }^{230} \mathrm{Th}$} & $2005 \mathrm{LIZZ}$ & $\begin{array}{l}\text { ATOMIC MASSES }{ }^{205} \mathrm{Tl},{ }^{220,221,224,225} \mathrm{At},{ }^{220,221,222,223} \mathrm{Rn},{ }^{223,224,227,225} \mathrm{Fr} \text {, } \\
\end{array}$ \\
\hline & & $\begin{array}{l}\text { measured masses. Schottky mass spectrometry, }{ }^{238} \mathrm{U} \text { fragmentation. } \\
\text { REPT GSI 2005-1,P79,Litvinov }\end{array}$ \\
\hline & 2005P002 & $\begin{array}{l}\text { RADIOACTIVITY }{ }^{234,235,238} \mathrm{U}(\alpha) \text {; measured } \mathrm{E} \alpha \text {, I } \alpha \text { from thick source. } \\
\text { Comparison with model predictions. JOUR RMEAE } 39565\end{array}$ \\
\hline${ }^{230} \mathrm{~Np}$ & 2002AS08 & $\begin{array}{l}\text { RADIOACTIVITY }{ }^{235,236} \mathrm{Am}(\mathrm{EC})\left[\mathrm{from}{ }^{233,235} \mathrm{U}\left({ }^{6} \mathrm{Li}, \mathrm{xn}\right)\right] \text {; measured } \\
\text { prompt and delayed } \mathrm{E} \gamma, \mathrm{I} \gamma, \gamma \gamma \text {-coin; deduced } \log \mathrm{ft} .{ }^{235,236} \mathrm{Pu} \text { deduced } \\
\text { levels, J, } \pi \text {, configurations. }{ }^{233,234,235,236} \mathrm{Am},{ }^{237,238} \mathrm{Cm}(\alpha)[\text { from } \\
\left.{ }^{233,235} \mathrm{U},{ }^{237} \mathrm{~Np}\left({ }^{6} \mathrm{Li} \text {, xn }\right)\right] ; \text { measured } \mathrm{E} \alpha, \mathrm{T}_{1 / 2} \text {. Isotope separator. } \\
\text { JOUR JNRSA 3.No } 1.187\end{array}$ \\
\hline
\end{tabular}

\section{$\mathrm{A}=231$}

${ }^{231} \mathrm{Ra} \quad 2005 \mathrm{HE} 26$

2005LIZZ

${ }^{231} \mathrm{Ac} \quad 2005 \mathrm{LIZZ}$

${ }^{231} \mathrm{Th} \quad$ 2005GA36

2005LIZZ

2005P002

${ }^{231} \mathrm{~Np} \quad$ 2002AS08

${ }^{232} \mathrm{Ra} \quad 2005 \mathrm{HE} 26$
ATOMIC MASSES ${ }^{229,230,231,232} \mathrm{Ra},{ }^{230} \mathrm{Fr}$; measured masses. Penning trap mass spectrometer. JOUR ZAANE 25 s01 17

ATOMIC MASSES ${ }^{205} \mathrm{Tl},{ }^{220,221,222} \mathrm{At},{ }^{220,221,222,223} \mathrm{Rn},{ }^{223,224,225} \mathrm{Fr}$, ${ }^{223,224,225,226,227,229,230,231} \mathrm{Ra},{ }^{227,229,230,231} \mathrm{Ac},{ }^{230,231} \mathrm{Th},{ }^{232} \mathrm{U}$; measured masses. Schottky mass spectrometry, ${ }^{238} \mathrm{U}$ fragmentation. REPT GSI 2005-1,P79,Litvinov ATOMIC MASSES ${ }^{205} \mathrm{Tl},{ }^{220,221,222} \mathrm{At},{ }^{220,221,222,223} \mathrm{Rn},{ }^{223,224,225} \mathrm{Fr}$, ${ }^{223,224,225,226,227,229,230,231} \mathrm{Ra},{ }^{227,229,230,231} \mathrm{Ac},{ }^{230,231} \mathrm{Th},{ }^{232} \mathrm{U}$; measured masses. Schottky mass spectrometry, ${ }^{238} \mathrm{U}$ fragmentation. REPT GSI 2005-1,P79,Litvinov RADIOACTIVITY ${ }^{235} \mathrm{U}(\alpha)$; measured $\mathrm{E} \alpha$, I $\alpha$; deduced emission probabilities. JOUR NIMAE 550581

ATOMIC MASSES ${ }^{205} \mathrm{Tl},{ }^{220,221,222} \mathrm{At},{ }^{220,221,222,223} \mathrm{Rn},{ }^{223,224,225} \mathrm{Fr}$, ${ }^{223,224,225,226,227,229,230,231} \mathrm{Ra},{ }^{227,229,230,231} \mathrm{Ac},{ }^{230,231} \mathrm{Th},{ }^{232} \mathrm{U}$; measured masses. Schottky mass spectrometry, ${ }^{238} \mathrm{U}$ fragmentation. REPT GSI 2005-1,P79,Litvinov RADIOACTIVITY ${ }^{234,235,238} \mathrm{U}(\alpha)$; measured $\mathrm{E} \alpha$, I $\alpha$ from thick source. Comparison with model predictions. JOUR RMEAE 39565 RADIOACTIVITY ${ }^{235,236} \mathrm{Am}$ (EC) [from ${ }^{233,235} \mathrm{U}\left({ }^{6} \mathrm{Li}, \mathrm{xn}\right)$ ]; measured prompt and delayed $\mathrm{E} \gamma, \mathrm{I} \gamma, \gamma \gamma$-coin; deduced $\log \mathrm{ft} .{ }^{235,236} \mathrm{Pu}$ deduced levels, J, $\pi$, configurations. ${ }^{233,234,235,236} \mathrm{Am},{ }^{237,238} \mathrm{Cm}(\alpha)$ [from $\left.{ }^{233,235} \mathrm{U},{ }^{237} \mathrm{~Np}\left({ }^{6} \mathrm{Li}, \mathrm{xn}\right)\right]$; measured $\mathrm{E} \alpha, \mathrm{T}_{1 / 2}$. Isotope separator. JOUR JNRSA 3,No 1,187

$$
\mathrm{A}=232
$$

ATOMIC MASSES ${ }^{229,230,231,232} \mathrm{Ra},{ }^{230} \mathrm{Fr}$; measured masses. Penning trap mass spectrometer. JOUR ZAANE 25 s01 17 


\section{$\mathrm{A}=232$ (continued)}

\begin{tabular}{|c|c|c|}
\hline${ }^{232} \mathrm{U}$ & 2005LIZZ & $\begin{array}{l}\text { ATOMIC MASSES }{ }^{205} \mathrm{Tl},{ }^{220,221,222} \mathrm{At},{ }^{220,221,222,223} \mathrm{Rn},{ }^{223,224,225} \mathrm{Fr}, \\
\end{array}$ \\
\hline & & $\begin{array}{l}\text { measured masses. Schottky mass spectrometry, }{ }^{238} \mathrm{U} \text { fragmentation. } \\
\text { REPT GSI 2005-1,P79,Litvinov }\end{array}$ \\
\hline${ }^{232} \mathrm{~Np}$ & 2002AS08 & $\begin{array}{l}\left.\text { RADIOACTIVITY }{ }^{235,236} \mathrm{Am}(\mathrm{EC}) \text { [from }{ }^{233,235} \mathrm{U}\left({ }^{6} \mathrm{Li}, \mathrm{xn}\right)\right] \text {; measured } \\
\text { prompt and delayed } \mathrm{E} \gamma, \mathrm{I} \gamma, \gamma \gamma \text {-coin; deduced } \log \mathrm{ft} .{ }^{235,236} \mathrm{Pu} \text { deduced } \\
\text { levels, J, } \pi \text {, configurations. }{ }^{233,234,235,236} \mathrm{Am},{ }^{237,238} \mathrm{Cm}(\alpha) \text { [from } \\
\left.{ }^{233,235} \mathrm{U},{ }^{237} \mathrm{~Np}\left({ }^{6} \mathrm{Li} \text {, xn }\right)\right] ; \text { measured } \mathrm{E} \alpha, \mathrm{T}_{1 / 2} \text {. Isotope separator. }\end{array}$ \\
\hline
\end{tabular}

\section{$\mathrm{A}=233$}

${ }^{233} \mathrm{Th}$ 2004ADZW NUCLEAR REACTIONS ${ }^{209} \mathrm{Bi}(\mathrm{n}, 4 \mathrm{n}),(\mathrm{n}, 5 \mathrm{n}),(\mathrm{n}, 6 \mathrm{n}),(\mathrm{n}, 7 \mathrm{n}),(\mathrm{n}$, 9n), ${ }^{232} \mathrm{Th}(\mathrm{n}, \gamma),{ }^{197} \mathrm{Au}(\mathrm{n}, 2 \mathrm{n}),(\mathrm{n}, 4 \mathrm{n}),(\mathrm{n}, 6 \mathrm{n}),(\mathrm{n}, 7 \mathrm{n}),(\mathrm{n}, \gamma),{ }^{115} \operatorname{In}(\mathrm{n}$, $5 \mathrm{n}),(\mathrm{n}, 6 \mathrm{n}),(\mathrm{n}, 7 \mathrm{n}),{ }^{59} \mathrm{Co}(\mathrm{n}, 2 \mathrm{n}),(\mathrm{n}, 3 \mathrm{n}),(\mathrm{n}, 4 \mathrm{n}),(\mathrm{n}, 5 \mathrm{n}),(\mathrm{n}, \gamma),(\mathrm{n}$, $\mathrm{p}),(\mathrm{n}, 6 \mathrm{n} 2 \mathrm{p}), \mathrm{E}=$ spectrum; measured $\mathrm{E} \gamma, \mathrm{I} \gamma$; deduced reaction rates. $\mathrm{Pb}(\mathrm{p}, \mathrm{nX}), \mathrm{E}=1 \mathrm{GeV}$; deduced spallation neutron spectrum. REPT JINR-E1-2004-16,Adam 2004HA64 NUCLEAR REACTIONS ${ }^{232} \mathrm{Th}(\mathrm{n}, \gamma), \mathrm{E}=0.05-2 \mathrm{MeV} ;{ }^{230} \mathrm{Th}$, ${ }^{231,233} \mathrm{~Pa}(\mathrm{n}, \mathrm{F}), \mathrm{E}=0.5-10 \mathrm{MeV}$; measured $\sigma$. Comparison with previous results. JOUR BJPHE 34814

2005AD01 NUCLEAR REACTIONS ${ }^{209} \mathrm{Bi}(\mathrm{n}, 4 \mathrm{n}),(\mathrm{n}, 5 \mathrm{n}),(\mathrm{n}, 6 \mathrm{n}),(\mathrm{n}, 7 \mathrm{n}),(\mathrm{n}$, 9n), ${ }^{232} \mathrm{Th}(\mathrm{n}, \gamma),{ }^{197} \mathrm{Au}(\mathrm{n}, 2 \mathrm{n}),(\mathrm{n}, 4 \mathrm{n}),(\mathrm{n}, 6 \mathrm{n}),(\mathrm{n}, 7 \mathrm{n}),(\mathrm{n}, \gamma),{ }^{59} \mathrm{Co}(\mathrm{n}$, 2n), (n, 3n), (n, 4n), (n, 5n), (n, p), (n, 6n2p), ${ }^{115} \operatorname{In}(\mathrm{n}, 5 \mathrm{n}),(\mathrm{n}, 6 \mathrm{n}),(\mathrm{n}$, $7 \mathrm{n}), \mathrm{E}=$ spectrum; measured $\mathrm{E} \gamma, \mathrm{I} \gamma$; deduced reaction rates. Activation technique, spallation neutrons from $1 \mathrm{GeV}$ proton beam, comparison with model predictions. JOUR ZAANE 2361

2005AEZZ NUCLEAR REACTIONS ${ }^{232} \mathrm{Th}(\mathrm{n}, \gamma), \mathrm{E}=0-1 \mathrm{MeV}$; measured capture $\sigma$; deduced resonance parameters. CONF Santa Fe (Nucl Data for Sci and Technol) Proc,Vol2,P1470

2005MAZO NUCLEAR REACTIONS ${ }^{232} \mathrm{Th}(\mathrm{n}, \gamma), \mathrm{E}=$ thermal; measured capture $\sigma$. Activation technique. CONF Santa Fe (Nucl Data for Sci and Technol) Proc,Vol2,P1466

${ }^{233} \mathrm{Pu}$ 2002AS08 RADIOACTIVITY ${ }^{235,236} \mathrm{Am}$ (EC) [from $\left.{ }^{233,235} \mathrm{U}\left({ }^{6} \mathrm{Li}, \mathrm{xn}\right)\right]$; measured prompt and delayed $\mathrm{E} \gamma, \mathrm{I} \gamma, \gamma \gamma$-coin; deduced log ft. ${ }^{235,236} \mathrm{Pu}$ deduced levels, J, $\pi$, configurations. ${ }^{233,234,235,236} \mathrm{Am},{ }^{237,238} \mathrm{Cm}(\alpha)$ [from $\left.{ }^{233,235} \mathrm{U},{ }^{237} \mathrm{~Np}\left({ }^{6} \mathrm{Li}, \mathrm{xn}\right)\right]$; measured $\mathrm{E} \alpha, \mathrm{T}_{1 / 2}$. Isotope separator. JOUR JNRSA 3,No 1,187

${ }^{233} \mathrm{Am}$ 2002AS08 RADIOACTIVITY ${ }^{235,236} \mathrm{Am}(\mathrm{EC})$ [from $\left.{ }^{233,235} \mathrm{U}\left({ }^{6} \mathrm{Li}, \mathrm{xn}\right)\right]$; measured prompt and delayed $\mathrm{E} \gamma, \mathrm{I} \gamma, \gamma \gamma$-coin; deduced log $\mathrm{ft} .{ }^{235,236} \mathrm{Pu}$ deduced levels, J, $\pi$, configurations. ${ }^{233,234,235,236} \mathrm{Am},{ }^{237,238} \mathrm{Cm}(\alpha)$ [from $\left.{ }^{233,235} \mathrm{U},{ }^{237} \mathrm{~Np}\left({ }^{6} \mathrm{Li}, \mathrm{xn}\right)\right]$; measured $\mathrm{E} \alpha, \mathrm{T}_{1 / 2}$. Isotope separator. JOUR JNRSA 3,No 1,187 


\section{$\mathrm{A}=234$}

${ }^{234} \mathrm{Th} \quad$ 2005CHZT NUCLEAR REACTIONS ${ }^{233} \mathrm{Th}(\mathrm{n}, \gamma), \mathrm{E}=$ thermal; measured E $\gamma, \mathrm{I} \gamma$; deduced effective $\sigma$, resonance integral. CONF Santa Fe (Nucl Data for Sci and Technol) Proc,Vol1,P664

2005 P002 RADIOACTIVITY ${ }^{234,235,238} \mathrm{U}(\alpha)$; measured E $\alpha$, I $\alpha$ from thick source. Comparison with model predictions. JOUR RMEAE 39565

${ }^{234} \mathrm{U} \quad 2004 \mathrm{KU} 35$ RADIOACTIVITY ${ }^{238} \mathrm{Pu},{ }^{226} \mathrm{Ra}(\alpha) ;{ }^{152} \mathrm{Eu}(\mathrm{EC})$; measured low-energy electron spectra, angular distributions, (electron) $\alpha-$, (electron) $\gamma-$, (electron)(X-ray)-coin. JOUR BRSPE 681358

2005P002 RADIOACTIVITY ${ }^{234,235,238} \mathrm{U}(\alpha)$; measured E $\alpha$, I $\alpha$ from thick source. Comparison with model predictions. JOUR RMEAE 39565

${ }^{234} \mathrm{Pu} \quad$ 2002AS08

RADIOACTIVITY ${ }^{235,236} \mathrm{Am}(\mathrm{EC})$ [from $\left.{ }^{233,235} \mathrm{U}\left({ }^{6} \mathrm{Li}, \mathrm{xn}\right)\right]$; measured prompt and delayed $\mathrm{E} \gamma, \mathrm{I} \gamma, \gamma \gamma$-coin; deduced log ft. ${ }^{235,236} \mathrm{Pu}$ deduced levels, J, $\pi$, configurations. ${ }^{233,234,235,236} \mathrm{Am},{ }^{237,238} \mathrm{Cm}(\alpha)$ [from $\left.{ }^{233,235} \mathrm{U},{ }^{237} \mathrm{~Np}\left({ }^{6} \mathrm{Li}, \mathrm{xn}\right)\right]$; measured $\mathrm{E} \alpha, \mathrm{T}_{1 / 2}$. Isotope separator. JOUR JNRSA 3,No 1,187

${ }^{234} \mathrm{Am} \quad$ 2002AS08 RADIOACTIVITY ${ }^{235,236} \mathrm{Am}(\mathrm{EC})$ [from $\left.{ }^{233,235} \mathrm{U}\left({ }^{6} \mathrm{Li}, \mathrm{xn}\right)\right]$; measured prompt and delayed $\mathrm{E} \gamma, \mathrm{I} \gamma, \gamma \gamma$-coin; deduced $\log \mathrm{ft} .{ }^{235,236} \mathrm{Pu}$ deduced levels, J, $\pi$, configurations. ${ }^{233,234,235,236} \mathrm{Am},{ }^{237,238} \mathrm{Cm}(\alpha)$ [from $\left.{ }^{233,235} \mathrm{U},{ }^{237} \mathrm{~Np}\left({ }^{6} \mathrm{Li}, \mathrm{xn}\right)\right]$; measured $\mathrm{E} \alpha, \mathrm{T}_{1 / 2}$. Isotope separator. JOUR JNRSA 3,No 1,187

\section{$\mathrm{A}=235$}

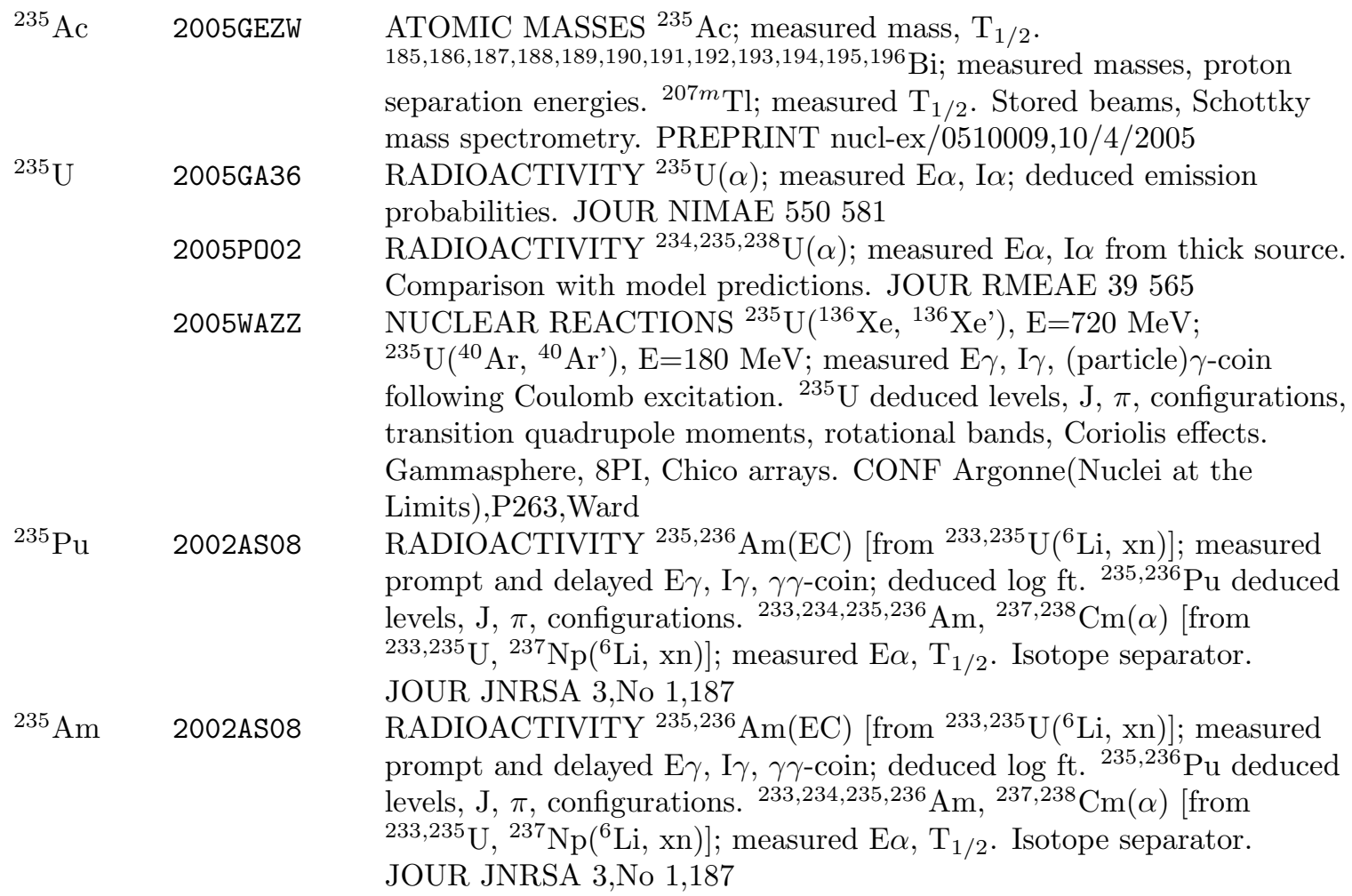




\section{$\mathrm{A}=236$}

${ }^{236} \mathrm{U}$ 2005CS01 NUCLEAR REACTIONS ${ }^{235} \mathrm{U}(\mathrm{d}, \mathrm{pF}), \mathrm{E}=9.73 \mathrm{MeV}$; measured Ep, prompt fission probability vs excitation energy. ${ }^{236} \mathrm{U}$ deduced hyperdeformed rotational bands, fission barrier features, resonant tunneling. JOUR PYLBB 615175

2005CSZZ NUCLEAR REACTIONS ${ }^{235} \mathrm{U}(\mathrm{d}, \mathrm{pF}), \mathrm{E}=13 \mathrm{MeV}$; measured Ep, fission fragment angular correlations. ${ }^{236} \mathrm{U}$ deduced hyperdeformed resonances. REPT MLL 2004 Annual,P19,Csige

2005RY03 NUCLEAR REACTIONS ${ }^{232} \mathrm{Th},{ }^{238} \mathrm{U}(\mathrm{n}, \mathrm{F}), \mathrm{E}=21-95 \mathrm{MeV}$; measured fission fragments angular distributions, anisotropy. ${ }^{232} \mathrm{Th},{ }^{238} \mathrm{U}(\mathrm{n}, \mathrm{F})$, $\mathrm{E}=0-95 \mathrm{MeV} ;{ }^{232} \mathrm{Th},{ }^{238} \mathrm{U}(\mathrm{n}, 2 \mathrm{n}),(\mathrm{n}, 3 \mathrm{n}),(\mathrm{n}, \mathrm{xnF}), \mathrm{E}=0-20 \mathrm{MeV}$; calculated $\sigma$, fission fragments angular anisotropy. ${ }^{238} \mathrm{U}(\mathrm{n}, \mathrm{pX})$, $\mathrm{E}=25-65 \mathrm{MeV}$; calculated $\sigma$. Multichance fission, saddle-point statistical model analysis. JOUR NUPAB 76019

${ }^{236} \mathrm{Pu} \quad$ 2002AS08 RADIOACTIVITY ${ }^{235,236} \mathrm{Am}(\mathrm{EC})\left[\right.$ from $\left.{ }^{233,235} \mathrm{U}\left({ }^{6} \mathrm{Li}, \mathrm{xn}\right)\right] ;$ measured prompt and delayed $\mathrm{E} \gamma, \mathrm{I} \gamma, \gamma \gamma$-coin; deduced $\log \mathrm{ft} .{ }^{235,236} \mathrm{Pu}$ deduced levels, J, $\pi$, configurations. ${ }^{233,234,235,236} \mathrm{Am},{ }^{237,238} \mathrm{Cm}(\alpha)$ [from $\left.{ }^{233,235} \mathrm{U},{ }^{237} \mathrm{~Np}\left({ }^{6} \mathrm{Li}, \mathrm{xn}\right)\right]$; measured $\mathrm{E} \alpha, \mathrm{T}_{1 / 2}$. Isotope separator. JOUR JNRSA 3,No 1,187

2005AS01 RADIOACTIVITY ${ }^{236,236 m} \mathrm{Am}(\mathrm{EC})$ [from ${ }^{235} \mathrm{U}\left({ }^{6} \mathrm{Li}, 5 \mathrm{n}\right)$ ]; measured $\mathrm{E} \gamma, \mathrm{I} \gamma, \gamma \gamma-,\left(\mathrm{X}\right.$-ray) $\gamma$-coin, $\mathrm{T}_{1 / 2}$; deduced log ft. ${ }^{236} \mathrm{Pu}$ deduced levels, $\mathrm{J}, \pi$, configurations, $\beta$-feeding intensities, $\mathrm{B}(\mathrm{E} 1), \mathrm{B}(\mathrm{M} 1) .{ }^{236} \mathrm{Am}$ deduced isomeric state $\mathrm{J}, \pi$, configuration. JOUR ZAANE 23395

2005QIZZ RADIOACTIVITY ${ }^{240} \mathrm{Cm}(\alpha)$ [from $\left.{ }^{232} \mathrm{Th}\left({ }^{12} \mathrm{C}, 4 \mathrm{n}\right)\right]$; measured $\mathrm{E} \alpha$. REPT GSI 2005-1,P75,Qin

${ }^{236} \mathrm{Am} \quad$ 2002AS08 RADIOACTIVITY ${ }^{235,236} \mathrm{Am}(\mathrm{EC})\left[\right.$ from $\left.{ }^{233,235} \mathrm{U}\left({ }^{6} \mathrm{Li}, \mathrm{xn}\right)\right]$; measured prompt and delayed $\mathrm{E} \gamma, \mathrm{I} \gamma, \gamma \gamma$-coin; deduced log $\mathrm{ft} .{ }^{235,236} \mathrm{Pu}$ deduced levels, J, $\pi$, configurations. ${ }^{233,234,235,236} \mathrm{Am},{ }^{237,238} \mathrm{Cm}(\alpha)$ [from $\left.{ }^{233,235} \mathrm{U},{ }^{237} \mathrm{~Np}\left({ }^{6} \mathrm{Li}, \mathrm{xn}\right)\right]$; measured $\mathrm{E} \alpha, \mathrm{T}_{1 / 2}$. Isotope separator. JOUR JNRSA 3, No 1,187

2005AS01 RADIOACTIVITY ${ }^{236,236 m} \mathrm{Am}(\mathrm{EC})$ [from ${ }^{235} \mathrm{U}\left({ }^{6} \mathrm{Li}, 5 \mathrm{n}\right)$ ]; measured $\mathrm{E} \gamma, \mathrm{I} \gamma, \gamma \gamma$-, (X-ray) $\gamma$-coin, $\mathrm{T}_{1 / 2}$; deduced $\log \mathrm{ft} .{ }^{236} \mathrm{Pu}$ deduced levels, $\mathrm{J}, \pi$, configurations, $\beta$-feeding intensities, $\mathrm{B}(\mathrm{E} 1), \mathrm{B}(\mathrm{M} 1) .{ }^{236} \mathrm{Am}$ deduced isomeric state $\mathrm{J}, \pi$, configuration. JOUR ZAANE 23395

\section{$\mathrm{A}=237$}

${ }^{237} \mathrm{U} \quad 2005 \mathrm{RY03}$

NUCLEAR REACTIONS ${ }^{232} \mathrm{Th},{ }^{238} \mathrm{U}(\mathrm{n}, \mathrm{F}), \mathrm{E}=21-95 \mathrm{MeV}$; measured fission fragments angular distributions, anisotropy. ${ }^{232} \mathrm{Th},{ }^{238} \mathrm{U}(\mathrm{n}, \mathrm{F})$, $\mathrm{E}=0-95 \mathrm{MeV} ;{ }^{232} \mathrm{Th},{ }^{238} \mathrm{U}(\mathrm{n}, 2 \mathrm{n}),(\mathrm{n}, 3 \mathrm{n}),(\mathrm{n}, \mathrm{xnF}), \mathrm{E}=0-20 \mathrm{MeV}$; calculated $\sigma$, fission fragments angular anisotropy. ${ }^{238} \mathrm{U}(\mathrm{n}, \mathrm{pX})$, $\mathrm{E}=25-65 \mathrm{MeV}$; calculated $\sigma$. Multichance fission, saddle-point statistical model analysis. JOUR NUPAB 76019

2005ZH20 NUCLEAR REACTIONS ${ }^{239} \mathrm{Pu}\left({ }^{207} \mathrm{~Pb},{ }^{207} \mathrm{~Pb}\right.$ '), E=1300 MeV; measured $\mathrm{E} \gamma, \mathrm{I} \gamma, \gamma \gamma$-coin following Coulomb excitation. ${ }^{238} \mathrm{U}\left({ }^{207} \mathrm{~Pb}\right.$, $\left.{ }^{208} \mathrm{~Pb}\right), \mathrm{E}=1400 \mathrm{MeV}$; measured $\mathrm{E} \gamma, \mathrm{I} \gamma, \gamma \gamma$-coin. ${ }^{239} \mathrm{Pu},{ }^{237} \mathrm{U}$ deduced high-spin levels, J, $\pi$, octupole correlation strength. JOUR PYLBB 61851 


\section{$\mathrm{A}=237$ (continued)}

${ }^{237} \mathrm{~Np} \quad 2005 \mathrm{IW01}$

2005MA90

2005PA56

${ }^{237} \mathrm{Cm}$

2002AS08

${ }^{238} \mathrm{U}$

2005P002

$2005 Y 012$

${ }^{238} \mathrm{~Np} \quad$ 2004KRZX

2005ADZZ

2005ESZZ

2005RE25

2005SH15

2005SHZT
RADIOACTIVITY ${ }^{65} \mathrm{Zn}\left(\beta^{+}\right)$, (EC); measured $\mathrm{E} \gamma, \mathrm{I} \gamma,(\mathrm{X}$-ray) $\gamma$-coin; deduced $\gamma$-ray emission probability. ${ }^{241} \operatorname{Am}(\alpha)$; measured $\mathrm{E} \gamma, \mathrm{I} \gamma$, $\alpha \gamma$-coin; deduced $\gamma$-ray emission probabilities. ${ }^{65} \mathrm{Cu},{ }^{237} \mathrm{~Np}$ deduced transitions. JOUR ARISE 63107

RADIOACTIVITY ${ }^{242} \mathrm{Am}\left(\beta^{-}\right)$, (EC) [from ${ }^{241} \mathrm{Am}(\mathrm{n}, \gamma)$ ]; measured $\beta \gamma$-coin; deduced source activity. ${ }^{241} \operatorname{Am}(\alpha)$; measured $\mathrm{E} \alpha$. JOUR NIMAE 553559 RADIOACTIVITY ${ }^{252} \mathrm{Cf}(\mathrm{SF})$; measured neutron emission rates. ${ }^{241} \operatorname{Am}(\alpha)$; measured neutron emission rates for Am-Be source. Manganese sulphate bath system. JOUR KPSJA 47603 RADIOACTIVITY ${ }^{235,236} \mathrm{Am}(\mathrm{EC})$ [from ${ }^{233,235} \mathrm{U}\left({ }^{6} \mathrm{Li}, \mathrm{xn}\right)$ ]; measured prompt and delayed $\mathrm{E} \gamma, \mathrm{I} \gamma, \gamma \gamma$-coin; deduced log $\mathrm{ft} .{ }^{235,236} \mathrm{Pu}$ deduced levels, J, $\pi$, configurations. ${ }^{233,234,235,236} \mathrm{Am},{ }^{237,238} \mathrm{Cm}(\alpha)$ [from $\left.{ }^{233,235} \mathrm{U},{ }^{237} \mathrm{~Np}\left({ }^{6} \mathrm{Li}, \mathrm{xn}\right)\right]$; measured $\mathrm{E} \alpha, \mathrm{T}_{1 / 2}$. Isotope separator. JOUR JNRSA 3,No 1,187

\section{$\mathrm{A}=238$}

RADIOACTIVITY ${ }^{234,235,238} \mathrm{U}(\alpha)$; measured $\mathrm{E} \alpha$, I $\alpha$ from thick source. Comparison with model predictions. JOUR RMEAE 39565

RADIOACTIVITY ${ }^{238} \mathrm{U}(\mathrm{SF})$; measured spontaneous fission decay constant. Solid-state nuclear track detectors. JOUR NIMAE 555386 NUCLEAR REACTIONS ${ }^{27} \mathrm{Al}(\mathrm{n}, \mathrm{n} 3 \mathrm{p}),{ }^{59} \mathrm{Co},{ }^{139} \mathrm{La},{ }^{129} \mathrm{I},{ }^{197} \mathrm{Au}$, ${ }^{237} \mathrm{~Np}(\mathrm{n}, \gamma),{ }^{59} \mathrm{Co},{ }^{127,129} \mathrm{I},{ }^{197} \mathrm{Au},{ }^{209} \mathrm{Bi}(\mathrm{n}, \mathrm{xn}),{ }^{235,238} \mathrm{U}(\mathrm{n}, \mathrm{F})$, $\mathrm{E}=$ spectrum; measured yields; deduced reaction rates. $\mathrm{Pb}(\mathrm{p}, \mathrm{nX})$, $\mathrm{E}=1.5 \mathrm{GeV}$; deduced neutron spectrum. REPT

JINR-E1-2004-79,Krivopustov NUCLEAR REACTIONS ${ }^{129} \mathrm{I}(\mathrm{n}, 7 \mathrm{n}),(\mathrm{n}, 6 \mathrm{n}),(\mathrm{n}, 4 \mathrm{n}),(\mathrm{n}, \gamma), \mathrm{E}=$ fast; ${ }^{237} \mathrm{~Np}(\mathrm{n}, \gamma), \mathrm{E}=$ fast; measured yields. ${ }^{237} \mathrm{~Np}(\mathrm{n}, \mathrm{F}){ }^{91} \mathrm{Sr} /{ }^{97} \mathrm{Zr} /{ }^{132} \mathrm{Te} /$ ${ }^{133} \mathrm{I} /{ }^{135} \mathrm{I}, \mathrm{E}=$ fast; ${ }^{238} \mathrm{Pu}(\mathrm{n}, \mathrm{F}){ }^{97} \mathrm{Zr} /{ }^{129} \mathrm{Sb} /{ }^{132} \mathrm{I} /{ }^{133} \mathrm{I} /{ }^{135} \mathrm{Xe} /$ ${ }^{105} \mathrm{Ru}, \mathrm{E}=$ fast; ${ }^{239} \mathrm{Pu}(\mathrm{n}, \mathrm{F}){ }^{88} \mathrm{Kr} /{ }^{91} \mathrm{Sr} /{ }^{92} \mathrm{Sr} /{ }^{92} \mathrm{Y} /{ }^{97} \mathrm{Zr} /{ }^{99} \mathrm{Mo} /$ ${ }^{103} \mathrm{Ru} /{ }^{105} \mathrm{Ru} /{ }^{128} \mathrm{Sb} /{ }^{129} \mathrm{Sb} /{ }^{132} \mathrm{Te} /{ }^{131} \mathrm{I} /{ }^{132} \mathrm{I} /{ }^{133} \mathrm{I} /{ }^{135} \mathrm{I} /{ }^{135} \mathrm{Xe}$ $/{ }^{143} \mathrm{Ce} /{ }^{140} \mathrm{Ba} /{ }^{140} \mathrm{La}, \mathrm{E}=$ fast; measured fission fragment yields. Secondary neutrons from proton irradiation. JINR nuclotron. CONF St Petersburg,P195,Adam NUCLEAR REACTIONS ${ }^{237} \mathrm{~Np}(\mathrm{n}, \gamma), \mathrm{E}=0.02-100 \mathrm{eV}$; measured $\sigma$. Comparison with previous results. CONF Santa Fe (Nucl Data for Sci and Technol) Proc,Vol1,P989

NUCLEAR REACTIONS ${ }^{237} \mathrm{~Np}(\mathrm{n}, \gamma), \mathrm{E}=0.01-10 \mathrm{eV}$; measured $\sigma$. Comparison with previous results. JOUR NIMBE 241176 NUCLEAR REACTIONS ${ }^{237} \mathrm{~Np}(\mathrm{n}, \gamma), \mathrm{E}=0.02-100 \mathrm{eV}$; measured average capture $\sigma$; deduced resonance integral. Comparison with previous results. JOUR JNSTA 42135

NUCLEAR REACTIONS ${ }^{237} \mathrm{~Np}(\mathrm{n}, \gamma), \mathrm{E}=0.02-100 \mathrm{eV}$; measured capture $\sigma$; deduced resonance integral. Comparisons with previous results. CONF Santa Fe (Nucl Data for Sci and Technol)

Proc,Vol1,P1007 


\section{$\mathrm{A}=238$ (continued)}

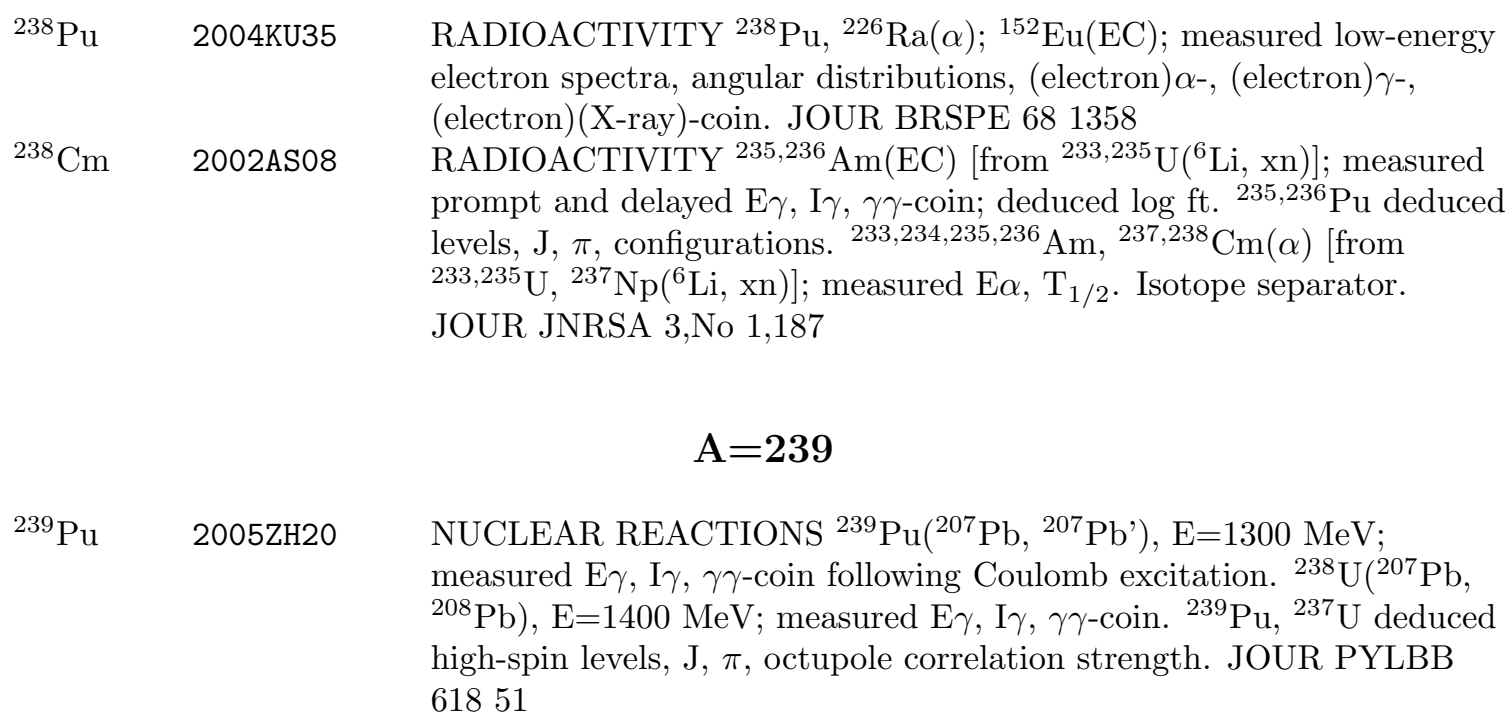

\section{$\mathrm{A}=\mathbf{2 4 0}$}

\begin{tabular}{|c|c|c|}
\hline${ }^{240} \mathrm{U}$ & 2005IS07 & $\begin{array}{l}\text { NUCLEAR REACTIONS }{ }^{238} \mathrm{U}\left({ }^{18} \mathrm{O},{ }^{16} \mathrm{O}\right), \mathrm{E}=200 \mathrm{MeV} ; \text { measured } \mathrm{E} \gamma \text {, } \\
\mathrm{I} \gamma \text {, (particle) } \gamma \text {-coin, } \gamma \text {-ray anisotropy. }{ }^{240} \mathrm{U} \text { deduced levels, J, } \pi \text {, } \\
\text { rotational bands, octupole correlations. JOUR PRVCA } 72021301\end{array}$ \\
\hline${ }^{240} \mathrm{Pu}$ & 2005GRZY & $\begin{array}{l}\text { NUCLEAR REACTIONS }{ }^{235} \mathrm{U},{ }^{239} \mathrm{Pu}(\mathrm{n}, \gamma),(\mathrm{n}, \mathrm{F}), \mathrm{E}=2-2150 \mathrm{eV} \\
\text { measured } \gamma \text {-ray multiplicities; deduced } \sigma \text { ratio, Doppler effect. CONF } \\
\text { Santa Fe (Nucl Data for Sci and Technol) Proc,Vol1,P928 }\end{array}$ \\
\hline & 2005THZZ & $\begin{array}{l}\text { NUCLEAR REACTIONS }{ }^{232} \mathrm{Th},{ }^{238} \mathrm{U}(\alpha, 2 \mathrm{n}), \mathrm{E}=20-27 \mathrm{MeV} \text {; } \\
\text { measured prompt and delayed fission fragment yields; deduced } \\
\text { excitation functions for isomeric and prompt fission. REPT MLL } 2004 \\
\text { Annual,P17,Thirolf }\end{array}$ \\
\hline${ }^{240} \mathrm{Cm}$ & 2005QIZZ & $\begin{array}{l}\left.\text { RADIOACTIVITY }{ }^{240} \mathrm{Cm}(\alpha) \text { [from }{ }^{232} \mathrm{Th}\left({ }^{12} \mathrm{C}, 4 \mathrm{n}\right)\right] \text {; measured } \mathrm{E} \alpha \text {. } \\
\text { REPT GSI 2005-1,P75,Qin }\end{array}$ \\
\hline
\end{tabular}

\section{$\mathrm{A}=\mathbf{2 4 1}$}

${ }^{241} \mathrm{Pu} \quad$ 2005CAZX NUCLEAR REACTIONS ${ }^{237} \mathrm{~Np},{ }^{240} \mathrm{Pu}(\mathrm{n}, \gamma), \mathrm{E}=0-300 \mathrm{keV}$; measured capture $\sigma$. CONF Santa Fe (Nucl Data for Sci and Technol) Proc, Vol2,P1442

${ }^{241} \mathrm{Am} \quad$ 2005IW01 RADIOACTIVITY ${ }^{65} \mathrm{Zn}\left(\beta^{+}\right),(\mathrm{EC}) ;$ measured $\mathrm{E} \gamma, \mathrm{I} \gamma,(\mathrm{X}$-ray) $\gamma$-coin; deduced $\gamma$-ray emission probability. ${ }^{241} \operatorname{Am}(\alpha)$; measured $\mathrm{E} \gamma, \mathrm{I} \gamma$, $\alpha \gamma$-coin; deduced $\gamma$-ray emission probabilities. ${ }^{65} \mathrm{Cu},{ }^{237} \mathrm{~Np}$ deduced transitions. JOUR ARISE 63107

2005MA90 RADIOACTIVITY ${ }^{242} \mathrm{Am}\left(\beta^{-}\right)$, (EC) [from ${ }^{241} \mathrm{Am}(\mathrm{n}, \gamma)$ ]; measured $\beta \gamma$-coin; deduced source activity. ${ }^{241} \operatorname{Am}(\alpha)$; measured $\mathrm{E} \alpha$. JOUR NIMAE 553559 


\section{$\mathrm{A}=241$ (continued)}

2005PA56 RADIOACTIVITY ${ }^{252} \mathrm{Cf}(\mathrm{SF})$; measured neutron emission rates. ${ }^{241} \operatorname{Am}(\alpha)$; measured neutron emission rates for Am-Be source. Manganese sulphate bath system. JOUR KPSJA 47603

${ }^{241} \mathrm{Cm}$ 2003ASZY RADIOACTIVITY ${ }^{241} \mathrm{Bk}(\mathrm{EC})\left[\right.$ from $\left.{ }^{239} \mathrm{Pu}\left({ }^{6} \mathrm{Li}, 4 \mathrm{n}\right)\right] ;$ measured $\mathrm{E} \gamma, \mathrm{I} \gamma$, (X-ray) $\gamma$-coin, $\mathrm{T}_{1 / 2}$; deduced log ft. ${ }^{241} \mathrm{Bk}$ deduced ground-state configuration. ${ }^{241} \mathrm{Cm}$ deduced levels, J, $\pi$. REPT JAERI-TV 2002 Annual,P29,Asai

${ }^{241} \mathrm{Bk}$ 2003ASZY NUCLEAR REACTIONS ${ }^{239} \mathrm{Pu}\left({ }^{6} \mathrm{Li}, 4 \mathrm{n}\right), \mathrm{E}=34-42 \mathrm{MeV}$; measured prompt and delayed $\mathrm{E} \gamma, \mathrm{I} \gamma$, (X-ray) $\gamma$-, (recoil) $\gamma$-coin; deduced evidence for ${ }^{241} \mathrm{Bk}$. Isotope separator. REPT JAERI-TV 2002 Annual,P29,Asai 2003ASZY RADIOACTIVITY ${ }^{241} \mathrm{Bk}(\mathrm{EC})$ [from $\left.{ }^{239} \mathrm{Pu}\left({ }^{6} \mathrm{Li}, 4 \mathrm{n}\right)\right]$; measured $\mathrm{E} \gamma$, I $\gamma$, (X-ray) $\gamma$-coin, $\mathrm{T}_{1 / 2}$; deduced log ft. ${ }^{241} \mathrm{Bk}$ deduced ground-state configuration. ${ }^{241} \mathrm{Cm}$ deduced levels, J, $\pi$. REPT JAERI-TV 2002 Annual,P29,Asai

\section{$\mathrm{A}=242$}

${ }^{242} \mathrm{Pu} \quad$ 2005MA90

RADIOACTIVITY ${ }^{242} \mathrm{Am}\left(\beta^{-}\right)$, (EC) [from ${ }^{241} \mathrm{Am}(\mathrm{n}, \gamma)$ ]; measured $\beta \gamma$-coin; deduced source activity. ${ }^{241} \operatorname{Am}(\alpha)$; measured $\mathrm{E} \alpha$. JOUR NIMAE 553559

${ }^{242} \mathrm{Am} \quad$ 2005MA90 RADIOACTIVITY ${ }^{242} \mathrm{Am}\left(\beta^{-}\right)$, (EC) [from $\left.{ }^{241} \mathrm{Am}(\mathrm{n}, \gamma)\right]$; measured $\beta \gamma$-coin; deduced source activity. ${ }^{241} \operatorname{Am}(\alpha)$; measured E $\alpha$. JOUR NIMAE 553559

20050HZY NUCLEAR REACTIONS ${ }^{241} \mathrm{Am}(\mathrm{n}, \gamma), \mathrm{E}=$ fast; measured isomer production ratio. ${ }^{237} \mathrm{~Np},{ }^{241,243} \mathrm{Am},{ }^{244} \mathrm{Cm}(\mathrm{n}, \mathrm{X}), \mathrm{E}=$ fast; measured residual isotopes yield ratios following reactor irradiation. Comparison with previous results. CONF Santa Fe (Nucl Data for Sci and Technol) Proc, Vol1,P472

${ }^{242} \mathrm{Cm}$ 2005MA90 RADIOACTIVITY ${ }^{242} \mathrm{Am}\left(\beta^{-}\right)$, (EC) [from $\left.{ }^{241} \mathrm{Am}(\mathrm{n}, \gamma)\right] ;$ measured $\beta \gamma$-coin; deduced source activity. ${ }^{241} \operatorname{Am}(\alpha)$; measured $\mathrm{E} \alpha$. JOUR NIMAE 553559

\section{$\mathrm{A}=243$}

$\begin{array}{ll}{ }^{243} \mathrm{Cf} & \text { 2005KUZZ } \\ { }^{243} \mathrm{Es} & 2005 \mathrm{HE} 27\end{array}$

RADIOACTIVITY ${ }^{216,216 m} \mathrm{Th}(\alpha)$, (IT) [from $\left.{ }^{170} \operatorname{Er}\left({ }^{50} \mathrm{Ti}, 4 \mathrm{n}\right)\right]$; ${ }^{251,251 m} \mathrm{No},{ }^{247,247 m} \mathrm{Fm}(\alpha)$ [from ${ }^{206} \mathrm{~Pb}\left({ }^{48} \mathrm{Ca}, 3 \mathrm{n}\right)$ and subsequent decay]; ${ }^{257,257 m} \mathrm{Db},{ }^{253,253 m} \mathrm{Lr},{ }^{249} \mathrm{Md}(\alpha)$ [from ${ }^{209} \mathrm{Bi}\left({ }^{50} \mathrm{Ti}, 2 \mathrm{n}\right)$ and subsequent decay]; measured $\mathrm{E} \alpha, \mathrm{E} \gamma, \alpha \gamma$-coin, $\mathrm{T}_{1 / 2}$. CONF Argonne(Nuclei at the Limits),P231,Kuusiniemi RADIOACTIVITY ${ }^{247,249,251,253,255} \mathrm{Md}(\alpha)$ [from ${ }^{209} \mathrm{Bi}\left({ }^{40} \mathrm{Ar}, \mathrm{xn}\right)$, $\left({ }^{50} \mathrm{Ti}, \mathrm{xn}\right),{ }^{207,207} \mathrm{~Pb},{ }^{209} \mathrm{Bi}\left({ }^{48} \mathrm{Ca}, \mathrm{xn}\right)$ and subsequent decay]; measured $\mathrm{E} \alpha, \mathrm{E} \gamma, \alpha \gamma$-coin; deduced branching ratios, hindrance factors. $243,245,247,249,251$ Es deduced levels, J, $\pi$, configurations, deformation. Comparison with model predictions. JOUR ZAANE 26233 


\section{$\mathrm{A}=244$}

${ }^{244} \mathrm{Cm} \quad$ 2004NA44 RADIOACTIVITY ${ }^{244} \mathrm{Cm}(\mathrm{SF})$; measured fission fragments isomeric yield ratios; deduced fragment angular momentum distributions. JOUR RAACA 921

2005RE06 RADIOACTIVITY ${ }^{244} \mathrm{Cm},{ }^{252} \mathrm{Cf}(\mathrm{SF})$; measured $\mathrm{E} \gamma$, I $\gamma$; deduced fission fragments isotopic yields. JOUR JRNCD 264243

2005VOZX RADIOACTIVITY ${ }^{252} \mathrm{Cf},{ }^{244,248} \mathrm{Cm}(\mathrm{SF})$; measured fission fragment mass distributions and kinetic energy spectra, prompt neutron multiplicity distributions vs fragment mass; deduced fission mechanism features. CONF Santa Fe (Nucl Data for Sci and Technol) Proc, Vol1,P613

\section{$\mathrm{A}=245$}

${ }^{245} \mathrm{Es} \quad$ 2005HE27 RADIOACTIVITY $247,249,251,253,255 \mathrm{Md}(\alpha)$ [from ${ }^{209} \mathrm{Bi}\left({ }^{40} \mathrm{Ar}, \mathrm{xn}\right)$, $\left({ }^{50} \mathrm{Ti}, \mathrm{xn}\right),{ }^{207,207} \mathrm{~Pb},{ }^{209} \mathrm{Bi}\left({ }^{48} \mathrm{Ca}, \mathrm{xn}\right)$ and subsequent decay]; measured $\mathrm{E} \alpha, \mathrm{E} \gamma, \alpha \gamma$-coin; deduced branching ratios, hindrance factors. $243,245,247,249,251$ Es deduced levels, J, $\pi$, configurations, deformation. Comparison with model predictions. JOUR ZAANE 26233 2005KUZZ RADIOACTIVITY ${ }^{216,216 m} \mathrm{Th}(\alpha)$, (IT) [from ${ }^{170} \mathrm{Er}\left({ }^{50} \mathrm{Ti}, 4 \mathrm{n}\right)$ ]; ${ }^{251,251 m} \mathrm{No},{ }^{247,247 m} \mathrm{Fm}(\alpha)$ [from ${ }^{206} \mathrm{~Pb}\left({ }^{48} \mathrm{Ca}, 3 \mathrm{n}\right)$ and subsequent decay]; ${ }^{257,257 m} \mathrm{Db},{ }^{253,253 m} \mathrm{Lr},{ }^{249} \mathrm{Md}(\alpha)$ [from ${ }^{209} \mathrm{Bi}\left({ }^{50} \mathrm{Ti}, 2 \mathrm{n}\right)$ and subsequent decay]; measured $\mathrm{E} \alpha, \mathrm{E} \gamma, \alpha \gamma$-coin, $\mathrm{T}_{1 / 2}$. CONF Argonne(Nuclei at the Limits),P231,Kuusiniemi

\section{$\mathrm{A}=246$}

No references found

\section{$\mathrm{A}=247$}

${ }^{247} \mathrm{Es} \quad 2005 \mathrm{GR} 36$

RADIOACTIVITY ${ }^{255} \mathrm{Lr},{ }^{251} \mathrm{Md}(\alpha)$ [from ${ }^{209} \mathrm{Bi}\left({ }^{48} \mathrm{Ca}, 2 \mathrm{n}\right)$ and subsequent decay]; measured $\mathrm{E} \alpha, \alpha \alpha$-coin; deduced excited state decay. JOUR ZAANE 25 s01 599

2005HE27 RADIOACTIVITY ${ }^{247,249,251,253,255} \mathrm{Md}(\alpha)$ [from ${ }^{209} \mathrm{Bi}\left({ }^{40} \mathrm{Ar}\right.$, xn), $\left({ }^{50} \mathrm{Ti}, \mathrm{xn}\right),{ }^{207,207} \mathrm{~Pb},{ }^{209} \mathrm{Bi}\left({ }^{48} \mathrm{Ca}, \mathrm{xn}\right)$ and subsequent decay]; measured $\mathrm{E} \alpha, \mathrm{E} \gamma, \alpha \gamma$-coin; deduced branching ratios, hindrance factors. $243,245,247,249,251$ Es deduced levels, J, $\pi$, configurations, deformation. Comparison with model predictions. JOUR ZAANE 26233

${ }^{247} \mathrm{Fm} \quad 2005 \mathrm{KUZZ}$ RADIOACTIVITY ${ }^{216,216 m} \mathrm{Th}(\alpha)$, (IT) [from ${ }^{170} \operatorname{Er}\left({ }^{50} \mathrm{Ti}, 4 \mathrm{n}\right)$ ]; ${ }^{251,251 m} \mathrm{No},{ }^{247,247 m} \mathrm{Fm}(\alpha)$ [from ${ }^{206} \mathrm{~Pb}\left({ }^{48} \mathrm{Ca}, 3 \mathrm{n}\right)$ and subsequent decay]; ${ }^{257,257 m} \mathrm{Db},{ }^{253,253 m} \mathrm{Lr},{ }^{249} \mathrm{Md}(\alpha)$ [from ${ }^{209} \mathrm{Bi}\left({ }^{50} \mathrm{Ti}, 2 \mathrm{n}\right)$ and subsequent decay]; measured $\mathrm{E} \alpha, \mathrm{E} \gamma, \alpha \gamma$-coin, $\mathrm{T}_{1 / 2}$. CONF Argonne(Nuclei at the Limits),P231,Kuusiniemi

2005SUZX RADIOACTIVITY ${ }^{255} \mathrm{Rf},{ }^{251} \mathrm{No}(\alpha)$ [from ${ }^{207} \mathrm{~Pb}\left({ }^{50} \mathrm{Ti}, 2 \mathrm{n}\right)$ and $\left.{ }^{206} \mathrm{~Pb}\left({ }^{48} \mathrm{Ca}, 2 \mathrm{n}\right)\right]$; measured $\mathrm{E} \gamma, \alpha \gamma$-coin. ${ }^{251}$ No deduced isomeric state. REPT GSI 2005-1,P74,Sulignano 


\section{$\mathrm{A}=247$ (continued)}

${ }^{247} \mathrm{Md}$ 2005HE27 RADIOACTIVITY ${ }^{247,249,251,253,255} \mathrm{Md}(\alpha)$ [from ${ }^{209} \mathrm{Bi}\left({ }^{40} \mathrm{Ar}, \mathrm{xn}\right)$, $\left({ }^{50} \mathrm{Ti}, \mathrm{xn}\right),{ }^{207,207} \mathrm{~Pb},{ }^{209} \mathrm{Bi}\left({ }^{48} \mathrm{Ca}, \mathrm{xn}\right)$ and subsequent decay]; measured $\mathrm{E} \alpha, \mathrm{E} \gamma, \alpha \gamma$-coin; deduced branching ratios, hindrance factors. $243,245,247,249,251$ Es deduced levels, J, $\pi$, configurations, deformation. Comparison with model predictions. JOUR ZAANE 26233

\section{$\mathrm{A}=248$}

${ }^{248} \mathrm{Cm} \quad$ 2005GA25

2005UR01

2005UR02

RADIOACTIVITY ${ }^{248} \mathrm{Cm}(\mathrm{SF})$; measured $\mathrm{E} \gamma, \mathrm{I} \gamma$; deduced 138,139,140,141,142 Xe fission fragment yields. JOUR FECLA 12544 RADIOACTIVITY ${ }^{248} \mathrm{Cm}(\mathrm{SF})$; measured $\mathrm{E} \gamma, \mathrm{I} \gamma, \gamma \gamma$-coin. ${ }^{109,110,111} \mathrm{Tc},{ }^{135} \mathrm{I}$ deduced transitions. ${ }^{111} \mathrm{Tc}$ deduced levels, J, $\pi$, configurations. Eurogam2 array. Level systematics in neighboring nuclides discussed. JOUR ZAANE 24161 correlations. ${ }^{107}$ Mo deduced high-spin levels, J, $\pi$, configurations. Eurogam2 array. JOUR PRVCA 72027302

2005VOZX RADIOACTIVITY ${ }^{252} \mathrm{Cf},{ }^{244,248} \mathrm{Cm}$ (SF); measured fission fragment mass distributions and kinetic energy spectra, prompt neutron multiplicity distributions vs fragment mass; deduced fission mechanism features. CONF Santa Fe (Nucl Data for Sci and Technol)

Proc, Vol1,P613

${ }^{248} \mathrm{Fm} \quad$ 2005NIZW
NUCLEAR REACTIONS ${ }^{238} \mathrm{U}\left({ }^{16} \mathrm{O}, 4 \mathrm{n}\right),\left({ }^{16} \mathrm{O}, 5 \mathrm{n}\right),\left({ }^{16} \mathrm{O}, 6 \mathrm{n}\right), \mathrm{E}(\mathrm{cm})$ $\approx 70-100 \mathrm{MeV}$; measured evaporation residue $\sigma$; deduced fusion probability. CONF Santa Fe (Nucl Data for Sci and Technol) Proc, Vol1,P977

\section{$\mathrm{A}=249$}

${ }^{249} \mathrm{Cm} \quad$ 2004AHZY

RADIOACTIVITY ${ }^{253} \mathrm{Es}(\alpha)$; measured $\mathrm{E} \gamma, \mathrm{I} \gamma, \gamma \gamma$-coin. ${ }^{249} \mathrm{Cm}\left(\beta^{-}\right)$ [from ${ }^{248} \mathrm{Cm}(\mathrm{n}, \gamma)$ ]; measured $\mathrm{E} \gamma, \mathrm{I} \gamma .{ }^{249} \mathrm{Bk}$ deduced transitions, proton single-particle states $\mathrm{J}, \pi$, configurations. REPT ANL-04/22,P45,Ahmad

2005AH03 RADIOACTIVITY ${ }^{253} \mathrm{Es}(\alpha)$; measured $\mathrm{E} \alpha, \mathrm{E} \gamma, \gamma \gamma$-, $\alpha \gamma$-coin. ${ }^{249} \mathrm{Cm}\left(\beta^{-}\right)$[from $\left.{ }^{248} \mathrm{Cm}(\mathrm{n}, \gamma)\right]$; measured $\mathrm{E} \gamma$, I $\gamma .{ }^{249} \mathrm{Bk}$ deduced levels, $\mathrm{J}, \pi$, configurations, $\mathrm{B}(\lambda)$, g factors. ${ }^{253} \mathrm{Es}(\mathrm{SF}) ;$ measured $\mathrm{E} \gamma, \mathrm{I} \gamma$ from fission fragment decay; deduced fission branching ratio. Gammasphere array, comparisons with model predictions. JOUR PRVCA 71054305

2005AHZZ RADIOACTIVITY ${ }^{253} \mathrm{Es}(\alpha)$; measured $\mathrm{E} \alpha, \mathrm{E} \gamma, \gamma \gamma$-, $\alpha \gamma$-coin. ${ }^{249} \mathrm{Cm}\left(\beta^{-}\right)$[from $\left.{ }^{248} \mathrm{Cm}(\mathrm{n}, \gamma)\right]$; measured $\mathrm{E} \gamma, \mathrm{I} \gamma .{ }^{249} \mathrm{Bk}$ deduced levels, $\mathrm{J}, \pi$, configurations. Gammasphere array. CONF Argonne(Nuclei at the Limits),P251,Ahmad

${ }^{249} \mathrm{Bk}$ 2002AH06 RADIOACTIVITY ${ }^{255} \mathrm{Fm},{ }^{253} \mathrm{Es}(\alpha)$; measured E $\gamma, \mathrm{I} \gamma, \gamma \gamma$-coin. ${ }^{251} \mathrm{Cf}$, ${ }^{249} \mathrm{Bk}$ deduced levels, J, $\pi$, single-particle states. JOUR JNRSA 3,No 1,179 


\section{$\mathrm{A}=249$ (continued)}

2004AHZY RADIOACTIVITY ${ }^{253} \mathrm{Es}(\alpha)$; measured $\mathrm{E} \gamma, \mathrm{I} \gamma, \gamma \gamma$-coin. ${ }^{249} \mathrm{Cm}\left(\beta^{-}\right)$ $\left[\right.$ from $\left.{ }^{248} \mathrm{Cm}(\mathrm{n}, \gamma)\right]$; measured $\mathrm{E} \gamma, \mathrm{I} \gamma .{ }^{249} \mathrm{Bk}$ deduced transitions, proton single-particle states $\mathrm{J}, \pi$, configurations. REPT ANL-04/22,P45,Ahmad

2005AH03 RADIOACTIVITY ${ }^{253} \operatorname{Es}(\alpha)$; measured E $\alpha, \mathrm{E} \gamma, \gamma \gamma$-, $\alpha \gamma$-coin. ${ }^{249} \mathrm{Cm}\left(\beta^{-}\right)$[from $\left.{ }^{248} \mathrm{Cm}(\mathrm{n}, \gamma)\right]$; measured $\mathrm{E} \gamma, \mathrm{I} \gamma .{ }^{249} \mathrm{Bk}$ deduced levels, $\mathrm{J}, \pi$, configurations, $\mathrm{B}(\lambda)$, g factors. ${ }^{253} \mathrm{Es}(\mathrm{SF}) ;$ measured $\mathrm{E} \gamma, \mathrm{I} \gamma$ from fission fragment decay; deduced fission branching ratio. Gammasphere array, comparisons with model predictions. JOUR PRVCA 71054305

2005AHZZ RADIOACTIVITY ${ }^{253} \operatorname{Es}(\alpha)$; measured E $\alpha, \mathrm{E} \gamma, \gamma \gamma-, \alpha \gamma$-coin. ${ }^{249} \mathrm{Cm}\left(\beta^{-}\right)$[from $\left.{ }^{248} \mathrm{Cm}(\mathrm{n}, \gamma)\right]$; measured $\mathrm{E} \gamma, \mathrm{I} \gamma .{ }^{249} \mathrm{Bk}$ deduced levels, $\mathrm{J}, \pi$, configurations. Gammasphere array. CONF Argonne(Nuclei at the Limits),P251,Ahmad

2005SE08 RADIOACTIVITY ${ }^{253,254} \mathrm{Es},{ }^{255} \mathrm{Fm}(\alpha)$ [from $\left.{ }^{252} \mathrm{Cf}(\mathrm{n}, \mathrm{X})\right]$; measured $\mathrm{E} \alpha$, angular distributions from decay of oriented nuclei; deduced anisotropies. Comparison with model predictions. JOUR PRVCA 71 044324

${ }^{249} \mathrm{Es} \quad 2005 \mathrm{HE} 27$ RADIOACTIVITY ${ }^{247,249,251,253,255} \mathrm{Md}(\alpha)$ [from ${ }^{209} \mathrm{Bi}\left({ }^{40} \mathrm{Ar}, \mathrm{xn}\right)$, $\left({ }^{50} \mathrm{Ti}, \mathrm{xn}\right),{ }^{207,207} \mathrm{~Pb},{ }^{209} \mathrm{Bi}\left({ }^{48} \mathrm{Ca}, \mathrm{xn}\right)$ and subsequent decay]; measured $\mathrm{E} \alpha, \mathrm{E} \gamma, \alpha \gamma$-coin; deduced branching ratios, hindrance factors. 243,245,247,249,251 Es deduced levels, J, $\pi$, configurations, deformation. Comparison with model predictions. JOUR ZAANE 26233

${ }^{249} \mathrm{Fm} \quad$ 2005NIZW NUCLEAR REACTIONS ${ }^{238} \mathrm{U}\left({ }^{16} \mathrm{O}, 4 \mathrm{n}\right),\left({ }^{16} \mathrm{O}, 5 \mathrm{n}\right),\left({ }^{16} \mathrm{O}, 6 \mathrm{n}\right), \mathrm{E}(\mathrm{cm})$ $\approx 70-100 \mathrm{MeV}$; measured evaporation residue $\sigma$; deduced fusion probability. CONF Santa Fe (Nucl Data for Sci and Technol) Proc, Vol1,P977

${ }^{249} \mathrm{Md}$ 2005HE27 RADIOACTIVITY ${ }^{247,249,251,253,255} \mathrm{Md}(\alpha)$ [from ${ }^{209} \mathrm{Bi}\left({ }^{40} \mathrm{Ar}, \mathrm{xn}\right)$, $\left({ }^{50} \mathrm{Ti}, \mathrm{xn}\right),{ }^{207,207} \mathrm{~Pb},{ }^{209} \mathrm{Bi}\left({ }^{48} \mathrm{Ca}, \mathrm{xn}\right)$ and subsequent decay]; measured $\mathrm{E} \alpha, \mathrm{E} \gamma, \alpha \gamma$-coin; deduced branching ratios, hindrance factors. $243,245,247,249,251$ Es deduced levels, J, $\pi$, configurations, deformation. Comparison with model predictions. JOUR ZAANE 26233 2005KUZZ RADIOACTIVITY ${ }^{216,216 m} \mathrm{Th}(\alpha)$, (IT) [from ${ }^{170} \mathrm{Er}\left({ }^{50} \mathrm{Ti}, 4 \mathrm{n}\right)$ ]; ${ }^{251,251 m} \mathrm{No},{ }^{247,247 m} \mathrm{Fm}(\alpha)$ [from ${ }^{206} \mathrm{~Pb}\left({ }^{48} \mathrm{Ca}, 3 \mathrm{n}\right)$ and subsequent decay]; ${ }^{257,257 m} \mathrm{Db},{ }^{253,253 m} \mathrm{Lr},{ }^{249} \mathrm{Md}(\alpha)$ [from ${ }^{209} \mathrm{Bi}\left({ }^{50} \mathrm{Ti}, 2 \mathrm{n}\right)$ and subsequent decay]; measured $\mathrm{E} \alpha, \mathrm{E} \gamma, \alpha \gamma$-coin, $\mathrm{T}_{1 / 2}$. CONF Argonne(Nuclei at the Limits),P231,Kuusiniemi

\section{$\mathrm{A}=\mathbf{2 5 0}$}

${ }^{250} \mathrm{Bk} \quad 2005 \mathrm{SE} 08$

RADIOACTIVITY ${ }^{253,254} \mathrm{Es},{ }^{255} \mathrm{Fm}(\alpha)$ [from ${ }^{252} \mathrm{Cf}(\mathrm{n}, \mathrm{X})$ ]; measured $\mathrm{E} \alpha$, angular distributions from decay of oriented nuclei; deduced anisotropies. Comparison with model predictions. JOUR PRVCA 71 044324

${ }^{250} \mathrm{Fm} \quad$ 2005NIZW NUCLEAR REACTIONS ${ }^{238} \mathrm{U}\left({ }^{16} \mathrm{O}, 4 \mathrm{n}\right),\left({ }^{16} \mathrm{O}, 5 \mathrm{n}\right),\left({ }^{16} \mathrm{O}, 6 \mathrm{n}\right), \mathrm{E}(\mathrm{cm})$ $\approx 70-100 \mathrm{MeV}$; measured evaporation residue $\sigma$; deduced fusion probability. CONF Santa Fe (Nucl Data for Sci and Technol) Proc, Vol1,P977 
$\mathrm{A}=\mathbf{2 5 1}$
${ }^{251} \mathrm{Cf} \quad 2002 \mathrm{AHO6}$

${ }^{251} \mathrm{Es}$

${ }^{251} \mathrm{Md}$

${ }^{251}$ No

2005SUZX

(2005

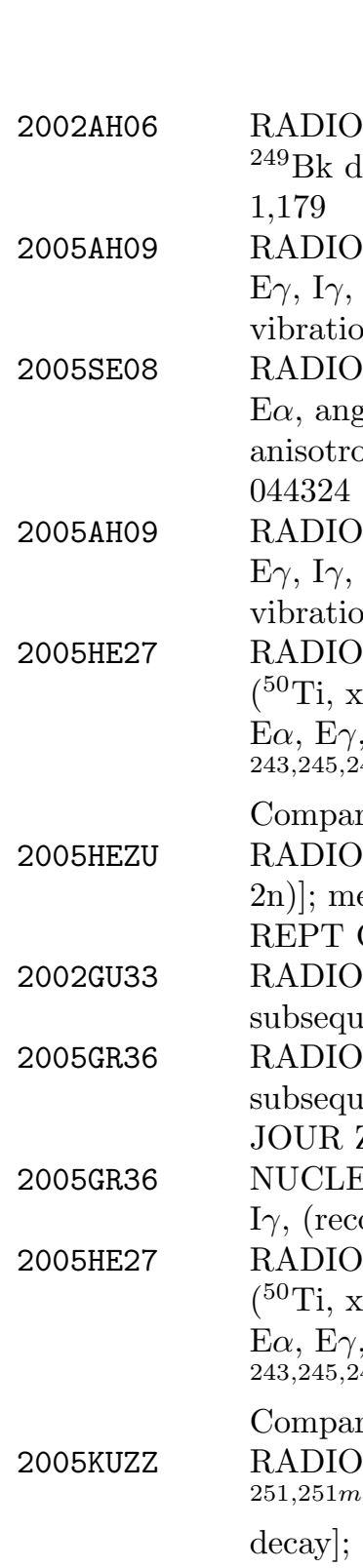

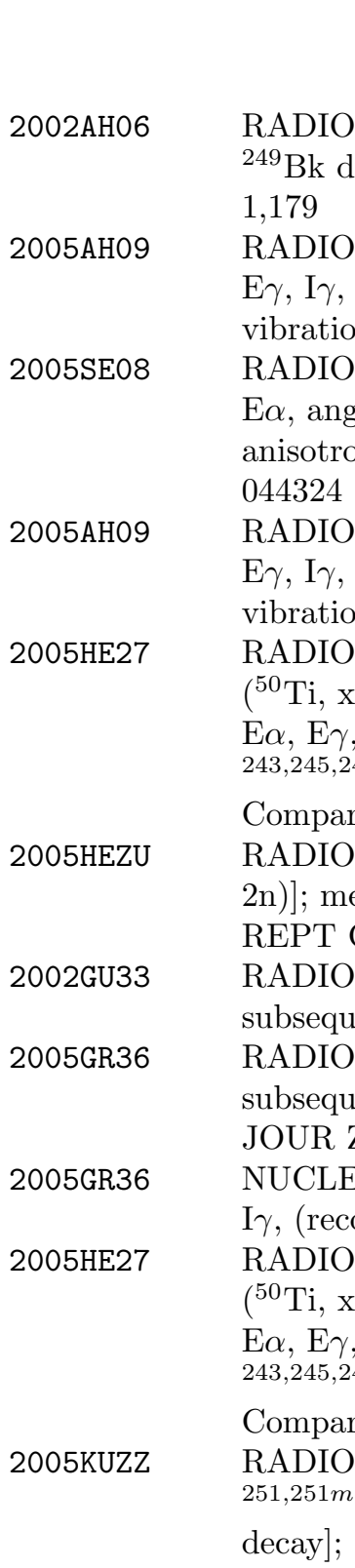

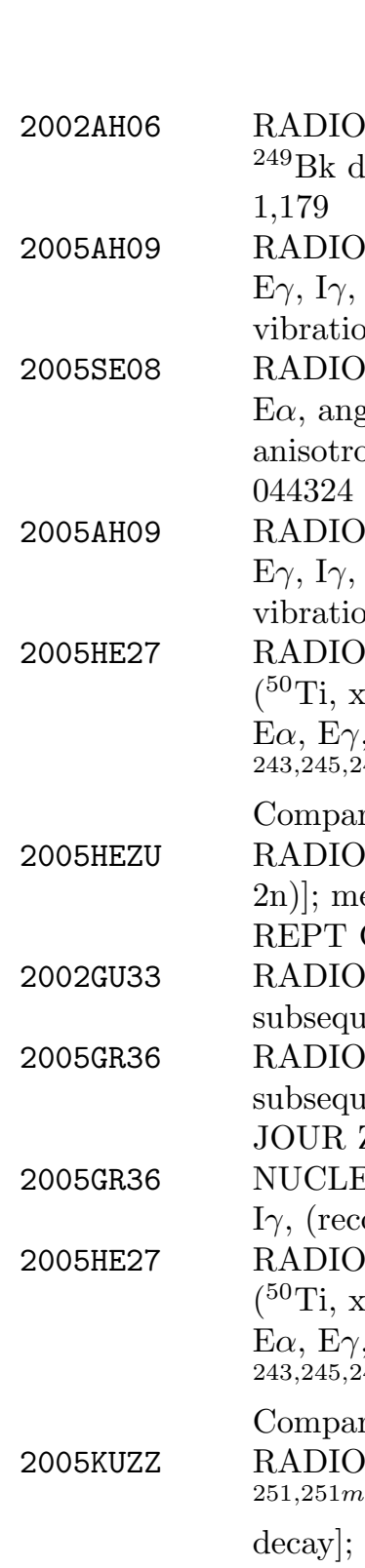

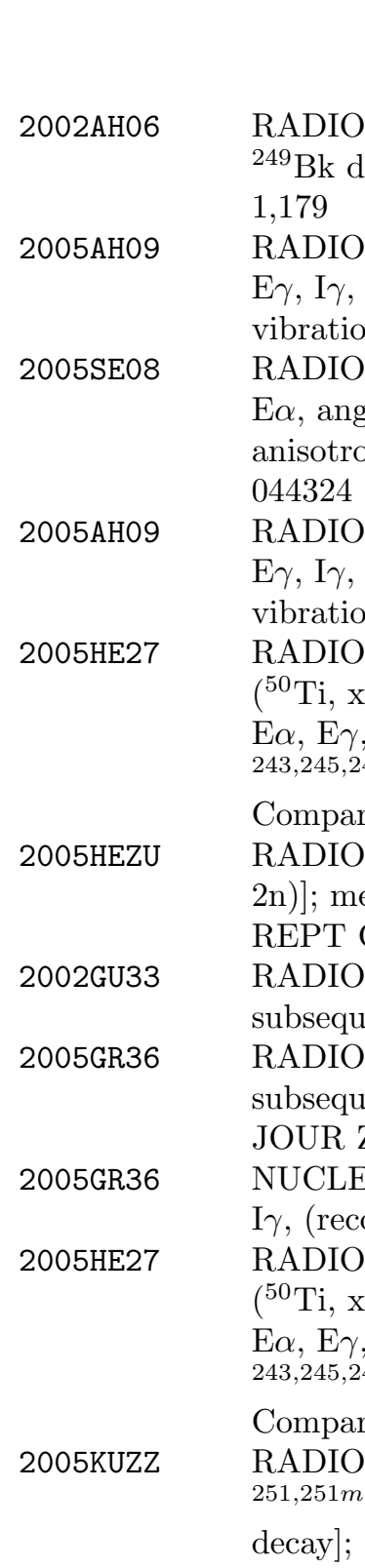

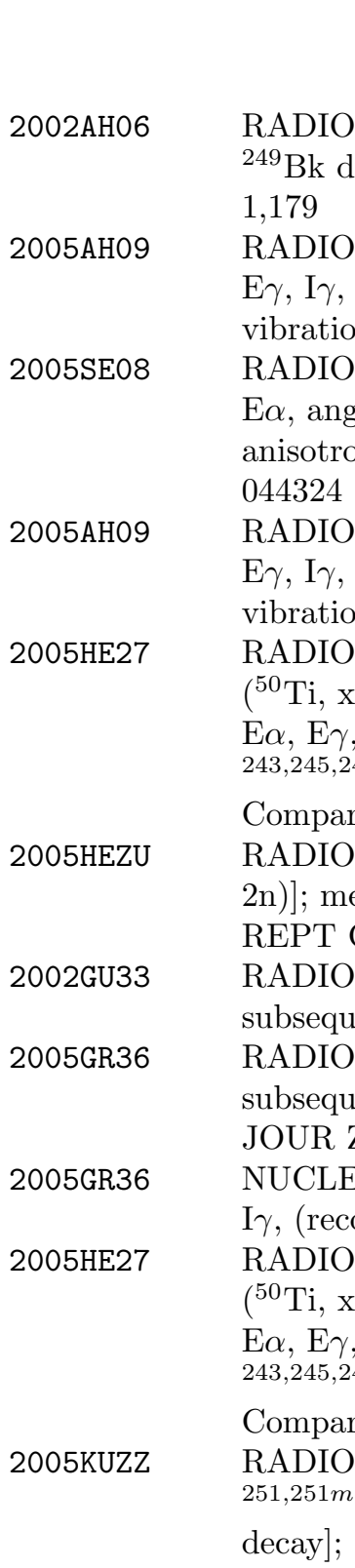

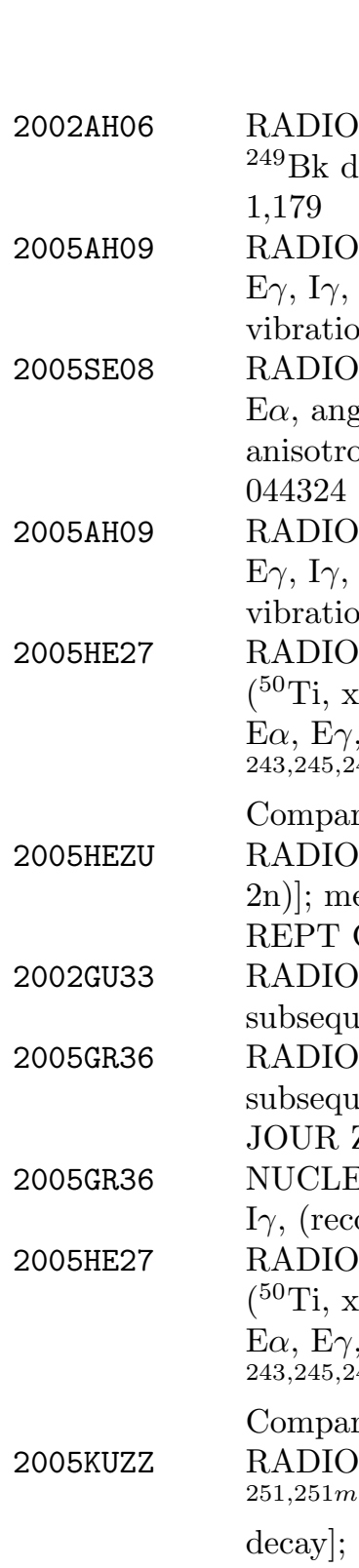

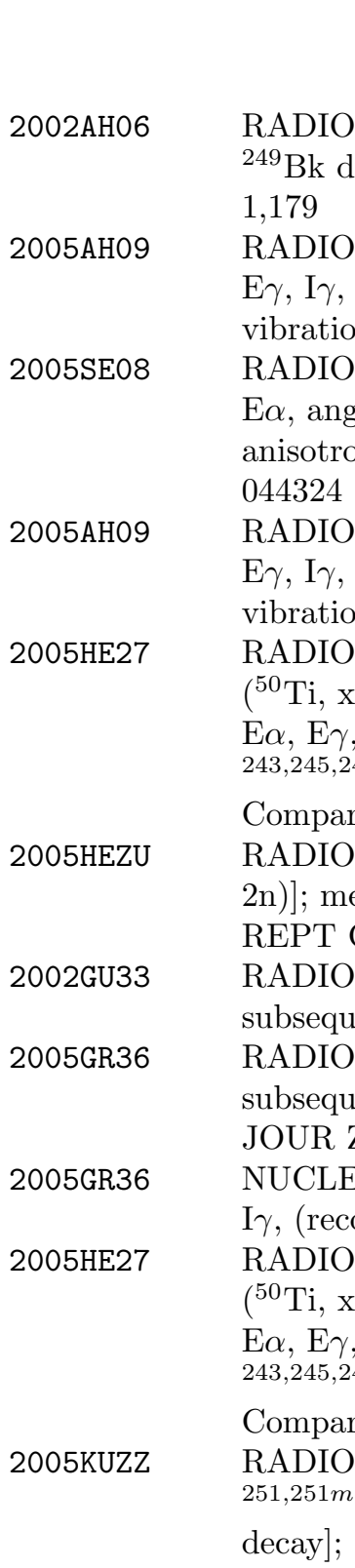

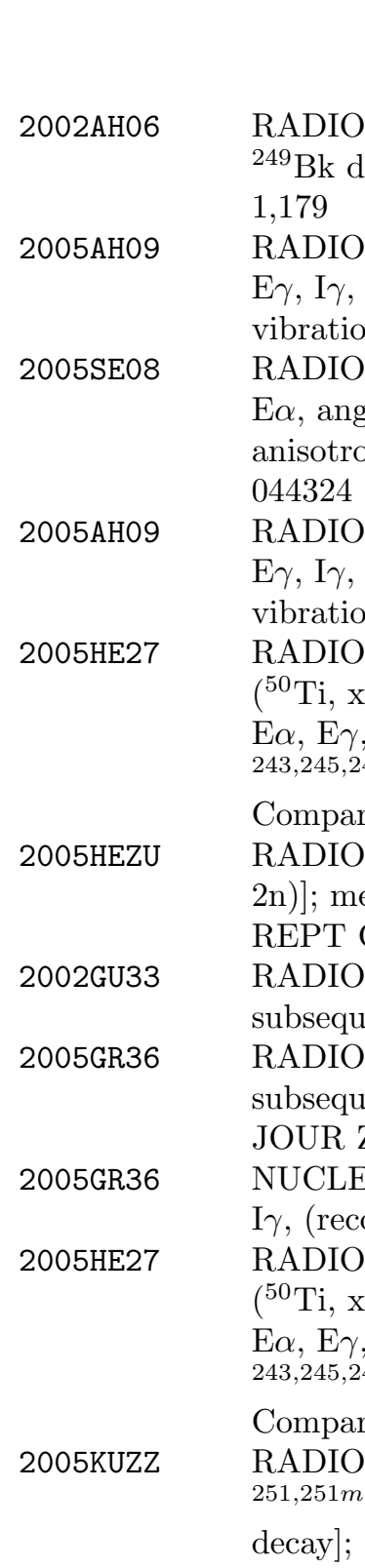

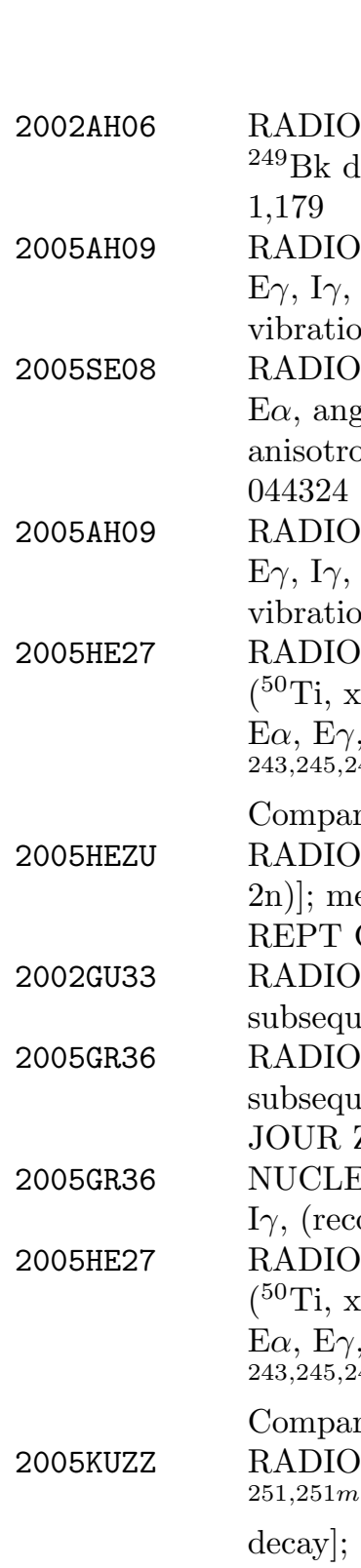

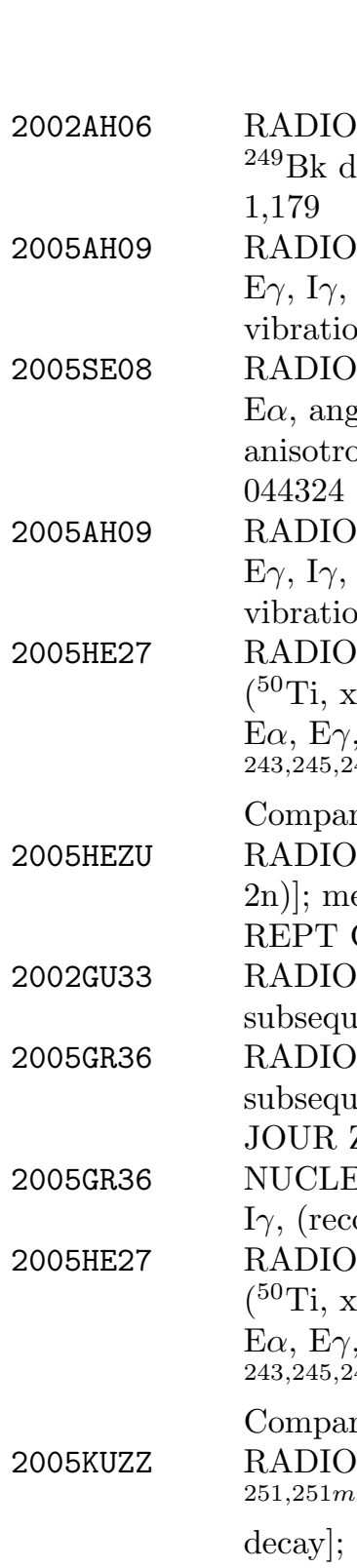

2005KUZZ
subsequent decay]; measured $\mathrm{E} \alpha, \mathrm{E} \gamma, \alpha \gamma$-coin, $\mathrm{T}_{1 / 2}$. CONF Argonne(Nuclei at the Limits),P231,Kuusiniemi RADIOACTIVITY ${ }^{255} \mathrm{Rf},{ }^{251} \mathrm{No}(\alpha)$ [from ${ }^{207} \mathrm{~Pb}\left({ }^{50} \mathrm{Ti}, 2 \mathrm{n}\right)$ and $\left.{ }^{206} \mathrm{~Pb}\left({ }^{48} \mathrm{Ca}, 2 \mathrm{n}\right)\right]$; measured $\mathrm{E} \gamma, \alpha \gamma$-coin. ${ }^{251}$ No deduced isomeric state. REPT GSI 2005-1,P74,Sulignano

\section{$\mathrm{A}=252$}

${ }^{252} \mathrm{Cf} \quad 2004 \mathrm{PYZZ}$
RADIOACTIVITY ${ }^{252} \mathrm{Cf}(\mathrm{SF})$; measured fission fragment mass distributions, neutron multiplicity; deduced ternary decay mode. REPT JINR-E15-2004-65,Pyatkov 


\section{$\mathrm{A}=252$ (continued)}

2005 BI02 RADIOACTIVITY ${ }^{252} \mathrm{Cf}(\mathrm{SF})$; measured Doppler-shifted E $\gamma$, I $\gamma$, (particle) $\gamma$-, $\gamma \gamma$-coin. ${ }^{142,144}$ Ba levels deduced $\mathrm{T}_{1 / 2}$, transition quadrupole moments. Euroball, Saphir arrays, differential Doppler shift method. JOUR PRVCA 71011301

2005F009 RADIOACTIVITY ${ }^{252} \mathrm{Cf}(\mathrm{SF})$; measured $\mathrm{E} \gamma, \mathrm{I} \gamma, \gamma \gamma$-coin. ${ }^{113,115,117} \mathrm{Pd}$ deduced levels, J, $\pi$. Gammasphere array. JOUR PRVCA 72014315

2005F017 RADIOACTIVITY ${ }^{252} \mathrm{Cf}(\mathrm{SF}) ;$ measured $\mathrm{E} \gamma, \mathrm{I} \gamma, \gamma \gamma$-coin. ${ }^{98} \mathrm{Sr}$, ${ }^{102,104} \mathrm{Zr},{ }^{137} \mathrm{Xe},{ }^{143} \mathrm{Ba},{ }^{152} \mathrm{Ce}$ levels deduced $\mathrm{T}_{1 / 2}$. Gammasphere array, time-gated triple-coincidence method. JOUR ZAANE 25 s01 465

2005HAZQ RADIOACTIVITY ${ }^{252} \mathrm{Cf}(\mathrm{SF})$; measured neutron spectra, fission fragment mass distribution. CONF Santa Fe (Nucl Data for Sci and Technol) Proc,Vol1,P644

2005HW06 RADIOACTIVITY ${ }^{252} \mathrm{Cf}(\mathrm{SF})$; measured E $\gamma, \mathrm{I} \gamma, \gamma \gamma$-coin. ${ }^{95,97} \mathrm{Sr},{ }^{99} \mathrm{Zr}$, ${ }^{108} \mathrm{Tc},{ }^{133,134} \mathrm{Te},{ }^{137} \mathrm{Xe}$ levels deduced $\mathrm{T}_{1 / 2}$. Gammasphere array, time-gated triple-coincidence method. JOUR ZAANE 25 s01 463

2005JA12 RADIOACTIVITY ${ }^{252} \mathrm{Cf}(\mathrm{SF})$; measured $\mathrm{E} \gamma, \mathrm{I} \gamma, \alpha \gamma-, \gamma \gamma$-coin for $\alpha$-accompanied ternary fission; deduced fission fragments average angular momentum. ${ }^{100,102} \mathrm{Zr},{ }^{106} \mathrm{Mo},{ }^{144,146} \mathrm{Ba},{ }^{138,140,142} \mathrm{Xe}$; deduced transition intensities. Gammasphere array. JOUR ZAANE 24373

2005JE04 RADIOACTIVITY ${ }^{252} \mathrm{Cf}(\mathrm{SF})$; measured $\mathrm{E} \alpha$, light charged particle yields, spectra, coincidences from quaternary fission; deduced fission mechanism features. JOUR ZAANE 24379

2005J024 RADIOACTIVITY ${ }^{252} \mathrm{Cf}(\mathrm{SF})$; measured $\mathrm{E} \gamma, \mathrm{I} \gamma, \gamma \gamma$-coin. ${ }^{162,164} \mathrm{Gd}$ deduced levels, J, $\pi$. Gammasphere array, level systematics in neighboring nuclides discussed. JOUR ZAANE 25 s01 467

2005LU21 RADIOACTIVITY ${ }^{252} \mathrm{Cf}(\mathrm{SF})$; measured E $\gamma$, I $\gamma, \gamma \gamma$-coin. ${ }^{99,101} \mathrm{Y}$, ${ }^{101,105} \mathrm{Nb}$ deduced levels, J, $\pi$, configurations, rotational bands, shape transition features. Gammasphere array, triaxial-rotor-plus-quasiparticle calculations. JOUR JPGPE 311303

2005LU24 RADIOACTIVITY ${ }^{252} \mathrm{Cf}(\mathrm{SF})$; measured E $\gamma$, I $\gamma, \gamma \gamma$-coin. ${ }^{99,101} \mathrm{Y}$, ${ }^{101,105} \mathrm{Nb}$ deduced levels, J, $\pi$, configurations, deformation.

Gammasphere array, triaxial-rotor-plus-particle calculations. JOUR ZAANE 25 s01 469

2005PA56 RADIOACTIVITY ${ }^{252} \mathrm{Cf}(\mathrm{SF})$; measured neutron emission rates. ${ }^{241} \mathrm{Am}(\alpha)$; measured neutron emission rates for Am-Be source. Manganese sulphate bath system. JOUR KPSJA 47603

2005RE06 RADIOACTIVITY ${ }^{244} \mathrm{Cm},{ }^{252} \mathrm{Cf}(\mathrm{SF})$; measured $\mathrm{E} \gamma$, I $\gamma$; deduced fission fragments isotopic yields. JOUR JRNCD 264243

2005SH49 RADIOACTIVITY ${ }^{252} \mathrm{Cf}(\mathrm{SF})$; measured Doppler-shifted E $\gamma, \mathrm{I} \gamma, \gamma \gamma$-, (fragment) $\gamma$-coin. ${ }^{144} \mathrm{Ba}$ deduced transitions $\mathrm{T}_{1 / 2}, \mathrm{~B}(\mathrm{E} 2)$, transition dipole, quadrupole, and octupole moments for alternating-parity band. Gammasphere array, cluster-model analysis. JOUR ZAANE 25387

2005SIZY RADIOACTIVITY ${ }^{252} \mathrm{Cf}(\mathrm{SF})$; measured neutron leakage spectrum from uranium sphere. CONF Santa Fe (Nucl Data for Sci and Technol) Proc, Vol1,P67 


\section{$\mathrm{A}=252$ (continued)}

2005SM08 RADIOACTIVITY ${ }^{252} \mathrm{Cf}(\mathrm{SF})$; measured $\mathrm{E} \gamma, \mathrm{I} \gamma(\theta, \mathrm{H}, \mathrm{t}), \gamma \gamma$-coin. ${ }^{96,100,102} \mathrm{Zr},{ }^{102,104,106,108} \mathrm{Mo},{ }^{106,108,110,112} \mathrm{Ru},{ }^{110,114,116} \mathrm{Pd}$ levels deduced $\mathrm{g}$ factors, $\mathrm{B}(\mathrm{E} 2)$. Gammasphere array, time-integral perturbed angular correlation technique. Comparison with interacting boson model predictions. JOUR JPGPE 31 S1433

2005TR06 RADIOACTIVITY ${ }^{252} \mathrm{Cf}(\mathrm{SF})$; measured iron-moderated photon and neutron spectra. Comparison with model predictions. JOUR AENGA 9854

2005VOZX RADIOACTIVITY ${ }^{252} \mathrm{Cf},{ }^{244,248} \mathrm{Cm}$ (SF); measured fission fragment mass distributions and kinetic energy spectra, prompt neutron multiplicity distributions vs fragment mass; deduced fission mechanism features. CONF Santa Fe (Nucl Data for Sci and Technol)

Proc, Vol1,P613

2005ZH36 RADIOACTIVITY ${ }^{252} \mathrm{Cf}(\mathrm{SF})$; measured E $\gamma$, I $\gamma, \gamma \gamma$-coin. ${ }^{106} \mathrm{Mo}$ deduced high-spin levels, $\mathrm{J}, \pi$, chiral vibrational bands. Gammasphere array, tilted-axis cranking model analysis. JOUR ZAANE 25 s01 459

${ }^{252} \mathrm{No} \quad 2002 \mathrm{SU} 35$ NUCLEAR REACTIONS ${ }^{206} \mathrm{~Pb}\left({ }^{48} \mathrm{Ca}, 2 \mathrm{n}\right),{ }^{186} \mathrm{~W}\left({ }^{34} \mathrm{~S}, 4 \mathrm{n}\right)$, E not given; measured yields, focal-plane position spectra in recoil separator. JOUR NIMAE 48171

2005YE02 RADIOACTIVITY ${ }^{252} \mathrm{No}(\mathrm{SF})$ [from ${ }^{206} \mathrm{~Pb}\left({ }^{48} \mathrm{Ca}, 2 \mathrm{n}\right)$ ]; measured fission fragments spectra, prompt neutron multiplicity. Recoil separator.

JOUR NIMAE 539441

\section{$\mathrm{A}=253$}

${ }^{253} \mathrm{Es} \quad 2002 \mathrm{AHO6}$

2004AHZY

2005AH03

2005SE08

${ }^{253} \mathrm{Fm}$
RADIOACTIVITY ${ }^{255} \mathrm{Fm},{ }^{253} \mathrm{Es}(\alpha)$; measured $\mathrm{E} \gamma, \mathrm{I} \gamma, \gamma \gamma$-coin. ${ }^{251} \mathrm{Cf}$, ${ }^{249} \mathrm{Bk}$ deduced levels, J, $\pi$, single-particle states. JOUR JNRSA 3,No 1,179

RADIOACTIVITY ${ }^{253} \mathrm{Es}(\alpha)$; measured $\mathrm{E} \gamma, \mathrm{I} \gamma, \gamma \gamma$-coin. ${ }^{249} \mathrm{Cm}\left(\beta^{-}\right)$ [from $\left.{ }^{248} \mathrm{Cm}(\mathrm{n}, \gamma)\right]$; measured $\mathrm{E} \gamma, \mathrm{I} \gamma .{ }^{249} \mathrm{Bk}$ deduced transitions, proton single-particle states $\mathrm{J}, \pi$, configurations. REPT ANL-04/22,P45,Ahmad RADIOACTIVITY ${ }^{253} \operatorname{Es}(\alpha)$; measured $\mathrm{E} \alpha, \mathrm{E} \gamma, \gamma \gamma-, \alpha \gamma$-coin. ${ }^{249} \mathrm{Cm}\left(\beta^{-}\right)\left[\right.$from $\left.{ }^{248} \mathrm{Cm}(\mathrm{n}, \gamma)\right]$; measured $\mathrm{E} \gamma, \mathrm{I} \gamma .{ }^{249} \mathrm{Bk}$ deduced levels, $\mathrm{J}, \pi$, configurations, $\mathrm{B}(\lambda)$, g factors. ${ }^{253} \mathrm{Es}(\mathrm{SF})$; measured $\mathrm{E} \gamma, \mathrm{I} \gamma$ from fission fragment decay; deduced fission branching ratio. Gammasphere array, comparisons with model predictions. JOUR PRVCA 71054305 RADIOACTIVITY ${ }^{253} \operatorname{Es}(\alpha)$; measured $\mathrm{E} \alpha, \mathrm{E} \gamma, \gamma \gamma-, \alpha \gamma$-coin.

${ }^{249} \mathrm{Cm}\left(\beta^{-}\right)\left[\right.$from $\left.{ }^{248} \mathrm{Cm}(\mathrm{n}, \gamma)\right]$; measured $\mathrm{E} \gamma, \mathrm{I} \gamma .{ }^{249} \mathrm{Bk}$ deduced levels, $\mathrm{J}, \pi$, configurations. Gammasphere array. CONF Argonne(Nuclei at the Limits),P251,Ahmad RADIOACTIVITY ${ }^{253,254} \mathrm{Es},{ }^{255} \mathrm{Fm}(\alpha)$ [from ${ }^{252} \mathrm{Cf}(\mathrm{n}, \mathrm{X})$ ]; measured $\mathrm{E} \alpha$, angular distributions from decay of oriented nuclei; deduced anisotropies. Comparison with model predictions. JOUR PRVCA 71 044324

RADIOACTIVITY ${ }^{269,270} \mathrm{Hs},{ }^{265,266} \mathrm{Sg},{ }^{257} \mathrm{No}(\alpha) ;{ }^{261,262} \mathrm{Rf}(\mathrm{SF})$ [from ${ }^{248} \mathrm{Cm}\left({ }^{26} \mathrm{Mg}, \mathrm{xn}\right)$ and subsequent decay]; measured $\mathrm{E} \alpha, \mathrm{T}_{1 / 2}$. JOUR RAACA 92855 


\section{$\mathrm{A}=253$ (continued)}

2005AS05 RADIOACTIVITY ${ }^{257} \mathrm{No}(\alpha)$ from $\left.{ }^{248} \mathrm{Cm}\left({ }^{13} \mathrm{C}, 4 \mathrm{n}\right)\right]$; measured $\mathrm{E} \gamma, \mathrm{E} \alpha$, $\mathrm{E}(\mathrm{ce}), \alpha \gamma-,\left(\right.$ ce) $\alpha$-coin; deduced branching ratios. ${ }^{253} \mathrm{Fm}$ deduced levels, J, $\pi$, ICC, configurations. ${ }^{257}$ No deduced ground-state J, $\pi$, configuration. JOUR PRLTA 95102502

${ }^{253} \mathrm{Md}$ 2005HE27 RADIOACTIVITY ${ }^{247,249,251,253,255} \mathrm{Md}(\alpha)$ [from ${ }^{209} \mathrm{Bi}\left({ }^{40} \mathrm{Ar}, \mathrm{xn}\right)$, $\left({ }^{50} \mathrm{Ti}, \mathrm{xn}\right),{ }^{207,207} \mathrm{~Pb},{ }^{209} \mathrm{Bi}\left({ }^{48} \mathrm{Ca}, \mathrm{xn}\right)$ and subsequent decay]; measured $\mathrm{E} \alpha, \mathrm{E} \gamma, \alpha \gamma$-coin; deduced branching ratios, hindrance factors.

243,245,247,249,251 Es deduced levels, J, $\pi$, configurations, deformation.

Comparison with model predictions. JOUR ZAANE 26233 $\begin{array}{ll}{ }^{253} \mathrm{No} & \text { 2005RE14 } \\ & \text { IUCLEAR REACTIONS }{ }^{207} \mathrm{~Pb}\left({ }^{48} \mathrm{Ca}, 2 \mathrm{n}\right), \mathrm{E}=219 \mathrm{MeV} \text {; measurecoil } \gamma \text {-coin. }{ }^{253} \mathrm{No} \text { deduced high-spin levels, J, } \pi, \\ & \text { configurations. Gammasphere array, fragment separator. JOUR }\end{array}$ PRLTA 95032501

${ }^{253} \mathrm{Lr} \quad$ 2005KUZZ RADIOACTIVITY ${ }^{216,216 m} \mathrm{Th}(\alpha)$, (IT) [from $\left.{ }^{170} \operatorname{Er}\left({ }^{50} \mathrm{Ti}, 4 \mathrm{n}\right)\right]$; ${ }^{251,251 m} \mathrm{No},{ }^{247,247 m} \mathrm{Fm}(\alpha)$ [from ${ }^{206} \mathrm{~Pb}\left({ }^{48} \mathrm{Ca}, 3 \mathrm{n}\right)$ and subsequent decay]; ${ }^{257,257 m} \mathrm{Db},{ }^{253,253 m} \mathrm{Lr},{ }^{249} \mathrm{Md}(\alpha)\left[\right.$ from ${ }^{209} \mathrm{Bi}\left({ }^{50} \mathrm{Ti}, 2 \mathrm{n}\right)$ and subsequent decay]; measured $\mathrm{E} \alpha, \mathrm{E} \gamma, \alpha \gamma$-coin, $\mathrm{T}_{1 / 2}$. CONF Argonne(Nuclei at the Limits),P231,Kuusiniemi

\section{$\mathrm{A}=254$}

\section{${ }^{254} \mathrm{Es} \quad$ 2005SE08}

${ }^{254} \mathrm{No}$

2004KHZY

2005EE01

2005EE02

2005MUZZ

${ }^{255} \mathrm{Fm} \quad 2002 \mathrm{AHO6}$

2005AH09 044324 JOUR ZAANE 26227

\section{$\mathrm{A}=\mathbf{2 5 5}$} 1,179
RADIOACTIVITY ${ }^{253,254} \mathrm{Es},{ }^{255} \mathrm{Fm}(\alpha)$ [from ${ }^{252} \mathrm{Cf}(\mathrm{n}, \mathrm{X})$ ]; measured $\mathrm{E} \alpha$, angular distributions from decay of oriented nuclei; deduced anisotropies. Comparison with model predictions. JOUR PRVCA 71

RADIOACTIVITY ${ }^{254 m} \mathrm{No}(\mathrm{IT})$ [from ${ }^{208} \mathrm{~Pb}\left({ }^{48} \mathrm{Ca}, 2 \mathrm{n}\right)$ ]; measured $\mathrm{E}(\mathrm{ce}), \mathrm{I}(\mathrm{ce})$ following decay of high-spin isomer. ${ }^{254}$ No level deduced $\mathrm{J}$, $\pi$, configuration. REPT ANL-04/22,P47,Khoo

NUCLEAR REACTIONS ${ }^{208} \mathrm{~Pb}\left({ }^{48} \mathrm{Ca}, 2 \mathrm{n}\right)$, E not given; measured $\mathrm{E} \gamma$, $\mathrm{I} \gamma$, (recoil) $\gamma$-coin. ${ }^{254}$ No deduced rotational band levels, J, $\pi$. Jurogam array, recoil-decay tagging. JOUR ZAANE 25 s01 605

NUCLEAR REACTIONS ${ }^{208} \mathrm{~Pb}\left({ }^{48} \mathrm{Ca}, 2 \mathrm{n}\right), \mathrm{E}=219,221 \mathrm{MeV}$; measured $\mathrm{E} \gamma, \mathrm{I} \gamma, \gamma \gamma$-, (recoil) $\gamma$-coin. ${ }^{254}$ No deduced levels, J, $\pi$, rotational band, non-yrast state. Jurogam array, recoil-decay tagging. RADIOACTIVITY ${ }^{254} \mathrm{No}$ (IT) [from ${ }^{208} \mathrm{~Pb}\left({ }^{48} \mathrm{Ca}, 2 \mathrm{n}\right)$ ]; measured $\mathrm{E}(\mathrm{ce})$, $\mathrm{I}(\mathrm{ce}), \mathrm{T}_{1 / 2} .{ }^{254}$ No deduced isomer $\mathrm{J}, \pi$, configuration. CONF Argonne(Nuclei at the Limits),P243,Mukherjee

RADIOACTIVITY ${ }^{255} \mathrm{Fm},{ }^{253} \mathrm{Es}(\alpha)$; measured $\mathrm{E} \gamma, \mathrm{I} \gamma, \gamma \gamma$-coin. ${ }^{251} \mathrm{Cf}$, ${ }^{249} \mathrm{Bk}$ deduced levels, J, $\pi$, single-particle states. JOUR JNRSA 3,No

RADIOACTIVITY ${ }^{255} \mathrm{Fm}(\alpha)$; measured $\mathrm{E} \gamma, \mathrm{I} \gamma \cdot{ }^{251} \mathrm{Es}(\mathrm{EC})$; measured $\mathrm{E} \gamma, \mathrm{I} \gamma, \mathrm{E}(\mathrm{ce}), \mathrm{I}(\mathrm{ce}) .{ }^{251} \mathrm{Cf}$ deduced levels, J, $\pi$, configurations, vibrational states. JOUR PRVCA 72054308 


\section{$\mathrm{A}=255$ (continued)}

2005SE08 RADIOACTIVITY ${ }^{253,254} \mathrm{Es},{ }^{255} \mathrm{Fm}(\alpha)$ [from ${ }^{252} \mathrm{Cf}(\mathrm{n}, \mathrm{X})$ ]; measured $\mathrm{E} \alpha$, angular distributions from decay of oriented nuclei; deduced anisotropies. Comparison with model predictions. JOUR PRVCA 71 044324

${ }^{255} \mathrm{Md}$ 2005HE27 RADIOACTIVITY ${ }^{247,249,251,253,255} \mathrm{Md}(\alpha)$ [from ${ }^{209} \mathrm{Bi}\left({ }^{40} \mathrm{Ar}, \mathrm{xn}\right)$, $\left({ }^{50} \mathrm{Ti}, \mathrm{xn}\right),{ }^{207,207} \mathrm{~Pb},{ }^{209} \mathrm{Bi}\left({ }^{48} \mathrm{Ca}, \mathrm{xn}\right)$ and subsequent decay]; measured $\mathrm{E} \alpha, \mathrm{E} \gamma, \alpha \gamma$-coin; deduced branching ratios, hindrance factors. $243,245,247,249,251$ Es deduced levels, J, $\pi$, configurations, deformation. Comparison with model predictions. JOUR ZAANE 26233

\begin{tabular}{|c|c|c|}
\hline${ }^{255} \mathrm{No}$ & 2005HEZU & $\begin{array}{l}\text { RADIOACTIVITY }{ }^{255} \mathrm{No}(\alpha)\left[\text { from }{ }^{208} \mathrm{~Pb}\left({ }^{48} \mathrm{Ca}, \mathrm{n}\right) \text { and }{ }^{209} \mathrm{Bi}\left({ }^{48} \mathrm{Ca} \text {, }\right.\right. \\
\text { 2n)]; measured } \mathrm{E} \alpha, \mathrm{E} \gamma, \alpha \gamma \text {-coin. }{ }^{251} \mathrm{Fm} \text { deduced levels, configurations. } \\
\text { REPT GSI 2005-1,P73,Hessberger }\end{array}$ \\
\hline${ }^{255} \mathrm{Lr}$ & 2002GU33 & $\begin{array}{l}\text { RADIOACTIVITY }{ }^{259} \mathrm{Db},{ }^{255} \operatorname{Lr}(\alpha)\left[\text { from }{ }^{241} \mathrm{Am}\left({ }^{22} \mathrm{Ne}, 4 \mathrm{n}\right) \text { and }\right. \\
\text { subsequent decay]; measured } \mathrm{E} \alpha, \mathrm{T}_{1 / 2} . \text { JOUR JNRSA } 3, \text { No } 1,183\end{array}$ \\
\hline & 2005 GR36 & $\begin{array}{l}\text { RADIOACTIVITY }{ }^{255} \mathrm{Lr},{ }^{251} \mathrm{Md}(\alpha) \text { [from }{ }^{209} \mathrm{Bi}\left({ }^{48} \mathrm{Ca}, 2 \mathrm{n}\right) \text { and } \\
\text { subsequent decay]; measured } \mathrm{E} \alpha, \alpha \alpha \text {-coin; deduced excited state decay. } \\
\text { JOUR ZAANE } 25 \text { s01 } 599\end{array}$ \\
\hline${ }^{255} \mathrm{Rf}$ & 2005SUZX & $\begin{array}{l}\text { RADIOACTIVITY }{ }^{255} \mathrm{Rf},{ }^{251} \mathrm{No}(\alpha)\left[\text { from }{ }^{207} \mathrm{~Pb}\left({ }^{50} \mathrm{Ti}, 2 \mathrm{n}\right) \text { and }\right. \\
\left.{ }^{206} \mathrm{~Pb}\left({ }^{48} \mathrm{Ca}, 2 \mathrm{n}\right)\right] ; \text { measured } \mathrm{E} \gamma, \alpha \gamma \text {-coin. }{ }^{251} \mathrm{No} \text { deduced isomeric state. } \\
\text { REPT GSI 2005-1,P74,Sulignano }\end{array}$ \\
\hline
\end{tabular}

$$
\mathrm{A}=256
$$

No references found

\section{$\mathrm{A}=257$}

${ }^{257} \mathrm{No} \quad 2004 \mathrm{~V} 024$

${ }^{257} \mathrm{Rf} \quad 2005 \mathrm{ST} 16$

${ }^{257} \mathrm{Db}$

$2005 \mathrm{KUZZ}$

RADIOACTIVITY ${ }^{269,270} \mathrm{Hs},{ }^{265,266} \mathrm{Sg},{ }^{257} \mathrm{No}(\alpha) ;{ }^{261,262} \mathrm{Rf}(\mathrm{SF})$ [from ${ }^{248} \mathrm{Cm}\left({ }^{26} \mathrm{Mg}, \mathrm{xn}\right)$ and subsequent decay]; measured $\mathrm{E} \alpha, \mathrm{T}_{1 / 2}$. JOUR RAACA 92855

RADIOACTIVITY ${ }^{257} \mathrm{No}(\alpha)$ [from ${ }^{248} \mathrm{Cm}\left({ }^{13} \mathrm{C}, 4 \mathrm{n}\right)$ ]; measured $\mathrm{E} \gamma, \mathrm{E} \alpha$, $\mathrm{E}(\mathrm{ce}), \alpha \gamma-$ - (ce) $\alpha$-coin; deduced branching ratios. ${ }^{253} \mathrm{Fm}$ deduced levels, J, $\pi$, ICC, configurations. ${ }^{257}$ No deduced ground-state J, $\pi$, configuration. JOUR PRLTA 95102502

RADIOACTIVITY ${ }^{277} 112,{ }^{273} \mathrm{Ds},{ }^{269} \mathrm{Hs},{ }^{265} \mathrm{Sg},{ }^{261} \mathrm{Rf}(\alpha)$ [from ${ }^{208} \mathrm{~Pb}\left({ }^{70} \mathrm{Zn}, \mathrm{n}\right)$ and subsequent decay]; measured $\mathrm{E} \alpha, \mathrm{T}_{1 / 2}$. REPT RIKEN 2004 Annual,P69,Morita

NUCLEAR REACTIONS ${ }^{208} \mathrm{~Pb}\left({ }^{50} \mathrm{Ti}, \mathrm{n}\right), \mathrm{E}=237 \mathrm{MeV}$; measured delayed $\alpha \alpha$-coin; deduced evidence for ${ }^{257} \mathrm{Rf}$. Gas-filled separator, fast liquid-liquid extraction system. JOUR NIMAE 543509

RADIOACTIVITY ${ }^{216,216 m} \mathrm{Th}(\alpha)$, (IT) [from ${ }^{170} \operatorname{Er}\left({ }^{50} \mathrm{Ti}, 4 \mathrm{n}\right)$ ]; ${ }^{251,251 m} \mathrm{No},{ }^{247,247 m} \mathrm{Fm}(\alpha)$ [from ${ }^{206} \mathrm{~Pb}\left({ }^{48} \mathrm{Ca}, 3 \mathrm{n}\right)$ and subsequent decay]; ${ }^{257,257 m} \mathrm{Db},{ }^{253,253 m} \mathrm{Lr},{ }^{249} \mathrm{Md}(\alpha)$ [from ${ }^{209} \mathrm{Bi}\left({ }^{50} \mathrm{Ti}, 2 \mathrm{n}\right)$ and subsequent decay]; measured $\mathrm{E} \alpha, \mathrm{E} \gamma, \alpha \gamma$-coin, $\mathrm{T}_{1 / 2}$. CONF Argonne(Nuclei at the Limits),P231,Kuusiniemi 


\section{$\mathrm{A}=258$}

No references found

$$
\mathrm{A}=259
$$

${ }^{259} \mathrm{Db} \quad 2002 \mathrm{GU} 33 \quad$ NUCLEAR REACTIONS ${ }^{241} \mathrm{Am}\left({ }^{22} \mathrm{Ne}, 4 \mathrm{n}\right), \mathrm{E}=118 \mathrm{MeV}$; measured delayed $\mathrm{E} \alpha, \alpha \alpha$-coin; deduced evidence for ${ }^{259} \mathrm{Db}$. JOUR JNRSA 3,No 1,183

2002GU33 RADIOACTIVITY ${ }^{259} \mathrm{Db},{ }^{255} \operatorname{Lr}(\alpha)$ [from ${ }^{241} \mathrm{Am}\left({ }^{22} \mathrm{Ne}, 4 \mathrm{n}\right)$ and subsequent decay]; measured $\mathrm{E} \alpha, \mathrm{T}_{1 / 2}$. JOUR JNRSA 3,No 1,183

\section{$\mathrm{A}=260$}

No references found

\section{$\mathrm{A}=261$}

${ }^{261} \mathrm{Rf} \quad$ 2002NA37

NUCLEAR REACTIONS ${ }^{248} \mathrm{Cm}\left({ }^{18} \mathrm{O}, 5 \mathrm{n}\right), \mathrm{E}=91,94,99 \mathrm{MeV}$;

${ }^{248} \mathrm{Cm}\left({ }^{19} \mathrm{~F}, 5 \mathrm{n}\right), \mathrm{E}=106 \mathrm{MeV}$; measured delayed $\mathrm{E} \alpha, \alpha \alpha$-coin; deduced production $\sigma$. JOUR JNRSA 3,No 1,85

2004MOZU RADIOACTIVITY ${ }^{277} 112,{ }^{273} \mathrm{Ds},{ }^{269} \mathrm{Hs},{ }^{265} \mathrm{Sg}(\alpha)$ [from ${ }^{208} \mathrm{~Pb}\left({ }^{70} \mathrm{Zn}, \mathrm{n}\right)$ and subsequent decay]; measured $\mathrm{E} \alpha, \mathrm{T}_{1 / 2} .{ }^{261} \mathrm{Rf}(\mathrm{SF})$; measured $\mathrm{T}_{1 / 2}$. PREPRINT Morita

2004 V024 RADIOACTIVITY ${ }^{269,270} \mathrm{Hs},{ }^{265,266} \mathrm{Sg},{ }^{257} \mathrm{No}(\alpha) ;{ }^{261,262} \mathrm{Rf}$ (SF) [from ${ }^{248} \mathrm{Cm}\left({ }^{26} \mathrm{Mg}, \mathrm{xn}\right)$ and subsequent decay]; measured $\mathrm{E} \alpha, \mathrm{T}_{1 / 2}$. JOUR RAACA 92855

2005MOZT RADIOACTIVITY ${ }^{277} 112,{ }^{273} \mathrm{Ds},{ }^{269} \mathrm{Hs},{ }^{265} \mathrm{Sg},{ }^{261} \mathrm{Rf}(\alpha)$ [from ${ }^{208} \mathrm{~Pb}\left({ }^{70} \mathrm{Zn}, \mathrm{n}\right)$ and subsequent decay]; measured $\mathrm{E} \alpha, \mathrm{T}_{1 / 2}$. REPT RIKEN 2004 Annual,P69,Morita

$$
\mathrm{A}=262
$$

${ }^{262} \mathrm{Rf} \quad$ 2004V024 RADIOACTIVITY ${ }^{269,270} \mathrm{Hs},{ }^{265,266} \mathrm{Sg},{ }^{257} \mathrm{No}(\alpha) ;{ }^{261,262} \mathrm{Rf}(\mathrm{SF})$ [from ${ }^{248} \mathrm{Cm}\left({ }^{26} \mathrm{Mg}, \mathrm{xn}\right)$ and subsequent decay]; measured $\mathrm{E} \alpha, \mathrm{T}_{1 / 2}$. JOUR RAACA 92855

${ }^{262} \mathrm{Db}$ 2002NA37 NUCLEAR REACTIONS ${ }^{248} \mathrm{Cm}\left({ }^{18} \mathrm{O}, 5 \mathrm{n}\right), \mathrm{E}=91,94,99 \mathrm{MeV}$;

${ }^{248} \mathrm{Cm}\left({ }^{19} \mathrm{~F}, 5 \mathrm{n}\right), \mathrm{E}=106 \mathrm{MeV}$; measured delayed $\mathrm{E} \alpha, \alpha \alpha$-coin; deduced production $\sigma$. JOUR JNRSA 3,No 1,85

2005MOZS RADIOACTIVITY ${ }^{278} 113,{ }^{274} \mathrm{Rg},{ }^{270} \mathrm{Mt},{ }^{266} \mathrm{Bh}(\alpha)$ [from ${ }^{209} \mathrm{Bi}\left({ }^{70} \mathrm{Zn}\right.$, n) and subsequent decay]; measured $\mathrm{E} \alpha, \mathrm{T}_{1 / 2}$. REPT RIKEN 2004 Annual,P70,Morita

\section{$\mathrm{A}=263$}

No references found 


\section{$\mathrm{A}=264$}

No references found

\section{$\mathrm{A}=265$}

${ }^{265} \mathrm{Sg} \quad$ 2004MOZU

2004V024

2005MOZT

RADIOACTIVITY ${ }^{277} 112,{ }^{273} \mathrm{Ds},{ }^{269} \mathrm{Hs},{ }^{265} \mathrm{Sg}(\alpha)$ [from ${ }^{208} \mathrm{~Pb}\left({ }^{70} \mathrm{Zn}, \mathrm{n}\right)$ and subsequent decay]; measured $\mathrm{E} \alpha, \mathrm{T}_{1 / 2} .{ }^{261} \mathrm{Rf}(\mathrm{SF})$; measured $\mathrm{T}_{1 / 2}$. PREPRINT Morita RADIOACTIVITY ${ }^{269,270} \mathrm{Hs},{ }^{265,266} \mathrm{Sg},{ }^{257} \mathrm{No}(\alpha) ;{ }^{261,262} \mathrm{Rf}(\mathrm{SF})$ [from ${ }^{248} \mathrm{Cm}\left({ }^{26} \mathrm{Mg}\right.$, xn $)$ and subsequent decay]; measured $\mathrm{E} \alpha, \mathrm{T}_{1 / 2}$. JOUR RAACA 92855

RADIOACTIVITY ${ }^{277} 112,{ }^{273} \mathrm{Ds},{ }^{269} \mathrm{Hs},{ }^{265} \mathrm{Sg},{ }^{261} \mathrm{Rf}(\alpha)$ [from ${ }^{208} \mathrm{~Pb}\left({ }^{70} \mathrm{Zn}, \mathrm{n}\right)$ and subsequent decay]; measured $\mathrm{E} \alpha, \mathrm{T}_{1 / 2}$. REPT RIKEN 2004 Annual,P69,Morita

\section{$A=266$}

${ }^{266} \mathrm{Sg} \quad 2004 \mathrm{VO} 24$

RADIOACTIVITY ${ }^{269,270} \mathrm{Hs},{ }^{265,266} \mathrm{Sg},{ }^{257} \mathrm{No}(\alpha) ;{ }^{261,262} \mathrm{Rf}(\mathrm{SF})$ [from ${ }^{248} \mathrm{Cm}\left({ }^{26} \mathrm{Mg}\right.$, xn $)$ and subsequent decay]; measured $\mathrm{E} \alpha, \mathrm{T}_{1 / 2}$. JOUR RAACA 92855

${ }^{266} \mathrm{Bh} \quad$ 2005MOZS

RADIOACTIVITY ${ }^{278} 113,{ }^{274} \mathrm{Rg},{ }^{270} \mathrm{Mt},{ }^{266} \mathrm{Bh}(\alpha)$ [from ${ }^{209} \mathrm{Bi}\left({ }^{70} \mathrm{Zn}\right.$, n) and subsequent decay]; measured $\mathrm{E} \alpha, \mathrm{T}_{1 / 2}$. REPT RIKEN 2004 Annual,P70,Morita

\section{$\mathrm{A}=267$}

${ }^{267} \mathrm{Rf} \quad 20040 \mathrm{G} 12$

RADIOACTIVITY ${ }^{271} \mathrm{Sg},{ }^{275} \mathrm{Hs},{ }^{279} \mathrm{Ds},{ }^{282,283,285} 112,{ }^{286,287,288,289} 114$, ${ }^{292,293} 116(\alpha) ;{ }^{267} \mathrm{Rf},{ }^{271} \mathrm{Sg},{ }^{279,281} \mathrm{Ds},{ }^{284} 112,{ }^{286} 114$ (SF) [from ${ }^{233,238} \mathrm{U}$, ${ }^{242} \mathrm{Pu},{ }^{248} \mathrm{Cm}\left({ }^{48} \mathrm{Ca}, \mathrm{xn}\right)$ and subsequent decay]; measured $\mathrm{E} \alpha, \mathrm{T}_{1 / 2}$. Comparison with model predictions. JOUR PRVCA 70064609

20040GZZ RADIOACTIVITY ${ }^{271} \mathrm{Sg},{ }^{275} \mathrm{Hs},{ }^{279} \mathrm{Ds},{ }^{282,283,285} 112,{ }^{286,287,288,289} 114$, ${ }^{292,293} 116(\alpha) ;{ }^{267} \mathrm{Rf},{ }^{271} \mathrm{Sg},{ }^{279,281} \mathrm{Ds},{ }^{284} 112,{ }^{286} 114$ (SF) [from ${ }^{233,238} \mathrm{U}$, ${ }^{242} \mathrm{Pu},{ }^{248} \mathrm{Cm}\left({ }^{48} \mathrm{Ca}, \mathrm{xn}\right)$ and subsequent decay]; measured $\mathrm{E} \alpha, \mathrm{T}_{1 / 2}$. Comparison with model predictions. REPT JINR-E7-2004-160,Oganessian 20050G03 RADIOACTIVITY ${ }^{294} 118,{ }^{290,291,292,293} 116,{ }^{287,288,289} 114,{ }^{285} 112$, ${ }^{275} \mathrm{Hs}(\alpha) ;{ }^{286} 114,{ }^{283} 112,{ }^{279} \mathrm{Ds},{ }^{271} \mathrm{Sg}(\alpha)$, (SF); ${ }^{282,284} 112,{ }^{281} \mathrm{Ds}$, ${ }^{267} \mathrm{Rf}(\mathrm{SF})$; measured $\mathrm{E} \alpha, \mathrm{T}_{1 / 2}$, branching ratios. JOUR ZAANE $25 \mathrm{~s} 01$ 589

${ }^{267} \mathrm{Db} \quad$ 20030GZY RADIOACTIVITY ${ }^{287,288} 115,{ }^{283,284} 113,{ }^{279,280} \mathrm{Rg},{ }^{275,276} \mathrm{Mt}$, ${ }^{272} \mathrm{Bh}(\alpha)$ [from ${ }^{243} \mathrm{Am}\left({ }^{48} \mathrm{Ca}, \mathrm{xn}\right)$ and subsequent decay]; measured $\mathrm{E} \alpha$, $\mathrm{T}_{1 / 2}$; deduced $\mathrm{Q} \alpha .{ }^{267,268} \mathrm{Db}(\mathrm{SF})\left[\right.$ from ${ }^{243} \mathrm{Am}\left({ }^{48} \mathrm{Ca}\right.$, xn) and subsequent decay]; measured $\mathrm{T}_{1 / 2}$. Comparison with model predictions. REPT JINR-E7-2003-178,Oganessian

20050G02 RADIOACTIVITY ${ }^{287,288} 115,{ }^{283,284} 113,{ }^{279,280} \mathrm{Rg},{ }^{275,276} \mathrm{Mt},{ }^{272} \mathrm{Bh}(\alpha)$ [from ${ }^{243} \mathrm{Am}\left({ }^{48} \mathrm{Ca}, \mathrm{xn}\right)$ and subsequent decay]; measured $\mathrm{E} \alpha, \mathrm{T}_{1 / 2}$; deduced $\mathrm{Q} \alpha .{ }^{267,268} \mathrm{Db}(\mathrm{SF})$; measured $\mathrm{T}_{1 / 2}$. JOUR PRVCA 72034611 


\section{$\mathrm{A}=\mathbf{2 6 8}$}

${ }^{268} \mathrm{Db}$

20030GZY

2004DMZZ

20050G02

${ }^{269} \mathrm{Hs}$

2004MOZU

$2004 \mathrm{VO} 24$

2004V024

2005MOZT

${ }^{270} \mathrm{Hs}$

2004V024

${ }^{270} \mathrm{Mt} \quad$ 2005MOZS

${ }^{271} \mathrm{Sg}$

20040G12
RADIOACTIVITY ${ }^{287,288} 115,{ }^{283,284} 113,{ }^{279,280} \mathrm{Rg},{ }^{275,276} \mathrm{Mt}$, ${ }^{272} \mathrm{Bh}(\alpha)$ [from ${ }^{243} \mathrm{Am}\left({ }^{48} \mathrm{Ca}, \mathrm{xn}\right)$ and subsequent decay]; measured $\mathrm{E} \alpha$, $\mathrm{T}_{1 / 2}$; deduced $\mathrm{Q} \alpha .{ }^{267,268} \mathrm{Db}(\mathrm{SF})$ [from ${ }^{243} \mathrm{Am}\left({ }^{48} \mathrm{Ca}\right.$, xn) and subsequent decay]; measured $\mathrm{T}_{1 / 2}$. Comparison with model predictions. REPT JINR-E7-2003-178, Oganessian RADIOACTIVITY ${ }^{268} \mathrm{Db}(\mathrm{SF})$ [from ${ }^{243} \mathrm{Am}\left({ }^{48} \mathrm{Ca}, 3 \mathrm{n}\right)$ and subsequent decay]; measured $\mathrm{T}_{1 / 2}$. REPT JINR-E12-2004-157,Dmitriev RADIOACTIVITY ${ }^{287,288} 115,{ }^{283,284} 113,{ }^{279,280} \mathrm{Rg},{ }^{275,276} \mathrm{Mt},{ }^{272} \mathrm{Bh}(\alpha)$ [from ${ }^{243} \mathrm{Am}\left({ }^{48} \mathrm{Ca}, \mathrm{xn}\right)$ and subsequent decay]; measured $\mathrm{E} \alpha, \mathrm{T}_{1 / 2}$; deduced $\mathrm{Q} \alpha .{ }^{267,268} \mathrm{Db}(\mathrm{SF})$; measured $\mathrm{T}_{1 / 2}$. JOUR PRVCA 72034611

\section{$\mathrm{A}=\mathbf{2 6 9}$}

RADIOACTIVITY ${ }^{277} 112,{ }^{273} \mathrm{Ds},{ }^{269} \mathrm{Hs},{ }^{265} \mathrm{Sg}(\alpha)$ [from ${ }^{208} \mathrm{~Pb}\left({ }^{70} \mathrm{Zn}, \mathrm{n}\right)$ and subsequent decay]; measured $\mathrm{E} \alpha, \mathrm{T}_{1 / 2} .{ }^{261} \mathrm{Rf}(\mathrm{SF})$; measured $\mathrm{T}_{1 / 2}$. PREPRINT Morita NUCLEAR REACTIONS ${ }^{248} \mathrm{Cm}\left({ }^{26} \mathrm{Mg}, \mathrm{xn}\right), \mathrm{E}=144-149 \mathrm{MeV}$; measured delayed $\alpha \alpha$-coin; deduced evidence for ${ }^{269,270} \mathrm{Hs}$. Radiochemical analysis. JOUR RAACA 92855 RADIOACTIVITY ${ }^{269,270} \mathrm{Hs},{ }^{265,266} \mathrm{Sg},{ }^{257} \mathrm{No}(\alpha) ;{ }^{261,262} \mathrm{Rf}(\mathrm{SF})$ [from ${ }^{248} \mathrm{Cm}\left({ }^{26} \mathrm{Mg}\right.$, xn $)$ and subsequent decay]; measured $\mathrm{E} \alpha, \mathrm{T}_{1 / 2}$. JOUR RAACA 92855 RADIOACTIVITY ${ }^{277} 112,{ }^{273} \mathrm{Ds},{ }^{269} \mathrm{Hs},{ }^{265} \mathrm{Sg},{ }^{261} \mathrm{Rf}(\alpha)$ [from ${ }^{208} \mathrm{~Pb}\left({ }^{70} \mathrm{Zn}, \mathrm{n}\right)$ and subsequent decay]; measured $\mathrm{E} \alpha, \mathrm{T}_{1 / 2}$. REPT RIKEN 2004 Annual,P69,Morita

$$
\mathrm{A}=\mathbf{2 7 0}
$$

NUCLEAR REACTIONS ${ }^{248} \mathrm{Cm}\left({ }^{26} \mathrm{Mg}\right.$, xn $), \mathrm{E}=144-149 \mathrm{MeV}$; measured delayed $\alpha \alpha$-coin; deduced evidence for ${ }^{269,270} \mathrm{Hs}$. Radiochemical analysis. JOUR RAACA 92855 RADIOACTIVITY ${ }^{269,270} \mathrm{Hs},{ }^{265,266} \mathrm{Sg},{ }^{257} \mathrm{No}(\alpha) ;{ }^{261,262} \mathrm{Rf}(\mathrm{SF})$ [from ${ }^{248} \mathrm{Cm}\left({ }^{26} \mathrm{Mg}\right.$, xn $)$ and subsequent decay]; measured $\mathrm{E} \alpha, \mathrm{T}_{1 / 2}$. JOUR RAACA 92855 RADIOACTIVITY ${ }^{278} 113,{ }^{274} \mathrm{Rg},{ }^{270} \mathrm{Mt},{ }^{266} \mathrm{Bh}(\alpha)$ [from ${ }^{209} \mathrm{Bi}\left({ }^{70} \mathrm{Zn}\right.$, $\mathrm{n})$ and subsequent decay]; measured $\mathrm{E} \alpha, \mathrm{T}_{1 / 2}$. REPT RIKEN 2004 Annual,P70,Morita

\section{$\mathrm{A}=\mathbf{2 7 1}$}

RADIOACTIVITY ${ }^{271} \mathrm{Sg},{ }^{275} \mathrm{Hs},{ }^{279} \mathrm{Ds},{ }^{282,283,285} 112,{ }^{286,287,288,289} 114$, ${ }^{292,293} 116(\alpha) ;{ }^{267} \mathrm{Rf},{ }^{271} \mathrm{Sg},{ }^{279,281} \mathrm{Ds},{ }^{284} 112,{ }^{286} 114$ (SF) [from ${ }^{233,238} \mathrm{U}$, ${ }^{242} \mathrm{Pu},{ }^{248} \mathrm{Cm}\left({ }^{48} \mathrm{Ca}\right.$, xn $)$ and subsequent decay]; measured $\mathrm{E} \alpha, \mathrm{T}_{1 / 2}$. Comparison with model predictions. JOUR PRVCA 70064609 


\section{$\mathrm{A}=271$ (continued)}

20040GZZ RADIOACTIVITY ${ }^{271} \mathrm{Sg},{ }^{275} \mathrm{Hs},{ }^{279} \mathrm{Ds},{ }^{282,283,285} 112,{ }^{286,287,288,289} 114$, ${ }^{292,293} 116(\alpha) ;{ }^{267} \mathrm{Rf},{ }^{271} \mathrm{Sg},{ }^{279,281} \mathrm{Ds},{ }^{284} 112,{ }^{286} 114$ (SF) [from ${ }^{233,238} \mathrm{U}$, ${ }^{242} \mathrm{Pu},{ }^{248} \mathrm{Cm}\left({ }^{48} \mathrm{Ca}, \mathrm{xn}\right)$ and subsequent decay]; measured $\mathrm{E} \alpha, \mathrm{T}_{1 / 2}$. Comparison with model predictions. REPT

JINR-E7-2004-160,Oganessian

20050G03 RADIOACTIVITY ${ }^{294} 118,{ }^{290,291,292,293} 116,{ }^{287,288,289} 114,{ }^{285} 112$, ${ }^{275} \mathrm{Hs}(\alpha) ;{ }^{286} 114,{ }^{283} 112,{ }^{279} \mathrm{Ds},{ }^{271} \mathrm{Sg}(\alpha),(\mathrm{SF}) ;{ }^{282,284} 112,{ }^{281} \mathrm{Ds}$, ${ }^{267} \mathrm{Rf}(\mathrm{SF}) ;$ measured $\mathrm{E} \alpha, \mathrm{T}_{1 / 2}$, branching ratios. JOUR ZAANE $25 \mathrm{~s} 01$ 589

${ }^{271} \mathrm{Bh} \quad$ 20030GZY RADIOACTIVITY ${ }^{287,288} 115,{ }^{283,284} 113,{ }^{279,280} \mathrm{Rg},{ }^{275,276} \mathrm{Mt}$, ${ }^{272} \mathrm{Bh}(\alpha)$ [from ${ }^{243} \mathrm{Am}\left({ }^{48} \mathrm{Ca}, \mathrm{xn}\right)$ and subsequent decay]; measured $\mathrm{E} \alpha$, $\mathrm{T}_{1 / 2}$; deduced $\mathrm{Q} \alpha .{ }^{267,268} \mathrm{Db}(\mathrm{SF})$ [from ${ }^{243} \mathrm{Am}\left({ }^{48} \mathrm{Ca}\right.$, xn) and subsequent decay]; measured $\mathrm{T}_{1 / 2}$. Comparison with model predictions. REPT JINR-E7-2003-178,Oganessian

20050G02 RADIOACTIVITY ${ }^{287,288} 115,{ }^{283,284} 113,{ }^{279,280} \mathrm{Rg},{ }^{275,276} \mathrm{Mt},{ }^{272} \mathrm{Bh}(\alpha)$ [from ${ }^{243} \mathrm{Am}\left({ }^{48} \mathrm{Ca}, \mathrm{xn}\right)$ and subsequent decay]; measured $\mathrm{E} \alpha, \mathrm{T}_{1 / 2}$;

deduced $\mathrm{Q} \alpha .{ }^{267,268} \mathrm{Db}(\mathrm{SF}) ;$ measured $\mathrm{T}_{1 / 2}$. JOUR PRVCA 72034611

\section{$\mathrm{A}=272$}

${ }^{272} \mathrm{Bh} \quad$ 20030GZY

RADIOACTIVITY ${ }^{287,288} 115,{ }^{283,284} 113,{ }^{279,280} \mathrm{Rg},{ }^{275,276} \mathrm{Mt}$, ${ }^{272} \mathrm{Bh}(\alpha)$ [from ${ }^{243} \mathrm{Am}\left({ }^{48} \mathrm{Ca}, \mathrm{xn}\right)$ and subsequent decay]; measured $\mathrm{E} \alpha$, $\mathrm{T}_{1 / 2}$; deduced $\mathrm{Q} \alpha .{ }^{267,268} \mathrm{Db}(\mathrm{SF})$ [from ${ }^{243} \mathrm{Am}\left({ }^{48} \mathrm{Ca}\right.$, xn) and subsequent decay]; measured $\mathrm{T}_{1 / 2}$. Comparison with model predictions. REPT JINR-E7-2003-178,Oganessian

20050G02 RADIOACTIVITY ${ }^{287,288} 115,{ }^{283,284} 113,{ }^{279,280} \mathrm{Rg},{ }^{275,276} \mathrm{Mt},{ }^{272} \mathrm{Bh}(\alpha)$ [from ${ }^{243} \mathrm{Am}\left({ }^{48} \mathrm{Ca}, \mathrm{xn}\right)$ and subsequent decay]; measured $\mathrm{E} \alpha, \mathrm{T}_{1 / 2}$; deduced $\mathrm{Q} \alpha .{ }^{267,268} \mathrm{Db}(\mathrm{SF})$; measured $\mathrm{T}_{1 / 2}$. JOUR PRVCA 72034611

\section{$\mathrm{A}=\mathbf{2 7 3}$}

${ }^{273} \mathrm{Ds} \quad$ 2004MOZU

RADIOACTIVITY ${ }^{277} 112,{ }^{273} \mathrm{Ds},{ }^{269} \mathrm{Hs},{ }^{265} \mathrm{Sg}(\alpha)$ [from ${ }^{208} \mathrm{~Pb}\left({ }^{70} \mathrm{Zn}, \mathrm{n}\right)$ and subsequent decay]; measured $\mathrm{E} \alpha, \mathrm{T}_{1 / 2} .{ }^{261} \mathrm{Rf}(\mathrm{SF})$; measured $\mathrm{T}_{1 / 2}$. PREPRINT Morita

2005MOZT RADIOACTIVITY ${ }^{277} 112,{ }^{273} \mathrm{Ds},{ }^{269} \mathrm{Hs},{ }^{265} \mathrm{Sg},{ }^{261} \mathrm{Rf}(\alpha)$ [from ${ }^{208} \mathrm{~Pb}\left({ }^{70} \mathrm{Zn}, \mathrm{n}\right)$ and subsequent decay]; measured $\mathrm{E} \alpha, \mathrm{T}_{1 / 2}$. REPT RIKEN 2004 Annual,P69,Morita

\section{$\mathrm{A}=\mathbf{2 7 4}$}

${ }^{274} \mathrm{Rg} \quad$ 2005MOZS

RADIOACTIVITY ${ }^{278} 113,{ }^{274} \mathrm{Rg},{ }^{270} \mathrm{Mt},{ }^{266} \mathrm{Bh}(\alpha)$ [from ${ }^{209} \mathrm{Bi}\left({ }^{70} \mathrm{Zn}\right.$, n) and subsequent decay]; measured $\mathrm{E} \alpha, \mathrm{T}_{1 / 2}$. REPT RIKEN 2004 Annual,P70,Morita 


\section{$\mathrm{A}=\mathbf{2 7 5}$}

${ }^{275} \mathrm{Hs} \quad 20040 \mathrm{G} 12$

20040GZZ

${ }^{275} \mathrm{Mt}$

20030GZY

20050G02

20050G02

${ }^{276} \mathrm{Mt} \quad$ 20030GZY

${ }^{277} 112 \quad$ 2004MOZU

2004MOZU

2005MOZT

2005MOZT
RADIOACTIVITY ${ }^{271} \mathrm{Sg},{ }^{275} \mathrm{Hs},{ }^{279} \mathrm{Ds},{ }^{282,283,285} 112,{ }^{286,287,288,289} 114$, ${ }^{292,293} 116(\alpha) ;{ }^{267} \mathrm{Rf},{ }^{271} \mathrm{Sg},{ }^{279,281} \mathrm{Ds},{ }^{284} 112,{ }^{286} 114$ (SF) [from ${ }^{233,238} \mathrm{U}$, ${ }^{242} \mathrm{Pu},{ }^{248} \mathrm{Cm}\left({ }^{48} \mathrm{Ca}, \mathrm{xn}\right)$ and subsequent decay]; measured $\mathrm{E} \alpha, \mathrm{T}_{1 / 2}$. Comparison with model predictions. JOUR PRVCA 70064609

RADIOACTIVITY ${ }^{271} \mathrm{Sg},{ }^{275} \mathrm{Hs},{ }^{279} \mathrm{Ds},{ }^{282,283,285} 112,{ }^{286,287,288,289} 114$, ${ }^{292,293} 116(\alpha) ;{ }^{267} \mathrm{Rf},{ }^{271} \mathrm{Sg},{ }^{279,281} \mathrm{Ds},{ }^{284} 112,{ }^{286} 114$ (SF) [from ${ }^{233,238} \mathrm{U}$, ${ }^{242} \mathrm{Pu},{ }^{248} \mathrm{Cm}\left({ }^{48} \mathrm{Ca}, \mathrm{xn}\right)$ and subsequent decay]; measured $\mathrm{E} \alpha, \mathrm{T}_{1 / 2}$. Comparison with model predictions. REPT

JINR-E7-2004-160,Oganessian RADIOACTIVITY ${ }^{294} 118,{ }^{290,291,292,293} 116,{ }^{287,288,289} 114,{ }^{285} 112$, ${ }^{275} \mathrm{Hs}(\alpha) ;{ }^{286} 114,{ }^{283} 112,{ }^{279} \mathrm{Ds},{ }^{271} \mathrm{Sg}(\alpha)$, (SF); ${ }^{282,284} 112,{ }^{281} \mathrm{Ds}$, ${ }^{267} \mathrm{Rf}(\mathrm{SF}) ;$ measured $\mathrm{E} \alpha, \mathrm{T}_{1 / 2}$, branching ratios. JOUR ZAANE $25 \mathrm{~s} 01$ 589 RADIOACTIVITY ${ }^{287,288} 115,{ }^{283,284} 113,{ }^{279,280} \mathrm{Rg},{ }^{275,276} \mathrm{Mt}$, ${ }^{272} \mathrm{Bh}(\alpha)$ [from ${ }^{243} \mathrm{Am}\left({ }^{48} \mathrm{Ca}\right.$, xn $)$ and subsequent decay]; measured $\mathrm{E} \alpha$, $\mathrm{T}_{1 / 2}$; deduced Q $\alpha .{ }^{267,268} \mathrm{Db}(\mathrm{SF})$ [from ${ }^{243} \mathrm{Am}\left({ }^{48} \mathrm{Ca}\right.$, xn $)$ and subsequent decay]; measured $\mathrm{T}_{1 / 2}$. Comparison with model predictions. REPT JINR-E7-2003-178,Oganessian RADIOACTIVITY ${ }^{287,288} 115,{ }^{283,284} 113,{ }^{279,280} \mathrm{Rg},{ }^{275,276} \mathrm{Mt},{ }^{272} \mathrm{Bh}(\alpha)$ [from ${ }^{243} \mathrm{Am}\left({ }^{48} \mathrm{Ca}\right.$, xn $)$ and subsequent decay]; measured $\mathrm{E} \alpha, \mathrm{T}_{1 / 2}$; deduced $\mathrm{Q} \alpha .{ }^{267,268} \mathrm{Db}(\mathrm{SF})$; measured $\mathrm{T}_{1 / 2}$. JOUR PRVCA 72034611

\section{$\mathrm{A}=\mathbf{2 7 6}$}

RADIOACTIVITY ${ }^{287,288} 115,{ }^{283,284} 113,{ }^{279,280} \mathrm{Rg},{ }^{275,276} \mathrm{Mt}$, ${ }^{272} \mathrm{Bh}(\alpha)$ [from ${ }^{243} \mathrm{Am}\left({ }^{48} \mathrm{Ca}\right.$, xn $)$ and subsequent decay]; measured $\mathrm{E} \alpha$, $\mathrm{T}_{1 / 2}$; deduced Q $\alpha .{ }^{267,268} \mathrm{Db}(\mathrm{SF})$ [from ${ }^{243} \mathrm{Am}\left({ }^{48} \mathrm{Ca}\right.$, xn $)$ and subsequent decay]; measured $\mathrm{T}_{1 / 2}$. Comparison with model predictions. REPT JINR-E7-2003-178,Oganessian RADIOACTIVITY $287,288115,{ }^{283,284} 113,{ }^{279,280} \mathrm{Rg},{ }^{275,276} \mathrm{Mt},{ }^{272} \mathrm{Bh}(\alpha)$ [from ${ }^{243} \mathrm{Am}\left({ }^{48} \mathrm{Ca}, \mathrm{xn}\right)$ and subsequent decay]; measured $\mathrm{E} \alpha, \mathrm{T}_{1 / 2}$; deduced $\mathrm{Q} \alpha$. ${ }^{267,268} \mathrm{Db}(\mathrm{SF})$; measured $\mathrm{T}_{1 / 2}$. JOUR PRVCA 72034611

\section{$\mathrm{A}=\mathbf{2 7 7}$}

NUCLEAR REACTIONS ${ }^{208} \mathrm{~Pb}\left({ }^{70} \mathrm{Zn}, \mathrm{n}\right), \mathrm{E}=349.5 \mathrm{MeV}$; measured delayed $\alpha \alpha$-coin; deduced production $\sigma$. PREPRINT Morita RADIOACTIVITY ${ }^{277} 112,{ }^{273} \mathrm{Ds},{ }^{269} \mathrm{Hs},{ }^{265} \mathrm{Sg}(\alpha)$ [from ${ }^{208} \mathrm{~Pb}\left({ }^{70} \mathrm{Zn}, \mathrm{n}\right)$ and subsequent decay]; measured $\mathrm{E} \alpha, \mathrm{T}_{1 / 2} \cdot{ }^{261} \mathrm{Rf}(\mathrm{SF})$; measured $\mathrm{T}_{1 / 2}$. PREPRINT Morita NUCLEAR REACTIONS ${ }^{208} \mathrm{~Pb}\left({ }^{70} \mathrm{Zn}, \mathrm{n}\right), \mathrm{E}=349.5 \mathrm{MeV}$; measured delayed $\alpha \alpha$-coin; deduced production $\sigma$. REPT RIKEN 2004 Annual,P69,Morita

RADIOACTIVITY ${ }^{277} 112,{ }^{273} \mathrm{Ds},{ }^{269} \mathrm{Hs},{ }^{265} \mathrm{Sg},{ }^{261} \mathrm{Rf}(\alpha)$ [from ${ }^{208} \mathrm{~Pb}\left({ }^{70} \mathrm{Zn}, \mathrm{n}\right)$ and subsequent decay]; measured $\mathrm{E} \alpha, \mathrm{T}_{1 / 2}$. REPT RIKEN 2004 Annual,P69,Morita 


\section{$\mathrm{A}=\mathbf{2 7 8}$}

${ }^{278} \mathrm{Ds}$

20040G12

${ }^{278} 113 \quad 2005 \mathrm{MOZS}$

2005MOZS

${ }^{279} \mathrm{Ds}$

20040G12

20040GZZ

20030GZY

20050G02

20050G03
20040GZZ

RADIOACTIVITY ${ }^{271} \mathrm{Sg},{ }^{275} \mathrm{Hs},{ }^{279} \mathrm{Ds},{ }^{282,283,285} 112,{ }^{286,287,288,289} 114$, ${ }^{292,293} 116(\alpha) ;{ }^{267} \mathrm{Rf},{ }^{271} \mathrm{Sg},{ }^{279,281} \mathrm{Ds},{ }^{284} 112,{ }^{286} 114(\mathrm{SF})$ [from ${ }^{233,238} \mathrm{U}$, ${ }^{242} \mathrm{Pu},{ }^{248} \mathrm{Cm}\left({ }^{48} \mathrm{Ca}, \mathrm{xn}\right)$ and subsequent decay]; measured $\mathrm{E} \alpha, \mathrm{T}_{1 / 2}$. Comparison with model predictions. JOUR PRVCA 70064609

RADIOACTIVITY ${ }^{271} \mathrm{Sg},{ }^{275} \mathrm{Hs},{ }^{279} \mathrm{Ds},{ }^{282,283,285} 112,{ }^{286,287,288,289} 114$, ${ }^{292,293} 116(\alpha) ;{ }^{267} \mathrm{Rf},{ }^{271} \mathrm{Sg},{ }^{279,281} \mathrm{Ds},{ }^{284} 112,{ }^{286} 114(\mathrm{SF})$ [from ${ }^{233,238} \mathrm{U}$, ${ }^{242} \mathrm{Pu},{ }^{248} \mathrm{Cm}\left({ }^{48} \mathrm{Ca}, \mathrm{xn}\right)$ and subsequent decay $]$; measured $\mathrm{E} \alpha, \mathrm{T}_{1 / 2}$. Comparison with model predictions. REPT

JINR-E7-2004-160,Oganessian

NUCLEAR REACTIONS ${ }^{209} \mathrm{Bi}\left({ }^{70} \mathrm{Zn}, \mathrm{n}\right), \mathrm{E}=349.0 \mathrm{MeV}$; measured delayed $\alpha \alpha$-coin; deduced production $\sigma$. REPT RIKEN 2004 Annual,P70,Morita RADIOACTIVITY ${ }^{278} 113,{ }^{274} \mathrm{Rg},{ }^{270} \mathrm{Mt},{ }^{266} \mathrm{Bh}(\alpha)$ [from ${ }^{209} \mathrm{Bi}\left({ }^{70} \mathrm{Zn}\right.$, n) and subsequent decay]; measured E $\alpha, T_{1 / 2}$. REPT RIKEN 2004 Annual,P70,Morita

\section{$\mathrm{A}=279$}

RADIOACTIVITY ${ }^{271} \mathrm{Sg},{ }^{275} \mathrm{Hs},{ }^{279} \mathrm{Ds},{ }^{282,283,285} 112,{ }^{286,287,288,289} 114$, ${ }^{292,293} 116(\alpha) ;{ }^{267} \mathrm{Rf},{ }^{271} \mathrm{Sg},{ }^{279,281} \mathrm{Ds},{ }^{284} 112,{ }^{286} 114(\mathrm{SF})$ [from ${ }^{233,238} \mathrm{U}$, ${ }^{242} \mathrm{Pu},{ }^{248} \mathrm{Cm}\left({ }^{48} \mathrm{Ca}, \mathrm{xn}\right)$ and subsequent decay]; measured $\mathrm{E} \alpha, \mathrm{T}_{1 / 2}$. Comparison with model predictions. JOUR PRVCA 70064609 RADIOACTIVITY ${ }^{271} \mathrm{Sg},{ }^{275} \mathrm{Hs},{ }^{279} \mathrm{Ds},{ }^{282,283,285} 112,{ }^{286,287,288,289} 114$, ${ }^{292,293} 116(\alpha) ;{ }^{267} \mathrm{Rf},{ }^{271} \mathrm{Sg},{ }^{279,281} \mathrm{Ds},{ }^{284} 112,{ }^{286} 114(\mathrm{SF})$ [from ${ }^{233,238} \mathrm{U}$, ${ }^{242} \mathrm{Pu},{ }^{248} \mathrm{Cm}\left({ }^{48} \mathrm{Ca}, \mathrm{xn}\right)$ and subsequent decay]; measured $\mathrm{E} \alpha, \mathrm{T}_{1 / 2}$. Comparison with model predictions. REPT

JINR-E7-2004-160,Oganessian RADIOACTIVITY ${ }^{294} 118,{ }^{290,291,292,293} 116,{ }^{287,288,289} 114,{ }^{285} 112$, ${ }^{275} \mathrm{Hs}(\alpha) ;{ }^{286} 114,{ }^{283} 112,{ }^{279} \mathrm{Ds},{ }^{271} \mathrm{Sg}(\alpha),(\mathrm{SF}) ;{ }^{282,284} 112,{ }^{281} \mathrm{Ds}$, ${ }^{267} \mathrm{Rf}(\mathrm{SF})$; measured $\mathrm{E} \alpha, \mathrm{T}_{1 / 2}$, branching ratios. JOUR ZAANE 25 s01 589

RADIOACTIVITY ${ }^{287,288} 115,{ }^{283,284} 113,{ }^{279,280} \mathrm{Rg},{ }^{275,276} \mathrm{Mt}$, ${ }^{272} \mathrm{Bh}(\alpha)$ [from ${ }^{243} \mathrm{Am}\left({ }^{48} \mathrm{Ca}, \mathrm{xn}\right)$ and subsequent decay]; measured $\mathrm{E} \alpha$, $\mathrm{T}_{1 / 2}$; deduced Q $\alpha .{ }^{267,268} \mathrm{Db}(\mathrm{SF})\left[\right.$ from ${ }^{243} \mathrm{Am}\left({ }^{48} \mathrm{Ca}, \mathrm{xn}\right)$ and subsequent decay]; measured $\mathrm{T}_{1 / 2}$. Comparison with model predictions. REPT JINR-E7-2003-178,Oganessian RADIOACTIVITY ${ }^{287,288} 115,{ }^{283,284} 113,{ }^{279,280} \mathrm{Rg},{ }^{275,276} \mathrm{Mt},{ }^{272} \mathrm{Bh}(\alpha)$ [from ${ }^{243} \mathrm{Am}\left({ }^{48} \mathrm{Ca}, \mathrm{xn}\right)$ and subsequent decay]; measured $\mathrm{E} \alpha, \mathrm{T}_{1 / 2}$; deduced $\mathrm{Q} \alpha .{ }^{267,268} \mathrm{Db}(\mathrm{SF})$; measured $\mathrm{T}_{1 / 2}$. JOUR PRVCA 72034611

\section{$\mathrm{A}=280$}

${ }^{280} \mathrm{Rg} \quad 20030 \mathrm{GZY}$

RADIOACTIVITY ${ }^{287,288} 115,{ }^{283,284} 113,{ }^{279,280} \mathrm{Rg},{ }^{275,276} \mathrm{Mt}$, ${ }^{272} \mathrm{Bh}(\alpha)$ [from ${ }^{243} \mathrm{Am}\left({ }^{48} \mathrm{Ca}, \mathrm{xn}\right)$ and subsequent decay]; measured $\mathrm{E} \alpha$, $\mathrm{T}_{1 / 2}$; deduced Q $\alpha .{ }^{267,268} \mathrm{Db}(\mathrm{SF})\left[\right.$ from ${ }^{243} \mathrm{Am}\left({ }^{48} \mathrm{Ca}, \mathrm{xn}\right)$ and subsequent decay]; measured $\mathrm{T}_{1 / 2}$. Comparison with model predictions. REPT JINR-E7-2003-178,Oganessian 
$\mathrm{A}=\mathbf{2 8 0}$ (continued)

20050G02 RADIOACTIVITY ${ }^{287,288} 115,{ }^{283,284} 113,{ }^{279,280} \mathrm{Rg},{ }^{275,276} \mathrm{Mt},{ }^{272} \mathrm{Bh}(\alpha)$ [from ${ }^{243} \mathrm{Am}\left({ }^{48} \mathrm{Ca}, \mathrm{xn}\right)$ and subsequent decay]; measured $\mathrm{E} \alpha, \mathrm{T}_{1 / 2}$; deduced $\mathrm{Q} \alpha .{ }^{267,268} \mathrm{Db}(\mathrm{SF})$; measured $\mathrm{T}_{1 / 2}$. JOUR PRVCA 72034611

\section{$\mathrm{A}=281$}

${ }^{281} \mathrm{Ds} \quad 20040 \mathrm{G} 12$

RADIOACTIVITY ${ }^{271} \mathrm{Sg},{ }^{275} \mathrm{Hs},{ }^{279} \mathrm{Ds},{ }^{282,283,285} 112,{ }^{286,287,288,289} 114$, ${ }^{292,293} 116(\alpha) ;{ }^{267} \mathrm{Rf},{ }^{271} \mathrm{Sg},{ }^{279,281} \mathrm{Ds},{ }^{284} 112,{ }^{286} 114$ (SF) [from ${ }^{233,238} \mathrm{U}$, ${ }^{242} \mathrm{Pu},{ }^{248} \mathrm{Cm}\left({ }^{48} \mathrm{Ca}, \mathrm{xn}\right)$ and subsequent decay]; measured $\mathrm{E} \alpha, \mathrm{T}_{1 / 2}$. Comparison with model predictions. JOUR PRVCA 70064609

20040GZZ RADIOACTIVITY ${ }^{271} \mathrm{Sg},{ }^{275} \mathrm{Hs},{ }^{279} \mathrm{Ds},{ }^{282,283,285} 112,{ }^{286,287,288,289} 114$, ${ }^{292,293} 116(\alpha) ;{ }^{267} \mathrm{Rf},{ }^{271} \mathrm{Sg},{ }^{279,281} \mathrm{Ds},{ }^{284} 112,{ }^{286} 114$ (SF) [from ${ }^{233,238} \mathrm{U}$, ${ }^{242} \mathrm{Pu},{ }^{248} \mathrm{Cm}\left({ }^{48} \mathrm{Ca}, \mathrm{xn}\right)$ and subsequent decay]; measured $\mathrm{E} \alpha, \mathrm{T}_{1 / 2}$. Comparison with model predictions. REPT

JINR-E7-2004-160, Oganessian

20050G03 RADIOACTIVITY ${ }^{294} 118,{ }^{290,291,292,293} 116,{ }^{287,288,289} 114,{ }^{285} 112$, ${ }^{275} \mathrm{Hs}(\alpha) ;{ }^{286} 114,{ }^{283} 112,{ }^{279} \mathrm{Ds},{ }^{271} \mathrm{Sg}(\alpha)$, (SF); ${ }^{282,284} 112,{ }^{281} \mathrm{Ds}$, ${ }^{267} \mathrm{Rf}(\mathrm{SF})$; measured $\mathrm{E} \alpha, \mathrm{T}_{1 / 2}$, branching ratios. JOUR ZAANE 25 s01 589

\section{$\mathrm{A}=\mathbf{2 8 2}$}

${ }^{282} 112$ 20030GzZ RADIOACTIVITY ${ }^{294} 118,{ }^{290} 116(\alpha),{ }^{286} 114(\alpha)$, (SF) [from ${ }^{249} \mathrm{Cf}\left({ }^{48} \mathrm{Ca}, 3 \mathrm{n}\right)$ and subsequent decay]; measured $\mathrm{E} \alpha, \mathrm{T}_{1 / 2}$, fission fragment spectra. Comparison with model predictions. REPT UCRL-ID-151619, Oganessian

$20040 \mathrm{G} 12$ RADIOACTIVITY ${ }^{271} \mathrm{Sg},{ }^{275} \mathrm{Hs},{ }^{279} \mathrm{Ds},{ }^{282,283,285} 112,{ }^{286,287,288,289} 114$, ${ }^{292,293} 116(\alpha) ;{ }^{267} \mathrm{Rf},{ }^{271} \mathrm{Sg},{ }^{279,281} \mathrm{Ds},{ }^{284} 112,{ }^{286} 114$ (SF) [from ${ }^{233,238} \mathrm{U}$, ${ }^{242} \mathrm{Pu},{ }^{248} \mathrm{Cm}\left({ }^{48} \mathrm{Ca}, \mathrm{xn}\right)$ and subsequent decay]; measured $\mathrm{E} \alpha, \mathrm{T}_{1 / 2}$. Comparison with model predictions. JOUR PRVCA 70064609

20040GZZ RADIOACTIVITY ${ }^{271} \mathrm{Sg},{ }^{275} \mathrm{Hs},{ }^{279} \mathrm{Ds},{ }^{282,283,285} 112,{ }^{286,287,288,289} 114$, ${ }^{292,293} 116(\alpha) ;{ }^{267} \mathrm{Rf},{ }^{271} \mathrm{Sg},{ }^{279,281} \mathrm{Ds},{ }^{284} 112,{ }^{286} 114$ (SF) [from ${ }^{233,238} \mathrm{U}$, ${ }^{242} \mathrm{Pu},{ }^{248} \mathrm{Cm}\left({ }^{48} \mathrm{Ca}, \mathrm{xn}\right)$ and subsequent decay]; measured $\mathrm{E} \alpha, \mathrm{T}_{1 / 2}$. Comparison with model predictions. REPT

JINR-E7-2004-160, Oganessian

$20050 G 03 \quad$ NUCLEAR REACTIONS ${ }^{238} \mathrm{U}\left({ }^{48} \mathrm{Ca}, 3 \mathrm{n}\right),\left({ }^{48} \mathrm{Ca}, 4 \mathrm{n}\right),{ }^{233} \mathrm{U}$, ${ }^{242} \mathrm{Pu}\left({ }^{48} \mathrm{Ca}, 2 \mathrm{n}\right),\left({ }^{48} \mathrm{Ca}, 3 \mathrm{n}\right),\left({ }^{48} \mathrm{Ca}, 4 \mathrm{n}\right), \mathrm{E} \approx 230-250 \mathrm{MeV}$; measured $\sigma$. JOUR ZAANE 25 s01 589

20050G03 RADIOACTIVITY ${ }^{294} 118,{ }^{290,291,292,293} 116,{ }^{287,288,289} 114,{ }^{285} 112$, ${ }^{275} \mathrm{Hs}(\alpha) ;{ }^{286} 114,{ }^{283} 112,{ }^{279} \mathrm{Ds},{ }^{271} \mathrm{Sg}(\alpha),(\mathrm{SF}) ;{ }^{282,284} 112,{ }^{281} \mathrm{Ds}$, ${ }^{267} \mathrm{Rf}(\mathrm{SF}) ;$ measured $\mathrm{E} \alpha, \mathrm{T}_{1 / 2}$, branching ratios. JOUR ZAANE $25 \mathrm{~s} 01$ 589 


\section{$\mathrm{A}=283$}

${ }^{283} 112$ 2003YA22 NUCLEAR REACTIONS ${ }^{238} \mathrm{U}\left({ }^{48} \mathrm{Ca}, 3 \mathrm{n}\right), \mathrm{E} \approx 233 \mathrm{MeV}$; measured radiochemical yield; deduced chemical properties. JOUR RAACA 91 433

$20040 G 12$ RADIOACTIVITY ${ }^{271} \mathrm{Sg},{ }^{275} \mathrm{Hs},{ }^{279} \mathrm{Ds},{ }^{282,283,285} 112,{ }^{286,287,288,289} 114$, ${ }^{292,293} 116(\alpha) ;{ }^{267} \mathrm{Rf},{ }^{271} \mathrm{Sg},{ }^{279,281} \mathrm{Ds},{ }^{284} 112,{ }^{286} 114$ (SF) [from ${ }^{233,238} \mathrm{U}$, ${ }^{242} \mathrm{Pu},{ }^{248} \mathrm{Cm}\left({ }^{48} \mathrm{Ca}, \mathrm{xn}\right)$ and subsequent decay]; measured $\mathrm{E} \alpha, \mathrm{T}_{1 / 2}$. Comparison with model predictions. JOUR PRVCA 70064609

20040GZZ RADIOACTIVITY ${ }^{271} \mathrm{Sg},{ }^{275} \mathrm{Hs},{ }^{279} \mathrm{Ds},{ }^{282,283,285} 112,{ }^{286,287,288,289} 114$, ${ }^{292,293} 116(\alpha) ;{ }^{267} \mathrm{Rf},{ }^{271} \mathrm{Sg},{ }^{279,281} \mathrm{Ds},{ }^{284} 112,{ }^{286} 114$ (SF) [from ${ }^{233,238} \mathrm{U}$, ${ }^{242} \mathrm{Pu},{ }^{248} \mathrm{Cm}\left({ }^{48} \mathrm{Ca}, \mathrm{xn}\right)$ and subsequent decay]; measured $\mathrm{E} \alpha, \mathrm{T}_{1 / 2}$. Comparison with model predictions. REPT

JINR-E7-2004-160,Oganessian

2005GR19 NUCLEAR REACTIONS ${ }^{238} \mathrm{U}\left({ }^{48} \mathrm{Ca}, 3 \mathrm{n}\right), \mathrm{E}=230.3,235.6 \mathrm{MeV}$; measured $\sigma$ upper limits; deduced no evidence for ${ }^{283} 112$. Comparison with previous results. JOUR PRVCA 72014605

2005HOZX NUCLEAR REACTIONS ${ }^{238} \mathrm{U}\left({ }^{48} \mathrm{Ca}, \mathrm{xn}\right), \mathrm{E}=233,236,239 \mathrm{MeV}$; measured fission fragment spectra; deduced evidence for ${ }^{283} 112$.

PREPRINT Hofmann

$20050 G 03 \quad$ NUCLEAR REACTIONS ${ }^{238} \mathrm{U}\left({ }^{48} \mathrm{Ca}, 3 \mathrm{n}\right),\left({ }^{48} \mathrm{Ca}, 4 \mathrm{n}\right),{ }^{233} \mathrm{U}$, ${ }^{242} \mathrm{Pu}\left({ }^{48} \mathrm{Ca}, 2 \mathrm{n}\right),\left({ }^{48} \mathrm{Ca}, 3 \mathrm{n}\right),\left({ }^{48} \mathrm{Ca}, 4 \mathrm{n}\right), \mathrm{E} \approx 230-250 \mathrm{MeV}$; measured $\sigma$. JOUR ZAANE $25 \mathrm{~s} 01589$

20050G03 RADIOACTIVITY ${ }^{294} 118,{ }^{290,291,292,293} 116,{ }^{287,288,289} 114,{ }^{285} 112$, ${ }^{275} \mathrm{Hs}(\alpha) ;{ }^{286} 114,{ }^{283} 112,{ }^{279} \mathrm{Ds},{ }^{271} \mathrm{Sg}(\alpha),(\mathrm{SF}) ;{ }^{282,284} 112,{ }^{281} \mathrm{Ds}$, ${ }^{267} \mathrm{Rf}(\mathrm{SF})$; measured $\mathrm{E} \alpha, \mathrm{T}_{1 / 2}$, branching ratios. JOUR ZAANE 25 s01 589

${ }^{283} 113$ 20030GZY RADIOACTIVITY ${ }^{287,288} 115,{ }^{283,284} 113,{ }^{279,280} \mathrm{Rg},{ }^{275,276} \mathrm{Mt}$, ${ }^{272} \mathrm{Bh}(\alpha)$ [from ${ }^{243} \mathrm{Am}\left({ }^{48} \mathrm{Ca}\right.$, xn $)$ and subsequent decay]; measured $\mathrm{E} \alpha$, $\mathrm{T}_{1 / 2}$; deduced $\mathrm{Q} \alpha .{ }^{267,268} \mathrm{Db}(\mathrm{SF})$ [from ${ }^{243} \mathrm{Am}\left({ }^{48} \mathrm{Ca}\right.$, xn $)$ and subsequent decay]; measured $\mathrm{T}_{1 / 2}$. Comparison with model predictions. REPT JINR-E7-2003-178,Oganessian

20050G02 RADIOACTIVITY ${ }^{287,288} 115,{ }^{283,284} 113,{ }^{279,280} \mathrm{Rg},{ }^{275,276} \mathrm{Mt},{ }^{272} \mathrm{Bh}(\alpha)$ [from ${ }^{243} \mathrm{Am}\left({ }^{48} \mathrm{Ca}, \mathrm{xn}\right)$ and subsequent decay]; measured $\mathrm{E} \alpha, \mathrm{T}_{1 / 2}$; deduced $\mathrm{Q} \alpha .{ }^{267,268} \mathrm{Db}(\mathrm{SF}) ;$ measured $\mathrm{T}_{1 / 2}$. JOUR PRVCA 72034611

\section{$\mathrm{A}=284$}

${ }^{284} 112 \quad 20040 G 12$

RADIOACTIVITY ${ }^{271} \mathrm{Sg},{ }^{275} \mathrm{Hs},{ }^{279} \mathrm{Ds},{ }^{282,283,285} 112,{ }^{286,287,288,289} 114$, ${ }^{292,293} 116(\alpha) ;{ }^{267} \mathrm{Rf},{ }^{271} \mathrm{Sg},{ }^{279,281} \mathrm{Ds},{ }^{284} 112,{ }^{286} 114$ (SF) [from ${ }^{233,238} \mathrm{U}$, ${ }^{242} \mathrm{Pu},{ }^{248} \mathrm{Cm}\left({ }^{48} \mathrm{Ca}, \mathrm{xn}\right)$ and subsequent decay]; measured $\mathrm{E} \alpha, \mathrm{T}_{1 / 2}$. Comparison with model predictions. JOUR PRVCA 70064609

20040GZZ RADIOACTIVITY ${ }^{271} \mathrm{Sg},{ }^{275} \mathrm{Hs},{ }^{279} \mathrm{Ds},{ }^{282,283,285} 112,{ }^{286,287,288,289} 114$, ${ }^{292,293} 116(\alpha) ;{ }^{267} \mathrm{Rf},{ }^{271} \mathrm{Sg},{ }^{279,281} \mathrm{Ds},{ }^{284} 112,{ }^{286} 114(\mathrm{SF})$ [from ${ }^{233,238} \mathrm{U}$, ${ }^{242} \mathrm{Pu},{ }^{248} \mathrm{Cm}\left({ }^{48} \mathrm{Ca}\right.$, xn $)$ and subsequent decay]; measured $\mathrm{E} \alpha, \mathrm{T}_{1 / 2}$. Comparison with model predictions. REPT

JINR-E7-2004-160,Oganessian 


\section{$\mathrm{A}=284$ (continued)}

20050G03 RADIOACTIVITY ${ }^{294} 118,{ }^{290,291,292,293} 116,{ }^{287,288,289} 114,{ }^{285} 112$, ${ }^{275} \mathrm{Hs}(\alpha) ;{ }^{286} 114,{ }^{283} 112,{ }^{279} \mathrm{Ds},{ }^{271} \mathrm{Sg}(\alpha)$, (SF); ${ }^{282,284} 112,{ }^{281} \mathrm{Ds}$, ${ }^{267} \mathrm{Rf}(\mathrm{SF}) ;$ measured $\mathrm{E} \alpha, \mathrm{T}_{1 / 2}$, branching ratios. JOUR ZAANE $25 \mathrm{~s} 01$ 589

${ }^{284} 113$ 20030GZY RADIOACTIVITY ${ }^{287,288} 115,{ }^{283,284} 113,{ }^{279,280} \mathrm{Rg},{ }^{275,276} \mathrm{Mt}$, ${ }^{272} \mathrm{Bh}(\alpha)$ [from ${ }^{243} \mathrm{Am}\left({ }^{48} \mathrm{Ca}\right.$, xn $)$ and subsequent decay]; measured $\mathrm{E} \alpha$, $\mathrm{T}_{1 / 2}$; deduced $\mathrm{Q} \alpha .{ }^{267,268} \mathrm{Db}(\mathrm{SF})\left[\right.$ from ${ }^{243} \mathrm{Am}\left({ }^{48} \mathrm{Ca}, \mathrm{xn}\right)$ and subsequent decay]; measured $\mathrm{T}_{1 / 2}$. Comparison with model predictions. REPT JINR-E7-2003-178,Oganessian 20050G02 RADIOACTIVITY ${ }^{287,288} 115,{ }^{283,284} 113,{ }^{279,280} \mathrm{Rg},{ }^{275,276} \mathrm{Mt},{ }^{272} \mathrm{Bh}(\alpha)$ [from ${ }^{243} \mathrm{Am}\left({ }^{48} \mathrm{Ca}, \mathrm{xn}\right)$ and subsequent decay]; measured $\mathrm{E} \alpha, \mathrm{T}_{1 / 2}$; deduced $\mathrm{Q} \alpha .{ }^{267,268} \mathrm{Db}(\mathrm{SF}) ;$ measured $\mathrm{T}_{1 / 2}$. JOUR PRVCA 72034611

\section{$\mathrm{A}=\mathbf{2 8 5}$}

$285112 \quad 20040 G 12$

RADIOACTIVITY ${ }^{271} \mathrm{Sg},{ }^{275} \mathrm{Hs},{ }^{279} \mathrm{Ds},{ }^{282,283,285} 112,{ }^{286,287,288,289} 114$, ${ }^{292,293} 116(\alpha) ;{ }^{267} \mathrm{Rf},{ }^{271} \mathrm{Sg},{ }^{279,281} \mathrm{Ds},{ }^{284} 112,{ }^{286} 114$ (SF) [from ${ }^{233,238} \mathrm{U}$, ${ }^{242} \mathrm{Pu},{ }^{248} \mathrm{Cm}\left({ }^{48} \mathrm{Ca}, \mathrm{xn}\right)$ and subsequent decay]; measured $\mathrm{E} \alpha, \mathrm{T}_{1 / 2}$. Comparison with model predictions. JOUR PRVCA 70064609

20040GZZ RADIOACTIVITY ${ }^{271} \mathrm{Sg},{ }^{275} \mathrm{Hs},{ }^{279} \mathrm{Ds},{ }^{282,283,285} 112,{ }^{286,287,288,289} 114$, ${ }^{292,293} 116(\alpha) ;{ }^{267} \mathrm{Rf},{ }^{271} \mathrm{Sg},{ }^{279,281} \mathrm{Ds},{ }^{284} 112,{ }^{286} 114$ (SF) [from ${ }^{233,238} \mathrm{U}$, ${ }^{242} \mathrm{Pu},{ }^{248} \mathrm{Cm}\left({ }^{48} \mathrm{Ca}, \mathrm{xn}\right)$ and subsequent decay]; measured $\mathrm{E} \alpha, \mathrm{T}_{1 / 2}$. Comparison with model predictions. REPT JINR-E7-2004-160,Oganessian 20050G03 RADIOACTIVITY ${ }^{294} 118,{ }^{290,291,292,293} 116,{ }^{287,288,289} 114,{ }^{285} 112$, ${ }^{275} \mathrm{Hs}(\alpha) ;{ }^{286} 114,{ }^{283} 112,{ }^{279} \mathrm{Ds},{ }^{271} \mathrm{Sg}(\alpha)$, (SF); ${ }^{282,284} 112,{ }^{281} \mathrm{Ds}$, ${ }^{267} \mathrm{Rf}(\mathrm{SF}) ;$ measured $\mathrm{E} \alpha, \mathrm{T}_{1 / 2}$, branching ratios. JOUR ZAANE $25 \mathrm{~s} 01$ 589

\section{$\mathrm{A}=\mathbf{2 8 6}$}

$20030 \mathrm{GZZ}$

RADIOACTIVITY ${ }^{294} 118,{ }^{290} 116(\alpha),{ }^{286} 114(\alpha)$, (SF) [from ${ }^{249} \mathrm{Cf}\left({ }^{48} \mathrm{Ca}, 3 \mathrm{n}\right)$ and subsequent decay]; measured $\mathrm{E} \alpha, \mathrm{T}_{1 / 2}$, fission fragment spectra. Comparison with model predictions. REPT UCRL-ID-151619, Oganessian $20040 \mathrm{G12}$ RADIOACTIVITY ${ }^{271} \mathrm{Sg},{ }^{275} \mathrm{Hs},{ }^{279} \mathrm{Ds},{ }^{282,283,285} 112,{ }^{286,287,288,289} 114$, ${ }^{292,293} 116(\alpha) ;{ }^{267} \mathrm{Rf},{ }^{271} \mathrm{Sg},{ }^{279,281} \mathrm{Ds},{ }^{284} 112,{ }^{286} 114$ (SF) [from ${ }^{233,238} \mathrm{U}$, ${ }^{242} \mathrm{Pu},{ }^{248} \mathrm{Cm}\left({ }^{48} \mathrm{Ca}, \mathrm{xn}\right)$ and subsequent decay]; measured $\mathrm{E} \alpha, \mathrm{T}_{1 / 2}$. Comparison with model predictions. JOUR PRVCA 70064609

20040GZZ RADIOACTIVITY ${ }^{271} \mathrm{Sg},{ }^{275} \mathrm{Hs},{ }^{279} \mathrm{Ds},{ }^{282,283,285} 112,{ }^{286,287,288,289} 114$, ${ }^{292,293} 116(\alpha) ;{ }^{267} \mathrm{Rf},{ }^{271} \mathrm{Sg},{ }^{279,281} \mathrm{Ds},{ }^{284} 112,{ }^{286} 114$ (SF) [from ${ }^{233,238} \mathrm{U}$, ${ }^{242} \mathrm{Pu},{ }^{248} \mathrm{Cm}\left({ }^{48} \mathrm{Ca}, \mathrm{xn}\right)$ and subsequent decay]; measured $\mathrm{E} \alpha, \mathrm{T}_{1 / 2}$. Comparison with model predictions. REPT JINR-E7-2004-160,Oganessian $20050 G 03 \quad$ NUCLEAR REACTIONS ${ }^{238} \mathrm{U}\left({ }^{48} \mathrm{Ca}, 3 \mathrm{n}\right),\left({ }^{48} \mathrm{Ca}, 4 \mathrm{n}\right),{ }^{233} \mathrm{U}$, ${ }^{242} \mathrm{Pu}\left({ }^{48} \mathrm{Ca}, 2 \mathrm{n}\right),\left({ }^{48} \mathrm{Ca}, 3 \mathrm{n}\right),\left({ }^{48} \mathrm{Ca}, 4 \mathrm{n}\right), \mathrm{E} \approx 230-250 \mathrm{MeV}$; measured $\sigma$. JOUR ZAANE 25 s01 589 


\section{$\mathrm{A}=286$ (continued)}

20050G03 RADIOACTIVITY ${ }^{294} 118,{ }^{290,291,292,293} 116,{ }^{287,288,289} 114,{ }^{285} 112$, ${ }^{275} \mathrm{Hs}(\alpha) ;{ }^{286} 114,{ }^{283} 112,{ }^{279} \mathrm{Ds},{ }^{271} \mathrm{Sg}(\alpha)$, (SF); ${ }^{282,284} 112,{ }^{281} \mathrm{Ds}$, ${ }^{267} \mathrm{Rf}(\mathrm{SF}) ;$ measured $\mathrm{E} \alpha, \mathrm{T}_{1 / 2}$, branching ratios. JOUR ZAANE $25 \mathrm{~s} 01$ 589

\section{$\mathrm{A}=287$}

$280040 G 12$

RADIOACTIVITY ${ }^{271} \mathrm{Sg},{ }^{275} \mathrm{Hs},{ }^{279} \mathrm{Ds},{ }^{282,283,285} 112,{ }^{286,287,288,289} 114$, ${ }^{292,293} 116(\alpha) ;{ }^{267} \mathrm{Rf},{ }^{271} \mathrm{Sg},{ }^{279,281} \mathrm{Ds},{ }^{284} 112,{ }^{286} 114$ (SF) [from ${ }^{233,238} \mathrm{U}$, ${ }^{242} \mathrm{Pu},{ }^{248} \mathrm{Cm}\left({ }^{48} \mathrm{Ca}, \mathrm{xn}\right)$ and subsequent decay]; measured $\mathrm{E} \alpha, \mathrm{T}_{1 / 2}$. Comparison with model predictions. JOUR PRVCA 70064609

20040GZZ RADIOACTIVITY ${ }^{271} \mathrm{Sg},{ }^{275} \mathrm{Hs},{ }^{279} \mathrm{Ds},{ }^{282,283,285} 112,{ }^{286,287,288,289} 114$, ${ }^{292,293} 116(\alpha) ;{ }^{267} \mathrm{Rf},{ }^{271} \mathrm{Sg},{ }^{279,281} \mathrm{Ds},{ }^{284} 112,{ }^{286} 114$ (SF) [from ${ }^{233,238} \mathrm{U}$, ${ }^{242} \mathrm{Pu},{ }^{248} \mathrm{Cm}\left({ }^{48} \mathrm{Ca}, \mathrm{xn}\right)$ and subsequent decay]; measured $\mathrm{E} \alpha, \mathrm{T}_{1 / 2}$. Comparison with model predictions. REPT

JINR-E7-2004-160, Oganessian

$20050 G 03 \quad$ NUCLEAR REACTIONS ${ }^{238} \mathrm{U}\left({ }^{48} \mathrm{Ca}, 3 \mathrm{n}\right),\left({ }^{48} \mathrm{Ca}, 4 \mathrm{n}\right),{ }^{233} \mathrm{U}$, ${ }^{242} \mathrm{Pu}\left({ }^{48} \mathrm{Ca}, 2 \mathrm{n}\right),\left({ }^{48} \mathrm{Ca}, 3 \mathrm{n}\right),\left({ }^{48} \mathrm{Ca}, 4 \mathrm{n}\right), \mathrm{E} \approx 230-250 \mathrm{MeV}$; measured $\sigma$. JOUR ZAANE 25 s01 589

20050G03 RADIOACTIVITY ${ }^{294} 118,{ }^{290,291,292,293} 116,{ }^{287,288,289} 114,{ }^{285} 112$, ${ }^{275} \mathrm{Hs}(\alpha) ;{ }^{286} 114,{ }^{283} 112,{ }^{279} \mathrm{Ds},{ }^{271} \mathrm{Sg}(\alpha),(\mathrm{SF}) ;{ }^{282,284} 112,{ }^{281} \mathrm{Ds}$, ${ }^{267} \mathrm{Rf}(\mathrm{SF}) ;$ measured $\mathrm{E} \alpha, \mathrm{T}_{1 / 2}$, branching ratios. JOUR ZAANE $25 \mathrm{~s} 01$ 589

${ }^{287} 115$ 20030GzY NUCLEAR REACTIONS ${ }^{243} \mathrm{Am}\left({ }^{48} \mathrm{Ca}, 3 \mathrm{n}\right),\left({ }^{48} \mathrm{Ca}, 4 \mathrm{n}\right), \mathrm{E}=253 \mathrm{MeV}$; measured (recoil) $\alpha-, \alpha \alpha$-coin following residual nucleus decay; deduced production $\sigma$. REPT JINR-E7-2003-178,Oganessian

20030GZY RADIOACTIVITY ${ }^{287,288} 115,{ }^{283,284} 113,{ }^{279,280} \mathrm{Rg},{ }^{275,276} \mathrm{Mt}$, ${ }^{272} \mathrm{Bh}(\alpha)$ [from ${ }^{243} \mathrm{Am}\left({ }^{48} \mathrm{Ca}\right.$, xn $)$ and subsequent decay]; measured $\mathrm{E} \alpha$, $\mathrm{T}_{1 / 2}$; deduced $\mathrm{Q} \alpha .{ }^{267,268} \mathrm{Db}(\mathrm{SF})$ [from ${ }^{243} \mathrm{Am}\left({ }^{48} \mathrm{Ca}, \mathrm{xn}\right)$ and subsequent decay]; measured $\mathrm{T}_{1 / 2}$. Comparison with model predictions. REPT JINR-E7-2003-178,Oganessian

20050G02 NUCLEAR REACTIONS ${ }^{243} \mathrm{Am}\left({ }^{48} \mathrm{Ca}, 3 \mathrm{n}\right),\left({ }^{48} \mathrm{Ca}, 4 \mathrm{n}\right), \mathrm{E}=248,253$ $\mathrm{MeV}$; measured delayed $\mathrm{E} \alpha, \alpha \alpha$-coin; deduced $\sigma$. JOUR PRVCA 72 034611

20050G02 RADIOACTIVITY ${ }^{287,288} 115,{ }^{283,284} 113,{ }^{279,280} \mathrm{Rg},{ }^{275,276} \mathrm{Mt},{ }^{272} \mathrm{Bh}(\alpha)$ [from ${ }^{243} \mathrm{Am}\left({ }^{48} \mathrm{Ca}, \mathrm{xn}\right)$ and subsequent decay]; measured $\mathrm{E} \alpha, \mathrm{T}_{1 / 2}$; deduced $\mathrm{Q} \alpha .{ }^{267,268} \mathrm{Db}(\mathrm{SF}) ;$ measured $\mathrm{T}_{1 / 2}$. JOUR PRVCA 72034611

\section{$\mathrm{A}=\mathbf{2 8 8}$}

${ }^{288} 114 \quad$ RADIOACTIVITY ${ }^{271} \mathrm{Sg},{ }^{275} \mathrm{Hs},{ }^{279} \mathrm{Ds},{ }^{282,283,285} 112,{ }^{286,287,288,289} 114$, ${ }^{292,293} 116(\alpha) ;{ }^{267} \mathrm{Rf},{ }^{271} \mathrm{Sg},{ }^{279,281} \mathrm{Ds},{ }^{284} 112,{ }^{286} 114$ (SF) [from ${ }^{233,238} \mathrm{U}$, ${ }^{242} \mathrm{Pu},{ }^{248} \mathrm{Cm}\left({ }^{48} \mathrm{Ca}, \mathrm{xn}\right)$ and subsequent decay]; measured $\mathrm{E} \alpha, \mathrm{T}_{1 / 2}$. Comparison with model predictions. JOUR PRVCA 70064609 


\section{$\mathrm{A}=288$ (continued)}

20040GZZ

\section{G03}

$288115 \quad 20030 G Z Y$

20030GZY

2004DMZZ

20050G02

20050G02

${ }^{289} 114$

20040G12

20040GZZ

20050G03
RADIOACTIVITY ${ }^{271} \mathrm{Sg},{ }^{275} \mathrm{Hs},{ }^{279} \mathrm{Ds},{ }^{282,283,285} 112,{ }^{286,287,288,289} 114$, ${ }^{292,293} 116(\alpha) ;{ }^{267} \mathrm{Rf},{ }^{271} \mathrm{Sg},{ }^{279,281} \mathrm{Ds},{ }^{284} 112,{ }^{286} 114$ (SF) [from ${ }^{233,238} \mathrm{U}$, ${ }^{242} \mathrm{Pu},{ }^{248} \mathrm{Cm}\left({ }^{48} \mathrm{Ca}, \mathrm{xn}\right)$ and subsequent decay]; measured $\mathrm{E} \alpha, \mathrm{T}_{1 / 2}$. Comparison with model predictions. REPT

JINR-E7-2004-160, Oganessian NUCLEAR REACTIONS ${ }^{238} \mathrm{U}\left({ }^{48} \mathrm{Ca}, 3 \mathrm{n}\right),\left({ }^{48} \mathrm{Ca}, 4 \mathrm{n}\right),{ }^{233} \mathrm{U}$, ${ }^{242} \mathrm{Pu}\left({ }^{48} \mathrm{Ca}, 2 \mathrm{n}\right),\left({ }^{48} \mathrm{Ca}, 3 \mathrm{n}\right),\left({ }^{48} \mathrm{Ca}, 4 \mathrm{n}\right), \mathrm{E} \approx 230-250 \mathrm{MeV}$; measured $\sigma$. JOUR ZAANE 25 s01 589 RADIOACTIVITY ${ }^{294} 118,{ }^{290,291,292,293} 116,{ }^{287,288,289} 114,{ }^{285} 112$, ${ }^{275} \mathrm{Hs}(\alpha) ;{ }^{286} 114,{ }^{283} 112,{ }^{279} \mathrm{Ds},{ }^{271} \mathrm{Sg}(\alpha)$, (SF); ${ }^{282,284} 112,{ }^{281} \mathrm{Ds}$, ${ }^{267} \mathrm{Rf}(\mathrm{SF})$; measured $\mathrm{E} \alpha, \mathrm{T}_{1 / 2}$, branching ratios. JOUR ZAANE 25 s01 589 NUCLEAR REACTIONS ${ }^{243} \mathrm{Am}\left({ }^{48} \mathrm{Ca}, 3 \mathrm{n}\right),\left({ }^{48} \mathrm{Ca}, 4 \mathrm{n}\right), \mathrm{E}=253 \mathrm{MeV}$; measured (recoil) $\alpha$-, $\alpha \alpha$-coin following residual nucleus decay; deduced production $\sigma$. REPT JINR-E7-2003-178,Oganessian RADIOACTIVITY ${ }^{287,288} 115,{ }^{283,284} 113,{ }^{279,280} \mathrm{Rg},{ }^{275,276} \mathrm{Mt}$, ${ }^{272} \mathrm{Bh}(\alpha)$ [from ${ }^{243} \mathrm{Am}\left({ }^{48} \mathrm{Ca}\right.$, xn $)$ and subsequent decay]; measured $\mathrm{E} \alpha$, $\mathrm{T}_{1 / 2}$; deduced $\mathrm{Q} \alpha .{ }^{267,268} \mathrm{Db}(\mathrm{SF})$ [from ${ }^{243} \mathrm{Am}\left({ }^{48} \mathrm{Ca}, \mathrm{xn}\right)$ and subsequent decay]; measured $\mathrm{T}_{1 / 2}$. Comparison with model predictions. REPT JINR-E7-2003-178,Oganessian NUCLEAR REACTIONS ${ }^{243} \mathrm{Am}\left({ }^{48} \mathrm{Ca}, 3 \mathrm{n}\right), \mathrm{E}=247 \mathrm{MeV}$; measured delayed fission fragment and neutron spectra following radiochemical separation; deduced $\sigma$, evidence for $\mathrm{Z}=115$ and $\mathrm{Z}=113$ production. REPT JINR-E12-2004-157,Dmitriev NUCLEAR REACTIONS ${ }^{243} \mathrm{Am}\left({ }^{48} \mathrm{Ca}, 3 \mathrm{n}\right),\left({ }^{48} \mathrm{Ca}, 4 \mathrm{n}\right), \mathrm{E}=248,253$ $\mathrm{MeV}$; measured delayed $\mathrm{E} \alpha, \alpha \alpha$-coin; deduced $\sigma$. JOUR PRVCA 72 034611 RADIOACTIVITY ${ }^{287,288} 115,{ }^{283,284} 113,{ }^{279,280} \mathrm{Rg},{ }^{275,276} \mathrm{Mt},{ }^{272} \mathrm{Bh}(\alpha)$ [from ${ }^{243} \mathrm{Am}\left({ }^{48} \mathrm{Ca}, \mathrm{xn}\right)$ and subsequent decay]; measured $\mathrm{E} \alpha, \mathrm{T}_{1 / 2}$; deduced $\mathrm{Q} \alpha$. ${ }^{267,268} \mathrm{Db}(\mathrm{SF})$; measured $\mathrm{T}_{1 / 2}$. JOUR PRVCA 72034611

\section{$\mathrm{A}=\mathbf{2 8 9}$}

RADIOACTIVITY ${ }^{271} \mathrm{Sg},{ }^{275} \mathrm{Hs},{ }^{279} \mathrm{Ds},{ }^{282,283,285} 112,{ }^{286,287,288,289} 114$, ${ }^{292,293} 116(\alpha) ;{ }^{267} \mathrm{Rf},{ }^{271} \mathrm{Sg},{ }^{279,281} \mathrm{Ds},{ }^{284} 112,{ }^{286} 114$ (SF) [from ${ }^{233,238} \mathrm{U}$, ${ }^{242} \mathrm{Pu},{ }^{248} \mathrm{Cm}\left({ }^{48} \mathrm{Ca}, \mathrm{xn}\right)$ and subsequent decay]; measured $\mathrm{E} \alpha, \mathrm{T}_{1 / 2}$. Comparison with model predictions. JOUR PRVCA 70064609

RADIOACTIVITY ${ }^{271} \mathrm{Sg},{ }^{275} \mathrm{Hs},{ }^{279} \mathrm{Ds},{ }^{282,283,285} 112,{ }^{286,287,288,289} 114$, ${ }^{292,293} 116(\alpha) ;{ }^{267} \mathrm{Rf},{ }^{271} \mathrm{Sg},{ }^{279,281} \mathrm{Ds},{ }^{284} 112,{ }^{286} 114$ (SF) [from ${ }^{233,238} \mathrm{U}$, ${ }^{242} \mathrm{Pu},{ }^{248} \mathrm{Cm}\left({ }^{48} \mathrm{Ca}, \mathrm{xn}\right)$ and subsequent decay]; measured $\mathrm{E} \alpha, \mathrm{T}_{1 / 2}$. Comparison with model predictions. REPT

JINR-E7-2004-160, Oganessian RADIOACTIVITY ${ }^{294} 118,{ }^{290,291,292,293} 116,{ }^{287,288,289} 114,{ }^{285} 112$, ${ }^{275} \mathrm{Hs}(\alpha) ;{ }^{286} 114,{ }^{283} 112,{ }^{279} \mathrm{Ds},{ }^{271} \mathrm{Sg}(\alpha)$, (SF); ${ }^{282,284} 112,{ }^{281} \mathrm{Ds}$, ${ }^{267} \mathrm{Rf}(\mathrm{SF})$; measured $\mathrm{E} \alpha, \mathrm{T}_{1 / 2}$, branching ratios. JOUR ZAANE $25 \mathrm{~s} 01$ 589 


\section{$\mathrm{A}=\mathbf{2 9 0}$}

${ }^{290} 116$ 20030GzZ RADIOACTIVITY ${ }^{294} 118,{ }^{290} 116(\alpha),{ }^{286} 114(\alpha)$, (SF) [from ${ }^{249} \mathrm{Cf}\left({ }^{48} \mathrm{Ca}, 3 \mathrm{n}\right)$ and subsequent decay]; measured $\mathrm{E} \alpha, \mathrm{T}_{1 / 2}$, fission fragment spectra. Comparison with model predictions. REPT UCRL-ID-151619, Oganessian 20050G03 RADIOACTIVITY ${ }^{294} 118,{ }^{290,291,292,293} 116,{ }^{287,288,289} 114,{ }^{285} 112$, ${ }^{275} \mathrm{Hs}(\alpha) ;{ }^{286} 114,{ }^{283} 112,{ }^{279} \mathrm{Ds},{ }^{271} \mathrm{Sg}(\alpha)$, (SF); ${ }^{282,284} 112,{ }^{281} \mathrm{Ds}$, ${ }^{267} \mathrm{Rf}(\mathrm{SF})$; measured $\mathrm{E} \alpha, \mathrm{T}_{1 / 2}$, branching ratios. JOUR ZAANE 25 s01 589

\section{$\mathrm{A}=\mathbf{2 9 1}$}

${ }^{291} 116$ 20040G12 NUCLEAR REACTIONS ${ }^{233,238} \mathrm{U},{ }^{242} \mathrm{Pu},{ }^{248} \mathrm{Cm}\left({ }^{48} \mathrm{Ca}, 2 \mathrm{n}\right),\left({ }^{48} \mathrm{Ca}\right.$, $3 \mathrm{n}),\left({ }^{48} \mathrm{Ca}, 4 \mathrm{n}\right),\left({ }^{48} \mathrm{Ca}, 5 \mathrm{n}\right), \mathrm{E}^{*} \approx 25-55 \mathrm{MeV}$; measured excitation functions. Comparison with model predictions. JOUR PRVCA 70 064609

20040GZZ NUCLEAR REACTIONS ${ }^{233,238} \mathrm{U},{ }^{242} \mathrm{Pu},{ }^{248} \mathrm{Cm}\left({ }^{48} \mathrm{Ca}, 2 \mathrm{n}\right),\left({ }^{48} \mathrm{Ca}\right.$, $3 \mathrm{n}),\left({ }^{48} \mathrm{Ca}, 4 \mathrm{n}\right),\left({ }^{48} \mathrm{Ca}, 5 \mathrm{n}\right), \mathrm{E}^{*} \approx 25-55 \mathrm{MeV}$; measured excitation functions. Comparison with model predictions. REPT

JINR-E7-2004-160, Oganessian

20050G03 RADIOACTIVITY ${ }^{294} 118,{ }^{290,291,292,293} 116,{ }^{287,288,289} 114,{ }^{285} 112$, ${ }^{275} \mathrm{Hs}(\alpha) ;{ }^{286} 114,{ }^{283} 112,{ }^{279} \mathrm{Ds},{ }^{271} \mathrm{Sg}(\alpha),(\mathrm{SF}) ;{ }^{282,284} 112,{ }^{281} \mathrm{Ds}$,

${ }^{267} \mathrm{Rf}(\mathrm{SF}) ;$ measured $\mathrm{E} \alpha, \mathrm{T}_{1 / 2}$, branching ratios. JOUR ZAANE $25 \mathrm{~s} 01$ 589

\section{$\mathrm{A}=292$}

292116 NUCLEAR REACTIONS ${ }^{233,238} \mathrm{U},{ }^{242} \mathrm{Pu},{ }^{248} \mathrm{Cm}\left({ }^{48} \mathrm{Ca}, 2 \mathrm{n}\right),\left({ }^{48} \mathrm{Ca}\right.$, $3 \mathrm{n}),\left({ }^{48} \mathrm{Ca}, 4 \mathrm{n}\right),\left({ }^{48} \mathrm{Ca}, 5 \mathrm{n}\right), \mathrm{E}^{*} \approx 25-55 \mathrm{MeV}$; measured excitation functions. Comparison with model predictions. JOUR PRVCA 70 064609

$20040 \mathrm{G} 12$ RADIOACTIVITY ${ }^{271} \mathrm{Sg},{ }^{275} \mathrm{Hs},{ }^{279} \mathrm{Ds},{ }^{282,283,285} 112,{ }^{286,287,288,289} 114$, ${ }^{292,293} 116(\alpha) ;{ }^{267} \mathrm{Rf},{ }^{271} \mathrm{Sg},{ }^{279,281} \mathrm{Ds},{ }^{284} 112,{ }^{286} 114$ (SF) [from ${ }^{233,238} \mathrm{U}$, ${ }^{242} \mathrm{Pu},{ }^{248} \mathrm{Cm}\left({ }^{48} \mathrm{Ca}, \mathrm{xn}\right)$ and subsequent decay]; measured $\mathrm{E} \alpha, \mathrm{T}_{1 / 2}$. Comparison with model predictions. JOUR PRVCA 70064609 20040GZZ NUCLEAR REACTIONS ${ }^{233,238} \mathrm{U},{ }^{242} \mathrm{Pu},{ }^{248} \mathrm{Cm}\left({ }^{48} \mathrm{Ca}, 2 \mathrm{n}\right),\left({ }^{48} \mathrm{Ca}\right.$, $3 \mathrm{n}),\left({ }^{48} \mathrm{Ca}, 4 \mathrm{n}\right),\left({ }^{48} \mathrm{Ca}, 5 \mathrm{n}\right), \mathrm{E}^{*} \approx 25-55 \mathrm{MeV}$; measured excitation functions. Comparison with model predictions. REPT JINR-E7-2004-160,Oganessian

20040GZZ RADIOACTIVITY ${ }^{271} \mathrm{Sg},{ }^{275} \mathrm{Hs},{ }^{279} \mathrm{Ds},{ }^{282,283,285} 112,{ }^{286,287,288,289} 114$, ${ }^{292,293} 116(\alpha) ;{ }^{267} \mathrm{Rf},{ }^{271} \mathrm{Sg},{ }^{279,281} \mathrm{Ds},{ }^{284} 112,{ }^{286} 114$ (SF) [from ${ }^{233,238} \mathrm{U}$, ${ }^{242} \mathrm{Pu},{ }^{248} \mathrm{Cm}\left({ }^{48} \mathrm{Ca}, \mathrm{xn}\right)$ and subsequent decay]; measured $\mathrm{E} \alpha, \mathrm{T}_{1 / 2}$. Comparison with model predictions. REPT

JINR-E7-2004-160,Oganessian

20050G03 RADIOACTIVITY ${ }^{294} 118,{ }^{290,291,292,293} 116,{ }^{287,288,289} 114,{ }^{285} 112$, ${ }^{275} \mathrm{Hs}(\alpha) ;{ }^{286} 114,{ }^{283} 112,{ }^{279} \mathrm{Ds},{ }^{271} \mathrm{Sg}(\alpha),(\mathrm{SF}) ;{ }^{282,284} 112,{ }^{281} \mathrm{Ds}$, ${ }^{267} \mathrm{Rf}(\mathrm{SF}) ;$ measured $\mathrm{E} \alpha, \mathrm{T}_{1 / 2}$, branching ratios. JOUR ZAANE $25 \mathrm{~s} 01$ 589 


\section{$A=293$}

${ }^{293} 116$ 20040G12 NUCLEAR REACTIONS ${ }^{233,238} \mathrm{U},{ }^{242} \mathrm{Pu},{ }^{248} \mathrm{Cm}\left({ }^{48} \mathrm{Ca}, 2 \mathrm{n}\right),\left({ }^{48} \mathrm{Ca}\right.$, $3 \mathrm{n}),\left({ }^{48} \mathrm{Ca}, 4 \mathrm{n}\right),\left({ }^{48} \mathrm{Ca}, 5 \mathrm{n}\right), \mathrm{E}^{*} \approx 25-55 \mathrm{MeV}$; measured excitation functions. Comparison with model predictions. JOUR PRVCA 70 064609

$20040 \mathrm{G} 12$ RADIOACTIVITY ${ }^{271} \mathrm{Sg},{ }^{275} \mathrm{Hs},{ }^{279} \mathrm{Ds},{ }^{282,283,285} 112,{ }^{286,287,288,289} 114$, ${ }^{292,293} 116(\alpha) ;{ }^{267} \mathrm{Rf},{ }^{271} \mathrm{Sg},{ }^{279,281} \mathrm{Ds},{ }^{284} 112,{ }^{286} 114(\mathrm{SF})$ [from ${ }^{233,238} \mathrm{U}$, ${ }^{242} \mathrm{Pu},{ }^{248} \mathrm{Cm}\left({ }^{48} \mathrm{Ca}, \mathrm{xn}\right)$ and subsequent decay]; measured $\mathrm{E} \alpha, \mathrm{T}_{1 / 2}$. Comparison with model predictions. JOUR PRVCA 70064609

20040GzZ NUCLEAR REACTIONS ${ }^{233,238} \mathrm{U},{ }^{242} \mathrm{Pu},{ }^{248} \mathrm{Cm}\left({ }^{48} \mathrm{Ca}, 2 \mathrm{n}\right),\left({ }^{48} \mathrm{Ca}\right.$, $3 \mathrm{n}),\left({ }^{48} \mathrm{Ca}, 4 \mathrm{n}\right),\left({ }^{48} \mathrm{Ca}, 5 \mathrm{n}\right), \mathrm{E}^{*} \approx 25-55 \mathrm{MeV}$; measured excitation functions. Comparison with model predictions. REPT

JINR-E7-2004-160,Oganessian

20040GZZ RADIOACTIVITY ${ }^{271} \mathrm{Sg},{ }^{275} \mathrm{Hs},{ }^{279} \mathrm{Ds},{ }^{282,283,285} 112,{ }^{286,287,288,289} 114$, ${ }^{292,293} 116(\alpha) ;{ }^{267} \mathrm{Rf},{ }^{271} \mathrm{Sg},{ }^{279,281} \mathrm{Ds},{ }^{284} 112,{ }^{286} 114(\mathrm{SF})$ [from ${ }^{233,238} \mathrm{U}$, ${ }^{242} \mathrm{Pu},{ }^{248} \mathrm{Cm}\left({ }^{48} \mathrm{Ca}, \mathrm{xn}\right)$ and subsequent decay]; measured $\mathrm{E} \alpha, \mathrm{T}_{1 / 2}$. Comparison with model predictions. REPT

JINR-E7-2004-160,Oganessian

20050G03 RADIOACTIVITY ${ }^{294} 118,{ }^{290,291,292,293} 116,{ }^{287,288,289} 114,{ }^{285} 112$, ${ }^{275} \mathrm{Hs}(\alpha) ;{ }^{286} 114,{ }^{283} 112,{ }^{279} \mathrm{Ds},{ }^{271} \mathrm{Sg}(\alpha)$, (SF); ${ }^{282,284} 112,{ }^{281} \mathrm{Ds}$, ${ }^{267} \mathrm{Rf}(\mathrm{SF})$; measured $\mathrm{E} \alpha, \mathrm{T}_{1 / 2}$, branching ratios. JOUR ZAANE 25 s01 589

\section{$\mathrm{A}=294$}

${ }^{294} 116$ NUCLEG12 2004 REACTIONS ${ }^{233,238} \mathrm{U},{ }^{242} \mathrm{Pu},{ }^{248} \mathrm{Cm}\left({ }^{48} \mathrm{Ca}, 2 \mathrm{n}\right),\left({ }^{48} \mathrm{Ca}\right.$, $3 \mathrm{n}),\left({ }^{48} \mathrm{Ca}, 4 \mathrm{n}\right),\left({ }^{48} \mathrm{Ca}, 5 \mathrm{n}\right), \mathrm{E}^{*} \approx 25-55 \mathrm{MeV}$; measured excitation functions. Comparison with model predictions. JOUR PRVCA 70 064609

20040GzZ NUCLEAR REACTIONS ${ }^{233,238} \mathrm{U},{ }^{242} \mathrm{Pu},{ }^{248} \mathrm{Cm}\left({ }^{48} \mathrm{Ca}, 2 \mathrm{n}\right),\left({ }^{48} \mathrm{Ca}\right.$, $3 \mathrm{n}),\left({ }^{48} \mathrm{Ca}, 4 \mathrm{n}\right),\left({ }^{48} \mathrm{Ca}, 5 \mathrm{n}\right), \mathrm{E}^{*} \approx 25-55 \mathrm{MeV}$; measured excitation functions. Comparison with model predictions. REPT

JINR-E7-2004-160,Oganessian

${ }^{294} 118 \quad$ NUC30GzZ NUEAR REACTIONS ${ }^{249} \mathrm{Cf}\left({ }^{48} \mathrm{Ca}, 3 \mathrm{n}\right), \mathrm{E}=245 \mathrm{MeV}$; measured E $\alpha$, fission fragment spectra following residual nucleus decay; deduced evidence for ${ }^{294} 118$. Gas-filled recoil separator. REPT UCRL-ID-151619,Oganessian

20030GzZ RADIOACTIVITY ${ }^{294} 118,{ }^{290} 116(\alpha),{ }^{286} 114(\alpha)$, (SF) [from ${ }^{249} \mathrm{Cf}\left({ }^{48} \mathrm{Ca}, 3 \mathrm{n}\right)$ and subsequent decay]; measured $\mathrm{E} \alpha, \mathrm{T}_{1 / 2}$, fission fragment spectra. Comparison with model predictions. REPT UCRL-ID-151619,Oganessian

20050G03 RADIOACTIVITY ${ }^{294} 118,{ }^{290,291,292,293} 116,{ }^{287,288,289} 114,{ }^{285} 112$, ${ }^{275} \mathrm{Hs}(\alpha) ;{ }^{286} 114,{ }^{283} 112,{ }^{279} \mathrm{Ds},{ }^{271} \mathrm{Sg}(\alpha)$, (SF); ${ }^{282,284} 112,{ }^{281} \mathrm{Ds}$, ${ }^{267} \mathrm{Rf}(\mathrm{SF})$; measured $\mathrm{E} \alpha, \mathrm{T}_{1 / 2}$, branching ratios. JOUR ZAANE 25 s01 589 


\section{References}

2002 AH06 I.Ahmad - J.Nucl.Radiochem.Sci. 3, No 1, 179 (2002)

Structure and Spectroscopy of Transcurium Nuclei

2002AS08 M.Asai, M.Sakama, K.Tsukada, S.Ichikawa, H.Haba, I.Nishinaka, Y.Nagame, S.Goto, K.Akiyama, A.Toyoshima, Y.Kojima, Y.Oura, H.Nakahara, M.Shibata, K.Kawade - J.Nucl.Radiochem.Sci. 3, No 1, 187 (2002)

Decay Studies of Neutron-deficient Am, Cm, and Bk Nuclei Using an On-line Isotope Separator

2002B066 V.A.Bondarenko, J.Honzatko, V.A.Khitrov, A.M.Sukhovoj, I.Tomandl -

Fizika(Zagreb) B 11, 83 (2002)

Cascade $\gamma$-decay of the ${ }^{193}$ Os compound nucleus and some aspects of dynamics of

change in nuclear properties below $\mathrm{B}_{n}$

2002B067 V.A.Bondarenko, J.Honzatko, V.A.Khitrov, A.M.Sukhovoj, I.Tomandl -

Fizika(Zagreb) B 11, 201 (2002)

Two-step cascades of the ${ }^{185} \mathrm{~W}$ compound nucleus $\gamma$-decay

2002 DU22 Ch.E.Dullmann, B.Eichler, R.Eichler, H.W.Gaggeler, D.T.Jost, D.Piguet, A.Turler Nucl.Instrum.Methods Phys.Res. A479, 631 (2002)

IVO, a device for In situ Volatilization and On-line detection of products from heavy ion reactions

2002 GU33 J.S.Guo, Z.Qin, Z.G.Gan, H.M.Fan, Y.B.Xu, J.J.He, X.G.Lei, X.L.Wu, H.Y.Liu, X.H.Zhou, S.G.Yuan, G.M.Jin - J.Nucl.Radiochem.Sci. 3, No 1, 183 (2002)

Preparation of Thick Americium Targets and Synthesis of ${ }^{259} \mathrm{Db}$

2002 LI68 C.J.Lin, H.Q.Zhang, Z.H.Liu, J.C.Xu, F.Yang - J.Nucl.Radiochem.Sci. 3, No 1, 27 (2002)

The Effects of Deformed Projectile in Threshold Anomaly and Fusion Reaction for ${ }^{19} \mathrm{~F}+{ }^{208} \mathrm{~Pb}$ System

2002NA37 Y.Nagame, M.Asai, H.Haba, S.Goto, K.Tsukada, I.Nishinaka, K.Nishio, S.Ichikawa, A.Toyoshima, K.Akiyama, H.Nakahara, M.Sakama, M.Schadel, J.V.Kratz, H.W.Gaggeler, A.Turler - J.Nucl.Radiochem.Sci. 3, No 1, 85 (2002)

Production Cross Sections of ${ }^{261} \mathrm{Rf}$ and ${ }^{262} \mathrm{Db}$ in Bombardments of ${ }^{248} \mathrm{Cm}$ with ${ }^{18} \mathrm{O}$ and ${ }^{19} \mathrm{~F}$ Ions

2002 SU35 K.Subotic, Yu.Ts.Oganessian, V.K.Utyonkov, Yu.V.Lobanov, F.Sh.Abdullin, A.N.Polyakov, Yu.S.Tsyganov, O.V.Ivanov - Nucl.Instrum.Methods Phys.Res. A481, 71 (2002)

Evaporation residue collection efficiencies and position spectra of the Dubna gas-filled recoil separator 
2003ASZY M.Asai, K.Tsukada, S.Ichikawa, M.Sakama, H.Haba, Y.Nagame, I.Nishinaka, K.Akiyama, A.Toyoshima, T.Kaneko, Y.Oura, Y.Kojima, M.Shibata - Japan Atomic Energy Res.Inst.Tandem VDG Ann.Rept., 2002, p.29 (2003); JAERI-Review 2003-028 (2003)

EC Decay of the New Isotope ${ }^{241} \mathrm{Bk}$

2003 G041 O.K.Gorpinich, O.M.Povoroznyk, O.O.Yachmenov - Ukr.J.Phys. 48, 1035 (2003) About the evidence for high excited levels of ${ }^{5} \mathrm{Li}$ above the $\mathrm{t}+2 \mathrm{p}$ threshold in the ${ }^{3} \mathrm{He}(\alpha, \mathrm{dt}){ }^{2} \mathrm{He}$ reaction

$2003 H I 23 \quad$ J.C.Hill, for the NA53 Collaboration - Ukr.J.Phys. 48, 1165 (2003)

Electromagnetic dissociation of relativistic heavy ions

2003KI26 N.Kinoshita, A.Yokoyama, T.Nakanishi - J.Nucl.Radiochem.Sci. 4, No 1, 5 (2003)

Half-Life of Samarium-147

2003MB03 V.M.Mazur, V.A.Zheltonozhsky, Z.M.Bigan - Ukr.J.Phys. 48, 403 (2003)

Excitation mechanism of isomer states of ${ }^{197} \mathrm{Pt}$ and ${ }^{197} \mathrm{Hg}$ nuclei in photoneutron reactions in the energy region of a giant E1-resonance

2003MOZP C.B.Moon, G.D.Dracoulis, R.A.Bark, A.P.Byrne, P.M.Davidson, A.N.Wilson, A.M.Baxter, T.Kibedi, G.J.Lane, J.C.Hazel, A.M.Bruce, N.Orce Gonzalez, F.Prados-Estevez, H.El-Masri, C.Wheldon, P.M.Walker, R.Wood - Australian National Univ., Dept. of Nuclear Physics 2002 Ann.Rept., p.17 (2003);

ANU-P/1564 (2003)

Quasi-collective bands in ${ }^{126} \mathrm{I}$

2003MOZQ C.B.Moon, G.D.Dracoulis, R.A.Bark, A.P.Byrne, P.M.Davidson, A.N.Wilson, T.Kibedi, G.J.Lane, A.M.Baxter - Australian National Univ., Dept. of Nuclear Physics 2002 Ann.Rept., p.15 (2003); ANU-P/1564 (2003)

Collective bands in ${ }^{124} \mathrm{I}$

2003MOZR C.B.Moon, G.D.Dracoulis, R.A.Bark, A.P.Byrne, P.M.Davidson, A.N.Wilson, T.Kibedi, G.J.Lane, A.M.Baxter - Australian National Univ., Dept. of Nuclear Physics 2002 Ann.Rept., p.13 (2003); ANU-P/1564 (2003)

The $\pi \mathrm{h}_{11 / 12}(\mathrm{x}) \nu \mathrm{h}_{11 / 2}$ band in the odd-odd nuclide ${ }^{122} \mathrm{I}$

2003MOZS C.B.Moon, G.D.Dracoulis, R.A.Bark, A.P.Byrne, P.M.Davidson, A.N.Wilson, T.Kibedi, G.J.Lane - Australian National Univ., Dept. of Nuclear Physics 2002 Ann.Rept., p.11 (2003); ANU-P/1564 (2003)

Candidates for Chiral Bands in ${ }^{120} \mathrm{I}$

20030GZY Yu.Ts.Oganessian, V.K.Utyonkov, Yu.V.Lobanov, F.Sh.Abdullin, A.N.Polyakov, I.V.Shirokovsky, Yu.S.Tsyganov, G.G.Gulbekian, S.L.Bogomolov, A.N.Mezentsev, S.Iliev, V.G.Subbotin, A.M.Sukhov, A.A.Voinov, G.V.Buklanov, K.Subotic, V.I.Zagrebaev, M.G.Itkis, J.B.Patin, K.J.Moody, J.F.Wild, M.A.Stoyer, N.J.Stoyer, D.A.Shaughnessy, J.M.Kenneally, R.W.Lougheed - JINR-E7-2003-178 (2003) Experiments on the synthesis of element 115 in the reaction ${ }^{243} \mathrm{Am}\left({ }^{48} \mathrm{Ca}\right.$, $\mathrm{xn})^{291-x} 115$ 
20030GzZ Y.T.Oganessian, V.K.Utyonkov, Y.V.Lobanov, F.S.Abdullin, A.N.Polyakov, I.V.Shirokovsky, Y.S.Tsyganov, A.N.Mezentsev, S.Iliev, V.G.Subbotin, A.M.Sukhov, O.V.Ivanov, A.A.Voinov, K.Subotic, V.I.Zagrebaev, M.G.Itkis, K.J.Moody, J.F.Wild, M.A.Stoyer, N.J.Stoyer, C.A.Laue, D.A.Shaughnessy, J.B.Patin, R.W.Lougheed - UCRL-ID-151619 (2003)

Results from the First ${ }^{249} \mathrm{Cf}+{ }^{48} \mathrm{Ca}$ Experiment

2003WIZU A.N.Wilson, R.A.Bark, P.M.Davidson - Australian National Univ., Dept. of Nuclear Physics 2002 Ann.Rept., p.18 (2003); ANU-P/1564 (2003)

High-spin study of ${ }^{127} \mathrm{Ce}$

2003 Ya22 A.B.Yakushev, I.Zvara, Yu.Ts.Oganessian, A.V.Belozerov, S.N.Dmitriev, B.Eichler, S.Hubener, E.A.Sokol, A.Turler, A.V.Yeremin, G.V.Buklanov, M.L.Chelnokov, V.I.Chepigin, V.A.Gorshkov, A.V.Gulyaev, V.Ya.Lebedev, O.N.Malyshev, A.G.Popeko, S.Soverna, Z.Szeglowski, S.N.Timokhin, S.P.Tretyakova, V.M.Vasko, M.G.Itkis - Radiochim.Acta 91, 433 (2003)

Chemical identification and properties of element 112

2004ADZW J.Adam, A.R.Balabekyan, V.S.Barashenkov, R.Brandt, V.M.Golovatyuk, V.G.Kalinnikov, K.Katovsky, M.I.Krivopustov, V.Kumar, H.Kumawat, R.Odoj, V.S.Pronskikh, A.A.Solnyshkin, V.I.Stegailov, V.M.Tsoupko-Sitnikov, W.Westmeier - JINR-E1-2004-16 (2004)

Spallation Neutron Spectrum on a Massive Lead / Paraffin Target Irradiated with 1 GeV Protons

2004AG09 Y.Agus, I.Celenk, A.Ozmen - Radiochim.Acta 92, 63 (2004)

Measurement of cross sections of threshold detectors with spectrum average technique

2004AHZW M.Ahmed, M.Blackston, A.S.Crowell, C.R.Howell, K.Joshi, N.Kalantar, S.O.Nelson, R.M.Prior, B.Perdue, A.L.Sabourov, K.Sabourov, M.Spraker, A.P.Tonchev, H.R.Weller - Triangle Univ.Nuclear Lab., Ann.Rept., p.28 (2004); TUNL-XLIII (2004) Measurement of the ${ }^{7} \mathrm{Li}(\mathrm{d}(\mathrm{pol}), \mathrm{n})^{8} \mathrm{Be}$ Reaction at Low Energies

2004AHZY I.Ahmad, E.F.Moore, R.R.Chasman, J.P.Greene, M.P.Carpenter, C.J.Lister, R.V.F.Janssens, T.Lauritsen, D.Seweryniak, F.G.Kondev, R.W.Hoff, J.E.Evans, R.W.Lougheed, C.E.Porter, L.K.Felker - ANL-04/22 (Physics Division Ann.Rept., 2003), p.45 (2004)

Proton Single-Particle States in ${ }^{249} \mathrm{Bk}$

2004AHzZ I.Ahmad, J.P.Greene, D.J.Henderson, C.L.Jiang, R.C.Pardo, T.Pennington, K.E.Rehm, R.Scott, S.Sinha, X.Tang, R.Vondrasek, H.Nassar, M.Paul, S.Ghelberg, S.Dababneh, M.Heil, F.Kappeler, R.Plag, R.Reifarth, A.Heger, H.Koivisto, P.Collon, S.O'Brien, M.Bettan, D.Berkovits, N.Patronis - ANL-04/22 (Physics Division Ann.Rept., 2003), p.15 (2004)

Stellar ${ }^{62} \mathrm{Ni}(\mathrm{n}, \gamma)^{63} \mathrm{Ni}$ Reaction 
2004 AL35 A.Algora, E.Nacher, B.Rubio, D.Cano-Ott, J.L.Tain, A.Gadea, J.Agramunt, M.Karny, Z.Janas, K.Rykaczewski, R.Collatz, M.Hellstrom, Z.Hu, R.Kirchner, E.Roeckl, M.Shibata, L.Batist, F.Moroz, V.Wittmann - Phys.Rev. C 70, 064301 (2004) $\beta$ decay of ${ }^{148}$ Dy: Study of the Gamow-Teller giant state by means of total absorption spectroscopy

2004AL43 J.A.Alcantara-Nunez, J.R.B.Oliveira, E.W.Cybulska, N.H.Medina, M.N.Rao, R.V.Ribas, M.A.Rizzutto, W.A.Seale, F.Falla-Sotelo, K.T.Wiedemann, V.I.Dimitrov, S.Frauendorf - Braz.J.Phys. 34, 999 (2004)

Chiral Bands in ${ }^{105} \mathrm{Rh}$

2004AL44 J.A.Alcantara-Nunez, J.R.B.Oliveira, E.W.Cybulska, N.H.Medina, M.N.Rao, R.V.Ribas, M.A.Rizzutto, W.A.Seale, F.Falla-Sotelo - Braz.J.Phys. 34, 1005 (2004) Rotational and Vibrational bands in ${ }^{108} \mathrm{Pd}$

2004AS13 N.I.Ashwood, M.Freer, J.C.Angelique, V.Bouchat, W.N.Catford, N.M.Clarke, N.Curtis, O.Dorvaux, F.Hanappe, Y.Kerckx, M.Labiche, J.L.Lecouey, F.M.Marques, T.Materna, A.Ninane, G.Normand, N.A.Orr, S.Pain, N.Soic, L.Stuttge, C.Timis, A.Unshakova, V.A.Ziman - Phys.Rev. C 70, 064607 (2004)

Measurements of the breakup and neutron removal cross sections for ${ }^{16} \mathrm{C}$

2004AZZW L.S.Azhgirey, S.V.Afanasiev, A.Yu.Isupov, V.I.Ivanov, A.N.Khrenov, V.P.Ladygin, N.B.Ladygina, A.G.Litvinenko, V.F.Peresedov, N.P.Yudin, V.N.Zhmyrov, L.S.Zolin - JINR-E1-2004-117 (2004)

Measurement of the tensor $\mathrm{A}_{y y}$ and vector $\mathrm{A}_{y}$ analyzing powers of the deuteron inelastic scattering on beryllium at $5.0 \mathrm{GeV} / \mathrm{c}$ and $178 \mathrm{mrad}$

2004AZZX L.S.Azhgirey, S.V.Afanas'ev, V.N.Zhmyrov, L.S.Zolin, V.I.Ivanov, A.Yu.Isupov, V.P.Ladygin, A.G.Litvinenko, V.F.Leresedov, A.N.Khrenov, N.L.Yudin JINR-P1-2004-118 (2004)

Tensor Analyzing Power of the Relativistic Deuteron Fragmentation as a Means of Study of Deuteron Structure in the Framework of Light-Front Dynamics

2004BE58 G.J.Beyer, S.K.Zeisler, D.W.Becker - Radiochim.Acta 92, 219 (2004)

The Auger-electron emitter ${ }^{165} \mathrm{Er}$ : excitation function of the ${ }^{165} \mathrm{Ho}(\mathrm{p}, \mathrm{n}){ }^{165} \mathrm{Er}$ process

2004BEZP E.Becheva - IPNO-T-04-17 (2004)

Etude de la reaction ${ }^{22} \mathrm{O}(\mathrm{p}, \mathrm{p}$ ') avec le detecteur Must + Developpement de l'etage CsI(Tl) de l'ensemble MUST II

2004B047 D.Bosnar, M.Makek, for the A1 Collaboration at MAMI - Fizika(Zagreb) B 13, 507 (2004)

Modifications of Delta in nuclear medium 
2004B0ZX V.R.Bom, A.M.Demin, D.L.Demin, C.W.E.van Eijk, M.P.Faifman, V.V.Filchenkov, A.N.Golubkov, N.N.Grafov, S.K.Grishechkin, K.I.Gritsaj, V.G.Klevtsov, A.D.Konin, A.V.Kuryakin, S.V.Medved, R.K.Musyaev, V.V.Perevozchikov, A.I.Rudenko, S.M.Sadetsky, Yu.I.Vinogradov, A.A.Yukhimchuk, S.A.Yukhimchuk, V.G.Zinov, S.V.Zlatoustovskii - JINR-E15-2004-132 (2004)

Experimental investigation of muon-catalyzed dt fusion in wide ranges of D / T mixture

2004BRzV V.B.Brudanin, N.I.Rukhadze, Sh.Brianson, P.Benesh, T.Vylov, N.Gusev, V.Egorov, A.A.Klimenko, V.E.Kovalenko, N.A.Korolev, A.V.Salamatin, V.V.Timkin, P.Cermak, M.V.Shirchenko, Yu.A.Shitov, I.Stekl - JINR-P6-2004-219 (2004)

Searching of Double Electron Capture in the TGV Experiment

2004BUZY B.D.Buck, B.J.Crowe III, A.S.Crowell, J.H.Esterline, C.R.Howell, J.H.Kelley, R.A.Macri, R.S.Pedroni, W.Tornow, R.L.Walter, G.J.Weisel, H.Witala - Triangle Univ.Nuclear Lab., Ann.Rept., p.20 (2004); TUNL-XLIII (2004)

Neutron-Deuteron Analyzing Power Data at $19.0 \mathrm{MeV}$

2004CAZW M.P.Carpenter, F.G.Kondev, R.V.F.Janssens, I.Ahmad, C.N.Davids, N.Hammond, T.L.Khoo, T.Lauritsen, C.J.Lister, G.Mukherjee, D.Seweryniak, S.Sinha, D.J.Jenkins, P.Raddon, R.Wadsworth, S.F.Freeman, S.M.Fischer, G.Jones, A.J.Larabee, A.Liechty - ANL-04/22 (Physics Division Ann.Rept., 2003), p.43 (2004) Alpha Decay of ${ }^{181} \mathrm{~Pb}$

2004C026 C.Cozzini, G.Angloher, C.Bucci, F.von Feilitzsch, D.Hauff, S.Henry, Th.Jagemann, J.Jochum, H.Kraus, B.Majorovits, V.Mikhailik, J.Ninkovic, F.Petricca, W.Potzel, F.Probst, Y.Ramachers, W.Rau, M.Razeti, W.Seidel, M.Stark, L.Stodolsky, A.J.B.Tolhurst, W.Westphal, H.Wulandari - Phys.Rev. C 70, 064606 (2004) Detection of the natural $\alpha$ decay of tungsten

2004C027 F.Confortola, D.Bemmerer, R.Bonetti, C.Broggini, P.Corvisiero, H.Costantini, J.Cruz, A.Formicola, Z.Fulop, G.Gervino, A.Guglielmetti, C.Gustavino, G.Gyurky, G.Imbriani, A.P.Jesus, M.Junker, A.Lemut, R.Menegazzo, P.Prati, V.Roca, C.Rolfs, M.Romano, C.Rossi Alvarez, F.Schumann, E.Somorjai, O.Straniero, F.Strieder, F.Terrasi, H.P.Trautvetter, S.Zavatarelli - Nuovo Cim. C 27, 423 (2004) The ${ }^{14} \mathrm{~N}(\mathrm{p}, \gamma){ }^{15} \mathrm{O}$ measurement at low energy

2004 C29 S.Courtin, P.Baumann, Ph.Dessagne, F.Marechal, Ch.Miehe, F.Perrot, E.Poirier, M.Ramdhane, and the ISOLDE Collaboration - Braz.J.Phys. 34, 850 (2004)

Beta Decay and Structure of Exotic Nuclei in the Mass Regions N=Z, A 70 and Near the $\mathrm{N}=20$ Closed Shell

2004CRZZ B.J.Crowe III, A.S.Crowell, J.H.Esterline, C.R.Howell, R.A.Macri, R.S.Pedroni, S.Tajima, W.Tornow, G.J.Weisel - Triangle Univ.Nuclear Lab., Ann.Rept., p.23 (2004); TUNL-XLIII (2004)

Neutron-Helion Analyzing Power Data at 4.02 and $5.54 \mathrm{MeV}$ 
2004DAZX C.N.Davids, D.Seweryniak, B.Blank, M.P.Carpenter, N.Hammond, R.V.F.Janssens, G.Mukherjee, S.Sinha, A.Robinson, P.J.Woods, T.Davinson, Z.Liu, S.J.Freeman, N.Hoteling, J.Shergur, W.B.Walters, A.Woehr, A.A.Sonzogni - ANL-04/22 (Physics Division Ann.Rept., 2003), p.29 (2004)

Recoil-Decay Tagging Study of ${ }^{146} \mathrm{Tm}$

2004DMZZ S.N.Dmitriev, Yu.Ts.Oganessian, V.K.Utyonkov, S.V.Shishkin, A.V.Yeremin, Yu.V.Lobanov, Yu.S.Tsyganov, V.I.Chepigin, E.A.Sokol, G.K.Vostokin, N.V.Aksenov, M.Hussonnois, M.G.Itkis, H.W.Gaggeler, D.Schumann, H.Bruchertseifer, R.Eichler, D.A.Shaughnessy, P.A.Wilk, J.M.Kenneally, M.A.Stoyer, J.F.Wild - JINR-E12-2004-157 (2004)

Results of the experiment on chemical identification of $\mathrm{Db}$ as a decay product of element 115

2004FI12 J.M.Finn, for the Jefferson Laboratory E93038 Collaboration - Fizika(Zagreb) B 13, $545(2004)$

Measurements of the electric form factor of the neutron at JLab via recoil polarimetry in the reaction $\mathrm{d}(\mathrm{e}(\mathrm{pol})$, e'n( $\mathrm{pol}))$

2004F009 B.Fornal, S.Zhu, R.V.F.Janssens, M.Honma, R.Broda, P.F.Mantica, B.A.Brown, M.P.Carpenter, P.J.Daly, S.J.Freeman, Z.W.Grabowski, N.J.Hammond,

F.G.Kondev, W.Krolas, T.Lauritsen, S.N.Liddick, C.J.Lister, E.F.Moore, T.Otsuka, T.Pawlat, D.Seweryniak, B.E.Tomlin, J.Wrzesinski - Phys.Rev. C 70, 064304 (2004) Development of shell closures at $\mathrm{N}=32,34$. II. Lowest yrast excitations in even-even Ti isotopes from deep-inelastic heavy-ion collisions

2004FOZY C.Fox, C.Iliadis, A.E.Champagne, A.Coc, J.Jose, R.Longland, J.Newton, J.Pollanen, R.Runkle - Triangle Univ.Nuclear Lab., Ann.Rept., p.32 (2004); TUNL-XLIII (2004) Explosive Hydrogen Burning of ${ }^{17} \mathrm{O}$ in Classical Nova

2004F0ZZ R.D.Foster, C.R.Gould, D.G.Haase, J.H.Kelley, D.M.Markoff, W.Tornow - Triangle Univ.Nuclear Lab., Ann.Rept., p.18 (2004); TUNL-XLIII (2004)

Longitudinal (n(pol)-d(pol)) Total Cross-Section Difference

2004FR34 M.Freer, M.P.Nicoli, S.M.Singer, C.A.Bremner, S.P.G.Chappell, W.D.M.Rae, I.Boztosun, B.R.Fulton, D.L.Watson, B.J.Greenhalgh, G.K.Dillon, R.L.Cowin, D.C.Weisser - Phys.Rev. C 70, 064311 (2004)

${ }^{8} \mathrm{Be}+{ }^{8} \mathrm{Be}$ decay of excited states in ${ }^{16} \mathrm{O}$

2004FU30 K.Furutaka, H.Harada, S.Raman - J.Nucl.Sci.Technol.(Tokyo) 41, 1033 (2004)

Prompt Gamma Rays Emitted in Thermal-neutron Capture Reaction by ${ }^{99} \mathrm{Tc}$ and Its Reaction Cross Section

2004FU34 M.Furic, E.V.Hungerford, for the E89-009 / HNSS Collaboration - Fizika(Zagreb) B $13,645(2004)$

High resolution spectroscopy of the ${ }_{\Lambda}^{12} \mathrm{~B}$ hypernucleus produced by the $\left(\mathrm{e}, \mathrm{e}^{\prime} \mathrm{K}^{+}\right)$ reaction 
2004FUZX T.Fukuchi, Y.Gono, A.Odahara, H.Watanabe, S.Tanaka, M.Inoue, Y.Wakabayashi, T.Sasaki, M.Kibe, N.Hokoiwa, T.Shinozuka, M.Fujita, A.Yamazaki, T.Sonoda,

C.S.Lee, Y.K.Kwon, J.H.Lee - CNS-REP-64, p.109 (2004)

High-Spin Isomer in ${ }^{93} \mathrm{Mo}$

2004GA57 Yu.P.Gangrsky, N.N.Kolesnikov, V.G.Lukashik, L.M.Melnikova -

Bull.Rus.Acad.Sci.Phys. 68, 187 (2004)

Isomeric ratios in crossing reactions yielding odd-odd ${ }^{184} \mathrm{Re},{ }^{190} \mathrm{Ir}$, and ${ }^{196} \mathrm{Au}$ nuclei

2004GA60 Yu.P.Gangrsky, V.I.Zhemenik, N.Yu.Maslova, G.V.Myshinsky, Kh.S.Norov,

Yu.E.Penionzhkevich - Bull.Rus.Acad.Sci.Phys. 68, 1298 (2004)

Independent yields of $\mathrm{Kr}$ and Xe fragments from ${ }^{237} \mathrm{~Np}$ photofission

2004GAZV Yu.L.Gangrsky, V.I.Zhemenik, G.V.Myshinsky, Yu.E.Lenionzhkevich JINR-P15-2004-119 (2004)

Independent Yields of Kr and Xe Fragments at Photofission of Odd Nuclei ${ }^{237} \mathrm{~Np}$ and ${ }^{243} \mathrm{Am}$

2004GE20 F.A.Genezini, C.B.Zamboni, M.T.F.da Cruz, J.Y.Zevallos-Chavez - Braz.J.Phys. 34, $722(2004)$

Angular Correlation Measurements in ${ }^{155} \mathrm{Eu}$ Nuclei

2004GL10 M.Glaser, D.B.Mackay, A.S.O.Ranicar, S.L.Waters, F.Brady, S.K.Luthra -

Radiochim.Acta 92, 951 (2004)

Improved targetry and production of iodine-124 for PET studies

2004G054 M.S.Golovkov, L.V.Grigorenko, A.S.Fomichev, S.A.Krupko, Yu.Ts.Oganessian, A.M.Rodin, S.I.Sidorchuk, R.S.Slepnev, S.V.Stepantsov, G.M.Ter-Akopian, R.Wolski, M.G.Itkis, A.A.Bogatchev, N.A.Kondratiev, E.M.Kozulin, A.A.Korsheninnikov, E.Yu.Nikolskii, P.Roussel-Chomaz, W.Mittig, R.Palit, V.Bouchat, V.Kinnard, T.Materna, F.Hanappe, O.Dorvaux, L.Stuttge, C.Angulo, V.Lapoux, R.Raabe, L.Nalpas, A.A.Yukhimchuk, V.V.Perevozchikov, Yu.I.Vinogradov, S.K.Grishechkin, S.V.Zlatoustovsky - Phys.Rev.Lett. 93, 262501 (2004)

Observation of Excited States in ${ }^{5} \mathrm{H}$

2004 G056 O.K.Gorpinich, O.M.Povoroznyk, O.O.Yachmenov - Ukr.J.Phys. 49, 16 (2004) Investigation of excited levels of ${ }^{6} \mathrm{Li}$ nucleus from the three-particle ${ }^{3} \mathrm{H}(\alpha, \mathrm{d} \alpha) \mathrm{n}$ reaction

2004 G058 C.Gordon, J.Melone, P.Cole, J.Kellie, F.Klein, K.Livingston, J.Mueller, J.C.Sanabria, D.Tedeschi, for the CLAS Collaboration - Fizika(Zagreb) B 13, 553 (2004)

Vector meson and associated strangeness production using a linearly polarised photon beam at Jefferson Lab

2004 G059 S.K.Godovikov, S.M.Nikitin - Bull.Rus.Acad.Sci.Phys. 68, 1335 (2004)

Controlled radioactive decay of ${ }^{125 m}$ Te nuclei

2004G0ZZ J.TM.Goon - Thesis, University of Tennessee, Knoxville (2004) 
Alpha and Gamma-ray Spectroscopic Studies of Au, Pt, and Ir Nuclei Near the Proton Dripline

2004GU21 V.Guimaraes - Braz.J.Phys. 34, 1012 (2004)

Nuclear Reaction Studies with Exotic Boron Beams

2004HA59 B.Hadinia, B.Cederwall, K.Lagergren, J.Blomqvist, T.Back, S.Eeckhaudt, T.Grahn, P.Greenlees, A.Johnson, D.T.Joss, R.Julin, S.Juutinen, H.Kettunen, M.Leino,

A.-P.Leppanen, R.J.Liotta, P.Nieminen, M.Nyman, J.Pakarinen, E.S.Paul, P.Rahkila, C.Scholey, J.Uusitalo, R.Wadsworth, D.R.Wiseman - Phys.Rev. C 70, 064314 (2004)

First identification of $\gamma$-ray transitions in ${ }^{107} \mathrm{Te}$

2004HA64 B.Haas - Braz.J.Phys. 34, 814 (2004)

Nuclear Data for New Fuel Cycles

2004HAZR Y.Hayashi, T.Tamae, K.Abe, R.Hashimoto, K.Hirose, T.Ishikawa, H.Kanda, O.Konno, K.Maeda, H.Miyase, M.Nanao, I.Nishikawa, T.Ohtsuki, Y.Sato, K.Takahashi, T.Takahashi, H.Tsubota, M.Wakamatsu, H.Yamazaki -

Res.Rep.Lab.Nucl.Sci., Tohoku Univ. 37, 1 (2004)

Comparison of the ${ }^{16} \mathrm{O}(\mathrm{e}, \mathrm{e}$ 'p) Cross Section at Low Momentum Transfer with

Relativistic Calculations Including MEC

2004HE35 A.Hermanne, S.Takacs, F.Tarkanyi, R.Bolbos - Radiochim.Acta 92, 215 (2004)

Cross section measurements of proton and deuteron induced formation of ${ }^{103} \mathrm{Ag}$ in natural palladium

2004HIZZ K.Hilgers, I.Spahn, Ye.Skakun, F.Tarkanyi, S.Takacs, S.M.Qaim, H.H.Coenen NEA/NSC/DOC(2004)14 (Jul-4151), p.15 (2004)

Cross sections for production of the therapeutic radionuclides ${ }^{103} \mathrm{Pd},{ }^{140} \mathrm{Nd},{ }^{169} \mathrm{Yb}$ and ${ }^{192} \mathrm{Ir}$

2004H025 J.Honzatko, I.Tomandl, A.M.Sukhovoi, V.A.Khitrov - Bull.Rus.Acad.Sci.Phys. 68, $1324(2004)$

Cascade $\gamma$-decay of compound states of ${ }^{71,74} \mathrm{Ge}$ nuclei

2004IszX T.Ishii, M.Asai, A.Makishima, M.Matsuda, S.Ichikawa, T.Kohno, M.Ogawa CNS-REP-64, p.27 (2004)

Nano-second isomer spectroscopy of the ${ }^{48} \mathrm{Ca}$ region through deep-inelastic collisions

2004J019 K.L.Jones, R.L.Kozub, C.Baktash, D.W.Bardayan, J.C.Blackmon, W.N.Catford, J.A.Cizewski, R.P.Fitzgerald, M.S.Johnson, R.J.Livesay, Z.Ma, C.D.Nesaraja, D.Shapira, M.S.Smith, J.S.Thomas, D.W.Visser - Phys.Rev. C 70, 067602 (2004) Study of the ${ }^{124} \mathrm{Sn}(\mathrm{d}, \mathrm{p})$ reaction in inverse kinematics close to the Coulomb barrier

2004Ka62 L.Kaubler, H.Schnare, R.Schwengner, H.Prade, F.Donau, P.von Brentano, J.Eberth, J.Enders, A.Fitzler, C.Fransen, M.Grinberg, R.-D.Herzberg, H.Kaiser, P.von Neumann-Cosel, N.Pietralla, A.Richter, G.Rusev, Ch.Stoyanov, I.Wiedenhover Phys.Rev. C 70, 064307 (2004)

Dipole and quadrupole excitations in ${ }^{88} \mathrm{Sr}$ up to $6.8 \mathrm{MeV}$ 
2004KA68 S.Kastleiner, Yu.N.Shubin, F.M.Nortier, T.N.Van der Walt, S.M.Qaim Radiochim.Acta 92, 449 (2004)

Experimental studies and nuclear model calculations on (p, xn) and (p, pxn) reactions on ${ }^{85} \mathrm{Rb}$ from their thresholds up to $100 \mathrm{MeV}$

2004KE15 A.Kelic, K.-H.Schmidt, T.Enqvist, A.Boudard, P.Armbruster, J.Benlliure, M.Bernas, S.Czajkowski, R.Legrain, S.Leray, B.Mustapha, M.Pravikoff, F.Rejmund, C.Stephan, J.Taieb, L.Tassan-Got, C.Volant, W.Wlazlo - Phys.Rev. C 70, 064608 (2004) Isotopic and velocity distributions of ${ }_{83} \mathrm{Bi}$ produced in charge-pickup reactions of ${ }_{82}^{208} \mathrm{~Pb}$ at $1 \mathrm{~A} \mathrm{GeV}$

2004KE18 J.J.Kelly, for the Jefferson Laboratory Hall A Collaboration - Fizika(Zagreb) B 13, $81(2004)$

Recoil polarization for neutral pion electroproduction near the delta resonance

2004KHZX T.L.Khoo, T.Lauritsen, I.Ahmad, I.Calderin, M.P.Carpenter, G.Hackman, R.V.F.Janssens, A.Lopez-Martens, T.Dossing, B.Herskind, M.Matsuo, K.Yoshida, F.Hannachi, A.Korichi - ANL-04/22 (Physics Division Ann.Rept., 2003), p.61 (2004) Narrow Spreading Widths of Excited Bands in a Superdeformed Well

2004KHZY T.L.Khoo, R.Blinstrup, D.Seweryniak, I.Ahmad, M.P.Carpenter, C.N.Davids, S.Freeman, J.Greene, N.Hammond, R.V.F.Janssens, F.G.Kondev, T.Lauritsen, C.J.Lister, G.Mukherjee, P.A.Butler, P.Chowdhury, J.A.Cizewski, R.Gramer, R.D.Herzberg, A.Heinz, P.Ikin, M.Johnson, G.D.Jones, E.Ngijoi-Yogo, P.Reiter ANL-04/22 (Physics Division Ann.Rept., 2003), p.47 (2004)

Electrons from a $0.3 \mathrm{~s}$ Isomer in ${ }^{254} \mathrm{No}$

$2004 K I 23$ H.Kim, J.K.Ahn, G.Kim, T.-I.Ro, W.-C.Chung, M.Igashira, T.Ohsaki, S.Mizuno J.Korean Phys.Soc. 45, 1474 (2004)

Measurement of Neutron Capture Cross-Sections and Capture $\gamma$-ray Spectra of $161,162,163,164$ Dy at $550 \mathrm{keV}$

2004KM01 M.Kmiecik, A.Maj, B.Million, M.Brekiesz, W.Krolas, W.Meczynski, J.Styczen, M.Zieblinski, A.Bracco, F.Camera, G.Benzoni, S.Leoni, O.Wieland, S.Brambilla, B.Herskind, M.Kicinska-Habior, N.Dubray, J.Dudek, N.Schunck - Phys.Rev. C 70, 064317 (2004)

Probing nuclear shapes close to the fission limit with the giant dipole resonance in ${ }^{216} \mathrm{Rn}$

2004KMZZ M.Kmiecik, A.Maj, J.Styczen, P.Bednarczyk, M.Brekiesz, J.Grebosz, M.Lach, W.Meczynski, M.Zieblinski, K.Zuber, A.Bracco, F.Camera, G.Benzoni, B.Million, S.Leoni, O.Wieland, B.Herskind, D.Curien, N.Dubray, J.Dudek, N.Schunck, K.Mazurek - nucl-ex/0412046,12/21/2004 (2004)

GDR Feeding of the Highly-Deformed Band in ${ }^{42} \mathrm{Ca}$ 
2004K064 A.Korff, P.Haefner, C.Baumer, A.M.van den Berg, N.Blasi, B.Davids, D.De Frenne, R.de Leo, D.Frekers, E.-W.Grewe, M.N.Harakeh, F.Hofmann, M.Hunyadi, E.Jacobs, B.C.Junk, A.Negret, P.von Neumann-Cosel, L.Popescu, S.Rakers, A.Richter, H.J.Wortche - Phys.Rev. C 70, 067601 (2004)

Deuteron elastic and inelastic scattering at intermediate energies from nuclei in the mass range $6 \leq \mathrm{A} \leq 116$

2004KRZX M.I.Krivopustov, J.Adam, A.R.Balabekyan, Yu.A.Batusov, M.Bielewicz, R.Brandt, P.Chaloun, D.Chultem, K.K.Dwivedi, A.F.Elishev, M.Fragopoulou, V.Henzl, D.Henzlova, V.G.Kalinnikov, M.K.Kievets, A.Krasa, F.Krizek, A.Kugler, M.Manolopoulou, I.I.Mariin, A.Nourreddine, R.Odoj, A.V.Pavliouk, V.S.Pronskikh, H.Robotham, K.Siemon, M.Szuta, V.I.Stegailov, A.A.Solnyshkin, A.N.Sosnin, S.Stoulos, V.M.Tsoupko-Sitnikov, Ts.Tumendelger, A.Wojciechowski, V.Wagner, J.S.Wan, W.Westmeier, M.Zamani-Valassiadou, H.Kumawat, V.Kumar, O.S.Zaveriokha, I.V.Zhuk - JINR-E1-2004-79 (2004)

Investigation of Neutron Spectra and Transmutation of ${ }^{129} \mathrm{I},{ }^{237} \mathrm{~Np}$ and Other Nuclides with 1.5 GeV Protons from the Dubna Nuclotron Using the Electronuclear Setup $<<$ Energy plus Transmutation $>>$

$2004 K U 30 \quad$ K.A.Kuterbekov, I.N.Kuchtina, B.M.Sadykov, E.I.Ismatov - Ukr.J.Phys. 49, 841 (2004)

Phenomenological and semimicroscopic analysis of $50-\mathrm{MeV}$ alpha particles scattering and structure of even tin isotopes

2004KU33 J.Kuhnhenn, U.Herpers, R.Michel - Radiochim.Acta 92, 233 (2004)

New determination of the half-life of ${ }^{205} \mathrm{Bi}$

2004KU35 V.T.Kupryashkin, L.P.Sidorenko, A.I.Feoktistov, I.P.Shapovalova -

Bull.Rus.Acad.Sci.Phys. 68, 1358 (2004)

Angular distributions of near-zero-energy electrons emitted from surface of source in radioactive decay

2004KU36 R.Kuramoto, R.Lichtenthaler, A.Lepine-Szily, V.Guimaraes, G.F.Lima, E.Benjamim, P.N.de Faria - Braz.J.Phys. 34, 933 (2004)

Spectroscopy of ${ }^{6} \mathrm{Li}$ Using the ${ }^{3} \mathrm{He}\left({ }^{7} \mathrm{Li}, \alpha\right){ }^{6} \mathrm{Li}$ Reaction

2004LAZV G.J.Lane, K.H.Maier, A.P.Byrne, G.D.Dracoulis, R.Broda, B.Fornal, M.P.Carpenter, R.M.Clark, M.Cromaz, R.V.F.Janssens, A.O.Macchiavelli, I.Wiedenhover, K.Vetter - ANU-P/1637 (2004)

High-Spin Isomers and Three-Neutron Valence Configurations in ${ }^{211} \mathrm{~Pb}$

2004LAZW

T.Lauritsen, I.Ahmad, M.P.Carpenter, A.M.Heinz, R.V.F.Janssens, D.G.Jenkins, T.L.Khoo, F.G.Kondev, C.J.Lister, D.Seweryniak, P.Fallon, A.O.Macchiavelli, D.Ward, R.M.Clark, M.Cromaz, A.Larabee, B.Herskind, T.Dossing, A.Lopez-Martens, A.Korichi, S.Siem - ANL-04/22 (Physics Division Ann.Rept., 2003), p.51 (2004)

Rotational Damping, Ridges, and the Quasicontinuum of $\gamma$ Rays in ${ }^{152}$ Dy 
2004 LI75 S.N.Liddick, P.F.Mantica, R.Broda, B.A.Brown, M.P.Carpenter, A.D.Davies, B.Fornal, T.Glasmacher, D.E.Groh, M.Honma, M.Horoi, R.V.F.Janssens, T.Mizusaki, D.J.Morrissey, A.C.Morton, W.F.Mueller, T.Otsuka, J.Pavan, H.Schatz, A.Stolz, S.L.Tabor, B.E.Tomlin, M.Wiedeking - Phys.Rev. C 70, 064303 (2004) Development of shell closures at $\mathrm{N}=32,34$. I. $\beta$ decay of neutron-rich Sc isotopes

2004 L22 Yu.E.Loginov, S.E.Malyutenkova - Bull.Rus.Acad.Sci.Phys. 68, 1292 (2004) $\gamma$-lines from ${ }^{183} \mathrm{~W}(\mathrm{n} \gamma){ }^{184} \mathrm{~W}$ reaction

2004MaZP H.Matsumura, T.Sanami, K.Masumoto, N.Nakao, A.Toyoda, M.Kawai, T.Aze, H.Nagai, M.Takada, H.Matsuzaki - KEK Preprint 2004-90 (2004)

Target Dependence of Beryllium Fragment Production in Neutron- and Alpha-induced Nuclear Reactions at Intermediate Energies

2004MB03 B.N.Markov, Z.Blaszczak, Yu.P.Gangrsky, S.G.Zemlyanoi, D.V.Karaivanov, K.P.Marinova, L.M.Melnikova - Bull.Rus.Acad.Sci.Phys. 68, 157 (2004)

Radii of magnetic moment distribution in atomic nuclei

2004MB08 G.V.Marti, A.J.Pacheco, J.E.Testoni, D.Abriola, O.A.Capurro, D.E.DiGregorio, J.O.Fernandez Niello, A.O.Macchiavelli, R.M.Clark, P.Fallon, A.Goergen, D.Ward, C.Y.Wu, A.Hayes, D.Cline, R.Teng - Braz.J.Phys. 34, 885 (2004)

Measurement of Inelastic-Scattering Cross Sections in the ${ }^{16} \mathrm{O}+{ }^{28} \mathrm{Si}$ System to Discriminate Regular and Chaotic Regimes

2004ME23 D.Mekterovic, I.Supek, for the Crystal Ball Collaboration - Fizika(Zagreb) B 13, 501 (2004)

Preliminary differential cross sections of the charge-exchange reaction

2004ME25 N.H.Medina, J.R.B.Oliveira, E.W.Cybulska, M.N.Rao, R.V.Ribas, M.A.Rizzutto, W.A.Seale, F.R.Espinoza-Quinones, D.Bazzacco, F.Brandolini, S.Lunardi, C.M.Petrache, Zs.Podolyak, C.Rossi-Alvarez, F.Soramel, C.A.Ur, M.A.Cardona, G.de Angelis, D.R.Napoli, P.Spolaore, A.Gadea, D.De Acuna, M.De Poli, E.Farnea, D.Foltescu, M.Ionescu-Bujor, A.Iordachescu - Braz.J.Phys. 34, 1002 (2004)

First Allowed Bandcrossing in Neutron Deficient Nucleus ${ }^{141} \mathrm{~Tb}$

2004MIZR S.Michimasa, S.Shimoura, H.Iwasaki, M.Tamaki, N.Aoi, H.Baba, N.Iwasa, S.Kanno, S.Kubono, K.Kurita, M.Kurokawa, T.Minemura, T.Motobayashi, M.Notani, H.J.Ong, S.Ota, A.Saito, H.Sakurai, S.Takeuchi, E.Takeshita, Y.Yanagisawa, A.Yoshida - CNS-REP-64, p.269 (2004)

In-beam Gamma-ray Spectroscopy of Neutron-rich Nucleus ${ }^{23} \mathrm{~F}$

2004MIZS R.Michel, W.Glasser, D.Kollar, M.A.M.Uosif, U.Herpers, P.Malmborg, A.V.Prokofiev, V.Dangendorf, R.Nolte, H.Schuhmacher, I.Ryzhov, A.N.Smirnov, D.Schumann, R.Weinreich, P.W.Kubik, H.-A.Synal, I.Leya, J.-P.Meulders NEA/NSC/DOC(2004)14 (Jul-4151), p.28 (2004)

Production of Residual Nuclides at Medium Energies- Finalizing the HINDAS Project 
2004M054 S.Mohammadi, Zs.Podolyak, G.de Angelis, M.Axiotis, D.Bazzacco, P.G.Bizzeti, F.Brandolini, R.Broda, D.Bucurescu, E.Farnea, W.Gelletly, A.Gadea, M.Ionescu-Bujor, A.Iordachescu, Th.Kroll, S.Longdown, S.Lunardi, N.Marginean, T.Martinez, N.H.Medina, B.Quintana, P.H.Regan, B.Rubio, C.A.Ur, J.J.Valiente Dobon, P.M.Walker, Y.H.Zhang - Braz.J.Phys. 34, 792 (2004)

High-Spin States Populated in Deep-Inelastic Reactions

2004MOZT C.B.Moon - Priv.Comm. (2004)

2004MOZU K.Morita - Priv.Comm. (to be published in Proc. EXON 2004) (2004)

Decay of an isotope ${ }^{277} 112$ produced by ${ }^{208} \mathrm{~Pb}+{ }^{70} \mathrm{Zn}$ reaction

2004NA42 B.S.Nara Singh, M.Hass, Y.Nir-El, G.Haquin - Phys.Rev.Lett. 93, 262503 (2004)

New Precision Measurement of the ${ }^{3} \mathrm{He}\left({ }^{4} \mathrm{He}, \gamma\right){ }^{7} \mathrm{Be}$ Cross Section

2004NA44 H.Naik, R.J.Singh, R.H.Iyer - Radiochim.Acta 92, 1 (2004)

Fission fragment angular momentum in the spontaneous fission of ${ }^{244} \mathrm{Cm}$

2004NE13 A.Neusser, H.Hubel, A.Al-Khatib, P.Bringel, A.Burger, N.Nenoff, G.Schonwasser, A.K.Singh, C.M.Petrache, G.Lo Bianco, I.Ragnarsson, G.B.Hagemann, B.Herskind, D.R.Jensen, G.Sletten, P.Fallon, A.Gorgen, P.Bednarczyk, D.Curien, G.Gangopadhyay, A.Korichi, A.Lopez-Martens, B.V.T.Rao, T.S.Reddy, N.Singh Phys.Rev. C 70, 064315 (2004)

Superdeformed band at very high spin in ${ }^{140} \mathrm{Nd}$

2004NI18 S.Niccolai, and the CLAS Collaboration - Phys.Rev. C 70, 064003 (2004)

Complete measurement of three-body photodisintegration of ${ }^{3} \mathrm{He}$ for photon energies between 0.35 and $1.55 \mathrm{GeV}$

20040DZZ A.Odahara, Y.Gono, Y.Wakabayashi, T.Fukuchi, N.Hokoiwa, M.Kibe, T.Teranishi, S.Kubono, M.Notani, Y.Yanagisawa, S.Michimasa, J.J.He, H.Iwasaki, S.Shimoura, H.Watanabe, T.Kishida, E.Ideguchi, H.Baba, S.Nishimura, M.Nishimura, J.Y.Moon, S.Kato, H.Sagawa - CNS-REP-64, p.289 (2004)

Gamma-Ray Spectroscopy by Secondary Fusion Reactions using CRIB

$20040 G 12$ Yu.Ts.Oganessian, V.K.Utyonkov, Yu.V.Lobanov, F.Sh.Abdullin, A.N.Polyakov, I.V.Shirokovsky, Yu.S.Tsyganov, G.G.Gulbekian, S.L.Bogomolov, B.N.Gikal, A.N.Mezentsev, S.Iliev, V.G.Subbotin, A.M.Sukhov, A.A.Voinov, G.V.Buklanov, K.Subotic, V.I.Zagrebaev, M.G.Itkis, J.B.Patin, K.J.Moody, J.F.Wild, M.A.Stoyer, N.J.Stoyer, D.A.Shaughnessy, J.M.Kenneally, P.A.Wilk, R.W.Lougheed, R.I.Ilkaev, S.P.Vesnovskii - Phys.Rev. C 70, 064609 (2004); Erratum Phys.Rev. C 71, 029902 (2005)

Measurements of cross sections and decay properties of the isotopes of elements 112, 114 , and 116 produced in the fusion reactions ${ }^{233,238} \mathrm{U},{ }^{242} \mathrm{Pu}$, and ${ }^{248} \mathrm{Cm}+{ }^{48} \mathrm{Ca}$ 
20040GZZ Yu.Ts.Oganessian, V.K.Utyonkov, Yu.V.Lobanov, F.Sh.Abdullin, A.N.Polyakov, I.V.Shirokovsky, Yu.S.Tsyganov, G.G.Gulbekian, S.L.Bogomolov, B.N.Gikal, A.N.Mezentsev, S.Iliev, V.G.Subbotin, A.M.Sukhov, A.A.Voinov, G.V.Buklanov, K.Subotic, V.I.Zagrebaev, M.G.Itkis, J.B.Patin, K.J.Moody, J.F.Wild, M.A.Stoyer, N.J.Stoyer, D.A.Shaughnessy, J.M.Kenneally, P.A.Wilk, R.W.Lougheed, R.I.Ilkaev, S.P.Vesnovskii - JINR-E7-2004-160 (2004)

Measurements of cross sections and decay properties of the isotopes of elements 112, 114 and 116 produced in the fusion reactions ${ }^{233,238} \mathrm{U},{ }^{242} \mathrm{Pu}$ and ${ }^{248} \mathrm{Cm}+{ }^{48} \mathrm{Ca}$

20040NZZ T.K.Onishi, A.Gelberg, H.Sakurai, K.Yoneda, N.Aoi, N.Imai, H.Baba, P.von Brentano, N.Fukuda, Y.Ichikawa, M.Ishihara, H.Iwasaki, D.Kameda, T.Kishida, A.F.Lisetskiy, H.J.Ong, M.Osada, T.Otsuka, M.K.Suzuki, K.Ue, Y.Utsuno, H.Watanabe - CNS-REP-64, p.235 (2004)

Gamow-Teller beta decay of ${ }^{46} \mathrm{Cr}$

2004PA42 Y.Parpottas, S.M.Grimes, S.Al-Quraishi, C.R.Brune, T.N.Massey, J.E.Oldendick, A.Salas, R.T.Wheeler - Phys.Rev. C 70, 065805 (2004); Erratum Phys.Rev. C 73, 049907 (2006)

Astrophysically important ${ }^{26} \mathrm{Si}$ states studied with the $\left({ }^{3} \mathrm{He}, \mathrm{n}\right)$ reaction and the

${ }^{25} \mathrm{Al}(\mathrm{p}, \gamma){ }^{26} \mathrm{Si}$ reaction rates in explosive hydrogen burning environments

2004 PE24 B.A.Perdue, M.W.Ahmed, A.P.Tonchev, H.R.Weller, G.Feldman, V.N.Litvinenko, I.V.Pinayev, B.E.Norum, R.M.Prior, M.C.Spraker, B.D.Sawatzky - Phys.Rev. C 70, 064305 (2004)

Compton scattering of polarized $\gamma$ rays by ${ }^{16} \mathrm{O}$ for $\mathrm{E}_{\gamma}=25-40 \mathrm{MeV}$

2004PEZW O.Perru - IPNO-T-05-02 (2004)

Etude des fermetures de couches $\mathrm{N}=40$ et $\mathrm{N}=50$ dans les noyaux riches en neutrons

2004PYZZ Yu.V.Pyatkov, D.V.Kamanin, W.Trzaska, S.R.Yamaletdinov, E.A.Sokol,

A.N.Tyukavkin, A.A.Aleksandrov, I.A.Aleksandrova, S.V.Denisov, V.P.Kraynov,

S.V.Khlebnikov, T.E.Kuzmina, E.A.Kuznetsova, S.V.Mitrofanov,

Yu.E.Penionzhkevich, Yu.V.Ryabov, V.G.Tishchenko, B.V.Florko -

JINR-E15-2004-65 (2004)

New Results in Study of the Collinear Cluster Tripartition of the ${ }^{252} \mathrm{Cf}$ Nucleus

2004QAZZ S.M.Qaim, S.Sudar, Ye.Skakun, A.Fessler - NEA/NSC/DOC(2004)14 (Jul-4151), p.11 (2004)

Fundamental Studies on Isomeric Cross Sections

2004RA28 P.M.Raddon, D.G.Jenkins, C.D.O'Leary, A.J.Simons, R.Wadsworth, A.N.Andreyev, R.D.Page, M.P.Carpenter, F.G.Kondev, T.Enqvist, P.T.Greenlees, P.M.Jones,

R.Julin, S.Juutinen, H.Kettunen, M.Leino, A.-P.Leppanen, P.Nieminen,

J.Pakarinen, P.Rahkila, J.Uusitalo, D.T.Joss - Phys.Rev. C 70, 064308 (2004)

$\alpha$ decay and recoil decay tagging studies of ${ }^{183} \mathrm{Tl}$

2004RA29 U.Ratzel, C.Arlandini, F.Kappeler, A.Couture, M.Wiescher, R.Reifarth, R.Gallino, A.Mengoni, C.Travaglio - Phys.Rev. C 70, 065803 (2004)

Nucleosynthesis at the termination point of the s process 
2004REZY K.E.Rehm, C.L.Jiang, J.P.Greene, D.Henderson, R.V.F.Janssens, E.F.Moore, G.Mukherjee, R.C.Pardo, T.Pennington, J.P.Schiffer, S.Sinha, X.D.Tang, R.H.Siemssen, L.Jisonna, R.E.Segel, A.H.Wuosmaa - ANL-04/22 (Physics Division Ann.Rept., 2003), p.3 (2004)

Studies of the ${ }^{8} \mathrm{~B}(\alpha, \mathrm{p}){ }^{11} \mathrm{C}$ Reaction

2004R047 C.L.Rodrigues, M.R.D.Rodrigues, T.Borello-Lewin, L.B.Horodynski-Matsushigue, J.L.M.Duarte, G.M.Ukita, G.N.Hanninger - Braz.J.Phys. 34, 760 (2004)

Coulomb-Nuclear Interference (CNI) Results of the Collective Quadrupolar

Excitations in Odd and Even Ru Isotopes

2004R048 M.R.D.Rodrigues, C.L.Rodrigues, T.Borello-Lewin, L.B.Horodynski-Matsushigue, J.L.M.Duarte, G.M.Ukita - Braz.J.Phys. 34, 777 (2004)

Coulomb Nuclear Interference with Deuterons in even Palladium Isotopes

2004SA61 K.Sabourov, M.W.Ahmed, R.S.Canon, B.Crowley, K.Joshi, J.H.Kelley, S.O.Nelson, B.A.Perdue, E.C.Schreiber, A.Sabourov, A.Tonchev, H.R.Weller, E.A.Wulf, R.M.Prior, M.C.Spraker, H.M.Hofmann, M.Trini - Phys.Rev. C 70, 064601 (2004) Experimental and theoretical study of the ${ }^{2} \mathrm{H}(\mathrm{d}(\mathrm{pol}), \gamma){ }^{4} \mathrm{He}$ reaction below $\mathrm{E}_{c . m .}=60 \mathrm{keV}$

2004SA64 M.E.Sadler, for the Crystal Ball Collaboration - Fizika(Zagreb) B 13, 405 (2004) Pion-nucleon charge exchange measurements in the region of the $\Delta(1232)$ resonance

2004SC48 B.Scholten, S.Takacs, F.Tarkanyi, H.H.Coenen, S.M.Qaim - Radiochim.Acta 92, 203 (2004)

Excitation functions of deuteron induced nuclear reactions on enriched ${ }^{78} \mathrm{Kr}$ with particular relevance to the production of ${ }^{76} \mathrm{Br}$

2004SCZU B.Scholten, H.E.Hassan, S.Spellerberg, D.Steyn, N.van der Walt, S.Takacs, F.Tarkanyi, S.M.Qaim, H.H.Coenen - NEA/NSC/DOC(2004)14 (Jul-4151), p.13 (2004) Cross sections and yields of nuclear processes for the production of the positron emitters ${ }^{76} \mathrm{Br},{ }^{124} \mathrm{I}$ and ${ }^{82} \mathrm{Sr}\left({ }^{82} \mathrm{Rb}\right)$

2004SEZW D.Seweryniak, C.N.Davids, M.P.Carpenter, N.Hammond, R.V.F.Janssens, T.-L.Khoo, G.Mukherjee, S.Sinha, A.Robinson, P.J.Woods, B.Blank, T.Davinson, S.J.Freeman, N.Hoteling, Z.Liu, J.Shergur, A.A.Sonzogni, W.B.Walters, A.Woehr ANL-04/22 (Physics Division Ann.Rept., 2003), p.27 (2004)

Ground-State Bands in the Proton Emitters ${ }^{145,147} \mathrm{Tm}$

2004SEzX D.Seweryniak, M.P.Carpenter, N.Hammond, A.Heinz, R.V.F.Janssens, T.L.Khoo, C.J.Lister, G.Mukherjee, E.Rehm, S.Sinha, P.J.Woods, B.Blank, T.Davinson, S.J.Freeman, J.Goerres, N.Hoteling, D.G.Jenkins, H.Mahmud, Z.Liu, F.Sarazin, J.Shergur, M.Shawcross, A.Woehr - ANL-04/22 (Physics Division Ann.Rept., 2003), p.12 (2004)

The Level Structured and Mass of ${ }^{22} \mathrm{Mg}$ 
2004SHZZ V.I.Sharov, N.G.Anischenko, V.G.Antonenko, S.A.Averichev, L.S.Azhgirey, V.D.Bartenev, N.A.Bazhanov, A.A.Belyaev, N.A.Blinov, N.S.Borisov, S.B.Borzakov, Yu.T.Borzunov, Yu.P.Bushuev, L.P.Chernenko, E.V.Chernykh, V.F.Chumakov, S.A.Dolgii, A.N.Fedorov, V.V.Fimushkin, M.Finger, M.Finger, Jr., L.B.Golovanov, G.M.Gurevich, A.Janata, A.D.Kirillov, V.G.Kolomiets, E.V.Komogorov, A.D.Kovalenko, A.I.Kovalev, V.A.Krasnov, P.Krstonoshich, E.S.Kuzmin, V.P.Ladygin, A.B.Lazarev, F.Lehar, A.de Lesquen, M.Yu.Liburg, A.N.Livanov, A.A.Lukhanin, P.K.Maniakov, V.N.Matafonov, E.A.Matyushevsky, V.D.Moroz, A.A.Morozov, A.B.Neganov, G.P.Nikolaevsky, A.A.Nomofilov, Tz.Panteleev, Yu.K.Pilipenko, I.L.Pisarev, Yu.A.Plis, Yu.P.Polunin, A.N.Prokofiev, V.Yu.Prytkov, P.A.Rukoyatkin, V.A.Schedrov, O.N.Schevelev, S.N.Shilov, R.A.Shindin, M.Slunecka, V.Sluneckova, A.Yu.Starikov, G.D.Stoletov, L.N.Strunov, A.L.Svetov, Yu.A.Usov, T.Vasiliev, V.I.Volkov, E.I.Vorobiev, I.P.Yudin, I.V.Zaitsev, A.A.Zhdanov, V.N.Zhmyrov - JINR-E1-2004-87 (2004)

Measurements of the Total Cross-Section Difference $\Delta \sigma_{L}(\mathrm{np})$ at $1.39,1.69,1.89$ and $1.99 \mathrm{GeV}$

2004SIZX S.Sinha, J.Greene, D.Henderson, R.V.F.Janssens, C.L.Jiang, E.F.Moore, R.C.Pardo, K.E.Rehm, J.P.Schiffer, X.Tang, A.Chen, L.Jisonna, R.Segel, R.H.Siemssen, A.H.Wuosmaa - ANL-04/22 (Physics Division Ann.Rept., 2003), p.8 (2004) Study of the Breakout Reaction ${ }^{18} \mathrm{Ne}(\alpha, \mathrm{p})^{21} \mathrm{Na}$

2004S035 N.Soic, S.Blagus, M.Bogovac, W.Catford, S.Cherubini, N.Clarke, E.Costanzo, L.Donadille, S.Fazinic, M.Freer, B.Fulton, B.Greenhalgh, K.Jones, M.Lattuada, P.Leask, D.Mahboub, M.Milin, D.Miljanic, D.Rendic, S.Romano, C.Spitaleri, T.Tadic, D.Watson, D.Weisser, M.Zadro - Fizika(Zagreb) B 13, 433 (2004) Experimental evidence for molecular structures in light nuclei

2004S036 F.A.Souza, A.Szanto de Toledo, M.G.Munhoz, J.Takahashi, N.Carlin, A.A.P.Suaide, M.M.de Moura, e.M.Szanto - Braz.J.Phys. 34, 907 (2004)

Study of the Effect of the Breakup on the Fusion Cross Section of the Systems ${ }^{6,7} \mathrm{Li}+{ }^{59} \mathrm{Co}$

2004SP06 I.Spahn, H.H.Coenen, S.M.Qaim - Radiochim.Acta 92, 183 (2004)

Enhanced production possibility for the therapeutic radionuclides ${ }^{64} \mathrm{Cu},{ }^{67} \mathrm{Cu}$ and ${ }^{89} \mathrm{Sr}$ via $(\mathrm{n}, \mathrm{p})$ reactions induced by fast spectral neutrons

2004ST32 S.Strauch, for the CLAS Collaboration - Fizika(Zagreb) B 13, 179 (2004) Helicity-dependent angular distributions in double-charged-pion photoproduction

2004 TA46 F.Tarkanyi, A.Hermanne, S.Takacs, Yu.N.Shubin, A.I.Dityuk - Radiochim.Acta 92, $223(2004)$

Cross sections for production of the therapeutic radioisotopes ${ }^{198} \mathrm{Au}$ and ${ }^{199} \mathrm{Au}$ in proton and deuteron induced reactions on ${ }^{198} \mathrm{Pt}$

2004TAZW X.Tang, K.E.Rehm, I.Ahmad, J.Greene, A.Hecht, D.Henderson, C.L.Jiang, E.F.Moore, M.Notani, R.C.Pardo, G.Savard, J.P.Schiffer, S.Sinha, M.Paul, R.E.Segel, L.Jisonna, C.Brune, A.Wuosmaa - ANL-04/22 (Physics Division Ann.Rept., 2003), p.5 (2004)

Measurement of the $\beta$-Delayed $\alpha$ Spectrum of ${ }^{16} \mathrm{~N}$ with a New Technique 
2004 TH15 M.Thoennessen - Acta Phys.Hung.N.S. 21, 379 (2004)

Pushing the Limits of Nuclear Stability

2004VA37 S.I.Vasiliev, Yu.G.Zdesenko, A.A.Klimenko, S.B.Osetrov, A.A.Smolnikov, V.I.Tretyak - Bull.Rus.Acad.Sci.Phys. 68, 1255 (2004)

Experimental limit on charge-nonconserving ${ }^{73} \mathrm{Ge} \beta$-decay probability

2004VE14 D.Verney, F.Ibrahim, O.Perru, O.Bajeat, C.Bourgeois, F.Clapier, E.Cottereau, C.Donzaud, S.Du, M.Ducourtieux, S.Essabaa, S.Gales, L.Gaudefroy, D.Guillemaud-Mueller, F.Hammache, F.Hosni, C.Lau, H.Lefort, F.Le Blanc, A.C.Mueller, N.Pauwels, J.C.Potier, F.Pougheon, J.Proust, B.Roussiere, J.Sauvage, O.Sorlin - Braz.J.Phys. 34, 979 (2004)

Study of the $\mathrm{N}=50$ Shell Closure Close to ${ }^{78} \mathrm{Ni}$

2004V021 A.N.Vodin, A.S.Kachan, L.P.Korda, V.M.Mishchenko, R.P.Slabospitsky, I.V.Ushakov, G.K.Khomyakov - Bull.Rus.Acad.Sci.Phys. 68, 210 (2004) Isobar-analogue resonances in ${ }^{22} \mathrm{Ne}(\mathrm{p} \gamma)^{23} \mathrm{Na}$ reaction

2004 V022 A.N.Vodin, A.S.Kachan, L.P.Korda, V.M.Mishchenko, R.P.Slabospitsky, I.V.Ushakov, G.K.Khomyakov - Bull.Rus.Acad.Sci.Phys. 68, 218 (2004)

Fine structure of analogue $\mathrm{d}_{5 / 2}$ resonance in ${ }^{31} \mathrm{P}$ nucleus

2004V024 A.von Zweidorf, R.Angert, W.Bruchle, S.Burger, K.Eberhardt, R.Eichler, H.Hummrich, E.Jager, H.-O.Kling, J.V.Kratz, B.Kuczewski, G.Langrock, M.Mendel, U.Rieth, M.Schadel, B.Schausten, E.Schimpf, P.Thorle, N.Trautmann, K.Tsukada, N.Wiehl, G.Wirth - Radiochim.Acta 92, 855 (2004)

Evidence for the formation of sodium hassate(VIII)

2004 WA35 H.L.Wang, Y.H.Zhang, X.H.Zhou, M.M.Ndontchueng, Y.X.Guo, X.G.Lei, M.L.Liu, L.Peng, C.Y.Xie, L.T.Song, H.P.Yu, Y.Zheng, W.T.Guo, S.X.Wen, L.H.Zhu, X.G.Wu, F.R.Xu - Phys.Rev. C 70, 064306 (2004)

Observation of the $\pi \mathrm{h}_{11 / 2}(\mathrm{X}) \nu \mathrm{i}_{13 / 2}$ band in odd-odd ${ }^{170} \mathrm{Re}$

2004WAZW H.Watanabe, H.Ueno, A.Yoshimi, Y.Kobayashi, T.Haseyama, T.Kishida, K.Asahi, D.Kameda, H.Miyoshi, K.Shimada, S.Emori, G.Kato, Y.Gono, A.Odahara CNS-REP-64, p.243 (2004)

g-Factor measurements of high-spin isomers and condensed matter studies with $\gamma$-TDPAD method

2004WE17 F.R.Wesselmann - Fizika(Zagreb) B 13, 531 (2004)

Measurement of the neutron's electric form factor $\mathrm{G}_{E}^{n}$ via doubly polarized, quasi-elastic scattering at Jefferson Lab

2004WIZX A.N.Wilson, G.D.Dracoulis, P.M.Davidson, A.P.Byrne, R.M.Clark, P.Fallon, A.Gorgen, G.J.Lane, A.O.Macchiavelli, D.Ward - ANU-P/1610 (2004)

Quadrupole Moment of the Yrast Superdeformed Band in ${ }^{192} \mathrm{~Pb}$

2004 WU08 C.Y.Wu, H.Hua, D.Cline, A.B.Hayes, R.Teng, R.M.Clark, P.Fallon, A.Goergen, A.O.Macchiavelli, K.Vetter - Phys.Rev. C 70, 064312 (2004) 
Multifaceted yrast structure and the onset of deformation in ${ }^{96,97} \mathrm{Sr}$ and ${ }^{98,99} \mathrm{Zr}$

2004 YU11 K.L.Yurkewicz, D.Bazin, B.A.Brown, C.M.Campbell, J.A.Church, D.-C.Dinca, A.Gade, T.Glasmacher, M.Honma, T.Mizusaki, W.F.Mueller, H.Olliver, T.Otsuka, L.A.Riley, J.R.Terry - Phys.Rev. C 70, 064321 (2004)

E2 excitation strength in ${ }^{55} \mathrm{Ni}$ : Coupling of the ${ }^{56} \mathrm{Ni} 2_{1}^{+}$collective core vibration to the $\mathrm{f}_{7 / 2}$ odd neutron hole

2004ZA15 G.S.Zahn, C.B.Zamboni, F.A.Genezini, M.P.Raele, M.T.F.da Cruz, J.Y.Zevallos-Chavez - Braz.J.Phys. 34, 719 (2004)

New Gamma Transitions in ${ }^{193}$ Ir From the Beta Decay of ${ }^{193}$ Os

2004ZH42 X.Zheng, and the Jefferson Lab Hall A Collaboration - Phys.Rev. C 70, 065207 (2004)

Precision measurement of the neutron spin asymmetries and spin-dependent structure functions in the valence quark region

2004ZH45 B.V.Zhuravlev, A.A.Lychagin, N.N.Titarenko, V.G.Demenkov, V.I.Trykova, A.Ya.Slekenichs - Bull.Rus.Acad.Sci.Phys. 68, 1319 (2004)

Level densities in ${ }^{90} \mathrm{Nb}$ and ${ }^{94} \mathrm{Nb}$ nuclei from neutron evaporation spectra

2005AB01 M.Abdel-Bary, A.Budzanowski, A.Chatterjee, J.Ernst, P.Hawranek, F.Hinterberger, V.Jha, K.Kilian, S.Kliczewski, D.Kirillov, D.Kolev, M.Kravcikova, T.Kutsarova, M.Lesiak, J.Lieb, H.Machner, A.Magiera, R.Maier, G.Martinska, S.Nedev, J.Niskanen, N.Piskunov, D.Prasuhn, D.Protic, P.von Rossen, B.J.Roy, I.Sitnik, R.Siudak, M.Smiechowicz, R.Tsenov, M.Ulicny, J.Urban, G.Vankova, C.Wilkin, and the GEM Collaboration - Phys.Lett. B 610, 31 (2005)

Detailed comparison of the pp $\rightarrow \pi^{+}$pn and pp $\rightarrow \pi^{+} \mathrm{d}$ reactions at $951 \mathrm{MeV}$

2005AB04 B.M.Abramov, Yu.A.Borodin, S.A.Bulychjov, I.A.Dukhovskoy, A.P.Krutenkova, V.V.Kulikov, M.A.Martemyanov, M.A.Matsyuk, V.E.Tarasov, E.N.Turdakina, A.I.Khanov - Yad.Fiz. 68, 503 (2005); Phys.Atomic Nuclei 68, 474 (2005)

Quasielastic Deuteron and Triton Knockout from Lithium Isotopes by Intermediate-Energy Pions

2005AB22 U.Abbondanno, for the n_TOF Collaboration - Nucl.Phys. A758, 501c (2005) Neutron capture cross section measurements for nuclear astrophysics at CERN n_TOF

2005AC22 P.Achenbach, for the A1 Collaboration - Eur.Phys.J. A 25, 177 (2005) Measurement of the Asymmetries in ${ }^{3}(\mathrm{He}(\mathrm{pol}))\left(\mathrm{e}(\mathrm{pol}), \mathrm{e}\right.$ p)d and ${ }^{3}(\mathrm{He}(\mathrm{pol}))(\mathrm{e}(\mathrm{pol})$, e'p)np

2005AD01 J.Adam, A.R.Balabekyan, V.S.Barashenkov, R.Brandt, V.M.Golovatyuk, V.G.Kalinnikov, K.Katovsky, M.I.Krivopustov, V.Kumar, H.Kumawat, R.Odoj, V.S.Pronskikh, A.A.Solnyshkin, V.I.Stegailov, V.M.Tsoupko-Sitnikov, W.Westmeier - Eur.Phys.J. A 23, 61 (2005)

Spallation neutron spectrum on a massive lead / paraffin target irradiated with 1 $\mathrm{GeV}$ protons 
2005 AD29 P.Adrich, A.Klimkiewicz, M.Fallot, K.Boretzky, T.Aumann, D.Cortina-Gil, U.Datta Pramanik, Th.W.Elze, H.Emling, H.Geissel, M.Hellstrom, K.L.Jones, J.V.Kratz, R.Kulessa, Y.Leifels, C.Nociforo, R.Palit, H.Simon, G.Surowka, K.Summerer, W.Walus, and the LAND-FRS Collaboration - Phys.Rev.Lett. 95, 132501 (2005) Evidence for Pygmy and Giant Dipole Resonances in ${ }^{130} \mathrm{Sn}$ and ${ }^{132} \mathrm{Sn}$

2005AD35 S.Adhikari, C.Samanta, C.Basu, S.Ray, A.Chatterjee, S.Kailas - Eur.Phys.J. A 25, Supplement 1, 299 (2005)

Entrance channel dependence in compound nuclear reactions with loosely bound nuclei

2005ADZV E.G.Adelberger, O.D.Biesel, A.Garcia, H.E.Swanson, S.Triambak - Univ. Washington Nucl.Phys.Lab., Ann.Rept., 2005, p.59 (2005)

Resonance energies in ${ }^{32} \mathrm{~S}(\mathrm{p}, \gamma)$ and calibration of ${ }^{33} \mathrm{Ar}$ beta-delayed spectrum

2005ADZW E.G.Adelberger, A.Garcia, S.A.Hoedl, S.K.L.Sjue, A.L.Sallaska, S.Triambak - Univ. Washington Nucl.Phys.Lab., Ann.Rept., 2005, p.58 (2005)

Mass determination of the lowest $\mathrm{T}=2$ state in ${ }^{32} \mathrm{~S}$ using the ${ }^{31} \mathrm{P}(\mathrm{p}, \gamma)$ reaction

2005ADZX P.Adrich, T.Aumann, K.Boretzky, D.Cortina-Gil, U.Datta Pramanik, Th.W.Elze, H.Emling, M.Fallot, H.Geissel, M.Hellstrom, K.L.Jones, A.Klimkiewicz, J.V.Kratz, R.Kulessa, Y.Leifels, C.Nociforo, R.Palit, H.Simon, G.Surowka, K.Summerer, W.Walus - GSI 2005-1, p.94 (2005)

Pygmy and Giant Dipole Resonances in ${ }^{130-132} \mathrm{Sn}$

2005ADZZ J.Adam, A.Balabekyan, R.Brandt, P.Caloun, D.Chultem, V.G.Kalinnikov, K.Katovsky, M.I.Krivopustov, A.Kugler, R.Odoj, A.V.Pavlyuk, V.S.Pronskikh, A.A.Solnyshkin, V.I.Stegailov, S.G.Stetsenko, V.M.Tsoupko-Sitnikov, V.Wagner, W.Westmeier - Book of Abstracts, LV National Conference on Nuclear Physics "Frontiers in the Physics of Nucleus", St.-Petersburg, p.195 (2005) Yields of Product Nuclei in Radioactive ${ }^{129} \mathrm{I},{ }^{237} \mathrm{~Np},{ }^{238} \mathrm{Pu}$ and ${ }^{239} \mathrm{Pu}$ Samples Exposed to the Secondary Neutrons of the U / Pb-Assembly of the Setup "Energy Plus Transmutation" Irradiated by $1 \mathrm{GeV}$ Protons from Nuclotron (JINR, Dubna)

2005AEZZ G.Aerts, for the n_TOF Collaboration - Proc.Intern.Conf.Nuclear Data for Science and Technology, Santa Fe, New Mexico, 26 September-1 October, 2004, R.C.Haight, M.B.Chadwick, T.Kawano, P.Talou, Eds., Vol.2, p. 1470 (2005); AIP Conf.Proc. $769(2005)$

Measurement of the ${ }^{232}$ Th Neutron Capture Cross Section at the CERN n_TOF Facility

2005AF02 M.Afzal Ansari, N.P.M.Sathik, D.Singh, M.H.Rashid - J.Phys.Soc.Jpn. 74, 1150 (2005) Measurement and Analysis of Alpha Particle Induced Reactions on Praseodymium

2005AG03 E.S.Ageev, and the COMPASS Collaboration - Phys.Lett. B 612, 154 (2005) Measurement of the spin structure of the deuteron in the DIS region 
2005AG04 M.Agnello, G.Beer, L.Benussi, M.Bertani, S.Bianco, E.Botta, T.Bressani, L.Busso, D.Calvo, P.Camerini, P.Cerello, B.Dalena, F.De Mori, G.D'Erasmo, D.Di Santo, F.L.Fabbri, D.Faso, A.Feliciello, A.Filippi, V.Filippini, E.M.Fiore, H.Fujioka, P.Gianotti, N.Grion, A.Krasnoperov, V.Lucherini, S.Marcello, T.Maruta, N.Mirfakhrai, O.Morra, A.Olin, E.Pace, M.Pallotta, M.Palomba, A.Pantaleo, A.Panzarasa, V.Paticchio, S.Piano, F.Pompili, R.Rui, G.Simonetti, H.So, S.Tomassini, R.Wheadon, A.Zenoni - Nucl.Phys. A752, 139c (2005)

First results from the FINUDA experiment

2005AG09 M.Agnello, G.Beer, L.Benussi, M.Bertani, S.Bianco, E.Botta, T.Bressani, L.Busso, D.Calvo, P.Camerini, P.Cerello, B.Dalena, F.De Mori, G.D'Erasmo, D.Di Santo, F.L.Fabbri, D.Faso, A.Feliciello, A.Filippi, V.Filippini, E.M.Fiore, H.Fujioka, P.Gianotti, N.Grion, A.Krasnoperov, V.Lucherini, S.Marcello, T.Maruta, N.Mirfakhrai, O.Morra, A.Olin, E.Pace, M.Palomba, A.Pantaleo, A.Panzarasa, V.Paticchio, S.Piano, F.Pompili, R.Rui, G.Simonetti, H.So, S.Tomassini, R.Wheadon, A.Zenoni - Nucl.Phys. A754, 399c (2005)

First results from the FINUDA experiment at DA $\Phi$ NE

2005AG11 M.Agnello, and the FINUDA Collaboration - Phys.Lett. B 622, 35 (2005)

First results on ${ }_{\Lambda}^{12} \mathrm{C}$ production at $\mathrm{DA} \phi \mathrm{NE}$

2005AG15 U.Agvaanluvsan, E.Algin, J.A.Becker, M.Guttormsen, G.E.Mitchell, S.Siem, A.Schiller, A.Voinov - Nucl.Instrum.Methods Phys.Res. B241, 180 (2005)

Investigation of the radiative strength function

2005AH01 J.Ahrens, V.M.Alexeev, J.R.M.Annand, H.J.Arends, R.Beck, G.Caselotti, S.N.Cherepnya, D.Drechsel, L.V.Filkov, K.Fohl, I.Giller, P.Grabmayr, T.Hehl, D.Hornidge, V.L.Kashevarov, M.Kotulla, D.Krambrich, B.Krusche, M.Lang, J.C.McGeorge, I.J.D.MacGregor, V.Metag, M.Moinester, R.Novotny, M.Pfeiffer, M.Rost, S.Schadmand, S.Scherer, A.Thomas, C.Unkmeir, Th.Walcher - Eur.Phys.J. A 23, 113 (2005)

Measurement of the $\pi^{+}$-meson polarizabilities via the $\gamma \mathrm{p} \rightarrow \gamma \pi^{+} \mathrm{n}$ reaction

2005AH03 I.Ahmad, F.G.Kondev, E.F.Moore, M.P.Carpenter, R.R.Chasman, J.P.Greene, R.V.F.Janssens, T.Lauritsen, C.J.Lister, D.Seweryniak, R.W.Hoff, J.E.Evans, R.W.Lougheed, C.E.Porter, L.K.Felker - Phys.Rev. C 71, 054305 (2005)

Energy levels of ${ }^{249} \mathrm{Bk}$ populated in the $\alpha$ decay of ${ }_{99}^{253} \mathrm{Es}$ and $\beta^{-}$decay of ${ }_{96}^{249} \mathrm{Cm}$

2005AH05 J.Ahrens, and the GDH and A2 Collaborations - Phys.Lett. B 624, 173 (2005) Intermediate resonance excitation in the $\gamma \mathrm{p} \rightarrow \mathrm{p} \pi^{0} \pi^{0}$ reaction

2005AH07 J.Ahrens, S.Altieri, J.R.M.Annand, H.-J.Arends, R.Beck, A.Braghieri, N.d'Hose, H.Dutz, S.Goertz, P.Grabmayr, S.Hasegawa, E.Heid, H.Holvoet, L.Van Hoorebeke, N.Horikawa, T.Iwata, O.Jahn, P.Jennewein, R.Kondratiev, J.Krimmer, M.Lang, B.Lannoy, K.Livingston, J.C.McGeorge, W.Meyer, A.Panzeri, P.Pedroni, T.Pinelli, I.Preobrajenski, G.Reicherz, G.Rosner, M.Rost, T.Rostomyan, D.Ryckbosch, M.Schumacher, B.Seitz, G.Tamas, A.Thomas, R.van de Vyver, Th.Walcher, F.Zapadtka - Eur.Phys.J. A 26, 135 (2005)

Measurement of the G asymmetry for the $\gamma \mathrm{p} \rightarrow \mathrm{N} \pi$ channels in the $\Delta(1232)$ resonance region 
2005AH09 I.Ahmad, J.P.Greene, E.F.Moore, F.G.Kondev, R.R.Chasman, C.E.Porter, L.K.Felker - Phys.Rev. C 72, 054308 (2005)

Energy levels of ${ }^{251} \mathrm{Cf}$ populated in the $\alpha$ decay of ${ }_{100}^{255} \mathrm{Fm}$ and EC decay of ${ }_{99}^{251} \mathrm{Es}$

2005 AHZZ I.Ahmad, F.G.Kondev, E.F.Moore, R.R.Chasman, M.P.Carpenter, J.P.Greene, R.V.F.Janssens, T.Lauritsen, C.J.Lister, D.Seweryniak, R.W.Hoff, J.E.Evans, R.W.Lougheed, C.E.Porter, L.K.Felker - Proc.Nuclei at the Limits, Argonne, Illinois, D.Seweryniak and T.L.Khoo, eds., p.251 (2005); AIP Conf. Proc 764 (2005) Proton Single-Particle States In The Heaviest Actinide Nuclei

2005AI06 A.Airapetian, and the HERMES Collaboration - Phys.Rev.Lett. 95, 242001 (2005) Measurement of the Tensor Structure Function $b_{1}$ of the Deuteron

2005AK09 A.Aktas, and the H1 Collaboration - Eur.Phys.J. C 44, 1 (2005) Measurement of deeply virtual Compton scattering at HERA

2005AL01 M.Altmeier, F.Bauer, J.Bisplinghoff, K.Busser, M.Busch, T.Colberg, L.Demirors, H.P.Engelhardt, P.D.Eversheim, K.O.Eyser, O.Felden, R.Gebel, M.Glende, J.Greiff, F.Hinterberger, E.Jonas, H.Krause, T.Lindemann, J.Lindlein, B.Lorentz, R.Maier, R.Maschuw, A.Meinerzhagen, D.Prasuhn, H.Rohdjess, D.Rosendaal, P.von Rossen, N.Schirm, V.Schwarz, W.Scobel, H.-J.Trelle, K.Ulbrich, E.Weise, A.Wellinghausen, R.Ziegler, and the EDDA Collaboration - Eur.Phys.J. A 23, 351 (2005)

Excitation functions of the analyzing power in elastic proton-proton scattering from 0.45 to $2.5 \mathrm{GeV}$

2005 AL03 J.J.S.Alves, P.R.S.Gomes, J.Lubian, L.C.Chamon, D.Pereira, R.M.Anjos, E.S.Rossi, Jr., C.P.Silva, M.A.G.Alvarez, G.P.A.Nobre, L.R.Gasques - Nucl.Phys. A748, 59 (2005)

Consistent analysis of peripheral reaction channels and fusion for the ${ }^{16,18} \mathrm{O}+{ }^{58} \mathrm{Ni}$ systems

2005AL15 D.V.Aleksandrov, E.Yu.Nikolskii, B.G.Novatskii, S.B.Sakuta, D.N.Stepanov - Pisma Zh.Eksp.Teor.Fiz. 81, 49 (2005); JETP Lett. 81, 43 (2005)

Search for Resonances in the Three- and Four-Neutron Systems in the ${ }^{7} \mathrm{Li}\left({ }^{7} \mathrm{Li}\right.$, $\left.{ }^{11} \mathrm{C}\right) 3 \mathrm{n}$ and ${ }^{7} \mathrm{Li}\left({ }^{7} \mathrm{Li},{ }^{10} \mathrm{C}\right) 4 \mathrm{n}$ Reactions

2005AL20

A.Al-Khatib, A.K.Singh, H.Hubel, P.Bringel, A.Burger, A.Neusser, G.Schoenwasser, G.B.Hagemann, C.R.Hansen, B.Herskind, G.Sletten, A.Algora, Zs.Dombradi, J.Gal, G.Kalinka, J.Molnar, B.M.Nyako, D.Sohler, J.Timar, L.Zolnai, M.Kmiecik, A.Maj, J.Styczen, K.Zuber, K.Hauschild, A.Korichi, A.Lopez-Martens, J.Roccaz, S.Siem, F.Hannachi, J.N.Scheurer, P.Bednarczyk, Th.Byrski, D.Curien, O.Dorvaux, G.Duchene, B.Gall, F.Khalfallah, I.Piqueras, J.Robin, K.Juhasz, S.B.Patel, A.O.Evans, G.Rainovski, A.Airoldi, G.Benzoni, A.Bracco, F.Camera, B.Million, P.Mason, A.Paleni, R.Sacchi, O.Wieland, C.M.Petrache, D.Petrache, G.La Rana, R.Moro, G.De Angelis, P.Fallon, I.-Y.Lee, J.C.Lisle, B.Cederwall, K.Lagergren, R.M.Lieder, E.Podsvirova, W.Gast, H.Jaeger, N.Redon, A.Gorgen - Acta Phys.Pol. B36, 1029 (2005)

High-spin states in ${ }^{124} \mathrm{Ba}$ 
2005AL25 J.A.Alcantara-Nunez, J.R.B.Oliveira, E.W.Cybulska, N.H.Medina, M.N.Rao, R.V.Ribas, M.A.Rizzutto, W.A.Seale, F.Falla-Sotelo, K.T.Wiedemann - Phys.Rev. C 71, 054315 (2005)

High-spin structures in ${ }^{108} \mathrm{Pd}$ : $\gamma$-vibrational band and two-quasineutron excitations

2005AL27 V.Kh.Alimov, M.Mayer, J.Roth - Nucl.Instrum.Methods Phys.Res. B234, 169 (2005) Differential cross-section of the $\mathrm{D}\left({ }^{3} \mathrm{He}, \mathrm{p}\right){ }^{4} \mathrm{He}$ nuclear reaction and depth profiling of deuterium up to large depths

2005AL37 G.D.Alkhazov, V.V.Astashin, A.G.Atamantchouk, W.Augustyniak, D.Bachelier, V.V.Baublis, M.Boivin, J.-L.Boyard, J.-F.Clavelin, P.Courtat, M.Denoit, R.Douet, C.Ellegaard, L.Farhi, C.Gaarde, R.Gacougnolle, V.Ya.Gerzenstein, V.L.Golovtsov, V.V.Golubev, T.Hennino, J.-C.Jourdain, A.V.Khanzadeev, B.G.Komkov, A.V.Kravtsov, L.G.Kudin, R.Kunne, J.Larsen, S.Lebon, L.V.Malinina, A.Maroni, J.-M.Martin, H.-P.Morsch, V.A.Mylnikov, E.M.Orischin, M.Osterlund, V.Poux, A.N.Prokofiev, P.Radvanyi, B.Ramstein, B.V.Razmyslovich, M.Roy-Stephan, V.M.Samsonov, R.Skowron, I.B.Smirnov, E.A.Strokovsky, I.I.Tkach, V.V.Vikhrov, S.S.Volkov, An.A.Vorobyov, A.A.Zhdanov, P.Zupranski - Nucl.Instrum.Methods Phys.Res. A551, 290 (2005)

SPES4- $\pi$ : installation for exclusive study of nuclear reactions

2005AL45 J.J.S.Alves, P.R.S.Gomes, J.Lubian, L.C.Chamon, R.M.Anjos, D.Pereira, E.S.Rossi, Jr., C.P.Silva, M.A.G.Alvarez, G.P.A.Nobre, L.R.Gasques - Braz.J.Phys. 35, 909 (2005)

Elastic, Inelastic Scatterings and Transfer Reactions for ${ }^{16,18} \mathrm{O}$ on ${ }^{58} \mathrm{Ni}$ Described by the Sao Paulo Potential

2005ALZX A.Alimeti, G.H.R.Kegel, J.J.Egan, D.J.DeSimone, T.M.McKittrick, C.Ji, S.E.Tremblay, C.Roldan, X.Chen, D.S.Kim - Proc.Intern.Conf.Nuclear Data for Science and Technology, Santa Fe, New Mexico, 26 September-1 October, 2004, R.C.Haight, M.B.Chadwick, T.Kawano, P.Talou, Eds., Vol.1, p. 1054 (2005); AIP Conf.Proc. 769 (2005)

Neutron Scattering Cross Section Measurements for ${ }^{169} \mathrm{Tm}$ via the (n, n') Technique

2005ALZY I.E.Alekseev, V.V.Lazarev - Book of Abstracts, LV National Conference on Nuclear Physics "Frontiers in the Physics of Nucleus", St.-Petersburg, p.181 (2005)

The Yields of ${ }^{186} \mathrm{Re}$ in Reaction ${ }^{186} \mathrm{~W}(\mathrm{~d}, 2 \mathrm{n})$

2005 ALzZ R.A.Aliev, A.N.Ermakov, B.S.Ishkhanov, I.M.Kapitonov, I.G.Konyukhov, K.Kyaw, I.V.Makarenko, T.N.Mineeva, K.A.Stopani - Book of Abstracts, LV National Conference on Nuclear Physics "Frontiers in the Physics of Nucleus", St.-Petersburg, p.56 (2005)

Excitation of $135.5 \mathrm{keV}$ Isomeric Level of ${ }^{92} \mathrm{Nb}$ Nucleus in Photonuclear Reactions

2005AM02 H.Amro, G.B.Hagemann, W.C.Ma, R.M.Diamond, J.Domscheit, P.Fallon, B.Herskind, H.Hubel, D.R.Jensen, Y.Li, A.O.Macchiavelli, D.Roux, G.Sletten, J.Thompson, I.Wiedenhover, J.N.Wilson, J.A.Winger - Phys.Rev. C 71, 011302 (2005)

Competing modes of excitations and the decay out of the triaxial strongly deformed well in ${ }^{167} \mathrm{Lu}$ 
2005AM04 Y.Amelin, W.J.Davis - Geochim.Cosmochim.Act. 69, 465 (2005)

Geochemical test for branching decay of ${ }^{176} \mathrm{Lu}$

2005 AM05 M.Ambrogiani, and the Fermilab E835 Collaboration - Phys.Lett. B 610, 177 (2005)

Measurement of the angular distribution in (p-bar)p $\rightarrow \psi(2 \mathrm{~S}) \rightarrow \mathrm{e}^{+} \mathrm{e}^{-}$

2005AN01 A.Andrighetto, O.Bajeat, A.E.Barzakh, S.Essabaa, D.V.Fedorov, A.M.Ionan, V.S.Ivanov, R.Leroy, G.Lhersonneau, K.A.Mezilev, F.V.Moroz, S.Yu.Orlov, V.N.Panteleev, L.Stroe, L.B.Tecchio, A.Villari, Yu.M.Volkov, X.F.Wang -

Eur.Phys.J. A 23, 257 (2005)

On-line production of $\mathrm{Rb}$ and $\mathrm{Cs}$ isotopes from uranium carbide targets

2005AN03 L.-L.Andersson, E.K.Johansson, J.Ekman, D.Rudolph, R.du Rietz, C.Fahlander, C.J.Gross, P.A.Hausladen, D.C.Radford, G.Hammond - Phys.Rev. C 71, 011303 (2005)

Identification of excited states in ${ }_{31}^{61} \mathrm{Ga}_{30}$ : Mirror nuclei in the upper fp shell

2005AN04 K.Andgren, Zs.Podolyak, A.Dewald, F.R.Xu, A.Algora, M.Axiotis, D.Bazzacco, P.G.Bizzeti, A.M.Bizzeti-Sona, B.Cederwall, G.de Angelis, E.Farnea, A.Fitzler, A.Gadea, W.Gelletly, S.Lunardi, O.Moller, N.Marginean, T.Martinez, T.Pissulla, C.Rusu, C.A.Ur, R.Venturelli, P.M.Walker, C.Wheldon - Phys.Rev. C 71, 014312 (2005)

Lifetime measurements of normal deformed states in ${ }_{71}^{165} \mathrm{Lu}$

2005AN15 A.Antusek, K.Jackowski, M.Jaszunski, W.Makulski, M.Wilczek - Chem.Phys.Lett. 411, $111(2005)$

Nuclear magnetic dipole moments from NMR spectra

2005An17 A.N.Andreyev, D.Ackermann, S.Antalic, I.G.Darby, S.Franchoo, F.P.Hessberger, S.Hofmann, M.Huyse, P.Kuusiniemi, B.Lommel, B.Kindler, R.Mann,

G.Munzenberg, R.D.Page, S.Saro, B.Sulignano, B.Streicher, K.Van de Vel, P.Van Duppen, D.R.Wiseman - Phys.Rev. C 72, 014612 (2005)

Cross section systematics for the lightest $\mathrm{Bi}$ and Po nuclei produced in complete fusion reactions with heavy ions

2005An23 C.Angulo, E.Casarejos, A.Coc, T.Davinson, N.Achouri, D.Cortina-Gil, M.Couder, P.Figuera, B.Fulton, J.Kiener, P.Leleux, I.Mukha, A.S.Murphy, A.Ninane, N.Orr, V.Tatischeff, F.Vanderbist - Nucl.Phys. A758, 775c (2005)

Study of ${ }^{7} \mathrm{Be}+\mathrm{d}$ reactions for Standard Big Bang Nucleosynthesis

2005An24 E.Angeli, A.Tartari, M.Frignani, D.Mostacci, F.Rocchi, M.Sumini -

Appl.Radiat.Isot. 63, 545 (2005)

Preliminary results on the production of short-lived radioisotopes with a Plasma

Focus Device 
2005 AN26 K.Andgren, S.F.Ashley, P.H.Regan, E.A.McCutchan, N.V.Zamfir, L.Amon, R.B.Cakirli, R.F.Casten, R.M.Clark, G.Gurdal, K.L.Keyes, D.A.Meyer, M.N.Erduran, A.Papenberg, N.Pietralla, C.Plettner, G.Rainovski, R.V.Ribas, N.J.Thomas, J.Vinson, D.D.Warner, V.Werner, E.Williams - J.Phys.(London) G31, S1563 (2005)

$\mathrm{RDM}$ lifetime measurements in ${ }^{107} \mathrm{Cd}$

2005An30 C.Angulo, E.Casarejos, M.Couder, P.Demaret, P.Leleux, F.Vanderbist, A.Coc, J.Kiener, V.Tatischeff, T.Davinson, A.S.Murphy, N.L.Achouri, N.A.Orr,

D.Cortina-Gil, P.Figuera, B.R.Fulton, I.Mukha, E.Vangioni - Astrophys.J. 630, L105 (2005)

The ${ }^{7} \mathrm{Be}(\mathrm{d}, \mathrm{p}) 2 \alpha$ Cross Section at Big Bang Energies and the Primordial ${ }^{7} \mathrm{Li}$ Abundance

2005ANZY A.N.Andreyev, S.Antalic, D.Ackermann, S.Franchoo, F.P.Hessberger, S.Hofmann, M.Huyse, I.Kojouharov, B.Kindler, P.Kuusiniemi, S.R.Lesher, B.Lommel, R.Mann, G.Munzenberg, K.Nishio, R.D.Page, J.Ressler, B.Streicher, B.Sulignano, P.Van Duppen, D.Wiseman - GSI 2005-1, p.77 (2005)

New isotopes ${ }^{186,187} \mathrm{Po}$ and ${ }^{192} \mathrm{At}$

2005ANZZ K.A.Aniol, and the HAPPEX Collaboration - nucl-ex/0506010,6/07/2005 (2005)

Parity-Violating Electron Scattering from ${ }^{4} \mathrm{He}$ and the Strange Electric Form Factor of the Nucleon

2005AR21 D.S.Armstrong, and the G0 Collaboration - Phys.Rev.Lett. 95, 092001 (2005) Strange-Quark Contributions to Parity-Violating Asymmetries in the Forward G0 Electron-Proton scattering Experiment

2005AR25 C.Arnaboldi, D.R.Artusa, F.T.Avignone III, M.Balata, I.Bandac, M.Barucci, J.W.Beeman, C.Brofferio, C.Bucci, S.Capelli, L.Carbone, S.Cebrian, O.Cremonesi, R.J.Creswick, A.de Waard, H.A.Farach, E.Fiorini, G.Frossati, E.Guardincerri, A.Giuliani, P.Gorla, E.E.Haller, R.J.McDonald, A.Morales, E.B.Norman, A.Nucciotti, E.Olivieri, M.Pallavicini, E.Palmieri, E.Pasca, M.Pavan, M.Pedretti, G.Pessina, S.Pirro, E.Previtali, L.Risegari, C.Rosenfeld, S.Sangiorgio, M.Sisti, A.R.Smith, L.Torres, G.Ventura - Phys.Rev.Lett. 95, 142501 (2005)

New Limit on the Neutrinoless $\beta \beta$ Decay of ${ }^{130} \mathrm{Te}$

2005AR27 R.Arnold, C.Augier, J.Baker, A.Barabash, G.Broudin, V.Brudanin, A.J.Caffrey, E.Caurier, V.Egorov, K.Errahmane, A.I.Etienvre, J.L.Guyonnet, F.Hubert, Ph.Hubert, C.Jollet, S.Jullian, O.Kochetov, V.Kovalenko, S.Konovalov, D.Lalanne, F.Leccia, C.Longuemare, G.Lutter, Ch.Marquet, F.Mauger, F.Nowacki, H.Ohsumi, F.Piquemal, J.L.Reyss, R.Saakyan, X.Sarazin, L.Simard, F.Simkovic, Yu.Shitov, A.Smolnikov, L.Stekl, J.Suhonen, C.S.Sutton, G.Szklarz, J.Thomas, V.Timkin, V.Tretyak, V.Umatov, L.Vala, I.Vanushin, V.Vasilyev, V.Vorobel, Ts.Vylov Phys.Rev.Lett. 95, 182302 (2005)

First Results of the Search for Neutrinoless Double-Beta Decay with the NEMO 3 Detector 
2005AS01 M.Asai, M.Sakama, K.Tsukada, S.Ichikawa, H.Haba, I.Nishinaka, Y.Nagame, S.Goto, Y.Kojima, Y.Oura, H.Nakahara, M.Shibata, K.Kawade - Eur.Phys.J. A 23, 395 (2005)

Proton-neutron configurations in ${ }^{236 g, m} \mathrm{Am}$ and its EC-decay daughter ${ }^{236} \mathrm{Pu}$

2005AS04 N.I.Ashwood, M.Freer, D.J.Millener, N.A.Orr, F.Carstoiu, S.Ahmed, J.C.Angelique, V.Bouchat, W.N.Catford, N.M.Clarke, N.Curtis, F.Hanappe, M.Horoi, Y.Kerckx, J.L.Lecouey, F.M.Marques, T.Materna, G.Normand, S.Pain, N.Soic, C.Timis, A.Unshakova, V.A.Ziman - Phys.Rev. C 72, 024314 (2005)

High-energy two-neutron removal from ${ }^{10} \mathrm{Be}$

2005AS05 M.Asai, K.Tsukada, M.Sakama, S.Ichikawa, T.Ishii, Y.Nagame, I.Nishinaka, K.Akiyama, A.Osa, Y.Oura, K.Sueki, M.Shibata - Phys.Rev.Lett. 95, 102502 (2005) Experimental Identification of Spin-Parities and Single-Particle Configurations in ${ }^{257}$ No and Its $\alpha$-Decay Daughter ${ }^{253} \mathrm{Fm}$

2005AT04 F.Atchison, B.van den Brandt, T.Brys, M.Daum, P.Fierlinger, P.Hautle, R.Henneck, S.Heule, M.Kasprzak, K.Kirch, J.A.Konter, A.Michels, A.Pichlmaier, M.Wohlmuther, A.Wokaun, K.Bodek, U.Szerer, P.Geltenbort, J.Zmeskal, Y.Pokotilovsky - Phys.Rev. C 71, 054601 (2005)

Production of ultracold neutrons from a cold neutron beam on a ${ }^{2} \mathrm{H}_{2}$ target

2005BA01 A.S.Barabash, and the NEMO Collaboration - Nucl.Phys. B(Proc.Supp.) S138, 207 (2005)

Nemo-3 double beta decay experiment: Present status

2005BA02 D.Bandyopadhyay, N.Warr, C.Fransen, N.Boukharouba, V.Werner, S.W.Yates, J.L.Weil, M.T.McEllistrem - Nucl.Phys. A747, 206 (2005)

Three-phonon excitations in ${ }^{124} \mathrm{Sn}$

2005BA10 G.Basar, G.Basar, I.K.Ozturk, F.G.Acar, S.Kroger - Phys.Scr. 71, 159 (2005)

Hyperfine Structure of High Lying Levels of Tm I

2005BA11 E.Kh.Bazarov, V.V.Glagolev, K.G.Gulamov, S.L.Lutpullaev, K.Olimov, A.A.Yuldashev, E.I.Ismatov, D.A.Karshiev, Kh.Sh.Khamidov, B.S.Yuldashev Ukr.J.Phys. 50, 16 (2005)

Fragmentation of oxygen nuclei into $\alpha$-particles and ${ }^{12} \mathrm{C}$ nuclei in ${ }^{16} \mathrm{Op}$-collisions at $3.25 \mathrm{~A} \mathrm{GeV} / \mathrm{c}$

2005BA13 M.D.L.Barbosa, T.Borello-Lewin, L.B.Horodynski-Matsushigue, J.L.M.Duarte, C.L.Rodrigues, M.R.D.Rodrigues, G.M.Ukita - Phys.Rev. C 71, 024303 (2005) Coulomb-nuclear interference with ${ }^{6} \mathrm{Li}$ : Isospin character of the $2_{1}^{+}$excitation in ${ }^{70,72,74} \mathrm{Ge}$

2005BA14 C.Baumer, A.M.van den Berg, B.Davids, D.Frekers, D.De Frenne, E.-W.Grewe, P.Haefner, M.N.Harakeh, F.Hofmann, S.Hollstein, M.Hunyadi, M.A.de Huu, E.Jacobs, B.C.Junk, A.Korff, K.Langanke, G.Martinez-Pinedo, A.Negret, P.von Neumann-Cosel, L.Popescu, S.Rakers, A.Richter, H.J.Wortche - Phys.Rev. C 71, 024603 (2005); Erratum Phys.Rev. C 71, 029903 (2005) 
Determination of the Gamow-Teller strength distribution from the odd-odd nucleus ${ }^{50} \mathrm{~V}$ measured through ${ }^{50} \mathrm{~V}\left(\mathrm{~d},{ }^{2} \mathrm{He}\right){ }^{50} \mathrm{Ti}$ and astrophysical implications

2005BA18 A.R.Balabekyan, A.S.Danagulyan, J.R.Drnoyan, N.A.Demekhina, J.Adam, V.G.Kalinnikov, M.I.Krivopustov, V.S.Pronskikh, V.I.Stegailov, A.A.Solnyshkin, P.Chaloun, V.M.Tsoupko-Sitnikov, G.Musulmanbekov - Yad.Fiz. 68, 195 (2005); Phys.Atomic Nuclei 68, 171 (2005)

Investigation of Spallation Reactions on ${ }^{120} \mathrm{Sn}$ and $(\mathrm{d}, \mathrm{xn}),(\mathrm{d}, \mathrm{pxn}),(\mathrm{p}, \mathrm{xn})$, and (p, pxn) Reactions on Enriched Tin Isotopes

2005BA25 S.Baunack, for the A4 Collaboration - Eur.Phys.J. A 24, Supplement 2, 35 (2005) Transverse spin asymmetry at the A4 experiment: Experimental results

2005BA30 M.S.Basunia, E.B.Norman, H.A.Shugart, A.R.Smith, M.J.Dolinski, B.J.Quiter Phys.Rev. C 71, 035801 (2005)

Measurement of cross sections for the ${ }^{63} \mathrm{Cu}(\alpha, \gamma){ }^{67} \mathrm{Ga}$ reaction from 5.9 to $8.7 \mathrm{MeV}$

2005BA33 A.S.Barabash, and the NEMO Collaboration - Yad.Fiz. 68, 443 (2005);

Phys.Atomic Nuclei 68, 414 (2005)

Investigation of Double-Beta-Decay Processes at the NEMO-3 Tracking Detector

2005BA34 Chr.Bargholtz, B.A.Chernyshev, L.Geren, V.N.Grebenev, Yu.B.Gurov, B.Hoistad, I.V.Laukhin, K.Lindberg, V.G.Sandukovsky, R.R.Shafigullin, P.-E.Tegner - Yad.Fiz. 68, 517 (2005); Phys.Atomic Nuclei 68, 488 (2005)

A Search for Deeply bound Pionic States of Xenon Produced in the ${ }^{136} \mathrm{Xe}(\mathrm{d}$, $\left.{ }^{3} \mathrm{He}\right){ }^{135} \mathrm{Xe}_{\pi-\text { bound }}$ Reaction

2005BA40 E.Kh.Bazarov, V.V.Glagolev, V.V.Lugovoy, S.L.Lutpullaev, K.Olimov, V.I.Petrov, A.A.Yuldashev, B.S.Yuldashev - Pisma Zh.Eksp.Teor.Fiz. 81, 174 (2005); JETP Lett. 81, 140 (2005)

Cross Sections for the Production of Stable and Unstable Isotopes with Charge Numbers from One to Eight in ${ }^{16} \mathrm{Op}$ Collisions at $3.25 \mathrm{~A} \mathrm{GeV} / \mathrm{c}$

2005BA43 C.Baumer, D.Frekers, E.-W.Grewe, P.Haefner, S.Hollstein, B.C.Junk, A.Korff, S.Rakers, R.Schmidt, A.M.van den Berg, B.Davids, M.N.Harakeh, M.Hunyadi, M.A.de Huu, H.J.Wortche, N.Blasi, D.De Frenne, E.Jacobs, A.Negret, L.Popescu, R.De Leo, F.Hofmann, P.von Neumann-Cosel, A.Richter - Phys.Rev. C 71, 044003 (2005)

Measurement of the ${ }^{2} \mathrm{H}\left(\mathrm{d},{ }^{2} \mathrm{He}\right)^{2} \mathrm{n}$ reaction at $\mathrm{E}_{d}=171 \mathrm{MeV}$ and implications for the neutron-neutron scattering length

2005BA50 F.Bauer, J.Bisplinghoff, K.Busser, M.Busch, T.Colberg, C.Dahl, L.Demirors, P.D.Eversheim, K.O.Eyser, O.Felden, R.Gebel, J.Greiff, F.Hinterberger, E.Jonas, H.Krause, C.Lehmann, J.Lindlein, R.Maier, A.Meinerzhagen, C.Pauly, D.Prasuhn, H.Rohdjess, D.Rosendaal, P.von Rossen, N.Schirm, W.Scobel, K.Ulbrich, E.Weise, T.Wolf, R.Ziegler, and the EDDA Collaboration - Phys.Rev. C 71, 054002 (2005) Excitation functions of spin correlation parameters $\mathrm{A}_{N N}, \mathrm{~A}_{S S}$, and $\mathrm{A}_{S L}$ in elastic $\mathrm{p}(\mathrm{pol}) \mathrm{p}(\mathrm{pol})$ scattering between 0.45 and $2.5 \mathrm{GeV}$ 
2005BA51 A.M.Baxter, A.P.Byrne, G.D.Dracoulis, P.M.Davidson, T.Kibedi, R.V.F.Janssens, M.P.Carpenter, C.N.Davids, T.L.Khoo, T.Lauritsen - Phys.Rev. C 71, 054302 (2005)

Spherical and deformed structures in ${ }^{189} \mathrm{~Pb}$

2005BA58 S.Baunack, for the A4 Collaboration - Nucl.Phys. A755, 249c (2005)

Single Spin Asymmetries from the Mainz A4 Experiment

2005BA60 A.M.Bakalyarov, A.Ya.Balysh, S.T.Belyaev, V.I.Lebedev, S.V.Zhukov - Part. and Nucl., Lett. 125, 21 (2005)

Results of the Experiment on Investigation of ${ }^{76} \mathrm{Ge}$ Double Beta Decay

2005BA64 A.E.Barzakh, D.V.Fedorov, A.M.Ionan, V.S.Ivanov, F.V.Moroz, K.A.Mezilev, S.Yu.Orlov, V.N.Panteleev, Yu.M.Volkov - Phys.Rev. C 72, 017301 (2005) Laser spectroscopic studies of ${ }^{145} \mathrm{Gd},{ }^{145} \mathrm{Gd}^{m}$, and ${ }^{143} \mathrm{Gd}^{m}$

2005BA82 D.W.Bardayan, J.C.Blackmon, J.Gomez del Campo, R.L.Kozub, J.F.Liang, Z.Ma, L.Sahin, D.Shapira, M.S.Smith - Nucl.Phys. A758, 737c (2005)

New ${ }^{19}$ Ne Level Observed with a Thick Target ${ }^{18} \mathrm{~F}(\mathrm{p}, \mathrm{p}){ }^{18} \mathrm{~F}$ Measurement

2005BA88 R.A.Bark, M.Lipoglavsek, S.M.Maliage, S.S.Ntshangase, A.Shevchenko J.Phys.(London) G31, S1747 (2005)

Aspects of nuclear physics research at iThemba LABS, South Africa

2005BA93 O.Bartholomy, and the CB-ELSA Collaboration - Phys.Rev.Lett. 94, 012003 (2005) Neutral-Pion Photoproduction off Protons in the Energy Range $0.3 \mathrm{GeV} \leq \mathrm{E} \gamma \leq 3$ $\mathrm{GeV}$

2005BA94 J.C.Batchelder, J.-C.Bilheux, C.R.Bingham, H.K.Carter, D.Fong, P.E.Garrett, R.Grzywacz, J.H.Hamilton, D.J.Hartley, J.K.Hwang, W.Krolas, W.D.Kulp, Y.Larochelle, A.Piechaczek, A.V.Ramayya, K.P.Rykaczewski, E.H.Spejewski, D.W.Stracener, M.N.Tantawy, J.A.Winger, J.L.Wood, E.F.Zganjar - Phys.Rev. C $72,044306(2005)$

New isomeric state in ${ }^{116} \mathrm{Ag}$

2005BA96 S.Barua, J.J.Das, A.Jhingan, N.Madhavan, T.Varughese, P.Sugathan, K.Kalita, S.Verma, B.Bhattacharjee, S.K.Datta, K.Boruah - Phys.Rev. C 72, 044602 (2005) Investigation of scattering between mirror nuclei ${ }^{7} \mathrm{Be}$ and ${ }^{7} \mathrm{Li}$

2005BAZP H.Baba, S.Shimoura, T.Minemura, Y.U.Matsuyama, A.Saito, H.Akiyoshi, N.Aoi, T.Gomi, Y.Higurashi, K.Ieki, N.Imai, N.Iwasa, H.Iwasaki, S.Kanno, S.Kubono, M.Kunibu, S.Michimasa, T.Motobayashi, T.Nakamura, H.Sakurai, M.Serata, E.Takeshita, S.Takeuchi, T.Teranishi, K.Ue, K.Yamada, Y.Yanagisawa CNS-REP-66, p.28 (2005) Isoscalar E0 and E1 Responses in ${ }^{14} \mathrm{O}$ 
2005BAZQ H.Baba, S.Shimoura, T.Minemura, Y.U.Matsuyama, A.Saito, H.Akiyoshi, N.Aoi, T.Gomi, Y.Higurashi, K.Ieki, N.Imai, N.Iwasa, H.Iwasaki, S.Kanno, S.Kubono, M.Kunibu, S.Michimasa, T.Motobayashi, T.Nakamura, H.Sakurai, M.Serata, E.Takeshita, S.Takeuchi, T.Teranishi, K.Ue, K.Yamada, Y.Yanagisawa - RIKEN Accelerator Progress Report 2004, p.48 (2005)

Isoscalar electric multipole strength in ${ }^{14} \mathrm{O}$

2005BAZR C.M.Baglin, E.B.Norman, R.Larimer, G.A.Rech - Proc.Intern.Conf.Nuclear Data for Science and Technology, Santa Fe, New Mexico, 26 September-1 October, 2004, R.C.Haight, M.B.Chadwick, T.Kawano, P.Talou, Eds., Vol.2, p. 1370 (2005); AIP Conf.Proc. 769 (2005)

Measurement of ${ }^{107} \mathrm{Ag}(\alpha, \gamma){ }^{111}$ In Cross Sections

2005BAZS M.S.Basunia, E.B.Norman, H.A.Shugart, A.R.Smith, M.J.Dolinski, B.J.Quiter Proc.Intern.Conf.Nuclear Data for Science and Technology, Santa Fe, New Mexico, 26 September-1 October, 2004, R.C.Haight, M.B.Chadwick, T.Kawano, P.Talou, Eds., Vol.2, p. 1366 (2005); AIP Conf.Proc. 769 (2005)

Measurement of Cross Sections for the ${ }^{63} \mathrm{Cu}(\alpha, \gamma){ }^{67} \mathrm{Ga}$ Reaction from 5.9-8.7 MeV

2005BAZU M.Baba - Proc.Intern.Conf.Nuclear Data for Science and Technology, Santa Fe, New Mexico, 26 September-1 October, 2004, R.C.Haight, M.B.Chadwick, T.Kawano, P.Talou, Eds., Vol.1, p. 884 (2005); AIP Conf.Proc. 769 (2005)

Experimental Studies on Particle and Radionuclide Production Cross Sections for Tens of MeV Neutrons and Protons

2005BAZV F.B.Bateman, S.I.Al-Quraishi, C.E.Brient, N.Boukharouba, A.D.Carlson, D.E.Carter, S.M.Grimes, R.C.Haight, T.N.Massey, R.T.Wheeler -

Proc.Intern.Conf.Nuclear Data for Science and Technology, Santa Fe, New Mexico, 26 September-1 October, 2004, R.C.Haight, M.B.Chadwick, T.Kawano, P.Talou, Eds., Vol.1, p. 834 (2005); AIP Conf.Proc. 769 (2005)

Measurement of the $\mathrm{H}(\mathrm{n}, \mathrm{n}) \mathrm{H}$ Elastic Scattering Angular Distribution at $\mathrm{E}_{n}=15$ $\mathrm{MeV}$

2005BazY A.M.Baxter, G.D.Dracoulis, A.P.Byrne, T.Kibedi, P.M.Davidson, R.V.F.Janssens, M.P.Carpenter, C.N.Davids, T.L.Khoo, T.Lauritsen - Proc.Nuclei at the Limits, Argonne, Illinois, D.Seweryniak and T.L.Khoo, eds., p.62 (2005); AIP Conf. Proc $764(2005)$

Spherical And Deformed Structures In ${ }^{189} \mathrm{~Pb}$

2005BAzZ A.M.Baxter, A.P.Byrne, G.D.Dracoulis, P.M.Davidson, T.Kibedi, R.V.F.Janssens, M.P.Carpenter, C.N.Davids, T.L.Khoo, T.Lauritsen - ANU-P/1634 (2005)

Spherical and deformed structures in ${ }^{189} \mathrm{~Pb}$

2005BB01 C.Bachelet, G.Audi, C.Gaulard, C.Guenaut, F.Herfurth, D.Lunney, M.De Saint Simon, C.Thibault - Eur.Phys.J. A 25, Supplement 1, 31 (2005)

Mass measurement of short-lived halo nuclides 
2005BB02 J.C.Batchelder, M.Tantawy, C.R.Bingham, M.Danchev, D.J.Fong, T.N.Ginter, C.J.Gross, R.Grzywacz, K.Hagino, J.H.Hamilton, M.Karny, W.Krolas, C.Mazzocchi, A.Piechaczek, A.V.Ramayya, K.P.Rykaczewski, A.Stolz, J.A.Winger, C.-H.Yu, E.F.Zganjar - Eur.Phys.J. A 25, Supplement 1, 149 (2005)

Study of fine structure in the proton radioactivity of ${ }^{146} \mathrm{Tm}$

2005BB05 D.W.Bardayan, J.C.Blackmon, J.Gomez del Campo, R.L.Kozub, J.F.Liang, Z.Ma, D.Shapira, L.Sahin, M.S.Smith - Eur.Phys.J. A 25, Supplement 1, 643 (2005)

New ${ }^{19} \mathrm{Ne}$ resonance observed using an exotic ${ }^{18} \mathrm{~F}$ beam

2005 BB06 L.Barron-Palos, E.Chavez, A.Huerta, M.E.Ortiz, G.Murillo, E.Aguilera, E.Martinez, E.Moreno, R.Policroniades, A.Varela - Eur.Phys.J. A 25, Supplement 1, 645 (2005)

${ }^{12} \mathrm{C}+{ }^{12} \mathrm{C}$ cross-section measurements at low energies

2005BE03 R.Bernabei, P.Belli, F.Cappella, F.Montecchia, F.Nozzoli, A.d'Angelo, A.Incicchitti, D.Prosperi, R.Cerulli, C.J.Dai, H.H.Kuang, J.M.Ma, Z.P.Ye - Eur.Phys.J. A 23, 7 (2005)

Search for spontaneous transition of nuclei to a superdense state

2005BE12 F.Benmokhtar, for the Jefferson Lab Hall A Collaboration - Phys.Rev.Lett. 94, 082305 (2005)

Measurement of the ${ }^{3} \mathrm{He}(\mathrm{e}, \mathrm{e}$ 'p)pn Reaction at High Missing Energies and Momenta

2005BE17 R.Bernabei, P.Belli, F.Cappella, F.Montecchia, F.Nozzoli, A.d'Angelo, A.Incicchitti, D.Prosperi, R.Cerulli, C.J.Dai, H.L.He, H.H.Kuang, J.M.Ma, Z.P.Ye, V.I.Tretyak Eur.Phys.J. A 24, 51 (2005)

A search for spontaneous emission of heavy clusters in the ${ }^{127}$ I nuclide

2005BE33 P.Bednarczyk, A.Banu, T.Beck, F.Becker, M.A.Bentley, G.Benzoni, A.Bracco, A.Burger, F.Camera, P.Doornenbal, C.Fahlander, H.Geissel, J.Gerl, M.Gorska, H.Grawe, J.Grebosz, G.Hammond, M.Hellstrom, H.Hubel, J.Jolie, M.Kmiecik, I.Kojouharov, N.Kurz, R.Lozeva, A.Maj, S.Mandal, W.Meczynski, B.Million, S.Muralithar, P.Reiter, D.Rudolph, N.Saito, T.R.Saito, H.Schaffner, J.Simpson, J.Styczen, N.Warr, H.Weick, C.Wheldon, O.Wieland, M.Winkler, H.J.Wollersheim Acta Phys.Pol. B36, 1235 (2005)

Status of the RISING project at relativistic energies

2005BE34 G.Benzoni, A.Bracco, S.Leoni, N.Blasi, F.Camera, C.Grassi, B.Million, A.Paleni, M.Pignanelli, E.Vigezzi, O.Wieland, M.Matsuo, T.Dossing, B.Herskind, G.B.Hagemann, J.Wilson, A.Maj, M.Kmiecik, G.Lo Bianco, C.M.Petrache, M.Castoldi, A.Zucchiati, G.De Angelis, D.Napoli, P.Bednarczyk, D.Curien Phys.Lett. B 615, 160 (2005)

Is the $\mathrm{K}$-quantum number conserved in the order-to-chaos transition region?

2005BE38 F.D.Becchetti, R.S.Raymond, D.A.Roberts, J.Lucido, P.A.DeYoung, B.Hilldore, J.Bychowski, A.J.Huisman, P.J.VanWylen, J.J.Kolata, G.Rogachev, J.D.Hinnefeld Phys.Rev. C 71, 054610 (2005)

The $\left({ }^{8} \mathrm{Li}, \alpha\right)$ reaction at low energy: Direct ${ }^{4} \mathrm{H}$ cluster transfer? 
2005BE40 G.Beer, A.M.Bragadireanu, M.Cargnelli, C.Curceanu Petrascu, J.-P.Egger, H.Fuhrmann, C.Guaraldo, M.Iliescu, T.Ishiwatari, K.Itahashi, M.Iwasaki, P.Kienle, T.Koike, B.Lauss, V.Lucherini, L.Ludhova, J.Marton, F.Mulhauser, T.Ponta, L.A.Schaller, R.Seki, D.L.Sirghi, F.Sirghi, J.Zmeskal, and the DEAR Collaboration Phys.Rev.Lett. 94, 212302 (2005)

Measurement of the Kaonic Hydrogen X-Ray Spectrum

2005BE43 A.C.Betker, S.Chang, E.J.Stephenson, A.D.Bacher, S.M.Bowyer, W.A.Franklin, J.Liu, C.Olmer, D.L.Prout, S.P.Wells, S.W.Wissink, C.Yu, R.A.Lindgren,

H.Baghaei, V.Gladyshev, J.A.Carr, S.K.Yoon, F.Petrovich, B.L.Clausen, J.Lisantti Phys.Rev. C 71, 064607 (2005)

Reaction mechanism for natural parity (p, p') transitions in ${ }^{10} \mathrm{~B}$

2005BE60 M.Belleguic, F.Azaiez, Zs.Dombradi, D.Sohler, M.J.Lopez-Jimenez, T.Otsuka, M.G.Saint-Laurent, O.Sorlin, M.Stanoiu, Y.Utsuno, Yu.-E.Penionzhkevich, N.L.Achouri, J.C.Angelique, C.Borcea, C.Bourgeois, J.M.Daugas, F.De Oliveira-Santos, Z.Dlouhy, C.Donzaud, J.Duprat, Z.Elekes, S.Grevy, D.Guillemaud-Mueller, S.Leenhardt, M.Lewitowicz, S.M.Lukyanov, W.Mittig, M.G.Porquet, F.Pougheon, P.Roussel-Chomaz, H.Savajols, Y.Sobolev, C.Stodel, J.Timar - Phys.Rev. C 72, 054316 (2005)

Search for neutron excitations across the $\mathrm{N}=20$ shell gap in ${ }^{25-29} \mathrm{Ne}$

2005BE61 N.Benczer-Koller, G.Kumbartzki, J.R.Cooper, T.J.Mertzimekis, M.J.Taylor, L.Bernstein, K.Hiles, P.Maier-Komor, M.A.McMahan, L.Phair, J.Powell, K.-H.Speidel, D.Wutte - Eur.Phys.J. A 25, Supplement 1, 203 (2005) First $\mathrm{g}$-factor measurement using a radioactive ${ }^{76} \mathrm{Kr}$ beam

2005BEZS

T.Behrens, R.Gernhauser, D.Habs, Th.Kroll, R.Krucken, R.Lutter, T.Morgan, M.Pasini, K.Rudolph, P.G.Thirolf, V.Bildstein, H.Scheit, J.Jolie, D.Martin, N.Warr, and the MINIBALL / REX-ISOLDE Collaboration - Maier-Leibnitz-Laboratorium 2004 Ann.Rept., p.14 (2005)

Coulomb Excitation of Neutron-rich Cd Isotopes

2005BEZT T.Belgya, P.Mutti, H.G.Borner, M.Jentschel, G.L.Molnar -

Proc.Intern.Conf.Nuclear Data for Science and Technology, Santa Fe, New Mexico, 26 September-1 October, 2004, R.C.Haight, M.B.Chadwick, T.Kawano, P.Talou, Eds., Vol.1, p. 1074 (2005); AIP Conf.Proc. 769 (2005)

Accurate Wavelength Measurement of High-Energy Gamma Rays from the ${ }^{35} \mathrm{Cl}(\mathrm{n}$, $\gamma)$ Reactions

2005BEZU L.A.Bernstein, J.T.Burke, J.A.Church, L.Ahle, J.R.Cooper, R.D.Hoffman, J.Punyon, A.Schiller, E.Algin, C.Plettner, H.Ai, C.W.Beausang, R.F.Casten, R.Hughes, E.Ricard-McCutchan, D.Meyer, J.J.Ressler, J.A.Caggiano, N.V.Zamfir, H.Amro, A.Heinz, P.Fallon, M.A.McMahan, A.O.Macchiavelli, L.W.Phair Proc.Intern.Conf.Nuclear Data for Science and Technology, Santa Fe, New Mexico, 26 September-1 October, 2004, R.C.Haight, M.B.Chadwick, T.Kawano, P.Talou, Eds., Vol.1, p. 890 (2005); AIP Conf.Proc. 769 (2005)

Surrogate Nuclear Reactions using STARS 
2005BEZV T.Belgya, G.L.Molnar, Zs.Revay, J.L.Weil - Proc.Intern.Conf.Nuclear Data for Science and Technology, Santa Fe, New Mexico, 26 September-1 October, 2004, R.C.Haight, M.B.Chadwick, T.Kawano, P.Talou, Eds., Vol.1, p. 744 (2005); AIP Conf.Proc. 769 (2005)

Determination of Thermal Neutron Capture Cross Sections using Cold Neutron Beams

2005BEZW O.A.Bezshyyko, I.M.Kadenko, V.M.Mazur, V.A.Plujko, N.V.Strilchuk, I.M.Vishnevsky, R.V.Yermolenko, V.A.Zheltonozhsky - Proc.Intern.Conf.Nuclear Data for Science and Technology, Santa Fe, New Mexico, 26 September-1 October, 2004, R.C.Haight, M.B.Chadwick, T.Kawano, P.Talou, Eds., Vol.1, p. 641 (2005); AIP Conf.Proc. 769 (2005)

Mean Angular Momenta of Primary Photofission Products

2005BEZX M.M.Be, M.-N.Amiot, C.Bobin, M.-C.Lepy, J.Plagnard, J.M.Lee, K.B.Lee, T.S.Park, A.Luca, M.Sahagia, A.-M.Razdolescu, L.Grigorescu, Y.Sato, Y.Hino, K.Kossert, R.Klein, M.H.K.Schneider, H.Schrader, P.Dryak, J.Sochorova, P.Kovar, P.Auerbach, M.Havelka, T.Altzitzoglou, A.Iwahara, M.A.L.da Silva, J.U.Delgado, C.J.Da Silva, L.Johansson, S.Collins, A.Stroak - CEA-R-6081 (2005)

Activity measurement and gamma emission intensities determination in the decay of ${ }^{65} \mathrm{Zn}$

2005BH06 T.Bhattacharjee, S.Chanda, S.Bhattacharyya, S.K.Basu, R.K.Bhowmik, S.Muralithar, R.P.Singh, N.S.Pattabiraman, S.S.Ghugre, U.Datta Pramanik, S.Bhattacharya - Nucl.Phys. A750, 199 (2005)

Structure of odd-odd ${ }^{136} \mathrm{La}$ at high spin

2005BI02 D.C.Biswas, A.G.Smith, R.M.Wall, D.Patel, G.S.Simpson, D.M.Cullen, J.L.Durell, S.J.Freeman, J.C.Lisle, J.F.Smith, B.J.Varley, T.Yousef, G.Barreau, M.Petit, Ch.Theisen, E.Bouchez, M.Houry, R.Lucas, B.Cahan, A.Le Coguie, B.J.P.Gall, O.Dorvaux, N.Schulz - Phys.Rev. C 71, 011301 (2005); Erratum Phys.Rev. C 71, $019901(2005)$

Lifetimes of excited states in octupole-collective ${ }^{142,144}$ Ba nuclei

2005BI24 C.R.Bingham, M.N.Tantawy, J.C.Batchelder, M.Danchev, T.N.Ginter, C.J.Gross, D.J.Fong, R.Grzywacz, K.Hagino, J.H.Hamilton, M.Karny, W.Krolas, C.Mazzocchi, A.Piechaczek, A.V.Ramayya, K.Rykaczewski, A.Stolz, J.A.Winger, C.-H.Yu, E.F.Zganjar - Nucl.Instrum.Methods Phys.Res. B241, 185 (2005)

Nuclear structure studies at the proton drip line via proton radioactivity studies

2005BI25 J.Billowes - Eur.Phys.J. A 25, Supplement 1, 187 (2005)

Developments in laser spectroscopy at the Jyvaskyla IGISOL

2005BIZY Z.M.Bigan, V.I.Kirischuk, V.M.Mazur, D.M.Simochko, V.A.Zheltonozhsky - Book of Abstracts, LV National Conference on Nuclear Physics "Frontiers in the Physics of Nucleus", St.-Petersburg, p.215 (2005)

Excitation of ${ }^{113 m} \mathrm{In},{ }^{195 m} \mathrm{Pt}$ and ${ }^{199 m} \mathrm{Hg}$ Isomers in the Reactions of Inelastic Gamma Scattering 
2005BIZZ Z.M.Bigan, V.A.Zheltonozhsky, V.M.Mazur, D.M.Simochko - Book of Abstracts, LV National Conference on Nuclear Physics "Frontiers in the Physics of Nucleus", St.-Petersburg, p.214 (2005)

Cross-Sections of $(\gamma, \mathrm{n})$ Reaction on ${ }^{81} \mathrm{Br}$ and ${ }^{121} \mathrm{Sb}$ Nuclei

2005BL09 Y.Blumenfeld - Nucl.Phys. A752, 279c (2005)

Reactions near the neutron drip-line

2005Bl15 B.Blank, A.Bey, G.Canchel, C.Dossat, A.Fleury, J.Giovinazzo, I.Matea, N.Adimi, F.De Oliveira, I.Stefan, G.Georgiev, S.Grevy, J.C.Thomas, C.Borcea, D.Cortina, M.Caamano, M.Stanoiu, F.Aksouh, B.A.Brown, F.C.Barker, W.A.Richter Phys.Rev.Lett. 94, 232501 (2005); Erratum Phys.Rev.Lett. 94, 249901 (2005) First Observation of ${ }^{54} \mathrm{Zn}$ and its Decay by Two-Proton Emission

2005BL23 J.C.Blackmon, F.Carstoiu, L.Trache, D.W.Bardayan, C.R.Brune, C.A.Gagliardi, U.Greife, C.J.Gross, C.C.Jewett, R.L.Kozub, T.A.Lewis, J.F.Liang, B.H.Moazen, A.M.Mukhamedzhanov, C.D.Nesaraja, F.M.Nunes, P.D.Parker, L.Sahin, J.P.Scott, D.Shapira, M.S.Smith, J.S.Thomas, R.E.Tribble - Phys.Rev. C 72, 034606 (2005)

Elastic scattering of the proton drip-line nucleus ${ }^{17} \mathrm{~F}$

2005Bl31 B.Blank, N.Adimi, A.Bey, G.Canchel, C.Dossat, A.Fleury, J.Giovinazzo, I.Matea, F.De Oliveira, I.Stefan, G.Georgiev, S.Grevy, J.C.Thomas, C.Borcea, D.Cortina, M.Caamano, M.Stanoiu, F.Aksouh - Eur.Phys.J. A 25, Supplement 1, 169 (2005) First observation of ${ }^{54} \mathrm{Zn}$ and its decay by two-proton emission

2005Blzz B.Blank, A.Bey, G.Canchel, C.Dossat, A.Fleury, J.Giovinazzo, I.Matea, N.Adimi, F.De Oliveira, I.Stefan, G.Georgiev, S.Grevy, J.C.Thomas, C.Borcea, D.Cortina, M.Caamano, M.Stanoiu, F.Aksouh, B.A.Brown, F.C.Barker, W.A.Richter nucl-ex/0505016,5/13/2005 (2005)

First observation of ${ }^{54} \mathrm{Zn}$ and its decay by two-proton emission

2005B010 M.L.Bonardi, F.Groppi, H.S.Mainardi, V.M.Kokhanyuk, E.V.Lapshina, M.V.Mebel, B.L.Zhuikov - J.Radioanal.Nucl.Chem. 264, 101 (2005)

Cross section studies on ${ }^{64} \mathrm{Cu}$ with zinc target in the proton energy range from 141 down to $31 \mathrm{MeV}$

2005B015 V.R.Bom, A.M.Demin, D.L.Demin, C.W.E.van Eijk, M.P.Faifman, V.V.Filchenkov, A.N.Golubkov, N.N.Grafov, S.K.Grishechkin, K.I.Gritsaj, V.G.Klevtsov, A.D.Konin, A.V.Kuryakin, S.V.Medved, R.K.Musyaev, V.V.Perevozchikov, A.I.Rudenko, S.M.Sadetsky, Yu.I.Vinogradov, A.A.Yukhimchuk, S.A.Yukhimchuk, V.G.Zinov, S.V.Zlatoustovskii - Zh.Eksp.Teor.Fiz. 127, 752 (2005);

J.Exper.Theo.Phys. 100, 663 (2005)

Experimental Investigation of Muon-catalyzed dt Fusion in Wide Ranges of D / T Mixture Conditions

2005B022 J.-L.Boyard, W.Augustyniak, R.Dahl, M.Drews, C.Ellegaard, L.Farhi, C.Gaarde, T.Hennino, J.A.Jensen, J.-C.Jourdain, M.Kagarlis, R.Kunne, J.S.Larsen, P.Radvanyi, B.Ramstein, M.Roy-Stephan, P.Zupranski - Nucl.Phys. A755, 507c (2005)

Coherent pion production in heavy ion charge exchange reactions 
2005B027 A.Borella, A.Moens, P.Schillebeeckx, R.Van Bijlen, G.L.Molnar, T.Belgya, Zs.Revay, L.Szentmiklosi - J.Radioanal.Nucl.Chem. 265, 267 (2005)

Determination of the ${ }^{209} \operatorname{Bi}(\mathrm{n}, \gamma)$ capture cross section at a cold neutron beam

2005B035 K.Bodek, G.Ban, M.Beck, A.Bialek, T.Brys, A.Czarnecki, W.Fetscher, P.Gorel, K.Kirch, St.Kistryn, A.Kozela, M.Kuzniak, A.Lindroth, O.Naviliat-Cuncic, J.Pulut, A.Serebrov, N.Severijns, E.Stephan, J.Zejma - J.Res.Natl.Inst.Stand.Technol. 110, $461(2005)$

Search for Time Reversal Violating Effects: R-Correlation Measurement in Neutron Decay

2005B036 P.Boutachkov, G.V.Rogachev, V.Z.Goldberg, A.Aprahamian, F.D.Becchetti, J.P.Bychowski, Y.Chen, G.Chubarian, P.A.DeYoung, J.J.Kolata, L.O.Lamm, G.F.Peaslee, M.Quinn, B.B.Skorodumov, A.Wohr - Phys.Rev.Lett. 95, 132502 (2005)

Doppler Shift as a Tool for Studies of Isobaric Analog States of Neutron-Rich Nuclei: Application to ${ }^{7} \mathrm{He}$

2005B039 H.G.Bohlen, R.Kalpakchieva, W.von Oertzen, T.N.Massey, A.A.Ogloblin, G.de Angelis, Ch.Schulz, Tz.Kokalova, C.Wheldon - J.Phys.(London) G31, S1461 (2005) Structure studies of excited states of ${ }^{17} \mathrm{C}$ and ${ }^{16} \mathrm{C}$

2005B045 D.Borremans, D.L.Balabanski, K.Blaum, W.Geithner, S.Gheysen, P.Himpe, M.Kowalska, J.Lassen, P.Lievens, S.Mallion, R.Neugart, G.Neyens, N.Vermeulen, D.Yordanov - Phys.Rev. C 72, 044309 (2005); Erratum Phys.Rev. C 72, 059902 (2005)

New measurement and reevaluation of the nuclear magnetic and quadrupole moments of ${ }^{8} \mathrm{Li}$ and ${ }^{9} \mathrm{Li}$

2005B047 V.Bondarenko, J.Honzatko, I.Tomandl, T.von Egidy, H.-F.Wirth, A.M.Sukhovoj L.A.Malov, L.I.Simonova, P.Alexa, J.Berzins, R.Hertenberger, Y.Eisermann, G.Graw - Nucl.Phys. A762, 167 (2005)

Low-spin mixed particle-hole structures in ${ }^{185} \mathrm{~W}$

2005B049 P.Boutachkov, G.V.Rogachev, V.Z.Goldberg, A.Aprahamian, F.D.Becchetti, J.P.Bychowski, Y.Chen, G.Chubarian, P.A.DeYoung, J.J.Kolata, L.O.Lamm, G.F.Peaslee, M.Quinn, B.B.Skorodumov, A.Wohr - Eur.Phys.J. A 25, Supplement 1, $259(2005)$

Isobaric analog states of neutron-rich nuclei. Doppler shift as a measurement tool for resonance excitation functions

2005BOZT A.Borella - Thesis, Gent Univ. Belgium (2005)

Determination of the neutron resonance parameters for ${ }^{206} \mathrm{~Pb}$ and of the thermal neutron capture cross section for ${ }^{206} \mathrm{~Pb}$ and ${ }^{209} \mathrm{Bi}$ 
2005BOZU D.Boutin, Yu.A.Litvinov, C.Scheidenberger, K.Beckert, P.Beller, F.Bosch, T.Faestermann, B.Franczak, B.Franzke, H.Geissel, E.Kaza, A.Kelic, P.Kienle, O.Klepper, C.Kozhuharov, L.Maier, M.Matos, G.Munzenberg, F.Nolden, Yu.N.Novikov, T.Ohtsubo, W.Plass, M.Portillo, K.-H.Schmidt, J.Stadlmann, M.Steck, H.Weick, M.Winkler, T.Yamaguchi - GSI 2005-1, p.81 (2005)

Study of production and decay of long-lived isomeric states using time-resolved Schottky Mass Spectrometry

2005B0ZV A.Borella, A.Brusegan, M.C.Moxon, G.Aerts, F.Gunsing, P.Siegler, P.Schillebeeckx Proc.Intern.Conf.Nuclear Data for Science and Technology, Santa Fe, New Mexico, 26 September-1 October, 2004, R.C.Haight, M.B.Chadwick, T.Kawano, P.Talou, Eds., Vol.2, p. 1539 (2005); AIP Conf.Proc. 769 (2005)

High-Resolution Neutron Total and Capture Cross-Section Measurements on ${ }^{206} \mathrm{~Pb}$

2005B0ZW A.Borella, P.Schillebeeckx, G.Molnar, T.Belgya, Zs.Revay, L.Szentmiklosi, E.Berthoumieux, F.Gunsing, A.Letourneau, F.Marie - Proc.Intern.Conf.Nuclear Data for Science and Technology, Santa Fe, New Mexico, 26 September-1 October, 2004, R.C.Haight, M.B.Chadwick, T.Kawano, P.Talou, Eds., Vol.1, p. 648 (2005); AIP Conf.Proc. 769 (2005)

The ${ }^{209} \mathrm{Bi}\left(\mathrm{n}_{t h}, \gamma\right){ }^{210} \mathrm{Bi}$ and ${ }^{209} \mathrm{Bi}\left(\mathrm{n}_{t h}, \gamma\right){ }^{210 m, g} \mathrm{Bi}$ Cross Sections Determined at the Budapest Neutron Centre

2005BR04 C.Broggini, and the LUNA Collaboration - Nucl.Phys. B(Proc.Supp.) S143, 60 (2005)

Neutrino Cross Sections for Astrophysics

2005BR10 A.Bracco, G.Benzoni, S.Leoni, N.Blasi, F.Camera, C.Grassi, B.Million, A.Paleni, M.Pignanelli, E.Vigezzi, O.Wieland, M.Matsuo, T.Dossing, B.Herskind, G.B.Hagemann, J.Wilson, A.Maj, M.Kmiecik, G.Lo Bianco, C.M.Petrache, M.Castoldi, A.Zucchiati, G.De Angelis, D.Napoli, D.Curien, P.Bednarczyk Nucl.Phys. A752, 227c (2005)

Conservation of the K-quantum number in warm nuclei

2005BR14 P.Bringel, G.B.Hagemann, H.Hubel, A.Al-khatib, P.Bednarczyk, A.Burger, D.Curien, G.Gangopadhyay, B.Herskind, D.R.Jensen, D.T.Joss, Th.Kroll, G.Lo Bianco, S.Lunardi, W.C.Ma, N.Nenoff, A.Neusser-Neffgen, C.M.Petrache, G.Schonwasser, J.Simpson, A.K.Singh, N.Singh, G.Sletten - Eur.Phys.J. A 24, 167 (2005)

Evidence for wobbling excitation in ${ }^{161} \mathrm{Lu}$

2005BR15 C.Broggini, the LUNA Collaboration - Nucl.Phys. B(Proc.Supp.) S145, 33 (2005)

Laboratory measurements of astrophysical factors

2005BR18 R.Broda, B.Fornal, W.Krolas, T.Pawlat, J.Wrzesinski, D.Bazzacco, S.Lunardi, G.de Angelis, A.Gadea, C.Ur, N.Marginean, R.V.F.Janssens, M.P.Carpenter, S.J.Freeman, N.Hammond, T.Lauritsen, C.J.Lister, F.Moore, D.Seweryniak, P.J.Daly, Z.W.Grabowski, B.A.Brown, M.Honma - Acta Phys.Pol. B36, 1343 (2005) Yrast states in $\mathrm{N}=30{ }^{50} \mathrm{Ca}$ and ${ }^{51} \mathrm{Sc}$ isotones studied with deep-inelastic heavy ion reactions 
2005BRZU F.Bringas, M.T.Yamashita, I.D.Goldman, P.R.Pascholati, V.Sciani Proc.Intern.Conf.Nuclear Data for Science and Technology, Santa Fe, New Mexico, 26 September-1 October, 2004, R.C.Haight, M.B.Chadwick, T.Kawano, P.Talou, Eds., Vol.2, p. 1374 (2005); AIP Conf.Proc. 769 (2005)

Measurement of Proton-Induced Reaction Cross Sections in Ti, Ni and Zr near the Threshold

2005BRZV A.Brusegan, E.Berthoumieux, A.Borella, F.Gunsing, M.Moxon, P.Siegler, P.Schillebeeckx - Proc.Intern.Conf.Nuclear Data for Science and Technology, Santa Fe, New Mexico, 26 September-1 October, 2004, R.C.Haight, M.B.Chadwick, T.Kawano, P.Talou, Eds., Vol.1, p. 953 (2005); AIP Conf.Proc. 769 (2005) Neutron Capture and Transmission Measurements on ${ }^{103} \mathrm{Rh}$ Down to Thermal Energies

2005BRZX V.B.Brudanin, N.I.Rukhadze, V.G.Egorov, Ch.Briancon, P.Benes, P.Cermak, K.N.Gusev, F.A.Danevich, A.A.Klimenko, V.E.Kovalenko, A.Kovalik, A.V.Salamatin, I.Stekl, V.V.Timkin, V.I.Tretyak, Ts.Vylov - Book of Abstracts, LV National Conference on Nuclear Physics "Frontiers in the Physics of Nucleus", St.-Petersburg, p.299 (2005)

Search for Double Electron Capture of ${ }^{106} \mathrm{Cd}$ in the Experiment TGV-2

2005BU02 D.G.Burke - Nucl.Phys. A747, 131 (2005)

Single-proton-transfer studies of ${ }^{153} \mathrm{Eu}$ levels

2005BU05 A.A.Burlon, A.J.Kreiner, A.A.Valda, D.M.Minsky, H.R.Somacal, M.E.Debray, P.Stoliar - Nucl.Instrum.Methods Phys.Res. B229, 144 (2005)

Optimization of a neutron production target and a beam shaping assembly based on the ${ }^{7} \operatorname{Li}(\mathrm{p}, \mathrm{n})^{7}$ Be reaction for $\mathrm{BNCT}$

2005BU07 D.G.Burke, G.Lovhoiden - Nucl.Phys. A750, 185 (2005)

$(t, p)$ reaction studies of ${ }^{165} \mathrm{Dy}$ and ${ }^{179} \mathrm{Hf}$

2005BU08 D.Bucurescu, Zs.Podolyak, C.Rusu, G.de Angelis, Y.H.Zhang, G.Cata-Danil, I.Cata-Danil, M.Ivascu, N.Marginean, R.Marginean, L.C.Mihailescu, G.A.Suliman, P.H.Regan, W.Gelletly, S.D.Langdown, J.J.Valiente-Dobon, D.Bazzacco, S.Lunardi, C.A.Ur, M.Axiotis, A.Gadea, E.Farnea, M.Ionescu-Bujor, A.Iordachescu, Th.Kroll, T.Martinez, P.G.Bizzeti, R.Broda, N.H.Medina, B.Quintana, B.Rubio - Phys.Rev. C 71, 034315 (2005)

High-spin states in the nuclei ${ }^{91} \mathrm{Y}$ and ${ }^{95} \mathrm{Nb}$

2005BU14 A.Burger, T.Saito, A.Al-Khatib, A.Banu, T.Beck, F.Becker, P.Bednarczyk, G.Benzoni, A.Bracco, P.Bringel, F.Camera, E.Clement, P.Doornenbal, H.Geissel, J.Gerl, M.Gorska, A.Gorgen, H.Grawe, J.Grebosz, G.Hammond, M.Hellstrom, H.Hubel, M.Kavatsyuk, O.Kavatsyuk, M.Kmiecik, I.Kojouharov, N.Kurz, R.Lozeva, A.Maj, S.Mandal, W.Meczynski, D.Mehta, B.Million, S.Muralithar, A.Neusser, Zs.Podolyak, T.S.Reddy, P.Reiter, N.Saito, H.Schaffner, A.K.Singh, H.Weick, O.Wieland, C.Wheldon, M.Winkler, H.J.Wollersheim - Acta Phys.Pol. B36, 1249 (2005)

Relativistic Coulomb excitations of ${ }^{54,56,58} \mathrm{Cr}$ 
2005BU20 D.Bucurescu, Y.Eisermann, G.Graw, R.Hertenberger, H.-F.Wirth, V.Yu.Ponomarev - Nucl.Phys. A756, 54 (2005)

Detailed spectroscopy of ${ }^{113} \mathrm{Cd}$ through transfer reactions

2005BU21 D.G.Burke, I.G.Nowikow - Nucl.Phys. A756, 308 (2005)

Nuclear structure studies and particle-rotor model tests for ${ }^{151,153} \mathrm{Sm}$ using ${ }^{149,151} \mathrm{Sm}(\mathrm{t}, \mathrm{p})$ reactions

2005BU29 A.Burger, and the RISING Collaboration - Phys.Lett. B 622, 29 (2005)

Relativistic Coulomb excitation of neutron-rich ${ }^{54,56,58} \mathrm{Cr}$ : On the pathway of magicity from $\mathrm{N}=40$ to $\mathrm{N}=32$

$2005 B U 33$ N.Burtebaev, M.K.Baktybaev, B.A.Duisebaev, R.J.Peterson, S.B.Sakuta - Yad.Fiz. 68, 1356 (2005); Phys.Atomic Nuclei 68, 1303 (2005)

Scattering of $\alpha$ Particles on ${ }^{11} \mathrm{~B}$ Nuclei at Energies 40 and $50 \mathrm{MeV}$

2005BU37 M.J.Burns, R.Chapman, K.M.Spohr, J.Ollier, M.Labiche, X.Liang, E.Farnea, M.Axiotis, T.Martinez, D.R.Napoli, C.A.Ur, Th.Kroll - J.Phys.(London) G31, S1827 (2005)

High spin studies of the Er and Tm isotopes around $\mathrm{A}=166$

2005BUZZ D.Bucurescu, H.-F.Wirth, R.Hertenberger, G.Graw, D.A.Meyer, R.F.Casten, S.Heinze, J.Jolie - Maier-Leibnitz-Laboratorium 2004 Ann.Rept., p.16 (2005) Investigation of $0^{+}$Excitations in a Deformed Nucleus: ${ }^{168} \mathrm{Er}$

2005BY03 J.Byrne - J.Res.Natl.Inst.Stand.Technol. 110, 395 (2005)

Determination of the Electron-Antineutrino Angular Correlation Coefficient $a_{0}$ in Unpolarized Neutron $\beta$-Decay

2005BY04 J.Byrne, R.U.Khafizov, Yu A.Mostovoi, O.Rozhnov, V.A.Solovei, M.Beck, V.U.Kozlov, N.Severijns - J.Res.Natl.Inst.Stand.Technol. 110, 415 (2005)

Search for Radiative $\beta$-Decay of the Free Neutron

2005CA02 M.Caamano, P.M.Walker, P.H.Regan, M.Pfutzner, Zs.Podolyak, J.Gerl, M.Hellstrom, P.Mayet, M.N.Mineva, A.Aprahamian, J.Benlliure, A.M.Bruce, P.A.Butler, D.Cortina Gil, D.M.Cullen, J.Doring, T.Enqvist, C.Fox, J.Garces Narro, H.Geissel, W.Gelletly, J.Giovinazzo, M.Gorska, H.Grawe, R.Grzywacz, A.Kleinbohl, W.Korten, M.Lewitowicz, R.Lucas, H.Mach, C.D.O'Leary, F.De Oliveira, C.J.Pearson, F.Rejmund, M.Rejmund, M.Sawicka, H.Schaffner, C.Schlegel, K.Schmidt, K.-H.Schmidt, P.D.Stevenson, Ch.Theisen, F.Vives, D.D.Warner, C.Wheldon, H.J.Wollersheim, S.Wooding, F.Xu, O.Yordanov - Eur.Phys.J. A 23, $201(2005)$

Isomers in neutron-rich $\mathrm{A} \approx 190$ nuclides from ${ }^{208} \mathrm{~Pb}$ fragmentation

2005CA03 C.M.Cattadori, M.De Deo, M.Laubenstein, L.Pandola, V.I.Tretyak - Nucl.Phys. A748, 333 (2005)

Observation of $\beta$ decay of ${ }^{115} \mathrm{In}$ to the first excited level of ${ }^{115} \mathrm{Sn}$ 
2005CA06 G.Canchel, B.Blank, M.Chartier, F.Delalee, P.Dendooven, C.Dossat, J.Giovinazzo, J.Huikari, A.S.Lalleman, M.J.Lopez Jimenez, V.Madec, J.L.Pedroza, H.Penttila, J.C.Thomas - Eur.Phys.J. A 23, 409 (2005)

Precision measurement of the half-life and the decay branches of ${ }^{62} \mathrm{Ga}$

2005CA14 S.A.Catlow, G.L.Troyer, D.R.Hansen, R.A.Jones - J.Radioanal.Nucl.Chem. 263, 599 (2005)

Half-life measurement of ${ }^{126} \mathrm{Sn}$ isolated from Hanford nuclear defense waste

2005CA23 F.Camera, M.Kmiecik, O.Wieland, G.Benzoni, A.Bracco, S.Brambilla, F.Crespi, P.Mason, A.Moroni, B.Million, S.Leoni, A.Maj, J.Styczen, M.Brekiesz, W.Meczynski, M.Zieblinski, F.Gramegna, S.Barlini, V.L.Kravchuk, A.L.Lanchais, P.F.Mastinu, M.Bruno, M.D'Agostino, E.Geraci, A.Ordine, G.Casini, M.Chiari Acta Phys.Pol. B36, 1145 (2005)

GDR in hot nuclei: new measurements

2005CA29 R.F.Carlson, A.Ingemarsson, M.Lantz, G.J.Arendse, A.Auce, A.J.Cox, S.V.Fortsch, N.M.Jacobs, R.Johansson, J.Nyberg, J.Peavy, P.-U.Renberg, O.Sundberg, J.A.Stander, G.F.Steyn, G.Tibell, R.Zorro - Nucl.Instrum.Methods Phys.Res. A547, $541(2005)$

A method for measuring light ion reaction cross-sections

2005CA42 G.Cazzoli, C.Puzzarini, J.Gauss - Astrophys.J.Suppl.Ser. 159, 181 (2005)

The rare isotopomers of $\mathrm{HCN}: \mathrm{HC}^{15} \mathrm{~N}$ and $\mathrm{DC}^{15} \mathrm{~N}$ rotational spectrum and resolved nuclear hyperfine structures due to ${ }^{15} \mathrm{~N}$ and $\mathrm{D}$

2005CA43 M.P.Carpenter, F.G.Kondev, R.V.F.Janssens - J.Phys.(London) G31, S1599 (2005) Studies of neutron-deficient nuclei near the $\mathrm{Z}=82$ shell closure via cold fusion reactions

2005CA44 W.N.Catford, C.N.Timis, R.C.Lemmon, M.Labiche, N.A.Orr, L.Caballero, R.Chapman, M.Freer, M.Chartier, H.Savajols, M.Rejmund, N.Amzal, N.I.Ashwood, T.D.Baldwin, M.Burns, N.Curtis, G.de France, W.Gelletly, X.Liang, S.D.Pain, V.P.E.Pucknell, B.Rubio, O.Sorlin, K.Spohr, Ch.Theisen, D.D.Warner J.Phys.(London) G31, S1655 (2005)

Nucleon transfer via $(\mathrm{d}, \mathrm{p})$ using TIARA with a ${ }^{24}$ Ne radioactive beam

2005CA50 W.N.Catford, R.C.Lemmon, M.Labiche, C.N.Timis, N.A.Orr, L.Caballero, R.Chapman, M.Chartier, M.Rejmund, H.Savajols, and the TIARA Collaboration Eur.Phys.J. A 25, Supplement 1, 245 (2005)

First experiments on transfer with radioactive beams using the TIARA array

2005CAZW C.M.Cattadori, M.De Deo, M.Laubenstein, L.Pandola, V.I.Tretyak nucl-ex/0509020,9/15/2005 (2005)

Beta decay of ${ }^{115}$-In to the first excited level of ${ }^{115} \mathrm{Sn}$ : Potential outcome for neutrino mass 
2005CAZX D.Cano-Ott, and the n_TOF Collaboration - Proc.Intern.Conf.Nuclear Data for Science and Technology, Santa Fe, New Mexico, 26 September-1 October, 2004, R.C.Haight, M.B.Chadwick, T.Kawano, P.Talou, Eds., Vol.2, p. 1442 (2005); AIP Conf.Proc. 769 (2005)

Measurements at n_TOF of the Neutron Capture Cross Section of Minor Actinides Relevant to the Nuclear Waste Transmutation

2005CAZY M.P.Carpenter, F.G.Kondev, R.V.F.Janssens - Proc.Intern.Conf.Nuclear Data for Science and Technology, Santa Fe, New Mexico, 26 September-1 October, 2004, R.C.Haight, M.B.Chadwick, T.Kawano, P.Talou, Eds., Vol.1, p. 894 (2005); AIP Conf.Proc. 769 (2005)

Shape Coexistence at the Outer Edges of Stability

2005CAzZ C.M.Campbell, N.Aoi, D.Bazin, M.D.Bowen, B.A.Brown, J.M.Cook, D.-C.Dinca, A.Gade, T.Glasmacher, S.Kanno, T.Motobayashi, W.F.Mueller, H.Olliver, H.Sakurai, K.Starosta, S.Takeuchi, J.R.Terry, K.Yoneda - Proc.Nuclei at the Limits, Argonne, Illinois, D.Seweryniak and T.L.Khoo, eds., p.127 (2005); AIP Conf. Proc $764(2005)$

Trends In Collectivity Approaching $\mathrm{N}=28$

2005CE02 J.Cerny, Z.Dlouhy, Z.Dolezal, M.P.Ivanov, P.Kubik, E.A.Kuznetsova, Yu.E.Penionzhkevich, E.A.Sokol, J.Svejda, I.Wilhelm - Nucl.Instrum.Methods Phys.Res. A540, 430 (2005)

Neutron response function for a detector with ${ }^{3} \mathrm{He}$ counters for the $0.39-1.54 \mathrm{MeV}$ neutron energy range

2005CH30 A.A.Chen, R.E.Azuma, S.Bishop, L.Buchmann, M.L.Chatterjee, J.M.D'Auria, S.Engel, D.Gigliotti, U.Greife, D.Hunter, A.Hussein, D.Hutcheon, C.C.Jewett, J.Jose, J.D.King, A.M.Laird, M.Lamey, R.Lewis, W.Liu, A.Olin, D.Ottewell, P.Parker, J.Rogers, C.Ruiz, M.Trinczek, C.Wrede - Nucl.Phys. A752, 510c (2005) The ${ }^{21} \mathrm{Na}(\mathrm{p}, \gamma){ }^{22} \mathrm{Mg}$ reaction in novae and $\mathrm{x}$-ray bursts

2005CH38 S.K.Chamoli, P.Joshi, A.Kumar, R.Kumar, R.P.Singh, S.Muralithar, R.K.Bhowmik, I.M.Govil - Phys.Rev. C 71, 054324 (2005)

Shape coexistence and lifetime measurement in ${ }^{187} \mathrm{Tl}$ nucleus

2005CH44 A.Chafa, V.Tatischeff, P.Aguer, S.Barhoumi, A.Coc, F.Garrido, M.Hernanz, J.Jose, J.Kiener, A.Lefebvre-Schuhl, S.Ouichaoui, N.de Sereville, J.-P.Thibaud Phys.Rev.Lett. 95, 031101 (2005); Erratum Phys.Rev.Lett. 96, 019902 (2006) Hydrogen Burning of ${ }^{17} \mathrm{O}$ in Classical Novae

2005CH49 L.V.Chulkov, H.Simon, I.J.Thompson, T.Aumann, M.J.G.Borge, Th.W.Elze, H.Emling, H.Geissel, L.V.Grigorenko, M.Hellstrom, B.Jonson, J.W.V.Kratz, R.Kulessa, K.Markenroth, M.Meister, G.Munzenberg, F.Nickel, T.Nilsson, G.Nyman, V.Pribora, A.Richter, K.Riisager, C.Scheidenberger, G.Schrieder, O.Tengblad, M.V.Zhukov - Nucl.Phys. A759, 23 (2005)

Three-body correlations in electromagnetic dissociation of Borromean nuclei: The ${ }^{6} \mathrm{He}$ case 
2005CH50 L.V.Chulkov, F.Aksouh, A.Bleile, O.V.Bochkarev, D.Cortina-Gil, A.V.Dobrovolsky, P.Egelhof, H.Geissel, M.Hellstrom, N.B.Isaev, O.A.Kiselev, B.G.Komkov, M.Matos, F.N.Moroz, G.Munzenberg, M.Mutterer, V.A.Mylnikov, S.R.Neumaier, V.N.Pribora, D.M.Seliverstov, L.O.Sergeev, A.Shrivastava, K.Summerer, S.Yu.Torilov, H.Weick, M.Winkler, V.I.Yatsoura - Nucl.Phys. A759, 43 (2005) Quasi-free scattering with ${ }^{6,8} \mathrm{He}$ beams

2005 CH53 J.A.Church, L.Ahle, L.A.Bernstein, J.Cooper, F.S.Dietrich, J.Escher, C.Forssen, H.Ai, H.Amro, M.Babilon, C.Beausang, J.Caggiano, A.Heinz, R.Hughes, E.McCutchan, D.Meyer, C.Plettner, J.Ressler, V.Zamfir - Nucl.Phys. A758, 126c (2005) Determining neutron capture cross sections with the Surrogate Reaction Technique: Measuring decay probabilities with STARS

2005CH60 M.Chartier, M.B.Gomez Hornillos, W.Mittig, A.Lepine-Szily, L.Caballero Ontanaya, C.E.Demonchy, G.Georgiev, N.A.Orr, G.Politi, M.Rousseau, P.Roussel-Chomaz, A.C.C.Villari - J.Phys.(London) G31, S1771 (2005)

Direct mass measurement of $\mathrm{N} \sim \mathrm{Z}$ nuclei with $\mathrm{A}=64-80$ using the CSS2 cyclotron

2005CH62 Y.Cheng, B.Xia, Y.-N.Liu, Q.-X.Jin - Chin.Phys.Lett. 22, 2530 (2005)

Rhodium Mossbauer Effect Generated by Bremsstrahlung Excitation

2005CH65 A.Chakraborty, Krishichayan, S.S.Ghugre, R.Goswami, S.Mukhopadhyay, N.S.Pattabiraman, S.Ray, A.K.Sinha, S.Sarkar, P.V.Madhusudhana Rao, U.Garg, S.K.Basu, M.B.Chatterjee, M.S.Sarkar, L.Chaturvedi, A.Dhal, R.K.Sinha, I.M.Govil, R.K.Bhowmik, A.Jhingan, N.Madhavan, S.Muralithar, S.Nath, R.P.Singh, P.Sugathan - Phys.Rev. C 72, 054309 (2005)

Spectroscopy of ${ }^{90} \mathrm{Nb}$ at high spin

2005 CH66 J.A.Church, C.M.Campbell, D.-C.Dinca, J.Enders, A.Gade, T.Glasmacher, Z.Hu, R.V.F.Janssens, W.F.Mueller, H.Olliver, B.C.Perry, L.A.Riley, K.L.Yurkewicz Phys.Rev. C 72, 054320 (2005)

Measurement of E2 transition strengths in ${ }^{32,34} \mathrm{Mg}$

2005CH67 R.S.Chakrawarthy, P.M.Walker, M.B.Smith, A.N.Andreyev, S.F.Ashley, G.C.Ball, J.A.Becker, J.J.Daoud, P.E.Garrett, G.Hackman, G.A.Jones, Y.Litvinov, A.C.Morton, C.J.Pearson, C.E.Svensson, S.J.Williams, E.F.Zganjar - Eur.Phys.J. A 25, Supplement 1, 125 (2005)

Discovery of a new $2.3 \mathrm{~s}$ isomer in neutron-rich ${ }^{174} \mathrm{Tm}$

2005CHZT H.Chatani - Proc.Intern.Conf.Nuclear Data for Science and Technology, Santa Fe, New Mexico, 26 September-1 October, 2004, R.C.Haight, M.B.Chadwick, T.Kawano, P.Talou, Eds., Vol.1, p. 664 (2005); AIP Conf.Proc. 769 (2005) Measurement of Effective Cross Section of Th-233(n, $\gamma$ )Th-234 Reaction Using the KUR

2005CHZW R.Chankova, A.Schiller, U.Agvaanluvsan, E.Algin, L.A.Bernstein, M.Guttormsen, F.Ingebretsen, T.Lonnroth, S.Messelt, G.E.Mitchell, J.Rekstad, S.Siem, A.C.Sunde, A.Voinov, S.Odegard - nucl-ex/0507007,7/04/2005 (2005)

Level densities and thermodynamical quantities of heated ${ }^{93-98}$ Mo isotopes 
2005CHZY C.Chandler, M.A.Bentley, M.P.Carpenter, C.N.Davids, R.Du Rietz, J.Ekman, S.J.Freeman, G.Hammond, R.V.F.Janssens, S.M.Lenzi, D.Seweryniak - Proc.Nuclei at the Limits, Argonne, Illinois, D.Seweryniak And T.L.Khoo, eds., p.199 (2005); AIP Conf. Proc 764 (2005)

First Observation Of Excited States In The T=-1, Odd-Odd Nucleus ${ }^{48} \mathrm{Mn}$

2005CHzz C.J.Chiara, D.G.Sarantites, M.Montero, J.O'Brien, O.L.Pechenaya, W.Reviol, R.M.Clark, P.Fallon, A.Gorgen, A.O.Macchiavelli, D.Ward, W.Satula - Proc.Nuclei at the Limits, Argonne, Illinois, D.Seweryniak and T.L.Khoo, eds., p.40 (2005); AIP Conf. Proc 764 (2005)

Discrete Linking Transitions For A Superdeformed Band In The A $\approx 80$ Region

2005CI07 J.A.Cizewski, K.L.Jones, S.D.Pain, J.S.Thomas, C.Baktash, D.W.Bardayan, J.C.Blackmon, C.Gross, J.F.Liang, D.Shapira, M.S.Smith, R.L.Kozub, B.H.Moazen, C.D.Nesaraja, H.K.Carter, M.S.Johnson, R.P.Fitzgerald, D.W.Visser, U.Greife, R.J.Livesay, W.Catford, Z.Ma - Nucl.Instrum.Methods Phys.Res. B241, 200 (2005) Neutron transfer reactions with neutron-rich radioactive ion beams

2005CL07 R.M.Clark, L.W.Phair, M.Descovich, M.Cromaz, M.A.Deleplanque, P.Fallon, I.Y.Lee, A.O.Macchiavelli, M.A.McMahan, L.G.Moretto, E.Rodriguez-Vieitez, S.Sinha, F.S.Stephens, D.Ward, M.Wiedeking, L.A.Bernstein, J.T.Burke, J.A.Church - Phys.Rev. C 72, 054605 (2005)

Population of nuclei via ${ }^{7} \mathrm{Li}$-induced binary reactions

2005CL08 J.A.Clark, R.C.Barber, B.Blank, C.Boudreau, F.Buchinger, J.E.Crawford, J.P.Greene, S.Gulick, J.C.Hardy, A.A.Hecht, A.Heinz, J.K.P.Lee, A.F.Levand, B.F.Lundgren, R.B.Moore, G.Savard, N.D.Scielzo, D.Seweryniak, K.S.Sharma, G.D.Sprouse, W.Trimble, J.Vaz, J.C.Wang, Y.Wang, B.J.Zabransky, Z.Zhou Eur.Phys.J. A 25, Supplement 1, 629 (2005)

Investigating the rp-process with the Canadian Penning trap mass spectrometer

2005Clzz E.Clement, A.Gorgen, E.Bouchez, A.Chatillon, W.Korten, Y.Le Coz, C.Theisen, C.Andreoiu, F.Becker, B.Blank, C.Borcea, A.Buta, P.Butler, J.M.Casandjian, W.N.Catford, T.Czosnyka, A.Emsallem, G.de France, J.Genevey, J.Gerl, F.Hannachi, K.Hauschild, R.-D.Herzberg, A.Hurstel, J.Iwanicki, D.Jenkins, G.Jones, M.Lewitowicz, R.Lucas, I.Matea, F.Negoita, F.de Oliveira Santos, D.Pantelica, J.Pinston, P.Rahkila, M.Rejmund, G.Sletten, M.Stanoiu, C.Timis, R.Wadsworth, J.N.Wilson, M.Zielinska - Proc.Nuclei at the Limits, Argonne, Illinois, D.Seweryniak and T.L.Khoo, eds., p.55 (2005); AIP Conf. Proc 764 (2005)

Shape Coexistence In Light Krypton Isotopes

2005C002 L.Corradi, B.R.Behera, E.Fioretto, A.Gadea, A.Latina, A.M.Stefanini, S.Szilner, M.Trotta, Y.Wu, S.Beghini, G.Montagnoli, F.Scarlassara, R.N.Sagaidak, S.N.Atutov, B.Mai, G.Stancari, L.Tomassetti, E.Mariotti, A.Khanbekyan, S.Veronesi - Phys.Rev. C 71, 014609 (2005)

Excitation functions for ${ }^{208-211} \mathrm{Fr}$ produced in the ${ }^{18} \mathrm{O}+{ }^{197} \mathrm{Au}$ fusion reaction 
2005C016 H.Costantini, C.Angulo, D.Bemmerer, R.Bonetti, C.Broggini, F.Confortola, P.Corvisiero, J.Cruz, P.Descouvemont, A.Formicola, Z.Fulop, G.Gervino, A.Guglielmetti, C.Gustavino, G.Gyurky, G.Imbriani, A.P.Jesus, M.Junker, A.Lemut, R.Menegazzo, P.Prati, V.Roca, C.Rolfs, M.Romano, C.Rossi Alvarez, F.Schumann, E.Somorjai, O.Straniero, F.Strieder, F.Terrasi, H.P.Trautvetter, A.Vomiero, S.Zavatarelli - Nucl.Phys. A758, 383c (2005)

Recent results of the ${ }^{14} \mathrm{~N}(\mathrm{p}, \gamma){ }^{15} \mathrm{O}$ measurement at LUNA

2005C017 M.Couder, C.Angulo, E.Casarejos, P.Demaret, P.Leleux, F.Vanderbist - Nucl.Phys. A758, 741c (2005)

Study of the ${ }^{19} \mathrm{Ne}(\mathrm{p}, \gamma){ }^{20} \mathrm{Na}$ reaction and subsequent improvements to ARES

2005C022 A.Costin, N.Pietralla, T.Koike, C.Vaman, T.Ahn, G.Rainovski - Phys.Rev. C 72, $054305(2005)$

Subnanosecond lifetime measurement for the $\mathrm{T}=0,3_{1}^{+}$state of odd-odd $\mathrm{N}=\mathrm{Z}^{58} \mathrm{Cu}$

2005 C024 D.Cortina-Gil, J.Fernandez-Vazquez, T.Aumann, T.Baumann, J.Benlliure, M.J.G.Borge, L.V.Chulkov, U.Datta Pramanik, C.Forssen, L.M.Fraile, H.Geissel, J.Gerl, F.Hammache, K.Itahashi, R.Janik, B.Jonson, S.Mandal, K.Markenroth, M.Meister, M.Mocko, G.Munzenberg, T.Ohtsubo, A.Ozawa, Y.Prezado, V.Pribora, K.Riisager, H.Scheit, R.Schneider, G.Schrieder, H.Simon, B.Sitar, A.Stolz, P.Strmen, K.Summerer, I.Szarka, H.Weick - Eur.Phys.J. A 25, Supplement 1, 343 (2005) One-neutron knockout of ${ }^{23} \mathrm{O}$

2005C025 L.Corradi, A.M.Stefanini, S.Szilner, S.Beghini, B.R.Behera, E.Farnea, A.Gadea, E.Fioretto, F.Haas, A.Latina, N.Marginean, G.Montagnoli, G.Pollarolo, F.Scarlassara, M.Trotta, C.Ur, and the PRISMA-CLARA Collaboration Eur.Phys.J. A 25, Supplement 1, 427 (2005)

Multinucleon transfer reactions studied with the heavy-ion magnetic spectrometer PRISMA

2005CR05 J.Cruz, Z.Fulop, G.Gyurky, F.Raiola, A.Di Leva, B.Limata, M.Fonseca, H.Luis, D.Schurmann, M.Aliotta, H.W.Becker, A.P.Jesus, K.U.Kettner, J.P.Ribeiro, C.Rolfs, M.Romano, E.Somorjai, F.Strieder, and the LUNA Collaboration Phys.Lett. B 624, 181 (2005)

Electron screening in ${ }^{7} \operatorname{Li}(\mathrm{p}, \alpha) \alpha$ and ${ }^{6} \operatorname{Li}(\mathrm{p}, \alpha){ }^{3} \mathrm{He}$ for different environments

2005CS01 M.Csatlos, A.Krasznahorkay, P.G.Thirolf, D.Habs, Y.Eisermann, T.Faestermann, G.Graw, J.Gulyas, M.N.Harakeh, R.Hertenberger, M.Hunyadi, H.J.Maier, Z.Mate, O.Schaile, H.-F.Wirth - Phys.Lett. B 615, 175 (2005)

Resonant tunneling through the triple-humped fission barrier of ${ }^{236} \mathrm{U}$

2005Cszz L.Csige, M.Csatlos, A.Krasznahorkay, P.G.Thirolf, T.Faestermann, Z.Gacsi, J.Gulyas, D.Habs, R.Hertenberger, M.Hunyadi, H.J.Maier, T.Morgan, O.Schaile, W.Schwerdtfeger, H.-F.Wirth - Maier-Leibnitz-Laboratorium 2004 Ann.Rept., p.19 (2005) Angular Distribution of Fission Fragments from the ${ }^{236} \mathrm{U}(\mathrm{d}, \mathrm{pf})$ Reaction

2005CU05 D.M.Cullen, R.Glover, L.K.Pattison, P.M.Walker, S.Frauendorf, D.Almehed J.Phys.(London) G31, S1709 (2005) 
Nuclear-tidal waves in the osmium nuclei

2005CU06 N.Curtis, N.I.Ashwood, W.N.Catford, N.M.Clarke, M.Freer, D.Mahboub, C.J.Metelko, S.D.Pain, N.Soic, D.C.Weisser - Phys.Rev. C 72, 044320 (2005) $\alpha+\mathrm{Li}$ and $\mathrm{H}+\mathrm{Be}$ decay of ${ }^{10,11,12} \mathrm{~B}$

2005CU07 X.Z.Cui, L.H.Zhu, X.G.Wu, Z.M.Wang, C.Y.He, Y.Liu, G.S.Li, S.X.Wen, Z.L.Zhang, R.Meng, R.G.Ma, P.Luo, Y.Zheng, M.M.Ndontchueng, J.D.Huo, C.X.Yang - Phys.Rev. C 72, 044322 (2005)

High-spin states and shell structure of the odd-odd nucleus ${ }^{90} \mathrm{Nb}$

2005DA03 F.A.Danevich, P.G.Bizzeti, T.F.Fazzini, A.Sh.Georgadze, V.V.Kobychev, B.N.Kropivyansky, P.R.Maurenzig, A.S.Nikolaiko, V.I.Tretyak, S.Yu.Zdesenko, Yu.G.Zdesenko - Nucl.Phys. B(Proc.Supp.) S138, 230 (2005)

Double $\beta$ decay of ${ }^{116} \mathrm{Cd}$. Final results of the Solotvina experiment and CAMEO project

2005DA12 V.M.Datar, S.Kumar, D.R.Chakrabarty, V.Nanal, E.T.Mirgule, A.Mitra, H.H.Oza Phys.Rev.Lett. 94, 122502 (2005)

Direct Observation of the $4^{+}$-to- $2^{+}$Gamma Transition in ${ }^{8} \mathrm{Be}$

2005DA16 P.Datta, S.Chattopadhyay, S.Bhattacharya, T.K.Ghosh, A.Goswami, S.Pal, M.S.Sarkar, H.C.Jain, P.K.Joshi, R.K.Bhowmik, R.Kumar, N.Madhavan, S.Muralithar, P.V.Madhusudhana Rao, R.P.Singh - Phys.Rev. C 71, 041305 (2005) Observation of antimagnetic rotation in ${ }^{108} \mathrm{Cd}$

2005DA20 Y.Danon, R.C.Block - Nucl.Instrum.Methods Phys.Res. A544, 659 (2005)

Minimizing the statistical error in capture cross-section measurements

2005DA29 A.Danagoulian, D.J.Hamilton, C.E.Hyde-Wright, V.H.Mamyan, A.M.Nathan, M.Roedelbronn, B.Wojtsekhowski - Nucl.Phys. A755, 281c (2005)

Real Compton Scattering on Proton at High Momentum Transfers

2005DA34 T.Datta, S.P.Dange, H.Naik - J.Radioanal.Nucl.Chem. 266, 79 (2005)

Single nucleon transfer reactions near Coulomb barrier on ${ }^{197} \mathrm{Au}$

2005DA38 U.Datta Pramanik, P.Adrich, T.Aumann, K.Boretzky, D.Cortina, Th.W.Elze, H.Emling, H.Geissel, M.Hellstrom, K.L.Jones, L.H.Khiem, J.V.Kratz, R.Kulessa, Y.Leifels, G.Munzenberg, C.Nociforo, R.Palit, H.Scheit, H.Simon, K.Summerer, S.Typel, W.Walus, H.Weick - J.Phys.(London) G31, S1583 (2005)

Coulomb breakup of psd-shell neutron-rich nuclei

2005DA40 D.Das, V.Natarajan - Europhys.Lett. 72, 740 (2005)

Hyperfine spectroscopy on the $6 \mathrm{P}_{3 / 2}$ state of ${ }^{133} \mathrm{Cs}$ using coherent control

2005DA41 J.J.Das, P.Sugathan, N.Madhavan, P.V.Madhusudhana Rao, A.Jhingan, T.Varughese, S.Barua, S.Nath, A.K.Sinha, B.Kumar, J.Zacharias Nucl.Instrum.Methods Phys.Res. B241, 953 (2005)

Production of light radioactive ion beams (RIB) using inverse kinematics 
2005DA42 M.Danchev, J.Pavan, N.J.Stone, A.E.Stuchbery, C.Baktash, J.Beene, N.Benczer-Koller, C.R.Bingham, J.Dupak, A.Galindo-Uribarri, C.J.Gross, G.Kumbartzki, D.C.Radford, J.R.Stone, C.L.Timlin, C.-H.Yu, N.V.Zamfir Nucl.Instrum.Methods Phys.Res. B241, 971 (2005)

g-Factor measurements of first $2^{+}$states of heavy Te isotopes based on nuclear spin deorientation for nuclei recoiling in vacuum

2005DA43 U.Datta Pramanik, T.Aumann, K.Boretzky, D.Cortina, Th.W.Elze, H.Emling, H.Geissel, M.Hellstrom, K.L.Jones, L.H.Khiem, J.V.Kratz, R.Kulessa, Y.Leifels, G.Munzenberg, C.Nociforo, R.Palit, H.Scheit, H.Simon, K.Summerer, S.Typel, W.Walus, H.Weick - Eur.Phys.J. A 25, Supplement 1, 339 (2005)

Studies of light neutron-rich nuclei near the drip line

2005DAZW J.M.D'Auria, for the DRAGON Collaboration - Proc.Intern.Conf.Nuclear Data for Science and Technology, Santa Fe, New Mexico, 26 September-1 October, 2004, R.C.Haight, M.B.Chadwick, T.Kawano, P.Talou, Eds., Vol.2, p. 1345 (2005); AIP Conf.Proc. 769 (2005)

Nuclear Astrophysics At ISAC With DRAGON

2005DAZX D.Dashdorj, P.E.Garrett, J.A.Becker, L.A.Bernstein, J.R.Cooper, M.Devlin, N.Fotiades, G.E.Mitchell, R.O.Nelson, W.Younes - Proc.Intern.Conf.Nuclear Data for Science and Technology, Santa Fe, New Mexico, 26 September-1 October, 2004, R.C.Haight, M.B.Chadwick, T.Kawano, P.Talou, Eds., Vol.1, p. 1035 (2005); AIP Conf.Proc. 769 (2005)

${ }^{48} \mathrm{Ti}(\mathrm{n}, \mathrm{xnypz} \alpha \gamma)$ Reactions for Neutron Energies up to $250 \mathrm{MeV}$

2005DE01 H.De Witte, A.N.Andreyev, S.Dean, S.Franchoo, M.Huyse, O.Ivanov, U.Koster, W.Kurcewicz, J.Kurpeta, A.Plochocki, K.Van de Vel, J.Van de Walle, P.Van Duppen - Eur.Phys.J. A 23, 243 (2005)

Alpha-decay of neutron-deficient ${ }^{200} \mathrm{Fr}$ and heavier neighbours

2005DE02 A.Y.Deo, S.K.Tandel, S.B.Patel, P.V.Madhusudhana Rao, S.Muralithar, R.P.Singh, R.Kumar, R.K.Bhowmik, Amita - Phys.Rev. C 71, 017303 (2005)

Lifetime measurements in ${ }^{112} \mathrm{Sb}$

2005DE12 G.de Angelis - Nucl.Phys. A751, 533c (2005)

Future Perspectives in Nuclear Structure: From high intensity stable to radioactive nuclear beams

2005DE15 F.de Oliveira Santos, P.Himpe, M.Lewitowicz, I.Stefan, N.Smirnova, N.L.Achouri, J.C.Angelique, C.Angulo, L.Axelsson, D.Baiborodin, F.Becker, M.Belleguic, E.Berthoumieux, B.Blank, C.Borcea, A.Cassimi, J.M.Daugas, G.de France, F.Dembinski, C.E.Demonchy, Z.Dlouhy, P.Dolegieviez, C.Donzaud, G.Georgiev, L.Giot, S.Grevy, D.Guillemaud-Mueller, V.Lapoux, E.Lienard, M.J.Lopez Jimenez, K.Markenroth, I.Matea, W.Mittig, F.Negoita, G.Neyens, N.Orr, F.Pougheon, P.Roussel-Chomaz, M.G.Saint-Laurent, F.Sarazin, H.Savajols, M.Sawicka, O.Sorlin, M.Stanoiu, C.Stodel, G.Thiamova, D.Verney, A.C.C.Villari - Eur.Phys.J. A 24, 237 (2005)

Study of ${ }^{19} \mathrm{Na}$ at SPIRAL 
2005DE16 P.A.DeYoung, P.J.Mears, J.J.Kolata, E.F.Aguilera, F.D.Becchetti, Y.Chen, M.Cloughesy, H.Griffin, C.Guess, J.D.Hinnefeld, H.Jiang, S.R.Jones, U.Khadka, D.Lizcano, E.Martinez-Quiroz, M.Ojaniega, G.F.Peaslee, A.Pena, J.Rieth, S.VanDenDriessche, J.A.Zimmerman - Phys.Rev. C 71, 051601 (2005)

Two-neutron transfer in the ${ }^{6} \mathrm{He}+{ }^{209} \mathrm{Bi}$ reaction near the Coulomb barrier

2005DE34 A.N.Deacon, S.J.Freeman, R.V.F.Janssens, F.R.Xu, M.P.Carpenter, I.R.Calderin, P.Chowdhury, N.J.Hammond, T.Lauritsen, C.J.Lister, D.Seweryniak, J.F.Smith, S.L.Tabor, B.J.Varley, S.Zhu - Phys.Lett. B 622, 151 (2005); Erratum Phys.Lett. B $625,375(2005)$

Changes in $\nu \mathrm{g}_{9 / 2}$ shape polarisation across the odd neutron-rich $\mathrm{Cr}$ isotopes

2005DE42 L.De Smet, C.Wagemans, G.Goeminne, J.Wagemans, J.Heyse, J.Van Gils Nucl.Phys. A758, 80c (2005)

Investigation of the $(\mathrm{n}, \mathrm{p})$ and $(\mathrm{n}, \alpha)$ reactions on ${ }^{26} \mathrm{Al}$ and ${ }^{36} \mathrm{Cl}$ and their astrophysical relevance

2005DE45 N.de Sereville, E.Berthoumieux, A.Coc - Nucl.Phys. A758, 745c (2005)

The ${ }^{18} \mathrm{~F}(\mathrm{p}, \alpha){ }^{15} \mathrm{O}$ reaction rate for application to nova $\gamma$-ray emission

2005DE48 A.Dewald, O.Moller, B.Saha, K.Jessen, A.Fitzler, B.Melon, T.Pissulla, S.Heinze, J.Jolie, K.O.Zell, P.von Brentano, P.Petkov, S.Harissopulos, G.De Angelis, T.Martinez, D.R.Napoli, N.Marginean, M.Axiotis, C.Rusu, D.Tonev, A.Gadea, Y.H.Zhang, D.Bazzacco, S.Lunardi, C.A.Ur, R.Menegazzo, E.Farnea J.Phys.(London) G31, S1427 (2005)

Test of the critical point symmetry $\mathrm{X}(5)$ in the mass $\mathrm{A}=180$ region

2005DE54 G.Della Mea, A.Patelli, S.Restello, V.Rigato, A.Vomiero - Nucl.Instrum.Methods Phys.Res. B240, 803 (2005)

${ }^{14} \mathrm{~N}(\alpha, \mathrm{p}){ }^{17} \mathrm{O}$ nuclear reaction cross-section at 4.9-6.1 MeV

2005DEZT H.Denz, P.Amaudruz, J.T.Brack, J.Breitschopf, P.Camerini, J.L.Clark, H.Clement, L.Felawka, E.Fragiacomo, E.F.Gibson, N.Grion, G.J.Hofman, B.Jamieson, E.L.Mathie, R.Meier, G.Moloney, D.Ottewell, O.Patarakin, J.D.Patterson, M.M.Pavan, S.Piano, K.Raywood, R.A.Ristinen, R.Rui, M.E.Sevior, G.R.Smith, J.Stahov, R.Tacik, G.J.Wagner, F.von Wrochem, D.M.Yeomans nucl-ex/0512006,12/3/2005 (2005)

$\pi^{ \pm} \mathrm{p}$ differential cross sections at low energies

2005DEZV A.M.Demidov, L.I.Govor, V.A.Kurkin, I.V.Mikhailov - Book of Abstracts, LV National Conference on Nuclear Physics "Frontiers in the Physics of Nucleus", St.-Petersburg, p.53 (2005)

Multipole Mixtures in ${ }^{154} \mathrm{Sm} \gamma$-Transitions from the (n, n' $\gamma$ ) Reaction

2005DEZW M.S.Dewey, E.G.Kessler, Jr., R.D.Deslattes, H.G.Borner, M.Jentschel, C.Doll, P.Mutti - nucl-ex/0507011,7/06/2005 (2005)

Precision Measurement of the ${ }^{29} \mathrm{Si},{ }^{33} \mathrm{~S}$, and ${ }^{36} \mathrm{Cl}$ Binding Energies 
2005DEZX M.A.Deleplanque, F.S.Stephens, I.Y.Lee, A.O.Macchiavelli, D.Ward, P.Fallon, M.Cromaz, R.M.Clark, M.Descovich, R.M.Diamond, E.Rodriguez-Vieitez -

Proc.Nuclei at the Limits, Argonne, Illinois, D.Seweryniak and T.L.Khoo, eds., p.303 (2005); AIP Conf. Proc 764 (2005)

Transition From Order To Chaos In Rotational Nuclei

2005DEZZ F.Della Vedova, S.M.Lenzi, M.Ionescu-Bujor, N.Marginean, E.Farnea, M.Nespolo, G.de Angelis, M.Axiotis, D.Bazzacco, A.Bizzeti-Sona, P.G.Bizzeti, F.Brandolini, D.Bucurescu, A.Iordachescu, S.Lunardi, R.Menegazzo, D.R.Napoli, C.Rossi Alvarez, C.A.Ur - Proc.Nuclei at the Limits, Argonne, Illinois, D.Seweryniak and T.L.Khoo, eds., p.205 (2005); AIP Conf. Proc 764 (2005)

Isospin Symmetry Along The N=Z Line In The sd Shell

2005DI03 P.Di Nezza - Eur.Phys.J. A 24, Supplement 1, 23 (2005)

Spin structure of the nucleon

2005DI05 D.-C.Dinca, R.V.F.Janssens, A.Gade, D.Bazin, R.Broda, B.A.Brown, C.M.Campbell, M.P.Carpenter, P.Chowdhury, J.M.Cook, A.N.Deacon, B.Fornal, S.J.Freeman, T.Glasmacher, M.Honma, F.G.Kondev, J.-L.Lecouey, S.N.Liddick, P.F.Mantica, W.F.Mueller, H.Olliver, T.Otsuka, J.R.Terry, B.A.Tomlin, K.Yoneda Phys.Rev. C 71, 041302 (2005)

Reduced transition probabilities to the first $2^{+}$state in ${ }^{52,54,56} \mathrm{Ti}$ and development of shell closures at $\mathrm{N}=32,34$

2005DI15 I.Dillmann, M.Heil, F.Kappeler, T.Rauscher, F.-K.Thielemann - Nucl.Phys. A758, 513c (2005)

The $(\mathrm{n}, \gamma)$ cross sections of the p-process nuclei ${ }^{74} \mathrm{Se}$ and ${ }^{84} \mathrm{Sr}$ at $\mathrm{kT}=25 \mathrm{keV}$

2005DI16 C.Aa.Diget, F.C.Barker, M.G.Borge, J.Cederkall, V.N.Fedosseev, L.M.Fraile, B.R.Fulton, H.O.U.Fynbo, H.B.Jeppesen, B.Jonson, U.Koster, M.Meister, T.Nilsson, G.Nyman, Y.Prezado, K.Riisager, S.Rinta-Antila, O.Tengblad, M.Turrion, K.Wilhelmsen, J.Aysto - Nucl.Phys. A760, 3 (2005)

Properties of the ${ }^{12} \mathrm{C} 10 \mathrm{MeV}$ state determined through $\beta$-decay

2005DIZY F.Ditroi, F.Tarkanyi, J.Csikai, M.S.Uddin, M.Hagiwara, M.Baba -

Proc.Intern.Conf.Nuclear Data for Science and Technology, Santa Fe, New Mexico, 26 September-1 October, 2004, R.C.Haight, M.B.Chadwick, T.Kawano, P.Talou, Eds., Vol.1, p. 1011 (2005); AIP Conf.Proc. 769 (2005)

Investigation of Activation Cross Sections of the Proton Induced Nuclear Reactions on Natural Iron at Medium Energies

2005DizZ D.C.Dinca, R.V.F.Janssens, A.Gade, B.Fornal, S.Zhu, D.Bazin, R.Broda, C.M.Campbell, M.P.Carpenter, P.Chowdhury, J.M.Cook, P.J.Daly, A.N.Deacon, S.J.Freeman, T.Glasmacher, Z.W.Grabowski, N.J.Hammond, F.G.Kondev, W.Krolas, T.Lauritsen, J.-L.Lecouey, S.N.Liddick, C.J.Lister, P.F.Mantica, E.F.Moore, W.F.Mueller, H.Olliver, T.Pawlat, D.Seweryniak, K.Starosta, J.R.Terry, B.E.Tomlin, J.Wrzesinski, K.Yoneda - Proc.Nuclei at the Limits, Argonne, Illinois, D.Seweryniak and T.L.Khoo, eds., p.131 (2005); AIP Conf. Proc 764 (2005)

Neutron-Rich Ti Isotopes And Possible $\mathrm{N}=32$ And $\mathrm{N}=34$ Shell Gaps 
2005D016 Zs.Dombradi, Z.Elekes, R.Kanungo, H.Baba, Zs.Fulop, J.Gibelin, A.Horvath, E.Ideguchi, Y.Ichikawa, N.Iwasa, H.Iwasaki, S.Kanno, S.Kawai, Y.Kondo, T.Motobayashi, M.Notani, T.Ohnishi, A.Ozawa, H.Sakurai, S.Shimoura, E.Takeshita, S.Takeuchi, I.Tanihata, Y.Togano, C.Wu, Y.Yamaguchi, Y.Yanagisawa, A.Yoshida, K.Yoshida - Phys.Lett. B 621, 81 (2005)

Decoupling of valence neutrons from the core in ${ }^{17} \mathrm{~B}$

2005D020 C.Dossat, A.Bey, B.Blank, G.Canchel, A.Fleury, J.Giovinazzo, I.Matea, F.de Oliveira Santos, G.Georgiev, S.Grevy, I.Stefan, J.C.Thomas, N.Adimi, C.Borcea, D.Cortina Gil, M.Caamano, M.Stanoiu, F.Aksouh, B.A.Brown, L.V.Grigorenko Phys.Rev. C 72, 054315 (2005)

Two-proton radioactivity studies with ${ }^{45} \mathrm{Fe}$ and ${ }^{48} \mathrm{Ni}$

2005D0ZW Zs.Dombradi, D.Sohler, T.Otsuka, F.Azaiez, M.Belleguic, M.J.Lopez-Jimenez, M.G.Saint-Laurent, O.Sorlin, N.L.Achouri, J.C.Angelique, C.Borcea, C.Bourgeois, J.M.Daugas, F.De Oliveira-Santos, Z.Dlouhy, C.Donzaud, J.Duprat, S.Grevy, D.Guillemaud-Mueller, S.Leenhardt, M.Lewitowicz, S.M.Lukyanov, W.Mittig, Y.Utsuno, Yu.-E.Penionzhkevich, M.G.Porquet, F.Pougheon, P.Roussel-Chomaz, H.Savajols, Y.Sobolev, C.Stodel, M.Stanoiu, J.Timar - ATOMKI 2004 Ann.Rept., p.10 (2005)

Neutron excitations across the $\mathrm{N}=20$ shell gap in ${ }^{26,28} \mathrm{Ne}$

2005D0ZX Zs.Dombradi, D.Sohler, F.Azaiez, M.Belleguic, M.J.Lopez-Jimenez, M.G.Saint-Laurent, O.Sorlin, N.L.Achouri, J.C.Angelique, C.Borcea, C.Bourgeois, J.M.Daugas, F.De Oliveira-Santos, Z.Dlouhy, C.Donzaud, J.Duprat, S.Grevy, D.Guillemaud-Mueller, S.Leenhardt, M.Lewitowicz, S.M.Lukyanov, W.Mittig, Yu.-E.Penionzhkevich, M.G.Porquet, F.Pougheon, P.Roussel-Chomaz, H.Savajols, Y.Sobolev, C.Stodel, M.Stanoiu, J.Timar - ATOMKI 2004 Ann.Rept., p.8 (2005) In beam $\gamma$-ray spectroscopy of the neutron rich isotope ${ }^{19} \mathrm{~N}$

2005DOZY C.Domingo-Pardo, and the n_TOF Collaboration - Proc.Intern.Conf.Nuclear Data for Science and Technology, Santa Fe, New Mexico, 26 September-1 October, 2004, R.C.Haight, M.B.Chadwick, T.Kawano, P.Talou, Eds., Vol.2, p. 1521 (2005); AIP Conf.Proc. 769 (2005)

New Measurement of the Capture Cross Section of Bismuth and Lead Isotopes

2005Dr05 G.D.Dracoulis, G.J.Lane, F.G.Kondev, A.P.Byrne, T.Kibedi, H.Watanabe, I.Ahmad, M.P.Carpenter, S.J.Freeman, R.V.F.Janssens, N.J.Hammond, T.Lauritsen, C.J.Lister, G.Mukherjee, D.Seweryniak, P.Chowdhury, S.K.Tandel Phys.Rev. C 71, 044326 (2005); Erratum Phys.Rev. C 73, 019901 (2006) Structure of two-, four-, and six-quasiparticle isomers in ${ }^{174} \mathrm{Yb}$ and K-forbidden decays

2005DRZW G.D.Dracoulis, G.J.Lane, T.M.Peatey, A.P.Byrne, A.M.Baxter, P.M.Davidson, A.N.Wilson, T.Kibedi, F.R.Xu - ANU-P/1662 (2005)

E3 strength of the $11^{-}$to $8^{+}$isomeric decays in ${ }^{194} \mathrm{~Pb}$ and ${ }^{196} \mathrm{~Pb}$ and oblate deformation 
2005DRZY G.D.Dracoulis, G.J.Lane, F.G.Kondev, A.P.Byrne, T.Kibedi, H.Watanabe, I.Ahmad, M.P.Carpenter, S.J.Freeman, R.V.F.Janssens, N.J.Hammond, T.Lauritsen, C.J.Lister, G.Mukherjee, D.Seweryniak, P.Chowdhury, S.K.Tandel ANU-P/1648 (2005)

Structure of two- four- and six-quasiparticle isomers in ${ }^{174} \mathrm{Yb}$ and $\mathrm{K}$-forbidden decays

2005DU14 C.Duweke, R.Emmerich, A.Imig, J.Ley, G.Tenckhoff, H.Paetz gen.Schieck, J.Golak, H.Witala, E.Epelbaum, W.Glockle, A.Nogga - Phys.Rev. C 71, 054003 (2005) The reaction ${ }^{2} \mathrm{H}(\mathrm{p}, \mathrm{pp}) \mathrm{n}$ in three kinematical configurations at $\mathrm{E}_{p}=16 \mathrm{MeV}$

2005 DU15 C.L.Duncan, K.S.Krane - Phys.Rev. C 71, 054322 (2005)

Neutron capture cross section of ${ }^{102} \mathrm{Pd}$

2005 DU19 R.du Rietz, S.J.Williams, D.Rudolph, J.Ekman, C.Fahlander, C.Andreoiu, M.Axiotis, M.A.Bentley, M.P.Carpenter, C.Chandler, R.J.Charity, R.M.Clark, M.Cromaz, A.Dewald, G.de Angelis, F.Della Vedova, P.Fallon, A.Gadea, G.Hammond, E.Ideguchi, S.M.Lenzi, A.O.Macchiavelli, N.Marginean, M.N.Mineva, O.Moller, D.R.Napoli, M.Nespolo, W.Reviol, C.Rusu, B.Saha, D.G.Sarantites, D.Seweryniak, D.Tonev, C.A.Ur - Phys.Rev. C 72, 014307 (2005)

Investigation of high-spin states in ${ }^{53} \mathrm{Fe}$

2005 DU23 Ch.E.Dullmann, C.M.Folden III, K.E.Gregorich, D.C.Hoffman, D.Leitner, G.K.Pang, R.Sudowe, P.M.Zielinski, H.Nitsche - Nucl.Instrum.Methods Phys.Res. A551, 528 (2005)

Heavy-ion-induced production and physical preseparation of short-lived isotopes for chemistry experiments

2005 DZ03 S.N.Dzhosyuk, A.Copete, J.M.Doyle, L.Yang, K.J.Coakley, R.Golub, E.Korobkina, T.Kreft, S.K.Lamoreaux, A.K.Thompson, G.L.Yang, P.R.Huffman -

J.Res.Natl.Inst.Stand.Technol. 110, 339 (2005)

Determination of the Neutron Lifetime Using Magnetically Trapped Neutrons

2005EE01 S.Eeckhaudt, N.Amzal, J.E.Bastin, E.Bouchez, P.A.Butler, A.Chatillon, K.Eskola, J.Gerl, T.Grahn, A.Gorgen, P.T.Greenlees, R.-D.Herzberg, F.P.Hessberger, A.Hurstel, P.J.C.Ikin, G.D.Jones, P.Jones, R.Julin, S.Juutinen, H.Kettunen, T.L.Khoo, W.Korten, P.Kuusiniemi, Y.Le Coz, M.Leino, A.-P.Leppanen, P.Nieminen, J.Pakarinen, J.Perkowski, A.Pritchard, P.Reiter, P.Rahkila, C.Scholey, Ch.Theisen, J.Uusitalo, K.Van de Vel, J.Wilson, H.J.Wollersheim - Eur.Phys.J. A 25, Supplement 1, 605 (2005)

In-beam gamma-ray spectroscopy of ${ }^{254} \mathrm{No}$

2005EE02 S.Eeckhaudt, P.T.Greenlees, N.Amzal, J.E.Bastin, E.Bouchez, P.A.Butler, A.Chatillon, K.Eskola, J.Gerl, T.Grahn, A.Gorgen, R.-D.Herzberg, F.P.Hessberger, A.Hurstel, P.J.C.Ikin, G.D.Jones, P.Jones, R.Julin, S.Juutinen, H.Kettunen, T.L.Khoo, W.Korten, P.Kuusiniemi, Y.Le Coz, M.Leino, A.-P.Leppanen, P.Nieminen, J.Pakarinen, J.Perkowski, A.Pritchard, P.Reiter, P.Rahkila, C.Scholey, Ch.Theisen, J.Uusitalo, K.Van de Vel, J.Wilson - Eur.Phys.J. A 26, 227 (2005)

Evidence for non-yrast states in ${ }^{254} \mathrm{No}$ 
2005EG01 A.I.Egorov, R.I.Krutova, Yu.E.Loginov, S.Eh.Malyutenkova - Nucl.Instrum.Methods Phys.Res. A545, 296 (2005)

Measurement of thermal neutron radiative capture cross-sections of the ${ }^{14} \mathrm{~N}$ and ${ }^{19} \mathrm{~F}$ by in-beam $\gamma$-spectroscopy method with reactor neutrons

2005EGZZ K.S.Egiyan, for the CLAS Collaboration - nucl-ex/0508026,8/24/2005 (2005)

Measurement of 2- and 3-Nucleon Short Range Correlation Probabilities in Nuclei

2005EK01 J.Ekman, L.-L.Andersson, C.Fahlander, E.K.Johansson, R.du Rietz, D.Rudolph Eur.Phys.J. A 25, Supplement 1, 363 (2005)

News on mirror nuclei in the sd and fp shells

2005EL07 Z.Elekes, Zs.Dombradi, R.Kanungo, H.Baba, Zs.Fulop, J.Gibelin, A.Horvath, E.Ideguchi, Y.Ichikawa, N.Iwasa, H.Iwasaki, S.Kanno, S.Kawai, Y.Kondo, T.Motobayashi, M.Notani, T.Ohnishi, A.Ozawa, H.Sakurai, S.Shimoura, E.Takeshita, S.Takeuchi, I.Tanihata, Y.Togano, C.Wu, Y.Yamaguchi, Y.Yanagisawa, A.Yoshida, K.Yoshida - Phys.Lett. B 614, 174 (2005)

Low-lying excited states in ${ }^{17,19} \mathrm{C}$

2005EL10 H.M.El-Masri, P.M.Walker, G.D.Dracoulis, T.Kibedi, A.P.Byrne, A.M.Bruce, J.N.Orce, A.Emmanouilidis, D.M.Cullen, C.Wheldon, F.R.Xu - Phys.Rev. C 72, $054306(2005)$

High-K states in the odd-odd nuclide ${ }^{180} \mathrm{Re}$

2005ER01 K.N.Ermakov, T.S.Oposhnyan, V.L.Rappoport, O.V.Rogachevsky, V.V.Sarantsev, S.G.Sherman - Eur.Phys.J. A 23, 345 (2005)

Study of the $\pi^{-} \mathrm{n} \rightarrow \pi^{-} \pi^{-}$reaction at $430 \mathrm{MeV}$ energy

2005ER03 K.Ermisch, H.R.Amir-Ahmadi, A.M.van den Berg, R.Castelijns, B.Davids, A.Deltuva, E.Epelbaum, W.Glockle, J.Golak, M.N.Harakeh, M.Hunyadi, M.A.de Huu, N.Kalantar-Nayestanaki, H.Kamada, M.Kis, M.Mahjour-Shafiei, A.Nogga, P.U.Sauer, R.Skibinski, H.Witala, H.J.Wortche - Phys.Rev. C 71, 064004 (2005) Systematic investigation of three-nucleon force effects in elastic scattering of polarized protons from deuterons at intermediate energies

2005ERZZ T.Eronen, V.Elomaa, U.Hager, J.Hakala, A.Jokinen, I.Moore, H.Penttila, S.Rahaman, S.Rinta-Antila, A.Saastamoinen, T.Sonoda, J.Aysto, A.Bey , B.Blank, G.Canchel, C.Dossat, J.Giovinazzo, I.Matea, N.Adimi - nucl-ex/0512010,12/12/2005 (2005)

Q-value of the superallowed $\beta$ decay of ${ }^{62} \mathrm{Ga}$

2005ESZZ E.-I.Esch, A.Alpizar-Vicente, E.M.Bond, T.A.Bredeweg, S.E.Glover, U.Greife, R.C.Haight, R.Hatarik, A.Kronenberg, J.M.O'Donnell, E.J.Pitcher, R.Reifarth, R.S.Rundberg, J.L.Ullmann, D.J.Vieira, S.A.Wender, J.M.Wouters -

Proc.Intern.Conf.Nuclear Data for Science and Technology, Santa Fe, New Mexico, 26 September-1 October, 2004, R.C.Haight, M.B.Chadwick, T.Kawano, P.Talou, Eds., Vol.1, p. 989 (2005); AIP Conf.Proc. 769 (2005)

Status of the Neutron Capture Measurement on ${ }^{237} \mathrm{~Np}$ with the DANCE Array at LANSCE 
2005FA06 P.Fallon - Nucl.Phys. A752, 231c (2005)

From Superdeformations to Hyperdeformations

2005FE11 J.O.Fernandez-Niello, A.Priller, A.Arazi, D.Djokic, R.Golser, W.Kutschera, P.Steier, C.Vockenhuber, A.Wallner - Nucl.Instrum.Methods Phys.Res. B240, 495 (2005) A study of the tandem-terminal-stripper reaction ${ }^{1} \mathrm{H}\left({ }^{12} \mathrm{C}, \gamma\right){ }^{13} \mathrm{~N}$ with accelerator mass spectrometry

2005FI01 R.Fitzgerald, E.Abbotoy, D.W.Bardayan, J.C.Blackmon, A.E.Champagne, A.A.Chen, U.Greife, D.W.Hill, A.N.James, R.L.Kozub, T.A.Lewis, R.Livesay, Z.Ma, S.L.Mahan, J.W.McConnell, W.T.Milner, B.H.Moazen, P.D.Parker, D.E.Pierce, M.E.Roettger, L.Sahin, D.Shapira, M.S.Smith, F.Strieder, K.B.Swartz, J.S.Thomas, D.W.Visser - Nucl.Phys. A748, 351 (2005)

Studies of $(\mathrm{p}, \gamma)$ reactions with the Daresbury Recoil Separator at ORNL'S HRIBF

2005FI10 S.M.Fischer, T.Anderson, P.Kerns, G.Mesoloras, D.Svelnys, C.J.Lister, D.P.Balamuth, P.A.Hausladen, D.G.Sarantites - Phys.Rev. C 72, 024321 (2005)

Shape coexistence in ${ }^{71} \mathrm{Br}$ and the question of the ground-state spin of ${ }^{71} \mathrm{Kr}$

2005FL02 F.Fleurot, A.M.van den Berg, B.Davids, M.N.Harakeh, V.L.Kravchuk, H.W.Wilschut, J.Guillot, H.Laurent, A.Willis, M.Assuncao, J.Kiener, A.Lefebvre, N.de Sereville, V.Tatischeff - Phys.Lett. B 615, 167 (2005)

${ }^{16} \mathrm{O}$ Coulomb dissociation: towards a new means to determine the ${ }^{12} \mathrm{C}+\alpha$ fusion rate in stars

2005F003 C.Fox, C.Iliadis, A.E.Champagne, R.P.Fitzgerald, R.Longland, J.Newton, J.Pollanen, R.Runkle - Phys.Rev. C 71, 055801 (2005)

Thermonuclear reaction rate of ${ }^{17} \mathrm{O}(\mathrm{p}, \gamma){ }^{18} \mathrm{~F}$

2005F005 N.Fotiades, J.A.Cizewski, R.Krucken, R.M.Clark, P.Fallon, I.Y.Lee, A.O.Macchiavelli, D.P.McNabb, J.A.Becker, L.A.Bernstein, W.Younes - Phys.Rev. C 71, $064312(2005)$

High-spin states in $\mathrm{N}=50{ }^{85} \mathrm{Br}$ and ${ }^{87} \mathrm{Rb}$ nuclei

2005 F006 N.Fotiades, R.O.Nelson, M.Devlin, K.Starosta, J.A.Becker, L.A.Bernstein, P.E.Garrett, W.Younes - Phys.Rev. C 71, 064314 (2005)

States in ${ }^{197} \mathrm{Au}$ from the (n, n' $\left.\gamma\right)$ reaction

2005F009 D.Fong, J.K.Hwang, A.V.Ramayya, J.H.Hamilton, Y.X.Luo, P.M.Gore, E.F.Jones, W.B.Walters, J.O.Rasmussen, M.A.Stoyer, S.J.Zhu, I.Y.Lee, A.O.Macchiavelli, S.C.Wu, A.V.Daniel, G.M.Ter-Akopian, Yu.Ts.Oganessian, J.D.Cole, R.Donangelo, W.C.Ma - Phys.Rev. C 72, 014315 (2005)

Negative parity bands of ${ }^{115} \mathrm{Pd}$ and band structures in ${ }^{113,115,117} \mathrm{Pd}$

2005F014 B.Fornal, S.Zhu, R.V.F.Janssens, M.Honma, R.Broda, B.A.Brown, M.P.Carpenter, S.J.Freeman, N.Hammond, F.G.Kondev, W.Krolas, T.Lauritsen, S.N.Liddick, C.J.Lister, S.Lunardi, P.F.Mantica, N.Marginean, T.Mizusaki, E.F.Moore, T.Otsuka, T.Pawlat, D.Seweryniak, B.E.Tomlin, C.A.Ur, I.Wiedenhover, J.Wrzesinski - Phys.Rev. C 72, 044315 (2005)

Yrast structure of neutron-rich ${ }^{53} \mathrm{Ti}$ 
2005F017 D.Fong, J.K.Hwang, A.V.Ramayya, J.H.Hamilton, C.J.Beyer, K.Li, P.M.Gore, E.F.Jones, Y.X.Luo, J.O.Rasmussen, S.J.Zhu, S.C.Wu, I.Y.Lee, P.Fallon, M.A.Stoyer, S.J.Asztalos, T.N.Ginter, J.D.Cole, G.M.Ter-Akopian, A.Daniel, R.Donangelo - Eur.Phys.J. A 25, Supplement 1, 465 (2005)

Investigations of short half-life states from $\mathrm{SF}$ of ${ }^{252} \mathrm{Cf}$

2005F0ZZ N.Fotiades, R.O.Nelson, M.Devlin, M.B.Chadwick, P.Talou, J.A.Becker, P.E.Garrett, W.Younes - Proc.Intern.Conf.Nuclear Data for Science and Technology, Santa Fe, New Mexico, 26 September-1 October, 2004, R.C.Haight, M.B.Chadwick, T.Kawano, P.Talou, Eds., Vol.1, p. 898 (2005); AIP Conf.Proc. 769 (2005)

Cross Sections for $\gamma$-Ray Production in the ${ }^{191} \operatorname{Ir}(\mathrm{n}, \mathrm{xn} \gamma)$ Reactions

2005FR14 M.Freer, I.Boztosun, C.A.Bremner, S.P.G.Chappell, R.L.Cowin, G.K.Dillon, B.R.Fulton, B.J.Greenhalgh, M.P.Nicoli, W.D.M.Rae, S.M.Singer, D.L.Watson, D.C.Weisser - Phys.Rev. C 71, 047305 (2005)

${ }^{8} \mathrm{Be}+{ }^{12} \mathrm{C}\left(0_{2}^{+}\right)$decay of excited states in ${ }^{20} \mathrm{Ne}$

2005FR17 C.Fransen, V.Werner, D.Bandyopadhyay, N.Boukharouba, S.R.Lesher, M.T.McEllistrem, J.Jolie, N.Pietralla, P.von Brentano, S.W.Yates - Phys.Rev. C 71, $054304(2005)$

Investigation of low-spin states in ${ }^{92} \mathrm{Zr}$ with the (n, n' $\left.\gamma\right)$ reaction

2005FR19 J.Fridmann, I.Wiedenhover, A.Gade, L.T.Baby, D.Bazin, B.A.Brown, C.M.Campbell, J.M.Cook, P.D.Cottle, E.Diffenderfer, D.-C.Dinca, T.Glasmacher, P.G.Hansen, K.W.Kemper, J.L.Lecouey, W.F.Mueller, H.Olliver,

E.Rodriguez-Vieitez, J.R.Terry, J.A.Tostevin, K.Yoneda - Nature(London) 435, 922 (2005)

'Magic' nucleus ${ }^{42} \mathrm{Si}$

2005FR29 S.J.Freeman, R.V.F.Janssens, A.N.Deacon, F.Xu, I.J.Calderin, M.P.Carpenter, P.Chowdhury, S.M.Fischer, N.J.Hammond, M.Honma, T.Lauritsen, C.J.Lister, T.L.Khoo, G.Mukherjee, D.Seweryniak, J.F.Smith, S.L.Tabor, B.J.Varley, S.Zhu J.Phys.(London) G31, S1465 (2005)

Structure of the neutron-rich Cr isotopes

2005FRZZ S.J.Freeman, R.V.F.Janssens, B.A.Brown, I.V.Calderin, M.P.Carpenter, P.Chowdhury, A.N.Deacon, S.M.Fischer, N.J.Hammond, M.Honma, T.Lauritsen, C.J.Lister, T.L.Khoo, G.Mukherjee, D.Seweryniak, J.F.Smith, S.L.Tabor, B.J.Varley, M.Whitehead, S.Zhu - Proc.Nuclei at the Limits, Argonne, Illinois, D.Seweryniak and T.L.Khoo, eds., p.142 (2005); AIP Conf. Proc 764 (2005) Structure Of The Neutron Rich Cr Isotopes: Inadequacy Of The fp Model Space And Onset Of Deformation

2005FU01 T.Fukuchi, Y.Gono, A.Odahara, S.Tanaka, M.Inoue, Y.Wakabayashi, T.Sasaki, M.Kibe, N.Hokoiwa, T.Shinozuka, M.Fujita, A.Yamazaki, T.Sonoda, C.S.Lee, Y.K.Kwon, J.Y.Moon, J.H.Lee - Eur.Phys.J. A 24, 249 (2005)

High-spin isomer in ${ }^{93} \mathrm{Mo}$ 
2005FU03 M.Fukada, M.Ohmura, F.Harima, K.Ogino, K.Takimoto, S.Ohkubo - Phys.Rev. C 71, $067602(2005)$

$\alpha$-cluster states in ${ }^{38} \mathrm{Ar}$ observed via the ${ }^{34} \mathrm{~S}\left({ }^{7} \mathrm{Li}, \mathrm{t} \alpha\right){ }^{34} \mathrm{~S}$ reaction

2005Fu13 Zs.Fulop, Gy.Gyurky, E.Somorjai, D.Schurmann, F.Raiola, F.Strieder, C.Rolfs, B.N.Limata, L.Gialanella, G.Imbriani, V.Roca, M.Romano, N.De Cesare, A.D’Onofrio, D.Rogalla, F.Terrasi - Nucl.Phys. A758, 697c (2005)

Decay of ${ }^{7} \mathrm{Be}$ in metallic environment

2005Fu16 Y.Fujita, T.Adachi, P.von Brentano, G.P.A.Berg, C.Fransen, D.De Frenne, H.Fujita, K.Fujita, K.Hatanaka, E.Jacobs, K.Nakanishi, A.Negret, N.Pietralla, L.Popescu, B.Rubio, Y.Sakemi, Y.Shimbara, Y.Shimizu, Y.Tameshige, A.Tamii, M.Yosoi, K.O.Zell - Phys.Rev.Lett. 95, 212501 (2005)

Gamow-Teller Strengths in Proton-Rich Exotic Nuclei Deduced in the Combined Analysis of Mirror Transitions

2005FUZV

T.Fukuchi, N.Hokoiwa, Y.Wakabayashi, Y.Gono, A.Odahara, T.Shinozuka, M.Fujita, A.Yamazaki, T.Sonoda, T.Suzuki - CNS-REP-66, p.17 (2005)

Study of High-Spin States in ${ }^{91} \mathrm{Zr}$

2005FUZW H.Fujikawa, S.Kubono, A.Saito, H.Yamaguchi, G.Amadio, J.J.He, L.H.Khiem, S.Nishimura, H.Ohta, A.Ozawa, Y.Tagishi, Y.Wakabayashi, M.Yamaguchi, T.Yasuno - CNS-REP-66, p.13 (2005)

Development of a High-Precision Method for Alpha Resonant Scattering Measurements for Nuclear Astrophysics

2005FUZX Zs.Fulop, H.Yamaguchi, Z.Elekes, S.Kubono, G.Amadio, H.Fujikawa, J.J.He, S.Michimasa, J.Niikura, S.Nishimura, A.Saito, T.Teranishi, Y.Wakabayashi, Y.Yanagisawa - CNS-REP-66, p.8 (2005)

Production of ${ }^{39} \mathrm{Ar}$ at the CRIB Facility

2005FUZY K.Furutaka, H.Harada, S.Raman - Proc.Intern.Conf.Nuclear Data for Science and Technology, Santa Fe, New Mexico, 26 September-1 October, 2004, R.C.Haight, M.B.Chadwick, T.Kawano, P.Talou, Eds., Vol.2, p. 1454 (2005); AIP Conf.Proc. $769(2005)$

Determination of the ${ }^{99} \mathrm{Tc}($ thermal $\mathrm{n}, \gamma){ }^{100} \mathrm{Tc}$ Reaction Cross Section from the Prompt $\gamma$-Ray Yields

2005GA01 L.Gaudefroy, O.Sorlin, C.Donzaud, J.C.Angelique, F.Azaiez, C.Bourgeois, V.Chiste, Z.Dlouhy, S.Grevy, D.Guillemaud-Mueller, F.Ibrahim, K.-L.Kratz, M.Lewitowicz, S.M.Lukyanov, I.Matea, J.Mrazek, F.Nowacki, F.de Oliveira Santos,

Yu.-E.Penionzhkevich, B.Pfeiffer, F.Pougheon, M.G.Saint-Laurent, M.Stanoiu -

Eur.Phys.J. A 23, 41 (2005)

Beta-decay studies of neutron-rich Sc-Cr nuclei

2005GA09 F.Garibaldi, on behalf of the Hall A Collaboration - Eur.Phys.J. A 24, Supplement 1, $91(2005)$

High resolution hypernuclear spectroscopy 
2005GA14 G.Gangopadhyay, S.Bhowal, R.K.Bhowmik, U.Datta Pramanik, P.Ghosh, A.Goswami, C.Petrache, A.Mukherjee, S.Muralithar, R.Raut, M.S.Sarkar, A.K.Singh, R.P.Singh, S.Bhattacharya - Eur.Phys.J. A 24, 173 (2005)

Levels in doubly odd ${ }^{138} \mathrm{Pr}$

2005GA15 A.Gade, D.Bazin, B.A.Brown, C.M.Campbell, J.A.Church, D.-C.Dinca, J.Enders, T.Glasmacher, P.G.Hansen, M.Honma, T.Mizusaki, W.F.Mueller, H.Olliver, T.Otsuka, L.A.Riley, J.R.Terry, J.A.Tostevin, K.L.Yurkewicz - Acta Phys.Pol. B36, $1227(2005)$

Nuclear spectroscopy with fast exotic beams

2005GA18 A.Gade, D.Bazin, C.A.Bertulani, B.A.Brown, C.M.Campbell, J.A.Church, D.C.Dinca, J.Enders, T.Glasmacher, P.G.Hansen, Z.Hu, K.W.Kemper, W.F.Mueller, H.Olliver, B.C.Perry, L.A.Riley, B.T.Roeder, B.M.Sherrill, J.R.Terry, J.A.Tostevin, K.L.Yurkewicz - Phys.Rev. C 71, 051301 (2005)

Knockout from ${ }^{46} \mathrm{Ar}: \mathrm{l}=3$ neutron removal and deviations from eikonal theory

2005GA20 A.Gadea, S.M.Lenzi, D.R.Napoli, M.Axiotis, C.A.Ur, G.Martinez-Pinedo, M.Gorska, E.Roeckl, E.Caurier, F.Nowacki, G.De Angelis, L.Batist, R.Borcea, F.Brandolini, D.Cano-Ott, J.Doring, C.Fahlander, E.Farnea, H.Grawe, M.Hellstrom, Z.Janas, R.Kirchner, M.La Commara, C.Mazzocchi, E.Nacher, C.Plettner, A.Plochocki, B.Rubio, K.Schmidt, R.Schwengner, J.L.Tain, J.Zylicz - Phys.Lett. B 619, 88 (2005) Hindered E4 decay of the $12^{+}$yrast trap in ${ }^{52} \mathrm{Fe}$

2005GA21 D.Galaviz, Zs.Fulop, Gy.Gyurky, Z.Mate, P.Mohr, T.Rauscher, E.Somorjai, A.Zilges - Phys.Rev. C 71, 065802 (2005)

Elastic $\alpha$ scattering on ${ }^{112} \mathrm{Sn}$ and ${ }^{124} \mathrm{Sn}$ at astrophysically relevant energies

2005GA22 A.Gade, D.Bazin, A.Becerril, C.M.Campbell, J.M.Cook, D.J.Dean, D.-C.Dinca, T.Glasmacher, G.W.Hitt, M.E.Howard, W.F.Mueller, H.Olliver, J.R.Terry, K.Yoneda - Phys.Rev.Lett. 95, 022502 (2005); Erratum Phys.Rev.Lett. 96, 189901 (2006) Quadrupole Deformation of the Self-Conjugate Nucleus ${ }^{72} \mathrm{Kr}$

2005GA25 Yu.P.Gangrsky, V.I.Zhemenik, N.Yu.Maslova, G.V.Mishinsky, Yu.E.Penionzhkevich - Part. and Nucl., Lett. 125, 44 (2005)

Yields of Fragments in the Spontaneous and Photofission of ${ }^{248} \mathrm{Cm}$

2005GA36 E.Garcia-Torano, M.T.Crespo, M.Roteta, G.Sibbens, S.Pomme, A.M.Sanchez, M.P.R.Montero, S.Woods, A.Pearce - Nucl.Instrum.Methods Phys.Res. A550, 581 (2005)

$\alpha$-particle emission probabilities in the decay of ${ }^{235} \mathrm{U}$

2005GA40 D.Galaviz, A.Kretschmer, S.Muller, K.Sonnabend, A.Zilges - Nucl.Phys. A758, 521c (2005)

Nuclear astrophysics with real photons 
2005GA44 A.Gadea, N.Marginean, L.Corradi, S.M.Lenzi, C.A.Ur, E.Farnea, G.de Angelis, E.Fioretto, D.R.Napoli, A.M.Stefanini, S.Szilner, M.Axiotis, B.R.Behera, A.Latina, C.Rusu, W.Zhimin, D.Bazzacco, S.Beghini, S.Lunardi, G.Montagnoli, R.Menegazzo, F.Scarlassara, F.Della Vedova, M.Nespolo, A.Bracco, F.Camera, S.Leoni, B.Million, M.Pignanelli, G.Pollarolo, M.Trotta, P.G.Bizzeti, A.M.Bizzeti-Sona, D.Curien, P.Medina, M.Chambit, R.Chapman, X.Liang, S.J.Freeman, A.Smith, B.J.Varley, V.Pucknell, R.Lemmon - J.Phys.(London) G31, S1443 (2005)

The CLARA-PRISMA setup installed at LNL: first results

2005GA45 L.Gaudefroy, O.Sorlin, D.Beaumel, Y.Blumenfeld, Z.Dombradi, S.Fortier, S.Franchoo, M.Gelin, J.Gibelin, S.Grevy, F.Hammache, F.Ibrahim, K.Kemper, K.L.Kratz, S.M.Lukyanov, C.Monrozeau, L.Nalpas, F.Nowacki, A.N.Ostrowski, Yu.-E.Penionzhkevich, E.Pollacco, P.Roussel-Chomaz, E.Rich, J.A.Scarpaci, M.G.St Laurent, D.Sohler, M.Stanoiu, E.Tryggestad, D.Verney - J.Phys.(London) G31, S1623 (2005)

Study of ${ }^{45}$ Ar through $(\mathrm{d}, \mathrm{p})$ reaction at SPIRAL

2005GA46 A.B.Garnsworthy, N.J.Thompson, Zs.Podolyak, P.M.Walker, S.J.Williams, G.D.Dracoulis, G.de France, G.J.Lane, K.Andgren, A.M.Bruce, A.P.Byrne, W.N.Catford, B.Cederwall, G.A.Jones, B.McGuirk, S.Mandal, E.S.Paul, V.Pucknell, N.Redon, B.Rosse, R.J.Senior, G.Sletten - J.Phys.(London) G31, S1851 (2005) Spectroscopy of ${ }^{212} \mathrm{Po}$ and ${ }^{213} \mathrm{At}$ using a ${ }^{8} \mathrm{He}$ radioactive beam and EXOGAM

2005GA47 P.E.Garrett, W.D.Kulp, J.L.Wood, D.Bandyopadhyay, S.Christen, S.Choudry, A .Dewald, A.Fitzler, C .Fransen, K.Jessen, J.Jolie, A.Kloezer, P.Kudejova, A.Kumar, S.R.Lesher, A.Linnemann, A.Lisetskiy, D.Martin, M.Masur, M.T.McEllistrem, O.Moller, M.Mynk, J.N.Orce, P.Pejovic, T.Pissulla, J.M.Regis, A.Schiller, D.Tonev, S.W.Yates - J.Phys.(London) G31, S1855 (2005)

Octupole and hexadecapole bands in ${ }^{152} \mathrm{Sm}$

2005GA50 Yu.P.Gangrsky, V.I.Zhemenik, G.V.Mishinsky, Yu.E.Penionzhkevich - Yad.Fiz. 68, 1475 (2005); Phys.Atomic Nuclei 68, 1417 (2005)

Independent Yields of Kr and Xe Fragments in the Photofission of ${ }^{237} \mathrm{~Np}$ and ${ }^{243} \mathrm{Am}$ Odd Nuclei

2005GA54 A.Gade, D.Bazin, B.A.Brown, C.M.Campbell, J.A.Church, D.-C.Dinca, J.Enders, T.Glasmacher, P.G.Hansen, Z.Hu, K.W.Kemper, W.F.Mueller, H.Olliver, B.C.Perry, L.A.Riley, B.T.Roeder, B.M.Sherrill, J.R.Terry, J.A.Tostevin, K.L.Yurkewicz -

Eur.Phys.J. A 25, Supplement 1, 251 (2005)

Spectroscopic factors in exotic nuclei from nucleon-knockout reactions

2005GA56

A.Gadea - Eur.Phys.J. A 25, Supplement 1, 421 (2005)

First results of the CLARA-PRISMA setup installed at LNL

2005GazT C.Gaulard, G.Audi, C.Bachelet, D.Lunney, M.de Saint Simon, C.Thibault, N.Vieira - nucl-ex/0511007,11/2/2005 (2005)

Accurate mass measurements of ${ }^{26} \mathrm{Ne},{ }^{26-30} \mathrm{Na},{ }^{29-33} \mathrm{Mg}$ performed with the MISTRAL spectrometer 
2005GAZU Ju.M.Gavriljuk, A.M.Gangapshev, V.V.Kuzminov, S.I.Panasenko, S.S.Ratkevich nucl-ex/0510071,10/26/2005 (2005)

Results of a search for $2 \beta$-decay of ${ }^{136} \mathrm{Xe}$ with high-pressure copper proportional counters in Baksan Neutrino Observatory

2005GAZV Ju.M.Gavriljuk, V.N.Gavrin, A.M.Gangapshev, V.V.Kazalov, V.V.Kuzminov, N.Ya.Osetrova, I.I.Pul'nikov, A.V.Ryabukhin, A.N.Shubin, G.M.Skorynin, S.I.Panasenko, S.S.Ratkevich - nucl-ex/0510070,10/26/2005 (2005)

Next stage of search for $2 \mathrm{~K}(2 \nu)$-capture of ${ }^{78} \mathrm{Kr}$

2005GAZW Yu.P.Gangrsky, N.N.Kolesnikov, V.G.Lukashik, G.V.Mishinsky, Yu.E.Penionzhkevich, V.I.Zhemenik - Book of Abstracts, LV National Conference on Nuclear Physics "Frontiers in the Physics of Nucleus", St.-Petersburg, p.66 (2005) The Ratio of ${ }^{135 m}$ Xe and ${ }^{135 g} \mathrm{Xe}$ Yields in the Photofission Reactions of Heavy Nuclei

2005GE06 W.Geithner, B.A.Brown, K.M.Hilligsoe, S.Kappertz, M.Keim, G.Kotrotsios, P.Lievens, K.Marinova, R.Neugart, H.Simon, S.Wilbert - Phys.Rev. C 71, 064319 (2005)

Nuclear moments of neon isotopes in the range from ${ }^{17} \mathrm{Ne}$ at the proton drip line to neutron-rich ${ }^{25} \mathrm{Ne}$

2005GE07 M.T.Gericke, J.D.Bowman, R.D.Carlini, T.E.Chupp, K.P.Coulter, M.Dabaghyan, D.Desai, S.J.Freeman, T.R.Gentile, R.C.Gillis, G.L.Greene, F.W.Hersman, T.Ino, S.Ishimoto, G.L.Jones, B.Lauss, M.B.Leuschner, B.Losowski, R.Mahurin, Y.Masuda, G.S.Mitchell, S.Muto, H.Nann, S.A.Page, S.I.Penttila, W.D.Ramsay, S.Santra, P.-N.Seo, E.I.Sharapov, T.B.Smith, W.M.Snow, W.S.Wilburn, V.Yuan, H.Zhu J.Res.Natl.Inst.Stand.Technol. 110, 215 (2005)

Commissioning of the NPDGamma Detector Array: Counting Statistics in Current Mode Operation and Parity Violation in the Capture of Cold Neutrons on $\mathrm{B}_{4} \mathrm{C}$ and ${ }^{27} \mathrm{Al}$

2005GE09 G.Georgiev, I.Matea, D.L.Balabanski, J.M.Daugas, F.de Oliveira Santos, S.Franchoo, F.Ibrahim, F.Le Blanc, M.Lewitowicz, G.Lo Bianco, S.Lukyanov, V.Meot, P.Morel, G.Neyens, Yu.E.Penionzhkevich, A.Saltarelli, O.Sorlin, M.Stanoiu, M.Tarisien, N.Vermeulen, D.Verney, D.Yordanov - J.Phys.(London) G31, S1439 (2005) g-factor measurement of the $9 / 2^{+}$isomeric state in ${ }^{65} \mathrm{Ni}$

2005GE10 H.Geissel, Yu.A.Litvinov - J.Phys.(London) G31, S1779 (2005)

Precision experiments with relativistic exotic nuclei at GSI

2005GEZW H.Geissel, Yu.A.Litvinov, B.Pfeiffer, F.Attallah, G.Audi, K.Beckert, P.Beller, F.Bosch, D.Boutin, T.J.Burvenich, L.Chen, T.Faestermann, M.Falch, B.Franzke, M.Hausmann, E.Kaza, T.Kerscher, P.Kienle, O.Klepper, R.Knobel, C.Kozhuharov, K.-L.Kratz, S.A.Litvinov, K.E.G.Lobner, L.Maier, M.Matos, F.Montes, G.Munzenberg, C.Nociforo, F.Nolden, Yu.N.Novikov, T.Ohtsubo, A.Ostrowski, Z.Patyk, W.Plass, M.Portillo, T.Radon, H.Schatz, C.Scheidenberger, J.Stadlmann, M.Steck, B.Sun, K.Takahashi, G.Vorobjev, H.Weick, M.Winkler, H.Wollnik, T.Yamaguchi - nucl-ex/0510009,10/4/2005 (2005) 
Present and Future Experiments with Stored Exotic Nuclei at Relativistic Energies

2005GEZY L.N.Generalov, A.G.Zvenigorodsky, S.N.Abramovich, I.A.Karpov, Yu.I.Vinogradov Book of Abstracts, LV National Conference on Nuclear Physics "Frontiers in the Physics of Nucleus", St.-Petersburg, p.172 (2005)

Investigation of the Excitation Function of ${ }^{11} \mathrm{~B}(\mathrm{t}, \mathrm{p}){ }^{13} \mathrm{~B}$ Reaction

2005GEZZ L.N.Generalov - Book of Abstracts, LV National Conference on Nuclear Physics

"Frontiers in the Physics of Nucleus", St.-Petersburg, p.171 (2005)

Total Cross Section of ${ }^{9} \mathrm{Be}(\mathrm{p}, \alpha)^{6} \mathrm{Li}^{*}(3.56 \mathrm{MeV})$ Reaction at $\mathrm{E}_{p}=3.1-5.24 \mathrm{MeV}$

2005GI03 G.Giorginis, V.Khriatchkov - Nucl.Instrum.Methods Phys.Res. A538, 550 (2005)

The effect of particle leaking and its implications for measurements of the (n, $\alpha)$ reaction on light elements by using ionisation chambers

2005GI07 L.Giot, P.Roussel-Chomaz, C.E.Demonchy, W.Mittig, H.Savajols, N.Alamanos, F.Auger, A.Gillibert, C.Jouanne, V.Lapoux, L.Nalpas, E.C.Pollacco, J.L.Sida, F.Skaza, M.D.Cortina-Gil, J.Fernandez-Vazquez, R.S.Mackintosh, A.Pakou, S.Pita, A.Rodin, S.Stepantsov, G.M.Ter-Akopian, K.Rusek, I.J.Thompson, R.Wolski -

Phys.Rev. C 71, 064311 (2005)

Investigation of ${ }^{6} \mathrm{He}$ cluster structures

2005GI15 J.Giovinazzo - J.Phys.(London) G31, S1509 (2005)

The two-proton radioactivity in the $\mathrm{A} \sim 50$ mass region

2005GI17 A.Giannatiempo, A.Perego, P.Sona, A.Nannini, H.Mach, B.Fogelberg, M.J.G.Borge, O.Tengblad, L.M.Fraile, A.J.Aas, K.Gulda - Phys.Rev. C 72, 044308 (2005)

Spectroscopy and lifetime measurements of states in ${ }^{76} \mathrm{Kr}$ populated in ${ }^{76} \mathrm{Rb}$ decay

2005GI18 L.Giot, P.Roussel-Chomaz, N.Alamanos, F.Auger, M.-D.Cortina-Gil,

Ch.E.Demonchy, J.Fernandez, A.Gillibert, C.Jouanne, V.Lapoux, R.S.Mackintosh,

W.Mittig, L.Nalpas, A.Pakou, S.Pita, E.C.Pollacco, A.Rodin, K.Rusek, H.Savajols, J.L.Sida, F.Skaza, S.Stepantsov, G.Ter-Akopian, I.Thompson, R.Wolski -

Eur.Phys.J. A 25, Supplement 1, 267 (2005)

Study of the ground-state wave function of ${ }^{6} \mathrm{He}$ via the ${ }^{6} \mathrm{He}(\mathrm{p}, \mathrm{t}) \alpha$ transfer reaction

2005GIZX J.Gibelin, N.Aoi, H.Baba, D.Beaumel, Y.Blumenfeld, Z.Elekes, N.Fukuda, T.Gomi, K.Ishikawa, Y.Kondo, T.Kubo, V.Lima, T.Motobayashi, T.Nakamura, A.Saito, Y.Satou, E.Takeshita, S.Takeuchi, T.Teranishi, Y.Togano, A.M.Vinodkumar, Y.Yanagisawa, K.Yoshida - RIKEN Accelerator Progress Report 2004, p.53 (2005) In-beam gamma spectroscopy of ${ }^{25} \mathrm{Ne}$ via ${ }^{26} \mathrm{Ne}$ breakup

2005GIZY G.Giorginis, V.Khryachkov - Proc.Intern.Conf.Nuclear Data for Science and Technology, Santa Fe, New Mexico, 26 September-1 October, 2004, R.C.Haight, M.B.Chadwick, T.Kawano, P.Talou, Eds., Vol.1, p. 816 (2005); AIP Conf.Proc. 769 (2005)

Particle Leaking, Cross-Section Ratio ${ }^{10} \mathrm{~B}(\mathrm{n}, \alpha) /{ }^{238} \mathrm{U}$ (n, fission), and Excitation Function of the Reaction ${ }^{10} \mathrm{~B}(\mathrm{n}, \alpha){ }^{7} \mathrm{Li}$ at $\mathrm{MeV}$ Energies 
2005GIZZ L.Giot, P.Roussel-Chomaz, C.E.Demonchy, W.Mittig, H.Savajols, N.Alamanos, F.Auger, A.Gillibert, C.Jouanne, V.Lapoux, L.Nalpas, E.C.Pollacco, J.L.Sida, F.Skaza, M.D.Cortina-Gil, J.Fernandez-Vazquez, R.S.Mackintosh, A.Pakou, S.Pita, A.Rodin, S.Stepantsov, G.M.Ter-Akopian, K.Rusek, I.J.Thompson, R.Wolski nucl-ex/0505007,5/04/2005 (2005)

Investigation of the ${ }^{6} \mathrm{He}$ cluster structures

2005GL03 D.I.Glazier, M.Seimetz, J.R.M.Annand, H.Arenhovel, M.A.Antelo, C.Ayerbe, P.Bartsch, D.Baumann, J.Bermuth, R.Bohm, D.Bosnar, M.Ding, M.O.Distler, D.Elsner, J.Friedrich, S.Hedicke, P.Jennewein, G.J.Manas, F.H.Klein, F.Klein, M.Kohl, K.W.Krygier, K.Livingston, I.J.D.MacGregor, M.Makek, H.Merkel, P.Merle, D.Middleton, U.Muller, R.Neuhausen, L.Nungesser, M.Ostrick, R.Perez Benito, J.Pochodzalla, Th.Pospischil, M.Potokar, A.Reiter, G.Rosner, J.Sanner, H.Schmieden, A.Sule, Th.Walcher, D.P.Watts, M.Weis, and the A1 Collaboration Eur.Phys.J. A 24, 101 (2005)

Measurement of the electric form factor of the neutron at $\mathrm{Q}^{2}=0.3-0.8(\mathrm{GeV} / \mathrm{c})^{2}$

2005 GL05 I.V.Glavanakov, Yu.F.Krechetov, O.K.Saigushkin, E.N.Shuvalov, V.M.Bystritskii Pisma Zh.Eksp.Teor.Fiz. 81, 546 (2005); JETP Lett. 81, 432 (2005)

Nucleon-Nucleon Correlations in the $\left(\gamma, \pi^{-} \mathrm{p}\right)$ and $\left(\gamma, \pi^{-} \mathrm{pp}\right)$ Reactions on the ${ }^{12} \mathrm{C}$ Nucleus

2005 GL09 K.A.Gladnishki, D.L.Balabanski, P.Petkov, A.Dewald, D.Tonev, M.Axiotis, A.Fitzler, M.Danchev, S.Harissopulos, S.Lalkovski, N.Marginean, T.Martinez, O.Moeller, G.Neyens, A.Spyrou, E.A.Stefanova, C.Ur - J.Phys.(London) G31, S1559 (2005)

Lifetime measurements in the Yrast magnetic band in ${ }^{193} \mathrm{~Pb}$

2005 G009 P.R.S.Gomes, M.D.Rodriguez, G.V.Marti, I.Padron, L.C.Chamon, J.O.Fernandez Niello, O.A.Capurro, A.J.Pacheco, J.E.Testoni, A.Arazi, M.Ramirez, R.M.Anjos, J.Lubian, R.Veiga, R.Liguori Neto, E.Crema, N.Added, C.Tenreiro, M.S.Hussein Phys.Rev. C 71, 034608 (2005)

Effect of the breakup on the fusion and elastic scattering of weakly bound projectiles on ${ }^{64} \mathrm{Zn}$

2005 G010 T.P.Gorringe, D.P.Corbin, T.J.Stocki - Phys.Rev. C 71, 035503 (2005) Recoil alignment in muon capture on ${ }^{14} \mathrm{~N}$

2005G011 A.Gorelov, D.Melconian, W.P.Alford, D.Ashery, G.Ball, J.A.Behr, P.G.Bricault, J.M.D'Auria, J.Deutsch, J.Dilling, M.Dombsky, P.Dube, J.Fingler, U.Giesen, F.Gluck, S.Gu, O.Hausser, K.P.Jackson, B.K.Jennings, M.R.Pearson, T.J.Stocki, T.B.Swanson, M.Trinczek - Phys.Rev.Lett. 94, 142501 (2005)

Scalar Interaction Limits from the $\beta-\nu$ Correlation of Trapped Radioactive Atoms

2005G014 O.K.Gorpinich, O.M.Povoroznyk - Ukr.J.Phys. 50, 327 (2005)

Determination of the energy parameters of the unbound states of ${ }^{6} \mathrm{Li}$ up to an excitation energy of $6 \mathrm{MeV}$ 
2005G015 A.Gorgen, E.Clement, E.Bouchez, A.Chatillon, W.Korten, Y.Le Coz, Ch.Theisen, C.Andreoiu, F.Becker, B.Blank, A.Burger, P.Butler, J.M.Casandjian, W.Catford, T.Czosnyka, P.Davies, S.P.Fox, G.de France, G.Georgiev, J.Gerl, H.Hubel, J.Iwanicki, D.G.Jenkins, F.Johnston-Theasby, P.Joshi, I.Matea, P.Napiorkowski, F.de Oliveira Santos, G.Sletten, C.Timis, R.Wadsworth, M.Zielinska - Acta Phys.Pol. B36, 1281 (2005)

Shape coexistence in light Krypton isotopes

2005 G030 V.Z.Goldberg, K.-M.Kallman, T.Lonnroth, P.Manngard, B.B.Skorodumov Yad.Fiz. 68, 1123 (2005); Phys.Atomic Nuclei 68, 1079 (2005)

Alpha-Cluster States in ${ }^{18} \mathrm{O}$

2005 G033 T.Gomi, T.Motobayashi, Y.Ando, N.Aoi, H.Baba, K.Demichi, Z.Elekes, N.Fukuda, Zs.Fulop, U.Futakami, H.Hasegawa, Y.Higurashi, K.Ieki, N.Imai, M.Ishihara, K.Ishikawa, N.Iwasa, H.Iwasaki, S.Kanno, Y.Kondo, T.Kubo, S.Kubono, M.Kunibu, K.Kurita, Y.U.Matsuyama, S.Michimasa, T.Minemura, M.Miura, H.Murakami, T.Nakamura, M.Notani, S.Ota, A.Saito, H.Sakurai, M.Serata, S.Shimoura, T.Sugimoto, E.Takeshita, S.Takeuchi, Y.Togano, K.Ue, K.Yamada, Y.Yanagisawa, K.Yoneda, A.Yoshida - Nucl.Phys. A758, 761c (2005)

Coulomb Dissociation of ${ }^{23} \mathrm{Al}$ for the stellar ${ }^{22} \mathrm{Mg}(\mathrm{p}, \gamma){ }^{23} \mathrm{Al}$ reaction

2005G034 T.Gomi, T.Motobayashi, Y.Ando, N.Aoi, H.Baba, K.Demichi, Z.Elekes, N.Fukuda, Zs.Fulop, U.Futakami, H.Hasegawa, Y.Higurashi, K.Ieki, N.Imai, M.Ishihara, K.Ishikawa, N.Iwasa, H.Iwasaki, S.Kanno, Y.Kondo, T.Kubo, S.Kubono, M.Kunibu, K.Kurita, Y.U.Matsuyama, S.Michimasa, T.Minemura, M.Miura, H.Murakami, T.Nakamura, M.Notani, S.Ota, A.Saito, H.Sakurai, M.Serata, S.Shimoura, T.Sugimoto, E.Takeshita, S.Takeuchi, Y.Togano, K.Ue, K.Yamada, Y.Yanagisawa, K.Yoneda, A.Yoshida - J.Phys.(London) G31, S1517 (2005)

Coulomb dissociation experiment for explosive hydrogen burning: study of the ${ }^{22} \mathrm{Mg}(\mathrm{p}, \gamma){ }^{23} \mathrm{Al}$ reaction

2005 G036 M.B.Gomez Hornillos, M.Chartier, W.Mittig, B.Blank, F.Chautard, C.E.Demonchy, A.Gillibert, B.Jacquot, B.Jurado, N.Lecesne, A.Lepine-Szily, N.A.Orr, P.Roussel-Chomaz, H.Savajols, A.C.C.Villari - J.Phys.(London) G31, S1869 (2005) Mass measurements with the CIME cyclotron at GANIL

2005G043 A.Gorgen, E.Clement, A.Chatillon, A.Dewald, W.Korten, Y.Le Coz, N.Marginean, B.Melon, R.Menegazzo, O.Moller, Ch.Theisen, D.Tonev, C.A.Ur, K.O.Zell -

Eur.Phys.J. A 26, 153 (2005)

Lifetime measurement in ${ }^{74} \mathrm{Kr}$ and ${ }^{76} \mathrm{Kr}$

2005G0ZX C.Goessling, M.Junker, H.Kiel, D.Muenstermann, S.Oehl, K.Zuber nucl-ex/0508016,08/12/2005 (2005)

Experimental study of ${ }^{113} \mathrm{Cd}$ beta decay using CdZnTe detectors 
2005GOZY M.S.Golovkov, L.V.Grigorenko, A.S.Fomichev, S.A.Krupko, Yu.Ts.Oganessian, A.M.Rodin, S.I.Sidorchuk, R.S.Slepnev, S.V.Stepantsov, G.M.Ter-Akopian, R.Wolski, A.A.Korsheninnikov, E.Yu.Nikolskii, P.Roussel-Chomaz, W.Mittig, R.Palit, C.Angulo, V.Bouchat, V.Kinnard, T.Materna, F.Hanappe, O.Dorvaux, L.Stuttge - Book of Abstracts, LV National Conference on Nuclear Physics "Frontiers in the Physics of Nucleus", St.-Petersburg, p.124 (2005) Correlation Studies of the ${ }^{5} \mathrm{H}$ Spectrum

2005G0ZZ A.Gorgen, G.B.Hagemann, I.Hamamoto, R.Bengtsson, R.M.Clark, M.Cromaz, P.Fallon, H.Hubel, I.Y.Lee, A.O.Macchiavelli, G.Sletten, D.Ward - Proc.Nuclei at the Limits, Argonne, Illinois, D.Seweryniak and T.L.Khoo, eds., p.9 (2005); AIP Conf. Proc 764 (2005)

Quadrupole Moments And Gamma Deformation Of Wobbling Excitations In ${ }^{163} \mathrm{Lu}$

2005GR07 G.F.Grinyer, C.E.Svensson, C.Andreoiu, A.N.Andreyev, R.A.E.Austin, G.C.Ball, R.S.Chakrawarthy, P.Finlay, P.E.Garrett, G.Hackman, J.C.Hardy, B.Hyland, V.E.Iacob, K.A.Koopmans, W.D.Kulp, J.R.Leslie, J.A.Macdonald, A.C.Morton, W.E.Ormand, C.J.Osborne, C.J.Pearson, A.A.Phillips, F.Sarazin, M.A.Schumaker, H.C.Scraggs, J.Schwarzenberg, M.B.Smith, J.J.Valiente-Dobon, J.C.Waddington, J.L.Wood, E.F.Zganjar - Phys.Rev. C 71, 044309 (2005)

High precision measurements of ${ }^{26} \mathrm{Na} \beta^{-}$decay

2005GR09 F.Gramegna, S.Barlini, V.L.Kravchuk, A.L.Lanchais, O.Wieland, A.Bracco, A.Moroni, G.Casini, G.Benzoni, N.Blasi, S.Brambilla, M.Brekiesz, M.Bruno, F.Camera, M.Chiari, F.Crespi, E.Geraci, B.Guiot, M.Kmiecik, S.Leoni, A.Maj, P.F.Mastinu, B.Million, A.Nannini, A.Ordine, G.Vannini - Acta Phys.Pol. B36, $1155(2005)$

Light charged particle emission and the giant dipole resonance in Ce nucleus

2005GR10 E.Grodner, I.Zalewska, T.Morek, J.Srebrny, Ch.Droste, M.Kowalczyk, J.Mierzejewski, M.Salata, A.A.Pasternak, J.Kownacki, M.Kisielinski, A.Kordyasz, P.Napiorkowski, M.Wolinska, S.G.Rohozinski, R.Kaczarowski, W.Plociennik, E.Ruchowska, A.Wasilewski, J.Perkowski - Int.J.Mod.Phys. E14, 347 (2005)

Lifetime measurements in ${ }^{128} \mathrm{Cs}$ and ${ }^{132} \mathrm{La}$ as a test of chirality

2005GR15 P.Grabmayr, for the GDH Collaboration - Prog.Part.Nucl.Phys. 55, 375 (2005) An experimental test of the GDH sum rule

2005GR19 K.E.Gregorich, W.Loveland, D.Peterson, P.M.Zielinski, S.L.Nelson, Y.H.Chung, Ch.E.Dullmann, C.M.Folden III, K.Aleklett, R.Eichler, D.C.Hoffman, J.P.Omtvedt, G.K.Pang, J.M.Schwantes, S.Soverna, P.Sprunger, R.Sudowe, R.E.Wilson, H.Nitsche - Phys.Rev. C 72, 014605 (2005)

Attempt to confirm superheavy element production in the ${ }^{48} \mathrm{Ca}+{ }^{238} \mathrm{U}$ reaction

2005GR22 C.Granja, S.Pospisil, R.E.Chrien, S.A.Telezhnikov - Nucl.Phys. A757, 287 (2005)

Levels of ${ }^{174} \mathrm{Yb}$ populated in average resonance neutron capture

2005GR25 C.J.Gross - J.Phys.(London) G31, S1639 (2005)

Recent results at HRIBF using neutron-rich radioactive ion beams 
2005GR26 I.Grabowska-Bold - Eur.Phys.J. C 44, S1 (2005)

Measurement of deeply virtual Compton scattering using the ZEUS detector at HERA

2005GR28 N.Grion, M.Bregant, P.Camerini, E.Fragiacomo, S.Piano, R.Rui, E.F.Gibson, G.Hofman, E.L.Mathie, R.Meier, M.E.Sevior, G.R.Smith, R.Tacik, for the CHAOS Collaboration - Nucl.Phys. A763, 80 (2005)

The $\pi \rightarrow \pi \pi$ process in nuclei and the restoration of chiral symmetry

2005GR30 S.Grevy, F.Negoita, I.Stefan, N.L.Achouri, J.C.Angelique, B.Bastin, R.Borcea, A.Buta, J.M.Daugas, F.De Oliveira, O.Giarmana, C.Jollet, B.Laurent, M.Lazar, E.Lienard, F.Marechal, J.Mrazek, D.Pantelica, Y.Penionzhkevich, S.Pietri, O.Sorlin, M.Stanoiu, C.Stodel, M.G.St-Laurent - Eur.Phys.J. A 25, Supplement 1, 111 (2005) Observation of the $0_{2}^{+}$state in ${ }^{44} \mathrm{~S}$

2005GR32 R.Grzywacz, M.Karny, K.P.Rykaczewski, J.C.Batchelder, C.R.Bingham, D.Fong, C.J.Gross, W.Krolas, C.Mazzocchi, A.Piechaczek, M.N.Tantawy, J.A.Winger, E.F.Zganjar - Eur.Phys.J. A 25, Supplement 1, 145 (2005)

Discovery of the new proton emitter ${ }^{144} \mathrm{Tm}$

2005GR35 T.Grahn, A.Dewald, O.Moller, C.W.Beausang, S.Eeckhaudt, P.T.Greenlees, J.Jolie, P.Jones, R.Julin, S.Juutinen, H.Kettunen, T.Kroll, R.Krucken, M.Leino, A.-P.Leppanen, P.Maierbeck, D.A.Meyer, P.Nieminen, M.Nyman, J.Pakarinen, P.Petkov, P.Rahkila, B.Saha, C.Scholey, J.Uusitalo - Eur.Phys.J. A 25, Supplement $1,441(2005)$

RDDS lifetime measurement with JUROGAM + RITU

2005GR36 P.T.Greenlees, N.Amzal, J.E.Bastin, E.Bouchez, P.A.Butler, A.Chatillon, O.Dorvaux, S.Eeckhaudt, K.Eskola, B.Gall, J.Gerl, T.Grahn, A.Gorgen, N.J.Hammond, K.Hauschild, R.-D.Herzberg, F.-P.Hessberger, R.D.Humphreys, A.Hurstel, D.G.Jenkins, G.D.Jones, P.Jones, R.Julin, S.Juutinen, H.Kankaanpaa, A.Keenan, H.Kettunen, F.Khalfallah, T.L.Khoo, W.Korten, P.Kuusiniemi, Y.Le Coz, M.Leino, A.-P.Leppanen, M.Muikku, P.Nieminen, J.Pakarinen, P.Rahkila, P.Reiter, M.Rousseau, C.Scholey, Ch.Theisen, J.Uusitalo, J.Wilson, H.-J.Wollersheim - Eur.Phys.J. A 25, Supplement 1, 599 (2005)

In-beam and decay spectroscopy of transfermium elements

2005GRZY Yu.V.Grigoriev, V.Ya.Kitaev, V.V.Sinitsa, Zh.V.Mezentseva, H.Faikov-Stanczyk, N.B.Janeva - Proc.Intern.Conf.Nuclear Data for Science and Technology, Santa Fe, New Mexico, 26 September-1 October, 2004, R.C.Haight, M.B.Chadwick, T.Kawano, P.Talou, Eds., Vol.1, p. 928 (2005); AIP Conf.Proc. 769 (2005) The Investigation of the Doppler-Effect of the $\alpha$-Value of ${ }^{235} \mathrm{U}$ and ${ }^{239} \mathrm{Pu}$ for Different Temperatures

2005 GU07 Yu.B.Gurov, D.V.Aleshkin, M.N.Behr, S.V.Lapushkin, P.V.Morokhov, V.A.Pechkurov, N.O.Poroshin, V.G.Sandukovsky, M.V.Telkushev, B.A.Chernyshev, T.D.Tschurenkova - Yad.Fiz. 68, 520 (2005); Phys.Atomic Nuclei 68, 491 (2005) Spectroscopy of Superheavy Hydrogen Isotopes in Stopped-Pion Absorption by Nuclei 
2005GU16 M.Guttormsen, R.Chankova, U.Agvaanluvsan, E.Algin, L.A.Bernstein, F.Ingebretsen, T.Lonnroth, S.Messelt, G.E.Mitchell, J.Rekstad, A.Schiller, S.Siem, A.C.Sunde, A.Voinov, S.Odegard - Phys.Rev. C 71, 044307 (2005)

Radiative strength functions in ${ }^{93-98} \mathrm{Mo}$

2005GU17 Yu.B.Gurov, M.N.Behr, D.V.Aleshkin, B.A.Chernyshev, S.V.Lapushkin, P.V.Morokhov, V.A.Pechkurov, N.O.Poroshin, V.G.Sandukovsky, M.V.Telkushev Eur.Phys.J. A 24, 231 (2005)

Spectroscopy of superheavy hydrogen isotopes ${ }^{4} \mathrm{H}$ and ${ }^{5} \mathrm{H}$

2005GU18 W.Guryn, for the pp2pp Collaboration - Nucl.Phys. B(Proc.Supp.) S146, 82 (2005) The First Measurement of $\mathrm{A}_{N}$ at $\sqrt{ } \mathrm{s}=200 \mathrm{GeV}$ in proton-proton elastic scattering at RHIC

2005 GU25 F.Q.Guo, J.Powell, D.W.Lee, D.Leitner, M.A.McMahan, D.M.Moltz, J.P.O'Neil, K.Perajarvi, L.Phair, C.A.Ramsey, X.J.Xu, J.Cerny - Phys.Rev. C 72, 034312 (2005)

Reexamination of the energy levels of ${ }^{15} \mathrm{~F}$ by ${ }^{14} \mathrm{O}+{ }^{1} \mathrm{H}$ elastic resonance scattering

2005GU27 C.Guenaut, G.Audi, D.Beck, K.Blaum, G.Bollen, P.Delahaye, F.Herfurth, A.Kellerbauer, H.-J.Kluge, D.Lunney, S.Schwarz, L.Schweikhard, C.Yazidjian J.Phys.(London) G31, S1765 (2005)

Mass measurements of ${ }^{56-57} \mathrm{Cr}$ and the question of shell reincarnation at $\mathrm{N}=32$

2005 GU28 G.Gurdal, H.Amro, C.W.Beausang, D.S.Brenner, M.P.Carpenter, R.F.Casten, C.Engelhardt, G.B.Hagemann, C.R.Hansen, D.J.Hartley, B.Herskind, H.Hubel, T.L.Khoo, T.Lauritsen, W.C.Ma, D.A.Meyer, E.F.Moore, A.Neusser, P.Bringel, D.G.Roux, G.Sletten, R.B.Yadav, Y.Zhang - J.Phys.(London) G31, S1873 (2005)

Quadrupole moment measurements of TSD1 and TSD2 bands in ${ }^{167} \mathrm{Lu}$

2005 GU29 B.Guo, Z.H.Li, W.P.Liu, X.X.Bai, G.Lian, S.Q.Yan, B.X.Wang, S.Zeng, J.Su, Y.Lu - Nucl.Phys. A761, 162 (2005)

The ${ }^{8} \mathrm{Li}(\mathrm{d}, \mathrm{p}){ }^{9} \mathrm{Li}$ reaction and astrophysical ${ }^{8} \mathrm{~B}(\mathrm{p}, \gamma){ }^{9} \mathrm{C}$ reaction rate

2005 GU32 P.Guazzoni, L.Zetta, B.F.Bayman, A.Covello, A.Gargano, G.Graw, R.Hertenberger, H.-F.Wirth, M.Jaskola - Phys.Rev. C 72, 044604 (2005)

${ }^{120} \mathrm{Sn}$ homologous levels via the ${ }^{123} \mathrm{Sb}(\mathrm{p}(\mathrm{pol}), \alpha){ }^{120} \mathrm{Sn}$ reaction: Experimental evidence and microscopic calculations

2005GU37 C.Guenaut, G.Audi, D.Beck, K.Blaum, G.Bollen, P.Delahaye, F.Herfurth, A.Kellerbauer, H.-J.Kluge, D.Lunney, S.Schwarz, L.Schweikhard, C.Yazidjian Eur.Phys.J. A 25, Supplement 1, 35 (2005)

Extending the mass "backbone" to short-lived nuclides with ISOLTRAP

2005GUZW P.Guazzoni, L.Zetta, F.Della Vedova, S.M.Lenzi, A.Vitturi, G.Graw, R.Hertenberger, H.-F.Wirth, M.Jaskola - Maier-Leibnitz-Laboratorium 2004 Ann.Rept., p.7 (2005)

The ${ }^{48} \mathrm{Sc}(\mathrm{p}(\mathrm{pol}), \alpha)^{42} \mathrm{Ca}$ Reaction and the Corresponding Homologous States 
2005GUZX P.Guazzoni, S.Russo, M.Sassi, L.Zetta, F.Della Vedova, S.M.Lenzi, A.Vitturi, G.Graw, R.Hertenberger, H.-F.Wirth, M.Jaskola - Maier-Leibnitz-Laboratorium 2004 Ann.Rept., p.6 (2005) The ${ }^{44} \mathrm{Ca}(\mathrm{p}(\mathrm{pol}), \alpha)^{41} \mathrm{~K}$ Reaction

2005GUZZ Yu.B.Gurov, D.V.Aleshkin, B.A.Chernyshev, S.V.Lapushkin, I.V.Laukhin, V.A.Pechkurov, N.O.Poroshin, V.G.Sandukovsky, M.V.Tel'kushev - Book of Abstracts, LV National Conference on Nuclear Physics "Frontiers in the Physics of Nucleus", St.-Petersburg, p.139 (2005)

The Search for the Superheavy Hydrogen Isotope ${ }^{7} \mathrm{H}$ in the Reaction ${ }^{9} \mathrm{Be}\left(\pi^{-}, \mathrm{pp}\right) \mathrm{X}$

2005GY02 Gy.Gyurky, Z.Elekes, Zs.Fulop, G.Kiss, E.Somorjai, A.Palumbo, M.Wiescher Phys.Rev. C 71, 057302 (2005)

Precise half-life measurement of ${ }^{110} \mathrm{Sn}$ and ${ }^{109} \mathrm{In}$ isotopes

2005GY03 Gy.Gyurky, Zs.Fulop, G.Kiss, Z.Mate, E.Somorjai, J.Gorres, A.Palumbo, M.Wiescher, D.Galaviz, A.Kretschmer, K.Sonnabend, A.Zilges, T.Rauscher Nucl.Phys. A758, 517c (2005)

A comprehensive study of the ${ }^{106} \mathrm{Cd}(\alpha, \gamma){ }^{110} \mathrm{Sn}$ reaction at energies relevant to the p-process

2005GYZY Gy.Gyurky, Z.Elekes, Zs.Fulop, G.G.Kiss, E.Somorjai, A.Palumbo, M.Wiescher, J.Gorres, W.Rapp, N.Ozkan, R.T.Guray, T.Rauscher - ATOMKI 2004 Ann.Rept., p.19 (2005)

Alpha capture cross section of ${ }^{106} \mathrm{Cd}$ for the astrophysical p-process

2005GYZZ Gy.Gyurky, Z.Elekes, Zs.Fulop, G.G.Kiss, E.Somorjai, A.Palumbo, M.Wiescher nucl-ex/0503012,3/18/2005 (2005)

Precise half-life measurement of ${ }^{110} \mathrm{Sn}$ and ${ }^{109} \mathrm{In}$ isotopes

2005HA03 M.Hagemann, C.Baumer, A.M.van den Berg, D.De Frenne, D.Frekers, V.M.Hannen, M.N.Harakeh, J.Heyse, M.A.de Huu, E.Jacobs, K.Langanke, G.Martinez-Pinedo, A.Negret, L.Popescu, S.Rakers, R.Schmidt, H.J.Wortche - Phys.Rev. C 71, 014606 (2005) Spin-isospin excitations in the medium-mass nucleus ${ }^{58}$ Co investigated with the $(\mathrm{d}$, ${ }^{2} \mathrm{He}$ ) reaction

2005HA05 D.J.Hartley, M.K.Djongolov, L.L.Riedinger, G.B.Hagemann, R.V.F.Janssens, F.G.Kondev, E.F.Moore, M.A.Riley, A.Aguilar, C.R.Bingham, D.B.Campbell, M.P.Carpenter, P.Chowdhury, M.Cromaz, D.M.Cullen, M.Danchev, G.D.Dracoulis, P.Fallon, J.Goon, R.A.Kaye, T.L.Khoo, R.W.Laird, T.Lauritsen, A.O.Macchiavelli, B.McClain, G.Mukherjee, E.Ngijoi-Yogo, H.I.Park, G.Sletten, S.K.Tandel, P.M.Walker, J.-Y.Zhang - Phys.Lett. B 608, 31 (2005)

Wobbling excitations in strongly deformed Hf nuclei?

2005HA07 R.Hartke, D.R.Symes, F.Buersgens, L.E.Ruggles, J.L.Porter, T.Ditmire Nucl.Instrum.Methods Phys.Res. A540, 464 (2005)

Fusion neutron detector calibration using a table-top laser generated plasma neutron source 
2005 HA16 J.W.Hammer, M.Fey, R.Kunz, J.Kiener, V.Tatischeff, F.Haas, J.L.Weil, M.Assuncao, C.Beck, C.Boukari-Pelissie, A.Coc, J.J.Correia, S.Courtin, F.Fleurot, E.Galanopoulos, C.Grama, F.Hammache, S.Harissopulos, A.Korichi, E.Krmpotic, D.Le Du, A.Lopez-Martens, D.Malcherek, R.Meunier, P.Papka, T.Paradellis, M.Rousseau, N.Rowley, G.Staudt, S.Szilner - Nucl.Phys. A752, 514c (2005) New determination of the ${ }^{12} \mathrm{C}(\alpha, \gamma){ }^{16} \mathrm{O}$ reaction rate from $\gamma$-ray angular distribution measurements

2005HA25 G.Hammond, M.A.Bentley, F.Becker, J.Grebosz, M.J.Taylor, A.Banu, C.J.Barton, T.Beck, P.Bednarczyk, A.Bracco, A.M.Bruce, L.C.Bullock, A.Burger, F.Camera, C.Chandler, P.Doornenbal, J.Gerl, H.Geissel, M.Gorska, M.Hellstrom, D.Judson, I.Kojouharov, N.Kurz, R.Lozeva, A.Maj, S.Mandal, B.McGuirk, S.Muralithar, E.S.Paul, Zs.Podolyak, W.Prokopowicz, D.Rudolph, N.Saito, T.R.Saito, H.Schaffner, J.Simpson, D.D.Warner, H.Weick, C.Wheldon, M.Winkler, H.-J.Wollersheim - Acta Phys.Pol. B36, 1253 (2005)

Spectroscopy of $\mathrm{T}=3$ / 2 mirror nuclei via two-step fragmentation using RISING

2005HA32 D.J.Hamilton, and the Jefferson Lab Hall A Collaboration - Phys.Rev.Lett. 94, 242001 (2005)

Polarization Transfer in Proton Compton Scattering at High Momentum Transfer

2005HA37 D.K.Hasell, for the BLAST Collaboration - Nucl.Phys. A755, 257c (2005)

Recent Results from BLAST

2005HA39 C.Hadjidakis, on behalf of the HERMES Collaboration - Nucl.Phys. A755, 557c (2005)

Exclusive pion production at HERMES

2005HA45 S.Harissopulos, J.Doring, M.La Commara, K.Schmidt, C.Mazzocchi, R.Borcea, S.Galanopoulos, M.Gorska, H.Grawe, M.Hellstrom, Z.Janas, R.Kirchner, E.Roeckl, I.P.Johnstone, R.Schwengner, L.D.Skouras - Phys.Rev. C 72, 024303 (2005)

$\beta$ decay of ${ }^{95} \mathrm{Ag}$

2005HA48 J.W.Hammer, M.Fey, R.Kunz, J.Kiener, V.Tatischeff, F.Haas, J.L.Weil, M.Assuncao, C.Beck, C.Boukari-Pelissie, A.Coc, J.J.Correia, S.Courtin, F.Fleurot, E.Galanopoulos, C.Grama, F.Hammache, S.Harissopulos, A.Korichi, E.Krmpotic, D.Le Du, A.Lopez-Martens, D.Malcherek, R.Meunier, P.Papka, T.Paradellis, M.Rousseau, N.Rowley, G.Staudt, S.Szilner - Nucl.Phys. A758, 363c (2005)

E1 and E2 capture cross section and astrophysical reaction rate of the key reaction ${ }^{12} \mathrm{C}(\alpha, \gamma){ }^{16} \mathrm{O}$

2005HA49 S.Harissopulos, A.Spyrou, A.Lagoyannis, Ch.Zarkadas, H.-W.Becker, C.Rolfs, F.Strieder, J.W.Hammer, A.Dewald, K.-O.Zell, P.von Brentano, R.Julin, P.Demetriou, S.Goriely - Nucl.Phys. A758, 505c (2005)

Systematic measurements of proton- and alpha-capture cross sections relevant to the modelling of the p process 
2005HA56 S.Harissopulos, A.Lagoyannis, A.Spyrou, Ch.Zarkadas, S.Galanopoulos, G.Perdikakis, H.-W.Becker, C.Rolfs, F.Strieder, R.Kunz, M.Fey, J.W.Hammer, A.Dewald, K.-O.Zell, P.von Brentano, R.Julin, P.Demetriou - J.Phys.(London) G31, S1417 (2005)

Proton and alpha-particle capture reactions at sub-Coulomb energies relevant to the p process

2005HA57 B.Hadinia, B.Cederwall, J.Blomqvist, E.Ganioglu, P.T.Greenlees, K.Andgren, I.G.Darby, S.Eeckhaudt, E.Ideguchi, P.M.Jones, D.T.Joss, R.Julin, S.Juutinen, S.Ketelhut, K.Lagergren, A.-P.Leppanen, M.Leino, M.Nyman, J.Pakarinen, E.S.Paul, M.Petri, P.Rahkila, M.Sandzelius, J.Saren, C.Scholey, J.Uusitalo, R.Wadsworth, R.Wyss - Phys.Rev. C 72, 041303 (2005)

First identification of excited states in ${ }^{106} \mathrm{Te}$ and evidence for isoscalar-enhanced vibrational collectivity

2005HA60 T.Hayakawa, T.Shizuma, T.Kajino, S.Chiba, N.Shinohara, T.Nakagawa, T.Arima Astrophys.J. 628, 533 (2005)

New s-Process Path and Its Implications for a ${ }^{187}$ Re$^{-187} \mathrm{Os}$

Nucleo-Cosmochronometer

2005HA64 M.Hatano, H.Sakai, T.Wakui, T.Uesaka, N.Aoi, Y.Ichikawa, T.Ikeda, K.Itoh, H.Iwasaki, T.Kawabata, H.Kuboki, Y.Maeda, N.Matsui, T.Ohnishi, T.K.Onishi, T.Saito, N.Sakamoto, M.Sasano, Y.Satou, K.Sekiguchi, K.Suda, A.Tamii, Y.Yanagisawa, K.Yako - Eur.Phys.J. A 25, Supplement 1, 255 (2005)

First experiment of ${ }^{6} \mathrm{He}$ with a polarized proton target

2005HAZN S.Harissopulos, H.W.Becker, J.W.Hammer, A.Lagoyannis, C.Rolfs, F.Strieder nucl-ex/0509014,9/09/2005 (2005)

The reaction ${ }^{13} \mathrm{C}(\alpha, \mathrm{n}){ }^{16} \mathrm{O}$ : a background for the observation of geo-neutrinos

2005HAZQ F.-J.Hambsch, N.Varapai, S.Zeinalov, S.Oberstedt, O.Serot -

Proc.Intern.Conf.Nuclear Data for Science and Technology, Santa Fe, New Mexico, 26 September-1 October, 2004, R.C.Haight, M.B.Chadwick, T.Kawano, P.Talou, Eds., Vol.1, p. 644 (2005); AIP Conf.Proc. 769 (2005)

Prompt Fission Neutron Emission in Resonance Fission of ${ }^{239} \mathrm{Pu}$

2005HAZU M.Hatano, H.Sakai, T.Wakui, T.Uesaka, N.Aoi, Y.Ichikawa, T.Ikeda, K.Itoh, H.Iwasaki, T.Kawabata, H.Kuboki, Y.Maeda, N.Matsui, T.Ohnishi, T.K.Onishi, T.Saito, N.Sakamoto, M.Sasano, Y.Satou, K.Sekiguchi, K.Suda, A.Tamii, Y.Yanagisawa, K.Yako - Proc.Nuclei at the Limits, Argonne, Illinois, D.Seweryniak and T.L.Khoo, eds., p.360 (2005); AIP Conf. Proc 764 (2005)

${ }^{6}$ He Scattering With Polarized Proton Target

2005HAZW C.R.Hansen, G.B.Hagemann, B.Herskind, D.R.Jensen, G.Sletten, J.N.Wilson, S.Odegard, P.Bringel, C.Engelhardt, H.Hubel, A.Neusser, A.K.Singh, G.Benzoni, A.Bracco, F.Camera, S.Leoni, A.Maj, T.Byrski, D.Curien, P.Bednarczyk, A.Korichi, J.Roccaz, J.C.Lisle, T.Steinhardt, O.Thelen, M.P.Carpenter, R.V.F.Janssens, T.L.Khoo, T.Lauritsen, R.M.Clark, P.Fallon - Proc.Nuclei at the Limits, Argonne, Illinois, D.Seweryniak and T.L.Khoo, eds., p.46 (2005); AIP Conf. Proc 764 (2005) Search For Hyperdeformation In Xe Nuclei 
2005HAZX D.J.Hartley, M.K.Djongolov, L.L.Riedinger, G.B.Hagemann, R.V.F.Janssens, F.G.Kondev, E.F.Moore, M.A.Riley, A.A.Aguilar, C.R.Bingham, D.B.Campbell, M.P.Carpenter, P.Chowdhury, M.Cromaz, D.M.Cullen, M.Danchev, G.D.Dracoulis, P.Fallon, J.Goon, R.A.Kaye, T.L.Khoo, R.W.Laird, T.Lauritsen, A.O.Macchiavelli, B.McClain, G.Mukherjee, E.Ngijoi-Yogo, H.I.Park, G.Sletten, S.K.Tandel, P.M.Walker, J.-y.Zhang - Proc.Nuclei at the Limits, Argonne, Illinois, D.Seweryniak and T.L.Khoo, eds., p.15 (2005); AIP Conf. Proc 764 (2005)

Search For Wobbling Excitations In Hf Nuclei: Are The SD Bands Triaxial?

2005HAzZ T.Hayakawa, T.Shizuma, T.Arima, T.Kajino, S.Chiba, N.Shinohara, H.Utsunomiya - Proc. Intern. Symposium on the Origin of Matter and Evolution of Galaxies, Riken, Saitama, Japan, 17-19 November 2003, World Scientific, Singapore, p.208 (2005)

${ }^{186}$ Re isomer contribution to ${ }^{187} \mathrm{Re}^{187}$ Os cosmochronometer

2005HE03 A.Herlert, D.Beck, K.Blaum, F.Carrel, P.Delahaye, S.George, C.Guenaut, F.Herfurth, A.Kellerbauer, H.-J.Kluge, D.Lunney, M.Mukherjee, L.Schweikhard, C.Yazidjian - New Journal of Physics 7, 44 (2005)

Mass spectrometry of atomic ions produced by in-trap decay of short-lived nuclides

2005HE04 M.Heil, S.Dababneh, A.Juseviciute, F.Kappeler, R.Plag, R.Reifarth, S.O'Brien Phys.Rev. C 71, 025803 (2005)

Quasistellar spectrum for neutron activation measurements at $\mathrm{kT}=5 \mathrm{keV}$

2005HE05 A.Hermanne, F.Tarkanyi, S.Takacs, Yu.N.Shubin - Nucl.Instrum.Methods Phys.Res. B229, 321 (2005)

Experimental determination of cross section of alpha-induced reactions on ${ }^{n a t} \mathrm{Pd}$

2005HE08 P.Heckman, B.B.Back, T.Baumann, M.P.Carpenter, I.Dioszegi, D.J.Hofman, T.L.Khoo, S.Mitsuoka, V.Nanal, T.Pennington, J.P.Seitz, M.Thoennessen,

E.Tryggestad, R.L.Varner - Nucl.Phys. A750, 175 (2005)

Temperature and spin dependence of the giant dipole resonance width

2005HE13 A.Hermanne, F.Tarkanyi, S.Takacs, Z.Szucs, Yu.N.Shubin, A.I.Dityuk Appl.Radiat.Isot. 63, 1 (2005)

Experimental study of the cross-sections of $\alpha$-particle induced reactions on ${ }^{209} \mathrm{Bi}$

2005HE19 M.Heil, S.Dababneh, F.Kappeler, R.Plag, A.Juseviciute, N.Winckler, R.Reifarth, S.O'Brien - Nucl.Phys. A758, 529c (2005)

A neutron source to measure stellar neutron capture cross sections at $\mathrm{kT}=5 \mathrm{keV}$

2005HE24 M.He, S.Jiang, Y.Nagashima, Y.Yang, T.Takahashi, K.Liu, K.Sasa, K.Dong, S.Wu, T.Matsuhiro, H.Tosaki, X.Ren, R.Seki, K.Sueki - Nucl.Instrum.Methods Phys.Res. B240, $612(2005)$

Measurement of the cross-section of ${ }^{14} \mathrm{~N}\left({ }^{16} \mathrm{O}, \alpha\right){ }^{26} \mathrm{Al}$ with AMS 
2005 HE26 F.Herfurth, G.Audi, D.Beck, K.Blaum, G.Bollen, P.Delahaye, S.George, C.Guenaut, A.Herlert, A.Kellerbauer, H.-J.Kluge, D.Lunney, M.Mukherjee, S.Rahaman, S.Schwarz, L.Schweikhard, C.Weber, C.Yazidjian - Eur.Phys.J. A 25, Supplement 1, 17 (2005)

Recent high-precision mass measurements with the Penning trap spectrometer ISOLTRAP

2005HE27 F.P.Hessberger, S.Antalic, B.Streicher, S.Hofmann, D.Ackermann, B.Kindler, I.Kojouharov, P.Kuusiniemi, M.Leino, B.Lommel, R.Mann, K.Nishio, S.Saro, B.Sulignano - Eur.Phys.J. A 26, 233 (2005)

Energy systematics of low-lying Nilsson levels in odd-mass einsteinium isotopes

2005HEZS J.J.He, S.Kubono, T.Teranishi, M.Notani, H.Baba, S.Nishimura, J.Y.Moon, M.Nishimura, S.Michimasa, H.Iwasaki, Y.Yanagisawa, N.Hokoiwa, M.Kibe, J.H.Lee, S.Kato, Y.Gono, C.S.Lee - CNS-REP-66, p.3 (2005)

Study of Proton Resonant States in ${ }^{23} \mathrm{Al}$ using a Radioactive Beam of ${ }^{22} \mathrm{Mg}$

2005HEZT J.J.He, S.Kubono, T.Teranishi, M.Notani, H.Baba, S.Nishimura, J.Y.Moon, M.Nishimura, S.Michimasa, H.Iwasaki, Y.Yanagisawa, N.Hokoiwa, M.Kibe, J.H.Lee, S.Kato, Y.Gono, C.S.Lee - CNS-REP-66, p.1 (2005)

Study of Proton Resonant States in ${ }^{22} \mathrm{Mg}$ using a Radioactive Beam of ${ }^{21} \mathrm{Na}$

2005HEZU F.P.Hessberger, S.Hofmann, D.Ackermann, S.Antalic, I.Kojouharov, P.Kuusiniemi, R.Mann, K.Nishio, S.Saro, B.Streicher, B.Sulignano, M.Venhart - GSI 2005-1, p.73 (2005)

Decay spectroscopy of ${ }^{255} \mathrm{No}$

2005HEZW A.Hermanne, F.Tarkanyi, S.Takacs, Yu.N.Shubin - Proc.Intern.Conf.Nuclear Data for Science and Technology, Santa Fe, New Mexico, 26 September-1 October, 2004, R.C.Haight, M.B.Chadwick, T.Kawano, P.Talou, Eds., Vol.1, p. 961 (2005); AIP Conf.Proc. 769 (2005)

Experimental Determination of Cross Section of Alpha-Induced Reactions on ${ }^{\text {nat }} \mathrm{Pd}$

2005HEZX A.Hermanne, F.Tarkanyi, S.Takacs, Z.Szucs - Proc.Intern.Conf.Nuclear Data for Science and Technology, Santa Fe, New Mexico, 26 September-1 October, 2004, R.C.Haight, M.B.Chadwick, T.Kawano, P.Talou, Eds., Vol.1, p. 957 (2005); AIP Conf.Proc. 769 (2005)

Experimental Study of the Cross Sections of alpha-Particle Induced Reactions on ${ }^{209} \mathrm{Bi}$

2005HEZZ J.J.He, S.Kubono, T.Teranishi, M.Notani, S.Michimasa, H.Baba, S.Nishimura, M.Nishimura, Y.Yanagisawa, N.Hokoiwa, M.Kibe, Y.Gono, J.Y.Moon, J.H.Lee, C.S.Lee, H.Iwasaki, S.Kato - Proc. Intern. Symposium on the Origin of Matter and Evolution of Galaxies, Riken, Saitama, Japan, 17-19 November 2003, World Scientific, Singapore, p.481 (2005)

Investigation of resonant states in ${ }^{23} \mathrm{Al}$ using a radioactive beam of ${ }^{22} \mathrm{Mg}$ on a proton target

2005HI03 Y.Hirayama, T.Shimoda, H.Izumi, A.Hatakeyama, K.P.Jackson, C.D.P.Levy, H.Miyatake, M.Yagi, H.Yano - Phys.Lett. B 611, 239 (2005) 
Study of ${ }^{11} \mathrm{Be}$ structure through $\beta$-delayed decays from polarized ${ }^{11} \mathrm{Li}$

2005HI04 S.F.Hicks, G.K.Alexander, C.A.Aubin, M.C.Burns, C.J.Collard, M.M.Walbran, J.R.Vanhoy, E.Jensen, P.E.Garrett, M.Kadi, A.Martin, N.Warr, S.W.Yates Phys.Rev. C 71, 034307 (2005)

Intruder structures observed in ${ }^{122} \mathrm{Te}$ through inelastic neutron scattering

2005HI08 K.Hilgers, S.Sudar, S.M.Qaim - Appl.Radiat.Isot. 63, 93 (2005)

Experimental study and nuclear model calculations on the ${ }^{192} \mathrm{Os}(\mathrm{p}, \mathrm{n}){ }^{192} \mathrm{Ir}$ reaction: Comparison of reactor and cyclotron production of the therapeutic radionuclide ${ }^{192} \mathrm{Ir}$

2005HIZX K.Hilgers, S.M.Qaim, H.H.Coenen - Proc.Intern.Conf.Nuclear Data for Science and Technology, Santa Fe, New Mexico, 26 September-1 October, 2004, R.C.Haight, M.B.Chadwick, T.Kawano, P.Talou, Eds., Vol.2, p. 1631 (2005); AIP Conf.Proc. $769(2005)$

New Cross Section Data for Production of the Therapeutic Radionuclides ${ }^{64} \mathrm{Cu}$, ${ }^{140} \mathrm{Nd}$, and ${ }^{192} \mathrm{Ir}$

2005HIZY A.Hildebrand, J.Blomgren, A.Atac, B.Bergenwall, C.Johansson, J.Klug, P.Mermod, L.Nilsson, S.Pomp, M.Esterlund, S.Dangtip, U.Tippawan, P.Phansuke, O.Jonsson, P.-U.Renberg, A.Prokofiev, P.Nadel-Turonski, K.Elmgren, N.Olsson, V.Blideanu, C.Le Brun, J.-F.Lecolley, F.-R.Lecolley, M.Louvel, N.Marie-Noury, C.Schweitzer, Ph.Eudes, F.Haddad, C.Lebrun, A.J.Koning - Proc.Intern.Conf.Nuclear Data for Science and Technology, Santa Fe, New Mexico, 26 September-1 October, 2004, R.C.Haight, M.B.Chadwick, T.Kawano, P.Talou, Eds., Vol.1, p. 853 (2005); AIP Conf.Proc. 769 (2005)

Elastic Neutron Scattering at $96 \mathrm{MeV}$

2005HIZZ S.F.Hicks, G.K.Alexander, C.A.Aubin, M.C.Burns, C.J.Collard, M.W.Walbran, J.R.Vanhoy, E.Jensen, P.E.Garrett, M.Kadi, A.Martin, N.Warr, S.W.Yates Priv.Comm. (2005)

Intruder Structures in ${ }^{122}$ Te through inelastic neutron scattering

2005H008 P.T.Hosmer, H.Schatz, A.Aprahamian, O.Arndt, R.R.C.Clement, A.Estrade, K.-L.Kratz, S.N.Liddick, P.F.Mantica, W.F.Mueller, F.Montes, A.C.Morton, M.Ouellette, E.Pellegrini, B.Pfeiffer, P.Reeder, P.Santi, M.Steiner, A.Stolz, B.E.Tomlin, W.B.Walters, A.Wohr - Phys.Rev.Lett. 94, 112501 (2005) Half-Life of the Doubly Magic r-Process Nucleus ${ }^{78} \mathrm{Ni}$

2005H010 F.Hofmann, C.Baumer, A.M.van den Berg, D.Frekers, V.M.Hannen, M.N.Harakeh, M.A.de Huu, Y.Kalmykov, P.von Neumann-Cosel, V.Yu.Ponomarev, S.Rakers, B.Reitz, A.Richter, A.Shevchenko, K.Schweda, J.Wambach, H.J.Wortche Phys.Lett. B 612, 165 (2005)

Polarized proton scattering on ${ }^{58} \mathrm{Ni}$ at small momentum transfer: A test of the microscopic optical model and effective interactions

2005 H015 J.Honzatko, V.Bondarenko, I.Tomandl, T.von Egidy, H.-F.Wirth, D.Bucurescu, V.Yu.Ponomarev, N.Marginean, R.Hertenberger, Y.Eisermann, G.Graw, L.Rubacek - Nucl.Phys. A756, 249 (2005) 
Nuclear structure of ${ }^{127}$ Te studied with $(\mathrm{n}, \gamma)$ and $(\mathrm{d}(\mathrm{pol}), \mathrm{p})$ reactions and interpreted with IBFM and QPM

2005H016 M.V.Hoshovsky, V.M.Mazur, Z.M.Bigan, V.I.Lyamayev, T.Yo.Marynets Ukr.J.Phys. 50, 649 (2005)

Excitation cross sections for the $13 / 2^{+}$isomer state of mercury-199 nucleus in the $\left(\gamma, \gamma^{\prime}\right)$ and $(\gamma, \mathrm{n})$ reactions

2005HOZW M.J.Hornish, L.De Braeckeleer, A.S.Barabash, V.I.Umatov nucl-ex/0512030,12/20/2005 (2005)

Double Beta Decay of ${ }^{100}$ Mo to Excited Final States

2005HozX S.Hofmann, D.Ackermann, S.Antalic, H.G.Burkhard, R.Dressler, F.P.Hessberger, B.Kindler, I.Kojouharov, P.Kuusiniemi, M.Leino, B.Lommel, R.Mann, G.Munzenberg, K.Nishio, A.G.Popeko, S.Saro, H.J.Schott, B.Streicher, B.Sulignano, J.Uusitalo, A.V.Yeremin - Priv.Comm. (2005)

Synthesis of SHE at ship

2005 HU08 R.O.Hughes, N.V.Zamfir, D.C.Radford, C.J.Gross, C.J.Barton, C.Baktash, M.A.Caprio, R.F.Casten, A.Galindo-Uribarri, P.A.Hausladen, E.A.McCutchan, J.J.Ressler, D.Shapira, D.W.Stracener, C.-H.Yu - Phys.Rev. C 71, 044311 (2005) $\gamma$-ray spectroscopy of ${ }^{132} \mathrm{Te}$ through $\beta$ decay of a ${ }^{132} \mathrm{Sb}$ radioactive beam

2005HU10 M.Hunyadi, A.M.van den Berg, M.Csatlos, L.Csige, B.Davids, U.Garg, J.Gulyas, M.N.Harakeh, M.A.de Huu, A.Krasznahorkay, D.Sohler, H.J.Wortche - Acta Phys.Pol. B36, 1115 (2005)

Overtones of isoscalar giant resonances studied in direct particle decay measurements

2005HU14 D.Huang, P.Wang, X.Chen, W.Tian, B.Ni, L.Zhang, G.Zhang, C.Liu, L.Liu J.Radioanal.Nucl.Chem. 265, 499 (2005)

Half-life of ${ }^{97} \mathrm{Zr}$, re-measured

2005HUZZ H.Hubel, A.Burger, T.R.Saito, H.Grawe, P.Reiter, F.Becker, J.Gerl, M.Gorska, H.J.Wollersheim, A.Al-Khatib, A.Banu, T.Beck, P.Bednarczyk, G.Benzoni, A.Bracco, S.Brambilla, P.Bringel, F.Camera, E.Clement, P.Doornenbal, H.Geissel, A.Gorgen, J.Grebosz, G.Hammond, M.Hellstrom, M.Kavatsyuk, O.Kavatsyuk, M.Kmiecik, I.Kojouharov, W.Korten, N.Kurz, R.Lozeva, A.Maj, S.Mandal, B.Million, S.Muralithar, A.Neusser, Zs.Podolyak, N.Saito, A.K.Singh, H.Weick, O.Wieland, M.Winkler, C.Wheldon - Proc. XLIII Intern.Winter Meeting on Nuclear Physics, Bormio, Italy, 14 - 19 March 2005, I.Iori and A.Bortolotti, eds., p.232 (2005)

Relativistic Coulomb Excitation of Neutron-Rich Cr Isotopes

2005Hw06 J.K.Hwang, A.V.Ramayya, J.H.Hamilton, D.Fong, C.J.Beyer, K.Li, P.M.Gore, E.F.Jones, Y.X.Luo, J.O.Rasmussen, S.J.Zhu, S.C.Wu, I.Y.Lee, M.A.Stoyer, J.D.Cole, G.M.Ter-Akopian, A.Daniel, R.Donangelo - Eur.Phys.J. A 25, Supplement $1,463(2005)$

Half-life measurement of excited states in neutron-rich nuclei 
2005HY04 B.Hyland, D.Melconian, G.C.Ball, J.R.Leslie, C.E.Svensson, P.Bricault, E.Cunningham, M.Dombsky, G.F.Grinyer, G.Hackman, K.Koopmans, F.Sarazin, M.A.Schumaker, H.C.Scraggs, M.B.Smith, P.M.Walker - J.Phys.(London) G31, S1885 (2005)

Precision half-life measurement of ${ }^{62} \mathrm{Ga}$

2005IC02 S.Ichikawa, M.Asai, K.Tsukada, H.Haba, Y.Nagame, M.Shibata, M.Sakama, Y.Kojima - Phys.Rev. C 71, 067302 (2005)

$\beta$-decay half-lives of new neutron-rich rare-earth isotopes ${ }^{159} \mathrm{Pm},{ }^{162} \mathrm{Sm}$, and ${ }^{166} \mathrm{Gd}$

2005ID03 E.Ideguchi, M.Niikura, C.Ishida, T.Fukuchi, H.Baba, N.Hokoiwa, H.Iwasaki, T.Koike, T.Komatsubara, T.Kubo, M.Kurokawa, S.Michimasa, K.Miyakawa, K.Morimoto, T.Ohnishi, S.Ota, A.Ozawa, S.Shimoura, T.Suda, M.Tamaki, I.Tanihata, Y.Wakabayashi, K.Yoshida, B.Cederwall - Eur.Phys.J. A 25,

Supplement 1, 429 (2005)

Study of high-spin states in the ${ }^{48} \mathrm{Ca}$ region by using secondary fusion reactions

2005IDZY E.Ideguchi, B.Cederwall, E.Ganioglu, B.Hadinia, K.Lagergren, T.Back, S.Eeckhaudt, T.Grahn, P.Greenlees, A.Johnson, D.T.Joss, R.Julin, S.Juutinen, H.Kettunen, M.Leino, A.-P.Leppanen, P.Nieminen, M.Nyman, J.Pakarinen, E.S.Paul, P.Rahkila, C.Scholey, J.Uusitalo, R.Wadsworth, D.R.Wiseman, R.Wyss CNS-REP-66, p.19 (2005)

A Rotational Band in ${ }^{107}$ In

2005IDzZ E.Ideguchi, M.Niikura, C.Ishida, T.Fukuchi, H.Baba, N.Hokoiwa, H.Iwasaki, T.Koike, T.Komatsubara, T.Kubo, M.Kurokawa, S.Michimasa, K.Miyakawa, K.Morimoto, T.Ohnishi, S.Ota, A.Ozawa, S.Shimoura, T.Suda, M.Tamaki, I.Tanihata, Y.Wakabayashi, K.Yoshida, B.Cederwall - Proc.Nuclei at the Limits, Argonne, Illinois, D.Seweryniak and T.L.Khoo, eds., p.136 (2005); AIP Conf. Proc 764 (2005)

Study Of High-Spin States In ${ }^{48}$ Ca Region Induced By Secondary Fusion Reactions

$2005 I 101$ H.Iimura, M.Asai, S.Ichikawa, J.Katakura, M.Magara, A.Osa, M.Oshima, N.Shinohara, H.Yamamoto - Eur.Phys.J. A 23, 33 (2005)

Levels in ${ }^{127}$ La fed by the ${ }^{127}$ Ce beta-decay

2005IL01 M.Iliescu, for the DEAR and SIDDHARTA Collaborations - Nucl.Phys. A755, 455c (2005)

Precision measurements on kaonic hydrogen and kaonic deuterium: DEAR and SIDDHARTA

2005 IL02 C.Iliadis, A.E.Champagne - Nucl.Phys. A758, 73c (2005)

Nuclear Astrophysics: Direct measurements with stable beams

2005 IL03 C.Iliadis, A.Champagne, J.Jose - J.Phys.(London) G31, S1785 (2005)

Explosive hydrogen burning of ${ }^{23} \mathrm{Na}$ in classical novae 
2005 Im02 G.Imbriani, H.Costantini, A.Formicola, A.Vomiero, C.Angulo, D.Bemmerer, R.Bonetti, C.Broggini, F.Confortola, P.Corvisiero, J.Cruz, P.Descouvemont, Z.Fulop, G.Gervino, A.Guglielmetti, C.Gustavino, Gy.Gyurky, A.P.Jesus, M.Junker, J.N.Klug, A.Lemut, R.Menegazzo, P.Prati, V.Roca, C.Rolfs, M.Romano, C.Rossi-Alvarez, F.Schumann, D.Schurmann, E.Somorjai, O.Straniero, F.Strieder, F.Terrasi, H.P.Trautvetter - Eur.Phys.J. A 25, 455 (2005)

S-factor of ${ }^{14} \mathrm{~N}(\mathrm{p}, \gamma){ }^{15} \mathrm{O}$ at astrophysical energies

2005ImZY G.Imbriani, H.Costantini, A.Formicola, A.Vomiero, C.Angulo, D.Bemmerer, R.Bonetti, C.Broggini, F.Confortola, P.Corvisiero, J.Cruz, P.Descouvemont, Zs.Fulop, G.Gervino, A.Guglielmetti, C.Gustavino, Gy.Gyurky, A.P.Jesus, M.Junker, J.N.Klug, A.Lemut, R.Menegazzo, P.Prati, V.Roca, C.Rolfs, M.Romano, C.Rossi Alvarez, F.Schumann, D.Schurmann, E.Somorjai, O.Straniero, F.Strieder, F.Terrasi, H.P.Trautvetter - nucl-ex/0509005,9/01/2005 (2005)

S-factor of ${ }^{14} \mathrm{~N}(\mathrm{p}, \gamma){ }^{15} \mathrm{O}$ at astrophysical energies

$2005 I M Z Z$ N.Imai, N.Aoi, H.J.Ong, H.Sakurai, K.Demichi, H.Kawasaki, H.Baba, Zs.Dombradi, Z.Elekes, N.Fukuda, Zs.Fulop, A.Gelberg, T.Gomi, H.Hasegawa, K.Ishikawa, H.Iwasaki, E.Kaneko, S.Kanno, T.Kishida, Y.Kondo, T.Kubo, K.Kurita, S.Michimasa, T.Minemura, M.Miura, T.Motobayashi, T.Nakamura, M.Notani, T.K.Onishi, A.Saito, S.Shimoura, T.Sugimoto, M.K.Suzuki, E.Takeshita, S.Takeuchi, M.Tamaki, K.Yoneda, H.Watanabe, M.Ishihara - RIKEN Accelerator Progress Report 2004, p.41 (2005)

Lifetime of the first excited state of ${ }^{12} \mathrm{Be}$

2005 I002 M.Ionescu-Bujor, A.Iordachescu, D.Bucurescu, N.Marginean - Phys.Rev. C 72, $044313(2005)$

New short-lived isomers in ${ }^{84} \mathrm{Y}$

2005IS07 T.Ishii, S.Shigematsu, M.Asai, A.Makishima, M.Matsuda, J.Kaneko, I.Hossain, S.Ichikawa, T.Kohno, M.Ogawa - Phys.Rev. C 72, 021301 (2005)

In-beam $\gamma$-ray spectroscopy of ${ }^{240} \mathrm{U}$ using the $\left({ }^{18} \mathrm{O},{ }^{16} \mathrm{O}\right)$ reaction

2005ISzZ H.Ishiyama, H.Miyatake, M.-H.Tanaka, Y.Watanabe, N.Yoshikawa, S.Jeong, Y.Matsuyama, Y.Fuchi, I.Katayama, T.Nomura, T.Hashimoto, T.Ishikawa, K.Nakai, S.K.Das, P.K.Saha, T.Fukuda, K.Nishio, S.Mitsuoka, H.Ikezoe, M.Matsuda, S.Ichikawa, T.Furukawa, H.Izumi, T.Shimoda, Y.Mizoi, M.Terasawa - Proc. Intern. Symposium on the Origin of Matter and Evolution of Galaxies, Riken, Saitama, Japan, 17-19 November 2003, World Scientific, Singapore, p.316 (2005)

Direct measurements of the astrophysical $(\alpha, \mathrm{n})$ and $(\mathrm{p}, \mathrm{n})$ reactions by using low-energy light neutron-rich RNB

2005Iw01 A.Iwahara, M.A.L.da Silva, A.E.Carvalho Filho, E.M.de Oliveira Bernardes, J.U.Delgado - Appl.Radiat.Isot. 63, 107 (2005)

Determination of disintegration rates and $\gamma$-ray emission probabilities of ${ }^{65} \mathrm{Zn}$ and ${ }^{241} \mathrm{Am}$ 
2005IW02 H.Iwasaki, T.Motobayashi, H.Sakurai, K.Yoneda, T.Gomi, N.Aoi, N.Fukuda, Zs.Fulop, U.Futakami, Z.Gacsi, Y.Higurashi, N.Imai, N.Iwasa, T.Kubo, M.Kunibu, M.Kurokawa, Z.Liu, T.Minemura, A.Saito, M.Serata, S.Shimoura, S.Takeuchi, Y.X.Watanabe, K.Yamada, Y.Yanagisawa, M.Ishihara - Phys.Lett. B 620, 118 (2005)

Quadrupole collectivity of ${ }^{28} \mathrm{Ne}$ and the boundary of the island of inversion

2005Iw03 H.Iwasaki, N.Aoi, S.Takeuchi, S.Ota, H.Sakurai, M.Tamaki, T.K.Onishi, E.Takeshita, H.J.Ong, N.Iwasa, H.Baba, Z.Elekes, T.Fukuchi, Y.Ichikawa, M.Ishihara, S.Kanno, R.Kanungo, S.Kawai, T.Kubo, K.Kurita, S.Michimasa, M.Niikura, A.Saito, Y.Satou, S.Shimoura, H.Suzuki, M.K.Suzuki, Y.Togano, Y.Yanagisawa, T.Motobayashi - Eur.Phys.J. A 25, Supplement 1, 415 (2005) Intermediate-energy Coulomb excitation of the neutron-rich Ge isotopes around $\mathrm{N}=$ 50

2005IWZX N.Iwasa, S.Bishop, Z.Elekes, J.Gibelin, M.Hosoi, K.Ieki, K.Ishikawa, H.Iwasaki, S.Kawai, S.Kubono, K.Kurita, M.Kurokawa, N.Matsui, T.Minemura, H.Morikawa, T.Nakamura, M.Niikura, M.Notani, S.Ota, A.Saito, H.Sakurai, S.Shimoura, K.Sugawara, T.Sugimoto, H.Suzuki, T.Suzuki, E.Takeshita, S.Takeuchi, I.Tanihata, T.Teranishi, Y.Togano, K.Yamada, K.Yamaguchi, Y.Yanagisawa, T.Motobayashi RIKEN Accelerator Progress Report 2004, p.58 (2005)

Coulomb excitation of ${ }^{20} \mathrm{Mg}$

2005JA03 Z.Janas, C.Mazzocchi, L.Batist, A.Blazhev, M.Gorska, M.Kavatsyuk, O.Kavatsyuk, R.Kirchner, A.Korgul, M.La Commara, K.Miernik, I.Mukha, A.Plochocki, E.Roeckl, K.Schmid - Eur.Phys.J. A 23, 197 (2005)

Measurements of ${ }^{110} \mathrm{Xe}$ and ${ }^{106} \mathrm{Te}$ decay half-lives

2005JA06 Z.Janas, L.Batist, J.Doring, M.Gierlik, R.Kirchner, J.Kurcewicz, H.Mahmud, C.Mazzocchi, A.Plochocki, E.Roeckl, K.Schmidt, P.J.Woods, J.Zylicz - Eur.Phys.J. A 23, 401 (2005)

Total absorption spectroscopy of the $\beta$-delayed proton emitter ${ }^{117} \mathrm{Ba}$

2005JA10 Z.Janas, L.Batist, R.Borcea, J.Doring, M.Gierlik, M.Karny, R.Kirchner, M.La Commara, S.Mandal, C.Mazzocchi, F.Moroz, S.Orlov, A.Plochocki, E.Roeckl, J.Zylicz - Eur.Phys.J. A 24, 205 (2005)

Lifetimes of proton unstable states in ${ }^{113}$ I measured by the particle-X-ray coincidence technique

2005JA12 M.Jandel, J.Kliman, L.Krupa, M.Morhac, J.H.Hamilton, J.Kormicki, A.V.Ramayya, J.K.Hwang, Y.X.Luo, D.Fong, P.Gore, G.M.Ter-Akopian, Yu.Ts.Oganessian, A.M.Rodin, A.S.Fomichev, G.S.Popeko, A.V.Daniel, J.O.Rasmussen, A.O.Macchiavelli, M.A.Stoyer, R.Donangelo, J.D.Cole - Eur.Phys.J. A 24, 373 (2005) Angular momenta of fission fragments in the $\alpha$-accompanied fission of ${ }^{252} \mathrm{Cf}$

2005JA17 Th.Jagemann, J.Jochum, F.V.Feilitzsch - Nucl.Instrum.Methods Phys.Res. A551, $245(2005)$

Neutron scattering facility for the measurement of nuclear recoil quenching factors 
2005JAzZ H.C.Jain, S.Lakshmi, P.K.Joshi - Proc.Nuclei at the Limits, Argonne, Illinois, D.Seweryniak and T.L.Khoo, eds., p.99 (2005); AIP Conf. Proc 764 (2005) Identification Of Chiral Bands In ${ }^{135} \mathrm{Ce}$

2005JE01 H.B.Jeppesen, and the ISOLDE and REX-ISOLDE Collaborations - Nucl.Phys. A748, 374 (2005)

Low energy reactions with radioactive ions at REX-ISOLDE-the ${ }^{9} \mathrm{Li}+{ }^{2} \mathrm{H}$ case

2005JE03 D.G.Jenkins, B.R.Fulton, J.Pearson, C.J.Lister, M.P.Carpenter, S.J.Freeman, N.J.Hammond, R.V.F.Janssens, T.L.Khoo, T.Lauritsen, A.H.Wuosmaa, P.Fallon, A.Gorgen, A.O.Macchiavelli, M.McMahan, M.Freer, F.Haas - Phys.Rev. C 71, $041301(2005)$

Doorway states as a principal decay pathway in ${ }^{12} \mathrm{C}\left({ }^{12} \mathrm{C}, \gamma\right)$ radiative capture

2005JE04 P.Jesinger, Yu.N.Kopatch, M.Mutterer, F.Gonnenwein, A.M.Gagarski, J.V.Kalben, V.Nesvizhevsky, G.A.Petrov, W.H.Trzaska, H.-J.Wollersheim - Eur.Phys.J. A 24, $379(2005)$

New experimental studies on the quaternary fission of ${ }^{233,235} \mathrm{U}\left(\mathrm{n}_{t h}, \mathrm{f}\right)$ and ${ }^{252} \mathrm{Cf}(\mathrm{sf})$

2005JE06 D.G.Jenkins, B.R.Fulton, C.J.Lister, R.V.F.Janssens, T.L.Khoo, E.F.Moore, K.E.Rehm, B.Truett, A.H.Wuosmaa, M.Freer, J.Jose - Nucl.Phys. A758, 749c (2005) Reevaluation of the ${ }^{22} \mathrm{Na}(\mathrm{p}, \gamma)$ reaction rate

2005JE07 D.G.Jenkins, C.J.Lister, M.P.Carpenter, P.Chowdhury, N.J.Hammond, R.V.F.Janssens, T.L.Khoo, T.Lauritsen, D.Seweryniak, T.Davinson, P.J.Woods, A.Jokinen, H.Penttila - Phys.Rev. C 72, 031303 (2005)

Mirror energy differences in the $\mathrm{A}=31$ mirror nuclei, ${ }^{31} \mathrm{~S}$ and ${ }^{31} \mathrm{P}$, and their significance in electromagnetic spin-orbit splitting

2005JEZY M.Jentschel, H.G.Borner, P.Mutti, E.G.Kessler, Jr., M.S.Dewey, A.Henins Proc.Intern.Conf.Nuclear Data for Science and Technology, Santa Fe, New Mexico, 26 September-1 October, 2004, R.C.Haight, M.B.Chadwick, T.Kawano, P.Talou, Eds., Vol.1, p. 617 (2005); AIP Conf.Proc. 769 (2005) Accurate Determination of Neutron Binding Energies

2005JEZZ D.G.Jenkins, B.R.Fulton, J.Pearson, C.J.Lister, M.P.Carpenter, S.J.Freeman, N.J.Hammond, R.V.F.Janssens, T.L.Khoo, T.Lauritsen, A.H.Wuosmaa, P.Fallon, A.Gorgen, A.O.Macchiavelli, M.McMahan, F.Haas, and the DRAGON E947 Collaboration - Proc.Nuclei at the Limits, Argonne, Illinois, D.Seweryniak and T.L.Khoo, eds., p.367 (2005); AIP Conf. Proc 764 (2005)

Doorway States As Principal Decay Pathway In ${ }^{12} \mathrm{C}\left({ }^{12} \mathrm{C}, \gamma\right)$ Radiative Capture

2005J002 C.Johansson, J.Blomgren, A.Atac, B.Bergenwall, S.Dangtip, K.Elmgren, A.Hildebrand, O.Jonsson, J.Klug, P.Mermod, P.Nadel-Turonski, L.Nilsson, N.Olsson, S.Pomp, A.V.Prokofiev, P.-U.Renberg, U.Tippawan, M.Osterlund Phys.Rev. C 71, 024002 (2005)

Forward-angle neutron-proton scattering at $96 \mathrm{MeV}$ 
2005J003 M.S.Johnson, J.A.Cizewski, M.B.Smith, J.S.Thomas, J.A.Becker, L.A.Bernstein, A.Schiller, D.P.McNabb, P.Fallon, A.O.Macchiavelli - Phys.Rev. C 71, 024317 (2005)

Population of superdeformed excitations in ${ }^{198} \mathrm{Po}$

2005J004 P.Joshi, A.R.Wilkinson, T.Koike, D.B.Fossan, S.Finnigan, E.S.Paul, P.M.Raddon, G.Rainovski, K.Starosta, A.J.Simons, C.Vaman, R.Wadsworth - Eur.Phys.J. A 24, $23(2005)$

First evidence for chirality in Tc isotopes: Spectroscopy of ${ }^{100} \mathrm{Tc}$

2005J010 M.S.Johnson, J.A.Cizewski, K.Y.Ding, N.Fotiades, M.B.Smith, J.S.Thomas, W.Younes, J.A.Becker, L.A.Bernstein, K.Hauschild, D.P.McNabb,

M.A.Deleplanque, R.M.Diamond, P.Fallon, I.Y.Lee, A.O.Macchiavelli, F.S.Stephens - Phys.Rev. C 71, 044310 (2005)

Quasicontinuous decay and properties of superdeformed excitations in ${ }^{195} \mathrm{~Pb}$

2005J011 G.A.Jones, Zs.Podolyak, N.Schunck, P.M.Walker, G.De Angelis, Y.H.Zhang, M.Axiotis, D.Bazzacco, P.G.Bizzeti, F.Brandolini, R.Broda, D.Bucurescu, E.Farnea, W.Gelletly, A.Gadea, M.Ionescu-Bujor, A.Iordachescu, Th.Kroll, S.D.Langdown, S.Lunardi, N.Marginean, T.Martinez, N.H.Medina, B.Quintana, P.H.Regan, B.Rubio, C.A.Ur, J.J.Valiente-Dobon, S.J.Williams - Acta Phys.Pol. B36, 1323 (2005)

Oblate collectivity in the yrast structure of ${ }^{194} \mathrm{Pt}$

2005J012 C.Jouanne, V.Lapoux, F.Auger, N.Alamanos, A.Drouart, A.Gillibert, G.Lobo, A.Musumarra, L.Nalpas, E.Pollacco, J.-L.Sida, M.Trotta, Y.Blumenfeld, E.Khan, T.Suomijarvi, T.Zerguerras, P.Roussel-Chomaz, H.Savajols, A.Lagoyannis, A.Pakou - Phys.Rev. C 72, 014308 (2005)

Structure of low-lying states of ${ }^{10,11} \mathrm{C}$ from proton elastic and inelastic scattering

2005J018 D.T.Joss, J.Simpson, D.E.Appelbe, K.Lagergren, C.J.Barton, B.Cederwall, S.Eeckhaudt, T.Grahn, P.M.Jones, R.Julin, S.Juutinen, B.Hadinia, H.Kettunen, M.Leino, A.-P.Leppanen, P.Nieminen, R.D.Page, J.Pakarinen, E.S.Paul, J.Perkowski, P.Rahkila, M.A.Riley, C.Scholey, J.Uusitalo, K.Van de Vel, D.D.Warner, D.R.Wiseman - J.Phys.(London) G31, S1715 (2005)

Yrast structures in the light $\mathrm{Pt}$ isotopes ${ }^{169-173} \mathrm{Pt}$

2005J019 G.A.Jones, Zs.Podolyak, P.M.Walker, P.H.Regan, G.de Angelis, M.Axiotis, D.Bazzacco, P.G.Bizzeti, F.Brandolini, R.Broda, D.Bucurescu, E.Farnea, W.Gelletly, A.Gadea, M.Ionescu-Bujor, A.Iordachescu, Th.Kroll, S.D.Langdown, S.Lunardi, N.Marginean, T.Martinez, N.H.Medina, B.Quintana, B.Rubio, C.A.Ur, J.J.Valiente-Dobon, S.J.Williams, Y.H.Zhang - J.Phys.(London) G31, S1891 (2005)

Population of yrast states in ${ }^{191}$ Os using deep-inelastic reactions

2005J020 P.Joshi, S.Finnigan, D.B.Fossan, T.Koike, E.S.Paul, G.Rainovski, K.Starosta, C.Vaman, R.Wadsworth - J.Phys.(London) G31, S1895 (2005)

Evidence for a new region of chirality around $\mathrm{A} \sim 104$

2005J021 K.Joo, for the CLAS Collaboration - Phys.Rev. C 72, 058202 (2005) 
Measurement of the polarized structure function $\sigma_{L T^{\prime}}$ for pion electroproduction in the Roper-resonance region

2005J022 A.Jokinen, T.Eronen, U.Hager, J.Hakala, S.Kopecky, A.Nieminen, S.Rinta-Antila, J.Aysto - Eur.Phys.J. A 25, Supplement 1, 27 (2005)

Ion manipulation and precision measurements at JYFLTRAP

2005J023 K.L.Jones, C.Baktash, D.W.Bardayan, J.C.Blackmon, W.N.Catford, J.A.Cizewski, R.P.Fitzgerald, U.Greife, M.S.Johnson, R.L.Kozub, R.J.Livesay, Z.Ma, C.D.Nesaraja, D.Shapira, M.S.Smith, J.S.Thomas, D.Visser - Eur.Phys.J. A 25, Supplement 1, $283(2005)$

Developing techniques to study $\mathrm{A} \sim 132$ nuclei with $(\mathrm{d}, \mathrm{p})$ reactions in inverse kinematics

2005J024 E.F.Jones, J.H.Hamilton, P.M.Gore, A.V.Ramayya, J.K.Hwang, A.P.deLima Eur.Phys.J. A 25, Supplement 1, 467 (2005)

Identification of levels in ${ }^{162,164} \mathrm{Gd}$ and decrease in moment of inertia between $\mathrm{N}=$ 98-100

2005J0ZX C.Johansson, J.Blomgren, A.Atac, B.Bergenwall, A.Hildebrand, J.Klug, P.Mermod, L.Nilsson, N.Olsson, M.Osterlund, S.Pomp, U.Tippawan, O.Jonsson, A.Prokofiev, P.-U.Renberg, K.Elmgren, S.Dangtip, P.Nadel-Turonski - Proc.Intern.Conf.Nuclear Data for Science and Technology, Santa Fe, New Mexico, 26 September-1 October, 2004, R.C.Haight, M.B.Chadwick, T.Kawano, P.Talou, Eds., Vol.1, p. 804 (2005);

AIP Conf.Proc. 769 (2005)

Forward-Angle Neutron-Proton Scattering at $96 \mathrm{MeV}$

2005JOZY

G.D.Jones, N.J.Hammond, C.J.Lister, K.Teh, E.F.Moore - Proc.Nuclei at the Limits, Argonne, Illinois, D.Seweryniak and T.L.Khoo, eds., p.348 (2005); AIP Conf. Proc 764 (2005)

Linear Polarization Measurements Of Gamma Rays Following Alpha Decay

2005J0ZZ K.L.Jones, C.Baktash, D.W.Bardayan, J.C.Blackmon, W.N.Catford, J.A.Cizewski, R.P.Fitzgerald, U.Greife, C.J.Gross, M.S.Johnson, R.L.Kozub, J.F.Liang, R.J.Livesay, Z.Ma, B.H.Moazen, C.D.Nesaraja, D.Shapira, M.S.Smith, J.S.Thomas, D.Visser - Proc.Nuclei at the Limits, Argonne, Illinois, D.Seweryniak And T.L.Khoo, eds., p.176 (2005); AIP Conf. Proc 764 (2005) Studies Of Neutron-Rich Nuclei With (d, p) Reactions In Inverse Kinematics At The HRIBF

2005JU03 A.R.Junghans, E.C.Mohrmann, K.A.Snover, T.D.Steiger, E.G.Adelberger, J.M.Casandjian, H.E.Swanson, L.R.Buchmann, A.Laird, S.Park, A.Y.Zyuzin Nucl.Phys. B(Proc.Supp.) S138, 112 (2005)

The new Seattle-TRIUMF ${ }^{7} \mathrm{Be}(\mathrm{p}, \gamma)^{8} \mathrm{~B}$ S-factor determination

2005JU12 D.S.Judson, A.M.Bruce, M.J.Taylor, G.D.Dracoulis, T.Kibedi, A.P.Byrne, K.H.Maier, P.Nieminen, J.N.Orce - J.Phys.(London) G31, S1899 (2005) Microsecond isomers in ${ }^{125} \mathrm{Sb}$ 
2005KA06 R.Kanungo, Z.Elekes, H.Baba, Zs.Dombradi, Zs.Fulop, J.Gibelin, A.Horvath, Y.Ichikawa, E.Ideguchi, N.Iwasa, H.Iwasaki, S.Kawai, Y.Kondo, T.Motobayashi, M.Notani, T.Ohnishi, A.Ozawa, H.Sakurai, S.Shimoura, E.Takeshita, S.Takeuchi, I.Tanihata, Y.Togano, C.Wu, Y.Yamaguchi, Y.Yanagisawa, A.Yoshida, K.Yoshida Phys.Lett. B 608, 206 (2005)

Excited states in neutron rich boron isotopes

2005Ka23 S.Kameoka, S.Ajimura, K.Aoki, A.Banu, H.C.Bhang, T.Fukuda, O.Hashimoto, J.I.Hwang, B.H.Kang, E.H.Kim, J.H.Kim, M.J.Kim, T.Maruta, Y.Miura, Y.Miyake, T.Nagae, M.Nakamura, S.N.Nakamura, H.Noumi, S.Okada, Y.Okayasu, H.Outa, H.Park, P.K.Saha, Y.Sato, M.Sekimoto, T.Takahashi, H.Tamura, K.Tanida, A.Toyoda, K.Tsukada, T.Watanabe, H.J.Yim - Nucl.Phys. A754, 173c (2005) Measurement of the $\pi^{-}$decay width of ${ }_{\Lambda}^{5} \mathrm{He}$

2005KA25

S.G.Karshenboim, V.G.Ivanov, Yu.I.Neronov, B.P.Nikolaev, Yu.N.Tolparov Can.J.Phys. 83, 405 (2005)

A new determination of the proton-to-deuteron ratio of magnetic moments

2005KA26

R.Kanungo, Z.Elekes, H.Baba, Zs.Dombradi, Zs.Fulop, J.Gibelin, A.Horvath, Y.Ichikawa, E.Ideguchi, N.Iwasa, H.Iwasaki, S.Kawai, Y.Kondo, T.Motobayashi, M.Notani, T.Ohnishi, A.Ozawa, H.Sakurai, S.Shimoura, E.Takeshita, S.Takeuchi, I.Tanihata, Y.Togano, C.Wu, Y.Yamaguchi, Y.Yanagisawa, A.Yoshida, K.Yoshida Nucl.Phys. A757, 315 (2005)

Search for an isomeric state in ${ }^{19} \mathrm{C}$

2005KA33 M.Karadag, H.Yucel - Nucl.Instrum.Methods Phys.Res. A550, 626 (2005)

Thermal neutron cross-section and resonance integral for ${ }^{164} \mathrm{Dy}(\mathrm{n}, \gamma){ }^{165}$ Dy reaction

2005KA34 O.Kavatsyuk, M.Kavatsyuk, L.Batist, A.Banu, F.Becker, A.Blazhev, W.Bruchle, J.Doring, T.Faestermann, M.Gorska, H.Grawe, Z.Janas, A.Jungclaus, M.Karny, R.Kirchner, M.La Commara, S.Mandal, C.Mazzocchi, I.Mukha, S.Muralithar, C.Plettner, A.Plochocki, E.Roeckl, M.Romoli, M.Schadel, R.Schwengner, J.Zylicz Eur.Phys.J. A 25, 211 (2005)

Beta decay of ${ }^{103} \mathrm{Sn}$

2005KA39 A.Kankainen, G.K.Vorobjev, S.A.Eliseev, W.Huang, J.Huikari, A.Jokinen, A.Nieminen, Yu.N.Novikov, H.Penttila, A.V.Popov, S.Rinta-Antila, H.Schatz, D.M.Seliverstov, Yu.P.Suslov, J.Aysto - Eur.Phys.J. A 25, 355 (2005) Isomers of astrophysical interest in neutron-deficient nuclei at masses $\mathrm{A}=81,85$ and 86

2005KA45 T.Kautzsch, A.Wohr, W.B.Walters, K.-L.Kratz, B.Pfeiffer, M.Hannawald, J.Shergur, O.Arndt, S.Hennrich, S.Falahat, T.Griesel, O.Keller, A.Aprahamian, B.A.Brown, P.F.Mantica, M.A.Stoyer, H.L.Ravn, for the ISOLDE IS333 and Rochester CHICO / Gammasphere Collaborations - Eur.Phys.J. A 25, Supplement 1, 117 (2005)

Structure of neutron-rich even-even ${ }^{124,126} \mathrm{Cd}$ 
2005KA46 A.Kankainen, S.A.Eliseev, T.Eronen, S.P.Fox, U.Hager, J.Hakala, W.Huang, J.Huikari, D.Jenkins, A.Jokinen, S.Kopecky, I.Moore, A.Nieminen, Yu.N.Novikov, H.Penttila, A.V.Popov, S.Rinta-Antila, H.Schatz, D.M.Seliverstov, G.K.Vorobjev, Y.Wang, J.Aysto, and the IS403 Collaboration - Eur.Phys.J. A 25, Supplement 1, $129(2005)$

Beta-delayed gamma and proton spectroscopy near the $\mathrm{Z}=\mathrm{N}$ line

2005KA47 M.Karny, L.Batist, A.Banu, F.Becker, A.Blazhev, K.Burkard, W.Bruchle, J.Doring, T.Faestermann, M.Gorska, H.Grawe, Z.Janas, A.Jungclaus, M.Kavatsyuk, O.Kavatsyuk, R.Kirchner, M.La Commara, S.Mandal, C.Mazzocchi, K.Miernik, I.Mukha, S.Muralithar, C.Plettner, A.Plochocki, E.Roeckl, M.Romoli, K.Rykaczewski, M.Schadel, K.Schmidt, R.Schwengner, J.Zylicz - Eur.Phys.J. A 25, Supplement 1, 135 (2005)

Beta-decay studies near ${ }^{100} \mathrm{Sn}$

2005KA48 M.Kavatsyuk, O.Kavatsyuk, L.Batist, A.Banu, F.Becker, A.Blazhev, W.Bruchle, K.Burkard, J.Doring, T.Faestermann, M.Gorska, H.Grawe, Z.Janas, A.Jungclaus, M.Karny, R.Kirchner, M.La Commara, S.Mandal, C.Mazzocchi, I.Mukha, S.Muralithar, C.Plettner, A.Plochocki, E.Roeckl, M.Romoli, M.Schadel, R.Schwengner, J.Zylicz - Eur.Phys.J. A 25, Supplement 1, 139 (2005)

Beta-decay spectroscopy of ${ }^{103,105} \mathrm{Sn}$

2005KA51 R.Kanungo, M.Chiba, B.Abu-Ibrahim, S.Adhikari, D.Q.Fang, N.Iwasa, K.Kimura, K.Maeda, S.Nishimura, T.Ohnishi, A.Ozawa, C.Samanta, T.Suda, T.Suzuki, Q.Wang, C.Wu, Y.Yamaguchi, K.Yamada, A.Yoshida, T.Zheng, I.Tanihata Eur.Phys.J. A 25, Supplement 1, 327 (2005)

Observation of a two-proton halo in ${ }^{17} \mathrm{Ne}$

2005KA52 S.A.Karamian - Yad.Fiz. 68, 1827 (2005); Phys.Atomic Nuclei 68, 1765 (2005) Comparative Analysis of the ${ }^{178 m 2} \mathrm{Hf}$ Yield at Reactions with Different Projectiles

2005KAZU T.Kawabata, H.Akimune, H.Fujita, Y.Fujita, M.Fujiwara, K.Hara, K.Hatanaka, M.Itoh, Y.Kanada-En'yo, S.Kishi, K.Nakanishi, H.Sakaguchi, Y.Shimbara, A.Tamii, S.Terashima, M.Uchida, T.Wakasa, Y.Yasuda, H.P.Yoshida, M.Yosoi nucl-ex/0512040,12/25/2005 (2005)

Indication of dilute $2 \alpha+\mathrm{t}$ cluster structure in ${ }^{11} \mathrm{~B}$

2005KAZV T.Kawabata, H.Akimune, H.Fujita, Y.Fujita, M.Fujiwara, K.Hara, K.Hatanaka, M.Itoh, Y.Kanada-Enyo, S.Kishi, K.Nakanishi, H.Sakaguchi, Y.Shimbara, A.Tamii, S.Terashima, M.Uchida, T.Wakasa, Y.Yasuda, H.P.Yoshida, M.Yosoi -

CNS-REP-66, p.40 (2005)

Quadrupole Excitation Strengths and Cluster Structures in ${ }^{11} \mathrm{~B}$

2005KazX V.G.Kalinnikov, Z.Hons, V.I.Stegailov, P.Chaloun - Book of Abstracts, LV National Conference on Nuclear Physics "Frontiers in the Physics of Nucleus",

St.-Petersburg, p.72 (2005)

Decay of ${ }^{160} \mathrm{Ho}(3 \mathrm{~s})$ 
2005KaZY V.G.Kalinnikov, V.I.Stegailov, A.V.Sushkov, P.Chaloun, Ya.V.Yushkevich - Book of Abstracts, LV National Conference on Nuclear Physics "Frontiers in the Physics of Nucleus", St.-Petersburg, p.58 (2005)

Measurement of Lifetimes of some ${ }^{156,158,160}$ Ho Levels by Delayed Coincidence Method

2005KAzZ O.R.Kakuee, M.A.G.Alvarez, M.V.Andres, S.Cherubini, T.Davinson, A.Di Pietro, W.Galster, J.Gomez-Camacho, A.M.Laird, M.Lamehi-Rachti, I.Martel, A.M.Moro, J.Rahighi, A.M.Sanchez-Benitez, A.C.Shotter, W.B.Smith, J.Vervier, P.J.Woods nucl-ex/0507024,7/18/2005 (2005)

Long range absorption in the scattering of ${ }^{6} \mathrm{He}$ on ${ }^{208} \mathrm{~Pb}$ and ${ }^{197} \mathrm{Au}$ at $27 \mathrm{MeV}$

2005KE05 W.Ketter, W.Heil, G.Badurek, M.Baron, R.Loidl, H.Rauch J.Res.Natl.Inst.Stand.Technol. 110, 241 (2005)

Measurement of the Coherent Neutron Scattering Length of ${ }^{3} \mathrm{He}$

2005KE07 K.C.Kelley, N.E.Hertel, E.J.Pitcher, M.Devlin, S.G.Mashnik - Nucl.Phys. A760, 225 (2005)

${ }^{148} \mathrm{Gd}$ production cross section measurements for $600-$ and $800-\mathrm{MeV}$ protons on tantalum, tungsten, and gold

2005KE08 K.L.Keyes, A.Papenberg, R.Chapman, J.Ollier, X.Liang, M.J.Burns, M.Labiche, K.M.Spohr, N.Amzal, C.Beck, P.Bednarczyk, F.Haas, G.Duchene, P.Papka, B.Gebauer, T.Kokalova, S.Thummerer, W.von Oertzen, C.Wheldon J.Phys.(London) G31, S1903 (2005)

Spectroscopy of $\mathrm{Ne}, \mathrm{Na}$ and $\mathrm{Mg}$ isotopes approaching the Island of Inversion

2005KE10 H.Kettunen, T.Enqvist, K.Eskola, T.Grahn, P.T.Greenlees, K.Helariutta, P.Jones, R.Julin, S.Juutinen, H.Kankaanpaa, A.Keenan, H.Koivisto, P.Kuusiniemi, M.Leino, A.-P.Leppanen, M.Muikku, P.Nieminen, J.Pakarinen, P.Rahkila, J.Uusitalo -

Eur.Phys.J. A 25, Supplement 1, 181 (2005)

Decay studies of neutron-deficient odd-mass At and Bi isotopes

2005KE11 K.L.Keyes, A.Papenberg, R.Chapman, J.Ollier, X.Liang, M.J.Burns, M.Labiche, K.-M.Spohr, N.Amzal, C.Beck, P.Bednarczyk, F.Haas, G.Duchene, P.Papka, B.Gebauer, T.Kokalova, S.Thummerer, W.von Oertzen, C.Wheldon - Eur.Phys.J. A 25, Supplement 1, 431 (2005)

Spectroscopy of Ne and Na isotopes: Preliminary results from a EUROBALL + Binary Reaction Spectrometer experiment

2005KEZZ K.Kettern, I.Spahn, S.Spellerberg, S.M.Qaim, H.H.Coenen -

Proc.Intern.Conf.Nuclear Data for Science and Technology, Santa Fe, New Mexico, 26 September-1 October, 2004, R.C.Haight, M.B.Chadwick, T.Kawano, P.Talou, Eds., Vol.1, p. 758 (2005); AIP Conf.Proc. 769 (2005)

Nuclear Reaction Cross-Section Measurements via Characterization of Soft Radiation Emitting Products

2005KH13 D.T.Khoa, H.G.Bohlen, W.von Oertzen, G.Bartnitzky, A.Blazevic, F.Nuoffer, B.Gebauer, W.Mittig, P.Roussel-Chomaz - Nucl.Phys. A759, 3 (2005) 
Study of refractive structure in the inelastic ${ }^{16} \mathrm{O}+{ }^{16} \mathrm{O}$ Image scattering at the incident energies of 250 to $1120 \mathrm{MeV}$

2005KHZX R.U.Khafizov, N.Severijns, O.Zimmer, H.-F.Wirth, D.Rich, S.V.Tolokonnikov, V.A.Solovei, M.R.Kolhidashvili - nucl-ex/0512001,12/1/2005 (2005)

Discovery of the neutron radiative decay

2005KHzZ D.T.Khoa, H.G.Bohlen, W.von Oertzen, G.Bartnitzky, A.Blazevic, F.Nuoffer, B.Gebauer, W.Mittig, P.Roussel-Chomaz - nucl-ex/0504020,4/22/2005 (2005) Study of refractive structure in the inelastic ${ }^{16} \mathrm{O}+{ }^{16} \mathrm{O}$ scattering at the incident energies of 250 to $1120 \mathrm{MeV}$

2005KI01 S.Kishimoto, Y.Yoda, Y.Kobayashi, S.Kitao, R.Haruki, M.Seto - Nucl.Phys. A748, 3 (2005)

Evidence for nuclear excitation by electron transition on ${ }^{193} \mathrm{Ir}$ and its probability

2005KI03 P.M.King, for the $\mathrm{G}^{0}$ Collaboration - Eur.Phys.J. A 24, Supplement 2, 39 (2005)

Normal beam spin asymmetries during the $\mathrm{G}^{0}$ forward angle measurement

2005KI09 J.-H.Kim, C.S.Lee, Y.K.Kwon, J.H.Lee - J.Korean Phys.Soc. 46, 1318 (2005)

Measurement of Deuterons Scattered from the ${ }^{12} \mathrm{C}(\mathrm{p}, \mathrm{d}){ }^{11} \mathrm{C}$ Reaction by Using a Double-sided Silicon Strip Detector

2005KI11 T.Kishimoto, T.Hayakawa, S.Ajimura, S.Minami, A.Sakaguchi, Y.Shimizu, R.E.Chrien, M.May, P.Pile, A.Rusek, R.Sutter, H.Noumi, H.Tamura, M.Ukai, Y.Miura, K.Tanida - Nucl.Phys. A754, 383c (2005)

Kaonic nuclei probed by the in-flight $\left(\mathrm{K}^{-}, \mathrm{n}\right)$ reaction

2005KI17 T.Kii, T.Shima, T.Baba, Y.Nagai - Nucl.Instrum.Methods Phys.Res. A552, 329 (2005)

A time projection chamber for the study of nuclear photodisintegration

2005KI19 St.Kistryn, E.Stephan, A.Biegun, K.Bodek, A.Deltuva, E.Epelbaum, K.Ermisch, W.Glockle, J.Golak, N.Kalantar-Nayestanaki, H.Kamada, M.Kis, B.Klos, A.Kozela, J.Kuros-Zolnierczuk, M.Mahjour-Shafiei, U.-G.Meissner, A.Micherdzinska, A.Nogga, P.U.Sauer, R.Skibinski, R.Sworst, H.Witala, J.Zejma, W.Zipper - Phys.Rev. C 72, 044006 (2005)

Systematic study of three-nucleon force effects in the cross section of the deuteron-proton breakup at $130 \mathrm{MeV}$

2005KI20 E.R.Kinney, J.L.Matthews, P.A.M.Gram, D.W.MacArthur, E.Piasetzky, G.A.Rebka, Jr., D.A.Roberts - Phys.Rev. C 72, 044608 (2005)

Inclusive pion double charge exchange in ${ }^{4} \mathrm{He}$ at intermediate energies

2005KI21 O.A.Kiselev, F.Aksouh, A.Bleile, O.V.Bochkarev, L.V.Chulkov, D.Cortina-Gil, A.V.Dobrovolsky, P.Egelhof, H.Geissel, M.Hellstrom, N.B.Isaev, B.G.Komkov, M.Matos, F.V.Moroz, G.Munzenberg, M.Mutterer, V.A.Mylnikov, S.R.Neumaier, V.N.Pribora, D.M.Seliverstov, L.O.Sergueev, A.Shrivastava, K.Summerer, H.Weick, M.Winkler, V.I.Yatsoura - Eur.Phys.J. A 25, Supplement 1, 215 (2005) 
Investigation of nuclear matter distribution of the neutron-rich He isotopes by proton elastic scattering at intermediate energies

2005KIZV G.G.Kiss, Zs.Fulop, Gy.Gyurky, Z.Mate, E.Somorjai, D.Galaviz, A.Zilges ATOMKI 2004 Ann.Rept., p.14 (2005)

Study of low energy $\alpha$-scattering on ${ }^{89} \mathrm{Y}$

2005KL02 H.V.Klapdor-Kleingrothaus, for the Heidelburg-Moscow / HDMS / Genius-TF Collaborations - Nucl.Phys. B(Proc.Supp.) S143, 229 (2005)

First evidence for neutrinoless double beta decay, with enriched ${ }^{76} \mathrm{Ge}$ in Gran Sasso 1990-2003

2005KLzZ T.Klechneva, C.Carasco, I.Goussev, M.Hauger, J.Jourdan, B.Krusche, H.Muehry, Ch.Normand, D.Rohe, D.Seliverstov, I.Sick, G.Testa, G.Warren, H.Wohrle, M.Zeier - nucl-ex/0509008,9/05/2005 (2005)

Vector and Tensor Analyzing Powers of the ${ }^{1} \mathrm{H}\left((\mathrm{d}(\mathrm{pol}), \gamma)^{3} \mathrm{He}\right.$-capture reaction

2005KM01 M.Kmiecik, A.Maj, J.Styczen, P.Bednarczyk, M.Brekiesz, J.Grebosz, M.Lach, W.Meczynski, M.Zieblinski, K.Zuber, A.Bracco, F.Camera, G.Benzoni, B.Million, S.Leoni, O.Wieland, B.Herskind, D.Curien, N.Dubray, J.Dudek, N.Schunck, K.Mazurek - Acta Phys.Pol. B36, 1169 (2005)

GDR feeding of the highly-deformed band in ${ }^{42} \mathrm{Ca}$

2005Kn02 H.H.Knudsen, H.O.U.Fynbo, M.J.G.Borge, R.Boutami, P.Dendooven, C.Aa.Diget, T.Eronen, S.Fox, L.M.Fraile, B.Fulton, J.Huikary, H.B.Jeppesen, A.S.Jokinen, B.Jonson, A.Kankainen, I.Moore, A.Nieminen, G.Nyman, H.Penttila, K.Riisager, S.Rinta-Antila, O.Tengblad, Y.Wang, K.Wilhelmsen, J.Aysto - Phys.Rev. C 72, $044312(2005)$

$\beta$-decay of ${ }^{13} \mathrm{O}$

2005K001 Tz.Kokalova, W.von Oertzen, S.Torilov, S.Thummerer, M.Milin, A.Tumino, G.de Angelis, E.Farnea, A.Gadea, D.R.Napoli, Th.Kroll, N.Marginean, T.Martinez, M.Axiotis, S.M.Lenzi, C.Ur, P.Papka, M.Rousseau - Eur.Phys.J. A 23, 19 (2005) Emission of unbound ${ }^{8} \mathrm{Be}$ and ${ }^{12} \mathrm{C}^{*}\left(0_{2}^{+}\right)$clusters in compound nucleus reactions

2005K002 K.Kobayashi, Y.Itow, M.Shiozawa, M.Yosoi, H.Toyokawa, H.Akimune, H.Ejiri, H.Fujimura, M.Fujiwara, K.Hara, K.Y.Hara, T.Ishikawa, M.Itoh, T.Kawabata, M.Nakamura, H.Sakaguchi, Y.Sakemi, H.Takeda, , M.Uchida, T.Yamada, Y.Yasuda, H.P.Yoshida, R.G.T.Zegers - Nucl.Phys. B(Proc.Supp.) S139, 72 (2005)

Detection of nuclear de-excitation gamma-rays in water Cherenkov detector

2005K009 R.L.Kozub, D.W.Bardayan, J.C.Batchelder, J.C.Blackmon, C.R.Brune, A.E.Champagne, J.A.Cizewski, T.Davinson, U.Greife, C.J.Gross, C.C.Jewett, R.J.Livesay, Z.Ma, B.H.Moazen, C.D.Nesaraja, L.Sahin, J.P.Scott, D.Shapira, M.S.Smith, J.S.Thomas, P.J.Woods - Phys.Rev. C 71, 032801 (2005)

New constraints on the ${ }^{18} \mathrm{~F}(\mathrm{p}, \alpha){ }^{15} \mathrm{O}$ rate in novae from the $(\mathrm{d}, \mathrm{p})$ reaction 
2005K011 W.Korten, E.Clement, E.Bouchez, A.Chatillon, A.Gorgen, Y.Le Coz, Ch.Theisen, J.M.Casandjian, G.de France, G.Sletten, T.Czosnyka, J.Iwanicki, M.Zielinska, F.Becker, J.Gerl, W.Catford, C.Timis, P.Butler, C.Andreoiu - Nucl.Phys. A752, $255 \mathrm{c}(2005)$

Investigation of heavy $\mathrm{N} \sim \mathrm{Z}$ nuclei using energetic radioactive ion beams

2005K013 Y.Kondo, T.Nakamura, N.Aoi, H.Baba, D.Bazin, N.Fukuda, T.Gomi, H.Hasegawa, N.Imai, M.Ishihara, T.Kobayashi, T.Kubo, M.Miura, T.Motobayashi, A.Saito, H.Sakurai, S.Shimoura, T.Sugimoto, K.Watanabe, Y.X.Watanabe, T.Yakushiji, Y.Yanagisawa, K.Yoneda - Phys.Rev. C 71, 044611 (2005) In-beam $\gamma$-ray spectroscopy of neutron-rich boron isotopes ${ }^{15,17} \mathrm{~B}$ via inelastic scattering on ${ }^{12} \mathrm{C}$

2005K031 R.L.Kozub, D.W.Bardayan, J.C.Batchelder, J.C.Blackmon, C.R.Brune, A.E.Champagne, J.A.Cizewski, T.Davinson, U.Greife, C.J.Gross, C.C.Jewet, R.J.Livesay, Z.Ma, B.H.Moazen, C.D.Nesaraja, J.P.Scott, L.Sahin, D.Shapira, M.S.Smith, J.S.Thomas, P.J.Woods - Nucl.Phys. A758, 753c (2005)

New Limits for the ${ }^{18} \mathrm{~F}(\mathrm{p}, \alpha){ }^{15} \mathrm{O}$ Rate in Novae

2005K032 C.Kohstall, D.Belic, P.von Brentano, C.Fransen, A.Gade, R.-D.Herzberg, J.Jolie, U.Kneissl, A.Linnemann, A.Nord, N.Pietralla, H.H.Pitz, M.Scheck, F.Stedile, V.Werner, S.W.Yates - Phys.Rev. C 72, 034302 (2005)

Low-lying dipole excitations in vibrational nuclei: The Cd isotopic chain studied in photon scattering experiments

2005K040 A.Korgul, H.Mach, B.A.Brown, A.Covello, A.Gargano, B.Fogelberg, R.Schuber, W.Kurcewicz, E.Werner-Malento, R.Orlandi, M.Sawicka - Eur.Phys.J. A 25, Supplement 1, 123 (2005)

On the structure of the anomalously low-lying $5 / 2^{+}$state of ${ }^{135} \mathrm{Sb}$

2005K041 M.Kowalska, D.Yordanov, K.Blaum, D.Borremans, P.Himpe, P.Lievens, S.Mallion, R.Neugart, G.Neyens, N.Vermeulen - Eur.Phys.J. A 25, Supplement 1, 193 (2005)

Laser and $\beta$-NMR spectroscopy on neutron-rich magnesium isotopes

2005K043 U.Koster, O.Arndt, U.C.Bergmann, R.Catherall, J.Cederkall, I.Dillmann, M.Dubois, F.Durantel, L.Fraile, S.Franchoo, G.Gaubert, L.Gaudefroy, O.Hallmann, C.Huet-Equilbec, B.Jacquot, P.Jardin, K.L.Kratz, N.Lecesne, R.Leroy, A.Lopez, L.Maunoury, J.Y.Pacquet, B.Pfeiffer, M.G.Saint-Laurent, C.Stodel, A.C.C.Villari, L.Weissman - Eur.Phys.J. A 25, Supplement 1, 729 (2005)

ISOL beams of neutron-rich oxygen isotopes

2005KR03 Ch.Kraus, B.Bornschein, L.Bornschein, J.Bonn, B.Flatt, A.Kovalik, B.Ostrick, E.W.Otten, J.P.Schall, Th.Thummler, Ch.Weinheimer - Eur.Phys.J. C 40, 447 (2005)

Final results from phase II of the Mainz neutrino mass search in tritium $\beta$ decay 
2005KR14 K.Kramer, D.S.Armstrong, T.D.Averett, W.Bertozzi, S.Binet, C.Butuceanu, A.Camsonne, G.D.Cates, J.-P.Chen, S.Choi, E.Chudakov, F.Cusanno, A.Deur, P.Djawotho, D.Dutta, J.M.Finn, H.Gao, F.Garibaldi, O.Gayou, R.Gilman, A.Glamazdin, V.Gorbenko, K.A.Griffioen, J.-O.Hansen, D.W.Higinbotham, W.Hinton, T.Horn, C.W.de Jager, X.Jiang, W.Korsch, J.LeRose, D.Lhuillier, N.Liyanage, D.J.Margaziotis, K.McCormick, Z.-E.Meziani, R.Michaels, B.Milbrath, B.Moffit, S.Nanda, C.F.Perdrisat, R.Pomatsalyuk, V.Punjabi, B.Reitz, J.Roche, R.Roche, M.Roedelbronn, N.Savvinov, J.Secrest, J.Singh, S.Sirca, K.Slifer, P.Solvignon, D.J.Steiner, R.Suleiman, V.Sulkosky, A.Tobias, A.Vacheret, Y.Xiao, X.Zheng, J.Zhou, L.Zhu, X.Zhu, P.A.Zolnierczuk - Phys.Rev.Lett. 95, 142002 (2005) $\mathrm{Q}^{2}$ Dependence of the Neutron Spin Structure Function $\mathrm{g}_{2}^{n}$ at Low $\mathrm{Q}^{2}$

2005KR15 A.Krishna, K.Pandey, A.Wasan, V.Natarajan - Europhys.Lett. 72, 221 (2005) High-resolution hyperfine spectroscopy of excited states using electromagnetically induced transparency

2005KRZY

A.Krasznahorkay, F.W.N.de Boer, J.Gulyas, Z.Gacsi, T.J.Ketel, M.Csatlos, L.Csige, M.Hunyadi, J.Van Klinken, A.Krasznahorkay, Jr., A.Vitez - ATOMKI 2004 Ann.Rept., p.3 (2005)

Internal pairs observed in a forbidden $0^{-} \rightarrow 0^{+}$transition: a sign for a neutral boson?

2005KSzZ R.Kshetri, I.Ray, P.Banerjee, R.Raut, A.Goswami, J.M.Chatterjee, S.Chattopadhyay, U.Datta Pramanik, A.Mukherjee, C.C.Dey, S.Bhattacharya, B.Dasmahapatra, M.S.Sarkar, S.Sarkar, S.Bhowal, G.Ganguly, K.S.Golda, R.Kumar, R.P.Singh, S.Muralithar - nucl-ex/0507019,7/13/2005 (2005)

Absence of M2 Retardation in ${ }^{35} \mathrm{Cl}$ : Evidence for Stronger Isospin-Mixing Effects in $\mathrm{A}=35$ Mirror Nuclei

2005KU01 T.Kutsarova, C.Schuck, E.Gueorguieva, A.Minkova, I.Zartova, F.Hannachi, A.Korichi, A.Lopez-Martens - Eur.Phys.J. A 23, 69 (2005)

Coexisting oblate and strongly triaxial structures in ${ }^{191} \mathrm{Pt}$

2005KU06 P.Kuusiniemi, F.P.Hessberger, D.Ackermann, S.Hofmann, I.Kojouharov Eur.Phys.J. A 23, 417 (2005)

Decay studies of ${ }^{210-214} \operatorname{Fr}$ using $\alpha$ - $\gamma$-coincidences

2005KU10 R.Kumar, K.Singh, D.Mehta, N.Singh, S.S.Malik, E.S.Paul, A.Gorgen, S.Chmel, R.P.Singh, S.Muralithar - Eur.Phys.J. A 24, 13 (2005)

Collective structures of the ${ }^{131} \mathrm{Cs}$ nucleus

2005KU17 W.D.Kulp, J.L.Wood, P.E.Garrett, J.M.Allmond, D.Cline, A.B.Hayes, H.Hua, K.S.Krane, R.-M.Larimer, J.Loats, E.B.Norman, P.Schmelzenbach, C.J.Stapels, R.Teng, C.Y.Wu - Phys.Rev. C 71, 041303 (2005)

Identification of a pairing isomeric band in ${ }^{152} \mathrm{Sm}$ 
2005KU27 S.Kubono, T.Teranishi, M.Notani, H.Yamaguchi, A.Saito, J.J.He, M.Wakabayashi, H.Fujikawa, G.Amadio, H.Baba, T.Fukuchi, S.Shimoura, S.Michimasa, S.Nishimura, M.Nishimura, Y.Gono, A.Odahara, S.Kato, J.Y.Moon, J.H.Lee, C.S.Lee, J.C.Kim, K.I.Hahn, T.Ishikawa, T.Hashimoto, H.Ishiyama, Y.X.Watanabe, M.H.Tanaka, H.Miyatake, Zs.Fulop, V.Guimaraes, R.Lichtenthaler - Nucl.Phys. A758, 733c (2005) Study of Stellar Reactions in Explosive Hydrogen Burning with CRIB

2005KU28 A.Kumar, J.N.Orce, S.R.Lesher, C.J.McKay, M.T.McEllistrem, S.W.Yates Phys.Rev. C 72, 034313 (2005)

Heterogeneous vibrations in ${ }^{112} \mathrm{Sn}$

2005KU31 P.Kuusiniemi, F.P.Hessberger, D.Ackermann, S.Hofmann, B.Sulignano,

I.Kojouharov, R.Mann - Eur.Phys.J. A 25, 397 (2005)

Decay studies of ${ }^{215-217}$ Th using ER- $\gamma-\alpha-\gamma$ coincidences

2005KU34 R.Kumar, A.Kumar, S.K.Chamoli, K.Singh, M.Sharma, D.Mehta, N.Singh, S.S.Ghugre, N.S.Pattabiraman, L.Chaturvedi, P.K.Joshi, H.C.Jain, Z.Naik, C.R.Praharaj, I.M.Govil - Phys.Rev. C 72, 044319 (2005)

Polarization measurement and $\gamma$-ray spectroscopy of ${ }^{122} \mathrm{Cs}$

2005KU36 S.Kumar, S.V.Kumar, G.L.N.Reddy, V.Kain, J.V.Ramana, V.S.Raju -

Nucl.Instrum.Methods Phys.Res. B240, 704 (2005)

Depth profiling of nitrogen using $429 \mathrm{keV}$ and $897 \mathrm{keV}$ resonances in the ${ }^{15} \mathrm{~N}(\mathrm{p}$, $\alpha \gamma)^{12} \mathrm{C}$ reaction

2005KU37 A.Kumar, J.N.Orce, S.R.Lesher, C.J.McKay, M.T.McEllistrem, S.W.Yates Eur.Phys.J. A 25, Supplement 1, 443 (2005)

Lifetime measurements and low-lying structure in ${ }^{112} \mathrm{Sn}$

2005KUZV P.Kuusiniemi, F.P.Hessberger, D.Ackermann, S.Hofmann, K.Nishio, B.Sulignano, I.Kojouharov, R.Mann - GSI 2005-1, p.76 (2005)

$\alpha$ - Decay Fine Structure of ${ }^{213} \mathrm{Ra}$ to ${ }^{209} \mathrm{Rn}$

2005KUZX W.D.Kulp, J.L.Wood, K.S.Krane, J.Loats, P.D.Schmelzenbach, C.J.Stapels, E.B.Norman - Proc.Intern.Conf.Nuclear Data for Science and Technology, Santa Fe, New Mexico, 26 September-1 October, 2004, R.C.Haight, M.B.Chadwick, T.Kawano, P.Talou, Eds., Vol.1, p. 830 (2005); AIP Conf.Proc. 769 (2005) An Investigation of ${ }^{154} \mathrm{Eu}$ as a High-Precision Multi- $\gamma$-Ray Intensity Calibration Standard for Detector Arrays

2005KUZZ P.Kuusiniemi - Proc.Nuclei at the Limits, Argonne, Illinois, D.Seweryniak and T.L.Khoo, eds., p.231 (2005); AIP Conf. Proc 764 (2005)

Nuclear Structure Investigations Of Heavy Nuclei And The Decay Of SHE

2005LA01 G.J.Lane, K.H.Maier, A.P.Byrne, G.D.Dracoulis, R.Broda, B.Fornal, M.P.Carpenter, R.M.Clark, M.Cromaz, R.V.F.Janssens, A.O.Macchiavelli, I.Wiedenhover, K.Vetter - Phys.Lett. B 606, 34 (2005)

High-spin isomers and three-neutron valence configurations in ${ }^{211} \mathrm{~Pb}$ 
2005LA07 S.Lalkovski, G.Rainovski, K.Starosta, M.P.Carpenter, D.B.Fossan, S.Finnigan, S.Ilieva, P.Joshi, T.Koike, E.S.Paul, N.Pietralla, C.Vaman, R.Wadsworth Phys.Rev. C 71, 034318 (2005)

Quasi- $\gamma$ band and odd-even staggering effect in ${ }^{102} \mathrm{Ru}$

2005LA13 A.Laptev, H.Harada, S.Nakamura, J.Hori, M.Igashira, T.Ohsaki, K.Ohgama Nucl.Instrum.Methods Phys.Res. A543, 502 (2005)

Baseline distortion effect on gamma-ray pulse-height spectra in neutron capture experiments

2005LA19 M.Lach, J.Styczen, W.Meczynski, P.Bednarczyk, A.Bracco, J.Grebosz, A.Maj, J.C.Merdinger, N.Schulz, M.B.Smith, K.M.Spohr, M.Zieblinski - Eur.Phys.J. A 25, $1(2005)$

Unnatural-parity states in ${ }_{21}^{44} \mathrm{Sc}_{23}$

2005LA25 M.La Cognata, A.Musumarra, C.Spitaleri, A.Tumino, C.Bonomo, S.Cherubini, P.Figuera, L.Lamia, M.G.Pellegriti, A.Rinollo, R.G.Pizzone, C.Rolfs, S.Romano, D.Schurmann, F.Strieder, S.Tudisco, S.Typel - Nucl.Phys. A758, 98c (2005)

Study of the ${ }^{3} \mathrm{He}(\mathrm{d}, \mathrm{p}){ }^{4} \mathrm{He}$ reaction through the Trojan Horse Method

2005LA28 M.Labiche, C.N.Timis, R.C.Lemmon, W.N.Catford, R.Chapman, N.Amzal, N.I.Ashwood, T.D.Baldwin, M.Burns, L.Caballero, M.Chartier, N.Curtis, G.de France, W.Gelletly, X.Liang, M.Freer, N.A.Orr, S.D.Pain, V.P.E.Pucknell, M.Rejmund, B.Rubio, H.Savajols, O.Sorlin, K.Spohr, Ch.Theisen, D.D.Warner J.Phys.(London) G31, S1691 (2005)

Study of transfer reactions in inverse kinematics with the TIARA array

2005LA29 S.Lakshmi, H.C.Jain, P.K.Joshi, I.Mazumdar, R.Palit, A.K.Jain, S.S.Malik Nucl.Phys. A761, 1 (2005)

High spin structure of ${ }^{136} \mathrm{Ce}$

2005LA30 V.P.Ladygin, L.S.Azhgirey, S.V.Afanasiev, V.V.Arkhipov, V.K.Bondarev, Yu.T.Borzunov, G.Filipov, L.B.Golovanov, A.Yu.Isupov, V.I.Ivanov, A.A.Kartamyshev, V.A.Kashirin, A.N.Khrenov, V.I.Kolesnikov, V.A.Kuznetsov, A.G.Litvinenko, S.G.Reznikov, P.A.Rukoyatkin, A.Yu.Semenov, I.A.Semenova, G.D.Stoletov, A.P.Tzvinev, N.P.Yudin, V.N.Zhmyrov, L.S.Zolin - Phys.Lett. B 629, $60(2005)$

Tensor analyzing power $\mathrm{A}_{y y}$ in deuteron inclusive breakup on hydrogen and carbon at $9 \mathrm{GeV} / \mathrm{c}$ and large proton transverse momenta

2005LA32 K.Lagergren, M.A.Riley, J.Simpson, D.E.Appelbe, P.Bednarczyk, D.B.Campbell, C.Chandler, P.T.W.Choy, D.Curien, D.T.Joss, E.S.Paul - Phys.Rev. C 72, 057303 (2005)

Observation of the second proton alignment in ${ }^{160} \mathrm{Tm}$

2005LAzZ T.Lauritsen, R.V.Janssens, T.L.Khoo, I.Ahmad, M.P.Carpenter, A.M.Heinz, D.G.Jenkins, F.G.Kondev, C.J.Lister, E.F.Moore, D.Seweryniak, S.Zhu, T.Dossing, B.Herskind, R.M.Clark, M.Cromaz, P.Fallon, G.Lane, A.O.Macchiavelli, D.Ward, A.Korichi, A.Lopez-Martens A.J.Larabee - Proc.Nuclei at the Limits, Argonne, Illinois, D.Seweryniak and T.L.Khoo, eds., p.34 (2005); AIP Conf. Proc 764 (2005) 
Rotational Damping, Ridges And The Quasi-Continuum Of $\gamma$ Rays In ${ }^{152}$ Dy

2005LE04 I.Leya, R.Wieler, J.-C.David, S.Leray, L.Donadille, J.Cugnon, R.Michel Nucl.Instrum.Methods Phys.Res. B229, 1 (2005)

Production of noble gas isotopes by proton-induced reactions on lead

2005LE12 J.Leske, K.-H.Speidel, S.Schielke, O.Kenn, D.Hohn, J.Gerber, P.Maier-Komor Phys.Rev. C 71, 034303 (2005)

Experimental g factors and $\mathrm{B}(\mathrm{E} 2)$ values of $2_{1}^{+}, 4,{ }_{1}^{+} 2_{2}^{+}$, and $33_{1}^{-}$states in ${ }^{64} \mathrm{Zn}$ and ${ }^{68} \mathrm{Zn}$ compared to shell model predictions

2005LE19 J.Leske, K.-H.Speidel, S.Schielke, O.Kenn, J.Gerber, P.Maier-Komor, S.J.Q.Robinson, A.Escuderos, Y.Y.Sharon, L.Zamick - Phys.Rev. C 71, 044316 (2005)

Nuclear structure of the first $2^{+}$state in radioactive ${ }^{68} \mathrm{Ge}$ based on $\mathrm{g}$ factor and lifetime measurements

2005LE21 S.Leoni, G.Benzoni, A.Bracco, N.Blasi, F.Camera, C.Grassi, B.Million, A.Paleni, M.Pignanelli, E.Vigezzi, O.Wieland, M.Matsuo, T.Dossing, B.Herskind,

G.B.Hagemann, J.Wilson, A.Maj, M.Kmiecik, G.Lo Bianco, C.M.Petrache, M.Castoldi, A.Zucchiati, G.De Angelis, D.Napoli, P.Bednarczyk, D.Curien - Acta Phys.Pol. B36, 1121 (2005)

Warm rotating nuclei: damping mechanism and the order-to-chaos transition

2005LE30 R.Lewis, J.Caggiano, A.Parikh, C.Deibel, D.Visser, K.Fear, P.Parker, W.R.Hix Nucl.Phys. A758, 84c (2005)

${ }^{26} \mathrm{Al}^{g, m}+\mathrm{p}$ resonances studied via proton decays of ${ }^{27} \mathrm{Si}$

2005LE34 F.Le Blanc, L.Cabaret, E.Cottereau, J.E.Crawford, S.Essabaa, J.Genevey, R.Horn, G.Huber, J.Lassen, J.K.P.Lee, G.Le Scornet, J.Lettry, J.Obert, J.Oms, A.Ouchrif, J.Pinard, H.Ravn, B.Roussiere, J.Sauvage, D.Verney - Phys.Rev. C 72, 034305 (2005)

Charge-radius change and nuclear moments in the heavy tin isotopes from laser spectroscopy: Charge radius of ${ }^{132} \mathrm{Sn}$

2005LE35 S.Leoni, G.Benzoni, A.Bracco, N.Blasi, F.Camera, C.Grassi, P.Mason, B.Million, A.Paleni, M.Pignanelli, E.Vigezzi, O.Wieland, M.Matsuo, T.Dossing, B.Herskind, G.B.Hagemann, J.Wilson, A.Maj, M.Kmiecik, G.Lo Bianco, C.M.Petrache, M.Castoldi, A.Zucchiatti, G.de Angelis, D.R.Napoli, P.Bednarczyk, D.Curien Phys.Rev. C 72, 034307 (2005)

Damping mechanisms and order-to-chaos transition in the warm rotating ${ }^{163} \mathrm{Er}$ nucleus

2005LE38 J.Leske, K.-H.Speidel, S.Schielke, J.Gerber, P.Maier-Komor, T.Engeland, M.Hjorth-Jensen - Phys.Rev. C 72, 044301 (2005)

Dominant $\left(\mathrm{g}_{9 / 2}\right)^{2}$ neutron configuration in the $4_{1}^{+}$state of ${ }^{68} \mathrm{Zn}$ based on new $\mathrm{g}$ factor measurements 
2005LE42 A.-P.Leppanen, J.Uusitalo, S.Eeckhaudt, T.Enqvist, K.Eskola, T.Grahn, F.P.Hessberger, P.T.Greenlees, P.Jones, R.Julin, S.Juutinen, H.Kettunen, P.Kuusiniemi, M.Leino, P.Nieminen, J.Pakarinen, J.Perkowski, P.Rahkila, C.Scholey, G.Sletten - Eur.Phys.J. A 25, Supplement 1, 183 (2005)

Alpha-decay study of ${ }^{218} \mathrm{U}$; a search for the sub-shell closure at $\mathrm{Z}=92$

2005LEZW A.I.Levon, I.B.Kovgar, Yu.V.Nosenko, V.A.Onischuk, A.A.Shevchuk - Book of Abstracts, LV National Conference on Nuclear Physics "Frontiers in the Physics of Nucleus", St.-Petersburg, p.81 (2005)

Nuclear g-Factors of High-Spin Isomers in ${ }^{190,192,194} \mathrm{Pt}$ and ${ }^{196,198} \mathrm{Hg}$

2005LEZX J.Leske, K.-H.Speidel, S.Schielke, J.Gerber, P.Maier-Komor, T.Engeland, M.Hjorth-Jensen - nucl-ex/0506006,6/05/2005 (2005)

Dominant $\left(\mathrm{g}_{9 / 2}\right)^{2}$ neutron configuration in the $4_{1}^{+}$state of ${ }^{68} \mathrm{Zn}$ based on new $\mathrm{g}$ factor measurements

2005LEzZ S.Leoni, M.Matsuo, A.Bracco, G.Benzoni, N.Blasi, F.Camera, C.Grassi, B.Million, A.Paleni, M.Pignanelli, E.Vigezzi, O.Wieland, T.Dossing, B.Herskind, G.B.Hagemann, J.Wilson, A.Maj, M.Kmiecik, G.LoBianco, C.M.Petrache, M.Castoldi, A.Zucchiati, G.De Angelis, D.Napoli, P.Bednarczyk, D.Curien -

Proc.Nuclei at the Limits, Argonne, Illinois, D.Seweryniak and T.L.Khoo, eds., p.309 (2005); AIP Conf. Proc 764 (2005)

Compound And Rotational Damping In Warm Nuclei

2005LH01 G.Lhersonneau, S.Brant - Phys.Rev. C 72, 034308 (2005)

Levels in ${ }^{99} \mathrm{Zr}$ observed in the decay of ${ }^{99} \mathrm{Y}$

2005LI02 Y.Li, S.-F.Shen, S-H.Shi, J.-H.Gu, J.-Y.Liu, H.Xu - Chinese Physics 14, 95 (2005) New levels and transitions in ${ }^{72} \mathrm{Ge}$ following the decay of ${ }^{72} \mathrm{Ga}$

2005 LI09 Y.Li, S.-F.Shen, J.-H.Gu, S.-H.Shi, J.-Y.Liu - Chinese Physics 14, 487 (2005)

A study on the decay of ${ }^{72} \mathrm{As}$

2005LI13 D.Li, K.Imasaki, S.Miyamoto, S.Amano, T.Mochizuki - J.Nucl.Sci.Technol.(Tokyo) 42, 259 (2005)

Experiment on Photonuclear Reaction Induced by Laser Compton Scattering Gamma-Ray

2005LI14 T.C.Li, N.Pietralla, C.Fransen, H.von Garrel, U.Kneissl, C.Kohstall, A.Linnemann, H.H.Pitz, G.Rainovski, A.Richter, M.Scheck, F.Stedile, P.von Brentano, P.von Neumann-Cosel, V.Werner - Phys.Rev. C 71, 044318 (2005)

One-phonon $2_{1, m s}^{+}$mixed-symmetry state of ${ }^{148} \mathrm{Sm}$ observed in nuclear resonance fluorescence

2005LI17 Z.Liu, J.Kurcewicz, P.J.Woods, C.Mazzocchi, F.Attallah, E.Badura, C.N.Davids, T.Davinson, J.Doring, H.Geissel, M.Gorska, R.Grzywacz, M.Hellstrom, Z.Janas, M.Karny, A.Korgul, I.Mukha, M.Pfutzner, C.Plettner, A.Robinson, E.Roeckl, K.Rykaczewski, K.Schmidt, D.Seweryniak, H.Weick - Nucl.Instrum.Methods Phys.Res. A543, 591 (2005) 
Decay spectroscopy of suburanium isotopes following projectile fragmentation of ${ }^{238} \mathrm{U}$ at $1 \mathrm{GeV} / \mathrm{u}$

$2005 L I 19$ Z.H.Li, W.P.Liu, X.X.Bai, B.Guo, G.Lian, S.Q.Yan, B.X.Wang, S.Zeng, Y.Lu, J.Su, Y.S.Chen, K.S.Wu, N.C.Shu, T.Kajino - Phys.Rev. C 71, 052801 (2005)

The ${ }^{8} \mathrm{Li}(\mathrm{d}, \mathrm{p}){ }^{9} \mathrm{Li}$ reaction and the astrophysical ${ }^{8} \mathrm{Li}(\mathrm{n}, \gamma){ }^{9} \mathrm{Li}$ reaction rate

2005LI29 A.G.Lipson, A.S.Rusetskii, A.B.Karabut, G.Miley - Zh.Eksp.Teor.Fiz. 127, 1334 (2005); J.Exper.Theo.Phys. 100, 1175 (2005)

DD Reaction Enhancement and X-ray Generation in a High-Current Pulsed Glow

Discharge in Deuterium with Titanium Cathode at 0.8-2.45 kV

2005 LI35 Z.H.Li, B.Guo, W.-P.Liu, X.-X.Bai, G.Lian, J.Su, S.-Q.Yan, B.-X.Wang, S.Zeng Chin.Phys.Lett. 22, 1870 (2005)

Radius of ${ }^{9} \mathrm{C}$ from the Asymptotic Normalization Coefficient

2005LI40 W.Liu, Z.Li, X.Bai, G.Lian, B.Guo, S.Zeng, S.Yan, B.Wang, N.Shu, K.Wu Y.Chen Nucl.Phys. A758, 110c (2005)

Nuclear Astrophysics Experiments at CIAE

2005LI47 Z.Liu, P.J.Woods, K.Schmidt, H.Mahmud, P.S.L.Munro, A.Blazhev, J.Doring, H.Grawe, M.Hellstrom, R.Kirchner, Z.K.Li, C.Mazzocchi, I.Mukha, C.Plettner, E.Roeckl, M.La Commara - Phys.Rev. C 72, 047301 (2005)

Reinvestigation of direct proton decay of ${ }^{105} \mathrm{Sb}$

2005LI53 S.N.Liddick, P.F.Mantica, R.Broda, B.A.Brown, M.P.Carpenter, A.D.Davies, B.Fornal, M.Horoi, R.V.F.Janssens, A.C.Morton, W.F.Mueller, J.Pavan, H.Schatz, A.Stolz, S.L.Tabor, B.E.Tomlin, M.Wiedeking - Phys.Rev. C 72, 054321 (2005) $\beta$-decay of odd-A ${ }^{57} \mathrm{Ti}$ and ${ }^{59} \mathrm{~V}$

2005LIZY Z.Liu, P.J.Woods, K.Schmidt, H.Mahmud, P.S.L.Munro, A.Blazhev, J.Doring, H.Grawe, M.Hellstrom, R.Kirchner, Z.K.Li, C.Mazzocchi, I.Mukha, C.Plettner, E.Roeckl, M.La Commara - GSI 2005-1, p.85 (2005)

Reinvestigation of direct proton decay of ${ }^{105} \mathrm{Sb}$

2005LIzZ Yu.A.Litvinov, D.Boutin, L.Chen, H.Geissel, E.Kaza, C.Kozhuharov, S.A.Litvinov, M.Matos, Yu.N.Novikov, W.Plass, C.Scheidenberger, G.Vorobjev, H.Weick, F.Attallah, K.Beckert, P.Beller, F.Bosch, L.Caceres, J.J.Carroll, R.S.Chakrawarthy, D.Cullen, B.Franczak, B.Franzke, J.Gerl, E.Greda, M.Hausmann, G.Jones, A.Kishada, O.Klepper, H.-J.Kluge, R.Knobel, R.Koyama, K.L.Kratz, E.Kulich, N.Kuzminchuk, Z.Liu, S.Mandal, F.Montes, G.Munzenberg, F.Nolden, T.Ohtsubo, A.Ostrowski, A.Ozawa, Z.Patyk, B.Pfeiffer, Z.Podolyak, M.Portillo, R.Propri, T.Radon, S.Rigby, N.Saito, T.Saito, H.Schatz, M.Shindo, J.Stadlmann, M.Steck, K.Summerer, T.Suzuki, P.Ugorowski, D.Vieira, P.M.Walker, S.Watanabe, S.Williams, M.Winkler, T.Winkler, H.-J.Wollersheim, H.Wollnik, T.Yamaguchi GSI 2005-1, p.79 (2005)

Direct Mass Measurements of Stored exotic Nuclei

2005 L006 S.J.Lokitz, G.E.Mitchell, J.F.Shriner, Jr. - Phys.Rev. C 71, 064315 (2005)

Test of ${ }^{45} \mathrm{Sc}$ level densities with proton resonances 
2005Lu06 Y.-W.Lui, D.H.Youngblood, H.L.Clark, Y.Tokimoto, B.John - Acta Phys.Pol. B36, $1107(2005)$

Giant monopole resonance in $\mathrm{Cd}$ and $\mathrm{Sn}$ isotopes

2005LU07 S.Lunardi - Acta Phys.Pol. B36, 1301 (2005)

Investigation of neutron-rich nuclei with the clover array and the PRISMA magnetic spectrometer

2005LU21 Y.X.Luo, J.O.Rasmussen, I.Stefanescu, A.Gelberg, J.H.Hamilton, A.V.Ramayya, J.K.Hwang, S.J.Zhu, P.M.Gore, D.Fong, E.F.Jones, S.C.Wu, I.Y.Lee, T.N.Ginter, W.C.Ma, G.M.Ter-Akopian, A.V.Daniel, M.A.Stoyer, R.Donangelo J.Phys.(London) G31, 1303 (2005)

Shape trends and triaxiality in neutron-rich odd-mass $\mathrm{Y}$ and $\mathrm{Nb}$ isotopes

2005LU24 Y.X.Luo, J.O.Rasmussen, J.H.Hamilton, A.V.Ramayya, A.Gelberg, I.Stefanescu, J.K.Hwang, S.J.Zhu, P.M.Gore, D.Fong, E.F.Jones, S.C.Wu, I.Y.Lee, T.N.Ginter, W.C.Ma, G.M.Ter-Akopian, A.V.Daniel, M.A.Stoyer, R.Donangelo - Eur.Phys.J. A 25, Supplement 1, 469 (2005)

Shape transitions and triaxiality in neutron-rich odd-mass $\mathrm{Y}$ and $\mathrm{Nb}$ isotopes

2005MA03 P.Mason, N.Marginean, S.M.Lenzi, M.Ionescu-Bujor, F.Della Vedova, D.R.Napoli, T.Otsuka, Y.Utsuno, F.Nowacki, M.Axiotis, D.Bazzacco, P.G.Bizzeti, A.Bizzeti-Sona, F.Brandolini, M.Cardona, G.de Angelis, E.Farnea, A.Gadea, D.Hojman, A.Iordachescu, C.Kalfas, Th.Kroll, S.Lunardi, T.Martinez,

C.M.Petrache, B.Quintana, R.V.Ribas, C.Rossi Alvarez, C.A.Ur, R.Vlastou, S.Zilio - Phys.Rev. C 71, 014316 (2005)

High spin structure of ${ }^{34} \mathrm{~S}$ and the proton-neutron coupling of intruder states

2005Ma10 H.-L.Ma - Chinese Physics 14, 511 (2005)

Measurement of hyperfine structure and isotope shifts in the $580.56 \mathrm{~nm}$ line of $142-145,146,148,150 \mathrm{Nd}^{+}$

2005MA13 F.E.Maas, K.Aulenbacher, S.Baunack, L.Capozza, J.Diefenbach, B.Glaser, Y.Imai, T.Hammel, D.von Harrach, E.-M.Kabuss, R.Kothe, J.H.Lee, A.Sanchez-Lorente, E.Schilling, D.Schwaab, G.Stephan, G.Weber, C.Weinrich, I.Altarev, J.Arvieux, M.Elyakoubi, R.Frascaria, R.Kunne, M.Morlet, S.Ong, J.Van de Wiele, S.Kowalski, R.Suleiman, S.Taylor - Phys.Rev.Lett. 94, 082001 (2005)

Measurement of the Transverse Beam Spin Asymmetry in Elastic Electron-Proton Scattering and the Inelastic Contribution to the Imaginary Part of the Two-Photon Exchange Amplitude

2005MA19 F.E.Maas, for the A4 Collaboration - Eur.Phys.J. A 24, Supplement 2, 47 (2005)

Parity violating electron scattering at the MAMI facility in Mainz The strangeness contribution to the form factors of the nucleon 
2005MA25 F.E.Maas, K.Aulenbacher, S.Baunack, L.Capozza, J.Diefenbach, B.Glaser, T.Hammel, D.von Harrach, Y.Imai, E.-M.Kabuss, R.Kothe, J.H.Lee, A.Lorente, E.Schilling, D.Schwaab, M.Sikora, G.Stephan, G.Weber, C.Weinrich, I.Altarev, J.Arvieux, M.El-Yakoubi, R.Frascaria, R.Kunne, M.Morlet, S.Ong, J.van de Wiele, S.Kowalski, B.Plaster, R.Suleiman, S.Taylor - Phys.Rev.Lett. 94, 152001 (2005) Evidence for Strange-Quark Contributions to the Nucleon's Form Factors at $\mathrm{Q}^{2}=0.108(\mathrm{GeV} / \mathrm{c})^{2}$

2005MA44 F.E.Maas, for the A4-Collaboration - Prog.Part.Nucl.Phys. 55, 320 (2005) The Mainz A4 parity violation experiment (single spin asymmetries in elastic scattering)

2005MA45 T.Maruta, S.Ajimura, K.Aoki, A.Banu, H.Bhang, T.Fukuda, O.Hashimoto, J.I.Hwang, S.Kameoka, B.H.Kang, E.H.Kim, J.H.Kim, M.J.Kim, Y.Miura, Y.Miyake, T.Nagae, M.Nakamura, S.N.Nakamura, H.Noumi, S.Okada, Y.Okayasu, H.Outa, H.Park, P.K.Saha, Y.Sato, M.Sekimoto, T.Takahashi, H.Tamura, K.Tanida, A.Toyoda, K.Tsukada, T.Watanabe, H.J.Yim - Nucl.Phys. A754, 168c (2005)

Proton asymmetry in non-mesonic weak decay of light hypernuclei

2005MA48 J.W.Martin, for the $G^{0}$ Collaboration - Nucl.Phys. A755, 245c (2005)

The Strange Form Factors of the Proton and the $G^{0}$ Experiment

2005MA51 G.K.Mabala, E.Gueorguieva, J.F.Sharpey-Schafer, M.Benatar, R.W.Fearick, K.I.Korir, J.J.Lawrie, S.M.Mullins, S.H.T.Murray, N.J.Ncapayi, R.T.Newman, D.G.Roux, F.D.Smit, R.Wyss - Eur.Phys.J. A 25, 49 (2005)

Shears band with a large dynamic moment of inertia in ${ }^{197} \mathrm{Bi}$

2005MA52 H.Makii, K.Mishima, M.Segawa, E.Sano, H.Ueda, T.Shima, Y.Nagai, M.Igashira, T.Ohsaki - Nucl.Instrum.Methods Phys.Res. A547, 411 (2005)

Measurement system of the $\gamma$-ray angular distributions of the ${ }^{12} \mathrm{C}(\alpha, \gamma){ }^{16} \mathrm{O}$ reaction

2005Ma55 N.Marginean, C.Rusu, D.Bucurescu, C.Rossi Alvarez, C.A.Ur, D.Bazzacco, S.Lunardi, P.Pavan, G.de Angelis, M.Axiotis, E.Farnea, A.Gadea, M.Ionescu-Bujor, A.Iordachescu, W.Krolas, Th.Kroll, S.M.Lenzi, T.Martinez, R.Menegazzo, D.R.Napoli, B.Quintana, P.Spolaore, Y.H.Zhang, J.Wrzesinski - Phys.Rev. C 72, 014302 (2005); Erratum Phys.Rev. C 72, 039903 (2005)

Identification of excited states and shell model description of the $\mathrm{N}=\mathrm{Z}+1$ nucleus ${ }^{91} \mathrm{Rh}$

2005MA59 C.Mazzocchi, R.Grzywacz, J.C.Batchelder, C.R.Bingham, D.Fong, J.H.Hamilton, J.K.Hwang, M.Karny, W.Krolas, S.N.Liddick, A.F.Lisetskiy, A.C.Morton, P.F.Mantica, W.F.Mueller, K.P.Rykaczewski, M.Steiner, A.Stolz, J.A.Winger Phys.Lett. B 622, 45 (2005)

Low energy structure of even-even $\mathrm{Ni}$ isotopes close to ${ }^{78} \mathrm{Ni}$

2005Ma69 H.Makii, Y.Nagai, T.Shima, M.Segawa, H.Ueda, T.Masaki, K.Mishima, M.Igashira, T.Ohsaki, S.Okabe - Nucl.Phys. A758, 371c (2005)

Measurement of the ${ }^{12} \mathrm{C}(\alpha, \gamma){ }^{16} \mathrm{O}$ Reaction Cross Section Using Pulsed $\alpha$ Beams

2005MA71 C.Matei, C.R.Brune - Nucl.Phys. A758, 403c (2005) 
Branching ratio measurements of the $7.12-\mathrm{MeV}$ state in ${ }^{16} \mathrm{O}$

2005MA73 S.Marrone, for the n_TOF Collaboration - Nucl.Phys. A758, 533c (2005) Measurement of the ${ }^{151} \mathrm{Sm}(\mathrm{n}, \gamma){ }^{152} \mathrm{Sm}$ cross section at n_TOF

2005MA81 H.Mach, P.M.Walker, R.Julin, M.Leino, S.Juutinen, M.Stanoiu, Zs.Podolyak, R.Wood, A.M.Bruce, T.Back, J.A.Cameron, B.Cederwall, J.Ekman, B.Fogelberg, P.T.Greenlees, M.Hellstrom, P.Jones, W.Klamra, K.Lagergren, A.-P.Leppanen, P.Nieminen, R.Orlandi, J.Pakarinen, P.Rahkila, D.Rudolph, G.Simpson, J.Uusitalo, C.Wheldon - J.Phys.(London) G31, S1421 (2005)

Application of ultra-fast timing techniques to the study of exotic and weakly produced nuclei

2005MA84 P.Mason, G.Benzoni, A.Bracco, F.Camera, B.Million, O.Wieland, S.Leoni, A.K.Singh, A.Al-Khatib, H.Hubel, P.Bringel, A.Burger, A.Neusser, G.Schonwasser, G.B.Hagemann, C.R.Hansen, B.Herskind, G.Sletten, A.Algora, Zs.Dombradi, J.Gal, G.Kalinka, J.Molnar, B.M.Nyako, D.Sohler, J.Timar, L.Zolnai, M.Kmiecik, A.Maj, J.Styczen, K.Zuber, F.Azaiez, K.Hauschild, A.Korichi, A.Lopez-Martens, J.Roccaz, S.Siem, F.Hannachi, J.N.Scheurer, P.Bednarczyk, Th.Byrski, D.Curien, O.Dorvaux, G.Duchene, B.Gall, F.Khalfallah, I.Piqueras, J.Robin, K.Juhasz, S.B.Patel, A.O.Evans, G.Rainovski, C.M.Petrache, D.Petrache, G.La Rana, R.Moro, G.De Angelis, P.Fallon, I.-Y.Lee, J.C.Lisle, B.Cederwall, K.Lagergren, R.M.Lieder, E.Podsvirova, W.Gast, H.Jager, N.Redon, A.Gorgen - J.Phys.(London) G31, S1729 (2005)

Octupole signatures in ${ }^{124,125} \mathrm{Ba}$

2005MA86 F.Marechal, D.L.Balabanski, D.Borremans, J.-M.Daugas, F.de Oliveira Santos, P.Dessagne, G.Georgiev, J.Giovinazzo, S.Grevy, P.Himpe, C.Jollet, I.Matea, G.Neyens, F.Perrot, E.Poirier, O.Roig, M.Stanoiu, C.Stodel, J.-C.Thomas, K.Turzo, D.Yordanov, E.Caurier, F.Nowacki, A.Poves - Phys.Rev. C 72, 044314 (2005) $\beta$ decay of ${ }^{31} \mathrm{Mg}$ : Extending the "island of inversion"

2005MA90 N.L.Maidana, M.N.Takeda, M.S.Dias, M.F.Koskinas, V.R.Vanin Nucl.Instrum.Methods Phys.Res. A553, 559 (2005)

Absolute measurement of ${ }^{242 g} \mathrm{Am}$ sources activities in the ${ }^{241} \mathrm{Am}(\mathrm{n}, \gamma)$ cross-section determination-Improvement by simulation

2005MA92 N.W.Makau, T.E.Derry - Nucl.Instrum.Methods Phys.Res. A555, 31 (2005) A new way of depositing thin targets of monolayer thickness for nuclear reaction analysis: A case of the $550 \mathrm{keV}{ }^{13} \mathrm{C}(\mathrm{p}, \gamma){ }^{14} \mathrm{~N}$ resonant nuclear reaction

2005MA93 P.F.Mantica, S.N.Liddick, B.E.Tomlin - Nucl.Instrum.Methods Phys.Res. B241, 195 (2005)

Beta-decay studies of neutron-rich nuclei produced using projectile fragmentation at the NSCL

2005MA95 C.Mazzocchi, R.Grzywacz, J.C.Batchelder, C.R.Bingham, D.Fong, J.H.Hamilton, J.K.Hwang, M.Karny, W.Krolas, S.N.Liddick, A.C.Morton, P.F.Mantica, W.F.Mueller, K.P.Rykaczewski, M.Steiner, A.Stolz, J.A.Winger - Eur.Phys.J. A 25, Supplement 1, 93 (2005) 
Beta-delayed $\gamma$ and neutron emission near the double shell closure at ${ }^{78} \mathrm{Ni}$

2005 MA96 H.Mach, L.M.Fraile, O.Tengblad, R.Boutami, C.Jollet, W.A.Plociennik, D.T.Yordanov, M.Stanoiu, M.J.G.Borge, P.A.Butler, J.Cederkall, Ph.Dessagne, B.Fogelberg, H.Fynbo, P.Hoff, A.Jokinen, A.Korgul, U.Koster, W.Kurcewicz, F.Marechal, T.Motobayashi, J.Mrazek, G.Neyens, T.Nilsson, S.Pedersen, A.Poves, B.Rubio, E.Ruchowska, and the ISOLDE Collaboration - Eur.Phys.J. A 25, Supplement 1, 105 (2005)

New structure information on ${ }^{30} \mathrm{Mg},{ }^{31} \mathrm{Mg}$ and ${ }^{32} \mathrm{Mg}$

2005MAZL M.Mahgoub, A.Bergmaier, D.Bucurescu, T.Behrens, G.Dollinger, T.Faestermann, R.Gernhauser, R.Hertenberger, Th.Kroll, R.Krucken, P.Maierbeck, F.Nebel, M.Schlarb, H.-F.Wirth - Maier-Leibnitz-Laboratorium 2004 Ann.Rept., p.9 (2005) One and two Neutron Transfer Reactions in Normal and Inverse Kinematics

2005MAZM P.Maierbeck, T.Behrens, A.Bergmaier, M.Bohmer, T.Faestermann, R.Gernhauser, R.Hertenberger, Th.Kroll, R.Krucken, R.Lutter, M.Mahgoub, L.Maier, S.Nimmrichter, B.Sailer, M.Schlarb, H.-F.Wirth, T.Nilsson, M.Pantea, A.Richter, G.Schrieder, S.Volz, N.Braun, B.Bruyneel, T.Striepling, P.Reiter, A.Wiens, N.Warr, and the MINIBALL Collaboration - Maier-Leibnitz-Laboratorium 2004 Ann.Rept., p.8 (2005)

Study of ${ }^{49} \mathrm{Ca}$ in One-Neutron Transfer Reactions

2005MAZN Y.Maeda, K.Fujita, M.B.Greenfield, Y.Hagiwara, K.Hatanaka, M.Hatano, K.Itoh, H.Kamada, J.Kamiya, T.Kawabata, H.Kuboki, T.Kudoh, Y.Nagasue, H.Okamura, T.Saito, H.Sakai, Y.Sakemi, M.Sasano, K.Sekiguchi, Y.Shimizu, K.Suda, A.Tamii, T.Wakasa, K.Yako - CNS-REP-66, p.38 (2005)

Measurements of the nd Elastic Scattering at $250 \mathrm{MeV}$ and Three Nucleon Force Effects

2005MAZ0 N.L.Maidana, V.R.Vanin, R.M.Castro, P.R.Pascholati, O.Helene, M.S.Dias, M.F.Koskinas - Proc.Intern.Conf.Nuclear Data for Science and Technology, Santa Fe, New Mexico, 26 September-1 October, 2004, R.C.Haight, M.B.Chadwick, T.Kawano, P.Talou, Eds., Vol.2, p. 1466 (2005); AIP Conf.Proc. 769 (2005)

${ }^{232} \mathrm{Th}(\mathrm{n}, \gamma)^{233} \mathrm{Th}$ Thermal Reaction Cross-Section Measurement

2005MAZP W.Mannhart, D.Schmidt - Proc.Intern.Conf.Nuclear Data for Science and Technology, Santa Fe, New Mexico, 26 September-1 October, 2004, R.C.Haight, M.B.Chadwick, T.Kawano, P.Talou, Eds., Vol.1, p. 609 (2005); AIP Conf.Proc. 769 (2005)

Measurement of Neutron Reaction Cross Sections between 8 and $14 \mathrm{MeV}$

2005MAZQ T.N.Massey, S.M.Grimes, M.T.Wenner, A.Haghighat, J.M.Adams, A.D.Carlson Proc.Intern.Conf.Nuclear Data for Science and Technology, Santa Fe, New Mexico, 26 September-1 October, 2004, R.C.Haight, M.B.Chadwick, T.Kawano, P.Talou, Eds., Vol.1, p. 480 (2005); AIP Conf.Proc. 769 (2005)

Measurement of Neutron Transmission Through Iron Spheres 
2005MAZX C.Mazzocchi, R.Grzywacz, J.C.Batchelder, C.R.Bingham, D.Fong, J.H.Hamilton, J.K.Hwang, M.Karny, W.Krolas, S.N.Liddick, P.F.Mantica, A.C.Morton, W.F.Mueller, K.P.Rykaczewski, M.Steiner, A.Stolz, J.A.Winger - Proc.Nuclei at the Limits, Argonne, Illinois, D.Seweryniak And T.L.Khoo, eds., p.164 (2005); AIP Conf. Proc 764 (2005)

Isomer And Beta-Decay Studies Of Nuclei Near ${ }^{78} \mathrm{Ni}$

2005MC01 E.A.McCutchan, N.V.Zamfir, R.F.Casten, M.A.Caprio, H.Ai, H.Amro, C.W.Beausang, A.A.Hecht, D.A.Meyer, J.J.Ressler - Phys.Rev. C 71, 024309 (2005) $\gamma$-ray spectroscopy of ${ }^{166} \mathrm{Hf}: \mathrm{X}(5)$ in $\mathrm{N}>90$ ?

2005MC12 P.McEwan, M.Freer, N.Ashwood, N.Curtis, D.Price, V.Ziman, H.G.Bohlen, Tz.Kokalova, Ch.Schulz, R.Torabi, W.von Oertzen, C.Wheldon, T.N.Massey, R.Kalpakchieva, W.Catford - J.Phys.(London) G31, S1921 (2005)

Investigation into the structure of ${ }^{14} \mathrm{C}$ using recoil co-incidence techniques

2005MC13 C.J.McKay, J.N.Orce, S.R.Lesher, D.Bandyopadhyay, M.T.McEllistrem, C.Fransen, J.Jolie, A.Linnemann, N.Pietralla, V.Werner, S.W.Yates - Eur.Phys.J. A 25, Supplement 1, 377 (2005); Eur.Phys.J. A 25, Supplement 1, 773 (2005)

Identification of mixed-symmetry states in odd-A ${ }^{93} \mathrm{Nb}$

2005ME01 A.K.M.M.H.Meaze, H.Ahmed, G.Kim, D.Son, Y.S.Lee, H.Kang, M-H.Cho, I.S.Ko, W.Namkung, T.-I.Ro, W.-C.Chung, Y.-A.Kim, K.J.Yoo, J.H.Chang - J.Korean Phys.Soc. 46, 401 (2005)

Measurement of the Total Neutron Cross-Sections and the Resonance Parameters of Natural Hafnium at the Pohang Neutron Facility

2005ME03 Z.E.Meziani, for the polarized 3He Collaboration - Eur.Phys.J. A 24, Supplement 1, $153(2005)$

$\mathrm{Q}^{2}$ evolution of ${ }^{3} \mathrm{He}$ spin structure functions moments

2005ME05 S.Yu.Mezhevych, A.T.Rudchik, K.Rusek, A.Budzanowski, B.Czech, J.Choinski, L.Glowacka, S.Kliczewski, E.I.Koshchy, V.M.Kyryanchuk, A.V.Mokhnach, A.A.Rudchik, S.B.Sakuta, R.Siudak, I.Skwirczynska, A.Szczurek - Nucl.Phys. A753, $13(2005)$

Excitation of ${ }^{14} \mathrm{C}$ by $45 \mathrm{MeV}{ }^{11} \mathrm{~B}$ ions

2005ME09 A.A.Mehmandoost-Khajeh-Dad, H.R.Amir-Ahmadi, J.C.S.Bacelar, A.M.van den Berg, R.Castelijns, A.Deltuva, E.D.van Garderen, W.Glockle, J.Golak, N.Kalantar-Nayestanaki, H.Kamada, M.Kis, R.Koohi-Fayegh-Dehkordi, H.Lohner, M.Mahjour-Shafiei, H.Mardanpour, J.G.Messchendorp, A.Nogga, P.Sauer, S.V.Shende, R.Skibinski, H.Witala, H.J.Wortche - Phys.Lett. B 617, 18 (2005) Spin observables in deuteron-proton radiative capture at intermediate energies

2005ME19 D.A.Meyer, G.Graw, R.Hertenberger, H.-F.Wirth, R.F.Casten, P.von Brentano, D.Bucurescu, S.Heinze, J.L.Jerke, J.Jolie, R.Krucken, M.Mahgoub, P.Pejovic, O.Moller, D.Mucher, C.Scholl - J.Phys.(London) G31, S1399 (2005)

Systematic exploration of $0^{+}$states in structurally diverse nuclei 
2005MEZY P.Mermod, J.Blomgren, B.Bergenwall, A.Hildebrand, C.Johansson, J.Klug, L.Nilsson, N.Olsson, M.Osterlund, S.Pomp, U.Tippawan, O.Jonsson, A.Prokofiev, P.-U.Renberg, P.Nadel-Turonski, Y.Maeda, H.Sakai, A.Tamii -

Proc.Intern.Conf.Nuclear Data for Science and Technology, Santa Fe, New Mexico, 26 September-1 October, 2004, R.C.Haight, M.B.Chadwick, T.Kawano, P.Talou, Eds., Vol.1, p. 688 (2005); AIP Conf.Proc. 769 (2005)

Investigation of Three-Body Force Effects in Neutron-Deuteron Scattering at 95 $\mathrm{MeV}$

2005MEZZ A.A.Mehmandoost-Khajeh-Dad, H.R.Amir-Ahmadi, J.C.S.Bacelar, A.M.van den Berg, R.Castelijns, A.Deltuva, E.D.van Garderen, W.Glockle, J.Golak, N.Kalantar-Nayestanaki, H.Kamada, M.Kis, R.Koohi-Fayegh-Dehkordi, H.Lohner, M.Mahjour-Shafiei, H.Mardanpur, J.G.Messchendorp, A.Nogga, P.Sauer, S.V.Shende, R.Skibinski, H.Witala, H.J.Wortche - nucl-ex/0501012,1/17/2005 (2005)

Spin observables in deuteron-proton radiative capture at intermediate energies

2005MI13 M.Milin, M.Zadro, S.Cherubini, T.Davinson, A.Di Pietro, P.Figuera, D.Miljanic, A.Musumarra, A.Ninane, A.N.Ostrowski, M.G.Pellegriti, A.C.Shotter, N.Soic, C.Spitaleri - Nucl.Phys. A753, 263 (2005)

Sequential decay reactions induced by a $18 \mathrm{MeV}^{6} \mathrm{He}$ beam on ${ }^{6} \mathrm{Li}$ and ${ }^{7} \mathrm{Li}$

2005MI19 Y.Miura, S.Ajimura, Y.Fujii, T.Fukuda, O.Hashimoto, H.Hotchi, K.Imai, W.Imoto, Y.Kakiguchi, S.Kameoka, A.Krutenkova, T.Maruta, A.Matsumura, K.Miwa, K.Mizunuma, S.N.Nakamura, T.Nagae, H.Nomura, H.Noumi, H.Outa, P.K.Saha, T.Saitoh, Y.Sato, M.Sekimoto, T.Takahashi, H.Tamura, K.Tanida, A.Toyoda, M.Ukai, H.Yamauchi - Nucl.Phys. A754, 75c (2005)

$\gamma$ spectroscopy of ${ }_{\Lambda}^{11} \mathrm{~B}$

2005MI20 K.Miwa, K.Tanida, H.Akikawa, Y.Fukao, E.Hiyama, H.Hotchi, K.Imai, Y.Miura, K.Mizunuma, S.N.Nakamura, M.Niiyama, S.Ota, P.K.Saha, H.Takahashi, T.Takahashi, H.Tamura, S.Terashima, M.Togawa, M.Ukai - Nucl.Phys. A754, 80c (2005) High-resolution $\gamma$-ray spectroscopy of hyperfragments produced by stopped $\mathrm{K}^{-}$ reactions

2005MI28 T.Mitsui, R.Masuda, S.Kitao, M.Seto - J.Phys.Soc.Jpn. 74, 3122 (2005) Nuclear Resonant Scattering of Synchrotron Radiation by ${ }^{158} \mathrm{Gd}$

2005MI32 S.Michimasa, S.Shimoura, H.Iwasaki, M.Tamaki, S.Ota, N.Aoi, H.Baba, N.Iwasa, S.Kanno, S.Kubono, K.Kurita, M.Kurokawa, T.Minemura, T.Motobayashi, M.Notani, H.J.Ong, A.Saito, H.Sakurai, S.Takeuchi, E.Takeshita, Y.Yanagisawa, A.Yoshida - Eur.Phys.J. A 25, Supplement 1, 367 (2005)

Study of single-particle states in ${ }^{23} \mathrm{~F}$ using proton transfer reaction

2005MIZT S.Michimasa, S.Shimoura, H.Iwasaki, M.Tamaki, S.Ota, N.Aoi, H.Baba, N.Iwasa, S.Kanno, S.Kubono, K.Kurita, M.Kurokawa, T.Minemura, T.Motobayashi, M.Notani, H.J.Ong, A.Saito, H.Sakurai, E.Takeshita, S.Takeuchi, Y.Yanagisawa, A.Yoshida - CNS-REP-67 (2005)

Proton Single-Particle States in the Neutron-rich ${ }^{23}$ F Nucleus 
2005MIZU S.Michimasa, S.Shimoura, H.Iwasaki, M.Tamaki, S.Ota, N.Aoi, H.Baba, N.Iwasa, S.Kanno, S.Kubono, K.Kurita, M.Kurokawa, T.Minemura, T.Motobayashi, M.Notani, H.J.Ong, A.Saito, H.Sakurai, E.Takeshita, S.Takeuchi, Y.Yanagisawa, A.Yoshida - CNS-REP-66, p.26 (2005)

Proton Single Particle States in ${ }^{23} \mathrm{~F}$

2005MIZV S.Michimasa, S.Shimoura, H.Iwasaki, M.Tamaki, S.Ota, N.Aoi, H.Baba, N.Iwasa, S.Kanno, S.Kubono, K.Kurita, M.Kurokawa, T.Minemura, T.Motobayashi, M.Notani, H.J.Ong, A.Saito, H.Sakurai, S.Takeuchi, E.Takeshita, Y.Yanagisawa, A.Yoshida - RIKEN Accelerator Progress Report 2004, p.51 (2005)

Proton single particle states in ${ }^{23} \mathrm{~F}$

2005MIZW K.Miernik, L.Batist, A.Blazhev, T.Faestermann, M.Gorska, H.Grawe, Z.Janas, M.Karny, M.Kavatsyuk, O.Kavatsyuk, R.Kirchner, M.La Commara, C.Mazzocchi, I.Mukha, C.Plettner, A.Plochocki, E.Roeckl, K.Rykaczewski, K.Schmidt - GSI 2005-1, p.84 (2005)

Beta decay studies of ${ }^{106} \mathrm{Sb}$ and ${ }^{107} \mathrm{Sb}$

2005MIZX I.Miyazaki, T.Shimizu, M.Shibata, K.Kawade, A.Taniguchi, H.Harada, K.Furutaka, H.Sakane - Proc.Intern.Conf.Nuclear Data for Science and Technology, Santa Fe, New Mexico, 26 September-1 October, 2004, R.C.Haight, M.B.Chadwick, T.Kawano, P.Talou, Eds., Vol.1, p. 996 (2005); AIP Conf.Proc. 769 (2005) Reliability of Prompt $\gamma$-Ray Intensities for the Measurement of Neutron Capture Cross Sections

2005MIZY L.C.Mihailescu, C.Borcea, A.J.M.Plompen - Proc.Intern.Conf.Nuclear Data for Science and Technology, Santa Fe, New Mexico, 26 September-1 October, 2004, R.C.Haight, M.B.Chadwick, T.Kawano, P.Talou, Eds., Vol.1, p. 973 (2005); AIP Conf.Proc. 769 (2005)

Measurement of Neutron Inelastic Scattering Cross Sections for ${ }^{209} \mathrm{Bi}$ from Threshold up to $18 \mathrm{MeV}$

2005MIZZ R.Michel, W.Glasser, U.Herpers, H.Schuhmacher, H.J.Brede, V.Dangendorf, R.Nolte, P.Malmborg, A.V.Prokofiev, A.N.Smirnov, I.Rishkov, D.Kollar, J.P.Meulders, M.Duijvestijn, A.Koning - Proc.Intern.Conf.Nuclear Data for Science and Technology, Santa Fe, New Mexico, 26 September-1 October, 2004, R.C.Haight, M.B.Chadwick, T.Kawano, P.Talou, Eds., Vol.1, p. 861 (2005); AIP Conf.Proc. 769 (2005)

Residual Nuclide Production from Iron, Lead, and Uranium by Neutron-Induced Reactions up to $180 \mathrm{MeV}$

2005 M004 S.A.Morrow, J.Arneil, E.C.Aschenauer, M.F.van Batenburg, H.P.Blok, D.J.Boersma, D.Branford, T.Davinson, G.DeMeyer, J.E.Ducret, W.H.A.Hesselink, D.Groep, K.Hicks, D.G.Ireland, N.Kalantar-Nayestanaki, L.Lapikas, M.Liang, J.Mackenzie, C.Marchand, R.Medaglia, J.Ryckebusch, M.van Sambeek, R.Starink, G.van der Steenhoven, M.A.van Uden, H.de Vries - Phys.Rev. C 71, 014607 (2005) Comparison between the transverse responses of the reactions ${ }^{12} \mathrm{C}\left(\mathrm{e}, \mathrm{e}^{\prime} \mathrm{p}\right){ }^{11} \mathrm{~B}$ and ${ }^{12} \mathrm{C}(\gamma, \mathrm{p}){ }^{11} \mathrm{~B}$ 
2005M007 K.Morita, H.Miyahara, Y.Ogata, K.Katoh - Nucl.Instrum.Methods Phys.Res. A540, $324(2005)$

Emission probability measurement of $\gamma$-ray of ${ }^{105} \mathrm{Rh}$

2005M020 O.Moller, N.Warr, J.Jolie, A.Dewald, A.Fitzler, A.Linnemann, K.O.Zell, P.E.Garrett, S.W.Yates - Phys.Rev. C 71, 064324 (2005)

E2 transition probabilities in ${ }^{114} \mathrm{Te}$ : A conundrum

2005M028 J.Y.Moon, C.S.Lee, J.H.Lee, C.C.Yun, J.C.Kim, M.Youn, S.Kubono, T.Teranishi, J.J.He, M.Notani, S.Nishimura, M.Nishimura, V.Guimaraes, R.F.Lichtenthaler, S.Kato - Nucl.Phys. A758, 158c (2005)

Study of proton resonances in ${ }^{26} \mathrm{Si}$ and ${ }^{27} \mathrm{P}$ by using the ${ }^{25} \mathrm{Al}$ and ${ }^{26} \mathrm{Si}$ radioactive ion beams

2005M030 F.Montes, H.Schatz, A.Aprahamian, O.Arndt, A.Estrade, K.-L.Kratz, S.N.Liddick, P.F.Mantica, W.F.Mueller, P.Hosmer, A.C.Morton, M.Ouellette, E.Pellegrini, B.Pfeiffer, P.Reeder, P.Santi, A.Stolz, B.E.Tomlin, W.B.Walters, A.Wohr Nucl.Phys. A758, 643c (2005)

$\beta$-Decay Studies Close to the $\mathrm{N}=82 \mathrm{r}$-process Path

2005M033 O.Moller, P.Petkov, B.Melon, A.Dewald, A.Fitzler, J.Jolie, D.Tonev, S.Christen, B.Saha, K.O.Zell, M.Heidemann - Phys.Rev. C 72, 034306 (2005)

Lifetimes of the first excited $2^{+}$states in ${ }^{176,178,180} \mathrm{Os}$

2005MOZR J.Y.Moon, C.S.Lee, J.H.lee, C.C.Yun, J.C.Kim, M.Youn, S.Kubono, T.Teranishi, J.J.He, M.Notani, S.Nishimura, M.Nishimura, V.Guimaraes, R.F.Lichtenthaler, S.Kato - CNS-REP-66, p.6 (2005)

Study of the Proton Resonance States in ${ }^{27} \mathrm{P}$ by using a Radioactive Beam of ${ }^{26} \mathrm{Si}$

2005MOZS K.Morita, K.Morimoto, D.Kaji, T.Akiyama, S.Goto, H.Haba, E.Ideguchi, R.Kanungo, K.Katori, H.Koura, H.Kudo, T.Ohnishi, A.Ozawa, T.Suda, K.Sueki, H.Xu, T.Yamaguchi, A.Yoneda, A.Yoshida, Y.-L.Zhao - RIKEN Accelerator Progress Report 2004, p.70 (2005)

Experiment on synthesis of element 113 in reaction ${ }^{209} \mathrm{Bi}\left({ }^{70} \mathrm{Zn}, \mathrm{n}\right){ }^{278} 113$

2005MOZT K.Morita, K.Morimoto, D.Kaji, T.Akiyama, S.Goto, H.Haba, E.Ideguchi, H.Koura, H.Kudo, T.Ohnishi, A.Ozawa, T.Suda, K.Sueki, H.Xu, T.Yamaguchi, A.Yoneda, A.Yoshida, Y.-L.Zhao - RIKEN Accelerator Progress Report 2004, p.69 (2005) Experiments on synthesis of isotope ${ }^{277} 112$ by ${ }^{208} \mathrm{~Pb}+{ }^{70} \mathrm{Zn}$ reaction

2005MOZU J.Y.Moon, C.-S.Lee, J.-H.Lee, C.C.Yun, J.C.Kim, M.Youn, S.Kubono, T.Teranishi, J.J.He, M.Notani, S.Nishimura, M.Kurata-Nishimura, S.Kato, V.Guimaraes, R.F.Lichtenthaler - RIKEN Accelerator Progress Report 2004, p.63 (2005) Study of astrophysically important resonance states in ${ }^{26} \mathrm{Si}$ and ${ }^{27} \mathrm{P}$ using radioactive ion beams

2005MOZV M.Mosconi, and the n_TOF Collaboration - Proc.Intern.Conf.Nuclear Data for Science and Technology, Santa Fe, New Mexico, 26 September-1 October, 2004, R.C.Haight, M.B.Chadwick, T.Kawano, P.Talou, Eds., Vol.2, p. 1335 (2005); AIP Conf.Proc. 769 (2005) 
Neutron Capture Cross Sections for the Re / Os Clock

2005MOZW C.Moreau, and the n-TOF Collaboration - Proc.Intern.Conf.Nuclear Data for Science and Technology, Santa Fe, New Mexico, 26 September-1 October, 2004, R.C.Haight, M.B.Chadwick, T.Kawano, P.Talou, Eds., Vol.1, p. 880 (2005); AIP Conf.Proc. 769 (2005)

Measurement of Capture Cross Sections of ${ }^{90,91,92,94,96} \mathrm{Zr}$ Isotopes at n_TOF

2005MozZ J.Y.Moon, C.S.Lee, J.H.Lee, C.C.Yun, J.C.Kim, M.Youn, S.Kubono, T.Teranishi, J.J.He, M.Notani, S.Nishimura, M.Nishimura, V.Guimaraes, R.F.Lichtenthaler, S.Kato - Proc. Intern. Symposium on the Origin of Matter and Evolution of Galaxies, Riken, Saitama, Japan, 17-19 November 2003, World Scientific, Singapore, p.505 (2005)

Study of proton resonances in ${ }^{26} \mathrm{Si}$ and ${ }^{27} \mathrm{P}$ by the elastic scattering of ${ }^{1} \mathrm{H}\left({ }^{25} \mathrm{Al}\right.$, p $)^{25} \mathrm{Al},{ }^{1} \mathrm{H}\left({ }^{26} \mathrm{Si}, \mathrm{p}\right){ }^{26} \mathrm{Si}$

2005MU15 I.Mukha, E.Roeckl, J.Doring, L.Batist, A.Blazhev, H.Grawe, C.R.Hoffman, M.Huyse, Z.Janas, R.Kirchner, M.La Commara, C.Mazzocchi, C.Plettner, S.L.Tabor, P.Van Duppen, M.Wiedeking - Phys.Rev.Lett. 95, 022501 (2005) Observation of Proton Radioactivity of the $\left(21^{+}\right)$High-Spin Isomer in ${ }^{94} \mathrm{Ag}$

2005MU21 G.Muhrer, E.J.Pitcher, G.J.Russell, F.Maekawa, Y.Kasugai, H.Takada Nucl.Instrum.Methods Phys.Res. A547, 555 (2005)

Comparison of calculated and measured high energy neutron reaction rates at the manual Jr. Lujan Center Spallation Source

2005MU22 S.Mukherjee, N.L.Singh, G.Kiran Kumar, L.Chaturvedi - Phys.Rev. C 72, 014609 (2005)

Measurement and analysis of isomeric cross section ratios in the reaction ${ }^{93} \mathrm{Nb}(\alpha$, 2n) ${ }^{95}$ Tc: Pre-equilibrium reaction mechanism

2005MU26 I.Mukha, M.Kavatsyuk, A.Algora, L.Batist, A.Blazhev, J.Doring, H.Grawe, M.Hellstrom, O.Kavatsyuk, R.Kirchner, M.La Commara, C.Mazzocchi, C.Plettner, E.Roeckl - Nucl.Phys. A758, 647c (2005)

The reaction of triple radiative capture $\alpha \alpha(\mathrm{n}, \gamma){ }^{9} \mathrm{Be}$ studied in a $\beta$ decay of ${ }^{9} \mathrm{Li}$

2005MUZX I.Mukha, E.Roeckl, L.Batist, A.Blazhev, J.Doring, H.Grawe, L.Grigorenko, C.Hoffman, M.Huyse, Z.Janas, R.Kirchner, M.La Commara, C.Mazzocchi, C.Plettner, S.L.Tabor, P.Van Duppen, M.Wiedeking - GSI 2005-1, p.87 (2005) Two-proton radioactivity of ${ }^{94 m} \mathrm{Ag}\left(21^{+}\right)$

2005MUZY P.Mutti, H.Beer, A.Brusegan, F.Corvi, R.Gallino - Proc.Intern.Conf.Nuclear Data for Science and Technology, Santa Fe, New Mexico, 26 September-1 October, 2004, R.C.Haight, M.B.Chadwick, T.Kawano, P.Talou, Eds., Vol.2, p. 1327 (2005); AIP Conf.Proc. 769 (2005)

New Kr Cross Sections and Astrophysical Constraints on Presolar Grains 
2005MUZZ G.Mukherjee, T.L.Khoo, R.Blinstrup, D.Seweryniak, I.Ahmad, P.A.Butler, M.P.Carpenter, P.Chowdhury, J.A.Cizewski, C.N.Davids, S.J.Freeman, J.P.Greene, N.J.Hammond, A.Heinz, R.D.Herzberg, P.J.C.Ikin, R.V.F.Janssens, M.S.Johnson, G.D.Jones, F.G.Kondev, T.Lauritsen, C.J.Lister, E.F.Moore, E.Ngijoi-Yogo, P.Reiter, S.Sinha - Proc.Nuclei at the Limits, Argonne, Illinois, D.Seweryniak and T.L.Khoo, eds., p.243 (2005); AIP Conf. Proc 764 (2005)

Electrons From A 0.3s Isomer In ${ }^{254}$ No

2005NA05 H.Naik, S.P.Dange, R.J.Singh - Phys.Rev. C 71, 014304 (2005)

Angular momentum of fission fragments in low energy fission of actinides

2005NA08 H.Nassar, M.Paul, I.Ahmad, D.Berkovits, M.Bettan, P.Collon, S.Dababneh, S.Ghelberg, J.P.Greene, A.Heger, M.Heil, D.J.Henderson, C.L.Jiang, F.Kappeler, H.Koivisto, S.O'Brien, R.C.Pardo, N.Patronis, T.Pennington, R.Plag, K.E.Rehm, R.Reifarth, R.Scott, S.Sinha, X.Tang, R.Vondrasek - Phys.Rev.Lett. 94, 092504 (2005)

Stellar $(\mathrm{n}, \gamma)$ Cross Section of ${ }^{62} \mathrm{Ni}$

2005NA14 B.Naranjo, J.K.Gimzewski, S.Putterman - Nature(London) 434, 1115 (2005)

Observation of nuclear fusion driven by a pyroelectric crystal

2005NA15 Y.Nagai, M.Igashira, T.Takaoka, T.Kikuchi, T.Shima, A.Tomyo, A.Mengoni, T.Otsuka - Phys.Rev. C 71, 055803 (2005)

${ }^{7} \mathrm{Li}(\mathrm{n}, \gamma)^{8} \mathrm{Li}$ reaction and the $\mathrm{S}_{17}$ factor at $\mathrm{E}_{c . m .}>500 \mathrm{keV}$

2005NA28 B.S.Nara Singh, M.Hass, G.Goldring, D.Ackermann, J.Gerl, F.P.Hessberger, S.Hofmann, I.Kojouharov, P.Kuusiniemi, H.Schaffner, B.Sulignano, B.A.Brown Phys.Rev. C 72, 027303 (2005)

Parity nonconservation in the $\gamma$ decay of polarized $17 / 2^{-}$isomers in ${ }^{93} \mathrm{Tc}$

2005NA30 H.Nassar, M.Paul, S.Ghelberg, A.Ofan, N.Trubnikov, Y.Ben-Dov, M.Hass, B.S.Nara Singh - Nucl.Phys. A758, 411c (2005)

Study of the supernova nucleosynthesis ${ }^{40} \mathrm{Ca}(\alpha, \gamma){ }^{44} \mathrm{Ti}$ reaction: progress report

2005NA31 Y.Nagai, A.Tomyo, Y.Temma, M.Segawa, T.Shima, H.Makii, M.Igashira, T.Ohsaki Nucl.Phys. A758, 537c (2005)

Measurement of the keV neutron capture cross section of ${ }^{62} \mathrm{Ni}$

2005NA32 B.S.Nara Singh, M.Hass, Y.Nir-El, G.Haquin - Nucl.Phys. A758, 689c (2005)

A New Precision Measurement of the ${ }^{3} \mathrm{He}\left({ }^{4} \mathrm{He}, \gamma\right){ }^{7} \mathrm{Be}$ Cross section

2005NA36

S.Nakayama, T.Yamagata, H.Akimune, Y.Arimoto, H.Daito, H.Ejiri, H.Fujimura, Y.Fujita, M.Fujiwara, K.Fushimi, M.B.Greenfield, H.Kohri, N.Koori, K.Takahisa, T.Takeuchi, M.Tanaka, K.Yonehara, H.P.Yoshida - Phys.Rev. C 72, 041001 (2005) M1 cross section for the photodisintegration of deuterium using the ${ }^{2} \mathrm{H}\left({ }^{7} \mathrm{Li},{ }^{7} \mathrm{Be}\right)$ reaction 
2005NA37 S.Naguleswaran, R.S.Chakrawarthy, U.Garg, K.L.Lamkin, G.Smith, J.C.Walpe, A.Galindo-Uribarri, V.P.Janzen, D.C.Radford, R.Kaczarowski, D.B.Fossan, D.R.Lafosse, P.Vaska, Ch.Droste, T.Morek, S.Pilotte, J.DeGraaf, T.Drake, R.Wyss Phys.Rev. C 72, 044304 (2005)

Magnetic and intruder rotational bands in ${ }^{113} \mathrm{In}$

2005NA43 B.S.Nara Singh, M.Hass, G.Goldring, D.Ackermann, J.Gerl, F.P.Hessberger, S.Hofmann, I.Kojouharov, P.Kuusiniemi, H.Schaffner, B.Sulignano, B.A.Brown Eur.Phys.J. A 25, Supplement 1, 703 (2005)

Parity non-conservation in the $\gamma$-decay of polarized $17 / 2^{-}$isomers in ${ }^{93} \mathrm{Tc}$

2005NAZW H.Nassar, M.Paul, I.Ahmad, Y.Ben-Dov, J.Caggiano, S.Ghelberg, S.Goriely, J.P.Greene, M.Hass, A.Heger, A.Heinz, D.J.Henderson, R.V.F.Janssens, C.L.Jiang, Y.Kashiv, B.S.Nara Singh, A.Ofan, R.C.Pardo, T.Pennington, K.E.Rehm, G.Savard, R.Scott, R.Vondrasek - nucl-ex/0509006,9/03/2005 (2005)

The ${ }^{40} \mathrm{Ca}(\alpha, \gamma){ }^{44} \mathrm{Ti}$ reaction in the energy regime of supernova nucleosynthesis

2005NAZY M.Nakao, J.Hori, K.Ochiai, S.Sato, M.Yamauchi, N.S.Ishioka, T.Nishitani Proc.Intern.Conf.Nuclear Data for Science and Technology, Santa Fe, New Mexico, 26 September-1 October, 2004, R.C.Haight, M.B.Chadwick, T.Kawano, P.Talou, Eds., Vol.2, p. 1489 (2005); AIP Conf.Proc. 769 (2005)

Measurements of Deuteron-Induced Activation Cross Sections for IFMIF Accelerator Structural Material

2005NAZZ T.Nakamura, N.Fukuda - Proc. Intern. Symposium on the Origin of Matter and Evolution of Galaxies, Riken, Saitama, Japan, 17-19 November 2003, World Scientific, Singapore, p.155 (2005)

Neutron capture cross section of ${ }^{14} \mathrm{C}$ studied by intermediate-energy Coulomb dissociation

2005NC01 N.J.Ncapayi, S.M.Mullins, M.Benatar, E.Gueorgueiva, J.J.Lawrie, G.K.Mabala, S.Mukherjee, S.H.T.Murray, K.P.Mutshena, R.T.Newman, J.F.Sharpey-Schafer, F.D.Smit, P.Vymers - Eur.Phys.J. A 26, 265 (2005)

Characterization of quasiparticle states at and beyond stability in ytterbium isotopes: Spectroscopy of ${ }^{175} \mathrm{Yb},{ }^{176} \mathrm{Yb}$ and ${ }^{177} \mathrm{Yb}$

2005NE01 G.Neyens, M.Kowalska, D.Yordanov, K.Blaum, P.Himpe, P.Lievens, S.Mallion, R.Neugart, N.Vermeulen, Y.Utsuno, T.Otsuka - Phys.Rev.Lett. 94, 022501 (2005) Measurement of the Spin and Magnetic Moment of ${ }^{31} \mathrm{Mg}$ : Evidence for a Strongly Deformed Intruder Ground State

2005NE05 A.Negret, T.Adachi, G.P.A.Berg, P.von Brentano, H.Fraiquin, D.De Frenne, H.Fujita, K.Fujita, Y.Fujita, K.Hatanaka, E.Jacobs, K.Nakanishi, L.Popescu, Y.Sakemi, Y.Shimbara, Y.Shimizu, Y.Tameshige, A.Tamii, M.Uchida, M.Yosoi Phys.Rev. C 71, 047303 (2005)

Level widths of ${ }^{14} \mathrm{O}$ states studied in the high-resolution ${ }^{14} \mathrm{~N}\left({ }^{3} \mathrm{He}, \mathrm{t}\right){ }^{14} \mathrm{O}$ reaction 
2005NE14 A.Negret, T.Adachi, C.Baumer, A.M.van den Berg, G.P.A.Berg, P.von Brentano, D.Frekers, D.De Frenne, K.Fujita, Y.Fujita, E.-W.Grewe, P.Haefner, K.Hatanaka, M.Hunyadi, E.Jacobs, A.Korff, K.Nakanishi, L.Popescu, S.Rakers, Y.Sakemi, Y.Shimbara, Y.Shimizu, Y.Tameshige, A.Tamii, M.Uchida, H.J.Wortche, M.Yosoi J.Phys.(London) G31, S1931 (2005)

High resolution study of the Gamow-Teller strength distribution starting from the ground state of ${ }^{14} \mathrm{~N}$ in the $\beta^{-}$and $\beta^{+}$directions

2005NEZY R.O.Nelson, N.Fotiades, M.Devlin, J.A.Becker, P.E.Garrett, W.Younes Proc.Intern.Conf.Nuclear Data for Science and Technology, Santa Fe, New Mexico, 26 September-1 October, 2004, R.C.Haight, M.B.Chadwick, T.Kawano, P.Talou, Eds., Vol.1, p. 838 (2005); AIP Conf.Proc. 769 (2005)

Cross-Section Standards for Neutron-Induced Gamma-Ray Production in the MeV Energy Range

2005NI09 O.Niedermaier, H.Scheit, V.Bildstein, H.Boie, J.Fitting, R.von Hahn, F.Kock, M.Lauer, U.K.Pal, H.Podlech, R.Repnow, D.Schwalm, C.Alvarez, F.Ames, G.Bollen, S.Emhofer, D.Habs, O.Kester, R.Lutter, K.Rudolph, M.Pasini, P.G.Thirolf, B.H.Wolf, J.Eberth, G.Gersch, H.Hess, P.Reiter, O.Thelen, N.Warr, D.Weisshaar, F.Aksouh, P.Van den Bergh, P.Van Duppen, M.Huyse, O.Ivanov, P.Mayet, J.Van de Walle, J.Aysto, P.A.Butler, J.Cederkall, P.Delahaye, H.O.U.Fynbo, L.M.Fraile, O.Forstner, S.Franchoo, U.Koster, T.Nilsson, M.Oinonen, T.Sieber, F.Wenander, M.Pantea, A.Richter, G.Schrieder, H.Simon, T.Behrens, R.Gernhauser, T.Kroll, R.Krucken, M.Munch, T.Davinson, J.Gerl, G.Huber, A.Hurst, J.Iwanicki, B.Jonson, P.Lieb, L.Liljeby, A.Schempp, A.Scherillo, P.Schmidt, G.Walter - Nucl.Phys. A752, $273 \mathrm{c}(2005)$

The neutron-rich Mg isotopes: first results from MINIBALL at REX-ISOLDE

2005NI11 O.Niedermaier, H.Scheit, V.Bildstein, H.Boie, J.Fitting, R.von Hahn, F.Kock, M.Lauer, U.K.Pal, H.Podlech, R.Repnow, D.Schwalm, C.Alvarez, F.Ames, G.Bollen, S.Emhofer, D.Habs, O.Kester, R.Lutter, K.Rudolph, M.Pasini, P.G.Thirolf, B.H.Wolf, J.Eberth, G.Gersch, H.Hess, P.Reiter, O.Thelen, N.Warr, D.Weisshaar, F.Aksouh, P.Van den Bergh, P.Van Duppen, M.Huyse, O.Ivanov, P.Mayet, J.Van de Walle, J.Aysto, P.A.Butler, J.Cederkall, P.Delahaye, H.O.U.Fynbo, L.M.Fraile, O.Forstner, S.Franchoo, U.Koster, T.Nilsson, M.Oinonen, T.Sieber, F.Wenander, M.Pantea, A.Richter, G.Schrieder, H.Simon, T.Behrens, R.Gernhauser, T.Kroll, R.Krucken, M.Munch, T.Davinson, J.Gerl, G.Huber, A.Hurst, J.Iwanicki, B.Jonson, P.Lieb, L.Liljeby, A.Schempp, A.Scherillo, P.Schmidt, G.Walter - Phys.Rev.Lett. 94, $172501(2005)$ "Safe" Coulomb Excitation of ${ }^{30} \mathrm{Mg}$

2005NI12 N.Nica, J.C.Hardy, V.E.Iacob, J.R.Montague, M.B.Trzhaskovskaya - Phys.Rev. C 71, 054320 (2005)

Precise measurement of K-shell fluorescence yield in iridium: An improved test of internal-conversion theory

2005NI13 J.S.Nico, M.S.Dewey, D.M.Gilliam, F.E.Wietfeldt, X.Fei, W.M.Snow, G.L.Greene, J.Pauwels, R.Eykens, A.Lamberty, J.Van Gestel, R.D.Scott - Phys.Rev. C 71, $055502(2005)$ 
Measurement of the neutron lifetime by counting trapped protons in a cold neutron beam

2005NI20 B.Nilsson, J.-O.Adler, B.-E.Andersson, J.R.M.Annand, I.Akkurt, M.J.Boland, G.I.Crawford, K.G.Fissum, K.Hansen, P.D.Harty, D.G.Ireland, L.Isaksson, M.Karlsson, M.Lundin, J.C.McGeorge, G.J.Miller, H.Ruijter, A.Sandell, B.Schroder, D.A.Sims, D.Watts - Phys.Lett. B 626, 65 (2005)

Near-threshold measurement of the ${ }^{4} \mathrm{He}(\gamma, \mathrm{n})$ reaction

2005NIZT M.Niikura, E.Ideguchi, T.Fukuchi, H.Baba, N.Hokoiwa, C.Ishida, H.Iwasaki, T.Koike, T.Komatsubara, T.Kubo, M.Kurokawa, S.Michimasa, K.Miyakawa, K.Morimoto, T.Ohnishi, S.Ota, A.Ozawa, S.Shimoura, T.Suda, M.Tamaki,

I.Tanihata, Y.Wakabayashi, K.Yoshida - CNS-REP-66, p.22 (2005)

Study of High-Spin States in ${ }^{49-51} \mathrm{Ti}$

2005NIZU S.Nishimura, M.Kurata-Nishimura, H.Fujikawa, A.Guilherume, J.J.He, S.Kubono, H.Yamaguchi, T.Teranishi, Y.Wakabayashi, S.Bishop, M.Kurokawa, T.Kishida, T.Motobayashi - CNS-REP-66, p.9 (2005)

Study of Low Energy Nuclear Reaction Measurements using Monolithic Silicon Telescope at CRIB

2005NIZV M.Niikura, E.Ideguchi, T.Fukuchi, H.Baba, N.Hokoiwa, C.Ishida, H.Iwasaki, T.Koike, T.Komatsubara, T.Kubo, M.Kurokawa, S.Michimasa, K.Miyakawa, K.Morimoto, T.Ohnishi, S.Ota, A.Ozawa, S.Shimoura, T.Suda, M.Tamaki, I.Tanihata, Y.Wakabayashi, K.Yoshida - RIKEN Accelerator Progress Report 2004, p.67 (2005)

Excitation function measurement of neutron-rich Ti isotope by secondary fusion reaction

2005NIZW K.Nishio, H.Ikezoe, Y.Nagame, M.Asai, K.Tsukada, S.Mitsuoka, K.Tsuruta, K.Satou, C.J.Lin, T.Ohsawa - Proc.Intern.Conf.Nuclear Data for Science and Technology, Santa Fe, New Mexico, 26 September-1 October, 2004, R.C.Haight, M.B.Chadwick, T.Kawano, P.Talou, Eds., Vol.1, p. 977 (2005); AIP Conf.Proc. 769 (2005)

Measurement of Evaporation Residue Cross Sections for the Sub-barrier ${ }^{16} \mathrm{O}+{ }^{238} \mathrm{U}$ Reaction

2005NIZX B.Nilsson, J.-O.Adler, B.-E.Andersson, J.R.M.Annand, I.Akkurt, M.J.Boland, G.I.Crawford, K.G.Fissum, K.Hansen, P.D.Harty, D.G.Ireland, L.Isaksson, M.Karlsson, M.Lundin, J.C.McGeorge, G.J.Miller, H.Ruijter, A.Sandell, B.Schroder, D.A.Sims, D.Watts - nucl-ex/0506001,6/01/2005 (2005)

Near-threshold measurement of the ${ }^{4} \mathrm{He}(\gamma, \mathrm{n})$ reaction

2005NIZZ S.Nishimura, Y.Nishi, M.Kurata-Nishimura, I.Tanihata, M.A.Famiano, M.E.Howard, D.Reitzner, E.E.Smith, R.N.Boyd - Proc. Intern. Symposium on the Origin of Matter and Evolution of Galaxies, Riken, Saitama, Japan, 17-19 November 2003, World Scientific, Singapore, p.304 (2005)

Half-life measurement of neutron-rich nuclei and future at RIBF 
2005N001 C.Nociforo, K.L.Jones, L.H.Khiem, P.Adrich, T.Aumann, B.V.Carlson, D.Cortina-Gil, U.Datta Pramanik, Th.W.Elze, H.Emling, H.Geissel, M.Hellstrom, J.V.Kratz, R.Kulessa, T.Lange, Y.Leifels, H.Lenske, E.Lubkiewicz, G.Munzenberg, R.Palit, H.Scheit, H.Simon, K.Summerer, S.Typel, E.Wajda, W.Walus, H.Weick, and the LAND-FRS Collaboration - Phys.Lett. B 605, 79 (2005)

Coulomb breakup of ${ }^{23} \mathrm{O}$

2005N004 E.B.Norman, A.R.Smith, A.F.Barghouty, R.C.Haight, S.A.Wender - Nucl.Phys. B(Proc.Supp.) S143, 508 (2005)

Cosmic-Ray Production of ${ }^{60}$ Co in Double Beta-Decay Source Materials

2005N013 T.Noro, T.Yonemura, S.Asaji, N.S.Chant, K.Fujita, Y.Hagihara, K.Hatanaka, G.C.Hillhouse, T.Ishida, M.Itoh, S.Kishi, M.Nakamura, Y.Nagasue, H.Sakaguchi, Y.Sakemi, Y.Shimizu, H.Takeda, Y.Tamesige, S.Terashima, M.Uchida, T.Wakasa, Y.Yasuda, H.P.Yoshida, M.Yosoi - Phys.Rev. C 72, 041602 (2005)

Analyzing powers for exclusive $1 \mathrm{~s}_{1 / 2}$ proton knockout from light nuclei

2005N015 W.Nortershauser, B.A.Bushaw, A.Dax, G.W.F.Drake, G.Ewald, S.Gotte, R.Kirchner, H.-J.Kluge, Th.Kuhl, R.Sanchez, A.Wojtaszek, Z.-C.Yan, C.Zimmermann - Eur.Phys.J. A 25, Supplement 1, 199 (2005)

Measurement of the nuclear charge radii of ${ }^{8,9} \mathrm{Li}$ : The last step towards the determination of the charge radius of ${ }^{11} \mathrm{Li}$

2005NY02 B.M.Nyako, F.Papp, J.Gal, J.Molnar, J.Timar, A.Algora, Zs.Dombradi, G.Kalinka, L.Zolnai, K.Juhasz, A.K.Singh, H.Hubel, A.Al-Khatib, P.Bringel, A.Burger, A.Neusser, G.Schonwasser, B.Herskind, G.B.Hagemann, C.R.Hansen, G.Sletten, J.N.Scheurer, F.Hannachi, M.Kmiecik, A.Maj, J.Styczen, K.Zuber, K.Hauschild, A.Korichi, A.Lopez-Martens, J.Roccaz, S.Siem, P.Bednarczyk, Th.Byrski, D.Curien, O.Dorvaux, G.Duchene, B.Gall, F.Khalfallah, I.Piqueras, J.Robin, S.B.Patel, A.O.Evans, G.Rainovski, A.Airoldi, G.Benzoni, A.Bracco, F.Camera, B.Million, P.Mason, A.Paleni, R.Sacchi, O.Wieland, G.La Rana, R.Moro, C.M.Petrache, D.Petrache, G.De Angelis, P.Fallon, I.-Y.Lee, J.C.Lisle, B.Cederwall, K.Lagergren, R.M.Lieder, E.Podsvirova, W.Gast, H.Jager, N.Redon, A.Gorgen - Acta Phys.Pol. B36, 1033 (2005)

Search for hyperdeformation in light Xe nuclei

20050B04 S.Oberstedt, A.Oberstedt, D.Rochman, F.Gonnenwein, I.Tsekhanovich, J.Becker, A.Sartz, H.Bax, F.-J.Hambsch, S.Raman - Nucl.Phys. A761, 173 (2005) Light charged particle emission in the reaction ${ }^{251} \mathrm{Cf}\left(\mathrm{n}_{t h}, \mathrm{f}\right)$

20050G02 Yu.Ts.Oganessian, V.K.Utyonkov, S.N.Dmitriev, Yu.V.Lobanov, M.G.Itkis, A.N.Polyakov, Yu.S.Tsyganov, A.N.Mezentsev, A.V.Yeremin, A.A.Voinov, E.A.Sokol, G.G.Gulbekian, S.L.Bogomolov, S.Iliev, V.G.Subbotin, A.M.Sukhov, G.V.Buklanov, S.V.Shishkin, V.I.Chepigin, G.K.Vostokin, N.V.Aksenov, M.Hussonnois, K.Subotic, V.I.Zagrebaev, K.J.Moody, J.B.Patin, J.F.Wild, M.A.Stoyer, N.J.Stoyer, D.A.Shaughnessy, J.M.Kenneally, P.A.Wilk, R.W.Lougheed, H.W.Gaggeler, D.Schumann, H.Bruchertseifer, R.Eichler Phys.Rev. C 72, 034611 (2005)

Synthesis of elements 115 and 113 in the reaction ${ }^{243} \mathrm{Am}+{ }^{48} \mathrm{Ca}$ 
20050 G03 Yu.Ts.Oganessian, V.K.Utyonkov, Yu.V.Lobanov, F.Sh.Abdullin, A.N.Polyakov, I.V.Shirokovsky, Yu.S.Tsyganov, G.G.Gulbekian, S.L.Bogomolov, B.N.Gikal, A.N.Mezentsev, S.Iliev, V.G.Subbotin, A.M.Sukhov, A.A.Voinov, G.V.Buklanov, K.Subotic, V.I.Zagrebaev, M.G.Itkis, J.B.Patin, K.J.Moody, J.F.Wild, M.A.Stoyer, N.J.Stoyer, D.A.Shaughnessy, J.M.Kenneally, P.A.Wilk, R.W.Lougheed -

Eur.Phys.J. A 25, Supplement 1, 589 (2005)

New elements from Dubna

20050H04 K.Ohgama, M.Igashira, T.Ohsaki - J.Nucl.Sci.Technol.(Tokyo) 42, 333 (2005) Measurement of keV-Neutron Capture Cross Sections and Capture Gamma-Ray Spectra of ${ }^{91,92} \mathrm{Zr}$

20050H08 T.Ohtsubo, F.Bosch, H.Geissel, L.Maier, C.Scheidenberger, F.Attallah, K.Beckert, P.Beller, D.Boutin, T.Faestermann, B.Franczak, B.Franzke, M.Hausmann, M.Hellstrom, E.Kaza, P.Kienle, O.Klepper, H.-J.Kluge, C.Kozhuharov, Yu.A.Litvinov, M.Matos, G.Munzenberg, F.Nolden, Yu.N.Novikov, M.Portillo, T.Radon, J.Stadlmann, M.Steck, T.Stohlker, K.Summerer, K.Takahashi, H.Weick, M.Winkler, T.Yamaguchi - Phys.Rev.Lett. 95, 052501 (2005); Erratum Phys.Rev.Lett. 95, 069901 (2005)

Simultaneous Measurement of $\beta^{-}$Decay to Bound and Continuum Electron States

20050HZX K.Ohgama, M.Igashira, T.Ohsaki - Proc.Intern.Conf.Nuclear Data for Science and Technology, Santa Fe, New Mexico, 26 September-1 October, 2004, R.C.Haight, M.B.Chadwick, T.Kawano, P.Talou, Eds., Vol.1, p. 945 (2005); AIP Conf.Proc. 769 (2005)

Systematic Measurement of keV-Neutron Capture Cross Sections and Capture Gamma-Ray Spectra of Zr Isotopes

20050HZY S.Ohki - Proc.Intern.Conf.Nuclear Data for Science and Technology, Santa Fe, New Mexico, 26 September-1 October, 2004, R.C.Haight, M.B.Chadwick, T.Kawano, P.Talou, Eds., Vol.1, p. 472 (2005); AIP Conf.Proc. 769 (2005)

Validation of MA Nuclear Data by Sample Irradiation Experiments with the Fast Reactor JOYO

20050 02 S.Okada, S.Ajimura, K.Aoki, A.Banu, H.C.Bhang, T.Fukuda, O.Hashimoto, J.I.Hwang, S.Kameoka, B.H.Kang, E.H.Kim, J.H.Kim, M.J.Kim, T.Maruta, Y.Miura, Y.Miyake, T.Nagae, M.Nakamura, S.N.Nakamura, H.Noumi, Y.Okayasu, H.Outa, H.Park, P.K.Saha, Y.Sato, M.Sekimoto, T.Takahashi, H.Tamura, K.Tanida, A.Toyoda, K.Tsukada, T.Watanabe, H.J.Yim - Nucl.Phys. A752, 196c (2005) Nucleon-nucleon coincidence measurement in the non-mesonic weak decay of ${ }_{L}^{5} \mathrm{He}$ and ${ }_{\Lambda}^{12} \mathrm{C}$ hypernuclei

20050K04 S.Okada, S.Ajimura, K.Aoki, A.Banu, H.C.Bhang, T.Fukuda, O.Hashimoto, J.I.Hwang, S.Kameoka, B.H.Kang, E.H.Kim, J.H.Kim, M.J.Kim, T.Maruta, Y.Miura, Y.Miyake, T.Nagae, M.Nakamura, S.N.Nakamura, H.Noumi, Y.Okayasu, H.Outa, H.Park, P.K.Saha, Y.Sato, M.Sekimoto, T.Takahashi, H.Tamura, K.Tanida, A.Toyoda, K.Tsukada, T.Watanabe, H.J.Yim - Nucl.Phys. A754, 178c (2005)

$\pi^{0}$ decay branching ratios of ${ }_{\Lambda}^{5} \mathrm{He}$ and ${ }_{\Lambda}^{12} \mathrm{C}$ hypernuclei 
20050KZZ H.Okada, I.G.Alekseev, A.Bravar, G.Bunce, S.Dhawan, R.Gill, W.Haeberli, O.Jinnouchi, A.Khodinov, A.Kponou, Y.Makdisi, W.Meng, A.Nass, N.Saito, H.Spinka, E.J.Stephenson, D.N.Svirida, T.Wise, A.Zelenski nucl-ex/0502022,2/25/2005 (2005)

Measurement of the analyzing power in pp elastic scattering in the peak CNI region at RHIC

20050L02 J.Ollier, R.Chapman, X.Liang, M.Labiche, K.-M.Spohr, M.Davison, G.de Angelis, M.Axiotis, T.Kroll, D.R.Napoli, T.Martinez, D.Bazzacco, E.Farnea, S.Lunardi, A.G.Smith, F.Haas - Phys.Rev. C 71, 034316 (2005)

Intruder configurations in neutron-rich ${ }^{34} \mathrm{P}$

20050L04 J.Ollier, A.Hodsdon, R.Chapman, X.Liang, M.Burns, K.Keyes, M.Labiche, A.Papenberg, K.-M.Spohr, M.Davison, G.de Angelis, M.Axiotis, B.Behera, L.Corradi, A.Gadea, A.Latina, T.Kroll, N.Marginean, T.Martinez, D.R.Napoli, A.Stefanini, M.Trotta, S.Beghini, D.Bazzacco, E.Farnea, S.Lunardi, G.Montagnoli, F.Scarlassara, A.Deacon, A.G.Smith, J.F.Smith, D.Curien, F.Haas, D.Verney, A.Jungclaus, F.Azaiez, F.Ibrahim, M.Stanoiu, Zs.Dombradi - J.Phys.(London) G31, S1935 (2005)

Intruder configurations in neutron-rich $\mathrm{P}$ and $\mathrm{S}$ isotopes

20050 N03 T.K.Onishi, A.Gelberg, H.Sakurai, K.Yoneda, N.Aoi, N.Imai, H.Baba, P.von Brentano, N.Fukuda, Y.Ichikawa, M.Ishihara, H.Iwasaki, D.Kameda, T.Kishida, A.F.Lisetskiy, H.J.Ong, M.Osada, T.Otsuka, M.K.Suzuki, K.Ue, Y.Utsuno, H.Watanabe - Phys.Rev. C 72, 024308 (2005)

Gamow-Teller decay of the $\mathrm{T}=1$ nucleus ${ }^{46} \mathrm{Cr}$

20050N04 H.J.Ong, N.Imai, N.Aoi, H.Sakurai, Zs.Dombradi, A.Saito, Z.Elekes, H.Baba, K.Demichi, Zs.Fulop, J.Gibelin, T.Gomi, H.Hasegawa, M.Ishihara, H.Iwasaki, S.Kanno, S.Kawai, T.Kubo, K.Kurita, Y.U.Matsuyama, S.Michimasa, T.Minemura, T.Motobayashi, M.Notani, S.Ota, H.K.Sakai, S.Shimoura, E.Takeshita, S.Takeuchi, M.Tamaki, Y.Togano, K.Yamada, Y.Yanagisawa, K.Yoneda - Eur.Phys.J. A 25, Supplement 1, 347 (2005)

Inelastic proton scattering on ${ }^{16} \mathrm{C}$

20050R02 J.N.Orce, T.Kibedi, G.D.Dracoulis, R.Julin, S.W.Yates - J.Phys.(London) G31, S1705 (2005)

Conversion-electron study of $0^{+}$excitations in ${ }^{208} \mathrm{~Pb}$

20050S02 W.H.Oskay, W.M.Itano, J.C.Bergquist - Phys.Rev.Lett. 94, 163001 (2005) Measurement of the ${ }^{199} \mathrm{Hg}^{+} 5 \mathrm{~d}^{9} 6 \mathrm{~s}^{22} \mathrm{D}_{5 / 2}$ Electric Quadrupole Moment and a Constraint on the Quadrupole Shift

20050002 H.Outa, S.Ajimura, K.Aoki, A.Banu, H.C.Bhang, T.Fukuda, O.Hashimoto, J.I.Hwang, S.Kameoka, B.H.Kang, E.H.Kim, J.H.Kim, M.J.Kim, T.Maruta, Y.Miura, Y.Miyake, T.Nagae, M.Nakamura, S.N.Nakamura, H.Noumi, S.Okada, Y.Okayasu, H.Park, P.K.Saha, Y.Sato, M.Sekimoto, T.Takahashi, H.Tamura, K.Tanida, A.Toyoda, K.Tsukada, T.Watanabe, H.J.Yim - Nucl.Phys. A754, 157c (2005) 
Hypernuclear weak decay experiments at KEK: n-n and n-p coincidence measurement

2005PA07 A.A.Pasternak, E.O.Podsvirova, R.M.Lieder, S.Chmel, W.Gast, Ts.Venkova, H.M.Jager, L.Mihailescu, G.de Angelis, D.R.Napoli, A.Gadea, D.Bazzacco, R.Menegazzo, S.Lunardi, W.Urban, Ch.Droste, T.Morek, T.Rzaca-Urban, G.Duchene, A.Dewald - Eur.Phys.J. A 23, 191 (2005)

Investigation of lifetimes in dipole bands of ${ }^{142} \mathrm{Gd}$

2005Pa22 J.Pal, S.Saha, C.C.Dey, P.Banerjee, S.Bose, B.K.Sinha, M.B.Chatterjee, S.K.Basu Phys.Rev. C 71, 034605 (2005)

Pre-equilibrium and equilibrium emission of neutrons in ${ }^{114} \mathrm{Cd}(\alpha, \mathrm{xn})$ reactions

2005PA23 E.Padilla-Rodal, A.Galindo-Uribarri, C.Baktash, J.C.Batchelder, J.R.Beene, R.Bijker, B.A.Brown, O.Castanos, B.Fuentes, J.Gomez del Campo, P.A.Hausladen, Y.Larochelle, A.F.Lisetskiy, P.E.Mueller, D.C.Radford, D.W.Stracener, J.P.Urrego, R.L.Varner, C.-H.Yu - Phys.Rev.Lett. 94, 122501 (2005)

$\mathrm{B}(\mathrm{E} 2) \uparrow$ Measurements for Radioactive Neutron-Rich Ge Isotopes: Reaching the $\mathrm{N}=50$ Closed Shell

2005PA30 E.S.Paul, P.T.W.Choy, C.Andreoiu, A.J.Boston, A.O.Evans, C.Fox, S.Gros, P.J.Nolan, G.Rainovski, J.A.Sampson, H.C.Scraggs, A.Walker, D.E.Appelbe, D.T.Joss, J.Simpson, J.Gizon, A.Astier, N.Buforn, A.Prevost, N.Redon, O.Stezowski, B.M.Nyako, D.Sohler, J.Timar, L.Zolnai, D.Bazzacco, S.Lunardi, C.M.Petrache, P.Bednarczyk, D.Curien, N.Kintz, I.Ragnarsson - Phys.Rev. C 71, 054309 (2005)

Highest spin discrete levels in ${ }^{131,132}$ Ce: Spin generation near the mesoscopic limit

2005PA31 A.Parikh, J.A.Caggiano, C.Deibel, J.P.Greene, R.Lewis, P.D.Parker, C.Wrede Phys.Rev. C 71, 055804 (2005)

Mass measurements of ${ }^{22} \mathrm{Mg}$ and ${ }^{26} \mathrm{Si}$ via the ${ }^{24} \mathrm{Mg}(\mathrm{p}, \mathrm{t}){ }^{22} \mathrm{Mg}$ and ${ }^{28} \mathrm{Si}(\mathrm{p}, \mathrm{t}){ }^{26} \mathrm{Si}$ reactions

2005PA33 S.R.Palvanov, G.Yu.Tadzhibaev, Sh.M.Ruzimov - At.Energ. 98, 238 (2005); At.Energy 98, 230 (2005)

Isomeric ratios of the yields of $(\gamma, \mathrm{n})$ reactions on ${ }^{85} \mathrm{Rb}$ and ${ }^{87} \mathrm{Rb}$ nuclei

2005PA39 K.D.Paschke, for the HAPPEx Collaborations - Nucl.Phys. A755, 241c (2005) The Second Generation HAPPEx Experiments

2005PA41 J.H.Park, C.S.Lee, Y.K.Kwon, J.H.Lee, J.Y.Kim, K.B.Lee, H.Park - J.Korean Phys.Soc. 47, 23 (2005)

Activation Cross Section Measurements of ${ }^{16} \mathrm{O}\left(\mathrm{n},{ }^{3} \mathrm{He}\right){ }^{14} \mathrm{C}$ and ${ }^{16} \mathrm{O}(\mathrm{n}, \mathrm{t}){ }^{14} \mathrm{~N}$ above Neutron Thresholds up to $18.1 \mathrm{MeV}$

2005PA42 J.Pakarinen, I.G.Darby, S.Eeckhaudt, T.Enqvist, T.Grahn, P.T.Greenlees, V.Hellemans, K.Heyde, F.Johnston-Theasby, P.Jones, R.Julin, S.Juutinen, H.Kettunen, M.Leino, A.-P.Leppanen, P.Nieminen, M.Nyman, R.D.Page, P.M.Raddon, P.Rahkila, C.Scholey, J.Uusitalo, R.Wadsworth - Phys.Rev. C 72, $011304(2005)$ 
Evidence for oblate structure in ${ }^{186} \mathrm{~Pb}$

2005PA48 D.Pantelica, I.Gh.Stefan, N.Nica, M.-G.Porquet, G.Duchene, A.Astier, S.Courtin, I.Deloncle, F.Hoellinger, A.Bauchet, N.Buforn, L.Donadille, O.Dorvaux, J.Duprat, B.J.P.Gall, C.Gautherin, T.Kutsarova, S.Lalkovski, R.Lucas, M.Meyer, A.Minkova, A.Prevost, N.Redon, N.Schulz, H.Sergolle, O.Stezowski, Ts.Venkova, A.Wilson Phys.Rev. C 72, 024304 (2005)

High-spin states in ${ }^{92-96} \mathrm{Zr}$ nuclei

2005PA50 Y.Parpottas, S.M.Grimes, S.Al-Quraishi, C.R.Brune, T.N.Massey, J.E.O'Donnell, J.E.Oldendick, A.Salas, R.T.Wheeler - Phys.Rev. C 72, 025802 (2005)

The ${ }^{17} \mathrm{~F}(\mathrm{p}, \gamma){ }^{18} \mathrm{Ne} 3^{+}$resonance state studied with the ${ }^{16} \mathrm{O}\left({ }^{3} \mathrm{He}, \mathrm{n}\right){ }^{18} \mathrm{Ne}$ reaction

2005Pa56 H.Park, K.-O.Choi, J.-M.Lee, K.B.Lee, M.S.Hahn, M.Kralik - J.Korean Phys.Soc. 47, $603(2005)$

Absolute Measurement of the Neutron Emission Rate with a Manganese Sulphate Bath System

2005PA68 S.D.Pain, W.N.Catford, N.A.Orr, J.C.Angelique, N.I.Ashwood, V.Bouchat, N.M.Clarke, N.Curtis, M.Freer, B.R.Fulton, F.Hanappe, M.Labiche, J.L.Lecouey, R.C.Lemmon, D.Mahboub, A.Ninane, G.Normand, N.Soic, L.Stuttge, C.N.Timis, J.A.Tostevin, J.S.Winfield, V.Ziman - Eur.Phys.J. A 25, Supplement 1, 349 (2005) Experimental evidence of a $\nu\left(1 \mathrm{~d}_{5 / 2}\right)^{2}$ component to the ${ }^{12}$ Be ground state

2005PA69 J.Pakarinen, I.Darby, S.Eeckhaudt, T.Enqvist, T.Grahn, P.Greenlees, F.Johnston-Theasby, P.Jones, R.Julin, S.Juutinen, H.Kettunen, M.Leino, A.-P.Leppanen, P.Nieminen, M.Nyman, R.Page, P.Raddon, P.Rahkila, C.Scholey, J.Uusitalo, R.Wadsworth - Eur.Phys.J. A 25, Supplement 1, 449 (2005)

Probing the three shapes in ${ }^{186} \mathrm{~Pb}$ using in-beam $\gamma$-ray spectroscopy

2005PAZV S.D.Pain, W.N.Catford, N.A.Orr, J.C.Angelique, N.I.Ashwood, V.Bouchat, N.M.Clarke, N.Curtis, M.Freer, B.R.Fulton, F.Hanappe, M.Labiche, J.L.Lecouey, R.C.Lemmon, D.Mahboub, A.Ninane, G.Normand, N.Soic, L.Stuttge, C.N.Timis, J.A.Tostevin, J.S.Winfield, V.Ziman - nucl-ex/0510048,10/16/2005 (2005) Structure of ${ }^{12} \mathrm{Be}$ : intruder d-wave strength at $\mathrm{N}=8$

2005PAZW A.Pavlik, P.Baumann, C.Borcea, E.Jericha, S.Jokic, M.Kerveno, S.Lukic, J.P.Meulders, L.C.Mihailescu, R.Nolte, A.J.M.Plompen, I.Raskinyte, G.Rudolf, and the $\mathrm{n}_{-} \mathrm{TOF}$ Collaboration - Proc.Intern.Conf.Nuclear Data for Science and Technology, Santa Fe, New Mexico, 26 September-1 October, 2004, R.C.Haight, M.B.Chadwick, T.Kawano, P.Talou, Eds., Vol.1, p. 876 (2005); AIP Conf.Proc. 769 (2005)

Cross-Section Measurements for (n, xn) Reactions by In-Beam Gamma-Ray Spectroscopy

2005PAZY Yu.N.Pavlenko, V.L.Shablov, F.I.Karmanov, V.N.Dobrikov, O.K.Gorpinich, V.O.Kyva, I.N.Kolomiets - Book of Abstracts, LV National Conference on Nuclear Physics "Frontiers in the Physics of Nucleus", St.-Petersburg, p.179 (2005)

On Excited States of ${ }^{5} \mathrm{He}$ in the Reactions ${ }^{6} \mathrm{Li}\left(\mathrm{d},{ }^{3} \mathrm{He}\right){ }^{5} \mathrm{He}$ and ${ }^{7} \mathrm{Li}\left(\mathrm{d},{ }^{4} \mathrm{He}\right){ }^{5} \mathrm{He}$ 
2005PAZZ S.D.Pain, W.N.Catford, N.A.Orr, J.C.Angelique, N.I.Ashwood, V.Bouchat, N.M.Clarke, N.Curtis, M.Freer, B.Fulton, F.Hanappe, M.Labiche, J.L.Lecouey, R.Lemmon, D.Mahboub, A.Ninane, G.Normand, N.Soic, L.Stuttge, C.N.Timis, J.A.Tostevin, V.Ziman, J.Winfield - Proc.Nuclei at the Limits, Argonne, Illinois, D.Seweryniak and T.L.Khoo, eds., p.373 (2005); AIP Conf. Proc 764 (2005) Evidence Of A $\left(1 \mathrm{~d}_{5 / 2}\right)^{2}$ Component To The ${ }^{12}$ Be Ground State

2005PE12 K.Perajarvi, J.Cerny, J.Hakala, J.Huikari, A.Jokinen, P.Karvonen, J.Kurpeta, D.Lee, I.Moore, H.Penttila, A.Popov, J.Aysto - Nucl.Instrum.Methods Phys.Res. A546, 418 (2005)

New ion-guide for the production of beams of neutron-rich nuclei between $Z=20$ 28

2005PE18 R.Peusquens, R.S.Chakrawarthy, A.Dewald, P.Petkov, P.von Brentano, R.Krucken, S.Kasemann, H.Tiesler, K.O.Zell, S.Lunardi, D.Bazzacco, F.Brandolini, N.H.Medina, P.Pavan, C.M.Petrache, C.Rossi-Alvarez, G.de Angelis, G.Maron, M.de Poli, D.R.Napoli - Phys.Rev. C 72, 031304 (2005)

Decay out of the highly deformed band in ${ }^{133} \mathrm{Nd}$

2005PE23 K.Perajarvi, J.Cerny, U.Hager, J.Hakala, J.Huikari, A.Jokinen, P.Karvonen, J.Kurpeta, D.Lee, I.Moore, H.Penttila, A.Popov, J.Aysto - Eur.Phys.J. A 25, Supplement 1, 749 (2005)

Production of beams of neutron-rich nuclei between $\mathrm{Ca}$ and $\mathrm{Ni}$ using the ion-guide technique

2005PEZZ M.G.Pellegriti, C.Spitaleri, A.Musumarra, L.Calabretta, S.Cherubini, A.Di Pietro, P.Figuera, R.G.Pizzone, S.Romano, S.Tudisco, A.Tumino - Proc. Intern. Symposium on the Origin of Matter and Evolution of Galaxies, Riken, Saitama, Japan, 17-19 November 2003, World Scientific, Singapore, p.513 (2005)

Quasi-free proton-proton elastic scattering in the Trojan Horse framework

2005 PI02 S.Pirro, C.Arnaboldi, D.R.Artusa, F.T.Avignone III, M.Balata, I.Bandac, M.Barucci, J.Beeman, C.Brofferio, C.Bucci, S.Capelli, F.Capozzi, L.Carbone, S.Cebrian, O.Cremonesi, R.J.Creswick, A.de Waard, H.A.Farach, A.Fascilla, E.Fiorini, G.Frossati, A.Giuliani, P.Gorla, E.E.Haller, I.G.Irastorza, R.J.McDonald, A.Morales, E.B.Norman, A.Nucciotti, E.Olivieri, V.Palmieri, E.Pasca, M.Pavan, M.Pedretti, G.Pessina, E.Previtali, C.Pobes, M.Pyle, L.Risegari, C.Rosenfeld, M.Sisti, A.R.Smith, L.Torres, G.Ventura - Nucl.Phys. B(Proc.Supp.) S138, 210 (2005)

First results of the CUORICINO experiment

2005PI08 J.Pinard, H.T.Duong, D.Marescaux, H.H.Stroke, O.Redi, M.Gustafsson, T.Nilsson, S.Matsuki, Y.Kishimoto, K.Kominato, I.Ogawa, M.Shibata, M.Tada, J.R.Persson, Y.Nojiri, S.Momota, T.T.Inamura, M.Wakasugi, P.Juncar, T.Murayama, T.Nomura, M.Koizumi, and the ISOLDE Collaboration - Nucl.Phys. A753, 3 (2005)

Precision hfs of ${ }^{126} \mathrm{Cs}\left(\mathrm{T}_{1 / 2}=1.63 \mathrm{~m}\right)$ by ABMR

2005PI13 J.A.Pinston, J.Genevey, R.Orlandi, A.Scherillo, G.S.Simpson, I.Tsekhanovich, W.Urban, H.Faust, N.Warr - Phys.Rev. C 71, 064327 (2005)

Shape coexistence in the very neutron-rich odd-odd ${ }^{96} \mathrm{Rb}$ 
2005PL04 R.Plag, M.Heil, F.Kappeler, R.Reifarth, K.Wisshak - Nucl.Phys. A758, 415c (2005) An independent measurement of the ${ }^{12} \mathrm{C}(\alpha, \gamma){ }^{16} \mathrm{O}$ cross section with the Karlsruhe $4 \pi \mathrm{BaF}_{2}$ detector

2005 P002 R.Pollanen, T.Siiskonen, P.Vesterbacka - Radiat.Meas. 39, 565 (2005)

High-resolution alpha spectrometry from thick sources

2005 P003 M.-G.Porquet, Ts.Venkova, R.Lucas, A.Astier, A.Bauchet, I.Deloncle, A.Prevost, F.Azaiez, G.Barreau, A.Bogachev, N.Buforn, A.Buta, D.Curien, T.P.Doan, L.Donadille, O.Dorvaux, G.Duchene, J.Durell, Th.Ethvignot, B.P.J.Gall, D.Grimwood, M.Houry, F.Khalfallah, W.Korten, S.Lalkovski, Y.Le Coz, M.Meyer, A.Minkova, I.Piqueras, N.Redon, A.Roach, M.Rousseau, N.Schulz, A.G.Smith, O.Stezowski, Ch.Theisen, B.J.Varley - Eur.Phys.J. A 24, 39 (2005)

High-spin structures of ${ }_{51}^{121,123,125,127} \mathrm{Sb}$ nuclei: Single proton and core-coupled states

2005P009 S.Pomme, T.Altzitzoglou, R.Van Ammel, G.Sibbens - Nucl.Instrum.Methods Phys.Res. A544, 584 (2005); Erratum Nucl.Instrum.Methods Phys.Res. A555, 459 (2005)

Standardisation of ${ }^{125} \mathrm{I}$ using seven techniques for radioactivity measurement

2005P010 A.R.Poletti, A.P.Byrne, G.D.Dracoulis, T.Kibedi, P.M.Davidson - Nucl.Phys. A756, $83(2005)$

High spin states in ${ }^{210} \mathrm{Rn}$ approaching the region of 3-particle-hole neutron excitations

2005P017 L.Popescu, T.Adachi, G.P.A.Berg, P.von Brentano, D.De Frenne, K.Fujita, Y.Fujita, K.Hatanaka, E.Jacobs, A.Negret, K.Nakanishi, Y.Sakemi, Y.Shimbara, Y.Shimizu, Y.Tameshige, A.Tamii, M.Uchida, M.Yosoi - J.Phys.(London) G31, S1945 (2005) Gamow-Teller transitions in the ${ }^{64} \mathrm{Ni}\left({ }^{3} \mathrm{He}, \mathrm{t}\right){ }^{64} \mathrm{Cu}$ reaction

2005POZZ

A.R.Poletti, A.P.Byrne, G.D.Dracoulis, T.Kibedi, P.M.Davidson - ANU-P/1649 (2005)

High spin states in ${ }^{210} \mathrm{Rn}$ approaching the region of 3-particle-hole neutron excitations

2005PR02 D.Protopopescu, and the CLAS Collaboration - Nucl.Phys. A748, 357 (2005) Survey of $\mathrm{A}_{L T^{\prime}}$ asymmetries in semi-exclusive electron scattering on ${ }^{4} \mathrm{He}$ and ${ }^{12} \mathrm{C}$

2005PR11 Y.Prezado, M.J.G.Borge, C.Aa.Diget, L.M.Fraile, B.R.Fulton, H.O.U.Fynbo, H.B.Jeppesen, B.Jonson, M.Meister, T.Nilsson, G.Nyman, K.Riisager, O.Tengblad, K.Wilhelmsen - Phys.Lett. B 618, 43 (2005)

Low-lying resonance states in the ${ }^{9} \mathrm{Be}$ continuum

2005PR16 S.Prakhov, and the Crystal Ball Collaboration - Phys.Rev. C 72, 025201 (2005) Measurement of the branching ratio for $\eta \rightarrow \pi^{0} \gamma \gamma$ decay 
2005PR20 P.Prati, D.Bemmerer, R.Bonetti, C.Broggini, F.Confortola, P.Corvisiero, H.Costantini, J.Cruz, A.Formicola, Z.Fulop, G.Gervino, A.Guglielmetti, C.Gustavino, G.Gyurky, G.Imbriani, A.P.Jesus, M.Junker, A.Lemut, R.Menegazzo, V.Roca, C.Rolfs, M.Romano, C.Rossi Alvarez, F.Schumann, E.Somorjai, O.Straniero, F.Strieder, F.Terrasi, H.P.Trautvetter - J.Phys.(London) G31, S1537 (2005)

Recent results from the LUNA facility at Gran Sasso

2005PU02 V.Punjabi, Jefferson Lab Hall A Collaboration - Phys.Rev. C 71, 055202 (2005);

Erratum Phys.Rev. C 71069902 (2005)

Proton elastic form factor ratios to $\mathrm{Q}^{2}=3.5 \mathrm{GeV}^{2}$ by polarization transfer

2005PYZZ I.Pysmenetska, S.Walter, J.Enders, H.von Garrel, O.Karg, U.Kneissl, C.Kohstall, P.von Neumann-Cosel, H.H.Pitz, V.Yu.Ponomarev, M.Scheck, F.Stedile, S.Volz nucl-ex/0512013,12/8/2005 (2005)

Two-phonon $1^{-}$state in ${ }^{112} \mathrm{Sn}$ observed in resonant photon scattering

2005QA01 I.A.Qattan, J.Arrington, R.E.Segel, X.Zheng, K.Aniol, O.K.Baker, R.Beams, E.J.Brash, J.Calarco, A.Camsonne, J.-P.Chen, M.E.Christy, D.Dutta, R.Ent, S.Frullani, D.Gaskell, O.Gayou, R.Gilman, C.Glashausser, K.Hafidi, J.-O.Hansen, D.W.Higinbotham, W.Hinton, R.J.Holt, G.M.Huber, H.Ibrahim, L.Jisonna, M.K.Jones, C.E.Keppel, E.Kinney, G.J.Kumbartzki, A.Lung, D.J.Margaziotis, K.McCormick, D.Meekins, R.Michaels, P.Monaghan, P.Moussiegt, L.Pentchev, C.Perdrisat, V.Punjabi, R.Ransome, J.Reinhold, B.Reitz, A.Saha, A.Sarty, E.C.Schulte, K.Slifer, P.Solvignon, V.Sulkosky, K.Wijesooriya, B.Zeidman Phys.Rev.Lett. 94, 142301 (2005)

Precision Rosenbluth Measurement of the Proton Elastic Form Factors

2005QIZZ Z.Qin, D.Ackermann, W.Bruchle, F.P.Hessberger, E.Jager, P.Kuusiniemi, G.Munzenberg, D.Nayak, E.Schimpf, M.Schadel, B.Schausten, A.Semchenkov, B.Sulignano, K.Eberhardt, J.V.Kratz, D.Liebe, P.Thorle, Yu.N.Novikov - GSI 2005-1, p.75 (2005)

Search for the "missing" $\alpha$-decay branch in ${ }^{239} \mathrm{Cm}$

2005RA09 D.C.Radford, C.Baktash, C.J.Barton, J.Batchelder, J.R.Beene, C.R.Bingham, M.A.Caprio, M.Danchev, B.Fuentes, A.Galindo-Uribarri, J.Gomez del Campo, C.J.Gross, M.L.Halbert, D.J.Hartley, P.Hausladen, J.K.Hwang, W.Krolas, Y.Larochelle, J.F.Liang, P.E.Mueller, E.Padilla, J.Pavan, A.Piechaczek, D.Shapira, D.W.Stracener, R.L.Varner, A.Woehr, C.-H.Yu, N.V.Zamfir - Nucl.Phys. A752, 264c (2005)

Coulomb excitation and transfer reactions with rare neutron-rich isotopes

2005RA13 S.Rakers, C.Baumer, A.M.van den Berg, B.Davids, D.Frekers, D.De Frenne, E.-W.Grewe, P.Haefner, M.N.Harakeh, S.Hollstein, M.Hunyadi, E.Jacobs, B.C.Junk, A.Korff, A.Negret, L.Popescu, H.J.Wortche - Phys.Rev. C 71, 054313 (2005) Low-lying $\mathrm{GT}^{+}$strength in ${ }^{116} \mathrm{In}$ from a $\left(\mathrm{d},{ }^{2} \mathrm{He}\right)$ reaction experiment and its implications for ${ }^{116} \mathrm{Cd}$ double $\beta$ decay

2005RA26 B.Rakvin, N.Maltar-Strmecki - Chem.Phys.Lett. 415, 161 (2005) 
Study of the first stable L-alanine paramagnetic center by 2D-HYSCORE spectroscopy: Detection of ${ }^{14} \mathrm{~N}$ hyperfine and quadrupole splitting

2005RA27 F.Raiola, B.Burchard, Z.Fulop, G.Gyurky, S.Zeng, J.Cruz, A.Di Leva, B.Limata, M.Fonseca, H.Luis, M.Aliotta, H.W.Becker, C.Broggini, A.D'Onofrio, L.Gialanella, G.Imbriani, A.P.Jesus, M.Junker, J.P.Ribeiro, V.Roca, C.Rolfs, M.Romano, E.Somorjai, F.Strieder, F.Terrasi, for the LUNA Collaboration - J.Phys.(London) G31, 1141 (2005)

Electron screening in $\mathrm{d}(\mathrm{d}, \mathrm{p}) \mathrm{t}$ for deuterated metals: temperature effects

2005RA29 D.R.Rao, K.V.Sai, M.Sainath, K.Venkataramaniah - Eur.Phys.J. A 26, 41 (2005) Precision electron-gamma spectroscopic measurements in ${ }^{75} \mathrm{As}$

2005RA30 M.A.Rahman, M.S.Chowdhury - Phys.Rev. C 72, 054303 (2005)

Level structure of ${ }^{95} \mathrm{Nb}$

2005RA32 D.C.Radford, C.Baktash, C.J.Barton, J.Batchelder, J.R.Beene, C.R.Bingham, M.A.Caprio, M.Danchev, B.Fuentes, A.Galindo-Uribarri, J.Gomez del Campo, C.J.Gross, M.L.Halbert, D.J.Hartley, P.Hausladen, J.K.Hwang, W.Krolas, Y.Larochelle, J.F.Liang, P.E.Mueller, E.Padilla, J.Pavan, A.Piechaczek, D.Shapira, D.W.Stracener, R.L.Varner, A.Woehr, C.-H.Yu, N.V.Zamfir - Eur.Phys.J. A 25, Supplement 1, 383 (2005)

Coulomb excitation and transfer reactions with neutron-rich radioactive beams

2005RA33 M.P.Raele, C.B.Zamboni, G.S.Zahn, F.A.Genezini - Braz.J.Phys. 35, 839 (2005) Decay of ${ }^{155} \mathrm{Sm}$

2005RAzZ A.Ray, P.Das, S.K.Saha, S.K.Das, J.J.Das, N.Madhavan, S.Nath, P.Sugathan, P.V.M.Rao, A.Jhingan - nucl-ex/0509021,9/15/2005 (2005)

Change of ${ }^{7} \mathrm{Be}$ decay rate in exohedral and endohedral $\mathrm{C}_{60}$ fullerene compounds and its implications

2005RE02 J.J.Ressler, C.W.Beausang, H.Ai, H.Amro, M.Babilon, J.A.Caggiano, R.F.Casten, G.Gurdal, A.Heinz, R.O.Hughes, E.A.McCutchan, D.A.Meyer, C.Plettner, J.Qian, M.J.S.Sciacchitano, N.J.Thomas, E.Williams, N.V.Zamfir - Phys.Rev. C 71, 014302 (2005)

Isomeric decay of ${ }^{208} \mathrm{Ra}$

2005RE06

E.L.Reber, R.J.Gehrke, R.Aryaeinejad, J.K.Hartwell - J.Radioanal.Nucl.Chem. 264, $243(2005)$

Measurement of the fission yields of selected prompt and decay fission product gamma-rays of spontaneously fissioning ${ }^{252} \mathrm{Cf}$ and ${ }^{244} \mathrm{Cm}$

2005RE09 P.Reimer, V.Avrigeanu, S.V.Chuvaev, A.A.Filatenkov, T.Glodariu, A.Koning, A.J.M.Plompen, S.M.Qaim, D.L.Smith, H.Weigmann - Phys.Rev. C 71, 044617 (2005)

Reaction mechanisms of fast neutrons on stable Mo isotopes below $21 \mathrm{MeV}$ 
2005RE11 P.H.Regan, C.Wheldon, A.D.Yamamoto, J.J.Valiente-Dobon, D.Cline, C.Y.Wu, A.O.Macchiavelli, F.R.Xu, J.F.Smith, K.Andgren, R.S.Chakrawarthy, M.Cromaz, P.Fallon, S.J.Freeman, A.Gorgen, A.Hayes, H.Hua, S.D.Langdown, I-Y.Lee, C.J.Pearson, Zs.Podolyak, R.Teng - Acta Phys.Pol. B36, 1313 (2005)

Vibrational and rotational sequences in ${ }^{101}$ Mo and ${ }^{103,4} \mathrm{Ru}$ studied via multinucleon transfer reactions

2005RE14 P.Reiter, T.L.Khoo, I.Ahmad, A.V.Afanasjev, A.Heinz, T.Lauritsen, C.J.Lister, D.Seweryniak, P.Bhattacharyya, P.A.Butler, M.P.Carpenter, A.J.Chewter, J.A.Cizewski, C.N.Davids, J.P.Greene, P.T.Greenlees, K.Helariutta, R.-D.Herzberg, R.V.F.Janssens, G.D.Jones, R.Julin, H.Kankaanpaa, H.Kettunen, F.G.Kondev, P.Kuusiniemi, M.Leino, S.Siem, A.A.Sonzogni, J.Uusitalo, I.Wiedenhover Phys.Rev.Lett. 95, 032501 (2005) Structure of the Odd-A, Shell-Stabilized Nucleus ${ }_{102}^{253} \mathrm{No}$

2005RE22 R.Reifarth, M.Heil, R.Plag, U.Besserer, S.Dababneh, L.Dorr, J.Gorres, R.C.Haight, F.Kappeler, A.Mengoni, S.O'Brien, N.Patronis, R.S.Rundberg, M.Wiescher, J.B.Wilhelmy - Nucl.Phys. A758, 787c (2005)

Stellar neutron capture rates of ${ }^{14} \mathrm{C}$

2005RE23 J.J.Ressler, C.W.Beausang, R.F.Casten, N.V.Zamfir, H.Ai, H.Amro, M.Babilon, R.B.Cakirli, J.A.Caggiano, G.Gurdal, A.Heinz, R.O.Hughes, S.D.Langdown, E.A.McCutchan, D.A.Meyer, C.Plettner, J.Qian, P.H.Regan, M.J.S.Sciacchitano, N.J.Thomas, E.Williams, A.Yamamoto - J.Phys.(London) G31, S1605 (2005) Isomers and seniority in the trans-Pb nuclei

2005RE25 R.Reifarth, E.-I.Esch, A.Alpizar-Vicente, E.M.Bond, T.A.Bredeweg, S.E.Glover, U.Greife, R.Hatarik, R.C.Haight, A.Kronenberg, J.M.O'Donnell, R.S.Rundberg, J.M.Schwantes, J.L.Ullmann, D.J.Vieira, J.B.Wilhelmy, J.M.Wouters Nucl.Instrum.Methods Phys.Res. B241, 176 (2005) $(\mathrm{n}, \gamma)$ measurements on radioactive isotopes with DANCE

2005RI11 L.A.Riley, M.A.Abdelqader, D.Bazin, M.J.Bojazi, B.A.Brown, C.M.Campbell, J.A.Church, P.D.Cottle, D.-C.Dinca, J.Enders, A.Gade, T.Glasmacher, M.Honma, S.Horibe, Z.Hu, K.W.Kemper, W.F.Mueller, H.Olliver, T.Otsuka, B.C.Perry, B.T.Roeder, B.M.Sherrill, T.P.Spencer, J.R.Terry - Phys.Rev. C 72, 024311 (2005) Thick-target inverse-kinematics proton scattering from ${ }^{46} \mathrm{Ar}$ and the $\mathrm{N}=28$ shell below ${ }^{48} \mathrm{Ca}$

2005RI13 A.Rinollo, S.Romano, C.Spitaleri, C.Bonomo, S.Cherubini, A.Del Zoppo, P.Figuera, M.La Cognata, L.Lamia, A.Musumarra, M.G.Pellegriti, R.G.Pizzone, C.Rolfs, D.Schurmann, F.Strieder, S.Tudisco, A.Tumino - Nucl.Phys. A758, 146c (2005) Measurement of cross section and astrophysical factor of the $\mathrm{d}(\mathrm{d}, \mathrm{p}) \mathrm{t}$ reaction using the Trojan Horse Method 
2005RI16 M.A.Riley, M.K.Djongolov, A.O.Evans, D.J.Hartley, R.V.F.Janssens, E.S.Paul, J.Simpson, A.A.Aguilar, D.E.Appelbe, C.R.Bingham, D.B.Campbell, M.P.Carpenter, P.Chowdhury, P.T.W.Choy, R.M.Clark, M.Cromaz, D.M.Cullen, M.Danchev, G.D.Dracoulis, P.Fallon, A.Gorgen, G.B.Hagemann, D.T.Joss, J.Goon, R.A.Kaye, T.L.Khoo, F.G.Kondev, R.W.Laird, K.Lagergren, T.Lauritsen, A.O.Macchiavelli, B.McClain, E.F.Moore, G.Mukherjee, E.Ngijoi-Yogo, P.J.Nolan, H.I.Park, A.Pipidis, L.L.Riedinger, G.Sletten, S.K.Tandel, P.M.Walker, D.Ward, I.Ragnarsson, F.Saric, J.Zhang - J.Phys.(London) G31, S1735 (2005)

Beyond band termination in ${ }^{157} \mathrm{Er}$ and the search for wobbling excitations in strongly deformed ${ }^{174} \mathrm{Hf}$

2005RI17 S.Rigby, D.M.Cullen, D.T.Scholes, C.Scholey, P.Rahkila, S.Eeckhaudt, T.Grahn, P.Greenlees, P.M.Jones, R.Julin, S.Juutinen, H.Kettunen, M.Leino, A.Leppanen, P.Nieminen, M.Nyman, J.Pakarinen, J.Uusitalo - J.Phys.(London) G31, S1949 (2005)

Mass-140 isomers near the proton dripline

2005RI19 S.Rinta-Antila, Y.Wang, P.Dendooven, J.Huikari, A.Jokinen, A.Kankainen, V.S.Kolhinen, G.Lhersonneau, A.Nieminen, S.Nummela, H.Penttila, K.Perajarvi, J.Szerypo, J.C.Wang, J.Aysto, and the ISOLDE Collaboration - Eur.Phys.J. A 25, Supplement 1, 119 (2005)

Structure of doubly-even cadmium nuclei studied by $\beta^{-}$decay

2005R002 P.Rossi, and the CLAS Collaboration - Phys.Rev.Lett. 94, 012301 (2005)

Onset of Asymptotic Scaling in Deuteron Photodisintegration

2005R011 T.Roth, O.Leupold, H.-C.Wille, R.Ruffer, K.W.Quast, R.Rohlsberger, E.Burkel Phys.Rev. B 71, 140401 (2005)

Coherent nuclear resonant scattering by ${ }^{61} \mathrm{Ni}$ using the nuclear lighthouse effect

2005R019 A.P.Robinson, P.J.Woods, D.Seweryniak, C.N.Davids, M.P.Carpenter, A.A.Hecht, D.Peterson, S.Sinha, W.B.Walters, S.Zhu - Phys.Rev.Lett. 95, 032502 (2005) Ground State Proton Radioactivity from ${ }^{121} \mathrm{Pr}$ : When Was This Exotic Nuclear Decay Mode First Discovered?

2005R021 T.Rostomyan, for the GDH and A2 Collaborations - Nucl.Phys. A755, 451c (2005) Helicity dependence of pion photoproduction on the proton: new results from Mainz

2005R029 C.D.Roper, W.Tornow, R.T.Braun, Q.Chen, A.Crowell, D.Gonzalez Trotter, C.R.Howell, F.Salinas, R.Setze, R.L.Walter, Z.Chen, H.Tang, Z.Zhou - Phys.Rev. C 72,024605 (2005)

Measurements of $\mathrm{A}_{y}(\theta)$ for ${ }^{12} \mathrm{C}(\mathrm{n}, \mathrm{n}){ }^{12} \mathrm{C}$ from $\mathrm{E}_{n}=2.2$ to $8.5 \mathrm{MeV}$

2005R035 S.D.Rosner, D.Masterman, T.J.Scholl, R.A.Holt - Can.J.Phys. 83, 841 (2005)

Measurement of hyperfine structure and isotope shifts in Nd II 
2005R037 G.V.Rogachev, A.A.Aprahamian, F.D.Becchetti, P.Boutachkov, Y.Chen, G.Chubarian, P.A.DeYoung, A.Fomichev, V.Z.Goldberg, M.S.Golovkov, J.J.Kolata, Yu.Ts.Oganessian, G.F.Peaslee, M.Quinn, A.Rodin, B.B.Skorodumov, R.S.Slepnev, G.Ter-Akopian, W.H.Trzaska, A.Wohr, R.Wolski - Nucl.Instrum.Methods Phys.Res. B241, 977 (2005)

Isobaric analog states as a tool for spectroscopy of exotic nuclei

2005R039 D.Rodriguez, V.S.Kolhinen, G.Audi, J.Aysto, D.Beck, K.Blaum, G.Bollen, F.Herfurth, A.Jokinen, A.Kellerbauer, H.-J.Kluge, M.Oinonen, H.Schatz, E.Sauvan, S.Schwarz - Eur.Phys.J. A 25, Supplement 1, 41 (2005)

Mass measurement on the rp-process waiting point ${ }^{72} \mathrm{Kr}$

2005R040 A.P.Robinson, C.N.Davids, D.Seweryniak, P.J.Woods, B.Blank, M.P.Carpenter, T.Davinson, S.J.Freeman, N.Hammond, N.Hoteling, R.V.F.Janssens, T.L.Khoo, Z.Liu, G.Mukherjee, C.Scholey, J.Shergur, S.Sinha, A.A.Sonzogni, W.B.Walters, A.Woehr - Eur.Phys.J. A 25, Supplement 1, 155 (2005)

Recoil decay tagging study of ${ }^{146} \mathrm{Tm}$

2005R042 M.Romoli, M.Mazzocco, E.Vardaci, M.Di Pietro, A.De Francesco, R.Bonetti, A.De Rosa, T.Glodariu, A.Guglielmetti, G.Inglima, M.La Commara, B.Martin, V.Masone, P.Parascandolo, D.Pierroutsakou, M.Sandoli, P.Scopel, C.Signorini, F.Soramel, L.Stroe, J.Greene, A.Heinz, D.Henderson, C.L.Jiang, E.F.Moore, R.C.Pardo, K.E.Rehm, A.Wuosmaa, J.F.Liang - Eur.Phys.J. A 25, Supplement 1, 289 (2005) The EXODET apparatus: Features and first experimental results

2005R0ZX D.Rohe, and the E97-006 Collaboration - nucl-ex/0506007,6/05/2005 (2005) Nuclear transparency from quasielastic ${ }^{12} \mathrm{C}(\mathrm{e}, \mathrm{e} p)$

2005ROZY A.P.Robinson, C.N.Davids, D.Seweryniak, P.J.Woods, B.Blank, M.P.Carpenter, T.Davinson, S.J.Freeman, N.Hammond, N.Hoteling, R.V.F.Janssens, Z.Liu, G.Mukherjee, C.Scholey, J.Shergur, S.Sinha, A.A.Sonzogni, W.B.Walters, A.Woehr - Proc.Nuclei at the Limits, Argonne, Illinois, D.Seweryniak and T.L.Khoo, eds., p.217 (2005); AIP Conf. Proc 764 (2005)

Recoil Decay Tagging Study Of Transitional Proton Emitters ${ }^{145,146,147} \mathrm{Tm}$

2005RU01 C.Ruiz, T.Davinson, F.Sarazin, I.Roberts, A.Robinson, P.J.Woods, L.Buchmann, A.C.Shotter, P.Walden, N.M.Clarke, A.A.Chen, B.R.Fulton, D.Groombridge, J.Pearson, A.S.Murphy - Phys.Rev. C 71, 025802 (2005)

Multichannel R-matrix analysis of elastic and inelastic resonances in the ${ }^{21} \mathrm{Na}+\mathrm{p}$ compound system

2005RU03 A.A.Rudchik, A.T.Rudchik, A.Budzanowski, A.Szczurek, B.Czech, T.Czosnyka, J.Choinski, L.Glowacka, S.Kliczewski, E.I.Koshchy, S.Yu.Mezhevych, A.V.Mokhnach, O.A.Momotyuk, Val.M.Pirnak, R.Siudak, I.Skwirczynska -

Eur.Phys.J. A 23, 445 (2005)

Mechanism of the ${ }^{12} \mathrm{C}\left({ }^{11} \mathrm{~B},{ }^{15} \mathrm{~N}\right){ }^{8} \mathrm{Be}$ reaction and ${ }^{8} \mathrm{Be}+{ }^{15} \mathrm{~N}$ optical-model potential

2005RU04 R.C.Runkle, A.E.Champagne, C.Angulo, C.Fox, C.Iliadis, R.Longland, J.Pollanen Phys.Rev.Lett. 94, 082503 (2005)

Direct Measurement of the ${ }^{14} \mathrm{~N}(\mathrm{p}, \gamma){ }^{15} \mathrm{O}$ S Factor 
2005 RU06 D.Rudolph, E.K.Johansson, L.-L.Andersson, J.Ekman, C.Fahlander, R.du Rietz Nucl.Phys. A752, 241c (2005)

Exotic Decay Modes in Rotating Nuclei

2005RU07 B.Rubio, E.Nacher, A.Algora, M.J.G.Borge, L.Caballero, D.Cano-Ott, S.Courtin, Ph.Dessagne, D.Escrig, L.M.Fraile, W.Gelletly, A.Jungclaus, G.Le Scornet, F.Marechal, Ch.Miehe, E.Poirier, J.L.Tain, O.Tengblad - Nucl.Phys. A752, 251c (2005)

Beta decay studies far from stability with the Total Absorption Technique: the case of ${ }^{76} \mathrm{Sr}$

2005RU14 G.Rusev, R.Schwengner, F.Donau, S.Frauendorf, L.Kaubler, L.K.Kostov, S.Mallion, K.D.Schilling, A.Wagner, E.Grosse, H.von Garrel, U.Kneissl, C.Kohstall, M.Kreutz, H.H.Pitz, M.Scheck, F.Stedile, P.von Brentano, J.Jolie, A.Linnemann, N.Pietralla, V.Werner - Phys.Rev.Lett. 95, 062501 (2005)

Decay of $1^{+}$States as a New Probe of the Structure of $0^{+}$Shape Isomers

2005RU15 C.Ruiz, M.Aliotta, R.E.Azuma, R.N.Boyd, L.Buchmann, A.Chen, N.M.Clarke, J.M.D'Auria, T.Davinson, B.R.Fulton, D.Groombridge, D.Hutcheon, A.M.Laird, A.S.Murphy, J.Pearson, I.Roberts, A.Robinson, F.Sarazin, A.C.Shotter, P.Walden, P.J.Woods - Nucl.Phys. A758, 166c (2005)

Multichannel R-matrix analysis of elastic and inelastic resonances in the ${ }^{20,21} \mathrm{Na}+\mathrm{p}$ compound systems

2005RU18 A.A.Rudchik, A.T.Rudchik, G.M.Kozeratska, O.A.Ponkratenko, E.I.Koshchy, A.Budzanowski, B.Czech, S.Kliczewski, R.Siudak, I.Skwirczynska, A.Szczurek, S.Yu.Mezhevych, K.W.Kemper, J.Choinski, T.Czosnyka, L.Glowacka - Phys.Rev. C $72,034608(2005)$

${ }^{7} \mathrm{Li}+{ }^{11} \mathrm{~B}$ elastic and inelastic scattering in a coupled-reaction-channels approach

2005RUZZ G.Rusev, E.Grosse, M.Erhard, A.Junghans, K.Kosev, K.-D.Schilling, R.Schwengner, A.Wagner - nucl-ex/0512027,12/20/2005 (2005)

Pygmy dipole strength close to particle-separation energies - the case of the Mo isotopes

2005RV01 M.M.Rvachev, and the Jefferson Lab Hall A Collaboration - Phys.Rev.Lett. 94, $192302(2005)$

Quasielastic ${ }^{3} \mathrm{He}(\mathrm{e} \text {, e'p })^{2} \mathrm{H}$ Reaction at $\mathrm{Q}^{2}=1.5 \mathrm{GeV}^{2}$ for Recoil Momenta up to 1 $\mathrm{GeV} / \mathrm{c}$

2005RY03 I.V.Ryzhov, M.S.Onegin, G.A.Tutin, J.Blomgren, N.Olsson, A.V.Prokofiev, P.-U.Renberg - Nucl.Phys. A760, 19 (2005)

Influence of multichance fission on fragment angular anisotropy in the ${ }^{232} \mathrm{Th}(\mathrm{n}, \mathrm{f})$ and ${ }^{238} \mathrm{U}(\mathrm{n}, \mathrm{f})$ reactions at intermediate energies 
2005RYZZ K.P.Rykaczewski, R.K.Grzywacz, D.R.Bingham, M.Danchev, C.Mazzocchi, M.N.Tantawy, C.J.Gross, C.H.Yu, J.C.Batchelder, M.Karny, W.Krolas, D.Fong, J.H.Hamilton, A.V.Ramayya, A.Piechaczek, E.Zganjar, J.A.Winger, T.N.Ginter, A.Stolz, K.Hagino - Proc.Nuclei at the Limits, Argonne, Illinois, D.Seweryniak and T.L.Khoo, eds., p.223 (2005); AIP Conf. Proc 764 (2005)

Structure Of Rare-Earth Nuclei Around The Proton Drip Line

2005SA03 P.Saha, T.Fukuda, W.Imoto, K.K.Ahn, A.Ajimura, K.Aoki, H.C.Bhang, H.Fujioka, H.Hotchi, J.I.Hwang, T.Itabashi, B.H.Kang, H.D.Kim, M.J.Kim, T.Kishimoto, A.Krutenkova, T.Maruta, Y.Miura, K.Miwa, T.Nagae, H.Noumi, H.Outa, T.Ohtaki, A.Sakaguchi, Y.Sato, M.Sekimoto, Y.Shimizu, H.Tamura, K.Tanida, A.Toyoda, M.Ukai, H.J.Kim - Phys.Rev.Lett. 94, 052502 (2005)

Production of the Neutron-Rich Hypernucleus ${ }_{\Lambda}^{10} \mathrm{Li}$ in the $\left(\pi^{-}, \mathrm{K}^{+}\right)$Double Charge-Exchange Reaction

2005SA04 Y.Sato, S.Ajimura, K.Aoki, H.Bhang, T.Hasegawa, O.Hashimoto, H.Hotchi, Y.D.Kim, T.Kishimoto, K.Maeda, H.Noumi, Y.Ohta, K.Omata, H.Outa, H.Park, M.Sekimoto, T.Shibata, T.Takahashi, M.Youn - Phys.Rev. C 71, 025203 (2005)

Mesonic and nonmesonic weak decay widths of medium-heavy $\Lambda$ hypernuclei

2005SA06 M.Sarsour, T.Peterson, M.Planinic, S.E.Vigdor, C.Allgower, B.Bergenwall, J.Blomgren, T.Hossbach, W.W.Jacobs, C.Johansson, J.Klug, A.V.Klyachko, P.Nadel-Turonski, L.Nilsson, N.Olsson, S.Pomp, J.Rapaport, T.Rinckel, E.J.Stephenson, U.Tippawan, S.W.Wissink, Y.Zhou - Phys.Rev.Lett. 94, 082303 (2005)

Measurement of the Absolute np Scattering Differential Cross Section at $194 \mathrm{MeV}$

2005SA07 X.Sarazin, on behalf of the NEMO Collaboration - Nucl.Phys. B(Proc.Supp.) S143, $221(2005)$

Search for neutrinoless double beta decay with the NEMO-3 detector: first results

2005SA12 A.Saha, for the Hall A Collaboration - Eur.Phys.J. A 24, Supplement 1, 81 (2005)

Detailed study of the few nucleon systems with (e, e'p) reactions

2005 SA15 D.Savran, S.Muller, A.Zilges, M.Babilon, M.W.Ahmed, J.H.Kelley, A.Tonchev, W.Tornow, H.R.Weller, N.Pietralla, J.Li, I.V.Pinayev, Y.K.Wu - Phys.Rev. C 71, 034304 (2005)

Parity assignments in ${ }^{172,174} \mathrm{Yb}$ using polarized photons and the $\mathrm{K}$ quantum number in rare earth nuclei

2005SA37 T.Saito, K.Yoshida, M.Oikawa, Y.Suga, K.Kino, T.Nakagawa, T.Tohei, K.Abe, H.Ueno - Phys.Rev. C 71, 064313 (2005)

Direct evidence of core excitation in the giant resonance through the (e, e'n) reaction

2005SA40 J.Sauvage, F.Ibrahim, B.Roussiere, J.Genevey, A.Gizon, G.Marguier, P.Kilcher, A.Knipper, R.Beraud, G.Cata-Danil, F.Le Blanc, J.Obert, J.Oms, J.C.Putaux, C.Richard-Serre, A.Wojtasiewicz, and the ISOLDE and NICOLE Collaborations Eur.Phys.J. A 25, 5 (2005)

Low-spin states of doubly odd ${ }^{184} \mathrm{Au}$ 
2005SA44 G.Savard, F.Buchinger, J.A.Clark, J.E.Crawford, S.Gulick, J.C.Hardy, A.A.Hecht, J.K.P.Lee, A.F.Levand, N.D.Scielzo, H.Sharma, K.S.Sharma, I.Tanihata, A.C.C.Villari, Y.Wang - Phys.Rev.Lett. 95, 102501 (2005)

Q Value of the Superallowed Decay of ${ }^{46} \mathrm{~V}$ and Its Influence on $\mathrm{V}_{u d}$ and the Unitarity of the Cabibbo-Kobayashi-Maskawa Matrix

2005SA52 A.M.Sanchez-Benitez, D.Escrig, M.A.G.Alvarez, M.V.Andres, C.Angulo, M.J.G.Borge, J.Cabrera, S.Cherubini, J.M.Espino, P.Figuera, M.Freer,

J.E.Garcia-Ramos, J.Gomez-Camacho, M.Gulino, O.R.Kakuee, I.Martel, C.Metelco, A.M.Moro, J.Rahighi, K.Rusek, D.Smirnov, O.Tengblad, P.Van Duppen, V.Ziman J.Phys.(London) G31, S1953 (2005)

Scattering of ${ }^{6} \mathrm{He}$ at energies around the Coulomb barrier

2005SAZS T.Saito, H.Sakai, T.Ikeda, K.Itoh, T.Kawabata, H.Kuboki, Y.Maeda, N.Matsui, M.Sasano, Y.Satou, K.Sekiguchi, K.Suda, A.Tamii, T.Uesaka, K.Yako -

CNS-REP-66, p.32 (2005)

Bell's Inequality Test via the ${ }^{1} \mathrm{H}\left(\mathrm{d},{ }^{2} \mathrm{He}\right) \mathrm{n}$ Reaction

2005SAZT S.Sakaguchi, T.Wakui, T.Uesaka, K.Itoh, T.Kawabata, H.Kuboki, Y.Maeda, H.Sakai, Y.Sasamoto, M.Sasano, K.Sekiguchi, K.Suda, Y.Takahashi, K.Yako RIKEN Accelerator Progress Report 2004, p.36 (2005)

Absolute calibration of proton polarization via $\mathrm{p}(\mathrm{pol})+{ }^{4} \mathrm{He}$ elastic scattering

2005SAZU H.Sakane, K.Furutaka, O.Shcherbakov, H.Harada, T.Fujii, H.Yamana Proc.Intern.Conf.Nuclear Data for Science and Technology, Santa Fe, New Mexico, 26 September-1 October, 2004, R.C.Haight, M.B.Chadwick, T.Kawano, P.Talou, Eds., Vol.1, p. 1000 (2005); AIP Conf.Proc. 769 (2005)

Development of a Method of Thermal-Neutron Capture Cross Section Measurement by Unfolding Prompt Gamma-Ray Spectra

2005SAZX A.Sanchez i Zafra, C.Beck, F.Haas, P.Papka, V.Rauch, M.Rousseau, F.Azaiez, P.Bednarczyk, S.Courtin, D.Curien, O.Dorvaux, A.Nourreddine, J.Robin, M.D.Salsac, W.von Oertzen, B.Gebauer, T.Kokalova, S.Thummerer, C.Wheldon, G.de Angelis, A.Gadea, S.Lenzi, D.R.Napoli, S.Szilner, W.N.Catford, D.Jenkins Proc. XLIII Intern.Winter Meeting on Nuclear Physics, Bormio, Italy, 14 - 19 March 2005, I.Iori and A.Bortolotti, eds., p.224 (2005)

Study of binary reaction channels in the ${ }^{24} \mathrm{Mg}+{ }^{12} \mathrm{C}$ collision

2005SAZY T.R.Saito, for the RISING Collaboration - Proc.Nuclei at the Limits, Argonne, Illinois, D.Seweryniak And T.L.Khoo, eds., p.151 (2005); AIP Conf. Proc 764 (2005) The RISING Project At GSI And Its First Results

2005SAzZ D.Savran, S.Muller, A.Zilges, M.Babilon, M.W.Ahmed, J.H.Kelley, A.Tonchev, W.Tornow, H.R.Weller, N.Pietralla, J.Li, I.V.Pinayev, Y.K.Wu nucl-ex/0501006,1/11/2005 (2005)

Parity assignments in ${ }^{172,174} \mathrm{Yb}$ using polarized photons and the $\mathrm{K}$ quantum number in rare earth nuclei

2005SC01 R.J.Scott, R.P.Rassool, M.N.Thompson, D.V.Webb - Nucl.Instrum.Methods Phys.Res. A539, 191 (2005) 
A new technique for precise measurement of the half-life of the Fermi $\beta$-decay of ${ }^{26 m} \mathrm{Al}$

2005SC17 D.Schurmann, A.Di Leva, N.De Cesare, L.Gialanella, G.Imbriani, C.Lubritto, A.Ordine, V.Roca, H.Rocken, C.Rolfs, D.Rogalla, M.Romano, F.Schumann, F.Strieder, F.Terrasi, H.-P.Trautvetter - Nucl.Phys. A758, 367c (2005)

Measurement of the cross section of ${ }^{12} \mathrm{C}(\alpha, \gamma){ }^{16} \mathrm{O}$ using the recoil mass separator ERNA

2005SC20 A.Schiller, T.Baumann, J.Dietrich, S.Kaiser, W.Peters, M.Thoennessen - Phys.Rev. C 72, $037601(2005)$

Search for particle-bound ${ }^{26} \mathrm{O}$ and ${ }^{28} \mathrm{~F}$ in p-stripping reactions

2005SC22 C.Scholey, M.Sandzelius, S.Eeckhaudt, T.Grahn, P.T.Greenlees, P.Jones, R.Julin, S.Juutinen, M.Leino, A.-P.Leppanen, P.Nieminen, M.Nyman, J.Perkowski, J.Pakarinen, P.Rahkila, P.M.Rahkila, J.Uusitalo, K.Van de Vel, B.Cederwall, B.Hadinia, K.Lagergren, D.T.Joss, D.E.Appelbe, C.J.Barton, J.Simpson, D.D.Warner, I.G.Darby, R.D.Page, E.S.Paul, D.Wiseman - J.Phys.(London) G31, S1719 (2005)

In-beam and decay spectroscopy of very neutron deficient iridium nuclei

2005SC26 P.Schury, G.Bollen, D.A.Davies, A.Doemer, D.Lawton, D.J.Morrissey, J.Ottarson, A.Prinke, R.Ringle, T.Sun, S.Schwarz, L.Weissman - Eur.Phys.J. A 25, Supplement 1, $51(2005)$

Precision experiments with rare isotopes with LEBIT at MSU

2005SC27 H.Scheit, O.Niedermaier, V.Bildstein, H.Boie, J.Fitting, R.von Hahn, F.Kock, M.Lauer, U.K.Pal, H.Podlech, R.Repnow, D.Schwalm, C.Alvarez, F.Ames, G.Bollen, S.Emhofer, D.Habs, O.Kester, R.Lutter, K.Rudolph, M.Pasini, P.G.Thirolf, B.H.Wolf, J.Eberth, G.Gersch, H.Hess, P.Reiter, O.Thelen, N.Warr, D.Weisshaar, F.Aksouh, P.Van den Bergh, P.Van Duppen, M.Huyse, O.Ivanov, P.Mayet, J.Van de Walle, J.Aysto, P.A.Butler, J.Cederkall, P.Delahaye, H.O.U.Fynbo, L.M.Fraile, O.Forstner, S.Franchoo, U.Koster, T.Nilsson, M.Oinonen, T.Sieber, F.Wenander, M.Pantea, A.Richter, G.Schrieder, H.Simon, T.Behrens, R.Gernhauser, T.Kroll, R.Krucken, M.Munch, T.Davinson, J.Gerl, G.Huber, A.Hurst, J.Iwanicki, B.Jonson, P.Lieb, L.Liljeby, A.Schempp, A.Scherillo, P.Schmidt, G.Walter - Eur.Phys.J. A 25, Supplement 1, 397 (2005)

Coulomb excitation of neutron-rich beams at REX-ISOLDE

2005SC28 H.Schatz, P.T.Hosmer, A.Aprahamian, O.Arndt, R.R.C.Clement, A.Estrade, K.-L.Kratz, S.N.Liddick, P.F.Mantica, W.F.Mueller, F.Montes, A.C.Morton, M.Ouellette, E.Pellegrini, B.Pfeiffer, P.Reeder, P.Santi, M.Steiner, A.Stolz, B.E.Tomlin, W.B.Walters, A.Wohr - Eur.Phys.J. A 25, Supplement 1, 639 (2005) The half-life of the doubly-magic r-process nucleus ${ }^{78} \mathrm{Ni}$

2005SC29 D.Schurmann, A.Di Leva, L.Gialanella, D.Rogalla, F.Strieder, N.De Cesare, A.D'Onofrio, G.Imbriani, R.Kunz, C.Lubritto, A.Ordine, V.Roca, C.Rolfs, M.Romano, F.Schumann, F.Terrasi, H.-P.Trautvetter - Eur.Phys.J. A 26, 301 (2005) First direct measurement of the total cross-section of ${ }^{12} \mathrm{C}(\alpha, \gamma){ }^{16} \mathrm{O}$ 
2005SCZT D.Schurmann, A.Di Leva, L.Gialanella, D.Rogalla, F.Strieder, N.De Cesare, A.D'Onofrio, G.Imbriani, R.Kunz, C.Lubritto, A.Ordine, V.Roca, C.Rolfs, M.Romano, F.Schumann, F.Terrasi, H.-P.Trautvetter - nucl-ex/0511050,11/29/2005 (2005)

First direct measurement of the total cross section of ${ }^{12} \mathrm{C}(\alpha, \gamma){ }^{16} \mathrm{O}$

2005SCZV W.Schwerdtfeger, B.Bruyneel, T.Faestermann, R.Gernhauser, D.Habs, Th.Kroll, R.Krucken, M.Lauer, R.Lutter, H.J.Maier, T.Morgan, M.Munch, O.Niedermaier, P.Reiter, O.Schaile, H.Scheit, P.G.Thirolf, W.von Oertzen, N.Warr, D.Weisshaar, H.Wolter - Maier-Leibnitz-Laboratorium 2004 Ann.Rept., p.4 (2005)

Towards Two-Neutron Transfer around the Island of Inversion

2005SCZW D.Schumann, R.Weinreich, R.Michel, H.-A.Synal, P.Kubik -

Proc.Intern.Conf.Nuclear Data for Science and Technology, Santa Fe, New Mexico, 26 September-1 October, 2004, R.C.Haight, M.B.Chadwick, T.Kawano, P.Talou, Eds., Vol.2, p. 1517 (2005); AIP Conf.Proc. 769 (2005)

Excitation Functions for the Production of ${ }^{10} \mathrm{Be},{ }^{26} \mathrm{Al},{ }^{129} \mathrm{I}$, and ${ }^{36} \mathrm{Cl}$ in the Reaction ${ }^{n a t} \mathrm{~Pb}(\mathrm{p}, \mathrm{xn}-\mathrm{yp})^{A} \mathrm{Z}$ with Proton Energies up to $2.6 \mathrm{GeV}$

2005SCZX F.Schumann, S.Typel, F.Hammache, K.Summerer, F.Uhlig, I.Bottcher, D.Cortina, A.Forster, M.Gai, H.Geissel, U.Greife, E.Grosse, N.Iwasa, P.Koczon, B.Kohlmeyer, R.Kulessa, H.Kumagai, N.Kurz, M.Menzel, T.Motobayashi, H.Oeschler, A.Ozawa, M.Ploskon, W.Prokopowicz, E.Schwab, P.Senger, F.Strieder, C.Sturm, Z.-Y.Sun, G.Surowka, A.Wagner, W.Walus - nucl-ex/0508014,08/11/2005 (2005)

Coulomb dissociation of ${ }^{8} \mathrm{~B}$ and the low-energy cross section of the ${ }^{7} \mathrm{Be}(\mathrm{p}, \gamma)^{8} \mathrm{~B}$ solar fusion reaction

2005SCZY

2005SE02

2005SE03

2005SE04
A.Schiller, T.Baumann, J.Dietrich, S.Kaiser, W.Peters, M.Thoennessen nucl-ex/0504007,4/5/2005 (2005)

Search for particle-bound ${ }^{26} \mathrm{O}$ and ${ }^{28} \mathrm{~F}$ in p-stripping reactions

A.Serebrov, V.Varlamov, A.Kharitonov, A.Fomin, Yu.Pokotilovski, P.Geltenbort, J.Butterworth, I.Krasnoschekova, M.Lasakov, R.Taldaev, A.Vassiliev, O.Zherebtsov - Phys.Lett. B 605, 72 (2005)

Measurement of the neutron lifetime using a gravitational trap and a low-temperature Fomblin coating

D.Seweryniak, P.J.Woods, M.P.Carpenter, T.Davinson, R.V.F.Janssens, D.G.Jenkins, T.Lauritsen, C.J.Lister, C.Ruiz, J.Shergur, S.Sinha, A.Woehr Phys.Rev.Lett. 94, 032501 (2005)

Level Structure of ${ }^{22} \mathrm{Mg}$ : Implications for the ${ }^{21} \mathrm{Na}(\mathrm{p}, \gamma){ }^{22} \mathrm{Mg}$ Astrophysical Reaction Rate and for the ${ }^{22} \mathrm{Mg}$ Mass

J.P.Seitz, B.B.Back, M.P.Carpenter, I.Dioszegi, K.Eisenman, P.Heckman, D.J.Hofman, M.P.Kelly, T.L.Khoo, S.Mitsuoka, V.Nanal, T.Pennington, R.H.Siemssen, M.Thoennessen, R.L.Varner - Nucl.Phys. A750, 245 (2005) Observation of the hot GDR in neutron-deficient thorium evaporation residues

R.Segel, and the JLab E01-001 Collaboration - Eur.Phys.J. A 24, Supplement 1, 55 (2005) 
Results from the super Rosenbluth separation experiment

2005SE05 H.R.Setze, C.R.Howell, W.Tornow, R.T.Braun, D.E.Gonzalez Trotter, A.H.Hussein, R.S.Pedroni, C.D.Roper, F.Salinas, I.Slaus, B.Vlahovic, R.L.Walter, G.Mertens, J.M.Lambert, H.Witala, W.Glockle - Phys.Rev. C 71, 034006 (2005)

Cross-section measurements of neutron-deuteron breakup at $13.0 \mathrm{MeV}$

2005SE08 N.Severijns, A.A.Belyaev, A.L.Erzinkyan, P.-D.Eversheim, V.T.Filimonov, V.V.Golovko, G.M.Gurevich, P.Herzog, I.S.Kraev, A.A.Lukhanin, V.I.Noga, V.P.Parfenova, T.Phalet, A.V.Rusakov, Yu.G.Toporov, C.Tramm, V.N.Vyachin, D.Zakoucky, E.Zotov - Phys.Rev. C 71, 044324 (2005)

Angular distribution of particles from oriented ${ }^{253,254} \mathrm{Es}$ and ${ }^{255} \mathrm{Fm}$ nuclei

2005SE11 D.Seweryniak, J.Uusitalo, P.Bhattacharyya, M.P.Carpenter, J.A.Cizewski, K.Y.Ding, C.N.Davids, N.Fotiades, R.V.F.Janssens, T.Lauritsen, C.J.Lister, A.O.Macchiavelli, D.Nisius, P.Reiter, W.B.Walters, P.J.Woods - Phys.Rev. C 71, 054319 (2005)

Multiparticle configurations in $\mathrm{N}=84$ isotones located at the proton drip line

2005SE14 N.Severijns, D.Venos, P.Schuurmans, T.Phalet, M.Honusek, D.Srnka, B.Vereecke, S.Versyck, D.Zakoucky, U.Koster, M.Beck, B.Delaure, V.Golovko, I.Kraev Phys.Rev. C 71, 064310 (2005)

Isospin mixing in the $\mathrm{T}=5 / 2$ ground state of ${ }^{71} \mathrm{As}$

2005SE16 M.Seimetz, for the A1 Collaboration - Nucl.Phys. A755, 253c (2005) Measurement of the Electric Form Factor of the Neutron at MAMI

2005SE17 A.Serebrov, V.Varlamov, A.Kharitonov, A.Fomin, Yu.Pokotilovski, P.Geltenbort, J.Butterworth, I.Krasnoschekova, M.Lasakov, R.Taldaev, A.Vassiliev, O.Zherebtsov - J.Res.Natl.Inst.Stand.Technol. 110, 333 (2005)

Measurement of the Neutron Lifetime Using a Gravitational Trap and a

Low-Temperature Fomblin Coating

2005SE19 M.Segawa, Y.Nagai, T.Shima, H.Makii, K.Mishima, H.Ueda, Y.Temma, T.Masaki, M.Igashira, T.Ohsaki, T.Shizuma, T.Hayakawa - Nucl.Phys. A758, 553c (2005)

Neutron experiment for the study of Re / Os cosmochronometer

2005SE22 K.Sekiguchi, H.Sakai, H.Witala, W.Glockle, J.Golak, K.Hatanaka, M.Hatano, K.Itoh, H.Kamada, H.Kuboki, Y.Maeda, A.Nogga, H.Okamura, T.Saito, N.Sakamoto, Y.Sakemi, M.Sasano, Y.Shimizu, K.Suda, A.Tamii, T.Uesaka, T.Wakasa, K.Yako - Phys.Rev.Lett. 95, 162301 (2005)

Resolving the Discrepancy of $135 \mathrm{MeV}$ pd Elastic Scattering Cross Section and Relativistic Effects

2005SE23 M.Segawa, A.Tomyo, Y.Nagai, Y.Temma, T.Masaki, T.Shima, J.Nishiyama, T.Ohsaki, M.Igashira - J.Phys.Soc.Jpn. 74, 2981 (2005)

New Approach for Measuring the $(n, \gamma)$ Cross Section of a Nucleus by a Few keV Neutron 
2005SE26 D.Seweryniak, C.N.Davids, A.Robinson, P.J.Woods, B.Blank, M.P.Carpenter, T.Davinson, S.J.Freeman, N.Hammond, N.Hoteling, R.V.F.Janssens, T.L.Khoo, Z.Liu, G.Mukherjee, J.Shergur, S.Sinha, A.A.Sonzogni, W.B.Walters, A.Woehr Eur.Phys.J. A 25, Supplement 1, 159 (2005)

Particle-core coupling in the transitional proton emitters ${ }^{145,146,147} \mathrm{Tm}$

2005SEZV K.Sekiguchi, H.Sakai, H.Witala, W.Gloeckle, J.Golak, K.Hatanaka, M.Hatano, K.Itoh, H.Kamada, H.Kuboki, Y.Maeda, A.Nogga, H.Okamura, T.Saito, N.Sakamoto, Y.Sakemi, M.Sasano, Y.Shimizu, K.Suda, A.Tamii, T.Uesaka, T.Wakasa, K.Yako - nucl-ex/0510005,10/3/2005 (2005)

Resolving the Discrepancy of $135 \mathrm{MeV}$ pd Elastic Scattering Cross Sections and Relativistic Effects

2005SEZW V.Semkova, A.J.M.Plompen, D.L.Smith - Proc.Intern.Conf.Nuclear Data for Science and Technology, Santa Fe, New Mexico, 26 September-1 October, 2004, R.C.Haight, M.B.Chadwick, T.Kawano, P.Talou, Eds., Vol.1, p. 1019 (2005); AIP Conf.Proc. $769(2005)$

Measurement of the ${ }^{58} \mathrm{Ni}(\mathrm{n}, \mathrm{t}){ }^{56} \mathrm{Co},{ }^{59} \mathrm{Co}(\mathrm{n}, \mathrm{p}){ }^{59} \mathrm{Fe}$, and ${ }^{63} \mathrm{Cu}(\mathrm{n}, \alpha){ }^{60} \mathrm{Co}$ Reaction Cross Sections from 14 to $20 \mathrm{MeV}$

2005SEZX V.Semkova, A.J.M.Plompen, D.L.Smith - Proc.Intern.Conf.Nuclear Data for Science and Technology, Santa Fe, New Mexico, 26 September-1 October, 2004, R.C.Haight, M.B.Chadwick, T.Kawano, P.Talou, Eds., Vol.1, p. 981 (2005); AIP Conf.Proc. 769 (2005)

Light Charged-Particle Production Activation Cross Sections of Zr Isotopes from 14 to $20 \mathrm{MeV}$

2005SH03 S.Shen, Y.Li, S.Shi, J.Gu, J.Liu - J.Phys.Soc.Jpn. 74, 299 (2005)

New Low-Spin Levels in ${ }^{72}$ Ge and Discussion of Its Yrast Band Structure

2005 SH15 O.Shcherbakov, K.Furutaka, S.Nakamura, H.Sakane, K.Kobayashi, S.Yamamoto, J.-I.Hori, H.Harada - J.Nucl.Sci.Technol.(Tokyo) 42, 135 (2005)

Measurement of Neutron Capture Cross Section of ${ }^{237} \mathrm{~Np}$ from 0.02 to $100 \mathrm{eV}$

2005 SH22 T.Shimizu, I.Miyazaki, K.Arakita, M.Shibata, K.Kawade, J.Hori, T.Nishitani Ann.Nucl.Energy 32, 949 (2005)

Measurement of (n, n') reaction cross-sections of ${ }^{79} \mathrm{Br},{ }^{90} \mathrm{Zr},{ }^{197} \mathrm{Au}$ and ${ }^{207} \mathrm{~Pb}$ with pulsed d-D neutrons

2005 SH23 J.Shergur, A.Wohr, W.B.Walters, K.-L.Kratz, O.Arndt, B.A.Brown, J.Cederkall, I.Dillmann, L.M.Fraile, P.Hoff, A.Joinet, U.Koster, B.Pfeiffer - Phys.Rev. C 71, $064321(2005)$

Level structure of odd-odd ${ }^{134} \mathrm{Sb}$ populated in the $\beta^{-}$decays of ${ }^{134,135} \mathrm{Sn}$

2005SH24 J.Shergur, D.J.Dean, D.Seweryniak, W.B.Walters, A.Wohr, P.Boutachkov, C.N.Davids, I.Dillmann, A.Juodagalvis, G.Mukherjee, S.Sinha, A.Teymurazyan, I.Zartova - Phys.Rev. C 71, 064323 (2005)

Identification of low-spin states in ${ }^{111} \mathrm{Sb}$ : Test of spin-orbit coupling in light nuclei 
2005 SH26 T.Shizuma, T.Hayakawa, S.Mitarai, T.Morikawa, T.Ishii - Phys.Rev. C 71, 067301 (2005)

Identification of the $\mathrm{K}^{\pi}=11 / 2^{+}$isomer in neutron-rich ${ }^{187} \mathrm{~W}$

2005 SH36 J.Shergur, A.Wohr, W.B.Walters, K.-L.Kratz, O.Arndt, B.A.Brown, J.Cederkall, I.Dillmann, L.M.Fraile, P.Hoff, A.Joinet, U.Koster, B.Pfeiffer - Phys.Rev. C 72, 024305 (2005)

Identification of shell-model states in ${ }^{135} \mathrm{Sb}$ populated via $\beta^{-}$decay of ${ }^{135} \mathrm{Sn}$

2005 SH37 T.Shizuma, H.Utsunomiya, P.Mohr, T.Hayakawa, S.Goko, A.Makinaga, H.Akimune, T.Yamagata, M.Ohta, H.Ohgaki, Y.-W.Lui, H.Toyokawa, A.Uritani, S.Goriely Phys.Rev. C 72, 025808 (2005)

Photodisintegration cross section measurements on ${ }^{186} \mathrm{~W},{ }^{187} \mathrm{Re}$, and ${ }^{188}$ Os:

Implications for the Re-Os cosmochronology

2005SH38 W.Shi, M.Redshaw, E.G.Myers - Phys.Rev. A 72, 022510 (2005)

Atomic masses of ${ }^{32,33} \mathrm{~S},{ }^{84,86} \mathrm{Kr}$, and ${ }^{129,132} \mathrm{Xe}$ with uncertainties $\leq 0.1 \mathrm{ppb}$

2005SH41 T.Shizuma, H.Utsunomiya, S.Goko, P.Mohr, T.Hayakawa, A.Makinaga, K.Y.Hara, H.Ohgaki, Y.-W.Lui, S.Goriely - Nucl.Phys. A758, 561c (2005)

Photoneutron cross section measurements on ${ }^{186} \mathrm{~W},{ }^{187} \mathrm{Re}$ and ${ }^{188} \mathrm{Os}$ for cosmochronology

2005SH46 S.Shimoura - J.Phys.(London) G31, S1759 (2005)

Single particle states in exotic nuclei via nucleon transfer reactions at $30-60 \mathrm{~A} \mathrm{MeV}$

2005SH49 T.M.Shneidman, R.V.Jolos, R.Krucken, A.Aprahamian, D.Cline, J.R.Cooper, M.Cromaz, R.M.Clark, C.Hutter, A.O.Macchiavelli, W.Scheid, M.A.Stoyer, C.Y.Wu - Eur.Phys.J. A 25, 387 (2005)

E2 transitions between positive- and negative-parity states of the ground-state alternating-parity bands

2005SH51 T.Shima, S.Naito, Y.Nagai, T.Baba, K.Tamura, T.Takahashi, T.Kii, H.Ohgaki, H.Toyokawa - Phys.Rev. C 72, 044004 (2005)

Simultaneous measurement of the photodisintegration of ${ }^{4} \mathrm{He}$ in the giant dipole resonance region

2005SH52 K.S.Sharma, J.Vaz, R.C.Barber, F.Buchinger, J.A.Clark, J.E.Crawford, H.Fukutani, J.P.Greene, S.Gulick, A.Heinz, J.K.P.Lee, G.Savard, Z.Zhou, J.C.Wang -

Eur.Phys.J. A 25, Supplement 1, 45 (2005)

Atomic mass ratios for some stable isotopes of platinum relative to ${ }^{197} \mathrm{Au}$

2005 SH53 J.Shergur, N.Hoteling, A.Wohr, W.B.Walters, O.Arndt, B.A.Brown, C.N.Davids, D.J.Dean, K.-L.Kratz, B.Pfeiffer, D.Seweryniak, and the ISOLDE Collaboration -

Eur.Phys.J. A 25, Supplement 1, 121 (2005)

New level information on $\mathrm{Z}=51$ isotopes, ${ }^{111} \mathrm{Sb}_{60}$ and ${ }^{134,135} \mathrm{Sb}_{83,84}$

2005SHZS A.Shrivastava, A.Navin, N.Keeley, K.Mahata, K.Ramachandran, V.Nanal, V.V.Parkar, A.Chatterjee, S.Kailas - nucl-ex/0512032,12/21/2005 (2005)

Evidence for transfer followed by breakup in ${ }^{7} \mathrm{Li}+{ }^{65} \mathrm{Cu}$ 
2005SHZT O.Shcherbakov, H.Harada, K.Furutaka, S.Nakamura, H.Sakane, K.Kobayashi, S.Yamamoto, J.-i.Hori - Proc.Intern.Conf.Nuclear Data for Science and Technology, Santa Fe, New Mexico, 26 September-1 October, 2004, R.C.Haight, M.B.Chadwick, T.Kawano, P.Talou, Eds., Vol.1, p. 1007 (2005); AIP Conf.Proc. 769 (2005)

Measurement of the Np-237 Neutron Capture Cross Section from Thermal to $100 \mathrm{eV}$

2005SHZU T.Shimizu, K.Arakita, I.Miyazaki, M.Shibata, K.Kawade, J.-i.Hori, K.Ochiai, T.Nishitani - Proc.Intern.Conf.Nuclear Data for Science and Technology, Santa Fe, New Mexico, 26 September-1 October, 2004, R.C.Haight, M.B.Chadwick, T.Kawano, P.Talou, Eds., Vol.1, p. 992 (2005); AIP Conf.Proc. 769 (2005) Measurement of Activation Cross Sections Producing Short-Lived Nuclei with Pulsed Neutron Beam

2005SHZW Yu.Shitov, on behalf of the NEMO Collaboration - Book of Abstracts, LV National Conference on Nuclear Physics "Frontiers in the Physics of Nucleus", St.-Petersburg, p.42 (2005)

Search for Neutrinoless Double Beta Decay with NEMO-3 and SUPERNEMO

2005SHzX T.Shizuma, H.Utsunomiya, P.Mohr, T.Hayakawa, S.Goko, A.Makinaga, H.Akimune, T.Yamagata, M.Ohta, H.Ohgaki, Y.-W.Lui, H.Toyokawa, A.Uritani, S.Goriely nucl-ex/0506027,6/30/2005 (2005)

Photo-disintegration cross section measurements on ${ }^{186} \mathrm{~W},{ }^{187} \mathrm{Re}$ and ${ }^{188} \mathrm{Os}$ : implications to the Re-Os cosmochronology

2005SHZY Y.Shimizu, K.Hatanaka, G.P.A.Berg, M.Wiescher, H.Schatz, T.Adachi, A.D.Bacher, C.C.Foster, K.Fujita, H.Fujita, Y.Fujita, J.Gorres, Ch.Herman, J.Kamiya, Y.Sakemi, Y.Shimbara, E.J.Stephenson, T.Wakasa, M.Yosoi - Proc. Intern. Symposium on the Origin of Matter and Evolution of Galaxies, Riken, Saitama, Japan, 17-19 November 2003, World Scientific, Singapore, p.367 (2005)

Resonance states in ${ }^{22} \mathrm{Mg},{ }^{26} \mathrm{Si}$ for reaction rates in the rp-process

2005SHZZ T.Shima, H.Makii, K.Mishima, Y.Nagai, M.Segawa, H.Ueda, T.Masaki, M.Igashira, T.Ohsaki, I.Sugai, Y.Takeda - Proc. Intern. Symposium on the Origin of Matter and Evolution of Galaxies, Riken, Saitama, Japan, 17-19 November 2003, World Scientific, Singapore, p.217 (2005)

Direct measurement of the E1 and E2 cross sections of the ${ }^{12} \mathrm{C}(\alpha, \gamma){ }^{16} \mathrm{O}$ reaction at $\mathrm{E}_{c . m}=1.3 \sim 1.5 \mathrm{MeV}$

2005 SI02 Z.Siketic, I.Bogdanovic Radovic, H.Muto, M.Jaksic - Nucl.Instrum.Methods Phys.Res. B229, 180 (2005)

$\mathrm{H}$ recoil cross-sections for ${ }^{7} \mathrm{Li}$ ions at 30 degrees and 45 degrees in the energy interval from 2.28 to $5.70 \mathrm{MeV}$

2005SI06 L.Simard, on behalf of the NEMO Collaboration - Nucl.Phys. B(Proc.Supp.) S145, $272(2005)$

Search for neutrinoless double beta decay with the NEMO-3 detector: first results 
2005SI13 A.J.Simons, P.Joshi, D.G.Jenkins, P.M.Raddon, R.Wadsworth, D.B.Fossan, T.Koike, C.Vaman, K.Starosta, E.S.Paul, H.J.Chantler, A.O.Evans, P.Bednarczyk, D.Curien - J.Phys.(London) G31, 541 (2005)

Evidence for chiral structures in ${ }^{130} \mathrm{Cs}$

2005SI14 J.M.Sisterson, J.Ullmann - Nucl.Instrum.Methods Phys.Res. B234, 419 (2005)

Measurements of energy integrated cross sections for reactions producing relatively short-lived radionuclides using neutron beams with an energy range of 0.1-750 MeV

2005SI21 N.L.Singh, S.Mukherjee, M.S.Gadkari - Int.J.Mod.Phys. E14, 611 (2005) Excitation functions of alpha induced reactions on natural nickel up to $50 \mathrm{MeV}$

2005 SI23 A.J.Simons, R.Wadsworth, D.G.Jenkins, R.M.Clark, M.Cromaz, M.A.Deleplanque, R.M.Diamond, P.Fallon, G.J.Lane, I.Y.Lee, A.O.Macchiavelli, F.S.Stephens, C.E.Svensson, K.Vetter, D.Ward, S.Frauendorf, Y.Gu - Phys.Rev. C 72, 024318 (2005) Investigation of antimagnetic rotation in light Cadmium nuclei: ${ }^{106,108} \mathrm{Cd}$

2005 SI28 M.A.G.Silveira, N.H.Medina, J.A.Alcantara-Nunez, E.W.Cybulska, H.Dias, J.R.B.Oliveira, M.N.Rao, R.V.Ribas, W.A.Seale, K.T.Wiedemann, B.A.Brown, M.Honma, T.Mizusaki, T.Otsuka - J.Phys.(London) G31, S1577 (2005)

Nuclear structure of the odd-odd nucleus ${ }^{58} \mathrm{Co}$

2005SI31 K.Singh, Z.Naik, R.Kumar, J.Goswamy, D.Mehta, N.Singh, C.R.Praharaj, E.S.Paul, K.P.Singh, R.P.Singh, S.Muralithar, N.Madhavan, J.J.Das, S.Nath, A.Jhingan, P.Sugathan, R.K.Bhowmik - Eur.Phys.J. A 25, 345 (2005)

Rotational structures in ${ }^{123} \mathrm{Cs}$

2005 SI32 J.M.Sisterson, F.D.Brooks, A.Buffler, M.S.Allie, D.T.L.Jones, M.B.Chadwick Nucl.Instrum.Methods Phys.Res. B240, 617 (2005)

Cross-section measurements for neutron-induced reactions in copper at neutron energies of 70.7 and $110.8 \mathrm{MeV}$

2005SI34 G.Sikler, G.Audi, D.Beck, K.Blaum, G.Bollen, F.Herfurth, A.Kellerbauer, H.-J.Kluge, D.Lunney, M.Oinonen, C.Scheidenberger, S.Schwarz, J.Szerypo, C.Weber - Nucl.Phys. A763, 45 (2005); Erratum Nucl.Phys. A768, 160 (2006) Mass measurements on neutron-deficient Sr and neutron-rich Sn isotopes with the ISOLTRAP mass spectrometer

2005 SI37 M.A.G.Silveira, N.H.Medina, J.R.B.Oliveira, J.A.Alcantara-Nunez, E.W.Cybulska, H.Dias, M.N.Rao, R.V.Ribas, W.A.Seale, K.T.Wiedemann - Braz.J.Phys. 35, 821 (2005)

In-Beam Gamma Ray Spectroscopy of ${ }^{58} \mathrm{Co}$

2005SIZY S.P.Simakov, M.G.Kobosev, A.A.Lychagin, V.A.Talalaev, D.Yu.Chuvilin, V.M.Maslov - Proc.Intern.Conf.Nuclear Data for Science and Technology, Santa Fe, New Mexico, 26 September-1 October, 2004, R.C.Haight, M.B.Chadwick, T.Kawano, P.Talou, Eds., Vol.1, p. 67 (2005); AIP Conf.Proc. 769 (2005) Benchmarking of Uranium-238 Evaluations against Spherical Transmission and (n, xn)-Reaction Experimental Data 
2005SK03 F.Skaza, N.Keeley, V.Lapoux, N.Alamanos, F.Auger, D.Beaumel, E.Becheva, Y.Blumenfeld, F.Delaunay, A.Drouart, A.Gillibert, L.Giot, K.W.Kemper, R.S.Mackintosh, L.Nalpas, A.Pakou, E.C.Pollacco, R.Raabe, P.Roussel-Chomaz, J.-A.Scarpaci, J.-L.Sida, S.Stepantsov, R.Wolski - Phys.Lett. B 619, 82 (2005) Important pickup coupling effect on ${ }^{8} \mathrm{He}(\mathrm{p}, \mathrm{p})$ elastic scattering

2005SK04 V.R.Skoy, T.Ino, Y.Masuda, S.Muto, G.Kim - J.Res.Natl.Inst.Stand.Technol. 110, $471(2005)$

On the Way to Experimental Test of the Time Reversal Invariance in the Nuclear Reactions

2005SkZZ Ye.Skakun, S.M.Qaim - Proc.Intern.Conf.Nuclear Data for Science and Technology, Santa Fe, New Mexico, 26 September-1 October, 2004, R.C.Haight, M.B.Chadwick, T.Kawano, P.Talou, Eds., Vol.2, p. 1634 (2005); AIP Conf.Proc. 769 (2005)

Excitation Functions of Helion-Induced Nuclear Reactions for the Production of the Medical Radioisotope ${ }^{103} \mathrm{Pd}$

2005SM04 D.Smirnov, F.Aksouh, S.Dean, H.De Witte, M.Huyse, O.Ivanov, P.Mayet, I.Mukha, R.Raabe, J.-C.Thomas, P.Van Duppen, C.Angulo, J.Cabrera, A.Ninane, T.Davinson - Nucl.Instrum.Methods Phys.Res. A547, 480 (2005)

Application of a thin double-sided microstrip detector for the registration of $\beta$-delayed charged particles: The ${ }^{6} \mathrm{He} \beta$ decay into the two-body continuum of ${ }^{6} \mathrm{Li}$

2005SM07 J.F.Smith, C.J.Chiara, M.P.Carpenter, H.J.Chantler, P.T.W.Choy, C.N.Davids, M.Devlin, J.L.Durell, D.B.Fossan, S.J.Freeman, R.V.F.Janssens, N.S.Kelsall, T.Koike, D.R.LaFosse, E.S.Paul, P.Reiter, D.G.Sarantites, D.Seweryniak, K.Starosta, R.Wadsworth, A.N.Wilson, P.-H.Heenen - Phys.Lett. B 625, 203 (2005) First observation of very neutron-deficient ${ }^{122} \mathrm{Ce}$

2005SM08 A.G.Smith, R.Orlandi, D.Patel, G.S.Simpson, R.M.Wall, J.F.Smith, O.J.Onakanmi, I.Ahmad, J.P.Greene, M.P.Carpenter, T.Lauritsen, C.J.Lister, R.V.F.Janssens,

F.G.Kondev, D.Seweryniak, B.J.P.Gall, O.Dorvaux, B.Roux - J.Phys.(London) G31, S1433 (2005)

The magnetic properties of collective states in $\mathrm{A} \sim 100$ fission fragments

2005SMZX A.B.Smith - ANL/NDM-161 (2005)

Neutron Scattering from the Standard ${ }^{197} \mathrm{Au}$

2005SMZZ A.N.Smirnov, V.P.Eismont, N.P.Filatov, S.N.Kirillov, J.Blomgren, H.Conde, N.Olsson, M.Duijvestijn, A.Koning - Proc.Intern.Conf.Nuclear Data for Science and Technology, Santa Fe, New Mexico, 26 September-1 October, 2004, R.C.Haight, M.B.Chadwick, T.Kawano, P.Talou, Eds., Vol.1, p. 637 (2005); AIP Conf.Proc. 769 (2005) Correlation of Intermediate Energy Proton- and Neutron-Induced Fission Cross Sections in the Lead-Bismuth Region

2005 S001 L.T.Song, X.H.Zhou, Y.H.Zhang, G.de Angelis, N.Marginean, A.Gadea, D.R.Napoli, M.Axiotis, C.Rusu, T.Martinez, Y.X.Guo, X.G.Lei, Y.Zheng, M.L.Liu - Phys.Rev. C 71, $017302(2005)$ 
High-spin level scheme of ${ }^{183} \mathrm{Au}$

2005 S006 D.Sohler, M.Palacz, Zs.Dombradi, M.Hjorth-Jensen, C.Fahlander, L.-O.Norlin, J.Nyberg, T.Back, K.Lagergren, D.Rudolph, A.Algora, C.Andreoiu, G.de Angelis, A.Atac, D.Bazzacco, J.Cederkall, B.Cederwall, B.Fant, E.Farnea, A.Gadea, M.Gorska, H.Grawe, N.Hashimoto-Saitoh, A.Johnson, A.Kerek, W.Klamra, J.Kownacki, S.M.Lenzi, A.Likar, M.Lipoglavsek, M.Moszynski, D.R.Napoli, C.Rossi-Alvarez, H.A.Roth, T.Saitoh, D.Seweryniak, O.Skeppstedt, J.Timar, M.Weiszflog, M.Wolinska - Nucl.Phys. A753, 251 (2005)

Maximally aligned states in the proton drip line nucleus ${ }^{106} \mathrm{Sb}$

2005S009 D.Sohler, J.Timar, G.Rainovski, P.Joshi, K.Starosta, D.B.Fossan, J.Molnar, R.Wadsworth, A.Algora, P.Bednarczyk, D.Curien, Zs.Dombradi, G.Duchene, A.Gizon, J.Gizon, D.G.Jenkins, T.Koike, A.Krasznahorkay, E.S.Paul, P.M.Raddon, J.N.Scheurer, A.J.Simons, C.Vaman, A.R.Wilkinson, L.Zolnai - Phys.Rev. C 71, $064302(2005)$

High-spin structure of ${ }^{102} \mathrm{Ru}$

2005 S013 N.Soic, M.Freer, L.Donadille, N.M.Clarke, P.J.Leask, W.N.Catford, K.L.Jones, D.Mahboub, B.R.Fulton, B.J.Greenhalgh, D.L.Watson, D.C.Weisser -

J.Phys.(London) G31, S1701 (2005)

Three-centre cluster structure in ${ }^{11} \mathrm{C}$ and ${ }^{11} \mathrm{~B}$

2005 S014 F.A.Souza, R.Liguori Neto, M.M.de Moura, M.G.Munhoz, A.A.P.Suaide, E.M.Szanto, J.Takahashi, A.Szanto de Toledo, N.Carlin - Braz.J.Phys. 35, 888 (2005)

Direct Measurement of the Breakup Process

2005SOZZ N.Soic, M.Freer, L.Donadille, N.M.Clarke, P.J.Leask, W.N.Catford, K.L.Jones, D.Mahboub, B.R.Fulton, B.J.Greenhalgh, D.L.Watson, D.C.Weisser nucl-ex/0504026,4/25/2005 (2005)

Three-centre cluster structure in ${ }^{11} \mathrm{C}$ and ${ }^{11} \mathrm{~B}$

2005SP01 N.F.Sparveris, R.Alarcon, A.M.Bernstein, W.Bertozzi, T.Botto, P.Bourgeois, J.Calarco, F.Casagrande, M.O.Distler, K.Dow, M.Farkhondeh, S.Georgakopoulos, S.Gilad, R.Hicks, M.Holtrop, A.Hotta, X.Jiang, A.Karabarbounis, J.Kirkpatrick, S.Kowalski, R.Milner, R.Miskimen, I.Nakagawa, C.N.Papanicolas, A.J.Sarty, Y.Sato, S.Sirca, J.Shaw, E.Six, S.Stave, E.Stiliaris, T.Tamae, G.Tsentalovich, C.Tschalaer, W.Turchinetz, Z.-L.Zhou, T.Zwart - Phys.Rev.Lett. 94, 022003 (2005)

Investigation of the Conjectured Nucleon Deformation at Low Momentum Transfer

2005SP02 D.T.Spayde, for the SAMPLE Collaboration - Eur.Phys.J. A 24, Supplement 2, 51 (2005)

Updated results from the SAMPLE experiment

2005SP04 I.Spahn, S.Takacs, Yu.N.Shubin, F.Tarkanyi, H.H.Coenen, S.M.Qaim Appl.Radiat.Isot. 63, 235 (2005)

Cross-section measurement of the ${ }^{169} \mathrm{Tm}(\mathrm{p}, \mathrm{n})$ reaction for the production of the therapeutic radionuclide ${ }^{169} \mathrm{Yb}$ and comparison with its reactor-based generation 
2005SPZY K.-H.Speidel, S.Schielke, J.Leske, C.C.Dey, J.Gerber, P.Maier-Komor, S.J.Q.Robinson, Y.Y.Sharon, L.Zamick - Maier-Leibnitz-Laboratorium 2004 Ann.Rept., p.5 (2005)

First Measurements of g Factors of the $2_{1}^{+}$States in ${ }^{36,38} \mathrm{Ar}$ and their Shell Model Interpretation

2005SPZZ S.Spataro, for the HADES Collaboration - Proc. XLIII Intern.Winter Meeting on Nuclear Physics, Bormio, Italy, 14 - 19 March 2005, I.Iori and A.Bortolotti, eds., p.305 (2005)

pp collisions at $2.2 \mathrm{GeV}$ with HADES

2005SR02 J.Srebrny, E.Grodner, T.Morek, I.Zalewska, Ch.Droste, J.Mierzejewski, A.A.Pasternak, J.Kownacki, J.Perkowski - Acta Phys.Pol. B36, 1063 (2005) Search for chirality in ${ }^{128} \mathrm{Cs}$ and ${ }^{132} \mathrm{La}$

2005ST03 F.S.Stephens, M.A.Deleplanque, I.Y.Lee, A.O.Macchiavelli, D.Ward, P.Fallon, M.Cromaz, R.M.Clark, M.Descovich, R.M.Diamond, E.Rodriguez-Vieitez Phys.Rev.Lett. 94, 042501 (2005)

Order-to-Chaos Transition in Rotational Nuclei

2005ST08 E.A.Stefanova, K.P.Lieb, I.Stefanescu, G.De Angelis, D.Curien, J.Eberth, E.Farnea, A.Gadea, G.Gersch, A.Jungclaus, T.Martinez, R.Schwengner, T.Steinhardt, N.Warr, D.Weisshaar, R.Wyss - Eur.Phys.J. A 24, 1 (2005)

Observation of negative-parity high-spin states of ${ }^{68} \mathrm{As}$

2005ST09 A.E.Stuchbery, A.N.Wilson, P.M.Davidson, A.D.Davies, T.J.Mertzimekis, S.N.Liddick, B.E.Tomlin, P.F.Mantica - Phys.Lett. B 611, 81 (2005)

Transient fields for $\mathrm{Mg}$ ions traversing gadolinium hosts at velocities above and below the K-shell electron velocity

2005ST16 L.Stavsetra, K.E.Gregorich, J.Alstad, H.Breivik, K.Eberhardt, C.M.Folden III, T.N.Ginter, M.Johansson, U.W.Kirbach, D.M.Lee, M.Mendel, L.A.Omtvedt, J.B.Patin, G.Skarnemark, R.Sudowe, P.A.Wilk, P.M.Zielinski, H.Nitsche, D.C.Hoffman, J.P.Omtvedt - Nucl.Instrum.Methods Phys.Res. A543, 509 (2005) Liquid-scintillation detection of preseparated ${ }^{257} \mathrm{Rf}$ with the SISAK-system

2005ST18 N.J.Stone, A.E.Stuchbery, M.Danchev, J.Pavan, C.L.Timlin, C.Baktash, C.Barton, J.Beene, N.Benczer-Koller, C.R.Bingham, J.Dupak, A.Galindo-Uribarri, C.J.Gross, G.Kumbartzki, D.C.Radford, J.R.Stone, N.V.Zamfir - Phys.Rev.Lett. 94, 192501 (2005)

First Nuclear Moment Measurement with Radioactive Beams by the Recoil-in-Vacuum Technique: The g Factor of the $2_{1}^{+}$State in ${ }^{132} \mathrm{Te}$

2005ST22 E.A.Stefanova, N.Benczer-Koller, G.J.Kumbartzki, Y.Y.Sharon, L.Zamick, S.J.Q.Robinson, L.Bernstein, J.R.Cooper, D.Judson, M.J.Taylor, M.A.McMahan, L.Phair - Phys.Rev. C 72, 014309 (2005)

Near spherical shell-model structure of the $2_{1}^{+}$state in ${ }^{40} \mathrm{Ar}$ from g-factor measurements

2005ST23 A.Starostin, for the Crystal Ball Collaboration - Phys.Rev. C 72, 015205 (2005)

Recent References: January 1, 2005 to December 31, $2005 \quad$ Page 460 
Measurement of $\pi^{-} \mathrm{p} \rightarrow \pi^{0} \mathrm{n}$ in the vicinity of the $\eta$ threshold

2005ST29 A.Stolz, T.Baumann, N.H.Frank, T.N.Ginter, G.W.Hitt, E.Kwan, M.Mocko, W.Peters, A.Schiller, C.S.Sumithrarachchi, M.Thoennessen - Phys.Lett. B 627, 32 (2005)

First observation of ${ }^{60} \mathrm{Ge}$ and ${ }^{64} \mathrm{Se}$

2005ST30 M.F.M.Steenbakkers, M.F.van Batenburg, Th.S.Bauer, H.P.Blok, T.Botto, G.E.Dodge, D.L.Groep, P.Heimberg, W.H.A.Hesselink, D.W.Higinbotham, E.Jans, Y.Jun, T.J.Ketel, L.Lapikas, D.J.J.de Lange, B.E.Norum, I.Passchier, R.Starink, L.Todor, H.de Vries - Phys.Rev.Lett. 95, 172501 (2005)

Quasifree $\pi^{0}$ and $\pi^{-}$Electroproduction on ${ }^{4} \mathrm{He}$ in the $\Delta$-Resonance Region

2005 ST33 N.J.Stone, A.E.Stuchbery, M.Danchev, J.Pavan, C.L.Timlin, C.Baktash, C.Barton, J.R.Beene, N.Benczer-Koller, C.R.Bingham, J.Dupak, A.Galindo-Uribarri, C.J.Gross, G.Kumbartzki, D.C.Radford, J.R.Stone, N.V.Zamfir - Eur.Phys.J. A 25, Supplement 1, 205 (2005)

First nuclear moment measurement with radioactive beams by recoil-in-vacuum method: g-factor of the $2_{1}^{+}$state in ${ }^{132} \mathrm{Te}$

2005ST34 A.Stolz, T.Baumann, N.H.Frank, T.N.Ginter, G.W.Hitt, E.Kwan, M.Mocko, W.Peters, A.Schiller, C.S.Sumithrarachchi, M.Thoennessen - Eur.Phys.J. A 25, Supplement 1, 335 (2005)

Discovery of ${ }^{60} \mathrm{Ge}$ and ${ }^{64} \mathrm{Se}$

2005SU07 A.C.Sunde, M.Guttormsen, R.Chankova, F.Ingebretsen, T.Lonnroth, S.Messelt, J.Rekstad, A.Schiller, S.Siem, N.U.H.Syed, A.Voinov, S.W.Odegard - Acta Phys.Pol. B36, 1197 (2005)

Thermal and electromagnetic properties of the light vanadium isotopes ${ }^{50,51} \mathrm{~V}$

2005SU25 T.Sumikama, T.Iwakoshi, T.Nagatomo, M.Ogura, Y.Nakashima, H.Fujiwara, K.Matsuta, T.Minamisono, M.Mihara, M.Fukuda, K.Minamisono, T.Yamaguchi Eur.Phys.J. A 25, Supplement 1, 709 (2005)

Alignment correlation term in mass $\mathrm{A}=8$ system and G-parity irregular term

2005SUZU A.C.Sunde, R.Chankova, M.Guttormsen, F.Ingebretsen, S.Messelt, J.Rekstad, S.Siem, N.U.H.Syed, S.W.Odegard, T.Lonnroth, A.Schiller, A.Voinov nucl-ex/0511054,11/30/2005 (2005)

Microcanonical entropies and radiative strength functions of ${ }^{50,51} \mathrm{~V}$

2005SUZV K.Suda, H.Mardanpour, H.R.Amir-Ahmadi, N.Kalantar-Nayestanaki, T.Kawabata, H.Kuboki, Y.Maeda, J.G.Messchendorp, S.Sakaguchi, H.Sakai, N.Sakamoto, Y.Sasamoto, K.Sekiguchi, Y.Takahashi, T.Uesaka, K.Yako - CNS-REP-66, p.34 (2005)

Determination of Vector and Tensor Analyzing Powers in Deuteron-Proton Elastic Scattering via the ${ }^{12} \mathrm{C}(\mathrm{d}(\mathrm{pol}), \alpha){ }^{10} \mathrm{~B}^{*}\left[2^{+}\right]$Reaction

2005SUZX B.Sulignano, F.P.Hessberger, S.Hofmann, D.Ackermann, S.Antalic, I.Kojouharov, P.Kuusiniemi, R.Mann, K.Nishio, S.Saro, B.Streicher, M.Venhart - GSI 2005-1, p.74 (2005) 
Evidence for an Isomeric State in ${ }^{251}$ No

2005SY01 A.Syntfeld, H.Mach, W.Kurcewicz, B.Fogelberg, N.Amzal, J.Galy, K.Gulda, A.Korgul, A.Lindroth, W.A.Plociennik, M.Sanchez-Vega, W.Urban - Eur.Phys.J. A $23,481(2005)$

The $\beta$-decay of ${ }^{147} \mathrm{Cs}$ to ${ }^{147} \mathrm{Ba}$

2005Sz02 F.Szelecsenyi, G.F.Steyn, Z.Kovacs, T.N.van der Walt, K.Suzuki, K.Okada, K.Mukai - Nucl.Instrum.Methods Phys.Res. B234, 375 (2005)

New cross-section data for the ${ }^{66} \mathrm{Zn}(\mathrm{p}, \mathrm{n}){ }^{66} \mathrm{Ga},{ }^{68} \mathrm{Zn}(\mathrm{p}, 3 \mathrm{n}){ }^{66} \mathrm{Ga},{ }^{n a t} \mathrm{Zn}(\mathrm{p}, \mathrm{x}){ }^{66} \mathrm{Ga}$, ${ }^{68} \mathrm{Zn}(\mathrm{p}, 2 \mathrm{n}){ }^{67} \mathrm{Ga}$ and ${ }^{n a t} \mathrm{Zn}(\mathrm{p}, \mathrm{x})^{67} \mathrm{Ga}$ nuclear reactions up to $100 \mathrm{Mev}$

2005 SZ03 Z.Szeglowski, B.Kubica, D.T.Lien, S.Timokhin, M.Tuteja-Krysa, H.Godunowa, R.Misiak, M.Stobinski - J.Radioanal.Nucl.Chem. 265, 367 (2005)

$\mathrm{A}{ }^{212} \mathrm{~Pb}$ generator for model experiments on aqueous chemistry of element 114

2005Sz04 F.Szelecsenyi, G.F.Steyn, Z.Kovacs, C.Vermeulen, N.P.van der Meulen, S.G.Dolley, T.N.van der Walt, K.Suzuki, K.Mukai - Nucl.Instrum.Methods Phys.Res. B240, 625 (2005)

Investigation of the ${ }^{66} \mathrm{Zn}(\mathrm{p}, 2 \mathrm{pn}){ }^{64} \mathrm{Cu}$ and ${ }^{68} \mathrm{Zn}(\mathrm{p}, \mathrm{x}){ }^{64} \mathrm{Cu}$ nuclear processes up to $100 \mathrm{MeV}$ : Production of ${ }^{64} \mathrm{Cu}$

2005 TA02 M.J.Taylor, N.Benczer-Koller, L.Bernstein, J.Cooper, K.Hiles, D.S.Judson, G.Kumbartzki, P.Maier-Komor, M.A.McMahan, T.J.Mertzimekis, L.Phair, S.J.Q.Robinson, Y.Y.Sharon, K.-H.Speidel, A.E.Stuchbery, L.Zamick - Phys.Lett. B $605,265(2005)$

Shell model configurations in the $2_{1}^{+}$state in ${ }^{46} \mathrm{Ca}$ from a g-factor measurement

2005TA19 H.Tamura, and the E930('01) / E509 / E518 Collaborations - Nucl.Phys. A754, 58c $(2005)$

$\gamma$-ray spectroscopy in $\Lambda$ hypernuclei

2005 TA23 G.Tagliente, for the $n_{-}$TOF Collaboration - Nucl.Phys. A758, 573c (2005)

Measurements of the ${ }^{90,91,92,94,96} \mathrm{Zr}(\mathrm{n}, \gamma)$ cross-sections at n_TOF

2005 TA26 F.Tarkanyi, B.Kiraly, F.Ditroi, S.Takacs, J.Csikai, A.Hermanne, M.S.Uddin, M.Hagiwara, M.Baba, T.Ido, Yu.N.Shubin, A.K.Dityuk - Nucl.Instrum.Methods Phys.Res. B239, 293 (2005)

Activation cross sections of proton induced nuclear reactions on iridium

2005TA27 M.J.Taylor, G.Hammond, M.A.Bentley, F.Becker, J.Grebosz, A.Banu, C.J.Barton, T.Beck, P.Bednarczyk, A.Bracco, A.M.Bruce, L.C.Bullock, A .Burger, F.Camera, C.Chandler, P.Doornenbal, J.Gerl, H.Geissel, M.Gorska, M.Hellstrom, D.S.Judson, I.Kojouharov, N.Kurz, R.Lozeva, A.Maj, S.Mandal, B.McGuirk, S.Muralithar, E.S.Paul, Z.Podolyak, W.Prokopowicz, D.Rudolph, N.Saito, T.R.Saito, H.Schaffner, J.Simpson, D.D.Warner, H.Weick, C.Wheldon, M.Winkler, H.J.Wollersheim J.Phys.(London) G31, S1527 (2005) Spectroscopy of nuclei approaching the proton drip-line using a secondary-fragmentation technique with the RISING detector array 
$2005 T$ T28 W.P.Tan, J.Gorres, J.Daly, M.Couder, A.Couture, H.Y.Lee, E.Stech, E.Strandberg, C.Ugalde, M.Wiescher - Phys.Rev. C 72, 041302 (2005)

Lifetime of the astrophysically important $4.03-\mathrm{MeV}$ state in ${ }^{19} \mathrm{Ne}$

$2005 T$ 31 M.N.Tantawy, C.R.Bingham, C.Mazzocchi, R.Grzywacz, W.Krolas, K.P.Rykaczewski, J.C.Batchelder, C.J.Gross, D.Fong, J.H.Hamilton, D.J.Hartley, J.K.Hwang, Y.Larochelle, A.Piechaczek, A.V.Ramayya, D.Shapira, J.A.Winger, C.-H.Yu, E.F.Zganjar - Eur.Phys.J. A 25, Supplement 1, 151 (2005)

Study of the $\mathrm{N}=77$ odd-Z isotones near the proton-drip line

2005 TA32 M.Takechi, M.Fukuda, M.Mihara, T.Chinda, T.Matsumasa, H.Matsubara, Y.Nakashima, K.Matsuta, T.Minamisono, R.Koyama, W.Shinosaki, M.Takahashi, A.Takizawa, T.Ohtsubo, T.Suzuki, T.Izumikawa, S.Momota, K.Tanaka, T.Suda, M.Sasaki, S.Sato, A.Kitagawa - Eur.Phys.J. A 25, Supplement 1, 217 (2005) Reaction cross-sections for stable nuclei and nucleon density distribution of proton drip-line nucleus ${ }^{8} \mathrm{~B}$

2005 TA33 K.Tanaka, M.Fukuda, M.Mihara, M.Takechi, T.Chinda, T.Sumikama, S.Kudo, K.Matsuta, T.Minamisono, T.Suzuki, T.Ohtubo, T.Izumikawa, S.Momota, T.Yamaguchi, T.Onishi, A.Ozawa, I.Tanihata, T.Zheng - Eur.Phys.J. A 25, Supplement 1, 221 (2005)

Nucleon density distribution of proton drip-line nucleus ${ }^{17} \mathrm{Ne}$

2005TAZS F.Tarkanyi, F.Ditroi, S.Takacs, I.Mahunka, J.Csikai, M.S.Uddin, M.Hagiwara, M.Baba, T.Ido, A.Hermanne, Yu.Shubin, A.I.Dityuk - Proc.Intern.Conf.Nuclear Data for Science and Technology, Santa Fe, New Mexico, 26 September-1 October, 2004, R.C.Haight, M.B.Chadwick, T.Kawano, P.Talou, Eds., Vol.2, p. 1662 (2005); AIP Conf.Proc. 769 (2005)

Excitation Functions of Proton-Induced Reactions on ${ }^{\text {nat }} \mathrm{Sn}$ and ${ }^{\text {nat }} \mathrm{Cd}$ : Relevance to the Production of ${ }^{111} \mathrm{In}$ and ${ }^{114 m}$ In for Medical Applications

2005TAZT F.Tarkanyi, F.Ditroi, S.Takacs, J.Csikai, I.Mahunka, M.S.Uddin, M.Hagiwara, M.Baba, T.Ido, A.Hermanne, M.Sonck, Yu.Shubin, A.I.Dityuk -

Proc.Intern.Conf.Nuclear Data for Science and Technology, Santa Fe, New Mexico, 26 September-1 October, 2004, R.C.Haight, M.B.Chadwick, T.Kawano, P.Talou, Eds., Vol.2, p. 1658 (2005); AIP Conf.Proc. 769 (2005)

Excitation Functions for Production of ${ }^{88} \mathrm{Zr}$ and ${ }^{88} \mathrm{Y}$ by Proton and Deuteron Irradiation of $\mathrm{Mo}, \mathrm{Nb}, \mathrm{Zr}$, and $\mathrm{Y}$

2005TAZV F.Tarkanyi, F.Ditroi, S.Takacs, B.Kiraly, A.Hermanne, M.S.Uddin, M.Hagiwara, M.Baba, Yu.N.Shubin, A.I.Dityuk - Proc.Intern.Conf.Nuclear Data for Science and Technology, Santa Fe, New Mexico, 26 September-1 October, 2004, R.C.Haight, M.B.Chadwick, T.Kawano, P.Talou, Eds., Vol.1, p. 1023 (2005); AIP Conf.Proc. 769 (2005)

Cross Sections of Proton Induced Nuclear Reactions on Iridium 
2005TAZW F.Tarkanyi, S.Takacs, F.Ditroi, J.Csikai, A.Hermanne, M.S.Uddin, M.Hagiwara, M.Baba, Yu.N.Shubin, A.I.Dityuk - Proc.Intern.Conf.Nuclear Data for Science and Technology, Santa Fe, New Mexico, 26 September-1 October, 2004, R.C.Haight, M.B.Chadwick, T.Kawano, P.Talou, Eds., Vol.1, p. 1015 (2005); AIP Conf.Proc. 769 (2005)

Measurement of Activation Cross Sections of the Proton, Deuteron, and Alpha Particle-Induced Nuclear Reactions on Platinum

2005TAZY X.Tang, A.Azhari, C.Fu, C.A.Gagliardi, A.M.Mukhamedzhanov, F.Pirlepesov, L.Trache, R.E.Tribble, V.Burjan, V.Kroha, F.Carstoiu, B.F.Irgaziev - Proc.Nuclei at the Limits, Argonne, Illinois, D.Seweryniak and T.L.Khoo, eds., p.329 (2005); AIP Conf. Proc 764 (2005)

A New ${ }^{13} \mathrm{~N}(\mathrm{p}, \gamma){ }^{14} \mathrm{O}$ Reaction Rate And Its Influence In Novae Nucleosynthesis

2005TAZZ

K.Takahisa, H.Akimune, H.Ejiri, H.Fujimura, M.Fujiwara, K.Hara, H.Hashimoto, K.Hatanaka, T.Itahashi, T.Kawabata, K.Kawase, Y.Koreeda, N.Maehara, S.Mordechai, Y.Nagai, K.Nakanishi, S.Ninomiya, T.Shima, M.Tanaka, S.Umehara, S.Umisedo, H.P.Yoshida, S.Yoshida, M.Yoshimura, M.Yosoi - Proc. Intern. Symposium on the Origin of Matter and Evolution of Galaxies, Riken, Saitama, Japan, 17-19 November 2003, World Scientific, Singapore, p.533 (2005)

The nuclear responses for double beta neutrinos and double spin isospin resonances by using of double charge exchange heavy ion reaction

2005TE04 S.A.Telezhnikov, C.Granja, H.T.Hiep, J.Honzatko, M.Kralik, M.-E.Montero-Cabrera, S.Pospisil - Nucl.Phys. A763, 31 (2005)

Primary gamma transitions in ${ }^{173,174} \mathrm{Yb}$ in neutron capture at isolated resonances

2005TE05 G.M.Ter-Akopian, A.S.Fomichev, M.S.Golovkov, L.V.Grigorenko, S.A.Krupko, Yu.Ts.Oganessian, A.M.Rodin, S.I.Sidorchuk, R.S.Slepnev, S.V.Stepantsov, R.Wolski, A.A.Korsheninnikov, E.Yu.Nikolskii, P.Roussel-Chomaz, W.Mittig, R.Palit, V.Bouchat, V.Kinnard, T.Materna, F.Hanappe, O.Dorvaux, L.Stuttge, C.Angulo, V.Lapoux, R.Raabe, L.Nalpas, A.A.Yukhimchuk, V.V.Perevozchikov, Yu.I.Vinogradov, S.K.Grishechkin, S.V.Zlatoustovskii - Eur.Phys.J. A 25, Supplement 1, 315 (2005)

New insights into the resonance states of ${ }^{5} \mathrm{H}$ and ${ }^{5} \mathrm{He}$

2005TEZX T.Teranishi, S.Kubono, H.Yamaguchi, J.J.He, A.Saito, Y.Wakabayashi, H.Fujikawa, G.Amadio, S.Nishimura, M.Nishimura, J.Y.Moon, C.S.Lee, A.Odahara, D.Sohler, M.Niikura, Z.H.Li, G.Lian, L.H.Khiem - CNS-REP-66, p.5 (2005)

Study of ${ }^{13} \mathrm{~N}+\mathrm{p}$ Resonance States

2005TEZY T.Teranishi, S.Kubono, J.J.He, M.Notani, T.Fukuchi, S.Shimoura, S.Nishimura, M.Nishimura, S.Michimasa, Y.Wakabayashi, N.Hokoiwa, Y.Gono, A.Odahara, H.Baba, J.Y.Moon, J.H.Lee, C.S.Lee, J.C.Kim, H.Ishiyama, Y.X.Watanabe, T.Hashimoto, T.Ishikawa, M.H.Tanaka, H.Miyatake, V.Guimaraes, R.Lichtenthaler, H.Sato, T.Kawamura, S.Kato - RIKEN Accelerator Progress Report 2004, p.59 (2005)

Elastic resonance scattering of ${ }^{23} \mathrm{Mg}+\mathrm{p}$ 
2005TEZZ T.Teranishi, S.Kubono, J.J.He, M.Notani, T.Fukuchi, S.Michimasa, S.Shimoura, S.Nishimura, M.Nishimura, Y.Wakabayashi, N.Hokoiwa, Y.Gono, A.Odahara, H.Ishiyama, Y.X.Watanabe, T.Hashimoto, T.Ishikawa, M.H.Tanaka, H.Miyatake, J.Y.Moon, J.C.Kim, C.S.Lee, V.Guimaraes, R.F.Lichtenthaler, H.Baba, K.Sato, T.Kawamura, S.Kato - Proc. Intern. Symposium on the Origin of Matter and Evolution of Galaxies, Riken, Saitama, Japan, 17-19 November 2003, World Scientific, Singapore, p.361 (2005)

Elastic resonance scattering of ${ }^{23} \mathrm{Mg}+\mathrm{p}$

2005 TH03 J.S.Thomas, D.W.Bardayan, J.C.Blackmon, J.A.Cizewski, U.Greife, C.J.Gross, M.S.Johnson, K.L.Jones, R.L.Kozub, J.F.Liang, R.J.Livesay, Z.Ma, B.H.Moazen, C.D.Nesaraja, D.Shapira, M.S.Smith - Phys.Rev. C 71, 021302 (2005)

First study of the level structure of the r-process nucleus ${ }^{83} \mathrm{Ge}$

2005 TH09 J.S.Thomas, D.W.Bardayan, J.C.Blackmon, J.A.Cizewski, R.P.Fitzgerald, U.Greife, C.J.Gross, M.S.Johnson, K.L.Jones, R.L.Kozub, J.F.Liang, R.J.Livesay, Z.Ma,

B.H.Moazen, C.D.Nesaraja, D.Shapira, M.S.Smith, D.W.Visser - Nucl.Phys. A758, $663 \mathrm{c}(2005)$

Neutron Single-Particle States in r-Process Nuclei Near Closed Shells

2005 TH12 J.S.Thomas, D.W.Bardayan, J.C.Blackmon, J.A.Cizewski, R.P.Fitzgerald, U.Greife, C.J.Gross, M.S.Johnson, K.L.Jones, R.L.Kozub, J.F.Liang, R.J.Livesay, Z.Ma, B.H.Moazen, C.D.Nesaraja, D.Shapira, M.S.Smith, D.W.Visser - Eur.Phys.J. A 25, Supplement 1, 371 (2005)

Single-neutron excitations in neutron-rich $\mathrm{N}=51$ nuclei

2005 THzZ P.G.Thirolf, D.Habs, R.Hertenberger, H.J.Maier, T.Morgan, O.Schaile, J.Schreiber, W.Schwerdtfeger, J.Szerypo - Maier-Leibnitz-Laboratorium 2004 Ann.Rept., p.17 (2005)

Search for a new Picosecond Fission Isomer in ${ }^{234} \mathrm{U}$

2005 T11 C.Timis, J.C.Angelique, A.Buta, N.L.Achouri, D.Baiborodin, P.Baumann, C.Borcea, S.Courtin, P.Dessagne, Z.Dlouhy, J.M.Daugas, S.Grevy, D.Guillemaud-Mueller, A.Knipper, F.R.Lecolley, J.L.Lecouey, M.Lewitowicz, E.Lienard, S.M.Lukyanov, F.M.Marques, C.Miehe, J.Mrazek, F.Negoita, F.Nowacki, F.de Oliveira, N.A.Orr, J.Peter, S.Pietri, Yu.E.Penionzhkevich, E.Poirier, M.Stanoiu, G.Walter - J.Phys.(London) G31, S1965 (2005) Spectroscopy around $\mathrm{N}=20$ shell closure: $\beta$-n decay study of ${ }^{35} \mathrm{Al}$

2005TIZX Yu.E.Titarenko, V.F.Batyaev, V.M.Zhivun, R.D.Mulambetov, S.V.Mulambetova, S.L.Zaitsev, S.G.Mashnik, R.E.Prael - Proc.Intern.Conf.Nuclear Data for Science and Technology, Santa Fe, New Mexico, 26 September-1 October, 2004, R.C.Haight, M.B.Chadwick, T.Kawano, P.Talou, Eds., Vol.1, p. 1070 (2005); AIP Conf.Proc. 769 (2005)

Excitation Functions of Products from ${ }^{208,207,206, n a t} \mathrm{~Pb}$ and ${ }^{209} \mathrm{Bi}$ (p, x) Reactions Measured in the 40-2600 MeV Energy Range and Predicted Theoretically

2005 TIZY Y.E.Titarenko, V.F.Batyaev, R.D.Mulambetov, V.M.Zhivun, V.S.Barashenkov, S.G.Mashnik, Y.N.Shubin, A.V.Ignatyuk - nucl-ex/0507009,7/05/2005 (2005) 
Excitation Functions of Product Nuclei from 40-2600 MeV Proton-Irradiated $206,207,208$, nat $\mathrm{Pb}$ and ${ }^{209} \mathrm{Bi}$

2005T011 Y.Togano, T.Gomi, T.Motobayashi, Y.Ando, N.Aoi, H.Baba, K.Demichi, Z.Elekes, N.Fukuda, Zs.Fulop, U.Futakami, H.Hasegawa, Y.Higurashi, K.Ieki, N.Imai, M.Ishihara, K.Ishikawa, N.Iwasa, H.Iwasaki, S.Kanno, Y.Kondo, T.Kubo, S.Kubono, M.Kunibu, K.Kurita, Y.U.Matsuyama, S.Michimasa, T.Minemura, M.Miura, H.Murakami, T.Nakamura, M.Notani, S.Ota, A.Saito, H.Sakurai, M.Serata, S.Shimoura, T.Sugimoto, E.Takeshita, S.Takeuchi, K.Ue, K.Yamada, Y.Yanagisawa, K.Yoneda, A.Yoshida - Nucl.Phys. A758, 182c (2005) Study of ${ }^{26} \mathrm{Si}(\mathrm{p}, \gamma){ }^{27} \mathrm{P}$ reaction using Coulomb dissociation method

2005 T014 A.Tomyo, Y.Temma, M.Segawa, Y.Nagai, H.Makii, T.Shima, T.Ohsaki, M.Igashira Astrophys.J. 623, L153 (2005)

${ }^{62} \mathrm{Ni}(\mathrm{n}, \gamma){ }^{63} \mathrm{Ni}$ reaction and overproduction of $\mathrm{Ni}$ isotopes

2005T015 B.S.Tomar, K.S.Babu, K.Sudarshan, R.Tripathi, A.Goswami - Pramana 64, 1 (2005) Angular momentum transfer in incomplete fusion

2005 T022 D.Tonev, G.de Angelis, P.Petkov, A.Dewald, A.Gadea, P.Pejovic, D.L.Balabanski, P.Bednarczyk, F.Camera, A.Fitzler, O.Moller, N.Marginean, A.Paleni, C.Petrache, K.O.Zell, Y.H.Zhang - Eur.Phys.J. A 25, Supplement 1, 447 (2005)

Check for chirality in real nuclei

2005T0ZY D.Tonev, G.de Angelis, P.Petkov, A.Dewald, A.Gadea, P.Pejovic, D.Balabanski, P.Bednarczyk, F.Camera, A.Fitzler, M.Axiotis, D.Bazzacco, E.Farnea, S.Lenzi, S.Lunardi, N.Marginean, T.Martinez, R.Menegazzo, O.Moller, D.R.Napoli, A.Paleni, C.Petrache, G.Prete, B.R.Behera, C.Rusu, C.Ur, K.O.Zell, Y.H.Zhang Proc.Nuclei at the Limits, Argonne, Illinois, D.Seweryniak and T.L.Khoo, eds., p.93 (2005); AIP Conf. Proc 764 (2005)

Transition Probabilities And Chiral Symmetry In ${ }^{134} \mathrm{Pr}$

2005T0ZZ Y.Togano, T.Gomi, T.Motobayashi, Y.Ando, N.Aoi, H.Baba, K.Demichi, Z.Elekes, N.Fukuda, Zs.Fulop, U.Futakami, H.Hasegawa, Y.Higurashi, K.Ieki, N.Imai, M.Ishihara, K.Ishikawa, N.Iwasa, H.Iwasaki, S.Kanno, Y.Kondo, T.Kubo, S.Kubono, M.Kunibu, K.Kurita, Y.U.Matsuyama, S.Michimasa, T.Minemura, M.Miura, H.Murakami, T.Nakamura, M.Notani, S.Ota, A.Saito, H.Sakurai, M.Serata, S.Shimoura, T.Sugimoto, E.Takeshita, S.Takeuchi, K.Ue, K.Yamada, Y.Yanagisawa, K.Yoneda, A.Yoshida - Proc. Intern. Symposium on the Origin of Matter and Evolution of Galaxies, Riken, Saitama, Japan, 17-19 November 2003, World Scientific, Singapore, p.549 (2005)

Study of the ${ }^{26} \mathrm{Si}(\mathrm{p}, \gamma){ }^{27} \mathrm{P}$ reaction by the Coulomb dissociation method

2005TR05 V.Tripathi, S.L.Tabor, P.F.Mantica, C.R.Hoffman, M.Wiedeking, A.D.Davies, S.N.Liddick, W.F.Mueller, T.Otsuka, A.Stolz, B.E.Tomlin, Y.Utsuno, A.Volya Phys.Rev.Lett. 94, 162501 (2005)

${ }^{29} \mathrm{Na}$ : Defining the Edge of the Island of Inversion for $\mathrm{Z}=11$

2005TR06 L.A.Trykov, A.A.Dubinin, V.A.Chernov - At.Energ. 98, 54 (2005); At.Energy 98, 50 (2005) 
Experimental and computed spectra of neutrons and photons emitted from spherical iron models with a ${ }^{252} \mathrm{Cf}$ source at the center

2005 Tr09 Yu.A.Troyan, A.V.Beljaev, A.Yu.Troyan, E.B.Plekhanov, A.P.Jerusalimov, G.B.Piskaleva, S.G.Arakelian - Part. and Nucl., Lett. 124, 36 (2005)

The Search and Study of the Baryonic Resonances with the Strangeness $\mathrm{S}=+1$ in the System on $\mathrm{nK}^{+}$from the Reaction $\mathrm{np} \rightarrow \mathrm{npK}^{+} \mathrm{K}^{-}$at the Momentum of Incident Neutrons $\mathrm{P}_{n}=(5.20 \pm 0.12) \mathrm{GeV} / \mathrm{c}$

2005TR11 M.Trinczek, C.C.Jewett, J.M.D'Auria, S.Bishop, L.Buchmann, A.A.Chen, S.Engel, D.Gigliotti, U.Greife, D.Hunter, A.Hussein, D.Hutcheon, J.Jose, A.M.Laird, M.Lamey, R.Lewis, A.Olin, D.Ottewell, P.Parker, M.M.Pavan, J.E.Pearson, J.Rogers, C.Ruiz, C.Wrede - Nucl.Phys. A758, 729c (2005)

Direct Measurement of the ${ }^{21} \mathrm{Na}(\mathrm{p}, \gamma)^{22} \mathrm{Mg}$ Reaction: Resonance Strengths and Gamma-Gamma Analysis

2005TR13 V.Tripathi, S.L.Tabor, P.F.Mantica, C.R.Hoffman, M.Wiedeking, A.D.Davies, S.N.Liddick, W.F.Mueller, A.Stolz, B.E.Tomlin, A.Volya - Eur.Phys.J. A 25, Supplement 1, 101 (2005)

Voyage to the "Island of Inversion": ${ }^{29} \mathrm{Na}$

2005TRZY M.J.Trbovich, D.P.Barry, R.E.Slovacek, Y.Danon, R.C.Block, J.A.Burke, N.Drindak, G.Leinweber, R.V.Ballad - Proc.Intern.Conf.Nuclear Data for Science and Technology, Santa Fe, New Mexico, 26 September-1 October, 2004, R.C.Haight, M.B.Chadwick, T.Kawano, P.Talou, Eds., Vol.1, p. 949 (2005); AIP Conf.Proc. 769 (2005)

Hafnium Resonance Parameter Analysis using Neutron Capture and Transmission Experiments

2005TRZZ R.Tramontano, O.Helene, P.R.Pascholati, N.L.Maidana, V.R.Vanin -

Proc.Intern.Conf.Nuclear Data for Science and Technology, Santa Fe, New Mexico, 26 September-1 October, 2004, R.C.Haight, M.B.Chadwick, T.Kawano, P.Talou, Eds., Vol.1, p. 261 (2005); AIP Conf.Proc. 769 (2005) Gamma Spectroscopy and Gamma-Ray Intensity Determination for ${ }^{48} \mathrm{~V}$

2005 TU09 A.Tumino, C.Spitaleri, C.Bonomo, S.Cherubini, P.Figuera, M.Gulino, M.La Cognata, L.Lamia, A.Musumarra, M.G.Pellegriti, R.G.Pizzone, A.Rinollo, S.Romano - Eur.Phys.J. A 25, Supplement 1, 649 (2005)

Quasi-free ${ }^{6} \mathrm{Li}(\mathrm{n}, \alpha)^{3} \mathrm{H}$ reaction at low energy from ${ }^{2} \mathrm{H}$ break-up

2005TUZz A.Tumino, C.Spitaleri, A.Musumarra, M.G.Pellegriti, R.G.Pizzone, A.Rinollo, S.Romano, L.Pappalardo, C.Bonomo, A.Del Zoppo, A.Di Pietro, P.Figuera, M.La Cognata, L.Lamia, S.Cherubini, C.Rolfs, S.Typel - Proc. Intern. Symposium on the Origin of Matter and Evolution of Galaxies, Riken, Saitama, Japan, 17-19 November 2003, World Scientific, Singapore, p.553 (2005)

The Trojan Horse method applied to the astrophysically relevant proton capture capture reactions on $\mathrm{Li}$ isotopes

2005UD01 M.S.Uddin, M.Hagiwara, M.Baba, F.Tarkanyi, F.Ditroi - Appl.Radiat.Isot. 62, 533 (2005) 
Experimental studies on excitation functions of the proton-induced activation reactions on silver

2005UD02 M.S.Uddin, M.Hagiwara, M.Baba, F.Tarkanyi, F.Ditroi - Appl.Radiat.Isot. 63, 367 (2005)

Experimental studies on excitation functions of the proton-induced activation reactions on yttrium

2005UE01 H.Ueno, D.Kameda, G.Kijima, K.Asahi, A.Yoshimi, H.Miyoshi, K.Shimada, G.Kato, D.Nagae, S.Emori, T.Haseyama, H.Watanabe, M.Tsukui - Phys.Lett. B $615,186(2005)$

Magnetic moments of ${ }_{13}^{30} \mathrm{Al}_{17}$ and ${ }_{13}^{32} \mathrm{Al}_{19}$

2005UEzZ T.Uesaka, T.Ikeda, T.Kawabata, H.Okamura, K.Itoh, H.Sakai, K.Yako, T.Saito, H.Kuboki, M.Sasano, K.Sekiguchi - RIKEN Accelerator Progress Report 2004, p.35 (2005)

Tensor analyzing power for the $\mathrm{d}(\mathrm{pol})+\alpha$ backward scattering

$2005 U$ C04 C.Ugalde, R.Azuma, A.Couture, J.Gorres, M.Heil, K.Scheller, E.Stech, W.Tan, M.Wiescher - Nucl.Phys. A758, 577c (2005)

The reaction rate for the destruction of fluorine in AGB stars

2005UK01 M.Ukai, O.Hashimoto, Y.Miura, T.Miyoshi, K.Mizunuma, S.N.Nakamura, Y.Okayasu, H.Tamura, S.Ajimura, T.Kishimoto, T.Hayakawa, S.Minami, H.Akikawa, K.Imai, H.Takahashi, D.E.Alburger, R.E.Chrien, H.Hotchi, M.May, P.Pile, A.Rusek, R.Sutter, T.Nagae, Y.Sato, A.Banu, G.B.Franklin, B.P.Quinn, J.Franz, K.Nakazawa, K.Tanida, L.Tang, L.Yuan, and the E930 '01 Collaboration Nucl.Phys. A754, 70c (2005)

Observation of hypernuclear fine structure in ${ }_{\Lambda}^{16} \mathrm{O}$

2005Un01 Unnati, M.K.Sharma, B.P.Singh, R.Prasad, S.Gupta, H.D.Bhardwaj, A.K.Sinha Int.J.Mod.Phys. E14, 775 (2005)

A study of excitation functions for some residues produced in the system ${ }^{14} \mathrm{~N}+$ ${ }^{128} \mathrm{Te}$ in the energy range $\approx 64-90 \mathrm{MeV}$

2005UOzZ M.A.M.Uosif, R.Michel, U.Herpers, P.-W.Kubik, M.Duijvestijn, A.Koning Proc.Intern.Conf.Nuclear Data for Science and Technology, Santa Fe, New Mexico, 26 September-1 October, 2004, R.C.Haight, M.B.Chadwick, T.Kawano, P.Talou, Eds., Vol.2, p. 1547 (2005); AIP Conf.Proc. 769 (2005)

Residual Nuclide Production by Proton-Induced Reactions on Uranium for Energies between 20 and $70 \mathrm{MeV}$

2005UR01 W.Urban, T.Rzaca-Urban, J.L.Durell, A.G.Smith, I.Ahmad - Eur.Phys.J. A 24, 161 (2005)

First observation of excited states in the 111Tc nucleus -A new region of deformation at $40 \leq \mathrm{Z} \leq 46, \mathrm{~N} \geq 68$ ?

2005UR02 W.Urban, T.Rzaca-Urban, J.A.Pinston, J.L.Durell, W.R.Phillips, A.G.Smith, B.J.Varley, I.Ahmad, N.Schulz - Phys.Rev. C 72, 027302 (2005)

Near-yrast, medium-spin structure of the ${ }^{107}$ Mo nucleus 
$2005 U 001$ Y.-N.U, S.J.Zhu, M.Sakhaee, L.M.Yang, C.Y.Gan, L.Y.Zhu, R.Q.Xu, X.L.Che, M.L.Li, Y.J.Chen, S.X.Wen, X.G.Wu, L.H.Zhu, G.S.Li, J.Peng, S.Q.Zhang, J.Meng - J.Phys.(London) G31, B1 (2005)

Search for the chiral doublet bands in ${ }^{122} \mathrm{Cs}$

$2005 U 002$ J.Uusitalo, M.Leino, T.Enqvist, K.Eskola, T.Grahn, P.T.Greenlees, P.Jones, R.Julin, S.Juutinen, A.Keenan, H.Kettunen, H.Koivisto, P.Kuusiniemi, A.-P.Leppanen, P.Nieminen, J.Pakarinen, P.Rahkila, C.Scholey - Phys.Rev. C 71, 024306 (2005) $\alpha$ decay studies of very neutron-deficient francium and radium isotopes

2005 UU03 J.Uusitalo, S.Eeckhaudt, T.Enqvist, K.Eskola, T.Grahn, P.T.Greenlees, P.Jones, R.Julin, S.Juutinen, H.Kettunen, P.Kuusiniemi, M.Leino, A.-P.Leppanen, P.Nieminen, M.Nyman, J.Pakarinen, P.Rahkila, C.Scholey - Eur.Phys.J. A 25, Supplement 1, 179 (2005)

Alpha-decay studies using the JYFL gas-filled recoil separator RITU

2005VA04 K.Van de Vel, A.N.Andreyev, D.Ackermann, H.J.Boardman, P.Cagarda, J.Gerl, F.P.Hessberger, S.Hofmann, M.Huyse, D.Karlgren, I.Kojouharov, M.Leino, B.Lommel, G.Munzenberg, C.Moore, R.D.Page, S.Saro, P.Van Duppen, R.Wyss Eur.Phys.J. A 24, 57 (2005)

Prolate structures in ${ }^{189} \mathrm{Po}$ and ${ }^{185} \mathrm{~Pb}$

2005VA09 J.J.Valiente-Dobon, C.E.Svensson, C.D.O'Leary, I.Ragnarsson, C.Andreoiu, D.E.Appelbe, R.A.E.Austin, G.C.Ball, J.A.Cameron, M.P.Carpenter, R.M.Clark, M.Cromaz, D.Dashdorj, P.Fallon, P.Finlay, S.J.Freeman, P.E.Garrett, A.Gorgen, G.F.Grinyer, D.F.Hodgson, B.Hyland, D.Jenkins, F.Johnston-Theasby, P.Joshi, N.S.Kelsall, A.O.Macchiavelli, F.Moore, G.Mukherjee, A.A.Phillips, W.Reviol, D.Sarantites, M.A.Schumaker, D.Seweryniak, M.B.Smith, J.C.Waddington, R.Wadsworth, D.Ward, S.J.Williams - Phys.Rev. C 71, 034311 (2005)

High-spin rotational structures in ${ }^{76} \mathrm{Kr}$

2005VA18 J.J.Valiente-Dobon, C.E.Svensson, C.D.O'Leary, I.Ragnarsson, C.Andreoiu, R.A.E.Austin, M.P.Carpenter, D.Dashdorj, P.Finlay, S.J.Freeman, P.E.Garrett, A.Gorgen, J.Greene, G.F.Grinyer, B.Hyland, D.Jenkins, F.Johnston-Theasby, P.Joshi, N.S.Kelsall, A.O.Macchiavelli, F.Moore, G.Mukherjee, A.A.Phillips, W.Reviol, D.Sarantites, M.A.Schumaker, D.Seweryniak, M.B.Smith, R.Wadsworth, D.Ward - Acta Phys.Pol. B36, 1339 (2005)

Lifetimes of High-spin states in ${ }^{76} \mathrm{Kr}$

2005VA19 J.Van Roosbroeck, H.De Witte, M.Gorska, M.Huyse, K.Kruglov, D.Pauwels, J.-Ch.Thomas, K.Van de Vel, P.Van Duppen, S.Franchoo, J.Cederkall, V.N.Fedoseyev, H.Fynbo, U.Georg, O.Jonsson, U.Koster, L.Weissman, W.F.Mueller, V.I.Mishin, D.Fedorov, A.De Maesschalck, N.A.Smirnova, K.Heyde - Phys.Rev. C 71, 054307 (2005)

Evolution of the nuclear structure approaching ${ }^{78} \mathrm{Ni}$ : $\beta$ decay of ${ }^{74-78} \mathrm{Cu}$

2005VA27 W.A.van Wijngaarden - Can.J.Phys. 83, 327 (2005)

Precision measurements of fine and hyperfine structure in lithium I and II 
2005VA30 J.J.Valiente-Dobon, T.Steinhardt, C.E.Svensson, A.V.Afanasjev, I.Ragnarsson, C.Andreoiu, R.A.E.Austin, M.P.Carpenter, D.Dashdorj, G.de Angelis, F.Donau, J.Eberth, E.Farnea, S.J.Freeman, A.Gadea, P.E.Garrett, A.Gorgen, G.F.Grinyer, B.Hyland, D.Jenkins, F.Johnston-Theasby, P.Joshi, A.Jungclaus, K.P.Lieb, A.O.Macchiavelli, E.F.Moore, G.Mukherjee, D.R.Napoli, A.A.Phillips, C.Plettner, W.Reviol, D.Sarantites, H.Schnare, M.A.Schumaker, R.Schwengner, D.Seweryniak, M.B.Smith, I.Stefanescu, O.Thelen, R.Wadsworth - Phys.Rev.Lett. 95, 232501 (2005)

Evidence for Nontermination of Rotational Bands in ${ }^{74} \mathrm{Kr}$

2005VA31 R.L.Varner, J.R.Beene, C.Baktash, A.Galindo-Uribarri, C.J.Gross, J.Gomez del Campo, M.L.Halbert, P.A.Hausladen, Y.Larochelle, J.F.Liang, J.Mas, P.E.Mueller, E.Padilla-Rodal, D.C.Radford, D.Shapira, D.W.Stracener, J.-P.Urrego-Blanco, C.-H.Yu - Eur.Phys.J. A 25, Supplement 1, 391 (2005)

Coulomb excitation measurements of transition strengths in the isotopes ${ }^{132,134} \mathrm{Sn}$

2005VAzZ S.I.Vasiliev, K.Ya.Gromov, A.A.Klimenko, Zh.K.Samatov, A.A.Smolnikov, V.I.Fominykh, V.G.Chumin - Book of Abstracts, LV National Conference on Nuclear Physics "Frontiers in the Physics of Nucleus", St.-Petersburg, p.320 (2005) Coincidence Summation in $\gamma$-Ray Spectra. Determination of Intensity for Weak Cross Over $\gamma$-Transitions

2005VE07 Ts.Venkova, W.Gast, R.M.Lieder, D.Bazzacco, G.de Angelis, E.O.Lieder, A.A.Pasternak, R.Menegazzo, S.Lunardi, C.Rossi Alvarez, C.Ur, T.Martinez, M.Axiotis, D.Napoli, W.Urban, T.Rzaca-Urban, S.Frauendorf - Eur.Phys.J. A 26, $19(2005)$

Suppression of band crossing in the neutron-rich nuclei ${ }^{172,173} \mathrm{Yb}$ due to the absence of a static pair field

2005VE08 V.A.Vesna, Yu.M.Gledenov, V.V.Nesvizhevsky, A.K.Petukhov, P.V.Sedyshev, T.Soldner, O.Zimmer, E.V.Shulgina - Pisma Zh.Eksp.Teor.Fiz. 82, 519 (2005); JETP Lett. 82, 463 (2005)

Discovery of a P-Odd Effect in Triton Emission from the reaction ${ }^{6} \operatorname{Li}(\mathrm{n}, \alpha)^{3} \mathrm{H}$

2005VEZZ C.Vermeulen, G.F.Steyn, F.M.Nortier, T.N.van der Walt, F.Szelecsenyi, Z.Kovacs, S.M.Qaim - Proc.Intern.Conf.Nuclear Data for Science and Technology, Santa Fe, New Mexico, 26 September-1 October, 2004, R.C.Haight, M.B.Chadwick, T.Kawano, P.Talou, Eds., Vol.2, p. 1650 (2005); AIP Conf.Proc. 769 (2005) Excitation Functions and Production Rates of Radionuclides Produced in the Proton Bombardment of ${ }^{n a t} \mathrm{Pr}$ and ${ }^{n a t} \mathrm{La}$

2005VIZY

S.E.Vigdor, for the CE71 Collaboration - Proc.Intern.Conf.Nuclear Data for Science and Technology, Santa Fe, New Mexico, 26 September-1 October, 2004, R.C.Haight, M.B.Chadwick, T.Kawano, P.Talou, Eds., Vol.1, p. 820 (2005); AIP Conf.Proc. 769 (2005) Measurement of Absolute np Scattering Differential Cross Sections with a Tagged Intermediate-Energy Neutron Beam 
2005VIZZ I.N.Vishnevsky, V.A.Zheltonozhsky, N.V.Strilchuk, S.S.Drapej - Book of Abstracts, LV National Conference on Nuclear Physics "Frontiers in the Physics of Nucleus", St.-Petersburg, p.65 (2005)

Investigation of Gamma-Transition's ICC in ${ }^{115} \mathrm{In},{ }^{117} \mathrm{Sn}$ and ${ }^{125} \mathrm{Te}$ Nuclei with a High Precision

2005V017 C.Vockenhuber, R.Golser, W.Kutschera, A.Priller, P.Steier, A.Wallner, M.Bichler Nucl.Phys. A758, 340c (2005)

${ }^{182} \mathrm{Hf}$ - from geophysics to astrophysics

2005V0ZX A.S.Vorobyev, V.N.Dushin, F.-J.Hambsch, V.A.Jakovlev, V.A.Kalinin, A.B.Laptev, B.F.Petrov, O.A.Shcherbakov - Proc.Intern.Conf.Nuclear Data for Science and Technology, Santa Fe, New Mexico, 26 September-1 October, 2004, R.C.Haight, M.B.Chadwick, T.Kawano, P.Talou, Eds., Vol.1, p. 613 (2005); AIP Conf.Proc. 769 (2005)

Distribution of Prompt Neutron Emission Probability for Fission Fragments in Spontaneous Fission of ${ }^{252} \mathrm{Cf}$ and ${ }^{244,248} \mathrm{Cm}$

2005WA06 T.J.Wasowicz, R.Drozdowski, J.Kwela - Phys.Scr. 71, 274 (2005)

Hyperfine Structure Study of Several Lines of ${ }^{207} \mathrm{~Pb}$ I

2005WA22 T.J.Wasowicz, R.Drozdowski, J.Kwela - Phys.Scr. 72, 200 (2005)

Hyperfine Structure Study of Several Lines of ${ }^{207} \mathrm{~Pb}$ I - Part II

2005WA23 S.Wang, H.Hua, J.Meng, Z.H.Li, S.Q.Zhang, F.R.Xu, H.L.Liu, Y.L.Ye, D.X.Jiang, T.Zheng, Q.J.Wang, Z.Q.Chen, C.E.Wu, G.L.Zhang, D.Y.Pang, J.Wang, J.L.Lou, B.Guo, G.Jin, S.G.Zhou, L.H.Zhu, X.G.Wu, G.S.Li, S.X.Wen, C.Y.He, X.Z.Cui, Y.Liu - Phys.Rev. C 72, 024317 (2005)

High-spin states in ${ }^{152} \mathrm{Gd}$

2005WA25 H.-L.Wang, Y.-H.Zhang, X-H.Zhou, Y.-X.Guo, X.-G.Lei, S.-W.Xu, Y.-X.Xie, M.-L.Liu, Y.Zheng, Y.-B.Xing, C.-Y.Xie, L.-T.Song, P.Luo, H.-P.Yu, W.-T.Guo Chin.Phys.Lett. 22, 2211 (2005)

Identification of New $\beta$-Delayed $\gamma$ Rays and a Low-Spin Isomer in ${ }^{176} \mathrm{Ir}$

2005WA29 J.Wang, X.Wang, T.Su - Phys.Rev. C 72, 037604 (2005)

Measurements of the ${ }^{71} \mathrm{Ga}(\mathrm{n}, 2 \mathrm{n})^{70} \mathrm{Ga}$ cross section in the neutron energy range of 13.5-14.7 MeV

2005WA31 A.Wagner, R.Beyer, M.Erhard, F.Donau, E.Grosse, A.Hartmann, A.R.Junghans, L.Kaubler, K.Kosev, S.Mallion, C.Nair, N.Nankov, G.Rusev, K.D.Schilling, W.Schulze, R.Schwengner - J.Phys.(London) G31, S1969 (2005)

The new bremsstrahlung facility at the superconducting electron accelerator ELBE

2005WA34 T.J.Wasowicz, R.Drozdowski, J.Kwela - Eur.Phys.J. D 36, 249 (2005)

Hyperfine structure and isotope shift study in a singly ionized lead 
2005WAZS Y.Wakabayashi, A.Odahara, Y.Gono, T.Fukuchi, T.Teranishi, S.Kubono, H.Yamaguchi, A.Saito, E.Ideguchi, S.Nishimura, J.J.He, H.Fujikawa, G.Amadio, S.Ota, J.Y.Moon, M.Notani, Y.Yanagisawa, S.Michimasa, S.Shimoura, H.Watanabe, T.Kishida, H.Baba, M.Nishimura, T.Ishii - CNS-REP-66, p.15 (2005)

Search for High-Spin Isomers in $\mathrm{N}=51$ Isotones using a Secondary ${ }^{17} \mathrm{~N}$ Beam

2005WAZU

2005WAZW

2005WAZX

2005WAZY

2005WAZZ

2005WE01

2005WE06
A.Wallner, R.Golser, W.Kutschera, A.Priller, P.Steier, C.Vockenhuber, H.Vonach, T.Faestermann, K.Knie, G.Korschinek - Proc.Intern.Conf.Nuclear Data for Science and Technology, Santa Fe, New Mexico, 26 September-1 October, 2004, R.C.Haight, M.B.Chadwick, T.Kawano, P.Talou, Eds., Vol.1, p. 621 (2005); AIP Conf.Proc. 769 (2005)

Potential of AMS for Quantifying Long-Lived Reaction Products

R.E.Warner, F.Carstoiu, J.A.Brown, F.D.Becchetti, D.A.Roberts, B.Davids, A.Galonsky, R.M.Ronningen, M.Steiner, M.Horoi, J.J.Kolata, A.Nadasen, C.Samanta, J.Schwarzenberg, K.Subotic - nucl-ex/0507025,7/18/2005 (2005) Reaction and proton-removal cross sections of ${ }^{6} \mathrm{Li},{ }^{7} \mathrm{Be},{ }^{10} \mathrm{~B},{ }^{9,10,11} \mathrm{C},{ }^{12} \mathrm{~N},{ }^{13,15} \mathrm{O}$ and ${ }^{17} \mathrm{Ne}$ on $\mathrm{Si}$ at 15 to $53 \mathrm{MeV} /$ nucleon

D.P.Watts, J.R.M.Annand, R.Beck, D.Branford, D.Glazier, P.Grabmayr, K.Livingston, I.J.D.MacGregor, J.C.McGeorge, R.O.Owens nucl-ex/0506018,6/14/2005 (2005)

Dependence of the ${ }^{12} \mathrm{C}(\gamma(\mathrm{pol})$, pd) reaction on photon linear polarization

W.B.Walters, M.A.Stoyer, J.Shergur, N.Hoteling, J.J.Ressler, J.Rikovska, K.-L.Kratz, A.Wohr, B.Pfeiffer, O.Arndt, P.F.Mantica, B.Tomlin, H.Schatz, F.Montes, B.A.Brown, D.Seweryniak, H.Ravn, V.Fedoseyev, U.Koster, C.Y.Wu, D.Cline, H.Hua, A.B.Hayes, R.Teng, and the ISOLDE Collaboration - Proc.Nuclei at the Limits, Argonne, Illinois, D.Seweryniak and T.L.Khoo, eds., p.335 (2005); AIP Conf. Proc 764 (2005)

Structure And Decay Of Neutron-Rich Nuclides In The $115 \leq \mathrm{A} \leq 138$ Mass Range And r-Process Nucleosynthesis

D.Ward, R.M.Clark, M.Cromaz, M.A.Deleplanque, R.M.Diamond, P.Fallon, G.J.Lane I.Y.Lee, A.Gorgen, A.O.Macchiavelli, F.S.Stephens, C.E.Svensson, K.Vetter, D.Cline, A.B.Hayes, R.Teng, C.-Y.Wu, T.Nakatsukasa - Proc.Nuclei at the Limits, Argonne, Illinois, D.Seweryniak and T.L.Khoo, eds., p.263 (2005); AIP Conf. Proc 764 (2005)

Aspects Of The Coriolis Interaction In ${ }^{235} \mathrm{U}$

Y.-B.Wei, Y.-G.Ma, X.-Z.Cai, C.Zhong, J.-G.Chen, H.-Y.Zhang, D.-Q.Fang, K.Wang, G.-L.Ma, W.Guo, W.-D.Tian, W.-Q.Shen, W.-L.Zhan, G.-Q.Xiao, H.-S.Xu, Z.-Y.Sun, J.-X.Li, Z.-Y.Guo, M.Wang, Z.-Q.Chen, Z.-G.Hu, L.-X.Chen, C.Li, R.-S.Mao, J.Bai - Chin.Phys.Lett. 22, 61 (2005)

Parallel Momentum Distribution of ${ }^{28} \mathrm{Si}$ Fragments from ${ }^{29} \mathrm{P}$

L.Weissman, A.F.Lisetskiy, O.Arndt, U.Bergmann, B.A.Brown, J.Cederkall, I.Dillmann, O.Hallmann, L.Fraile, S.Franchoo, L.Gaudefroy, U.Koster, K.-L.Kratz, B.Pfeiffer, O.Sorlin, and the ISOLDE Collaboration - J.Phys.(London) G31, 553 (2005) 
$\beta$-decay of ${ }^{22} \mathrm{O}$

2005WE11 C.Weber, G.Audi, D.Beck, K.Blaum, G.Bollen, F.Herfurth, A.Kellerbauer, H.-J.Kluge, D.Lunney, S.Schwarz - Phys.Lett. A 347, 81 (2005)

Weighting excited nuclear states with a Penning trap mass spectrometer

2005WH04 C.Wheldon, G.D.Dracoulis, A.N.Wilson, P.M.Davidson, A.P.Byrne, D.M.Cullen, L.K.Pattison, S.V.Rigby, D.T.Scholes, G.Sletten, R.Wood - Nucl.Phys. A763, 1 (2005) Gamma-ray spectroscopy of the doubly-odd nuclide ${ }^{184} \mathrm{Re}$

2005WI01 A.N.Wilson, G.D.Dracoulis, P.M.Davidson, A.P.Byrne, R.M.Clark, P.Fallon, A.Gorgen, G.J.Lane, A.O.Macchiavelli, D.Ward - Nucl.Phys. A748, 12 (2005) Quadrupole moment of the yrast superdeformed band in ${ }^{192} \mathrm{~Pb}$

2005WI05 M.Wiedeking, S.L.Tabor, J.Pavan, A.Volya, A.L.Aguilar, I.J.Calderin, D.B.Campbell, W.T.Cluff, E.Diffenderfer, J.Fridmann, C.R.Hoffman, K.W.Kemper, S.Lee, M.A.Riley, B.T.Roeder, C.Teal, V.Tripathi, I.Wiedenhover - Phys.Rev.Lett. 94, 132501 (2005)

p-sd Shell Gap Reduction in Neutron-Rich Systems and Cross-Shell Excitations in ${ }^{20} \mathrm{O}$

2005WI10 A.N.Wilson, G.D.Dracoulis, A.P.Byrne, P.M.Davidson, G.J.Lane, R.M.Clark, P.Fallon, A.Gorgen, A.O.Macchiavelli, D.Ward - Eur.Phys.J. A 24, 179 (2005) Observation of a superdeformed band in ${ }^{190} \mathrm{~Pb}$

2005WI17 F.E.Wietfeldt, M.S.Dewey, D.M.Gilliam, J.S.Nico, X.Fei, W.M.Snow, G.L.Greene, J.Pauwels, R.Eykens, A.Lamberty, J.Van Gestel - J.Res.Natl.Inst.Stand.Technol. $110,327(2005)$

Measurement of the Neutron Lifetime by Counting Trapped Protons

2005WI19 O.Wieland, S.Barlini, V.L.Kravchuk, A.Bracco, F.Camera, F.Gramegna, A.Maj, G.Benzoni, N.Blasi, S.Brambilla, M.Brekiesz, M.Bruno, G.Casini, M.Chiari, E.Geraci, A.Giussani, M.Kmiecik, S.Leoni, A.Lanchais, P.Mastinu, B.Million, A.Moroni, A.Nannini, A.Ordine, G.Vannini, L.Vannucci - J.Phys.(London) G31, S1973 (2005)

The $\gamma$-decay of the GDR in highly excited Ce nuclei

2005WI20 S.J.Williams, P.M.Jenneson, P.E.Garrett, P.H.Regan, C.Andreoiu, G.C.Ball, R.S.Chakrawarthy, J.J.Daoud, G.F.Grinyer, G.Hackman, B.Hyland, N.A.Mlwilo, A.C.Morton, C.J.Pearson, J.J.Ressler, M.A.Schumaker, M.B.Smith, C.E.Svensson, J.J.Valiente Dobon - J.Phys.(London) G31, S1979 (2005)

Testing the integration of $\mathrm{BaF}_{2}$ detectors into the $8 \pi$ array: fast-timing measurements at TRIUMF

2005WI21 A.N.Wilson, A.K.Singh, H.Hubel, P.M.Davidson, A.Gorgen, D.Rossbach, A.Korichi, A.Astier, F.Azaiez, D.Bazzacco, C.Bourgeois, N.Buforn, A.P.Byrne, G.D.Dracoulis, F.Hannachi, K.Hauschild, W.Korten, T.Kroll, G.J.Lane, A.Lopez-Martens, N.Redon, P.Reiter, C.Rossi-Alvarez, G.Schonwasser, O.Stezowski, P.G.Thirolf Phys.Rev.Lett. 95, 182501 (2005) 
Excitation Energies of Superdeformed States in ${ }^{196} \mathrm{~Pb}$ : Towards a Systematic Study of the Second Well in Pb Isotopes

2005WI23 K.T.Wiedemann, N.H.Medina, J.R.B.Oliveira, J.A.Alcantara-Nunez, W.A.Seale, R.V.Ribas, E.W.Cybulska, M.A.G.Silveira - Braz.J.Phys. 35, 898 (2005)

Yield Ratios in Particle- $\gamma$ Coincidence as a Spectroscopic Tool

2005WIZY A.N.Wilson, A.K.Singh, H.Hubel, P.M.Davidson, A.Gorgen, D.Rossbach, A.Korichi, A.Astier, F.Azaiez, D.Bazzacco, C.Bourgeois, N.Buforn, A.P.Byrne, G.D.Dracoulis, F.Hannachi, K.Hauschild, W.Korten, T.Kroll, G.J.Lane, A.Lopez-Martens, N.Redon, P.Reiter, C.Rossi-Alvarez, G.Schonwasser, O.Stezowski, P.G.Thirolf ANU-P/1667 (2005)

Excitation energies of superdeformed states in ${ }^{196} \mathrm{~Pb}$ : towards a systematic study of the second well in $\mathrm{Pb}$ isotopes

2005WIZZ K.Wisshak, F.Voss, F.Kappeler, L.Kazakov, M.Krticka - Proc.Intern.Conf.Nuclear Data for Science and Technology, Santa Fe, New Mexico, 26 September-1 October, 2004, R.C.Haight, M.B.Chadwick, T.Kawano, P.Talou, Eds., Vol.2, p. 1315 (2005); AIP Conf.Proc. 769 (2005)

Stellar Neutron Capture Cross Sections of the Lu and Hf Isotopes

2005W001 H.J.Wollersheim, D.E.Appelbe, A.Banu, R.Bassini, T.Beck, F.Becker, P.Bednarczyk, K.-H.Behr, M.A.Bentley, G.Benzoni, C.Boiano, U.Bonnes, A.Bracco, S.Brambilla, A.Brunle, A.Burger, K.Burkard, P.A.Butler, F.Camera, D.Curien, J.Devin, P.Doornenbal, C.Fahlander, K.Fayz, H.Geissel, J.Gerl, M.Gorska, H.Grawe, J.Grebosz, R.Griffiths, G.Hammond, M.Hellstrom, J.Hoffmann, H.Hubel, J.Jolie, J.V.Kalben, M.Kmiecik, I.Kojouharov, R.Kulessa, N.Kurz, I.Lazarus, J.Li, J.Leske, R.Lozeva, A.Maj, S.Mandal, W.Meczynski, B.Million, G.Munzenberg, S.Muralithar, M.Mutterer, P.J.Nolan, G.Neyens, J.Nyberg, W.Prokopowicz, V.F.E.Pucknell, P.Reiter, D.Rudolph, N.Saito, T.R.Saito, D.Seddon, H.Schaffner, J.Simpson, K.-H.Speidel, J.Styczen, K.Summerer, N.Warr, H.Weick, C.Wheldon, O.Wieland, M.Winkler, M.Zieblinski - Nucl.Instrum.Methods Phys.Res. A537, 637 (2005) Rare ISotopes INvestigation at GSI (RISING) using gamma-ray spectroscopy at relativistic energies

2005W003 M.Wolinska-Cichocka, J.Kownacki, W.Urban, E.Ruchowska, W.A.Plociennik, B.Bekman, Ch.Droste, W.Gast, R.Lieder, W.Meczynski, M.Kisielinski, A.Kordyasz, M.Kowalczyk, P.Kowina, J.Iwanicki, T.Morek, J.Perkowski, J.Srebrny, A.Stolarz, J.Styczen - Eur.Phys.J. A 24, 259 (2005)

Gamma-ray spectroscopy in ${ }^{110} \mathrm{Sn}$ and ${ }^{111} \mathrm{Sn}$

2005W004 H.J.Worner, M.Grutter, E.Vliegen, F.Merkt - Phys.Rev. A 71, 052504 (2005); Erratum Phys.Rev. A 73, 059904 (2006)

Role of nuclear spin in photoionization: Hyperfine-resolved photoionization of Xe and Multichannel Quantum defect theory analysis

2005W006 A.Wolf, Z.Berant, N.V.Zamfir, D.S.Brenner, E.A.McCutchan, H.Ai, R.F.Casten, K.Dusling, A.Heinz, D.A.Meyer, E.A.Millman, N.Pietralla, C.Plettner, J.Qian, P.H.Regan, D.Swanson, J.Vinson, E.Williams, R.Winkler - Phys.Rev. C 72, 027301 (2005) 
$\mathrm{g}$ factor of the $2_{1}^{+}$state of ${ }^{160} \mathrm{Er}$

2005WR01 K.Wrzosek, M.Zielinska, J.Choinski, T.Czosnyka, J.Iwanicki, M.Kisielinski, M.Kowalczyk, P.Napiorkowski, L.Reissig, J.Srebrny, I.Ushakov, K.Zajac Int.J.Mod.Phys. E14, 359 (2005)

Search for shape coexistence in ${ }^{100}$ Mo using Coulomb excitation

2005WRZZ A.Wronska, V.Hejny, C.Wilkin, S.Dymov, C.Hanhart, V.Komarov, H.R.Koch, A.Kulikov, A.Magiera, T.Mersmann, S.Mikirtytchiants, H.Ohm, D.Prasuhn, R.Schleichert, H.J.Stein, H.Stroher - nucl-ex/0510056,10/20/2005 (2005) Near threshold $\eta$ meson production in the $\mathrm{dd} \rightarrow{ }^{4} \mathrm{He} \eta$ reaction

2005 WU02 C.Wu, J.Barth, W.Braun, J.Ernst, K.-H.Glander, J.Hannappel, N.Jopen, H.Kalinowsky, F.J.Klein, F.Klein, E.Klempt, R.Lawall, J.Link, D.Menze, W.Neuerburg, M.Ostrick, E.Paul, H.van Pee, I.Schulday, W.J.Schwille, B.Wiegers, F.W.Wieland, J.Wisskirchen - Eur.Phys.J. A 23, 317 (2005)

Photoproduction of $\rho^{0}$-mesons and $\Delta$-baryons in the reaction $\gamma \mathrm{p} \rightarrow \mathrm{p} \pi^{+} \pi^{-}$at energies up to $\sqrt{ } \mathrm{s}=2.6 \mathrm{GeV}$

2005WU03 A.H.Wuosmaa, K.E.Rehm, J.P.Greene, D.J.Henderson, R.V.F.Janssens, C.L.Jiang, L.Jisonna, E.F.Moore, R.C.Pardo, M.Paul, D.Peterson, S.C.Pieper, G.Savard, J.P.Schiffer, R.E.Segel, S.Sinha, X.Tang, R.B.Wiringa - Phys.Rev.Lett. 94, 082502 (2005)

Neutron Spectroscopic Factors in ${ }^{9} \mathrm{Li}$ from ${ }^{2} \mathrm{H}\left({ }^{8} \mathrm{Li}, \mathrm{p}\right){ }^{9} \mathrm{Li}$

2005WUZZ A.H.Wuosmaa, K.E.Rehm, J.P.Greene, D.J.Henderson, R.V.F.Janssens, C.L.Jiang, E.F.Moore, R.C.Pardo, D.Peterson, S.C.Pieper, G.Savard, J.P.Schiffer, S.Sinha, X.Tang, R.B.Wiringa, L.Jisonna, R.E.Segel - Proc.Nuclei at the Limits, Argonne, Illinois, D.Seweryniak and T.L.Khoo, eds., p.393 (2005); AIP Conf. Proc 764 (2005) Excited States In ${ }^{7} \mathrm{He}$ From d $\left({ }^{6} \mathrm{He}, \mathrm{p}\right){ }^{7} \mathrm{He}$

$2005 X 106$ C.Y.Xie, X.H.Zhou, Y.H.Zhang, Y.X.Guo, X.G.Lei, Y.Zheng, M.L.Liu, L.T.Song, H.L.Wang, W.T.Guo, H.P.Yu, L.H.Zhu, X.G.Wu, F.R.Xu - Phys.Rev. C 72, 044302 (2005)

Low-spin signature inversion in the $\pi \mathrm{h}_{9 / 2}(\mathrm{x}) \nu \mathrm{i}_{13 / 2}$ oblate band of ${ }^{190} \mathrm{Tl}$

$2005 X U 04 \quad$ S.-W.Xu, Z.-K.Li, Y.-X.Xie, Q.-Y.Pan, W.-X.Huang, X.-D.Wang, Y.Yu, Y.-B.Xing, N.-C.Shu, Y.-S.Chen, F.-R.Xu, K.Wang - Phys.Rev. C 71, 054318 (2005)

$\beta$-delayed proton decays near the proton drip line

2005 YA05 S.Yaschenko, S.Dymov, A.Kacharava, V.Komarov, G.Macharashvili, F.Rathmann, S.Barsov, R.Gebel, M.Hartmann, A.Khoukaz, P.Kulessa, A.Kulikov, V.Kurbatov, N.Lang, I.Lehmann, B.Lorentz, T.Mersmann, S.Merzliakov, S.Mikirtytchiants, A.Mussgiller, M.Nioradze, H.Ohm, D.Prasuhn, R.Schleichert, H.Seyfarth, E.Steffens, H.J.Stein, H.Stroher, Yu.Uzikov, B.Zalikhanov, N.Zhuravlev Phys.Rev.Lett. 94, 072304 (2005) Measurement of the Analyzing Power in $(\mathrm{p}(\mathrm{pol}) \mathrm{d}) \rightarrow(\mathrm{pp}) \mathrm{n}$ with a Fast Forward ${ }^{1} \mathrm{~S}_{0}$ Proton Pair 
$2005 Y$ Y11 K.Yako, H.Sakai, M.B.Greenfield, K.Hatanaka, M.Hatano, J.Kamiya, H.Kato, Y.Kitamura, Y.Maeda, C.L.Morris, H.Okamura, J.Rapaport, T.Saito, Y.Sakemi, K.Sekiguchi, Y.Shimizu, K.Suda, A.Tamii, N.Uchigashima, T.Wakasa - Phys.Lett. B 615, 193 (2005)

Determination of the Gamow-Teller quenching factor from charge exchange reactions on ${ }^{90} \mathrm{Zr}$

2005YA12 T.Yamagata, H.Akimune, S.Nakayama, M.Fujiwara, K.Fushimi, M.B.Greenfield, K.Hara, K.Y.Hara, H.Hashimoto, K.Ichihara, K.Kawase, M.Kinoshita, Y.Matsui, K.Nakanishi, M.Ohta, A.Shiokawa, M.Tanaka, H.Utsunomiya, M.Yosoi - Phys.Rev. C 71, $064316(2005)$

Di-trinucleon cluster resonances in $\mathrm{A}=6$ isobar nuclei

2005 Y17 T.Yamagata, S.Nakayama, M.Tanaka, T.Itahashi - J.Phys.Soc.Jpn. 74, 2640 (2005)

${ }^{7} \mathrm{Li}$-Excitation of a Resonance at $\mathrm{E}_{x}=15.5 \mathrm{MeV}$ in ${ }^{208} \mathrm{~Pb}$

2005YA26 K.Yamada, T.Motobayashi, N.Aoi, H.Baba, K.Demichi, Z.Elekes, J.Gibelin, T.Gomi, H.Hasegawa, N.Imai, H.Iwasaki, S.Kanno, T.Kubo, K.Kurita, Y.U.Matsuyama, S.Michimasa, T.Minemura, M.Notani, T.K.Onishi, H.J.Ong, S.Ota, A.Ozawa, A.Saito, H.Sakurai, S.Shimoura, E.Takeshita, S.Takeuchi, M.Tamaki, Y.Togano, Y.Yanagisawa, K.Yoneda, I.Tanihata - Eur.Phys.J. A 25, Supplement 1, 409 (2005)

Reduced transition probabilities for the first $2^{+}$excited state in ${ }^{46} \mathrm{Cr},{ }^{50} \mathrm{Fe}$, and ${ }^{54} \mathrm{Ni}$

2005YAZW S.W.Yates - Priv.Comm. (2005)

2005 YE01 Y.L.Ye, D.Y.Pang, D.X.Jiang, T.Zheng, Q.J.Wang, Z.H.Li, X.Q.Li, Y.C.Ge, C.Wu, G.L.Zhang, Q.Y.Hu, J.Wang, Z.Q.Chen, A.Ozawa, Y.Yamaguchi, R.Kanungo, I.Tanihata - Phys.Rev. C 71, 014604 (2005)

Quasielastic scattering of ${ }^{6} \mathrm{He}$ on ${ }^{9} \mathrm{Be}$ at $25 \mathrm{MeV} /$ nucleon

2005 YE02 A.V.Yeremin, A.V.Belozerov, M.L.Chelnokov, V.I.Chepigin, V.A.Gorshkov, A.P.Kabachenko, O.N.Malyshev, A.G.Popeko, R.N.Sagaidak, A.V.Shutov, E.A.Sokol, A.I.Svirikhin - Nucl.Instrum.Methods Phys.Res. A539, 441 (2005) Neutron detector at the focal plane of the set up VASSILISSA

2005 YE05 Y.L.Ye, D.Y.Pang, G.L.Zhang, D.X.Jiang, T.Zheng, Z.H.Li, X.Q.Li, Q.J.Wang, Y.C.Ge, C.E.Wu, J.Wang, Z.Q.Chen, Y.Ai, A.Ozawa, Y.Yamaguchi, D.Q.Fang, I.Tanihata - J.Phys.(London) G31, S1647 (2005)

Study of the halo nucleus ${ }^{6} \mathrm{He}$ through the direct nuclear reactions

2005 Y012 T.Yoshioka, T.Tsuruta, H.Iwano, T.Danhara - Nucl.Instrum.Methods Phys.Res. A555, 386 (2005); Erratum Nucl.Instrum.Methods Phys.Res. A557, 689 (2006) Spontaneous fission decay constant of ${ }^{238} \mathrm{U}$ determined by SSNTD method using CR-39 and DAP plates

2005 YU04 D.-Q.Yuan, Y.-N.Zheng, D.-M.Zhou, Y.Zuo, E.-P.Du, X.Duan, C.-H.Wang, Q.Luo, X.-G.Wu, G.-S.Li, S.-X.Wen, G.-J.Xu, Z.-C.Gao, Y.-S.Chen, S.-Y.Zhu -

Chin.Phys.Lett. 22, 1628 (2005) 
Measurements of g-Factor of Rotational Levels in ${ }^{83} \mathrm{Y}$

2005 YU06 H.-P.Yu, Y.-X.Gou, X.-H.Zhou, Y.-H.Zhang, X-G.Lei, P.Luo, M.-L.Liu, L.-T.Song, H.L.Wang, C.-Y.Xie, Y.Zheng, W.-T.Guo, L.-H.Zhu, X.-G.Wu - Chin.Phys.Lett. 22, $1873(2005)$

High-Spin States in Odd-Odd ${ }^{140}$ Pr Nucleus

2005 YU07 C.-H.Yu, C.Baktash, J.C.Batchelder, J.R.Beene, C.Bingham, M.Danchev, A.Galindo-Uribarri, C.J.Gross, P.A.Hausladen, W.Krolas, J.F.Liang, E.Padilla, J.Pavan, D.C.Radford - Eur.Phys.J. A 25, Supplement 1, 395 (2005)

Coulomb excitation of odd-A neutron-rich radioactive beams

2005ZA14 N.V.Zamfir, R.O.Hughes, R.F.Casten, D.C.Radford, C.J.Barton, C.Baktash, M.A.Caprio, A.Galindo-Uribarri, C.J.Gross, P.A.Hausladen, E.A.McCutchan, J.J.Ressler, D.Shapira, D.W.Stracener, C.-H.Yu - Eur.Phys.J. A 25, Supplement 1, $389(2005)$

${ }^{132} \mathrm{Te}$ and single-particle density-dependent pairing

2005ZA15 G.S.Zahn, C.B.Zamboni, F.A.Genezini, M.P.Raele, J.Y.Zevallos-Chavez, M.T.F.da Cruz - Braz.J.Phys. 35, 843 (2005)

Investigation of Excited Levels in ${ }^{193}$ Ir From the Beta Decay of ${ }^{193}$ Os

$2005 Z$ D02 Yu.G.Zdesenko, F.T.Avignone III, V.B.Brudanin, F.A.Danevich, V.V.Kobychev, B.N.Kropivyansky, S.S.Nagorny, V.I.Tretyak, T.S.Vylov - Astropart.Phys. 23, 249 (2005)

CARVEL experiment with ${ }^{48} \mathrm{CaWO}_{4}$ crystal scintillators for the double $\beta$ decay study of ${ }^{48} \mathrm{Ca}$

$2005 Z$ Z03 S.Zeng, W.-P.Liu, Z.-H.Li, X.-X.Bai, S.-Q.Yan, B.-X.Wang, G.Lian, J.Su Chin.Phys.Lett. 22, 2219 (2005)

Measurement of ${ }^{2} \mathrm{H}\left({ }^{8} \mathrm{Li},{ }^{9} \mathrm{Be}\right) \mathrm{n}$ Reaction Relevant to Primordial Nucleosynthesis

2005ZE04 R.G.T.Zegers, A.L.Cole, H.Akimune, S.M.Austin, D.Bazin, A.M.van den Berg, G.P.A.Berg, J.Brown, I.Daito, Y.Fujita, M.Fujiwara, K.Hara, M.N.Harakeh, G.W.Hitt, M.E.Howard, J.Janecke, T.Kawabataj T.Nakamura, H.Ueno, H.Schatz, B.M.Sherrill, M.Steiner - Nucl.Phys. A758, 67c (2005)

Weak interaction strengths for supernovae calculations via the $\left(t,{ }^{3} \mathrm{He}\right)$ reaction on medium-heavy nuclei

2005ZEZZ R.G.T.Zegers, H.Akimune, Sam M.Austin, D.Bazin, A.M.van den Berg, G.P.A.Berg, B.A.Brown, A.L.Cole, I.Daito, Y.Fujita, M.Fujiwara, S.Gales, M.N.Harakeh, H.Hashimoto, R.Hayami, G.W.Hitt, M.E.Howard, M.Itoh, J.Janecke, T.Kawabata, K.Kawase, M.Kinoshita, T.Nakamura, K.Nakanishi, S.Nakayama, S.Okamura, W.A.Richter, D.A.Roberts, B.M.Sherrill, Y.Shimbara, M.Steiner, M.Uchida, H.Ueno, T.Yamagata, M.Yosoi - nucl-ex/0512025,12/20/2005 (2005)

The $\left(\mathrm{t},{ }^{3} \mathrm{He}\right)$ and $\left({ }^{3} \mathrm{He}, \mathrm{t}\right)$ reactions as probes of Gamow-Teller strength

2005ZH09 S.-H.Zhou, Z.-Y.Liu, J.Zhou, Q.-Y.Meng, C.-B.Li, G.Lian, B.-X.Wang X.-X.Bai Chin.Phys.Lett. 22, 565 (2005)

Large Decay Rate Variation of ${ }^{7} \mathrm{Be}$ in $\mathrm{Pd}$ and $\mathrm{Au}$ 
2005ZH14 L.Y.Zhu, and the Jefferson Lab Hall A Collaboration and Jefferson Lab E94-104 Collaboration - Phys.Rev. C 71, 044603 (2005)

Cross section measurements of charged pion photoproduction in hydrogen and deuterium from 1.1 to $5.5 \mathrm{GeV}$

$2005 Z$ Z16 S.J.Zhu, S.D.Xiao, X.L.Che, Y.N.U, M.L.Li, Y.J.Chen, L.H.Zhu, G.S.Li, S.X.Wen, X.G.Wu - Eur.Phys.J. A 24, 199 (2005)

High-spin states and band structures in the doubly odd ${ }^{136}$ La nucleus

2005ZH20 S.Zhu, R.V.F.Janssens, G.J.Lane, I.Wiedenhover, M.P.Carpenter, I.Ahmad, A.P.Byrne, P.Chowdhury, D.Cline, A.N.Deacon, G.D.Dracoulis, S.J.Freeman, N.J.Hammond, G.D.Jones, T.L.Khoo, F.G.Kondev, T.Lauritsen, C.J.Lister, A.O.Macchiavelli, E.F.Moore, D.Seweryniak, J.F.Smith, C.Y.Wu - Phys.Lett. B $618,51(2005)$

Strength of octupole correlations in the actinides: contrasting behavior in the isotones ${ }^{237} \mathrm{U}$ and ${ }^{239} \mathrm{Pu}$

2005ZH30 Y.H.Zhang, Y.D.Fang, G.de Angelis, N.Marginean, A.Gadea, D.R.Napoli, M.Axiotis, C.Rusu, T.Martinez, H.L.Wang, X.H.Zhou, W.T.Guo, M.L.Liu, Y.X.Guo, X.G.Lei, M.Oshima, Y.Toh, F.R.Xu - J.Phys.(London) G31, S1545 (2005) High-spin states of an odd-odd ${ }^{184} \mathrm{Au}$ nucleus

$2005 Z H 31$ X.H.Zhou, C.Y.Xie, Y.H.Zhang, Y.X.Guo, X.G.Lei, Y.Zheng, M.L.Liu, L.T.Song, H.L.Wang, W.T.Guo - J.Phys.(London) G31, S1985 (2005)

First observation of low-spin signature inversion in the $\pi \mathrm{h}_{9 / 2}(\mathrm{X}) \nu \mathrm{i}_{13 / 2}$ oblate band

2005ZH32 Y.-H.Zhang, S.Guo, X.-H.Zhou, L.Ma, M.Oshima, Y.Toh, M.Koizumi, A.Osa, A.Kimura, Y.Hatsukawa, M.Sugawara, H.Kusakari - Chin.Phys.Lett. 22, 2788 (2005)

Observation of High-j Two-Quasiparticle Bands in Odd-Odd ${ }^{174} \mathrm{Re}$

$2005 Z$ Z36 S.J.Zhu, J.H.Hamilton, A.V.Ramayya, P.M.Gore, J.O.Rasmussen, V.Dimitrov, S.Frauendorf, R.Q.Xu, J.K.Hwang, D.Fong, L.M.Yang, K.Li, Y.J.Chen, X.Q.Zhang, E.F.Jones, Y.X.Luo, I.Y.Lee, W.C.Ma, J.D.Cole, M.W.Drigert, M.Stoyer, G.M.Ter-Akopian, A.V.Daniel - Eur.Phys.J. A 25, Supplement 1, 459 (2005) Soft chiral vibrations in ${ }^{106} \mathrm{Mo}$

2005ZHZZ B.V.Zhuravlev, N.V.Kornilov, A.A.Lychagin, N.N.Titarenko, V.I.Trykova Proc.Intern.Conf.Nuclear Data for Science and Technology, Santa Fe, New Mexico, 26 September-1 October, 2004, R.C.Haight, M.B.Chadwick, T.Kawano, P.Talou, Eds., Vol.1, p. 931 (2005); AIP Conf.Proc. 769 (2005)

Nuclear Level Densities of ${ }^{56} \mathrm{Co},{ }^{57} \mathrm{Co},{ }^{90} \mathrm{Nb}$, and ${ }^{94} \mathrm{Nb}$ from Neutron Evaporation Spectra in the $(\mathrm{p}, \mathrm{n})$ Reaction

$2005 Z 102$ M.Zielinska, T.Czosnyka, K.Wrzosek, J.Choinski, Y.Hatsukawa, J.Iwanicki, M.Koizumi, H.Kusakari, M.Matsuda, T.Morikawa, P.J.Napiorkowski, A.Osa, M.Oshima, T.Shizuma, J.Srebrny, M.Sugawara, K.Zajac - Acta Phys.Pol. B36, 1289 (2005)

Shape coexistence in even-even Mo isotopes studied via Coulomb excitation 
2005ZU01 K.Zuber - Nucl.Phys. B(Proc.Supp.) S138, 236 (2005)

COBRA - A new approach to double beta decay

2006B001 Ch.Bobin, J.Bouchard - Appl.Radiat.Isot. 64, 124 (2006)

A $4 \pi(\mathrm{LS}) \beta-\gamma$ coincidence system using a TDCR apparatus in the $\beta$ channel

2006DI01 F.Ditroi, F.Tarkanyi, J.Csikai, M.S.Uddin, M.Hagiwara, M.Baba, Yu.N.Shubin, S.F.Kovalev - Nucl.Instrum.Methods Phys.Res. B243, 20 (2006)

Excitation functions of long lived products in deuteron induced nuclear reactions on platinum up to $40 \mathrm{MeV}$

2006DR01 Ch.Droste, B.Czajkowska, Z.Marcinkowska, R.M.Lieder, T.Morek, T.Rzaca-Urban, W.Gast - Nucl.Instrum.Methods Phys.Res. A556, 182 (2006)

Polarisation sensitivity of the CLUSTER detector used in EUROBALL array

2006HA01 K.F.Hassan, S.M.Qaim, Z.A.Saleh, H.H.Coenen - Appl.Radiat.Isot. 64, 101 (2006)

Alpha-particle induced reactions on ${ }^{n a t} \mathrm{Sb}$ and ${ }^{121} \mathrm{Sb}$ with particular reference to the production of the medically interesting radionuclide ${ }^{124} \mathrm{I}$

2006LE01 P.Leleux, P.Lipnik, S.wa Kitwanga, J.Vanhorenbeeck - Nucl.Instrum.Methods Phys.Res. A556, 397 (2006)

About the neutron yield from the $\mathrm{p}+{ }^{13} \mathrm{C}$ interaction at $30 \mathrm{MeV}$ 